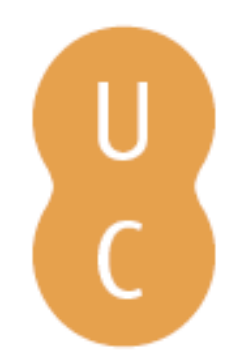

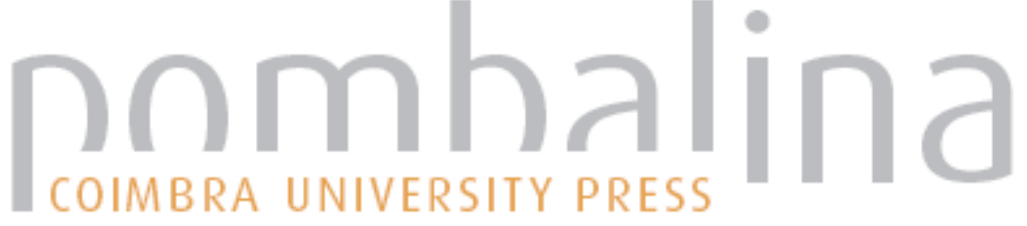

\section{Advances in forest fire research 2018}

Autor(es): $\quad$ Viegas, Domingos Xavier (ed.)

Publicado por: Imprensa da Universidade de Coimbra

URL

persistente: URI:http://hdl.handle.net/10316.2/44517

DOI: $\quad$ DOI:https://doi.org/10.14195/978-989-26-16-506

Accessed : $\quad$ 26-Apr-2023 15:25:19

A navegação consulta e descarregamento dos títulos inseridos nas Bibliotecas Digitais UC Digitalis, UC Pombalina e UC Impactum, pressupõem a aceitação plena e sem reservas dos Termos e Condições de Uso destas Bibliotecas Digitais, disponíveis em https://digitalis.uc.pt/pt-pt/termos.

Conforme exposto nos referidos Termos e Condições de Uso, o descarregamento de títulos de acesso restrito requer uma licença válida de autorização devendo o utilizador aceder ao(s) documento(s) a partir de um endereço de IP da instituição detentora da supramencionada licença.

Ao utilizador é apenas permitido o descarregamento para uso pessoal, pelo que o emprego do(s) título(s) descarregado(s) para outro fim, designadamente comercial, carece de autorização do respetivo autor ou editor da obra.

Na medida em que todas as obras da UC Digitalis se encontram protegidas pelo Código do Direito de Autor e Direitos Conexos e demais legislação aplicável, toda a cópia, parcial ou total, deste documento, nos casos em que é legalmente admitida, deverá conter ou fazer-se acompanhar por este aviso. 


\section{ADVANCES IN}

\section{FOREST FIRE RESEARCH}

\section{8}

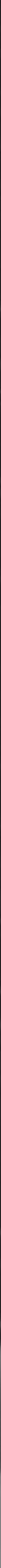


EDIÇão

Imprensa da Universidade de Coimbra

Email: imprensa@uc.pt

URL: http//www.uc.pt/imprensa_uc

Vendas online: http://livrariadaimprensa.uc.pt

COORDENAC̄̃̃O EDITORIAL

Imprensa da Universidade de Coimbra

IMAGEM DA CAPA

ADAI/CEIF

Composição

Luís Mário Ribeiro

ISBN Digital

978-989-26-16-506

DOI

https://doi.org/10.14195/978-989-26-16-506 

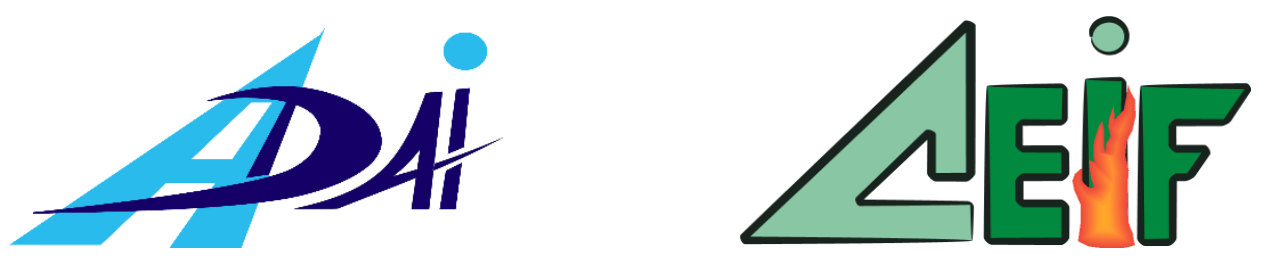

\author{
ADAI/CEIF \\ Associação para o Desenvolvimento da Aerodinâmica Industrial (ADAI) \\ Centro de Estudos sobre Incêndios Florestais (CEIF) \\ Rua Pedro Hispano ${ }^{\circ} 12$ \\ 3030 - 289 Coimbra \\ Telf: +351239708580 | Fax: +351239708589 \\ www.adai.pt/ceif \\ https://facebook.com/ceif.adai \\ https://twitter.com/adai_ceif
}

Coimbra 2018

All rights reserved.

This publication may not be reproduced in whole or in part, stored in a retrieval system or transmitted, in any form or by any means without the permission of the Publisher, ADAI.

\title{
Composition
}

Luís Mário Ribeiro 



\section{Advances in}

\section{Forest Fire Research \\ 2018}

Edited by

Domingos Xavier Viegas

ADAI/CEIF, University of Coimbra, Portugal 



\section{Contents}

Contents 7

Preface 14

Chapter 1 Fire Risk Management 16

A model for identifying blow-up fire potential 17

Assessing the increase in wildfire occurrence with climate change and the uncertainties associated with this projection.

Calibration of the Canadian FWI System for the Territory of Europe. 33

Evaluation of wildfire danger in the Peruvian Andes: First step for its reduction and adaptation .......... 44

Fires in Portugal on 15th October 2017: a Catastrophic Evolution ....................................................... 57

Generation and evaluation of ensemble simulations of wildfire spread for probabilistic forecast .......... 71

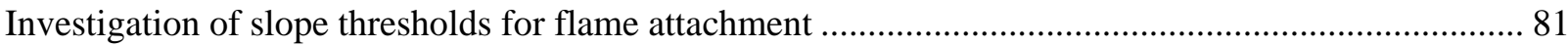

IPA Adriatic Holistic Forest Fire Protection Project - the year after ................................................... 88

Modelling of the rate of fire spread in heterogeneous fuel beds based on experimental data ................ 99

Monitoring of forest fire risk conditions in Portugal with NOAA/NESDIS satellite-based Vegetation

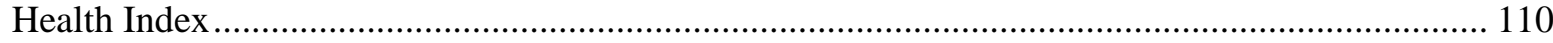

Protecting lives and property: testing tanker crew protection systems .............................................. 119

Temporal variability of the Haines index and its relationship with forest fire in Portugal.................... 127

The extreme weather conditions behind the destructive fires of June and October 2017 in Portugal ... 138

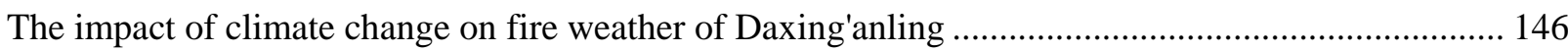

The organisation of fire protection in disaster areas using as an example the case of Bialowieza Forest 156

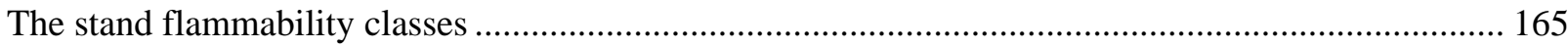

Understanding the changing fire environment of south-west Western Australia ................................ 173

Veld fire mitigation strategy: A vision for an innovative and integrated approach to managing risks in land reform farms, a case of land reform beneficiaries in South Africa ........................................ 183

Wildfire occurrence estimation in Spanish regions based on Land Use-Land Cover interfaces and

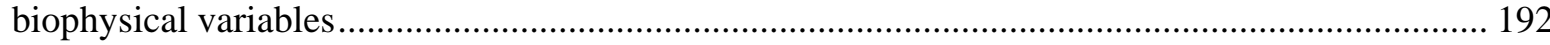

Wildfire susceptibility mapping in Liguria (Italy). Comparison of Statistical Driven Partitioning and

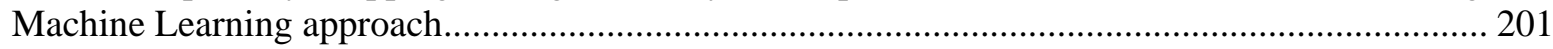

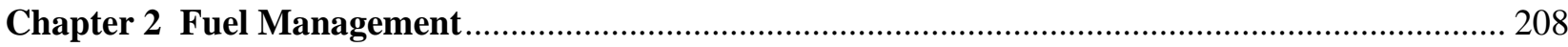

Climatic conditions and fire regime affect vegetation recovery after large wildfires in Pinus forest

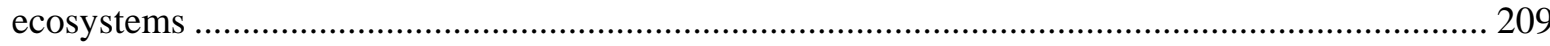

Estimation of live fuel moisture content of shrubland using MODIS and Sentinel-2 images ............... 218

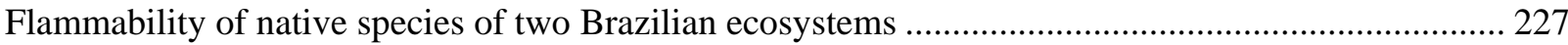

Influence of fire frequency and epoch of prescribed burn on topkill rates in vegetation of Cerrado in

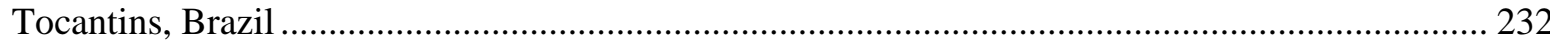

Influence of thermal treatment on the bioavailability of $\mathrm{Cu}, \mathrm{Cd}$ and $\mathrm{Zn}$ in polluted forest soils........... 238

Live fuel moisture content: variability, predictability and impact on fire behavior and activity ........... 246 
Management of firebrand potential through the candling of bark fuel ............................................... 254

Optimisation of fuel treatments at landscape level in NW Spain ..................................................... 262

Plasticity of fire related traits of some obligate seeder species in different fire-prone areas of SE France:

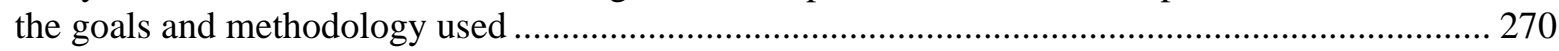

Quantifying effects on fire propagation in woody fuel models using the thin fuels assumption ........... 278

Soil resilience under different scenarios of fire recurrence and severity in Pinus forest ecosystems affected

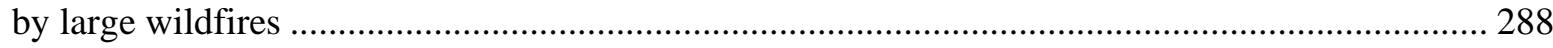

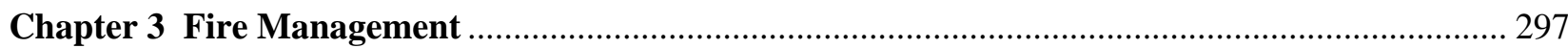

17 years of wildfire detection and management supported by an optical sensor system: Field report from Germany's Brandenburg national forest, one of Europe's top wildfire regions at risk ................... 298

A project to measure and model pyrolysis to improve prediction of prescribed fire behavior ............... 308

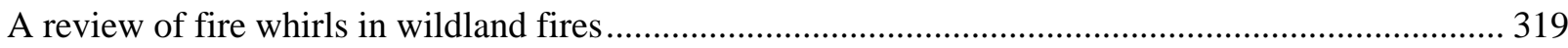

A study of the structure of a turbulent line fire subjected to cross-flow using large eddy simulations . 325

A two-dimensional reaction-advection-diffusion model of the spread of fire in wildlands .................. 334

An experimental investigation of the effect of grass fuel load on grassfire behaviour.......................... 343

Assessment of a human body thermoregulation software to predict the thermophysiological response of

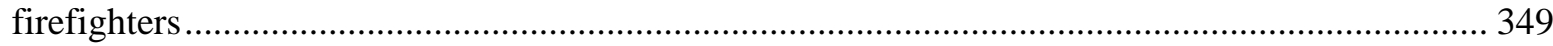

Cfd study of effect of water drop by aerial firefighting to protect forest fire growth ............................ 359

Circulation weather types and their influence on the fire regime in Portugal...................................... 372

Concurrent multi-scale physical parametrization of fire-spotting: A study on the role of macro- and meso-

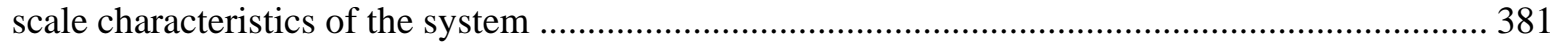

Does prescribed burning reduce the amount of inflorescences in conifer forests? Implications for

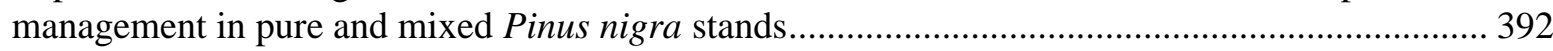

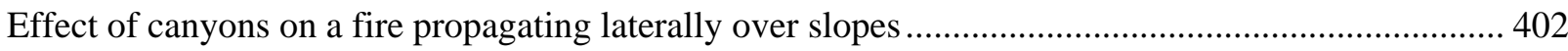

Effective efficiency index (IEE): methodology for analyzing the efficiency of fire retardants in laboratory 410

Effects of aerodynamic lift on firebrand trajectories: Numerical investigations based on simplified models. 418

Experimental characterization and analysis on fire whirls using forest fuels ...................................... 429

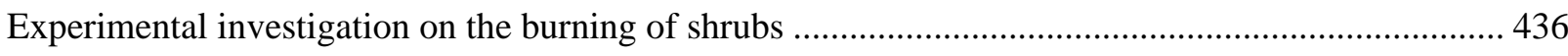

Fire growth patterns in the 2017 mega fire episode of October 15, central Portugal ............................ 447

Fire intensity, individual protective clothing and firefighting safety ................................................... 454

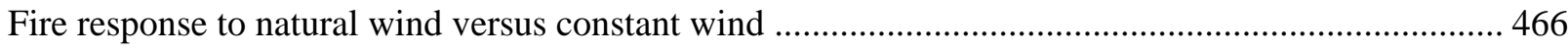

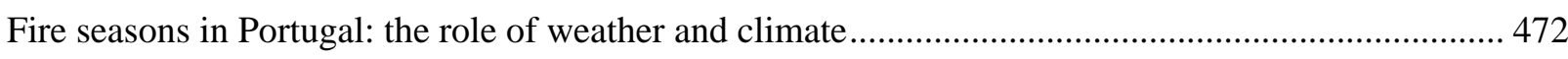

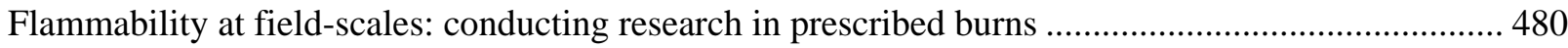

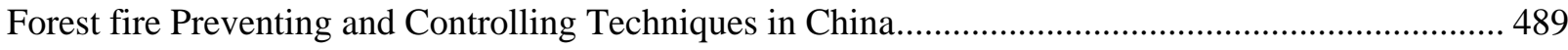

Identifying pyroregions by means of Self Organizing Maps and hierarchical clustering algorithms in

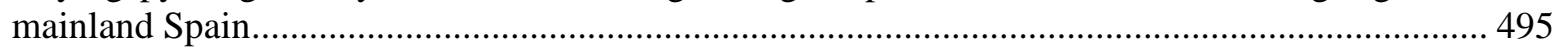

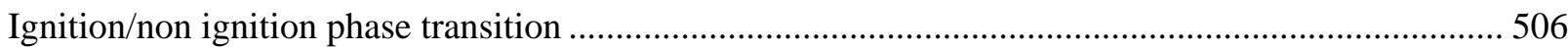

Improvement of Forest Fire Danger Rating in Russia ....................................................................... 514

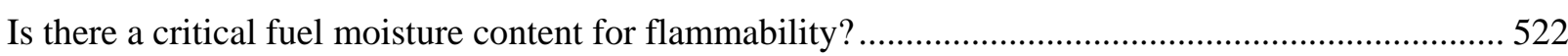

Literature review on the transition from smouldering to flaming fires and its application to peat fires 529 
Measurement of pyrolysis products from mixed fuel beds during fires in a wind tunnel...................... 534

Measurement of three-dimensional flow speed and direction in wildfires ........................................... 542

Modelling of junction fires with analytical and numerical analysis of the phenomena......................... 549

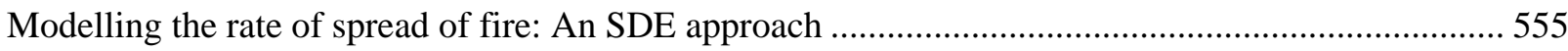

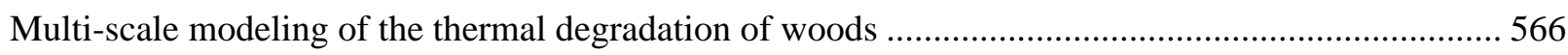

New Lagrangian surface forest fire propagation model.................................................................. 577

Observations on wildfire spotting occurrence and characteristics in Greece....................................... 588

Past fire practices and new steps towards an effective fire management approach in the Brazilian savannas 598

Prescribed fire experiences on crop residue removal for biomass exploitations and cost-benefits analysis, in mediterranean ecosystems.

Radiative and convective heat exchanges between a fireman and the fire front and wind .................... 617

RPAS and nighttime wildfire fighting: The NITROFIREX PROJECT

Semi-empirical fire spread model for chamise and big sagebrush shrubs with spatially-defined fuel elements and flames.

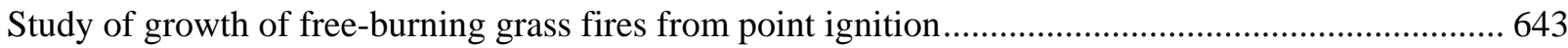

The effect of interstitial flow on the burning dynamics of porous fuel beds

The fires of Mount Hymettus near Athens Greece (1996-2017): history and fire behavior characteristics 661

The role of forest fires in land use/land cover changes in Portugal 670

The role of the terrain-modified wind on driving the fire behaviour over hills - an Experimental and Numerical Analysis. 677

Understanding the dynamics of inclined and wind-driven flames in wildland fires. 695

Understanding unburned patches patterns in extreme wildfire events: a new approach........................ 700

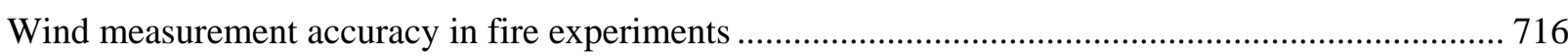

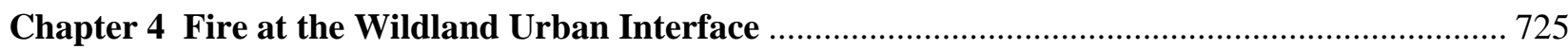

Carcaixent fire impact against a self-protected WUI zone. Lessons learned ........................................ 726

Coupling multi-criteria analysis and GLM for modelling houses vulnerability to forest fires at WUI. 736

Defining and Mapping the Wildland-Urban Interface in Portugal ...................................................... 742

Developement of a Metodology to Assess School Bushfire Risk....................................................... 750

Development of a spatial analysis tool for radiant heat flux predictions .............................................. 761

Estimating net heat flux from surrogate firebrand accumulations using an inverse heat transfer approach 769

Fire Risk Assessment of UNESCO Historic Villages by Heat Flux from Forest Fire ........................... 780

Flammability and combustibility of potential species for use as fuel breaks........................................ 788

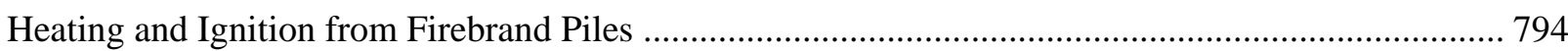

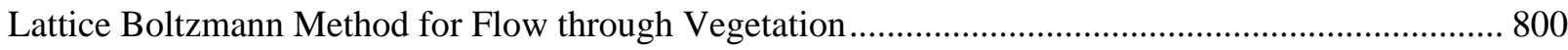

Mathematical modeling of the forest fires initiation, spread and impact on buildings .......................... 808

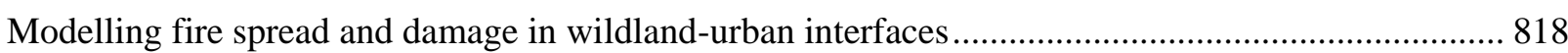

On the use of time-resolved three-dimensional diagnostics to characterize firebrand showers in the WUI 
Proper Width Calculation to a firebreak line to protect flame spread from forest fire 837

Terpene-containing species vs terpeneless species: what best drives flammability of ornamental species

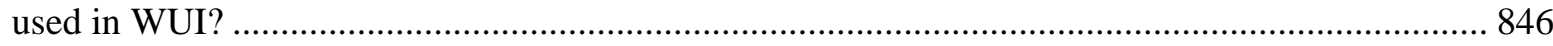

The large fire of Pedrógão Grande (Portugal) and its impact on structures......................................... 852

The urban side of the Wildland-urban interface - a new fire audience identified following an extreme wildfire event in Aotearoa/New Zealand 859

Wildland Urban Interface assessment and prediction in relation to land use and land cover changes. The

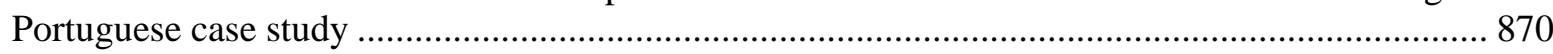

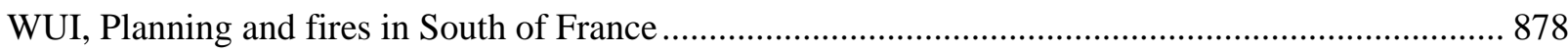

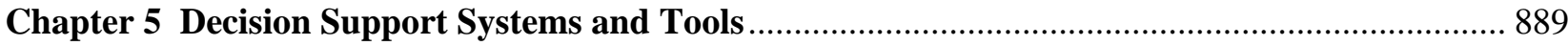

AdriaFirePropagator and AdriaFireRisk - user friendly Web based wildfire propagation and wildfire risk

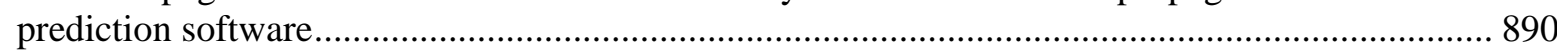

An upper non-reflecting boundary condition for atmospheric compressible flow ................................ 900

Applying GPU Parallel Technology to Accelerate FARSITE Forest Fire Simulator ............................ 913

Atmospheric flow and a Large fire interaction: the unusual case of Pedrogão Grande, Portugal

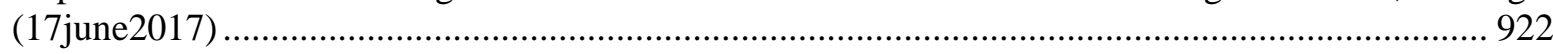

Biomass burning emissions using the Sentinel 2 Small Fire Database ............................................... 933

CeaseFire: a website to assist fire managers in Portugal .................................................................. 941

Coupled fire-atmosphere-smoke forecasting: current capabilities and plans for the future................... 950

Data Likelihood of Active Fires Satellite Detection and Applications to Ignition Estimation and Data

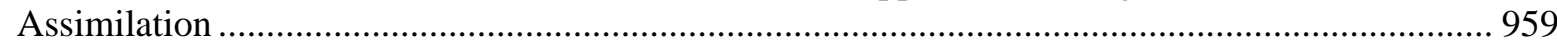

Detecting Peatland Combustion using Shortwave and Thermal Infrared Landsat-8 data .................... 969

Development of multilingual network resource for comparative-contrastive dictionary of fire science

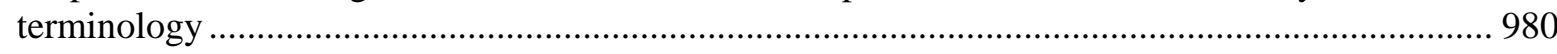

Do we need weather prediction models to account for local weather modifications by wildland fires?987

Examination of the forest fires detection - The relationship between the fire and the detection ........... 995

Factors Contributing to High and Low Risk Fires in the United States: An Analysis of the Relative Risk

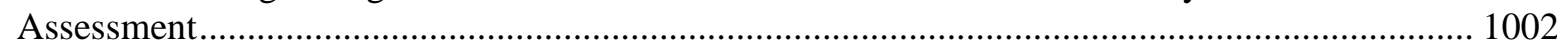

Fast computational models of dynamic fire propagation using near-field techniques.......................... 1009

Feature Selection for Burned Area Classification in the Castelo de Paiva Region.............................. 1015

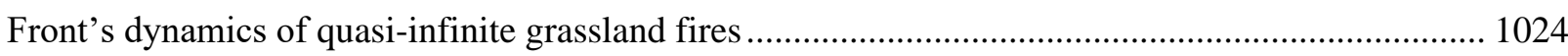

Generation of a global burned area product from satellite data: the ESA Fire_cci project ................. 1035

Integration of the emissions and smoke dispersion models in the European Forest Fire Information System

Mapping fire severity levels of burned areas in Galicia (NW Spain) by Landsat images and the dNBR index: Preliminary results about the influence of topographical, meteorological and fuel factors on the highest severity level.

Mean atmospheric flow pattern and forest fire risk on the Adriatic coast of Croatia .......................... 1061

Non-Rigid Feature Extraction Methods in Real Time Forest Fire Detection Algorithms ................... 1069

On the use of compact thermal cameras for quantitative wildfire monitoring.................................... 1077

Predictive distribution modeling of forest fire in pine zone of Uttarakhand Himalayas of India ........ 1087

System for personnel training in decision making of wildfires fighting ............................................ 1095 


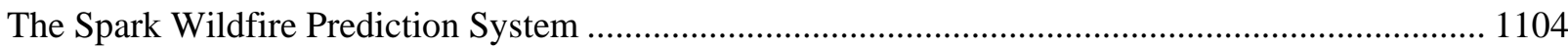

Tracking of uncertainty when aggregating from fine to coarse spatial resolution .............................. 1110

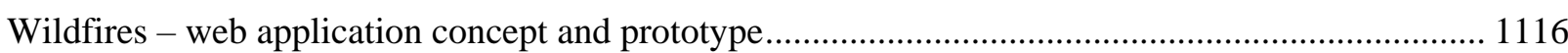

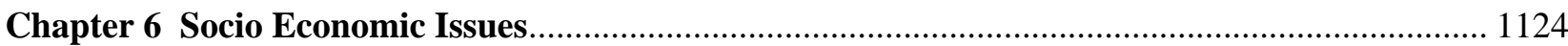

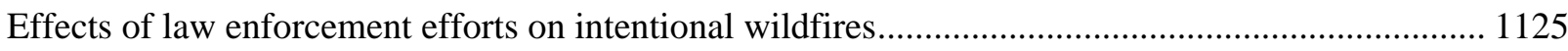

Human perception of fire hazard in wildland urban interface areas - a Portuguese survey analysis of spot

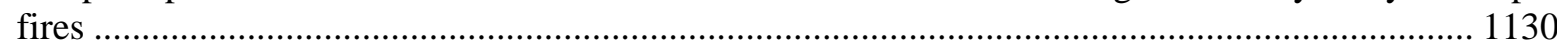

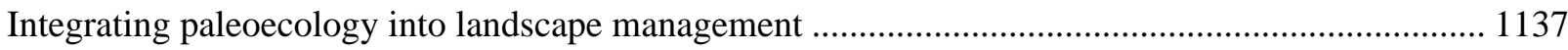

Modeling the productivity of forest fire suppression operations using production functions. A

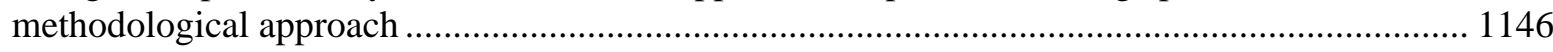

Residents' experiences of the 2016 Fort McMurray Wildfire, Alberta ................................................ 1155

Risk assessment and reflections on socio-economic perception of wildfires at the fireshed level ...... 1160

The consideration of arson for forest fires caused by traditional uses of fire prohibited in Spain. A problem

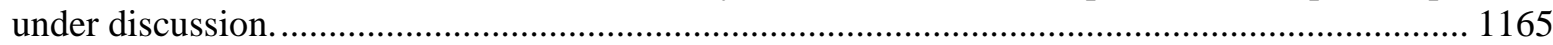

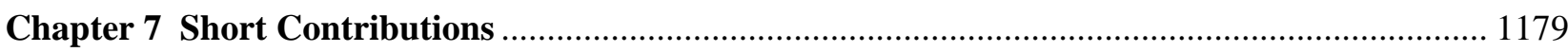

A universal rate of spread index for Australian fuel types............................................................ 1180

Assessment of wildfire exposure and vulnerability factors in Alvares, Góis: integration of structural and

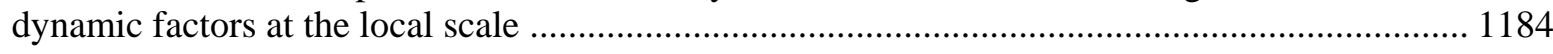

Causes of forest fires in Federal Conservation Units of Brazil from 2006 to 2012 ........................... 1189

Climate-induced variations in global severe fire weather conditions ................................................ 1193

Cohesive forest fire management within an imperfect information environment: a review of risk handling

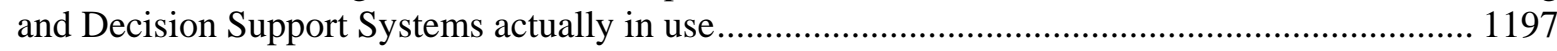

Extreme weather conditions: The role of an heat wave on wildfires in Portugal .............................. 1200

High Resolution Seasonal Forest Fire Danger mapping using WRF forecasts for Greece: A tool for forest fires prevention planning and fire risk management support ...................................................... 1205

Simulating the effectiveness of prescribed burning at altering wildfire behaviour in Tasmania ......... 1209

The role of fire size, geometry, and intensity, in "extreme" plume development ............................... 1212

A rapid technique to quantify bark fuel hazard with smartphones ..................................................... 1216

Aliens \& Flames — a new research initiative joining fire behaviour and invasion ecology ............... 1219

Bench-scale measurement of pyrolysis products from intact live fuels........................................... 1223

Fire in wet eucalypt forests: rethinking fuel-accumulation models for Tasmania's most unique fuel type

Fire, landscape pattern and biodiversity: Using fire for conservation in a south-eastern Australian

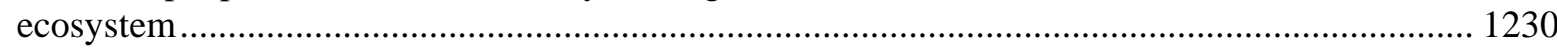

FireCaster Decision Support System: on the need for a new fuel description approach .................... 1233

Mid-term effects of thinning on canopy variables related to crown fire hazard on pure, even-aged pine

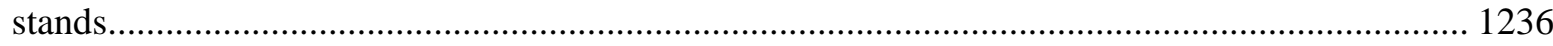

Multidisciplinary fire science research at the Sycan Marsh, Oregon................................................ 1240

A comparison of in-situ fire energy measurements to remote sensed thermography using Unmanned

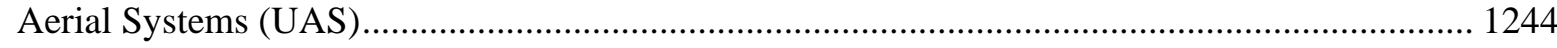

Assessing fire severity using charcoal reflectance following a recent heathland wildfire on Carn Brea, Cornwall, UK 
Carbon dioxide emission on recurrent burnt peat swamp forest in Raja Musa Forest Reserve, Selangor, Malaysia

Characterization of long term retardants

Data mining techniques in the assessment of usability and effectiveness of forest fire video surveillance 1262

Drivers of wildland fire behaviour variation across the Earth 1267

Effect of moisture content and ventilation on the burning rate of porous fuel beds

Evaluating ecological tipping points across levels of wildfire suppression under various climate and fuel treatment scenarios on US northern Rocky Mountain landscapes using landscape simulation..... 1274

Field-tested laboratory-derived models to predict forest fire front spread rate. 1278

Flexible design of a helipad network for forest firefighting helicopters, applied to the case of Sardinia 1280

Influence of moisture and inorganic contents on smouldering spread rates in the lateral and in-depth directions.

New Zealand prescribed fire experiments to test convective heat transfer in wildland fires 1288

Numerical investigations of coupled fire-atmosphere feedbacks influencing fire behavior on slopes 1293

Numerical simulation of low-intensity fire spread in pine litter 1296

Out of context: fire background temperature and methods for its calculation..... 1300

Simulations of surface fire propagating under a canopy: flame angle and intermittency 1303

Spontaneous ignition of vertically positioned wood samples under time-dependent heat flux 1308

Toward safer firefighting strategies and tactics

Towards more resilient and productive forest landscapes: tradeoffs in a recently burned area in Portugal

Vegetation fire management in strict nature reserves.

1320

Wind and slope effects on flame scaling in laboratory fires

Aerodynamic study of the airflow around a scale triangular prismatic hill for Low Reynolds Number 1326

Burnt area and the wildland-urban interface: A first approach to the Galician wildfires occurred in October 2017. 1334

Burnt severity and satellite data: a first approach in the ornemental vegetation in wildland urban interface 1337

Critical conditions for the ignition of cedar needle fuel bed as a result of firebrands accumulation ... 1340

Institutional engagement and public involvement at the WUI. A case study in the South of Spain: Córdoba municipality

Mapping Canadian Interface Areas. 1346

Risk analysis of LPG tanks at the wildland-urban interface: are regulated safety distances large enough?

Analysis of the temporal behaviour of the number of forest fires in some countries of Europe 1354

Building the prototype for a new National Fire Danger Rating System for Australia.

Fire and land use in recent years in the Atlantic Forest in Brazil

FireCCI50: a global burned area mapping algorithm based on MOD09GQ within Fire_cci project .. 1366

Global generation of long-term burned area with AVHRR-LTDR data 
High resolution weather forecasting applied to forest fire behaviour simulation 1373

Laboratory measurement of transient carbon emissions from controlled smouldering peat fire. 1376

Management of an infrared imaging system for wildfire monitoring. 1379

Modeling fuels and treatment effects in 3D with STANDFIRE 1384

Modeling the probability of impact from wildland fires: a near real-time approach 1389

Modelling the performance of forest and wildland fire aerial detection systems 1391

Rate of spread in coupled models: fireline curvature and pyrogenic potential 1393

RPI Engine: Visualization in a web environment of post-fire regeneration using Landsat time series 1396

The determinants of crown fire runs during extreme wildfires in broadleaf forests in Australia 1401

The relative contributions of climate drivers on extreme Australian fire weather. 1406

Understanding fire, weather and land cover interactions from long-term terrestrial observations and satellite data on a transect from Europe to North Africa. 1408

Does it pay to invest in better suppression resources? - policy analysis of alternative scenarios with simulation..... 1411 


\section{Preface}

In spite of all the efforts from the governments and the operational agencies, and the contribution from the scientific and technical communities, the impact of forest fires persists and, at least in some regions, even with an increasing trend. The tragic fires of Portugal in 2017 and of Greece in 2018, with unprecedented numbers of fatalities among the civil population in modern times, drive our attention to the fact that what is at stake in forest fires is not only the natural environment, the climate, the landscape, the economy or the ecology. In these and in other episodes from the recent years, all over the World, forest fires have threatened the safety and wellbeing of people and have shown how capable they are of destroying the greatest value that we have to protect, that of human life.

After the fires of 2017 in Portugal, in which we had more than one hundred fatalities, mostly of common citizens, we felt that everything must have failed for this to be possible. Instead of blaming one or other agency, or person, we have to recognize that the society was not up to the challenge posed by these uncommon but not unrepeatable circumstances and events. We from the scientific community have also to ask ourselves the level of understanding that we have on these processes and about our ability to provide assistance to prevent, predict and control them.

In our judgement we have to recognize that we are still very far from even a satisfactory point. There is so much that we ignore and do not understand about the very complex processes that the management of forest fires involve that we must accept that much more has to be done if we wish to, at least, grasp the immense power and overwhelming capacity that nature has with the scourge of wildfires.

As a scientific community we have to face this challenge and be even more focused and collaborative to deal with it. The International Conference on Forest Fire Research that is now on its eight edition is open to become a platform to support this effort, as it has always been.

This eBook compiles the valuable contributions to this Conference, made by authors from the scientific community all around the world. Each one of the six major themes of the conference includes different topics in the entire range of fields related to the problem of wildfires management. They compose the first six chapters, as follows:

Chapter I) Fire Risk Management

Topics covered: Fire Risk Assessment, Fire Risk Communication, Climate Change, Cascade effects, Firefighting training

Chapter II) Fuel Management

Topics covered: Fire Ecology, Evaluation and Management of Burned Areas, Fuel Management, Fuel Modelling, Fire Prevention

Chapter III) Fire Management

Topics covered: Fire Management, Fire Prevention, Fire Preparedness, Fire Behaviour, Fire Suppression, Large Fires, Fire Safety, International cooperation

Chapter IV) Fire at the Wildland Urban Interface

Topics covered: Fuel Management at the WUI, Fire Management at the WUI, Risk Assessment 
Chapter V) Decision Support Systems and Tools

Topics covered: Fire Detection and Monitoring, Remote Sensing, Fire Behaviour Prediction Systems, Meteorology and other support information, New Technologies

\section{Chapter VI) Socio Economic Issues}

Topics covered: Human and Institutional Factors, Economic Issues, Fire impacts on society

Chapter VII includes other short contributions that have under 1500 words (excluding acknowledgments and references) and that provide insights to more complete work that authors are still finishing or have published in other scientific journals.

We hope that this Conference and eBook may serve the purpose of keeping a record of the recent research in these fields and to illustrate the role that is played by the scientific community in the face of the challenges that these new times bring to fire science. We are sure that it will inspire and provoke more investigation and collaboration.

This e-book, published by the Coimbra University Press, is considered as a publication per se, independent from the Conference, and each paper is considered to be part of a chapter.

In the name of the Organizing Committee we wish to thank all the authors for their valuable work and for participating in the Conference.

We wish to thank Dr. Mike Flannigan for having accepted, once more, the role of Head of the Scientific Committee, who with the help of a group of selected experts reviewed and revised the submitted abstracts. Their work was very important to keep the high level of quality that has been the standard of this Conference.

We wish to thank to all the distinguished individuals that accepted to be members of the Honorary Committee.

We would like to express our personal thanks to all the members of the Organizing Committee and in particular to our colleagues of the Local Committee and the Secretariat for all their support, work and dedication during the preparation of the Conference. They carried the largest part of the work load that included the performance of large and small tasks that are required to put this Conference together and therefore they deserve thanks from all of us.

Coimbra, 24 August 2018

Domingos Xavier Viegas, Chairman of the Conference

Luís Mário Ribeiro, Co-Chairman of the Conference 


\section{Chapter 1 \\ Fire Risk Management}




\title{
A model for identifying blow-up fire potential
}

\author{
Richard H.D. McRae ${ }^{1}$; Jason J. Sharples ${ }^{2 *}$; Rachel L. Badlan ${ }^{2}$ \\ ${ }^{1}$ ACT Emergency Services Agency, Canberra, Australia \\ ${ }^{2}$ School of Physical, Environmental and Mathematical Sciences; UNSW Australia \\ \{j.sharples@adfa.edu.au*\}
}

\begin{abstract}
Australia's fire services mitigate the risk to the community from wildfires in a manner that leaves minimal residual risk. This work is founded on forecasts of fire behaviour based on fuel, terrain and weather. Using steady-state fire behaviour equations, any given set of conditions permits a single expected fire behaviour prediction. Since 2001, Australia has been impacted with rapidly increasing frequency by a class of fires driven by a coupling of the fire with the atmosphere above the mixed layer. In their most extreme form, these fires produced pyroCbs. The continental climatology has four events in the satellite era up to 2001, but since then 56 further confirmed events. This change in frequency is considered to be due to climate change. Blowup events were seen to occur when a fire fire switches from normal linear flaming to areal flaming - over $5 \mathrm{~km}$ flaming depth has been observed. If this happens in an unstable atmosphere, the plume forms a cloud before it mixes out. The latent heat of condensation then released allows a rapid escalation of fire intensity on the surface. Arising from the extreme wildfires that hit Canberra on 18 January 2003, a series studies have shown how these fires develop. These insights were used to develop a predictive model for these events. The Blow Up Fire Outlook (BUFO) model incorporates these in a process model that has been formally trialled. An extreme wildfire is defined here as one where one or more blow-up events occur before it is suppressed. Deep flaming events are known to be caused by: two steady-state processes (strong winds and wind changes), a set of dynamic processes (vorticity-driven lateral spread, eruptive spread, dense spotting), inappropriate use of ignition devices, and reburning of areas as new fuel becomes flammable with time. The BUFO model was run in trial mode in the Australian Capital Territory and New South Wales over three summers. The results indicated that the trial was successful, despite the small sample size involved. The POD was $100 \%$, the FAR was $33 \%$ and the TNR was $100 \%$. The model utilised weather forecast data from the Bureau of Meteorology, weather observations, terrain analysis data from the HighFire Risk project (www.highfirerisk.com.au) and fire information. It makes heavy use of remotely-sensed information. The model is designed to start if there is an uncontained wildfire with raised fire danger, and to ask questions that are either true or false. The answers form a path that either loops back (for the model to be re-run at a different time) or yields a prediction of the potential for a blow-up fire event. Most of the nine questions are required to assess the potential for the formation of deep flaming. So, given that most of the damage is caused by fires not covered by the standard toolbox used by fire services, BUFO is designed to augment the work of Fire Behaviour Analysts. We are working to increase its uptake across the sector.
\end{abstract}

Keywords: Extreme wildfire, blow-up fire, dynamic fire spread, deep flaming, atmospheric instability, vorticity-driven lateral spread.

\section{Introduction}

Since the turn of the century, extreme wildfires, although still largely uncommon, have become more frequent in Australia, and in other countries. These fires include the many of the most damaging and high profile fires of recent times, including Canberra in 2003, Tasmania in 2013 and Victoria in 2003, 2006 and 2009. They manifest as large, unstoppable fires that couple with the atmosphere. They are also the source of pyrocumulonimbus clouds (pyroCbs or fire thunderstorms). Since 2001 there have been 56 confirmed pyroCb events in Australia.

We have formally defined an extreme wildfire (Sharples, et al., 2016) as one where one or more 
blow-up events occur before it is suppressed. Blow-up events are defined as instances when the fire switches from normal linear flaming to areal, or 'deep', flaming - over $5 \mathrm{~km}$ flaming depth has been observed. If this happens in an unstable atmosphere the fire can couple with the atmosphere (Potter, 2002), modifying the fire's behaviour. An example of deep flaming occurring during the 2013 Wambelong Fire can be seen in Figure 1. If deep flaming occurs in an atmosphere conducive to enhanced plume development, the plume can form a cloud before it mixes out. The release of the latent heat of condensation then further exacerbates the coupling between the fire and upper levels of the atmosphere, which can produce more erratic and unpredictable winds, facilitate the mixing down of drier air, increase the potential for ember transport, and produce dry lightning.

Arising from the extreme wildfires that hit Canberra on 18 January 2003 (Fromm, et al., 2006), a series of studies have provided insights into how these fires develop (Mills, 2005, 2008a \& 2008b, Fromm, et al. 2012, McRae, et al. 2015). In particular, they have highlighted the role of deep flaming events in combination with atmospheric conditions conducive to violent plume development. These insights have been drawn upon to develop a predictive model for blow-up fire events (McRae \& Sharples, 2013 \& 2014). The Blow Up Fire Outlook (BUFO) model incorporates insights into the triggers of deep flaming with measures of atmospheric instability. Deep flaming events are known to be caused by: two steady-state processes (strong winds and wind changes), a set of dynamic processes (vorticity-driven lateral spread, eruptive spread, dense spotting and fire coalescence), inappropriate use of ignition devices, and reburning of areas as new fuel becomes flammable with time.

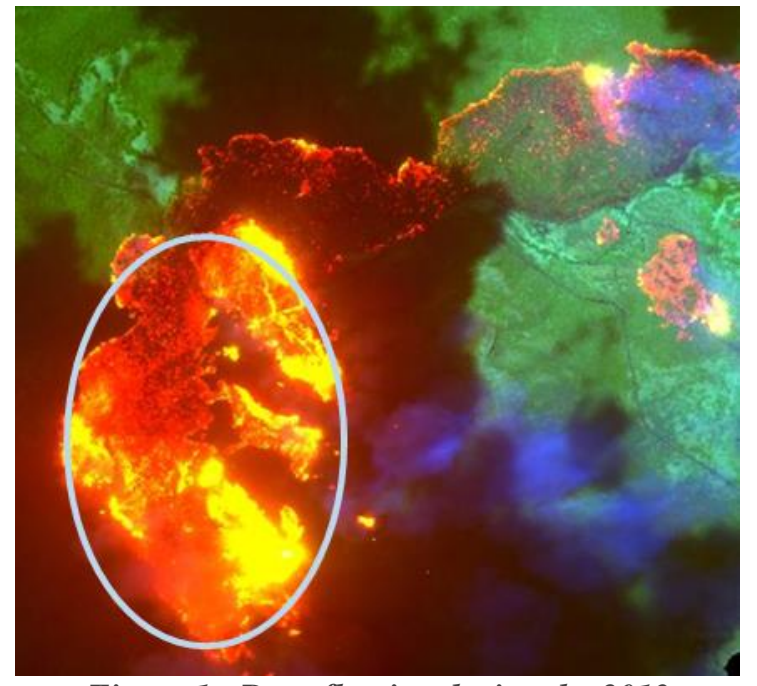

Figure 1 - Deep flaming during the 2013 Wambelong fire in NSW. The circled area exhibits widespread flaming in contrast to the more typical frontal fire behaviour that can be seen on other parts of the fire.

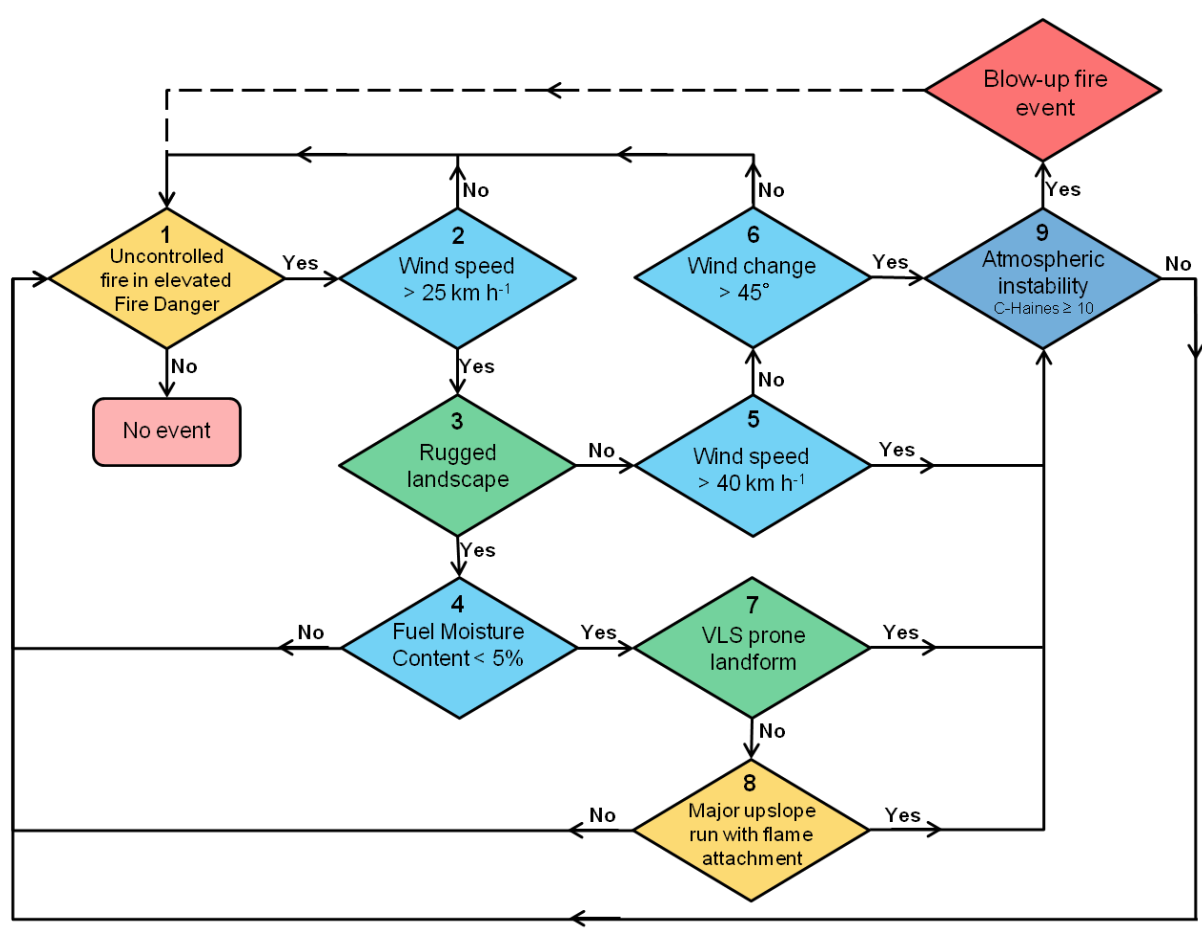

Figure 2 - Blow-Up Fire Outlook model flowchart. 
BUFO takes the form of a process model, which has recently been formally trialled. In this paper we provide an overview of the BUFO model and report on the outcome of the formal trial.

\section{The Blow-Up Fire Outlook (BUFO) model}

The BUFO model (Figure 2) takes the form of a decision tree, or flowchart. It comprises a series of questions leading from the start point, which concerns the occurrence of an uncontrolled fire on a day of elevated fire danger (i.e. hot, dry and windy surface weather). Each of the subsequent questions considers the setting of the fire and are formulated to provide a path dichotomy, with the "yes" option leading to further questions or to inferred occurrences of interest. The "no" option is used to loop back to allow re-running of the model at a future time (either to a schedule or when new information becomes available). The ultimate, inferred occurrence is that of a blow-up fire-event, and from there the model may be re-run at a future time. This loop-back is essential as some fire complexes may repeatedly blow-up. Examples of this include the 2003 and 2006 Victorian Alpine Complex and the West Fork (Colorado) Complex of July 2013.

In essence the model considers the likelihood of the fire exhibiting deep flaming in an atmospheric environment that is conducive to violent pyroconvective development of the plume. At present the atmospheric component of the model is assessed based on the continuous Haines index, with values over 10 being considered necessary for blow-up fire occurrence. However, in the future this component will likely be refined as a more comprehensive understanding of the influence of detailed frontal dynamics on plume development becomes available.

The model utilises weather forecast data from the Bureau of Meteorology, weather observations, terrain analysis data (e.g. available at www.highfirerisk.com.au), and fire information such as aerial linescans. It also makes heavy use of remotely-sensed information.

\section{Results of a formal trial}

The BUFO model was run in trial mode in the Australian Capital Territory and New South Wales between December 2013 and March 2016 (inclusive).

Table 1 - BUFO trial activity timeline. Only the last twelve months looked at areas outside of NSW/ACT.

\begin{tabular}{cccc} 
Month & $\begin{array}{c}\text { Assessments } \\
\text { undertaken }\end{array}$ & $\begin{array}{c}\text { NSW/ACT } \\
\text { Warnings }\end{array}$ & $\begin{array}{c}\text { NSW/ACT } \\
\text { Events }\end{array}$ \\
\hline Dec-13 & 1 & 0 & 0 \\
Jul-14 & 3 & 0 & 0 \\
Sep-14 & 2 & 0 & 0 \\
Oct-14 & 2 & 0 & 0 \\
Nov-14 & 4 & 1 & 0 \\
Dec-14 & 1 & 0 & 0 \\
Oct-15 & 2 & 0 & 0 \\
Nov-15 & 4 & 1 & 1 \\
Dec-15 & 3 & 0 & 0 \\
Jan-16 & 2 & 1 & 1 \\
Feb-16 & 1 & 0 & 0 \\
Mar-16 & 1 & 0 & 0 \\
\hline
\end{tabular}

Over that period there were 23 days when the initial conditions for the model were met, or nearly met, and so warranted analysis. Overall, three alerts were issued. In two of these instances blow-up events were observed, while in one instance no blow-up eventuated. Therefore, the Probability of 
Detection was $100 \%$ and the False Alarm Rate was 33\%. The True Negative Rate, based on assessments made without an alert being issued and subsequently no blow-up event occurring, was $100 \%$. A future automated system would remove potential biases in the latter statistic, as all days could be assessed.

\subsection{Yarriman Road Fire (Pillaga), 26 November $2015\left(149.6^{\circ},-30.1^{\circ}\right)$}

A fire started in the Pilliga Scrub in New South Wales in the early afternoon. It was assessed with the BUFO model, as it was an uncontained wildfire under elevated Forest Fire Danger Index. The wind was over $25 \mathrm{~km} / \mathrm{hr}$, allowing various dynamic effects to potentially come into play. The landscape was flat, suggesting a wind driven elliptical fire with limited wind-terrain interactions. The winds were generally under $50 \mathrm{~km} / \mathrm{hr}$, but gusts exceeded that. A wind change was forecast to arrive midafternoon, which could produce deep flaming if a flank became a new, broader headfire. However gust winds could also produce deep flaming through high rates of spread. For either mechanism, the forecast Continuous Haines Index was 12, suggesting enough instability and dry air above the LCL to permit the fire to couple with the atmosphere.

This event shows the potential for a warning being issued with a useful lead-time being provided:

- 14:50 BUFO alert issued, with a review flagged for 17:00.

- 15:10 fire plume starts to show in Namoi weather radar.

- 15:30 radar reflectivity indicates enhanced pyroconvective activity, confirming a blow-up event (Figure 3).

- It is likely that the wind change arrived at 15:40.

- 18:40 radar reflectivity declines.

Therefore, the alert was able to be issued 40 minutes before the blow-up occurred. This was a true positive for the model. There were other fires in the trial area that caused operational concern, but for which the BUFO model did not indicate blow-up potential. None of these fires formed blow-ups events.

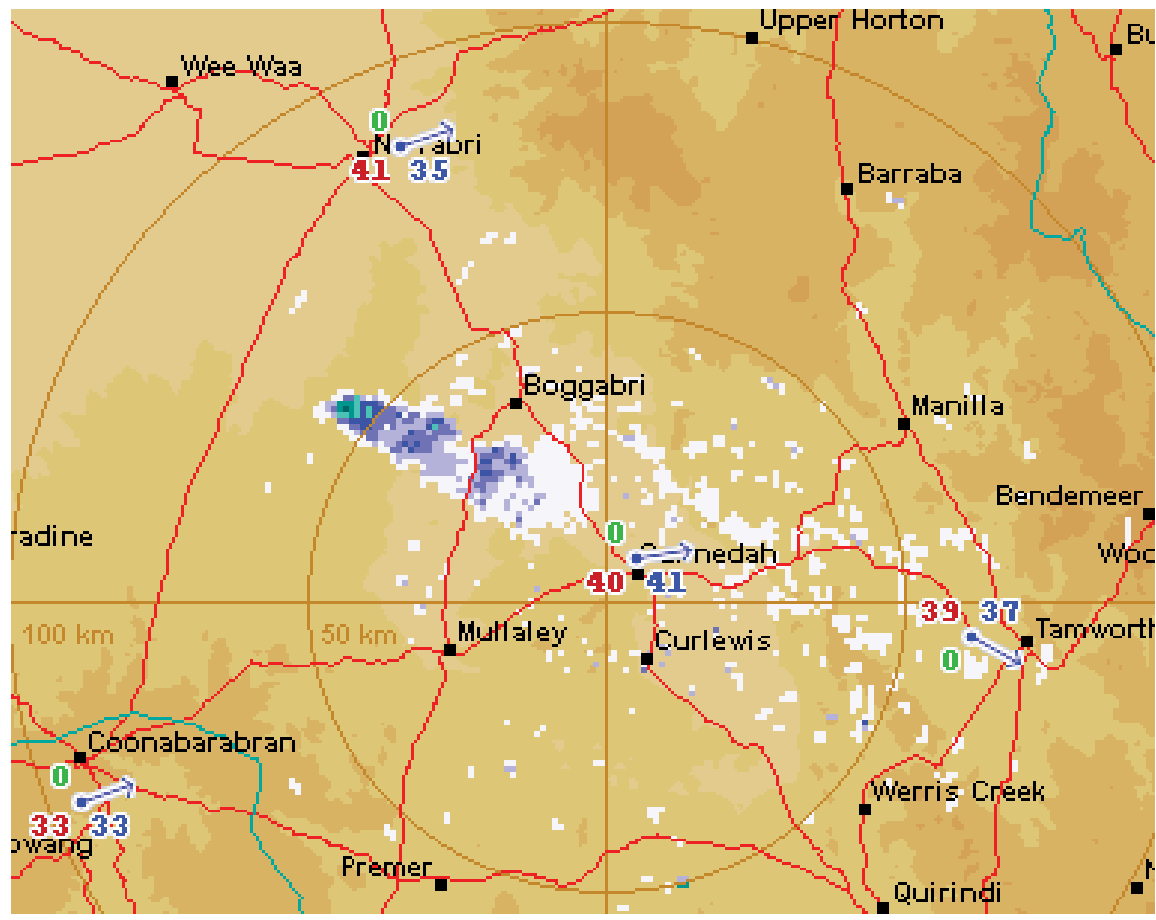

Figure 3 - Radar image of the plume of the Yarriman Road Fire showing partial $50 \mathrm{~km}$ and $100 \mathrm{~km}$ range rings. Image time: 16:00 AEDT (UTC+11); radar: Bureau of Meteorology, Namoi reflectivity. 


\subsection{Sir Ivan Fire, 11 February $2017\left(149.6^{\circ},-32.0^{\circ}\right)$}

After the trial had concluded, an operatonal BUFO alert was issued for a fire that went on to form a pyroCb the next day. It is believed that vorticity-driven lateral spread (Sharples, et al., 2012) was initiated along numerous sandstone cliffs in the area, further excerbated by passage of a trough overhead. The use of Himawari-8 satellite imagery at this fire was improtant, and suggested new aspects of violent pyro-convection that might be detected.

\section{Conclusion}

A useful level of skill has been demonstrated for anticipating fires that become blow-up events. The context, a landscape with elevated fire danger and a number of fires, can be analysed to identify where risks to the community and fire crews are most elevated. Subsequent work, outside the formal trial domain, indicates that broader implementation of the BUFO model will increase the likelihood of capturing future major wildfires and the threats that they pose. A holistic approach will provide more experience for practitioners and the ability to train on remote fires that pose little or no risk to the local community.

Most importantly, operational BUFO modeling promises to improve the ability of fire agencies to warn the community and their crews of potential threats from the most dangerous class of wildfires. Current best practice involves warnings to entire regions. There is promise of an ability to warn in a far more targeted manner, both spatially and temporally. Benefits of this could be far-reaching: avoiding loss-of-life (including avoiding tragedies on roads during evacuation); increasing the chance that warnings reflect real threats; and lessened community and economic impacts on a regional scale.

\section{Acknowledgements}

The authors are indebted to Laurence McCoy, NSW Rural Fire Service, who helped facilitate the operational trial of the model.

\section{References:}

Fromm M, Tupper A, Rosenfeld D, Servranckx R, McRae R, (2006) Violent pyro-convective storm devastates Australia's capital and pollutes the stratosphere. Geophysical Research Letters 33, L05815.

Fromm MJ, McRae RHD, Sharples JJ, Kablick GP (2012) Pyrocumulonimbus pair in Wollemi and Blue Mountains National Parks, 22 November 2006. Australian Meteorlogical and Oceanographic Journal, 62(3): 117-126.

McRae RHD, Sharples JJ (2013) A process model for forecasting conditions conducive to blow-up fire events. Proceedings, 2013 MODSIM Conference, Adelaide.

McRae RHD, Sharples JJ (2014) Forecasting conditions conducive to blow-up fire events. CAWCR Research Letters, 11, 14-19.

McRae R H D, Sharples JJ, Fromm M (2015) Linking local wildfire dynamics to pyroCb development. Natural Hazards and Earth Systems Sciences, 15, 417-428.

Mills, G.A. (2005) On the sub-synoptic scale meteorology of two extreme fire weather days during the Eastern Australian fires of January 2003. Australian Meteorological Magazine V54, pp: 265290.

Mills, G.A. (2008a). Abrupt surface drying and fire weather Part 1: overview and case study of the South Australian fires of 11 January 2005. Aust. Met. Mag. 57: 299-309. 
Mills, G.A. (2008b). Abrupt surface drying and fire weather Part 2: a preliminary synoptic climatology in the forested areas of southern Australia. Aust. Met. Mag. 57: 311-328.

Potter BE (2002) A dynamics based view of atmosphere-fire interactions. International Journal of Wildland Fire, 11, 247-255.

Sharples JJ, Cary GJ, Fox-Hughes P et al. (2016) Natural hazards in Australia: extreme bushfire. Climatic Change 139. doi:10.1007/s10584-016-1811-1.

Sharples, J.J., McRae, R.H.D. \& Wilkes, S.R. (2012). Wind-terrain effects on the propagation of wildfires in rugged terrain: fire channelling. International Journal of Wildland Fire, 21, 282-296 


\title{
Assessing the increase in wildfire occurrence with climate change and the uncertainties associated with this projection
}

\author{
Hélène Fargeon ${ }^{1 *}$; Nicolas Martin-StPaul ${ }^{1}$; François Pimont ${ }^{1}$; Miquel De Cáceres ${ }^{2}$; Julien \\ Ruffault $^{3}$; Thomas Opitz ${ }^{4}$; Denis Allard ${ }^{4}$; Jean-Luc Dupuy ${ }^{1}$ \\ 1*URFM INRA, 84914 Avignon, France. \{helene.fargeon@inra.fr*,nicolas.martin@inra.fr, \\ nicolas.martin@inra.fr, jean-luc.dupuy@inra.fr \} \\ ${ }^{2}$ CTFC, 25280 Solsona, Spain. \{miquelcaceres@gmail.com\} \\ 3IMBE, 13290 Aix-en-Provence France. \{julien.ruff@gmail.com\} \\ ${ }^{4}$ BioSP INRA, 84914 Avignon, France. \{thomas.opitz@inra.fr, denis.allard@inra.fr \}
}

\begin{abstract}
According to climate projections, global warming is associated with increasing temperatures and dry spells in some parts of the world, especially the Mediterranean area. This climate change has already triggered increases in wildfire danger and fire season length in Southern Europe and is expected to amplify in the forthcoming decades. However, it is quite challenging for the scientific community to assess the intensity of these changes, because (i) the trend relies on the greenhouse gases (GHG) emission scenario and (ii) fire occurrence depends on multiple factors (including climate, but not only). A proper assessment of the trend in terms of fire occurrence and of uncertainties associated with this increasing trend, still lacks, especially for the French territory.

Our study refines traditional approaches of fire risk projection under climate change on two aspects: (i) the impact of climate prediction uncertainties on the prediction of fire danger, and (ii) the translation of a danger index into a fire occurrence (per size classes).
\end{abstract}

Keywords: climate change, uncertainties, fire danger

\section{Introduction}

The impact of climate change on wildfire danger has been extensively studied in different parts of the world. The traditional approach consists in projecting climatic danger indices like the Fire Weather Index (FWI) or its subcomponents. The FWI System is a fire danger rating system designed by the Canadian Forestry Service (Van Wagner 1987). It models the moisture content of three classes of forest fuel and combines it with the effect of wind on fire behaviour. It is noticeable that the FWI System refers primarily to a standard fuel pine type but has been widely used as a general measure of forest fire danger, even in areas with climate and vegetation markedly differing from that in Canada. Yet, a link between high FWI values and observed fire occurrences has been shown in the Mediterranean context (Good et al. 2008; Viegas et al. 1999). Regarding climate change, some studies have projected FWI at Europe scale for various GHG emission scenarios (Moriondo et al. 2006; Bedia et al. 2014a) and various climate models, predicting an increase in overall FWI values and a change in the length of the fire season (Moriondo et al. 2006). Many FWI projections have also been produced at country or regional scale in Europe. However, few studies address the origin of the uncertainties in these projections: can we separate the uncertainty coming from the climate model and the uncertainty coming from the scenario choice, and which one is predominant? Does this partition vary during the time period of interest? In a first step, we project future FWI and attempt to evaluate the resulting uncertainties for France. The interpretation of FWI projections in terms of future fire activity with metrics such as expected fire number or burnt area (e.g. Amatulli et al. 2013) is challenging. Indeed, FWI is not a straightforward proxy for fire activity, as its response function is often unknown and as a 
lot of other factors interact to determine fire activity (Bedia et al. 2014b): ignition patterns and fuel availability are linked to land use and land cover, to vegetation adaptation to climate change, to population density and awareness to the risk, fire prevention policies, etc. As a result, interpreting temporal and spatial averages of FWI (or of derived metrics of the FWI such as number of days above a certain threshold) is challenging. For example, it is not straightforward to interpret the exact meaning of a 10\% increase in the average of FWI over given territories and time periods, in terms of quantification of fire activity evolution. In the present study, we develop a statistical model to predict fire occurrence as a function of daily FWI and a few other factors for Southern France and project the fire occurrence under future climate, in order to facilitate the interpretation of FWI projection under future climate.

\section{Assessing future climatic fire danger and its uncertainties}

\subsection{Increasing droughts and temperatures}

To simulate the evolution of climate over France during the coming century, we used simulations output from five couples of global and regional climate models (GCM-RCM), with two IPCC scenarios (rcp4.5 and rcp8.5) at ca. $0.5^{\circ}$ spatial resolution. GCM-RCM pairs were chosen according to the synthesis of McSweeney et al 2014, to be the most realistic while providing a contrasted range of projections for temperature and precipitation amount, two climate variables especially involved in fire probability. This was done in order to encompass the whole range of predictions with a minimum of models.

A bias correction and downscaling was performed using quantile mapping and anomaly method with R package "meteoland" (De Cáceres et al. 2017). The 8-km-resolution-SAFRAN reanalysis (Vidal et al. 2010) was used as reference data. The FWI, widely used to forecast climatic fire danger, was then computed for all the daily climate series for the period 2005-2100, using package "cffdrs" (Wang et al. 2017).

All models converged toward an increase in FWI during the $21^{\text {st }}$ century (Figure 1). The projections nevertheless differed in terms of intensity, with some models predicting harder conditions than others in accordance with our prior model selection.

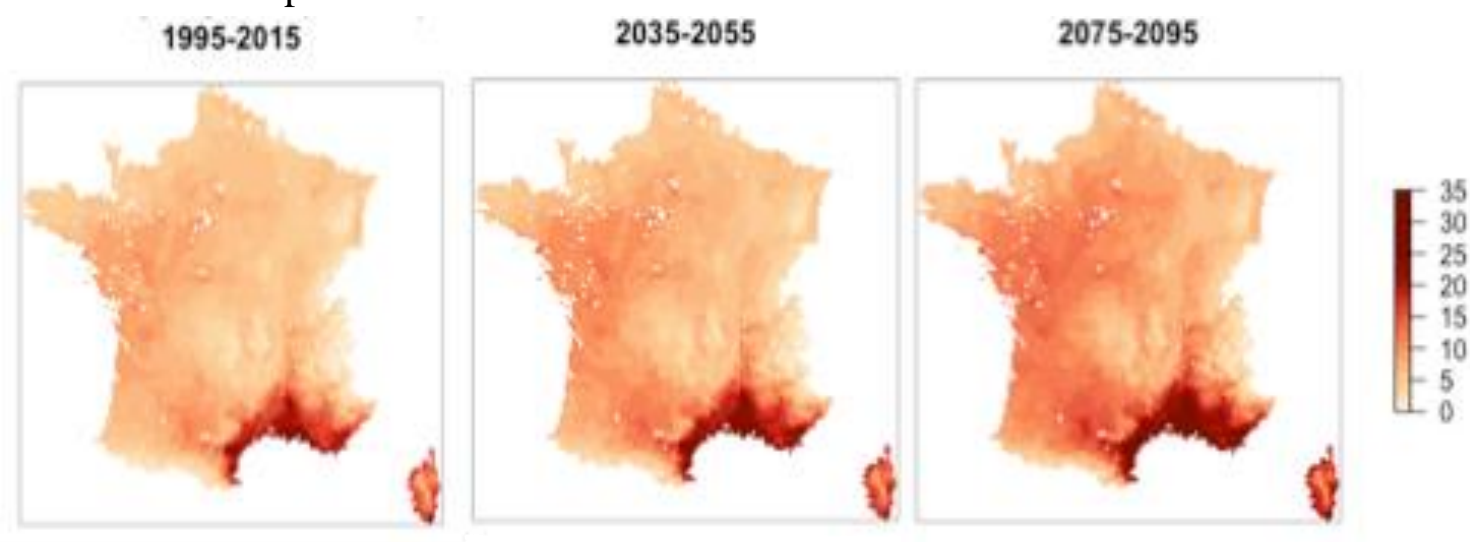

Figure 1 - Evolution of the mean FWI during fire season (June-September) for different time horizons. Each map corresponds to an average between models and scenarios. They show an overall increase in FWI values over the period.

\subsection{Uncertainties about the projections}

We distinguish three different sources of uncertainties, following the approach developed in (Hawkins and Sutton 2009). The inter-annual variability of the climatic danger, calculated as the variation of annual mean values with respect to a trend defined as a 30-year moving average, is called "internal variability". We consider this internal variability as constant during the whole time-period. 
A second source of uncertainty, called "model uncertainty", comes from the differences between the trends of the different models. Finally, the last source of uncertainty we considered derived from differences in GHG emission scenarios and is calculated as the difference between the multimodel mean values given respectively by scenario rcp 4.5 and by scenario rcp8.5 ("scenario uncertainty").

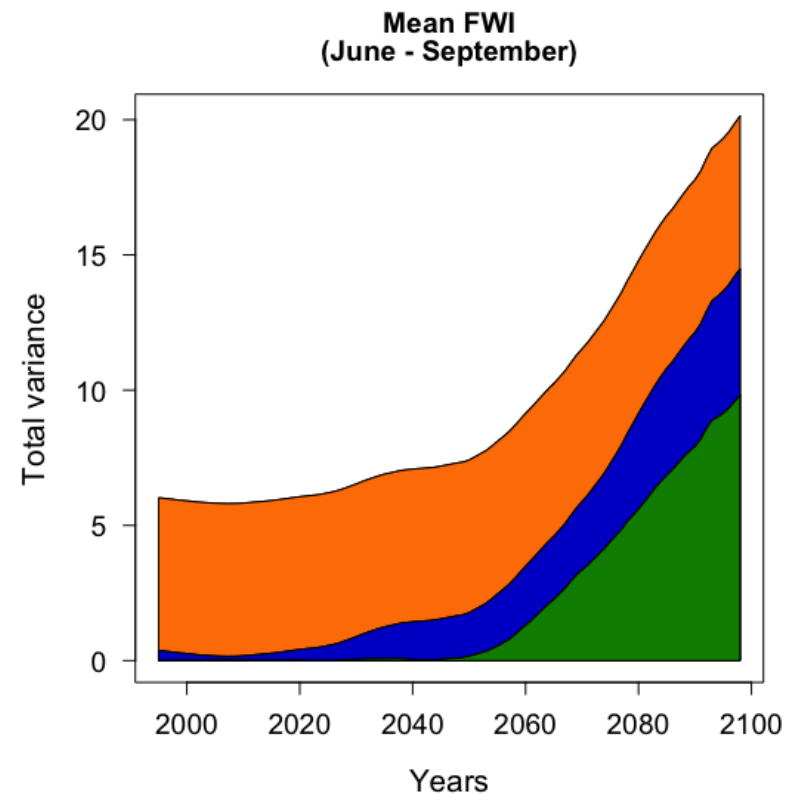

Figure 2 - Proportion of the different contribution to total variance in mean future annual FWI predictions during fire season. Internal variability contribution is in orange, model uncertainty in blue, and scenario uncertainty in green.

Internal variability remains the major source of overall variance in the prediction until the 2050s (Figure 2). In the second half of the century, overall variance begins to increase at an important pace, thanks to growing model and scenario uncertainty. This almost leads to a fourfold increase of the total variance in 2100 as compared to 2000.

\section{Projecting the fire occurrence in Southern France}

\subsection{Linking past fires with past weather conditions through a statistical model}

The next step of our approach consists in transforming FWI projections into expected wildfire occurrence, i.e. number of fires (per size classes, i.e. above some threshold for burnt area) per day and $\mathrm{km}^{2}$. In the fire-prone French Mediterranean area, data showed that observed fire occurrence (derived from the Prométhée database) clearly increased with daily FWI (computed with SAFRAN reanalysis), which confirms the relevance of FWI projection for the evaluation of climate change impacts on fire risk. However, the wildfire occurrence does not increase linearly with FWI and is also strongly affected by other temporal and spatial factors (Figure 3), which raises some concerns regarding the interpretation of projections. 


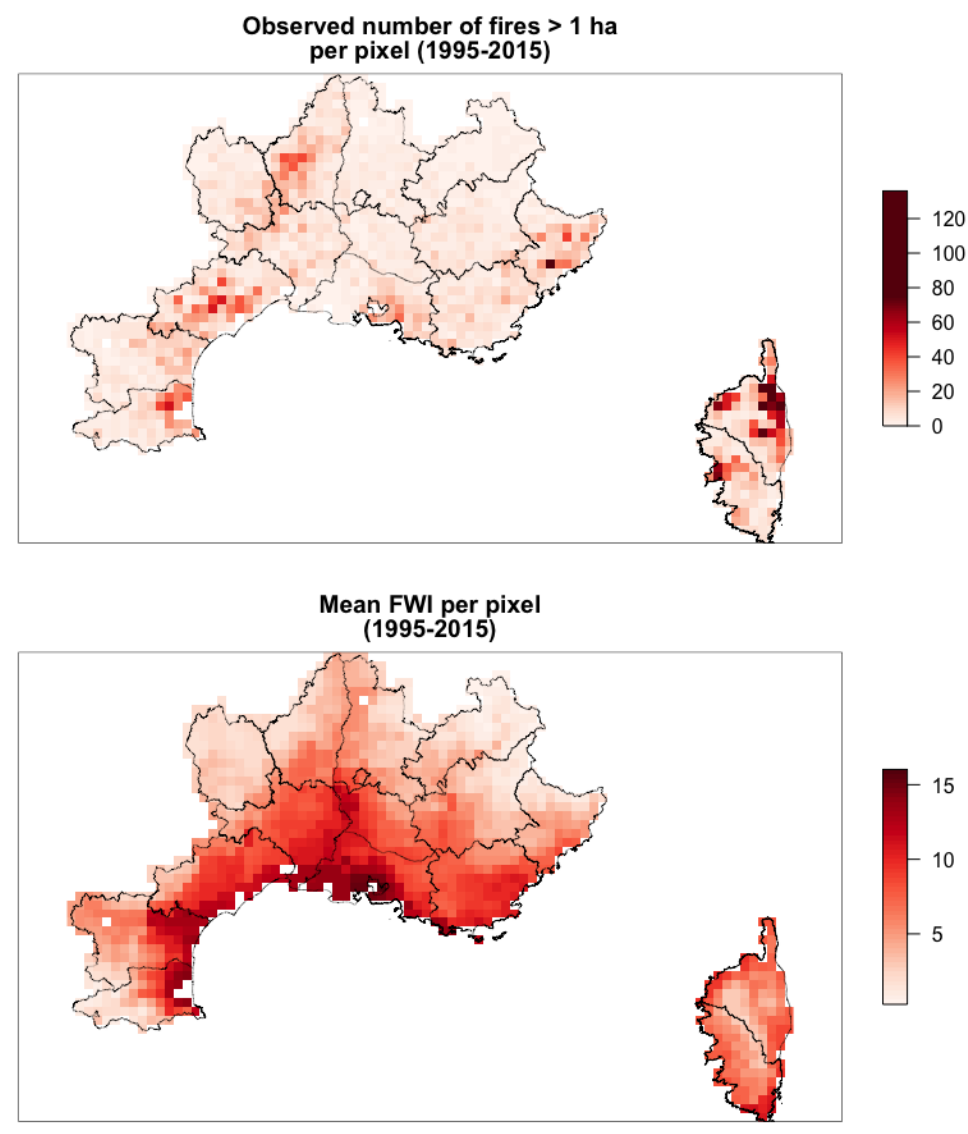

Figure 3 - Spatial distribution of observed fires above 1 ha burnt area in French Mediterranean area (Prométhée database), compared with the distribution of mean annual $F$ WI values per pixel during the same time period. Wildfire occurrence appears to be strongly affected by spatial factors.

With this in mind, we developed a probability-based model for predicting the daily expected number of forest fires above 1 ha burnt area as a function of explanatory variables like FWI, season and location. The approach is adapted from the framework developed to model the probability of fire occurrence in (Brillinger et al. 2003; Preisler et al. 2004). Here we had to group our data into spatialtemporal cells $\left(64 \mathrm{~km}^{2}\right.$-day cell level), so that the logistic model used in the original studies was replaced by the Poisson model, fitting a non-parametric Poisson model with log-additive intensity to the data. Aside from the FWI, we estimate partial multiplicative effects of other variables, selected to better represent data features that are not well represented (e.g. seasonal pattern), or not represented at all (e.g. spatial effects such as proximity to urban interfaces and networks) in the definition of the FWI. The form of the model is described in Equation (1), where $S$ refers to the forested area in the pixel. The model is adjusted using integrated nested Laplace approximations (INLA) described in (Rue et al. 2009; Lindgren and Rue 2015).

$$
\text { Fire number }=\text { Intercept } * S * f(S) * g(F W I) * h(\text { Week }) * i(\text { Pixel })
$$

The partial effects of each variable on fire occurrence according to the statistical model are presented in Figure 4 and Figure 5. 

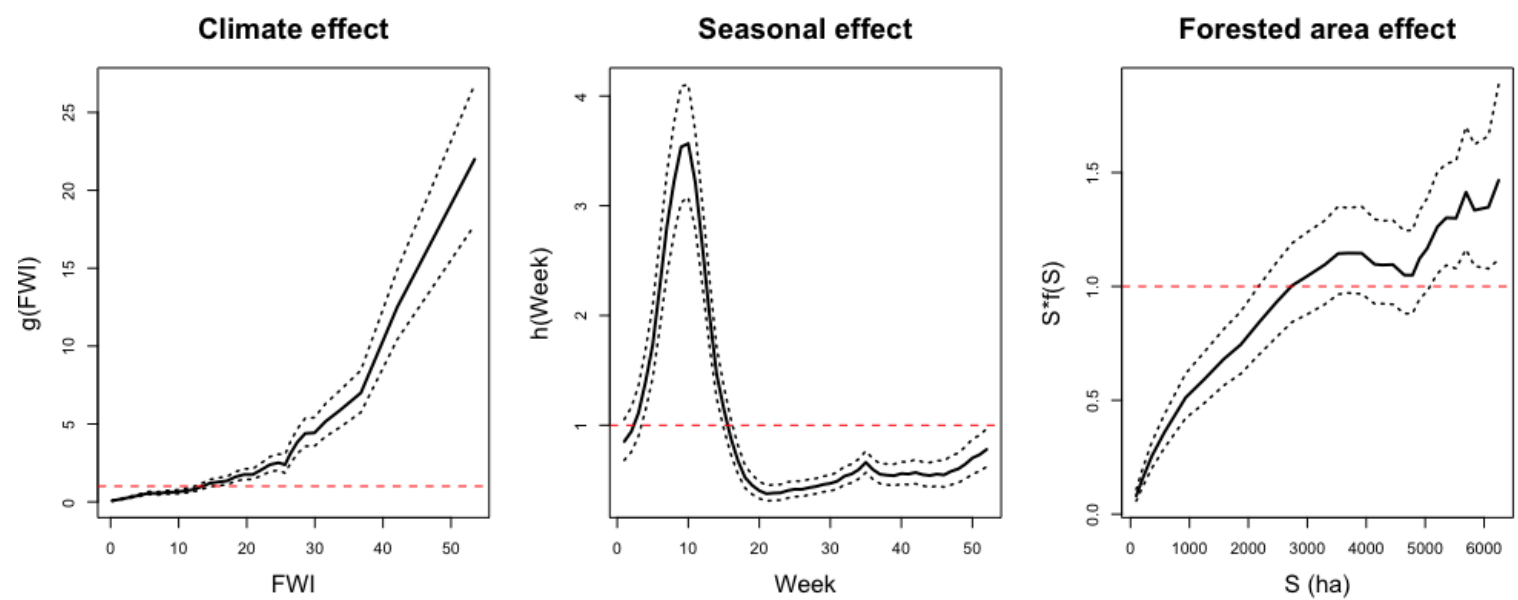

Figure 4 - Partial effects of the statistical climate-fire model. From left to right, we show the effects of daily $F$ WI (surrogate for climate effect), season (week of the year) and forested area on the number of fires above 1 ha burnt area per day and per $\mathrm{km}^{2}$. Low values indicate an inhibition effect on fire occurrence, while high values show an enhancement.

The link between high FWI and high fire occurrence is confirmed and exhibits a clear non-linear response function. Importantly, such non-linear shape of the function makes it tricky to interpret the FWI directly in terms of fire occurrence: it is not the same to have one day at FWI $=5$ and one day at $\mathrm{FWI}=45$, compared to 2 days at $\mathrm{FWI}=25$. The expected number of fire will be much larger in the first than in the second scenario, because of the convexity of the response function.

Concerning seasonal relative fire risk, a peak in fires associated to early spring is noticeable, not well addressed by the FWI alone. This seasonal effect is probably related to the seasonality of live fuel moisture. An FWI of 10 in March will correspond to a much larger number of actual fires than in June, which again raises the question of the significance of the FWI.

Fire occurrence increases with increasing forested area, as expected since the surface area available for ignition increases. However, a saturation of occurrence is observed for high forested areas and can be interpreted as an effect of lower population and infrastructure densities in densely forested pixels, resulting in a lower human ignition pressure.

\section{Spatial effect}
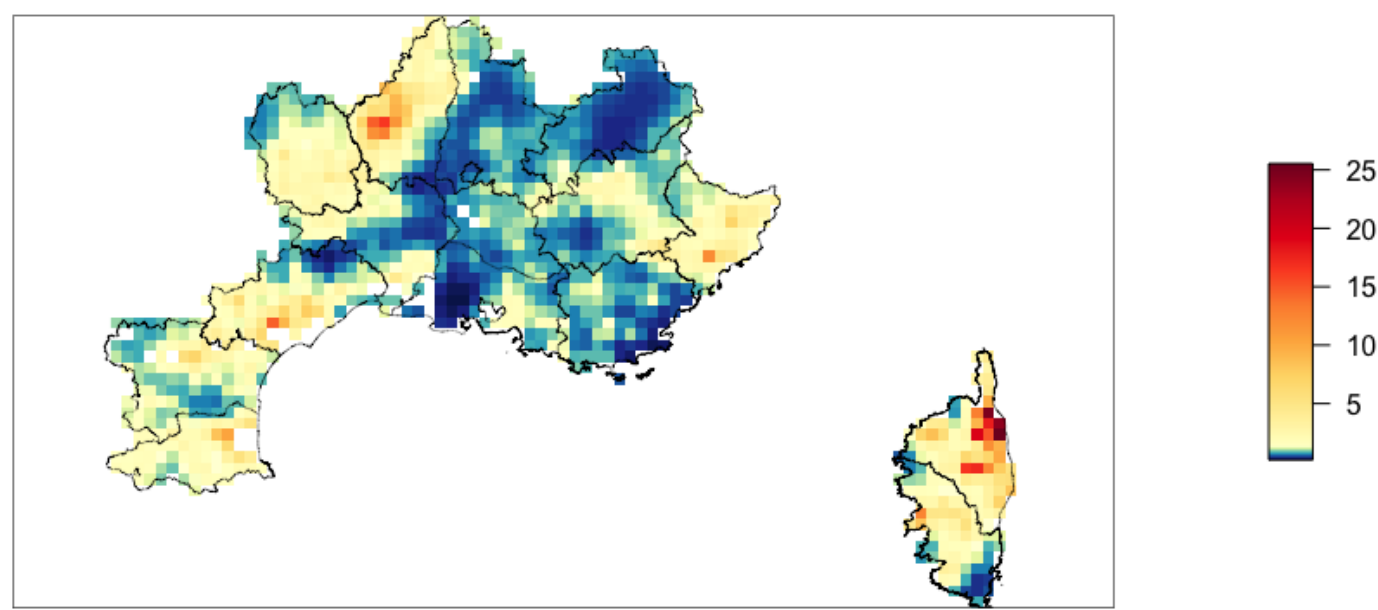

Figure 5 - Effect of spatial location on the number of fires above 1 ha burnt area per day and per $\mathrm{km}^{2} . \mathrm{High}$ variability is visible, with some hotspots of expected fires that cannot be explained by the other explanatory variables included in the model. 
A remaining spatial pattern, which accounts for regional variations that are not explained by the other factors, also exhibits a strong influence on fire occurrence. Indeed, the geographic position is the only other explanatory variable which explains variation in fire occurrence with amplitudes comparable to the FWI effect.

Using this generative model to simulate expected fires under past conditions, we can try to unveil hidden explanatory variables like human action. This has been done in Figure 6.

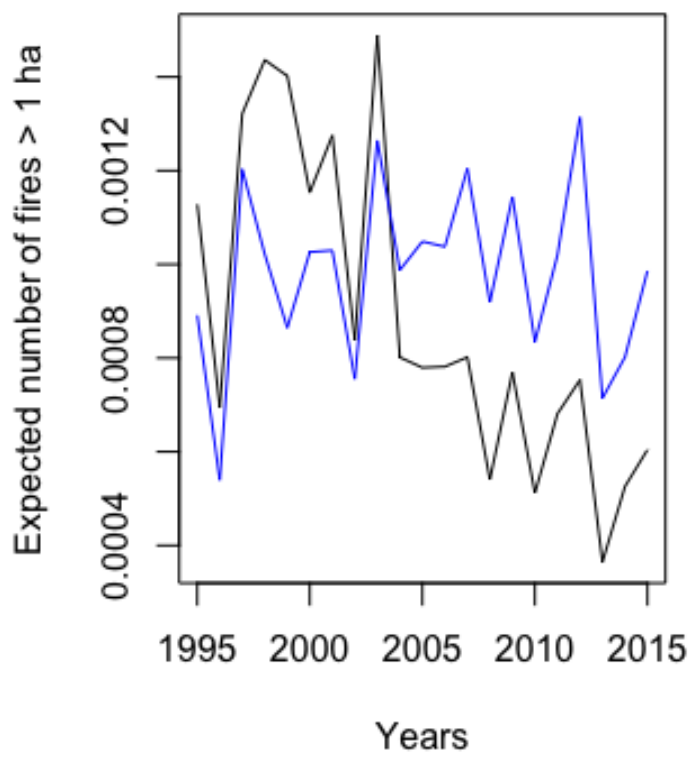

Figure 6 - Expected mean number of fires above 1 ha burnt area over the full Prométhée zone, per year and per pixel. Observed values are in black while simulated values are in blue.

We see that the model reproduces well the interannual pattern attributable to climate variations, as peaks and valleys of the simulated occurrence (in blue) most often occur for the same years than the actual data (in black). However, the model fails to take into account the evolution between pre- and post-2003 periods. The observed decrease in actual fire densities over time while simulated data remained fairly constant suggests an improvement in prevention and fire suppression policies in France since 2003, a historical year in terms of wildfires in France. This improvement in the last decade could be otherwise falsely interpreted as a result of less fire-prone climatic period.

\subsection{Projecting potential wildfire occurrence increase in the future}

The last step of our approach consists in projecting the expected fire occurrence (trend and uncertainty) in future climate using the statistical model we built for climate-fire relation. The benefit of this approach is that the fire number (occurrence) is an additive meaningful metric of fire activity, which can readily be integrated over time and space, contrary to FWI which requires the definition of subjective thresholds for its interpretation.

We used daily FWI series for the period 2080-2100 given by climatic models to predict the expected numbers of fires according to our model. The first results integrated over the Mediterranean area partly reproduce the intra annual distribution of wildfires observed in the past, with a first peak of fires in early spring and a second one in summer (Figure 7). The summer fire occurrence increases remarkably, probably because of the sharper increase of FWI values expected in summer (compared to the other seasons). 


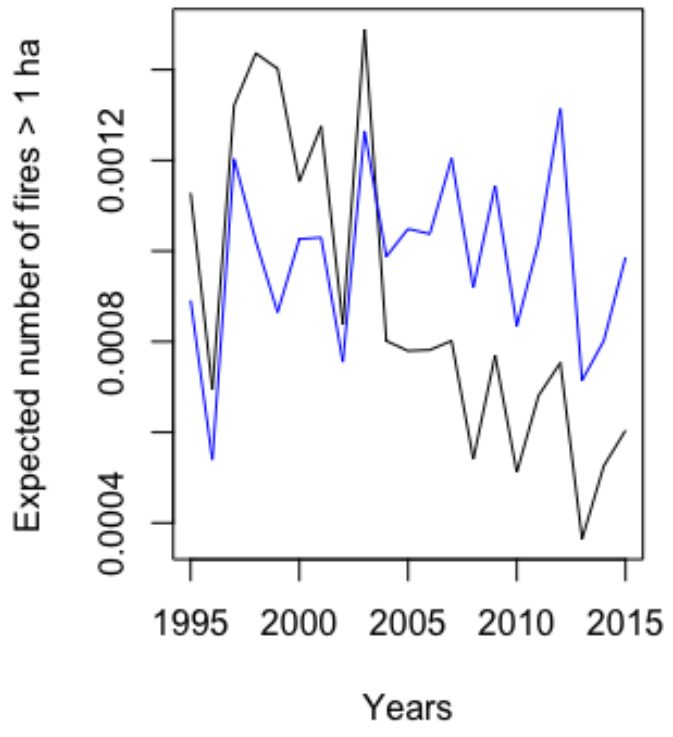

Figure 7 - Expected mean number of fires above 1 ha burnt area over all Promethee zone per year and per pixel $\left(8 * 8 \mathrm{~km}^{2}\right)$. Current observed values (1995-2015) are in black while projected values (model CNRM RCA4, scenario rcp8.5, 2080-2100) are in red. The model predicts an increase in summer fire occurrence. Winter fire occurrence remains stable for this time horizon.

\section{Discussion and perspectives}

Figure 8 shows the contributions of the different sources of uncertainty associated with climate projections of the seasonal mean FWI to the total variance (Figure 8, left) and compares them to the results that Hawkins and Sutton (2009) reported, for the global, decadal mean air temperature (Figure 8 , right). It is worth noting that the scenario uncertainty at the end of the century dominates for both variables, but that the air temperature exhibits far less intrinsic variability (and negligible from 2050) and far more model spread (dominant until 2050) than the FWI. In other words, it is not appropriate to use uncertainties on weather variables provided by climatologists when looking at projections of a specific climate impact such as wildfire danger. The FWI is a non-linear combination of weather variables, hence we should expect such differences to arise.
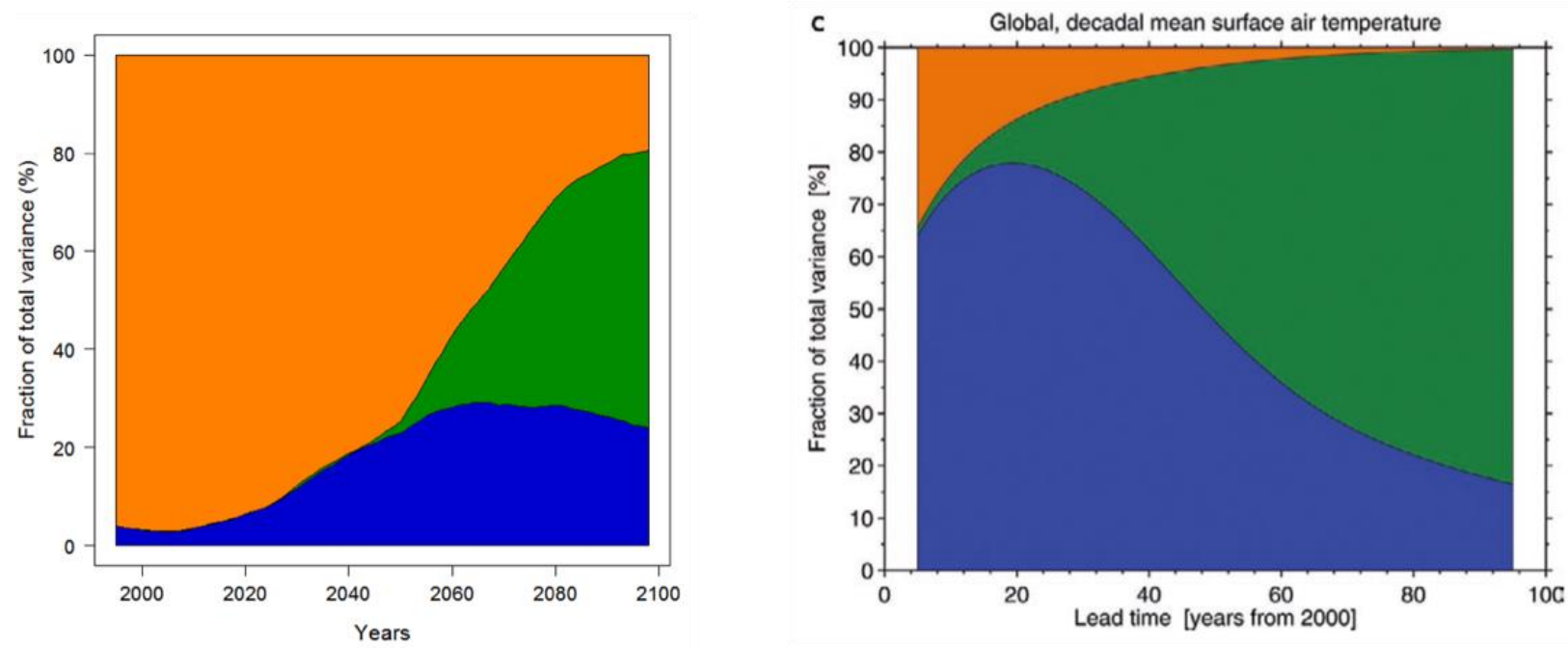

Figure 8 - Contributions of the different sources of uncertainty associated with climate projections of the seasonal mean FWI values (left) and global decadal mean temperatures (right) to the variance.

The fact that for FWI projections the scenario uncertainty overwhelms the other sources of uncertainty at the end of the century, demonstrates the importance of implementing climate policies to avoid a dangerous path in terms of wildfire danger. The result is particularly important because, in 
contrast, intrinsic variability dominates during the first decades up to 2050 and could hide the reality of the mean growing fire danger trend.

Our model performs well to highlight the explanatory power of the FWI as a predictor of past fire occurrence. The non-linear form of the curve confirms the issue arising when averaging FWI over space or time as a metric of future fire danger. This issue is related to, but should not be confounded with, the issue addressed in the FWI System with the proposed Daily Severity Rating (DSR). The DSR is an exponential transformation of the FWI and is supposed to be in more direct proportion to the work required to suppress a fire, but it does not correct a statistical bias. Yet, the Seasonal Severity Rating (SSR), which has been used in some projection studies (e.g. Amatulli et al. 2013; Bedia et al. 2014a), is the average of the DSR over the fire season and is more suitable for averaging than the FWI (Van Wagner 1987). In other words, it makes sense to average fire control effort, not fire danger rates.

Ongoing work integrates the development of a proper metric to compare the uncertainties arising from the climate model projections with those arising from the statistical model of expected fire occurrence.

Among the limitations of our projection method, it is applied here to fire occurrence which is not the best metric for fire risk interpretation. Similar work on burnt area is much more challenging, as burnt areas are more variable than occurrences. Another limitation is the fact that the occurrence model is fitted on the South-East of France in which fire datasets are available and in which fire occurs more frequently than in other regions. Projecting the model to whole France would be of high interest as fire-prone areas are expected to expand to the north owing to climate warming (Chatry et al. 2010), but this raises several problems, since many factors strongly vary among French territory, like land use and land cover, forest structure, population density, fire suppression policies. Yet we could project a potential fire number using the FWI partial effect $g(F W I)$ estimated in our occurrence model and bracket the projected values by uncertainties associated with other fire drivers.

To get more mechanistic insights into the drivers of fire that are hidden behind the FWI, it would be relevant to assess the relative contribution of the different sub-component of the FWI (DC, DMC, FFMC...) to the fire occurrence and burnt area. This would also help to evaluate spatially which components of climate change are responsible for the increase in FWI and fire danger and thus help to develop appropriate mitigation/adaptation strategies.

Finally, the statistical model for fire occurrence was initially developed for climate change impact studies, but it could be used for operational purposes at the scale of the South-East France as well, after cross-validation and testing over some evaluation period. Indeed, daily forecasts of fire numbers would be of a higher value to fire managers than a fire danger rate.

\section{Conclusion}

We propose a methodology to assess the impact of climate change on forest fire occurrence in French Mediterranean area. This method allows a quantitative assessment of the different uncertainties associated with the climate projection. The statistical model we develop enables us to transform predictions of FWI into expected numbers of fires and reproduces accurately observed patterns of past fire activity. Disentangling the effects of climate and other spatial-temporal effects has seldom been done in the French Mediterranean area and provides insights on the FWI limitations as well as on interesting spatial effects. The predictive ability of the model under changing climate is still to be considered with caution, due to the correlation existing between the different factors governing fire occurrence and severity, that might change under a changing climate and time period. The model for fire occurrence, after proper testing, could provide daily forecasts of fire numbers in the French Mediterranean area. 


\section{Acknowledgements}

Acknowledgements are expressed to the French Ministry of Agriculture which funded the research activities.

\section{References}

Amatulli, G., A. Camia, and J. San-Miguel-Ayanz. 2013. 'Estimating Future Burned Areas under Changing Climate in the EU-Mediterranean Countries'. Science of The Total Environment 450-451 (April): 209-22.

Bedia, J., S. Herrera, A. Camia, J. M. Moreno, and J. M. Gutiérrez. 2014. 'Forest Fire Danger Projections in the Mediterranean Using ENSEMBLES Regional Climate Change Scenarios'. Climatic Change 122 (1-2): 185-99.

Bedia, J., S. Herrera, and J. M. Gutiérrez. 2014. 'Assessing the Predictability of Fire Occurrence and Area Burned across Phytoclimatic Regions in Spain'. Natural Hazards and Earth System Science 14 (1): 53-66.

Brillinger, D. R., H. K. Preisler, and J. W. Benoit. 2003. 'Risk Assessment: A Forest Fire Example'. In Institute of Mathematical Statistics Lecture Notes - Monograph Series, 177-96. Beachwood, OH: Institute of Mathematical Statistics.

Chatry C., Le Quentrec M., Laurens D., Le Gallou J-Y., Lafitte J-J., Creuchet B. 2010. Rapport de la mission interministérielle 'Changement climatique et extension des zones sensibles aux feux de forêts'. CGAER-CGEDD-IGA. 89 p.

De Cáceres, M., N. Martin-StPaul, V. Granda, and A. Cabon. 2017. Meteoland: Landscape Meteorology Tools. R Package Version 0.6. 4.

Good, P., M. Moriondo, C. Giannakopoulos, and M. Bindi. 2008. 'The Meteorological Conditions Associated with Extreme Fire Risk in Italy and Greece: Relevance to Climate Model Studies'. International Journal of Wildland Fire 17 (2): 155.

Hawkins, E., and R. Sutton. 2009. 'The Potential to Narrow Uncertainty in Regional Climate Predictions'. Bulletin of the American Meteorological Society 90 (8): 1095-1108.

Lindgren, F., and H. Rue. 2015. 'Bayesian Spatial Modelling with R - INLA'. Journal of Statistical Software 63 (19).

McSweeney, C. F., Jones, R. G., Lee, R. W., \& Rowell, D. P. 2015. Selecting CMIP5 GCMs for downscaling over multiple regions. Climate Dynamics, 44 (11-12), 3237-3260.

Moriondo, M., P. Good, R. Durao, M. Bindi, C. Giannakopoulos, and J. Corte-Real. 2006. 'Potential Impact of Climate Change on Fire Risk in the Mediterranean Area'. Climate Research 31 (June): $85-95$.

Preisler, H. K., D. R. Brillinger, R. E. Burgan, and J. W. Benoit. 2004. 'Probability Based Models for Estimation of Wildfire Risk'. International Journal of Wildland Fire 13 (2): 133-42.

Rue, H., S. Martino, and N. Chopin. 2009. 'Approximate Bayesian Inference for Latent Gaussian Models by Using Integrated Nested Laplace Approximations'. Journal of the Royal Statistical Society: Series B (Statistical Methodology) 71 (2): 319-92.

Van Wagner, C. E. 1987. Development and Structure of the Canadian Forest Fire Weather Index System. Forestry Technical Report 35. Ottawa: Canada Communication Group Publ.

Vidal, J.-P., E. Martin, L. Franchistéguy, M. Baillon, and J.-M. Soubeyroux. 2010. 'A 50-Year HighResolution Atmospheric Reanalysis over France with the Safran System'. International Journal of Climatology 30 (11): 1627-44. 
Viegas, D. X., G. Bovio, A. Ferreira, A. Nosenzo, and B. Sol. 1999. 'Comparative Study of Various Methods of Fire Danger Evaluation in Southern Europe'. International Journal of Wildland Fire 9 (4): 235.

Wang, Xianli, B. M. Wotton, A. S. Cantin, M.-A. Parisien, K. Anderson, B. Moore, and M. D. Flannigan. 2017. 'Cffdrs: An R Package for the Canadian Forest Fire Danger Rating System'. Ecological Processes 6 (1). 


\title{
Calibration of the Canadian FWI System for the Territory of Europe
}

\author{
Daniela Alves*; Luís Mário Ribeiro; Domingos Xavier Viegas \\ Forest Fire Research Centre (CEIF), ADAI-LAETA, University of Coimbra. Coimbra 3030-289, \\ Portugal.\{danielaalves@adai.pt*\}
}

\begin{abstract}
The role played by the meteorology in the development of a forest fire is widely recognized. There are several models to assess the risk of forest fires used by the authorities to define the state of readiness of the fire-fighting resources to suppress them in their early stages. The Fire Weather Index (FWI) of the Canadian Fire Danger System, widely used in several parts of the world, is a composite index that represents fire weather conditions quite well.

An important aspect in the application of the FWI is the definition of the threshold values to determine the fire danger classes for a given region and day. One of the simplest methods to define the classes is to use percentiles based on historical data, but this method lacks information regarding wildfire history and its relation to FWI. To obtain a credible assessment of the fire danger using the FWI it is important to perform a calibration to determine the limiting values of FWI for each class. Probabilities of fire duration exceeding specified thresholds are then used to calibrate FWI leading to the definition of fire danger classes. Although the Canadian Fire Danger System proposes an indicative table of limiting values for the five original fire danger classes that are usually adopted, these limiting values are not universal and have to be determined for a given region and season.

This study that was requested by the Joint Research Centre of Europe (JRC), aims to produce a calibration for the FWI based on historical wildfire data, for the territory of Europe, providing an updated table of threshold values to have a better definition of the risk classes of each region. The spatial division of the various countries and regions covered by the daily analysis of fire risk was defined to be the level 3 of the Nomenclature of territorial units for statistics (NUTS3). The methodology to calibrate the fire danger classes based on the statistical data of daily FWI, number of fires and burned area for each NUTS3, was performed between 2006 and 2015.
\end{abstract}

Keywords: fire danger, fire meteorology, Canadian Fire Danger System, Fire Weather Index

\section{Introduction}

Forest fire danger is greatly dependent on meteorological conditions, although the fire risk depends on other factors like vegetation cover and socio-economic parameters, namely those related to fire ignition, fire prevention and fire suppression activities.

In forest fire management, the need for understanding and predicting fire ignition probability and difficulty of control is the main reason for collecting fire statistics and for the development and use of fire danger rating systems (Xanthopoulos, et al., 2014). These systems are usually translated into a general risk scale with 3 to 6 fire danger classes. There are multiple methods for estimating the fire danger classes, based on meteorological parameters, which are used around the world. Several methods were developed with the aim of being applied to a given region or country, considering the specific fire regime; others were developed considering a more general application. In some countries, several systems are used to estimate the index, leaving the decision to adopt the most appropriate level of risk in each situation to the users (Viegas, et al., 2011). 
The Fire Weather Index (FWI) of the Canadian Fire Danger System has a very broad use not only in Canada but also in other parts of the world including Europe (Stocks et al. 1989, Forestry Canada Fire Danger Group, 1992). The Fire Weather Index (FWI) component of this system was evaluated favourably, among others, by Viegas et al. (1999) in comparison with four other methods of fire danger evaluation. More information on the existing methods can be found in Viegas et al. (1994) and Reis (1998).

An important aspect in the application of the FWI is the definition of the threshold values to determine the fire danger classes for a given region and day. Although the Canadian system proposes an indicative table of limiting values for the five original fire danger classes that are usually adopted, these limiting values are not universal and have to be determined for a given region and season. One of the simplest methods to present classes is to use percentiles based on historical data, but this method lacks information regarding wildfire history and its relation to FWI. To obtain a credible assessment of the fire danger using the FWI it is important to perform a calibration to validate the limiting values of FWI for each class.

In 1999, ADAI/CEIF team proposed and applied an original calibration of the FWI index which was developed in collaboration with the Portuguese Institute of Meteorology (Viegas, et al., 2004) and was performed for each district of Portugal. A recent work (Rocha, 2014), extended this calibration to the council level using more recent data, showing that the methodology used is quite robust, and can help to identify differences between and within regions.

By solicitation of the Joint Research Centre of Europe, ADAI/CEIF performed a calibration, based on the mentioned methodology, for the European Countries that are present in the European Forest Fire Information System (EFFIS - http://effis.jrc.ec.europa.eu/).

\subsection{Canadian Fire Danger System}

The Canadian Fire Danger System characterized by its FWI, is the result of years of research applied in Canada since 1968, culminating in the presentation of the system in a document presented by Van Wagner (1987). The structure of the Canadian system succinctly presents 3 levels: the meteorological observations, the fire moisture codes and the fire behaviour indexes.

The FWI is an indicator of the fire behaviour and fire danger and is the output parameter of the system which is more directly related to the possibility of occurrence of fires and with the respective danger and represents only a part of the complex role of meteorology in forest fires. For a more detailed information on the meaning of the different components of the Canadian system, as well as for obtaining the mathematical formulas which relate the variables involved, see Van Wagner (1987).

\subsection{Objectives}

Fire Weather Index does not consider only weather parameters, excluding aspects such as socio economic factors, topography, vegetation or fuel cover. For these reasons the interpretation of FWI values can be different from region to region. FWI is used in Portugal and in other countries of Europe with differences in its assessment: different input data, different calibration system and different interpretation of results.

In this work we applied a calibration method to the territory of Europe to provide the JRC with an updated table of threshold values to better define the class of risk of each region considering, not only the meteorological conditions, but also the history of fire occurrence in terms of daily fires and burned area.

\section{Methodology - Calibration of the Canadian System}

The conceptual basis of the Canadian system uses physical parameters, like the equilibrium moisture content and its relationship with meteorological parameters. Therefore its application can be 
extended to other ecosystems beyond those for which it was initially developed. This application, however, requires that a system calibration is performed for each specific region.

The proposed calibration process is based on fire history of the region in which it will be applied, and its relation to FWI. Therefore, the first and crucial step, was to define the optimal physical or spatial unit to be used in the process. The only limitation for choosing this unit lays on the existence of input data, both related to FWI and fire records. In agreement with the JRC, the spatial unit of the various countries and regions covered by the daily analysis of fire risk was defined to be NUTS3, and the period selected for calibration was 2006 to 2015. The NUTS classification (Nomenclature of Territorial Units for Statistics) is a hierarchical system for dividing up the economic territory of the EU (http://ec.europa.eu). The current NUTS 2016 classification is valid from 1 January 2018 and lists 104 regions at NUTS 1, 281 regions at NUTS 2 and 1348 regions at NUTS 3 level (European Commission, 2018). The $3^{\text {rd }}$ level has the smallest dimension.

For the proposed calibration for a region and time, the parameters used were:

- The daily FWI values;

- The number of forest fires $(\mathrm{NbF})$

- The burned area (BA);

Assuming that there are different fire patterns across Europe, depending on the time of year, it was decided to perform the calibration process for two different seasons. The first includes the months with higher fire activity, usually late Spring and Summer. The second corresponds to the rest of the year. A preliminary analysis (Section 2.2.1) was performed to define these periods: the "Summer Season" was considered to be the period from May $15^{\text {th }}$ to September $30^{\text {th }}$ and "Winter Season" from October $1^{\text {st }}$ to May $14^{\text {th }}$.

This section describes the methodology used to calibrate the FWI. The process was the same for all NUTS3, obviously using the individual records for each region and some general considerations, identified below. This process can be repeated with other sets of data (spatial or temporal).

\subsection{General considerations}

According to the methodology proposed in Viegas et al. (2004), the concept of high or low risk has a relative character and can be different across various territories. Fire history, prevention actions and available suppression resources in each region can largely influence this classification.

The result of the calibration is translated into risk levels, usually on a five level scale. For the Summer season we considered the existence of five fire danger classes while for the Winter season we considered only three. In regions or periods of the year, such as in winter and spring, where the occurrence of fires is lower, a scale of only three levels can be sufficient (Viegas et al., 2011).

The main fire seasons were defined using the fire occurrence data which were observed in each country during the years of analysis. The proposed risk levels and the respective classes for each season are presented in Section 2.2.9 (Table ). It is considered that the days of maximum risk (very high class) should not be many, on average, to avoid distorting the meaning of this term. In the calibration methodology design it was established that this number should not exceed 3 to $5 \%$ of the total days in the considered period.

This methodology is based on the existence of a good correlation between the daily value of FWI in a given region and the corresponding value of burned area and number of fires. For most regions with strong fire activity (like the Mediterranean Countries) we have checked that this assumption is correct in the large majority of cases. When developing the work, we checked regularly the validity of this assumption for various territorial units and found that it is usually better for the relationship between FWI and the number of daily fires, but this was not always the case. 


\subsection{Calibration steps}

\subsubsection{Collecting, preparing and analyzing fire records data}

Fire records for Europe were provided by JRC. A preliminary analysis of the data to retain records where the following fields were known:
i. Burned area
ii. NUTS 3
iii. Date

In this analysis we found that some of the European NUTS3 regions do not have fire records. This is shown in Figure .

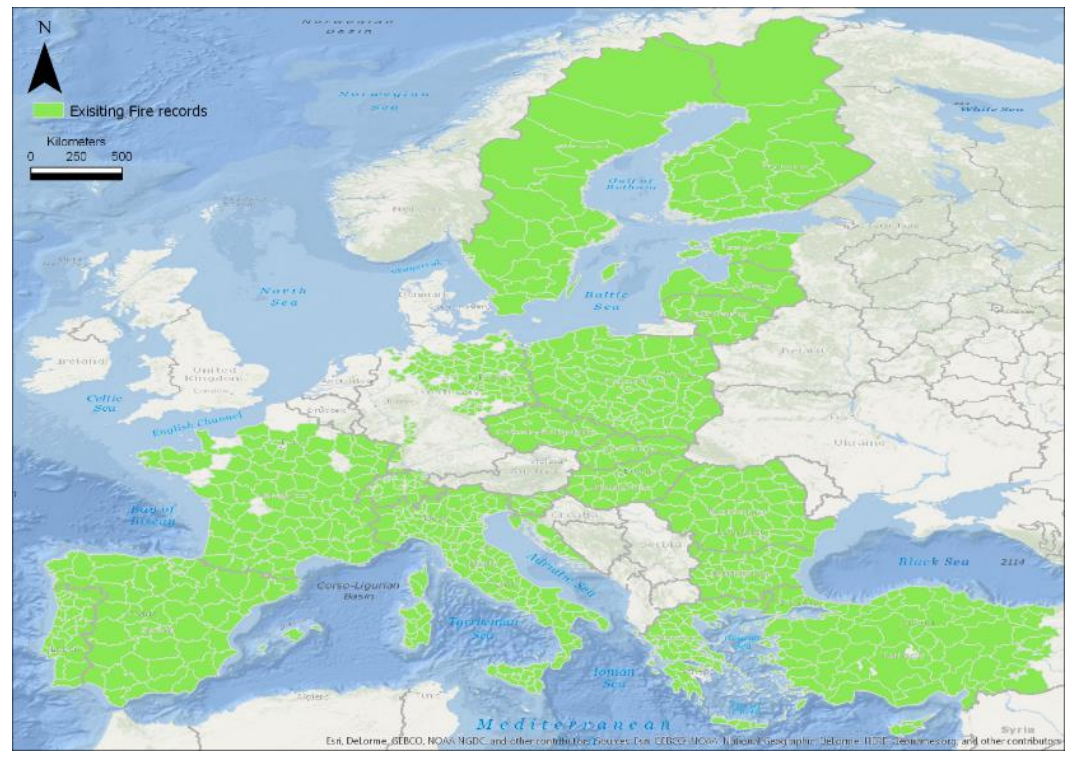

Figure 1 - Map depicting the extent of the provided fire records

In a second stage, we analyzed the number of fires per country and their respective burned area. Figure represents these results per year. This gives a simple indication of wildfires average burned area in each country. It may not be representative in some cases, as countries with very few fires can have one large fire that conditions this result.

In Figure 3 we represent the annual average burned area in relation to the respective country total area. It is clear that, in general, the Mediterranean Basin countries like Portugal (PT), Spain (ES), Italy (IT) or Greece (EL) are the most affected ones, either in number of fires or burned area. Portugal has an average of $0.83 \%$ of its area burned by wildfires each year.

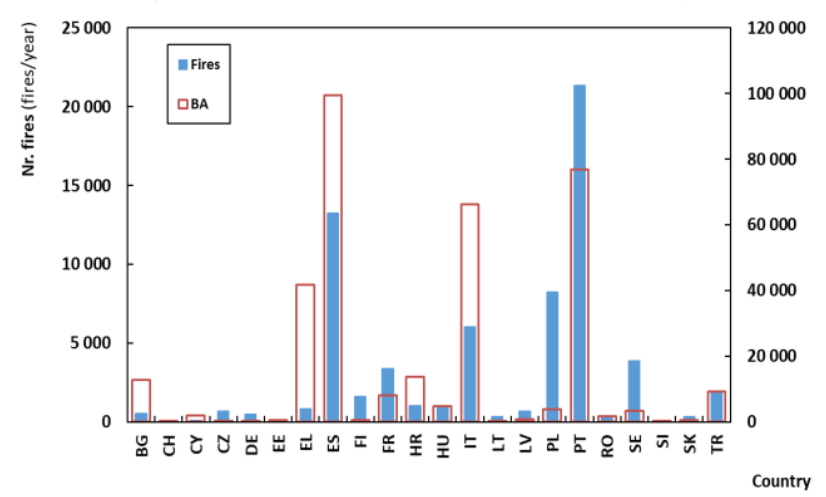

Figure 2 - Number of fires and Burned area

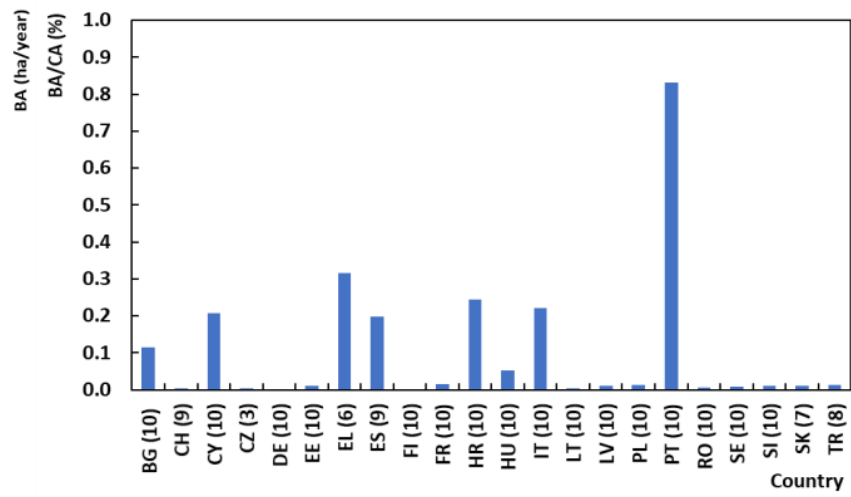

Figure 3 - Annual average of burned area/Country area $(\%)$ 
In Figure and Figure the number of fires and burned area is shown, globally, for the two proposed seasons (Summer and Winter).

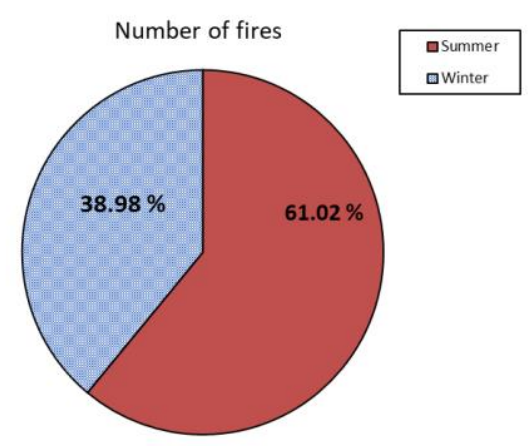

Figure 4 - Total number of fires, in percent, in Summer and Winter seasons

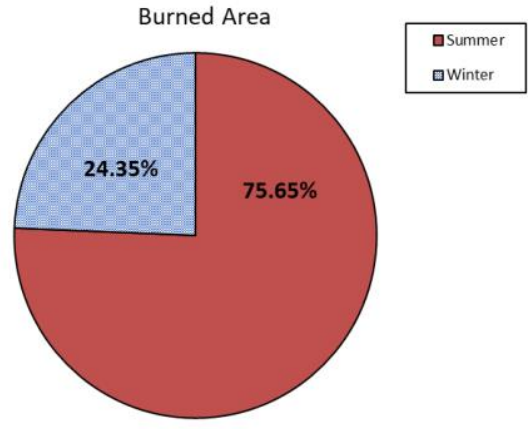

Figure 5 - Total burned area, in percent, in Summer and Winter seasons

The selected dates for the two different seasons seem to be representative, as two thirds of the number of fires and three quarters of the burned area occur in the Summer period, although this may be different for some regions.

After this preliminary analysis, we found no evidence or need to have different seasons in different NUTS3 areas. The same periods were therefore used for all the regions. Nevertheless, we recognize that in some regions where fires occur mostly in the Winter period this criterium may have to be revisited.

\subsubsection{Evaluating FWI (provided by JRC)}

Evaluation of the daily value of FWI is made by estimation of an average daily value of the relevant meteorological parameters: air temperature, relative humidity, wind velocity and precipitation. In this work FWI was provided directly by JRC in the form of an evenly spaced grid of points covering Europe. For the period of 2006-2015, the following fields were provided:

$\begin{array}{llll}\text { i. } & \text { Point ID; } & \text { v. } & \text { DMC; } \\ \text { ii. } & \text { X and Y coordinates; } & \text { vi. } & \text { DC; } \\ \text { iii. } & \text { FWI; } & \text { vii. } & \text { ISI; } \\ \text { iv. } & \text { FFMC; } & \text { viii. } & \text { BUI. }\end{array}$

For the proposed calibration process, only the FWI value was used. As the spatial unit used for the calibration process is NUTS3, FWI values were spatially interpolated and a mean value was assigned to each NUTS3 polygon. ArcMap was used for this step of the process. The interpolations were done using the Thiessen polygons method. When the process ended, each NUTS 3 polygon had the FWI values for every day of each year of the study period.

\subsubsection{Defining Study Area or Area of Interest (AoI)}

As stated before, the Area of Interest (AoI) needs to meet the requisites of having both historical fire and FWI data at the defined spatial division of NUTS 3 that were provided by JRC for the period chosen for the calibration (2006-2015). FWI was made available for all the territory. Unfortunately, the fire data was not homogeneously distributed in time and/or space. The main constrain was the absence of fire data in certain countries in some periods and the complete absence in others. Table 1 presents the countries for which fire records existed, the respective number of NUTS3, and the period for which it was available. 
Table 1 - Countries analyzed - study area

\begin{tabular}{|l|c|c|c|c|c|c|}
\hline \multirow{2}{*}{ Country } & \multicolumn{2}{c|}{ Number of NUTS3 } & \multicolumn{3}{c|}{ Fire Data period } \\
\cline { 3 - 7 } & & All country & With data & From & To & Years \\
\hline Bulgaria & BG & 28 & 28 & 2006 & 2015 & 10 \\
\hline Croatia & HR & 21 & 7 & 2006 & 2015 & 10 \\
\hline Cyprus & CY & 1 & 1 & 2006 & 2015 & 10 \\
\hline Czech Republic & CZ & 14 & 14 & 2006 & 2008 & 3 \\
\hline Estonia & EE & 59 & 5 & 2006 & 2015 & 10 \\
\hline Finland & FI & 19 & 18 & 2006 & 2015 & 10 \\
\hline France & FR & 100 & 84 & 2006 & 2015 & 10 \\
\hline Germany & DE & 402 & 101 & 2006 & 2015 & 10 \\
\hline Greece & EL & 52 & 50 & 2006 & 2011 & 6 \\
\hline Hungary & HU & 20 & 20 & 2006 & 2015 & 10 \\
\hline Italy & IT & 110 & 110 & 2006 & 2015 & 10 \\
\hline Latvia & LV & 6 & 6 & 2006 & 2015 & 10 \\
\hline Lithuania & LT & 10 & 10 & 2006 & 2015 & 10 \\
\hline Poland & PL & 72 & 66 & 2006 & 2015 & 10 \\
\hline Portugal & PT & 30 & 28 & 2006 & 2015 & 10 \\
\hline Romania & RO & 42 & 42 & 2006 & 2015 & 10 \\
\hline Slovakia & SK & 8 & 8 & 2006 & 2012 & 7 \\
\hline Slovenia & SI & 12 & 12 & 2006 & 2015 & 10 \\
\hline Spain & ES & 59 & 52 & 2006 & 2014 & 9 \\
\hline Sweden & SE & 21 & 21 & 2006 & 2015 & 10 \\
\hline Switzerland & CH & 26 & 23 & 2006 & 2014 & 9 \\
\hline Turkey & TR & 81 & 63 & 2006 & 2013 & 8 \\
\hline & & & & & & \\
\hline
\end{tabular}

Therefore, the definition of the AoI was constrained by the fire records availability, and it coincides with the areas already identified in Figure .

\subsubsection{Merging datasets}

After AoI definition, FWI and Fire records datasets were merged into one single alphanumeric dataset. Table shows an example of final merged dataset.

Table 2 - Final merged full dataset

\begin{tabular}{|c|c|c|c|c|c|c|c|c|c|c|c|}
\hline NUTS_ID & Day & NbF & BA & FWI & FFMC & DMC & DC & ISI & BUI & Num_date & Date_nuts_join \\
\hline PT111 & $31 / 07 / 2006$ & 3 & 43.1 & 21.6 & 83.3 & 80.7 & 605.2 & 5.1 & 121.1 & 38929 & $38929 P T 111$ \\
\hline PT112 & $31 / 07 / 2006$ & 9 & 1.1 & 21.6 & 83.3 & 80.8 & 605.2 & 5.1 & 121.1 & 38929 & $38929 P T 112$ \\
\hline
\end{tabular}

\subsubsection{Harmonization and calculation}

From this step forward, all calculations were done for each NUTS 3 and for the Summer and Winter periods in separate, which required the full dataset to be split accordingly: ${ }^{\text {st }}$ ) Split full dataset by NUTS 3 and $2^{\text {nd }}$ ) Split each NUTS 3 into Summer and Winter periods

This resulted in a set of 769 individual tables for Summer and the same number for Winter (corresponding to the sum of the NUTS 3 regions with fire records, as identified in Table 1). The process of calibration for each NUTS 3 region is described next.

\subsubsection{Reordering FWI by ascending order and add cumulative days}

At this point all it matters is the value of FWI, the number of days in the analysis and their relationship with fire records. Maintaining the correspondence between all fields the table is rearranged by ordering the FWI column in an ascending order. Afterwards, the "Date" field is replaced by a 
numerical incremental field, with the value of " 1 " being attributed to the record with the lowest value of FWI, and consecutively adding "1" to the following record. Table 3 schematically represents this procedure.

Table 3 - Sorting FWI Values and adding incremental days field

\begin{tabular}{|c|c|c|c|}
\hline \multicolumn{5}{|c|}{ Original } \\
\hline Date & FWI & $\begin{array}{c}\text { Number } \\
\text { of fires }\end{array}$ & $\begin{array}{c}\text { Burned } \\
\text { area (ha) }\end{array}$ \\
\hline Date_1 & FWI_3 & NbF_1 & BA_1 \\
\hline Date_2 & FWI_4 & NbF_2 & BA_2 \\
\hline Date_3 & FWI_2 & NbF_3 & BA_3 \\
\hline Date_4 & FWI_1 & NbF_4 & BA_4 \\
\hline Date_n & FWI_n & NbF_n & BA_5 \\
\hline
\end{tabular}

$\rightarrow$\begin{tabular}{c|c|c|c|}
\hline \multicolumn{5}{|c|}{ After re-ordering } \\
\hline $\begin{array}{c}\text { Incremental } \\
\text { day }\end{array}$ & FWI & $\begin{array}{c}\text { Number } \\
\text { of fires }\end{array}$ & $\begin{array}{c}\text { Burned } \\
\text { area (ha) }\end{array}$ \\
\hline $\mathrm{d} 1$ & FWI_1 & NbF_4 & BA_4 \\
\hline $\mathrm{d} 2$ & FWI_2 & NbF_3 & BA_3 \\
\hline d3 & FWI_3 & NbF_1 & BA_1 \\
\hline d4 & FWI_4 & NbF_2 & BA_2 \\
\hline dTotal & FWI_n & NbF_n & BA_n \\
\hline
\end{tabular}

\subsubsection{Adding probability field}

A new field called "Probability" was then added. This field reflects the weight that a given day (and its respective FWI) has in respect to the total number of days. The higher the FWI, the higher the "incremental day" and the higher the respective "probability" of occurrence of values of FWI lower than the specified value. The probability field is calculated by dividing the "incremental day" number, by the total number of days:

$$
\text { Probability }=\frac{\text { Incremental day }\left(d_{n}\right)}{\text { Incremental day total }\left(d_{\text {Total }}\right)}
$$

Table represents the resulting table after the addition and calculation of the probability field.

Table 4. Adding the probability field

\begin{tabular}{|c|c|c|c|c|}
\hline $\begin{array}{c}\text { Incremental } \\
\text { day }\end{array}$ & Probability & FWI & Number of fires & Burned Area (ha) \\
\hline$d 1$ & d1/dTotal & FWI_1 & NbF_4 & BA_4 \\
\hline$d 2$ & d2/dTotal & FWI_2 & NbF_3 & BA_3 \\
\hline$d 3$ & d3/dTotal & FWI_3 & NbF_1 & BA_1 \\
\hline$d 4$ & d4/dTotal & FWI_4 & NbF_2 & BA_2 \\
\hline$d$ Total & $\mathbf{1}$ (=dTotal/dTotal) & FWI_n & NbF_n & BA_n \\
\hline
\end{tabular}

Adding the number of fires and burned area, allows a graphical representation of the cumulative probability, as the example for PT111 region, shown in Figure .
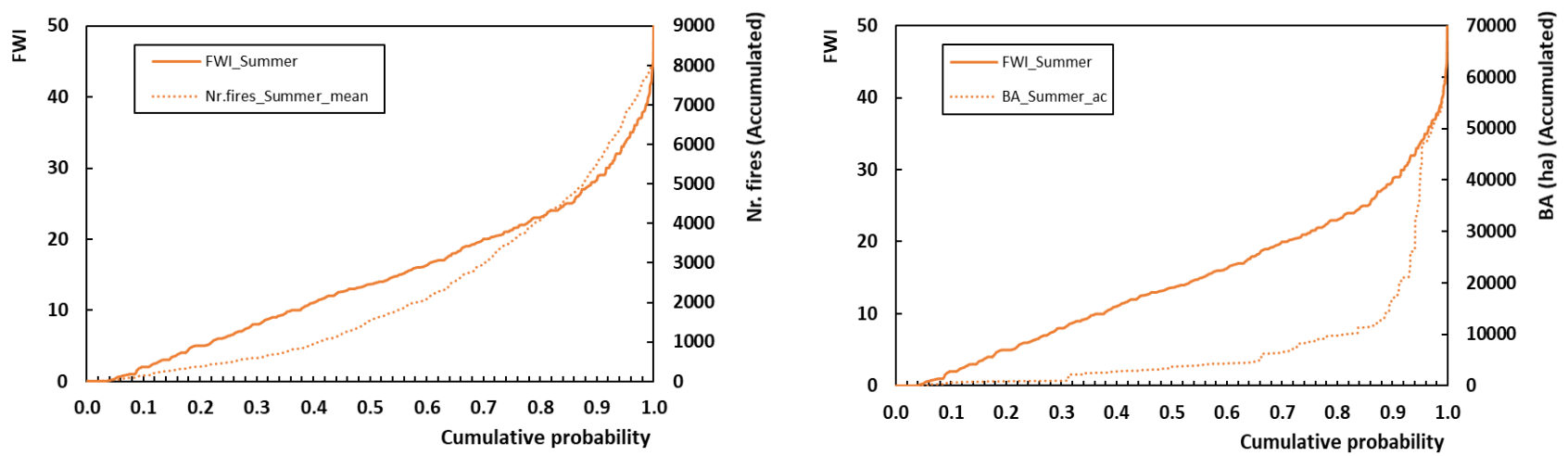

Figure 6 - Plotting FWI distribution in function of the probability - Graphic example for PT111 NUTS3 in Summer. Left-number of accumulated fires. Right-accumulated burned area

These graphics show that the probability of having larger number of fires and burned area increases as FWI increases. 


\subsubsection{Grouping by probability classes and calculating statistics}

From the analysis of the previous graphics (Figure ) we infer the need to categorize the probability into classes. Grouping it into classes is equivalent to splitting the results by percentiles. The breaks used to split classes were $0.1,0.2,0.3,0.4,0.5,0.6,0.7,0.8,0.9,0.95,0.97$. For each class the following parameters were calculated, although the proposed calibration only needs the maximum FWI value of each class:

- Maximum FWI value, limiting the class

- $\quad$ Average FWI value;
- Average number of fires

- Average burned area

Table shows an example of these calculations for PT111 NUTS3 Region, in the Summer period.

Table 5 - Example of results for PT111 NUTS3 region

\begin{tabular}{|c|c|c|c|c|}
\hline \multirow{2}{*}{$\begin{array}{c}\text { Probability } \\
\text { class }\end{array}$} & $\begin{array}{c}\text { Maximum } \\
\text { FWI (limit of } \\
\text { the class) }\end{array}$ & FWI & NbF & BA \\
\hline 0.10 & 2.0 & 0.6 & 1.2 & 5.3 \\
\hline 0.20 & 5.0 & 3.4 & 1.8 & 1.8 \\
\hline 0.30 & 8.0 & 6.4 & 1.7 & 0.8 \\
\hline 0.40 & 11.0 & 9.5 & 2.8 & 14.6 \\
\hline 0.50 & 13.7 & 12.5 & 4.9 & 7.4 \\
\hline 0.60 & 16.3 & 14.9 & 4.4 & 5.0 \\
\hline 0.70 & 20.0 & 18.1 & 7.2 & 18.4 \\
\hline 0.80 & 23.1 & 21.4 & 8.9 & 25.5 \\
\hline 0.90 & 28.4 & 25.4 & 11.6 & 55.4 \\
\hline 0.95 & 33.6 & 30.8 & 20.0 & 366.9 \\
\hline 0.97 & 36.1 & 34.9 & 17.3 & 381.8 \\
\hline 1.00 & 51.2 & 39.4 & 23.7 & 295.0 \\
\hline
\end{tabular}

\subsubsection{Defining risk classes}

The fire risk classes definition, the main goal of this work, was performed by dividing FWI values in groups, according to its historical probability of occurrence. After the individual analysis of a series of random NUTS3 results, calculated in the previous step (2.2.8), it was decided to use, for the Summer period five classes, and for the Winter three classes, with the probability grouped as identified in Table

Table 6 - Proposed "Risk levels" and respective classes in (a) Summer and (b) Winter

\begin{tabular}{|c|c|c|c|c|}
\hline \multirow{2}{*}{ Risk Level } & \multicolumn{2}{|c|}{ a. Summer } & \multicolumn{2}{c|}{ b. Winter } \\
\cline { 2 - 5 } & Risk class & $\begin{array}{c}\text { Historical } \\
\text { probability }\end{array}$ & Risk class & $\begin{array}{c}\text { Historical } \\
\text { probability }\end{array}$ \\
\hline $\mathbf{1}$ & Very Low & $\mathrm{P}<0.30$ & Low & $\mathrm{P}<0.50$ \\
\hline $\mathbf{2}$ & Low & $0.30 \leq \mathrm{P}<0.60$ & Moderate & $0.50 \leq \mathrm{P}<0.90$ \\
\hline $\mathbf{3}$ & Moderate & $0.60 \leq \mathrm{P}<0.90$ & High & $\mathrm{P} \geq 0.90$ \\
\hline $\mathbf{4}$ & High & $0.90 \leq \mathrm{P}<0.97$ & - & - \\
\hline $\mathbf{5}$ & Very High & $\mathrm{P} \geq 0.97$ & - & - \\
\hline
\end{tabular}

\subsubsection{Merging final calibration tables}

The result of the described process consists of two tables for each NUTS3 region, one for Summer and one for Winter. 
The final step of the methodology is the merging of all individual tables for each season: one calibration table for European NUTS3 regions for Summer and another for Winter. To accomplish this, the "Probability" lines need to be transposed into columns, maintaining the FWI Maximum values filling the line for the respective NUTS3 region.

A simple merge of all the NUTS3 individual results produces the final Calibration Tables, like in the example given below (Table and Table ).

\section{Results}

The entire process, performed for all NUTS3 regions in the defined AoI, resulted in two calibration tables, one for Summer and other for Winter, as defined before.

\subsection{Calibration tables}

The calibration table for Summer has 6 columns:
i. COUNTRY
iv. Break_Prob_60
ii. NUTS_ID
v. Break_Prob_90
iii. Break_Prob_30
vi. Break_Prob_97

The first two are self-explanatory, as they represent the country and the NUTS3 identification code. The last 4 represent the breaks used to define the five risk classes, as explained earlier (Table ).

The calibration table for Winter has 4 columns:
i. COUNTRY
iii. Break_Prob_50
ii. NUTS_ID
iv. Break_Prob_90

Again, the first two are self-explanatory and the last 2 represent the breaks used to define the three risk classes.

In Table and Table the calibration table with the threshold values just for 22 from 769 NUTS3, for Summer and for Winter, respectively is presented.

Table 7 - Calibration table for Summer (22 from 769 NUTS3)

\begin{tabular}{|c|c|c|c|c|c|c|}
\hline & Country & NUTS_ID & Break_P_30 & Break_P_60 & Break_P_90 & Break_P_97 \\
\hline 1 & BG & BG312 & 7.8 & 22 & 39.5 & 51 \\
\hline 2 & CH & CH023 & 0.9 & 5.6 & 13.9 & 19.6 \\
\hline 3 & CY & CY000 & 25.5 & 32 & 44.1 & 50.5 \\
\hline 4 & CZ & CZ041 & 2.8 & 9.6 & 20.6 & 27.6 \\
\hline 5 & DE & DE122 & 4.7 & 13 & 24.7 & 30.7 \\
\hline 6 & EE & EE001 & 1 & 4.3 & 11 & 19 \\
\hline 7 & EL & EL111 & 19.8 & 33.8 & 51.4 & 58.2 \\
\hline 8 & ES & ES411 & 28 & 39.2 & 52.8 & 62.5 \\
\hline 9 & FI & FI195 & 0.7 & 4.2 & 13.7 & 25 \\
\hline 10 & FR & FR213 & 6 & 15 & 29 & 38.7 \\
\hline 11 & HR & HR035 & 15.7 & 29.4 & 47.5 & 56 \\
\hline 12 & HU & HU222 & 8.4 & 19.1 & 35.3 & 45.6 \\
\hline 13 & IT & ITC34 & 0.6 & 6.4 & 17.4 & 22.9 \\
\hline 14 & LT & LT001 & 2.5 & 8.8 & 20.7 & 29.5 \\
\hline 15 & LV & LV003 & 2.1 & 7.4 & 19.4 & 29.4 \\
\hline 16 & PL & PL114 & 5.7 & 15 & 28 & 40.3 \\
\hline 17 & PT & PT114 & 6 & 13.8 & 24.1 & 32.5 \\
\hline 18 & RO & RO116 & 5.9 & 17 & 31.7 & 38.8 \\
\hline 19 & SE & SE122 & 1.6 & 8 & 21 & 29.2 \\
\hline 20 & SI & SI012 & 5 & 14.9 & 29 & 40 \\
\hline 21 & SK & SK022 & 6.1 & 16.3 & 29 & 37.5 \\
\hline 22 & TR & TR411 & 28.4 & 45.1 & 60.8 & 68.6 \\
\hline
\end{tabular}


Table 8 - Calibration table for Winter (22 from 769 NUTS3)

\begin{tabular}{|c|c|c|c|c|}
\hline & Country & NUTS_ID & Break_P_50 & Break_P_90 \\
\hline 1 & BG & BG312 & 1.0 & 16.3 \\
\hline 2 & CH & CH023 & 0.4 & 6.0 \\
\hline 3 & CY & CY000 & 12.4 & 30.2 \\
\hline 4 & CZ & CZ041 & 0.4 & 8.6 \\
\hline 5 & DE & DE122 & 0.7 & 9.1 \\
\hline 6 & EE & EE001 & 0.5 & 4.3 \\
\hline 7 & EL & EL111 & 2.2 & 15.2 \\
\hline 8 & ES & ES411 & 5.9 & 23.3 \\
\hline 9 & FI & FI195 & 0.2 & 1.0 \\
\hline 10 & FR & FR213 & 0.8 & 12 \\
\hline 11 & HR & HR035 & 3.3 & 18 \\
\hline 12 & HU & HU222 & 2.3 & 18.3 \\
\hline 13 & IT & ITC34 & 0.3 & 4.2 \\
\hline 14 & LT & LT001 & 0.3 & 8.0 \\
\hline 15 & LV & LV003 & 0.4 & 5.1 \\
\hline 16 & PL & PL114 & 0.8 & 12.6 \\
\hline 17 & PT & PT114 & 1.6 & 10.1 \\
\hline 18 & RO & RO116 & 0.9 & 15.9 \\
\hline 19 & SE & SE122 & 0.4 & 7.0 \\
\hline 20 & SI & SI012 & 1.0 & 13.3 \\
\hline 21 & SK & SK022 & 0.7 & 13.1 \\
\hline 22 & TR & TR411 & 4.6 & 23.5 \\
\hline
\end{tabular}

Two scripts in Python language were written to facilitate the calibration process and allow integration with other tools. The scripts classify FWI values into calibrated fire risk classes, according to the proposed methodology.

\section{Final considerations}

One of the main constrains inherent to the proposed calibration was the lack of consistent data. Meteorological data was available for the entire EU area, but fire records were not. The absence of these records has a strong influence in the significance of the calibration values. The longer the data series used, the higher the confidence in the results. Assuming this, we advise that the calibration is recalculated whenever larger series of data are compiled.

In our opinion, at an European level it is worthy using NUTS 3 as the minimum spatial unit, as it highlights differences between and within larger regions.

The full process of calibration was achieved using ArcGIS models that allow "scripting" for users who are not familiar with programing in python language. Models allowed the splitting of the somehow complex set of operations and the easy iteration of multiple tables at once. Although the set of models built allows for the replication of the calibration process, they use specific ArcGIS tools and commands, but a ready-to-use python script to be used by other platforms was not done yet.

This calibration is based on the assumption of the existence of a good correlation between fire occurrence expressed by the number of daily fires and burned area. In a similar work to JRC, we calculated the correlation coefficient between FWI and number of fires and burned area, separately for Summer and Winter period in order to confirm our assumption. Then we checked if the calibration that was produced worked well to predict days with very large burned area both in Summer and Winter periods. We found that there is a good correlation in areas with important fire activity while in the others the correlation may not be so good. We also found that in some regions the FWI may not be sufficient to predict days with large fire activity and possibly other indices should be used to 
complement it and in areas in which winter fires are relatively important it may be necessary to introduce five fire danger classes.

\section{Acknowledgments}

The authors acknowledge JRC for the support provided by the Contract JRC-FWI (JRC/G02/E/rc/Ares (2016)). The partial support provided by projects FIREXTR (PTDC/ATPGEO/0462/2014), FIREWHIRL (PTDC/EMS-ENE/2530/2014) is also gratefully acknowledged.

\section{References}

European Commission. (2018). Eurostat. Cbtained from https://ec.europa.eu/eurostat/web/nuts/background

Forestry Canada Fire Danger Group. (1992). Development and structure of the Canadian Forest Fire. (p. 63). Ottawa: Forestry Canada.

Reis, R. M. (1998). Scales for the assessment of forest fire danger. In Proc. of III International Conf. on Forest Fire Research/14th Conf. on Fire and Forest Meteorology, I, pp. 1235-1247. Luso.

Rocha, C. F. (2014). Validação do Índice de Risco de Incêndio Florestal - ICRIF. Master thesis, University of Coimbra.

Stocks, B.J., Lawson, B.D., Alexander, M.E., Van Wagner, C.E., McAlpine, R.S., Lynham, T.J., Dube, D.E., 1989. The Canadian forest fire danger rating system: an overview. The Forestry Chronicle 65, 258-265

Van Wagner, C. (1987). Development and structure of the Canadian Forest Fire Weather Index System. Otawa: Canadian Forestry Service, Forestry Technical Report 35.

Viegas, D. X., Bovio, G., Ferreira, A., Nosenzo, A., \& Sol, B. (1999). Comparative study of various methods of fire danger evaluation in Southern Europe. International Journal of Wildland Fire, 9(4): 235-246.

Viegas, D. X., Reis, R. M., Cruz, M. G., \& Viegas, M. T. (2004). Calibração do Sistema Canadiano de Perigo de Incêndio para Aplicação em Portugal. 77-94. EFN, Lisboa: Silva Lusitana.

Viegas, D. X., Rossa, C., \& Ribeiro, L. M. (2011). Incêndios Florestais. Lisboa: Verlag Dashofer.

Viegas, D. X., Sol, B., Bovio, G., Nosenzo, A., \& Ferreira, A. D. (1994). Comparative study of various methods of fire danger evaluation in Southern Europe. In Proceedings of the II International Conference on Forest Fire Research, (pp. 571-590). Coimbra.

Xanthopoulos, G., Roussos, A., Giannakopoulos, C., Karali, A., \& Hatzaki, M. (2014). Investigation of the weather conditions leading to large forest fires in the area around Athens, Greece. In D. X. Viegas, \& ADAI-CEIF (Ed.), Advances in Forest Fire Research (pp. 1207-1212). Coimbra: Imprensa da Universidade de Coimbra. doi:http://dx.doi.org/10.14195/978-989-26-0884-6 


\title{
Evaluation of wildfire danger in the Peruvian Andes: First step for its reduction and adaptation
}

\author{
Maria I. Manta ${ }^{1}$; Roberto Kometter ${ }^{2}$; Alexis Navia ${ }^{1}$ \\ ${ }^{1}$ Universidad Nacional Agraria La Molina. Avenida La Universidad S/N, Apartado 12-056, Lima, \\ Peru, ORCID 0000-0002-4467-9778 \{mmanta@lamolina.edu.pe*, 20081514@lamolina.edu.pe\} \\ ${ }^{2}$ Helvetas Swiss Intercooperation. Av. Ricardo Palma 857, Miraflores - Lima, Peru, \\ \{roberto.kometter@helvetas.org\}
}

\begin{abstract}
Recent events of extreme wildfires are occurring in different parts of the world. In Peru, climate of fire has also occurred and has caused material and environmental services losses. The population and the ecosystems from the Peruvian Andes have been directly affected by the fire and a greater reduction in the water supply could affect $95 \%$ of the Peruvian population. Taking into account that there are limitations to estimate the fire danger index and aware the population about wildfire occurrences, here wildfire statistics were used to analyze its spatial and temporal trends from 1973 to 2014 and map fire danger for the Peruvian Andes. Wildfires are spread in all departments of the Peruvian highlands (18) and their fire season length has increased from 4 to 12 months in a year: August, September and October are months with the highest occurrence of wildfire, currently. Four departments of the Southern Andes have a very high fire danger class; while two departments of the Northern Andes have a high fire danger class. In these six departments, the total area burned in 42 years has been 201278 ha (93\% of the total burned area). The ecosystems most affected by the fire were grassland (the Puna, the Páramo and Peatlands), natural forests (Dry Forest in Inter-Andean valleys, Dry Mountain Forest and Tropical Montane Cloud forests) and the Andean shrubs. If the government organizations do not prioritize the implementation of strategies to reduce and adapt to wildfires and its occurrence continues, the severity of the fire could intensify on the Andean natural vegetation and its role in climate and hydrological cycle regulation may change dramatically, affecting the socioeconomic development and the welfare of the population.
\end{abstract}

Keywords: Fire danger map, spatial and temporal distribution, material and environmental losses, disasters, Peru

\section{Introduction}

It is well known the role that weather plays in the occurrence of wildfires because it determines the ease of ignition, propagation rates and severity (Benson, et al., 2009, Fujioka et al., 2008). Övergaard et al. (2007) and FAO (2007) complement that wildfires are usually caused by man accidentally or on purpose all over the world, where fire is widely used for land use change, to improve grazing, and to remove cropping residues and other wastes. However, the magnitude of these fires is related to weather conditions, and exacerbated by drought, high air temperatures and wind. Likewise, extreme weather/climatic conditions that occur over a period of time will affect the moisture content of the fuels and therefore the activity of wildfires.

The knowledge of meteorology constitutes the foundation for the development of fire danger weather indices (FDWI). Fire managers around the world use a variety of systems to track and predict fire danger and fire behaviour, at spatial scales that span from local to global extents, and temporal scales ranging from minutes to seasons (Fujioka, et al., 2008).

In all scenarios the representative concentration pathways of greenhouse gas emissions, a warmer climate is predicted by the end of the century; the magnitude of daily maximum temperatures will increase in the near future, and the frequency of this increase will occur every year, instead of 2 to 3 years as calculated in previous studies (IPCC, 2014). Higher temperatures have already been 
recorded in Sydney $\left(47,8^{\circ}\right.$ C) this year, Spain $\left(47,3^{\circ}\right.$ C) in 2017 (Portillo, 2018), and Russia $\left(43,8^{\circ}\right.$ $\mathrm{C}$, national maximum) in 2010 (Wahlstron, 2010). The increase of temperature has caused a greater effective

evapotranspiration producing a depletion of the water in the city of Cabo Blanco and Sao Paulo in 2017 (Portillo, 2018) which would affect the wildfire extinction systems. On the other hand, at a regional level, it is expected that droughts will intensify in dry localities due to the reduction of precipitation and / or the increase in evapotranspiration with a medium confidence level; while at a global level the confidence level is low, due to the lack of direct observations (IPCC, 2014). However, unprecedented droughts have been recorded in very humid ecosystems such as those of the Amazon Basin in 2005 and 2010 (Marengo et al., 2012). In addition, extremely dry, warm and strong winds have contributed to numerous wildfires in Australia (Oliveira, 2010).

As the average global surface temperature increases, extreme precipitation is likely to occur in most mid-latitude places; while in the humid tropical regions the events of extreme daily precipitation will increase in the near future. Similarly, on a regional scale, the variability of precipitation related to El Niño-Southern Oscillation (ENSO) is likely to intensify (IPCC, 2014); situation that would increase the amount of live fuel that will then form the litter ready to burn. The large wildfires in Canada (McGrath, 2016), in Indonesia in 2016, 2009 and 1997-1998 (Jolly et al., 2015), in the Northwest of the United States 2016 (Sullivan, 2016), and in Brazil and Peru in 2005, 1997-1998 occurred in relation to ENSO (FAO, 2007); while big wildfires such as Alaska indoor fire complex (2004), Cedro Fire in Southern California (2003), Biscuit Fire in Southwest Oregon (2002) have occurred in a hot and dry climate (Makenzi, 2009).

Other wildfires with catastrophic consequences in the last two decades have been recorded in Portugal (Ferreira, et al. (2017), Russia 2002 (Chubarova, et al., 2009), Russia 2010 (Jolly, et al., 2015), Australia 2013 (Jolly, et al., 2015) and 2009 (Oliveira, 2010), in Chile 2016-2017 and Peru 2016 (Manta, 2018), all of them associated with climate change.

Given the effect of climate change on the activity of wildfires, the Canadian Fire Weather Index "FWI", part of the Canadian Forest Fire Danger Rating System "CFFDRS", has been used to quantify changes in fire activity in various future climate scenarios. Fujioka, et al. (2008) and Camia et al. (2008;) consider that the FWI is a very precise index to identify the current fire danger weather and their projections in the context to climate change due to their physical foundations, which allows it to define the generality, almost universal of the ignition and propagation of wildfires

The study carried out by Mata Reis (1998) determined that there were several dozens of FDWI among static, dynamic, cumulative and non-cumulative in the European regional association of the World Meteorological Organisation (WMO). According to the study, 31 countries had an FWD Index, 21 of them used it in their operating systems, 13 made forecasts, and only one country spreaded the index using mass media. The study also reported that $59 \%$ of the countries (18) did not evaluate the behaviour of the FDWI in the field.

On the other hand, Moffat and Pearce (2013) analyzed the FDWI of New Zealand and England and determined that in some cases these were discredited in the eyes of its users. They pointed out that it was an erroneous and laborious guide especially when the weather data were not to hand. The same authors found that the scientific model of the indices was unknown by the managers, so their results had little value from a perspective of managing the danger information and there was a limited guidance on the applications of the resulting danger classes. Equally disturbing was the lack of use of FDW Index in some sensitive and relatively fire-prone districts where fire danger was assessed, preferably by "gut feeling". In addition, Manta (2003) mentioned that many FDWI had not been validated with wildfire statistics (area of fires and number of fires) so the warnings should be taken with caution. She adds that some indices require computation programs that are not friendly to managers, and the use of different indices prevented the generalization of FWI use. 
Currently, many countries do not have an FDW Index, despite, the recommendations of the WMO (1998) to adopt the FWI. Mexico uses the FWI, but not in an operating system (SEMARNAT, 2002); while countries of Central America do not have one. In South America, some provinces in Argentina use the FWI (Dentoni, et al., 2007); Public Universities of Brazil (Sampaio, 1998, Soares and Paez, 1974) and Peru (Lau, 2017) have researched in the adaptation of the FWI, but its use has not been implemented in an operational way.

In a warm climate the fire season would become extreme and frequent and the less accurate FDWI would present little variation in the high fire danger class throughout fire season. They could also exceed the maximum value on the highest danger class. The misperception that would generate the extreme fire climate, could lead to inadequate warnings about the large-scale fire danger. This was the case of Russia's FWD Index in 2002 (Chubarova et al., 2010) and Australia in 2009 (Tolhurst, 2010).

Unlike fires of natural origin where it is difficult to determine the number of ignitions and their spatial distribution, wildfires caused by man are not random events; they are often located near inhabited nuclei and tend to follow seasonal patterns, so that they can be known with some accuracy. Consequently, it is possible to assess the trends of fire danger on the geographical history of the occurrence of wildfires characterizing the geographical location of human populations and their production systems. Thus new definition of fire danger comes from the conceptual model of disaster risk management of Hyogo Framework for Action (ISDR, 2005) and the unified procedure for global risk management (ISO 31000, 2009).

The Andes is the longest mountain range on the Earth and is home to more than $75 \%$ of the world's tropical peaks and forms part of the structure and functioning of climate and hydrological cycle. The glaciers store, maintain and bring out the availability of fresh water for human life, natural vegetation and also produce hydroelectric power. The melting of the Andean glaciers contributes to the water flow of 62 rivers of the Pacific slope that supply with water to $95 \%$ of the Peruvian population. Likewise, the Amazon river (was born in the Andes) provides water to the population of the Amazon basin because it is the widest and the largest river on the planet. The Andean natural vegetation has different water yield and maintains infiltration capacity and moisture retention of soils. This is the case of wetlands (tropical Peatlands) that provide $7000 \mathrm{M}$ of $\mathrm{m} 3$ of water per year. Also, Andean soils constitute a natural reservoir of water (Vonk, 1988). Unfortunately, the Peruvian glaciers have fallen by $42 \%(871 \mathrm{~km} 2)$ in a period of 40 years (Santillán, 2015) as a possible result of the increase in air temperature and transport of black coal produced by burning of biomass from the Amazon to the Central Andes (Andrade et al., 2015).

Taking into consideration that government administrations in Peru have limitations in the estimation of FDW Index; which occupies the 23rd position in exposure to climate change (Corporación Andina de Fomento, 2014) and the occurrence of wildfires of disastrous consequences in 2016, the objectives of this study were:

a) Determine the spatial distribution of wildfires in 18 departments of the Peruvian highlands in the last 42 years.

b) Determine the temporal distribution of wildfires in the last 42 years, in 18 departments of the Peruvian highlands.

c) Determine the consequences of wildfires on man and their livelihoods, and on Andean forest ecosystems, and

d) Map out fire danger in the Peruvian Andes.

The knowledge of fire danger distribution in the Peruvian Andes must contribute to planning the strategies of reduction and adaptation to wildfires, guaranteeing that resources will be directed to areas with the greatest danger. 


\section{Methodology}

\subsection{Area of study}

The study area includes 18 departments of Peru with altitudes above 2000 m.a.s.l. The study area was $39,2 \mathrm{M}$ ha (31,8\% of the national territory) where approximately $16,8 \mathrm{M}$ inhabitants live (Instituto Nacional de Estadística e Información "INEI", 2015). The population that lives in five of the departments studied (Amazonas, Apurimac, Ayacucho, Cajamarca, and La Libertad) is characterized by having the highest incidence of poverty in the Peruvian Andes.

According to the Ministry of Environment (2015), natural vegetation of the Andes covers 26,2\% of the national surface. The predominant landscape is the Andean Puna (14,2\%), followed by the Andean Shrub (5,9\%), the Tropical Montane Cloud Forest (3,2\%), the Cardonales (2\%), tropical Peatland $(0,4 \%)$, the Dry Forest of Inter-Andean Valley $(0,34 \%)$ and the Páramo $(0,2 \%)$. Unfortunately, these ecosystems, legally protected or not, are intentionally burned to change the use of forest and protection lands to agricultural, mining and illicit crops, mainly.

\subsection{Main definitions}

Using the Regulation of Natural Land Use Capacity ( ${ }^{\circ}$ 062/1975-AG), Manta and León (2004) define wildfire as the uncontrolled fire that affects the wild vegetation that grows on forest and on protection lands, anywhere within the national territory.

In this research fire danger was defined as the potential occurrence of an event or physical tendency of wildfires of human or natural origin, which can cause loss of life, injury, impairment of livelihoods and negative effects on human health; loss of ecosystems, environmental resources, provision of services, as well as damages and losses in properties and infrastructure (modified from ISDR, 2005). In different circumstances, a generalized interruption and damage to a community that exceeds its ability to cope through the use of its resources is defined as catastrophe or disaster (Mayner and Arbon, 2015).

\subsection{To identify fire danger, the following procedure was followed}

a) The background of wildfires in 18 departments of the Peruvian Highlands was collected, reviewed, and a study period of 42 years was defined (1973 to 2014). The information came from government institutions with competencies in wildfire, the Universidad Nacional Agraria La Molina, other research centres, as well as news from local and national newspapers, radio and virtual networks.

b) The information regarding to wildfires occurrence was ordered and classified in order to create a wildfire database in Excel (2000).

c) To know the spatial distribution of the number of wildfires, the extent of area affected by fire, the consequences on man and their livelihoods, on the Andean vegetation, as well as the causes of wildfires, a record format was designed in Excel (2000).

d) To know the temporal distribution of wildfires, the date of wildfires occurrences was analyzed.

e) The geographic location of populations within of each department was established, through the use of information from the INEI (2015).

\subsection{The fire danger rating}

The fire danger rating across the studied departments was determined on the basis of area of wildfires occurred in each department and its historical-geographical location in relation to the total area affected by fire in the period studied (Table 1). 
Table 1 - Threshold values of area burned defining fire danger classes

\begin{tabular}{lcl}
\hline Class & Area affected by wildfires (ha) & \multicolumn{1}{c}{ De finition } \\
\hline Very high & $\geq 25000$ and $<75000$ & $\begin{array}{l}\text { The department has from } 10 \text { to } 35 \% \text { of total area } \\
\text { affected by wildfires over } 42 \text { years }\end{array}$ \\
High & $\geq 10000$ and $<25000$ & $\begin{array}{l}\text { Ihe department has trom } 5 \text { to }<10 \% \text { of total area } \\
\text { aftected by wildfires over } 42 \text { years }\end{array}$ \\
Medium & $\geq 1000$ and $<10000$ & $\begin{array}{l}\text { The department has from } 1 \text { to }<5 \% \text { of total area } \\
\text { affected by wildfires over } 42 \text { years }\end{array}$ \\
Low & $\geq 0,01$ and $<1000$ & $\begin{array}{l}\text { The department has from } 0,01 \text { to }<1 \% \text { of total area } \\
\text { aftected by wildtres over } 42 \text { years }\end{array}$ \\
\hline
\end{tabular}

\section{Results and discussion}

Wildfires are spatially distributed in all departments studied (18) in the period 1973-2014. In departments of Tacna and San Martín, wildfires occurred, but it could not be quantified due to lack of written evidence. Wildfires occurred in all provinces of departments of Cusco and Apurímac; in 10 departments, wildfires have occurred in more than $50 \%$ of their provinces. At the district level, in departments of Cusco, Apurímac and Puno in 63\% (68), 63,3\% (50) and 26\% (28) of their districts respectively, wildfires have occurred; while in 13 departments, from $20 \%$ to 2,3\% of theirs districts have had wildfires (Table 2). The wide spatial distribution of wildfires could be the fact that the main cause of wildfires is livestock activity (60\%), followed by agriculture (30\%) and mining. In these activities, fire is used to clear lands due to it is the most economical tool.

Table 2 - Spatial distribution of wildfires in the Peruvian Andes. Period 1973-2014

\begin{tabular}{|c|c|c|c|c|c|c|c|}
\hline \multirow[t]{2}{*}{$\mathbf{N}^{\circ}$} & \multirow[t]{2}{*}{ Department } & \multirow{2}{*}{$\begin{array}{r}\text { Provinces } \\
\left(N^{\circ}\right)\end{array}$} & \multicolumn{2}{|c|}{ Provinces with wildfires } & \multirow{2}{*}{$\begin{array}{r}\text { Districts } \\
\left(N^{\circ}\right)\end{array}$} & \multicolumn{2}{|c|}{ Districts with wildfires } \\
\hline & & & $\left(\mathrm{N}^{\circ}\right)$ & $(\%)$ & & $\left(\mathrm{N}^{\circ}\right)$ & $(\%)$ \\
\hline 1 & Cusco & 13 & 13 & 100,0 & 108 & 68 & 62,0 \\
\hline 2 & Puno & 13 & 10 & 76,9 & 108 & 28 & 25,9 \\
\hline 3 & Arequipa & 8 & 6 & 75,0 & 108 & 22 & 20,4 \\
\hline 4 & Apurimac & 7 & 7 & 100,0 & 79 & 50 & 63,3 \\
\hline 5 & Cajamarca & 13 & 8 & 61,5 & 127 & 19 & 15,0 \\
\hline 6 & Piura & 8 & 3 & 37,5 & 62 & 5 & 8,1 \\
\hline 7 & Ancash & 20 & 10 & 50,0 & 166 & 12 & 7,2 \\
\hline 8 & Ayacucho & 11 & 7 & 63,6 & 111 & 15 & 13,5 \\
\hline 9 & Junin & 9 & 6 & 66,7 & 123 & 8 & 6,5 \\
\hline 10 & Amazonas & 7 & 4 & 57,1 & 83 & 7 & 8,4 \\
\hline 11 & Pasco & 3 & 2 & 66,7 & 28 & 3 & 10,7 \\
\hline 12 & Huancavelica & 7 & 4 & 57,1 & 93 & 4 & 4,3 \\
\hline 13 & La Libertad & 12 & 2 & 16,7 & 82 & 2 & 2,4 \\
\hline 14 & Huámuco & 11 & 3 & 27,3 & 75 & 3 & 4,0 \\
\hline 15 & Lima & 10 & 4 & 40,0 & 171 & 4 & 2,3 \\
\hline 16 & Moquegua & 3 & 2 & 66,7 & 20 & 4 & 20,0 \\
\hline
\end{tabular}

Every year fire danger is present in Cusco since 1973 (42 years); while in Apurímac the danger would have been present for 20 years (Table 3). Furthermore, Huánuco has the lowest number of years 
with wildfire activity and in five departments fire occurrence is five years old. However, this result should be taken with caution because the lack of written records does not mean that there have not been wildfires. National awareness of wildfire began by the 2016 fire season because the high-impact wildfire, which threatened critical communities and infrastructure. This is the reason why they have not record the incidents of wildfire systematically and no have a complete statistic at the national level.

Table 3 - Characteristics of wildfires in departments of the Peruvian Andes

\begin{tabular}{rlrrrrrrr}
\hline $\mathbf{N}^{\circ}$ & Department & $\begin{array}{c}\text { Years with } \\
\text { wildfires }\end{array}$ & \multicolumn{1}{c}{$\begin{array}{c}\mathbf{N}^{\circ} \text { of } \\
\text { fires }\end{array}$} & \multicolumn{2}{c}{$\begin{array}{c}\text { Area of fires } \\
\text { (ha) }\end{array}$} & \multicolumn{1}{c}{ (\%) } & \multicolumn{2}{c}{$\begin{array}{c}\text { Population } \\
\text { Poor }\end{array}$} \\
\hline Extrem poor & \multicolumn{1}{c}{ Total } \\
\hline 1 & Cusco & 42 & 313 & 71040,29 & 32,79 & 646838 & 262026 & 1265827 \\
2 & Puno & 11 & 132 & 48535,90 & 22,34 & 815136 & 347237 & 1340684 \\
3 & Arequipa & 12 & 63 & 27682,90 & 12,74 & 222984 & 33749 & 1205317 \\
4 & Apurimac & 20 & 249 & 26078,36 & 12,0 & 312274 & 179013 & 444202 \\
5 & Cajamarca & 8 & 42 & 14905,58 & 6,86 & 836169 & 371797 & 1493159 \\
6 & Piura & 5 & 10 & 13035,01 & 6,00 & 694897 & 168460 & 1754791 \\
7 & Ancash & 12 & 81 & 6633,48 & 3,05 & 349602 & 91008 & 1109849 \\
8 & Ayacucho & 11 & 22 & 4189,53 & 1,93 & 402500 & 168459 & 642972 \\
9 & Junin & 12 & 506 & 2549,01 & 1,17 & 443269 & 129233 & 1292330 \\
10 & Amazonas & 10 & 19 & 1874,04 & 0,86 & 245804 & 102761 & 411043 \\
11 & Pasco & 13 & 112 & 392,03 & 0,18 & 160928 & 69425 & 290483 \\
12 & Huancavelica & 4 & 5 & 210,03 & 0,1 & 364168 & 220765 & 471720 \\
13 & La Libertad & 3 & 3 & 60,01 & 0,03 & 671054 & 222535 & 1725075 \\
14 & Huánuco & 2 & 3 & 40,01 & 0,02 & 528628 & 267182 & 819578 \\
15 & Lima & 3 & 5 & 27,51 & 0,01 & 1246745 & 64766 & 8095747 \\
16 & Moquegua & 5 & 9 & 25,33 & 0,01 & 32687 & 6267 & 169365 \\
17 & San Martin & N.D. & N.D. & N.D. & N.D. & 340020 & 92523 & 771021 \\
18 & Tacna & N.D. & N.D. & N.D. & N.D. & 55218 & 5049 & 315534 \\
Total Andes & & $\mathbf{1 5 7 4}$ & $\mathbf{2 1 7 2 7 9 , 0 2}$ & $\mathbf{1 0 0 , 0 0}$ & $\mathbf{8 3 6 8 9 2 2}$ & $\mathbf{2 8 0 2 2 5 3}$ & \\
\hline
\end{tabular}

N. D.: No Data

The annual occurrence of wildfire shows an accelerated increase over 42 years in almost all the departments studied. The annual increase, both in the number of wildfires and area of fires (Figure 1), can be explained by a greater use of fire in livestock and agricultural activities (two annual harvests) generated by market demand (Defries, et al., 2010), as well as the change in the use of forest and protection lands for agricultural use. But, the higher temperatures associated with the humidity deficit generated a greater flammability of dead and living biomass, with the consequent increase in size of wildfires. Also, this increase would be the result of the ENSO of 1994 and 1998, as well as the droughts that occurred in 2005 and 2010. The average burned area in each decade confirm this trend (Figure 2). 


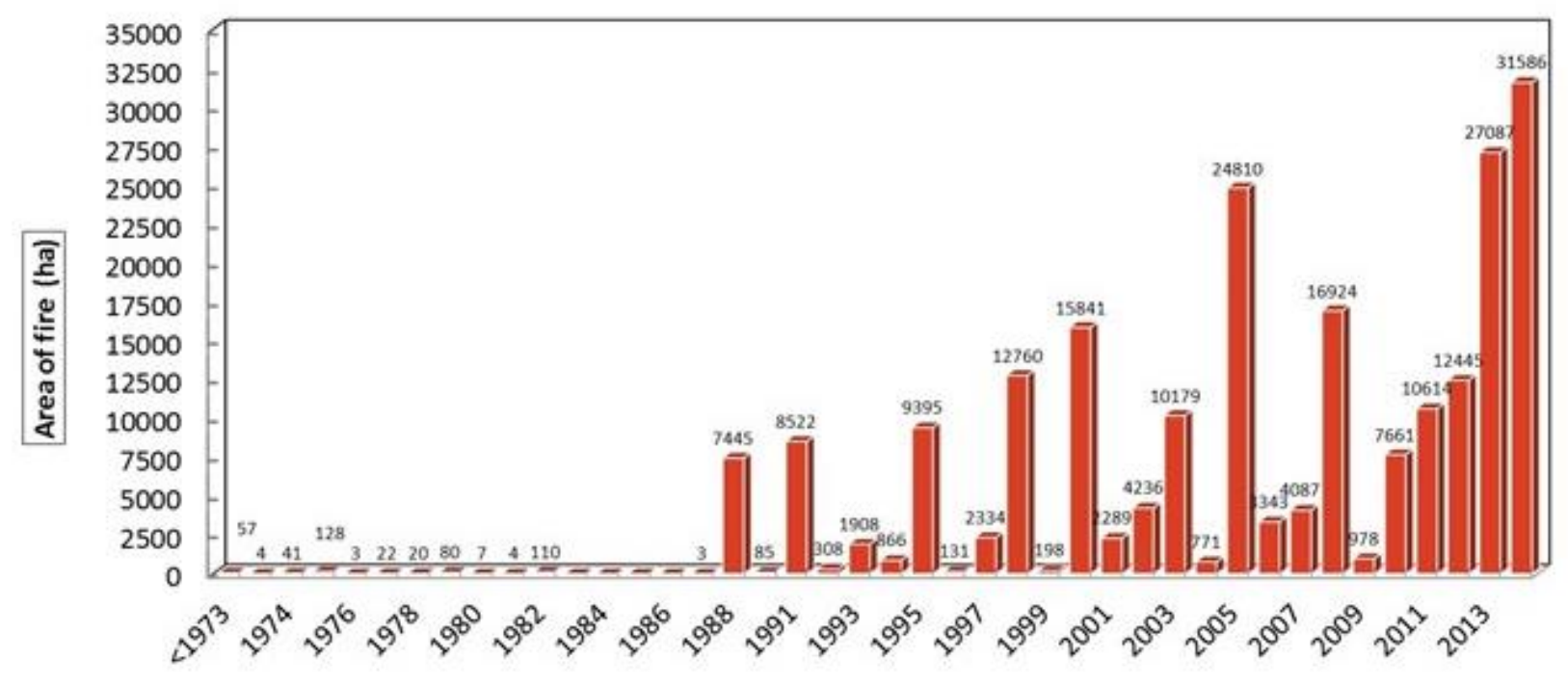

Figure 1 - Distribution of the annual area of fire in the Peruvian Andes

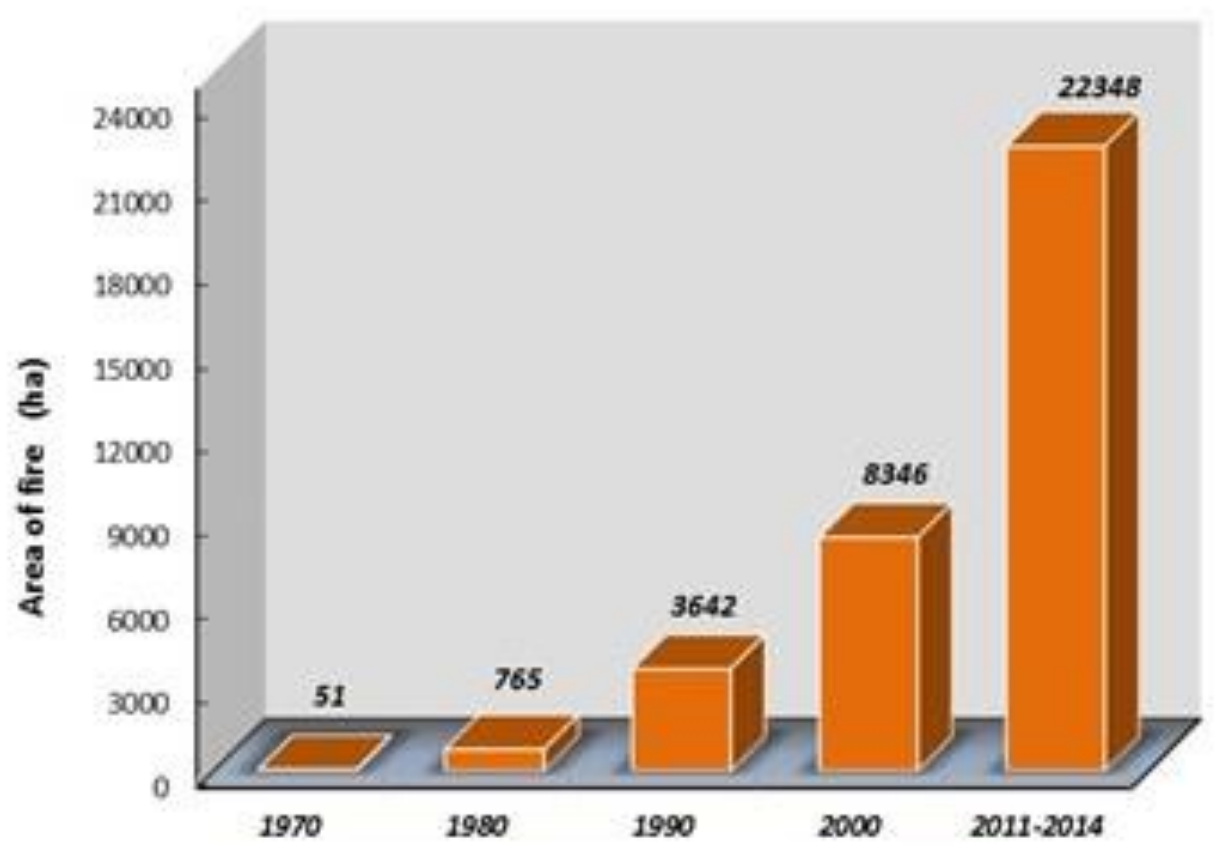

Figure 2 - Average of area burned by decades. Period: 1973-2014.

The temporal analysis of the number of wildfires in a calendar year, determined an increase in the number of months with wildfires. During 1973 to 2004 the fire season length comprised four months (from July to October); during the period 2005 to 2007 the season lasted seven months (from May to November). In the period from 2008 to 2014, the wildfire season length was extended to 12 months; although in December and January, as well as from February to May, the number of wildfires and the area of fire was low. This result suggests a collapse in the resources of extinction available to departments. On the other hand, this result reveals the consistency of the forecasts made by Jolly, et al. (2015) on the global wildfire danger.

The spatial and temporal distribution of wildfires in 18 departments threatens 11,2 million poor and extreme poverties Peruvians. The total Lima's population has been excluded because they are living in the capital of the department (Table 3). The threatened population has a low Human Development 
Index (between 0,2 to 0,4); they do not know the means to stop causing IF, to prevent them, as well as to handle the consequences of them. This situation makes them vulnerable to FIs and other natural hazards that they face year after year. The geographic location of the occurrences of the FIs will make it possible to take protective measures, carry out preventive actions, pre-suppression, suppression and rehabilitation of the affected areas in the localities, districts and provinces where IF occurs.

The spatial and temporal establishment of wildfires in the Peruvian Andes has produced material and environmental losses. During 1995-2014, 24 farmers died mainly, when they were trying to put out wildfires. In addition, on average each year, 7 people suffered accidents in the extinction activities; in the same way, 2317 people lost their homes and 6593 people were left with homes partially because the fire. Since medical statistics are not available in most departments, it has not been possible to estimate the number of people treated for respiratory diseases. Nevertheless, each year $\mathrm{CO}_{2}$ emissions affect human health, climate and contribute to glaciers retreat.

In the last 42 years, at least 1574 wildfires have occurred in the Peruvian highlands, causing loss and deterioration of $0,2 \mathrm{M}$ ha of natural Andean vegetation cover. The effect of fire on the Andean vegetation has depended on their sensitivity to fire, the frequency of wildfires, as well as the duration and severity of the fire. Similarly, the degree of deterioration of Andean soils depended on the type of fire, the severity and frequency of the fire, the associated production practices, as well as climate. The ecosystems most affected by the fire were: The Andean Puna and the Páramo (61\%), the Natural Forests (9\% of (Dry Forest in Inter-Andean valleys, Dry Mountain Forest and Tropical Montane Cloud forests), the Andean shrubs (1,4\%) and the tropical Peatland (1,3\%), as shown in the Figure 3 . However, $26 \%$ of the area of fire could not be classified due to lack of information. The scarce forest plantations in the country were also affected in $0,8 \%$ (1726 ha) of its surface.

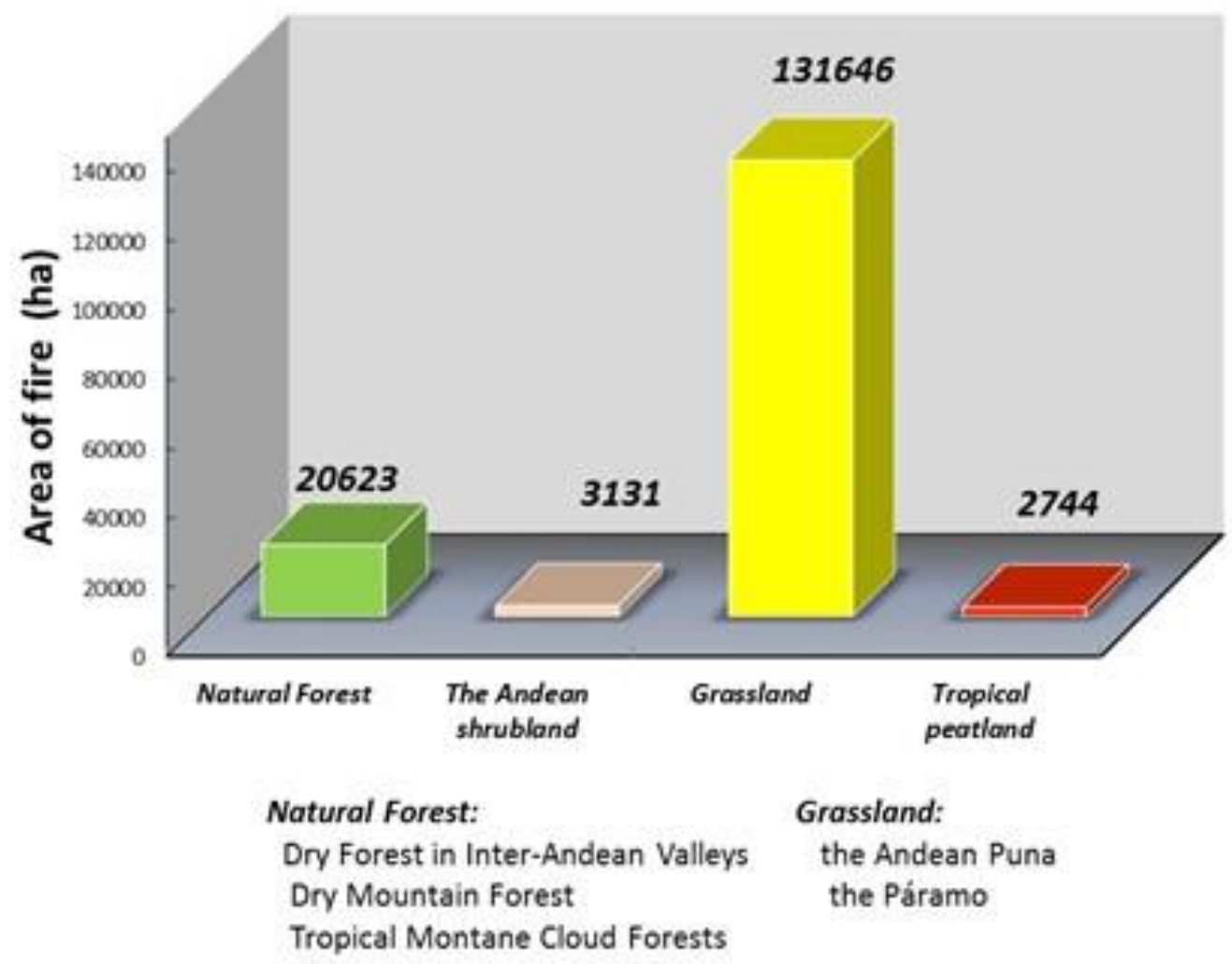

Figure 3 - Different vegetation types affected by wildfires in the Peruvian Andes

Loss of vegetation cover and forest and protection soils implies loss of $\mathrm{CO} 2$ retention capacity, contributing to global warming. Likewise, the reduction of the Andean Puna, the Páramo and the 
tropical Peatland would be generating decrease of rivers flow in Apurímac, Huancavelica and Ayacucho, in the last years. According to Arroyave (2007), the Páramo is the ecosystem with the highest average water yield (63\% of total flow/precipitation); next in importance is the Andean forest with 57\% average water yield (Tobón and Arroyave, 2008); while Murphy and Lugo (1986) affirmed that the tropical dry forest had the lowest water yield (19\%). Rapid assessment of the economic valuation of material losses and environmental services let SERFOR and AGRORURAL (2016) affirm that it reaches an average annual loss per department of US \$2,1 M and US \$ 4,6 M respectively.

Unfortunately, it is believed that the Andean ecosystems have the capacity of recover to the fire and other types of disturbious. The Tropical Montane Cloud Forests, Dry Forest in Inter-Andean valleys and Dry Mountain Forest, are not adapted to fire (Páramo, 2011), so the regeneration capacity and return to original conditions is very low. Meanwhile, The Andean Puna and the Páramo are adapted to fire because they have lived with it for centuries (Myers, 2007); however, frequent and severe wildfires and other human disturbances are altering their resilience.

This study presents, for the first time, the fire danger map for the Peruvian Andes (Figure 4). The very high danger class is found in four departments in the south of the country (Cusco, Puno, Arequipa and Apurímac) that represent $80 \%$ of the area affected by fire in 42 years; two of them have a high fire danger class and represent $13 \%$ of the burned area (Cajamarca and Piura); three departments have a medium fire danger class and represent $6 \%$ of the burned area (Ancash, Ayacucho and Junín); while, seven departments have a low fire danger class since the area affected by the fire reached $1 \%$ of the total burned area (Amazonas Pasco, Huancavelica, La Libertad, Huánuco, Lima and Moquegua).

The fire danger in the Peruvian Andes can be explained through the sociological, weather and vegetation cover variables. The use of fire in livestock and agricultural activities is a major component of fire danger; the weather variable contributes to explain a greater severity of wildfires due to the decrease in rainfall from North to South which is manifested in the area affected by the fire. When the vegetation map is overlaid with the fire danger map, it is observed that the surface most affected by fire corresponds to the Andean Puna of the Southern Highlands due to the flammability of dead and living biomass and the rate of fire spread in the large and rugged area that it occupies.

According to the fire danger map, it is expected that the next fire seasons, severity of fire will intensify over the same localities, for which, the managers involved should make sure to prevent and prepare the society and the territory to respond and recover from wildfires.

\section{Conclusion}

The statistics of the area affected by the fire have allowed us to show spatially and temporally wildfires occurrences and establish four fire danger classes in the Peruvian Andes.

According to the fire danger map, 56,2\% (9) of the departments studied have values that are in a very high, high and medium classes. These departments should be of interest in the implementation of strategies to reduce and adapt to fire danger. Priority should be given to six departments with very high and high classes of fire danger, which reach $93 \%$ of total area of fire in the period studied. The very high fire danger class is present in four 


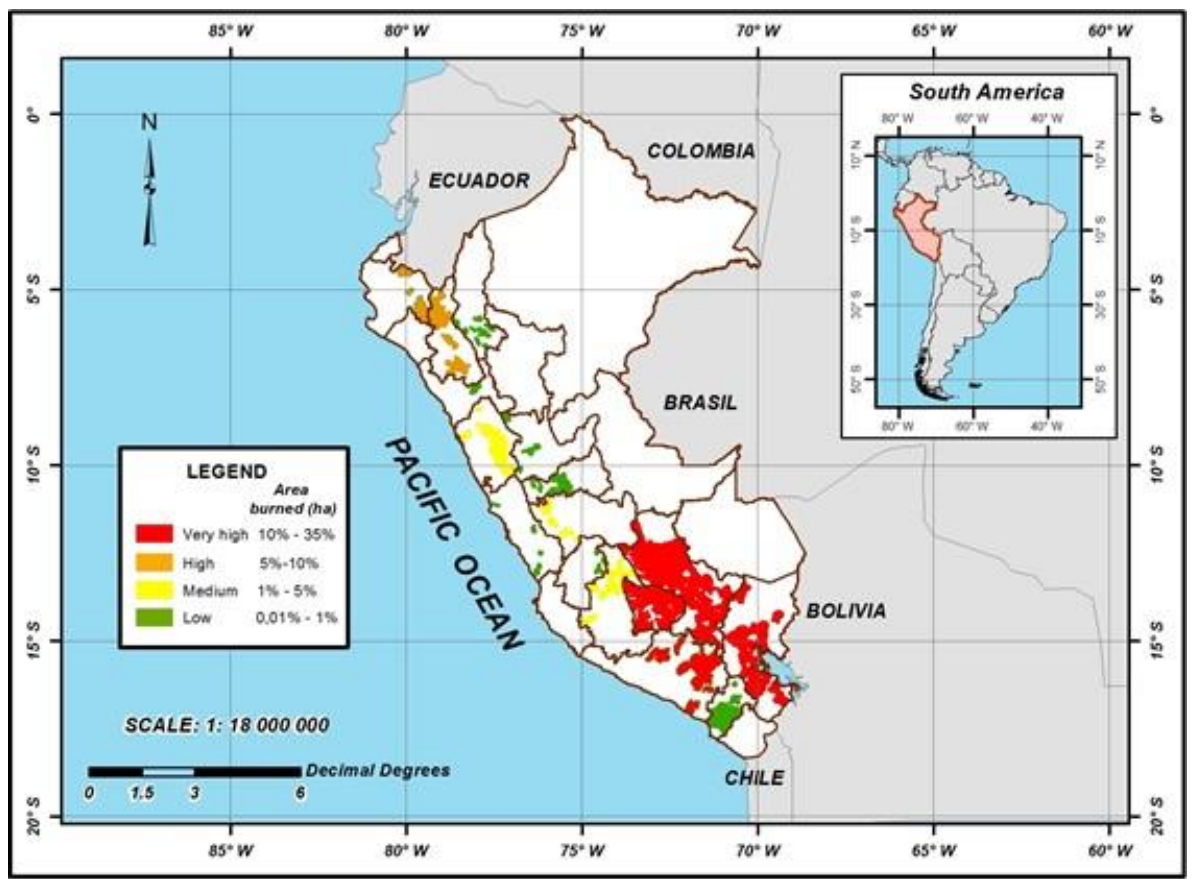

Figure 4 - Fire danger map for the Peruvian Andes

departments located in the Southern Highlands of the country, while the high fire danger class is present in two departments of the North Highlands of the country.

The vegetation most threatened by wildfires is the grasslands. The danger increases in the Páramo and the Peatland because they have a small surface and because they lose their water efficiency. If the occurrence of wildfires continues, the Andean vegetation will disappear and with this, the regulation of climate and hydrological cycle will have changed, affecting socio-economic development and wellbeing of Peruvians.

\section{References}

Arroyave, F. 2007. Contribución de la precipitación horizontal al ciclo hidrológico de los páramos, con referencia inicial al páramo de Guerrero. Tesis Mg. Sc. Universidad Nacional de Colombia. Medellín. 112 p.

Andrade, M., Zaratti, F., Laja, P., Wiedensohler, A., Krejci, R., Whiteman, N., Ramonet, M., Calzolari, F., Vuillermoz, E., Rose, C. 2015. Transporte de carbón negro hacia los glaciares centrales Andinos. In Andes regional conference: mitigating open agricultural burning. International Criosfera climatic initiative (ICCI), Molina Center, Autoridad Nacional del Agua and CONDESAN. 12 y 13 de febrero, Lima, Perú.

Camia, A., Amatulli, G., San-Miguel-Ayanz, J. 2008. Past and future trends of forest fire danger in Europe. Luxembourg: Office for Official Publications of the European Communities. UR Scientific and Technical Research Series - ISSN 1018-5593.

Benson, R., Roads, J., Weise, D. 2009. Climatic and weather factors affecting fire occurrence and behavior. Developments in Environmental Science 8, 37-60.

Chubarova, N., Prilepsky, N., Rublev, A., Riebau, A. 2009. A Mega-fire event in Central Russia: fire weather, radiative, and optical properties of the atmosphere, and consequences for Sub Boreal forest plants. Developments in Environmental Science, 8, 247-264.

Corporación Andina de Fomento. 2014. Índice de vulnerabilidad y adaptación al cambio climático en la región de América Latina y el Caribe. 211 p. 
DeFries, R.; Rudel, T., Uriarte, M., Hansen, M. 2010. Deforestation driven by urban population growth and agricultural trade in the twenty first century. Nature geosciences 3;178-181.

Dentoni, M. Muñoz, M., Epele, F. 2007. Implementación de un sistema nacional de evaluación de peligro de incendios: la experiencia argentina. In 4ta Conferencia Internacional de Incendios Forestales. 2007. Editado por R. Veléz. Sevilla, España.

Estrategia Internacional para la Reducción de Desastres (EIRD). 2005. Marco de Acción de Hyogo para 2005-2015. In Conferencia Mundial sobre la Reducción de los Desastres. 18 a 22 de enero de 2005, Kobe, Hyogo, Japón. Naciones Unidas, Ginebra. Suiza. 22 p.

FAO. 2007. Fire Management Global Assessment 2006. FAO, Forestry Paper $N^{\circ} 151$. A Thematic study prepared in the framework of the Global Forest Resource Assessment 2005. Rome, Italy. 121 p. ISSN.0258-6150.

Ferreira-Leite, F., Ganho, N., Bento-Gonçalves, A., Botelho, F. 2017. Iberian atmospheric dynamics and large forest fires in mainland Portugal. Agricultural and Forest Meteorology 247 (15):551-559.

Fujioka, F, Malcolm, A, Viegas, d., Mike, b. 2008. Fire Danger and Fire Behavior Modeling Systems in Australia, Europe, and North America. Developments in Environmental Science, 8, 471-497.

INEI. 2015. Perú: Anuario de Estadísticas Ambientales 2014. Oficina de Impresiones del Instituto Nacional de Estadística e Informática. 573 p.

IPCC, 2014: Climate Change 2014: Synthesis Report. Contribution of Working Groups I, II and III to the Fifth Assessment Report of the Intergovernmental Panel on Climate Change [Core Writing Team, R.K. Pachauri and L.A. Meyer (eds.)]. Geneva, Switzerland. 151 p.

Jolly, W., Cochrane, M., Freeborn, F., Holden, Z., Brown, T., Williamson, G., Bowman, A. 2015. Climate-induced variations in global wildfire danger from 1979 to 2013. Nature Communication, 6,7537 doi: 10.1038/ncomms8537 (2015).

Lau, J. 2017. Utilización del índice meteorológico de incendios forestales (Fire Weather Index) en el departamento de Cusco. Tesis Ing. Meteorológo. Facultad de Ciencias. Universidad Nacional Agraria La Molina 136 p.

McGrath, M. 2016. "Perfect storm" of El Niño and warming boosted Alberta fires". BBC News. May 5 BBC. https://en.wikipedia.org/wiki/2016_Fort_McMurray_wildfire Retrieved Feb 7, 2018.

McKenzie, D., Peterson, D., Littell, J. 2009. Global warming and stress complexes in forests of western North America. Developments in Environmental Science 8.,319-338.

Manta, M. I. 2018. El desafío de preparar a la sociedad ante los incendios forestales. El Ingeniero de Lima (Perú) XXV (83):32-33

Manta, M.I., León, H. 2004. Los Incendios Forestales del Perú: Grave problema por resolver. Floresta (Brasil) 34(2), 179-189.

Manta, M.I. 2003. Estudio de la estructura y funcionamiento de dos índices de peligro meteorológico de incendios forestales. Aplicación a tres zonas climáticas de España peninsular. Tesis $\mathrm{Ph}$. D. Escuela Técnica Superior de Ingenieros de Montes. Universidad Politécnica de Madrid. Madrid, España. 329 p.

Marengo, J., Tomasella, J., Alves, L., Soares, W., Rodriguez, A. 2011. The drought of 2010 in the context of historical droughts in the Amazon region, Geophys. Res. Lett.0 38, L12703, doi:10.1029/2011GL047436.

Marengo, J., Nobre, C., The Drought of Amazonia in 2005. Journal of Climate 21(3), 495-516. 175/2007JCLI1600.1

Mata Reis, R. 1998. Scales for the assessment of forest fire danger. In III International Conference on Forest Fire Research. 14 ${ }^{\mathrm{a}}$. Conference on Fire and Forest Meteorology. Editado por D. Viegas. Luso; Coimbra, Portugal. ADAI. p. 1.235-1.243. 
Mayner, L.; Arbon, P. 2015. Defining disaster: The need for harmonisation of terminology. Australas. J. Disaster. Trauma Stud. 19, 21-26

Ministerio del Ambiente. 2015. Mapa nacional de cobertura vegetal: Memoria descriptiva. Dirección General de Evaluación, Valoración y Financiamiento del Patrimonio Natural. Lima, Perú. 108 p.

Moffat, A., Pearce, H. 2013. Harmonising approaches to evaluation of forest fire risk. NZ TRANZFOR Visit: Final Report. Forest Research, Farnham. 42 p.

Murphy, P.; Lugo, A. 1986. Ecology of tropical dry forest. Annals Review of Ecology and Systematics 17, 67-68

Myers, R. 2007. Conceptos básicos en el manejo integral del fuego. In Taller Internacional Nuevas Estrategias para el Manejo Integral del Fuego en Ecosistemas Tropicales Prioritarios para la Conservación. The Nature Conservancy y ProNaturaleza. Puerto Maldonado, Perú, del 18 al 22 de junio.

Oliveira, R. 2010. Um estudo sobre os incêndios florestais ocorridos no estado de Vitória (Austrália), em fevereiro de 2009. Tese de Mestrado Interdisciplinar em Dinâmicas Sociais, Riscos Naturais e Tecnológicos. Faculdade de Ciências e Tecnologia Departamento de Engenharia Mecânica. Universidade de Coimbra, Portugal. 98 p.

Övergaard, R., Gemmel, P. and Karlsson, M. 2007. Effects of weather conditions on mast year frequency in beech (Fagus sylvatica L.). Sweden. Forestry 80, 555-565.

Páramo, G. 2011. Susceptibilidad de las coberturas vegetales de Colombia al fuego. In Incendios de la Cobertura vegetal en Colombia. Tomo I. Ed. por Álvaro del Campo Parra Lara. Universidad Autónoma de Occidente. Cali, Colombia. p. 73-144.

Portillo, G. 2018. Ciudadanos propone cambios en el Plan Hidrológico Nacional para adaptarse al cambio climático en España. www.meteorologiaenred.com/ciudadanos-propone-cambios-phnadaptarse-al-cambio-climatico.htm. Retrieved January 19, 2018.

Sampaio, O.; Soares, R.; Viegas, D. 1998. Evaluation and test of four methods of forest fire danger in Sao Paulo, Brazil. In III International Conference on Forest Fire Research. 14a ${ }^{\mathrm{a}}$. Conference on Fire and Forest Meteorology. Editado por D. Viegas. Luso; Coimbra, Portugal. ADAI. p.1.249-1.262.

Santillán, N. 2015. Impacto del Cambio Climático en los Glaciares Regionales: Contexto Regional y Nacional. In Andes regional conference: mitigating open agricultural burning. International Criosfera climatic initiative (ICCI), Molina Center, Autoridad Nacional del Agua and CONDESAN. 12 y 13 de febrero, Lima, Perú

Secretaría de Medio Ambiente y Recursos Naturales. 2002. Curso Protección contra incendios Forestales. Formación de instructores. Módulo índice de peligro. México/USAID.

SERFOR, AGRORURAL. 2016. Diagnóstico sobre la situación actual de los incendios forestales en el Perú, con especial referencia a los bosques Andinos. Consultora María Isabel Manta Nolasco, Docente de la Facultad de Ciencias Forestales, UNALM. Financiado por el Programa bosques Andinos del Helvetas Swiss Intercooperation. 168 p.

Soares, R.; Paez, G. 1974. Uma nova formula para determiner o grau de peligro de incedios florestais na Regi ao Centro Paranense. Revista Floresta. Trabajo presentado en el II Congreso Forestal de Brasil.

Sullivan, B. 2016. El Nino added to Alberta's fire woes before Fort McMurray Burned. News. https://en.wikipedia.org/wiki/2016_Fort_McMurray_wildfire .Retrieved April 6, 2018.

Tobón, C., Arroyave, F. 2008. Hidrología de los bosques alto-andinos. En: Ecología de Bosques Andinos. Editado por: León. Universidad Nacional de Colombia. 213 p.

Tolhurst, K. 2010. Report on fire danger ratings and public warning. Department of Forest and Ecosystem Science University of Melbourne, Australia. 26p. 
Vonk, J. 1988. Soil conservation in Peru. In: Conservation Farming on Steep Lands. Edited by Ankeny, IA; Moldenhauer W. and N. Hudson. Soil and Water Conservation Society and World Association of Soil and Water Conservation. New York, USA.

Wahlstron, M. 2010. Ola de calor Rusia y Pakistán, Desastres naturales 2010. http://www.bbc.com/mundo/noticias/2010/12/101221_desastres_naturales_2010_lh. Retrieved May 7,2018.

World Meteorological Organisation. 1994. Systems for evaluation and predicting the effects of weather and climate on Wildland fires. Special Environment Report $N^{\circ} 11$. WMO No 496. Geneva, Switzerland. Secretariat of the World Meteorological Organisation. 34 p. 


\title{
Fires in Portugal on 15th October 2017: a Catastrophic Evolution
}

\author{
Ilda Novo*; Paulo Pinto; João Rio; Célia Gouveia \\ Instituto Português do Mar e da Atmosfera (IPMA), Lisbon, 1749-077; Portugal \\ \{ilda.novo@ipma.pt*\}
}

\begin{abstract}
In 2017 Portugal mainland was affected by two events with major forest fires, that caused a large number of casualties and extensive property loss. Both events happened outside the typical forest fire season, which goes between July 1st to September 30th. Severe drought since the early spring 2017 and extreme heat in the summer are factors that may lead enhance the size, severity and spread rate of forest fires (Pereira et al, 2015). On October 15th, a number of factors joined together simultaneously: very low humidity in both the boundary layer and cumulated biomass (Gouveia et al., 2012), unseasonably high temperatures and a tropical cyclone (Ophelia) located to the west of Iberia, enhancing a south-easterly flow over mainland Portugal. The combination of these factors leads to a record number of forest fires in a single day with serious consequences, including the loss of lives and property.
\end{abstract}

Keywords: Weather Pattern, Anomaly, Hurricane, Forest Fire Risk, Pyroconvective Plume

\section{Introduction}

On $15^{\text {th }}$ October 2017 mainland Portugal was affected by a major forest fire event that occurred outside the typical forest fire season ( $1 \mathrm{~s}^{\mathrm{t}} \mathrm{July}$ and $30^{\text {th }}$ September). Severe drought in spring and hot, long lasting summer conditions, are among those factors that may enhance the size, intensity and rate of spread of forest fires (Pereira et al, 2005) and cumulated biomass (Gouveia et al., 2012). Besides these long term factors, a combination of weather conditions and orography lead to a record number of forest fires on a single day, in Portugal. The goals of this study are to find out which weather factors did play an important role in the event and which was the relevancy of a particular flow-orography driven interaction, to the onset of hazardous conditions, in the scope of forest fire propagation and intensity. This event has caused a large number of casualties and an extensive property loss, having been one of the worse ever recorded over territory.

\section{Data}

For the study of the weather pattern, ECMWF (European Centre for Medium-Range Weather Forecasts) model analysis and forecasts were used, as well as other numerical products, DWD (Deutscher Wetterdienst) $500 \mathrm{hPa}$ chart, HYSDPLITT Trajectory Model, information about Hurricane Ophelia from NOAA (National Ocean Atmospheric Administration, USA) and satellite images from EUMETSAT (European Organization for the Exploitation of Meteorological Satellites). To analyze the meteorological conditions on a mesoscale, it was used information from two IPMA radars systems, one located in northern Portugal, Porto radar (A/ PG), and another one located near Lisbon (C/CL). Upper air sounding at Lisbon weather station (Lx/GC), ground observations from IPMA weather network and indexes of risk and fire danger, computed at IPMA on a daily basis, were used.

\section{Weather Patterns}

\subsection{Anomaly and Extremes}

The upper-level $(500 \mathrm{hPa})$ circulation geopotencial anomaly over Northern Hemisphere on October (first half of the month), shows west-east trough/ridge pairs with a highly meridional state, being 
positive anomalies associated with upper-level ridges at mid latitudes and negative anomalies associated with upper-level troughs (Figure1a). Over North America and Western Europe there was a strong positive anomaly related with a strong ridge over mid Atlantic there was a strong negative anomaly related with a deep trough (Figure $1 b$ ).

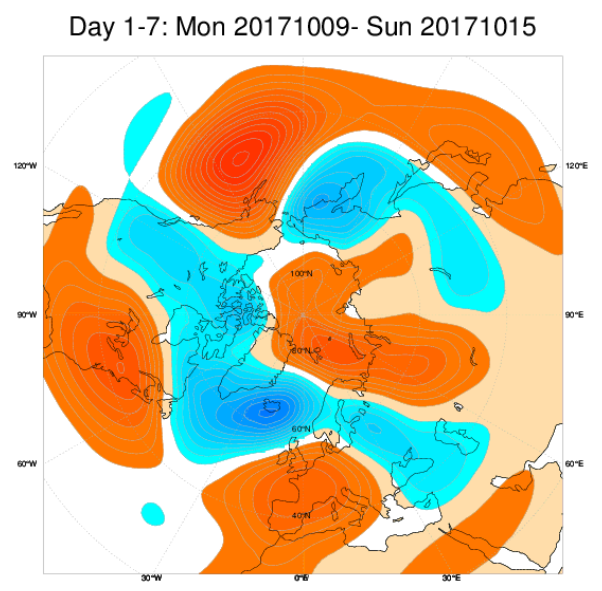

(a)

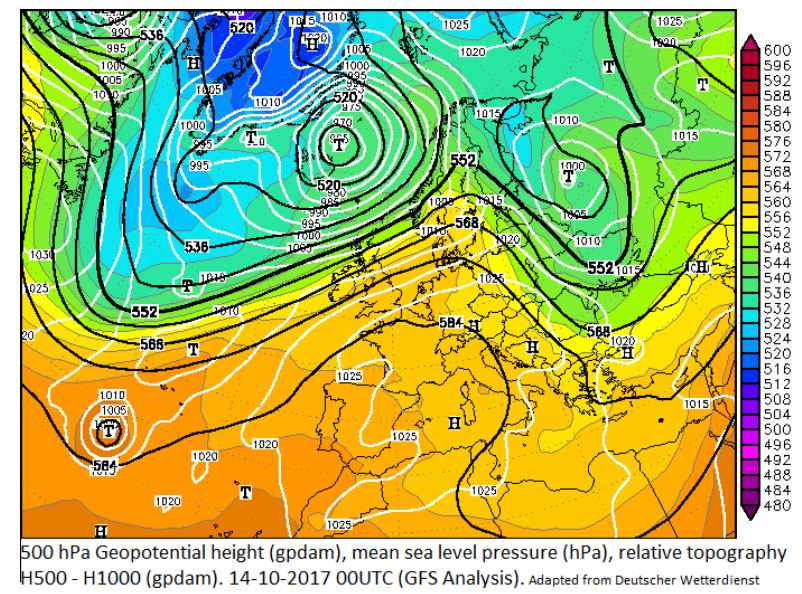

(b)

Figure 1- (a) ECMWF weekly-mean 500hPa geopotential anomaly for the ensemble mean (contours of 2 gpdam), orange positive anomaly, blue negative anomaly, (b) 500hPa geopotencial analysis 20171014 OOUTC, adapted from DWD.

Over Iberian Peninsula (IP), particularly in Portugal, this meridional weather pattern brought a very dry and hot October, reaching the peak by $15^{\text {th }}$ October. The geopotential height anomalies clearly show a positive value over PI and the advection of very warm, $+10^{\circ} \mathrm{C}$ anomaly at the $850 \mathrm{hPa}$ level, and dry air, below $30 \%$ anomaly of relative humidity (Figure 2).

At surface level, extremely high temperatures were recorded over mainland Portugal with $2 \mathrm{~m}$ air temperatures above $35^{\circ} \mathrm{C}$ in coastal areas. The exceptionality was such that the maximum absolute high records for October were, during this day, exceeded in the vast majority of coastal weather stations. On the other hand, the low temperature value records for October 15th were extremely high, exceeded in some inland weather stations the climatological value.
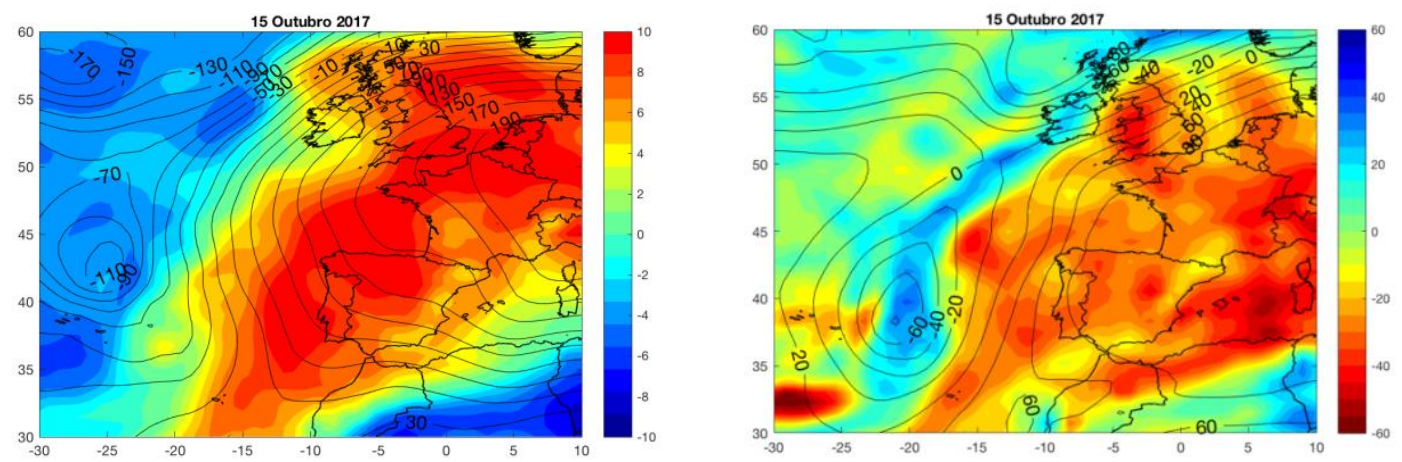

Figure 2 - Left panel: geopotential height anomaly (lines, in $\mathrm{m}$ ) at the $500 \mathrm{hPa}$ level and temperature at the 850 level (shaded, ${ }^{\circ} \mathrm{C}$ ). Right panel: geopotential height anomaly (lines, in $\mathrm{m}$ ) and relative humidity at the 850 hPa (shaded, \%).

On the $15^{\text {th }}$ October, it was also reached the highest level of meteorological drought in the territory for the entire year of 2017, as estimated by the Palmer Drought Severity Index (PDSI): 100\% of the territory was in severe or extreme drought conditions. 


\subsection{Synoptic Analysis}

Since early October a block ridge with a large and intense high settled over Iberia and the Atlantic adjacent area was settled in. By $15^{\text {th }}$ October, (Figure 3b), the high was located over the Alpine region, extending to North Africa (Morocco, Algeria), thus promoting a more intense flow from south/southeast over IP, as well as an increase in the transport of warmer and drier air (Figure 3b, c). Figure $3 \mathrm{~d}$ reveals the trajectory of air parcels at levels of $500 \mathrm{~m}, 1500 \mathrm{~m}$ and $3000 \mathrm{~m}$, crossing North Africa and southern Spain before being transported over Portuguese territory. For levels above 4000m the trajectories the air particles were across Atlantic (not shown).

In this synoptic configuration the usual Iberian thermal low does not establish, neither any sea breeze circulation over the west coast. This weather pattern is, in fact, a non-characteristic summer pattern over Iberia.

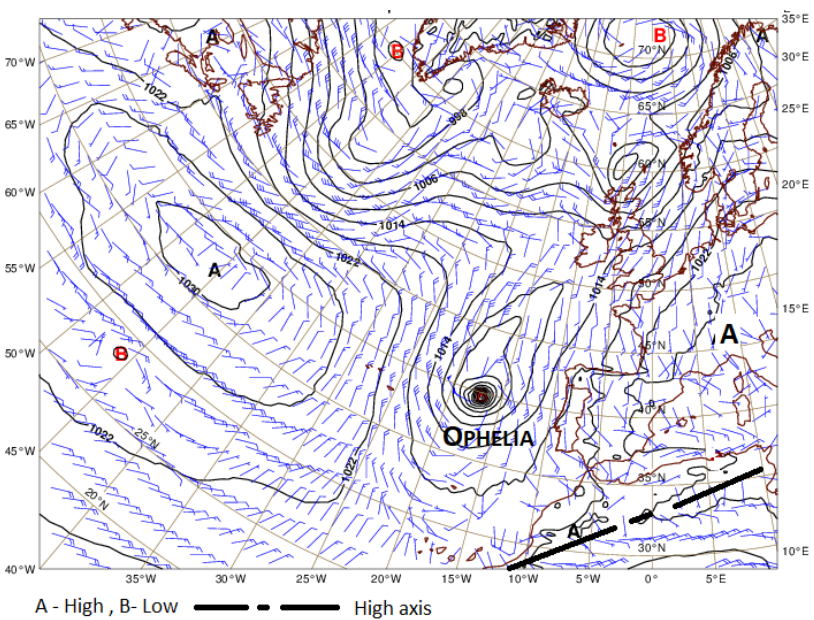

(a)

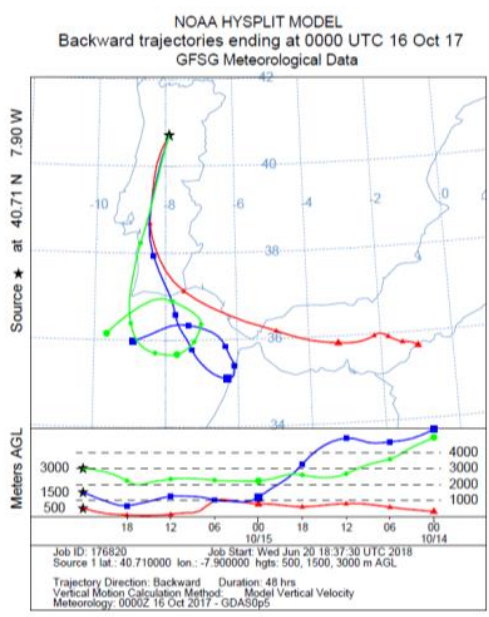

(b)

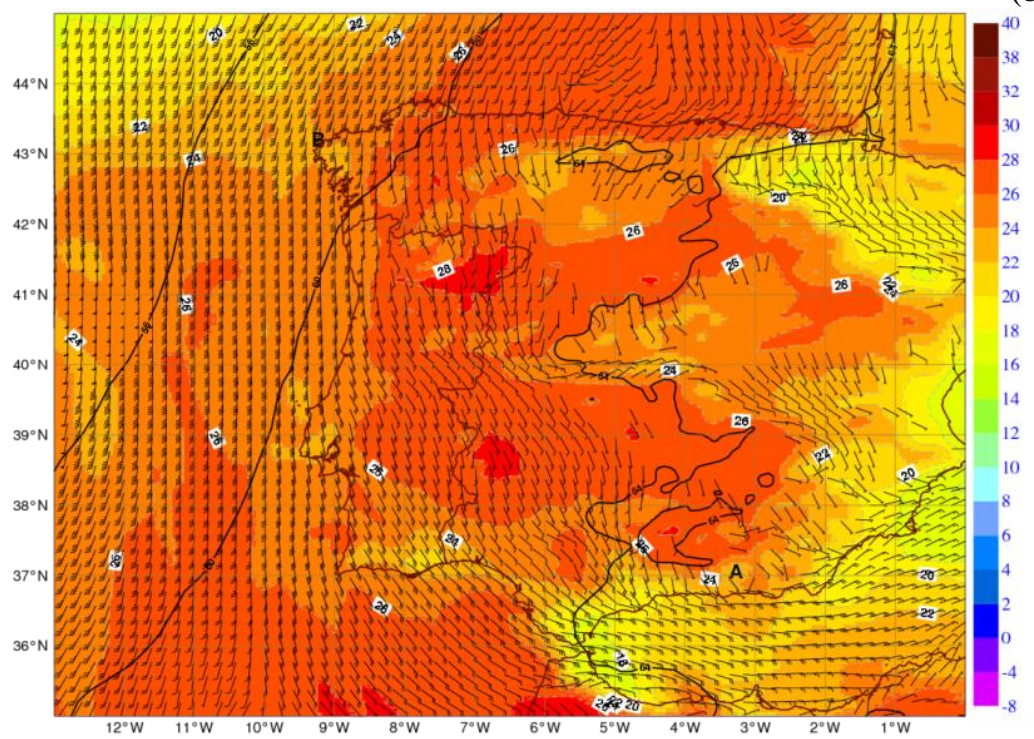

(c)

Figure 3 - ECMWF Analysis valid at 12UTC, 20171015 (a) pressure at mean sea level (pnmm, lines, hPa), wind barbs at 10m (kt), (b) HYSPLITT Trajectory Model, NOAA, backward trajectory from 2017/10/14 at 00 UTC to 2017/10/16 at 00 UTC, level: $500 \mathrm{~m}, 1500 \mathrm{~m} 3000 \mathrm{~m}$, (c) ECMWF H+12 forecast valid at 12 UTC, 20171015, geopotential height at the 950hPa (lines, gpdam) at the 950hPa, temperature at 950hPa (shadow, $\left.{ }^{\circ} \mathrm{C}\right)$, wind barbs at 950hPa $(\mathrm{kt})$. 


\subsubsection{The role of hurricane Ophelia}

Hurricane Ophelia was born as an extratropical low in the mid Atlantic, west-southwest of Azores, during the first week of October. A sea surface temperature (SST) near $27^{\circ} \mathrm{C}$ and a weak wind shear across the troposphere, held the system to become a tropical storm, on the 9th October, and a hurricane, by $18 \mathrm{UTC}$ of the $11^{\text {th }}$ October, when it was located about $1200 \mathrm{~km}$ southwest of the Azores (National Hurricane Centre, NHC). On $14^{\text {th }}$ October, a broad mid-latitude trough over North Atlantic moved east-southeastwards. The southwesterly flow ahead of the trough and associated cold front, turned Ophelia's track to the northeast, followed by its forward speed increase and intensification. It became a Hurricane 3 category during the afternoon of the $14 \mathrm{t}^{\mathrm{h}}$ October, when was located $900 \mathrm{~km}$ southwest of the Azores.

As Ophelia continued its track to the northeast, embedded in the stated trough, decreased its strength, being located about $460 \mathrm{~km}$ west-northwest of the northern coast of Portugal by $15^{\text {th }}$ October, 18UTC (Figure 4). At this time, maximum wind speed was about $80 \mathrm{kt}$ and minimum pressure 959 hPa. By 00UTC of $16^{\text {th }}$ October, over Ireland, Ophelia turned into an extratropical low, as it merged with the cold front.

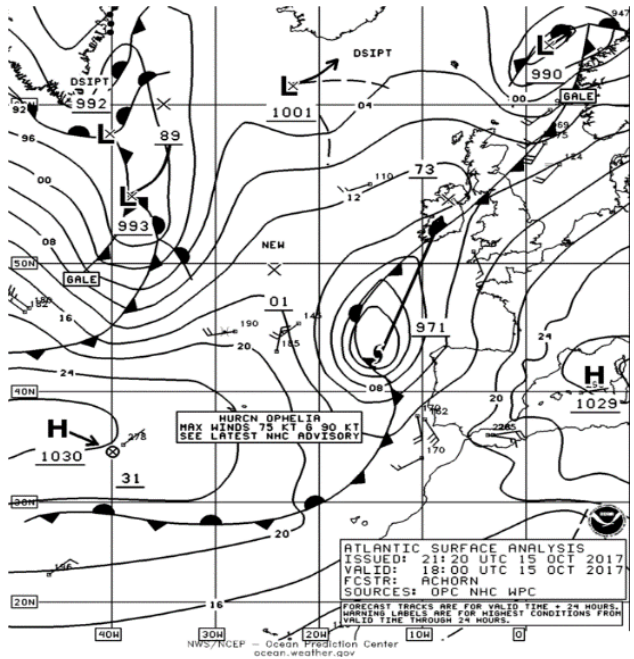

(a)

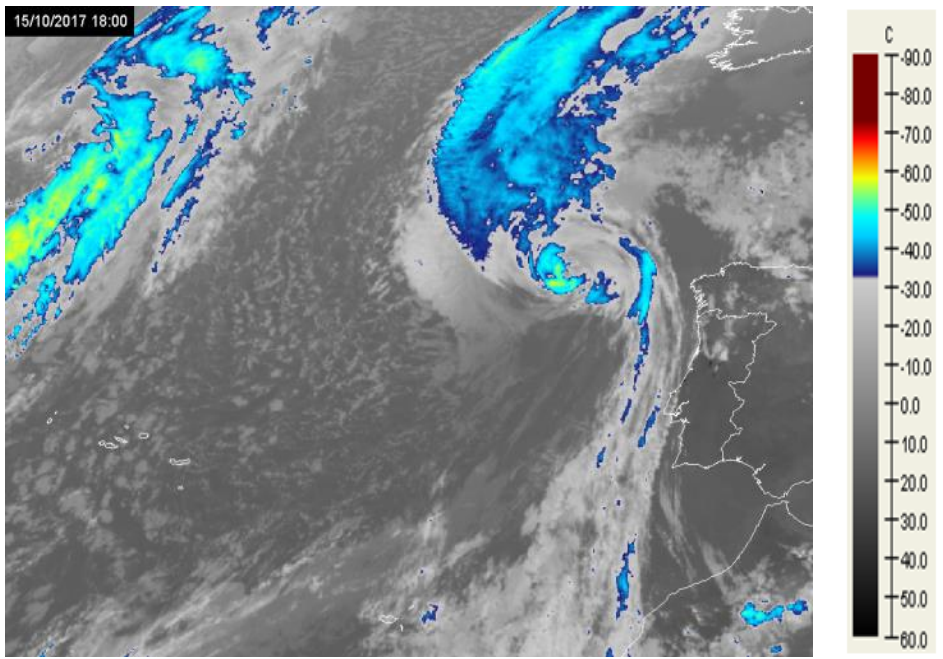

(b)

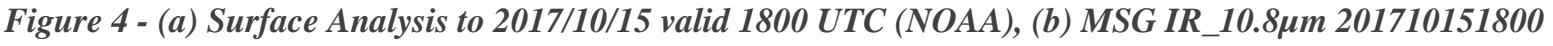
UTC.

On satellite imagery (Figure 4b) the cold tops (light blue and green) mark the centre of Ophelia and a stratiform cloudiness band, associated to the cold front (lower tops, grey shades), is organized close to the west coast of IP. In Figure 4a, the mean sea level pressure field, $18 \mathrm{UTC}$ of $15^{\text {th }}$ October, shows the high pressure system over the western Mediterranean. Hurricane Ophelia and this high pressure system generated a strong pressure gradient over IP, forcing an increase in flow intensity, as shown in Figure 5. During the morning there was an intensification of the south-southeast flow (Figure 5a) while during the afternoon the flow turned south, and intensified slightly (Figure 5b, c), as the hurricane was crossing the Atlantic near Iberia west coast. Afterwards it gradually turned to the southwest, slightly decreasing (Figure 5d). 


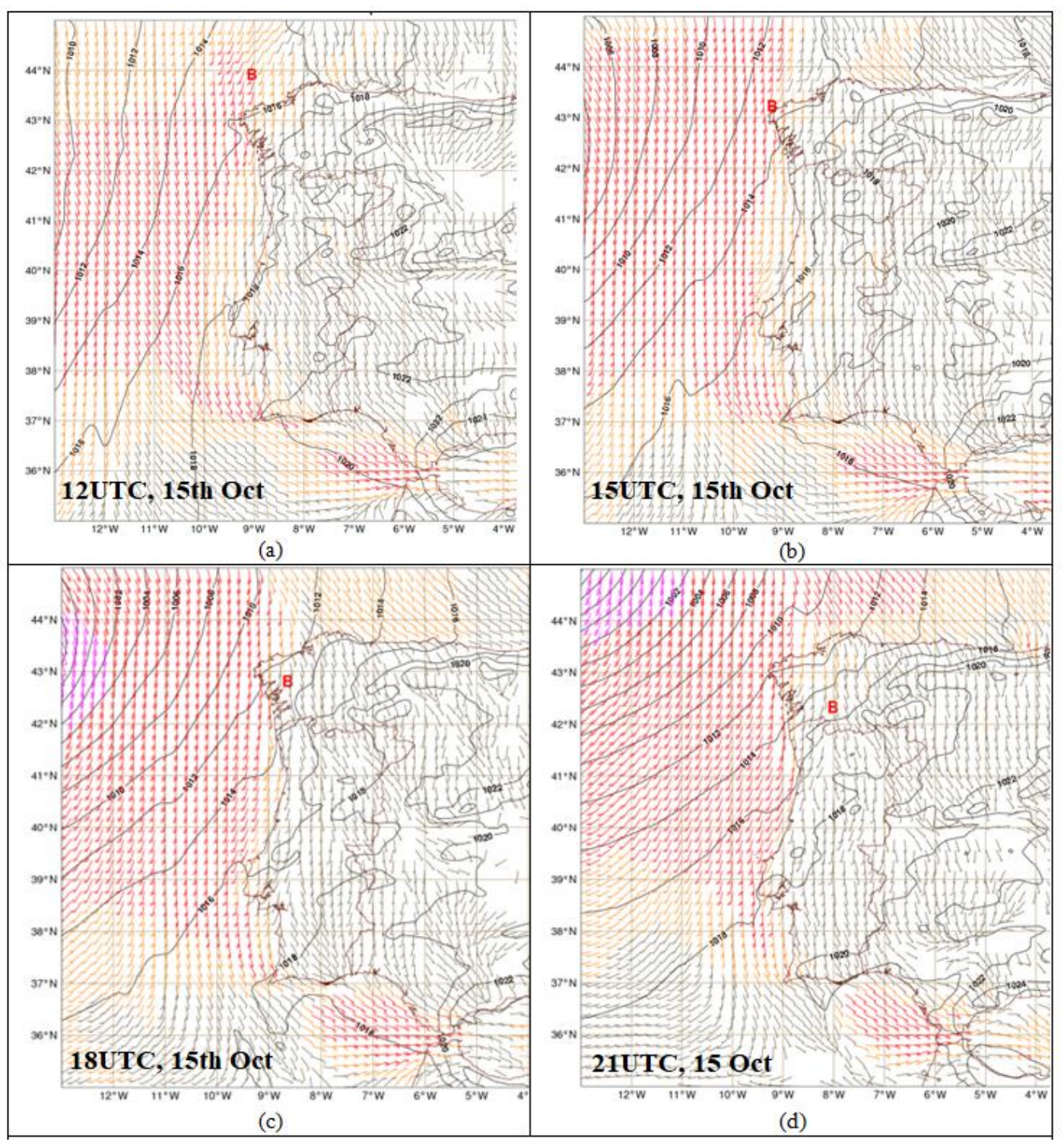

Figure 5- ECMWF forecasts pressure at medium sea level (pnmm, lines, hPa), barbs wind (kt): equal 5 kt and less than $15 \mathrm{kt}$, grey, equal $15 \mathrm{kt}$ and less than $20 \mathrm{kt}$, yellow, equal $20 \mathrm{kt}$ and less than $30 \mathrm{kt}$, red, equal or greater $30 \mathrm{kt}$, pink. (a) H+12 valid 12UTC 2017/10/15, (b) H+15 valid 12UTC 2017/10/15, (c) H+18 valid 18 UTC 2017/10/15, (d) H+24 valid 00 UTC 2017/10/16.

The flow intensification was mainly felt on the west coast and over central and southern mainland Portugal. In the northern region, however, a weaker flow persisted during almost the entire period, as Figure 5 illustrates, as well as ground station observations (not shown). The cause for this behaviour is not clear. There is some radar observational evidence suggesting that a certain obstacle effect (Austin, 1969) may have been produced by the presence of extensive pyroconvective plumes located in the atmospheric volumes, upwind of the northern region. This effect may have joined other undetermined causes.

\subsubsection{The Ophelia Cold Front}

Over IP, the airmass exhibited very low values of dew point (below $6^{\circ} \mathrm{C}$ ), associated to a very dry airmass (Figure 6a). On the other hand, over the Atlantic area adjacent to western Iberia, dew point values of $18-20^{\circ} \mathrm{C}$ (Figure 6a) and total precipitable water of $30-35 \mathrm{~mm}$ (even about $50 \mathrm{~mm}$ close to the centre of Ophelia, Figure 6b) were typical of a Tropical Maritime airmass. So, an extremely warm and moist airmass was available close to the coast, in the circulation of Ophelia and associated cold frontal 
system. This cold front moved slowly to the east-northeast, approaching the west coast of IP in the evening of $15^{\text {th }}$ and the early morning of $16^{\text {th }}$ October. At the same time, the pressure field over eastern Iberia intensified and blocked the westerly transport of the moist and unstable (Jefferson stability index $>30^{\circ} \mathrm{C}$, Figure $6 \mathrm{~d}$ ) air band associated with the cold front, making it stationary (Figure $6 \mathrm{c}$ ). Thus, the cold frontal system barely affected the coastal area and slowly propagated inland during the beginning of day $16^{\text {th }}$, while dissipating, also due to some pressure build up in the post-frontal sector (Figure 6c). This situation did not allow expressive convective developments and only the formation of low and stratified cloudiness was observed during the morning of $16^{\text {th }}$ October.

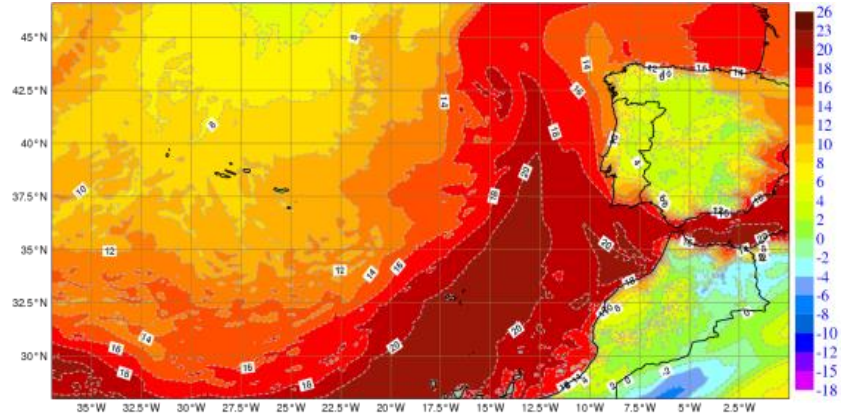

(a)

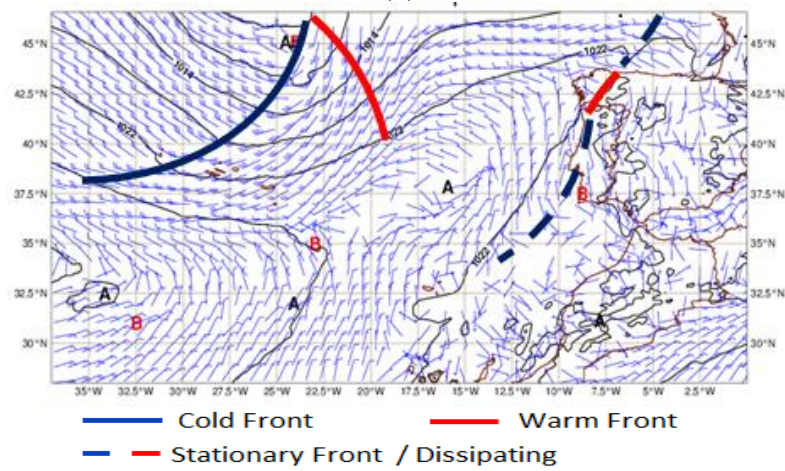

(c)

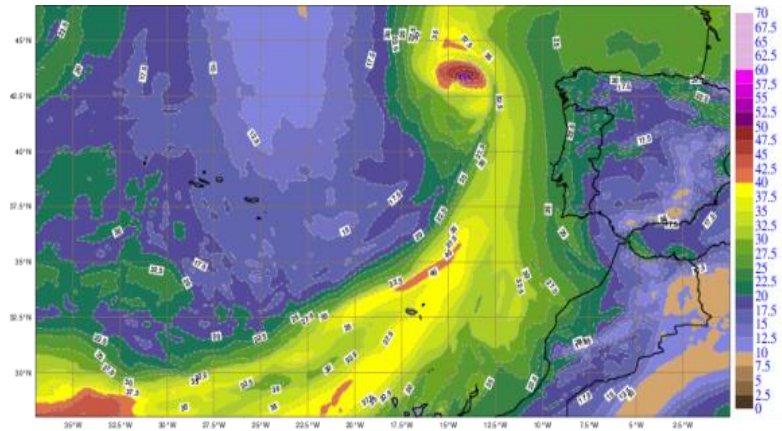

(b)

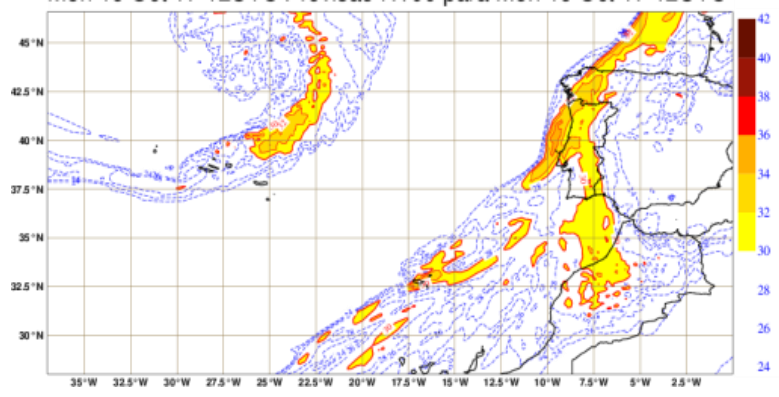

(d)

Figure 6 -, (a) ECMWF forecast H+18 valid 18 UTC $201710152 m$ dew point temperature (shadow, in ${ }^{\circ}$ C), (b) ECMWF forecast H+6 valid 12 UTC 20171015 Total Water Precipitation (shadow, in mm). (c) ECMWF analysis valid 12 UTC 2017/10/15 mean sea level pressure (pnmm, lines, hPa), wind barbs at 10m (kt), fronts are marked, (d) ECMWF analysis valid 12 UTC 20171016 Jefferson stability index (shadow, ${ }^{\circ}$ C). (adapted from Novo, I. and Pinto, P. 2017, in Portuguese).

So, in spite of this warm and moist airmass near the coast, the referred factors have prevented a significant change of the existing environment. A sharp change in weather conditions did, in fact, occur just by the end of $16^{\text {th }}$, beginning of $17^{\text {th }}$ October, when another frontal system propagated from the west (frontal system west of IP, Figure 6c), causing widespread precipitation from early morning onwards (Figure 7). 


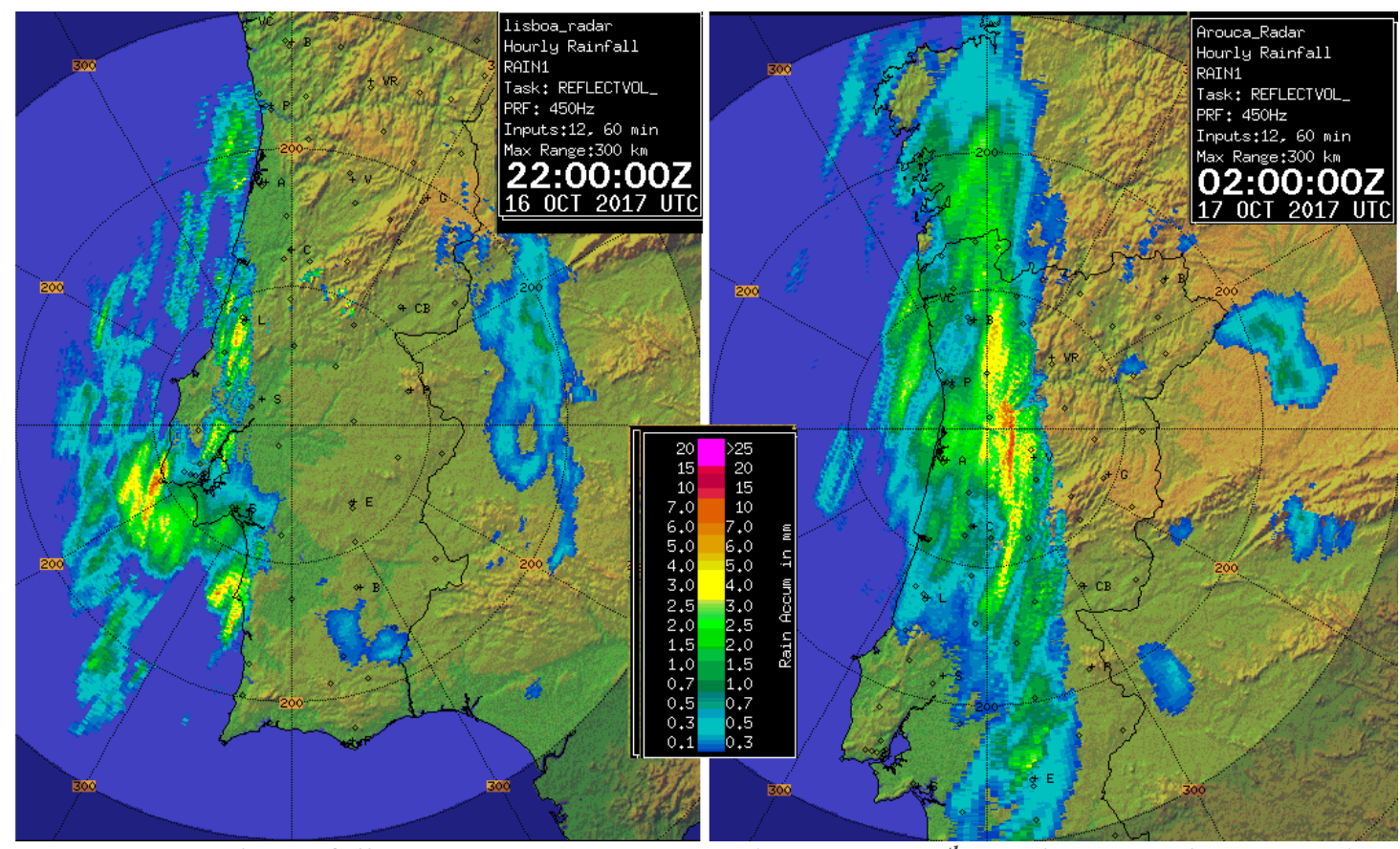

Figure 7- Hourly rainfall, RAIN1 (mm), (a) C/CL radar, 22UTC 16 ${ }^{\text {th }}$ October 2017, (b) A/PG radar, 02UTC $17^{\text {th }}$ October 2017 (adapted from Novo, I. and Pinto, P. 2017, in Portuguese).

\subsection{Low level weather conditions on 15 th October}

On $15^{\text {th }}$ October, as previously discussed, it was in place a widespread transport of hot and dry air, caused by an intense advection from North Africa. The involved anticyclonic circulation has contributed to extensive air subsidence and, thus, further dryness and heating of the air at the lower levels, with the onset of temperature inversion layers that were specially noted by the surface, at the sea level (Figure 8). A temperature inversion for Aveiro (ECMWF profile, at 00UTC) and a very dry atmosphere up to mid levels is shown in Figures $8 \mathrm{a}, \mathrm{b}$. As an example, the lifting condensation level (LCL) is about $3000 \mathrm{~m}$ high in the Lisbon sounding (Lx/GC, at 12 UTC). These profiles also show an increasing in moisture by mid-upper troposphere, but the value of the lift index (LI), at 00UTC, in Aveiro, was about $5^{\circ} \mathrm{C}$ and showing no CAPE (Convective Available Potential Energy). These values are indicative of a stable atmosphere. On both profiles the flow was from the southern quadrants at middle levels, being stronger by 12 UTC (up to 20 to $40 \mathrm{kt}$ ). This was confirmed by radar (not shown).

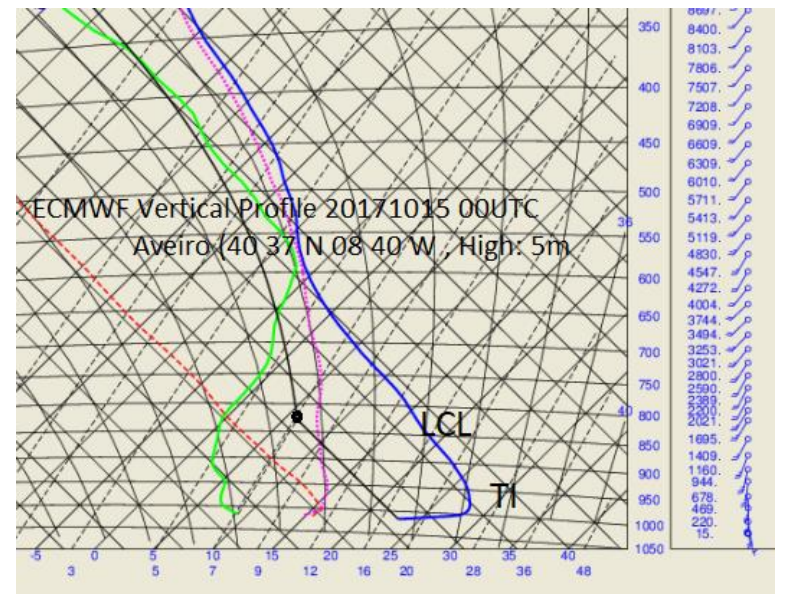

(a)

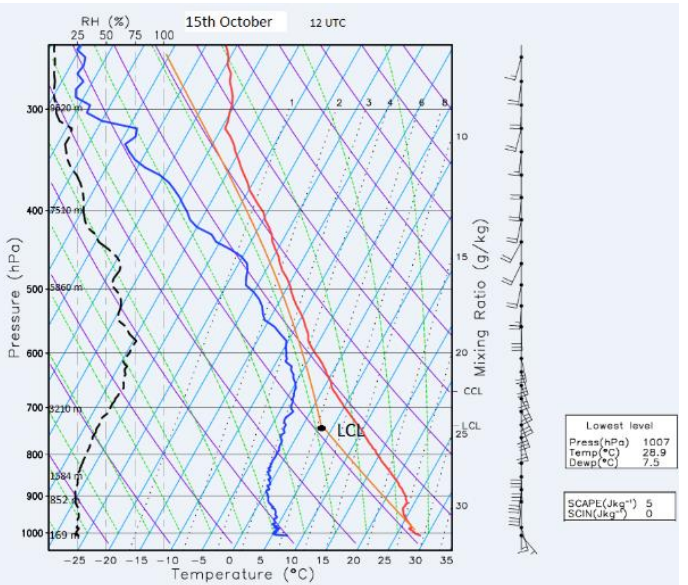

(b)

Figure 8 - ECMWF Vertical profile 20171015 00UTC (Extract) in Aveiro, (b) Lisbon air sounding 2017151012 UTC. (adapted from Novo, I. and Pinto, P. 2017, in portuguese). 
During this day, high values of maximum temperature and very low values of relative humidity were recorded over almost the entire territory (Figure 9). By the coast, in the central part of mainland Portugal, air temperatures exceeding $35^{\circ} \mathrm{C}$ coexisted with very low (10-20\%) relative humidity records by 15 UTC (Figure 9).

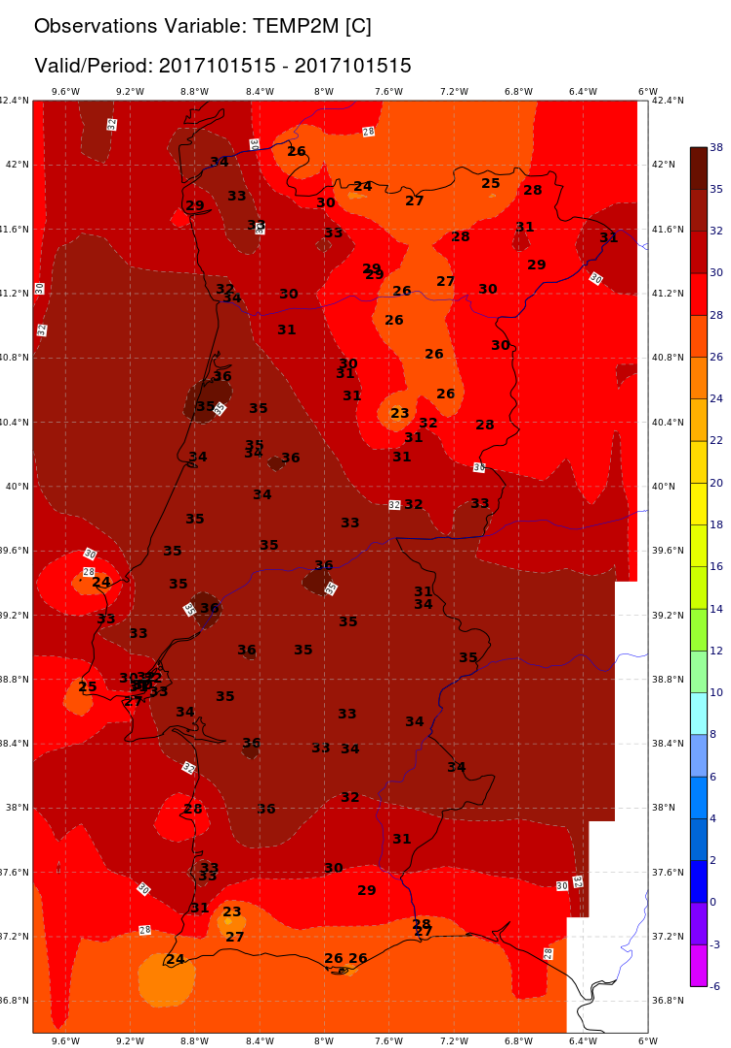

(a)

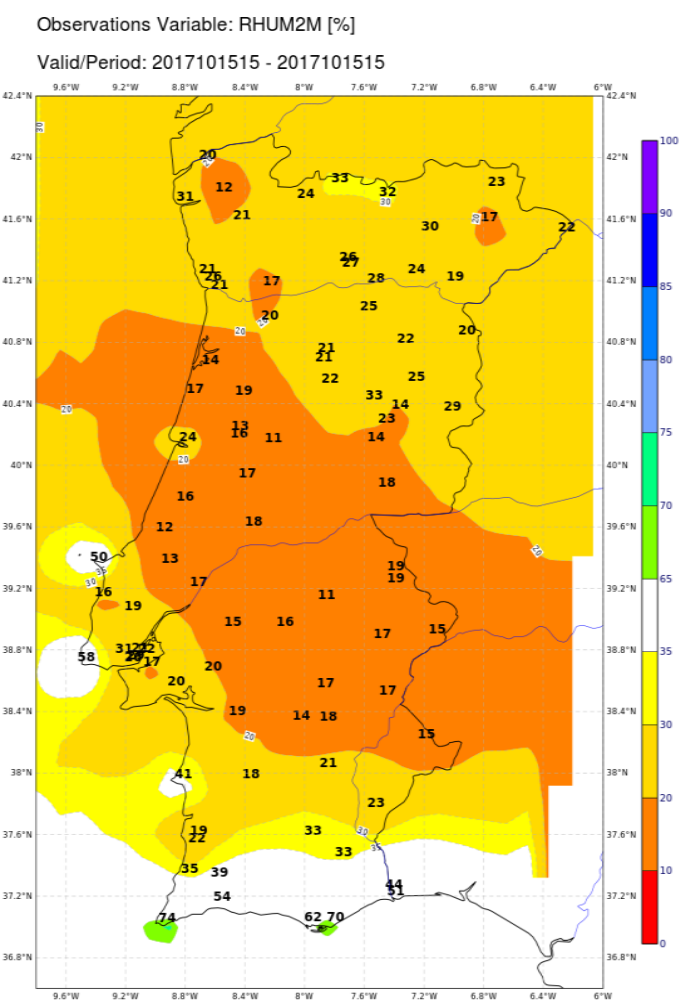

(b)

Figure 9 - (a) $2 m$ temperature, (b) and relativity humidity at 15UTC, 15 ${ }^{\text {th }}$ October 2017. (adapted from Novo, I. and Pinto, P. 2017, in Portuguese).

The largest mean wind and gust values were recorded near the coast and high elevations, from the early afternoon of the 15th October until the evening of the same day.

Alcobaça and Viseu AWS were chosen as representative of air temperature, relative humidity, average wind and gust observations, for each corresponding area: Alcobaça for west coastal areas and Viseu for inland areas. Figure 10 illustrates a relevant wind behaviour directly related with the wind regime. 


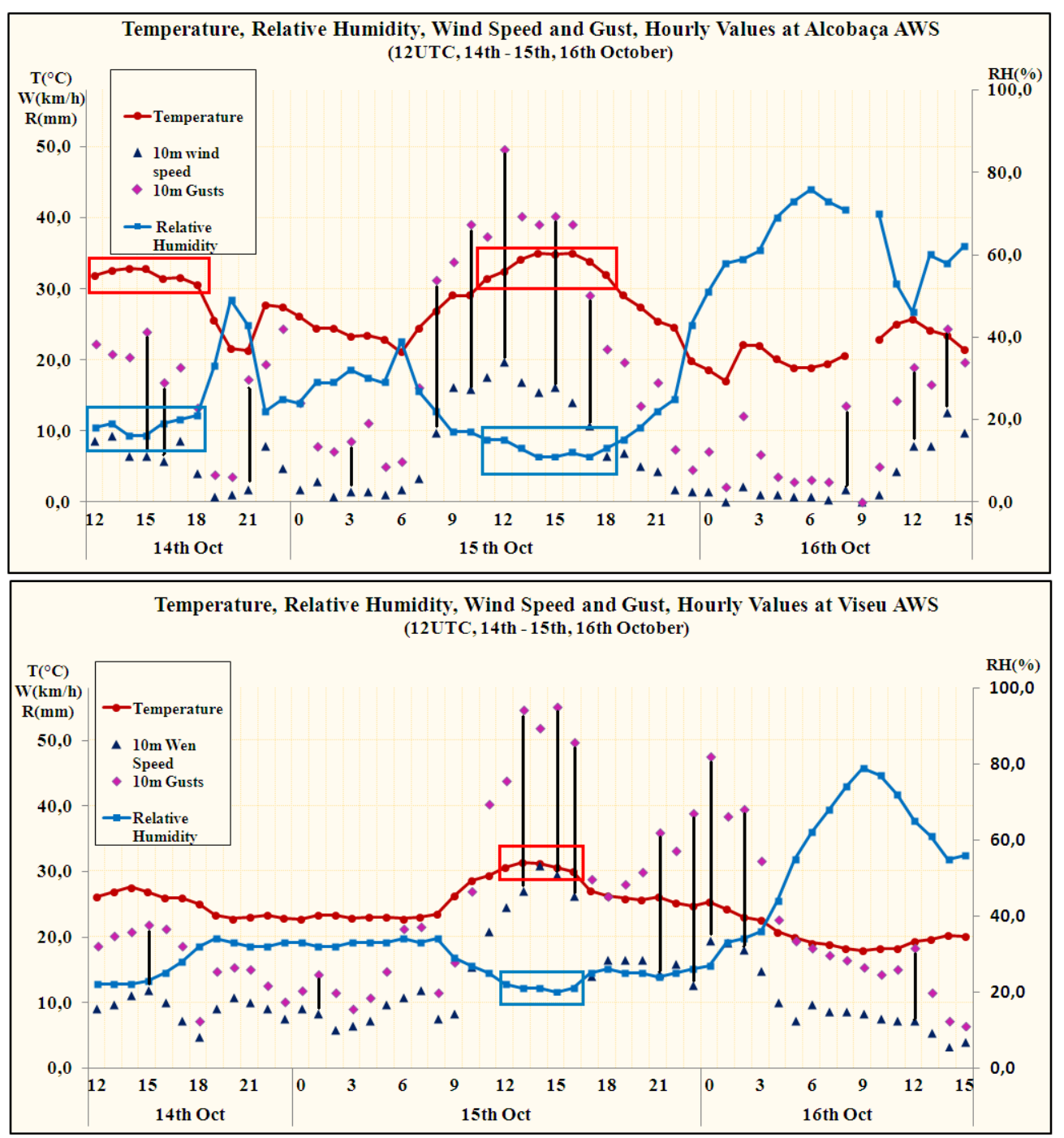

Figure 10 - Hourly values recorded of $2 m$ temperature $\left(T,{ }^{\circ} \mathrm{C}\right), 2 \mathrm{~m}$ relativity humidity $\mathrm{RH}(\%), 10 \mathrm{~m}$ wind speed $(\mathrm{V}$, $\mathrm{km} / \mathrm{h}), 10 \mathrm{~m}$ wind speed $(\mathrm{V}, \mathrm{km} / \mathrm{h}), 12 U T C 14^{\mathrm{st}}-15 \mathrm{UTC}$, 16th October 2017. Upper panel: Viseu AWS, Lower panel: Alcobaça AWS (adapted from Novo, I. and Pinto, P. 2017, in Portuguese).

In fact, the gustiness (relationship between mean wind and gust for a 10 min average period) at each station, clearly increased from the $14^{\text {th }}$ October until the central hours of the $15^{\text {th }}$, decreasing thereafter. The vertical black segments marked in Figure 10 materialize the gustiness, being evident that this was at a maximum during the early afternoon of the 15th October. So, it was during this period that the turbulence of the regime was at a maximum level and, simultaneously, minimum relative humidity and maximum temperature values were being recorded. Similar behaviour was observed on other locations (not shown).

Mesoscale phenomena (spatial scale in the range 200-2000 km, alpha scale), such as sea breeze fronts or other low level convergence fronts, may have strong influence on fire propagation intensity. Weather radars did not show any sea breeze fronts, on these days. Nevertheless, the C/CL radar enabled the identification of thin reflectivity lines usually corresponding to wind surface discontinuities. During the early afternoon several of these lines, exhibiting spectral width patterns suggestive of large horizontal wind shear values (not shown), were captured, being consistent with the turbulent nature of the flow, already detected on AWS. The turbulence nature of the regime was a supplementary adverse factor regarding fires propagation. 
As already mentioned, the anticyclonic circulation has caused air subsidence in the large scale, thus enhancing further dryness and heating of the air towards low levels. On the other hand, the MontejuntoEstrela mountainous system, predominantly southwest-northeast oriented, may also have played a role in interacting with a predominant southeasterly flow. The classical Foehn effect, described in literature, involves condensation in upwind slopes. However, there are cases involving dry airmasses without condensation, which was the current situation, in which the air, while plunging from higher levels, dries and warms due to adiabatic compression, as it descends the downwind slope. This effect is called "dry Foehn" (Sharples et al, 2010) and was suggested by comparing dewpoint depression and wind gust records at two AWS (Figure 11). One of them, located upwind (Tomar AWS) and the other one, downwind (Lousã AWS) of the referred mountainous system. At Lousã AWS, the air was drier than at Tomar, for nearly 30 successive hours (Figure 11, dew point reached about $-1^{\circ} \mathrm{C}$ at Lousã whereas Tomar reached $+7^{\circ} \mathrm{C}$ ) and the southerly wind was stronger (wind gust about $60 \mathrm{~km} / \mathrm{h}$ at Lousã whereas it reached $45 \mathrm{~km} / \mathrm{h}$ at Tomar). This long lasting behaviour was also observed on another AWS.

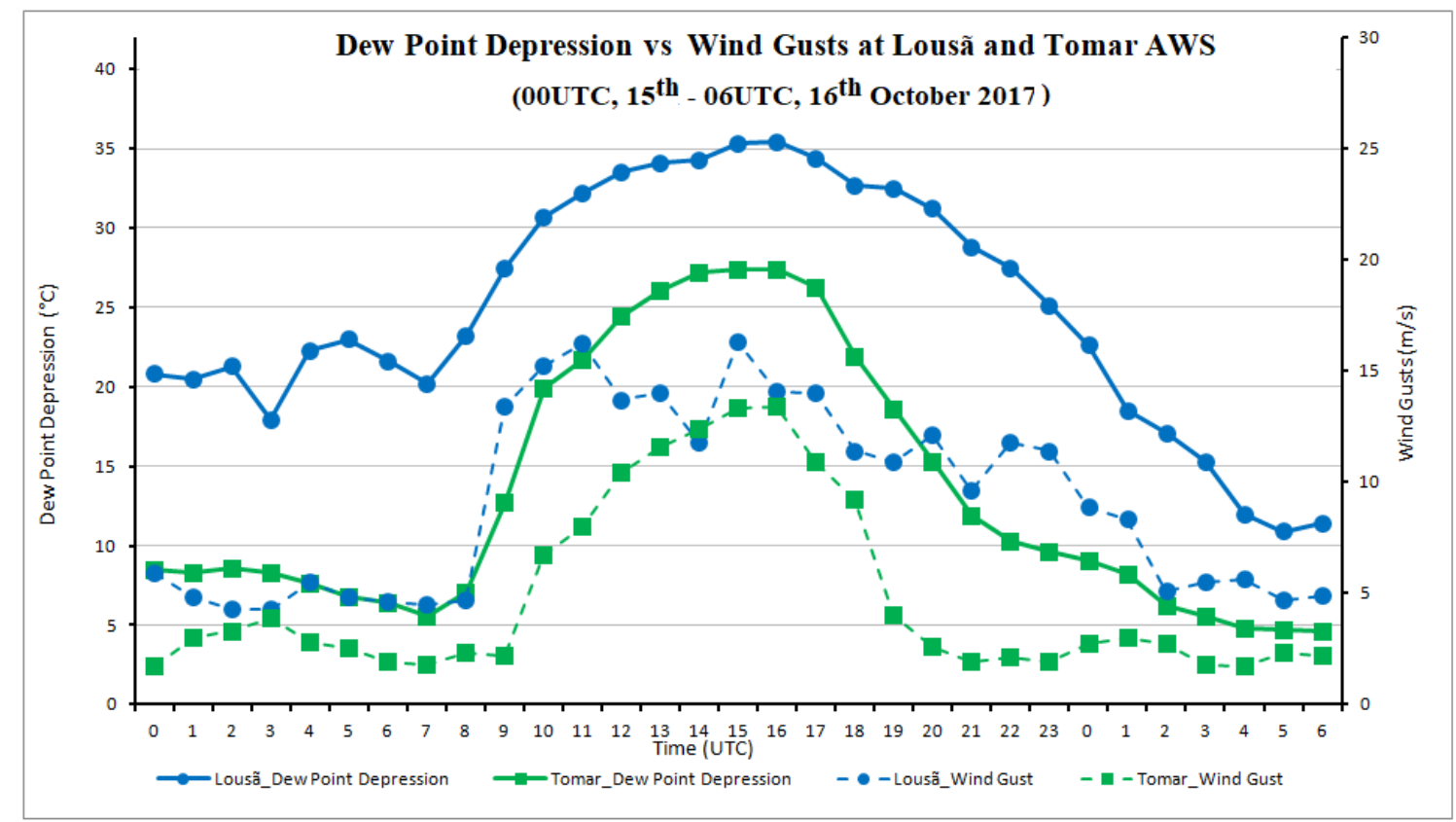

Figure 11 - Hourly Dew point depression $\left({ }^{\circ} \mathrm{C}\right)$ and Wind gust $(\mathrm{m} / \mathrm{s})$ records in Lousã (blue colour) and Tomar (green colour), 00UTC, $15^{\text {th }}$ October - 06UTC, $16^{\text {th }}$ October 2017).

\section{Forest Fire Risk}

The meteorological conditions on $15^{\text {th }}$ October, bringing great adversity in the fight against forest fires, were reflected in danger and fire risk index values, operationally computed on a daily basis at IPMA, namely the six components of the Canadian Forest Fire Weather Index (FWI) System. The FWI values were extremely high over the majority of the country (98th percentile), breaking a record in the historical period since 1999 (Figure 12a, b). 


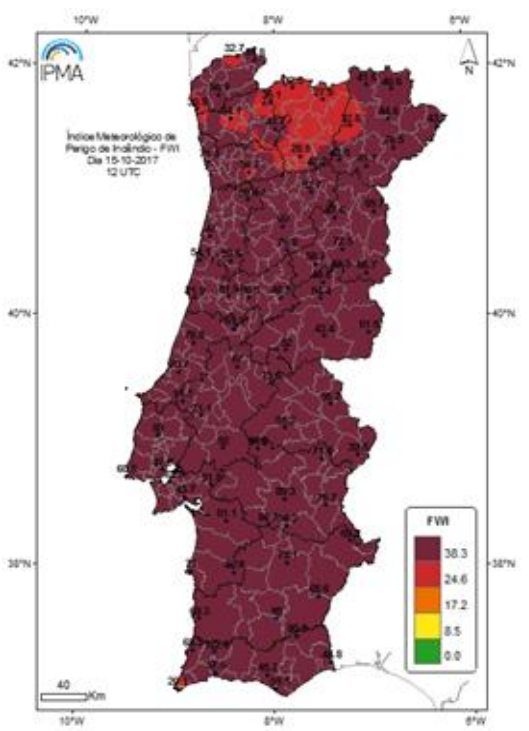

(a)

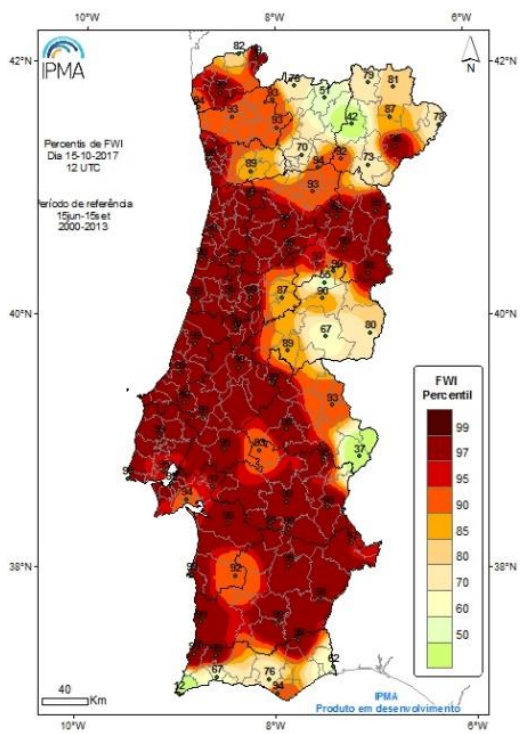

(b)

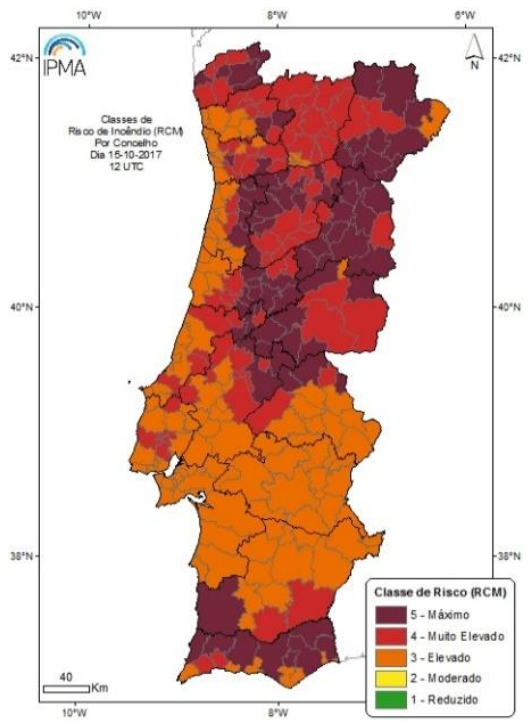

(c)

Figure 12 - Forest fire risk on $15^{\text {th }}$ October 2017, (a) FWI, (b) FWI percentile, (c) RCM. Risk classes: Low ("1- Reduzido"), Moderate ("2-Moderado"), High ("3-Elevado"), Very High ("4-Muito Elevado") and Maximum ("5-Máximo"). (adapted from Novo, I. and Pinto, P. 2017, in Portuguese).

The fire risk index disseminated daily by IPMA, RCM ("Risco Conjuntural e Meteorológico") is a combination of FWI index with a structural risk. This structural risk includes burnt areas, fire climatology, land cover and land use. This structural risk is annually updated by the National Forests Service, ICNF. Risk index values are divided into five risk classes: Low, Moderate, High, Very High and Maximum, computed for a county area. On $15^{\text {th }}$ October this risk index was the highest since 2006 (Figure 12c), with all the administrative areas in mainland Portugal at their maximum class of risk.

\section{Pyroconvective plumes identified on radar}

In the scope of weather radar observation, the term pyroconvective plume refers to a radar reflectivity pattern associated to a certain location (approximate fire area), corresponding to a relative maximum of radar reflectivity, revealing a divergent structure downwind of the fire area spot. Gravity tends to sort out smoke particles in a way such that a greater concentration of larger particles tends to be located closer to the fire spot. Thus, it is admissible that a relative maximum of reflectivity tends to be observed closer to the fire spot. Nevertheless, it is to be noted that this procedure was just intended to follow up the general evolution of the fire plumes, both in space and time. It was not meant to identify the approximate number of fires. Since 1) several plumes may be below the minimum radar observation height during their entire life cycle (beam overshooting) and 2) radar beam resolution issues and the coexistence of smoke scatterers and spurious echoes, it is possible that the number of fires may have exceeded the number of detected independent plumes. In the following discussion, the time each plume was detected refers to its first detection. As an example, by 16:10 UTC of $15^{\text {th }}$ October, several plumes were being identified (Figure 13). Using both mentioned radars, it was possible to identify a large number of plumes. Plumes were identified between 5 UTC, $15^{\text {th }}$ October, and 00UTC, $16^{\text {th }}$ October.

Using the subjective technique, thirty one (31) plumes were identified. The graphic (Figure 14) shows, on an hourly basis, the number of plumes detected (first detection) during that specific hour. 


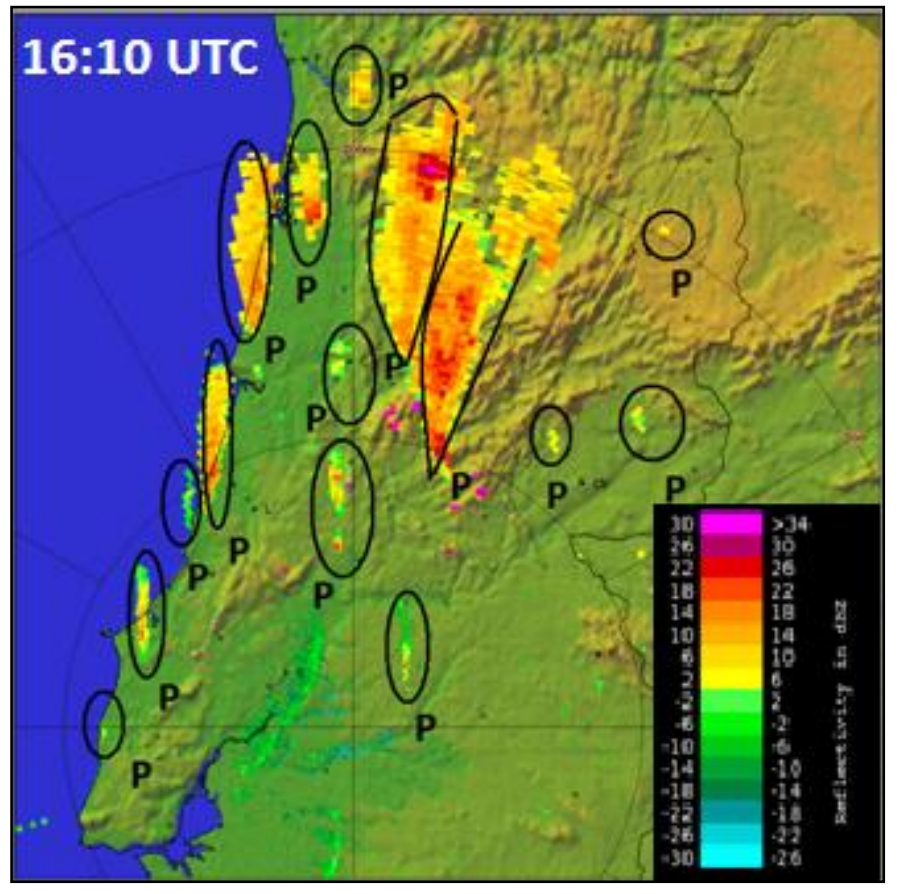

Figure 13 - Plane Position Indicator of Z (dBZ), $0.1^{\circ}$ elevation, 16:10UTC, $15^{\text {th }}$ October 2017, radar. Black segments mark 15 plumes that were identified on radar $(C / C L)$, by this time (adapted from Novo, I. and Pinto, P. 2017, in Portuguese).

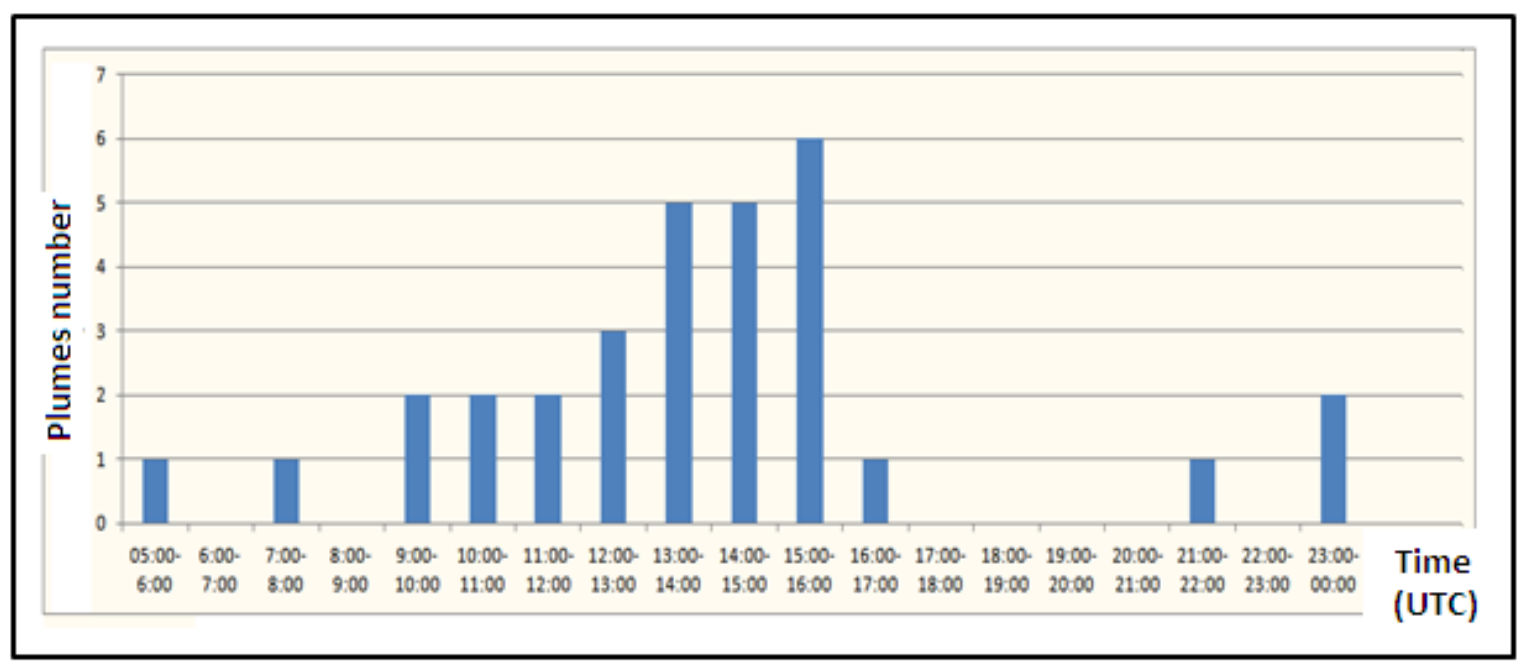

Figure 14 - Number of plumes (first detection instant) between 5 UTC, $15^{\text {th }}$ October and 00utc, $16^{\text {th }}$ October, 2017. Diagnose based on A/PG and C/CL radars (adapted from Novo, I. and Pinto, P. 2017, in Portuguese).

It is impressive to see how the plumes detection rate, along the day, incremented substantially by early afternoon. It is noteworthy to realize that in a short period of just 4 hours (12-16 UTC), 19 plumes were detected, corresponding to more than $60 \%$ of the total amount that was detected during the entire 18 hours considered period. Thus, this short period has proven to be very favorable to fire development, and with no surprise. In fact, it was observed, then, that factors such as high air temperature, low relative humidity and a low level turbulent wind regime (see 3.3) have combined across a major part of north and centre mainland Portugal. 


\section{Pyroconvective plumes and glaciation}

The dual polarization $\mathrm{A} / \mathrm{PG}$ radar system processes an interesting parameter regarding the microphysical characterization of scatterers in the atmosphere. In fact, the correlation coefficient ( $\left.\rho_{\mathrm{hv}}\right)$ has been considered as a fine particle type discriminator. Lang et al (2014) and LaRoche and Lang (2017) have found that a mixture of fire ash and rimed ice particles uses to have $\rho$ hv values larger than or equal to 0.8 , whereas pure ash uses to have $\rho$ hv values lower than 0.8 . Observational experience collected in Portugal, still to be published, sustains similar opinion. Thus, $\rho$ hv values less than 0.8 were considered as typical from dry pyroconvective plumes whereas larger than 0.8 were considered as characteristic of a mixture of ash and ice particles. On this concern, it is shown a pyroconvective formation example, as observed by 22:50 UTC of $15^{\text {th }}$ October 2017. It was possible to detect a pattern of phv values larger than 0.8 (yellowish pixels, Figure 15), meaning that part of the top of this pyroconvective cloud had a glaciated (or partially glaciated) area.

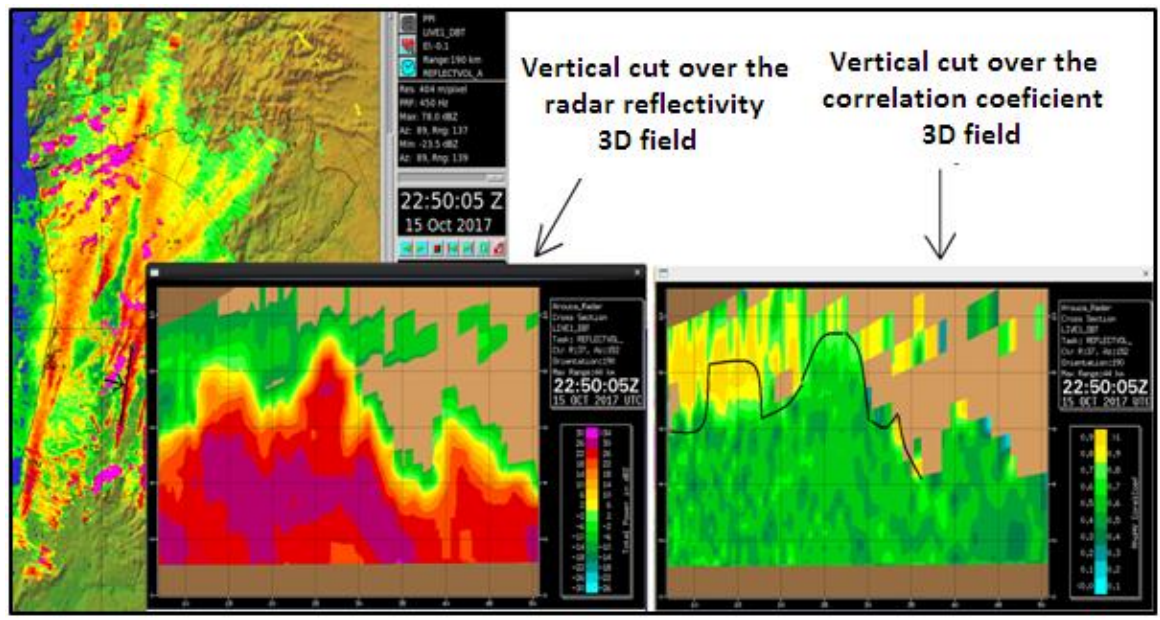

Figure 15 - Vertical cuts over $3 D$ reflectivity field (Z, dBZ, left) and over 3D correlation coefficient field (no dimensions, right), 22:50UTC, $15^{\text {th }}$ October 2017, A/PG radar. Transect cut and observer position marked with black line and arrow over PPI of reflectivity (left image in background). Cloud tops as detected by radar are marked with black contour lines in Vertical cut over correlation coefficient. Vertical scale discretization: $2 \mathrm{~km}$. (adapted from Novo, I. and Pinto, P. 2017, in Portuguese).

This example shows that during the evening of $15^{\text {th }}$ October 2017 , there existed conditions that favoured the genesis of pryroconvective clouds. As stated in the specific literature, these developments are frequently seen as hazardous in respect to fire evolution in the short term.

\section{Final Remarks}

A catastrophic forest fire event occurred over mainland Portugal on the $15^{\text {th }}$ October 2017, outside the typical forest fire season.

An accentuated meridional flow pattern over the Atlantic has caused a ridge settling over Iberia, promoting the extension of an already hot and dry summer throughout October. At the same time, the anomalous track of hurricane Ophelia, in the surroundings of western Iberia coast by the $15^{\text {th }}$ October, has intensified the southerly flow and its turbulency. Furthermore, it has reinforced the advection of an unseasonal hot and dry airmass over mainland Portugal. Air turbulence, heat and extreme air dryness have combined with a $4^{\text {th }}$ factor that, is suggested, has played a significant role in the hazardous environment. In fact, according to ground observations, a "dry Foehn" phenomenon has, likely, 
reinforced the large scale adiabatic compression effects. This was especially noted in the dryness of the air and flow intensity in downwind slope areas.

\section{References and bibliography}

Balakrishnan, N., and Zrnic, D., 1990: Use of Polarization to Characterize Precipitation and Discriminate Large Hail, Journal of the Atmospheric Sciences, Volume 47, No. 13, 1525-1540.

Gouveia C. M., Bastos A., Trigo R. M., DaCamara C.C. ,2012: Drought impacts on vegetation in the and post-fire events over Iberian Peninsula, Natural Hazards Earth System Sciences,12,3123-3137, 2012, doi:10.5194/nhess-12-3123-2012.

IPMA, 2017: Boletim Climatológico outubro 2017, Portugal Continental, ISSN 2183-1076 (in portuguese).

Novo, I., Pinto, P., 2018: Os Incêndios Florestais de 14 a 16 de Outubro de 2017 em Portugal Continental Caraterização Meteorológica. Relatório, DivMV, IPMA, 79pp. (in Portuguese)

Jason J. Sharples, Graham A. Mills, Richard H. D. McRAe, Rodney O. Weber, 2010: Foenh-Like Winds and Elevated Fire Danger Conditions in Southeastern Australia, Journal of Applied Meteorology and Climatology, volume 49, 1067- 1095.

Lang, T., B. Dolan, P. Krehbiel, W. Rison, and D. T. Lindsey, 2014: Lightning in wildfire smoke plumes observed in Colorado during summer 2012. Monthly Weather Review, 142, 489-507.

LaRoche, K., and Lang, T., 2017: Observations of Ash, Ice, and Lightning within Pyrocumulus Clouds Using Polarimetric NEXRAD Radars and the National Lightning Detection Network, Monthly Weather Review, Volume 145, 4899-4910.

Pereira, M.G., Trigo, R. M., DaCamara, C. C., Pereira, J.M.C., Leite, S. M., 2005: Synoptic patterns associated with large summer forest fires in Portugal, Agriculture and Forest Meteorology, 129, 11 25.

Stacy R. Stewart, 2017: Hurricane Ophelia, Tropical Cyclone Report, National Hurricane Center, 27 March 2018, NOAA.

Van Wagner, C. E., 1987: Development and Struture of the Canadian Forest Fire Weather Index System, Cabadian Forestry Service, and Forestry Technical Report 35 Ottawa 1987. 


\title{
Generation and evaluation of ensemble simulations of wildfire spread for probabilistic forecast
}

\author{
Frédéric Allaire $^{1 *}$; Jean-Baptiste Filippi ${ }^{2}$; Vivien Mallet ${ }^{1}$ \\ ${ }^{1}$ Inria, 2 rue Simone Iff, Paris, France and CEREMA, Sorbonne Universités, UPMC, Lab. \\ Jacques-Louis Lions UMR CNRS 7598. \{frederic.allaire@inria.fr*,vivien.mallet@inria.fr\} \\ ${ }^{2}$ SPE - UMR 6134 CNRS. Université de Corse, France. \{filippi@univ-corse.fr\};
}

\begin{abstract}
Typical simulation of a large wildfire requires semi-empirical models for rate of spread (ROS) such as the Rothermel model. ROS models usually take into account several physical parameters such as slope, wind speed, moisture content and other fuel properties (load, height, surface-to-volume ratio, heat of combustion, etc.). However, these parameters can be highly uncertain. In addition, the model itself is far from perfect and the solver is subject to numerical approximations. A promising direction for the prediction of wildfire spread is to rely on an ensemble of simulations instead of a single deterministic simulation.

The generation of an ensemble may follow a Monte Carlo strategy where the input parameters are sampled according to given discrete or continuous probability distributions. Each sample is independent from each other and is used to run one wildfire simulation. For a wildfire starting at a given location and time, we can use the outputs from the ensemble of simulations to estimate a burn probability map. One main challenge of the method lies in the choice of the distributions of the input parameters. Depending on the sensitivity of the model to the input parameters, small changes on the probability distributions could yield highly different outputs. At this stage, a suggestion of distributions is introduced, although calibration should be applied in order to improve simulation outputs.

Similar probabilistic approaches have already been developed in wildland fire and particularly in forest fire behavior models such as the FSPro system. A significant difficulty regarding the evaluation of such systems is to properly take into account their probabilistic aspects. We propose to perform ensemble evaluation considering all the burn probabilities that are predicted at the scale of a specific fire event. It also requires the observed burned surfaces corresponding to the specific event. Then, probabilistic scores that are commonly used in meteorological applications, such as the Brier Score, can be computed. The whole methodology is applied to a wildfire that occurred in Corsica in summer 2017 and could be extended to more fire cases.
\end{abstract}

Such probabilistic framework could then be completed with a description of ignition probability and vulnerability in order to assess fire risk over a region like Corsica.

Keywords: simulation ; wildfire ; uncertainty quantification ; Monte Carlo ; probabilistic score

\section{Introduction}

In an operational context, being able to accurately forecast how a wildfire will spread would provide invaluable help in decision making, not only in a crisis situation where a fire just ignited but also in the assessment of fire risk. In the latter case, one goal is to identify areas that require high-priority land planning actions in the long run or the mobilization of firefighting resources in the short term. In spite of the significant efforts in modeling to provide better and faster-to-compute forecasts of fire spread, systematically making an accurate prediction remains a distant goal (Gollner et al., 2015). One of the reasons lies in the difficulty to predict and describe the weather conditions and the state of the vegetation that will drive the fire spread. In this uncertain context, one may think that a range of likely outcomes might be more useful than a single forecast. This can be achieved by running many 
simulations. This strategy is not new to the fire community as an approach relying on several runs of likely fire scenarios was already implemented for assessment of long-term fire risk in systems such as BURN-P3 (Parisien et al., 2005) and FSim (Finney et al., 2011a) where a probability of a zone burning during a year is computed (among others) over a whole region. In the case of a fire that has just ignited, which is the focus of this study, the FSPro (Finney et al., 2011b) system uses an ensemble of simulations based on a time series approach to define several weather inputs and provide a map of burn probability, that is to say, an estimate of the chance that a zone close to ignition point will burn after a given time. Evaluation in FSPro is then performed in two ways. The first method consists in comparing the mean area of the simulated burned surfaces to the observed burned area. The second method, relies on the predicted burn probabilities obtained at the vertices of the polygon describing the observed burned surface.

We propose another method for ensemble simulation in this context. One main difference with FSPro lies in the approach used when generating the inputs for each simulation. Here, we used perturbations on weather forecasts, but also on other input data such as fuel parameters. In addition, we present tools for evaluation that are commonly used in meteorological applications. Indeed, judging the quality of an ensemble implies the confrontation against observed data with appropriate scores. This methodology is applied to the Calenzana fire that occurred in Corsica in August 2017.

The method for generating an ensemble of wildfire simulations is introduced in the first section. In the second section, we define scores for ensemble evaluation. In the third section, we describe the data and perturbations used in the simulations of the fire event. The results are presented and discussed in the last section.

\section{Generation of an ensemble}

\subsection{Running one simulation of wildfire spread}

In this study, we used the ForeFire code, described in (Filippi et al., 2009). ForeFire is a front propagation solver based on discrete event simulation together with a Lagrangian front-tracking method where the fire line is described by markers linked to each other. Each marker represents a point in a 2D space that may move according to a given speed vector. The direction is determined by the local geometry of the fire line. On the other hand, the speed norm depends on a formula for rate of spread (ROS) that can be based on local weather, slope, and fuel properties, in addition to fire line geometry.

To start a simulation, the user needs to select not only a ROS formulation, ignition date, time and location, but also elevation, weather and fuel data so that ROS can be computed at any time of the fire spread and at any point in space around ignition point. However, when a fire starts, knowledge regarding these input variables is limited. This uncertainty makes the prediction of wildfire spread especially difficult as a relatively small change on some of the inputs could lead to significantly different results. Here, we decided to focus only on the inputs, but it should be noted that uncertainty also stems from numerical approximation and from the ROS model which is based on simplification assumptions and therefore does not describe perfectly the physics of the phenomenon.

\subsection{Running an ensemble of simulations}

In a context of high uncertainty, only one simulation may give a unreliable prediction, even if the forecaster chooses the most likely input variables. Another strategy is to make a choice based on several simulations of fire spread that were computed with different inputs, even though these inputs are not all the most likely ones.

Here, we assume that we have a nominal value $x$ for a given input variable. Depending on our knowledge on the variable, we might want to try a simulation with another value $x_{p}$ (the underscript $p$ stands for "perturbed") that remains relatively close to $x$. Also, given the physical parameter described 
by the variable, some values are not admissible for $x_{p}$. One obvious constraint is that many variables cannot be negative. Therefore, choosing a perturbation of wind speed norm in a range of $+/-5 \mathrm{~m} / \mathrm{s}$ when the nominal value (obtained by a weather forecast, for instance) is $3 \mathrm{~m} / \mathrm{s}$ is not possible.

In this study we define 3 main classes of perturbations: "additive", "percentage" and "multiplicative". Let $z$ be our perturbation coefficient. The perturbed value $x_{p}$ will be computed based on $x$ and $z$ according to the perturbation class.

For an additive perturbation, we have $x_{p}=x+z$ where $z$ has the same unit as $x$ and is real (i.e., it can be either positive or negative).

For a percentage perturbation, we have $x_{p}=x *(1+z)$ where $z$ has no unit and must be between 1 and $1(z=-0.2$ corresponds to $-20 \%$, for instance $)$.

For a multiplicative perturbation, we have $x_{p}=x * z$ where $z$ has no unit and must be positive.

The sampling of perturbations for one input variable is made according to some probability distribution. A given $z$ is therefore conceived as a realization of a random variable $Z$. In most cases, $Z$ can be described by a probability density function (PDF). In this study, we decided to use mainly "centered uniform" PDFs, which are described by only one parameter. Let $z$ max be the maximum value that $z$ can take. For the additive and percentage classes, $z$ is directly sampled according to a uniform distribution in $\left[-z_{\max }, z_{\max }\right]$. In the multiplicative case, we have $z=\exp (u)$, where $u$ is sampled uniformly in the interval $\left[-\log \left(z_{\max }\right), \log \left(z_{\max }\right)\right]$. This way, $z$ is in $\left[1 / z_{\max }, z_{\max }\right]$. We can talk about a (centered) "log-uniform" distribution by analogy to the log-normal distribution.

In this framework, a perturbation $\mathrm{Z}$ associated to a given input variable $x$ is entirely described by its class and one parameter zmax.

To run an ensemble of simulations, for a given input variable we simply draw $M$ independent samples $z l, \ldots, z M$ following the distribution of $Z$. Then, for $m$ in $\{1, \ldots, M\}$, we run the fire spread simulation with $x$ perturbed by $z m$.

In the case of wildfires, we define a fourth perturbation class specifically for fuel types: "transition". Indeed, physical parameters of the fuel are usually determined by its type. This perturbation allows a given fuel type to be changed to another type (and therefore, receive new values of physical fuel parameters) with some probability $p$ in $[0,1]$. We have the constraint that all transition probabilities for a given fuel type (including transition towards itself, i.e., "no change") are nonnegative and sum to 1 .

Choosing the probability distributions of the perturbations is a way of quantifying the uncertainty regarding the input variables.

Outputs of the ensemble of simulations

At the end of a simulation, ForeFire may return the geometry of the fire line, potentially at any time during the fire event, as well as an arrival time of the fire at any point in space. Since the observed data available when studying a fire event consists mostly of a 2D burned surface observed after the fire has completely stopped, we decided to focus on the fire line obtained at the end of the simulation. In the following, a simulation domain is described by a set of $\mathrm{N}$ points $x_{1}, \ldots, x_{N}$ where each $x_{i}$ is associated to a number $b_{i}$ that takes the value 1 if $x_{i}$ is burned, 0 otherwise.

In a probabilistic framework, a set $\left(b_{1}, \ldots, b_{N}\right)$ associated to a given domain can be understood as a realization of a random vector $\left(\mathrm{B}_{1}, \ldots, \mathrm{B}_{\mathrm{N}}\right)$ where each component $\mathrm{B}_{\mathrm{i}}$ follows a Bernoulli law of parameter $\mathrm{P}\left[\mathrm{B}_{\mathrm{i}}=1\right]$, which represents the burn probability (conditionally to fire ignition).

With our ensemble of $\mathrm{M}$ simulations, since the inputs are generated independently, we can give a Monte Carlo estimate of $\mathrm{P}\left[\mathrm{B}_{\mathrm{i}}=1\right]$ :

$$
p_{i}=\left(\text { Number of simulations where } b_{i}=1\right) / \mathrm{M} \text {. }
$$


We define the burn probability map for a given fire as the set $\left\{\left(x_{i}, p_{i}\right) \mid \mathrm{i}=1, \ldots, \mathrm{N}\right\}$. It is one of the main products of the ensemble of simulations. An example is given in Figure 1.

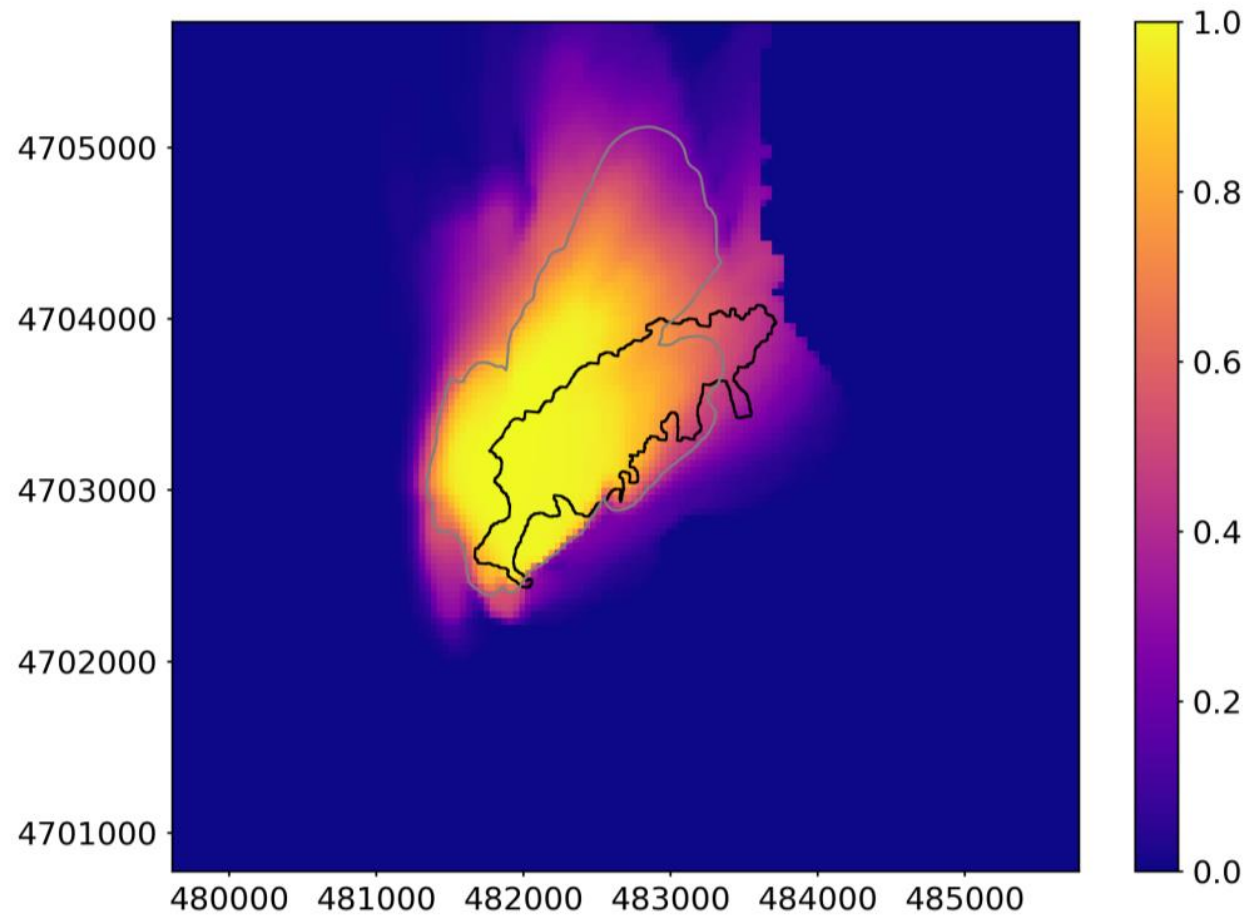

Figure 1 - Burn probability map obtained for Calenzana (2017.08.05) wildfire.

The colorbar gives the value of the burn probability $p_{i}$. Axes give the point coordinates $x_{i}$ in EPSG:32632 (m). Black line: contour of observed burned area (obtained from Office de l'Environnement de la Corse).

Grey line: contour of the burned area returned by a simulation without perturbation.

\section{Evaluation}

Now that we have proposed a method for ensemble generation, we need to define desirable properties of an ensemble.

The ultimate goal of a forecaster is to give a perfect prediction of what is to come. For an ensemble, this would mean that all simulation results are the same as the observations, in any case. However, not only is it hard (if not impossible) to come close to this goal, but making an ensemble of simulations would be irrelevant in that case as only one forecast would be enough.

A significant difficulty when dealing with an ensemble is that one needs to compare a given observation to a set of simulations that yields a probability. The comparison between two items of different nature, namely the realization of an event and a corresponding probability, is not trivial. That is why in this section we define scores commonly used in meteorology that enable to evaluate the quality of an ensemble.

\subsection{Brier score}

In order to compare an observation with one (or more) forecast(s), numerical scores can be computed. If we had just one forecast, some surface comparison scores such as those defined in Filippi et al. (2013) could be employed. However, we cannot compare a burn probability map with a burned surface by the means of such scores as they are meant for comparison with one simulation. For a numerical comparison with an ensemble forecast, we need to use probabilistic scores. 
A score that seems appropriate in the case of a wildfire which is essentially binary (i.e., "burn or no burn") is the Brier score (BS). Let us consider the burn event : "A given elementary surface burns", we note $o=1$ if the event occurred, 0 otherwise. Let $p$ be the probability of the burn event that was predicted by our ensemble. In this case, the Brier score is defined as $\mathrm{BS}=(o-p)^{2}$.

In the general case, if we have $\mathrm{N}$ observations $o_{1}, \ldots, o_{N}$ for the burn event and $\mathrm{N}$ associated predicted probabilities $p_{1}, \ldots, p_{N}$, then $\mathrm{BS}$ is defined as follows:

$$
B S=\frac{1}{N} \sum_{i=1}^{N}\left(o_{i}-p_{i}\right)^{2} .
$$

This score is positive and negatively oriented: the closer it is to 0 , the better the ensemble forecast. For a detailed presentation of probabilistic scores, we refer the reader to Brocker (2009). In particular, the Brier score has the advantage of being strictly proper.

Using the notations of section 2.3, $o$ i will be the value of the observed $b_{i}$. $\mathrm{N}$ is the number of points in the study area and $p_{i}$ is the Monte Carlo estimate of the burn probability $\mathrm{P}\left[\mathrm{B}_{\mathrm{i}}=1\right]$ obtained with the ensemble.

The attentive reader might notice that proceeding this way, we have one observation for each one of the $\mathrm{N}$ burn events and not $\mathrm{N}$ observations of one burn event. The Brier score was introduced with the latter view, which corresponds to a probabilistic expectation. The way we propose to compute it, the Brier score corresponds to a spatial average.

\subsection{Rank histogram}

Another tool used in ensemble forecasting is the rank histogram. The rank is a variable used to test whether the observation is distinguishable from the predictions or not. When the distinction cannot be made, we say that the ensemble prediction system is consistent.

Let us define a variable $y$ that corresponds to the observation and $y_{1}, \ldots, y_{M}$ that correspond to the predictions obtained from the simulations. The rank $\mathrm{R}(y)$ of $y$ among $y_{1}, \ldots, y_{M}$ is usually defined as follows: if there are exactly $m$ of the $y_{j}$ that are strictly lower than $y$ (and therefore the remaining $\mathrm{M}$ $m$ of the $y_{j}$ are equal to or higher than $y$ ), then $\mathrm{R}(y)=m$. The rank can therefore take values in $\{0,1 \ldots$, $\mathrm{M}$ \}. Let us give a few examples. If all the $y_{j}$ are strictly lower than $y$, then $\mathrm{R}(\mathrm{y})=\mathrm{M}$. Conversely, if $\mathrm{y}$ is lower than all the $y_{j}$, then $\mathrm{R}(\mathrm{y})=0$. If only $y_{3}$ and $y_{5}$ are strictly lower than $y$, then $\mathrm{R}(y)=2$. One interesting property of the rank is that if we evaluate it on many occasions and if the ensemble prediction system is consistent, each value of the rank (i.e., each integer in the set $\{0,1 \ldots, M\}$ ) should be obtained approximately $100 /(\mathrm{M}+1) \%$ of the time.

In most applications, $y, y_{1}, \ldots, y_{M}$ are defined in a real interval and the case where $y$ is equal to one or more of the $y_{j}$ is not problematic. However, in the context of forest fires we may consider the burn event and the variables can only take two values : 0 or 1 . For instance, in the case where $M=10$, if 7 of the $y_{j}$ are equal to 0 (the point is not burned in 7 out of 10 simulations) and $\mathrm{y}=0$ (we observe that the fire did not reach this point), one may wonder which rank to pick in $\{0, \ldots, 7\}$. In fact, it is not satisfactory to pick one rather than the others. This is why, in the binary case, we follow a different strategy. Instead of considering a single value for the rank, we define a weight for all the possible ranks in $\{0,1, \ldots, M\}$.

The weights of the rank of $y$ among $y_{1}, \ldots, y_{M}$ are given by the vector $\left(w_{0}, w_{1}, \ldots, w_{M}\right)$ of size $\mathrm{M}+1$. Assuming that the variables $y, y_{1}, \ldots, y_{M}$ can only take the value 1 or 0 ("burned" or "not burned"), this vector is defined as follows: 
Let us assume that $\sum_{\mathbf{j}=1}^{\mathrm{M}} \mathrm{y}_{\mathrm{j}}=\mathrm{m}$ (i.e., exactly $m$ of the $y_{j}$ are equal to 1 ).

Then if $y=0, \forall k \in\{0, \ldots, M-m\}, w_{k}=\frac{1}{M+1-m}$.

Else (if $y=1$ ), $\forall k \in\{M-m, \ldots, M\}, w_{k}=\frac{1}{m+1}$.

In both cases, for the remaining values of $k, w_{k}=0$.

In practice, we proceed with this calculation for each point $x_{i}$, which yields the corresponding weight vectors $\left(w o^{i}, w_{1}^{i}, \ldots, w_{M}^{i}\right)$. For each rank $k$ in $\{0, \ldots, \mathrm{M}\}$, we define the total weight $\mathrm{W}_{\mathrm{k}}$ as

$$
W_{k}=\sum_{i=1}^{N} w_{k}^{i} \text {, }
$$

and we obtain the total weight vector $\left(\mathrm{W} 0, \mathrm{~W}_{1}, \ldots, \mathrm{W}_{\mathrm{M}}\right)$. We define the rank histogram as the histogram where the total weight vector $\left(\mathrm{W}_{0}, \ldots, \mathrm{W}_{\mathrm{M}}\right)$ is plotted against the rank vector $(0, \ldots, \mathrm{M})$. An example is given in Figure 2.

If ideally we have a consistent ensemble prediction system and $\mathrm{N}$ is high enough, then each of the $\mathrm{W}_{\mathrm{j}}$ should be approximately equal to $\mathrm{N} /(\mathrm{M}+1)$. It implies that the rank histogram will be flat. When the ensemble over-predicts burn probability, there will be higher bars on the left and lower bars on the right (compared to the ideal flat histogram). Conversely, under-predicting the burn probability will lead to higher bars on the left and lower bars on the right.

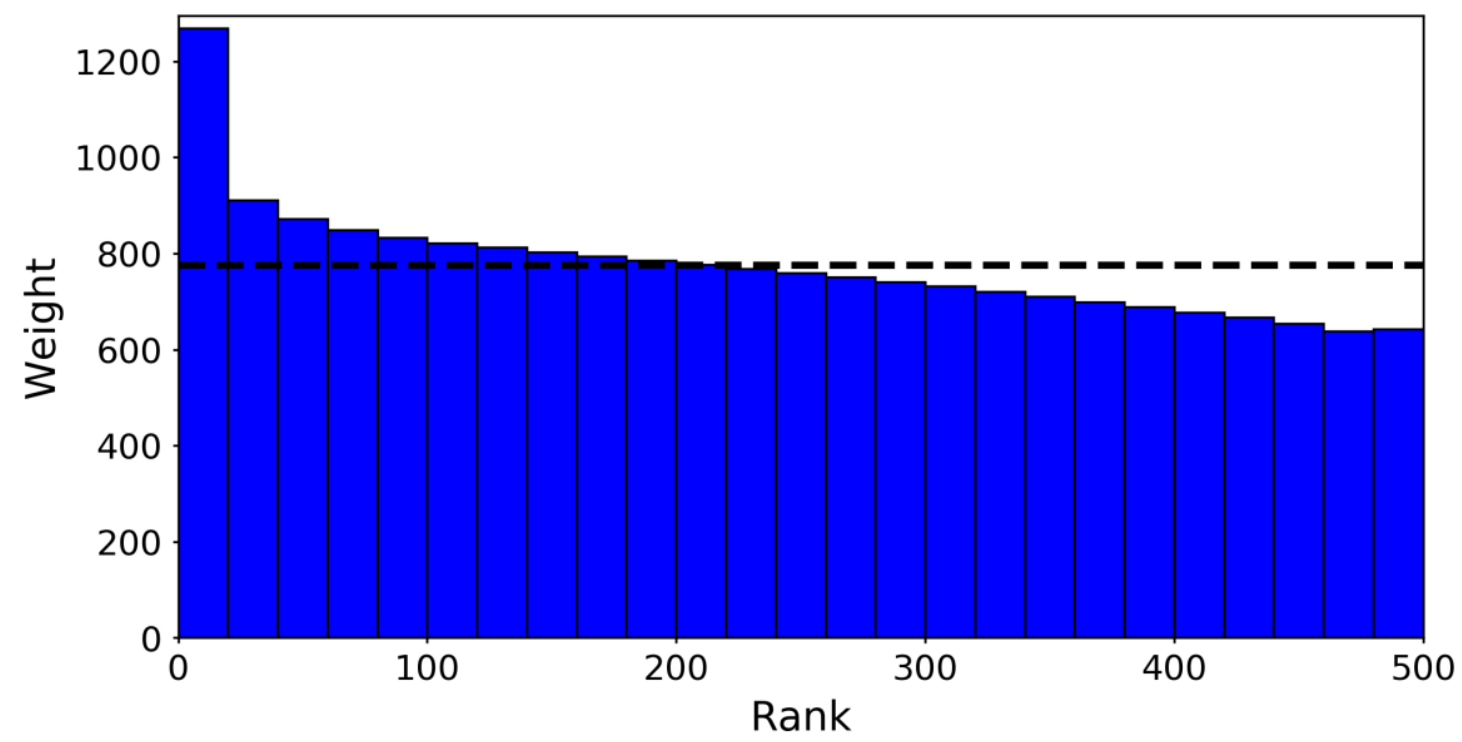

Figure 2 Rank histogram obtained for Calenzana (2017.08.05) wildfire.

The 501 ranks are regrouped in 25 bins of 20 consecutive ranks (21 for the first bin).

The dashed line indicates the flat histogram that would be obtained with a consistent ensemble. The shape of the diagram indicates that the ensemble overestimates burn probability.

\section{Application}

\subsection{ROS model and data sources}

The area of study is Corsica island (France). Spatial data consist of an elevation field combined with a field of fuel type. The latter was obtained from Corine Land Cover (CLC) land use maps. Both fields are at raster format with a resolution of $80 \mathrm{~m}$.

In this study, we decided to use the semi-empirical model of Rothermel (Rothermel, 1972), although any ROS model can be implemented (we could use the one described in (Balbi et al., 2009), for instance). A few points on the implementation of the model in this study must be noted. First, the 
mineral damping coefficient is always 1 . Second, all fuel types are assumed to have a moisture of extinction of 0.3 .

For a given CLC fuel type, all ROS input parameters, except for wind speed, wind direction, and slope, are the same everywhere. Although the discrete event simulation method allows for change of any ROS input parameter in time, only wind speed, wind direction and dead fuel moisture were allowed to change during a fire simulation.

Spatial wind fields for the cardinal and intermediate directions were precomputed to take into account the elevation field and to save computational time. Wind speed, wind direction and dead fuel moisture data for a given wildfire are obtained from weather forecasts of the atmospheric model MesoNH. Forecasts are run everyday with an origin time of 00:00 UTC over a range of 42 hours with a time step of 3 hours. Spatial resolution of the fields is around $600 \mathrm{~m}$.

\subsection{Fire case}

The simulations were run based on a fire that occurred in Corsica on August $8^{\text {th }} 2017$. Fire started near the town of Calenzana, at approximately 17:42 (local time). Consequently, the MesoNH forecasts with origin date and time 2017.08.05, 00:00 UTC were used. The observed burned surface after the event was around $120 \mathrm{ha}$. The burned surface together with the corresponding (assumed) ignition time and location were provided by Office de l'Environnement de la Corse. We decided to quantify uncertainty regarding ignition time with an additive uniform perturbation of +/- 10 min for ignition time. For ignition location, we first defined a square of $200 \mathrm{~m}$ side length centered around the reference point. The ignition locations used in the simulations were randomly chosen among the centers of the CLC squares that were contained in the $200 \mathrm{~m} \times 200 \mathrm{~m}$ square.

In general, one of the major sources of uncertainty regarding a fire event is its duration. Since we do not take into account the firefighting actions, ForeFire simulates a fire with free spread. Therefore, the only way for a simulation to end was either to reach the end of the input fire duration, or to have a ROS of almost 0 at every point of the fire line (which did not happen in our simulations). With this method, it is important to note that the simulation returns the burned surface of a fire that could continue to spread while the observation represents the burned surface of a fire that is not burning anymore. An important implication is that evaluation will highly depend on the prescribed fire duration. One way to address this difficulty is to choose a fire duration that accounts for most of the burned area. In the Calenzana case, although the fire spread was almost stopped 3 hours after ignition time, it seems that most of the surface was burned during the first hour. Therefore, we chose a reference duration of 1 hour with a uniform perturbation of 10 minutes.

\subsection{Perturbation parameters}

The perturbations on ignition time, ignition location and fire duration were addressed in the previous section because they depend on our knowledge of the event, which may differ in another fire case.

For the other inputs, we used constant perturbation coefficients. It means for example that in a given simulation, even though wind speed may change in time, the same wind speed perturbation will be applied at the different steps of the simulation. Similarly, a perturbation on fuel parameters will be the same for all fuel types. Finally, a fuel type transition affects all the CLC cells with fuel type. For instance, it means that if a fuel transition from vineyards to sclerophyllous plants is applied in a simulation (which happens with a probability $p$ ), ForeFire will behave as if all vineyard cells (and not just a proportion $p$ of these cells) had become sclerophyllous plant cells.

For a given simulation, the different perturbation coefficients were sampled independently. Still, the same (percentage) perturbation was applied on the following fuel parameters: height, load, heat of combustion (positively) and particle density (negatively). For example, if we obtain a value of $+15 \%$ for this global perturbation, then height, load and heat of combustion undergo a $15 \%$ increase while 
the particle density decreases by $15 \%$. From a physical perspective, there is no reason for the uncertainties regarding these four fuel parameters to be fully correlated. The motivation behind this choice is more pragmatic. Indeed, increasing either fuel height, fuel load or heat of combustion should increase the ROS, while increasing the particle density should decrease the ROS. Consequently, with that global fuel coefficient, we expect to obtain an upper bound on the uncertainty regarding the area of the simulated burned surfaces.

The range of the perturbations on wind was chosen after the assumed uncertainties listed in (Hanna et al., 1998) with the difference that we chose uniform PDFs instead of normal ones. As for perturbation on fuel parameters, it was based on common values encountered in the literature and expert judgment. The implementation of a fuel transition from vineyards (CLC type 221) to sclerophyllous plants (CLC type 323) was motivated by the presence of vineyards in our fuel data near the ignition point, where the ROS is much lower than for other fuel types. In this case, data were outdated, since there was no vineyard in this zone at the time of the fire. Transition allows to account for the classification error.

The distributions of the main perturbations that were used in this study are given in Table 1 . We stress that at the moment the distributions are just an a priori suggestion. To obtain more reliable distribution parameters, some calibration method based on the Brier score and/or a Bayesian approach could be implemented. Calibration based on just one fire is not likely to give good evaluation results for other events, therefore it is out of the scope of this paper.

Table 1 - Main perturbations used in the ForeFire simulations with the classification described in section 2.2. *For these parameters, the perturbation may differ depending on the fire event.

\begin{tabular}{|c|c|c|}
\hline Perturbation & Type & $\mathbf{z}_{\max } / \mathbf{p}$ \\
\hline Wind direction & Additive & $40^{\circ}$ \\
\hline Wind speed norm & Multiplicative & 1.5 \\
\hline Dead fuel & Percentage & $30 \%$ \\
\hline Surface-volume & Multiplicative & 2 \\
\hline Fuel global & Percentage & $20 \%$ \\
\hline Ignition time* & Additive & $10 \mathrm{~min}$ \\
\hline Fire duration* & Additive & $10 \mathrm{~min}$ \\
\hline $221 \rightarrow 323$ & Fuel transition & 0.8 \\
\hline
\end{tabular}

Following these distributions, an ensemble of 500 simulations was run.

\section{Results and discussion}

The simulations were run on a Linux kernel with 8 cores, allowing for parallel computations. The whole computational process took about 5 minutes. It should be noted that given the relatively short fire duration, one simulation took a few seconds. But if we wanted to obtain a 3-hour probability map, simulation time would have increased. Also, in other fire events, higher fire duration and higher average ROS could lead to larger burned area, and therefore runtime could reach one minute or more for one simulation. In any case, this indicates that with appropriate computational resources to account for the simulation of potentially large fires (e.g., more cores to run the simulations in parallel), this 
method has potential for use in an operational context. For instance, hourly burn probability maps over a few hours could be computed in a few minutes.

The burn probability map with coordinates in EPSG:32632 Mercator conformal projection system is represented in Figure 1 together with two lines, one encompassing the observed burned surface while the other encompasses the simulated burned surface obtained when there is no perturbation on the inputs. Qualitatively, the burn probability seem to have a better coverage of the observed burned area than the unperturbed contour. This analysis is supported by the Brier score, that we computed for the ensemble of 500 simulations and for an ensemble where the only member is the unperturbed simulation. It is much better in the ensemble case (0.055) than in the latter (0.072). It also appears that the predicted probability might be too high in some areas outside of the burned surface.

The rank histogram is represented in Figure 2. An aggregation was carried out so that each one of the 25 rectangles represents 20 ranks, except for the first one which is associated to 21 ranks. Without aggregation, the overall shape of the histogram remains similar. It appears that the histogram has higher bars on the left, which means that the burn probability is overestimated in several locations.

In spite of those promising results, a few difficulties regarding the methodology need to be discussed.

First, the choice of the number $M$ of members in the ensemble is not obvious. Since we used a Monte Carlo method, we know that a given burn probability estimator $p_{i}$ converges as $\mathrm{M}$ grows and confidence intervals can be computed for this estimator. However, to our knowledge, there is no reference for confidence levels in this context. Also, as $\mathrm{M}$ increases, computational time increases as well, which will limit $\mathrm{M}$ and the narrowness of the confidence intervals.

Second, many points in the study area were not burned and obtained a predicted burn probability of 0 . If we were to extend the study area, the number of such points would increase. One implication is that the rank histogram would be flattened. Similarly, the Brier score would get lower because N would increase while the sum of the errors would be unchanged. In some sense, it is possible to cheat with a configuration where the burn probability is easy to predict over most of the domain (since most points are far from the ignition point), because in this case, evaluation will lead to better scores.

Finally, we noticed that some points outside of the observed burned surface obtained a burn probability of 1 . A very plausible explanation is that the simulations did not model the firefighting actions. Still, this case can be viewed as a "failure" of the prediction system. The same can be said of the case where a point of the observed burned area receives a burn probability of 0 , although it did not happen in this study. This raises the question of the relevance of extreme probabilities (i.e., close to 0 or close to 1) obtained via the ensemble. A possible way of circumventing this issue could be the use of probability thresholds (e.g., 0.1 and 0.9 ) on the burn probability maps. These thresholds could be interpreted as a consequence of a part of uncertainty that is not explained by the perturbations.

An application of this method on several cases of bigger fires is currently in process to make the evaluation more robust.

\section{Acknowledgement}

This research was developed within the ANR-16-CE04-0006 FIRECASTER.

\section{References}

Balbi J-H, Morandini F, Silvani X, Filippi J-B, Rinieri F (2009) A physical model for wildland fires. Combustion Flame 156 (12), 2217-2230.

Brocker J, (2009) Reliability, sufficiency, and the decomposition of proper scores. Quarterly Journal of the Royal Meteorological Society 135 (643), 1512-1519. 
Filippi J-B, Morandini F, Balbi J-H, Hill D (2009) Discrete event front-tracking simulation of a physical fire spread model. Simulation 86 (10), 629-644.

Filippi J-B, Mallet V, Nader B (2013) Representation and evaluation of wildfire propagation of wildfire simulations. International Journal of Wildland Fire 2 (34), 2633-2640.

Finney MA, McHugh CW, Grenfell IC, Karin LR, Short KC (2011a) A simulation of probabilistic wildfire risk components for the continental United States. Stochastic Environmental Research and Risk Assessment 25 (7), 973-1000.

Finney MA, Grenfell IC, McHugh CW, Seli RC, Trethewey D, Stratton RD, Brittain S (2011b) A method for ensemble wildland fire simulation. Environmental Modeling and Assessment 16 (2), 153-167.

Gollner M, Trouve A Altintas I, Block J, De Callafon R, Clements C, Cortés A, Ellicott E, Filippi JB, Finney M, Ide K, Jenkins MA, Jimenez D, Lautenberger C, Mandel J, Rochoux M, Simeoni A (2015) Towards Data-Driven Operational Wildfire Spread Modeling : A Report of the NSF-Funded WIFIRE Workshop. DOI: 10.13016/M2Z70R.

Hanna SR, Chang JC, Fernau ME (1998) Monte Carlo estimates of uncertainties in predictions by a photochemical grid model (UAM-IV) due to uncertainties in input variables. Atmosoheric Environment 32 (21), 3619-3628.

Parisien MA, Kafka VG, Hirsch KG, Todd JB, Lavoie SG, Maczek PD (2005) Mapping wildfire susceptibility with the BURN-P3 simulation model. Natural Resources Canada, Canadian Forest Service, Northern Forestry Center, Information Report NOR-X-405 (Edmonton, Alberta).

Rothermel R (1972) A mathematical model for predicting fire spread in wildland fuels. USDA Forest Service, Intermountain Forest and Range Experiment station, Research Paper RP-INT-115 (Ogden, UT). 


\title{
Investigation of slope thresholds for flame attachment
}

\author{
Jason J. Sharples*; Ross Edgar; Harvinder S. Sidhu \\ School of Physical, Environmental and Mathematical Sciences, UNSW Australia. \\ \{j.sharples@adfa.edu.au*\}
}

\begin{abstract}
It is well known that wildfires, burning in the landscape, spread more rapidly as the slope of the terrain increases. Typically rules of thumb are employed to provide guidance on expected rates of spread on slopes up to about twenty degrees. For slopes inclined above twenty degrees, however, fire behaviour is more difficult to predict. Currently, the reasons for this are not well understood and there is no definitive model (operational or otherwise) that can account for the types of fire behaviour observed on very steep or confined topography. This behaviour includes so called eruptive fire behaviour, which is characterized by dynamic fire propagation - the fire can accelerate up the slope to very high rates of spread, sometimes with tragic consequences. In this paper we present the results of computational fluid dynamics analyses of convective plume dynamics in a rectangular trench similar to those involved in the Kings Cross Underground disaster. We demonstrate the existence of a threshold angle of inclination, above which the convective plume attaches to the floor of the trench. We discuss the implications of our results for wildland fire safety.
\end{abstract}

Keywords: Eruptive fire behaviour, plume attachment, flame attachment, computational fluid dynamics, slope, dynamic fire propagation.

\section{Introduction}

It is well known that wildfires, burning in the landscape, spread more rapidly as the slope of the terrain increases. Consequently, knowledge of how a fire will spread on slopes in the landscape is an important aspect of wildfire risk management. However, there have been very few systematic studies of slope-driven landscape fires, and no definitive account of the driving processes has been forthcoming. Nevertheless, a number of methods for accounting for the effects of slope on rate of wildfire spread are employed in modelling frameworks and there has been considerable effort put into laboratory-scale studies.

Laboratory-scale studies have also been utilised in examining the nature and causes of fire spread in building structures. Examples include those performed as part of the investigation into a disastrous fire that engulfed an escalator in Kings Cross, London and killed 31 people. Despite initial assessments, which revealed nothing to suggest that it would rapidly develop (Crossland 1992), the fire suddenly spread with extreme ferocity up the escalator trench and into the ticket hall and surrounding areas. Investigations into the King's Cross fire revealed that the extreme fire growth was primarily caused by a distinct phenomenon, subsequently termed 'flame attachment', whereby the buoyant convective plume containing the flames and combustion products attaches to the surface. In a number of instances eyewitnesses and fire investigators described the fire behaviour associated with flame attachment as 'eruptive' (Drysdale et al. 1992).

The term 'eruptive' has also been used to describe certain instances of wildfire behaviour (Viegas 2006). Such instances exemplify a very dangerous aspect of wildland fire behaviour; they pose a significant threat to the safety of wildland fire-fighters and complicate the broader problem of wildfire risk management in landscapes prone to their occurrence. Currently, there is no definitive model (operational or otherwise) that accounts for eruptive fire behaviour. However, studies addressing the incidence of eruptive fire behaviour have revealed that eruptive events are more likely to occur on steep slopes and in canyons. In particular, Viegas and Pita (2004) indicated that eruptive fire behaviour occurred whenever a canyon was sufficiently steep or sufficiently closed. Similarly, the vast majority of eruptive wildfire events have taken place in canyons and on steep slopes. Thus, observations of 
eruptive fire behaviour have suggested the existence of a set of geometric precursors that are necessary for, or at least enhance the likelihood of, an eruptive event.

Similarly, experimental and theoretical investigations conducted after the King's Cross fire indicated that flame attachment was strongly dependent on the surface geometry. Indeed, it was found to more rapidly cover surfaces that were steeper, and to occur more readily in more enclosed, 'trenchlike' configurations. In particular, flame attachment was investigated using state-of-the-art computational fluid dynamics modelling by Simcox et al. (1992), who investigated the phenomenon in a rectangular trench inclined at $30^{\circ}$. In doing so, Simcox et al. (1992) were able to confirm that flame attachment did indeed occur in such a configuration. However, Simcox et al. (1992) did not consider similar trench geometries inclined at angles below $30^{\circ}$, and so were unable to confirm the existence of a slope threshold for flame attachment similar to that which had been identified in experimental studies.

In this paper, we therefore report on a numerical study of the flow of convective plumes in a trenchlike configuration similar to that studied by Simcox et al. (1992), but at lower angles of inclination and various energy intensities.

\section{Computational Fluid Dynamics (CFD) Model}

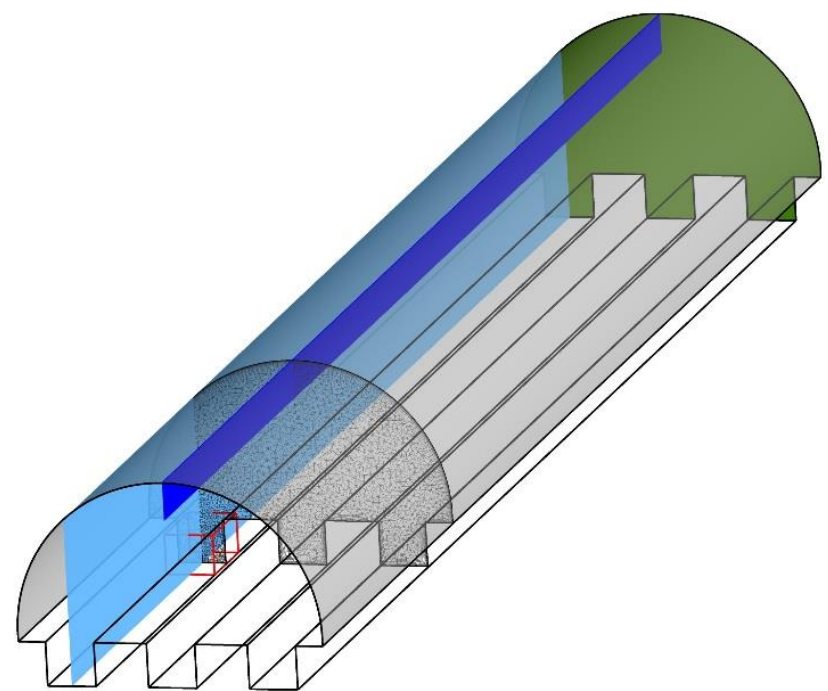

Figure 1 - The flame attachment domain of the tunnel with three escalator trench numeric model. The upper tunnel openning is shaded green and the roof is shaded grey with a semi-transparent rendering. The fire source volume is outlined in red and later figures, i.e. the flow field results in Figures 2 and 3, show results on the light and dark blue two dimensional virtual planes viewed from a normal angle. Another virtual plane orthogonal to the latter pair is shown cutting through the fire volume to reveal the size of three dimensional mesh element crosections like fine lace through the interior of the domain.

The interaction of variables within a dynamic flow field are well described by the Navier-Stokes equations that were derived during the first half of ninteenth century and are today generally understood to include the mass, momentum and energy conservation relations. Being highly non-linear second order partial differential equations, a systemmatic method of finding analytic solutions to describe turbulent regions of fluid problems have not been found other than for a few exceptional situations far removed from fire driven convective states of relevance to this study. By contrast, computational methods are able to replace the intractable differential continuum relations with discrete linear algebraic approximations that are then solved simultaneously for the discetised geometry and then repeatedly for the required sequence of discrete timesteps needed to deterministically evolve the transient flow field from its initial to subsequent states. 
We employ a computational model domain based on a subset of the King's Cross underground complex as shown in Figure 1. In particular we use only the tunnel section of the domain used by Simcox et al. (1992).

Identical geometry and mesh were used for all tunnel domain inclinations, which was defined by the alignment of the geometry with the gravity vector. Simulations were run with tunnel inclinations of $18^{\circ}, 20^{\circ}, 22^{\circ}, 24^{\circ}, 26^{\circ}, 28^{\circ}$ and $30^{\circ}$.

The fluid within the model domain is defined as an ideal gas having the physical and thermal properties of air at $25^{\circ} \mathrm{C}$ and $1 \mathrm{~atm}$ pressure.

The fire is modelled as a thermal energy source that fills the volume of the left escalator trench to a height of $0.9 \mathrm{~m}$ and extends from $10 \mathrm{~m}$ to $12 \mathrm{~m}$ inside the lower entrance of the $42 \mathrm{~m}$ long tunnel. To mimic flames, the fire source volume was divided tunnelwise into eight sections with alternating sets of four sections releasing the fire's energy and swapping with each other at $50 \mathrm{~Hz}$.

The tunnel surfaces were all defined as smooth, no-slip adiabatic walls. The tunnel ends were both defined as openings which had defined zero average relative pressures and turbulence gradients. Air could freely move in or out of the openings however when entering was constrained to travel normal to the boundary and have a temperature defined according to the adiabatic lapse rate of the system.

All simulations started from a condition of zero flow.

The model initialisation conditions, boundaries and discretisation scales were validated by special simulations, e.g. no flow developed when the fire energy source was $0 \mathrm{~W}$ and smaller discretisation of the spatial and temporal scales reinforced the observed outcomes associated with the baseline model.

The resulting convectively-driven flow was calculated using the CFD package ANSYS CFX (ver. 17) with empirically derived "baseline Reynolds Stress Model (RSM)" equations approximating turbulence details (ANSYS, 2015). The RSM is superior to the standard Reynolds Averaged NavierStokes (RANS) models because it has individual transport equations for all six independent components of the Reynolds stress tensor instead of assuming it is isotropic with one. The RSM is thus well suited to model fire plumes which are turbulent and exhibit significant directional differences in viscosity proportional to their directional rates of strain.

As with the most advanced SST two equation RANS models, the baseline RSM also solves the wall distance of geometry elements and transforms from a turbulence-dissipation (or $\varepsilon$ in the $k-\varepsilon$ RANS model where $k$ is turbulence-energy) to turbulence-frequency (or $\omega$ in the $k$ - $\omega$ RANS model) representation of the conservation equations to better capture the influence of the viscous sub-layer on the flow field variables close to walls.

\section{Results}

The simulated flows had significant features in common despite the broad range of tunnel inclinations and fire energy levels examined. The hot plume from the fire source initially rose vertically towards the tunnel ceiling. At later stages, and significantly much later stages for simulations with lower fire source energy and/or tunnel slope angles, the base of the vertically rising plume started to attach to the floor of the trench and work its way along that surface with a laminar flow pattern for about $10 \mathrm{~m}$ (in the case of the geometry studied) until being dissipated or squeezed out to the trench sides.

Figure 2 shows simulation results for the fire source energy equal to $431 \mathrm{~kW}$ runs at typical later stages of flow field development. Parameters of the simulations are listed in individual text headers and a pair of vertical planes of the three dimensional geometry are shown for each: i.e. through the centre of the fire trench escalator (at the bottom of each pair with fine black line showing top of the escalator trench); and for the top part of the pair the top part of the tunnel centre plane. The planes are 
coloured by the velocity component in the tunnelwise direction with superimposed grey shaded temperature contours.

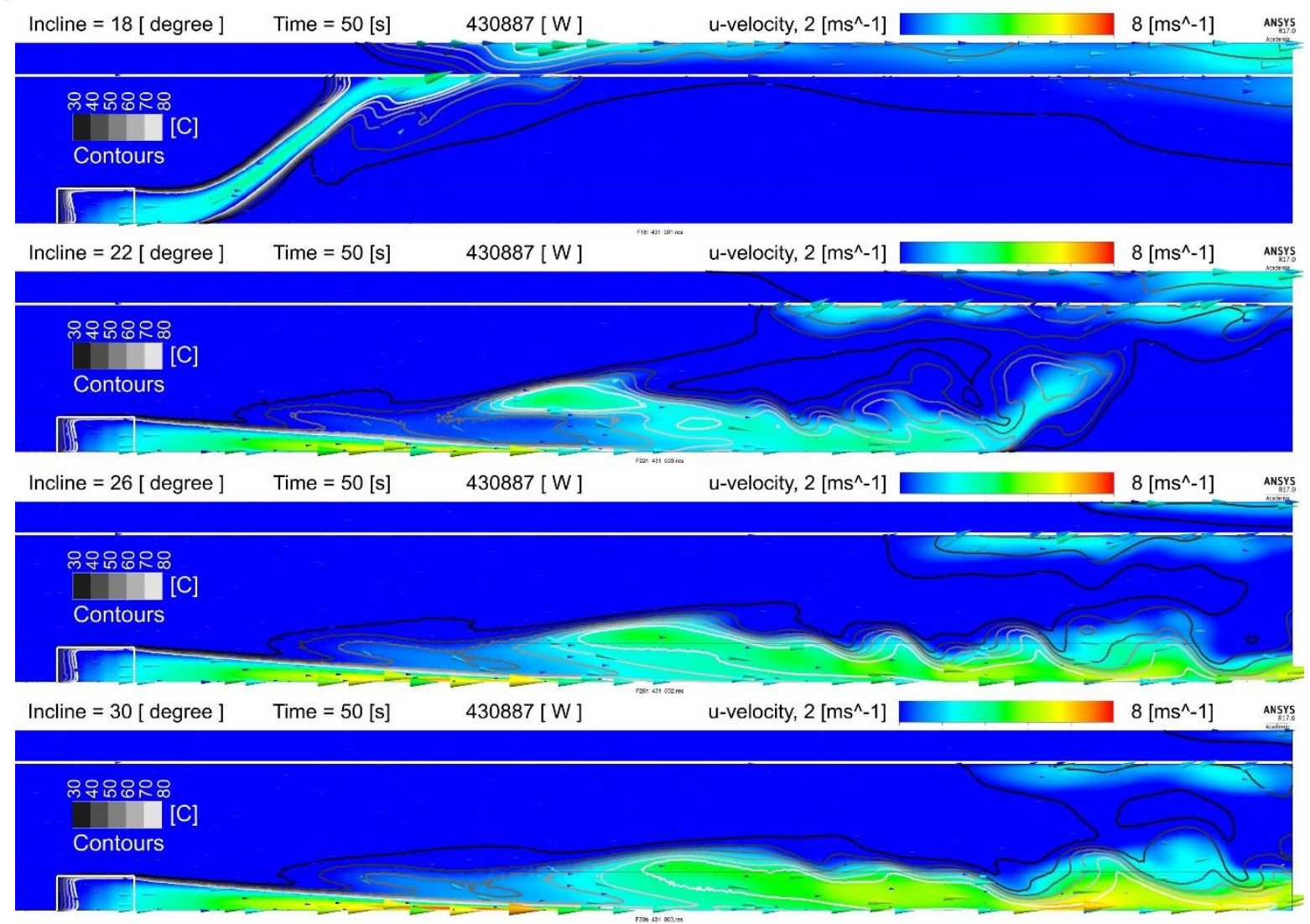

Figure 2 - Flame attachment in trenches has similar characteristics over the broad range of tunnel slopes simulated. The tapering laminar flow from the fire source runs about $10 \mathrm{~m}$ towards the trench floor, given a longer time for the lower slopes, before being squeezed out to the trench sides and continuing along the full length of the trench, however with increasing turbulence at lower slope angles.

Figure 3 shows the laminar boundary layer of flows in the $26^{\circ}$ slope tunnel 26 heading towards the escalator trench floor over the significant range of fire source energies simulated. The laminar boundary layer is eventually squeezed or pushed out to the sides after about $10 \mathrm{~m}$ just as it was for the tunnel slope range shown in Figure 2. Also similarly, the flame attachment along the sides of the escalator trench eventually reached the end of the tunnel however took longer and was associated with less turbulence for lower fire energy source levels. 


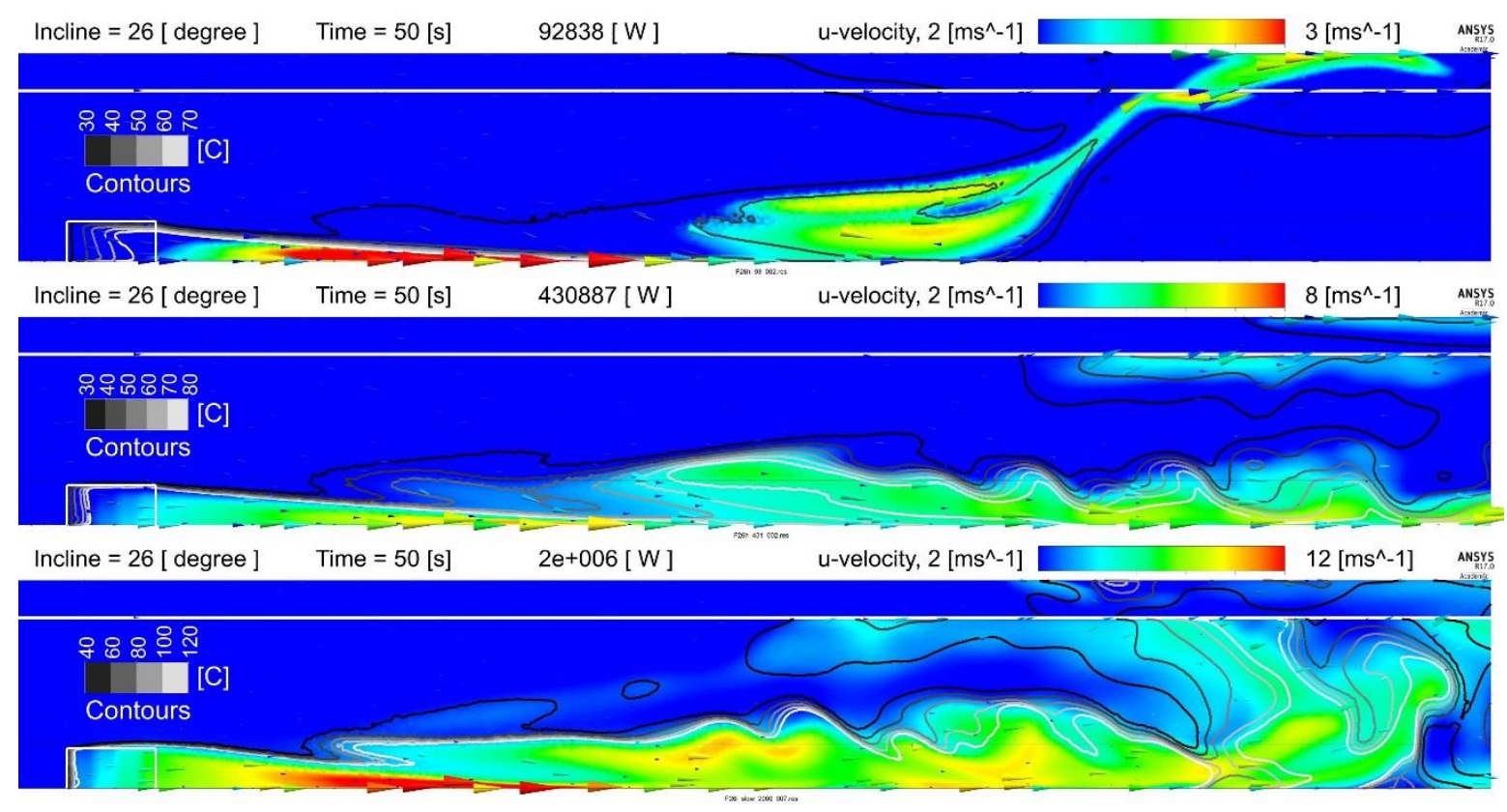

Figure 3 - Flame attachment in the trenches has similar characteristics over the broad range of fire source energies simulated. The tapering laminar flow from the source typically runs about $10 \mathrm{~m}$ towards the trench floor, given a longer time for lower energies, before being squeezed out to the trench sides and continuing with increasing turbulence at higher energy levels. The plume of the $92838 \mathrm{~W}$ source would be expected to attach along the full length of the tunnel's trench and be less turbulent than the higher fire energy sources shown if the simulation time were extended.

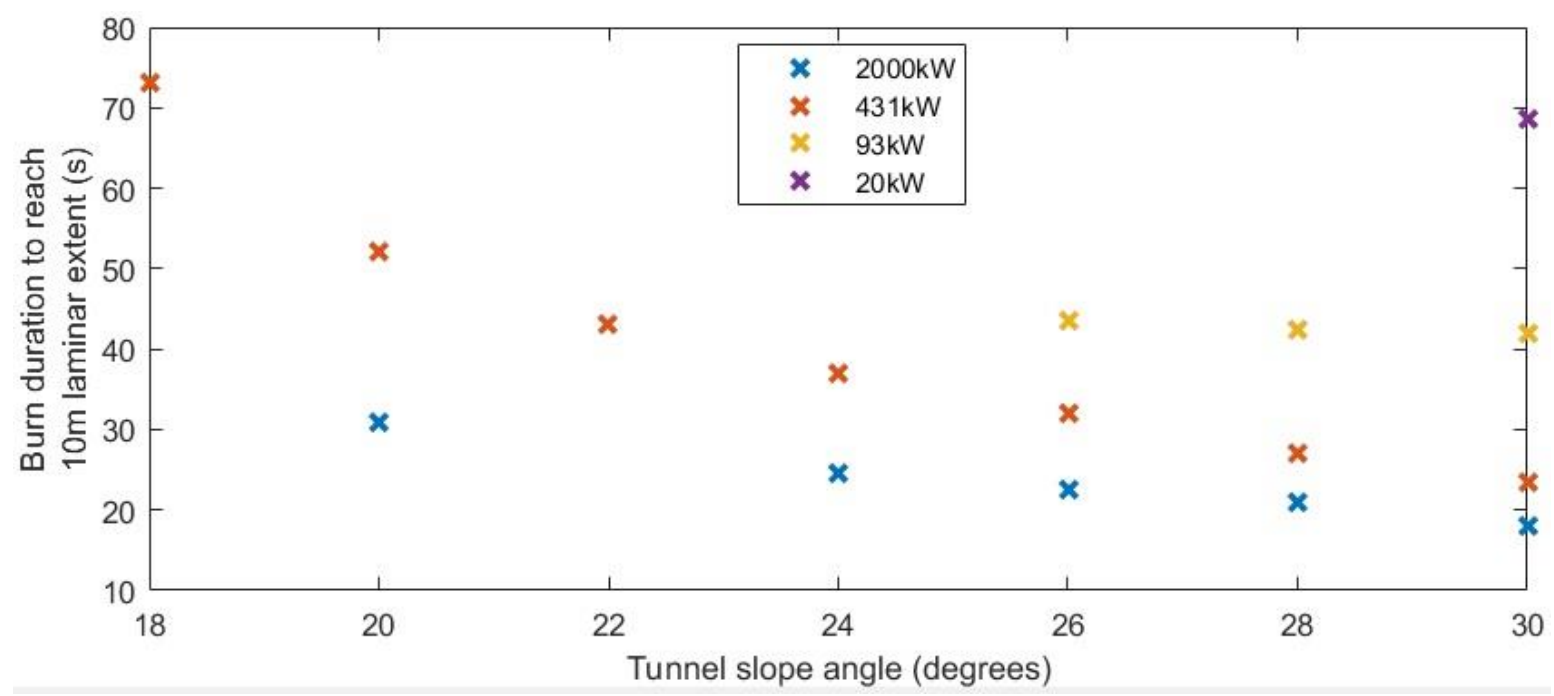

Figure 4 - Simulation time taken for flame attachment to work its way up the escalator trench to point when its laminar boundary layer depth was squeezed out to the trench sides, or $10 \mathrm{~m}$ for the geometry of the tunnel trench considered.

Figure 4 shows the time taken for the flame attachment, or hot plume, to work its way along the floor of the trench to the point of its laminar boundary layer depth being squeezed out to the sides, generally $10 \mathrm{~m}$ as stated earlier for the tunnel trench geometry considered. The escalator trench was $1.0 \mathrm{~m}$ high and $1.1 \mathrm{~m}$ wide (i.e. out of the page in the sense of Figures 2 and 3).

Depending on the combination of tunnel slope and fire source energy, the hot plume subsequently either remained attached to the trench sides, reinforcing a laminar flow across the entire trench section, 
or broke away from the top corners of the trench in turbulent waves rising to the tunnel roof. The latter occurs when the tunnel slope is too low to keep the buoyancy force in check and hot plumes are able to rise with less momentum loss when detaching from the upper corners of the trench despite the implicit turbulence that entails.

In terms of simplistic analogies, drawn from the well studied Coandă effect, it may be useful to think of the surfaces of the trench as the most efficient hot plume transport channel available for the flow from low to upper tunnel heights because of the laminar flow conditions that dominate (and involve far less momentum loss than turbulent flow) for a significant boundary layer depth there. However, if the vertical buoyancy force overwhelms the inertial advantage of remaining within the trench surface flow's laminar sublayer, then hot plumes will detach from surfaces to better placate the buoyancy drivers.

Because the physics of the flow along the surfaces of the tunnel and trench have a determinant influence on the behaviour of the plume, it is imperative that the simulation's manner of modelling the wall flow properties is robust. To ensure this was the case:

- independent transport equations were solved, using the RSM, for each of the independent Reynolds stress tensor components and so the viscous sublayer stress of flows near surfaces varied directionally to reflect that surface's gradient;

- twenty-two prism element inflation layers were placed on all tunnel surfaces, when discretising the fluid's geometry (expanding at the rate of 1.2 from a first layer height of $0.75 \mathrm{~mm}$ ), to produce a smooth transition to the maximum $200 \mathrm{~mm}$ side length unstructured tetrahedral elements of the free stream and to keep the critical $y^{+}$first element centroid's wall distance below the critical threshold of 30 required to properly use the ANSYS CFX wall model equations (ANSYS 2017);

- the maximum root mean square average Courant number of the flow in the simulations, which measures the number of discrete element lengths the flow would traverse in one discrete timestep duration, was kept near one so that the properties of each element along each flow path had the opportunity to influence the flow field's development in the sequential manner a physically meaningful evolution of the flow requires (Courant et al, 1928).

In the simulation space, the upper corner of the escalator trench represents an extremely sharp discontinuity where two surfaces meet at a right angle. Flow there is difficult for the model to capture accurately since element sizes are minute and the courant number rises well above one. In order to accurately simulate the influence of this sharp edge on the flow, a much shorter discretisation time scale, i.e. timestep, needed to be used for the faster $2000 \mathrm{~kW}$ fire energy source conditions. For example, the $30 \mathrm{~ms}$ time steps used for the simulations generally needed to be shortened to $3 \mathrm{~ms}$ for the $2000 \mathrm{~kW}$ fire energy source tunnels to show the flow was consistently drawn $10 \mathrm{~m}$ towards the trench floor before breaking away from trench sides in plumes. At the longer discretisation time scale the hot plumes would break away earlier and disrupt the otherwise consistent laminar flow from the height of the source to the escalator floor $10 \mathrm{~m}$ further up the tunnel.

\section{Conclusion}

The results of this study provide further support for the notion that plume attachment is essentially a geometric phenomenon - it arises as a consequence of the interaction between the convective plume and the geometry of the inclined surface.

Wu et al. (2000) reported finding a critical angle of attachment between $24^{\circ}-26^{\circ}$ when considering flames on inclined planar surfaces. In CFD simulations by Simcox et al (1992) flame attachment in a $30^{\circ}$ sloping tunnel trench was found at the upper range of the energy levels we studied and for various 
pressure driven inlet flow conditions; however, other slopes were not considered. In a separate study we considered the effect of the intensity of the convective plume and found very similar results to those reported here (Edgar et al, 2015). Overall, the indication is that the plume attachment phenomenon is independent of the convective intensity of the plume, at least for intensities up to the order of a megawatt per cubic metre. Despite this, the rapidity with which the fire progresses up the slope does vary, and is significantly faster for higher intensities at least up to that at which wildfires could be expected to burn. As such, plume attachment should be considered as a distinct likelihood for fires burning in landscapes that are sufficiently inclined and more so when also enclosed; for example, like a canyon or gully. As such, these findings have strong implications for fire safety in wildland fire environments.

With the developed simulation model confidently working following validation of the discretisation scales used, it may now be readily extended to the study of other geometry trenches in tunnels and open fields more consistent with conditions encountered during wildfires.

\section{Acknowledgement:}

This research was funded by an Australian Research Council (IN140100011), and undertaken with the assistance of resources provided at the NCI National Facility under the Australian Government National Computational Merit Allocation Scheme.

\section{References:}

ANSYS Inc. ANSYS CFX Modelling Guide - release 17.0. 2015.

Courant R, Friedrichs K, Lewy H. Uber die patiellen differenzengleichungen der mathematischen physic 1928; Translation: on the partial difference equations of mathematical physics 1967; Mathematische Annalen 100 (1): 32-74; Translation - IBM Journal March 1967: 215-234.

Crossland, B. (1992) The King's Cross Underground Fire and the setting up of the investigation. Fire Safety Journal 18, 3-11.

Drysdale, D.D., Macmillan, A.J.R. (1992) Flame spread on inclined surfaces. Fire Safety Journal 18, 245-254.

Edgar RA, Sharples JJ, Sidhu HS. Revisiting the King's Cross Underground disaster with implications for modelling wildfire eruption, 21st International Congress on Modelling and Simulation Modelling and Simulation Society of Australia and New Zealand 2015: 215-221.

Simcox S, Wilkes N, and Jones L. Computer simulation of the flows of hot gases from the fire at King's Cross underground station. Fire Safety Journal 1992. 18 (1): 49-73.

Viegas, D.X., Pita, L.P. (2004) Fire spread in canyons. International Journal of Wildland Fire 13, 253 274.

Viegas, D.X. (2006a) Parametric study of an eruptive fire behaviour model. International Journal of Wildland Fire 15, 169-177.

Wu, Y., Xing, H.J., Atkinson, G. (2000) Interaction of fire plume with inclined surface. Fire Safety Journal 35, 391-403. 


\title{
IPA Adriatic Holistic Forest Fire Protection Project - the year after
}

\author{
Darko Stipaničev*1; Ljiljana Šerić ${ }^{1}$; Damir Krstinić ${ }^{1}$; Marin Bugarić1; Anđelka Vuković ${ }^{2}$ \\ ${ }^{1}$ University of Split Faculty of Electrical Engineering, Mechanical Engineering and Naval \\ Architecture in Split, Ruđera Boškovića 32, 21000 Split, Croatia, \{darko.stipanicev@fesb.hr*, \\ ljiljana.seric@fesb.hr;damir.krstinic@fesb.hr;marin.bugaric@fesb.hr\} \\ ${ }^{2}$ Split-Dalmatia County, Domovinskog rata 2, 21000 Split, Croatia, \\ \{Andelka.Vukovic@dalmacija.hr\}
}

\begin{abstract}
The regions around Adriatic Sea have high risk of wildfires, especially during summer due to very warm climate with low level of humidity, densely spaced Mediterranean vegetation, particularly conifer forests and lot of tourists. During fire season that is usually between July 1 and October 31, there are lot of wildfires in this region. Therefore, the biggest Croatian county Split-Dalmatia County has initiated in 2007 preparation of strategic project target to Adriatic IPA funds, based on Split-Dalmatia County holistic approach to forest fires protection and prevention. The HOLISTIC Project with full name Adriatic Holistic Forest Fire Protection has been finally accepted in 2013 as Adriatic IPA project and ended in December 2016. HOLISTIC project was focuses on the prevention and damages mitigation of the natural risks, with special focus on wildfire and earthquake risks, aiming at improvement, promotion and strengthening of institutional capabilities in implementing policies and actions to reducing the causes and damages in regions facing the Adriatic Sea. HOLISTIC project was not focused on research and development of new technologies in wildfire prevention, protection and fighting, but rather toward the application of existing technologies in fire fighters everyday tasks. Projects outputs, results and particularly pilot projects, are the main topic of this paper, including the analysis of project influences on fire fighters practice, one year after the project ending.

Note: Authors from FESB were, during HOLISTIC Project realisation, Key Experts for Work Packages connected with implementation of Information-Communication Technologies (ICT) in wildfire protection and fighting for various Dalmatian Counties.
\end{abstract}

Keywords: EU project, wildfires, forest fires, wildfire protection, ICT in wildfires, sustainable forest management.

\section{Introduction}

The regions around Adriatic Sea have high risk of wildfires, especially during the summer due to very warm climate with low level of humidity, densely spaced Mediterranean vegetation, particularly conifer forests and lot of tourists. During fire season that is usually between July 1 and October 31, there are lot of wildfires in this region. For example in Republic Croatia located on the east side of Adriatic Sea in the last 10 years 2.320 wildfires have been recoded that affected 84.250 hectares of forests and forest land.

Therefore, the biggest Croatian county Split-Dalmatia County has initiated in 2007 preparation of strategic project target to Adriatic IPA funds, based on Split-Dalmatia County holistic approach to forest fires protection and prevention (Stipanicev et al, 2007). The HOLISTIC Project has been finally accepted in 2013 as Adriatic IPA project under priority 2 Natural and Cultural Resources and Risk Prevention, measure 2.2 Natural and cultural resource management and prevention of natural and technological risks. HOLISTIC project, with full name Adriatic Holistic Forest Fire Protection, ended in December 2016 (HOLISTIC, 2013). HOLISTIC project was focused on the prevention and damages mitigation of the natural risks, with special focus on wildfire and earthquake risks, aiming at improvement, promotion and strengthening of institutional capabilities in implementing policies and actions to reducing the causes and damages in regions facing the Adriatic Sea. It has gathered 20 
partners from Greece, Albania, Montenegro, Bosnia and Herzegovina, Croatia, Slovenia, Italy and Serbia, leaded and coordinated by Split-Dalmatia County. The total project budget was about 9.3 million $€$. HOLISTIC project was not focused on research and development of new technologies in wildfire prevention, protection and fighting, but rather toward the application of existing technologies in fire fighters everyday tasks. It gathered together almost all regions around Adriatic Sea, trying to find common, sustainable approach to forest fire prevention, protection and fighting.

The main topics of this paper are outputs and results of HOLISTIC project, particularly pilot projects, but the paper also includes the analysis of project influences on fire fighters practice, one year after the project ending.

\section{HOLISTIC Project overview}

HOLISTIC project has been focused on the prevention and damages mitigation of the natural risks, particularly on wildfire and earthquake risks, aiming at improvement, promotion and strengthening of institutional capabilities in implementing policies and actions to reducing the causes and potential start of natural breakdowns to improve reaction and intervention readiness, to reduce the damages in areas of regions facing the Adriatic.

The project's most important results and the respective outputs were:

1. Developed common guidelines and standard procedures for:

- Policy and institutional enhancement for the implementation of updated and sustainable fire protection and prevention policies and mechanisms.

- Legislative and institutional regulations for the implementation of integral and sustainable public buildings protection policies.

- Territorial analysis of set-up methodology for fire safety.

- Forest sustainable management aimed at reducing wildfire risks.

- Prevention and fire fighting management, particularly in cross-border fires. Territorial analysis of set-up methodology for public and historical buildings protection policies.

2. Survey of actually applied procedures, policies and measures for:

- Fire protection, prevention and previsions.

- Public buildings protection from earthquakes.

3. Established static and dynamic spatial database about terrestrial, meteorological and sociological features, either measured, estimated and simulated, related to fire hazards or fire fighting policy.

4. Developed post fire monitoring and damage assessment methods integrating multi-source spatial information for land management support.

5. Harmonized fire risks data and maps.

6. Implemented up-to-date ICT solutions in pre-fire, fire and post-fire activities.

7. Staff trained in the use of ICT technologies in wild land fire prevention, protection and fighting.

8. Established network of scientific and research capacities for wildfire prevention and protection research and education based on modern technologies. 
9. Application of GIS and remote sensing technologies for prevention of fire hazard risks, advanced fire fighting and post-fire recuperation.

10. Developed and experimented model of:

- Monitoring, surveillance and fire outbreaks early warning integrated system.

- Financial, social and eco sustainable wood life cycle (silviculture, wood waste treatment, biomass production and marketing).

- Indirect prevention campaign, diversified according to the type of user.

- Fire risks predictive models on micro-location scale adapted and tuned for Adriatic region.

- Fire behaviour modelling and spread simulation adapted and tuned for Adriatic region.

- Early warning monitoring and surveillance system adapted and tuned for Adriatic region.

- Buildings' real-time seismic vulnerability inspection and monitoring.

As usual, special work package was reserved for awareness raising activities to be undertaken by local stakeholders with the aim to achieve local ownership of the project and its results. With aims to aggregate significant added value and gain synergies, the project has accounted the experiences and results from previous EU projects and the experience made by many Beneficiaries who participated in projects addressed to the same theme of Holistic project.

All together 20 partners participate in HOLISTIC project (Figure 1). Project coordinator and lead beneficiary was Split Dalmatia County from Croatia. The project has started in October 2013 and ended in December 2016 and the total project budget was 9.363.801,29€.

\section{Holistic Project Partnership}
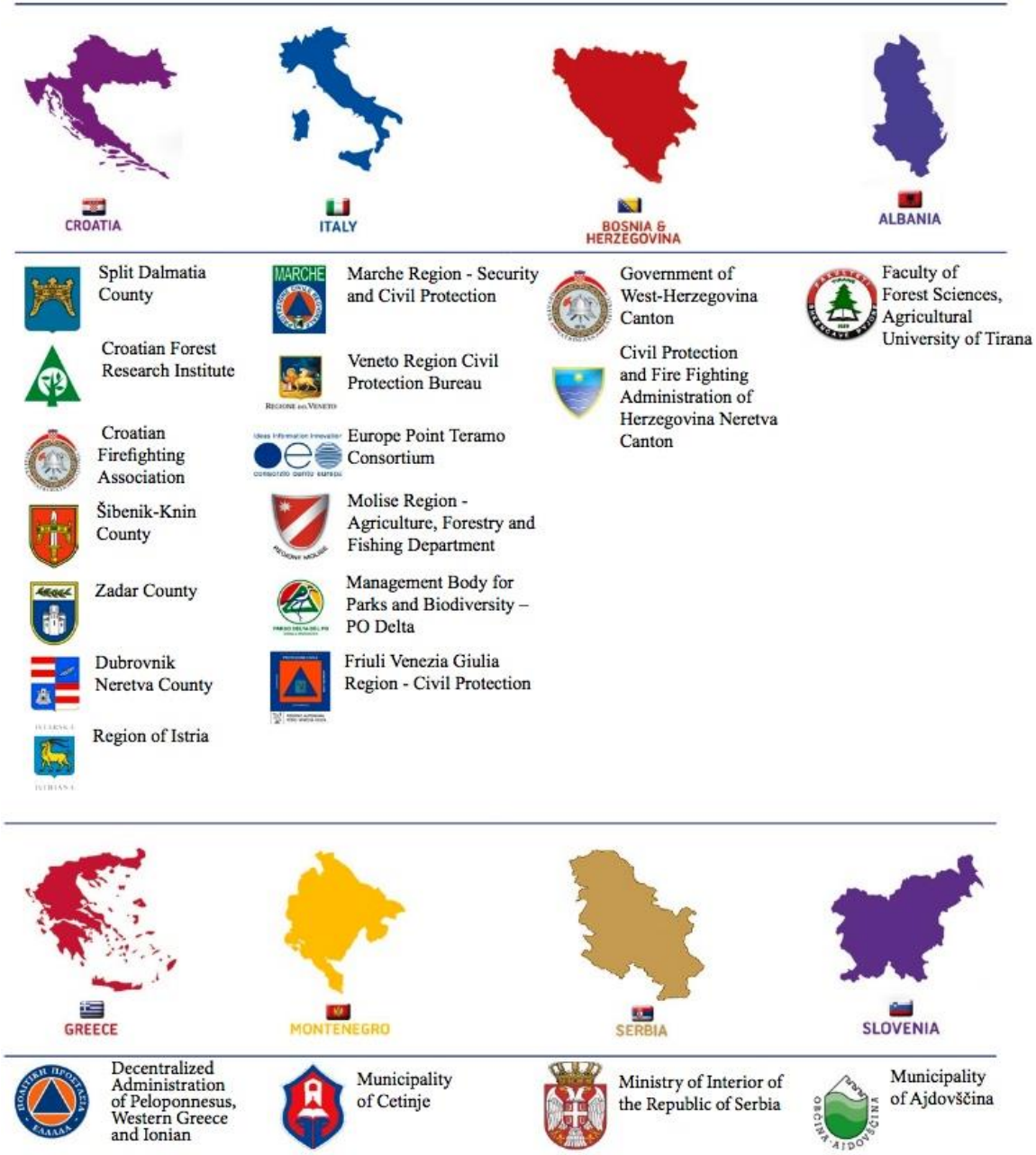

Figure 1-IPA Adriatic HOLISTIC project partnership. 


\section{HOLISTIC Project outputs and results}

HOLISTIC Project has intended to reduce the number and the impact of forest fires, to protect people, natural environment and properties, and in particular to promote fire prevention among rural communities in fire prone areas of regions facing the Adriatic. In addition it has intended to promote awareness and stable fire risk mitigation activities and technological improvement of territory monitoring and surveillance through the development of shared models, methods and tools for integrated and sustainable natural risk management, protection and prevention. iscep:

The project was organized in 8 work packages.

WP0 - covers preparatory activities of the partnership and project drafting and WP1 - Project Management and Coordination ensures availability of human and technological resources, project reliability and monitoring of targets' achievement;

\section{WP 2 - Communication and Dissemination}

The aim of this Workpackage was to assure the dissemination and promotion of the project activities and achievements towards target groups inside and outside the involved territories, essential for the dissemination of the results, the capitalization of the activities and the transparency of the funds allocated to the project, assuring that the planned information and dissemination activities are able to achieve visibility among relevant target groups. The WEB Site, press conferences, press releases, newspaper articles, regional and transnational conferences and participation at International exhibition and conferences with presentations, in accord with projects milestones, represent the communication and dissemination activities. To complete them a network of stakeholders from all participating countries is built using national and thematic international conferences and the final conference, presenting the project outcomes. Figure 2 shows home page of the project Web site (HOLISTIC, 2013) that has achieved average of 509 hits per month.

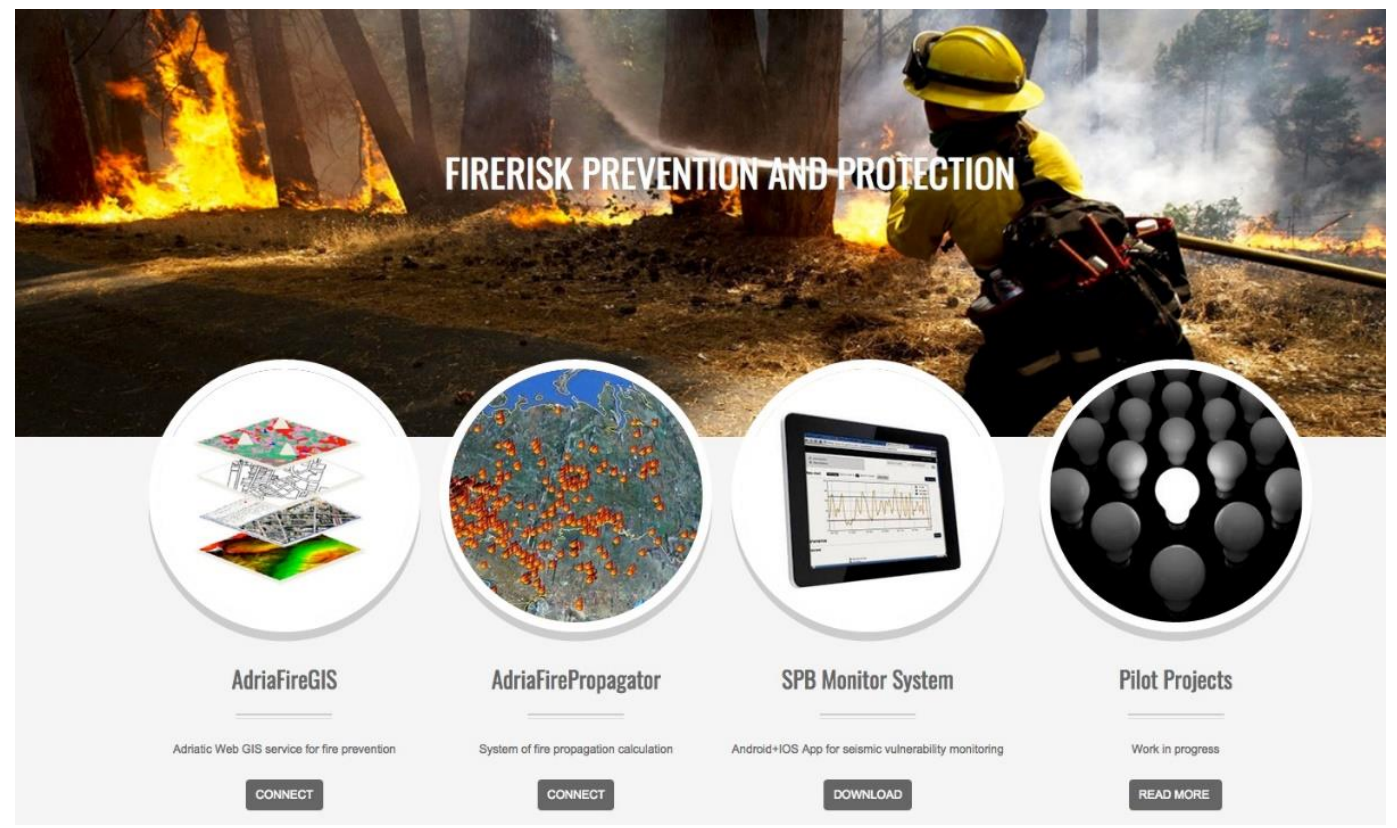

Figure 2 - IPA Adriatic HOLISTIC project Web site - http://www.adriaholistic.eu

The final results were 6 main Project meetings, 195 articles/appearances published in the press and in other media (including online media, TV, radio), 13 press conferences, 29 publications produced, 54 national/regional events and 5 international seminars. It is estimated that almost 20000 individuals were directly reached via dissemination outputs. 


\section{WP 3 - Capitalization and sustainability}

Within WP 3 one of the major achievements was production of Joint plan of capitalization. This document was based on previously identified common problems and aimed to identify common challenges and propose future actions in each specific thematic topic. The purpose of the capitalization process was to propose effective answers based on the best practices that have been analyzed through adopted methodological approach: analyzing post and current experiences in order to collect lessons learned and put in place the acquired good practice and sharing achievements in order to learn mutually. 36 case study reports have been investigated and 4 best practices selected during 10 thematic workshops and 9 regional stakeholders roundtables. Join plan of capitalization provides proposals, but their future implementation is directly linked with financial possibilities and institutional support and interest of each partner.

\section{WP 4 - Joint management policies}

WP 4 has provided a screening of an existing situation of protocols/actions regarding pre-fire, fire and post-fire activities in IPA countries that could be useful for future standardization of protocols/actions in order to have better preparedness when wildfires or earthquakes appear. Four reports has been prepared: Report of existing policies in region countries concerning pre-fire, fire and post-fire activities, Technical report with recommendation for pre-fire prevention and protection activities, Technical report with recommendation for common protocols for fire monitoring, alarming and evacuation, Technical report with recommendation for common protocols for fire fighting intervention and Technical report with recommendation for common protocols for post fire recuperation, as well as Catalogue of the state of the art in forest fire management policies in pre-fire, fire and post-fire local policies, practices and coordination mechanisms, Harmonization and standardization of pre-fire activities concerning wildland/urban interfaces, vulnerable natural and manmade infrastructures based on forest management and fire protected activities, Harmonization and standardization of pre-fire and fire activities concerning fire monitoring, alarming and evacuation and Harmonization and standardization of fire activates concerning fire fighting intervention.

\section{WP 5 - Territorial analysis concerning wildfire prevention and fire fighting}

WP 5 was dedicated to development of regional GIS system adapted for wildfire activates demands and tuned to Adriatic region and particularly to cross border cooperation in order to harmonized and improve accessibility of spatial - temporal data about landscape characteristics, vegetation, meteorological and sociological data important for wildfire fighting. Three main outcomes of WP5 package where user-friendly regional GIS service, wildfire propagation and wildfire risk prediction simulation tools. Those tools are:

- AdriaFireGIS - a multi-user wildfire Web based service accessible by all partners in region. It has collected regional GIS layers in the form suitable to be used through appropriate WMS servers in all Web based applications developed through this project (static terrestrial layers, static vegetation layers, dynamic meteorological data, wildfire sociological layers).

- AdriaFirePropagator - a multi-user, Web based service for wildfire behavior and spread simulation, useful for all firefighting activities, and

- AdriaFireRisk and AdriaFireEruptiveRisk - fire risk index based on climatological, meteorological parameters, vegetation, terrain configuration and various anthropogenic parameters that run as additional module with AdriaFirePropagator, as well as prediction of potential dangerous wildfire eruptive behavior based on terrain and meteorological parameters.

Simulations in AdriaFirePropagator can easily be run on a single click of a button after choosing the ignition point or area. In this case the simulation is run in "during wildfire" mode, where current meteorological conditions are automatically obtained (several times a day, with hourly forecast) and 
used as input to simulation. In such cases, AdriaFirePropagator can be helpful to organize firefighting actions, such as unit arrangement, number of vehicles, etc.

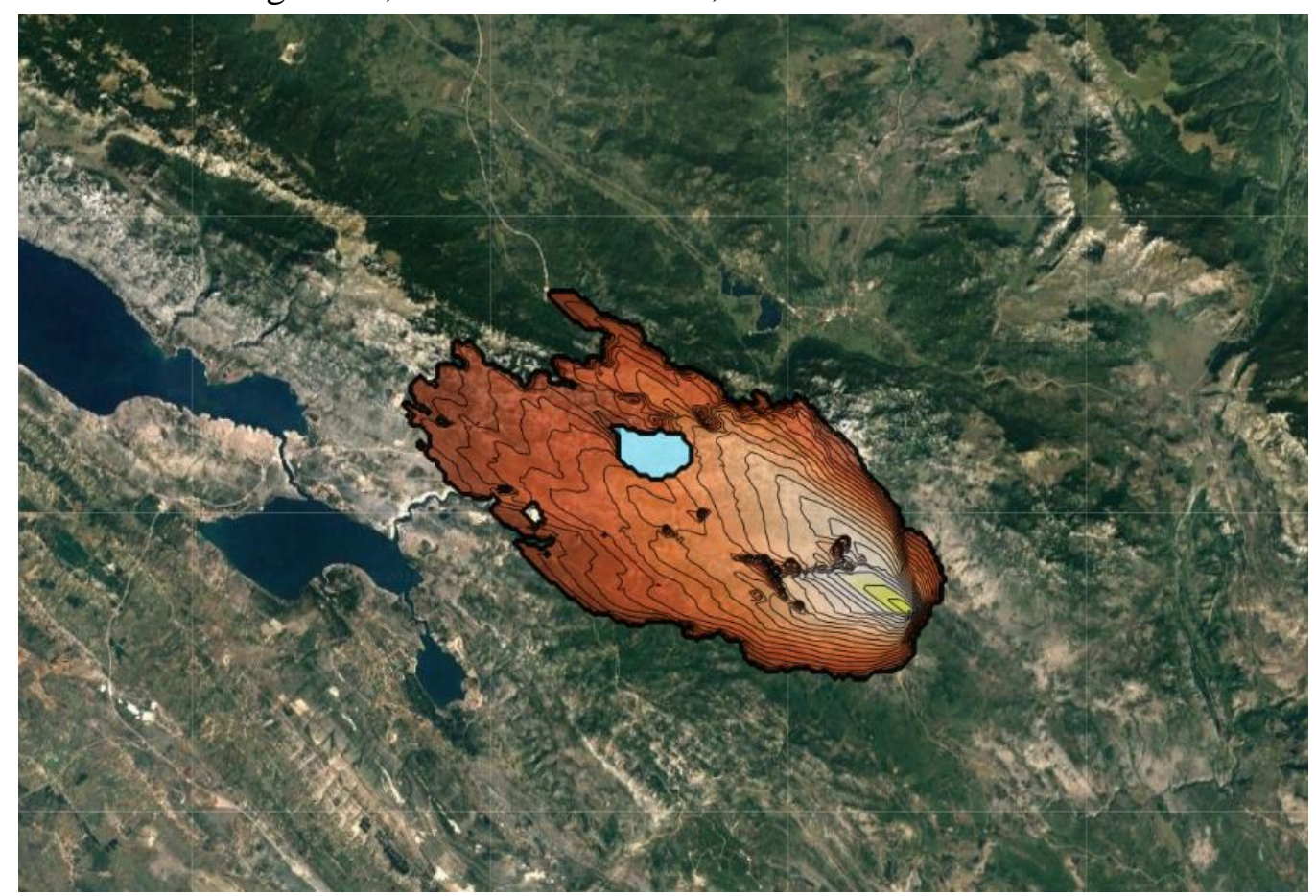

Figure 3 - AdriaFirePropagator simulation - blue area represents fire barrier (non burnable area).

On the other hand, AdriaFirePropagator is also a highly customizable tool where most of the simulation inputs can be modified and adjusted, allowing the simulations in "before wildfire" mode. In this mode, users can adjust wind, moisture, fuel and other parameters to set conditions in which simulation will run. All this is of great importance as it allows AdriaFirePropagator to be used for training and educating firefighting personnel, simulation of different scenarios that can occur in the case of wildfire, investigation of previous wildfires, etc. Important is to emphasize that users can specify fire barriers, areas on the map that will be ignored during simulation (non-burnable areas), as well as fire fronts - areas describing initially ignited (already burning) area for the simulation.

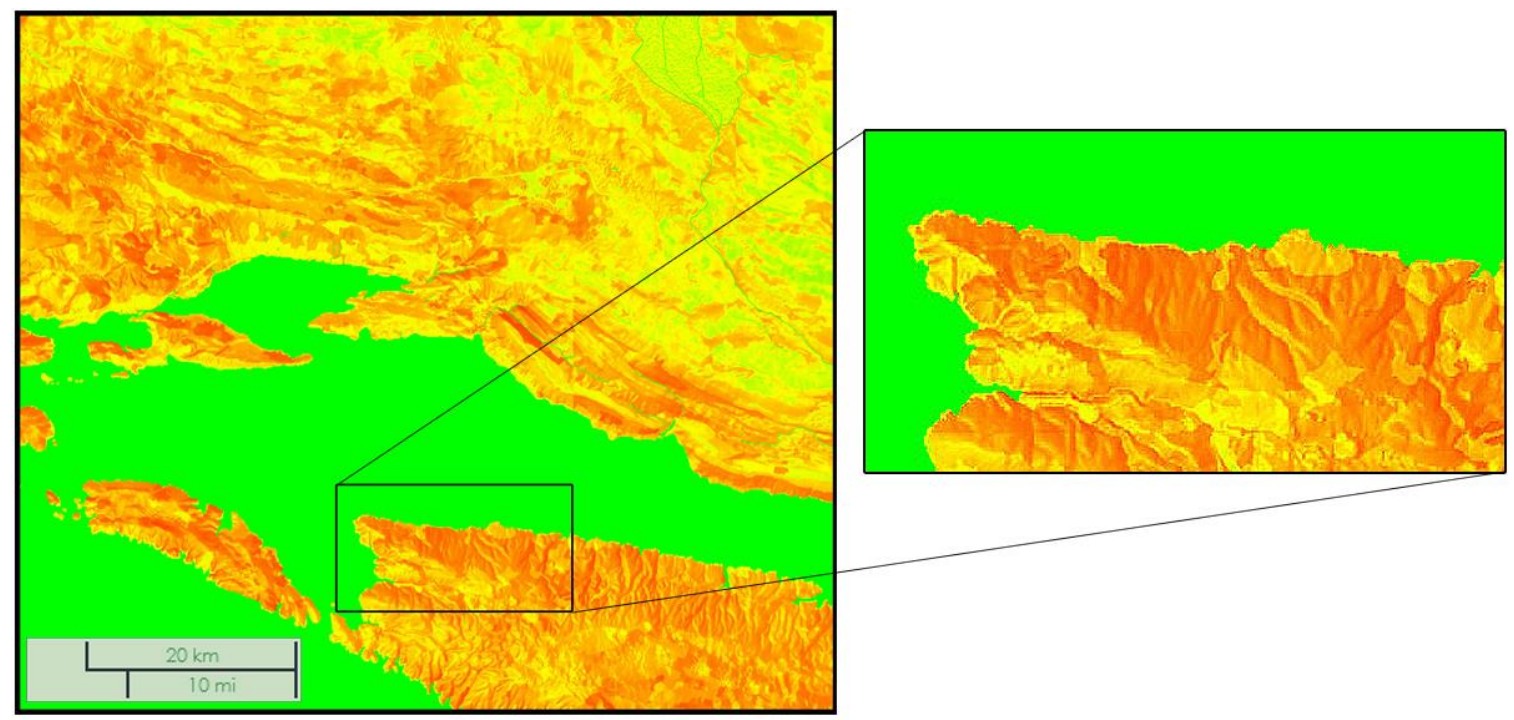

Figure 4 - AdriaFireRisk - wildfire risk index for Adriatic region during spring months 
AdriaFireRisk is a numerical indicator that defines the level of fire risk at certain geographical area calculated as additional module to AdriaFirePropagator. It is based on climatological and meteorological parameters, vegetation, terrain configuration and anthropogenic parameters. Climatological and meteorological parameters give AdriaFireRisk its dynamics, while the other parameters belong to the set of static indicators. The main advantage of AdriaFireRisk over other indexes is that it is better adapted to the micro-location, that is, it is site-specific wildfire risk index. Panels have been placed on spots of important public interest sensitive to wildfires, such as national parks, city forests, protected islands. Those panels are connected to AdriaFireRisk system and show current risk at that area in real time. Along with AdriaFireRisk, eruptive wildfire risk index (AdriaFireEruptiveRisk) is also calculated using terrain and meteorological parameters as inputs in order to detect zones where dangerous eruptive fire behavior could happen.

\section{WP 6 - Application of ICT in fire prevention and protection}

In WP 6 cross border cooperation was realized through information exchange about common guidelines and standard procedures for installation of wildfire monitoring and surveillance systems and networks. Detail analysis of all commercial ground based wildfire monitoring and surveillance systems from Italy, France, Portugal, Croatia, Turkey/US, South Africa, Germany, Spain and Russia has been done. The main focus was on functional system integrated in standard wildfire monitoring services and particularly located in Mediterranean countries. Experimental and research system, although they were lot of them, have not been analyzed, because from research to real life implementation is a long way. Based on this analysis a new regional model named AdriaFireMonitor has been developed tuned and adapted for specific Adriatic surroundings.

Final outcomes od this WP 6 were establishment of Technical guidelines and specifications of wildfire video monitoring unit (wildfire observer) and wildfire video monitoring network (wildfire observer network) adapted for Adriatic region, Technical guidelines for systematic wildfire monitoring locations determinations, Emergency intervention code of conduct report with distribution of tasks, definition of coordinating bodies and User rights report with definition of accessibility and rights to use for different level users. Based on this reports partners involved in this Workpackage have identified monitoring locations for video surveillance system set-up in their regions, provide all necessary procedures for installation of advanced 3 cameras visible spectra wildfire video monitoring and surveillance systems and additionally in Istria County and Split - Dalmatia County installation of advanced 1 camera IR spectra wildfire video monitoring and surveillance systems integrated with visible spectra monitoring. Part of tis Workpackage was installation of advanced GPS tracking systems for fire fighters vehicles (each system with 5 GPS trackers) and installation of tablet based advanced system for burned area mapping, as well as supply of mobile monitoring unit to Split - Dalmatia County as Figure 5 shows and acquisition of various drone and UAV monitoring units.

Finally WP 6 has organized few regional conference concerning application of advanced ICT technologies in forest fire prevention and protection and assessment of pilot projects' achievements, but also establish a network of scientific and research capacities in region capable for forest fire research by reuse of existing research capabilities in systematic forest fire research and initiate a regional research and education activities concerning forest fire prevention, protection and fighting by strengthen research and education capabilities in region concerning fire prevention and fighting. 


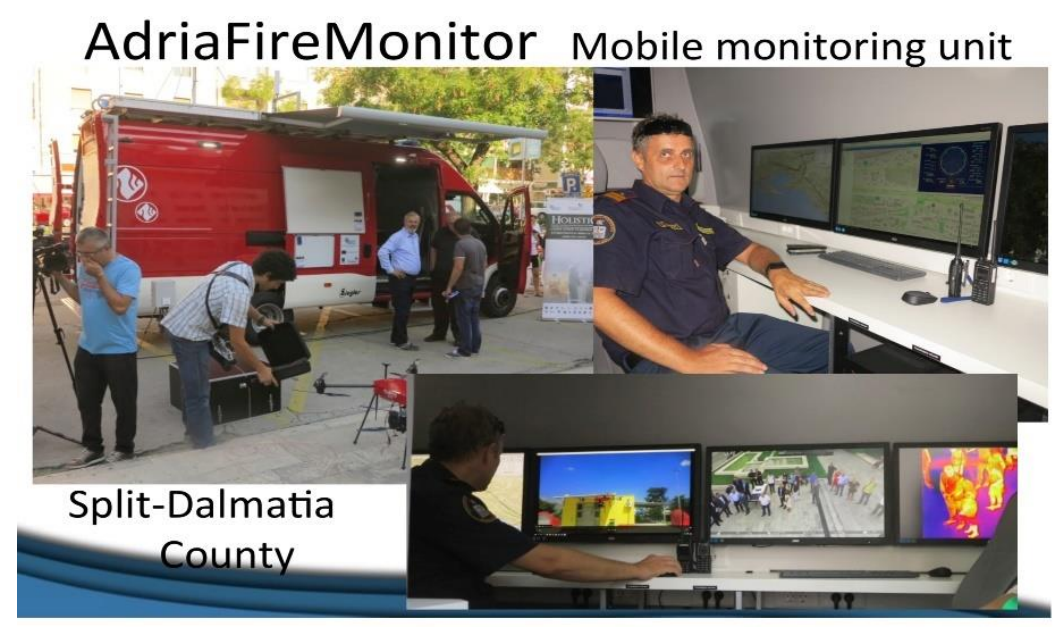

Figure 5 - AdriaFireMonitor - Split-Dalmatia County mobile monitoring unit

\section{WP 7 - Reduction of fire risk by sustainable forest management}

Major achievements of WP 7 have been cooperation between beneficiaries devoted to the forest sustainable management and use of forest biomass in sustainable wildfire prevention and protection. The collaboration has achieved the systematic collection, analysis and capitalization of previously implemented projects and studies and preparation of important documents useful for the utilization of forest biomass for bioenergy production. Furthermore WP 7 has implemented appealing pilot actions - the installation of six demonstrative heating plants in public buildings (primarily schools) fed with wood biomass (wood chips and wood-pellet).

\section{WP 8 - Target Groups Education and Awareness}

Thanks to efforts from all project partners and associative partners, all of them have been involved in the activities related to WP 8. Preciously 11.186 participants between the groups targeted by the project activities, in particular land owners, farmers, shepherds, hunters, tourists and inhabitants, schoolchildren and students, Civil Protection Volunteers and firefighting associations, local stakeholders and representatives of the institutional bodies of the project areas has been involved in target groups education concerning wildfire prevention and protection. 27 targeted campaigns training \& info days for schoolchildren and student have been organized, as well as 27 targeted campaigns training courses for civil Protection Volunteers and 38 targeted campaigns for general public advertising - posters, radio trail and TV appearances.

\section{HOLISTIC Project influences on fire fighters practice}

After the end of the project HOLISTIC project outcomes and results introduced great improvement in fire fighting policies and practices. In this section we will describe some of the most visible project outcomes and how their introduction changed and improved the practices and procedures in fire preparedness, fire fighting and fire reporting. The influence of the project will be described qualitatively in the form of good practices description. I this section we will also mention a methodology for evaluation of AdriaFireMonitor usefulness that is introduced in another paper.

The HOLISTIC project offered systematic introduction for fire fighting community to tools and applications of Information Communication Technologies that can be used to improve the overall communication, information dissemination and fire fighting. Before this, each organization used ICT tools in some aspect of their work (like using panoramic video cameras for fire surveillance, or GPS tracking for fuel consumption analysis) but without integration of those tools with one another or with other organizations. Specific outcomes of the project that will be discussed here are: AdriaFireGIS, 
AdriaFireGPS, AdriaFirePropagator, AdriaFireRisk, AdriaFireMonitor and AdriaFireBurnedAreaMapping.

AdriaFireGIS provided integrated interface for spatial information presentation in the form of standardized GIS map.

Majority of fire fighting vehicles used in operative actions are equipped with the GPS tracking devices. The devices are interconnected with interface for GPS tracking maintained by Croatian Fire fighting Association (CFA). The GPS tracking routes are even made visible within GIS system maintained by Croatian National Rescue and Protection Directorate (DUZS)- The ZEOS system that overtook the task of AdriaFireGIS, due to the large amount of useful data collected and stored.

During the project HOLISTIC fire fighting commanders were trained for using AdriaFirePropagator. The usefulness of the tool showed itself when fire fighting commander of Šibenik-Knin County has used the tool for training before the season and run the simulation on location Promina were, according to his experience the wildfire was expected. He run simulations with different meteorological conditions and expected scenarios to analyse possible outcomes when the fire occurs. In August 2017 wildfire on location Promina occurred. It is reported that one of the expected scenarios happened and the wildfire lasted for almost 10 days, but fire fighters were prepared and no casualties happened. Also during the fire in Skradin region in September the AdriaFirePropagator was used to help decision for evacuation (Figure 6).

AdriaFireMonitor was the most visible project outcome. Almost each project partner installed the pilot forest fire video monitoring and surveillance system on 3 locations. The system was installed at the end of 2016 fire season so the operators had time to familiarize themselves with the system before the next season. During the 2017 fire season video surveillance and monitoring system provided fire fighting commanders video signal directly from the field and helped them make better and informed decisions. In some situations commanders reported that they had better overview of the fire with the use of the camera then the fire fighters on the field. Many fires were spotted in its ignition stage by the system, even several hours before citizens reported them. When the citizens report fire, it is easily confirmed by inspecting the area with the camera. The location of fire reported is more accurately determined because of integration with the GIS system. Also professional person can determine extent and severity of the fire that can them make better decision on number of troops and vehicles necessary for fire fighting. All operators' actions are logged and stored in the system, and these logs and records were used in assessment of usefulness of the monitoring system that is described in another paper.

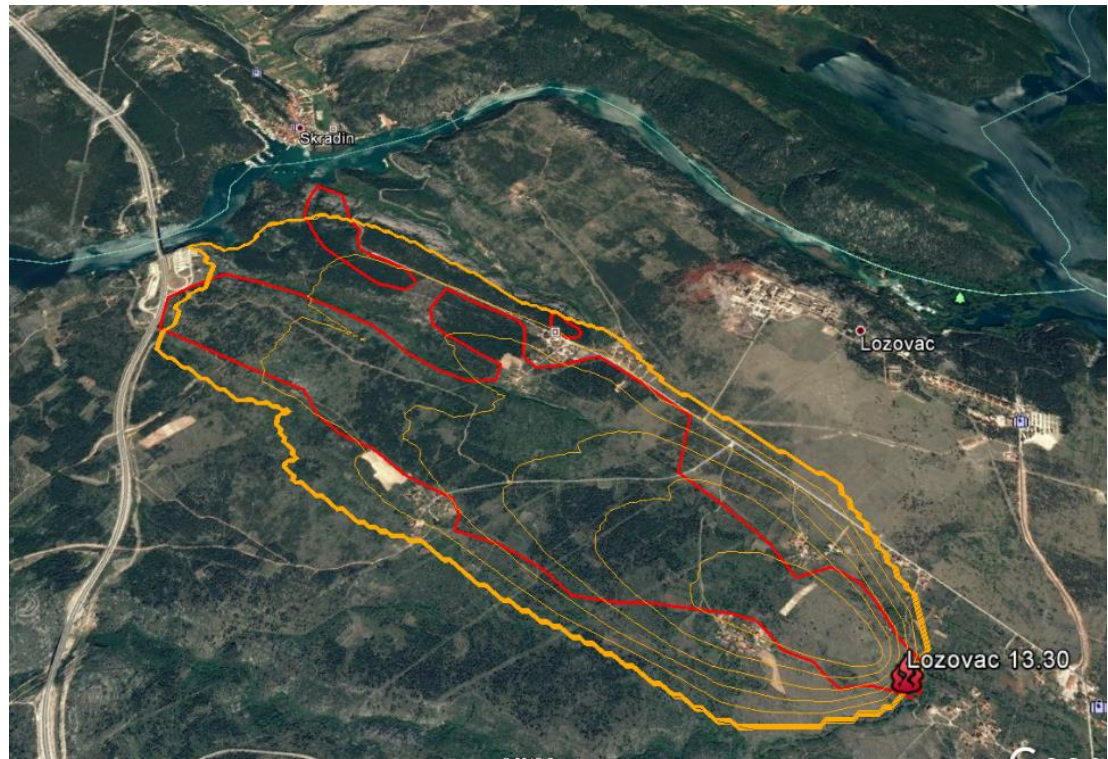

Figure 6 - Finally Comparison of fire simulation (yellow) and real fire contours (red) for the fire near Skradin in September 2017 
Having in mind good experience from the pilot installation, Croatian Forests ltd invested almost 3 million EUR in the forest fire video-communication system that is extension to AdriaFireMonitor.

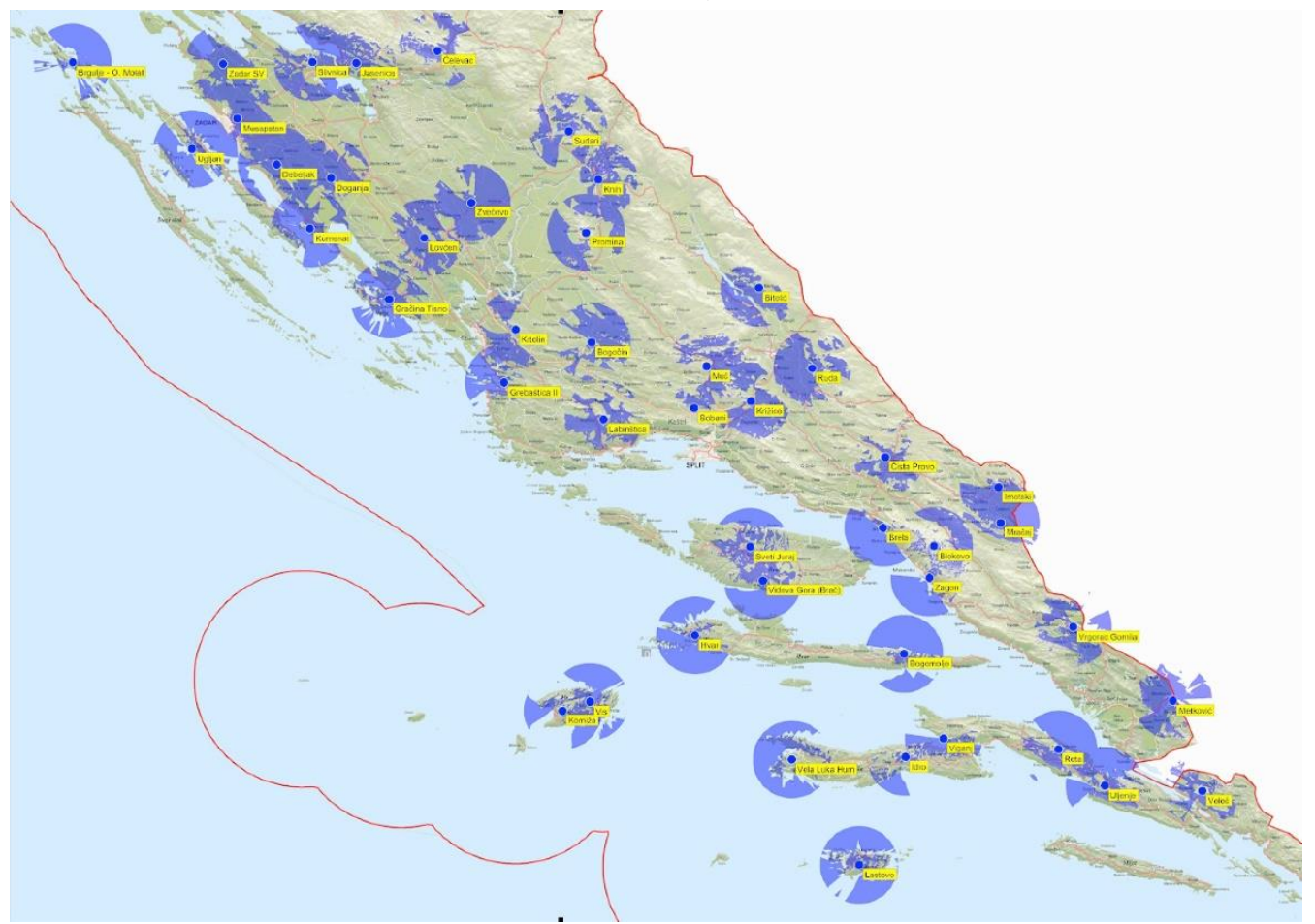

Figure 7 - Area covered by the forest fire video-communication system STRIBOR installed in summer 2018 thanks to a good practice of HOLISTIC experimental monitoring system

The system is installed in 4 coastal Counties in Croatia at the beginning of the fire season 2018. This system consists of 46 monitoring locations covering over $80 \%$ of the forests area in these, mostly endangered area.

The main leading idea embodied in specifications of HOLISTIC projects outcomes and results was using open standards and open interfaces. Following this made it possible to utilize the HOLISTC project outcomes in other crisis management situations. A Situation Headquarter was organized with the goal of being central headquarter for crisis situation management during summer fire season and put in use in the beginning of summer season of 2018. All information generated by HOLISTIC project outcomes are present at the centre and used in crisis situation for better decision making.

\section{Conclusion}

IPA Adriatic HOLISTIC project was more successful than we have planned in his preparation from 2007 to 2013. The project brought together almost all regions surrounding Adriatic Sea focused on the prevention and damages mitigation of the natural risks, with special focus on wildfires and earthquake risks, aiming for improvement, promotion and strengthening of institutional capabilities in implementing policies and actions for reducing the causes and potential start of natural breakdowns to improve reactions and intervention readiness to reduce the damage in areas of regions facing the Adriatic.

Implementation of HOLISTIC project activities combined in 9 WPs resulted in successful implementation of almost all planned outputs and results and achievement of main project objectives. Although number of partners was quite large (20 partners), communication between the partners went smoothly during the project implementation. Project outputs and results were very well communicated and disseminated via project Website, Facebook, YouTube and other media channels, as well as via different seminars and workshops. Last but not least the HOLISTIC project has initiated a major 
breakdown in implementation of advanced ICT technologies in wildfire fighting, at least in eastern part of Adriatic. Due to the successful implementation of HOLISTIC pilot projects, in 2018, Croatian Forests invested in expansion of terrestrial wildfire video based monitoring with 46 new locations and equipment of 4 monitoring centres, integrated with wildfire behavior and spread simulation systems and advanced site specific wildfire risk index and eruption fire risk index estimation.

\section{References}

(HOLISTIC, 2013) HOLISTIC Project official Web site, http://www.adriaholistic.eu

(Stipaničev et al, 2007) Stipaničev, D., Hrastnik, B., Vujčić, R. Holistic Approach to Forest Fire Protection in Split and Dalmatia County of Croatia // Wildfire 2007 Int. Conference. Sevilla, Spain, 2007 


\title{
Modelling of the rate of fire spread in heterogeneous fuel beds based on experimental data
}

\author{
Miguel Almeida ${ }^{1 *}$; Domingos Xavier Viegas ${ }^{1,2}$; Filipe Mendonça ${ }^{2}$; Cinzia Fois ${ }^{2}$; Abel Lopes ${ }^{2}$ \\ ${ }^{1}$ Forest Fire Research Centre (CEIF), ADAI-LAETA, University of Coimbra, 3030 - 289 \\ Coimbra, \{miguelalmeida@adai.pt*\} \\ ${ }^{2}$ Departamento de Engenharia Mecânica, Faculdade de Ciências e Tecnologia da Universidade \\ de Coimbra. Pólo II da Universidade de Coimbra. Rua Luís Reis Santos. 3030-788 Coimbra
}

\begin{abstract}
195 laboratory tests were performed to analyse the effect of either slope and wind in the fire spreading in mixed fuel beds composed by one live fuel and one dead fuel - live Pinus pinaster needles and straw were the fuels used, respectively.

Several models were built by other researchers to predict the fire spread in mixture fuel beds. Many of these models were produced using data achieved for conditions of no wind nor slope. In these tests, the effect of the airflow/wind and the effect of slope in the fire spread in mixed fuel beds was analysed. It was found that the presence of wind or slope do not clearly affect the value of the critical mass fraction $\mathrm{xc}$ that correspond to the minimum percentage of the dead fuel required to sustain the fire propagation.

The experimental results were modelled using exponential decay law applied to mixed fuel beds and the concept of degree of curing. These models show a good fit to the experimental results hereby presented so they can be extended to conditions of wind and slope.

In the modelling of surface forest fire spread, the prediction of the rate of spread (ROS) of a fire front, or of part of it, is the main goal that is attempted in order to be able to estimate the advance of the fire front in the course of time. It is commonly accepted that the ROS at a given section of the fire perimeter depends on the fuel bed properties, local topography and atmospheric conditions, namely air flow intensity and direction (e.g. Linn et al., 2007; Cavard et al., 2015). Although this concept can be challenged in several situations when the dynamic behaviour of the fire changes its environment and modifies its ROS properties (Hilton et al., 2016). In this work, we shall assume, as it is commonly done, that fire spreads in a quasi-steady state and that average values of the ROS can be established and determined at least during short periods of time. This work is a follow up of previous works on ROS in heterogeneous fuel beds (Viegas et al., 2010 and 2013) performed by some of the authors of the present publication.
\end{abstract}

Keywords: combustibility, fuel mixtures, heterogeneous fuels, moisture content, rate of spread

\section{Introduction}

In the modelling of surface forest fire spread, the prediction of the rate of spread (ROS) of a fire front, or of part of it, is the main goal that is attempted in order to be able to estimate the advance of the fire front in the course of time. It is commonly accepted that the ROS at a given section of the fire perimeter depends on the fuel bed properties, local topography and atmospheric conditions, namely air flow intensity and direction (e.g. Linn et al., 2007; Cavard et al., 2015). However, this concept can be challenged in several situations when the dynamic behaviour of the fire changes its environment and modifies its ROS properties (Hilton et al., 2016). In this work, we shall assume, as it is commonly done, that fire spreads in a quasi-steady state and that average values of the ROS can be established and determined at least during short periods of time. This work is a follow up of previous works on ROS in heterogeneous fuel beds (Viegas et al., 2010 and 2013) performed by some of the authors of the present publication.

Almost 200 experimental tests were carried out aiming at the determination of the ROS in 
heterogenous fuel beds composed by two different fuels - one fuel of higher combustibility (e.g, dead fuel) and other fuel of lower combustibility (e.g., live fuel). The concept of degree of curing (Anderson et al., 2005) of the fuel bed was considered. Properties such as the fuel moisture content, the slope and the air flow velocity were used as independent variables. The effect of the mentioned properties on the ROS was evaluated for sequences of tests in which the fraction $x$ of the higher combustibility fuel was varied from a fuel bed uniquely composed by the higher combustibility fuel $(x=1)$ to a fuel bed composed by the larger fraction of lower combustibility fuel $(1-x)$ from which the combustion was no longer sustained. This threshold of $x$ below which fire stops spreading was designated by critical mass fraction $x_{c}$. The fuels used in this work were straw and dead and live Pinus pinaster needles. Tests were performed for a range of airflow velocity from 0 to $4 \mathrm{~m} \cdot \mathrm{s}^{-1}$ and slope up to $40^{\circ}$.

The experimental results were modelled following the same approach of Viegas et al. (2013) and it was found that the exponential decay law can be used with reasonable accuracy to estimate the ROS of a fire front for the range of the parameters analyzed.

\section{Methodology}

The fuel beds were composed by straw or by a mixture of straw and live Pinus pinaster needles. The straw was obtained directly from the producer and thus it was collected during the later Spring or during the Summer, much time before the experiments. Regarding the pine needles, several branches of diversified pine trees were collected in the field in Lousã-Coimbra no more than two days before the experiments. In the day of the experiments the needles were detached from the pine branches. The storage of the straw, the pine branches and needles was made in the laboratory at a temperature around $20^{\circ} \mathrm{C}$ and relative humidity of about $45 \%$.

The fuel moisture content was determined using a moisture analyser $(A \& D M X-50$ resolution $0.01 \% \operatorname{Max}=51 \mathrm{~g}$ ), previously calibrated by the gravimetric method, exposing a sample of the fuel at $105^{\circ} \mathrm{C}$ during 15 minutes. After the determination of the $F M C$, the amount of each fuel was weighted according the specifications of the test in order to have a total fuel load of $0.8 \mathrm{~kg} \cdot \mathrm{m}^{-2}$ on a dry basis. The two amounts of fuels were mixed in a box taking care not to crumble the fuel particles and then the mixed fuels were homogeneously distributed in the combustion table. In the tests using uniquely straw the mixture was not performed and the fuel was distributed directly in the fuel bed after being weighted.

Every $20 \mathrm{~cm}$ of the fuel bed, cotton threads were stretched transversally to the expected fire spread. This procedure aimed at the determination of the rate of spread by the elapsed time between the cut by the fire of two consecutive threads. The linear ignition was carried out using a woollen yarn soaked in a mixture of gasoline and diesel. The woollen yarn was extended in the beginning of the fuel bed, transversally to the expected fire spread, and than ignited to produce a fire front that spread to the fuel bed. In the slope effect tests, the slope was set before the ignition. In the airflow effect tests, the airflow turned on immediately after the ignition. The tests were considered finished when the fire stopped propagating, either because it reached the end of the fuel bed or because the higher fraction of live fuel, and consequently the higher value of FMC, did not allow a sustainable fire spread.

The slope effect tests were carried out in a combustion table with a dimension of $2.95 \times 2.95 \mathrm{~m}^{2}$ allowing a variation of the slope angle in the direction of the intended fire spread. The fuel beds invariably had a dimension of $1.0 \mathrm{~m}$ width and $2.0 \mathrm{~m}$ length. The slope angles varied from $0^{\circ}$ to $40^{\circ}$. The airflow effect tests were carried out in the wind combustion tunnel existing in the Forest Fires Studies laboratory of ADAI. The fuel beds' size was of $2.0 \mathrm{~m}$ width and $6.0 \mathrm{~m}$ length and the airflow velocity varied from $0 \mathrm{~m} \cdot \mathrm{s}^{-1}$ to $4 \mathrm{~m} \cdot \mathrm{s}^{-1}$.

Since the tests were carried out in different days with slightly different conditions, the resulting data were harmonized aiming at a more accurate comparison. The harmonization of the results was based in the designated "reference tests" using the same methodology described in Viegas et al. (2013). The 
reference tests carried out in a combustion table of $1 \mathrm{~m}^{2}$ area consist on tests with a fuel bed composed uniquely by $0.8 \mathrm{~kg} \cdot \mathrm{m}^{-2}$ of straw, in the absence of wind and slope. These rate of spread in the reference tests was measured using the same methodology based on cotton threads stretched previously described. Two reference tests were performed per day of experiments - one test during the morning and other test during the afternoon. The rate of spread obtained in the slope or airflow effect tests were dimensionless dividing the basic rate of spread of a test by the rate of spread obtained in the reference test performed in the same period of the day.

In Table 1 and Table 2 the parameters of the tests are presented. The acronym "SL" in the reference is used to specify the slope effect tests and "AF" is used to indicate the airflow effect tests.

Table 1 - Parameters of the series SL on the role of slope on the rate of spread $R$ of a linear fire line in a fuel bed composed by a mixture of live Pinus pinaster needles (LPP) and straw (ST) with different values of Fuel Moisture Content (FMC). Date is presented in the format yymmdd. The reference tests are highlighted in bold.

\begin{tabular}{|c|c|c|c|c|c|c|c|c|c|c|c|c|c|}
\hline Refer. & Day & $\begin{array}{c}F M C \\
S T(\%) \\
\end{array}$ & $\begin{array}{c}F M C \\
L P P(\%) \\
\end{array}$ & $\begin{array}{c}\text { Slope } \\
\left({ }^{\circ}\right)\end{array}$ & $x$ & $\begin{array}{c}R \\
\left(\mathrm{~cm} \cdot \mathrm{s}^{-1}\right)\end{array}$ & Refer. & Day & \begin{tabular}{|c|}
$F M C$ \\
$S T(\%)$ \\
\end{tabular} & $\begin{array}{c}F M C \\
L P P(\%) \\
\end{array}$ & $\begin{array}{c}\text { Slope } \\
\left({ }^{\circ}\right)\end{array}$ & $x$ & $\begin{array}{c}R \\
\left(\mathrm{~cm} \cdot \mathrm{s}^{-1}\right) \\
\end{array}$ \\
\hline SL01 & 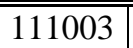 & $\begin{array}{l}10.13 \\
\end{array}$ & 110.08 & 0 & 1 & 0.93 & SL47 & 111228 & $\begin{array}{c}5.01 \\
\end{array}$ & $\begin{array}{l}79.53 \\
\end{array}$ & 0 & 1 & 1.14 \\
\hline SL02 & 111003 & 10.13 & 110.08 & 0 & 0.8 & 0.66 & SL48 & 111228 & 5.22 & 79.53 & 0 & \begin{tabular}{|l|}
0.6 \\
\end{tabular} & 0.66 \\
\hline SL03 & 111003 & 10.13 & 110.08 & 0 & 0.6 & 0.40 & SL49 & 111228 & 5.18 & 79.53 & 0 & 0.5 & 0.00 \\
\hline SL04 & 111003 & 10.13 & 110.08 & 0 & 0.5 & 0.00 & SL50 & 111228 & 5.22 & 79.53 & 0 & \begin{tabular}{|l|}
0.8 \\
\end{tabular} & 0.99 \\
\hline SL05 & 111003 & 10.13 & 110.08 & 0 & 1 & 0.99 & SL51 & 120102 & 4.97 & 79.53 & 0 & 1 & 1.03 \\
\hline SL06 & 111003 & 10.13 & 110.08 & 0 & 1 & 0.92 & SL52 & 120102 & 6.59 & 82.15 & 20 & 1 & 2.44 \\
\hline SL07 & 111011 & 4.82 & 52.44 & 0 & 1 & 0.53 & SL53 & 120102 & 6.37 & 82.15 & 0 & 1 & 1.25 \\
\hline SL08 & 111011 & 4.82 & 52.44 & 30 & 1 & 3.31 & SL54 & 120102 & 6.63 & 82.15 & 20 & \begin{tabular}{|l|}
0.8 \\
\end{tabular} & 1.51 \\
\hline SL09 & 111011 & 4.82 & 52.44 & 30 & 0.6 & 0.95 & SL55 & 120102 & 6.46 & 82.15 & 20 & \begin{tabular}{|l|}
0.6 \\
\end{tabular} & 0.94 \\
\hline SL10 & 111011 & 4.82 & 52.44 & 30 & 0.4 & 0.00 & SL56 & 120102 & 6.55 & 82.15 & 20 & \begin{tabular}{|l|}
0.5 \\
\end{tabular} & 0.73 \\
\hline SL11 & 111011 & 4.82 & 52.44 & 30 & 0.5 & 1.71 & SL57 & 120109 & 6.42 & 82.15 & 0 & 1 & 1.21 \\
\hline SL12 & 111011 & 4.82 & 52.44 & 30 & 0.8 & 2.24 & SL58 & 120109 & 7.38 & 108.77 & 40 & 1 & 6.35 \\
\hline SL13 & 111020 & 4.82 & 52.44 & 0 & 1 & 0.93 & SL59 & 120109 & 6.31 & 108.77 & 0 & 1 & 1.07 \\
\hline SL14 & 111020 & 13.77 & 143.31 & 20 & 1 & 1.94 & SL60 & 120109 & 6.72 & 108.77 & 40 & \begin{tabular}{|l|}
0.6 \\
\end{tabular} & 0.82 \\
\hline SL15 & 111020 & 13.77 & 143.31 & 0 & 1 & 0.59 & SL61 & 120109 & 6.84 & 108.77 & 40 & \begin{tabular}{|l|}
0.8 \\
\end{tabular} & 3.88 \\
\hline SL16 & 111020 & 13.77 & 143.31 & 20 & 0.8 & 0.45 & SL62 & 120109 & 8.70 & 108.77 & 0 & 1 & 1.13 \\
\hline SL17 & 111128 & 13.77 & 143.31 & 20 & 0.6 & 0.00 & SL63 & 120112 & 8.70 & 108.77 & 40 & \begin{tabular}{|l|}
0.5 \\
\end{tabular} & 0.58 \\
\hline SL18 & 111128 & 21.07 & 60.00 & 20 & 1 & 1.14 & SL64 & 120112 & 5.68 & 107.47 & 40 & 1 & 6.16 \\
\hline SL19 & 111128 & 21.07 & 60.00 & 0 & 1 & 0.55 & SL65 & 120112 & 5.39 & 107.47 & 0 & 1 & 1.24 \\
\hline SL20 & 111128 & 21.07 & 60.00 & 20 & 0.6 & 0.42 & SL66 & 120112 & 6.52 & 107.47 & 40 & \begin{tabular}{|l|}
0.6 \\
\end{tabular} & 1.58 \\
\hline SL21 & 111128 & 21.07 & 60.00 & 20 & 0.7 & 0.40 & SL67 & 120112 & 6.32 & 107.47 & 40 & \begin{tabular}{|l|}
0.5 \\
\end{tabular} & 0.69 \\
\hline SL22 & 111128 & 21.07 & 60.00 & 20 & 0.8 & 0.60 & SL68 & 120112 & 6.27 & 107.47 & 40 & \begin{tabular}{|l|}
0.8 \\
\end{tabular} & 3.58 \\
\hline SL23 & 111130 & 21.07 & 60.00 & 0 & 1 & 0.57 & SL69 & 120118 & 5.68 & 107.47 & 40 & \begin{tabular}{|l|}
0.4 \\
\end{tabular} & 0.72 \\
\hline SL24 & 111130 & 16.55 & 105.47 & 30 & 1 & 1.50 & SL70 & 120118 & 6.09 & 100.80 & 40 & 1 & 5.83 \\
\hline SL25 & 111130 & 16.55 & 105.47 & 0 & 1 & 0.54 & SL71 & 120118 & 5.35 & 100.80 & 0 & 1 & 1.25 \\
\hline SL26 & 111130 & 16.55 & 105.47 & 30 & 0.6 & 0.55 & SL72 & 120118 & 6.39 & 100.80 & 40 & \begin{tabular}{|l|}
0.8 \\
\end{tabular} & 3.57 \\
\hline SL27 & 111130 & 16.55 & 105.47 & 30 & 0.8 & 0.00 & SL73 & 120118 & 6.02 & 100.80 & 40 & \begin{tabular}{|l|}
0.5 \\
\end{tabular} & 0.43 \\
\hline SL28 & 111212 & 16.55 & 105.47 & 30 & 0.9 & 0.84 & SL74 & 120208 & 6.32 & 100.80 & 40 & \begin{tabular}{|l|}
0.7 \\
\end{tabular} & 2.53 \\
\hline SL29 & 111212 & 6.28 & 52.91 & 0 & 1 & 0.78 & SL75 & 120208 & 6.61 & 108.77 & 20 & 1 & 2.53 \\
\hline SL30 & 111212 & 6.08 & 52.91 & 0 & 1 & 0.86 & SL76 & 120208 & 5.14 & 108.77 & 0 & 1 & 1.24 \\
\hline SL31 & 111212 & 6.71 & 52.91 & 0 & 0.6 & 0.22 & SL77 & 120208 & 5.95 & 108.77 & 20 & \begin{tabular}{|l|}
0.8 \\
\end{tabular} & 1.92 \\
\hline SL32 & 111212 & 6.94 & 52.91 & 0 & 0.6 & 0.00 & SL78 & 120208 & 5.95 & 108.77 & 20 & \begin{tabular}{|l|}
0.5 \\
\end{tabular} & 0.89 \\
\hline SL33 & 111212 & 17.51 & 52.91 & 0 & 1 & 0.51 & SL79 & 120208 & 5.22 & 108.77 & 0 & 1 & 1.53 \\
\hline SL34 & 111215 & 17.51 & 52.91 & 0 & 0.8 & 0.26 & SL80 & 120210 & 5.87 & 108.77 & 20 & \begin{tabular}{|l|}
0.6 \\
\end{tabular} & 1.06 \\
\hline SL35 & 111215 & 8.62 & 73.01 & 20 & 1 & 1.93 & SL81 & 120210 & 4.96 & 80.51 & 30 & 1 & 3.79 \\
\hline SL36 & 111215 & 8.22 & 73.01 & 0 & 1 & 1.06 & SL82 & 120210 & 4.75 & 80.51 & 0 & 1 & 0.92 \\
\hline SL37 & 111215 & 8.54 & 73.01 & 20 & 0.6 & 0.57 & SL83 & 120210 & 4.96 & 80.51 & 30 & 0.5 & 0.66 \\
\hline
\end{tabular}




\begin{tabular}{|c|c|c|c|c|c|c||c|c|c|c|c|c|c|}
\hline SL38 & 111215 & 8.62 & 73.01 & 20 & 0.7 & 0.93 & SL84 & 120210 & 5.15 & 80.51 & 30 & 0.6 & 1.05 \\
\hline SL39 & 111215 & 8.98 & 73.01 & 20 & 0.8 & 0.98 & SL85 & 120210 & 4.75 & 80.51 & 0 & 1 & 0.97 \\
\hline SL40 & 111219 & 8.38 & 73.01 & 0 & 1 & 1.07 & SL86 & 120214 & 5.06 & 80.51 & 30 & 0.4 & 0.65 \\
\hline SL41 & 111219 & 5.58 & 94.55 & 30 & 1 & 3.62 & SL87 & 120214 & 8.15 & 65.02 & 0 & 1 & 0.85 \\
\hline SL42 & 111219 & 5.35 & 94.55 & 0 & 1 & 1.26 & SL88 & 120214 & 7.16 & 65.02 & 0 & 0.5 & 0.00 \\
\hline SL43 & 111219 & 5.96 & 94.55 & 30 & 0.7 & 1.91 & SL89 & 120214 & 7.16 & 65.02 & 0 & 0.6 & 0.56 \\
\hline SL44 & 111219 & 5.77 & 94.55 & 30 & 0.6 & 0.85 & SL90 & 120214 & 7.63 & 65.02 & 0 & 0.8 & 0.72 \\
\hline SL45 & 111228 & 5.58 & 94.55 & 30 & 0.5 & 0.77 & SL91 & 120214 & 6.74 & 65.02 & 0 & 1 & 0.79 \\
\hline SL46 & 111228 & 5.39 & 79.53 & 0 & 1 & 1.16 & & & & & & & \\
\hline
\end{tabular}

Table 2 - Parameters of the series $A F$ on the role of airflow on the rate of spread $R$ of a linear fire line in a fuel bed composed by a mixture of live Pinus pinaster needles (LPP) and straw (ST) with different values of fuel moisture content (FMC). Date is presented in the format yymmdd. The reference tests are highlighted in bold.

\begin{tabular}{|c|c|c|c|c|c|c|c|c|c|c|c|c|c|}
\hline Refer. & Day & $\begin{array}{c}F M C \\
S T(\%)\end{array}$ & $\begin{array}{l}F M C \\
L P P \\
(\%)\end{array}$ & $\begin{array}{c}\boldsymbol{U} \\
\left(\boldsymbol{m} \cdot \mathrm{s}^{-1}\right)\end{array}$ & $x$ & $\begin{array}{c}R \\
\left(\mathrm{~cm} \cdot \mathrm{s}^{-1}\right)\end{array}$ & Refer. & Day & $\begin{array}{c}\text { FMC } \\
S T \\
(\%)\end{array}$ & $\begin{array}{c}F M C \\
L P P \\
(\%)\end{array}$ & $\begin{array}{c}\boldsymbol{U} \\
\left(\boldsymbol{m} \cdot \mathrm{s}^{-1}\right)\end{array}$ & $x$ & $\begin{array}{c}R \\
\left(\mathrm{~cm} \cdot \mathrm{s}^{-1}\right)\end{array}$ \\
\hline AF001 & 130319 & 20,10 & --- & 1 & 1 & 0,91 & AF053 & 130626 & 11,10 & 65,50 & 3 & 0,5 & 0,72 \\
\hline AF002 & 130319 & 20,19 & --- & 0 & 1 & 0,52 & AF054 & 130626 & 9,40 & --- & 0 & 1 & 0,98 \\
\hline AF003 & 130417 & 15,60 & --- & 1 & 1 & 1,42 & AF055 & 130626 & 11,10 & --- & 0 & 1 & 1,07 \\
\hline AF004 & 130417 & 15,60 & 73,61 & 1 & 0,8 & 0,83 & AF056 & 130627 & 10.00 & $\begin{array}{l}94,90 \\
\end{array}$ & 3 & 0,4 & 0,00 \\
\hline AF005 & 130417 & 11,48 & 51,51 & 1 & 0,7 & 1,10 & \begin{tabular}{|l} 
AF057 \\
\end{tabular} & 130627 & 10.00 & 94,90 & 2 & 0,4 & 0,00 \\
\hline AF006 & 130417 & 15,60 & --- & 0 & 1 & 0,62 & \begin{tabular}{|l|} 
AF058 \\
\end{tabular} & 130627 & 9,10 & 112,70 & 2 & 0,8 & 1,20 \\
\hline AF007 & 130417 & 11,48 & --- & 0 & 1 & 0,70 & \begin{tabular}{|l|} 
AF059 \\
\end{tabular} & 130627 & 10,20 & --- & 2 & 1 & 2,30 \\
\hline AF008 & 130417 & 14,10 & --- & 1,5 & 1 & 1,71 & AF060 & 130627 & 10.00 & --- & 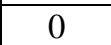 & 1 & 0,83 \\
\hline AF009 & 130418 & 14,10 & 94,17 & 1,5 & 0,8 & 0 & \begin{tabular}{|l|} 
AF061 \\
\end{tabular} & 130627 & 9,29 & --- & 0 & 1 & 0,70 \\
\hline AF010 & 130418 & 11,60 & 94,17 & 1,5 & 0,6 & 0,00 & \begin{tabular}{|l} 
AF062 \\
\end{tabular} & 130703 & 15,70 & 49,25 & 2 & 0,6 & 0,54 \\
\hline AF011 & 130418 & 14,10 & --- & 0 & 1 & 0,50 & \begin{tabular}{|l|l} 
AF063 \\
\end{tabular} & 130703 & 11,20 & 101,20 & 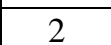 & 0,5 & 0,68 \\
\hline AF012 & 130419 & 11,60 & --- & 2 & 1 & 2,37 & \begin{tabular}{|l|l} 
AF064 \\
\end{tabular} & 130703 & 11,40 & 56,49 & 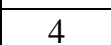 & 0,8 & 3,16 \\
\hline AF013 & 130419 & 13,20 & 58,22 & 2 & 0,8 & 0,57 & \begin{tabular}{|l} 
AF065 \\
\end{tabular} & 130703 & 15,70 & --- & 0 & 1 & 0,89 \\
\hline AF014 & 130419 & 9,50 & 85,52 & 2 & 0,7 & 0,00 & AF066 & 130703 & 11,20 & --- & 0 & 1 & 0,95 \\
\hline AF015 & 130419 & 9,50 & 85,52 & 1,5 & 0,7 & 1,81 & AF067 & 130705 & 9,80 & --- & 4 & 1 & 6,38 \\
\hline AF016 & 130419 & 13,20 & --- & 0 & 1 & 0,60 & \begin{tabular}{|l} 
AF068 \\
\end{tabular} & 130705 & 8,90 & 81,40 & 4 & 0,6 & 0 \\
\hline AF017 & 130420 & \begin{tabular}{|l|}
11,80 \\
\end{tabular} & --- & 1 & 1 & 1,48 & \begin{tabular}{|l} 
AF069 \\
\end{tabular} & 130705 & 9,52 & 88,30 & 4 & 0,7 & 1,33 \\
\hline AF018 & 130420 & 11,80 & 69,70 & 1 & 0,8 & 0,57 & AF070 & 130705 & 9,80 & --- & 0 & 1 & 1,24 \\
\hline AF019 & 130420 & 9,29 & 73.00 & 1 & 0,6 & 0,78 & AF071 & 130705 & 9,50 & --- & 0 & 1 & 0,93 \\
\hline AF020 & 130420 & 11,80 & --- & 0 & 1 & 0,85 & AF072 & 130709 & 9,70 & --- & 4 & 1 & 3,52 \\
\hline AF021 & 130423 & 12,70 & 56,49 & 2 & 0,4 & 0,00 & AF073 & 130709 & 10,90 & 93,70 & 4 & 0,8 & 2,81 \\
\hline AF022 & 130423 & 12,70 & 67,70 & 1 & 0,5 & 0,00 & \begin{tabular}{|l} 
AF074 \\
\end{tabular} & 130709 & 8,90 & 86,90 & 4 & 0,7 & 1,72 \\
\hline AF023 & 130423 & 12,70 & 67,70 & 2 & 0,5 & 0,00 & \begin{tabular}{|l} 
AF075 \\
\end{tabular} & 130709 & 9,70 & --- & 0 & 1 & 1,01 \\
\hline AF024 & 130603 & 12,70 & --- & 0 & 1 & 0,82 & \begin{tabular}{|l} 
AF076 \\
\end{tabular} & 130709 & 8,90 & --- & 0 & 1 & 1,27 \\
\hline AF025 & 130603 & $\begin{array}{l}11, \\
\end{array}$ & --- & 1,5 & 1 & 2, & \begin{tabular}{|l} 
AF077 \\
\end{tabular} & 130716 & 12,80 & --- & 3 & 1 & 2,49 \\
\hline AF026 & 130603 & 10 & 85,52 & 1 , & 0,8 & 1 , & AF078 & 130716 & 10,90 & 72,70 & 3 & 0,7 & 66 \\
\hline AF027 & 130603 & 9,40 & 90,10 & 1,5 & 0,6 & 0,00 & \begin{tabular}{|l} 
AF079 \\
\end{tabular} & 130716 & 10,90 & 78,50 & 3 & 0,6 & 0,00 \\
\hline AF028 & 130603 & 9,40 & 90,10 & 1,5 & 0,7 & 1,15 & \begin{tabular}{|l} 
AF080 \\
\end{tabular} & 130716 & 12,8 & --- & 0 & 1 & 0,63 \\
\hline AF029 & 130603 & 11,10 & -- & 0 & 1 & 0,88 & \begin{tabular}{|l} 
AF081 \\
\end{tabular} & 130716 & 10,90 & --- & 0 & 1 & 1,10 \\
\hline AF030 & 130603 & 9,40 & --- & 0 & 1 & 0,93 & AF082 & 130717 & 10,80 & --- & 1 & 1 & 1,16 \\
\hline AF031 & 130604 & 10.00 & --- & 2 & 1 & 2,88 & \begin{tabular}{|l} 
AF083 \\
\end{tabular} & 130717 & 10,80 & 94,90 & 1 & 0,8 & 0,79 \\
\hline AF032 & 130604 & 10.00 & 73.00 & 2 & 0,8 & 2,89 & \begin{tabular}{|l} 
AF084 \\
\end{tabular} & 130717 & 10,80 & 106,10 & 1 & 0,6 & 0,00 \\
\hline AF033 & 130604 & 8,81 & 73.00 & 2 & 0,6 & 1,46 & \begin{tabular}{|l} 
AF085 \\
\end{tabular} & 130717 & 10,80 & 106,10 & 4 & 0,6 & 0,00 \\
\hline AF034 & 130604 & 8,81 & 75,40 & 2 & 0,5 & 0,89 & \begin{tabular}{|l} 
AF086 \\
\end{tabular} & 130717 & 10,80 & --- & 0 & 1 & 0,89 \\
\hline AF035 & 130604 & 10.00 & --- & 0 & 1 & 1,03 & \begin{tabular}{|l} 
AF087 \\
\end{tabular} & 130717 & 10,80 & --- & 0 & 1 & 0,90 \\
\hline
\end{tabular}




\begin{tabular}{|c|c|c|c|c|c|c||c|c|c|c|c|c|c|}
\hline AF036 & 130604 & 8,81 & --- & 0 & 1 & 0,90 & AF088 & 130718 & 11,50 & --- & 4 & 1 & 3,84 \\
\hline AF037 & 130605 & 10,8 & --- & 3 & 1 & 4,50 & AF089 & 130718 & 10,80 & --- & 2 & 1 & 2,34 \\
\hline AF038 & 130605 & 10,80 & 100,10 & 3 & 0,8 & 1,88 & AF090 & 130718 & 10,10 & 129,80 & 2 & 0,7 & 0,84 \\
\hline AF039 & 130605 & 13,80 & 75,70 & 3 & 0,6 & 0,00 & AF091 & 130718 & 12,90 & 67,20 & 2 & 0,6 & 1,00 \\
\hline AF040 & 130605 & 13,80 & 75,70 & 1,5 & 0,6 & 0,00 & AF092 & 130718 & 12,90 & -- & 0 & 1 & 0,78 \\
\hline AF041 & 130605 & 13,80 & 75,70 & 3 & 0,7 & 0,55 & AF093 & 130718 & 10,10 & --- & 0 & 1 & 1,02 \\
\hline AF042 & 130605 & 10,80 & --- & 0 & 1 & 1,02 & AF094 & 130723 & 12,70 & 73,60 & 3 & 0,6 & 0,00 \\
\hline AF043 & 130605 & 13,80 & --- & 0 & 1 & 0,80 & AF095 & 130723 & 12,80 & 85,80 & 3 & 0,7 & 2,45 \\
\hline AF044 & 130612 & 11,10 & --- & 2 & 1 & 2,29 & AF096 & 130723 & 11,60 & --- & 3 & 1 & 4,81 \\
\hline AF045 & 130612 & 13,20 & 106,60 & 2 & 0,8 & 1,24 & AF097 & 130723 & 11,60 & --- & 1 & 1 & 1,34 \\
\hline AF046 & 130612 & 13,20 & 86,50 & 2 & 0,6 & 0,00 & AF098 & 130723 & 12,70 & --- & 0 & 1 & 0,84 \\
\hline AF047 & 130612 & 13,20 & 108,30 & 2 & 0,7 & 0,80 & AF099 & 130725 & 13,80 & --- & 1 & 1 & 0,70 \\
\hline AF048 & 130612 & 11,10 & --- & 0 & 1 & 0,67 & AF100 & 130725 & 13,80 & 84,50 & 1 & 0,7 & 0,00 \\
\hline AF049 & 130612 & 13,20 & --- & 0 & 1 & 0,83 & AF101 & 130725 & 9,40 & 82,80 & 1 & 0,75 & 0,73 \\
\hline AF050 & 130626 & 9,40 & --- & 3 & 1 & 4,48 & AF102 & 130725 & 11,80 & --- & 4 & 1 & 3,87 \\
\hline AF051 & 130626 & 11,10 & 68,60 & 3 & 0,8 & 2,27 & AF103 & 130725 & 13,80 & --- & 0 & 1 & 0,82 \\
\hline AF052 & 130626 & 11,10 & 65,50 & 3 & 0,6 & 1,26 & AF104 & 130725 & 9,40 & -- & 0 & 1 & 0,85 \\
\hline
\end{tabular}

\section{Results and discussion}

\subsection{Rate of spread and critical mass fraction}

In this section, the rate of spread will be analysed considering it in the dimensional or dimensionless form. In Figure 1 the rate of spread $\mathrm{R}$ directly obtained from the experiments as a function of the percentage of straw $\mathrm{x}$ in the mixture of the fuels is presented for the series of airflow effect tests (Figure 1a) and for the slope effect tests (Figure 1b). The lines in the plots correspond to the linear tendency curves for each class of airflow velocity or slope demonstrating the effect of the airflow velocity and slope in the rate of spread. The tendency lines also allow the analysis of the critical mass fraction $x_{c}$ i.e., the fraction of the fuel with lower combustibility (straw) from which the combustion is no longer sustained - the final part of this section will be dedicated to the analysis of $x_{c}$.

(a)

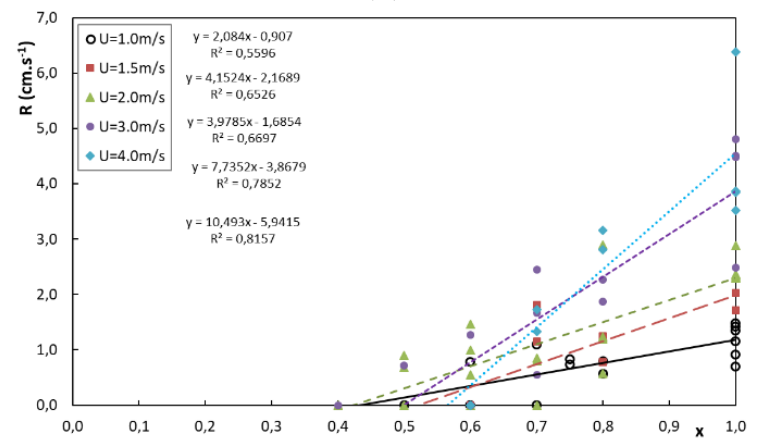

(b)

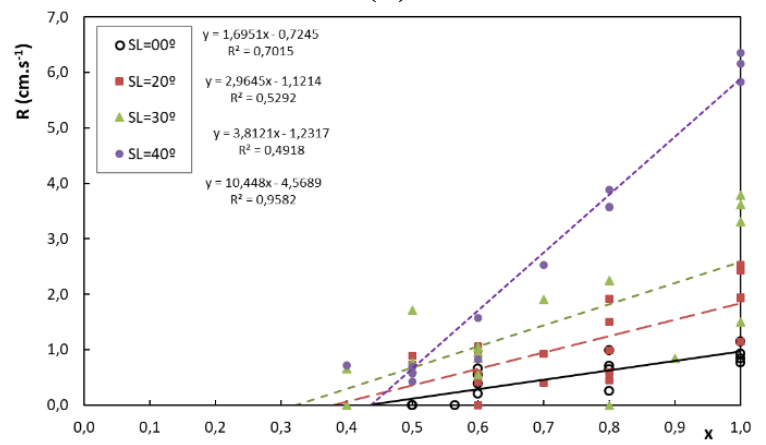

Figure 1 - Rate of spread (R) of the fire front as a function of the mass fraction ( $x$ ) of the dead fuel (straw): a) airflow effect tests; b) slope effect tests.

The dimensionless rate of spread $R^{\prime}$ presented in Figure 2 is determined by the quotient between the basic rate of spread obtained in the ordinary tests (airflow or slope effect tests) and the rate of spread obtained in the reference tests (Equation 1), providing harmonized results allowing a more accurate data analysis.

$$
R^{\prime}=R / R O \quad[E q .1]
$$


(a)

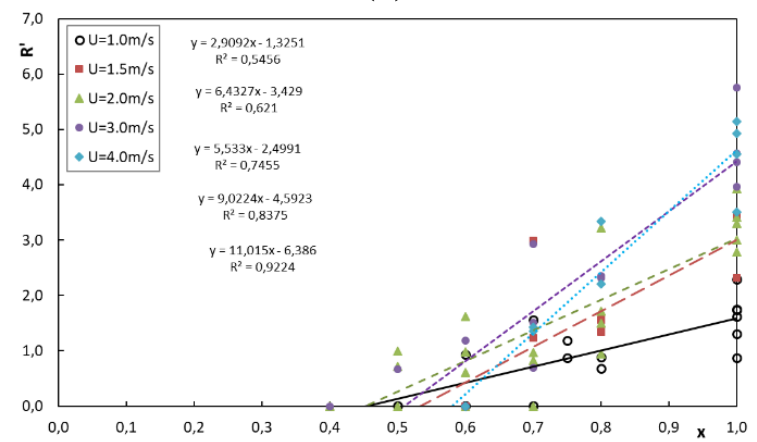

(b)

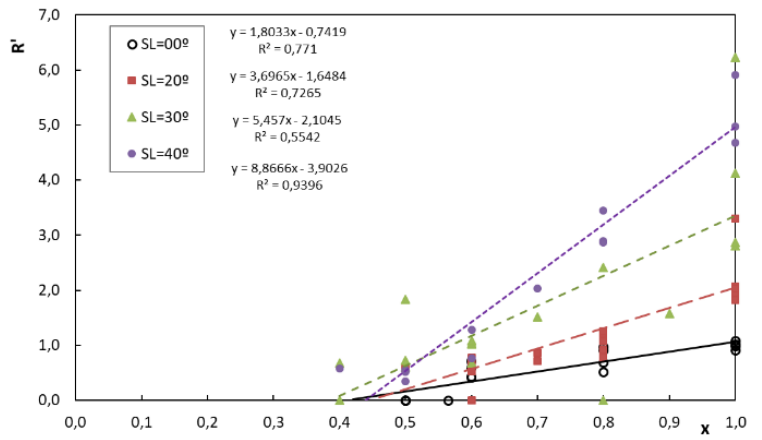

Figure 2 - Non-Dimensional rate of spread $\left(R / R_{0}\right)$ of the fire front as a function of the mass fraction $(x)$ of the dead fuel (straw): a) airflow effect tests; b) slope effect tests.

In Figure 3, the normalized rate of spread $R^{\prime \prime}$ is presented. $R^{\prime \prime}$ is determined by the quotient between the basic rate of spread $R$ and the rate of spread $R_{l}$ obtained in tests with fuel beds uniquely composed by straw $(x=1)$ with the same conditions of slope or airflow velocity (Equation 2$)$. Theoretically all the lines should intercept the point $\left(R^{\prime \prime}=1, x=1\right)$ but that was not observed due to the deviations in the results that led to a correlation coefficient $r^{2} \neq 1$.

$$
R^{\prime \prime}=R / R_{1}
$$

(a)

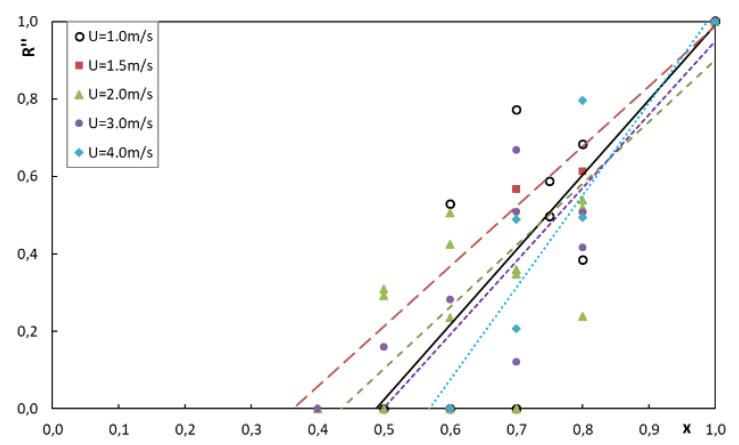

[Eq. 2]

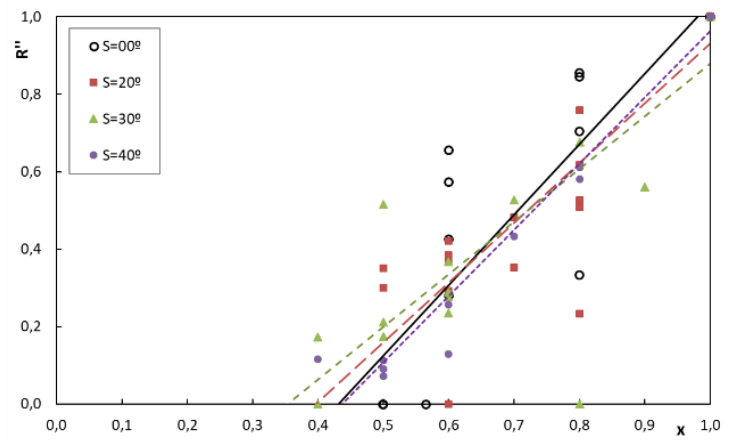

Figure 3 - Normalized rate of spread (R) of the fire front as a function of the mass fraction $(x)$ of the dead fuel (straw): a) airflow effect tests; b) slope effect tests.

The values presented in Table 3 are referred to the tendency lines showed in. Figure 1 to Figure 3. The right side of the table is referred to the critical mass factor $x_{c}$ analysed by two perspectives. The $x_{c}$ (linear) corresponds to the interception of the tendency line in the $\mathrm{x}$-axis for $y=0$. One value of $x_{c}$ was obtained for each form of the rate of $\operatorname{spread}\left(R, R^{\prime}\right.$ and $\left.R^{\prime}\right)$. The $x c$ (experimental) is based on the direct experimental results considering the range of $x_{c}$ for which the fire propagation was not sustained $\left(R=0 m \cdot s^{-1}\right)$ and tests with $x$ immediately above for which the fire propagation was observed $\left(R>0 m \cdot s^{-1}\right)$. 
Table 3 - Values the gradient " $b$ " of the tendency line $(y=a+b x)$ followed by the correlation coefficient $r^{2}$ between parentheses; critical mass fraction $x_{c}$ deducted from the linear tendency from the figures 1-3 for no fire propagation $\left(R / R^{\prime} / R^{\prime \prime}=0\right)$; range of $x_{c}$ obtained from the experimental data considering the percentage of straw $(x)$ when the fire front did not propagate $\left(R=0 m-s^{-1}\right)$ and the value of $x$ of the immediate test with fire propagation $\left(R>0 m-s^{-1}\right)$.

\begin{tabular}{|c|c|c|c|c|c|c|c|c|}
\hline & \multicolumn{3}{|c|}{$b\left(r^{2}\right)$} & \multicolumn{3}{|c|}{$\mathrm{x}_{\mathrm{c}}$ (linear) } & \multirow{2}{*}{$\begin{array}{l}\mathrm{x}_{\mathrm{c}}(\text { experimental }) \\
{[\mathrm{R}=0 ; \mathrm{R}>0] \mathrm{m} \cdot \mathrm{s}^{-1}}\end{array}$} \\
\hline & & $\mathrm{R}$ & $\mathrm{R}^{\prime}$ & $\mathrm{R}^{\prime \prime}$ & $\mathrm{R}$ & $\mathrm{R}^{\prime}$ & $\mathrm{R}^{\prime \prime}$ & \\
\hline \multirow{5}{*}{ 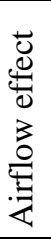 } & $\mathrm{U}=1.0 \mathrm{~m} \cdot \mathrm{s}^{-1}$ & $2.084(0.56)$ & $2.909(0.55)$ & $1.936(0.76)$ & 0.4 & 0.5 & 0.5 & {$[0.402 ; 0.348]$} \\
\hline & $\mathrm{U}=1.5 \mathrm{~m} \cdot \mathrm{s}^{-1}$ & $4.152(0.65)$ & $6.433(0.62)$ & $1.550(0.96)$ & 0.5 & 0.5 & 0.4 & {$[0.416 ; 0.330]$} \\
\hline & $\mathrm{U}=2.0 \mathrm{~m} \cdot \mathrm{s}^{-1}$ & $3.979(0.67)$ & $5.533(0.75)$ & $1.595(0.77)$ & 0.4 & 0.5 & 0.4 & {$[0.500 ; 0.492]$} \\
\hline & $\mathrm{U}=3.0 \mathrm{~m} \cdot \mathrm{s}^{-1}$ & $7.735(0.79)$ & $9.022(0.84)$ & $1.894(0.83)$ & 0.5 & 0.5 & 0.5 & {$[0.609 ; 0.383]$} \\
\hline & $\mathrm{U}=4.0 \mathrm{~m} \cdot \mathrm{s}^{-1}$ & $10.493(0.82)$ & $11.015(0.92)$ & $2.385(0.92)$ & 0.6 & 0.6 & 0.6 & {$[0.434 ; 0.327]$} \\
\hline \multirow{4}{*}{ 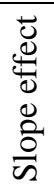 } & $\mathrm{SL}=0^{\circ}$ & $1.695(0.70)$ & $1.803(0.77)$ & $1.821(0.79)$ & 0.4 & 0.4 & 0.4 & {$[0.462 ; 0.269]$} \\
\hline & $\mathrm{SL}=20^{\circ}$ & $2.964(0.53)$ & $3.696(0.73)$ & $1.543(0.77)$ & 0.4 & 0.4 & 0.4 & {$[0.656 ; 0.509]$} \\
\hline & $\mathrm{SL}=30^{\circ}$ & $3.812(0.49)$ & $5.457(0.55)$ & $1.357(0.70)$ & 0.3 & 0.4 & 0.4 & {$[0.334 ; 0.503]$} \\
\hline & $\mathrm{SL}=40^{\circ}$ & $10.448(0.96)$ & $8.867(0.94)$ & $1.710(0.96)$ & 0.4 & 0.4 & 0.4 & {$[\mathrm{ND} ; 0.668]$} \\
\hline
\end{tabular}

As can be seen in the figures and table above, the increase of slope or the airflow velocity drives to higher values of the rate of spread either in the dimensional and dimensionless forms, as could be expect. This statement is most evident for values of higher values of $x$. When the percentage of straw is closer to the critical value $x_{c}$ for which the fire does not spread sustainably, the variations in the slope or airflow velocity do not drive to higher deviations in the rate of spread until $x=x_{c}$ that by definition implies a rate of spread equal to zero. This statement gains relevance as the value of $x_{c}$ is very similar, around 0.45 , to all the series of tests as can be seen in Table 3 and Table 4.

Table 4 - Average values (Av.) and Standard deviation $(S d)$ of the critical mass fraction values $x_{c}$ obtained for the airflow effect tests and slope effect tests, considering the tendency lines of figures 1 to 3.

\begin{tabular}{|c|c|c|c|c|c|c|}
\hline & \multicolumn{2}{|c|}{$\mathrm{R}$} & \multicolumn{2}{c|}{$\mathrm{R}^{\prime}$} & \multicolumn{2}{c|}{$\mathrm{R}^{\prime \prime}$} \\
\hline Airflow effect tests & 0.49 & 0.054 & 0.51 & 0.048 & 0.47 & 0.069 \\
\hline Slope effect tests & 0.39 & 0.045 & 0.42 & 0.024 & 0.40 & 0.034 \\
\hline
\end{tabular}

These results are very similar to the values of $x_{c}$ presented by Viegas et al. (2013) where a range of $0.3<x_{c}<0.5$ was found for tests with no slope and no airflow conditions. This similarity of results indicates that the critical values for fire propagation depends mainly on the fuel bed composition instead of external factor like the slope or the wind.

In Figure the range of $x$ values in which $x_{c}$ is included, presented in Table 4 as " $x_{c}$ (experimental)", are analysed as a function of the fuel moisture content (dry basis) of the mixture of fuels. The arrowhead in this figure indicates the upper limit of the $x_{c}$ (experimental) range, i.e. the values of the tests with lower $x$ with fire spread sustainably.

(a)

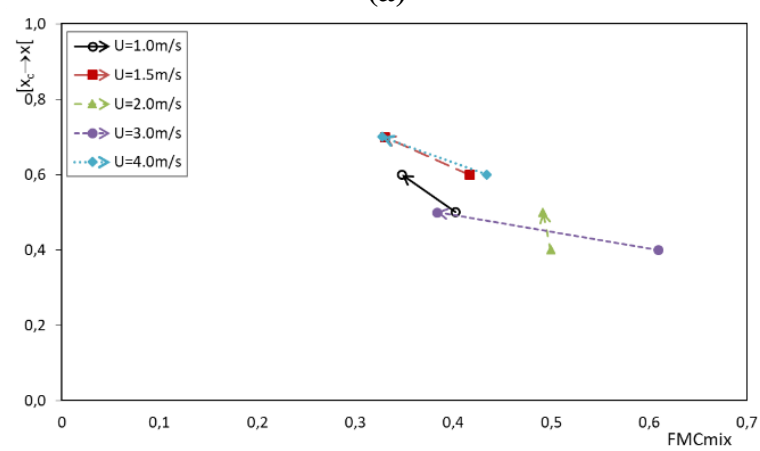

(b)

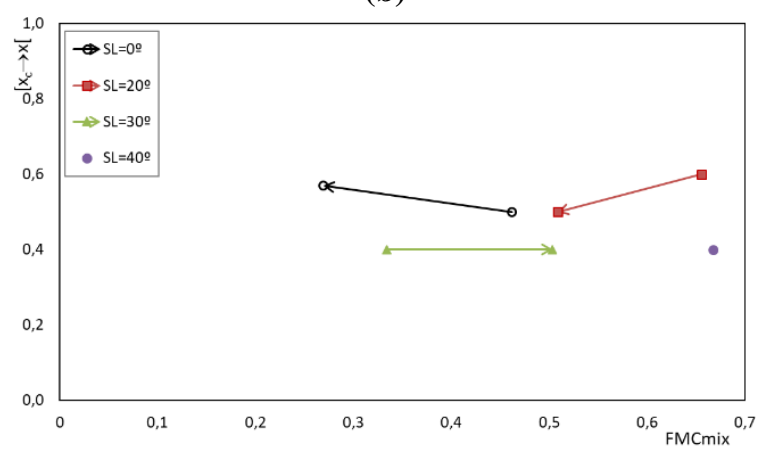

Figure 4 - Critical mass fraction as function of the fuel moisture content of the mixture of fuels for the airflow effect tests $(a)$ and slope effect tests $(b)$. The arrowhead indicates the tests with $R>0 m . s^{-1}$ and the arrow tail indicates the tests for which the fire propagation was not sustained $\left(R=0 \mathrm{~m} . \mathrm{s}^{-1}\right)$. As mentioned in Table, the lower limit of $x_{c}$ for $40^{\circ}$ slope was not determined. 
As could be expected, the decrease from $x$ to $x_{c}$ drives to a higher values of $F M C$ as the mass percentage of the dry fuel (straw) decreases relatively to the live fuel (live pine needles). In the slope effect tests, that was not so obvious with two tests $S L=30^{\circ}$ with $x=0.4$ presenting surprising results the test with $F M C=0.50$ led to a sustained fire propagation and the test with $F M C=0.33$ did not sustained the fire spread. A different result occurs also for $S L=20^{\circ}$ with a sustained combustion verified for $x=0.5$, while the value of $x=x_{c}=0.6$ was obtained for other experiments - this case was not so surprising as the $F M C$ value was much lower in the tests with sustained combustion. All these results show the high importance that the $F M C$ have in the definition of the critical mass value. However, the consistency of the $x_{c}$ range resulting from the large number of tests performed and the many other tests presented by Viegas et al. (2013), for different values of $F M C$, show that the $F M C$ is not the only factor affecting this parameter. The composition of the fuel bed with dead and alive fuels also have an important role in the fire propagation that can be explained by the different chemical composition of the fuels with different release of flammable volatile organic compounds during the burning. The presence of live fuels in relation to the dead fuels is associated to the degree of curing that will be analysed in the next section.

\subsection{Degree of curing}

The dimensionless normalized rate of spread $\mathrm{R}^{\prime \prime}$ was analysed as a function of the degree of curing C (\%) defined by Luke and McArthur (1978) and Cheney and Sullivan (1997, 2008) as the percentage of vegetation in grasslands that present physical damages or variations in colour related to its natural green state. Hereby, the degree of curing is highly dependent on the percentage of straw in the mixed fuel bed. This analysis is presented in Figure where the dotted line represents the experimental results obtained by Barber (1990), which was also used as a comparison term in Viegas et al. (2013). In this plots, two models for the determination of the degree of curing were used - the Formulation of Barber (1990) (Eq. 3) and the Formulation of Anderson (2005) (Eq. 4).

$$
\begin{gathered}
C(\%)=-6.295 \times 10^{-6} \times F M C^{3}+4.4 \times 10^{-3} \times F M C^{2}-1.0721 \times F M C+109.6758 \\
C(\%)=90 \times \exp \left(1.0439 \times 10^{-3} \times F M C^{1.335}\right)+10 \\
{[E q .}
\end{gathered}
$$

(a)

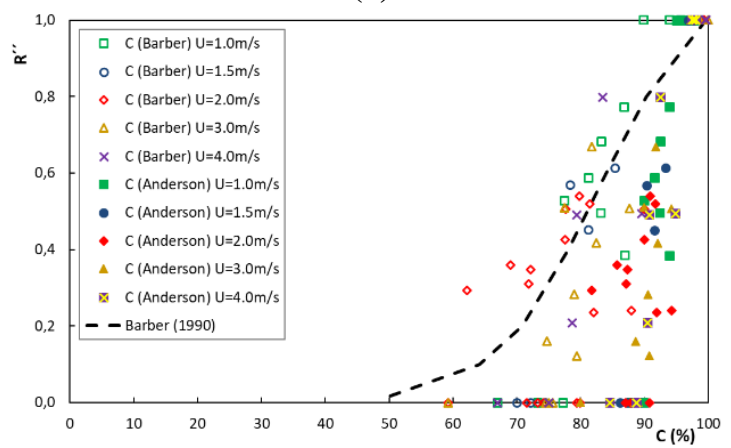

(b)

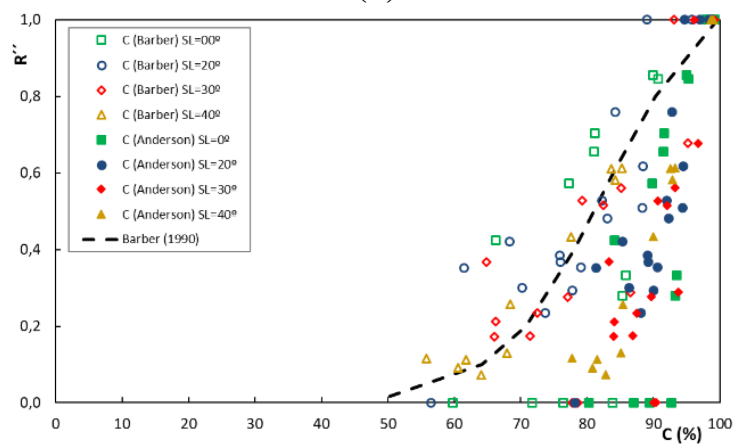

Figure 5 - Comparison between present results for dimensionless normalized rate of spread ( $\left.R^{\prime \prime}\right)$ hereby presented and those obtained by Barber (1990)(dotted line) as a function of degree of curing $C(\%)$, using the formulations proposed by Barber (Eq. 3) and Anderson et al. (2005) (Eq. 4): (a) airflow effect tests and (b) slope effect tests.

As can be observed the experimental results hereby presented and those obtained by Barber (1990) follow reasonably the same tendency. The formulation proposed by Anderson (Eq. 4) drives to values of $\mathrm{C}(\%)$ a little larger than those determined by the Formulation of Barber (Eq. 3) which fit better to the experimental results obtained by the same author. These were the same conclusions of Viegas et al. (2013). 


\subsection{Exponential Decay Law}

In Wilson (1990), an exponential decay model to describe the moisture content damping effect on fuel bed combustibility properties was proposed (Eq. 5 and Eq. 6) with $\xi$ as the parameter to be analysed and FMC $^{*}$ a reference value of the fuel bed moisture content. In Viegas et al. (2013) the parameter $\xi$ was the basic rate of spread of the mixed fuel bed with the definition presented in Eq. 7 with $\mathrm{R}^{*} 0$ as a reference value of the basic rate of spread of the mixture that corresponds to a value of $\mu=0$. In this section we will follow the same approach of Viegas et al. (2013).

$$
\begin{array}{ll}
\xi=\exp (\mu) & {[E q .5]} \\
\mu=F M C / F M C^{*} & {[E q .6]} \\
\xi=R 0_{0} / R^{*} & {[E q .7]}
\end{array}
$$

The plots of Figure were used to determine the values of " $a$ " and " $b$ " used by Viegas et al. (2013) to apply the exponential decay law according Eq. 8. In Table, the summary of the fuel bed moisture content damping law parameters is presented. These parameters were used to produce the plots of Figure .

$$
R=a \times \exp \left(-b \times F M C_{m i x}\right) \quad[E q .8]
$$

(a)

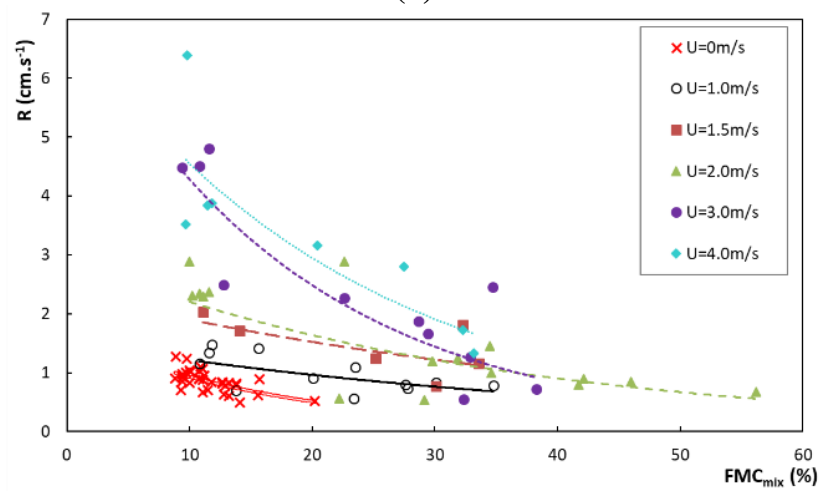

(b)

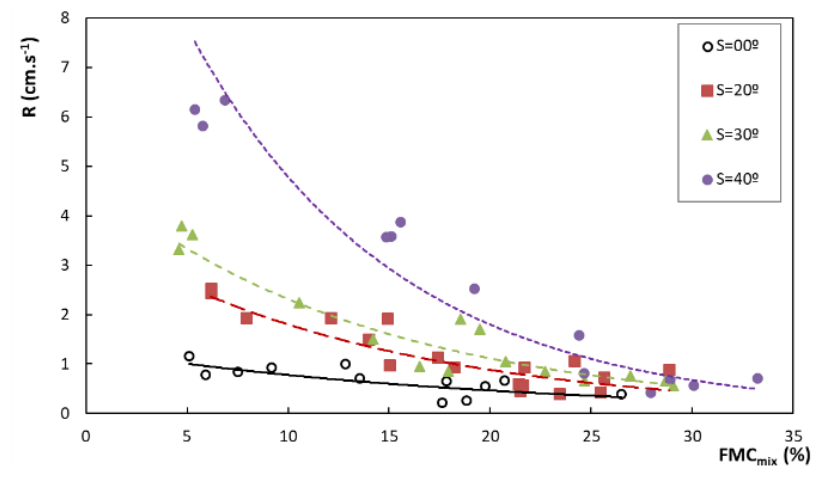

\begin{tabular}{|c|c|c|c|c|c|c|c|c|c|}
\hline \multicolumn{5}{|c|}{ Airflow effect tests } & \multicolumn{5}{|c|}{ Slope effect tests } \\
\hline $\mathrm{U}\left(\mathrm{m} \cdot \mathrm{s}^{-1}\right)$ & $\begin{array}{l}\mathrm{a}=\mathrm{R}^{*}{ }_{0} \\
\left(\mathrm{~cm} \cdot \mathrm{s}^{-1)}\right.\end{array}$ & b & $r^{2}$ & $\begin{array}{l}\mathrm{FMC}_{\text {mix }} \\
(\%)\end{array}$ & $\operatorname{SL}\left({ }^{\circ}\right)$ & $\begin{array}{c}\mathrm{a}=\mathrm{R}^{*}{ }_{0} \\
\left(\mathrm{~cm} \cdot \mathrm{s}^{-1}\right)\end{array}$ & b & $r^{2}$ & $\begin{array}{l}\mathrm{FMC}_{\text {mix }} \\
(\%)\end{array}$ \\
\hline 0 & 1.700 & 0.060 & 0.431 & 16.67 & 0 & 1.303 & 0.052 & 0.434 & 19.23 \\
\hline 1.0 & 1.533 & 0.023 & 0.378 & 43.48 & 20 & 3.681 & 0.072 & 0.676 & 13.89 \\
\hline 1.5 & 2.374 & 0.022 & 0.351 & 45.45 & 30 & 4.812 & 0.073 & 0.868 & 13.70 \\
\hline 2.0 & 2.968 & 0.030 & 0.543 & 33.33 & 40 & 12.744 & 0.098 & 0.903 & 10.20 \\
\hline 3.0 & 7.385 & 0.054 & 0.638 & 18.52 & & & & & \\
\hline 4.0 & 7.020 & 0.043 & 0.804 & 23.26 & & & & & \\
\hline
\end{tabular}

Figure 6 - Basic rate of spread $(R)$ as a function of the fuel moisture content of the mixture of fuels for the airflow effect tests (a) and the slope effect tests (b).

Table 5 - Summary of the fuel bed moisture content damping law parameters. 
(a)

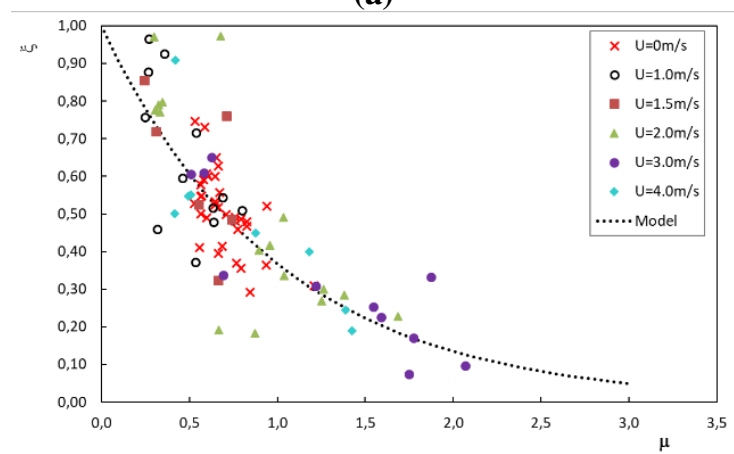

(b)

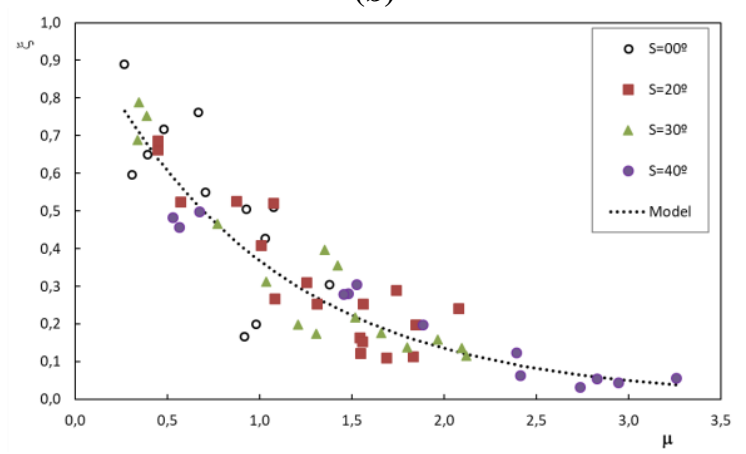

Figure 7 - Non-dimensional rate of spread decay ( $\xi$ ) of mixed fuel beds as a function of the ratio of fuel moisture contents FMC and FMC* (Eq. 6) for the airflow effect tests (a) and the slope effect tests (b). The dotted line corresponds to the model given by Eq. 5 .

As can be observed the exponential decay model fits well to the experimental results allowing a good estimation of the rate of spread in mixed fuel beds in conditions of wind or slope. It is important to highlight that the tests with no sustained fire propagation were not included in this analysis since in Viegas et al. (2013) this model is not applied for tests with $\mathrm{x} \leq \mathrm{x}_{\mathrm{c}}$.

\section{Conclusions}

195 tests were carried out to analyse the effect of slope and wind in the rate of spread in mixed fuel beds composed by one live fuel (live Pinus pinaster needles) with higher fuel moisture content and one dead fuel (straw) with lower FMC. The results clearly show that the increase of the straw percentage in the mixture drives to high values of the rate of spread. As could be expected, the increase of either slope and wind/airflow also lead to higher values of the basic rate of spread $R$ and for the harmonized rate of spread $R^{\prime}$. The hereby designated calibrated rate of spread $R$ ', did not show that tendency with the results following approximated values for the same fuel bed composition (same $x$ ).

These experiments showed that either the slope or the wind do not have an important role in the definition of the critical mass fraction parameter $x_{c}$, from which the fire does not spread sustainably, since all the sequence of tests performed resulted in values of $x_{c}$ very similar around 0.45 . These results are very consistent with the results of Viegas et al. (2013) which obtained an average value of $x_{c}=0.4$ for tests in mixed fuel beds with no wind and no slope.

The concept of the degree of curing $C(\%)$ was also analysed in this study and the formulations of Barber (1990) and Anderson (2005) were tested. Both formulations showed a good fitting to the experimental results.

The Exponential Decay Law described by Wilson (1990) and adapted by Viegas et al (2013) for mixed fuel beds was tested in these experiments with airflow and slope do not considering the tests where the fire did not propagate sustainably. A good fitting of this law to the experimental results was observed.

\section{List of acronyms}

$\begin{array}{ll}a, b & \text { Parameters of Equation } 8 \\ A v & \text { Average } \\ C(\%) & \text { Degree of curing in percentage } \\ F M C & \text { Fuel moisture content } \\ F M C_{m i x} & \text { Fuel moisture content of the fuel bed } \\ F M C^{*} & \text { Reference value of the fuel bed moisture content in Eq. } 6 \\ L P P & \text { Live Pinus pinaster needles }\end{array}$




$\begin{array}{ll}R & \text { Basic rate of spread } \\ R^{\prime} & \text { Harmonized rate of spread (Eq. 1) } \\ R^{\prime \prime} & \text { Normalized rate of spread (Eq. 2) } \\ R O S & \text { Rate of spread } \\ S d & \text { Standard deviation } \\ S L & \text { Slope } \\ S T & \text { Straw } \\ U & \text { Airflow velocity } \\ x & \text { Mass percentage of straw in the fuel bed } \\ x_{c} & \text { Critical mass value } \\ \xi & \text { Combustibility property in analysis } \\ \mu & \text { Ratio between FMC and FMC* }\end{array}$

\section{Acknowledgements}

The authors acknowledge the financial support given by Fundação para a Ciência e Tecnologia (FCT) through the project Project "Firewhirl - Vorticity Effects in Forest Fires" (Ref. PTDC/EMSENE/2530/2014). The first author work is financial supported by a funded project coordinated by FCT.

\section{References}

Anderson SAJ, Anderson WR, Hines F, Fountain A (2005). "Determination of field sampling methods for the assessment of curing levels in grasslands". Bushfire CRC Report. Project A1.4. Improved methods for the assessment and prediction of grassland curing, Australia. Available at http://www.bushfirecrc.com/projects/a14/grassland-curing

Barber JR (1990) "Monitoring the curing of grassland fire fuels in Victoria, Australia with sensors in satellites and aircraft".(Country Fire Authority: Melbourne)

Cavard X, Boucher JF and Bergeron Y (2015). "Vegetation and topography interact with weather to drive the spatial distribution of wildfires in the eastern boreal forest of Canada". International Journal of Wildland Fire 24(3) 391-406 http://dx.doi.org/10.1071/WF13128

Hilton JE, Miller C, Sharples JJ and Sullivan AL (2016). "Curvature effects in the dynamic propagation of wildfires". International Journal of Wildland Fire 25(12) 1238-1251 http://dx.doi.org/10.1071/WF16070

Linn R, Winterkamp J, Edminster C, Colman JJ and Smith WS (2007). "Coupled influences of topography and wind on wildland fire behaviour". International Journal of Wildland Fire 16(2) 183195 http://dx.doi.org/10.1071/WF06078

Viegas DX, Almeida M, Miranda A, Ribeiro LM (2010). "Linear model for spread rate and mass loss rate for mixed-size fuel beds". International Journal of Wildland Fire 19(5) 531-540 http://dx.doi.org/10.1071/WF09007

Viegas DX, Soares J and Almeida M (2013). "Combustibility of a mixture of live and dead fuel components". International Journal of Wildland Fire 22(7) 992-1002 http://dx.doi.org/10.1071/WF12031 


\title{
Monitoring of forest fire risk conditions in Portugal with NOAA/NESDIS satellite-based Vegetation Health Index
}

\author{
Lourdes Bugalho*1; Natália Camara ${ }^{1}$; F.N. Kogan ${ }^{2}$ \\ ${ }^{1}$ Instituto Português do Mar e da Atmosfera, Rua C do Aeroporto, Lisboa, 1749-077, Portugal. \\ \{lourdes.bugalho@ipma.pt*; natalia.camara@ipma.pt\} \\ ${ }^{2}$ NOAA/NESDIS Center for Satellite Applications and Research, Washington DC, USA
}

\begin{abstract}
NOAA/NESDIS satellite-based Vegetation Health Index (VHI) was applied to study wildfire favourable environmental conditions for period 2001-2017.VHI consists of two terms: Vegetation Condition Index (VCI), and Temperature Condition Index (TCI), which reflect vegetation moisture conditions and vegetation thermal conditions. Joint analysis of drought conditions (VHI $<40)$ and vegetation stress $(\mathrm{VCI}, \mathrm{TCI}<40)$ on the one hand and burned area and number of fires on the other hand showed, that $88 \%$ of big fires in Portugal have relation with droughts or vegetation stress.
\end{abstract}

Keywords: Forest Fire Risk monitorization, Vegetation Health Index, vegetation stress, droughts

\section{Intoduction}

In the Mediterranean region forest fires are usual phenomenon in conditions summer or hot and dry spring / autumn, being one of the severe natural disasters during the summer months. Portugal is one of the European countries most affected by wildfire. So it is very important to effectuate weather and environmental monitoring of wildfire risks conditions. Unfortunately, there are no regular observations of soil water content and density of meteorological stations is not enough for this purpose. At present time, remote sensing of earth surface from operational satellites have become powerful tools to realize fire risk monitoring. Thus, some satellite-based vegetation indices, like Normalized Difference Vegetation Index (NDVI) and Enhanced Vegetation Index (EVI), have been generally used to model forest fire danger (Illera, P. et al, 2007, Bisquert, Mar et al, 2014).

Fire favourable conditions and fire regime characteristics (burned area, frequency, and severity) are controlled by weather and climate. Numerous studies indicate the relationship between droughts and wild fires. Drought determines forest productivity and fuel state and thus affects fire intensity and severity.

In this work we intend to show the application of the NOAA/NESDIS satellite-based Vegetation Health Index (VHI) for monitoring of wildfire risk conditions in Portugal. During the last 20 years Vegetation Health ( $\mathrm{VH}$ ) indices method was developed, tested, validated and applied globally and regionally (https://www.star.nesdis.noaa.gov/smcd/emb/vci/VH/vh_ftp.php). The VHI represents the moisture-thermal vegetation health and combines two indices: Temperature Condition Index (TCI) and Vegetation Condition Index (VCI). The VCI reflects the vegetation moisture conditions and TCI reflects the vegetation thermal conditions. (Kogan 1997). VCI and TCI reflect vegetation response to weather changes, because climatic signals are removed, using climatic extremes. These indices were successfully used for monitoring droughts, environmentally-based vegetation stress, fire risk, soil saturation and other natural hazards around the world.This study is focused on the relationship of the VHI and its terms (VCI and TCI) to the most severe forest fires ( $\geq 1000 \mathrm{ha})$ in Portugal in period 20012017. 
Droughts events have important impact on the forest fires conditions, since precipitation deficit reduces soil and fuel moisture, that lead to ease ignition and spread of forest fire (Shoennagel et al.,2004, Jurdao et al.,2012).

The relationship between drought and forest fires conditions seems simple, but it is difficult analyse because the fire favourable conditions depend on human impact as well as environmental. Many of the causes of forest fires are results of human activities or negligence. On the other hand, the forest fires have an impact on the human habitat, altering type of the soil cover and vegetation, leading to degradation of the soils.

\section{Vegetation Healthy Index (VHI) and Droughts}

The NOAA/NESDIS Vegetation Health (VH) method is based on the properties of green vegetation to reflect sunlight and emit absorbed solar radiation.

Pigmentation of the plants leaves, chlorophyll, strongly absorbs light in a visible part (VIS) of the spectrum $(0.4$ to $0.7 \mu \mathrm{m})$ for use in the photosynthesis process. On the other hand, the cell structure of the leaves strongly reflects the light in part of the near infrared spectrum (NIR). Healthy and without water stress vegetation has a reduced VIS and enlarged NIR, resulting in higher NDVI. Healthy vegetation absorbs and emits less thermal IR radiation, resulting in lower BT and cooler canopy. Drought depresses vegetation greenness, decreases vigor and makes the canopy hot due to an increase in VIS (following chlorophyll depletion), decrease in NIR (following a drop in water content), a reduction of NDVI and an increase in thermal IR and BT values (Cracknell 1997; Kogan 2001).

NDVI and BT indices contain three environmental signals: ecosystem, climate and real-time weather (precipitation, temperature etc.). Unfortunately, the ecosystem/climate signal has much stronger contribution in the NDVI and BT values, compared to the weather. Therefore, in order to estimate weather impacts on land surface, the values of climate/ecosystem were removed from these indices, normalizing them to their climatology. This procedure was performed by calculating a NDVI and BT climatology following three biophysical laws: the Leibig's Low-of-Minimum, the Shelford's Low-of-Tolerance and the Principal of Carrying Capacity (Kogan 1995, 2001, Kogan et al., 2003).

Thus, NDVI and BT values were expressed as deviations from climatologic extremes and became:

-Vegetation Conditions Index (VCI) - a proxy of vegetation moisture conditions

$$
\text { VCI }=100 *(\text { NDVI-NDVImin) } /(\text { NDVImax-NDVImin) }
$$

-Temperature Conditions Index (TCI) - a proxy of the thermal conditions of vegetation

$$
\text { TCI }=100 *(\text { BTmax }-B T) /(\text { BTmax-BTmin })
$$

-Vegetation Health Index (VHI), which presents a combination of the first two

$$
\mathrm{VHI}=\alpha^{*} \mathrm{VCI}+(1-\alpha) * \mathrm{TCI}, \alpha=0.5
$$

BTmax, NDVImax, BTmin, NDVImin are the maximum and minimum values of NDVI and BT for each week during the observation period of 36 years. They represent the extreme climatologic thresholds of the weekly values of the NDVI and BT variations due to the change in time. Differences (NDVImax-NDVImin) and (BTmax-BTmin) reflect the level of climate variability.

Thus, expressions for VCI and TCI show the percentage of deviation of the NDVI and BT values of extreme climatologic thresholds and reflects vegetation stress due to lack of soil water and excessive heating.

Each index (VCI, TCI and VHI) varies from zero, characterizing the extreme stress of vegetation, up to 100 , characterizing optimal conditions for vegetation. For many agricultural crops, a reduction of $\mathrm{VH}$ indices below 40 correlates with a reduction of crop yield below long-term mean or trend 
(Kogan et al., 1998, Kogan and Guo 2014, 2015). Therefore, the value of indices below 40 was accepted as the beginning of a drought (Kogan 2001). Drought intensifies when the indices decrease from 40 (mild drought) to 0 (exceptional drought). The criterion for drought intensity was established based on crop yield correlation with VH indices (Hayas and Decker 1996. Kogan et al., 1998, Kogan and Guo 2014). The VH indices have been validated in 29 countries and used globally since the 2000s, approximating vegetation condition. VH-based drought assessments include drought area, intensity, duration and origination (either from moisture or thermal or both), crop and pasture losses, wildfire risk, and drastic changes in conditions over time.

\section{Data e methodology.}

NOAA/NESDIS Advanced Very High Resolution Radiometer's (AVHRR) data were used in this study. The Advanced Very High Resolution Radiometer (AVHRR) was installed in NOAA polar orbiting satellites during 1981-2017. The AVHRR sensor measured in the visible (VIS 0.58-0.68 $\mu \mathrm{m}$, band1), near infrared (NIR,0.72-1.00 $\mu \mathrm{m}$, band2) and infrared (IR, 10.3-11.3 $\mu \mathrm{m}$ band4 and 11.3-12.3 $\mu \mathrm{m}$, band5). These data are global, with the resolution of $4 \mathrm{~km}$ and are composed of 7 days.

TCI, VCI and VHI-indices were collected for Portugal Continental for period 2000-2017. (ftp://ftp.star.nesdis.noaa.gov/pub/corp/scsb/wguo/data/VHP 4km/VH ). From collected data and using VHI method for each of 18 districts of Portugal were calculated: 1) average values of VHIindices, averaging the values of the VHI indices within the district area and 2) percentage of area of districts with the droughts, detected with VHI indices.

The fires information (burned areas and number of occurrences of forest fires) is the responsibility of the Institute of Nature Conservation and Forests, (http://www.icnf.pt ), which provide daily fire occurrence for each of the 18 districts of Portugal. Data were obtained from January 1, 2001 to December 31, 2017 and for each year were accumulated for periods of 7 days between January 1 to December 31

The processed VHI data were matched with data of wild forest fires (number of fires and burned areas). The goal was to investigate how vegetation conditions and local droughts, detected with VHImethod, coincide with occurred fires.

In fires researches there is no direct and simple relation among natural causes and their consequences due to human and socioeconomic impact. By these reasons it is difficult to obtain good results in statistical analyses of impact of droughts, vegetation stress or other natural conditions which favor forest fires.

Two methods were used to relate drought and vegetation stress with burned areas (or number of events):

1. Graphical use to visualize the dynamic of average values of VHI, VCI, TCI and percentage of drought and the burned area or number of occurrences. The visual analysis was done weekly by district for the period 2001 to 2017.

2. For this study, the relationships between the burned area with values equal to or higher 1000 ha and the percentage of dry area of the district with values equal to or higher 20 were analyzed, that means more than $20 \%$ of the district in a dry condition. For this purpose, the analysis by contingency table was used and the probabilities of detection (POD), the false alarm rate (FAR), and the miss ratio, were calculated. 


\section{Contingency Table}

\begin{tabular}{|l|c|c|c|}
\hline \multirow{4}{*}{$\begin{array}{l}\text { \%drought } \geq 20 \\
\text { \%drought }<20\end{array}$} & burntarea $\geq 1000$ ha & \multicolumn{2}{|c|}{ burntarea $<1000$ ha } \\
\cline { 2 - 3 } & $\mathrm{a}$ & $\mathrm{b}$ & $\mathrm{a}+\mathrm{b}$ \\
& $\mathrm{c}$ & $\mathrm{d}$ & $\mathrm{c}+\mathrm{d}$ \\
\cline { 2 - 4 } & $\mathrm{a}+\mathrm{c}$ & $\mathrm{b}+\mathrm{d}$ \\
\hline
\end{tabular}

The probability of detection (POD) is: $P O D=\frac{a}{a+c}$

The false alarm rate (FAR) is: $F A R=\frac{b}{a+b}$

The miss ratio: $M R=\frac{c}{c+d}$

To complete the study it was also analyzed the relationship between the number of weeks with burned area equal or exceeding 1000 ha and the percentile of dryness for each district.

\section{Results and Discussion}

Droughts are part of climate of Portugal. The deep, severe and extreme droughts always start with local droughts, which can transform later in a severe. In Portugal local droughts occur every year and can be detected using VHI-method (Figure1).

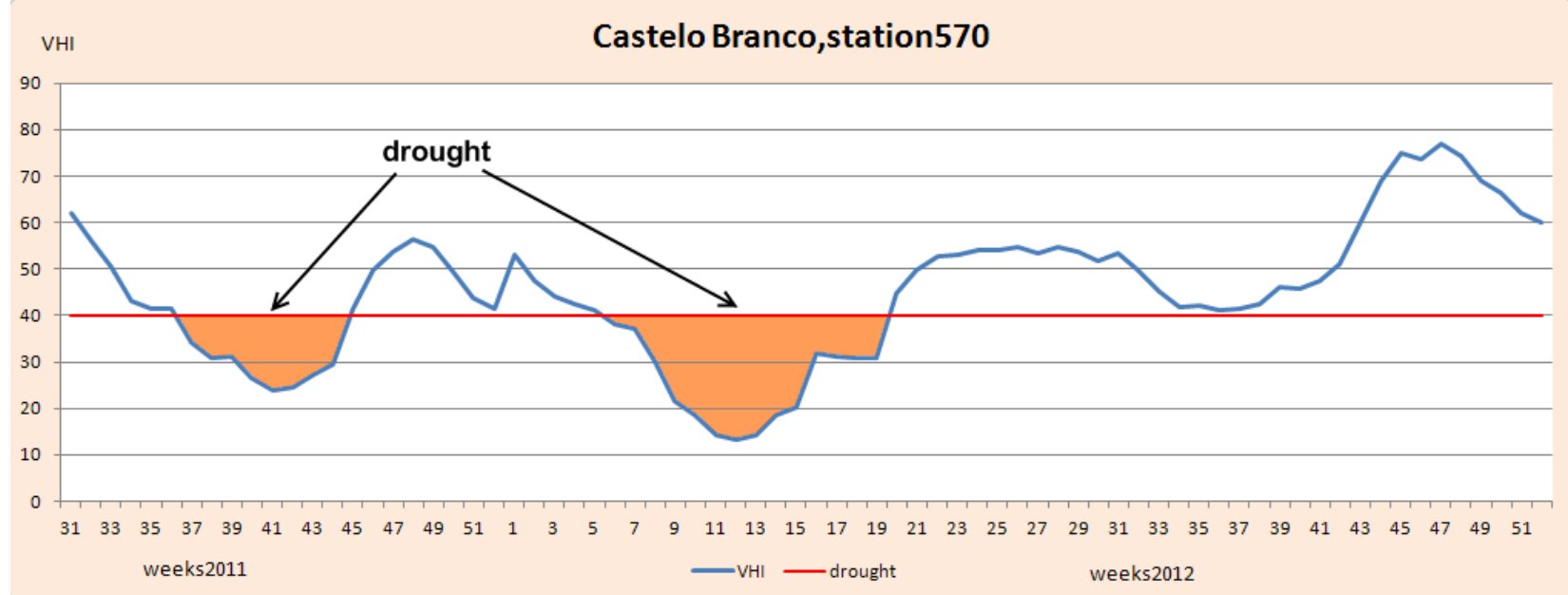

Figure 1 - Local droughts, detected by VHI-method.

The particularity of climate of Portugal is hot summer with low amount precipitation. According to "Iberian Climate Atlas" in July and August climate normal values of precipitation (1971-2000) are less then 30mm almost for all territory of Portugal. By this reason second part of July-August is the period of fire activity, independently of amount of precipitation and drought occurrence. (Figure2) 

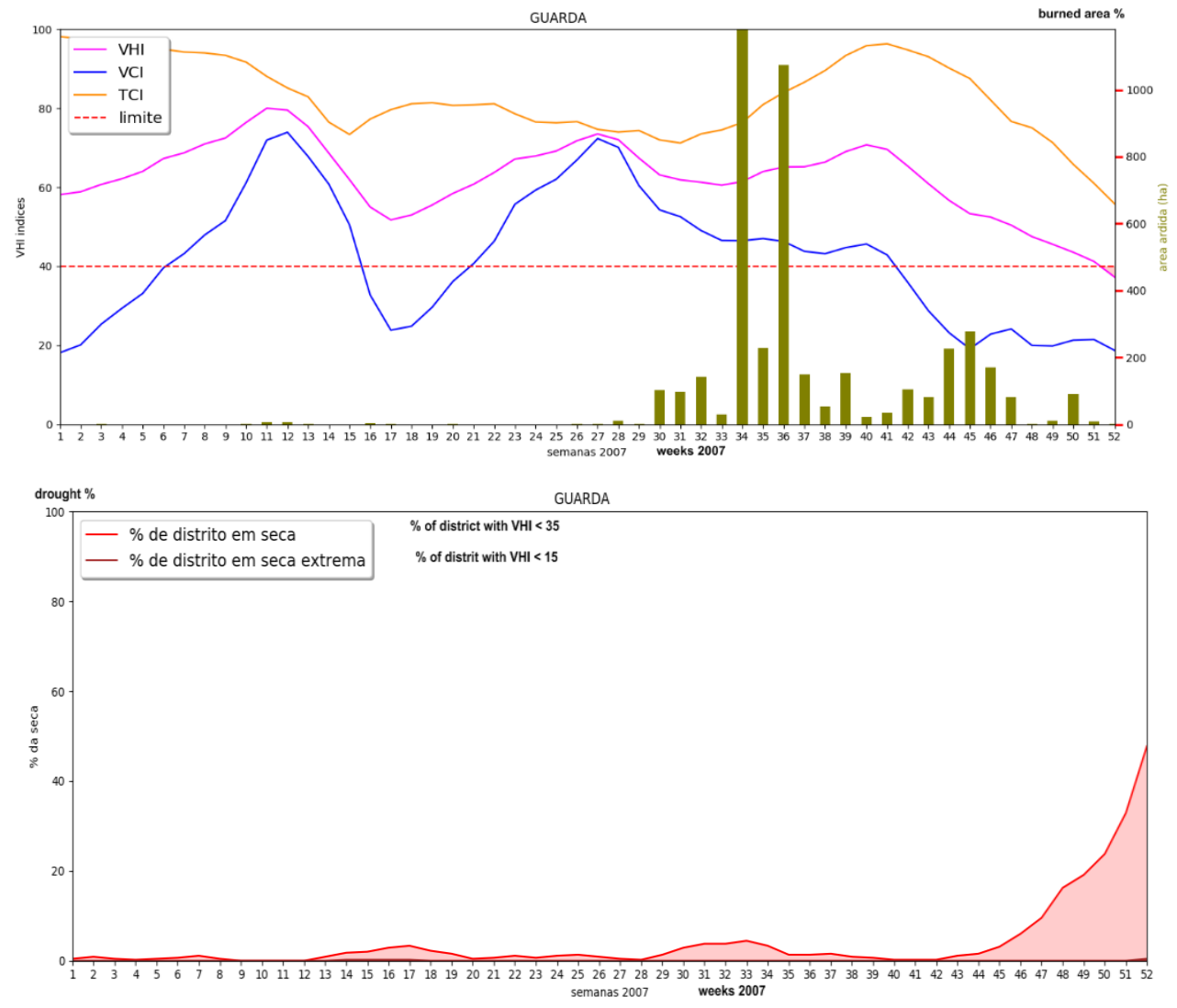

Figure 2 - Fire occurrence in summer 2007 and VHI analysis.

Visual analysis showed that local droughts, detected with VHI-method, are associated with fires activity frequently, especially in spring and autumn (Figure 3).
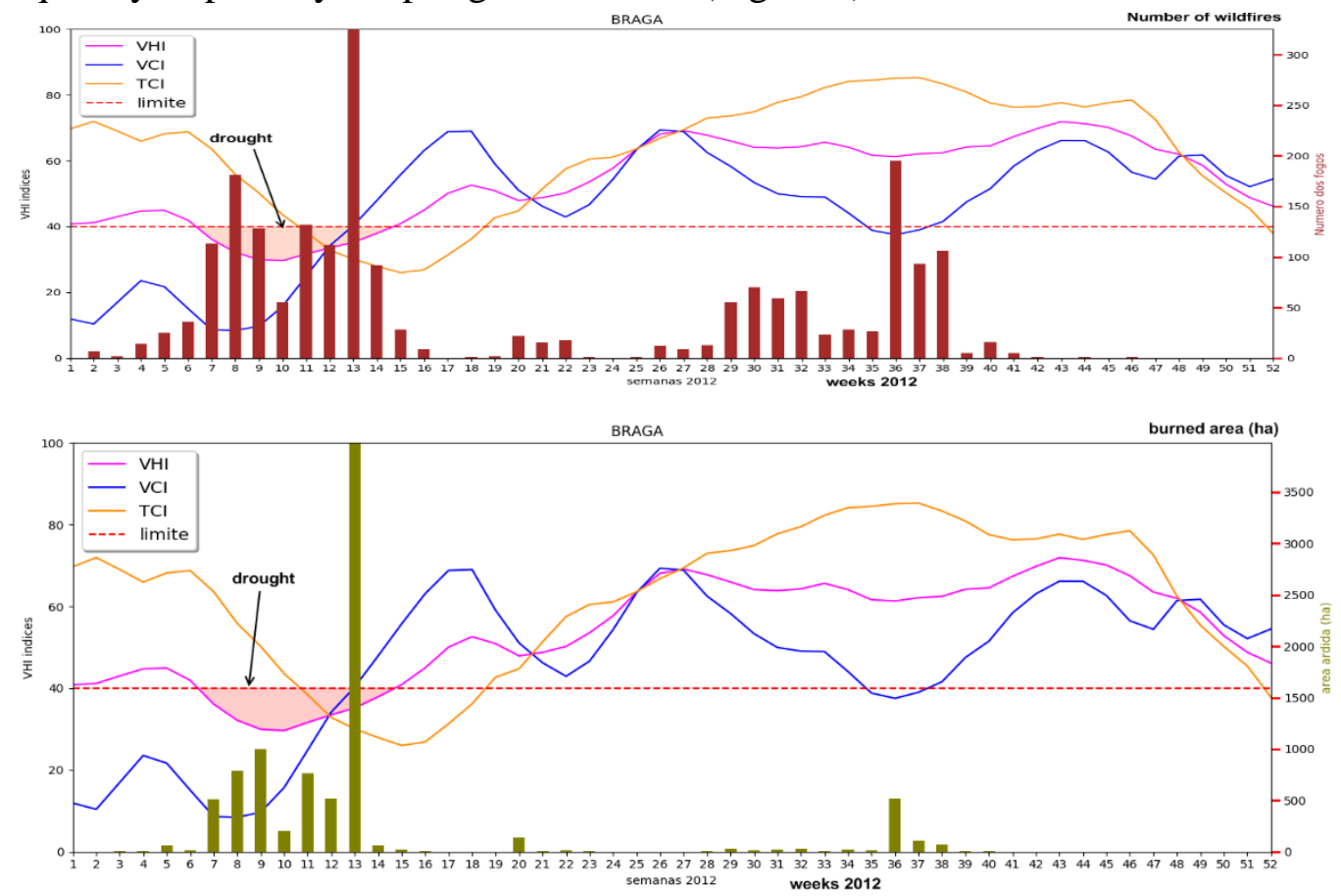


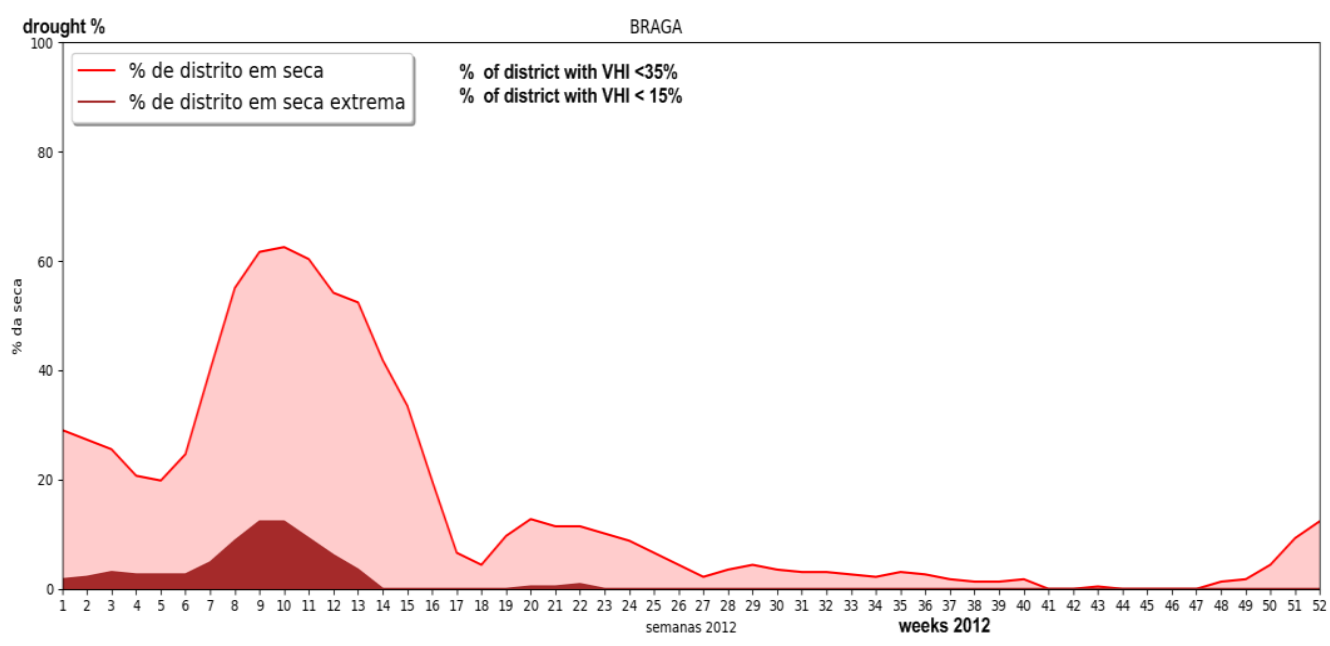

Figure 3 - Local drought, detected by VHI-method and fire occurrence in spring 2012

Fire conditions, controlled by climate and weather, which affect both fuel moisture (direct effect) and fuel structure (indirect effect). Fuel moisture determines availability to burn and fuel structure refers to the amount of burnable resources. It is well known, that fuel flammability and fire hazard increase in dry and warm years (Flannigan \& Harrington, 1988; Piñol et al., 1998; Founda \& Giannakopoulos, 2009). Fire activity may also increase when moist conditions precede the fire season, creating potential fuel (Keeley, 2004; Pausas, 2004; Littell et al., 2009; Archibald et al., 2010). In conditions of Mediterranean climate with dry, hot summer vegetation stress due to the lack of soil water or because of leaves excessive heating can lead to increasing fine fuel and flammable conditions.

By these reasons we compared correspondence of big fires (burned area $\geq 1000 \mathrm{ha}$ ) to the next conditions: 1) vegetation stress because of lack of soil water (VCI < 40.0); 2) leaves overheating (TCI $<40.0)$;3) percentage of area with droughts, detected by VHI-method, exceeded $20 \%$ of district.

Effectuated analyses showed, that for all districts, $88 \%$ of big fires have relation with at least one of the mentioned above conditions (Table1.)

Table 1 - A-number of wildfires with burned area > 1000ha;

B-number of wildfires, which correspond to drought or conditions of vegetation stress.

\begin{tabular}{|l|r|r|}
\hline DISTRICT & A & \multicolumn{1}{|c|}{ B } \\
\hline Aveiro & 12 & 11 \\
\hline Braga & 38 & 37 \\
\hline Bragança & 35 & 29 \\
\hline CBranco & 30 & 28 \\
\hline Coimbra & 22 & 14 \\
\hline Faro & 11 & 8 \\
\hline Guarda & 53 & 46 \\
\hline Leiria & 14 & 13 \\
\hline Porto & 27 & 25 \\
\hline Santarem & 23 & 20 \\
\hline Viana & 29 & 24 \\
\hline VilaReal & 48 & 45 \\
\hline Viseu & 52 & 47 \\
\hline & & \\
\hline total: & 394 & 347 \\
\hline
\end{tabular}

As was referred above, for each districts we calculated POD, FAR and Miss Ratio. Table 2 shows the results for the districts with frequent large forest fires.

We can see that false alarms (FAR) have very high percentages in all districts, which means that the district has a percentage of drought $20 \%$ or more, but there have been no forest fires with areas of 1000ha or higher. Such situations occur often when assessing forest fire hazards. The ideal would be 
to decrease the value of false alarms. For this purpose it would have to increase the percentage of drought in the district, for example, to put $30 \%$ instead of $20 \%$, but also reduce the POD and increase the Miss Ratio.

The Miss Ratio aims to respond to how often it is expected that there is no event but it happens. The perfect value would be the null. As we can see, the value of miss ratio is very small, close to zero, for all districts.

The POD (probability of detection) indicates how many times the event occurred under the expected conditions (in this case drought percentage equal to or greater than 20). As we can see POD values are high for almost all districts, showing a good relation between the percentage of drought and big forest fires. With exception of Faro, Viana do Castelo and Coimbra, where this value is lower than 0.5, due to the high number of big fires in situations when the district's percentage of drought was less than $20 \%$.

Table2 - Probability of Detection (P.O.D), False Alarm Rate (F.A.R.) and Miss Ratio calculated from contingency table for Burned Area $>=1000$ ha and \%drought $>=20 \%$

\begin{tabular}{|l|rrr|}
\hline & P.O.D. & F.A.R. & MissRatio \\
\hline Aveiro & 0,69 & 0,93 & 0,01 \\
Braga & 0,74 & 0,85 & 0,01 \\
Bragança & 0,55 & 0,88 & 0,02 \\
Cbranco & 0,87 & 0,89 & 0,01 \\
Coimbra & 0,43 & 0,90 & 0,02 \\
Faro & 0,36 & 0,98 & 0,01 \\
Guarda & 0,79 & 0,85 & 0,02 \\
Leiria & 0,71 & 0,92 & 0,01 \\
Porto & 0,68 & 0,90 & 0,01 \\
Santarém & 0,78 & 0,93 & 0,01 \\
Viana & 0,38 & 0,88 & 0,02 \\
VilaReal & 0,80 & 0,82 & 0,02 \\
Viseu & 0,77 & 0,75 & 0,02 \\
\hline
\end{tabular}

In order to analyze the relationship between the number of weeks with burned area exceeding 1000 ha and the percentage of drought of each district:

- the percentiles for the district's weekly percentage of drought were calculated ;

- class were constructed: between the value of the $0^{\text {th }}$ percentile and $10^{\text {th }}$ percentile, between the value of the $10^{\text {th }}$ percentile and that of the $20^{\text {th }}$ percentile, etc .;

- for each class the number of weekly forest fires with burned areas equal or greater than $1000 \mathrm{ha}$ was calculated.

It was observed, that for all districts there is a relation between the highest values of the percentile of the district's drought percentage and the number of big forest fires. 

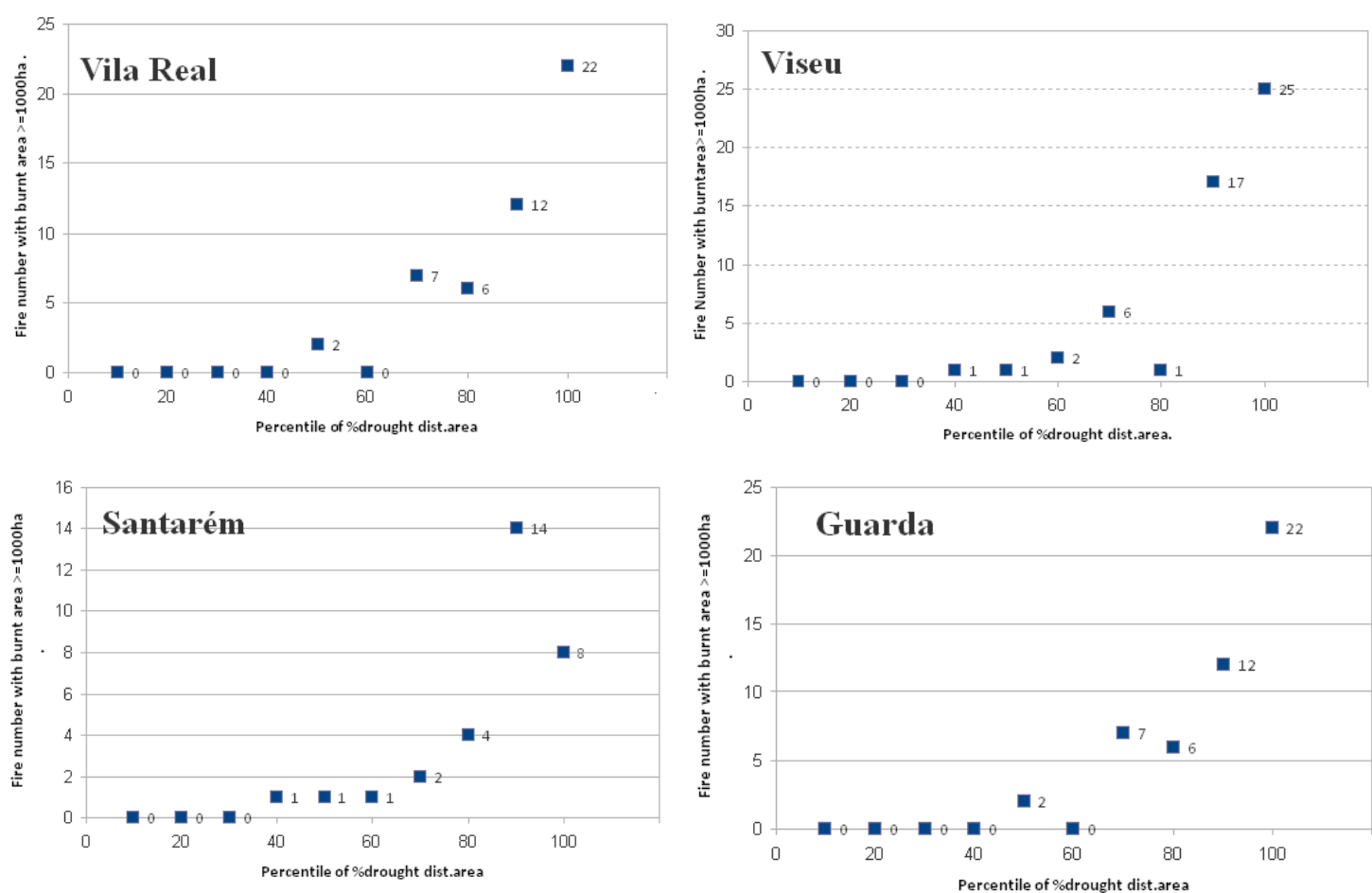

Figure 3 - Plot of the total number of weekly fires with burned area $\geq 1000 h a$, summed for each percentile class of the percentage of drought area of the district. Each point represents the total number of fires $(\geq 1000 h a)$ occurring in the class defined in this percentile

\section{Conclusions}

It is very difficult to establish the relationship between forest fires (area, number of events of big forest fires) and weather conditions, because the results depend on causes of another nature (such as population density and activity, socioeconomic conditions, cultural aspects, etc.)

The VHI index, as well as the sub-indexes VCI and TCI, show a strong relation both to the burned areas and to the number of events of forest fires. Graphs with the representation of these relations for some districts showed, as examples, on Figure 2 and Figure 3.

The analyses results showed, that for all districts, $88 \%$ of big fires have relation with at least one of next conditions, detected with VHI-method:

- vegetation stress because of lack of soil water (VCI <40.0);

- vegetation stress because the leaves overheating (TCI <40.0);

- percentage of drought by district exceeded $20 \%$.

Also, the analysis with the contingency tables shows a close relationship between the percentage of drought area of a district and the burned area $\geq 1000$ ha, that is to say, that preferably the large forest fires occur if the district has a percentage of drought area $\geq 20$. This observation can also be made by analyzing the graphs of the number of fires with burned area $\geq 1000$ ha for each district in each class of percentage of drought area made based on the percentiles.

\section{References}

Archibald, S.,Nickless, A., Govender, N., Scholes, R.J. \& Lehsten,V. (2010) Climate and the interannual variability of fire in southern Africa: a meta-analysis using long-term field data and satellitederived burnt area data. Global Ecology and Biogeography, 19, 794-809. 
Cracknell AP. 1997. The Advanced Very High Resolution Radiometer (AVHRR). London: Taylor \& Francis. 534 p.

Flannigan, M.D. \& Harrington, J.B. (1988) A study of the relation of meteorological variables to monthly provincial area burned by wildfire in Canada (1953-80). Journal of Applied Meteorology, $27,441-452$.

Founda, D. \& Giannakopoulos, C. (2009) The exceptionally hot summer of 2007 in Athens, Greece a typical summer in the future climate? Global and Planetary Change, 67, 227-236.

Hayas, M.J. and Decker W.L. 1996. Using NOAA AVHRR data to estimate maize production in the United States Corn Belt. International Journal of Remote Sensing, 17, pp 3189-3200.

Iberian Climate Atlas (1971-2000), Instituto Portugues de Meteorologia, 2011, Lisboa, http://www.ipma.pt/resources.www/docs_pontuais/ocorrencias/2011/atlas_clima_iberico.pdf

Jurdao S, Chuvieco E, Arevalillo JM (2012) Modelling fire ignition probability from satellite estimates of live fuel moisture content. Fire Ecology 8(1). 77-97.

Keeley, J.E. (2004) Impact of antecedent climate on fire regimes in coastal California. International Journal of Wildland Fire, 13, 173-182.

Kogan F. 1997, Global Drought Watch from Space, Bulletin American Meteorological Society 78:621-636.

Kogan F. and Guo W. 2015. 2006-2015 mega-drought in the western USA and its monitoring from space data. Geomatic, Natural Hazards and Risk, DOI:10/1080/19475705.2015.1079265. http://dx.doi.org/10/1080/19475705.2015.1079265

Kogan F., GitelsonA., Zakarin E. and Lebed E., AVHRR-Based Spectral Vegetation Index for Quantitative Assessment of Vegetation State and Productivity: Calibration and Validation, 2003. $\begin{array}{lllll}\text { Photogrammetric Engineering \& } \quad \& \quad \text { Remote } & \text { 69(8) }\end{array}$ https://digitalcommons.unl.edu/natrespapers/278

Kogan F., GitelsonA., Zakarin E. and Lebed E., Using AVHRR Data for Quantitative Estimation of Vegetation Conditions: Calibration and ValidationhttpsAdv. Space Res.,1998, Vol. 22, No.5. pp. 673--676.

Kogan F.N. 1995. Droughts of the Late 1980s in the United States as Derived from NOAA PolarOrbiting Satellite Data. Bulletin of the American Meteorological Society, 76 (5), pp. 655-667.

Kogan FN. 2001. Operational space technology for global vegetation assessment. Bulletin of the American Meteorological Society 82: 1949-1964.

Kogan, F. and Guo W. 2014, Early twenty-first-century droughts during the warmest climate. Geomatics, Natural Hazards and Risk http://dx.doi.org/10.1080/19475705.2013.878399

Littell, J.S., McKenzie, D., Peterson, D.L. \& Westerling, A.L. (2009) Climate and wildfire area burned in western US ecoprovinces, 1916-2003. Ecological Applications, 19, 1003-1021.

Mar Bisquert, Juan Manuel Sánchez and Vicente Caselles, 2014, Modeling Fire Danger in Galicia and Asturias (Spain) from MODIS ImagesRemote Sens. 2014, 6, 540-554.

P. Illera, A. Fernández \& J. A. Delgado (2007) Temporal evolution of the NDVI as an indicator of forest fire danger, International Journal of Remote Sensing, 17:6, 1093-1105.

Pausas, J.G. (2004) Changes in fire and climate in the eastern Iberian Peninsula (Mediterranean Basin). Climatic Change, 63, 337-350.

Piñol, J., Terradas, J. \& Lloret, F. (1998) Climate warming, wildfire hazard, and wildfire occurrence in coastal eastern Spain. Climatic Change, 38, 345-357. 


\title{
Protecting lives and property: testing tanker crew protection systems
}

\author{
Rachel Bessell*1; David Nichols ${ }^{1}$; Justin Leonard ${ }^{2}$; Miguel G. Cruz ${ }^{3}$; Alen Slijepcevic ${ }^{1}$ \\ ${ }^{1}$ Country Fire Authority. Victoria, Australia, \{r.bessell@cfa.vic.gov.au*\} \\ ${ }^{2}$ CSIRO Bushfire Research, Land and Water, Clayton, Australia \\ ${ }^{3}$ CSIRO Bushfire Behaviour and Risks, Land and Water, Canberra, Australia
}

\begin{abstract}
Vehicle entrapment and fire burn over is a life-threating situation for fire fighters. In Australia, significant crew protection research commenced following the 1998 Linton wildfire (in Victoria, Australia) where five CFA volunteers perished when their tanker was entrapped and burnt over. Since the Linton fire burn over, CFA crew protection research has aimed to reduce the probability of loss of life in a tanker burn over. This involves increasing the number of protection layers between fire fighters and the fire including: personal protective blankets, drop-down radiant heat shielding and water spray deluge systems with pump protection.

From 2004 to 2017, a series of experimental burns were conducted to test the safety of fire fighters in tankers. Initial system validation tests were conducted in a gas-fuelled flame front simulator at Mogo, New South Wales, Australia. Subsequently, successful field validation tests were conducted in forested environments at low intensities. This successful testing resulted in all new CFA medium to heavy tankers (upwards of 2000 litre water capacity) being fitted with a crew protection system from 2006, and all existing tankers retro-fitted from 2013.

Due to restricted water capacity, ultra-light tankers (less than 800 litre water capacity) need a different system. Two new water enhancing methods were designed and tested, a Compressed Air Foam System (CAFS) and a polymer gel system.

In March 2014, the medium tanker and gel ultra-light crew protection systems were tested in grass fire field experiments in Wangaratta, Victoria; both crew protections systems met survivability objectives.

In March 2017, a high intensity experimental fire was conducted in Brucknell, Victoria, in order to validate the crew protection systems retrofitted onto a CFA medium tanker and to test the two new water enhancing methods on two ultra-light tankers. The Brucknell fire had an average intensity of $19,000 \mathrm{~kW} / \mathrm{m}^{2}$ (twice that of the Linton wildfire) with a peak intensity of $31,000 \mathrm{~kW} / \mathrm{m}^{2}$. All crew protection systems met survivability objectives.

Ten years since the 1998 Linton wildfire, on 7 February 2009, Victoria experienced unprecedented extremes in fire weather resulting in the Black Saturday wildfires. 173 people perished, over 2000 properties were lost and over 430,000 hectares were burnt. Of the hundreds of fire fighting vehicles involved, a number were caught out in burn over situations, yet no fire fighters were seriously injured. This remarkable result can be credited to the tanker crew protection systems.
\end{abstract}

Keywords: firefighter safety, vehicle crew protection, vehicle entrapment, burn over

\section{Introduction}

'It must be understood that safety, in the environment of a wildfire can be a complex issue, which needs constant attention and vigilance, by those agencies responsible for its management.'

Extract from the Corners Court of Victoria (2002), Inquest into the Linton Wildfire, page 572.

Wildfires are a common phenomenon in the state of Victoria, Australia. In trying to control these fires, the Country Fire Authority (CFA) has experienced multiple firefighting tanker burn overs resulting in serious injuries and fatalities. Two of the most serious instances in Victoria were (1) during the Ash Wednesday wildfires in 1983 where twelve firefighters were killed during a burn over 
of two tankers in Belgrave South/Beaconsfield Upper (CFA, 1983) and (2) in the Linton wildfire in 1998 where a tanker became entrapped and burnt over killing all five fire fighters (CFA and NRE, 1999). In both of these instances, fire fighters were working away from the edge of the fire and were caught off guard with a sudden change in wind direction and fire behaviour. Known as the 'dead-man zone', fire fighters have very little time to egress or seek refuge before the fire overwhelms them (Cheney et al, 2001).

Since these tragedies, CFA made substantial safety improvements to its fire fighting tanker fleet to ensure the safety and protection of its fire fighters. CFA tanker crew protection systems are designed to increase the level of protection by maintaining the integrity of the cabin, particularly the windows, to reduce the temperatures inside the cabin and increase the number of protective layers between the fire fighters and the fire.

Over the initial stages of the crew protection system testing (pre-2010), various system elements were designed, tested and refined. Some of these improvements include: personal protective blankets, low level water indicators, water spray deluge systems, internal cabin drop down radiant heat shielding curtains, additional heat shielding around the water pump system and vulnerable tanker components, a reduction of plastic material on the external surfaces of the tankers, metal air cleaner filters, flame resistant hose and cabling, upgraded communication systems and all diesel engines. This work has resulted in the installation of crew protection systems on the entire fleet of CFA medium and heavy tankers. Crew protection systems have been installed on all newly built tankers since 2006 while all older tankers were retro-fitted prior to the 2013-2014 summer fire season.

The focus of this paper is to highlight the crew protection system validation and testing that has occurred since 2013. To validate the crew protection systems on a retro-fitted medium (2000L) tanker, the tanker was burnt in experimental fires in both grass and forest, and at different intensities in a gaspropelled flame front simulator. Additionally, two ultra-light tankers (less than 500L capacity) systems have been designed and tested in experimental fires and in the gas-propelled flame front simulator.

\section{Experimental burns}

From 2004 to 2017, multiple experimental burns have been conducted in Victoria and New South Wales (NSW) to test the safety of fire fighters in tankers. As there are no occupational exposure standards that exist for fire smoke, air toxic tenability levels have been determined using Safe Work Australia short-term (15-minute) exposure guidelines (Leonard et al., 2017). Also there are no guidelines for survivability levels, they have been estimated at five times the tenability limits. The respirable particle survivability limit is set at $30 \mathrm{mg} / \mathrm{m}^{3}$, however fire fighters are often exposed to much higher levels during prescribed burning actitivies (Reisen and Brown, 2009). A criterion has been used for the studies as it is important to identify that some firefighters may be more susceptable to fire smoke than others at these respirable particle levels. Different strategies, such a respiratory protection could be established to minimise health risks (Leonard et al., 2017).

Pre-2010, crew protection systems installed on a medium tanker had undergone repeatable tests at the New South Wales Rural Fire Service (NSW RFS) Hot Fire Training Facility south of Mogo, NSW, Australia. The flame front simulator design and subsequent testing was as described by Nichols (2010). During this testing the medium tanker met crew survivability objectives at $5000 \mathrm{~kW} / \mathrm{m}^{2}$.

Field testing was also conducted at Tumbarumba, NSW in February 2004. Of these two experimental fires, both fell well below the intended intensity objectives, however validated the crew protection system at medium intensities, less than $2000 \mathrm{~kW} / \mathrm{m}^{2}$ (Nichols, 2010).

In order to validate the crew protection system installed on the fleet of CFA medium and heavy tankers, further field testing was conducted in both grass and forest environments. 


\subsection{Grass fire - Wangaratta, Victoria, March 2014}

The aim of the Wangaratta tests were to see how the medium tanker and ultra-light gel crew protection systems performed under grass fire conditions. The tests were conducted in March 2014 on a rural property south of Wangaratta, Victoria. The tankers were burnt in seperate experiments, within grass plots of $35 \times 35 \mathrm{~m}$.

The full details of the experiment are detailed in Leonard et al. (2014). Temperatures both inside and outside the cabin were measured, and radiometers were utilised to determine fire intensities. Air toxic exposures were also measured. The ambient temperature during the testing was between 27 and $30^{\circ} \mathrm{C}$. Unfortuntely on the day conditions were not optimal to conduct high intensity grass fires, due to overnight due formation and light and variable winds (Cruz et al., 2014).

The medium tanker tests were replicated (two experiments) as the wind shifted and the headfire did not directly impact the tanker in the first experiment. During the second test, flame heights of $2.5 \mathrm{~m}$ were recorded, with the flame front directly impacting the tanker. The water deluge spray system was activated from the start of the test, and it took the flames approximately 45 seconds to reach the tanker (Leonard et al, 2014). Maximum radiant heat, recorded on the passenger side door was $25 \mathrm{~kW} / \mathrm{m}^{2}$. The inside air temperature remained unchanged throughout the test at $40^{\circ} \mathrm{C}$. The peak temperature recorded on the outside passenger door was around $180^{\circ} \mathrm{C}$ noting that this temperature is within the water mist spray and hence does not reflect the true flame temperature (Leonard et al., 2014). Air toxics were only measured for the first medium tanker experiment, and recorded values were well below tenability and survivability thresholds.

Overall there was no reported damage to the tanker, with the spray system providing adequate protection to the tanker as well as grass surrounding and beneath the tanker (Figure 1).

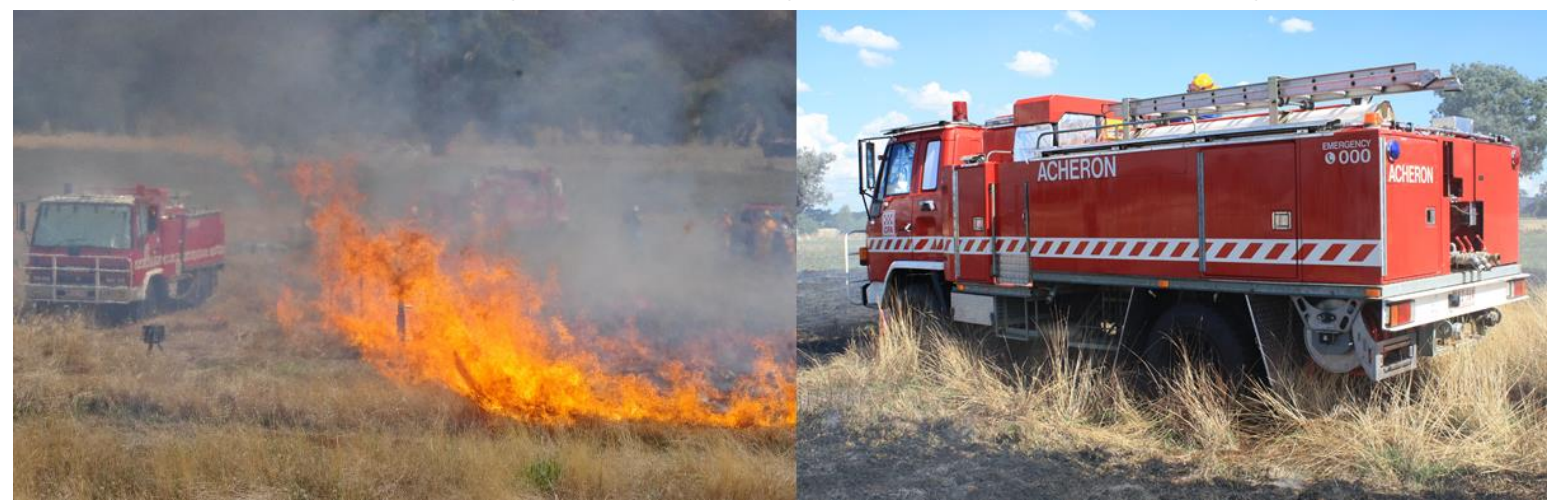

Figure 1 - CFA Medium tanker during and following the second experimental burn, Wangaratta, Victoria.

The gel ultra-light tanker test was observed to be the most severe of the three experiments conducted on the day. Time from ignition to impact was approximately 40 seconds, and flame heights of $3 \mathrm{~m}$ were observed. The polymer gel was pre-applied before ignition. The radiant heat profile peak was $95 \mathrm{~kW} / \mathrm{m}^{2}$ on the passenger side. Outside air temperature peak was at $330^{\circ} \mathrm{C}$, yet the inside air temperature was a constant $40^{\circ} \mathrm{C}$. No in-cabin pollutant concentrations exceeded the tenability or survivability thresholds. There was no significant damage to the tanker (Leonard et al., 2014).

\subsection{Forest fire - Brucknell, Victoria, March 2016}

An experimental burn block of 0.9 ha at Brucknell, Victoria was selected for the forest fire experiment. The block was north facing, had a $7^{\circ}$ slope, and a fuel complex appropriate to achieve a high intensity fire. The overstorey was dominated by Stringybark eucalypts and had not been burnt since the Ash Wednesday wildfires 33 years earlier. In the days preceeding the burn, a number of 
small prescribed burns were conducted in mild conditions surrounding the burn block to limit the likelihood of the experimental fire escaping.

The objectives of the experiment were:

(i) To conduct a high intensity experimental fire of at least $10,000 \mathrm{~kW} / \mathrm{m}^{2}$ to replicate conditions of the 1998 Linton wildfire.

(ii) Field test the following CFA crew protection systems:

a. A retro-fitted crew protection system on a medium tanker (2000L capacity)

b. A polymer gel delivery system on an ultra-light tanker (400L capacity)

c. A Compressed Air Foam Systems (CAFS) on an ultra-light tanker (400L capacity)

A full report on the fuels, weather and fire behaviour aspects of the Brucknell experimental burn is documented in Cruz et al., (2016).

The Brucknell fire was ignited at 12:59:00 on 17 March 2016. The preceeding months of January and February were drier than usual. The nearby weather stations had an average Keetch-Byram Drought Index of 132, and a Drought Factor of 10. The prevalent weather conditions at 13:00 were:

Temperature $=33^{\circ} \mathrm{C}$

Relative Humidity $=25 \%$

$10 \mathrm{~m}$ open wind speed $=33 \mathrm{~km} / \mathrm{h}$ (average); gusts to $50 \mathrm{~km} / \mathrm{h}$

These conditions resulted in a Forest Fire Danger Index (FFDI) of 34.5 representating a Very High Fire Danger Rating.

The fireline was ignited with two pairs of two igniters with hand-held drip torches, lighting an approximately $100 \mathrm{~m}$ long ignition line. A high intensity flame front developed right after igniton, and a psuedo-steady state rate of spread was observed after $20 \mathrm{~m}$. Flame heights estimated to be $5-6 \mathrm{~m}$, with bark fuels allowing it to climb $10 \mathrm{~m}$ up tree trunks. There were multiple spot fire ignitions occuring in front of the vehicles. It took 3.5 minutes from ignition for the fire to reach the tankers (Figure 2). Water sprays on the medium tanker, and CAFS on one of the ultra-light tankers were activated 2 minutes after ignition. The gel delivery system was still yet to be refined, as such the gel was pre-applied to the ultra-light tanker approximately 20 minutes before ignition. Considering the estimated fuel consumed, the average rate of spread was $31 \mathrm{~m} /$ minute, which gives a fireline intensity of approximately $19,000 \mathrm{~kW} / \mathrm{m}^{2}$ (Cruz et al., 2016).

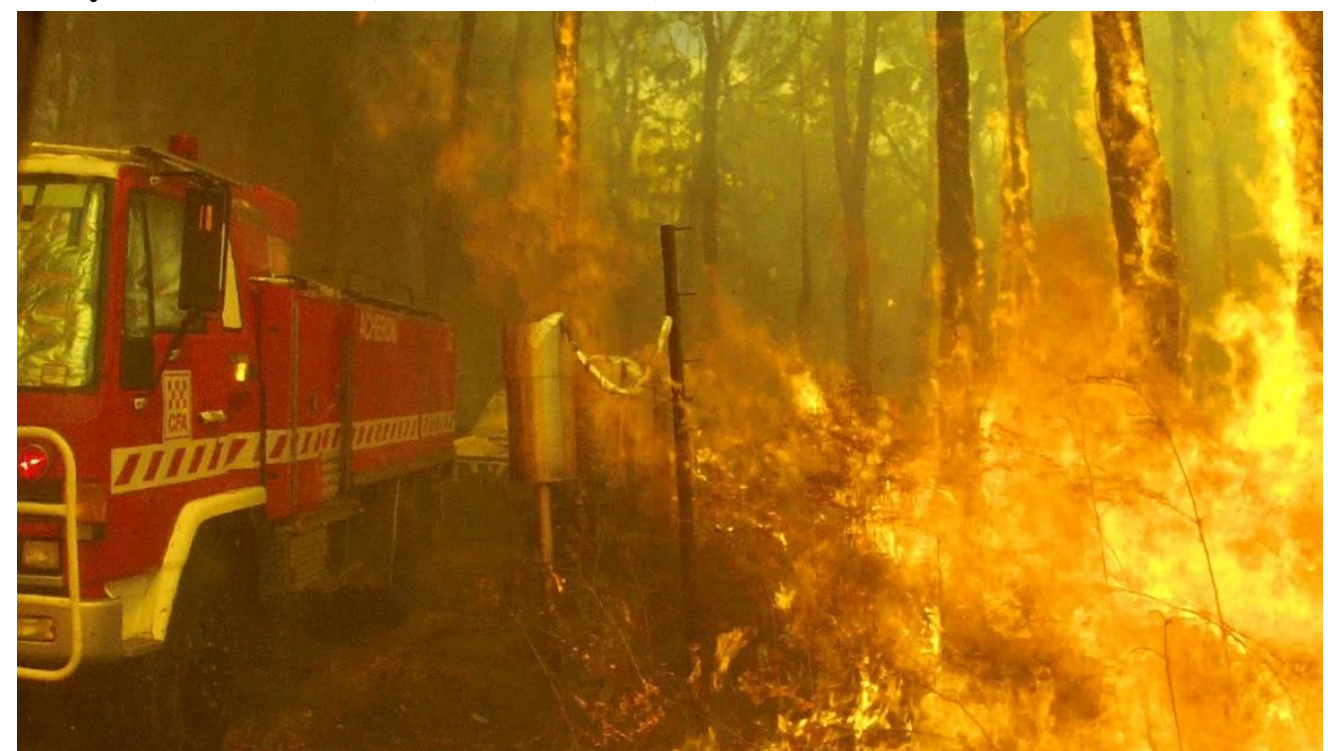

Figure 2. Brucknell experimental burn impacting the CFA medium tanker, CAFS and Gel ultra-light tankers 
A full report of the tanker tenability and survivability objectives is documented by Leonard et al., (2016). The internal cabins of all three tankers were instrumented for temperature and air toxics.

\subsubsection{Gel protected ultra-light tanker results}

The gel ultra-light tanker had a radiant heat peak of $60 \mathrm{~kW} / \mathrm{m}^{2}$. Outside air temperature above the passenger door peaked at $350^{\circ} \mathrm{C}$, yet the internal air temperature remained unchanged at $33^{\circ} \mathrm{C}$ until after the fire front had passed around four minutes from start of the test. Fourteen minutes after ignition the passenger front wheel showed elevated temperatures consistent with tyre combustion. From air toxicity results, the cabin exceeded the carbon monoxide tenability limit (of 100ppm) 4.5 minutes after direct flame contact. Respirable particle concentrations exceeded survivability concentrations two minutes after flame contact. Volatile Organic Compounds (VOC) levels were well below detection levels 30 seconds after flame impact; however 3.5 minutes later, aromatic VOC contentrations were much higher than external concentrations and chlorinated VOCs were observed indicating polyvinyl chloride (PVC) in the gel ultra-light cabin had ignited (Leonard et al., 2016).

\subsubsection{CAFS protected ultra-light tanker results}

No radiant heat measurements were taken from the CAFS ultra-light tanker due to lack of an effective system to protect the radiometer from the foam. Outside air temperature near the cabin peaked between 200 and $250^{\circ} \mathrm{C}$. The inside air temperature remained unchanged at $38^{\circ} \mathrm{C}$ until after the fire front has passed. Temperature gradually increased over time, but remained below $60^{\circ} \mathrm{C}$ eight minutes after the front has passed. There was no significant observable damage to the tanker. No tenablility or survivablility criteria were exceeded in the CAFS ultra-light tanker cabin except for smoke ingress (Leonard et al., 2016).

\subsubsection{Medium tanker results}

The radiant heat peaked at $35 \mathrm{~kW} / \mathrm{m}^{2}$, four minutes after ignition. The outside temperature of the vehicle dropped once the spray system was turned on, and increased rapidly when the fire front reached the tanker (depicted in Figure 2), at $300^{\circ} \mathrm{C}$. The inside air temperature remained unchanged at $33^{\circ} \mathrm{C}$ until after the fire front had passed, then it slowly increased over time as the water spray system was completed, but remained around $50^{\circ} \mathrm{C}$. The crew compartment in the rear of the tanker started to rise following the fire front reaching $70^{\circ} \mathrm{C}$ for 90 seconds before decling in line with the internal temperatures. There was no visible damage to the tanker with the water deluge spray system also protecting the remnant fuels surrounding and beneath the tanker (Figure 3). No tenablility or survivablility criteria were exceeded in the medium tanker cabin except for smoke ingress (Leonard et al., 2016).

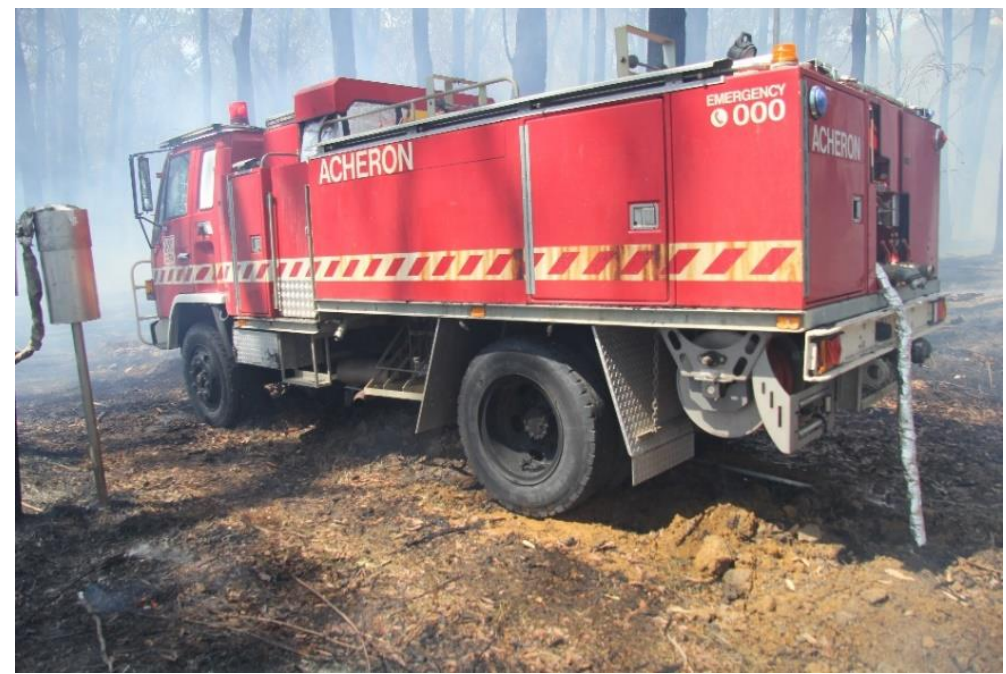

Figure 3. CFA Medium tanker after Brucknell experimental burn 


\subsection{Mogo - flame front simulator, November 2017}

In Mogo, NSW, in November 2017, the medium tanker and the CAFS protected ultra-light tanker were tested for different objectives. The flame front simulator test of the medium tanker was performed at three different intensity levels to understand how the system would perform at elevated exposure levels. The full report of this experiment is documented in Leonard et al., (2018).

The objective of the CAFS ultra-light tanker experiment was to test the survivability of the ultralight tanker with the CAFS crew protection system installed at a simulated fireline intensity of $5000 \mathrm{~kW} / \mathrm{m}^{2}$. The full report of this experiment is documented in Leonard et al., (2017).

The gel protected ultra-light tanker had previously been tested at Mogo and passed tenability and survivability objectives (Leonard et al., 2013).

\subsubsection{Medium tanker tests}

The medium tanker retrofitted with the current CFA crew protection system was subject to three experimental burns under three different intensities: $10,000 \mathrm{~kW} / \mathrm{m}^{2}, 15,000 \mathrm{~kW} / \mathrm{m}^{2}$ and $25,000 \mathrm{~kW} / \mathrm{m}^{2}$. As per previous experiments, internal and external temperatures were recorded as well as air toxics to determine if the system meets tenability and surviviability requirements.

Outside temperatures at $1 \mathrm{~m}$ peaked at around $800^{\circ} \mathrm{C}$ for all three experiments. The inside air temperature peaked at around $50^{\circ} \mathrm{C}$ for the $10,000 \mathrm{~kW} / \mathrm{m}^{2}$ and $15,000 \mathrm{~kW} / \mathrm{m}^{2}$ experiments and $70^{\circ} \mathrm{C}$ at the cabin ceiling $\left(50^{\circ} \mathrm{C}\right.$ at seat height $)$ in the $25,000 \mathrm{~kW} / \mathrm{m}^{2}$ experiment.

For the medium tanker at $10,000 \mathrm{~kW} / \mathrm{m}^{2}$ and $15,000 \mathrm{~kW} / \mathrm{m}^{2}$ resulted in no survivabilty criteria being exceeded for the truck cabin with the exception of respirable particles. For the $25,000 \mathrm{~kW} / \mathrm{m}^{2}$ test, the tanker was compromised by the earlier experiments (Figure 4) and exhibited more failures to tenability and survivability, though a less extensive range of air toxics were measured.

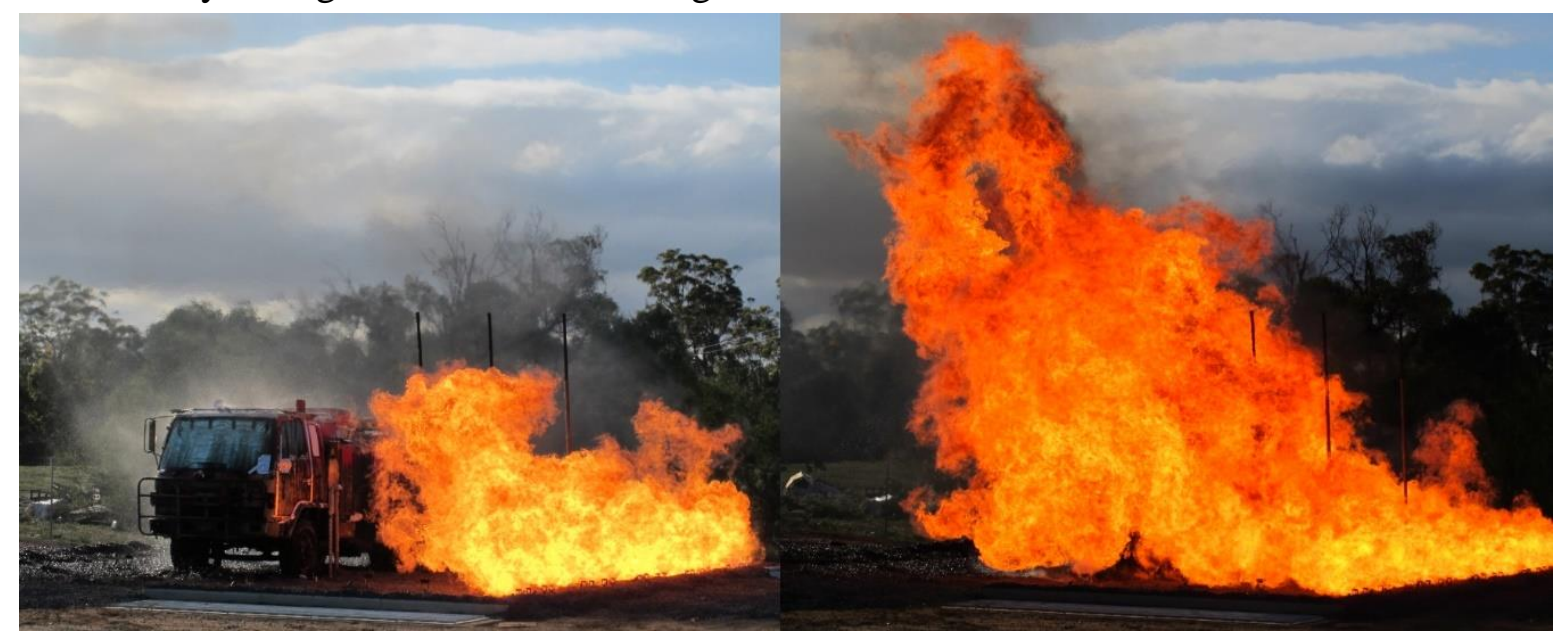

Figure 4. CFA Medium tanker on the flame front simulator during the 25,000k W/m2 Mogo, NSW, experiment

\subsubsection{CAFS ultra-light tanker test}

The outside temperatures at $1 \mathrm{~m}$ high peaked at $800^{\circ} \mathrm{C}$, while inside the cabin peaked at $50^{\circ} \mathrm{C}$. For air toxics, respirable particles increased at flame immersion, and exceeded tenability and survivability criteria. There was a significant fire that developed in the engine compartment following flame immersion which would have caused an increase to carbon monoxide levels and respirable particles. While there was no visible damage to the cabin interior, the delayed air toxic build up was evident and consistent with a late-stage engine compartment fire (Leonard et al., 2017). 


\section{Summary of results}

\subsection{Wangaratta grass fire experiments}

The medium tanker and gel ultra-light crew protection systems passed all tenability and survivability objectives.

\subsection{Brucknell forest fire experiment}

The medium tanker and CAFS ultra-light tankers passed all tenabilty and survivability objectives. The gel ultra-light passed the immediate test, however the cabin did become untenable during the latter stage of the experiment. However, cabins only need to be tenable as the fire front passes through, at this stage fire fighters can leave the cabin and seek refuge on burnt ground.

\subsection{Mogo flame front simulator}

The test confirmed that the medium tanker crew protection systems are robust enough at even the highest level of $25,000 \mathrm{~kW} / \mathrm{m}^{2}$ fireline intensity with cabin air temperatures remaining at survivable levels. At $25,000 \mathrm{~kW} / \mathrm{m}^{2}$ the cabin was compromised before the experiment began due to damage in earlier tests; and the truck exhibited more significant failures of air toxicity tenability and survivability. At the lower intensities $\left(10,000 \mathrm{~kW} / \mathrm{m}^{2}\right.$ and $\left.15,000 \mathrm{~kW} / \mathrm{m}^{2}\right)$ no survivability criteria was exceeded with the exception of respirable particles.

For the CAFS ultra-light tanker experiment at $5,000 \mathrm{~mW} / \mathrm{m}^{2}$, the cabin air temperature remained survivable throughout the test. No air toxicity survivablility criteria was exceeded within the cabin with the exception of respirable particles.

\section{Discussion}

On 7 February 2009, Victoria experienced unprecedented extremes in fire weather resulting in the Black Saturday wildfires. 173 people perished, over 2000 properties were lost and over 430,000 hectares were burnt. Twenty-nine fire fighting vehicles were burn over by wildfire, but remarkably no fire fighters were seriously injured (Nichols, 2010). A number of the fire fighting crews caught out in these burn overs praised the equipment and safety measures made available to them (Royal Commission into Victoria's Bushfires, 2010).

In 2014 the Australasian Fire and Emergency Services Authority Council (AFAC) identified three principles in the guideline for Rural Fire fighting Vehicles Burn over Protection (AFAC, 2015):

1. Ensure the fire fighting vehicle is designed to keep moving so that it does not become disabled or otherwise prevented from escaping to a safe zone.

2. Ensure that the operational functionality of the fire fighting vehicle is not compromised and that the safety support systems and other critical equipment continue to function.

3. Use the fire fighting vehicle to provide a refuge area for the fire fighters operating it.

These guidelines have been derived from the Australian research and development invested by CFA, Commonwealth Scientific and Industrial Research Organisation (CSIRO) and NSW RFS.

\section{Acknowledgements}

There were a number of people involved in muliple facets of this research. From CSIRO Land and Water: Lyndon Macindoe, Steve Brown and Glenn Newnham, who were responsible for the instrumentation and measurements at the Wangaratta, Brucknell and Mogo fire experiments. Richard Hurley, CSIRO Bushfire Research, for his weather and thermal instrumentation, and helpful direction of suppression crews. 
The EVTA Group consisting of Ross Luke, Brenton Luke and Mitch Luke whom designed and developed the Red Door Gel 'Crew Protection' System, alongside retired CFA engineer Robert Rankin. CFA engineer Andrew Webb and mechanic Peter Hill worked with Bell Engineering to design the Compressed Air Foam (CAFS) ultra-light crew protection system.

NSW RFS for the use of the Mogo facility. And to all CFA members who assisted with finding the land and providing appropriate fire suppression support to enable the experimental burns to occur.

\section{References}

Australiasian Fire and Emergency Services Authority Council (2015) Rural Firefighting Vehicles Burn-over Protection Guideline Version 1.0 March, 2015.

Cheney, N.P., Gould, J.S., and McCaw, L. (2001) The Dead-Man Zone - A neglected area of firefighter safety Australian Forestry 64(1), 45-50.

Corners Court of Victoria (2002) Report on the investigation and inquest into a wildfire and deaths of five firefighters at Linton on 2 December 1998. Report by the State of Victoria, January 2002.

Country Fire Authority (1983) The major fires originiating 16th February 1983. Report by CFA, December 1983.

Country Fire Authority and Natural Resource and Environment (1999) Report on the operations review of Linton fire/Midland fire \#15 on Wednesday 2nd December 1998. Operations review team report, March 1999.

Cruz, M.G., Gould, J., Kidnie, S., Nichols, D., Anderson, W., Bessell, R., Hurley, R., Koul, V., (2014) Grass Curing and Fire Behaviour. CSIRO Report (EP146628) prepared for CFA, August 2014.

Cruz, M.G., Hurley, R., Bessell, R., Nichols, D. (2016) Brucknell vehicle burn trial - report on fuels, weather and fire behaviour. CSIRO Report prepared for CFA, June 2016.

Leonard, J., Macindoe, L., Brown, S.K. (2013) Ultra-light Fire Tanker Gel Burnover Assessment May 2013. CSIRO Report prepared for CFA, May 2013.

Leonard, J., Macindoe, L., Brown, S.K. (2014) Fire Tanker Grassfire Burnover Assessments May 2014. CSIRO Report prepared for CFA, May 2014.

Leonard, J., Macindoe, L., Brown, S.K., Newnham, G. (2016) Fire Tanker Bushfire Assessment 2016. CSIRO Report prepared for CFA, March 2016.

Leonard, J., Newnham, G., Blanchi, R., Brown, S., McIndoe, L. (2017) CFA Ultra-light Fire Tanker CAFS Burnover Assessment. Mogo NSW - 23rd November 2017. CSIRO Report (EP181372) prepared for CFA, November 2017.

Leonard, J., Newnham ,G., Blanchi, R., Brown, S., MacIndoe, L. (2018) CFA ISUZU Fire Tanker Burnover Spray Assessment 2017, 1 CSIRO Report (EP183195) prepared for CFA, June 2018.

Nichols, D. (2010) Wildfire Vehicle Crew Protection. Conference Proceedings VI International Conference on Forest Fire Research, November 2010.

Reisen, F. \& Brown, S.K. (2009) Australian firefighters' exposure to air toxics during bushfire burns of autumn 2005 and 2006. Environment International 35 (2), 342-352.

Royal Commission into Victoria's Bushfires, McLeod, R. N., Pascoe, S. M., \& Teague, B. G. (2010). Final report. [Melbourne]: Government Printer for the State of Victoria. 


\title{
Temporal variability of the Haines index and its relationship with forest fire in Portugal
}

\author{
Lourdes Bugalho \\ Instituto Português do Mar e da Atmosfera, Rua C do Aeroporto, Lisboa, ORCID 0000-0001- \\ 7034-0623, \{lourdes.bugalho@ipma.pt\}
}

\begin{abstract}
The above-ground atmospheric conditions, such as atmospheric stability and moisture content, play a critical role in fire behaviour, especially for larger fire. The Haines Index and Continuous Haines Index are widely used as a measure of above ground conditions relevant to wild land fire, especially in Australia. This work presents the temporal variability of the Haines index and the continuous Haines index, and its relationship with the large forest fires in Portugal in the period from 2011 to 2017.
\end{abstract}

Keywords: Forest Fire Risk, Haines Index, Continuous Haines Index

\section{Introduction}

Forest fires are one of the most important natural hazards affecting Portugal, especially during summer time, with enormous economic and social impact. They are also indirectly responsible for fast changes on the land cover. Wildfires typically occur during periods of increased temperature and drought.

Among the fire risks that are dependent on meteorological parameters, the most usual and known one is the FWI (Canadian Fire Weather Index), used in several countries since the mid-1990s. However, the above-ground atmospheric conditions, such as atmospheric stability, also play a critical role in fire behaviour, especially for larger fires. In 1988 Haines (Haines, DA 1988) developed the Lower Atmosphere Stability Index, or Haines Index (HI). It is used to indicate the potential for wildfire growth, given ignition has occurred. It measures the stability and dryness of the air over the fire. The $\mathrm{HI}$ is widely used as a measure of above ground conditions relevant to wild land fire (Potter, BE et al, 2003, Potter, BE et al, 2008, Taitl, H. et al, 2014) and it is derived from the stability (temperature difference between different levels of the atmosphere) and moisture content (dew point depression) of the lower atmosphere. The Continuous Haines Index (CHI) was developed in Australia (Mills, GA et al, 2010), since climatology conditions in Australia were considerably different compare with the original used to develop the Haines Index. For the climatology conditions in Portugal, the CHI seems also more appropriate than the HI.

Management decisions for wild fires incorporate a vast array of factors that are weighted differently. These factors can be infrastructure at risk, resources available, fuel conditions, weather conditions, firefighter safety, public safety from fire and smoke. The relative weights of the factors are highly dependent on the local specific situation, such as population density or the type and stress of the vegetation, and the uncertainty or reliability of any data used in the decisions is an important piece of information. This knowledge includes the description of the climatological characteristics of the indices $\mathrm{HI}$ and $\mathrm{CHI}$ and their sub-indices, stability term (term A) and moisture term (term B). In this work the $\mathrm{HI}$ and $\mathrm{CHI}$ indices are analyzed in their temporal variability, annual and monthly, and in their relationship with periods of large forest fires. The results are presented for the period from 2011 to 2017. The main objective of this work is to understand the behavior of $\mathrm{HI}$ and $\mathrm{CHI}$ in Portugal. 


\section{Method}

\subsection{Haines and Continuous Haines Index}

The stability and moisture content of different atmospheric levels are generally used terms to assess the risk in the propagation and evolution of a forest fire, assuming ignition occurs. The HI combines these two terms to determine the environmental potential for the growth of forest fires. Knowledge of typical HI values, together with the weather conditions that generate them, can be extremely useful in prevention and planning specific fire-fighting strategies. The calculation of the HI is performed according to Table 1, where the risk values associated to the stability term (term A) and values of risk associated with the term of humidity (term B) are found. The sum of the two terms is the value of the HI.

The higher the value of term A, the more unstable the atmosphere is, and the higher the probability of fires dominated by convective feathers. Similarly, drier atmospheric conditions, that are higher values of term B, favor the propagation of forest fires. Thus, HI values of 5 or 6 indicate critical conditions for the formation of convective feathers and high propagation speeds.

Table 1 - Variables and scales for calculating the Haines index according to the altitude of the place (Haines, 1988; Choi et al., 2006, Potter et al., 2008)

\begin{tabular}{|c|c|c|c|c|c|c|}
\hline $\begin{array}{c}\text { Surface } \\
\text { Elevations }\end{array}$ & $\begin{array}{c}\text { Pressure } \\
\text { Level }\end{array}$ & $\begin{array}{c}\text { Stability } \\
(T P 1-T P 2)\end{array}$ & $A$ & $\begin{array}{c}\text { Humidity } \\
(T P 3-T d P 3)\end{array}$ & $B$ & $H I=A+B$ \\
\hline \multirow{3}{*}{$\begin{array}{c}<305 \mathrm{~m} \\
(\text { low })\end{array}$} & \multirow{3}{*}{$\begin{array}{c}\mathrm{P} 1=950 \mathrm{hPa} \\
(925 \mathrm{hPa}) \\
\mathrm{P} 2=850 \mathrm{hPa} \\
\mathrm{P} 3=850 \mathrm{hPa}\end{array}$} & $<4^{\circ} \mathrm{C}\left(2,7^{\circ} \mathrm{C}\right)$ & 1 & $<6^{\circ} \mathrm{C}$ & 1 & \multirow{9}{*}{$\begin{array}{l}\text { Potential for } \\
\text { large fires } \\
\text { 2-3: very low } \\
\text { 4: low } \\
\text { 5: mid } \\
\text { 6: high }\end{array}$} \\
\hline & & $\begin{array}{l}\geq 4^{\circ} \mathrm{C}\left(2,7^{\circ} \mathrm{C}\right) \\
<8^{\circ} \mathrm{C}\left(6,7^{\circ} \mathrm{C}\right)\end{array}$ & 2 & $\begin{array}{l}\geq 6^{\circ} \mathrm{C} \\
<10^{\circ} \mathrm{C}\end{array}$ & 2 & \\
\hline & & $\geq 8^{\circ} \mathrm{C}\left(6,7^{\circ} \mathrm{C}\right)$ & 3 & $\geq 10^{\circ} \mathrm{C}$ & 3 & \\
\hline \multirow{3}{*}{$\begin{array}{c}305-914 \mathrm{~m} \\
\text { (mid) }\end{array}$} & \multirow{3}{*}{$\begin{array}{l}\mathrm{P} 1=850 \mathrm{hPa} \\
\mathrm{P} 2=700 \mathrm{hPa} \\
\mathrm{P} 3=850 \mathrm{hPa}\end{array}$} & $<6^{\circ} \mathrm{C}$ & 1 & $<6^{\circ} \mathrm{C}$ & 1 & \\
\hline & & $\begin{array}{l}\geq 6^{\circ} \mathrm{C} \\
<11^{\circ} \mathrm{C}\end{array}$ & 2 & $\begin{array}{l}\geq 6^{\circ} \mathrm{C} \\
<13^{\circ} \mathrm{C}\end{array}$ & 2 & \\
\hline & & $\geq 11^{\circ} \mathrm{C}$ & 3 & $\geq 13^{\circ} \mathrm{C}$ & 3 & \\
\hline \multirow{3}{*}{$\begin{array}{l}>914 \text { m } \\
\text { (high) }\end{array}$} & \multirow{3}{*}{$\begin{array}{l}\mathrm{P} 1=700 \mathrm{hPa} \\
\mathrm{P} 2=500 \mathrm{hPa} \\
\mathrm{P} 3=700 \mathrm{hPa}\end{array}$} & $<18^{\circ} \mathrm{C}$ & 1 & $<15^{\circ} \mathrm{C}$ & 1 & \\
\hline & & $\begin{array}{l}\geq 18^{\circ} \mathrm{C} \\
<22^{\circ} \mathrm{C}\end{array}$ & 2 & $\begin{array}{l}\geq 15^{\circ} \mathrm{C} \\
<21^{\circ} \mathrm{C}\end{array}$ & 2 & \\
\hline & & $\geq 22^{\circ} \mathrm{C}$ & 3 & $\geq 21^{\circ} \mathrm{C}$ & 3 & \\
\hline
\end{tabular}

Both $\mathrm{HI}$ and $\mathrm{CHI}$ combine a term of humidity and a term of stability to predict the potential for fire propagation given ignition occurs. The difference between the two is that instead of assigning a score to each term resulting in a value between 2 and 6 , the $\mathrm{CHI}$ ranges any value between 0 and approximately 14 . The $\mathrm{CHI}$ index eliminates the abrupt transitions between the categories and provides greater discrimination at high values. It also allows a more realistic assessment of the contributions of atmospheric instability and dew point depression to the overall score. For this reason, the CHI index is now included, together with other indices, in operational activities in Portugal.

The calculation of the $\mathrm{CHI}$ index is performed using the following equations:

$$
\begin{aligned}
& \mathrm{CA}=0.5(\mathrm{~T} 850-\mathrm{T} 700)-2 .\text { (continuous stability term, } \mathrm{CA}) \\
& \mathrm{CB}=0.3333(\mathrm{~T} 850-\mathrm{DP} 850)-1 \quad(\text { continuous moisture term, } \mathrm{CB}) \\
& \mathrm{CHI}=\mathrm{CA}+\mathrm{CB}
\end{aligned}
$$

Subject to the following conditions:

If $\left(\mathrm{T} 850\right.$ - DP850)> $30{ }^{\circ} \mathrm{C}$, then $(\mathrm{T} 850-\mathrm{DP} 850)=30^{\circ} \mathrm{C}$

If $\mathrm{CB}>5$., then $\mathrm{CB}=5 .+(\mathrm{CB}-5) /$.2 . 
Here, T850 is the temperature for the $850 \mathrm{hPa}$ level, T700 is the temperature for the $700 \mathrm{hPa}$ level and DP850 is the dew point temperature for the $850 \mathrm{hPa}$.

As mentioned, one of the features of the $\mathrm{CHI}$ was to obtain greater discrimination at higher values of risk. Terms CA and CB have an upper limit of approximately 6.5 and 7, respectively. Thus, the upper limit of the proposed $\mathrm{CHI}$ is approximately 14 . There is no specified lower limit, but that is not the range of interest. In the datasets developed in this study, the values are limited so not to be less than zero.

\subsection{Data}

The meteorological data were obtained from the analysis of the European Centre for MediumRange Weather Forecasts (ECMWF) atmospherics' model, with grid spacing of approximately $16 \mathrm{~km}$ ( $0.125^{\circ}$ latitude and longitude) from 2011 to 2017 , at 00, 06, 12 and 18 UTC.

The computation of $\mathrm{HI}$ and $\mathrm{CHI}$ require temperature values at the 950, 850, 700 and $500 \mathrm{hPa}$ pressure levels, and dew point temperatures at the 850 and $700 \mathrm{hPa}$ levels. In the absence of information for the $950 \mathrm{hPa}$ level, the required data, namely the temperature, was obtained by interpolation between two levels containing the $950 \mathrm{hPa}$. This level is only required for land altitudes lower than 305m (see Table 1).

The HI and CHI statistical values, such as monthly averages, percentiles, number of days with value above a certain threshold, were calculated for each pixel in a rectangle containing Portugal, between latitudes $41.875^{\circ} \mathrm{N}$ and $36.25^{\circ} \mathrm{N}$ and longitudes $-6.0 \mathrm{~W}$ and $-9.375 \mathrm{~W}$. The calculations were made on a monthly basis, considering the values for each hour (00, 06, 12 and 18 UTC) and for each day. One of the most important information is the number of days with $\mathrm{CHI}$ value above threshold 10, considered to be a very high-risk value. If the CHI exceeds 10 at any of the times $00,06,12$ or $18 \mathrm{UTC}$, in any pixel point, of a certain day, then that day is accounted as a day with CHI value above 10. Indeed, Gwangyong Choi showed that daily high HI and its monthly frequency have statistically significant correlations with the frequency of the wildland fire occurrences during the fire season (DecemberApril) in South Korea, (Gwangyong Choi et al, 2006).

The forest fire data was obtained from the Instituto da Conservação da Natureza e das Florestas (ICNF). Monthly data were obtained for the period 2011 to 2017.

\section{Climatological Characterization of the Haines Index and Continuous Haines Index in Portugal}

\subsection{Climatology of the Haines Index (HI) in Portugal}

The average monthly values of HI for mainland Portugal are shown in Figure 1. Here, the HI daily value is the average of all pixels. for 00, 06, 12 and 18 UTC. The average monthly values of HI show lower values in January, February, November and December, ranging approximately from 3.6 to 3.7. The higher of HI are in July and August, ranging between 4.4 (at 06UTC) and 5.0 (at 18 UTC). HI values range from 2 to 6 , hence mean values of 4.5 or 5.0 in one month indicate that high daily values, namely above 5, occur with high frequency in almost all the country's pixels. 


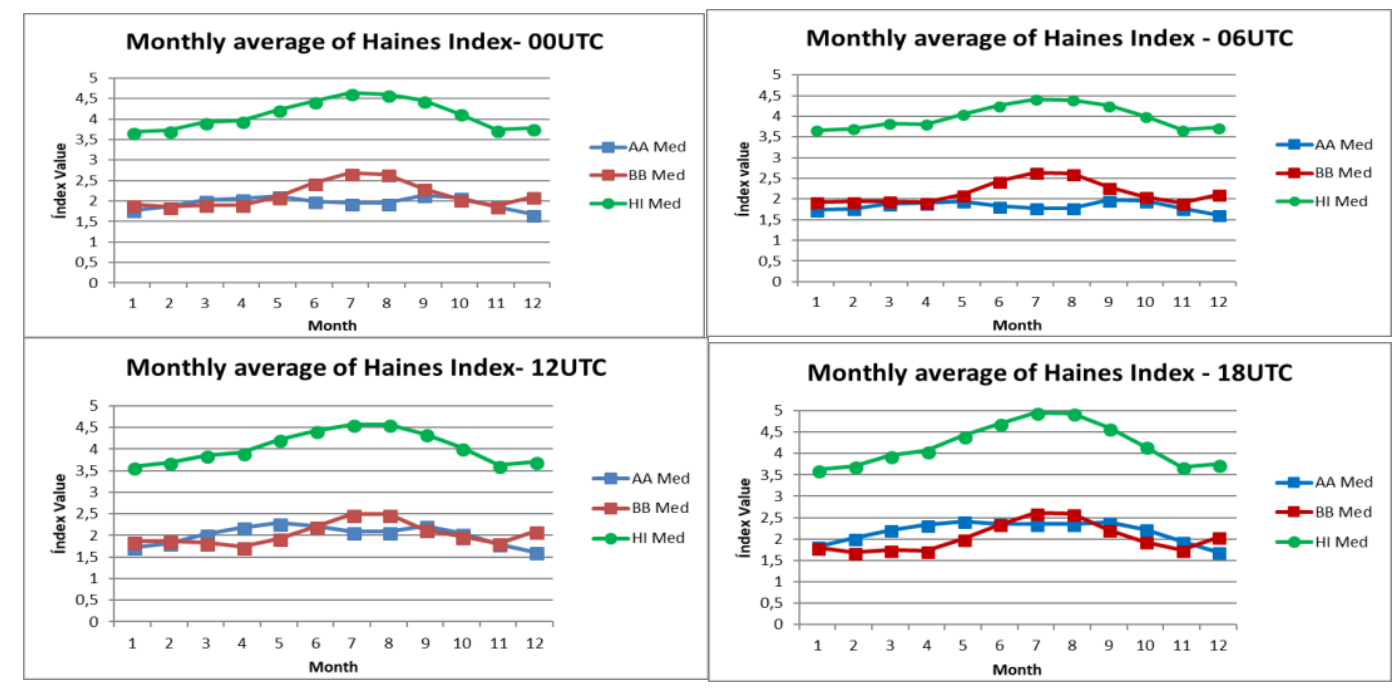

Figure 1 - Average monthly value of the Haines Index (HI) and the sub index A (term associated with stability) and B (term associated with moisture content), at 00, 06,12 and 18 UTC for Continental Portugal in the period 2011-2017

Between 00 UTC and 18UTC, Figure 1, the average monthly values show a slight difference with the highest average values (July and August), around 5.0, attained at 18UTC, while the lowest values, approximately 4.4 , occur at 06UTC and at 12 UTC. The monthly average values of the sub indices, ranging between 1 and 3, do not vary much. The sub index values associated with atmospheric stability (term A) do not change much on a monthly basis, with slightly higher values in May and September, which are months of frequent instability. The sub index values associated with the moisture content (term B) do not vary much either. This sub-index has its lower values in April and November, which are months that are usually associated with higher rainfall and humidity. On the other hand, its higher values occur in July and August, which are months climatologically characterized by low precipitation and dry conditions.

In Figure 2 are presented the values of HI for August at 12 UTC in mainland Portugal. August is one of the months with highest monthly values of the HI. It can be observed that great part of the country presents an average monthly value of 4 , with some parts of Alentejo region reaching 5 . For August at 12 UTC, the 95th percentile is 6 (HI maximum value) throughout the country, except for the coastal zones (see Figure 2). This spatial distribution of the $95^{\text {th }}$ percentile was observed for other hours.
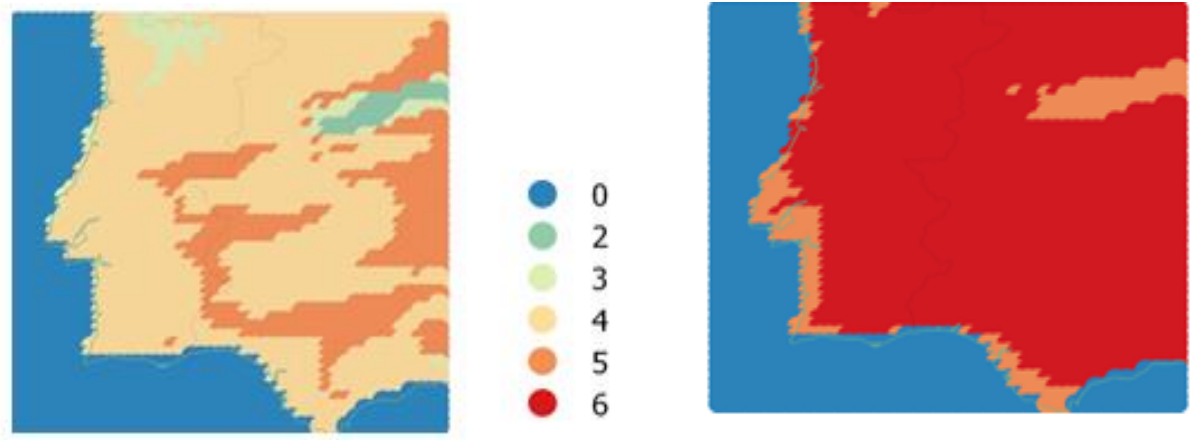

Figure 2 - Maps of the spatial distribution (per pixel) of the monthly average HI value (left) and the 95th HI percentile (right) for August at 12 UTC.

\subsection{Climatology of the Continuous Haines Index (CHI) in Portugal}

The average monthly values of CHI are presented in Figure 3 for 00, 06, 12 and 18 UTC. We observe that the lower monthly average values occur in January, February, March, April, November 
and December, corresponding to winter months, early spring and late fall. The highest monthly average values are observed in July and August. It can also be seen from Figure 3 that the average monthly values at 18UTC are generally slightly higher than at other hours, especially in July.

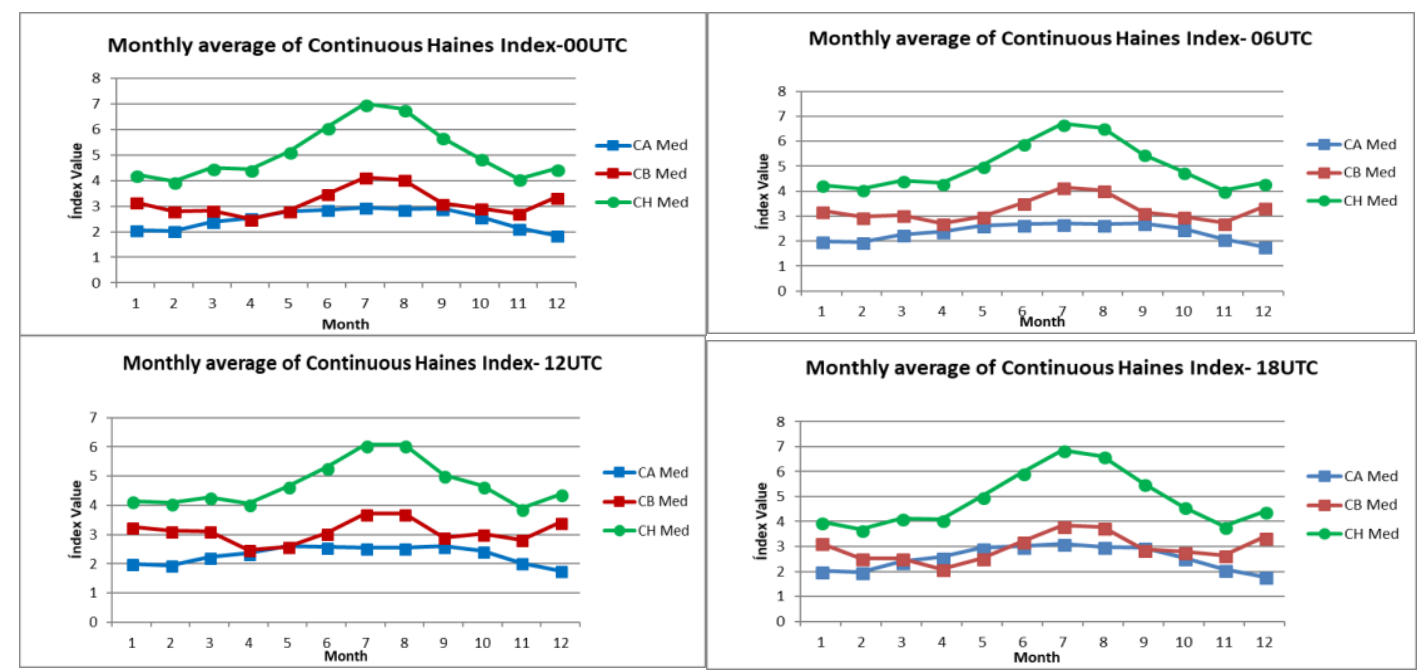

Figure 3 - Average monthly value of the Continuous Haines Index (CHI) and the sub index CA (term associated with stability) and $\mathrm{CB}$ (term associated with moisture content), at 00, 06, 12 and 18 UTC for mainland Portugal.

As expected, the term associated with moisture content (CB) exhibits higher monthly average values in the summer, followed by the winter. In spring and autumn, slightly lower monthly average values are observed. Regarding the monthly average values of the term associated with stability (CA), they present a small monthly variation but with slightly higher values from May to September. For what concerns the monthly values of the 95th percentile of the $\mathrm{CHI}$, it is observed that these are high throughout the country. Furthermore, they do not vary much from January to December, with slightly higher values from June to August and with lower values in November and December. This variation is most pronounced at 18UTC. The term CA associated with the atmospheric stability in the 850-700 hPa layer presents slightly higher 95th percentile values between May and October, compared to the remaining months of the year.

For a better understanding of the behavior of CHI, it is necessary to analyze the extreme cases, looking at the maximum values of the index. Figure 4 illustrates the maximum value of $\mathrm{CHI}$ for mainland Portugal in the period from 2011 to 2017.

Monthly Maximum Value for 00, 06, 12 and 18 UTC

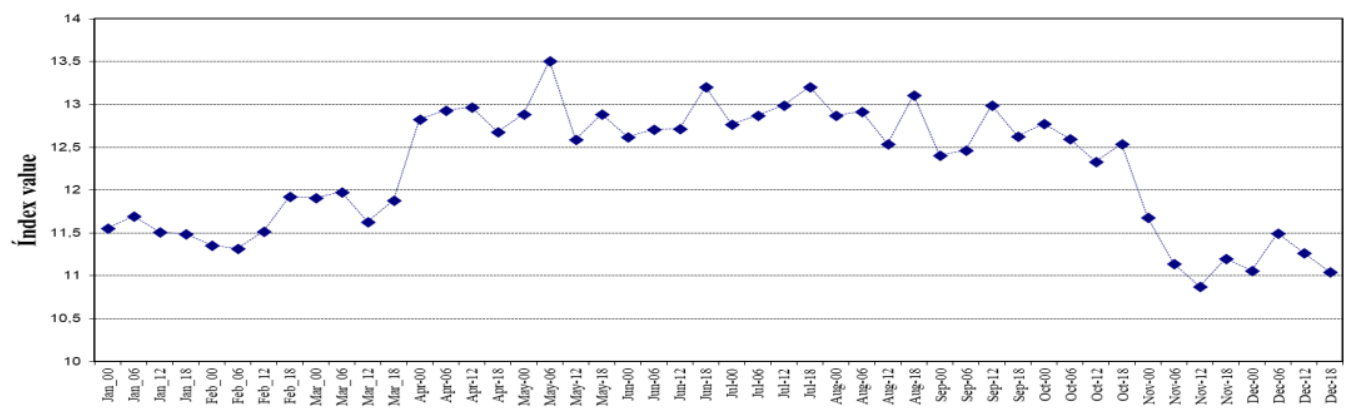

Figure 4 - Maximum monthly value of CHI at 00, 06, 12 and 18 UTC, from 2011 to 2017, for Portugal.

From Figure 4, CHI monthly maximum values are always greater than 10, for all months, for all the considered hours $(00,06,12$ and 18 UTC), and in the study period from 2011 to 2017. In months 
from January to March, the monthly maximum values for the considered hours were between 11.3 and 12. In months from April to October the maximum values of range from 12.3 to 13.5, above 12, which is an extremely high value. The maximum values for the study period are higher than 13 for May at 06UTC and for June, July and August at 18 UTC.

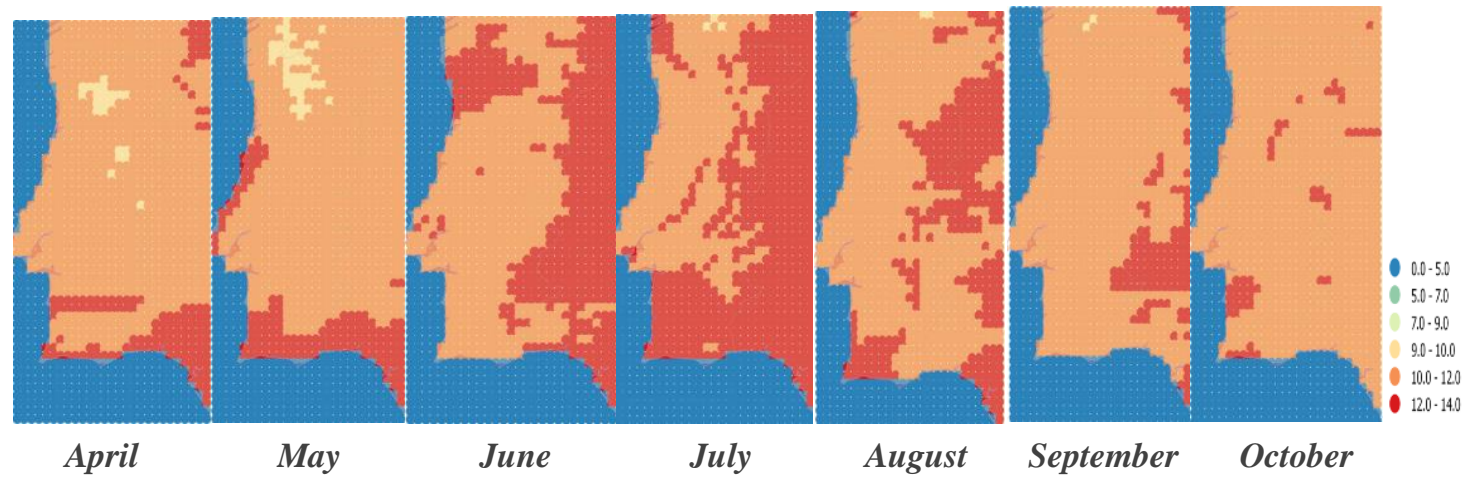

Figure 5 - Map of the monthly maximum CHI value at 18 UTC, for months from April to October, in the period from 2011 to 2017.

The spatial CHI distribution of the monthly maximum values at 00, 06, 12 and 18 UTC was also analyzed. In particular, Figure 5 shows the monthly maximum values per pixel at 18UTC from April to October, in the study period ranging from 2011 to 2017. From April to October, at 18UTC, some pixels with $\mathrm{CHI}$ peak values, between 12 and 14, are observed. In April and May the highest peak values of CHI, above 12, are observed in the southern coast region. In May, peak values above 12 are also observed in the coastal region, north of Cabo da Roca. From June to August, maximum values of $\mathrm{CHI}$ higher than 12 are observed in general in the interior regions. This spatial distribution of CHI peak values is consistent with the fact that in the summer the conditions of great dryness are frequent, as well as instability conditions. This dryness and instability conditions are particularly noticeable in the southern and interior regions in the period from middle afternoon to night's beginning.

The number of days per month with CHI values above 10 was also analyzed and it is shown in Figure 6. From the results, it can be seen that:

- The months of May to October usually have a greater number of days with a $\mathrm{CHI}$ index greater or equal to 10;

- July and August show the highest values (25 in July 2015 and 25 in August 2016);

- 2017 had the highest number of days with CHI greater or equal to 10 of March, April and October months from 2011 to 2017;

- 2015 had the highest number of days with CHI greater or equal to 10 of June and July months from 2011 to 2017 ;

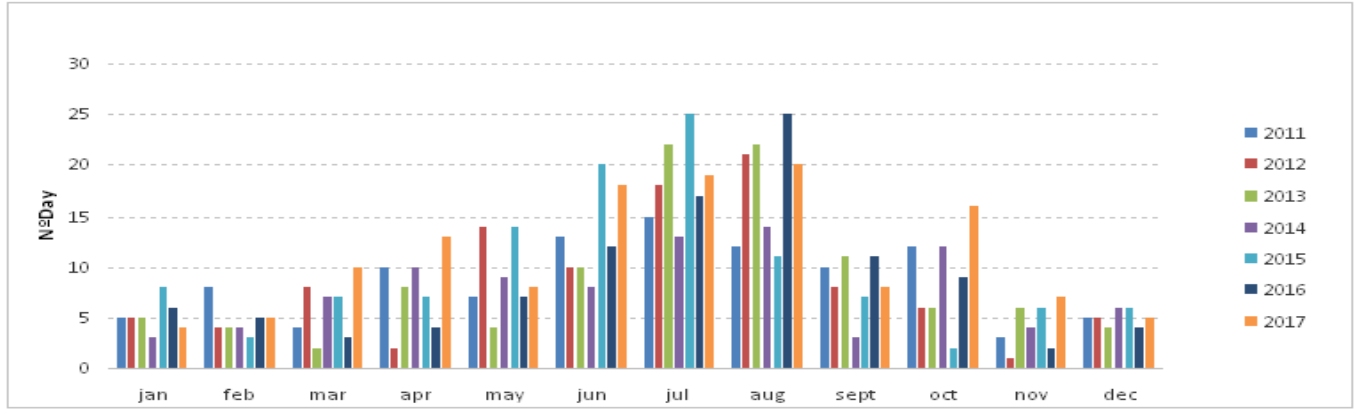

Figure 6 - Number of days with CHI value equal to or greater than 10, per month and per year, from 2011 to 2017.

It is interesting to note that in March 2012, when large forest fires and several fire events occurred, the number of days with CHI larger or equal to 10 was almost has high as in 2017. Also, the large 
fires, with convective feathers, that occurred in 2017 happened in June and October, when the number of days with $\mathrm{CHI}$ greater or equal to 10 was also very high.

The spatial distribution of the number of days with CHI greater or equal to 10 can be seen in Figures 7, 8 and 9 for June, August and October months, respectively. From these figures we can observe the regions with the highest frequencies of high $\mathrm{CHI}$ values, that is, with the higher number of days with CHI larger or equal to 10.

From Figure 7, regarding June, we can see that in 2015 and 2017 the frequency of days with CHI higher or equal to 10 was very high, compared with the remaining years. Namely, in the central and southern interior regions and the north and central coast. In 2017 this was also observed in the central coast regions, where the June fires occurred. 2016 and 2014 were the years with the lower values, between 1 and 3 days, in almost all the country.

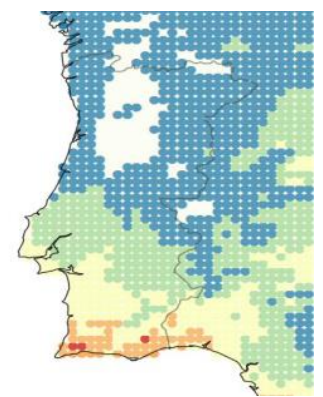

2011

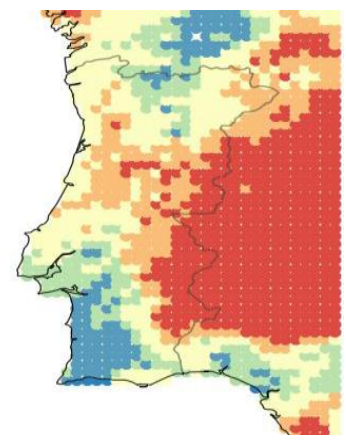

2015

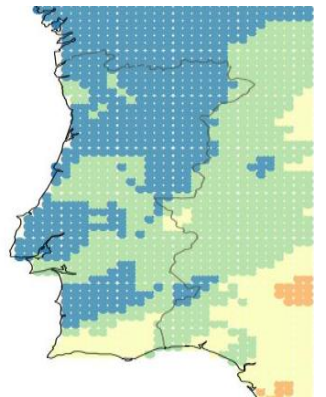

2012

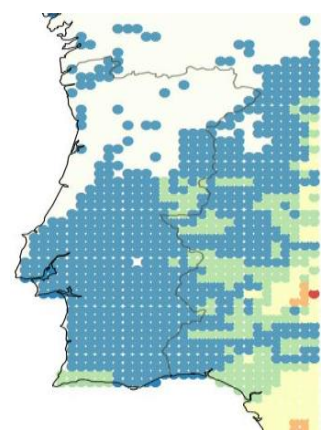

2016

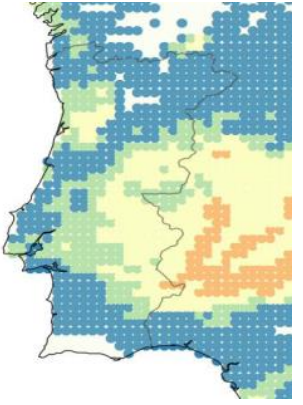

2013

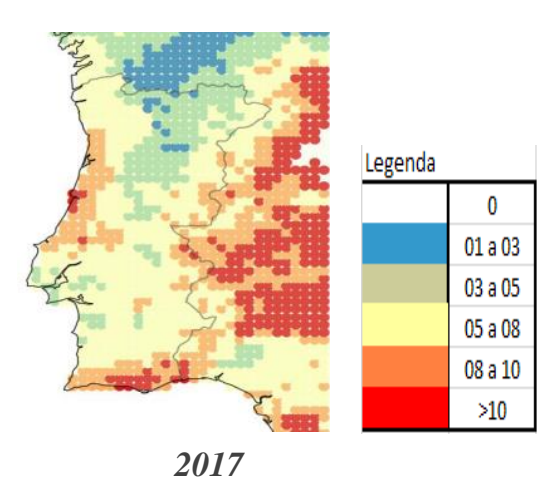

2017

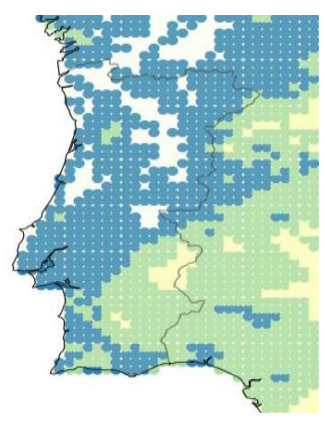

2014

Figure 7 - Maps of the spatial distribution of the number of days with a CHI value greater or equal to 10 in June, in the period from 2011 to 2017 


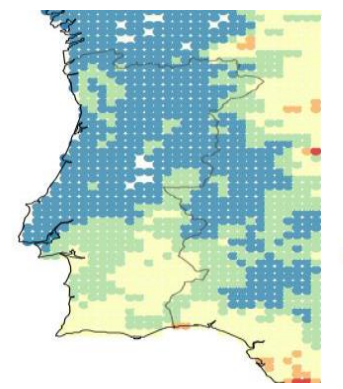

2011

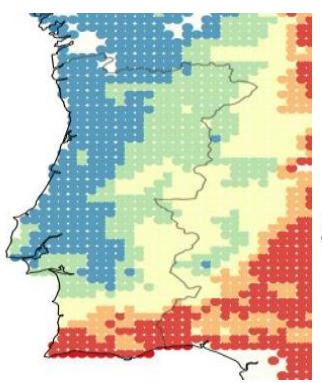

2012

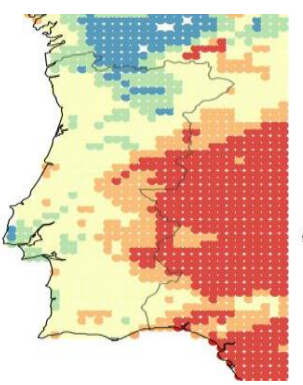

2013

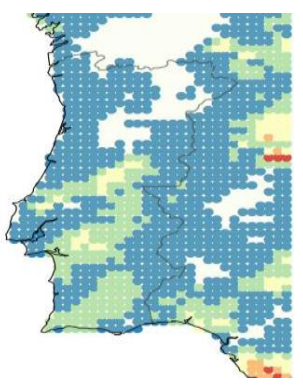

2014

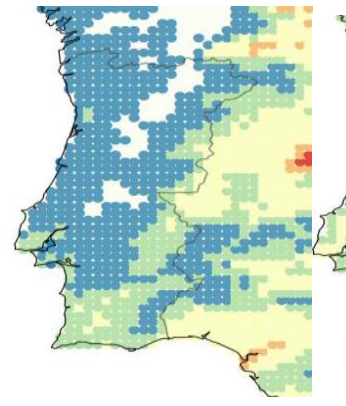

2015

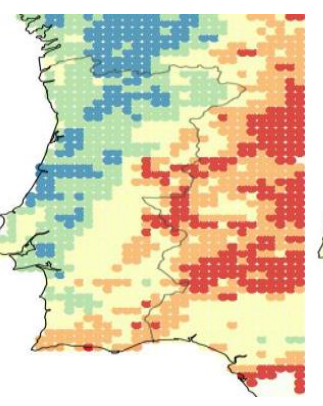

2016

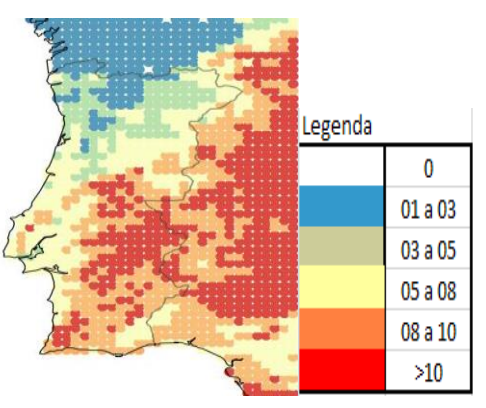

2017

Figure 8 - Maps of the spatial distribution of the number of days with a CHI value greater or equal to 10 in August, in the period from 2011 to 2017

From Figure 8, regarding August, which is a month generally associated with forest fires, we find that the regions with the higher number of days with CHI greater or equal to 10 are the interior and south. The years with the highest values were 2012, especially in the southern region, 2013 and 2016, in the interior, central and southern regions, and 2017, in the entire central and southern region. We also observe from Figure 8 that in 2013 the number of days with $\mathrm{CHI}$ greater or equal to 10 was abnormally high throughout the country, including the western coastal regions. This situation was again observed in 2017, where values were abnormally high throughout the central and southern regions. It should be noted that even in the coastal region north of Cabo da Roca, the values were the highest in the period from 2011 and 2017.

From Figure 9, concerning October, very low values are observed in general, with very few days with values of the CHI larger or equal to 10. Exceptions were the years of 2014, especially in the regions of the south, 2011 throughout the country, and especially 2017, also throughout the country. In 2017 some regions, such as near Aveiro and in some regions of the center, had a high frequency, more than 10 days, with $\mathrm{CHI}$ greater or equal to 10 . This represents an extremely high value for October month, and corresponds to the regions and time of the largest forest fires. 

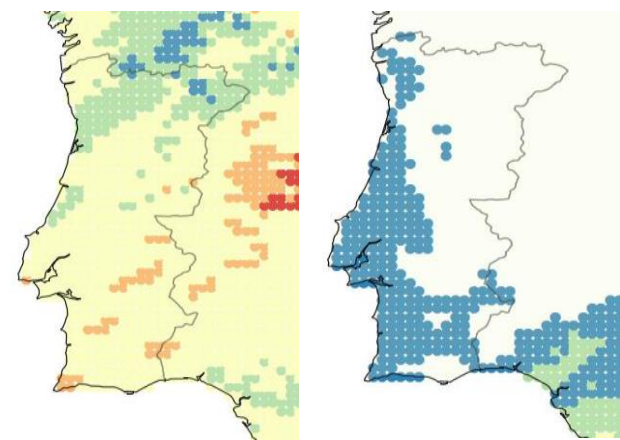

2011

2012

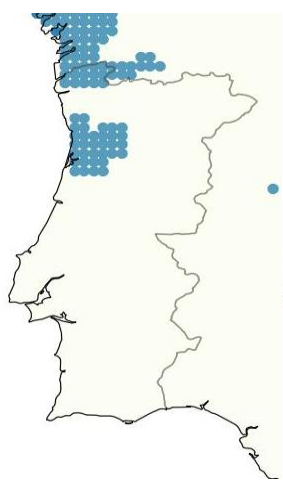

2015

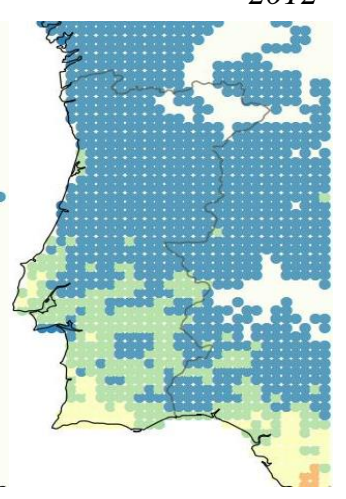

2016

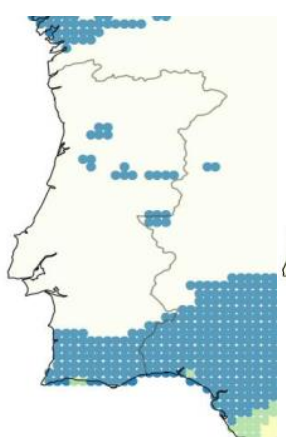

2013

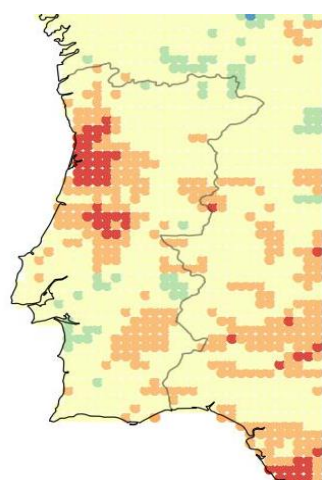

2017

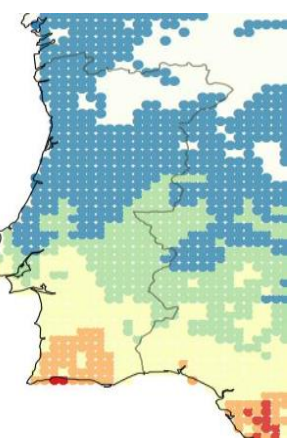

2014

Figure 9 - Maps of the spatial distribution of the number of days with a CHI value greater or equal to 10 in October, in the period from 2011 to 2017

\subsection{Continuous Haines Index (CHI) and Forest Fire Data}

The value of the Pearson correlation between the number of days with $\mathrm{CHI}$ larger or equal to 10 and the monthly burned area data, in the period from 2011 to 2017, is 0.46 . Also, the Pearson correlation between the number of days with CHI larger or equal to 10 and the number of occurrences of forest fires is 0.63 , in the same period. The years with the highest burned areas of the study period were 2017, followed by 2016 and 2013. The correlation for these three years were $0.56,0.81$ and 0.77 , respectively for 2017, 2016 and 2013, for the burnt area, and 0.81, 0.84 and 0.93, respectively for 2017, 2016 and 2013, for the number of fire events. The behavior of the burnt area and number of fire events for these three years are depicted in Figures 10, 11 and 12, for years 2013, 2016 and 2017, respectively.
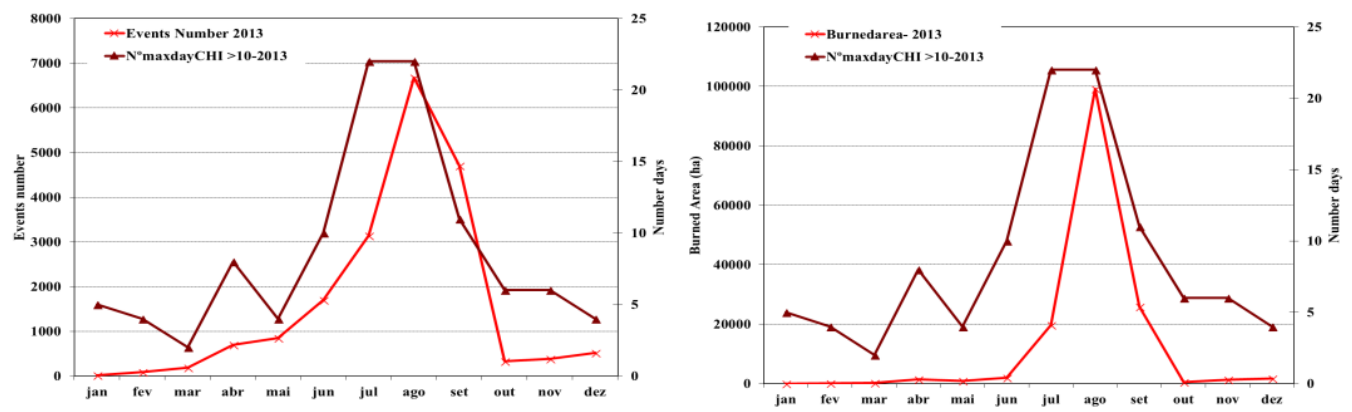

Figure 10 - Monthly values of the number of events (left) and burnt area in ha (right) of the forest fires and number of days with values of $C H I \geq 10$, in 2013. 

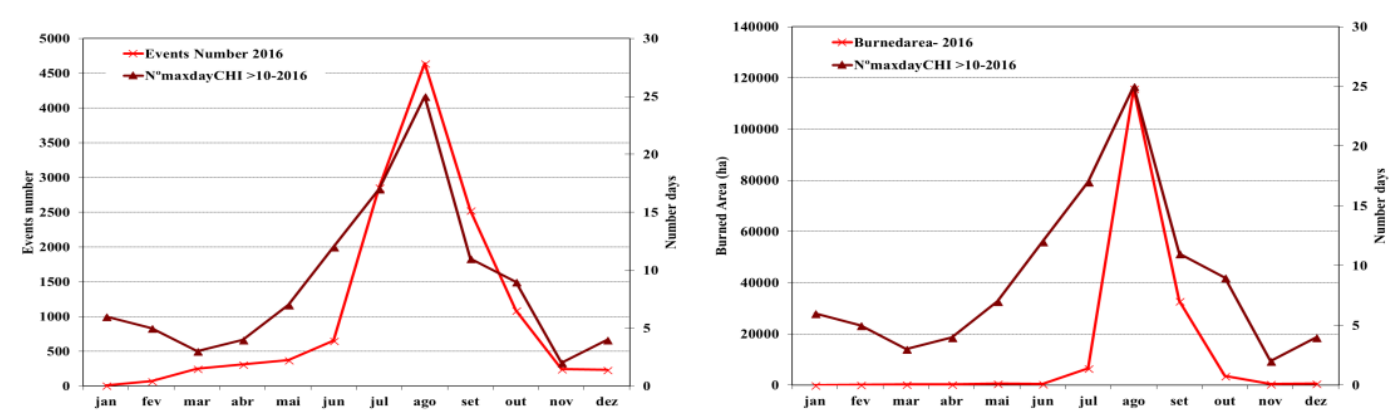

Figure 11 - Monthly values of the number of events (left) and burnt area in ha (right) of the forest fires and number of days with values of $C H I \geq 10$, in 2016.
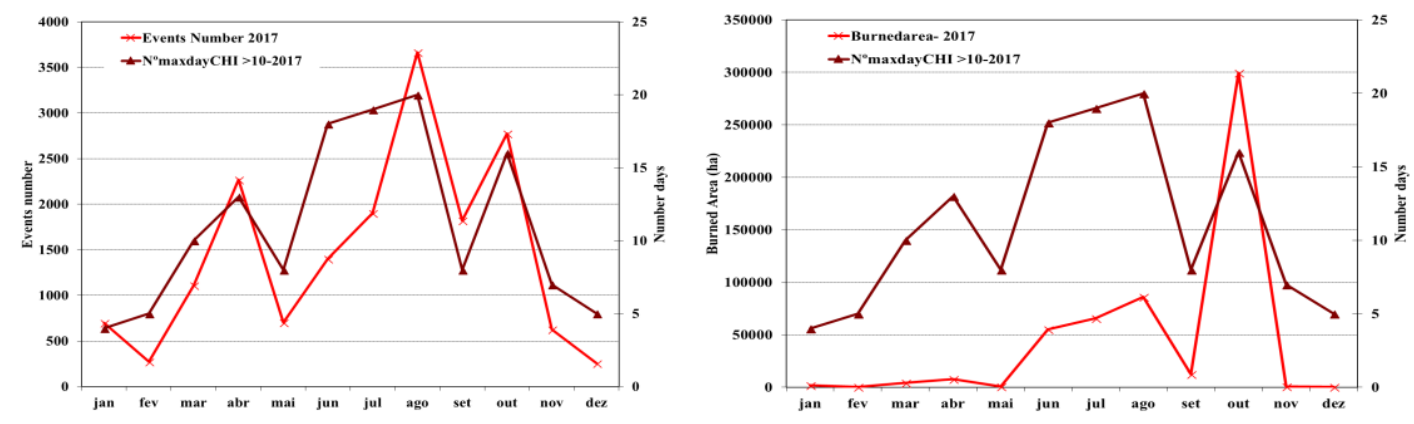

Figure 12 Monthly values of the number of events (left) and burnt area in ha (right) of the forest fires and number of days with values of $\mathrm{CHI} \geq 10$, in 2017.

\section{Conclusions}

In recent years there has been increasing awareness of the importance of the vertical structure of the low atmosphere in the formation of catastrophic forest fires. All forest fire risk indices provide information that may help in prevention and firefighting strategies. The importance of the Continuous Haines Index (CHI) is that it provides information on the stability conditions and the moisture content, generally above the boundary layer of the atmosphere, between 850 and $700 \mathrm{hPa}$, completing the information given by calculated risk indices with surface data.

The study period, from 2011 to 2017 , may be small given the variability of favorable weather conditions for forest fires. It should be noted that the year 2017, having been a year with very burdensome conditions for forest fires and abnormally high areas burned in forest fires, has a great influence on statistical values.

In relation to the climatology of $\mathrm{CHI}$ in Portugal we can conclude the following.

- The highest values of CHI occur from April to October, with maximum values occurring at 18 UTC from June to August;

- The regions of the south coast and the interior are where the highest values of CHI are reached. Namely, CHI peak values, ranging from 12 to 14, are observed in the July and August in the southern regions and at all hours;

- The maximum values of CHI are observed in general in the interior regions at 18 UTC, especially in July and August;

- The months from May to October usually have a greater number of days with a CHI index greater or equal to 10, with July and August having the highest frequency. 
The frequency of high values of this index, given by the number of days with high values, relates well to the areas burned and to the number forest fire events. This is particularly observed for years with larger burnt areas. We can hence conclude that the number of days with CHI larger or equal to 10 is a relevant indicator for characterizing conditions favorable to forest fires.

\section{References}

Gwangyong Choi, Junsu Kim, and Myoung-Soo Won, (2006) - Spatial Patterns and Temporal Variability of the Haines Index related to the Wildland Fire Growth Potential over the Korean Peninsula, Journal of the Korean Geographical Society, Vol. 41, No. 2, 2006(168 187)

Haines, D.A. 1988. A lower atmospheric severity index for wildland fire. National Weather Digest. Vol 13. No. 2:23-27.

Littell, J. S., McKenzie, D. , Peterson, D. L. and Westerling, A. L. (2009), Climate and wildfire area burned in western U.S. ecoprovinces, 1916-2003. Ecological Applications, 19: 1003-1021. doi:10.1890/07-1183.1

Mills, Graham A. and Lachlan McCaw, 2010: Atmospheric Stability Environments and Fire Weather in Australia - extending the Haines Index CAWCR Technical report No. 20 March 2010

Potter BE. 1996. Atmospheric properties associated with large wildfires. Int. J. Wildland Fires 6: 7176.

Potter BE, Goodrick S. 2003. Performance of the Haines Index during August 2000 for Montana. In Proceedings of the 4th Symposium on Fire and Forest Meteorology, 13-15 November 2001, Reno, NV . American Meteorological Society: Boston, MA; 233-236.

Potter BE, Winkler JA, Dwight FW, Ryan PS, Xindi B. 2008.Computing the low-elevation variant of the Haines Index for fire weather forecasts. Weather Forecast. 23: 159-167.

Pyne, S. J., Andrews, P. L., and Laven, R. D.: Introduction to wildland fire, 2 edition, Wiley, New York, 769 pp., 1996

Tatli, H. and Murat T"urkes, (2014):"Climatological evaluation of Haines forest fire weather índex over the Mediterranean Basin" Meteorol. Appl. 21: 545-552 (2014) Published online 22 February 2013 in Wiley Online Library (wileyonlinelibrary.com) DOI: 10.1002/met.1367

Vázquez A, Moreno JM (1993) Sensitivity of fire occurrence to meteorological variables in Mediterranean and Atlantic areas of Spain. Landscape and Urban Planning 24,129-142.

Williams, R. J., Gill, A. M., and Moore, P. H. R.: Seasonal Changes in Fire Behaviour in a Tropical Savanna in Northern Australia, Int. J. Wildland Fire, 8, 227-239, 2001

Williams, A., Karoly, D., and Tapper, N.: The sensitivity of Australian fire danger to climate change, Climatic Change, 49, 171-191, 2001.

Viegas, D., Bovio, G., Ferreira, A., Nosenzo, A., and Sol, B.: Comparative study of various methods of fire danger evaluation in southern Europe, Int. J. Wildland Fire, 9, 235-246, 1999. 


\title{
The extreme weather conditions behind the destructive fires of June and October 2017 in Portugal
}

\author{
Miguel M. Pinto ${ }^{1}$; Alexandra Hurduc $^{1}$; Ricardo M. Trigo ${ }^{1}$; Isabel F. Trigo ${ }^{2}$; Carlos C. DaCamara ${ }^{1}$ \\ ${ }^{1}$ Instituto Dom Luiz (IDL), Faculdade de Ciências, Universidade de Lisboa, Lisbon, 1749-016, \\ Portugal. \{mnpinto@fc.ul.pt*; ahurduc@fc.ul.pt; rmtrigo@fc.ul.pt; cdcamara@fc.ul.pt\} \\ ${ }^{2}$ Instituto Português do Mar e da Atmosfera (IPMA), Lisbon, 1749-077, Portugal. \\ \{isabel.trigo@ipma.pt\}
}

\begin{abstract}
The 2017 fire season in Portugal was marked by two tragic events leading to a toll of 116 fatalities and serious impacts at social, economic, and ecological levels. The first of the two events started on June 17 and the second on October 15. With the aim to better understand and predict future similar situations, we conducted an analysis of the meteorological conditions in which the two fire events took place. First, we analyzed the events in a high-resolution meteorological context, using simulations by the Weather Research and Forecasting Model (WRF) and then within a climatic context with resource to ERA-Interim reanalysis. Fire Weather Index (FWI) was used to evaluate the meteorological conditions at the surface level while the Continuous Haines Index (CHI) was employed to assess the atmospheric instability conditions. WRF simulations show high temperature and low relative humidity for both fire events. Conversely, the October 15 event presents strong winds whereas the June 17 event show weak winds at synoptic level. However, for the June event, the model simulates several downburst phenomena, associated with a mesoscale convective system, in agreement with the Doppler radar observations that show several gust fronts associated to the convective system that developed during the evening, reinforcing the need for the CHI to assess the vertical instability conditions. Monthly climatology of daily FWI and CHI were computed for the period spanning 1979-2016. Results show that for October 15, FWI is the new absolute maximum in the daily time-series, and CHI is at the percentile 90 for the extended fire season (June to September). On the other hand, for June 17, FWI is near the percentile 75 for the extended fire season and CHI is close to the percentile 99 . We conclude that both fire events were associated to extreme meteorological conditions, however their characteristics are very distinct. For the June 17 event the atmospheric instability played a main role in the fast spread of the fire, whereas for the October 15 the strong winds associated with the close passage of hurricane Ophelia, together with extreme drought conditions were the main drivers.
\end{abstract}

Keywords: wildfires, mega-fires, weather models, Fire Weather Index, Continuous Haines Index.

\section{Introduction}

Forest fires are an important characteristic of Mediterranean climate. Portugal is regularly affected by large and destructive wildfires leading to serious impacts at social, economic, and ecological levels (Amraoui et al. 2015). The 2017 fire season was particularly conspicuous in several ways, with a record total burned area of about 500000 hectares and tragic toll of 116 fatalities. Furthermore, the unusual extent of the 2017 fire season implied that the two most tragic events occurred prior (17-20 June) and after (15-17 October) the official fire season window used by the Portuguese authorities.

The case of June was marked by a heat wave that affected western and central Europe leading to very unusual conditions for that month (Sánchez-Benítez et al. 2018). The largest fires in central Portugal (Pedrogão-Grande and Gois) started on June 17, a day characterised by anomalous high temperatures and low relative humidity, associated to very unstable atmospheric conditions that favoured the formation of convective cells and thunderstorms. According to the report from the Portuguese Weather Service (IPMA) this instability, further strengthened by local fires, also led to 
several downburst events and associated gust fronts that played an important role in the fast spread of the fire (Moreira et al. 2017).

The case of October was marked by strong and persistent southerly winds caused by the close passage of hurricane Ophelia moving northward. The winds and the very dry vegetation due to the extreme drought situation were the main ingredients for the tragic events of October 15.

In this study we explore the two events, first in a high-resolution meteorological context using simulations by the Weather Research and Forecasting Model (WRF) initialized with ERA5 reanalysis and running on a grid covering Portugal with a spatial resolution of $4 \mathrm{~km}$ and then within a climatic context with resource to ERA-Interim reanalysis (Dee et al. 2011) for the period 1979-2017. Both ERA-Interim and ERA5 reanalysis are produced by the European Centre for Medium-Range Weather Forecasts (ECMWF), being the ERA5 the most recent state-of-the-art dataset, featuring hourly analysis fields with $0.3^{\circ}$ spatial resolution.

\section{Data and Methods}

This study focuses on the region comprising mainland Portugal paying close attention to the subregion delimited by the green box in Figure 1.

To assess the mesoscale weather conditions, we used WRF version 3.9.1 simulations on a $4 \mathrm{~km}$ spatial resolution grid, covering mainland Portugal, initialized with ERA5 reanalysis data as boundary conditions, with 64 vertical levels.

Meteorological fire danger conditions at the surface level were evaluated using the Fire Weather Index (FWI) that is part of the Canadian Fire Weather Index System (CFFWIS, Van Wagner 1974; Van Wagner 1987) and is a widely used fire danger rating system in Mediterranean Europe (e.g.: San-Miguel-Ayanz et al. 2012; DaCamara et al. 2014; Pinto et al. 2018). This index measures the severity due to the present meteorological conditions but has also a memory from the past conditions based on a measure of fuel moisture. The Continuous Haines Index (CHI) is an extension of a discrete index proposed by Haines (1988) to measure atmospheric instability conditions that may allow for erratic fire behaviour (Mills and McCaw 2010).

The climatology of both FWI and CHI was computed using data from ERA-Interim reanalysis for the period spanning 1979 to 2017. Average FWI values for the defined sub-region are computed using 2 metre temperature and relative humidity, 10 metre wind speed and $24 \mathrm{~h}$ accumulated precipitation all referring to the $12 \mathrm{~h} \mathrm{UTC}$. Average $\mathrm{CHI}$ for the subregion are also computed at $12 \mathrm{~h}$ UTC using the temperature in degrees Celsius at 850 and $700 \mathrm{hPa}$ and the dew point temperature at $850 \mathrm{hPa}$ (Mills and McCaw, 2010).

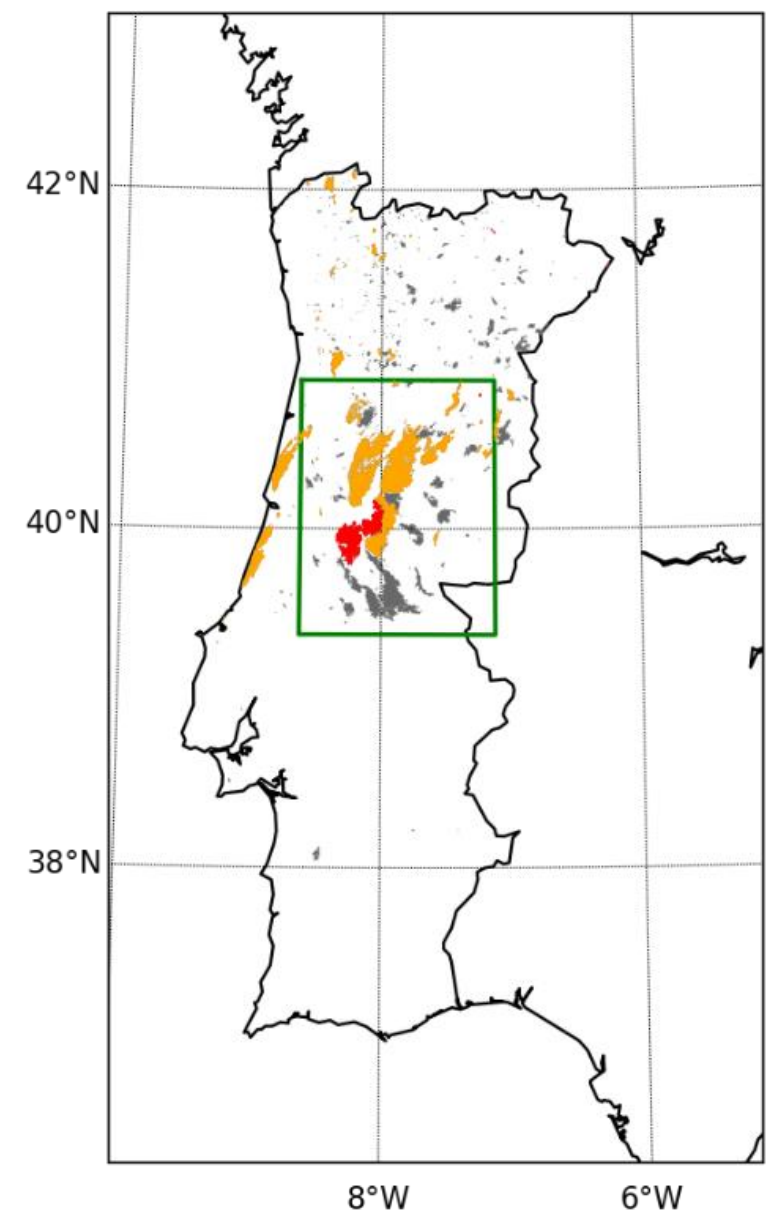

Figure 1 - The map shows the burned areas for 2017 in mainland Portugal for the October case (orange), the June case (red) and the remaining period (gray). The green rectangle delimits the sub-region of study. 
In order to evaluate the sensitivity of FWI to meteorological variables, the average of each input variable is calculated for the respective month during the study period and FWI is then computed replacing each variable by the climatology of the month, one at the time. This allows evaluating how much FWI increases or decreases due to the positive or negative anomalies observed for each variable and to accordingly rank the contribution of each meteorological variable.

\section{Results and discussion}

High-resolution simulations produced by the WRF model at a $4 \mathrm{~km}$ spatial and hourly temporal resolutions allow for a better understanding of mesoscale characteristics that are not neither represented in the coarser grids of reanalyses nor in global circulation models. Figure 2 shows the WRF forecasts for 2 metre temperature, relative humidity and wind speed (left to right panels) valid at 15 UTC of 17 June (top panels) and 15 October, 2017 (bottom panels).

We can see that for June 17 (Fig 2, top panels) values of temperature were very high in most of the region, reaching anomalies of more than $+12^{\circ} \mathrm{C}$ (as marked by black contours) when comparing to the average for June for the period 1979-2017. As for relative humidity, very dry conditions were observed, with values more than $20 \%$ below average over most of the country. For wind speed, the picture is quite distinct, with negative anomalies being observed in most of the study region although the WRF shows several round-shaped regions of strong winds, up to $70 \mathrm{~km} / \mathrm{h}$ at the considered hour. These concentric diverging strong wind regions are consistent with phenomena of downbursts and convective gust fronts that are simulated by the model in association with a convective system (Fujita 1985). Moreover, the location, spatial extent and wind velocity achieved are fairly in agreement with satellite and radar observations that show the development of strong convective storms and the occurrence of multiple convective gust fronts during the afternoon (Moreira et al. 2017). Figure 3 (left panel) presents the average vertical profile for the sub-region of study as simulated by the WRF model at 15 UTC. Dry conditions at the lower atmospheric levels are observed followed by a more humid region that became saturated in places where the convective system developed. Rain or ice droplets falling start to evaporate when passing through the dry region, leading to evaporative cooling that results in downwards acceleration until the air mass reaches the ground and spreads horizontally with strong, sometimes damaging, wind gusts (Fujita 1985).

As shown in the bottom panels of Figure 2, the October 15 event is characterized by high temperatures, with anomalies above $+10^{\circ} \mathrm{C}$ and very low relative humidity values, more than $40 \%$ below average in a large part of central and north Portugal. However, it must be stressed that wind was the main driver of this event. The last panel of Figure 2 shows strong winds with anomalies greater than $10 \mathrm{~km} / \mathrm{h}$. These strong winds with southerly orientation were mainly caused by the nearby passage of hurricane Ophelia which was centred at about $485 \mathrm{~km}$ from Viana do Castelo $\left(41.7^{\circ} \mathrm{N}, 8.82^{\circ} \mathrm{W}\right)$ on the considered day (IPMA, 2017). As shown in Figure 3 a dry region at the lower layers of the atmosphere is present in both June and October cases, superimposed by a more humid layer (at about $500 \mathrm{hPa}$ ), the main difference being the presence of a drier layer above $400 \mathrm{hPa}$ in the October event. It is also important to notice that, in October, besides the strong wind, the ongoing extreme drought conditions were a major key ingredient for the unprecedented dimension of burned area observed in Portugal in a single day. 

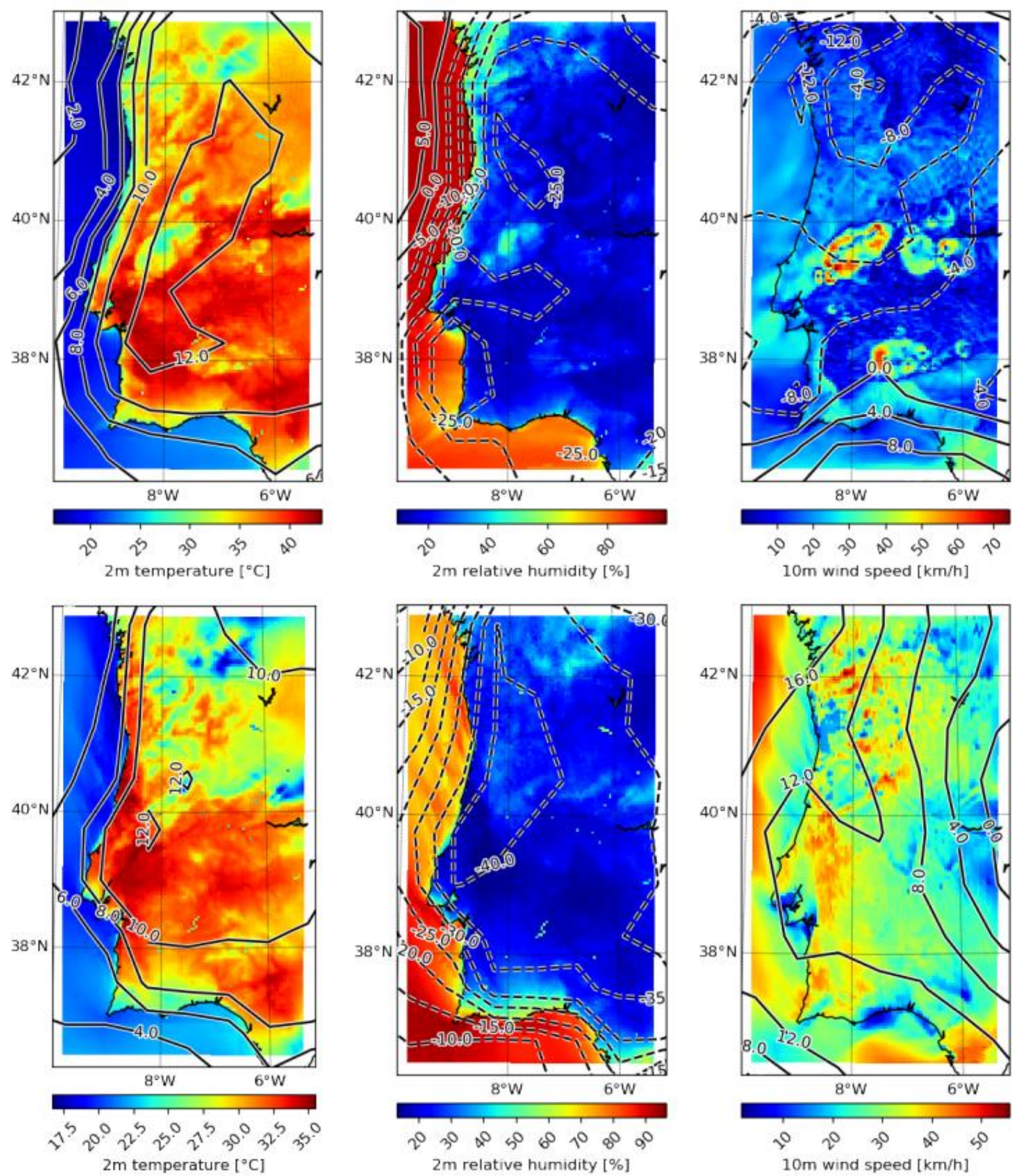

Figure 2-15 UTC fields of 2-metre temperature and relative humidity and of wind speed (left to right panels) as simulated by the WRF model for June 17 (top panels) and October 15, 2017 (bottom panels). Fields are coloured according to the respective colour bars and black contours represent ERA-Interim anomalies, with dashed lines indicating negative anomalies. 

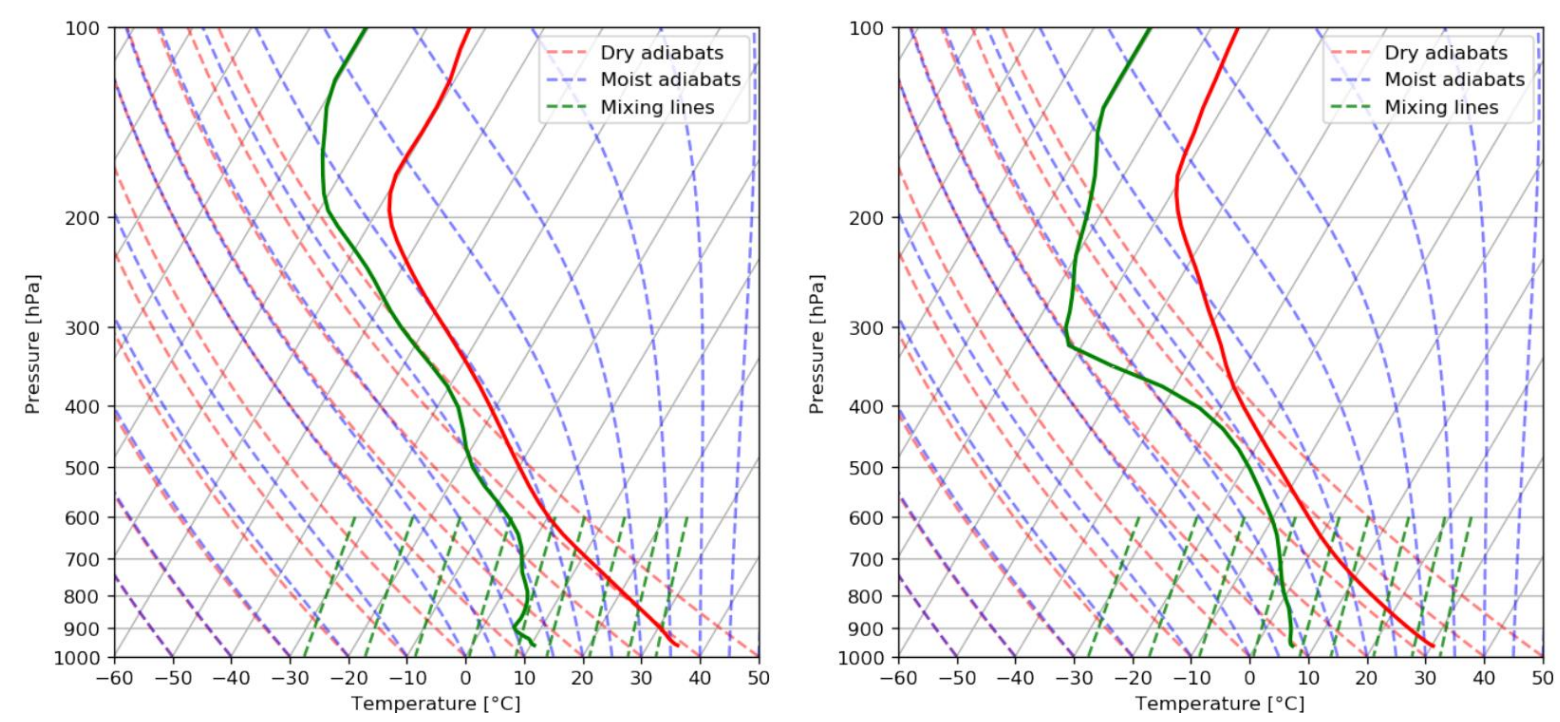

Figure 3 - Vertical profiles of temperature (red line) and dew point temperature (green line) in ${ }^{\circ} \mathrm{C}$, as simulated by the WRF model, at 15 UTC of June 17 (left panel) and October 15 (right panel). Dashed red, blue and green lines respectively represent the dry adiabats, moist adiabats and saturating mixing ratio.

It is also worth mentioning that while the October case is very well characterized by means of largescale conditions, in the case of June, high resolution information provided by the mesoscale model is fundamental to resolving the mesoscale convective storms and better understand the situation. This predisposition of the atmosphere for instability conditions can be measured by CHI (Mills and McCaw 2010). For that we computed both CHI and FWI for each day since 1979 using ERA-Interim reanalysis data. Results (Figure 4) show that the October case was indeed an absolute extreme in FWI while the June case was characterised by values above percentile 75 for the month of June and just below percentile 75 when considering the extended fire season (from June to September). However, when looking for CHI we see that the June case shows a CHI above 10, very close to percentile 99 for the extended fire season, while the October case corresponds to percentile 90 . This reinforces the idea that looking to the vertical instability conditions provides additional information that is necessary in order to obtain a more in-depth characterization of the extreme event at the local to regional scales. Moreover, instability indices such as CHI that are fairly simple to compute can be used for a more complete information about fire danger. According to Mills and McCaw (2010), values of CHI above 10 imply an extremely difficult setting to extinguish fires and a high risk of under-predicted behaviour. This is in agreement with the observations for June 17 where fast fire spread was observed with the development of pyrocumulonimbus (Moreira et al. 2017).
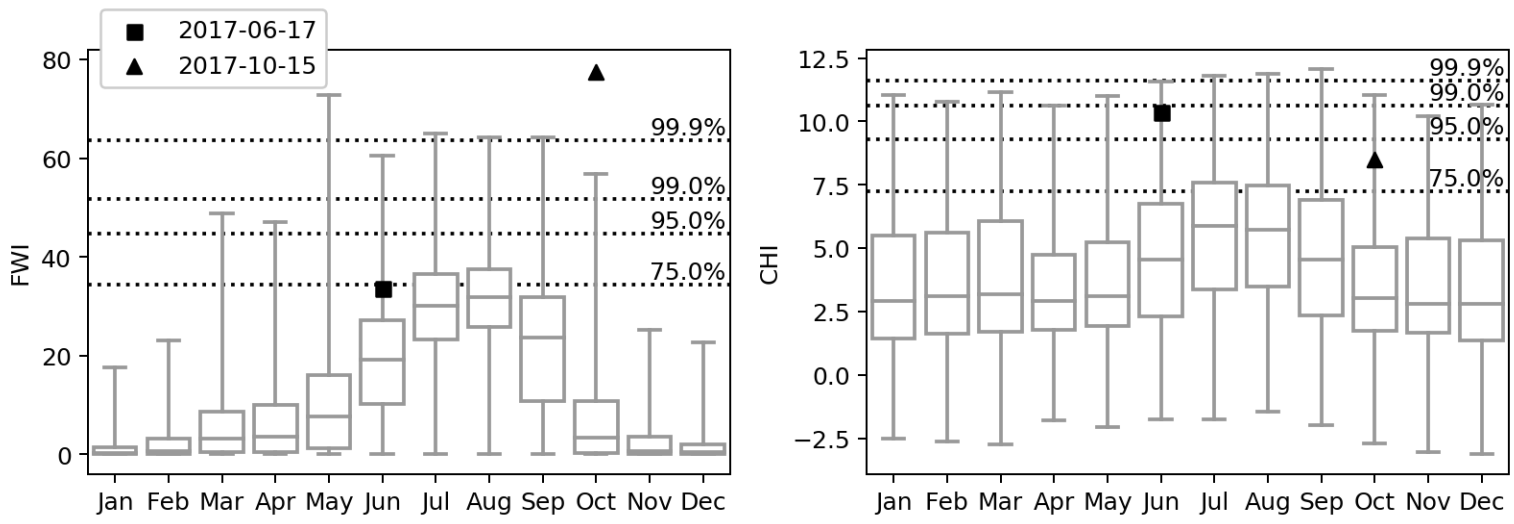

Figure 4 - Boxplots of monthly distributions of FWI (left panel) and CHI (right panel) for the period 1979-2016). The horizontal dashed lines indicate percentiles for the extended fire season (June to September) whereas the black squares and triangles refer to the values for June 17 and October 15, 2017. 
Table 1 presents a comparison of values of FWI for June 17 and October 15 with values of the same index computed with temperature, relative humidity, and wind intensity, respectively replaced by the respective monthly means over the period 1979-2017.

Considering the case of June 17, when replacing the wind speed by the climatological mean there is a small increase of $3 \%$ of FWI (from 34 to 35) whereas decreases of 18\% and 24\%, (from 34 to 28 and from 34 to 26) are obtained when replacing temperature and relative humidity by climatological means. This is to be expected, since the large-scale wind speed is below average whereas the other two variables are above average (Figure 2). Relative humidity was accordingly the most important factor contributing to the observed value of FWI, closely followed by temperature. However as discussed earlier, the mesoscale wind associated with the convective system has played a main role that is not captured by FWI at such coarse scale and the effect is better quantified by $\mathrm{CHI}$.

Table 1: FWI observed and modelled (replacing observed temperature, wind and relative humidity by respective monthly means in 1979-2017) for June 17 and October 15, 2017. In brackets, the relative change (\%) of each modelled value from the respective observed value.

\begin{tabular}{|c|c|c|c|}
\hline & \multicolumn{2}{|c|}{ FWI } \\
\hline & & 17 Jun & 15 Oct \\
\hline \multicolumn{2}{|r|}{ Observed } & 34 & 78 \\
\hline \multirow{3}{*}{$\overline{\bar{e}}_{\bar{c}}^{\bar{e}} \overline{\mathrm{d}}$} & Wind & $35[+3 \%]$ & $41[-47 \%]$ \\
\hline & Temperature & $28[-18 \%]$ & $66[-15 \%]$ \\
\hline & Relative humidity & $26[-24 \%]$ & $46[-41 \%]$ \\
\hline
\end{tabular}

Analysing the case of October 15, it may be noted that FWI reached a value of 78 (more than double of June 17). In this case all parameters present a positive contribution. The most notorious change occurs when considering the average wind speed, followed by the relative humidity. When modelling FWI replacing wind speed by the monthly average for October, the resulting FWI value drops to 41, almost half of the observed value. The contribution of relative humidity closely follows that of wind whereas temperature has the smallest contribution.

\section{Conclusions}

Analysis of FWI and CHI in a climate context shows that both June and October fire events were associated to extreme meteorological conditions. However, the characteristics of these two events are very distinct. The June event happened very early in the fire season in a context of a heat wave and a very unstable atmosphere that allowed for a fast and unpredictable spread of the fires. The October case, in turn, took place late in the fire season in a situation of extreme drought and in a day marked by strong winds associated with the close passage of hurricane Ophelia that led to a very fast fire spread.

A closer look at the sensitivity of FWI to meteorological conditions shows that for the June 17 case temperature and relative humidity were the main contributors, the large-scale wind speed being below average. For the October 15 case, all meteorological variables considered show a positive contribution. Wind speed and relative humidity were the most relevant contributing variables that led to a record FWI value of 78 for the considered region, indicating an extreme potential fire intensity associated with a high rate of fire spread due to the strong winds.

It is also shown that the WRF model initialized with boundary conditions given by state-of-the-art reanalyses, in this case ERA5, can reasonably well simulate the development of the convective systems and the atmospheric instability. Furthermore, the incorporation of $\mathrm{CHI}$ as a measure of atmospheric 
instability can represent an added value when rating fire danger, a topic that is currently being addressed in our research.

\section{Acknowledgments}

Research by Miguel M. Pinto was supported by the Portuguese Science Foundation (FCT) through $\mathrm{PhD}$ grant PD/BD/142779/2018. Research by Alexandra Hurduc was supported by a grant in the framework of Project "Reabilitação das Áreas Queimadas na Freguesia de Alvares", financed by donation of Observador on time SA.

\section{References}

Amraoui M, Pereira MG, DaCamara, CC, Calado TJ (2015) Atmospheric conditions associated with extreme fire activity in the Western Mediterranean region. Science of the Total Environment 524, 32-39.

DaCamara CC, Calado TJ, Ermida SL, Trigo IF, Amraoui M, Turkman KF (2014) Calibration of the Fire Weather Index over Mediterranean Europe based on fire activity retrieved from MSG satellite imagery. International Journal of Wildland Fire 23(7), 945-958.

Dee DP, Uppala SM, Simmons AJ, Berrisford P, Poli P, Kobayashi S, Andrae U, Balmaseda MA, Balsamo G, Bauer P, Bechtold P, Beljaars ACM, van de Berg L, Bidlot J, Bormann N, Delson C, Dragani R, Fuentes M, Geer AJ, Haimberger L, Healy SB, Hersbach H, Hólm EV, Isaksen L, Kallberg P, Köhler M, Matricardi M, McNally AP, Monge-Sanz BM, Morcrette J-J, Park B-K, Peubey C, de Rosnay P, Tavolato C, Thépaut J-N, Vitart F (2011) The ERA-Interim reanalysis: Configuration and performance of the data assimilation system. Quarterly Journal of the Royal Meteorological Society 137, 553-597.

Fujita TT (1985) The Downburst: Microburst and Macroburst. SMRP Research Paper 210, 112.

Haines DA (1988) A lower atmosphere severity index for wildlife fires. National Weather Digest 13, 23-27.

IPMA (2017) Boletim Climatológico outubro 2017 Portugal continental. Instituto Português do Mar e da Atmosfera. (Lisbon)

Mills GA, McCaw WL (2010) Atmospheric stability environments and fire weather in Australia extending the Haines Index. Centre for Australian Weather and Climate Research Technical Report number 20. (Melbourne)

Moreira N, Silva A, Novo I, Rio J, Pinto P, Correia S, Barbosa S, Cota T (2017) Condições meteorológicas associadas ao incêndio de Pedrógão Grande de 17 junho 2017. Instituto Português do Mar e da Atmosfera. (Lisbon)

Pinto MM, DaCamara CC, Trigo IF, Trigo RM, Turkman KF (2018) Fire danger rating over Mediterranean Europe based on fire radiative power derived from Meteosat. Natural Hazards and Earth System Sciences 18(2), 515-529.

San-Miguel-Ayanz J, Schulte E, Schmuck G, Camia A, Strobl P, Liberta G, Giovando C, Boca R, Sedano F, Kempeneers P, McInerney D, Withmore C, Oliveira SS, Rodrigues M, Durrant T, Corti P, Oehler F, Vilar L, Amatulli G (2012) Comprehensive Monitoring of Wildfires in Europe: The European Forest Fire Information System (EFFIS). In 'Approaches to Managing Disaster Assessing Hazards, Emergencies and Disaster Impacts'. (Ed. J Tiefenbacher) (InTech: Croatia)

Sánchez-Benítez A, García-Herrera R, Barriopedro D, Sousa PM, Trigo RM (2018) June 2017: The Earliest European Summer Mega-heatwave of Reanalysis Period. Geophysical Research Letters 45(4), 1955-1962. 
Van Wagner CE (1974) Structure of the Canadian Forest Fire Weather Index. Canadian Department of Environment. Canadian Forestry Service, Publication 1333. (Ottawa, ON)

Van Wagner CE (1987) The development and structure of the Canadian Forest Fire Weather Index System. Canadian Forest Service, Petawawa National Forestry Institute, Forestry Technical Report FTR-35. (Chalk River, ON) 


\title{
The impact of climate change on fire weather of Daxing'anling
}

\author{
Xiaorui Tian*, Lifu Shu, Mingyu Wang, Fengjun Zhao \\ Research Institute of Forest Ecology, Environment and Protection, Chinese Academy of Forestry, \\ State Forestry Administration's Key Open Laboratory of Forest Protection Beijing 100091, \\ China, \{tianxr@caf.ac.cn*\}
}

\begin{abstract}
There are amount of natural forests in Daxing'anling, and wildfires occurred in the region every year. Assessing the changes in fire weather in the future under multi-climate scenarios would contribute to our understanding of the influences of climate change for the region and provide a reference for applying adaptation measures for fire management. This study analysed the changes in fire weather indices and the fire season under four climate scenarios (RCP2.6, RCP4.5, RCP6.0, RCP8.5) for 2021-2050 using data from five global climate models together with observation data. The results showed that the analog data could project the average state of the climate for a given period but were not effective for simulating extreme weather conditions. Compared with the baseline period (1971-2000), the average temperature and annual precipitation were predicted to increase by $2.02^{\circ} \mathrm{C}-2.65{ }^{\circ} \mathrm{C}$ and $25.4-40.3 \mathrm{~mm}$ during 2021-2050, respectively. Multiple comparisons revealed that all of the indices would increase in 2021-2050 except for DC. The average FWI (fire weather index) was 4.53 at baseline, which would increase by $6.2 \%, 11.3 \%$, $10.6 \%$, and $11.2 \%$ under scenarios RCP2.6, RCP4.5, RCP6.0, and RCP8.5, respectively. SSR (seasonal severity rating) would increase by $5.5 \%, 16.2 \%, 15.1 \%$, and $17.2 \%$ in $2021-2050$ under scenarios RCP2.6, RCP4.5, RCP6.0, and RCP8.5, respectively. The DMC (duff moisture code), ISI (initial spread index), BUI (build-up index), FWI and SSR were predicted to increase significantly under scenarios RCP 4.5, RCP 6.0, and RC P8.5. Furthermore, days with high or higher fire danger rating were predicted to prolong 3-6 days in 2021-2050.
\end{abstract}

Keywords: climate change; forest fire; fire season; Daxing'anling

\section{Introduction}

The main features of climate change are warming and drying, and wildfires are closely linked these features (Hu et al. 2012; Flannigan et al. 2013; Sun et al. 2014). Fire activity is expected to increase in most regions worldwide with increasing temperature (Pechony and Shindell 2010). Fire is a natural disturbance in most forest ecosystems. The boreal forest is exhibiting responses to climate change. An average of 5-15 million hectares of boreal forest are burned each year in northern Siberia, Alaska and Canada, and serious fire years are occurring more frequently (Flannigan et al. 2009). Global warming will increase the potential for prolonged fire storms, causing fuel dryness (via increased evapotranspiration and drying of litter) and leading to more wildfires; i.e., fire severity is sensitive to global warming (Flannigan et al., 2013). Landscape fires are expected to change during the 21st century in response to multiple agents of global change. Important controlling factors include climate controls on the length and intensity of the fire season, fuel availability, and fire management, which are already anthropogenically perturbed at present and are predicted to change further in the future (Kloster et al. 2012). By 2030 and the end of the century, the number of forest fires in northern regions of Canada will increase by $30 \%$ and $75 \%$, respectively (Wotton \& Flannigan 2010). The fire season in western North America would prolong due to the advance of the spring snowmelt date and rising temperatures in spring and summer (Westerling et al. 2006; Running 2006). Spracklen et al. (2009) predicted that by the 2050s, the average annual burned area in the western United States will increase by 54\%, and forest fires in this region and in the Pacific Northwest area of the Rocky Mountains will increase by $78 \%$ and $175 \%$, respectively. Wildfires would increase by $179 \%$ in Portugal under a 
scenario of $2 \times \mathrm{CO}_{2}$, and burned areas would increase by $378 \%$, with an annual growth rate of 1.4 7.8\% (Carvalho et al. 2010).

The fire regime has been altered in Daxing'anling in the past fifty years and is expected to change continuously in the future. The fire season are getting prolonged and more lighting fires in the region. From 1961 to 2010, the annual rate of the increase in temperature in northeastern Inner Mongolia was $0.375^{\circ} \mathrm{C} / 10 \mathrm{a}$, and lightning fires increased significantly ( $\mathrm{Li}$ et al. 2011). Forest fire danger also increased for the Greater Xing'an Mountains in Inner Mongolia, and the fire season has been extended, with more fires occurring in the forest in summer (Zhao et al. 2009). Fire control will become more difficult in the future (Di et al. 2011). The potential for fires in northeast China was expected to increase 10-18\% during the period 2071 - 2100, with the fire season being extended by 21-25 days (Tian et al. 2011). Liu et al. (2012) estimated that the density of fires in the boreal forest in northeastern China in the period from 2081 to 2100 will increase by $30 \%$ (CGCM3 B1 scenario) to $230 \%$ (HadCM3 A2 scenario). Additionally, the annual days with high and very high fire danger ratings are expected to increase by 43 and 36 days under scenarios A2a and B2a in 2040-2069, respectively (Yang et al. 2010; 2012).

The RCP (Representative Concentration Pathways) climate scenario was adopted in the IPCC (Intergovernmental Panel on Climate Change) Fifth Assessment Report, which included four scenarios, RCP2.6, RCP4.5, RCP6.0, and RCP8.5. Each of these scenarios represents different conditions of forced radiation levels, $\mathrm{CO} 2$ concentrations, and their pattern of change (Van Vuuren $e t$ al., 2011). Scenarios RCP2.6, RCP4.5, RCP6.0, and RCP8.5 indicate increases in forced radiation of 2.6, 4.5, 6.0, and $8.5 \mathrm{~W} / \mathrm{m}^{2}$ in 2100 . RCP2.6 is a warming mitigation scenario (with forced radiation tending to decrease in 2100), and RCP4.5 and RCP6.0 represent stable situations (with the variation of forced radiation being relatively stable in 2100), while RCP8.5 is a scenario involving high greenhouse gas emissions (with forced radiation continuing to increase before 2100). In 2100, the $\mathrm{CO}_{2}$ concentration would reach 421, 538, 670, and 936 ppm under scenarios RCP 2.6, RCP 4.5, RCP 6.0, and RCP 8.5, respectively. Studying the effects on forest fire danger in Daxing'anling under the four RCP scenarios will contribute to a better understanding of fire activities in the future and provide a scientific reference for adaptation measures on fire management.

\section{Study area}

The study area is located in Daxing'anling, China (Figure 1), with a geographical range of 119.71 ${ }^{\circ}-127.02^{\circ} \mathrm{E}, 48.48^{\circ}-3.56^{\circ} \mathrm{N}$. The main forest type is a deciduous needle-leaved mixed forest dominated by Larix gemelini. Other common tree species that may be present include Pinus sylvestris, Betula platyphylla, Quercus mongolicus, Populus davidiana, and Salix matsudan.

There is a cold temperate continental monsoon climate. The winter is cold and long, while the summer is hot and short. The annual average temperature is $-2.8^{\circ} \mathrm{C}$, and annual precipitation is 450 $500 \mathrm{~mm}$ (Xu 1998). The soil is typically classified as a brown taiga coniferous forest soil. The topography is undulating, with an average elevation of $573 \mathrm{~m}$ ASL; however, higher hill tops can reach $1,528 \mathrm{~m}$ ASL. The region has an active fire season from March to October. 


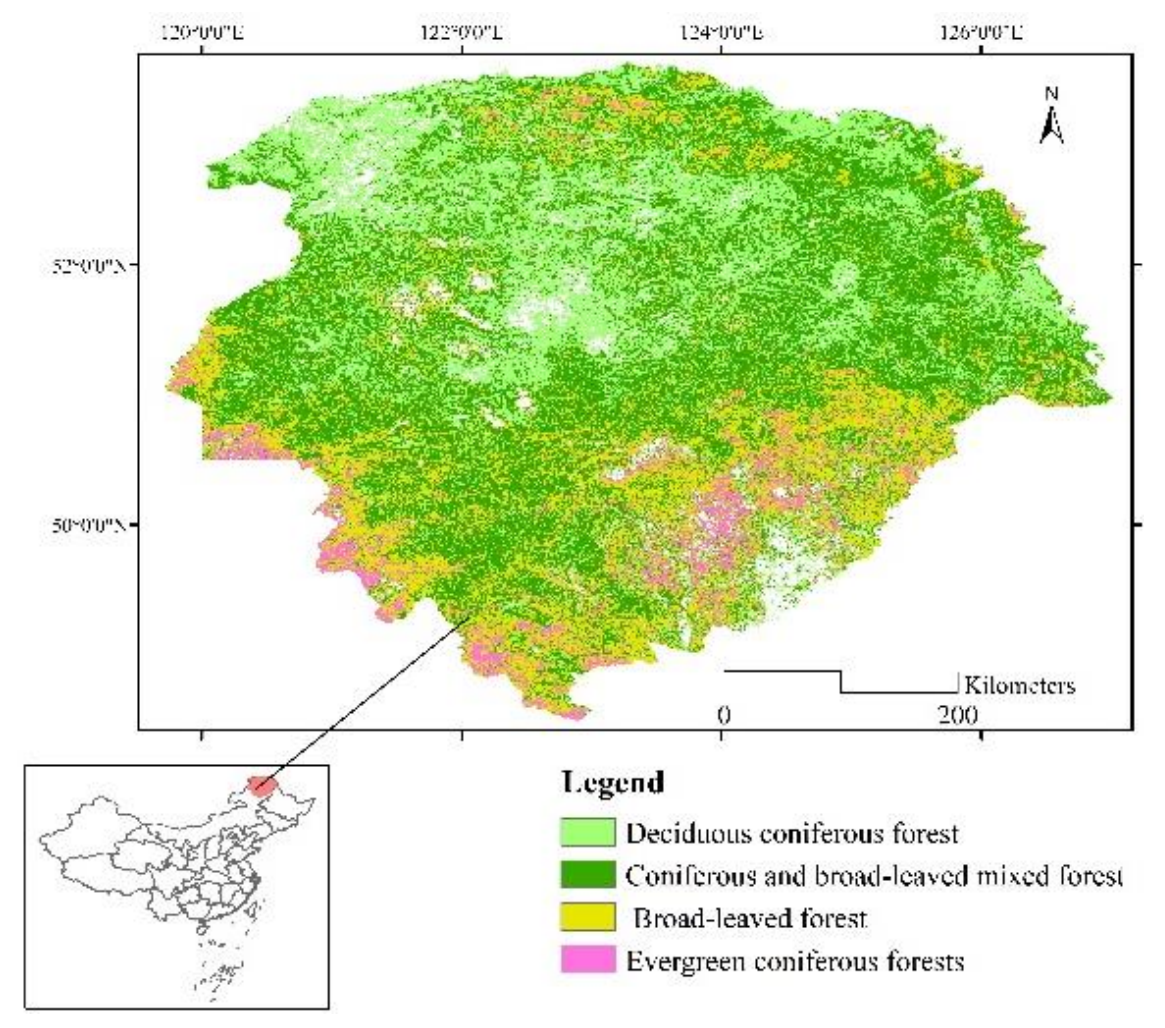

Figure 1 - Location and vegetation types of the study area

\section{Methods}

\subsection{Climate data for the four scenarios}

Climate data for the four scenarios under 1951-2050 were obtained from the Inter-Sectoral Impact Model Intercomparison Project (ISI-MIP) (http://www.isi-mip.org). These data were extracted by the Institute of Environment and Sustainable Development of Agriculture of the Chinese Academy of Agricultural Science and converted to ASCII code. The daily data included five meteorological elements (average temperature, maximum temperature, precipitation, average relative humidity, and average wind speed near the ground) for a global climate model (HadGEM2-ES) with four scenarios (RCP2.6, RCP4.5, RCP6.0, RCP8.5). The horizontal resolution of the data was $0.5^{\circ} \times 0.5^{\circ}$. The bilinear interpolation method was used to handle the original data, and statistical bias was processed based on the probability distribution (Piani et al. 2010; Hagemann et al. 2011; ISI-MIP 2013). Daily surface observation data from 10 weather stations for the baseline period (1971-2010) were used to adjust the simulation data (1951-2050).

\subsection{Forest fire danger index calculation}

The fire weather index (FWI) system requires inputs of data on the air temperature, relative humidity, precipitation and wind speed at noon. However, the regional climate model outputs only provided daily data. Therefore, the adjusted noon temperature, relative humidity, daily rainfall and average wind speed were used as the inputs to calculate fire weather indices for each grid. The system outputs three fuel moisture codes and three fire behavior indices. The fuel moisture codes include the fine fuel moisture code (FFMC), the duff moisture code (DMC), and the drought code (DC). The three fire behavior indices are the initial spread index (ISI), which is an indicator of the potential rate of fire spreading; the build-up index (BUI), representing total fuel available to a fire, which is used to perform correlations with fuel consumption; and the FWI which is a combination of the ISI and BUI, representing the intensity of a spreading fire. The FWI is used to indicate the overall fire danger to the public. The daily severity rating (DSR) is converted from FWI (Van Wagner 1970), which we averaged 
to obtain seasonal values (SSR, Seasonal Severity Rating) to assess fire severity for a fire season. The software ArcInfo 10.0 and ANUSPLIN (Hutchinson 1998) were used to interpolate the FWI system data to spatial surfaces at a $1 \times 1-\mathrm{km}$ resolution.

\subsection{Fire danger ratings}

The fire danger ratings for each FWI System component were classified as low, moderate, high, very high, or extreme based on the classification of danger classes for the FWI System. According to the distribution of the fire danger indices during 1987-2006 in Daxing'anling, the low, medium, high, very high and extreme fire danger ratings were found to correspond to FWI ranges of 0-2.5, 2.6-10.0, 10.1-18.0, 8.1-31.0 and >31.0, respectively (Tian et al. 2009).

\section{Results}

\subsection{Temperature and precipitation changes in the fire season}

Compared with the baseline period, the average annual temperature and precipitation in 2021-2050 were predicted to increase by $2.02-2.65{ }^{\circ} \mathrm{C}$ and $25.4-40.3 \mathrm{~mm}$ under scenarios RCP2.6, RCP4.5, RCP6.0, and RCP8.5 (Table 1). For 1951-2050, the annual mean temperature showed a gradual upward trend, but no significant trend for the precipitation (Figure 2). Compared with the observation data (1961-2010), the outputs from the global climate models exhibited less fluctuation, especially for precipitation. This indicated that regarding the analogy extreme values of temperature and precipitation in certain years, the projected values were lower than the observed values.

Compared with the baseline period, the daily average and maximum temperature and precipitation in fire season in $2021-2050$ would increase by $1.77-2.40^{\circ} \mathrm{C}, 1.72-2.28^{\circ} \mathrm{C}$ and $15.8-32.3 \mathrm{~mm}$. These results represented significant differences, and the corresponding $\mathrm{P}$ values for the t-test were $0.00,0.00$ and 0.01 , respectively.

Table 1 Changes in temperature and precipitation under four climate scenarios

\begin{tabular}{cccccc}
\hline Scenario & Period & $\begin{array}{c}\text { Annual } \\
\text { average } \\
\text { temperature }\end{array}$ & $\begin{array}{c}\text { Average } \\
\text { temperature in } \\
\text { fire season }\end{array}$ & $\begin{array}{c}\text { Maximum } \\
\text { temperature in fire } \\
\text { season }\end{array}$ & $\begin{array}{c}\text { Annual } \\
\text { precipitation } \\
\text { fire season }\end{array}$ \\
\hline Observations & $1971-2000$ & -3.9 & 9.79 & 17.40 & 53 \\
Rcp2.6 & $2021-2050$ & -1.71 & 11.71 & 19.32 & 507.3 \\
Rcp4.5 & $2021-2050$ & -1.69 & 11.77 & 19.28 & 543.0 \\
Rcp6.0 & $2021-2050$ & -1.88 & 11.56 & 19.12 & 535.8 \\
Rcp8.5 & $2021-2050$ & -1.25 & 12.19 & 19.68 & 532.7 \\
\hline
\end{tabular}
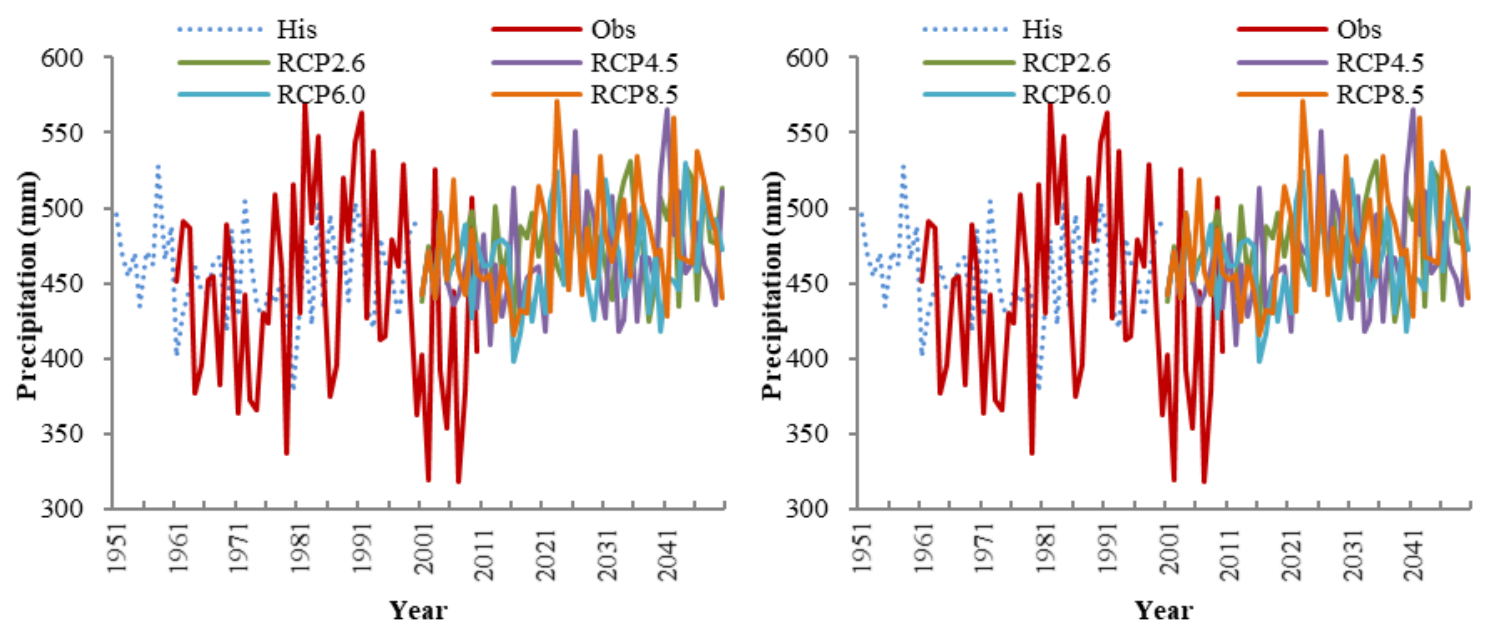

Figure 2 - Temperature and precipitation during the fire season in 1951-2050. (a) Average temperature in the fire season; (b) precipitation in the fire season. 


\subsection{Changes in fire weather indices}

The calculated average values for FFMC, DMC, DC, BUI, and FWI from the simulated climate data were higher than those from the observed data (Table 2), with DMC, DC, and BUI showing significant differences (with two-tailed $\mathrm{P}$ values from t-tests of $0.0216,0.0000$, and 0.0002, respectively). However, FFMC and FWI did not show significant changes (with two-tailed P values from t-tests of 0.1597 and 0.6840 , respectively). The average ISI and SSR values based on the simulated climate data were lower than the values calculated from the observed data, with ISI showing a significant difference $(\mathrm{P}=0.0073)$, whereas that for $\mathrm{SSR}$ was no significant $(\mathrm{P}=0.4847)$. The coefficients of variation $(\mathrm{CV})$ for all of the fire danger indices calculated based on historical observations were greater compared with those based on simulated climate data. This indicated that the simulated data could describe the average state of the climate and fire danger in a given period very well, but not for extreme years. Therefore, the averages for 30 years are considered reliable to describe the possible status of fire danger in the future.

The curves for each fire weather index for 1971 - 2050 also showed that the volatility of the calculations based on simulated data was lower compared with that for observed data (Figure 3). In 2007, the indices calculated from observed data were significantly higher than in any other year because there were higher temperatures and less rainfall in that fire season. The average temperature was higher $14 \%\left(+1.4^{\circ} \mathrm{C}\right)$, and rainfall was $30 \%$ lower than the baseline values in 2000 , which was another anomalous season.

Table 2 - Comparison of the results calculated from the simulated and observed data for 1976-2000

\begin{tabular}{ccccccc}
\hline Index & Data & Average & Minimum & Maximum & Range & Coefficient of variation \\
& Source & & & & & \\
\hline \multirow{2}{*}{ BUI } & Observed & 29.7 & 17.2 & 41.6 & 24.4 & 0.185 \\
& Simulated & 35.8 & 32.1 & 41.2 & 9.1 & 0.063 \\
DC & Observed & 197.8 & 131.6 & 320.0 & 188.4 & 0.219 \\
& Simulated & 309.0 & 263.5 & 371.2 & 107.7 & 0.100 \\
DMC & Observed & 20.5 & 12.0 & 29.8 & 17.8 & 0.213 \\
& Simulated & 22.7 & 19.9 & 26.4 & 6.5 & 0.064 \\
FFM & Observed & 0.8 & 0.3 & 1.6 & 1.3 & 0.407 \\
& Simulated & 0.8 & 0.6 & 1.1 & 0.5 & 0.132 \\
FWI & Observed & 69.3 & 64.0 & 73.6 & 9.5 & 0.028 \\
& Simulated & 68.7 & 67.1 & 71.0 & 3.9 & 0.014 \\
& Observed & 4.2 & 2.2 & 6.5 & 4.3 & 0.240 \\
& Simulated & 4.3 & 3.7 & 4.9 & 1.3 & 0.071 \\
& Observed & 1.9 & 1.3 & 2.7 & 1.4 & 0.181 \\
\hline
\end{tabular}

Note: Here, we only used the data for 1976-2000, due to missing relative humidity data in 1971-1975. 

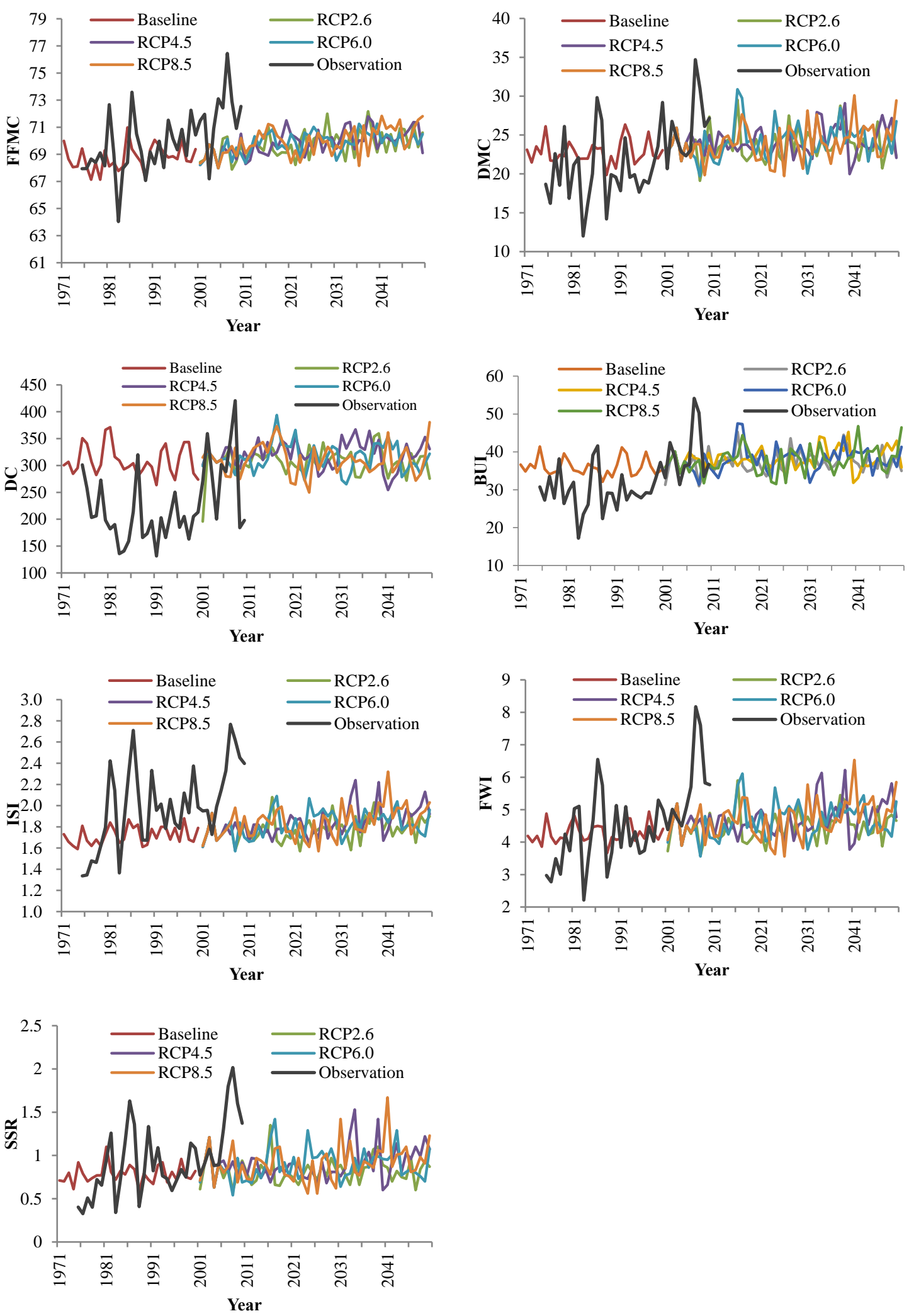

Figure 3 - The fire weather indices in 1971 - 2050

Based on the consistency of the data, we used the results calculated from the simulated data from the GCM at baseline to analyse the changes in the fire weather indices for 2021 - 2050. Multiple comparisons revealed that all of the indices would increase in 2021 - 2050 except for DC (Table 3). FFMC would increase significantly for all four scenarios, but DMC, ISI, BUI, FWI and SSR would 
increase significantly only for scenarios RCP4.5, RCP6.0, and RCP8. DC would not change significantly under any scenario.

Table 3 - Multiple comparisons of the fire weather indices for 2021-2050 and the baselinefor the four climate scenarios

\begin{tabular}{cllll}
\hline Fire weather indices & Rcp2.6 & Rcp4.5 & Rcp6.0 & Rcp8.5 \\
\hline FFMC & $1.3360^{*}$ & $1.4807^{*}$ & $1.4000^{*}$ & $1.3953^{*}$ \\
DMC & 0.9713 & $1.4637^{*}$ & $1.5767^{*}$ & $1.4457^{*}$ \\
DC & -0.7983 & 11.3537 & 4.3327 & -2.4017 \\
ISI & $0.0867^{*}$ & $0.1613^{*}$ & $0.1367^{*}$ & $0.1440^{*}$ \\
BUI & 1.4873 & $2.4080^{*}$ & $2.2763^{*}$ & $1.9697^{*}$ \\
FWI & 0.2603 & $0.5223^{*}$ & $0.4673^{*}$ & $0.4847^{*}$ \\
SSR & 0.042 & $0.1453^{*}$ & $0.1360^{*}$ & $0.1487^{*}$ \\
\hline
\end{tabular}

Note: * indicates that the mean difference was significant at $\alpha=0.05$.

The average FWI was 4.53 (range, 1.68 to 6.78 ) at baseline, which would increase by $6.2 \%, 11.3 \%$, $10.6 \%$, and $11.2 \%$ under scenarios RCP2.6, RCP4.5, RCP6.0, and RCP8.5, respectively (Figure 4). For scenario RCP2.6, FWI would increase significantly in central areas, whereas it would increase significantly in the southwest under the other three scenarios. FWI would increase by more than $15 \%$ in some areas under scenario RCP8.5.

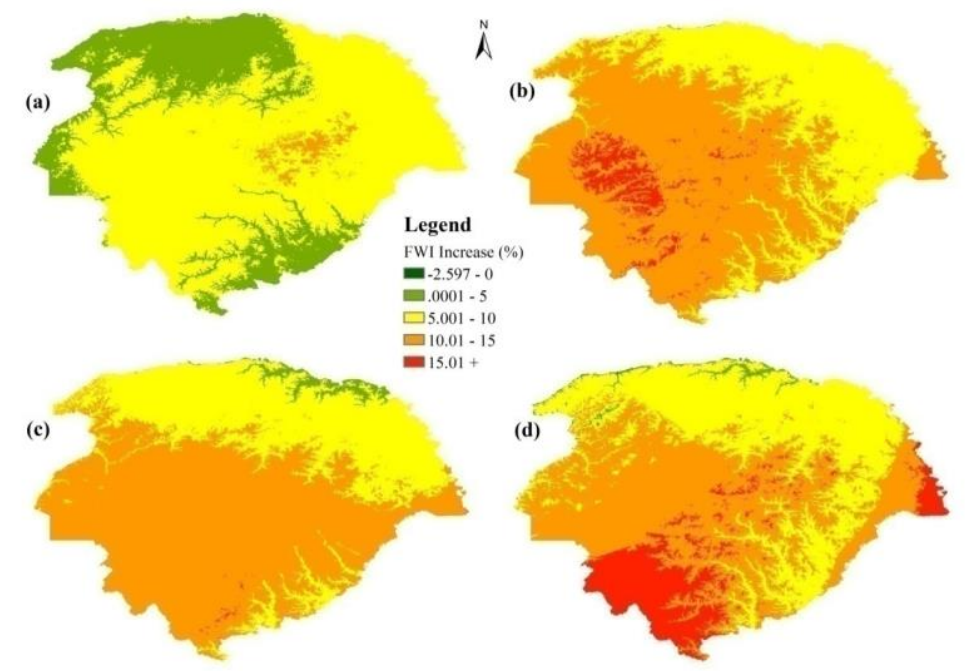

Figure 4 - Increment of FWI for 2021- 2050. (a), (b), (c) and (d) are maps of the FWI increment (\%) under scenarios RCP2.6, RCP4.5, RCP6.0, and RCP8.5, respectively.

\subsection{Fire weather severity analysis}

At baseline, the average SSR was 0.86 (range from 0.03 to 1.61 ), with SSR increasing gradually from the northeast to the southwest. SSR would increase by $5.5 \%, 16.2 \%, 15.1 \%$, and $17.2 \%$ in 2021 2050 under scenarios RCP2.6, RCP4.5, RCP6.0, and RCP8.5, respectively.

There were small parts of the central region where SSR would increase by over $10 \%$ under scenario RCP2.6, while an SSR increment of greater than $10 \%$ was predicted in most of the region under the other three scenarios, with this change being more significant (over 20\%) in the southwest (Figure 5). Under scenarios RCP4.5, RCP6.0, and RCP8.5, SSR would increase more in 2021-2050 where the higher SSR values occurred at baseline, though a different result was obtained for scenario RCP2.6. 


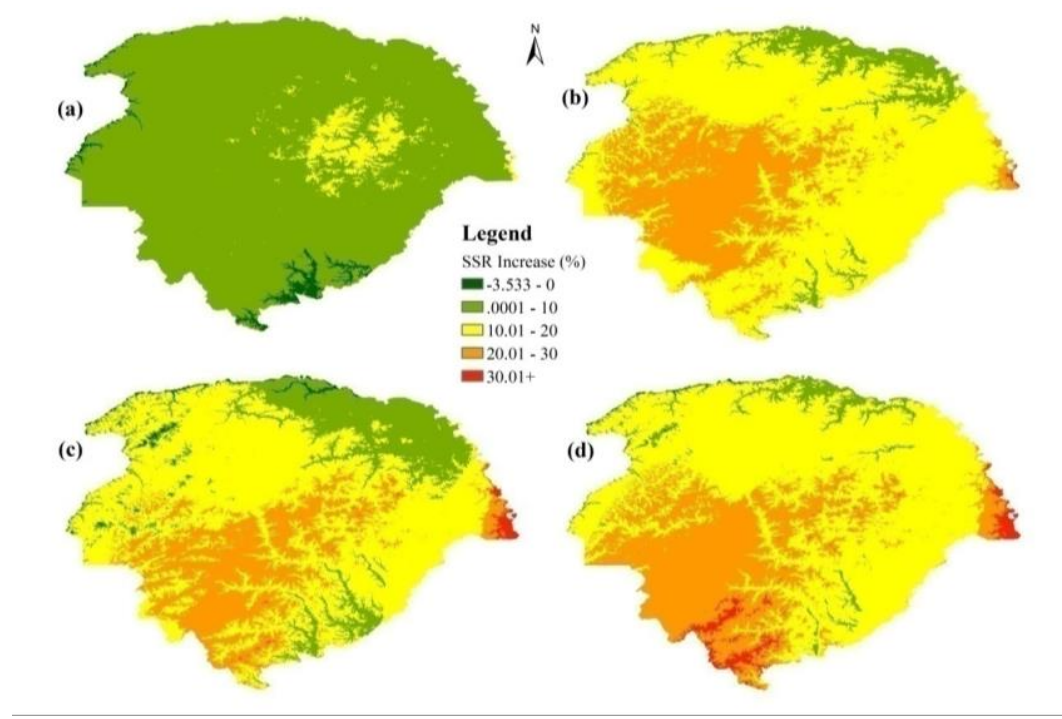

Figure 5 - SSR increment in 2021-2050. (a) Scenario RCP2.6; (b) Scenario RCP4.5; (c) Scenario RCP6.0; (d) Scenario RCP8.5.

\subsection{Changes in the number of days with high or higher fire danger ratings}

At baseline, the number of days with high, very high, and extreme fire danger ratings was 37.7 (range from 13 to 59 days), with this number being smaller in the north and greater in the south. Compared with the baseline period, the number of days with high or higher fire danger rating would increase by 3.3, 5.6, 5.6 and 5.7 days in 2021- 2050 under scenarios RCP2.6, RCP4.5, RCP6.0, and RCP8.5, respectively. The corresponding ranges were 0.8-5.8, 2.2-9.1, 0-10.6, and 2.4-11.8 days (Figure 6). The number of days with high or higher fire danger rating would increase least under scenario RCP2.6 and most under scenario RCP8.5. Under scenario RCP4.5, the change in the number of days would increase gradually from the northeast to the southwest, while increasing from north to south for scenarios RCP6.0 and RCP8.5.

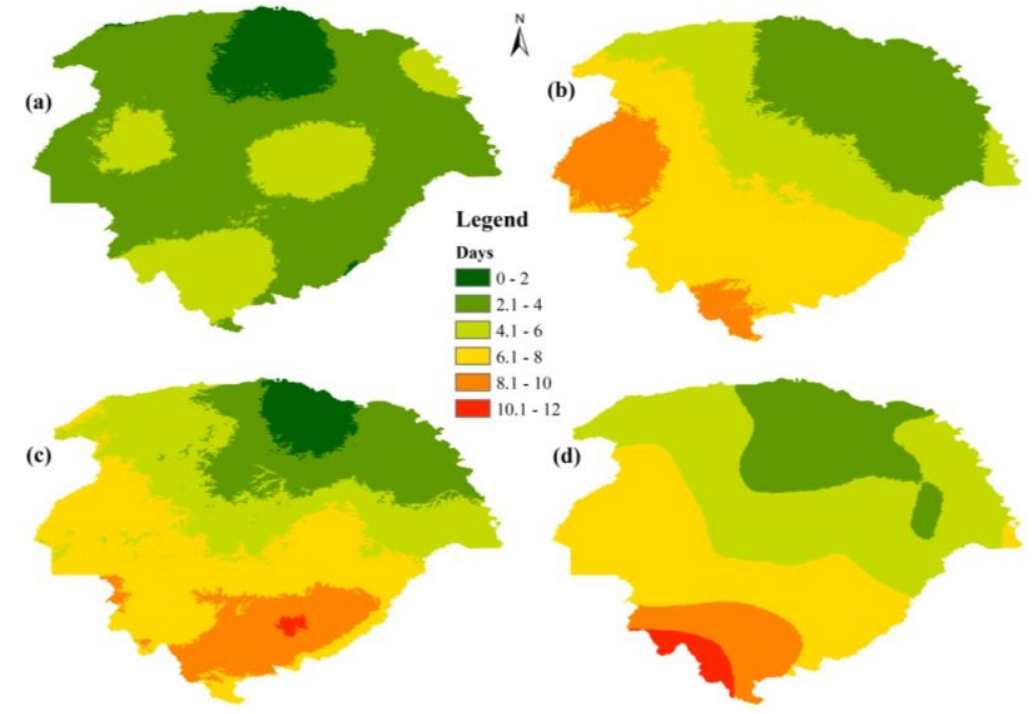

Figure 6 - The change in the number of days with high or higher fire danger ratings in 2021 - 2050. (a) Scenario RCP2.6; (b) Scenario RCP4.5; (c) Scenario RCP6.0; (d) Scenario RCP8.5.

\section{Discussion}

Previous studies have shown that there is significant positive correlation between the burned area and DMC, ISI, and FWI in Daxing'anling (Tian et al. 2013). The relationship between the FWI ratio 
and potential burned areas is 1:1, and an increase in the FWI ratio would cause a similar increase in the burned area (Flannigan et al. 2001). Therefore, if it is assumed that the fire management capacity will not change significantly in the near future, we can conclude that the potential burned areas in Daxing'anling will increase by 6-11\% in 2021 -2050 compared with baseline. Past studies for the region have indicated that fire danger will increase to some degree (Yang et al. 2010; Tian et al. 2011; Liu et al. 2012). However, these studies were based on SEARS scenarios. Van Vuuren et al. (2011) considered scenario RCP2.6 to be the most likely scenario in the future. Under this scenario, the seasonal severity rating for the region would increase by more than $5 \%$.

It was found that $77.5 \%$ of all fires occurred during weather associated with a high or higher fire danger class in Daxing'anling (Tian et al. 2009). Thus, the length of the period of weather associated with a high or higher fire danger would have a greater impact on fire activity for the region. We projected that this period would increase by 3-6 days (8.7-15.1\%) in 2021-2050. Our results showed a large difference from those of Yang et al. (2010; 2012), who projected that the average number of days with a high and very high fire danger would increase by 43 and 36 days in 2040-2069 for scenarios A2a and B2a, respectively. The main reasons for this difference were the differences in the climate scenarios, the threshold values for fire danger ratings, and the adjusted methods for the climate data. In this study, the thresholds of the fire danger ratings were derived from historical observations (Tian et al. 2009), and the simulated data were adjusted with the observations. As the simulation data did not reflect extreme weather well, the number of days with a high or higher fire danger rating may be fewer than indicated by the actual data.

We did not consider changes in fuel, ignition sources, and fire management capabilities in this study. However, these factors are currently considered to be unlikely to undergo fundamental changes in the next 30 years. Therefore, the uncertainty caused by these factors would have a limited influence on the conclusion.

\section{Conclusion}

GCM-simulated data can describe the average climate state for a given period well, but they cannot simulate extreme weather conditions. It would be reliable to describe the state of the climate and forest fire danger over an average of 30 years. Compared with the baseline period, temperature is predicted to increase significantly in 2021-2050, whereas precipitation is not predicted to show a significant change. FWI is predicted to increase by $6.2-11.2 \%$ in 2021- 2050. DMC, ISI, BUI, FWI, and SSR are predicted to increase significantly under scenarios RCP4.5, RCP6.0, and RCP8.5. The number of days with a high or higher fire danger rating are predicted to increase by 3-6 days, and the increase is predicted to be remarkable in the south for scenarios RCP4.5, RCP6.0, and RCP8.5.

\section{Acknowledgements}

This study received financial support from Project (31270695) of the National Natural Science Foundation of China and the National Science and Technology Support Plan (2018YFD0600205).

\section{References}

Carvalho A, Flannigan M, Logan K, Gowman L, Miranda A, Borrego C (2010) The impact of spatial resolution on area burned and fire occurrence projections in Portugal under climate change. Climate Change 98:177-197

Di X, Li Y, Sun J, Yang G (2011) Dynamics of forest fire weather indices in Tahe County of Great Xing'an Mountains region, Heilongjiang Province. Chinese Journal of Applied Ecology 22(5):1240-1246 
Flannigan MD, Krawchuk MA, de Groot WJ, Wotton BM, Gowman LM (2009) Implications of changing climate for global wildland fire. International Journal of Wildland Fire 18: 483-507.

Flannigan MD, Wotton BM (2001) Climate, weather and area burned. Forest Fires: Behavior \& Ecological Effects (Eds. Johnson EA, Miyanishi K), Academic Press, New York, 335-357

Hagemann S, Chen C, Haerter JO, Heinke J, Gerten D, Piani C (2011) Impact of a Statistical Bias Correction on the Projected Hydrological Changes Obtained from Three GCMs and two Hydrology Models. Journal of Hydrometrorology 12:556-578.

$\mathrm{Hu} \mathrm{H}$, Wei S, Sun L (2012) Estimation of carbon emissions due to forest fire in Daxing'an Mountains from 1965 to 2010. Chinese Journal of Plant Ecology 36(7): 629-644

ISI-MIP (2013) The inter-sectoral impact model intercomparison project design and simulation protocol (V2.3). http://www.ncbi.nlm.nih.gov/pmc/articles/PMC3948262/pdf/pnas.201312330.pdf

Li X, Wu W, Zhang C, Guo R (2011) Influence of climate change on northeastern of Inner Mongolia grassland forest fire. Journal of Arid Land Resources and Environment 25(11): 114-119

Piani C, Weedon GP, Best M, Gomes SM, Viterbo P, Hagemann S, Haerter JO (2010) Statistical bias correction of global simulated daily precipitation and temperature for the application of hydrological models. Journal of Hydrometrorology 395: 199-215

Running SW (2006) Climate change : Is global warming causing more larger wildfires. Science 313(5789): 927-928

Spracklen DV, Mickley LJ, Logan JA, Hudman RC, Yevich R, Flanning MD, Westerling AL (2009) Impacts of climate change from 2000 to 2050 on wildfire activity and carbonaceous aerosol concentrations in the western United States. Journal of Geophysical Research 114, D20301, DOI:10.1029/2008JD010966

Sun Long, Wang Q, Wei S (2014) Response Characteristics and Prospect of Forest Fire Disasters in the Context of Climate Change in China. Journal of Catastrophology 29(1): 12-17

Tian X, Shu L, Zhao F, Wang M, Mcrae DJ (2011) Future Impacts of Climate Change on Forest Fire Danger in Northeastern China. Journal of forestry research 22(3): 437-446

Tian X, Shu L, Wang M, Zhao F (2009) Spatial and Temporal Distribution of Lightning Fire and Forecasting Model for Daxing'anling Region. Forest Research 22(1): 14-20

van Vuuren DP, Edmonds J, Kainuma M, Riahi K, Thomson A, Hibbard K, Hurtt GC, Kram T, Krey V, Lamarque JF, Masui T, Meinshausen M, Nakicenovic N, Smith SJ, Rose SK (2011) The representative concentration pathways: an overview. Climatic Change 109: 5-31

Westerling AL, Hidalgo HG, Cayan DR, Swetnam TW (2006) Warming and earlier spring increase western U.S. forest wildfire activity. Science 313(5789): 940-943

Wittrock V, Wheaton E (1997) Climate Variations, Fire Characteristics and Budget Implications: Preliminary Analysis of their Relationships. Saskatchewan Research Council (SRC), Saskatoon, Saskatchewan. SRC Publication No. R-1550-2-E-97

Yang G, Di X, Zeng T, Shu Z, Wang C, Yu H (2010) Prediction of area burned under climatic change scenarios: A case study in the Great Xing'an Mountains boreal forest. Journal of Forestry Research 2l(2): 213-218

Yang G, Shu L, Di X (2012) Prediction on the changes of forest fire danger rating in Great Xing'an Mountain region ofNortheast China in the 21 st century under effects of climate change. Chinese Journal of Applied Ecology 23(12): 3236 -3242

Zhao F, Shu L, Di X, Tian X, Wang M (2009) Changes in the occurring date of forest fires in the inner Mongolia Daxing'anling forest region under global warming. Scientia Silvae Sinicae 45(6):166172 


\title{
The organisation of fire protection in disaster areas using as an example the case of Bialowieza Forest
}

\author{
Ryszard Szczygiel, Miroslaw Kwiatkowski, Bartlomiej Kolakowski* \\ Forest Research Institute. Sękocin Stary, 3, Braci Leśnej Street, 05-090 Raszyn, Poland, \\ \{r.szczygiel@ibles.waw.pl,m.kwiatkowski@ibles.waw.pl,b.kolakowski@ibles.waw.pl*\}
}

\begin{abstract}
The Bialowieza Forest is particularly precious, considering environmental aspects, forest land where recently an outbreak of bark beetle (Ips typograpus) took place on an unprecedented scale leading to disintegration of spruce stands. This phenomenon of a calamitous nature led to the increase of forest fire risk level due to appearance of large volume of dead wood and grassy cover as the results of the canopy openness. The grassy cover, when over-dried, is extremely flammable and conducive to fire outbreak and rapid fire behaviour. Before the outbreak of bark beetle (June 2012) the whole area of Bialowieza Forest (Forest District Białowieża, Brwosk, Hajnówka and Bialowieza National Park) was characterised by a low fire risk (on a three-level scale). The three forest districts manage their forest using the regular management plan for the rest of Poland while Bialowieza National Park is under strict protection. The forest fire protection system in place was a standard one, in compliance with the legal requirements laid down for this level of risk to managed forests. It does not include the current risk and the adaptation of the existing fire protection system for Bialowieza Forest is then indispensable and must be adequate to the actual risk. The unique environmental value of that region must be considered as well during that process.
\end{abstract}

Keywords: deadwood, natural habitats, bark beetle outbreak, fire risk, Bialowieza, fuel load

\section{Forest fire risk analysis for Bialowieza Forest}

\subsection{Occurrence of forest fires}

In the years 2000 - 201783 forest fires broke out in Bialowieza Forest (including 14 since the bark beetle outbreak - covering $3.83 \mathrm{ha}$ ) covering 38.66 ha of forest land. The mean burnt area was then 0.47 ha and it was higher than the mean burnt area for State Forests in Poland (amounting to 0.30 ha). The soil cover forest fires were the most frequent (amounting to 62.5\%) and covered as much as 99.2\% total burnt area. The very numerous, comparing to the average of less than $1 \%$, were single tree fires amounting to $33.3 \%$. Ground fire occurred only once while there was no total forest fire registered. Among the causes of fires, the domination of arson incidents was observed (37.5\%), and those of which the cause is undefined (33.3\%). Negligence was a cause of $16.7 \%$ of fire outbreaks. Attention should be given to the high percentage of fires due to lighting $(8.3 \%)$ as the result the large number of high trees in that forest. Spot fires from adjacent ground constituted up to $4.2 \%$. The coniferous stands (fresh coniferous forest and fresh mixed coniferous forest, covering 19.7\% of the total area of Bialowieza Forest) together with the stands less than 40 years old (amounting to $14.3 \%$ of the total area of Bialowieza Forest) mostly determined the forest fire risk of that region before the bark beetle outbreak. The forest fire risk was also increased by communication trails, fuel bases, freight terminals and intensified tourism. Since the middle of 2012, the rapidly increasing amount of dead wood was the amplifying factor for fire risk followed by the appearance of the abundant grassy cover as the result of spruce dieback and unlimited access of the sun to the forest floor. 


\subsection{Forest fire risk - dead wood}

The appearance of the dead wood due to a natural, gradual process does not cause any significant increase of the fire risk as the result of an excessive amount of fuel. From the environmental point of view dead wood, regardless of the amount, does not cause any fire risk, although standing dead trees can be dangerous for people in the forest, especially when they are located along the forest roads, touristic trails. Moreover, if located along the communication trails, a large amount of dead wood will increase fire risk. According to the data of the Large-scale inventory of the State of Forests in years 2012 - 2016 (BFMG, 2017), the average volume of dead wood, both standing and lying, of all property types in Poland amounting to $6.3 \mathrm{~m}^{3} / \mathrm{ha}$. Considering that coniferous species constitute around $54 \%$ of all dead wood, the mean fuel load was estimated at 4 ton/ha. Forest fire risk linked with dead wood depends mostly on the size, type and decomposition stage. In general the greater the size of the dead wood, the lower the risk is. The highest risk is caused by fine fuel (diameter up to $7 \mathrm{~cm}$ ). Larger diameters, between 7 and $40 \mathrm{~cm}$ (medium size) and more than $40 \mathrm{~cm}$ (large size); so called coarse fuels because of their slow drying process and relative stability of moisture content close to the flammability threshold or even higher do not present a real fire risk. Including decomposition classes of dead wood by Maser (Maser, et al. 1979), from the fire risk perspective lying dead wood in decomposition class I creates the highest fire risk, concerning both ignition and spreading velocity. The main reasons for that are small branches with diameter up to $3 \mathrm{~cm}$, often with dried needles or leaves at the beginning, intact bark and incompact structure when trunk with branches rests on the ground. The fire risk decreases when small branches fall off, texture is still intact or only insignificantly and the trunk has not touched the ground yet. When dead wood reaches 3 and 4 class of decomposition its density decreases due to better air access, especially when a trunk is uncovered and exposed to sunlight and there has been no precipitation for a long time in such a case decomposing wood can smoulder very slowly. There is almost no fire risk when dead wood reaches 5 class of decomposition. In the case of standing dead wood, the highest risk is created by trees that are still alive or already declining ( 1 and 2 phase), which is the result of fuel continuity of small, dried branches, which enables transformation from soil cover fire into crown fire. In the next phases, $(3-8)$, fire risk decreases gradually but definitely. In the last phase (9) stumps smoulder and it is a long-lasting process. In general, higher fire risk is associated with fallen dead wood rather than standing, which is the result of no fuel continuity already in phase 3 of decomposition.

\subsection{Dead wood of Bialowieza Forest}

The amount of fuel load and its type are the basic criteria for the fire risk estimation as the fire energy balance, fire behaviour and size of loses are driven by it. The dead fuel load plays a particular role, not only during the initial phase of burning but also during fire spreading. The forest dead wood inventory was conducted in Bialowieza Forest in the years 2010 - 2011 in the framework of revision of the Forest Management Plans for Promotional Complex Bialowieza Forest, before the bark beetle outbreak. The mean amount of deadwood in the Forest Districts situated in the Forest was $24.9 \mathrm{~m}^{3} / \mathrm{ha}$. Including mean density of wood at the moisture content amounting to $15 \%$ (spruce, pine, oak and hornbeam, the main trees species of Bialowieza Forest) the mean fuel load is $15.8 \mathrm{ton} / \mathrm{ha}\left(1.58 \mathrm{~kg} / \mathrm{m}^{2}\right)$. For the purpose of comparison, mean volume of the dead wood in State Forests is $5.3 \mathrm{~m}^{3} / \mathrm{ha}$, private forest $-4.2 \mathrm{~m}^{3} / \mathrm{ha}$ and national parks $-37.3 \mathrm{~m}^{3} / \mathrm{ha}$ according to Bureau for Forest Management and Geodesy in years 2007 - 2011 (BFMG, 2007). Since June 2012, the volume of deadwood has increased twice up to $50.2 \mathrm{~m}^{3} / \mathrm{ha}$ and in 2017 it was already $231.8 \mathrm{~m}^{3} / \mathrm{ha}$. Table 1 presents collective data (source: Regional Directorate of State Forests in Bialystok) regarding volume of infestation of spruce trees and its area with stands disintegration and also the volume of dead wood per unit of area before and after partial removal of infested trees. 
Table 1 - The volume of infested trees and the area of dead stands in years 2012 - 2017

\begin{tabular}{|c|c|c|c|c|c|}
\hline \multirow{3}{*}{ Year } & \multirow{3}{*}{$\begin{array}{c}\begin{array}{c}\text { Volume of } \\
\text { planted spruce } \\
\text { trees }\end{array} \\
\mathrm{m}^{3}\end{array}$} & \multirow{3}{*}{$\begin{array}{c}\text { Volume of } \\
\begin{array}{c}\text { removed spruce } \\
\text { trees }\end{array} \\
\mathrm{m}^{3}\end{array}$} & \multirow[b]{2}{*}{$\begin{array}{c}\text { Area of dead tree } \\
\text { stands }\end{array}$} & \multicolumn{2}{|c|}{ Volume of dead wood } \\
\hline & & & & before removal & after removal \\
\hline & & & ha & \multicolumn{2}{|c|}{$\mathrm{m}^{3} / \mathrm{ha}$} \\
\hline $2012 *$ & 23289 & 6412 & 464 & 50.2 & 36.4 \\
\hline 2013 & 102592 & 19610 & 689 & 148.9 & 120.4 \\
\hline 2014 & 198123 & 25488 & 1482 & 133.7 & 116.5 \\
\hline 2015 & 267080 & 42288 & 2088 & 127.9 & 107.6 \\
\hline 2016 & 483683 & 39148 & 2498 & 193.6 & 1779 \\
\hline 2017 & 366982 & 162020 & 1583 & 231.8 & 129.5 \\
\hline
\end{tabular}

*since June

In the year 2017 the total volume of infested spruce trees was equal to $1.414 .749 \mathrm{~m}^{3}$ on the area of 8804 ha. Removal of the dead wood (totally $294.966 \mathrm{~m}^{3}$ ) enabled slight (on average $20 \%$ ) reduction of the volume of the dead wood during the analysed period. Changes in the average amount of the fuel load were calculated based on the presented data for particular years and also calculated in a cumulatively including total volume of infested spruce trees and its area, presented on the figure 1. The density of spruce wood at the moisture content of $15 \%$ was adopted as equal to $470 \mathrm{~kg} / \mathrm{m}^{3}$.

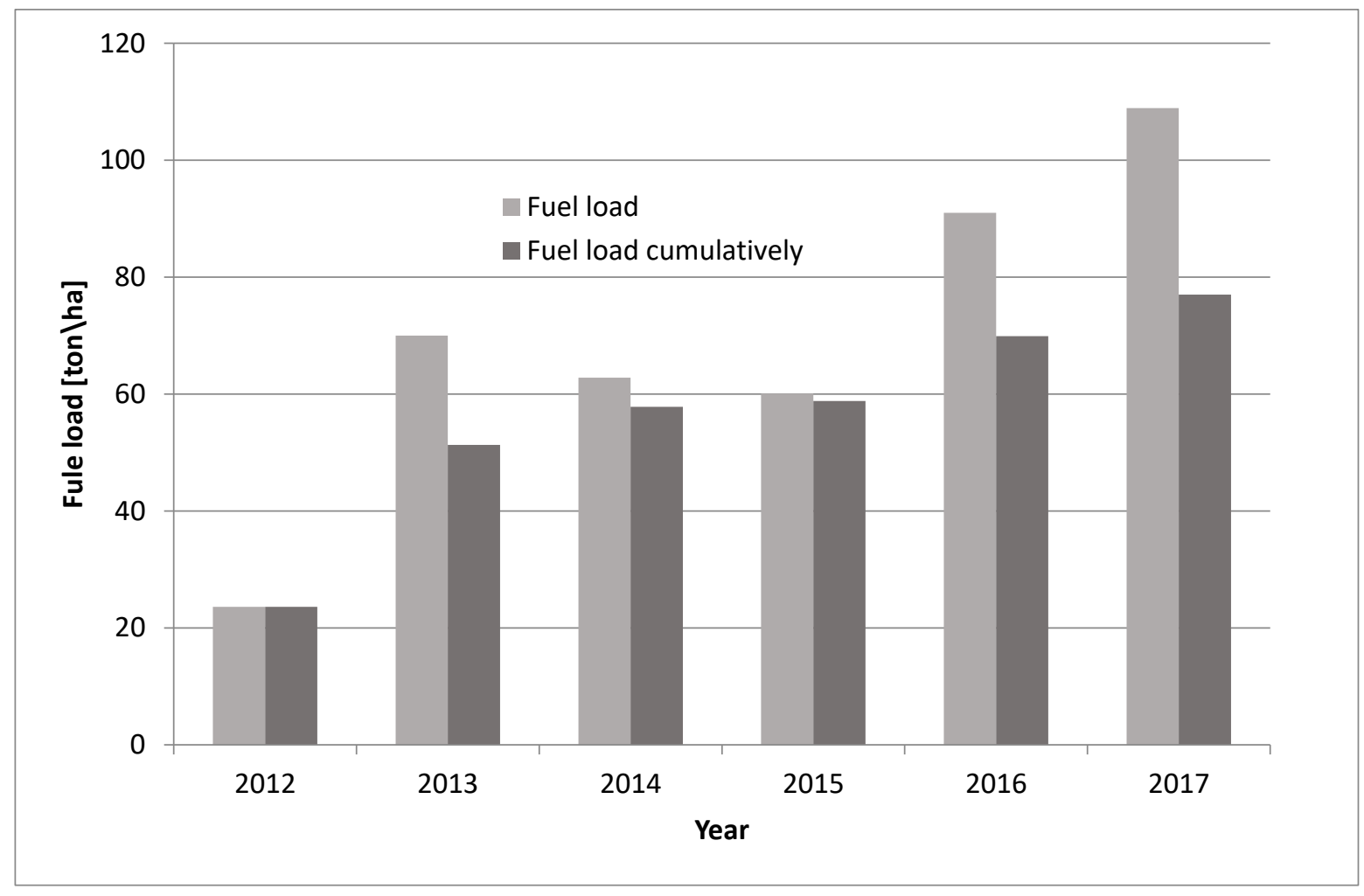

Figure 1 - Changes in the volume of fuel load in years 2012 - 2017 in Bialowieza Forest

The smallest amount of fuel load, equal to 23.6 ton/ha, was at the beginning of the bark beetle outbreak in 2012. The next years brought an increase in fuel load of up to 60-70 t/ha with the maximum of 108.9 ton/ha in year 2017. According to the precise data the maximum amount of dead wood in some sub-compartments was equal to $629 \mathrm{~m}^{3} / \mathrm{ha}$, which corresponds to $295.6 \mathrm{ton} / \mathrm{ha}$ of fuel load. 
Figure 2 presents spatial distribution of standing dead spruce wood in Bialowieza Forest in year 2017 determined by airborne laser scanning (ALS), conducted as part of the Life+ ForBioSensing project.

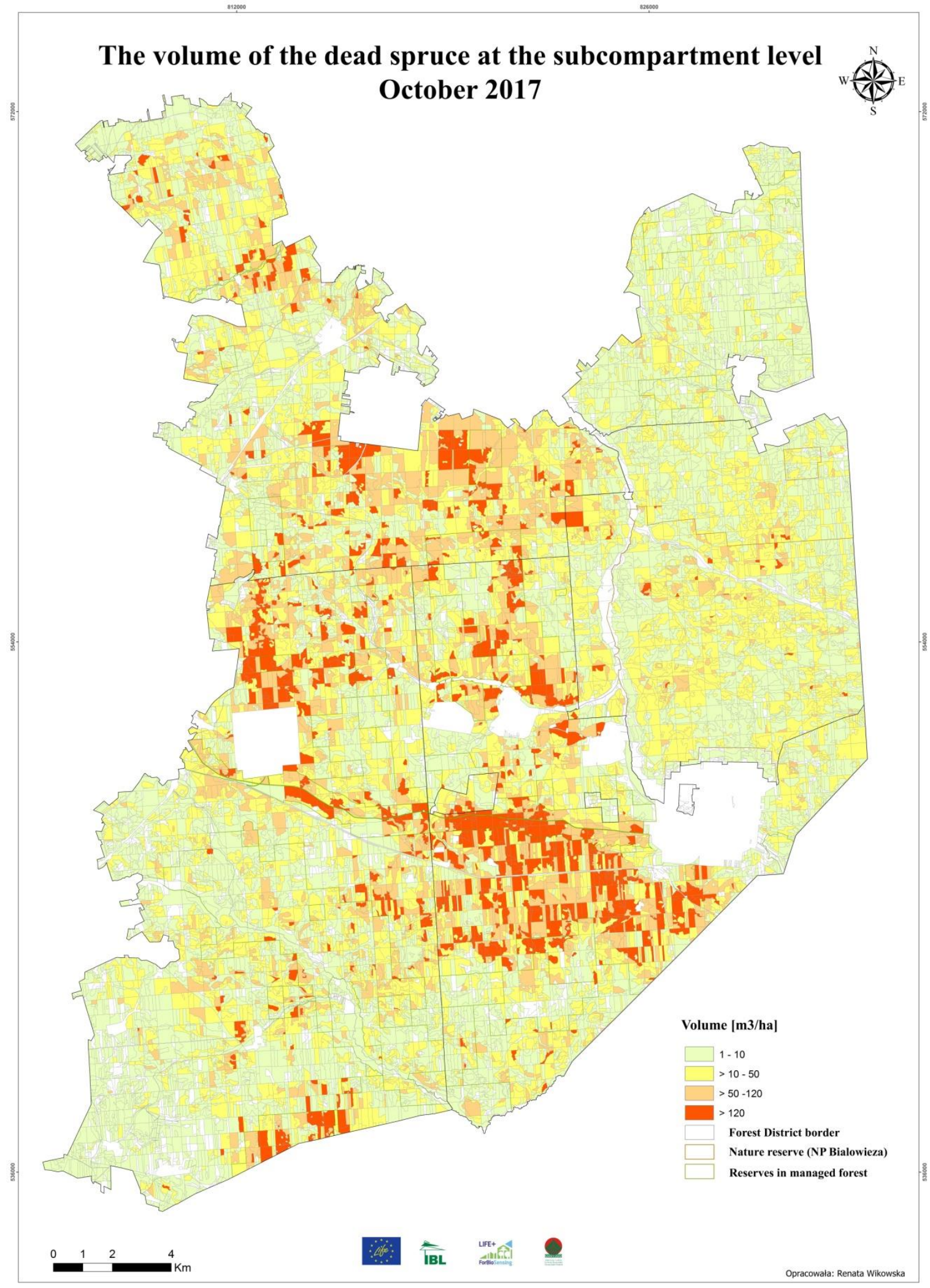

Powyższa mapa została sporządzona na podstawie klasyfikacji gatunkowej wykonanej na podstawie Lotniczego Skaningu Laserowego z 2015 roku oraz ze zdjęć lotniczych wykonanych w pażdzierniku 2017 roku.

Figure 2 - The volume of the dead spruce at the subcompartment level - October 2017 
Because dead fine fuel is the most risky, fuel load field measurements were conducted, choosing randomly 5 areas with the greatest amount of fallen dead wood for estimation of the maximum amount. Each plot was separated (average area $16 \mathrm{~m}^{2}$ ) and all the lying down dead wood was weighted. The amounts of dead wood differ from 86.76 up to 139.88 ton/ha, on average 105.77 ton/ha air-dried. That amount of dead wood intensifies the risk of fire outbreak with increased dynamic in its behaviour. The risk increases particularly where dead trees with dried branches lying on the forest floor. Their incompact structure stimulates ignition and the intensity of fire enables the transition of fire into the crowns of still living trees and dead trees with dried crown. Standing dead spruce will fall down with time or break (it will take between 3 and 6 years according to the experts), which leads to an increase of fallen fuel load, it is more dangerous than a standing fuel load. Trunks lying on the forest floor, because of their density, present lower risk due to less susceptibility to ignition compared to other forest materials. As the decomposition of dead wood continues with time, its role will change and in the case of fire outbreak these trunks will be more susceptible for ignition and the fire will be prolonged. Fallen dead wood creates obstacles for conducting rescue action by blocking fire trails and the access to fire spots.

\subsection{Soil cover}

Grassy cover occurred massively on the areas with bark beetle outbreak, mainly reed grass but also fern and raspberry. This type of vegetation, in dried stage (early spring, summer droughts, early autumn), creates high fire risk, conducive to fire outbreak and its rapid behaviour. The inventory of the grassy areas (area and cover percentage) was conducted in 2016 for forest districts of Bialowieza Forest including infested areas. The results of the collective inventory are presented in table 2 together with the list of areas by dominant type of vegetation and its cover percentage.

Table 2 - Grassy cover inventory results

\begin{tabular}{|c|r|r|r|r|r|}
\hline \multirow{2}{*}{ Dominant type of vegetation } & \multicolumn{5}{|c|}{ Cover percentage [\%] } \\
\cline { 2 - 6 } & 10 & 20 & 30 & $40-100$ & \multicolumn{1}{c|}{ total } \\
\hline Heather and juniper & 159.73 & 219.28 & 218.96 & 20.48 & 618.45 \\
\hline Fern & 288.90 & 440.34 & 269.99 & 216.39 & 1215.62 \\
\hline Grass & 1539.50 & 1446.66 & 1290.54 & 1416.99 & 5693.69 \\
\hline Total & 1988.13 & 2106.28 & 1779.49 & 1653.86 & 7527.76 \\
\hline
\end{tabular}

Total area with the vegetation type of high fire risk equals 7527.76 ha, which constitutes $15 \%$ of the total forest land of Bialowieza Forest. The largest area was covered by grass, followed by mixture of raspberry and bracken, and lastly heather with juniper.

For the purpose of fuel load estimation field measurements were conducted on the area with canopy openness and on the reference area in the case of grass. All the vegetation on $1 \mathrm{~m}^{2}$ was weighted for the purpose of calculation of dry fuel load. All together measurements were conducted on total area of 35 plots, including 15 with grass, 10 with fern, 5 with raspberry and 5 as reference plots. Mean values of the fuel load of risky vegetation type are presented in table 3.

Table 3 - Mean fuel load for selected high risk vegetation types

\begin{tabular}{|c|c|c|}
\hline Vegetation type & Mean height [cm] & Mean fuel load[ton/ha] \\
\hline grass & 129 & 2,50 \\
\hline fern & 116 & 3,67 \\
\hline raspberry & 89 & 2,87 \\
\hline grass/reference & 15 & 0,97 \\
\hline
\end{tabular}


Measured fuel load for grassy cover varied between 1.17 up to 4.56 ton/ha, for raspberry between 2.36 to 3.27 ton/ha and for fern between 1.92 up to 7.99 ton/ha. It shows the variety of flammable biomass for all the vegetation types. On the reference area the mean fuel load for grassy cover was 2.5 times less compared to the area with open canopy and varied between 0.44 to $1.36 \mathrm{ton} / \mathrm{ha}$. The fuel load for previously measured lowland forest is equal to the average $0.5 \mathrm{~kg} / \mathrm{m}^{2}$. It was 5 times less compared to the stands with open canopy. The highest risk is presented by grass cover when it is dry due to its loose spatial structure, height of the fuel bed and the fact it is found in open terrain, which influences its rapid drying. According to model calculations the fire behaviour in these conditions shall be characterised by a 6 times faster spreading of the fire front and at the same time the burnt area will be 30 time larger compared to the situation before the bark beetle infestation outbreak and disintegration of the stands.

\subsection{Fire risk classification}

According to the existing rules, Forest District Bialowieza, Browsk and Hajnowka together with Bialowieza National Park are marked as III, the lowest forest fire risk category considering the frequency of forest fires, stands conditions, climate, and anthropogenic pressure. Figure 3 presents the fire risk according to the forest fire risk category (in accordance with existing regulations) for three forest districts of Bialowieza Forests - Browsk, Hajnowka and Bialowieza.

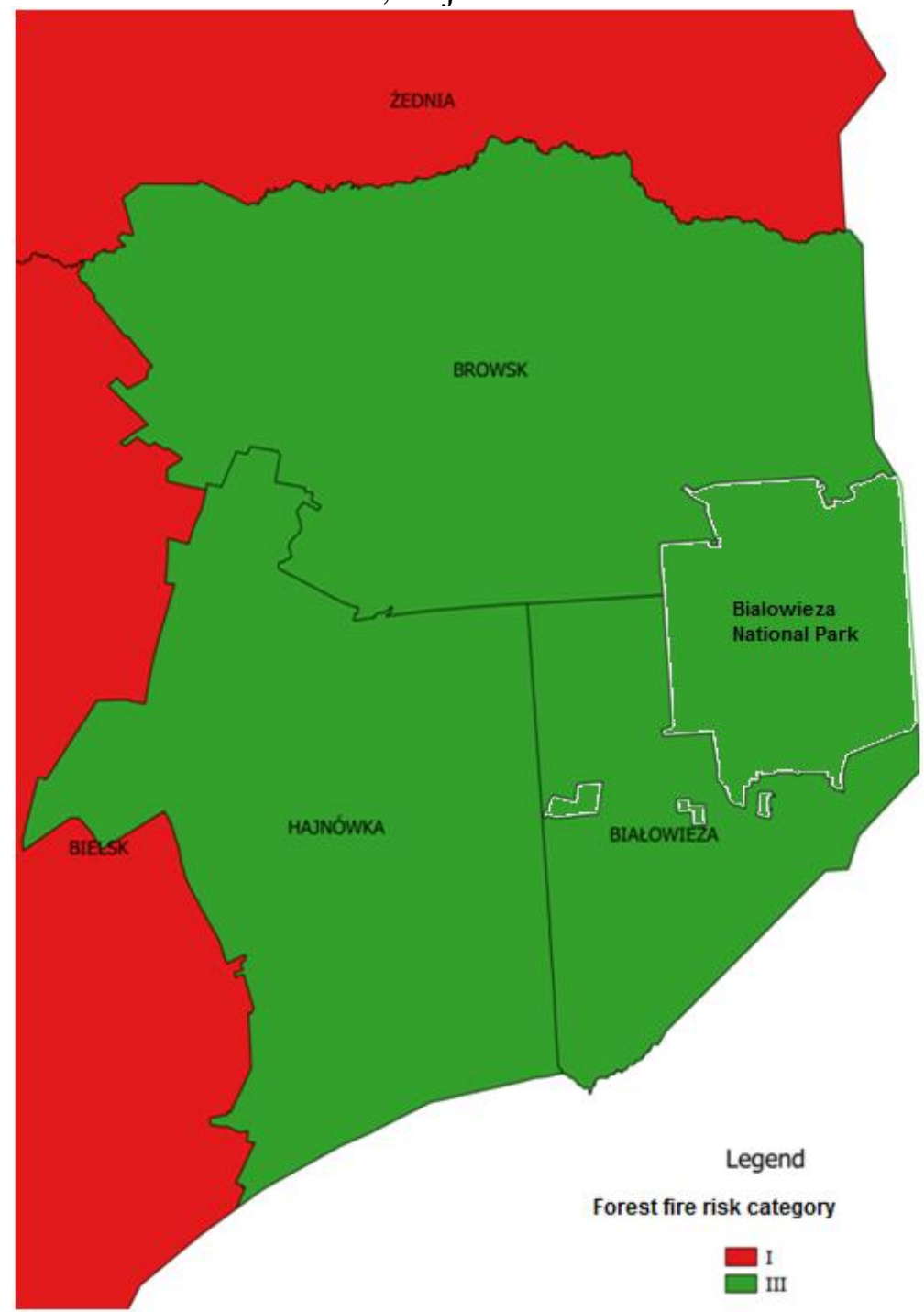

Figure 3 - Forest fire risk according to the forest fire risk category for three forest districts of Bialowieza Forests Browsk, Hajnowka and Bialowieza. 
Forest fire risk category determines the organisation of the forest fire protection system, including formation of forest structure in case of fire. The applied macro-scale method for fire risk estimation corresponds with the total classified area of forest district or national park, excluding local risk. The new method (presented in paper "The stand flammability classes" included in conference materials) applied to estimate fire risk of Bialowieza Forest enables determination of the risk at the level of subcompartment, compartment and forest range. Flammability class (three-stage scale) is established on the basis of forest habitat type and soil cover type and expresses the susceptibility of forest flammable materials to fire, with flammability indices based on statistical calculation. The above mentioned method expresses more accurately the real fire risk in the forest - figure 4.

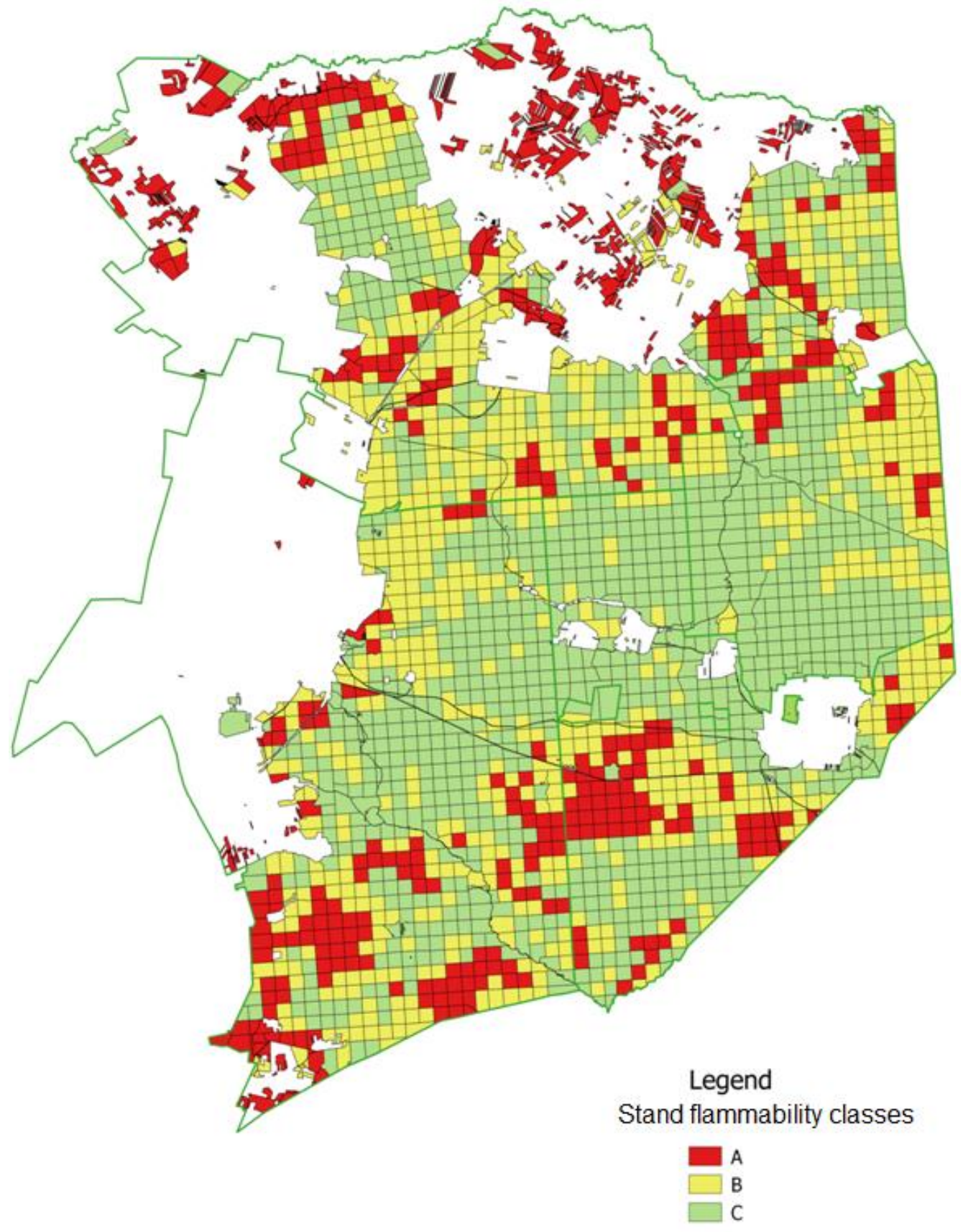

Figure 4 - The stand flammability classes of Bialowieza Forest - compartment level - 2017 


\section{Forest fire protection system of Bialowieza Forest}

The analysis of the fire risk and existing fire protection system, including the result of forest inventory of selected elements of fire infrastructure, were the base for modification of fire protection system of Bialowieza National Park according to the current risk. Due to the limited volume of this paper all the rules are presented only in an abbreviated version.

\subsection{Activities limiting fuel load}

Fuel load should be limited, if possible, where the possibility of ignition is high; for example along the communication trails (also considering the probability of blocking roads by fallen trees and other obstacles to the rescue service), and touristic paths and places. Formation of the piles made of dried wood (especially small dimensions) creates high fire risk and its rapid spread to the adjacent land and should therefore be forbidden.

\subsection{Alarm and communication system}

The existing communication system in the forest districts of Bialowieza and Bialowieza National Park (mobile) does not guarantee the expected quality and possibility of communication and early alarm together with the lack of coverage for the whole area of Bialowieza Forest. Some areas have limited communication or even work without it. The communication should be provided over the whole area of Bialowieza Forest, which is obviously connected with financial support and new organisation of the communication.

\subsection{Observation network}

Due to lack of the coverage from lookout towers estimated up to $30 \%$ - including areas with A and B flammability classes located on southern part of Forest District Bialowieza and Hajnowka - it is necessary to built a new lookout tower. By the time of incorporating new lookout tower into the system ground patrolling should be launched covering areas with high forest fire risk when the calculated forest fire risk degree is 3 (the highest).

\subsection{Fire trails}

The possible localization of the new fire trails was elaborated basing on the results of terrain inventory, taking into account current and future forest fire risk and the needs derived from existing fire protection rules. For the purpose of providing access to the most endangered areas, $127 \mathrm{~km}$ of new fire trails were appointed. For the purpose of improving access for the rescue services renovations were ordered including stabilization of road surface and its extension where needed. The fire trails leading to forest areas marked as A and B flammability class will be renovated first, especially parts with destroyed surface and very narrow ones.

\subsection{Water supplying}

Basing on the results of terrain inventory potential localization of two new water supplying points was chosen. Only the objects most adopted for the fire engines requirements were selected. Taking into account that water supplying for Bialowieza Forest is seasonally insufficient (summer and droughts) and limited possibility of water collection (ground water level too low) it was decided to use deep wells located near forestry lodge.

\subsection{Fire fighting equipment}

Due to improvement of the effectiveness of fire suppression using airplanes, it was suggested to use private airfield (after signing of the appropriate agreement), located on the edge of Bialowieza Forest and which shall be used as operational airfield. It will shorten the distance needed for the airplane to reach the target (before it was $80 \mathrm{~km}$ ). The retrofitting of the existing fire units was suggested as well 
including foldable water tanks with minimum capacity of $8 \mathrm{~m}^{3}$ and replacement of fire fighting equipment. In case of buying new fire engines, four wheel drive should be preferred.

\subsection{Forest fire risk forecasting}

For the purpose of improving accuracy of the forecasting forest fire risk degree it is necessary to separate new smaller prognostic zones for Bialowieza Forest with the prognostic and auxiliary meteorological points located in place. Forest fire risk degree determines everyday activities related to forest fires and operational readiness of rescue services.

\subsection{Education and prevention}

It was recommended to prepare educational campaign regarding current fire risk in Bialowieza Forest including rules while visiting forest and for limitation of ignition risk. Effective campaign should be professionally designed, with long term perspective and target groups, especially local people. The problem of increasing fire risk of that region should be the part of everyday work of people working in education and webmasters of official web pages and Facebook profiles of forest districts in Bialowieza Forest.

\subsection{Activities related to planning and organization of rescue action}

Actualization of the rescue plans, prepared by the forest and fire service, for the region of Bialowieza Forest was commissioned including current situation with bark beetle outbreak. It was recommended to organize common manoeuvres of forest service, park rangers and other units involved in fire suppression on the area directly affected by the bark beetle outbreak. In the framework of transboundary agreement between Poland and Belarus regarding mutual help in case of fire outbreak it is necessary to elaborate fire procedures and rules for crossing the border.

The above recommendations for improvement of fire protection system of Bialowieza Forest are gradually implemented by forest administration and State Fire Service and should lead to decrease in fire risk and limit effects of potential outbreaks.

\section{Literature}

BFMG. Bureau for Forest Management and Geodesy. Large scale forest inventory in years 2012 2016. 2017..Sękocin Stary.

Maser C., Anderson R.G., Cromack K., Williams J.T., Martin R.E. 1979. Dead and down woody material. USDA Forest Service, Agriculture Handbook No 553, Portland-Washington D.C.

BFMG. Bureau for Forest Management and Geodesy. Large scale forest inventory in years 2007 2011. 2012. Sękocin Stary. 


\title{
The stand flammability classes
}

\author{
Miroslaw Kwiatkowski; Ryszard Szczygiel; Bartlomiej Kolakowski* \\ Forest Research Institute. Sękocin Stary, 3, Braci Leśnej Street, 05-090 Raszyn, Poland. \\ \{m.kwiatkowski@ibles.waw.pl,r.szczygiel@ibles.waw.pl,b.kolakowski@ibles.waw.pl*\}
}

\begin{abstract}
The fire risk classification now applied in Poland to forest areas is based on the frequency of fire outbreaks and the stand-related, climatic and anthropogenic conditions. The minimum area for which the forest risk category is determined is the area of a forest district (on average seventeen thousand five hundred ha), which reaches a maximum area of up to thirty-odd thousand hectares. The classification enables the determination of the fire risk for a county, sub-region or province, or the Regional Directorate of State Forests. Thus, this method enables identification of the potential fire risk on the macro scale. However, it is impossible to identify the fire risk on the micro scale.

The classification of stand flammability according to forest habitat types, which has been developed as a result of the implementation of the project and fuel models, shall enable the mapping of forests in terms of their fire characteristics at the sub-compartment level.
\end{abstract}

Keywords: forest fire risk categories, stand flammability classes, forest habitat types, soil cover types

\section{Introduction}

The fire risk classification now applied in Poland to forest areas is based on the frequency of fire outbreaks and the stand-related, climatic and anthropogenic conditions. The minimum area, for which the forest risk category is determined, is the area of a forest district (on average seventeen thousand five hundred ha), which has a maximum area of up to thirty-odd thousand hectares. The classification enables the determination of the fire risk for a county, sub-region or province, or the Regional Directorate of State Forests. Thus, this method enables identification of the potential fire risk on the macro scale. However, it is impossible to identify the fire risk on the micro scale.

The classification of stand flammability according to forest habitat types, which has been developed as a result of the implementation of the project and fuel models, shall enable the mapping of forests in terms of their fire characteristics at the sub-compartment level. This shall meet the requirements of Directive 2007/2/EC of the European Parliament and of the Council of 14 March 2007 establishing an Infrastructure for Spatial Information in the European Community (INSPIRE), which is implemented in Poland by the Ministry of the Environment. One of the expected effects of the implementation of the Directive is the possibility of mapping forest fire risks. At present, forest areas are mapped in terms of fire risk at two levels:

1. The national level, the classification of the Regional Directorates of State Forests (provinces, sub-regions) according to forest fire risk categories,

2. The regional level, the classification of forest districts (counties) according to forest fire risk categories.

The project involved the separation of a third forest fire risk mapping level, the local level of a forest district, considering flammability classes determined by a flammability analysis of forest habitat types and soil cover types. The fire risk mapping method provided for the use of necessary valuation data from the Information System of State Forests (SILP). Since a sub-compartment was a basic mapping unit, this led to very detailed, but too meticulous and fuzzy mapping, which could limit its practical usefulness in certain situations. Therefore, data generalisation rules were developed to enable 
the determination of the flammability class for larger areas, a compartment and a forest district. It was decided that a generalised class should be a weighted mean of the classes of individual subcompartments, with the qualification that where the area of the stands of any class exceeded $50 \%$ of the surface of a given area, it was assigned as a whole to that class.

\section{Methodology}

The stand flammability classification according to forest habitat types was based on an analysis of fire outbreaks in State Forests in the period from 2007 to 2017. In elaborating it, when calculating the flammability index consideration was given to the number of fires and the burnt area relative to the area occupied by particular forest habitat types and soil cover types. This index was the quotient of the proportion of fires or the burnt area and the proportion of a given stand type. In relation to the number of fires, consideration was only given to those sub-compartments where a fire had occurred, whereas in relation to the burnt area the total area affected by a fire was taken into account. These data were referred to current information about the areas of stands growing on particular sites and the soil cover types identified from detailed data on all the sub-compartments found in the Forest Data Bank. The classification of stands in terms of fire risk shall enable assessing it at the sub-compartment level and to create an LMN (Forest Numeric Map) layer with precise fire risk mapping. In elaborating the final classification method, the weights of individual indices were dependent on the correlation strength of the density of fires or the burnt area and the presence of selected stand types.

\section{Results}

\subsection{Classification of stand flammability according to forest habitat type and soil cover type.}

The analysis of the forest fire occurrence, which the classification of the stands is based on, distinctly shows the domination of the tree habitat types, considering both the number of forest fires and the burnt area. Regarding the volume of this publication, authors focused only at the results for lowland habitats covering almost $90 \%$ of Polish forests. These are: fresh coniferous, fresh mixed coniferous and fresh mixed broadleaved forest. The domination of these habitat types is mostly linked to the area covered by them. More accurate information regarding stands flammability is derived from flammability indices - both for number of forest fires and burnt area (table 1). In terms of the number of fires, dry coniferous, moist mixed coniferous, moist coniferous, fresh coniferous, and fresh mixed coniferous forest sites should be considered as the most flammable (with flammability indices higher than 1), whereas in the case of the burnt area these are, respectively, dry coniferous, moist coniferous, bog coniferous, moist mixed coniferous, fresh coniferous, fresh mixed coniferous and fresh mixed broadleaved forest sites. It is noticeable that for the wet sites flammability indices of burnt area is frequently higher than the one for the number of fires.

Table 1 - The occurrence of fires depending on forest habitat types and flammability indices.

\begin{tabular}{|c|c|c|c|c|c|}
\hline \multirow{2}{*}{$\begin{array}{c}\text { Forest habitat type } \\
\text { Stand area [\%] }\end{array}$} & $\begin{array}{c}\text { Share of number of } \\
\text { forest fires [\%] }\end{array}$ & $\begin{array}{c}\text { Share of burnt } \\
\text { area [\%] }\end{array}$ & $\begin{array}{c}\text { Flammability index according } \\
\text { to habitat type }\end{array}$ \\
\cline { 4 - 6 } & $\begin{array}{c}\text { For number of } \\
\text { fires }\left(W_{S L}\right)\end{array}$ & $\begin{array}{c}\text { For burnt } \\
\text { area }\left(W_{S P}\right)\end{array}$ \\
\hline $\begin{array}{c}\text { Bb-bog coniferous } \\
\text { forest }\end{array}$ & 0.15 & 0.06 & 0.34 & 0.39 & 2.28 \\
\hline $\begin{array}{c}\text { BMb-bog mixed } \\
\text { coniferous forest }\end{array}$ & 0.63 & 0.39 & 0.51 & 0.62 & 0.80 \\
\hline $\begin{array}{c}\text { BMśw - fresh mixed } \\
\text { coniferous forest }\end{array}$ & 23.66 & 32.99 & 26.31 & 1.39 & 1.11 \\
\hline BMw & 4.44 & 7.06 & 9.56 & 1.59 & 2.15 \\
\hline Bs -dry coniferous & 0.17 & 0.58 & 2.25 & 3.40 & 13.17 \\
\hline forest
\end{tabular}

Advances in Forest Fire Research 2018 - Page 166 


\begin{tabular}{|c|c|c|c|c|c|}
\hline $\begin{array}{c}\text { Bśw - fresh coniferous } \\
\text { forest }\end{array}$ & 19.72 & 28.90 & 28.22 & 1.47 & 1.43 \\
\hline $\begin{array}{c}\mathrm{Bw}-\text { moist coniferous } \\
\text { forest }\end{array}$ & 0.61 & 0.93 & 4.42 & 1.53 & 7.27 \\
\hline Lł-riparian forest & 0.47 & 0.31 & 0.38 & 0.65 & 0.80 \\
\hline $\begin{array}{c}\text { LMb - bog mixed } \\
\text { broadleaved forest }\end{array}$ & 0.73 & 0.15 & 0.14 & 0.20 & 0.20 \\
\hline $\begin{array}{c}\text { LMśw - fresh mixed } \\
\text { broadleaved forest }\end{array}$ & 19.07 & 14.54 & 11.79 & 0.76 & 0.62 \\
\hline $\begin{array}{c}\text { LMw - moist mixed } \\
\text { broadleaved forest }\end{array}$ & 3.82 & 3.55 & 3.97 & 0.93 & 1.04 \\
\hline $\begin{array}{c}\text { Lśw - fresh broadleaved } \\
\text { forest }\end{array}$ & 9.79 & 3.52 & 3.93 & 0.36 & 0.40 \\
\hline $\begin{array}{c}\text { Lw - moist broadleaved } \\
\text { forest }\end{array}$ & 1.95 & 0.62 & 0.73 & 0.32 & 0.38 \\
\hline Ol-alder forest & 179 & 0.41 & 0.55 & 0.23 & 0.30 \\
\hline OlJ-alder-ash forest & 0.81 & 0.12 & 009 & 0.14 & 0.11 \\
\hline
\end{tabular}

It is clearly visible that estimating the occurrence of the same group of fires in relation to soil cover around $50 \%$ occur in stands with turf (table 2). However, when the share of stands with turf is close to $60 \%$, both for number of fires and burnt area, flammability indices are close to 0.8 . The highest values of flammability index, in both cases, occur for stands with bare ground. Still, considering insignificant (less than 1\%) share of stands with bare ground, it does not influence the characteristic of fires occurrence.

Table 2 - The occurrence of fires depending on the soil cover types and forest flammability indices.

\begin{tabular}{|c|c|c|c|c|c|}
\hline \multirow{2}{*}{ Soil cover type } & \multirow{2}{*}{$\begin{array}{l}\text { Share of total } \\
\text { stand area }[\%]\end{array}$} & \multirow{2}{*}{$\begin{array}{l}\text { Share of number of } \\
\text { forest fires [\%] }\end{array}$} & \multirow{2}{*}{$\begin{array}{c}\text { Share of burnt } \\
\text { area }[\%]\end{array}$} & \multicolumn{2}{|c|}{$\begin{array}{l}\text { Flammability index } \\
\text { according to habitat type }\end{array}$} \\
\hline & & & & $\begin{array}{l}\text { For number of } \\
\text { fires }\left(W_{P L}\right)\end{array}$ & $\begin{array}{c}\text { For burnt } \\
\text { area }\left(W_{P P}\right)\end{array}$ \\
\hline MSZ-moss & 15.43 & 18.78 & 13.38 & 1.22 & 0.87 \\
\hline MSZC - moss/bilberry & 11.53 & 5.33 & 5.16 & 0.46 & 0.45 \\
\hline NAGA - bare & 0.42 & 0.65 & 1.03 & 1.53 & 2.44 \\
\hline SZAD - turf (dense) & 16.34 & 1289 & 19.29 & 0.79 & 1.18 \\
\hline $\mathrm{SZCH}$ - weed (dense) & 8.65 & 5.12 & 6.93 & 0.59 & 0.80 \\
\hline ŚCIO - litter & 4.01 & 4.47 & 4.36 & 1.11 & 1.09 \\
\hline ZAD - turf & 57.09 & 48.18 & 45.81 & 0.84 & 0.80 \\
\hline ZIEL-herbaceous & 13.48 & 4.60 & 4.04 & 0.34 & 0.30 \\
\hline
\end{tabular}

Another stage involved an attempt to assess the importance of particular indices for establishing the method for classifying the stand flammability where the flammability index determined for the number of fires was higher than 1 . In relation to the number of fires, the investigation concerned the correlation of the density of fires (the number of fires in 10,000 ha of a forest area) in forest districts with their proportion of stands growing on the sites BMśw, BMw, Bs, Bśw, Bw and also with their proportion of stands growing on the sites with moss, bare ground and litter.

In relation to the burnt area, the investigation concerned the correlation of its density (the burnt area in 10,000 ha of a forest area) with their proportion of stands growing on the sites Bb, BMśw, BMw, Bs, Bśw, Bw, LMw and also with their proportion of stands growing on the sites with bare ground, dense turf and litter.

Figure 1 illustrates the relation between share of forest habitat types and density of fires. 


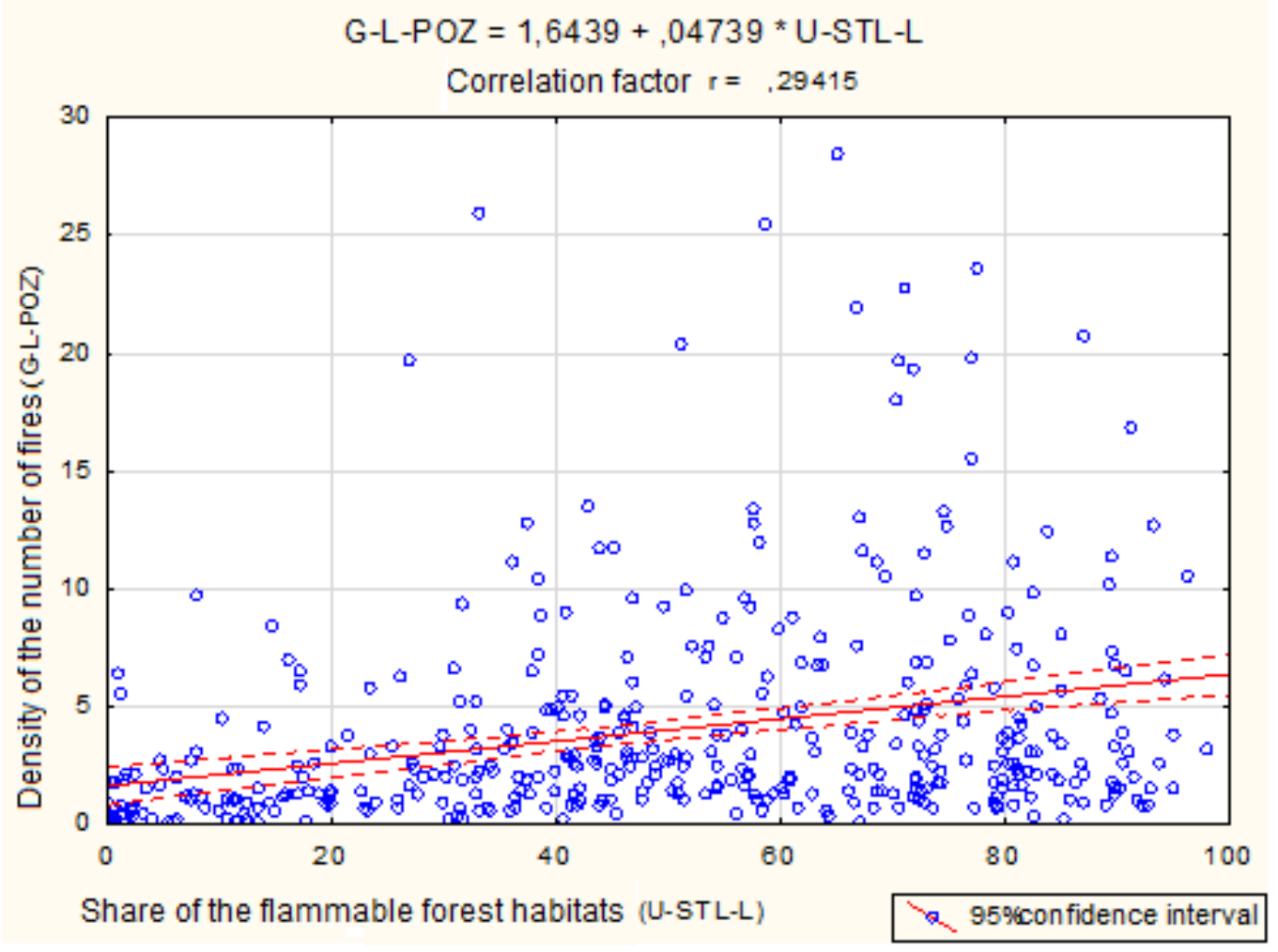

Figure 1 - The correlation between density of fires (GL - POZ) and share of forest habitat types (U-STL-L)

In all the cases, this correlation was poor, with the highest correlation coefficient found for the density of the number of fires depending on the proportion of forest habitat types $(r=0.2941)$, followed by the density of the burnt area depending on the proportion of forest habitat types $(r=0.2158)$.

In relation to the proportion of soil cover types, this was, respectively, $r=0.1710$ for the burnt area and $\mathrm{r}=0.1660$ for the number of fires.

Taking the above into account, the formula for the cumulative flammability index was derived:

where:

$$
W_{S}=0.8 \cdot W_{S L}+0.4 \cdot W_{S P}+\text { if }\left(W_{P P}>W_{P L}\right) 0.3
$$

$W_{S L}$ - the flammability index of forest habitat types for the number of fires,

$W_{S P}$ - the flammability index of forest habitat types for the burnt area,

$W_{P L}$ - the flammability index of forest soil cover types for the number of fires,

$W_{P P}$ - the flammability index of forest soil cover types for the burnt area.

On this basis, it is possible to determine the stand risk class, in accordance with Table 3.

Table 3 - The intervals of the cumulative flammability index for the stand flammability classes.

\begin{tabular}{|c|c|}
\hline Value of cumulative flammability index $W_{S}$ & Flammability classes \\
\hline$\geq 1,7$ & A (high) \\
\hline $0,7 \div 1,7$ & B (medium) \\
\hline$\leq 0,7$ & C (low) \\
\hline
\end{tabular}

According to the assumption above, all the stands (considering forest habitat type and soil cover) should be included in one of the below flammability classes (Table 4). 
Table 4 - The stand flammability classes in relation to the forest habitat type and soil cover

\begin{tabular}{|c|c|c|c|c|c|c|c|c|}
\hline \multirow{2}{*}{$\begin{array}{c}\text { Forest habitat } \\
\text { type }\end{array}$} & \multicolumn{8}{|c|}{ Soil cover } \\
\hline & $M S Z$ & $M S Z C$ & $N A G A$ & $S Z A D$ & $\mathrm{SZCH}$ & $S \mathrm{CIO}$ & $Z A D$ & ZIEL \\
\hline$B b$ & $B$ & $B$ & $B$ & $B$ & $B$ & $B$ & $B$ & $B$ \\
\hline$B M b$ & $B$ & $B$ & $B$ & $B$ & $B$ & $B$ & $B$ & $B$ \\
\hline$B M s^{\prime} w$ & $B$ & $B$ & $\bar{A}$ & $\bar{A}$ & $\bar{A}$ & $B$ & $B$ & $B$ \\
\hline$B M w$ & $\bar{A}$ & $\bar{A}$ & $A$ & $\bar{A}$ & $\bar{A}$ & $\bar{A}$ & $\bar{A}$ & $\bar{A}$ \\
\hline Bs & $A$ & $A$ & $A$ & $A$ & $A$ & $A$ & $A$ & $A$ \\
\hline$B s ́ w$ & $\bar{A}$ & $\bar{A}$ & $A$ & $\bar{A}$ & $A$ & $A$ & $A$ & $A$ \\
\hline$B w$ & $A$ & $A$ & $\bar{A}$ & $A$ & $A$ & $A$ & $A$ & $A$ \\
\hline$L t$ & $B$ & $B$ & $B$ & $B$ & $B$ & $B$ & $B$ & $B$ \\
\hline$L \nmid G$ & $C$ & $C$ & $C$ & $C$ & $C$ & $C$ & $C$ & $C$ \\
\hline$L M b$ & $C$ & $C$ & $C$ & $C$ & $C$ & $C$ & $C$ & $C$ \\
\hline$L M s ́ w$ & $B$ & $B$ & $B$ & $B$ & $B$ & $B$ & $B$ & $B$ \\
\hline$L M w$ & $B$ & $B$ & $B$ & $B$ & $B$ & $B$ & $B$ & $B$ \\
\hline$L s ́ w$ & $C$ & $C$ & $B$ & $B$ & $B$ & $C$ & $C$ & $C$ \\
\hline$L w$ & $C$ & $C$ & $B$ & $B$ & $B$ & $C$ & $C$ & $C$ \\
\hline $\mathrm{Ol}$ & $C$ & $C$ & $C$ & $C$ & $C$ & $C$ & $C$ & $C$ \\
\hline OlJ & $C$ & $C$ & $C$ & $C$ & $C$ & $C$ & $C$ & $C$ \\
\hline
\end{tabular}

According to the elaborated method flammability classes were established for all sub-compartments in Poland based on the data derived from Forest Data Bank, as on 31.12.2016. The largest area is covered by the stands with "B" class, which constitute $51.5 \%$ of the total forest area, followed by "A" class stands covering $27.9 \%$ of forest land. The less numerous are the stands marked as "C" class, covering $20.6 \%$ of forest land in Poland. This data is close to the share of the forest land according to the forest fire risk categories, which constitute (as on 31.12.2016) 34.7\% for I category (highest risk), $46.79 \%$ II category (medium risk), and $18.4 \%$ for III category (low risk).

\subsection{Mapping of forest fire risk according to flammability classes and fuel type.}

The way of mapping forest risk is presented using part of the Regional Directorate in Katowice. The forest area of the presented part (figure 2) is more than 65000 ha and includes forest districts marked as I and II forest fire risk category. Figure 3 illustrates the classification of the stands using flammability classes. The greatest share constitutes "B" class with $49.5 \%$ of forest land, followed by "A" class with $34.3 \%$ of forest land and " $\mathrm{C}$ " with $16.2 \%$ of forest land. Figure 4 illustrates flammability classes generalised to the level of compartment. In the last case the share of the "B" class is bigger than above $(50.8 \%)$, while the remaining classes constitute less than above ("A" $-34.1 \%$ and "C" $15.1 \%$ ). The third, the most general level of spatial accuracy, is the level of forest range and it is illustrated on figure 5 . In this case "B" share equals to $53.1 \%$, followed by "A" class with $32.5 \%$ of forest land and "C" class with only $14.4 \%$. 


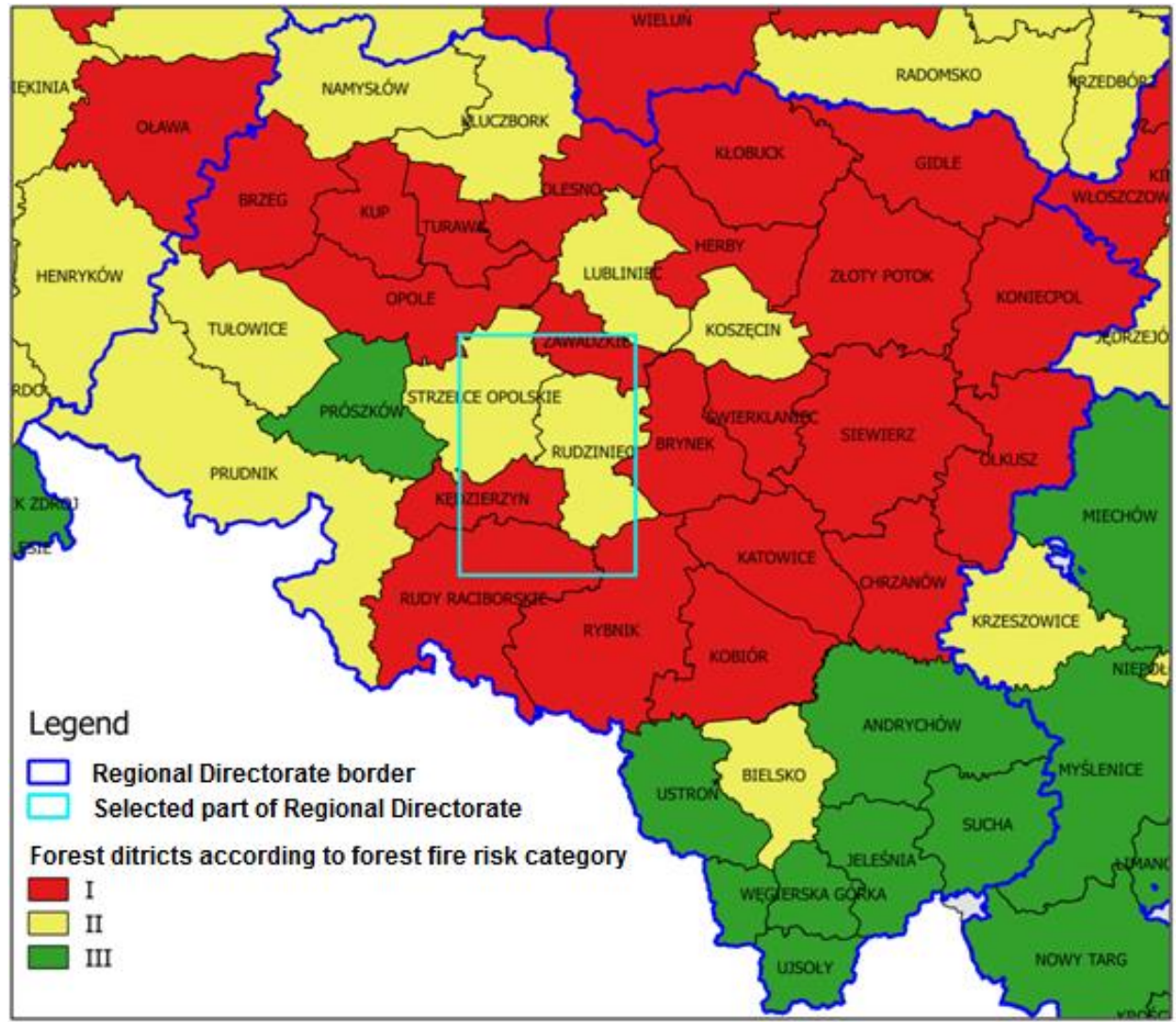

Figure 3 - The forest districts of Regional Directorate in Katowice according to the forest fire risk category with the selected part for conducting forest fire danger mapping.

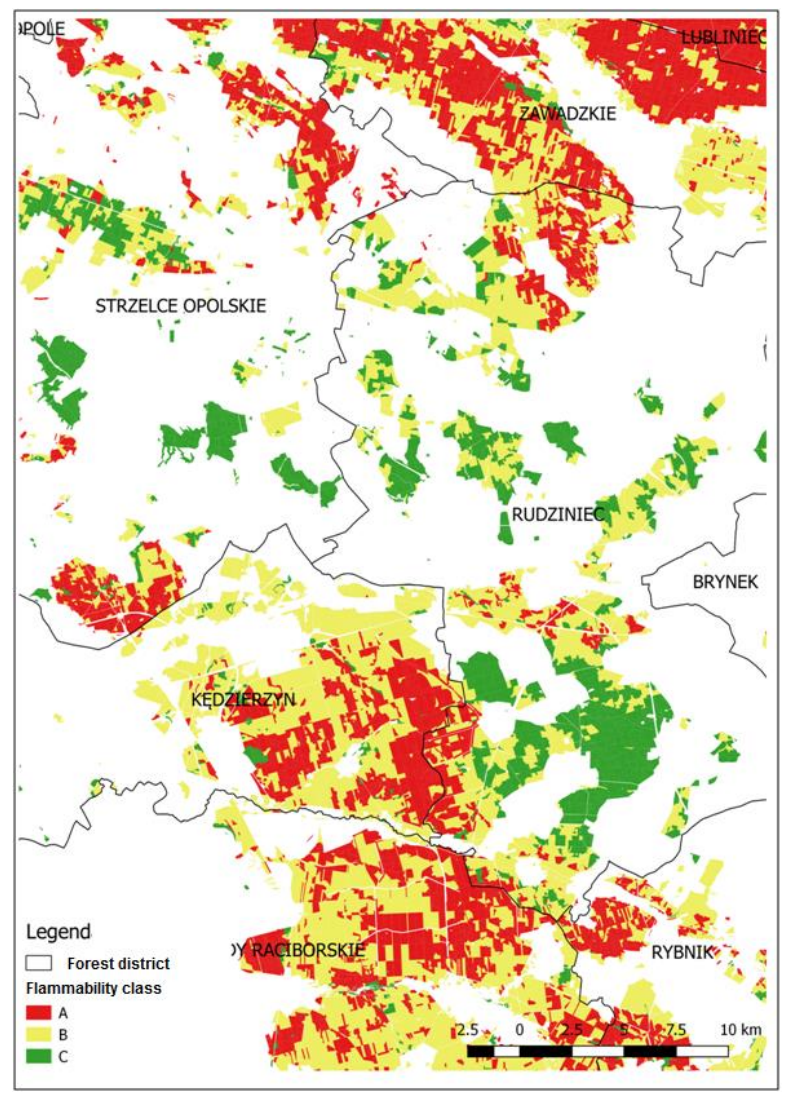

Figure 3 - Forest fire danger map for Regional Directorate in Katowice - flammability classes at the sub-compartment level. 


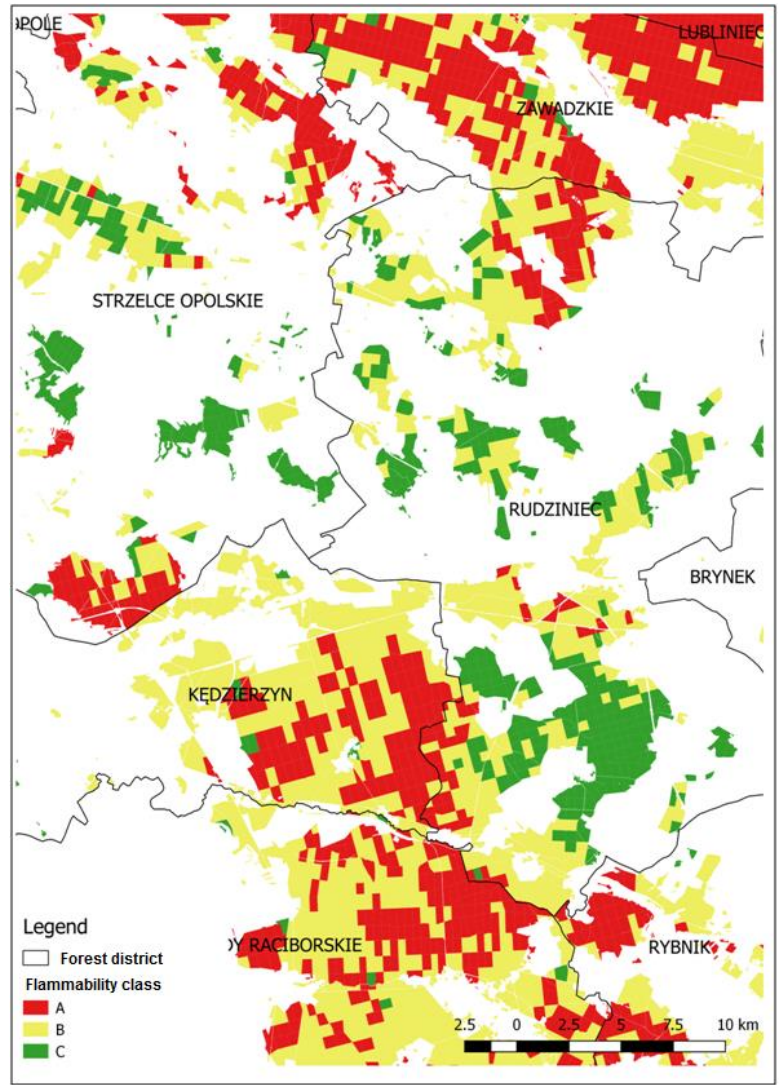

Figure 4 - Forest fire danger map for Regional Directorate in Katowice - flammability classes at the compartment level.

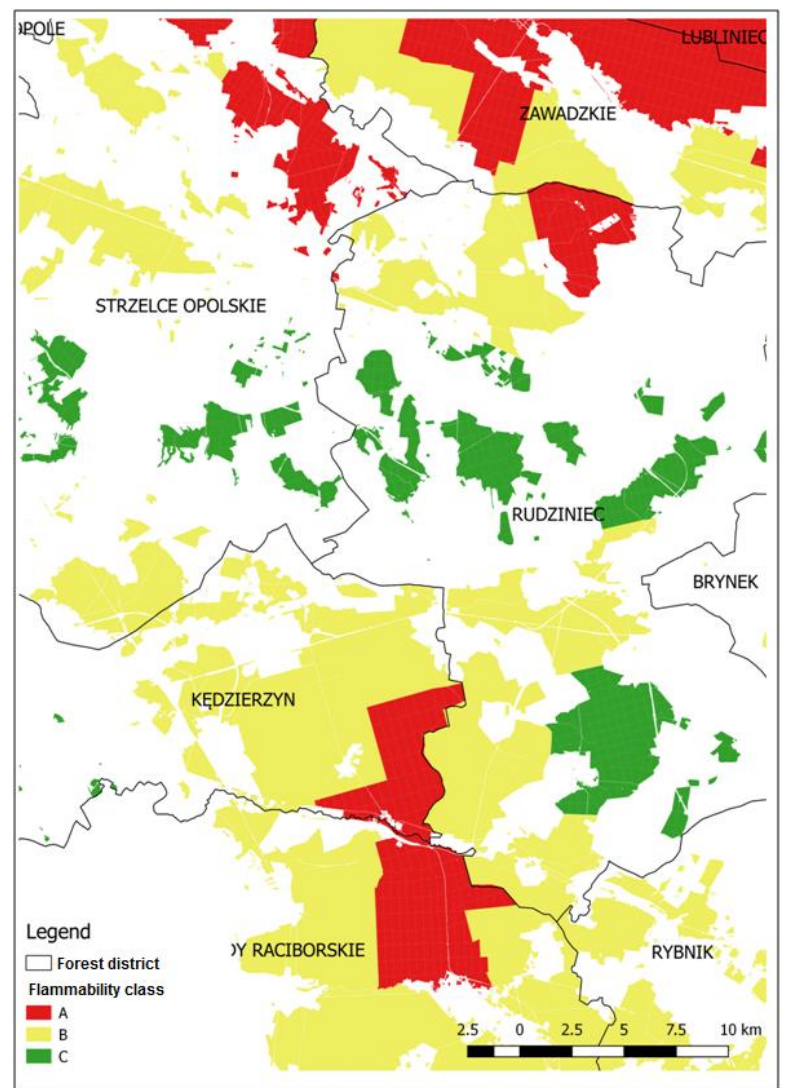

Figure 5 - Forest fire danger map for Regional Directorate in Katowice - flammability classes at the forest range level. 


\section{Summary}

The current forest fire risk classification method enables determination of the forest fire risk category in the macro scale; the smallest classified area is the forest district. There was no possibility of estimating forest fire risk in the micro scale. It would be the significant premise to organise a more efficient fire protection system, including creating the infrastructure and operational planning for rescue services. It would be the fulfilment of the INSPIRE directive, which requires classification and mapping of forest risk at that level. The elaborated method adopts forest habitat type as a base for classification. Forest habitat types determine the type of soil cover fuel, which is measure of forest fire susceptibility expressed by the number of forest fire and the burnt area. The sub-compartment is the classified unit and it is possible to generalise it, according to the elaborated rules, up to the level of compartment or forest range. The elaborated pattern, which includes flammability indices for the number of forest fires and the burnt area between 2007 - 2017, enables determination of the flammability class of forest stands as A - high class, B - medium class and C - low class, using forest habitat type and soil cover type. The method presented in this paper can be used in practice. The most detailed classification at the sub-compartment level might be useful for operational planning and organisation of the rescue action. The compartment level could be used for planning forest fire trails, water supply points and during suppression of large forest fires. The most generalised level, the level of forest range, can be useful during elaboration of procedures in the event of forest fire, lookout towers or equipment base localisation. 


\title{
Understanding the changing fire environment of south-west Western Australia
}

\author{
Lachlan McCaw \\ Department of Biodiversity, Conservation and Attractions, Manjimup 6258, Western Australia \\ \{lachie.mccaw@dbca.wa.gov.au\}
}

\begin{abstract}
Fire environment is the resultant effect of factors that influence the ignition, behaviour and extent of fires in a landscape. This paper synthesises information available from a variety of sources to evaluate evidence for changes over the past three decades in components of the fire environment for south-west Western Australia including climate and weather, fuel, and ignition. A combination of land use, socio-economic and organisational factors has resulted in more widespread extent of lands unburnt for several decades, increasing the risk of high severity fires with adverse impacts on the community and environment. The Mediterranean type climate of the region has become warmer and significantly drier since the 1970s, with associated changes in the timing and duration of the traditional fire season. Lightning ignition has become more common, either as result of greater summer thunderstorm activity or increased opportunity for ignitions to sustain and spread. Increased lightning ignition is significant because of the dis-proportionately large proportion of area burnt by lightning caused fires in SWWA. Adapting to a changing fire environment has implications for all aspects of the fire management business, and a variety of new products and services are available to inform fire managers about temporal and spatial trends in components of the fire environment.
\end{abstract}

Keywords: fire environment, climate, eucalypt forest, Mediterranean ecosystems

\section{The fire environment concept}

Fire environment is the resultant effect of factors that influence the ignition, behaviour and extent of fires in a landscape. Countryman (1966) defined the fire environment as comprising the fuel, topographic and airmass factors that influence or modify the inception, growth and behaviour of fire. In this paper ignition is also considered a component of the fire environment for the reason that the cause, frequency, spatial pattern and timing of ignition are characteristic of a region and a period of time. The fire environment provides a setting within which a variety of fire regimes defined by season, frequency and intensity of burning are enacted in response to a combination of chance events and human actions.

The focus of this paper is the south-west corner of Western Australia (SWWA), a region characterised by ancient landscapes, a Mediterranean type climate and a unique flora and fauna that have evolved in prolonged isolation from other parts of the Australian continent. Defining features of region include the densely populated and extensively cleared Swan coastal plain, a relatively continuous zone of open eucalypt forest comprising State forest and various categories of conservation reserve, and an extensively cleared dryland farming zone known as the Western Australian Wheatbelt (Fig. 1). Public lands occupy about $6 \mathrm{M}$ ha of this region in the form of national park, multiple-use State forest and other categories of reserved land. The eastern margin of the Wheatbelt interfaces with a vast, remote and sparsely populated semi-arid zone where mining and pastoralism are the dominant economic activities. Much of this area remains as unallocated crown land, with a renewed emphasis on the role of traditional owners as custodians and managers of the land. 


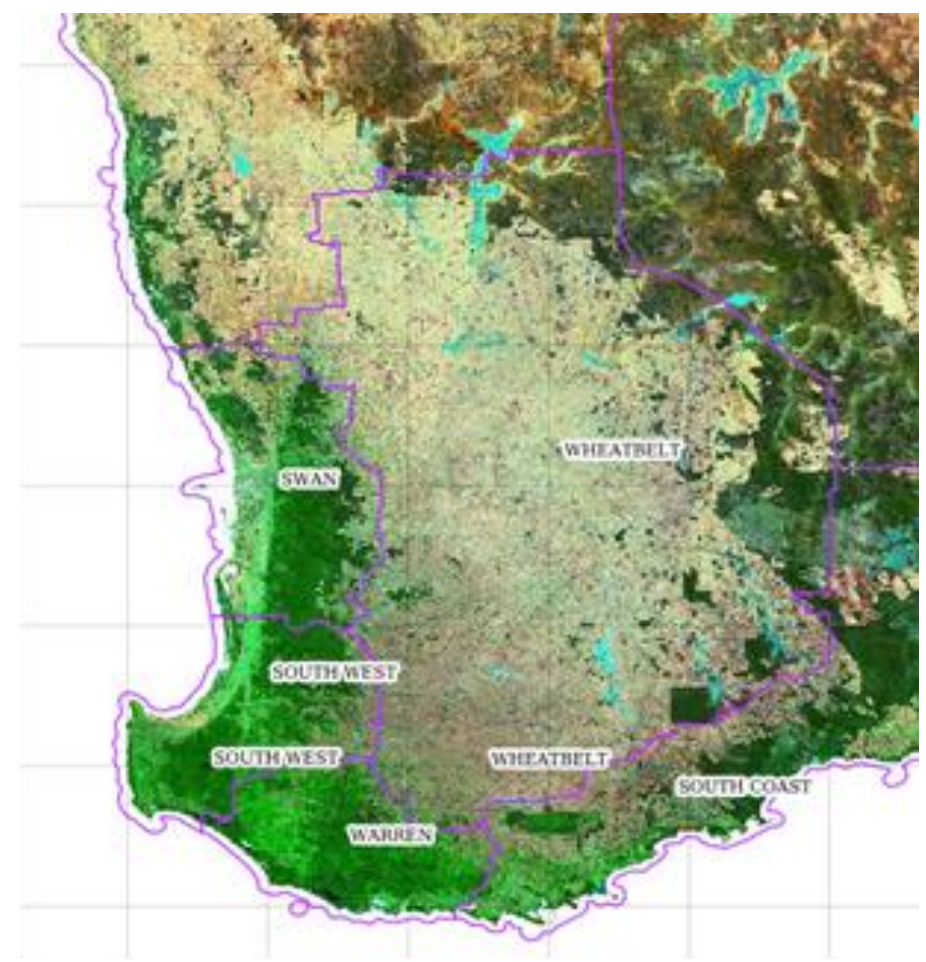

Figure 1 - Satellite image showing broad land use zones within south-west Western Australia. Names indicate regional administrative areas for management of public land by the Department of Biodiversity, Conservation and Attractions Western Australia.

Essential features of the fire environment of SWWA have been described by McCaw and Hanstrum (2003). Weather factors exerting a significant influence on the fire evironment include coastal sea breezes, strong dry winds blowing from the continental interior, abrupt wind changes associated with pre-frontal troughs, and periodic incursion of tropical cyclones south of latitude $30^{\circ} \mathrm{S}$. The 15 year period since the publication of McCaw and Hanstrum (2003) has seen a reduction in the area burnt by planned (prescribed) fire and a substantial increase in the area burnt by uplanned bushfire across SWWA, including a number of large and destructive fires (eg. Ferguson 2016). This period has also seen significant advances in numerical weather prediction that have allowed temporal and spatial patterns of weather and other environmental variables (eg. soil dryness) to be analysed independently of the observational network which is limited across much of SWWA. Re-analysis data now provide a robust method for establishing climatological norms for meteorological variables and derived values of fire danger and potential fire behaviour (Jones et al. 2009, Dowdy and Pepper 2018).

The intent of this paper is to synthesise information available from a variety of sources and products to evaluate evidence for change over the past three decades in each of the core components of the fire environment:

- climate and weather

- ignition,

- the landscapes in which fires are burning.

\section{Fire and planned burning activity in SWWA}

Over the 15 year period from 2002-2017 the Department of Biodiversity, Conservation and Attractions (and previous public land management agencies) responded to more than 7000 bushfires on public land across SWWA (Fig. 2). The total number of fires that occurred during this period would 
be considerably greater once fires on private property attended by volunteer brigades and fires in gazetted fire districts attended by career Fire and Rescue personnel are accounted for. Around half of the fires on public land were lit deliberately, with lightning being the second largest cause of ignition (1200 fires). Lightning ignition was the dominant cause of area burnt, accounting for $1.43 \mathrm{M}$ ha of the total $1.94 \mathrm{M}$ ha burned by bushfires during the period. During this period bushfires ignited by lightning burnt around $23 \%$ of the public land in SWWA.
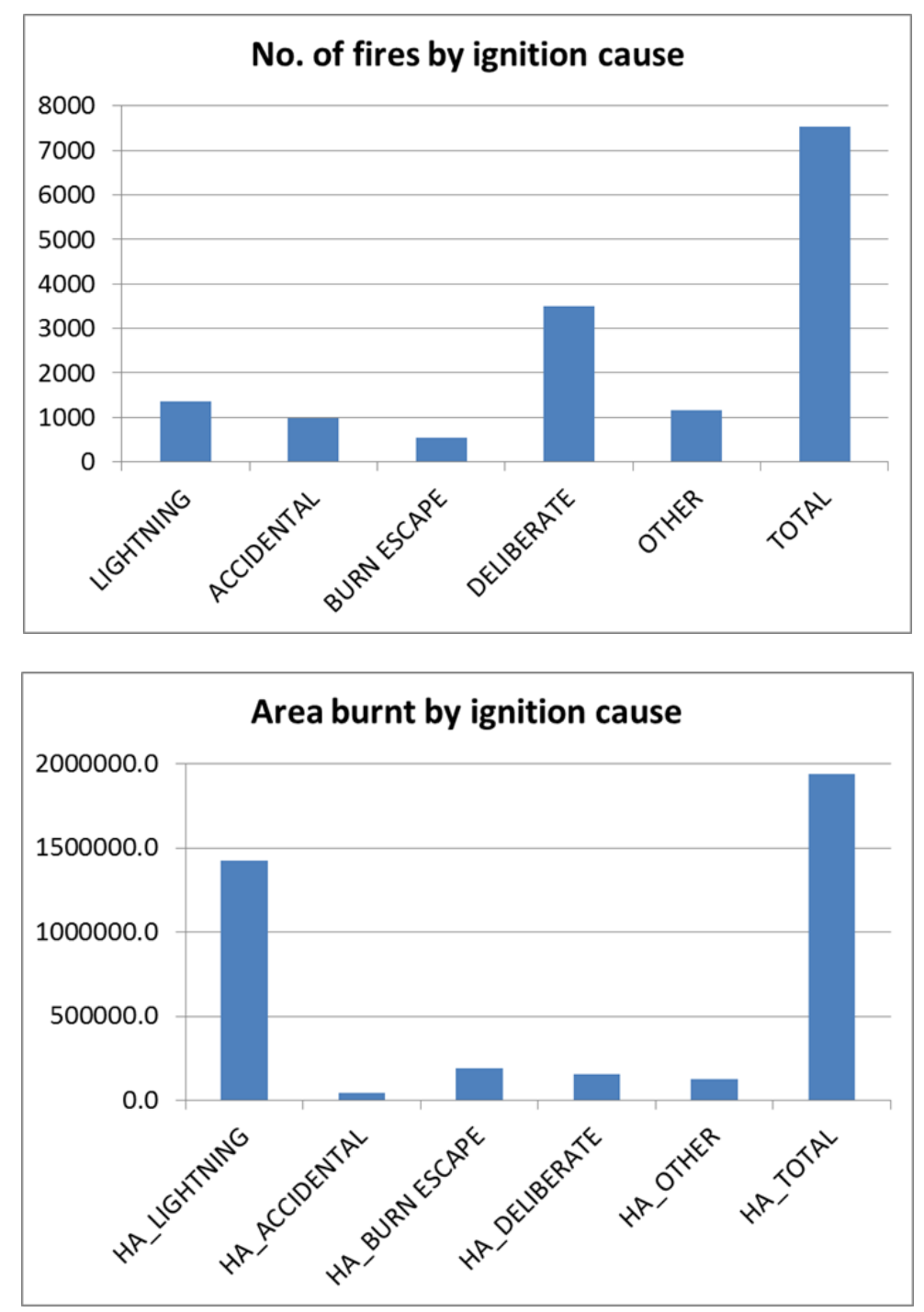

Figure 2 - Number of bushfires (upper panel) and area burnt by bushfires (lower panel) categorised by ignition cause for the period 2002-2017. Data extracted from fire reports for SWWA regional administrative areas shown in Fig. 1.

Prescribed fire has been used extensively in the predominantly forested regions of SWWA since the late 1950s to manage fuels and achieve a range of land management objectives (Burrows and McCaw 2013). Following a period of relatively stable planned burning activity through the 1980s and 1990s the period since 2000 has seen increased variability and an overall reduction in the scale of the planned burning program (Fig. 3). 


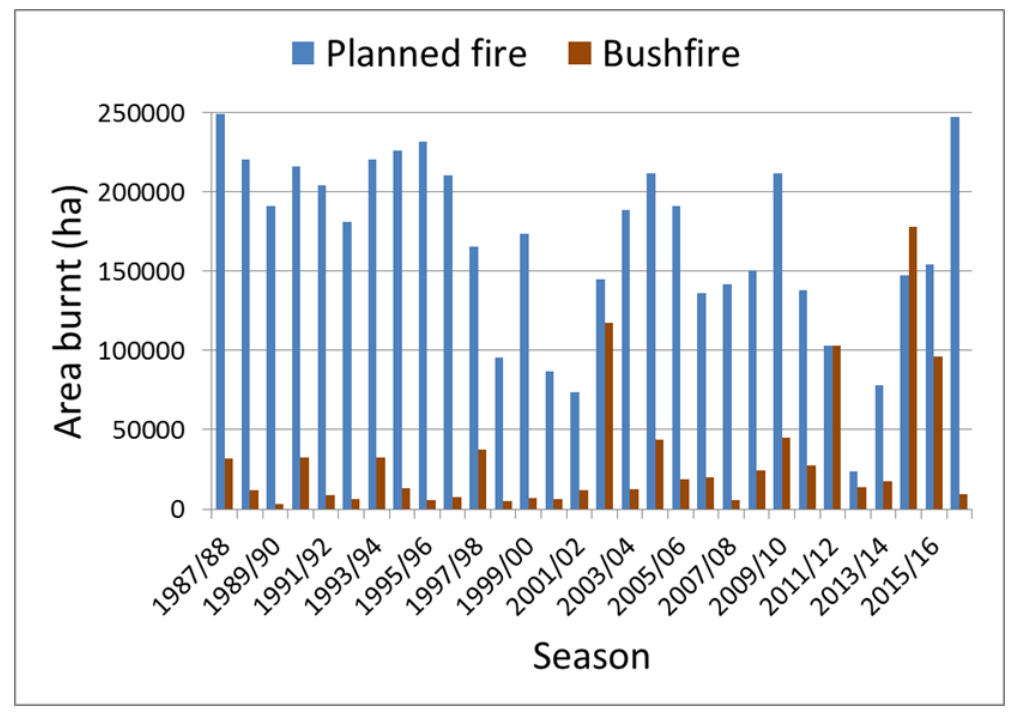

Figure 3 - Trend in area burnt by planned and unplanned fire for Swan, South West and Warren regional administrative areas for the period 1987-2017.

In the predominantly forested regions bushfire activity exhibited a relatively stable pattern during the 15 year period from 1987 to 2002 with less than 10000 ha burnt in most years, and more active bushfire seasons every third or fourth with up to 40000 ha burnt (Fig. 3). However, the 2002/03 bushfire season marked a turning point with a major increase in the area burnt by bushfire, mostly due ignition by lightning. The trend of increased area burnt by bushfire has been maintained for the following 15 years with notably large areas burnt in 2011/12, 2014/15 and 2015/16. Damaging bushfires requiring an extended suppression response also occurred in Swan Region in 2005, 2007 and 2009.

Reduction in the extent of prescribed burning after 2000 would partially explain the increased area burnt by bushfire, with a study by Boer et al. (2009) demonstrating a strong inverse relationship between the extent of prescribed burning and unplanned fire in the Warren Region over a period of five decades. A variety of factors have contributed to the reduced area of prescribed burning achieved since 2000. These factors include greater sensitivity to smoke by the community and the wine industry, constraints on burning imposed by Government following significant escapes from prescribed burning in spring 2011, temporary restrictions on operations arising from a fatal burn-over incident during a bushfire in spring 2012 and increasing complexity of managing prescribed fire in landscapes fragmented by mining and intensive timber harvesting. Burning opportunities in the tall forests of the Warren Region (Fig. 1) have been foregone because of the need to deploy personnel and equipment to bushfire suppression operations in other parts of the south-west. In particular, this has impacted on the achievement of prescribed burning in tall forests dominated by karri (Eucalyptus diversicolor) where conditions suitable for burning typically occur in early summer (December, January), coinciding with the peak period of bushfire activity in other parts of SWWA.

\section{Land use}

Land use activities have a major influence on the condition of vegetation and the ability to manage fuels through planned burning in a safe and cost-effective way. Bauxite mining has been underway on State forest lands in the Swan and South West regions for around 50 years and is an important economic activity for the state. A total of about 25000 ha have been cleared to date of which 20000 ha have been rehabilitated with trees and shrubs native to the local area. Since only 20-30 percent of each catchment is cleared for mining, the total area impacted is about three to five times larger (75000- 
100000 ha, Batini 2016). Fragmentation of the landscape caused by mining operations (Fig. 4) means that planned burning is deferred for an extended period while mining takes place, and then as the rehabilitated pits are left to grow on to a stage where the vegetation becomes fire tolerant. Fuel management of residual native forest within the mining envelope is technically possible but the complexity, risk and unit cost of operations is much higher than in undisturbed forest landscapes. In practice, the combined effect of these factors has led to prescribed fire being excluded from large areas of forest for several decades resulting in high connectivity of heavy fuels at the landscape scale. This situation was exemplified by the 2016 Waroona bushfire which exhibited two violent pyro-convective events including a massive convective plume that developed within 12 hours of ignition (Ferguson 2016, Peace et al. 2018). Lightning activity associated with this plume ignited further fires that caused unexpectedly rapid spread overnight. Atmospheric conditions were clearly favourable for convective activity, with long unburnt fuels on steep slopes and extreme dryness of the fine and woody fuels contributing to intense fire behaviour.

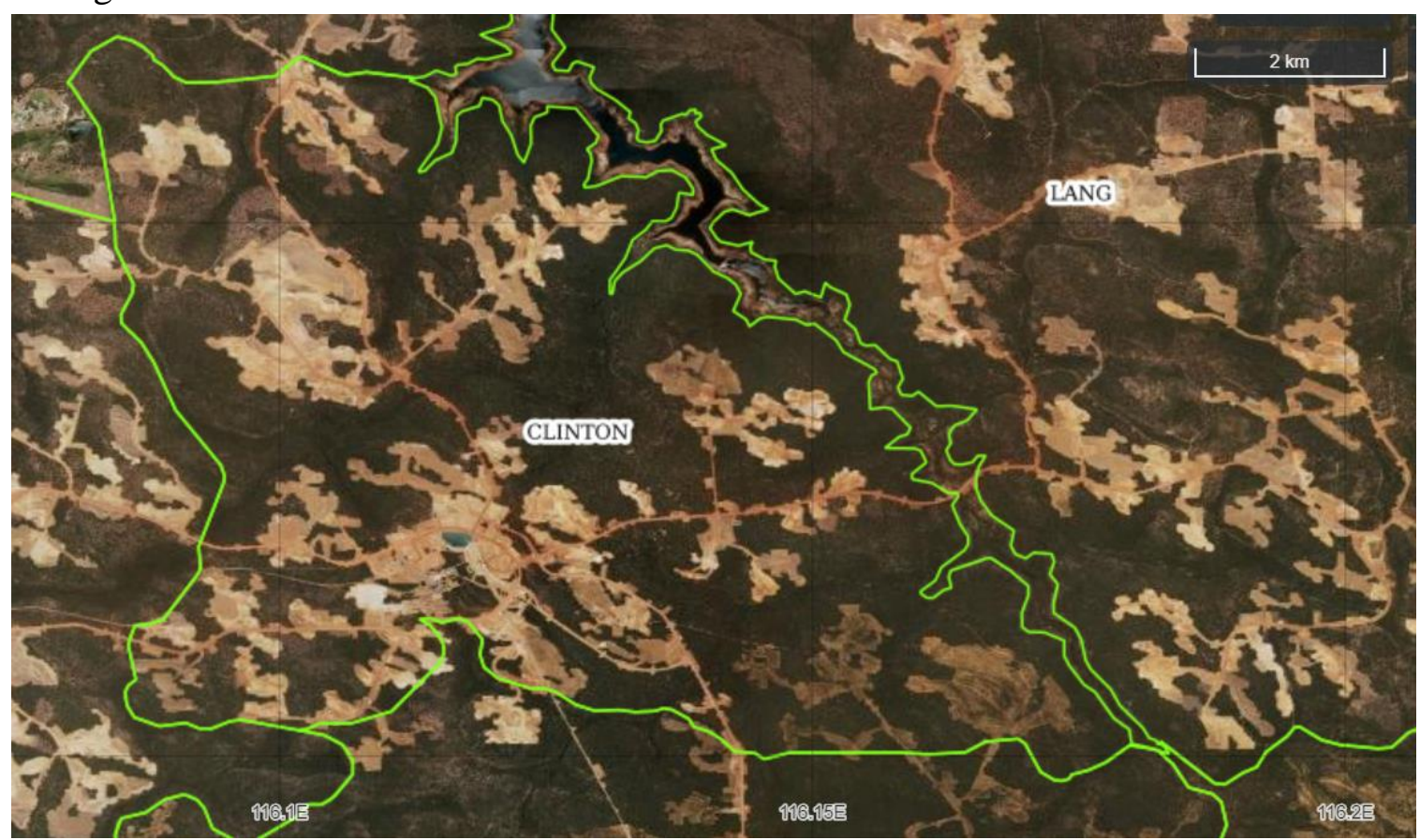

Figure 4 - Aerial image showing the extent of current (2017) bauxite mining operations on State forest in Clinton and Lang forest blocks in the Swan Region. Green lines show the management boundary of each forest block. Note the scale bar shown at top right of image.

Similar issues exist in areas of forest that have been subject to intensive timber harvesting and regeneration of even-aged stands. Forest managers must consolidate young regeneration into management units of sufficient scale and homogeneity to allow cost effective use of planned burning for fuel management. This may require multiple ignitions over a period of weeks, possibly longer, to achieve desired burning conditions for stands in different developmental stages.

\section{Trends in climate, weather and fuel dryness}

The Mediterranean type climate of SWWA results in a prolonged dry period during the Austral summer and autumn with the fire season typically extending from October to May in most years. Since the 1970s SWWA has undergone a persistent drying trend that is most pronounced along the west coast where annual rainfall has declined by 20 to $40 \mathrm{~mm}$ per decade (Bureau of Meteorology 2018). Rainfall decline has been predominantly during the autumn and early winter period, and days of very heavy rainfall have become less common (Indian Ocean Climate Initiative 2012). Notably dry years 
include 2006, 2010 and 2015. A warming trend is also evident from the 1970s onwards, becoming persistent from the mid-1990s with very few below normal summers to offset the trend (Fig. 5).

Summer mean temperature anomaly - Southwestern Australia (1910-2016)

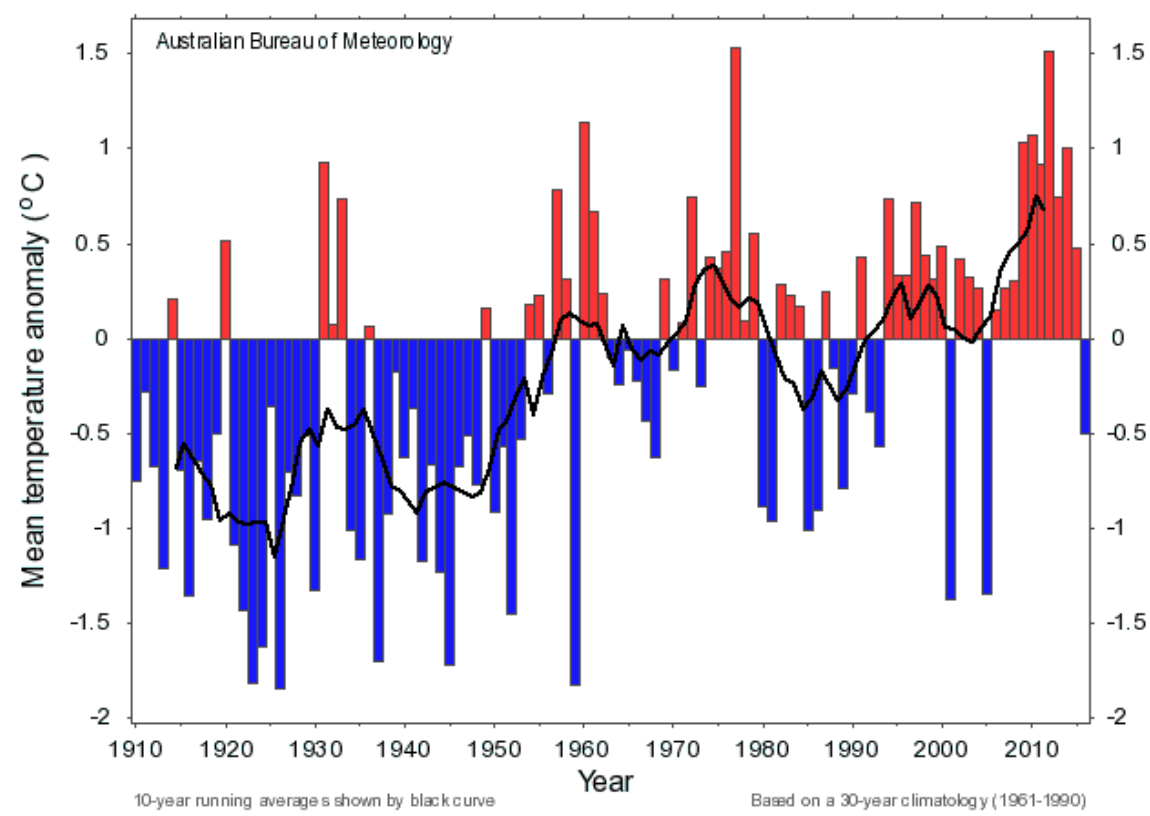

Figure 5 - Trend in summer mean temperature anomaly 1910-2016.

Source: Bureau of Meteorology (www.bom.gov.au)

Consistent with the trend to a warmer and drier climate Dowdy (2018) reported a significant increase in seasonal mean values of the Forest Fire Danger Index (FFDI; McArthur 1967) during winter (JJA) and spring (SON) across most of SWWA, particularly for the period from 2000-2016. No long term trend in mean FFDI was detected for the peak bushfire season spanning summer (DJF) and autumn (MAM). The number of days exceeding the $90^{\text {th }}$ percentile value for the 56 year study period also increased significantly during spring, with some evidence of more localised increases during summer particulalry along the Indian Ocean coast. This signal was stronger for the period 2000-2016 than for earlier periods. The trends identified by Dowdy (2018) point to a lengthening of the bushfire season more than an intensification of mean fire danger during the peak summer months.

Other empirical evidence of a lengthening of the fire season is provided by Soil Dryness Index (SDI; Mount 1972, Burrows 1987) which is a numerical value derived from daily rainfall and maximum temperature. The SDI reflects the dryness of soil, deep layers of surface fuel, woody fuel and some components of living vegetation. It is one of several indices used to guide selection of conditions for prescribed burning and to indicate the potential severity and difficulty of suppression of forest fires. Typically, the SDI rises rapidly during spring with the onset of warm dry weather, reaches a peak during early autumn and then falls, often abruptly, with the arrival of the strong cold fronts in mid autumn. Operational guidance in the Forest Fire Behaviour Tables for Western Australia (Sneeuwjagt and Peet 1985) recommends a fall of 400 units ( $\mathrm{mm} \times 10)$ of SDI prior to commencement of prescribed burning in autumn. Based on SDI values calculated for Manjimup, a location broadly representative of the Warren Region, the median date for a fall of 400 units has shifted almost three weeks later into autumn since the decade 1975-84 (Table 1). This trend has implications for the window of suitable weather conditions available for autumn burning, as deep litter and woody fuel remain dry later into autumn while other factors that also determine the success of prescribed burning, particularly solar angle and day length, remain unchanged. In order to retain a realistic window of opportunity for autumn prescribed burning operational guidance has been revised to adopt a more riskbased approach where the consequences of burning at various values of SDI are specified in relation 
to the likelihood of achievement of burn objectives. This approach permits fire practitioners to make an informed decision based on risk and likely outcome.

Table 1 - Date for an autumn fall of 400 units ( $m m x$ 10) in the Soil Dryness Index at Manjimup by decadal periods from 1975 to 2014

\begin{tabular}{cccc}
\hline Period & $\begin{array}{c}\text { Median date for } 400 \\
\text { unit fall }\end{array}$ & $\begin{array}{c}\text { Earliest date for } 400 \\
\text { unit fall }\end{array}$ & $\begin{array}{c}\text { Latest date for } 400 \\
\text { unit fall }\end{array}$ \\
\hline $1975 / 84$ & 25 April & 3 April & 28 May \\
$1985 / 94$ & 6 May & 1 March & 23 May \\
$1995 / 04$ & 13 May & 14 April & 31 May \\
$2005 / 14$ & 8 May & 1 April & 26 May
\end{tabular}

Recent developments in process based hydrological modelling have assisted in understanding and interpreting trends at the landscape scale. The Australian Landscape Water Balance is a daily $0.05^{\circ}$ grid-based, distributed water balance model that simulates the flow of water through the landscape from rainfall entering the grid cell, through the vegetation and soil moisture stores, and then out of the grid cell through evapotranspiration, runoff and deep drainage to the groundwater (Smith et al. 2015). The AWRA-L model provides values for three soil layers (upper: $0-10 \mathrm{~cm}$, lower: $10-100 \mathrm{~cm}$, and deep: 1-6 m) expressed either as the actual percentage of available water content or as a percentile value relative to a calibration period from 1911-2016. Figure 6 shows the trend in annual deep soil moisture over the period 2005-2017 for the Warren River catchment which includes the town of Manjimup. During this period deep soil moisture declined below the 1 percentile value for the historical reference period in 2010 , and remained below the $10^{\text {th }}$ percentile value until the winter of 2016.

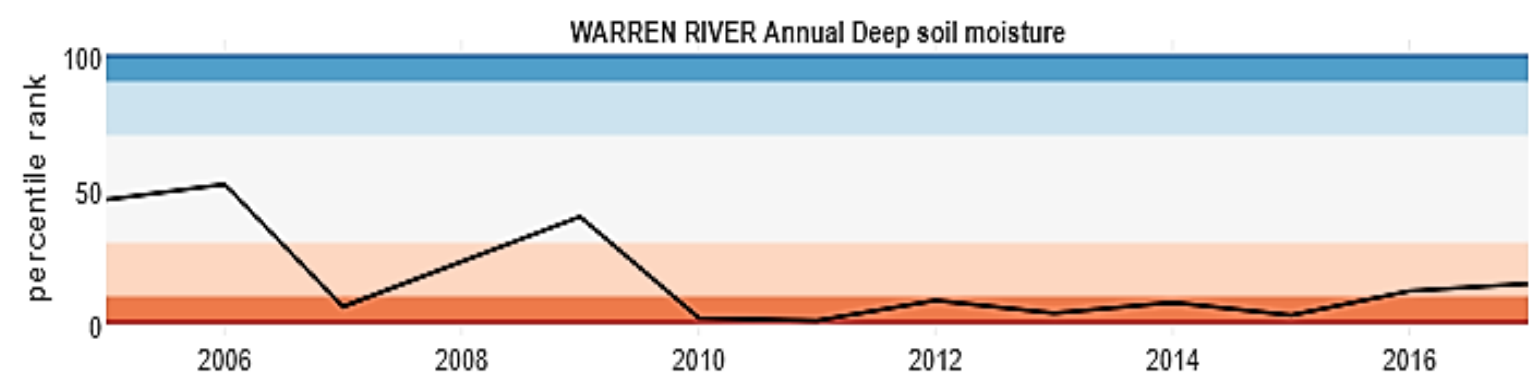

Figure 6 - Trend in annual deep soil moisture over the period 2005-2017 for the Warren River catchment expressed as a percentile for the calibration period 1911-2016. Orange band represents values below the 10th percentile and the red band values are below 1 percentile. Source: Bureau of Meteorology (www.bom.gov.au/water/landscape/)

Drier landscapes have important implications for fire management. Lack of moisture recharge means that swamps and riparian vegetation are less likely to act as temporary barriers to fire spread in spring and early summer. Peat soils, which are common on the coastal plain, may ignite and burn for extended periods with adverse consequences for smoke emissions and ecological values including plant and animal species that rely on peat swamps as habitat.

Lack of moisture recharge also means that woody fuels ignite more readily and at an earlier time in the fire season. In open eucalypt forests in SWWA woody fuel loads consisting of stumps and fallen $\operatorname{logs}$ will typically be in the range from $75-100 \mathrm{t} \mathrm{ha}^{-1}$, more than four times the equilibrium fine fuel load of 10-15 $\mathrm{t} \mathrm{ha}^{-1}$ of dead leaves and twigs (McCaw 2011). Under dry summer conditions at least half this woody material will burn away representing up to four times the quantity of fine fuel consumed (Hollis et al. 2016). This increase in total heat release potentially adds to convective activity 
above the fire, and increases direct thermal effects on soil and vegetation leading to higher fire severity. Total emissions to the atmosphere will also be greater, with a higher proportion originating from smouldering combustion. Mop-up operations to extinguish burning logs and dead trees also take longer and are more costly when a large proportion of the woody fuel is ignited.

\section{Trends in lightning ignition}

Lightning is an important cause of unplanned fires in SWWA causing 1200 ignitions on public land in the period from 2002 to 2017 (Fig. 2). Fires ignited by lightning burned an area of 1.43 Million ha during this period, exceeding the total area burned by all other causes combined. Lightning caused fires account for a dis-proportionately large percentage of area burnt because of their occurrence in large spatial and temporal clusters, and in remote areas not otherwise prone to ignition by human causes.

Analysis of reliable fire report data over a 41 year period reveal a statistically significant increase in the number of lightning ignitions for the Warren Region particularly from 2002 onwards (Fig. 7, Bates et al. in press). The amplitude and length of the seasonal cycle also appear to have changed, with an increase in lightning ignition during October and November.

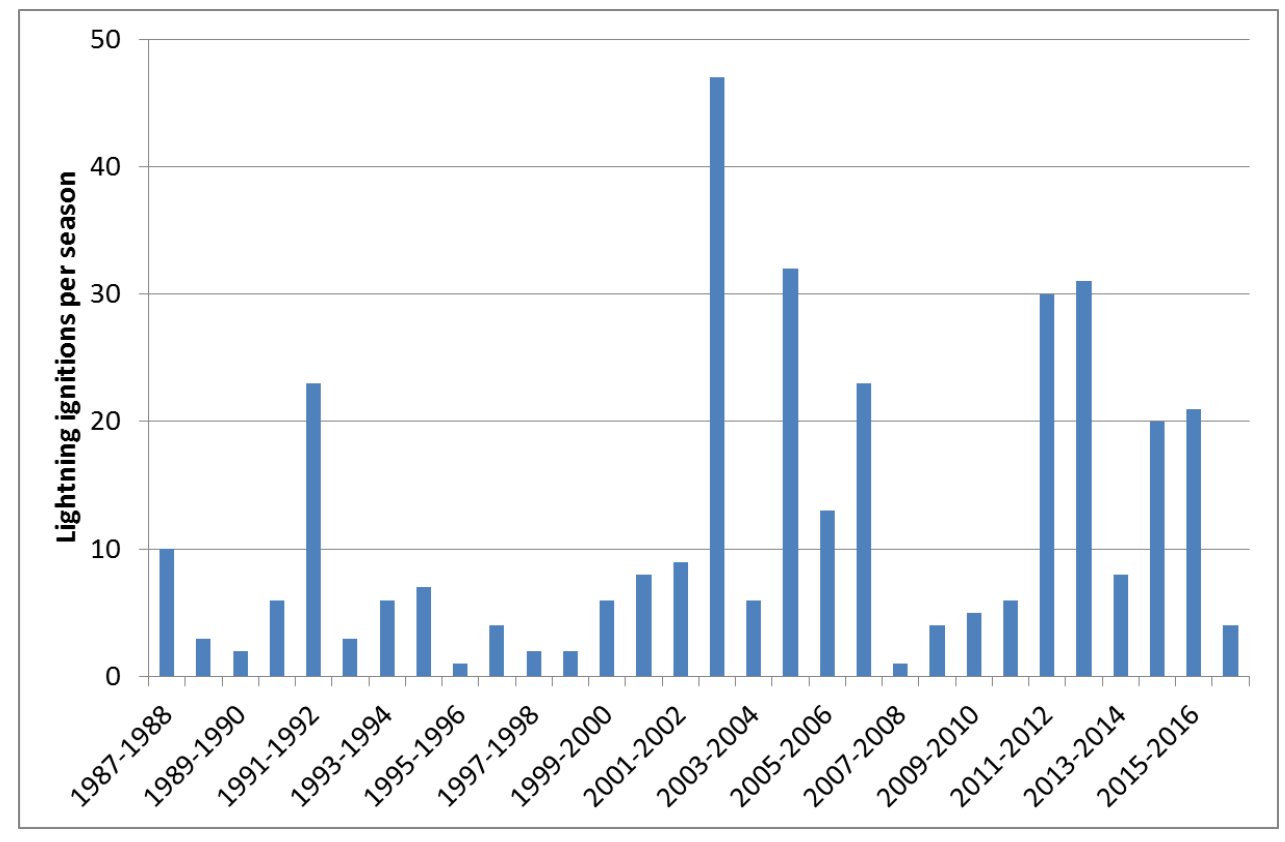

Figure 7 - 30 year trend in lightning ignition for the Warren Region, SWWA.

Ongoing investigations seek to establish the relative influence of changes in thunderstorm activity and increased opportunity for ignitions to sustain and spread in a drier climate. A climatological analysis of the Continuous Haines Index derived from the ERA-Interim reanalysis indicated little evidence of change over the period 1979-2016. The role of regional scale climate drivers is also being investigated to determine whether seasonal lightning activity can be forecast at timescales of weeks to a few months ahead.

\section{Discussion}

Evidence from a variety of sources points to significant changes in the fire environment of SWWA during the current century. The trend to a drier climate has been reinforced by a series of low rainfall years that have resulted in declining streamflow and very low levels of moisture in the deep soil profile. 
This has increased the connectivity between vegetation types allowing fires to spread extensively across the landscape at an earlier time in the fire season than would have been the case several decades ago. Shorter and drier winters have reduced the opportunity for moisture uptake by large woody fuels and organic soils making them prone to ignition at an earlier time in the fire season.

The trend towards increased fire danger and lightning ignition activity during the spring months indicates a lengthening of the fire season, as does the later arrival of rain bearing cold fronts in the autumn. Fuels and vegetation now remain dry for several weeks longer in autumn than was normal during the 1980s. There is little evidence of a trend of increasing fire danger during the peak summer fire season, albeit that SWWA already experiences the highest mean number of January days $(\geq 12)$ when the FFDI is above the annual $90^{\text {th }}$ percentile value (Dowdy 2018).

The increased area burnt by large bushfires, most of which have been ignited by lightning, is consistent with previous research showing an inverse relationship between prescribed burning effort and area burnt by bushfire. Land use activities have made it difficult to undertake safe and costeffective prescribed burning at a landscape scale over significant parts of the public forest estate in SWWA leading to increased extent and connectivity of areas where fuels have accumulated for several decades. Addressing the backlog of areas in need of fuel reduction will require a clearly focussed effort, a substantial commitment of resources, and a preparedness to adapt work processes and operational guidance to meet changing circumstances. Potential changes in bushfire risk arising from land use activities need to be dealt with explicity as part of planning processes. Land managers can also exercise options to manage vegetation structure in ways that make it more resilient to fire, for example through silvicultural thinning to promote larger and more widely spaced trees.

Adapting to a changing fire environment has implications for all aspects of the fire management business, and a variety of new products and services are available to inform fire managers about temporal and spatial trends in components of the fire environment.

\section{References}

Bates BC, McCaw L, Dowdy AJ (in press) Exploratory analysis of lightning-ignited wildfires in the Warren Region, Western Australia. Journal of Environmental Management

Batini F (2016) The impact of bauxite mining on water yield. Paper presented at a workshop on managing forested catchments, Perth, 30 September 2016 (Institute of Foresters of Australia) Available at https://www.forestry.org.au/resources/managing-forested-catchments-threats-andopportunities. [Verified 29 June 2018]

Boer MM, Sadler RJ, Wittkuhn RS, McCaw WL, Grierson PF (2009) Long-term impacts of prescribed burning on regional extent and incidence of wildfires - evidence from 50 years of active fire management in SW Australian forests. Forest Ecology and Management 259, 132-142. http://dx.doi.org/10.1016/j.foreco.2009.10.005

Bureau of Meteorology (2018) Climate change and variability. http://www.bom.gov.au/climate/change/ [Verified 29 June 2018]

Burrows ND (1987) The Soil Dryness Index for use in fire control in the south-west of Western Australia. Technical Report No 17. (Department of Conservation and Land Management Western Australia, Perth).

Burrows N, McCaw L (2013) Prescribed burning in southwestern Australian forests. Frontiers in Ecology and Environment 11 (Online Issue 1): e25-e34.

Countryman CM (1966) The concept of fire environment. Fire Control Notes 27, 8-10.

Dowdy AJ (2018) Climatological variability of fire weather in Australia. Journal of Applied Meteorology and Climatology. doi: 10.1175/JAMC-D-17-0167.1 
Dowdy AJ, Pepper A (2018) Pyroconvection risk in Australia: climatological changes in atmospheric stability and surface fire weather conditions. Geophysical Research Letters 45, 2005-2013. doi: 10.1002/2017GL076654

Ferguson E (2016) Reframing rural fire management - report of the Special Inquiry into the January 2016 Waroona fire. (Government of Western Australia, Perth).

Hollis JJ, McCaw WL, Whitford KR, Cruz MG (2016) Woody fuel consumption by fires in open eucalypt forest in south-west Western Australia. In: Proceedings of the 5th International Fire Behaviour and Fuels Conference April 11-15, 2016, Melbourne, Australia. (International Association of Wildland Fire, Missoula, Montana, USA).

Indian Ocean Climate Initiative (2012) Western Australia's weather and climate: a synthesis of Indian Ocean Climate Initiative Stage 3 Research (Eds. B Bates, C Fredericksen, J Wormworth) (CSIRO and Bureau of Meteorology, Australia).

Jones D, Wang W, Fawcett R (2009) High-quality spatial climate datasets for Australia. Australian Meteorological Magazine 58, 233-248.

McArthur AG (1967) Fire behaviour in eucalypt forests. Forestry and Timber Bureau Leaflet 107. (Commonwealth Department of National Development, Canberra).

McCaw WL (2011) Characteristics of jarrah (Eucalyptus marginata) forests at Forestcheck monitoring sites in south-west Western Australia: stand structure, litter, woody debris, soil and foliar nutrients. Australian Forestry 74, 254-265.

McCaw L, Hanstrum B (2003) Fire environment of Mediterranean south-west Western Australia. In: 'Fire in ecosystems of south-west Western Australia: Impacts and management' (Eds. I Abbott, N Burrows) pp 87-106. (Backhuys Publishers, Leiden, The Netherlands).

Mount AB (1972) Derivation and testing of a soil dryness index using run-off data. Bulletin No 4 (Forestry Commission Tasmania, Hobart).

Peace M, McCaw L, Santos B, Kepert JD, Burrows N, Fawcett RJB (2017) Meteorological drivers of extreme fire behaviour during the Waroona bushfire, Western Australia, January 2016. Journal of Southern Hemisphere Earth Systems Science 67, 79-106. doi: 10.22499/3.6702.002

Smith A, Hafeez M, Rahman J, Baron-Hay S, Shipman D (2015) A new web based water information service leveraging the Australian Water Resources Assessment Modelling System [online]. In: '36th Hydrology and Water Resources Symposium: The art and science of water' pp 1276-1283. (Engineers Australia, Barton, ACT).

Sneeuwjagt RJ, Peet GB (1985) Forest fire behaviour tables for Western Australia. (Western Australian Department of Conservation and Land Management, Perth) 


\title{
Veld fire mitigation strategy: A vision for an innovative and integrated approach to managing risks in land reform farms, a case of land reform beneficiaries in South Africa
}

\author{
Siviwe Zukile Shwababa ${ }^{1}$; Agoston Restas ${ }^{2}$ \\ ${ }^{1}$ University of Free State, \{shwababasz@gmail.com\} \\ ${ }^{2}$ National University of Public Service \{restas.agoston@uni-nke.hu\}
}

\begin{abstract}
South Africa has a diverse climate and an equally diverse range of vegetation types, many of which are veld fire-prone. Veld fires form an integral process in many of these ecosystems, and indeed are often necessary for the maintenance of healthy ecosystems and biodiversity. However, in land reform farms headed by land reform beneficiaries veld fires are increasingly viewed and experienced as a destructive force, impacting on crops, livestock, pastures, forestry plantations, human habitation and even on human life.

Land reform beneficiaries are part of the state's programme which seeks to provide the poor and the previously disadvantaged population with land to improve their livelihoods, and also use the land for establishing farming enterprises. These beneficiaries are mostly black emerging farmers, farming with crop and livestock and are largely dependent on the government's recapitalisation programme for the support of their agricultural enterprises. These beneficiaries are faced with various trade-offs and constraints as veld fires expose them to greater risk and renders some of their current practices unworkable. Their vulnerability to veld fires, mitigation, adaptation and coping practices towards veld fires has never been researched before. This study therefore is an attempt to fill this gap.

The management of veld fires within the South African land reform programme, leaves much to be desired and calls for new institutional arrangements and a coordinated approach. This research paper seeks to facilitate veld fire management in land reform farms, through the introduction of measures to combat veld, forest and mountain fires throughout the land reform farms in South Africa and thereby reducing the damage and losses to natural vegetation, life and property. The unequivocal evidence presented in this paper balanced the social, environmental, and economic aspects of veld fires through a risk management strategy that emphasises hazard mitigation, preparedness, and recovery, as well as efficient veld fire response and suppression.
\end{abstract}

Keywords: risk management, veld fires, a veld fire mitigation strategy, hazard mitigation, eco systems, land reform, land degradation

\section{Introduction}

\subsection{Historical context of the land reform programme in South Africa}

The origins of the South African land reform programme can be traced back to 1913, where the previous apartheid government enacted the Native Land Act which became law and served to limit African land ownership to $7 \%$ of the land, consequently increased to $13 \%$ through the 1936 Native Trust and Land Act of South Africa. These legislations restricted indigenous black people from buying or occupying land and gave white people ownership of $87 \%$ of the land. The application of these statutes entrenched discriminatory practices that resulted in land being taken away from black people, until the Commission of Restitution was established in 1994 in order to provide the equitable redress to victims of racially motivated land dispossession (Boudreaux, 2010).

Since 1994, the South African government has been designing and developing a land reform programme that aims to bring about a fair and equitable land dispensation in the country in an orderly 
manner. The land redistribution aspect of the land reform programme focuses on redistributing land to poor black South Africans who need access to land for residential and productive uses, in order to improve their income, quality of life and participation in agricultural production. The Department of Rural Development and Land Reform (DRDLR) is charged with the responsibility of providing previously disadvantaged people access to agricultural land to improve their livelihood, food security, and their quality of life. The department further ensures that beneficiaries placed in land reform farms use farms productively by providing sufficient post settlement support (DRDLR, 2012).

\subsection{Justification and contextualisation of the study}

Almost every winter season, the news media carry stories of veld fires raging across the landscape of the Eastern Cape Province, threatening rural communities, causing evacuations, and sometimes burning public and private property. This portrayal of veldfires as a threat to society is often accurate but it is only part of the story. Veld fires have always and will always be a natural and important phenomenon in environmental systems. However, because of an intensification brought on through anthropogenic activities, fires are having a negative impact on the environment and more so on society and the economy. A large percentage of South Africa's population is located in rural areas; these rural areas are generally situated in fire-prone regions of the country, making them vulnerable to fires (Agri SA, 2017).

Veld fires lead to severe environmental degradation resulting into diminished land cover exposing the land to agents of accelerated soil erosion, changes in the hydrological cycle, increase in overland flow or surface run-off and modifications in various ecological processes. Soil erosion leads to the siltation of rivers and dams, thus reducing their water carrying capacity. This was likely to induce floods in low-lying areas (Kruger, 2006). Severe fires have been revealed to remove surface vegetation and influence soil organic material and peat. This loss of vegetation effectively declines the surface's ability to infiltrate surface run-off after a precipitation event. Fires on mountain slopes have been shown to decrease infiltration by as much as $50 \%$, thereby increasing the catchment's risk of flash flooding (Agri SA, 2016). Much of the Eastern Cape Province where this study was conducted is vulnerable to severe land degradation because of improper agricultural practices such as overgrazing.

Land degradation in already vulnerable areas may be exacerbated through increased veld fire activity. There are also mountainous regions within the province which are also vulnerable because of the steepness of slopes and the high fire frequencies associated with berg winds (AgriSETA. 2010).

The above-mentioned negative impacts of veld fires posed severe environmental consequences and strain on natural resources. By identifying these areas, those in authority may be able to combat further degradation by limiting veld fire activity and ensuring the prevention of uncontrollable veld fires. Veld fires can also cause injuries to the victims; loss of lives, loss of livelihood assets, health hazards, and moreover a threat to the tourism industry.

The Eastern Cape Province of South Africa is endowed with rich biodiversity where a multitude of fauna and flora exist; fire plays a vital role in the conservation and extinction of many of these species and at times veld fires are a consequence of nature's primary way of keeping the environment (including forests, grasslands, and parks) healthy and productive (Forsyth et al., 2010). Accordingly, policymakers and practitioners are faced with the complex and difficult task of managing veld fires so that their environmental benefits are maximised and simultaneously the risk to people and property is minimised.

Recognising that the contemporary veld fire challenges and those of the future cannot be solved by simply using the thinking and methods of the past. This study proposes a veld fire mitigation strategy which addresses the root causes and the symptoms of veld fires amongst land reform beneficiaries who are farming on land reform farms. It is envisaged that the application of this strategy will increase safety on the land reform farms, improve the health and well-being of the beneficiaries and enhance agricultural production. 


\subsection{The veld fire risk}

A total of about 2500 veld fires were reported during the 2017 fire season in the Eastern Cape province of South Africa, with a majority of fires originating from open flames during waste, grass or bush burnings; some of these fires led to close to fatalities and more than ZAR2 million in financial losses (ECUFPA, 2018). Extensive analysis conducted by the Provincial Disaster Management Centre has found that the vulnerability of people, property, and natural resources to veld fires has reached an unprecedented level and is projected to continue to rise rapidly.

This can be attributed to more frequent and intense veld fires resulting from severe droughts that leave dead and highly flammable fuel load. Meanwhile, current veld fire re-suppression capacity is eroding as facilities and equipment age and experienced fire fighting professionals retire. It is only a matter of time until another major fire season occurs again in the Eastern Cape and the greatest concern is that next time the tragic consequences may include the loss of human lives as seen recently in other parts of the country (PDMC, 2018).

\section{Study area}

The study focused on land reform beneficiaries located and farming in three district municipalities of the Eastern Cape Province, namely Joe Gqabi, Cacadu and OR Tambo District Municipalities.

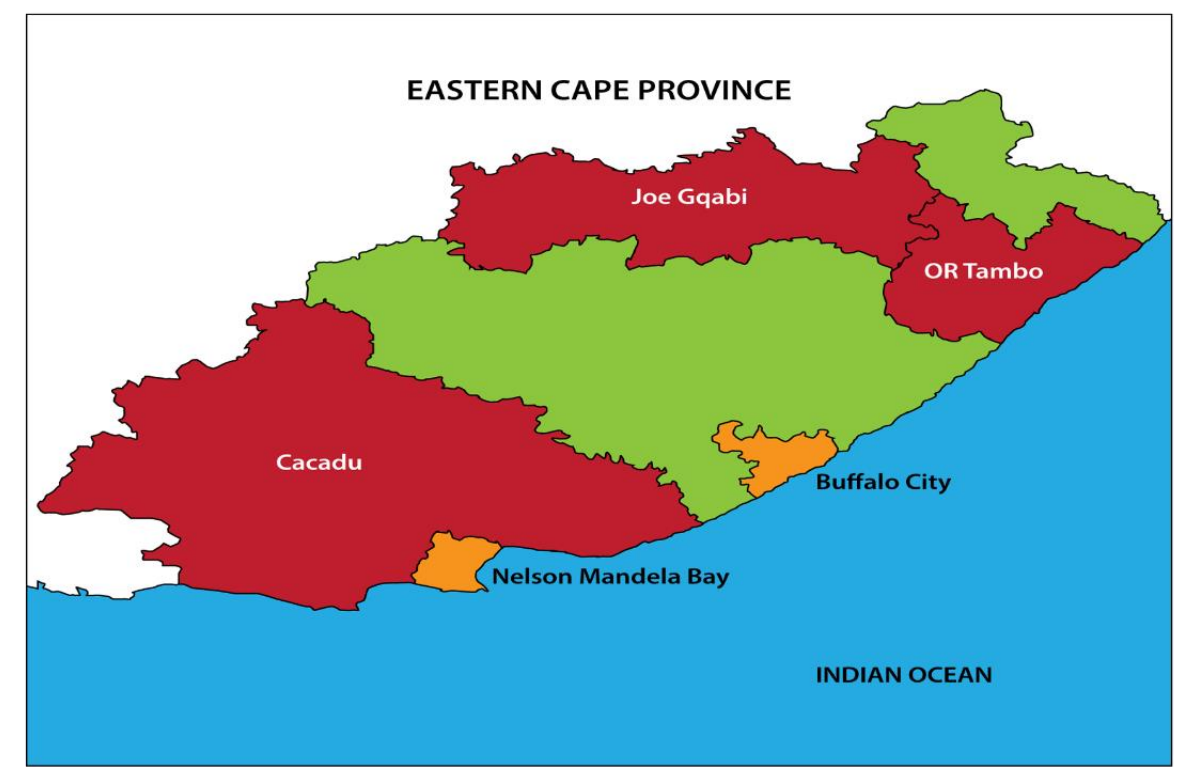

Figure 1 - Map showing the Eastern Cape district municipalities Source: DRDLR (2013)

\section{Methodology}

The aim of this study was to research the effects of veld fires on livelihood assets and agricultural production of land reform beneficiaries and inspire the Department of Rural Development and Land Reform (DRDLR) to develop its own veld fire mitigation strategy that would incorporate preparedness, prevention and mitigation measures for veld fires occurring in land reform farms.

The study was carefully chosen because of practical veld fire related problems encountered within the Land Reform Programme (LRP). For several years, land reform beneficiaries in the Eastern Cape Province have grappled with the far-reaching consequences of various veld fires with no effective plans and mitigation measures. The DRDLR has made no provision for a standard approach to mitigating the effects of veld fires on land reform farms. The current traditional approach towards veld fires is that of crisis management, which is reactive in nature and void of proactive risk reduction 
measures and strategies that will mitigate the devastating impacts of future veld fire events (DRDLR, 2013).

The LRP has no documented model to strengthen the absorptive, adaptive and transformative capacities of vulnerable beneficiaries to cope with and recover from the effects of veld fires (Mock et al., 2015).

The Participatory Learning Action (PLA) technique was used as a data collection tool in this study. Workshops were conducted in all of the three study sites where land reform beneficiaries participated. Through the PLA technique the respondents acquired a platform to, for the first time, articulate their own narratives (in their own language) regarding the extent of veld fires they experienced and the impact these had on their livelihoods and agricultural production. The usage of the PLA technique empowers local people by giving them opportunities to express and enhance their knowledge and take action (Chambers, 2008; Bunce et al., 2010). Through the PLA technique respondents were afforded an opportunity to draw a mental map reflecting shocks which had a negative effect on their livelihoods and agricultural production. Mental maps are useful tools which help to 'visualise' the complex reality people deal with in their day-to-day lives (SEAGA, 2001; Wilde 2001). In these mental maps the respondents were also able to highlight the causes and effects of the said shocks. A word frequency analysis method was used as part of the PLA technique and it revealed the extent and severity of veld fires in each study site. The analysis was carried out using correspondence analysis by Abdi and Béra (2014). The observation of the veld fire phenomenon in some of the study sites was a useful data collection method, which culminated into a successful research project.

\section{Results}

In drawing up their mental map, the respondents specified veld fires as one of the shocks which have an effect on their farming enterprises. The root cause was attributed to the lack of firebreaks and dry weather conditions, with loss of livestock and damage to property as resultant effects. Drought was also depicted as a shock alongside veld fires, thus endorsing a commonly held view by several scientists that there is a very close relationship between drought and veld fires (Wilhite et al., 2014). Drought conditions make the vegetation to dry out quicker and with no rains due to drought before a fire, the rate of spread for veld fires becomes considerably higher.

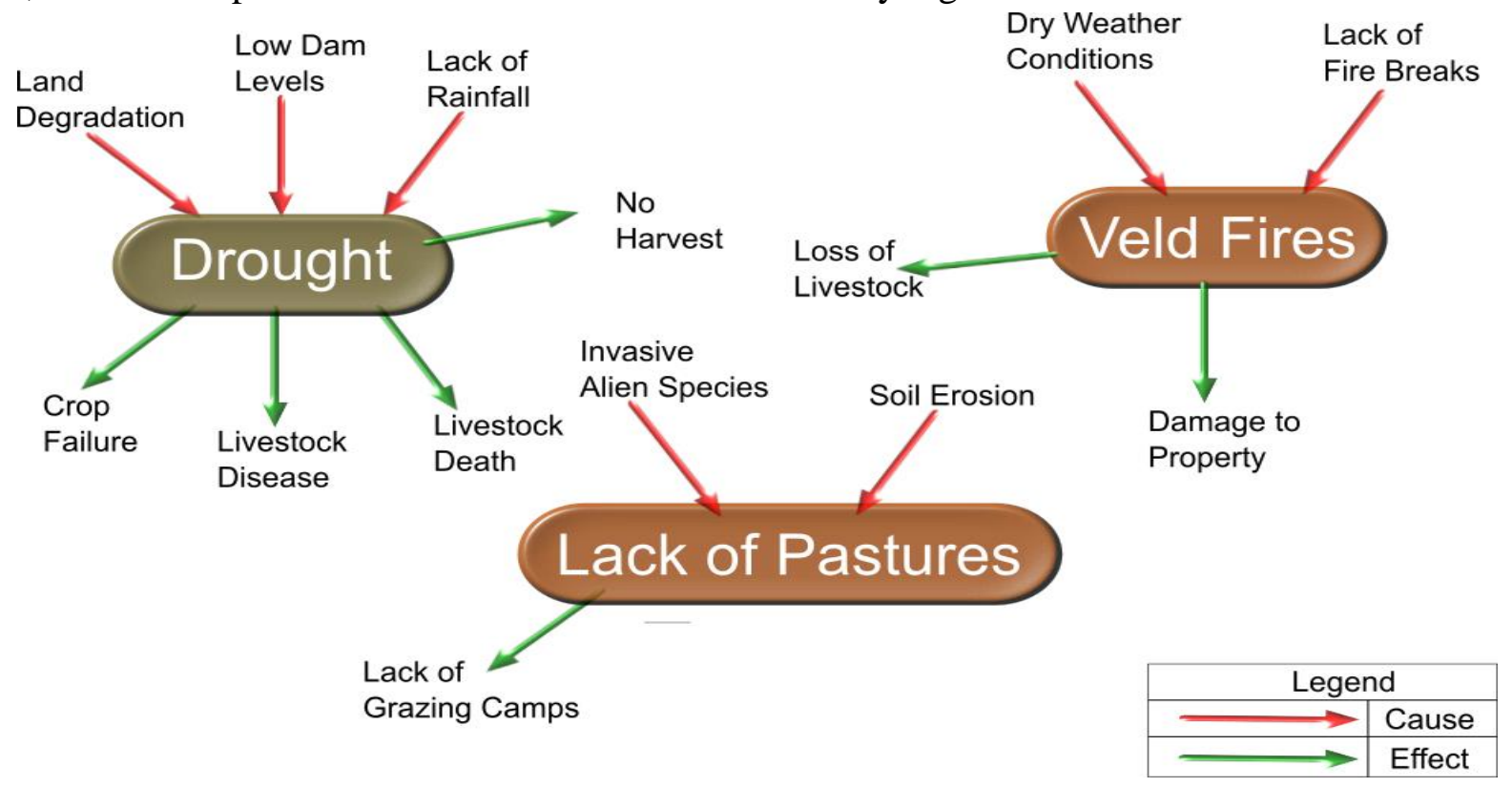

Figure 2 - Mental map 
Another finding portrayed by respondents through mental maps was lack of pastures caused by soil erosion and invasion of alien species. This finding concurs with the IPCC (2014) who pointed out that wherever veld fires occur, they turn to culminate into to severe environmental degradation. More explicitly, veld fires lessen land cover, thus uncovering the land to agents of fast-tracked soil erosion, changes in the hydrological cycle, escalation in overland flow or surface run off and alterations in a number of ecological processes (Lynn, 2014). The presence of alien species perpetuates the spread of veld fires, reducing the aesthetic value of the natural land and causing a strain on natural resources. Fires do provide an environmental service and are an integral part in the maintenance of the ecosystem; however, increased veld fire activity, brought about by anthropogenic action, leads to adverse effects in the environment.

The word frequency analysis method contributed to revealing the extent and severity of veld fires in each study site. Through the ranking process it further exposed the importance associated with the mentioned shock. This gave a clear overview in terms of the manner in which veld fires affected the livelihoods and agricultural production of the land reform beneficiaries in the study sites.

\section{Shocks: Veld Fires}

Frequency Mentioned (1-4)
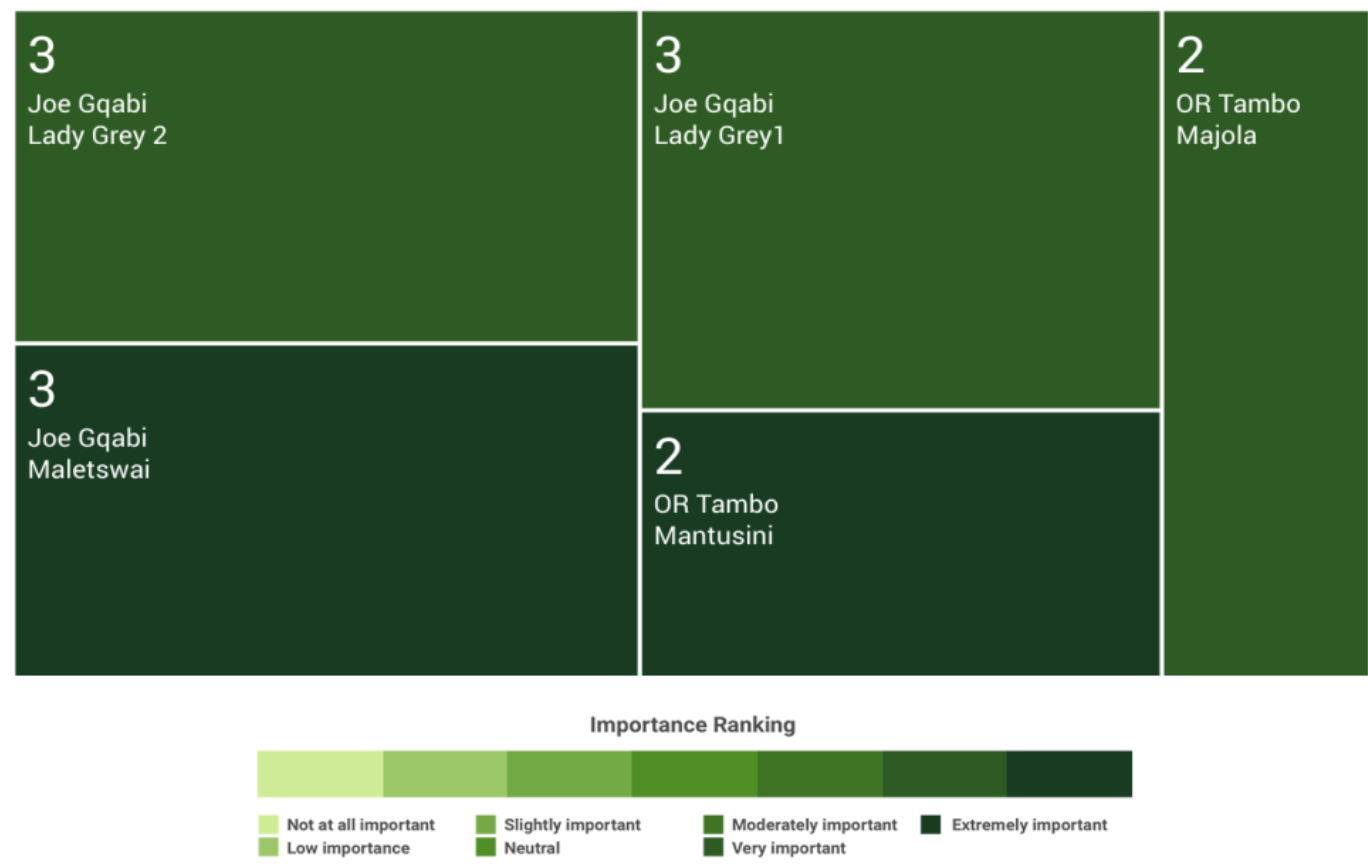

Figure 3 - Tree map word frequency analysis of 'veld fires'

As illustrated in Figure 3, the shock of 'veld fires' was of major concern in Maletswai, based on the number of times they were mentioned and ranked in terms of importance. In Lady Grey 1 and Lady Grey 2 veld fires were of a concern, although at a lower ranking, compared to Maletswai, Mantusini and the Majola Tea Estate that shared the value in terms of the frequency that the shock was cited; however, it was different in terms of how it was ranked. In Mantusini, veld fires were of a significantly higher ranking compared to that of the Majola Tea Estate.

During the 2016 fire season, the land reform beneficiaries of the Majola Tea Estate were captured by the local press extinguishing veld fires without the necessary fire fighting equipment. The article entitled "Majola Tea Estate workers spent about five hours trying to extinguish a fire which engulfed at least 35 hectares of the tea fields, resulting in a loss estimated to run into millions of rands" (Ntshobane, 20 July 2016). 


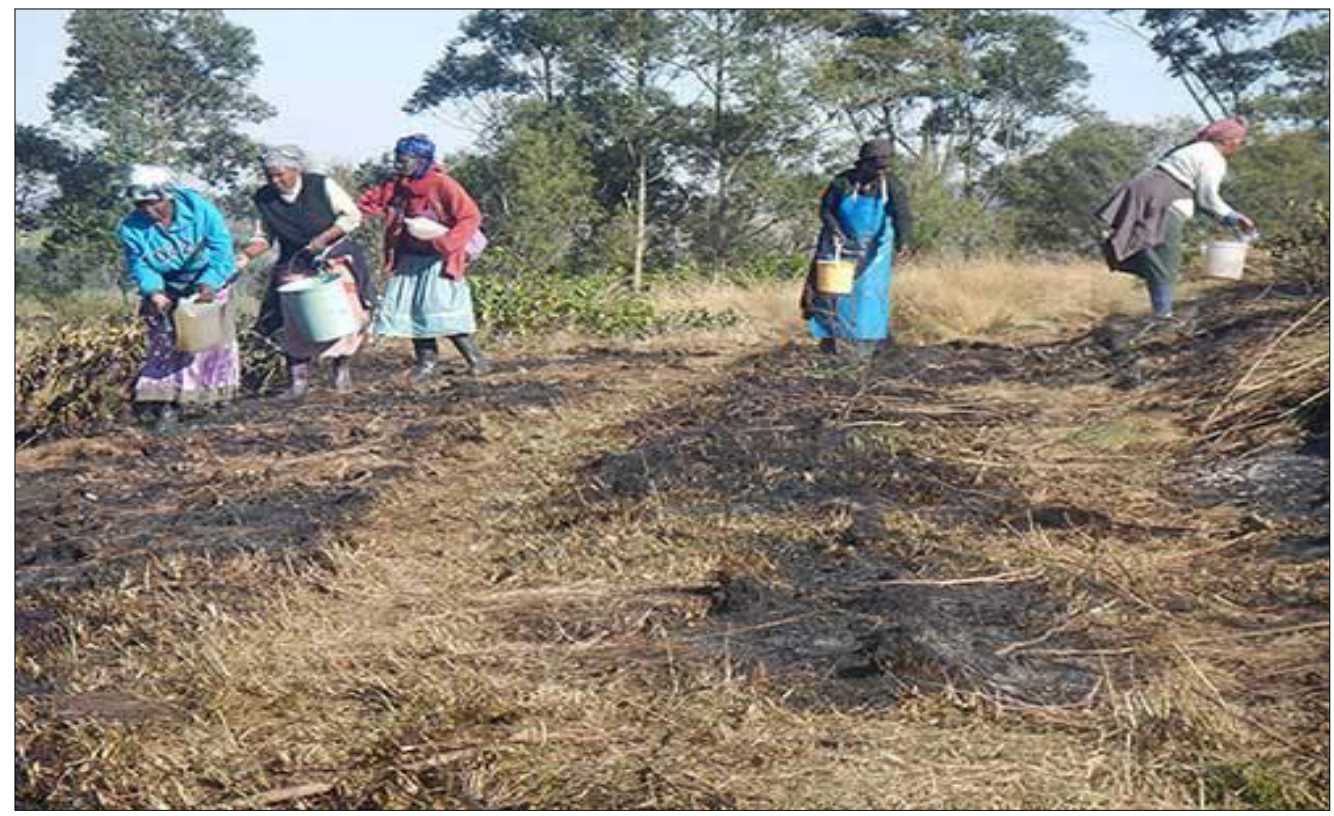

Figure 4 - Majola Tea Estate beneficiaries trying to extinguish a veld fire. Source: Ntshobane (2016)

The above finding depicts land reform beneficiaries attempting to extinguish veld fires without the necessary equipment and apparatus, thus exposing their health to the adverse effects of veld fires. This is because veld fires are comprised of carbon dioxide and water vapour, combined with the hazardous chemicals released from the burning of land fuels. The inhalation of smoke from a veld fire could therefore cause a significant health hazard (NOAA, 2014).

Veld fires have been classified as one of South African hazards which often lead to disasters, resulting in both loss of human and animal life and loss of property. The results of this study provide an insight into the effect of veld fires on livelihoods and agricultural production of land reform beneficiaries. In order for fire disasters to be mitigated within the land reform programme, the DRDLR as the landowner and government decision-makers need to ensure an efficient and effective allocation of resources, including human resources, to limit the spread of veld fires and to ensure that veld fires do not result in adverse effects on the environment and society. In order for the land reform beneficiaries to be resilient against future veld fire disasters, there needs to be a better understanding of high risk areas. This study has made way for further investigation into high risk vulnerable areas of the South African land reform programme.

\section{Proposed innovative veld fire mitigation strategy}

Figure 5 depicts the proposed innovative veld fire mitigation strategy for land reform beneficiaries. The strategy consists of five themes, the collective application of these themes will result into proper management of veld fire risks within land reform farms and sustainable livelihoods and agricultural production. 


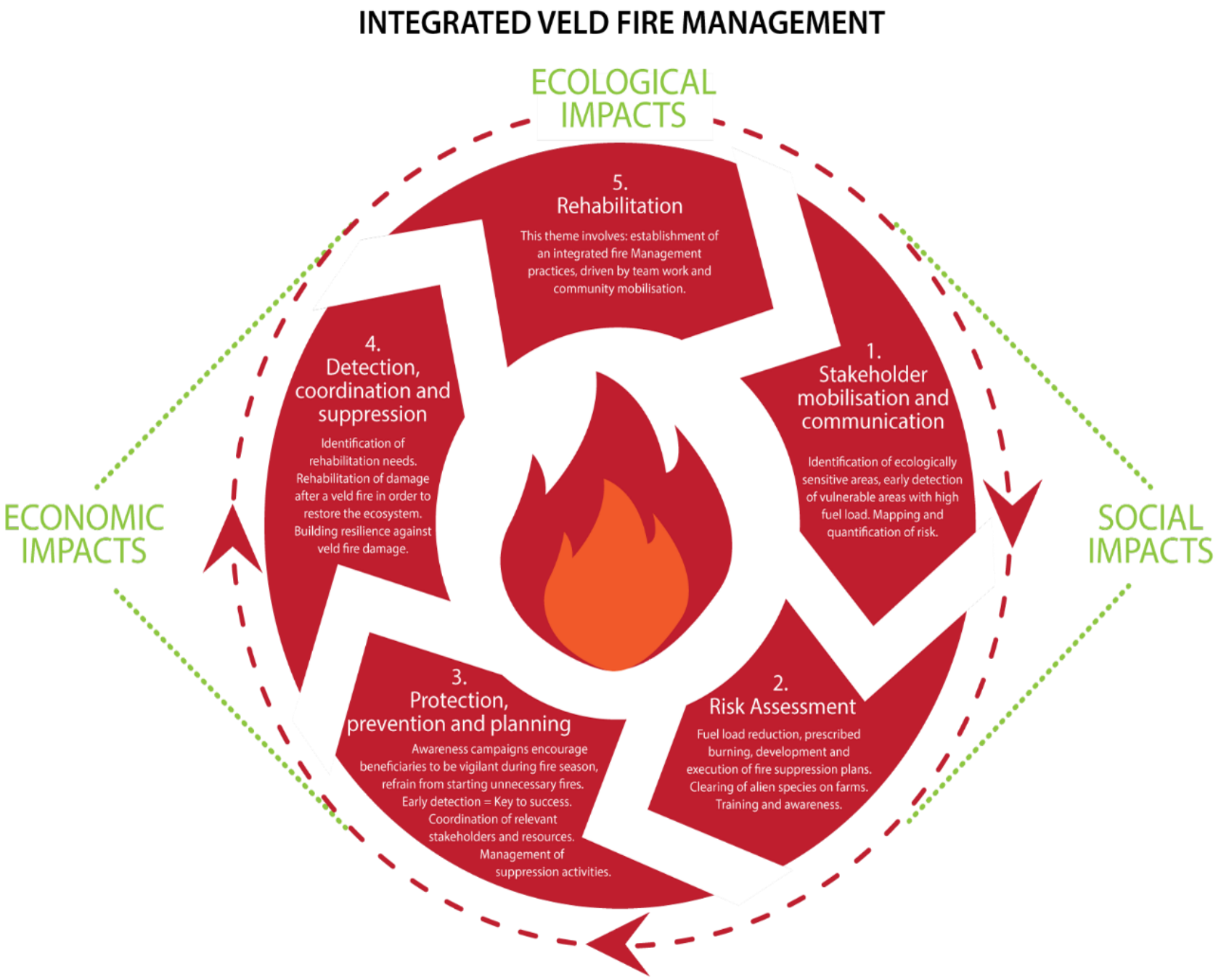

Figure 5 - Proposed innovative veld fire mitigation strategy

\section{Conclusion}

The results provided confirm the need for a veld fire mitigation strategy within the land reform programme. The strategy will boost active involvement thus strengthening capacity to cope with veld fires in land reform farms and improve livelihood security. Additionally, the strategy will assist in determining on farm infrastructure under threat from veld fires and vulnerable areas where there is an abundance of fuel that can start veld fires.

\section{References}

Abdi, H. \& Béra, M. 2014. Correspondence analysis. In R. Alhajj \& J. Rokne (eds.), Encyclopedia of social networks and mining (275-284). New York: Springer Verlag. (Preprint available at https://www.utdallas.edu/ herve/abdi-AB2014_CA.pdf)

Agri SA. 2016. A raindrop in the drought. Report to the multi-stakeholder task team on the drought. Agri SA's status report on the current drought crisis. Available at http://www.nstf.org.za/wpcontent/uploads/2016/06/Agri-SA-Drought-Report_CS4.pdf (Accessed 3 May 2018). 
Agri SA. 2017. Land audit: A transactions approach. Pretoria. Available at https://www.agrisa.co.za/wp-content/uploads/2017/11/AgriSA_Land-Audit_November-2017.pdf (4 June 2018)

AgriSETA. 2010. Sector analysis: Agriculture. Pretoria. Available at http://www.agriseta.co.za/downloads/news/AGRISETA_Sector_Analysis_290610-version_2.pdf (5 June 2018...)

Boudreaux, K. 2010. Land reform as social justice. Institute of Economic Affairs, 30(1):13-20.

Bunce, M., Rosendo, S., Brown, K. 2010. Perceptions of climate change, multiple stressors and livelihoods on marginal African coasts. Environment, Development and Sustainability, 12:407-440.

Chambers, R. 2008. PRA, PLA and pluralism: practice and theory. In P. Reason \& H. Bradbury (eds.). The Sage handbook of action research: participative inquiry and practice. London: SAGE.

CSIR. 2010. Natural resources and the environment, national veldfire risk assessment: Analysis of exposure to social, economic and environmental assets to veldfire in South Africa, Stellenbosch.

DLRLR (Department of Rural Development and Land Reform). 2012. Land Reform Policy Discussion Document. Pretoria.

DRDLR (Department of Rural Development and Land Reform). 2013. Policy for the Recapitalisation and Development Programme of the Department of Rural Development and Land Reform. Pretoria.

ECUFPA. 2018. Integrated Fire Management Report. Easter Cape Umbrella Fire Protection Association. Stutterheim.

Forsyth, G., Kruger, F.G. \& Le Montre, C.G. 2010. National veldfire risk assessment: Analysis of exposure of social, economic and environmental assets to veldfire hazards. Available at: http://www.ehs.unu.edu/file/get/5254 (Accessed 26 June 2018)

Intergovernmental Panel on Climate Change (IPCC). Summary for policy makers. In: Climate change 2014: Impacts, adaptions, and vulnerability. Part A: Global and sectoral aspects. Contribution of Working Group II to the Fifth Assessment Report of the IPCC. Cambridge, UK: Cambridge University Press; 2014. p. 1-32. http://dx.doi.org/10.1017/CBO9781107415416.005

Kruger, F.J., Forsyth, G.G., M Kruger, L.G., Slater, K., Le Maitre, D.C. \& J Matshate, J. 2006.

International Conference on forest fire research: Classification of Veldfire Risk in South Africa for the Administration of the Legislation regarding Fire Management [Online]. Retrieved from: http://www.ehs.unu.edu/file/get/5254 html [2018, June 26].

Mock, N., Béné, C., Constas, M. \& Frankenberger, T. 2015. Systems cluster paper: Systems analysis in the context of resilience. For discussion at the meeting on Resilience Measurement Technical Briefings. Resilience Measurement Technical Working Group. Rome: FSIN.

NASA image courtesy Jeff Schmaltz, MODIS Rapid Response Team. Caption: NASA/Goddard, Lynn Jenner with information from the Volunteer Wildfire Services in South Africa, and Weather South Africa. [Online] Retrieved from: http://www.nasa.gov/content/goddard/veld-fires-in-south-africa/ html (Accessed 28 May 2018)

National Oceanic and Atmospheric Administration (NOAA). Climate at a glance. National Climate Data Centre. Available at: http://www.ncdc.noaa.gov/cag/time-series/global (Accessed 11 May 2018)

Ntshobane, S. 2016. Workers battle to kill fire at Majola tea estate. Dispatch LIVE, 20 July 2016. Available online at https://www.dispatchlive.co.za/news/2016-07-20-workers-battle-to-kill-fire-atmajola-tea-estate/

PDMC (Provincial Disaster Management Centre). 2018. Provincial fire status quo report, 2018 fire season. Provincial Disaster Management Centre. Bhisho. 
Socio-economic and Gender Analysis (SEAGA) Programme. 2001. Field level handbook. Food and Agriculture Organization (FAO) of the United Nations, Italy, Rome.

Wilde, V. 2001. Socio-economic and gender analysis (SEAGA) programme. Gender and Development Service. Handbook. Published by FAO (Food and Agriculture Organisation of the United Nations).

Wilhite, D.A., Sivakumar, M.V. and Pulwarty, R. 2014. Managing drought risk in a changing climate: The role of national drought policy. Weather and Climate Extremes, 3:4-13. 


\title{
Wildfire occurrence estimation in Spanish regions based on Land Use- Land Cover interfaces and biophysical variables
}

\author{
Lara Vilar $^{1 *}$, J. Garrido ${ }^{1}$, S. Herrera ${ }^{2}$, J. Bedia ${ }^{2}$, M. Yebra. ${ }^{3,4}$, P. Echavarría ${ }^{1}$, M.P. Martín ${ }^{1}$ \\ 1* Institute of Economics, Geography and Demography, Centre for Human and Social Sciences, \\ Spanish National Research Council (CSIC), Albasanz 26-28, 28037, Madrid, Spain, \\ \{lara.vilar@cchs.csic.es*\} \\ ${ }^{2}$ Meteorology Group. Dpto. De Matemática Aplicada y C.C. University of Cantabria, Avda de los \\ Castros sn, 39005, Santander, Spain \\ ${ }^{3}$ Fenner School of Environment and Society, The Australian National University, Acton, ACT, \\ Australia \\ ${ }^{4}$ Bushfire and Natural Hazards Cooperative Research Centre, Melbourne, Australia.
}

\begin{abstract}
This study aims to estimate wildfire occurrence in two representative Spanish regions with different territorial, social and environmental characteristics as well as fire history; Zamora and Madrid. Land-Use Land Cover (LULC) interfaces derived from ESA Climate Change Iniciative-Land Cover (CCI-LC) 2005 (covering 2003-2007 period at 300m resolution) were used as indirect indicator of socio-economic drivers associated with human-caused wildfire ignition sources. Three general interfaces were used; forestagricultural interface (FAI), grassland-forest interface (GFI) and wildland urban interface (WUI), as well as other more specific ones derived from a disaggregated LULC legend. A set of biophysical variables including orientation, monthly precipitation, temperature (mean, minimum and maximum) as well as live fuel moisture content (FMC) were also considered in the model. Monthly precipitation and temperature were obtained from a regional climate dataset developed for the study sites by the University of Cantabria (Spain) at $1 \mathrm{~km}^{2}$ spatial resolution while FMC were calculated from MODIS images following Yebra et al. (2018) methodology. Orientation was obtained from the Digital Terrain Model at 25m available for Spain at the Spanish National Geographic Institute (CNIG).

Generalized Linear Models (GLMs) were used to obtain four sub-models to estimate wildfire occurrence in the two study regions during the 2000-2010 period. Two models included only LULC interfaces at two different disaggregation levels, and the other two models also included biophysical drivers. Results showed that disaggregated interfaces helped to improve the spatial characterization of probability of occurence. FAI was the LULC general interface that contributed the most to the models. Biophysical drivers had larger contribution to the models than the interfaces but the later still had importance explaining the spatial distribution of the fire occurrence probability. The accuracy of the models improved when biophysical variables were included achieving a sensitivity of $42 \%$ and omission errors of $\sim 58 \%$ in Zamora and $\sim 6 \%$ and $90 \%$, respectively in Madrid. The results for Madrid were less accurate mainly due to the small number of fire events to fit the models. Future works will deal with modeling in areas with small but of importance number of fires as in this last site. This study indicates that the human component of wildfires represented by LULC interfaces and its disaggregation can be integrated into wildfire occurrence estimation.
\end{abstract}

Keywords: CCI-Land Cover, LULC interfaces, MODIS Hotspots, wildfires

\section{Introduction}

Reported changes in the use of land and in climate are affecting the fire cycle (Pausas and Fernández-Muñoz 2012) increasing the frequency and severity of wildland fires (Moreno et al. 2013) and leading to threats to the ecosystem stability, the provision of services, habitat conservation as well as properties and human lives. Most of these fires are human-caused so an effort is necessary to identify and monitor fire risk associated to human activity. Previous works to predict wildfire occurrence have considered different types of factors that represent the human activities that can lead to the ignition of 
a fire i.e. roads, electric lines, population, natural protected areas (Martínez et al. 2009) (Rodrigues 2014) (Vilar et al. 2016b). Among all the considered factors, the contact areas between the forest and other land cover (the so-called interfaces) have been also included because of the high implication for the security of the people living close to these areas as in the Wildland Urban Interface (WUI). However, in these contact areas human activities cannot only suffer the effects but also to lead to fire ignitions either by accident, negligence or deliberate actions (pasture or agricultural burnings, recreational activities close to urban areas, etc.). Consequently, Land Use Land Cover (LULC) interfaces can be considered as a proxy to human activities associated to potential sources of wildfires ignition (Gallardo et al. 2015) (Vilar et al. 2016a). In this work we consider different LULC interfaces at various thematic disaggregation levels in combination with other biophysical parameters associated to wildfire ignition risk, to estimate fire occurrence at a regional scale. The study sites are two Spanish provinces, Zamora and Madrid, because of their differences in terms of territorial characteristics, LULC and fire history. Generalized Linear Models (GLMs) were applied for the period 2000-2010 and four sub-models at $1 \mathrm{~km}^{2}$ grid cell resolution were calculated for each province by using (1) LULC interfaces (general and specific) and (2) a combination of LULC interfaces and biophysical variables (precipitation, temperature and Fuel Moisture Content-FMC-).

\section{Methods}

\subsection{Study sites}

The study sites are two Spanish provinces, Zamora and Madrid (Figure 1). The two regions are quite similar in size (Zamora area is $\sim 10,500 \mathrm{~km}^{2}$ and Madrid $\sim 8,400 \mathrm{~km}^{2}$ ) and are dominated by Mediterranean continental climate (mean temperature $12-14^{\circ} \mathrm{C}$ and $400-1000 \mathrm{~mm}$ annual precipitation (AEMET 2011)). However, both regions have substantial differences in population density (17 hab/ $\mathrm{km}^{2}$ in Zamora and $800 \mathrm{hab} / \mathrm{km}^{2}$ in Madrid) (INE 2018). Regarding natural vegetation forest, mainly evergreen occupy the $25 \%$ of the total area in both provinces. Important zones are covered by shrublands in Zamora, and only less than the $2 \%$ is covered by grassland, while Madrid is mainly dominate by mixed shrubland-trees, deciduous forest and grasslands, with the latest representing approximately a $14 \%$ of the surface (CCI-LC 2010).

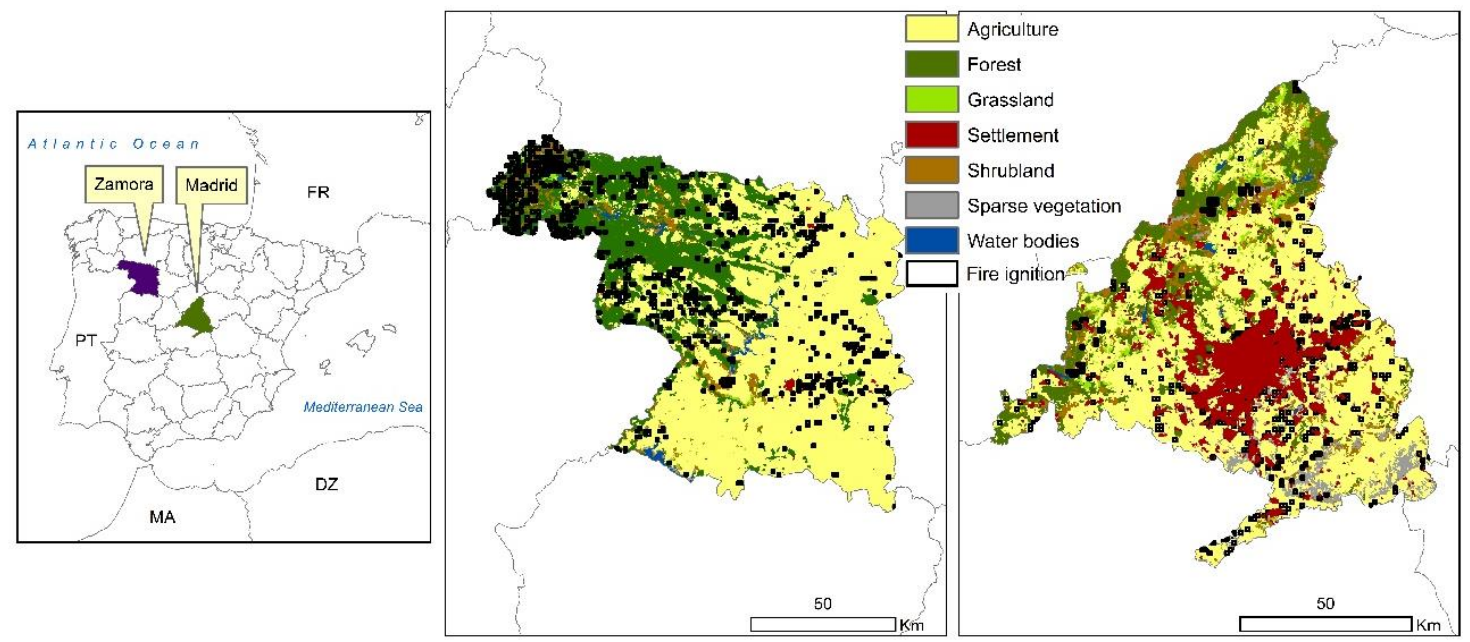

Figure 1 - Location of the study sites and their corresponding reclassified Land Cover categories from ESA Climate Change Iniciative-Land Cover (CCI-LC) and obtained ignition points 2001-2010 (MODIS Burned Area MCD64A1 and MODIS Hotspots products combination)

Agricultural areas cover $\sim 64 \%$ and $\sim 40 \%$ of the territory in Zamora and Madrid respectively. Urban areas represent $\sim 9 \%$ of the territory in Madrid while are really scarce in Zamora $(<1 \%)$. Concerning wildfires 5,200 events happened in Zamora between 2001 and 2010 period, burning 14,000 ha. 1,000 fire events affected 25,000 ha in Madrid. Deliberately caused wildfires are more than $75 \%$ in Zamora 
while in Madrid accidents cause the $22 \%$ of the total wildfires being $\sim 50 \%$ classified as unknown causes (Cubo María et al. 2012).

\subsection{Data}

\subsubsection{Fire data}

Fire data used for this work was the result of combining two data sources, (1) MODIS Terra and Aqua Burned Area (BA) MCD64A1 product, which is a global and monthly gridded $500 \mathrm{~m}$ resolution product (Giglio and Justice 2015) and (2) MODIS Hotspots (HS) from MCD14DL and the Visible Infrared Imaging Radiometer Suite (VIIIRS) 375 m (VNP14IMGTDL_NRT) (Giglio L et al. 2003). BA was downloaded from LP-DAAC NASA Land Products and Services (https://lpdaac.usgs.gov/dataset_discovery/modis/modis_products_table/mcd64a1_v006) and HS from Active Fire Data (https://firms.modaps.eosdis.nasa.gov/) for the period 2000-2010. The BA polygons with less than $50 \mathrm{~m}$ distance between them were assumed to be the same fire event and therefore were grouped. Afterwards, the pixel with the earliest date was selected from the group and considered the ignition point. Spatial coincidence between the HS and the BA was checked to confirm the location of the fire ignition point. All the HS detected at the same date and less than 1,500 m distance from the BA polygons were considered to belong to the same fire (Hantson et al. 2013; Vilar et al. 2015). Also, groups of HS with no BA associated but with a distance less than $1,500 \mathrm{~m}$ among them were assumed to be another fire event. Afterwards those selected BA pixel or HS were placed within the $1 \mathrm{~km}^{2}$ cell grid of reference. The response variable was finally established as the presence or absence of fires in the period 2000-2010 in each $1 \mathrm{~km}^{2}$ grid cell.

\subsubsection{Independent variables of the model}

Several LULC Interfaces and biophysical variables were considered independent vatiables of the model (Table 2). LULC interfaces were derived from ESA CCI LC products (Defourny et al. 2016). The main source of input Earth Observation (EO) data for the global LC maps is the full archive (20032012) of MERIS instrument (Bontemps et al. 2015) at $300 \mathrm{~m}$ resolution. To obtain the LULC interfaces the original CCI-LC 2005 epoch (2003-2007 period) legend was reclassified as showed in Table 2. The three general interfaces were defined as follows forest (class 2)- agricultural (class 1) interface (FAI), forest (class 2)- grassland (class 3) interface (FGI) and wildland (class 2)-urban (class 5) interface (WUI). Other 23 specific LULC interfaces were obtained (6 urban, 12 agricultural and five grasslands types). LULC interfaces were spatially defined as the contact pixels among uses that formed each interface type; then, they were overlaid with the $1 \mathrm{~km}^{2}$ cell grid and thus the density value of each interface in each cell was calculated, obtaining the LULC independent variables by $1 \mathrm{~km}^{2}$.

Table 1 - CCI-LC legend reclassification to define LULC interfaces

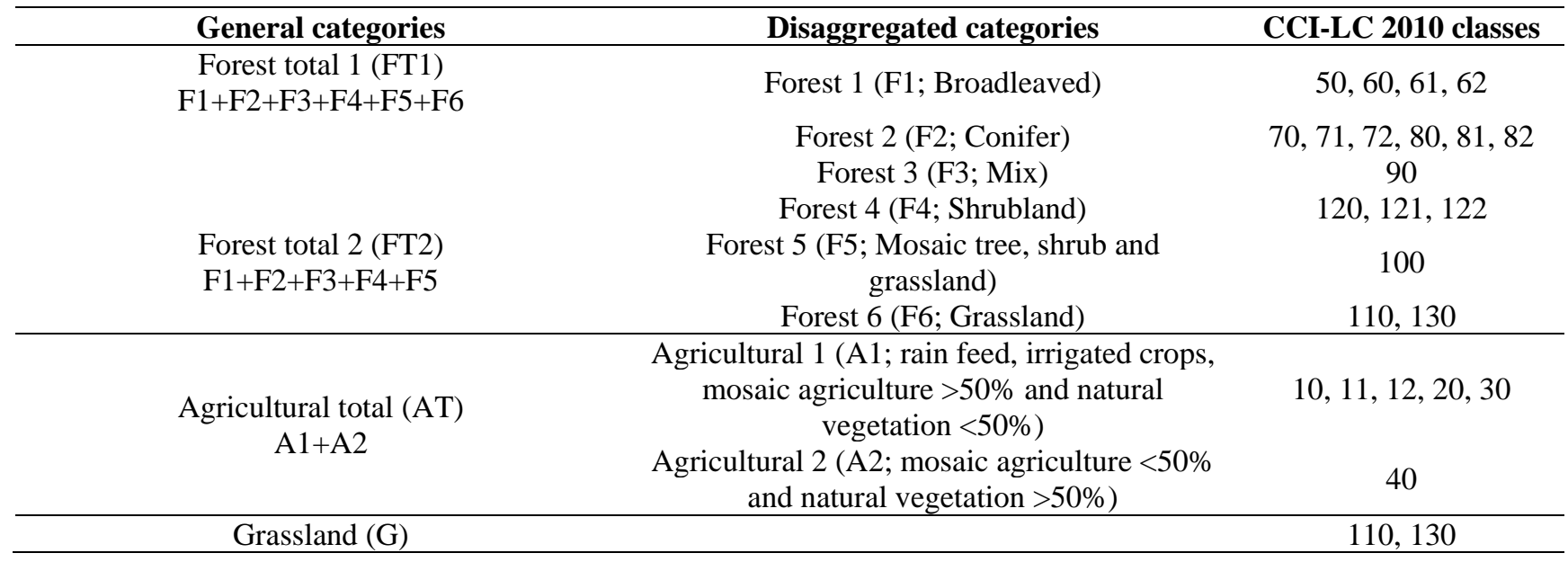


Forest total 1 (FT1) was the sum of all forest categories while Forest total 2 (FT2) excluded forest grassland (F6). Agricultural total (AT) was the sum of A1 and A2. Figure 32 shows the spatial location of the general LULC interfaces.

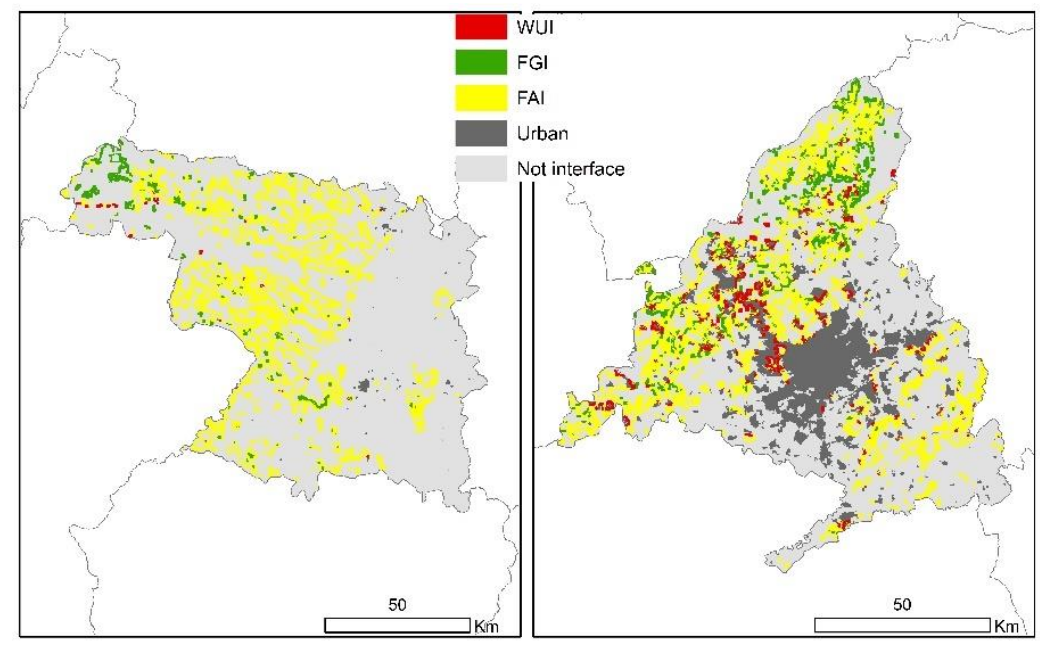

Figure 2 - LULC Interfaces Forest-Grassland Interface (FGI), Forest-Agriculture Interface (FAI) and WildlandUrban Interface (WUI) at $1 \mathrm{~km} 2$ grid cell resolution for Zamora (left) and Madrid (right)

As biophysical parameters were considered orientation, maximum, minimum and mean temperature, accumulated precipitation and live Fuel Moisture Content (FMC). Regarding the meteorological variables (maximum, minimum and mean temperature, and accumulated precipitation) used for this study, they have been built based on a quality controlled weather stations network belonging to the Spanish Meteorology Agency (AEMET). A two-step regression kriging (Hengl et al. 2007) interpolation method has been applied to the monthly values. First, a regression model was defined for each region considering as predictors several orographic variables including elevation, distance to coastline and topographic blocking effects (Bedia et al. 2013) to interpolate the observations to the high-resolution grid of $1 \mathrm{~km}^{2}$ spatial resolution. Then, the monthly residuals obtained from the regression model were interpolated to the target resolution using Ordinary Kriging. Finally, the interpolated values were obtained by combining both interpolated values using an additive and multiplicative functions for temperatures and precipitation, respectively. As a result, the weatherrelated biophysical parameters (Table 2) were defined as the climatologies for the period considered in the analysis (2000-2010).

Live FMC was obtained using a physically-based retrieval model and MODIS reflectance data (MCD43A4 Collection 6) using radiative transfer model Look-Up Table inversion (Yebra et al. 2018).

Table 2 - Independent variables of the model

\begin{tabular}{|c|c|c|c|}
\hline Variable type & Variable & Description & Abbreviation \\
\hline \multirow[t]{4}{*}{ Biophysical } & $\begin{array}{l}\text { Mean accumulated } \\
\text { precipitation }\end{array}$ & $\begin{array}{l}\text { Interpolated monthly } \\
\text { accumulated precipitation } \\
(\mathrm{mm}) . \text { Mean of } 2000-2010\end{array}$ & pr_m \\
\hline & $\begin{array}{l}\text { Mean monthly } \\
\text { maximum, } \\
\text { minimum and } \\
\text { mean temperature }\end{array}$ & $\begin{array}{l}\text { Interpolated monthly } \\
\text { maximum, minimum and } \\
\text { mean temperature }\left({ }^{\circ} \mathrm{C}\right) \text {. } \\
\text { Mean of } 2000-2010\end{array}$ & $\begin{array}{l}\text { tmax_m } \\
\text { tmin_m } \\
\text { tas_m }\end{array}$ \\
\hline & $\begin{array}{c}\text { Live Fuel Moisture } \\
\text { Content }\end{array}$ & Mean of 2000-2010 & LFMC_m \\
\hline & Orientation & & or \\
\hline $\begin{array}{l}\text { LULC main } \\
\text { interfaces }\end{array}$ & $\begin{array}{l}\text { Forest Agricultural } \\
\text { Interface }\end{array}$ & $\begin{array}{c}\text { Agricultural1+Agricultural } 2 \\
\text { || Forest total } 1\end{array}$ & FAI \\
\hline
\end{tabular}




\begin{tabular}{|c|c|c|c|}
\hline 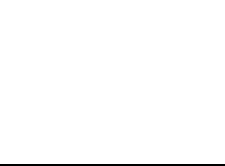 & $\begin{array}{l}\text { Forest Grassland } \\
\text { Interface } \\
\text { Wildland Urban } \\
\text { Interface }\end{array}$ & $\begin{array}{c}\text { Grassland \| Forest total } 2 \\
\text { Urban } \| \text { Forest total } 1\end{array}$ & $\begin{array}{l}\text { FGI } \\
\text { WUI }\end{array}$ \\
\hline $\begin{array}{c}\text { LULC specific } \\
\text { interfaces }\end{array}$ & & $\begin{array}{c}\text { Urban || Forest } 1 \\
\text { Urban \|| Forest } 2 \\
\text { Urban \|| Forest } 3 \\
\text { Urban \|| Forest } 4 \\
\text { Urban \|| Forest } 5 \\
\text { Urban \|| Forest } 6 \\
\text { Agricultural } 1 \text { || Forest } 1 \\
\text { Agricultural } 1 \text { || Forest } 2 \\
\text { Agricultural } 1 \text { || Forest } 3 \\
\text { Agricultural } 1 \text { || Forest } 4 \\
\text { Agricultural } 1 \text { || Forest } 5 \\
\text { Agricultural } 1 \text { || Forest } 6 \\
\text { Agricultural } 2 \text { || Forest } 1 \\
\text { Agricultural } 2 \text { || Forest } 2 \\
\text { Agricultural } 2 \text { || Forest } 3 \\
\text { Agricultural } 2 \text { || Forest } 4 \\
\text { Agricultural } 2 \text { || Forest } 5 \\
\text { Agricultural } 2 \text { || Forest } 6 \\
\text { Grassland || Forest } 1 \\
\text { Grassland || Forest } 2 \\
\text { Grassland || Forest } 3 \\
\text { Grassland || Forest } 4 \\
\text { Grassland }|| \text { Forest } 5 \\
\end{array}$ & $\begin{array}{l}\text { U-F1 } \\
\text { U-F2 } \\
\text { U-F3 } \\
\text { U-F4 } \\
\text { U-F5 } \\
\text { U-F6 } \\
\text { A1-F1 } \\
\text { A1-F2 } \\
\text { A1-F3 } \\
\text { A1-F4 } \\
\text { A1-F5 } \\
\text { A1-F6 } \\
\text { A2-F1 } \\
\text { A2-F2 } \\
\text { A2-F3 } \\
\text { A2-F4 } \\
\text { A2-F5 } \\
\text { A2-F6 } \\
\text { G-F1 } \\
\text { G-F2 } \\
\text { G-F3 } \\
\text { G-F4 } \\
\text { G-F5 }\end{array}$ \\
\hline
\end{tabular}

\subsection{Statistical analysis: Generalized Linear Models (GLMs)}

Wildfire occurrence was estimated using Generalized Linear Models (GLMs). GLMs are extensions of linear regression models that support dependent variables with non-normal distributions, such as binomials (Guisan et al. 2002). Fire events are a rare event and therefore the number of cells with fire absence is highly superior to the cells with fire presence. A random sample of the absencefire cells was selected as model input to retain enough covariate information on the non-ignitions for modelling as in Preisler (2004). This introduces a deterministic offset term of $-\log \left(\pi_{\mathrm{xyt}}\right)$ that does not bias the analysis (Vilar et al. 2010). $\pi_{\mathrm{xyt}}$ denotes the response-specific sampling rate. When $\pi_{\mathrm{xyt}}=1, \pi_{\mathrm{xyt}}$ is also 1 , and when $\pi_{\mathrm{xyt}}=0, \pi_{\mathrm{xyt}}=\pi$. In this work a sample of $10 \%$ (Zamora) and $5 \%$ (Madrid) of the zero-fire cells was selected. See Preisler et al. for further details (2004). The resulting dataset was randomly divided into two groups, $75 \%$ for model calibration and $25 \%$ for validation. The lowest Akaike's Information Criterion (AIC) value was use to select the best model. As regression models assume uncorrelated independent variables, a multicollinearity analysis was performed before running GLM using the Spearman correlation among variables and by Variance Inflation Factor (VIF). VIF can distinguish the degree of multicollinearity when variables are not centred (Freund et al. 2003). Spearman correlations higher than 0.7 and/or VIF higher than 10 (Hair et al. 1995) indicated multicollinearity and affected independent variables were not included in the analysis.

\section{Results}

Four sub-models to estimate wildfire occurrence were obtained for each province (Table 4 and Table 5). Exploratory analysis showed high Spearman correlations between tmin_m and tmax_m $(0.77)$ and between tas_m with tmax_m and tmin_m ( 0.9) with VIF>100 in Zamora. So for multicollinearity problems tmin_m and tas_m were not included into the analysis. 
Table 3 - Estimated coefficients and significances (Wald test) for each of the GLM predictors for Zamora

\begin{tabular}{|c|c|c|c|c|}
\hline & \multicolumn{2}{|c|}{ LULC } & \multicolumn{2}{|c|}{ LULC + Biophysical } \\
\hline & \multicolumn{2}{|c|}{ LULC general } & \multicolumn{2}{|c|}{ LULC general } \\
\hline Predictor & Estimated coefficient & Probability & Estimated coefficient & Probability \\
\hline (Intercept) & -2.1127 & $<2 \mathrm{e}-16 * * *$ & -6.3841 & $1.62 \mathrm{e}-06 * * *$ \\
\hline FAI & 0.3815 & $0.0307 *$ & 0.7239 & $0.000153 * * *$ \\
\hline WUI & 9.2816 & $0.0408 *$ & - & - \\
\hline FGI & 2.5393 & $9.65 \mathrm{e}-08 * * *$ & - & - \\
\hline tmax_m & - & - & 0.1058 & 0.100256 \\
\hline pr_m & - & - & 0.0433 & $<2 \mathrm{e}-16 * * *$ \\
\hline \multirow[t]{2}{*}{ LFMC_m } & - & - & -0.0291 & $0.000115 * * *$ \\
\hline & \multicolumn{2}{|c|}{ LULC specific } & \multicolumn{2}{|c|}{ LULC specific } \\
\hline (Intercept) & -2.0403 & $<2 \mathrm{e}-16 * * *$ & -7.6508 & $3.49 \mathrm{e}-08 * * *$ \\
\hline A1-F1 & 3.02733 & 0.074423 . & - & - \\
\hline A1-F2 & 2.76241 & $0.003724 * *$ & 1.5750 & 0.1353 \\
\hline A1-F3 & & & 16.4596 & 0.2393 \\
\hline A1-F5 & - & - & 1.2283 & $0.0018 * *$ \\
\hline A2-F1 & 28.13043 & 0.26208 & 54.2354 & 0.1163 \\
\hline A2-F2 & & & -3.7303 & $0.0092 * *$ \\
\hline A2-F4 & - & - & 1.7752 & 0.0627 \\
\hline A2-F5 & 4.4464 & $0.00021 * * *$ & 2.0049 & 0.1270 \\
\hline A2-F6 & -2.6657 & $0.02520 *$ & -3.0915 & $0.0182 *$ \\
\hline U-F1 & & & -76.6902 & 0.2126 \\
\hline U-F3 & & & -5.6318 & 0.0538 \\
\hline U-F6 & 64.5968 & 0.1569 & - & - \\
\hline G-F2 & 2.5017 & 0.05652 & - & - \\
\hline G-F4 & 7.6999 & $0.00015 * * *$ & - & - \\
\hline tmax_m & - & - & 0.17048 & $0.0110 *$ \\
\hline pr_m & - & - & 0.04573 & $<2 \mathrm{e}-16 * * *$ \\
\hline LFMC_m & - & - & -0.02834 & $0.00023 * * *$ \\
\hline
\end{tabular}

In Zamora province, the model using as independent variables only general LULC interfaces (FAI, FGI and WUI) showed that the three of them were significant, however where the biophysical variables were also included the only significant LULC was the FAI along with the mean precipitation and the live FMC. In those models that included all 23 specific LULC interfaces, the ones that contributed to the model were the Agricultural (with no natural vegetation, A1)-Conifer forest (F2), Mosaic agriculture (A2)- Mosaic forest (F5), Mosaic agriculture (A2)-Forest grassland (F6) and GrasslandShrub (F4) interface. Both mean precipitation and live FMC were significant when the biophysical variables were included in the model. A2-F6 was also contributing to the model as well as two more specific agricultural-forest related interfaces. The global accuracy of the models improved when the biophysical variables were included with a sensitivity a omission error of $42.78 \%$ and $57.22 \%$ and $41.18 \%$ and $58.82 \%$ in the LULC general and specific model, respectively.

Table 4 - Estimated coefficients and significances (Wald test) for each of the GLM predictors for Madrid

\begin{tabular}{|c|c|c|c|c|}
\hline & \multicolumn{2}{|c|}{ LULC } & \multicolumn{2}{|c|}{ LULC + Biophysical } \\
\hline & \multicolumn{2}{|c|}{ LULC general } & \multicolumn{2}{|c|}{ LULC general } \\
\hline Predictor & Estimated coefficient & Probability & Estimated coefficient & Probability \\
\hline (Intercept) & -1.3870 & $<2 \mathrm{e}-16 * * *$ & -0.0081 & 0.9850 \\
\hline FAI & -2.1279 & $4.62 \mathrm{e}-12 * * *$ & -1.8267 & $1.65 \mathrm{e}-08 * * *$ \\
\hline WUI & - & - & - & - \\
\hline FGI & - & - & & \\
\hline $\mathrm{pr}$ & - & - & -0.0350 & $0.0011 * *$ \\
\hline
\end{tabular}




\begin{tabular}{|c|c|c|c|c|}
\hline & \multicolumn{2}{|c|}{ LULC specific } & \multicolumn{2}{|c|}{ LULC specific } \\
\hline (Intercept) & -1.4321 & $<2 \mathrm{e}-16 * * *$ & 0.0380 & 0.9329 \\
\hline A1-F3 & -21.2314 & 0.1652 & -19.3975 & 0.1682 \\
\hline A1-F5 & -3.5485 & $0.0191 *$ & -3.4152 & $0.0272 *$ \\
\hline A1-F6 & -1.9583 & $0.02594 * *$ & -2.422 & $0.0019 * *$ \\
\hline A2-F3 & 36.6788 & 0.3786 & 33.1694 & 0.3575 \\
\hline A2-F5 & -3.5859 & $0.0014 *$ & -3.0695 & $0.0064 * *$ \\
\hline U-F2 & 4.7141 & 0.1728 & 6.0985 & 0.1009 \\
\hline G-F1 & -28.0549 & 0.2770 & -28.9154 & 0.2360 \\
\hline G-F2 & & - & 6.2943 & $0.0479 *$ \\
\hline G-F5 & -8.8926 & $0.0001 * * *$ & -9.8587 & $0.0001 * * *$ \\
\hline $\mathrm{pr}$ & & - & -0.0382 & $0.0006 * * *$ \\
\hline
\end{tabular}

In Madrid the model with the general LULC interfaces showed FAI was significant and with negative sign. When the biophysical variables were added, the mean precipitation also contributed to the model. Regarding the models that included the specific LULC interfaces the significant variables were the Agricultural (with no natural vegetation, A1)- Forest Mosaic forest (F5) and Forest grassland (F6), and Grassland- Mosaic forest (F5) (negative trend). When biophysical variables were included, mean precipitation was significant as well as three agricultural-related LULC specific interfaces (A1F5, A1F6, A2F5), G-F2 and G-F5. Similarly to Zamora, the global accuracy of the models also improved in Madrid when the biophysical variables were included with a sensitivity of $\sim 6 \%$ and a large omission error of $\sim 90 \%$. However, the general fit of the model was not satisfactory for this site.

\section{Discussion and conclusions}

LULC interfaces have been used as predictor factors to estimate wildfire occurrence in previous works (Syphard et al. 2007); (Padilla and Vega-García 2011); (Vilar et al. 2016b); (Martínez et al. 2009). In this work include LULC disaggregated interfaces were included to consider different forest types regarding tree species (conifer, deciduous, mixed) and different vegetation formations (shrubs and pastures). Two very different Spanish provinces (Zamora and Madrid) have been analyzed with the aim to obtain predictive wildfire models that gathered those territorial and fire-related differences. The inclusion of the biophysical variables allowed to quantify the improvement on the predictive ability of the proposed models in comparison to models only calibrated with LULC.

Biophysical factors contributed the most in both sites. Mean precipitation and LFMC were the stronger contributors in Zamora while precipitation in Madrid. Regarding the LULC interfaces, agricultural-forest related interfaces explained fire occurrence in Zamora with the expected trends (positive), coincident with Rodrigues et al. (2014). The authors used Geographic Weighted Regression (GWR) model for the estimation of fire occurrence probability in Spain and FAI interface also showed an important contribution in Zamora. Also, FGI contributed to the general model, possibly related fires associated with accidents or negligences derived from traditional agricultural practices as pasture burning. In Madrid, none of the specific WUI related interfaces contributed to the model and most of the variables had a negative trend, contrary as expected about fire ignition in this region (Vilar et al. 2016b).

In general, the models performed better in Zamora than in Madrid. We hypothesize that the spatial distribution of the response variable might be influencing in this result as MODIS is mainly detecting the agricultural fires but not the forest fires due to the small area that these fires usually have in this region where firefighting is quite effective in extinguishing the fires in their earlier phases.

The use of indicators of human-related ignition potential is a key issue in fire risk estimation in European Mediterranean areas where a high percentage of fires are due to human causes. As proven in this work and also demonstrate by other authors, LULC interfaces indirectly represent human 
activities associated with fire ignition. This information can be obtained from Land Cover (LC) maps globally available at appropriate management scales as is the case for the CCI-LC. This availablility of global LC maps will allow applying the proposed methodology to other study sites and temporal periods. The combination of specific LULC interfaces along with the biophysical variables might help to obtain a more detailed wildfire occurrence explanation at a global scale too.

\section{References}

AEMET (2011) 'Atlas Climático Ibérico.' (Ministerio de Agricultura, Alimentación y Medio Ambiente: Spain)

Bedia, J, Herrera, S, Gutiérrez, JM (2013) Dangers of using global bioclimatic datasets for ecological niche modeling. Limitations for future climate projections. Global and Planetary Change 107, 112.

Bontemps, S, Boettcher, M, Brockmann, C, Kirches, G, Lamarche, C, Radoux, J, Santoro, M, Van Bogaert, E, Wegmüller, U, Herold, M, Achard, F, Ramoino, F, Arino, O, Defourny, P (2015) 'Multiyear global land cover mapping at $300 \mathrm{~m}$ and characterization for climate modelling: achievements of the land cover component of the ESA Climate Change Initiative, 36th International Symposium on Remote Sensing of Environment.' 11-15 May 2015. (The International Archives of the Photogrammetry, Remote Sensing and Spatial Information Sciences: Berlin, Germany)

Cubo María, JE, Enríquez, E, Gallar Pérez-Pastor, JJ, Jemes Díaz, V, López García, M, Mateo Díez, ML, Muñoz Correal, A, Parra Orgaz, PJ (2012) Los Incendios Forestales en España. Decenio 20012010. Madrid.

Defourny, P, Kirches, G, Brockmann, C, Boettcher, M, Peters, M, Bontemps, S, Lamarche, C, Schlerf, M, M., S (2016) Land Cover CCI: Product User Guide Version 2. UCL-Geomatics, Louvain-laNeuve, Belgium.

Freund, RJ, Littell, RC, Creighton, L (2003) 'Regression Using JMP.' Cary, NC: SAS Institute, Inc. )

Gallardo, M, Gómez, I, Vilar, L, Martínez-Vega, J, Martín, MP (2015) Impacts of future land use/land cover on wildfire occurrence in the Madrid region (Spain). Regional Environmental Change 16, 1047-1061.

Giglio L, Kendall JD, R, M (2003) A multi-year active fire dataset for the tropics derived from the TRMM VIRS. Internarional Journal of Remote Sensing 24, 4505-4525.

Giglio, L, Justice, C, 2015. MCD64A1 MODIS/Terra+Aqua Burned Area Monthly L3 Global 500m SIN Grid V006

Guisan, A, Edwards, TC, Hastie, T (2002) Generalized linear and generalized additive models in studies of species distributions: setting the scene. Ecological Modelling 157, 89-100.

Hair, JFJ, Anderson, RE, Tatham, RL, Black, WC (1995) 'Multivariate Data Analysis.' (Macmillan Publishing Company: New York)

Hantson, S, Padilla, M, Corti, D, Chuvieco, E (2013) Strengths and weaknesses of MODIS hotspots to characterize global fire occurrence. Remote Sensing of Environment 131, 152-159.

Hengl, T, Heuvelink, G, Rossiter, D (2007) About regression-kriging: from equations to case studies. Computers and Geosciences 33, 1301-1315.

INE (2018) 'Cifras de población y censos demográficos. http://www.ine.es/.' Available at http://www.ine.es/dyngs/INEbase/es/categoria.htm?c=Estadistica_P\&cid=1254735572981

Martínez, J, Vega-García, C, Chuvieco, E (2009) Human-caused wildfire risk rating for prevention planning in Spain. Journal of Environmental Management 90, 1241-1252.

Moreno, JM, Vallejo, R, Chuevico, E (2013) Current Fire Regimes, Impacts and the Likely Changes - VI: Euro Mediterranean. In 'Vegetation Fires and Global Change - Challenges for Concerted 
International Action A White Paper directed to the United Nations and International Organizations.' (Ed. JG Goldammer.) (Global Fire Monitoring Center (GFMC): Kessel Publishing House)

Padilla, M, Vega-García, C (2011) On the comparative importance of fire danger rating indices and their integration with spatial and temporal variables for predicting daily human-caused fire occurrences in Spain. International Journal of Wildland Fire 20, 46-58.

Pausas, JG, Fernández-Muñoz, S (2012) Fire regime changes in the Western Mediterranean Basin: from fuel-limited to drought-driven fire regime. Climatic Change 110, 215-226.

Preisler, HK, Brillinger, DR, Burgan, RE, Benoit, JW (2004) Probability bases models for estimation of wildfire risk. International Journal of Wildland Fire 13, 133-142.

Rodrigues, M, de la Riva, J., Fotheringham, S. (2014) Modeling the spatial variation of the explanatory factors of human-caused wildfires in Spain using geographically weighted logistic regression. Applied Geography 48, 52-63.

Syphard, AD, Radeloff, VC, Keeley, JE, Hawbaker, TJ, Clayton, MK, Stewart, SI, Hammer, RB (2007) Human influence in California Fire regimes. Ecological Applications 17, 1388-1402.

Vilar, L, Camia, A, San-Miguel-Ayanz, J (2015) A comparison of remote sensing products and forest fire statistics for improving fire information in Mediterranean Europe. European Journal of Remote Sensing 48, 345-364.

Vilar, L, Camia, A, San-Miguel-Ayanz, J, Martín, MP (2016a) Modeling temporal changes in humancaused wildfires in Mediterranean Europe based on Land Use-Land Cover interfaces. Forest Ecology and Management 378, 68-78.

Vilar, L, Gómez, I, Martínez-Vega, J, Echavarría, P, Riaño, D, Martín, MP (2016b) Multitemporal Modelling of Socio-Economic Wildfire Drivers in Central Spain between the 1980s and the 2000s: Comparing Generalized Linear Models to Machine Learning Algorithms. PLoS ONE 11, e0161344.

Vilar, L, Woolford, DG, Martell, DL, Martín, MP (2010) A model for predicting human-caused wildfire occurrence in the region of Madrid, Spain. International Journal of Wildland Fire 19, 325337.

Yebra, M, Quan, X, Riaño, D, Rozas Larraondo, P, van Dijk, AIJM, Cary, GJ (2018) A fuel moisture content and flammability monitoring methodology for continental Australia based on optical remote sensing. Remote Sensing of Environment 212, 260-272. 


\title{
Wildfire susceptibility mapping in Liguria (Italy). Comparison of Statistical Driven Partitioning and Machine Learning approach
}

\author{
Guido Biondi ${ }^{1}$, Lorenzo Campo ${ }^{1}$; Mirko D’Andrea ${ }^{1}$; Silvia Degli Esposti ${ }^{1}$; Paolo Fiorucci ${ }^{1 *}$; Marj \\ Tonini $* 2$ \\ ${ }^{1}$ CIMA Research Foundation. Via A. Magliotto 2, 17100, Savona, \\ \{paolo.fiorucci@cimafoundation.org*\} \\ ${ }^{2}$ Institute of Earth Surface Dynamics (IDYST), Faculty of Geosciences and Environment, \\ University of Lausanne, Lausanne, Switzerland, \{marj.tonini@unil.ch*\}
}

\begin{abstract}
The identification of areas most vulnerable to fire risk is a key tool in wildfire management, particularly in view of the limited availability of fire risk management resources, most of which are used for national and regional air services. The few resources available can thus be used on a yearly basis to mitigate problems in the areas at highest risk by defining a program of interventions.

In this contest, the main objective of the present study is to elaborate wildfire susceptibility mapping for Liguria region (Italy), and to compare results obtained by a statistical driven partitioning models with the ones obtaining by appling a machine learnig approach, based on Random Forest (RF) algorithm.

The availability of a mapping of fire perimeters spans almost 20 years (1996-2016), and this, combined with a detailed knowledge of topography, climate and land cover, allowed to understand the main features involved in wildfire occurrences and their behavior. The seasonality of the fire regime was also considered, partitioning the analysis in two macro season (November-April and May- October).

The analysis was based on a recursive-quantiles subdivision of the territory in classes based on the different available information layers: elevation, slope, aspect, rainfall height, temperature (the latter subdivided in winter and summer periods). The algorithm was designed in order to assure the equal representation of each class, in which the number of fires occurred in the period of analysis is considered, in order to have an estimation of the fire hazard with a constant statistical confidence.

The resulting wildfire susceptibility map was then compared with the map obtained applying a machine learning (ML) approach. ML explores the conception of algorithms capable of learning from and make predictions on data, through the modeling of the hidden relationships between a set of input and output variables (i.e. the predisposing factors and the occurrence of the phenomenon). The main benefit is that these approaches are data driven, meaning that they do not need a priori knowledge of the process. Moreover, RF directly provides the measurement of the importance of each variable.

The results show that both the approaches identify shrubs as the vegetation most affected by wildfire both in summer and winter season. RF algorithm seems to perform better, detecting $90 \%$ of the of the BA within $10 \%$ of the entire territory instead of $60 \%$ of burned area within the $20 \%$ of the territory for the statistical driven partitioning. These results are preliminary and need to be further evaluated in detail by establishing a working plan accounting for a common validation dataset for the two approaches.
\end{abstract}

Keywords: Wildfire susceptibility mapping, Machine learning, Random Forest, Statistical partitioning, forest management plan

\section{Introduction}

Susceptibility maps are a modern tool to support forest protection plans and to address fuel management strategies in order to reduce fires' consequences. Wildfire susceptibility mapping allows to identify areas subject to fires, assessed defining a rank from low to high. These are elaborated taking into account two aspects: where the wildfires occur and which are the predisposing factors. Distance to transport networks and distance to urban or recreation areas are the most frequently used human 
factors. As regards environmental variables, those related to weather, fuel and topography result to be the most significant drivers of ignition of wildfires, especially in Mediterranean-type regions (Ganteaume et al., 2013). A number of models for wildfire hazard mapping and risk analyses were recently developed. These are often GIS oriented, including statistical analyses and modeling to assess the variables' importance (Eugenio et al., 2016; Parente and Pereira, 2016; Pourghasemi et al., 2016; Pourtaghi et al., 2016; Rodrigues et al.,2014; Fiorucci et al., 2008), and often relie on expert knowledge to estimate predisposing factors. Comparison of phisically based and stochastics approach highligt the benefit of using a data driven method (Leuenberger et al., 2018), which do not need a priori knowledge of the process.

Wildfire risk is particularly significant in Italy, both in summer and winter season due to the high topographic and vegetation heterogeneity of the territory. Liguria is one of the few regions in Italy affected by wildfires both in summer and winter. Most of the fires in Italy occur in summer season and the burned area is largely greater than in winter season. The seasonal fire regime in Liguria differs from this behaviour and is charactetized by a number of wildfires and burned area higher in winter than in summer (Fig.1). Winter fire regime is mainly due to frequent extremely dry winds from the north in condition of curing for most of the herbaceous species.
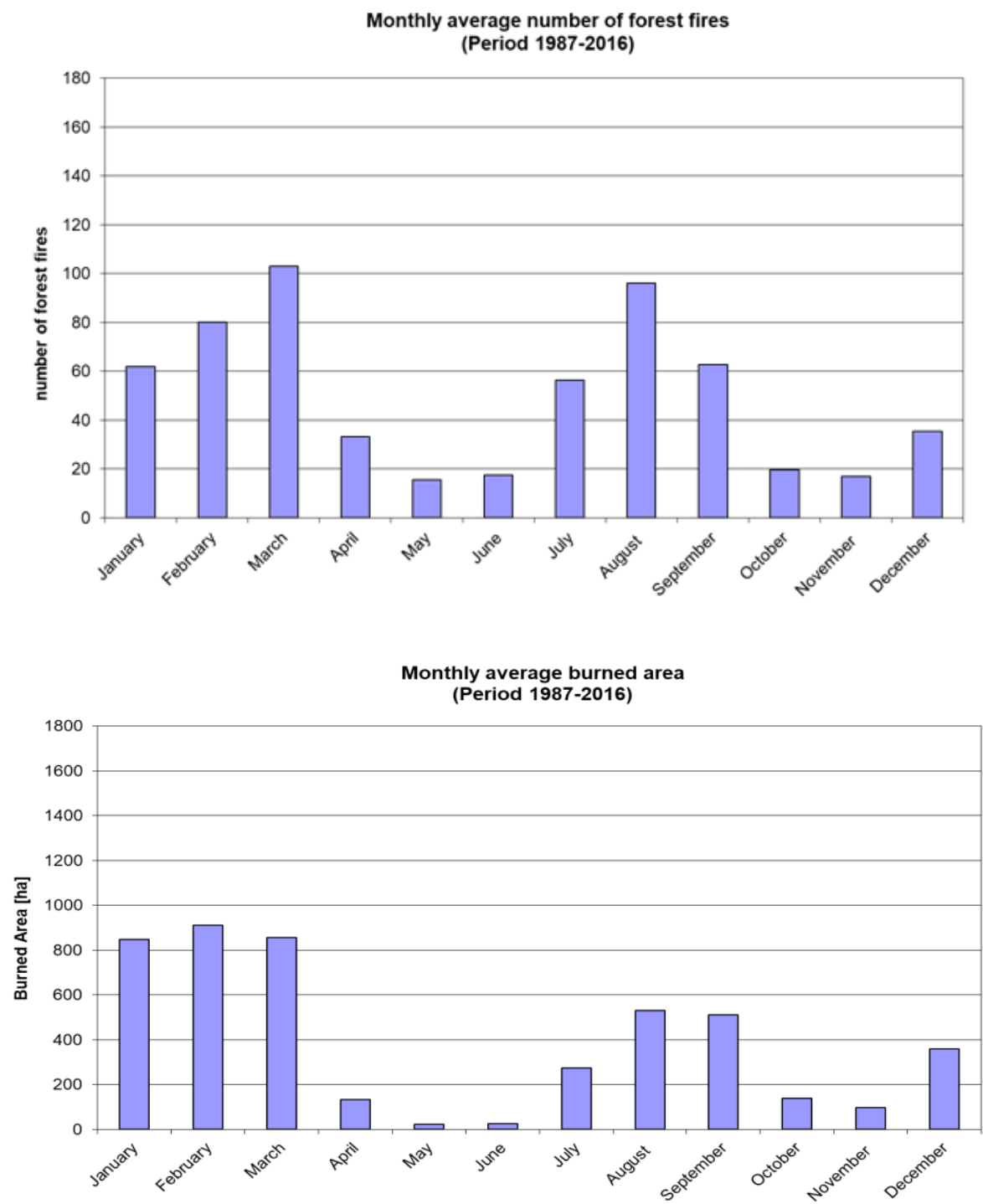

Figure 1 - Monthly frequency of forest fires and burned area in Liguria region (Italy) in the last two decades (1987 2016) 
In view of the limited availability of fire risk management resources, most of which are used in the management of national and regional air services, it is necessary to precisely identify the areas most vulnerable to fire risk. The few resources available can thus be used on a yearly basis to mitigate problems in the areas at highest risk by defining a program of forest management interventions.

The main objective of the present study is to elaborate wildfire susceptibility mapping for Liguria region using two approaches, the first needing of a priori knowledge of the fires behaviour within the investigated area, and the second based on a machine learning stochastic algoriothm. Namely, we compared results obtained by a statistical driven partitioning models with the ones obtaining by appling Random Forest.

\section{Materials and methods}

The availability of a mapping of fire perimeters spans almost 20 years (1996-2016), and this, combined with a detailed knowledge of topography, climate and land cover, allowed to understand the main features involved in wildfire occurrences and their behavior. The seasonality of the fire regime was also considered, partitioning the analysis in two macro season (November-April and MayOctober). Total precipitation and average air temperature obtained from the interpolation of 30 yearslong time series from 164 raingauges and 127 thermometers series were considered. The analysis was carried out at a spatial resolution of $20 \mathrm{~m}$ on the Liguria region territory $\left(5,400 \mathrm{~km}^{2}\right)$ by using a dataset of fires occurrences that spans from 1996 to 2016.

The statistical driven partitioning analysis relied on a recursive-quantiles subdivision of the territory in classes based on the different available information layers: elevation, slope, aspect, type of vegetation (Table 1), rainfall height, temperature (these two latter subdivided in winter and summer periods). The algorithm was designed in order to assure the equal representation of each class, in which the number of fires occurred in the period of analysis is considered, in order to have an estimation of the fire hazard with a constant statistical confidence.

Table 1 - Type of vegetation cover considered in the models

\begin{tabular}{|l|l|}
\hline Code & Type of Vegetation \\
\hline 10 & Non-vegetated area \\
\hline 21 & Other cultivated area \\
\hline 22 & Grasslands \\
\hline 23 & Olive groves \\
\hline 32 & Pine forests \\
\hline 34 & Other deciduous forest \\
\hline 35 & Chestnut groves \\
\hline 37 & Shrub vegetation \\
\hline 333 & Less-burnable forest \\
\hline
\end{tabular}

The wildfire susceptibility map obtained with the described approach has been compared with the map obtained with a machine learning (ML) method. ML include a class of algorithm for the analysis, modelling and visualization of environmental data, and perform particularly well to model environmental and anthropogenic hazard, which naturally present a complex and non-linear behavior. ML explores the conception of algorithms capable of learning from and make predictions on data, through the modeling of the hidden relationships between a set of input and output variables (i.e. the predisposing factors and the occurrence of the phenomenon). After a training procedure, allowing to calibrate the parameters of the model, susceptibility maps can be displayed. In this study, we used Random Forest (RF) (Breiman, 2001), an ensemble ML algorithm based on decision trees (Fig.2). The 
number of decision trees generated (ntree), and the number of variables randomly sampled as candidates at each split (mtry) are the only hyperparameters that need to be specified. Then the algorithm generates ntree subsets of the training dataset (counting about two-third of the observations) by bootstrapping (i.e. random sampling with replacement). For each subset, a decision tree is generated by iterating the following rules up to the maximum level, when each node contains less than a fixed number of data points: at each split, the algorithm select randomly mtry variables and computes the Gini index to select the best variable. The prediction of new data points is finally computed taking the average value of all decision tress for regression and the maximum voting for classification. The prediction error is assessed by evaluating prediction on those observations which were not used in the subset, that is the remaining one-third defined "out-of-bag" (OOB)
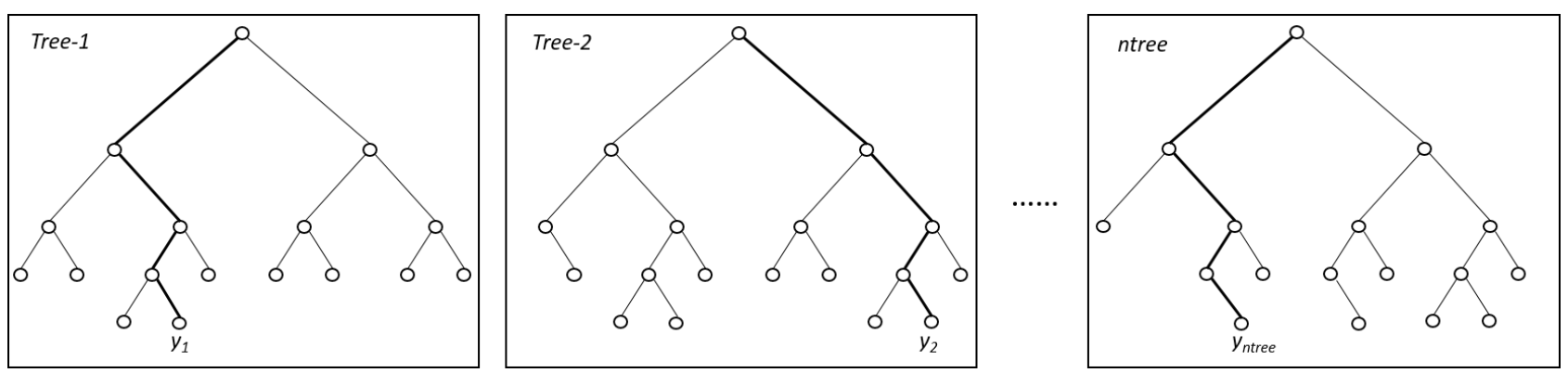

Figure 2 - Structure of Random Forest based on an ensamble of single decision trees

The main benefit of using ML algorith in general is that these approaches are data driven, meaning that no a priori knowledge of the process is required. Another specific advantage of RF is that it allows to measure the relative importance of each variable on the prediction. This is obtained by looking at how much the tree nodes, which use that variable, reduce impurity across all the trees in the forest.

\section{Results}

Results of statistical driven partitioning method show a very high correlation with the topographic aspects, both in winter and summer. Rainfall is almost uncorrelated in both season. Shrubs results the vegetation most affected by fires both in summer and winter. In winter grassland burn quite often in the internal part characterized by high elevation and slope.

Random forest gives as output a prediction value, expressed as the probability for each pixel of burning under the assumpition of a set af predisposing variables. This value is then projected on the training and testing datasets, allowing to produce a susceptibility maps and to validate the procedure. The testing and the OOB mean squared error are in our case both equal to about 0.2 for winter season and to 0.17 for summer summer. This let us to conclude that the RF setted model is robust and good for generalisation, since it performs well on testing data which were never used during the learing process.

As regards the variable importance measurement, it results that shrub vegetation is by far the most important variable in predicting burnig area (Fig.3), both in summer and in winter season. Grassland is the second most important type of vegetation. Neverthless this one and all the other type of vegetation are less important than raifall, altitute and slope and temperature. 

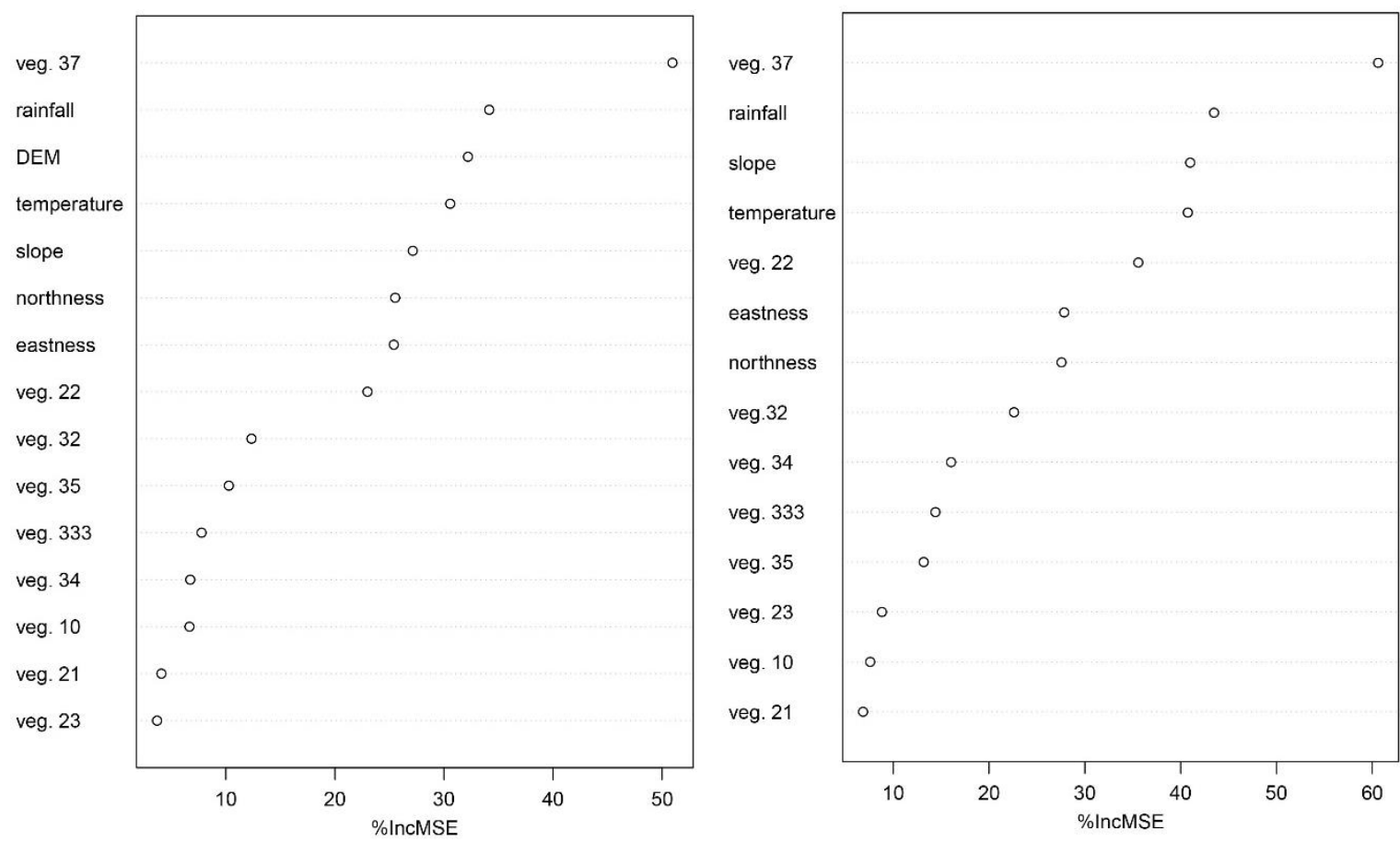

Figure 3 - Variable importance diagrams resulting from Random Forest, evaluated by computing the percentage increase of mean square error (\%IncMSE). Left side: summer season; right side: winter season

Susceptibility maps for winter and summer were elaborated as resulting from the two approach (Fig.4). In Tab. 2 a numerical comparison of the statistical driven partitioning (PA) and machine learning approach, namely random forest (RF) is reported. Results are classified in 5 classes $(\mathrm{Cl})$ of susceptibility to wildfires, using the same thresholds for both approaches. The total area (TA) classified in each class and the burned area (BA) occurred in each class is reported considering the two approaches in summer and winter.

Table 2 - Comparison of statistical driven partitioning (PA) and random forest algorithm (RF)

\begin{tabular}{|l|l|l|l|l|l|l|l|l|}
\cline { 2 - 9 } \multicolumn{1}{c|}{} & \multicolumn{4}{l}{ Summer } & \multicolumn{2}{l|}{ Winter } \\
\cline { 2 - 9 } \multicolumn{1}{c|}{} & PA & \multicolumn{2}{l|}{ RF } & \multicolumn{2}{l|}{ RA } \\
\cline { 2 - 9 } \multicolumn{1}{c|}{} & TA [\%] & BA [\%] & TA [\%] & BA[\%] & TA [\%] & BA[\%] & TA [\%] & BA[\%] \\
\hline C11 & 0,26 & 0,007 & 0,50 & 0,020 & 0,14 & 0,006 & 0,13 & 0,003 \\
\hline C12 & 0,11 & 0,011 & 0,09 & 0,009 & 0,11 & 0,015 & 0,11 & 0,004 \\
\hline C13 & 0,33 & 0,131 & 0,25 & 0,048 & 0,43 & 0,191 & 0,41 & 0,044 \\
\hline C14 & 0,14 & 0,147 & 0,06 & 0,032 & 0,15 & 0,175 & 0,14 & 0,045 \\
\hline C15 & 0,15 & 0,703 & 0,10 & 0,890 & 0,17 & 0,614 & 0,20 & 0,904 \\
\hline
\end{tabular}




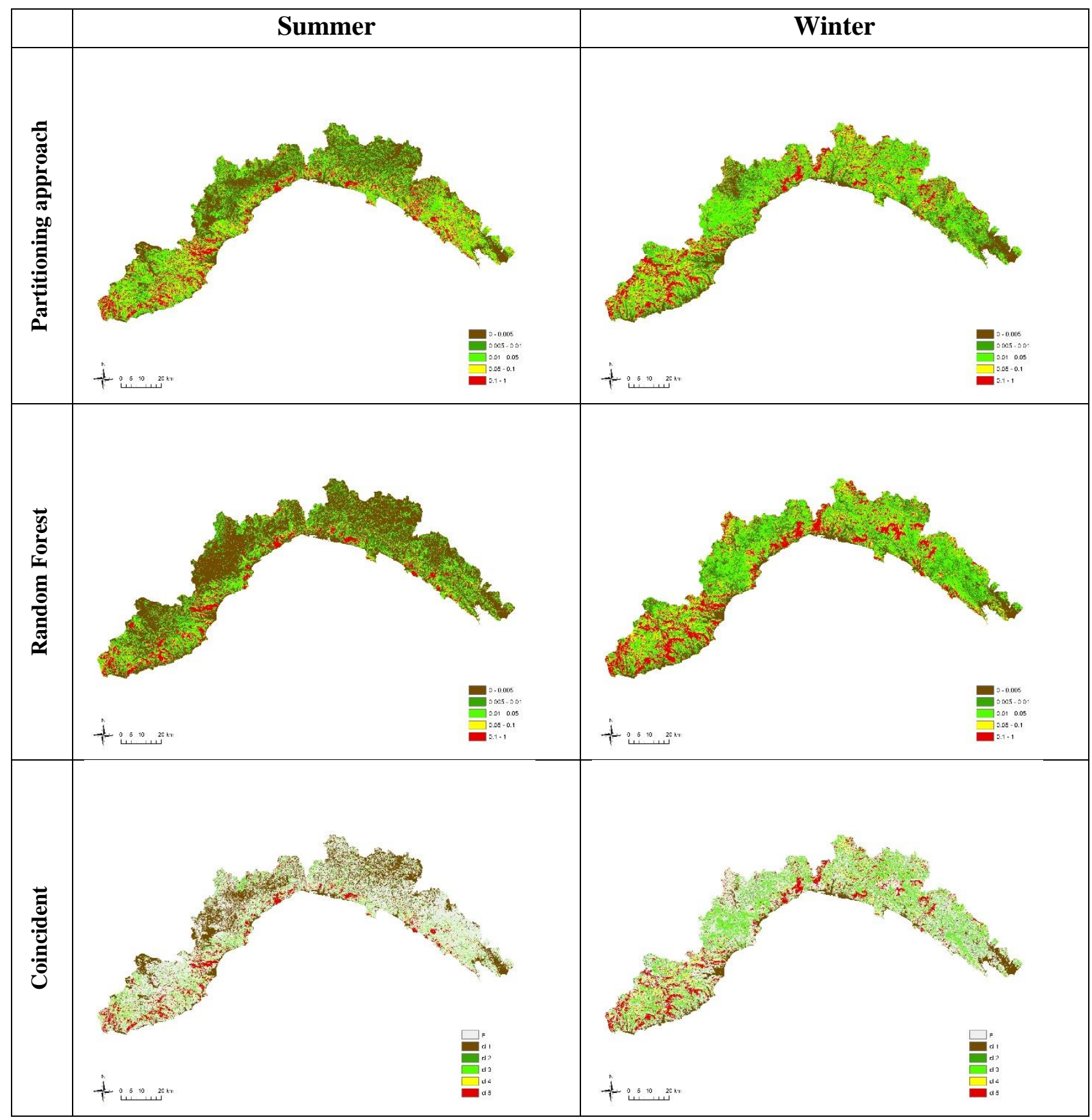

Figure 4 - Wildfires susceptibility maps for the two methods based on 5 generated classes from hevy hight (red) to very low (brown). The map of coincidences is also shown on the bottom.

Susceptibility maps obtained by applying the two methods are broadly similar and show higher class matching with observed burned area, which are closer to the coast in summer and develop along the interior in winter. The RF algorithm seems to perform better, detecting about $90 \%$ of the BA within $10 \%$ of the entire territory, in summer, and $90 \%$ within $20 \%$ in winter VS $70 \%$ of BA within the $15 \%$ of the territory detected by the statistical driven partitioning in summer, and about $60 \%$ of BA within $17 \%$ of the territory in winter. These results are preliminary and need to be further evaluated in detail by establishing a working plan accounting for a common validation dataset for the two approaches.

In summary, RF seems to be a promising alternative to deterministic or statistical expert- based method for wildfire susceptibility mapping. 


\section{References}

Breiman L, (2001). Random forests. Machine Learning, 45, 5-32

Eugenio F C, dos Santos A R, Fiedler N C, Ribeiro G A, da Silva A G, dos Santos Á B, Paneto G G, Schettino V R, (2016). Applying GIS to develop a model for forest fire risk: a case study in Espírito Santo, Brazil. Journal of Environmental Management, 173, 65-71

Fiorucci P., Gaetani F., Minciardi R., (2008). Regional partitioning for wildfire regime characterization. Journal of Geophysical Research: Earth Sciences, V. 113, Issue F2. https://doi.org/10.1029/2007JF000771.

Ganteaume A, Camia A, Jappiot M, San-Miguel-Ayanz J, Long-Fournel M, Lampin C, (2013). A review of the main driving factors of forest fire ignition over Europe. Environmental Management, 51, 651-662

Leuenberger M, Parente J, Tonini M, Pereirac M G, Kanevski M, (2018) Wildfire susceptibility mapping: Deterministic vs. stochastic approaches. Environmental Modelling \& Software, 101, 194203

Parente J, Pereira M G, (2016). Structural fire risk: the case of Portugal. Science of the Total Environment, 573, 883-893

Pourghasemi H R, (2016). GIS-based forest fire susceptibility mapping in Iran: a comparison between evidential belief function and binary logistic regression models. Scandinavian Journal of Forest Research, 31, 80-98

Pourtaghi Z S, Pourghasemi H R, Aretano R, Semeraro T, (2016). Investigation of general indicators influencing on forest fire and its susceptibility modelingusing different data mining techniques. Ecological Indicators, 64, 72-84

Rodrigues M, de la Riva J, Fotheringham S, (2014). Modeling the spatial variation of the explanatory factors of human-caused wildfires in Spain using geographically weighted logistic regression. Applied Geography, 48, 52-63 


\section{Chapter 2}

Fuel Management 


\section{Climatic conditions and fire regime affect vegetation recovery after large wildfires in Pinus forest ecosystems}

Leonor Calvo ${ }^{1 *}$, Reyes Tárrega ${ }^{1}$, Luz Valbuena ${ }^{1}$, Elena Marcos ${ }^{1}$, Angela Taboada ${ }^{1}$, Víctor Fernández-García $^{1}$, José Manuel Fernández-Guisuraga ${ }^{1}$, Alfonso Fernández Manso ${ }^{2}$, Carmen Quintano $^{3}$, Estanislao de Luis ${ }^{1}$, Otilia Reyes ${ }^{4}$, M. Jaime Baeza ${ }^{5}$, Susana Suárez-Seoane ${ }^{1}$.

${ }^{1}$ Area of Ecology. Faculty of Biological and Environmental Sciences. University of León, 24071 León. \{leonor.calvo@unileon.es*\}

${ }^{2}$ Agrarian Engineering Department, University of León, Av. Astorga s/n, Ponferrada, Spain

${ }^{3}$ Electronic Technology Department, Sustainable Forest Management Research Institute, University of Valladolid, Spanish National Institute for Agriculture and Food Research and Technology (INIA), C/Francisco Mendizábal s/n, 47014 Valladolid, Spain.

${ }^{4}$ Área de Ecología. Dpto. de Biología Funcional. Fac. de Biología. Universidad de Santiago de Compostela. Campus Vida. 15782 Santiago de Compostela. Spain.

${ }^{5}$ Department of Ecology. Faculty of Sciences. University of Alicante. Carretera de San Vicente del Raspeig s/n. E-03690 San Vicente del Raspeig. Alicante. Spain.

\section{Abstract}

Fire is one of the most important disturbance processes in Pinus forest ecosystems in the Mediterranean Basin. These ecosystems differ markedly in their response to this disturbance. They rely on climatic conditions in the area, fire regime and biological traits of the vegetation. Thus, understanding the influence of climatic conditions and fire characteristics in shaping postfire vegetation recovery will help to identify the most appropiate post-fire management strategies.

During recent decades, wildfire ocurrence in fire-prone ecosysems in the Mediterranean Basin has increased because of changes in land use, increased fuel load and continuity in the landscape. Moreover, these socio-ecological changes are related to the increase in burn severity and the surface affected. These large wildfires usually result in high heterogenous spatial mosaics with different degrees of severity and recurrence, which clearly influence vegetation regeneration capacity. However, fire regime characteristics not only affect vegetation regeneration after wildfire but also climatic conditions and their functional traits. Many authors recognise that in situations of high recurrence, resprouter species have an advantage over seeders.

To determine how vegetation regeneration in a Pinus fire-prone ecosystem may be related to climatic gradients, fire regime characteristics and plant traits, we selected three large wildfires that occurred in the Iberian Peninsula during 2012 and 2013: the Carnota wildfire in Atlantic climate conditions (Galicia), the Sierra del Teleno wildfire in transition climatic conditions (León) and the Cortes de Pallás wildfire in Mediterranean climatic conditions (Valencia). In each study area, a burn severity map of two levels (low and high) was obtained from a classification (threshold) of a Landsat-based differenced Normalized Burn Ratio image. Moreover, in each fire scar we evaluated recurrence in the last 20 years and differentiated two recurrence situations: low (1 wildfire) and high ( 2 or more wildfires). As a result, four scenarios were differentiated: low recurrence-low severity, low recurrence-high severity, high recurrence- low severity and high recurrence-high severity.

In each scenario, a proportional number of $2 \mathrm{~m} \times 2 \mathrm{~m}$ plots were established. In each plot, we sampled the visual cover percentage of all plant taxa in 4 subplots of $1 \mathrm{~m}^{2}$ three years after the wildfire. Mean cover values of Pinus saplings and resprouter and seeder shrubs were differentiated and compared among fire regime scenarios and climatic conditions. We observed diferences in the regeneration patterns in the three study sites, with more similarity between the Transition and Mediterranean ones. The common pattern of regeneration among the three climatic conditions has a significant negative effect on Pinus recruitment and cover in scenarios of high recurrence with low and high severity. At the same time, the resprouter shrubs species were favored by high recurrence.

Keywords: climatic gradients, Pinus forest, post-fire regeneration, vegetation functional traits, wildfire recurrence, wildfire severity 


\section{Introduction}

Fire is a major and frequent disturbance in forest ecosystems, especially in Mediterranean Basin countries (Hosseini et al. 2018), where it shapes plant communities and landscapes. During recent decades, the natural fire regime has been affected by several factors, mainly by changes in land use, increased fuel load and continuity, global warming, changes in forest policies and an increase in invasive plant species (Pausas and Keeley 2014). Therefore, the main changes in the Mediterranean Basin in fire regimes characteristics include an increase in the number of fires (San-Miguel-Ayanz et al. 2016; Fernandez-García et al. 2018) and also in burn severity and the surface affected (Gonzálezde Vega et al. 2018). These large wildfires usually result in high heterogenous spatial mosaics with different degrees of severity and recurrence, which clearly influence vegetation regeneration capacity. However, fire regime characteristics not only affect vegetation regeneration after wildfire but also climatic conditions and their functional traits.

Fire affects all plants in burned ecosystems, but its effects strongly depend on the functional traits of the species, which determine their responses to disturbances, and reflect the mechanisms underlying these responses (Lavorel et al. 1997). Hence, changes in the abundance of plants with different functional traits could be used to assess the effects of fire. In addition, species sharing similar traits are expected to show similar responses after fire (Pausas 1999). For instance, many authors recognise that in situations of high recurrence, resprouter species have an advantage over seeders (Diez Delgado and Pons 2001; Calvo et al. 2012). However, in scenarios of high severity fires, there is not a common pattern in the behaviour of the resprouter versus seeder species. The reason for this could be because it depends on the type of resprouting mechanism or on the ability to survive inside serotine cones or fruit and the soil seed bank.

In general, fire-prone ecosystems, such as Mediterranean Pinus forest, are characterized by good resilience of vegetation to fire throughout the two regeneration strategies: resprouters (buds in soil or protected by tissues) or obligate seeders (seeds buried in the soil bank or enclosed in aerial banks) (Calvo et al. 2003, 2008, 2012). In some cases, seed germination can be initiated or improved by heat, presence of burnt wood or ash as scarifying agents (Alvarez et al. 2005, 2007; Calvo et al. 2013, 2016). High temperatures can also facilitate the opening of serotinous cones or fruits to release seeds after a fire (Calvo et al. 2008).

Several studies have been carried out on the effects of fire on Pinus pinaster (Maritime pine) forests in the western Mediterranean Basin after one fire (Calvo et al. 2003, 2008, 2012, 2016). However, the importance of this study lies in the effects of wildfire in maritime pine forest under different scenarios of recurrence and severity of fire. This species is one of the best-adapted tree species to fire. It is an obligate seeder with a dual-life strategy, early reproduction and both serotinous and non-serotinous cones, with a high percentage of serotinity, mainly in the north-western populations of the Iberian Peninsula very frequently affected by fire (Tapias et al. 2001).

The objective of this research was to determine how vegetation regeneration in a Pinus fire-prone ecosystem may be related to climatic gradients, fire regime characteristics and plant traits. Our goal was to improve knowledge of fire recurrence and severity effects on vegetation regeneration as a support tool to be used in post-fire planning and decision making for the recovery of ecosystem services provided by these forest.

\section{Materials and Methods}

We selected three study sites along an Atlantic-Transition-Mediterranean climatic gradient within Spain (Figure 1). The Atlantic study site is located in La Coruña Province (Carnota), within the perimeter of a large forest fire that occurred in September 2013, which burned 2,523 ha mostly covered by Pinus pinaster forest. The soils are acidic. The annual average rainfall is $1655 \mathrm{~mm}$ and the average

Advances in Forest Fire Research 2018 - Page 210 
annual temperature is $13{ }^{\circ} \mathrm{C}$, and there were no drought months during the summer. The Transition site (Sierra del Teleno) is a mega-fire that occurred in August 2012 located in the Southwest of León Province (Spain). The burned surface was 11,602 ha, predominantly covered by a Pinus pinaster forest. The mean annual rainfall is/was? $640 \mathrm{~mm}$ and average annual temperature $10{ }^{\circ} \mathrm{C}$, presenting two months of summer drought. The Mediterranean site is a megafire in June 2012 in the West of Valencia province (Cortes de Pallás) that burned 29,752 ha. The surface affected by the fire was represented by reforestation stands of Pinus pinaster and Pinus halepensis. The soils are basic. Annual average rainfall is $582 \mathrm{~mm}$ and average annual temperature $16{ }^{\circ} \mathrm{C}$, with three months of summer drought (Ninyerola et al. 2005).

In each study area, a burn severity map of two levels (low and high) was obtained from a classification (threshold) of a Landsat-based differenced Normalized Burn Ratio image (FernandezGarcía et al. 2018). Moreover, in each fire scar we evaluated recurrence in the last 20 years and differentiated two recurrence situations: low (1 wildfire) and high ( 2 or more wildfires). As a result, four scenarios were differentiated: low recurrence-low severity, low recurrence-high severity, high recurrence- low severity and high recurrence-high severity (Fig. 1).

A set of 120 plots of $2 \times 2 \mathrm{~m}$ were randomly established in each study site. These plots were proportionally distributed among the four scenarios. In each plot, we sampled the visual cover percentage of all plant taxa in 4 subplots of $1 \mathrm{~m}^{2}$ three years after the wildfire. Mean cover values of Pinus saplings, resprouter and seeder shrubs, and herbaceous species were differentiated and compared among fire regime scenarios and climatic conditions.
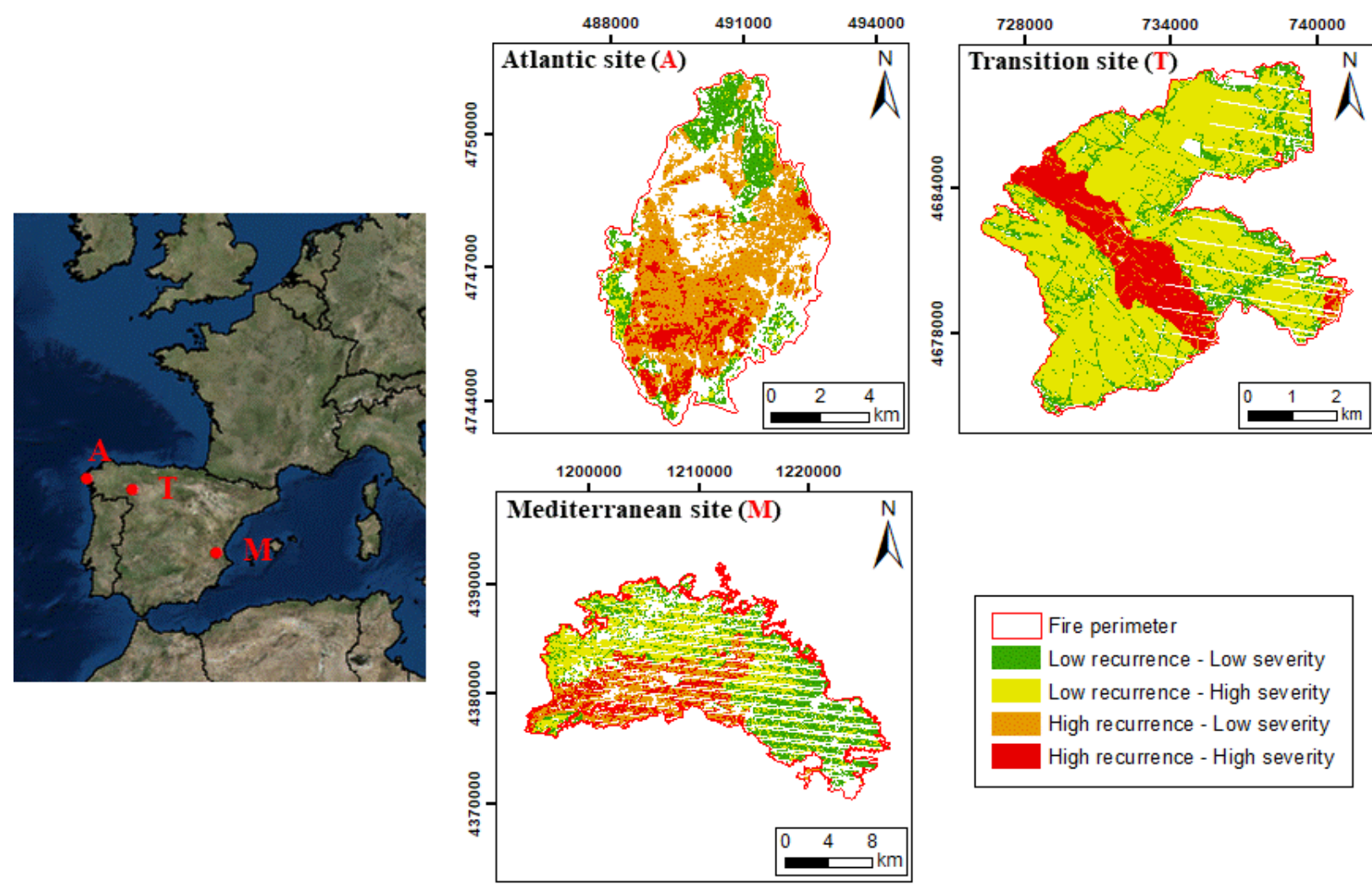

Figure 1 - Location of the three study sites in Spain across an Atlantic (A)-Transition (T)-Mediterranean (M) climatic gradient. Fire perimeter and four scenarios of recurrence (low= one fire in the last 20 years, high= more than one fire in the last 20 years) and severity (low and high) 
A Principal Component Analysis (PCA) was carried out to distinguish the effects of study sites and recurrence-severity scenarios in the vegetation response (Pine saplings cover, resprouter shrubs cover, seeder shrubs cover and herbs cover).

Principle Component Analysis (PCA), which included all the studied variables (Pine saplings cover, resprouter shrubs cover, seeder shrubs cover and herbs cover), were performed for the comparison among the three study sites (Atlantic-Transition-Mediterranean) and in each site to identify the behaviour of each recurrence-severity scenario.

All data analyses were carried out with $\mathrm{R}$ (R Core Team, 2016), using the vegan package (Oksanen et al. 2016).

\section{Results}

In the analysis of the ordination of the three study areas together in relation to the plant functional traits we observed a clear differentiation (Fig. 2) between the Atlantic site, characterized by the dominance of herbaceous cover and resprouter woody cover, mainly dominated by Ulex europaeus and Rubus sp., and the other two study sites. Both the Transition and Mediterranean sites were more similar in terms of vegetation functional traits, which were characterised by the abundance of seeder shrub species and the highest cover of pine seedlings.

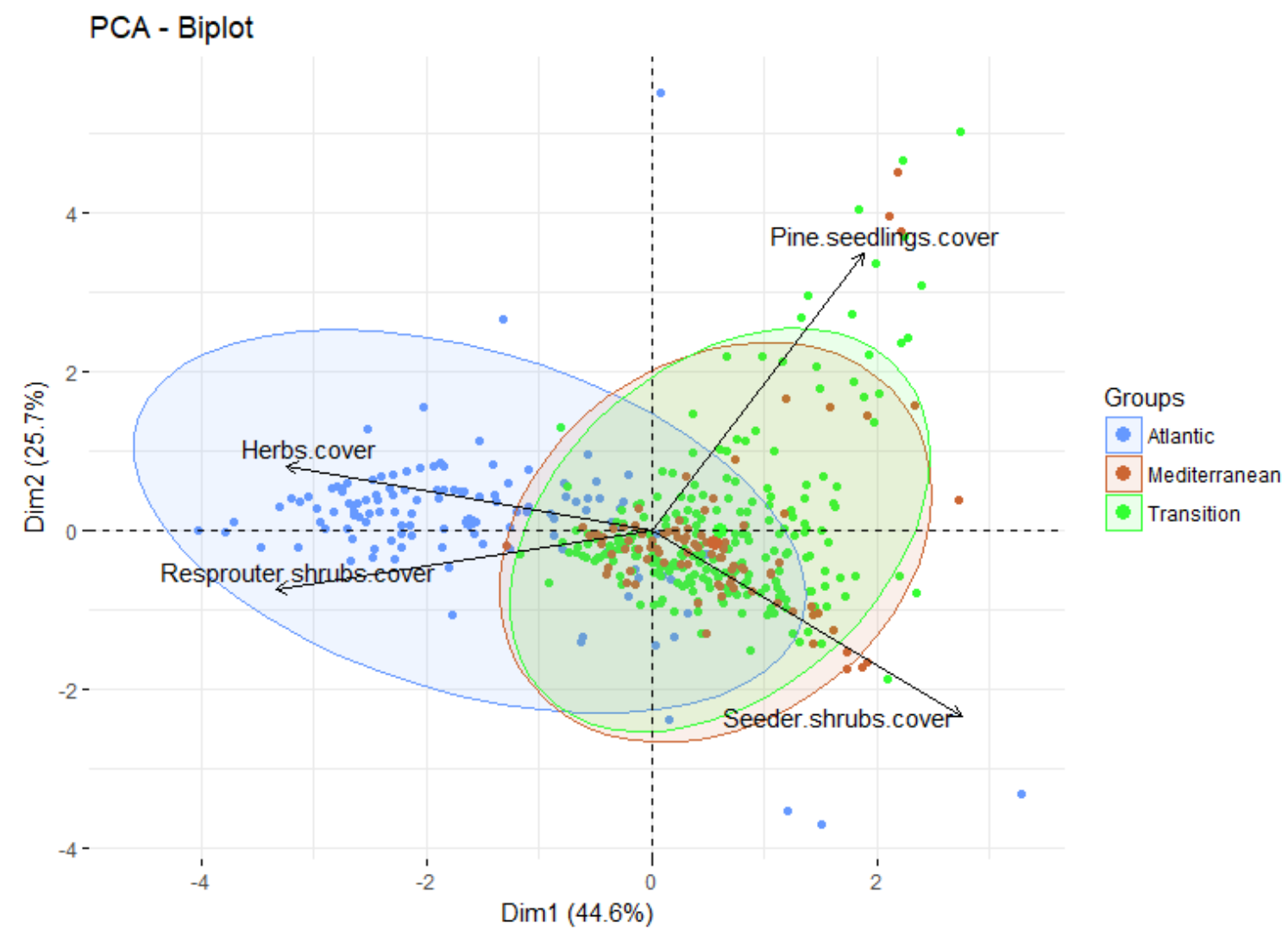

Figure 2 - PCA biplot showing relationships between recurrence-severity scenarios in the three study sites (Atlanticblue dots, Transition- green dots and Mediterranean- brown dots) and vegetation functional traits (Herbaceous cover, Resprouter shrubs cover, Seeder shrub cover and Pinus seedlings cover). 
There is no clear effect of the different fire regime attributes in the abundance of the different plant functional traits after fire in the Atlantic site (Fig. 3). We observed that in the scenario of high recurrence and high severity herbs cover predominated, under high recurrence and low severity there was higher cover of shrubs species, both resprouters (Ulex europaeus) and seeders (Halimium lasianthum, Erica cinerea and Erica ciliaris) .Pine seedlings were abundant in environments of low recurrence and low severity.

However, in the Transition site, recurrence represents the main fire regime attribute that affected vegetation regeneration. Under the scenario of high recurrence, more than two fires in 20 years, resprouter woody species, such as Erica australis and Pterospartum tridentatum showed better adaptation to regeneration. Whereas, in scenarios of low recurrence, Pinus pinaster seedlings have a better opportunity to germinate.

In the Mediterranean site there was a common pattern of vegetation traits regeneration behaviour, with clear dominance of resprouters in a high recurrence scenario, regardless of severity. Quercus coccifera is the resprouter species with highest abundance. However, in scenarios of low recurrence, there was a clear effect of fire severity. So, low severity favoured the increase in the cover of Pinus halepensis and Pinus pinaster seedlings, while high severity positively affected the dominance of shrub seeder species such us Ulex parviflorus.

\section{Discussion}

A better understanding of the ecological role of fire regime attributes in the regeneration of ecosystems is a matter of importance, given the evident increase in both the number and severity of wildfires in the Mediterranean Basin (Moreno and Chuvieco 2013). The field study revealed marked differences in the type of plant functional traits regeneration in relation to fire regime attributes and climatic conditions. In general, areas with wetter climatic conditions, such as the Atlantic site, show faster regeneration of the resprouter and seeder shrubs and herbaceous species than much drier climate areas, such as the Transition and Mediterranean study sites. This is in accordance with findings obtained by other authors (Arnan et al. 2007; Acácio et al. 2009).

Vegetation from fire-prone ecosystems is fire-adapted, and is therefore highly resilient, meaning that almost all perennial species can recover after a fire (Calvo et al. 2008), though, they could be constrained by the fire regime attributes. Post-fire vegetation cover depends not only on the number of fires, but also on the time interval between them and severity. Some authors have indicated that the time since the last fire has a stronger effect than the number of fires, provided the former is sufficiently long to allow some vegetation to recover (Espelta et al. 2008; Fernandes and Rigolot 2007).

The results of the current study indicated that the fire recurrence has a greater impact on the regeneration of the different plant functional traits than fire severity. Many authors have observed that high recurrence and short intervals between successive fire events may lead to substantial changes in vegetation (Tessler et al. 2014, 2016). In general, the impact of recurrent fires on vegetation composition produce an increase in the dominance of resprouter shrubs (Delitti et al. 2005; Schaffhauser et al. 2011, 2012). So, resprouters can regenerate efficiently up to a certain fire recurrence threshold. In the case of the Transition site, Erica australis is one of the most favoured resprouters by fire recurrence. The buds of this species, Erica australis, are found in the lignotuber (Calvo et al. 2002). The presence of a lignotuber gives a great advantage in their response to recurrent fires (Moreno et al. 1999). The presence of this type of storage mechanism is probably associated with recurring perturbations that eliminate all the aboveground biomass. Another shrub species with great ability to resprout in the Transition site is Pterospartum tridentatum, mainly from the bank of shoots situated in the root (Tárrega et al. 1992). This species undergoes a process of progressive ageing of the aerial biomass, only the outer edge of each branch remaining green. Therefore, recurring perturbations favour rejuvenation (Calvo et al. 2002). However, a decrease in pine tree cover was documented after high 
recurrence in the three study sites. A fire regime of low recurrence allows the pines to reach reproductive age (Reyes and Casal 2002).

Lloret et al. (2003) pointed out that high fire recurrence produced an increase in grasses, and a decrease in abundance of Quercus, and disappearance of Pinus. In the same way, Tessler et al. (2014) showed that a higher number of fires led to a decrease in $P$. halepensis density in Israel. A similar tendency was found by Herman (2009) and Santana et al. (2010) in studies carried out in Spain.

In the current study, the effects of burn severity were only observed in the low recurrence scenario, where low severity favoured the increase in Pinus halepensis cover and Pinus pinaster seedlings, while high severity increased the cover of seeder species, such us Ulex parviflorus, in the Mediterranean site. The last species are characterised by hard coat seeds, which allow them to resist the high temperature reached under high severity fires (Santana et al. 2012). Similarly, the position of these seeds in the soil seed bank is another factor that controls their ability to germinate after fire. Baeza et al. (2002) in prescribed fires carried out in the south of Spain, observed that Ulex parviflorus showed the highest percentage of germination when situated $3 \mathrm{~cm}$ deep, which seems to evidence that very intense temperature pulses in the first centimeters of soil profile could produce seed death, while at deeper situation $(3 \mathrm{~cm})$ stimulate seed germination. This seed dormancy-breaking behavior would guarantee their capacity to regenerate in areas affected by high severity fire (Baeza et al. 2002).
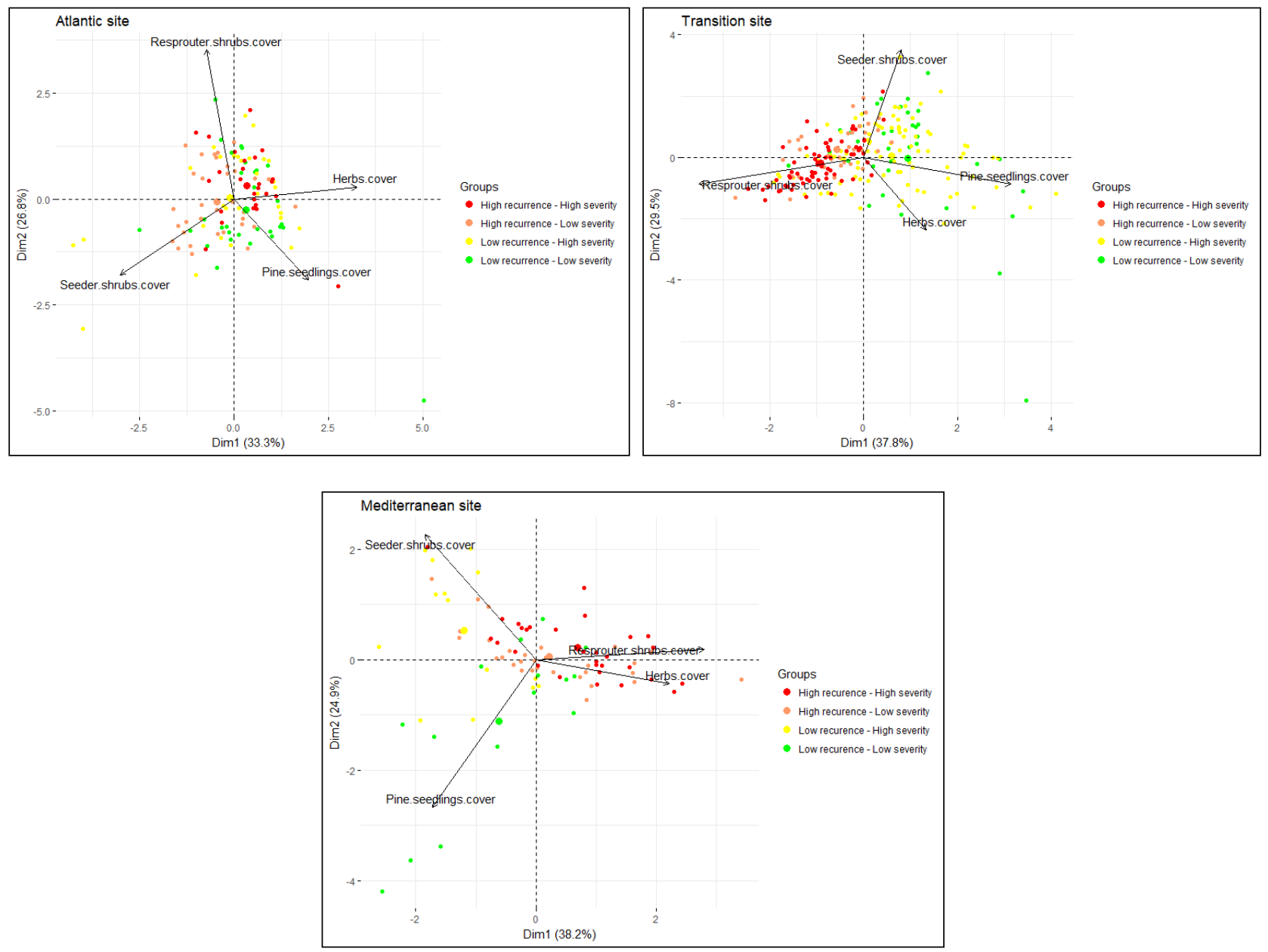

Figure 3 - PCA biplot showing relationships between recurrence-severity scenarios ( high recurrence and high severity (red dots); high recurrence and low severity (orange dots), low recurrence and high severity (yellow dots) and low recurrence and low severity (green dots)) and vegetation functional traits (Herbaceous cover, Resprouter shrubs cover, Seeder shrub cover and Pinus seedlings cover) in each of the three study sites (Atlantic, Transition and Mediterranean). 
Adaptive strategies developed by plants that have evolved in Mediterranean climates provide resilience to regenerate after fire during a short-term period. However, it is important to acquire knowledge about the ecological effects of fire severity and recurrence to apply proper adaptive forest management in changing scenarios in which a fire regime is not natural. According to the results obtained in this study, we conclude that the regeneration of the different plant functional traits after wildfire depends on the climate in the study area, with greater vegetation regeneration speed under wetter climatic conditions.

Among the fire regime attributes, apparently fire recurrence showed more impact on the vegetation regeneration with a clear dominance of resprouter species and a decrease in the cover of pine seedlings. The main effect of severity was observed in the Mediterranean study site and with low recurrence, where seeder species and pine seedlings were favored.

\section{Acknowledgements}

The study was financially supported by the Spanish Ministry of Economy and Competitiveness, and the European Regional Development Fund (ERDF), within the framework of the GESFIRE projects (AGL2013-48189-C2-1-R and AGL2013-48189-C2-2-R); and by the Regional Government of Castile and León within the framework of the SEFIRECYL project (LE001P17).

\section{References}

Acácio V, Holmgren M, Rego F, Moreira F, Mohren GMJ (2009) Are drought and wildfires turning Mediterranean cork oak forests into persistent shrublands? Agroforestry Systems 76, 389-400.

Alvarez R, Valbuena L, Calvo L. (2005) The influence of tree age on germination response to environmental factors and inhibitory substances in Pinus pinaster. International Journal of Wildland Fire 14, 277-284.

Alvarez R, Valbuena L, Calvo L. (2007) Effect of high temperature on seed germination, survival, and growth of three species of pine: Pinus pinaster, Pinus sylvestris and Pinus nigra. International Journal of Wildland Fire 16, 63-70.

Arnan X, Rodrigo A, Retana J, Collins B (2007) Post-fire regeneration of Mediterranean plant communities at a regional scale is dependent on vegetation type and dryness. Journal of Vegetation Science 18, 111-122.

Baeza MJ, Raventós J, Escarré A. (2002) Ulex parviflorus germination after experimental burning: effects of temperature and soil depth. In 'Fire and Biological Processes'. (Eds L Trabaud, R. Prodon) pp. 83-91. (Backhuys Publishers: Leiden)

Calvo L, Tárrega R, Luis E (2002) The dynamics of Mediterranean shrubs species over 12 years following perturbations. Plant Ecology 160, 25-42.

Calvo L, Santalla S, Marcos E, Valbuena L, Tárrega R, Luis E (2003) Regeneration after wildfire in communities dominated by Pinus pinaster, an obligate seeder, and others dominated by Quercus pyrenaica, a typical resprouter. Forest Ecology and Management 184, 209-223

Calvo L, Santalla S, Valbuena L, Marcos E, Tárrega R, Luis-Calabuig E (2008) Post-fire natural regeneration of a Pinus pinaster forest in NW Spain. Plant Ecology 197, 81-90.

Calvo L, Baeza J, Marcos E, Santana V, Papanastasis VP (2012) Post-Fire Management of Shrublands. In 'Post-Fire Management and Restoration of Southern European Forests, Managing Forest Ecosystems 24'. (Eds F Moreira, M Arianoutsou, P Corona, J De las Heras) pp. 293-319. (Springer Science+Business Media: Brussels)

Calvo L, Torres O, Valbuena L, Luis-Calabuig E (2013) Recruitment and early growth of Pinus pinaster seedlings over five years after a wildfire in NW Spain. Forest Systems 22, 582-586. 
Calvo L, Hernández V, Valbuena L, Taboada A (2016) Provenance and seed mass determine seed tolerance to high temperatures associated to forest fires in Pinus pinaster. Annals of Forest Science 73, 381-391.

Díaz-Delgado R, Lloret P, Pons X, Terradas J (2002) Satellite evidence of decreasing resilience in Mediterranean plant communities after recurrent wildfires. Ecology 83, 2293-2303.

Espelta JM, Verkaik I, Eugenio M, Lloret F (2008) Recurrent wildfires constrain long-term reproduction ability in Pinus halepensis Mill. Int. Journal of Wildland Fire, 17, 579-585.

Fernández-García V, Santamarta M, Fernández-Manso A, Quintano C, Marcos E, Calvo L (2018) Burn severity metrics in fire-prone pine ecosystems along a climatic gradient using Landsat imagery. Remote Sensing of Environment 206, 2015-217

Fernandes PM, Rigolot E (2007) The fire ecology and management of maritime pine (Pinus pinaster Ait.). Forest Ecology and Management 241, 1-13.

González-De Vega S, de las Heras J, Moya D (2018) Post-Fire Regeneration and Diversity Response to Burn Severity in Pinus halepensis Mill. Forests 9(6), 299.

Lavorel S, McIntyre S, Landsberg J, Forbes TDA (1997) Plant functional classifications: from general groups to specific groups based on response to disturbance. Trends in Ecology and Evolution 23, 2.

Lloret F, Pausas J, Vila M (2003) Responses of Mediterranean plant species to different fire frequency in Garraf Natural Park (Catalonia, Spain): field observations and modeling predictions. Plant Ecology 167, 223-235.

Moreno JM, Cruz A, Oechel WC (1999) Allometric relationships in two lignotuberous species from Mediterranean-type climate areas of Spain and California. Journal of Mediterranean Ecology 1, 49-60.

Moreno MV, Chuvieco E (2013) Characterising fire regimes in Spain from fire statistics. International Journal of Wildland Fire 22, 296-305.

Ninyerola M, Pon, X, Roure JM, (2005) ‘Atlas Climático Digital de la Península Ibérica. Metodología y aplicaciones en bioclimatología y geobotánica' (Universidad Autónoma de Barcelona: Bellaterra)

Pausas JG (1999) Mediterranean vegetation dynamics: modelling problems and functional types. Plant Ecology 140, 27-39.

Pausas JG, Keeley JE (2009) A burning story: the role of fire in the history of life. BioScience 59, 593601.

Pausas JG, Keeley JE (2014) Abrupt climate-independent fire regime changes. Ecosystems 17, 11091120.

R Core Team (2016) 'R: a Language and Environment for Statistical Computing'. R Foundation for Statistical Computing, Vienna, Austria. URL: http://www.R-project.org/

Reyes O, Casal, M (2002) Effect of high temperatures on cone opening and on the release and viability of Pinus pinaster and P. radiata seeds in NW Spain. Annals of Forest Science 59, 327-334.

Schaffhauser A, Curt T, Véla E, Tatoni T (2012) Recurrent fires and environment shape the vegetation in Quercus suber L. woodlands and maquis. Comptes Rendus Biologies 335, 424-434.

San-Miguel-Ayanz J, Durrant T, Boca R, Libertà G, Bocacci F, Di Leo M, López-Pérez J, Schulte E (2016) Forest Fires in Europe, Middle East and North Africa 2015. Joint Research Center of the European Commission Technical Reports, EUR 28158EN.

Santana VM, Baeza MJ, Marrs RH, Vallejo VR (2010) Old-field secondary suc-cession in SE Spain: can fire divert it? Plant Ecology 211, 337-349.

Santana VM, Baeza MJ, Maestre FT (2012) Seedling establishment along post-fire succession in Mediterranean shrublands dominated by obligate seeders. Acta Oecologica 39, 51-60. 
Taboada A, Fernández-García V, Marcos E, Calvo L (2018) Interactions between large high-severity fires and salvage logging on a short return interval reduce the regrowth of fire-prone serotinous forests. Forest Ecology and Management 414, 54-63.

Tapias R, Gil L, Fuentes-Utrilla P, Pardos J A (2001) Canopy seed banks in Mediterranean pines of south-eastern Spain: a comparison between Pinus halepensis Mill.; P. pinaster Ait.; P. nigra Arn. and $P$. pinea L. Journal of Ecology 89(4), 629-638.

Tárrega R, Calvo L, Trabaud L (1992) Effect of high temperatures on seed germination of two woody Leguminosae. Vegetatio 102, 139-147.

Tessler N, Wittenberg L, Provizor E, Greenbaum N (2014) The influence of short interval recurrent forest fires on the abundance of Aleppo pine (Pinus halepensis Mill.) on Mount Carmel, Israel. Forest ecology and Management 324, 109-116.

Tessler N, Wittenberg L, Greenbaum N (2016) Vegetation cover and species richness after recurrent forest fires in the Eastern Mediterranean ecosystem of Mount Carmel, Israel on Mount Carmel, Israel. Science of the Total environment 572, 1395-1402. 


\title{
Estimation of live fuel moisture content of shrubland using MODIS and Sentinel-2 images
}

\author{
Eva Marino $^{1 *}$; Mariluz Guillén-Climent ${ }^{1}$; Nur Algeet ${ }^{1}$; José Luis Tomé ${ }^{1}$; Carmen Hernando ${ }^{2}$ \\ ${ }^{1}$ AGRESTA Sociedad Cooperativa. C/Duque de Fernán Núñez 2, 28012 Madrid. \\ \{emarino@agresta.org*\} \\ ${ }^{2}$ INIA, Forest Research Centre, Department of Silviculture and Forest Management, Forest fire \\ laboratory. Crta. A Coruña Km 7.5, 28040 Madrid, Spain
}

\begin{abstract}
Live fuel moisture content (LFMC) is a critical parameter affecting vegetation flammability and fire behaviour. Reliable and updated estimations of LFMC are needed by fire managers for operational wildfire risk assessment. However, detailed and constant monitoring of LFMC in the field is costly and timeconsuming. Remote sensing technologies are an important source of geospatial data that can provide spectral information related to LFMC at different temporal and spatial resolution. In this study, we used a database of LFMC monitoring sampled during 2016 and 2017 (n=81) in a monospecific Cistus ladanifer L. shrubland in Madrid region (Central Spain). C. ladanifer is a representative shrub species commonly found in Mediterranean fire-prone areas, and has been already identified as an indicator species for wildfire risk assessment by different regional fire services. A set of spectral indices (SI) derived from MODIS images (MOD09GA) were calculated at $500 \mathrm{~m}$ resolution and compared with field data. We also used Sentinel-2 images for SI retrieval at $20 \mathrm{~m}$ resolution with the aim of addressing the scale problem between field sampling site and the low spatial resolution of MODIS data. The same SI were calculated adapting formulations to Sentinel-2 spectral resolution. The timelag between images and field sampling date was limited to a maximum of 2 days for operational purposes. Multiple linear regression analysis was used to assess the potential of SI for LFMC estimation, comparing results for both type of images. Most of the SI tested showed a significant correlation with LFMC data derived from MODIS $(n=62)$ and Sentinel-2 $(n=35)$. For MODIS, the best indices were EVI, VARI, and VIGREEN $\left(\mathrm{R}^{2}=0.82\right.$, MAE $\left.=12 \%\right)$, followed by NDVI and SAVI $\left(\mathrm{R}^{2}=0.76\right.$, $\mathrm{MAE}=14 \%)$. For Sentinel-2, the best indices were VARI $\left(\mathrm{R}^{2}=0.72, \mathrm{MAE}=13 \%\right)$, EVI $\left(\mathrm{R}^{2}=0.71, \mathrm{MAE}=13 \%\right)$, VIGREEN $\left(\mathrm{R}^{2}=0.67, \mathrm{MAE}=14 \%\right)$ and $\mathrm{NDVI}\left(\mathrm{R}^{2}=0.62, \mathrm{MAE}=14 \%\right)$. In both cases, a significant multivariate model was found including NDVI and VARI, with a slight increase in prediction accuracy compared to simple regression models $\left(\mathrm{R}^{2}=0.85\right.$ with $\mathrm{MAE}=11 \%$ for MODIS, and $\mathrm{R}^{2}=0.76$, MAE $=12 \%$ for Sentinel-2). Our findings indicate that MODIS and Sentinel-2 images provide similar results for the SI tested, and that both satellites can be used for near real-time estimation of LFMC in C. ladanifer shrubland. The proposed models can be used to improve monitoring of the variability of LFMC during the year, as well as helping the integration of remote sensing data on wildfire danger rating systems.
\end{abstract}

Keywords: Live fuel moisture content; MODIS; Sentinel-2; remote sensing; Cistus ladanifer

\section{Introduction}

Live fuel moisture content (LFMC) is a critical parameter affecting vegetation flammability and fire behaviour (Denisson and Moritz 2009, Marino et al. 2012). Moisture of both live and dead components of vegetation are often required as input to predict fire behaviour in wildfire simulation models. Reliable and updated estimations of LFMC are also needed by fire managers for wildfire danger rating (Chuvieco et al. 2014).

Extreme weather is becoming more frequent due to climate change thus extending the fire season in most Mediterranean areas (Ruffault et al. 2018), where fire regimes have shifted from fuel-limited 
to drougth-driven in the last decades (Pausas and Fernández-Múñoz, 2012). Dead fuel moisture, especially fine fuels, is generally more easily determined from weather variables as it relies on fuel size, local atmospheric conditions and precipitation (Viney et al. 1991, Nolan et al. 2016). Conversely, LFMC is more difficult to estimate as it strongly depends on physiological and phenological characteristics of each species that may be mostly driven by medium-term meteorological conditions (Yebra et al. 2013). Fire management services are spending important amount of resources on sampling vegetation for LFMC estimation to be used in pre-fire alert system and fire-fighting operations. However, detailed and constant monitoring of LFMC in the field is costly and timeconsuming. Therefore, fire management services often focus on indicator species that are relevant for wildfire prevention systems.

Previous studies demonstrate the ability of remote sensing data for LFMC estimation (Chuvieco et al. 2004, Garcia et al. 2008, Yebra et al. 2008). Different espectral, spatial and temporal resolutions are available depending on the type of sensor used. MODIS is one of most commonly used optical sensors due to its high temporal resolution. Empirical models were proposed by many authors to estimate LFMC from a combination of different espectral indices derived from MODIS images (Dennison et al. 2005, Stow et al. 2006, Peterson et al. 2008, Caccamo et al. 2012). Other authors used radiative transfer models (RTM), a more complex method based on physical approaches from simulations that can provide more robust LFMC estimations independent of site specificities (Hao and Qu 2007, Yebra et al. 2008, 2013, Yebra and Chuvieco 2009, Jurdao et al. 2013). Both methods applied on MODIS images provide spectral information at a coarse spatial resolution, which may limit its use in small scale sampling areas or heterogenenous vegetation. More recently, some authors assessed the potential of microwave remote sensing as an alternative for LFMC estimation, obtaining moderate results compared to optical indices derived from MODIS (Tanase et al. 2015, Fan et al. 2018). Hence, further research is still needed to provide fire managers with operational models and tools for accurate LFMC prediction that could be included in operational fire danger rating systems (Yebra et al. 2013). The new generation of Sentinel-2 sensors provide similar spectral information to MODIS images but at higher spatial resolution, which offers an opportunity to improve LFMC estimations for operational purposes.

The objetive of this work was to compare the ability of two different type of satellites, MODIS and Sentinel-2, for LFMC estimation. Research is focused on monospecific Cistus ladanifer shrubland. $C$. ladanifer is a representative shrub species commonly found in Mediterranean fire-prone areas, and has been already identified as an indicator species for wildfire risk assessment by different regional fire services.

\section{Methods}

\subsection{Field samples and laboratory protocol}

A database of LFMC monitored in C. ladanifer shrubland was used as reference data. The study area is a 45 ha of monospecific shrubland located in Madrid region (Central Spain) where samples of C. ladanifer were systematically collected during 2016 and $2017(\mathrm{n}=81)$. Samples of live fine fuels, including leaves and terminal twigs, were collected according to a field protocol defined by INIA forest fire laboratory. Sampling frequency varied along the year, starting in spring 2016, with an increased frequency of up to three days during the summer, weekly during spring and autum, and biweekly in winter.

Field samples were immediately conducted to INIA forest fire laboratory in sealed plastic pots to prevent moisture losses after cutting. Fresh samples were weighted daily and then oven-dried (24h, $100^{\circ}$ ) for moisture content estimation. LFMC was calculated as the percentage of water content of vegetation on a dry-weight basis following the equation: 


$$
L F M C=\left(\frac{W_{f}-W_{d}}{W_{d}}\right) \times 100
$$

where $\mathrm{W}_{\mathrm{f}}$ is the fresh weight and $\mathrm{W}_{\mathrm{d}}$ the dry weight of each sample.

\subsection{Remote sensing data}

\subsubsection{Image selection and preprocessing}

Images from two different type of sensors were used: MODIS and Sentinel-2. Terra MODIS images corresponded to MOD09GA, a daily product available at $500 \mathrm{~m}$ and $1000 \mathrm{~m}$ resolution. Sentinel-2 images corresponded to both satellites $2 \mathrm{~A}$ and $2 \mathrm{~B}$, providing information from $10 \mathrm{~m}$ to $60 \mathrm{~m}$ depending on the spectral band.

Time series from april 2016 to october 2017 were used in this study for both types of images. Since MODIS and Sentinel-2 products used are 1-day images, i.e. not composite products, the timelag between images and sampling date was limited to a maximum of 2 days in order to compare available images with field data. A total of 62 cloud-free MODIS images were selected for 2016-2017. As Sentinel-2 is a more recent satellite, only 8 images were available for 2016 compared to 27 for 2017, resulting in a total of 35 for the study period.

The MOD09GA product obtained by the Terra MODIS satellite corresponded to atmospherically corrected surface reflectance. The images were downloaded from the NASA Land Processess Distributed Active Archive Center (LP DAAC, https://lpdaac.usgs.gov/).

Level 1C Sentinel-2 imagery was downloaded from the European Space Agency (ESA) and preprocessed with Sen2Cor software (Louis et al. 2016) developed by ESA. This software performs the tasks of Atmospheric Correction and Scene Classification of Level 1C input data. Level 2A outputs are Bottom-Of-Atmosphere (BOA) corrected reflectance images.

\subsubsection{Spectral indices}

A set of spectral indices (SI) derived from MODIS images were calculated at $500 \mathrm{~m}$ resolution (Table 1), including Normalized Difference Vegetation Index (NDVI), Normalized Difference Infrared Index with band 6 and 7 (NDII6, NDII7), Global Vegetation Moisture Index (GVMI), Normalized Difference Water Index (NDWI), Enhanced Vegetation Index (EVI), Soil Adjusted Vegetation Index (SAVI), Visible Atmospherically Resistant Index (VARI), Vegetation Index Green, or Normalized Green Red Difference (VIGREEN).

The Sentinel-2 satellites include a high-resolution optical sensor that operates in 13 bands between the visible and the SWIR. Spatial resolution is $10 \mathrm{~m}$ for visible and NIR bands, $20 \mathrm{~m}$ for red-edge and SWIR bands, and $60 \mathrm{~m}$ for atmospheric bands. The same MODIS indices (except GVMI and NDWI) were calculated for Sentinel-2 images at a $20 \mathrm{~m}$ pixel resolution, adapting formulation to the spectral resolution of Sentinel-2 when needed.

For MODIS images, SI values corresponded to the weighted mean of pixel values according to each pixel surface inside the sampling area. For Sentinel-2, SI values were the average values of the pixels completely included inside the sampling area. 
Table 1 - Spectral indices used to estimate LFMC from MODIS data. $\rho_{x}$ is reflectance in MODIS band $x$.

\begin{tabular}{|c|c|c|}
\hline Index & Formulation & Reference \\
\hline $\begin{array}{l}\text { Normalized Difference Vegetation } \\
\text { Index }\end{array}$ & $N D V I=\frac{\rho_{2}-\rho_{1}}{\rho_{2}+\rho_{1}}$ & Rouse et al. (1974) \\
\hline $\begin{array}{l}\text { Normalized Difference Infrared Index } \\
\text { (band 6) }\end{array}$ & $N D I I 6=\frac{\rho_{2}-\rho_{6}}{\rho_{2}+\rho_{6}}$ & Hardisky et al. (1983) \\
\hline $\begin{array}{l}\text { Normalized Difference Infrared Index } \\
\text { (band 7) }\end{array}$ & $N D I I 7=\frac{\rho_{2}-\rho_{7}}{\rho_{2}+\rho_{7}}$ & Hardisky et al. (1983) \\
\hline Global Vegetation Moisture Index & $G V M I=\frac{\left(\rho_{2}+0.1\right)-\left(\rho_{6}+0.02\right)}{\left(\rho_{2}+0.1\right)+\left(\rho_{6}+0.02\right)}$ & Ceccato et al. (2002) \\
\hline Normalized Difference Water Index & $N D W I=\frac{\rho_{2}-\rho_{5}}{\rho_{2}+\rho_{5}}$ & Gao (1996) \\
\hline Enhanced Vegetation Index & $E V I=\frac{2.5 \times\left(\rho_{2}-\rho_{1}\right)}{\left(\rho_{2}+6 \times \rho_{1}-7.5 \times \rho_{3}+1\right)}$ & Huete et al. (2002) \\
\hline Soil Adjusted Vegetation Index & $S A V I=(1+0.5) \frac{\left(\rho_{2}-\rho_{1}\right)}{\left(\rho_{2}+\rho_{1}+0.5\right)}$ & Huete (1988) \\
\hline $\begin{array}{l}\text { Visible Atmospherically Resistant } \\
\text { Index }\end{array}$ & $V A R I=\frac{\rho_{4}-\rho_{1}}{\rho_{4}+\rho_{1}-\rho_{3}}$ & Gitelson et al. (2002) \\
\hline Vegetation Index - Green & VIGREEN $=\frac{\rho_{4}-\rho_{1}}{\rho_{4}+\rho_{1}}$ & Tucker (1979) \\
\hline
\end{tabular}

\subsection{Statistical analysis}

The temporal variability of LFMC monitored during the field sampling was assessed and compared with the temporal profiles of the SI derived from satellites images. Linear regression was used to assess the potential of each SI for LFMC estimation, analysing the perfomance of spectral information derived from MODIS and Sentinel-2 independently.

In addition, multiple regression models combining different SI were assessed, comparing the results obtained from both sensors. Colinearity was checked, rejecting models with variance inflation factor (VIF) $>5$ for any variable included in the model. Evaluation metrics included coefficiente of determination $\left(\mathrm{R}^{2}\right)$, mean absolute error (MAE), and root mean square error (RMSE). The adjusted $\mathrm{R}^{2}$ value was used in order to compared goodness-of-fit between models with different number of input variables.

\section{Results}

\subsection{MODIS images}

Most of the SI derived from MODIS ( $\mathrm{n}=62$ ) showed a significant correlation with LFMC data (Table 2). The best indices were EVI, VARI, and VIGREEN $\left(\mathrm{R}^{2}=0.83, \mathrm{MAE}=12 \%, \mathrm{RMSE}=15 \%\right)$, followed by NDVI and SAVI $\left(\mathrm{R}^{2}=0.76\right.$, MAE $=14 \%$, RMSE $\left.=18 \%\right)$. NDWI showed a moderate correlation with field data $\left(\mathrm{R}^{2}=0.61, \mathrm{MAE}=17 \%\right.$, RMSE $\left.=22 \%\right)$, whereas NDII6, NDII7 and GVMI had the lower correlation and higher errors $\left(\mathrm{R}^{2}<0.56\right.$, MAE $>20 \%$, RMSE $\left.>24 \%\right)$.

A significant multivariate model was found including NDVI and VARI, with a slight increase in prediction accuracy compared to simple regression models $\left(\mathrm{R}^{2}=0.85\right.$ with $\mathrm{MAE}=11 \%$ and RMSE $=14 \%$ ). 
Table 2 - Results of the most significant linear regression models to estimate LFMC from MODIS and Sentinel-2 data. MAE, mean absolute error; RMSE, root mean square error.

\begin{tabular}{llcccc}
\hline Sensor & Model & $\mathbf{R}^{\mathbf{2}}$ (adjusted) & p-value & MAE (\%) & RMSE (\%) \\
\hline \multirow{6}{*}{ MODIS } & 0.756 & $<0.0001$ & 14.190 & 17.816 \\
& NDVI & 0.553 & $<0.0001$ & 20.356 & 24.142 \\
& NDII6 & 0.469 & $<0.0001$ & 22.286 & 26.314 \\
& GDII7 & 0.553 & $<0.0001$ & 20.356 & 24.142 \\
& NDWI & 0.611 & $<0.0001$ & 17.218 & 22.509 \\
& EVI & 0.823 & $<0.0001$ & 12.611 & 15.202 \\
& SAVI & 0.756 & $<0.0001$ & 14.190 & 17.816 \\
& VARI & 0.832 & $<0.0001$ & 12.220 & 14.799 \\
& VIGREEN & 0.823 & $<0.0001$ & 12.477 & 15.179 \\
& NDVI, VARI & 0.847 & $<0.0001$ & 11.283 & 13.987 \\
\hline \multirow{5}{*}{ Sentinel-2 } & NDVI & 0.619 & $<0.0001$ & 14.457 & 18.119 \\
& NDII6 & 0.565 & $<0.0001$ & 15.302 & 19.362 \\
& EVI & 0.712 & $<0.0001$ & 12.539 & 15.758 \\
& SAVI & 0.588 & $<0.0001$ & 14.897 & 18.840 \\
& VARI & 0.717 & $<0.0001$ & 13.433 & 15.616 \\
& VIGREEN & 0.670 & $<0.0001$ & 14.144 & 16.864 \\
& NDVI, VARI & 0.764 & $<0.0001$ & 12.045 & 14.054 \\
\hline
\end{tabular}

\subsection{Sentinel-2 images}

For Sentinel-2 $(\mathrm{n}=35)$, the best indices were VARI $\left(\mathrm{R}^{2}=0.72\right.$, MAE $=13 \%$, RMSE $\left.=16 \%\right)$, EVI $\left(\mathrm{R}^{2}=0.71, \mathrm{MAE}=13 \%, \mathrm{RMSE}=16 \%\right)$, VIGREEN $\left(\mathrm{R}^{2}=0.67, \mathrm{MAE}=14 \%, \mathrm{RMSE}=17 \%\right)$ and NDVI $\left(\mathrm{R}^{2}=0.62, \mathrm{MAE}=14 \%\right.$, $\left.\mathrm{RMSE}=18 \%\right)$. The lower correlations were found for NDII6 and SAVI $\left(\mathrm{R}^{2}<0.59\right.$, MAE $=15 \%$, RMSE $\left.=19 \%\right)$.

Similarly to MODIS data, the combination of NDVI and VARI resulted in a significant multivariate model that increased the performance of simple regression models $\left(\mathrm{R}^{2}=0.76, \mathrm{MAE}=12 \%\right.$, RMSE $=14 \%$ ). Figure 1 depicts NDVI and VARI values derived from both satellites compared to LFMC during the study period.

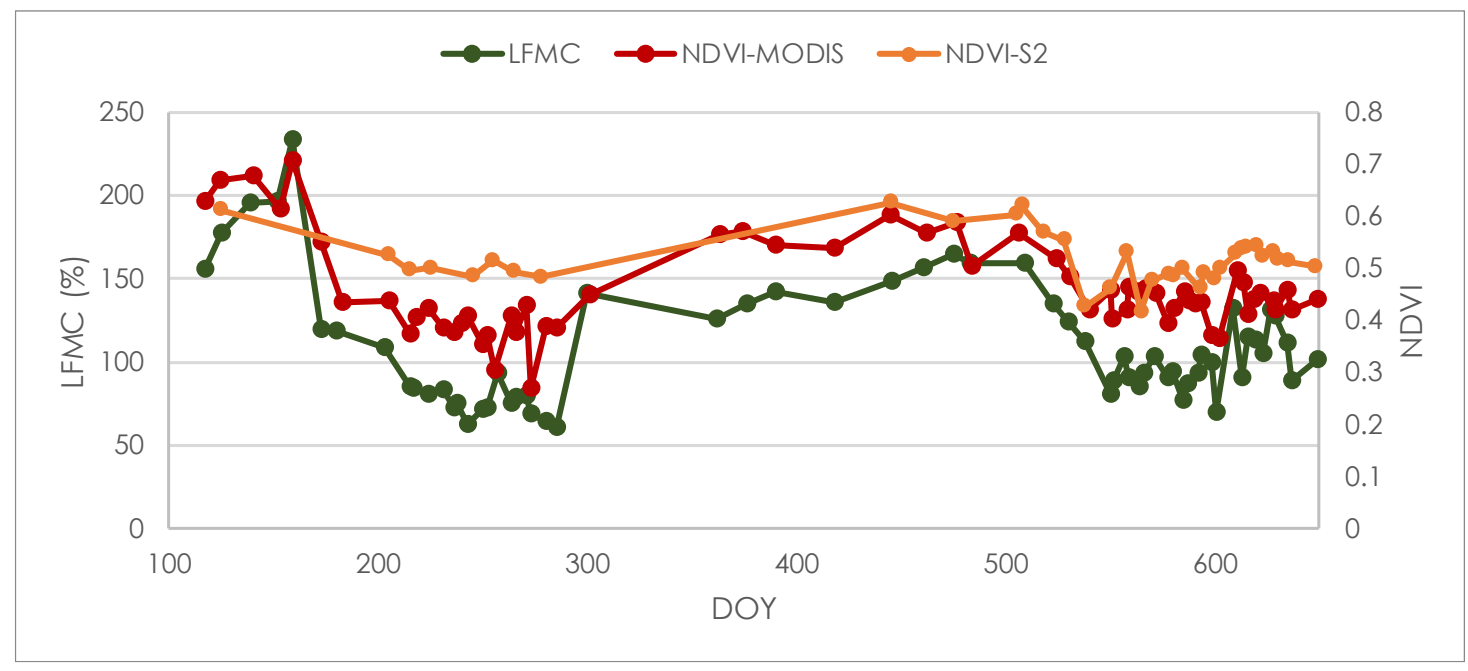




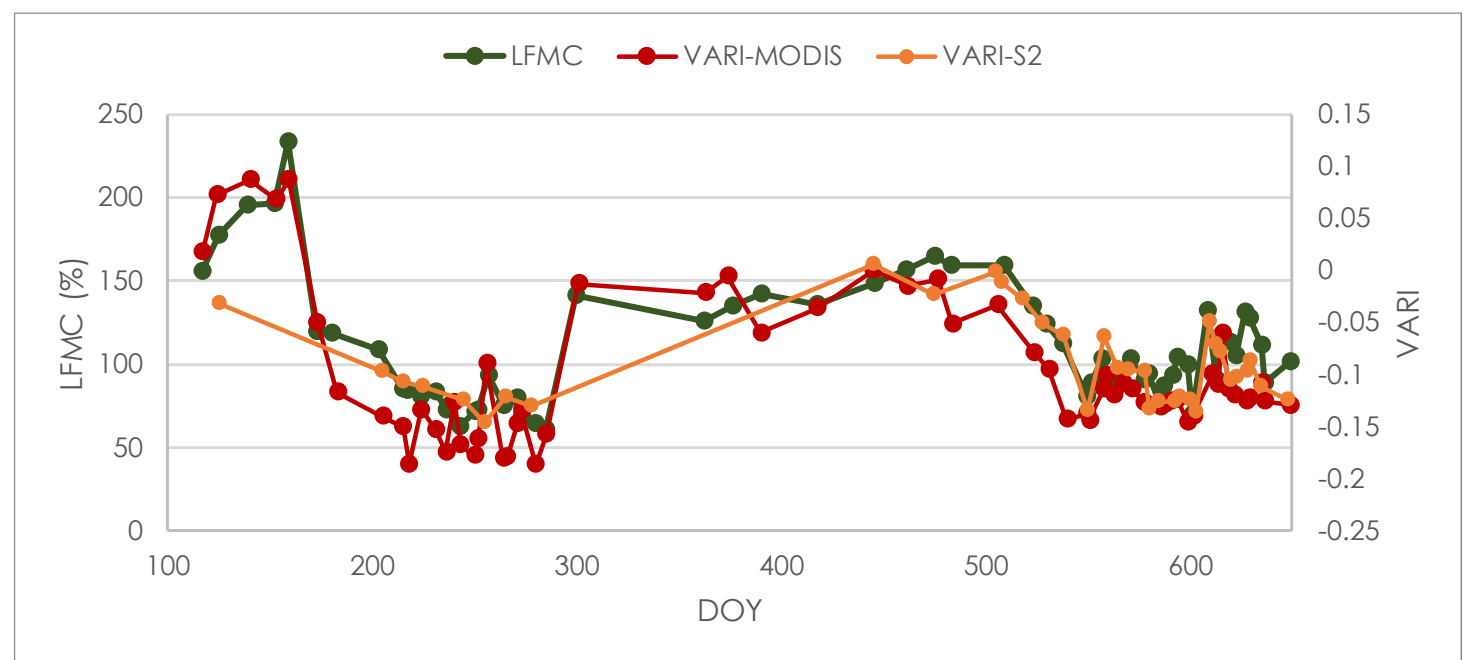

Figure 1 - Comparison of LFMC vith NDVI and VARI derived from MODIS and Sentinel-2 in Cistus ladanifer shrubland, from 24 April 2016 (DOY = 117) to 10 October 2017 (DOY = 649).
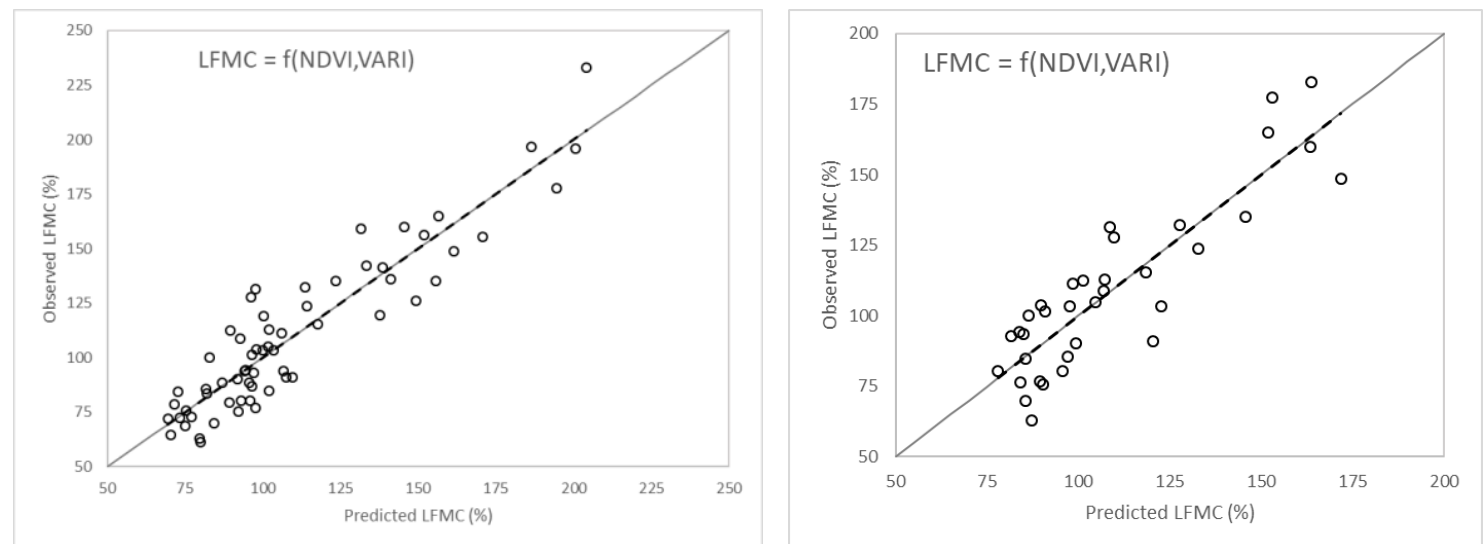

Figure 2 - Observed vs predicted values of LFMC for the best models obtained for MODIS (left, n=62) and Sentinel-2 (right, $n=35$ ) data.

\section{Discussion}

As reported in previous works, this study shows that spectral vegetation indices are an efficient means of obtaining empirical information related to LMFC from multispectral sensors (Yebra et al. 2013). Our results indicate that MODIS and Sentinel-2 images provide similar results for the SI tested (Table 2, Figure 2). The slightly better performance of most SI in the coarser resolution images could be partly due to the lower number of images available from Sentinel-2 for the study period. This limitation specially affected 2016 dataset, with only 8 cloud-free images found compared to 26 from MODIS. However, 27 images from Sentinel-2 were available in 2017 compared to 36 from MODIS. Our results suggest that longer time series may be used in future Sentinel-2 models to better account for the temporal variability of LFMC. This should not prevent the use of spectral information derived from Sentinel-2 images as, with both satellites 2A and 2B fully operational, it is currently possible to produce LFMC estimations every 5 days. However, MODIS still have higher potential in terms of temporal resolution compared to Sentinel-2, which may be a constraint when trying to achieve daily operational products at a finer spatial resolution.

We found that VARI was the SI showing the higher correlation with field measurements of LFMC (Table 2). Regarding MODIS images, previous authors also highlight VARI as the best indicator of LFMC variability in shrubland species compared to other SI (Stow et al. 2006, Peterson et al. 2008, 
Caccamo et al. 2012, Fan et al. 2018). Stow et al. (2006) and Peterson et al. (2008) reported $\mathrm{R}^{2}$ between 0.74 and 0.93 depending on the chaparral sampling site. Caccamo et al. (2012) found lower prediction capacity of VARI with only 0.42 , but mixing different types of vegetation. Our results are in general agreement with these authors, finding $\mathrm{R}^{2}$ of 0.83 for MODIS data. However, it should be highlighted that all these authors used MODIS composite products (generally 8-days composites) compared to the 1-day images used in the present study. Avoiding the use of a composite product in our study reduced the number of available cloud-free images to compare field data $(n=81)$ with spectral information $(\mathrm{n}=62$ for MODIS). However, this choice was deliberately done in order to test the ability of near realtime daily images (only up to two days timelag to sampling date) for LFMC estimation, which is a critical aspect for models to be include in any operational system for wildfire risk assessment.

The best multivariate models combined the same spectral indices (VARI and NDVI) in both type of images. In Australia, Caccamo et al. (2012) suggested NDII6 for improving performance in a multivariate model derived to estimate LFMC from MODIS in shrubland and heathland. Stow et al. (2006) found the best multivariate model combining VARI with NDWI in California chaparral, whereas Peterson et al.(2008) reported better results with VARI and VIGREEN for the same type of shrubland. In our case, VIGREEN also had a strong correlation in the simple models, but NDII6 and NDWI were among the indices with lower correlation with LFMC data. Both VARI and NDVI measure greenness variability, whereas NDWI and NDII6 directly account for water content variations. Although greenness indices do not include water absorption bands, they can be used as an indirect estimation of water content since moisture variations affect chlorophyll activity, which is the case of C.ladanifer (Yebra et al. 2008).

Despite Sentinel-2 provided a slightly lower accuracy, LFMC prediction errors were very similar in the best multivariate model, with mean absolute error of $12 \%$ compared to $11 \%$ of MODIS (Table 2). According to fire management services, these are good results taking into account that $10 \%$ errors in LFMC estimation from field measurements are generally acceptable. Nevertheless, the proposed models should be validated with 2018 data in the same sampling area, in order to assess the sensitivity of both sensors to monitor seasonal and interannual variability of LFMC. Empirical models are also known to have limitations in terms of its ability for extrapolationg accurate results to different sites compared to RTM models (Yebra et al. 2008). However some authors reported moderately good model results for different sites with the same type of vegetation (Stow et al. 2006, Peterson et al. 2008, Caccamo et al. 2012). Hence, further comparison with similar shrubland areas would be highly recommended in order to test limitations when applying these models in other monospecific $C$. ladanifer shrubland.

\section{Conclusions}

This work compares the capacity of empirical models for LFMC estimation in Cistus ladanifer shrubland from two different type of satellite sensors: MODIS and Sentinel-2. MODIS provides daily estimates at a coarser spatial resolution whereas Sentinel-2 can provide higher spatial accuracy every 5 days. Despite both sensors provide images at different spatial and temporal resolution, this study showed similar results in terms of the most relevant spectral indices and model performance. The combination of VARI and NDVI provided the best models for both sensors tested. The proposed models derived from 1-day images should be validated with indepedent datasets and with longer time series. However, results suggest that both sensors could be used for near real-time estimation of LFMC in monospecific $C$. ladanifer shrubland. This study contributes to improve monitoring of the variability of LFMC during the year based on non-composite products, helping the integration of remote sensing data on wildfire danger rating systems. 


\section{Acknowledgements}

This study is part of a broader research performed within the frame of GEPRIF project (RTA201400011-C06-06) co-funded by INIA (Spanish National Research Institute for Agriculture) and the EU through the FEDER program. Eva Marino's participation is supported by a postdoctoral grant from the Spanish Ministry of Economy and Competitiveness (Torres-Quevedo program), which is partially funded by the European Social Fund (ESF) from the European Commission. We acknowledge the firefighting service from Comunidad de Madrid for providing field data.

\section{References}

Caccamo G, Chisholm LA, Bradstock RA, Puotinen ML, Pippen BG (2012) Monitoring live fuel moisture content of heathland, shrubland and sclerophyll forest in south-eastern Australia using MODIS data. International Journal of Wildland Fire 21, 257-269.

Ceccato P, Flasse S, Gregoire JM (2002) Designing a spectral index to estimate vegetation water content from remote sensing data: Part 2. Validation and applications. Remote Sensing of Environment 82, 198-207.

Chuvieco E, Cocero D, Riaño D, Martín MP, Martínez-Vega J, de la Riva J, Pérez F(2004) Combining NDVI and surface temperature for the estimation of live fuel moisture content in forest fire danger rating. Remote Sensing of Environment 92, 322-331.

Chuvieco E, Aguado I, Jurdao S, Pettinari ML, Yebra M, Salas J, Hantson S, de la Riva J, Ibarra P, Rodrigues M, Echevarría M, Azqueta D, Román MV, Bastarrika A, Martínez S, Redondo C, Zapico E, Martínez-Vega FJ (2014) Integrating geospatial information into fire risk assessment. International Journal of Wildland Fire 23, 606-619.

Dennison PE, Roberts DA, Peterson SH, Rechel J (2005) Use of Normalized Difference Water Index for monitoring live fuel moisture. International Journal of Remote Sensing 26, 1035-1042.

Fan L, Wigneron JP, Xiao Q, Al-Yaari A, Wen J, Martin-St Paul N, Dupuy JL, Pimont F, Al Bitar A, Fernandez-Moran R, Kerr YH (2018) Evaluation of microwave remote sensing for monitoring live fuel moisture content in the Mediterranean region. Remote Sensing of Environment 205, 210-223.

Gao BC (1996) NDWI. A normalized difference water index for remote sensing of vegetation liquid water from space. Remote Sensing of Environment 58, 257-266.

Garcia M, Chuvieco E, Nieto H, Aguado I (2008) Combining AVHRR andmeteorological data for estimating live fuelmoisture content. Remote Sensing of Environment 112, 3618-3627.

Gitelson A, Kaufmam JY, Stark R, Rundquist D (2002) Novel algorithms for remote estimation of vegetation fraction. Remote Sensing of Environment 80, 76-87.

Hao XJ, Qu JJ (2007) Retrieval of real-time live fuel moisture content using MODIS measurements. Remote Sensing of Environment 108, 130-137.

Hardisky MA, Klemas V, Smart RM (1983) The influence of soil salinity, growth form, and leaf moisture on the spectral radiance of Spartina alterniflora canopies. Photogrammetric Engineering and Remote Sensing 49, 77-83.

Huete AR (1988) A soil-adjusted vegetation index (SAVI). Remote Sensing of Environment 25, 295309.

Huete A, Didan K, Miura T, Rodriguez EP, Gao X, Ferreira LG (2002) Overview of the radiometric and biophysical performance of the MODIS vegetation indices. Remote Sensing of Environment 83, 195-213.

Jurdao S, Yebra M, Guerschman JP, Chuvieco E (2013) Regional estimation of woodland moisture content by inverting Radiative Transfer Models. Remote Sensing of Environment 132, 59-70. 
Louis J, Debaecker V, Pflug B, Main-Knorn M, Bieniarz J, Mueller-Wilm U, Cadau E, Gascon F (2016) Sentinel-2 Sen2Cor: L2A Processor for Users. In: L. Ouwehand L (ed), Proceedings of the Living Planet Symposium, 9-13 May 2016, Prague, Czech Republic. ESA-SP Volume 740, p.91. ISBN: 978-92-9221-305-3.

Marino E, Dupuy JL, Pimont F, Guijarro M, Hernando C, Linn R (2012) Fuel bulk density and fuel moisture content effect on fire rate of spread: a comparison between FIRETEC model predictions and experimental results in shrub fuels. Journal of Fire Sciences 30(4), 277-299.

Nolan RH, Resco de Dios V, Boer MM, Caccamo G, Goulden ML, Bradstock RA (2016) Predicting dead fine fuel moisture at regional scales using vapour pressure deficit from MODIS and gridded weather data. Remote Sensing of Environment 174, 100-108.

Pausas JG, Fernández-Muñoz S (2012) Fire regime changes in the Western Mediterranean Basin: from fuel-limited to drought-driven fire regime. Climatic Change 10, 215-226.

Peterson S, Roberts DA, Dennison PE (2008) Mapping live fuel moisture with MODIS data: A multiple regression approach. Remote Sensing of Environment 112, 4272-4284.

Rouse Jr JW, Haas RW, Schell JA, Deering DH, Harlan JC (1974) Monitoring the vernal advancement and retrogradation (Greenwave effect) of natural vegetation. Type III final report. Greenbelt, MD, USA: NASA/GSFC.

Ruffault J, Curt T, Martin-StPaul NK, Moron V, Trigo RM (2018) Extreme wildfire events are linked to global-change-type droughts in the northern Mediterranean. Natural Hazards and Earth System Sciences 18(3), 847-856.

Stow D, Madhura N, Kaiser J (2006) Time series of chaparral live fuel moisture maps derived from MODIS satellite data. International Journal of Wildland Fire 15, 347-360.

Tanase MA, Panciera R, Lowell K, Aponte C (2015) Monitoring live fuel moisture in semiarid environments using L-band radar data. International Journal of Wildland Fire 24, 560-572.

Tucker CJ (1979) Red and photographic infrared linear combinations for monitoring vegetation. Remote Sensing of Environment 8, 127-150.

Viney NR (1991) A review of fine fuel moisture modelling. International Journal of Wildland Fire 1, 215-234.

Yebra M, Chuvieco E (2009) Linking ecological information and radiative transfer models to estimate fuel moisture content in the Mediterranean region of Spain: Solving the ill-posed inverse problem. Remote Sensing of Environment 113, 2403-2411.

Yebra M, Chuvieco E, Riaño D (2008) Estimation of live Fuel Moisture Content from MODIS images for fire risk assessment. Agricultural and Forest Meteorology 148, 523-536. doi:10.1016/J.AGRFORMET.2007.12.005

Yebra M, Dennison PE, Chuvieco E, Riaño D, Zylstra P, Hunt ER Jr, Danson FM, Qi Y, Jurdao S (2013) A global review of remote sensing of live fuel moisture content for fire danger assessment: Moving towards operational products. Remote Sensing of Environment 136, 455-468. 


\title{
Flammability of native species of two Brazilian ecosystems
}

\author{
João Francisco Labres dos Santos; Igor Eloi Silva Machado; Ronaldo Viana Soares*; Antonio \\ Carlos Batista; Pedro Cordeiro Neves; Alexandre França Tetto \\ Universidade Federal do Paraná. Avenida Prefeito Lothario Meissner, 900, 80210-170 - Curitiba \\ - Paraná - Brasil, \{joaolabres@ufpr.br,igeloi@hotmail.com,rvsoares02@gmail.com*, \\ batistaufpr@gmail.com,pedrocn@gmail.com,tetto@ufpr.br\}
}

\begin{abstract}
The Cerrado (Brazilian savanna) presents species with adaptations to fire and, therefore, is considered a fire dependent ecosystem. On the other hand, the Mixed Ombrophilous Forest (FOM), characterized by the presence of higher levels of moisture, is considered a fire sensitive ecosystem. Flammability is defined as the ability of the fuel to ignite after being subjected to an increase of caloric energy, until the material reaches the ignition stage, after the reduction in the moisture content. The objective of this research was to test and classify species from the Cerrado and the Araucaria Forest (FOM) according to their flammability. The following species from the Cerrado were tested: Vatairea macrocarpa, Myrcia splendens, Davilla elliptica, Ouratea hexasperma, Copaifera langsdorffi, Diospyros hispida. The tested species from the FOM were: Ocotea puberula, Araucaria angustifolia, Allophylus edulis, Cupania vernalis and Luehea divaricata. Using an epirradiator, 50 replications of burning for each species were carried out. Each tested sample consisted of $1.0 \pm 0.1 \mathrm{~g}$ of green fuel material less than $0.7 \mathrm{~cm}$ of diameter, containing leaves and branches. The measures parameters were: Time of ignition (Ti), combustion duration (Dc), ignition frequency (Fi) and flame height $(\mathrm{Hc})$. The species were classified according to their flammability rating value (Vi). To validate the classification and determine which parameters were the main flammability inductors, a Principal Component Analysis (PCA) was carried out. Araucaria angustifolia presented poor flammability, due to the higher ignition time. Allophylus edulis presented the higher flammability among the FOM species, and the ignition frequency was the decisive parameter to this classification. The species from the Cerrado showed similar values of combustion duration, except Davilla elliptica and Myrcia splendens, which presented intermediate values of ignition time. Vatairea macrocarpa and Copaifera langsdorffi, were highly and extremely flammable, respectively. The flammability values validated the hypothesis that the Cerrado species present higher flammability when compared to the FOM species. The Principal Components Analysis was effective in the results validation and allowed to explain what parameters determined the flammability.
\end{abstract}

Keywords: Principal component analysis; ignition, Cerrado, Araucaria forest.

\section{Introduction}

The Cerrado (Brazilian savanna) presents species with adaptations to fire and, therefore, is considered a fire dependent ecosystem. On the other hand, the Mixed Ombrophilous Forest (FOM), characterized by the presence of higher levels of moisture, is considered a fire sensitive ecosystem (Myers, 2006). The forest fuel is essential to the occurrence of fire, along with the moisture condition which controls the level of flammability (Soares et al., 2017).

Mutch (1970) affirms that plant communities dependent on the fire burn more easily than nondependent communities of fire, because natural selection has favored the development of characteristics that make it more flammable. Pausas et al. (2017), adds that there are several strategies of flammability selected in different evolutionary contexts and confer benefits of suitability to plants that live under the regime of recurrent fires, in addition to being able to act as a means by which the plants can modify fire regimes to create favorable conditions for development (Schwick, 2003).

According to Vallete (1990), the flammability is defined as the ability of the fuel to ignite after being subjected to an increase of caloric energy, until the material reaches the ignition stage, after the 
reduction in the moisture content. However, this characteristic does not necessarily represents how well a plant burning under field conditions, and to a lesser extent, the flammability depends on the size, thickness and shape of the plant, which can be evaluated directly by laboratory tests. (Pausas et al., 2016).

Considering the characteristics of each ecosystem and the lack of research related to the flammability of native species in Brazil, the hypothesis that the species from the Cerrado are more flammable than those from the Araucaria Forest (FOM) was tested. The objective of this research was to test and classify species from the Cerrado and Mixed Ombrophilous Forest and determine which parameters present higher influence in the species flammability.

\section{Methodology}

The tests were developed at the "Centro de Monitoramento Ambiental e Manejo do Fogo - CeMAF, Universidade Federal do Tocantins" and the "Laboratório de Incêndios Florestais do Departamento de Ciências Florestais da Universidade Federal do Paraná", in accordance with the methodology presented by Valette (1990) and Petriccione (2006). Eleven species, five from the FOM (Ocotea puberula, Araucaria angustifolia, Allophylus edulis, Cupania vernalis e Luehea divaricata) and six from the Cerrado (Vatairea macrocarpa, Myrcia splendens, Davilla elliptica, Ouratea hexasperma, Copaifera langsdorffi, Diospyros hispida) were tested.

For each evaluated species, 50 replications of burning were carried out. Each sample consisted of $1.0 \pm 0.1 \mathrm{~g}$ of green combustible material less than $0.7 \mathrm{cmdiameter}$, containing leaves and branches. The tests were conducted up to two hours after the material collection to prevent loss of moisture. The experimental burnings were performed in an epirradiator, with controlled temperature range of $250^{\circ} \mathrm{C}$ to $350^{\circ} \mathrm{C}$, to prevent highly flammable fuels from being classified as poorly flammable (Kauf et al. 2014), and with the aid of a pilot flame $4 \mathrm{~cm}$ above the center of the disc in a place free of air currents (Figure 1).

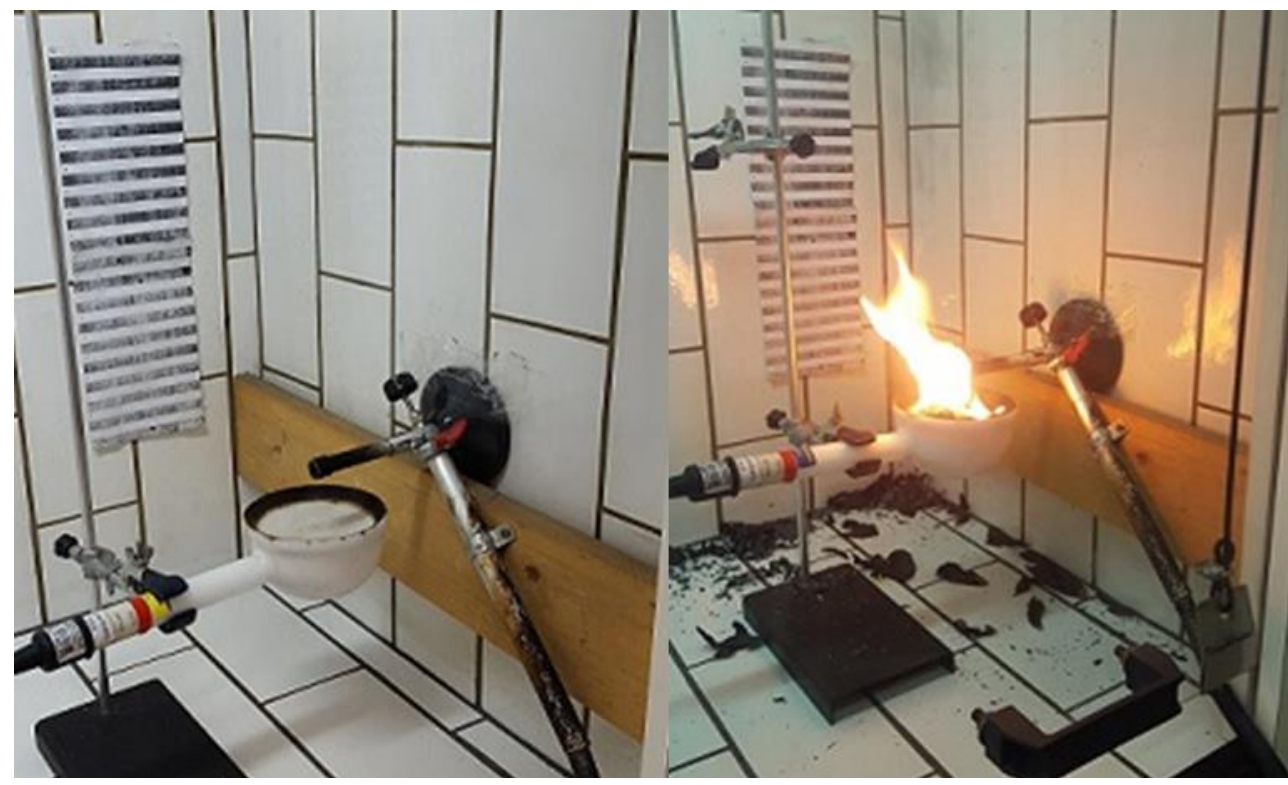

Figure 1 - Epirradiador in the test chamber and in operation.gure

The samples collected were dried in an oven for a period of 48 hours at $75{ }^{\circ} \mathrm{C}$ and the moisture content was determined by the gravimetric method by the following equation:

$$
M C=(W M-D M) / D M * 100
$$


Where MC is the moisture content of combustible material in percentage, WM is the mass (wet) of combustible material at the time of collection and the DM mass (dry) of combustible material after drying in the oven.

The measured variables were time of ignition (Ti) and combustion duration (Dc), in seconds; flame height $(\mathrm{Hc})$, in centimeters; and ignition frequency $(\mathrm{Fi})$. The data obtained were submitted to the flammability rating value (Vi) and Principal Components Analysis (PCA), with the objective of validate the classification and identify the variables that present higher influence in the species flammability.

\section{Results and discussion}

The flammability rating values are presented in Table 1.

Table 1 - Values of the flammability variables of the tested species and the final classification.

\begin{tabular}{|c|c|c|c|c|c|}
\hline Espécies & $\mathrm{Ti}(\mathrm{s})$ & $\mathrm{Dc}(\mathrm{s})$ & $\mathrm{Hc}(\mathrm{cm})$ & $\mathrm{Fi}$ & $\mathrm{Vi}$ \\
\hline Ocotea puberula & 35,14 & 11,65 & 24,03 & 37 & $\mathrm{I}$ \\
\hline Araucaria angustifolia & 47,25 & 15,25 & 13,50 & 4 & I \\
\hline Cupania vernalis & 31,03 & 16,48 & 18,31 & 29 & I \\
\hline Luehea divaricata & 23,21 & 14,50 & 13,50 & 14 & I \\
\hline Myrcia splendens & 25,30 & 7,60 & 11,16 & 32 & I \\
\hline Allophylus edulis & 28,10 & 14,51 & 19,44 & 41 & II \\
\hline Davilla elliptica & 21,63 & 7,86 & 12,19 & 22 & II \\
\hline Ouratea hexasperma & 19,00 & 8,00 & 15,20 & 38 & II \\
\hline Diospyros hispida & 20,35 & 11,45 & 15,21 & 32 & II \\
\hline Vatairea macrocarpa & 12,71 & 13,42 & 19,88 & 50 & $\mathrm{~V}$ \\
\hline Copaifera langsdorffi & 9,45 & 11,45 & 19,20 & 50 & VI \\
\hline
\end{tabular}

Vi: Flammability rating value of Valette (1990); I: Low flammability; II: Slightly flammable; III: Moderately flammable; IV: flammable; V: highly flammable; VI: Extremely flammable.

Figure 1 shows the results of the Principal Component Analysis, in which the species with similar parameters are forthcoming in the plan, while those with very different parameters are distant (Petriccione, 2006).

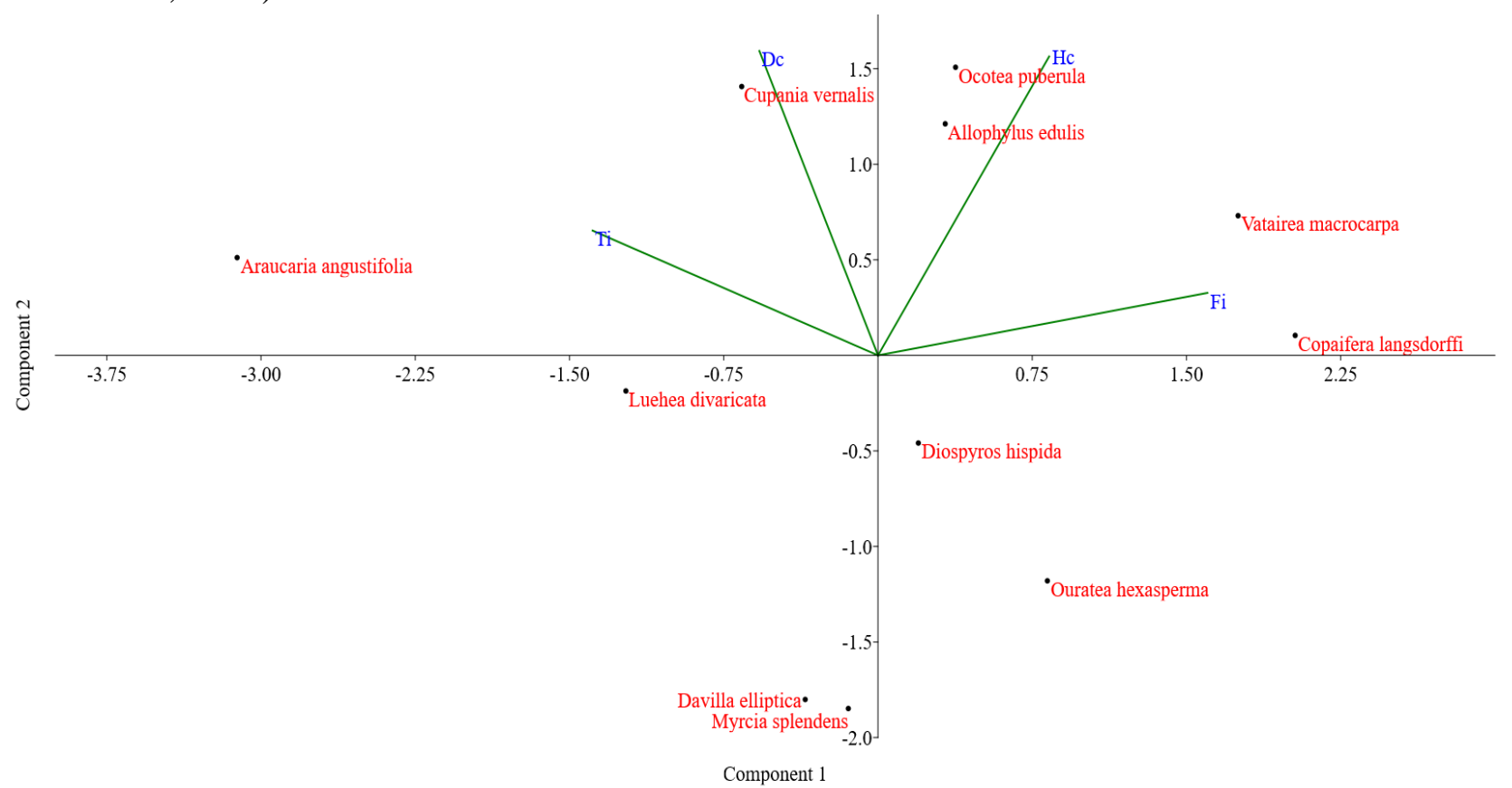

Figure 1 - Principal Components Analysis based on the flammability variables of the tested species. 
Analyzing the PCA, along with the table 1 it can be observed that ignition time and frequency are opposites in the plan, corroborating, therefore, with the Vi classification (Vallete, 1990), while the variables Dc and Hc, when plotted on biplot, explain the behavior of the species regarding to fire (Petriccione, 2006; Vélez, 2009).

White and Zipperer (2010) emphasize that the moisture content is a preponderant factor for flammability by increasing the thermal capacity of the material and hamper the start of combustion. According to Hernando (2009), the variables related to the fire are directly related to the moisture content of fuels, especially the time of ignition and the height of the flame. Therefore, among the species examined for the cerrado, V. macrocarpa and $C$. langsdorffi were those that showed a lower content of moisture, $89.23 \%$ and $76.38 \%$, respectively, and consequent higher flammability, getting next to the right side of the plan, with higher Fi and lower Ti. It is important to remember that, in addition to the moisture, characteristics such as lignin content, mineral salts and compounds also affect the flammability of forest fuels (Batista and Biondi, 2009).

It was found that the A. edulis was the most flammable of species of FOM, although classified as poorly flammable, with the frequency of ignition being the determining parameter for this classification, even with moisture content of $253.73 \%$, a result similar to that found by Molina et al. (2017) for the specie Ailanthus altissima, with moisture content of $297.5 \%$, in a study conducted in Spain. The specie L. divaricata presented $80.76 \%$ of moisture content, being that has lower moisture content among the species of FOM but was classified as poorly flammable due to low frequency of ignition.

Araucaria angustifolia showed a moisture content of $123.45 \%$ and proved to be poorly flammable, due to the high ignition time, positioning itself in the left quadrant of the graph. The Ocotea puberula showed a similar behavior, with $110.12 \%$ moisture content, even with the greatest value of flame height, being classified as poorly flammable, due to the fact it has a relatively high, although with higher values of Dc and Hc. These results corroborate those found by Weise et al. (2005), evaluating ornamental plants of low flammability, found moisture contents of 180 to 304\%. Hachimi et al. (2011) in a study of the flammability of forest fuels, found a high correlation between moisture content and the parameters related to the flammability of fire. Petriccione (2006), in evaluation of species of the Mediterranean, stated that it had found a correlation between moisture and time of ignition or duration of combustion.

Generally, in areas with forest formations in advanced stage, for example, the fire has difficulties to enter due to the high humidity, however, Nepstad et al. (1999) clarify that the higher the frequency of fires, the greater becomes the flammability of the forest, because there is a substitution of species by an increase in the density of grasses, under the risk of the forest becoming a savanna.

Only fires of medium to high intensity may consume the plants that usually have moisture from 75 to $150 \%$ of its dry weight (Rodriguez, 2011). The meteorological variables, such as relative humidity and air temperature, wind and solar radiation are factors that directly affect the moisture content of a plant tissue, and, therefore, the living material is more stable in relation to the moisture that the dead material, because this does not require great energy to ignite (Fernandes and Cruz. 2012). Therefore, much of the initial heat of the combustion, before starting the combustion itself, is spent primarily to vaporize the water present in the fuel and water vapor resulting will reduce the oxygen near reducing the combustion of flammable gases (Soares et al. 2017).

All species showed similar values of Dc, with the exception of Davilla elliptica and Myrcia splendens, with intermediate values of Thee, positioning itself more to the center, in the lower left quadrant of the PCA. 


\section{Conclusions}

The hypothesis that the Cerrado species present higher flammability was confirmed through the flammability rating values. The Principal Components Analysis was effective in the results validation and allowed to explain what variables determined the species flammability.

\section{References}

Batista AC, Biondi D (2009) Avaliação da inflamabilidade de Ligustrum licidum Aiton (Oleaceae) para uso potencial em cortinas de segurança na região sul do Brasil. Revista Brasileira de Ciências Agrárias 4, 435-439.

Fernandes PM, Cruz, MG (2012) Plant flammability experiments offer limited insight into vegetationfire dynamics interactions. New Phytologist 194, 606-609.

Hachmi MH, Sesbou A, Benjelloun H, El Handouz N, Bouanane F (2011) A Simple technique to estimate the flammability index of Moroccan forest fuels. Journal of combustion 1, 1-11.

Hernando C (2009) Combustibles forestales: inflamabilidad. In 'La defensa contra incendios forestales. Fundamentos y experiencias'. (Coord. R Vélez) pp. 6.3-6.15. (McGraw-Hill: Madrid)

Kauf Z, Fangmeier A, Rosavec R, Španjo Ž. (2014) Testing vegetation flammability:the problem of extremely low ignition frequency and overall flammability score. Journal of combustion 1, 1-10.

Molina JR, Martín T, Silva FR, Herrera MA (2017) The ignition index based on flammability of vegetation improves planning in the wildland-urban interface: A case study in Southern Spain. Landscape and urban planning 158, 129-138.

Myers RL (2006) 'Convivendo com o fogo: manutenção dos ecossistemas \& subsistência com o manejo integrado do fogo.' (The Nature Conservancy: Tallahassee)

Nepstad DC, Moreira A, Alencar AA. (1999) 'A Floresta em Chamas: Origens, Impactos e Prevenção de Fogo na Amazônia. Programa Piloto para a Proteção das Florestas Tropicais do Brasil.' (Binagri: Brasília).

Petriccione M (2006) 'Infiammabilità della lettiera di diverse specie vegetali di ambiente Mediterraneo.' (Università Degli Studi Di Napoli Federico II: Napoli)

Rodríguez MPR. (2010). 'Manejo del Fuego.' (Editorial Felix Varela: Havana)

Soares RV, Batista AC, Tetto AF (2017) 'Incêndios florestais: controle, efeitos e uso do fogo.' (UFPR: Curitiba)

Soares RV, Batista AC, Tetto AF (2017) 'Incêndios florestais: controle, efeitos e uso do fogo.' (UFPR: Curitiba)

Valette JC (1990) lnflammabilités des espèces forestières méditerranénnes: conséquences sur la combustibilité des formations forestières (Mediterranean Forest Species Flammability, Consequences on Forest Communities Combustibility) Revue Forestière Française 42, 76-92.

Vélez R. Combustibiles forestales: combustibilidad. In 'La defensa contra incendios forestales. Fundamentos y experiencias'. (Coord. R Vélez) pp. 7.1-7.16. (McGraw-Hill: Madrid)

Weise DR., White, RH, Beall FC, Etlinger M. (2005) Use of the cone calorimeter to detect seasonal differences in selected combustion characteristics of ornamental vegetation. International Journal of Wildland Fire. 14, 321-338.

White RH, zipperer WC. (2010) Testing and classification of individual plants for fire behaviour: plant selection for the wildland-urban interface. International Journal of Wildland Fire. 19, -338.

White RH, zipperer WC. Testing and classification of individual plants for fire behaviour: plant selection for the wildland-urban interface. International Journal of Wildland Fire, v.19, n.2, p. 213 $22,2010$. 


\title{
Influence of fire frequency and epoch of prescribed burn on topkill rates in vegetation of Cerrado in Tocantins, Brazil
}

\author{
Allan Deyvid Pereira da Silva ${ }^{1}$; Antonio Carlos Batista ${ }^{1}$; Marcos Giongo ${ }^{3}$; Micael Moreia Santos ${ }^{1}$; \\ Eduardo Ganassoli Neto ${ }^{1}$; Edmar Vinícius de Carvalho ${ }^{2}$; Igor Eloi Silva Machado ${ }^{1}$; Jader Nunes \\ Cachoeira ${ }^{1}$. \\ ${ }^{1}$ Federal University of Paraná, \{allanuft@gmail.com, batistaufpr@gmail.com, \\ moreirasmicael@gmail.com,eduardo.florestal@mail.uft.edu.br,igeloi@hotmail.com, \\ jadernunes@mail.uft.edu.br\} \\ ${ }^{2}$ Federal University of Tocantins, \{giongo@uft.edu.br, carvalho.ev@uft.edu.br\}
}

\begin{abstract}
The severity of fire can be understood as the level of change caused by a fire to a location. Considering the importance of fire in the Cerrado biome, the objective of this work was to evaluate the fire severity, expressed through suppression of the aerial part of the vegetation (topkill), in Cerrado environment. The research was performed at the ecological station Estação Ecológica Serra Geral do Tocantins, Jalapão region, Brazil. At areas with two, three and four years without occurrence of forest fires, identified by satellite images, prescribed burns were performed in the months of May, June, August and September of 2017. The fire severity was determined through the variation of the total number of individuals before and after the fire passage. At areas with four years with no occurrence of fires, regardless of the month in which the prescribed firing occurred, topkill values did not differ statistically (45 to 56\%). The three-year areas presented higher topkill values in September (66\%); and the areas of two years presented the highest rates in August (62.8\%). At conditions with greater accumulation of fuel material, due to the time without occurrence of fires, the period of the controlled burn (beginning of the drought or end of this season) did not influence the topkill rate of the vegetable individuals. Topkill rates among areas with different periods without burning varied only in September. The values of three and four-year areas (66 and 52.3\%, respectively) did not differ from each other $(p>0,05)$ and were higher than rates at areas of two years with no occurrence of fire $(38 \%)$. The distinction of topkill rates at locations with different periods without occurrence of fires was only possible when the prescribed burn was performed at the end of the dry season in Cerrado; that is, the period that the climatic conditions contribute to lower air moisture content and forest fuels, thus increasing the burning efficiency.
\end{abstract}

Keywords: burn severity; fire severity; forest fires.

\section{Introduction}

The consumption of forest fuels, below and above the ground, is commonly titled as fire severity; and largely reflect fire intensity (Knox; Clarke, 2016).

The fire severity may reflect the variation of the dynamics of plant population and vegetation mortality; therefore, its measurement and recording are important (Keeley, 2009; Knox; Clarke, 2016).

The main factors that determine the intensity and severity of a forest fire are associated with the topography of the terrain, fuel characteristics and meteorological conditions at burning moment (Clarke et al., 2014; Lecina-Diaz et al., 2014; Soares et al., 2017).

In Cerrado, the drought commonly intensifies itself in early May and extends until mid-October, and the fuel accumulation is associated to the frequency of fires. In general, in Savanna environments, fire intervals of two to three years are common (Coutinho, 1990; Russell-Smith et al., 1997). 
The drought and fuel accumulation are important regarding level of fire severity. The suppression of the aerial part of the vegetable (topkill) has been used as a measure to evaluate the level of severity of forest fires in diverse researches in the world (Hoffmann; Solbrig, 2003; Nicholson et al., 2017).

The objective of this work was to evaluate the influence of the fire frequency and the period of burning about the variation of topkill rates in Cerrado of dirty field type.

\section{Methods}

The work was developed at the ecological station Estação Ecológica Serra Geral do Tocantins (EESGT), Jalapão region, Brazil. The area is a domain area of the Cerrado biome, composed, predominantly, by Campo Limpo and Campo Sujo. There are predominance of soils of the type Entisols; they can be leptsols (lithic entisol), fluvisols (fluvent entisol) and regosols (Psamment and Orthent entisol). The relief is relatively flat, with declivity equal or superior to 5\%; annual average temperature of $26^{\circ} \mathrm{C}$; annual mean rainfall of $1500 \mathrm{~mm}$ (Tocantins, 2012; MAPA, 2018b).

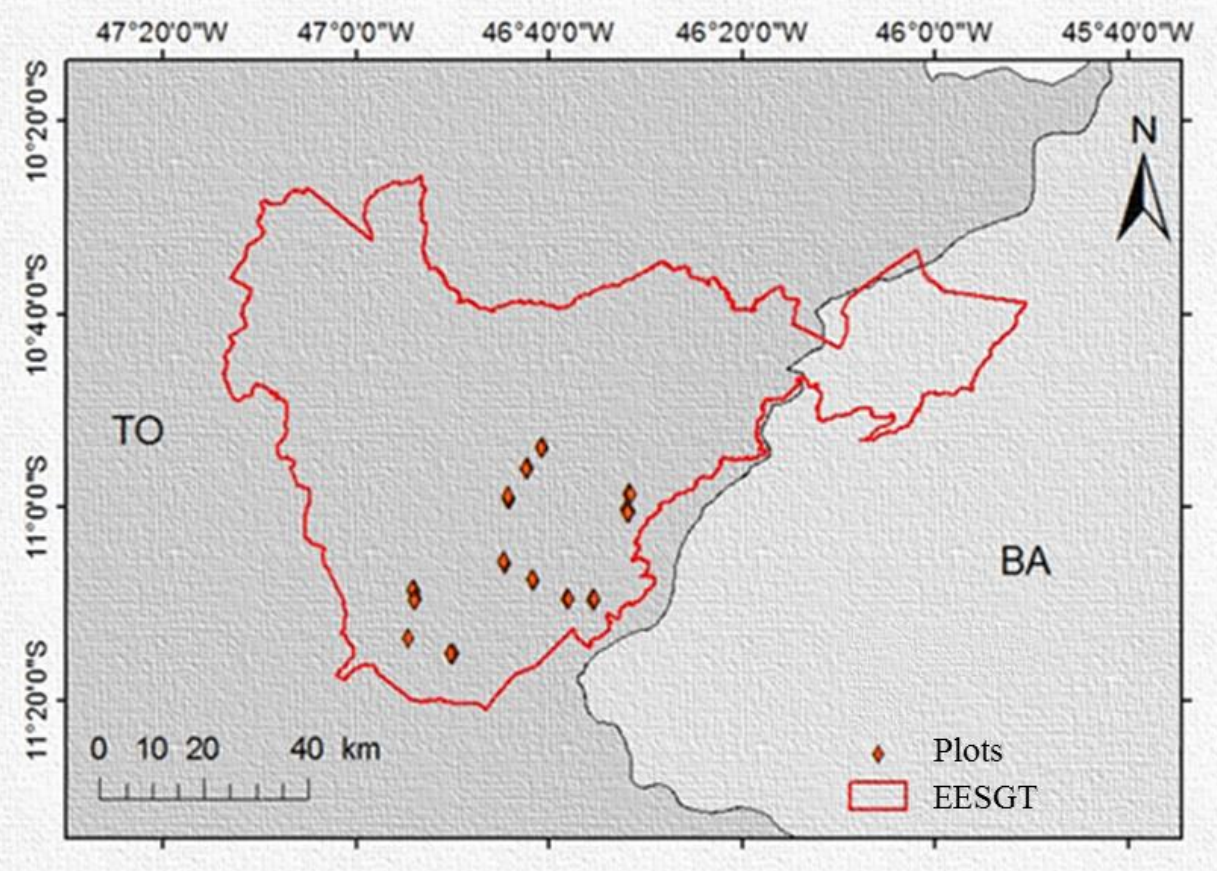

Figure 3 - Distribution of Experimental Areas at EESGT.

For the experiment, plots were established in areas with two, three and four years without occurrence of forest fires, identified through satellite images, where were performed prescribed burns in the months of May, June, August and September of 2017.

The treatments were composed of two factors: years without occurrence of fires versus season of prescribed burns). They were evaluated in four plots of $30 \times 30 \mathrm{~m}$, totaling 48 sample units randomly distributed at burning locations.

At each plot, for 20 possibilities, four sub-plots of $1 \mathrm{~m}^{2}$ were randomly drawn (Figure 2) and all vegetables up to $20 \mathrm{~mm}$ of diameter were counted - before and after the forest fire. The percentage of individuals whose aerial part was consumed by fire was considered as Topkill Rate - which was calculated with the following equation:

$$
\mathrm{TR}=\frac{(\mathrm{IBF}-\mathrm{IAF})}{\mathrm{IBF}} 100
$$


Being: TR - Topkill rate; IBF - number of individuals before fire; IAF - number of individuals after fire.

The forest fuel available for combustion (up to $1,8 \mathrm{~m}$ ) was quantified in 8 samples of $0,25 \mathrm{~m}^{2}$, randomly established at plot; and classified as living and dead material and as type (herbaceous). The material collected was dried in an oven at $70^{\circ} \mathrm{C}$ for 72 hours, weighed and its value converted to kg.m2.

The speed of fire propagation and the flame length were registered at the moment of fire front passage, based on 2,5 m height rulers, fixed to the plot and equidistant $3 \mathrm{~m}$ one from the other, perpendicular to the line of fire.

In order to statistically analyze the data, the test of normality was applied, followed by the factorial analysis of variance (years without fires versus season of prescribed burns) and the Scott-Knott test (p $>0,05)$.

\section{Results}

\subsection{Forest fuel and fire behavior}

The total biomass and herbaceous biomass available at areas with 2, 3 and 4 years without fires follow a pattern: load increase throughout time without occurrence of burning; and besides that, they present a predominance of dead vegetation (Table 1).

Table 1 - Characteristics of biomass among areas with different historical of the last burning and the fire behavior at each month of prescribed burns.

\begin{tabular}{|c|c|c|c|}
\hline \multicolumn{4}{|c|}{ Fuels characteristics } \\
\hline \multirow{2}{*}{ Biomass $\left(\mathrm{kg} \cdot \mathrm{m}^{-2}\right)$} & \multicolumn{3}{|c|}{ Fire frequency } \\
\hline & 2 years & 3 years & 4 years \\
\hline Total & $0,55(0,051)$ & $0,62(0,032)$ & $0,64(0,039)$ \\
\hline Living & $0,26(0,025)$ & $0,25(0,019)$ & $0,28(0,031)$ \\
\hline Dead & $0,30(0,038)$ & $0,37(0,024)$ & $0,37(0,020)$ \\
\hline Total herbaceous & $0,24(0,024)$ & $0,24(0,014)$ & $0,27(0,019)$ \\
\hline Living herbaceous & $0,10(0,010)$ & $0,08(0,006)$ & $0,09(0,010)$ \\
\hline Dead herbaceous & $0,14(0,019)$ & $0,16(0,013)$ & $0,18(0,012)$ \\
\hline \multicolumn{4}{|c|}{ Fire behavior } \\
\hline Season & Speed of prop & gation $\left(\mathrm{m} . \mathrm{s}^{-1}\right)$ & Flame length (m) \\
\hline 1 (Maio) & 0,20 & ,047) & $2,51(0,390)$ \\
\hline 2 (Junho) & 0,20 & $, 031)$ & $2,60(0,282)$ \\
\hline 3 (Agosto) & 0,17 & $, 045)$ & $2,26(0,264)$ \\
\hline 4 (Setembro) & 0,41 & $, 036)$ & $3,68(0,211)$ \\
\hline
\end{tabular}

Values in parenthesis correspond to the standard error of the mean.

Among the different burning steps, the speed of propagation presented values from 0,17 to 0,41 m.s-1 (Table 1), being classified as extreme speed (Botelho; Ventura, 1990); and the flame length during burnings had values from 2,26 to $3,68 \mathrm{~m}$.

\subsection{Climate during research}

The monthly averages of meteorological variables considering the research period (Table 2) show that climate conditions varied among months with controlled burns, with May and June being both the most humid and with the lowest temperatures; August and September were both the hottest and the 
driest months. At Corredor Ecológico da Região do Jalapão (Ecological Corridor of Jalapão Region), the aforementioned characteristics are the normal conditions of beginning (May and June) and end (August and September) of the dry season, respectively (MMA, 2013).

Table 2 - Meteorological conditions during the period of the research (MAPA, 2018a).

\begin{tabular}{lccccc}
\hline Month/2017 & $\begin{array}{c}\text { Temperature } \\
\left({ }^{\circ} \mathrm{C}\right)\end{array}$ & $\begin{array}{c}\text { Relative } \\
\text { humidity } \\
(\%)\end{array}$ & $\begin{array}{c}\text { Wind } \\
\left(\mathrm{m} \cdot \mathrm{s}^{-1}\right)\end{array}$ & $\begin{array}{c}\text { Radiation } \\
\left(\mathrm{kJ} . \mathrm{m}^{2}\right)\end{array}$ & $\begin{array}{c}\text { Rainfall } \\
(\mathrm{mm})\end{array}$ \\
\hline May & 25,71 & 69,22 & 161,39 & 821,28 & 0,02 \\
June & 24,38 & 59,22 & 151,13 & 801,36 & 0,01 \\
August & 26,66 & 39,76 & 135,01 & 888,65 & 0,00 \\
September & 29,48 & 34,40 & 96,08 & 1380,42 & 0,00 \\
\hline
\end{tabular}

Only the average monthly precipitation did not differ by among months, being, actually, minimum and typical for the period at the study area. The climatic variability and forest fuel are the main determining factors of the fire behavior and, thus, are important for the dynamic comprehension of severity of fire under distinct conditions (Kane et al., 2015; Soares; Batista; Tetto, 2017).

\subsection{Topkill}

The evaluation of severity of fire in vegetation through topkill rate and the percentage of reduction of the number of vegetable individuals after burning show that (Table 2), among areas with different years without burning, the variation occurred only in September. The values of areas of three and four years without forest fires $(66,0$ and $52,3 \%$, respectively) did not differ by among them $(\mathrm{p}>0,05)$ and were higher than rate at areas with two years without fires $(37,8 \%)$. At the end of dry season, considering an area with seven yours without burnings, Hoffman and Solbrig (2003) verified topkill rates of $100 \%$. Thus, it is observed that in burnings performed at the end of dry season, topkill rates tend to increase at areas with higher period of fire suppression.

Table 3 - Topkill rate of vegetation (\%)

\begin{tabular}{lccc}
\hline \multirow{2}{*}{ Month } & \multicolumn{3}{c}{ Fire frequency } \\
\cline { 2 - 4 } & 2 years & 3 years & 4 years \\
\hline May & $29,5 \mathrm{Ab}$ & $32,3 \mathrm{Ab}$ & $45,0 \mathrm{Aa}$ \\
\hline June & $38,0 \mathrm{Ab}$ & $43,5 \mathrm{Ab}$ & $56,0 \mathrm{Aa}$ \\
\hline August & $62,8 \mathrm{Aa}$ & $43,8 \mathrm{Ab}$ & $48,8 \mathrm{Aa}$ \\
\hline September & $37,8 \mathrm{Bb}$ & $66,0 \mathrm{Aa}$ & $52,3 \mathrm{Aa}$ \\
\hline Septem & the statistical difference at $5 \%$ significance through Scott-Knott test; being capital letters \\
paring years without fires and lowercase letters the months of burning.
\end{tabular}

This variation observed only in September may be associated to the higher fuel load at areas of three and four years without burning (Table 1) and the meteorological conditions more propitious to burning of higher severity: greater air temperature $\left(29,48{ }^{\circ} \mathrm{C}\right)$, lower relative air humidity $(34,40 \%)$ and higher incidence of solar radiation $\left(1380,42 \mathrm{~kJ} / \mathrm{m}^{2}\right)$ (Table 2). These factors contributed for higher speed of fire propagation $(0,41 \mathrm{~m} / \mathrm{s})$ and larger flame length $(3,68 \mathrm{~m})$ (Table 1$)$.

At areas with two years without burning, the topkill rate was higher in August $(62,8 \%)$. This condition may be associated to a possible higher residence time of fire, in function of reduction of wind speed in this period (Table 1). According to Cochrane et al., (1999), the residence time contributes for increase on damage to vegetation. Besides that, the areas with two years without burning presented part of the vegetation with tender and thin stems, great part sprouted from the last 
fire, facilitating the consumption by fire at conditions of higher residence time. On the contrary, individuals with larger diameter stems and thicker bark tend to be more resilient to fire (Hoffmann; Solbrig, 2003; Hoffmann et al., 2009; Moreira et al., 2009).

At areas with three years without burning, the highest topkill rates occurred in September (66\%). These values are linked to the extreme drought conditions in this period (Table 2) which contributed for the largest flame length (Table 1).

Areas with four years without burning presented values between $45 \%$ and $56 \%$, which did not differ by statistically among them ( $>>0,05)$. These results show that at areas with higher accumulation of fuel material, due to period of time without burning occurrences, the period of burning (beginning or end of the dry season) was not capable of differentiating topkill rates. From the fire triangle (fuel, heat and oxygen), the fuel is, probably, the most complex component, since it varies in quantity, geometry, density, continuity and chemical characteristics (Parsons et al., 2016; Soares et al., 2017). May the homogeneity of topkill rates at areas of four years without burning be associated to the higher fuel quantity in comparison to the areas with three and two years (Table 1). These factors favored a more homogeneous behavior of fire in these areas.

\section{Conclusion}

Only at areas with four years of fuel accumulation there was not variation in topkill rates throughout the months of burning. Regarding the comparison among areas with two, three and four years of fuel accumulation, difference was only observed in September - the end of the dry season.

\section{References}

Botelho, H. S., \& Ventura, J. (1990). Modelos de comportamento do fogo. A técnica do fogo controlado. Universidade de Trás-os-Montes e Alto Douro: Vila Real, 49-55.

Clarke, P. J., Knox, K. J., Bradstock, R. A., Munoz-Robles, C., \& Kumar, L. (2014). Vegetation, terrain and fire history shape the impact of extreme weather on fire severity and ecosystem response. Journal of vegetation science, 25(4), 1033-1044. Doi:10.1111/jvs.12166

Cochrane, M. A., Alencar, A., Schulze, M. D., Souza, C. M., Nepstad, D. C., Lefebvre, P., \& Davidson, E. A. (1999). Positive feedbacks in the fire dynamic of closed canopy tropical forests. Science, 284(5421), 1832-1835. Doi:10.1126/science.284.5421.1832

Coutinho, L.M. (1990). Fire in the ecology of the Brazilian cerrado. In: Goldhammer, J.G. (Ed.), Fire in the Tropical Biota. Springer, Berlin, pp. 82-105. Doi: 10.1007/978-3-642-75395-4_6

Hoffmann, W. A., \& Solbrig, O. T. (2003). The role of topkill in the differential response of savanna woody species to fire. Forest ecology and management, 180(1-3), 273-286. Doi:10.1016/S03781127(02)00566-2

Hoffmann, W. A., Adasme, R., Haridasan, M., De Carvalho, M.T., Geiger, E. L., Pereira, M. A.B., Gotsch, S.G., \& Franco, A. C. (2009). Tree topkill, not mortality, governs the dynamics of savannaforest boundaries under frequent fire in central Brazil. Ecology, 90(5), 1326-1337. Doi:10.1890/080741.1

Kane, V. R., Cansler, C. A., Povak, N. A., Kane, J. T., McGaughey, R. J., Lutz, J. A., Churchill, D. J. \& North, M. P. (2015). Mixed severity fire effects within the Rim fire: relative importance of local climate, fire weather, topography, and forest structure. Forest Ecology and Management, 358, 6279. Doi:10.1016/j.foreco.2015.09.001

Keeley, J. E. (2009). Fire intensity, fire severity and burn severity: a brief review and suggested usage. International Journal of Wildland Fire, 18(1), 116-126. Doi:10.1071/WF07049 
Knox, K. J., \& Clarke, P. J. (2016). Measuring fire severity: Are canopy, understorey and belowground measures coupled in sclerophyll forest fires?. Plant ecology, 217(6), 607-615. Doi:10.1007/s11258-016-0609-6

Lecina-Diaz, J., Alvarez, A., \& Retana, J. (2014). Extreme fire severity patterns in topographic, convective and wind-driven historical wildfires of Mediterranean pine forests. PloS one, 9(1), e85127. Doi:10.1371/journal.pone.0085127

MAPA - Ministério da Agricultura, Pecuária e Abastecimento. (2018a). Banco de Dados Meteorológicos para Ensino e Pesquisa - BDMEP. Instituto Nacional de Meteorologia. Disponível em: <http://www.inmet.gov.br/projetos/rede/pesquisa/>, acesso em: 27 de março de 2018.

MAPA - Ministério da Agricultura, Pecuária e Abastecimento. (2018b). Embrapa Solos. Correspondência entre classes do SiBCS, WRB/FAO e Soil Taxo. Disponível em: <https://www.embrapa.br/solos/sibcs/correlacao-com-wrb-fao-e-soil-taxonomy>, acesso em: $27 \mathrm{de}$ março de 2018.

MMA - Ministério do Meio Ambiente. (2013). Atlas do corredor ecológico da região do Jalapão. Instituto Chico Mendes de Conservação da Biodiversidade - ICMBio, 2.

Moreira, F., Catry, F., Duarte, I., Acácio, V., \& Silva, J. S. (2009). A conceptual model of sprouting responses in relation to fire damage: an example with cork oak (Quercus suber L.) trees in Southern Portugal. Plant Ecology, 201(1), 77-85. Doi:10.1007/s11258-008-9476-0

Nicholson, Á., Prior, L. D., Perry, G. L., \& Bowman, D. M. (2017). High post-fire mortality of resprouting woody plants in Tasmanian Mediterranean-type vegetation. International Journal of Wildland Fire, 26(6), 532-537. Doi:10.1071/WF16211

Parsons, R., Jolly, W. M., Hoffman, C., \& Ottmar, R. (2016). The Role of Fuels in Extreme Fire Behavior. Synthesis of Knowledge of Extreme Fire Behavior, 55-82.

Russell-Smith, J., Ryan, P. G., \& Durieu, R. (1997). A LANDSAT MSS-Derived fire history of kakadu national park, monsoonal northern australial, 1980-94: Seasonal extent, frequency and patchiness. Journal of Applied Ecology, 34 (3), 748-766. Doi:10.2307/2404920

Soares, R., V., Batista, A., C.; \& Tetto, A.F. (2017). Incêndios florestais: controle, efeitos e uso do fogo. Ronaldo Viana Soares and Antonio Carlos Batista independent publisher. Curitiba, PR (Brazil). 255 p.

Tocantins (2012). Atlas do Tocantins: subsídios ao planejamento da gestão territorial. Secretaria do Planejamento e da Modernização da Gestão Pública-SEPLAN, 6. 


\title{
Influence of thermal treatment on the bioavailability of $\mathrm{Cu}, \mathrm{Cd}$ and $\mathrm{Zn}$ in polluted forest soils
}

\author{
Xiufeng Xia ${ }^{1}$; Yanyan $\mathrm{Liu}^{1 *}$; Kai Xu² ${ }^{2}$ Xianfeng Chen ${ }^{1}$; Xinyan Huang ${ }^{3}$; Shaoxian Song ${ }^{1}$ \\ ${ }^{1}$ School of Resources and Environmental Engineering, Wuhan University of Technology, Wuhan, \\ China,430070 \{wulengheiyin@whut.edu.cn*\} \\ ${ }^{2}$ Center for Material Research and Analysis, Wuhan University of Technology, Wuhan, China, \\ 430070 \{kaix@whut.edu.cn\} \\ ${ }^{3}$ Research Centre for Fire Engineering, The Hong Kong Polytechnic University, Kowloon, Hong \\ Kong \{xy.huang@polyu.edu.hk\}
}

\begin{abstract}
The role of fire on the soil is a complex and comprehensive process. Fire has influences on soil physiochemical properties and hence the soil ecosystem. Meanwhile, forest soil faces an increasingly severe pollution problem due to the use of pesticide and fertilizer. The heavy metals transfer into soil and underground water and bio-accumulate in flora and fauna, which is of great concern due to the immediate risks to human and ecosystems health. This work aims to investigate the factor of heating temperature on the heavy metals in forest soils by using thermal treatment microscopically, in order to establish the correlation between the macro-scale and micro-scale research on the investigation of fire effect on heavy metals in soils; disclose the mechanisms of individual fire factor on heavy metals transformation by micro-scale experiments. Soil samples were taken from the upper layer $(0-5 \mathrm{~cm})$ of the forest soil that situated in Huangshi Mining National Park, China where used to be a mining site and now reserved as a national park with vegetation recovery. However, the soils are still in the pollution state with high concentration of heavy metals, such as $\mathrm{Cu}, \mathrm{Cd}, \mathrm{Zn}, \mathrm{As}$, etc. The thermal interaction of heat is achieved by controlling the temperature and reservation time. In addition, the heating atmosphere with and without oxygen is also compared by using vacuum furnace and muffle furnace, respectively. Concentrations of heavy metals, compositions of soils are determined by ICP-MS and ICP-AES. Soils are dried and sieved. Other characterizing techniques include thermogravimetric analysis, dynamic laser light scattering, elemental analysis. The original concentrations of $\mathrm{Cu}, \mathrm{Cd}, \mathrm{Zn}$ were determined as $1303.41 \mathrm{mg} / \mathrm{kg}, 6.91 \mathrm{mg} / \mathrm{kg}, 927.1 \mathrm{mg} / \mathrm{kg}$ and $118.3 \mathrm{mg} / \mathrm{kg}$, respectively. Effect of heating on heavy metals displayed difference roles. Furthermore, the absence of oxygen may also change the fractions of heavy metals and reduce their bioavailability. The results of the effect of heating on heavy metals in soils by thermal treatment may shed light on the understanding of the mechanisms of wildfire effect on heavy metals in forest polluted soils which may provide the bias for the prediction of heavy metals transformation in the post-fire ecosystem and the exposure assessment of health risk. These mechanisms may shed light on the theory of forest fire ecology and provide measures for forest fire management and soil restoration.
\end{abstract}

Keywords: heat; heavy metals; bioavailability; soil pollution; fire ecology;

\section{Introduction}

Wildfire is a unique disturbance factor that plays a significant role on the evolution of earth ecosystem (DeBano et al, 1998). It can reduce the forest area and affect the biodiversity of flora and fauna in the burnt area. Soil experiences transformation in its physical, chemical and microbial properties, especially the modification of heavy metals and organic components. The organic matter can be decreased when temperature of soil reached to $300^{\circ} \mathrm{C}$.

Work of macro-scale fire on soil has been drawn great attention in various publications. Debano et al., (2000) and Malkinson et al. (2011) discussed the mechanisms of fire and soil heating on water 
repellency in wildland environments. Hydrophobic compounds were generated and migrated into soil deeper during the fire event, which may increase the water repellency. Meanwhile, the higher water content can provide a positive role on the protection for the soil against fire (Badia et al, 2017). Soil temperature and heat duration as a function of soil moisture and soil texture were identified to indicate that $20 \%$ volumetric moisture or greater was an effective means for limiting lethal heating in soils (Busse et al 2010). In spite of the damage by the fire, wildfire is considered that it can improve the soil quality (Stankov et al, 2011). Fire can increase soil $\mathrm{pH}$ and the bioavailability of N, P, K, Ca, nutrtion elements. However, few of the work were focused on the effect of fire on $\mathrm{Fe}, \mathrm{Mn}, \mathrm{Cu}, \mathrm{Zn}, \mathrm{Co}$, $\mathrm{B}$, et al., especially the heavy metals in polluted soils. Transformation of forms of $\mathrm{Mn}$ in burnt soils were observed (Parra and Lopes, 1996). The transformation of Fe, $\mathrm{Mn}, \mathrm{Cu}, \mathrm{Zn}$, were showed of irregularity. Macroscopic wildfire research under filed conditions is defective with various factors (vegetation, litter depth, soil water content, soil pore network, et al.) that may affect the soil properties comprehensively during fire conditions (Busse et al 2010; Archibold et al, 1998; Campbell and Jungbauer, 1995). Furthermore, literature has reported that the thermal remediation can alter the soil properties (O'Brian et al, 2018), indicating that the thermal treatment may change the forms of heavy metals in the soils.

Thus, this work aims to investigate the effect of temperature on the fractions of heavy metals in forest soils by using thermal treatment microscopically, in order to establish the correlation between the macro-scale and micro-scale research on the investigation of fire effect on heavy metals in soils; disclose the mechanisms of individual fire factor on heavy metals transformation by micro-scale experiments.

\section{Materials and Methods}

\subsection{Soil description and soil sampling}

Soil samples were taken from the upper layer $(0-5 \mathrm{~cm})$ of the forest soil that situated in Huangshi Mining National Park, China were historically under long-term mining operation. Concentrations of heavy metals, such as $\mathrm{Cu}, \mathrm{Cd}$ and $\mathrm{Ni}$, in the Huangshi Mine National Park were determined exceeding the criteria concentration in 2007 (Han, 2007).The main vegetation in this area are schima superba, locust tree and camphor tree. The soil type can be classified to red earth. Samples with 11 sampling points were taken in "S" shape and contained in the polyethylene box. Subsequently, soil samples were air-dried, grounded, sieved by $150 \mu \mathrm{m}$ particle size, and stored in a drying vessel before sample characterization and thermal treatment.

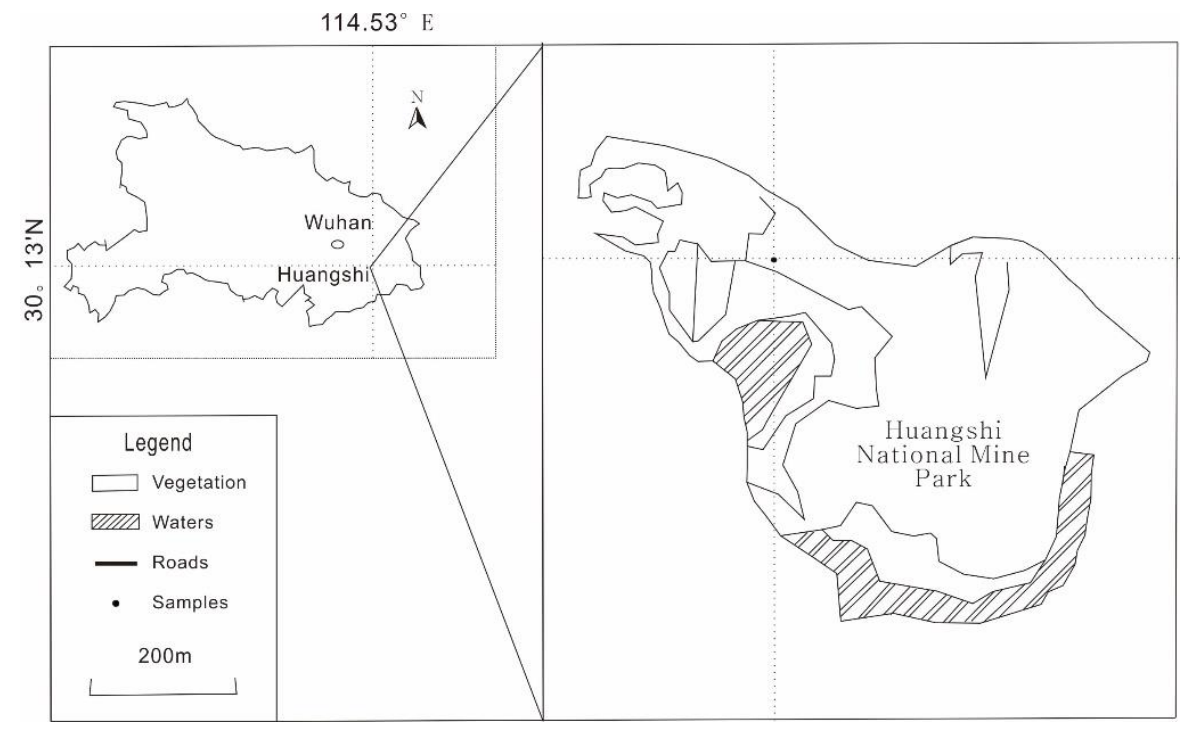

Figure 1 - Location of study area 
Chemical reagents include acetic acid, hydroxylamine hydrochloride, ammonium acetate, $\mathrm{HClO}_{4}$, $\mathrm{HCl}, \mathrm{HNO}_{3}$ etc. All the reagents are analytic. Mili-Q water $(18 \mathrm{M} \Omega \cdot \mathrm{cm})$ was used for all solution preparation.

\subsection{Thermal treatment of soil samples}

$8 \mathrm{~g}$ soil sample in the crucible was put in the tube furnace (MXG1200-S40), under $\mathrm{N}_{2}$ atmosphere. The parameters included heating temperature and reservation time, which were set at 200, 300, 400, $500{ }^{\circ} \mathrm{C}$ and $30,60,90 \mathrm{~min}$, respectively. As a contrast, the group of muffle furnace was set at $500{ }^{\circ} \mathrm{C}$. The soil samples after heating were cooled to room temperature and reserved in drying vessel for further characterization.

\subsection{BCR extraction method}

In this study, modified four-step BCR method were applied to extract the heavy metals in the soil samples. Accordingly, $0.5 \mathrm{~g}$ of dry soil was extracted in a $50-\mathrm{mL}$ centrifuge tube with the corresponding extracting solution. The first fraction (exchangeable fraction) was considered as most soluble and $0.11 \mathrm{~mol} / \mathrm{L}$ acetic acid was used as the extractant. The second extraction (Reducible fraction), from which the reducible elements were obtained, was performed using $0.5 \mathrm{~mol} / \mathrm{L}$ hydroxylamine hydrochloride. In the third extraction (Oxidizable fraction), the oxidizable fraction was obtained and $8.8 \mathrm{~mol} / \mathrm{L}$ ammonium acetate $(\mathrm{pH}=2)$ was used following oxidation with $8.8 \mathrm{~mol} / \mathrm{L} \mathrm{H}_{2} \mathrm{O}_{2}$. Finally, the fourth extraction (residual fraction) was perform by using Milli-Q water. All the samples were agitated at room temperature by using end-over-end shaking. Subsequently, all the supernate of soil samples were determined by inductively coupled plasma emission spectrometer(ICP).

\subsection{Charactersation methods}

TGA (Thermogravimetric Analysis, Perkinelmer sta6000) was used to analysis the weight loss of soil samples. ICP (Inductively Coupled Plasma Emission Spectrometer, Agilent 5100) was applied to determine the concentration of the heavy metals in the soil sample before and after heat treatment. Other equipment used included centrifugal machine, table concentrator, etc..

\section{Results and discussion}

\subsection{Fractions of heavy metals in soil samples before thermal treatment}

Total concentrations of $\mathrm{Cu}, \mathrm{Cd}, \mathrm{Zn}$ in the soil were determined to be $1303.41 \mathrm{mg} / \mathrm{kg}, 6.91 \mathrm{mg} / \mathrm{kg}$, $927.1 \mathrm{mg} / \mathrm{kg}$ and $118.3 \mathrm{mg} / \mathrm{kg}$, respectively, which far exceeded the soil criteria of China. The heavy metal fractions of original samples are shown in Table 1. The percentage of the fraction distribution of heavy metals displays in Figure 1. The main form of $\mathrm{Cd}$ is residual, so $\mathrm{Cd}$ is very stable in the soil sample. The order of concentrations of different fractions of $\mathrm{Cu}$ is as following: the reducible > oxidation $>$ the residual $>$ the acid soluble. $\mathrm{Cu}$ in the sample has a relatively high bioavailability, The order of concentration of different fractions of $\mathrm{Zn}$ shows: the residual $>$ the reducible $>$ oxidation $>$ the acid soluble. The available fractions of heavy metal are similar to $\mathrm{Cu}$, both of which can cause a risk to ecosystem and human health. 


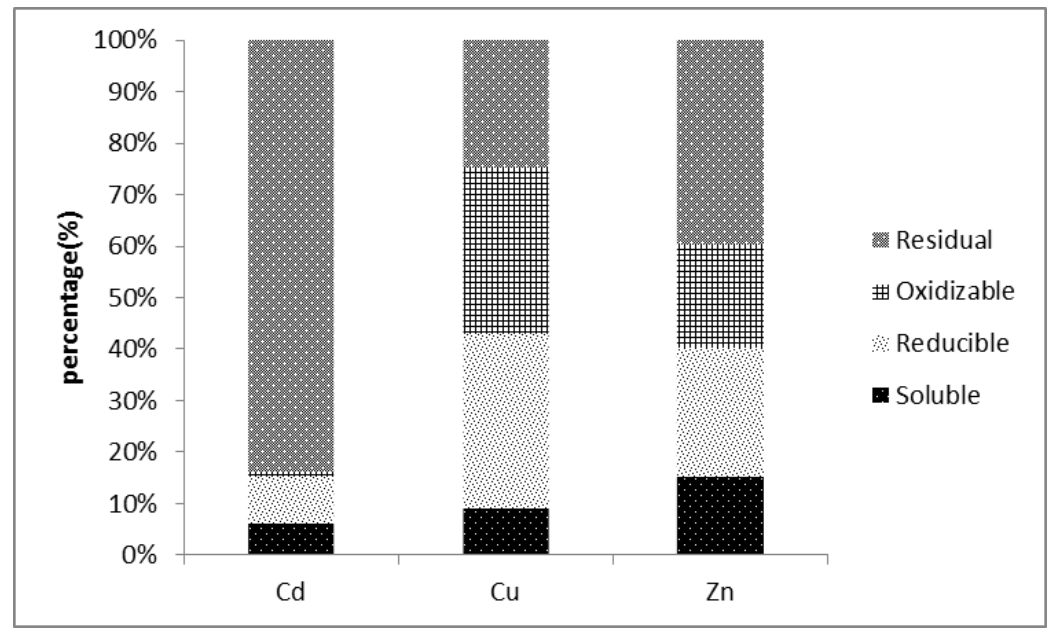

Figure 2 - The fraction distribution of $\mathrm{Cd}, \mathrm{Cu}$ and $\mathrm{Zn}$

Table 1 concentrations of copper, cadmium, zinc in the soil samples

\begin{tabular}{|c|c|c|c|c|c|c|}
\hline $\begin{array}{c}\text { Heavy } \\
\text { metals }\end{array}$ & $\begin{array}{c}\text { Soluble } \\
(\mathrm{mg} / \mathrm{kg})\end{array}$ & $\begin{array}{c}\text { Reducible } \\
(\mathrm{mg} / \mathrm{kg})\end{array}$ & $\begin{array}{c}\text { Oxidizable } \\
(\mathrm{mg} / \mathrm{kg})\end{array}$ & $\begin{array}{c}\text { Residual } \\
(\mathrm{mg} / \mathrm{kg})\end{array}$ & $\begin{array}{c}\text { Total } \\
(\mathrm{mg} / \mathrm{kg})\end{array}$ & $\begin{array}{c}\text { Total in } \\
\text { Criteria } \\
(\mathrm{mg} / \mathrm{kg})\end{array}$ \\
\hline $\mathrm{Zn}$ & 17.43 & 28.98 & 23.08 & 46.02 & 927.1 & 500 \\
\hline $\mathrm{Cu}$ & 116.45 & 442.75 & 421.25 & 322.95 & 1303.41 & 400 \\
\hline $\mathrm{Cd}$ & 0.42 & 0.63 & 0.07 & 5.79 & 6.91 & 0.3 \\
\hline
\end{tabular}

\subsection{Effects of temperature on heavy metal fractions}

Distribution of $\mathrm{Cu}, \mathrm{Cd}$ and $\mathrm{Zn}$ in the aqueous extracts and solid residue from sequential extraction of soil samples are shown in Figure 2. Concentrations of available fraction are shown in Figure 3. It can be easily found that the trend of avaiable fraction concentration of three heavy metals is decreased with the increase of temperature, which is same with Yang's results [13]. The increase of $\mathrm{Cu}$ and $\mathrm{Cd}$ from $200^{\circ} \mathrm{C}$ to $300^{\circ} \mathrm{C}$ may due to the weight loss during temperature increasing, which can be obviously seen in Figure 4. The changes of fraction distribution is rather complex, but compared to at $200^{\circ} \mathrm{C}$, the percent of available fraction in $\mathrm{Cd}, \mathrm{Cu}$ and $\mathrm{Zn}$ is lower when the constant heating temperature was set at $500^{\circ} \mathrm{C}$, which can indicate that the relative high temperature is able to reduce the bioavailability of heavy metals.

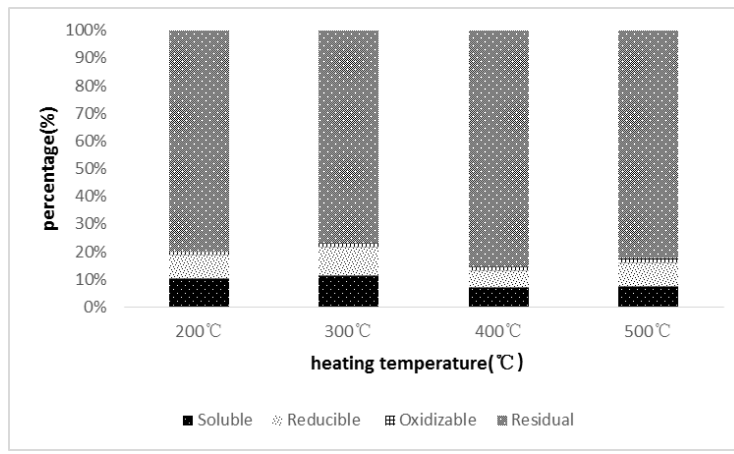

(a)

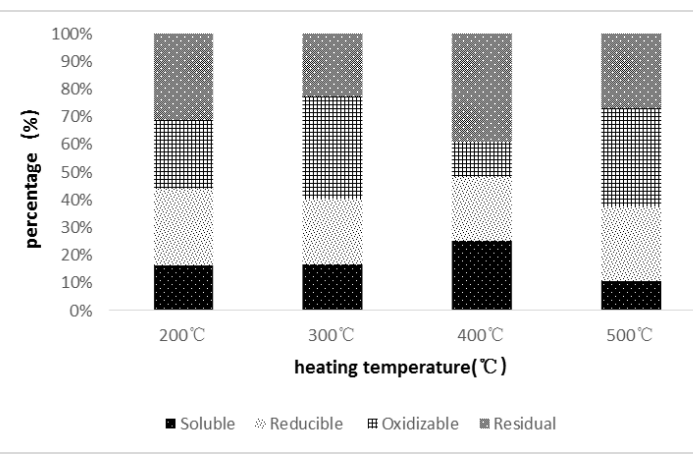

(b) 


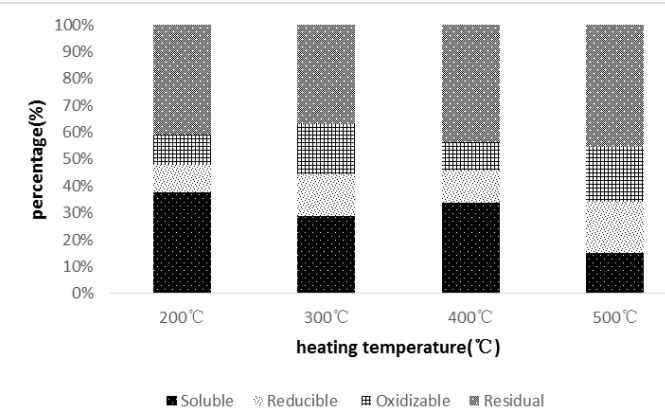

(c)

Figure 2 - Effects of temperature on the distribution of $\mathrm{Cd}(\mathrm{a}), \mathrm{Cu}(\mathrm{b})$, and $\mathrm{Zn}(\mathrm{c})$.

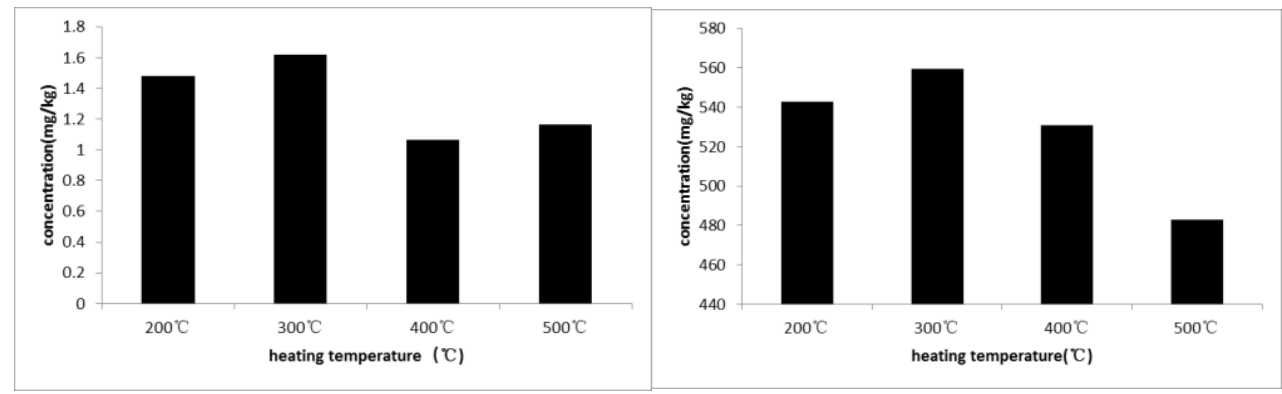

(a)

(b)

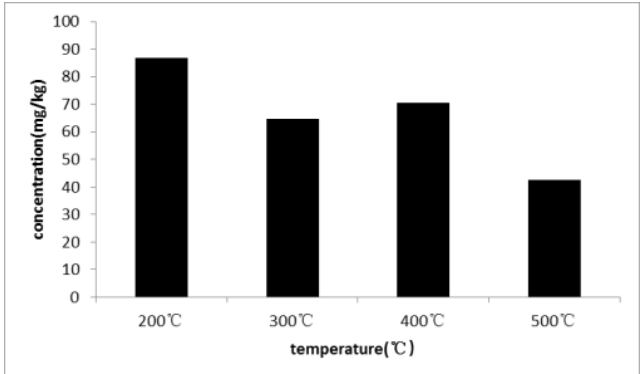

(c)

Figure 3 - Effects of temperature on available fraction concentration of $\mathrm{Cd}(\mathrm{a}), \mathrm{Cu}(\mathrm{b})$, and $\mathrm{Zn}(\mathrm{c})$.

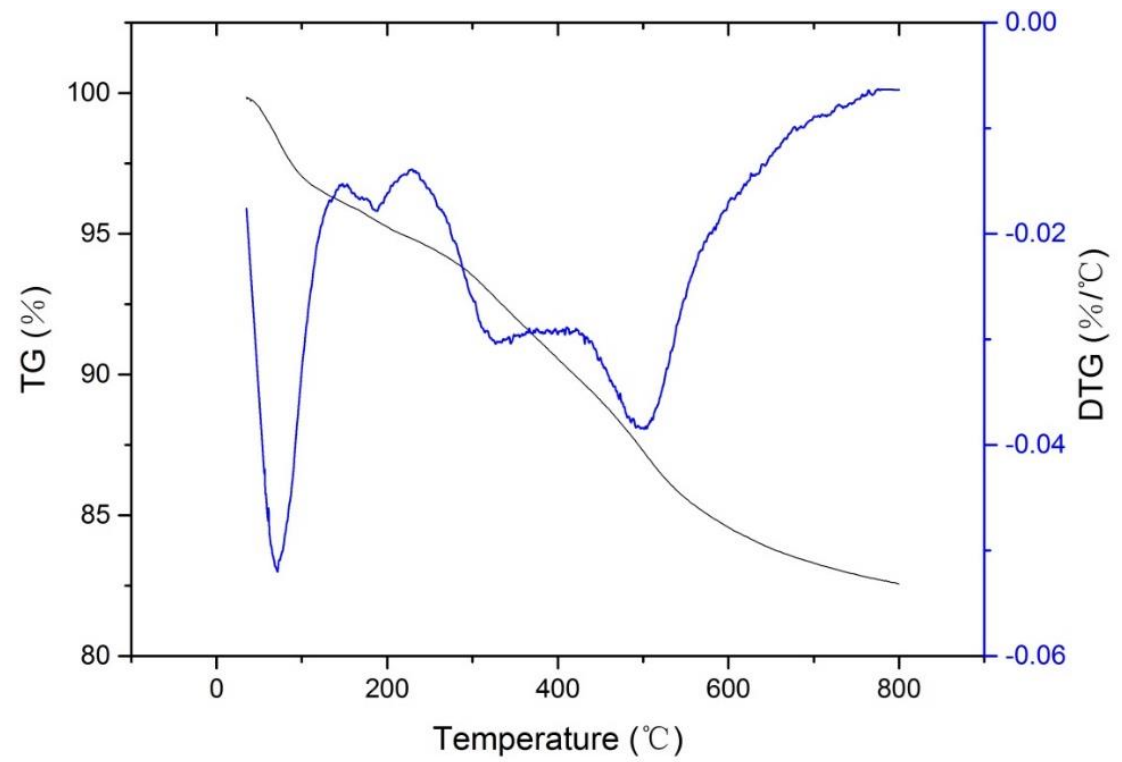

Figure 4 - The result of TGA(Thermogravimetric Analysis) 


\subsection{Effects of heating time on heavy metal fractions}

Figure 5, 6 show the effects of reservation heating time on heavy metal fraction distribution. The trend of available fraction of $\mathrm{Cu}$ and $\mathrm{Zn}$ was decreased when the heating time increased. In addition, the distribution of $\mathrm{Cd}$ was almost constant because the residual fraction of $\mathrm{Cd}$ was very high in original soil sample. The available concentrations of heavy metals were decreased with the heating time shown in Fig..., which indicated that long-time heating might benefit for decreasing heavy metal bioavailability. Besides, compared to $\mathrm{Cd}$ and $\mathrm{Zn}$, the change of $\mathrm{Cu}$ was not obvious, which may be the result of that $\mathrm{Cu}$ possesses a relatively high boiling point.

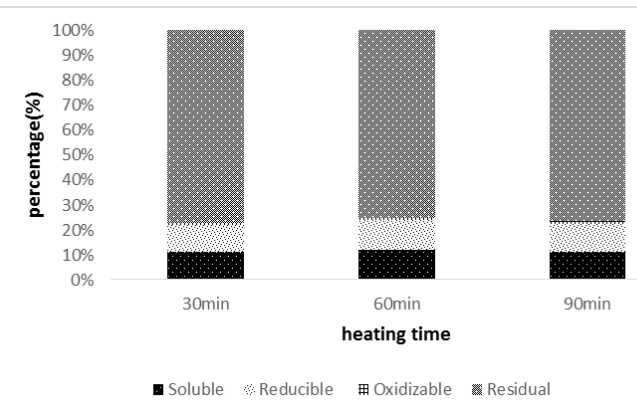

(a)

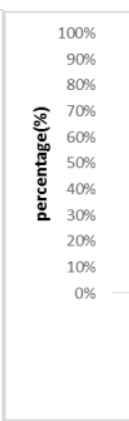

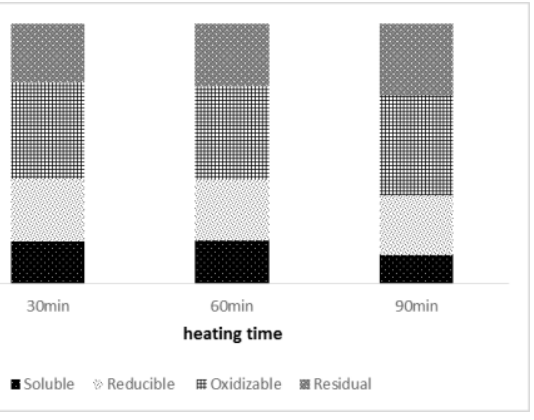

(b)

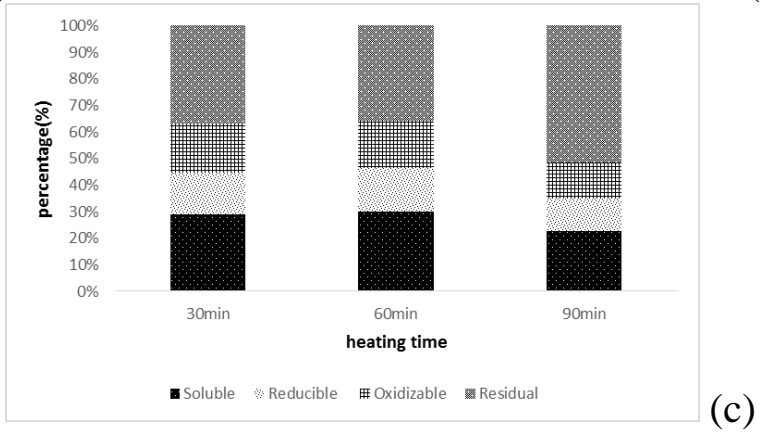

Figure 5 - Effects of temperature on the distribution of $\mathrm{Cd}(\mathrm{a}), \mathrm{Cu}(\mathrm{b})$, and $\mathrm{Zn}(\mathrm{c})$.

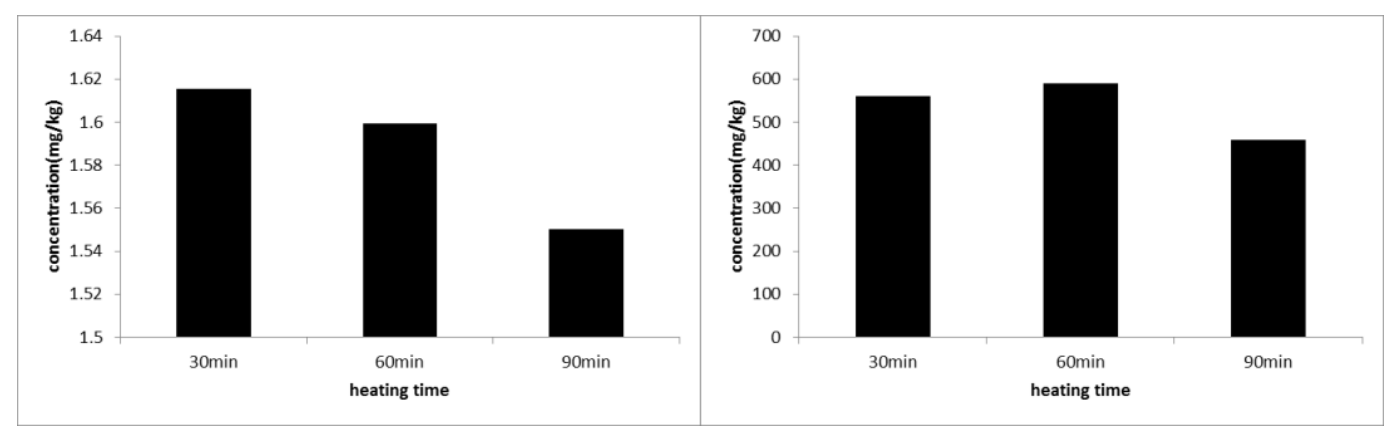

(a)

(b)

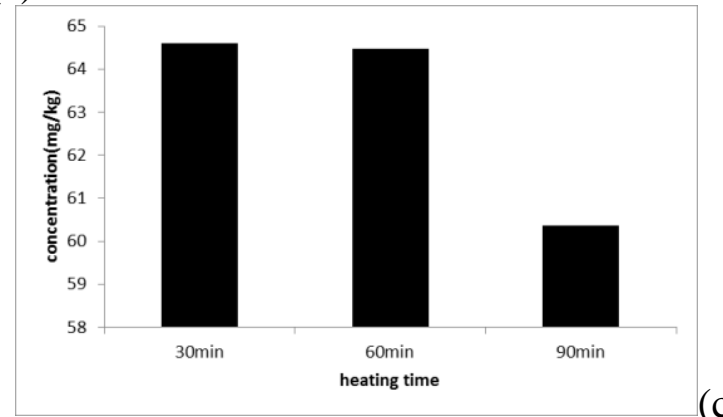

(c)

Figure 6 -The influence of heating time on available $\mathrm{Cd}(\mathrm{a}), \mathrm{Cu}(\mathrm{b}), \mathrm{Zn}(\mathrm{c})$ concentration 


\subsection{Oxygen effect}

Oxygen effect on the heavy metals in soils were performed and compared by muffle furnace and tube furnace, shown in Figure 7. The available $\mathrm{Cd}$ concentrations of the tube furnace and muffle furnace showed little difference with values of 1.16 and $1.27 \mathrm{mg} / \mathrm{kg}$ respectively. However, there was an obvious difference for $\mathrm{Cu}$ and $\mathrm{Zn}$. The available $\mathrm{Cd}$ concentrations of tube furnace and muffle furnace are $483.1046,596.5688 \mathrm{mg} / \mathrm{kg}$ respectively. For $\mathrm{Zn}$, the concentration was 42.56 and $72.20 \mathrm{mg} / \mathrm{kg}$, respectively. Such results indicated that absence of oxygen may play a role on reducing the heavy metal bioavailibity.

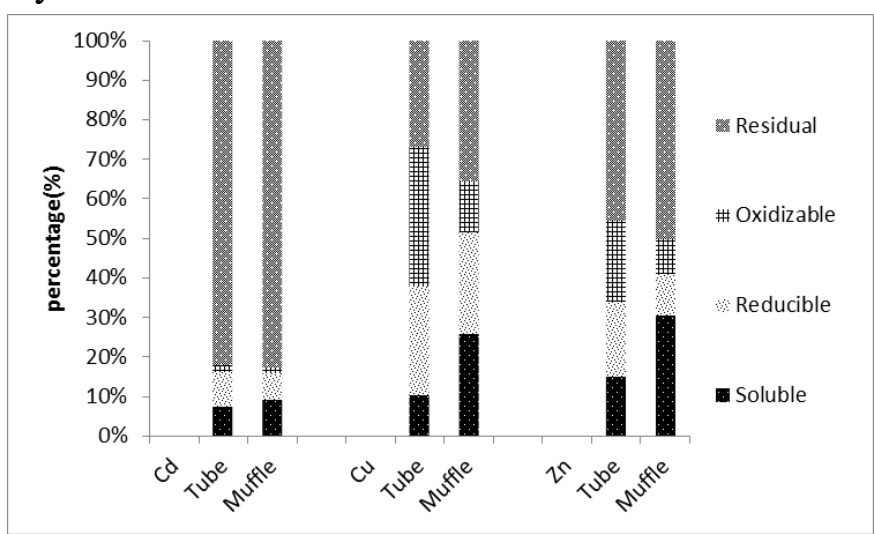

Figure 7 - The comparision between tube furnace and muffle furnace

\section{Conclusions}

The work investigated the effect of heating time and reservation time on heavy metals in forest polluted soils by using thermal treatment method. The original concentrations of $\mathrm{Cu}, \mathrm{Cd}, \mathrm{Zn}$ were determined as $1303.41 \mathrm{mg} / \mathrm{kg}, 6.91 \mathrm{mg} / \mathrm{kg}, 927.1 \mathrm{mg} / \mathrm{kg}$ and $118.3 \mathrm{mg} / \mathrm{kg}$, respectively. Effect of heating on heavy metals displayed difference roles. After thermal treatment, concentration of Cd was increased after $200,300,400,500{ }^{\circ} \mathrm{C}$. which may due to the weight loss during heating. Percentage of fractions the bioavailability of $\mathrm{Cu}, \mathrm{Cd}, \mathrm{Zn}$ was no noticeable. Furthermore, the absence of oxygen may also change the fractions of heavy metals and reduce their bioavailability. The results of the effect of heating on heavy metals in soils by thermal treatment may shed light on the understanding of the mechanisms of wildfire effect on heavy metals in forest polluted soils which may provide the bias for the prediction of heavy metals transformation in the post-fire ecosystem and the exposure assessment of health risk.

\section{Acknowledgement}

This work is financially supported by the National Natural Science Foundation of China (51504174) and Open Foundation of Center for Material Research and Analysis (KFJJ201812).

\section{References:}

DeBano, L.F., Neary, D.G. \& Ffolliott, P.F. (1998) Fire's Effects on Ecosystems. New York: John Wiley \& Sons, Inc., .

DeBano, L.F. (2000) The role of fire and soil heating on water repellency in wildland environments: a review. Journal of Hydrology 231-232, 195-206.

Malkinson, D. and Wittenberg, L. (2011) Post fire induced soil water repellency-Modeling short and long-term processes. Geomorphology 125(1), 186-192. 
Badía, D., López-García, S., Martí,C.,Ortíz-Perpiñá, O., Girona-García, C. (2017) Burn effects on soil properties associated to heat transfer under contrasting moisture content. Science of The Total Environment 601-602,1119-1128.

Busse, M.D., Shestak, C.J.,Hubbert,K.R.,Knapp,E.E. (2010) Soil Physical Properties Regulate Lethal Heating during Burning of Woody Residues.Soil Science Society of America Journal 74,947-955.

V.P. Stankov Jovanovic, M.D.I., M.S. Markovic, V.D. Mitic, S.D. Nikolic Mandic, G.S. Stojanovic (2011) Wild fire impact on copper, zinc, lead and cadmium distribution in soil and relation with abundance in selected plants of Lamiaceae family from Vidlic Mountain (Serbia). Chemosphere 84,1584-1591.

Parra JG, R.V., Lopez TI (1996) Forms of Mn in soils affected by a forest fire. . Science of the Total Environment, 231-236.

Archibold, O.W.,Nelson L.J.,Ripley E.A.,Delanoy L.(1998) Fire temperatures in plant communities of the northern mixed prairie. Canadian Field-Naturalist 112(2), 234-240.

Campbell, G.S., JUNGBAUER,JR J. D. (1995) Soil temperature and water content beneath a surface fire. Soil Science 159(6), 363-374.

O'Brien, P.L., Desutter, T.M. Fxm, C., Khan E.,Wick A.F. (2018) Thermal remediation alters soil properties - a review. Journal of Environmental Management 206, 826-835.

Ming Han, H.L (2007) Analysis of Changing Trend on Heavy Metal Pollution in Huangshi City. Environmental Science and Technology 30,75-76. 


\title{
Live fuel moisture content: variability, predictability and impact on fire behavior and activity
}

\author{
Martin-StPaul Nicolas ${ }^{1 *}$, Ruffault Julien ${ }^{2}$, François Pimont ${ }^{1}$, Jean-Luc Dupuy ${ }^{1}$ \\ ${ }^{1}$ URFM INRA. 84914 Avignon, France, \\ \{nicolas.martin@inra.fr*,francois.pimont@inra.fr,jean-luc.dupuy@inra.fr\} \\ ${ }^{2} I M B E$, Aix Marseille Université, CNRS, IRD, Avignon Université, Marseille, France, \\ \{julien.ruff@gmail.com\}
}

\begin{abstract}
Live Fuel Moisture Content (LFMC) - the ratio of water mass to the dry mass of live fuel - is a critical factor of fire behavior and hazard. This parameter is largely controlled by weather conditions and is affected by climate changes. There is therefore an increasing need, to understand its variability, to improve its predictability and its impact on fire behavior and activity. This would enhance the development of tools for operational fire risk management and wildfire research. Here we compile several recent findings regarding these issues, most of them were based on the French LFMC database collected for operational purposes and containing more than 20,000 measurement dates during 22 fire seasons on 30 sites and 25 species distributed over the French Mediterranean. First we evaluated the predictability of LFMC by fitting linear relationships between LFMC and various daily empirical drought indices (Drought Code=DC, Keetch-Byram Drought Index $=$ KBDI, Dead Moisture Code=DMC) and a water balance model describing the Relative Water Content of the soil (RWC). We found a limited explanatory power of the drought indices based on climate only due to large differences between sites and species. This support the view that predictions can be improved by accounting for stand and species specific parameters by developing more process-based approaches. The RWC model is a first step toward this direction, and can account for stand level parameterization of soil properties and leaf area index of the vegetation if data are available. To account for species specific traits involved in plant dehydration, we suggest an adaptation of a plant hydraulic model to simulate LFMC response to water potential according to plant hydraulic traits. This is promising with regard to the development of a fully-mechanistic approach of the prediction of LFMC, which would include the responses of both plant and soil. Second, we used an existing dataset reporting shrub fire experiments to investigate the response function of fire rate of spread (ROS) to LFMC. We found a very significant effect of LFMC below $100 \%$. However, because most LFMC values in the ROS database are higher than those prevailing in the French database, large uncertainties in ROS estimation were obtained below LFMC of ca. 70\%. Finally, we explored the relationship between and fire occurrence using a French database of fire activity and the French LFMC database. We found that the response of the fire occurrence of more than tha was very similar to the response of rate of spread. Our global approach drawing on long-term field LFMC data constitutes an important step forward in weaving the mechanistic links between climate, vegetation functioning and fire activities.
\end{abstract}

Keywords: LFMC, fire occurrence; drought index;

\section{Introduction}

Live Fuel Moisture Content (LFMC) - the ratio of water mass to the dry mass of live fuel - is increasingly recognized as a critical factor of fire behavior (Rossa et al. 2016) and hazard (Dennison and Moritz 2009; Nolan et al. 2016; Ruffault et al. 2018). This fuel parameter is obviously affected by drought and thus climate change, but its dynamic remains poorly understood and predicted. 
While field measurement remains the reference method for evaluating LFMC, it is generally limited for obvious practical constraints. Remote sensing is an appealing alternative to field measurements but requires extensive calibration and validation (Yebra et al. 2013). Another widespread approach is the use of empirical relationships between meteorological drought indices (Drought Code, KeetchByramDrought Index, etc.) and LFMC (Viegas et al. 2001) (Viegas et al., 2001), which enables forecast and projection, since such indices implement simple functions of basic daily weather data (temperature, precipitation, etc.).

A 20,000 multispecies and multisite dataset of LFMC measurements in Mediterranean France over 20 years has recently been released (referred to as "RH" for "Réseau Hydrique", Martin-StPaul et al. 2018). This dataset provides new opportunities to increase knowledge regarding 1) the predictability of LFMC through meteorological drought indices and remote sensing products; 2) The basis for a mechanistic modelling of LFMC, which could account for both soil and vegetation properties; 3) its impact on fire activity, through comparisons between LFMC dynamics and actual fire activity.

In the present study, we address these three points, with the exception of remote sensing predictability, which has already been evaluated on this dataset (Fan et al. 2018). The basis In order to evaluate the impact of LFMC on fire behavior, we also use the shrub fire experiment dataset collected in Anderson et al. (2015) to assess the response of the fire rate of spread (ROS) to LFMC.

\section{Live Fuel Moisture predictability and variability}

\subsection{Empirical indices to predict Live Fuel Moisture Content}

We evaluated the predictability of LFMC by fitting linear relationships between LFMC and various empirical drought indices (Drought Code=DC, Keetch-Byram Drought Index=KBDI, Dead Moisture Code $=$ DMC) and a process-based water balance model describing the Relative Water Content of the soil (RWC). Climatic data required (including temperature, rainfall, radiation, relative humidity) were extracted for each site from the French reanalysis SAFRAN. The species were classified in two groups according to their seasonal variability: high and low responding species. We found that the performance was similar for all drought indices, with RMSE on the order of $18 \%$ and determination coefficients on the order of 0.3 (Figure 1). As pointed out in Yebra et al. (2013), such an error is too large for operational and research purposes, as the ignition probability corresponding to this error might range from low to high levels (from 19-60\%). Most of the factor of variability were due to site and species differences (Ruffault et al. under review)

Those results suggest that empirical drought indices should be used with caution for LFMC prediction and that such indices could probably be improved, using more mechanistic approaches that could account for site other than climate and species properties (Ruffault et al. under review). RWC is a first step toward this direction, but requires adequate parameterization of soil properties and leaf area index of the vegetation. We are currently consolidating a large database of soil properties and leaf area index for the different RH site where LFMC is currently measured in the French Mediterranean. This will help to elucidate to what extent soil and LAI contribute to defining LFMC dynamics during drought. 

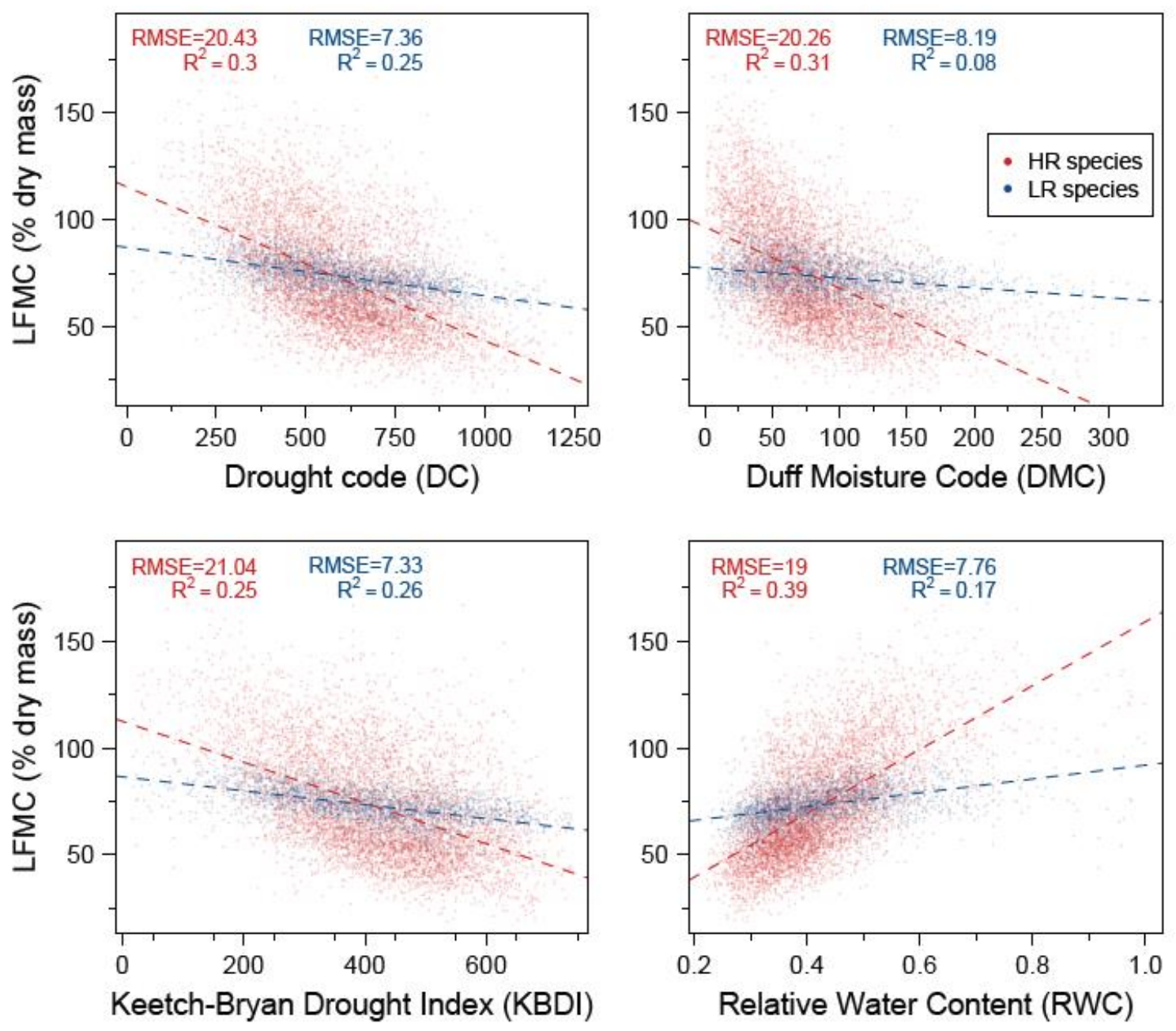

Figure 1 - Linear models between live fuel moisture content (LFMC) and different drought indices (DC, DMC, KDBI and $\left.R W C_{H}\right)$ for two different groups of species: High responding $(H R)$ and Low responding (LR) species. LFMC was measured during the fire season on 20 different sites for the period from 2000 to 2013.

\subsection{A physiological hydraulic basis for predicting species dynamics of LFMC}

We found that the predictability of LFMC including the low and high responding strategies- were linked to certain physiological traits related to plant hydraulic and plant regulation of water potential. This suggests that the physiology of water transport in plants, namely plant hydraulic, can provide insights regarding LFMC response to drought. In this context, we suggest a mechanistic framework to derive LFMC from plant water potential $(\psi, \mathrm{MPa})$. This framework is based on the plant hydraulic model SurEau presented in (Martin-StPaul et al. 2017). SurEau simulates two processes involved in plant desiccation: (i) the dehydration of the living cells (symplastic tissue) giving the relative water content of the symplast (RWCs) and (ii) the cavitation of the xylem (apoplastic tissue) giving the relative water content of the apoplast (RWCa). Both values can then be used to predict LFMC, if the specific dry mass content and the apoplastic fraction of the tissue considered are known. Fig. 2 illustrates a typical dynamic of symplastic and apoplastic RWC as well as LFMC as a function of plant water potential, for a set of species-specific parameters indicated in the legend. Further details about the equations and parameters can be found in the description of the SurEau model in Martin-StPaul et al (2017). 


\section{LFMC vs water potential}

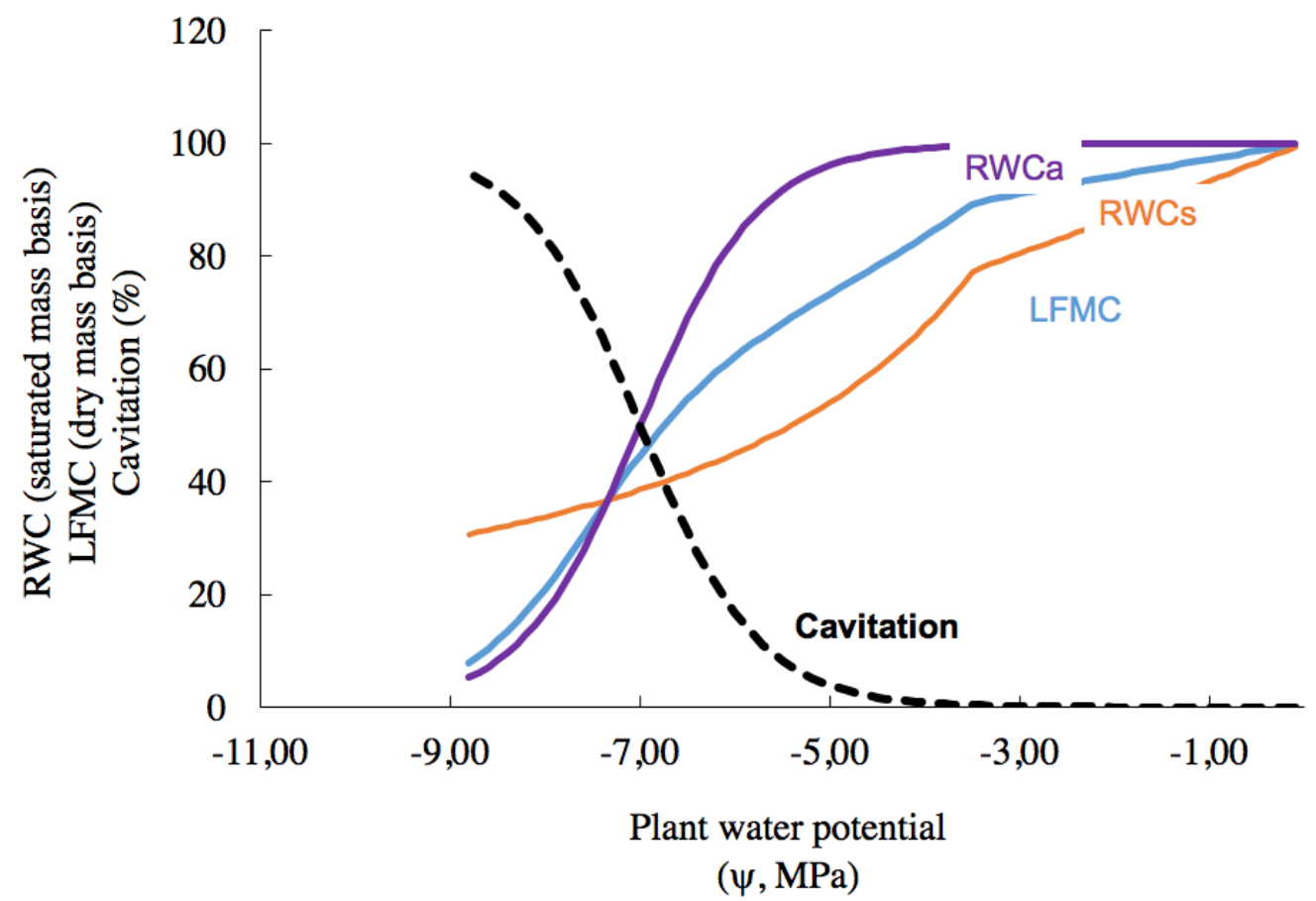

Figure 2 - Theoretical response of LFMC to plant water potential simulated with SurEau describing the dynamic of relative water content in both the symplastic compartment (RWCs) and the apoplastic compartment (RWCa). The dynamic of cavitation is shown in black. For these simulations we used a cavitation resistance (taken as the water potential causing $50 \%$ cavitation) of $-7 \mathrm{MPa}$, a turgor loss point of $-3.5 \mathrm{MPa}$, a leaf dry matter content of 500mg/g, and an apoplastic fraction of 0.4. Further details about the equations and parameters can be found in the description of the SurEau model in Martin-StPaul et al (2017).

\section{Live Fuel Moisture impact on fire behavior}

In this subsection, we use a shrub fire experiment dataset (Anderson et al. 2015) to fit a model for rate of spread (ROS) as a function of LFMC. The model is the following generalized additive model (GAM, Hastie et al. 2009):

$$
R O S=a U^{b} e^{-c D F M C} H^{d} S(L F M C)
$$

where $\mathrm{s}$ is smooth function. The model fit, using a "log" link and assuming a gamma distribution of the residuals, is described in details in Pimont et al. (2018a).

The response of ROS to LFMC is a multiplicative function showing a strong increase of fire behavior when LFMC is below 100\%, but negligible above it (Fig. 2). The green, orange and red arrows show the different ROS increases (in \%) associated with the three thresholds used by French fire prevention and fighting managers. Our analysis shows that these thresholds effectively correspond to strong increase in fire behavior. However, the range of the confidence intervals strongly increases below $67 \%$, as the experimental fires were hold in LFMC conditions above 67\% (between 67 and 256 $\%)$. 


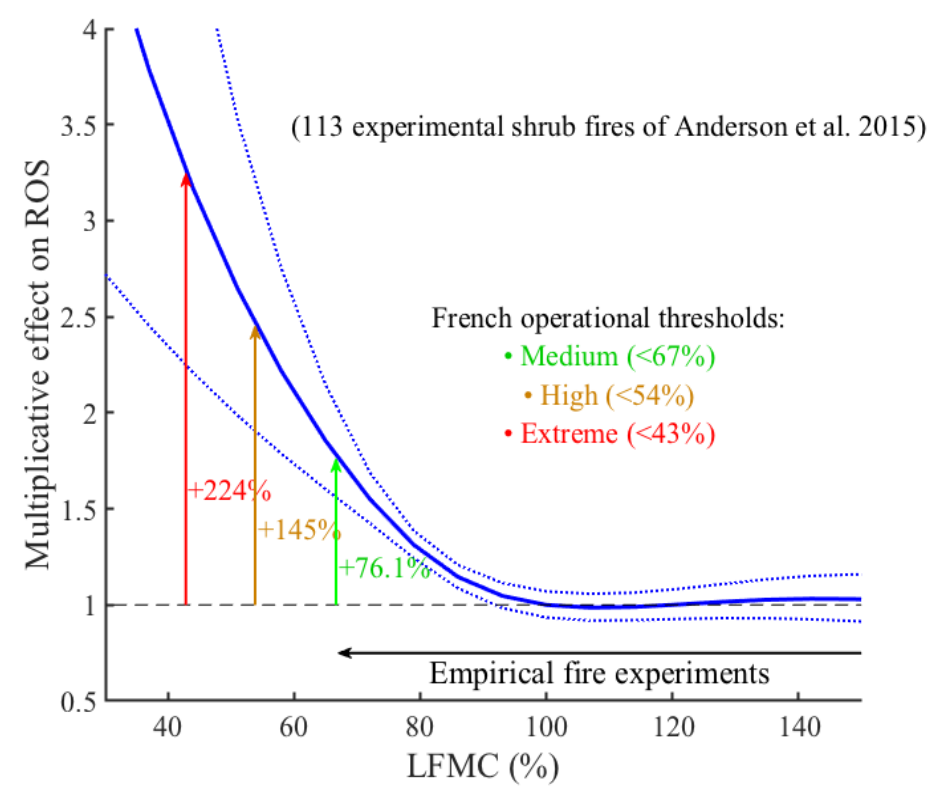

Figure 3 - Response of fire rate of spread as a function of LFMC, derived from Eq. 1, fitted over the fire experiments described in Anderson et al. (2015). A strong increase in ROS is observed below 100\%, even if the confidence intervals strongly increase below $67 \%$ because of the lack of experimental data in this range.

Comparing the range of LFMC in fire experiments and in the $\mathrm{RH}$ dataset confirms this strong discrepancy between LFMC in fire experiments and the conditions prevailing during the actual fire season.

\section{Live Fuel Moisture impact on fire activity}

We used the LFMC dataset and a French database reporting fire activity in order to evaluate how LFMC relates to fire occurrence, based on fires that occurred at less than $10 \mathrm{~km}$ to the LFMC measurement sites. In order to study how fire activity relates to LFMC, we first used the approach of Dennison and Moritz (2009), but found that the method was very sensitive to the fire frequency distribution (Pimont et al. 2018b). Instead, we used a GAM model to study the response function for fire activity to LFMC in an approach derived from (Preisler et al. 2004). We first fitted the number of fires larger than 1 ha $\left(\mathrm{N}_{>1 \text { ha }}\right)$ as a function of LFMC, using a quasi Poisson family and a "log" link.

$$
\mathrm{N}_{>1 \text { ha }}=\mathrm{S}_{\mathrm{f}} \mathrm{s}(\mathrm{LFMC}) \text { Site }
$$

where $\mathrm{s}$ is smooth function, $\mathrm{S}_{\mathrm{f}}$ is the forest area in $10 \mathrm{~km}$ buffer zone around the LFMC measurement sites (in which fires were observed) and "Site" a factor to account for an eventual effect of the LFMC sampling location on fire density. Figure 4a represents the multiplicative response to LFMC of the number of fire larger than 1 ha, in a similar manner than Fig. 3. Interestingly, the shape of this response function, as well as its magnitude was very similar to the one reported for fire behavior on a completely different dataset and at a different scale. 

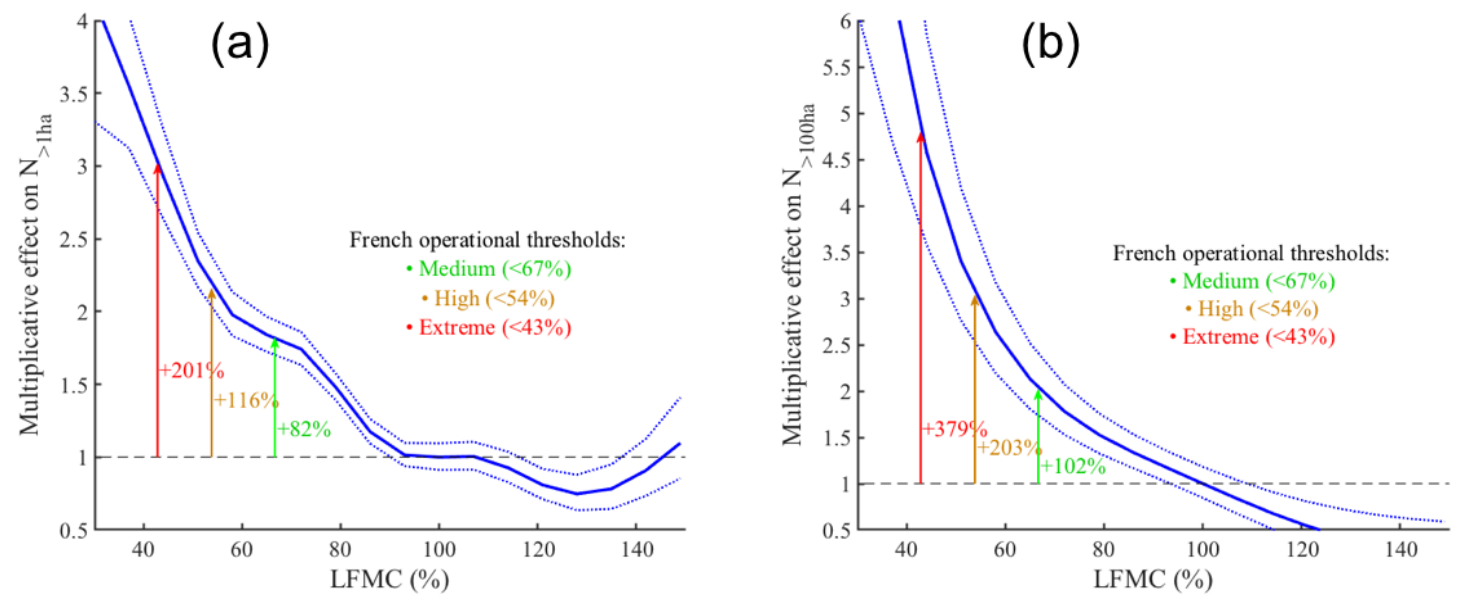

Figure 4 - (a) Response of the number of fires larger than 1ha as a function of LFMC, derived from Eq. 2, fitted over the RH dataset. A strong increase in fire number is observed below 100\%, exhibiting a response function very similar to the one of the ROS (Fig. 2). (b) Response of the number of fires larger than 1ha as a function of LFMC, as in Fig 3. The shape of the curve is sharper than for the >1ha fires and still decay below $100 \%$.

We applied the same approach to the number of fires larger than 100ha $\left(\mathrm{N}_{>100 \mathrm{ha}}\right)$. However, the dataset is to sparse in this context to use the quasi binomial distribution and to account for the site effect. The model fit which assumes the basic binomial distribution (which does not account for over dispersion of the fire numbers, and hence might underestimate the confidence intervals) is shown in Fig. 4b.

The response function was much sharper than the one of the fire behavior and the number of fires larger than 1ha, which can be considered as an evidence of a stronger effect of LFMC on large fires. Also the effect of LFMC did not saturate below $100 \%$, suggesting that the probability to get such a large fire is much lower in humid conditions than in standard "LFMC $=100 \%$ " conditions.

\section{Discussion and conclusion}

This work presents new insights regarding the variability, predictability, physiological drivers and impact of LFMC on fire hazard based on extensive analysis applied to a large LFMC dataset. First, the variability between sites and species considerably limit the accuracy of drought indices. Comparison with calibration of remote sensing products suggest that the accuracy is relatively similar (Fan et al., 2018).

Improvements of LFMC modelling is nevertheless possible by accounting for site specific parameters in water balance models (e.g. soil water capacity and leaf area index) and species traits driving desiccation dynamic through plant hydraulic model (cavitation resistance, turgor loss). Our analyses also suggest that LFMC modelling can be significantly improved in the future on the basis of ecophysiological models (Ruffault et al. under review) which account for soil and vegetation properties, which is in line with a recent publication (Jolly and Johnson 2018). Here we suggested the use of the SurEau model for plant hydraulics (Martin-StPaul et al., 2017) to simulate LFMC with a mechanistic approach including traits. A major advantage of this approach is that is can be readily applied to a large variety of species thanks to the emergence of large databases of pressure volume curves and vulnerability curve to cavitation (Bartlett et al. 2012; Choat et al. 2012; Martin-StPaul et al. 2017). Ongoing research aims at coupling this approach to a water budget model (Ruffault et al. 2013) and to validate the approach over LFMC measurements in situ, thanks to the RH database and the ongoing data acquisition of complementary data (LAI, soil depth, weather data). However, there are still important limitations and research needs both in terms of data and model structure. For 
instance, the prediction of plant water potential and leaf shedding due to drought is not straightforward (Choat et al. 2018). Furthermore, accounting for phenology and decoupling the effects of water status from seasonal dry matter changes would likely strengthen these studies (Jolly et al. 2014).

The reanalyze of the empirical shrub fire dataset previously collected shows that the effect of LFMC on fire behavior is much stronger than previously estimated (Anderson et al. 2015), especially in the range below $100 \%$. Unfortunately, there is still a lack of empirical data in dry conditions corresponding to high and extreme fire sensitivity (Fig. 2), so that the confidence intervals are very large in this range. This argues for field experiments closer to the actual fire conditions, even if such experiments would be clearly very demanding for economic and safety reasons.

The comparison between the $\mathrm{RH}$ database and the fire database shows that fire occurrence is strongly dependent on LFMC. Fires of more than 1 ha exhibit a response to LFMC which is very similar to fire spread, which would suggest that fires can reach 1 ha only when they spread fast enough to be caught out by fire fighters. Larger fires are even more strongly determined by LFMC suggesting a fire frequency on the order of 5 times higher when LFMC is shifted from $100 \%$ to $43 \%$. It would be even 10 times higher when shifting from $120 \%$.

Most of the large-scale evidences of link between LFMC and fire activities were obtained through remote sensing and drought indices, which have shown to be limited in their ability to predict LFMC (Ruffault et al. 2018). Our on-going study of the influence of LFMC on fire behavior and activity from a local scale results in a better understanding of the role of LFMC.

\section{References}

Bartlett MK, Scoffoni C, Sack L (2012) The determinants of leaf turgor loss point and prediction of drought tolerance of species and biomes: A global meta-analysis. Ecology letters 15, 393-405. doi:10.1111/j.1461-0248.2012.01751.x.

Choat B, Brodribb TJ, Brodersen CR, Duursma RA, López R, Medlyn BE (2018) Triggers of tree mortality under drought. Nature 558, 531-539. doi:10.1038/s41586-018-0240-x.

Choat B, Jansen S, Brodribb TJ, Cochard H, Delzon S, Bhaskar R, Bucci SJ, Feild TS, Gleason SM, Hacke UG, Jacobsen AL, Lens F, Maherali H, Martínez-Vilalta J, Mayr S, Mencuccini M, Mitchell PJ, Nardini A, Pittermann J, Pratt RB, Sperry JS, Westoby M, Wright IJ, Zanne AE (2012) Global convergence in the vulnerability of forests to drought. Nature 491, 752-755. doi:10.1038/nature11688.

Dennison PE, Moritz MA (2009) Critical live fuel moisture in chaparral ecosystems: A threshold for fire activity and its relationship to antecedent precipitation. International Journal of Wildland Fire 18, 1021-1027. doi:10.1071/WF08055.

Fan L, Wigneron JP, Xiao Q, Al-Yaari A, Wen J, Martin-StPaul N, Dupuy JL, Pimont F, Al Bitar A, Fernandez-Moran R, Kerr YH (2018) Evaluation of microwave remote sensing for monitoring live fuel moisture content in the Mediterranean region. Remote Sensing of Environment 205, 210-223. doi:10.1016/j.rse.2017.11.020.

Hastie T, Tibshirani R, Friedman J (2009) ‘The Elements of Statistical Learning.' (Springer New York: New York, NY) doi:10.1007/b94608.

Jolly WM, Hadlow AM, Huguet K (2014) De-coupling seasonal changes in water content and dry matter to predict live conifer foliar moisture content. International Journal of Wildland Fire 23, 480-489. doi:10.1071/WF13127.

Jolly WM, Johnson DM (2018) Pyro-Ecophysiology: Shifting the Paradigm of Live Wildland Fuel Research. Fire 1, 8. doi:10.3390/fire1010008. 
Martin-StPaul N, Delzon S, Cochard H (2017) Plant resistance to drought depends on timely stomatal closure (H Maherali, Ed.). Ecology Letters 1-23. doi:10.1111/ele.12851.

Martin-StPaul N, Pimont F, Dupuy JL, Rigolot E, Ruffault J, Fargeon H, Cabane E, Duché Y, Savazzi R, Toutchkov M (2018) Live fuel moisture content (LFMC) time series for multiple sites and species in the French Mediterranean area since 1996. Annals of Forest Science 75, doi:10.1007/s13595-018-0729-3.

Nolan RH, Boer MM, Resco de Dios V, Caccamo G, Bradstock RA (2016) Large-scale, dynamic transformations in fuel moisture drive wildfire activity across southeastern Australia. Geophysical Research Letters 43, 4229-4238. doi:10.1002/2016GL068614.

Pimont F, Ruffault J, Martin-StPaul N, Dupuy JL (2018b) Why is the effect of live fuel moisture content on fire rate of spread underestimated in field experiments in shrublands? Submitted to International Journal of Wildland Fire.

Pimont F, Ruffault J, Martin-StPaul N, Dupuy JL (2018b) A cautionary note regarding the use of cumulative burnt areas for the determination of fire danger index breakpoints. Under review in International Journal of Wildland Fire.

Preisler HK, Brillinger DR, Burgan RE, Benoit JW (2004) Probability based models for estimation of wildfire risk *. International Journal of Wildland Fire 13, 133. doi:10.1071/WF02061.

Rossa CG, Veloso R, Fernandes PM (2016) A laboratory-based quantification of the effect of live fuel moisture content on fire spread rate. International Journal of Wildland Fire 25, 569. doi:10.1071/WF15114.

Ruffault J, Curt T, Martin-StPaul NK, Moron V, Trigo RM (2018) Extreme wildfire events are linked to global-change-type droughts in the northern Mediterranean. Natural Hazards and Earth System Sciences 18, 847-856. doi:10.5194/nhess-18-847-2018.

Ruffault J, Martin-StPaul NK, Rambal S, Mouillot F (2013) Differential regional responses in drought length, intensity and timing to recent climate changes in a Mediterranean forested ecosystem. Climatic Change 117, 103-117. doi:10.1007/s10584-012-0559-5.

Viegas DX, Piñol J, Viegas MT, Ogaya R (2001) Estimating live fine fuels moisture content using meteorologically-based indices. International Journal of Wildland Fire 10, 223-240. doi:10.1071/WF01022.

Yebra M, Dennison PE, Chuvieco E, Riaño D, Zylstra P, Hunt ER, Danson FM, Qi Y, Jurdao S (2013) A global review of remote sensing of live fuel moisture content for fire danger assessment: Moving towards operational products. Remote Sensing of Environment 136, 455-468. doi:10.1016/j.rse.2013.05.029. 


\title{
Management of firebrand potential through the candling of bark fuel
}

\author{
Thomas J. Duff*; Penny Richards; Jane G. Cawson \\ University of Melbourne, Burnley, Australia \\ \{tjduff@unimelb.edu.au*,p.richards@student.unimelb.edu.au, jane.cawson@unimelb.edu.au\}
}

\begin{abstract}
Aerially suspended fuels play an important role in forest fire behaviour. They can act as a ladder to flames, increasing the potential for crownfire, and can ignite and act as firebrands. When large accumulations of these fuels are present, wildfires may spread more rapidly, be more difficult to suppress and be more likely to impact assets such as houses. However, as these fuels are suspended above the ground, their moisture status is predominantly a function of atmospheric humidity. As a result, bark and suspended fuels may become flammable at times when the remainder of the fuel bed is too wet to burn due to high soil moisture levels. This means that these fuels can be reduced by burning when conditions are unfavourable for prescribed burning using the practice candling. Candling is the deliberate ignition of bark and other dead fine ladder fuels under conditions where surface fires are unlikely to spread. We compared the number of days available for prescribed burning and candling for a locality in South Eastern Australia and found that in the period 2012 - 2016, candling could be undertaken for an average 124 days per year, 48 days more than the window available for prescribed burning (76 days). As each accumulation of aerial fuel must be individually lit during candling, the practice is labour intensive and inefficient over large areas relative to prescribed burning, so it is best used for targeted risk reduction such as near control lines or assets. However, it can be used to reduce risk with low chance of escape in locations where prescribed burning is difficult such as the Wildland Urban Interface. The practice is applied operationally in South Eastern Australia, however to date there has been limited research into its effects on wildfire spread and intensity. Given its suitability for strategic use near highly vulnerable assets, we believe further investigation into its utility is warranted.
\end{abstract}

Keywords: burn window; embers; firebrand; fuel management; hazard; prescribed burn, torching; spotting

\section{Introduction}

In forests, aerially held dead fine fuels can play important roles in fire behaviour. During wildfires these fuels can ignite rapidly, act as ladder between the surface fuels and tree canopy and dislodge to become firebrands (Billing and Bywater 1982; Hines et al. 2010; Ellis 2011).

Aerially held dead fine fuels can increase the intensity of wildfires by allowing a greater amount of biomass to be encompassed in the flaming front. They do this by providing a ladder from burning surface / near surface fuels to the crowns of trees. This allows flames to bridge the gap between the overstorey and understorey, facilitating the occurrence of crownfires (Jenkins et al. 2012). During crown fires, fire spread rate is enhanced, suppression becomes ineffective and the likelihood of detrimental ecological impacts is increased (Stephens and Finney 2002; Alexander and Cruz 2011; Hoffman et al. 2016).

Firebrands - burning fragments of material - can enhance wildfire progression where they are carried by winds ahead of the fire area to start new spotfires (McArthur 1967). Under extreme conditions the spread of fire via the coalescence of spotfires can be the primary driver of wildfire spread (Cheney and Bary 1969; Cruz et al. 2012). Firebrands also allow fires to traverse fuel discontinuities, limiting the effectiveness of fire suppression (Koo et al. 2010). Additionally, they are the dominant ignition source for houses during wildfires (Blanchi and Leonard 2005). 
The bark of one genus of trees is recognised for its contribution to spotting in damaging wildfires worldwide, Eucalyptus. Eucalypts typically either have smooth bark that is shed annually or fibrous 'stringy' bark that is retained (Blanchi and Leonard 2005). The fibrous trunks of species such as $E$. obliqua are easily ignited, and the loosely held nature of its bark means that they produce copious amounts of small firebrands (Fig 1).

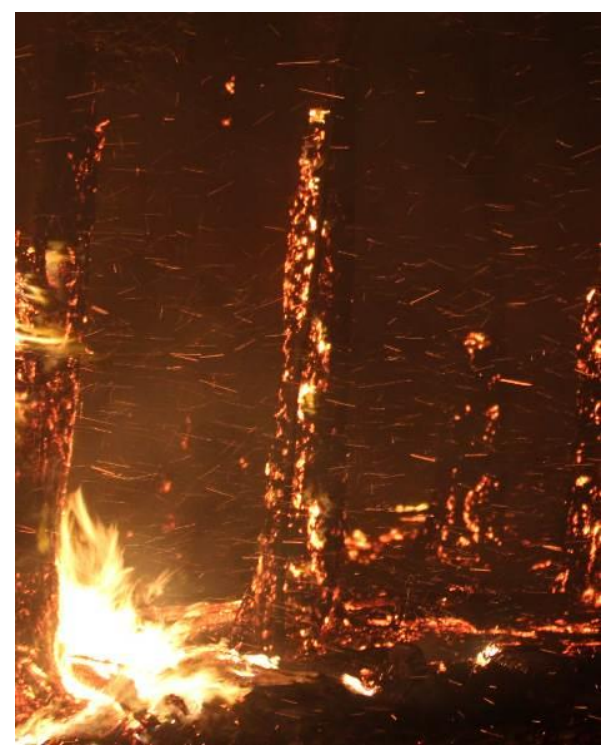

Figure 1 - Firebrands from Eucalyptus obliqua trees during an autumn prescribed burn adjacent to a control line

Dead aerial fuels have typically been managed in conjunction with other fuels. This has predominantly been through prescribed burning. Prescribed burning can be an effective way to modify dead aerial fuels in forests, reducing the total amount, altering properties and reducing bark ignitability by creating a layer of char on tree boles (Chatto et al. 2003; Penman et al. 2017). Effective prescribed burning can only be done within a particular range of weather conditions - the burn window - whereby a forested site is dry enough to be flammable, but not so dry that a burn is likely to escape control and become a wildfire (Slijepcevic et al. 2015). Burn windows typically occur at the fringes of the dry season, but can vary greatly interannually. The timing and lighting patterns of burns can be used to manage fire behaviour, allowing large areas to be burnt with few material inputs. Prescribed burning comes with a risk of escape (Tolhurst and Cheney 1999); escaped burns can have adverse impacts, particularly where there are concentrations of high value assets such as in the Wildland Urban Interface (WUI). As a result, prescribed burning requires high levels of planning, investment in specialised firefighting equipment and the need to have crews at hand when weather conditions are favourable. The unpredictability of weather may also mean that particular burns may not be completed when scheduled.

Given the importance of aerially suspended dead fine fuels to fire behaviour, it is important to be able to effectively reduce its hazard. In particular, in the WUI, aerially suspended fuels pose a high risk to assets, but are difficult to manage using prescribed burning due to the high potential losses in the event of fires escaping. Here we describe and evaluate an alternative method, Candling. Candling is a method of burning bark and other dead fine fuels on the tree bole and branches under conditions where fire spread will be limited by high levels of landscape moisture. We compare the conditions under which candling can be carried out relative to prescribed burning to evaluate its suitability for use in temperate climates such as South-Eastern Australia. 


\section{Methods}

\subsection{The practice of candling}

Candling is the practice of igniting bark or dead material held on individual tree boles in a manner that allows flames to climb the trunk and burn out the majority of dead aerial fine fuel (Billing and Bywater 1982). To effectively burn this material, it must be in a dry enough state to burn. As it is aerially suspended, its moisture status is predominantly a function of atmospheric humidity which can vary greatly over short periods. Surface fuels are in contact with the ground and consequently are strongly influenced by soil moisture which varies more gradually. As a result, aerial fine fuels can be flammable under conditions where the remainder of the fuel bed is too wet to burn. Ideal candling conditions are where the suspended fuels are flammable, but high surface fuel moistures mean that there is little or no potential for fire spread. Draft prescriptions for undertaking candling in South Eastern Australia are presented in Table 1. These have been developed collaboratively by the Victorian Department of Environment, Land, Water and Planning (DELWP), and the Country Fire Authority.

Table 1 - Draft prescriptions to undertake candling and prescribed burning of fibrous tree bark when in natural forest with a shrubby understorey in Victoria, Australia

\begin{tabular}{|c|c|c|}
\hline Parameter & Candling & Prescribed burning \\
\hline Forest Fire Danger Index ${ }^{1}$ & $<10$ & $5-11$ \\
\hline FFDI following day & $<12$ & $<12$ \\
\hline Bark Fuel Moisture Content (\%) & $12-14 \%$ & \\
\hline Surface Fuel Moisture (\%) & $>18 \%$ & $11-16$ \\
\hline Profile Fuel Moisture (\%) & $>20 \%$ & \\
\hline Wind speed $(\mathbf{k m} / \mathbf{h})$ & $<20$ (forecast) $<15$ subcanopy & $<20$ Forecast $<15$ subcanopy \\
\hline Relative Humidity (\%) & $35-65$ for at least 4 hours & $30-70$ \\
\hline Keetch Byram Drought Index ${ }^{2}$ & $<80$ & $<50$ \\
\hline
\end{tabular}

${ }^{1}$ (McArthur 1967), ${ }^{2}$ (Keetch and Byram 1968)

Candling can be done where there are large accumulations of suspended materials where there is sufficiently connectivity to allow burning or on trees with loose fibrous bark on the entire tree bole (Fig 2A). Ignitions can be done using hand held drip torches (Fig 2B) or vehicular flame throwers. The lighting of rings of fire around the base of trees will promote fire driven convection and will allow the fire to climb the bole of the tree. As there is no spread between trees or fuel accumulations, each needs to be lit separately, so the process is labour intensive. 


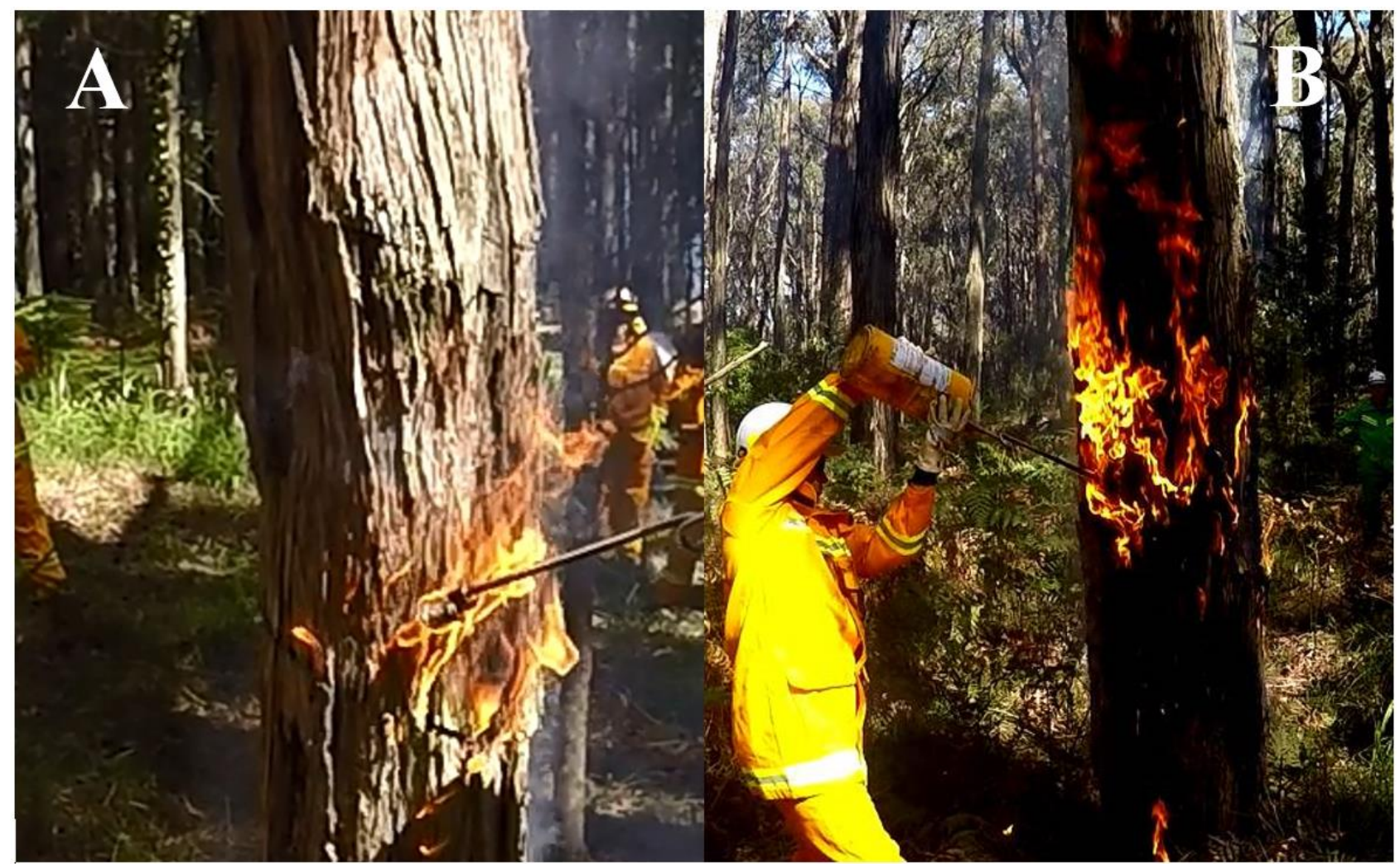

Figure 2 - A) Fibrous bark of Eucalyptus Obliqua. B) Candling of E. obliqua during winter in South Eastern Australia

\subsection{Analysis of data}

We compared the conditions under which prescribed burning and candling can be done for a forested site in Victoria, Australia using historic Automatic Weather Station Data. We used the Scoresby Research Institute weather station http://www.bom.gov.au/climate/averages/tables/cw_086104.shtml), as it is in an area that has a strong presence of Eucalyptus obliqua (Messmate stringybark), a species notorious for firebrands. Data were obtained representing daily weather conditions for the period from June 30, 2011 to December 30, 2016. We used burn prescriptions to compare the proportion of the year under which candling can be completed in comparison to prescribed burning. The prescriptions used for candling are presented in Table 1, however as daily data were used, RH and fuel moisture constraints were excluded, and a maximum $3 \mathrm{pm}$ wind speed of $20 \mathrm{~km} / \mathrm{h}$ was used instead of FFDI. The prescriptions used to represent prescribed burning are those for dry sclerophyll forest (regrowth, without wiregrass) obtained from DELWP. As the prescriptions are intended to represent the maximum safe burning conditions, the conditions where it was too wet to undertake prescribed burning were determined to be these where the McArthur Drought Factor was less than 6 as determined by Duff et al. (2018). The summer months (from December to March) were excluded from the analysis since during this period there are fire restrictions to limit the potential for wildfires. The first six months of the KBDI data for the period analysed were excluded from analysis to allow the algorithm to equilibrate, since KBDI calculations are done using an account keeping process.

\section{Results and Discussion}

We found that candling can be done for a much larger proportion of the year than prescribed burning. Over the study period, on average 124 days per year were suitable for candling, 48 days more 
than the 76 day window available for prescribed burning. Much of the time suitable for candling was coincident with that for burning (Fig 3), however candling could also be done after rain events when it was too wet for effective prescribed burning. Candling could be done for the entire winter period, whereas prescribed burning was limited to the autumn and spring.

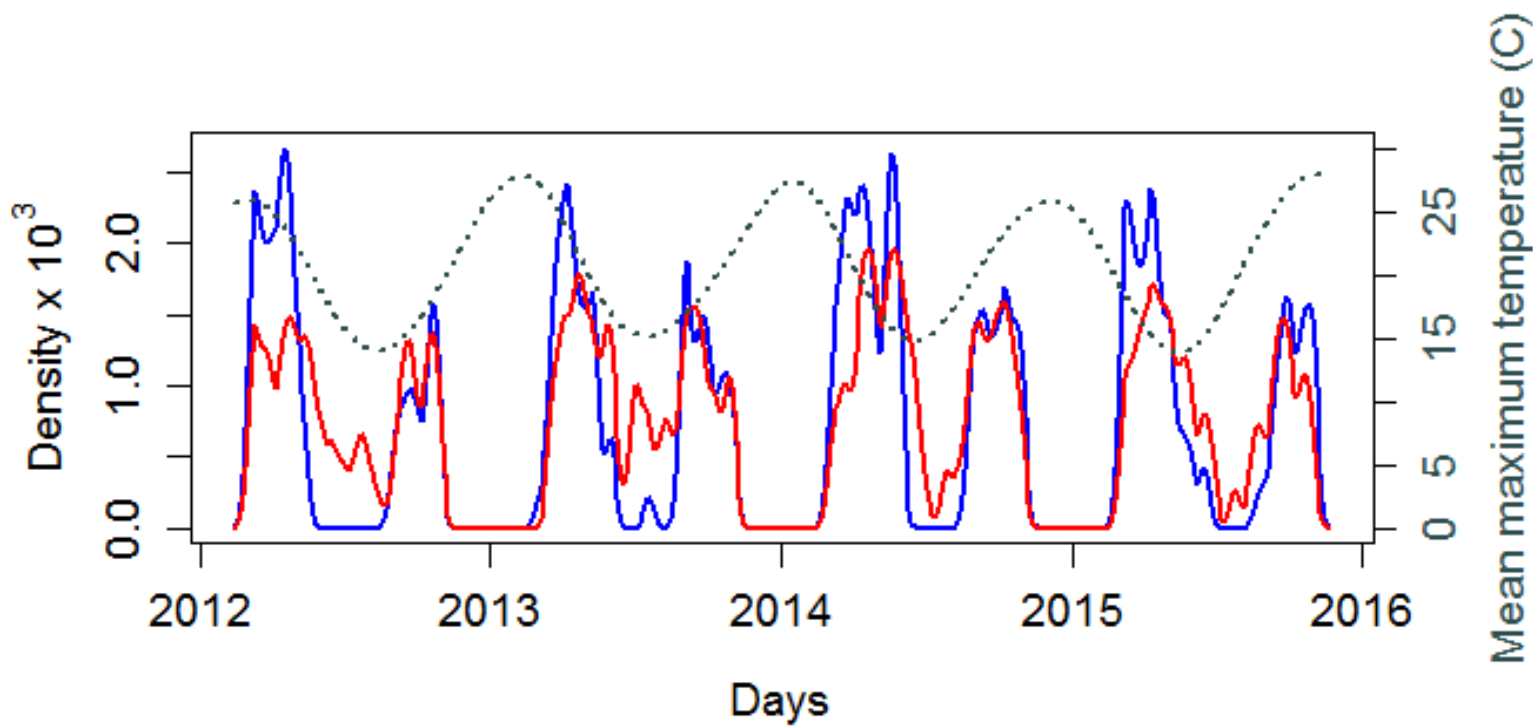

Figure 3 - A) Density plots of the days available for prescribed burning (blue) and candling (red). To facilitate plotting, a density bandwidth of 6 days was used. Maximum temperature is shown using a grey dotted line that has been smoothed with a 25 day moving window.

This means that candling has the potential to allow wildfire risk reduction activities to be undertaken at times when fire crews are not currently active. At these times, due to the non-flammable landscape there is little to no chance of fire escape. In the WUI, bark fuels pose a major issue for house loss, and so prescribed burns are difficult to implement due the high levels of consequences if there is an escape. Candling is potentially an effective means to safely and cheaply reduce asset exposure to firebrands during wildfires and prescribed burning. As it has limited effect on vegetation structure, it may also be more socially acceptible than burning.

As burn escapes are commonly a result of firebrands (Dether and Black 2006), candling can be applied as a complimentary measure to burning by reducing the likelihood of escape by pre-treating trees around the burn perimeter. Such an approach could also be used adjacent to existing firebreaks in the landscape to reduce the likelihood that they are breached during fires and enhance the safety of fire suppression crews.

Candling is one of a number of approaches that can be used to manage aerial dead fire fuels that contribute to crowning and spotting. It has advantages in contrast to other approaches that warrant its consideration in fire risk planning, although its limitations need to be recognised (Table 2). 
Table 2 - Advantages and disadvantages of ladder fuel treatment approaches

\begin{tabular}{|c|c|c|}
\hline Practice & Advantages & Disadvantages \\
\hline \multirow{6}{*}{$\begin{array}{l}\text { Prescribed } \\
\text { burning }\end{array}$} & Reduce a wide variety of fuels & The chance of escape and loss \\
\hline & Complex to safely undertake & Limited seasonal window \\
\hline & Efficient over large areas & Cannot be done in all places \\
\hline & Can have desirable ecological outcomes & High biomass consumption \\
\hline & & Potential for unwanted ecological outcomes \\
\hline & & May have high canopy mortality \\
\hline Mechanical & Reduces live and dead ladder fuels & Requires specialised equipment \\
\hline \multirow[t]{4}{*}{ Treatment } & Does not depend on a burn window & Expensive over large areas \\
\hline & & May increase surface fuels \\
\hline & & May have unwanted ecosystem outcomes \\
\hline & & $\begin{array}{l}\text { Not a suitable method for reducing bark fuels unless } \\
\text { entire tree removed }\end{array}$ \\
\hline \multirow[t]{7}{*}{ Candling } & Reduces fine dead ladder and bark fuels & Labour intensive \\
\hline & Low chance of escape & Limited research on how fire behaviour is affected \\
\hline & Low likelihood of ecological damage & Not suited for all vegetation types \\
\hline & Requires no specialised equipment & Limited research on ecological impacts \\
\hline & $\begin{array}{l}\text { Can be done where it is too dangerous to burn } \\
\text { (i.e. WUI) }\end{array}$ & \\
\hline & $\begin{array}{l}\text { Has a wider seasonal window than prescribed } \\
\text { burning }\end{array}$ & \\
\hline & Low biomass consumption & \\
\hline
\end{tabular}

Candling is currently used operationally in the state of Victoria, Australia. It has had limited operational adoption outside this state or elsewhere. The effectiveness of candling has been inferred from the effect it has on dead aerial fine fuels, particularly bark. However, to-date there has been no empirical research into how candling modifies fire behaviour, fire brand production and the relative levels of exposure that assets and firebreaks will be exposed to during wildfires. As a result, it is unclear how much effort and investment fire agencies should direct towards candling.

To effectively integrate candling as a treatment into a fire risk management program, further research is warranted. This should include:

- Quantifying the effect candling has on fuel properties in different vegetation communities;

- Determining differences in fire behaviour and ember production in treated areas;

- Developing models of fuel recovery after treatment;

- Determining the environmental factors that influence candling outcomes;

- Determining the optimal spatial configurations of candling in the landscape to have the greatest moderating effect on fire behaviour;

- Evaluating the ecological impacts of candling;

- Comparison of the outcomes of different candling techniques (including conditions and methods); and

- Consideration of how to account for candled areas in landscape fire simulation systems used in risk planning.

As its application has had limited use outside a single jurisdiction, potential applications may have not been considered. For example, it may be effective in North American systems to reduce dead canopy needles in locations where there has been widespread death of conifers due to bark beetles (Jenkins et al. 2012). 


\section{Conclusion}

Candling is a technique that can be used to manage dead fine aerial fuels, particularly fibrous bark. Its adoption for operational use has been extremely limited and is restricted to South Eastern Australia. Its practice requires few material resources and it can be undertaken for wider burning window than is available for prescribed burning. However, while its efficacy can be inferred from the way it modifies fuels, there is currently no empirical research describing how it modifies wildfire risk or modifies ecosystem processes. Given the challenges posed to fire managers worldwide by eucalyptus firebrands, candling is a technique that warrants consideration and further investigation.

\section{Acknowledgements}

This work was funded in-part by the Country Fire Authority of Victoria, and the Victorian Department of Environment, Land, Water and Planning. We gratefully acknowledge their contributions.

\section{References}

Alexander, ME, Cruz, MG (2011) What are the safety implications of crown fires? . In 'Proceedings of 11th International Wildland Fire Safety Summit. Missoula, Montana, USA.', April 4-8, 2011. ( International Association of Wildland Fire)

Billing, P, Bywater, JV (1982) Using fire to reduce aerial fuels in first thinned radiata pine. Forest Commission, Victoria No. 16, Melbourne, Australia.

Blanchi, R, Leonard, J (2005) Investigation of bushfire attack mechanisms resulting in house loss in the ACT bushfire 2003. Bushfire CRC, East Melbourne, Australia.

Chatto, K, Bell, TL, Kellas, JD (2003) Effects of repeated low-intensity fire on tree growth and bark in a mixed Eucalypt foothill forest in south-eastern Australia. Department of Sustainability and Environment No. 66, East Melbourne, Victoria, Australia.

Cheney, NP, Bary, GAV (1969) The propagation of mass conflagrations in a standing eucalypt forest by the spotting process. In ' Mass fire symposium: collected papers. Canberra', 10-12 February 1969. (Commonwealth of Australia, Defence Standard Laboratories: Melbourne)

Cruz, MG, Sullivan, AL, Gould, JS, Sims, NC, Bannister, AJ, Hollis, JJ, Hurley, RJ (2012) Anatomy of a catastrophic wildfire: The Black Saturday Kilmore East fire in Victoria, Australia. Forest Ecology and Management 269-285.

Dether, D, Black, A (2006) Learning from escaped prescribed fires - lessons for high reliability. Fire Management Today 66, 50-56.

Duff, T, J., Cawson, J, G., Harris, S (2018) Dryness thresholds for fire occurrence vary by forest type along an aridity gradient: evidence from Southern Australia. Landscape Ecology

Ellis, PFM (2011) Fuelbed ignition potential and bark morphology explain the notoriety of the eucalypt messmate 'stringybark' for intense spotting. International Journal of Wildland Fire 20, 897-907.

Hines, F, Tolhurst, K, G., Wilson, AG, McCarthy, GJ (2010) Overall fuel hazard assessment guide, 4th Edition. Department of Sustainability and Environment Victoria, Melbourne, Melbourne.

Hoffman, CM, Canfield, J, Linn, RR, Mell, W, Sieg, CH, Pimont, F, Ziegler, J (2016) Evaluating crown fire rate of spread predictions from physics-based models. Fire Technology 221-237.

Jenkins, MJ, Page, WG, Hebertson, EG, Alexander, ME (2012) Fuels and fire behavior dynamics in bark beetle-attacked forests in Western North America and implications for fire management. Forest Ecology and Management 275, 23-34. 
Keetch, JJ, Byram, GM (1968) A drought index for forest fire control. Forest Service, U.S. Department of Agriculture, Ashville, North Carolina.

Koo, E, Pagni, PJ, Weise, DR, Woycheese, JP (2010) Firebrands and spotting ignition in large-scale fires. International Journal of Wildland Fire 19, 818-843.

McArthur, AG (1967) Fire behaviour in Eucalypt forests. Forestry and Timber Bureau, Department of National Development, Canberra, Australia.

Penman, TE, Cawson, JG, Murphy, S, Duff, TJ (2017) Messmate Stringybark: bark ignitability and burning sustainability in relation to fragment dimensions, hazard and time since fire. International Journal of Wildland Fire

Slijepcevic, A, Anderson, WR, Matthews, S, Anderson, DH (2015) Evaluating models to predict daily fine fuel moisture content in eucalypt forest. Forest Ecology and Management 335, 261-269.

Stephens, SL, Finney, MA (2002) Prescribed fire mortality of Sierra Nevada mixed conifer tree species: effects of crown damage and forest floor combustion. Forest Ecology and Management $162,261-271$.

Tolhurst, KG, Cheney, NP (1999) Synopsis of the knowledge used in prescribed burning in Victoria. Department of Natural Resources and Environment, East Melbourne, Victoria, Australia. 


\title{
Optimisation of fuel treatments at landscape level in NW Spain
}

\author{
Enrique Jiménez*; José M. Fernández-Alonso; Cristina Fernández; Teresa Fontúrbel; José A. \\ Vega \\ Centro de Investigación Forestal-Lourizán, Xunta de Galicia \\ \{enrique.jimenez.carmona@xunta.es*\}
}

\begin{abstract}
In the current framework of forest fires incidence, climate change and forest management budget constraints, fuel treatment activities should be located in strategically areas to meet a set of prevention, economic and ecological criteria. Characterizing potential forest fire behavior is critical to develop effective fuel treatment plans in order to reduce the negative impacts of wildfires. This study was carried out in the District XIX Caldás - Salnés (Pontevedra, Spain) with 132004 ha, an area with high occurrence of wildfires. Fire behavior characteristics at landscape level were obtained with Flammap fire modeling software using as inputs spatially explicit data. This software requires as inputs weather, terrain, stand and fuel characteristics. Simulations were carried out under extreme fire weather conditions. Fuel layers used for the simulations were obtained combining the Spanish National Forest Map (4th Spanish National Forest Inventory) and a new fuel classification developed for forest fuels in Galicia. The software WindNinja was employed to include in the simulations the spatial variability of wind speed and direction as a consequence of topography. After computing output data from fire behavior simulation, Landscape Treatment Designer (LTD) was the software employed to obtain the optimal treatment locations. LTD creates a sequence of spatially defined project areas that maximizes the objectives with the provided constraints and treatment thresholds. Flame length was selected as treatment threshold variable. Priority stands for treatment were defined as a function of a set of preventive, ecological and economic parameters (presence of human infrastructures, fire risk according to fire frequency and ecological value, fuel model and stand structure). The maximum area to treat was constraint to 3000 ha for the whole scenario. After obtaining flame length for each stand with Flammap, LTD creates both aggregated and dispersed treatment schedules, each representing a different spatial treatment scenario, taking into account the priority functions.
\end{abstract}

The results highlight the potential of this methodology as a very useful tool for fuel treatments at landscape level, taking into account that the effective allocation of resources is critical.

Keywords: FlammMap; Lanscape Treatment Designer; fuel treatments

\section{Introduction}

Fuel load and continuity increased notably in southern Europe during the second half of the twentieth century leading to more intense wildfires (Moreira et al. 2011). Annually, some 51,200 forest fires burn approximately 477,400 ha in southern European countries (from 1980 to 2013, in Portugal, Spain, France, Italy and Greece; Rodriguez-Aseretto et al. 2014). Moreover, the Mediterranean forests are expected to suffer higher frequency of fire in the future (Turco et al. 2014). In the current framework of forest fires incidence, climate change and forest management budget constraints, fuel treatment activities should be located in strategically areas to meet a set of prevention, economic and ecological criteria. The efficacy of fuel treatments decreasing fire severity and size has been previously elucidated (Agee and Skinner, 2005; Jiménez et al. 2016). It has been also found that the strategic allocation of fuel treatments reduced the predicted growth rates of modeled fires more effectively than random placement (Finney et al. 2006).

Characterizing potential forest fire behavior is critical to develop effective fuel treatment plans in order to reduce the negative impacts of wildfires. The great complexity of wildfire risk assessment and fuel treatment planning has resulted in the increase in the employment of different fire behavior 
simulation software in research and operational context(Ager et al. 2011; Calkin et al. 2011). Wildfire modeling can be employed as general indicators of wildfire exposure and fuel treatments planning for optimization of fire prevention activities (Salis et al. 2013). There is an increased interest in the use of decision support tools to mitigate wildfire risk (Matin et al. 2016). These tools include wildfire simulation models allow managers to quantitatively evaluate management decisions in fuel treatment activities planning (Martin et al. 2016).

The aim of this study was to simulate fire behavior (Flammap) within an entire landscape and use a decision support tool (Landscape Treatment Designer, LTD - Ager et al. 2012) to spacially allocate fuel treatments taking into account a set of preventive, ecological and economic parameters.

\section{Data and methods}

\subsection{Study area}

This study was carried out in the District XIX Caldas - Salnés (Pontevedra, Spain) with 132,004 ha (Figure 1). It covers 28 municipalities. Topography is dominated by uplands and lowlands, ranging from 0 m.a.s.l (at the coastal areas) to 1010 m.a.s.l. The climate is oceanic, with a mean annual precipitation between $1200-1800 \mathrm{~mm}$ and a dry period of 2 months. The mean annual temperature is between $12-14^{\circ} \mathrm{C}$. North-east winds are those associated with the period of higher fire frequency.

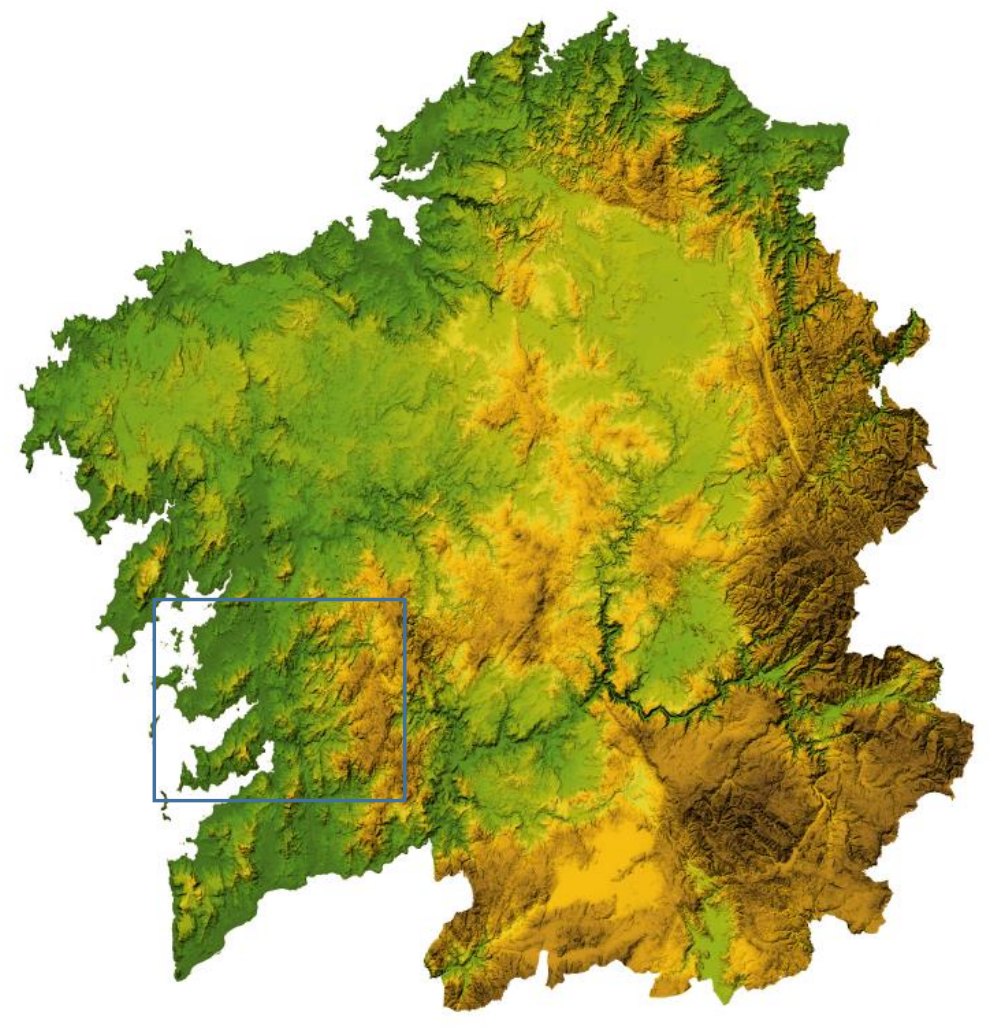

Figure 1 - Location of the study area, in Galicia (NW Spain)

The forest covers $65.25 \%$ of the total area. The main forest land cover type is eucalypt (26.9\%), followed by shrublands (18.8\%), mixed pine and eucalypt forest (17.9\%) and pine forest (13.1\%).

The District XIX Caldas-Salnés is an area with high occurrence of wildfires. From 2002-2007, the mean annual number of fires was 709, burning 4097 ha. Wildfires occurred mainly in summer season (August). Most of these wildfires are man-caused, with a high level of intentionality. The preventive and fire fighting plan of the District XIX Caldás-Salnés define every year the areas with higher fire risk according to fire frequency and ecological value. 


\subsection{Fire behaviour simulations}

Fire behavior characteristics at landscape level were obtained with Flammap fire modeling software (Finney 2006) using as inputs spatially explicit data. Flammap was selected because it is the single available option to estimate maximum fire potential for any given point (pixel) of a landscape (Botequim et al. 2017). We analyzed FlamMap outputs for flame length. This software requires as inputs weather, terrain, stand and fuel characteristics (Figure 2). Terrain inputs consisted of elevation, aspect and slope extracted from a digital elevation model (DEM) at horizontal resolution of $25 \mathrm{~m}$ was obtained for the study area from the Spanish Geographic National Institute. Fuel-related data layers used for the simulations were obtained combining the Spanish National Forest Map (4th Spanish National Forest Inventory) - standard fire behavior fuel models (Anderson 1982) -, and a new fuel classification developed for forest fuels in Galicia based on main species, age class and understory fuel characteristics - canopy fuels (Arellano et al. 2016).

Simulations were carried out under extreme fire weather conditions. Extreme wildfire risk conditions were defined as wind speed of $30 \mathrm{~km} \mathrm{~h}^{-1}$ - value proposed by Mitsopoulos and Dimitrakopoulos (2007) and Fernández-Alonso et al. (2013) in other Mediterranean areas - and dead fine fuel moisture of $6 \%$. Crown and undercanopy live fuel moisture was established as $100 \%$. The previous DEM was employed to simulate the wind fields within the fire perimeters with WindNinja (Forthofer 2007). WindNinja is a mass-consistent fluid flow dynamics models that estimates the modifying effects of topography on synoptic winds.

\subsection{Optimal treatment locations}

After computing output data from fire behavior simulation, Landscape Treatment Designer (LTD) was the software employed to obtain the optimal treatment locations (Ager et al. 2012, Ager et al. 2013, Vogler et al. 2015). LTD creates a sequence of spatially defined project areas that maximizes the objectives with the provided constraints and treatment thresholds. Flame length was selected as treatment threshold variable. Fuel treatments were triggered when a stand exceeded a flame length of 1.5 meters. Any classifiable stand feature, such as habitat value, proximity to human communities, and wildfire hazard, may be combined in prioritizing stands for treatment. Priority stands for treatment were defined as a function of a set of preventive, ecological and economic parameters (presence of human infrastructures, fire risk according to fire frequency and ecological value, following the fire fighting plan of the District XIX Caldás-Salnés, fuel model and stand structure) - Table 1. Prior to LTD runs, each stand was assigned numeric rating scores according to these parameters (Table 1). The maximum area to treat was constraint to 3000 ha for the whole scenario. LTD can create both aggregated and non-aggregated project areas. In this study we only employed the non-aggregated method allowing the model to distribute selected stands over the landscape. LTD creates a map of treatment priority resulting in a sequence of project areas and respective priorities.

Table 1 - Presence of human infrastructures, fire risk according to fire frequency and ecological value, fuel model and stand structure, and assigned wildfire hazard ratings for LTD runs

\begin{tabular}{cc|cc|cc|cc}
\hline $\begin{array}{c}\text { Presence of } \\
\text { infrastructure }\end{array}$ & Value & Fire risk & Value & $\begin{array}{c}\text { Fuel } \\
\text { model }\end{array}$ & Value & Stand structure & Value \\
\hline Yes & 0 & Low & 1 & $1,2,8,9$ & 0 & Shrub & 0 \\
No & 2 & Medium & 2 & 3,5 & 1 & Seedlings/saplings & 0 \\
& & High & 3 & 6,7 & 2 & Pole & 1 \\
& & Very High & 4 & $4,10,11$ & 3 & Saw & 3 \\
\hline
\end{tabular}




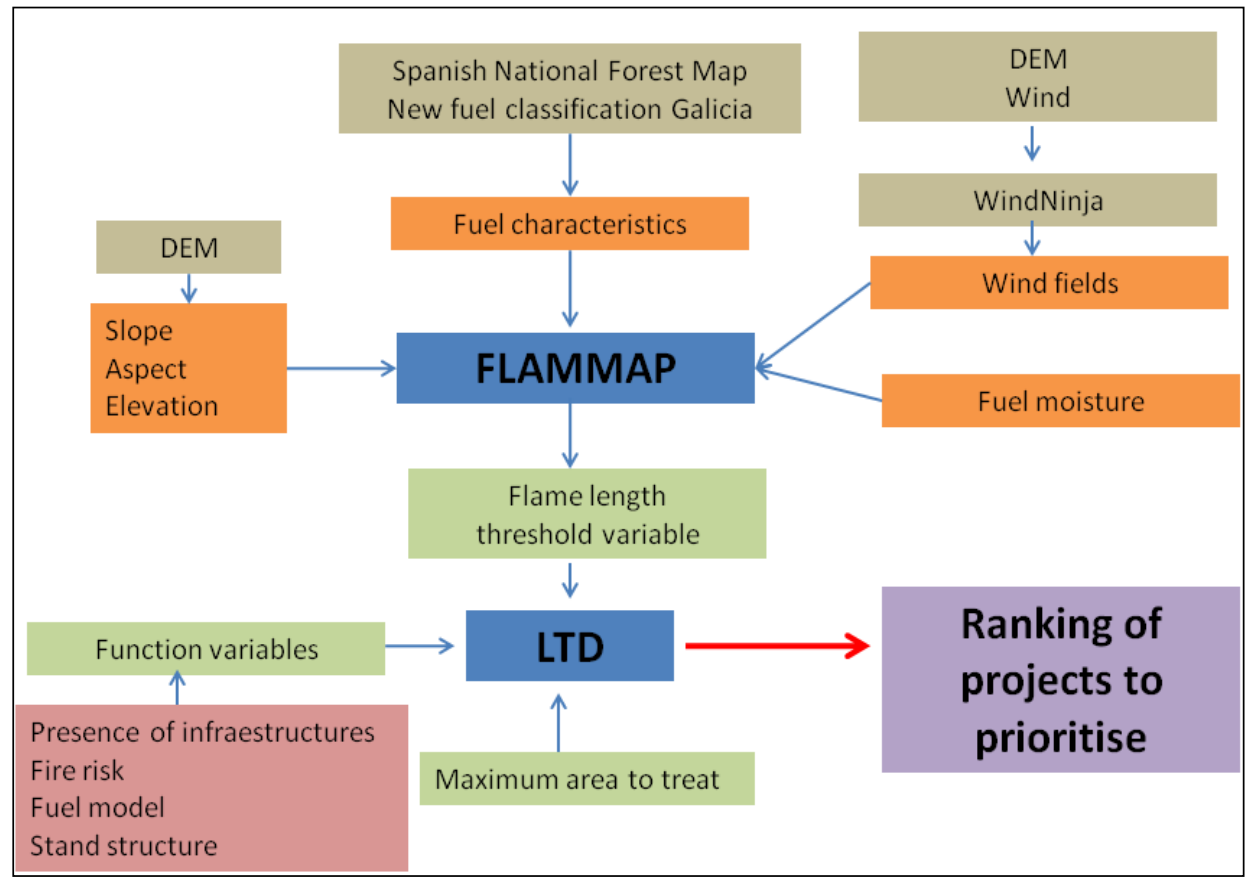

Figure 2 - Scheme of inputs employed for fire behavior simulations (FLAMMAP) and optimal treatment locations by LTD.

\section{Results and discussion}

The Figure 3 shows the spatial variation of flame length for the whole landscape obtained from FLAMMAP under the extreme meteorological conditions.

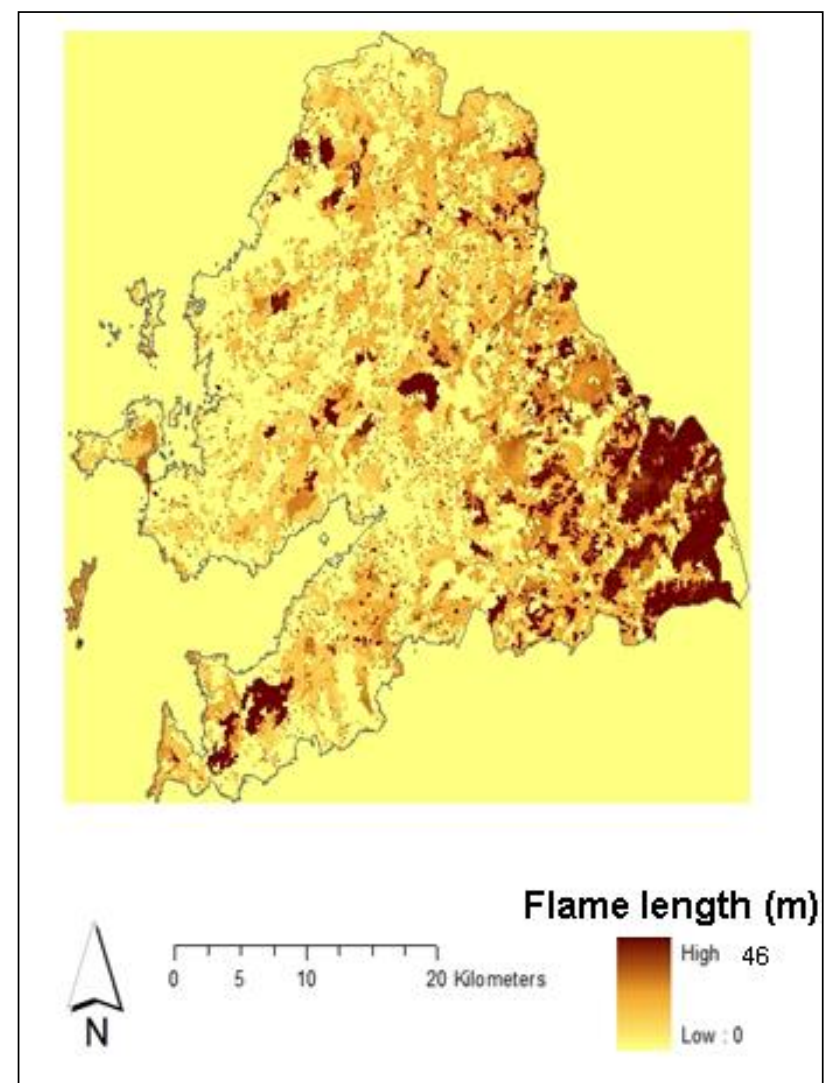

Figure 3 - Spatial variation of flame length of the study area obtained from FLAMMAP 
Taking into account the standard fuel models for each stand defined in the Spanish National Forest Map, mean flame length ranged from $0.90 \mathrm{~m}$ (fuel model 8) to $3.17 \mathrm{~m}$ (fuel model 4) - Table 2. If we consider the main species of the stand, mean flame length ranged from $0.70 \mathrm{~m}$ (Agrostis curtisii areas) to $1.50 \mathrm{~m}$ (Ulex sp. areas) for pastures and shrubland stands, and from $0.75 \mathrm{~m}$ (Pinus radiata stands) to $1.27 \mathrm{~m}$ (Castanea sativa stands).

Table 2 - Mean flame length (range between brackets) obtained from FLAMMAP of the study area stands as a function standard fuel models and main species

\begin{tabular}{cc|cc}
\hline $\begin{array}{c}\text { Standard fuel } \\
\text { model }\end{array}$ & Flame length $(\mathrm{m})$ & Main species & Flame length $(\mathrm{m})$ \\
\hline 1 & $0.93(0.01-2.20)$ & Agrostis curtisii & $0.70(0.01-1.70)$ \\
2 & $1.09(0.03-8.53)$ & Cytisus multiflorus & $0.90(0.08-2.22)$ \\
3 & $1.47(0.02-2.75)$ & Pteridium aquilinum & $1.26(0.66-2.55)$ \\
4 & $3.17(0.01-10.22)$ & Ulex sp. & $1.50(0.01-8.38)$ \\
5 & $1.06(0.00-14.73)$ & Betula sp. & $1.14(0.06-7.32)$ \\
6 & $1.32(0.00-8.53)$ & Castanea sativa & $1.27(0.05-8.28)$ \\
7 & $1.13(0.00-9.46)$ & Eucalyptus sp. & $1.22(0.00-14.73)$ \\
8 & $0.90(0.30-2.94)$ & Pinus pinaster & $1.16(0.00-9.96)$ \\
9 & $1.02(0.40-8.19)$ & Pinus radiata & $0.75(0.00-3.06)$ \\
10 & $1.77(0.05-3.12)$ & Quercus robur & $1.23(0.00-8.19)$ \\
11 & $2.29(0.59-7.84)$ & & \\
\hline
\end{tabular}

The non-aggregate option of LTD created a dispersed treatment schedule based on fire hazard and fire risk (Figure 4). This option creates a ranking of projects to priorities the whole landscape into a sequence of project areas. The project labeled with number 1 represents the highest priority area for the given objectives, and the project labeled with the number 7 represents the lowest priority.

For the project 1 (highest priority), the fuel model 5 represented the $54.6 \%$ of the 3000 ha to treat, followed by the fuel model $6(31.7 \%)$ and fuel model $4(11.9 \%)$. The high percentage of area to treat covered by fuel model 5 could be consequence of the high surface covered by this fuel model in the entire area (23.3\%). Taking into account the main species, Ulex sp. areas represented $48.5 \%$ of the area to treat, followed by Eucalyptus sp. stands (26.9\%) and Pinus pinaster stands (16.0\%). Although Ulex sp. areas only cover $9.8 \%$ of the entire landscape, the high flame length obtained with simulation for these areas could explain the high percentage of area to treat represented by this species. 


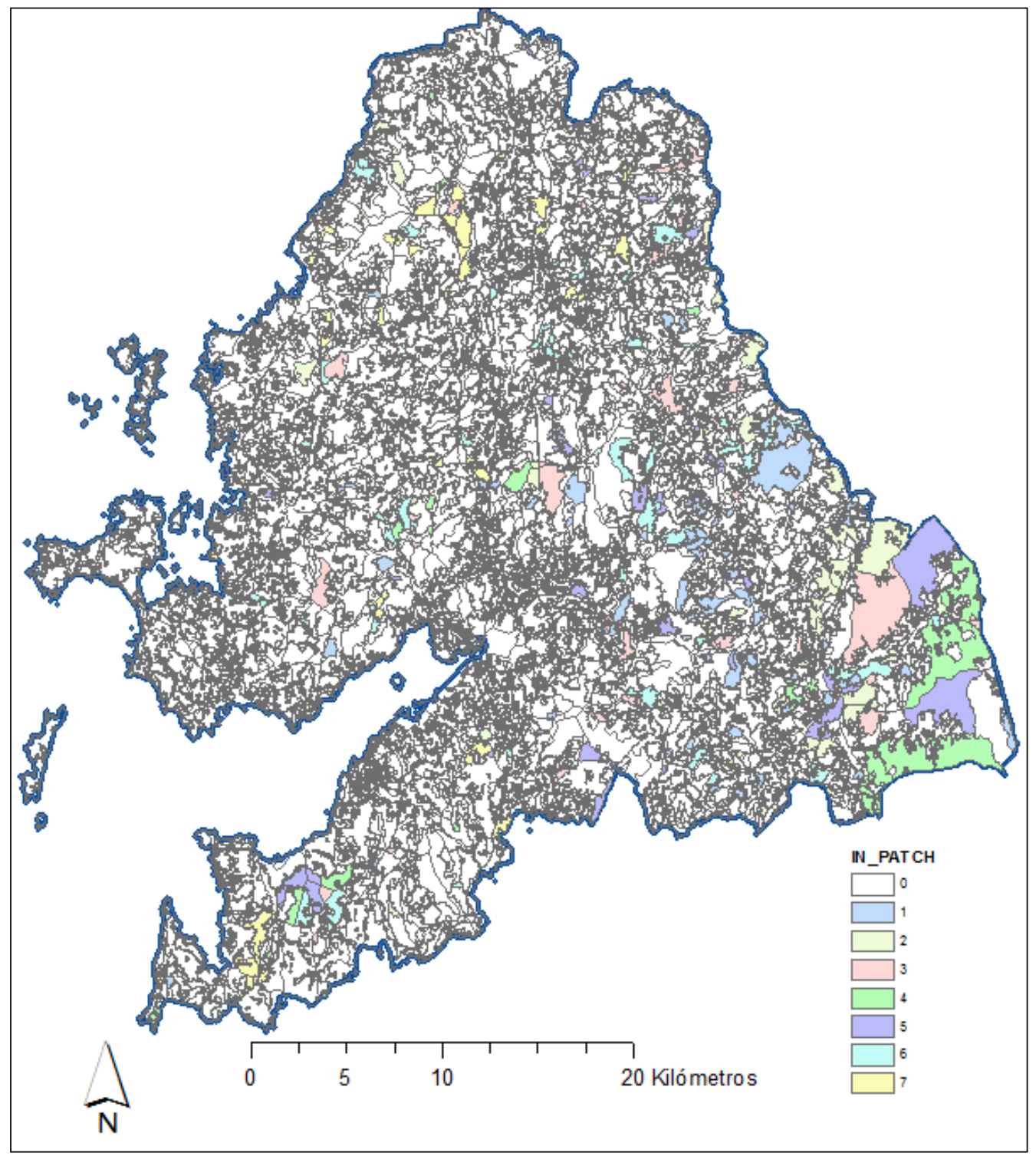

Figure 4 - Ranking of projects to priorities the whole landscape into a sequence of project areas.

Table 3 - Percentage of area to treat for the project of highest priority as a function of standard fuel model and main species

\begin{tabular}{cc|cc}
\hline $\begin{array}{c}\text { Standard fuel } \\
\text { model }\end{array}$ & $\begin{array}{c}\text { Percentage of area to } \\
\text { treat }(\%)\end{array}$ & Main species & $\begin{array}{c}\text { Percentage of area to } \\
\text { treat }(\%)\end{array}$ \\
\hline 1 & 0 & Agrostis curtisii & 0 \\
2 & 1.6 & Cytisus multiflorus & 0 \\
3 & 0 & Pteridium aquilinum & 0 \\
4 & 11.9 & Ulex sp. & 48.5 \\
5 & 54.6 & Betula sp. & 2.3 \\
6 & 31.7 & Castanea sativa & 4.8 \\
7 & 0.2 & Eucalyptus sp. & 26.9 \\
8 & 0 & Pinus pinaster & 16.0 \\
9 & 0 & Pinus radiata & 0 \\
10 & 0 & & 1.5 \\
11 & 0 & & \\
\hline
\end{tabular}


Although it has been suggested limitations of wildfire models (Mell et al. 2007; Alexander and Cruz, 2013), the results highlight the potential of this methodology as a very useful tool for strategically locate fuel treatments at landscape level, taking into account the cost of carrying out preventive activities.

\section{Acknowledgments}

This research was funded by the INTERREG-SUDOE PLURIFOR project.

\section{References}

Agee JK, Skinner CN, 2005. Basic principles of forest fuel reduction treatments. Forest Ecol Manag 211: 83-96.

Ager AA, Vaillant NM, Finney MA, 2011. Integrating fire behavior models and geospatial analysis for wildland fire risk assessment and fuel management planning. Journal of Combustion, $19 \mathrm{pp}$.

Ager AA, Vaillant NM, Owens DE, Brittain S, Hamann J, 2012. Overview and example application of the Landscape Treatment Designer. Gen. Tech. Rep. PNW-GTR-859, USDA Forest Service, Pacific Northwest Research Station, Portland, OR.

Ager AA, Vaillant NM, McMahan A, 2013. Restoration of fire in managed forests: a model to prioritize landscapes and analyze tradeoffs. Ecosphere 4: 29.

Alexander ME, Cruz MG, 2013. Are the applications of wildland fire behaviour models getting ahead of their evaluation again? Environ Modell Softw 41: 65- 71.

Anderson HE, 1982. Aids to determining fuel models for estimating fire behavior. Gen. Tech. Rep. INT-122. USDA For. Serv. Intermountain Forest and Range Experiment Station. Ogden. UT, 22 p.

Arellano S, Vega JA, Ruiz AD, Arellano A, Álvarez JG, Vega DJ, Pérez E, 2016. Foto-guía de combustibles forestales de Galicia. Andavira. Santiago de Compostela, 248 pp.

Botequim B, Fernandes PM, García-Gonzalo J, Silva A, Borges JG, 2017. Coupling fire behaviour modelling and stand characteristics to assess and mitigate fire hazard in a maritime pine landscape in Portugal. Eur. J. For. Res. 136: 527-542.

Cain DC, Finney MA, Ager AA, Thompson MP, Gebert KM, 2011. Progress towards and barriers to implementation of a risk framework for US federal wildland fire policy and decision making. Forest Policy and Economics 13: 378-389.

Fernández-Alonso JM, Alberdi I, Alvarez-González JG, Vega JA,Cañellas I, Ruiz-González AD, 2013. Canopy fuel characteristics in relation to crown fire potential in pine stands: analysis, modelling and classification. Eur J For Res 132:363-377

Finney MA, 2006. An Overview of FlamMap Fire Modeling Capabilities. In Fuel Management-How to Measure Success: Conference Proceedings RMRS-P-41. 28-30 March, Portland, Oregon. pp. 213-220.

Finney MA, Seli RC, Mchugh CW, Ager AA, Bahro B, Agee JK, 2006. Simulation of Long-Term Landscape-Level Fuel Treatment Effects on Large Wildfires. In USDA Forest Service Proceeding RMRS-P-41, pp. 125-147.

Forthofer JM, 2007. Modeling wind in complex terrain for use in fire spread prediction. Masters thesis. Colorado State University, USA. 123 pp.

Jiménez E, Vega-Nieva D, Rey E, Fernández C, Vega JA, 2016. Midterm fuel structure recovery and potential fire behaviour in a Pinus pinaster Ait. forest in northern central Spain after thinning and mastication. Eur. J. For. Res. 135: 675-686. 
Martin A, Botequim B, Oliveira TM, Ager A, Pirotti F, 2016. Temporal optimisation of fuel treatment design in blue gum (Eucalyptus globulus) plantations. Forest Systems 25, 9 pp.

Mell W, Jenkins MA, Gould J, Cheney P, 2007. A physics-based approach to modelling grassland fires. Int J Wild Fire 16: 1-22.

Mitsopoulos ID, Dimitrakopoulos AP, 2007. Canopy fuel characteristics and potential crown fire behaviour in Aleppo pine (Pinus halepensis Mill.) forests. Ann For Sci 64:287-299

Moreira F, Viedma O, Arianoutsou M, Curt T, Koutsias N, Rigolot F, Barbati A, Corona P, Vaz P, Xanthopoulos G, Mouillot F, Bilgili E, 2011. Landscape-wildfire interactions in southern Europe: implications for landscape management. J Environ Manag 92:2389-2402.

Rodriguez-Aseretto RD, Boca R, San-Miguel-Ayanz J, Liberta G, Schmuck G, Camia A, Schulte E, Petroliagkis T, Durrant T, Boccacci F, Di-Leo M, 2014. Forest fires in Europe, Middle East and North Africa 2013. Publications Office of the European Union, Luxemburg

Salis M, Ager AA, Arca B, Bacciu V, Duce P, Spano D, 2013.Assessing exposure of human and ecological values to wildfire in Sardinia, Italy. Int J Wildland Fire 22: 549-565.

Turco M, Llasat MC, von Hardenberg J, Provenzale A, 2014. Climate change impacts on wildfires in a Mediterranean environment. Clim Change 125:369-380

Vogler KC, Ager AA, Day MA, Jennings M, Bailey JD, 2015. Prioritization of forest restoration projects: tradeoffs between wildfire protection, ecological restoration and economic objectives. Forests 6: 4403-4420. 


\title{
Plasticity of fire related traits of some obligate seeder species in different fire-prone areas of SE France: the goals and methodology used
}

\author{
Bastien Romero*, Anne Ganteaume \\ Irstea.3275 Route de Cezanne, 13100 Aix-en-Provence, France \{bastien.romero@irstea.fr*, \\ anne.ganteaume@irstea.fr\}
}

\begin{abstract}
Species having traits adapted to a given fire regime can be endangered when the fire regime changes if these traits do not vary at the same time, especially with the increase in fire recurrence following the global change. In this framework, an on-going $\mathrm{PhD}$ work studies plant fire-traits and flammability according to the fire regime to highlight a possible adaptation of these traits which would confer a better flammability when the fire recurrence increases to the resilient species Pinus halepensis and a better resistance to fire to the resistant species Pinus sylvestris. The study area encompasses two locations located on North-South gradient of the Provence area (SE France) to verify is the trend is the same within the biogeographical range of the species and at its boundaries. The current paper presents the goals of this work and methodology implemented to achieve them
\end{abstract}

Keywords: Fire Regime, Fire-related Traits, Plasticity, Flammability, Mediterranean Region, Climate Change

\section{Introduction}

Wildfires have been shaping landscapes in the Mediterranean regions for thousands years (Keeley et al. 2011) as climate conditions in these areas favour fire ignition and propagation, and most species have adapted because of this high selection pressure (Pausas \& Keeley 2009, Moreno \& Oechel 2012). However, for decades, the increase in temperature has led to an increase in fire activity all over the world (Westerling et al. 2006; Pausas 2004; Oliveira et al. 2012). At the global scale as at regional scale (i.e. Mediterranean basin), this trend is likely to be strengthened (Moritz et al. 2012; Pausas et al. 2004) leading to an increase in risks such as the forest fire risk. Moreover, changes in the boundaries of bio-climatic areas are expected, especially in the northern part of the French Mediterranean region and at higher elevation that should also lead to an increase in the fire risk towards the North. Some species could be impacted by this change, as, for instance, the European mountain trees that are unequally exposed to an increasing fire risk according to their niche flammability (Fréjaville 2015), or species present in several bio-climatic areas that are adapted to specific conditions, such as fire regimes (Bradshaw et al. 2011; Keeley et al. 2011; Pausas 2015).

Plant flammability is an important driver that plays a fundamental role in terrestrial ecosystems (Bond \& Keeley 2005). Fire-related traits enhancing flammability differ between species (Papió \& Trabaud 1991; Behm et al. 2004) and affect different components of flammability (Santana et al. 2011; Schwilk \& Caprio 2011); some plant communities are thus more likely to burn than others (Bond \& Van Wilgen 1996). Different adaptation strategies are used by species depending on the fire regime, inducing different fire-related traits. For low to medium intensity surface fires, these traits enable species to survive in case of fire (resistant species) in decreasing the fire impact (thick bark, selfpruning, and large height) and being less flammable (i.e. higher leaf or litter bulk density). On the 
contrary, resilient species have adapted to high intensity crown fires favouring their post fire regeneration capacity (developing functional traits such as serotiny) and thus enhancing their capability to burn (with structural and chemical traits such as high dead fuel retention i.e.no selfpruning, high contents of chemical compounds i.e. terpenes in leaves and litter, or a low fuel bulk density, etc.) that allow these species to create their own ecological niche according to Bond \& Midgley's “Born to Burn” hypothesis (1995).

Previous works on plant flammability in fire-prone ecosystems have mostly focused on differences between species, especially between different regeneration strategies (Cowan \& Ackerly 2010; SauraMas et al. 2010) and suggested different genetic mechanisms explaining the evolution of flammability (Kerr et al. 1999; Schwilk \& Kerr 2002). However, the functional traits such as resprouting, serotiny are adaptative in fire-prone environments even if the plants are more adapted to a fire regime than to the fire itself (Keeley et al. 2011) and their traits linked to flammability could vary with a change in the fire regime. However, few works, such as that of Pausas et al. (2012), studied the intraspecific variability and its relation to fire regime but these works generally focused on one type of fuel to assess flammability and on one geographical area (generally within the biogeographical range of the species studied). As stated by Keeley et al. (2011), species having traits adapted to a given fire regime can be endangered when the fire regime changes if these traits do not vary at the same time, especially with the increase of the fire recurrence following the global change, but also with the implementation, in some place, of recurrent prescribed burnings to control the fuel biomass. Identifying if there is a variation of the fire-related traits and of the flammability (and to what extent) according to the fire regime, within intraspecific populations of species will be necessary to predict ecosystem responses to on-going global change.

The aims of this on-going PhD work are (i) to compare the fire-related traits and fuel flammability in different populations sampled in areas submitted to different fire regimes (high fire regime vs low fire regime), in two geographical areas of SE France and (ii) to highlight possible micro-evolutional responses at the different fire regimes, looking for the traits heritability. The current paper focuses on the methodology used to achieve these goals.

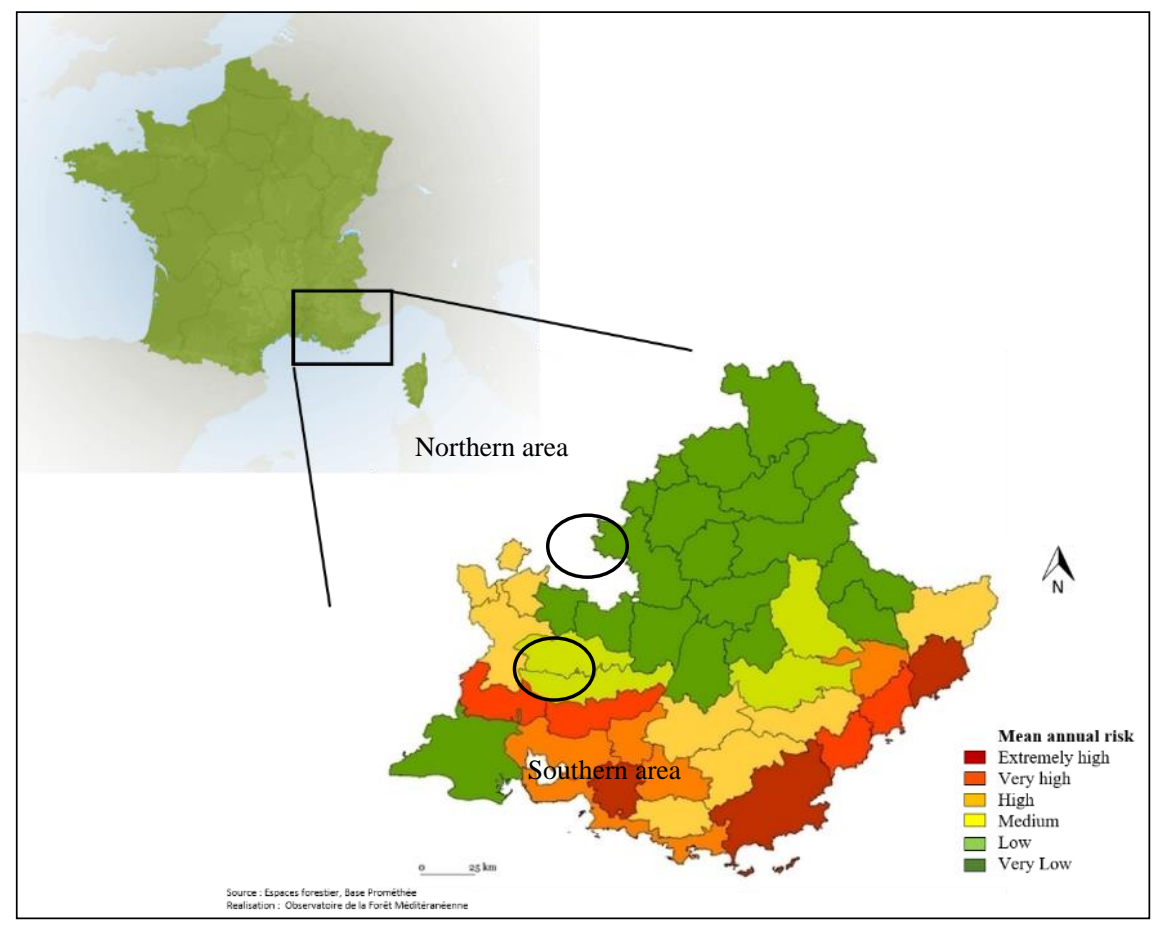

Figure 1 - Map of the fire risk in Provence, SE France (according to fire occurrence and burned area) and the two study areas. 


\section{Methodology}

\subsection{Study areas}

In France, the study areas are located in Provence (Southeastern France). This region is one of the most affected by wildfires (Forest Fires Database of Direction Départementale du Territoire, DDT) with a fire history recorded since 1960 and therefore presents a wide range of fire-prone ecosystems and provides a large gradient of bioclimatic conditions from Thermo-Mediterranean to OroMediterranean, acting on the fire risk. . The work is carried out in two different biogeographical areas, one located in the South of Provence where the fire regime is high and the other in the North of this region (regional natural park of Baronnies Provençales), where the fire frequency and intensity are less severe (Fig. 1), in order to show if the possible trends of adaptation remain the same when the species is sampled within its biogeographical range and at the boundaries of this range.

\subsection{Species studied}

As stated by Pausas et al. (2014), obligate seeders that are species that lack resprouting ability and for whom post-fire regeneration relies only on germination from the seed bank (Bond \& Van Wilgen 1996; Pausas et al. 2004), have shorter and no overlapping generations as well as higher population turnover than resprouters (Verdú et al. 2007). Thus, this species should be more sensitive to fire regime changes. In the current work, two obligate seeder species that present different adaptive strategies to fire, i) Pinus halepensis (resilient species) and ii) Pinus sylvestris (resistant species), are studied.

\subsubsection{The resilient species Pinus halepensis}

$P$. halepensis is a typically fire-resilient species adapted to high intensity crown fire regime and is dominant in the region, especially in its southern part and at low elevation. Post-fire $P$. halepensis strategies are based on sexual reproduction and seed development and are related to seed storage in long-closed cones that stay closed pending the arrival of a fire; the high temperature causing the opening of the cones and the seed release (Lamont et al. 1991; Tapias et al. 2004). This post fire regeneration depends totally upon this species' canopy-stored seed bank (Ne'eman et al. 2004), mechanism called serotiny. Serotiny and early flowering in Pinus halepensis reflect its resilient strategy in relation to fire as this character is advantageous to survive frequent crown fires and to attain successful post-fire recruitment (Tapias et al. 2004).

\subsubsection{The resistant species Pinus sylvestris}

Contrary to the previous pine species, Pinus sylvestris is more adapted to low/medium intensity surface fire regime (Agee 1998; Keeley 2012) and is present especially in the northern part of the region and at higher elevation. Non-serotinous pines, like Pinus sylvestris, once they are burned in an intense fire, are dependent for their recovery on seed dispersal from adjacent unburned patches. In the case of a light or moderate surface fires, trees are protected by their thick bark and because of the selfpruning of the dead branches, their canopy is not affected, allowing seed germination after fire (Retana et al. 2002). Late flowering and absence of serotinous cones in this pine species indicate that their natural forest did not evolve under frequent crown fires (Tapias et al 2004). Phylogenetic studies indicate a strong correlation between heights, self-pruning, and bark thickness in this pine species (Schwilk and Ackerly 2001).

\subsection{Sampling}

The main challenge of this work is to assess the intraspecific variations of the fire-related traits and flammability in pine populations submitted to different fire regimes avoiding, as much as possible, differences in environmental conditions. In order to measure the actual variations due to the fire regime, the populations of both species are sampled according to many environmental similarities (i.e. 
altitude, exposure, aspect, age of the trees, understorey floristic composition, past land use, etc. ; Fig. 2).

In order to sample the populations according to two different fire regimes, a map of the fire perimeters (fire size $>2 \mathrm{ha}$ ) has been performed using the regional fire database of DDT and satellite images before and after each fire (Fig. 3).

For each species, three (in the North) to five populations are sampled in zones where fires have never occurred (NoFi) and three to five others in zones where several fires (one at least in the northern part of the study area which is less impacted by fires) occurred (HiFi) since the last 60 years.

In each area, climatic conditions of HiFi populations should be within the range of the conditions of NoFi populations, that is, there is no geographical segregation between HiFi and NoFi populations. Indeed, the sampling of different populations (of same age) of obligate seeder species must be carried out in a way that the differences of phenotypes observed between different populations are not related to environmental conditions, but are a result of the difference in fire regime (populations coming from old-field colonization for low fire regime vs populations coming from post-fire colonization for high fire regime).

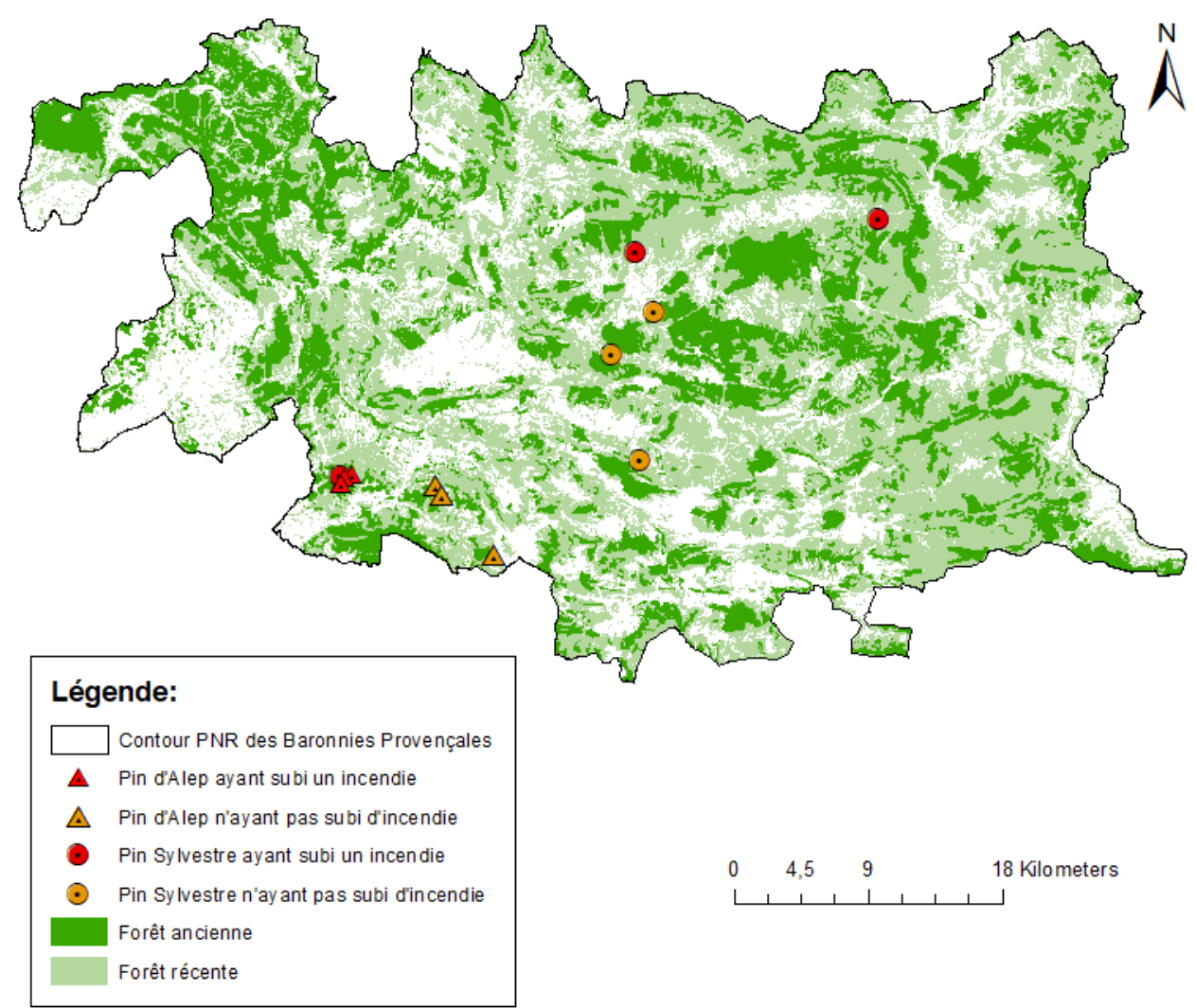

Figure 2 - Map of old forests (1850, dark green) and young forests (2014, light green) with site locations in Regional Natural Park of Baronnies Provençales (triangle: P. halepensis, circle: P. sylvestris and red: HiF, orange: NoF) 


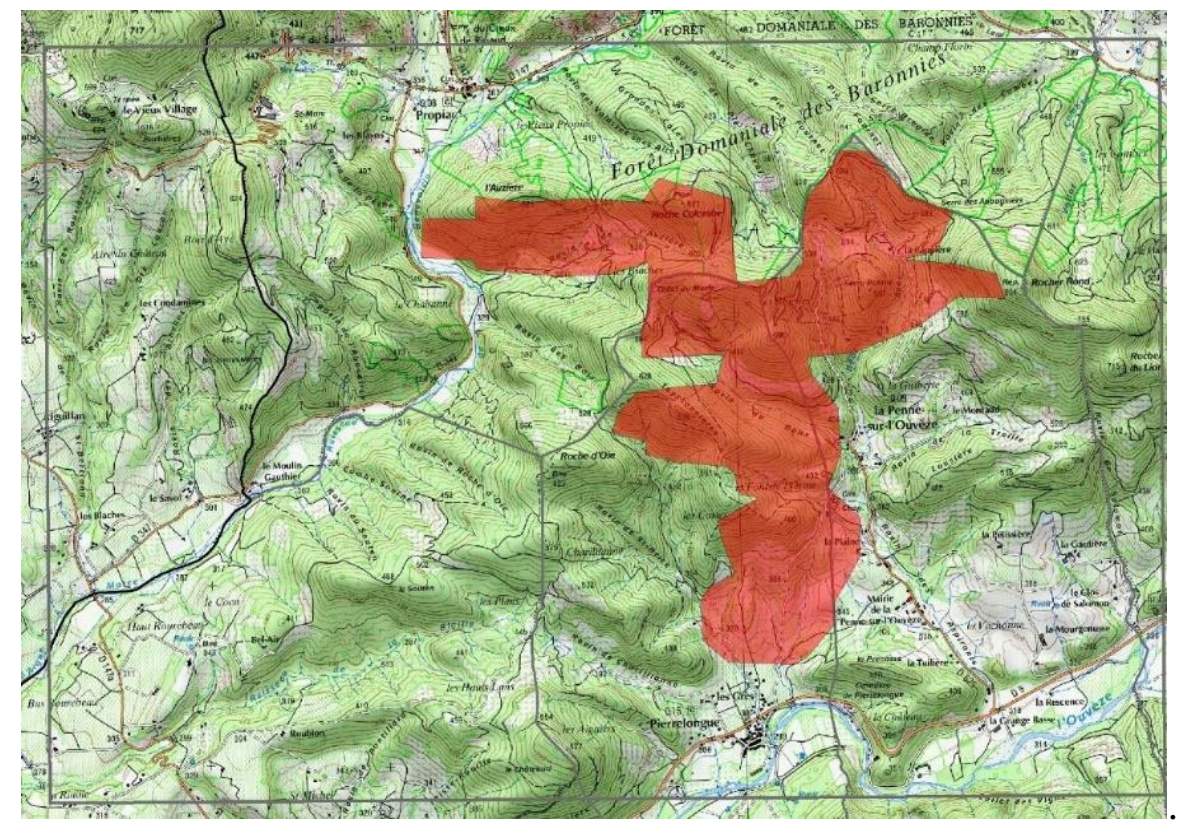

Figure 3 - Map of a fire that occurred in 1986 in Buis-les-Baronnies (Regional Natural Park of Baronnies Provençales, France)

\subsection{Fire-related traits measured}

The fire related traits taken into account in this work are functional, structural or chemical but depend on the species studied and on the adaptation strategy to fire. They are measured on 10 trees per populations.

Functional trait:

- Serotiny: based on Budde et al. (2014) the percentage of serotinous cones will be estimated using 300 cones per populations (10 for each tree sampled), these cones being located on two opposite branches. Serotiny will be assessed during summer to avoid counting errors as cones tend to remained closed in winter when the relative humidity is higher.

Structural traits:

- Bark thickness: for each individual, four measurements were carried out using a drill and a steel ruler.

- Self-pruning: height of the first branch.

- Branch bulk density (weight-to-volume ratio of the branch)

- Needle physical characteristics: for each tree sampled, 10 needles are selected randomly and their weight, volume, surface, surface to volume ratio (Hachmi et al. 2011), length are measured.

Chemical traits:

The terpene content is often studied in the assessment of flammability because of their low flash point (Owens et al. 1998; Alessio et al. 2008; Pausas et al. 2016). In this work, monoterpenes, sesquiterpenes, and diterpenes are analyzed (molecule diversity and concentration).

\subsection{Flammability assessment}

According to Anderson (1970) and Martin et al. (1993), flammability is defined by four components, i) ignitability (fuel capacity to ignite), ii) sustainability (how well the fuel continues to burn), iii) combustibility (how rapidly or intensely a material burns), and iv) consumability (amount of fuel that is consumed). In this study, we assess branch and litter flammability with adapted protocols for each species derived from previous works (Ganteaume et al. 2011, 2014). 


\subsubsection{Dead surface fuel flammability}

Two different protocols are used depending on the species but in each case, we used only pure pine litter demanding a time-consuming sorting of the litter samples collected in the field which contained different types of particles of different species. Regarding P. halepensis, $20 \mathrm{~g}$ of litter (3 burnings per tree) are burned in a circular aluminum tray placed on a weighing scale (Mettler Toledo scale with an accuracy of $0.01 \mathrm{~g}$.) in order to record the mass loss during the burning (Fig. 4). Four thermocouples (k-thermocouples Omega, $25 \mathrm{~mm}$ ) are placed $10 \mathrm{~cm}$ above the sample centre for recording the temperature variation (Mean temperature, Maximal Temperature) during the burning. Just after the ignition (the ignition source is a flaming calibrated firebrand placed at the centre of the sample), timeto-ignition, rate of spread, flaming duration are recorded with a chronometer.

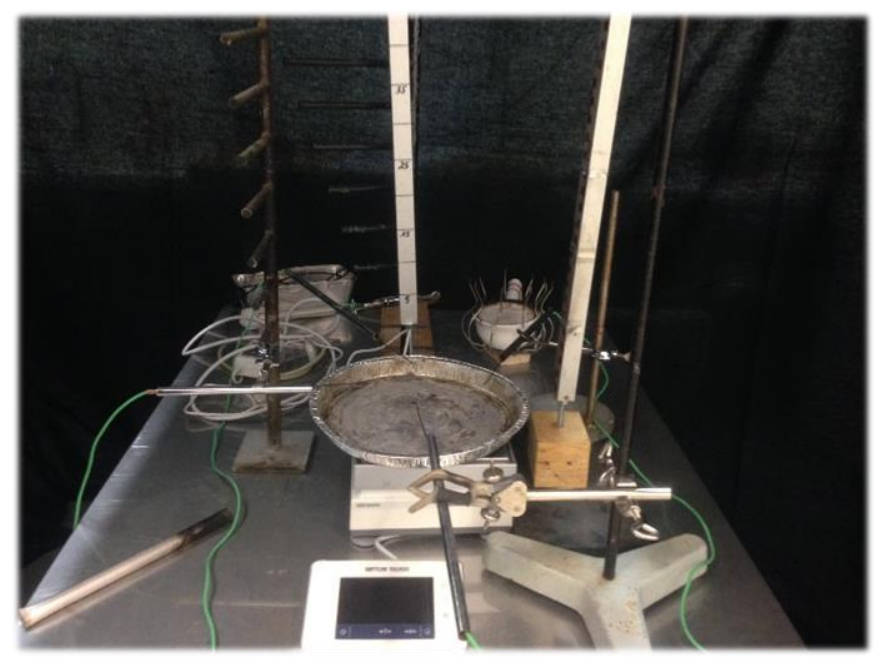

Figure 4 - P. halepensis' litter burning device.

Regarding $P$. sylvestris, $1 \mathrm{~g}$-needle samples are burned using an epiradiator as the amount of litter found under the trees was very low and did not allow designing $20 \mathrm{~g}$-samples as for the previous species. Only time-to-ignition, ignition frequency, flaming duration and maximum (and mean) temperature are recorded using this device according to Ganteaume et al. (2013).

\subsubsection{Branch flammability}

Thirty calibrated fresh branches of each species are burned on the same fire bench as the one used for assessing $P$. halepensis' litter flammability. The branches are ignited by a flame front generated by an excelsior fuel bed located $10 \mathrm{~cm}$ below the branch.

\subsection{Terpene analysis}

The content of the three main terpene classes (monoterpenes, sesquiterpenes, diterpenes) is screened in the llive and dead leaves of the two species studied. The analyses are conducted on $10 \mathrm{~g}$ samples using a GC-MS (GC System 7890B - Agilent Technologies®).

\subsection{Molecular genetics}

Population structure, genetic diversity, and gene flow are assessed using molecular markers. A test of the correlation between genetic patterns and fire-related traits among populations focusing on serotiny is used to address the evolution of this trait. Microsatellites, or simple sequence repeats (SSRs), are regions the genome that contain short repeated stretches of nucleotides. Their high mutation rate, thought to result primarily from slippage during DNA replication, is extremely useful for measuring differences among populations. 


\section{Acknowledgements}

This work is funded by an European Feder programme POIA \#PA0005333.

\section{Reference}

Agee JK (1998) The landscape ecology of western forest fire regimes Northwest Science 72:24

Alessio G, Peñuelas J, Llusià J, Ogaya R, Estiarte M, De Lillis M (2008) Influence of water and terpenes on flammability in some dominant Mediterranean species International Journal of Wildland Fire 17:274-286

Anderson H (1970) Forest fuel ignitibility Fire technology 6:312-319

Behm AL, Duryea ML, Long AJ, Zipperer WC (2004) Flammability of native understory species in pine flatwood and hardwood hammock ecosystems and implications for the wildland-urban interface International Journal of Wildland Fire 13:355-365

Bond W, Van Wilgen B (1996) Fire and Plants (population and community biology series 14) Population and Community Biology 263

Bradshaw SD, Dixon KW, Hopper SD, Lambers H, Turner SR (2011) Little evidence for fire-adapted plant traits in Mediterranean climate regions Trends in plant science 16:69-76

Budde KB, Heuertz M, Hernández-Serrano A, Pausas JG, Vendramin GG, Verdú M, GonzálezMartínez SC (2014) In situ genetic association for serotiny, a fire-related trait, in Mediterranean maritime pine (Pinus pinaster) New Phytologist 201:230-241

Cowan PD, Ackerly DD (2010) Post-fire regeneration strategies and flammability traits of California chaparral shrubs International Journal of Wildland Fire 19:984-989

Frejaville T (2015) Vulnérabilité des forêts de montagne des Alpes occidentales au changement de régime d'incendie. Aix-Marseille

Ganteaume A, Jappiot M, Lampin C, Curt T, Borgniet L (2014) Flammability of litter sampled according to two different methods: comparison of results in laboratory experiments. International Journal of Wildland Fire 23, 1061-1075

Ganteaume A, Jappiot M, Lampin C (2013) Assessing the flammability of surface fuels beneath ornamental vegetation in wildland-urban interfaces in Provence (south-eastern France) International journal of wildland fire 22:333-342.

Ganteaume A, Jappiot M, Lampin-Maillet C, Curt T, Borgniet L (2011) Effects of vegetation type and fire regime on flammability of undisturbed litters in Southeastern France. Forest Ecology and Management 261, 2223-2231

Hachmi M, Sesbou A, Benjelloun H, Bouanane F (2011) Alternative equations to estimate the surfaceto-volume ratio of different forest fuel particles International Journal of Wildland Fire 20:648-656

Keeley JE (2012) Ecology and evolution of pine life histories Annals of Forest Science 69:445-453

Keeley JE, Pausas JG, Rundel PW, Bond WJ, Bradstock RA (2011) Fire as an evolutionary pressure shaping plant traits Trends in plant science 16:406-411

Kerr B, Schwilk DW, Bergman A, Feldman MW (1999) Rekindling an old flame: a haploid model for the evolution and impact of flammability in resprouting plants Evolutionary Ecology Research 1:807-833

Lamont BB, Le Maitre D, Cowling R, Enright N (1991) Canopy seed storage in woody plants The Botanical Review 57:277-317

Martin R et al. Assessing the flammability of domestic and wildland vegetation. In: Proceedings of the 12th conference on fire and forest meteorology, 1993. pp 26-28 
Moreno JM, Oechel WC (2012) The role of fire in Mediterranean-type ecosystems vol 107. Springer Science \& Business Media,

Moritz MA, Parisien M-A, Batllori E, Krawchuk MA, Van Dorn J, Ganz DJ, Hayhoe K (2012) Climate change and disruptions to global fire activity Ecosphere 3:1-22

Ne'eman G, Goubitz S, Nathan R (2004) Reproductive traits of Pinus halepensis in the light of fire-a critical review Plant Ecology 171:69-79

Oliveira S, Oehler F, San-Miguel-Ayanz J, Camia A, Pereira JM (2012) Modeling spatial patterns of fire occurrence in Mediterranean Europe using Multiple Regression and Random Forest Forest Ecology and Management 275:117-129

Owens MK, Lin C-D, Taylor CA, Whisenant SG (1998) Seasonal patterns of plant flammability and monoterpenoid content in Juniperus ashei Journal of Chemical Ecology 24:2115-2129

Papió C, Trabaud L (1991) Comparative study of the aerial structure of five shrubs of Mediterranean shrublands Forest Science 37:146-159

Pausas J, Alessio G, Moreira B, Segarra-Moragues J (2016) Secondary compounds enhance flammability in a Mediterranean plant Oecologia 180:103-110

Pausas JG (2004) Changes in fire and climate in the eastern Iberian Peninsula (Mediterranean basin) Climatic change 63:337-350

Pausas JG (2015) Bark thickness and fire regime Functional Ecology 29:315-327

Pausas JG, Alessio GA, Moreira B, Corcobado G (2012) Fires enhance flammability in Ulex parviflorus New Phytologist 193:18-23

Pausas JG, Keeley JE (2009) A burning story: the role of fire in the history of life BioScience 59:593601

Pausas JG, Keeley JE (2014) Evolutionary ecology of resprouting and seeding in fire-prone ecosystems New Phytologist 204:55-65

Retana J, Maria Espelta J, Habrouk A, Luis OrdoÑEz J, de Solà-Morales F (2002) Regeneration patterns of three Mediterranean pines and forest changes after a large wildfire in northeastern Spain Ecoscience 9:89-97

Santana VM, Baeza MJ, Vallejo VR (2011) Fuel structural traits modulating soil temperatures in different species patches of Mediterranean Basin shrublands International Journal of Wildland Fire 20:668-677

Saura-Mas S, Paula S, Pausas J, Lloret F (2010) Fuel loading and flammability in the Mediterranean Basin woody species with different post-fire regenerative strategies International Journal of Wildland Fire 19:783-794

Schwilk DW, Ackerly DD (2001) Flammability and serotiny as strategies: correlated evolution in pines Oikos 94:326-336

Schwilk DW, Caprio AC (2011) Scaling from leaf traits to fire behaviour: community composition predicts fire severity in a temperate forest Journal of Ecology 99:970-980

Schwilk DW, Kerr B (2002) Genetic niche-hiking: an alternative explanation for the evolution of flammability Oikos 99:431-442

Tapias R, Climent J, Pardos JA, Gil L (2004) Life histories of Mediterranean pines Plant ecology 171:53-68

Verdú M, Pausas JG, Segarra-Moragues JG, Ojeda F (2007) Burning phylogenies: fire, molecular evolutionary rates, and diversification Evolution 61:2195-2204

Westerling AL, Hidalgo HG, Cayan DR, Swetnam TW (2006) Warming and earlier spring increase western US forest wildfire activity science 313:940-943 


\title{
Quantifying effects on fire propagation in woody fuel models using the thin fuels assumption
}

\author{
David Blasen $^{1 *}$; Jesse Johnson ${ }^{1}$; William Jolly ${ }^{2}$; Russell Parsons ${ }^{2}$ \\ ${ }^{1}$ University of Montana. Interdisciplinary Science Building 406 Missoula, MT 59801 \\ \{david.blasen@umontana.edu*,jesse.johnson@mso.umt.edu\} \\ ${ }^{2}$ Missoula Fire Sciences Laboratory. 5775 US West Highway 10 Missoula, MT 59808, \\ \{mjolly@fs.fed.us,rparsons@fs.fed.us\}
}

\begin{abstract}
In this paper, we quantify the effects of thermally thin fuel prescriptions commonly made in numerical models that eliminate temperature gradients within a heated object. This assumption affects the modeled ignition and burn behavior but there is little research on its impact, particularly in larger fuels.

We begin by comparing modeled to observed ignition times and burn rates. To control for the variability in the material properties of wood, as opposed to the modeled variability due to the thin-fuel assumption, we conduct experiments using thermogravimetric analysis (TGA) for samples of lodgepole pine. From this data, we derive material properties via optimization with genetic algorithms. We also perform burnout experiments on large, woody fuels to confirm ignition time and mass loss rates for a range of fuel specimens. These experiments are then repeated with a numerical modeling platform to validate the model. Once validated, we use the model to explore the significance of thermally thin fuel assumptions by performing the same analyses on fuels with both thermally thick and thermally thin fuel treatments. We quantify the above phenomena but also examine how the composition of fuels varies spatially and temporally.
\end{abstract}

Keywords: physics simulation, numerical model, experimental, pyrolysis, ignition delay, burn rate, propagation

\section{Introduction}

Physics-based simulation of fire has become a valuable tool in the study of wildland fire behavior (Mell et al, 2007). The decreasing cost of computation and improvements in model fidelity present an experimental platform for hypothesis testing that is far less constrained by limitations of physical laboratories. In addition, the precise measurements available within a computational model simplify the study of processes that are difficult to measure experimentally. A growing selection of toolkits for designing and carrying out computational experiments presents many opportunities to scientists curious about fire behavior but unable to carry out experiments on the desired scale.

However, among the many opportunities offered by these toolkits are pitfalls that unwary users may encounter. Building even relatively simple models requires diligent observation of good design principles and skeptical examination of their results; it is trivially easy to get interesting results from a nonsensical model. These pitfalls are not intended to confound new users-- they are natural byproducts of the enormous flexibility of the platforms faithfully carrying out the user's instructions whether they accurately prescribe the scenario or not. It is plausible that a user could use kinetic parameters for a fuel that they found but have not validated for their case. There are also many ways to prescribe sources of heat with significant differences in their behavior. The user is expected to know where these tools are valid.

One such example of an assumption with a limited range of validity lies in the prescription of thermally thin fuels. By definition, a thermally thin fuel has no temperature gradient (Quientiere,2006). This assumption requires that the object be small as described in Appendix A and is generally stated to be valid for most fuels under $1 \mathrm{~mm}$ thick (Quientiere, 2006). 
In spite of the limited range of validity, simulation toolkits like FDS (McGrattan et al, 2016) allow prescription of thermally thin fuel treatments. Models like FIRETEC (Linn et al, 2002) use them exclusively, possibly surpassing their range of validity. While thermally thin fuels may be very appropriate representations for certain objects, it is ultimately up to the user to ensure that they are utilized in the right scenarios. These models place no restriction on the application of thermally thin fuel prescriptions.

The ignition time for thermally thin fuels has been studied (Quientiere, 2006) but other relevant properties such as the mass loss rates of ignited fuels are not well characterized. Thus there is a need to quantify other consequences of thermally thin fuel treatments.

In this paper, we begin by creating an appropriate model for heating cylindrical fuel elements over a fire. We validate the model by comparing to previous experimental work then proceed to test a variety of fuels treated as thermally thick and thermally thin to quantify differences in their ignition times and mass loss rates. We also examine the composition of the fuels as they undergo heating and pyrolysis. The fuels are prescribed with differing moisture contents and size and are burned in fire environments of varying intensity. We contrast the behaviors of thermally thick and thermally thin fuel treatments to illustrate their appropriateness in various scenarios.

\section{Methods}

\subsection{Description}

Our model aims to capture relevant physical processes by emulating an experimental design that is appealing due to its simplicity and the utility of the data it gathered (Albini \& Reinhardt, 1995). We specify a $1 \mathrm{~m}^{2}$ open-flame burner and place wooden fuels $60 \mathrm{~cm}$ above where they absorb a fraction of the burner's heat flux. We model a sensor inside of a ball of ceramic cement and tune our burner to roughly match the average recorded fire environment temperature from the experiment $\left(\sim 928{ }^{\circ} \mathrm{K}\right)$. We specify the parameters of our wood fuels to match those derived by numerical approximation of experimental results described below. We describe the chemistry of the fuel produced by the pyrolyzing fuel by referring to existing work that identifies heat release rate of burning wood rather than bench-scale determinations (Ritchie, 1997). The computational domain is $2 \mathrm{~m}$ by $2 \mathrm{~m}$ by $1 \mathrm{~m}$ with a $10 \mathrm{~cm}$ mesh scale; this resolution was determined through mesh refinement to be the point at which results cease to depend on mesh resolution. The boundaries, aside from the ground, are treated as open air. The resulting model exhibits much of the desired behavior.

\subsection{Material Parameters}

One area of considerable uncertainty in the formulation of the model lay in the material parameters of our fuels. While there are numerous examples of woody fuel materials in FDS, they vary considerably in their parameterizations and it's unclear which description might be appropriate for a new fuel. A shortage of validated material parameters is in fact a lingering concern of FDS; while the project's modeling of the physics in solid fuels is believed to be reasonably good, the lack of validated material parameters for many solid fuels of interest remains an area of active work (McDermott, 2017).

Our solution to this problem is to derive material parameters from experimental data. We conducted thermogravimetric analysis (TGA) on samples of lodgepole pine (Pinus contorta) and used this data as input to gpyro, a fine-resolution pyrolysis model that has a module for numerically approximating material parameters (Lautenberger and Fernandez-Pello, 2009). By doing this we were able to obtain the kinetic parameters for dried samples of lodgepole pine wood which we then used in our model. The parameters we identified are enumerated in Table 1 in Appendix B along with a description of their relationships. The fit between our experimental data and a simultation using these parameters can be seen in Figure 1f. 


\subsection{Thin Fuel Support}

Another important consideration for this model is that it must allow for simple characterization of both thin and thick fuels. The platform used in this project, FDS Version 6, permits both fine and coarse sub-mesh fuel description of temperature and material composition. Individual fuels in the mesh have their own sub-grids that are smaller than that used for the computational fluid dynamics of the mesh (McGrattan, 2017). A user may impose a coarse representation upon the fuel to prescribe thermally thin treatment.

\subsection{Model Assumptions}

The model operates under a few key assumptions imposed by the simulation framework:

1) Only one gaseous reaction (the combustion of wood gasses) is significant.

2) Mass transfer of moisture and flammable gases inside of fuel is insignificant.

3) The fuel is homogeneous.

To determine if the physical parameters describing fuels we found by fitting TGA data with gpyro are appropriate for the numerical treatment used in FDS, we compared numerical experiments to observations from experimental work (Albini and Reinhardt, 1995). Agreement was excellent for intermediate moisture content $(21 \%)$ but deteriorated for low (4\%) and high (60\%) moisture content. Agreement was excellent for fuels of smaller diameter $(\sim 5 \mathrm{~cm})$ but suffered on those of larger diameter $(\sim 10 \mathrm{~cm})$. In all cases differences in mass loss rate between model and observations were less than $5 \%$ of total mass lost with insignificant differences on smaller diameter, intermediate moisture content fuels.

\section{Experiments}

\subsection{Overview}

With a model that matches acceptably to previous experimental data, we move on to discuss experiments relevant to assessing the validity of thin fuel treatments. As mentioned above, we consider time until ignition and mass loss rate. We also examine the material composition of fuels as a function of time and space.

Each set of experiments compares a series of $30 \mathrm{~cm}$ long cylindrical fuels ranging from $1.0 \mathrm{~mm}$ to $5.0 \mathrm{~mm}$ in diameter at $4 \%, 21 \%$, and $60 \%$ moisture content are simulated. We also change the flux of the heat source to examine its significance at $112.5,225,450$, and $900 \mathrm{~kW} / \mathrm{m}^{2}$.

When we refer to heat flux we are referring to that of the burner and not that at the surface of the fuel. The fuel will only absorb a small fraction of the heat flux from the burner.

Because of model sensitivity to initial conditions we conduct each experiment ten times with slight $\left(1 \mathrm{~W} / \mathrm{m}^{2}\right)$ perturbations in the burner's heat flux. The resulting experimental data is averaged or regressed. This is mentioned in more detail in the discussion.

\subsection{Ignition Time}

We record the times at which each fuel reaches an ignition temperature of $350{ }^{\circ} \mathrm{C}$ at its surface. This is measured directly from the surface of the fuel in the model.

\subsection{Mass Loss Rate}

We record the mass loss rates of fuels starting at the time of heating. We record the mass of the fuel at every time step. 


\subsection{Fuel Composition}

We also gather detailed sub-grid measurements of temperature and composition throughout the fuel. Composition is determined by mass fraction of each solid phase material component: water, wood, and char.

\section{Experiment Discussion}

\subsection{Ignition time}

We find that thermally-thin fuels ignite more slowly than thermally-thick fuels but that the difference varies with moisture content, heat flux, and fuel diameter. A direct comparison of ignition times for fuel of different sizes, moisture contents, and under different heat fluxes can be seen in Figure 2. Values from all 10 experiments are regressed for trend lines. The total increase in ignition time caused by thermally thin fuel prescriptions for all of our experiments may be found in Figures 1a, 1b, and 1c. These plots show averages of the 10 experiments with slightly perturbed heat fluxes in order to smooth out the sensitivity of the experiment at low heat flux.

As can be seen in any plot in Figure 2, increasing fuel diameter increases the disparity between the ignition times of thermally thick and thermally thin fuel prescriptions. This is entirely expected. We can see that near $1 \mathrm{~mm}$ diameter, the difference in ignition time in all experiments is never more than a fraction of a second. This supports the idea that thermally thin fuel prescriptions are generally appropriate for fuels under $1 \mathrm{~mm}$ in thickness (Quientiere, 2006).

Figure 2 demonstrates that fuels with higher moisture content are more appropriately modeled by thermally thin prescriptions than fuels with lower moisture content. It is easier to see this in the lower heat flux experiments in the left column but it is also present in the higher heat flux experiments in the right. This finding is somewhat less intuitive but could be explained by increased thermal conductivity in the fuel moving heat away from the surface and delaying ignition. This would impact thermally thick fuel treatments but not thermally thin fuel treatments because they are effectively a point in space. In Figure 1a, we note that the ignition time disparity for $4 \%$ moisture content fuels heated at 112.5 $\mathrm{kW} / \mathrm{m}^{2}$ increases with size, but that in Figure $1 \mathrm{~b}$ it increases more gradually for $21 \%$ moisture content fuels and, in Figure 1c, it appears to not significantly increase at all for $60 \%$ moisture content fuels.

Figure 1 shows that changes in ignition time from thermally thin prescriptions are susceptible to variations in heat flux. Lower heat fluxes cause less pronounced differences in ignition time between thermally thick and thermally thin fuel prescriptions. This agrees with existing models (Quientiere, 2006). This could be explained by lower heat flux slowly heating fuels of both prescriptions to temperature instead of rapidly heating the outer surface of fuels treated as thick.

We see above that ignition time is affected by thermally thin fuel prescriptions quite significantly. When the fuels are as small as $1 \mathrm{~mm}$ in diameter, they generally ignite very similarly to thermally thick fuels regardless of moisture content or the heat of the fire; as seen in Figure 2, very small fuels can see a change in ignition delay of well under a second. But even a slight increase in fuel size will quickly cause the ignition times of these fuels to diverge. Once fuels are $5 \mathrm{~mm}$ in diameter, we see more than a doubling in ignition time for heating rates higher than $450 \mathrm{~kW} / \mathrm{m}^{2}$. We see that fuels much larger than a few millimeters in size will suffer from unrealistic ignition times. This is evident in many models that utilize the thin-fuels assumption; grass, needles, and other fuels rapidly ignite and are consumed but slightly larger fuels are left completely unaffected. 

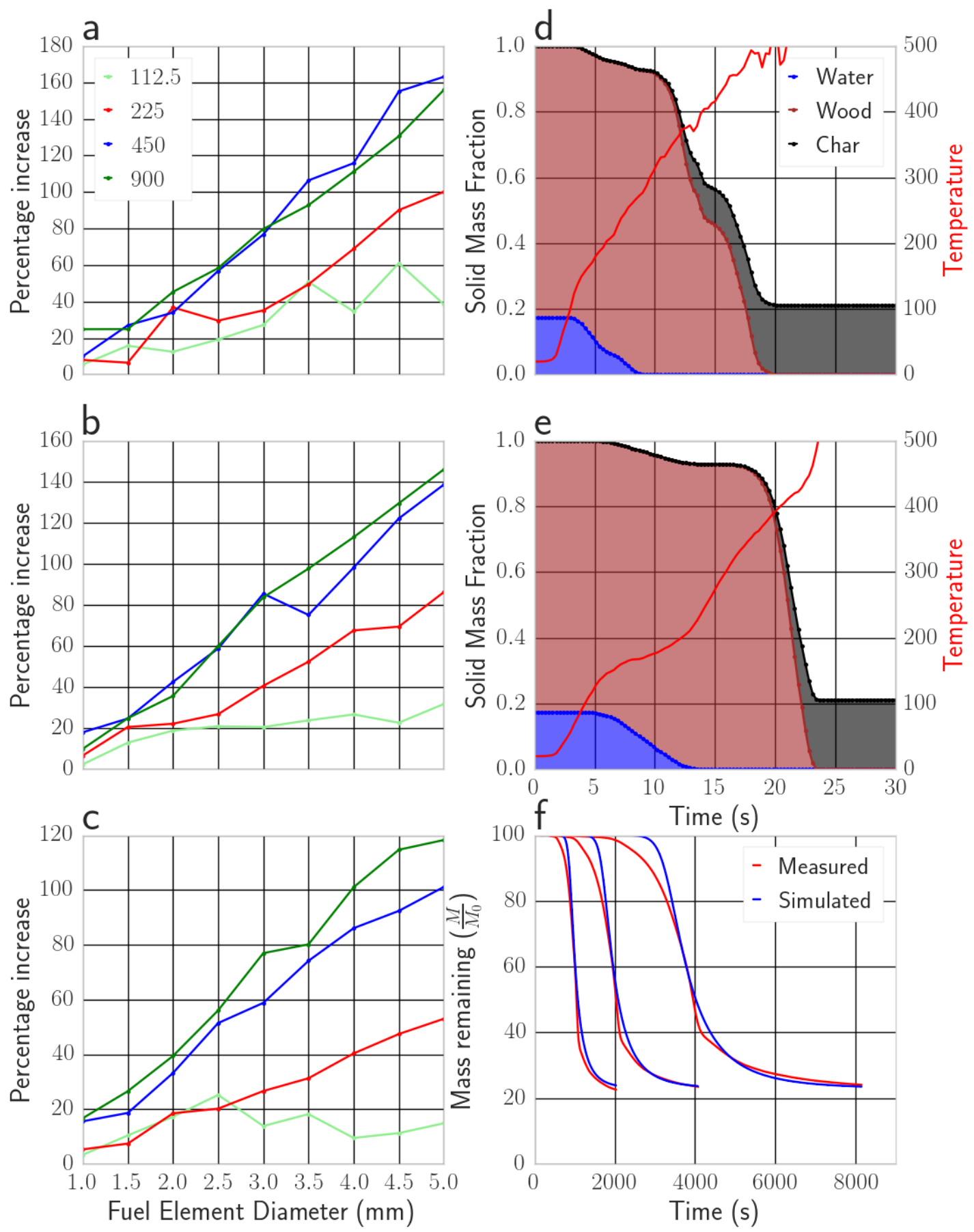

Figure 1 - a,b,c: Ignition time delay increase in thermally thin fuel treatment over thermally thick fuel treatment as percentage of total time at heat fluxes of $112.5,225,450$, and $900 \mathrm{~kW} / \mathrm{m}^{2} .4 \%, 21 \%$, and $60 \%$ moisture content top to bottom, respectively. d,e: Composition of $5 \mathrm{~mm}$ fuels at depth $=0.5 \mathrm{~mm}$, heat flux $=450 \mathrm{~kW} / \mathrm{m}^{2} ;$ d: thick treatment, e: thin treatment. $f$ : Simulated and measured TGA plots for lodgepole pine samples. $20^{\circ} \mathrm{C} / \mathrm{min}, 10^{\circ} \mathrm{C} / \mathrm{min}$, and $5^{\circ} \mathrm{C} / \mathrm{min}$ heating rates left to right, respectively. 

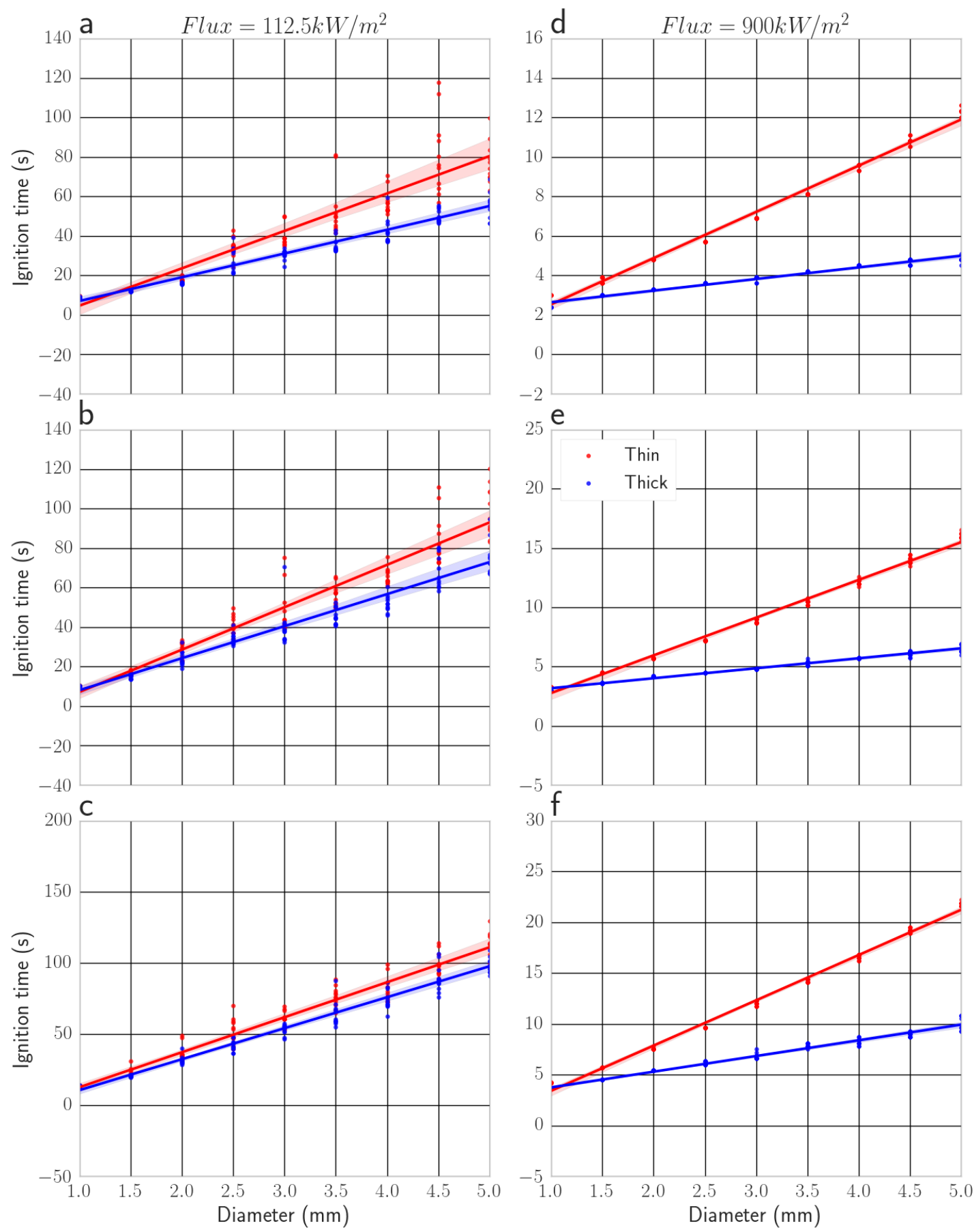

Figure 2 - Ignition time delays. Left column: heat flux $=112.5 \mathrm{~kW} / \mathrm{m} 2$. Right column: heat flux $=900 \mathrm{~kW} / \mathrm{m} 2$. Top row: $4 \%$ moisture content. Middle row: $21 \%$ moisture content. Bottom row: $60 \%$ moisture content. 

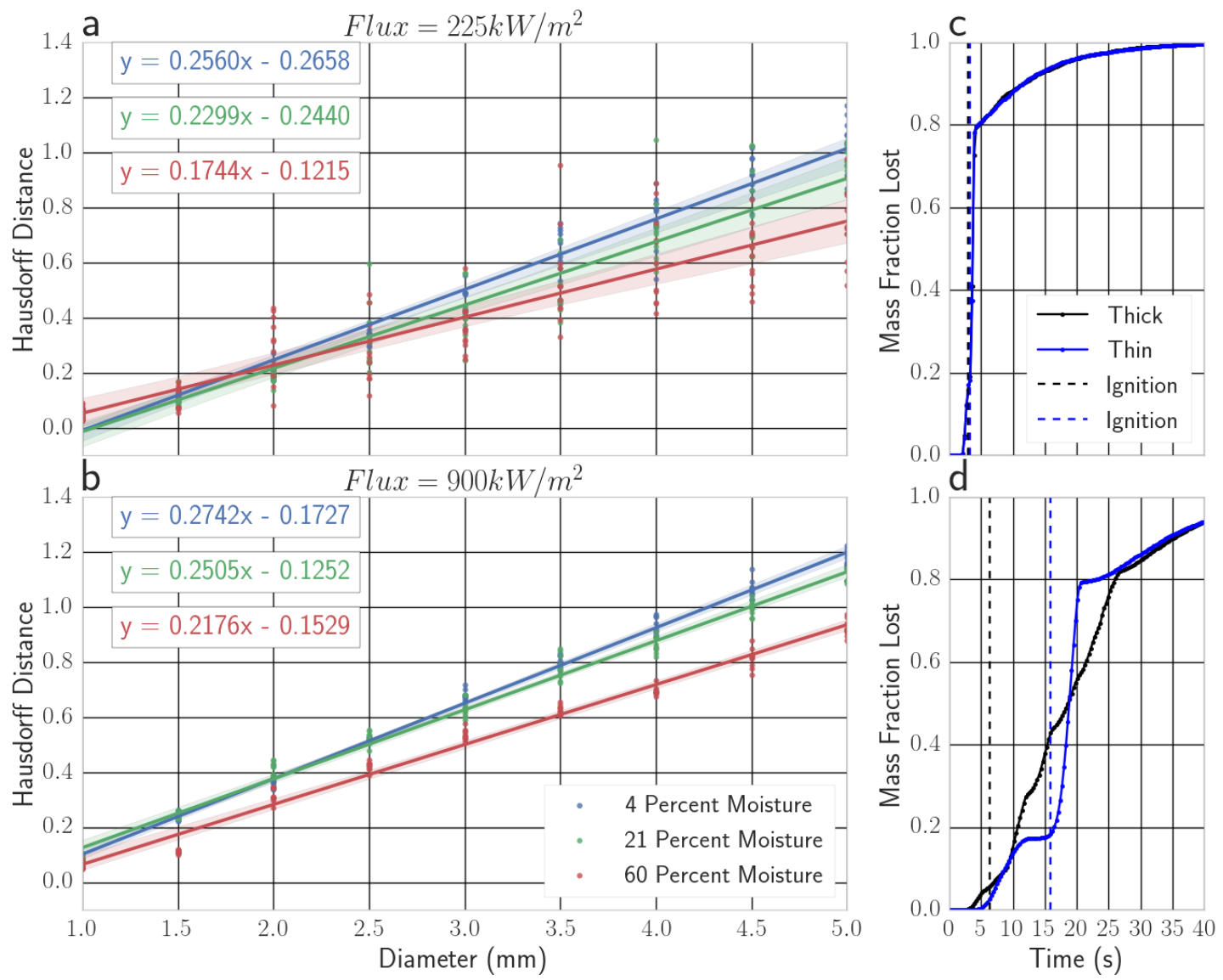

Figure 3 - Mass loss rates. a,b: Hausdorff distance as function of fuel diameter. Top: heat flux $=225 \mathrm{~kW} / \mathrm{m}^{2}$. Bottom: heat flux $=900 \mathrm{~kW} / \mathrm{m}^{2} . c, d$ : mass loss of $21 \%$ moisture content thermally thick and thermally thin treated fuels of diameter $1 \mathrm{~mm}$ (top) and $5 \mathrm{~mm}$ (bottom) at heat flux $=900 \mathrm{~kW} / \mathrm{m}^{2}$.

\subsection{Mass Loss Rate}

We find that mass loss rates of fuels of thermally thick and thermally thin prescriptions vary depending on moisture content, heat flux, and diameter. We see some direct mass loss rate comparisons in Figures $3 \mathrm{c}$ and $3 \mathrm{~d}$. We compute the Hausdorff distance between thermally-thick and thermally-thin treatments for fuels. This measures distances between subsets in a metric space: in our case, these are observations for an experiment in the metric space of time (Rockafeller and Wets, 2005). With this test, we have a metric to compare dissimilarity between mass loss rate curves. Hausdorff distances are regressed for trend lines. These can be seen in Figures $3 \mathrm{a}$ and $3 \mathrm{~b}$.

In Figure 3c we see that fuels of $1 \mathrm{~mm}$ in diameter burn up in virtually identical fashion whether they are prescribed as thermally thick or thermally thin. But in Figure 3d it's clear that fuels that are $5 \mathrm{~mm}$ in diameter show interesting differences based on their prescription. Thus, as expected, fuel diameter is a significant factor in mass loss rate differences between thermally thick and thermally thin treatments. The distinctive 'step' in the thermally thin prescribed fuel is where all moisture has been driven off but the wood has not yet started combusting; in Figure $3 \mathrm{c}$ we see that these processes occur with some overlap for thermally thick fuel treatments; fuels prescribed as thermally thin must have all of their moisture driven off before they can ignite.

We can clearly see that moisture content has a marked effect on mass loss rate in Figures $3 \mathrm{a}$ and $3 b$. We see that higher moisture contents decrease the difference in mass loss rates between thermally 
thick and thermally thin prescriptions for fuels. This is possibly also caused by increased thermal conductivity in fuels with higher moisture content as described above.

Heat flux is significant as well. Comparing Figure $3 a$ to $3 b$, we can see that Hausdorff distances between the mass loss rate curves of thermally thick and thermally thin prescriptions are uniformly greater with increased heat flux. This is likely influenced by the reduced disparity in ignition times mentioned above.

\subsection{Fuel Composition}

We find that the composition of a fuel is significantly influenced by whether the fuel is prescribed as thermally thick or thermally thin. These may be seen in Figure 1d and 1e. This finding makes sense given the different way that these fuels heat and thus pyrolyze. In particular, we see that moisture must be entirely purged from fuels treated as thermally thin before the wood may ignite.

The temperatures in the fuels also differ; we see that the fuel treated as thermally thin is heating more slowly than the other and that its temperature climb markedly slows as moisture is driven off. In the other fuel, temperature is more erratic due to its internal gradient and the process of driving off moisture is presumably less sudden since no such stall exists in the temperature data.

\section{Conclusion}

We established and validated a model for burning cylindrical fuels and recording their ignition times, mass loss rates, and material compositions. We numerically determined physical parameters for lodgepole pine fuel using experimental data. We conducted many experiments to establish the significance of fuel size, fuel moisture content, and fire intensity in the precision of thermally thin fuel prescriptions.

We found that thermally thin fuel prescriptions have insignificant effects on both ignition time delay and mass loss rate at fuel diameters around $1 \mathrm{~mm}$ but that both are significantly effected at fuel diameters approaching $5 \mathrm{~mm}$. We found that lower heat flux and higher fuel moisture content reduce the significance of these differences to a limited extent but that fuel diameter remains the single most important determinant in whether a thermally thin fuel prescription is appropriate.

We conclude that thermally thin fuel prescriptions are entirely appropriate under the commonly accepted thickness of $1 \mathrm{~mm}$ and suggest that, in cases where fuels are characterized by moderate to high moisture contents and are subjected to lower flux heat sources, they may be appropriate in slightly larger fuels. However, even with these conditions, they do suffer from loss of precision quite soon after. Researchers should carefully consider both their fuel and fire environment before using thermally thin fuel prescriptions.

\section{Acknowledgements}

This research was funded through agreement \#17-JV-1221637-129 with the United States Forest Service Rocky Mountain Research Station with underlying support from the Western Wildlands Environmental Threat Assessment Center. We would like to thank the ICFFR reviewers for their time and consideration of our submitted abstract. We would like to thank the members of the FDS project for incorporating a feature request on our behalf.

\section{Appendices}

\subsection{Appendix A: Smallness of Thermally Thin fuels}

Thermally thin objects are defined as being sufficiently small that no internal temperature gradient is formed under heating. Generally, it must be the case that the physical thickness, $d$, is less than the 
thermal penetration depth. For the temperature gradient to be small over region $d$, it must be the case that

$$
B i \equiv \frac{d h_{\mathrm{c}}}{k} \ll \frac{h_{\mathrm{c}}\left(T_{\mathrm{s}}-T_{\mathrm{o}}\right)}{\dot{q}^{\prime \prime}}
$$

where $B i$ is the object's Biot number, $h_{c}$ is the effective heat transfer coefficient, $k$ is the thermal conductivity, $T_{s}$ is the surface temperature of the object, T0 is the initial temperature of the object, and q'' is the heat flux (Quintiere, 2006). If these conditions are satisfied then the ignition time for a fuel prescribed as thermally thin should be very similar to that of a fuel prescribed as thermally thick.

\subsection{Appendix B: Gpyro Formulations}

Gpyro is used to numerically approximate material parameters described above in addition to parameters of a single step, heterogeneous reaction as:

$$
\frac{\mathrm{d} \alpha}{\mathrm{d} t}=Z \exp \left(-\frac{E}{R T}\right)(1-\alpha)^{n}
$$

Where $\alpha$ is the dimensionless conversion, $Z$ - called $A$ in the paper above- is the pre-exponential factor $\left(\mathrm{s}^{-1}\right), E$ is the activation energy $(\mathrm{kJ} / \mathrm{mol}), n$ is the dimensionless reaction order (Lautenberger, 2009), $T$ is temperature in ${ }^{\circ} \mathrm{K}$, and $R$ is the gas constant $(8.314 \mathrm{~J} / \mathrm{mol})$.

Gpyro assumes that the density and thermal conductivity of each condensed phase species vary with temperature. In the case of conductivity:

$$
k_{i}(T)=k_{s, i}(T)+k_{r, i}(T)=k_{0, i}\left(\frac{T}{T_{r}}\right)^{n_{k, j}}+\gamma_{i} \sigma T^{3}
$$

where $k_{0, i}$ is the conductivity at reference temperature $T_{r}, n_{k, i}$ is the exponent that scales the conductivity, $\gamma_{i}$ is the radiative portion of conductivity (for radiation crossing pores in the substrate) (Lautenberger, 2009), and $\sigma$ is the Stefan-Boltzmann constant $\left(5.67 * 10^{-8} \mathrm{~W}^{*} \mathrm{~m}^{-2 *} \mathrm{~K}^{-4}\right)$. Thus Gpyro establishes materials may change in conductivity with respect to temperature.

The parameters we numerically approximated are in Table 1.

Table 1 - Material and reaction parameters for lodgepole pine wood found through numerical approximation with Gpyro.

\begin{tabular}{|l|l|l|}
\hline Name & Unit & Value \\
\hline A (wood) & $\mathrm{s}^{-1}$ & $2.45 \mathrm{E}+13$ \\
\hline $\mathrm{E}($ wood) & $\mathrm{kJ} / \mathrm{mol}$ & 178 \\
\hline Order & - & 4.5 \\
\hline Rho initial (char) & $\mathrm{kg} / \mathrm{m}^{3}$ & 134 \\
\hline Conductivity initial (wood) & $\mathrm{W} /(\mathrm{m} * \mathrm{~K})$ & 0.19 \\
\hline Conductivity initial (char) & $\mathrm{W} /(\mathrm{m} * \mathrm{~K})$ & 0.095 \\
\hline Conductivity exp (wood) & - & 0.038 \\
\hline Conductivity exp (char) & - & 0.14 \\
\hline Specific Heat Cap. (wood) & $\mathrm{J} /(\mathrm{kg} * \mathrm{~K})$ & 2845 \\
\hline Specific Heat Cap. (char) & $\mathrm{J} /(\mathrm{kg} * \mathrm{~K})$ & 1734 \\
\hline
\end{tabular}




\section{References}

Albini, F. and Reinhardt, E. (1995). Modeling Ignition and Burning Rate of Large Woody Natural Fuels. International Journal of Wildland Fire, 5(2):81-91

Lautenberger, C. and Fernandez-Pello, A.C. (2009). Generalized Pyrolysis Model for Combustible Solids. Fire Safety Journal, 44:819-839

Linn, R.R., Reisner, J. Colman J., Winterkamp J., 2002. "Studying wildfire behavior using FIRETEC." International Journal of Wildland Fire 11(3-4): 233-246.

McDermott, R., McGrattan, K., Floyd, D. (2017). FDS Road Map. https://github.com/firemodels/fds/wiki/FDS-Road-Map

McGrattan, K., McDermott, R., Weinschenk, C, and Forney, G. (2016). Sixth Edition Fire Dynamics Simulator Technical Reference Guide. NIST Special Publication 1018.

McGrattan, K., Hostikka, S., McDermott, R. (2017) Sixth Edition Fire Dynamics Simulator Technical Reference Guide Volume 1: Mathematical Model. NIST Special Publication 1018.

Mell W.E., Jenkins M.A., Gould J., Cheney P. (2007) A physics-based approach to modelling grassland fires. International Journal of Wildland Fire 16, 1-22.

Quintiere, J. (2006) Fundamentals of Fire Phenomena. John Wiley \& Sons Ltd.

Ritchie, S., Steckler, K., Hamins, A., Cleary, T., Yang, G. and Kashiwagi, T. (1997). The Effect of Sample Size on the Heat Release Rate of Charring Materials. Fire Safety Science 5: 177-188.

Rockafeller, R. T. and Wets, R. JB (2005). Variational Analysis. Springer-Verlag. p. 117. 


\title{
Soil resilience under different scenarios of fire recurrence and severity in Pinus forest ecosystems affected by large wildfires
}

\author{
Elena Marcos ${ }^{1 *}$; Victor Fernández-García ${ }^{1}$; José Manuel Fernández-Guisuraga ${ }^{1}$; Alfonso \\ Fernández-Manso $^{2}$; Carmen Quintano ${ }^{3}$; Leonor Calvo ${ }^{1}$ \\ ${ }^{1}$ Area of Ecology. Faculty of Biological and Environmental Sciences. University of León, 24071 \\ León. \{elena.marcos@unileon.es*\} \\ ${ }^{2}$ Agrarian Engineering Department, University of León, Av. Astorga s/n, Ponferrada, Spain \\ ${ }^{3}$ Electronic Technology Department, Sustainable Forest Management Research Institute, \\ University of Valladolid, Spanish National Institute for Agriculture and Food Research and \\ Technology (INIA), C/Francisco Mendizábal s/n, 47014 Valladolid, Spain.
}

\begin{abstract}
Fire is as a global phenomenon that represents one of the main disturbances in the Mediterranean Pinus forest ecosystem. It is also considered to be a soil forming factor and temporary modifier of soil properties. The degree of this impact depends on different factors such as pre-fire conditions, the type of soils affected, topographic and metereological characteristics and fire-regime attributes (e.g. fire size, recurrence, burn severity). In the last few decades, the fire regime is changing in some regions of the world including the Iberian Peninsula. This is the result of land abandonment, fire suppression policies and climate change. These factors generate a more favourable environment for the occurrence of large forest fires that may play a key role in affecting the natural rates and patterns of soils structure and functioning. As a consequence, both resiliency and sustainability of Mediterranean Pinus forest could be engaged. This survey is aimed at getting to know the effects of combined fire-regime attributes (recurrence and severity) on soil biochemical properties from a medium-term perspective (four years).

The study was conducted in the Sierra del Teleno mountain range (León province; NW Iberian Peninsula). The climate is Mediterranean with 2-3 months' dryness in summer. Soils are acidic (pH around 4.8) and mainly classified as Cambisol and Leptosol. A large wildfire occurred on August $19^{\text {th }} 2012$ affected an area of $119 \mathrm{~km}^{2}\left(103 \mathrm{~km}^{2}\right.$ being occupied by Pinus pinaster Ait. forests). In this wildfire, a fire recurrence-severity map was elaborated by remote sensing methods, and validated using ground truth and with the information provided by the Regional Administration. We characterized fire recurrence throughout a 16-year period (1998-2014). Fire severity was measured by the dNBR (difference of the Normalized Burn Ratio) spectral index, and classified according to the ground reference values of the CBI (Composite Burn Index). We differentiated four scenarios of recurrence (low and high) and severity (low and high), and established a minimum of five $30 \mathrm{~m} * 30 \mathrm{~m}$ field plots in each of the four recurrence-severity scenarios. In each field plot, we collected soil samples from a depth of $0-3 \mathrm{~cm}$ four years after the fire, and analysed the enzymatic activities $\beta$-glucosidase, urease, and acid-phosphatase and microbial biomass carbon.

Four years after the fire, it can be observed that fire-regime attributes have a great influence on the resilience of soil properties. In the scenario of low recurrence-high severity, which means the highest intensity fire, decreased phosphatase and urease activities and soil microbial biomass carbon. This scenario showed a slight resilience to $\beta$-glucosidase activity but marked depletion in the other soil properties. High recurrence scenarios favour the recovery of soil biochemical and microbial properties. Soil enzymatic activities and microbial biomass could be a proxy to identify the general state of soil health after large fires.
\end{abstract}

Keywords: enzymatic activities, medium-term, post-fire, recovery, soil microbial biomass, wildfires

\section{Introduction}

Fire is an important process which causes severe disturbances in many ecosystems, mainly in Mediterranean forest ecosystem. In this region, forests most affected by fire are pine ecosystems 
(Moreira et al., 2008) with Pinus pinaster Ait. and Pinus halepensis Mill. as the dominant species. Fire impact on the ecosystem depends on different factors such as forest structure (Jain \& Graham 2007; Shive et al., 2013), topographic and meteorological characteristics (Fang et al., 2018; Moreno \& Chuvieco, 2016) and fire-regime attributes (Fernández et al., 2018).

In the last few decades, fire regime is changing in some regions of the world such as the Iberian Peninsula. This is the result of land abandonment, fire suppression policies and climate change (Moreno et al., 2014). These factors generate a more favourable environment for the increase in number and size of fires and thus in fire frequency (Guénon et al., 2013) and severity. As a consequence, natural rates and patterns of soil structure and functioning could be altered. Fire severity, defined as the loss of or change in biomass of the ecosystem caused by fire (Keeley, 2009), is used as an indicator of the fire's indirect impacts on the hydrological response of burned areas (Vieira et al., 2015). This is associated with negative effects mainly in soil physical properties such as water repellency and aggregate stability, and it is attributed to the changes in soil organic matter (Varela et al., 2015; Merino et al., 2018). Likewise, fire severity is a dominant factor in explaining changes in soil microbial communities and soil enzyme activity (Knelman et al., 2015), constraining the post-fire functioning of the ecosystem. On the other hand, fire recurrence continuously impoverishes soil especially in terms of organic matter and nutrients (Knicker, 2007) which would negatively affect to the recovery of microbial activities and increased the loss of ecosystem resilience in the long-term (Guénon et al., 2013).

On the other side, soil microbial biomass and enzyme activities are considered good indicators of soil quality and the impact of fire on soil (López-Poma \& Bautista, 2014; Hinojosa et al., 2016). These properties are more sensitive to soil management practices or disturbances than chemical and physical ones (Dilly et al., 2018). The highest soil microbial content is found in natural ecosystems (woods and pastures) while this content decreases in managed ecosystems (crops and plantations) (Dilly et al., 2018) or in severely burned soils (Vega et al., 2013). The activity of soil enzyme is generally reduced immediately after fire (Vega et al., 2013; López-Poma \& Bautista, 2014). The persistence of this impact can be ephemeral or persist several years according to the capacity of establishment of plant communities (Knelman et al., 2015) and the resilience of soil enzyme activity (López-Poma \& Bautista, 2014).

Although different research has evaluated the effects of fire recurrence (Guénon et al., 2013) or fire severity (Vega et al., 2013) in both biochemical and microbiological soil properties, there are not studies analyzing the effects of the combination of fire regime attributes (recurrence and severity) in them. For this reason, the present survey is aimed at determining to know the role of combined fireregime attributes (recurrence and severity) in soil resilience of biochemical and microbiological properties from a medium-term perspective (four years).

\section{Material and methods}

\subsection{Study area}

The study was conducted in the Sierra del Teleno mountain range (León province; NW Spain) The climate is Mediterranean temperate with an annual precipitation of 650-900 mm (Quintano et al., 2015) and an average temperature of around $11^{\circ} \mathrm{C}$. The area is characterised by a heterogeneous orography, with an elevation above sea level ranging from 850 to $2100 \mathrm{~m}$. The lithologies are siliceous, and dominated by stones, silts, clays and conglomerates in the flat areas, and slate, sandstone, and quartzite in the mountainous ones (IGME, 1982). The soils are classified as Cambisol and Leptosol (Forteza et al., 1987) with a $\mathrm{pH}$ around 5, low soil organic carbon content $(\sim 2 \%)$, total nitrogen $(\sim 0.1 \%)$ and available phosphorous $\left(<5 \mathrm{mg} \mathrm{kg}^{-1}\right)$. The vegetation present before fire was covered mainly by Pinus 
pinaster Ait. with an understorey layer dominated by Erica australis L., Pterospartum tridentatum (L.) Willk., and Halimium lasianthum spp. alyssoides (Lam.) Greuter (Santalla et al., 2002)

Fires caused by dry spring-summer storms occur frequently in this forest ecosystem, but usually affect small areas. Nevertheless, a large fire occurred on 19th August 2012. This fire burned $119 \mathrm{~km}^{2}$ being $103 \mathrm{~km}^{2}$ being occupied by $P$. pinaster forests, with a tree age of 35-95 years old. The weather conditions during the first day were $32^{\circ} \mathrm{C}$ and $27 \%$ relative humidity. There was a very significant accumulated drought, with Haines index values of 6 (Quintano et al., 2015).

\subsection{Field data collection and soil analysis}

In this wildfire, a fire recurrence-severity map was elaborated by remote sensing methods, and validated using ground truth and with the information provided by the Regional Administration (Fernández-Manso et al., 2015) (Figure 1). To determine fire recurrence we considered the period 1998-2014 and classified as low (1 fire) and high (2 fires) recurrence. In order to characterise fire severity, we calculated the dNBR spectral index (Key, 2006) from the Landsat 7 ETM+ scenes of September 20th, 2011 (pre-fire situation) and September 6th, 2012 (post-fire). Four years after wildfire, a total of 44 field plots of $30 \mathrm{~m}$ x $30 \mathrm{~m}$ were established in four recurrence scenarios (low and high) and burn severity (low and high). The plots were randomly distributed in these scenarios with a minimum of 5 plots per recurrence-severity category: 10 plots for low recurrence-low severity; 10 plots for low recurrence-high severity; 10 plots for high recurrence-low severity and 14 plots for high recurrence-high severity.

To evaluate soil resilience, we collected two soil composite samples from each 30 x $30 \mathrm{~m}$ plot. Each soil sample was made up of four subsamples. The soil subsamples were collected along two $15 \mathrm{~m}$ perpendicular transects (N-W and S-W), after removing litter and plant debris from the surface, using an auger ( $5 \mathrm{~cm}$ diameter $\mathrm{x} 3 \mathrm{~cm}$ depth). This approach would allow the variability of the $30 \times 30$ plot to be captured. The soil samples were mixed, air-dried, sieved $(<2 \mathrm{~mm})$ and stored at ambient temeperature for further analysis.

In these samples, we analysed three soil extracellular enzymatic activities corresponding to the biogeochemical cycles of $\mathrm{C}, \mathrm{N}$ and $\mathrm{P}$. Soil acid phosphatase and $\beta$-glucosidase activities were determined colorimetrically as the amount of p-nitrophenol (p-NP) produced after incubation of 0.5 and $1 \mathrm{~g}$ of soil $\left(37^{\circ} \mathrm{C}, 1 \mathrm{~h}\right)$ with p-nitrophenyl-phosphate and p-nitrophenyl- $\beta$-D-glucopyranoside substrates, respectively (Tabatabai and Bremner, 1969; Tabatabai, 1982). The p-NP formed was determined in a spectrophotometer at $400 \mathrm{~nm}$ (UV-1700 PharmaSpec, Shimadzu, Kyoto, Japan). Urease activity was determined following Kandeler \& Gerber (1988) as the amount of N-NH4+ released from $1 \mathrm{~g}$ of soil after incubation $\left(37^{\circ} \mathrm{C}, 2 \mathrm{~h}\right)$ with urea substrate. The $\mathrm{N}-\mathrm{NH} 4+$ released was measured colorimetrically at $690 \mathrm{~nm}$. Two sample blanks for each soil sample were used.

Soil microbial biomass $\mathrm{C}$ content was determined by the fumigation-extraction method (Vance et al., 1987). This procedure is based on a Walkley-Black dichromate digestion to calculate the difference $\left(\mathrm{E}_{\mathrm{C}}\right)$ in organic $\mathrm{C}$ between filtered extracts of chloroform fumigated $(\mathrm{CHCl} 3,24 \mathrm{~h})$ and non-fumigated soil samples. Then, we used an extraction efficiency coefficient ( $\mathrm{k}_{\mathrm{EC}}$ ) of 0.38 (Vance et al., 1987; Joergensen, 1996) to calculate the microbial biomass $\mathrm{C}$ following the formula: microbial biomass $\mathrm{C}=$ $\mathrm{EC} / \mathrm{keC}$. 


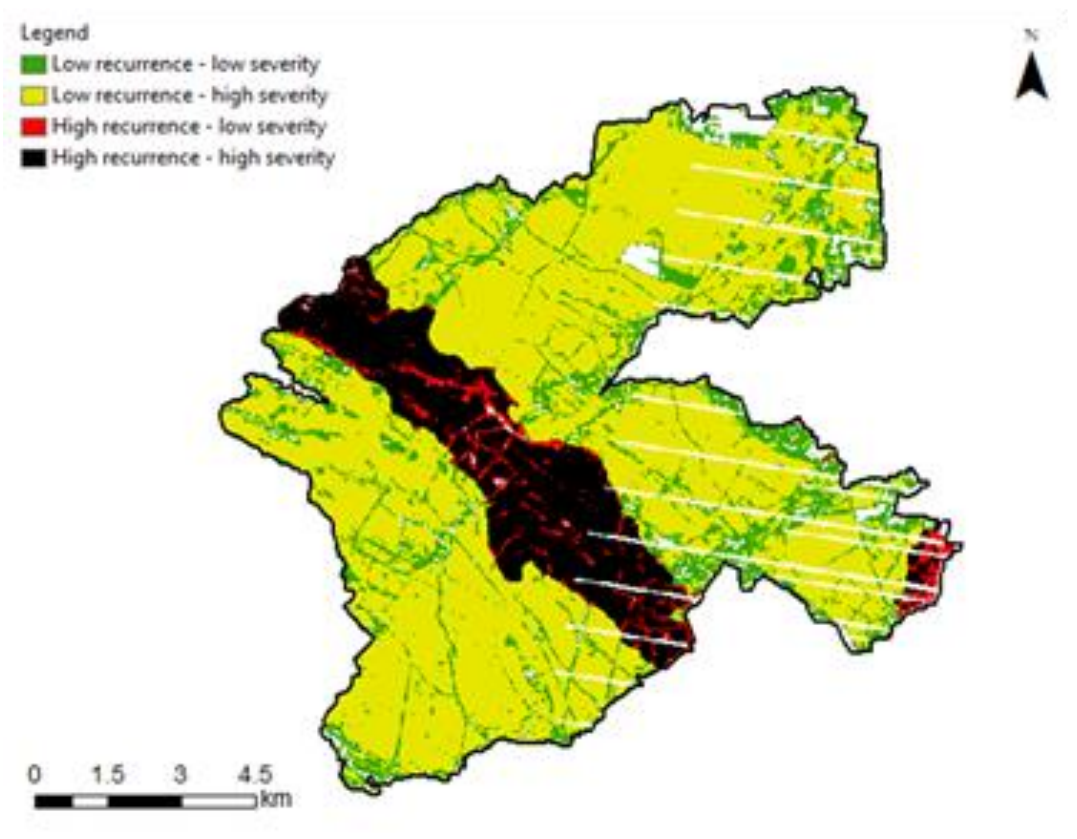

Figure 1 - Map of recurrence-burn severity levels in the study area

\subsection{Resilience index}

The soil enzymatic and microbial biomass resilience to different recurrence and severity scenarios was calculated using the resilience index developed by Banning \& Murphy (2008):

$$
R x=-100\left[\frac{C x-P x}{P x}\right]
$$

Where $\mathrm{Rx}$ is the reslience index; $\mathrm{Cx}$ is the value in the response variable in the low recurrence-low severity scenario, which was considered as a control value; $\mathrm{Px}$ is the value for the response variable in the other scenarios four years after fire. A Rx index value of zero indicates complete recovery at a given time $\mathrm{x}$. Positive values indicate resilience of the response variable, while an Rx value of -100 indicates a further degradation of soil response variable.

\subsection{Data analysis}

In order to analyse the effects of recurrence-severity situations on soil biochemical and microbiological properties, we performed an ANOVA of the Generalised Linear Models (GLMs). GLMs were fitted using Gamma error distribution with the "log" link function. The goodness of fit of the models was assessed by visual analysis of homoscedasticity and normality of residuals. Differences in treatment levels were identified through a pairwise multiple comparison of means (Tukey HSD).

All data analyses were carried out with R software, version 3.4.0, using the "multcomp" package (Hothorn et al.,2008).

\section{Results}

Medium-term enzyme activities of $\beta$-glucosidase and acid phosphatase and urease showed a different pattern among the scenarios of recurrence and severity. $\beta$-glucosidase activity increased significantly (Table 1; Figure 2;) from the best scenario (low recurence-low severity) to the worst (high recurrence-high severity). 
Table 1 - Results of the generalized linear models (GLMs) ['Anova()' outputs] showing the effects of the factor recurrence-severity (Low-Low, Low-High, High-Low and High-High) on each soil property ( $\beta$-glucosidase, urease, phosphatase and microbial biomass $C$ ). Df are degrees of freedom. Significant $P$-values are in bold face.

\begin{tabular}{|c|c|c|c|c|c|c|}
\hline Response variable & Model term & Df & Deviance & $\begin{array}{l}\text { Residual } \\
\text { deviance }\end{array}$ & $\mathrm{F}$ & $P$ \\
\hline \multirow{2}{*}{$\beta$-glucosidase } & Null & & & 13.440 & & \\
\hline & Recurrence-Severity & 3 & 1.486 & 11.954 & 3.010 & 0.035 \\
\hline \multirow{2}{*}{ Urease } & Null & & & 32.648 & & \\
\hline & Recurrence-Severity & 3 & 5.270 & 27.378 & 5.864 & 0.001 \\
\hline \multirow{2}{*}{ Phosphatase } & Null & & & 37.344 & & \\
\hline & Recurrence-Severity & 3 & 6.315 & 31.029 & 4.655 & 0.004 \\
\hline \multirow{2}{*}{ Soil microbial C } & Null & & & 26.189 & & \\
\hline & Recurrence-Severity & 3 & 4.321 & 21.869 & 5.457 & 0.002 \\
\hline
\end{tabular}

A different pattern was observed in urease activity with a significant decrease (Table 1; Figure 2) in the low recurrence-high severity scenario. In the case of acid phosphatase, significant differences were detected among high recurrence scenarios, and the low recurrence-high severity situation (Table 1; Figure 2) which presented the highest activity values.

Soil microbial carbon showed the lowest significant value in the low recurrence-high severity scenario (Figure 2; Table 1). We did not find significant differences amongst the remaining situations.
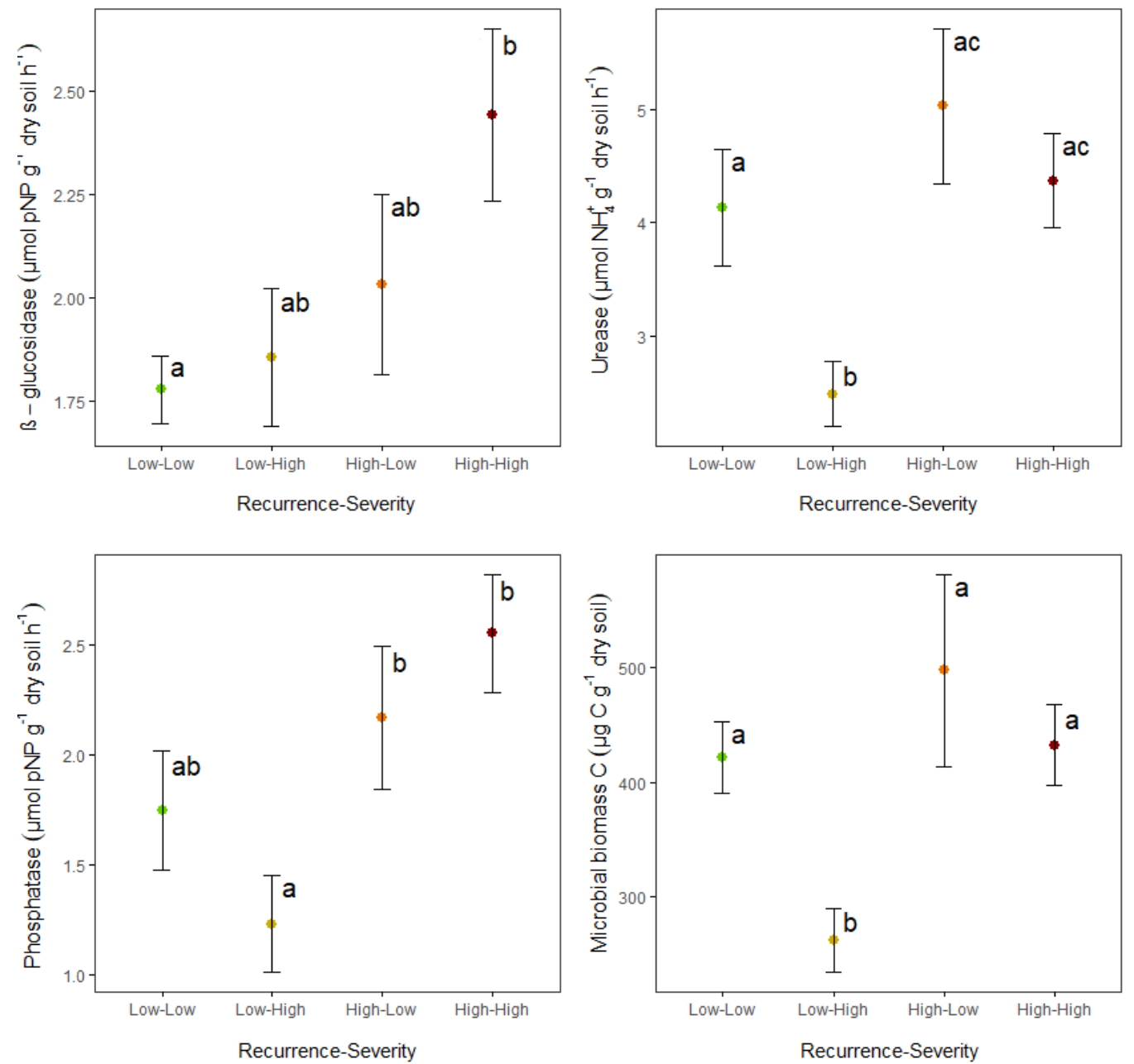

Figure 2 - Mean values \pm standard error of soil properties ( $\beta$-glucosidase, urease, phosphatase and microbial biomass

$C$ ) in relation to the fire recurrence-severity (low recurrence-low severity, low recurrence-high severity, high recurrence-low severity and high recurrence-high severity). Different letters above the error bars $(a, b, c)$ denote statistically significant differences between mean values $(p<0.05)$. 
Soil enzymes showed different resilience capacity in relation different scenarios of recurrenceseverity. $\beta$-glucosidase activity recovered well in all situations, reaching always higher values than low recurrence-low severity (Figure 2). Ureasa and acid phosphatase activities presented negative resilience values in low recurrence-high severity scenario (39\% and $27 \%$ respectively) but good resilience in relation both high recurrence situations (Table 2). The same pattern was observed in soil microbial carbon, with an important degradation in low recurrence-high severity scenario (Table 2) but a good recovery in the other ones, mainly if the severity is low.

Table 2- Percentage of resilience (Rx) of soil enzymatic activities and microbial biomass in different scenarios of fire recurrence-severity scenarios (Low-High, High-Low and High-High)

\begin{tabular}{|c|c|c|c|c|c|}
\hline $\begin{array}{l}\text { Scenarios fire } \\
\text { severity scenarios }\end{array}$ & recurrence- & $\beta$-glucosidase & Urease & Phosphatase & $\begin{array}{l}\text { Soil microbial } \\
\text { C }\end{array}$ \\
\hline Low-High & & $3.0(13)$ & $-39.4(10)$ & $-27.7(18)$ & $-37.7(9)$ \\
\hline High-Low & & $12.8(12)$ & $22.7(23)$ & $27.3(27)$ & $18.0(28)$ \\
\hline High-High & & $35.6(13)$ & $6.6(12)$ & $50.0(18)$ & $2.6(10)$ \\
\hline
\end{tabular}

Values are means for each recurrence-severity scenario ( $\mathrm{n}=10$ for Low-High and High-Low and $\mathrm{n}=20$ for High-High) with standard errors in parenthesis.

\section{Discussion}

This study examined the resilience of soil biochemical and microbiological properties to a combination of fire regime attributes: recurrence and severity. Our results demonstrated that the low recurrence-high severity scenario was the worst situation for the recovery of biochemical and microbiological properties throghout time. In this scenario (at least 14 years without fire), both the fuel load and the intensity and severity of fire. This could influence in the post-fire regeneration traits of plant species which modulate soil enzymatic activity recovery (López-Poma \& Bautista, 2014). In this study area, we found that high severities negatively affected the cover of resprouter species but positively affected seeder species (Fernández-García et al., 2017). However, in the high recurrence scenarios the highest cover was due to resprouter shrubs, which rapidly increased their cover after fire. We found greater enzyme activity in those situations where resprouter species are dominant (high recurrence), because after a fire, the root system of the resprouter species is scarcely affected by fire, the microbiological rhyzosphere hotspots are more durable, and enzyme inputs to soils from root exudates are not interrupted (Mayor et al., 2016). Furthermore, the regeneration of resprouter species is very rapid and presents more nutrient demand and rhizosphere activity (López-Poma \& Bautista, 2014).

On the other hand, enzyme activity showed a different response with regard to resilience. While $\beta$ glucosidase activity showed a positive recovery trend in all scenarios, acid phosphatase and urease showed a positive recovery under high recurrence scenarios but a degradation in low recurrence-high severity scenarios. Some authors have pointed out that the highest increase in $\beta$-glucosidase activity is related to greater plant density (Guénon et al., 2013), since there is an increase in enzyme substrate due to litter deposition and this produces an increase in the number of microorganisms (Mayor et al., 2016). The decrease in acid phosphatase and urease activity in the low recurrence-high severity scenario can be the result of of great availability of inorganic $\mathrm{N}$ and $\mathrm{P}$, which often persist at mediumterm after fire (Dzwonko et al., 2015), as in our study area (data unpublished). If the concentrations of nutrients is high, micro-organisms do not need to produce these extracellular enzymes (Pourreza et al., 2014), since they are substrate-dependent.

Although microbial biomass is markedly reduced by fire (Banning \& Murphy, 2008), four years after it showed a good resilience except in the low recurrence-high severity scenario. This low resilience would be related with a decrease in the quality of soil organic matter, because of the 
emergence of new particulate $\mathrm{C}$ forms highly resistant to oxidation and biological degradation (González-Pérez et al., 2004) that act by limiting soil microbial recovery. However, frequent wildfires can enable the recovery of net nitrification and nitrate content, resulting in a recovery of C-substrate utilisation capabilities of microbial communities (Guénon et al., 2013) .Microbial biomass recovery was assessed by Prieto-Fernández et al. (1998) in a soil under Pinus spp. four years after fire, and was attributted to the addition of cellulose to the burnt soil favouring fungal mycelium development and the speed of plant recolonisation (Certini, 2005) and the litter layer.

The combination of fire regime attributes (recurrence and severity) had an important effect on soil resilience. High recurrence scenarios favour the recovery of soil biochemical and microbial properties, due to the rapid regeneration of resprouter species. Under these conditions urease activity and soil microbial biomass $\mathrm{C}$ do better if the severity is low, while an opposite pattern showed the other two enzyme activities. The low recurrence-high severity scenario negatively affects soil resilience, which results in a depletion of enzyme activities (except $\beta$-glucosidase) and soil microbial biomass $\mathrm{C}$.

\section{Acknowledgements}

This study was financially supported by the Spanish Ministry of Economy and Competitiveness, and the European Regional Development Fund (ERDF), in the framework of the GESFIRE (AGL2013-48189-C2-1-R) and FIRESEVES (AGL2017-86075-C2-1-R) projects; and by the Regional Government of Castile and León in the framework of the FIRECYL (LE033U14) and SEFIRECYL (LE001P17) projects.

\section{References}

Banning NC, Murphy DV (2008) Effect of heat-induced disturbance on microbial biomass and activity in forest soil and the relationship between disturbance effects and microbial community structure. Applied Soil Ecology 40, 109-119.

Certini G (2005) Effects of fire on properties of forest soils: a review. Oecologia 143, 1-10.

Dilly O, Pompili L, Benedetti A (2018) Soil micro-biological indicators separated land use practices in contrast to abiotic soil properties at the $50 \mathrm{~km}$ scale under summer warm Mediterranean climate in nirthern Italy. Ecological Indicators 84, 298-303.

Dzwonko Z, Loster S, Gawronski S (2015) Impact of fire severity on soil properties and the development of tree and shrub species in a Scots pine moist forest site in southern Poland. Forest Ecology and Management 342, 56-63.

Fang L, Yang J, White M, Liu Z (2015) Predicting potential fire severity using vegetation, topography and surface moisture availability in a Eurasian Boreal Forest Landscape. Forests 9, 130.

Fernández-García V, Quintano C, Taboada A, Marcos E, Calvo L, Fernández-Manso A (2018) Remote sensing applied to the study of fire regime attributes and their influence on post-fire greenness recovery in pine ecosystems. Remote Sensing 10, 733.

Fernández-García V, Baeza J, Santana VM, Tárrega R, Valbuena L, Taboada A, Luis-Calabuig E, Fernández-Guisuraga JM, Suárez-Seoane S, Marcos E, Calvo L (2017) ¿Son diferentes los rasgos biológicos de las plantas en relación con la severidad de incendios en pinares propensos al fuego? Actas del $7^{\circ}$ Congreso Forestal Español, Plasencia. .pp. 1-10.

Fernández-Manso F, Fernández-García V, Quintano C, Marcos E, Calvo L (2015) Cartografía de recurrencia-severidad en grandes incendios forestales utilizando técnicas de teledetección. Actas del XVI Congreso de la AET. Sevilla, Spain. 
Forteza J, Lorenzo L, Najac N, Cuadrado S, Ingelmo F, Hernández J, García P, Prat L, Mulez C, Macarro C, Rivas D (1987) Mapa de suelos de Castilla y León, Scale 1:500000, Dirección General de Medio Ambiente y Urbanismo, Junta de Castilla y León, Valladolid.

González-Pérez JA, González-Vila FJ, Almendros G, Knicker H (2004) The effect of fire on soil organic matter - a review. Environment International 30, 855-870.

Guénon R, Vennetier M, Dupuy N, Roussosi S, Pailleri A, Gros R (2013) Trends in recovery of Mediterranean soil chemical properties and microbial activities after infrequent and frequent wildfires. Land Degradation and Development 24, 115-128.

Hinojosa MB, Parra A, Laudicina VA, Moreno JM (2016) Post-fire soil functionality and microbial community structure in a Mediterranean shrubland subjected to experimental drought. Science of Total Environment 573, 1178-1189.

Hothorn T, Bretz F, Westfall P (2008). Simultaneous Inference in General Parametric Models. Biometrical Journal 50(3), 346--363.

IGME (1982) Instituto Geológico y Minero de España, Mapa Geológico de España, Scale 1:50000. Sheets 230 and 231. Ministerio de Industria y Energía, Madrid.

Jain TB, Graham RT (2007) The relarion between tree burn severity and forest structure in the Rocky Mountains. General Technical Report PSW-GTR-203. USDA Forest Service, Rocky Mountain Research Sattion, Ogden, UT.

Joergensen JG (1996) The fumigation-extraction method to estimate soil microbial biomass: calibration of the kEC value. Soil Biology and Biochemestry 28, 25-31.

Kandeler E, Gerber H (1988) Short term assay of soil urease activity using colorimetric determination of ammonium. Biology and Fertility of Soils 6, 68-72.

Keeley JE (2009) Fire intensity, fire severity and burn severity: A brief review and suggested usage. International Journal of Wildland Fire 18, 116-126.

Key CH (2006) Ecological and sampling constraints on defining landscape fire severity. Fire Ecology 2, 34-59.

Knelman JE, Graham EB, Trahan NA, Schmidt SK, Nemergut DR (2015) Fire severity shapes plant colonization effects on bacterial community structure, microbial biomass, and soil enzyme activity in secondary succession of a burned forest. Soil Biology \& Biochemistry 90, 161-168.

Knicker H (2007) How does fire affect the nature and stability of soil organic nitrogen and carbon? A review. Biogeochemistry 85, 91-118.

López-Poma R, Bautista S (2014) Plant regeneration functional groups modulate the response to fire of soil enzyme activities in a Mediterranean shrubland. Soil Biology and Biochemistry 79, 5-13.

Mayor AG, Goirán SB, Vallejo VR, Bautista S (2016) Variation in soil enzyme activity as a function of vegetation amount, type, and spatial structure in fire-prone Mediterranean shrublands. Science of Total Environment 573, 1209-1216.

Merino A, Fonturbel MT, Fernández C, Chávez-Vergara B, García-Oliva F, Vega JA (2018) Inferring changes in soil organic matter in post-wildfire soil burn severity levels in a temperate climate. Science of Total Environmental 627, 622-632.

Moreira F, Arianoutsou M, Corona, P, De las Heras J (2012) 'Post-Fire Management and Restoration of Southern European Forests, Managing Forest Ecosystems'. pp. 319 (Springer Science + Business Media B.V.: Brussels).

Moreno MV, Chuvieco E (2016) Fire regime characteristics along environmental gradients in Spain. Forests 7, 262

Moreno MV, Conedera M, Chuvieco E, Boris G (2014) Fire regime changes and major driving forces in Spain from 1968 to 2010. Environmental Science Policy 37, 11-22. 
Pourreza M, Hosseini SM, Sinegani AAS, Matinizadeh M, Dick WA (2014) Soil microbial activity in response to fire severity in Zagros oak (Quercus brantii Lindl.) forests, Iran, after one year. Geoderma 213, 95-102.

Prieto-Fernández A, Acea MJ, Carballas T (1998) Soil microbial and extractable C and N after wildfire. Biology and Fertility of Soils 27, 132-142.

Quintano C, Fernández-Manso A, Calvo L, Marcos E, Valbuena L (2015) Land surface temperature as potential indicator of burn severity in forest Mediterranean ecosystems. International Journal of Applied Earth Observation 36, 1-12.

Santalla S, Salgado JM, Calvo L, Fernández M (2002) Changes in the Carabidae community after a large fire in a Pinus pinaster stand. In 'Fire and Biological Processes' (Eds L Trabaud, R Prodon, R) pp 215-231 (Backhuys Publishers. Leiden).

Shive KL, Sieg CH., Fulé PZ (2013) Pre-wildfire management treatments interact with fire severity to have lasting effects on post-wildfire vegetation response. Forest Ecology and Management 297, 75-83.

Tabatai MA (1982) Soil enzymes. In 'Methods of Soil Analyses, Part 2, Chemical and Microbiological Properties’. (Eds AL Page, EM Miller, DR Keeney) pp. 903-947 (American Society of Agronomy, Madison WI.)

Tabatai MA, Bremmer JM (1969) Use of p-nitrophenyl phosphate for assay of soil phosphatase activity. Soil Biology and Biochenistry 1, 301-307.

Vance ED, Brookes PC, Jenkinson DS, (1987) An extraction method for measuring soil microbial biomass C. Soil Biology and Biochemistry 19, 703-707.

Varela ME, Benito E, Keizer JJ (2015) Influence of wildfire severity on soil physical degradation in two pine forest stands of NW Spain. Catena 133, 342-348.

Vega JA, Fontúrbel T, Merino A, Fernández C, Ferreiro A, Jiménez E (2013) Testing the ability of visual indicators of soil burn severity to reflect changes in soil chemical and microbial properties in pine forests and shrubland. Plant and Soil 369, 73-91.

Vieira DCS, Fernández C, Vega JA, Keizer JJ (2015) Does soil burn severity affect the post-fire runoff and interrill erosion response? A review based on meta-analysis of field rainfall simulation data. Journal of Hydrology 523, 452-464. 


\section{Chapter 3}

Fire Management 


\title{
17 years of wildfire detection and management supported by an optical sensor system: Field report from Germany's Brandenburg national forest, one of Europe's top wildfire regions at risk
}

\author{
Raimund Engel \\ Chief Forester \& Wildfire Protection Officer of the state of Brandenburg \\ State Forest Administration Brandenburg (Landesbetrieb Forst Brandenburg) Heinrich-Mann- \\ Allee 103, Building 5, 14473 Potsdam, Germany, \{raimund.engel@lfb.brandenburg.de\}
}

\begin{abstract}
When it comes to wildfires in Europe, people usually think about southern countries, such as Spain, Portugal or Italy. However, parts of northern and northeastern Germany have been classified by the European Union as top regions at risk of wildfires. The state of Brandenburg has more than one million hectares of forest, which is equal to $37 \%$ of the state area. Dry and sandy soil means poor growth conditions, and only undemanding plants may be cultivated with reasonable effort. That's why monoculture pine forests make up $78 \%$ of all woods in this area. Precipitation easily permeates the sandy soil while fires burn the extremely dry ground, fueled by liquid resin, needles and forest litter that does not decompose. This is particularly problematic since Brandenburg was a battleground of the final phase of World War II and a military training ground for more than 100 years. The region was contaminated by hundreds of thousands of tons of unexploded artillery grenades and bombs. About $36 \%$ of the entire state and $50 \%$ of the woodland is still heavily polluted with old weapons posing major risks to the civilian population and particularly to fire fighters. Early wildfire detection is crucial under these circumstances, and generations of human fire spotters worked for decades using a dense network of watchtowers. Due to extremely high maintenance costs of these watchtowers and due to difficult labor legislation, the automatic remote surveillance system FireWatch was introduced in 2001. Today human fire spotting is history; 108 sensors are operating successfully and have proven to be technologically efficient. Plenty of detailed statistics haven been collected for decades giving evidence that the burnt area per hectare of a fire has been reduced tremendously since the installation of FireWatch and that the system has clearly outperformed the human spotter from a long-term perspective.
\end{abstract}

Keywords: early wildfire detection; forest fire management; climate; wildfire suppression; forest fire risk; monoculture forests; optical sensors; weapons and ammunition pollution; preservation of environment, safety and order

\section{The Threat of Wildfires}

\subsection{Climate and Geography}

Brandenburg is the most eastern state of the federal republic of Germany sharing a border of 252 $\mathrm{km}$ with the republic of Poland. Brandenburg is one of the most forested states of Germany encompassing 1.1 million hectares of forest of which pine trees dominate more than three quarters.

The sandy soil is characterized by the glacial period. The moraine landscape is slightly hilly, yet the flat outwash plains dominate the area, intermittent by numerous aquiferous drainage and by glacial valleys. Because of its eastern border location, the majority of the state is continentally influenced by climate. Only the northern and northwestern part of Brandenburg is characterized an oceanic climate (sub-Atlantic). Low precipitation and extreme temperature differences affect the continental climate. In the long-term average, precipitation in Brandenburg is below $500 \mathrm{~mm}$ per year. Influenced by location and climate, vast pine tree forests dominate the landscape. Based on these prerequisites, the wildfire risk in Germany is the highest in Brandenburg.

In the beginning of the 1990's, the European Commission categorized the forests of Brandenburg to be of high and highest wildfire risk, which is comparable to many Mediterranean regions. 


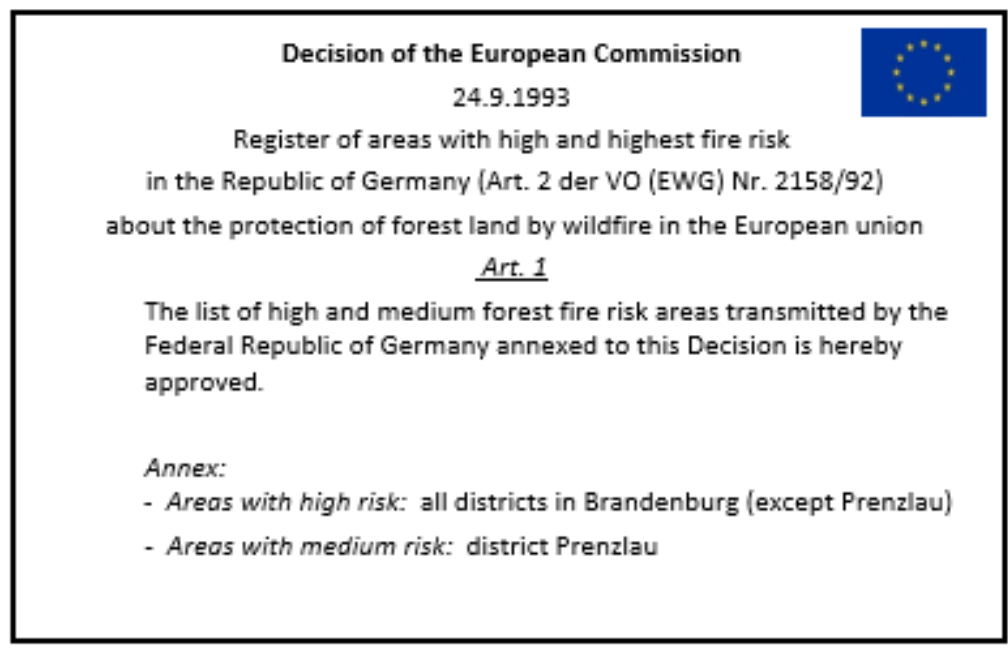

Figure 1 - Wildfire risk areas in Germany, decision of the European Commission

In a nationwide comparison, records of the last decades show that the Brandenburg forests burn most frequently. Even though not as many wildfires burned in the last years as in 2003 during the hottest summer of the century, still a yearly average of 230 wildfires burned in Brandenburg alone. Additionally, the many field and wasteland fires are not included in the forest administration's statistics.

Table 1 - Wildfire Statistics of the state of Brandenburg (1975-2016)

\begin{tabular}{|c|c|c|c|c|c||}
\hline Year & Quantity & Area (ha) & $\begin{array}{c}\text { Average Area } \\
\text { (ha) }\end{array}$ & $\begin{array}{c}\text { Fires > 100 ha: } \\
\text { Quantity }\end{array}$ & $\begin{array}{c}\text { Fires > 100 ha: } \\
\text { Area (ha) }\end{array}$ \\
\hline 1975 & 487 & 923 & 1.9 & 1 & 144 \\
\hline 1976 & 1259 & 2296 & 1.82 & 3 & $115 ; 279 ; 365$ \\
\hline 1977 & 106 & 56 & 0.53 & & \\
\hline 1978 & 434 & 367 & 0.85 & & \\
\hline 1979 & 323 & 194 & 0.6 & & \\
\hline 1980 & 407 & 302 & 0.74 & & 173 \\
\hline 1981 & 278 & 276 & 0.99 & & $184 ; 273 ; 478$ \\
\hline 1982 & 1161 & 1251 & 1.08 & & \\
\hline 1983 & 641 & 1688 & 2.63 & 3 & \\
\hline 1984 & 284 & 317 & 1.12 & & \\
\hline 1985 & 300 & 184 & 0.61 & & \\
\hline 1986 & 384 & 207 & 0.54 & & 196 \\
\hline 1987 & 147 & 74 & 0.51 & & 121 \\
\hline 1988 & 549 & 381 & 0.69 & & $115 ; 242$ \\
\hline 1989 & 653 & 337 & 0.52 & & $136 ; 295$ \\
\hline 1990 & 477 & 450 & 0.94 & & \\
\hline 1991 & 471 & 414 & 0.88 & 1 & \\
\hline 1992 & 1024 & 1296 & 1.27 & 2 & \\
\hline 1993 & 510 & 656 & 1.29 & 2 & \\
\hline 1994 & 532 & 582 & 1.11 & & \\
\hline 1995 & 406 & 139 & 0.34 & & \\
\hline 1996 & 434 & 248 & 0.57 & & \\
\hline 1997 & 452 & 161 & 0.36 & & \\
\hline 1998 & 315 & 83 & 0.26 & & \\
\hline 1999 & 375 & 134 & 0.36 & & \\
\hline & & & & & \\
\hline
\end{tabular}




\begin{tabular}{|l|c|c|c|c|c||}
\hline 1999 & 375 & 134 & 0.36 & & \\
\hline 2000 & 422 & 186 & 0.44 & & \\
\hline 2001 & 174 & 35 & 0.2 & & \\
\hline 2002 & 200 & 34 & 0.17 & & \\
\hline 2003 & 679 & 600 & 0.88 & & \\
\hline 2004 & 197 & 87 & 0.44 & & \\
\hline 2005 & 163 & 50 & 0.31 & & \\
\hline 2006 & 368 & 312 & 0.85 & & \\
\hline 2007 & 201 & 45 & 0.22 & & 197 \\
\hline 2008 & 321 & 353 & 1.1 & 1 & \\
\hline 2009 & 243 & 86 & 0.35 & & 237 \\
\hline 2010 & 208 & 295 & 1.42 & & \\
\hline 2011 & 221 & 42.9 & 0.19 & & \\
\hline 2012 & 234 & 76 & 0.32 & & \\
\hline 2013 & 161 & 45.3 & 0.28 & & \\
\hline 2014 & 115 & 27.4 & 0.24 & & \\
\hline 2015 & 315 & 321 & 1.02 & & \\
\hline 2016 & 232 & 92.3 & 0.4 & & \\
\hline \hline
\end{tabular}

\subsection{Pollution: Left-Over Military Ammunition, Bombs and Grenades}

But that's not all when it comes to forest fire danger in Brandenburg. Aside from the climatic and geographic extremes and, consequently, the occurrence of pure pine stand, there is another variable at play: the pollution of ammunition in forests, fields and waters as well as in villages and cities. Nationwide, the state of Brandenburg shows the highest number of regions laden with ammunition and weapons.

Today, approximately 392,000 hectares of Brandenburg's total area size are still categorized as "polluted." Especially the regions of the former $1^{\text {st }}$ and $2^{\text {nd }}$ front lines of German and Soviet armed conflicts during World War II along the river Oder, at Seelow Heights, in Halbe and in close vicinity to the city of Berlin are affected. In terms of counties, this especially applies to Märkisch-Oderland, Oder-Spree, Dahme-Spreewald and Teltow-Fläming. On the following map, areas suspected of weapon pollution are depicted in red. However, the assumption that other areas are laden with ammunition and weapons is not ruled out. 


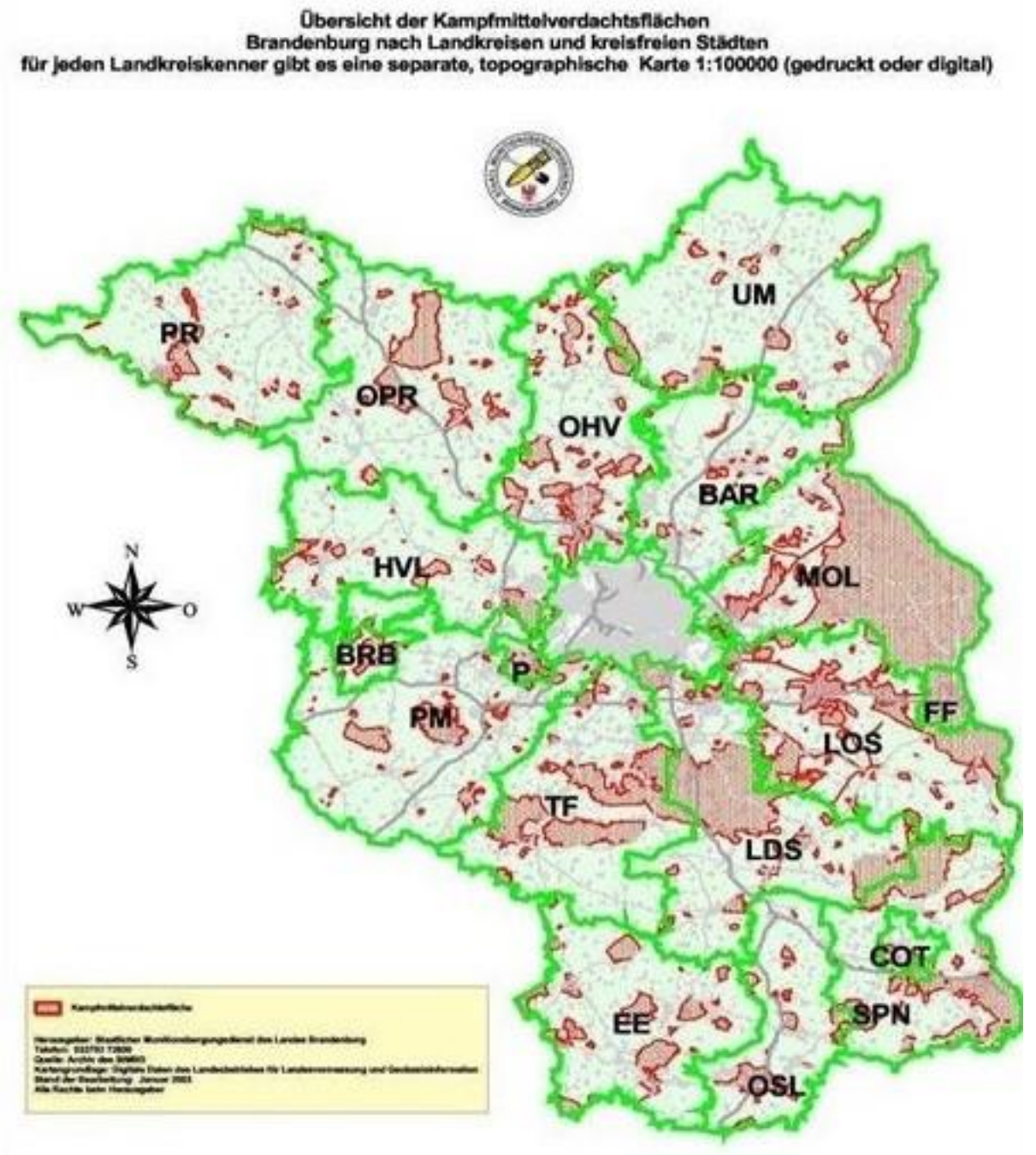

Figure 2 - Areas suspected of weapon pollution (red) in the state of Brandenburg

Located in Märkisch-Oderland, the Seelow Heights are mainly used for agriculture, but the suspected areas in the counties of Teltow-Fläming, Dahme-Spreewald and Oder-Spree are heavily forested. Additionally, ammunition from World War I as well as from the many Soviet military training grounds can be found here. In conclusion, almost all of Brandenburg is categorized as vulnerable.

Even though the weapons disposal service (Kampfmittelbeseitigungsdienst, KMBD) of Brandenburg collects, transports and disposes of hundreds of tons of different weapons every year, such as explosive and incendiary bombs, mines, grenades, and a vast amount of ammunition, the wildfires still pose a high threat.

Plenty of small caliber bullets covered with phosphor on their tips can be found. These are called tracer bullets because they helped trace the shooter's trajectory. Many bullets however did not burn down or are left over from trenches, bomb craters or simply in the woods.

With temperatures of only $23^{\circ} \mathrm{C}$ and an influx in oxygen, the phosphorous tip can easily ignite. If enough fire load (brushwood, dead grass) is available, it could lead to a wildfire in areas with no roads and pathways, even in early spring. Especially in polluted woods, plenty of wildfires occur that ignite due to the left-over weapons. In the south, the percentage of weapons as a cause of wildfires is in the two-digits every year. 


\subsection{Firefighting in Polluted Woods}

Forest fire fighting in areas that are heavily polluted with left-over weapons is considered as difficult and as too dangerous for ground-based firefighting. Even a surface fire can lead to small caliber weapons detonating and exploding.

If the region in question is polluted in such a way that a detonation is expected or inevitable, the firefighting forces will not be deployed for the sake of the fighters' safety. Only the nearest official safety zones would be accessed in order to fight the blaze with fire monitors (water cannons).

Yet, as a consequence the areas inside the safety zones burn less controlled. The damaged and partially destroyed wooded sections in those areas increase due to the fire, hence, the usage of fire fighting forces and resources is prolonged to multiple days if not weeks.

For example, an area the size of 160 hectares burned down near Jüterbog (County Teltow-Fläming) in 2015, and 250 hectares burned in Lieberose in 2017.

At the same time, firefighting helicopters are not an alternative as slivers of detonating ammunition can be disseminated in any direction. As a result, the aerial crew could equally be in danger above the blaze.

For a few years now, the county Teltow-Fläming has been using a privately-owned firefighting tank for areas heavily polluted with ammunition. Due to two fire monitors, the tank allows the firefighting crew to effectively attack the fire.

Managers will continuously be challenged to fight forest fires. However, one thing is clear: safety first. In areas that are heavily polluted with ammunition, risky firefighting is never justified.

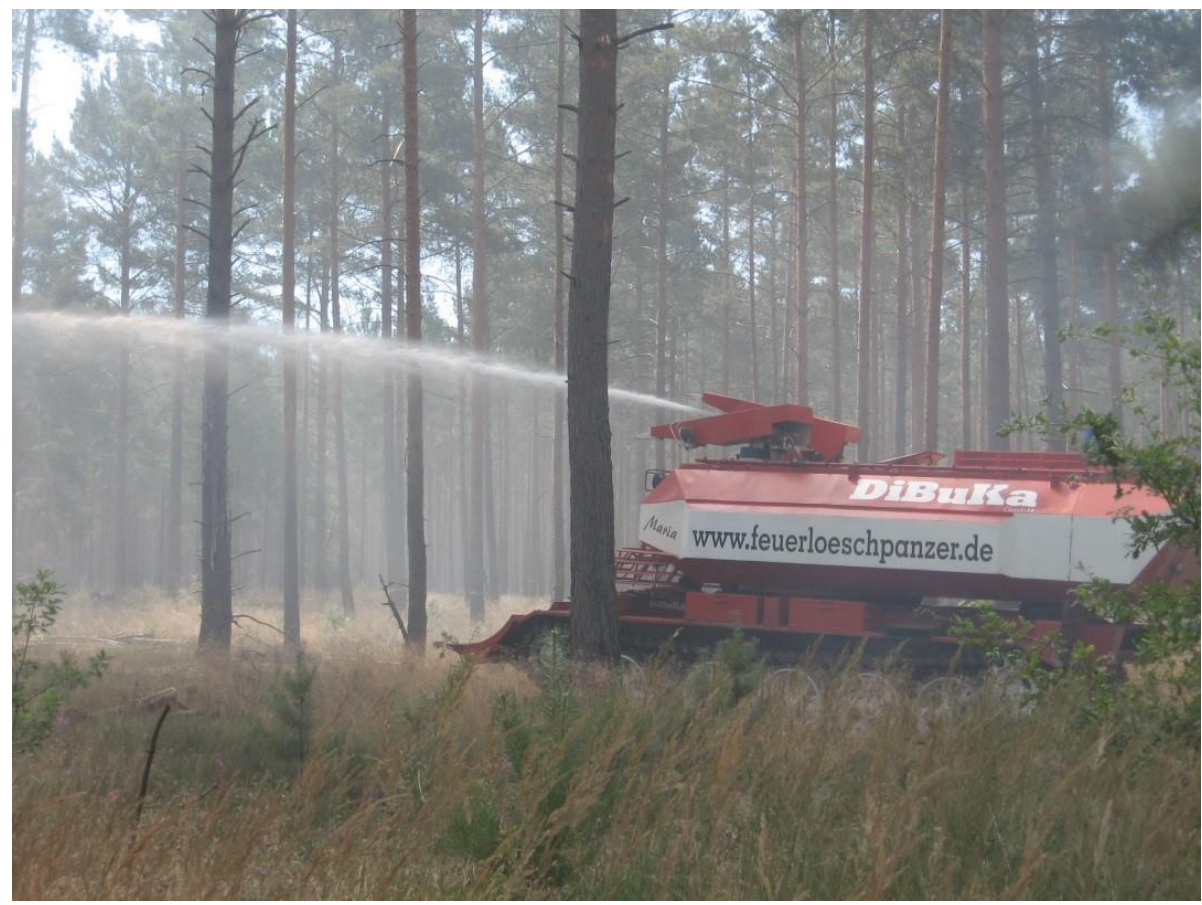

Figure 3 - Fire tank in action

\section{Wildfire Detection Methods: Then and Now}

\subsection{Human Fire Spotting}

While wildfire fighting as a method of wildfire protection is part of the municipalities' managerial obligations, the Brandenburg forest legislature decided that preventative wildfire protection is under direct obligation of the forest owners. This includes managing firebreaks of 30 meters wide and of 300 
meters wide, as well as installations and upkeep of extinguishing water sites. Forest owners are also obligated to control forests that are vulnerable to wildfires, especially after a fire that spread onto neighboring properties. The European Union, the federation and the state of Brandenburg support forest owners with adequate aids and funds for preventative wildfire protection.

While wildfire fighting as a method of wildfire protection is part of the municipalities' managerial obligations, the Brandenburg forest legislature decided that preventative wildfire protection is under direct obligation of the forest owners. This includes managing firebreaks of 30 meters wide and of 300 meters wide, as well as installations and upkeep of extinguishing water sites. Forest owners are also obligated to control forests that are vulnerable to wildfires, especially after a fire that spread onto neighboring properties. The European Union, the federation and the state of Brandenburg support forest owners with adequate aids and funds for preventative wildfire protection.

Early wildfire detection has been and continues to be the states' obligation no matter whose property the forests are.

Until 1989, Brandenburg had a network of 133 fire lookout towers; employees of the forest administration would work these towers on days marked with high fire danger. Under sometimes extreme working conditions, these employees would spend up to 12 hours on top of fire lookout towers at heights of 36 meters and in direct sun. Their job was to spot smoke plumes on the horizon with their binoculars and to pin-point the location by using iron sights. By telephone or radio, they would pass on the degrees to the nearest forest office where administrative employees would try to determine the closest tower to perform a cross bearing, resulting in pin-pointing the exact fire location on a map.

As early as 1995, the Brandenburg forest administration was contemplating how to organize and implement early wildfire detection more efficiently.

It soon showed that the system of staff-intensive fire watch towers would not be economically feasible in the long-term. Additionally, working these towers constituted an intense health concern for employees. The structural preservation of those towers would be a continuous financial burden for the forest administration that would be incalculable. An alternative solution for the early wildfire detection was needed soon.

\subsection{Automated Early Wildfire Detection: FireWatch}

In 1994, the Brandenburg state forest administration initiated first pilot projects revealing how wildfires could be detected faster and more efficiently by automated early wildfire detection systems. The German Aerospace Center (Deutsches Zentrum für Luft- und Raumfahrt e.V. (DLR)) was won as partner of the project. Researchers of the DLR relied on space technology that was already developed. Cameras that detect smoke and that were originally built for the exploration of Mars and other planets, were reconstructed and combined with a specialized image processing software. Starting in 1997 in the Lausitz border region in the southeast of the state of Brandenburg, an extensive pilot project funded by the INTERREG program of the European Union was installed.

The early wildfire detection optical sensors that detect smoke rising right above treetops clearly showed signs of technological possibilities and were a right step into the future.

It was clear for every long-term human spotter: the optical sensor works better than any human and could never become tired. Day after day, the sensors deliver consistent high-quality images and the specifically-programmed smoke detection software allows for timely and safe wildfire detection.

In 2003, the state forest administration had a complete FireWatch surveillance network installed. At the same time, the Brandenburg forest legislation was amended, and the statutory basis for the protection against wildfires added that from then on, the state of Brandenburg would use an early wildfire detection system in vulnerable forested areas. Forest owners had to comply with the 
installation and operation of the early wildfire detection system (see: $\S 20$ section 3 of forest legislature state of Brandenburg ${ }^{1}$ ).

In 2006, after only 3 years of operation, Brandenburg's forests (over 1 million hectares) were surveilled by 108 optical sensors. Neighboring states, such as Saxony, Saxony-Anhalt and Mecklenburg-Western Pomerania followed and installed the early wildfire detection system FireWatch. Now, this system consists of a cross-border network of 178 sensors in regions of the Federal Republic of Germany with the highest wildfire danger.

The sensor rotates $360^{\circ}$ every 4-6 minutes and scans an area of up to $700 \mathrm{~km}^{2}$. Up to 16.000 grey scale values are compared in sections. A smoke plume that derives from burned organic material has a unique pattern, unique coloration, and dynamics of development that are all detected by the software. If incoming values equate to those of a smoke plume, the system will signal an alarm at the control center. The data of each of the optical sensors are transmitted in real time via radio relay to the control center, of which there are currently 6 in the state of Brandenburg. An operator, a specially qualified forest administration employee of the state, can watch up to 8 systems and those incoming alarms.

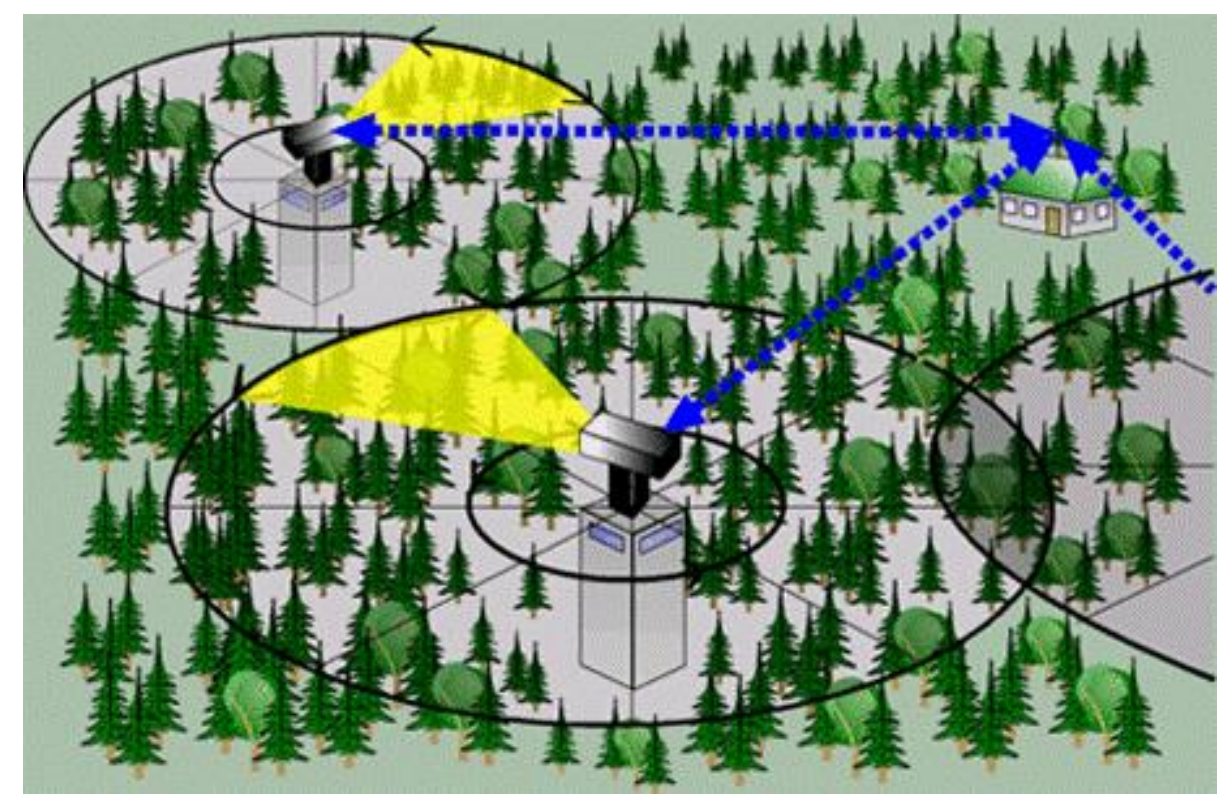

Figure 4 - Animated simulation of forest covered by FireWatch - 360 degree rotation

In the following two years, Brandenburg will be upgrading the existing systems of automated early wildfire detection to the newest generation of optical sensors and smoke detection software. The technological infrastructure of data transmission will also be updated. In order to effectively work and use synergistic effects, it seems plausible to equally reduce the numbers of control centers. Starting in 2020, alarms of all 108 optical sensors will be analyzed in only two interconnected control centers. The field-tested optimization of the software makes it possible to watch additional sensors at one work station. From then on, up to 18 systems can be watched by one operator, but the usual workload for one operator would be 12 systems.

${ }^{1}$ Waldgesetz des Landes Brandenburg (LWaldG) vom 20. April 2004 (GVB1. I S. 137), zuletzt geändert durch Art. 1 des Gesetzes vom 10. Juli 2014 (GVB1. Nr. 33) 
Incoming alarms of each sensor appear in a panorama image. At the same time, the location of the potential fire is optically depicted by a signal beam on a topographic map on a second monitor. Once another sensor registered a detection, an automatic cross bearing follows aiding in pin-pointing the exact location of the fire.

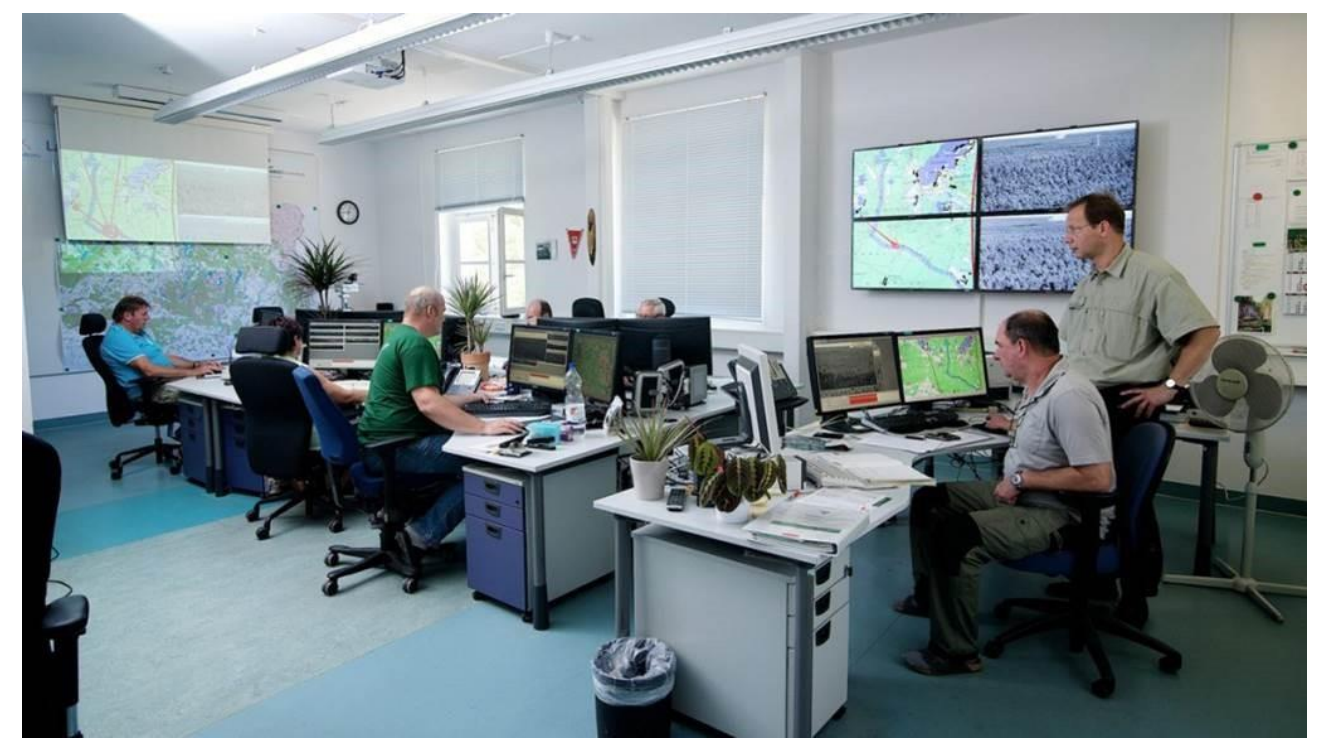

Figure 5 - Wildfire control center in Wünsdorf, Brandenburg

If the detected smoke is confirmed to be a forest fire, the regional headquarters of the fire and emergency services are digitally informed. Aside from the basic data (date, time and forest address), headquarters also receive the exact coordinates (in all common coordinate formats) as well as a current image derived from the sensors along with a map showing the cross bearing.

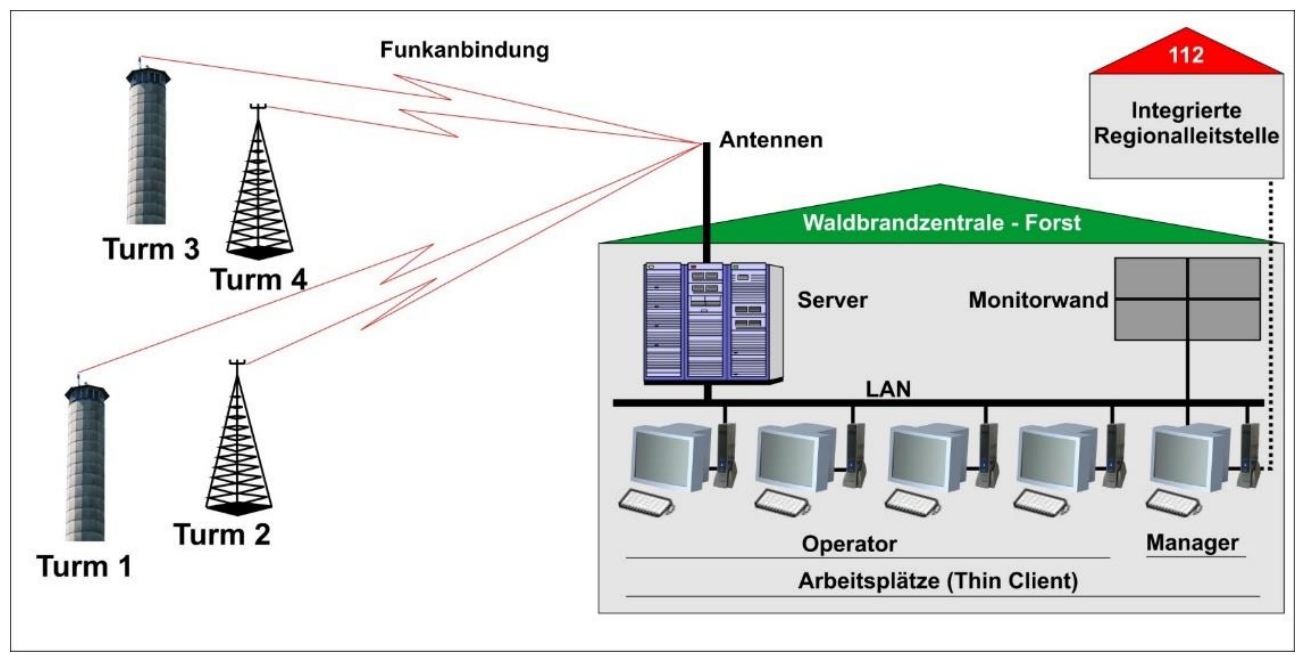

Figure 6 - Network of sensors, wildfire control center \& emergency and fire services

For more details, the map also shows all available extinguishing water sites and the trails that are open year-round for trucks. All forested areas in the state of Brandenburg have an official forest address, which is comparable to a regular street address. That way, a wildfire can be located not only geographically by a map but its location can also be verbally described.

It is probably only a matter of time, or better a matter of technological development, until wildfire detection data can be transferred from regional headquarters to the emergency vehicles. The 
coordinates could then be implemented into the navigation systems. The Brandenburg state forest administration has already now gathered all passable trails in a trail information system. Therefore, navigation of all emergency vehicles outside the public road network would be viable.

An essential component in the process of evaluating incoming automated alarms of the early wildfire detection is the so-called "mini clip." Within seconds, the operator at the control center receives a moving image showing the smoke development so he can visualize the direction and the speed of fire propagation. This information helps the forest employees and the fire department to be prepared for the situation before having arrived at the site of fire.

The season of wildfire surveillance usually starts on March $1^{\text {st }}$ and ends on September $30^{\text {th }}$ of every year. Working shifts for each employee of the states' forest administration are posted in the control center. Weather pattern and forecast determine every wildfire danger level and therefore, also determine working shifts at the control center. When the danger rating is at 3, mid-level, employees at the control center work from $10-18$ o'clock. Once the rating increases, the working hours also increase. When it is at level 4 - high danger - working hours are extended until 19 o'clock, and when it is level 5 - highest danger - hours are until 20 o'clock. With extreme weather conditions working shifts can even be extended until dark.

While IQ wireless GmbH, manufacturer of the system, have developed and released day and night detection technology onto the market already, Brandenburg is only focused on detection during the day. Out of 100 wildfires, 80 ignite during the day between 10 and 18 o'clock. With only a few exceptions per year, humidity as well as ground moisture both decrease the chances of a wildfire igniting at night. Statistics currently don't justify the addition of a night system to the existing day detection system in Brandenburg.

\section{Conclusion and Look to the Future}

After 17 years of using FireWatch in Brandenburg, the wildfire areas have been reduced successfully due to this special surveillance technology. In the last years, the average wildfire area has decreased to under $3000 \mathrm{~m}^{2}$ in size. The earlier the wildfire is detected and the earlier the rescue services are alerted with an exact fire location, the earlier the targeted firefighting can be initiated. Reasonable and available usage of forces and resources can get an initial ground fire under control and extinguish it. If a wildfire has developed into a full fire blaze with crown fires, it is clear that the fire department would have to work at it for days.

With the early wildfire detection system FireWatch, the Brandenburg state forest administration is contributing substantially to the protection of forests.

By now, climate change has also impacted Europe in many ways. Increasing temperatures and longlasting heat waves will increase in the foreseeable future. Temperatures of $40^{\circ} \mathrm{C}$ will most likely not be an isolated incident. Wildfire danger will also rise. Therefore, it is crucial to try everything so that wildfires do not turn into catastrophes. Wildfires need to be detected and located as early as possible. Only then, catastrophes can be prevented.

Early wildfire detection - a substantial contribution to preserving our environment and to maintaining public safety and order.

\section{References}

Deutsches Zentrum für Luft- und Raumfahrt e.V. (2005) Wie DLR-Forscher Arbeitsplätze in der Wirtschaft schaffen. DLR Nachrichten 112, 12.

Engel R (2009) Brandenburg setzt flächendeckend auf „Fire Watch.“ AFZ-Der Wald 12, 632-634. 
Engel R (2016) Wenn es brennt, zählt jede Minute. Feuerwehr-Magazin, Sonderheft Wald- und Flächenbrände 1, 14-19.

Engel R (2016) Brände auf militärischen Flächen. Feuerwehr - Magazin, Sonderheft Wald- und Flächenbrände 1, 93-95.

Engel R (2017) 15 Jahre Waldbrandfrüherkennung Fire Watch im Land Brandenburg. AFZ - Der Wald 18, 21-22.

Feldmann M (2015) Früh zum Forst gekommen - Der Waldbrandschutzbeauftragte Brandenburgs. Behörden-Spiegel 11, 3.

Goodrich D (2010) FireWatch Australia Stage 2 Trial Proposal: Proven Automatic Bushfire Detection for the $21^{\text {st }}$ Century. FireWatch Australia, Australian National FireWatch Early Detection Network. (Sydney, Australia)

Gräser G (1996) AWFS: Elektronik für den Wald. Berliner Feuerwehr. (Berlin, Germany)

Hartung W, Erlemeier T (2001) Automatisiertes Waldbrandfrüherkennungssystem statt konventioneller Feuerwachtürme. Forsttechnische Informationen (FTI) 4, 48-49.

Hartung W, Gruner R (2000) Weltraumtechnologie für die Waldbrandfrüherkennung. AFZ - Der Wald 7, 388-389.

IQ wireless GmbH (2010) FireWatch - Produktinformation. IQ wireless GmbH. (Berlin, Germany)

IQ wireless GmbH (2012) Wo Rauch ist, ist auch ein Feuer: FireWatch - ein Waldbrandfrüherkennungssystem. IQ wireless GmbH. (Berlin, Germany)

Lachmann M (2012) Waldbrandstatistik der Bundesrepublik Deutschland für das Jahr 2011. Bundesanstalt für Landwirtschaft und Ernährung. (Bonn, Germany)

Landesvermessung und Geobasisinformation Brandenburg LGB (2007) Land Brandenburg in Zahlen und Karten. Landesvermessung und Geobasisinformation Brandenburg. (Potsdam, Germany)

Ministerium für Infrastruktur und Landwirtschaft MIL (2013) Daten zu Wald und Forstwirtschaft in Brandenburg. Ministerium für Infrastruktur und Landwirtschaft Land Brandenburg MIL. (Potsdam, Germany)

Ministerium für Ländliche Entwicklung, Umwelt und Landwirtschaft des Landes Brandenburg (2007) FireWatch - Erfahrungen und Ausblicke. Brandenburgische Forstnachrichten 130, 8-9.

Turnham J (2013) FireWatch Texas Product Information. FireWatch Texas, LLC. (Austin, Texas) 


\title{
A project to measure and model pyrolysis to improve prediction of prescribed fire behavior
}

David R. Weise ${ }^{1 *}$; Thomas H. Fletcher ${ }^{2}$, Timothy J. Johnson ${ }^{3}$, WeiMin Hao ${ }^{4}$, Mark Dietenberger ${ }^{5}$, Marko Princevac ${ }^{6}$, Bret Butler ${ }^{4}$, Sara McAllister ${ }^{4}$, Joseph O’Brien ${ }^{7}$, Louise Loudermilk ${ }^{7}$, Roger Ottmar $^{8}$, Andrew Hudak ${ }^{4}$, Akira Kato ${ }^{9}$, Babak Shotorban ${ }^{10}$, Shankar Mahalingam ${ }^{10}$, William E. Mell $^{8}$

${ }^{1}$ USDA Forest Service, PSW Research Station. 4955 Canyon Crest Drive, Riverside, California, USA 92507, \{dweise@fs.fed.us*\}

${ }^{2}$ Chemical Engineering Dept., Brigham Young University. Provo, Utah, USA, \{tom_fletcher@byu.edu\}

${ }^{3}$ Pacific Northwest National Laboratory. Richland, Washington, USA,

\{timothy.johnson@pnnl.gov\}

${ }^{4}$ USDA Forest Service, Rocky Mountain Research Station, \{whao@fs.fed.us\}, \{bwbutler@fs.fed.us\}, \{smcallister@fs.fed.us, ahudak@fs.fed.us\}

${ }^{5}$ USDA Forest Service, Forest Products Laboratory, Madison, Wisconsin, USA, \{mdietenberger@fs.fed.us\}

${ }^{6}$ Mechanical Engineering Dept., University of California-Riverside, Riverside, California, USA, \{marko.princevac@ucr.edu\}

${ }^{7}$ USDA Forest Service, Southern Research Station, Athens, Georgia, USA, \{jjobrien@fs.fed.us\}, \{elloudermilk@fs.fed.us\}

${ }^{8}$ USDA Forest Service, Pacific Northwest Research Station, Seattle, Washington, USA, \{rottmar@fs.fed.us,wemell@fs.fed.us\}

${ }^{9}$ Graduate School of Horticulture, Chiba University, Chiba, Japan, \{akiran@faculty.chiba-u.jp\}

10 The University of Alabama in Huntsville, Huntsville, Alabama, USA,

\{Babak.Shotorban@uah.edu,Shankar.Mahalingam@uah.edu\}

\begin{abstract}
Laboratory and field experiments focused on pyrolysis and ignition coupled with sufficient description of fuel characteristics and physics-based modeling are being used to improve our understanding of combustion processes in mixed (heterogeneous) fuel beds managed with prescribed fire in the southern United States. Previous pyrolysis work has typically used ground-up samples, thus eliminating any effects caused by moisture content, fuel particle shape, or heating mode. We are measuring pyrolysis at bench, laboratory and field-scale using intact fuels from living plants. Pyrolysis products being measured include light gases and tars using off-line and real-time spectroscopic instruments. 3-D fuel description, heat transfer to the fuels, and air flow around the fuels are being determined using a variety of intrusive and nonintrusive methods. The ability of high fidelity physics-based FDS, WFDS, and GPYRO3D to reproduce experimental results and to study conditions outside the range of the experimental data is being examined. This presentation will present an overview of the project and the questions being addressed. Preliminary results from initial attempts to measure pyrolysis at field-scale and results from early modeling of pyrolysis of live leaves using coupled GPYRO3D and FDS models are presented.
\end{abstract}

Keywords: FTIR spectroscopy, gas sampling, pyrolysis, convection, gas chromatography, flaming, smoldering, wildland fuels 


\section{Objective}

Wildland fire is an important component of many North American ecosystems and has been used by humans to accomplish various objectives for several thousand years. Prescribed burning in the southern United States is an important tool used by the Department of Defense and other land managers to accomplish several objectives including hazardous fuel reduction, wildlife habitat management, critical training area maintenance, ecological forestry and infrastructure protection. Vegetation is heterogeneous unlike the homogeneous fuel beds assumed by current operational fire spread models. These models do not contain fundamental descriptions of chemical reactions and heat transfer processes necessary to predict fire spread and energy release needed for process-based fire effects models. To improve prescribed fire application, an improved understanding is needed of the fundamental processes related to pyrolysis and ignition in heterogeneous fuel beds. The objective of this project is to address several fundamental questions to improve our understanding and modeling capability of fire propagation in natural fuel beds including 1) detailed description of pyrolysis and the evolution of its products for a greater variety of southern fuels than is currently known, 2) how convective and radiative heat transfer from flames to live fuel particles influences pyrolysis and ignition at laboratory and field scales, and 3) more detailed insight into pyrolysis, combustion and heat transfer processes in wildland fire spread through the use of high-fidelity physics-based models. The questions are being tested with data derived from three activities: 1) characterization of pyrolysis products by measurement for a variety of live and dead foliar fuel particles in laboratory and smallscale field experiments, 2) determination of the effects of convection and radiation heat transfer on pyrolysis, and 3) performance of high-fidelity physics-based modeling of pyrolysis and ignition for bench-scale, wind tunnel and small-scale field experiments. This paper describes the overall project.

\section{Methods}

Laboratory and field experiments focused on pyrolysis and ignition coupled with sufficient description of fuel characteristics and physics-based modeling are being used to improve our understanding of combustion processes in mixed (heterogeneous) fuel beds managed with prescribed fire on DoD installations. Previous pyrolysis work has typically used ground-up samples, thus eliminating any effects caused by moisture content, fuel particle shape, or heating mode. Thermal gravimetric analysis (TGA) coupled with gas chromatography-mass spectrometry (GC-MS) and evolved gas analysis (EGA) have been used for decades to describe the composition and energy content of pyrolysis products. We are measuring pyrolysis at three scales - bench-scale measurements at BYU and the Forest Products Laboratory (FPL), laboratory burn-scale in a wind tunnel at RFL, and small field-scale burns $\left(1600 \mathrm{~m}^{2}\right)$ at Fort Jackson in South Carolina using intact fuels from living plants in natural fuelbeds beneath southern pine forests. 14 plant species were selected (Table 7). One of the dominant shrub species in the pine stands at Ft. Jackson is sparkleberry. Since this plant is deciduous, it causes a significant change to prescribed fire behavior when its foliage is present during the growing season. Sparkleberry was chosen to be the live plant species to link bench-scale measurements to fieldscale measurements. 
Table 1 - Southern plant species used in pyrolysis experiments.

\begin{tabular}{lll}
\hline Common name & Scientific name & Experiment $^{\mathbf{2}}$ \\
\hline Wiregrass & Aristida stricta Michx. & $\mathrm{B}$ \\
Little bluestem & Schizachyrium scoparium (Michx.) Nash & $\mathrm{B}$ \\
Inkberry & Ilex glabra (L.) A. Gray & $\mathrm{B}, \mathrm{W}$ \\
Yaupon & Ilex vomitoria Aiton 'Schelling Dwarf' & $\mathrm{B}$ \\
Fetterbush & Lyonia lucida (Lam.) K. Koch & $\mathrm{B}, \mathrm{W}$ \\
Wax myrtle & Morella cerifera (L.) Small & $\mathrm{B}$ \\
Swamp bay & Persea palustris (Raf.) Sarg. & $\mathrm{B}$ \\
Sparkleberry & Vaccinium arboreum Marshall & $\mathrm{B}, \mathrm{W}, \mathrm{F}$ \\
Darrow's blueberry & Vaccinium darrowii Camp “Rosa's Blush" & $\mathrm{B}, \mathrm{W}$ \\
Longleaf pine foliage & Pinus palustris Mill. & $\mathrm{B}$ \\
Longleaf pine litter & Pinus palustris Mill. & $\mathrm{B}, \mathrm{W}, \mathrm{F}$ \\
Water oak & Quercus nigra L. & $\mathrm{B}$ \\
Live oak & Quercus virginiana Mill. & $\mathrm{B}$ \\
Dwarf palmetto & Sabal minor (Jacq.) Pers. & $\mathrm{B}$ \\
Saw palmetto & Serenoa repens (W. Bartram) Small & $\mathrm{B}$ \\
\hline
\end{tabular}

1. USDA, NRCS. 2018. The PLANTS Database (http://plants.usda.gov, 10 January 2018). National Plant Data Team, Greensboro, NC 27401-4901 USA; Radford, A.E., Ahles, H.E., Bell, C.R. 1968. Manual of the vascular flora of the Carolinas, University of North Carolina Press, Chapel Hill, NC

2. B - bench, $\mathrm{W}$ - wind tunnel, $\mathrm{F}$ - field

\subsection{Bench-scale}

In order to perform calculations related to gas and tar composition as well as modeling the plants with physical fire behavior models, many chemical and physical properties of the plant material in Table 7 were determined using a variety of methods. Because some live leaves will undergo enzymatic hydrolysis when plucked and some live leaves will emit significant combustible volatiles at elevated temperatures below the usual oven dry temperatures of $105{ }^{\circ} \mathrm{C}$, samples were collected and immediately vacuum dried at $45{ }^{\circ} \mathrm{C}$. Proximate and ultimate analysis, and compositional content of live leaves were determined by various standard methods. A special holder equipped with fine thermocouples is being used in the cone calorimeter to determine properties such as thermal conductivity (Figure 53).

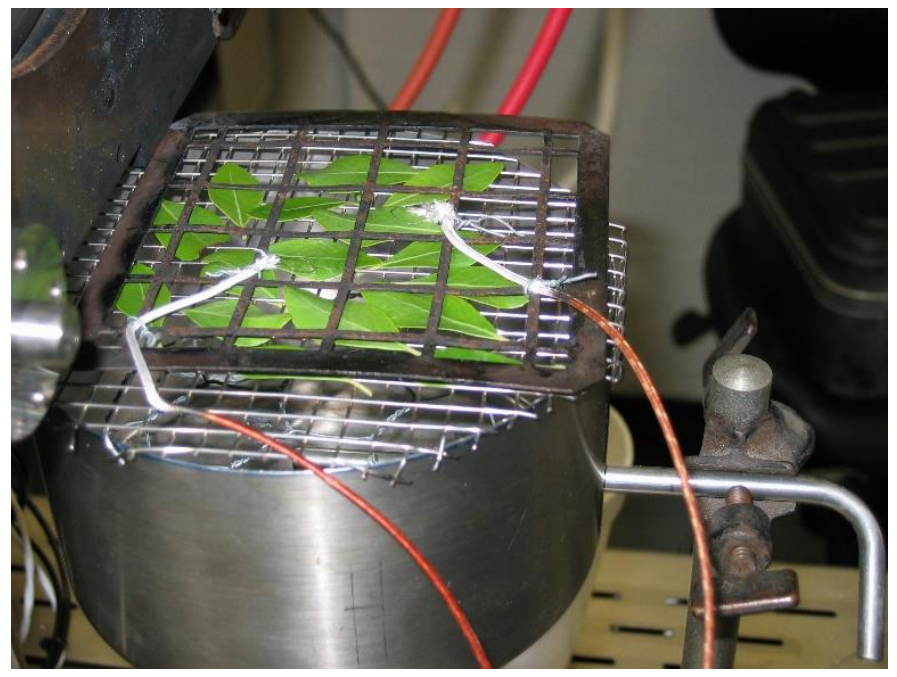

Figure 1 - Specialized holder used for foliage samples in cone calorimeter. 
A flat-flame burner (FFB) apparatus was used to study pyrolysis of foliar samples from the plants in Table 7. (Details of the apparatus and initial results of the tests will be presented by Fletcher et al. (2018) in this conference.) The interested reader is referred to Safdari et al. (2018). In addition to the offline analysis associated with the FFB, a nitrogen purged Bruker Tensor 37 FTIR spectrometer ${ }^{1}$ was deployed to provide real-time measurement of gaseous pyrolysis products by collecting a gas sample every 1.46 seconds (Figure 54). The spectrometer was equipped with a glow bar source, potassium bromide $(\mathrm{KBr})$ beamsplitter and a liquid nitrogen cooled mercury cadmium telluride (MCT) detector. The spectral resolution was $1 \mathrm{~cm}^{-1}$ with the number of scans set to 1 for flowing measurements and 1024 for static measurements. The acquisition mode was set to double-sided, forward-backward. For the Fourier transform, the apodization function was Blackman-Harris 3-Term with a zerofilling factor of 4, and the phase correction mode was Mertz (Mertz 1967). The gas sample was pumped into a White cell (Bruker Optics, A136/2-L) with an effective path length of $8 \mathrm{~m}$. FTIR spectroscopy has been used extensively to characterize smoke emissions from wildland fuels (e.g. Yokelson et al. 1996). Briefly, spectra analysis was carried out using the program Malt5 (Griffith 1996) with $50^{\circ} \mathrm{C}$ reference spectra from the PNNL database (Johnson et al. 2006) and absorption lines from HITRAN. The FTIR method allows for the simultaneous quantification of multiple analytes at the same time; up to two dozen species can have their absolute concentration (and hence fluxes) quantified.

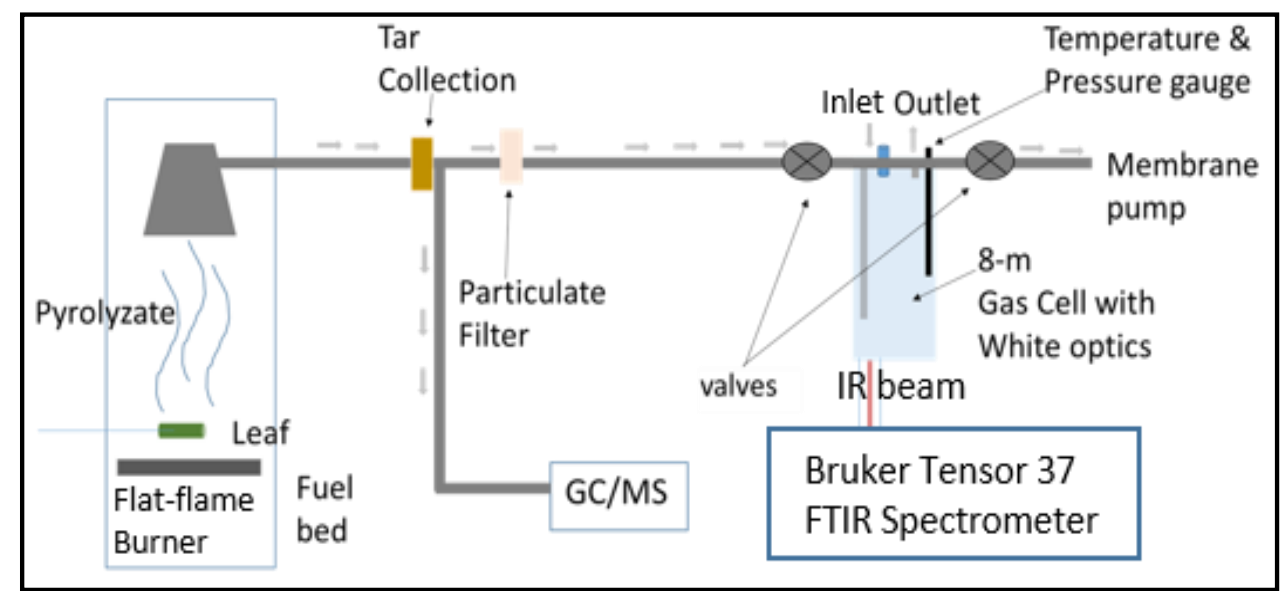

Figure 2 - FTIR spectrometer setup used in conjunction with flat-flame burner to provide real-time measurement of gaseous pyrolysis products.

\subsection{Wind tunnel}

Seventy-three experimental fires have been burned in a low speed wind tunnel located at the Riverside Fire Laboratory (Cobian-Iñiguez et al. 2017). Fuel beds $2 \mathrm{~m}$ long and approximately $1 \mathrm{~m}$ wide composed of longleaf pine needles and various combinations of fetterbush, sparkleberry, and inkberry (Table 7) were burned with nominal wind speeds of 0 or $1 \mathrm{~m} \mathrm{~s}^{-1}$. Pyrolysis gases are sampled in real-time using a variety of methods and instruments (Figure 55, Figure 56). Note that the Bruker Tensor 37 (T37) is the same device used to determine composition of gases at BYU. For Riverside experiments, the resolution was set to $4 \mathrm{~cm}^{-1}$, the acquisition mode was set to double sided, forwardbackward, the apodization function was Blackman-Harris 3-Term, and phase correction mode was Mertz (1967) with a zerofilling factor of 2. In order to increase temporal resolution, the number of

${ }^{1}$ The use of trade or firm names in this publication is for reader information and does not imply endorsement by the U.S. Department of Agriculture of any product or service. 
scans was set to 1 with continuous measurements to obtain a spectral time resolution of 0.2 seconds. This instrument configuration analyzed gases extracted with a $3 / 8$ " metal tubing probe above a plant during measurements (Figure 56).

The other FTIR instrument used for gas phase measurements was the Bruker OPAG-22, which is an open path gas analyzer. The OPAG was mounted on a tripod on one side of the wind tunnel with the glow bar IR source on the opposite side. The path in between the OPAG and the source was 1.2 meters and it was directly in line with a row of plants (Figure 55). The OPAG is equipped with a $\mathrm{KBr}$ beamsplitter and a Stirling-cycle cooled mercury cadmium telluride (MCT) detector. Interferograms were collected in the range of 4000 to $0 \mathrm{~cm}^{-1}$ at a resolution of $2 \mathrm{~cm}^{-1}$ and at an acquisition mode set to double-sided forward-backward. The number of scans per measurement was set to 1 and the measurements were repeatedly collected to obtain a time profile with a resolution (time between spectra) of 0.53 seconds. Gas samples were pumped into canisters for offline analysis using gas chromatography. An array of 9 stainless steel tubes were inserted vertically into the fuel bed. A sample of the flaming gases was collected in one canister initially from one tube and a sample of pyrolysis gases was collected from the other 6 tubes sequentially as the flame front moved horizontally along the fuel bed.

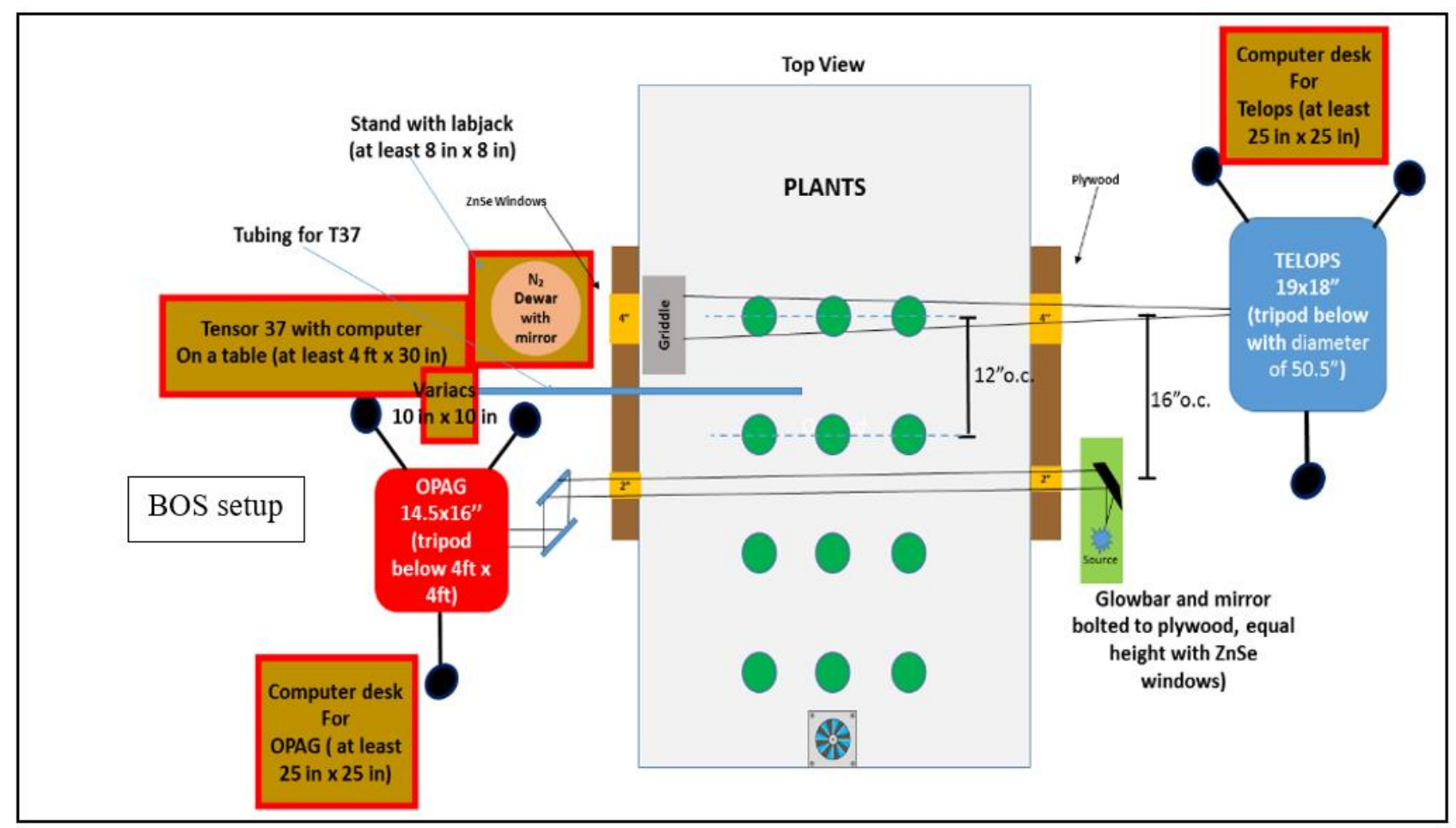

Figure 3 - Setup of 3 instruments used to measure composition of gaseous pyrolysis products in mixed fuel beds of longleaf pine needles and small shrubs.

Mass of a single plant, air temperature and relative humidity within the wind tunnel and in the larger combustion room were sampled at $1 \mathrm{hz}$ during each experimental fire. While we have used a variety of techniques previously to describe the flow field around experimental laboratory fires, the use of smoke tracers and introduced particles was precluded by the gas sampling objective. The TPIV technique (Zhou et al. 2003) could not be used because the large glass windows in the wind tunnel are opaque to the infrared spectrum. The backgound-oriented Schlieren (BOS) approach was selected to determine the flow field in the flame and surrounding the plants (Raffel 2015). The BOS setup (Figure ) was located upwind of the PNNL gas sampling instruments (Figure 55). Total and radiant heat fluxes at the top of the fuel bed were measured using a Schmidt-Boelter type of sensor. A stereo pair of vertical photographs of each fuel bed were taken before and after each experimental fire to develop 3- 
D images of the fuel bed. A longwave infrared (LWIR) camera provided a nadir view of the fuel bed and measured radiance from the fuel bed will be converted into temperature.

\subsection{Pilot Tests - Field}

A trial deployment was performed in May 2017 at Ft. Jackson (Figure ). The forest structure was characterized from Ft. Jackson's existing airborne LiDAR data using a canopy height model (Silva et al. 2016) for the tree overstory and rasterized metrics for the shrub understory (Hudak, Dickinson, et al. 2016; Hudak, Bright, et al. 2016). Destructive fuel sampling methods, 3D measurement of forest and understory vegetation structure using an unmanned autonomous system (UAS), heat flux measurement, and pyrolysis gas sampling were tested during the trial deployment. Burn plots were characterized non-destructively in 3D using terrestrial lidar scans (TLS) and UAS remote sensing tools and destructively in adjacent small fuel sample plots to describe the spatial distribution of fuel elements. TLS of the small fuel plots were collected from multiple view perspectives to characterize $3 \mathrm{D}$ fuel distributions in 10-cm voxels (volumetric pixels), before and after burning. A digital camera mounted on a UAS collected stereo color imagery from overhead perspectives. These metrics serve as independent variables for predicting fuel loads measured in the traditional destructive sample plots pre- and post-fire in units of $\mathrm{kg} \mathrm{m}^{-2}$.
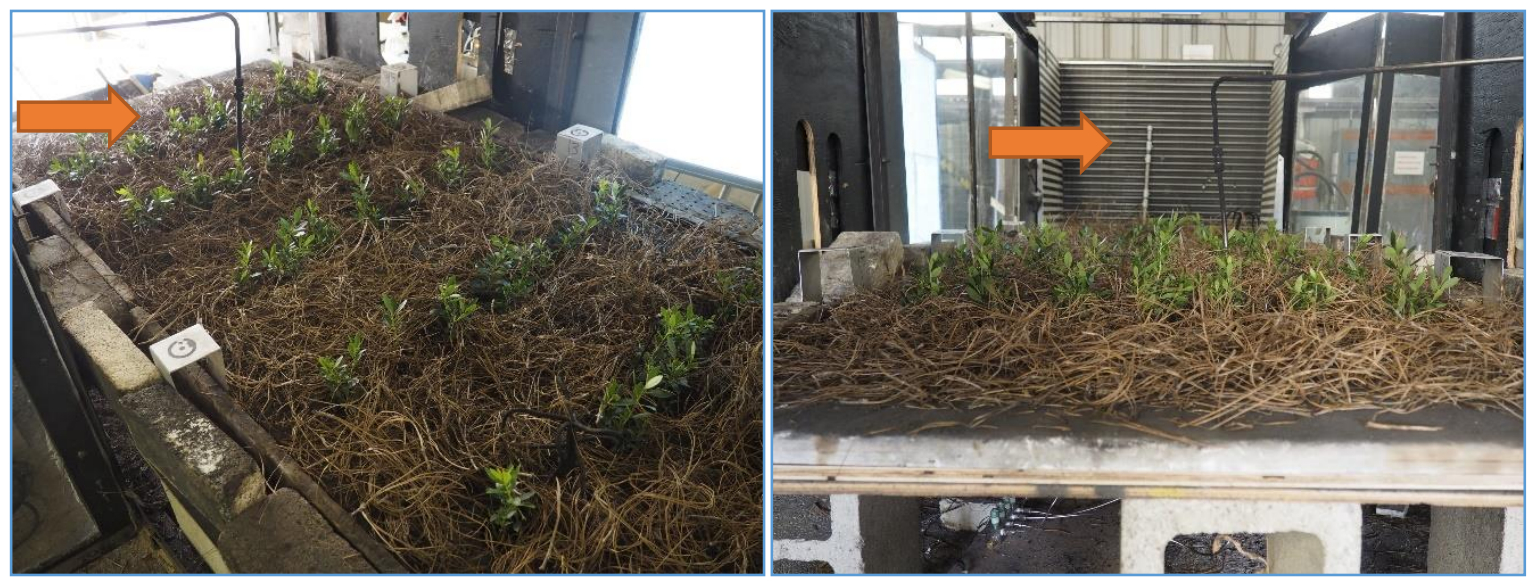

Figure 4 - Fuel bed composed of longleaf pine needles and Lyonia lucida plants. Sample probe to collect pyrolysis gases for the Bruker Tensor 37 is indicated by the arrow.

As pyrolysis gases have never been measured to our knowledge in a field setting, we used a metal probe to sample emitted pyrolysis products very close to the fire. The probe is a variable length (1-4 m) stainless steel tube connected by flexible stainless steel hose to a manifold, from which a canister system, real time gas sensors, and particulate matter (PM) filter samplers draw pyrolysis emissions downstream from the flame. $\mathrm{CO}_{2}$ and $\mathrm{CO}$ were measured in-situ at a $1 \mathrm{~Hz}$ sample rate by their respective sensors. Hydrogen, methane and $\mathrm{C}_{2}-\mathrm{C}_{6}$ alkanes, alkenes and aromatic compounds of smoke samples collected in canisters will be measured by gas chromatography. For the trial deployment, the focus was determining how close personnel could get to the fire in personal protective equipment to capture samples of pyrolysis gases (Figure 57).

\subsection{High-fidelity Physics Modeling}

Prior work by the modeling team has examined the ability of the Fire Dynamics Simulator (FDS) coupled with the GPyro pyrolysis model to simulate pyrolysis, ignition and burning of single leaf particles (Yashwanth et al. 2015, 2016) and the ability of the Wildland Urban Interface extension of FDS (WFDS) to model ignition of many leaf particles (Anand et al. 2017). Significant improvements have been made to the modeling of convective ignition of leaves in the BYU flat-flame burner by Gpyro3D-FDS (Shotorban et al. 2018). One of the improvements included a new rigorous formulation for the calculation of the mean porosities in the computational cells of Gpyro to account for the net 
volume reduction that the condensed (solid) phase experiences within the computational cells during moisture evaporation and pyrolysis. Another improvement was the use of a more advanced pyrolysis mechanism in Gpyro to include the main constituents of biomass, i.e., cellulose, hemicellulose and lignin at virgin and active states in the dry mass. In addition, the modeled leaf now matches the measured manzanita leaf dimensions much better than in the previous modeling work (Yashwanth $e t$ al. 2016). These improvements were necessary in order to model the pyrolysis experiments that are being performed at BYU.

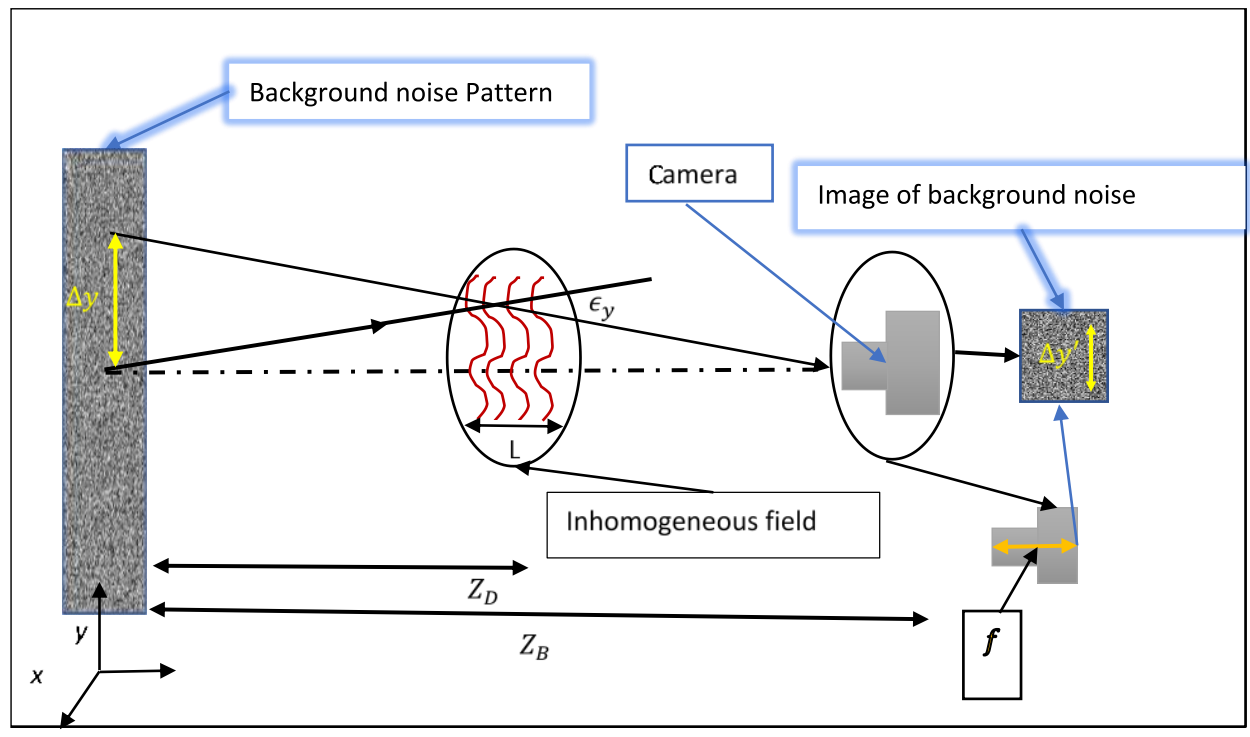

Figure 5 - Simple schematic of background-oriented Schlieren configuration used to nonintrusively estimate the flow field surrounding pyrolyzing plants. $Z_{D}$ is the distance of the flame from the background noise pattern, $Z_{B}$ is the distance of the camera lens from the background, $\epsilon_{y}$ represents the deflection angle caused by the flame-generated distortion, $L$ is the flame zone width, $f$ is the focal length of the camera, $\Delta y^{\prime}$ represents displacement in the camera sensor plane and $\Delta y$ represents displacement in the background plane.

The computational configuration resembles the experimental setup of (Pickett et al. 2010). The burner and the gas phase combustion within the burner was not modeled; the convective heating effect is modeled through the introduction of heated air at $1100{ }^{\circ} \mathrm{C}$ which reduces to around $1000{ }^{\circ} \mathrm{C}$ at $5 \mathrm{~cm}$ above the burner which is consistent with experimental measurements. As the initial condition, the leaf was assumed to consist of moisture and dry mass with standard air in the pores. The initial mass fraction of moisture was determined by the fuel moisture content. Initial dry mass consisted of 33\% cellulose, $33 \%$ hemicellulose and 34\% lignin which differ by $1 \%$ from the experiments. These slight differences had barely observable effect on the simulation results. The initial temperature was set to an ambient temperature of $300 \mathrm{~K}$. A 10-step reaction mechanism (Miller and Bellan 1997) was used for the dry fuel; each of the dry species underwent three primary reactions of conversion from a virgin state to an active state, from the active state to char and pyrolyzate gases, and from the active state to tar, and a secondary reaction for breakdown of tar to pyrolyzate. A single step reaction was used for moisture evaporation (Bryden and Hagge 2003). A stoichiometric reaction between methane and air was used to model the combustion of the pyrolyzate gas in FDS.

\section{Abbreviated Results}

As previously stated, details of the fast pyrolysis experiments are presented elsewhere. Preliminary results from the wind tunnel experiments are presented as a poster in this conference (Weise et al. 2018). From the pilot field experiment, fuel loading was dominated by litter and duff ranging from 2.8 to 8.7 and from 3.2 to $10.2 \mathrm{Mg} \mathrm{ha}^{-1}$, respectively. The consumption of the fuel bed categories followed 
similar trends with the most consumption occurring in the litter and duff categories. Fuel moisture content ranged from $8.6 \%$ for the litter to $175.2 \%$ for the shrub fuels bed categories just prior to ignition. It was a challenge in the field to sample the point and time of pyrolysis - the flame is not an even front when it hits the plant but a matrix of different branches catching fire (Figure 58). Also the time of capture for the pyrolysis emissions is short so the actual volume of a good sample is very small. Some of the pyrolysis samples collected had very high $\mathrm{H}_{2}$ and $\mathrm{CO}$ levels indicating that we probably did measure some pyrolysis emissions. These measurements will be compared with the regular smoke samples of flaming combustion that were collected.

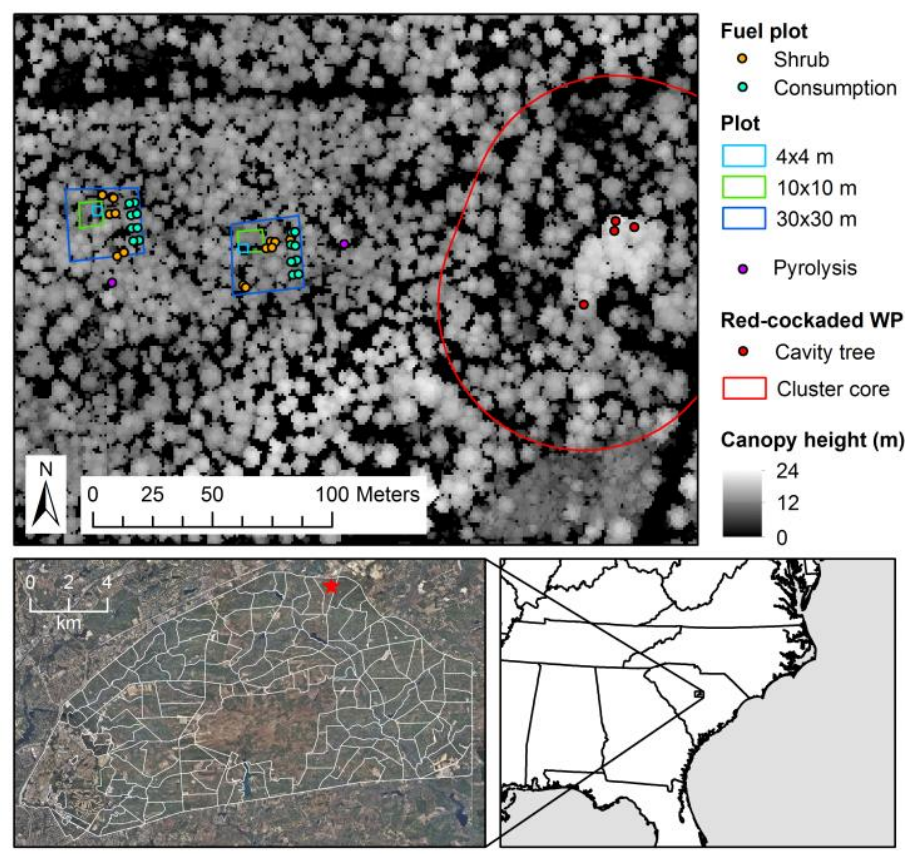

Figure 6 - Individual tree crowns in and around the May 2017 trial deployment plots derived from airborne LiDAR data.

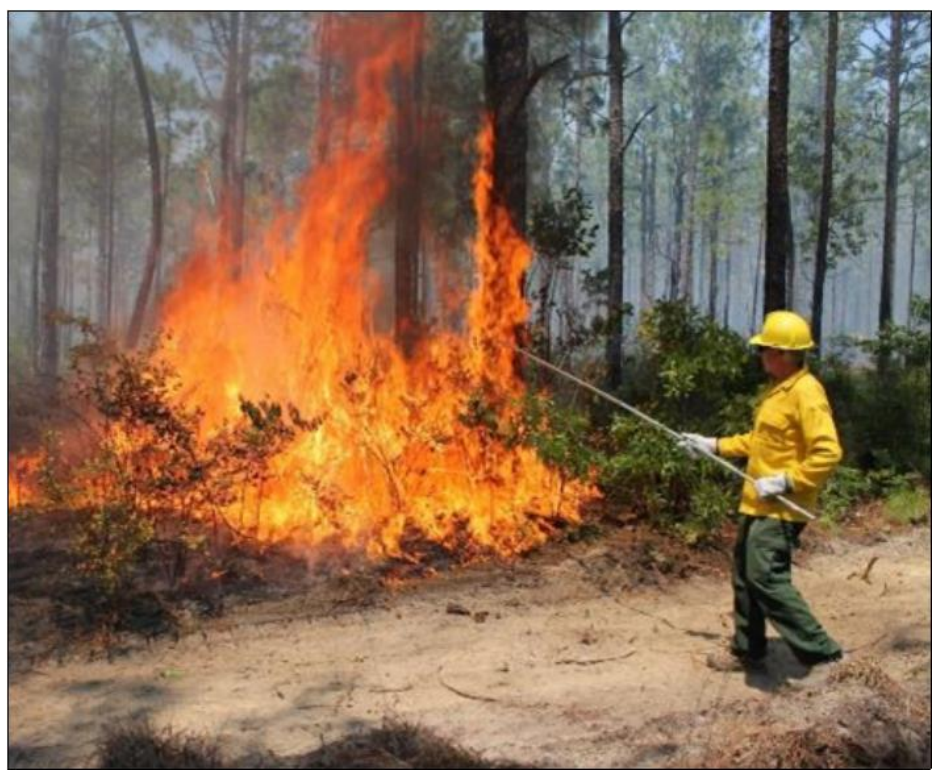

Figure 7 - Rigid tubing ("fishing pole") used to sample pyrolysis gases produced by heated sparkleberry during May 2017 trial deployment at Ft. Jackson, South Carolina, USA. 


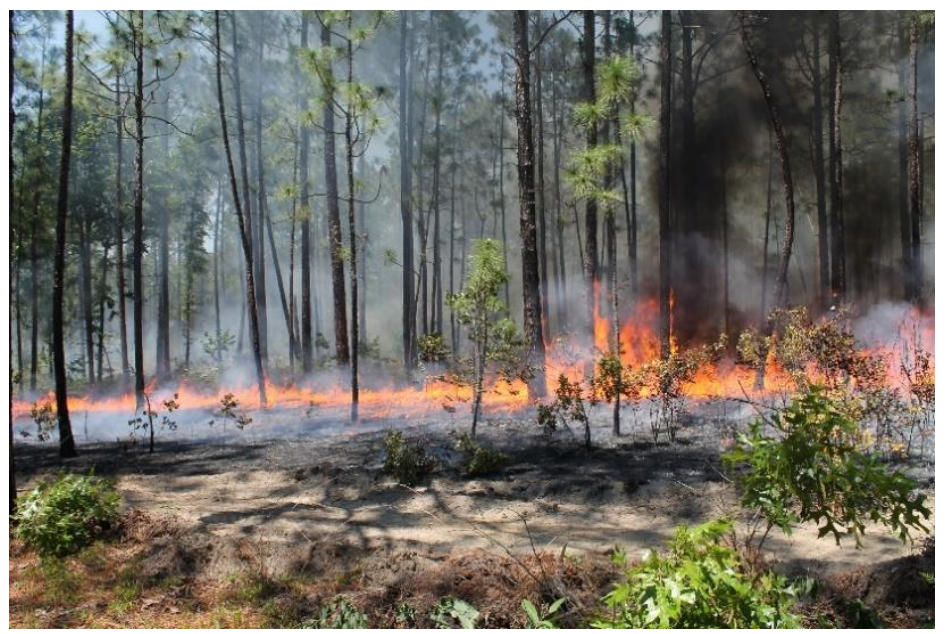

Figure 8 - Typical range in fire behavior observed on low density plot burned May 2017 at Ft. Jackson, SC.

The pyrolysis mechanism included in Gpyro was initially validated against published experimental and computational TGA results. The burning of leaf-like fuels with three initial fuel moisture content (FMC) of $40 \%, 76 \%$, and $120 \%$ was modeled. The time evolutions of the normalized mass were good for the modeled fuels with $76 \%$ and $120 \%$ FMCs and fair for the one with a $40 \%$ FMC when compared to the experimental burning results of four manzanita leaves with unspecified FMCs. The computed ignition time was also in good agreement with the measurement. The computed burnout time was somewhat shorter than the measurement. Modeling revealed the formation of unsteady flow structures including vortices and regions with high strain rates near the fuel that acted as a bluff body against the stream of the burner exit (Figure 59). These structures played a significant role in the spatial distribution of gas phase temperature and species around the fuel, which in turn, had an impact on the ignition location. Fuel moisture content primarily affected the temperature response of the fuel and solid phase decomposition. Full details of these results can be found in (Shotorban et al. 2018).
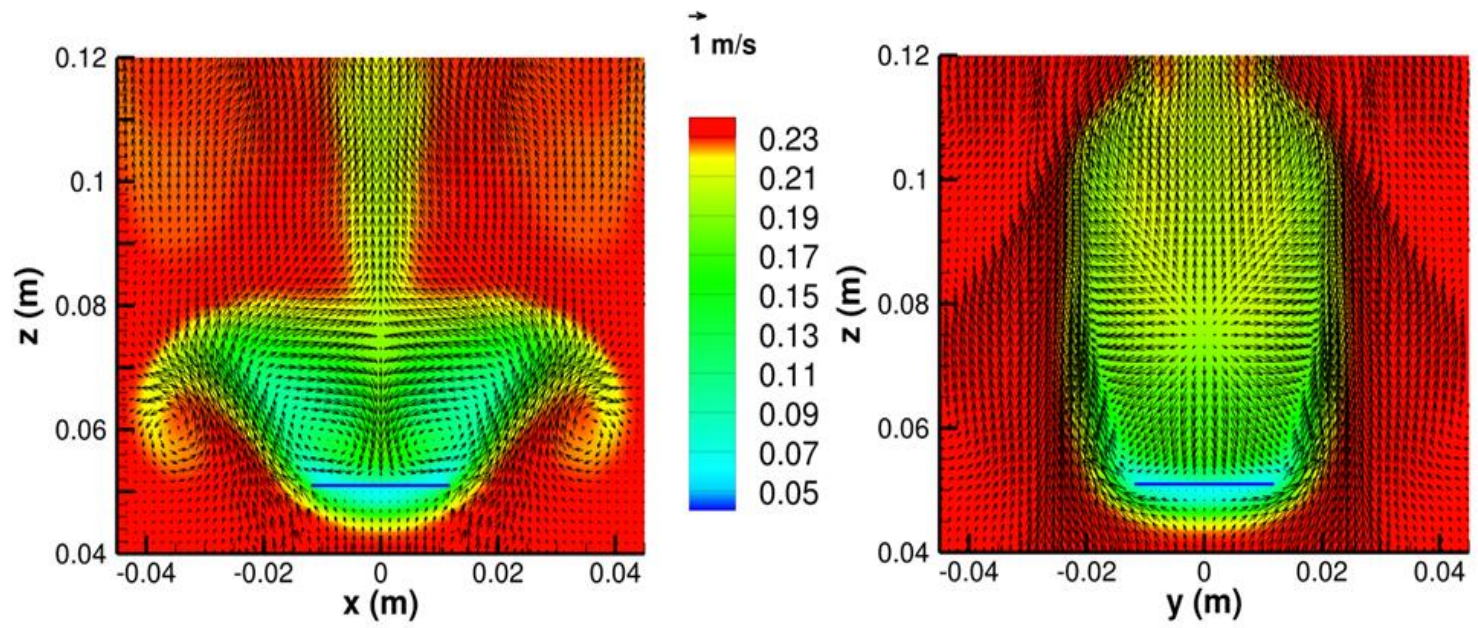

Figure 9 - Contours of oxygen mass fraction and velocity vectors on an $x z$ slice passing $y=0$ (left) and yz slice passing $x=0$ (right) at $t=11.5 \mathrm{~s}$ for an initial FMC of 76\%. See Erro! A origem da referência não foi encontrada. for locations of the slices.

\section{Acknowledgement}

This work was supported was the DOD/EPA/DOE Strategic Environmental Research and Development Program project RC-2640. 


\section{Literature Cited}

Anand C, Shotorban B, Mahalingam S, McAllister S, Weise DR (2017) Physics-based modeling of live wildland fuel ignition experiments in the forced ignition and flame spread test apparatus. Combustion Science and Technology 189, 1551-1570. doi:10.1080/00102202.2017.1308357.

Bryden KM, Hagge MJ (2003) Modeling the combined impact of moisture and char shrinkage on the pyrolysis of a biomass particle弥. Fuel 82, 1633-1644. doi:10.1016/S0016-2361(03)00108-X.

Cobian-Iñiguez J, Aminfar A, Chong J, Burke G, Zuniga A, Weise DR, Princevac M (2017) Wind tunnel experiments to study chaparral crown fires. Journal of Visualized Experiments 1-14. doi:10.3791/56591.

Fletcher TH, Safdari M-S, Amini E, Weise DR (2018) Bench-scale measurement of pyrolysis products from intact live fuels. In 'Adv. For. Fire Res. 2018', Coimbra, Portugal. 3. (Coimbra University Press: Coimbra, Portugal)

Griffith DWT (1996) Synthetic calibration and quantitative analysis of gas-phase FT-IR spectra. Applied Spectroscopy 50, 59-70. doi:10.1366/0003702963906627.

Hudak AT, Bright BC, Pokswinski SM, Loudermilk EL, O’Brien JJ, Hornsby BS, Klauberg C, Silva CA (2016) Mapping Forest Structure and Composition from Low-Density LiDAR for Informed Forest, Fuel, and Fire Management at Eglin Air Force Base, Florida, USA. Canadian Journal of Remote Sensing 42, 411-427. doi:10.1080/07038992.2016.1217482.

Hudak AT, Dickinson MB, Bright BC, Kremens RL, Loudermilk EL, O'Brien JJ, Hornsby BS, Ottmar RD (2016) Measurements relating fire radiative energy density and surface fuel consumption RxCADRE 2011 and 2012. International Journal of Wildland Fire 25, 25-37.

Johnson TJ, Sharpe SW, Covert MA (2006) Disseminator for rapid, selectable, and quantitative delivery of low and semivolatile liquid species to the vapor phase. Review of Scientific Instruments 77, 094103. doi:10.1063/1.2349298.

Mertz L (1967) Auxiliary computation for Fourier spectrometry. Infrared Physics 7, 17-23. doi:10.1016/0020-0891(67)90026-7.

Miller RS, Bellan J (1997) A generalized biomass pyrolysis model based on superimposed cellulose, hemicellulose and liqnin kinetics. Combustion Science and Technology 126, 97-137. doi:10.1080/00102209708935670.

Pickett BM, Isackson C, Wunder R, Fletcher TH, Butler BW, Weise DR (2010) Experimental measurements during combustion of moist individual foliage samples. International Journal of Wildland Fire 19, 153-162. doi:10.1071/WF07121.

Raffel M (2015) Background-oriented schlieren (BOS) techniques. Experiments in Fluids 56,. doi:10.1007/s00348-015-1927-5.

Safdari M-S, Rahmati M, Amini E, Howarth JE, Berryhill JP, Dietenberger M, Weise DR, Fletcher TH (2018) Characterization of pyrolysis products from fast pyrolysis of live and dead vegetation native to the Southern United States. Fuel 229, 151-166. doi:10.1016/j.fuel.2018.04.166.

Shotorban B, Yashwanth BL, Mahalingam S, Haring DJ (2018) An investigation of pyrolysis and ignition of moist leaf-like fuel subject to convective heating. Combustion and Flame 190, 25-35. doi:10.1016/j.combustflame.2017.11.008.

Silva CA, Hudak AT, Vierling LA, Loudermilk EL, O’Brien JJ, Hiers JK, Jack SB, Gonzalez-Benecke C, Lee H, Falkowski MJ, Khosravipour A (2016) Imputation of individual longleaf pine ( Pinus palustris Mill.) tree attributes from field and LiDAR data. Canadian Journal of Remote Sensing $\mathbf{4 2}$, 554-573. doi:10.1080/07038992.2016.1196582.

Weise DR, Johnson TJ, Hao WM, Princevac M, Scharko N, Oeck A, Myers TL, Baker S, Lincoln E, Aminfar AH (2018) Measurement of pyrolysis products from mixed fuel beds during fires in a wind 
tunnel. In 'Adv. For. Fire Res. 2018', Coimbra, Portugal.(Coimbra University Press: Coimbra, Portugal)

Yashwanth BL, Shotorban B, Mahalingam S, Lautenberger CW, Weise DR (2016) A numerical investigation of the influence of radiation and moisture content on pyrolysis and ignition of a leaflike fuel element. Combustion and Flame 163, 301-316. doi:10.1016/j.combustflame.2015.10.006.

Yashwanth BL, Shotorban B, Mahalingam S, Weise DR (2015) An investigation of the influence of heating modes on ignition and pyrolysis of woody wildland fuel. Combustion Science and Technology 187, 780-796. doi:10.1080/00102202.2014.973948.

Yokelson RJ, Griffith DWT, Ward DE (1996) Open-path Fourier transform infrared studies of largescale laboratory biomass fires. Journal of Geophysical Research 101, 21067. doi:10.1029/96JD01800.

Zhou X, Sun L, Mahalingam S, Weise DR (2003) Thermal particle image velocity estimation of fire plume flow. Combustion Science and Technology 175, 1293-1316. doi:10.1080/00102200302376. 


\title{
A review of fire whirls in wildland fires
}

\author{
Michael Gollner*1; Ali Tohidi²; Huahua Xiao ${ }^{3}$ \\ ${ }^{1}$ University of Maryland, College Park, Department of Fire Protection Engineering. 3106 J.M. \\ Patterson Bldg., College Park, Maryland 20742, United States \{mgollner@umd.edu*\} \\ ${ }^{2}$ One Concern, Inc., 1699 University Avenue, Palo Alto, CA 94301, United States \\ \{ali@oneconcern.com\} \\ ${ }^{3}$ University of Maryland, College Park, Department of Aerospace Engineering. 3179 Glenn L. \\ Martin Hall, College Park, Maryland 20742, United States \{hhxiao@umd.edu\}
}

\begin{abstract}
Fire whirls have long fascinated the research community, however their destructive power has hidden many features of their formation, growth and propagation. Most of what is known about fire whirls, therefore, comes from scale modeling experiments in the laboratory. Both the methods of formation, dominated by wind and geometry, and the inner structure of the whirl, including velocity and temperature fields, have been studied at this scale. The particular case of quasi-steady fire whirls directly over a fuel source forms the bulk of current experimental knowledge, despite many other cases existing in nature. This presentation will review the state of knowledge concerning the fluid dynamics of fire whirls, including the conditions for their formation, their structure and the mechanisms which control their unique state. Particular emphasis will be given to the formation of fire whirls during wildland fires, especially during extreme fire events. Finally, recent discoveries will be highlighted as well as potential research avenues for the future.
\end{abstract}

Keywords: fire whirl, fire tornado, vortex

\section{Introduction}

The fire whirl is one of the most dramatic structures which arises at the intersection of combustion and fluid mechanics. Throughout the literature, fire whirls have been identified by a variety of names such as devil, tornado, twister, whirlwind, or even dragon twist (Japanese). Regardless of the name, when the right combination of wind and fire interact, the result is an intensification of combustion with whirling flames which we call the fire whirl. While the fire whirl or "fire tornado" shares some features with its atmospheric counterparts, it remains distinct in its source of buoyancy, combusting fuel, structure, and formation patterns. In nature, fire whirls are most often observed in mass fires. These include both large wildland (also known as forest or bush fires) and urban conflagrations such as the burning of cities or towns. Due to the diversity of topography, wind, and fire conditions that can occur in wildland fires, fire whirls are a frequent phenomenon.

Fire whirls have mostly been studied in the context of fire safety, as their erratic movement and ability to loft burning firebrands contribute to the rapid ignition of new fires, presenting significant hazards to nearby firefighting personnel (Countryman 1971; Forthofer and Goodrick 2011).

Despite the incredible interest they can garner, fire whirls remain a relatively poorly understood phenomenon due to their convoluted dynamics and difficulties in obtaining quantitative data (Morton 1970; Albini 1985; Soma and Saito 1991). Hence, many details of the fire whirl, whether formed in the laboratory or by natural means, remain elusive. A review of the literature on fire whirls related to wildland fires, beginning with description of important parameters governing their dynamics followed by review of the various formation mechanisms will be presented. Finally, the processes governing the fire whirl will be reviewed along with concluding remarks and avenues for future research. 


\section{Essential Conditions for Formation}

Fire whirls emerge when terrain/domain features (obstructions), and wind coalesce over a strong self-sustaining source of buoyancy (fire plume) and form a concentrated flaming vortex column. A fire whirl is not necessarily comprised of swirling flames within the vortex column (Countryman 1971), as many cases have been reported to form from hot gases at downwind of large fires (McRae and Flannigan 1990; Zhou and Wu 2007). Hence, given the collective body of evidence on fire whirls, they can be classified in two main types: on-source and off-source. When the whirling flame (vortex column) forms directly over the fuel source, the fire whirl is defined as on-source, and when it forms with an offset from the fuel surface, it is considered off-source (Hartl and Smits 2016). Both on-source and off-source types can be found in a quasi-steady or unsteady state. We shall use this classification to describe documented instances of fire whirls in the remaining sections.

Regardless of this classification, fire whirls can also be categorized based on their characteristic length scale, which is most often chosen to be height of the vortex column. Fire whirls with flame heights between 0.1-1 m are defined as small scale (Snegirev et al. 2004), which have been abundantly studied both experimentally and numerically (Emori and Saito 1982; Battaglia et al. 2000; Snegirev et al. 2004; Zhou and Wu 2007; Chuah et al. 2009; Lei et al. 2015; Hartl and Smits 2016). Whirls with flame heights between 1-10 m and $10-(\mathrm{O})^{\prime} 100 \mathrm{~m}$ are categorized as medium and large-scale cases, respectively (Snegirev et al. 2004; Hartl and Smits 2016). Even larger events, of the order of kilometers, are documented in the literature that have occurred during large urban conflagrations (Soma and Saito 1991) or bushfires. For instance, a fire-atmospheric event termed pyro-tornadogenesis has been described by (McRae et al. 2013).

\section{Fire Whirl Formation}

For all different types and scales of fire whirls, three crucial factors are essential in their formation, namely a thermally-driven fluid sink, an eddy (vorticity) generation mechanism, and a surface drag force to create a radial boundary layer, such that it facilitates air entrainment to the generated vortex column (Byram, G.M. ; Martin 1962; Byram and Martin 1970). The fire acts as a fluid sink, where the generated plume naturally drives horizontal flows radially towards the vortex column. Therefore, the most substantial element in fire whirl formation is the presence of an eddy-generating mechanism.

During wildland fires, the possibility of having strong eddies coalescing with fluid sinks and shear forces at the base is high. In these extreme events a variety of natural means exist that can generate the required eddy, such as flow channeled by topological features (Countryman 1971), the interaction of multiple fires or plumes (Liu et al. 2007), the wake of a hill, ridge or large fire plume (Emori and Saito 1982), and generally the transformation of horizontal vorticity into the vertical direction (Church et al. 1980; Forthofer and Goodrick 2011).

Most known open fire whirl configurations, including on and off-source, quasi-steady and unsteady, can all be observed over an L-shaped fire in cross-flow. This configuration is similar to the Hifukushoato fire whirl (HAFW), which occurred following a devastating earthquake in Tokyo in 1923, killing almost 38,000 people (Soma and Saito 1991; Kuwana et al. 2007). Three types of whirls are found in this configuration; a stable on-source fire whirl on both ends of the ' $L$ ', unsteady fire whirls which travel along the edge or flanks of the fire and shed periodically in its wake, and a stable off-source whirl which forms within the unburnt region between the ' $L$ '. The first type, Type $I$, is most like enclosed fire whirls extensively studied in the laboratory, being both on-source and quasi-steady (Satoh et al. 1997; Hassan et al. 2005; Liu et al. 2007; Chuah et al. 2009; Zhou et al. 2013; Dobashi et al. 2015; Hartl and Smits 2016; Wang et al. 2016; Xiao et al. 2016). These types could also be formed by array of multiple fires, with or without cross-flow, where any asymmetry in the flow or geometry may cause/amplify the swirl which generates a whirl (Zhou and Wu 2007). This behavior is thought to have been observed following the deliberate fire-bombing of Dresden and Hamburg during World

Advances in Forest Fire Research 2018 - Page 320 
War II (Soma and Saito 1991). The second type, which is off-source and periodic in nature, is also seen on the flanks of large wildfires as well as in the wake following large bent-over plumes. These have been called "Dessens" fire whirls (DFW) in the past due to their similarity to whirls observed downstream of large-scale experiment by Dessens (1962); a schematic of such cases is shown in the figure below) (Dessens 1962). Finally, fire whirls known as Type III are formed in off-source regions with either flames or merely hot cases under cross-flow, similar to the HAFW whirl described above.

Other fire whirls include those seen to form on the lee side of a hill following a wildfire. These whirls are similar to Type II or III whirls, depending on whether they are quasi-steady, the location of the fuel and obstruction, and the orientation of ambient flow. In general, strong vorticity is generated behind an obstruction in cross-flow, such as a hill, which has been known to create re-circulation regions that can drastically modify fire spread at the top of hills; however, when vorticity becomes tilted upward, one or more fire whirls may form. Fire whirls have also been seen to arise from interactions between a line fire and cross-flow, where an unsteady, on-source whirl can form under specific velocity, orientation and fire sizes. These fire whirls may travel along a line fire similar to Type II fire whirls which form on the flanks of larger fires (Kuwana et al. 2013; Zhou et al. 2016).

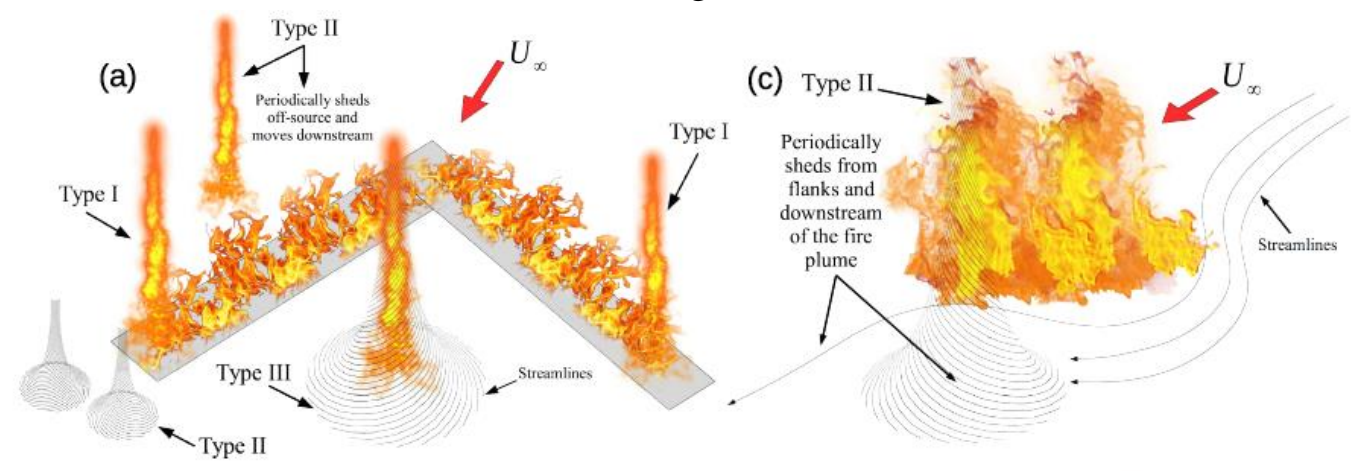

(b)

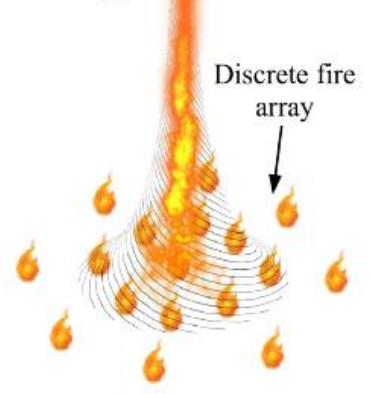

(d)

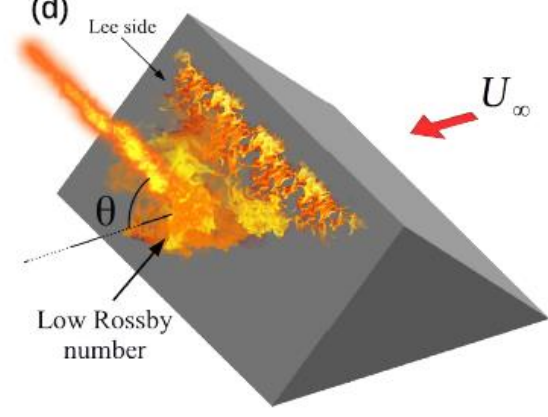

(e)

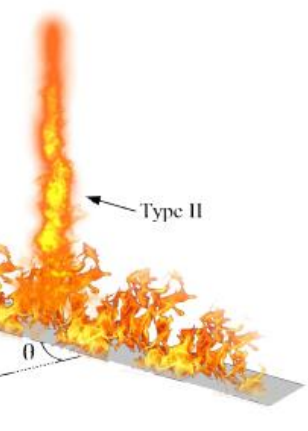

Figure 1 - Possible methods for fire whirls to form in the open. All of these are seen in wildland fires. From Tohidi et al., 2018.

\section{Conclusions and Directions for Future Research}

Despite many years of study, fire whirls continue to fascinate the scientific community and present challenges for fire safety, especially in wildland and wildland-urban interface fires. Without a definitive theory of the flow structure for a fire whirl, many gaps in our fundamental understanding remain. Much progress has been made on on-source, quasi-steady fire whirls, such as those formed in enclosed laboratory apparatuses. However, some open questions remain. First and foremost, the underlying process governing entrainment is not well known. In Emmons \& Ying's (1967) early work, they raised clear issues with regard to the treatment of entrainment as a function of height, and although some detailed PIV measurements have assisted in this understanding, these issues have not yet been fully resolved (Emmons and Ying 1967). This will help researchers better formulate a model of the fire whirl. 
Other types of fire whirls continue to challenge our understanding, particularly those that occur off source or are nonsteady, most resembling those occurring in wildland fires. Certainly, the velocity and temperature fields within these whirls may differ, but exactly how remains to be seen. A reliable method for generating these whirls within the laboratory, as well as detailed measurements of their structures, would be very beneficial to our understanding. Even stationary fire whirls over a fuel source precess around the source, causing them to move and wander, triggering unknown effects to the structure of the fire whirl, especially when they are near the limits of their stability (e.g., fuel rich or high swirl). The effects of the level of circulation (e.g., Rossby number) on the structure of fire whirls are not well known, as most experiments have been performed only under a limited range of circulations and scales.

Scaling laws that describe the formation of these complex whirls have highlighted the dependence on ambient cross flow; however, there is no general form that describes the conditions for the generation of a fire whirl. This knowledge would certainly be useful in the operational modeling of wildfires, where resolutions are too coarse to resolve the complicated flow dynamics leading to fire whirls, but predictions of critical conditions could be used to send a warning to firefighters who might be dangerously near potential fire whirl formation. Still, scale modeling between laboratory wind tunnels and large-scale observations has been relatively successful in predicting the limiting conditions under which wind-driven fire whirls will form. As large-scale measurements of the fire whirl structure are, for the most part, not available, it is not yet known how this may change with increasing scale. Further measurements or numerical modeling should be performed to better understand whether the mechanisms governing the structure of fire whirls change at increasing scales.

Compared to related areas of fluid dynamics including combustion and fire phenomena, the use of numerical modeling has been relatively lacking in this field; this may be due to the complex interactions that occur during the generation and growth of a fire whirl. However, numerical models could provide invaluable information if validated against experimental measurements. Continued development of these models, particularly for cases other than stationary, on-source fire whirls, is highly encouraged. This may also be useful in understanding the formation of fire whirls, especially under wind, allowing researchers to visualize flow structures that cannot be easily measured experimentally.

\section{Acknowledgements}

This extended abstract is an excerpt from the paper by Tohidi et al. (2018) published in the Annual Review of Fluid Mechanics. The authors would like to thank Forman Williams, Antonio Sanchez, Alexander Smits, Katie Hartl, and Elaine Oran for interesting discussions that improved the original manuscript. Support for this work came from the National Science Foundation awards CBET-1507623 and CBET-1554026, and from the collaborative agreement 13-CS-11221637-124 from the National Fire Decision Support Center and the USDA Forest Service Missoula Fire Sciences Laboratory. Any opinions, findings, conclusions, or recommendations expressed in this material are those of the author(s) and do not necessarily reflect the views of the National Science Foundation or the USDA Forest Service.

\section{References}

Albini FA (1985) A Model for Fire Spread in Wildland Fuels by-Radiation†. Combustion Science and Technology 42, 229-258. doi:10.1080/00102208508960381.

Battaglia F, McGrattan KB, Rehm RG, Baum HR (2000) Simulating fire whirls. Combustion Theory and Modelling 4, 123-138. doi:10.1088/1364-7830/4/2/303.

Byram, G.M. ; Martin R. (1962) Fire whirlwinds in the laboratory. Fire Control Notes 23, 13-17. 
Byram G, Martin R (1970) The modeling of fire whirlwinds. Forest Science. http://www.ingentaconnect.com/content/saf/fs/1970/00000016/00000004/art00003.

Chuah KH, Kuwana K, Saito K (2009) Modeling a fire whirl generated over a 5-cm-diameter methanol pool fire. Combustion and Flame 156, 1828-1833. doi:10.1016/j.combustflame.2009.06.010.

Church CR, Snow JT, Dessens J (1980) Intense Atmospheric Vortices Associated with a 1000 MW Fire. Bull Amer Meteor Soc 61, 682-694. doi:10.1175/15200477(1980)061<0682:IAVAWA>2.0.CO;2.

Countryman CM (1971) Fire Whirls... why, when and where. Pacific Southwest Forest and Range Experimental Station, Forest Service, U.S. Department of Agriculture,

Dessens J (1962) Man-made tornadoes. Nature. doi:10.1038/193013a0.

Dobashi R, Okura T, Nagaoka R, Hayashi Y, Mogi T (2015) Experimental Study on Flame Height and Radiant Heat of Fire Whirls. Fire Technology. doi:10.1007/s10694-015-0549-z.

Emmons HW, Ying S-J (1967) The fire whirl. Symposium (International) on Combustion 11, 475488.

Emori RI, Saito K (1982) Model experiment of hazardous forest fire whirl. Fire Technology 18, 319327. doi:10.1007/BF02473115.

Forthofer JM, Goodrick SL (2011) Review of Vortices in Wildland Fire. Journal of Combustion 2011, 1-14. doi:10.1155/2011/984363.

Hartl KA, Smits AJ (2016) Scaling of a small scale burner fire whirl. Combustion and Flame 163, 202-208. doi:10.1016/j.combustflame.2015.09.027.

Hassan MI, Kuwana K, Saito K, Wang F (2005) Flow structure of a fixed-frame type fire whirl. Fire Safety Science 951-962.

Kuwana K, Sekimoto K, Minami T, Tashiro T, Saito K (2013) Scale-model experiments of moving fire whirl over a line fire. Proceedings of the Combustion Institute 34, 2625-2631. doi:10.1016/j.proci.2012.06.092.

Kuwana K, Sekimoto K, Saito K, Williams FA, Hayashi Y, Masuda H (2007) Can We Predict the Occurrence of Extreme Fire Whirls? AIAA Journal 45, 16-19.

Lei J, Liu N, Zhang L, Satoh K (2015) Temperature, velocity and air entrainment of fire whirl plume: A comprehensive experimental investigation. Combustion and Flame 162, 745-758. doi:10.1016/j.combustflame.2014.08.017.

Liu N, Liu Q, Deng Z, Kohyu S, Zhu J (2007) Burn-out time data analysis on interaction effects among multiple fires in fire arrays. Proceedings of the Combustion Institute 31, 2589-2597. doi:10.1016/j.proci.2006.08.110.

McRae DJ, Flannigan MD (1990) Development of large vorticies on perscirbed fires. Can J For Res 1878-1887.

McRae RHD, Sharples JJ, Wilkes SR, Walker A (2013) An Australian pyro-tornadogenesis event. Natural Hazards 65, 1801-1811. doi:10.1007/s11069-012-0443-7.

Morton BR (1970) The Physics of Fire Whirls. Fire Research Abstracts and Reviews, National Academy of Sciences 12, 1-19.

Satoh K, Yang KT, Dame N (1997) Simulations of Swirling Fires Controlled by Channeled Selfgenerated Entrainment Flows. Fire Safety Science 5, 201-212.

Snegirev AY, Marsden JA, Francis J, Makhviladze GM (2004) Numerical studies and experimental observations of whirling flames. Int J Heat Mass Transfer 47, 2523-2539.

Soma S, Saito K (1991) Reconstruction of fire whirls using scale models. Combustion and Flame 86, 269-284. doi:10.1016/0010-2180(91)90107-M. 
Wang P, Liu N, Hartl K, Smits A (2016) Measurement of the Flow Field of Fire Whirl. Fire Technology 52, 263-272. doi:10.1007/s10694-015-0511-0.

Xiao H, Gollner MJ, Oran ES (2016) From fire whirls to blue whirls and combustion with reduced pollution. Proceedings of the National Academy of Sciences 113, 9457-9462. doi:10.1073/pnas.1605860113.

Zhou K, Liu N, Lozano JS, Shan Y, Yao B, Satoh K (2013) Effect of flow circulation on combustion dynamics of fire whirl. Proceedings of the Combustion Institute 34, 2617-2624. doi:10.1016/j.proci.2012.06.053.

Zhou K, Liu N, Yuan X (2016) Effect of Wind on Fire Whirl Over a Line Fire. Fire Technology 52, 865-875. doi:10.1007/s10694-015-0507-9.

Zhou R, Wu Z-N (2007) Fire whirls due to surrounding flame sources and the influence of the rotation speed on the flame height. Journal of Fluid Mechanics 583, 313. doi:10.1017/S0022112007006337. 


\title{
A study of the structure of a turbulent line fire subjected to cross-flow using large eddy simulations
}

\author{
Arnaud Trouvé*; Salman Verma \\ Department of Fire Protection Engineering, University of Maryland. College Park, MD, 20742, \\ USA, \{atrouve@umd.edu*,salman.iaal@gmail.com\}
}

\begin{abstract}
The general objective of this project is to provide a basic understanding of the transition between the different flame regimes observed in fires with cross-flow and/or fires along inclined surfaces. We consider here a simplified configuration corresponding to a methane-air, buoyancy-driven, turbulent line flame stabilized on top of a horizontal floor surface and subjected to different air cross-flow velocities. At high values of the cross-flow velocity, the flame features a horizontal shape and develops as a boundary layer flame in the vicinity of the floor surface; the flow downstream of the flame is attached to the floor surface and air entrainment into the flame is one-sided. In contrast, at low values of the cross-flow velocity, the flame features a tilted vertical shape and develops as a pool-like flame; the flow downstream of the flame separates from the floor surface and air entrainment into the flame is two-sided. In the present study, we analyze the transition from an attached flame to a lifted flame using wall-resolved large eddy simulations (LES). Simulations are performed with an LES solver developed by FM Global and called FireFOAM. The simulated line burner is $50-\mathrm{cm}$ wide and $5-\mathrm{cm}$ long; the flame power is $50 \mathrm{~kW}$; and the air cross-flow velocities range between 0.75 and $3 \mathrm{~m} / \mathrm{s}$. The LES simulations provide a detailed description of the different contributions to flow kinetic energy in the horizontal and vertical directions and thereby provide unique insights into the competing effects of the external momentum of the horizontal cross-flow and the internal momentum of the vertical buoyant motions produced by the combustion heat release and the resulting unstable thermal stratification. A new criterion is proposed to measure the relative strength of external/cross-flow-driven versus internal/buoyancy-driven motions and to thereby predict the transition from an attached to a lifted flame regime.
\end{abstract}

Keywords: Buoyant turbulent diffusion flame; Wind-driven flames; Boundary layer flame; Pool flame; Air entrainment; Large Eddy Simulation

\section{Introduction}

Developing a fundamental understanding of the effects of cross-flow on fires is of considerable interest in fire science; because of the ubiquity and importance of such effects in wildland, urban and industrial fires. One basic way in which cross-flow affects a fire is by changing the flame geometry: as the external momentum of the horizontal cross-flow increases, the flame transitions from a vertical pool-like flame to a horizontal flame attached to the downstream surface (Tang et al. 2017). Among other things, attachment of the flame increases the convective heat transfer to the downstream surface; this in turn increases the rate of flame spread (in cases for which the downstream surface contains fresh fuel).

The variations in flame geometry in fires subjected to cross-flow have been studied both experimentally (Putnam 1965; Oka et al. 2000; Oka et al. 2003; Cole et al. 2011; Hu et al. 2013; Lam and Weckman 2015) and numerically (Albini 1981; Sinai and Owens 1995; Morvan et al. 1998; Porterie et al. 2000; Morvan et al. 2001; Nmira et al. 2010; Vasanth et al. 2013) over the past several decades. However, past experimental studies typically use a limited range of diagnostics, primarily video imaging and temperature measurements, while past numerical studies typically use low-order approaches, for instance Reynolds-Averaged Navier-Stokes simulations (Sinai and Owens 1995; 
Morvan et al. 1998; Porterie et al. 2000; Morvan et al. 2001; Nmira et al. 2010; Vasanth et al. 2013), that do not resolve the relevant turbulent flow and flame scales. Thus, a basic understanding of the physics associated with variations in wind-driven flame geometry is lacking and is the primary motivation behind the present study.

In the present study, a methane-fueled, buoyancy-driven, turbulent line flame stabilized on top of a horizontal floor surface and subjected to different cross-flow velocities is simulated using wallresolved Large Eddy Simulations (LES). The primary objective is to gain fundamental insights into the transition from a vertical to a horizontal flame resulting from increases in the cross-flow velocity. In what is seen as an intermediate step, the present study considers a gas-fueled turbulent flame that is non-spreading, is statistically stationary and that therefore lends itself to simplified analysis. Configurations that are closer to wildland fire configurations and that feature representative vegetation fuel and spreading flames will be considered in future work.

\section{Numerical Solver and Configuration}

Numerical simulations are performed using FireFOAM (FireFOAM 2018), a fire modeling solver developed by FM Global and based on an open-source, general-purpose, Computational Fluid Dynamics (CFD) software package called OpenFOAM (OpenFOAM 2018). FireFOAM is a secondorder accurate, finite volume solver with implicit time integration; the solver features advanced meshing capabilities (structured/unstructured polyhedral mesh); it also features a massively parallel computing capability using Message Passing Interface protocols.

FireFOAM uses a Favre-filtered, compressible-flow, LES formulation and provides a choice between several modeling options for the treatment of turbulence, combustion and thermal radiation. In the present study: subgrid-scale (SGS) turbulence is described using the WALE (Wall-Adapting Local Eddy-viscosity) model (Nicoud and Ducros 1999); combustion is described using the classical concept of a global combustion equation combined with the Eddy Dissipation Concept (EDC) model (Magnussen and Hjertager 1977); radiation is described by solving the radiative transfer equation (RTE) using a discrete-ordinates, finite-volume method and by assuming a non-scattering, nonabsorbing, optically-thin medium and using a prescribed global radiative loss fraction $\chi_{\text {rad }}$.

The numerical configuration is presented in Fig. 1. The computational domain is $780-\mathrm{cm}$-long in the streamwise $x$-direction, $50-\mathrm{cm}$-wide in the spanwise $y$-direction and $250-\mathrm{cm}$-high in the vertical $z$ direction. The line burner face is 5-cm-long in the $x$-direction and 50-cm-wide in the $y$-direction; and the spanwise center of its leading edge is placed at the origin $(x, y, z)=(0,0,0)$. The burner is flushmounted on a 50-cm-wide horizontal solid plate that starts at $x=(-20) \mathrm{cm}$ and ends at $x=205 \mathrm{~cm}$. The cross-flow air is injected through a $50-\mathrm{cm}$-wide and 50-cm-high wind tunnel whose outlet is located at the inlet boundary of the computational domain, at $x=(-30) \mathrm{cm}$. A 5-mm-long, 50-cm-wide and 5mm-high trip wire is placed at $x=(-10.5) \mathrm{cm}$ in order to perturb the incoming flow and promote laminar-to-turbulent transition. 


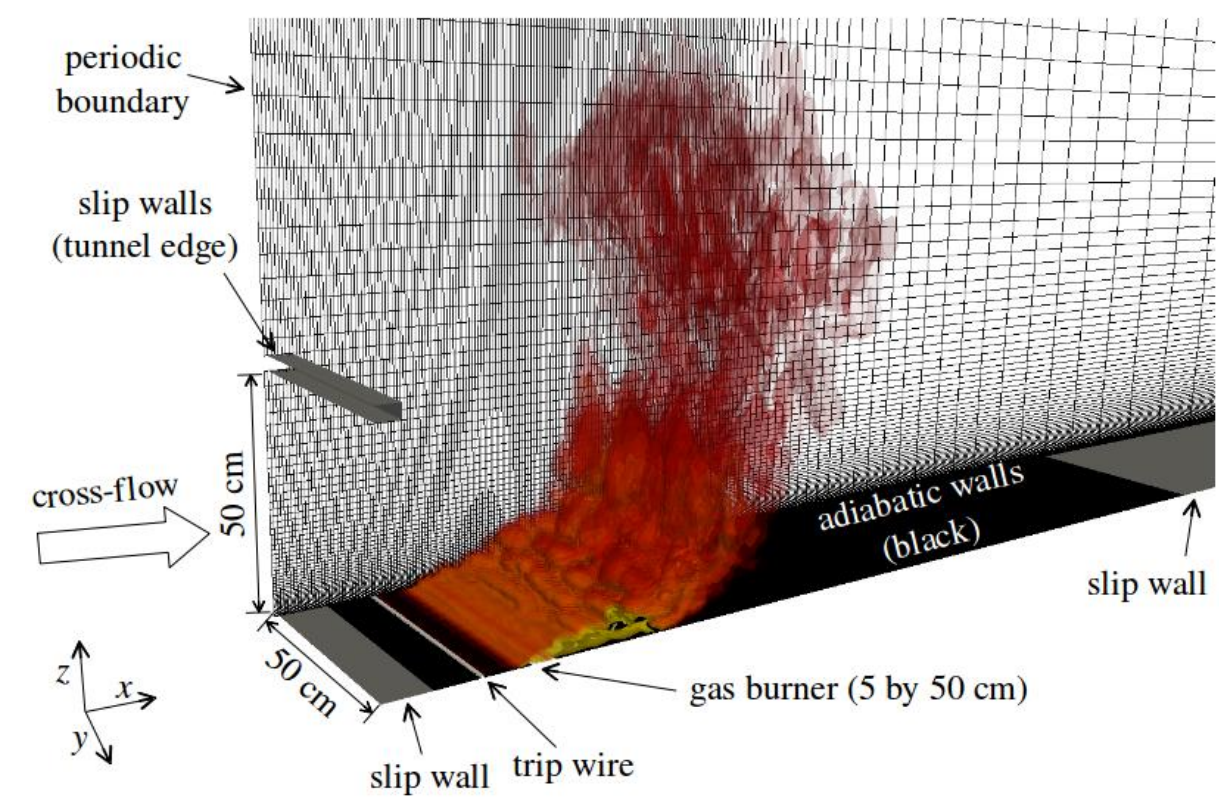

Figure 1 - Illustration of the FireFOAM numerical configuration; the $50 \mathrm{~kW}$ methane diffusion flame is visualized using isocontours of instantaneous volumetric heat release rate $(0.1,1,10,100,1000,10000 \mathrm{~kW} / \mathrm{m3})$.

The computational grid is a rectangular Cartesian mesh. Grid spacing in the streamwise $x$-direction is uniform and is equal to $5 \mathrm{~mm}$ for $x \leq 100 \mathrm{~cm}$; beyond that location, the $x$-grid is stretched with a stretch factor equal to 1.06. Grid spacing in the spanwise $y$-direction is uniform and is equal to $5 \mathrm{~mm}$. This streamwise (spanwise) resolution corresponds to 10 (100) grid cells across the burner length (width). Grid spacing in the vertical $z$-direction is non-uniform: the $z$-grid spacing is $1.2 \mathrm{~mm}$ at $z=0$ (i.e, the first cell center is $0.6 \mathrm{~mm}$ above the south boundary of the computational domain) and is 20 $\mathrm{mm}$ at $z=50 \mathrm{~cm}$ with a stretching factor of 1.04 . For $z \geq 50 \mathrm{~cm}$, the $z$-grid is stretched with a stretch factor equal to 1.06. Note that with the present resolution, the trip wire is under-resolved and is described with 1 (4) grid cell(s) in the $x$ - $\left(z^{-}\right)$direction. The total number of cells is 3.5 million.

The methane mass flow rate is prescribed at the burner boundary and the air velocity is fixed at the tunnel outlet. The horizontal solid plate and the trip wire are both treated as no-slip adiabatic solid walls. The surface located at $z=0$ between the tunnel outlet and the leading edge of the solid plate is treated as a slip wall. The surface located at $z=0$ beyond the solid plate, at $x \geq 205 \mathrm{~cm}$, is also treated as a slip wall. The side boundaries at $y=(-25)$ and $25 \mathrm{~cm}$ correspond to periodic conditions. Other boundaries are treated as boundaries with open flow conditions.

In all cases, the methane mass flow rate is linearly increased from 0 to $1 \mathrm{~g} / \mathrm{s}$ during the first five seconds and is then held constant for the remainder of the simulations. This is done to allow the crossflow to establish itself over the line burner before the fire reaches its nominal value of the heat release rate equal to $50 \mathrm{~kW}$. All simulations are performed for a duration of $30 \mathrm{~s}$. Turbulent statistics are collected for the final $15 \mathrm{~s}$ of each simulation, after the flow and flame become statistically stationary and long enough for the statistics to be converged (to improve convergence, statistics are computed using both temporal- and spanwise-averaging). The time step is controlled by a classical CourantFriedrichs-Lewy (CFL) condition and is approximately equal to $0.35 \mathrm{~ms}$. Each simulation is run using 200 processors on a large-scale Linux cluster with a typical simulation requiring 40,000 CPU-hours.

\section{Results and Discussion}

We first consider the mean flame shape for a range of cross-flow velocities (Fig. 2). As $U_{\infty}$ increases from 0.75 to $3 \mathrm{~m} / \mathrm{s}$, the flame transitions from a lifted (vertically tilted) flame to an attached (horizontal) 
flame. The vertical elevation of the flame decreases (from 50 to $10 \mathrm{~cm}$ ); the flame length (loosely defined as the distance from the burner to the flame tip) increases (from 50 to $100 \mathrm{~cm}$ ); and the flame attachment length (defined as the $x$-wall-distance downstream of the burner within the flame region) increases (from 30 to $90 \mathrm{~cm}$ ). In the present configuration the transition from a vertical tilted flame to a horizontal flame is gradual and happens between $U_{\infty}=1$ and $1.5 \mathrm{~m} / \mathrm{s}$.

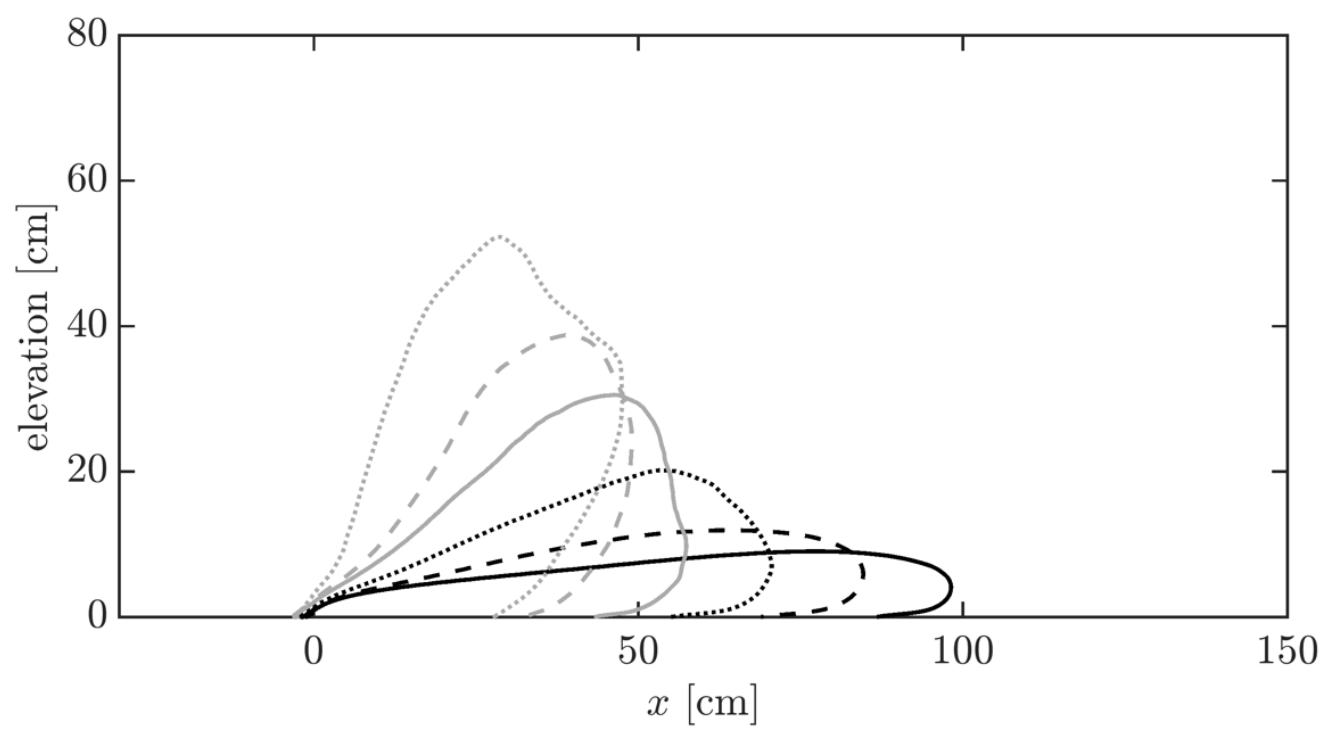

Figure 2 - Mean flame shape visualized using a particular isoline of the mean heat release rate per unit volume (50 $\mathrm{kW} / \mathrm{m}^{3}$ ). From left to right: $U_{\infty}=0.75,1,1.25,1.5,2,3 \mathrm{~m} / \mathrm{s}$.

We then consider the mean plume shape for the six different cases (Fig. 3). The plume, in contrast to the flame, transitions much more abruptly; for instance, the plume shape changes drastically (from vertically tilted to horizontal) when $U_{\infty}$ is increased from 1.5 to $2 \mathrm{~m} / \mathrm{s}$.

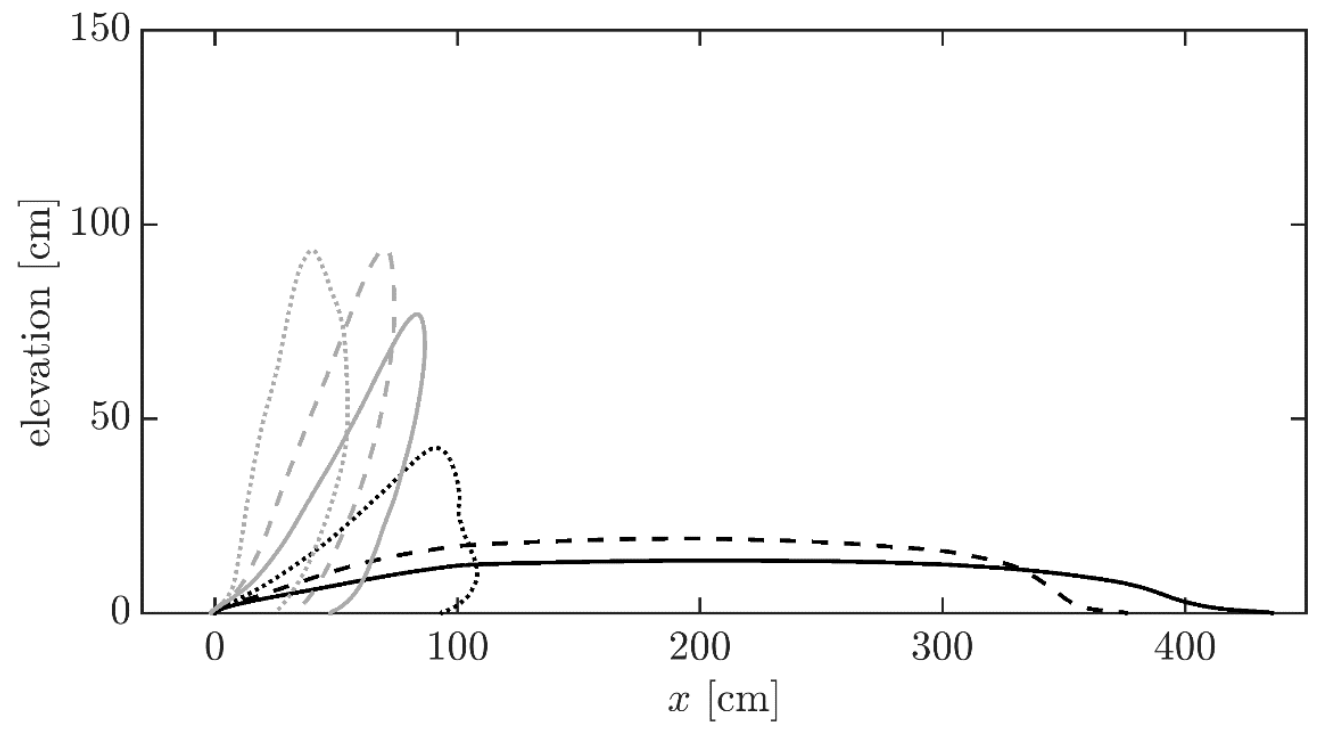

Figure 3 - Mean plume shape visualized using a particular isoline of the mean temperature (400 K). From left to right: $U_{\infty}=0.75,1,1.25,1.5,2,3 \mathrm{~m} / \mathrm{s}$.

We now consider the spatial variations of mean velocity vector $(\bar{U}, \bar{W})$ for two cases, $U_{\infty}=0.75$ and $3 \mathrm{~m} / \mathrm{s}$ (Fig. 4). In the $0.75-\mathrm{m} / \mathrm{s}$ case, the cross-flow is strongly deflected upwards; in addition, a reversed flow is induced by the flame on the downwind side leading to two-sided entrainment similar to pool 
fires. In contrast, in the 3-m/s case, the cross-flow is only weakly affected by the presence of the flame and entrainment is one-sided due to the flame being attached to the downstream surface. These differences in flow pattern are known to be key ingredients in flame spread mechanisms (Dold and Zinoviev 2009).
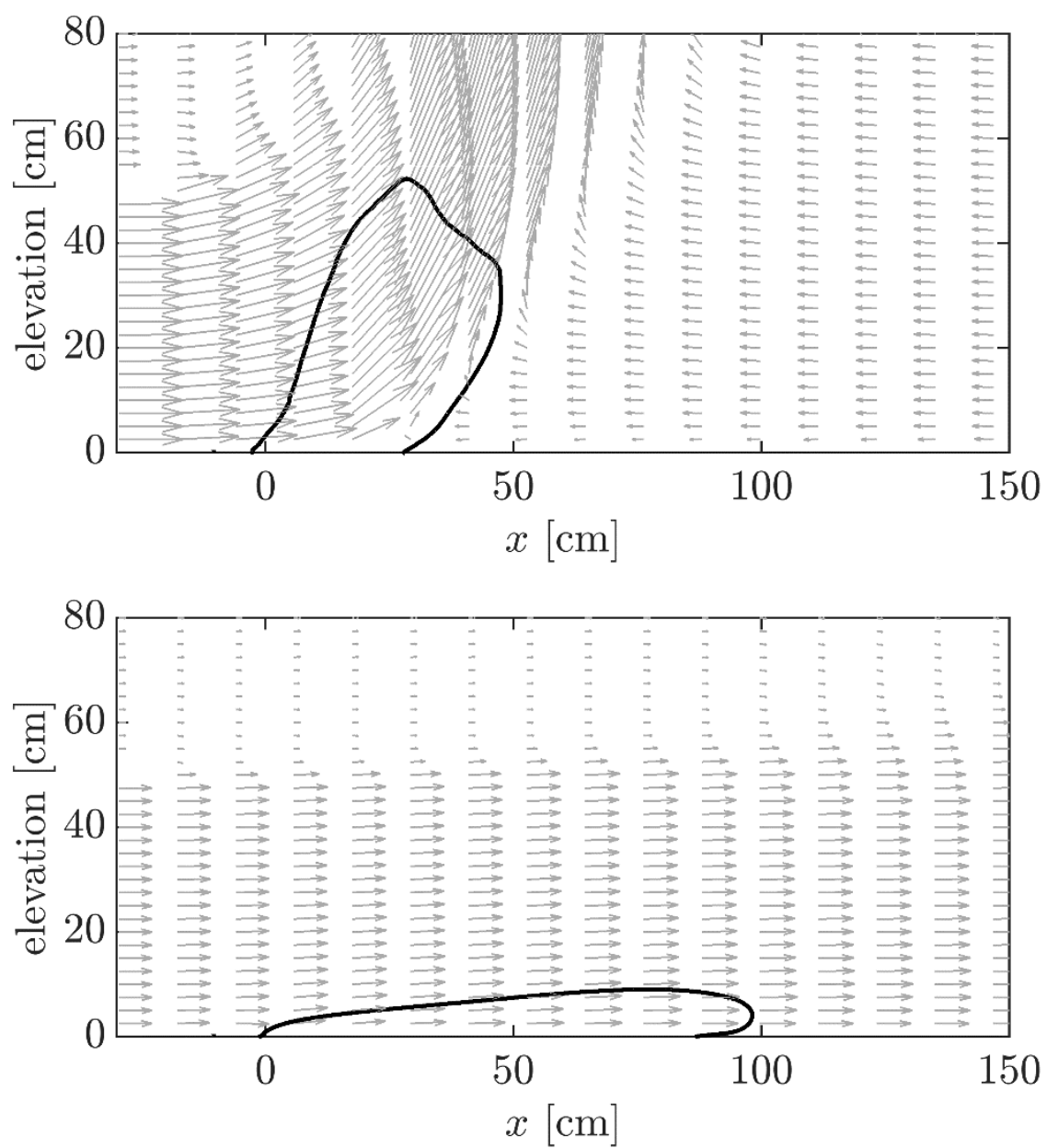

Figure 4 - Spatial variations of the mean flow velocity vector $(\bar{U}, \bar{W}): U \infty=0.75 \mathrm{~m} / \mathrm{s}$ (top); $U \omega_{\infty}=3 \mathrm{~m} / \mathrm{s}$ (bottom). Solid lines are isolines of the mean heat release rate per unit volume $(50 \mathrm{~kW} / \mathrm{m} 3)$ and are used for marking the flame region.

Figure 5 presents $z$-profiles of different quantities of interest $\left(\bar{U}, \bar{W}, U_{r m s}, W_{r m s}, B_{z}\right.$ and $\left.S_{x z}\right)$ across the flame zone, at $x=50 \mathrm{~cm}$ and for $U_{\infty}=1.5 \mathrm{~m} / \mathrm{s}$. $U_{r m s}\left(W_{r m s}\right)$ is defined as the mean amplitude of temporal fluctuations of the grid-resolved streamwise (vertical) flow velocity component; and $B_{z}$ and $S_{x z}$ are the source terms responsible for producing turbulent kinetic energy in the $z$ - and $x$ directions, respectively, $B_{z}=\left(-\bar{w}^{\prime \prime}(\partial \bar{p} / \partial z)\right)$ and $S_{x z}=\left(-\overline{\rho u} w^{\prime \prime}(\partial \tilde{u} / \partial z)\right)$, where $\bar{q}(\tilde{q})$ designates a straight (mass-weighted) temporal mean of a grid-resolved quantity $q$, and $q^{\prime \prime}=(q-\widetilde{q}) . B_{z}$ represents production of (vertical) turbulence by buoyancy whereas $S_{x z}$ represents production of (horizontal) turbulence by shear.

The case with $U_{\infty}=1.5-\mathrm{m} / \mathrm{s}$ is important because this flame is close to the point of transition between the horizontal and vertical flame regimes. Figure 5 shows that: turbulence production in the flame zone is dominated by buoyancy ( $B_{z}$ is much larger than $S_{x z}$ ); the flow activity in the vertical direction is strongly turbulent $\left(W_{r m s}>\bar{W}\right)$; and the vertical turbulent velocities take values that have the same order of magnitude as those of the cross-flow velocity $\left(W_{r m s}=\mathrm{O}\left(U_{\infty}\right)\right)$. These results suggest that flow deflection and the establishment of a vertically-oriented flame is a consequence of the vertical turbulent motions produced by buoyancy. 

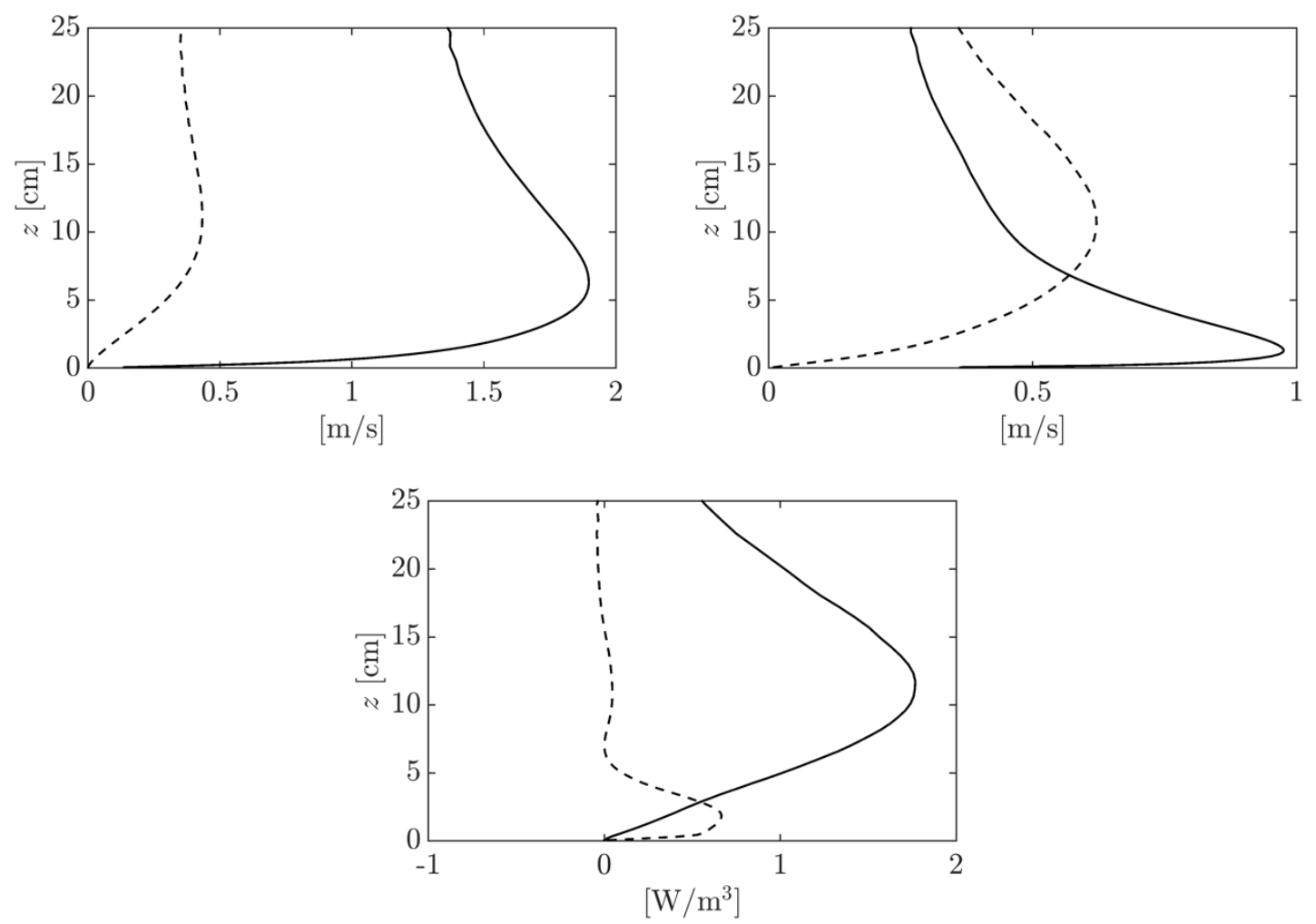

Figure 5 - Vertical profiles for $U_{\infty}=1.5 \mathrm{~m} / \mathrm{s}$ at $x=50 \mathrm{~cm}: \bar{U}$ and $\bar{W}$ (top left); $U_{r m s}$ and $W_{r m s}$ (top right); $B_{z}$ and $S_{x z}$ (bottom). $\bar{U}, U_{r m s}, B_{z}\left(\bar{W}, W_{r m s}, S_{x z}\right)$ are plotted using solid (dashed) lines.

The magnitude of this effect can be measured globally by spatially-integrating the source term $B_{z}$ and by comparing the resulting integrated value to the flow rate of kinetic energy (or equivalently the power) of the incoming cross-flow. We write:

$$
R=\frac{\left(\int_{0}^{L_{x}}\left(\int_{0}^{L_{z}} B_{z} d z\right) d x\right) L_{y}}{\frac{1}{2} \rho_{\infty} U_{\infty}^{3} L_{z} L_{y}}=\frac{\left(\int_{0}^{L_{x}}\left(\int_{0}^{L_{z}} B_{z} d z\right) d x\right)}{\frac{1}{2} \rho_{\infty} U_{\infty}^{3} L_{z}}
$$

where $R$ is the ratio of the power of the production of $z$-turbulence due to buoyancy divided by the power of the incoming cross-flow. In Eq. $1, \rho_{\infty}$ is the air mass density, and $L_{x}, L_{y}$ and $L_{z}$ are the $x$-, $y$ and $z$-sizes of the control volume under consideration (due to periodicity in the $y$-direction, $L_{y}$ is simply dropped).

Figure 6 presents the variations of the power ratio $R$ with streamwise distance using $L_{x}=x$ and $L_{z}=$ $H_{t}\left(H_{t}=50 \mathrm{~cm}\right.$ is the wind tunnel height $)$. With these choices for $L_{x}$ and $L_{z}, R$ represents the cumulative effects of production of vertical turbulence by buoyancy up to a given location $x$ compared to the total power of the flow delivered by the wind tunnel. We expect significant flow deflection and a transition from an upwind horizontal flame to a downwind vertical flame when $R$ takes values close to or above 1 prior to the end of the flame zone. Figure 6 shows that the $1.25-\mathrm{m} / \mathrm{s}-$ flame reaches a peak value of $R$ equal to 0.4 and that the $1-\mathrm{m} / \mathrm{s}$-flame reaches a peak value above 1 . These estimates suggest that the $1.25-\mathrm{m} / \mathrm{s}-\mathrm{flame}$ is in the transitional regime while the $1-\mathrm{m} / \mathrm{s}$ flame is in the vertical flame regime. 


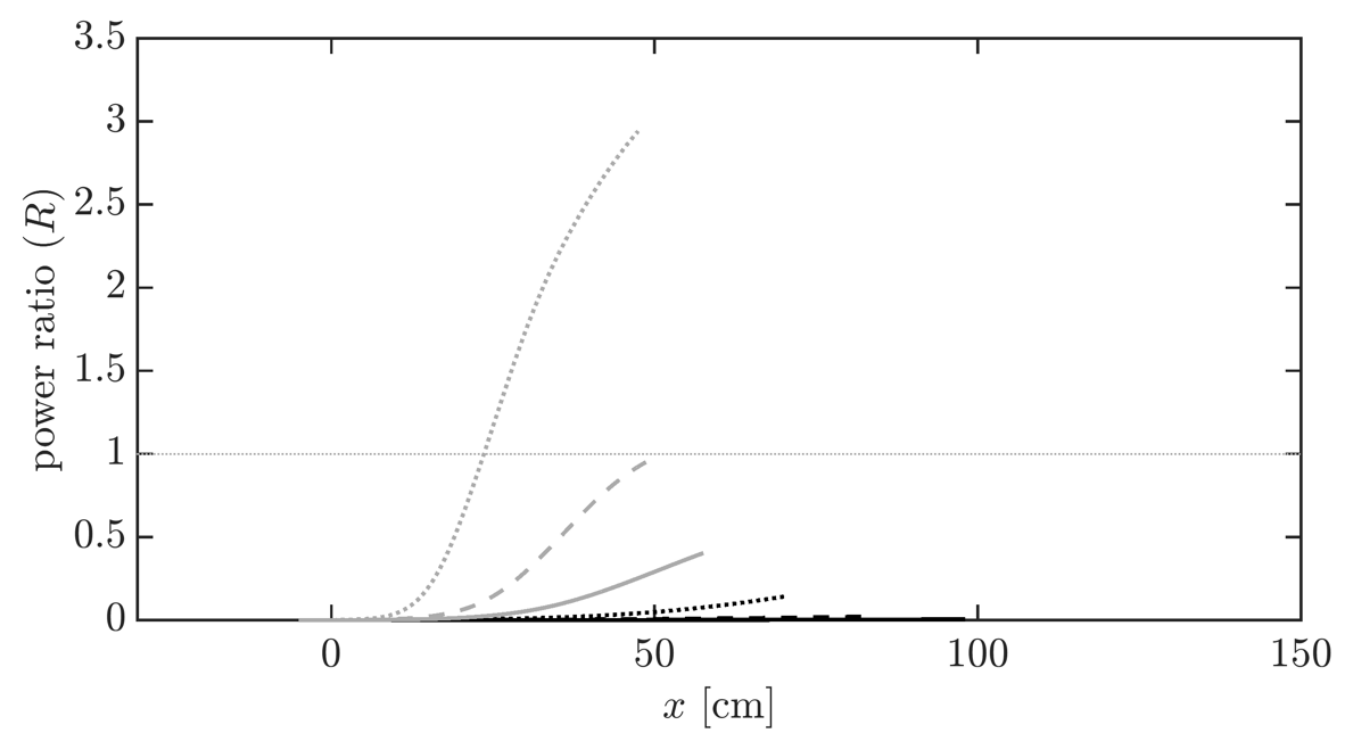

Figure 6 - Streamwise evolution of the power ratio $R$ (see Eq. 1), plotted from $x=0$ to the downstream $x$-location of the flame tip. From left to right: $U \infty=0.75,1,1.25,1.5,2,3 \mathrm{~m} / \mathrm{s}$; note that the 2-and 3-m/s curves correspond to low values of $R$ and are horizontal.

It is worth noting that the power ratio $R$ introduced in the present study is similar to, but different from Byram's convection number $N_{C}$, defined as:

$$
N_{C}=\frac{g I}{\frac{1}{2} \rho_{\infty} U_{\infty}^{3} c_{p} T_{\infty}}
$$

where $g$ is the acceleration of gravity, $I$ the fireline intensity (in the present configuration $I=100$ $\mathrm{kW} / \mathrm{m}), c_{p}$ the specific heat of ambient air at constant pressure and $T_{\infty}$ the ambient temperature. Like $R, N_{C}$ is a power ratio that compares the effect of buoyancy to that of the cross-flow (Nelson 1993); compared to $R, N_{C}$ has the advantage to explicitly bring out the effect of the fire power through $I$ (in the expression of $R$, this effect is implicit through $B_{z}$ ); but unlike $R, N_{C}$ does not account for $x$-variations and therefore cannot predict the streamwise location where a possible change in flame or plume structure may occur.

Using standard scaling arguments as well as some guidance from the simplified flame/plume models proposed by Albini (1981) and Nelson et al. (2012), a possible expression for $R$ is as follows:

$$
R=C_{R} N_{C}^{\frac{1}{2}}\left(\frac{g\left(\Delta T_{\text {flame }} / T_{\infty}\right) x}{U_{\infty}^{2}}\right)
$$

where $\Delta T_{\text {flame }}$ denotes the mean excess flame temperature and $C_{R}$ is a model coefficient. In the following, we use $\Delta T_{\text {flame }}=305 \mathrm{~K}$ and $C_{R}=0.057$.

Figure 7 presents a comparison of the model expression for $R$ given in Eq. (3) with its definition given in Eq. (1), as obtained from the LES simulations. It is found that the model expression for $R$ has some limitations: it varies linearly with $x$ and is not able to represent the higher order streamwise variations observed in the LES data. It is also found, however, that the expression in Eq. (3) is capable of capturing the changes in the values of the power ratio associated with changes in the cross-flow velocity. Work is currently in progress in order to determine whether the model expression for $R$ can be used to predict the streamwise location where a change in flame or plume structure will occur. 


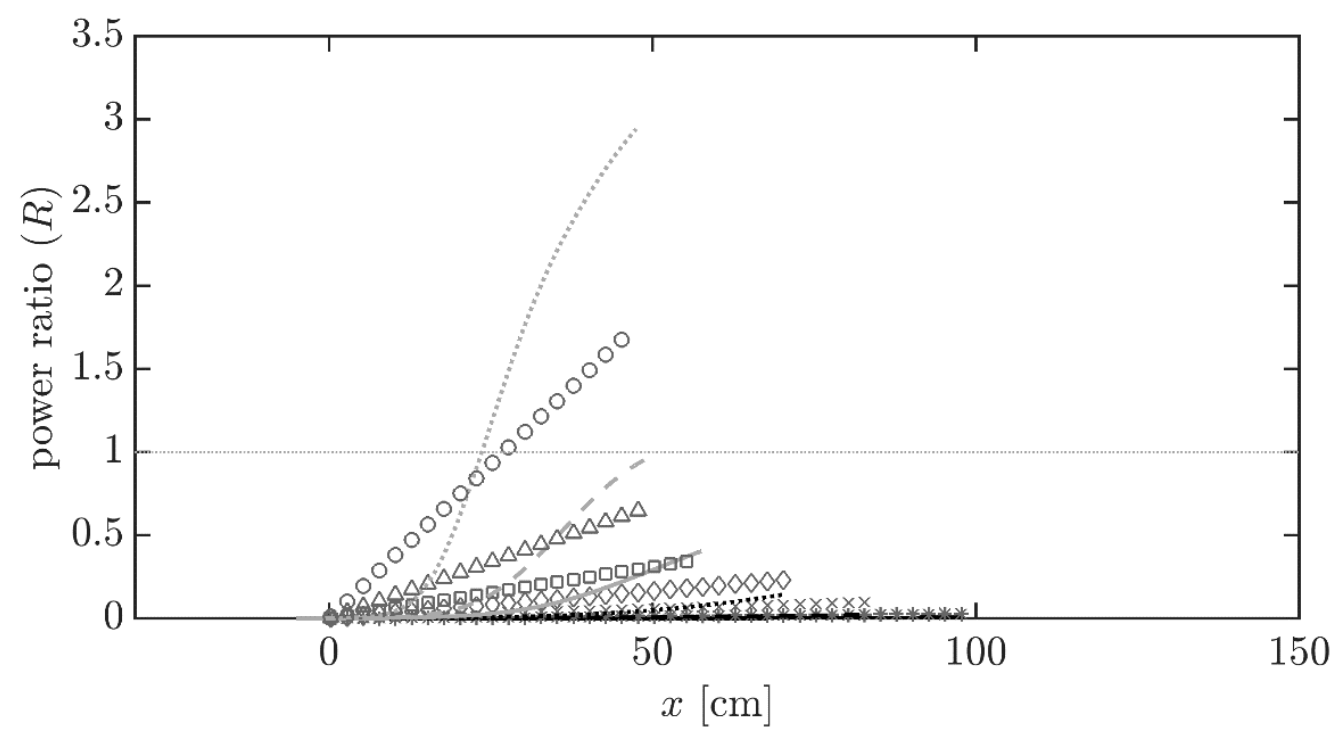

Figure 7 - Streamwise evolution of the power ratio $R$, plotted from $x=0$ to the downstream $x$-location of the flame tip. From left to right: $U \infty=0.75,1,1.25,1.5,2,3 \mathrm{~m} / \mathrm{s}$. Comparison between Eq. (1) (solid lines) and Eq. (3) (symbols).

\section{Conclusions}

Fine-grained LES are performed to bring fundamental insight into the effects of cross-flow velocity on the structure of a methane-air, buoyancy-driven, turbulent line flame $(50 \mathrm{~kW})$ stabilized on top of a horizontal floor surface. As the cross-flow velocity is increased, the flame transitions from a poollike flame characterized by a tilted vertical shape to a boundary layer flame characterized by a horizontal shape. The pool-like flame strongly deflects the incoming cross-flow upwards and features downwind flow separation and two-sided air entrainment into the flame. In contrast, the boundary layer flame does not significantly deflect the incoming cross-flow and features downwind flow attachment and one-sided air entrainment into the flame.

The present simulations are analyzed in terms of production of mean flow and turbulent flow kinetic energy. Results suggest that the transition between the (vertical) pool-like and (horizontal) boundary layer flame regimes is controlled by the strength of the vertical turbulent motions produced by buoyancy. A new non-dimensional number that measures the ratio of the power of the production of $z$-turbulence due to buoyancy divided by the power of the incoming cross-flow is introduced to explain the transition.

\section{Acknowledgments}

This project is financially supported by the U.S Forest Service, Rocky Mountain Research Station, with Dr. Mark Finney as Program Manager, and by FM Global, with Dr. Yi Wang as Program Manager. The project is also supported by supercomputing resources made available by the University of Maryland (http://hpcc.umd.edu) and by the U.S. National Science Foundation (XSEDE Program, Grant \# TG-CTS140046).

\section{References}

Albini FA (1981) A model for the wind-blown flame from a line fire. Combustion and Flame 43, 155174. 
Cole WJ, Dennis MH, Fletcher TH, Weise DR (2011) The effects of wind on the flame characteristics of individual leaves. International Journal of Wildland Fire 20, 657-667.

Dold JW, Zinoviev A (2009) Fire eruption through intensity and spread rate interaction mediated by flow attachment. Combustion Theory and Modelling 13, 763-793.

FireFOAM (2018) Developed by FM Global, available at: https://github.com/fireFoam-dev.

$\mathrm{Hu}$ L, Wu L, Liu S (2013) Flame length elongation behavior of medium hydrocarbon pool fires in cross air flow. Fuel 111, 613-620.

Lam CS, Weckman EJ (2015) Wind-blown pool fire, Part I: Experimental characterization of the thermal field. Fire Safety Journal 75, 1-13.

Magnussen BF, Hjertager BH (1977). On mathematical modeling of turbulent combustion with special emphasis on soot formation and combustion. Symposium (international) on Combustion 16, 719 729.

Morvan D, Porterie B, Larini M, Loraud JC (1998) Numerical simulation of turbulent diffusion flame in cross flow. Combustion Science and Technology 140, 93-122.

Morvan D, Porterie B, Loraud JC, Larini M (2001) A numerical investigation of cross wind effects on a turbulent buoyant diffusion flame. Combustion Science and Technology 164, 1-35.

Nelson RM (1993) Byram's derivation of the energy criterion for forest and wildland fires. International Journal of Wildland Fire 3, 131-138.

Nelson RM, Butler BW, Weise DR (2012) Entrainment regimes and flame characteristics of wildland fires. International Journal of Wildland Fire 21, 127-140.

Nicoud F, Ducros F (1999) Subgrid-scale stress modelling based on the square of the velocity gradient tensor. Flow, Turbulence and Combustion 62, 183-200.

Nmira F, Consalvi JL, Boulet P, Porterie B (2010) Numerical study of wind effects on the characteristics of flames from non-propagating vegetation fires. Fire Safety Journal, 45, 129-141.

Oka Y, Kurioka H, Satoh H, Sugawa O (2000) Modelling of unconfined flame tilt in cross-winds. Fire Safety Science 6, 1101-1112.

Oka Y, Sugawa O, Imamura T, Matsubara Y (2003) Effect of cross-winds to apparent flame height and tilt angle from several kinds of fire source. Fire Safety Science 7, 915-926.

OpenFOAM (2018) Developed by the OpenFOAM foundation, available at: http://www.openfoam.org.

Porterie B, Morvan D, Loraud JC, Larini M (2000) Firespread through fuel beds: modeling of windaided fires and induced hydrodynamics Physics of Fluids 12, 1762-1782.

Putnam AA (1965) A model study of wind-blown free-burning fires. Symposium (International) on Combustion 10, 1039-1046.

Sinai YL, Owens MP (1995) Validation of CFD modelling of unconfined pool fires with cross-wind: flame geometry. Fire Safety Journal 24, 1-34.

Tang W, Miller CH, Gollner MJ (2017) Local flame attachment and heat fluxes in wind-driven line fires. Proceedings of the Combustion Institute 36, 3253-3261.

Vasanth S, Tauseef SM, Abbasi T, Abbasi SA (2013) Assessment of four turbulence models in simulation of large-scale pool fires in the presence of wind using computational fluid dynamics (CFD). Journal of Loss Prevention in the Process Industries 26, 1071-1084. 


\title{
A two-dimensional reaction-advection-diffusion model of the spread of fire in wildlands
}

\author{
Paolo Grasso; Mauro S. Innocente \\ Smart Vehicles Control Laboratory (SVeCLab), Coventry University. UK. \\ \{grassop@uni.coventry.ac.uk, Mauro.S.Innocente@coventry.ac.uk\}
}

\begin{abstract}
The aim of this research is to develop a model of the spread of wildfires that is computationally efficient and easy-to-use, yet comprehensive enough to capture the major phenomena that govern the behaviour of a real fire. Namely, the pyrolysation of wood; the combustion of a mono-phase medium composed of premixed gas of fuel and air; the heat transferred by diffusion, convection and radiation considering emission and absorption of hot semi-transparent gases; and the thermal energy transport due to atmospheric wind and spatial distribution of vegetation. The model consists of two coupled partial differential equations, one representing the mass formation of each chemical species involved in the combustion, and the other ensuring the balance of enthalpy. The dimensionality reduction sought by modelling these three-dimensional (3D) phenomena in two-dimensional (2D) space is an intricate problem that has been overcome by means of pseudo-3D terms in the energy balance equation. For instance, the convection in the direction perpendicular to the 2D domain of the model has been represented as heat loss whose magnitude is linearly dependent on the temperature difference between the ambient and the simulation domain. Thus, the pseudo-3D convection term acts as a sink of thermal energy. It is important to note, however, that some environment properties have necessarily been disregarded in the interest of efficiency. Thus, the effect of topography on the spread of fire has been ignored in this model. In addition, the balance of momentum in the simulation plane is not included in the formulation as air density is considered independent of temperature and the wind velocity field is steady-state and uniform. Furthermore, the diffusion coefficient is augmented to account for the energy transport due to convection inside the flattened vegetal substrate, which is abstractly represented as a premixed gaseous layer. This augmentation has been calibrated for particular environment conditions such as the porosity of the fuel and its spatial distribution so that the model can be used as a tool for prediction of fire propagation. Making use of the $4^{\text {th }}$-Order Runge-Kutta method and running simulations for different constraints and boundary-initial conditions, results appear realistic. The presented fire-spread model is aimed at supporting the design of innovative fire management and suppression technologies and strategies, as well as to function as a decision-support tool to assist fire fighters in the use of current technology. Hence it must be both realistic and computationally efficient. Nonetheless, a more advanced 3D high-fidelity model is currently under development at the other end of the spectrum of the accuracy/efficiency trade-off with the aim to provide better insight into the fire dynamics.
\end{abstract}

Keywords: wildfire propagation, modelling, reaction, advection, diffusion, fuel, combustion, radiation, suppression.

\section{Introduction}

Modelling wild fires is a real challenge because of its complex combination of several interdependent phenomena acting at different length scales. Few researchers have attempted to handle this cumbersome problem. According to the methodologies utilised, the different fire models can be grouped in classes. Based on the mathematical formulation adopted, models can be classified as either continuous or cellular automata based (Sullivan, 2009, p.349; Sullivan, 2009, p.369; Papadopoulos and Pavlidou, 2010; Séro-Guillaume et al., 2008). The first group consists of models based on the laws of physics, with mathematical formulations in terms of coupled partial differential equations typically including balances of mass, momentum and energy. Instead, the cellular automata approach models the domain as a set of nodes that can have only a discrete number of states - e.g. unburnt, burning or 
burnt. In (Anez et al., 2017), a multilayer cellular automaton was used to model smouldering using three layers: the fuel (ash, char, dry fuel, wet fuel), the heat layer (heat release, no heat, heat losses), and oxygen (oxygen or nitrogen). This allowed a far more detailed estimation of the fire dynamics compared with the simpler burnt/burning/unburnt scheme. The evolution in time of the automata is determined by probabilistic transition laws that involve the state of the neighbouring nodes. The advantage of the cellular automata approach over the physics-based one is its considerably faster computation.

One of the most eminent continuous models of wildfire that can be found in the literature was developed by members of the Laboratoire d'Energetique et de Mechanique Theorique et Appliquee (LEMTA) (Séro-Guillaume and Margerit, 2002; Margerit and Séro-Guillaume, 2002). It is a threedimensional (3D) multiphase and multiscale wildfire model which elegantly depicts the fire phenomena from the smallest to the largest scale. In fact, they believe the repertoire of fire models should be classified according to the length-scale to be investigated: microscopic scale, where vaporisation and pyrolysis of the solid fuel are the main involved phenomena; mesoscopic scale, at which the geometry of the vegetation (leaves, branches, etc.) heavily affects the heat fluxes from solid to surrounding air; macroscopic scale, where the combustion occurs; and gigascopic scale, where the fire is driven by atmospheric wind and topography. A way to overcome this complexity and modelling at large scales in order to have a faster simulation is upscaling through averaging and by the convenient use of Large Eddy Simulation (LES) for turbulence modelling. But one of the most severe limitations of large-scale models are the errors associated with point-to-point comparison, which is critical given than the rate of spread is very sensitive to small variations (Houssami et al., 2018).

Furthermore, the available freeware simulators of wildfire can also be differentiated between continuous and cellular automata based models. The most well known simulators world-wide are FARSITE (Finney, 1998), Prometheus (Tymstra et al., 2010) and the Fire Dynamics Simulator (FDS) (McGrattan and Forney, 2004; McGrattan, 2006). The first two are based on the same Huygens principle for the determination of the fire front perimeter, which consists of the envelope contour of ellipses whose focuses are located in the previous step's fire-front, and whose shapes depend on many environmental parameters (e.g. wind) and fuel properties (e.g. moisture level). These fire simulators are mostly focused on determining the fire spread efficiently in order to assist fire management operations. On the other hand, FDS is more focused on capturing as many detailed features of fire phenomena as possible. It can be both 2D and 3D and makes use of the direct physics approach. SéroGuillaume et al. (Séro-Guillaume et al., 2008) acknowledge two main types of continuous approaches to modelling fire. The one adopted by Margerit et al. (Margerit and Séro-Guillaume, 2002) simplifies and averages from the most complex and detailed mathematical formulation to the most practical one that can be more easily implemented numerically. Conversely, the FDS approach starts from the simplest 3D gaseous-phase combustion model and adds complexity through the development of several submodels that are superposed to the main simulation and interact with it - e.g. volume-less Lagrangian particles (McGrattan, 2006) used to model solid firebrand particles floating in the air and exchanging mass, momentum and energy with the fluid while reacting with it. These submodels usually have mostly empirical bases, steming from extensive and expensive experimental observations. Some recent submodels either developed or under development are as follows:

- Correlation between the firebrand generation and the fire behaviour in order to determine fluxes, sizes and separation distances (Thomas et al., 2017, p. 864-871).

- Development of smouldering models (combustion of solid microporous media), both numerical (Anez et al., 2017) and empirical ones.

- Wildland-urban interface Fire Dynamics Simulator (WFDS), which is and extension of the American National Institute of Standards and Technology's (NIST) FDS. 
- Regarding upscaling techniques: determining the heat transfer coefficient between solid and fluid phases at macroscopic level using a surrogate model based on numerical experiments of the heat transfer throughout a fractal model of vegetation geometry (Collin and Lamorlette, 2012).

- Radiation, which is a very complex problem, especially if the whole spectrum is considered.

- Incomplete combustion models (mostly empirical) due to variation of oxygen flux (Thomas et al., 2017, p.855-863).

There is extensive debate upon which fire modelling approach is the best in capturing its dynamics. For instance, Papadopoulos (Papadopoulos and Pavlidou, 2010) states that FARISTE "is considered to be the most precise fire propagation simulation model by most of the researchers around the world", whilst Sullivan (Sullivan, 2009) claims that still FARSITE and Prometheus are the best simulators found in literature that can reproduce historical fires. At the same time, there are other research centres that focus their attention on the direct physics approach, proudly acclaiming its rigour in detecting the fire dynamics. For example, FDS is the result of 25 years of collaboration between many researchers across the world, whilst LEMTA's fire-spread models have been developed during 15 years of work. In line with (Séro-Guillaume et al., 2008), we are interested in classifying fire models according to their purpose, for instance to achieve a fast (possibly real-time) prediction or to capture some microscale phenomena.

The aim of this research is to model the spread of forest fire efficiently in order to support the design of innovative fire management and suppression technologies and strategies, while also comprising a decision-support tool to assist fire-fighters in their use of current technology. Therefore, the model must be at the same time efficient and comprehensive enough to capture the major phenomena that govern the behaviour of a real fire.

The proposed fire-spread model is given by a $2 \mathrm{D}$ reaction-advection-diffusion equation that describes the combustion of a mono-phase medium composed of premixed gas of fuel and air. It can be placed in the 2D direct-physics semi-empirical reaction-diffusion class of fire spread models, and it is similar to the Ferragut's (Ferragut et al., 2007) and Margerit's (Margerit and Séro-Guillaume, 2002) 2D models. By reducing dimensionality, some phenomena of fire dynamics are disregarded, e.g. the buoyancy due to density changes of the medium immersed in a gravitational field. The energy transport due to atmospheric wind is modelled, though the wind field is limited at this stage to be steady-state and uniform throughout the 2D space considered. The effect of the slope and that of the water vapour pressure are left to be accounted for in a 3D model that is currently being developed. Another simplifying assumption is that $2 \mathrm{D}$ convection is negligible, which is reasonable since the velocities induced by density variation in the horizontal plane are very small, with the resulting energy transport comprising less than $1 \%$ of the total energy fluxes. Considering that buoyancy is not modelled and assuming that the chemical reaction rate depends mostly on temperature and mole fractions of fuel and oxygen, the density variation due to temperature changes has no strong effect on the fire dynamics modelled. Hence density is assumed to be constant, and the momentum balance and mass conservation are not formulated. Thus, the mixed gases are confined within their original location, just as if pyrolysis gasses were to be burnt exactly where they had been released. The diffusion coefficient is corrected in this efficient model in order to account for the energy transport due to $2 \mathrm{D}$ convection inside the premixed layer - i.e. in the vegetation stratum. Furthermore, two pseudo-3D terms are added into the energy balance equation with the intention of modelling the energy losses due to convection and radiation in the direction perpendicular to the $2 \mathrm{D}$ domain being modelled (vertical direction). Horizontal radiation is modelled so as to affect only the neighbouring cells, since the cell size in the discretised domain can be set larger than the optical thickness or radiation absorption length. The heat capacity at constant pressure of each chemical species is considered to be constant 
and equal to an average value in the considered temperature range, from $T_{\mathrm{amb}}=293 \mathrm{~K}$ (ambient temperature) to approximately $1200 \mathrm{~K}$ (maximum temperature). Although, in the eventuality of strong wind, the temperature may rise to peaks of $1500 \mathrm{~K}$.

\section{The 2D Wildfire Spread Model}

The considered irreversible chemical reaction in (2.1) represents the combustion of the pyrolysis gasses in air, which is composed of oxygen, carbon dioxide, vapour and nitrogen.

$$
\mathrm{CH}_{4}+2 \mathrm{O}_{2} \rightarrow \mathrm{CO}_{2}+2 \mathrm{H}_{2} \mathrm{O}
$$

The fire-spread model can be represented by a system of five coupled partial differential equations, namely the enthalpy balance in (2.2) and the four equations in (2.3) for the consumption or formation of each chemical species (Fuel, $\left.\mathrm{O}_{2}, \mathrm{CO}_{2}, \mathrm{H}_{2} \mathrm{O}\right)$, i.e. $j=1, \ldots, 4$.

$$
\begin{gathered}
\frac{\partial T}{\partial t}=F_{T}\left(T, X_{j}\right) \\
\frac{\partial X_{j}}{\partial t}=F_{X_{j}}\left(T, X_{j}\right)
\end{gathered}
$$

Equation (2.2) is expanded in (2.4) where the phenomenon modelled by each term is clearly labelled, namely: 1) the combustion energy term including the combustion enthalpy $\left(h_{c}\right)$ and the reaction rate; 2) the transport term accounting for atmospheric wind; 3) the diffusion terms; 4) the pseudo-3D convection term accounting for energy loss by vertical convection; the $2 D$ radiation terms; and the pseudo-3D radiation term modelling vertical emission.

$$
\begin{gathered}
\rho c_{p} \frac{\partial T}{\partial t}=\overbrace{-\rho h_{c} \frac{M}{M_{1}} r}^{\text {Combustion }} \overbrace{-\rho c_{p} u_{i}^{*} \frac{\partial T}{\partial x_{i}}}^{\text {Diffusion }}+\ldots \\
\overbrace{k \frac{\partial}{\partial x_{i}}\left(\frac{1}{c_{p}} \frac{\partial c_{p} T}{\partial x_{i}}\right)+k \frac{\partial}{\partial x_{i}}\left(\frac{1}{c_{p}} \frac{\partial h_{c} T}{\partial x_{i}}\right)}^{\text {Transport }}+\ldots \\
\overbrace{\sigma \varepsilon\left[4 d x_{i} \frac{\partial}{\partial x_{i}}\left(T^{3} \frac{\partial T}{\partial x_{i}}\right)\right]}^{\overbrace{C_{a}\left(T_{\text {amb }}-T\right)}}+\ldots \overbrace{\sigma \varepsilon\left[\frac{T_{\text {amb }}^{4}-T^{4}}{d x_{3}}\right]}^{\text {pseudo3D-Radiation }} \\
\frac{\partial X_{i}}{\partial t}=-\frac{\theta_{i}}{\theta_{\text {fuel }}} \frac{M}{M_{\text {fuel }} r} ;
\end{gathered}
$$

In order to close the system composed by (2.4) and (2.5), additional closure equations are required, in particular for:

- The molar mass of the mixture $(M)$ in (2.6), which is the weighted summation of each chemical species molar mass $\left(M_{i}\right)$ by their mass fractions $\left(X_{i}\right)$.

- The constant pressure heat capacity of the mixture $\left(c_{\mathrm{p}}\right)$ in (2.7), which is the weighted summation of all the partial heat capacities $\left(c_{\mathrm{p} i}\right)$.

- The combustion rate $(r)$ following the Arrhenius law in (2.8), where the subscripts ' 1 ' stands for fuel and ' 2 ' for oxygen. The formulation is the empirical formula related to wood pyrolysis 
in (Ragland et al., 1991). Bear in mind the pre-exponential coefficient $\left(A_{\mathrm{r}}\right)$ for following discussion.

- The specific combustion enthalpy $\left(h_{\mathrm{c}}\right)$ in (2.9), which is the summation of all the formation enthalpies at the local temperature $(T)$.

$$
\begin{gathered}
M=\sum_{i=1}^{5} X_{i} M_{i} \\
c_{p}=\sum_{i=1}^{5} X_{i} \frac{M_{i}}{M} c_{p i} \\
r\left(T, X_{1}, X_{2}\right)=-\delta_{\left(T, X_{1,2}\right)}^{+} A_{r} T X_{1}^{0.5} X_{2} \exp \left(-\frac{T_{\mathrm{a}}}{T}\right) \\
h_{c}=\frac{H_{c}(T)}{M}=-\frac{1}{M} \sum_{i=1}^{5} \theta_{i} H_{i}(T)=\frac{1}{M} \sum_{i=1}^{5} \theta_{i}\left(H_{i, \mathrm{ref}}+M_{i} c_{p i}\left(T_{\mathrm{ref}}-T\right)\right)
\end{gathered}
$$

The conductivity $(k)$, the pre-exponential coefficient $\left(A_{r}\right)$ and the optical absorption length $\left(\mathrm{d} x_{i}\right)$ have been calibrated (see Table 2) using the results obtained by FARSITE simulator (Finney, 1998), not having any experimental data available at the moment. The representative values considered are the burnt area, radius, and the maximum temperature. Given that the burning (a.k.a. flaming) area can be smaller than the burnt area, the latter has been chosen for calibration purposes as the flaming area is very dynamic and its estimation affected by the numerical model used.

For instance, we performed two exemplar calibrations for two types of fuels structurally different: the values in Table 2 have been obtained for the fuel model FM3 (tall coarse grass) and FM10 (coniferous trees) (Scott and Burgan, 2005). More precisely, FM10 consists of $15 \mathrm{~m}$ trees with $4 \mathrm{~m}$ base, whose canopy coverage has been set to $100 \%$ in the presented simulations, with a density of $0.2 \mathrm{~kg} \cdot \mathrm{m}^{-}$ 3 . Moreover, the selected elevation is $300 \mathrm{~m}$ and the moisture level about $20 \%$. The remaining known parameters and some characteristic variables are listed in Table 1.

Please note that the optical absorption length, for radiation modelling, has to be smaller than or equal to the cell size in order for the model to be valid. Moreover, the asymptotic wind magnitude, above the vegetation stratum, has to be reduced in order to take the drag into account. Hence the modified wind velocity $\left(u_{i}^{*}\right)$ in $(2.4)$ is obtained by multiplying the wind velocity by a reduction coefficient $(w r)$, which is a function of the fuel type and of the canopy coverage. The higher the canopy coverage and the denser the fuel type, the smaller the reduction coefficient. For instance, by our calculations during calibration phase, $10 \mathrm{~m} / \mathrm{s}$ for FM10 corresponds to approximately $1.3 \times 10^{-3} \mathrm{~m} / \mathrm{s}$ in the premixed gas layer.

\section{Results and Discussion}

The simulations have been performed on a $100 \mathrm{~m} \times 100 \mathrm{~m}$ domain, and their results appear realistic from both qualitative and quantitative point of views. However, in order to use this simulation tool for prediction of fire propagation, the parameters of the model must be calibrated for particular environmental conditions, including fuel properties and spatial distribution. 
Table 1 - General settings for simulation of fire spread with fuel model FM10. (* calibrated parameters)

\begin{tabular}{l|l|r|l} 
Symbol & Description & Value & Units \\
\hline$R$ & Universal gas constant & 8.314 & $\mathrm{~J} \cdot \mathrm{mol}^{-1} \cdot \mathrm{K}^{-1}$ \\
\hline$T_{\mathrm{amb}}\left(T_{\mathrm{ref}}\right)$ & Ambient (reference) temperature & 298.15 & $\mathrm{~K}$ \\
\hline$T_{\mathrm{ig}}$ & Ignition temperature & 573 & $\mathrm{~K}$ \\
\hline$T_{\mathrm{a}}$ & Activation temperature & 684 & $\mathrm{~K}$ \\
\hline$P_{\mathrm{amb}}\left(P_{\mathrm{ref}}\right)$ & Ambient (reference) pressure & 101325 & $\mathrm{~Pa}$ \\
\hline$\rho$ & Gas mixture density & 1.2172 & $\mathrm{~kg} \cdot \mathrm{m}^{-3}$ \\
\hline$A_{\mathrm{r}}$ & Pre-exponential coefficient (Arrhenius) & $(*)$ & - \\
\hline$C_{\mathrm{a}}$ & Turbulent convection coefficient in atmosphere & 0.065 & $\mathrm{~J} \cdot \mathrm{m}^{-3} \cdot \mathrm{K}^{-1} \cdot \mathrm{s}^{-1}$ \\
\hline$k$ & Thermal conductivity & $(*)$ & $\mathrm{W} \cdot \mathrm{m}^{-1} \cdot \mathrm{K}^{-1}$ \\
\hline$c_{\mathrm{p}}$ & Specific heat capacity at constant pressure & calculated & $\mathrm{J} \cdot \mathrm{kg}^{-1} \cdot \mathrm{K}^{-1}$ \\
\hline$X_{\mathrm{f}}$ & Initial fuel molar fraction & 0.1 & - \\
\hline$\sigma$ & Stefan-Boltzmann constant & $5.6704 \mathrm{e}-8$ & $\mathrm{~W} \cdot \mathrm{m}^{-2} \cdot \mathrm{K}^{-4}$ \\
\hline$\varepsilon$ & Emissivity factory (<1) & 0.55 & - \\
\hline $\mathrm{d} x_{i}$ & Optical absorption length & $(*)$ & $\mathrm{M}$ \\
\hline $\mathrm{d} x_{3}$ & Optical absorption length in z direction & 1 & $\mathrm{M}$ \\
\hline$h_{c}$ & Specific combustion enthalpy & calculated & $\mathrm{J} \cdot \mathrm{kg}^{-1}$ \\
\hline$w r$ & Wind reduction coefficient & $(*)$ & - \\
\hline$\Delta t$ & Time step & 7.5 & $\mathrm{~S}$ \\
\hline$\Delta x=\Delta y$ & Cell-size & 1 & $\mathrm{M}$
\end{tabular}

Table 2 - Values of parameters describing the fuel model FM3 and FM10.

\begin{tabular}{c|c|c|c} 
Symbol & FM3 & FM10 & Units \\
\hline$k$ & $5.5703 \mathrm{e}-01$ & $6.3750 \mathrm{e}-02$ & $\mathrm{~W} \cdot \mathrm{m}^{-1} \cdot \mathrm{K}^{-1}$ \\
\hline$A_{\mathrm{r}}$ & $4.1699 \mathrm{e}-05$ & $6.6250 \mathrm{e}-06$ & - \\
\hline $\mathrm{d} x_{i}$ & 0.4 & 0.1 & $\mathrm{~m}$ \\
\hline$w r$ & $7.5106 \mathrm{e}-5$ & $1.3075 \mathrm{e}-04$ & -
\end{tabular}

Figure 1.a shows the classical ring-shaped flame front, which has been used for calibration purposes, spreading radially from the ignition points at the same rate for uniformly distributed fuel and no wind. In case of both single and multiple ignition points and zero wind condition, the estimation error of the burnt area (with respect to FARSITE simulation) is less than $1 \%$.

In turn, Figure 1.b shows the energising effect of wind: the flame front is distorted, the area affected by the fire is enlarged, and the maximum temperature increases (from $1215 \mathrm{~K}$ to about $1297 \mathrm{~K}$ in this case). The presence of wind results in a 'teardrop' shape of the fuel consumption due to the asymmetric spread of the fire, as can be observed in Figure 2.b. In presence of wind and with single/multiple ignition points, the estimation error of the burnt area oscillates between less than $1 \%$ to approximately $30 \%$ (for very intense wind of up to $80 \mathrm{~m} / \mathrm{s}$ speed). The stronger the wind, the larger the discrepancies between FARSITE simulations and the presented model simulations. It is hard to assess whether one or the other are the most correct simulator of reality, but for sure a large part of the error is inherent to the mathematical formulation and the numerical methods that have been used, let alone the dimensionality reduction. It is interesting to note that in those regions where flames persist in the same location, for instance at zero wind condition or in particular stagnation points, the fuel mass fraction 
tends to zero (Figure 2.a) whereas the fuel tends to be only partially consumed in the presence of wind (Figure 2.b) as the flame is blown away from the original ignition points.
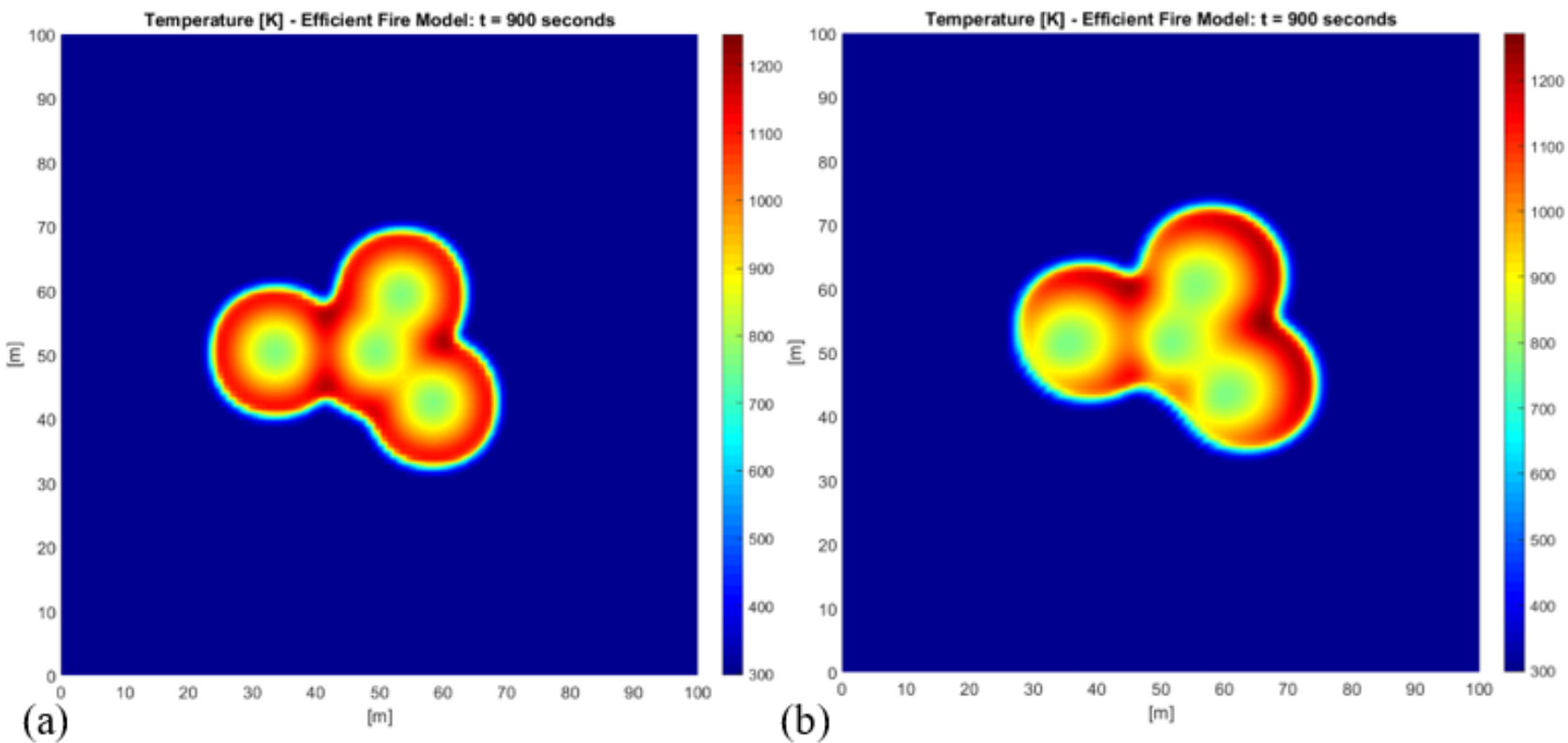

Figure 2 - Flaming Area: Temperature field at 15 min after four-points random ignition (a) without wind and (b) with strong wind of $40 \mathrm{~m} / \mathrm{s}$ for FM10.
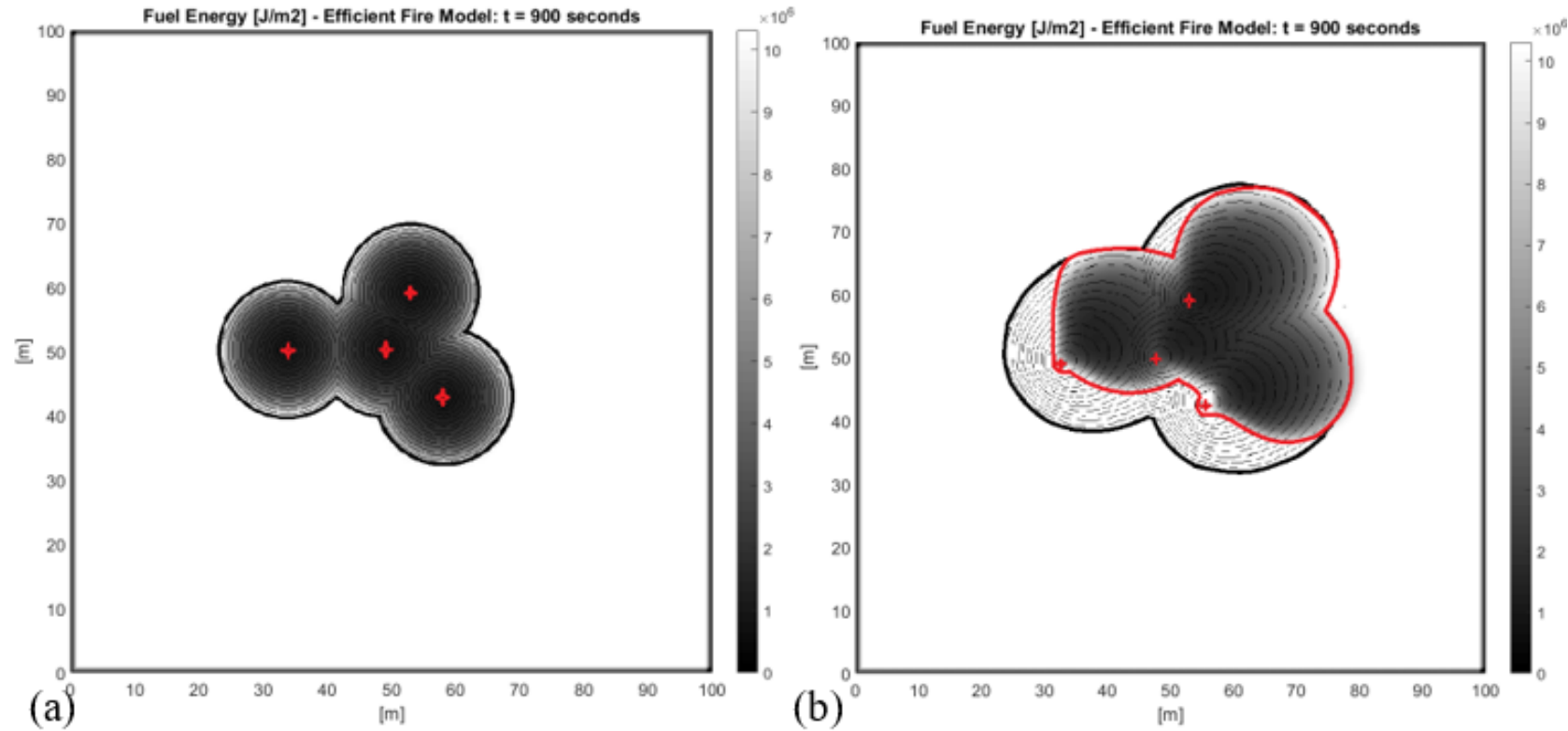

Figure 3 - Burnt Area: Distribution of fuel energy at 15 min after four-points random ignition (a) without wind and (b) with strong wind of $40 \mathrm{~m} / \mathrm{s}$ for FM10. The red stars represent the ignition points (the same of Figure 1), and black areas correspond to zero-fuel condition. The thick black contour corresponds to FARSITE prediction, while the red one is obtained by the presented model.

\section{Conclusion}

The output of the presented research is a successful attempt to simulate efficiently and with a high level of accuracy the spread of wildfires with the use of a macroscopic two-dimensional reaction- 
advection-diffusion model. The developed model shows its versatility to be calibrated for any type of fuel models, tackling any environmental condition (except for topography) - e.g. moisture and wind. Thanks to its simplicity and computational lightweight (e.g. on a standard quad-core i7 computer, it predicts $1 \mathrm{~min}$ of fire dynamics in approximately $1 \mathrm{sec}$ computational time for $10^{4}$ unit cells), this model has been coupled with a swarm of drones (Innocente and Grasso, 2018) in order to develop an autonomous technology to either extinguish or mitigate wildfires. The use of this robust technology aims to considerably lower the risks to human lives during firefighting operations. Thus, the presented model is both realistic and computationally efficient. Nonetheless, a more advanced and intensive 3D high-fidelity model is currently under development with the aim to provide more accurate insight into the fire dynamics.

\section{References}

Anez NF, Christensen K and Rein G (2017) Two-dimensional model of smouldering combustion using multi-layer cellular automaton: The role of ignition location and direction of airflow. IAFFS $12^{\text {th }}$ Symposium. Fire Safety Journal.

Collin A and Lamorlette A (2012) Quantification of convective heat transfer inside tree structures. Journal of Physiscs: Conference Series, IOP Publishing, 395, 012145

Ferragut L, Asensio M and Monedero S (2007) A numerical method for solving convection-reactiondiffusion multivalued equations in fire spread modelling. Advances in Engineering Software, vol. 38, no. 6, pp. 366-371

Finney MA (1998) FARSITE: Fire Area Simulator-Model development and evaluation. USDA Forest Service, Rocky Mountain Research Station Research Paper RMRS-RP-4. (Ogden, UT)

Houssami ME, Lamorlette A, Morvan D, Hadden RM and Simeoni A (2018) Framework for submodel improvement in wildfire modeling. Combustion and Flame, Elsevier BV, 190, 12-24

Innocente MS, Grasso P (2018) Swarms of autonomous drones self-organised to fight the spread of wildfires. Proceedings of the RSFF'18 Workshop, L'Aquila, Italy

Margerit J and Séro-Guillaume O (2002) Modelling forest fires. Part II: reduction to two-dimensional models and simulation of propagation. International Journal of Heat and Mass Transfer, vol. 45, no. 8, pp. 1723-1737, 2002.

McGrattan KB and Forney GP (2004) Fire dynamics simulator User's Guide (version 4). VTT \& NIST, National Institute of Standards and Technology

McGrattan KB (2006) Fire dynamics simulator Technical Reference Guide Volume 1: Mathematical Model (version 4). VTT \& NIST, National Institute of Standards and Technology

Papadopoulos GD and Pavlidou FN (2010) A Comparative Review on Wildfire Simulators. International Scientific Journal

Ragland KW, Aerts DJ and Baker AJ (1991) Properties of Wood for Combustion Analysis. Bioresource Technology

Sullivan AL (2009) Wildland surface fire spread modelling, 1990-2007. 1: Physical and quasiphysical models. International Journal of Wildland Fire, CSIRO Publishing, 18 , 349

Sullivan AL (2009) Wildland surface fire spread modelling, 1990-2007. 2: Empirical and quasiempirical models. International Journal of Wildland Fire, CSIRO Publishing, 18 , 369

Scott JH and Burgan RE (2005) Standard Fire Behavior Fuel Models: A Comprehensive Set for Use with Rothermel's Surface Fire Spread Model. USDA Forest Service, Rocky Mountain Research Station Research Paper RMRS-GTR-153. (Ogden, UT) 
Séro-Guillaume O and Margerit J (2002) Modelling forest fires. Part I: a complete set of equations derived by extended irreversible thermodynamics. International Journal of Heat and Mass Transfer, vol. 45, no. 8, pp. 1705-1722

Séro-Guillaume O, Ramezani S, Margerit J and Calogine D (2008) On large scale forest fires propagation models. International Journal of Thermal Sciences

Thomas JC, Mueller EV, Santamaria S, Gallagher M, Houssami ME, Filkov A, Clark K, Skowronski N, Hadden RM, Mell W and Simeoni A (2017) Investigation of firebrand generation from an experimental fire: Development of a reliable data collection methodology. Fire Safety Journal, Elsevier BV, 91 , 864-871

Thomas JC, Hadden RM and Simeoni A (2017) Experimental investigation of the impact of oxygen flux on the burning dynamics of forest fuel beds. Fire Safety Journal, Elsevier BV, 2017 , 91, 855863

Tymstra C, Bryce RW, Wotton BM, Taylor SW and Armitage OB (2010) Development and Structure of Prometheus: the Canadian Wildland Fire Growth Simulation Model - Information Report NORX-417. Northern Forestry Centre \& Canadian Forest Service, Northern Forestry Centre \& Canadian Forest Service 


\title{
An experimental investigation of the effect of grass fuel load on grassfire behaviour
}

\author{
Miguel G. Cruz*; Andrew L. Sullivan; James S. Gould, Richard J. Hurley, Matt P. Plucinski \\ CSIRO. GPO Box 1700, Canberra, ACT 2601, Australia, \{miguel.cruz@csiro.au*\}
}

\begin{abstract}
The effect of grass fuel load on fire propagation, behaviour and fire danger has been a contentious issue for some time in Australia. Various implementations of fire behaviour and fire danger formulations have placed different emphases on the effect of fuel load on model outputs, which has created uncertainty in the operational assessment of fire potential and has led to user and public distrust in the model outputs.

A field-based experimental burning program was conducted to quantify the effect of fuel load on the rate of spread and behaviour of fire in grasslands. A total of 58 experimental fires ( $33 \times 33 \mathrm{~m}$ plots) conducted at six sites across Australia were analysed. Mean fuel loads ranged between $0.17 \mathrm{~kg} / \mathrm{m}^{2}$ and $1.05 \mathrm{~kg} / \mathrm{m}^{2}$. Fire spread rate and fireline intensity ranged between 12.5 and $150 \mathrm{~m} / \mathrm{min}$ and 1260 and $18700 \mathrm{~kW} / \mathrm{m}$, respectively.

Contrary to current modelling assumptions used in Australia, we found an inverse relationship between fuel load and fire spread rate in grasslands; i.e. as fuel load increases, fire rate of spread decreases. This result is valid for grasslands where fuel load is not a limiting factor for fire propagation (e.g., load $>0.05 \mathrm{~kg} / \mathrm{m}^{2}$ ). We model the effect of fuel load on the rate of fire spread through regression analysis and an iterative backtracking method to produce a fuel load function that can be applied to current recommended operational grassfire spread models used in Australia.

We found that areas with standing continuous grass have the potential to sustain very high rates of fire spread, regardless of the fuel load. An increase in fuel load does not result in an increase in rate of fire spread, as assumed in certain modelling frameworks and some long held beliefs in Australia.
\end{abstract}

Keywords: fire behaviour modelling, fire prediction, fire danger rating

\section{Introduction}

The effect of grass fuel load on fire behaviour and grassfire danger index has been a contentious issue for many years in Australia. Early grassfire behaviour prediction tools used in Australia assumed the rate of forward spread of a fire to be directly proportional to fuel quantity (McArthur 1960). This effect was later removed (McArthur 1966) due to the (1) absence of direct evidence and; (2) the dependence of the biomass quantity in a grass sward on the structural properties of the grass species, i.e., higher fuel load grasses are typically characterized by coarser, lower surface area-to-volume ratio, fuel particles (Luke and McArthur 1978). Luke and McArthur (1978, p. 117) suggested that the effects of these two variables might cancel each other, and a direct effect of fuel load on fire spread rate might not be observable. In the early 1980s, a fuel load effect was retro-fitted to the Mk 4 Grassland Fire Danger Meter (Purton 1982) which confused the issue further. Cheney et al. (1993) undertook a large field experiment and found that fuel load did not have a significant effect on grassfire rate of spread. Despite these findings, a fuel load effect has continued to be applied in operational decision support tools in Australia (e.g. Purton 1982; Setterfield et al. 2014).

The objective of our study was to quantify the effect of grass fuel bed structure and biomass across a wide range of values on the rate of forward spread and flame characteristics of free-burning grassfires in Australia. 


\section{Methods}

Experimental fires were conducted at six locations across eastern Australia. Each site was characterised by a continuous cover of standing grass. Experimental fires were conducted in plots 33 $\times 33 \mathrm{~m}$, separated by a $3 \mathrm{~m}$ wide mown grass fuel breaks. All experimental fires used in the analysis were conducted in fully cured grasses.

Fires were ignited on the upwind edge of each plot with a line ignition. This was achieved by two people with hand-held drip-torches starting from the adjacent corners and travelling towards the centre of the plot edge. Ignition lines were offset when wind direction was not perpendicular to the plot boundary, but maintaining the ignition line length. It generally took 10-12 sec to set a continuous flame front that would ensure a well-developed headfire and pseudo-steady state propagation by the time the fire reached half of its intended run (Fig. 1).

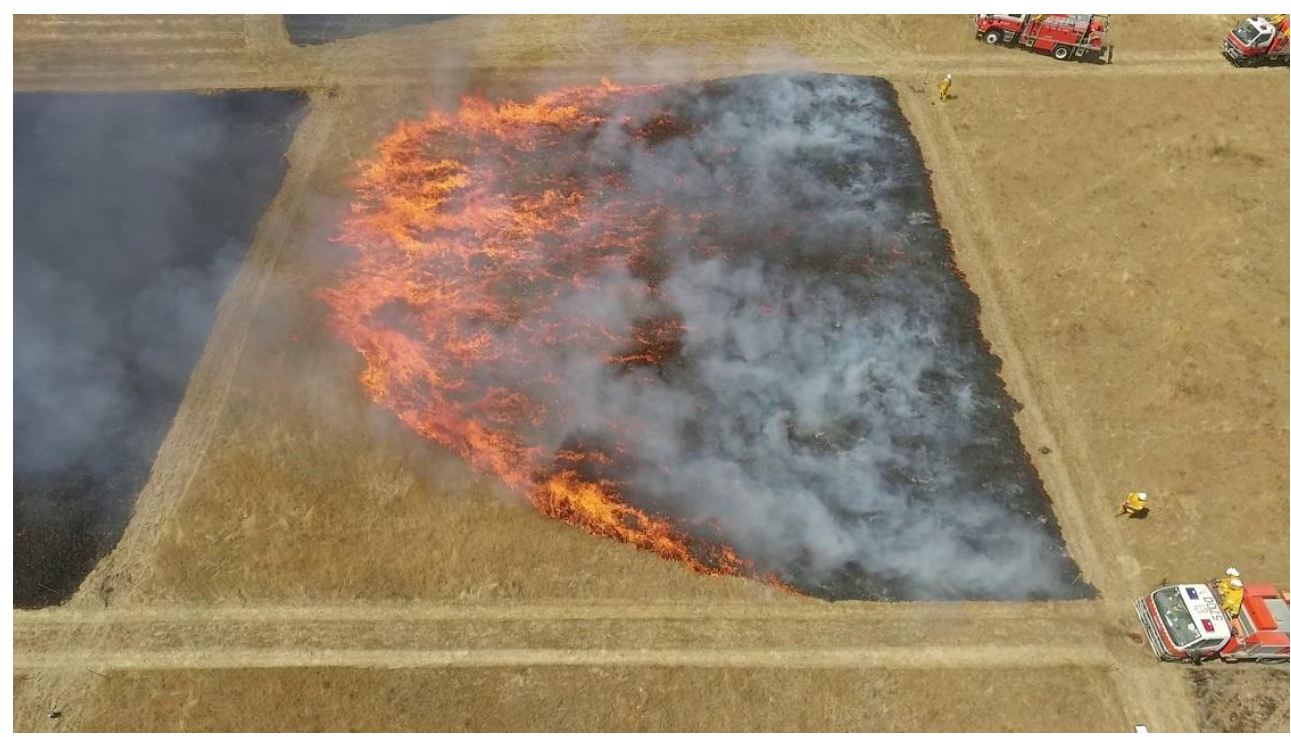

Figure 1 - Typical high intensity fire propagation from a line source ignition in a grassland fire experimental plot. Photos was taken 30 seconds after the ignition start.

Grass fuels were sampled for physical structure (load, height, compactness). An automatic weather station measuring air temperature, relative humidity and solar radiation at $1.5 \mathrm{~m}$ above ground and at 10-min intervals was established at each experimental site. At this location wind speed and direction at 2 and 10-m height were measured with 2-D sonic anemometers sampled and logged at a frequency of $10 \mathrm{~Hz}$. For each individual burn, one 2-D sonic anemometer at 2- $\mathrm{m}$ height was located approximately $35 \mathrm{~m}$ up-wind of the ignition line, with wind speed and direction sampled and logged at $10 \mathrm{~Hz}$. Prior to each experimental fire, three fuel moisture content samples (20-30 g) were collected and sealed in airtight metal tins. The behaviour of each experimental fire was monitored using in-situ instrumentation, ground-based and aerial videography and visual observations by experienced fire behaviour observers. The observers in close proximity to the fire monitored various flame front characteristics (i.e. depth, height and angle), fuel responsible for propagation and the duration of indraught winds (i.e. lulls in fire propagation) throughout the duration of each experimental fire.

Rate of fire spread (R, m/min) was determined from measurements of the time of fire arrival at grid points (grid spacing $7.5 \times 7.5 \mathrm{~m}$ or $10 \times 10 \mathrm{~m}$ ) within each experimental plot and video imagery. Time of fire arrival was assumed to coincide with a temperature of $320^{\circ} \mathrm{C}$ measured by $1.5-\mathrm{mm}$ diameter, type $\mathrm{K}$ metal-sheathed thermocouples connected to small dataloggers logging at $1 \mathrm{~Hz}$. Video imagery 
captured from remotely piloted aircraft (i.e. drones) or from the top of 5- or 10-m poles were used to determine flame movement from which rate of spread could be visually estimated.

Statistical analysis used the R 2.13.1 software package (R Development Core Team 2012). The effect of fuel load and other fuel characteristics in rate of fire spread was explored using two distinct approaches. The first approach was based on linear and nonlinear regression analysis. Because the fuel variable effect on grassland rate of fire spread determined through this approach cannot be readily incorporated into existing fire spread models used in Australia a second approach was used to develop a multiplicative function that (1) described the effect of fuel load or bulk density on rate of fire spread; and (2) could be implemented into the current operational model for undisturbed grasslands (Cheney et al. 1998). In this approach the Cheney et al. (1998) model was used to predict the experimentally observed rate of fire spread, and a fuel effect function added to the model to explain the effect of fuel load or bulk density. From the possible functional forms, an iterative process was used to find the formulation that result on the minimisation of both the root mean square error and bias while still producing adequate results outside the bounds of the experimental data.

\section{Results}

A total of 58 experimental fires were available for the analysis. Total fuel load at the plot level ranged from a minimum of $0.17 \mathrm{~kg} / \mathrm{m}^{2}$ to $1.05 \mathrm{~kg} / \mathrm{m}^{2}$. Observed rate of fire spread varied between 12.5 and $150 \mathrm{~m} / \mathrm{min}$. The dataset had 16 fires ( $25 \%$ of the data) with a fireline intensity above 10000 $\mathrm{kW} / \mathrm{m}$.

Rate of fire spread was significantly correlated with wind speed $(\mathrm{p}<0.001$; Table 3$)$ and had a significant inverse relationship with fuel load $(\mathrm{r}=-0.50 ; \mathrm{p}<0.001)$. It was poorly correlated with bulk density $(r=-0.19 ; p>0.05)$ and sampled dead fuel moisture $(r=0.12 ; p=0.37)$.

Multiple linear regression analysis was used to develop a model using 2-m wind speed, dead fuel moisture content and a fuel structure metric, fuel load, fuel bed bulk density or fuelbed height. For this modelling approach wind speed was highly significant ( $\mathrm{p}$ 0.001). Sampled dead fuel moisture was not a significant predictor $(\mathrm{p}=0.76)$, but the estimated dead fuel moisture was marginally significant $(p=0.09)$. Fuel load was a significant predictor of rate of fire spread $(p=0.02)$ through an inverse relationship (i.e. an increase in fuel load results in a reduction in rate of fire spread). This model explained $71 \%$ of the variation in the dataset. Fuel bulk density was also a significant predictor of rate of spread $(\mathrm{p}=0.02)$. A similar model with fuel bed height found this variable not to be significant $(\mathrm{p}$ $=0.41)$.

Non-linear regression analysis identified both fuel load $\left(w, \mathrm{~kg} / \mathrm{m}^{2}\right)$ and fuel bed bulk density $\left(\rho_{b}\right.$, $\left.\mathrm{kg} / \mathrm{m}^{3}\right)$ in isolation as significant $(\mathrm{p}<0.05)$ predictors of rate of fire spread. The best model form that had these variables incorporated through a power function:

$$
\begin{aligned}
& R=5.03 U_{2}^{0.89} \exp \left(-0.016 M_{e}\right) w^{-0.23} \\
& R=3.62 U_{2}^{1.05} \exp \left(-0.005 M_{e}\right) \rho_{b}{ }^{-0.32}
\end{aligned}
$$

Where $U_{2}$ is wind speed $(\mathrm{km} / \mathrm{h})$ measured at 2-m height and $M_{e}$ is the dead fuel moisture content (\%) estimated using Cheney et al. (1998) model parameterization of McArthur (1960) fuel moisture tables. The analysis showed both fuel parameters to have a negative effect on the rate of fire spread. Both models had a wind exponent close to 1 . The estimated dead fuel moisture was not significant in either model, although a stronger effect was found for the fuel load model (Eq. 1). The model with 
bulk density (Eq. 2) had a comparable fit as indicated by the residual standard error (14.99 and 15.27, respectively) and the mean absolute error (11.8 and $11.7 \mathrm{~m} / \mathrm{min})$.

Given the comparable effect of fuel load and fuel bed bulk density on rate of fire spread, we decided to use fuel load, a variable of simpler estimation, to develop a fuel structure effect function for operational applications. For this modelling approach we first applied the Cheney et al. (1998) undisturbed grassland model to the experimental dataset. For this simulation $U_{2}$ was converted to $U_{10}$ using a wind reduction factor of 0.81 , as determined from vertical wind profiles (Cruz et al. 2017). The fuel moisture content used in the simulations is the one estimated by Cheney et al. (1989) used in Cheney et al. (1998) model parameterization. The model predicted the observed rate of fire spread with a mean absolute error of $19.3 \mathrm{~m} / \mathrm{min}$, and a mean bias error of $1.28 \mathrm{~m} / \mathrm{min}$. Residual analysis revealed a relatively high over-prediction bias for fuel loads greater than $0.6 \mathrm{~kg} / \mathrm{m}^{2}$ and an under prediction bias for fires burning with estimated fuel moisture contents greater than $10 \%$.

To develop a fuel load function we tested several functional forms available. From the ones tested we choose to model the fuel load effect with a Ricker function due to its form, with an initial growth up to a local maximum followed by a gradual decrease:

$$
\Phi(\mathrm{w})=\mathrm{r}_{1}+\mathrm{r}_{2} w \exp \left(-\mathrm{r}_{3} w\right)
$$

where $\Phi(w)$ is the fuel load effect function, and $\mathrm{r}_{2}$ and $\mathrm{r}_{3}$ are the two parameters in the Ricker function. To find the best estimates for $r_{1}, r_{2}$ and $r_{3}$ we apply the fuel load function to the Cheney et al. (1998) model as a multiplicative factor.

The model was then run iteratively with coefficients $r_{2}$ and $r_{3}$ varying systematically between 1 and 5 (increments of 0.1) and 2 and 4 (increments of 0.1), respectively. The best results (lower RMSE and lowest bias error) were found for an $r_{1}$ of $0.7, r_{2}$ of 2.4 and $r_{3}$ of 3.4 (Fig. 2). These coefficients resulted in a root mean square error of 1.31 ; a mean absolute error of $17.7 \mathrm{~m} / \mathrm{min}$ and mean bias error of -1.4 $\mathrm{m} / \mathrm{min}$.

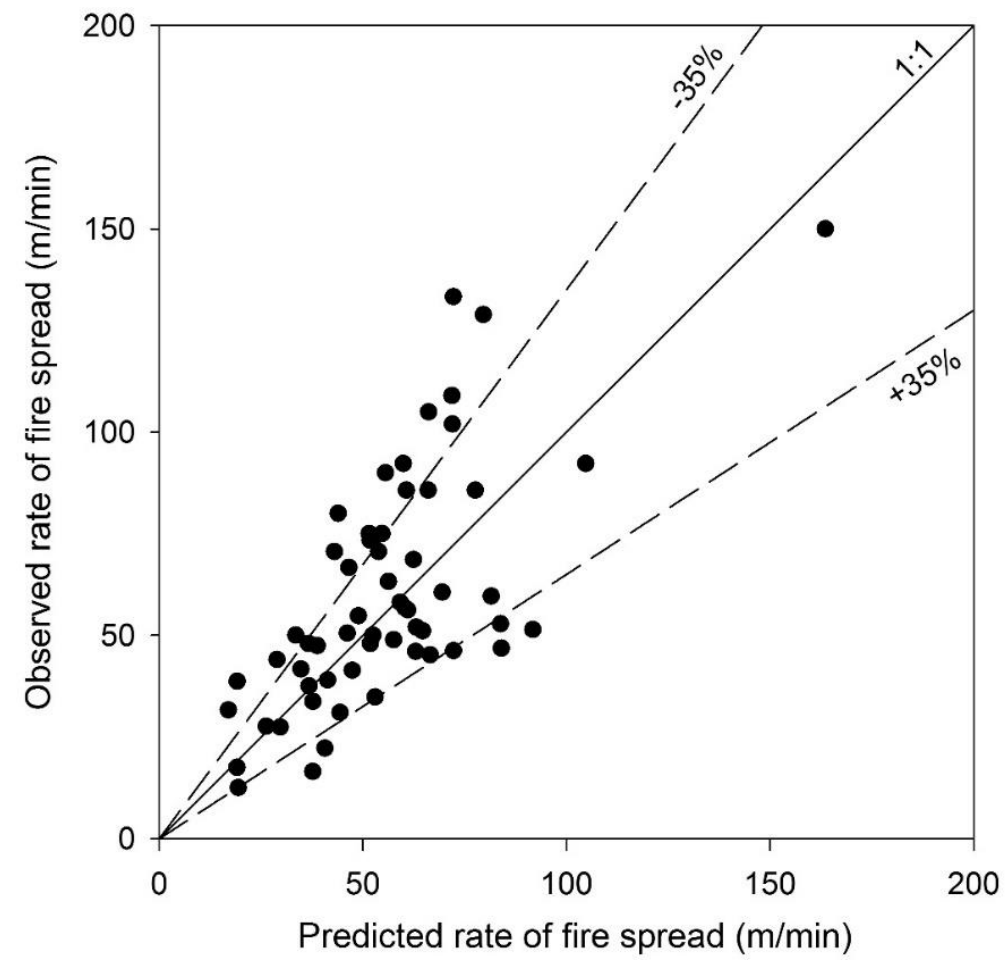

Figure 2 - Observed vs predicted rate of fire spread by Cheney et al. (1998) undisturbed model with the fuel load effect function (Eq. 3). 
Figure 3 presents the relative effect of the fuel load function over the range 0.1 to $1.5 \mathrm{~kg} / \mathrm{m}^{2}$. The relative effect has a low value of 0.92 for a fuel load of $0.1 \mathrm{~kg} / \mathrm{m}^{2}$ and peaks to 1.0 for fuel loads between 0.25 and $0.4 \mathrm{~kg} / \mathrm{m} 2$. At extremes of the fuel load range, the relative effect is 0.76 for a low fuel load value of $0.01 \mathrm{~kg} / \mathrm{m}^{2}$ and asymptotes for fuel load values above $1.4 \mathrm{~kg} / \mathrm{m}^{2}$, with a relative of effect of 0.74 for an extreme grass fuel load value of $2.0 \mathrm{~kg} / \mathrm{m}^{2}$. Figure 3 also shows the advantages of the Ricker function over the nonlinear power function used in Eq. 1. Although the fit statistics were similar for the datasets, the regression models provide unsatisfactory results for the lower $(\mathrm{w}<0.3$ $\mathrm{kg} / \mathrm{m}^{2}$ ) range of fuel load. Similar situation, but not as dramatic occurs for the upper range of fuel load (e.g. $\left.\mathrm{w}>1.2 \mathrm{~kg} / \mathrm{m}^{2}\right)$.

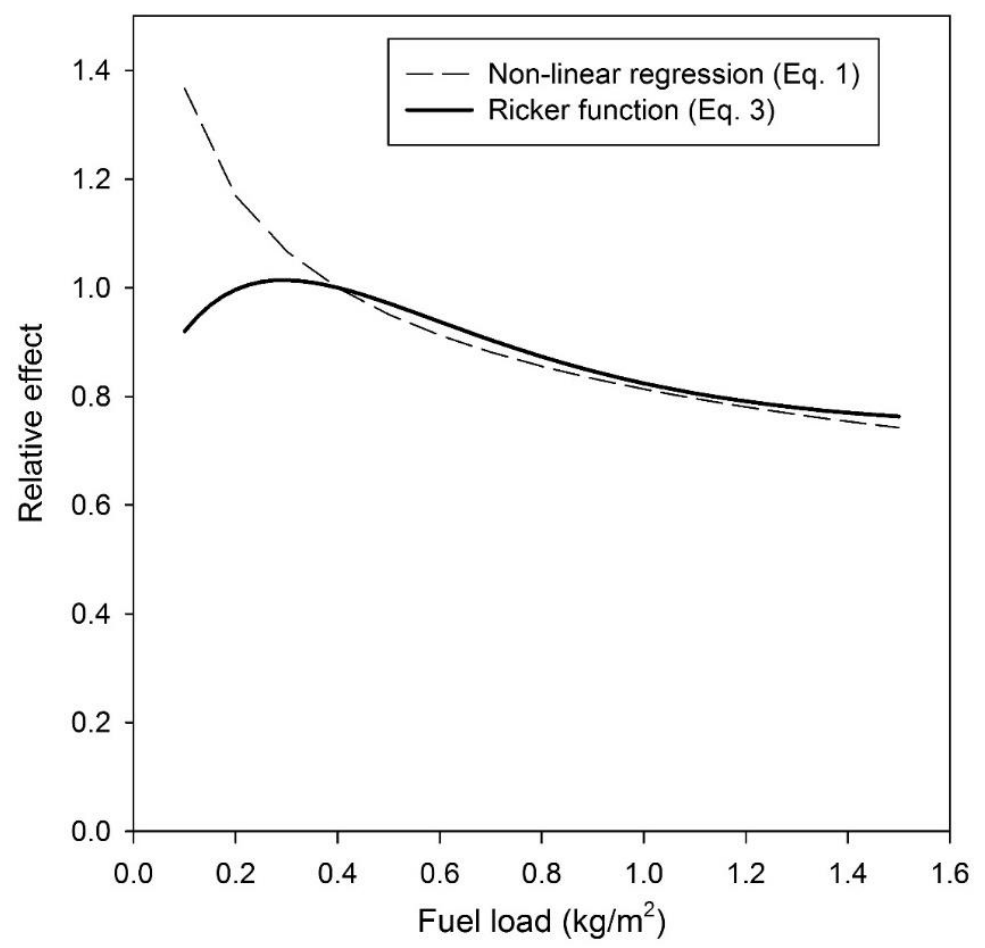

Figure 3 - Relative effect of the tested fuel load functions on the rate of spread of grassfires

\section{Discussion and conclusions}

Our analysis showed that there is a statistically significant, inverse relationship between grass fuel load and rate of fire spread, i.e., an increase in fuel load leads to a decrease in rate of fire spread. Although it might seem surprising, our results follow the trend from Cheney et al. (1993) study. In that study, conducted over a narrower range of fuel loads than in our study $\left(0.17-1.05 \mathrm{~kg} / \mathrm{m}^{2} \mathrm{cf} .0 .23\right.$ $0.57 \mathrm{~kg} / \mathrm{m}^{2}$ for Cheney et al. (1993)) the authors found a non-significant influence of fuel load on rate of fire spread, which had the same trend as that found in our study. The results from Cheney et al. (1993) and ours suggest that the effect is not linear, with minimal effect within the 0.2 to $0.4 \mathrm{~kg} / \mathrm{m}^{2}$ range, and a stronger effect for fuel loads between 0.5 and $1.1 \mathrm{~kg} / \mathrm{m}^{2}$. In the absence of data beyond this range, the fuel load effect function given here asymptotes to a value of approximately $74 \%$ of the maximum rate of spread at a fuel load of $2.0 \mathrm{~kg} / \mathrm{m}^{2}$. Beyond this value the model yields negligible changes for increasing fuel loads. These results are applicable to environments where fuel load is not a limiting factor. In sparse cover grasslands found in arid and semi-arid regions of Australia, fuel load, as a surrogate of cover, is a limiting factor where decreases in fuel load might constrain the forward propagating flux to unburned fuels and limiting flank propagation. 
Our results are contrary to the current Australian practice of assuming a direct positive effect of fuel load on rate of fire spread (Tolhurst et al. 2008) and fire danger rating (Purton 1982). Methods relying on this effect should be revised in light of this and previous (Cheney et al. 1993) scientific evidence.

\section{Acknowledgements}

The authors are grateful to the Queensland Fire and Emergency Services (QFES), and in particular Andrew Sturgess, for supporting this research project. The authors also thank the Country Fire Authority (CFA) of Victoria, in particular David Nichols and Alen Slijepcevic, and the NSW Rural Fire Service (RFS), in particular Simon Heemstra and Laurence McCoy, for their contributions to this research. We also acknowledge the important collaborations of Susan Kidnie, Rachel Bessell, Musa Kilinc, Nigel Buchanan and Tim Wells from CFA, Victoria. At each experimental burning site, suppression support was provided by local brigades who were mainly volunteers and are too numerous to mention individually. Their critical support for the safe and effective conduct of the experimental fires is greatly appreciated.

\section{References}

Cheney NP, Gould JS, Catchpole WR (1993) The influence of fuel, weather and fire shape variables on fire-spread in grasslands. International Journal of Wildland Fire 3, 31-44.

Cheney NP, Gould JS, Catchpole WR (1998) Prediction of fire spread in grasslands. International Journal of Wildland Fire 8, 1-13.

Cheney NP, Gould JS, Hutchings PT (1989) Prediction of fire spread in grassland. CSIRO, Canberra, ACT.

Cruz MG, Sullivan AL, Hurley RJ, Plucinski MP, Gould JS (2017) The effect of fuel load and structure on grassland fire behaviour and fire danger. CSIRO.

Luke RH, McArthur AG (1978) 'Bushfires in Australia.' (Australian Government Publishing Service: Canberra)

McArthur AG (1960) Fire danger rating tables for annual grasslands. Forestry and Timber Bureau, Australia Mimeograph Report, Canberra.

McArthur AG (1966) Weather and grassland fire behaviour. Commonwealth of Australia, Forestry and Timber Bureau Leaflet 100, Canberra, ACT.

Purton C (1982) 'Equations for the McArthur Mark 4 grassland fire danger meter.' (Bureau of Meteorology: Melbourne, VIC)

R Development Core Team (2012) 'R: A Language and environment for statistical computing.' (R Foundation for Statistical Computing: Vienna, Austria).

Setterfield SA, Rossiter-Rachor N, Douglas MM, McMaster D, Adams V, Ferdinands K (2014) 'The impacts of Andropogon gayanus (gamba grass) invasion on the fire danger index and fire management at a landscape scale, 19th Australasian Weeds Conference, Hobart, Tasmania'.(Ed. M. Baker.).

Tolhurst K, Shields B, Chong D (2008) Phoenix: development and application of a bushfire risk management tool. The Australian Journal of Emergency Management 23, 47-54. 


\title{
Assessment of a human body thermoregulation software to predict the thermophysiological response of firefighters
}

\author{
António M. Raimundo ${ }^{1 *}$; A. Virgílio M. Oliveira ${ }^{2}$; Divo A. Quintela ${ }^{1}$ \\ ADAI-LAETA, Department of Mechanical Engineering, Faculty of Sciences and Technology of the \\ University of Coimbra, Portugal. \{antonio.raimundo@dem.uc.pt*\} \\ 2 Department of Mechanical Engineering, Coimbra Institute of Engineering of Polytechnic \\ Institute of Coimbra, Portugal, \{avfmo@mail.isec.pt\}
}

\begin{abstract}
Firefighting requires hard work in very hot environments that often lead to a continuous increase of the heat stored in the human body, promoting heat stress and heat-related illnesses. Due to safety reasons of the individuals involved, a complete characterization of the evolution of the thermal stress state of firefighters is only possible through numerical simulations of the thermophysiological behavior of the human body.

The present work has two main objectives: (i) the assessment of the effectiveness of some body cooling techniques capable to mitigate the risk of hyperthermic stress; and (ii) the evaluation, test and validation of the ability of a human body thermoregulation software to predict in detail the thermophysiological response of firefighters.

The software under evaluation, the HuTheReg program, was developed by the authors. This tool is composed by several modules, namely for the calculation of the human body thermophysiological response, the heat and water transport through clothing, the heat and mass exchange between the external surface of clothing (or skin) and the environment, the start and evolution of skin injuries (pain and burn) and the detection of specific incidents.

In the field of body cooling methodologies, the cases selected embrace 3 different classes of scenarios: (i) body cooling during both exercise and recovery phases (by use of an ice jacket, by intake of very cold water or ice slurry, by both); (ii) body cooling only during the exercise phases (by use of an ice jacket, by intake of very cold water or ice slurry, by both); and (iii) body cooling only during the recovery phases by exposure to a cold environment (passive exposure, with hands and forearms immersion in cold water, with forced air movement using fans, with the use of an ice jacket, with the intake of very cold water or ice slurry, with a combination of these cooling methodologies).

The comparison between the experimental and the predicted values shows a good prediction ability of the HuTheReg program, which is a good indication of its capability to reproduce the human body thermoregulatory responses over the range of climatic conditions investigated. Although with very different effectiveness, all body cooling techniques considered proved to be capable to mitigate the level of hyperthermic stress of firefighters engaged in characteristic activities.
\end{abstract}

Keywords: Safety firefighting; Human thermoregulation modelling; Software validation; Body cooling techniques

\section{Introduction}

Firefighting requires long periods of hard work in very hot environments which often lead to a continuous increase of the heat stored in the human body, promoting heat stress and heat-related illnesses [Raimundo and Figueiredo 2009; Barr et al. 2010].

Firefighting may lead to increases in deep body temperature to values higher than $39^{\circ} \mathrm{C}$ which may have serious consequences or be even fatal [Carter et al. 1999; Raimundo and Figueiredo 2009]. Temperatures above $39^{\circ} \mathrm{C}$ represent acute hyperthermic stress. When attained, the person physical and 
mental performance substantially decreases while the risk of developing heat-related illnesses increases [Mündel et al. 2006, Lopez et al. 2008, Raimundo and Figueiredo, 2009].

The range of possible hyperthermic disorders reported in the literature [Raimundo \& Figueiredo 2009] are muscle cramping, heat exhaustion (collapse during or after exercise), introversion (violent sweating, loss of judgment, amnesia, delusions, etc.), superficial skin damage (pain and first-degree burns), heat-stroke (fainting, cessation of sweating, central nervous system alteration, etc.) and permanent injuries (burn degree greater than first, brain damage or, in more serious cases, death).

Wearing highly thermal insulating protective clothing reduces the gain of external heat, but also blocks the release of the heat produced and restricts movements. Hence, safety firefighting requires the wearing of appropriated clothing. Even so, frequently, this is not enough. In these cases, to increase the safety of firefighters, cooling techniques capable of mitigating hyperthermia should be applied [Carter et al. 1999; Selkirk et al. 2004; Giesbrecht et al. 2007; Chou et al. 2008; Lopez et al. 2008; Barr et al. 2009 \& 2010; Hostler et al. 2010; Colburn et al. 2011; Abreu et al. 2014].

Experimental studies concerning the thermophysiological response of firefighters carrying out typical firefighting activities (both structural and wildland) already exist. In these works, the recommended protective clothing and the exposure to representative environments are considered. However, due to safety reasons of the individuals involved, these trials always terminate before firefighters reach levels of hyperthermic stress with the potential to cause heat-related illnesses. Therefore, a complete characterization of the evolution of the thermal stress state of firefighters is only possible through numerical simulations of the thermophysiological behavior of the human body.

This work has two main objectives, both related to firefighters performing firefighting activities: (i) the assessment of the effectiveness of some body cooling techniques capable to mitigate the risk of hyperthermic stress; and (ii) the evaluation, test and validation of the ability of a human body thermoregulation software to predict in detail the thermophysiological response of firefighters.

\section{The Simulation Tool}

The software under test and validation, the HuTheReg program, was implemented by the authors [Raimundo and Figueiredo, 2009; Raimundo et al. 2012]. This software is composed by several modules. However, for present purposes only the ones for the calculation of the human body thermophysiological response, the heat and water transport through clothing, the heat and mass exchange between the external surface of clothing (or skin) and the environment, the start and evolution of skin injuries (pain and burn) and the detection of specific incidents within the individual are used. Due to its interdependency, all modules run iteratively in each time step until a specific convergence criterion is reached.

The simulation of the thermophysiological response is based on the Stolwijk (1971) 25 nodes thermoregulation model, improved with knowledge found in the literature [e.g., Henriques 1947; Konz et al. 1977; Wissler 1985; Huizenga et al. 1999; Fiala et al. 1999; Tanabe et al. 2002]. This 111-node model considers the human body divided in 22 segments (face, scalp, neck, chest, abdomen, upper back, lower back, pelvis, left shoulder, right shoulder, left arm, right arm, left forearm, right forearm, left hand, right hand, left thigh, right thigh, left leg, right leg, left foot and right foot). Each body segment is composed by 5 layers (core, muscle, fat, skin and clothing), the $111^{\text {th }}$ node being the central blood compartment. The model was implemented for an average man of $1.72 \mathrm{~m}$ tall, weighing $74.43 \mathrm{~kg}$ and with $14 \%$ of body fat $\left(1.869 \mathrm{~m}^{2}\right.$ of skin). For other anthropometric data, the appropriated coefficients are proportionally changed as a function of the body weight, skin area and body fat. The loss of heat by respiration is supposed to occur across the elements of the pulmonary tract. The repartition coefficients proposed by Fiala et al. (1999) were considered. 
The determination of the outer surface temperature of the clothing (or of the skin of nude segments) involves the model of simulation of the human thermophysiological response and simultaneously the heat and mass balances of the garment both considering the flow and the storage of heat and water in the clothing layer [Raimundo et al. 2012]. The global algorithm is applied to each specific human body segment, but always considering its influence and interdependence with the global thermal state of the body. Thus, for each of the 22 human body segments, individual values of clothing properties must be specified, namely of mass $\left(m_{c l}\right)$, of insulation $\left(I_{c l}\right)$, of vapor permeability efficiency $\left(i_{v p}\right)$ and of radiative reflectivity $(\rho)$. Each section is either completely clothed or nude. To consider the reduction of insulation due to body movements, the $I_{c l}$ value at each human body segment is adjusted according to the person activity using the relations proposed by Oliveira et al. (2011).

The convective heat and mass transfer phenomena are predicted using the empirical relations proposed by Quintela et al. (2004) for natural convection and by Havenith et al. (2002) for forced convection. The exchange of heat by radiation between the external surfaces of the segments of the human body and between them and the surroundings, is determined by a set of expressions established by Raimundo et al. (2004).

With the HuTheReg software a significant number of data can be obtained, both for the human body as a whole and for each of the segments, namely: (i) core, muscle, fat, skin and clothing temperatures; (ii) metabolism, heat stored and flux-rates of heat, of sweat, of water and of work; (iii) thermal comfort evaluation and indexes; (iv) detection of probable appearing of heat-related disorders within the person; (v) skin pain, burn areas and corresponding degree; and (vi) a wide range of other thermophysiological parameters.

Each run can simulate up to 20 consecutive scenarios (phases), each one representing different conditions in terms of: posture, orientation, activity, intake of food/drinks, clothing, thermal environment characteristics and impinging of thermal radiation from 6 directions (cardinal, top and bottom).

\section{Material}

The scientific literature seldom presents a complete description of experimental tests, condition that is required to accurately validate mathematical models. To overcome this difficulty, the missing information was replaced by the values suggested by Raimundo and Figueiredo (2009), namely gender (man), height $\left(h^{*}=1.72 \mathrm{~m}\right)$ and weight $\left(w^{*}=74.43 \mathrm{~kg}\right)$, the clothing specific heat $\left(c_{p}{ }^{*}=1000\right.$ $\left.\mathrm{J} /\left(\mathrm{kg} .{ }^{\circ} \mathrm{C}\right)\right)$, water vapor permeability $\left(i_{v p}{ }^{*}=1\right.$ for nude body parts, $i_{v p}{ }^{*}=0$ for the body parts dressed with impermeable clothing, $i_{v p}{ }^{*}=0.15$ for boots and shoes and $i_{v p}{ }^{*}=0.38$ for "normal" clothing) and radiative reflectivity $\left(\rho^{*}=0.10\right.$ for "normal" clothing and $\rho^{*}=0.07$ for human skin). Note that the "*" symbol indicates that this value was assumed only when it was not found in the scientific paper with the report of the experiments.

Furthermore, for situations involving human thermoregulation, the clothing is rarely characterized with the necessary detail. Therefore, for the HuTheReg program, which requires the characteristics of the clothing in each body part, it was necessary to distribute the garment characteristics over the 22 body parts and for this purpose the studies of Oliveira et al. (2008) and Raimundo \& Figueiredo (2009) were considered.

\subsection{Problem Description}

The generic protocol of the cases selected for this study is composed by the following phases: (i) preliminary thermal stabilization at neutral temperature; (ii) muscle warming; (iii) exercise in a hot environment; (iv) transition period; and (v) recovering period. In some cases, phases (iii) to (v) were repeated more than once. A normal ingestion of water is also considered. 
Due to lack of essential information only some of the cases reported in the literature are suitable for the present purposes. In terms of body cooling methodology, the cases selected can be gathered in 3 different scenarios: (i) body cooling during both exercise and recovery phases (by use of an ice jacket, by intake of very cold water or ice slurry, by both); (ii) body cooling only during the exercise phases (by use of an ice jacket, by intake of very cold water or ice slurry, by both); and (iii) body cooling only during the recovery phases by exposure to a cold environment (passive exposure, with hands and forearms immersion in cold water, with forced air movement using fans, with the use of an ice jacket, with the intake of very cold water or ice slurry, with a combination of these cooling methodologies).

\subsection{Validation}

The evaluation, test and validation of the software ability to predict the human body thermophysiological behavior is performed by comparing the program predictions with experimental results from the scientific literature. For this purpose, a wide-range of conditions was considered, which included different types of thermal environment (severe cold, cold, cool, neutral, warm, hot and very hot), of exposures (uniform, sudden change and cyclical), of exercise intensities (0.8 to 4 met) and of clothing ( 0 to 4 clo). The experimental results used for validation embrace core temperatures, average and local skin temperatures and mean body temperature as well as metabolic heat production and loss.

The comparison between the experimental and the predicted values was done using the statistical parameters mean relative difference $(\delta)$ and respective standard deviation $(\sigma)$, mean square deviation $(M S D)$ and Pearson coefficient $(r)$.

\section{Results and Discussion}

Several tests were conducted but, for the present purpose and due to the lack of space, only some of them are presented.

\subsection{Exposure to a uniform environment of semi-nude individuals}

The comparisons between measured values for rectal, tympanic and skin average temperatures and predicted data for rectal, hypothalamus and skin average temperatures are shown in Figure 1. The measured and predicted values of metabolic heat production are also presented. The experimental data represent averages for semi-nude, seated individuals after 1 hour exposure to a range of environment temperatures between 5 and $50^{\circ} \mathrm{C}$, obtained for 44 persons by Wyndham et al. (1964) and for 86 individuals by Werner and Reents (1980). Comparing the predicted values with the measured ones, a good prediction ability of the program is revealed, which is a good indication of its capability to reproduce the body thermoregulatory responses of semi-nude humans over the range of climatic conditions investigated. 


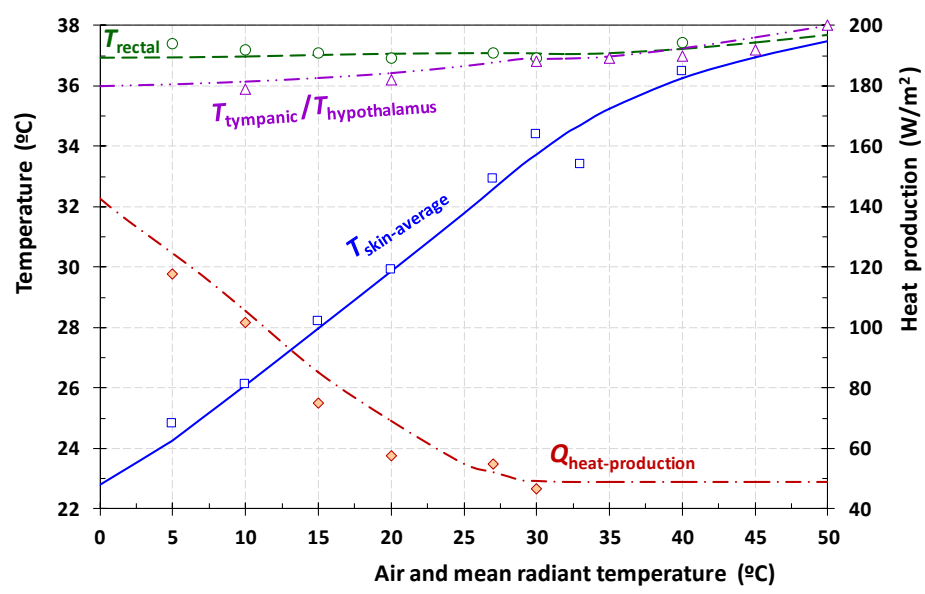

\begin{tabular}{|ccccc|}
\hline Value & $\delta$ & $\sigma$ & MSD & Pearson $(r)$ \\
\hline$T_{\text {re }}(\stackrel{\circ}{ } \mathrm{C})$ & 0.106 & 0.226 & 0.089 & 0.740 \\
\hline$T_{\text {hyp }}\left({ }^{\circ} \mathrm{C}\right)$ & -0.189 & 0.137 & 0.086 & 0.980 \\
\hline$T_{\text {sk }}(\stackrel{\circ}{ } \mathrm{C})$ & 0.088 & 0.606 & 0.203 & 0.990 \\
\hline$Q_{\text {heat-prod }}\left(\mathrm{W} / \mathrm{m}^{2}\right)$ & -5.397 & 5.307 & 2.961 & 0.985 \\
\hline
\end{tabular}

Figure 1 - Comparison of measured (points) and predicted (lines) values for rectal, tympanic (exp.) / hypothalamus (calc.) and skin average temperatures and metabolism heat production, for 1 hour exposure with $T_{\text {air }}=T_{m r}, v_{\text {air }}=0.2 \mathrm{~m} / \mathrm{s}, \mathrm{RH}_{\text {air }}=50 \%$, $I_{c l}=0.1$ clo and $M=0.8$ met.

\subsection{Exposure to a passive cold environment during recovery}

To check the capabilities of the program for simulation of sudden alterations of environmental conditions, a large range of comparisons were made for abrupt changes from hot to cold and from cold to hot. As an example, in Figure 2 a comparison is shown between some of the measured and predicted values representative of the human thermo-physiological response to a rapid change from a hot environment to a cold environment and back again. The experimental data were obtained from Hardy and Stolwijk (1966), representing average values of rectal and skin average temperatures, metabolic heat production and evaporation heat loss for three men exposed to four phases: initial thermo-neutral equilibrium, 1 hour in a hot environment $\left(T_{\text {air }}=T_{\mathrm{mr}}=43^{\circ} \mathrm{C}\right), 2$ hours in a cold environment $\left(17^{\circ} \mathrm{C}\right)$ and 1 hour again in hot $\left(43^{\circ} \mathrm{C}\right)$. The reasonably good agreement achieved indicates an interesting capacity of the program to predict the thermo-physiological reaction of human body in highly dynamic conditions.

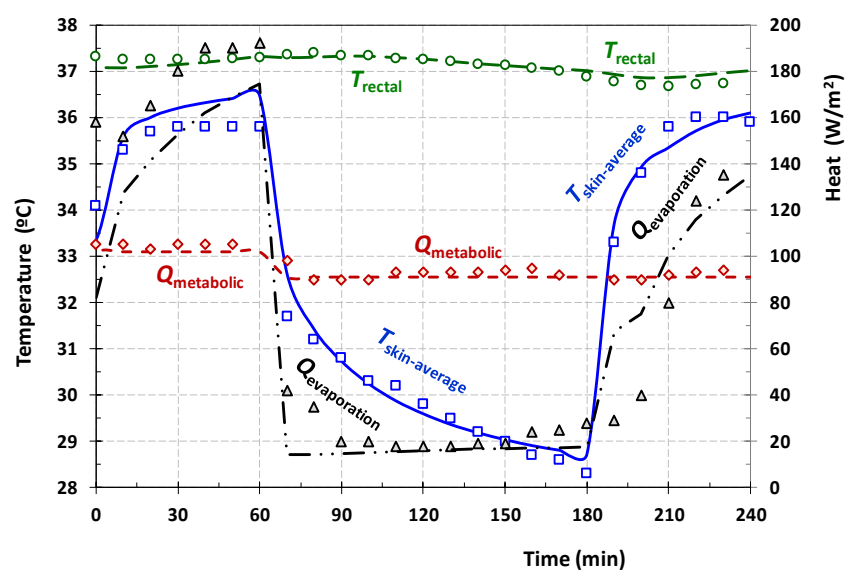

\begin{tabular}{|ccccc|}
\hline Value & $\delta$ & $\sigma$ & $M S D$ & Pearson $(r)$ \\
\hline$T_{\text {re }}(\stackrel{\circ}{ } \mathrm{C})$ & -0.005 & 0.120 & 0.024 & 0.905 \\
\hline$T_{\text {sk }}(\stackrel{\circ}{ } \mathrm{C})$ & -0.125 & 0.381 & 0.079 & 0.993 \\
\hline$Q_{\text {metab }}\left(\mathrm{W} / \mathrm{m}^{2}\right)$ & 1.912 & 2.012 & 0.585 & 0.945 \\
\hline$Q_{\text {evap }}\left(\mathrm{W} / \mathrm{m}^{2}\right)$ & 42.438 & 40.319 & 11.830 & 0.950 \\
\hline
\end{tabular}

Figure 2 - Measured (points) and predicted (lines) values of rectal and skin average temperatures and evaporation heat loss, for sudden ambient temperature changes from a hot environment of $43^{\circ} \mathrm{C}$ to a cold environment of $17^{\circ} \mathrm{C}$ and back again, with $T_{\text {air }}=T_{m r}, v_{\text {air }}=0.2 \mathrm{~m} / \mathrm{s}, \mathrm{RH}_{\text {air }}=30 \%$, $I_{c l}=0.1$ clo and $M=1.0$ met.

\subsection{Use of an ice jacket during exercise and recovery}

In the laboratory study of Chou el al. (2008), the effectiveness of the body cooling technique of wearing an ice jacket both during the exercise and the recovery phases was analyzed. For this purpose, 8 individuals (all males, $h^{(\text {a) }}=1.68 \mathrm{~m}, w^{(\text {a) }}=62.5 \mathrm{~kg},{ }^{(\text {a) }}$ mean values) were dressed in a firefighting personal protective ensemble. These individuals were submitted to the following protocol: (i) preliminary thermal stabilization; (ii) muscle warming $\left(t=10 \mathrm{~min}, T_{\text {air }}=T_{m r t}=25^{\circ} \mathrm{C}, R H_{\text {air }}=50 \%, v_{\text {air }}{ }^{*}=0.2 \mathrm{~m} / \mathrm{s}, M^{*}=1.2 \mathrm{met}, I_{c l}{ }^{*}=2.54 \mathrm{clo}, i_{v p}{ }^{*}=0.28\right.$ and $\left.\rho^{*}=0.10\right)$; (iii) transition $\left(t=10 \mathrm{~min}\right.$, the rest equal except $\left.T_{\text {air }}=T_{m r t}=28^{\circ} \mathrm{C}\right)$; (iv) exercise $(t=30 \mathrm{~min}$, 
$T_{\text {air }}=T_{m r t}=30^{\circ} \mathrm{C}, R H_{\text {air }}=50 \%, v_{\text {air }}{ }^{*}=0.2 \mathrm{~m} / \mathrm{s}, M^{*}=3.0$ met, $I_{c l}{ }^{*}=2.54 \mathrm{clo}, i_{v p}{ }^{*}=0.28$ and $\left.\rho^{*}=0.10\right)$; and (v) recovery $\left(t=10 \mathrm{~min}, T_{\text {air }}=T_{m r t}=30^{\circ} \mathrm{C}, R H_{\text {air }}=50 \%, v_{\text {air }}{ }^{*}=0.2 \mathrm{~m} / \mathrm{s}, M^{*}=0.8 \mathrm{met}, I_{c l}{ }^{*}=2.18 \mathrm{clo}, i_{v p}{ }^{*}=0.37\right.$ and $\left.\rho^{*}=0.11\right)$. Figure 3 shows the experimental results obtained by Chou et al. (2008) for the rectal $\left(T_{r e}\right)$ and mean skin $\left(T_{s k}\right)$ temperatures as well as the predicted values. A very good agreement was achieved for both temperatures, particularly for the rectal temperature.

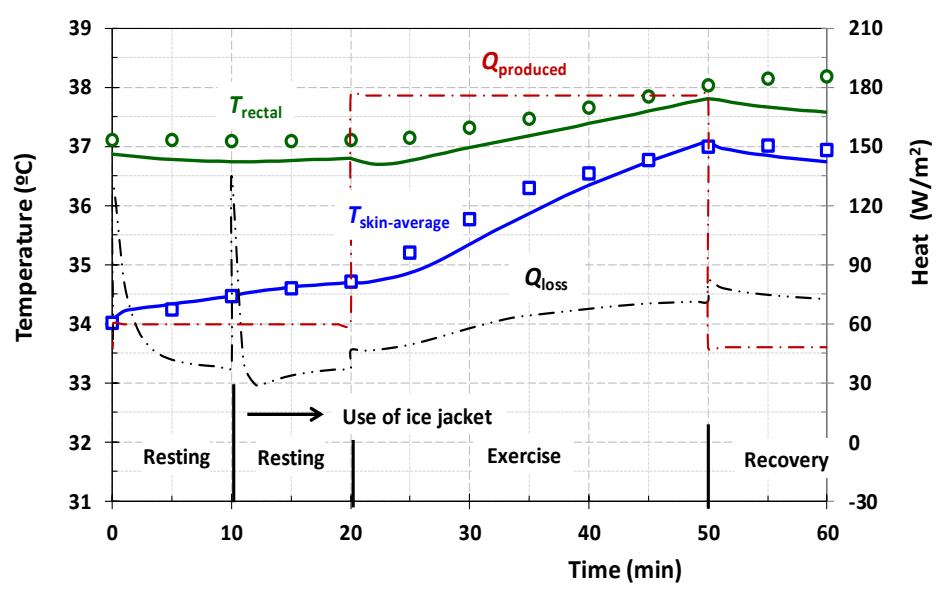

\begin{tabular}{|ccccc|}
\hline Value & $\delta$ & $\sigma$ & MSD & Pearson $(r)$ \\
\hline$T_{\text {re }}(\mathrm{O} C)$ & 0.108 & 0.038 & 0.032 & 0.996 \\
\hline$T_{\text {sk }}(\mathrm{O})$ & 0.149 & 0.198 & 0.067 & 0.986 \\
\hline
\end{tabular}

\subsection{Passive cooling reinforced by use of an ice jacket during recovery}

Figure 4 shown the experimental values (points) obtained by Lopez et al. (2008) and predicted values (lines) for a situation where, only during recovery phase, passive cooling is reinforced using an ice jacket.

Experimental results were obtained for 10 people preforming a protocol with: (i) preliminary thermal stabilization; (ii) muscle warming $\left(t=10 \mathrm{~min}, T_{\text {air }}{ }^{*}=T_{m r t}{ }^{*}=33^{\circ} \mathrm{C}, R H_{\text {air }}{ }^{*}=50 \%\right.$, $v_{\text {air }}{ }^{*}=0.2 \mathrm{~m} / \mathrm{s}, M^{*}=1.2 \mathrm{met}, I_{c l}{ }^{*}=0.263 \mathrm{clo}, i_{v p}{ }^{*}=0.38$ and $\left.\rho^{*}=0.10\right)$; (iii) exercise $(t=68 \mathrm{~min}$, $T_{\text {air }}=T_{m r t}=33.1^{\circ} \mathrm{C}, \quad R H_{\text {air }}=55.1 \%, \quad v_{\text {air }}=0.6 \mathrm{~m} / \mathrm{s}, \quad M=5.6 \mathrm{met}, \quad I_{c l}=0.263 \mathrm{clo}, i_{v p}{ }^{*}=0.38$ and $\left.\rho^{*}=0.10\right)$; and (iv) recovery $\left(t=35 \mathrm{~min}, T_{\text {air }}=T_{m r t}=26.6^{\circ} \mathrm{C}, R H_{\text {air }}=55.1 \%, \quad v_{\text {air }}=0.2 \mathrm{~m} / \mathrm{s}\right.$, $M=1.0$ met, $I_{c l}=0.445$ clo, $i_{v p}{ }^{*}=0.38$ and $\left.\rho^{*}=0.10\right)$. The results presented in Figure 4 for the core ( $T_{\text {core }}$ ) and mean skin $\left(T_{s k}\right)$ temperatures reveal a reasonable agreement between the measured and the predicted values for both temperatures.

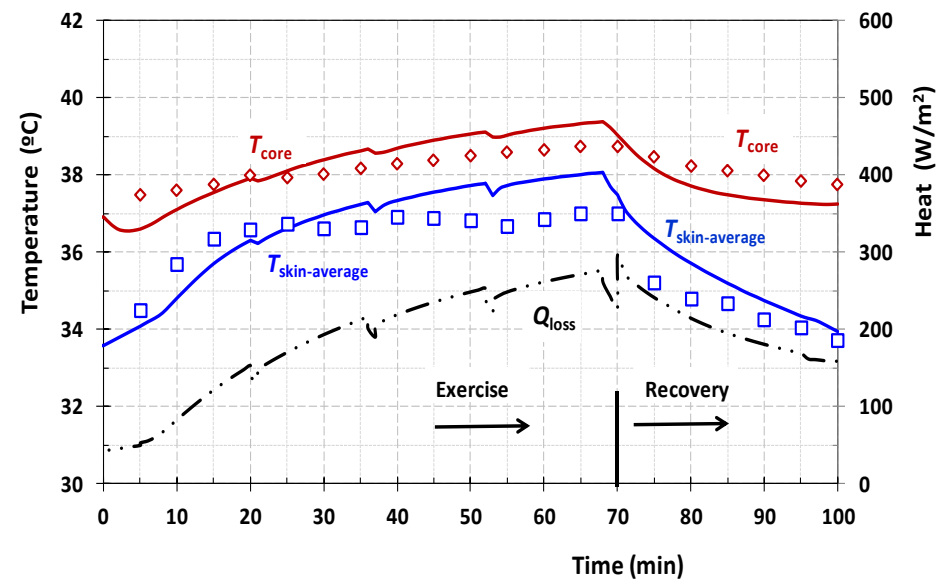

\begin{tabular}{|ccccc|}
\hline Value & $\delta$ & $\sigma$ & MSD & Pearson $(r)$ \\
\hline$T_{\text {core }}(\stackrel{\circ}{ }(\mathrm{C})$ & 0.049 & 0.457 & 0.103 & 0.968 \\
\hline$T_{\text {sk }}(\stackrel{\circ}{ } \mathrm{C})$ & 0.503 & 0.130 & 0.312 & 0.922 \\
\hline
\end{tabular}

Figure 4 - Experimental values (points) obtained by Lopez et al. 2008 and predicted values (lines) for body passive cooling reinforced using an ice jacket during the recovery phase. 


\subsection{Passive cooling reinforced with hands and forearms immersion in cold water during recovery}

The study of Selkirk et al. (2004) aimed to verify the effectiveness of the passive human body cooling technique in reducing thermal stress in a hot thermal environment and where it was necessary to wear personal protective clothing. Fifteen male firefighters participated in the study, for which in average $h=1.81 \mathrm{~m}$ and $w=86.9 \mathrm{~kg}$.

All the steps of the tests were performed in a room with controlled thermal conditions ( $T_{\text {air }}=T_{m r \mathrm{r}}=35^{\circ} \mathrm{C}, R H_{\text {air }}=50 \%$, vair $\left.^{*}=0.1 \mathrm{~m} / \mathrm{s}\right)$. The protocol included the following phases: (i) thermal stabilization in a neutral environment; (ii) muscle warming ( $t^{*}=3 \mathrm{~min}, M^{*}=1.2 \mathrm{met}$ ); (iii) exercise ( $\left.t=20 \mathrm{~min}, M^{*}=2.8 \mathrm{met}\right)$; (iv) transition ( $\left.t=10 \mathrm{~min}, M^{*}=1.5 \mathrm{met}\right)$; (v) exercise $(t=20 \mathrm{~min}$, $M^{*}=2.8 \mathrm{met}$ ); (vi) transition ( $t=5 \mathrm{~min}, M^{*}=1.2 \mathrm{met}$ ); (vii) application of passive recovery reinforced with hands and forearms immersion in water at $T_{\text {water }}=17^{\circ} \mathrm{C}$ ( $\left.t=20 \mathrm{~min}, M^{*}=0.8 \mathrm{met}\right)$; (viii) transition $\left(t=5 \mathrm{~min}, M^{*}=1.2 \mathrm{met}\right)$; (ix) exercise $\left(t=20 \mathrm{~min}, M^{*}=2.8 \mathrm{met}\right)$. During the exercise phases the subjects wear a typical forest firefighting personal protective ensemble, including individual breathing apparatus $\left(I_{c l}=1.55 \mathrm{clo}, i_{v p}{ }^{*}=0.28\right.$ and $\left.\rho^{*}=0.16\right)$. During the muscle warming and the recovery phases, they remove the helmet, the flash hood, the gloves, the jacket and the individual breathing apparatus $\left(I_{c l}{ }^{*}=1.06 \mathrm{clo}, i_{v p}{ }^{*}=0.56\right.$ and $\left.\rho^{*}=0.10\right)$.

Figure 5 shows that the rectal $\left(T_{r e}\right)$ and average skin $\left(T_{s k}\right)$ temperatures experimental results obtained by Selkirk et al. (2004) are in good agreement with the predicted ones, particularly for the rectal temperature.

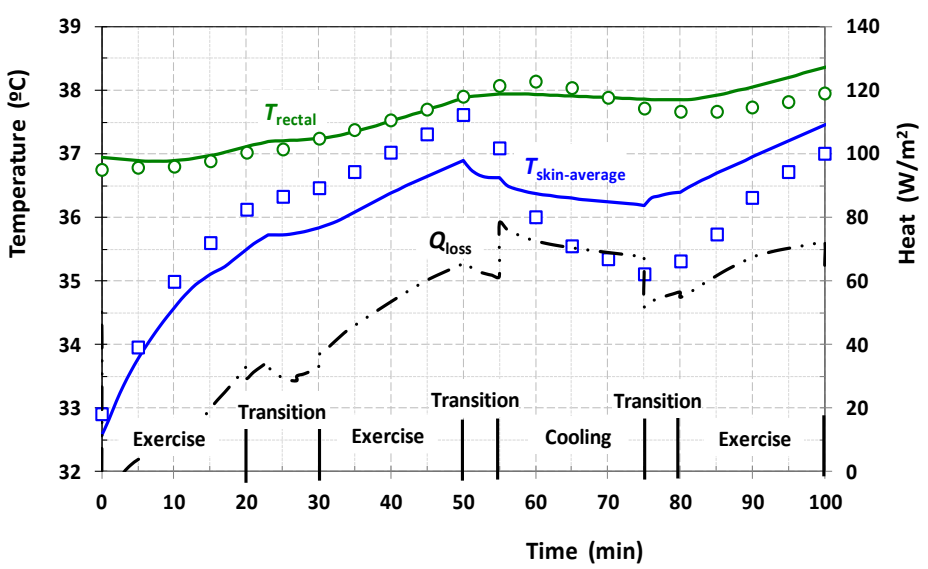

\begin{tabular}{|ccccc|}
\hline Value & $\delta$ & $\sigma$ & MSD & Pearson $(r)$ \\
\hline$T_{\text {re }}(\stackrel{\circ}{ } \mathrm{C})$ & -0.205 & 0.248 & 0.052 & 0.946 \\
\hline$T_{\text {sk }}(\stackrel{\circ}{ } \mathrm{C})$ & -0.500 & 0.862 & 0.162 & 0.731 \\
\hline
\end{tabular}

Figure 5 - Experimental values (points) obtained by Selkirk et al. 2004 and predicted values (lines) for body cooling by immersion of hands and forearms in cold water during the recovery phase.

\subsection{Recovery with passive cooling reinforced by use of an ice jacket and by immersion of hands and forearms in cold water}

Figure 6 shown the experimental values (points) obtained by Barr et al. (2009) and predicted values (lines) for a situation where, only during recovery phase, passive cooling is reinforced by the use of an ice jacket and by the immersion of hands and forearms in water at $T_{\text {water }}=19^{\circ} \mathrm{C}$.

Experimental values were obtained with 9 firefighters wearing firefighting personal protective clothing and performing the following protocol: (i) preliminary thermal stabilization; (ii) muscle warming; (iii) exercise; (iv) recovery; and (v) exercise. The exercise phases are characterized by $t=20 \mathrm{~min}, T_{\text {air }}=T_{m r t}=50^{\circ} \mathrm{C}, R H_{\text {air }}=13 \%$, vair $=0.2 \mathrm{~m} / \mathrm{s}, M=5.0$ met, $I_{c l}=2.46 \mathrm{clo}, i_{v p}{ }^{*}=0.34$ and 
$\rho^{*}=0.10$. The recovery phase is characterized by $t=15 \mathrm{~min}, T_{a i r}=T_{m r t}=21^{\circ} \mathrm{C}, R H_{a i r}=60 \%$, $v_{\text {air }}=0.5 \mathrm{~m} / \mathrm{s}, M=0.8 \mathrm{met}, I_{c l}=1.58 \mathrm{clo}, i_{v p}{ }^{*}=0.30, \rho^{*}=0.10$ and $T_{\text {water }}=19^{\circ} \mathrm{C}$.

The results presented in Figure 6 for the core $\left(T_{\text {core }}\right)$ and mean skin $\left(T_{s k}\right)$ temperatures reveal a satisfactory agreement between the measured and the predicted values for both temperatures.
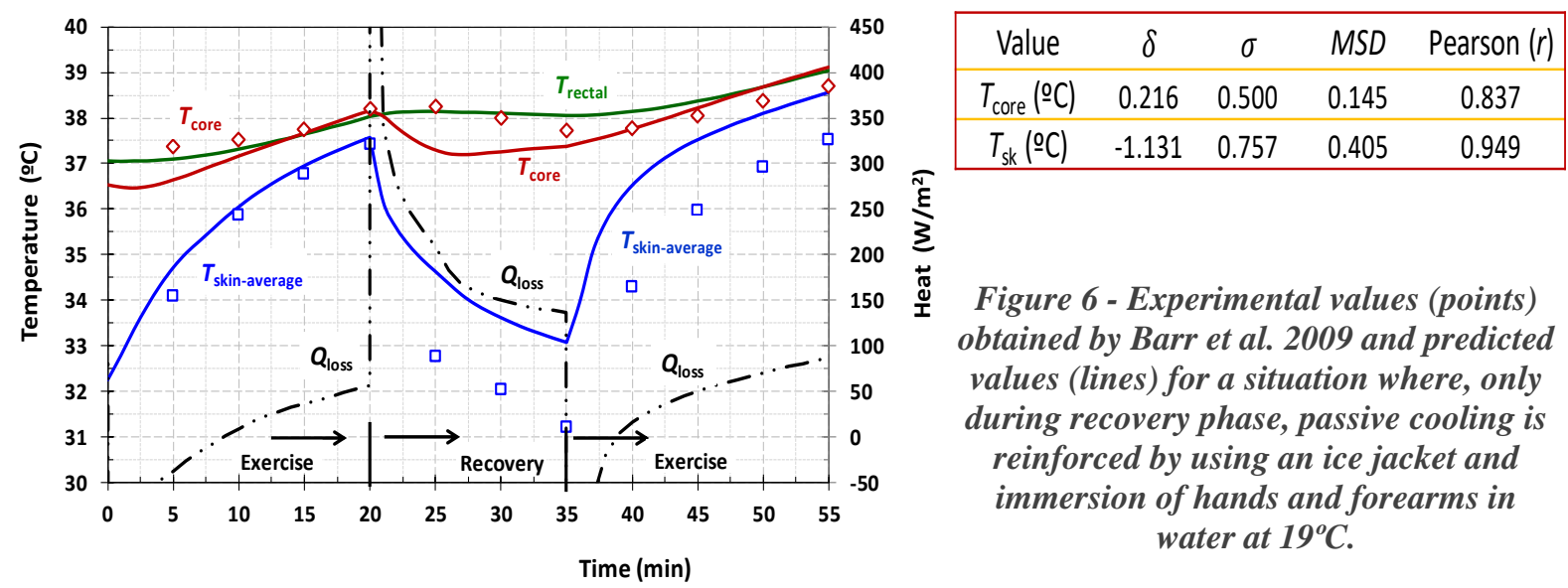

\section{Discussion}

In the present paper, the applicability of the HuTheReg program was tested with several cases, each involving different periods (phases) with distinct levels of metabolic activity, clothing thermal insulations, environmental conditions and body cooling techniques.

This paper presents different human body cooling techniques. The ones that can be used during the exercise phases have the advantage of dissipating the excess of heat as it is being generated. However, the use of ice jackets during exercise periods is not always possible. The application of body cooling techniques during recovery periods is usually easier to accomplish. All of them have shown to be beneficial in terms of reducing hyperthermic stress, and their efficiency can be even enhanced by simultaneous application of more than one technique.

To cover a wide range of situations, the applicability of the program was tested by applying it to several different cases, each involving several periods (phases) with distinct levels of body metabolic activity, clothing characteristics, environment conditions and human body cooling actions. No limitations were found on the program's ability to predict the thermophysiological behavior of the human body when exposed to a wide range of situations, even in cases involving periods with very different conditions.

In all the assessments tested the quality of the program's forecasts can be classified as very acceptable. This statement is based on the statistical comparison between experimental and predicted values. Some predictions were not as good as expected. That shall not be exclusively attributed to the pro-gram but most probably to the number of estimations that were necessary to perform due to the insufficient detail and lack of data found in the scientific papers related to this subject.

The results obtained with this study put in evidence the importance of simulation programs to carry out detailed evaluations of the thermophysiological response of the human body, which allows the anticipation of actions to attenuate thermal stress and thus avoid the advent of heat-related illnesses. 


\section{Conclusions}

A software for the simulation of the human body thermoregulation, the HuTheReg program, was tested against experimental results from the literature, which were obtained using real persons. At the same time, an assessment of the effectiveness of some human body cooling techniques capable to mitigate the risk of heat stress by hyperthermia was done.

Although with very different effectiveness, all body cooling techniques considered proved to be capable to mitigate the level of hyperthermic stress of firefighters engaged in firefighting activities.

No limitations were found on the program's ability to predict the human body thermophysiological behavior. A very acceptable outcome was obtained in all cases, being excellent in several of them.

The results show that it is reasonable to use simulation programs to carry out detailed evaluations of the human body thermophysiological response, allowing the anticipation of actions to attenuate thermal stress and thus avoiding of the advent of heat-related disorders.

\section{References}

Abreu RM, Raimundo AM, Quintela DA (2014). Analysis of the thermophysiological response to cooling techniques in firefighters, ICFFR $2014-7^{\text {th }}$ International Conference on Forest Fire Research, paper H01, Coimbra, Portugal, 14 - 21 november 2014.

Barr D, Gregson W, Sutton L, Reilly T (2009). A practical cooling strategy for reducing the physiological strain associated with firefighting activity in the heat, Ergonomics 52(4): 413-420.

Barr D, Gregson W, Reilly T (2010). The thermal ergonomics of firefighting reviewed, Applied Ergonomics, 41: 161-172.

Carter JB, Banister EW, Morrison JB (1999). Effectiveness of rest pauses and cooling in alleviation of heat stress during simulated fire-fighting activity, Ergonomics 42: 299-313.

Chou C, Tochihara Y, Kim T (2008). Physiological and subjective responses to cooling devices on firefighting protective clothing, European J Applied Physiology 104: 367-374.

Colburn D, Suyama J, Reis S (2011). A comparison of cooling techniques in firefighters after a live burn evolution, Prehospital Emergency Care 15: 226-232.

Fiala D, Lomas KJ, Stohrer M (1999). A computer model of human thermoregulation for a wide range of environmental conditions - the passive system, J Applied Physiology 87: 1957-1972.

Giesbrecht GG, Jamieson C, Cahill F (2007). Cooling hyper-thermic firefighters by immersing forearms and hands in $10^{\circ} \mathrm{C}$ and $20^{\circ} \mathrm{C}$ water, Aviation Space Environmental Medicine 78: 561-567.

Hardy JD, Stolwijk JAJ (1966). Partitional calorimetric studies of responses man during exposures to thermal transients, J Applied Physiology 21: 967-977.

Havenith G, Holmér I, Parsons K (2002). Personal factors in thermal comfort assessment - clothing properties and metabolic heat production, Energy and Buildings 34: 581-591.

Henriques F (1947). Studies of thermal injury V. The predictability and the significance of thermally induced rate processes leading to irreversible epidermal injury, Archives of Pathology 43: 489-502.

Hostler D, Reis S, Bednez JC, Kerin S, Suyama J (2010). Comparison of active cooling devices with passive cooling for rehabilitation of firefighters performing exercise in thermal protective clothing: a report from the Fireground Rehab Evaluation (FIRE) trial, Prehospital Emergency Care 14: 300309.

Huizenga C, Zhang H, Duan T, Arens E (1999). An improved multinode model of human physiology and thermal comfort, Proceedings of IBPSA Building Simulation 99, Kyoto, Vol. 1, pp 353-359. 
Konz S, Hwang C, Dhiman B, Duncan J, Masud A (1977). An experimental validation of mathematical simulation of human thermoregulation, Computers in Biology and Medicine 7: 71-82.

Lopez RM, Cleary MA, Jones LC, Zuri RE (2008). Thermoregulatory Influence of a Cooling Vest on Hyperthermic Athletes. J Athletic Training 43(1): 55-61.

Mündel T, King J, Collacott E, Jones DA (2006). Drink temperature influences fluid intake and endurance capacity in men during exercise in a hot dry environment, Experimental Physiology 91(5): 925-933.

Oliveira AVM, Gaspar AR, Quintela, DA (2008). Measurements of clothing insulation with a thermal manikin operating under the thermal comfort regulation mode: comparative analysis of the calculation methods, European J Applied Physiology 104: 679-688.

Oliveira AVM, Gaspar AR, Quintela DA (2011). Dynamic Clothing insulation. Measurements with a thermal manikin operating under the thermal comfort regulation mode, Applied Ergonomics 42(6): 890-899.

Quintela DA, Gaspar AR, Borges C (2004). Analysis of sensible heat exchanges from a thermal manikin, European J Applied Physiology 92(6): 663-668.

Raimundo AM, Gaspar AR, Quintela DA (2004). Numerical modelling of radiative exchanges between the human body and surrounding surfaces, Climamed $2004-1$ st Mediterranean congress of climatization, 16-17 of April, Lisbon, Portugal, paper 8/1.

Raimundo AM, Figueiredo AR (2009). Personal protective clothing and safety of firefighters near a high intensity fire front, Fire Safety Journal 44: 514-521.

Raimundo AM, Quintela DA, Gaspar AR, Oliveira AVM (2012). Development and validation of a computer program for simulation of the human thermophysiological response, Portuguese chapter of IEEE-EMBS, ISBN:978-1-4673-4524-8.

Selkirk G, McLellan TM \& Wong J (2004). Active versus passive cooling during work in warm environments while wearing firefighting protective clothing, J. Occupational Environment and Hygiene 1: 521-531.

Stolwijk JAJ (1971). A mathematical model of physiological temperature regulation in man. NASA Contractor Report CR-1855, NASA, Washington, DC.

Tanabe S, Kobayashi K, Nakano J, Ozeki Y, Konishi M (2002). Evaluation of thermal comfort using combined multi-node thermoregulation $(65 \mathrm{MN})$ and radiation models and computational fluid dynamics (CFD), Energy and Buildings 34: 637-646.

Werner J, Reents T (1980). A contribution to the topography of temperature regulation in man, European J Applied Physiology 45: 87-94.

Wissler EH (1985). Mathematical simulation of human thermal behaviour using whole body models, In: Shitzer A, Eberhart RC, (Eds), Heat Transfer in Medicine and Biology - Analysis and Applications. Plenum, New York London, pp 325-373.

Wyndham C, Ward J, Strydom N, Morrison J, Williams C, Bredell G, Peter J, Von Rahden M, Holdsworth L, Van Graan C, Van Rensburg A, Munro A (1964). Physiological reactions of caucasian and bantu males in acute exposure to cold, J Applied Physiology 19: 538-592. 


\title{
Cfd study of effect of water drop by aerial firefighting to protect forest fire growth
}

\author{
Koyu Satoh ${ }^{1 *}$; Domingos Viegas ${ }^{1}$; Naian $\mathrm{Liu}^{2}$; Xiaodong Xie ${ }^{2}$; Ran $\mathrm{Tu}^{3}$ \\ ${ }^{1}$ University of Coimbra, Coimbra 3000-370, Portugal. \{CF60420@ gmail.com*\} \\ ${ }^{2}$ University of Science and Technology of China, 96 Jinzhai Road, Hefei, Anhui 230026, China, \\ \{liunai@ustc.edu.cn\} \\ ${ }^{3}$ Huaqiao University, Xiamen, Fujian 361021, \{turan@hqu.edu.cn\}
}

\begin{abstract}
Wildfires destroy the natural environment, cause property damages and cause victims, therefore great research effort is dedicated to improve protection against wildfires. As fire growth depends on many parameters to examine behavior relevant to fire protection methods systematically, we used CFD simulations to analyze the role of aerial fire-fighting. CFD simulations of the fire spread in relation to the water drop effect in aerial fire-fighting were done using the $(15 \times 15)$ fuel array in windy conditions. A single fuel cell is $8 \mathrm{~m} \times 8 \mathrm{~m} \times 2 \mathrm{~m}$, varying the space distance between the adjacent fuel cells. The water drop effect is examined as a function of the heat release rate, varying the wind speed. The results of our previous studies showed that it is difficult to extinguish the burning area in wildfires by directly dropping water over the flames and also it is less effective to drop water in the fringe areas of burning fires parallel to the wind direction. Therefore, the way selected in this study is to drop water in the downwind area slightly away from the fire front, making a water belt to reduce the radiative heat transfer. However, if the fuel distributes densely, even the water belt dropped in the area of $20 \mathrm{~m} \times 60 \mathrm{~m}$ may not sufficient to stop the fire spread, since the fires may detour both sides of the water belt or the fires jump over the water belt. However, water dropped belts are very effective to delay the fire spreading speed. If the space distance between adjacent fuel cells is larger than $2 \mathrm{~m}$, the water belt can protect or highly delay the fire spread. One problem is the amount of water to realize such a water belt to stop the fire spread, since helicopters carry too few water and it is dangerous for helicopters to fly in the hot and smoky air. Large aircrafts carrying large amount of water are needed to make such a large water belt.
\end{abstract}

Keywords: CFD Study, Forest Fire, Aerial Firefighting, Water Drop, Fire Spreading Speed, Wind speed

\section{Introduction}

Wildfires destroy property, cause victims and emit large quantities of greenhouse gases, which may contribute to the climate change and enhance large-scale wildfires. Over recent years wildfires are globally increasing. Therefore, many researches (Viegas, 2006; Finney, 2003; Abatzoglou et al, 2018; Liu et al, 2007) have been made to protect and mitigate the damages by forest fires, examining the fire growth behavior. As the fire spread depends on many parameters and so it is difficult to examine the fire spread behavior in relation to the fire protection methods systematically, we used CFD simulation methods. The authors (Satoh et al, 2000; Satoh et al, 2003; Satoh et al, 2005; Satoh et al, 2008; McGrattan et al, 2000) have studied the fire protection methods in relation to the wind speed affecting the fire spread, together with the water drop effect by an aerial fire-fighting. The objective of this study is to examine the water drop effect upon the fire protection, particularly how large area is needed to protect the fire spread by the aerial water drop, using CFD simulation methods based on FDS by NIST (Floyd et al, 2006; McGrattan et al, 2001). CFD simulations of the fire spread are done using the (15 $x$ 15) fuel arrays. The results of our previous studies (Satoh et al, 2000; Satoh et al, 2005) showed that it is difficult to extinguish the burning area by directly dropping water over the fire and also it is less effect to drop water in the fringe areas of burning fires parallel to the wind direction. 
Therefore, the way selected in this study is to drop water in the downwind area, slightly away from the fire front, making a water belt to reduce the radiative and convective heat transfer.

\section{Schematics and Simulation Method}

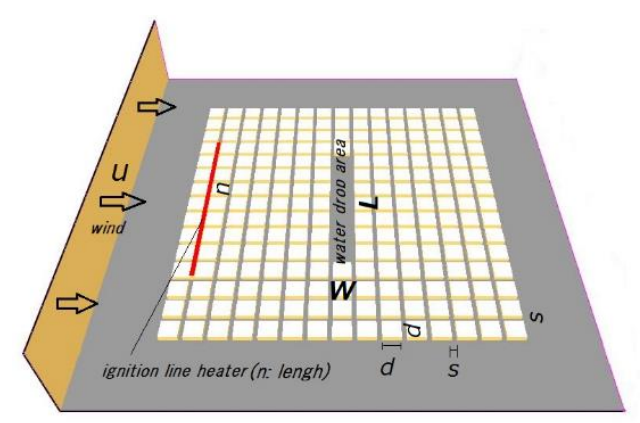

Figure 1 - Schematics of $(15 \times 15)$ fuels in CFD simulations

Figure 1 shows the schematics of the $(15 \times 15)$ array with an equal apace distance $(s)$ between adjacent square fuels. The space distance $(s)$ varies from $0 \mathrm{~m}$ to $4 \mathrm{~m}$. The dimensions of each fuel cell are $d \times \mathrm{x} \mathrm{x} h$, where $d$ is the length and $h$ is the height of each square fuel cell. This study deals only with one case of $d=8 \mathrm{~m}$ and $h=2 \mathrm{~m}$. Other configurations will be studied in the future. At one vertical boundary, uniform wind blows with the wind speed of $u$. An ignition line heater with the length of $n$ and the width of $1 \mathrm{~m}$ or a square $(2 \mathrm{~m} \times 2 \mathrm{~m})$ heater is used, giving the heat release rate per area $(q)$, but no fire brands are considered. The relationship between the total heat release rate $(Q)$ and the wind speed $(u)$, varying the ignition heat source length $(n)$ and the space distance $(s)$, under the water dropped conditions in the aerial fire-fighting or no water dropped. Here, "water dropped area" means no fuel exists in one fuel cell and the water dropped area has the width $W$ (in the wind direction) and the length $L$ (perpendicular to the wind direction), locating at $18 \mathrm{~m}$ (for small scale fires) to $72 \mathrm{~m}$ (for larger scale fires) apart from the ignition heat source. In this simulation study, using the software FDS by NIST (McGrattan et al, 2000; Floyd et al, 2006; McGrattan et al, 2001), the combustion of fuels ignited by a heater is based on a mixing-controlled chemical reaction model. After the reaction of fuel and oxygen, $\mathrm{CO}_{2}$ and soot are produced, together with the convective heat, conductive heat and radiative heat affecting the fuel combustion in the downwind area. Radiative heat transfer is based on the solution of the radiation transport equation for a gray gas and the details are found in the user's guide of FDS (McGrattan et al, 2001). Difficulty was the choice of the fuel material. As a fuel, several materials are tested in the preliminary simulations, but the fuel in this study has no porous structure like real forest materials. Simulations of some whole solid materials with the size of $8 \mathrm{~m} \times 8 \mathrm{~m} \times 2 \mathrm{~m}$ needed so long computing time and some soft materials burned out too short. Thus, the "black PMMA" shown in the user's guide of FDS (Floyd et al, 2006), having the heat of combustion of $25200 \mathrm{~kJ} / \mathrm{kg}$, the density of $1100 \mathrm{~kg} / \mathrm{m}^{3}$ and the conductivity of $0.20 \mathrm{~W} /(\mathrm{m} \mathrm{K})$, was used in this study. Except for the ground, all other boundaries are open, but a uniform wind is blowing in one vertical boundary. The four additional areas around the fuel array with the width of $30 \mathrm{~m}$ as shown in Figure 1 are added, so the total ground area is $180 \mathrm{~m} \times 180 \mathrm{~m}$ for $s=0 \mathrm{~m}$ and $240 \mathrm{~m} \times 240 \mathrm{~m}$ for $s=4 \mathrm{~m}$. Grid sizes used in the simulations largely affect the simulation results. However, the maximum number of grid cells used in the computations is limited due to the computer memory limit and the total computing time. Therefore, one uniform grid cell size of $0.5 \mathrm{~m} \times 0.5 \mathrm{~m} \times 0.5 \mathrm{~m}$ is used in this simulation, where one case computation reaching the whole area combustion during several thousand seconds needed two or three months by a single personal computer. More than 200 cases of simulations, varying the wind speed and the water drop area, were done systematically. 


\section{Results}

\subsection{Behavior of fire spread and effect of water drop to protect fire spread}

The fire spread behavior is highly affected by the distance between fuel cells. Therefore, we investigated the relationship between the total heat release rate produced by the fuel combustion $(Q)$ and the space distance $(s)$, with varying wind speed $(u)$. Preliminary simulations showed that the heat release rate per area $(q)$ of ignition heat source, between $q=1500$ and $2500 \mathrm{~kW} / \mathrm{m}^{2}$, against the fire spreading behavior had only small effect upon the fuel combustion and the fire spreading patterns. However, the heating time had considerable effect on the fire spreading patterns, since the ignition source assists it. Therefore we employed values of the ignition heat source of $q=2000 \mathrm{~kW} / \mathrm{m}^{2}$, heating time $t=1000 \mathrm{sec}$ and the size of ignition source is $(1 \mathrm{~m} \times n(\mathrm{~m}))$ or $(2 \mathrm{~m} \times 2 \mathrm{~m})$.

\subsubsection{Case of $s=0 m$ (no space between fuel cells) and $n=2 m$}

Figure 2 shows the fire spreading profiles at $u=2 \mathrm{~m} / \mathrm{s}$ and $10 \mathrm{~m} / \mathrm{s}$, where there is no space between fuel cells and no water is dropped, keeping the ignition source size at $(2 \mathrm{~m} \times 2 \mathrm{~m})$ and the heat release rate per area $q=2000 \mathrm{~kW} / \mathrm{m}^{2}$. In the case of $u=2 \mathrm{~m} / \mathrm{s}$, the fire spreading pattern of fuels with no spaces becomes round and spreading into the lateral direction, too, but in the case of $u=10 \mathrm{~m} / \mathrm{s}$ the fire is spreading mainly in the downwind direction.

(A) $u=2 \mathrm{~m} / \mathrm{s}$,

(1) $t=504 \mathrm{~s}$

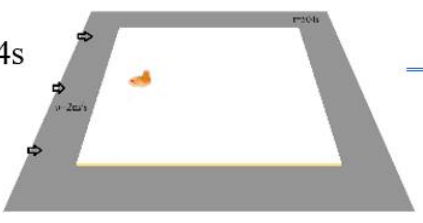

(2)

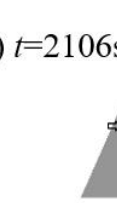

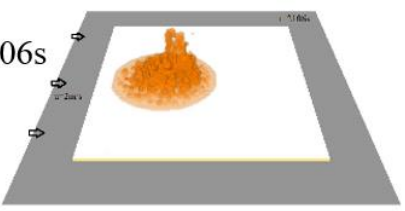

(3)

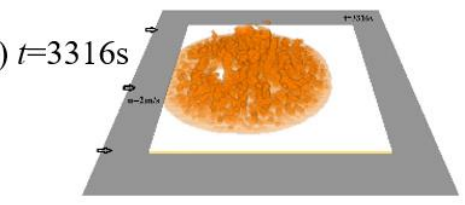

(B) $u=10 \mathrm{~m} / \mathrm{s}$

(1) $t=400 \mathrm{~s}$

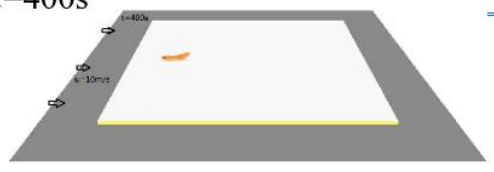

(2)

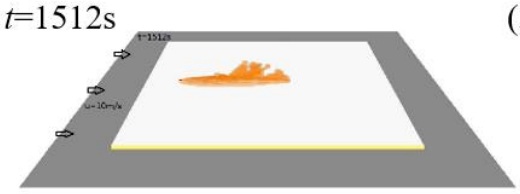

(3) $t=1912 \mathrm{~s}$

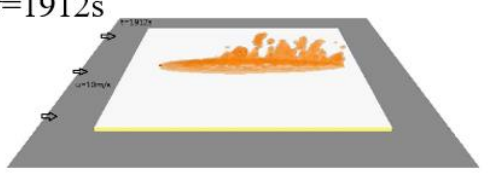

Figure 2 - Fire spreading profile of the case at $s=0 m$, with no water drop.

Next, the effect of water drop in the downwind area to limit the fire spread is examined, comparing with no water drop case, using the ignition heat of $(2 \mathrm{~m} \times 2 \mathrm{~m})$ under the wind speeds of $u=2 \mathrm{~m} / \mathrm{s}$ and $4 \mathrm{~m} / \mathrm{s}$. Figure 3 shows the total heat release rate as a function of time for Case (A) $s=0 \mathrm{~m}$ and $u=2 \mathrm{~m} / \mathrm{s}$ and Case (B) $s=0 \mathrm{~m}$ and $u=4 \mathrm{~m} / \mathrm{s}$. Figure 4 shows the fire spreading patterns for Cases (A) and (B). As shown in Figure 3, the heat release rate $(Q)$ begins to increase suddenly and rapidly, which may be dangerous for firefighters. The water dropped areas in Figure $3(\mathrm{~A})$ are $(W=10 \mathrm{~m} \times L=20 \mathrm{~m})$ and $(W=20 \mathrm{~m}$ $\mathrm{x} L=40 \mathrm{~m})$. In the case of water dropped area of $(10 \mathrm{~m} \times 20 \mathrm{~m})$, the fire can jump over the water belt and the fire can spread in the downwind area, as shown in Figure 3(C)-(2), although the fire spread is highly weakened. The water drop belt of $(20 \mathrm{~m} \mathrm{x} 40 \mathrm{~m})$ is effective to stop the fire spread, where the fire cannot jump over the belt with the width of $W=20 \mathrm{~m}$ and also cannot detour both sides of the belt, since the delay time is too long, the radiative heat transfer becomes weak and the spreading fires gradually burn out. Therefore, the water dropped belt of $(20 \mathrm{~m} \times 40 \mathrm{~m})$ in the downwind of the fire caused by a small ignition heat source in a weak wind can stop the fire spread. 
(A)

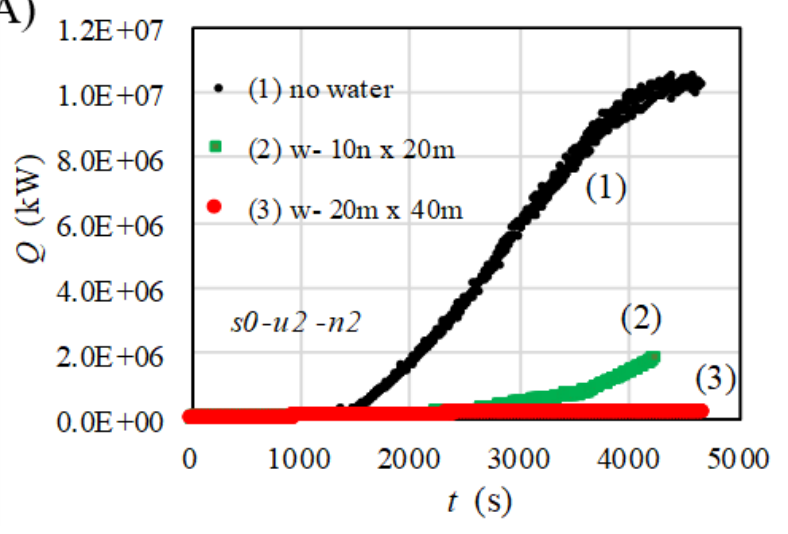

(B)

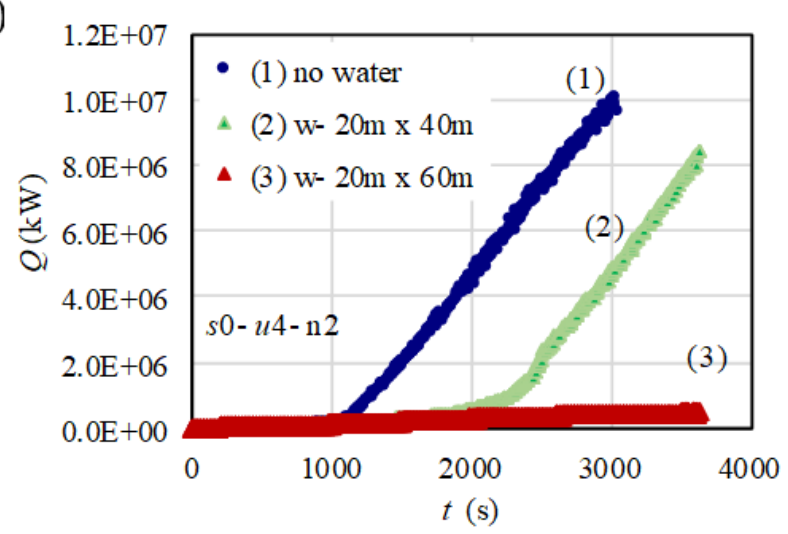

(C) $s=0 \mathrm{~m}, u=2 \mathrm{~m} / \mathrm{s}, n=2 \mathrm{~m}$

(1) no water,

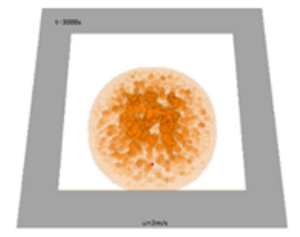

$t=3000 \mathrm{~s}$,

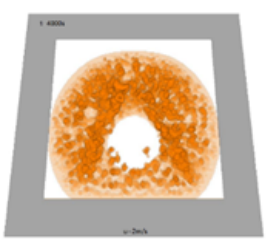

$t=4000$ s,
(2) water drop (10m x 20m),

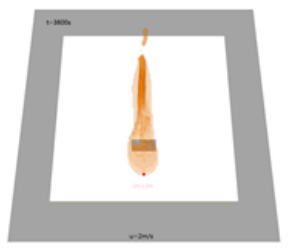

$t=3400 \mathrm{~s}$

(3) water drop (20m x 40m)

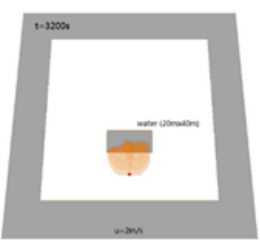

$t=3200 \mathrm{~s}$,

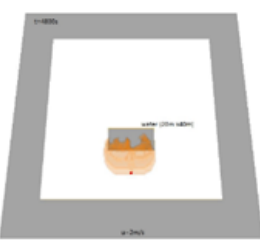

$t=4800 \mathrm{~s}$

(D) $s=0 \mathrm{~m}, u=4 \mathrm{~m} / \mathrm{s}, n=2 \mathrm{~m}$

(1) no water,

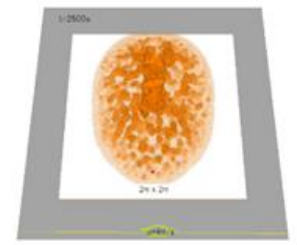

$t=2500 \mathrm{~s}$,
(2) water drop (20 m x 40m),

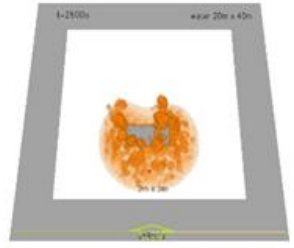

$t=2500 \mathrm{~s}$,

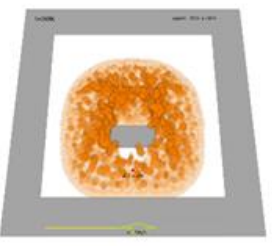

$t=3400$ s,
(3) water drop (20 m x 60m)

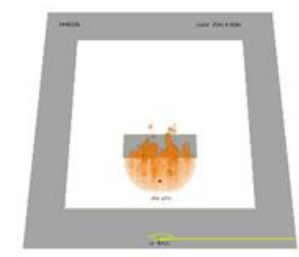

$t=4500 \mathrm{~s}$

Figure 3 - (A) and (B) total heat release of fuels as a function of time, $(C)$ and $(D)$ burning profiles, comparing water drop effect with no water drop, $(A)$ and $(C): s=0 m, u=2 m / s, n=2 m,(B)$ and $(D): s=0 m, u=4 m / s, n=2 m$

For the case of $u=4 \mathrm{~m} / \mathrm{s}$ at $s=0 \mathrm{~m}$, Figure 3(B) shows the water drop effect in the area of (20m x 40m) and $(20 \mathrm{~m} \times 60 \mathrm{~m})$ comparing with the case of no water drop. The case of water drop of $(20 \mathrm{~m} \times 40 \mathrm{~m})$ cannot allow the fire jumping over the water belt, but cannot stop the fire spread, since the fire soon begins to detour both sides of the water belt and spreads into the downwind area, which can however delay the fire spreading time. The water drop of $(20 \mathrm{~m} \times 60 \mathrm{~m})$ in the downwind area can stop the fire spread, as shown in Figure 3(D)-3, but gradually begins to detour both sides of the water dropped area, extending toward the downwind direction. Therefore, a much longer water belt may be needed to stop or to delay the fire spread much more. Extending the wind up to $12 \mathrm{~m} / \mathrm{s}$ and the water dropped area up to $80 \mathrm{~m}$, the effect of water dropped area against the fire spread is examined. Figure 4 shows the relationship between the total heat release rate of fuels and time for $s=0 \mathrm{~m}$ and $u=8 \mathrm{~m} / \mathrm{s}$, varying the water dropped area. The heat release rates quickly increase at a time when the fire begins to detour, which we called the "critical time $\left(t_{q}\right)$ " in this paper, defined as the time when the total heat release 
rate exceeds $Q=1 \mathrm{E}+06 \mathrm{~kW}$. More critical times are examined, varying the wind speed between $u=4 \mathrm{~m} / \mathrm{s}$ and $12 \mathrm{~m} / \mathrm{s}$. Figure 5 shows the relationship between the critical time $\left(t_{q}\right)$ and the length $(L)$ of water dropped area with the width $W=20 \mathrm{~m}$, varying the wind speed. The critical time $\left(t_{q}\right)$ is linearly proportinal to the length $(L)$ of water dropped area. At the width of water belt of $W=20 \mathrm{~m}$, the fires do not jump over the water belt, but the fires can detour both sides of the water belt as shown in Figure 6, which shows the burning profiles of fuels.

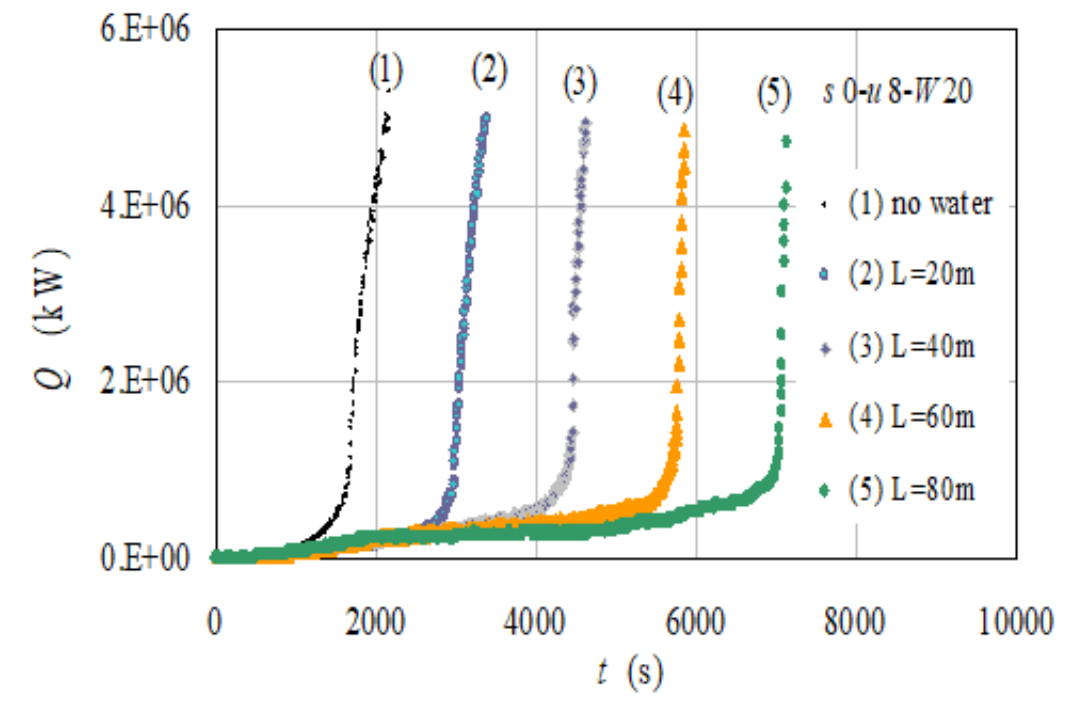

Figure 4 - Relationship between total heat release rate of fuels and time

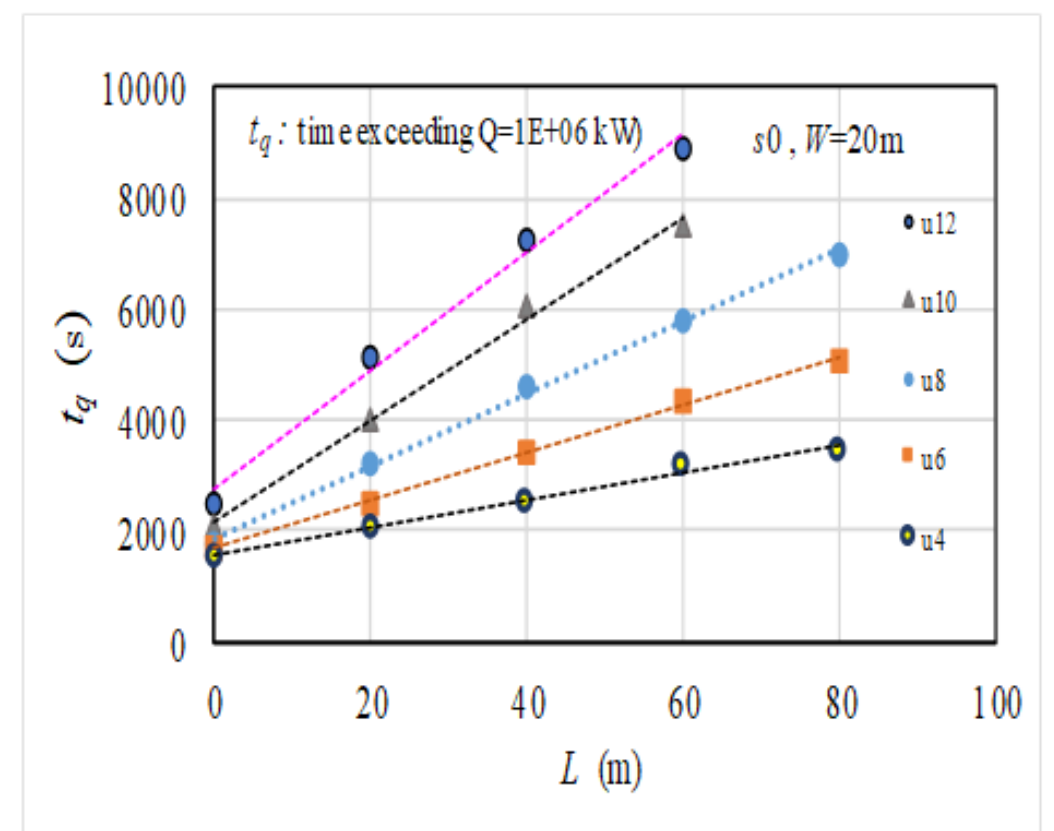

Figure 5 - Relationship between total heat release rate of fuels and heat length 
(1) No water,

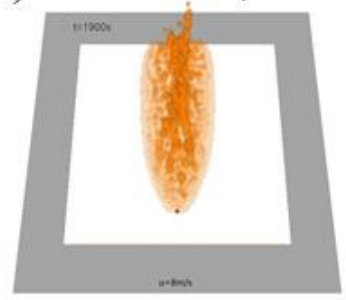

$t=1800 \mathrm{~s}$,
(2) $L=20 \mathrm{~m}$,

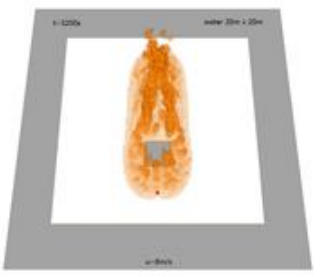

$t=3200$ s,
(3) $L=40 \mathrm{~m}$,

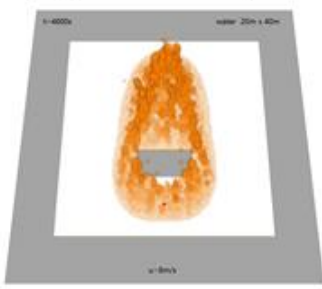

$t=4600 \mathrm{~s}$,
(4) $L=60 \mathrm{~m}$

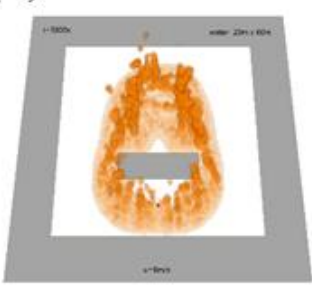

$t=5800 \mathrm{~s}$

Figure 6 - Burning profiles of fuels at $s=0 \mathrm{~m}, u=8 \mathrm{~m} / \mathrm{s}$ and $W=20 \mathrm{~m}$

Table 1 shows the critical time divided by the wind speed $(u)$. Except for some cases of no water $(L=0 \mathrm{~m})$, the divided values $\left(t_{q} / u\right)$ are almost similar for each length $L$. The average values of $\left(t_{q} / u\right)$ are plotted in Figure 7, as a function of $L$. Thus, those relationships shown in Figure 5 can be correlated by the following equation:

$$
t_{q} / u=7.4 L+281
$$

Table 1 - The critical time divided by the wind speed $\left(t_{q} / u\right)$

\begin{tabular}{r|rrrrrr}
\hline$L(\mathrm{~m})$ & $u=12 \mathrm{~m} / \mathrm{s}$ & $u=10 \mathrm{~m} / \mathrm{s}$ & $u=8 \mathrm{~m} / \mathrm{s}$ & $u=6 \mathrm{~m} / \mathrm{s}$ & $u=4 \mathrm{~m} / \mathrm{s}$ & average \\
\hline 0 & 208 & 209 & 216 & 287 & 375 & 259 \\
20 & 425 & 401 & 400 & 417 & 513 & 431 \\
40 & 604 & 603 & 575 & 573 & 625 & 596 \\
60 & 742 & 752 & 725 & 725 & 788 & 746 \\
80 & & & 869 & 842 & 858 & 856 \\
\hline
\end{tabular}

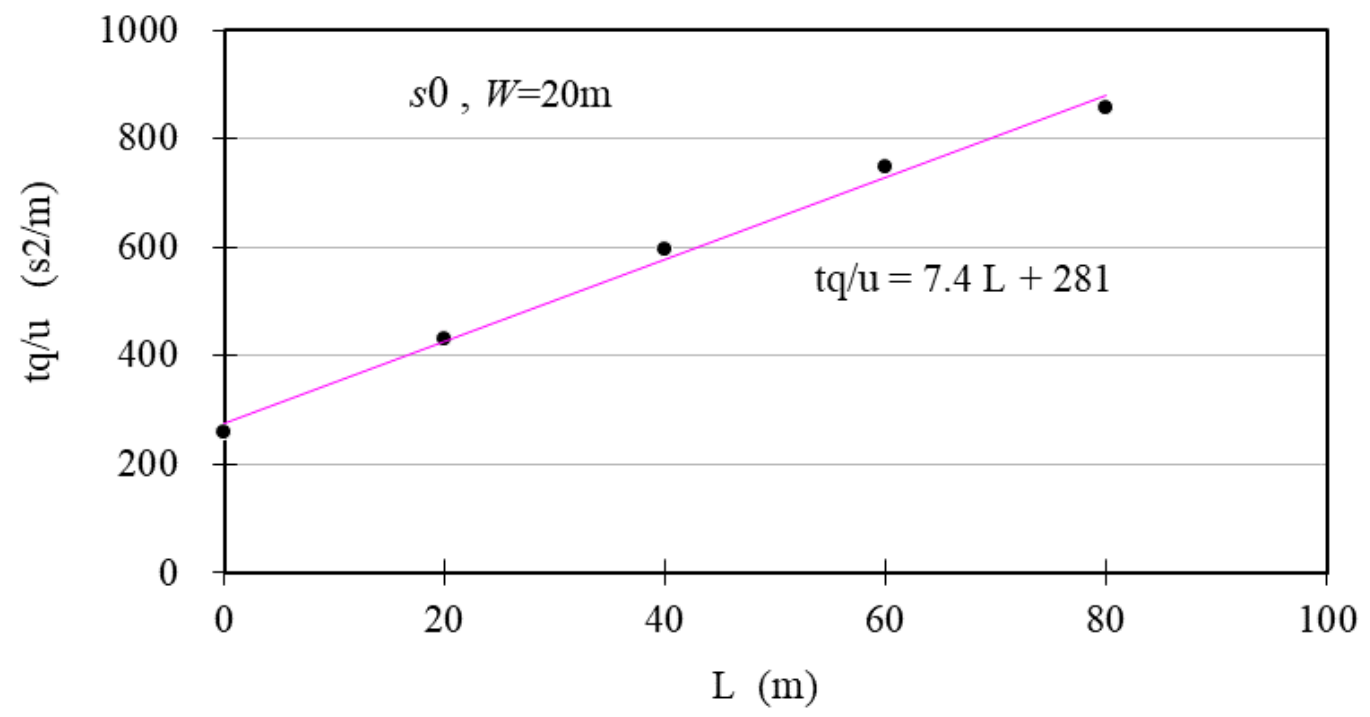

Figure 7 - Relationship between the average critical time divided by the wind speed and length of water dropped area. 


\subsubsection{Heat release rate using a line heater, $s=0 m$}

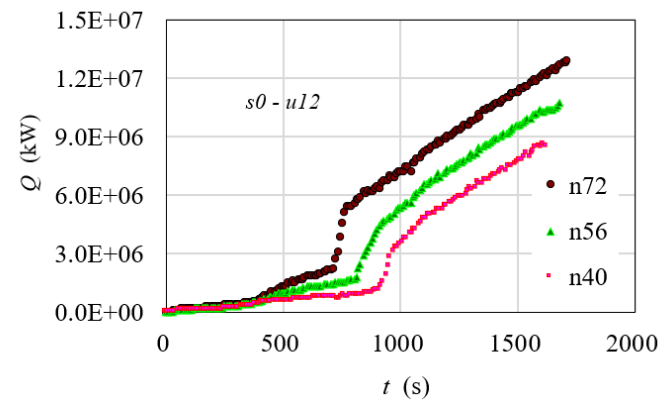

Figure 8 - Total heat release rate of fuels as a function of time, varying the length of line heater, $s=0 \mathrm{~m}, u=12 \mathrm{~m} / \mathrm{s}$

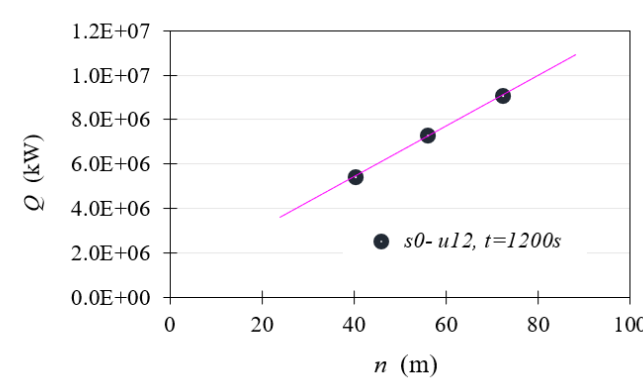

Figure 9 - Relationship between heat release rate and length of water drop area $(n)$ at $t=1200 \mathrm{sec}, \mathrm{s}=0 \mathrm{~m}, u=12 \mathrm{~m} / \mathrm{s}$

Figure 8 shows the total heat release of fuels as a function of time, varying the length (n) of line heater, for $\mathrm{s}=0 \mathrm{~m}$ and $\mathrm{u}=12 \mathrm{~m} / \mathrm{s}$, where the relationships are parallel between 1000 to $1500 \mathrm{sec}$. The heat release rates at $\mathrm{t}=1200 \mathrm{sec}$ are plotted as a function of the ignition heat source length (n) in Figure 9, where the heat release rate is linearly proportional to the ignition heat source length. This linear relationship shown in Figure 9 between the heat release rate and the wind speed are found in many cases varying the fuel space and the ignition source length. Figure 10(A) shows the heat release rate vs. time for $\mathrm{s}=0 \mathrm{~m}, \mathrm{u}=8 \mathrm{~m} / \mathrm{s}$ and $\mathrm{u}=12 \mathrm{~m} / \mathrm{s}$ at $\mathrm{n}=72 \mathrm{~m}$ and Figure $10(\mathrm{~B})$ shows the fire spreading patterns. At $\mathrm{u}=12 \mathrm{~m} / \mathrm{s}$ and the water drop area of $\mathrm{W}=18 \mathrm{~m} \times \mathrm{L}=80 \mathrm{~m}$, the fire spread is protected at $\mathrm{t}=1200 \mathrm{sec}$, but at $\mathrm{u}=8 \mathrm{~m} / \mathrm{s}$ and the water drop area of $\mathrm{W}=16 \mathrm{~m} \times \mathrm{L}=80 \mathrm{~m}$, the fire jumped over the water drop area at $\mathrm{t}=800 \mathrm{sec}$. Fires at $\mathrm{s}=0 \mathrm{~m}$ and ignited by a long heater can spread very fast and strong. The width $\mathrm{W}=18 \mathrm{~m}$ of the water drop area is effective to protect the fire jumping over the water dropping, but fires can detour from both sides of the water drop area. Therefore, it is generally difficult to protect the fire spread at $s=0 \mathrm{~m}$, even by a large water dropped area.

(A)

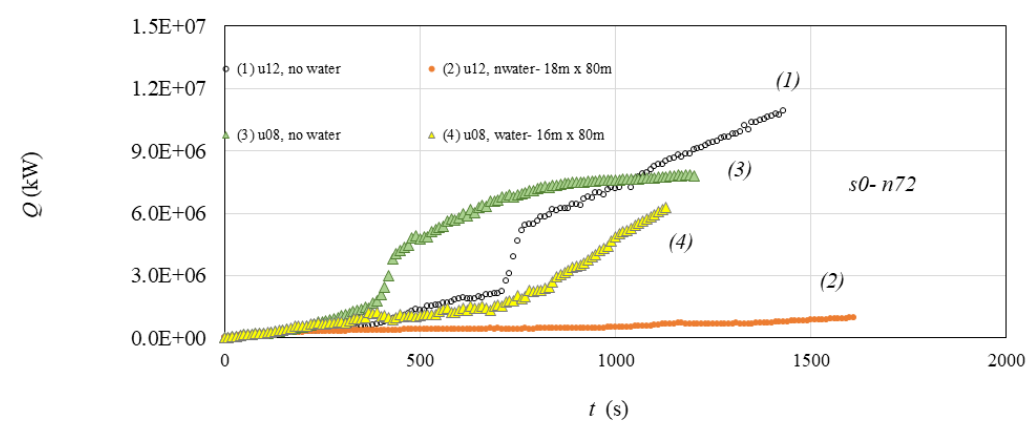

(B)

(1)

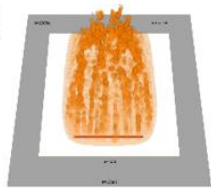

(2)

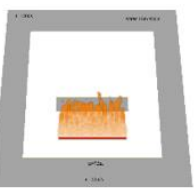

(3)

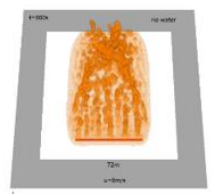

(4)

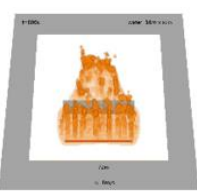

Figure 10 - (A) Relationship between heat release rate and time, (B) burning profiles at $t=800 \mathrm{~s}, n=72 \mathrm{~m},(1) u=12 \mathrm{~m} / \mathrm{s}$, no water, (2) $u=12 \mathrm{~m} / \mathrm{s}$, water $-W=18 \mathrm{~m} \times \mathrm{L}=80 \mathrm{~m}$, (3) $u=8 \mathrm{~m} / \mathrm{s}$, no water, (4) $u=8 \mathrm{~m} / \mathrm{s}$, water $-W=16 \mathrm{~m} \times \mathrm{L}=80 \mathrm{~m}$ 


\subsubsection{Case of $s=1 m$}

(A)

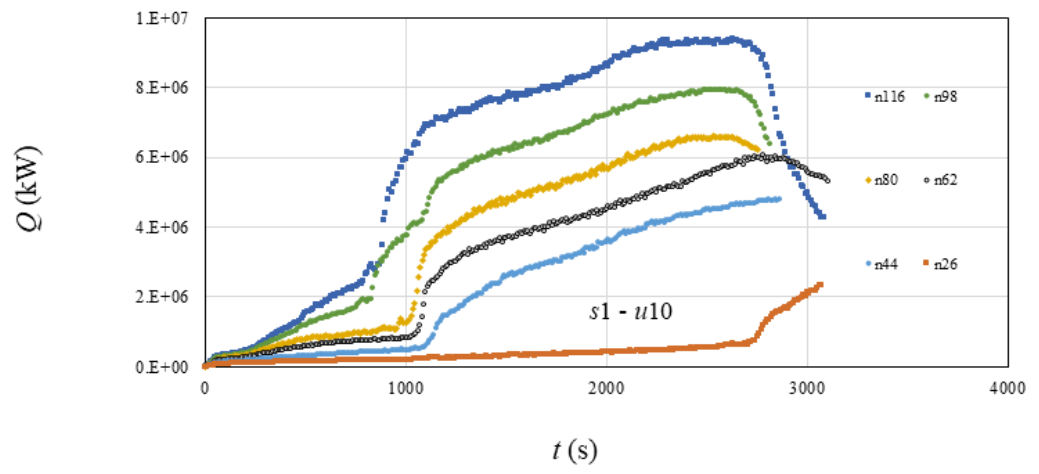

(B)

(1) $n=44 \mathrm{~m}$,

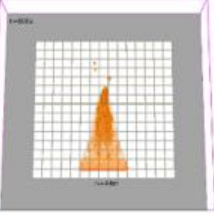

(2) $n=62 \mathrm{~m}$,

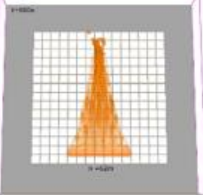

(3) $n=80 \mathrm{~m}$,

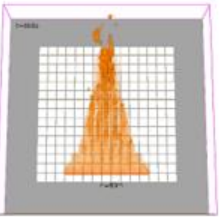

(4) $n=116 \mathrm{~m}$

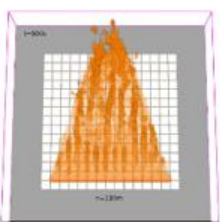

Figure 11 - (A) Relationship between heat release rate and time and (B) burning profiles of fuels at $t=800 \mathrm{~s}$, varying the ignition fire source length $(n), s=1 m, u=10 \mathrm{~m} / \mathrm{s}$.

Figure 11(A) shows the relationship between the total heat release rate and time for $s=1 \mathrm{~m}$ and $u=10 \mathrm{~m} / \mathrm{s}$, varying the ignition fire source length $(n)$. Figure 11(B) shows the burning profiles of fires. Figure 12 shows the relationship between the heat release rate and the length of water drop area $(n)$ at 1500 and 2000sec, where the heat release rate is linearly proportional to the ignition heat source length. As mentioned above, this linear relationshp between the heat release rate and the line heater length are observed in the cases of $s=2 \mathrm{~m}, 3 \mathrm{~m}$ and $4 \mathrm{~m}$.

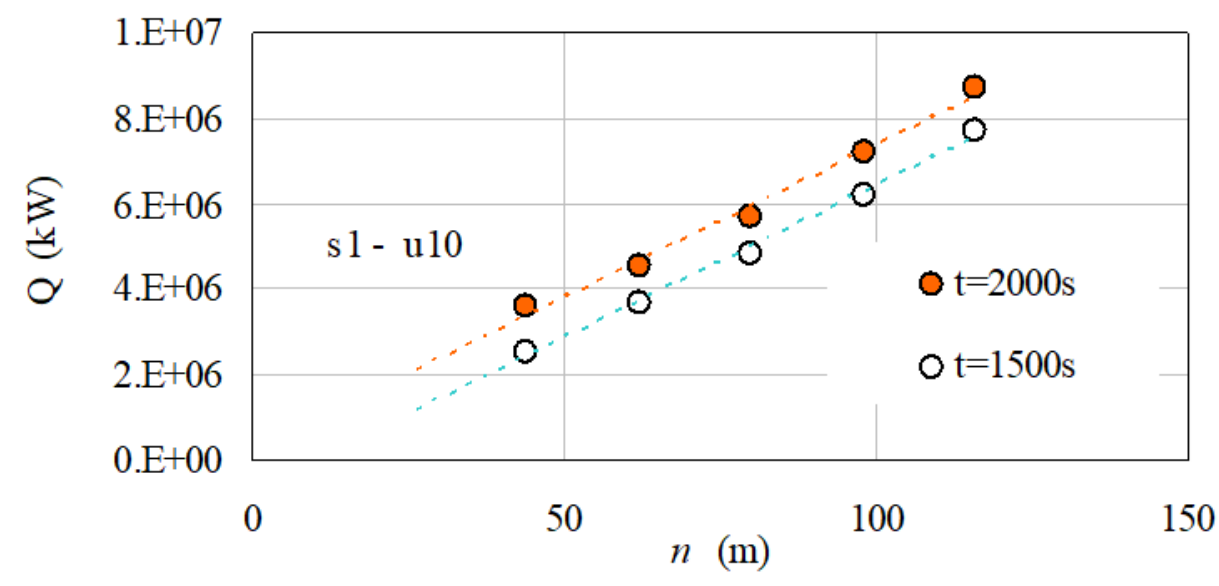

Figure 12 - Relationship between heat release rate and length of water drop area (n) at time 1500 and $2000 \mathrm{sec}, \mathrm{s}=1 \mathrm{~m}$, $u=10 \mathrm{~m} / \mathrm{s}$. 
(A)

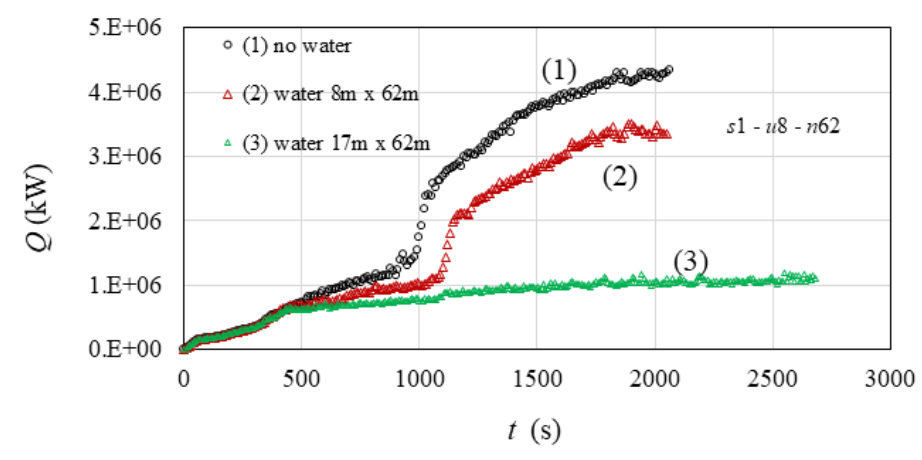

(B)

(1)

$t=1200$ s

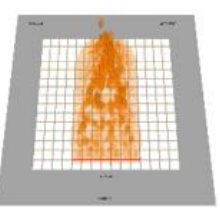

(2)

$t=1500$ s

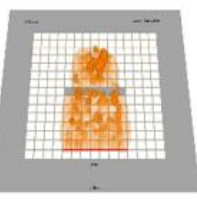

(3)

$t=2400$ s

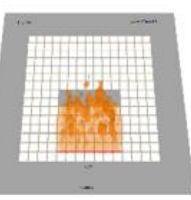

Figure 13 (A) - Relationship between heat release rate and time, (B) burning profiles of fires, for $s=1 \mathrm{~m}, u=8 \mathrm{~m} / \mathrm{s}$, $n=62 m$, (1) no water, (2) water $-W=8 m \times L=62 m$, (3) water $-W=17 m \times L=62 m$.

Figure 13(A) shows the relationship between heat release rate and time for $s=1 \mathrm{~m}$ and $u=8 \mathrm{~m} / \mathrm{s}$, comparing the effect of water drop area, keeping the ignition fire source $n=62 \mathrm{~m}$. Figure 13 (B) shows the fire spreading profiles. For $s=1 \mathrm{~m}$, the width $W=17 \mathrm{~m}$ of the water drop area is effective to protect the fire jumping over the water drop area, while the width $W=8 \mathrm{~m}$ allowed to jumping over the fire spread. Figure 14(A) shows the relationship between heat release rate and time for $s=2 \mathrm{~m}$ and $n=128 \mathrm{~m}$, warying the wind speed. Figure 14(B) shows the fire spreading profiles at $t=2100 \mathrm{~s}$, varying the wind speed. As shown in Figure 15, the heat release rates begin to increase from $u=4 \mathrm{~m} / \mathrm{s}$ and reach almost constant at $u=10 \mathrm{~m} / \mathrm{s}$. This relationship curve can be found in other cases of $s=1 \mathrm{~m}, 3 \mathrm{~m}$ and $4 \mathrm{~m}$, too.

\subsubsection{Case of $s=2 m$}

(A)

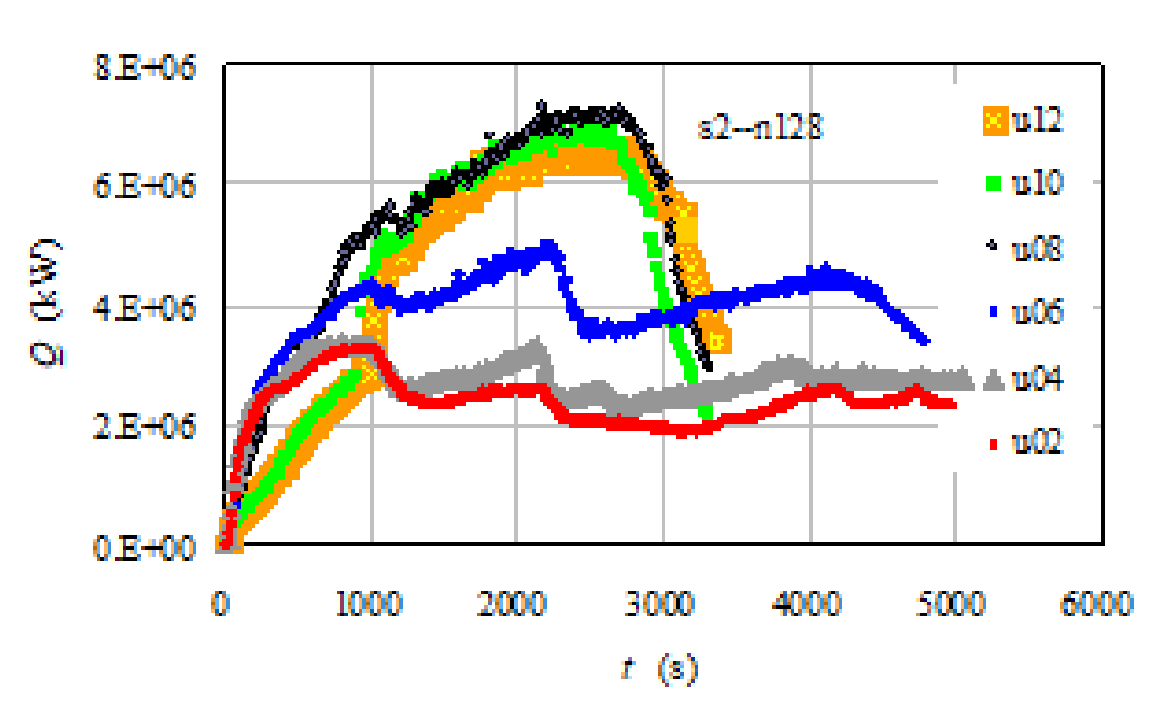


(B)

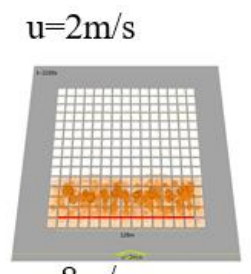

$\mathrm{u}=8 \mathrm{~m} / \mathrm{s}$

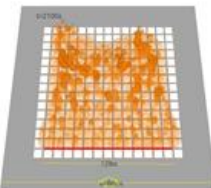

$\mathrm{u}=4 \mathrm{~m} / \mathrm{s}$

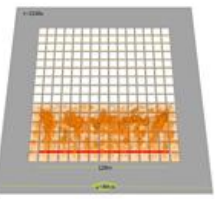

$\mathrm{u}=10 \mathrm{~m} / \mathrm{s}$

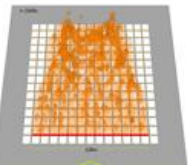

$\mathrm{u}=6 \mathrm{~m} / \mathrm{s}$

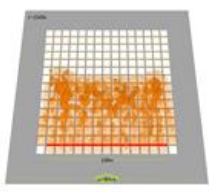

$\mathrm{u}=12 \mathrm{~m} / \mathrm{s}$

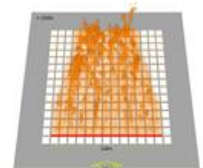

Figure 14 - (A) Relationship between heat release rate and time, (B) burning profiles of fires at $t=2100 s, s=2 m$, $n=128 \mathrm{~m}$, varying the wind speed

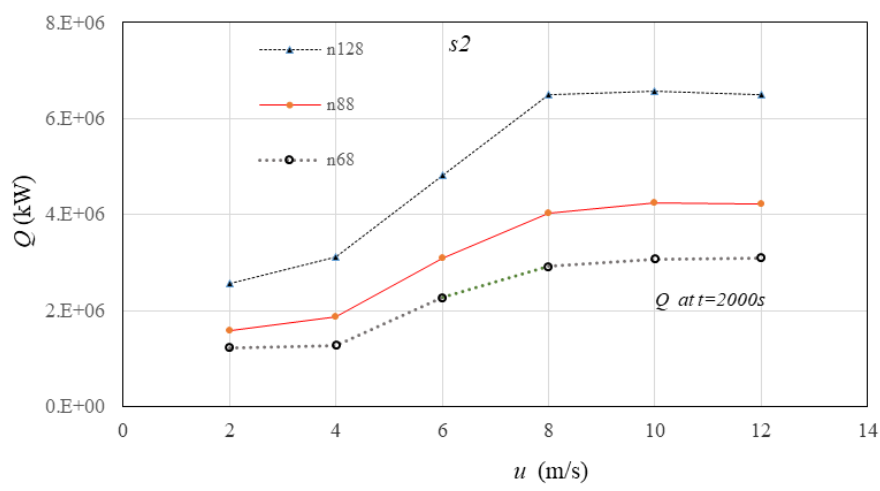

Figure 15 - Relationship between heat release rate and wind speed at $t=2000 \mathrm{sec}, \mathrm{s}=2 \mathrm{~m}$, varying the ignition source length (n)

Figure 16(A) shows the relationships between heat release rate and time for $s=2 \mathrm{~m}, \mathrm{u}=8 \mathrm{~m} / \mathrm{s}$ and $n=68 \mathrm{~m}$ and Figure 16(B) for $s=2 \mathrm{~m}, u=10 \mathrm{~m} / \mathrm{s}, n=88 \mathrm{~m}$, varying the water drop area. Figure 16(C) shows the fire spreading profiles at $t=1400 \mathrm{~s}$ and Figure $16(\mathrm{C})$ at $\mathrm{t}=1500 \mathrm{~s}$, varying the wind speed. The fire spread is protected by the water drop at $\mathrm{W}=18 \mathrm{~m} \times \mathrm{L}=68 \mathrm{~m}$, while the water drop area at $W=8 \mathrm{~m}$ cannot stop the jumping over the fire spread. Since the fires at $s=2 \mathrm{~m}$ cannot detour both sides of the water drop area, the width $W$ is important to protect the fire spread. Therefore, the water drop length $(L)$ larger than the ignition source length $(n)$ can protect the fire spread at $s=2 \mathrm{~m}$. 
(A)

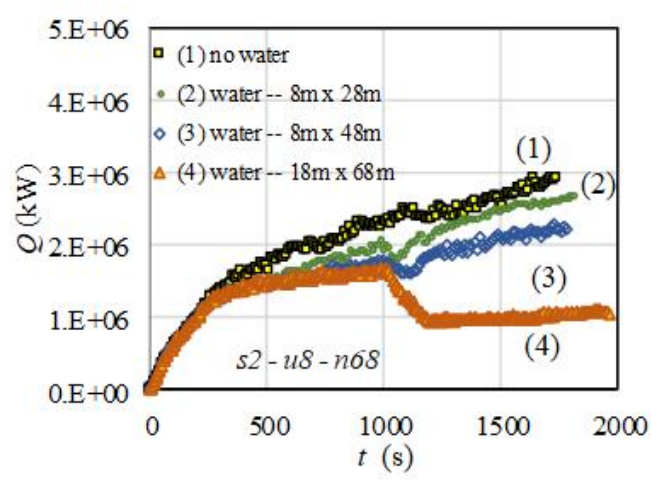

(C) no water,

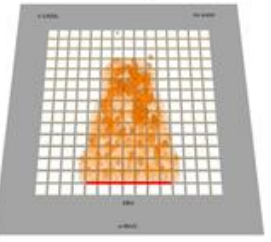

(D)

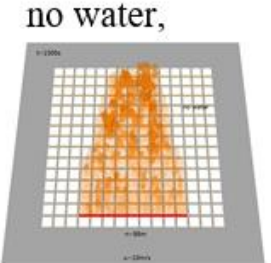

(B)

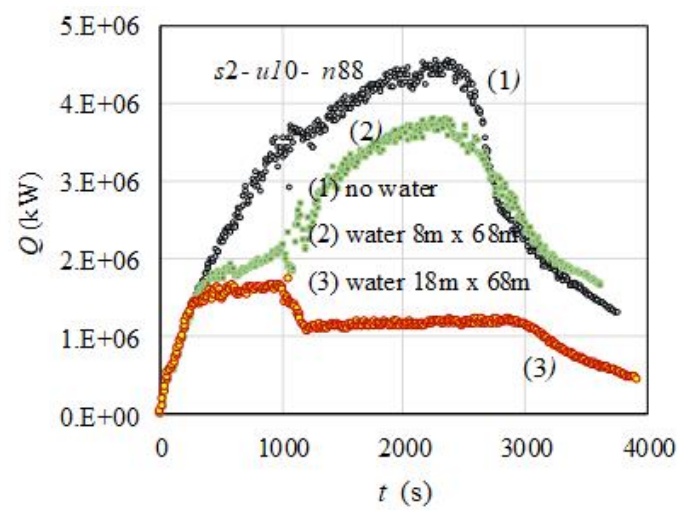

water $-8 \mathrm{~m} \times 28 \mathrm{~m}$,

water $-18 \mathrm{~m} \times 68 \mathrm{~m}$

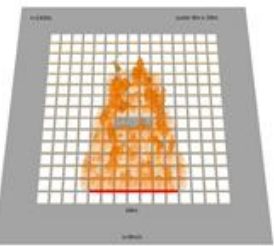

water $-8 \mathrm{~m} \times 68 \mathrm{~m}$,

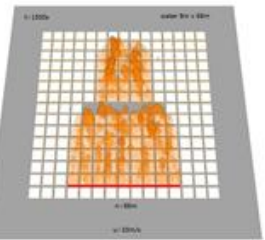

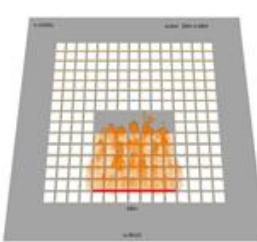

water $-18 \mathrm{~m} \mathrm{x} 68 \mathrm{~m}$

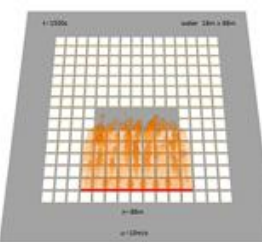

Figure 16 - (A), (B) Relationship between heat release rate and time, varying water drop area, $(A) s=2 m, u=8 m / s$ and $n=68 \mathrm{~m},(B) s=2 \mathrm{~m}, u=10 \mathrm{~m} / \mathrm{s}, n=88 \mathrm{~m},(C),(D)$ burning profiles of the fuels, $(C) s=2 \mathrm{~m}, u=8 \mathrm{~m} / \mathrm{s}$ and $n=68 \mathrm{~m}, t=1400 \mathrm{~s}$,

(D) $s=2 m, u=10 \mathrm{~m} / \mathrm{s}, \mathrm{n}=88 \mathrm{~m}, \mathrm{t}=1500 \mathrm{~s}$

\subsubsection{Cases of $s=3 m$ and $s=4 m$}

Figure 17 shows the relationship between heat release rate and time for Case (A) $s=3 \mathrm{~m}, u=10 \mathrm{~m} / \mathrm{s}$ and $n=52 \mathrm{~m}$, and Case (B) $s=4 \mathrm{~m}, u=6 \mathrm{~m} / \mathrm{s}$ or $8 \mathrm{~m} / \mathrm{s}, n=104 \mathrm{~m}$, varying the water drop area. Figures $17(\mathrm{C})$ and (D) show the fire spreading profiles of Case (A) at $t=3000 \mathrm{~s}$ and Case (B) at 3200s, respectively. The fire spread is protected by the water drop of Case A-(2) $W=8 \mathrm{~m} \times L=30 \mathrm{~m}$, Case A-(3) $W=8 \mathrm{~m} \times$ $L=52 \mathrm{~m}$, Case B-(2) $W=8 \mathrm{~m} \times L=80 \mathrm{~m}$, Case B-(4) $W=8 \mathrm{~m} \times L=104 \mathrm{~m}$. The fires at $s=3 \mathrm{~m}$ and $s=4 \mathrm{~m}$ do not jump over the water drop area and detour both sides of the water drop area. Therefore, if the spaces $(s)$ are much larger, much fewer water dropping can stop the fire spread.

(A)

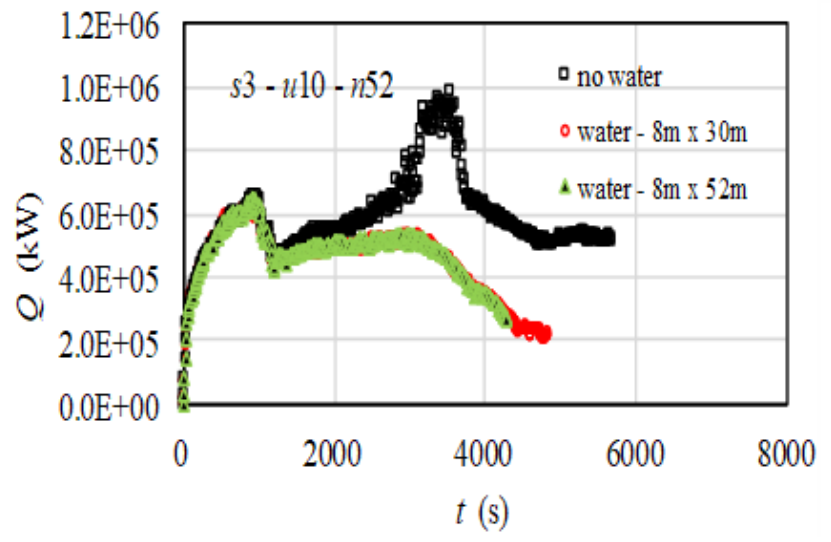

(B)

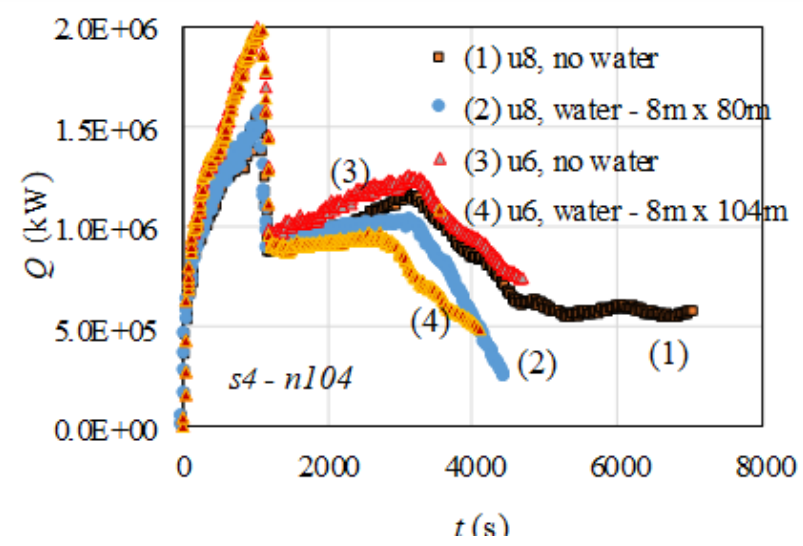


(C) Fire spreading patterns, $s=3 \mathrm{~m}, u=10 \mathrm{~m} / \mathrm{s}, n=52 \mathrm{~m}, t=3200 \mathrm{~s}$

(1) no water,

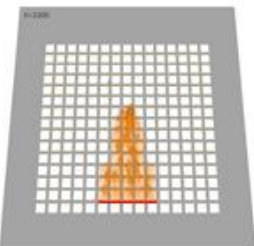

(2) water $-W=8 \mathrm{~m} \times L=30 \mathrm{~m}$,

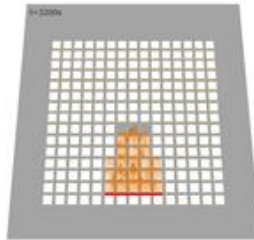

(3) water $-W=8 \mathrm{~m} \times L=52 \mathrm{~m}$

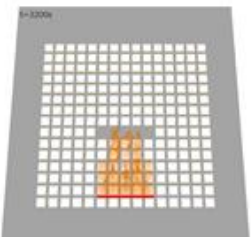

(D) Fire spreading patterns, $s=4 \mathrm{~m}, n=104 \mathrm{~m}, t=3000 \mathrm{~s}$

(1)-1

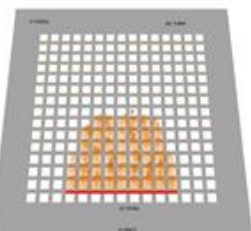

(1)-2

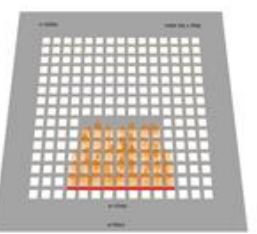

(2)-1

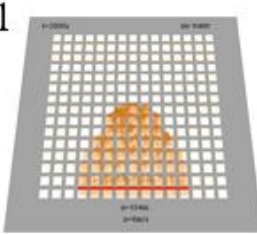

(2)-2

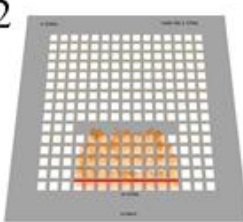

Figure 17 - (A) and (B): Relationship between heat release rate and time, varying water drop area, and $(C)$ and $(D)$ : Fire spreading patterns, varying the water drop area, $(A) s=3 \mathrm{~m}, u=10 \mathrm{~m} / \mathrm{s}, n=52 \mathrm{~m}, n=52 \mathrm{~m},(B) s=4 \mathrm{~m}, u=6 \mathrm{~m} / \mathrm{s}$ or $8 \mathrm{~m} / \mathrm{s}$, $n=104 \mathrm{~m},(C) s=3 \mathrm{~m}, u=10 \mathrm{~m} / \mathrm{s}, n=52 \mathrm{~m}, t=3200 \mathrm{~s},(D) s=4 \mathrm{~m}, n=104 \mathrm{~m}, t=3000 \mathrm{~s},(1)-1: u=8 \mathrm{~m} / \mathrm{s}$, no water, (1)-2: $u=8 \mathrm{~m} / \mathrm{s}$, water- $W=8 m \times L=80 m,(2)-1: u=6 m / s$, no water, $(2)-2: u=6 m / s$, water- $W=8 m \times L=80 m$.

\section{Discussion}

If the fuel distributes very densely like $s=0 \mathrm{~m}$, even the water belt dropped in the area of $20 \mathrm{~m} \times 60 \mathrm{~m}$ may not be sufficient to stop the fire spread perfectly, since fires may detour both sides of the water belt or jump over the water belt. To stop such fire spread, much larger water belt may be needed. If the fuels distribute discretely such as $s=3 \mathrm{~m}$ or $4 \mathrm{~m}$, the water belt of $20 \mathrm{~m}$ x $40 \mathrm{~m}$ may be able to stop the fire spread. However, helicopters carry only about 0.5 tons $=500 \mathrm{~kg}$ of water in the suspending bucket, but it is too few, since the water density on the ground is only $500 \mathrm{~kg} / 20 \mathrm{~m} / 40 \mathrm{~m}=0.6 \mathrm{~kg} / \mathrm{m}^{2}$ [7]. An aircraft carrying the water of 20tons can drop the water with the density of $25 \mathrm{~kg} / \mathrm{m}^{2}$ in the area of $20 \mathrm{~m} \times 40 \mathrm{~m}$, which is far better than those by helicopters. In this study, the selected way is to drop water in the downwind area slightly away from the fire front, eventually on a strongly burning area. It is dangerous for helicopter to fly in the hot and smoky sky, just above the fires. If helicopters fly at higher altitude and higher flight speed like larger aircrafts, it may be safe, but too few water drop may be disperse into mists in the sky. Therefore, large aircrafts carrying large amount of water, flying much higher altitude than helicopters, and passing through the smoke quickly above the burning fires, are needed to make such a large water belt. In the future, the water drop in an aerial firefighting may be done in much more effective ways based on the combination of the infrared reconnaissance of burning areas and the real-time CFD simulations of the burning profile of forest fires.

\section{Conclusion}

CFD simulations have been made to examine the effect of water dropped belt by an aerial firefighting to protect the fire spread. Some relationships are found, such as linear relationship between the heat release rate and the wind speed or the length of ignition heat source. The selected way in this study is to drop water in the downwind area slightly far from the fire front which may be more strongly burning place, making a water belt to reduce the radiative heat transfer to the fuels in the downwind area. However, if the fuel distributes densely, the water belt dropped in the area of $20 \mathrm{~m} \times 60 \mathrm{~m}$ may not be sufficient to stop the fire spread, since the fires may detour both sides of the water belt ort jump over the water belt. However, the water drops are very effective to delay the fire spreading speed. If 
the space distance between adjacent fuels is larger than $3 \mathrm{~m}$ or $4 \mathrm{~m}$, the water belt may be able to protect or extremely delay the fire spread. One problem is the amount of water to realize a water belt to protect the fire spread, since helicopters carry about 0.5 tons of water in the suspended buckets, which is too few and it is dangerous for helicopter to fly in the hot and smoky sky. If helicopters fly at higher altitude, it may be safe, but too few water drop may be disperse into mists in the sky. Large aircrafts carrying large amount of water such as 20tons are needed to make such a large water belt.

\section{References}

Viegas, D.X., , "A Mathematical Model For Forest Fires Blowup", Combustion Science and Technology, Vol. 177, pp.27-51, (2006)

Finney, M.A.,"Calculation of fire spread rates across random landscapes", International Journal of Wildland Fire, Vol.12, pp.167-174, (2003)

Abatzoglou, J.T., J. K. Balch, B. A. Bradley and C. A. Kolden, "Human-related ignitions concurrent with high winds promote large wildfires across the USA", International Journal of Wildland Fire, 27(6), pp.377-386, (2018)

Liu, N., Liu, Q., Deng, Z, Satoh, K., Zhu, J., "Burn-out time data analysis on interaction effects among multiple fires in fire arrays“, Proc. Comb. Inst., 31, pp. 2589-2597, (2007)

Satoh, K., S. Kitamura, K. Kuwahara, K.T.Yang, "An Analysis to Predict Forest Fire Danger and Fire Spread", ASME Proceedings of Heat Transfer, 2003 ASME Summer Heat Transfer Conference, Las Vegas, HT2003-47357, pp. 111-118 (2003)

Satoh K, K. Kuwahara, Yang K.T., "Experimental and Numerical Simulations of Flow Patterns of Dropping Water from Fire-fighting Helicopters", vol 5, ASME-HTD, Orlando, pp 57-64 (2000)

Satoh, K., Maeda, I., Kuwahara, K. and Yang, K.T., "A Numerical Study of Water Dump In Aerial Fire Fighting", IAFSS Fire Safety Science 8, Beijin, pp.777-787 (2005)

Satoh, K., N. Liu, Q. Liu, K.T.Yang, "Numerical and Experimental Study of Firewhirl Generated in 15x15 Array Fires Placed in Cross Wind", Proc. ASME 2008, Boston, pp.1-8, (2008)

McGrattan, K.B., Baum, H.R., Rehm, R.G., Hamins, A., and Forney, G.P., "Fire Dynamics Simulator: Technical Reference Guide," Technical Report NISTIR 6467, NIST, (2000).

Floyd, J., Forney, G., Hostikka, S., Korhonen, T., McDermott, R., and McGrattan, K., "Fire Dynamics Simulator User's Guide,” NIST Special Publication 1019, NIST, (2006).

McGrattan, K.B., Floyd, J., Forney, G., Baum, H.R., Hostikka, S., "Development of Combustion and Radiation Models for Large Scale Fire Simulation," Proceedings of Third Technical Symposium on Computer Applications in Fire Protection Engineering, pp.2001-2011, (2001). 


\title{
Circulation weather types and their influence on the fire regime in Portugal
}

\author{
Carlos C. DaCamara*; Ricardo M. Trigo \\ Instituto Dom Luiz (IDL), Faculdade de Ciências, Universidade de Lisboa, Lisbon, 1749-016, \\ Portugal, \{cdcamara@fc.ul.pt*,rmrigo@fc.ul.pt\}
}

\begin{abstract}
The fire regime in Portugal presents a marked annual cycle that is here characterized based on values of radiant energy released by wildfires as detected by Meteosat geostationary satellites. The role played by meteorological conditions in the fire regime is then analyzed from the point of view of synoptic climatology. For this purpose, we use $10 \mathrm{Lamb}$ circulation weather types (CWTs) that are grouped into 8 directional types, associated to the cardinal and intercardinal directions of the geostrophic flow, and 2 rotational types (cyclonic and anticyclonic), associated to the positive and negative signs of geostrophic vorticity.

The study covers the 14-year period from January 2004 to December 2017. The monthly distribution of total fire radiative energy presents large values during the fire season (defined by the months spanning from July to October), with a very strong peak located in August. A secondary peak, located in March, is also clearly visible. The number of fire days (i.e. days where there is a recorded release of fire energy in Portugal) during the fire season represents $56 \%$ of the total number of fire days but contributes to $92 \%$ of the total Fire Radiative Energy (FRE) released by wildfires.

When analyzing the fire season from the point of view of CWTs, results indicate that $61 \%$ of the FRE released in 2004-2017 is observed in days characterized by directional types NE and E that just occur in $28 \%$ of the fire days. The contrast is especially marked when restricting to the E type that occurs only in $8 \%$ of the fire days but contributes to $25 \%$ of the energy released. In turn, the dominant anticyclonic type, that occurs in $31 \%$ of the fire days, contributes to just $15 \%$ of the total FRE. The role played by the different CWTs is also noticeable in March, where fire days represent $36 \%$ of all March days and contribute to $2 \%$ of the total energy released in 2004-2017. Types E and SE that occur only in 14\% of the fire days are responsible for $71 \%$ of the total FRE in March and more than half (37\%) of this amount is released in days of type SE that are observed only in $5 \%$ of the days.

The importance of the contribution of NE and E types for the fire regime in Portugal is enhanced when restricting to the days with extreme release of energy. The distribution of daily FRE is long tailed with 5\% of the extreme fire days (113 days) accounting for 69\% of the total FRE. A very large proportion (96\%) of these extreme days took place in the fire season, namely in August (62\%), July (20\%), September (9\%) and October $(6 \%)$. Almost two thirds (66\%) of the released energy by extreme events are associated to directional types $\mathrm{NE}(36 \%)$ and $\mathrm{E}(30 \%)$.
\end{abstract}

Keywords: fire regime, fire radiative energy, circulation weather types, fire meteorology

\section{Introduction}

The tragic dimension of the fire events of 2017 in Portugal, both in terms of loss of human lives and of economic and social harm, has put at stake long-term established policies of fire management and has uncovered the vulnerabilities at the structural and operational levels of the current system of fire prevention and combat. Today, more than ever, there is a crucial need for reliable meteorological information, namely on the impact of prevailing weather conditions on fire behavior that will assist decision makers in taking preventive measures (e.g. prescribed fires) and in allocating resources to be employed.

The establishment of an optimal system of fire prevention and combat requires, in particular, having a thorough understanding of the factors playing a major role in extreme fire seasons characterized by 
an anomalously high or low number of ignitions, by very large or small amounts of burned area or by catastrophic or negligible impacts on the landscape or socio-economic activities. In this work, the fire regime in Portugal will be analyzed using daily values of fire radiative energy (FRE) released by wildfires as detected from radiometers on-board of geostationary satellite platforms. FRE is a physical property that has the advantage of being related to the amount of fuel burned and has been used as a proxy of fire line intensity in fire management and firefighting studies (Johnston et al., 2017).

Meteorological and climatic factors play a crucial role in fire behavior in Portugal. For instance, more than $2 / 3$ of the inter-annual variability of burned area is explained by meteorological factors namely i) the temperature and precipitation regimes of the spring preceding the fire season and ii) the occurrence during the fire season of circulation patterns of short-duration that induce extremely hot and dry spells over western Iberia (Pereira et al., 2005; Pereira et al., 2013).

The aim of this work is to assess the role played by meteorological conditions in the fire regime in Portugal. For this purpose, we adopt the point of view of synoptic climatology and use circulation weather types (CWTs) originally developed for the British Isles and later adapted to Portugal (Trigo and DaCamara, 2000). The versatility of this tool has already proven successful in a wide range of studies in Portugal and the Iberian Peninsula that include the precipitation regime (Trigo and DaCamara, 2000) and lightning activity (Ramos et al., 2011) in Portugal, and, for Iberia, the spatial variability of precipitation (Ramos et al., 2014) and drought (Russo et al., 2015), wildfire activity (Trigo et al., 2013) and upwelling activity along the western coast of Iberia (Ramos et al., 2013). Here, the impact of weather on wildfire activity is assessed by systematically comparing the contributions of the different CWTs to the FRE released by wildfires, special attention being devoted to CWTs associated to extreme fire days, defined as the top 5\% of days with largest released FRE.

\section{Data and methods}

Daily values of radiative energy released by wildfires in Portugal covering the 14-year period 20042017 were extracted from the Fire Radiative Power (FRP) product (Wooster et al., 2015) of EUMETSAT Satellite Application Facility on Land Surface Analysis (LSA SAF) that is based on information provided by the SEVIRI radiometer on-board Meteosat Second Generation (MSG) series of geostationary satellites operated by EUMETSAT (Trigo et al. 2011).

Classification of daily weather is based on Lamb circulation weather types (CWTs) as derived from sea level pressure fields covering the same period. Daily values of surface pressure were extracted from the ERA Interim reanalysis dataset (Dee et al., 2011) generated by the European Centre for Medium-Range Weather Forecasts (ECMWF).

As for the Lamb CWTs developed for the UK, the CWTs for Portugal are grouped into 8 directional (NE, E, SE, S, SW, W, NW and N) associated to the cardinal and intercardinal directions of the geostrophic flow, and 2 rotational types (cyclonic and anticyclonic) according to the two signs of geostrophic vorticity (Trigo and DaCamara, 2000).

\section{Results}

The monthly distribution of fire days (i.e. days where there is a recorded release of FRE by wildfires in Portugal) presents large values (above 200 fire days) during the period from July to October, hereafter referred to as the fire season (Figure 1, upper panel); the peak in August is closely followed by July. There is a secondary peak, located in March, with the number of fire days slightly above 150 and the remaining months present values below this threshold. 

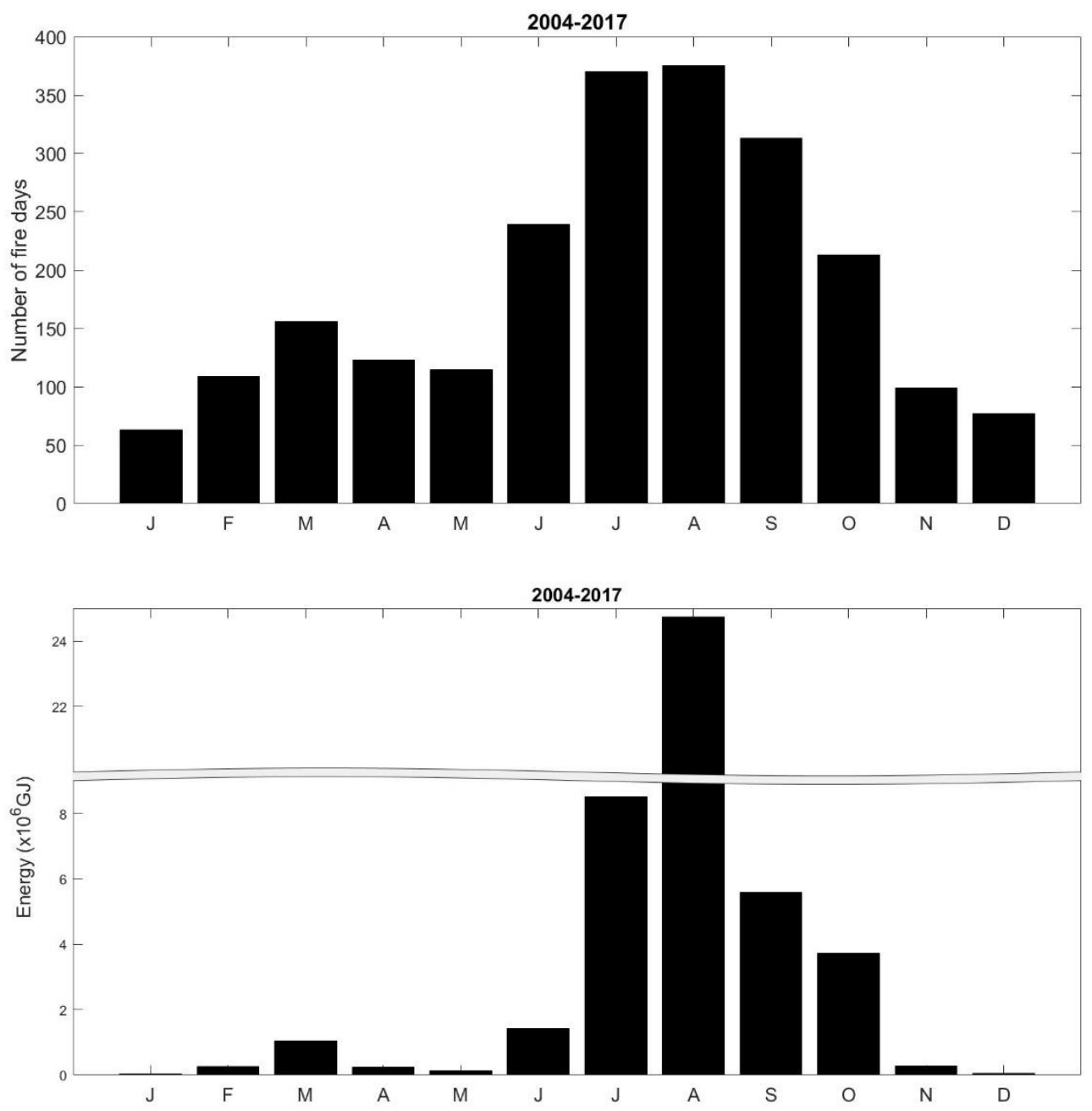

Figure 1 - Monthly distribution of fire days (upper panel) and of associated total fire radiative energy (lower panel) during the period 2004-2017.

The monthly distribution of total FRE (Figure 1, lower panel) is consistent with that of fire days with the higher values occurring during the fire season with a peak in August, and with a secondary peak in March; however, the peak in August of FRE is conspicuously high.

It is worth noting that the annual cycle of FRE is much more pronounced than that of fire days. For instance, the fire season contains $56 \%$ of all fire days in 2004-2017 but contributes to $92 \%$ of the total FRE $\left(4.6 \times 10^{7} \mathrm{GJ}\right)$, with August representing $17 \%$ of all fire days (30\% days of the fire season) and contributing to $54 \%$ of total FRE (and 58\% of FRE in the fire season).

The role played by meteorological conditions in the fire regime was analysed by comparing the relative frequency of occurrences of each CWT in each month with the relative contribution of that CWT to the total FRE of the considered month (Figure 2).

From June to October the number of fire days in each month represents always more than $49 \%$ of the total number of days of that month, with July and August having each more than $85 \%$ of fire days. Fire days in March represent 36\% of days and, in the remaining months, they represent less than $30 \%$ of the days of the respective month (a minimum of $15 \%$ being observed in January).

The anticyclonic (A) type is the most frequent CWT in fire days of all months except August when the NE type presents a slightly higher frequency of occurrence; however, the relative contribution of A to FRE is always lower that the relative frequency indicating that fire events associated to the A type tend to release lower values of radiative energy. The same happens with CWTs characterized by winds 
with a westerly component (SW, W and NW) as well as with the $\mathrm{N}$ type that is more frequent from May to August and, together with the A type is associated to days with a moderate release of FRE.

An opposite behavior is observed with CWTs characterized by winds with an easterly component (NE, E and SE) where the relative contributions to the monthly total of FRE are almost always larger than the respective relative frequency of occurrence, namely the E type in all months, the NE type from December to January and from May to August, and the SE type from December to June. The C type presents larger contributions to FRE than the relative frequency of occurrences in March, June and July and the same happens in April, June and September.

When analysing the whole fire season (Figure 3), results indicate that $61 \%$ of FRE released in the fire season is observed in days characterized by directional types NE and $\mathrm{E}$ that occur in less than half that fraction $(29 \%)$ of the days. When considering type E alone, one fourth $(25 \%)$ of the energy released is associated to less than three times that fraction (8\%) of days of type E. On the other hand, the dominant A type occurs in $31 \%$ of the days but contributes to only $15 \%$ of the total FRE. Directional type $\mathrm{N}$ that ranks third (after NE) occurs in $14 \%$ of the days and account for $11 \%$ of the released energy. Directional types with a westerly component (SW, W and NW) representing $16 \%$ of the days contribute to $6 \%$ of FRE.

March, being the month with secondary peaks in fire days and released energy is also worth being analyzed in detail. As shown in Figure 1, the total FRE released in March is $1 \times 10^{6}$ GJ, representing $2 \%$ of the total energy released in 2004-2017. Types E and SE that occur only in $14 \%$ of the days are responsible for $71 \%$ of the total FRE more than half (37\%) of this amount being released in days of type SE that are observed only in about $5 \%$ of the days.
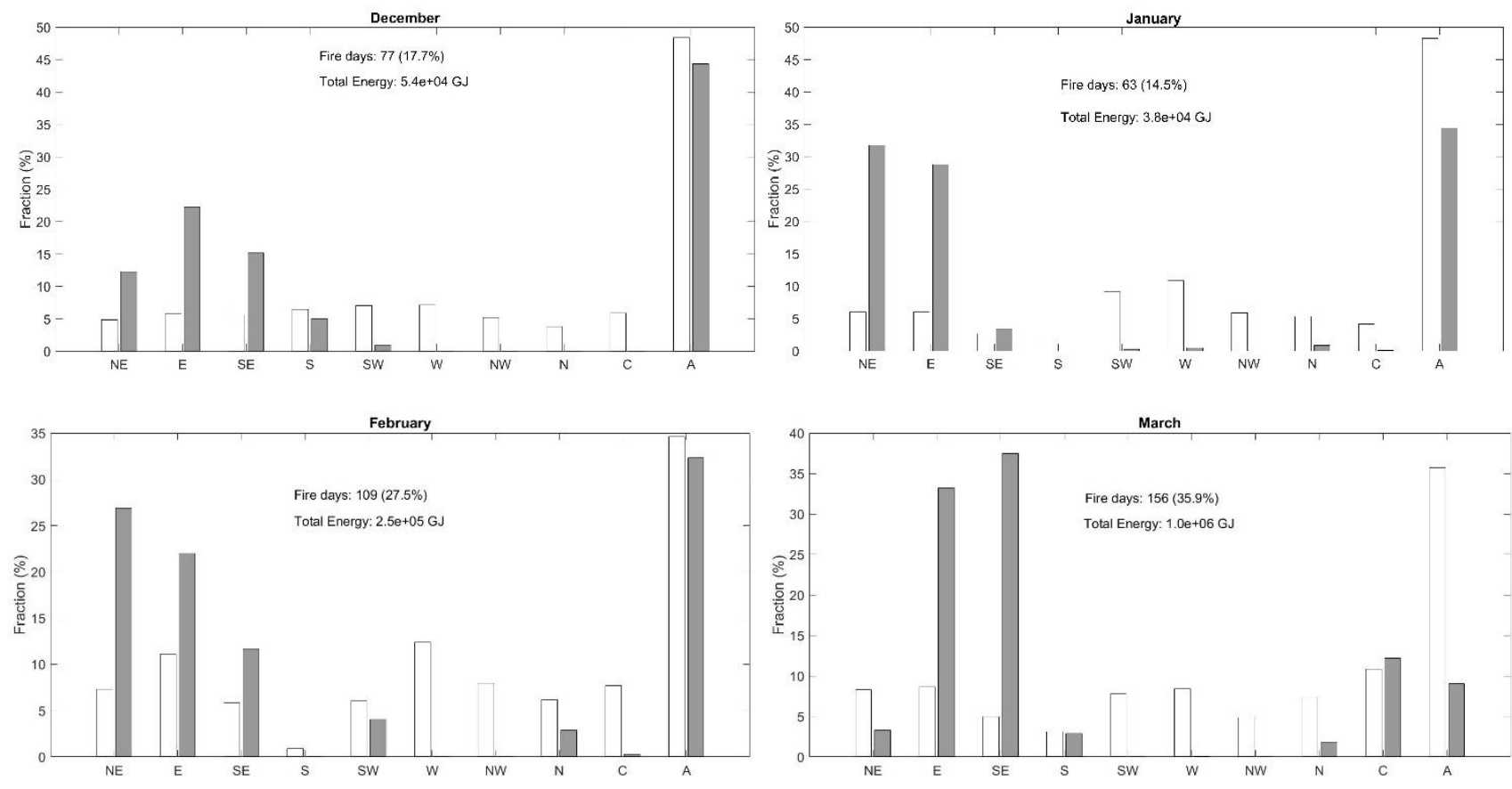

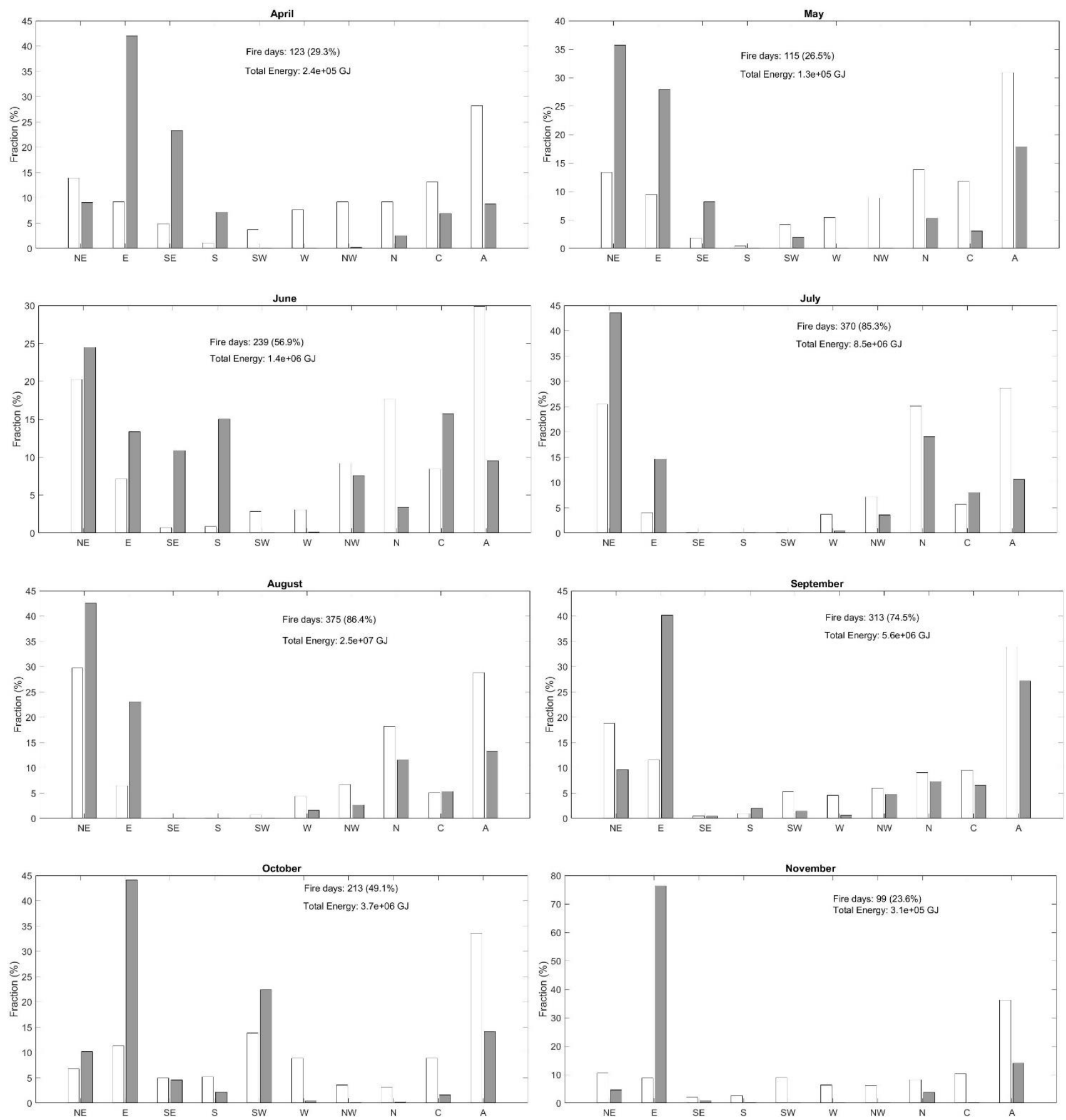

Figure 2 - Monthly analysis of contributions of CWTs to FRE during the period 2004-2017. For each CWT, the white column represents the fraction of days of that type during the considered period and the dark grey column represents the corresponding associated fraction of $F R E$ 


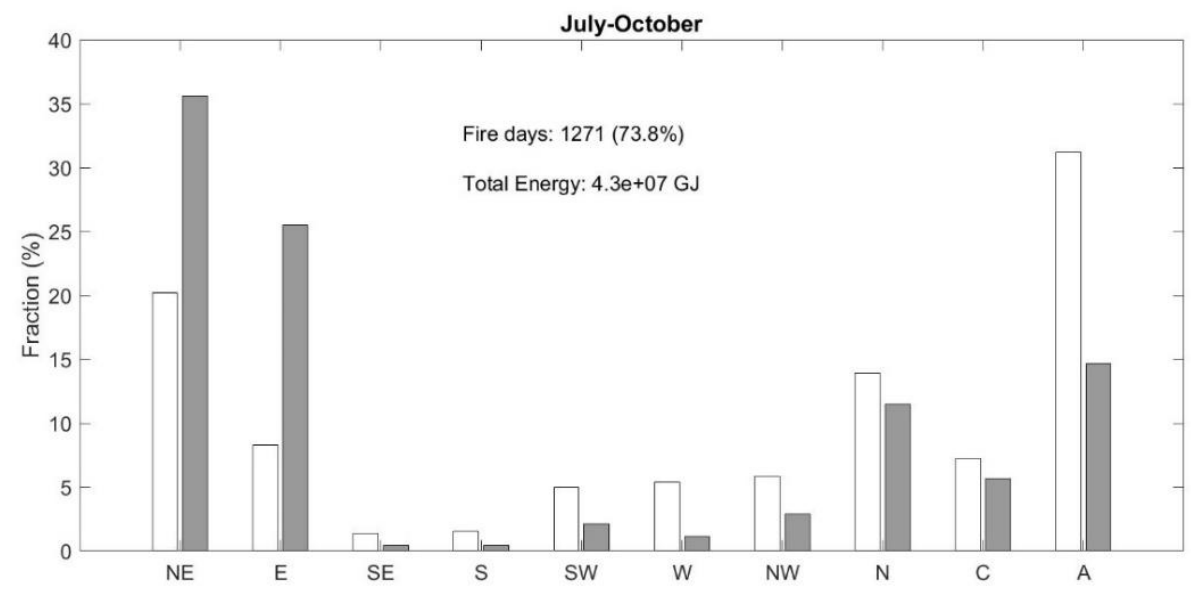

Figure 3 - As in Figure 2 but for the fire season (July to October).

It is a well-known fact that a relatively small number of wildfires are responsible for a very high proportion of the total energy released (Pinto et al., 2018). The distribution of daily FRE is therefore long tailed and this is put into evidence by means of the extreme proportion function (Figure 4) that is defined as the proportion of FRE attributable to the largest percentage of the fires (Strauss et al., 1989). The long-tailed character of the distribution of daily FRE translates into the very sharp increase of the extreme proportion curve for values of fraction of fire days within the range from 0 to about 0.1 . For instance, it may be noted that $1 \%$ of fire days (23 days) account for $31 \%$ of the total FRE, $2 \%$ of fire days (45 days) account for $45 \%$ of the total FRE and 5\% of fire days (113 days) account for $69 \%$ of the total FRE.

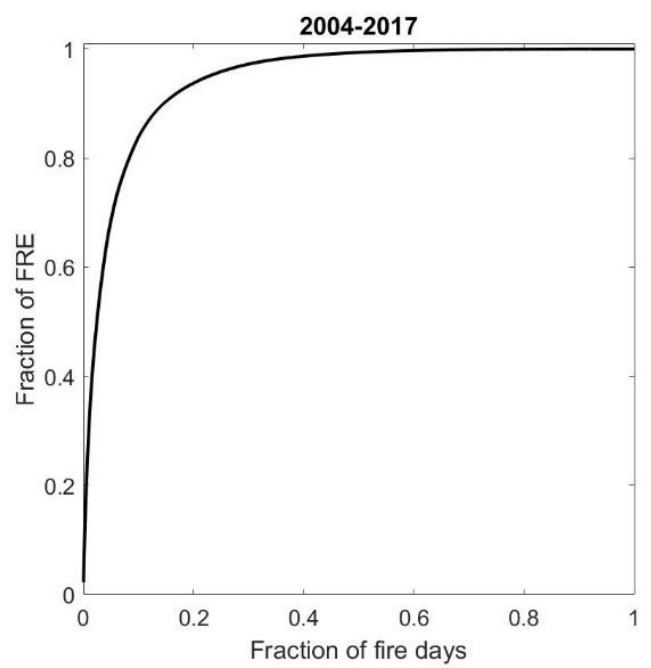

Figure 4 - The extreme proportion function for daily FRE released by wildfires during 2004-2017.

These results strongly suggest analysing in further detail the role played by meteorological conditions in days characterized by extremely high amounts of released energy by wildfires, e.g. restricting the sample to the top 5\% of fire days with the largest values of FRE. The clear majority $(96 \%)$ of these extreme fire days takes place during the fire season, with $62 \%$ occurring in August, followed by July (19\%), September (9\%) and October (6\%), and the remaining $4 \%$ of events are evenly distributed in March and June. Concerning the relative frequency of occurrence and the relative contribution to the FRE (Figure 5), it is worth emphasizing that two thirds (66\%) of the released energy in extreme days are associated to directional types NE (36\%) and E (30\%) with a frequency of 
occurrence of $62 \%$. These results put into evidence the role of $\mathrm{NE}$ and $\mathrm{E}$ types in days of extreme fire events.

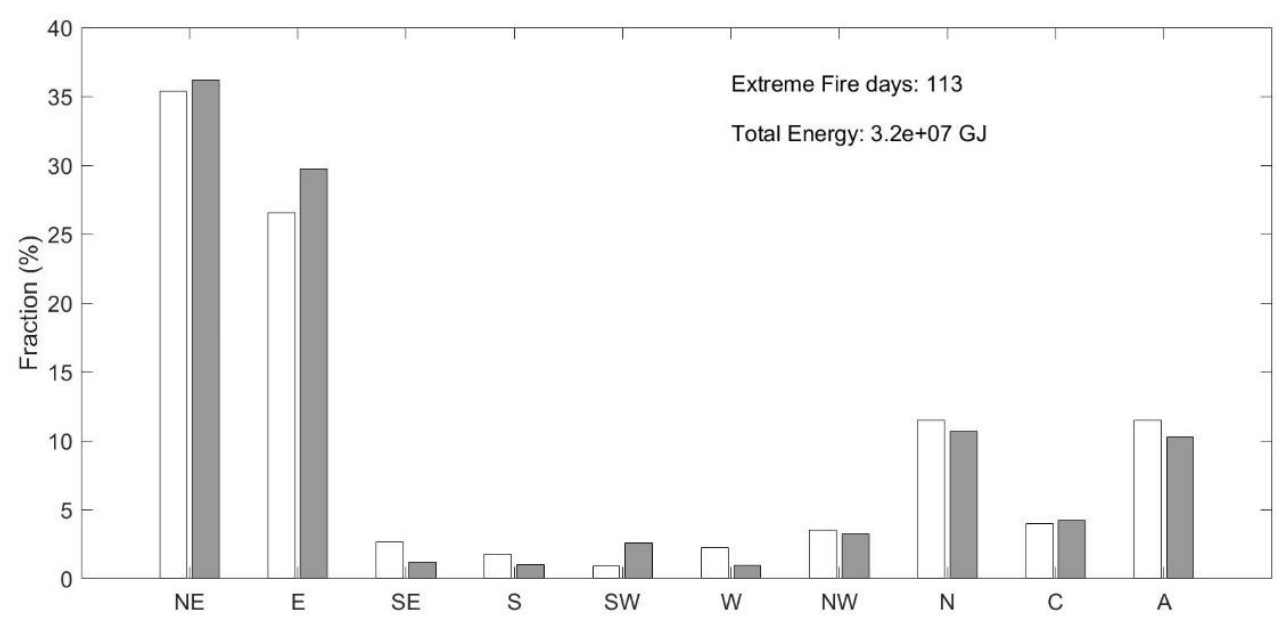

Figure 5 - As in Figure 2 but for the extreme fire days (top 5\%).

\section{Concluding remarks}

The fire regime in Portugal presents a marked annual cycle both in terms of number of fire days and in the amount of energy released by wildfires. The role of meteorological conditions in modulating the fire regime was assessed using the approach of synoptic climatology where daily weather conditions are stratified into eight directional weather types (associated to the prevailing wind direction) and two rotational types (associated to the sign of vorticity, i.e. cyclonic $\mathrm{C}$ or anticyclonic A).

The analysis encompassed the period 2004-2017 and results obtained showed that despite being the most frequently occurring Circulation Weather Type (CWT) in all months but August (where it ranks very close to first), the anticyclonic (A) type always contributes in a lower proportion to the Fire Radiative Energy (FRE) released by fires. On the contrary, the relative contribution of type E to FRE is larger than the frequency of occurrence in all months and the same happens with the remaining two CWTs characterized by an easterly wind component, namely the NE type from December to January and from May to August, and the SE type from December to June. The role played by CWTs characterized by east wind is further emphasized when restricting to the top 5\% of days with largest release of FRE; in this case, two thirds of the released energy are associated to directional types NE and $\mathrm{E}$.

These results open an interesting perspective for future studies involving seasonal forecasts and model simulations of present climate and future scenarios where approaches based on weather types have proven to be a useful tool for model validation (Ramos et al., 2015). This is especially important given that according to different scenarios of future climate in the Mediterranean, extreme fire events are likely to become more frequent (Flannigan et al., 2013; Pereira et al., 2013; Sousa et al., 2015).

\section{Acknowledgments}

Research performed is in the framework of Brazilian Fire-Land-Atmosphere System (BrFLAS) Project financed by the Portuguese and Brazilian science foundations, FCT and FAPESP (project references FAPESP/1389/2014 and 2014/20042-2) and of EUMETSAT Satellite Application Facility for Land Surface Analysis (LSA SAF). 


\section{References}

Dee DP, Uppala SM, Simmons AJ, Berrisford P, Poli P, Kobayashi S, Andrae U, Balmaseda MA, Balsamo G, Bauer P, Bechtold P, Beljaars ACM, van de Berg L, Bidlot J, Bormann N, Delson C, Dragani R, Fuentes M, Gee AJ, Haimberger L, Healy SB, Hersbach H, Hólm EV, Isaksen L, Kallberg P, Köhler M, Matricardi M, McNally AP, Monge-Sanz BM, Morcrette J-J, Park B-K, Peubey C, de Rosnay P, Tavolato C, Thépaut J-N, Vitart F (2011) The ERA-Interim reanalysis: Configuration and performance of the data assimilation system, Quarterly Journal of the Royal Meteorological Society, 137, 553-597. doi: 10.1002/qj.828

Flannigan M, Cantin AS, de Groot WJ, Wotton M, Newbery A, Gowman LM (2013) Global wildland fire season severity in the 21st century. Forest Ecology and Management 294, 54-61. https://doi.org/10.1016/j.foreco.2012.10.022.

Johnston JM. Wooster MJ, Paugam R, Wang X, Lynham TJ, Johnston L M (2017) Direct estimation of Byram's fire intensity from infrared remote sensing imagery, International Journal of Wildland Fire, 26, 668-684. doi:10.1071/WF16178

Pereira MG, Calado TJ, DaCamara CC, Calheiros T (2013) Effects of regional climate change on rural fires in Portugal. Climate Research 57, 187-200. doi: 10.3354/cr01176

Pereira MG, Trigo RM, DaCamara CC, Pereira J.M.C., Leite SM (2005). Synoptic patterns associated with large summer forest fires in Portugal, Agricultural and Forest Meteorology, 129, 11-25. doi:10.1016/j.agrformet.2004.12.007

Pinto MM, DaCamara CC, Trigo IF, Trigo RM, Turkman KF (2018) Fire danger rating over Mediterranean Europe based on fire radiative power derived from Meteosat. Natural Hazards Earth System Science 18, 515-529, https://doi.org/10.5194/nhess-18-515-2018.

Ramos AM, Cordeiro Pires A, Sousa PM, Trigo RM (2013) The use of circulation weather types to predict upwelling activity along the Western Iberian Peninsula coast. Continental Shelf Research 69, 38-51. doi: 10.1016/j.csr.2013.08.019

Ramos AM, Barriopedro D, Dutra E (2015) Circulation weather types as a tool in atmospheric, climate, and environmental research. Frontiers of Environmental Science 3:44. doi: 10.3389/fenvs.2015.00044

Ramos AM, Cortesi N, Trigo RM (2014) Circulation weather types and spatial variability of daily precipitation in the Iberian Peninsula. Frontiers of Earth Science 2:25. doi: 10.3389/feart.2014.00025

Ramos AM, Ramos R, Sousa P, Trigo RM, Janeira M, Prior V (2011) Cloud to ground lightning activity over Portugal and its association with circulation weather types. Atmospheric Research 101, 84-101. doi: 10.1016/j.atmosres.2011.01.014

Russo AC, Gouveia CM, Trigo RM, Liberato MLR, DaCamara CC (2015) The influence of circulation weather patterns at different spatial scales on drought variability in the Iberian Peninsula. Frontiers in Environmental Science 3:1. doi: 10.3389/fenvs.2015.00001

Sousa PM, Trigo RM, Pereira MG, Bedia J, Gutiérrez JM (2015) Different approaches to model future burnt area in the Iberian Peninsula. Agricultural and Forest Meteorology 202, 11-25. doi: http://dx.doi.org/10.1016/j.agrformet.2014.11.018

Strauss D, Bednar L, Mees R (1989) Do one percent of forest fires cause ninety nine percent of the damage? Forest Science 35, 319-328.

Trigo IF, DaCamara CC, Viterbo P, Roujean J -L, Olesen F, Barroso C, Camacho de Coca F, Freitas SC , García-Haro J, Geiger B , Gellens-Meulenberghs F, Meliá J, Pessanha L, Siljamo N (2011) The Satellite Application Facility for Land Surface Analysis, International Journal of Remote Sensing, 32, 2725-2744. doi:10.1080/01431161003743199 
Trigo RM, DaCamara CC (2000) Circulation weather types and their Influence on the precipitation regime in Portugal. International Journal of Climatology 20, 1559-1581. doi: 10.1002/10970088(20001115)20:13<1559::AID-JOC555>3.0.CO;2-5

Trigo RM, Sousa P, Pereira M, Rasilla D, Gouveia CM (2013) Modelling wildfire activity in Iberia with different Atmospheric Circulation Weather Types, International Journal of Climatology. doi: 10.1002/joc.3749

Wooster MJ, Roberts G, Freeborn PH, Xu W, Govaerts Y, Beeby R, He J, Lattanzio A, Fisher D, Mullen R (2015) LSA SAF Meteosat FRP products - Part 1: Algorithms, product contents, and analysis. Atmos. Chem. Phys. 15, 13217-13239. https://doi.org/10.5194/acp-15-13217-2015. 


\title{
Concurrent multi-scale physical parametrization of fire-spotting: A study on the role of macro- and meso-scale characteristics of the system
}

\author{
Vera N. Egorova ${ }^{1}$; Andrea Trucchia ${ }^{1,2}$; Gianni Pagnini ${ }^{1,3}$ \\ ${ }^{1}$ BCAM-Basque Center for Applied Mathematics, Alameda de Mazarredo, 14, E-48009, Bilbao, \\ Basque Country, Spain.\{vegorova@bcamath.org, atrucchia@bcamath.org, \\ gpagnini@bcamath.org*\} \\ ${ }^{2}$ Ikerbasque - Basque Foundation for Science, Calle de María Díaz de Haro, 3, E-48013, Bilbao, \\ Basque Country, Spain \\ ${ }^{3}$ University of the Basque Country UPV/EHU, Barrio Sarriena, s/n, 48940, Leioa, Basque \\ Country, Spain
}

\begin{abstract}
The strong impact of wildfires in terms of lives and homes lost and of damage to ecosystems, calls for an urgent improvement in the risk management. The aim of the present research is the improvement of these software codes by proposing a complete physical characterization of fire-spotting within an approach that is ready to be implemented as a post-processing routine of standard outputs. The main feature of the proposed method is that the effects of random fluctuations are included in a way that preserves the existing structure of the operational and industrial codes and can be implemented directly. The operational code WRF-SFIRE have been used to test the proposed post-processing routine. Results show the suitability of the approach for simulating random effects due to turbulent convection and fire-spotting, which are cases not resolved by standard operational codes. Results of simulations including response analysis with test cases are shown and discussed.
\end{abstract}

Keywords: Fire-spotting, Simulation, Multi-scale characteristics

\section{Introduction}

Propagation of a wildfire is a multiscale phenomenon involving processes from the scale of the combustion chemistry to the fire-atmosphere coupling including effects due to the flame geometry (Sullivan, 2017a, 2017b). One of the key aspects of fire propagation is the so-called fire-spotting (Fernandez-Pello, 2017). It occurs when burning embers tear off from the main fuel source and cause new independent ignitions. It accelerates significantly the fire spread causing dangerous consequences and increasing the damage. Moreover, the fire-spotting is a challenging issue in wildfire science due to its unpredictable nature.

Here we study the role for the emergence of fire-spotting phenomena and the ignition of secondary fires of both a macro-scale factor as the atmospheric stability, and of a meso-scale factor as the flame length.

Because of the interactions among scales, we follow a concurrent multiscale modelling that means estimating parameters related to aspects occurring in a very large range of scales and implementing them into the model for the macroscopic fire perimeter, see Figure 1. 


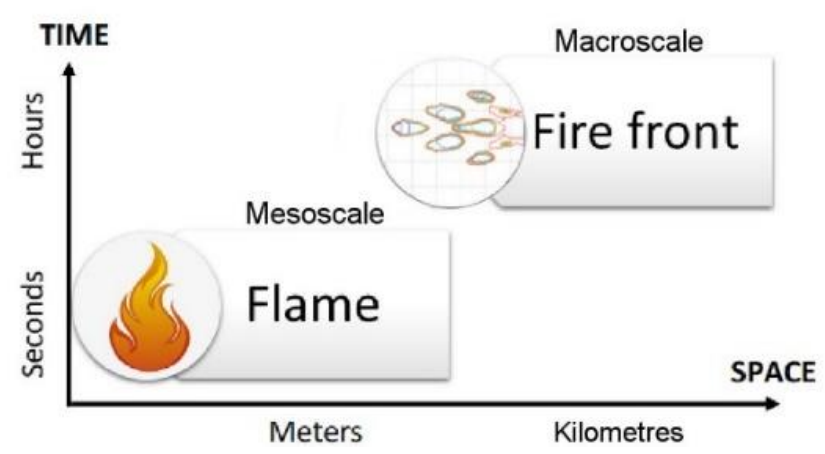

Figure 1 - Multiscale modelling of wildland fires

The adopted modelling approach (Pagnini and Mentrelli, 2014; Kaur et al., 2016) is based on the idea to split the motion of the front into a drifting part and a fluctuating part. The drifting part represents the main front motion as a free boundary problem. Thus, it can be treated by any existing method, for instance, based on the level-set method (LSM) (Sethian and Smereka, 2003) in analogy with WRFSFIRE model (Mandel et al., 2011). The fluctuating part, that is the result of a comprehensive statistical description of the physics of the system and includes the random effects, is here physically parametrized to include turbulent hot-air transport and firebrand landing distance.

The rest of the text is organized as follows. In Section 2 we propose a parametrization of the firebrand-landing distribution including macro-scale and meso-scale parameters. In Section 3 the model is proposed and in Section 4 the response analysis is performed by using the LSM. In Section 5 we show few scenarios obtained by the implementation of the proposed model into the operational code WRF-SFIRE. Conclusions are given in Section 6.

\section{Firebrand landing distribution}

\subsection{Lognormal probability density}

Fire-spotting can be introduced into operational codes as a post-processing routine. Fire-spotting can be describes via the firebrand landing distribution $q(\ell)$, that is here assumed to be lognormal distributed following (Sardoy et al., 2008; Himoto and Tanaka, 2005),

$$
q(\ell)=\frac{1}{\sqrt{2 \pi} \sigma \ell} e^{\frac{-\left(\ln \frac{\ell}{\mu}\right)^{2}}{2 \sigma^{2}}},
$$

where $\mu$ is the ratio between the square of the mean of landing distance $\ell$ and its standard deviation $\sigma$. The parametrisation stated in (Kaur and Pagnini, 2016) is based on the assumption that each firebrand is spherical with constant size. Following Kaur and Pagnini (2016), the lognormal parameters $\mu$ and $\sigma$ in (1) result to be

$$
\mu=H\left(\frac{3 \rho C_{d}}{2 \rho_{f}}\right)^{1 / 2}, \quad \sigma=\frac{1}{z_{p}} \ln \left(\frac{U}{\sqrt{r g}}\right),
$$

where $U$ is the wind speed and $H$ is the maximum loftable height of firebrands, $g$ is gravitational acceleration, $\rho$ is the ambient air mass density, $\rho_{f}$ and $r$ are the density and the radius of the firebrand. The dimensionless ratio

$$
F r=U^{2} /(r g)
$$


known as the Froude number is a measure of the balance between the inertial and the gravitational forces experienced by the burning ember. In terms of the Froude number the standard deviation $\sigma$ can be written as follows

$$
\sigma=\frac{1}{2 z_{p}} \ln F r
$$

In the following we parametrize firebrand landing distribution (1)-(2) taking into account atmospheric stability conditions and the flame characteristics.

\subsection{Macro-scale parametrs}

At the macroscopic scale, fire-spotting is affected by atmospheric conditions. In particular, we plug the depth of the atmospheric boundary layer (ABL), that is related to the atmospheric stability, into the estimation of the smoke-injection height including the uplift against the atmospheric stratification and the plume widening due to entrainment of the surrounding air (Sofiev et al., 2012). Later, we estimate the firebrand-injection height as an approximated lift for inertial particles flowing into the fire plume.

We assume that firebrands lofted by a smoke column are similar to inertial particles moving into a fluid, thus it is found that the maximum loftable height is a fraction of the smoke height $H^{*}$, i.e., the maximum height reached by a smoke particle, here we use $H \simeq 0.4 H^{*}$. According to Sofiev et al. (2012), $H^{*}$ is defined as follows

$$
H^{*}=\alpha H_{A B L}+\beta\left(\frac{I}{P_{f_{0}} \cdot d}\right)^{\zeta} \exp \left(-\frac{\delta N_{F T}^{2}}{N_{0}^{2}}\right),
$$

where $N^{2}$ is the Brünt-Väsäla frequency, sub-index $F T$ means free troposphere, $H_{\mathrm{ABL}}$ is the height of ABL. Note that, according to Sofiev et al. (2012), the parameters are chosen as follows: $\alpha<1, \beta>$ $0, \zeta<0.5$ and $\delta \geq 0$.

Atmospheric conditions affect both the firebrand landing distribution and the turbulent diffusion coefficient $\mathcal{D}$, that is estimated by the following formula (Kaur et al., 2016)

$$
\mathcal{D} \simeq \chi(0.1 R a-1)^{\beta \mathcal{D}}, \quad R a=\gamma_{\mathcal{D}} \Delta T g H_{A B L}^{3} v^{-1} \chi^{-1},
$$

where $\chi$ is the thermal diffusivity of the air at ambient temperature, $R a$ is the Rayleigh number, $\gamma_{\mathcal{D}}$ is the thermal expansion coefficient, $\Delta T$ is the temperature difference between the bottom and the top of the convective cell, and the vertical dimension of the convection cell is $H_{\mathrm{ABL}}, v$ is the kinematic viscosity. Parameter $\beta_{\mathcal{D}}$ is the exponent of the power law relating the Nusselt number $N u$ and the Rayleigh number $R a$, i.e., $N u=0.1 R a^{\beta_{\mathcal{D}}}$ (Niemela et al., 2000; Niemela and Sreenivasan, 2006). Previous experimental results suggested $\beta_{\mathcal{D}} \approx 2 / 7$ instead of $1 / 3$ ( Wu and Libchaber, 1992).

\subsection{Meso-scale parameters}

Let us consider the geometrical model of the flame, as it is presented in Figure 2. It is natural that the flame characteristics are strongly affected by the wind and the fire intensity. Moreover, it can be easily found that the flame length $L_{f}$ and the flame height $H_{f}$ are connected by the following trigonometric relation

$$
H_{f}=L_{f} \cos \theta \text {, }
$$

where $\theta$ is the flame angle, or flame tilt. Thus, in the no-wind case, $H_{f}=L_{f}$, since $\theta=0$. 


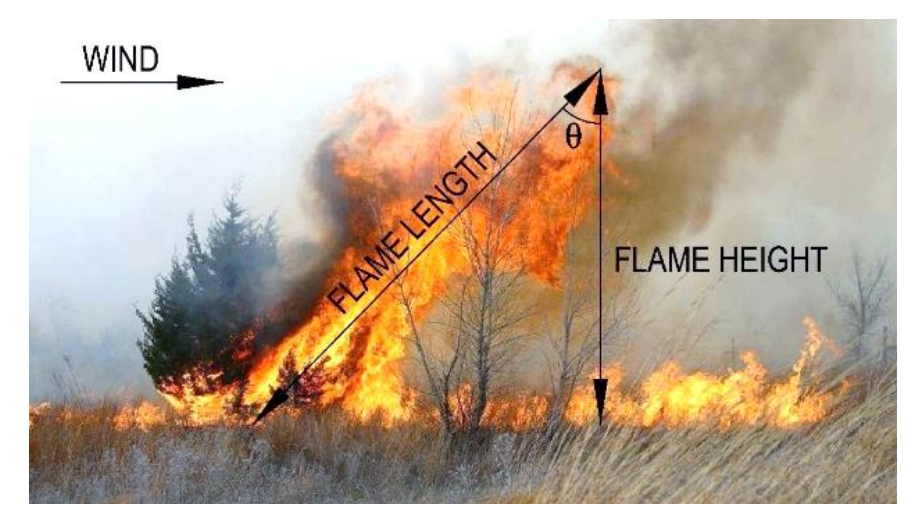

Figure 2 - Flame geometry in real wildland fire.

The flame height is leaded by the buoyancy and by the mean wind. Adopting the formula proposed by Albini (1981), one gets

$$
H_{f}=\left(\frac{1}{2 g\left(\rho c_{p} T_{a}\right)^{2}}\right)^{1 / 3} \mathrm{I}^{2 / 3} \cos \theta,
$$

where $c_{p}$ is the specific heat of fuel, I is the fireline intensity and the ambient temperature is denoted by $T_{\mathrm{a}}$.

Inserting (8) into (7), the flame length can be derived in term of the fireline intensity as follows

$$
L_{f}=\left(\frac{1}{2 g\left(\rho c_{p} T_{a}\right)^{2}}\right)^{1 / 3} \mathrm{I}^{2 / 3} .
$$

In order to take into consideration the flame length (9), the maximum travel distance for a spherical firebrand used in (1) is written in the following form (Wang, 2011):

$$
\ell_{\max }=H\left(\beta_{2} \cot \theta+U\left(\frac{3 \rho C_{d}}{2 \rho_{f} r g}\right)^{2}\right),
$$

where $\beta_{2}=0.7$ is a correction factor. Note that the angle of flame can be estimated by an empirical correlation $\cot \theta=1.35 U\left(g L_{f}\right)^{-1 / 2}$.

According to Kaur et al. (2016), the landing distance can be represented by the $p^{\text {th }}$ percentile of the lognormal distribution, such that

$$
\ell_{\max }=\mu e^{z_{p} \sigma} .
$$

The value that corresponds to this $p^{\text {th }}$ percentile can be defined in terms of $\kappa$ - the ratio between the maximum and minimum travel distance and the Froude number as follows:

$$
z_{p}=\frac{\ln F r}{\sqrt{2 \ln ^{\kappa} / F r}} \text {. }
$$

Thus, from (10) and (11) one gets

$$
\sigma=\frac{1}{z_{p}} \ln \left(F r^{1 / 2}+\beta_{3}\left(\frac{2 \rho_{f} r g U^{2}}{3 \rho C_{d} g L_{f}}\right)^{1 / 2}\right),
$$

where $\beta_{3}=0.945$ is a correcting factor from Wang (2011), and the flame length $L_{f}$ is defined by general formula (9). 


\section{Model description}

The motion of wildfire front can be split into a drifting part and a fluctuating part. After the splitting, the drifting part can be treated by any existing operational code such as WRF-SFIRE (Mandel et al., 2011). The fluctuating part, that is independent of the drifting part, describes the effects of turbulence and fire-spotting as random phenomena and they are implemented into the model through a postprocessing routine (Pagnini and Mentrelli, 2014; Kaur et al., 2016). Here we provide a short description of this model.

For a given computational domain $S$ the fire front contour is represented by a closed curve $\Gamma$. The region bounded by $\Gamma$ is denoted by $\Omega(t)$ and represents the burnt area at time $t$. Let us introduce an indicator function $\varphi(x, t)$ :

$$
\varphi(x, t)= \begin{cases}1, & \text { if } x \in \Omega(t), \\ 0, & \text { otherwise. }\end{cases}
$$

In order to treat the fluctuating part of the front motions, the following method is used. The random front contour caused by the turbulence and fire-spotting phenomena, can be defined by the effective indicator function (Pagnini and Mentrelli, 2014):

$$
\varphi_{e}(x, t)=\int_{s} \varphi(\bar{x}, t) f(x ; t \mid \bar{x}) d \bar{x}=\int_{\Omega(t)} f(x ; t \mid \bar{x}) d \bar{x},
$$

where $f(x ; t \mid \bar{x})$ is the probability density function (PDF) that accounts for turbulence and firespotting effects. Note that the point is labelled as burnt, if $\varphi_{e}(x, t)$ exceeds a threshold value $\varphi_{e}^{t h}$.

Denoting by $\omega$ the angle between the wind direction and the vector from some point of the computational domain to the point where the PDF is computed, the minimum acceptable for firespotting angle $\omega_{0}$ has to be established as follows

$$
\cos \omega_{0}=\max \left\{\frac{1}{\kappa}, F r^{-\frac{1}{2}}\right\},
$$

where $\kappa$ is the ratio between the maximum and minimum travel distance of a firebrand.

Taking into account (16) and assuming that the downwind phenomenon of fire-spotting is given by the turbulence and fire-spotting, the random process handled by $f(x ; t \mid \bar{x})$ in $(15)$ is defined as follows

$$
f(x ; t \mid \bar{x})=\left\{\begin{array}{cc}
\int_{0}^{\infty} G(x-\bar{x}-\ell n ; t) q(\ell) d \ell, & \text { if } \cos \omega>\cos \omega_{0}, \\
G(x-\bar{x} ; t), & \text { otherwise. }
\end{array}\right.
$$

where $q(\ell)$ is a lognormal distribution (1) with parameters $\mu$ and $\sigma_{\omega}$, given in (2) and (4), and $\cos \omega_{0}$ is defined by (16).

As it is mentioned above, the point is marked as burned if the effective indicator exceeds the threshold value $\varphi_{e}^{t h}$. However, there exists an additional criterion associated with an ignition delay due to pre-heating action of the hot air or to the landing of firebrands. This delay is considered as a heating-beforeburning mechanism due to the hot air (Pagnini and Massidda, 2012a, 2012b) and it can be generalized to include fire-spotting (Pagnini and Mentrelli, 2014). Since the fuel can burn because of two pathways - hot-air heating and firebrand landing, the resistance analogy suggests that the resulting ignition delay can be approximatively computed as resistances acting in parallel. Let $\tau_{h}$ and $\tau_{f}$ be the ignition delay due to hot air and firebrands landing, respectively, the joint ignition delay $\tau$ is

$$
\frac{1}{\tau}=\frac{1}{\tau_{h}}+\frac{1}{\tau_{f}}=\frac{\tau_{h}+\tau_{f}}{\tau_{h} \tau_{f}}
$$

The heating-before-burning mechanism is depicted as the persistence in time of the effective fire front, i.e., 


$$
\psi(x, t)=\int_{0}^{t} \varphi_{e}(x, \eta) \frac{d \eta}{\tau}
$$

where $\psi(x, t)=0$ corresponds to the unburned initial condition, and ignition at the point $x$ at the moment $t$ occurs, if $\psi(x, t)=1$.

\section{Response analysis}

In this section,we show the effects of the macro- and meso-scale parameters described above by simulations done performed with the LSM that is at the basis of WRF-SFIRE. All the factors and parameters, except those that are to be studied, are fixed. We set a wind speed $U=4.47 \mathrm{~ms}^{-1}$, that corresponds to the average wind speed in Madrid during 2017 (https://www.worldweatheronline.com/madridweather-averages/madrid/es.aspx), and fire intensity $I_{f}=20 \mathrm{MWm}^{-1}$. The changes in the values of $H_{\mathrm{ABL}}$ are reflected into the changes of the values of the maximum travel distance of firebrands $\ell_{\max }$ as well as into the values of the turbulent diffusion coefficient $\mathcal{D}$, see Table 1 . From (6) we have that the turbulent diffusion coefficient depends on $H_{\mathrm{ABL}}$ with the third power and the resulting changes affects strongly the propagation of the fire with an important effect on the merging of the secondary fires generated by the fire-spotting. In fact, during stable conditions a larger number of fires with respect to unstable conditions is observed but at the same time a lower burned area is computed, showing that in unstable conditions the turbulent heat transport is stronger with the double effects of a faster fire propagation and a more efficient merging.

Table 1 - Effects of atmospheric stability on the fire-spotting parameters.

\begin{tabular}{|l|rrrr|rrr|}
\hline & \multicolumn{3}{|c|}{ Case 1: Stable } & \multicolumn{3}{c|}{ Case 2: Unstable } \\
\hline$H_{A B L}[\mathrm{~m}]$ & 100 & 200 & 400 & 800 & 1000 & 1200 & 2000 \\
$\mu[\mathrm{m}]$ & 4.0325 & 4.3879 & 5.0985 & 6.5198 & 7.2304 & 7.9411 & 10.7836 \\
$\mathcal{D}\left[\mathrm{m}^{2} \mathrm{~s}^{-1}\right]$ & 0.0533 & 0.0995 & 0.1858 & 0.3467 & 0.4238 & 0.4993 & 0.7908 \\
\hline
\end{tabular}

Figure 3 shows the total number of burned points at different times for different atmospheric conditions. What is evident from the simulations is that, after an initial period of quiescence with no visible contributions, the burned area rapidly increases as a power law. This power law seems to hold at stable atmospheric conditions, but the quiescence period ends first in unstable conditions such that the burned area during unstable conditions is always larger than during stable conditions.

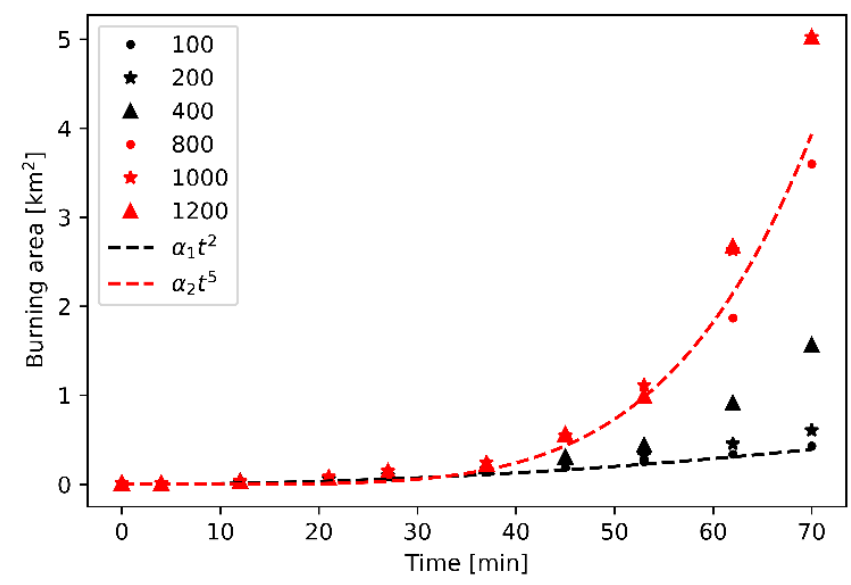

Figure 3 - A comparison of the burned area in different time moments in stable (black) and unstable (red) atmospheric boundary conditions for various $H_{A B L}$. 
In order to check if the rapid increasing of the burned area is mainly due to turbulence or firespotting we performed a series of simulations with fixed diffusion coefficient $\mathcal{D}$, i.e., independent of the atmospheric conditions. The results for stable $\left(H_{A B L}=100 \mathrm{~m}\right)$ and unstable $\left(H_{A B L}=1000 \mathrm{~m}\right)$ atmospheric conditions are shown in Figure 5. All the parameters are set equal to the previous simulations, but with fixed $\mathcal{D}=0.0387 \mathrm{~m}^{2} \mathrm{~s}^{-1}$. Note that this fixed value of $\mathcal{D}$ is less than any other value followed by the dependency on the $H_{A B L}$. Fire front propagates in the same way in both atmospheric conditions, displaying an equal number of secondary fires and equal burned area (see Figure 4). Thus, the atmospheric conditions affect the propagation of wildfires through the heat turbulent transfer. In particular, during unstable conditions we observe that turbulence pushes the front resulting into a faster propagation causing an increasing of the burned area and a more rapid merging of independent fires such that during unstable conditions the number of independent fires is less than during stable conditions in spite of the fact that the burned area is larger.

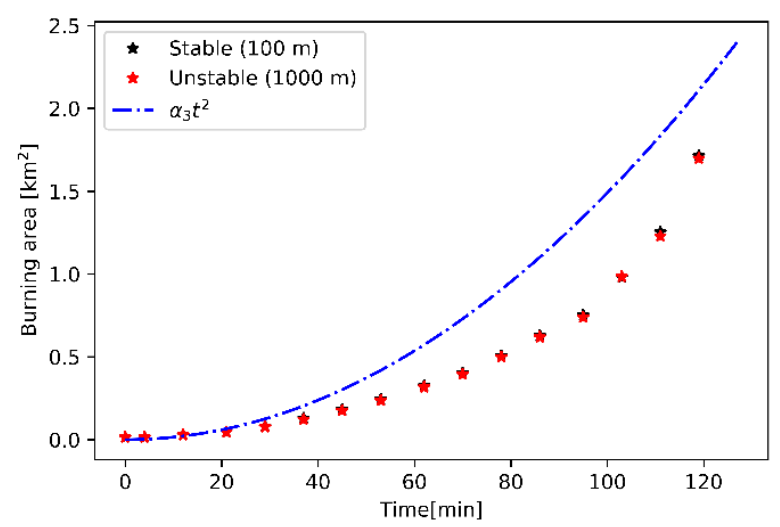

Figure 4 - A comparison of the total burning area in time in stable (black) and unstable (red) atmospheric boundary conditions in simulations with fixed diffusion coefficient $\mathcal{D}=0.0387 \mathrm{~m}^{2} \mathrm{~s}^{-1}$.

Now, let us study the impact of meso-scale factors, such as flame geometry. In formulation of the flame length $L_{f}=\beta_{0} I_{f}^{\beta_{1}}$, that in the case $\beta_{1}=2 / 3$ can be written in form (9), empirical values of $\beta_{0}$ proposed by various authors are given in Table 2 . We set the diffusion coefficient $\mathcal{D}=0.4238 \mathrm{~m}^{2} \mathrm{~s}^{-1}$, that corresponds to $H_{A B L}=1000 \mathrm{~m}$.

Table 2 - Empirical parameters $\beta_{0}$ and corresponding values of $\sigma$.

\begin{tabular}{|l|c|c|}
\hline References & $\beta_{0}$ & $\sigma$ \\
\hline Fons, 1963 & 0.1270 & 5.846 \\
Anderson et al. (Douglas-fir slash), 1966 & 0.0447 & 6.191 \\
Wang, 2011 & 0.0264 & 6.415 \\
Butler, 2004 & 0.0175 & 6.615 \\
\hline
\end{tabular}

The flame length does not affect to $\mu$, thus, $\mu=8.419$ is defined for the set of parameters independently of the chosen formulation of the flame length. Different values of $\sigma$ that correspond to various empirical values $\beta_{0}$ are collected in Table 2 as well. Decreasing factor $\beta_{0}$ leads to increasing value of the standard deviation $\sigma$. In fact, smaller flames produce smaller firebrands, i.e. the shortdistance spotting takes place with further merging and propagating as a joint fire front. However, fire propagation with higher flames is characterised by the long-distance spotting that occurs later, but there is no merging of secondary fires.

Smaller flame length leads to the short-distance spotting, usually, merging of spot-fires. It occurs in real life in many types of fuel and at any fire intensity. These spot-fires may generate new fire- 
spotting, that results in a very fast-moving flaming zone (see Figure 5, the solid line represents the burning area growth in the case of $\beta_{0}=0.127$, when the fire-spotting occurs.

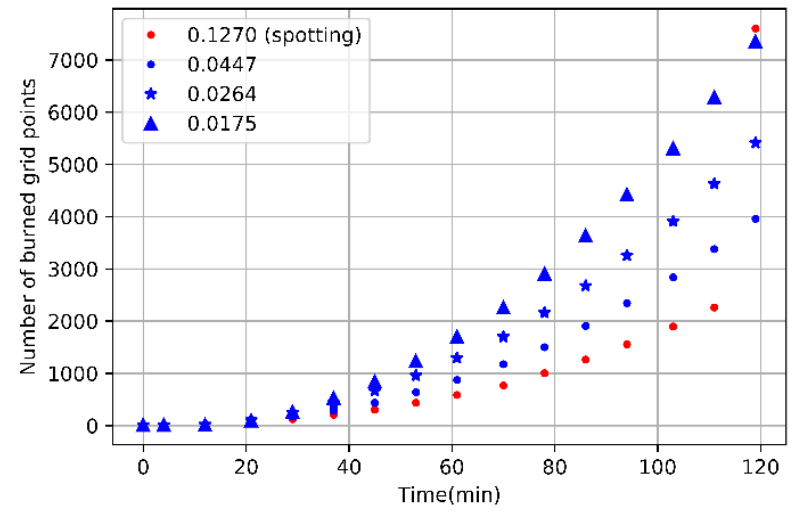

Figure 5 - Burning area with respect to time for various Bo in flame length formulation.

\section{Implementation into WRF-SFIRE}

In order to make the presented model useful for the point of view of the practitioners, it is strongly advised its implementation into full fledged operational codes. Equations (14) - (19) have been implemented in the framework of WRF-SFIRE simulator (Coen et al., 2012; Mandel et al., 2014).

We recall that the wildland fire behavior module WRF-SFIRE is fully integrated into a well known public domain numerical weather prediction model Weather Research and Forecasting (WRF) (Skamarock et al., 2008). The fire module is a surface fire behaviour model that adopts a two-way coupling with the atmospheric model. That is, near-surface winds from the atmospheric model are interpolated to a finer fire grid and are used, with fuel properties and local terrain gradients, to determine the fire's spread rate and direction used for the front propagation routines by the means of the LSM. Fuel consumption, in turn, releases sensible and latent heat fluxes into the atmospheric model's lowest layers, playing a role in boundary layer circulations. Recently, the model has been coupled with a fuel-moisture model, and chemical transport of emissions (WRF Chem) that contribute more and more to the close binding between forest fires and atmospheric observables. The interactions between different modules of the WRF model are shown in Figure 6.

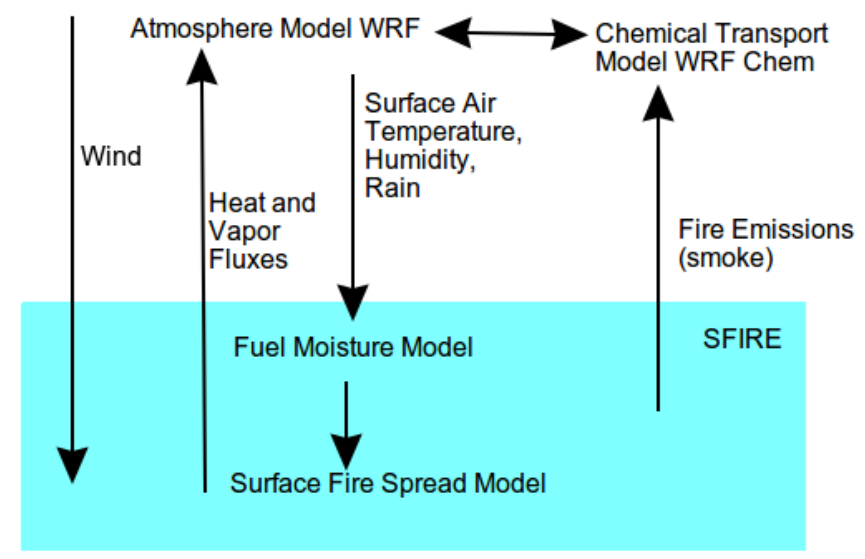

Figure 6 - WRF model

The results of the implementation of the proposed post-processing routine to the WRF-Sfire model are presented in Figures 7-9 at the different times. The main difference to the previous simulations is that fire-spotting parameter $\sigma$ is a space field due to the computed wind field that varies in space and 
time, see Figures 7-9 (a). In Figures 7-9 (b) and (c) the wind horizontal components are plotted, in Figure 7-9 (d) - the squared norm of the wind velocity.

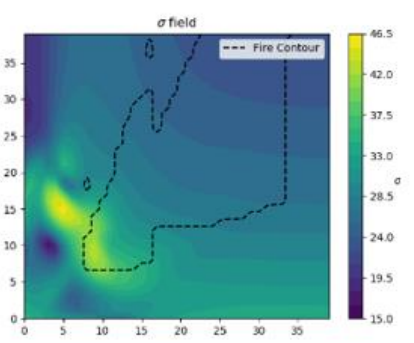

a)

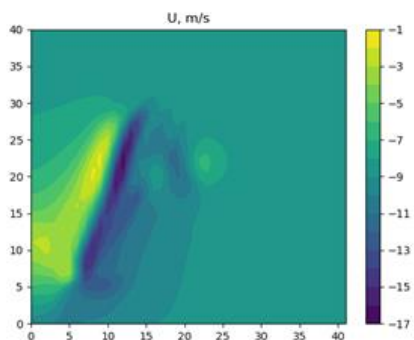

b)

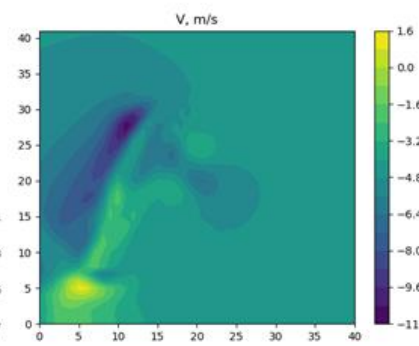

c)

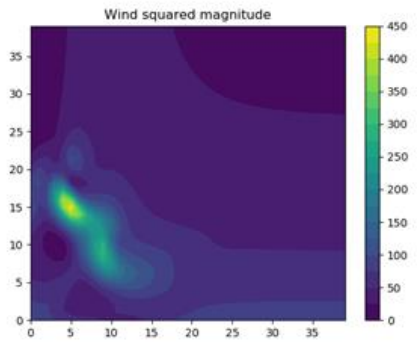

d)

Figure 7 - Space fields of (a) fire-spotting parameter $\sigma$, (b) horizontal and (c) vertical wind components, (d) the squared norm of the wind velocity.

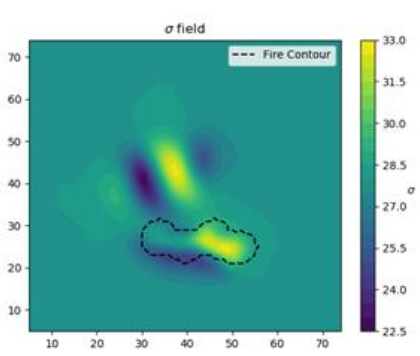

a)

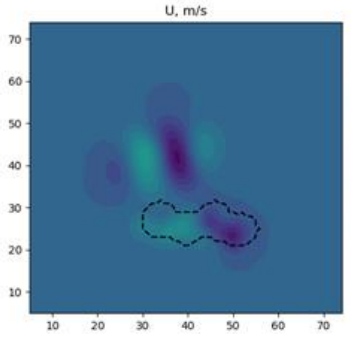

b)
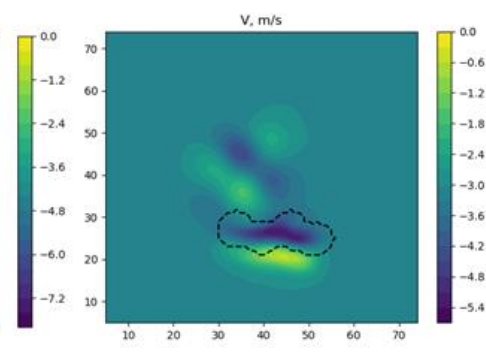

c)

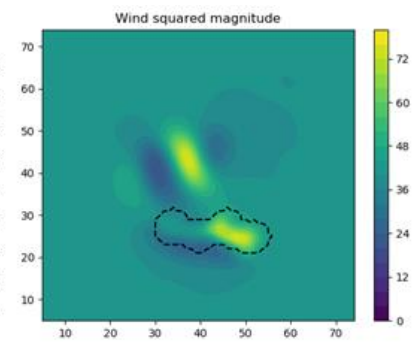

d)

Figure 8 - Space fields of (a) fire-spotting parameter $\sigma$, (b) horizontal and (c) vertical wind components, (d) the squared norm of the wind velocity after 20 minutes of burning.

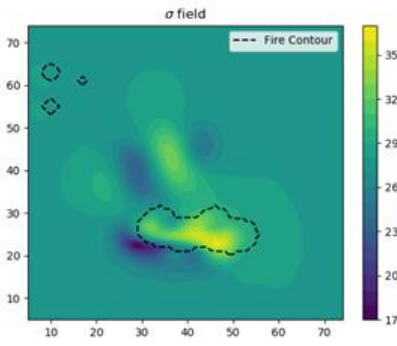

a)

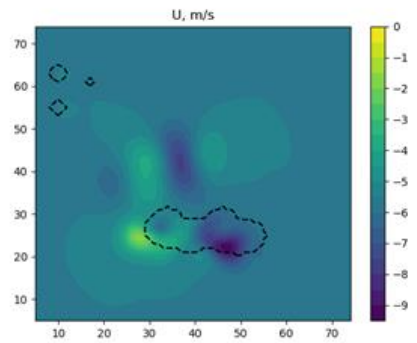

b)

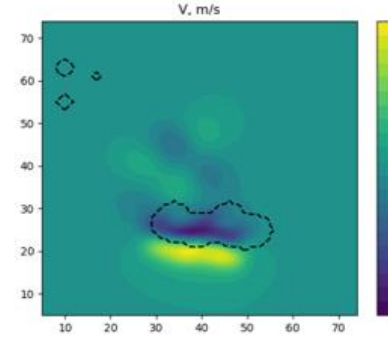

c)

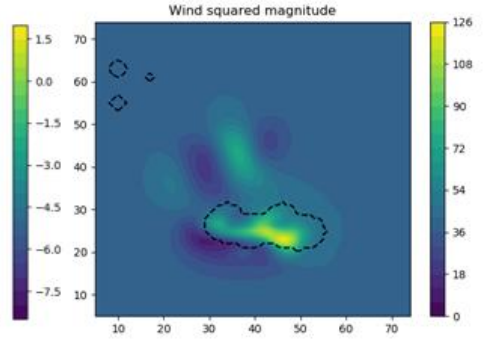

d)

Figure 9 - Space fields of (a) fire-spotting parameter $\sigma$, (b) horizontal and (c) vertical wind components, (d) the squared norm of the wind velocity after 20 minutes of burning.

\section{Conclusion}

The proposed fire-spotting model is based on the firebrand landing lognormal distribution with two parameters $\mu$ and $\sigma$. The response analysis to the parameters has been performed by using the LSM for test cases. Parameter $\mu$ is mainly related to the atmospheric stability conditions taking into account factors as wind speed, ambient air temperature and fire intensity. These ambient parameters affect slightly the firebrand landing distribution, but are found to be significant for effect of turbulent heat transfer on the fire-front propagation. Parameter $\sigma$ takes into account the flame geometry and the surround vegetation. It is shown that small flames provoke short-distance fire-spotting, that leads to the soon merging with the main fire front. Large flames provoke long-distance spotting. Thus, in such 
case the risk and damage increase due to the acceleration of the fire spread. The vegetation feeds the fire and it affects the fire intensity and, consequently, the flame length.

Furthermore, the propose parametrization of fire-spotting has been implemented into the operational code WRF-SFIRE and simulations in this realistic scenarios have been performed.

\section{Acknowledgement}

The research is supported by the Basque Government through the BERC 2018-2021 program and by the Spanish Ministry of Economy and Competitiveness through BCAM Severo Ochoa excellence accreditation SEV-2013-0323 and through project MTM2016-76016-R "MIP" and by the PhD grant "La Caixa 2014".

\section{References}

Albini FA (1981) A model for the wind-blown flame from a line fire. Combustion and Flame 43, 155174.

Coen JL, Cameron M, MichalakesJ, Patton EG, Riggan PJ, Yedinak KM (2012) WRF-Fire: coupled weather-wildland fire modeling with the weather research and forecasting model. Journal of Applied Meteorology and Climatology 52, 16-38.

Fernandez-Pello AC (2017) Wildland fire spot ignition by sparks and firebrands. Fire Safety Journal 91, 2-10.

Himoto K, Tanaka T (2005) Transport of disk-shaped firebrands in a turbulent boundary layer. In 'The Eighth International Symposium on Fire Safety Science, 1823 September 2005, Beijing, China' (Eds Gottuk D, Lattimer B), 433-444.

Kaur I, Mentrelli A, Bosseur F, Filippi JB, Pagnini G (2016) Turbulence and fire-spotting effects into wild-land fire simulators. Communications in Nonlinear Science and Numerical Simulation 39, 300-320.

Kaur I, Pagnini G (2016) Fire-spotting modelling and parametrisation for wildland fires. In 'Proceedings of the 8th International Congress on Environmental Modelling and Software (iEMSs2016); Toulouse, France, 10-14 July (2016)' (Eds Sauvage S, Sánchez-Pérez JM, Rizzoli AE), 384-391 (ISBN: 978-88-9035-745-9)

Mandel J, Amram S, Beezley JD, Kelman G, Kochanski AK, Kondratenko VY, Lynn BH, Regev B, VejmelkaM (2014) Recent advances and applications of WRF-SFIRE, Natural Hazards and Earth System Sciences 14, 2829-2845.

Mandel J, Beezley JD, Kochanski AK (2011) Coupled atmosphere-wildland fire modeling with WRF 3.3 and SFIRE 2011. Geoscientific Model Development 4, 591-610.

Niemela JJ, Skrbek L, Sreenivasan KR, Donnelly RJ (2000) Turbulent convection at very high Rayleigh numbers, Nature 404, 837-840.

Niemela JJ, Sreenivasan KR (2006) Turbulent convection at high Rayleigh numbers and aspect ratio 4. Journal of Fluid Mechanics 557, 411-422.

Pagnini G, Massidda L (2012a) The randomized level-set method to model turbulence effects in wildland fire propagation. In 'Modelling Fire Behaviour and Risk. Proceedings of the International Conference on Fire Behaviour and Risk. ICFBR 2011, Alghero, Italy, October 4-6 2011, 2012' (Eds Spano D, Bacciu V, Salis M, Sirca C), 126-131 (ISBN 978-88-904409-7-7)

Pagnini G, Massidda L (2012b) Modelling turbulence effects in wildland fire propagation by the randomized level-set method, Tech. Rep. 2012/PM12a, CRS4, Pula (CA), Sardinia, Italy, revised version: 
http://publications.crs4.it/pubdocs/2012/PM12a/pagnini_massiddalevelset.pdf and arXiv:1408.6129 (July 2012)

Pagnini G, Mentrelli A (2014) Modelling wildland fire propagation by tracking random fronts. Natural Hazards and Earth System Sciences 14, 2249-2263.

Sardoy N, Consalvi J, Kaiss A, Fernandez-Pello A, Porterie B (2008) Numerical study of ground-level distribution of firebrands generated by line fires. Combustion and Flame 154, 478-488.

Sethian JA, Smereka P (2003) Level set methods for fluid interfaces. Annual Review of Fluid Mechanics 35, 341-372.

Skamarock WC, Klemp JB, Dudhia J, Gill DO, Barker DM, Duda MG, Huang XY, Wang W, Powers JG (2008) A Description of the Advanced Research WRF Version 3. NCAR Technical Note NCAR/TN-475+STR, doi:10.5065/D68S4MVH

Sofiev M, Ermakova T, Vankevich R (2012) Evaluation of the smoke-injection height from wild-land fires using remote-sensing data. Atmospheric Chemistry and Physics 12, 1995-2006.

Sullivan AL (2017a) Inside the inferno: Fundamental processes of wildland fire behaviour. Part 1: Combustion chemistry and heat release. Current Forestry Reports 3, 132-149.

Sullivan AL (2017b) Inside the inferno: Fundamental processes of wildland fire behaviour. Part 2: Heat transfer and interaction. Current Forestry Reports 3, 150-171.

Wang HH (2011) Analysis on downwind distribution of firebrands sourced from a wildland fire. Fire Technology 47, 321-340.

Wu XZ, Libchaber A (1992) Scaling relations in thermal turbulence: The aspect-ratio dependence. Physical Review A 45, 842-845. 


\title{
Does prescribed burning reduce the amount of inflorescences in conifer forests? Implications for management in pure and mixed Pinus nigra stands
}

\author{
J. Espinosa ${ }^{1 *}$; A.C. De La Cruz ${ }^{1}$ J. Madrigal ${ }^{1,2} ;$ M. Guijarro ${ }^{1,2} ;$ C. Carrillo ${ }^{1}$; C. Diez ${ }^{1}$; C. \\ Hernando ${ }^{1,2}$ \\ ${ }^{1}$ INIA, Forest Research Centre, Department of Silviculture and Forest Management, Forest Fire \\ Laboratory. Ctra. Coruña Km 7,5 28040 Madrid, Spain. \{espinosa.juncal@inia.es*\} \\ ${ }^{2}$ iuFOR, University Institute for Sustainable Forest Management, uVA-INIA.
}

\begin{abstract}
Predictions of climate change project a future with an increasing incidence of fires. Prescribed burning, as a valuable tool for silvicultural management, might successfully reduce the severity and the intensity of subsequent wildfires, although with different effects on the individual tree. This is the reason why short term effect of prescribed burning in litterfall biomass is being evaluated in a network of experimental plots (18 plots of $50 \mathrm{~m} \times 50 \mathrm{~m}$ ) in pure (Pinus nigra Arn. ssp. salzmannii) and mixed (Pinus nigra $89 \%$ and Pinus pinaster Ait. 11\%) stands in two sites (El Pozuelo and Beteta) of Cuenca Mountains. An experimental design with 3 treatments (with 3 replicates) per site were performed (spring and autumn burning and control) and 8 litterfall collectors per plot were installed after burning.

Immediately after burning it was shown a short-term effect of prescribed burning in Beteta with an increase in amount of total litterfall in burned plots regarding unburned plots, although this effect was not significant in El Pozuelo there was an increase in needles. The increasing of total biomass (especially the amount of needles) could be an important effect determining the longevity of prescribed burning, especially in more productive ecosystems. Although, during the second year, the mean litterfall in burned plots is still higher than in control plots in Beteta, the differences have been reduced and the effect of fire has been weakened. The differences in the findings obtained between El Pozuelo and Beteta might be because of differences in pure and mixed stand structure.

Spanish black pine (Pinus nigra) is a not serotonin species, and the persistence of the ecosystem is related to the productions of inflorescences and the later establishment. Some studies reported that prescribed burning reduces de emergence and increase the mortality of seedling during the first year. In addition, Pinus nigra presents regular cycles of fructification every 6-8 years which generate discontinuous pulses of natural regeneration. Prescribed burning two years before a pulse of fructification (maturation period of cones) could be critical to guarantee the regeneration. In fact, during the first and second year, and in the two zones, it was detected a decrease in the amount of inflorescences in the burned plots regarding unburned plots.

In view of our findings prescribed burnings must be carefully planned in forest management, in particular when are aimed at regeneration in Pinus nigra pure and mixed stands, to avoid a negative impact of fire on the canopy. The possible dampening effect of the mixed stand is being evaluated too. The litterfall continues being monitored in and a complete database of litterfall in pure and mixed stands is being generated. Scarce information of the impact of fire in foliar and non-foliar biomass is available in Europe and more research is needed to confirm these preliminary results.
\end{abstract}

Keywords: litterfall, prescribed burning, Cuenca Mountains, defoliation, fire severity

\section{Introduction}

Fuels treatments reduce the quantity and continuity of fuels (vertical and horizontal) to mitigate fire severity (Graham et al., 2004). The effectiveness of treatments may vary depending on type, amount, size, spatial distribution and intensity of treatments, time since implementation, ecosystem type, topography, geographic location and weather conditions at the time of fire (Cochrane et al., 2012). 
Prescribed burning is a kind of treatment that removes surface fuels, kills small trees and shrubs, and scorches lower limbs (Finney, 2005). Hence, burning under controlled conditions allows reduce temporarily potential fire intensity (Fernandes and Botelho, 2003; Agee and Skinner, 2005) and reduces the severity of wildfire (Wimberly et al., 2009; Arkle et al., 2012). However, their effects (in both the short and long terms) on the vegetation are complexes and they vary affecting different parts of the individual tree (roots, bark, stem, crown, etc). In this study, special attention is given to the effect of the prescribed burning on crown trees. For this reason, monthly monitoring of litterfall has been carried out in unburned and burned plots. This information may be important in management decisions to determinate time between prescribed burnings. Furthermore, the negative impact of fire on forests is expected to increase in the future since model predictions indicate increased frequency, intensity and severity of forest fires due to land-use change and climate change (Flannigan et al., 2009).

For the purpose of this study pure stands of Pinus nigra (Spanish black pine) and mixed stands of Pinus nigra and Pinus pinaster (maritime pine) have been chosen. Spanish black pine is resistant to low intensity surface fires due to the thick bark characteristic of the species (Fulé et al., 2008; Touchan et al., 2012; Pausas, 2015). However, recurrent large wildfires and total lack of capacity of this nonserotinous pine to regenerate after fire (Espelta et al., 2003; Ordoñez et al., 2005; Fernandes et al., 2008; Lucas-Borja et al., 2017; Christopoulou et al., 2013) threatening the permanence of P. nigra forests in some Mediterranean areas. In addition, the species releases its seeds early in the year, at the beginning of spring, and therefore no seed bank is maintained after summer wildfires (Ordoñez et al., 2005). In Spain, the total area occupied by maritime pine that was burned between 1974 and 2010 was 674055 ha, representing $31 \%$ of the total burned forest area. Provenance of maritime pine of Cuenca Mountains does not produce serotinous cones (Alía et al. 1996) but has a thick bark that may allow trees survival after low intensity fires. Therefore, it is important to determine the level of impact of prescribed burnings in the production of inflorescences with the aim of guaranteeing the persistence of stands. In addition, several authors have reported higher stability of mixed species stands than of monocultures (e.g. Schütz et al., 2006; Felton et al., 2010). In fact, species composition is an important consideration for managers wanting to develop resilient stand with prescribed burning.

This study is a first attempt to compare litterfall patterns in pure stands of P.nigra and mixed stands of P.nigra and P. pinaster after execution of prescribed burning to prevent forest fires. Although in this paper only the effect of spring burning will be analyzed, the study is part of a more comprehensive research study on the impacts of prescribed burning (soil, vegetation, tree heating of trunks and growing) conducted under tree canopies, in two different seasons (spring and autumn).

\section{Material and methods}

\subsection{Study site}

Two sites in the Cuenca Mountains, El Pozuelo (40 33' 36" N / $\left.002^{\circ} 15^{\prime} 56^{\prime \prime} \mathrm{W}\right)$ and Beteta (40 $33^{\prime}$ $\left.06^{\prime \prime} \mathrm{N} / 002^{\circ} 06^{\prime} 32^{\prime \prime} \mathrm{W}\right)$, were chosen for the study. Both areas have slopes between 3 and $10 \%$ and altitude between 1015 and 1294 m a.s.l. The climate in the area is humid Mediterranean (Allué, 1990), with mean annual temperature of $10.7^{\circ} \mathrm{C}$ and an average precipitation in summer months (maximum litterfall season) of $56 \mathrm{~mm}$. The soil is calcareous (Lucas-Borja et. al., 2017). The mean shrub cover in both zones is in a range of 5-20\% and pine regenerated has irregular distribution. El Pozuelo has a mean density of 627 trees ha $^{-1}$ (mixed stand of Pinus nigra and Pinus pinaster) and an average height of $12.2 \mathrm{~m}$ with the first living branch appearing at $6.4 \mathrm{~m}$. The mean breast diameter is $19.8 \mathrm{~cm}$. The basal area is $25.4 \mathrm{~m}^{2} \mathrm{ha}^{-1}$. Beteta has a mean density of 1286 trees ha $^{-1}$ (pure stand of Pinus nigra) and an average height of $13.2 \mathrm{~m}$, with the first living branch appearing at $8.2 \mathrm{~m}$. The mean breast diameter is $18.8 \mathrm{~cm}$. The basal area is $36.6 \mathrm{~m}^{2} \mathrm{ha}^{-1}$. 


\subsection{Experimental design}

As mentioned, the study is part of a wider study being carried out in El Pozuelo and Beteta. A network of experimental plots (12 plots: $\mathrm{n}=6$ in El Pozuelo and $\mathrm{n}=6$ in Beteta) of $50 \mathrm{~m} \times 50 \mathrm{~m}$ were established. Two treatments (spring burning and control) with a three replicates per treatment were applied. Subplots of $30 \mathrm{~m}$ x $30 \mathrm{~m}$ were established in the centre of each plot to avoid the edge effect. Data collection is being carried out in subplots. The plots are fairly homogeneous in terms of vegetation, density, orientation, etc. Plots $\mathrm{P} 2 \mathrm{C}, \mathrm{P} 5 \mathrm{C}$, P9C represent the control plots and plots $\mathrm{P} 3 \mathrm{~B}$, P4B, P6B represent the burned plots in El Pozuelo. Plots B1C, B3C, B9C represent the control plots and $\mathrm{B} 2 \mathrm{~B}, \mathrm{~B} 5 \mathrm{~B}, \mathrm{~B} 6 \mathrm{~B}$ represent the burned plots in Beteta.

All the trees in each of the 12 plots were identified, and the following measurements were made: total height, height to the first live branch, diameter at heights of $0.3,0.6$ and $1.3 \mathrm{~m}$ from the base and maximum and minimum bark thickness at $0.6 \mathrm{~m}$ from the base

\subsection{Burning}

Prescribed burnings were carried out taking into account the usual season in the region (spring burning were carried out in May 2016). The strip ignition technique was applied at distances of 1-2 m, at downhill and with a head wind. During burnings, precipitation (Ortrat, S.L.; KW 3-02), wind speed (Casella; 178031C-3), temperature and relative humidity (Geonica; STH-5031) were recorded every 10 minutes at a meteorological station. The temperatures of the cambial (inner bark) and bark region (outer bark) of 15 randomly selected trees were monitored at a height of $0.6 \mathrm{~m}$ (height of maximum heating according to prescription) with type $\mathrm{K} 1 \mathrm{~mm}$ diameter inconel-sheathed thermocouples $(0.3 \mathrm{~s}$ of response time). The thermocouples were connected to data loggers (DT-USB TCDirect ${ }^{\circledR}$ ), which recorded the data with a frequency of 1 second. Maximum and minimum stem char height, percentage of crown scorch and scorch height were measured after burning.

The characteristics of prescribed burning in each plot are shown in Table 1 . The flame convection (chimney effect) generated a moderate mean maximum stem char height $(70 \mathrm{~cm}$ in El Pozuelo and 160 $\mathrm{cm}$ in Beteta). Thermocouples connected in inner bark recorded a mean temperature of $50{ }^{\circ} \mathrm{C}$ in $\mathrm{El}$ Pozuelo and $41{ }^{\circ} \mathrm{C}$ in Beteta. Temperature was higher $60^{\circ} \mathrm{C}$ during $27.7 \mathrm{~s}$ in El Pozuelo and $27.9 \mathrm{~s}$ in Beteta. It is commonly accepted that cambial temperatures above $60{ }^{\circ} \mathrm{C}$ may cause necrosis of the cambium. In outer bark, the mean temperature in El Pozuelo was $209^{\circ} \mathrm{C}$ and $279^{\circ} \mathrm{C}$ in Beteta.

\subsection{Litterfall}

The litterfall collection system was designed in accordance with the recommendations outlined in the Manual of the United Nations Economic Commission for Europe under the project entitled "International Co-operative Program on Assessment and Monitoring of Air Pollution Effects on Forests " (ICP Forests) (Ukonmaanaho et al., 2016), to guarantee the quality and quantity of the sample.

After prescribed burnings a total of 8 litterfall collectors per plot were at regular intervals installed in both study sites. The catchment area $\left(0.38 \mathrm{~m}^{2}\right)$ of collectors is horizontal to correct the effect of slope. The bag is $0.75 \mathrm{~m}$ deep to prevent the sample from being lost as a result of wind action. The collectors were placed at a height of $1.2 \mathrm{~m}$ from the ground to enable adequate drainage by gravity and to prevent capture of biomass from shrub strata. They were anchored firmly to the ground to provide greater resistance to the inclement conditions or other types of disturbance. Fiberglass mesh (pore size $2 \mathrm{~mm}$ ) provides resistance against external weather conditions and also ensures drainage while preventing loss of smaller elements, such as needles. The material was collected monthly to prevent decomposition of the biomass or the chemical leachate. This frequency of collection also ensures the easy identification of the fine elements which are rapidly compressed. Samples were taken to the laboratory on the same day and oven-dried at $65^{\circ} \mathrm{C}$ to constant weight (i.e. for at least 48 hours). The samples from each plot were then combined and the main fractions separated (needles, cones, 
inflorescences, miscellaneous, branches of diameter $<2 \mathrm{~cm}$ and bark) other fractions are also separated (but the biomass are not significant for this study). For the purpose of this paper, special attention is being put into the results obtained in the collected inflorescences after spring burnings.

\subsection{Statistical analysis}

The data were tested for normality and when necessary were transformed (logarithmic and angular transformation). The General Linear Model (GLM) repeated measures procedure was used to perform an impact analysis of burning and control treatments, to identify the effect of prescribed burning on the above parameters. The between-subjects factor (treatment) included two levels (control and burning) and the within-subjects factor was the date (12 levels). Comparison of parameters between treatments was conducted using the Bonferroni test. All above analyses were carried out with STATISTICA 10.0 (Statsoft Inc, Tulsa, USA).

Table 1 - Main traits of fire behavior and weather conditions of spring burnings in El Pozuelo and Beteta.

\begin{tabular}{cccccccccc}
\hline $\mathrm{Z}$ & $\mathrm{PN}$ & $\mathrm{T}$ & $\mathrm{RH}$ & $\mathrm{WS}$ & LHFc & SCHmm & Tmi & Tmo & Tmi $>60$ \\
\hline-- & -- & ${ }^{\circ} \mathrm{C}$ & $\%$ & $\mathrm{~m} / \mathrm{s}$ & $\%$ & $\mathrm{~cm}$ & ${ }^{\circ} \mathrm{C}$ & ${ }^{\circ} \mathrm{C}$ & $\mathrm{s}$ \\
\hline El Pozuelo & P3B & 20.17 & 53.12 & 0.17 & 16.9 & 32 & 32.8 & 139.8 & 24.5 \\
El Pozuelo & P4B & 21.97 & 47.47 & 0.81 & 25.8 & 66 & 61.9 & 312.9 & 17.5 \\
El Pozuelo & P6B & 22.42 & 42.61 & 1.32 & 38.2 & 113 & 54.6 & 175.3 & 41 \\
Beteta & B2B & 18.80 & 34.77 & 0.79 & 22.4 & 151 & 32.2 & 199.3 & 11.5 \\
Beteta & B5B & 20.65 & 33.10 & 0.71 & 18.3 & 150 & 52.4 & 285.0 & 60 \\
Beteta & B6B & 21.69 & 30.15 & 0.82 & 21.4 & 178 & 37.4 & 352.8 & 12.1 \\
\hline
\end{tabular}

Note = Z: zone; $P N$ : plot number; T: temperature air; $R H:$ relative humidity; WS: mean wind speed; LHFc: percentage of fuel load consumed (LHF); SCHmm: mean maximum stem char height; Tmi: mean temperature of inner bark; Tmo: mean temperature of outer bark; tmi>60: mean time that the temperature in inner bark was higher than $60^{\circ} \mathrm{C}$.

\section{Results}

\subsection{Litterfall}

The mean accumulated litterfall collected in control and burned plots in El Pozuelo and Beteta during the first and second year are shown in Table 2.

Table 2 - The mean accumulated litterfall.

\begin{tabular}{cccc}
\hline P & PT & El Pozuelo & Beteta \\
\hline-- & -- & $\mathrm{kg} \mathrm{ha}^{-1}$ & $\mathrm{~kg} \mathrm{ha}^{-1}$ \\
\hline \multirow{2}{*}{ May 2016 - April 2017 } & \multirow{2}{*}{ Control } & $\begin{array}{l}3171 \\
(649)\end{array}$ & $\begin{array}{c}2028 \\
(530)\end{array}$ \\
\hline \multirow{2}{*}{ May 2016 - April 2017 } & \multirow{2}{*}{ Burned } & $\begin{array}{l}3257 \\
(598)\end{array}$ & $\begin{array}{l}3520 \\
(135)\end{array}$ \\
\hline \multirow{2}{*}{ May 2017 - April 2018 } & \multirow{2}{*}{ Control } & 3537 & 2585 \\
& & $(583)$ & $(762)$ \\
\hline \multirow{2}{*}{ May 2017 - April 2018 } & \multirow{2}{*}{ Burned } & 2991 & 3120 \\
& & $(191)$ & $(327)$ \\
\hline
\end{tabular}

Note $=$ P: Period of time; PT: Plot treatment.

In El Pozuelo, litterfall peak took place in September during the first year, both in unburned and burned plots (670 kg ha-1 y $525 \mathrm{~kg} \mathrm{ha}^{-1}$ respectively). In the second year the maximum took place in August for both unburned and burned treatments (917 kg ha-1 and $684 \mathrm{~kg} \mathrm{ha}^{-1}$ respectively). The minimum peak occurred in December of the first year for the two treatments $\left(37 \mathrm{~kg} \mathrm{ha}^{-1}\right.$ y $28 \mathrm{~kg} \mathrm{ha}^{-1}$ 
in unburned and burned plots). In the second year, the minimum took place in January and February for unburned and burned plots ( $84 \mathrm{~kg} \mathrm{ha}^{-1}$ and $82 \mathrm{~kg} \mathrm{ha}^{-1}$ respectively).

In Beteta, during the first year, litterfall peak took place in August in untreated plots $\left(420 \mathrm{~kg} \mathrm{ha}^{-1}\right)$ and in August-September in treated plots (574-577 $\left.\mathrm{kg} \mathrm{ha}^{-1}\right)$. The same situation in August was repeated during the second year $\left(785 \mathrm{~kg} \mathrm{ha}^{-1}\right.$ in untreated plots and $632 \mathrm{~kg} \mathrm{ha}^{-1}$ in treated). Minimum amount of biomass collected occurred during December in the first year for both treatments $\left(55 \mathrm{~kg} \mathrm{ha}^{-1}\right.$ and 31 $\mathrm{kg} \mathrm{ha}^{-1}$ in unburned and burned plots) and in February for both treatments in the second year ( $81 \mathrm{~kg}$ $\mathrm{ha}^{-1}$ and $55 \mathrm{~kg} \mathrm{ha}^{-1}$ in unburned and burned plots respectively).

In El Pozuelo, in control plots, $47 \%$ and $45 \%$ of annual litterfall, respectively for the first and second year, were collected during the summer (July, August and September). A similar percentage took place during that period, in burned plots, collecting $45 \%$ the first year and 52\% the second one. But, litterfall came early in the second year (amount of litterfall was collected in June), reaching percentages of $60 \%$ and 57\% in control and burning plots respectively, from June to August.

A similar situation occurred in Beteta. In control plots during the summer, it was produced $51 \%$ of annual litterfall the first year and $38 \%$ the second year. Similar percentages were obtained in burned plots: $45 \%$ the first year and $40 \%$ the second year. As well, the second year, the level of litterfall collected in June was representative, reaching percentages of 48 and $45 \%$ in control and uncontrol plots respectively.

In control plots from El Pozuelo, needles represented $41 \%$ and $42 \%$ of annual litterfall during the first and second year respectively. Similar percentage appeared in burned plots during the first and second year $(51 \%$ and $49 \%)$.

In control plots from Beteta, needless represented 59\% and 39\% of annual litterfall during the first and second year respectively. In burned plots this percentage was 55\% and 35\% in the first and second year.

In El Pozuelo, inflorescences showed the same pattern during the first and second year (Fig. 1). The amount of inflorescences in burned plot decreased regarding to unburned plots especially during the months of maximum litterfall in this fraction (May, June and July). In fact, $46 \%$ less inflorescences were trapped in burned plots during the first year. This percentage was slightly less during the second year $(45 \%)$. After the months of maximum litterfall, the differences between burned and unburned plots were barely noticeable. This same pattern was repeated in Beteta during the two years for which data are currently available ( $42 \%$ less inflorescences in burned plots during the first year and $18 \%$ less in the second year).
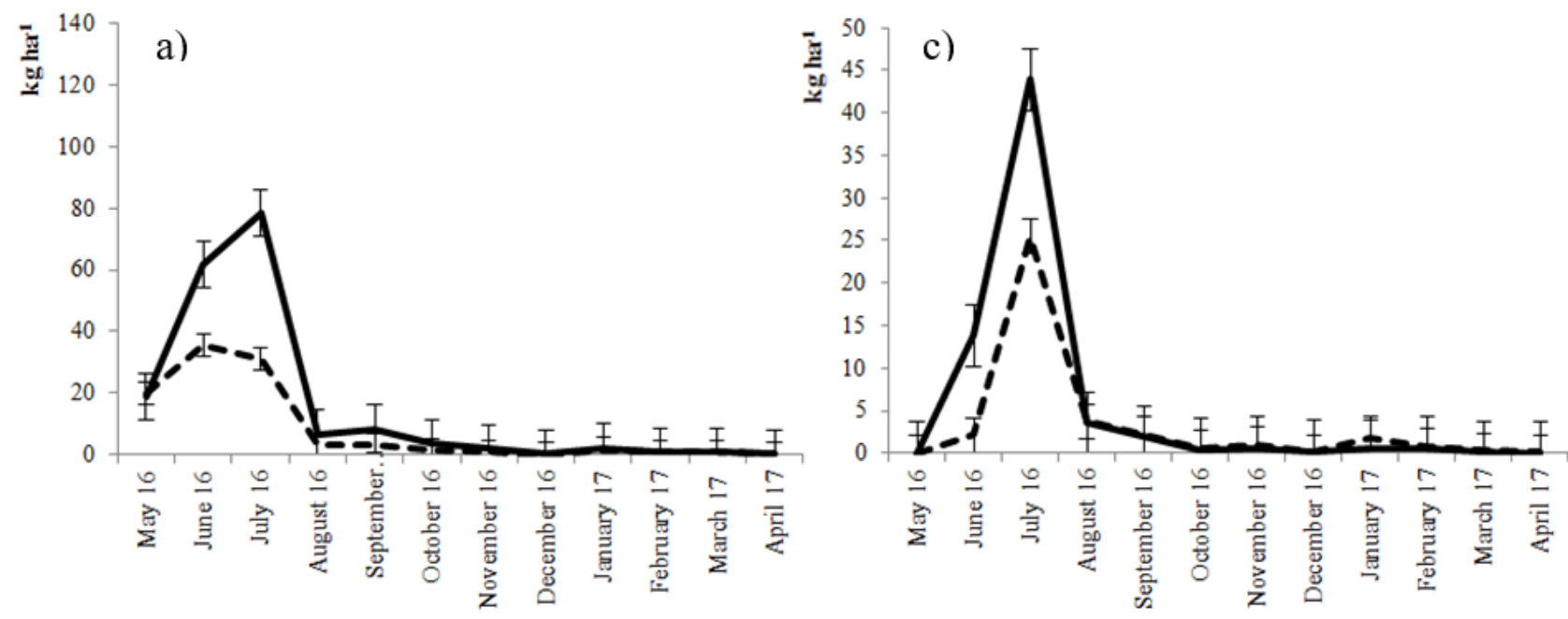

Advances in Forest Fire Research 2018 - Page 396 

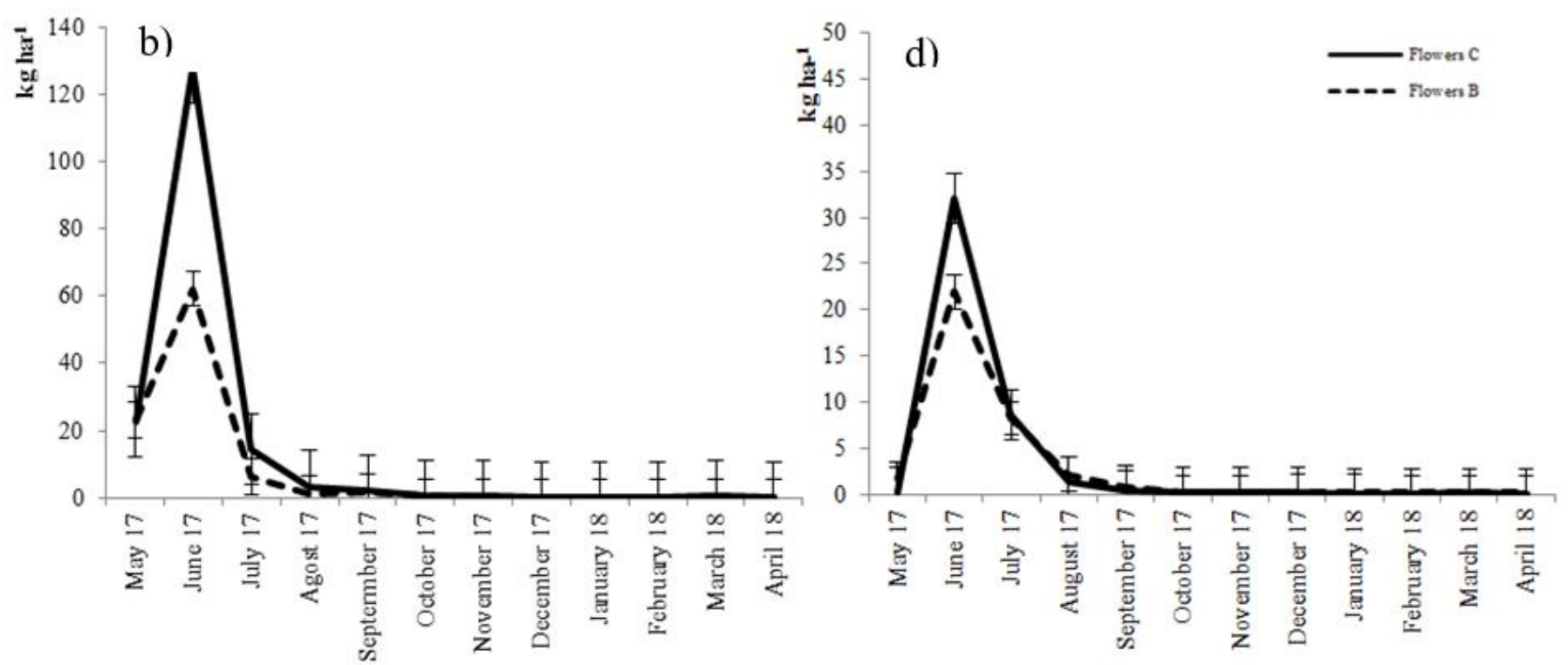

Figure 1 - Inflorescences $\left(\mathrm{kg} \mathrm{ha}^{-1}\right)$ collected in El Pozuelo during $1^{\text {st }}$ year (a), in El Pozuelo during $2^{\text {nd }}$ year (b) in Beteta $1^{\text {st }}$ year (c) and in Beteta 2 nd year (d). Control in solid line and burned in dashed line. Control plots (n=3), burned (n=3). Bars show standard errors. Significant differences (ANOVA repeated measurements, Bonferroni test p<0,05) are detected in June-July (El Pozuelo) and June (Beteta) for 1st and 2nd year after burning.

\section{Discussion}

Litterfall is one of the main components of net primary production (NPP) (Hansen et al., 2009) and along with root recycling, more than half of the nutrients absorbed by all are represented (Binkley, 1986). Our findings suggest that prescribed burning might change the patterns and regimes of litterfall biomass, at least in the short time (Espinosa et al., 2017). In fact, although the amount of total litterfall in burning plots of El Pozuelo was not significant regarding control plots (only $\approx 3 \%$ more litterfall in burning plots) an increase in needles in the two months after prescribed burning was observed. Beteta there was a short-term effect of prescribed burning in the amount of total litterfall with an increase of $\approx 74 \%$ in burning plots regarding control plots. The increasing of total biomass (especially the amount of needles) may be an important effect determining the longevity (time since last burning) of prescribed burning, especially in more productive ecosystems. Thus, the short term impact of treatment and the recovery of fuel and structure may result decisive to evaluate the effectiveness of prescribed burnings (Mirra et al., 2017).

During the second year, in maximum litterfall season (June-August) in El Pozuelo it was observed that the mean litterfall is higher in control plots than in burned plots. In Beteta, although the mean litterfall in burned plots was still higher than in control plots, the differences have been reduced and the effect of fire has been weakened. This may be due to the immediate effect of fire that dampen over the years and it is expected that the amount of biomass collected will be stabilized to ordinary values in subsequent years. Although there is scarce information in Europe assessing the effect of fire on litterfall biomass, there are some studies that evaluate the effect of silvicultural treatments, such as thinning, with similar results (e.g. Roig et al., 2005, Blanco et al., 2006). The differences in the findings obtained between El Pozuelo and Beteta might be because of differences in pure and mixed stand structure. Natural disturbances, including fires, disrupt ecosystem structure or function for time periods longer than the current seasonal vegetation cycle (Jactel et al., 2009). Mixed stand might contribute to maintain the stability of ecosystems and biogeochemical cycles. In fact, there is consensus that a minimum number of species is essential for ecosystem functioning under constant conditions (Loreau et al., 2001). In the second year, the total amounts of biomass collected increased with respect to the first year, which may be due to extraordinarily dry summer registered that year in the study area. In some Mediterranean species, the greatest flow of litterfall is gradual due to factors such as the longer 
duration of the light period (which leads to an increase in growth hormones) as well as temperature increases and factors such as water stress. In fact, Pausas et al., (1994) found high intrannual variability in the analysis of litterfall due to climatic variability along a bioclimatic gradient.

One of the effects observed during the first year from May to July (months just after the burns) and in the two zones, was a decrease in the inflorescences in the burned plots regarding the unburned ones (Fig. 1a, 1c). This effect has been maintained in the second year also in the two zones (Fig. 1b, 1d). In Beteta, $42 \%$ less quantity of inflorescences was collected in burned plots than in unburned plots and this percentage was maintained in the second year (46\%). In the case of El Pozuelo during the first year it was $46 \%$ less and in the second year this amount was $45 \%$ less in burned plots. Pinus nigra is a species able to tolerate low surface fires (Fernandes et al., 2008; Fulé et al., 2008). Despite this, its distribution has been reduced dramatically in mountains areas of the Mediterranean region (López Serrano et al., 2009). Spanish black pine is not serotonin, and the persistence of the ecosystem is related to the productions of inflorescences and the later establishment. In fact, many factors are cited hindering the regeneration in Pinus nigra stands (including forest fires), masting, dry summers over consecutive years or excessive grazing (Lucas-Borja et al., 2017). As well, Lucas-Borja et al. (2016) reported that prescribed burning reduces de emergence and increase the mortality of seedlings during the first year as regards control plots. In addition, Pinus nigra presents regular cycles of fructification every 6-8 years which generate discontinuous pulses of natural regeneration. Consequently, burns two years before a pulse of fructification (maturation period of cones) could be critical to guarantee the regeneration in some managed areas. In view of our findings prescribed burnings must be carefully planned in forest management, in particular when are aimed at regeneration in Pinus nigra pure and mixed stands.

\section{Conclusion and management aplications}

Pinus nigra is a non-serotype species, to guarantee the stand survival depends on the production of inflorescences and the success of their implantation. A decrease in the production of inflorescences in burned plots with respect to the unburned plots has been observed during the first and the second year in the two study zones. The litterfall continues being monitored in order to assess the effect of prescribed burning in the medium term (3-4 years) and a complete database of litterfall in pure and mixed stands is being generated. Moreover, the next evaluation of the number of cones two years after burning will ratify or not these results: if the amount of fructification were similar between plots, the fall of inflorescences would not be a good index of fructification potential, probably because prescribed burnings only affect to male flowers sited in the lower parts of the crown. But, if the number of cones were higher in the control plots, we could assume that prescribed burning significantly affects the regeneration potential in Pinus nigra stands. It is well known that prescribed burning is a tool for forest fuel management that can contribute to the reduction of severity of forest fires. But, its inclusion must take into account the peculiarities of each forest ecosystem, evaluating the affections to each part of tree. Litterfall is an important phenological indicator of climate change. Some studies have shown the importance of climate conditions on the litter production. In our work located in Central Spain, an increase in litterfall biomass has been seen after a dry period. Thus a careful planning of prescribed burning must be done under extreme weather conditions.

Scarce information of the impact of fire in foliar and non-foliar biomass is available in Europe and more research is needed to confirm the preliminary results.

\section{Acknowledgments}

This study was supported by the GEPRIF project (Fire Severity Reduction through New Tools and Technologies for Integrated Forest Fire Protection Management), Spanish R\&D project (RTA201400011-C06-01). GEPRIF is co-funded by the EU through the FEDER program. This study was also 
co-financed by INIA (FPI-SGIT 2015) and European Social Fund through a grant awarded to Juncal Espinosa. We thank Cristina Carrillo, Carmen Díez and Ángeles Tirado from INIA-CIFOR for the assistance during the experiment; Fire Extinguishing Service in Cuenca (SEIF Cuenca) responsible for the execution and conduct of prescribed fires and ICP Forests organization and their countries collaborators for the litterfall data base.

\section{Conflicts of Interest}

The authors declare no conflict of interest.

\section{References}

Agee, J.K., Skinner, C.N., 2005. Basic principles of forest fuel reduction treatments. Forest ecology and management, 211(1-2), 83-96. doi:10.1016/j.foreco.2005.01.034.

Alía, R., Martín, S., De Miguel, J., Galera, R., Agúndez D., Gordo, J., Salvador, L., Catalán G., Gil, L., 1996. Las regiones de procedencia de Pinus pinaster, Organismo Autónomo de Parques Nacionales y ETSI de Montes, Madrid.

Allué, J.L., 1990. Atlas Fitoclimático de España. Taxonomías. Ministerio de Agricultura, Pesca y Alimentación. Instituto Nacional de Investigaciones Agrarias, Madrid.

Arkle, R.S., Pilliod, D.S., Welty, J.L., 2012. Pattern and process of prescribed fires influence effectiveness at reducing wildfire severity in dry coniferous forests. Forest Ecology and Management, 276, 174-184. doi:10.1016/j.foreco.2012.04.002.

Binkley, D., 1986. Forest nutrition management. John Wiley \& Sons.

Blanco, J., Imbert, J., Castillo, F., 2006. Influence of site characteristics and thinning intensity on litterfall production in two Pinus sylvestris L. forests in the western Pyrenees. Forest Ecology and Management, 237(1-3), pp.342-352. https://doi.org/10.1016/j.foreco.2006.09.057.

Christopoulou, A., Fulé, P., Andriopoulos, P., Sarris, D., Arianoutsou, M., 2013. Dendrochronologybased fire history of Pinus nigra forests in Mount Taygetos, Southern Greece. Forest Ecology and Management, 293, pp.132-139. https://doi.org/10.1016/j.foreco.2012.12.048.

Cochrane, M.A., Moran, C.J., Wimberly, M.C., Baer, A.D., Finney, M.A., Beckendorf, K.L., ... Zhu, Z., 2012. Estimation of wildfire size and risk changes due to fuels treatments. International Journal of Wildland Fire, 21(4), 357-367. doi:10.1071/WF11079.

Espelta, J.M., Retana, J., Habrouk, A., 2003. An economic and ecological multi-criteria evaluation of reforestation methods to recover burned Pinus nigra forests in NE Spain. Forest Ecology and Management, 180(1), 185-198. https://doi.org/10.1016/S0378-1127(02)00599-6.

Espinosa, J., Madrigal, J., De La Cruz, A.C., Guijarro, M., Jimenez, E., Hernando, C., 2018. Shortterm effects of prescribed burning on litterfall biomass in mixed stands of Pinus nigra and Pinus pinaster and pure stands of Pinus nigra in the Cuenca Mountains (Central-Eastern Spain). Science of The Total Environment, 618, 941-951.

Felton, A., Lindbladh, M., Brunet, J., Fritz, Ö., 2010. Replacing coniferous monocultures with mixedspecies production stands: an assessment of the potential benefits for forest biodiversity in northern Europe. Forest ecology and management, 260(6), 939-947.

Fernandes, P.M., Botelho, H.S., 2003. A review of prescribed burning effectiveness in fire hazard reduction. International Journal of wildland fire, 12(2), 117-128. https://doi.org/10.1071/WF02042.

Agee, J.K., and Skinner, C.N. 2005. Basic principles of forest fuel reduction treatments. Forest ecology and management, 211(1-2), 83-96. doi:10.1016/j.foreco.2005.01.034.

Fernandes, P., Vega, J., Jiménez, E., Rigolot, E., 2008. Fire resistance of European pines. Forest Ecology and Management, 256(3), pp.246-255. https://doi.org/10.1016/j.foreco.2008.04.032. 
Finney, M.A., McHugh, C.W., Grenfell, I.C., 2005. Stand-and landscape-level effects of prescribed burning on two Arizona wildfires. Canadian Journal of Forest Research, 35(7), 1714-1722. doi:10.1139/x05-090.

Flannigan, M.D., Krawchuk, M.A., de Groot, W.J., Wotton, B.M., Gowman, L.M., 2009. Implications of changing climate for global wildland fire. International journal of wildland fire, 18(5), 483-507.

Fulé, P.Z., Ribas, M., Gutiérrez, E., Vallejo, R., Kaye, M.W., 2008. Forest structure and fire history in an old Pinus nigra forest, eastern Spain. Forest Ecology and Management, 255(3-4), 1234-1242.

Graham, R.T., McCaffrey, S., Jain, T.B., 2004. Science basis for changing forest structure to modify wildfire behavior and severity. The Bark Beetles, Fuels, and Fire Bibliography, 167. doi:10.2737/RMRS-GTR-120.

Hansen, K., Vesterdal, L., Schmidt, I.K., Gundersen, P., Sevel, L., Bastrup-Birk, A., ... Bille-Hansen, J., 2009. Litterfall and nutrient return in five tree species in a common garden experiment. Forest Ecology and Management, 257(10), 2133-2144.

Jactel, H., Nicoll, B.C., Branco, M., González-Olabarría, J.R., Grodzki, W., Långström, B., Moreira, F., et al., 2009. The influences of forest stand management on biotic and abiotic risks of damage. Ann. For. Sci. 66 (7), 701.

López-Serrano, F.R., Rubio Caballero, E., Andrés Abellán, M., Del Cerro Barja, A., García Morote, F.A., De las Heras Ibañez, J., Lucas-Borja, M.E., Moya Navarro, D., Odi Lara, M., 2009. Efectos del Cambio climático en los bosques castellanomanchegos. In: Rodríguez Torres, A., Fernández Castro, H., Rojano Saura, I. (Eds.), Impactos del Cambio climático en Castilla La Mancha. Fundación General de Medio Ambiente, Castilla-La Mancha, Spain.

Loreau, M., 2001. Biodiversity and ecosystem functioning: current knowledge and future challenges. Science 294 (5543):804-808. https://doi.org/10.1126/science.1064088.

Lucas-Borja, M., Madrigal, J., Candel-Pérez, D., Jiménez, E., Moya, D., Heras, J., Guijarro, M., Vega, J., Fernández, C., Hernando, C., 2016. Effects of prescribed burning, vegetation treatment and seed predation on natural regeneration of Spanish black pine (Pinus nigra Arn. ssp. salzmannii) in pure and mixed forest stands. For. Ecol. Manag. 378: 24-30.

Lucas-Borja, M., Candel-Pérez, D., Onkelinx, T., Fule, P., Moya, D., Gómez, R., De las Heras, J., 2017. Early Mediterranean pine recruitment in burned and unburned Pinus nigra Arn. ssp. salzmannii stands of central Spain: Influence of species identity, provenances and post-dispersal predation. Forest Ecology and Management, 390, pp.203-211. https://doi.org/10.1016/j.foreco.2017.01.026.

Mirra, I.M., Oliveira, T.M., Barros, A.M., and Fernandes, P.M., 2017. Fuel dynamics following fire hazard reduction treatments in blue gum (Eucalyptus globulus) plantations in Portugal. Forest ecology and management, 398, 185-195. https://doi.org/10.1016/j.foreco.2017.05.016.

Ordóñez, J., Retana, J., Espelta, J., 2005. Effects of tree size, crown damage, and tree location on postfire survival and cone production of Pinus nigra trees. Forest Ecology and Management, 206(1-3), pp.109-117. https://doi.org/10.1016/j.foreco.2004.10.067.

Pausas, J.G., Carceller, F., Vallejo, V.R., 1994. El desfronde en bosques de Pinus sylvestris L. en relación con las características climáticas. Ecología, (8), 167-176.

Pausas, J.G., 2015. Bark thickness and fire regime. Funct. Ecol. 29, 315-327. https://doi.org/10.1111/1365-2435.12372.

Roig, S., del Río, M., Cañellas, I., Montero, G., 2005. Litter fall in Mediterranean Pinus pinaster Ait. stands under different thinning regimes. Forest Ecology and Management, 206(1-3), pp.179-190. https://doi.org/10.1016/j.foreco.2004.10.068. 
Schütz, J., Götz, M., Schmid, W., Mandallaz, D., 2006. Vulnerability of spruce (Picea abies) and beech (Fagus sylvatica) forest stands to storms and consequences for silviculture. European Journal of Forest Research, 125(3), pp.291-302. https://doi.org/10.1007/s10342-006-0111-0.

Touchan, R., Baisan, C., Mitsopoulos, I., Dimitrakopoulos, A., 2012. Fire History in European Black Pine (Pinus nigra Arn.) Forests of the Valia Kalda, Pindus Mountains, Greece.Tree-Ring Research, 68(1), pp.45-50. https://doi.org/10.3959/2011-12.1.

Ukonmaanaho, L., Pitman R, Bastrup-Birk A., Breda N, Rautio P., 2016: Part XIII: Sampling and Analysis of Litterfall. In: UNECE ICP Forests Programme Co-ordinating Centre (ed.): Manual on methods and criteria for harmonized sampling, assessment, monitoring and analysis of the effects of air pollution on forests. Thünen Institute for Forests Ecosystems, Eberswalde, Germany, 15 p.

Wimberly, M.C., Cochrane, M.A., Baer, A.D., Pabst, K. 2009., Assessing fuel treatment effectiveness using satellite imagery and spatial statistics. Ecological Applications, 19(6), 1377-1384. https://doi.org/10.1890/08-1685.1. 


\title{
Effect of canyons on a fire propagating laterally over slopes
}

\author{
André Rodrigues *1; Domingos Xavier Viegas ${ }^{1,2}$; Abdelrahman Abouali ${ }^{1}$; Jorge Raposo ${ }^{1,2}$; Miguel \\ Almeida $^{1}$; Luís Mário Ribeiro ${ }^{1}$; Xieshang Yuan ${ }^{3}$ \\ ${ }^{1}$ Association for the Development of the Industrial Aerodynamics. Rua Pedro Hispano, $n^{\circ} 12,3030-$ \\ 289 Coimbra, Portugal, \{andrerodrigues@adai.pt*\} \\ ${ }^{2}$ University of Coimbra, Rua Luís Reis dos Santos, Coimbra 3030-788, Portugal \\ ${ }^{3}$ State Key Laboratory of Fire Science, University of Science and Technology of China, 230026, \\ Hefei, Anhui, China
}

\begin{abstract}
Forest fires often cause fatal losses, human lives and resources in wildfire-prone regions. Several accidents were registered in canyons with a scenario in which the fire propagates laterally over a slope then enters the canyon causing a rapid increase in the fire's rate of spread (Viegas, 2004; Lahaye et al. 2018; Pimont et al. 2012). This behaviour is known as eruptive fire behaviour (blow-up) - Viegas et al. 2011 - which usually happens over slopes or in canyon configurations (Viegas, 2005; Viegas, 2006; Viegas and Simeoni, 2011). The behaviour surprised fire management teams in several recorded events and caused loss of lives in some of them. In 2013, at Serra do Caramulo, two firefighters died on a sloped terrain when a flank fire entered a canyon (Viegas et al. 2013). This particular accident motivated us to study the change in the flank fire behaviour over slopes when it enters canyons with the aim of improving the safety of the personnel involved in the combat of wildfires.

In this study, we analyse results of laboratory-scale experiments that model a fire spreading latterly over a slope then enters a canyon from its base. We considered various conditions of terrain slope, canyon's axis angle along with altered canyon configurations. Also, we tested a number of fire approaching angle scenarios. Based on the analysis, we are proposing a mathematical model for predicting the fire's rate of spread in this configuration along with suggested personnel safety measures under these conditions.
\end{abstract}

Keywords: Forest Fires; Fire safety; Extreme Fire Behaviour; Eruptive Behaviour; Fire in canyons.

\section{Introduction}

The knowledge on fire behaviour is an essential tool to fire suppression and increase the personnel safety. Thus, research has contributed to the advancement of the scientific knowledge on forest fires. According to Viegas et al. (2011), the fire behaviour can be classified as normal and extreme. Accidents related to forest fires are often due to insufficient knowledge about fire and its behaviour (Viegas, 2006), especially in the case of Extreme Fire Behaviour (EFB).

Most of the behaviour models are based on the assumption that the fire propagation properties are almost static and can be determined by considering three essential factors: topography, fuel and meteorology, which is called the "fire triangle" (Byram, 1959). However, Viegas (2006) proposed, as an alternative to this classic concept, the concept of "fire square", adding a fourth factor: time.

A low and little time-dependent rate of spread is a characteristic of Normal Fire Behaviour. This type of behaviour doesn't pose a great risk to the operational, instead that happens with the Extreme Fire Behaviour. In Extreme Fire Behaviour there is a dynamic change of the characteristics of the fire over time, as shown by the studies on slopes of Dold and Zionviev (2009) and in canyons of Viegas and Pita (2004). According to Viegas et al. (2011), the Extreme Fire Behaviour is defined as the set of characteristics of propagation and properties that call into question the possibility the control in safety using the available technology and knowledge. One of the examples of Extreme Fire Behaviour is the 
fire spread in canyons. The rate of spread of the fire front has a dynamic behaviour and its properties depend on the canyon's geometry and the fire spread, suffering this acceleration by the variation of rate of spread and energy release rate (Viegas, 2005, 2006; Viegas et al., 2017). The induced fire convection becomes stronger due to the specific topography of the terrain which promotes the occurrence of the eruptive fire behaviour (Velez, 2000; Raposo et al., 2018). The rate of spread of the fire front increases steadily, even in the absence of wind or any other feature (Pyne et al., 1996; Dold, 2010). Canyons are associated with a large percentage of fatal accidents during forest fires worldwide (Viegas, 2005; Schemel et al., 2008; Lahaye et al., 2018). The eruptive effect created by this topographical configuration induces a spreading of increased velocity surprising experienced firefighters causing death (Viegas and Pita, 2004). In 2013 at Serra do Caramulo two firefighters died on a slope when the fire reached the canyon (Viegas et al., 2013).

The main objective of this paper is a detailed and exhaustive analysis on the phenomenon of the interaction on lateral spread of fire on a slope with a canyon. The intent to understand the variation of fire propagation velocity and direction and the values of the energy release rate, contributing towards the increase of scientific knowledge in this case of EFB. Fuel moisture, relative humidity and air temperature will be taken into account. Different ignition modes will be tested to evaluate their influence on the development in the forest fires. The acquisition of images in the visible range and infrared range to determine the velocity and direction of fire propagation, the flame geometry and the energy release rate is the purpose of this work. This study also aims to propose behaviour models and operation rules in safety in the fight against forest fires on slopes with canyons, based on previous studies and in the research that will be carried out.

\section{Conceptual Model}

For the experimental study a test table was built, which allows to change the inclination and rotation of the slope, the angle and the geometry of the canyon. The testing table (figure 1) allows to change the angle of inclination of the slope between $0^{\circ}$ and $45^{\circ}$ and the angle of rotation between $0^{\circ}$ and $360^{\circ}$. Various canyon configurations (figure 2 ) will be studied by changing their opening angle, width and depth.

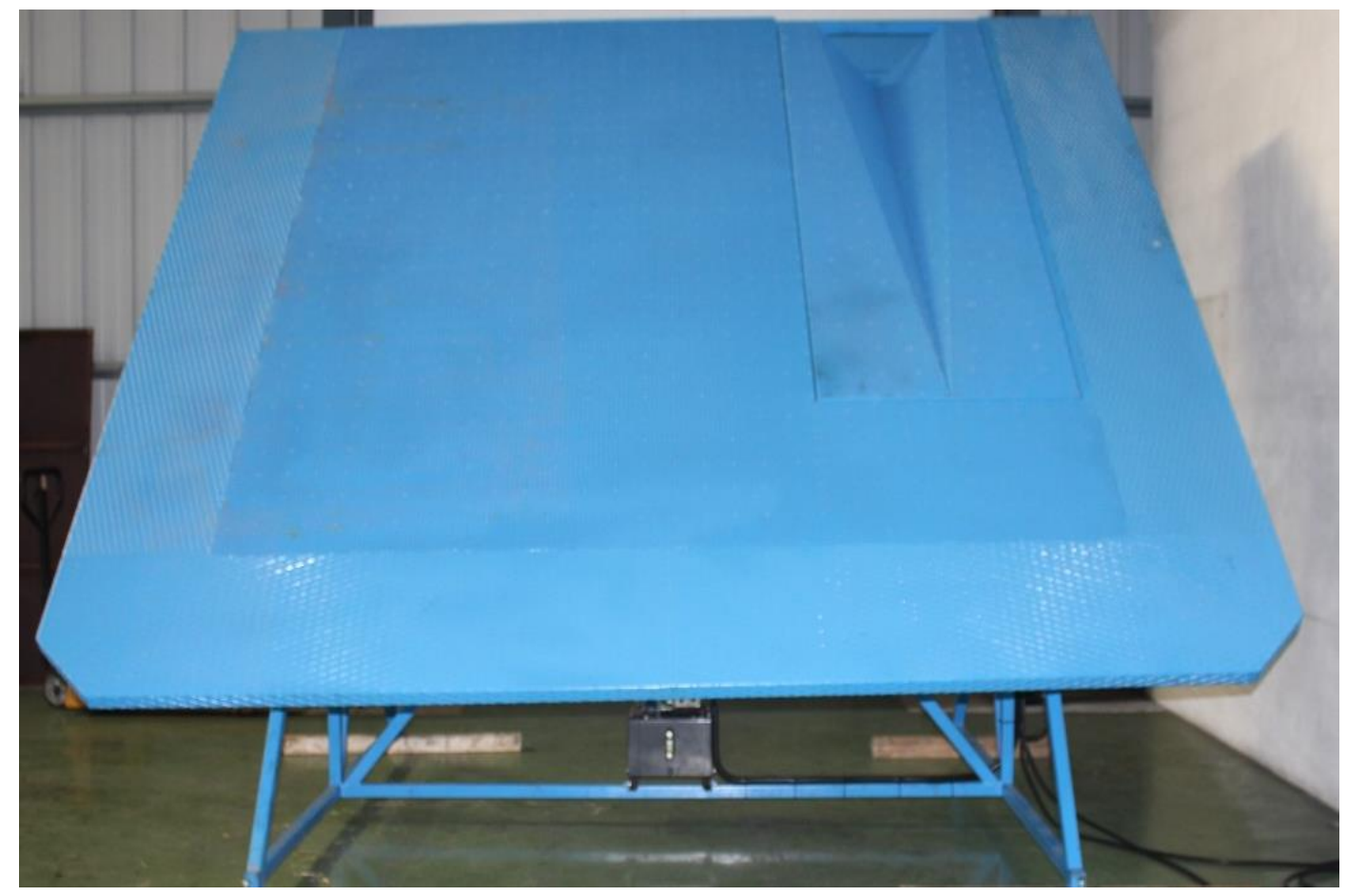

Figure 1 - Table of laboratory tests. 


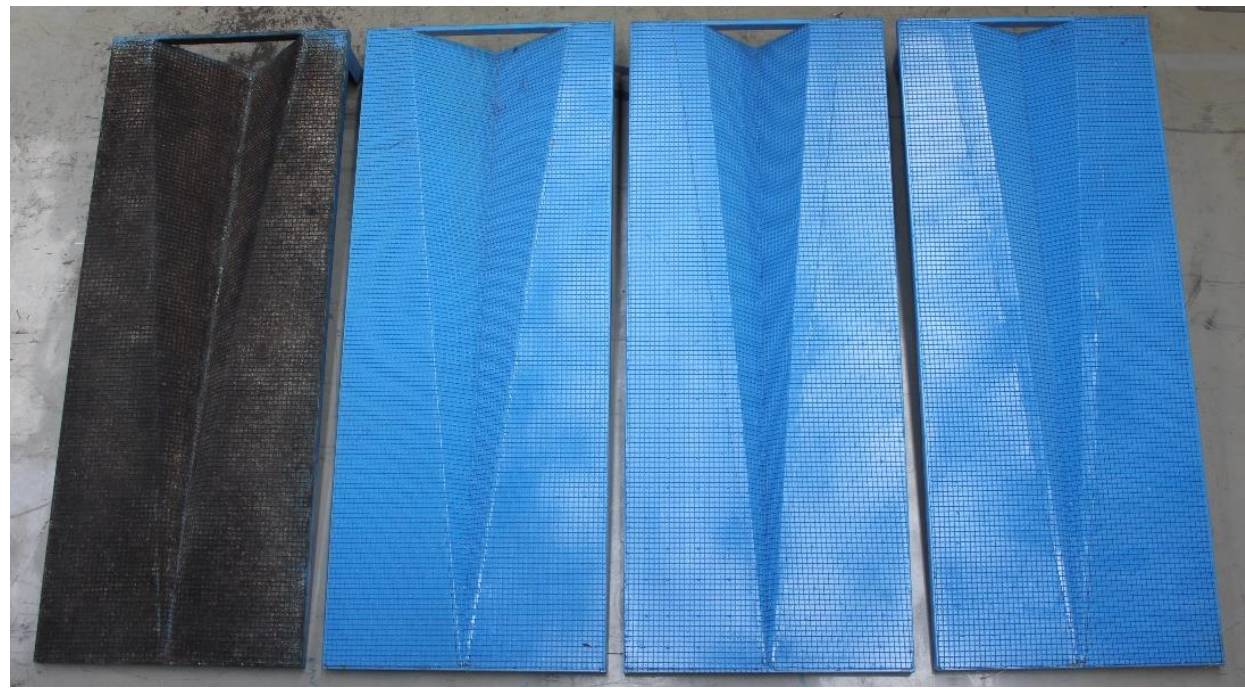

Figure 2 - Various canyon configurations, changing their opening angle, width and depth.

\section{Methodology}

A testing table was prepared as a flat surface with a canyon structure inserted within this surface. The table has an allowance to change its inclination $(\alpha)$, its rotation $(\gamma)$ and the opening angle of the canyon $(\delta)$, according to figure 3 .
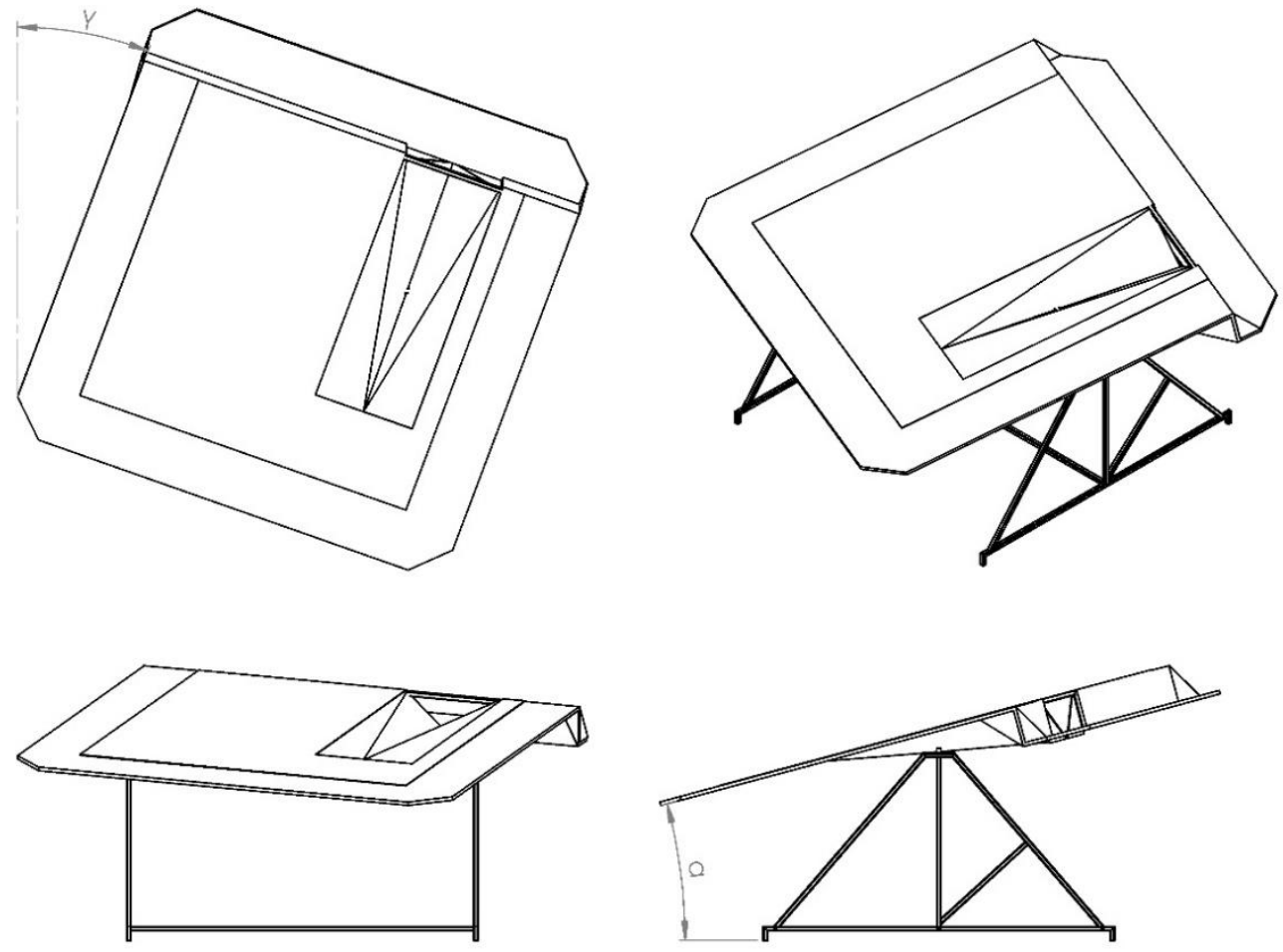

Figure 3 - The table and the variation of its geometry.

The flat surface of the table and the canyon covered with a fuel bed. A linear ignition is applied with different angles and locations, which are: A Total Ignition, which is a lateral ignition line with the full width of the fuel bed (parallel to the axis of the canyon); Partial Ignition, which is similar to the Total Ignition but the ignition line extends over only half of the width; finally, a Diagonal Ignition, which is a line forming an angle with the base side of the fuel bed. In the first experimental tests only 
half of the table (shaded area) was used. The figure 4 shows the different ignition modes and the shaded area. For the diagonal ignition an angle of $30^{\circ}$ was used.

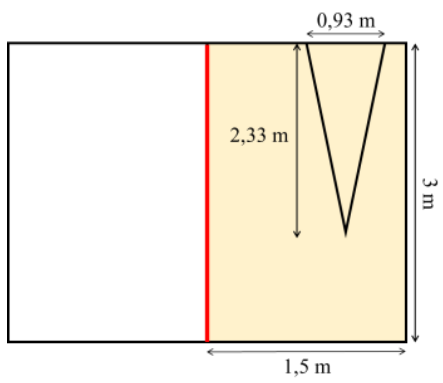

(a)

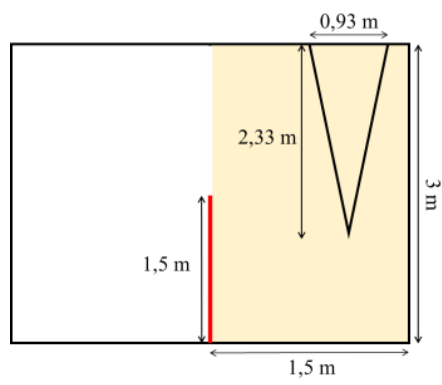

(b)

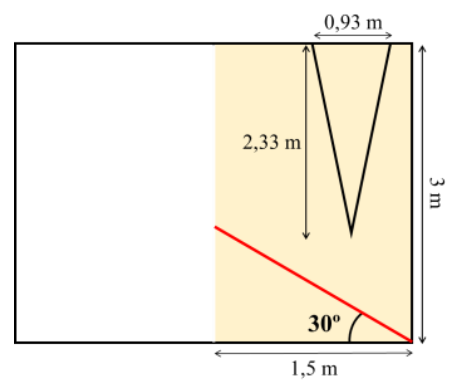

(c)

Figure 4 - Ignition modes: (a) total linear; (b) partial linear; (c) diagonal.

The conditions of fuel moisture, relative humidity, air temperature and the fuel loading were controlled. The objective of this study is to determine the fire's rate of spread and the propagation behaviour of the fire. The tests were monitored using a photographic camera, two video cameras - one in the frontal plane and one in the lateral plane - and an infrared camera. Data such as the time of preparation of the test, air temperature, relative humidity, fuel bed moisture and height were collected. In the carried out tests, the fuel used was Pinus Pinaster, with a load of $0.6 \mathrm{~kg} / \mathrm{m}^{2}$.

We analysed the infrared recorded frames and obtained the fire's rate of spread and propagation contours; the frames were taken from the recording with time intervals determined for each test, in order to follow the evolution of the fire front, following the methodology referred in Raposo (2016).

\section{Results and conclusions}

Pre-tests were performed with a slope angle $(\alpha)$ of $30^{\circ}$, a canyon opening angle of $45^{\circ}$ and three rotation angle of the table, which are: $-20^{\circ}, 0^{\circ}$ and $20^{\circ}$. For each angle of rotation of the table, the three types of ignition described above were tested.

Table 2 - Data of the tests performed.

\begin{tabular}{cccccc}
\hline Ref. & Designation & $\begin{array}{c}\mathrm{mf} * 1 \\
\%\end{array}$ & $\begin{array}{c}\text { R0*2 } \\
\text { cm.s-1 }\end{array}$ & $\begin{array}{c}\square \\
\mathrm{o}\end{array}$ & Ignition \\
\hline 1 & AVE_01 & 17.23 & 0.36 & 0 & Total \\
2 & AVE_02 & 9.82 & 0.35 & 0 & Partial \\
3 & AVE_03 & 12.36 & 0.33 & 20 & Total \\
4 & AVE_04 & 12.11 & 0.31 & 20 & Partial \\
5 & AVE_05 & 13.38 & 0.38 & -20 & Total \\
6 & AVE_06 & 13.77 & 0.34 & -20 & Partial \\
7 & AVE_07 & 12.49 & 0.34 & -20 & Diagonal \\
8 & AVE_08 & 10.74 & 0.25 & 20 & Diagonal \\
9 & AVE_09 & 10.74 & 0.55 & 0 & Diagonal \\
Tuel moisture Content $*{ }^{2} \boldsymbol{R}_{\boldsymbol{0}}-$ Basic rate of spread of linear fire in the absence of slope and wind.
\end{tabular}

In figure 5, we can observe the test "AVE_08" recorded using the infrared camera with frames every 30 seconds, from instant 0 s to 270 s. 

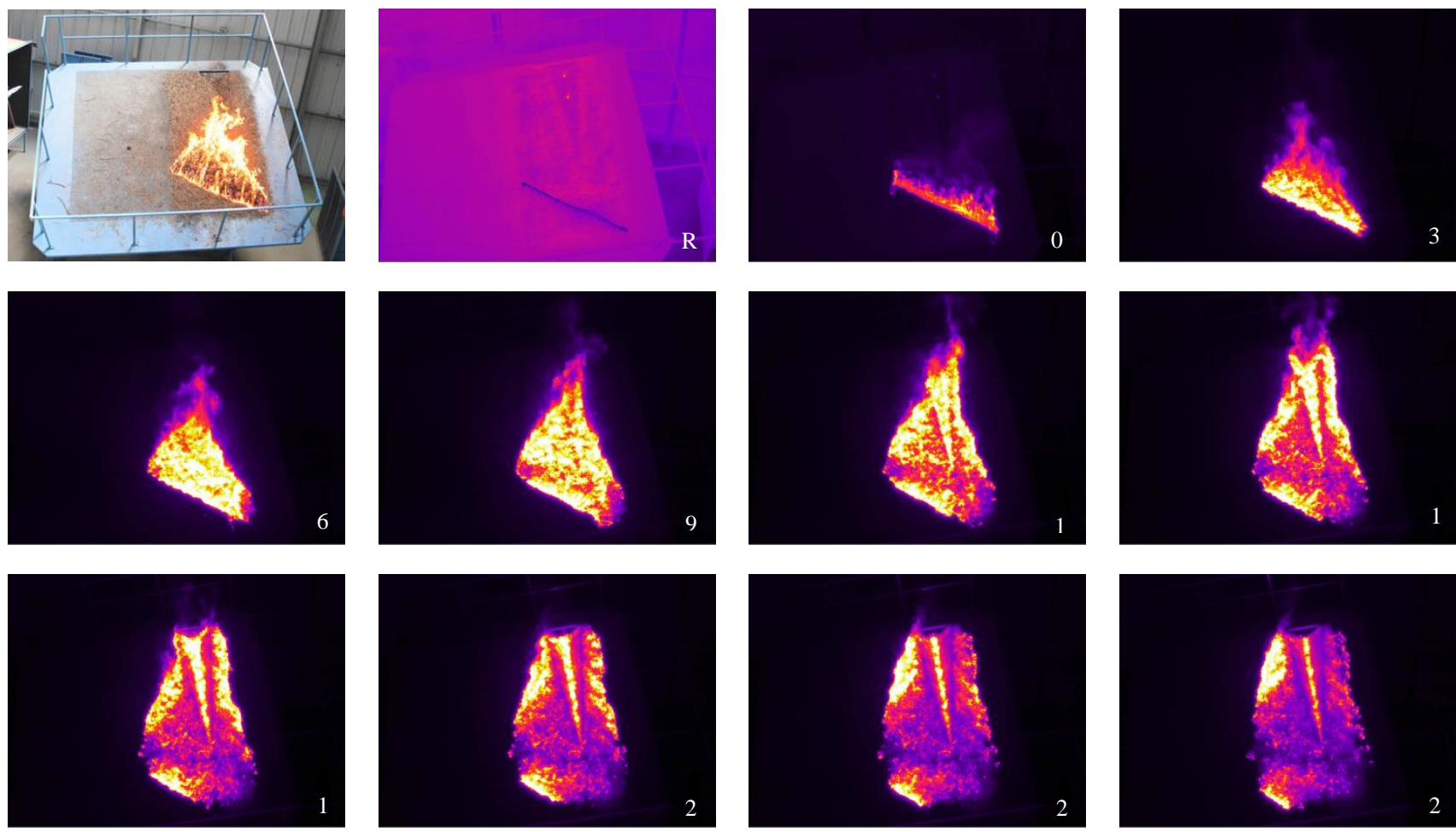

Figure 5 - Test $\mathrm{AVE0}\left(\alpha=30^{\circ} ; \gamma=20^{\circ} ; \delta=45^{\circ}\right)$ : visible picture of the table, reference frame and frames every 30 seconds, from instant 0 s to 270 s.

In figure 6 , we can observe the propagation contours of the fire front as a function of time for one of the tests with a Diagonal Ignition. It should be noted that, initially, the fire propagation isochronous are mostly parallel to the ignition line (red line in the figure). With the evolution of the time, it is observed that the direction of propagation changes from this initial direction to align with the maximum slope direction on the left of the canyon. However, the canyon also interferes with the direction of the propagation where the fire spreads with a higher rate of spread in the direction of the canyon's waterline affecting the adjacent points.

Figure 6 - Fire spread propagation contours of the AVE 08 test $\left(\alpha=30^{\circ} ; \gamma=20^{\circ} ; \delta=45^{\circ}\right)$ as a function of time (interval between 30-second contours).

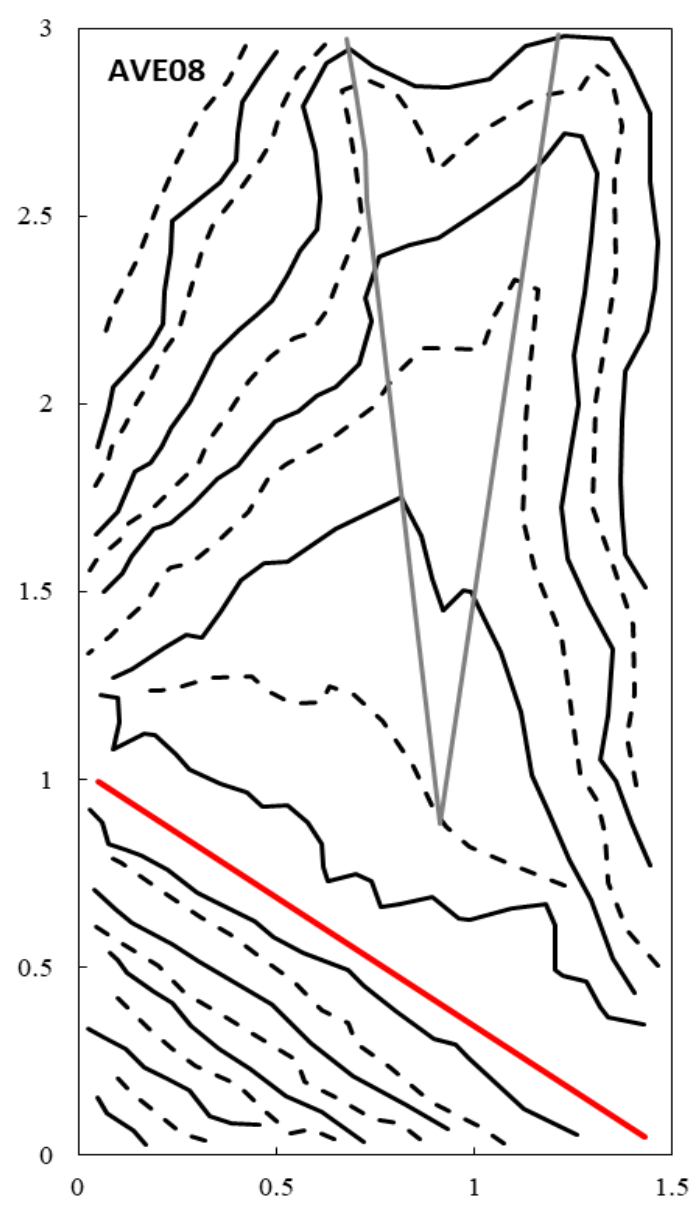


In figures 7 and 8, we can find the resultant rate of spread of the fire along the axis of the canyon (the waterline) for AVE07 $\left(\alpha=30^{\circ}, \gamma=-20^{\circ}, \delta=45^{\circ}\right), \operatorname{AVE08}\left(\alpha=30^{\circ}, \gamma=20^{\circ}, \delta=45^{\circ}\right)$ and AVE09 $\left(\alpha=30^{\circ}, \gamma=0^{\circ}, \delta=45^{\circ}\right)$. On these three tests, we used a Diagonal Ignition, and we changed only the rotation angle $(\gamma)$.

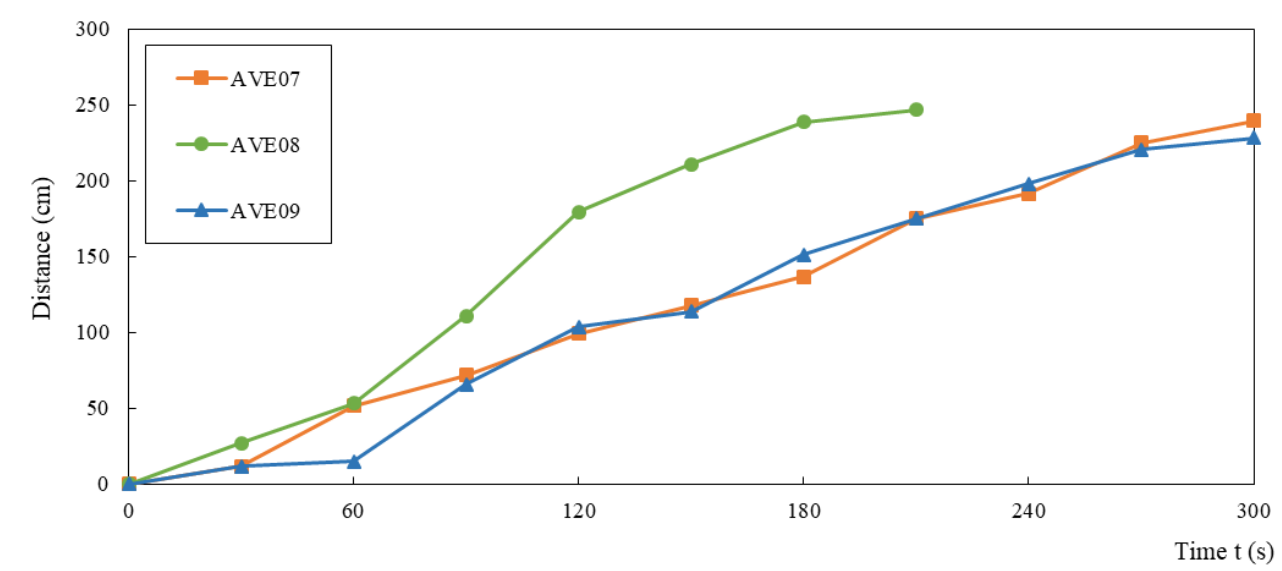

Figure 7 - Distance travelled by the fire front of the tests AVE07 ( $\left.\alpha=30^{\circ}, \gamma=-20^{\circ}, \delta=45^{\circ}\right), A V E 08\left(\alpha=30^{\circ}, \gamma=20^{\circ}\right.$, $\left.\delta=45^{\circ}\right)$ and $A V E 09\left(\alpha=30^{\circ}, \gamma=0^{\circ}, \delta=45^{\circ}\right)$ as a function of time.

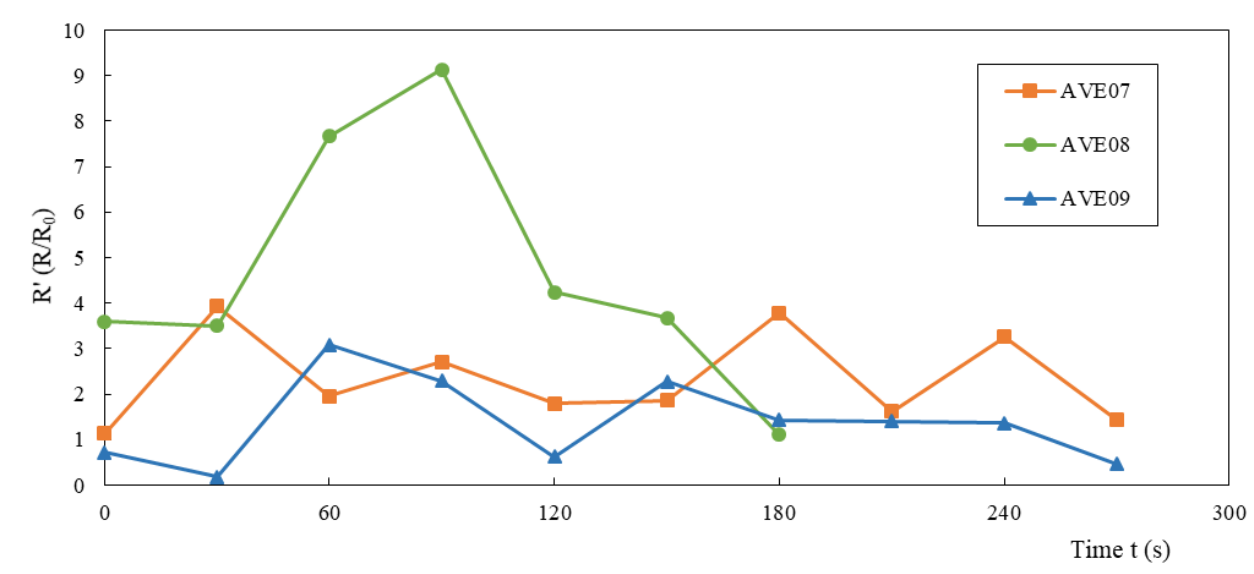

Figure 8 - Non-dimensional rate of spread along the axis of the canyon of the tests AVE07 $\left(\alpha=30^{\circ}, \gamma=-20^{\circ}, \delta=45^{\circ}\right)$, $A V E 08\left(\alpha=30^{\circ}, \gamma=20^{\circ}, \delta=45^{\circ}\right)$ and $A V E 09\left(\alpha=30^{\circ}, \gamma=0^{\circ}, \delta=45^{\circ}\right)$ as a function of time.

Based on the previous figures, it is concluded that the test AVE08 $\left(\alpha=30^{\circ} ; \gamma=20^{\circ} ; \delta=45^{\circ}\right)$ shows a higher fire front propagation velocity than the other two tests. This result is due to the fact that this test is performed with a table rotation angle of $+20^{\circ}$. On this test, the fire has entered the canyon from its base initially while the fire front tends to align with the maximum slope direction. After the fire has entered the canyon, an evident eruptive behaviour takes place, which is a common behaviour associated with this type of terrain configuration. In the other two tests, the fire's rate of spread was lower as the fire front entered the canyon from its left side and the eruptive behaviour did not occur intensity as on AVE08.

The tests carried out with total and partial ignition did not reveal the Extreme Fire Behaviour because the fire enters the canyon laterally, which does not lead to the eruptive behaviour, as it happens when the fire reach the canyon at the base.

The greater or less inclination of the slope has a decisive influence on the propagation of forest fires, since the more inclined it is the greater the effect of the convection that heats the fuel above the fire, increasing the upward propagation velocity. Thus on a slope the fire spread is much faster in the 
ascending than in the descending direction. Another very important aspect of the topography is manifested in the canyon. On an inclined slope with a canyon the Extreme Fire Behaviour is to be expected. Thus, it's important to note that the fight against forest fires in this configuration (slope with canyon) must have rules, such as: not to fight the fire by the top of the slope or the half slope; to have someone watching to see if there is fire below the position of the operational that can progress quickly and surprise them; to anchor the fire and make its fight from the rear, going through the flanks until it reaches the head; to establish a communications plan for all operational so that dangerous situations can be anticipated; before the fight define more than an escape route that everyone knows; to define a safety zone that can protect the operational in case of need.

\section{Acknowledgements}

This work was prepared under my $\mathrm{PhD}$ in Mechanical Engineering. I want to thank the FCTFoundation for Science and Technology for the PhD Grant (SFRH/BD/138235/2018) and for the Project Firewhirl (PTDC/EMS-ENE/2530/2014) that allowed this work to be done. I particularly wish to express my sincere gratitude to my scientific advisers Professor Domingos Xavier Viegas and Doctor Miguel Almeida for the guidance and support. I would like to express my thanks to Doctor Jorge Raposo, Abdelrahman Abouali, PhD candidate Xieshang Yuan and Nuno Luís for their support in laboratory tests. I also would like to express my thanks to MsC Luís Mário Ribeiro for his availability and for reviewing my paper.

\section{References}

Altintas I, Block J, De Callafon R, Crawl D, Cowart C, Gupta A, Nguyen M, Braun HW, Schulze J, Gollner M, Trouve A, Smarr L (2015) Towards an integrated cyberinfrastructure for scalable datadriven monitoring, dynamic prediction and resilience of wildfires. Procedia Computer Science 51, 1633-1642. doi:10.1016/j.procs.2015.05.296.

Byram GM (1959) Some Principles of Combustion And Their Significance In Forest Fire Behaviour. Reprinted from: Fire Control Notes 18, 47-57.

Dold J (2010) Flow attachment in eruptive fire growth. VI International Conference on Forest Fire Research.

Dold JW, Zinoviev a. (2009) Fire eruption through intensity and spread rate interaction mediated by flow attachment. Combustion Theory and Modelling 13, 763-793. doi:10.1080/13647830902977570.

Finney MA (2004) FARSITE: Fire Area Simulator-Model Development and Evaluation. Res Pap RMRS-RP-4, Ogden,UT: US Department of Agriculture, Forest Service, Rocky Mountain Research Station. https://www.fs.fed.us/rm/pubs/rmrs_rp004.pdf.

Finney MA (2006) An Overview of FlamMap Fire Modeling Capabilities. Fuel Management-How to Measure Success: Conference Proceedings 28-30 March 213-220. doi:U.S. Forest Serice Research Paper RMRS-P-41.

Kalabokidis K, Ager A, Finney M, Athanasis N, Palaiologou P, Vasilakos C (2016) AEGIS: A wildfire prevention and management information system. Natural Hazards and Earth System Sciences 16, 643-661. doi:10.5194/nhess-16-643-2016.

Lahaye S, Sharples J, Matthews S, Heemstra S, Price O, Badlan R (2018) How do weather and terrain contribute to firefighter entrapments in Australia? International Journal of Wildland Fire 27, 8598. https://doi.org/10.1071/WF17114. 
Mcferren G, Frost P (2009) The Southern African Advanced Fire Information System. http://researchspace.csir.co.za/dspace/bitstream/handle/10204/3631/McFerren_d1_2009.pdf?sequ ence $=1 \&$ is Allowed $=\mathrm{y}$.

Morvan D, Hoffman C, Rego F, Mell WE (2009) Numerical Simulation of the Interaction Between Two Fire Fronts in the Context of Suppression Fire Operations. 8th Symposium on Fire and Forest Meteorology.

Pyne SJ, Andrews PL, Laven RD (1996) 'Introduction to wildland fire.' (John Wiley and Sons: New York)

Raposo JR, Viegas DX, Xie X, Almeida M, Figueiredo AR, Porto L, Sharples J (2018) Analysis of the physical processes associated with junction fires at laboratory and field scales. International Journal of Wildland Fire 27, 52-68. doi:10.1071/WF16173.

Santoni PA, Balbi JH, Dupuy JL (1999) Dynamic modelling of upslope fire growth. International Journal of Wildland Fire 9, 285-292. http://www.publish.csiro.au/paper/WF00004.

Sharples JJ, McRae RHD, Wilkes SR (2012) Wind-terrain effects on the propagation of wildfires in rugged terrain: Fire channelling. International Journal of Wildland Fire 21, 282-296. doi:10.1071/WF10055.

Sullivan AL (2009a) Wildland surface fire spread modelling, 1990-2007. 1: Physical and quasiphysical models. Int. J. Wildl. Fire 18, 349-368. http://dx.doi.org/10.1071/WF06143.

Sullivan AL (2009b) Wildland surface fire spread modelling, 1990-2007. 3: Simulation and mathematical analogue models. International Journal of Wildland Fire 18, 387. doi:10.1071/WF06144.

Velez Muñoz R (2000) 'La Defensa contra incendios forestales : fundamentos y experiencias.'

Viegas DX (2005) A Mathematical Model For Forest Fires Blowup. Combustion Science and Technology 177, 27-51. doi:10.1080/00102200590883624.

Viegas DX (2006) Parametric study of an eruptive fire behaviour model. Int. J. Wildl. Fire 15, 169177. http://dx.doi.org/10.1071/WF05050.

Viegas DX, Pita LP (2004) Fire spread in canyons. International Journal of Wildland Fire 13, 253 274. doi:10.1071/WF03050.

Viegas DX, Ribeiro LM, Almeida MA, Oliveira R, Viegas MTP, Reva V, Figueiredo AR, Lopes S (2013) Os Grandes Incêndios Florestais e os Acidentes Mortais Ocorridos em 2013. (Coimbra)

Viegas DX, Rossa C, Ribeiro LM (2011) 'Incêndios Florestais.' (DX Viegas, Ed.). (Verlag Dashöfer Edições profissionais Unip., Lda.: Lisboa)

Weber RO (1991) Toward a Comprehensive Wildlife Spread Model. International Journal of Wildland Fire 1, 245-248. https://doi.org/10.1071/WF9910245.

Xie X, Liu N, Lei J, Shan Y, Zhang L, Chen H, Yuan X, Li H (2017) Upslope fire spread over a pine needle fuel bed in a trench associated with eruptive fire. Proceedings of the Combustion Institute 36, 3037-3044. doi:10.1016/j.proci.2016.07.091. 


\title{
Effective efficiency index (IEE): methodology for analyzing the efficiency of fire retardants in laboratory
}

\author{
Alexandre Beutling ${ }^{1 *}$; Antonio Carlos Batista ${ }^{2}$; Ronaldo Viana Soares ${ }^{2}$; Lorena Stolle ${ }^{1}$; Felipe \\ Beutling ${ }^{3}$ \\ ${ }^{1}$ Universidade Federal de Mato Grosso do Sul. Rodovia MS-306 - Zona Rural, Chapadão do Sul - \\ MS, 79560-000, \{beutling.a@gmail.com*,lorenastolle@yahoo.com.br\} \\ ${ }^{2}$ Universidade Federal do Paraná. CIFLOMA. Av. Pref. Lothário Meissner, 632, 80210-170 - \\ Jardim Botânico - Curitiba - PR \{batistaufpr@ufpr.br; rvsoares02@gmail.com\} \\ ${ }^{3}$ Eng. Florestal Felipe Beutling. Rua 7 de Setembro, 1865 - Sala 3 - Centro, Blumenau - SC, \\ 89012-401, \{felipe.beutling@gmail.com\}
}

\begin{abstract}
In Brazil, fire retardants for forest fire prevention and suppression are seldom used. However, these products can be found in the market, and there are some records of their use. There are also many researches dealing with efficiency evaluations of retardants, using different methodologies. Thus, this research aims to present a standard protocol which can be used by different researchers and orient the professionals who use this type of product in their daily activities. This protocol derives from an improvement of a methodology for evaluating the efficiency of retardants, based on fire behavior analysis in tests carried out in the laboratory. The Effective Efficiency Index (IEE) can be used in different assessment scenarios. With the aid of a preconfigured data processing worksheet, the IEE will facilitate the evaluation of fire retardants in laboratory, as well as to provide a way to compare different formulations and concentrations of many products available in the market.

In addition, to refine the results and establish the level of approval, "classification criteria" were created, which provides a distinguished interpretation and allows the comparison of different retardants and concentrations, regardless of their "retardant" or "suppressor " effects (i.e. a retardant that provides only "retardant" effect in the laboratory does not necessarily means that it will be less effective than a product that provides a "fire suppressor" result).

To develop the methodology, more than 1000 replicates were conducted in laboratory, using different fire retardants formulations, between 2006 and 2010. In 2018, for the improvement of the methodology (called IEE) and its validation, 250 replicates were conducted with different retardants, concentrations and densities, plus control (plain water). Results showed that the values of the efficiency indices resulting from the tests allows a good estimation of the action (or response) of the fire retardants in field conditions for forest fires control activities.
\end{abstract}

Keywords: Fire retardant, forest fire, fire control, forest protection

\section{The Use of Fire Retardants in Brazil}

In Brazil, the use of long term chemical retardants in the control of forest fires is still incipient, occurring only in some private companies and in emergency situations from the federal government. Although fire retardants have been heavily used in many regions of the world, being considered a fundamental tool in fire control activities, in Brazil there is no regulations or legal provisions which establish criteria to evaluate such products, as well as standard procedures for their use. This gap makes difficult the use of these products and restrains the refinement of the suppression techniques aimed to reduce the extension of damages and burned areas in the country.

Fire retardants are chemical products that reduce or impede the fuel combustion, increasing the water efficiency in fire suppression activities (Ribeiro et al, 2006). Many researchers (Pastor, 2004; 
Ribeiro et al., 2006; Ribeiro, 2006; Batista, 2008; Beutling et al, 2008; Vázquez, 2011; Vieira, 2011; Machado Filho et al, 2012; Canzian, 2013; Fiedler et al, 2015) have dedicated efforts performing laboratory and field tests in order to evaluate the effectiveness of retardants from different companies.

The objective of this research was to improve a standard methodology to evaluate the efficiency of fire retardants in laboratory conditions, as a tool to help forest managers to take decisions when dealing with fire control activities.

\section{Methodology}

The proposed methodology included three steps, as follows:

1 - Utilizes an adaptation of the Global Efficiency Index (Ie), proposed by Ribeiro et. al. (2006) - evaluation of the "retardant" effect, which describes the outcome of flames high and fire speed reduction, without extinguishing the fire front.

2 - Utilizes a variable called "hammer effect" (EfM), which evaluates the retardant efficiency to suppress the fire front as soon as it reaches the area where the retardant was applied. This variable takes in account two factors: extinction time (TE) and the average penetration distance (P) of the fire in the retardant application area, both in percentage. The calculation of these two factors was based in the measured time and the dimension of the application area.

3 - For the calculation of the "Effective Efficiency Index (IEE)", which establishes a relationship between (Ie) and (EfM), a multiplication factor called "result valorization" and a "scale adjustment" were used, in such way that "retardant" results never exceeds $70 \%$ in the (IEE) scale, and the results "Fire suppressor" can use the full scale range (from 0 to 100).

In addition, to refine the results and establish the level of approval, "classification criteria" were created, which provides a distinguished interpretation and allows the comparison of different retardants and concentrations, regardless of their "retardant" or "suppressant " effects $(i . e$. a retardant that provides only "retardant" effect in the laboratory does not necessarily means that it will be less effective than a product that provides a "fire suppressant" result). Figure 1 show the flow chart designed to evaluate the (IEE).

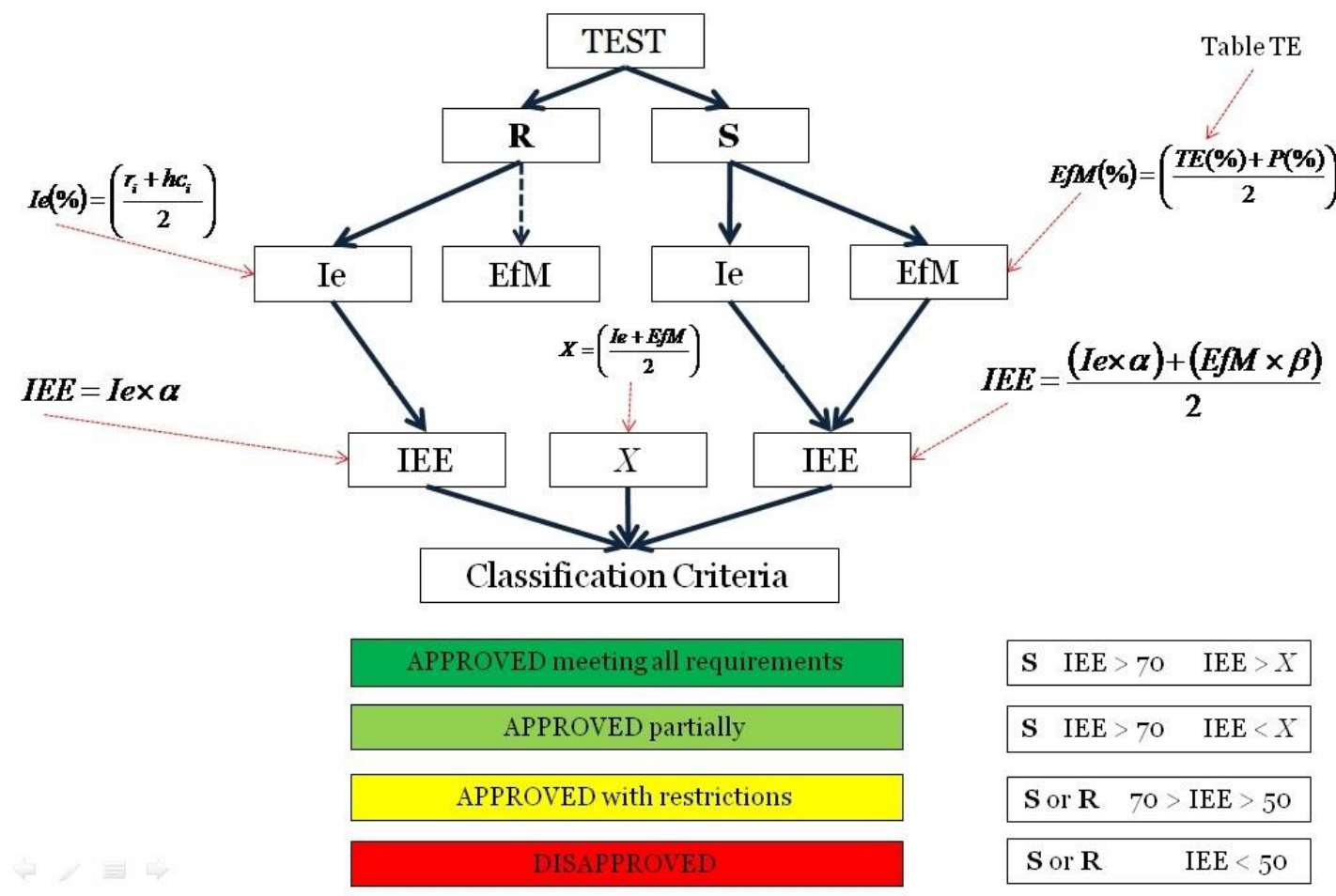

Figure 1 - Flow chart designed to evaluate the Effective Efficiency Index 
Descriptively, a test-formulation (FTn) in certain concentration, is submitted to the efficiency evaluation procedure (TESTE) in a standard burning (QP), whose result could be a retardant (R) or a suppressing (S) effect. The IEE value was calculated according the two following conditions:

a. When the effect is retardant $(R)$ : In this case, there is no suppressant effect and, consequently, the "hammer effect" (EfM) is zero. The index that evaluates the retardant potential of the product is the (Ie), which analyses the product efficiency on the flames height and rate of spread reduction in the following way:

$$
\begin{gathered}
\frac{\mathrm{VPMC}}{\mathrm{VPMS}}=\mathrm{RVP} \\
(1-\mathrm{RVP}) * 100=\mathrm{r}_{\mathrm{i}} \\
\frac{\mathrm{HMCC}}{\mathrm{HMCS}}=\mathrm{RHC} \\
(1-\mathrm{RHC}) * 100=\mathrm{hc}_{\mathrm{i}} \\
\mathrm{Ie}=\frac{\mathrm{r}_{\mathrm{i}}+\mathrm{hc}_{\mathrm{i}}}{2}
\end{gathered}
$$

Where:

VPMC $=$ Average rate of spread in plots treated with retardant;

VPMS = Average rate of spread in plots without retardant;

$\mathrm{RVP}=$ Rate of spread reduction;

$r_{i}=$ Percentage of rate of spread reduction;

$\mathrm{HMCC}=$ Average flames height in plots treated with retardant;

HMCS = Average flames height in plots without retardant;

RHC = Flame height reduction;

$\mathrm{hc}_{\mathrm{i}}=$ Percentage of flames height reduction;

$\mathrm{Ie}=$ Efficiency index;

b. When the effect is suppressant (S): In this case, the hammer effect (EfM) is calculated as follows:

$$
\frac{\% \mathrm{TE}+\% \mathrm{P}}{2}=\mathrm{EfM}
$$

Where:

$\% \mathrm{TE}=$ Extinction time in percentage - it is the time for complete extinction of the fire front inside the area where the product was applied. It is based in a relationship between time intervals (in seconds) and a percentage value of efficiency loss for each 10 seconds of active fire front, i.e., that the fire front remains lit.

$\% \mathrm{P}=$ Penetration distance in percentage - refers to the distance traveled by the fire front inside the area where the product was applied. It is the relationship between the linear distance (in $\mathrm{cm}$ ) and a percentage value of efficiency loss (for each centimeter travelled by the fire front inside the area, $1 \%$ efficiency loss is accounted). If $\mathrm{P}$ is equal to the total area length (which must be $100 \mathrm{~cm}$ maximum), $\% \mathrm{P}$ is equal to zero.

An average $(\mathrm{X})$ between the (Ie) and the (EfM) values should be calculated to help in the (FTn) framing in the classification criteria: 


$$
\frac{\mathrm{Ie}+\mathrm{Efm}}{2}=\mathrm{X}
$$

Based on the calculations presented before, the Effective Efficiency Index (IEE) is estimated, considering also:

i. Utilization of coefficients $\alpha$ e $\beta$ for adjusting the scale (rule) to the Classification Criteria, in order to enhance the suppressant values (EfM), and lower the retardant (Ie) values. Those coefficients should be multiplied to the (Ie) and to the (EfM).

ii. If the test result indicates a Retardant effect, the (IEE) will be the product of the (Ie) multiplied by the coefficient $\alpha$. If the (IEE) is higher than $50 \%$ the product is "Approved with Restrictions (YELLOW), otherwise it will be Reproved (RED)

$$
I e \times \alpha=I E E
$$

iii. If the test result indicates a Suppressant effect, the (IEE) is calculated as follows:

$$
\frac{(I e \times \alpha)+(E f M \times \beta)}{2}=I E E
$$

Where:

$\alpha=0,714285714285714 \ldots$

Obs: $\mathrm{Ie}=70 \%$ is the lower value for a product be approved as a Retardant ( $70 * \alpha=50$ ).

$\beta=1,285714285714285 \ldots$

$\beta=1+(1-\alpha)$

When the (IEE) is equal or higher than $70 \%$, the product will be declared "Approved considering partially the exigencies (LIGHT GREEN) if the (IEE) is lower than (X), or "Approved considering all the exigencies (DARK GREEN) if the (IEE) is higher than (X)

Summarizing, the Classification Criteria were established in the following way:

i. Suppressant, with (IEE) higher than (X): Approved considering all the exigencies (DARK GREEEN);

ii. Suppressant, with (IEE) lower than (X): Approved considering partially the exigencies (LIGHT GREEN);

iii. Suppressant or Retardant, with 70>(IEE) $>50$ : Approved with restrictions (YELLOW);

iv. Suppressant or Retardant, with (IEE) lower than 50: "Reproved (RED);

\section{Additional comments:}

The laboratory test result "Approved considering all the exigencies" indicates a strong possibility that the fire retardant presents suppressing effects in field conditions.

The (IEE) is very sensible to concentration and formulation variations, and directly dependent upon good laboratory practices (regarding the procedures related to the fuel bed assemblages, the product application, and the measurements made). For all those reasons, it needs a well trained professional for its execution.

To develop the methodology, more than 1000 replicates were conducted in laboratory, using different fire retardants formulations, between 2006 and 2010 (Beutling, 2007). For the improvement 
of the methodology (called IEE) and its validation, 250 replicates were conducted with different retardants, concentrations and densities, plus control (plain water). Fire behavior data from the areas with and without retardants were stored and processed using the softwares Excel 2003 and Statgraphics Centurion XV.

The IEE could be used in different scenarios and evaluations. Using a data processing customized platform, the IEE would make easier the retardant evaluation in laboratory environment. The IEE would be valuable also to compare different formulations and concentrations of the several products found in the market.

\subsection{Characteristics of the "Standard-Burn (QP)"}

The proposed laboratory conditions to carry out the burning tests are:

a) Fuel bed dimensions: for the IEE methodology, the fuel bed size does not interfere in the results. However, considering favorable logistic questions, it is recommended fuel bed with $1.5 \mathrm{~m}$ long, $0.75 \mathrm{~m}$ wide, divided in $10 \mathrm{~cm}$ intervals. The initial $10 \mathrm{~cm}$ are reserve to the ignition (fire start), and the final $10 \mathrm{~cm}$ to the fire stopping; in the central area, the first $80 \mathrm{~cm}$ are considered the area of free propagation (A.S - without product), and the following $50 \mathrm{~m}$ are reserved to the application of the tested retardant, called application area (A.C - with product) (Figure 2).

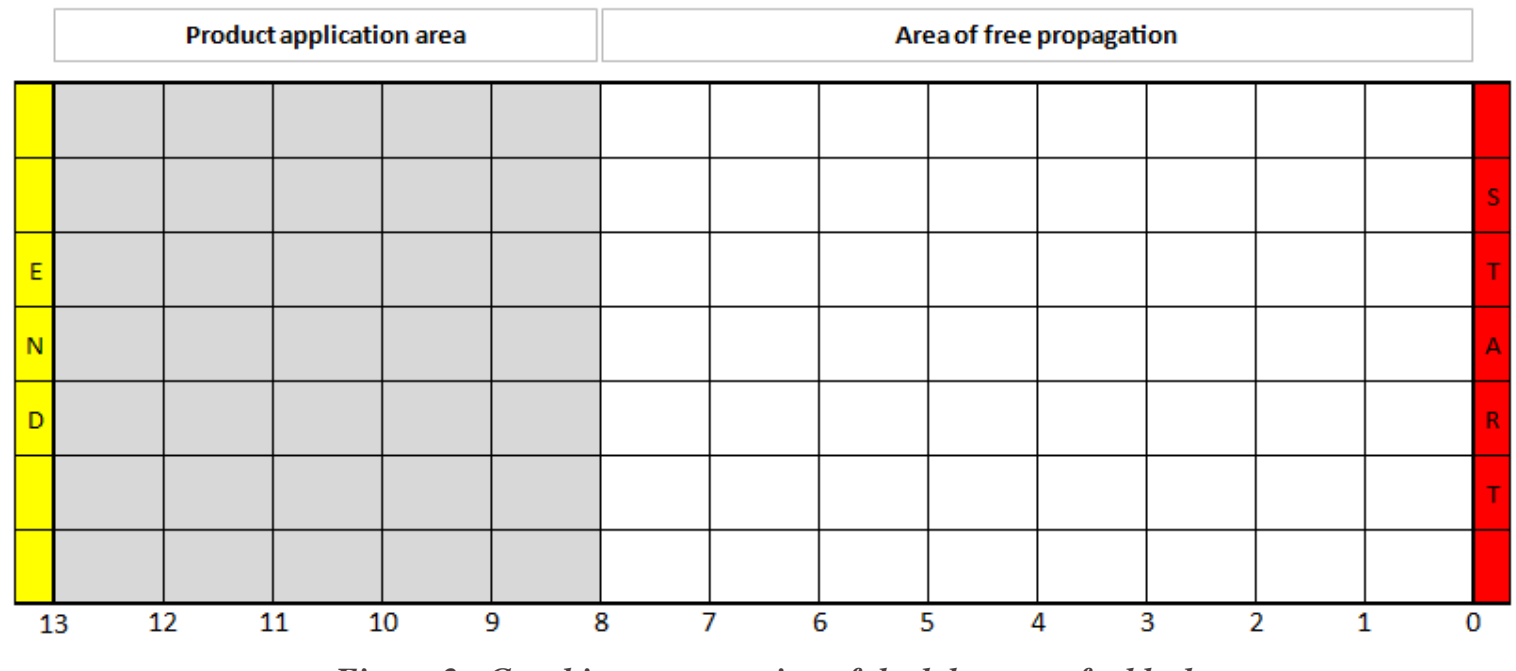

Figure 2 - Graphic representation of the laboratory fuel bed

b) Successive burns: it is a succession of 5 replications of each treatment that should be burned sequentially in the same day.

c) Time of burning: the burns should start after 10:00AM and, preferentially, not progress after 4:00PM (in the proposed fuel beds dimensions the total time to carry out the 5 burns may not take more than 3 hours).

d) Ideal environment: the tests should be carried out in laboratory environment, with exhaustion system, without the interference of external climatic conditions. The local must be closed, in order to maintain a minimum thermal and relative humidity variation and avoid wind interference. Laboratory doors and windows must be closed during the tests. Usually, the first burning tends to be slower, due to the lower temperature and higher relative humidity. Those conditions may change during the burnings. For this reason it is advisable to carry out calibration burns that would balance the laboratory environment.

e) Fuel: the best combustible material is probably "tifton" hay, (it can be easily found in agriculture shops). The fuel must be stove dried at $80^{\circ} \mathrm{C}$ for at least 12 hours before utilization. Twenty four hours would be even better, because when stored in great volumes the process of drying is not homogeneous, interfering in the moisture content: 
i. The fuel load should be $1.0 \mathrm{~kg} \cdot \mathrm{m}^{2}$, evenly distributed over the fuel bed;

ii. The recommended thickness of the litter (hay) is 8.0 to $9.0 \mathrm{~cm}$. Thickness lower than $7.0 \mathrm{~cm}$ increases the density and affects the product application. Besides that, it interferes in the fire behavior; flames height is lower, and fire spread too slow;

f) The recommended volume of the product to be tested is $0.51 . \mathrm{m}^{2}$, regardless the concentrations to be evaluated.

g) Application equipment: a pressurized manual atomizer, of low operational cost, is recommended. It should permit evaluating characteristics like viscosity and density of the solution during the application.

h) General procedure: the burning should be carried out the day after the fuel assemblage and product application. This is necessary to avoid the influence of water in the test result; in preliminary tests using only water the observed (IEE) was 9.8\%. Thus, in the day before the burning, the following procedures should be observed:

i. Take the fuel (hay) out from the oven;

ii. The fuel is weighted and evenly distributed over the fuel bed;

iii. A sample of the oven dried fuel is taken, weighted, and placed in a tray to be weighted again in the next day, before the burning. It is recommended to weigth 5 trays to estimate the moisture increment from the fuel assemblage in the fuel beds up to the burning time.

iv. The product (retardant) must be applied always the same way, according to the following sequence (position - direction):

- left side - from the front line to the end zone;

- end zone - from left to right;

- right side - from the end zone to the front line;

- midle - in zigzag from the right to the left, moving from the end zone to the front line;

- during the application, in each position-direction, the product should be applied only once, up to the end of the passages. If a gap is detected, it could be covered later, with the remaining product in the atomizer (it is not advisable to go back to cover the gap before finishing the application, because it could fail short of product to cover the whole area).

\subsection{Application of the IEE: a case study}

In order to exemplify the IEE application the efficiency of a fire retardant in concentrations of 5, 10,15 and $20 \%$ was carried out. The burnings were conducted in a fuel bed $1.48 \mathrm{~m}$ long and $0.76 \mathrm{~cm}$ wide. The measurements were made every $10.0 \mathrm{~cm}$ of fire progress, in a total of 40 replications (10 for each concentration). All fire behavior information was stored in the (IEE) data processing platform. The results of the retardant efficiency according to the used concentration are presented in Figure 3. 


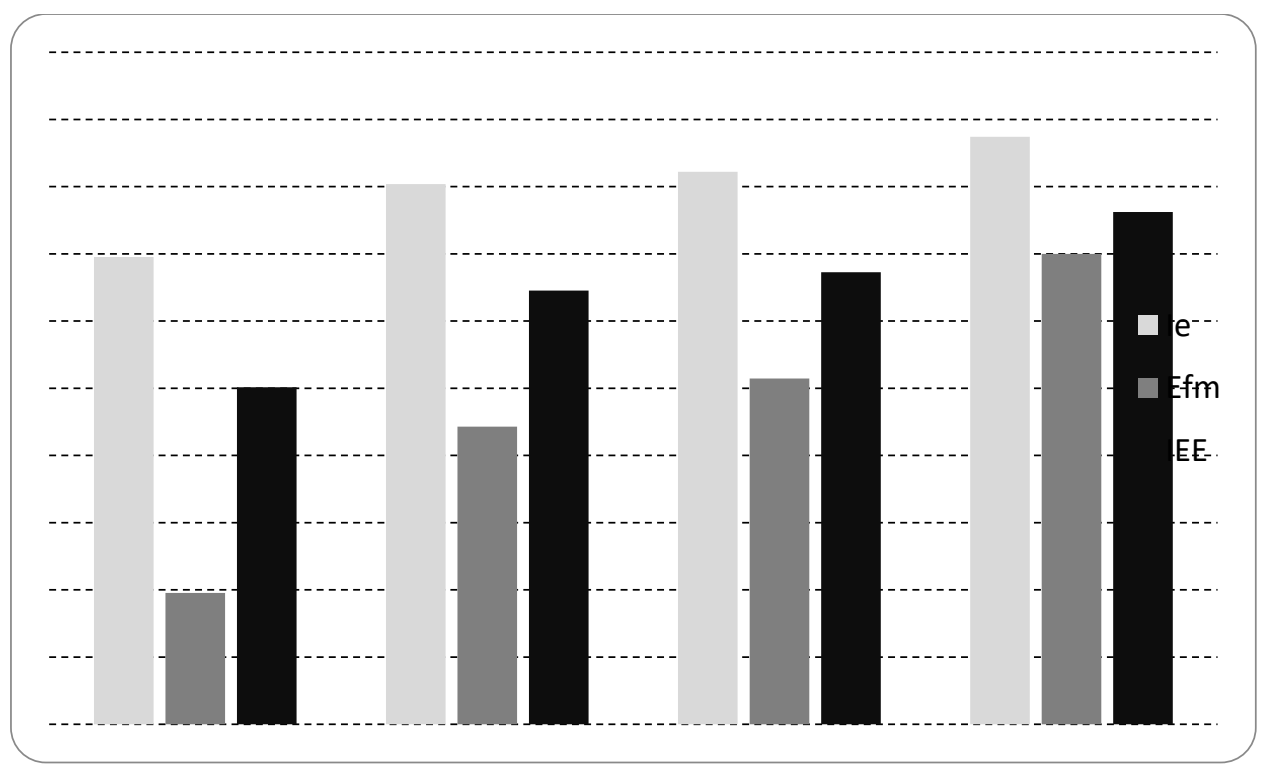

Figure 3 - Effective Efficiency Index (IEE) for the different concentrations of the fire retardant

The analyzed fire retardant presented good efficiency, even in the concentration of $5 \%$, reducing the flame height in $60.29 \%$ and the fire speed in $78.79 \%$, with an (IEE) equal to $50.15 \%$. In the $20 \%$ concentration, the (IEE) was $76.22 \%$, corresponding to $84.46 \%$ reduction in the flame height and $90.39 \%$ reduction in the fire speed. Regarding the relationship "retardant x suppressant", only the $20 \%$ concentration was $100 \%$ suppressor.

\section{Conclusions}

\subsection{Regarding the IEE}

The (IEE) is quite sensible to the efficiency variation of the retardants, and because it presents a finite scale ( 0 to $100 \%$ ), makes possible the comparison of different products, as well as the efficiency rating due to different concentrations.

The index allows the analysis of retardants efficiency along the time, i.e., several days after the application, what helps in the determination of how long the product maintains its efficiency.

The IEE can be useful on the developing of different retardant formulations, tests of raw materials, and quality control.

\subsection{Regarding the case study}

For direct attack to the fire, the $5 \%$ concentration of the tested retardant is recommended;

For indirect attack or fire prevention, the $20 \%$ concentration is recommended.

\section{References}

Batista, A. C. et al. Avaliação da eficiência de um retardante de longa duração, à base de polifosfato amônico, em queimas controladas em condições de laboratório. Scientia Forestalis, Piracicaba, v.36, n.79, p. 223-229, set. 2008.

Beutling, A. Índice de eficiência efetiva (i). Manual do Laboratório de Pesquisa e Controle de Incêndios (LPCI Rio Sagrado). Não publicado. 2007.

Beutling, A. et al. Propuesta de metodologia de evaluación de retardantes químicos en unidades de muestreo artificiales de campo. In: V Simposio Internacional sobre Manejo Sostenible de los 
Recursos Forestales - SIMFOR, 2008, Pinar del Río. Memórias del V Simposio Internacional sobre Manejo Sostenible de los Recursos Forestales-SIMFOR 2008, 2008. v. 1. p. 1-11.

Canzian, W. P. Análise da eficiência de retardantes de fogo em testes de laboratório. Monografia. Universidade Federal do Espírito Santo. 16p.

Fiedler, N. C. et al. Intensidade de queima de diferentes retardantes de fogo. Revista Árvore, Viçosa, Minas Gerais, v. 39, n. 4, p. 691-696, 2015.

Machado Filho, C. et al. Eficiência de um retardante de fogo de longa duração utilizado em incêndios florestais. Ciência Florestal, Santa Maria, v.22, n.2, p. 365-371, abr-jun. 2012.

Pastor F. E. Contribució a l'estudi dels efectes dels retardants en l'extinció d'incendis forestals. 2004. 304 p. Tese (Doutorado) - Centre de Estudis del Risc tecnològic, Departament d'Enginyeria Química, Escola Tècnica Superior d'Enginyeria Industrial de Barcelona, Barcelona, 2004

Ribeiro, L.M. Viegas, D.X. batalha, M. Assessment of fire retardant efficiency. Portugal, 2006. $11 f$. in "V International Conference on Forest Fire Research". CD.

Ribeiro, G. A. et al. Eficiência de um retardante de longa duração na redução da propagação do fogo. Revista Árvore, Viçosa, Minas Gerais, v. 30, n. 6, p. 1025-1031, nov./dez. 2006.

Vásquez, A. C. El ciclo del $\mathrm{N}$ em sistemas agroforestales: Evaluación isotópica $\left({ }^{15} \mathrm{~N}\right)$ em tres tipos de manejo. Santiago de Compostela, 2011. Tesis. Universidade de Santiago de Compostela. 241p.

Vieira, F. I. Combate a incêndio florestal: determinação do índice de eficiência global de retardantes químicos de curta e de longa duração e avaliação de seus efeitos sobre a redução da intensidade do fogo em vegetação em condições de laboratório. Monografia. Florianópolis: CEBM, 2011. 78 f. 


\title{
Effects of aerodynamic lift on firebrand trajectories: Numerical investigations based on simplified models.
}

\author{
Hangxu Zhou'; Yi Li ${ }^{2}$; Yang Zhang*1 \\ ${ }^{1}$ Department of Mechanical Engineering, University of Sheffield, Sheffield, S1 4DF, UK, \\ \{hzhou1@sheffield.ac.uk, yz100@sheffield.ac.uk*\} \\ ${ }^{2}$ School of Mathematics and Statistics, University of Sheffield, Sheffield, S3 7RH, UK, \\ \{yili@sheffield.ac.uk\}
}

\begin{abstract}
Spotting distance of a firebrand lofted in flow is an important mechanism of fire spread in large scale fires such as forest fires. Many models have been developed to study the propagation of a firebrand, but only a few focused on analyzing the aerodynamic lift on firebrand trajectories. From the modelling perspective, it is important to ascertain the relative importance of the lift compared with the drag force. However, no such study has been reported. In this paper, simplified models are developed to illustrate the effects of the lift on the maximum potential propagation distances of rectangular-shaped firebrands. The effects are firstly demonstrated clearly for non-burning firebrands. The effects of size regression and mass loss on burningparticle are then considered. A variable-thickness-burning model is developed to account for these effects. Two lift coefficient models, suitable for different Reynolds numbers, are considered. The results demonstrate that the lift force can have very significant effects on the firebrand trajectories. Using the high Reynolds number lift model, the spotting distance can be increased up to 14 times of the traveling distances calculated without the lift. Positive effects are found over whole ranges of parameters. On the other hand, when the low Reynolds number model is used, occasionally the lift can have very small negative effects, especially for the firebrands at large ambient flow velocities. By comparing with the results of variable-thickness-burning model, it is obtained that lift effects are sensitive to the inertia of a firebrand at small Reynolds and large flow velocity. Finally, for the parameters involved in this study, attempts are made to compare their importance to the effects of the lift. The main factor is found to be the ambient flow velocity which can significantly enhance or weaken the lift effects. Another main factor is the incident angle. The lift effects are strengthened at negative angles but weakened at positive ones. The lift effects are relatively weaker for higher particle density, whereas they are mostly independent of particle initial velocity. We conclude that the lift force has very significant effects on particle propagations, and should be considered in order to model extreme spotting distances.
\end{abstract}

Keywords: firebrands, trajectory, aerodynamic lift, numerical models.

\section{Introduction}

Spotting ignition has been found to be a significant mechanism of fire spreads since at least the 1960s (Tarifa et al, 1965). The flaming or glowing pieces of wood, branches, or charcoal etc., commonly called firebrands, are lofted by a fire plume and transported by wind to cause new ignition in large-scale fire. It has been shown that firebrands contribute to the spread of large-scale fires such as the 1666 London Fire, the 1871 Chicago Fire, the 1923 Tokyo Fire, the 2006 Iversen Fire, etc (Koo, 2010). The short-distance spotting firebrands, such as barks, needles, leaves and small wood particles, can continuously keep the fire spreading, while the long-distance spots, such as glowing charcoal, may result in new spot ignition which is kilo meters ahead of the main fire. The latter also is the main cause that decreases the efficiency of forest fire prevention methods used nowadays, such as firebreaks and fire resistance barriers. Predicting the spotting of firebrands is considered one of the most difficult, yet important problems in the study of fire spread, since it involves a board range of physical processes including generation of firebrands, transport, and ignition of fuel at the landing position. To predict 
firebrand transport, it requires the analyses of the aerodynamics and combustion of firebrands under various fluid and weather conditions, and the associated convective plume with different fire intensities. The present work focuses on the transport of firebrands, in particular the effects of aerodynamic lift. The mechanisms of the other two processes, i.e., the generation of firebrands and the potential ignition of fuel at the landing position, are not considered.

In 1965, the transport of firebrands in combustion were firstly studied by Tarifa et al (Tarifa et al, 1965). For burning particles tested in wind tunnels, including spherical and cylindrical shapes, the lifetime, the laws of variation of weight, and aerodynamic drag were determined as functions of time. Flight paths of firebrands were calculated for prescribed wind conditions. Parameters of particles, including initial shape, size, kind of wood and moisture content, were deduced in theoretical calculations. Models for the transport of spherical firebrands with convective plume were developed by Lee and Hellman in 1970 (Lee and Hellman, 1970). The trajectories of wood spheres in axisymmetric swirling convective plume were analysed and compared with Tarifa's results. Similar paths but shorter spotting distances were found. Models of cylindrical firebrands released in a fire swirl were developed by Muraszew et al (Muraszew et al, 1977). The aerodynamic behaviours of cylindrical firebrands were modelled differently in three regions, and the rotation of particles were calculated. The density variation of the cylindrical firebrands was found to be exponential in time and the diameter regression rate. Basing on the work of Muraszew, the burning rates of various shapes were modelled by Albini (Albini, 1979). Diameter regression rate was determined as a constant $(K=$ 0.0064). A linear equation was developed to describe the change of terminal velocity due to the changes of the density and the diameter of falling flaming firebrands. Models of cylindrical firebrands lofted by torching trees were developed to predict the trajectories and maximum spotting distance. Further, he studied the transport of firebrands by line thermal and modelled the behaviour of a strong line thermal.

Lift effects on the propagation of firebrands were firstly considered by Woycheese et al (1999). The maximum spotting distances of disk-shaped firebrands over Baum and McCaffrey plume were calculated. Constant angle of attack between disk and relative wind was assumed. The enhancement of firebrand propagation by the lift was observed. Besides, a large number of experiments were conducted by Woycheese et al to investigate the behaviour of lofted firebrands, including the trajectories of nonburning and combusting spheres, and wood brands of various geometries. However, the lift force was included in the integration of the aerodynamic trajectories of firebrands only in a few works (Himoto et al, 2005; Sardoy, 2007). A transport model for a disk-shaped firebrand in 3D space was developed by Himoto, which was integrated alongside a Large Eddy Simulation of a buoyancy flow. In his work, the lift coefficient was calculated by an empirical formula valid at Reynolds numbers around 40000. Sardoy developed a numerical model for the transport of burning brands lofted in a crown fire. A combustion model was discussed in detail including the analyses of both pyrolysis rate and char oxidation rate.

The above brief review shows that the aerodynamic lift, as an important factor of particle transport, has been largely missing in the models for the motion of the firebrands. The research focused on the effect of aerodynamic lift force during particle propagations is especially scarce since the enhancement effects from lift on spotting was found by Woycheese et al. This paper focuses on using simplified models to highlight the effects of aerodynamic lift force on the motion of both non-burning and burning firebrands, in particular their relative importance compared with the drag force. The effects of a broad range of properties, including the Reynolds number, the ambient flow velocity, the particle initial velocity and the incident angle of particle relative to ambient flow, are investigated and compared with each other. Based on the maximum potential spotting distance determined, the lift effects are discussed in details. 


\section{Conceptualisation of models}

\subsection{Transport of firebrands}

In the transport phase of firebrands spotting phenomenon, a mathematical model for determining the trajectory and predicting the maximum potential spotting distance is developed. When the firebrand exits main fire region and then glides in a constant flow field, shown in Fig. 1, it is no longer affected by the plume from the main fire but supported by aerodynamic drag and lift forces. The coordinate system can be simplified into two-dimensional with the assumptions that a rectangular-shaped firebrand obeys axisymmetric aerodynamic behaviours during propagation.

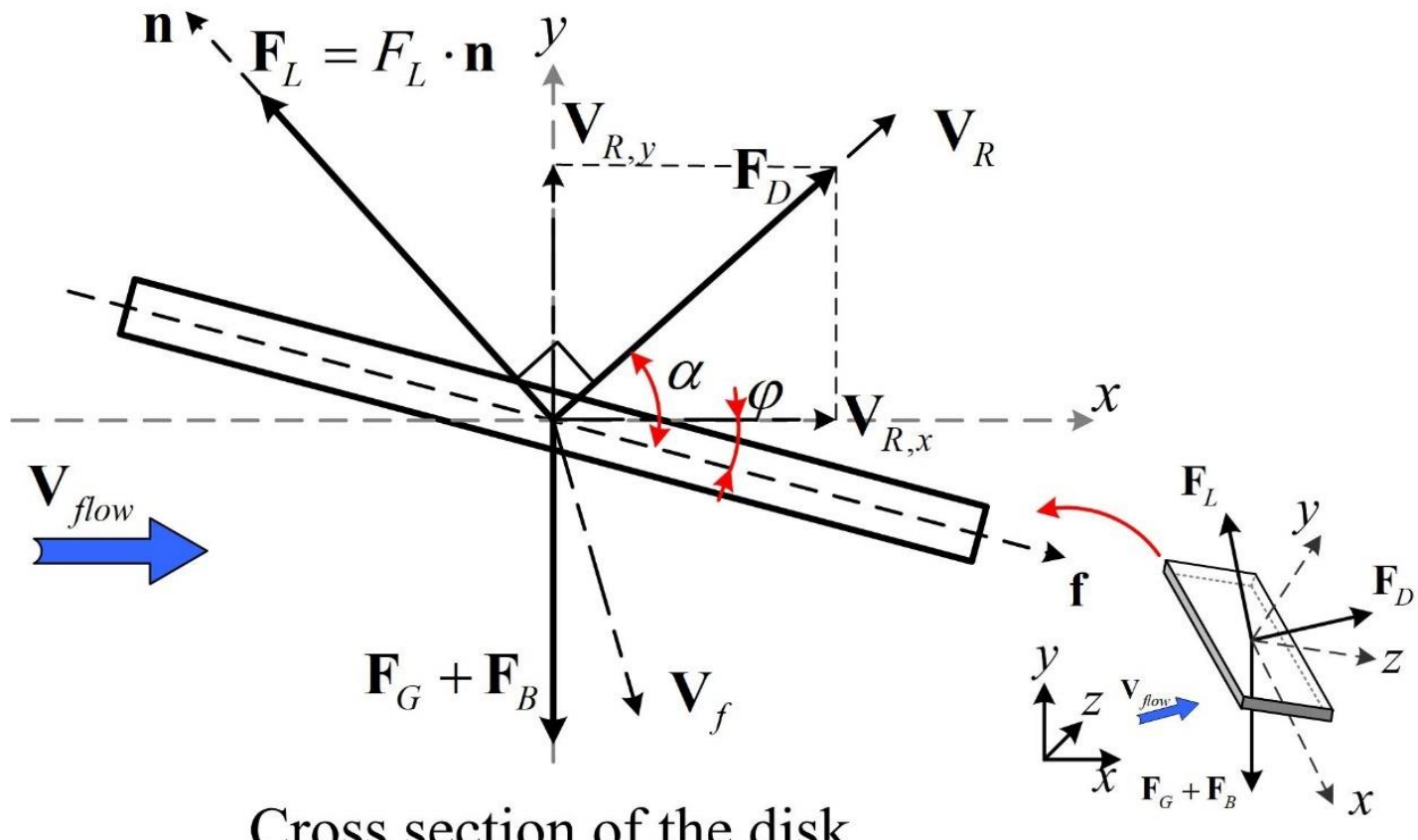

Figure 1 - Schematic representation of the transport model of a disk-shaped firebrand in the flow field.

$\mathbf{V}_{\mathrm{f}}, \mathbf{V}_{\text {flow }}$ and $\mathbf{V}_{\mathrm{R}}$ indicate the velocity of firebrands, ambient flow velocity due to wind, and the relative velocity between disk and air flow, respectively. The angle of attack is denoted by, $\alpha$, which is the angle between disk and relative velocity, whereas the incident angle, $\varphi$, indicates the angle between the disk and the horizontal direction. The direction vectors $\mathbf{f}$ and $\mathbf{n}$ represent the directions along the firebrand and the direction of lift force respectively.

This numerical model is based on the following assumptions:

1) This work focuses on determining the trajectories of firebrands in the transport phase of the spotting phenomenon of firebrands. The particle is assumed to have already exited the main fire zone and is released from $100 \mathrm{~m}$ above ground in air. The plume due to the forest fire in main fire zone is not concluded, and the ambient flow field due to wind is assumed to be constant in each testing cases but a board range of flow velocities are tested.

2) Firebrands are idealized as rectangular-shape disks with aspect ratio 1.6, and it is assumed that the orientation of the disk remains the same during the propagation. That is, the rotation and vibrations of the firebrand, such as tumbling and wobbling, are neglected.

3) As a simplified model to determine maximum spotting distance of firebrands, the aerodynamic effects of combustion on firebrands are beyond the scope of this paper. These include the effects on aerodynamic lift and drag coefficients due to flaming, which are neglected. According to the study by Tse and Fernande-Pello (Tse and Fernandez-Pello, 1998), the surface reaction of burning particles were enhanced at high Reynolds number and were not susceptible 
to be extinguished. The situation of flame extinction is thus not considered. The life time of a burning particle is determined based on size regression and mass loss rate.

4) It is assumed that the brands hold the shapes unchanged in combustion. Two models of burning particles have been used based on different assumptions on the changes of particle thickness in combustion. In the first one the thickness is assumed to be constant (Anthenien et al, 2006), whereas in the other it is variable over time and is calculated basing on the burning constant.

5) This work aims to obtain the trajectory and the maximum sporting distance of firebrand by considering limiting cases. The upper bonds of the parameter in this study are, for ambient flow velocity, $10 \mathrm{~m} / \mathrm{s}$, for density, $950 \mathrm{~kg} / \mathrm{m}^{3}$, and for initial particle velocity, $1.0 \mathrm{~m} / \mathrm{s}$.

\subsubsection{Aerodynamic forces}

Aerodynamic forces exerted on the disk-shaped firebrand transporting in flow include the drag force, $\mathbf{F}_{\mathrm{D}}$, the lift force, $\mathbf{F}_{\mathrm{L}}$, and the gravity combined with buoyancy, $\mathbf{F}_{\mathrm{G}}+\mathbf{F}_{\mathrm{B}}$, as shown in Fig. 1 . The net force on the firebrand thus can be expressed as

$$
\boldsymbol{F}=\boldsymbol{F}_{D}+\boldsymbol{F}_{L}+A \tau\left(\rho_{f}-\rho_{\text {flow }}\right) \boldsymbol{g}
$$

where $\mathbf{F}_{\mathrm{D}}$ and $\mathbf{F}_{\mathrm{L}}$ indicate the aerodynamic drag and lift forces respectively. The virtual mass force and the time-history effects (Yin et al, 2003) are both neglected. In terms of the relative velocity, $\mathbf{V}_{R}$, and the vector along the firebrand, $\mathbf{f}$, the lift force can be expressed as $\mathbf{V}_{R} \cdot \mathbf{f}$. Taking into account the fact that the agnle of attak isin the range of $\left[-90^{\circ}, 90^{\circ}\right]$, the direction vecotor of the lift force, $\mathbf{n}$, points in the same direction as the following vector:

$$
\left(\boldsymbol{V}_{R} \cdot \boldsymbol{f}\right)\left(\boldsymbol{f} \times \boldsymbol{V}_{R}\right) \times \boldsymbol{V}_{R}
$$

The movment in the third direction is negelcted. The full expression of $\mathbf{n}$ can be presented based on the incident angle, $\varphi$, shown as:

$$
\boldsymbol{n}=\left(V_{R, x} \cos \varphi+V_{R, y} \sin \varphi\right)\left(V_{R, y} \cos \varphi-V_{R, x} \sin \varphi\right) \cdot \frac{-v V_{R, y} \boldsymbol{i}+V_{R, x} \boldsymbol{j}}{\sqrt{V_{R, x}{ }^{2}+V_{R, y}{ }^{2}}}
$$

where the sign of the first term on the right hand side characterises the direction of $\mathbf{n}$. Meanwhile, the angle of attack, $\alpha$, can be calculated based on both vectors, as follows:

$$
\alpha=\arccos \frac{\left|\boldsymbol{V}_{R} \cdot \boldsymbol{f}\right|}{\left|\boldsymbol{V}_{R}\right|}
$$

Note that both the drag and lift forces will be zero if the relative velocity is parallel to the direction of the disk (when $\alpha=0^{\circ}$ ), due to the relatively small thicknesses of the firebrands tested. Finally, the aerodynamic drag and lift forces extered on the firebrand then can be expressed as

$$
\begin{aligned}
& \boldsymbol{F}_{D}=\frac{1}{2} C_{D} \rho_{\text {flow }} A\left|\boldsymbol{V}_{R}\right| \boldsymbol{V}_{R} \\
& \boldsymbol{F}_{L}=\frac{1}{2} C_{L} \rho_{\text {flow }} A\left(\boldsymbol{V}_{R}\right)^{2} \boldsymbol{n}
\end{aligned}
$$

where $A$ is the objective area of the firebrand. $C_{D}$ and $C_{L}$, respectively, denote the aerodynamic coeffcients of drag and lift. Considering the range of Reyonlds number can be as large as $2 \times 10^{5}$, different aerodynamic coeffcients have been used to check the sensitivity of the calculations. For relatively low Reynolds number, $\operatorname{Re}<10^{3}$, the formular given in Zastawny et al (Zastawny et al, 2012), are used: 


$$
\begin{aligned}
& C_{D}(\alpha)=\frac{5.82}{R e^{0.44}}+\frac{15.56}{R e^{1.068}}+\left(\frac{35.41}{R e^{0.96}}+\frac{3.63}{R e^{0.05}}-\frac{5.82}{R e^{0.44}}+\frac{15.56}{R e^{1.068}}\right) \sin ^{1.96}(\alpha) \\
& C_{L}=\left(\frac{12.111}{R e^{1.036}}+\frac{3.887}{R e^{0.109}}\right) \sin (\alpha)^{0.812+0.249 R e^{-0.198}} \cos (\alpha)^{5.821-4.7179 R e^{0.007}}
\end{aligned}
$$

Meanwhlie, the aerodynamic drag and lift coeffcients for higher Reynolds number, up to $2 \times 10^{5}$, was determined by Ortiz et al (Ortiz et al, 2015). The comparison with Zastawny's data showed that the drag coeffcients showed similar trends with increasing angle of attack although with slightly different values. The proposed emprical fits can be expressed as:

$$
\begin{aligned}
& C_{D}(\alpha)=-a\left(\frac{\alpha-45}{26.413}\right)^{3}-b\left(\frac{\alpha-45}{26.413}\right)^{2}+c\left(\frac{\alpha-45}{26.413}\right)+d \\
& C_{L_{\alpha \leq 35^{\circ}}}=-0.0014086 \times \alpha^{2}+0.068014 \times \alpha-5.8984 \times 10^{-16} \\
& C_{L_{35^{\circ}<\alpha \leq 50^{\circ}}}=-e\left(\frac{\alpha-42.5}{6.455}\right)^{2}-f\left(\frac{\alpha-42.5}{6.455}\right)+g \\
& C_{L_{50^{\circ}<\alpha \leq 90^{\circ}}}=-h\left(\frac{\alpha-42.5}{6.455}\right)^{2}-k\left(\frac{\alpha-42.5}{6.455}\right)+n
\end{aligned}
$$

where the constants $\mathrm{a}, \mathrm{b}, \mathrm{c}$ and $\mathrm{d}$ are given as $0.02829,0.04068,0.37165$ and 0.68864 respectively. For the lift coefficient, the constants $\mathrm{e}, \mathrm{f}, \mathrm{g}, \mathrm{h}, \mathrm{k}$ and $\mathrm{n}$ are $0.03125,0.01097,0.68219,6.711 \times 10^{5}$, 0.00632 and 1.1113 .

\subsubsection{Equations of motion}

Trajectories of the firebrands are determined from Newton's second law of motion, shown here as:

$$
m_{f} \frac{d V_{f}}{d t}=F_{D}+F_{L}+A \tau\left(\rho_{f}-\rho_{\text {flow }}\right) g
$$

The equation above can be resolved, respectively, in the $\mathrm{x}$ - and $\mathrm{y}$-directions as:

$$
\begin{aligned}
& m_{f} \frac{d V_{f, x}}{d t}=\frac{1}{2} C_{D} \rho_{\text {flow }} A\left|\boldsymbol{V}_{R}\right|\left|\boldsymbol{V}_{R, x}\right|+\frac{1}{2} C_{L} \rho_{\text {flow }} A \boldsymbol{V}_{R}{ }^{2}\left|\boldsymbol{n}_{x}\right| \\
& m_{f} \frac{d V_{f, y}}{d t}=\frac{1}{2} C_{D} \rho_{\text {flow }} A\left|\boldsymbol{V}_{R}\right|\left|\boldsymbol{V}_{R, y}\right|+\frac{1}{2} C_{L} \rho_{\text {flow }} A \boldsymbol{V}_{R}{ }^{2}\left|\boldsymbol{n}_{y}\right|-A \tau\left(\rho_{f}-\rho_{\text {flow }}\right) g
\end{aligned}
$$

A range of initial velocities is tested to determine the effects of particle initial velocity on spotting distance. The vertical component of the initial velocity is set as zero.

\subsection{Simplified combustion models of firebrands}

Two simplified combustion models, based on different assumptions on the behaviours of the thickness of the firebrands, are used to simulate the size regression rate and mass loss rate of the disks outside of the main fire zone. Since no published experimental data are available for the burning of rectangular-shaped disks, the most relevant calculations are found from Tarifa et al (1965), where the burning behaviours of spherical-shaped disks were presented in terms of the burning constant and the 'd-squared law', which can be written as:

$$
\frac{d\left(D_{e f f}^{2}\right)}{d t}=-\beta
$$


where $\beta$ is the dimensionless burning constant and $D_{\text {eff }}$ denotes the effective mass diameter of firebrands. Further, the modified burning constant was derived by Williams (Williams, 1985) as a function of the Reynolds number and the Prandtl number, shown below:

$$
\beta=\beta_{0}\left(1+0.276 \operatorname{Re}^{\frac{1}{2}} \operatorname{Pr}^{\frac{1}{3}}\right)
$$

where the value of $\beta_{0}$ has been found to be $1.8 \times 10^{-7}$. With the assumption that the thickness of the disk is constant, the proposed mass loss rate based the derivations above can be found from Anthenien et al (Anthenien et al, 2006) as:

$$
\frac{d(m)}{d t}=-\frac{\pi}{4} \tau \beta_{0} \rho_{f}\left(1+0.276 \operatorname{Re}^{\frac{1}{2}} \operatorname{Pr}^{\frac{1}{3}}\right)
$$

On the other hand, if we consider the change of thickness in combustion, the equation of the size regression rate can be derived as:

$$
\frac{d(m)}{d t}=-\frac{\pi}{4} \rho_{f}\left(\frac{6 m}{\rho_{f} \pi}\right)^{\frac{1}{3}} \beta_{0}\left(1+0.276 \operatorname{Re}^{\frac{1}{2}} \operatorname{Pr}^{\frac{1}{3}}\right)
$$

Although the geometry utilised in this model is a rectangular-shaped disk, other than the spherical disk in Anthenien's work, the reaction is still diffusion controlled, which justifies our derivation.

\section{Results and discussions}

\subsection{Computational details}

Over 4122 cases from 72 sets of simulations are investigated in this work to determine the lift effects on the firebrand for various working parameters, including the ambient flow velocity, the particle initial velocity, the incident angle and the density of firebrands. The maximum spotting distance of each brand, released from $100 \mathrm{~m}$ in height, is determined by its horizontal displacement once it hits the ground. Meanwhile, the position and the velocity of the tested firebrand as function of time are calculated from the equations of motion discussed above. The initial dimension of the tested firebrand is set as $80 \mathrm{~mm} \times 50 \mathrm{~mm} \times 5 \mathrm{~mm}$ based on the data of real firebrands found from Oakland Hill Fire (Koo, 2010). The tested range of wind speed is $[0,10](\mathrm{m} / \mathrm{s})(\approx 22.4 \mathrm{mph})$, the initial velocities of the firebrands are in the interval $[0,1](\mathrm{m} / \mathrm{s})$ and the range of the incident angle is $\left[-90^{\circ}, 90^{\circ}\right]$. Considering the various possible wood species in real forest fire, a broad range of density is used, from $250 \mathrm{~kg} / \mathrm{m}^{3}$, typical of wood charcoal, to $950 \mathrm{~kg} / \mathrm{m}^{3}$, typical of satinwood. 


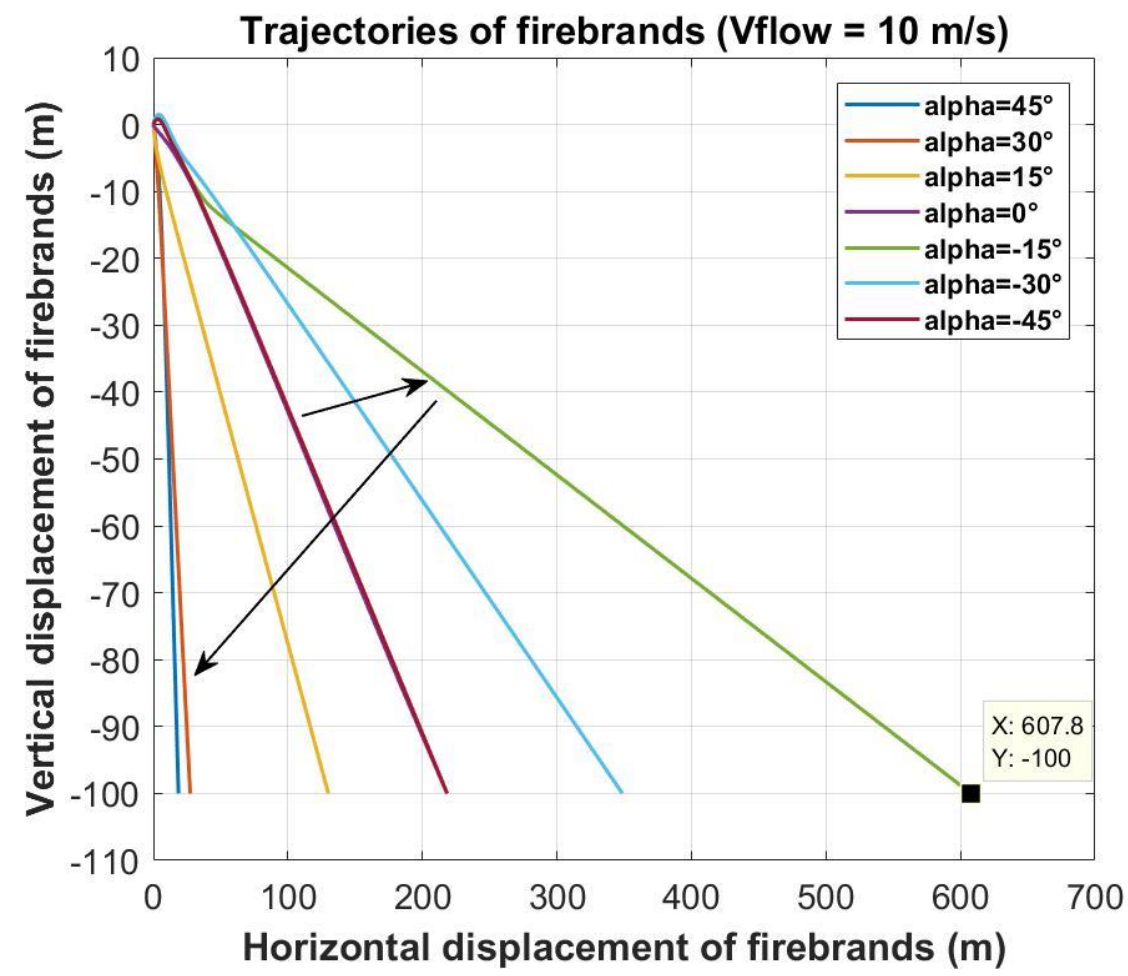

Figure 2 - Trajectories of firebrands at various incident angles with ambient wind velocity of $10 \mathrm{~m} / \mathrm{s}$.

Fig. 2 presents an example of the trajectories of the firebrand at various incident angles with $\mathrm{V}_{\text {flow }}$ $=10 \mathrm{~m} / \mathrm{s}$. It can be seen that the spotting distance and trajectory of a firebrand are highly dependent on the incident angle. The peak spotting distance occurs at the incident angle $-15^{\circ}$, which is a common feature covering most cases, except for non-burning firebrands and burning firebrands with constant thickness, where the peaks are found at $-30^{\circ}$. The 'lift-up', up to $2 \mathrm{~m}$, can be found at the early stage of propagation for all cases of negative incident angle. This behaviour is because the resultant force on upward direction is greater than the gravity, which quickly decreases with the change of angle of attack due to the increasing velocities on both directions.

\subsection{Effects of models}

A dimensionless 'Glide ratio' is used to describe and compare the spotting distance for each tested firebrand. The Glide ratio is the ratio of horizontal displacement over vertical displacement at the firebrand's landing. As shown in Table 1, a broad range of Glide ratios are found, from about 0 to 6.091. Very similar results are obtained from the non-burning and constant-thickness-burning models. This is because of the very small value of area regression rate in constant-thickness-model, which is around $7 \times 10^{-6} \mathrm{~m}^{2} / \mathrm{s}$ when Re is around $1 \times 10^{5}$. Little change of firebrand's propagation is observed due to such small size change. However, enhanced propagation distances are found for the variablethickness-burning models, which average about 0.01 larger with the large Reynolds number models and 0.134 larger with the small Reynolds number model. It proves that the considerations of thickness change can have significant impact on the calculations of firebrands propagations, especially for the cases with moderate Reynolds numbers.

More importantly, the very significant effects of aerodynamic lift on firebrands propagation are demonstrated by the results in the table, that the lift force can drastically increase the propagation distances of the firebrands with or without combustion, for different Reynolds numbers. The increase in GR can be as large as $3.845(171.19 \%)$, as is shown in the $4^{\text {th }}$ column of the table, which means 384.5 meters further in horizontal displacement for a firebrand falling from 100 meters in height. The vertical falling speed of a firebrand is decreased by lift force while the horizontal speed is increased, which result in the firebrands having not only larger horizontal speed but also longer flight time. 
Furthermore, it can be observed that the positive lift effects are increased in the variable-thicknessburning model. For a burning firebrand, although the magnitude of lift force is reduced due to area regression, the lose of weight creates a chance to glide further. However, it is also found that lift force has negative effects on propagations when the lift formula for lower Reynolds number is used, shown with the negative values highlighted in blue in Table 1. This is due to the combination of two effects. Firstly, stronger effects from the drag force are found for smaller Reynolds numbers (smaller coefficient of lift comparing to drag). Secondly, the change of angle of attack due to the increases in velocities is more sensitive (the direction of the lift force could quickly change from pointing upward to pointing downward), resulting in longer flight time when the lift is absence even though velocities are smaller.

Table 1 - Maximum Glide ratios of tested firebrands with different variables

\begin{tabular}{|c|c|c|c|c|c|c|}
\hline \multirow{3}{*}{$\begin{array}{c}\text { Independent } \\
\text { Variables }\end{array}$} & \multicolumn{6}{|c|}{ Max Glide Ratio (GR = horizontal displacement / vertical displacement) } \\
\hline & \multicolumn{3}{|c|}{ Large Reynolds Number Model } & \multicolumn{3}{|c|}{ Small Reynolds Number Model } \\
\hline & Lift + Drag & Drag only & Lift effect & Lift + Drag & Drag only & Lift effect \\
\hline \multicolumn{7}{|c|}{ Non-burning firebrands } \\
\hline $\begin{array}{l}\text { Flow velocity, } V_{\text {flow }} \& \\
\text { Incident angle, } \varphi\end{array}$ & 6.078 & 2.269 & 3.809 & 4.062 & 4.095 & $-\mathbf{0 . 0 3 3}$ \\
\hline $\begin{array}{l}\text { Flow velocity, } V_{\text {flow }} \& \\
\text { Initial velocity, } V_{f, 0}\end{array}$ & 6.078 & 2.250 & 3.828 & 4.062 & 4.009 & -0.053 \\
\hline $\begin{array}{c}\text { Flow velocity, } \mathbf{V}_{\text {flow }} \& \\
\text { Density, } \rho\end{array}$ & 6.078 & 2.246 & 3.832 & 4.062 & 4.006 & 0.056 \\
\hline $\begin{array}{c}\text { Incident angle, } \varphi \& \\
\text { Initial velocity, } V_{f, 0}\end{array}$ & 2.430 & 0.019 & 2.411 & 0.919 & 0.026 & 0.893 \\
\hline $\begin{array}{c}\text { Incident angle, } \varphi \& \\
\text { Density, } \rho\end{array}$ & 2.379 & $<0.001$ & 2.379 & 0.904 & $<0.001$ & 0.904 \\
\hline $\begin{array}{c}\text { Density, } \rho \& \\
\text { Initial velocity, } V_{\mathrm{f}, 0}\end{array}$ & 2.430 & 0.014 & 2.416 & 0.173 & $<0.001$ & 0.173 \\
\hline \multicolumn{7}{|c|}{ Burning firebrands (constant thickness) } \\
\hline $\begin{array}{l}\text { Flow velocity, } V_{\text {flow }} \& \\
\text { Incident angle, } \varphi\end{array}$ & 6.078 & 2.269 & 3.809 & 4.062 & 4.095 & -0.033 \\
\hline $\begin{array}{c}\text { Flow velocity, } V_{\text {flow }} \& \\
\text { Initial velocity, } V_{f, 0}\end{array}$ & 6.078 & 2.250 & 3.828 & 4.062 & 4.009 & -0.053 \\
\hline $\begin{array}{c}\text { Flow velocity, } V_{\text {flow }} \& \\
\text { Density, } \rho\end{array}$ & 6.078 & 2.246 & 3.832 & 4.062 & 4.006 & 0.056 \\
\hline $\begin{array}{l}\text { Incident angle, } \varphi \& \\
\text { Initial velocity, } V_{\mathrm{f}, 0} \\
\end{array}$ & 2.430 & 0.019 & 2.411 & 0.919 & 0.026 & 0.893 \\
\hline $\begin{array}{c}\text { Incident angle, } \varphi \& \\
\text { Density, } \rho\end{array}$ & 2.379 & $<0.001$ & 2.379 & 0.904 & $<0.001$ & 0.904 \\
\hline $\begin{array}{c}\text { Density, } \rho \& \\
\text { Initial velocity, } V_{\mathbf{f}, 0} \\
\end{array}$ & 2.430 & 0.014 & 2.416 & 0.173 & $<0.001$ & 0.173 \\
\hline \multicolumn{7}{|c|}{ Burning firebrands (variable thickness) } \\
\hline $\begin{array}{l}\text { Flow velocity, } V_{\text {flow }} \& \\
\text { Incident angle, } \varphi\end{array}$ & 6.091 & 2.269 & 3.822 & 4.101 & 4.114 & -0.013 \\
\hline $\begin{array}{c}\text { Flow velocity, } V_{\text {flow }} \& \\
\text { Initial velocity, } V_{f, 0}\end{array}$ & 6.091 & 2.250 & 3.841 & 4.101 & 4.026 & -0.075 \\
\hline $\begin{array}{c}\text { Flow velocity, } V_{\text {flow }} \& \\
\text { Density, } \rho\end{array}$ & 6.091 & 2.246 & 3.845 & 4.101 & 4.023 & -0.078 \\
\hline $\begin{array}{l}\text { Incident angle, } \varphi \& \\
\text { Initial velocity, } V_{\mathrm{f}, 0} \\
\end{array}$ & 2.431 & 0.019 & 2.412 & 1.254 & 0.022 & 1.232 \\
\hline $\begin{array}{c}\text { Incident angle, } \varphi \& \\
\text { Density, } \rho\end{array}$ & 2.381 & $<0.001$ & 2.381 & 1.221 & $<0.001$ & 1.221 \\
\hline $\begin{array}{c}\text { Density, } \rho \& \\
\text { Initial velocity, } V_{\mathrm{f}, 0}\end{array}$ & 2.431 & 0.014 & 2.417 & 0.207 & 0.008 & 0.199 \\
\hline
\end{tabular}




\subsection{Effects of control parameters}

Firebrands transport is a complex proceedure combining both aerodynamic and combustion behaviours. Without considering the effects of combustion, the control parameters can be simplified into four: ambient flow velocity, initial particle velocity, density and incident angle. Building on the results presented in the previous section, the effects of these parameters are further investigated. Due to the limited space in this paper, the discussions focus on the trajectories from the variable-thicknessburning model with large Reynolds numbers, shown in Fig. 3.
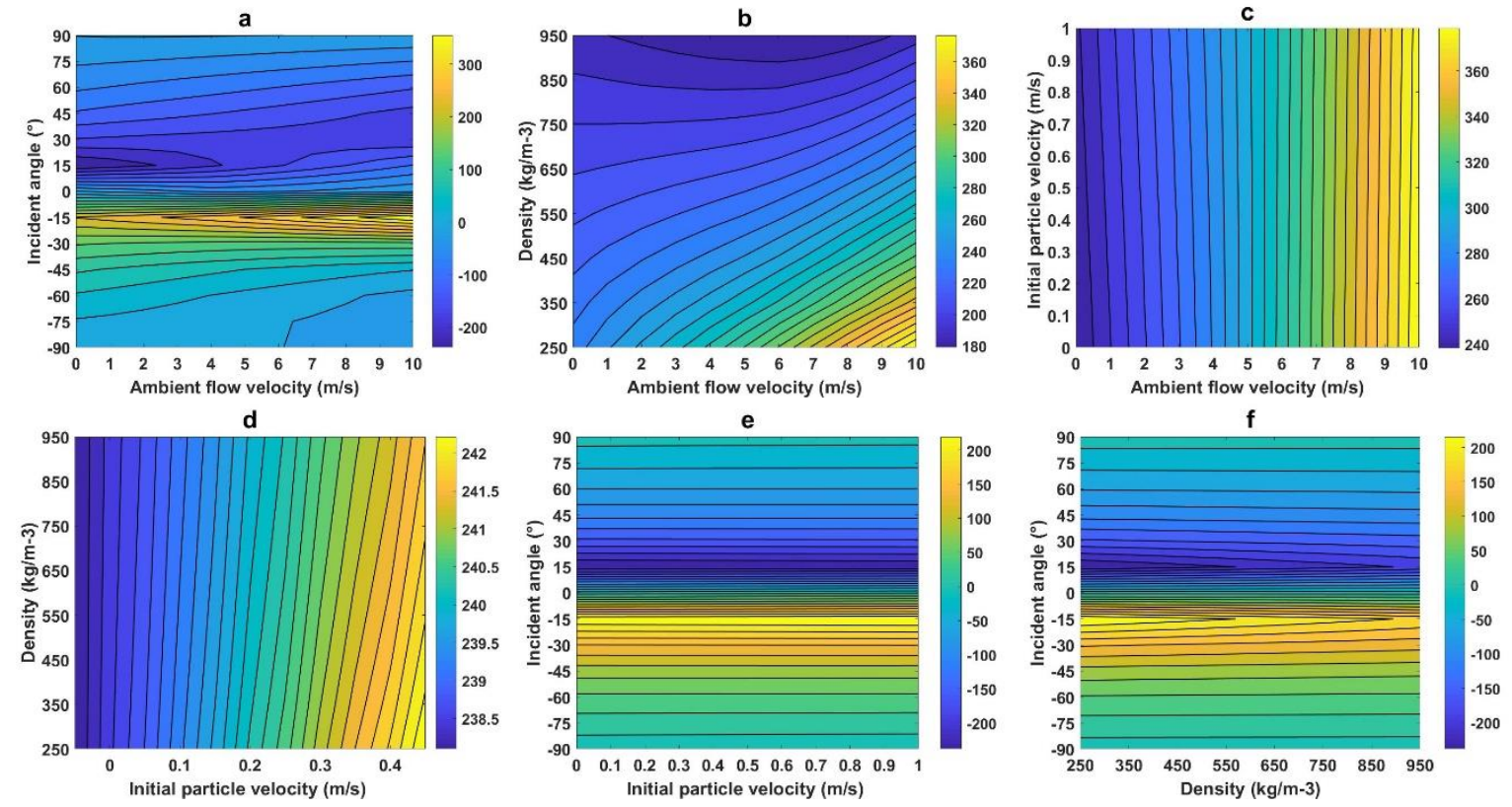

Figure 3 - Contour plots of lift effects of non-burning firebrands with various parameters.

Fig. 3 presents the contour plots for the differences in horizontal displacements for firebrands calculated with and without lift force, as functions of different parameters. Apart from the independent variables in each figure, the values for the other two parameters are given in Table 2. A complex dependence on the flow velocity and the incident angle can be observed in Fig. 3a, which shows the difference in the displacement is highly dependent on the incident angle, especially when it falls in the interval $\left[-30^{\circ}, 30^{\circ}\right]$. The propagations of firebrands are very sensitive to the incident angle and are increased for negative incident angles. With the increasing inertia due to larger density of a firebrand, the effects of the lift become more independent of the flow velocity, shown in Fig. 3b. However, it is clear that the effects of the lift depends more strongly on the flow velocity than the initial velocity, as is shown in Fig. 3c, and the latter in turn has stronger effects than the density, which can be observed from Fig. 3d. Above all, the incident angle has the most important effects. Apart from negative incident angles, large flow velocity and small firebrands density also enhance the lift effects on propagations.

Table 2 - Testing values of control parameters.

\begin{tabular}{cc}
\hline \hline Control Parameters & Testing values \\
\hline \hline Flow velocity, $V_{\text {flow }}$ & $10 \mathrm{~m} / \mathrm{s}$ \\
\hline Incident angle, $\varphi$ & $-15^{\circ}$ \\
\hline Initial velocity, $V_{\mathrm{f}, 0}$ & $1.0 \mathrm{~m} / \mathrm{s}$ \\
\hline Density & $250 \mathrm{~kg} / \mathrm{m}^{3}$ \\
\hline \hline
\end{tabular}




\subsection{Effects of the lift on trajectories}

Based on the above discussion, the effects of the ambient flow velocity and the incident angle deserve further investigation. The left panel of Fig. 4 shows the ratio between the horizontal displacements of the particle modelled with or without the lift force, calculated by the large Reynolds number model coupled with the variable-thickness burning model. It can be found that the result depends strongly on the incident angle. The lift increases the travel distances when the incident angle is negative (meaning the disk is tilted forward). The ratio peaks at an incident angle around $-15^{\circ}$. The peak value reaches up to 14 for small wind speed. It decreases to around 3 when the flow velocity is increased to $10 \mathrm{~m} / \mathrm{s}$. Even though the ratio decreases with the wind speed, the right panel in Fig. 4 shows that the absolute difference actually increases with the latter for incident angle between $-30^{\circ}$ and $0^{\circ}$, while it decreases between $-90^{\circ}$ and $-30^{\circ}$. Thus, at large $\mathrm{Re}$, the lift force has the potential to significantly increase the modelled propagation distances.
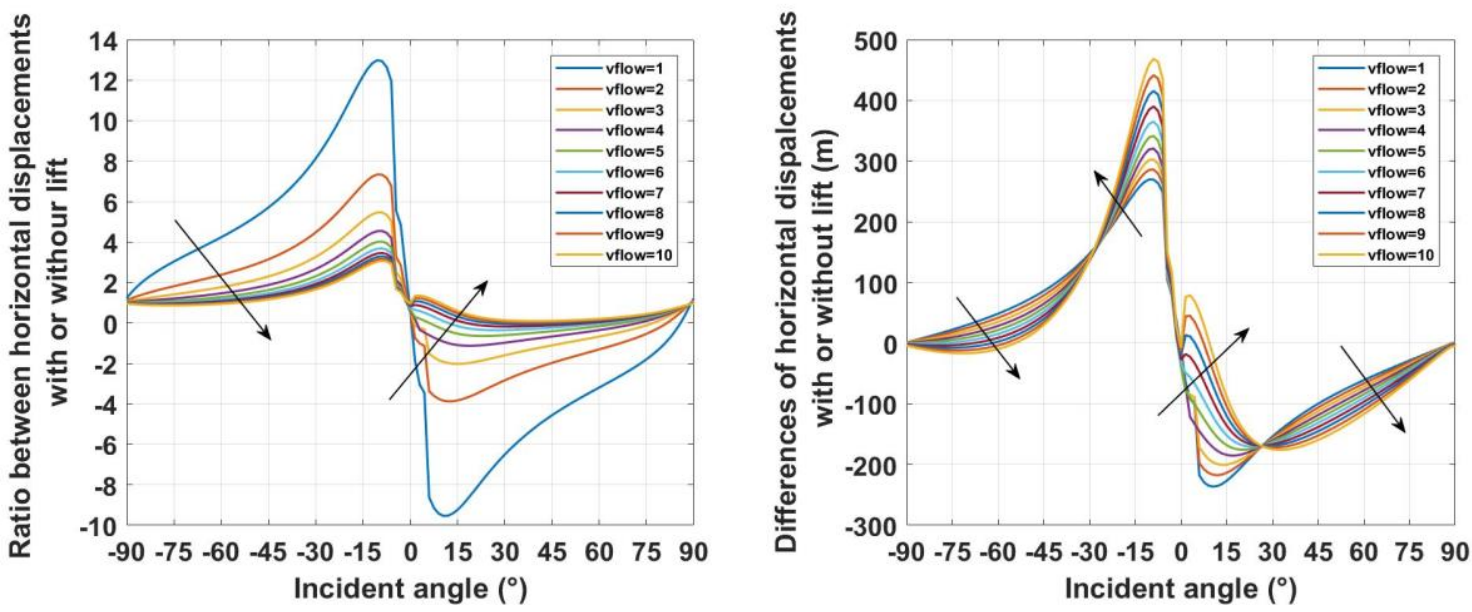

Figure 4 - Left: Ratio between horizontal displacement with or without lift; Right: Effects of lift on maximum distances.

\section{Conclusion}

The trajectories of both non-burning and burning firebrands are determined for three simplified models. A burning model is investigated where the variation of the thickness of the firebrand during combustion is considered. Parametric studies are performed to determine the effects of aerodynamic lift force. The maximum potential spotting distances at various parameters are calculated and expressed in terms of the Glide Ratios. The importance of the parameters involved, including Reynolds number, ambient flow velocity, incident angle, particle initial velocity and density of firebrand, are obtained and compared with each other.

It is found that the lift force has very significant effects on firebrand propagations. At large Reynolds numbers, the maximum spotting distance can be increased by up to $170 \%$ by the lift force for a firebrand released from $100 \mathrm{~m}$ in height. The lift effect strongly depends on incident angle. The peak value of the ratio between horizontal displacement with or without lift is found at $-15^{\circ}$ with a 14 times increase. The results highlight the importance of the aerodynamic lift force, and suggest that it should be included in order to estimate extreme spotting distances.

\section{Reference}

Albini, F. (1979). Spot fire distance from burning trees. Ogden, Utah: Intermountain Forest and Range Experiment Station, Forest Service, U.S. Dept. of Agriculture. 
Anthenien, R., Tse, S. and Carlos Fernandez-Pello, A. (2006). On the trajectories of embers initially elevated or lofted by small scale ground fire plumes in high winds. Fire Safety Journal, 41(5), pp.349-363.

Himoto, K. and Tanaka, T. (2005). Transport Of Disk-shaped Firebrands In A Turbulent Boundary Layer. Fire Safety Science, 8, pp.433-444.

Koo, E., Pagni, P., Weise, D. and Woycheese, J. (2010). Firebrands and spotting ignition in large-scale fires. International Journal of Wildland Fire, 19(7), p.818.

Lee, S. and Hellman, J. (1970). Firebrand trajectory study using an empirical velocity-dependent burning law. Combustion and Flame, 15(3), pp.265-274.

Muraszew, A., Fedele, J. and Kuby, W. (1977). Trajectory of firebrands in and out of fire whirls. Combustion and Flame, 30, pp.321-324.

Ortiz, X., Rival, D. and Wood, D. (2015). Forces and Moments on Flat Plates of Small Aspect Ratio with Application to PV Wind Loads and Small Wind Turbine Blades. Energies, 8(4), pp.24382453.

Sardoy, N., Consalvi, J., Porterie, B. and Fernandezpello, A. (2007). Modeling transport and combustion of firebrands from burning trees. Combustion and Flame, 150(3), pp.151-169.

Tarifa, C., Notario, P. and Moreno, F. (1965). On the flight paths and lifetimes of burning particles of wood. Symposium (International) on Combustion, 10(1), pp.1021-1037.

Tse, S. and Fernandez-Pello, A. (1998). On the flight paths of metal particles and embers generated by power lines in high winds - a potential source of wildland fires. Fire Safety Journal, 30(4), pp.333356.

Williams, F. (1985). Combustion theory. 2nd ed.

Woycheese, J., Pagni, P. and Liepmann, D. (1999). Brand Propagation From Large-Scale Fires. Journal of Fire Protection Engineering, 10(2), pp.32-44.

Yin, C., Rosendahl, L., Knudsen Kær, S. and Sørensen, H. (2003). Modelling the motion of cylindrical particles in a nonuniform flow. Chemical Engineering Science, 58(15), pp.3489-3498.

Zastawny, M., Mallouppas, G., Zhao, F. and van Wachem, B. (2012). Derivation of drag and lift force and torque coefficients for non-spherical particles in flows. International Journal of Multiphase Flow, 39, pp.227-239. 


\title{
Experimental characterization and analysis on fire whirls using forest fuels
}

\author{
Cláudia Pinto*; Jorge Raposo; Domingos Xavier Viegas \\ Forest Fire Research Centre (CEIF), ADAI-LAETA, University of Coimbra. Coimbra 3030-289, \\ Portugal, \{claudia.pinto@adai.pt*\}
}

\begin{abstract}
During a forest fire the formation of fire whirls can be observed but their characteristics and evolution are still difficult to predict. Due to the high thermal energy released, fire whirls can become a danger to firefighters and are, by their complexity and dangerousness, a way of extreme fire behavior that is poorly understood.

This work presents a study on the formation of fire whirls with vertical axis on wildfires at laboratory scale that analyses the influence of the variation of the following parameters: fuel load, density, fuel configuration, imposition of forced flow with different velocities through the use of fans. Comprehensive measurements were performed in order to establish correlations of the burning rate, heat release rate, flame height, temperature and velocity of fire whirls, and to clarify the similarities and differences between a fire whirl and a 'normal' fire, i.e., one in which vortex formation is not observed. Fire whirl experiments were performed in a vertical channel with a quadrangular section of $1 \times 1 \mathrm{~m} 2$ with a height of $7.8 \mathrm{~m}$, the fire whirl generator, using dried shrubs, which is a fuel mainly composed by heather (Erica australis) and gorse (Pterospartum tridentatum) quite common in forested areas in Central Portugal and in several Mediterranean climate regions.

Given the transient nature of the experiments relationships between time correlated parameters or with average or extreme values were established. Comparison with similar experiments showed that the properties of the flames generated with the present configuration of the fire whirl generator are similar to fire whirls produced in other laboratory studies and with full scale fire whirls. The maximum heat release power was of the order of $1 \mathrm{MW}$ which is higher than the reported values for similar laboratory tests. The results show that forced flow increases dramatically the burning rate and reduces the time needed to achieve a high rate of energy release. On the other hand, from a certain value of forced flow it is observed that the values of the burning rate and flame height decrease, thus existing a critical wind velocity for optimal fire whirl development. Comparison with results of other sources show that the flames that are generated in the present fire whirl generator are in a transition from fire whirl to pool fire regime and that it is possible to scale up some flow and thermal properties of field scale fire whirls and to derive predictive models on the basis of laboratory scale experiments.
\end{abstract}

Keywords: Fire whirl, Extreme fire behavior, Forest fires, Forest fuels, Burning rates, Fire safety

\section{Introduction}

In certain circumstances, fires can cause great destruction and endanger the population and those who fight them, particularly in situations where the fire spreads under extreme conditions due to weather, fuel or topographical factors, which are likely to potentiate accidents (Viegas et al., 2017). Under these conditions fires with strong vorticity phenomena, associated to rapidly changing fire spread properties, as in the case of fire whirls, are among the most dangerous and difficult to control. We consider a fire whirl a vertical axis flow structure having a rotating flame in its core that can release very high amounts of energy and produce intense spotting.

As the amount of energy produced at laboratory scale vortices is small compared to those in wildland fires the scale up of results is of great importance when attempting to apply information from small-scale experiments to full-scale fire whirls. Several authors have examined this problem (see e.g 
Kwuana et al., 2011 and Kwuana et al., 2013), but no universal scaling parameters exist for air intake velocity, burning rate, flame base size, etc

The types of fuels used to conduct experimental studies are mostly liquid and gaseous, and it is therefore important to approach the study of fire whirls behavior through the use of forest fuels (Pinto et al., 2017).

The influence of the lateral wind velocity was studied by Kuwana et al. (2008) and a scaling law that predicts the critical wind velocity at which the most intense fire whirl was generated was developed and validated. It was observed too that in actual mass fires the occurrence of fire whirls is time-dependent, in contrast to well controlled small-scale laboratory experiments, but this finding was not explored.

The present work aims contributing to a better understanding on the formation of fire whirls and characterization of this phenomenon through the continuation of the previous work (Pinto et al., 2017). Comparison with results from other laboratory scale experiments and full size fire whirls and pool fires showed that it is possible to derive predictive models for this type of fire whirls in a wide range of scales.

\section{Experimental Methodology}

The tests were performed at Laboratory for Forest Fire Studies (LEIF) in the Fire Whirl Generator (FWG) apparatus (Pinto et al., 2017) consisting of a vertical channel with a quadrangular section of $1 \times 1 \mathrm{~m}^{2}$ with a height of $6 \mathrm{~m}$ with two sides made of tempered glass and the other two made of steel sheet. The base of the FWG has a section of $2 \times 2 \mathrm{~m}^{2}$ and $1.8 \mathrm{~m}$ height tapering to the $1 \times 1 \mathrm{~m}^{2}$ section of the main channel section that is open at the top (see Figure 1). Each corner of the channel has a vertical opening $10 \mathrm{~cm}$ wide to induce tangential air entrainment. Inside the base of FWG there is a platform of $1 \times 1 \mathrm{~m}^{2}$ covered by ceramic tiles to support the fuel container. A set of four axial fans Rosenberg DR 630-4.6LA was attached to the base of the FWG to induce a tangential flow to create a forced vortex inside the combustion chamber. In the present experiments the inlet velocities, $v_{i}$, value in the range from $0 \mathrm{~m} / \mathrm{s}$ until $5 \mathrm{~m} / \mathrm{s}$ were used. The fuel containers were made of a metallic grid welded to form a cylinder open on the top and five different containers with $0.350 \mathrm{~m}, 0.500 \mathrm{~m}, 0.800 \mathrm{~m}$ and $1.120 \mathrm{~m}$ diameter were used. The solid porous fuel used consisted of dried shrubs, mainly composed by heather (Erica australis) and gorse (Pterospartum tridentatum) quite common in forested areas in Central Portugal and in several Mediterranean climate regions. The fuel in the container was placed on the plate inside the FWG, the ignition was initiated along the perimeter basis of the container using a gas burner and the fan frequency was tuned in order to achieve the desired flow velocity. The tests were made assuring the same conditions of ambient temperature and preparation methodology. In these tests the fuel load was $4 \mathrm{~kg}$ (dry mass) for all fuels.

The mass loss values were recorded directly to an Excel sheet with a time interval of one second, by RSKey v.1.40 software, using a laptop connected to the electronic scale. The velocities were measured using four Pitot tubes type $\mathrm{S}$, two of them measuring the radial direction flow velocity (one at $2.70 \mathrm{~m}$ height and the other at $4.20 \mathrm{~m}$ height) and the other two measuring the tangential direction flow velocity, at the same heights. All measuring points were located $10 \mathrm{~cm}$ from the axis of the vertical channel. Temperatures were measured using four thermocouples type $\mathrm{K}$ placed in the same locations of the Pitot tubes. A data acquisition system (LabVIEW software and National Instruments hardware) was used to register the values of temperature and airflow velocity during the experiments.

Two cameras were used for recording images: a Sony high definition video camera and a Cannon photographic high performance digital camera. The video camera was always in the same place and the recorded images were used to measure flame diameter and flame height. 

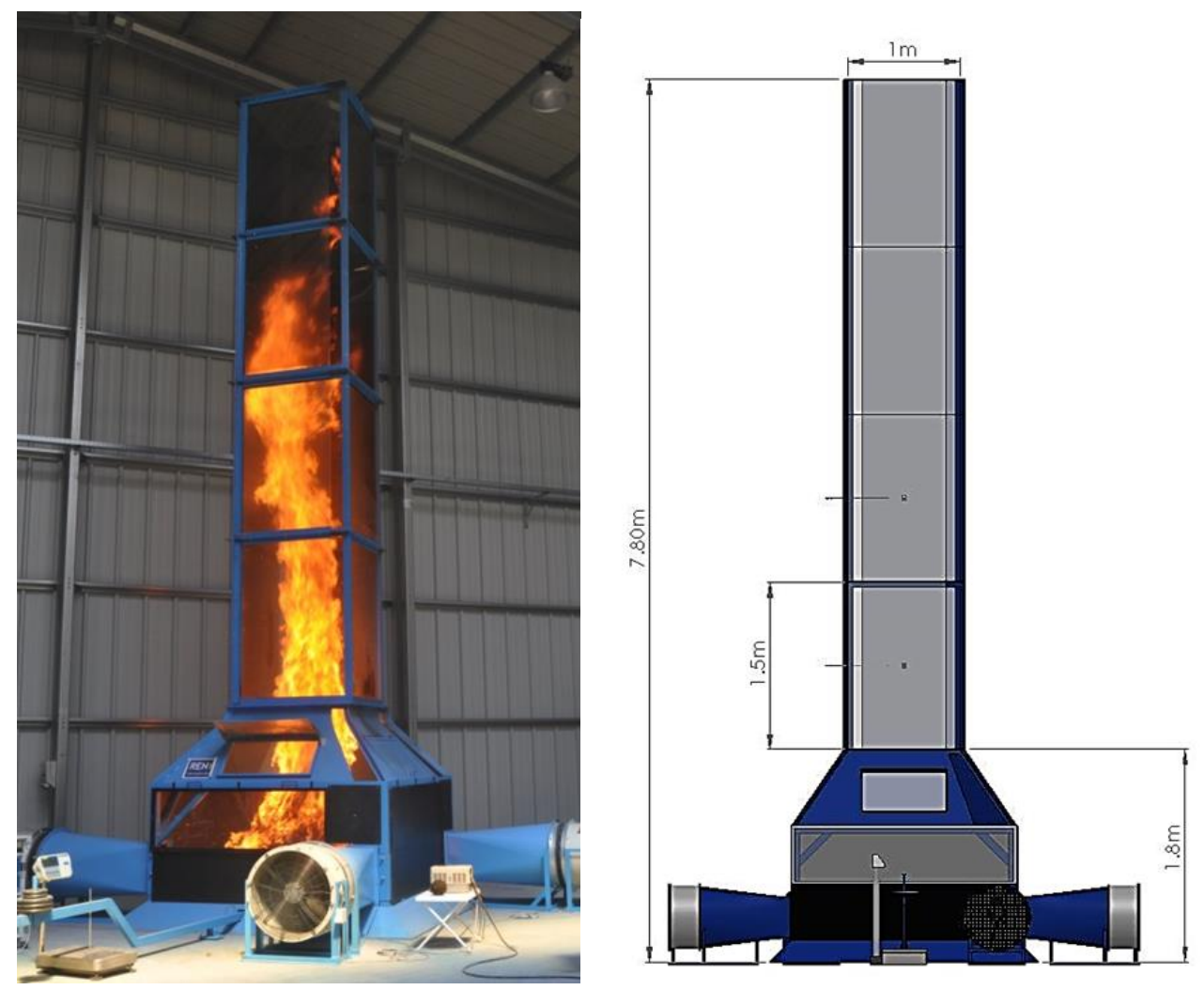

Figure 1 - Fire Whirl Generator (FWG) apparatus

\section{Results}

\subsection{Use of Forest fuels}

The use of natural forest fuels, namely shrubs, makes the study and visualization of the fire whirls more realistic and similar to fire whirls produced in forest fires. The amount of heat released, the mass loss rate, the flame height and diameter, the velocities and temperature change in the course of time as the mass loss rate is not constant during each test as can be observed in Figure 2a. Therefore, time dependent phenomena can be studied in these experiments and the variation of one parameter with another one can be checked using either instantaneous, average or the maximum values of the relevant parameters. Assuming that the behavior is quasi static correlations between time dependent variables can be made in a single test for a range of values of the relevant parameters (e.g., see Figure 2b).

For some parameters the maximum value was not well defined; in these cases, the average of five higher values in the test was considered as the maximum value. The maximum values of the parameters analysed here are higher than those obtained in similar size tests performed with liquid fuels, as for example, in our study heat power released by these fire whirls is higher than $1 \mathrm{MW}$ (see Table 2 of (Pinto et al., 2017)) in comparison with $600 \mathrm{~kW}$ from fire whirls produced with liquid fuels by Lei et al. (2011) for containers with $500 \mathrm{~mm}$ diameter in both studies.

\subsection{Burning rate and Forced flow}

The burning rates of fire whirls depend on diameter of the container, $d_{c}$, similarly to those of general pool fires, but with a much higher increasing rate versus the diameter of the container. As can be seen in Figures. $2 \mathrm{a}$ and $2 \mathrm{~b}$, larger diameter containers, $\mathrm{d}_{\mathrm{c}}$, correspond to higher values of mass loss rate, $\dot{m}$, and lower values of burning duration. These results are in agreement with other author's studies (Lei et al., 2011 and Martin et al., 1976). 
a)

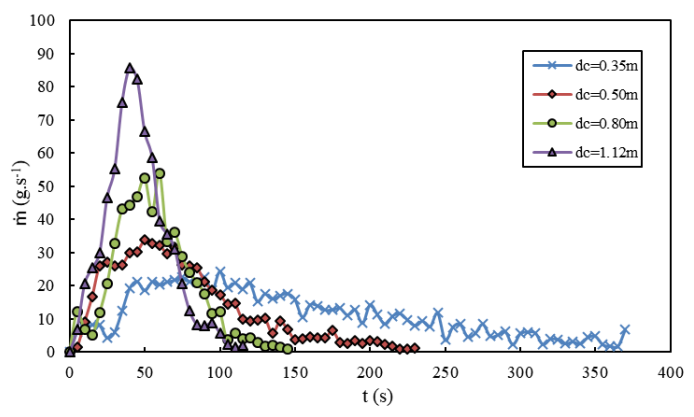

b)

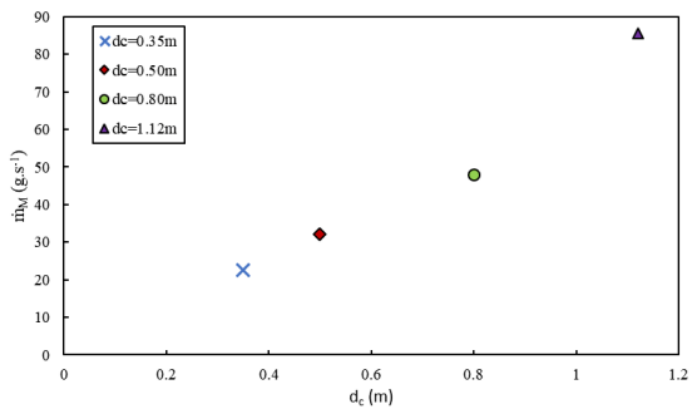

Figure 2 - (a) burning rate variation for different diameter containers $\left(d_{c}\right)$ as a function of time; b) maximum mass loss rate $\left(\dot{\boldsymbol{m}}_{M}\right)$ as a function of diameter containers

Following the previous work (Pinto et al., 2017), in the present study the potential of the FWG was explored to extend the range of parameters (burning rate, heat release rate and flame height) tested through the use of other sensors, Pitot tubes and thermocouples, to analyse the flow and temperature, respectively. As the repetition of tests in other conditions yielded similar results we will present the results of only one test. The test was performed with no forced flow and using the container with 500 $\mathrm{mm}$ diameter. Figures $3 \mathrm{a}$ and $3 \mathrm{~b}$ show the temperatures, $T$, the velocities, $U$, and the mass loss rate, $\dot{m}$, as a function of time. It can be observed that like in Figure $2 \mathrm{a}$ the behavior of these parameters are time dependent in which temperatures, velocities and fuel mass loss rates are initially low and constant, since they coincide with the initial combustion of the shrubs, when the formation of the fire whirl has not yet occurred; the formation and development of the fire whirl starts from approximately 50 seconds after ignition, causing the increase of temperatures, velocity values and mass loss rate up to the peak; finally the values decrease until the fire whirl extinguishes. The values of the velocity remain non-null and constant after 300 seconds, at values of approximately $1.5 \mathrm{~m} / \mathrm{s}$, due to the heat released by the nonflaming residual combustion of shrubs.

The values of fuel mass loss rate and velocity were acquired at the same time instants and so can be related to each other, as shown in Figure 3c. It is interesting to notice the story of the fire whirl which can be divided in three phases: 1) formation and development for $\dot{m}$ between 0 and $40 \mathrm{~g} \cdot \mathrm{s}^{-1}$ and rising $\mathrm{U}$ from the still condition (lower branch of the curve), 2) maximum development for $\dot{m} \approx 40 \mathrm{~g} / \mathrm{s}$ and $U \approx 7-8 \mathrm{~m} / \mathrm{s}$ (right upper corner of the curve), and 3 ) for decreasing $\dot{m}$ and $U$ (upper branch of the curve).

a)

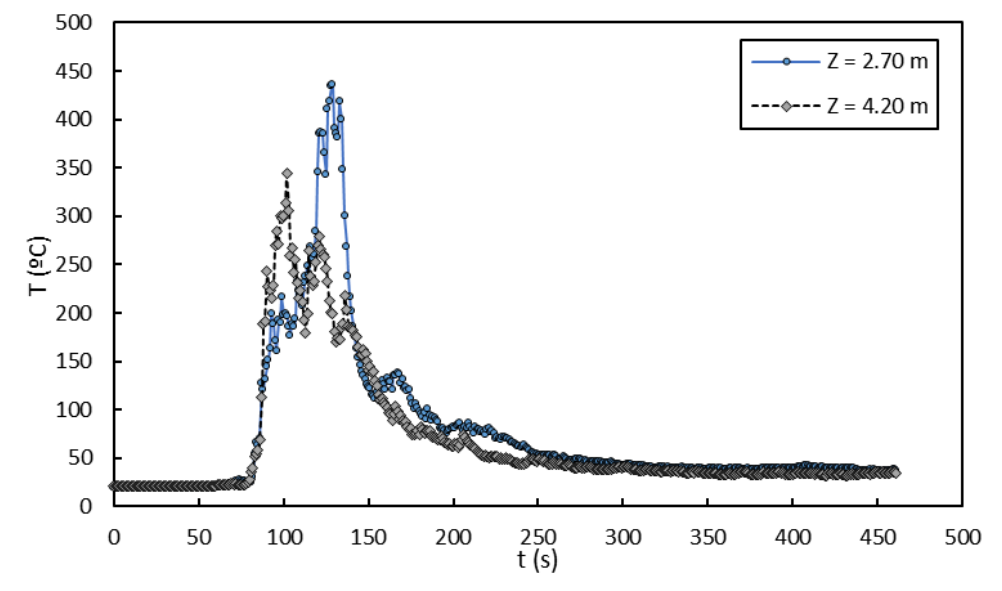

b) 

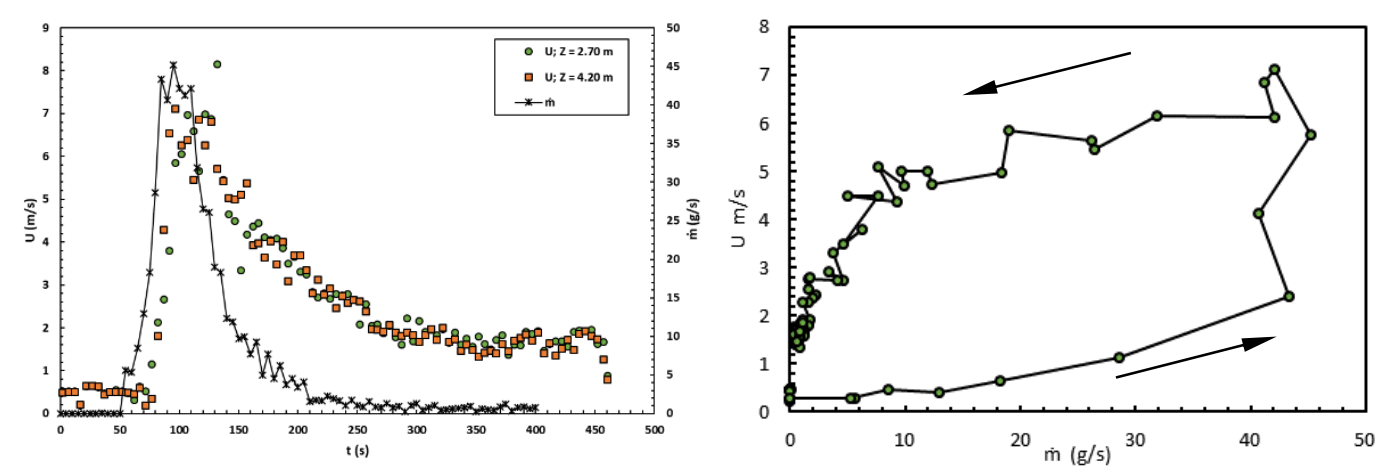

Figure 3 - Temperature, flow velocities and fuel mass loss rate measurements for different heights $(Z=2.70 \mathrm{~m}$ and $Z$ $=4.20 \mathrm{~m}$ ): (a) temperature in function of time; $b$ ) velocity and mass loss rate in function of time; $c$ ) velocity as a function of mass loss rate for the $4.20 \mathrm{~m}$ height Pitot tube

The experimental results of the tests with forced flow, $v_{i}$, were compared with other tests in similar conditions in terms of fuel load with no forced flow $\left(\mathrm{v}_{\mathrm{i}}=0 \mathrm{~m} \cdot \mathrm{s}^{-1}\right)$. It was possible to evaluate the positive effect of vorticity on several parameters like flame height, $L_{M}$, mass burning rate and fire intensity. The results show that forced flow increases dramatically the burning rate and reduces the time needed to achieve high values of energy release. In Figures $4 \mathrm{a}$ and $4 \mathrm{~b}$ the maximum value of mass loss rate increases generally with the forced flow velocity, at the same time the average flame height increases a little $(+11 \%)$ and the average flame diameter, $d_{m}$, decreases significantly $(-33 \%)$. On the other hand, it is observed that there is a value of forced flow for which the flame height reaches its maximum (for $\mathrm{vi}_{\mathrm{i}}=2.74 \mathrm{~m} \cdot \mathrm{s}^{-1}$ ), decreasing for higher values of forced flow, meaning that fire whirls formation and development can be dependent of the amount of air entrainment or the ambient vorticity. This effect must be studied more carefully as it may be an indication of the existence of either a change of regime in the fire whirls development.

a)

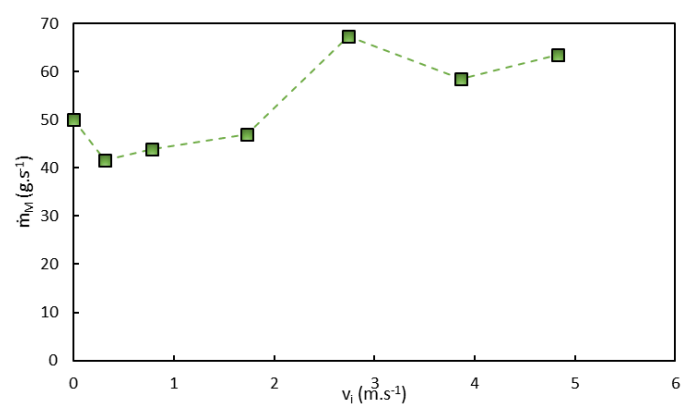

b)

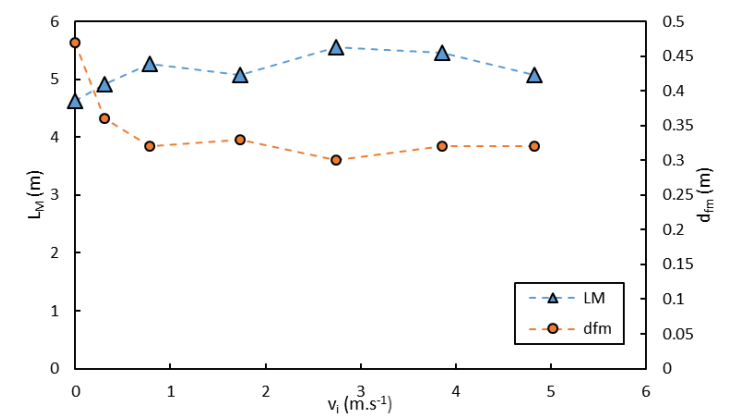

Figure 4 - Results for several air forced flow: (a) maximum values of mass loss rate; $b$ ) maximum values of height flame and diameter flame of fire whirl

\subsection{From laboratory scale to full scale fire whirls}

Using the present research, a relationship between the maximum flame height, $L_{M}$, and the size of the fuel bed, $d_{c}$, is proposed given by:

$$
L_{M}=a_{5} d_{c}^{b_{5}}
$$

Photos or video images of fire whirls can be used to obtain data on the two parameters that are used in Equation 1. Provided that there is some scale factor to assess vertical and horizontal dimensions and assuming that the flaming area at the base of the fire whirl - that is usually a circle - can be assimilated to the fuel bed or fuel container of the laboratory experiments we can use Equation 1 to estimate the properties of real scale fire whirls based on laboratory scale experiments. 
Our results together with those found in the literature are shown in Figure 5. The numbering used in Figure 5 is described in Table 1. Comparison with similar experiments showed that the properties of the flames generated with the FWG are similar to fire whirls produced in other laboratory studies. Our tests show that there is a transition from fire whirl to pool fire regime. Results from our tests with no forced flow and with forced flow (fire whirls) are presented with numbers 1 and 3, respectively. Results from fire whirls with liquid fuel (heptane) obtained by other authors are shown in this figure and fitted by curves 4 and 5; results from pool fires also with heptane are fitted by curves 6 and 7, and results from real fire whirls are shown by curve 9. Our results are in the middle of this range and seem to be in a transition between small laboratory scale tests and large scale field tests.

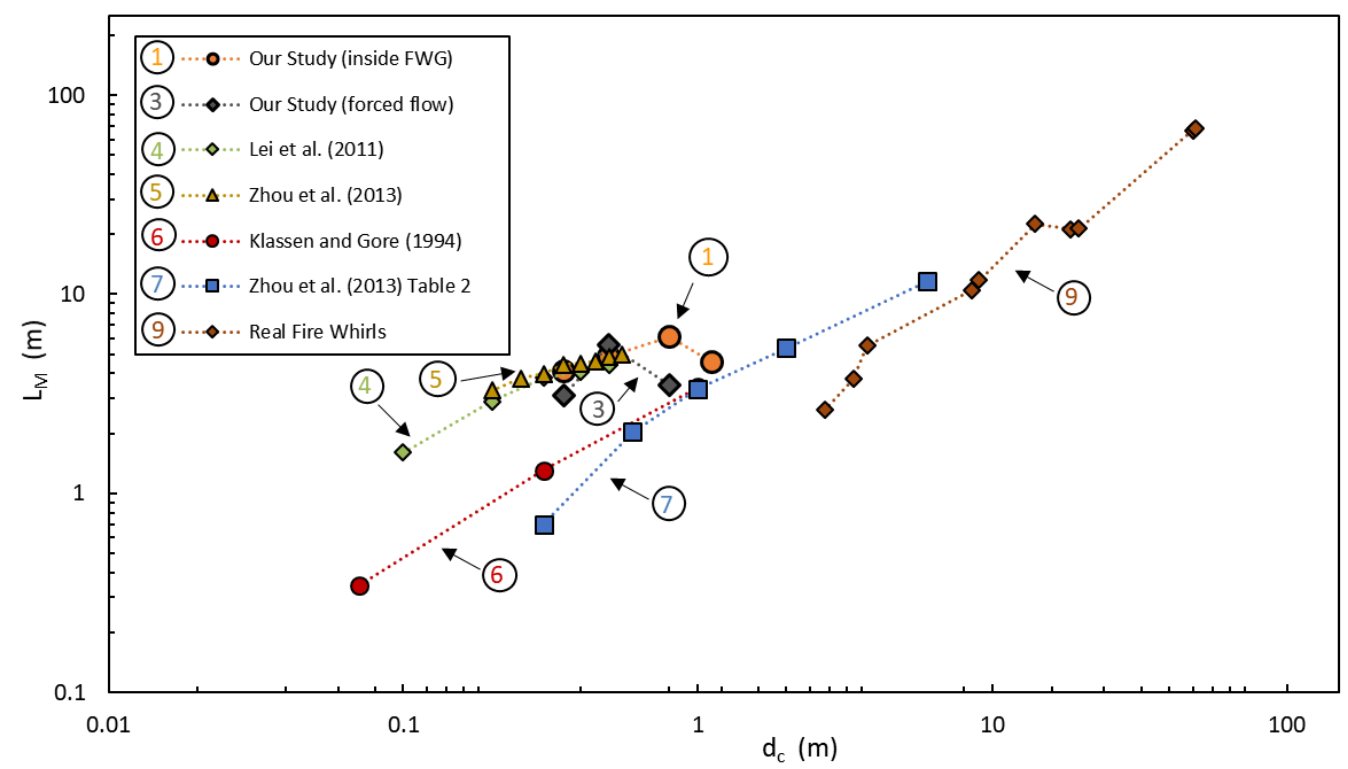

Figure 5 - Results of different studies of maximum flame height $\left(L_{M}\right)$ versus diameter of containers or the diameter of the base of the fire whirls. The sources of data that are indicated by a number are given in Table 1.

Table 1 - The coefficients that characterize the flame height vs the diameter of containers.

\begin{tabular}{lllll}
\hline Ref. & Description & $\mathrm{a}_{5}$ & $\mathrm{~b}_{5}$ & $\mathrm{R}_{5}{ }^{2}$ \\
& & & & \\
\hline (1) & Our Study (inside FWG) & 5.11 & 0.10 & 0.20 \\
(3) Our Study (forced flow) & 4.86 & 0.33 & 0.02 \\
(1) Lei et al. (2011) & 7.48 & 0.64 & 0.96 \\
(5) Zhou et al. (2013) & 7.48 & 0.44 & 0.97 \\
(6) Klassen and Gore (1994) & ${ }^{\mathrm{a}}$ & 3.48 & 0.86 & 0.99 \\
(7) Zhou et al. (2013) [Table 2] & 2.72 & 0.90 & 0.96 \\
(9) Real Fire Whirls & 1.09 & 1.06 & 0.98 \\
\hline${ }^{\mathrm{a}}$ Values from Table 2 of Lei et al. & & & \\
bValues from Table 2 of Zhou et al. & & &
\end{tabular}

\section{Conclusions}

In the present laboratory study medium-scale fire whirls were investigated. The major results are summarized as follows: 
- The parameters analysed change in the course of time as the mass loss is not constant during each test due to the use of natural fuels.

- The maximum values of the parameters analysed here are higher than those obtained in similar size tests performed with liquid fuels, as for example, heat power released by these fire whirls is higher than $1 \mathrm{MW}$ in comparison with $600 \mathrm{~kW}$ from fire whirls produced with liquid fuels.

- The burning rates of fire whirls depend on diameter of the container similarly to those of general pool fires, but with a much higher increasing rate versus the diameter of the container.

- The instantaneous values of fuel mass loss rate and flow velocity can be related to each other and it is possible to identify the process of formation and development of the fire whirl.

- The maximum value of mass loss rate and the average flame height increase generally with the forced flow velocity $(+11 \%)$. On the other hand, the average flame diameter decreases significantly $(-33 \%)$ with flow velocity.

- Our tests show that there is a transition from fire whirl to pool fire regime.

- Comparison with similar experiments showed that the properties of the flames generated with the FWG are similar to fire whirls produced in other laboratory studies and with full scale fire whirls. Relationship between the flame height and the size of the fuelbed is proposed.

\section{Acknowledgements}

This work was sponsored by the FCT under Projects FIREWHIRL "Vorticity Effects in Forest Fires" (PTDC/EMS - ENE/2530/2014) and Project FIREXTR "Prevent and prepare society for extreme fire events: the challenge of seeing the "forest" and not just the "trees" (PTDC/ATPGEO/0462/2014). The collaboration given to the experimental program that supports this paper by several collaborators is gratefully acknowledged.

\section{References}

Kuwana K, Sekimoto K, Saito K, Williams FA (2008) Scaling Fire Whirls, Fire Safety J., 43, 252 257.

Kuwana K, Morishita S, Dobashi R, Chuah KH, Saito K (2011) The burning rate's effect on the flame length of weak fire whirls. Proc. Combust. Inst. 33, 2425-2432.

Kuwana K, Sekimoto K, Minami T, Tashiro T, Saito K (2013) Scale-model experiments of moving fire whirl over a line fire, Proc. Combust. Inst. 34 (2), 2625- 2631.

Lei J, Liu N, Zhang L, Chen H, Shu L, Chen P, Deng Z, Zhu J, Satoh K, De Ris JL (2011) Experimental research on combustion dynamics of medium-scale fire whirl. Proc. Combust. Inst. 33, 2407-2415.

Martin R, Pendleton D, Burgess W (1976) Effect of fire whirlwind formation on solid fuel burning rates. Fire Technol. 12, 33-40.

Pinto C, Viegas DX, Almeida M, Raposo J (2017) Fire whirls in forest fires: An experimental analysis, Fire Safety Journal, Vol. 87, 37-48. (Doi:10.1016/j.firesaf.2016.11.004).

Viegas DX, Almeida M, Ribeiro LM, Raposo J, Viegas MT, Oliveira R, Alves D, Pinto C, Jorge H, Rodrigues A, Lucas D, Lopes S, Silva LF (2017) O complexo de incêndios de Pedrógão Grande e concelhos limítrofes, iniciado a 17 de junho de 2017. Centro de Estudos sobre Incêndios Florestais (CEIF/ADAI/LAETA), Universidade de Coimbra, Coimbra.

Zhou K, Liu N, Lozano JS, Shan Y, Yao B, Satoh K (2013) Effect of flow circulation on combustion dynamics of fire whirls. Proc. Combust. Inst. 34, 2617-2624. 


\title{
Experimental investigation on the burning of shrubs
}

\author{
J.B. Tramoni; F. Morandini; P.A. Santoni; V. Tihay-Felicelli; G. Gerandi \\ CNRS UMR 6134 SPE, Campus Grimaldi, BP 5220250 Corte, France, \{tramoni_jb@univ- \\ corse.fr,morandini_f@univ-corse.fr,santoni_p@univ-corse.fr, tihay@univ-corse.fr, \\ gerandi_g@univ-corse.fr\}
}

\begin{abstract}
This study aims to improve the knowledge on the combustion of Maquis shrubland for providing scientific bases for landscape management. The combustion dynamics of natural and isolated shrub was investigated. A typical shrub of Mediterranean vegetation, rockrose (cistus monspeliensis), was considered. This work was conducted in two steps. A structural characterization of shrubs of rockrose was firstly performed. The masses of the different class of particles composing the shrub, classified by range of thickness, were determined. Attention was paid to the composition of the crown and particularly to the thin particles (leaves, 0-2 mm diameter, more than $2 \mathrm{~mm}$ of diameter), which participate the most in the combustion process. The average mass distribution of these classes of particles along the height as well as their mass proportion in the crown were obtained. Then, the combustion dynamics of shrubs of rockrose was studied experimentally. Fire experiments were carried out with a Large Scale Heat Release apparatus which allows the measurement of the Heat Release Rate based on oxygen consumption principle. Two sets of two radiant panels were used to preheat and perform the ignition of the vegetation. The combustion study was based on the flammability criteria previously defined in the literature. The four criteria composing the flammability (ignitability, sustainability, combustibility and consumability) and their associated measurable parameters were investigated distinctively. New measurable parameters were proposed to study the combustibility and consumability. The study highlighted the influence of the moisture content and the ignition position on the parameters associated to the four criteria. Finally, the flammability was investigated as the combination of these criteria. A principal component statistical analysis was performed that revealed the relationships between the studied parameters. This analysis highlighted the presence of four flammability regimes depending on the position of ignition, time to ignition and the proportion of the thin particles within the crown.
\end{abstract}

Keywords: Wildland fire, calorimetry, flammability, vegetation characterization

\section{Introduction}

Different landscape managements are set-up to reduce the intensity and the impact of wildland fires. The treatment of these vegetation areas is based on the reduction of the amount of fuel at the ground level. However, the related practices are mainly performed from empirical knowledge and a quantitative approach relying on physical quantities is required by managers. Some experimental (Morvan and Dupuy 2004; Cruz et al. 2011) and numerical works have been devoted to the understanding of the combustion of shrubland but there remains a lack in measurement of physical quantities like the heat release rate to clearly understand their burning. More particularly the study of the combustion of isolated shrubs was poorly investigated (Tachajapong et al. 2014; Li et al. 2017). The influence of the Moisture content (MC) on the combustion dynamics was observed for Natural Douglas fir (Babrauskas 2006; Mell et al. 2009). A decrease of the effective heat of combustion was observed with an increase of MC. The effects of the ignition method, MC and bulk density on the rate of spread were studied for reconstructed chamise (Tachajapong et al. 2014; Li et al. 2017). An increase

Advances in Forest Fire Research 2018 - Page 436 
of the rate of spread within the crown was highlighted with increasing of bulk density or the decreasing MC.

In order to study the combustion dynamics of vegetative fuels, Anderson (Anderson 1970) introduced the flammability, which corresponds to the ability of vegetation to burn. The flammability was defined as a combination of three criteria: the ignitability, sustainability and combustibility. The ignitability is the capability of a material of being ignited. The sustainability represents how well the fire continues to burn once ignited. The combustibility is the intensity with which a fire burns. Martin et al. (Martin et al. 1993) added a fourth criterion to the flammability. The consumability corresponds to the mass of vegetation consumed during the combustion. Generally, the studies on the flammability focus on few criteria but they rarely consider all of them. Some physical quantities were used to characterize these four criteria. Thus, ignitability is usually studied with the time to ignition (Anderson 1970; Guijarro et al. 2002; Ganteaume et al. 2011). Sustainability is described by the flame duration (Plucinski and Catchpole 2001; Ganteaume et al. 2011). The flame temperature (Ganteaume et al. 2011; Santana and Marrs 2014), the flame height (Behm et al. 2004; Santana and Marrs 2014) and the rate of the fire spread (Guijarro et al. 2002; Ganteaume et al. 2011) are used to study the combustibility. Finally, the consumability is associated to the fuel consumption ratio (Guijarro et al. 2002; Santana and Marrs 2014) or the mass loss rate (Fonda et al. 1998; Santana and Marrs 2014). Various factors affect the parameters related to these criteria but the $\mathrm{MC}$ is the most influencing one. The increase of the time to ignition was observed with increasing MC (Weise et al. 2005; Pickett et al. 2010). Conversely, the decrease of consumption ratio (Dahale et al. 2013; Santana and Marrs 2014) and heat released (Babrauskas 2006) were observed with increasing MC. Furthermore, an increase in the proportion of thin particles, which participate actively in the combustion (Tihay et al. 2014; Barboni et al. 2017), lead to increases both the consumption ratio (Burrows 2001) and the heat released (Behm et al. 2004).

In this paper, the flammability of shrubs of rockrose (cistus monspeliensis) was investigated. A characterization of the distribution of the thin particles was performed to identify the potential mass of fuel involved during the flaming combustion processes. A particular attention was paid to the composition of the crown which is mainly composed of leaves and small diameter twigs. Experiments were then carried out on 41 shrubs to assess their flammability. The criteria introduced by Anderson (Anderson 1970) and Martin et al. (Martin et al. 1993) were first studied independently. The flammability was then considered as the combination of the four criteria through a statistical analysis (Principal Component Analysis).

\section{Materials and Methods}

\subsection{Vegetation Caracterization}

A shrub of rockrose is composed of particles with different thickness which participate in different ways in the combustion. The thin particles (thickness and diameter thinner than $2 \mathrm{~mm}$ ) are mainly involved in fires (Tihay et al. 2014) while larger particles (diameter greater than $6 \mathrm{~mm}$ ) are less prone to the thermal degradation. A characterization of the composition of shrub of rockrose in terms of class of particle was necessary for a better understanding of the combustion. Three shrubs of rockrose (cistus monspeliensis) with similar mass and dimensions were characterized. These shrubs were collected at Corte (Corsica, France) during autumn. In this study, the bases of the rockroses $(0.25 \mathrm{~m}$ above the roots) were not considered since they are composed of thick classes of particles that do not participate in the combustion (twigs with diameter in the range 6-25 mm) during wildfires. The mean height of the three rockroses was $1.23 \pm 0.12 \mathrm{~m}$, with a mean crown height of $0.35 \pm 0.07 \mathrm{~m}$ for a mean diameter of $0.68 \pm 0.06 \mathrm{~m}$. The mean dry mass of the shrubs was $0.76 \pm 0.03 \mathrm{~kg}$. Then, in order to quantify the different size of classes of particles constituting the rockroses, the shrubs were placed within a $1.45 \mathrm{~m}$ high, $0.9 \mathrm{~m}$ long and $0.9 \mathrm{~m}$ wide metallic frame spatially divided into 252 small cubes with sides of 
$15 \mathrm{~cm}$ (Figure 1). The position of each cube was indexed following the height. The vegetation contained in these small cubes was cut and oven dried at $60^{\circ} \mathrm{C}$ for $48 \mathrm{~h}$. Finally, for each cube, the vegetation particles were ranged according to the following diameter classes (leaves, twigs with diameter in between 0 and $2 \mathrm{~mm}, 2$ and $4 \mathrm{~mm}, 4$ and $6 \mathrm{~mm}, 6$ and $25 \mathrm{~mm}$ ) and weighed.

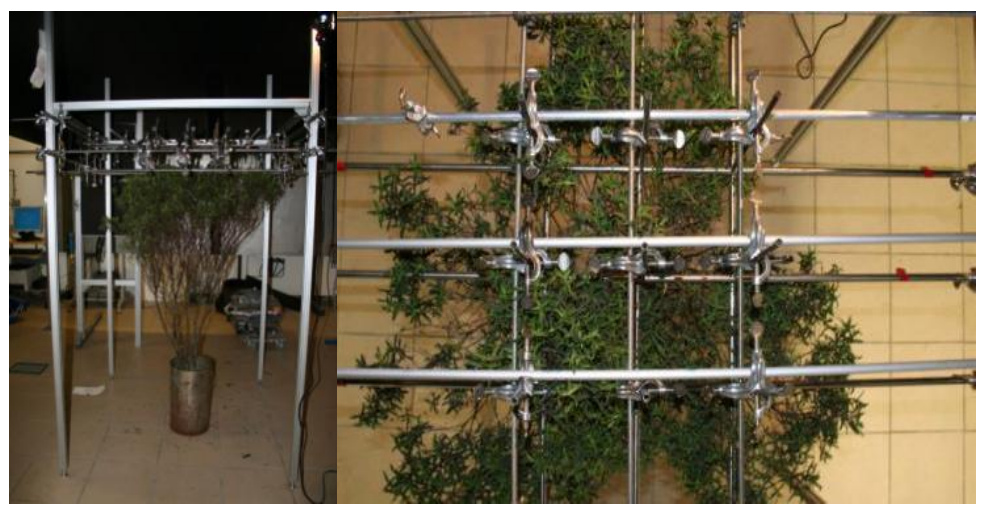

Figure 1 - Characterization device for the class of particles

\subsection{Combustion study}

A series of 41 shrubs of rockrose with similar dimensions and weight were collected within the same vegetation stand during spring, summer and autumn. Their average properties were: a $1.25 \mathrm{~m}$ height, a crown height of $0.3 \mathrm{~m}$, a crown diameter in the range of $0.6-0.8 \mathrm{~m}$ and a mass of $1.5 \mathrm{~kg}$ (excluding the $0.25 \mathrm{~m}$ base). The samples were conditioned $48 \mathrm{~h}$ in a room with an ambient air temperature of about $25^{\circ} \mathrm{C}$ and a relative air humidity of about $50 \%$ in order to obtain the required MC. This process allowed obtaining a leaf MC in the range of 3-20\% depending on the initial moisture content of the samples. It should be noted that the MC of the other classes of particles was higher (10$45 \%$ for the $0-2 \mathrm{~mm}$ diameter class).

The experiments were conducted with a $1 \mathrm{MW}$ Large Scale Heat Release apparatus (LSHR). A layout of the experimental design is provided in Figure 2. Live shrubs were placed under a $3 \mathrm{~m} \times 3 \mathrm{~m}$ hood with a $1 \mathrm{~m}^{3} / \mathrm{s}$ smoke extraction system. Two set of two radiant panels, heated at $773 \mathrm{~K}$, were used to preheat the vegetation samples up to ignition. To maximize the radiation impinging on the shrub samples, the two sets of radiant panels were used in orthogonal configuration. The emitted heat flux was close to $20 \mathrm{~kW} / \mathrm{m}^{2}$. The time to ignition was recorded for each test. The measurement of the heat release rate (HRR) was determined following the formulation derived by Parker (Parker 1984) using gas volume fraction and mass flow rate of exhausted gas. The samples were positioned on a load cell in order to record the mass loss during the thermal degradation. The measurements sampling rate was $1 \mathrm{~Hz}$.

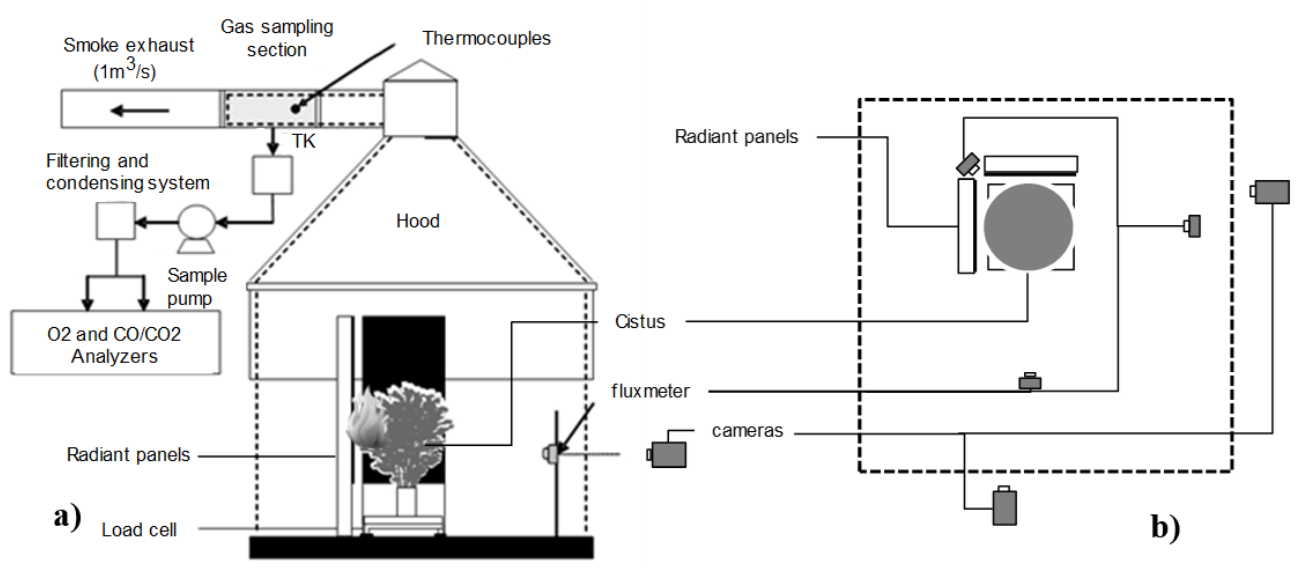

Figure 2 - Layout of the experimental set-up, a) side view b) top view 


\section{Results and discussion}

\subsection{Vegetation characterisation}

A particular attention was paid to the proportion of thin particles (leaves and twigs with diameter between 0-2 $\mathrm{mm}$ ) composing the shrub. As a first step, the mass proportion of the different classes of particles was estimated. It was defined for a class $k$ as:

$$
\Gamma_{k}=\frac{m_{k d}}{m_{d}}
$$

where $m_{k d}$ represents the dry mass of the $k$ class and $m_{d}=\sum_{k} m_{k d}$ is the total dry mass of vegetation sampled. Experimental results show a similar distribution of particles for the three shrubs of rockroses characterized. It can be considered that a shrub of rockrose is composed of $22.1 \pm 0.8 \%$ of leaves, $26.1 \pm 2.1 \%$ of twigs with a diameter between 0 and $2 \mathrm{~mm}$ and $51.8 \pm 2.0 \%$ of twigs with a diameter greater than $2 \mathrm{~mm}$.

In addition, during the burning experiments, the crown was the main part of the shrub that was consumed. Its composition was thus methodically measured in order to estimate the consumption of each class of particles. The crown represents $35.0 \pm 0.4 \%$ of the mass of the shrub of rockrose. It contains $83 \%$ of the total mass of leaves and $41 \%$ of the total mass of $0-2 \mathrm{~mm}$ diameter twigs. In addition, it should be highlighted that $72.0 \pm 8.0 \%$ of the total mass of particles thinner than $2 \mathrm{~mm}$ (participating actively in the combustion) is located in the crown. These results were used to assess the consumption ratio.

A second characterization was carried out on shrub of rockroses sampled in summer. A change in the mass proportion of leaves was observed with a decrease of the leaves mass ( $2 / 3$ less). Furthermore, flowers containing seeds, have grown during this season. Their mass was almost equal to that of fallen leaves.

\subsection{Ignitability}

Ignitability was defined as the time needed to ignite a shrub submitted to the heat flux emitted by the radiant panels. When the vegetation is exposed to a heat flux from an external source (radiant panels in this study), its temperature increases and the thermal degradation begins. For the experimental setup used in the present study, the ignition occurred at the edge of the shrubs, where the concentration of combustible gases near an incandescent particle is rich enough. Then, the fire spreads mainly horizontally across the crown. The leaves firstly ignited. The flame then engulfed the other classes of particles. Therefore, relationship between the leaves $\mathrm{MC}\left(M C_{l}\right)$ and time to ignition was investigated. Figure 3 shows the time to ignition as a function of $M C_{l}$ for different seasons.

Three tends were observed. For shrubs of rockroses sampled in autumn and summer, $t_{i g n}$ increases with increasing $M C_{l}$. However, the quantity of leaves is lower in the crown during summer, which causes a decrease in the mass of pyrolysis products. Thus, an increase of $M C_{l}$ during summer led to a more significant increase in time to ignition than in autumn. Finally, very long ignition times are obtained for shrubs of rockroses sampled in spring for which $M C_{l}$ was the lowest. In this case, juvenile leaves (absent in summer and winter) seem to have a flame retardant effect (probably related to their composition), significantly delaying the inflammation of the sample. 


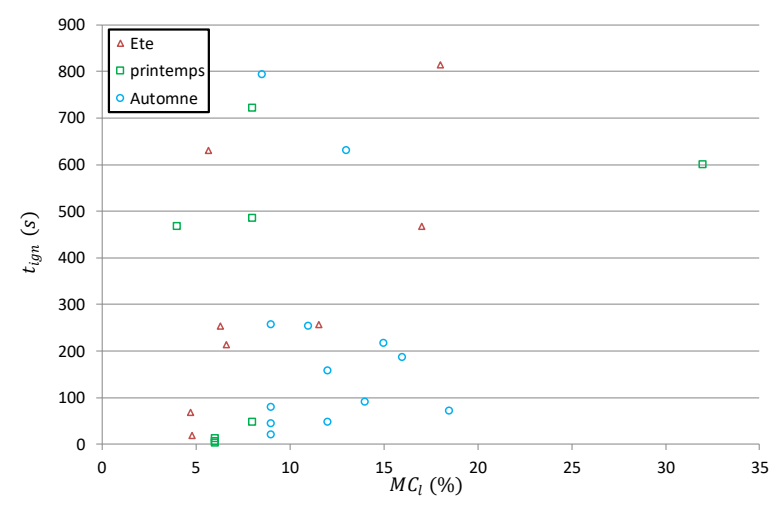

Figure 3 - Time to ignition in function of the leaves moisture content

\subsection{Combustibility}

Combustibility was defined as the intensity of the combustion. The combustion dynamic was analyzed with the following two characteristics of the HRR curves over time: the growth rate just after ignition and the established burning phase. It should be pointed out that summer rockroses were not considered in this section. Indeed, the presence of flowers end seeds alters the combustion dynamics and cannot be studied using this method.

\section{Growth phase}

To study the growth phase (HRR $>10 \mathrm{~kW}$ ), the HRR was fitted to the following relationship over time (NFPA $72[22])$ :

$$
H R R=\alpha t^{2}
$$

where $\alpha$ is the growth factor of the combustion.

The analysis of the data allowed to distinguish three combustibility types according to the value of $\alpha$ (Figure 4). For low values of $\alpha(\alpha<0.2)$, the combustion regime was slow and the fire propagated step by step across the crown. An intermediate combustion regime could be observed for medium values of $\alpha(0.2<\alpha<0.4)$. The fire spread was horizontal from the ignition zone towards the opposite edge of the crown. Finally, for high values of $\alpha(\alpha>0.4)$, the fire spread horizontally at a fast rate throughout the entire fine particles of the crown. The fire then spread vertically downward consuming larger diameter particles. The behavior of summer shrubs of rockrose is not presented here because their phenological characteristics lead to a very different combustion dynamic that will be presented hereafter.

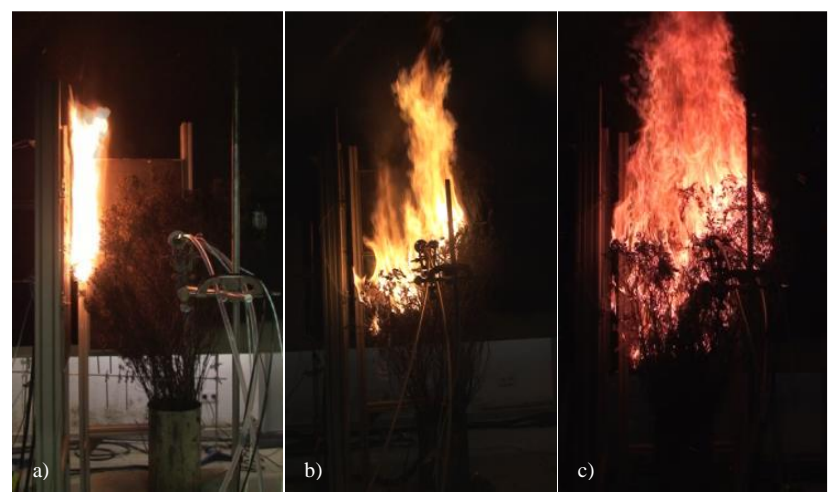

Figure 4 - Regimes of combustion 30s after the ignition for a) $\alpha<0.2$; b) $0.2<\alpha<0.4$; c) $0.4<\alpha$

\section{Established burning phase}

Two parameters were used to characterize the established burning phase: the peak HRR (obtained from the HRR over time) and the duration of the flaming combustion (obtained from observation). 
Different combustion regimes and peaks of HRR were observed as a function of the growth factor (Figure 5). The slow combustion rate $(\alpha<0.2)$ was obtained for $19 \%$ of the experiments. This combustion regime was characterized by a low value of the average peak HRR of $100 \pm 5 \mathrm{~kW}$ and long times of combustion of $100 \pm 14 \mathrm{~s}$. The intermediate combustion regime $(0.2<\alpha<0.4)$, corresponding to $38 \%$ of the tests, showed a medium value of the peak HRR of $188 \pm 27 \mathrm{~kW}$ and combustion times of the order of $77 \pm 10 \mathrm{~s}$. Finally, the fast combustion was obtained for $43 \%$ of the experiments and it exhibited

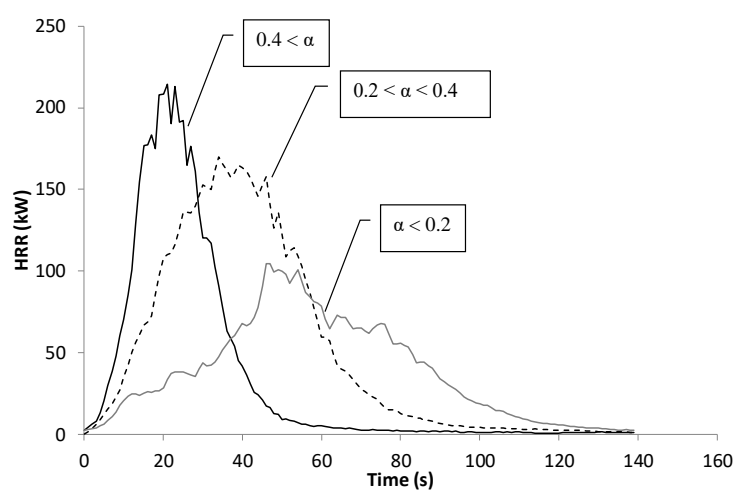

Figure 5 - Typical curve of HRR as function of time for the three regimes of combustion

HRR peaks of the order of $257 \pm 63 \mathrm{~kW}$ and short combustion times of $60 \pm 19 \mathrm{~s}$. In order to understand the cause of the different combustion regimes, a particular attention was paid to the ignition zone. We found that the growth coefficient strongly depends on the amount of fuel present above the ignition zone. When ignition occurred in the upper layer of the crown, without fuel above, a low growth coefficient and a slow combustibility was observed. When ignition occurred in between the middle and the top of the crown, with a significant presence of fuel, a more intense flaming was observed at ignition time and a moderate combustibility ensued. The greater the amount of fuel, the greater the growth coefficient. Finally, when ignition occurred in between the bottom and the middle of the crown, the convective heat transfers, by contact of the flame with the crown, caused a high growth coefficient, leading to the fast combustion regime. The different ignition zones are shown in Figure 6 . In the following, an ignition that occurred in between the bottom and the middle of the crown will be called a favorable ignition whereas an ignition located above this last zone will be called as unfavorable.

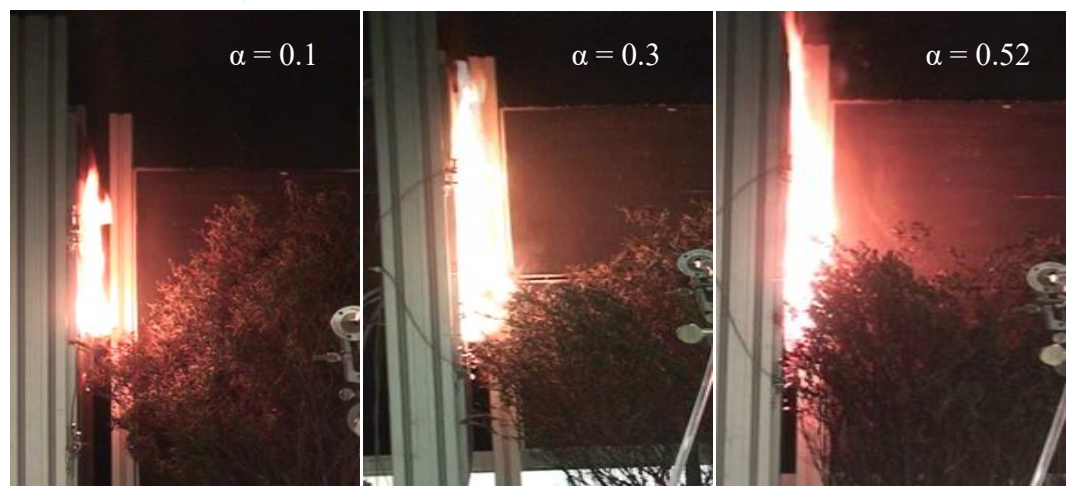

Figure 6 - Position of the ignition point in the shrub a) top of the crown, b) in the crown, c) at the bottom of the crown

\subsection{Sustainability}

Sustainability was defined by how well a fire will continue to burn once ignited. The main parameter used to characterize this criterion was the flame duration, $t_{f l}$. The results presented in the previous section suggested the existence of a relationship between the flame duration and the peak HRR. Flame durations greater than $80 \mathrm{~s}$ and up to $120 \mathrm{~s}$ were obtained for low peaks of HRR $(\leq 150$ 
$\mathrm{kW}$ ), while the shortest durations (around $40 \mathrm{~s}$ ) were obtained for high peaks of HRR ( $\geq 210 \mathrm{~kW}$ ). Figure 7 shows that the flame duration decreases with increasing peak HRR. These results can be explained by analyzing the fire spread within the crown for the different positions of ignition. When ignition occurred in the lower part of the crown, the heat transferred by convection and radiation from the ignition zone to the vegetation above involved the entire height of the crow. The fire quickly engulfed the crown of the shrub leading to a short duration of flame and a high HRR ensued. An ignition at the edge and middle of the crown height led to a flame that impacted a fewer amount of fuel located above. It resulted in a lower rate of spread, a longer residence time of flame and a weaker HRR. Finally, when the ignition occurred in the upper part of the crown, the fire spread mainly dominated by radiation. The fire was stopped when it encountered a small fuel discontinuity. The propagation was slow and combustion times were long. Thus, the sustainability is linked to the HRR and subsequently to the combustibility.

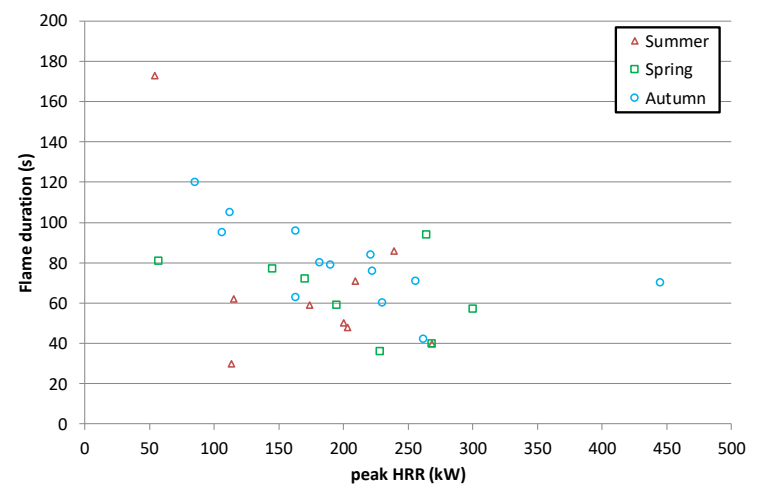

Figure 7 - Flame duration as function of the HRR peak for the different seasons

In addition, Figure 7 shows a shift in the trend of flame duration as function of peak HRR with the seasons. For a given peak HRR, the flame duration decreases when going from autumn to spring and then summer. This shift can be explained by the mass decrease of the finer classes of particles during spring and summer.

\subsection{Consumability}

Consumability was measured by the quantity of fuel consumed by the fire. From the characterization study, the amount of initial and remaining fuel of each particles class was estimated (taking into account the moisture content of the different classes). But for the sake of clarity only the whole burned mass fraction $\eta$, will be presented here. It defined as:

$$
\eta=1-\frac{m_{r d}}{m_{d}}
$$

where $m_{r d}$ and $m_{d}$ are the residual dry mass and initial mass on dry basis, respectively. The average mass lost for these specific experiments was $0.52 \pm 0.2 \mathrm{~kg}$ which represents $25 \pm 8 \%$ of the mass of the shrub. The results displayed in Figure 8 suggest a decrease of the fuel consumed with increasing $\mathrm{MC}$ of the leaves for shrub rockroses sampled during autumn. The results concerning the shrub of rockroses collected in summer do not follow this trend because of the presence of seeds that alter the combustion dynamics (see section combustibility). 


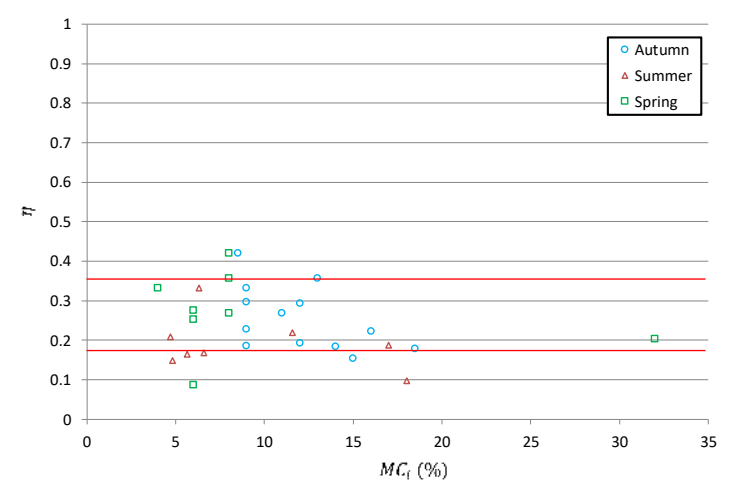

Figure 8 - Burnt mass fraction in function of the leaf moisture content for the different seasons

Experimental results showed that the mass lost was quasi exclusively located in the crown which is mainly composed of fine particles (leaves and 0-2 mm diameter twigs) representing 35\% of the total dry mass of the shrub. We recall that the characterization study revealed that the leaves and the $0-2$ $\mathrm{mm}$ diameter twigs in the crown represent $18 \%$ and $10 \%$ of the total mass of the rockrose, respectively. Therefore, a burned mass fraction below $18 \%$ indicates that the leaves in the crown have not all been consumed and the fire did not spread across the whole crown. For a burned mass fraction between 18 and $35 \%$, the measurements shown that all the leaves and part (or totality for 35\%) of the 0-2 mm particles class in the crown were consumed. Finally, a burned mass fraction greater than $33 \%$ indicates a consumption of the crown and a part of the classes of particles in the range of 0-6mm (mainly 0-2 $\mathrm{mm}$ and 2-4 $\mathrm{mm}$ particles classes) within the intermediate part of the rockrose. The classes of particles larger than $4 \mathrm{~mm}$ were partly burned only when the peak of HRR was high enough $(250 \pm 10 \mathrm{~kW})$. It should be noticed that the shrub of rockrose was burned wet and the initial dry mass was not measured. It was estimated from the wet mass, moisture content and distribution of each class of particles. Thus, the uncertainty related to the estimation of the dry mass affect directly the calculation of the burned mass fraction.

The current results allowed distinguishing three regimes for the consumability criterion: a low consumability regime for which only a part of the leaves inside the crown was consumed; a medium consumability regime for which the leaves and part of the 0-2 mm diameter twigs were consumed in the crown and a high consumability regime for which the crown was totally burned. Furthermore, for this last regime it should be noticed that some of the fine particles also burned under the crown of the shrub as well as some of the large diameter particles when HRR was high.

\subsection{Flammability}

Flammability was finally considered as the combination of the four criteria studied hereabove. In order to observe the impact of the different combustibility and consumability regimes on the flammability, a Principal Component Analysis (PCA) was performed. The parameters considered to study the criteria (ignition position, flame duration, HRR peak, growth coefficient and burnt mass fraction) were used as input parameters of the statistical analysis. It should be pointed out that the combustion dynamics of summer shrubs of rockroses was not considered in this statistical analysis between their reaction to fire was much more different that for the shrubs collected during the other seasons. The PCA provides information on the possible links and correlations between the input parameters. It allowed to cluster the experiments according to the parameter values in order to assess the flammability. Results extracted three main components that explain $87 \%$ of the total variance. The main component (PC 1) explains 54\% of the variance while the components PC 2 and PC 3 explain $19 \%$ and $14 \%$ of the variance, respectively. The projection of the combustion experiments on the factorial map in the plane (PC 1, PC 2) highlighted four types of flammability (Figure 9). The first group (circles) corresponds to the low flammability with $\alpha<0.2$, a low HRR peak $(83.9 \pm 20 \mathrm{~kW})$, a very long flame duration $(100 \pm 14 \mathrm{~s})$ and a weak burnt mass fraction $(18.9 \pm 3.0 \%)$, mainly involving 
leaves and 0-2 mm twigs diameter. The second group (squares) corresponds to a medium flammability with a value of $\alpha$ between 0.2 and 0.4 , a medium peak of HRR $(188 \pm 27 \mathrm{~kW})$, a long flame duration $(77 \pm 10 \mathrm{~s})$ and a moderate fuel consumption $(23 \pm 5 \%)$ including leaves and larger particles up to 2 $\mathrm{mm}$ in diameter. A high and fast flammability was associated to the third group (diamonds), characterized by $\alpha$ greater than 0.4 , a high peak of HRR $(228 \pm 15 \mathrm{~kW})$, a short flame duration (44 \pm $9 \mathrm{~s})$ and a moderate fuel consumption $(25 \pm 6 \%)$ involving the same classes of particles as the medium flammability. Finally, the last group (triangles) corresponds to the high and mass consuming flammability with $\alpha$ greater than 0.4 , a very high HRR peak $(384 \pm 99 \mathrm{~kW})$, a long flame duration $(70$ $\pm 14 \mathrm{~s}$ ) and a high mass consumption $(32 \pm 4 \%)$. It should be noted that, for the fourth group, twigs with diameter greater than $4 \mathrm{~mm}$ were burned while for the other groups, only thinner particles were involved in combustion.

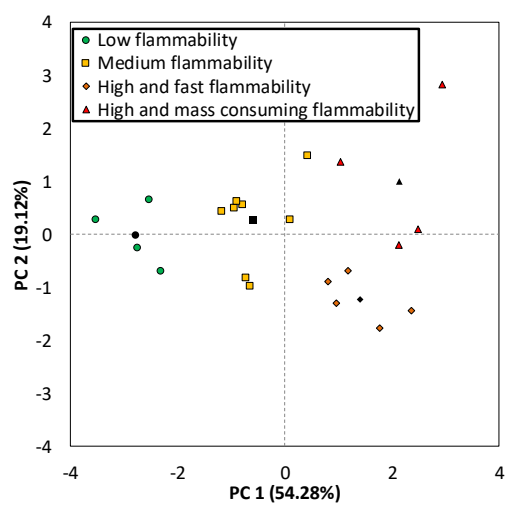

Figure 9 - Projection of the fire tests on the factorial plane (PC1 and PC2) of the PCA

The different flammability could be explained (Figure 10) by the ignition position, the time to ignition as well as the proportion of the thin particles within the crown. Favorable ignition (see combustibility section) results in a high flammability, while unfavorable ignition causes a low or medium flammability. In the case of high flammability, the difference between "high and fast" and "high and consuming" is caused by long time to ignition. For long time to ignition, the moisture content of the various particle classes is considerably reduced when ignition occurs, resulting in high mass consumption, including twigs classes with a diameter greater than $4 \mathrm{~mm}$. For shorter ignition times (high and fast flammability), large diameter twigs (greater than $4 \mathrm{~mm}$ ) do not receive enough heat to achieve desiccation and reach ignition. The differentiation of low and medium flammability is due to the structure of the vegetation. Indeed, among the 41 collected samples, some of them had a lower bulk density than the selected reference type of shrub. We noted a lower value of bulk density of leaves $\left(0.9 \pm 0.2 \mathrm{~kg} / \mathrm{m}^{3}\right)$ for the low flammability regime in comparison to the medium flammability one $\left(1.4 \pm 0.3 \mathrm{~kg} / \mathrm{m}^{3}\right)$. The low flammability was obtained for this type of shrubs. The reduction in the mass of thin particles (leaves and 0-2 $\mathrm{mm}$ ) leads to the decrease of the heat released, which limits the spread of the fire in the shrub.

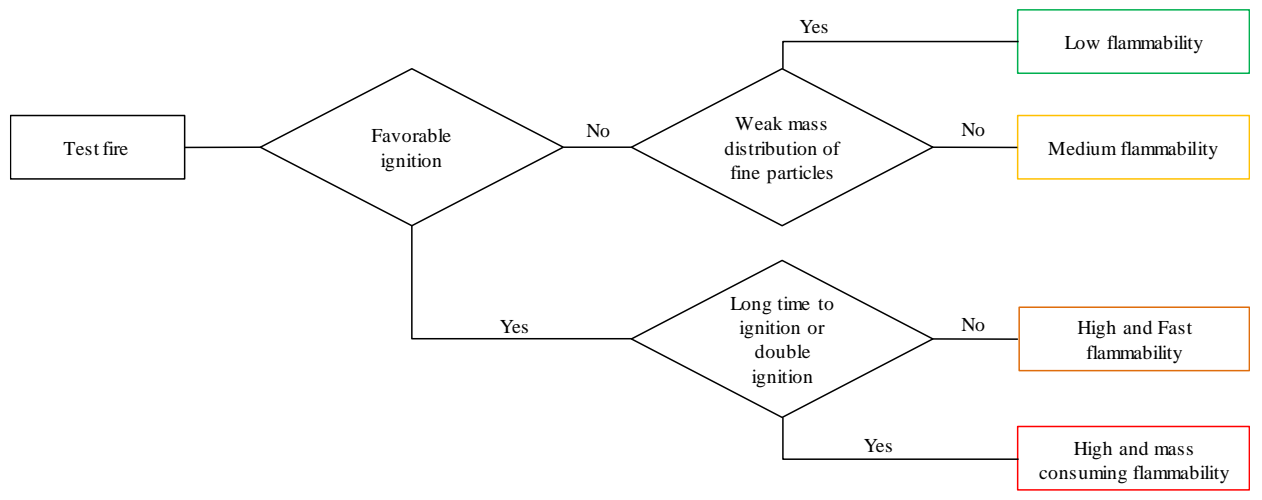

Figure 10 - Types of flammability 


\section{Conclusion}

In this study, the structure and flammability of shrubs of rockrose were investigated. Theses shrubs were characterized in order to determine their structure and the mass proportion of the different particle classes. A particular attention was paid to the composition of the crown which is the main part of the shrubs to be consumed. The characterization highlighted a very close repartition of the particles for similar shrubs. A $1.5 \mathrm{~m}$ high shrub of rockrose is composed of $48.2 \pm 2.9 \%$ of thin particles mainly located in the crown.

The flammability of the shrub of rockrose was then analyzed using the ignitability, sustainability, combustibility and consumability criteria and the associated measurable parameters. The ignitability, linked to the time to ignition, decreases with the leave MC. Combustibility and sustainability, studied with the HRR during the combustion and the flame duration, are influenced by the ignition position. The lower the position of ignition in the crown, the higher the peak HRR and the shorter the flame duration. The consumption ratio, corresponding to the consumability, increases with the decrease of the leave MC. Finally, a statistical analysis was carried out on the studied parameters in order to observe the global flammability of the shrubs of rockroses. The PCA highlighted four types of flammability depending on the ignition zone, the ignition HRR and the burned mass of thin particles.

It should be noted that more experiments with summer shrubs of rockroses are needed to further assess their behavior for this season. Indeed, the presence of a non-negligible dead leaves litter was observed during plant sampling in the field resulting in a different mass distribution of the leaves. Due to the presence of falling firebrands generated by flaming seeds, the interactions between fire spreading across the shrub's crown and bed of leaves need to be taken into account. Finally, a study of the combustion dynamics of simultaneously several burning shrubs will be necessary in order to get closer to real fire conditions.

\section{Bibliography}

Anderson HE (1970) Forest fuel ignitibility. Fire Technology 6, 312-319. doi:10.1007/BF02588932.

Babrauskas V (2006) Effective heat of combustion for flaming combustion of conifers. Canadian Journal of Forest Research 36, 659-663. doi:10.1139/x05-253.

Barboni T, Leonelli L, Santoni P-A, Tihay-Felicelli V (2017) Influence of particle size on the heat release rate and smoke opacity during the burning of dead Cistus leaves and twigs. Journal of Fire Sciences 35, 259-283. doi:10.1177/0734904117709964.

Behm AL, Duryea ML, Long AJ, Zipperer WC (2004) Flammability of native understory species in pine flatwood and hardwood hammock ecosystems and implications for the wildland-urban interface. International Journal of Wildland Fire 13, 355-365.

Burrows ND (2001) Flame residence times and rates of weight loss of eucalypt forest fuel particles. International Journal of Wildland Fire 10, 137-143.

Cruz MG, Butler BW, Viegas DX, Palheiro P (2011) Characterization of flame radiosity in shrubland fires. Combustion and Flame 158, 1970-1976. doi:10.1016/j.combustflame.2011.03.002.

Dahale A, Ferguson S, Shotorban B, Mahalingam S (2013) Effects of distribution of bulk density and moisture content on shrub fires. International Journal of Wildland Fire 22, 625. doi:10.1071/WF12040.

Dungan, KW (2003) Performance-Based Approach to Designing and Analyzing Fire Detection Systems. In 'NFPA 72® National Fire Alarm Code®(2007 ed)' pp.72-194

Fonda RW, Belanger LA, Burley LL (1998) Burning characteristics of western conifer needles. Northwest science (USA). 
Ganteaume A, Marielle J, Corinne L-M, Thomas C, Laurent B (2011) Effects of vegetation type and fire regime on flammability of undisturbed litter in Southeastern France. Forest Ecology and Management 261, 2223-2231. doi:10.1016/j.foreco.2010.09.046.

Guijarro M, Hernando C, Díez C, Martínez E, Madrigal J, Lampin-Cabaret C, Blanc L, Colin PY, Pérez-Gorostiaga P, Vega JA (2002) Flammability of some fuel beds common in the SouthEuropean ecosystems. In 'IV Int. Conf. For. Fire Res.',

Li J, Mahalingam S, Weise DR (2017) Experimental investigation of fire propagation in single live shrubs. International Journal of Wildland Fire 26, 58. doi:10.1071/WF16042.

Martin R, Gordon D, Gutierrez MA, Lee D, Molina-Terrén DM, Schroeder RA, Sapsis DB, Stephens SL (1993) Assessing the flammability of domestic and wildland vegetation. Unpublished, doi:10.13140/RG.2.1.3999.3680.

Mell W, Maranghides A, McDermott R, Manzello SL (2009) Numerical simulation and experiments of burning douglas fir trees. Combustion and Flame 156, 2023-2041. doi:10.1016/j.combustflame.2009.06.015.

Morvan D, Dupuy JL (2004) Modeling the propagation of a wildfire through a Mediterranean shrub using a multiphase formulation. Combustion and Flame 138, 199-210. doi:10.1016/j.combustflame.2004.05.001.

Parker WJ (1984) Calculations of the Heat Release Rate by Oxygen Consumption for Various Applications. Journal of Fire Sciences 2, 380-395. doi:10.1177/073490418400200505.

Pickett BM, Isackson C, Wunder R, Fletcher TH, Butler BW, Weise DR (2010) Experimental measurements during combustion of moist individual foliage samplesA. International Journal of Wildland Fire 19, 153-162.

Plucinski MP, Catchpole WR (2001) Predicting ignition thresholds in litter layers. In 'MODSIM 2001 Int. Congr. Model. Simul. Dec.', 967-971

Santana VM, Marrs RH (2014) Flammability properties of British heathland and moorland vegetation: Models for predicting fire ignition. Journal of Environmental Management 139, 88-96. doi:10.1016/j.jenvman.2014.02.027.

Tachajapong W, Lozano J, Mahalingam S, Weise DR (2014) Experimental modelling of crown fire initiation in open and closed shrubland systems. International Journal of Wildland Fire 23, 451. doi:10.1071/WF12118.

Tihay V, Santoni P-A, Barboni T, Leonelli L (2014) Experimental and theoretical study of diameter effect on the ignition of cistus twigs. 'Adv. For. Fire Res.' pp. 179-189. (Imprensa da Universidade de Coimbra) doi:10.14195/978-989-26-0884-6_19.

Weise DR, White RH, Beall FC, Etlinger M (2005) Use of the cone calorimeter to detect seasonal differences in selected combustion characteristics of ornamental vegetation. International Journal of Wildland Fire 14, 321-338. 


\title{
Fire growth patterns in the 2017 mega fire episode of October 15, central Portugal
}

\author{
Marc Castellnou ${ }^{1}$; Nuno Guiomar²; Francisco Rego ${ }^{3}$; Paulo M. Fernandes*4 \\ ${ }^{1}$ Cos de Bombers Generalitat. DGPEiS. Crta Cerdanyola Vallés 08029.Barcelona (España), \\ \{incendi@yahoo.com\} \\ ${ }^{2}$ Institute of Mediterranean Agricultural and Environmental Sciences, University of Évora, Núcleo \\ da Mitra. Apt.94, 7002-554 Évora (Portugal), \{nunogui@uevora.pt \} \\ ${ }^{3}$ Centro de Ecologia Aplicada Baeta Neves, Universidade de Lisboa. Tapada da Ajuda, Lisboa \\ (Portugal), \\ ${ }^{4}$ Centro de Investigação e Tecnologias Agroambientais e Biológicas, Universidade de Trás-os- \\ Montes e Alto Douro. Quinta de Prados, 5001-801 Vila Real (Portugal) \{pfern@utad.pt*\}
}

\begin{abstract}
Increasingly large fires are occurring in southern Europe, especially at its westernmost part, where a humid Mediterranean climate combines with rough topography, flammable vegetation types, undermanaged forest and high ignition rates. These fires have huge socioeconomic and environmental impacts, including the potential loss of human lives. Portugal was struck by an exceptionally severe fire season in 2017 that included a multiple mega fire event ( $200 \mathrm{kha})$ in mid-October, unprecedented in Europe in terms of season and the extent of area burned in a very short period. This new phenomenon, arguably caused by climate change, needs to be characterized and understood to inform future fire management. We describe how five mega fires (or fire complexes) have developed in central Portugal on October 15-16, 2017, and assess their major drivers. We described fire weather through the Canadian FWI System, the C-Haines index and atmospheric profiles, retrieved weather data, and estimated fine dead fuel moisture content. Seven extremely large fire scars resulted from the October 15 event. We selected five of them (18,503-48,462 ha) for fire growth reconstruction. To describe fire spread we compiled data from various sources, including satellite imagery, official reports and fire suppression data, photos and videos, and people accounts. When satellite hotspots presented high density, the Fire Radiative Power of these points was interpolated to identify the approximate location and shape of the active fire front. We built a fire location chronology and interpolated the resulting points to support the final production of a map of hourly fire isochrones. For each hour period we calculated burned area and fire growth rate. Fire danger rating was Extreme, with most weather stations displaying FWI $>50$, expressing the joint effects of critical fuel aridity and hurricane Ophelia and its advection of warm and dry air from Africa. C-Haines index reached 10-11 (on a maximum of 13) and the moisture content of fine dead fuels was 3-6\%. Forests comprised most (78\%) of the burned surface, with Pinus pinaster generally dominating. Among fires, an average of $73 \%$ of the area burned for the first time since 1975 or had not experienced fire for the last 19 years or more, indicating potentially high fuel load. Fires spread followed the SW-NE axis and often crowned, with spotting as an important spread mechanism that allowed fire percolation through fragmented forest landscapes to impact important wildland-urban interfaces. Estimates of maximum hourly rates of spread varied from 5 to $9 \mathrm{~km} \mathrm{hr}-1$ between fires, corresponding to 50-90 MW m-1 for a typical fuel load. Initial fire growth was fast and dominated by strong winds and very low fuel moisture content. Wind subsided subsequently, relative humidity increased, and a sequence of pyro-convection events developed, coinciding with the fastest, and also erratic, fire spread periods. Overall, an average of 10,000 ha burned per hour between 16:00 and 05:00 of the following day. The findings from this reconstruction and the explanation of the events will assist in improved fire management policies and operational guidelines, from fuel management to fire preparedness and suppression.
\end{abstract}

Keywords: extreme fire behaviour, fire weather, pyroconvection, rate of spread 


\section{Introduction}

Increasingly large fires are occurring in southern Europe, especially at its westernmost part, where a humid Mediterranean climate combines with rough topography, flammable vegetation types, undermanaged forest and high ignition rates. These fires have huge socioeconomic and environmental impacts, including the potential loss of human lives. Portugal was struck by an exceptionally severe and prolonged fire season in 2017 that included the deadly Pedrogão Grande fire in June, a significant number of very large fires (>5000 ha) throughout summer, and a multiple mega fire event ( 200 kha) in mid-October, which was unprecedented in Europe in terms of season and the extent of area burned in a very short period. Comparable, but less severe previous events did occur in Spain (146 kha from the $2^{\text {nd }}$ to the $9^{\text {th }}$ of July 1994), Portugal ( $100 \mathrm{kha}, 1^{\text {st }}$ to $2^{\text {nd }}$ of August 2003), and Greece (63 kha in six days of August 2007). This is a new phenomenon, arguably caused by climate change, and needs to be documented, characterized and understood to inform future fire management policies and practices.

Here we describe how five mega fires (or fire complexes) have developed in central Portugal on October 15-16, 2017 and assess their major drivers. Implications for fire management are discussed.

\section{Methods}

We described fire weather through the indices of the Canadian FWI System (12:00 UTC), the CHaines index and atmospheric profiles, retrieved air temperature, relative humidity and wind (speed and direction) data from the IPMA network of automatic weather stations, and estimated hourly fine dead fuel moisture content. Seven extremely large fire scars resulted from the October 15 event. We selected five of them for fire growth reconstruction, respectively the inland fires of Lousã (45,505 ha), Arganil-Seia complex (48,462 ha) and Sertã (32,356 ha), and the coastal fires of Pataias (18,600 ha) and Quiaios (18,503 ha). To describe fire spread over time we compiled and cross-referenced data from a variety of sources, including satellite imagery (MODIS, VIIRS), official reports and fire suppression data, georeferenced photos and videos, and people accounts. When satellite hotspots presented high density (typically because of strong wind or high flame residence time), the Fire Radiative Power of these points was interpolated to identify the approximate location and shape of the active fire front. We built a fire location chronology on Google Earth and interpolated the resulting points using Radial Basis Functions to support the final production of a map of hourly fire isochrones. For each hour period we calculated burned area and fire growth rate and estimated fireline intensity (FLI, $\mathrm{kW} \mathrm{m}^{-1}$ ) using observed meteorology and the fire spread rate resulting from those isochrones. Finally, to understand better driving factors of pyroconvection, we calculated the Pf and Pw numbers (Rothermel 1991) to assess weather fire was plume dominated or wind driven.

\section{Results}

Fire danger rating was Extreme on the $15^{\text {th }}$ of October, with most weather stations displaying FWI $>50$, and a few at FWI $>80$, expressing the joint effects of critical fuel aridity and hurricane Ophelia and its advection of warm and dry air from northern Africa. C-Haines index reached 10-11 (on a maximum of 13) and the moisture content of fine dead fuels was 3-6\%. Forests comprised most (78\%) of the burned surface, with maritime pine (Pinus pinaster) dominating (50-97\% cover), except in the Lousã fire, where blue gum (Eucalyptus globulus) prevailed (45\%). Among fires, an average of $73 \%$ of the area burned for the first time since 1975 or had not experienced fire for the last 19 years or more, indicating potentially high fuel load.

Fires spread following initially the SSE- NNW axis to turn gradually to the SW-NE axis, when their maximum growth occurred. They often crowned, with spotting as an important spread mechanism that allowed fire percolation through fragmented forest landscapes to impact significant wildland-urban

Advances in Forest Fire Research 2018 - Page 448 
interfaces. Estimates of maximum hourly rates of spread varied from 5 to $9 \mathrm{~km} \mathrm{hr}^{-1}$ between fires, corresponding to $50-90 \mathrm{MW} \mathrm{m}^{-1}$ for a typical fuel load of $20 \mathrm{tha}^{-1}$.

Initial fire growth was fast and dominated by strong winds and very low fuel moisture content (Figure 1). Wind subsided subsequently (after 16:00), relative humidity increased, and a sequence of pyro-convection events developed, especially after 19:00, that coincide with the fastest, and also erratic, fire spread periods (Figure 1). Overall, an average of 10,000 ha burned per hour between 16:00 and 05:00 of the following day.

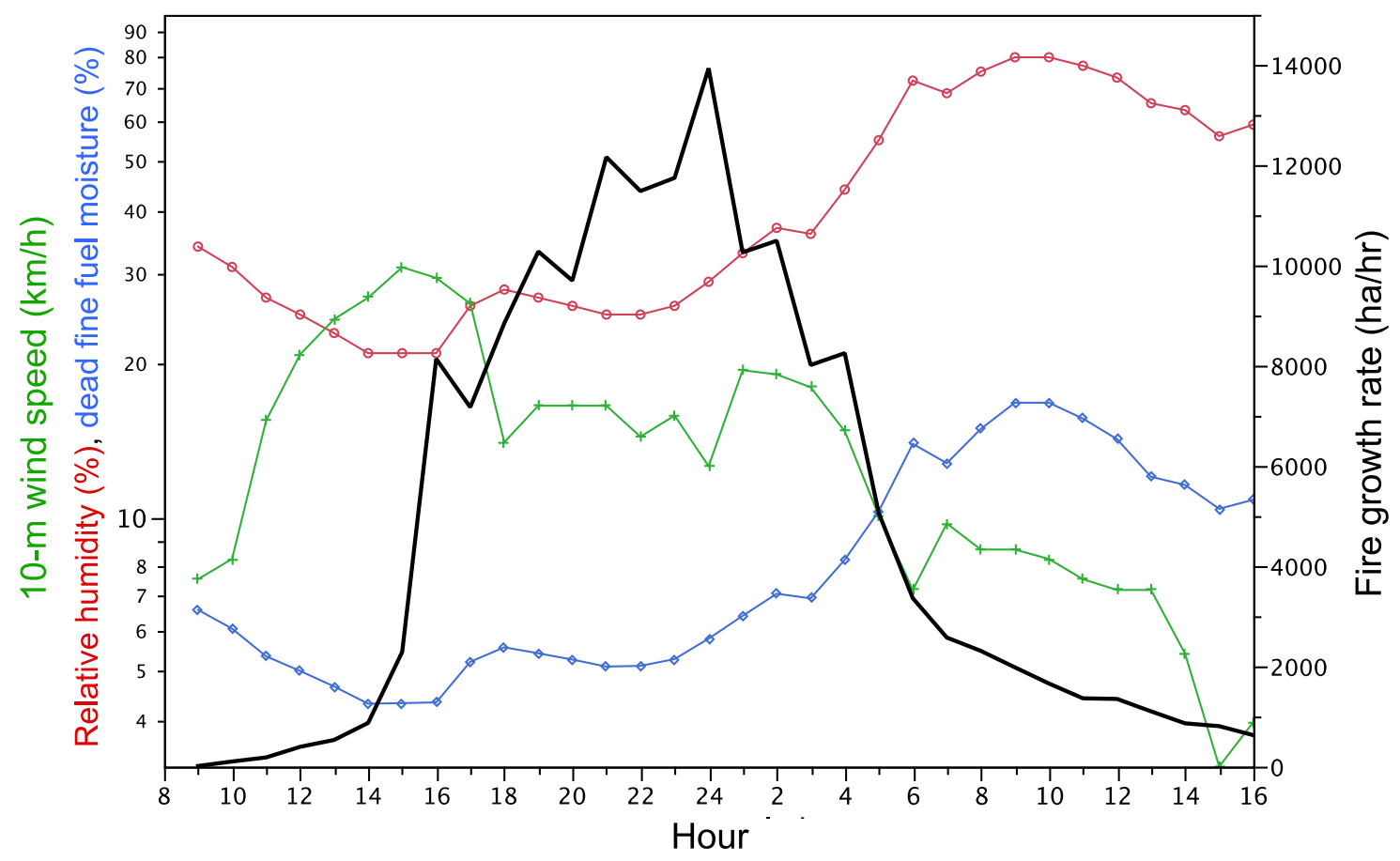

Figure 1 - Combined fire growth rate of the studied fires and fire weather elements at Viseu from 9:00 of October 15 to 16:00 of October 16, 2016. The Viseu weather station was selected because it simultaneously reflects the Ophelia effect on wind speed and the downdraft after midnight.

Looking at FLI fire by fire we found two types of fire spread patterns on the afternoon-evening of the 15th of October. On the coastal region, fires were of the 'wind driven' type at their peak in the afternoon, in contrast with the inland fires, which were 'plume dominated' (Rothermel, 1991) when they made their major growth late on the $15^{\text {th }}$ or early in the $16^{\text {th }}$. Figure 2 shows the pattern of coastal fires. Wind driven fires peaked around 16:00 to decline sharply after 19:00 with the slowing down of wind and the increase in relative humidity, displaying the expected pattern. The inland Lousã fire in Figure 3 shows a totally different pattern. It also peaks initially around 16:00 with the wind, but it is under weaker wind and increasing relative humidity that its higher FLI and subsequent growth peak occur. This corresponds to a plume dominated fire and a transition from PyroCu to PyroCb and the subsequent and associated downdraft phenomenon that allows the generation of firestorm-type fires that dramatically increase the burned surface (Fromm et al 2010). 


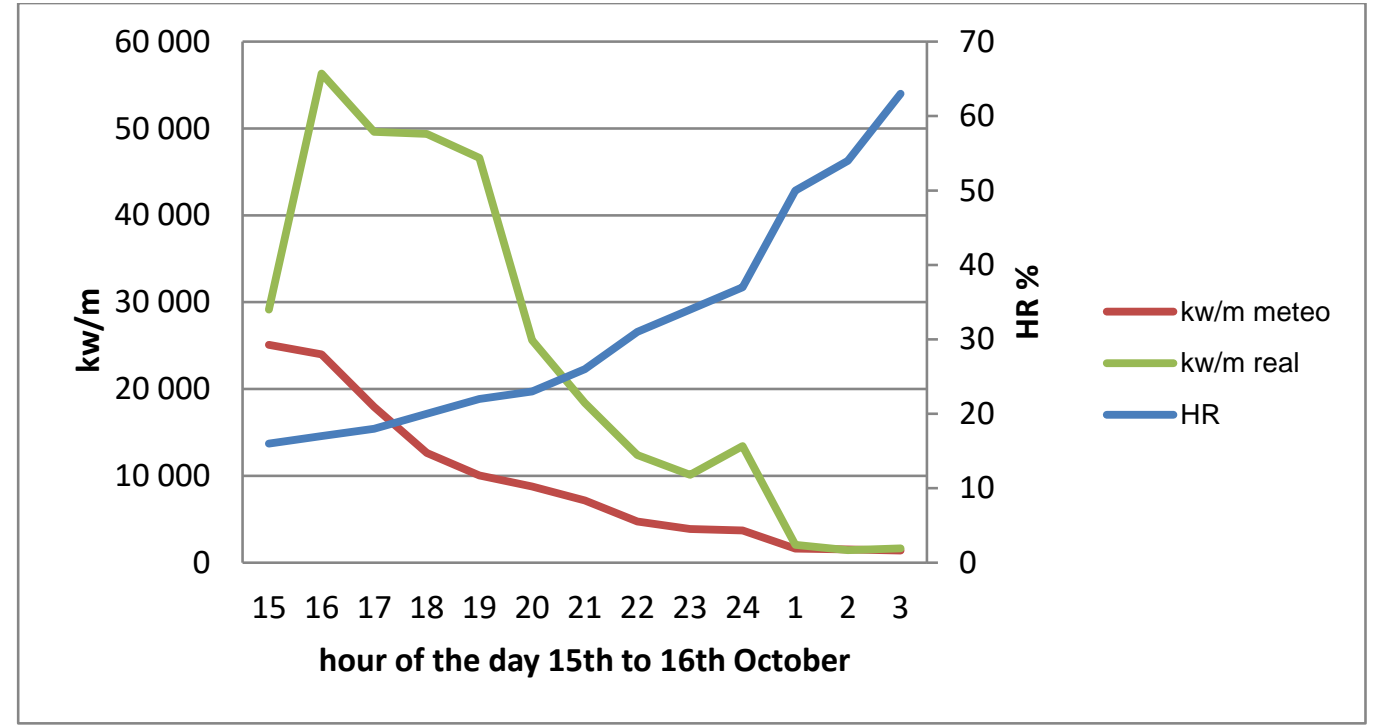

Figure 2 - Relation between reconstructed and simulated FLI for the coastal fire of Quiaios. HR is the air relative humidity.

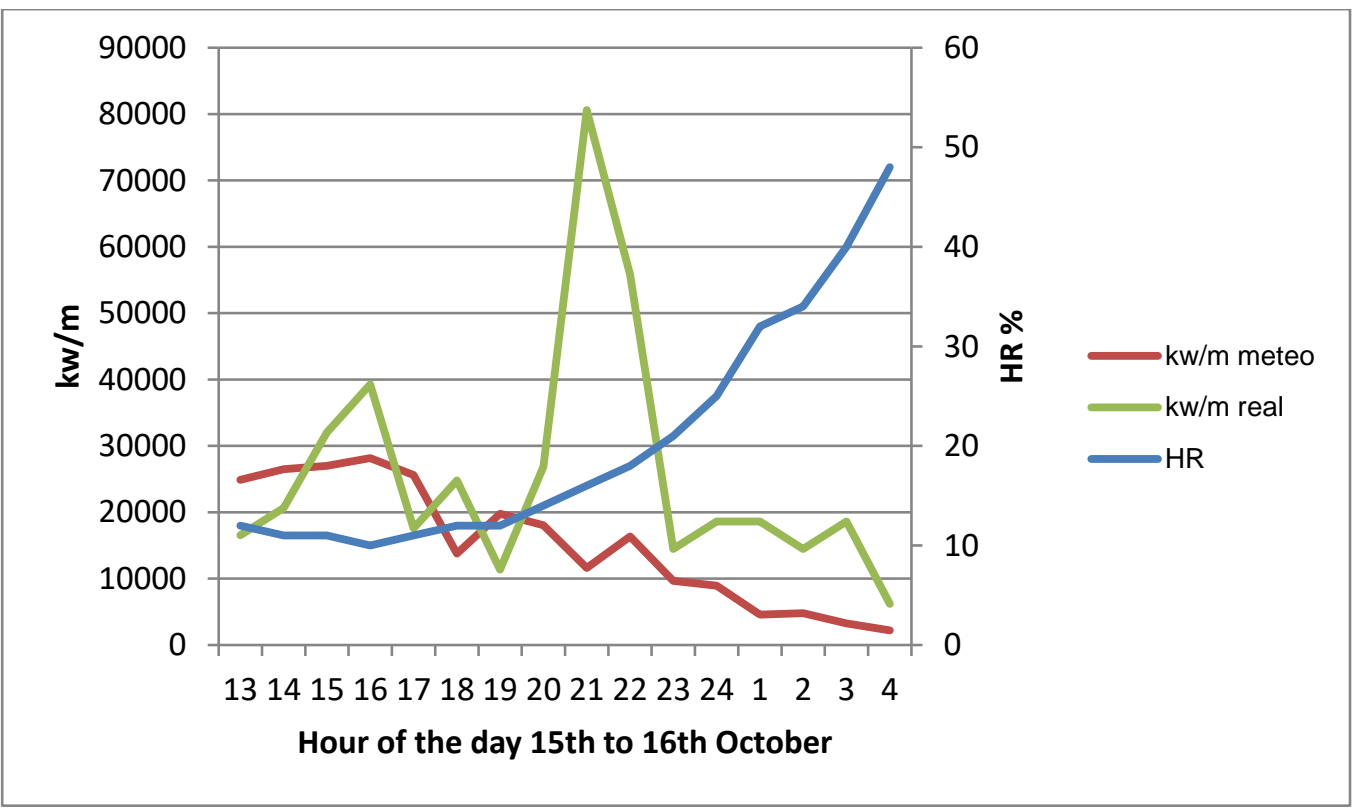

Figure 3 - Relation between reconstruted and simulated FLI for the inland fire of Lousã. HR is the air relative humidity.

\section{Discussion}

The burning afternoon and evening of the $15^{\text {th }}$ of October 2017 was driven by different factors which combined to create what is seen in Figure 1, favouring different types of spread at each moment and increasing because that unpredictability among population and fire service. That huge amount of surface burned was achieved by a chronology of events that included the hurricane-like storm Ophelia first and weaker winds with higher instability later. This created the already explained different types of fire behaviour, but it is the piling-up and especially the large and interacting land surfaces on fire that produced the huge difference. This is described in Table 1 and explains how the wind first expanded long narrow fires and then a series of transitions of PyroCu to PyroCb created indraft and downdraft events to expand those fires with gale strong winds and erratic movements mainly on the 
axis from SW to NE. In fact, what was seen in Pedrogão Grande in June was repeated here after Ophelia's passage for three large fires and was sustained from 19:00 on the $15^{\text {th }}$ to $04: 00$ on the $16^{\text {th }}$.

Table 1 Description of the moments of fire behaviour found when reconstructing the fire events.

\begin{tabular}{|c|c|c|c|}
\hline Hour & & Coastal & Inland \\
\hline $\begin{array}{l}11: 00 \text { to } \\
14: 00\end{array}$ & \multirow[b]{2}{*}{ : } & & Wind driven fires \\
\hline $\begin{array}{l}14: 00 \text { to } \\
15: 00\end{array}$ & & $\begin{array}{l}\text { Narrow long wind driven fires } \\
\text { Fires in Quiaios and Pataias show } \\
\mathrm{Pw}>\mathrm{Pf} \text { and sometimes } \mathrm{Pw}=\mathrm{Pf} \text {. }\end{array}$ & $\begin{array}{l}\text { Arganil and Lousã fires are shadowed by Sertã fire } \\
\text { where instability increases. They start to show the } \\
\text { first PyroCb. They create a huge indraft that } \\
\text { 'attracts' Sertã to them. Sertã has Pw }>\text { Pf due to that. }\end{array}$ \\
\hline $\begin{array}{l}16: 00 \text { to } \\
19: 00\end{array}$ & 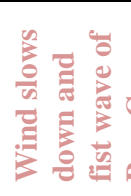 & $\begin{array}{l}\text { Wind is slowing down and Pw keeps } \\
\text { close to Pf. PyroCu development but } \\
\text { still not evolving to PyroCb, peaking at } \\
5000-6000 \mathrm{~m} \text {. Long distance spotting. }\end{array}$ & $\begin{array}{l}\text { Wind is slowing down and Pw keeps close but is } \\
\text { slightly lower than Pf. We have PyroCu going on } \\
\text { that still not evolves to PyroCb, peaking at 5000- } \\
6000 \mathrm{~m} \text {. Long distance spotting. }\end{array}$ \\
\hline $\begin{array}{l}\text { 19:00 to } \\
23: 00\end{array}$ & 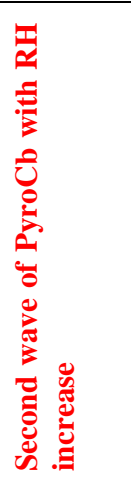 & $\begin{array}{l}\text { PyroCu are huge and spotting distance } \\
\text { increases. Low jet is disappearing and } \\
\text { this avoids transition to PyroCb. High } \\
\text { dispersion of PyroCu. With RH } \\
\text { increasing fires remain wind driven } \\
\text { and start slowing down but they are } \\
\text { already extremely large } \\
\text { The south fire is creating a small } \\
\text { transition to PyroCb when the first } \\
\text { humidity comes in. The northern } \\
\text { convection plume, shadowed by the } \\
\text { south plume is not registering this } \\
\text { moment. }\end{array}$ & $\begin{array}{l}\text { Increase of humidity allows PyroCu transition to } \\
\text { PyroCb and Pf doubles Pw. Big indrafts and fire to } \\
\text { fire interactions are generated. Huge fire storm } \\
\text { builds up its momentum. } \\
\text { From 19:00 to 23:00 the three fires have huge pyro } \\
\text { convective activity. This is a feedback of sequential } \\
\text { and sustained PyroCb outflows. }\end{array}$ \\
\hline $\begin{array}{l}\text { 00:00 to } \\
\text { 04:00 }\end{array}$ & 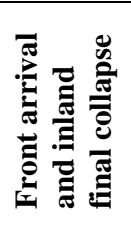 & $\begin{array}{l}\text { Wind driven fires. RH is high and FLI } \\
\text { relatively low. Fire spread is totally } \\
\text { impelled by the Pw }\end{array}$ & $\begin{array}{l}\text { The Lousã PyroCb, the last and northernmost one, } \\
\text { collapses and drives all fires to NE. It has been the } \\
\text { longer lasting fire of the event. This downdraft } \\
\text { changes the nearby Arganil fire into a wind driven } \\
\text { fire with } \mathrm{Pw} \gg>\mathrm{Pf} \text {, just when the general wind is at } \\
\text { its lowest. }\end{array}$ \\
\hline
\end{tabular}

It is important to note that the wind created long and fast-growing fires with substantial pyroconvective activity that favoured long distance spotting. This pyroconvective wind driven activity dominated fires in the first two stages. Under those wind driven plumes, higher instability could appear and that pushed convection columns higher and higher, creating huge PyroCu than then were rolling down, spreading and spotting away. Those typical and well know columns induced fast fire growth due to long distance and massive spotting, as observed during those moments in central coastal and inland Portugal.

Later, with Ophelia fading away and instability increasing while wind was calming, and relative humidity was increasing, it is when third stage appears. Fires on the coast were huge and unstoppable but they were starting their decline, unlike inland. Here, fires were able to access free convection in their atmosphere, so they transitioned into PyroCb, increasing indraft and downdraft or outflows that kept pushing the fires further away. Out of those fires, Sertã was being pushed by the indraft of Lousã. 


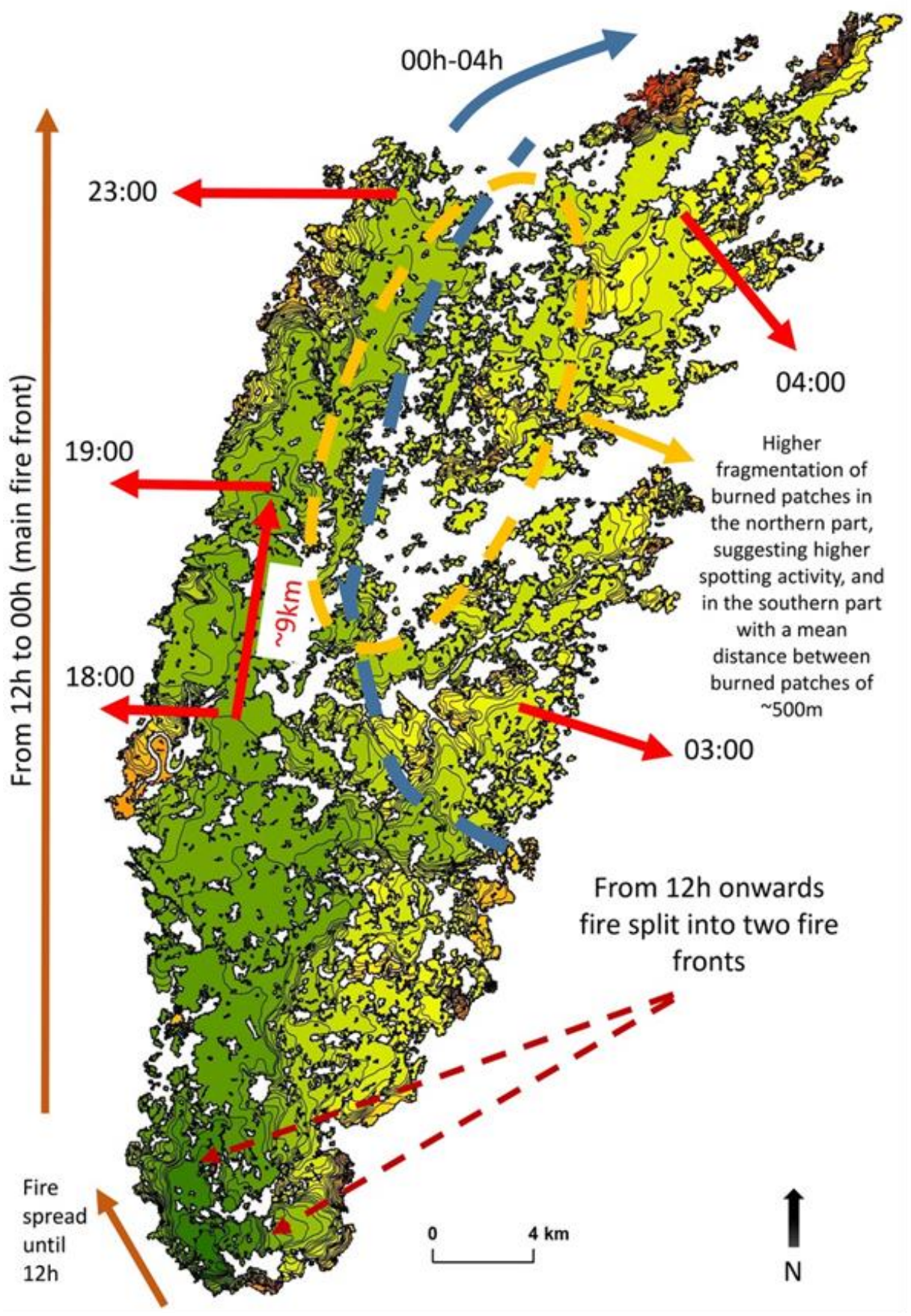

Figure 4 - Spread of the Vilarinho (Lousã) fire. Note the growth after 00:00 (blue line) into patchy fuels. This was possible because at that moment the outflows of pyroCb collapse were pushing the spread opening to the ENE.

Finally, the last moment developed about midnight, when the largest PyroCb (Lousã) collapsed, expanding itself and then ending the event. Those events of interacting outflow and indraft from different moments and the collapse of PyroCb can explain the high percolation of those fires in areas with low fuel connectivity (Figure 4). 


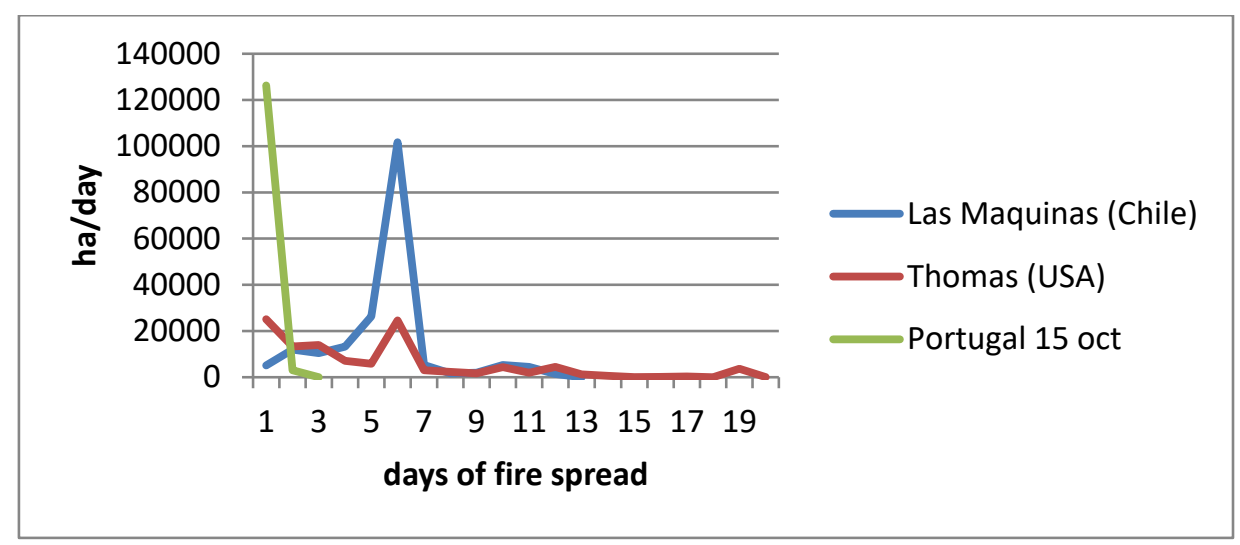

Figure 5 - Daily growth rate for three of the largest fires globally during 2017.

\section{Conclusion}

Some have argued that we are not facing new fire management challenges and that those were already present in other places. In Figure 5 we can see how Portugal's fires rate as the fastest growing among the largest of the 2017's season globally. This is really bad news for Europe, as our unmanaged landscapes are becoming a threat.

What we saw in Portugal during 2017 is a change on Europe's fire behaviour concept in terms of rate of spread, FLI and fire growth rate. Out of the large fires described, the second type, which matches the so-called 'plume dominated wildfire' is the most dangerous for European citizens and countries due to its unpredictability in terms of when the event will occur and where it will go. This is not new in terms of the phenomenon and had been seen in 2003 in Portugal, 2007 in Greece, and 2012 in Spain on a substantially smaller scale. Having those events in an overcrowded country implies pressure upon the forest firefighting culture to change to a civil protection approach. It is not anymore a matter of forest and landscape; it is a matter of national security and civil protection. It is within this view that forest management resources need to be fostered to protect our society and economy.

Given the observed fire behaviour and how it developed, the Atlantic facade and western central Europe are under pressure for those types of fires and phenomenon. It means the characteristic high net primary production turns into extreme fuel availability due to heat and drought extremes and this is the type of fire behaviour, with PyroCu to Pyro $\mathrm{Cb}$ transition, that is expected under the new fuel aridity that climate change is bringing in. For years to come, the approach to forest management and firefighting must change and be perceived from this new perspective. Landscape management rather than fuel management is needed to impact fire spread at such a large scale. Most importantly is that other countries will have conditions to reproduce such fire behaviour but will be inexperienced at this new reality. More than ever, no time should be wasted in thinking that fire suppression will 'make it' and a valuable lesson has been learnt and should be disseminated.

\section{References}

Fromm, M., Lindsey, D. T., Servranckx, R., Yue, G., Trickl, T., Sica, R., Godin-Beekmann, S. 2010. The untold story of pyrocumulonimbus. Bulletin of the American Meteorological Society, 91, 11931209, doi:10.1175/2010BAMS3004.1

Rothermel, R.C. 1991. Predicting behavior and size of crown fires in the northern Rocky Mountains. USDA Forest Service Research Paper INT-438. 


\title{
Fire intensity, individual protective clothing and firefighting safety
}

\author{
António M. Raimundo ${ }^{1 *}$; A. Virgílio M. Oliveira ${ }^{2}$; Divo A. Quintela ${ }^{1}$; A. Rui Figueiredo ${ }^{1}$ \\ ${ }^{1}$ ADAI-LAETA, Department of Mechanical Engineering, Faculty of Sciences and Technology of \\ the University of Coimbra, Portugal. \{antonio.raimundo@dem.uc.pt*\} \\ ${ }^{2}$ Department of Mechanical Engineering, Coimbra Institute of Engineering of Polytechnic Institute \\ of Coimbra,Portugal. \{avfmo@mail.isec.pt\}
}

\begin{abstract}
The main objective of this work is to establish useful guide-lines for secure firefighting, namely the evaluation of the influence of fire intensity, personal protective clothing properties and application of body cooling techniques on safety during firefighting operations. The physiological reaction of men exposed to these conditions is obtained by numerical simulation using a software implemented by the authors.

The protocol adopted submits the firefighter to four phases: neutral, pre-firefighting, firefighting and recovering. On the fire-fighting phase, the properties of the firefighter protective clothing depend on the test settings (values of intrinsic insulation between 0 and 9 clo were considered). In this phase, the firefighter, doing hard work $(M=3.0 \mathrm{met})$, is exposed to an impinging radiation in an environment with $T_{a i r}=T_{m r}=50^{\circ} \mathrm{C}$. Incident radiation fluxes $\left(I_{r a d}\right)$ between 0 and $40 \mathrm{~kW} \mathrm{~m}^{-2}$ were considered. On the recovering period, the firefighter is far from the fire and has a low level of activity $(M=1.2 \mathrm{met})$. Six alternative body cooling techniques were analyzed, which are applied only during the recovering period: passive cooling; use of an ice jacket; immersion of hands and forearms in water at $20^{\circ} \mathrm{C}$ and at $10^{\circ} \mathrm{C}$; use of an ice jacket reinforced by immersion of hands and forearms in water at $10^{\circ} \mathrm{C}$; and all body (except head and neck) immersion in water at $25^{\circ} \mathrm{C}$.

From a safety point of view, the most important indicator seems to be the time delay for the onset of heat stroke (fainting). The threshold for introversion is a good indicator to assess the exposure periods that must not be exceeded.

As expected, the predicted results indicate that the firefighter thermal stress level depends primarily on the intensity of the incoming radiation, on the time of exposure to it and on the clothing thermal insulation. Time safety intervals decrease with the increase of the impinging thermal radiation and increase with the insulation of the ensemble. However, clothing high thermal insulation can hide dangerous situations. Thus, a convenient control of the duration of firefighting periods it is crucial.

For the situation of $I_{\text {rad }}=5 \mathrm{~kW} \mathrm{~m}^{-2}$ and an active fighting period of 20-minutes, the cooling techniques tested can be ordered from the less effective to the highest one as: $6^{\text {th }}$ ) passive cooling; $5^{\text {th }}$ ) use of an ice jacket; $4^{\text {rd }}$ ) immersion of hands and forearms in water at $20^{\circ} \mathrm{C} ; 3^{\text {rd }}$ ) the same but with water at $10^{\circ} \mathrm{C} ; 2^{\text {nd }}$ ) use of an ice jacket reinforced by immersion of hands and forearms in water at $10^{\circ} \mathrm{C}$; and $1^{\text {st }}$ ) all body (except head and neck) immersion in water at $25^{\circ} \mathrm{C}$. Among the tested, the strategy of immersion the entire body in water at $25^{\circ} \mathrm{C}$ is undoubtedly substantially much more effective than the others.
\end{abstract}

Keywords: Safety firefighting; Human thermoregulation modelling; Times for the onset of heat-related illness

\section{Introduction}

The high level of physical work required during firefighting leads to high rates of metabolic heat production. Additionally, the heat fluxes gained by the body due to the impinging of thermal radiation from flames, the exchange of infrared radiation with the surroundings, the convection with surrounding hot gas (air and smoke), the conduction from heated grounds and the solar radiation should also be considered. If, as a result of this combination of heat gains, the hypothalamus temperature exceeds certain values, important and undesirable incidents can affect firefighters (Carter et al. 1999; Mündel 
et al. 2006; Lopez et al. 2008; Barr et al. 2009; Raimundo and Figueiredo 2009), namely introversion (violent sweating, misleading, amnesia, etc.), superficial skin damages (pain and burn degree 1), heat stroke (fainting, stop of sweating, central nervous system alteration, etc.) and permanent injuries (burn degree greater than 1, brain damage or, in the more serious cases, death).

Wearing high thermal insulating protective clothing reduces the gain of external heat, but also blocks the release of the heat produced and restricts movements. Therefore, firefighting safety demands the wearing of clothing with thermophysical properties appropriated to each firefighting feature, which include the assessment of the required activity level and of the thermal environment. These matters are described in the literature and are known by the firefighters which are aware of the individual protective clothing that should be used in each kind of fire (Sharkey 1999; McLellan \& Selkirk 2005; Bröde et al. 2010; Carballo-Leyenda et al. 2017; McQuerry et al. 2018).

To satisfy all the demands of the firefighting activity, it might be necessary to use recovery strategies for physical recuperation and for body cooling as a way to attenuate the thermal stress (Carter et al. 1999; Selkirk et al. 2004; Giesbrecht et al. 2007; Chou et al. 2008; Lopez et al. 2008; Barr et al. 2009; Hostler et al. 2010; Colburn et al. 2011; Abreu et al. 2014). The purpose of the body cooling process after the firefighting activity is to restore the thermophysiological balance of the body in the shortest time possible and thus avoid harmful health effects and, at the same time, try to help the recovery of the individual for any subsequent activity.

Nevertheless, despite the significant knowledge regarding the fire intensity, the level of activity required during firefighting, the individual protective clothing to be used by the firefighter and the application of recovering techniques, some topics still remain unanswered, namely what is the maximum time of exposure for safe firefighting? The objective of this work is to contribute to this issue.

Experimental studies concerning the thermophysiological response of firefighters carrying out typical firefighting activities (both structural and wildland), wearing the recommended protective clothing and exposed to environments that are representative of these fires have already been published (Carballo-Leyenda et al. 2017; Fontana et al. 2017; McQuerry et al. 2018; among others). However, due to safety reasons of the individuals involved, these trials always terminate before firefighters reach levels of hyperthermic stress with the potential to cause heat-related illnesses. This drawback is overcome by using validated models to simulate the human body thermophysiological response when exposed to very hot environments, with high levels of impinging thermal radiation.

\section{The Calculation Tool}

To obtain the results presented in this study, a computer program implemented by the authors (Raimundo and Figueiredo, 2009; Raimundo et al. 2012 \& 2015) is used for the simulation of heat and mass transfer and thermophysiological response of a male firefighter exposed to extreme environmental conditions, such as those occurring in the proximity of a high intensity fire front. This program is composed by several modules. However, for present purposes only the following ones are used ( $i$ ) person thermophysiological response, (ii) heat and water transport through the clothing, (iii) heat (by conduction, convection and radiation) and mass exchange between the external surface of clothing (or skin) and the environment and surroundings, (iv) start and evolution of skin injuries (pain and burn) and ( $v$ ) detection of specific incidents within the human being. Due to its interdependency, all modules run iteratively in each time step until a specific convergence criterion is reached.

The module for simulation of human's thermophysiological response is based on the Stolwijk (1971) thermoregulation model of 25 nodes, improved with knowledge found in the literature (e.g., Konz et al. 1977; Fiala et al. 1999; Tanabe et al. 2002). The thermoregulation model represents the human body by 111 nodes, corresponding to 22 segments, each one formed by 5 layers (core, muscle, fat, skin and clothing) and the central blood compartment (the $111^{\text {th }}$ node). The model was 
implemented for an average man with $74.43 \mathrm{~kg}$ and $1.72 \mathrm{~m}\left(1.869 \mathrm{~m}^{2}\right.$ of skin). For an individual with other anthropometric data, the appropriated coefficients are proportionally changed as a function of its body weight and skin area. The loss of heat by respiration is supposed to occur across the elements of the pulmonary tract. The repartition coefficients proposed by Fiala et al. (1999) were considered.

The simulation of heat and water transport through the clothing is based on the formulation proposed by Havenith et al. (2002). The calculations are made for each specific human body segment. Then, individual values of the local clothing parameters (intrinsic insolation, mass, specific heat, vapour permeability and radiation emissivity) must be specified for each of the 22 human body segments considered. Each section is either entirely clothed or completely nude.

The convective heat and mass transfer phenomena are predicted using the empirical relations proposed by Quintela et al. (2004) for natural convection and by Havenith et al. (2002) for forced convection. The exchange of heat by radiation between the external surfaces of the segments of the human body and between them and the surroundings, is determined by a set of expressions established by Raimundo et al. (2004).

The modeling of skin burn process implemented in the software is based on Henriques' theory [Henriques, 1947], which represents the skin thermal damage as chemical rate phenomena. As they do not start at the same instant, the prediction of the skin pain threshold is also useful. This is important because, as reported by Stoll and Greene (1959), when the pain is felt, it is often too late to avoid a $2^{\text {nd }}$ degree skin burn or, in some extreme situations, a heat-stroke or even death. Empirical correlations have been developed that allow the prediction of the time until pain is felt, some of them reported in Wenger (1988) and SFPE guide (2000). In the present program, the onset of skin pain is marked when epidermis-dermis interface temperature $\left(T_{\text {edi }}\right)$ reaches $45^{\circ} \mathrm{C}$ (Havenith and Heus 2000) or when Henriques (1947) integral injury parameter $(\Omega$ ) equals 0.53 , depending on what occurs first (generally, $\left.T_{\text {edi }}=45^{\circ} \mathrm{C}\right)$.

Each run can simulate up to 20 consecutive scenarios (phases), each one representing different conditions in terms of: posture, orientation, activity, intake of food/drinks, clothing, thermal environment characteristics and impinging of thermal radiation from 6 directions (cardinal, top and bottom). The global algorithm is applied to each specific human body segment, but always considering its influence and interdependence with the global thermal state of the body.

To understand the significance of the calculated body temperatures, it is necessary to identify the values for which there is a probability of occurrence of specific incidents in the human body. Normally, the rectal temperature is used to identify the risk of thermal injuries. Nevertheless, the temperature of blood reaching the hypothalamus is regarded as a major afferent stimulus for the intensity of the effective response of sweating, vasomotor activity and shivering. Then, a better relationship may be expected between hypothalamus temperature $\left(T_{\text {hyp }}\right)$ and the various physiological and sensory states (Pascoe et al. 1994; Kenney et al. 2004; Raimundo and Figueiredo 2009; Raimundo et al. 2012). In the program's module for detection of specific incidents, the temperature limits are set as function of the hypothalamus temperature ( $T_{\text {hyp}}$ ) following the Pascoe et al. (1994) scale: $\leq 25^{\circ} \mathrm{C} \rightarrow$ death, $\leq 28^{\circ} \mathrm{C} \rightarrow$ ventricular fibrillation, $\leq 30^{\circ} \mathrm{C} \rightarrow$ stop shivering and fainting, $\leq 34^{\circ} \mathrm{C} \rightarrow$ introversion and violent shivering, $34<T_{\text {hyp }}<39^{\circ} \mathrm{C} \rightarrow$ normal thermoregulation, $\geq 39^{\circ} \mathrm{C} \rightarrow$ introversion and violent sweating, $\geq 41^{\circ} \mathrm{C} \rightarrow$ heat-stroke (stop sweating and fainting), $\geq 42^{\circ} \mathrm{C} \rightarrow$ brain damage (permanent injuries) and $\geq 44^{\circ} \mathrm{C} \rightarrow$ death.

The evaluation, test and validation of the ability of HuTheReg software to predict the human body thermophysiological behavior was performed by comparing the program's predictions with experimental results. The validation process spanned a wide-range of conditions, which included different kinds of thermal environments, of exposures, of exercise intensities and of clothing. As stated in previous papers (Raimundo and Figueiredo 2009; Raimundo et al. 2015), a good agreement was

Advances in Forest Fire Research 2018 - Page 456 
achieved, which indicates an interesting capacity of the program to predict the thermophysiological response of the human body to a wide variety of conditions.

\section{Problem Description}

The protocol adopted in the present work submits the firefighter to 3 or 4 phases: neutral (stabilization), pre-firefighting, firefighting and recovering. The last one is considered only in the simulations to evaluate human body cooling techniques.

The neutral phase aims to ensure that all firefighters subjected to the protocol depart equally and in a state of thermal neutrality.

In the pre-firefighting phase, that starts 1 hour before the beginning of the thermal impact, the individual is exposed to an external environment with $T_{\text {air }}=T_{m r}=35^{\circ} \mathrm{C}$, vair $=5 \mathrm{~m} / \mathrm{s}, R H_{\text {air }}=50 \%$, performs moderate work $(M=1.5$ met: this task involves making preparations, moving towards the fire scene, making supporting activities, etc.) and wears a suit (composed by shirt, briefs, pants, socks and boots) with a global intrinsic insulation $I_{c l}=1.0$ clo, a vapor permeability $i_{v p}=0.47$, a radiative reflectivity $\rho=0.1$ and a total mass $m_{c l}=3.08 \mathrm{~kg}$ (boots included).

On the fire-fighting phase, the firefighter, doing hard work for which $M=3.0$ met, is exposed to an impinging radiation, in an environment with $T_{\text {air }}=T_{m r}=50^{\circ} \mathrm{C}, v_{\text {air }}=5 \mathrm{~m} / \mathrm{s}$ and $R H_{\text {air }}=50 \%$. In this phase, incident radiation fluxes $\left(I_{\mathrm{rad}}\right)$ between 0 and $40 \mathrm{~kW} \mathrm{~m}^{-2}$ were tested and the properties of the firefighter protective clothing depend on the test settings. Namely, they were considered clothing's with global values between 0 and 9 clo for $I_{c l}$, between 0 and 1 for $i_{v p}$ and between 0.1 and 1.0 for $\rho$.

The recovering phase (time break and cooling) aims to lower the heat stored in firefighter, then also his body temperatures. On this phase, the firefighter: is far from the fire front $\left(I_{\text {rad }}=0\right)$; has a low level of activity $(M=1.2 \mathrm{met})$; is wearing his protective clothing, but without the helmet, the balaclava, the coat, and the gloves (in one case only in shorts); and is in a shaded location with $T_{a i r}=T_{m r}=30^{\circ} \mathrm{C}$, $v_{\text {air }}=3 \mathrm{~m} / \mathrm{s}$ and $\mathrm{RH}_{\text {air }}=50 \%$.

\section{Results and Discussion}

As reported elsewhere [Rossi et al. 2011; Fontana et al. 2017; among others], during firefighting activities the firefighters can be exposed to radiant heat fluxes of up to $40 \mathrm{~kW} \mathrm{~m}^{-2}$ in environments with temperatures greater than $250^{\circ} \mathrm{C}$ and high relative humidities. Furthermore, levels of metabolic rates up to 8 met can be required during large periods of time. These conditions cause considerable thermoregulatory strain and represent a substantial risk for the onset of health-related incidents within the firefighter [Fontana et al. 2017]. As far as safety is concerned, the most important indicators seem to be the times delays for the onset of heat-stroke (fainting) and of skin pain, mainly the first one. The threshold for introversion is a good indicator for exposure limits that must not be exceeded.

Almost all existing regulations and standards on fire safety stipulate, as main risk assessment parameter for fire exposure, the thermal incident radiation on the human body coming directly from the fire $\left(I_{\mathrm{rad}}\right)$ [Raj 2008, EN 1473/2016]. That does not includ solar radiation and other thermal radiation exchanges. It is from $I_{\text {rad }}$ that all the other parameters are defined, such as the safety distance, the maximum exposure times, the level of protection required, etc.

Normally, regulations and standards postulate that an incident radiation flux up to $I_{\text {rad }}=1.5 \mathrm{~kW} \mathrm{~m}^{-}$ 2 is tolerable for persons not wearing fire protective clothing. For people dressed in fire protective ensembles, exposures to incident radiation fluxes up to $I_{\text {rad }}=5 \mathrm{~kW} \mathrm{~m}^{-2}$ are considered of acceptable risk. An exposure to an incident radiation flux higher than $I_{r a d}=8 \mathrm{~kW} \mathrm{~m}^{-2}$ is only considered minimally safe if a suitable fire protective ensemble is used. 
The aforesaid stipulations are too simplistic because, in order to establish the risk of potential heatrelated illness, the knowledge of $I_{\text {rad }}$ it is not enough; moreover it is necessary to take into account many other factors such as: the initial thermal state of the person; its anthropological characteristics; the area of the body exposed to the incident radiation; the intensity of the physical activity exerted; the thermo-hygrometric characteristics of clothing $\left(I_{c l}, m_{c l}, i_{v p}\right.$ and $\left.\rho\right)$; and the remaining environmental conditions ( $I_{\text {solar }}, T_{m r}, T_{\text {air }}, v_{\text {air }}, \mathrm{RH}_{\text {air }}$ and smoke level). In addition, it should be taken into consideration the fact that the regulations and the norms establish the human risk level based on the ratio between the expected exposure time threshold of fainting due to heat-stroke and / or of appearance of permanent injuries (skin burn degree 2, permanent brain damage) and the time required to take evasive actions and/or to initiate mitigation measures. Then, since they were not specifically postulated for firefighting activities, the aforesaid stipulations can hide situations of potential risk to firefighters, particularly the cases $I_{\mathrm{rad}}=1.5$ and $I_{\mathrm{rad}}=5 \mathrm{~kW} \mathrm{~m}^{-2}$. Therefore, in this work particularly attention is given to situations involving these two impinging thermal radiations.

The onset of heat-related diseases within the firefighter are show in Figures 1 and 2 for impinging thermal radiations of $I_{\mathrm{rad}}=1.5 \mathrm{~kW} \mathrm{~m}^{-2}$ and $I_{\mathrm{rad}}=5 \mathrm{~kW} \mathrm{~m}^{-2}$, respectively. In Figures 1 and $2(a, b$ and $c$ ) the evolutions of hypothalamus, rectal and maximum skin temperatures with time are represented for 3 different levels of clothing insulation, namely: $I_{c l}=1.21$ clo (complete ensemble with weak insulation), $I_{c l}=1.59$ clo (current for wildland firefighting) and $I_{c l}=2.63$ clo (current for structural firefighting). The four horizontal lines identify the threshold values for the beginning of introversion, of heat stroke, of lethal and of skin pain temperatures, as specified before. The start of the fire thermal impact occurs at instant zero on the time axis. Structural firefighting includes the fight of building and factories fires. Figures $1 d$ and $2 d$ shown the time (after fire impact) for the beginning of skin pain and burn degree 1, of introversion, of heat stroke, of brain damage and of death as function of the firefighter clothing insulation. For $I_{\text {rad }}=1.5 \mathrm{~kW} \mathrm{~m}^{-2}$ skin pain and skin burn degree 2 only "occur" after death, then they are not shown in Figure $1 d$. As presented in Figure 2, for $I_{\text {rad }}=5 \mathrm{~kW} \mathrm{~m}^{-2}$ skin pain starts before heat-stroke until $I_{c l}<1.21$ clo (incomplete covering of firefighter skin) and "after" death for $I_{c l} \geq 1.21$ clo (complete clothing); skin burn degree 2 only "occur" after death, then it is not shown in Figure $1 d$.
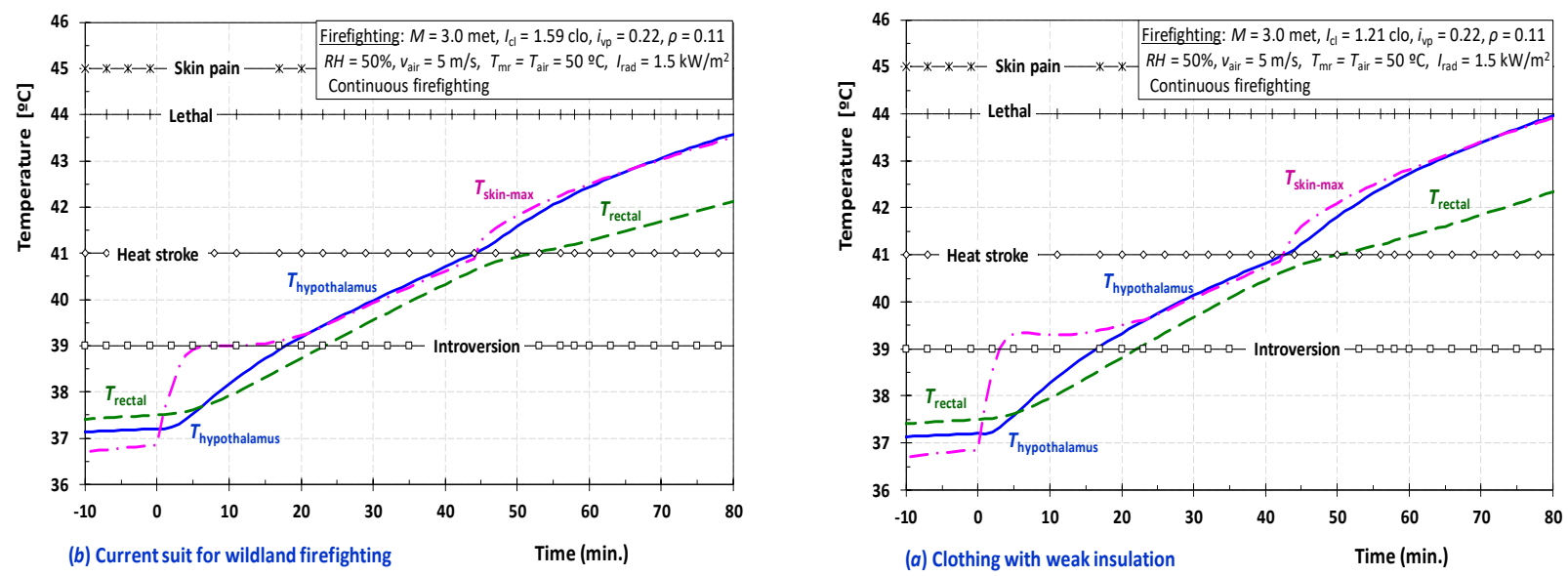

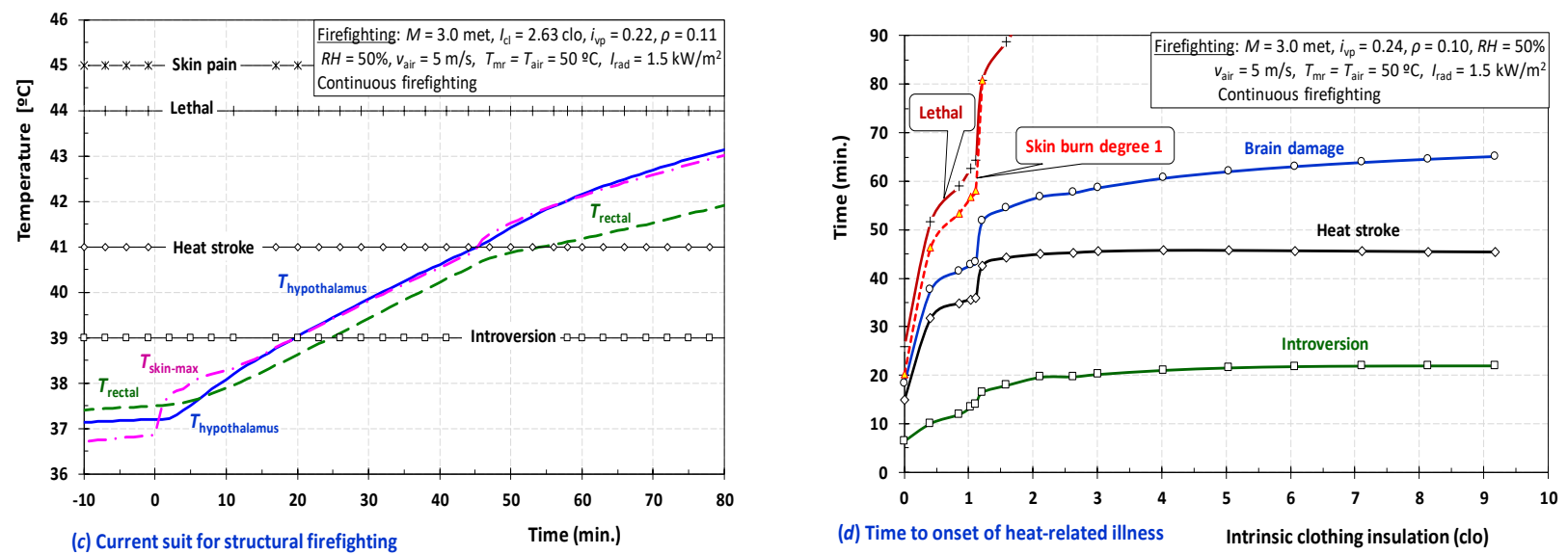

Figure 1 - Incident thermal radiation $I_{r a d}=1.5 \mathrm{~kW} \mathrm{~m}^{-2}:(a, b$ and c) Time evolution of temperatures on hypothalamus ( - ), rectal ( - - ) and maximum value of skin (-:- -) and limiting temperatures for the beginning of introversion, heat stroke, lethal and skin pain, for a complete ensemble with: a) $I_{c l}=1.21$ clo), b) $I_{c l}=1.59$ clo and c) $I_{c l}=2.63 \mathrm{clo}$; and (d) Time (after fire impact) for the beginning of skin pain, of introversion, of heat stroke, of brain damage and of death as function of clothing insulation.

It is possible to observe from Figures 1 and $2(a, b$ and $c)$ that the increasing of clothing thermal insulation implies more temperature uniformity between human body parts. For normal thermal environments, the rectal temperature is one of the highest in the human body and is often taken as representative of the body core temperature. This is no longer valid for situations where human body is exposed to extreme thermal conditions, where rectal temperature is a bad indicator for the maximum body core temperature. This is the reason why it was not used in this work as a criterion for the detection of specific undesired reactions within the man; instead the hypothalamus temperature is considered a more appropriated threshold.
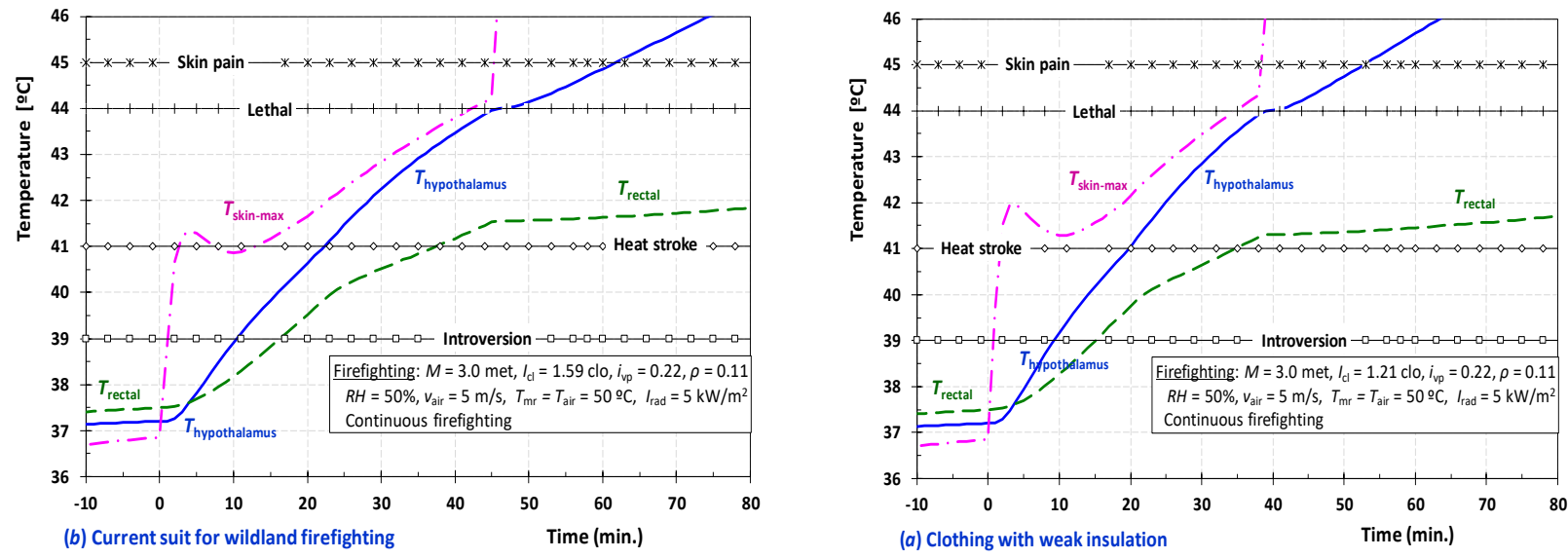

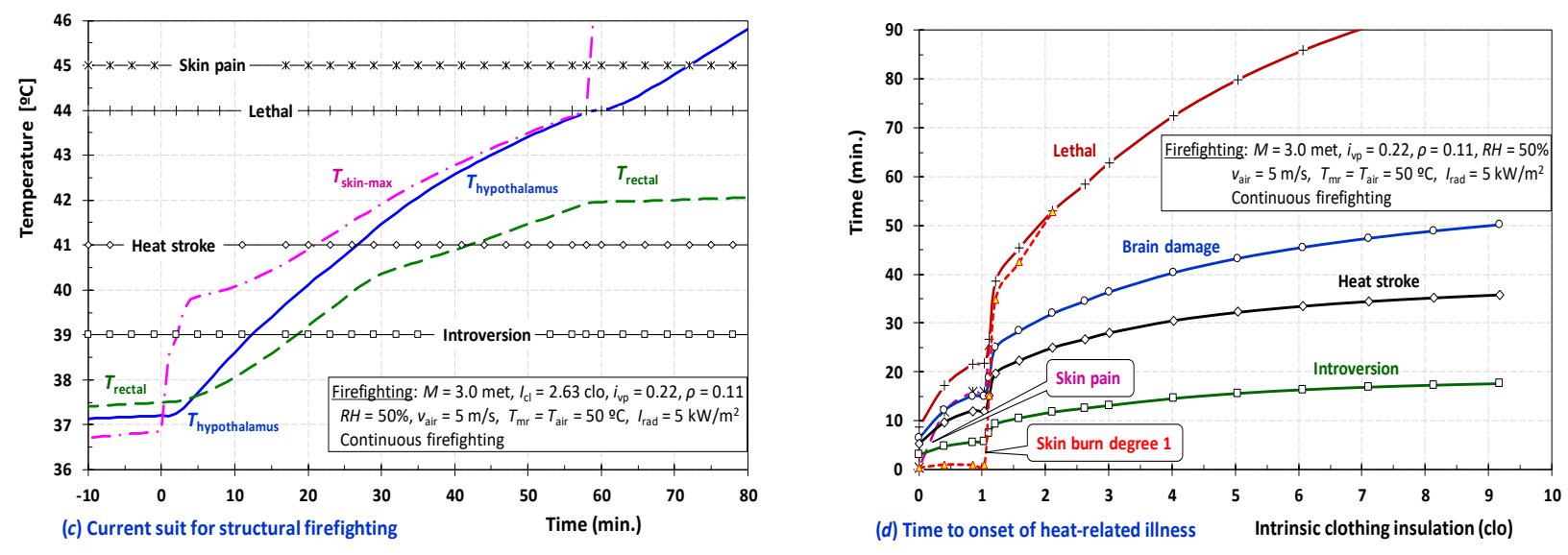

Figure 2 - Incident thermal radiation $I_{r a d}=5 \mathrm{~kW} \mathrm{~m}^{-2}:(a, b$ and $c)$ Time evolution of temperatures on hypothalamus ( -), rectal ( - - -) and maximum value of skin (- - - -) and limiting temperatures for the beginning of introversion, heat stroke, lethal and skin pain, for complete ensemble with: a) $I_{c l}=1.21 \mathrm{clo}$ ), $\left.b\right) I_{c l}=1.59 \mathrm{clo}$ and c) $I_{c l}=2.63 \mathrm{clo}$; and (d) Time (after fire impact) for the beginning of skin pain, of introversion, of heat stroke, of brain damage and of death as function of clothing insulation.

Figures $1 d$ and $2 d$ present the instants of probable onset of heat-related illness as functions of the global value of clothing insulation. In both situations these periods increase with global value of clothing thermal insulation, namely for the heat-stroke and death threshold. This effect is evident in the range between 1 to 3 clo, becoming less pronounced for higher values. Moreover, protective clothing ensembles with insulating values above 3 clo will restrict firefighter movements and then they are not practical to use.

In both situations shown in Figures $1 d$ and $2 d$, whatever clothing insulation, skin burn degree 1 starts before skin pain. For $I_{\mathrm{rad}}=1.5 \mathrm{~kW} \mathrm{~m}^{-2}$ and independently of clothing insulation, skin pain and skin burn degree 2 only "occur" after death, then, for fires of this intensity, skin pain is not a useful alert in terms of human safety. For $I_{r a d}=5 \mathrm{~kW} \mathrm{~m}^{-2}$ and if the skin is not complete covered $\left(I_{c l}<1.21 \mathrm{clo}\right)$, skin pain starts in very short exposure times after fire impact and before heat-stroke. The time interval between the onset of skin pain and of heat-stroke is also very short. In these conditions skin pain works as an alert signal, inducing the firefighter to escape quickly from the fire front line to avoid fainting and to be trapped by the fire. Then, without a complete covering of skin, is not possible to fight fires from which the impinging radiation is $I_{\mathrm{rad}} \geq 5 \mathrm{~kW} \mathrm{~m}^{-2}$. Nevertheless, this cannot be taken as a safety rule, as for complete firefighter ensembles the heat-stroke can be reached before skin pain, which obviously poses a crucial problem for personal safety.

From the values presented in Figures 1 and 2, it can be stated that firefighting activities requires the wearing of complete protective ensembles with $I_{c l} \geq 1.21$ clo. For fires with $I_{\text {rad }} \leq 1.5 \mathrm{~kW} \mathrm{~m}^{-2}$ it is enough to wear clothing with insulation equal or lower $I_{c l}=1.59$ clo (current for wildland firefighting) and for fires with $I_{\text {rad }} \leq 5 \mathrm{~kW} \mathrm{~m}^{-2}$ an insulation equal or lower than $I_{c l}=2.63$ clo (current suit for structural firefighting) it is enough.

The onset of heat-related diseases within the firefighter as function of the impinging thermal radiation $\left(I_{r a d}\right)$ is show in Figure $3 a$ for an ensemble with $I_{c l}=1.59$ clo (current for wildland firefighting) and in Figure $3 b$ for a suit with $I_{c l}=2.63$ clo (current for structural firefighting). If $I_{c l}=2.63$ clo skin burn degree 2 only "occur" after death, then it is not shown in Figure $3 b$. 

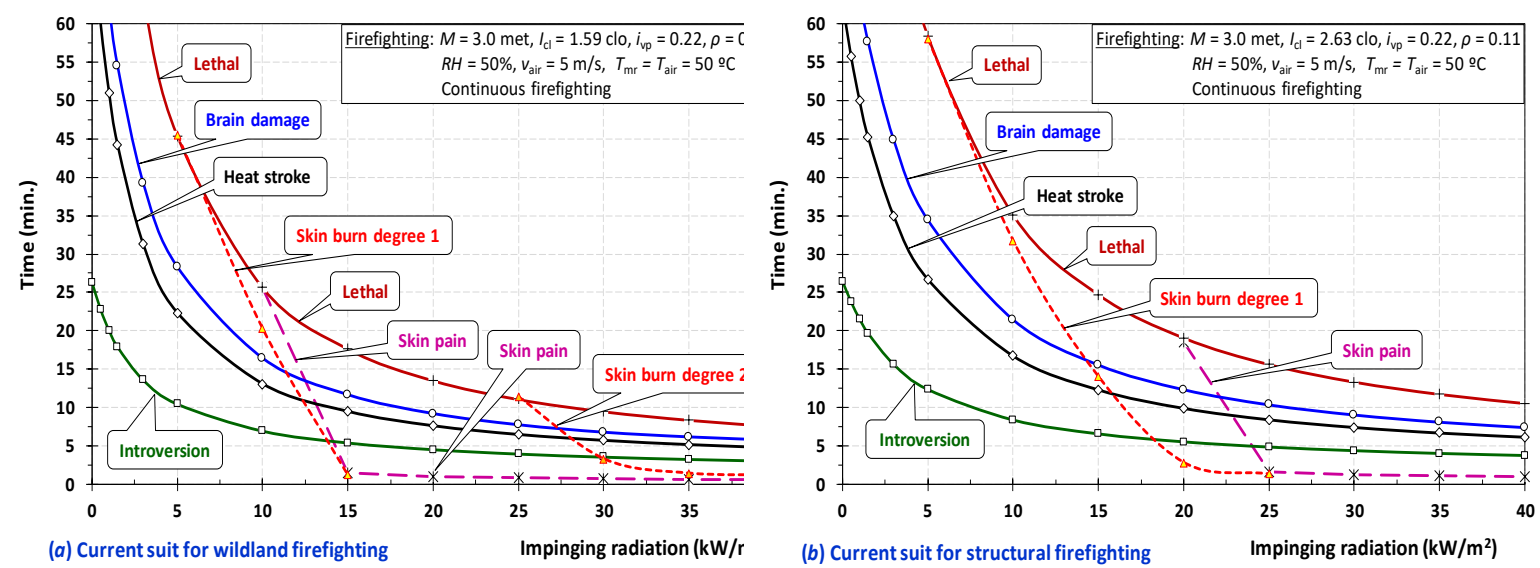

Figure 3 - Time for the onset of skin pain, of introversion, of heat stroke, of brain damage and of death as function of impinging radiation, for a complete ensemble with: (a) $I_{c l}=1.59 \mathrm{clo}$; and (b) $I_{c l}=2.63 \mathrm{clo}$.

Figure 3 show that the time (after fire impact) for the onset of heat-related illness decreases very rapidly with the increase of the incident radiation (up to about $10 \mathrm{~kW} \mathrm{~m}^{-2}$ ). For higher values of impinging radiation, these times decrease less and less until practically stabilize in a kind of minimum value. Regardless the fire intensity, and if protected with a full ensemble with $I_{c l} \geq 1.59$ clo, it will be possible to resist up to about 5 minutes without fainting. However, skin burn degree 2 can happen for ensembles with $I_{c l}=1.59$ clo if $I_{r a d} \geq 25 \mathrm{~kW} \mathrm{~m}^{-2}$. In addition, skin pains start too soon for high levels of impinging radiation. For instance, the onset of skin pain is below one minute of exposure in the case of wearing full clothing with $I_{c l}=1.59$ clo for $I_{r a d} \geq 15 \mathrm{~kW} \mathrm{~m}^{-2}$ and in the case of $I_{c l}=2.63$ clo for $I_{r a d} \geq 25 \mathrm{~kW} \mathrm{~m}^{-2}$. In short, the increase of thermal insulation of the ensemble amplify the range of impinging radiation where fainting occurs before the start of skin pain. Therefore, it is not possible to state that the increasing of the protective clothing thermal insulation will enhance firefighter's safety. In fact, a convenient control of the duration of firefighting periods and / or of the temperature of the hypothalamus (which has practically the same value as the tympanum) is crucial and therefore recommended.

A good clothing insulation protects against the incoming of external heal but also can allow the firefighter to stay in dangerous and extreme environments, and remain there for unsafe periods without any warning. Then, to avoid undesired pathologies the firefighting period must be of limited duration and followed by a recovering period.

After a firefighting period and to allow the firefighter to be able to return, as quickly as possible, safely to firefighting his, initial thermal state must be restored within a relatively short period. This is only possible through the application of an appropriate body cooling technique during the recovering time-break.

For the purposes of this work, it was selected the case of a fireman dressed in a protective clothing with $I_{c l}=2.63$ clo and exposed to a fire from which the thermal incident radiation is $I_{\text {rad }}=5 \mathrm{~kW} \mathrm{~m}^{-2}$. Considering the results presented in Figure 2, a 20-minute firefighting period was selected. Therefore, the following phase sequence was assumed: neutral (60 minutes), pre-firefighting (60 minutes), firefighting (20 minutes) and recovering (unlimited time).

On the recovering period, the firefighter is far from the fire front $\left(I_{r a d}=0\right)$, has a low level of activity $(M=1.2 \mathrm{met})$ and drinks $0.33 \mathrm{~kg}$ of water at $15^{\circ} \mathrm{C}$. Six alternative body cooling techniques were analyzed.

PC - Passive cooling: the firefighter rest in a shaded environment with $T_{a i r}=T_{m r}=30^{\circ} \mathrm{C}$, vair $=3 \mathrm{~m} / \mathrm{s}$ and $R H_{\text {air }}=50 \%$, and is dressed in his protective clothing, but without the helmet, the balaclava, the coat and the gloves $\left(I_{c l}=1.0\right.$ clo, $i_{v p}=0.47, \rho=0.1$ and $\left.m_{c l}=3.08 \mathrm{~kg}\right)$. 
HFIW20: The previous situation reinforced with the immersion of hands and forearms in water at $T_{\text {water }}=20^{\circ} \mathrm{C}$.

HFIW10: The passive cooling reinforced with the immersion of hands and forearms in water at $T_{\text {water }}=10^{\circ} \mathrm{C}$.

UIJ: The passive cooling reinforced with the using of an ice jacket.

UIJ + HFIW10: The passive cooling reinforced with the using of an ice jacket and the immersion of hands and forearms in water at $T_{\text {water }}=10^{\circ} \mathrm{C}$.

ABIW25: All body (except head and neck) immersion in water at $T_{\text {water }}=25^{\circ} \mathrm{C}$. In this case $I_{c l}=0.1$ clo, $i_{v p}=0, \rho=0.1$ and $m_{c l}=0.2 \mathrm{~kg}$.

To serve as a reference, Figure $4 a$ shows the predicted results for the case of passive cooling (PC) where the evolution with time of hypothalamus, rectal, body average and maximum skin temperatures is presented. The two horizontal lines identify the threshold values for the beginning of introversion and of heat-stroke. The start of the fire thermal impact occurs at instant zero on the time axis. For comparative evaluation, Figure $4 b$ shows that the evolution of the heat stored in the human body [in $\mathrm{kJ} / \mathrm{kg}$ ], as function of time, for all body cooling techniques tested.
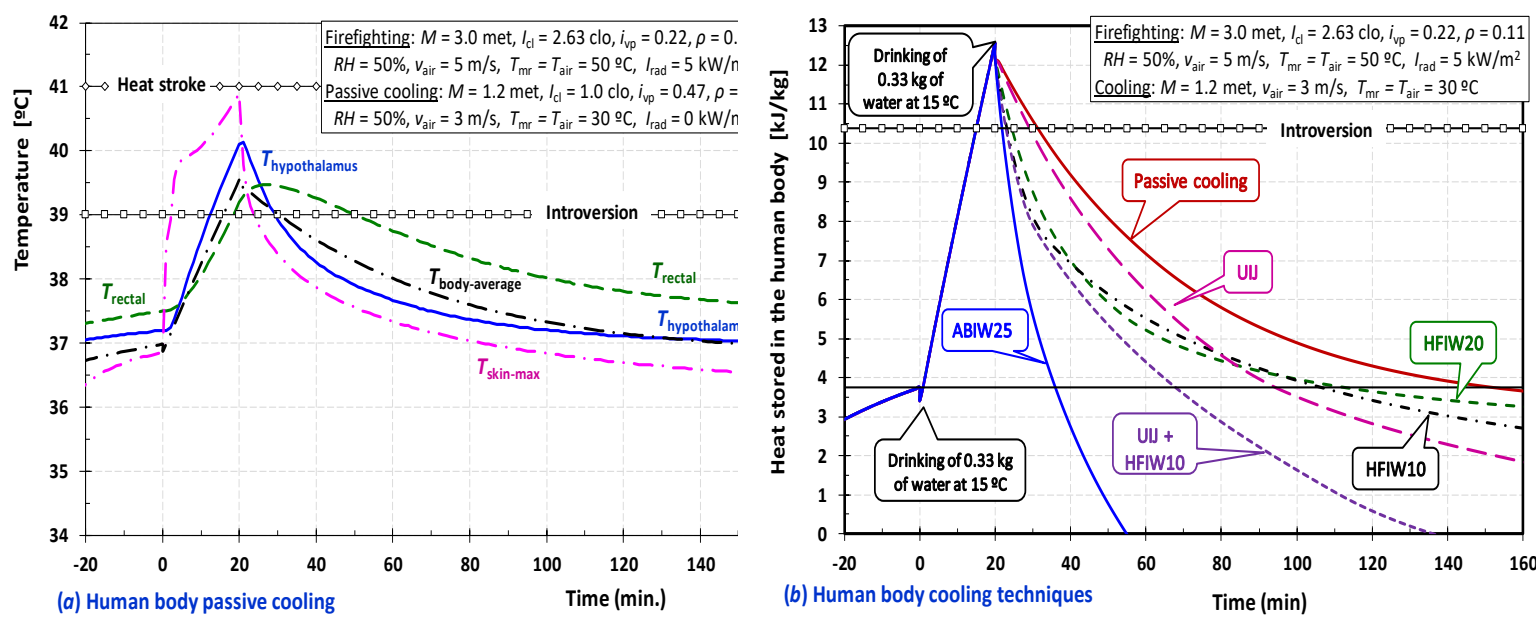

Figure 4 - Time evolution of human body temperatures and heat stored: (a) Passive cooling; and (b) heat stored in the human body for all body cooling techniques tested.

As can be observed from Figure 4, the drinking of cold water has only a very small influence on the reduction of firefighter thermal stress. However, drinking water is important for hydration purposes.

Although with very different effectiveness, all body cooling techniques considered proved to be capable to mitigate the level of hyperthermic stress of firefighters. In the situation considered, the passive cooling attenuates the heat stress but it is the less effective of all. Both cooling techniques where the passive cooling is reinforced by immersion of the hands and of the forearms in water (HFIW20 and HFIW10) have similar effectiveness. However, a slightly advantage exist when $T_{\text {water }}=10^{\circ} \mathrm{C}$, despite the appearance of vasoconstriction and eventually shivering when the water is at $10^{\circ} \mathrm{C}$.

For the situation considered, the cooling techniques tested can be ordered from the less effective to the highest one as: $6^{\text {th }}$ ) PC - passive cooling; $5^{\text {th }}$ ) UIJ - use of an ice jacket; $4^{\text {rd }}$ ) HFIW20 - immersion of hands and forearms in water at $T_{\text {water }}=20^{\circ} \mathrm{C} ; 3^{\text {rd }}$ ) HFIW $10-$ the same but with $T_{\text {water }}=10^{\circ} \mathrm{C} ; 2^{\text {nd }}$ ) UIJ + HFIW10 - use of an ice jacket reinforced by immersion of hands and forearms in water at $T_{\text {water }}=10^{\circ} \mathrm{C}$; and $1^{\text {st }}$ ) ABIW25 - all body (except head and neck) immersion in water at $T_{\text {water }}=25^{\circ} \mathrm{C}$. 
Among the tested, the strategy of immersion the entire body in water at $25^{\circ} \mathrm{C}$ is undoubtedly substantially more effective than the others. This is the one with the highest cooling rate, the lowest temperature values and lowest heat stored in the human body. Therefore, whenever feasible, it should be selected.

\section{Conclusions}

The protocol adopted in the present work submits the firefighter to four phases: neutral (stabilization), pre-firefighting, firefighting and recovering. Six alternative body cooling techniques were analyzed, which are applied during the recovering period.

From a safety point of view, the introversion threshold is a good indicator for exposure limits that must not be exceeded. Due to the nearest instantaneous onset of skin pain, only fires with incident radiation fluxes $I_{\text {rad }}<1.5 \mathrm{~kW} \mathrm{~m}^{-2}$ can be fought without wearing ensembles that completely cover the firefighter skin. The time to the onset of heat-related illness decreases very rapidly with the increase of the incident radiation. On the contrary, time safety intervals increase with the clothing thermal insulation. This is evident when passing from the value of 1 to 3 clo. For higher values, the effect is less pronounced. However, protective clothing ensembles with intrinsic insulating values above 3 clo are not practical to use. Regardless the fire intensity, and if protected with a full ensemble with $I_{c l} \geq 1.59$ clo, it will be possible to resist up to about 5 minutes without fainting. Even so, skin pain starts too soon for high levels of impinging radiation and burn degree 2 can occur. Thus, high thermal insulation may or not enhance safety. Therefore, a convenient control of the duration of firefighting periods is crucial

For the situation of $I_{r a d}=5 \mathrm{~kW} \mathrm{~m}^{-2}$ and an active fighting period of 20 minutes, the cooling techniques tested can be ordered from the less effective to the highest one as: $6^{\text {th }}$ ) passive cooling; $5^{\text {th }}$ ) use of an ice jacket; $4^{\text {rd }}$ ) immersion of hands and forearms in water at $20^{\circ} \mathrm{C} ; 3^{\text {rd }}$ ) the same but with water at $10^{\circ} \mathrm{C} ; 2^{\text {nd }}$ ) use of an ice jacket reinforced by immersion of hands and forearms in water at $10^{\circ} \mathrm{C}$; and $1^{\text {st }}$ ) all body (except head and neck) immersion in water at $25^{\circ} \mathrm{C}$. Among the tested, the strategy of immersion the entire body in water at $25^{\circ} \mathrm{C}$ is undoubtedly more effective than the others.

\section{References}

Abreu RM, Raimundo AM, Quintela DA (2014). Analysis of the thermophysiological response to cooling techniques in firefighters, ICFFR 2014 - 7th International Conference on Forest Fire Research, paper H01 (pp. 1-3), Coimbra, Portugal, 14 - 21 november 2014.

Barr D, Gregson W, Sutton L, Reilly T (2009). A practical cooling strategy for reducing the physiological strain associated with firefighting activity in the heat, Ergonomics 52(4): 413-420.

Bröde P, Kuklane K, Candas V, DenHartog EA, Griefahn B, Holmér I, Meinander H, Nocker W, Richards M, Havenith G (2010). Heat gain from thermal radiation through protective clothing with different insulation, reflectivity and vapour permeability, International J. Occupational Safety and Ergonomics 16(2): 231-244.

Carballo-Leyenda B, Villa JG, Lopez-Satué J, Jose A. Rodríguez-Marroyo JA (2017), Impact of different personal protective clothing on wildland firefighters' physiological strain, Frontiers in Physiology 8: Article 618.

Carter JB, Banister EW \& Morrison JB (1999). Effectiveness of rest pauses and cooling in alleviation of heat stress during simulated fire-fighting activity, Ergonomics 42: 299-313.

Colburn D, Suyama J, Reis S (2011). A comparison of cooling techniques in firefighters after a live burn evolution, Prehospital Emergency Care 15: 226-232. 
Chou C, Tochihara Y, Kim T (2008). Physiological and subjective responses to cooling devices on firefighting protective clothing, European J Applied Physiology 104: 367-374.

EN 1473 (2016). Installation and equipment for liquefied natural gas - design of onshore installations, European Standard, European Committee for Standardization.

Fiala D, Lomas KJ, Stohrer M (1999). A computer model of human thermoregulation for a wide range of environmental conditions - the passive system, J Applied Physiology 87: 1957-1972.

Fontana P, Saiani F, Grütter M, Croset J-P, Capt A, Camenzind M, Morrissey M, MacRae BA, Rossi RM, Annaheim S (2017). Thermophysiological impact of different firefighting protective clothing ensembles in a hot environment, Textile Research Journal 88(7): 744-753.

Giesbrecht GG, Jamieson C, Cahill F (2007). Cooling hyperthermic firefighters by immersing forearms and hands in $10^{\circ} \mathrm{C}$ and $20^{\circ} \mathrm{C}$ water, Aviation Space Environmental Medicine 78: 561-567.

Havenith G, Heus R (2000). Ergonomics of protective clothing, Proc. of Nokobetef 6 and 1st European Conference on protective clothing, Stockholm, Sweden, May 7-10.

Havenith G, Holmér I, Parsons K (2002). Personal factors in thermal comfort assessment - clothing properties and metabolic heat production, Energy and Buildings 34: 581-591.

Henriques F (1947). Studies of thermal injury V. The predictability and the significance of thermally induced rate processes leading to irreversible epidermal injury, Archives of Pathology 43: 489-502.

Hostler D, Reis S, Bednez JC, Kerin S, Suyama J (2010). Comparison of active cooling devices with passive cooling for rehabilitation of firefighters performing exercise in thermal protective clothing: a report from the Fireground Rehab Evaluation (FIRE) trial, Prehospital Emergency Care 14: 300309.

Kenney W, DeGroot D, Holowatz L (2004). Extremes of human heat tolerance - life at the precipice of thermoregulatory failure, J Thermal Biology 29: 479-485.

Konz S, Hwang C, Dhiman B, Duncan J, Masud A (1977). An experimental validation of mathematical simulation of human thermoregulation, Computers in Biology and Medicine 7: 71-82.

Lopez RM, Cleary MA, Jones LC, Zuri RE (2008). Thermoregulatory influence of a cooling vest on hyperthermic athletes. J Athletic Training 43(1): 55-61.

McLellan TM, Selkirk GA (2005), The management of the heat stress for the firefighter, DRDC, Canada.

McQuerry M, Barker R, DenHartog E (2018). Relationship between novel design modifications and heat stress relief in structural firefighters' protective clothing, Applied Ergonomics 70: 260-268.

Mündel T, King J, Collacott E, Jones DA (2006). Drink temperature influences fluid intake and endurance capacity in men during exercise in a hot dry environment, Experimental Physiology 91(5): 925-933.

Pascoe DD, Bellingar TA, McCluskey BS (1994). Clothing and exercise II. Influence of clothing during exercise/work in environmental extremes, Sports and Medicine 18: 94-108.

Quintela DA, Gaspar AR, Borges C (2004). Analysis of sensible heat exchanges from a thermal manikin, European J Applied Physiology 92(6): 663-668.

Raimundo AM, Gaspar AR, Quintela DA (2004). Numerical modelling of radiative exchanges between the human body and surrounding surfaces. Climamed $2004-1^{\text {st }}$ Mediterranean congress of climatization, 16-17 of April, Lisbon, Portugal, paper 8/1.

Raimundo AM, Figueiredo AR (2009). Personal protective clothing and safety of firefighters near a high intensity fire front, Fire Safety Journal 44: 514-521.

Raimundo AM, Quintela DA, Gaspar AR, Oliveira AVM (2012). Development and validation of a computer program for simulation of the human body thermophysiological response, Portuguese chapter of IEEE-EMBS, ISBN:978-1-4673-4524-8. 
Raimundo AM, Pereira CD, Quintela DA, Oliveira AVM (2015). Human body thermoregulation - test and validation of a computer program, SHO 2015 - International Symposium on Occupational Safety and Hygiene, paper 25, Guimarães, 12 - 13 February.

Raj PK (2008). A review of the criteria for people exposure to radiant heat flux from fires, J Hazardous Materials 159: 61-71.

Rossi JL, Simeoni A, Moretti B, Leroy-Cancellieri V (2011). An analytical model based on radiative heating for the determination of safety distances for wildland fires, Fire Safety Journal 46: 520 527.

Sharkey BJ (1999), Heat stress - wildland firefighter health and safety recommendations, April 1999 Conference, Missoula Technologic and Development Center, USDA Forest Service, Montana.

Selkirk G, McLellan TM \& Wong J (2004). Active versus passive cooling during work in warm environments while wearing firefighting protective clothing, J. Occupational Environment and Hygiene 1: 521-531.

SFPE Task Group on Engineering Practices (2000). Predicting 1st and 2nd degree skin burns from thermal radiation, Society of Fire Protection Engineers, Bethesda, MD.

Stoll A, Greene L (1959). Relationship between pain and tissue damage due to thermal radiation, J Applied Physiology 14: 373-382.

Stolwijk JAJ (1971). A mathematical model of physiological temperature regulation in man. NASA Contractor Report CR-1855, NASA, Washington, DC.

Tanabe S, Kobayashi K, Nakano J, Ozeki Y, Konishi M (2002). Evaluation of thermal comfort using combined multi-node thermoregulation $(65 \mathrm{MN})$ and radiation models and computational fluid dynamics (CFD), Energy and Buildings 34: 637-646.

Wenger CB (1988). Human heat acclimatization, In: Pandolf K, Sawka M, Gonzalez R, eds, Human performance physiology and environmental medicine at terrestrial extremes. Indianapolis Benchmark Press (now: Cooper Publishing Group), pp. 153-197. 


\title{
Fire response to natural wind versus constant wind
}

\author{
BW Butler ${ }^{1}$, D Jimenez ${ }^{1}$, S Quarles ${ }^{2} ;$ J. Obrien $^{3}$ \\ ${ }^{1}$ US Forest Service, Missoula Fire Sciences Laboratory, Missoula, MT, USA, \\ \{bwbutler@fs.fed.us\} \\ ${ }^{2}$ Insurance Institute for Business and Home Safety, Rock Hill, SC USA, \\ ${ }^{3}$ US Forest Service, Southern Research Station, \{jobrien@fs.fed.us\}
}

\begin{abstract}
While the relation between wildland fire spread rate and wind has been a topic of study for over a century, very few previous studies report measurements of fire rate of spread in controlled winds exceeding $5 \mathrm{~m} / \mathrm{s}$. In this study measurements of fire rate of spread, flame residence time, and energy release are reported for fires burning under controlled atmospheric conditions in a shallow bed of pine needles subject to winds ranging from 0 to $27.2 \mathrm{~m} / \mathrm{s}(6 \mathrm{~m} \mathrm{agl})$. The data suggest that when winds are less than $5 \mathrm{~m} / \mathrm{s}$ fire rate of spread increases linearly with wind speed. As wind speed exceeds $5 \mathrm{~m} / \mathrm{s}$ the fire rate of spread response increases at a rate approximately 5 times greater. When fires are burned in winds replicating natural spatiotemporal variability in speed and direction the rate of spread is 20 to $90 \%$ greater than when burned under constant steady winds.
\end{abstract}

Keywords: fire behavior, wind

\section{Introduction}

Wind is a dominant environmental variable in wildland fire spread and intensity. The relation between wildland fire rate of spread and wind speed (aligned with the direction of fire spread) used in the US and other fire behavior decision support systems was developed from experiments in wind tunnels and open burn experiments (Fang 1969). For the most part these facilities have been limited to approximately $3 \mathrm{~m} / \mathrm{s}$ is maximum speed; however there have been some exceptions. Weise and Bigging (1996) report measurements of fire spread rate in open wind tunnel at wind speeds up to 1.1 $\mathrm{m} / \mathrm{s}$. Catchpole et al (1998) present results from a large number of experiments conducted in a closed wind tunnel with controlled air temperature and humidity at winds up to $3.3 \mathrm{~m} / \mathrm{s}$. Their work exhibits a monotonically increasing correlation between fire rate of spread and wind speed over the range of winds explored.

Others report observations from naturally burning fires. McArthur $(1966,1969)$ relates fire rate of spread in natural fuels to average wind velocity in the open for winds of $60 \mathrm{~km} / \mathrm{h}$ as observed from several fires in Australia. Winds in the natural environment fluctuate in speed and direction on relatively small temporal and spatial scales. Beer (1991) evaluates the physics underlying fire/wind interaction and proposes that the three dimensional nature of buoyant plumes above fires forms peaks and troughs that interact with local wind fields through complicated mechanisms that enhance energy transport to the unburned fuel ahead of the fire front. He posits that fluctuations in wind speed enhance the fluctuations in flame size and structure thereby enhancing advective heating of the fuels and increased spread rates. In a theoretical study Albini (1982) concludes that the only effect of wind fluctuations on fire spread rate is further fluctuations in the smoke plume above the fire. Others report a power relation between wind and fire spread rate with the wind raised to an exponent of 1.5 (Nelson and Adkins 1987; Nelson Jr and Adkins 1988). Beer (1993) examines data for winds up to $9.2 \mathrm{~m} / \mathrm{s}$ and posits a power-law relation with two regimes (winds less than $2.5 \mathrm{~m} / \mathrm{s}$ and winds greater than $2.5 \mathrm{~m} / \mathrm{s}$ ) he proposes that the relative contribution of radiant and convective energy to the fire spread depends on wind speed, with increased convective heating for increased winds (Beer 1991). 
In this study the rate of spread and intensity of fires burning over a bed of pine needles for a wind speeds from 0.4 to $27 \mathrm{~m} / \mathrm{s}$ are measured. The winds speeds cover a much higher range than has been reported previously for any laboratory based study. To our knowledge no laboratory-based studies exist that explore the relation between fire rate of spread and a "natural" wind history under laboratory controlled conditions.

\section{Methods}

Here we report the results of twelve experiments completed at The Insurance Institute for Building and Home Safety (IBHS) located in Richburg, SC. This facility includes a large wind tunnel (approximate footprint of experimental floor space 35 by $50 \mathrm{~m}$ ) that has the capability to generate three wind conditions: 1) a constant wind speed (fan speeds held constant to generate a wind speed specified with the representative typical logarithmic boundary layer profile and 2) a nominally "semi-natural" wind history with fluctuations in the longitudinal wind direction and 3) a fully "natural" wind field with fluctuations in both the longitudinal and lateral components. Here we compare results from seven fires conducted under wind condition 1, one fire burned under wind condition 2 and five fires burned under wind condition 3 . The facility has the capability to generate winds between 5 and $63 \mathrm{~m} / \mathrm{s}$ (see Figure 1 left). Longitudinal speed is controlled by modulating fan rotational speed at the plenum inlet to replicate a wind speed record collected from a sensor in an open field. Wind direction is controlled by modulating vanes at the exit of each plenum to redirect the wind speed over a nominal range of \pm 20 degrees, replicating a directional wind record measured in an open field. Through these two features the wind field in the test chamber can be controlled to exactly replicate a wind field measured in the natural environment.

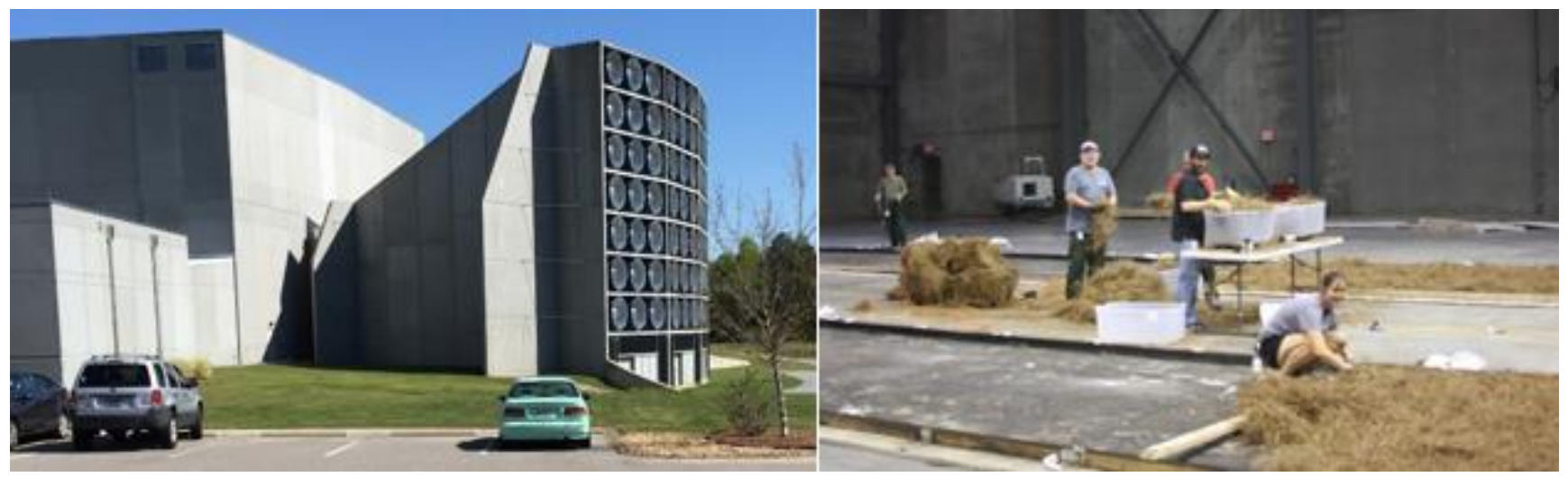

Figure 1 - Exterior photo of IBHS facility with fans (left image) and experimental chamber (right image) where staff are constructing fuel beds in preparation for a burn.

For this study two parallel fuel trays nominally $3 \mathrm{~m}$ wide by $24 \mathrm{~m}$ long were constructed on the floor of the IBHS facility (Figure 1 right). Fuel trays were equally spaced laterally along the longitudinal axis of the wind flow. The bed width was selected to minimize the effect of fire front width on rate of spread (Wotton et al. 2000a, 2000b). The fuel trays consisted of a double layer of 1.58 $\mathrm{cm}$ thick $244 \mathrm{~cm}$ long by $122 \mathrm{~cm}$ wide gypsum boards (total thickness $3.16 \mathrm{~cm}$ ) placed on the floor of the tunnel. The fuel tray sides were $8.9 \mathrm{~cm}$ tall. The lateral edges of the trays were separated by approximately $3 \mathrm{~m}$. Burn number, wind speed, fuel moisture, average rate of spread, and residence time are presented in table 1. 
Table 1 - Experimental conditions and key measurements averaged over both beds

\begin{tabular}{|c|c|c|c|c|c|c|}
\hline $\begin{array}{c}\text { Burn } \\
\#\end{array}$ & $\begin{array}{c}\text { Wind } 5 \mathrm{~m} \\
\text { above floor at } \\
\text { exit of } \\
\text { plenum }(\mathrm{m} / \mathrm{s})^{3}\end{array}$ & $\begin{array}{l}\text { Ave wind at } \\
\text { fuel surface } \\
\qquad(\mathrm{m} / \mathrm{s})\end{array}$ & $\begin{array}{l}\text { Flow } \\
\text { type }\end{array}$ & $\begin{array}{c}\text { Average fuel } \\
\text { moisture content } \\
(\% \text { dry mass })^{3}\end{array}$ & $\begin{array}{c}\text { Fire ROS } \\
\text { for both } \\
\text { beds } \\
(\mathbf{m} / \mathbf{s})^{3} \\
\end{array}$ & $\begin{array}{c}\text { Average } \\
\text { residence time } \\
(\mathbf{s})^{3}\end{array}$ \\
\hline 4 & $14.7(1.3)$ & $10.5(0.25)$ & $\mathrm{C}$ & $6.5(1.2)$ & $0.80(0.11)$ & $24.7(4.4)$ \\
\hline 5 & $14.7(1.3)$ & $10.7(1.4)$ & RW & $5.1(1.1)$ & $1.02(0.07)$ & $25.7(3.7)$ \\
\hline 6 & $14.3(1.0)$ & $10.2(0.44)$ & $\mathrm{C}$ & $7.8(2.4)$ & $0.64(0.08)$ & $30.2(3.0)$ \\
\hline 7 & $14.1(1.0)$ & $10.1(0.34)$ & $\mathrm{C}$ & $5.2(0.3)$ & $0.66(0.07)$ & $26.5(4.2)$ \\
\hline 8 & $10.8(2.2)$ & $8.24(0.88)$ & $\mathrm{R}$ & $4.2(0.8)$ & $0.84(0.06)$ & $24.0(2.4)$ \\
\hline 9 & $12.0(1.1)$ & $9.0(0.34)$ & $\mathrm{C}$ & $6.9(0.5)$ & $0.57(0.06)$ & $27.0(5.3)$ \\
\hline 10 & $11.7(1.2)$ & $8.8(0.51)$ & $\mathrm{C}$ & $7.5(2.7)$ & $0.67(0.084)$ & $23.0(2.6)$ \\
\hline 11 & $11.9(2.5)$ & $9.2(0.89)$ & $\mathrm{R}$ & $6.2(0.6)$ & $0.83(0.04)$ & $25.8(3.2)$ \\
\hline 13 & $8.7(1.4)$ & $7.4(0.81)$ & $\mathrm{R}$ & $8.7(0.3)$ & $0.32(0.05)$ & $25.8(4.6)$ \\
\hline 14 & $10.4(0.9)$ & $7.7(0.33)$ & $\mathrm{C}$ & $8.5(1.8)$ & $0.34(0.05)$ & $25.2(2.7)$ \\
\hline 15 & $15.3(3.3)$ & $12.3(0.97)$ & $\mathrm{R}$ & $7.4(2.6)$ & $0.91(0.13)$ & $19.7(5.2)$ \\
\hline 16 & $20.8(1.6)$ & $15.3(0.34)$ & $\mathrm{C}$ & $7.2(0.3)$ & $1.69(0.13)$ & $20.3(2.4)$ \\
\hline
\end{tabular}

${ }^{1}$ sensor located $28 \mathrm{~cm}$ above fuel tray surface $(20.4 \mathrm{~cm}$ above upper surface of fuel) at location $20 \mathrm{~m}$ along length of bed.

${ }^{2} C$-constant wind speed and direction, $R$ - "natural wind" with speed and direction varying in time, $R W$-speed varying in time but direction held constant.

3 average followed by standard deviation in parentheses.

A common landscaping material in this region of the United States consists of bales dead pine needles (primarily from Slash (Pinus elliottii) and long leaf (Pinus palustris) pine trees), this material is commonly referred to as pine straw. Taking advantage of the availability of this material, a supply of pine straw bales was purchased and stored in a climate controlled container. Immediately prior to each burn sufficient amounts of bales were removed from storage, separated into weighed amounts and distributed on the fuel trays to a target fuel depth $(8.9 \mathrm{~cm})$ and bulk density (packing ratio) per unit area of 0.02 . Fuel loading was nominally $0.84 \mathrm{~kg} / \mathrm{m}^{2}$ adjusted for moisture content. Samples of fuels were collected immediately prior to ignition from nominally $3 \mathrm{~m}$ and $20 \mathrm{~m}$ along the longitudinal axis of each fuel bed to determine fuel moisture content. Moisture content was nominally $15 \%$ for burns 1 and 2,12\% for burn 3, and between 5 and $8 \%$ for all other burns.

In a typical burn, pine needles were distributed at specified depth and bulk density, data loggers were started, fuel moisture samples were collected, wind fans were set to the idle setting $(\sim 5 \mathrm{~m} / \mathrm{s})$. Once all preparations were complete the leading edge of the fuel beds was ignited manually using handheld propane torches, as soon as the flame front was established personnel left the area. At this time the fans would be sped up to the target wind speed and profile. Time between ignition and fan ramp up was less than 3 seconds. Time for fans to accelerate from idle to full speed was less than 3 seconds. After a burn was complete the ash residue was removed and a new fuel bed constructed. 


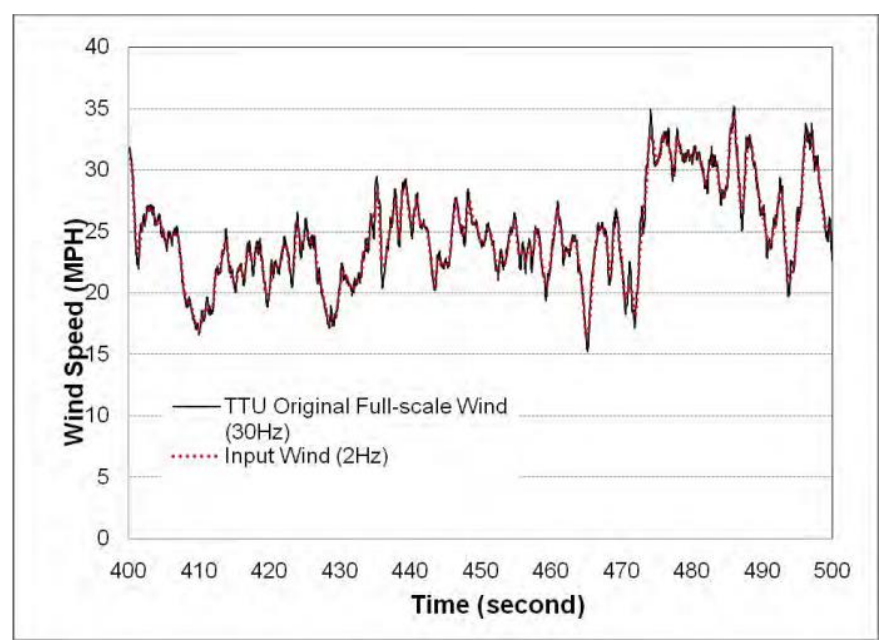

Figure 2 - Wind speed as measured in open terrain and replicated by the IBHS facility. Source http://disastersafety.org/wp-content/uploads/2015/07/wind-flow-validation-report_IBHS.pdf

Fire rate of spread was characterized from analysis of overhead visual video records. For this method visible targets were placed on the rail forming the edge of the fuel tray and on the floor between the two fuel beds. These targets had a length designation as well as a unique identifier that was visible in the video image (a number constructed of aluminum foil). Overall rate of spread was calculated by recording the fire front transit time between 5 and $24 \mathrm{~m}, 10$ and $20 \mathrm{~m}$, and 15 and $24 \mathrm{~m}$ along the bed tray length except for the zero wind burn where rate of spread was calculated as the time to spread from 0 to 3,1 to 2 and 2 to $3 \mathrm{~m}$.

Flame residence was calculated from overhead video record as the time for the flame front to spread over a specified distance. Ending time was defined as that which the majority of flaming combustion was complete; as for many cases at the higher wind speeds the partially burned fuel bed was blown down the fuel tray, essentially forming a secondary flaming and smoldering rolling flame front.

For a subset of experiments, radiant and convective heating were measured by sensors located approximately $0.5 \mathrm{~m}$ high at 5 and $25 \mathrm{~m}$ along the longitudinal axis of the fuel tray. These sensors have been described elsewhere (Butler et al. 2010; Frankman et al. 2013a) and are designed to measure heating from the flame incident on the sensor as the flames approach and burn around the sensor. Sensors were sampled at $10 \mathrm{hz}$. Reported values represent $3 \mathrm{~s}$ averages of the measured signal (Frankman et al. 2013b).

\section{Results and Discussion}

The experiments were performed with winds varying in both speed and direction. The relevant data are grouped by target wind speed $6.8,7.9,9,11.3 \mathrm{~m} / \mathrm{s}(15,17.5,20$ and $25 \mathrm{mph})$ and are shown in figure 3. In all cases the average rates of spread for the natural wind field driven fires were 20 to $90 \%$ greater than the fires burning under the constant speed wind over the range of average wind speeds of 9 to $15 \mathrm{~m} / \mathrm{s}$.

The time averaged wind speeds within a specific target wind speed group varied slightly between experiments, for example two separate burns of two beds each were conducted for the 20-C group with a slightly different average speed between burns. This variation is due to slight differences in the burning environment such as winds around the exterior of the facility and relative humidity; but in all cases the average rate of spread for the "natural" wind driven fires was faster than that for the constant speed wind driven fires across all target wind speed groups even when the average speed for the "natural" wind was slightly lower than that of the constant wind burns (i.e. 15-C and 15-R data). Of particular interest is the single burn set that was conducted at a target of $11.3 \mathrm{~m} / \mathrm{s}(25 \mathrm{mph})$ with 
constant speed but varying direction (data set 25-C-R). The rate of spread for these fires was greater than the constant wind case and similar to the fully "natural" wind case. While this is just one data point it implies that the higher fire spread rates associated with the natural winds are not due to the pulses in wind speed that exceed the average speed, but are rather due to turbulence introduced by the directional variations. This observation has implications to modeling of fire spread and warrants further exploration. The range of wind speeds explored in this study was not sufficient to explore the presence of the two regimes for natural winds as was observed for the larger data set.

While not shown here, measurements of radiant and total energy release were collected for a subset of the experiments. Radiant energy release was nominally two to ten times greater than the convective heating for the constant speed wind driven fires and five to ten times greater than the convective heating for the "natural" wind driven fires suggesting that turbulence and convective heating events are more common in fires burning under "natural" winds.

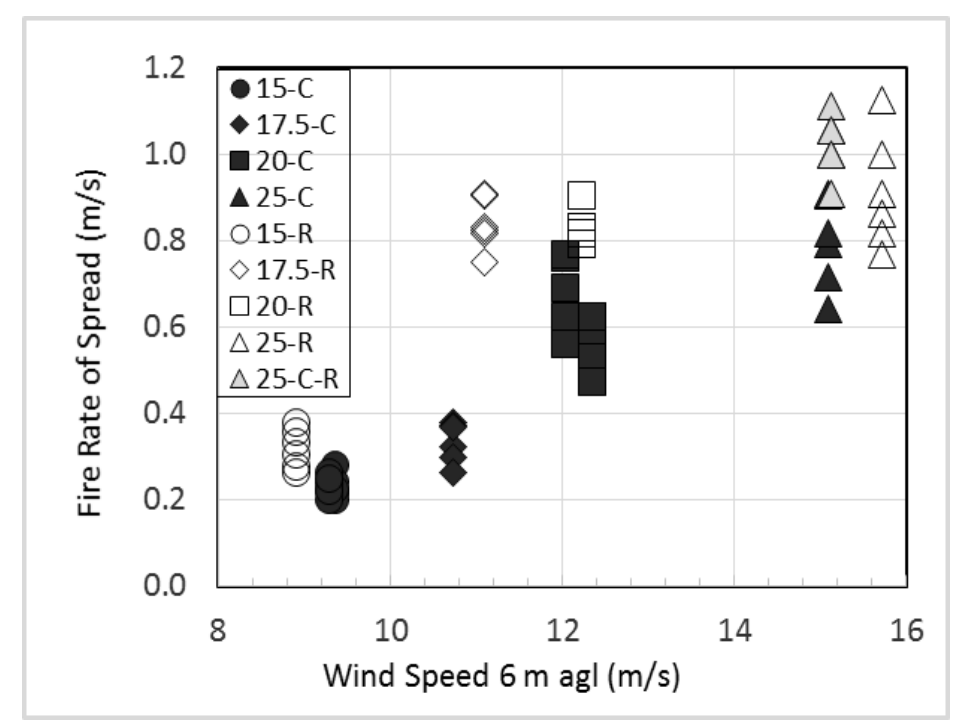

Figure 3 - Fire rate of spread under constant and naturally varying wind fields. The data are arranged by target average wind speed and wind type (e.g. 15-R indicates $15 \mathrm{mph}(6.8 \mathrm{~m} / \mathrm{s})$ target speed at "real" wind conditions, 20-C represents target average wind speed of $20 \mathrm{mph}(9 \mathrm{~m} / \mathrm{s})$ at constant speed and direction, 25-C-R represents a constant fan speed and varying direction wind condition).

\section{Conclusions}

This work explores the relation between wind speed and fire spread rate in fine dead fuels for a wider range of winds speeds than any previously reported efforts. The effort includes a limited exploration of the response of fire to natural winds (that is winds that vary in speed and direction). The results suggest that natural winds result in faster fire spread rates than a constant wind at the same average speed. This finding has significant implications to the development of models simulating fire growth. In the context of the wind limit observed in the MacArthur (1969) data and imposed by Rothermel (1972) in his original model, an optimum wind speed can be postulated based on the expectation that at some high wind flow convective cooling will dominate any heating thus the flame will essentially be "blown" out. As concluded by others (Andrews et al. 2013) these data did not exhibit a wind limit, at least up to a wind speed of $27 \mathrm{~m} / \mathrm{s}(60 \mathrm{mph})$. However, the data do show a nonlinear relation between wind speed and fire rate of spread. The increased dependency as wind exceeds $6 \mathrm{~m} / \mathrm{s}$ and the indication that fires spread faster under natural winds than constant winds has implications to both fire behavior and fire danger rating systems.

Advances in Forest Fire Research 2018 - Page 470 


\section{Acknowledgements}

This work was supported through funding provided from the office of the US Forest Service Deputy Chief for Research. The gracious assistance of the IBHS staff during the experiments is also appreciated as are the comments from the anonymous reviewers.

\section{References}

Albini, FA (1982) Response of free-burning fires to nonsteady wind. Combustion Science \& Technology 29, 225-241.

Andrews, PL, Cruz, MG, Rothermel, RC (2013) Examination of the wind speed limit function in the Rothermel surface fire spread model. International Journal of Wildland Fire 22, 959-969.

Beer, T (1991) The interaction of wind and fire. Boundary-Layer Meteorology 54, 287-308.

Beer, T (1993) The speed of a fire front and its dependence on wind speed. International Journal of Wildland Fire 3, 193-202.

Butler, BW, D Jimenez, J Forthofer, K Shannon, and P Sopoko. (2010) A Portable System for Characterizing Wildland Fire Behavior. Paper presented at the Proceedings of the 6th International Conference on Forest Fire Research.[CD-ROM]. Coimbra, Portugal: University of Coimbra.

Catchpole, WR, Catchpole, EA, Butler, BW, Rothermel, RC, Morris, GA, Latham, DJ (1998) Rate of spread of free-burning fires in woody fuels in a wind tunnel. Combustion Science Technology 131, 1-37.

Fang, JB (1969) An investigation of the effect of controlled wind on the rate of fire spread. University of New Brunswick.

Frankman, D, Webb, BW, Butler, BW, Jimenez, D, Forthofer, JM, Sopko, P, Shannon, KS, Hiers, JK, Ottmar, RD (2013a) Measurements of convective and radiative heating in wildland fires. International Journal of Wildland Fire 22, 157-167.

Frankman, D, Webb, BW, Butler, BW, Jimenez, D, Harrington, M (2013b) The effect of sampling rate on interpretation of the temporal characteristics of radiative and convective heating in wildland flames. International Journal of Wildland Fire 22, 168-173.

McArthur, AG (1966) Weather and grassland fire behaviour. Commonwealth of Australia No. No. 100, Canberra, Australia.

McArthur, AG (1969) The Tasmanian bushfires of 7 February 1967 and associated fire characteristics. In 'Mass Fire Symposium. Canberra ACT Australia'. (Defence Standards Laboratories, Maribymong, Victoria:

Nelson Jr, RM, Adkins, CW (1988) A dimensionless correlation for the spread of wind-driven fires. Canadian Journal of Forest Research 18, 391-397.

Nelson, RM, Jr, Adkins, CW (1987) A dimensionless correlation for the spread of wind-driven fires.

Rothermel, RC (1972) A mathematical model for predicting fire spread in wildland fuels. No. USDA Forest Service Research Paper INT-115, Ogden, UT.

Weise, DR, Biging, GS (1996) Effects of wind velocity and slope on flame properties. Canadian Journal of Forest Research 26, 1849-1858.

Wotton, B, McAlpine, R, Hobbs, M (2000a) The effect of fire front width on surface fire behaviour. International Journal of Wildland Fire 9, 247-253.

Wotton, BM, McAlpine, RS, Hobbs, MW (2000b) The Effect of Fire Front Width on Surface Fire Behavior. International Journal of Wildland Fire 9, 247-253. 


\title{
Fire seasons in Portugal: the role of weather and climate
}

\author{
Malik Amraoui ${ }^{1 *}$; Joana Parente ${ }^{1}$; Mário G. Pereira ${ }^{1,2}$ \\ ${ }^{1}$ Centro de Investigação e Tecnologias Agroambientais e Biológicas, Universidade de Trás-os- \\ Montes e Alto Douro, Quinta de Prados, 5000-801 Vila Real, Portugal, \{malik@utad.pt*, \\ joanaparente@utad.pt,gpereira@utad.pt\} \\ ${ }^{2}$ Instituto Dom Luiz, Faculdade de Ciências da Universidade de Lisboa, Lisboa, Portugal, \\ \{gpereira@utad.pt\}
}

\begin{abstract}
Forest fires (FFs) are critical elements of Earth system at the global, regional and local scales and, independently of their causes may have a high environmental, social, economic and climatological effects as well as cause loss of lives and injured people. Weather and climate control, directly and indirectly, most of the aspects of FFs. This study aims to identify: (i) the temporal variability of FFs, with special attention to the differences between the spring and summer fire seasons; and, (ii) the weather conditions during the days and months of higher and lower fire incidence. The fire database used is the Portuguese Rural Fire Dataset that comprises relevant information about each fire event such as: ignition date, fire duration, location ignition and total burnt area (BA). The meteorological dataset used is the ERA-Interim which covers the climatological 35-years period (1980 - 2014) and consists of daily fields at 12 UTC, with a spatial resolution of $0.125^{\circ} \times 0.125^{\circ}$ latitude/longitude grid, over a spatial domain $\left(10^{\circ} \mathrm{W}-6^{\circ} \mathrm{E}, 36.5^{\circ} \mathrm{N}-42.5^{\circ} \mathrm{N}\right)$ centred in Portugal. The role played by the meteorological conditions over a given region is assessed by analysing the associated patterns of thermo-hydro-dynamical fields at different atmospheric levels. Analysed patterns are anomaly composites, consisting of arithmetic means (performed over all days associated with very high number of FFs) of daily departures of 12 UTC meteorological fields from the respective average for that day over the reference period $(1980-2014)$. Temporal variability of fires (number of forest fires, NFF) in Portugal unveil two fire seasons: (i) the first one, during winter-spring with highest activity in March (part of the non-critical period, NCP); and, (ii) the second, in summer with pronounced peak in July and August (during the critical period, CP). Atmospheric thermodynamic and circulation patterns confirm the existence of anomalous conditions observed over the affected areas, on daily and monthly scales. This atmospheric circulation is characterized by predominant easterlies crossing that advect continental air into the sub-region. During summer, the circulation is characterized by a predominant hot and dry flow from continental Europe and north Africa, contributing to values of air temperature (relative humidity) well above (below) average. During winter, the extremely low values of relative humidity are the most noticeable feature, which result from the anomalous Eastward circulation over the cold and dry Western Europe. The findings of this study will contribute to a better fire prevention, firefighting and crisis management.
\end{abstract}

Keywords: Temporal variability; Fire Season; Forest Fires; Weather conditions; Portugal

\section{Introduction}

The natural vegetation in most of Europe is essentially forest and woodland from the Mediterranean through the deciduous forests of Central and Western Europe to the boreal forests in Fennoscandia (Bengtsson et al., 2000). Over the last 20 years, the forested area expanded in all European regions and has gained 0.8 million hectares each year (Forest Europe, 2011) except in southern European countries, where fires burn large areas every year (Schmuck et al, 2012).

Forest fires (FFs) are critical elements of Earth system at the global, regional and local scales and, independently of their causes (lightning/natural, negligent or intentional) may have a high environmental, social, economic and climatological effects as well as cause loss of lives and injured 
people. (Amraoui et al. 2015; Parente et al. 2016). Fires affect a larger area over a wider variety of biomes across the globe than any other natural disturbance to land-based eco-systems (Ichoku et al., 2008). The Mediterranean region, including Portugal, occupies a prominent position, given the occurrence of devastating fires that burn hundreds of thousands of hectares of forest, scrublands and grasslands every year (Barbosa et al., 2007). In this region, fire ignition is strongly conditioned by human behaviour and socioeconomic activities (Costa et al., 2010) but natural factors like the morphology of the landscape, land use, land cover, and meteorological conditions have also to be taken into account (Amraoui et al., 2013, Parente et al., 2018a).

Despite its smaller land area in comparison with other Mediterranean countries, Portugal is the European country with highest total number of FFs and the second largest total BA (San-MiguelAyanz et al., 2016). In Portugal, fire incidence (number of FFs and BA) presents a high spatial and temporal variabilities, with weather and climate conditions as the main drivers of the temporal distribution (Pereira et al., 2005; Parente et al. 2017) having a profound influence at all stages of biomass burning from ignition, spread and behaviour up to severity and effects. In this context, the aims of this study are to identify: (i) the temporal variability of FFs, namely the intra-annual variability, (ii) with special attention to the differences between the spring and summer fire seasons; and, (iii) the weather conditions during the periods of higher and lower fire incidence.

\section{Data and Methodology}

\subsection{Study Area}

Mainland of Portugal is located on the south-western coast of the Iberian Peninsula just at a few hundred kilometres of North Africa with a land area of about $90,000 \mathrm{~km}^{2}$. The Tagus River divides the territory into two regions with similar area but very different in terms of several biophysical and human drivers (Parente et al. 2016), which combined with the occurrence of extreme hot spells and drought periods promote the occurrence and the variability of FFs.

\subsection{Fire dataset}

Information about the FFs was obtained from the Portuguese Rural Fire Dataset (PRFD) originally available in Portuguese Institute for the Conservation of Nature and Forests portal. PRFD comprises relevant information about each fire event such as: ignition date, fire duration, location ignition and total BA. This study relied on the recent 14-years period (2001 - 2014) and based on fires having burnt area greater or equal than 0.1 ha for homogeneity reasons.

\subsection{Meteorological dataset}

The meteorological dataset used in this study corresponds to the ERA-Interim which covers the 35years climatological period $(1980-2014)$ and consists of multiple daily horizontal fields of the low and the medium atmosphere, at $12 \mathrm{UTC}$, with a spatial resolution of $0.125^{\circ} \times 0.125^{\circ}$ latitude/longitude grid, over a spatial domain $\left(10^{\circ} \mathrm{W}-6^{\circ} \mathrm{E}, 36.5^{\circ} \mathrm{N}-42.5^{\circ} \mathrm{N}\right)$ centred in Portugal for a set of the following variables: (i) mean sea level pressure, MSLP; (ii) air temperature at $2 \mathrm{~m}$ height, T2m; (iii) wind speed and direction at $10 \mathrm{~m}, \mathrm{~W} 10 \mathrm{~m}$; (iv) relative air humidity at $850 \mathrm{hPa}, \mathrm{RH} 850$; and, (v) geopotential height at $500 \mathrm{hPa}, \mathrm{Z500}$. Detailed additional information about the ERA-Interim product may be found in Dee et al. (2011).

\subsection{Methodology}

The role played by the meteorological conditions, during periods of high and low fire activity, over mainland Portugal were assessed by analysing the associated patterns of meteorological fields at different atmospheric levels, similar to what was done in previous works such as Pereira et al. (2005), Amraoui et al (2013 and 2015) and Trigo et al., (2013). Analysed patterns are composites and anomaly composites, consisting of arithmetic means (performed for a subset of days of very high/low number 
of FFs) of daily values, in the former case, and departures of 12 UTC meteorological fields from the respective average for that day over the reference period of $1980-2014$, in the latter case.

\section{Results}

\subsection{Temporal distribution of fires}

During the 14-year study period, a grand total of 145,616 FFs were observed over the study region and corresponding to $1.8 \mathrm{Mha}$ of BA, approximately. The temporal distribution of FFs reveals high inter-annual variability (Figure 1, upper panel), with the years of 2001, 2002, 2005, 2009 and 2011 presenting above-average number of FFs. In particular, the years of 2005 and 2001, correspond to the years with greater number of FFs, accounting of about 16,600 (11.4\% of total) and 15,000 (10.3\% of total), respectively. In contrast, 2014 and 2008 represent the years of very weak fire activity with only 3,100 (2.2\% of total) and 5,900 (4.1\% of total), respectively.

Regarding the intra-annual variability (Figure 1, lower panel), there is a prominent annual cycle of fire activity, showing two fire seasons in Portugal. The first one begins in late spring and lasts until early autumn (June to October), totalizing about $75 \%$ of annual FFs, with pronounced peak in AugustSeptember and corresponding to the critical period. The second season, counting about $20 \%$ of annual FFs, is observed in winter and early spring (February to April) with a less prominent peak in March and corresponding to the non-critical period. The remaining 5\% of annual FFs are registered during the months of November, December and January, corresponding to the period with lowest fire activity.

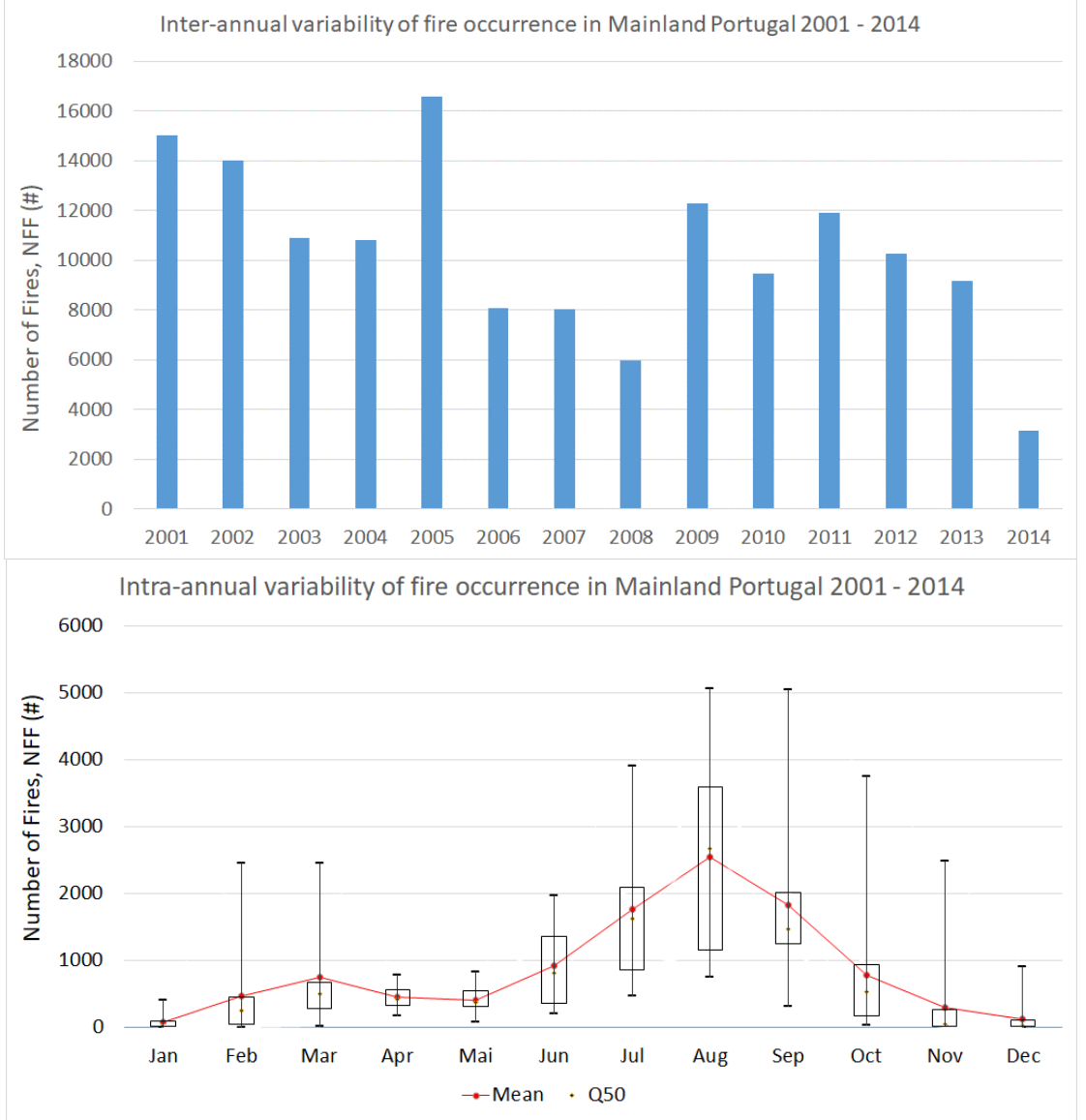

Figure 1 - Inter-annual (upper panel) and intra-annual (lower panel) variability of fire occurrence in mainland Portugal. The used data in the figure refers to all fires, with BA greater than 0.1 ha, observed in the country during the 2001-2014 study period. In the lower panel and for the monthly FFs, the black (red) dots represent the median (mean) value, the lower (upper) box limits represent the first (third) quartile and the lower (upper) whisker represents the minimum (maximum) value.

Advances in Forest Fire Research 2018 - Page 474 


\subsection{Atmospheric conditions associated with high fire activity}

Results obtained in the previous section suggest performing an assessment of the role played by meteorological conditions on the onset and spreading of fire events in mainland Portugal, during the two fire seasons. The weather conditions during periods with very low fire activity were also analysed. Anomaly composites fields of meteorological variables for periods of very high and very low fire activity in the region and season will be presented. Each plot includes results obtained for more than one meteorological field and for each case, two figures of anomalies are presented. The first one respecting to meteorological fields near the surface (T2m, MSLP and W10m) and the other at $500 \mathrm{hPa}$ and $850 \mathrm{hPa}$ levels (Z500 and RH850).

The vast majority of burned area in Portugal is due to a reduced number of extreme events that occur during a short period of time and are associated with several atmospheric processes interacting at different temporal and spatial scales. In general, when high fire incidence is registered, atmospheric thermodynamic and circulation patterns confirm the existence of anomalous conditions observed over the affected areas, on daily and even monthly scales.

\subsubsection{High fire activity during winter}

When FFs occur in the winter-spring season (e.g. Figure 2), the atmospheric surface circulation over Portugal is generally characterized by predominant easterlies that advect continental (cold and very dry) air into the region (Figure 2, lower panel), causing extremely low values of RH850 (Figure 2 , upper panel). These patterns are a usually a consequence of a strong omega blocking configuration (not shown) located west of Europe, enhancing the effect of the anticyclone over Western and Northern Iberia (Amraoui et al., 2015).
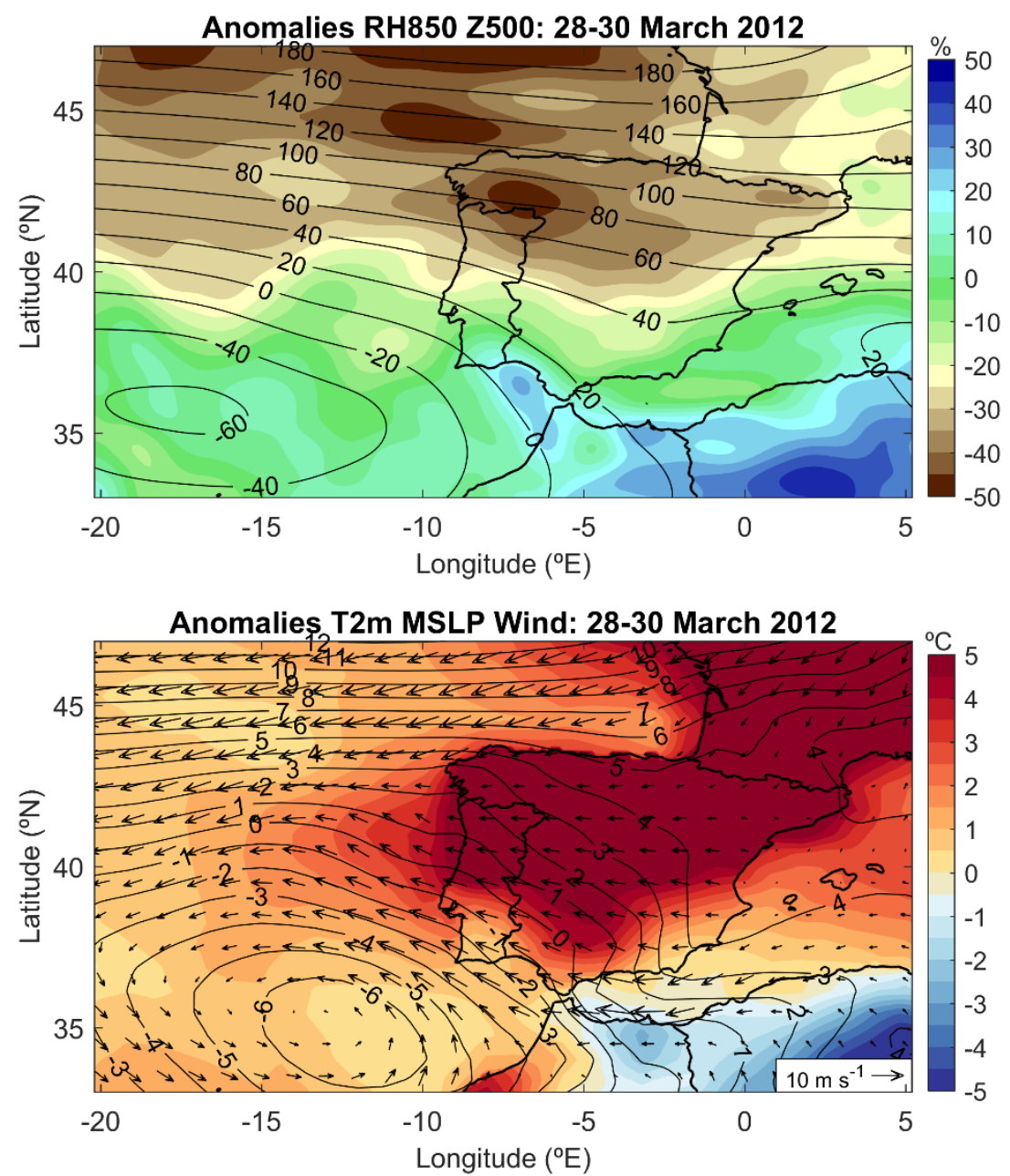

Figure 2 - Anomaly composites of relative air humidity (\%) at $850 \mathrm{hPa}$ level (RH850) and geopotential height (gpm) at $500 \mathrm{hPa}$ (Z500) (upper panel) as well as of air temperature $\left({ }^{\circ} \mathrm{C}\right)$ at $2 \mathrm{~m}(\mathrm{~T} 2 \mathrm{~m})$, mean sea level pressure (hPa) (MSLP), wind speed $\left(\mathrm{m} \cdot \mathrm{s}^{-1}\right)$ and direction at $10 \mathrm{~m}$ (W10m) (lower panel) over Continental Portugal for the period between 28 and 30 of March 2012. 
The anomalous circulation patterns at the $500 \mathrm{hPa}$ level during periods of high fire activity in the winter-spring season are dominated by positive departures of Z500 reaching the impressive value of about $180 \mathrm{gpm}$ (Figure 2, upper panel) over the British Isles. On the other hand, anomalies of meteorological parameters show higher values of T2m than normal over all Iberia and south of France (Figure 2, lower panel) and very low values of RH850, reaching the impressive value of $-50 \%$ in some of the cases (Figure 2, upper panel).

\subsection{High fire activity during summer}

During the summer-autumn season (e.g. Figure 3), the circulation pattern leads to strong NorthEastern, from continental Europe, and South-Eastern, from Northern Africa, advections of continental dry and very warm air over mainland Portugal, contributing to values of air temperature (relative humidity) well above (below) average. This atmospheric circulation is the result from the amplification of the anticyclone of Azores, extending from the Atlantic to Central Europe and by a sub-Saharan thermal low centred at South Algeria (not shown).
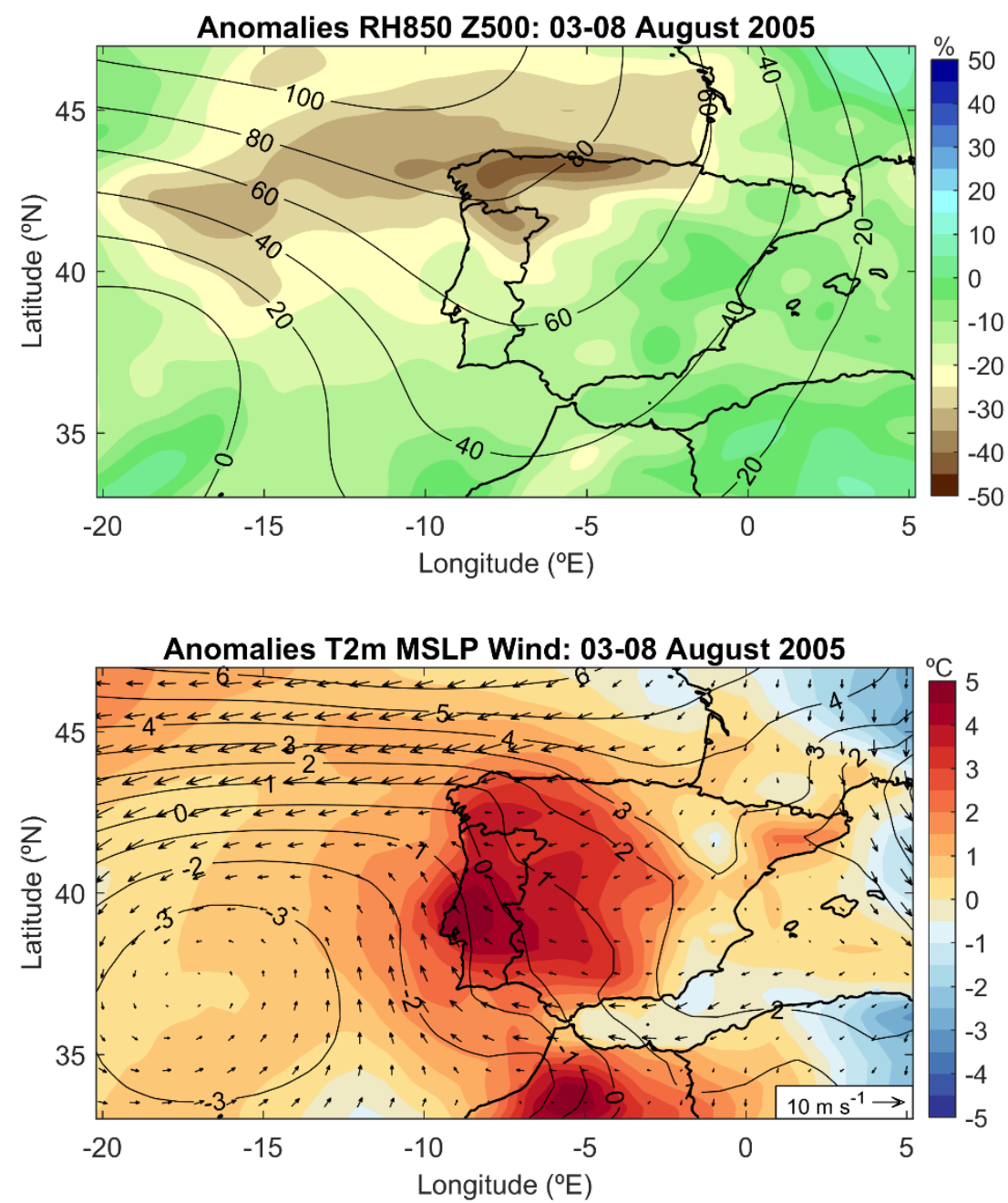

Figure 3 - Anomaly composites of air relative humidity (\%) at 850 hPa level (RH850) and geopotential height (gpm) at $500 \mathrm{hPa}$ (Z500) (left panel) as well as of air temperature $\left({ }^{\circ} \mathrm{C}\right)$ at $2 \mathrm{~m}(\mathrm{~T} 2 \mathrm{~m})$, mean sea level pressure (hPa) (MSLP), wind speed $(\mathrm{m} \cdot \mathrm{s}-1)$ and direction at $10 \mathrm{~m}(\mathrm{W10m})$ (right panel) over Continental Portugal for the period between 03 and 08 of August 2005.

The atmospheric flow at $500 \mathrm{hPa}$ (not shown) generally presents the synoptic baroclinic activity with a pronounced ridge with axes in the Southeast to Northwest direction over the Atlantic Ocean and West of Iberia, forcing warmer and drier air mass into the region. The case presented in figure 3 
corresponds to the Arouca extreme fire event that occurred between 03 and 08 of August 2005. In the above-mentioned case, the 500 level composite anomaly patterns exhibit positive departures of 80 gpm (Figure 3, upper panel) and, consequently, enhancing the subsidence of air into the region, reinforcing the increase of air temperature. At surface level, anomalies of meteorological parameters present low values of relative humidity (Figure 3, upper panel) and very hot air temperature (Figure 3, lower panel).

\subsubsection{Low fire activity}

On the other hand, when low fire incidence is observed (e.g. Figure 4) the atmospheric circulation is mostly dominated by western advection of mild wet air from the Atlantic over mainland Portugal, resulting in positive departures and very high values of RH850.
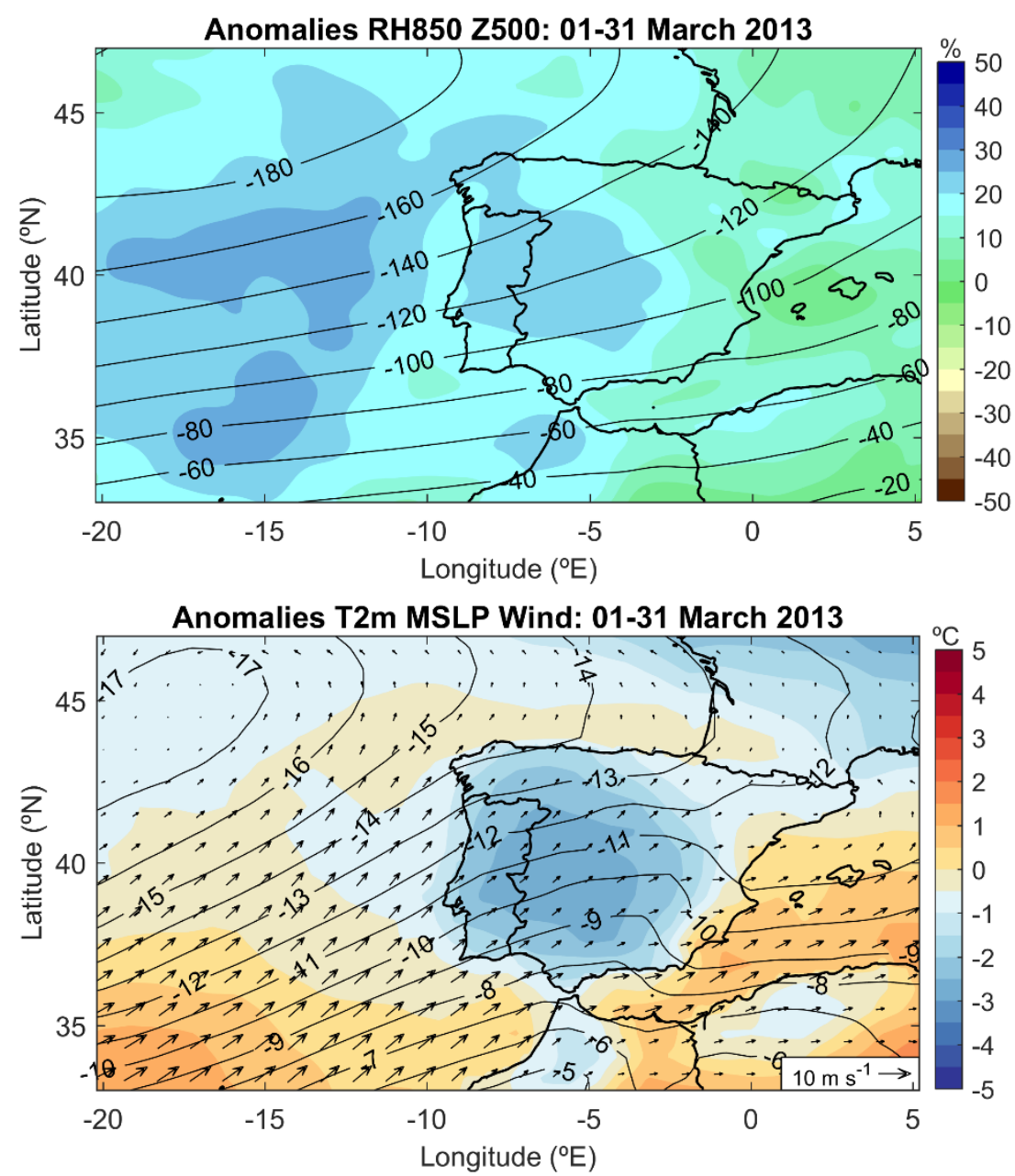

Figure 4 - Anomaly composites of relative air humidity (\%) at 850 hPa level (RH850) and geopotential height (gpm) at $500 \mathrm{hPa}$ (Z500) (left panel) as well as of air temperature $\left({ }^{\circ} \mathrm{C}\right)$ at $2 \mathrm{~m}(\mathrm{~T} 2 \mathrm{~m})$, mean sea level pressure (hPa) (MSLP), wind speed $\left(\mathrm{m} \cdot \mathrm{s}^{-1}\right)$ and direction at $10 \mathrm{~m}(\mathrm{~W} 10 \mathrm{~m})$ (right panel) over Continental Portugal for March of 2013

The case presented in figure 4 corresponds to March 2013 where a few number of fires was recorded. The atmospheric conditions in the region during the referred month are associated with cyclonic configurations located over the Atlantic Ocean, west, northwest or southwest of the Iberian Peninsula favouring precipitation over Portugal through strong advection of maritime air masses.

\section{Conclusions}

The intra- and inter-annual variability of fire activity in Mainland Portugal is strongly associated with the type of climate (Costa et al., 2010) and the occurrence of extreme weather conditions (Pereira 
et al., 2005; Amraoui et al., 2015). However, there is not a cause-effect relationship between atmospheric conditions and fire activity in the sense that fire ignition in Portugal, in particular, and Mediterranean region, in general, is mainly due to human activities (San-Miguel-Ayanz et al., 2013). The temperate type of the Mediterranean basin climate, characterized by wet and mild winters, favour the growth of vegetation, followed by warm and dry weather during summer, promote severe vegetation hydric and thermal stress (Pereira et al., 2013). In Portugal, and during the summer season, the regional heatwaves are very frequent and highly correlated with the occurrence of the most devastating fires in Portugal (Parente et al., 2018b). In addition, recent studies have shown that the occurrence of major droughts in southern Europe during the preceding winter and spring seasons may enlarge the amplitude of heatwaves in the following summer, which in turn, should be viewed as a primary factor of fire danger in this region because of their influence on fuel availability and vegetation hydric and thermal stress.

The vast majority of modelling studies agrees in that the Mediterranean will become hotter and drier in the future favouring the increase in frequency and amplitude of heatwaves over the Mediterranean basin (Fischer and Schär, 2010; Parente et al., 2018b) and, consequently, the intensification of catastrophic fire events and the resulting very high annual burnt area (Trigo et al., 2006, Amraoui et al, 2013). In this sense, knowing the spatial configuration of the meteorological fields associated with highest fire activity is of paramount importance for the planning of fire prevention activities and management of fire suppression resources (Amraoui et al. 2015). In this sense, the findings of this study will contribute to a better fire prevention, firefighting and crisis management.

\section{Acknowledgements}

This work was funded by the R\&D Project FIREXTR - Prevent and prepare society for extreme fire events: the challenge of seeing the "forest" and not just the "trees", with reference POCI-01-0145FEDER-016702 and PTDC/ATP-GEO/0462/2014, financed by the European Regional Development Fund (ERDF) through COMPETE 2020 - Operational Program for Competitiveness and Internationalization (POCI) and by the Foundation for Science and Technology (FCT). The study was also supported by Project Interact - Integrative Research in Environment, Agro-Chain and Technology, NORTE-01-0145-FEDER-000017, research line BEST, co-funded by FEDER/NORTE 2020.

\section{References}

Amraoui, M., Liberato, M.L.R., Calado, T.J., DaCamara, C.C., Pinto Coelho, L., Trigo, R.M., Gouveia, C.M., 2013. Fire activity over Mediterranean Europe based on information from Meteosat-8. For. Ecol. Manag. 294, 62-75.

Amraoui M, Pereira MG, DaCamara CC, Calado TJ, 2015. Atmospheric conditions associated with extreme fire activity in the Western Mediterranean region. Sci Total Environ 524:32-39.

Barbosa, P., Amatulli, G., Boca, R., Camia, A., Kucera, J., Libertà, G., San-Miguel Ayanz, J., Schmuck, G., Schulte, E., Dierks, H.-H., 2007. Forest fires in Europe 2006. EUR 22931 EN Joint Research Centre - Institute for Environment and Sustainability. EUR, Scientific and Technical Research seriesOffice for Official Publications of the European Communities, Luxembourg

Bengtsson J, Nilsson SG, Franc A, Menozzi P, 2000. Biodiversity, disturbances, ecosystem function and management of European forests. Forest Ecology and Management 132: 39-50

Costa, L., Thonicke, K., Poulter, B., Badek, F.W., 2010. Sensitivity of Portuguese forest fires to climatic, human, and landscape variables: subnational differences between fire drivers in extreme fire years and decadal averages. Reg. Environ. Chang. 11 (3), 543-551. 
Dee, D.P., Uppala, S.M., Simmons, A.J., Berrisford, P., Poli, P., Kobayashi, S., Andrae, U., Balmaseda, M.A., Balsamo, G., Bauer, P., Bechtold, P., Beljaars, A.C.M., van de Berg, L., Bidlot, J., Bormann, N., Delsol, C., Dragani, R., Fuentes, M., Geer, A.J., Haimberger, L., Healy, S.B., Hersbach, H., Hólm, E.V., Isaksen, L., Kållberg, P., Köhler, M., Matricardi, M., McNally, A.P., Monge-Sanz, B.M., Morcrette, J.-J., Park, B.-K., Peubey, C., de Rosnay, P., Tavolato, C., Thépaut, J.-N., Vitart, F., 2011. The ERA-Interim reanalysis: configuration and performance of the data assimilation system. Q. J. R. Meteorol. Soc. 137, 553-597.

Fischer, E.M., Schär, C., 2010. Consistent geographical patterns of changes in high impact European heatwaves. Nature Geosci.

Forest Europe U. FAO, 2011. State of Europe's forests 2011. Status and trends in sustainable forest management in Europe. In: Ministerial Conference on the Protection of Forests in Europe. pp: 978982.

Ichoku C, Giglio L, Wooster MJ, Remer LA, 2008. Global characterization of biomass-burning patterns using satellite measurements of fire radiative energy. Remote Sensing of Environment 112: 2950-2962.

Parente J, Pereira MG, Tonini M, 2016. Space-time clustering analysis of wildfires: The influence of dataset characteristics, fire prevention policy decisions, weather and climate. Sci Total Environ 559:151-165.

Parente J, Pereira MG, Amraoui M, Tedim F, 2017. Negligent and intentional fires in Portugal: Spatial distribution characterization. Sci Total Environ 624:424-437.

Parente J, Pereira MG, Amraoui M., Tedim, F., 2018a. Negligent and intentional fires in Portugal: Spatial distribution characterization. Science of The Total Environment, 624, 424-437.

Parente J, Pereira MG, Amraoui M., Fischer E.M., 2018b. Heat waves in Portugal: Current regime, changes in future climate and impacts on extreme wildfires. Science of The Total Environment, 631-632, 534-549.

Pereira, M.G., Trigo, R.M., DaCamara, C.C., Pereira, J.M.C., Leite, S.M., 2005. Synoptic patterns associated with large summer forest fires in Portugal. Agric. For. Meteorol. 129, 11-25

Pereira, M.G., Calado, T.J., DaCamara, C.C., Calheiros, T., 2013. Effects of regional climate change on rural fires in Portugal. Clim. Res. 57, 187-200.

San-Miguel-Ayanz, J., Moreno, J.M., Camia, A., 2013. Analysis of large fires in European Mediterranean landscapes: lessons learned and perspectives. For. Ecol. Manag. 294, 11-22.

San-Miguel-Ayanz, J., Durrant, T.H., Boca, R., Libertá, G., Boccacci, F., Di Leo, M., Pérez, J.L., Schulte, E., Benchikha, A., Abbas, M., 2016. Forest Fires in Europe, Middle East and North Africa 2015.

Schmuck, G., San-Miguel-Ayanz, J., Camia, A., Durrant, T., Boca, R., Libertá, G., Schulte, E., 2013. Forest Fires in Europe, Middle East and North Africa 2012. Publications Office of the European Union, Luxembourg.

Trigo, R.M., Pereira, J.M., Pereira, M.G., Mota, B., Calado, M.T., DaCamara, C.C., Santo, F.E., 2006. The exceptional fire season of summer 2003 in Portugal. Int. J. Climatol. 26, 1741-1757.

Trigo, R.M., Sousa, P.M., Pereira, M.G., Rasilla, D., Gouveia, C.M., 2013. Modelling wildfire activity in Iberia with different atmospheric circulation weather types. Int. J. Climatol. 


\title{
Flammability at field-scales: conducting research in prescribed burns
}

\author{
Jane Cawson*; Thomas Duff \\ University of Melbourne, Burnley, VIC, Australia \\ \{jane.cawson@unimelb.edu.au*,tjduff@unimelb.edu.au\}
}

\begin{abstract}
To better understand the role of plant flammability in driving landscape-scale fire behaviour and fire regimes, field-scale flammability research needs to occur. Yet, experimental fires are costly to implement and research within wildfires is both logistically challenging and potentially dangerous. As an alternative, we propose that operational prescribed burns undertaken for land management purposes should be exploited for flammability research.. In some parts of the world, large areas are burnt annually, providing extensive opportunities for research. In this paper we describe three broad methods that can be used to measure different facets of flammability in prescribed burns. We compare the strengths and potential limitations of each method before finally providing ten principles for conducting effective flammability research in prescribed burns. We conclude that operational prescribed burns are a largely untapped resource that could be used to better understand links between plant flammability and landscape-scale fire behaviour.
\end{abstract}

Keywords: burn observation; fire agency; fire management; flammability; fire behaviour; forest; fuel; ignition; prescribed burn

\section{Introduction}

Plant flammability is an important determinant of fire behaviour and fire regimes (Gill and Zylstra 2005; Pausas et al. 2017). Flammability is broadly defined as the capacity of vegetation to burn and is often measured in terms of the specific combustion characteristics of the vegetation (ignitability, combustibility, consumability and sustainability) (White and Zipperer 2010; Varner et al. 2015). Research shows that the flammability of a plant or plant part (e.g. leaf) depends on a combination of plant traits (Varner et al. 2015; Pausas et al. 2017). Yet, there is very little understanding about how these plant traits interact and combine to influence the flammability of whole vegetation communities (Schwilk 2015; Varner et al. 2015; Cawson et al. 2018). Such knowledge is needed to better predict fire behaviour, manage fire risk across landscapes and predict future changes to fire regimes and landscape flammability.

A large body of flammability research has been conducted in laboratories (e.g. Dickinson and Kirkpatrick 1985; Dimitrakopoulos and Papaioannou 2001; Plucinski and Anderson 2008; Ganteaume et al. 2013; Possell and Bell 2013). Laboratory-based flammability studies have several advantages over field-based studies (Table 1) - confounding variables are better controlled; large quantities of data can be collected in relatively short time frames; research can proceed irrespective of seasonal climatic conditions; and costs may be less. The challenge is in translating research results derived in the laboratory to field-scale fire behaviour. Practicalities of working within a laboratory mean that the scale of research is often restricted to individual leaves, plant parts or reconstructed fuel beds and the heat source applied to the vegetation may not be representative of wildfire conditions (White and Zipperer 2010). As such, laboratory-based flammability research has been criticised for having limited applicability to field-scale flammability and fire behaviour (Fernandes and Cruz 2012). 
Table 1 - Evaluation of research environments for measuring flammability. The research environments are evaluated against criteria from 1 (highest performing) to 4 (lowest performing).

\begin{tabular}{lcccc}
\hline Evaluation criteria & \multicolumn{4}{c}{ Research environment } \\
\cline { 2 - 5 } & Laboratory & $\begin{array}{c}\text { Experimental } \\
\text { fires }\end{array}$ & Wildfires & $\begin{array}{c}\text { Prescribed } \\
\text { burns }\end{array}$ \\
\hline Safety & 1 & 3 & 4 & 2 \\
\hline Cost & 2 & 4 & 3 & 1 \\
\hline $\begin{array}{l}\text { Ability to control for confounding } \\
\text { variables }\end{array}$ & 1 & 2 & 4 & 3 \\
\hline Ability to replicate measurements & 1 & 3 & 4 & 2 \\
\hline Ability to represent wildfire conditions & 4 & 2 & 1 & 3 \\
\hline $\begin{array}{l}\text { Vulnerability of study outcomes to } \\
\text { weather }\end{array}$ & 1 & 3 & 4 & 2 \\
\hline $\begin{array}{l}\text { Control over timeframes for data } \\
\text { collection }\end{array}$ & 1 & 3 & 4 & 2 \\
\hline Bureaucratic complexity & 1 & 3 & 4 & 2 \\
\hline Average ranking & $\mathbf{1}$ & $\mathbf{3}$ & $\mathbf{4}$ & $\mathbf{2}$ \\
\hline
\end{tabular}

At a field-scales, experimental fires have historically been an important component of fire behaviour research with the data yielded from these experiments the foundation of widely used empirical fire behaviour models (Alexander and Quintilio 1990; Cruz et al. 2015). Plant flammability is generally considered in these experimental programs in terms of total biomass and dead fine fuel moisture content, with most other plant traits not considered. Experimental fire programs could be extended to better address research questions relating to community-level plant flammability (e.g. Schwilk 2003; Fraser et al. 2016). However, such experimental fires can be expensive to implement, difficult to replicate and their success is highly dependent on weather conditions during the experimental period (Cruz and Gould 2009). Another field-based alternative is research conducted within active wildfires. Such research would certainly be representative wildfire conditions. However, safety concerns, bureaucratic hurdles and logistical issues make this research difficult to design and implement (Table 1). Even the collation of wildfire statistics and observations from fire agencies can be challenging (Duff et al. 2014).

An alternative for obtaining field-scale flammability measurements is to conduct flammability research in operational prescribed burns in partnership with fire agencies. Prescribed burns are carried out by fire agencies for wildfire mitigation or ecological purposes in a range of vegetation types. Globally, the extent of prescribed burning varies widely by region depending on the risk of wildfire, political and social acceptance of fire in the landscape, fire ecology of the ecosystem and government budgets. However, in some parts of the world prescribed burning programs are well established (Fernandes et al. 2013; McCaw 2013; Forest Fire Management Victoria 2017; US Forest Service 2018). These prescribed burning programs provide many potential opportunities for flammability research to occur, even when the research budget is modest. For example, in Victoria in south-eastern Australia, on average 900 prescribed burns are conducted annually (data from 2012 to 2016) (Figure 1) (Department of Environment Land Water and Planning 2016). The main limitation of this research is that the most extreme fire conditions will not be captured. Additionally, there are several limitations of working within prescribed burns that may impede research success if not well managed.

The purpose of this paper is to provide guidance for planning and executing flammability research in operational prescribed burns. We reflect on our experiences of conducting research in prescribed burns in south-eastern Australia by outlining three broad approaches for obtaining data about field- 
scale flammability. We then outline 10 key principles for maximising the chance of a successful research project in an operational prescribed burn.

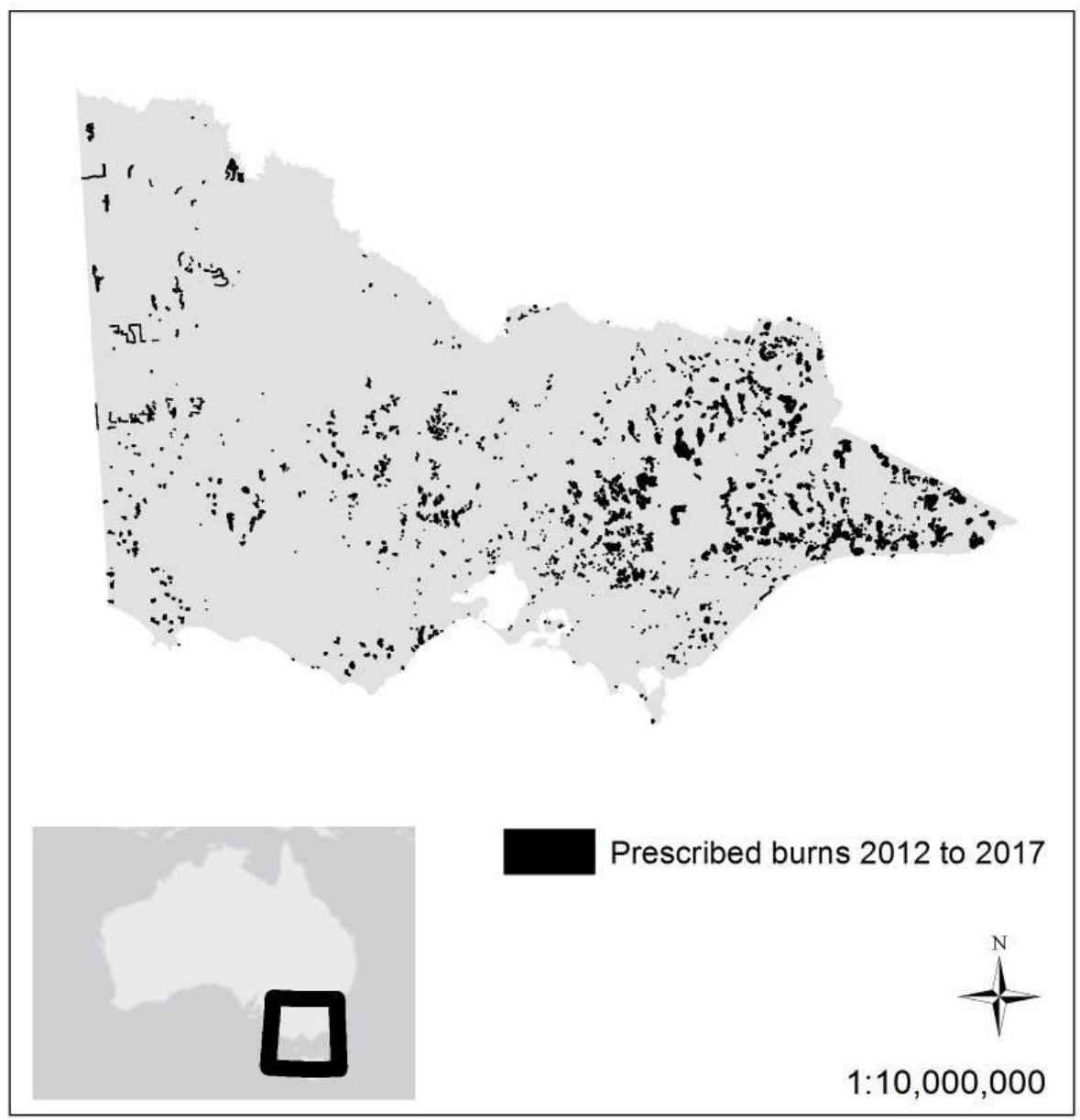

Figure 1 - Prescribed burns in Victoria, south-eastern Australia over a five-year period from 2012 to 2017. Data source: data.vic.gov.au

\section{Methods for quantifying flammability at field scales}

Here we describe three broad approaches to quantify flammability within prescribed burns:

1. Direct observation of burning - fuel, weather and burning characteristics are observed directly as researchers work alongside the lighting crew

2. Pre-and post-burn plots - plots established pre-burn to assess vegetation condition prior to the burn and the same plots are reassessed after the burn

3. Analysis of maps of burn outcomes - maps of burn extent or fire severity (often derived by the fire agency) are used to analyse burn outcomes at landscape scales

Table 2 outlines the suitability of each method to quantify the flammability (ignitability, combustibility, consumability and sustainability) of vegetation at field-scales. 
Table 2 - Suitability of methods to measure field-scale flammability in prescribed burns. 'Yes' indicates the method quantifies the flammability variable. 'No' indicates the method does not quantify the flammability variable.

\begin{tabular}{lccc}
\hline Flammability variable & $\begin{array}{c}\text { Direct observation } \\
\text { of burning }\end{array}$ & $\begin{array}{c}\text { Pre- and post- } \\
\text { burn plots }\end{array}$ & $\begin{array}{c}\text { Analysis of maps } \\
\text { of burn outcomes }\end{array}$ \\
\hline $\begin{array}{l}\text { Ignitability (ease of ignition) } \\
\begin{array}{l}\text { Combustibility (flame spread rate or mass } \\
\text { loss rate of fuel during burning) }\end{array}\end{array}$ & Yes & Yes & Yes \\
$\begin{array}{l}\text { Consumability (proportion of fuel } \\
\text { consumed, correlated with heat release) }\end{array}$ & Yes & Yes & No \\
$\begin{array}{l}\text { Sustainability (duration of flaming and/or } \\
\text { smouldering) }\end{array}$ & Yes & No & No \\
\hline
\end{tabular}

\subsection{Direct observation of burning}

Direct observation of burning involves measuring ignition success and burning characteristics while working alongside a lighting crew on the day of the burn and/or using sensors to record burning characteristics. Fuel attributes (fuel moisture content, fuel hazard, surface fuel load, dominant species, plant architecture, live-to-dead ratio) can be measured immediately ahead of the fire fighters and then the ignition process and resultant fire behaviour can be observed. Ignitability can be measured in terms of ignition success or failure with ignition deemed successful if burning is sustained beyond the initial points(s) of ignition. Combustibility can be quantified by recording the rate of spread; in the context of a prescribed burn this is usually the rate of spread of backing fire. Sustainability can be estimated by the flaming duration. Consumability can be determined by destructively sampling the litter bed preand post-burn in small plots (e.g. $0.1 \mathrm{~m}^{2}$ ).

Table 3 outlines the strengths and limitations of this research method. A key strength is fuel moisture content can be measured directly, rather than relying on predictions, because the researchers are within the plots immediately prior to burning. Additionally, fire behaviour can be measured directly, rather than inferred from char heights or crown scorch. A potential limitation is that the fire behaviour may be influenced by edge effects, as plots usually need to be situated on the burn boundary to enable safe access in dense vegetation. Additionally, burning characteristics (i.e. flames heights and rate of spread) may be a function of the lighting pattern in addition to the fuel, weather and topography.

Table 3 - Direct observation of burning - strengths and limitations

\begin{tabular}{|c|c|}
\hline Strengths & Limitations \\
\hline $\begin{array}{l}\text { Fuel moisture content is measured directly, rather } \\
\text { than relying on predictions }\end{array}$ & $\begin{array}{l}\text { Fire behaviour influenced by ignition technique } \\
\text { and edge effects as well as the fuel condition. }\end{array}$ \\
\hline $\begin{array}{l}\text { Fire behaviour is measured directly rather than } \\
\text { relying on reconstructions based on char height }\end{array}$ & $\begin{array}{l}\text { Measurements are done under time-pressure, so } \\
\text { must use rapid assessment techniques }\end{array}$ \\
\hline $\begin{array}{l}\text { Minimal pre-burn field work is required, though } \\
\text { pre-burn reconnaissance is valuable }\end{array}$ & $\begin{array}{l}\text { Pseudo-replication is a potential issue. Temporal } \\
\text { or spatial separation of plots necessary }\end{array}$ \\
\hline \multirow[t]{3}{*}{$\begin{array}{l}\text { Flexible approach - can be done at short notice in } \\
\text { any burn rather than being limited to burns } \\
\text { selected in advance }\end{array}$} & $\begin{array}{l}\text { Daily number of plots achieved is highly variable } \\
\text { depending on rate of ignition (from } 1 \text { to } 10 \text { plots } \\
\text { per day for a single research group) }\end{array}$ \\
\hline & $\begin{array}{l}\text { Method may not be suitable for observing } \\
\text { ignitions or fire behaviour that is not near the burn } \\
\text { perimeter or areas of safety }\end{array}$ \\
\hline & $\begin{array}{l}\text { The ability to travel between parts of the burn } \\
\text { being ignited may be limited by operational and } \\
\text { safety constraints }\end{array}$ \\
\hline
\end{tabular}


(a)

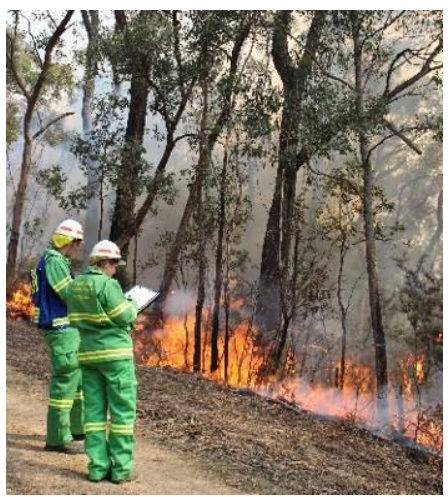

(b)

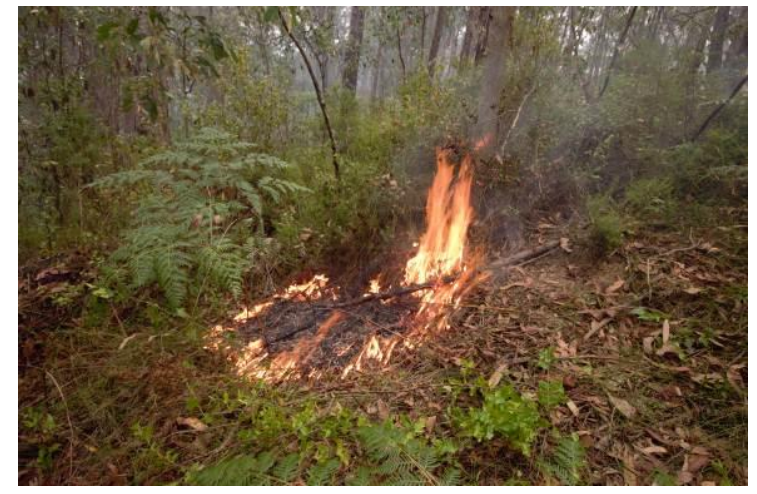

Figure 2 - Direct observation of burning in prescribed burns in Victoria, Australia. (a) Observing ignition success from burn edge. (b) Observing fire development from spot ignition.

Technologies such as fire proof camera boxes, thermocouples and drones can be used in Pre-and post-burn plots to provide more detail about fire behaviour, timing of ignition and duration of burning (Figure 3). However, such technologies are in their infancy and can be difficult to deploy in steep, densely vegetated terrain. Battery operated devices such as cameras within fire proof boxes and thermocouples attached to loggers can be difficult to switch on when the plots are far from a road. Replication may also be an issue since these devices are expensive and therefore a research team may only be able to access a small number. There are often restrictions on the use of drones within prescribed burns if other aircraft are also being used by the fire agencies. Furthermore, dense canopies and thick smoke limit the usefulness of drones as visibility is greatly reduced.

(a)

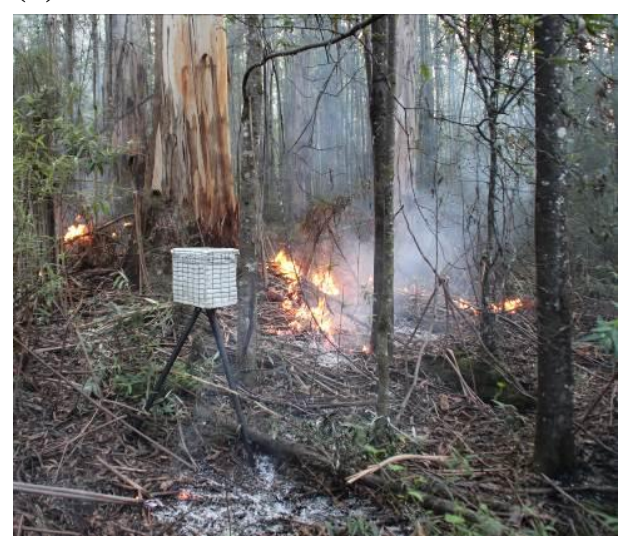

(b)

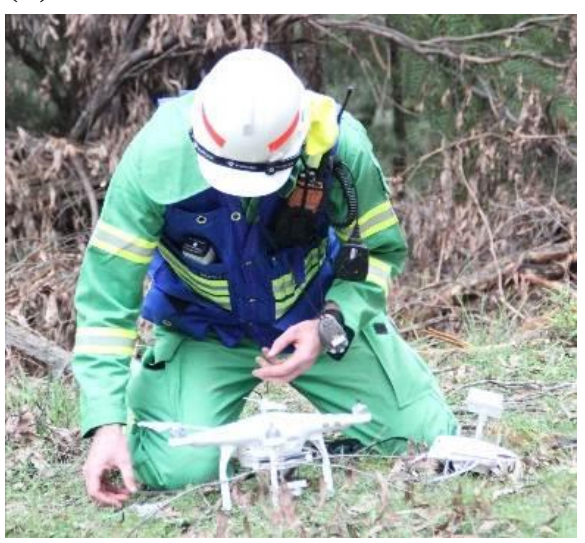

Figure 3 - New technologies for flammability studies in prescribed burns. (a) Fire proof boxes for camera and data loggers to observe flaming inside the burn. (b) Drones to observe rate of spread inside burn.

\subsection{Pre- and post-burn plots}

Vegetation condition within each plot can be measured in advance of the burn (e.g. fuel hazard, species composition, plant architecture, live-to-dead ratio) and indicators of fire behaviour after the burn (char height, scorch height and leaf freeze). Ignitability may be estimated based on the proportion of the plot burnt, for plots where there is evidence that there had been an ignition attempt. Destructive fuel sampling can be used to measure consumability.

Advantages of using pre- and post-burn plots are that researchers do not need to attend the burn (Table 4). Additionally, plots can be located further from the edge of the burn, reducing edge effects. However, the trade-off is that fuel moisture and fire behaviour are not measured directly, which means inferences must be made using measures such as canopy cover or predictive models for fuel moisture 
and char heights for fire behaviour. Additionally, because plots need to be installed in advance of the burn, there is a greater likelihood that some effort will be wasted because burn plans inevitably change.

A variation of this method is where there is some experimental manipulation within the plots, e.g. lighting techniques are modified to achieve different fire intensities (Cawson 2012) or fuel structure is modified to isolate the effects of different fuel components on flammability (Schwilk 2003). Such experimental manipulations may enable a better insight into causal mechanisms but can also add to the complexity of the research design. In the context of fire behaviour studies conducted within prescribed burns, any added complexity introduces further risk that the study will not succeed.

Table 4 - Pre- and post-burn plots - strengths and limitations

\begin{tabular}{ll}
\hline Strengths & Limitations \\
\hline Plots can be in the middle of the burn, to avoid edge & $\begin{array}{l}\text { Investment of time and effort in pre-burn } \\
\text { effects }\end{array}$ \\
measurements may be wasted if the plots are not \\
$\begin{array}{l}\text { Plot layout can be planned to achieve a balanced } \\
\text { besign across fuel types and terrain features }\end{array}$ \\
$\begin{array}{ll}\text { Researchers do not need to be present during burning } \\
\text { therefore relate weather to fire behaviour } \\
\text { Difficult to determine the nature of fire at a plot (i.e. } \\
\text { backing, flanking, head fire) } \\
\text { Plots cannot be accessed during the burn, so there is } \\
\text { an inability to measure fuel moisture or fire } \\
\text { behaviour directly }\end{array}$ \\
\hline
\end{tabular}

\subsection{Analysis of maps of burn outcomes}

Post-burn maps may be coupled with vegetation and fuel moisture maps in flammability studies to determine thresholds for ignition and fire behaviour under different weather and fuel moisture conditions (e.g. Nolan et al. 2016; Duff et al. 2018 in wildfire studies). These retrospective analyses are useful for obtaining broad-scale measures of ignitability and consumability (using fire severity as a proxy), but other flammability variables cannot be quantified with this data.

Advantages of using this method are that a landscape scale analysis can be done with data that are often freely available. However, without specific details about each burn the use of the data is limited.

Table 5 - Analysis of maps of burn outcomes - strengths and limitations

\begin{tabular}{ll}
\hline Strengths & Limitations \\
\hline Data are often freely available from fire agencies & Typically lacking specific details about the burn such \\
No field work required, reducing data collection costs & $\begin{array}{l}\text { as ignition patterns, pre-burn vegetation and moisture } \\
\text { conditions and when the burn occurred }\end{array}$ \\
for research project & $\begin{array}{l}\text { Only able to quantify ignitability, not combustibility } \\
\text { or sustainability. } \\
\text { incorporating many burns }\end{array}$ \\
& $\begin{array}{l}\text { No direct measures of fuel measure or fire behaviour } \\
\text { Often mapping accuracy is not assessed and may vary } \\
\text { between burns }\end{array}$ \\
\hline
\end{tabular}

\section{Key principles for effective flammability research in prescribed burns}

Drawing on our experiences working within prescribed burns, we have devised ten key principles for getting the best outcome from research in prescribed burns. 
1. Safety first. Don't compromise on safety when designing and executing your method. Falling trees, hot ashes and the fire itself can make some methods unsafe to implement.

2. Collaborate with fire managers. Research will be best supported if it can be shown to have relevance to operational practice. Managers can be valuable for obtaining insights to support research design and application.

3. Work within operational objectives. Managers have different priorities to researchers, and need to consider burn effectiveness, logistics and safety. Designing projects that do not disrupt operational activity are more likely to succeed. It is important to work within burn protocols and recognise when it is appropriate to leave the fire ground.

4. Focus your efforts on burns that are high priority for management. Not all planned burns are likely to be completed in any one year. Undertaking pre-burn assessments in burns that are of high management priority will boost the chances that research plots are burned. It can also be valuable to consider the parts of burns that will be higher priority for ignition.

5. Spread the risk. Burns may be cancelled or altered at short notice. Hedge the risk of sites not being burnt by choosing multiple burns rather than a single burn and undertaking higher numbers of plots with less intensive measurements.

6. Get appropriate firefighter training and approvals. Not only is it safer to be properly trained, but it makes it easier for fire agencies to allow researchers into the burn.

7. Stay informed. Ensure communication channels are maintained with fire managers before, during and after the burn. Use contacts within the fire agencies and public notification systems to know when your burn is likely to occur. Ensure that you fit within the burn's communication plan.

8. Be on standby for rapid deployment. Often there is little notice before a burn is ignited (less than 24 hours), so equipment and people need to be ready to go.

9. Be a help on the fire ground. Make sure your work is not impeding the burn effort and if possible, find ways to contribute to the burn.

10. Give something back. Provide your findings to those you worked with to show the value of your collaboration. It can be helpful to do this informally.

\section{Conclusion}

Operational prescribed burns provide valuable opportunities for researchers to quantify plant flammability at field-scales, even with a modest research budget. However, for this research to be effective the potential limitations of working within prescribed burns must be managed. Central to a successful research program is the maintenance of mutually beneficial working relationships with the fire agencies conducting the burns. Such relationships may also enhance the operational applicability of the research to fire management.

\section{Acknowledgements}

We gratefully acknowledge fire management staff from the Department of Environment, Land, Water and Planning, who have supported us to work within their prescribed burning program.

\section{References}

Alexander, ME, Quintilio, D (1990) Perspectives on experimental fires in Canadian forestry research. Mathematical Computer Modelling 13, 17-26. 
Cawson, JG (2012) Effects of prescribed burning on surface runoff and erosion.

Cawson, JG, Duff, TJ, Swan, MH, Penman, TD (2018) Wildfire in wet sclerophyll forests: the interplay between disturbances and fuel dyanmics. Ecosphere $\mathbf{9}$,

Cruz, MG, Gould, J (2009) Field-based fire behaviour research: past and future roles. In '18th World IMACS / MODSIM Congress. Cairns, Australia 13-17 July 2009'.

Cruz, MG, Gould, JS, Alexander, ME, Sullivan, AL, McCaw, WL, Matthews, S (2015) Empiricalbased models for predicting head-fire rate of spread in Australian fuel types. Australian Forestry 78, 118-158.

Department of Environment Land Water and Planning (2016) Fire history records of fires primarily on public land. Victorian State Goverment (data.vic.gov.au), Melbourne.

Dickinson, KJM, Kirkpatrick, JB (1985) The flammability and energy content of some important plant species and fuel components in the forests of southeastern Tasmania. Journal of Biogeography 12, 121-134.

Dimitrakopoulos, AP, Papaioannou, KK (2001) Flammability Assessment of Mediterranean Forest Fuels. Fire Technology 37, 143-152.

Duff, TJ, Cawson, JG, Harris, S (2018) Dryness thresholds for fire occurrence vary by forest type along an aridity gradient: evidence from Southern Australia. Landscape Ecology Online early,

Duff, TJ, Chong, DM, Cirulis, BA, Walsh, SF, Penman, TD, Tolhurst, KG (2014) Gaining benefits from adversity: the need for systems and frameworks to maximise the data obtained from wildfires. In 'Advances in Fire Research.' (Ed. DX Viegas.) pp. 766-774. (Imprensa da Universidade de Coimbra: Coimbra, Portugal)

Fernandes, PM, Cruz, MG (2012) Plant flammability experiments offer limited insight into vegetationfire dynamics interactions. New Phytologist 194, 606-609.

Fernandes, PM, Davies, GM, Ascoli, D, Fernández, C, Moreira, F, Rigolot, E, Stoof, CR, Vega, JA, Molina, D (2013) Prescribed burning in southern Europe: developing fire management in a dynamic landscape. Frontiers in Ecology and Environment 11,

Forest Fire Management Victoria (2017) Reducing Victoria's bushfire risk. Fuel management report 2016-17. Department of Environment, Land, Water and Planning, Melbourne.

Fraser, IP, Williams, R, J., Murphy, BP, Camac, JS, Vesk, PA (2016) Fuels and landscape flammability in an Australian alpine environment. Austral Ecology 41, 657-670.

Ganteaume, A, Jappiot, M, Lampin, C (2013) Assessing the flammability of surface fuels beneath ornamental vegetation in wildland-urban interfaces in Provence (south-eastern France). International Journal of Wildland Fire 22, 333-342.

Gill, AM, Zylstra, P (2005) Flammability of Australian forests. Australian Forestry 68, 87-93.

McCaw, WL (2013) Managing forest fuels using prescribed fire - A perspective from southern Australia. Forest Ecology and Management 294, 217-224.

Nolan, RH, Boer, MM, de Dios, VR, Caccamo, G, Bradstock, RA (2016) Large-scale, dynamic transformations in fuel moisture drive wildfire activity across southeastern Australia. Geophysical Research Letters 43, 4229-4238.

Pausas, JG, Keeley, JE, Schwilk, DW (2017) Flammability as an ecological and evolutionary driver. Journal of Ecology 105, 289-297.

Plucinski, MP, Anderson, WR (2008) Laboratory determination of factors influencing successful point ignition in the litter layer of shurbland vegetation. International Journal of Wildland Fire 17, 628637.

Possell, M, Bell, TL (2013) The influence of fuel moisture content on the combustion of Eucalyptus foliage. International Journal of Wildland Fire 22, 343-352. 
Schwilk, DW (2003) Flammability is a niche construction trait: Canopy architecture affects fire intensity. American Naturalist 162, 725-733.

Schwilk, DW (2015) Dimensions of plant flammability. New Phytologist 206, 486-488.

US Forest Service (2018) 'Hazardous Fuels Reduction and Landscape Restoration Accomplishments Fiscal Years (FY) 2001-2016. .' Available at

Varner, JM, Kane, JM, Kreye, JK, Engber, E (2015) The Flammability of Forest and Woodland Litter: a Synthesis. Current Forestry Reports 1, 91-99.

White, RH, Zipperer, WC (2010) Testing and classification of individual plants for fire behaviour: plant selection for the wildland-urban interface. International Journal of Wildland Fire 19, 213 227. 


\title{
Forest fire Preventing and Controlling Techniques in China
}

\author{
Shu Lifu ${ }^{1 *}$, Yangguang2, Hecheng ${ }^{3}$, Tian Xiaorui ${ }^{1}$, Zhaofengjun ${ }^{1}$, Wang Mingyu ${ }^{1}$ \\ ${ }^{1}$ Institute of Forest Ecology, Environment \& Protection, Chinese Academy of Forestry $(C A F)$ No. 2 \\ Dongxiaofu, XiangYi Road, Haidian, Beijing, 100091, China. \\ \{slfhxk@126.com*;shulf@caf.ac.cn*\}. \\ ${ }^{2}$ School of Forestry, Northeast Forestry University, No.26, Hexing Road, Harbin,150040, \\ Heilongjiang province, China. \{yangguang@nefu.edu.cn\}. \\ ${ }^{3}$ Forest Fire Research Center, Nanjing Forest Police College, Nanjing, 210023, \\ China.\{hech_eng@163.com\}
}

\begin{abstract}
The area of China is large and the natural environment is very complex. The average forest cover of per person is very low. Planted and secondary forest account for much proportion of overall forest, and forest fire is very severe. The occurrence of forest fire is impacted by many factors, as the forest fire in China is impacted by inter-annual variability of weather and the regional distribution of fuel, the forest fire in China is characterized by distinct spatial and temporal distribution. The provinces that have more number and burned area concentrate on the Northeast and Southwest of China, and impacted by atmosphere current and seasonal monsoon, the fire season of the two regions have distinct seasonal variance. Human caused fires dominate the most parts of all the fires.Since 1987, the Chinese government attaches great importance to fire management. But there are still many fires occurrence in China every year. For the period 200-2009, the yearly average fires were 9493 , which caused burned areas 333796 ha. The yearly cost of fighting fires was 171220000 RMB (about 26341538 US\$). Human activities were mainly attributable to the forest fire, occupying more than $98 \%$. Most forest fires occurred in spring and autumn. Most fires occurred in southern China, accounting for $52 \%$ of the total each year, $37 \%$ in the southwest, $6 \%$ in northwest, and $4 \%$ in northeast and inner Mongolia, and $1 \%$ in other areas. It is expected the average temperature in northeastern China could increase $2.22^{\circ} \mathrm{C}$ in scenario $\mathrm{A} 2$ and $2.55^{\circ} \mathrm{C}$ in the $2040 \mathrm{~s}$ in scenario B2. That may cause the potential burned area will increase by an average $3 \%$ and $20 \%$ in 2040s under scenario A2 and B2, respectively. The fire season will prolong on average by five days and 18 days in the 2040s under scenario A2 and B2, respectively. Measures were made for reducing the fires, such as improving the organization system, fire equipments, strengthening fire monitoring and developing the fire research, ect.
\end{abstract}

Keywords: Fire prevention; China

\section{Introduction}

Fire plays an important role in forest ecosystem. After the great fires occurred in 1987 in northeastern China, the government pays more attention to the fire management. The fire agencies were founded in local government at all levels. The measures were made on fire prevention and extinguishment. From 1988 till now, statistics show that the average number of forest fire is 7,935, and the affected forest area is 92,000 hectares. Chinese government attaches great importance to forest cultivation and prevention, especially grasps the intensification of prevention of forest fire as an important measure of the construction of the ecological and forestry prevention.

\section{Causes of forest fires}

\subsection{Human's activities}

Most fires are due to man's fault while inappropriately using fires in production and daily life. According to statistical analysis for 2001-2010, human activities were mainly attributable to the forest 
fire, occupying more than 98\%. Take 2010 for instance, charcoaling, burning paper as sacrificial offerings, smoking in forest areas, or fires caused by children and the mentally handicapped account for $34.1 \%, 18.1 \%, 5.9 \%, 4.3 \%$, and $3.5 \%$ respectively. In Daxing'anling, northeastern China, the lightning fires accounted for about $30 \%$. In southwestern and southern China, many fire caused from prescribed fires in farmland. In Northeast China, hunter's casual smoking and cooking fires by medical herb pickers or mushroom pickers often cause forest fires.

\section{Fires distribution}

\subsection{Fire peak seasons}

The occurrence of forest fires in China showed two peaks in a year (Fig. 1). In northern China, the fire season includes two periods, which are spring (from March to June) and autumn (from September to November). But in southern China, most fire occurred in dry season (from November to next May). In general, there are two peaks of fire occurrences. For example, in 2005 the most fires occurred in April (4 604 ha, 40\%), and the most burned areas in October (128 662 ha, 40\%) (Fig 1).

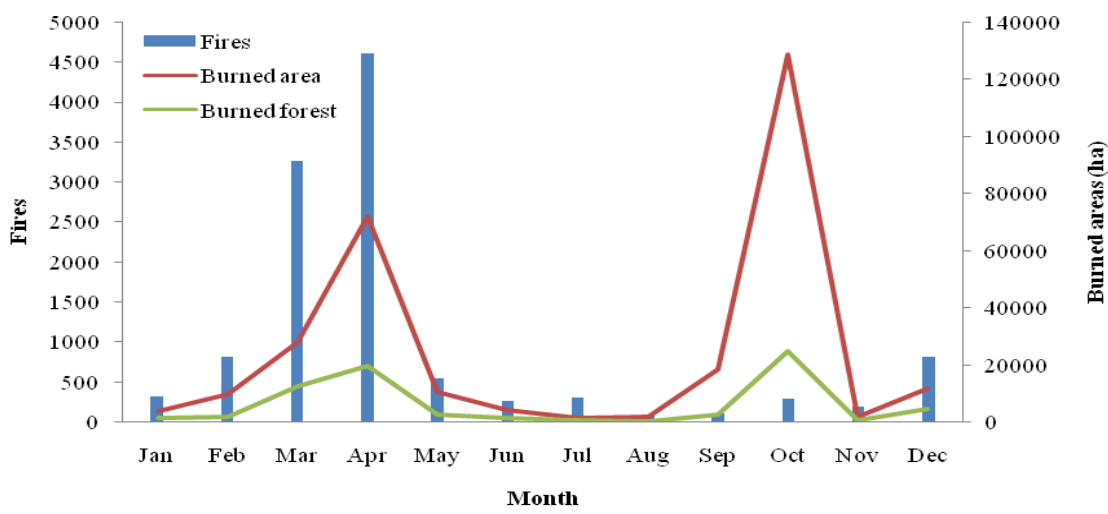

Figure 1 - Fire occurrence by month in 2005

\subsection{Regional distribution}

Most fires occurred in Southern forest, accounting for 52\% of the total each year, $37 \%$ in the southwest, $6 \%$ in northwest, and $4 \%$ in northeast and inner Mongolia, and $1 \%$ in other areas. For example, in 2005 the Hunan province had most fires (3,204 fires, accounted for $28 \%$ of the country) and Heilongjiang had most burned areas (132,248 ha, 46\%) and burned forests (24,307 ha, 33\%) (Fig. 2).

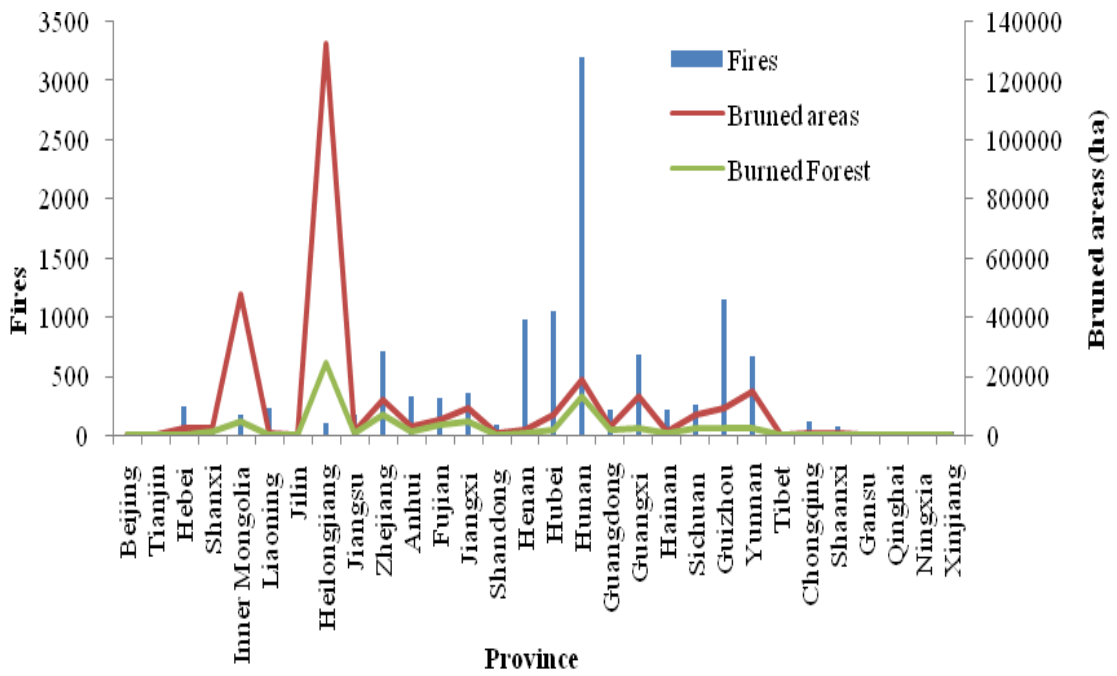

Figure 2 - Fire distribution in provinces in 2005 


\section{Recent fire situation}

For the period 200-2009, the yearly average fires was 9493 , in which fire alarm $(<1$ ha), average fire (1-100 ha), big fire (100-1000 ha) and conflagration (>1000 ha) were 5452 (57\%), 4014 (42\%), $23(0.2 \%)$ and $4(0.04 \%)$, respectively. The fires caused burned areas 333796 ha in each year, in which 138712 ha (42\%) was occupied by forests. In the period, the most burned areas occurred in 2003 (1 $123751 \mathrm{ha}$ ) and most fires (14 144) in 2008 (Fig 3).

The yearly cost of fighting fires was $171220000 \mathrm{RMB}$ (about 26341538 US\$), and brought the other losses 131257500 RMB (20 193460 US\$). Meanwhile, the wildfires caused 69 people died and 68 wounded in each year.

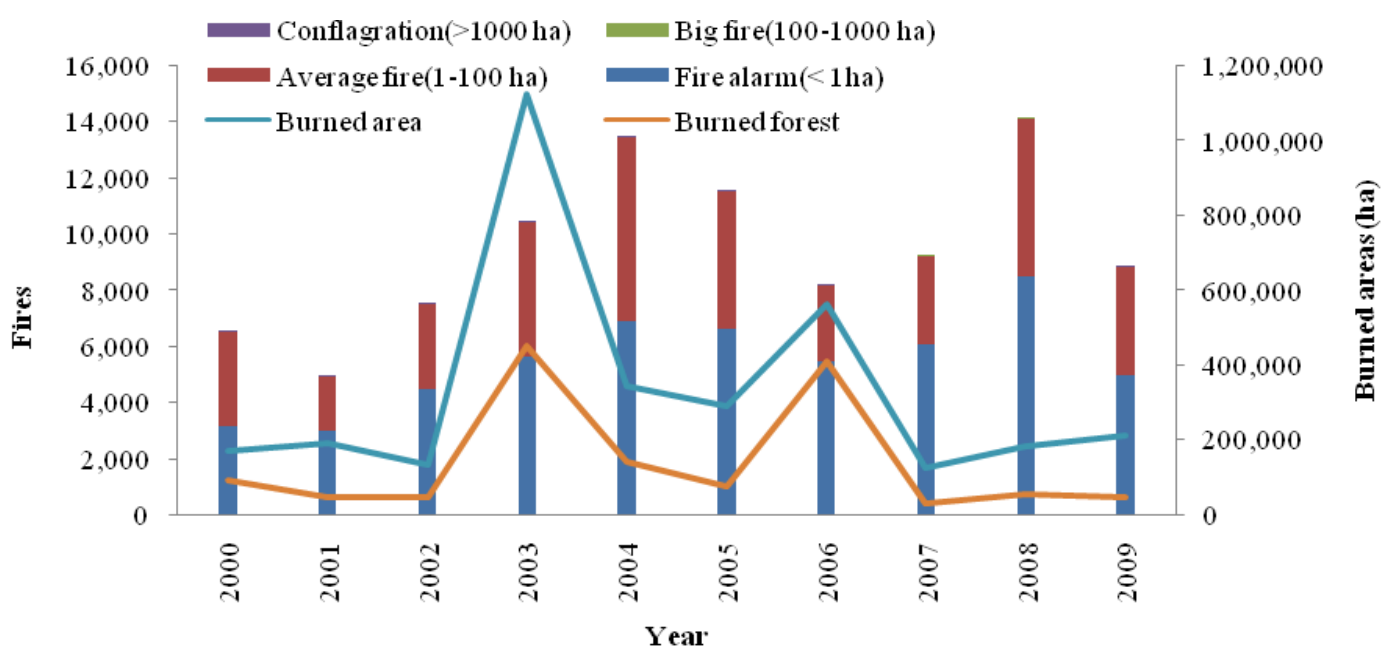

Figure 3 - Fire occurrence and burned area in China for 2000-2009

\section{Efforts to reduce the fires}

Effective measures are adopted to enhance the management of forest fire control, and minimize the number and losses of forest fires.

\subsection{Laws and regulations}

Since 2004, the Chinese central government has issued a series of documents, schemes, and regulations. Such as "Notification From the State Council on Further Implementing the Forest Fire Prevention", "State Emergency Preplan on Handling Particularly Serious Forest Fires", and "Forest Fire Regulations". These drafts and regulations greatly enhanced the scientificity and standardization of forest fire prevention career in China.

\subsection{Organization system}

At present, China government sets up the National Forest Fire Prevention office, which is composed of 19 member organizations. State council also holds meeting to deploy forest fire management. Officials of governments at all levels take their own responsibilities. Till 2010, 3326 forest fire command headquarters with 63,000 member staff and 3544 administrative offices with 20000 member staff have been built in China. There are fire agencies in the government at all levels.

\subsection{Fire sources management and education}

Fire sources were strictly controlled in key forest regions. Personals entering woodlands need to be checked and notified the fire sources management in the region. Billboards and posters are located in 
places. Broadcast, TVs, magazines, and networks were fully used to publicize the importance of forest fire prevention in key forest regions. So the public's awareness of the importance of fire prevention has been reinforced in past twenty years.

\subsection{Fire monitoring}

In China, the satellites and airplanes were used in fire detection except monitoring on the ground. There are 18 air bases in northeastern and southwestern China used in fire season. Each year more than 70 aircraft are rented, including more than 40 helicopters. Many satellites were used in fire season for fire detection, such as FY-1C, FY-1D, NOAA Series (NOAA-12, NOAA-14, NOAA-15, NOAA-16, NOAA-17) , EOS/MODIS (terra, aqua).

\subsection{Fire equipments}

There are 16,000 forest fire brigades in the whole country, which include 510,000 fire fighters. There also exists a forest armed police army (more than 10,000 in total). Since 2006, the central government has invested 2.6 billion RMB for fire prevention construction work. Over 200 projects were developed in key forest region to reduce the fire danger by using the comprehensive measures. So the infrastructures and facilities in high-risk environments and key point's areas are greatly improved. Funds on forest fire prevention and fighting are also included in the government budgets. Those were built of 16,000 fire watchtowers, 1,310,000 km highways, 1,190,000 km fire belts or fuel breaks. 63,000 special vehicles and 6700,000 fire machinery are equipped. There are 3 state-owned warehouses and 11,000 regional warehouses. More than 100 air-bombers are used in distinguishing fires in 15 provinces, flying more than 10,000 hours annually.

\subsection{Fire danger rating system}

A national fire danger rating system are founding and a net of fire weather stations will finish for better monitoring the fire danger. Some research projects are progressing on basic fire sciences and technologies application.

\subsection{Forest fire challenge}

It makes fire fighters further understand the nature of the forest fire.

In the past they thought their job was only to put out it once there is a fire, but now they have realized that it is more important than putting out the fire that they need to learn to manage the forest and then the fire. It shows that we have changed our strategy of fire fighting.

It improves the technology of forest fire forecast.

Though we haven't set up a fire forecast system around the whole country, we have started doing it in some areas of the country. And we have moved into a new stage of fire behavior prediction rather than the past metreological prediction of fire danger.

It raises the level of fire monitoring.

We have successfully built a network of fire monitoring through out the country. The network now has shown it significant effects. Plane's x-ray monitoring technology has been fully and widely adopted.

\subsection{Commanding fire fighting through information}

The transmission of message on forest fire must make it possible for fire fighters to get messages whenever and wherever they need. (for example when they are fighting the fire, when they want to prevent the fire or when they want to know how to use the fire safely). Especially as soon as defecting the fire, the message should be sent to the commanding center so that the center can arrange to send in time fire fighters and materials it needs to put out the fire to reduce the damage as much as possible. To achieve this, the message must be transmitted without stop between spots on land, between spots

Advances in Forest Fire Research 2018 - Page 492 
in the air, and between the air and the land. So we need to further the present system of communicative networks, at the same time we need to further develop the communicative technology in the fire field. We need to usse the short wave and ultra short wave to solve the communicative problems in the remote areas where message is difficult to reach.

\subsection{Fire extinguishers and fire fighting products}

It is not enough to have only scientific theories to prevent the forest fire. We need to have the advanced tools also. So we should not neglect the study of fire fighting facilities

\subsection{Isolating technology and the management of the burnable substances}

The management of the forest fire is not simply to prevent the fire passively but to actively prepared ourselves to prevent fire or to put out fire or even to use the fire through the technology of isolating the big fire and by managing the burnable substances. In future we need to do work in designing the fire preventing line and managing the forest by use fire.

\subsection{Fire fighters' training}

Knowing the importance of fire fighters' training is the basis to reduce the casualties and raise the efficiency of fire fighting. At present we badly need to strengthening our skill training of fire fighting and textbook compiling, and adding the number of our fire fighting facilities. In addition, we need to formulate our regulation of fire fighting training. In our training we need to use the multi-media resource, the computer network resource and some other facilities.

\section{Conclusion}

Although the government made great efforts on fire management, the serious fire situation is still a problem faced by the government. Most fires are caused by human activities. It is expected the average temperature will increase in most areas of China, and the potential burned area also increase in the future under the climate change scenarios. Measures should be made on fire prevention, monitoring, and fire fighting.

Forest fire management have make some progress in predicting and forecasting the forest fire causing weather, the cause of fire and fire behavior. Some works have done in the planned fire burning either inside or outside the forest.

Monitoring system is composed of the application of Meteorological satellites in monitoring and predicting forest fire and the application of distance satellites for emergency in communication.

In general forest fire study both at theoretical level and practical level, is mainly focused on the north part of the country while in other areas especially in the south and southwest areas has a relatively short history and backword.

\section{References}

Tian X.R., Shu L.F., Wang M.Y., Zhao F.J., Chen L.G. 2012A. The fire danger and fire regime for Daxing'anling region within 1987-2010. The 9th Asia-Oceania Symposium on Fire Science and Technology. Procedia Engineering, (in press)

Tian X.R., Douglas J.M., Jin J.Z., Shu L.F., Zhao F.J., Wang M.Y. 2011A, Wildfire and the Canadian forest fire weather index system for the Daxing'anling region of China. International Journal of Wildland Fire, 20, 963-973.

Tian X.R., Shu L.F., Zhao F.J., Wang M.Y. 2012B. Analysis of the conditions for lighting fire occurrence in Daxing'anling Region. Scientia Silvae Sinicae, 48(7): 96-103 (in Chinese) 
Zhao F.J., Shu L.F., Tiao X.R., Wang M.Y. 2009A. Change trends of forest fire danger in Yunnan Province in 1957-2007. Chinese Journal of Ecology, 28(11):2333-2338 (in Chinese)

Zhao F.J., Shu L.F., Di X.Y., Tian X.R., Wang M.Y. 2009B. Changes in the occurring date of forest fires in the Inner Mongolia Daixng'anling forest region under global warming. Scientia Silvae Sinicae, 45(6): 166-172 (in Chinese)

Zhao F.J., Shu L.F., Tian X.R., Wang M.Y. 2009C. The changes of forest fuel dryness conditions under global warming in Inner Mongolia Daixng'anling forest region. Acta Ecologica Sinica, 29(4): 1914-1920 (in Chinese)

Tian X.R., Shu L.F., Wang M.Y., Zhao F.J. 2011B. Forest fire danger ratings in the 2040s for northeastern China. Forestry Studies in China, 13(2): 85-96

Tian X.R., Shu L.F., Zhao F.J., Wang M.Y., Douglas J.M. 2011C. Future impacts of climate change on forest fire danger in northeastern China. Journal of Forestry Research, 22(3): 437-446

Tian X.R., Shu L.F., Zhao F.J., Wang M.Y. 2012C. Forest fire danger changes for southwest China under future scenarios. 48(1): 121-125 (in Chinese) 


\title{
Identifying pyroregions by means of Self Organizing Maps and hierarchical clustering algorithms in mainland Spain
}

\author{
Adrián Jiménez-Ruano ${ }^{1,3 *}$; Marcos Rodrigues Mimbrero ${ }^{1,2,3}$; Juan de la Riva Fernández ${ }^{1,3}$ \\ ${ }^{1}$ Department of Geography and Land Management, University of Zaragoza. Pedro Cerbuna, 12, \\ 50009, Zaragoza, Spain \{jimenez@unizar.es*\} \\ ${ }^{2}$ Department of Agriculture and Forest Engineering, University of Lleida. Alcalde Rovira Roure \\ 191,25198, Lleida, Spain \{rmarcos@unizar.es\} \\ ${ }^{3}$ GEOFOREST Group, IUCA, University of Zaragoza, Zaragoza, Spain \{delariva@unizar.es\}
}

\begin{abstract}
Defining pyro-regions, i.e., of homogenous zones of fire activity, is an on-going task in Spain with few case studies in the literature. Their characterisation and understanding is a crucial step towards improving forest fire management and prevention. It is widely agreed that fire activity is non-stationary. Several works already report temporal dynamics in fire frequency and burned area. In this work we propose a spatialtemporal approach to define pyro-regions considering both structural and temporal fire behaviour using historical fire records from the EGIF database. A combination of Self Organizing Maps (SOM) and hierarchical clustering is applied to time series (1974-2015) of fire regime features: number/burned area of summer fires, number/burned area of large fires ( $>500 \mathrm{ha})$, number/burned area of natural fires, number/burned area of winter fires and number of small fires $(<1 \mathrm{ha})$. The structural component of fire activity is computed as the average value whereas the temporal evolution is addressed by means of Sen's slope.

Prior to cluster analysis, fire features were submitted to Principal Component Analysis with Varimax rotation. Eigenvalues were then pre-classified using SOM. Subsequently, hierarchical clustering was applied to SOM outputs. We obtained a set of 4 structural clusters relating to increased number of fires; low fire incidence, slightly linked to winter season; large and natural fires; and moderate impact of human-related large fires mainly. The process was repeated using Sen's slope to build the dynamic component, ultimately characterised by: highly dynamic winter with increased in summer frequency; increased summer burned area and natural fires; and small fires; and no trend.
\end{abstract}

Keywords: forest fires, pyro-regions, Sen's Slope, SOM, hierarchical clustering, Spain

\section{Introduction}

Forest fires are a highly complex phenomenon affecting most ecosystems worldwide. Fire is known as a natural process responsible for the evolution of wild communities, but nowadays it has been altered, with potential undesired effects on vegetation structure, composition and ecosystemic functions. Fire activity is controlled by multiple factors such as climate, fuel, physiography and human activity. Humans influence fire incidence acting as both initiators and suppressors, increasing the complexity of the phenomena. Thus, understanding fire regime's components and behaviour (both temporal and spatial) may improve our current knowledge. Mapping fire regimes may contribute enhancing fire planning or risk assessment; as well as diminishing undesired ecological impacts (Morgan et al. 2001). In this sense, one of the most promising lines of study lies in the definition and characterization fire regime itself. Fire regime is usually described using several quantifiable parameters such as affected area, fire frequency, cause, seasonality, fire size, etc. (Boulanger et al. 2014). Currently, there still is an open debate on the definition of the concept itself, with slight variations depending on the scale of analysis, the length of the study period or the available information. 
Several attempts to define fire regimes from different approaches are already found in the literature. Without being exhaustive we find some analyses using remote sensing data (Chuvieco et al. 2008) or climate information (Boulanger et al. 2013, 2014; DaCamara et al. 2014). Others employ fire weather danger indexes coupled to fuel and environmental conditions (Perera and Cui 2010). Despite of the success in the characterisation of fire regime, most works still rely on existent zoning schemes to spatialize their boundaries and extent: administrative units (Pereira et al. 2015), ecoregions (Malamud et al. 2005; Kasischke and Turetsky 2006; Perera and Cui 2010; Mori and Johnson 2013) or a combination of both (Wotton et al. 2010).

In the case of Spain, examples of fire regime zoning are really scarce, with Moreno and Chuvieco (2013) as the most representative effort. We find other examples in Vázquez de La Cueva et al. (2006) and more recently in Montiel Molina and Galiana-Martín (2016). These approaches are mostly based on cluster analysis, the most used and well-known zoning approach. They are a flexible multivariate technique with different available implementations, widely used to analyse ignition points distribution (Wang and Anderson 2010; Serra et al. 2013; Pereira et al. 2015; Parente et al. 2016) or occurrence large fire linked to synoptic climatology (Rasilla et al. 2010). Nevertheless, all of them provide a static picture of fire regime, i.e., disregarding the evolution of fire features over time and space. For this reason, a temporal perspective is extremely necessary.

In this work we propose and exemplify a method to outline homogenous fire regime zones (the socalled pyroregions) in mainland Spain. We combine average information of fire features with their temporal evolution (trend detection) during the study period (1974-2015). The method is based on PCA and Self Organizing Maps coupled to hierarchical clustering. Such combination of methods is applied to the averaged values of fire features and their respective trends, separately. By doing so we are able to discriminate static and dynamic pyroregions.

\section{Materials and methods}

\subsection{Study area}

The study area encompasses the whole mainland Spain covering a surface of around $498,000 \mathrm{~km}^{2}$. Climate distribution in the region allows to differentiate two regions: Mediterranean and Oceanic. The first one is characterized by high annual thermal amplitude with hot-summer in the inner region and milder conditions towards the coast. Precipitation is irregularly distributed both in terms of time and space, with maximums peaking in autumn and spring. In addition, the driest areas are located in the southeast region and the Ebro Valley (inner Mediterranean region). On the other hand, Oceanic climate is notable by milder temperature values during summer-winter with high precipitation values regularly distributed throughout the year (average values over $1,000 \mathrm{~mm}$ ) with maximum during winter. From the biogeographical point of view, the Oceanic area is covered by diverse types of vegetation from deciduous to evergreen oak woodlands (Quercus robur, Fraximus excelsior or Fagus sylvatica) and large areas of scrubland and grassland, as well as areas with afforestation of fast-growing species such as Pinus radiata and Eucaliptus globulus. The Mediterranean vegetation coexists with complex mosaics of agricultural systems and plant communities, such as sclerophyllous and evergreen vegetation. Oak (Quercus ilex) and pine (mainly Pinus halepensis, the most widespread of the species introduced by afforestation) forest, and thermophilous scrubland, dominate the region. In addition, altitudinal belts exist within the highest ridges such as the Pyrenees along the French border or Sierra Nevada on the southern Mediterranean coast, being home to a large variety of tree species which are common in central Europe (deciduous species, beech, oak, and some mountain pines: Pinus uncinata, Pinus sylvestris). 


\subsection{Fire data}

Fire records in the period 1974-201 5 were collected from the General Wildfire Statistics (EGIF). Selected fire records were on a 10x10 km UTM reference grid. Then, fire frequency, total burned area (ha), ignition date and source were extracted from the database. Is it important to note, that only those grids with at least a $25 \%$ of forest cover were retained for analysis. Therefore, 3,308 out of 5200 grids were finally considered in the analyses.

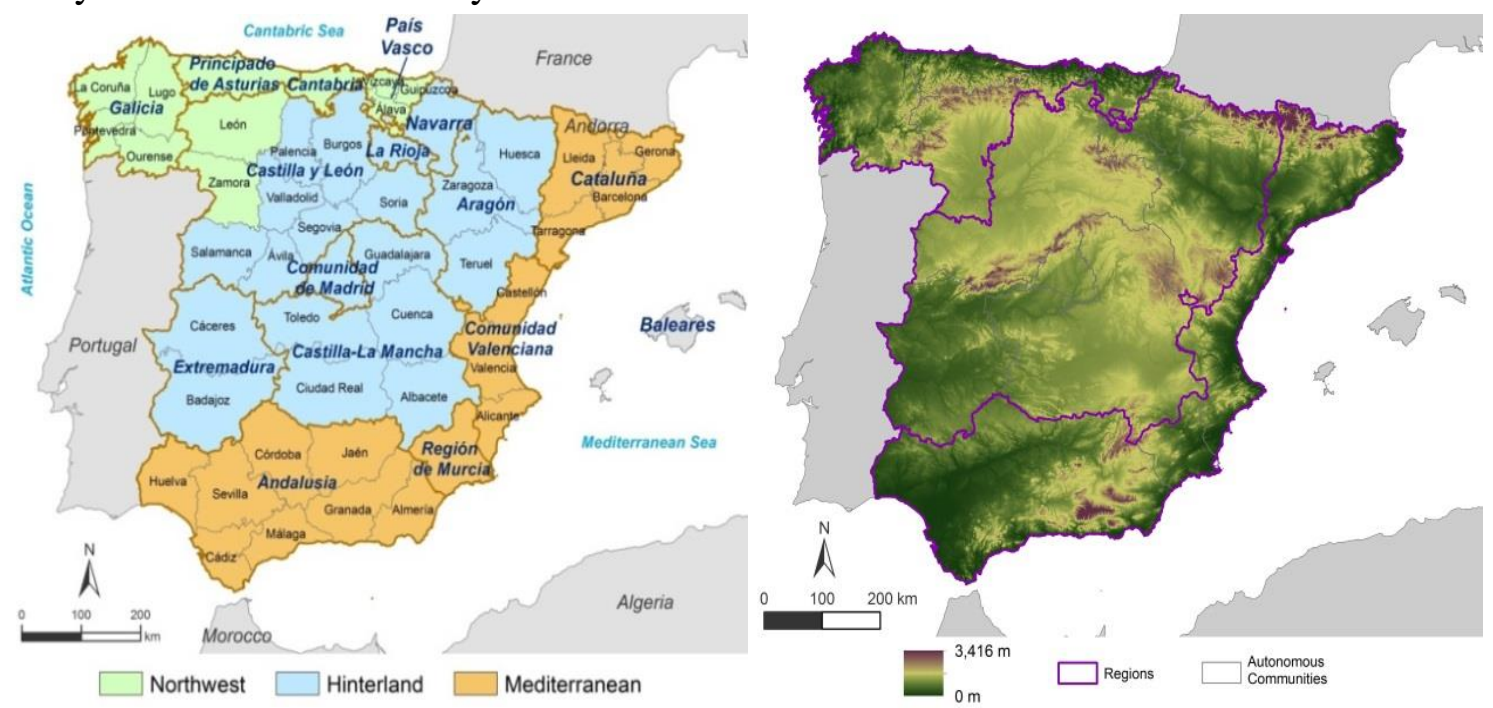

Figure 1 - Spatial distribution of the three regions (Northwest, Hinterland and Mediterranean) also NUTS3 and NUTS2 units in mainland Spain (left) and digital elevation model (right)

Two fire seasons were defined with the aim of differentiating the intra-annual peaks of fire activity (August and March). So, annual fire data were split into spring - summer season (S), from April to September; and autumn-winter season (W) from October to March. From all available fire information, we computed 9 fire features: number of fires and burned area during summer (NS-BAS), summer frequency and burnt area of large fires -above 500 ha- (N500, B500), summer frequency and burnt area of natural fires (NL-BL), number of fires and burnt area during winter (NW-BAW) and total number of small fires $(\mathrm{N}<1 \mathrm{ha})$.

\subsection{Temporal evolution of fire features}

In order to account for the temporal dimension of fire activity during the analyzed time span we estimated the magnitude of the temporal change using Sen's slope (Sen 1968) test. This allows to outline fire zones according to the temporal behavior of fire features rather than address the average 'structural' pattern alone.

\subsection{Environmental and human factors}

To characterize the final pyroregions we used data related with environmental and human factors. Temperature and precipitation data in the period 1974-2010 were extracted from MOTEDAS (González-Hidalgo et al. 2015) and MOPREDAS (González-Hidalgo et al. 2011) datasets (Figure 3). Additionally, forest communities were derived from the Forest Map of Spain. Finally, the Human Pressure Index (Figure 2) was calculated according to Jiménez-Ruano et al. (2017). 


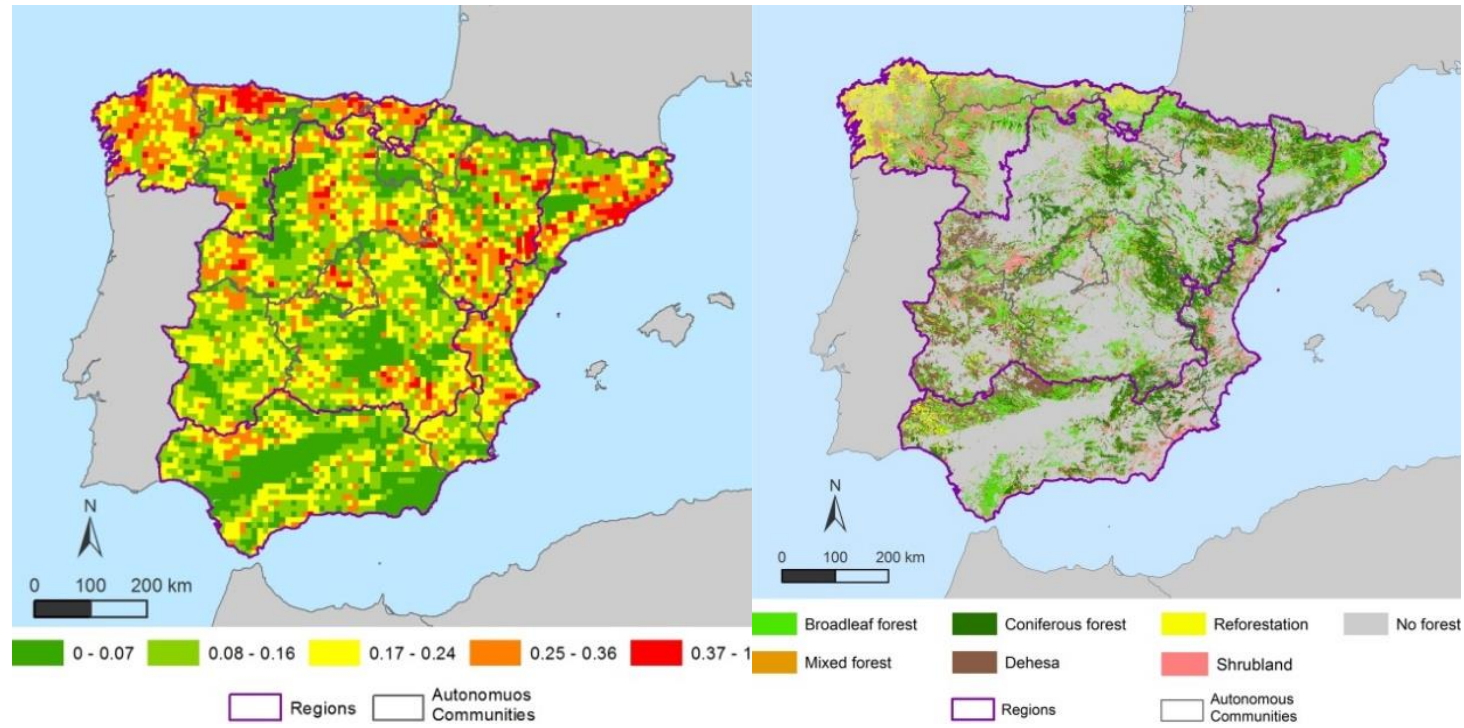

Figure 2 - Spatial distribution of the Human Pressure Index (left) and main forest formations from National Forest Map (right).

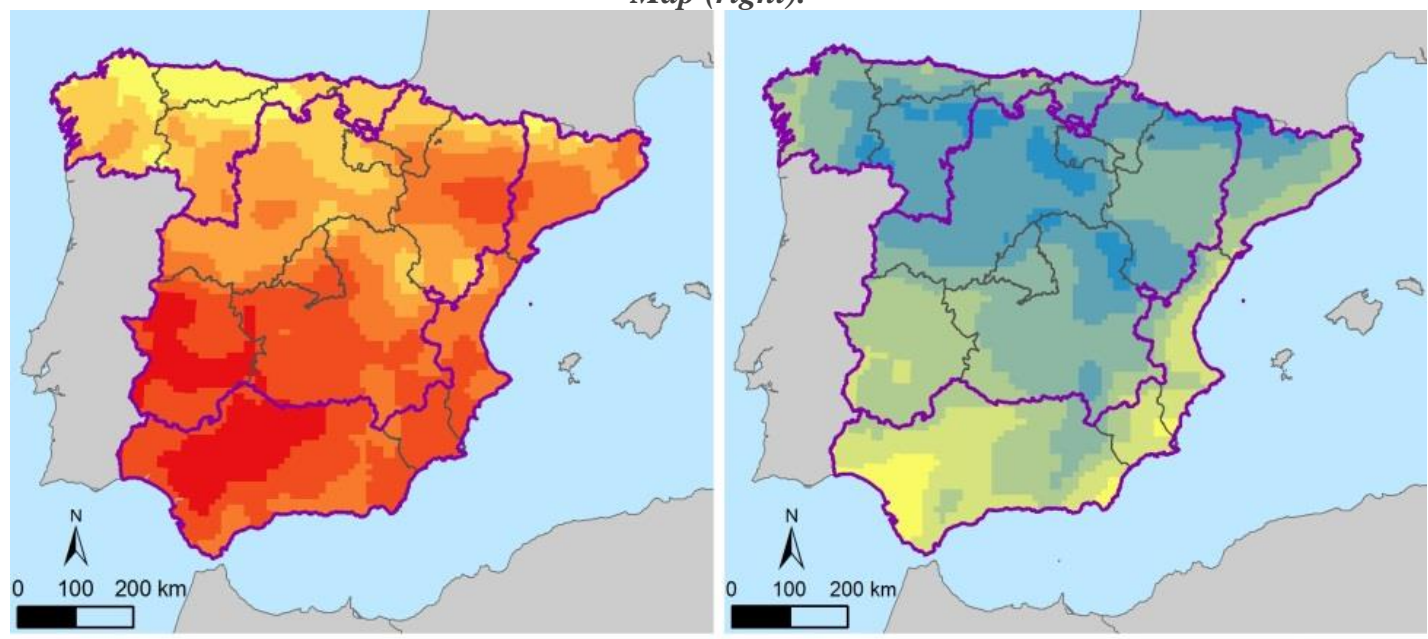

\begin{tabular}{|l|l|l|l|l|l|l|l|l|}
\hline 9-11 & $11.1-13$ \\
\hline
\end{tabular}

$\square$ Regions $\square$ Communities

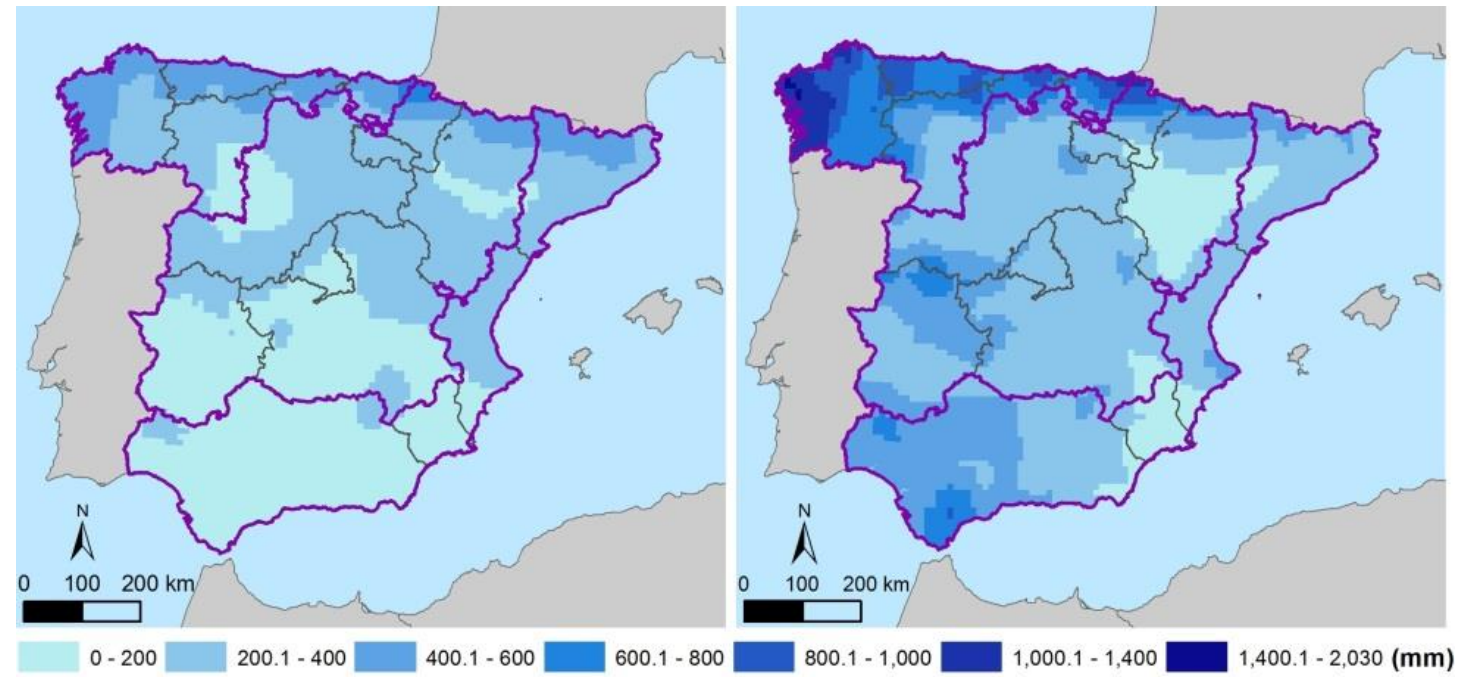

Figure 3 - Climate factors. Top-left, average summer temperature; top-right, average winter temperature; bottom-left, summer mean annual precipitation; bottom-left, winter mean annual precipitation 


\subsection{Principal Component Analysis and Varimax rotation}

Prior to submit fire data to cluster analysis, a PCA with varimax rotation was applied to reduce the amount of information. All fire features (both structural and dynamic) were scaled before applying PCA. Principal Components (PC) were selected according to the Kaiser Criterion, i.e., only those PC with standard deviation over 1 were retained.

\subsection{Clustering overview}

The objective of clustering analysis is grouping objects into categories such that objects within one cluster share more in common with one another than they do with the objects of other clusters (Gore 2000). Many clustering algorithms do exist. The most basic variants resort to data partition and minimizing the distance between points of a same group from another assigned as center. Among all the clustering methods, we selected hierarchical clustering coupled to Self-Organizing Maps to delineate our pyroregions.

The purpose of hierarchical clustering is determinate the best clustering scheme from different results obtained. It is proceeded with the application of various combinations of number of clusters, distance measures and clustering methods. This algorithm routinely produce a series of solutions ranging from $n$ clusters to a solutions with only one cluster present (Charrad et al. 2014). It requires a dissimilarity measure (or distance) and an agglomeration criterion. Many distances area available (Manhattan, Euclidean, etc.) as well as several agglomeration methods (Ward, single, centroid, etc.). In our case, we employed all methods available in the NbClust function from RStudio, the Canberra distance and the Ward D2 method (Murtagh and Legendre 2014), which minimizes the total withincluster variance and the dissimilarities are squared before cluster updating.

SOM is a neural-network algorithm that implements an orderly mapping whose main strength lies in converting complex and non-linear relationships between high-dimensional data (Kohonen 1998). In other words, it compresses information while keeping topological and metric relationships of the input data. The algorithm consists of a two-dimensional model of regular grid of nodes, where some data are associated with each node. In each iteration, the SOM algorithm computes all the models to best describe the domain of the observations. The idea is to group the similar models that are closer to each other in the grid than the more dissimilar ones.

As aforementioned the cluster approach was applied both to 'structural' and 'dynamic' components, thus 2 sets of cluster were obtained. In a final step we overlay all clusters (structural and dynamic) to into the final pyroregions.

\section{Results}

Figures 4 and 6 show the spatial distribution of the structural (4) and dynamic (3) clusters, and their description, respectively. First structural cluster characterises by high fire activity but no large fires; it extends across the Northwest region. In turn, cluster 2 comprises areas of moderate winter activity, in the remaining territory. Cluster 3 brings together summer large fires (>500 ha) caused by lightning. This cluster covers mostly mountain ranges. Finally, cluster 4 brings together medium-size humancaused fires.

Dynamic clusters depict a different behaviour. Tendencies were grouped into clusters 1 and 3 . In the first case, winter trends and the increase in summer small fires are grouped in cluster 1. Geographically, these trends are located in the north-western end, some locations of the inland mountain ranges and few spots of the Mediterranean basin. Remaining trends depict an increase in overall area burned during summer and decreased incidence of natural fires (Table 1), occupying an area that mainly extends over the northern and northwest hinterlands. 
When combining both cluster approaches into a single product we obtain a final set of 8 pyroregions (Figures 5 and 6). Generally speaking, three main groups of pyroregions can be distinguished: (1) those experiencing increase in the fire activity, especially small fires; (2) regions with no noticeable trend; and (3) those characterised by increased summer burnt area and lightning-triggered wildfires.

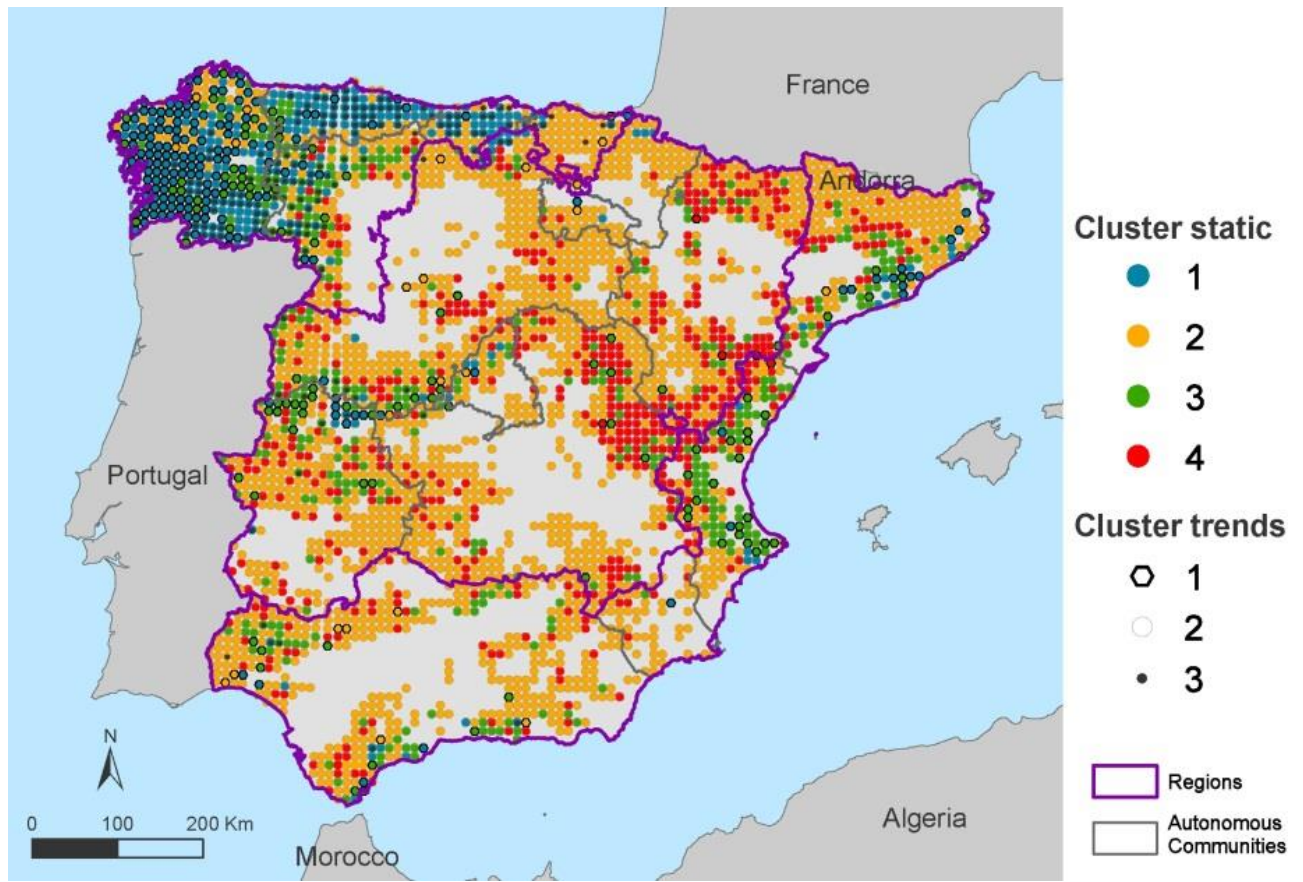

Figure 4 - Spatial distribution of the clusters structural (colour codes) and dynamic (shape codes).

Table 1 - PCA-Varimax eigenvectors of the first two components of static (fire features averages) and the first three components for trends in fire features

\begin{tabular}{|c|c|c|c|c|c|c|c|c|c|c|}
\hline & Fire features & NS & BAS & N500 & B500 & NL & BL & NW & BAW & N <1ha \\
\hline \multirow{2}{*}{ Static } & PC1 & $\mathbf{0 . 5 2 6}$ & 0.148 & & & & -0.152 & $\mathbf{0 . 5 2 6}$ & 0.328 & $\mathbf{0 . 5 2 9}$ \\
\cline { 2 - 12 } & PC2 & & $\mathbf{- 0 . 5 0 8}$ & -0.436 & $\mathbf{- 0 . 5 8 1}$ & -0.212 & -0.408 & & & \\
\hline \multirow{3}{*}{ Trends } & PC1 & $\mathbf{0 . 5 3 4}$ & -0.271 & & & & & $\mathbf{0 . 5 1 2}$ & 0.262 & $\mathbf{0 . 5 5 7}$ \\
\cline { 2 - 11 } & PC2 & & & & & $\mathbf{- 0 . 7 0 2}$ & $-\mathbf{0 . 7 0 5}$ & & & \\
\cline { 2 - 11 } & PC3 & & $\mathbf{0 . 6 7 4}$ & & & & & 0.230 & $\mathbf{0 . 6 7 2}$ & -0.203 \\
\hline
\end{tabular}
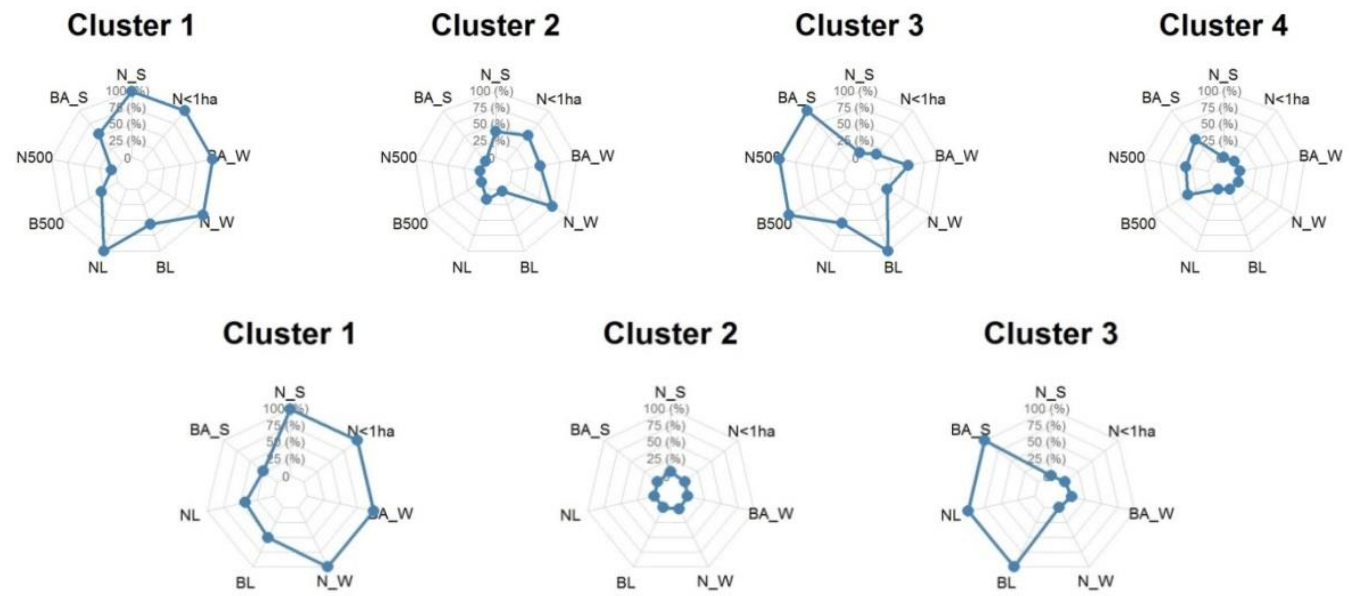

\section{Cluster 2}

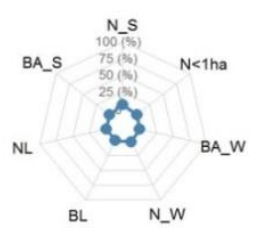

Cluster 3

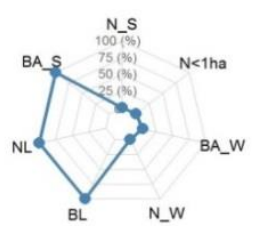

Figure 5 - Description of the contribution percentage for each fire feature in each cluster static (four on the top) and in each cluster of trends (three on the bottom) 


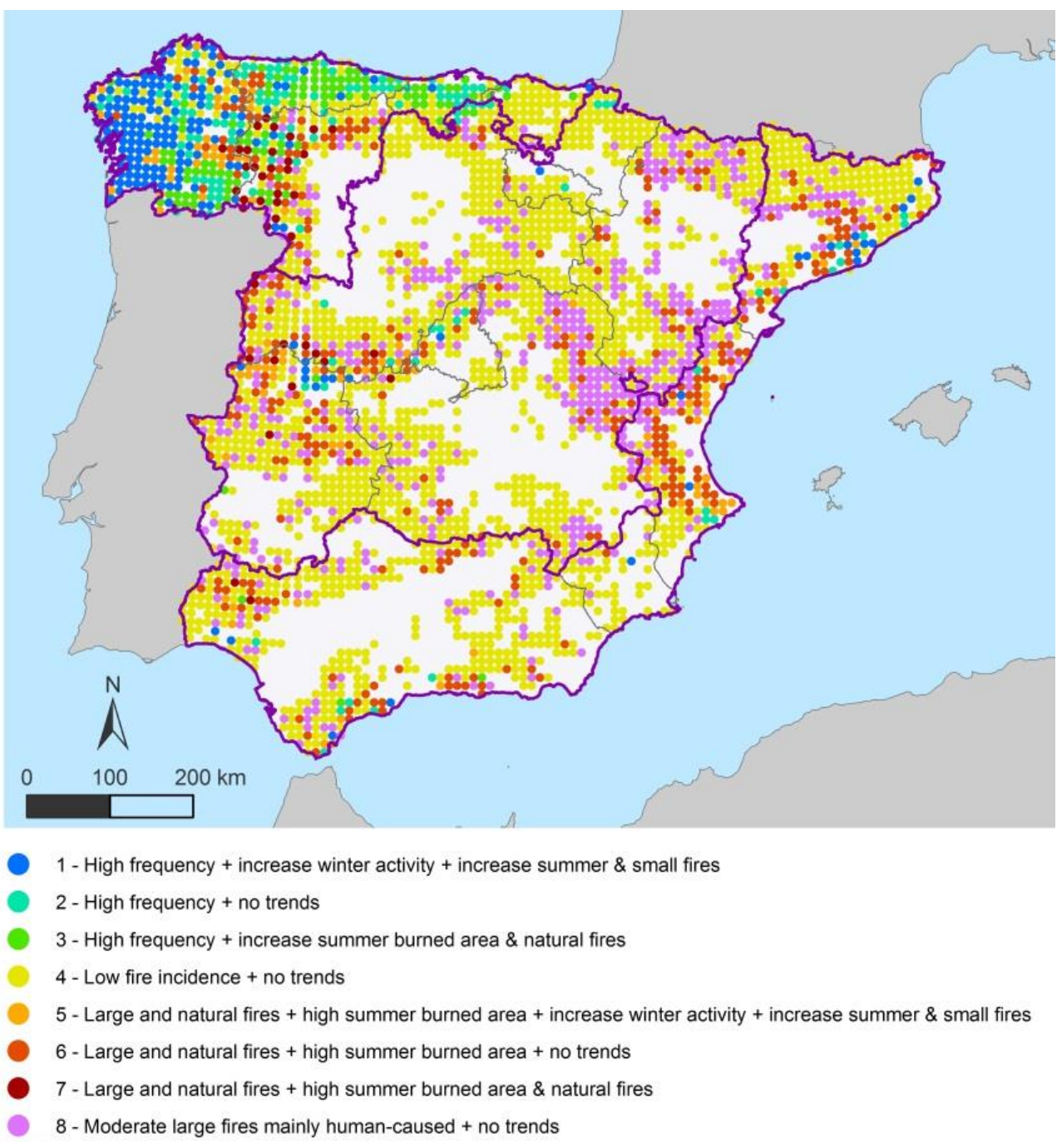

Figure 6 - Spatial distribution of the final pyroregions

The most relevant pyroregion in terms of spatial extent is $4(55.1 \%)$, characterized by a low fire incidence without trends. Secondly, pyroregion 8 , covering $16.4 \%$ of the territory, is represented by medium-sized fires mainly anthropogenic. With a $10.2 \%$ of the study area, pyroregion 6 combines large and natural fires with an increase in summer burned area. Pyroregion 1 (5.4\% area) is mainly located in the Northwest region. It shows a high fire frequency linked to winter dynamics, as well as an increase in summer and small fires. Remaining pyroregions account for just over $1 \%$ and less than $4 \%$ individually. In summary, they would reach roughly $11.6 \%$ of the territory. These are characterized by a high frequency with no trends (2), or with an increase in summer burnt area and natural fires (3). In addition, large fires with an increase in summer-winter activity and small fires (5) and large fires associated with an increase in summer burnt area and natural fires (7).

\subsection{Characterization of pyroregions based on environmental and human variables}

The inclusion of climate-and-human variables enables deeper insights into the characterisation of the pyroregion (Figure 7):

Pyroregion 1: small winter fires and increased summer fire activity. It covers conifer and reforested communities with large rainfall and moderate warm winters and low human pressure.

Pyroregion 2: low fire activity in areas with moderate rainfall, temperate winters and summers. 
Pyroregion 3: increasing winter fire incidence in shrubland communities linked to increased human pressure, large precipitation and moderate temperatures.

Pyroregion 4: low fire activity in isolated warm regions with conifer and mixed forest.

Pyroregion 5: low fire activity increasing during summer. It covers warm and dry regions with a variety of forest communities. Low human pressure.

Pyroregion 6: large natural fires with moderate-low human pressure, high temperature and low rainfall; affecting the whole spectrum of forest communities.

Pyroregion 7: very low fire activity in shrubland communities.

Pyroregion 8: natural fires in warm and dry locations affecting tree communities.

\section{Discussion}

The proposed methodology enabled identifying 8 pyroregions providing a more complete picture than previous attempts. We took a step further, not only by bringing in the dynamic component of fire incidence but digging into more sophisticated zoning techniques. Our contribution further deepens into fire features while complementing them with their main trends such as the rise in summer and winter activity or the increase in small fires.
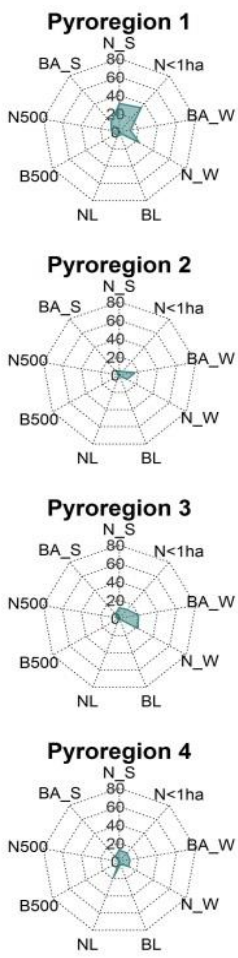
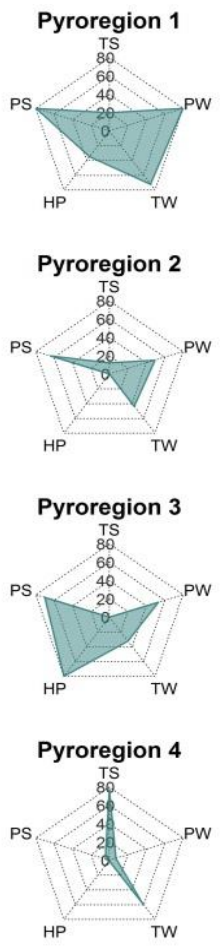
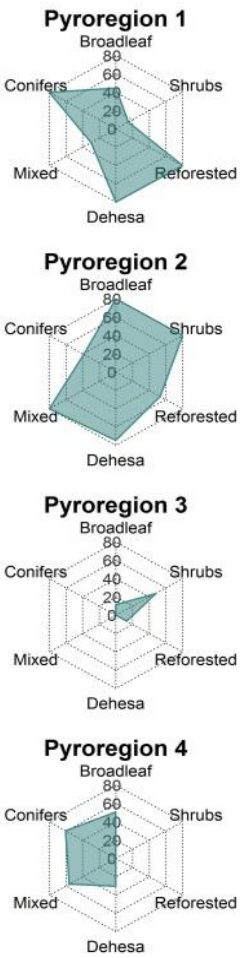
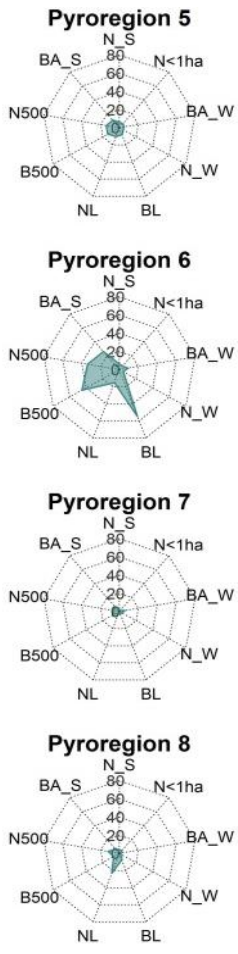
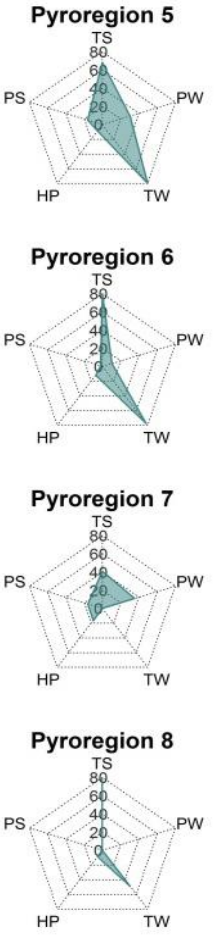
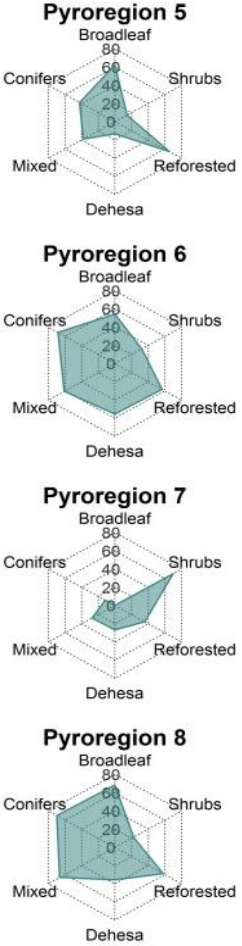

Figure 7 - Pyroregion description. Left column, fire features; center, environmental and human drivers; right, forest communities.

When combining the resulting pyroregions with climatic and human factors, we deliver deeper insights into what factors may be driving fire regimes. Our findings suggest coincidence between temporal clusters of increased fire activity (except for natural fires) dominated by pine woodland and reforestation communities (Vázquez et al. 2015). In many areas of Spain, plantations for timber harvesting and pine tree forests were promoted over the last decades (Pausas et al. 2004). This factor is known to increase flammability in the event of favourable weather conditions (Shakesby 2011).

Regarding climatic factors, fire-prone conditions along the Mediterranean coast seem to promote larger human-cause fires, especially during summer. However, the correspondence of climate with 
trend clusters is not clear. In this sense, the human impact (represented here as the combination of the length of wildland-urban and wildland-agricultural, WUI-WAI interfaces and demographic potential) seems to be more closely related with fire activity (Rodrigues and de la Riva 2014).

\section{Conclusions}

In this work we propose a pyrogeographical characterization of fire behaviour using averaged of fire features and their main temporal trends in mainland Spain. We submitted fire data in the period 1974-2015 to PCA and cluster analysis.

Our findings suggest 8 different pyroregions in mainland Spain, depicting by three structural fire regimes (high fire frequency, large-natural fires and medium size human-cause wildfires) and two main trends (overall increase in fire activity) and decrease in the incidence of natural fires. The implications of the delimited pyroregions play a crucial role in better understanding fire regimes in a broad context, not only in terms of their structural patterns but also of its main trends. Moreover, assessing the environmental and human conditions in the proposed pyroregions improved our understanding of the underlying drivers of fire regimes.

\section{References}

Boulanger Y, Gauthier S, Burton PJ (2014) A refinement of models projecting future Canadian fire regimes using homogeneous fire regime zones. Canadian Journal of Forest Research 44, 1-44. doi:10.1139/cjfr-2013-0372.

Boulanger Y, Gauthier S, Gray DR, Le Goff H, Lefort P, Morissette J (2013) Fire regime zonation under current and future climate over eastern Canada. Ecological Applications 23, 904-923. doi:10.1890/12-0698.1.

Charrad M, Ghazzali N, Boiteau V, Niknafs A (2014) NbClust : An $R$ Package for Determining the Relevant Number of Clusters in a Data Set. Journal of Statistical Software 61,. doi:10.18637/jss.v061.i06.

Chuvieco E, Giglio L, Justice C (2008) Global characterization of fire activity: toward defining fire regimes from Earth observation data. Global Change Biology 14, 1488-1502. doi:10.1111/j.13652486.2008.01585.x.

DaCamara CC, Pereira MG, Calado TJ, Calheiros T (2014) Impacts of climate change on the fire regime in Portugal. 'Adv. For. Fire Res.' (Ed DX Viegas) pp. 1193-1206. (Coimbra) doi:http://dx.doi.org/10.14195/978-989-26-0884-6_130.

González-Hidalgo JC, Brunetti M, de Luis M (2011) A new tool for monthly precipitation analysis in Spain: MOPREDAS database (monthly precipitation trends December 1945-November 2005). International Journal of Climatology 31, 715-731. doi:10.1002/joc.2115.

González-Hidalgo JC, Peña-Angulo D, Brunetti M, Cortesi N (2015) MOTEDAS: a new monthly temperature database for mainland Spain and the trend in temperature (1951-2010). International Journal of Climatology 35, 4444-4463. doi:10.1002/joc.4298.

Gore PA (2000) 'Cluster Analysis.' (HEA Tinsley and SD Brown, Eds.). (Academic Press) doi:10.1016/B978-012691360-6/50012-4.

Jiménez-Ruano A, Rodrigues Mimbrero M, de la Riva Fernández J (2017) Understanding wildfires in mainland Spain. A comprehensive analysis of fire regime features in a climate-human context. Applied Geography 89, 100-111. doi:10.1016/j.apgeog.2017.10.007.

Kasischke ES, Turetsky MR (2006) Recent changes in the fire regime across the North American boreal region - Spatial and temporal patterns of burning across Canada and Alaska. Geophysical Research Letters 33, 1-5. doi:10.1029/2006GL025677. 
Kohonen T (1998) The self-organizing map. Neurocomputing 21, 1-6. doi:10.1016/S09252312(98)00030-7.

Malamud BD, Millington JD a, Perry GLW (2005) Characterizing wildfire regimes in the United States. Proceedings of the National Academy of Sciences of the United States of America 102, 46944699. doi:10.1073/pnas.0500880102.

Montiel Molina C, Galiana-Martín L (2016) Fire Scenarios in Spain: A Territorial Approach to Proactive Fire Management in the Context of Global Change. Forests 7, 273. doi:10.3390/f7110273.

Moreno MV, Chuvieco E (2013) Characterising fire regimes in Spain from fire statistics. International Journal of Wildland Fire 22, 296-305. doi:10.1071/WF12061.

Morgan P, Hardy CC, Swetnam TW, Rollins MG, Long DG (2001) Mapping fire regimes across time and space: Understanding coarse and fine-scale fire patterns. International Journal of Wildland Fire 10, 329-342. doi:doi:10.1071/WF01032.

Mori AS, Johnson EA (2013) Assessing possible shifts in wildfire regimes under a changing climate in mountainous landscapes. Forest Ecology and Management. doi:10.1016/j.foreco.2013.09.036.

Murtagh F, Legendre P (2014) Ward's Hierarchical Agglomerative Clustering Method: Which Algorithms Implement Ward ' s Criterion? Journal of Classification 31, 274-295. doi:10.1007/s00357-.

Parente J, Pereira MG, Tonini M (2016) Space-time clustering analysis of wildfires: The influence of dataset characteristics, fire prevention policy decisions, weather and climate. The Science of the total environment 559, 151-165. doi:10.1016/j.scitotenv.2016.03.129.

Pausas JG, Bladé C, Valdecantos A, Seva JP, Fuentes D, Alloza JA, Vilagrosa A, Bautista S, Cortina J, Vallejo R, Ecology SP, Blad C, Seva JR, Alloza A, Vallejo R, Darwin CC (2004) Pines and oaks in the restoration of Mediterranean landscapes of Spain: New perspectives for an old practice - a review. Plant Ecology 171, 209-220.

Pereira MG, Caramelo L, Orozco C V., Costa R, Tonini M (2015) Space-time clustering analysis performance of an aggregated dataset: The case of wildfires in Portugal. Environmental Modelling and Software 72, 239-249. doi:10.1016/j.envsoft.2015.05.016.

Perera AH, Cui W (2010) Emulating natural disturbances as a forest management goal: Lessons from fire regime simulations. Forest Ecology and Management 259, 1328-1337. doi:10.1016/j.foreco.2009.03.018.

Rasilla DF, García-Codron JC, Carracedo V, Diego C (2010) Circulation patterns, wildfire risk and wildfire occurrence at continental Spain. Physics and Chemistry of the Earth 35, 553-560. doi:10.1016/j.pce.2009.09.003.

Rodrigues M, de la Riva J (2014) An insight into machine-learning algorithms to model human-caused wildfire occurrence. Environmental Modelling \& Software 57, 192-201. doi:10.1016/j.envsoft.2014.03.003.

Sen PK (1968) Estimates of the Regression Coefficient Based on Kendall's Tau. Journal of the American Statistical Association 63, 1379-1389.

Serra L, Juan P, Varga D, Mateu J, Saez M (2013) Spatial pattern modelling of wildfires in Catalonia, Spain 2004-2008. Environmental Modelling and Software 40, 235-244. doi:10.1016/j.envsoft.2012.09.014.

Shakesby R (2011) Post-wildfire soil erosion in the Mediterranean: Review and future research directions. Earth-Science Reviews 105, 71-100. doi:10.1016/j.earscirev.2011.01.001. 
Vázquez de la Cueva A, Del Barrio JMG, Quero MO, Palomares OS (2006) Recent fire regime in peninsular Spain in relation to forest potential productivity and population density. International Journal of Wildland Fire 15, 397-405. doi:10.1071/WF05071.

Vázquez de la Cueva A, Climent JM, Casais L, Quintana JR (2015) Current and future estimates for the fire frequency and the fire rotation period in the main woodland types of peninsular Spain: a case-study approach. Forest Systems 24, 13. doi:10.5424/fs/2015242-06454.

Wang Y, Anderson KR (2010) An evaluation of spatial and temporal patterns of lightning- and humancaused forest fires in Alberta, Canada, 1980-2007. International Journal of Wildland Fire 19, 10591072. doi:10.1071/WF09085.

Wotton BM, Nock CA, Flannigan MD (2010) Forest fire occurrence and climate change in Canada. International Journal of Wildland Fire 19, 253-271. doi:10.1071/WF09002. 


\title{
Ignition/non ignition phase transition
}

\author{
F. Z. Sabi ${ }^{1}$; M. S. Terrah ${ }^{1}$; O. Mosbah ${ }^{1}$; A. Dilem ${ }^{1}$; N. Hamamousse ${ }^{1,2}$; A. Sahila ${ }^{1}$; O. Harrouz ${ }^{1}$; \\ N. Zekri' ${ }^{*}$; A. Kaiss ${ }^{2}$; J-P. Clerc ${ }^{2}$; O. Rahli ${ }^{2}$; F. Giroud ${ }^{3}$; C. Picard ${ }^{3}$ \\ ${ }^{1}$ Université des Sciences et de la Technologie d'Oran, LEPM BP 1505 El Mnaouer Oran, Algeria, \\ \{nzekri@yahoo.com*\} \\ ${ }^{2}$ Aix Marseille Université. CNRS, IUSTI UMR 7343, 13453 Marseille, France \\ ${ }^{3}$ CEREN, Domaine de Valabre, 13120 Gardanne, France
}

\section{Abstract}

The flammable/non flammable transition is investigated both experimentally and numerically within the framework of statistical mechanics and percolation-type phase transitions. The heat flux threshold for ignition is usually estimated deterministically by fire research community. It is defined actually by ASTM standards as the average between the minimum flux allowing ignition and the maximum one which does not allow it. The probabilistic aspect of the ignition transition is demonstrated here, and a new method for the estimation of the ignition critical heat flux is proposed.

Dead wheat straw and live pinus halepensis needles are investigated experimentally using a cone calorimeter and numerically using a simple physical model. The experimental data on ignition time are different from those obtained numerically. This difference is due to the fact that the numerical model assumes auto-ignition whereas experimental data are obtained by using a pilot flame. It is found that the variation of ignition time with the incident heat flux obeys a universal power-law behaviour near the heat flux threshold. The critical heat flux for ignition estimated from this law is also obtained using the ignition probability method. This critical heat flux is as low as it cannot be obtained using the ASTM standards. The power-law exponent is found compatible for both dead straw and pinus halepensis suggesting a universal phase transition.

Further discussions on the comparison between flammability transition and spread/non spread transition are provided.

Keywords: Critical heat flux, flammability, phase transition

\section{Introduction}

The critical condition for ignition is an important property that allows land managers and fire operationals to control its spread. The most common criterion is based on the assumption that ignition occurs when a critical temperature at the surface, $T_{i g n}$, is reached. Piloted ignition occurs when the lower flammability limit is reached in the fuel-air mixture around the pilot flame. An increasing number of works were devoted to the study of the critical heat flux of fuel ignition (see Quintiere and Harkleroad 1984, Dietenberger 1996, Mikkola and Wichman 1989, Delichatsios and Chen 1993, Mindykowski et al. 2011). On the other hand, a still unexplained empirical observation is the "moisture of extinction" (Cohen and Bradshaw, 1986; Rothermel, 1972; Weise et al., 2005). Wildland fires in dead fuels will not spread above some threshold of fuel moisture content, typically assumed to be between 10\% and 40\% (Rothermel, 1972). Terrah et al. (Terrah et al. 2018) have shown no critical moisture content for ignition, and that phase transition is due to the critical heat flux for ignition. In a wildland fire, the heat flux produced by a flame depends on the mass of the flaming fuel. A proportion of this flux is received by the fuel exposed to the flame. The critical received heat flux for ignition corresponds then to a critical mass of the decomposing fuel. As the fuel is heterogeneous, it induces the emission of multiple heat fluxes and various flaming durations. Then, there is an effective heat flux induced by the heterogeneous decomposing fuel. Furthermore, to ensure spreading of wildland fires,

Advances in Forest Fire Research 2018 - Page 506 
the residence time of the burning fuel must be larger than the ignition time of the one which is exposed to the flame. As the flame residence time is always finite (e.g. around 30s for pine needles and straw if arranged in non-compacted beds), two transition thresholds appear: propagation and ignition thresholds. The critical heat flux for the propagation transition is thus larger than that of ignition transition. It is useful to express these transitions in terms of the ignition and combustion energies. Fig. 1 shows a schematic behaviour of the ignition and combustion energies as a function of the incident heat flux for the same fuel sample. As the received heat flux increases, the ignition energy (or ignition time) decreases, but the combustion energy (or residence time) increases (Chandler et al. 1983, Trabaud 1992). For simplicity the trends are shown here linear. The ignition transition occurs when the energy received from a heat flux, even during an infinite time, reaches the fuel ignition energy. Propagation/non propagation transition occurs if the flaming fuel combustion energy reaches the ignition energy of the exposed fuel (see the arrow of Fig.1). In 2005, (Zekri et al. 2005) have distinguished this transition from the percolation transition induced by the fuel inhomogeneity, and called its threshold the dynamical threshold. In the present work, we focus on the ignition transition, where the sample is exposed to a heat flux during a sufficient time for its ignition, as realised with a cone calorimeter.

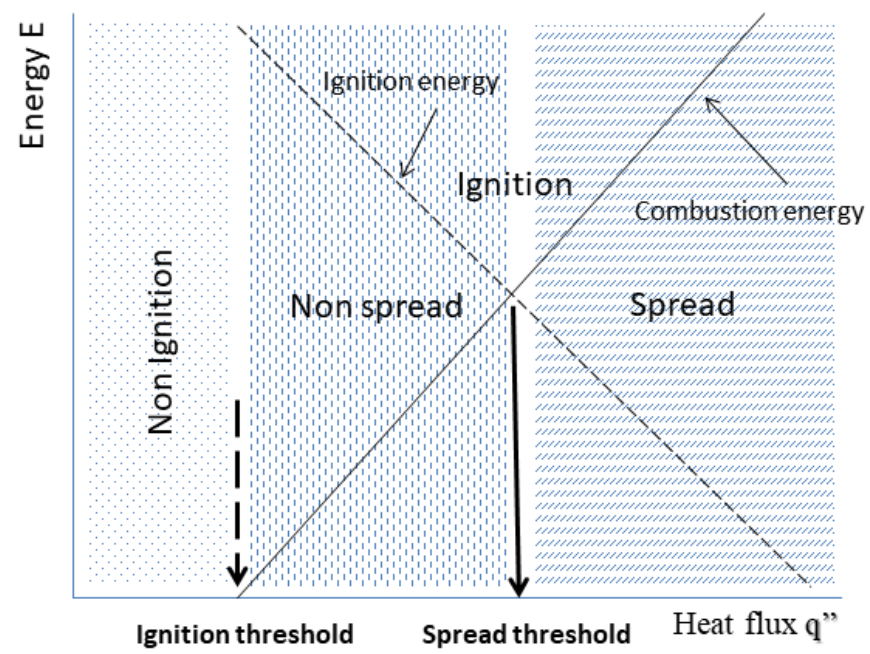

Figure 1 - A schematic representation of the ignition and combustion energies vs. the incident heat flux. Ignition and spreading thresholds are clearly shown.

Most of the studies using a cone calorimeter estimated the ignition critical heat flux from a linear fit of the inverse of ignition time as a function of the incident heat flux (Khan et al. 2008, Mindykowski 2011). However, the linear behaviour of the inverse of ignition time is observed only for high heat fluxes, whereas fluctuations and nonlinearity appear in the limit of low fluxes near the critical one. Therefore, the critical heat flux for ignition could be overestimated by such a fitting. On the other hand, the ASTM 1354 standards define the critical heat flux as the average value between the maximum flux where ignition cannot occur and the minimum one where it occurs. This definition induces an inherent uncertainty in the estimation of the critical heat flux.

Phase transitions, as observed in various fields such as conductor/insulator (Stauffer 1992), liquid/gas (Blundell et al. 2006) and paramagnetic/ferromagnetic (Stanley 1971, Pokrovskii 1999), are characterized by a strong disorder near the threshold. They are also characterized by diverging or vanishing quantities following a universal power-law formula near the critical region (Stanley 1971, Yeomans 1992). In the case of ignition/non ignition (or exothermic/endothermic) phase transition, ignition time must diverge as:

$$
t_{i g n} \sim\left(q^{\prime \prime}{ }_{i n c}-q^{\prime \prime}\right)^{-\gamma}
$$


Where $q{ }^{\prime \prime}{ }_{i n c}$ is the incident heat flux, $q{ }^{\prime \prime}$ the critical heat flux for ignition and $\gamma$ the critical exponent. This exponent expresses the rate of increase of the ignition time near the critical region. There should be also a probability of ignition near the critical region because of the disorder.

In the present work, the critical heat flux is estimated within the framework of phase transitions. Numerical simulations using a simple model (Koo et al. 2005) and experimental data on pinus halepensis and wheat straw using a cone calorimeter are considered.

\section{Experimental setup}

Two different species of vegetation have been used: wheat straw (dead fuel) at around $12 \%$ moisture content (august 2017) and pinus halepensis needles (live fuel) at 52\% moisture content (January 2018). Samples of Pinus halepensis are picked and burned on the same day to guarantee their properties of live fuel. This ensures that all the burned samples contain the same organic volatile composition.

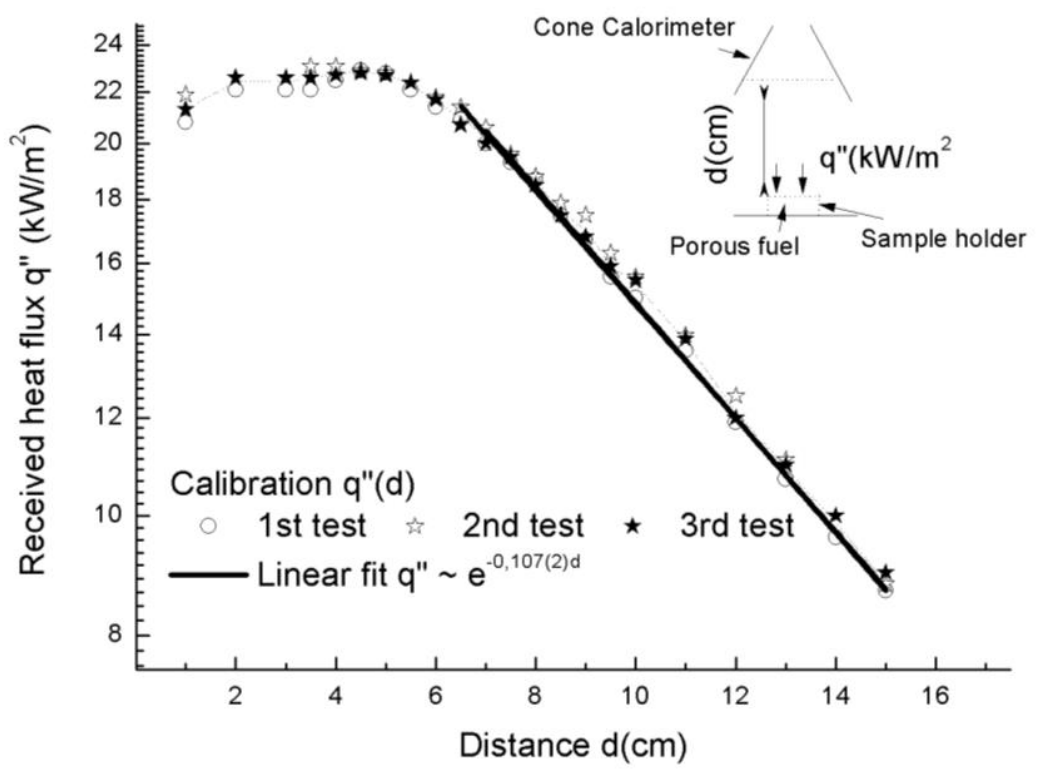

Figure 2 - A schematic representation of the calibration flux and the experimental setup.

Samples of $10 \mathrm{~g}$ mass were placed in a cylindrical holder of $10 \mathrm{~cm}$ diameter of a mesh shape, corresponding to a load of $1.27 \mathrm{~kg} / \mathrm{m}^{2}$. A cone calorimeter with an electrical resistance of a power of $3000 \mathrm{~W}$ as a heat source provides a heat flux to the samples. The samples are placed at different distances from the heat source so as to receive radiation heat fluxes $q^{\prime \prime}=q^{\prime \prime}{ }_{\text {inc }}$ of magnitudes ranging from 9 to $20 \mathrm{~kW} / \mathrm{m}^{2}$. The variation of the received heat flux is realized by changing the distance between the sample top surface and the heat source of the cone calorimeter. The experimental device and the incident heat flux calibration are shown schematically in Fig.2. The incident heat flux at the top surface position of the sample is calibrated by using a water cooled heat flux sensor of type Hukseflux SBG 01 working in the range $0-200 \mathrm{~kW} / \mathrm{m}^{2}$. The ignition is controlled by a pilot flame located $1 \mathrm{~cm}$ above the sample top surface, and the ignition time is recorded. In order to compare the experimental data with the numerical results where ignition process is auto- ignition, a test for wheat straw was realized by removing the pilot flame and closing the cone calorimeter on its top side. This allows us to get closer to auto-ignition, which is difficult to realize in all tests. Because of the heterogeneity of the sample structure, up to five (5) tests are repeated in order to determine the time to ignition as defined by ASTM standards (only one test is realized for wheat straw). The tests were conducted in a draft-free room with room temperatures and relative humidities in the ranges $13-19^{\circ} \mathrm{C}$, and $50-60 \%$, respectively for pine needles and $20-30^{\circ} \mathrm{C}$ and $45-55 \%$ for wheat straw. 


\section{Model description}

As stated by Drysdale (Drysdale 2011), the flame is a gas phase phenomenon and, clearly, flaming combustion of liquid and solid fuels must involve their conversion to a gaseous form. Oxygen/flammable gas mixture ignits at an ignition temperature when it reaches its flammability limit. It is difficult to find a simple model describing the fuel solid/gas and liquid/gas transitions and gas flammability. The present model assumes autoignition, and uses the criterion of ignition temperature at the surface of the fuel. It is assumed that the sample receives a radiative heat flux $q^{\prime \prime}=q^{\prime \prime}{ }_{\text {inc }}$ from the cone calorimeter. The effective heat flux $q^{\prime \prime}{ }_{\text {eff }}$ absorbed by the sample is:

$$
\mathrm{q}^{\prime \prime}{ }_{\text {eff }}=\mathrm{q}^{\prime \prime} \text { inc }-\mathrm{q}^{\prime \prime} \text { lost }
$$

Where $q$ "lost is the heat flux lost by the sample. The lost heat flux increases as the sample temperature increases. If we assume that the whole heat lost is radiative, we can express it as:

$$
\mathrm{q}_{\text {lost }}=\sigma \varepsilon_{\mathrm{fb}}\left(\mathrm{T}^{4}-\mathrm{T}_{0}^{4}\right)
$$

Where $\varepsilon_{f b}$ is the sample emissivity, $\sigma$ the stephen-Boltzman constant and $T_{0}$ the ambient temperature. The effective energy received during the ignition time is used to increase the sample temperature up to ignition temperature in three steps: $i$ ) increase of the moist sample temperature up to boiling $\left.\left(373^{\circ} \mathrm{K}\right), i i\right)$ evaporation of the water mass $m_{w}$ from the sample, iii) increase of the dry sample temperature until it reaches the ignition temperature. If we neglect the heat of desorption of water, the energy conservation becomes:

$$
\int_{0}^{\mathrm{t}_{\mathrm{ign}}} \mathrm{q}_{\text {eff }} \mathrm{dt}=\left\{\begin{array}{c}
\int_{\mathrm{T}_{0}}^{373^{\circ} \mathrm{K}}\left(\mathrm{m}_{\mathrm{dry}} \mathrm{c}_{\mathrm{p}}^{\mathrm{fb}}+\mathrm{m}_{\mathrm{w}} \mathrm{c}_{\mathrm{p}}^{\mathrm{w}}\right) \mathrm{dT}+ \\
\int_{\mathrm{m}_{\mathrm{w}}}^{0} \mathrm{~L}_{\mathrm{v}}\left(373^{\circ} \mathrm{K}\right) \mathrm{dM}+ \\
\int_{373^{\circ} \mathrm{K}}^{\mathrm{T}_{\mathrm{ign}}} \mathrm{m}_{\mathrm{dry}} \mathrm{c}_{\mathrm{p}}^{\mathrm{fb}} \mathrm{dT}
\end{array}\right.
$$

Here $c_{p}^{f b}, c_{p}^{w}$ are the specific heat of the dry fuel and water respectively $\left(c_{p}^{f b}=1827 \mathrm{~J} / \mathrm{kg} / \mathrm{K}\right.$ for pinus halepensis needles, $1700 \mathrm{~J} / \mathrm{kg} / \mathrm{K}$ for wheat straw and $4180 \mathrm{~J} / \mathrm{kg} / \mathrm{K}$ for water), $L_{v}=2257 \mathrm{~kJ} / \mathrm{kg}$ the water latent heat at $373^{\circ} \mathrm{K}$, and $m_{d r y}$ and $m_{w}$ are the dry fuel and water masses exposed to the incident flux. The fuel moisture constant is defined as:

$$
h(\%)=\frac{m_{w}}{m_{d r y}+m_{w}} \times 100
$$

In order to be coherent with the experimental setup, the fuel mass can be expressed as:

$$
m_{\text {fuel }}=\phi \cdot \rho_{\text {fuel }} . S . e
$$

Where the fuel density $\rho_{\text {fuel }}$ is around $500 \mathrm{~kg} / \mathrm{m}^{3}$ for the wheat straw and $789 \mathrm{~kg} / \mathrm{m}^{3}$ for pinus halepensis needles, $S$ the surface occupied by the fuel (in $m^{2}$ ) and $e$ the fuel thickness (in $m$ ). The packing ratio $\phi$ is the ratio of solid fuel volume to fuel bed volume. As the effective heat flux in the left side of (4) is usually expressed in $\mathrm{kW} / \mathrm{m}^{2}$, it is useful to introduce the fuel load $\sigma_{\text {fuel }}=m_{f u e l} / S$ (in $\mathrm{kg} / \mathrm{m}^{2}$ ). In order to compare with the experimental results, the fuel load is taken $1.27 \mathrm{~kg} / \mathrm{m}^{2}$ in all simulations.

\section{Results and discussions}

In order to understand the temperature behaviour near the critical region, let us consider the simulation model described in the previous section. When exposed to a heat flux, the fuel surface temperature increases according to (4), but the lost heat flux increases according to (3), and the 
received effective flux is reduced as a consequence. If the heat flux is not sufficient to ignite the fuel, its surface temperature reaches asymptotically a maximum temperature $T_{\max }$ (obviously smaller than $\mathrm{T}_{\text {ign }}$ ), so that the whole incident flux is lost. Thus, the surface temperature becomes nearly constant near $\mathrm{T}_{\max }$ (see Fig.3a). For the experimental tests on wheat straw, where the heat flux is not sufficient, the surface temperature was shown to reach a maximum value, then it decreases and becomes constant. This is due to the change in the specific heat of the sample because of the straw thermal degradation. The maximum temperature $\mathrm{T}_{\max }$ increases as the incident flux increases. Hence, the ignition critical flux corresponds to the condition $\mathrm{T}_{\text {ign }}=\mathrm{T}_{\max }$. In this limit, the fuel reaches the ignition temperature after an infinite time $\left(t_{\text {ign }} \rightarrow \infty\right)$. The ignition time becomes finite above the critical heat flux (Fig.3b).

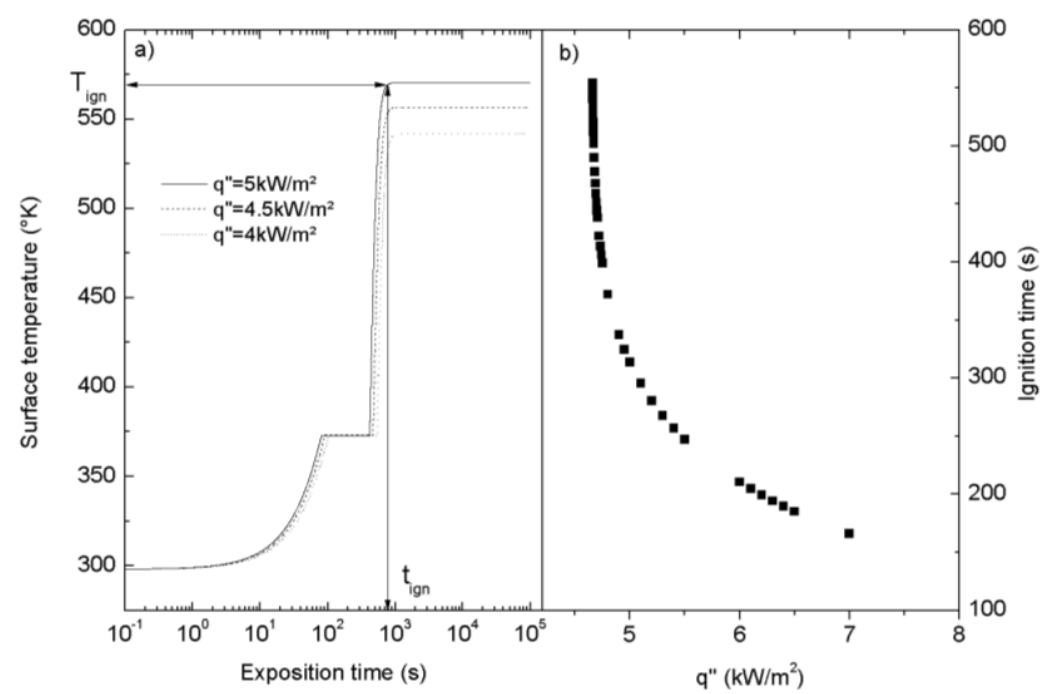

Figure 3 - Simulation for wheat straw of: a) Surface temperature vs. time of exposition for various incident fluxes. b) Ignition time vs. heat flux.

Note in Fig.3b, the strong variation of the ignition time near the critical heat flux (few $\mathrm{W} / \mathrm{m}^{2}$ ). This variation of the heat flux is impossible to realize experimentally. Furthermore, the fluctuations of ignition time are observed experimentally because of the heterogeneity of the sample shape, thickness, porosity etc. The simulation model can be considered as a mean field one. Assuming that the ignition time divergence in Fig.3b obeys the phase transition universal law (1), experimental and numerical data are presented in Fig.4 in a double logarithmic scale as a function of the incident heat flux in reference to the critical flux. A linear behaviour in Fig.4 corresponds to (1). The value of the ignition critical heat flux is estimated so as to obtain the best linear fit.

The ignition critical heat flux is clearly much larger for wheat straw $\left(\mathrm{q}^{\prime \prime} \sim 10 \mathrm{~kW} / \mathrm{m}^{2}\right)$ than for pinus halepensis $\left(\mathrm{q}_{\mathrm{c}} \sim 6 \mathrm{~kW} / \mathrm{m}^{2}\right)$. This confirms the large fire susceptibility index of pinus halepensis in the mediterranean wildlands (Alexandrian and Rigolot 1992). The simulation results of ignition critical heat flux $\left(q^{\prime \prime}{ }_{c} \sim 4.6 \pm 0.5 \mathrm{~kW} / \mathrm{m}^{2}\right)$ and ignition time for wheat straw using auto-ignition are very different from those obtained from experimental data for this fuel with piloted ignition (where the critical heat flux is $q{ }^{\prime \prime}=10.3 \pm 0.5 \mathrm{~kW} / \mathrm{m}^{2}$ ). This is due to the difference in the ignition process. As described above, the auto-ignition considered for the simulation model is difficult to realize experimentally, since it assumes solid fuel ignition. In fact, as the fuel temperature increases, a solidgas or a solid-liquid-gas phase transition occurs. The flammable gas/air mixture must reach ignition temperature at the fuel surface while it escapes from it. A mechanism to simplify the ignition process is to include a pilot flame or a hot spot. Then, there is no need for the gas/air mixture, when it reaches its lower flammability limit, to wait for the ignition temperature to be reached at the surface, since the pilot flame provides it (Torero 2016). 
In order to be closer from the auto-ignition condition and be able to compare with the simulations results, the pilot flame is removed, and the cone calorimeter closed at its top side. The ignition time is much larger than that of the pilot flame for the same heat flux (see star in Fig.4), but is also larger than the expected ignition time from the simulation model. In addition, the ignition did not occur at the fuel surface (auto-ignition), but inside the closed cone which plays the role of a hot source. The model clearly fails to reproduce experimental data. Obviously, it does not account for the fuel gas emission and flow at the sample surface. This kind of models is often used to describe wildland fire spread (Adou et al. 2010). Hence, these models must be improved to explain some unexpected situations such as junction fires (Viegas et al. 2012).

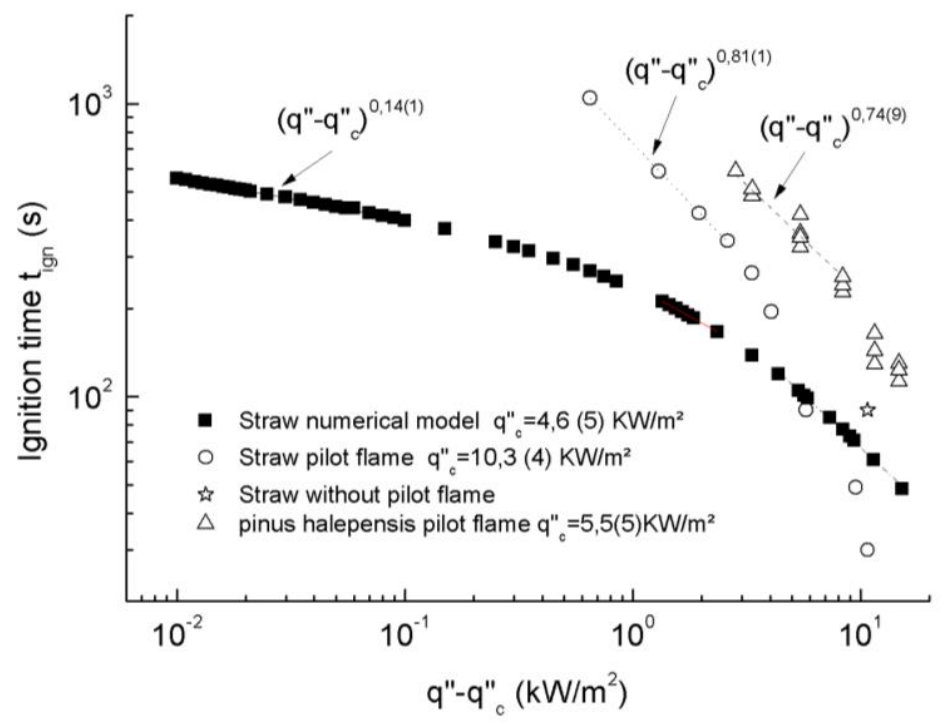

Figure 4 - Ignition time vs. $q "-q^{\prime \prime}{ }_{c}$ for the simulation model and experimental data on dead straw and live pinus halepensis. One data without pilot flame is presented for comparison.

In the recent experimental investigation of the ignition critical heat flux (Mindykowski et al. 2011), using a fire propagation aparatus, a linear behaviour of the inverse of ignition time was assumed. This behaviour corresponds to (1) with an exponent $\boldsymbol{\gamma}=\mathbf{1}$. However, the linear behaviour of the inverse of ignition time was observed for incident heat fluxes far from the critical region. In the present results (Fig.4), the exponent is around $\boldsymbol{\gamma}=\mathbf{0 . 1 4}$ for numerical simulation and $\boldsymbol{\gamma} \mathbf{0 . 7 5}$ for experimental results both for dead wheat straw and live pinus halepensis. Therefore, near the critical region the inverse of ignition time behaves nonlinearly in contrast to the assumption of Mindykowski et al. As for the ignition critical heat flux and ignition time, the difference between the values of exponents for numerical and experimental results can be attributed to the ignition process difference (auto-ignition and pilot flame). The equality of the exponents for wheat straw and pinus halepensis suggests a universal ignition phase transition (Stanley 1971, Yeomans 1992).

The results of Fig. 4 revealed an increasing number of tests that failed to ignite as the critical region is approached. This means that a percolation-type probabilistic behaviour occurs near the critical region (Stauffer 1992). In ASTM standards, only five (05) tests are repeated for each heat flux, which means that the minimum estimated ignition probability is $20 \%$. If for a given heat flux, all the five tests fail to ignite, it is decided that the flux threshold is not reached according to ASTM standards. Nevertheless, for the critical heat flux estimated from (1), the ignition probability might be much smaller than $20 \%$. Therefore, the ignition critical heat flux is overestimated when ASTM standards are used. As a consequence, such standards must be re-examined for fire safety.

Fire management at the wildland-urban interface requires fuel-breaks of width depending on front fire intensity. In order to stop the propagation of wildland fire to the urban zone, the heat flux at this region must be smaller than the critical spread flux. Therefore, an overestimation of this critical flux 
(underestimation of the width of fuel-break) leads to a fire spread in the urban zone. The determination of the ignition critical heat flux from Figs 1 and 3 allows us to have a better estimation of the heat flux threshold for fire spread. This will help us to preserve the urban habitat from fire propagation.

\section{Conclusion}

The ignition phase transition was investigated both experimentally using a cone calorimeter and numerically using a simple physical model. Wheat straw and pinus halepensis fuel samples were used for this end. Unlike ASTM standards for which ignition threshold is estimated deterministically, the probabilistic aspect of the ignition transition was pointed out, and a new method for the estimation of the ignition critical heat flux was proposed using the framework of statistical mechanics and percolation-type phase transitions.

The variation of ignition time with the received heat flux was found to obey a universal power-law behaviour near the ignition heat flux threshold (see Eq.1). The flux threshold estimated from this law is lower than the ASTM standards estimation.

A further study of the universality of the ignition transition and its connection to the spread/non spread transition is necessary.

\section{References}

Adou JK, Billaud Y, Brou DA, Clerc JP, Consalvi JL, Fuentes A, Kaiss A, Nmira F, Porterie B, Zekri L., Zekri N (2010), Simulating wildfire patterns using a small-world network model, Ecol.Modelling, 221, 1463-1471.

Alexandrian D and Rigolot E (1992), Sensibilité du pin d'Alep à l'incendie, Forêt méditerranéenne, 3, 185-198.

Blundell SJ and Blundell KM (2006), 'Concepts in Thermal Physics', (Oxford University Press, Oxford).

Chandler C, Cheney P, Thomas P, Trabaud L, Williams D (1983) 'Fire in forestry. Vol. I. Forest fire behaviour and effects.' (John Wiley \& Sons: New York)

Cohen J and Bradshaw B (1986) Fire behavior modeling - A decision tool. In 'Proceedings Prescribed Burning in the Midwest: State of the Art ' (Ed. A. L. Koonce), University of Wisconsin at Stevens Point, Stevens Point, WI, 1-5.

Dietenberger M (1996), Ignitability Analysis using the Cone Calorimeter and LIFT Apparatus, Proceedings of the International Conference on Fire Safety, Columbus,OH, 22, 189-197.

Delichatsios M, Chen Y(1993) Asymptotic, Approximate, and Numerical Solutions for the Heatup and Pyrolysis of Materials Including Reradiation Losses, Combustion and Flame, 92, 292-307.

Khan MM, De Ris JL, Ogden SD (2008) Effect of Moisture on Ignition Time of Cellulosic Materials, Fire Safety Science-proceedings of the Ninth International Symposium, 167- 178.

Koo E, Pagni P, Woycheese P, Stephens S, Weise D, Huff J (2005) A Simple Physical Model for Forest Fire Spread Rate, Fire Safety Science-proceedings of the Eighth International Symposium, 851-862

Mikkola E, Wichman I (1989), On the Thermal Ignition of Combustible Materials, Fire and Materials, 14, 87-96.

Mindykowski P, Fuentes P, Consalvi JL, Porterie B (2011), Piloted Ignition of Wildland Fuels, Fire Safety Journal 46 (1-2), 34-40.

Pokrovskii VL, (1999), Two-dimensional magnetic phase transitions, J.Magnet.Magnet. Mat, 200, 515-531.

Advances in Forest Fire Research 2018 - Page 512 
Quintiere J, Harkleroad M (1984), New Concepts for Measuring Flame Spread Properties, National Bureau of Standards, Gaithersburg, MD NBSIR 84-2943.

Rothermel RC (1972), A mathematical model for predicting fire spread in wildland fuels. USDA For. Serv. Res. Pap. INT-115. 40.

Stauffer D, Aharony A (Eds) (1992), Introduction to Percolation Theory', (Taylor and Francis, London).

Terrah SM, Zekri N (2018), Is there a critical fuel moisture content for flamability? (This conference)

Torero J (2016), Flaming ignition of solid fuels In 'SFPE Handbook of fire protection engineering' (Ed MJ Hurley) pp 633-661 (Springer: Heidelberg)

Trabaud L (1992), 'Les feux de forêt: mécanismes, comportement et environnement', (France selection, Paris)

Stanley HE (1971) 'Introduction to phase transitions and critical phenomena' (Clarendon Press Oxford)

Viegas DX, Raposo J, Davim D, Rossa C (2012), Analysis of the physical processes associated with junction fires at laboratory and field scales, Int. J.Wildland Fire, 21, 843-856

Weise DR, White RH, Beall FC, and Etlinger M (2005), Use of the cone calorimeter to detect seasonal differences in selected combustion characteristics of ornamental vegetation. Int. J.Wildland Fire, $14,321-338$.

Yeomans JM (1992) 'Statistical mechanics of phase transitions' (Clarendon Press Oxford)

Zekri N, Porterie B, Clerc J-P, Loraud J-C (2005) Propagation in a two-dimensional weighted local small-world network, Phys. Rev.E 71, 046121. 


\title{
Improvement of Forest Fire Danger Rating in Russia
}

\author{
Alexandra V. Volokitina ${ }^{1}$; Tatiana M. Sofronova*2; Mikhail A. Korets ${ }^{3}$ \\ ${ }^{1}$ Sukachev Institute of Forest SB RAS, ul. Akademgorodok, 50/28, Krasnoyarsk 660036, Russia, \\ \{volokit@ksc.krasn.ru\} \\ ${ }^{2}$ Astafiev Krasnoyarsk State Pedagogical University, ul. A. Lebedevoy,89, Krasnoyarsk 660049, \\ Russia, \{tmsofronova@gmail.com*\} \\ ${ }^{3}$ Sukachev Institute of Forest SB RAS, ul.Akademgorodok,50/28, Krasnoyarsk 660036, Russia, \\ \{mik@ksc.krasn.ru\}
}

\begin{abstract}
The paper analyses three kinds of forest fire danger rating (FDR) in the Russian forest fire protection: 1) FDR according to weather conditions (fire weather danger rating); 2) FDR according to local (regional) scales (a combination of fire weather and fire hazard adjusted to local conditions), and 3) FDR according to vegetation fuel complexes (the so called fire hazard). Unfortunately, all the enumerated FDRs are realized via techniques of 1940s - 1970s in spite of the fact that there are improved techniques developed in the country. The constant personnel perturbations in the Federal Forestry Service of Russia and absence of qualified forestry experts there make it impossible to keep an eye and timely consider the methodical recommendations (practical guidelines) received from scientists and aimed at improvement of forest fire protection in Russia. The main focus is on methods of earth remote sensing and increase of technical power though the world experience shows that this is a dead-end way in resolving the vegetation fire issues.

Analysis of the available elaborations has shown that the most forward-looking are the recommendations realized on the basis of a long-term fundamental research in the leading forest fire science laboratory of Russia at the Sukachev Institute of Forest SB RAS. For the FDR according to weather conditions it is suggested that an index should be used which takes into account not only air temperature and dew point but also humidity and hygroscopicity of vegetation fuels and "works" even under negative air temperatures. Making local (regional) FDR scales is based on prediction of probable density of active fires per each day. And the basis of the FDR according to vegetation fuels (fire hazard rating) consists of the developed classification of vegetation fuels (VF) and methods of their mapping.

The results of our fundamental fire science studies suggest improvement of forest fire danger rating in protected areas and in areas with valuable indigenous coniferous stands of Siberian pine, fir, pine, larch, and spruce. Recommendations are given for more accurate assessment of a fire weather index and fire hazard, and for making improved comparable local (regional) fire danger scales. The recommendations are aimed at fire science specialists, fire protection specialists in protected areas, forest offices, and air patrol, emergency services employees, faculty and university students.
\end{abstract}

Keywords: forest fire danger, fire danger rating according to weather conditions, local (regional) scales of fire danger rating, method of assessing fire hazard

\section{Introduction}

Forest fire danger is impacted by a large number of factors, direct and indirect, which differ by their role and dynamics. The major factors are those which are indispensible for ignition to start. Firstly, there are vegetation fuels (VF) as complexes, with the available fuel load being most important characteristic. Secondly, there are weather conditions (relative air moisture and air temperature, drought level, precipitation) which predetermine occurrence and dynamics of the available VF load. Thirdly, there are ignition sources (probability of their occurrence).

In Russia, forest fire protection fulfills the following major tasks: fire detection and daily regulation of forest fire protection services; planning and conducting fire preventive activities; direct fighting of

Advances in Forest Fire Research 2018 - Page 514 
occurring and active fires and their management. According to the forest fire protection tasks the following three kinds of fire danger are distinguished in practice:

1) fire danger according to weather conditions expressed as an index, e.g., the Nesterov and PV1 Indices (Nesterov, 1949; Vonsky et al., 1975, 1976);

2) fire danger according to VF complexes (the so called fire hazard) defined by special scales that determine the degree of ease of ignition there (Melekhov, 1947; Ovsyannikov, 1978);

3) fire danger rating according to local (regional) scales which is a combination of fire weather and fire hazard adjusted to local conditions (Kurbatsky, 1954, 1963; Zhdanko, 1965).

All the enumerated kinds of forest fire danger require improvement. Possible ways of their improvement are considered in this paper.

\section{Improvement of Forest Fire Danger Rating According to Weather Conditions}

Russian fire danger rating according to weather conditions was realized via the comprehensive Nesterov Index starting from 1949 and since 1967 - via the meteorological Nesterov Index with rough account of precipitation where the precipitation sum of less than $3 \mathrm{~mm}$ is ignored and the precipitation sum equal to $3 \mathrm{~mm}$ and over resets the index to zero and the calculation starts over again (Kats et al., 1975). Since 1976 one more index, the PV-1 Index, was introduced with amore differentiated account of precipitation (Vonsky, Zhdanko, 1976).

As for foreign fire danger rating systems, their detailed analysis and conclusion about inexpediency of application in Russia were made by T.M. Sofronova with coathors (Sofronova et al., 2007). Performance tests showed no advantages among the tested foreign methods of fire danger rating according to weather conditions (Viegas et al., 1999).

Analysis of the available elaborations (not yet implemented in practice) has shown that the most forward-looking is the index developed by the leading forest fire science laboratory of Russia at the Sukachev Institute of Forest SB RAS. This index as a further development of the Nesterov and PV-1 indices takes into account not only air temperature and dew point but also humidity and hygroscopicity of vegetation fuels and "works" even under negative air temperatures that is very important for such Russian regions as Zabaikalie and the Far East where spring and autumn fires can occur and spread under negative air temperatures.

The base of Nesterov Index and PV-1 Index reflect conditions of gradual total evaporation from the free water surface. However, evaporation from hygroscopic bodies (moss, lichen, litter et al.) is partial till the level of equilibrium moisture content depending upon air temperature and relative air humidity. This is the reason why fruticose lichens and moss Schreberi (Pleurozium Schreberi Mitt.) do not dry up to burnable state under relative air humidity higher than $85 \%$ and $60 \%$, respectively. Without rain, long increase of relative air humidity can moisten mosses, lichens and litter into a burnable state while the fire weather index is growing. To overcome this disparity, the index base is suggested to be compensated by the hygroscopicity of fuels, namely: $-5^{\circ} \mathrm{C}$ iss added to the difference $\left(\mathrm{t}-\mathrm{t}_{\mathrm{d}}\right)$. Therefore, when the difference $\left(t-t_{d}\right)$ is less than $5^{\circ}$, i.e. under the relative air humidity less than $85 \%$ in the afternoon, the index base becomes negative and the index itself decreases even without rain. For the new base to remain within the same level as the previous one, $+10^{\circ} \mathrm{C}$ is added to the first multiplier. This makes it possible to use almost the same scales. The new base acquires the following formula: $(t$ $\left.+10^{\circ}\right)\left(\mathrm{t}-\mathrm{t}_{\mathrm{d}}-5^{\circ}\right)$. The formulae for Nesterov (PN), PV-1 and PVG Indices are as follows:

$$
\mathrm{PN}_{\mathrm{n}}=\mathrm{PN}_{\mathrm{n}-1} \mathrm{~K}_{\mathrm{r}}+\left[\mathrm{t}\left(\mathrm{t}-\mathrm{t}_{\mathrm{d}}\right)\right]_{\mathrm{n}}
$$

$(\mathrm{PV}-1)_{\mathrm{n}}=\left\{(\mathrm{PV}-1)_{\mathrm{n}-1}+\left[\mathrm{t}\left(\mathrm{t}-\mathrm{t}_{\mathrm{d}}\right)\right]_{\mathrm{n}-1}\right\} \mathrm{K}_{\mathrm{r}} ;$

$\left.(\mathrm{PVG})_{\mathrm{n}}=\left[(\mathrm{PVG})_{\mathrm{n}-1}+\left(\mathrm{t}+10^{\circ}\right)_{\mathrm{n}}\left(\mathrm{t}-\mathrm{t}_{\mathrm{d}}-5^{\circ}\right)\right]_{\mathrm{n}}\right]\left(\mathrm{K}_{\mathrm{r}}\right)_{\mathrm{n}}$, 
where $\mathrm{t}$ - air temperature at 2-3 $\mathrm{pm},{ }^{\circ} \mathrm{C} ; \mathrm{t}_{\mathrm{d}}$ - dew point temperature at 2-3 $\mathrm{pm},{ }^{\circ} \mathrm{C} ; \mathrm{n}$ - day for which index is calculated; $\mathrm{n}-1$ - previous day; $\mathrm{K}_{\mathrm{r}}$ - precipitation coefficient for the sum of precipitation during previous 24 hours.

Testing the bases of indices in terms of the strength of their relationship with equilibrium moisture of wood showed that the correlation ratio of an old base $t\left(t-t_{d}\right)$ is equal to 0.91 and a new one $\left(t+10^{\circ}\right)(t$ $\left.-t_{d}-5^{\circ}\right)$ is equal to 0.99 . Thus, the index with a new base (PVG) is a bit higher in spring and lower in summer if compared with the PV-1 Index. Additional advantage of the PVG Index is that it can be calculated even under negative air temperature (up to $-10^{\circ} \mathrm{C}$ ) that is very important for Zabaikalie and the Far East where spring and autumn fires can occur and spread under negative air temperatures.

Precipitation account isalso suggested to be improved. When calculating the Nesterov Index, the sum of precipitation less than $3 \mathrm{~mm}$ corresponds to the precipitation coefficient equal to 1 . If the precipitation sum is more than $3 \mathrm{~mm}$, the precipitation coefficient is equal to 0 . The PV-1 index has a more differentiated account of precipitation starting from $0.5 \mathrm{~mm}$, special tables being made to facilitate calculations.

Analysis of existing precipitation coefficients and distribution of precipitation under canopies of different stands (Volokitina, 1979) lead to an improved precipitation coefficient (Sofronov et al., 2005; Sofronova et al., 2007):

$\mathrm{K}_{\mathrm{r}}=1.8 / \mathrm{R}+1$,

where R-sum of precipitation for the last 24 hours; if $\mathrm{R}<0.6 \mathrm{~mm} \mathrm{Kr}=1$.

Analysis of duration and sum of precipitation on the Obninsk rainfall-measuring ground (Dmitriyev, Isayev, 1970) showed that $1 \mathrm{~mm}$ of precipitation falls approximately within 1.3 hours (i.e. $0.8 \mathrm{~mm}$ of precipitation per hour). Therefore, there is another option for the precipitation coefficient:

$\mathrm{K}_{\mathrm{r}}=1.8 /(1.3 \mathrm{~T}+1)$,

Where $\mathrm{T}$ - precipitation duration within 24 hours, $\mathrm{h}$; if $\mathrm{T}<0.5 \mathrm{~h}, \mathrm{~K}_{\mathrm{r}}=1$.

There is a table of precipitation coefficients for calculation of the PVG Index (Table 1).

Table 1 - Precipitation coefficients $\left(K_{r}\right)$ for actual precipitation $(\boldsymbol{R}, \mathrm{mm})$ (Sofronov et al., 1992)

\begin{tabular}{|c|c|c|c|c|c|}
\hline $\mathrm{R}, \mathrm{mm}$ & $0.6-1.5$ & $1.6-2.5$ & $2.6-3.5$ & $3.6-4.5$ & $4.6-5.5$ \\
\hline $\mathrm{K}_{\mathrm{r}}$ & 0.90 & 0.60 & 0.45 & 0.36 & 0.30 \\
\hline $\mathrm{R}, \mathrm{mm}$ & $5.6-6.5$ & $6.6-7.5$ & $7.6-8.5$ & $8.6-9.5$ & $9.6-10.5$ \\
\hline $\mathrm{K}_{\mathrm{oc}}$ & 0.26 & 0.22 & 0.20 & 0.18 & 0.16 \\
\hline $\mathrm{R}, \mathrm{mm}$ & $10.6-11.5$ & $11.6-12.5$ & $12.6-13.5$ & $13.6-14.5$ & $14.6-16.0$ \\
\hline $\mathrm{K}_{\mathrm{r}}$ & 0.15 & 0.14 & 0.13 & 0.12 & 0.11 \\
\hline $\mathrm{R}, \mathrm{mm}$ & $16.1-18.0$ & $18.1-20.0$ & $20.1-24.0$ & $24.1-28.0$ & $28.1-32.0$ \\
\hline $\mathrm{K}_{\mathrm{r}}$ & 0.10 & 0.09 & 0.08 & 0.07 & 0.06 \\
\hline $\mathrm{R}, \mathrm{mm}$ & $32.1-40.0$ & $40.1-50.0$ & $50.1-60.0$ & - & - \\
\hline $\mathrm{K}_{\mathrm{r}}$ & 0.05 & 0.04 & 0.03 & - & - \\
\hline
\end{tabular}

Thus, the proposed PVG Index has the following advantages: 1) takes into account hygroscopicity of vegetation fuels, namely primary fire carriers (moss, lichen, litter); 2) works under negative temperatures (up to $-10^{\circ} \mathrm{C}$ ) that is very important for regions with spring and autumn fires spreading under negative temperature in the areas with grass and cured grass type of primary fire carrier; 3) uses an improved precipitation coefficient; 4) is calculated twice as an index of the previous day and a predicted index of a coming day.

\section{Improvement of forest fire danger rating using local (regional) scales}


The link between weather and daily fire danger level is not straightforward because of the impact of other fire danger factors. At present this link is defined through a system of regional scales which formerly were called "local scales of fire danger according to weather conditions".

The currently used technique of making local (regional) scales of forest fire danger rating developed by N.P. Kurbatsky (1954) generates fire danger classes incomparable even in adjacent regions with different forest vegetation conditions. For instance, under the same fire danger class in different forest offices the number of active fires per unit of area can be different. This prevents effective maneuvering of the available (usually insufficient) forces and means of forest fire protection.

The improved method of making local (regional) fire danger rating scales implies prediction of probable density of active fires per each day. Examples of such scales are made for the Chunsky Forest Office (Krasnoyarsky krai) which is most prone to wildfires. An algorithm for automatized creation of regional scales has been developed together with a beta version of the software (Volokitina et al., 2018).

Ten-year fire and weather data (2005-2014) of the Chunsky Forest Office (Krasnoyarsk krai) was obtained from the Krasnoyarsk Forest Fire Center for development of a technique for making improved regional scales of fire danger rating. An earlier developed principle method of making regional scales was applied (Kurbatsky, 1963), i.e. the empirical dependence between the long-term data on fire weather indices and the daily number of fires during a fire season in a specific area (a forest office). Moreover, an absolute criterion, fire density (fires per million ha), was introduced for distinguishing comparable classes of fire danger in regional scales (Sofronov, 1985). However, unlike the previously offered density of occurring fires, we used density of active fires, since its number is approximately twice higher than that of occurring fires according to the comparative analysis of long-term fire data (30 years). Therefore the recommended earlier gradations of fire density for fire danger classes were increased by the factor of 2 . We assumed that fire density changes by the factor of 4 from class to class in order to cover the whole range of possible fire densities in the given area (forest office) (Table 2) (Sofronova et al., 2007).

Table 2 - Gradation of fire danger classes by fire density

\begin{tabular}{|l|l|l|}
\hline \multirow{2}{*}{ Fire danger classes } & \multicolumn{2}{|l|}{ Fire density for a given day, fires / million ha } \\
\cline { 2 - 3 } & ocurring fires & active fires \\
\hline I & up to 0.10 & up to 0.20 \\
\hline II & $0.10-0.4$ & $0.20-0.8$ \\
\hline III & $0.41-1.6$ & $0.81-3.2$ \\
\hline IV & $1.61-6.4$ & $3.21-12.8$ \\
\hline V & over 6.4 & over 12.8 \\
\hline
\end{tabular}

It is very important to know the distribution of fires within a fire season (from April till September in the area of our study) for arrangement of forest fire protection. The considered 10-year period showed a sharp increase in number and area of fires starting from the second 10-day period in May and reaching its peak in the third 10-day period in June.

To elaborate optimal regional scales for the Chunsky Forest Office, we analyzed a mean daily number of active forest fires in relation with drought classes and their dynamics within a fire season for 10-year period (2005-2014) (Fig. 1). 


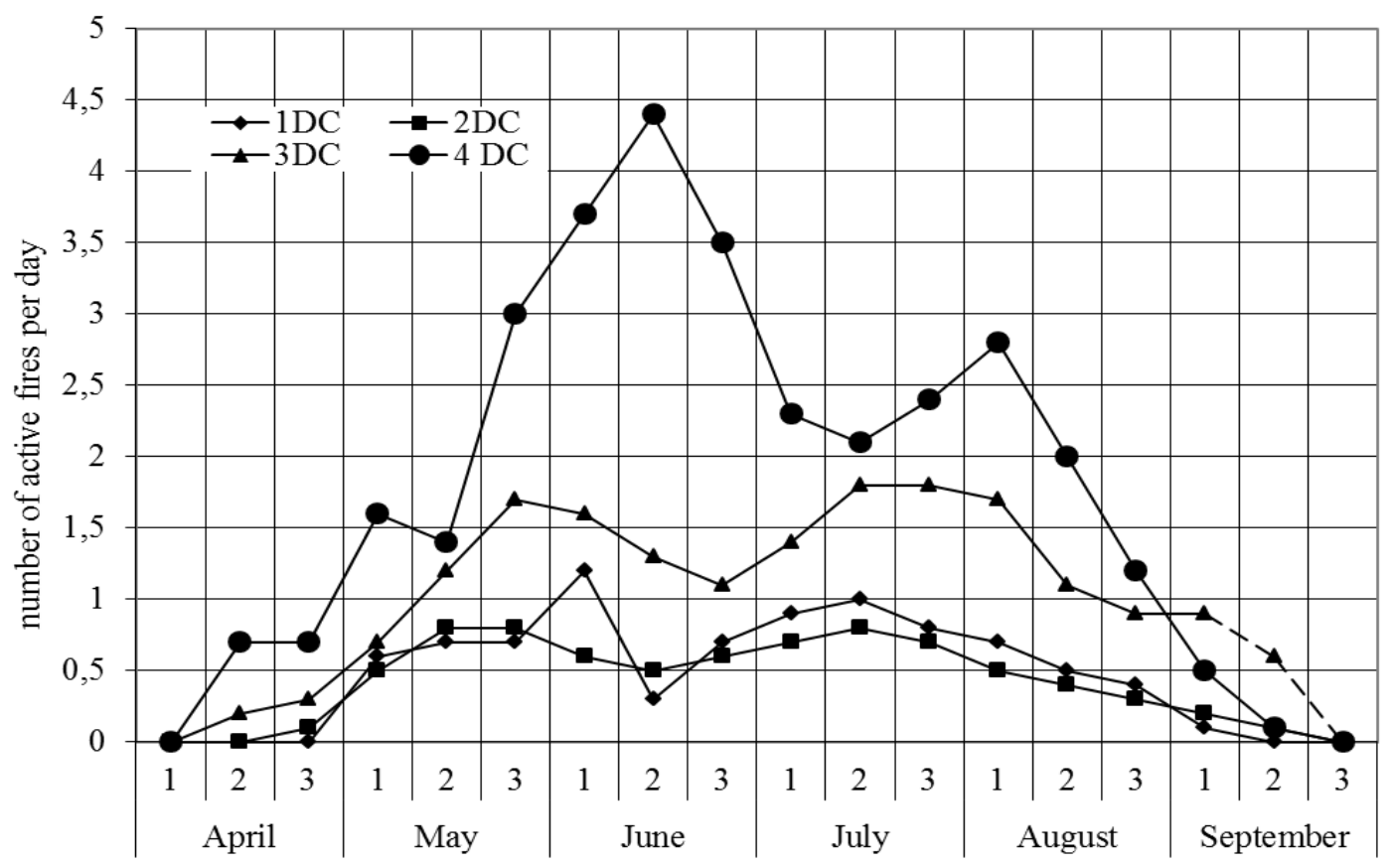

Figure 1 - Mean daily number of active forest fires in relation with drought classes $(D C)$ and their dynamics within a fire season (based on 2005-2014 data, Chunsky Forest Office). DCs correspond to the following gradation of the PV-1 and PVG Indices: $1 D C-$ up to 300, 2 DC-301-1000, 3 DC-1001-3000, $4 D C-3001-10000$, etc.).

Taking into account the dynamics of active fires which differs within a fire season, regional scales were made seperately for two periods of a fire season (from April 20 till June 30 and from July 1 till September 20) (Tables 3 and 4). Since the density of active fires for the period from 2005 till 2014 did not exceed the fourth fire danger class, the fifth class was not defined. However, the fifth class for this area can be theoretically assessed in case of extreme droughts.

Table 3 - Regional scales for the Chunsky Forest Office for the period from April 20 till June 30 (based on data from 2005 till 2014)

\begin{tabular}{|c|c|c|}
\hline Fire danger class & PV-1 Index value & Fire density per million ha \\
\hline I & up to 100 & up to 0.2 \\
\hline II & $101-800$ & $0.2-0.8$ \\
\hline III & $801-5200$ & $0.81-3.2$ \\
\hline IV & over 5200 & $3.21-12.8$ \\
\hline V & - & over 12.8 \\
\hline
\end{tabular}

Table 4 - Regional scales for the Chunsky Forest Office for the period from July 1 till September 20 (based on data from 2005 till 2014)

\begin{tabular}{|c|c|c|}
\hline Fire danger class & PV-1 Index value & Fire density per million ha \\
\hline I & up to 400 & up to 0.2 \\
\hline II & $401-1600$ & $0.20-0.8$ \\
\hline III & $1601-6000$ & $0.81-3.2$ \\
\hline IV & over 6000 & $3.21-12.8$ \\
\hline V & - & over 12.8 \\
\hline
\end{tabular}


Making regional fire danger scales manually takes considerable time. Having all the necessary information it is possible to automatize this process (Sofronova et al., 2013).

The following input data are needed to develop an programme algorithm: 1) area, million ha; 2) value of PV-1 or PVG Index per each day of a fire season for the last 10 years; 3) number of active fires per each day of a fire season for the last 10 years; 4) periods of a fire season.

The programme algorithm includes the following operations:

1. Group fire data within gradually expanding gradation of fire weather index values.

2. Calculate mean number of daily active fires within each gradation and assess density of daily active fires per million ha.

3. Make a correlation graph (crossing zero) between mean daily fire density and gradation of a fire weather index and define gradations of a fire weather index according to the scale with comparable fire danger classes: I FDC - up to 0.2; II FDC - 0.2-0.8; III FDC - 0.81-3.2; IV FDC - 3.2-12.8; V FDC - over 12.8 fires per million ha.

4. Make a regional scale as a table or a diagram.

The proposed improved regional scales of fire danger rating in the forest can help to assess more accurately a propbable daily density of active fires under each fire danger class. This will facilitate timely detection of occurring fires and more efficient manipulating of suppression forces and resources not only among forest offices (air patrol departments, regions) but also within one forest office (region) during different periods of a fire season as the tension of a fire situation in each area and in each period of a fire season will become comparable.

To help automatize regional assessment of daily fire danger we developed a program for making improved regional fire danger scales and made a beta version of the software (Sofronova et al., 2013).

\section{Improvement of fire danger rating according to vegetation fuel complexes (fire hazard)}

According to N.P. Kurbatsky (1972), the fire danger according to vegetation fuel (VF) complexes is a fire danger which is predetermined by the features of the area and related to a long-term period (under the assumption of constant availability of ignition sources which may cause a wildfire).

Russia still uses the scale for assessing forest plots by the degree of fire occurrence danger (Melekhov, 1947; Ovsyannikov, 1978) when planning fire prevention of the forest office territories. This scale only roughly assesses the integrated fire hazard.

The improved fire hazard rating is suggested to be based on the VF maps which contain information about all VF complexes of a given area and their characteristics. The main characteristic is the rate with which primary fire carriers become ready to burn. Methods of making VF maps have been developed. On their basis it is possible to make more accurate maps of fire hazard as well as maps of current fire hazard depending upon the class of drought. The information database for making such maps in GIS is developed for the Chunsky Forest Office (Krasnoyarsky krai) with area of 1 million ha. Besides fire hazard rating, the VF maps make it possible to predict behavior of an occurred fire, i.e. its spread rate, possible development (i.e. from the surface fire into the crown or ground fire), and its effects.

\section{Conclusion}

As a result of the carried out research the background was analyzed on forest fire danger according to weather conditions, according to VF complexes and according to regional scales. 
It is suggested that improvement of forest fire danger rating should be based on the results of fundamental research of the rate with which primary fire carriers become ready to burn in relation to the weather conditions and on the developed VF classification and methods of VF mapping.

Each kind of fire danger rating currently used in Russia is provided with recommendation on improvement of its performance (e.g., more accurate fire weather index calculation, improved comparable regional fire danger scales, etc.).

The proposed improvement will modernize fire danger rating according to weather conditions and regional scales as well as according to VF complexes.

\section{Acknowledgements}

The study was done within the framework of the basic project at the Sukachev Institute of Forest SB RAS \# VI.52.2.2 under partial support of the Russian Foundation For Basic Research grant \# 1805-00781A.

\section{References}

Dmitriyev AA, Isayev AA (1970) Evaluation of the possibility for extending the results of observation on the reference station over total daily precipitation to the surrounding area. Meteorologiya $i$ gidrologiya 9, 46-53.

Kats AL, Gusev VA, Shabunina TA (1975) 'Methodical guidelines for forest fire danger prediction according to weather conditions.' (Gidrometeoizdat: Moscow)

Kurbatsky NP (1954) Methodical guidelines for experimental development of local scales of fire danger.' (TsNIILH: Leningrad)

Kurbatsky NP (1963) Forest fire danger and its assessment by local scales. In 'Forest fires and their fighting'. pp. 5-30. (AN SSSR: Moscow)

Kurbatsky NP (1972) Terminology of forest fire science. In 'Issues of forest fire science'. pp. 171231. (ILiD: Krasnoyarsk)

Melekhov IS (1947) 'Nature offorest and forest fires.' (OGIZ: Archangelsk)

Nesterov VG (1949) 'Forest fire occurrence and methods of its assessment.' (Goslesbumizdat: Moscow)

Ovsyannikov IV (1978) 'Fire prevention arrangement of forests.' (Lesnaya promyshlennost: Moscow)

Sofronov MA (1985) 'Methodical guidelines for using standard regional scales of forest fire danger.' (ILiD SO RAN SSSR: Krasnoyarsk)

Sofronov MA, Volokitina AV, Fomina OA, Tartakovskaya TM (1992) 'Methodical guidelines for current fire danger assessment and prediction based on maps of forest fuels and weather forecasts.' (ILiD: Krasnoyarsk)

Sofronov MA, Volokitina AV, Korets MA, Sofronova TM, Mikhaylova IA (2005) 'Fire danger in natural conditions.' (IL SO RAN: Krasnoyarsk)

Sofronova TM, Volokitina AV, Pershin KS (2013) Automatized creation ofimproved local scales of fire danger. Vestnik KrasGAU 3, 157-163.

Sofronova TM, Volokitina AV, Sofronov MA (2007) 'Improvement of fire danger rating according to weather conditions in mountain forests of the South Baikal Lake basin.' (IL SO RAN, KUPU: Krasnoyarsk)

Viegas X, Bovio G, Ferreira A, Nosenzo A, Sol B (1999) Comparative study of various methods of fire danger evaluation in southern Europe. International Journal of Wildland Fire 9, 235-246. 
Volokitina AV (1979) Specifics of rain precipitation distribution under forest canopy. Lesovedenie 2, 40-48.

Volokitina AV, Sofronova TM, Korets MA (2018) 'Improvement of forest fire danger rating.' (IL SO RAN, KGPU: Krasnoyarsk)

Vonskiy SM, Zhdanko VA (1976) 'Principles for development of forest fire weather indices.' (LenNIILH: Leningrad)

Vonsky SM, Zhdanko VA, Korbut VI, et al. (1975) 'Assessment of forest fire hazard.' (LenNIILH: Leningrad)

Zhdanko VA (1965) 'Scientific fundamentals of making regional scales and their importance in fire prevention.’ (Lesnaya promyshlennost: Moscow) 


\title{
Is there a critical fuel moisture content for flammability?
}

\author{
S. M. Terrah ${ }^{1}$; F. Z. Sabi ${ }^{1}$; O. Mosbah ${ }^{1}$; A. Dilem ${ }^{1}$; N. Hamamousse ${ }^{1,2}$; A. Sahila ${ }^{1}$; O. Harrouz ${ }^{1}$; \\ N. Zekri' ${ }^{*}$; A. Kaiss ${ }^{2}$; J-P. Clerc ${ }^{2}$; O. Rahli ${ }^{2}$; F. Giroud ${ }^{3}$; C. Picard ${ }^{3}$ \\ ${ }^{1}$ Université des Sciences et de la Technologie d'Oran, LEPM BP 1505 El Mnaouer Oran, Algeria, \\ \{nzekri@yahoo.com*\} \\ ${ }^{2}$ Aix Marseille Université. CNRS, IUSTI UMR 7343, 13453 Marseille, France \\ ${ }^{3}$ CEREN, Domaine de Valabre, 13120 Gardanne, France
}

\section{Abstract}

The moisture threshold for ignition has attracted land managers and has been the main subject of discussion for several decades. The critical moisture of extinction is still a subject of extensive studies, and an endothermic/exothermic phase transition is expected to occur near the critical region. Ignition tests were realized for live pinus halepensis needles using a cone calorimeter. In order to ensure the live character of the fuel and avoid seasonal effects and changes in their chemical structure during ignition, the samples were picked the same day from the same tree, then partially dried using a microwave and a desiccator. By definition, phase transitions occur when a small variation of an external control parameter leads to a qualitative modification of the system. In order to determine whether the moisture content is the control parameter of the endothermic/exothermic phase transition or not, we had to verify the existence of a moisture content threshold for ignition by analysing the dependence of ignition time on moisture content. The experimental data have shown no critical moisture for the fuel ignition. We pointed out that the observed critical region corresponds to an ignition critical heat flux rather than a critical moisture.

Keywords: Critical moisture, flammability, pinus halepensis needles.

\section{Introduction}

Fuel moisture content is one of the most important variables affecting fire behaviour. It is known that moist fuels are relatively non-flammable, and even if the flammability occurs, the rate of combustion is reduced to less intense fires in contrast to the dry fuels that burn more fiercely (Pompe and Vines 1966, Van Wagner 1967, Trabaud 1976, Catchpole and Catchpole 1991). Jervis et al. (Jervis et al. 2015) showed that the most flammable samples are fresh dead and aged needles followed by dry dead and dry live needles. The least flammable are the fresh live needles. This confirms the importance of moisture content in the burning behaviour of pine needles, but the differences between live and dead samples cannot be explained solely in terms of moisture. It requires also the consideration of plant chemistry and sample drying. Live needles (green colour) are part of the foliage. Dead pine needles (red colour) are on the ground.

Not all studies have had success when correlating flammability measures with moisture content (Trabaud 1976, Fletcher 2007). Ignition fluctuations are caused by the dispersion of volatiles, moisture and the exposition of fuel particles in the sample. Recently, McAllister and Weise (McAllister and Weise 2017) pointed out that not only the moisture affects the fuel flammability, but also the chemical composition of volatiles. Live and dead fuels can have very different volatile components and then different ignition and combustion properties. The moisture threshold for ignition has been the main subject of discussion for several decades and has attracted land managers. A still unexplained empirical observation is the "moisture of extinction" (Cohen and Bradshaw, 1986; Rothermel, 1972; Weise et al., 2005). Wildland fires in dead fuels will not spread above some threshold of fuel moisture content, typically assumed to be between 10\% and 40\% (Rothermel, 1972). Actually, Trabaud 1976 has examined the effect of moisture content on the flammability and combustibility of various wildland

Advances in Forest Fire Research 2018 - Page 522 
dead fuels using an epiradiator at $620^{\circ} \mathrm{C}$. He proposed the following hyperbolic behaviour of the ignition time $\left(t_{i g n} \propto 1 /\left(h_{c}-h\right)\right)$. The moisture content $h$ is defined as:

$$
h=\left(m-m_{d r y}\right) / m
$$

with $m_{d r y}$ the dried mass and $m$ the mass of the moist sample. $h_{c}$ is the maximum moisture above which ignition cannot occur.

Near the critical moisture of ignition, an endothermic/exothermic phase transition is expected to occur. Phase transitions, as observed in various fields such as conductor/insulator (Stauffer 1992), liquid/gas (Blundell et al. 2006) and paramagnetic/ferromagnetic (Pokrovskii 1979), are characterized by a strong disorder near the threshold. They are also characterized by diverging or vanishing quantities following a universal power-law formula near the critical region (Stanley 1971, Yeomans 1992). In the case of ignition/non ignition phase transition, ignition time must diverge near the critical moisture as:

$$
t_{i g n} \sim\left(h_{c}-h\right)^{-\gamma}
$$

Where $h$ is the moisture content defined by (1), $h_{c}$ is the maximum moisture content allowing ignition estimated by Trabaud. Thus, it is defined as the critical moisture. The power-law formula (2) is characterized by a critical exponent $\gamma$ which distinguishes different phase transitions. The hyperbolic formula proposed by Trabaud is equivalent to (2) with a critical exponent $\gamma=1$, and the maximum moisture allowing ignition can be identified as the critical moisture content.

However, these tests were realized during several seasons or years, and the fuel was dried naturally. The fuel chemical composition changes from a season to another, leading to a variation in flammability and combustibility properties.

In the present work, ignition tests are realized for live pinus halepensis needles using a cone calorimeter. In order to ensure the live character of the fuel during ignition, the samples are picked the same day from the same tree, then partially dried to avoid seasonal effects. The ignition time dependence on moisture content (Eq. 2) is analysed to determine whether or not there is a critical moisture content for ignition.

\section{Experimental setup}

During the period from January to March 2018, pine needles (pinus halepensis) were picked from the same tree early in the morning (at $8.00 \mathrm{am}$ ). Immediately after the harvest, samples of live needles of 15 and $25 \mathrm{gr}$ were prepared for the determination of the moisture content of the vegetation. For this purpose, two drying techniques were used; a SAMSUNG household microwave at 800 watts and an infrared radiation dryer (Kern type MLS 3C). The samples are completely dried in the microwave $(25 \mathrm{~g}$ for 3 minutes), and in the desiccator $\left(15 \mathrm{~g}\right.$ at $80{ }^{\circ} \mathrm{C}$ for 24 hours). The holding time of the samples in the microwave and the desiccator determines the dry mass $\left(m_{d r y}\right)$, the moisture content $h_{\max }$ of the fresh samples and the maximum drying time for the two devices. The preparation of the samples at various intermediate moistures is realized by varying sample holding times $t$ in the two drying devices so as to cover the entire range of moistures ( $t \leq 24 h$ for the radiation dryer, and $t \leq 3 \mathrm{mn}$ for the microwave). Hence, the wet mass is obtained after the holding time $t(m=m(t))$, and the moisture content relative to this mass is defined by (1) with $h=h(t)$. Once dried, the samples are placed in a cylindrical mesh sample holder of $10 \mathrm{~cm}$ diameter. Then, these samples are exposed to a constant radiation heat flux provided by a cone calorimeter whose heat source is an electrical resistance of a power of $3000 \mathrm{~W}$. The experimental device is shown schematically in Fig.1. The incident heat flux at the top surface position of the sample $q^{\prime \prime}$ (which depends on the distance from the cone heater) is measured by a water cooled heat flux sensor of type Hukseflux SBG 01 working in the range $0-$ 
$200 \mathrm{~kW} / \mathrm{m}^{2}$ and its magnitude is fixed to $17 \mathrm{~kW} / \mathrm{m}^{2}$. This magnitude of the heat flux is far from the critical heat flux for pinus halepensis needles, which is about $10 \mathrm{~kW} / \mathrm{m}^{2}$ (Sabi et al. 2018). The ignition is controlled by a pilot flame located $1 \mathrm{~cm}$ above the sample surface (Torero 2016), and the ignition time is recorded. To take into account the heterogeneity of the fuel structure arrangement in the sample, each test is repeated three (3) times. All the measurements are made in the same day to guarantee that the tests are done on a live fuel with the same composition of organic flammable volatiles. All tests were conducted in a draft-free room with room temperatures and relative humidities in the ranges $13-19^{\circ} \mathrm{C}$, and $50-60 \%$, respectively.

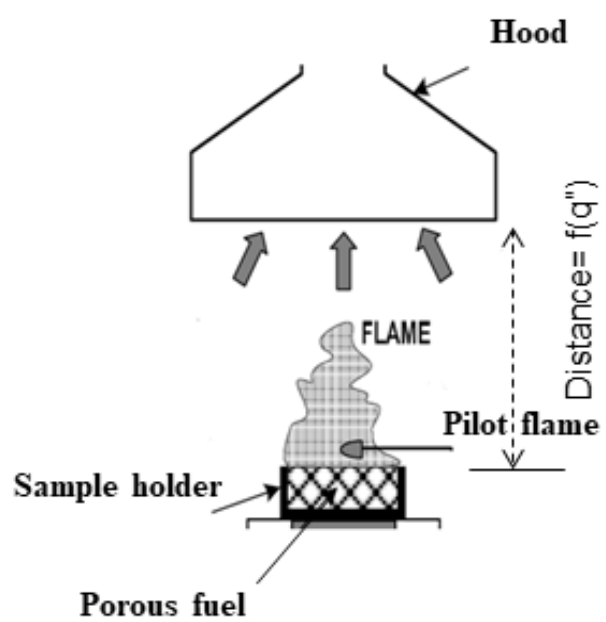

Figure 1 - A schematic representation of the experimental setup.

It is worth noticing that carrying out ignition tests on a constant mass of the sample after drying (e.g . 10g) may lead to significant errors on the ignition and combustion times. Indeed, once the sample has been dried (either partially or totally), its mass decreases, but the amount of volatile components responsible of ignition remains nearly constant in it. The drying techniques are assumed to evaporate mostly water. The release of volatile organic components (VOC) was found to increase as the temperature increases (Jervis and Rain 2015). Hence, it is expected that the MLS dryer at $80^{\circ} \mathrm{C}$ enhances the ignition time fluctuations contrary to the microwave device. Indeed, the microwave device emits radiations at a frequency of $2.45 \mathrm{GHz}$, which corresponds to the water characteristic dielectric frequency. Thus, it is mostly absorbed by the molecules of water leading to their evaporation. Then, it is expected that the release of VOC is significantly reduced with the microwave device.

If a constant part of the dried mass $m \leq m(t)$ is ignited, the remaining amount of volatiles decreases proportionally to $\mathrm{m} / \mathrm{m}(\mathrm{t})=1-\mathrm{h}$ from (1), and ignition is thus influenced by the drying process. In order to guarantee the same quantity of volatiles in the sample for each test independently of the moisture content, it is necessary to burn all the dried mass.

\subsection{The ignition process}

When a solid material, initially at ambient temperature, is subject to an external source of energy, the temperature of the exposed surface starts to increase. This moment is defined as the onset of the ignition process $(t=0)$. A series of physical and chemical phenomena are initiated as the energy reaches the surface of the material. The sample is composed of fine fuel particles arranged randomly. This yields a porosity distribution with an average value defined as the ratio of solid fuel volume to the fuel bed volume. When exposed to a heat flux, the particles are heated and loose energy by conductive and convective mechanisms (Torero et al. 2016). Thus, a solid-liquid-gas transformation occurs inducing a flammable gas/oxygen mixture at a low flammability limit and ignition temperature (Drysdale 2011). High porosity renders the overall thermal conductivity of a solid quite low making the ignition easier to happen. Therefore, auto-ignition is very difficult to occur as the condition for the

Advances in Forest Fire Research 2018 - Page 524 
emitted gas/air mixture to reach the low flammability limit and, simultaneously, the surface temperature to reach ignition temperature has a finite probability to not be realized. By definition, in the presence of a pilot source (such as a small flame, a heated wire or an electric spark) in the reactive fuel/air mixture flow, we have a piloted (or forced) ignition. A pilot source is not meant to heat the solid to generate the fuel gases nor to enhance the mixing of the fuel gases with air, but to locally induce the combustion reaction which would propagate into the mixture.

As an event, ignition occurs at a certain pronounced instant in the history of the exposure. The time to ignition in a given situation depends on three broadly grouped factors: 1) the degradative thermal response of the solid to yield the combustible gases, 2) the mixing of these gases with the oxygen (generally the oxygen of the normal air), and 3) the induction of the temperature and composition-dependent rate of the combustion reaction to a sufficiently high level to be measurable and self-supporting (Kanury 2008). The same ignition process occurs for a moist sample exposed to a heat flux, but the water evaporation complicates it. In the case of a thick fuel bed, water evaporation decreases the surface temperature and ignition can be delayed.

\section{Results and discussions}

The effect of moisture content on the ignition time is shown in Figs. 2 for fuels dried by microwave and also by the desiccator (for the data of January, only $10 \mathrm{~g}$ of the dried sample were burned). The present data are also compared with those of Trabaud (1976) for pinus halepensis. As discussed above, the MLS dryer induces a larger fluctuations of the ignition time compared to the microwave device, because of the influence of VOC release during the drying process. It is also obvious from Fig.2a that burning the full sample dried by microwave reduces signficantly the fluctuations of ignition time. Compared to the results of Trabaud where burning could not occur above a moisture of $20 \%$, the present samples were ignited even for moisture contents above $50 \%$. The heat flux received by the fuel from the epiradiator used by Trabaud might be much smaller than $17 \mathrm{KW} / \mathrm{m}^{2}$ (provided by our cone calorimeter). The heat flux provided by the epiradiator could be smaller than the critical flux for ignition, which is around $5.5 \mathrm{~kW} / \mathrm{m}^{2}$ for pinus halepensis needles (see Sabi et al. 2018). In order to answer this question, we assume the critical moisture as an exothermic/endothermic phase transition. Therefore, near the critical moisture, ignition time diverges according to (2). In Fig.2b, the same data of Fig.2a are presented in the double logarithmic plot as a function of $h_{c}-h$. The value of the critical moisture $h_{c}$ is estimated so as to obtain a linear fit with the best correlation coefficient $\left(\mathrm{R}^{2}\right)$.

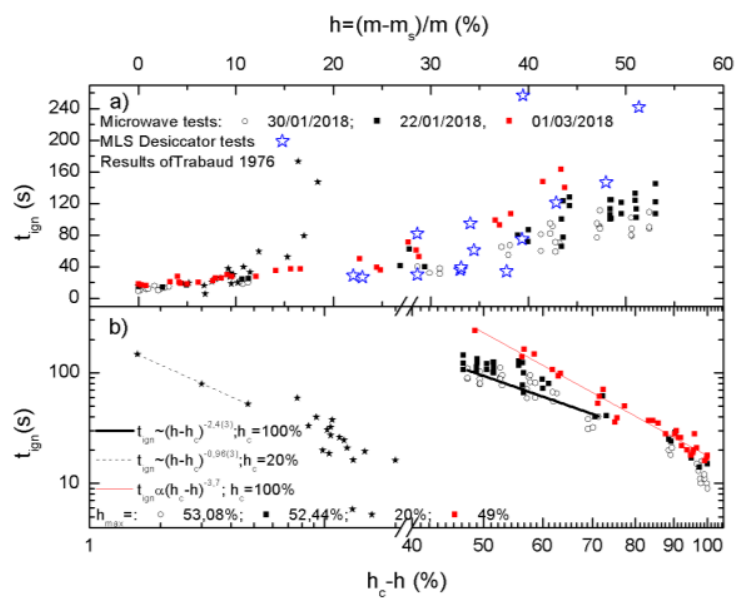

Figure 2 - a) tign vs. h for various moistures obtained by microwave and desiccator, and Trabaud's results. b) same as Fig.2a with log-log plot.

The best linear fit in Fig.2b is obtained for $h_{c}$ around $100 \%$. This means that the endothermic/exothermic phase transition occurs when the fuel mass vanishes, and only water 
evaporation occurs. Clearly, there is no critical moisture for ignition. The non flammability in the results of Trabaud at a moisture of about $\mathbf{2 0 \%}$ means that the samples were exposed to a heat flux around the critical flux for this moisture content. In addition, the power-law (2) is fitted in this figure only for three points located near the critical moisture. For each of these moisture contents, only one ignition test was successful, whereas far from the moisture threshold, in all tests, the ignition process occured. Therefore, a probabilistic behaviour occurs near the moisture threshold, which should correspond to the critical heat flux (Sabi et al. 2018).

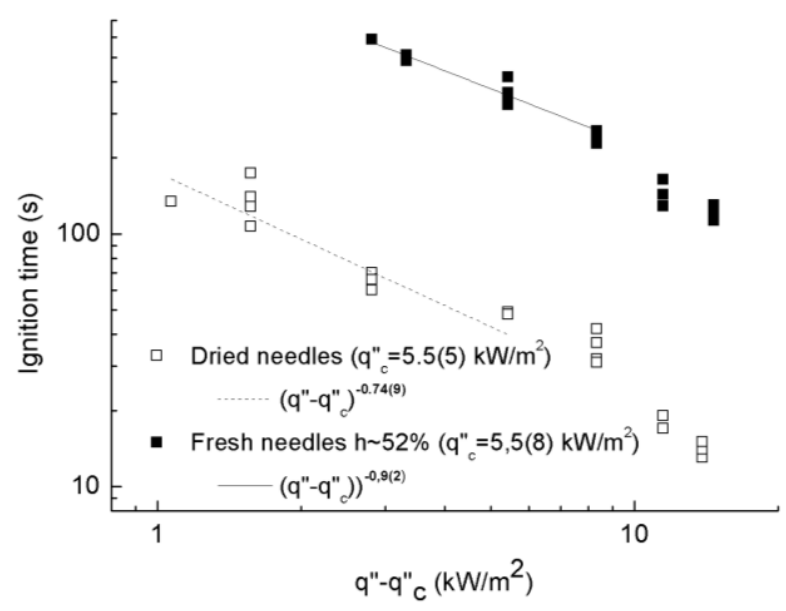

Figure 3 - A double logarithmic plot of tign $v s . q^{\prime \prime}-q^{\prime \prime}{ }_{c}$ for fresh and dry pinus halepensis needles.

In order to check whether the ignition threshold is due to the heat flux or the moisture content, a set of tests with fresh and dry samples were realized for different values of the incident heat flux $\boldsymbol{q}^{\prime \prime}$. The critical heat flux for ignition defines the endothermic /exothermic transition (Sabi et al. 2018). The ignition time behaves near this transition as:

$$
\operatorname{tign} \alpha\left(q "-q "{ }^{\prime}\right)^{-\alpha}
$$

The power exponent in Fig. 3 seems to be around -0.8 independently of the moisture content and of the fuel nature (see Sabi et al. 2018), indicating a universal phase transition (Stanley 1971). Therefore, for any moisture content, there exists a sufficiently large heat flux allowing the fuel ignition.

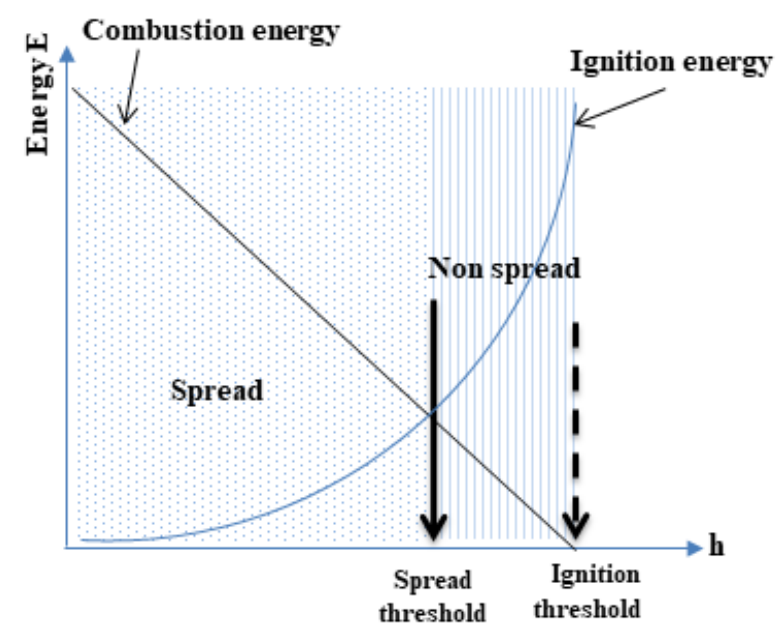

Figure 4 - A schematic representation of the ignition and combustion energies vs. the moisture content h. Ignition and spreading thresholds are clearly shown.

Advances in Forest Fire Research 2018 - Page 526 
In wildland fires, fire residence time is finite (30-40s for fine fuels) in contrast to that provided by the cone calorimeter where the radiant heat flux is maintained until the fuel ignition. Therefore, the comparison between the combustion and ignition energies leads to either spread or no spread of fire. The critical moisture for fire spread can be defined as the moisture content for which combustion and ignition energies coincide, as shown in Fig.4. This spread condition is not realistic since only a proportion of the released combustion energy is received by the fuel. This makes a much smaller critical moisture for spread. Therefore, the existence of a critical moisture is expected for wildfire spread, although it is considered as a retardant. Indeed, the moisture can make the ignition time as large as it becomes greater than the residence time of the flames to which the fuel is exposed. This corresponds to a dynamical threshold of spread as found by Zekri et al. (Zekri et al. 2005) using a Small World Network model. This model was validated by historical and experimental fires (Adou et al. 2010). Obviously, fire do not spread during winter, when the fuel is moist. Hence, the effect of moisture on the spreading phase transition is necessary to investigate both experimentally (experimental burning in fields) and theoretically (using physical models involving water desorption and evaporation).

\section{Conclusion}

The moisture threshold for ignition is examined by using the endothermic/exothermic phase transition analysis of the ignition time dependence on moisture content. Ignition tests of live pinus halepensis needles were realized using a cone calorimeter. The fuel samples were partially dried using a microwave and a desiccator. In order to avoid seasonal effects and changes in their chemical structure, live fuels were picked the same day from the same tree. The power law fit of the ignition phase transition showed no critical moisture for the fuel ignition. The critical region for ignition observed by Trabaud in 1976 corresponds to the ignition critical heat flux for the corresponsing moisture content. Further studies for other fuels wil be realized to confirm this result.

\section{References}

Adou JK, Billaud Y, Brou DA, Clerc JP, Consalvi JL, Fuentes A, Kaiss A, Nmira F, Porterie B, Zekri L., Zekri N (2010), Simulating wildfire patterns using a small-world network model, Ecol.Modelling, 221, 1463-1471.

Blundell SJ and Blundell KM (2006), 'Concepts in Thermal Physics', (Oxford University Press, Oxford).

Catchpole EA, Catchpole WR (1991), Modelling Moisture Damping for Fire Spread in a Mixture of Live and Dead Fuels, International Journal of Wildland Fire 1, 101-106.

Chandler C, Cheney P, Thomas P, Trabaud L, Williams D (1983) 'Fire in forestry. Vol. I. Forest fire behaviour and effects' (John Wiley \& Sons: New York)

Cohen J and Bradshaw B (1986) Fire behavior modeling - A decision tool. In 'Proceedings Prescribed Burning in the Midwest: State of the Art' (Ed. A. L. Koonce), University of Wisconsin at Stevens Point, Stevens Point, WI, 1-5.

Drysdale D (2011), An Introduction to Fire Dynamics, (3rd Ed. John Wiley and

Sons, Chichester)

Fletcher TH, Picket BM, Smith SG, Spittle GS, Woodhouse MM, Haake E, Weise DR (2007), Effect of moisture on ignition behaviour of moist California chaparral and Utah leaves, Combust. Sci. Technol., 179, 1183-1203.

Jervis FX and Rein G (2015), Experimental study on the burning behaviour of pinus halepensis needles using small-scale fire calorimetry of live, aged and dead samples, Fire Mater. 40, 385-395 
Kanury AM (2008), Flaming Ignition of Solid Fuels. In 'The SFPE handbook of fire protection engineering'. (3rd Eds Society of Fire Protection Engineers). (National Fire Protection Association), section 2 Chap 11.

McAllister S and Weise DR (2017) Effect of season on ignition of live wildland fuels using the forced ignition and flame spread test apparatus. Comb Sc. \& Technol., 185, 231-247

Pokrovskii V. (1979) Properties of ordered, continuously degenerate systems, Adv.Phys. 28, 595-656

Pompe A, Vines RG (1966), The influence of moisture on the combustion of leaves, Australian forestry, 30, 231-241.

Rothermel RC (1972), A mathematical model for predicting fire spread in wildland fuels. USDA For. Serv. Res. Pap. INT-115. 40.

Sabi FZ, Zekri N (2018), Ignition/non ignition phase transition (This conference)

Stanley HE (1971) 'Introduction to phase transitions and critical phenomena' (Clarendon Press Oxford)

Stauffer D, Aharony A (1992), 'Introduction to Percolation Theory', (Taylor and Francis, London).

Trabaud L (1976), Inflammabilité et combustibilité des principales espèces des garrigues de la région méditerranéenne, Oecol. Plant. 11, 117-136

Torero J (2016), Flaming ignition of solid fuels. In 'SFPE Handbook of fire protection engineering' . (Ed MJ Hurley) (Springer: Heidelberg) pp 633-661.

Van Wagner CE (1967), Seasonal variation in moisture content of eastern canadian tree foliage and the possible effect on crown fires, Canada Dept. Forestry, For.Res.Br.-1204 (Edmonton).

Weise DR, White RH, Beall FC, and Etlinger M (2005), Use of the cone calorimeter to detect seasonal differences in selected combustion characteristics of ornamental vegetation. Int. J.Wildland Fire, $14,321-338$.

Yeomans JM (1992) 'Statistical mechanics of phase transitions' (Clarendon Press Oxford)

Zekri N, Porterie B, Clerc J-P, Loraud J-C (2005) Propagation in a two-dimensional weighted local small-world network, Phys. Rev.E 71, 046121 


\title{
Literature review on the transition from smouldering to flaming fires and its application to peat fires
}

\author{
Muhammad Agung Santoso', Jiuling Yang ${ }^{2}$, Haixiang Chen ${ }^{2}$ and Guillermo Rein*1 \\ ${ }^{1}$ Department of Mechanical Engineering, Imperial College London, UK \{g.rein@imperial.ac.uk*\} \\ ${ }^{2}$ State Key Laboratory of Fire Science, University of Science and Technology of China, P.R. China
}

\begin{abstract}
Smoldering combustion is the slow, low temperature, flameless burning of porous fuels and the most persistent type of combustion phenomena. Overall, smoldering is responsible for up to $50 \%$ or more of the total burned biomass during wildfires, and it is the driving phenomenon of wildfires in peatlands. Once ignited, peat fires burn for very long periods of time (e.g., months, years) despite extensive rains, weather changes or fire-fighting attempts. In wildland fires, smouldering and flaming combustion often occur simultaneously, and one can lead to the other. Despite being a fundamental question in combustion phenomenon, firm understanding on transition from smouldering to flaming is not yet accomplished due to its complexity. At best, literatures are sparsely connected with each literature reported different variables as transition criteria (e.g. sample particle size, air velocity, oxygen concentration, and external heating). This review compiles these informations and proposes hyprothesis that feasible to be tested. Since most of the samples in the literatures represent synthetic porous fuels, the implication of proposed hypothesis of transition mechanism on natural porous fuels (e.g. peat) will be discussed.
\end{abstract}

Keywords: Smouldering, Transition to flaming, porous fuels, peatlands,

\section{Introduction}

Smouldering combustion is the slow, low temperature, flameless burning of porous fuels and the most persistent type of combustion phenomena. Overall, smouldering is responsible for up to $50 \%$ or more of the total burned biomass during wildfires, and it is the driving phenomenon of wildfires in peatlands. Once ignited, peat fires burn for very long periods of time (e.g., months, years) despite extensive rains, weather changes or fire-fighting attempts. Typical in wildland fires, smouldering and flaming combustion often occur simultaneously, and one can lead to the other. Despite being a fundamental question in the science of combustion, firm understanding is not yet accomplished due to its complexity. At best, literature on transition from smouldering to flaming are sparsely connected with each literature reported different variables as transition criteria (e.g. sample particle size, air velocity, oxygen concentration, and external heating). This review compiles these information and proposes hyprothesis that feasible to be tested. Since most of the samples in the literature represent synthetic porous fuels, the implication of proposed hypothesis of transition mechanism on natural porous fuels (e.g. peat) will be discussed.

Figure 1 shows schematic on the possible concurrent events of smouldering peat and flaming surface vegetation. The complex interaction between these two combustion mechanisms and surrounding conditions could lead to the transition from smouldering to flaming combustion, and vice versa. 


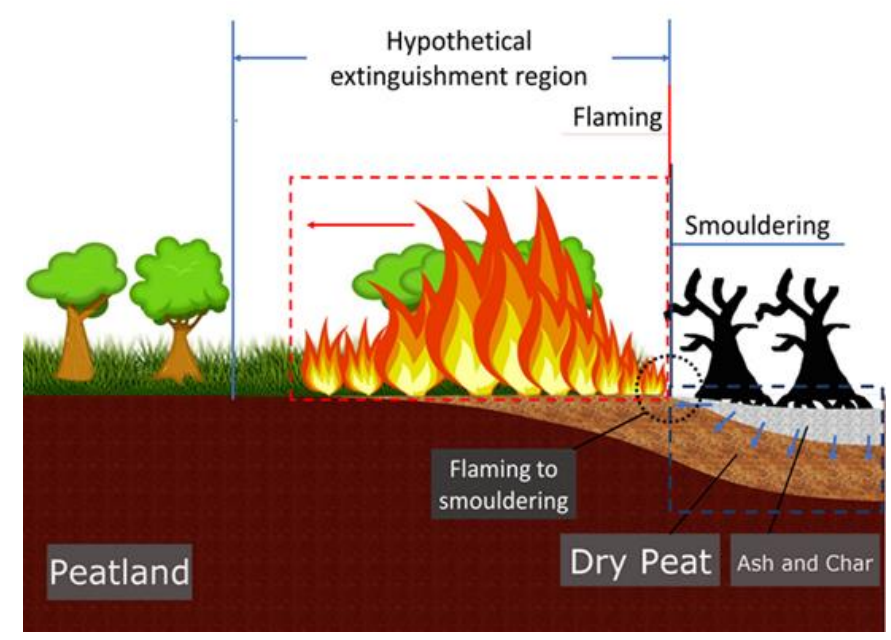

Figure 1 - Schematic on occurrence of smouldering initiation by flaming surface fires in peatland

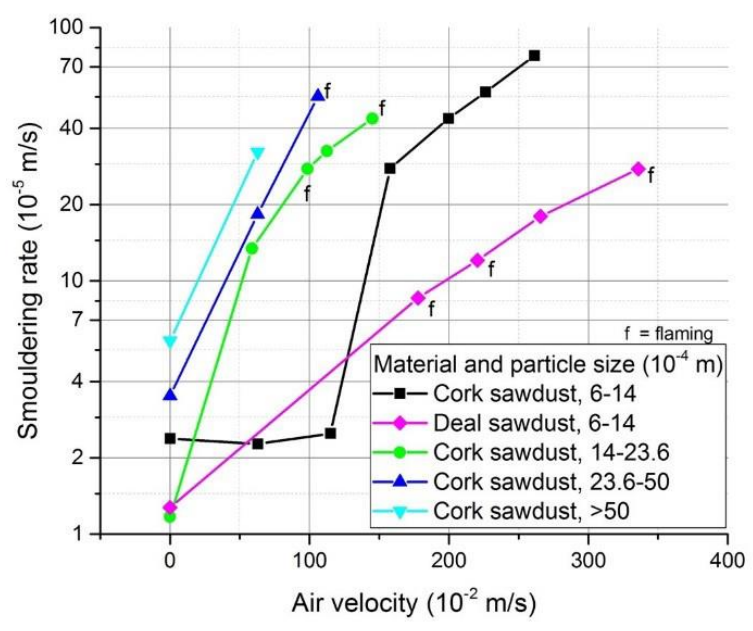

Figure 2 - Smouldering rate as a function of air velocity in a forward smouldering in Cork and Deal sawdust with different particle size (Palmer 1957)

The persistent smouldering of thick fuels (ie, tree branches, trunks) typically observed for days after a flaming wildfire has passed by is called residual smouldering combustion and has received some attention in the literature, e.g. Bertschi et al. (2003). On the other hand, transition to flaming offers the means for flaming fronts to re-establish in unexpected locations (e.g., across a fire break) and at unexpected times (e.g., long after burn out of the initial flaming front). Even if the transition to flaming is less often observed in wildfires than residual smouldering combustion because it requires less usual thermodynamic conditions, the transition to flaming is possible for example under enhanced oxygen supply (i.e., strong winds)

\subsection{Smouldering and Flaming Combustion}

The core of any combustion process is a global exothermic reaction that results in the release of heat, and both gaseous and solid products. In its simplest form, solid combustion can be approximated by two lumped chemical pathways: pyrolysis (Eq. 1) followed by oxidation (Eq. 2 or 3).

Pyrolysis:

Biomass (solid) + Heat $\rightarrow$ Pyrolyzate (gas) + Char (solid) + Ash (solid)

Heterogeneous oxidation:

Char (solid) $+\mathrm{O}_{2} \rightarrow$ Heat $+\mathrm{CO}_{2}+\mathrm{H}_{2} \mathrm{O}+$ other gases + Ash (solid)

Gas-phase oxidation:

Pyrolyzate (gas) $+\mathrm{O}_{2} \rightarrow$ Heat $+\mathrm{CO}_{2}+\mathrm{H}_{2} \mathrm{O}+$ other gases

Pyrolysis results in gaseous and solid products. Both the pyrolysate and the char are susceptible to subsequent oxidation. Char oxidation (Eq. 2) is a heterogeneous reaction and takes place on the char produced by the pyrolysis reaction and leads to smouldering combustion. Pyrolysate oxidation (Eq. 3) is airborne and takes place in the gas phase, which is a homogenous reaction and leads to flaming combustion. 


\section{Early Findings of transition to flaming}

One of the first recorded results on transition to flaming was conducted by Palmer (1957) with several types of dust as sample material. In his experiments, flaming did not develop from smouldering experiments with mean particle diameter less than $0.1 \mathrm{~cm}$ (Figure 2).

The influences of ambient oxygen concentration on transition phenomenon was studied by OrtizMolina et al. (1979). A synthetic porous fuel were placed in a chamber in which gas composition and pressure can be varied. Transition to flaming occurred at oxygen concentration ranged from 0.17 to 0.37 mole fraction for samples of relatively large size $(50 \times 120 \times 450 \mathrm{~mm})$. The samples in the form of small cylinders (18 $\mathrm{mm}$ in diameter) did not experienced transition. In another study by Roger and Ohlemiller (1980), the importance of oxygen supply on transition to flaming was confirmed. They investigated the opposed smouldering of flexible synthetic porous fuel in variable oxygen concentration. From their experiment, the transition occurred in oxygen mole fraction more than 0.6. The higher oxygen concentration was related to the weak response of opposed smouldering to air flow velocity. Ohlemiller (1990) investigated the influence of air flow velocity in forward and opposed smouldering of cellulosic insulation. He found that the opposed smouldering has weak response to air velocity increase, while forward smouldering increases significantly with flow velocity. Moreover, the transition to flaming was identified to only occur in forward smouldering, in range of air flow velocity up to $5 \mathrm{~m} / \mathrm{s}$.
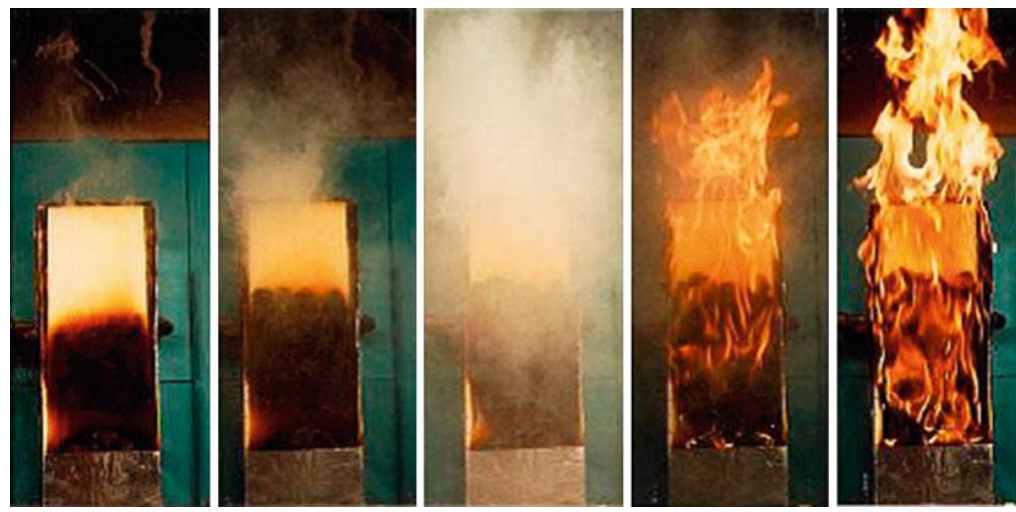

Figure 3 - Series of photographs of a combustion experiment illustrating the transition to flaming in a smouldering foam slab 40 cm high under external forced flow (Photo by group of Prof. Carlos Fernandez-Pello, University of California at Berkeley). After 1 h of smouldering only half the sample has burned (Photo 1, far left). When the rapid transition to flaming takes place (Photos 2 and 3), the whole sample is engulfed in flames in just a few seconds (Photos 4 and 5 , far right)

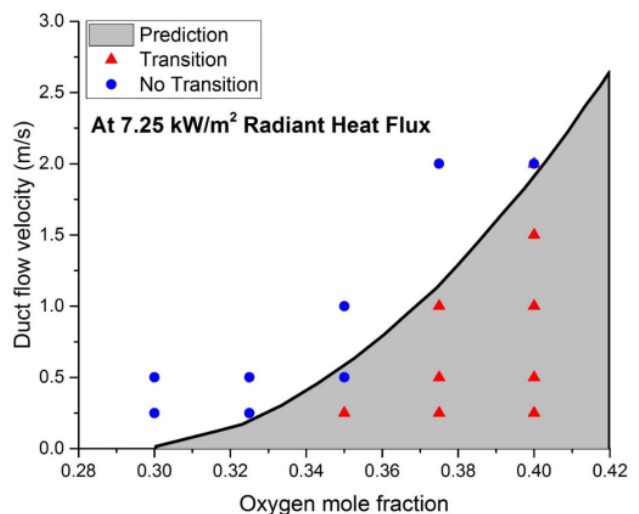

Figure 4 - Comparison of experimental results with the energy balance analysis prediction of transition/no transition regions as a function of oxygen mole fraction (Bar-Ilan et al. 2005)

\section{Current understanding of transition to flaming}

The most recent findings on the mechanism of transition from smouldering to flaming were mainly derived from the collective works of the Combustion and Fire Processes Lab of the University of California at Berkeley, USA. The works were carried out on syntethic porous media (i.e. PU foam) with variable oxidizer supply and radiant heat flux. Figure 3 shows snapshots of an experiment on the transition to flaming in a foam slab.

Bar-Ilan et al. (2005) carried out a simplified energy balance analysis by viewing the transition to flaming as an autoignition process. This analysis well predict the boundary between transition and notransition occurrences from experiments (Figure 4). Later, experiments by Bar-Ilan et al. (2005) and Putzeys et al. (2007) were reproduced computationally by Dodd et al. (2012) using two dimensional numerical transport model with eight-step reaction mechanism. Thermal non-equilibrium approach 
was taken to consider distinct temperature of solid and gas species. Even if there are some difference between experimental and simulation results, they relatively comparable and provide the basis for the development of predictive model of transition to flaming.

Char oxidation has been considered to lead to transition to flaming. This is from the argument that char oxidation produced the required heat to initiate combustion of gaseous fuel produced by solid reactions. Aldushin et al. (2006) numerically investigate the role of char oxidation in triggering transition to flaming. When smouldering front has been steadily propagate to a critical distance, char oxidation caused the sudden increase of temperature at reaction front. This increase is argued to be the initiation of flaming. In other study, char oxidation also considered to be an essential triggering mechanism of transition to flaming (Torero and Fernandez-Pello 1995, Tse et al. 1996, Putzeys et al. 2007, Dodd et al. 2012). In some literature, char oxidation also considered to produce the required gaseous fuel (Tse et al. 1996, Putzeys et al. 2007, Hagen et al. 2015). Investigation on which reaction that produce the gaseous fuel is where kinetics come into play. This is where further test on previous proposed mechanism is needed to fill the gap. It is whether pyrolysis or char oxidation is the primary source of gaseous fuel. By considering the different endothermicity and oxygen dependency of those reactions, this investigation could contribute to a better understanding of kinetics that lead to transition to flaming.

Experimental study on transition to flaming on cotton was conducted by Hagen et al. (2015). The boundary conditions used were open boundary and a lightweight concrete block covering one side of the sample. The occurrence of transition resulted from the difference in the smouldering velocity in the cotton. This difference exist due to the cover of the lightweight concrete. Thus, the existence of cover is important in the occurrence of transition in this study, implying the asymmetrical boundary condition.

Recent computational study on transition to flaming was carried out by Yang et al. (in press). They computationally reproduced forward horizontal smouldering of cellulosic insulation experiments of Ohlemiller (1990). The proposed computational model is two dimensional multiphase formulations with three-step solid decomposition reaction and one-global gas phase reaction. Three condensed phase species (solid sample, char, and ash) and five gas species (pseudo fuel gas species, $\mathrm{O}_{2}, \mathrm{~N}_{2}, \mathrm{CO}_{2}$, and $\mathrm{H}_{2} \mathrm{O}$ ) were used in the model. The model shows the transition to flaming occurence that located on the sample surface, in which the hotter surface char layer acts as an ignition source while the combustible gas fuel produced by surface char oxidation and solid sample pyrolysis. This work agrees well with Ohlemiller (1990) in that it well predicts smouldering velocity responses to wind velocity and bulk density.

\section{References}

Aldushin, A. P., Bayliss, A., Matkowsky, B. J., "On the transition from smoldering to flaming," Combustion and Flame 145, p. 579-606, 2006.

Bar-Ilan, A., Putzeys, O. M., Rein, G., Fernandez-Pello, C., Urban, D. L., "Transition from forward smoldering to flamingin small polyurethane foam samples," in Proceedings of the Combustion Institute, 2005.

Bertschi, I., Yokelson, R. J., Ward, D. E., Babbitt, R. E., Susott, R. A., Goode, J. G., Hao, W. M., "Trace gas and particle emissions from fires in large diameter and belowground biomass fuels," Journal of Geophysical Research, Vol. 108, No. D13, 8472, doi:10.1029/2002JD002100, 2003.

Dodd, A. B., Lautenberger, C. , Fernandez-Pello, A. C., "Computational modeling of smolder combustion and spontaneous transition to flaming," Combustion and Flame, vol. 159, p. 448, 2012. 
Hagen, B. C., Frette, V., Kleppe, G., Arntzen, B. J., "Transition from smoldering to flaming fire in short cotton samples with asymmetrical boundary conditions," Fire Safety Journal, vol. 71, p. 69, 2015.

Ohlemiller, T. J., "Forced Smolder Propagation and the Transition to Flaming in Cellulosic Insulation," Combustion and Flame, vol. 81, p. 354, 1990.

Ortiz-Molina, M. G., Toong, T.-Y., Moussa, N. A., Tesoro, G. C., "Smoldering Combustion of Flexible Polyurethane Foams and Its Transition to Faming or Extinguishment," Colloquium on Fire and Explosion, 1979

Palmer, K. N., "Smouldering Combustion in Dusts and Fibrous Materials," Combustion and Flame, vol. 1, no. 2, p. 129, 1957.

Putzeys, O., Bar-Ilan, A., Rein, G., Fernandez-Pello, A. C., Urban, D. L., "The role of secondary char oxidation in thetransition from smoldering to flaming," in Proceedings of the Combustion Institute, 2007.

Rogers, F. E. and Ohlemiller, T. J., "Smolder Characteristics of Flexible Polyurethane Foams,” Journal of Fire and Flammability, vol. 11, pp. 32-44, 1980.

Torero, J. L., Fernandez-Pello, A. C., "Natural Convection Smolder of Polyurethane Foam, Upward Propagation," Fire Safety Journal 24, pp. 35-52, 1995.

Tse, S. D., Fernandez-Pello, A. C., Miyasaka, K., "Controlling Mechanisms in the Transition from Smoldering to Flaming of Flexible Polyurethane Foam," Twenty-Sixth Symposium (International) on Combustion/The Combustion Institute, pp. 1505-1513, 1996.

Yang, J., Liu, N., Chen, H., "Smoldering and Spontaneous Transition to Flaming over Cellulosic Insulation," Proceedings of The Combustion Institute, in press. 


\title{
Measurement of pyrolysis products from mixed fuel beds during fires in a wind tunnel
}

\author{
David R. Weise ${ }^{1 *}$; Timothy J. Johnson ${ }^{2}$, WeiMin $\mathrm{Hao}^{3}$, Marko Princevac ${ }^{4}$, Nicole Scharko ${ }^{2}$, \\ Ashley Oeck ${ }^{2}$, Tanya L. Myers ${ }^{2}$, Steve Baker ${ }^{3}$, Emily Lincoln $^{3}$, AmirHessam Aminfar ${ }^{4}$ \\ ${ }^{1}$ USDA Forest Service, PSW Research Station. 4955 Canyon Crest Dr., Riverside, California, USA \\ 92507,\{dweise@fs.fed.us*\} \\ 2 Pacific Northwest National Laboratory, Richland, Washington, USA 99354, \\ \{timothy.johnson@pnnl.gov\} \\ ${ }^{3}$ USDA Forest Service, Rocky Mountain Research Station, Missoula, Montana, , \\ \{whao@fs.fed.us\} \\ ${ }^{4}$ Mechanical Engineering Dept., University of California-Riverside, Riverside, California, USA, \\ \{marko.princevac@ucr.edu,aamin006@ucr.edu\}
}

\begin{abstract}
Pyrolysis of intact wildland fuels in the southern United States is being measured at bench, wind tunnel, and field scales as part of a larger research project to measure and model pyrolysis of wildland fuels to improve models used to predict prescribed fire behavior. Traditional pyrolysis experiments typically use dried, ground samples in either an inert or oxidizing environment subject to uniform heating and heat transfer. Fletcher and others are presenting results of pyrolysis experiments using a flat-flame burner to heat intact foliage from 14 species of plants native to the southern U.S. at this conference. The fuel beds in prescribed burns in southern pine forests are composed of a mixture of dead pine needles and a large variety of herbaceous and woody plants. We have burned 73 fuel beds composed of Pinus palustris needles and mixtures of Lyonia lucida, Ilex glabra, and Vaccinium darrowii plants in a wind tunnel facility with and without a moderate wind speed of $\sim 1 \mathrm{~m} \mathrm{~s}-1$. The flame from the spreading fire is the heat source for the pyrolysis experiment. Canister samples of pyrolysis and flaming combustion gases within the fuel bed have been collected for offline analysis. Open path spectrometers nonintrusively measured composition of pyrolysis gases in real-time. Thermocouples, a Schmidt-Boelter heat flux sensor, a nadir thermal IR camera, and background-oriented Schlieren photography estimated heat transfer and air flow around the plants. This presentation will present results of this ongoing work.
\end{abstract}

Keywords: pyrolysis, longleaf, fetterbush, inkberry, sparkleberry, background-oriented Schlieren

\section{Introduction}

Wildland fire is an important component of many North American ecosystems and has been used by humans to accomplish various objectives for several thousand years. Prescribed burning in the southern United States is an important tool used by the Department of Defense and other land managers to accomplish several objectives including hazardous fuel reduction, wildlife habitat management, critical training area maintenance, ecological forestry and infrastructure protection. The vegetation on Department of Defense installations is heterogeneous, unlike the homogeneous fuel beds assumed by current operational fire spread models. These models do not contain fundamental descriptions of chemical reactions and heat transfer processes necessary to predict fire spread and energy release needed for process-based fire effects models. To improve prescribed fire application to accomplish desired fire effects and limit potential escapes, an improved understanding is needed of the fundamental processes related to pyrolysis and ignition in heterogeneous fuel beds of live and dead fuels. A large project consisting of measurement and modeling of pyrolysis from common wildland fuels of the southern United States is currently underway (Weise et al. 2018). Details of the bench-

Advances in Forest Fire Research 2018 - Page 534 
scale measurement of pyrolysis products from these fuels (individual leaves) can be found in (Fletcher et al. 2018; Safdari et al. 2018). A subset of the fuels have been burned as fuel beds approximating natural fuel beds in a small wind tunnel where similar pyrolysis measurements have been made. This paper presents details of the wind tunnel experiment and some preliminary results.

\section{Methods}

Approximately 100 experiments are planned for the low speed wind tunnel located at the Riverside Fire Laboratory (RFL) (Figure 1). The initial construction and subsequent modification to provide precise control of temperature and humidity within the test section are described elsewhere (Lozano 2011; Bartolome 2014). Fuel beds $2 \mathrm{~m}$ long and approximately $1 \mathrm{~m}$ wide composed of longleaf pine needles and various combinations of fetterbush, sparkleberry, and inkberry (Table 1) will be burned with nominal wind speeds of 0 and $1 \mathrm{~m} \mathrm{~s}^{-1}$. Pyrolysis gases were sampled in real-time using a variety of methods and instruments (Figure 2). A Bruker Tensor 37 FTIR spectrometer ${ }^{1}$ was deployed; note that this is the same device used in conjunction with the flat-flame burner measurements performed at BYU (Fletcher et al. 2018; Safdari et al. 2018). For the RFL experiments, the resolution was set to 4 $\mathrm{cm}^{-1}$, the acquisition mode was set to double sided, forward-backward, the apodization function was Blackman-Harris 3-Term, and phase correction mode was Mertz (1967) with a zerofilling factor of 2. In order to increase temporal resolution, the number of scans was set to 1 with continuous measurements to obtain a spectral time resolution of 0.2 seconds. This instrument configuration is referred to as an "extractive FTIR" because it consists of a probe with $3 / 8$ " metal tubing connected to extract the gas into the cell using a roughing pump. The probe was placed above a plant during measurements (Figure 3) and gases were extracted from the wind tunnel into the gas cell before, during and after the flame had passed the plant. Absorbance spectra $(A)$ were collected in real time where $I_{S}$ represents the intensity of the single beam spectrum of the sample during smoke measurement and $I_{0}$ is the single beam spectrum of the of the reference measurement (Eq. (1)). The reference measurement was collected when the instrument and gas cell were purged with high purity nitrogen gas; $\mathrm{N}_{2}$ does not absorb in the IR.

Table 1 - Southern plant species used in wind tunnel pyrolysis experiments.

\begin{tabular}{ll}
\hline Common name & Scientific name $^{1}$ \\
\hline Inkberry & Ilex glabra $(\mathrm{L}$.$) A. Gray$ \\
Fetterbush & Lyonia lucida (Lam.) K. Koch \\
Sparkleberry & Vaccinium arboreum Marshall \\
Darrow's blueberry & Vaccinium darrowii Camp "Rosa's Blush" \\
Longleaf pine litter & Pinus palustris Mill. \\
\hline
\end{tabular}

1. USDA, NRCS. 2018. The PLANTS Database (http://plants.usda.gov, 10 January 2018). National Plant Data Team, Greensboro, NC 27401-4901 USA; Radford, A.E., Ahles, H.E., Bell, C.R. 1968. Manual of the vascular flora of the Carolinas, University of North Carolina Press, Chapel Hill, NC

${ }^{1}$ The use of trade or firm names in this publication is for reader information and does not imply endorsement by the U.S. Department of Agriculture of any product or service. 


$$
A=-\log \left(\frac{I_{S}}{I_{0}}\right)
$$

The other FTIR instrument used for gas phase measurements was a Bruker OPAG-22, an open path gas analyzer previously used to measure smoke from prescribed burns at Ft. Jackson, South Carolina (Akagi et al. 2014). Unlike the extractive FTIR, this instrument is non-disrupting since it does not remove any of the gases from the wind tunnel, i.e. it does not perturb the sample. The OPAG was mounted on a tripod on one side of the wind tunnel with the glow bar IR source on the opposite side. The path between the OPAG and the source was 1.2 meters and it was directly in line with a row of plants (Figure 5). The OPAG is equipped with a $\mathrm{KBr}$ beamsplitter and a Stirling-cycle cooled mercury cadmium telluride (MCT) detector. Interferograms were collected in the range of 4000 to $0 \mathrm{~cm}^{-1}$ at a resolution of $2 \mathrm{~cm}^{-1}$ and at an acquisition mode set to double-sided forward-backward. To achieve better time resolution between measurements, interferograms were converted to spectra via post process procedures. The apodization function and phase correction mode were as above. The number of scans per measurement was set to 1 and the measurements were repeatedly collected to obtain a time profile with a resolution (time between spectra) of 0.53 seconds. The third instrument deployed was the TELOPS, an infrared hyperspectral imaging system that has high spectral and spatial resolution. Due to interference from the flame, the TELOPS will not be discussed further. Canister gas samples were collected for offline analysis using gas chromatography. An array of 9 stainless steel tubes were inserted vertically into the fuel bed (Figure 4). A sample of the flaming gases was collected in one canister and a sample of pyrolysis gases was collected from the other 6 tubes sequentially as the flame front moved horizontally along the fuel bed.

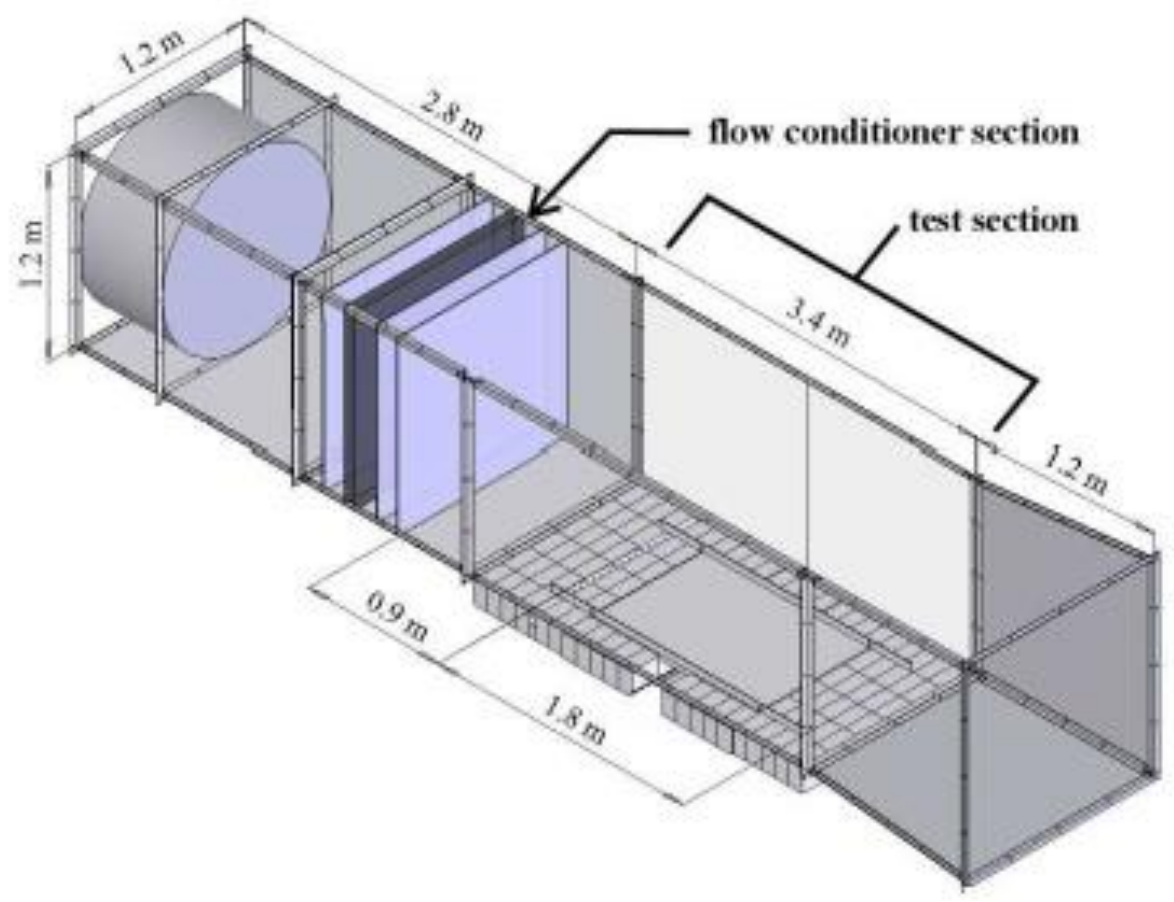

Figure 1 - Low speed wind tunnel at Riverside Fire Laboratory. 


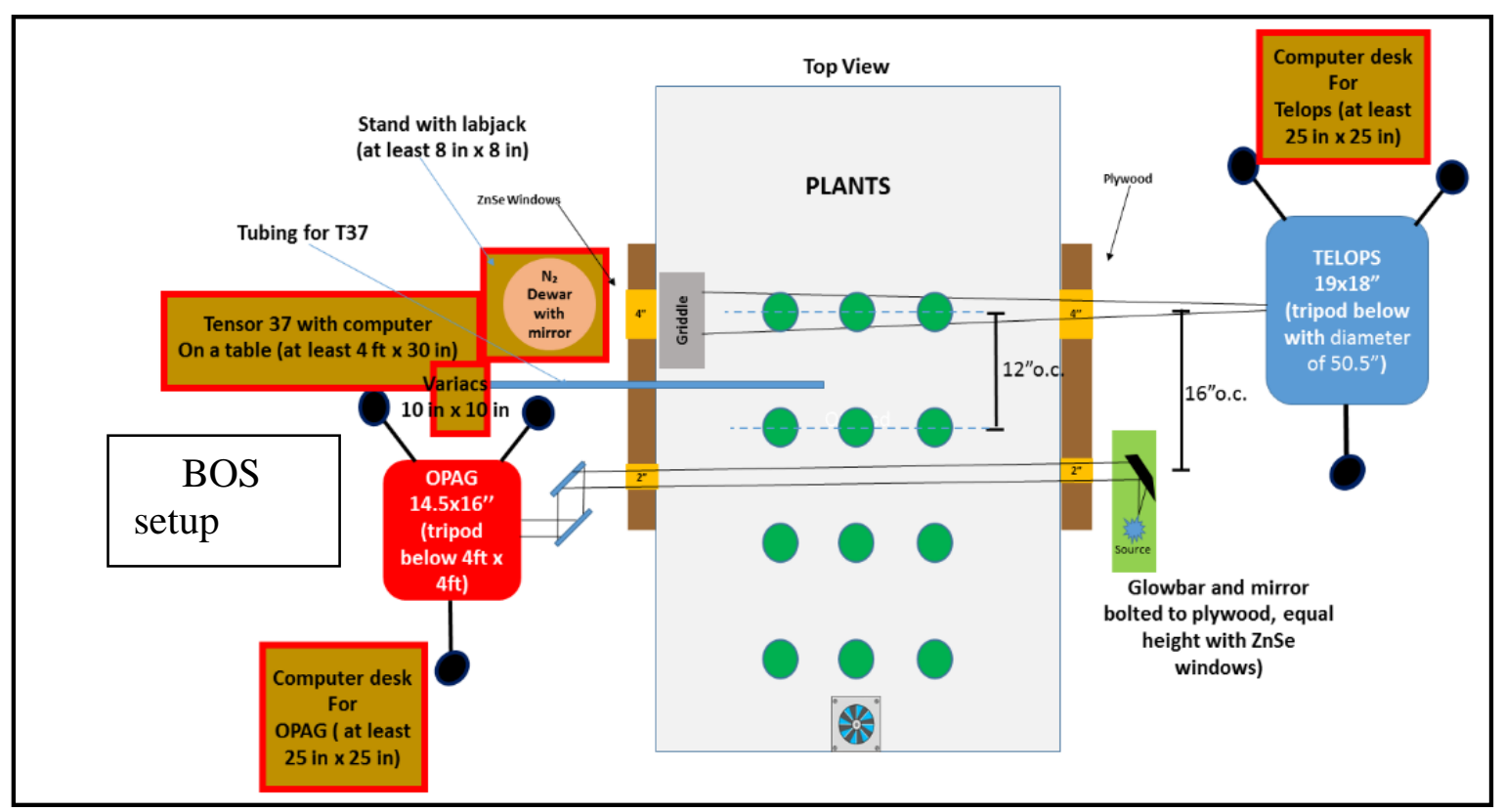

Figure 2 - Setup of 3 instruments used to measure composition of gaseous pyrolysis products in mixed fuel beds of longleaf pine needles and small shrubs.

In order to describe the environment and fuel properties as the plants were pyrolyzing, mass of a single plant, air temperature and relative humidity within the wind tunnel and in the larger combustion room were sampled at $1 \mathrm{hz}$ during each experimental fire. While we have used a variety of techniques to describe the flow field around experimental laboratory fires (Zhou et al. 2003; Lozano et al. 2010; Maynard et al. 2016), the use of smoke tracers and introduced particles in the present experiment was precluded by the gas sampling objective. The TPIV technique could not be used because the large glass windows in the wind tunnel are opaque to the infrared spectrum. The backgound-oriented Schlieren (BOS) approach was selected to determine the flow field in the flame and surrounding the plants (Richard and Raffel 2001; Meier 2002; Raffel 2015). The BOS setup (Figure 5) was located upwind of the PNNL gas sampling instruments. Total and radiant heat fluxes at the top of the fuel bed were measured using a Schmidt-Boelter type of sensor. A stereo pair of vertical photographs of each fuel bed were taken before and after each experimental fire to develop 3-D images of the fuel bed (Figure 6). A longwave infrared (LWIR) camera provided a nadir view of the fuel bed and the measured radiance from the fuel bed will be converted into a fuel surface temperature (Figure 7).
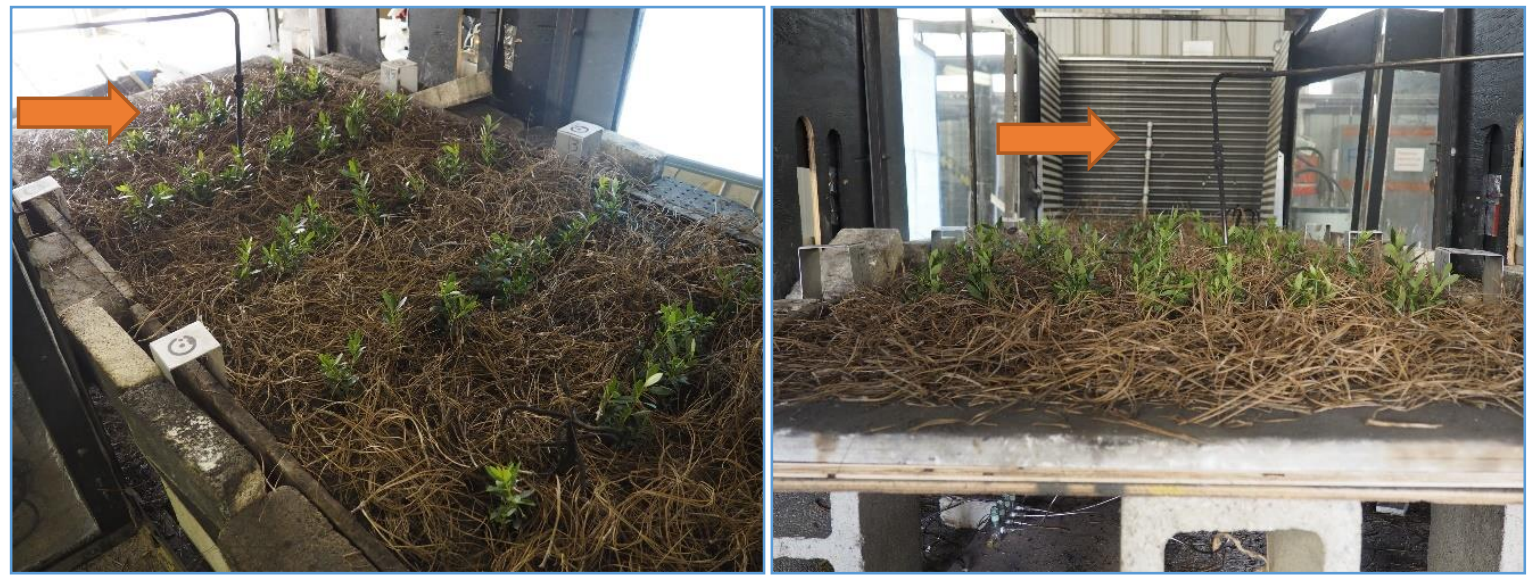

Figure 3 - Fuel bed composed of longleaf pine needles and Lyonia lucida plants. Sample probe to collect pyrolysis gases for the Bruker Tensor 37 is indicated by the arrow. 

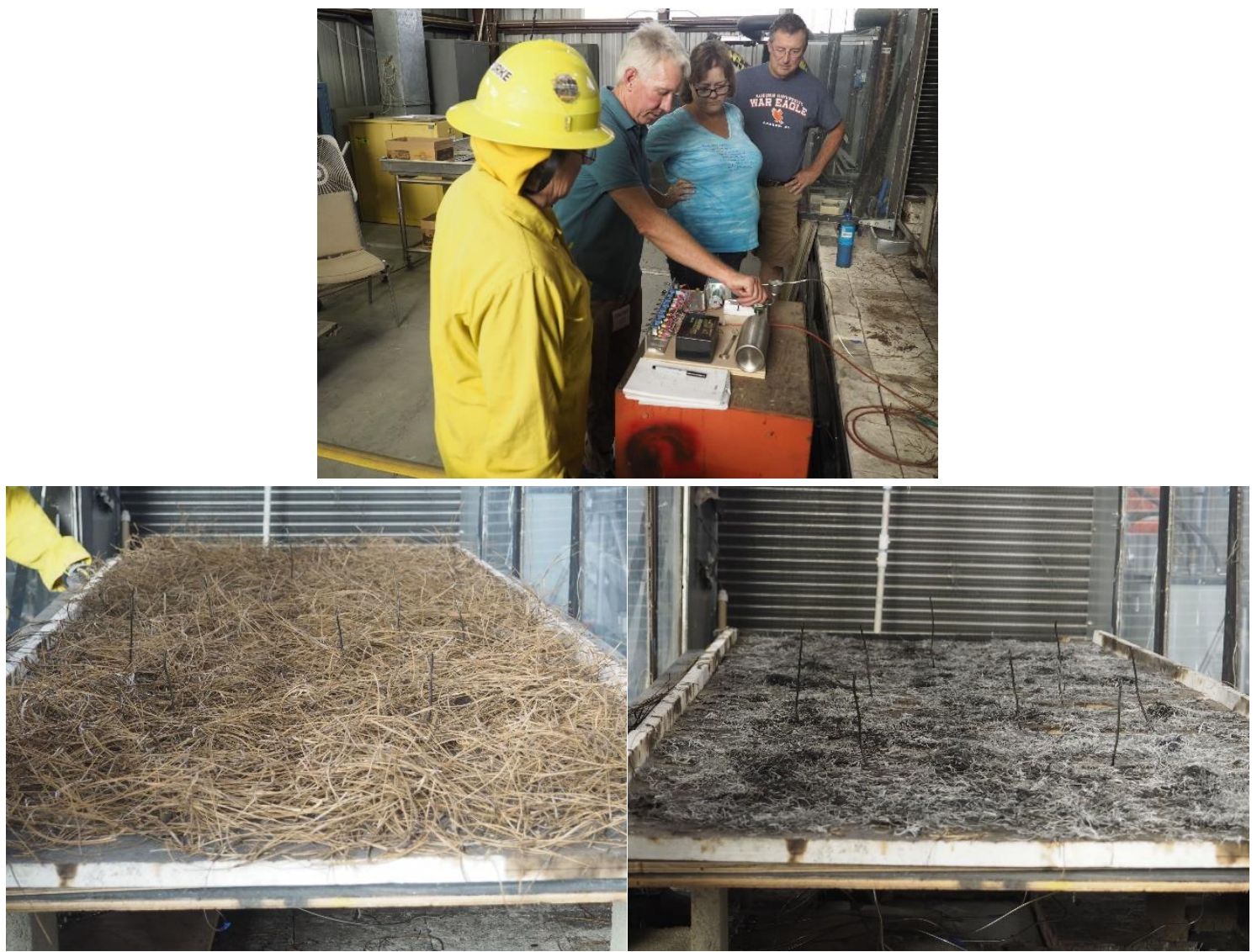

Figure 4 - Canister gas-sampling system with manifold (top) used to sample pyrolysis and flaming combustion gases from longleaf pine needle fuel beds through stainless steel tubes (bottom).

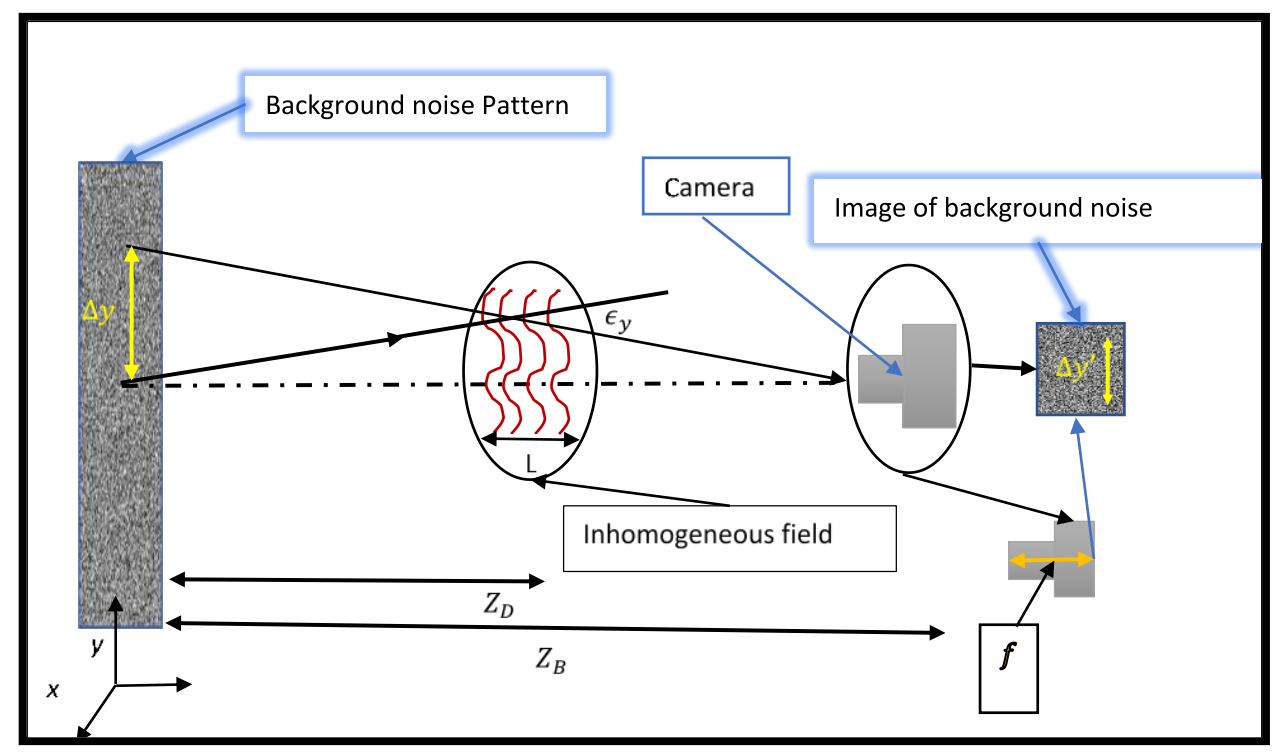

Figure 5 - Simple schematic of background-oriented Schlieren configuration used to nonintrusively estimate the flow field surrounding pyrolyzing plants. $Z_{D}$ is the distance of the flame from the background noise pattern, $Z_{B}$ is the distance of the camera lens from the background, $\epsilon_{y}$ represents the deflection angle caused by the flame-generated distortion, $L$ is the flame zone width, $f$ is the focal length of the camera, $\Delta y^{\prime \prime}$ represents displacement in the camera sensor plane and $\Delta y$ represents displacement in the background plane. 


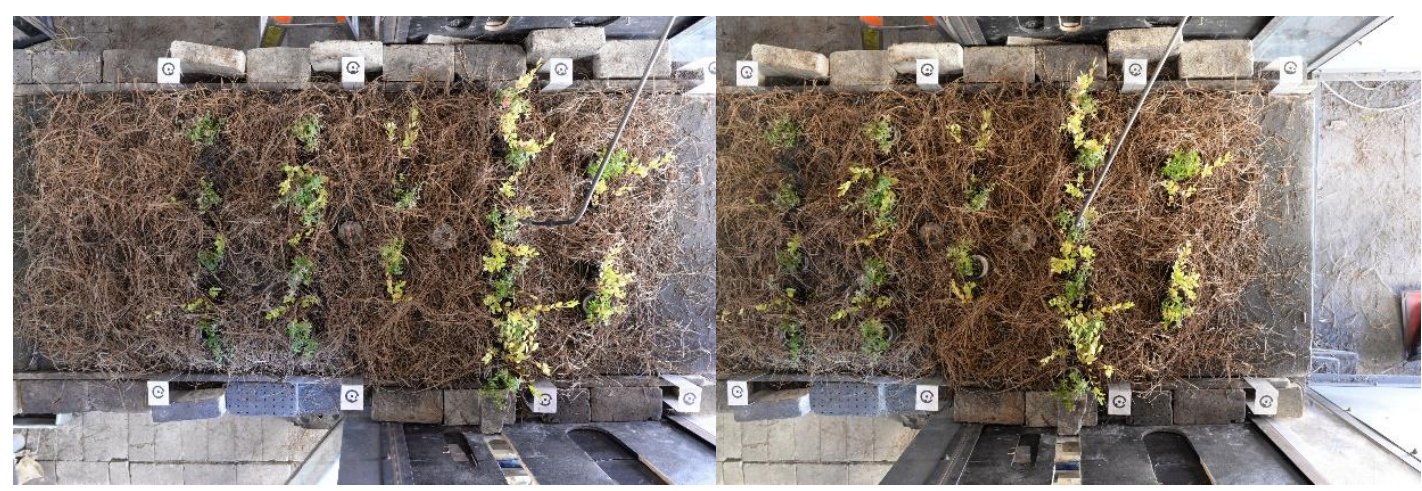

Figure 6 - Vertical stereo photographs of wind tunnel fuel bed used to provide 3D image.

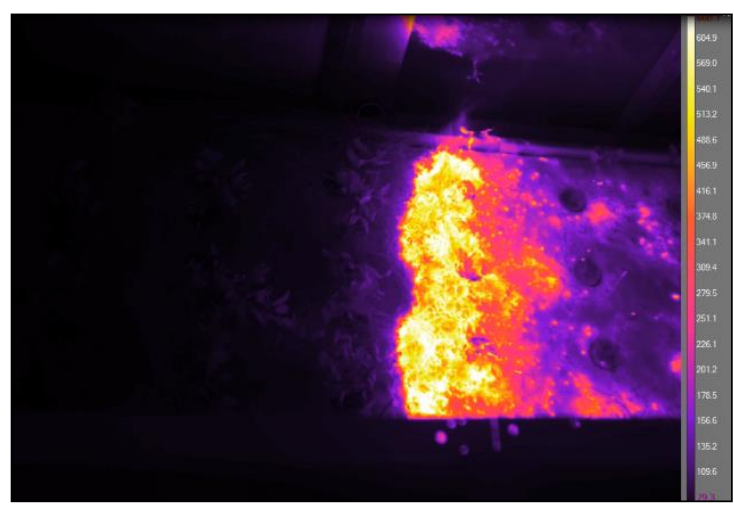

Figure 7 - Thermal infrared nadir view of fire spreading through fuel bed of longleaf pine needles and fetterbush shrubs in the RFL wind tunnel. Estimated temperature in ${ }^{\circ} \mathrm{C}$.

\section{Preliminary Results}

Prior to beginning the wind tunnel experiments, the canister gas sampling setup and protocol was tested. Fires 3 and 4 consisted of longleaf pine needles only; needles and live plants comprised the fuel bed of fires 5 and 6 . The composition of the gases from 6 canisters and the phase (pyrolysis or flaming combustion) was measured in ppm and converted to proportions to facilitate comparison since the data are compositional in nature (van den Boogaart and Tolosana-Delgado 2013). The proportions of CO, $\mathrm{H}_{2}, \mathrm{CH}_{4}$ and $\mathrm{C}_{2} \mathrm{H}_{6}$ were generally higher in the pyrolysis phase (Table 2) which is consistent with current understanding and indicated that the sampling system and protocol were appropriate. The modified combustion efficiency $\left(\Delta \mathrm{CO}_{2} /\left(\Delta \mathrm{CO}_{2}+\Delta \mathrm{CO}\right)\right)$ of the pyrolysis samples were much lower than is typical for flaming combustion--another indication of elevated pyrolyzate concentrations. The use of this efficiency in the pyrolysis environment is questionable; however, the ratio is still useful. The flaming samples had typical emissions concentrations for the gases measured. The pine needle test fire results provide good background gas concentrations for that component of the fuel beds.

Table 2 - Sampled pyrolysis and combustion gases during preliminary testing of canister gas sampling system for wind tunnel experiment. Gas composition expressed as proportions.

\begin{tabular}{|l|l|l|l|l|l|l|l|}
\hline Fire & Phase & $\mathrm{CO}_{2}$ & $\mathrm{H}_{2}$ & $\mathrm{CO}$ & $\mathrm{CH}_{4}$ & $\mathrm{C}_{2} \mathrm{H}_{6}$ & $\mathrm{MCE}$ \\
\hline 5 & Pyro & 0.770755 & 0.039321 & 0.172521 & 0.016297 & 0.001105 & 0.82 \\
\hline 6 & Pyro & 0.786460 & 0.036779 & 0.161488 & 0.014368 & 0.000905 & 0.83 \\
\hline 5 & Flam & 0.924032 & 0.009898 & 0.061951 & 0.003959 & 0.000160 & 0.94 \\
\hline 6 & Flam & 0.964003 & 0.003368 & 0.031151 & 0.001421 & 0.000058 & 0.97 \\
\hline 3 & Flam & 0.898100 & 0.015933 & 0.080057 & 0.005687 & 0.000223 & 0.92 \\
\hline 4 & Flam & 0.852222 & 0.030290 & 0.108157 & 0.008982 & 0.000349 & 0.89 \\
\hline
\end{tabular}


To date, seventy-one wind tunnel experiments were conducted in November 2017 and Janaury 2018 at RFL (Table 3). Forty-two of the fires were burned without wind. Wind increased the rate of spread appreciably which reduced the amount of time available to capture the pyrolysis gases. Gas samples were collected in canisters and are being processed at the Missoula Fire Sciences Lab. PNNL is analyzing the data collected by the two FTIR spectrometers.

Preliminary results from the BOS analysis to describe the fluid flow around the plants are promising. The images below are for the flame burning the pine needles fuels (Figure 8). The poster will contain more imagery and measurements resulting from this technique as well as information on the pyrolysis and flaming gases.

Table 3 - Brief description of wind tunnel experimental fires completed to date.

\begin{tabular}{|c|c|c|c|c|}
\hline Month & $\begin{array}{c}\text { NoWind/ } \\
\text { Wind }\end{array}$ & $\begin{array}{c}\text { Rate of spread } \\
(\mathrm{m} / \mathrm{s})\end{array}$ & $\begin{array}{c}\text { Needle } \\
\text { FMC }(\%)\end{array}$ & $\begin{array}{c}\text { Foliage } \\
\text { FMC }(\%)\end{array}$ \\
\hline Nov. 2017 & $42 / 8$ & 0.0057 & 10.3 & 112.9 \\
\hline Feb. 2018 & $0 / 21$ & 0.0184 & 10.6 & 122.9 \\
\hline
\end{tabular}
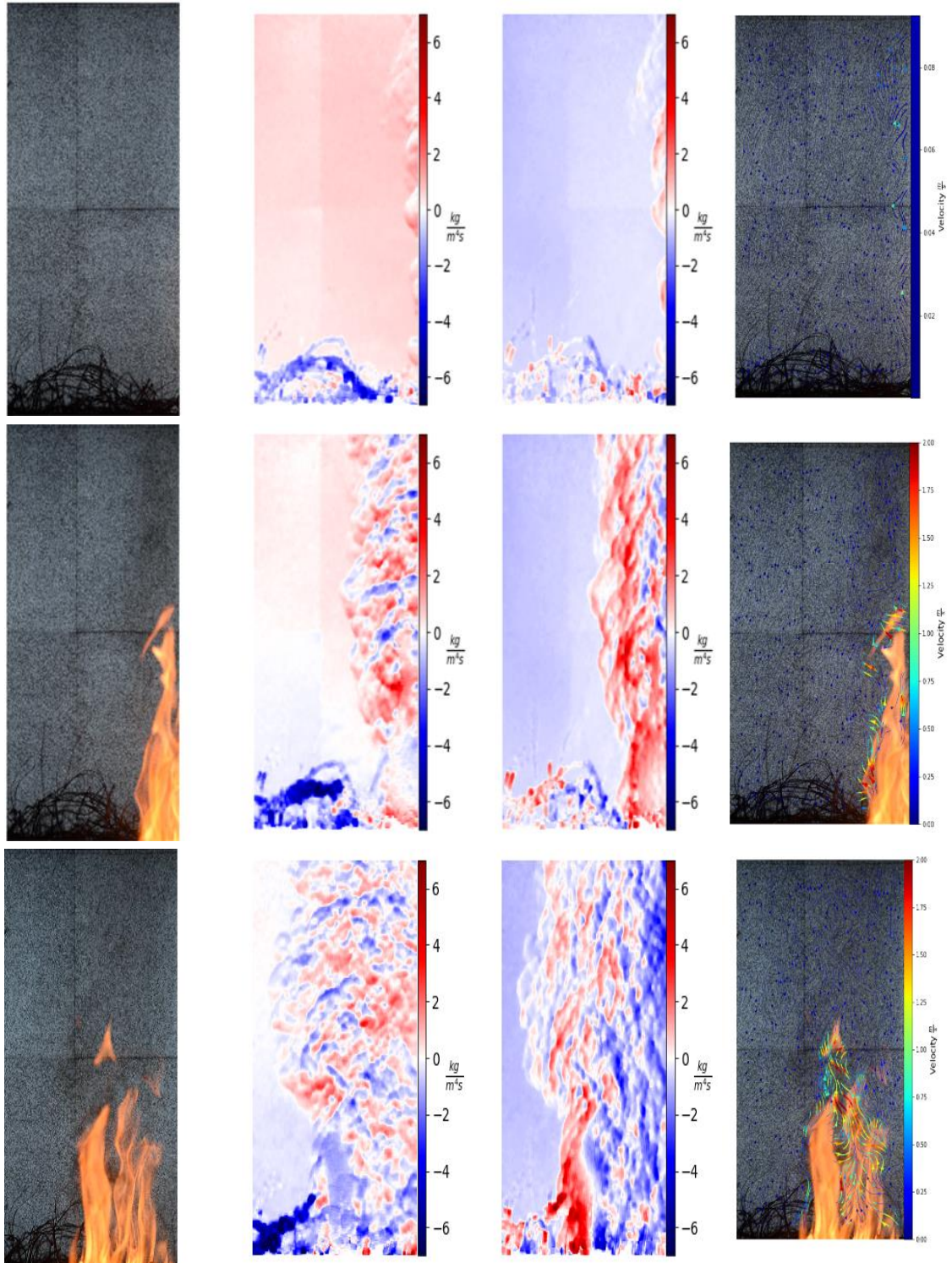

Figure 8 - Flow visualization of density gradients at three different times using a Background-oriented Schlieren technique. Time since ignition increases from the top frame to the bottom. The left column shows the actual image, the left middle column is the vertical density gradient $(\partial \rho \mathrm{V} \partial \mathrm{y})$ the right middle column is the horizontal density gradient $(\partial \rho \vee \partial x)$, and the right column shows the estimated velocity vectors. 


\section{Acknowledgement}

This work was supported was the DOD/EPA/DOE Strategic Environmental Research and Development Program project RC-2640.

\section{Literature Cited}

Akagi SK, Burling IR, Mendoza A, Johnson TJ, Cameron M, Griffith DWT, Paton-Walsh C, Weise DR, Reardon J, Yokelson RJ (2014) Field measurements of trace gases emitted by prescribed fires in southeastern US pine forests using an open-path FTIR system. Atmospheric Chemistry and Physics 14, 199-215. doi:10.5194/acp-14-199-2014.

Bartolome CJ (2014) Formation of superfog from wildland fires: theoretical and physical modeling. Dissertation, University of California - Riverside, Riverside, CA. http://scotty.ucr.edu/record=b4766864 S5.

van den Boogaart KG, Tolosana-Delgado R (2013) 'Analyzing compositional data with R.' (Springer: Heidelberg)

Fletcher TH, Safdari M-S, Amini E, Weise DR (2018) Bench-scale measurement of pyrolysis products from intact live fuels. In 'Adv. For. Fire Res. 2018', Coimbra, Portugal. 3. (Coimbra University Press: Coimbra, Portugal)

Lozano JS (2011) An investigation of surface and crown fire dynamics in shrub fuels. Dissertation, University of California, Riverside, CA.

Lozano J, Tachajapong W, Weise DR, Mahalingam S, Princevac M (2010) Fluid dynamic structures in a fire environment observed in laboratory-scale experiments. Combustion Science and Technology 182, 858-878. doi:10.1080/00102200903401241.

Maynard T, Princevac M, Weise DR (2016) A study of the flow field surrounding interacting line fires. Journal of Combustion 2016, 1-12. doi:10.1155/2016/6927482.

Meier G (2002) Computerized background-oriented schlieren. Experiments in Fluids 33, 181-187. doi:10.1007/s00348-002-0450-7.

Mertz L (1967) Auxiliary computation for Fourier spectrometry. Infrared Physics 7, 17-23. doi:10.1016/0020-0891(67)90026-7.

Raffel M (2015) Background-oriented schlieren (BOS) techniques. Experiments in Fluids 56,. doi:10.1007/s00348-015-1927-5.

Richard H, Raffel M (2001) Principle and applications of the background oriented schlieren (BOS) method. Measurement Science and Technology 12, 1576-1585. doi:10.1088/0957-0233/12/9/325.

Safdari M-S, Rahmati M, Amini E, Howarth JE, Berryhill JP, Dietenberger M, Weise DR, Fletcher TH (2018) Characterization of pyrolysis products from fast pyrolysis of live and dead vegetation native to the Southern United States. Fuel 229, 151-166. doi:10.1016/j.fuel.2018.04.166.

Weise DR, Fletcher TH, Johnson TJ, Hao WM, Dietenberger M, Princevac M, Butler B, McAllister S, O’Brien J, Loudermilk L, Ottmar R, Hudak A, Kato A, Shotorban B, Mahalingam S, Mell WE (2018) A project to measure and model pyrolysis to improve prediction of prescribed fire behavior. 'Adv. For. Fire Res. 2018'. (Coimbra University Press: Coimbra, Portugal)

Zhou X, Sun L, Mahalingam S, Weise DR (2003) Thermal particle image velocity estimation of fire plume flow. Combustion Science and Technology 175, 1293-1316. doi:10.1080/00102200302376. 


\title{
Measurement of three-dimensional flow speed and direction in wildfires
}

\author{
Torben P. Grumstrup*, Jason M. Forthofer, Mark A. Finney \\ Missoula Fire Sciences Laboratory, U. S. Forest Service. Missoula, Montana, U. S. A. \\ \{tgrumstrup@fs.fed.us*\}
}

\begin{abstract}
We report on the ongoing development of a new style of instrument package to measure three-dimensional flow speed and direction in wildland fires. The all-metal construction and lack of moving parts enables the instrument to survive immersion in flames for measurements in all fuel types. Disk style probes are used instead of the more common bi-directional probe to improve angle sensitivity of pressure measurement. Improved angular discrimination will permit calculation of fully three dimensional flow direction in addition to flow speed. An instrument package incorporating the disk anemometry system was developed and underwent preliminary testing. While the disk-style probe shows some improvement in angular sensitivity over a bi-directional probe, further improvement will result in greater tolerance in small inaccuracies in pressure measurement.
\end{abstract}

Keywords: convective heat transfer, instrumentation, wind speed, wind direction, flow velocity

\section{Introduction}

Convective heating of unburned fuel by adjacent flames has been shown to be a critical factor in the spread rate of wildfires (Finney et al. 2013; Finney et al. 2015). Calculation of the rate of heating of a given fuel element of a given shape requires knowledge of the fluid flow field (speed and direction) and temperature. For example, consider a fine fuel element like a pine needle or a small twig, which can be modeled as a cylinder. The convective heat transfer coefficient, $h$, can be determined using $h=k \cdot \mathrm{Nu} \cdot l^{-1}$, where $k$ is thermal conductivity, $l$ is a characteristic length scale (in this case, the diameter of the fuel element), and $\mathrm{Nu}$ is the Nusselt number from whose definition $\left(\mathrm{Nu} \equiv h l k^{-1}\right)$ we obtained the above relation. The Nussel number is estimated using empirical relations specific to convective heating of cylinders (e.g., see chapter 6 of (Holman 1997)). Having calculated $h$ for the fuel element, the rate of convective heat transfer per unit area is determined simply by applying Newton's law of cooling: $\dot{q}^{\prime \prime}=h\left(T_{s}-T_{\infty}\right)$ where $T_{s}$ and $T_{\infty}$ are surface temperature (of the fuel element) and ambient temperature, respectively.

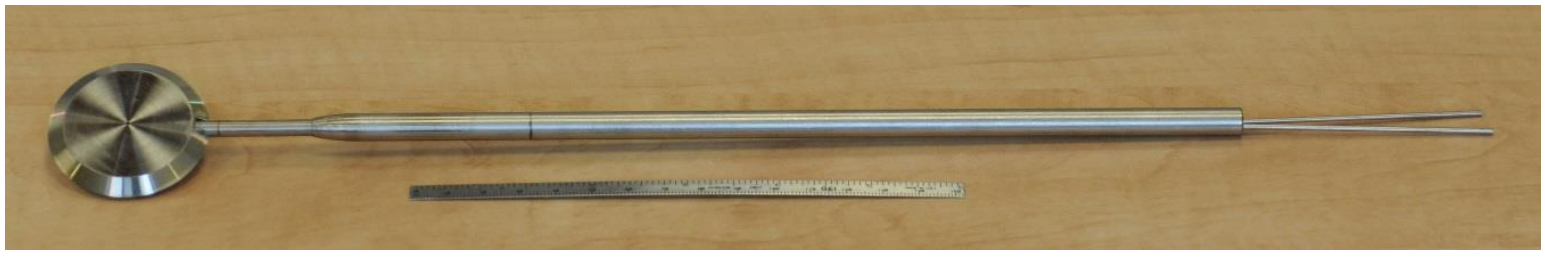

Figure 1 - A disk assembly (prior to support tube bending) showing the disk on the left, support tubing (center), and pressure tap tubes extending to the right. All materials are 304 stainless steel. A 150 mm straightedge is shown for scale.

In the course of calculating the rate of convective heating in a fire of fine fuel particles like grass, conifer needles, and small twigs, the calculations described above are simple enough. However, measurement of the flowfield velocity components and temperature in a wildland fire are more 
challenging. Instruments must be robust because they are subjected to intense heat, soot, and flying debris created by the passing fire front. They must also be rugged to withstand rough handling during transportation and deployment. Temperature measurement is easily implemented with fine-gauge thermocouples, but determination of flow speed and direction are more challenging. The meteorology research community depends on vane-and-cup and sonic anemometers for measurement of wind speed and direction. Unfortunately, they are unsuitable for fire applications because of the use of moving parts in the case of the former, and heat-sensitive electronic components in the latter. The wildland fire community needs a probe that is free of moving parts and has no exposed electronics (and, hopefully, inexpensive as well).

The bi-directional pressure probe has been a mainstay of velocity measurement in structural fire and wildfire research since it was introduced by McCaffrey and Heskestad in 1976 (McCaffrey and Heskestad 1976). While unarguably suitable for flow speed measurement, the bi-directional probe is (by design) markedly insensitive to the angle of the probe axis to the flow, making it unsuitable for determining three-dimensional flow direction. Motivated by a need to measure convective heating very near a spreading fire front, we are in the process of adapting a new type of probe that possesses the speed measurement capability of a bi-directional probe, but with improved sensitivity to angle: the disk anemometer (see Fig. 1).

The disk anemometer, like the bi-directional probe, is a differential pressure anemometer. That is, flow velocity is determined by measuring the difference in pressure between the side facing the flow and the side facing away from the flow. When three orthogonally oriented probes are used, a previously-determined calibration curve is consulted and, through a short series of iterations where Reynolds number is initially guessed then refined, flow speed and direction is determined (Green $e t$ al. 2001). The disk geometry and beveled edges make the pressure differential more strongly a function of flow angle.

The disk anemometer was invented by researchers from the University of British Columbia (Green and Rogak 1999; Green et al. 2001) who were working to develop a low cost 3D anemometer for maritime and other harsh meteorological environments. We adapted their disk anemometer concept into our heat flux instrument packages, which are fireproof and field-deployable (Fig. 2a). All components external to the electronics enclosure are stainless steel. The electronics enclosure is aluminum for light weight and can be insulated to the extent required for any given fuel type. Each disk contains a pressure tap on either face. Small stainless steel tubes communicate pressure from each tap down through the support tubes and into the electronics enclosure. There pressure is measured by differential pressure transducers (Fig. 2b) for the three disks respectively. Gas temperature is measured by a pair of fine gauge Type $\mathrm{K}$ thermocouples and radiation by a radiant heat flux gauge. Data from the three pressure transducers, two thermocouples, and the heat flux gauge are acquired by a dedicated in-house-designed logger, which also resides in the enclosure (Fig. 2c). 


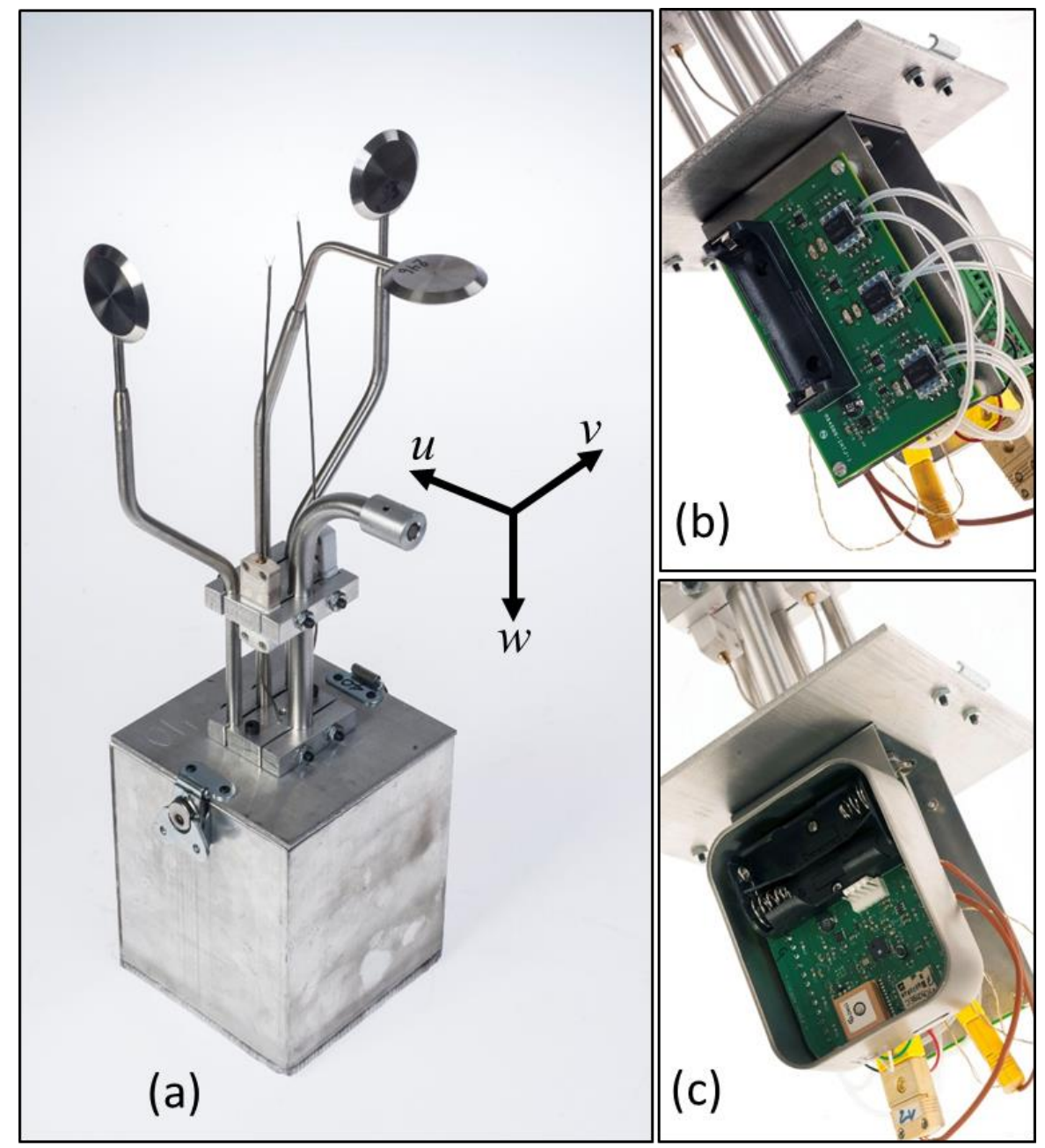

Figure 2 - (a) Fully assembled heat flux package showing disks of the anemometry system, thermocouples, radian heat flux gauge, and electronics enclosure; (b) Pressure signal conditioner; (c) Data logger. For a sense of scale, the heat flux package is $47 \mathrm{~cm}$ tall.

Disk anemometry works on the principle that the differential pressure measured on opposite faces of a disk is solely a function of the wind velocity and the angle the wind vector makes with the normal of the disk. Consider the disk schematic in Fig. 3 wherein the wind vector $\vec{u}$ is at angle $\alpha$ relative to the disk normal. The azimuthal angle $\phi$ is irrelevant to differential pressure measurement because of the rotational symmetry of the disk. Ideally, the differential pressure $\Delta p$ would vary strongly and linearly with both $\vec{u}$ and $\alpha$ so the methodology would be tolerant of small measurement inaccuracies.

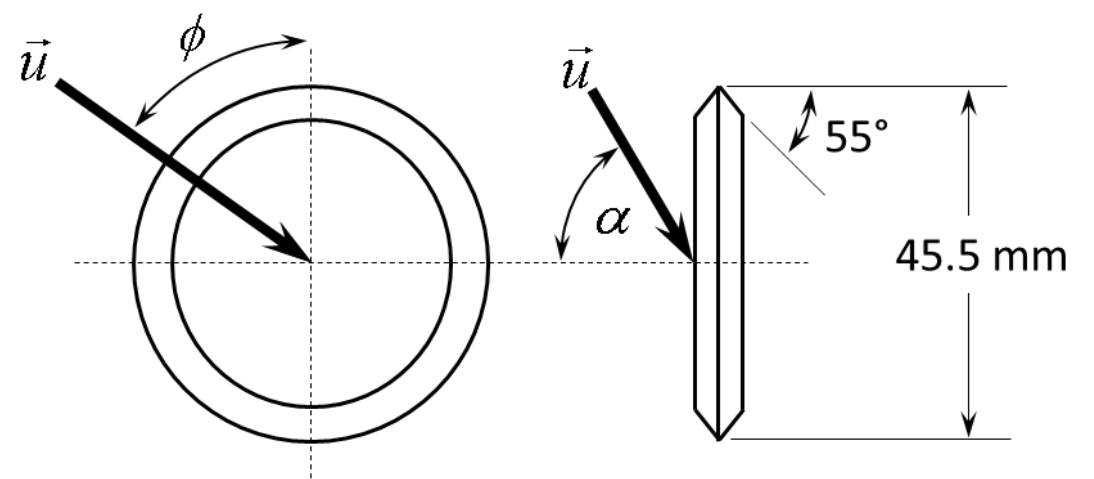

Figure 3 - Diagram of the front and side views of disks with some arbitrary wind vector. 
However, in practice $\Delta p$ is a weak function of $\vec{u}$ (or, more generally, Reynolds number $\mathrm{Re}$ ) and the relationship with $\alpha$ is nonlinear.

\section{Description of the Heat Flux Package}

\subsection{Heat Flux Package Design}

The heat flux package (Fig. 2a) is an instrument that is in ongoing development. Its purpose is to fulfil a need for acquiring the data needed to calculate the convective heating that fine fuels experience just ahead of a wildland fire front - namely, flow speed, flow direction, and gas temperature. The heat flux package also has the ability to measure radiant heat flux over a broad field of view.

The instrument contains three principle measurement capabilities: anemometry of local wind flows, thermometry of local gas temperature, and measurement of radiant heat flux. Here, we are concerned only with the first two since both wind speed, wind direction, and gas temperature are required for calculating convective heat transfer. Anemometry is accomplished with three disks that experience a differential pressure between the two faces of the respective disk that varies as a function of wind speed and incident angle relative to the disk faces. The differential pressure is measured by pressure transducers that communicate with the disk faces through internal plumbing connected to pressure taps on either face of the disk. Measurement of winds coming from any direction is made theoretically possible by the positioning of the disks in the three mutually orthogonal directions. The disk diameter $(44.5 \mathrm{~mm})$ and thickness $(6.1 \mathrm{~mm})$ were determined by scaling up the design in Green (Green et al. 2001) by a factor of 1.75 . The $55^{\circ}$ bevel on the disk edge as recommended by Green (Green and Rogak 1999) was maintained in our design. We decided to scale up the disk geometry to mitigate some of the disk manufacturing challenges.

The disks were machined with a CNC lathe by a local shop from 304 stainless steel round stock. The $1.588 \mathrm{~mm}(1 / 16 \mathrm{in}$.) holes required for internal pressure tubing proved to be the most challenging step - requiring careful attention to feeds and speeds to avoid breaking the diminutive drill bits. We plan to use 303 stainless steel, a more readily machinable alloy, for future versions.

The support tubes positioned the disks away from the electronics enclosure and also protected the small diameter pressure tubes from damage. Support tubes were made from seamless heavy-walled stainless steel tubing and bent into the design shape. Rather than

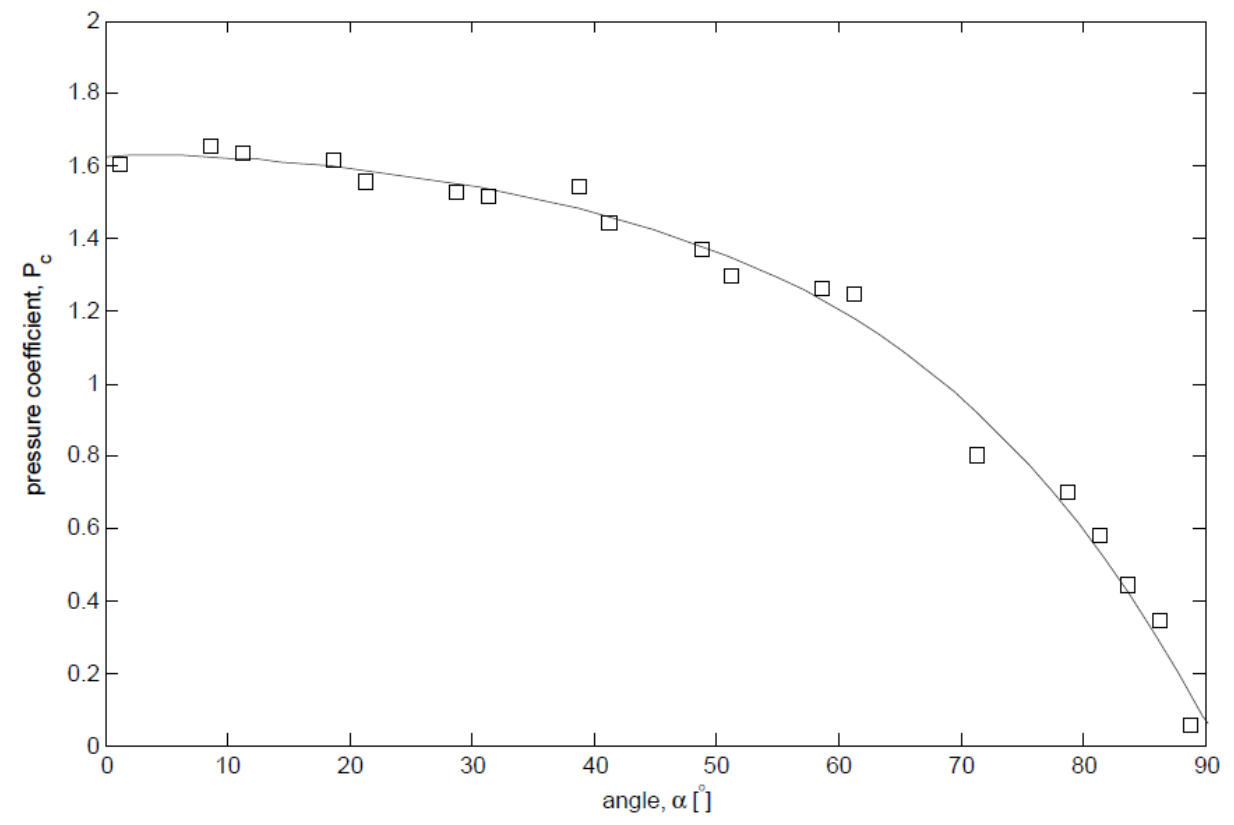

Figure 4 - Plot showing the sensitivity of pressure to angle. 
welding, the support tubes, pressure tubes, and disk were bonded together with high-temperature metallic adhesive (Durabond 954OD, Cotronics Corp.).

Local air and combustion gas temperature was measured by a pair of fine gauge thermocouples. The thermocouples consisted of a ceramic male connector, approximately $15 \mathrm{~cm}$ of stainless-steel-clad extension wire (Omega Engineering Omegaclad ${ }^{\mathrm{i}}$ ), and the actual thermocouple junctions were 50 micron welded junctions from the same vendor.

\subsection{Pressure Electronics and Data Logger}

The pressure gradient between one face of a disk relative to the opposite face was communicated to the electronics enclosure (Fig. 2a) by small diameter stainless steel tubing that was routed through the support tubes. Once inside the enclosure, the tubes were attached to three differential pressure transducers (Honeywell HSCDRRN001NDAA $5^{\mathrm{i}}$ ) which have a range of \pm 249 Pascal ( \pm 1 inch of water column). The pressure transducers were soldered to a signal conditioning circuit board (Fig. $2 \mathrm{~b}$ ) which converted the single-ended 3.3 volt output signal to a 2.5 volt differential signal that is compatible with the data logger. The pressure transducer signal conditioning circuit board was designed in-house and was powered by a single AA battery.

The three pressure transducers, two thermocouples, and two radiant heat flux gauge circuits constitute seven differential voltage signals. These data are recorded by a data logger (Fig $2 \mathrm{c}$ ) that was also designed in-house for the heat flux package and other field-going wildland fire research instruments. The logger acquires data from eight, 24-bit differential channels at $50 \mathrm{~Hz}$ for up to 20 hours when powered by a pair of high-quality alkaline batteries. The internal clock is set from Global Positioning System (GPS) satellite signals so that multiple loggers can be synchronized prior to deployment on a fire. The acquired data is stored on a removable microSD card. The compact logger design fits within a $92 \times 73 \times 32 \mathrm{~mm}$ steel tin (including batteries).

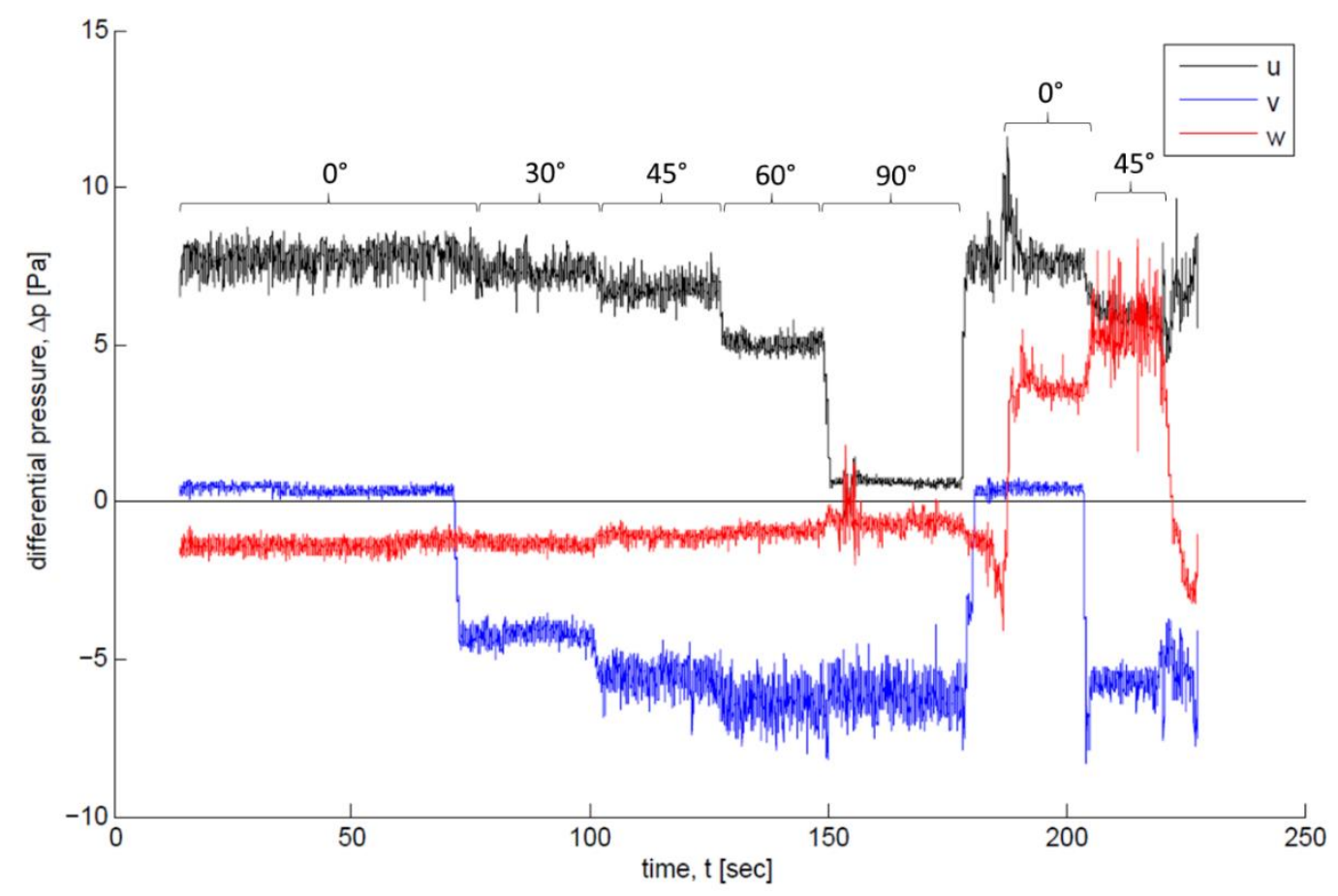

Figure 5 - Pressure traces from the three orthogonal components for a range of flow angles.

\subsection{Field Deployment of Heat Flux Package}

The heat flux packages are set up in the field with the disks approximately level with the height of the fuel. The heat flux packages are positioned vertically using a round bubble level. The azimuthal 
direction is determined by the wind direction expected at the time of ignition. A magnetic compass is used to ensure all the heat flux packages are pointed in the correct direction. ${ }^{1}$ Once all the heat flux packages are properly buried and oriented in a burn plot, the loggers are activated by inserting batteries. The loggers are given time to acquire signals from GPS satellites to synchronize their clocks, then the packages are closed up and thermocouples inserted into the plugs. There is no need to start logging immediately prior to ignition since the loggers can function all day. This method avoids the complication of triggering the logger to begin acquiring just as the fire approaches. Following the passing of the fire, the loggers are shut off and data cards collected.

\section{Results and Discussion}

Wind tunnel testing of the heat flux packages revealed aerodynamic properties of the anemometry system. This section contains results and interpretation of this testing, and plans for development moving forward.

Fig. 4 shows how pressure (in the form of pressure coefficient) varies as a function of angle $\alpha$ at $\mathrm{Re}=14000$, where the length scale is the $44.5 \mathrm{~mm}$ disk diameter. Between $0^{\circ}$ and approximately $60^{\circ}$ the relatively flat trend shows that pressure is a weak function of $\alpha$, while for angles $60^{\circ}$ to $90^{\circ}$ the dependence is stronger. The implications of this relationship are that small uncertainties in the measurement of pressure when the disk is at an angle of $0^{\circ}$ to $60^{\circ}$ to the wind will cause disproportionately large inaccuracies in the angle determined from the measured pressure. For flow angles beyond $60^{\circ}$, the trend is more favorable because the dependency is much stronger. The plot in Fig. 4 is representative of the pressure coefficient trend with angle for all Reynolds numbers tested (up to 45000). While this level of angular sensitivity is an improvement over bi-directional probes, it is not sufficient to ensure adequate tolerance of small inaccuracies of pressure measurement.

Fig. 5 shows other results from placing the heat flux package in the wind tunnel. The vertical axis shows differential pressure across the two faces of the respective disks. The signs of the pressures correspond to the $u, v, w$ axes illustrated in Fig. 2a. The numerical angles and brackets indicate the direction of the wind to which the heat flux package was subjected. For example, from 14 to 70 seconds, the heat flux package was positioned to face straight into the wind, generating a maximal signal in the $u$ component and nearly zero in the $v$ component. The heat flux package was tilted slightly backward to create a small negative $w$ component. The flow angle was systematically varied over the indicated angles from 17 to 180 seconds. From 180 to 220 seconds, the heat flux package was tilted forward approximately 25 degrees, creating a significant positive $w$ component. The Reynolds number with respect to the disk diameter is 10000 .

The plot in Fig. 5 demonstrates the difficulty in implementing the disk anemometry technique. Note that over the range of $0^{\circ}$ to $45^{\circ}$, there is little change in the pressure measured in the $u$ component. This range of angles corresponds to the region in the plot in Fig. 4 in which pressure has a weak dependence on angle. If angle is unknown (as it would be in a field deployment), it would be difficult to distinguish between, for example, wind coming from a bearing of $0^{\circ}$ or $20^{\circ}$. It is apparent that the differential pressure measured on the disks - as designed - does not have sufficient angular discrimination to be useful in the field. An improvement in disk design is warranted.

${ }^{1}$ Since the basic function of the disk anemometry system is to measure a three dimensional flowfield, the orientation of the heat flux packages would normally be irrelevant. However, the instrument is faced towards the expected fire front to ensure maximum converage by the radiant heat flux gauge acceptance angle $\left(\sim 110^{\circ}\right)$. 
A further source of error arises from the physics of the problem. Consider the pitot tube equation: $u=\left(2 \Delta p \rho^{-1}\right)^{0.5}$. The quadratic relationship between $u$ and $\Delta p$ means that when $\Delta p$ is near zero (i.e., velocity near zero), small inaccuracies in the measurement of $\Delta p$ results in disproportionately large inaccuracies in the calculated velocity (McCaffrey and Heskestad 1976). This effect compounds error that arises from angular insensitivity.

Wind tunnel testing has shown that there are significant limitations to using the disks for anemometry as they are presently designed. The weak angular sensitivity for angles over the range $0^{\circ}$ to approximately $60^{\circ}$ means that inaccuracy in pressure measurement can be magnified when calculating the corresponding velocity. Green and Rogak (1999) noted that improving the design of the disk requires an understanding of the boundary layer attachment and separation that occurs on the surface of the disk at relevant Reynolds numbers. To that end, we plan to carry out CFD simulations of various styles of disk designs. We will seek a design that is more sensitive to changes in angle so that it is more tolerant of small inaccuracies in pressure measurement.

\section{Conclusion}

We report on the ongoing development of a new style of instrument package to measure threedimensional flow speed and direction in wildland fires. The all-metal construction and lack of moving parts enables the instrument to survive immersion in flames for measurements in all fuel types. Disk style probes are used instead of the more common bi-directional probe to improve angle sensitivity of pressure measurement. Improved angular discrimination will permit calculation of fully three dimensional flow direction in addition to flow speed. An instrument package incorporating the disk anemometry system was developed and underwent preliminary testing. While the disk-style probe shows some improvement in angular sensitivity over a bi-directional probe, further improvement will result in greater tolerance in small pressure measurement inaccuracies.

\section{Acknowledgements}

Significant help in design, production, and assembly of heat flux packages was provided by our technical staff: Randy Pryhorocki, Josh Deering, Chelsea Phillips, Andrew Gorris, and Jon Bergroos. This work was supported by U. S. Forest Service Research and the National Fire Decision Support Center.

\section{References}

Finney, MA, Cohen, JD, Forthofer, JM, McAllister, SS, Gollner, MJ, Gorham, DJ, Saito, K, Akafuah, NK, Adam, BA, English, JD (2015) Role of buoyant flame dynamics in wildfire spread. Proceedings of the National Academy of Sciences 112, 9833-9838.

Finney, MA, Cohen, JD, McAllister, SS, Jolly, WM (2013) On the need for a theory of wildland fire spread. International Journal of Wildland Fire 22, 25-36.

Green, SI, Ostafichuk, P, Rogak, SN (2001) Measurement of three-dimensional unsteady flows using an inexpensive multiple disk probe. Journal of Atmospheric and Oceanic Technology 18, 883-891.

Green, SI, Rogak, SN (1999) A multiple disk probe for inexpensive and robust velocimetry. Journal of Fluids Engineering 121, 446-449.

Holman, JP, 1997. Heat transfer. McGraw-Hill Inc,

McCaffrey, B, Heskestad, G (1976) A robust bidirectional low-velocity probe for flame and fire application. Combustion and Flame 26, 125-127. 


\title{
Modelling of junction fires with analytical and numerical analysis of the phenomena
}

\author{
Jorge Raposo*1,2; Domingos Viegas ${ }^{1,2}$; Abdelrahman Abouali ${ }^{1}$; André Rodrigues ${ }^{1}$; Miguel \\ Almeida ${ }^{1}$, Jorge André, J. ${ }^{2}$; Xiaodong $\mathrm{Xie}^{3}$ \\ ${ }^{1}$ Association for the Development of Industrial Aerodynamics. Rua Pedro Hispano, $n^{\circ} 12,3030$ - \\ 289 Coimbra, Portugal, \{jorge.raposo@dem.uc.pt*\} \\ ${ }^{2}$ University of Coimbra, Rua Luís Reis dos Santos, Coimbra 3030-788, Portugal \\ ${ }^{3}$ State Key Laboratory of Fire Science, University of Science and Technology of China, Hefei, \\ China
}

\begin{abstract}
The concept of junction fires was introduced in Viegas et al. 2012, as two approaching lines of fire, making a small angle between them. In the approach, the fires interact and induce convective flows that modifies the fire behaviour mainly its rate of spread. This process induces very strong fields of vorticity, high rates of fire spread or a large number of spot fires. Since the beginning, an exhaustive parametric study of the phenomena of the junction fires was carried out. Results from laboratory and field-scale experiments, using various fuel beds and slope angles and from large-scale fires show that the processes are similar at a wide range of scales with little dependence on the initial boundary conditions. These results correspond to a large amount of data collected. With this data, a generalization of phenomena for different conditions was made through semiempirical models that can predict with a good agreement the sudden evolution of the rate of spread of the fire front, which results of the interaction of the fire lines, as function of the independent parameters namely time $\mathrm{t}$, distance $\mathrm{x}$, slope a and initial angle between the fire fronts. In addition, an effort to simulate the evolution of this kind of fires using CFD based numerical models was performed.
\end{abstract}

Keywords: fire behaviour; extreme fire behaviour; dynamic fire behaviour; accelerating fires; merging fires;

\section{Introduction}

The concept of junction fires was introduced in Viegas et al. 2012, when two approaching lines of fire make a small angle between them. In the approach, the fires interact and induce convective flows that modify the fire behaviour, mainly its rate of spread (ROS). This process induces very strong fields of vorticity, high rates of fire spread or a high number of spot fires. Since the beginning, an exhaustive parametric study of the phenomena of the junction fires was carried out. The results from laboratory and field-scale experiments, using various fuel beds and slope angles and from large-scale fires show that the processes are similar at a wide range of scales with little dependence on the initial boundary conditions. To characterize the effects of wind on junction fires, a set of experimental tests were performed, in CEIF's wind tunnel, using pine needles (Pinus pinaster), varying wind speed between 1 and $5 \mathrm{~m} / \mathrm{s}$

\section{Problem analysis}

The characteristics of a junction fire and IR images of various stages of its development were presented in Viegas et al. (2012) and in Viegas et al. (2013). In these studies, it was confirmed that two fire fronts merge at the intersection point D. A very complex and dynamic set of processes starts to develop in which the geometrical and physical properties of the fire fronts change continuously. A unique feature of this fire is the rapid advance of point $\mathrm{D}$ that causes the triangular shaped fuelbed to 
burn, maintaining its general shape but with increasing values of $\theta$. The rapid acceleration of the fire front near $\mathrm{D}$ caused by the convective flow induced by the fire is like the eruptive fire behaviour. Unlike the eruptive fire in a canyon (cf. (Viegas and Pita 2004); (Viegas 2006); (Viegas and Simeoni 2011)), the rate of spread increase has a break as if the fire and the mechanisms that support its acceleration are reduced and then undergo an inversion in their contributing effects. In this article one analysed the problem of junction fires under the wind effect, considering that the phenomena of junction fires under the effect of the slope was already addressed in previous works as well the effect of fuels and scale. The interaction between wind and slope can produce a set effects on the fire propagation. (Sharples et al. 2012). Commonly it is stated that the wind has a similar effect in the slope during the fire propagation, with the difference that the wind can achieve higher intensity compared with the slope (Viegas 1998). Several times the fire propagation is driven by the slope and wind together as referred in (Viegas 2004) Nevertheless it is common to study separately each factor, as it will be done in this article, here the focus is on the effect of wind. One of the firsts studies about the role of the wind in the forest fires was carried out by (Grumer and Strasser 1965), in the work it was proved trough laboratorial experiments that large scales fires rise with winds able of increasing the burning rate similar to firestorms. Rothermel (1972) proposed a model that includes the contribution of wind and slope in the fire propagation trough out adimensional coefficients. Morandini et al. (2006) investigated the effects of wind on the properties of a fire front, using Mediterranean shrubs as fuel. Sharples et al. (2010) study fire behaviour in atypical situations due to the interaction of wind and topography with fire. In the work (Viegas et al. 2012) is presented a general law of behaviour for junction fires. It was observed that the junction fires follow a pattern of evolution characterized by an acceleration phase, that persists during a certain time $t_{M}$, until achieve the maximum ROS ( $\left.R_{M}\right)$ followed by a deceleration phase, as presented in figure 1

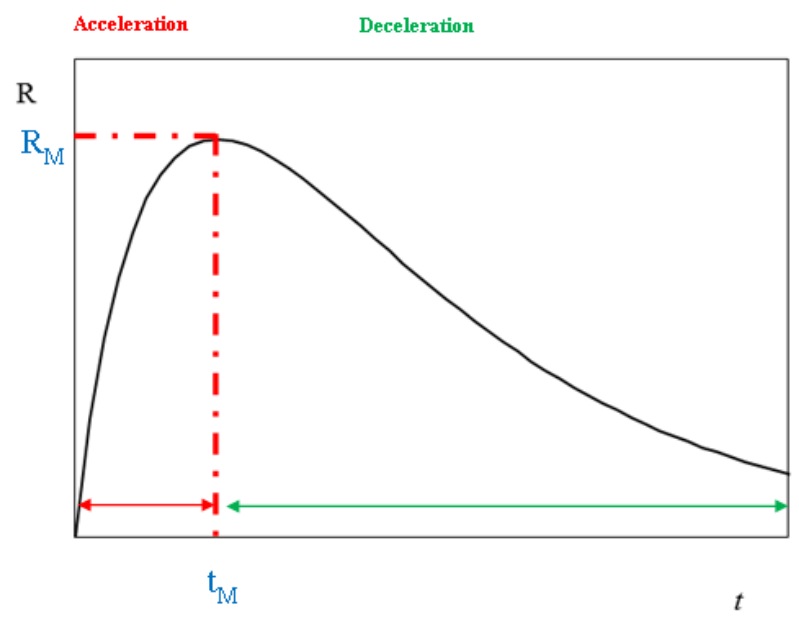

Figure 1 - Pattern of evolution of a junction fire

In (Raposo et al. 2018) general empirical laws of fire behaviour, which can be used to predict the evolution of a junction fire episodes, namely the evolution of non-dimensional rate of spread as a function of the independent slope angle $\alpha$ and angle between fire fronts $\theta_{\mathrm{o}}$ were presented. The same kind of analysis will be used for the tests with wind relating the different wind speeds with the evolution of the ROS.

\section{Methodology}

The tests were performed in the wind tunnel of LEIF. An image of the equipment can be observed in the figure 2.

Advances in Forest Fire Research 2018 - Page 550 


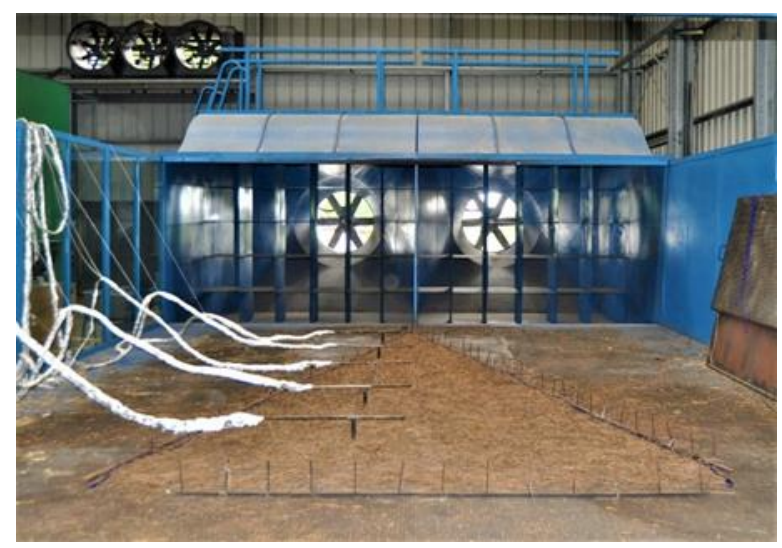

Figure 2 - wind tunnel of LEIF

In all of the tests dead pine needles of Pinnus Pinaster (PP) were used as fuel bed with a load of 0.6 $\mathrm{kg} / \mathrm{m}^{2}$, the wind speed experiments were $1,2,3,4$ and $5 \mathrm{~m} / \mathrm{s}$. Below is presented a table with the set of tests performed with an alphanumeric code to identify them, the first numeric digit refers to the order of realization of the test and the second digit is referred to the wind speed in $\mathrm{m} / \mathrm{s}$. They were randomly performed to avoid some bias in the data.

Table 1 - Set of tests performed

\begin{tabular}{|c|c|c|c|c|c|}
\hline Ref. & Designation & $\begin{array}{c}\text { Wind Speed } \\
\mathrm{m} / \mathrm{s}\end{array}$ & Fuel & $\alpha$ & $\theta$ \\
\hline 1 & CFW1-1 & 1 & \multirow{7}{*}{ PP } & \multirow{7}{*}{0} & \multirow{7}{*}{30} \\
\hline 4 & CFW4-1 & 1 & & & \\
\hline 2 & CFW2-2 & 2 & & & \\
\hline 5 & CFW5-3 & 3 & & & \\
\hline 3 & CFW3-4 & 4 & & & \\
\hline 7 & CFW7-4 & 4 & & & \\
\hline 6 & CFW6-5 & 5 & & & \\
\hline
\end{tabular}

To prepare the fuel bed in a V shape auxiliary lines were placed in the base of the wind tunnel demarking the shape of the fuel bed with $5 \mathrm{~m}$ length and a base of $2.68 \mathrm{~m}$, so that the angle of $30^{\circ}$ of angle between the fire fronts desired for the tests was respected.

The moisture content of the fuel $\left(\mathrm{m}_{\mathrm{f}}\right)$ was always registered as well as the values of the relative humidity and temperature of the air. The ignition of the fuel bed was made using two wool string lines sucked in a mixture of gasoline and diesel to ensure an instantaneous ignition. After the ignition the funs of the wind tunnel were turned on to set the wind speed. In every test, the spatial distribution of the temperature along with the fuel-bed infrared images were recorded, using an infra-red camera FLIR ThermaCam SC640. The acquisition rate was also 7Hz, the presence of the fire front was considered for values of temperature above $350^{\circ} \mathrm{C}$, which was considered a sign of the existence of flame in the place or time of measurement. Using IR methodology, the position of the fire perimeter at given time frames was assessed and from these images the ROS at various positions of the fire perimeter namely at point $\mathrm{D}$ was measured.

\section{Results and discussion}

From the tests performed in the experimental program the following data of the rate of spread of the fire front in the interception point was plotted for the five different wind speeds used. Also, a test performed with no slope and no wind in a V shape configuration was plotted together. 


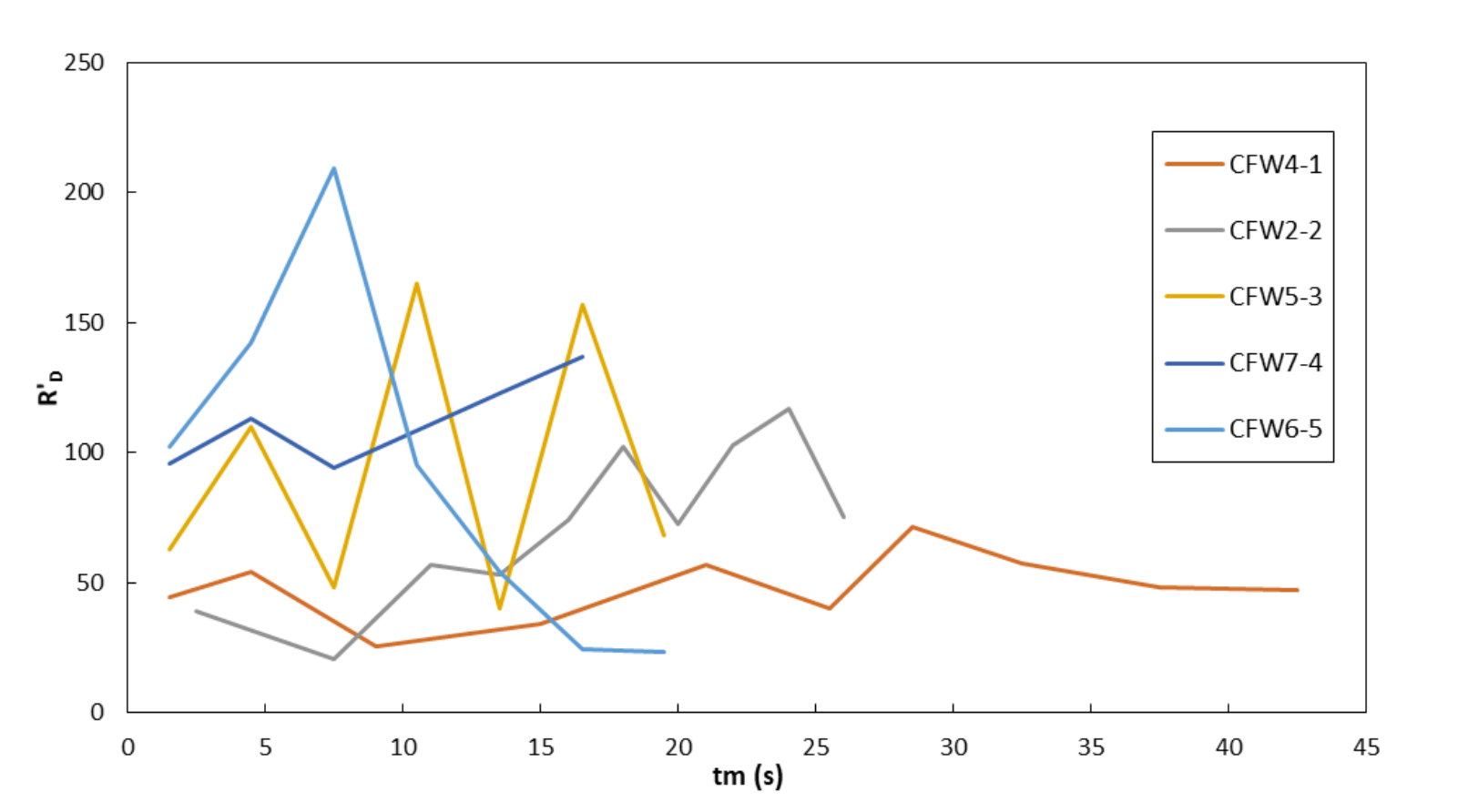

Figure 3 - ROS R'D as function of time, for the tests with wind and no slope

From the figure one can observe that the higher the wind speeds, the faster is the duration of the test until the complete burnt of the fuel bed, meaning that the average ROS in the intersection point D increases with the increase of wind speed.

The tests CFW3-4 and CFW1-1 were not used due to problems in their analysis.

Also, the tendency of the evolution of the ROS is of increasing with the differences between acceleration and deceleration phases, becoming less evident with the increasing of the wind speed, with exception of the test CFW6-5,

The data also allows to say that in the presence of wind the deceleration phase loses expression giving space to almost only a continuous acceleration of the fire. The explanation to this can be given by the fact that the introduction of the forced convection of the wind can overlap the natural buoyancy convection induced by the fire itself in a junction fire phenomenon. By the analysis of the plot, it is also possible to affirm that the maximum value of the ROS increases with the increase of the wind speed.

In figure 4 is showed the evolution of the ROS R'D as function of the distance XD. From the plot is observed that the distance of occurrence of the maximum ROS increases with the increase of the wind speed. The rate of variation of the maximum ROS from the test with $1 \mathrm{~m} / \mathrm{s}$ relatively to the test with $2 \mathrm{~m} / \mathrm{s}$ is considerably bigger than the increase from the tests with $2 \mathrm{~m} / \mathrm{s}$ to $4 \mathrm{~m} / \mathrm{s}$ which indicates a kind of saturation level of the effect of the wind in the propagation of a junction fire. 


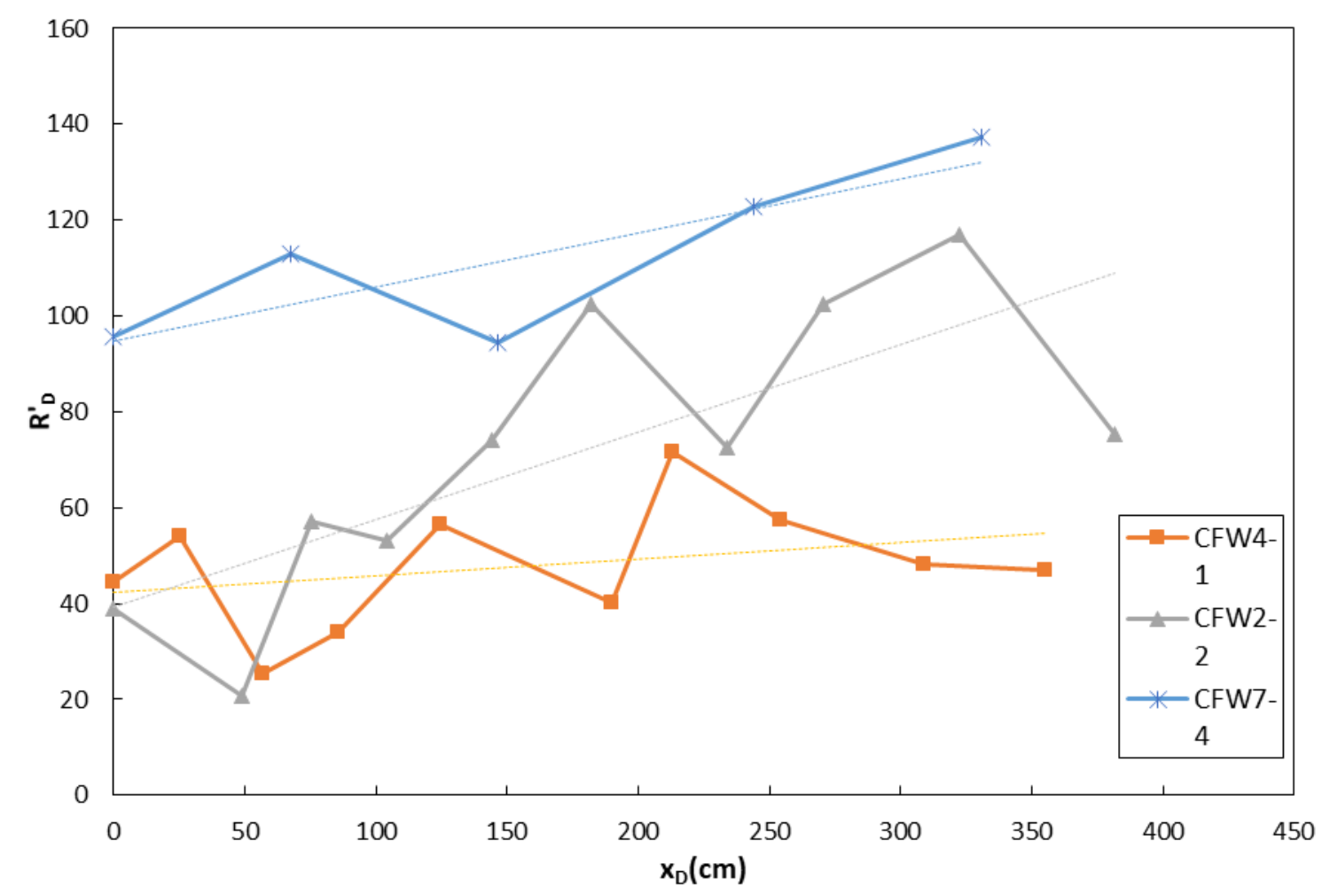

Figure 4 - ROS R'D as function of the distance $x_{D}$.

\section{Conclusion}

This is a preliminary study about the wind effect on the phenomena of the junction fires with a limited number of tests performed. Nevertheless, from the data obtained they seem to indicate that the maximum ROS of a junction fire increases with the increase of the wind speed until to a threshold value, that demands a more exhaustive work for its definition, but clearly it is observed by the saturation of the increment of the ROS as result of the rise of the wind speed.

\section{Acknowledgements}

This work was prepared under the framework namely of projects "Firewhirl - Vorticity Effects in Forest Fires" (Ref. PTDC/EMS-ENE/2530/2014), financed by national funds through the Portuguese Foundation for Science and Technology - FCT and project "Firextr - Prevent and prepare society for extreme fire events: the challenge of seeing the "forest" and not just the "trees" (Ref: PTDC/ATPGEO/0462/2014), co-financed by the European Regional Development Fund (ERDF) through the COMPETE 2020-Operational Program Competitiveness and Internationalization (POCI Ref: 16702) and national funds by FCT-Foundation for Science and Technology. It was also prepared under the framework of Projects "Fireprotect - Protection Systems for the protection of persons and elements exposed to the fire", Region (Centro 2020, Centro-01-0246-FEDER-000015) and project "ReNATURE - Valorisation of the Natural Endogenous Resources of the Centro Region" (Centro 2020, Centro-01-0145-FEDER-000007) supported by European Investment Funds by FEDER.)

The authors wish to thank their colleagues Luis Ribeiro and Nuno Luis for their support in the analysis of data and in the preparation of some work related with the article. 


\section{References}

Grumer J, Strasser A (1965) Uncontrolled fires - Specific burning rates and induced air velocities. Fire Technol ogy 1, 256-268.

Raposo J, Viegas DX, Xie X, Almeida M, Porto L, Sharples J, Figueiredo A (2018) Analysis of the physical processes associated to Junction Fires at Laboratory and Field Scales. International Journal of Wildland Fire.

Sharples JJ, McRae RHD, Wilkes SR (2012) Wind-terrain effects on the propagation of wildfires in rugged terrain: Fire channelling. International Journal of Wildland Fire 21, 282-296. doi:10.1071/WF10055.

Viegas DX (1998) Forest fire propagation. Philosophical Transactions of the Royal Society of London Series A: Mathematical, Physical and Engineering Sciences 356, 2907 - 2928.

Viegas DX (2004) Slope and wind effects on fire propagation. International Journal of Wildland Fire 13, 143-156. doi:10.1071/WF03046.

Viegas DX (2006) Parametric study of an eruptive fire behaviour model. Int. J. Wildl. Fire 15, 169177. http://dx.doi.org/10.1071/WF05050.

Viegas DX, Pita LP (2004) Fire spread in canyons. International Journal of Wildland Fire 13, 253 274. doi:10.1071/WF03050.

Viegas DX, Raposo JR, Davim D a., Rossa CG (2012) Study of the jump fire produced by the interaction of two oblique fire fronts. Part 1. Analytical model and validation with no-slope laboratory experiments. International Journal of Wildland Fire 21, 843-856. doi:http://dx.doi.org/10.1071/WF10155.

Viegas DX, Simeoni A (2011) Eruptive Behaviour of Forest Fires. Fire Technology 47, 303-320. doi:10.1007/s10694-010-0193-6. 


\title{
Modelling the rate of spread of fire: An SDE approach
}

\author{
Zlatko Jovanoski $^{1 *}$; Jason J. Sharples ${ }^{1}$; A. Malcolm Gill ${ }^{2}$; Simon Watt ${ }^{1}$; Harvinder S. Sidhu ${ }^{1}$; Isaac \\ N. Towers ${ }^{1}$; Smaila Sanni ${ }^{1}$ \\ ${ }^{1}$ School of Physical, Environmental and Mathematical Sciences; UNSW Canberra, ACT 2600, \\ Australia. \{z.jovanoski@adfa.edu.au*\} \\ ${ }^{2}$ Fenner School of the Environment and Society; ANU, ACT 0200, Australia
}

\begin{abstract}
From a point source, landscape fires accelerate until they reach a quasi-equilibrium rate of spread. The rate at which a fire accelerates from its ignition affects the time first responders have to attack a fire in its initial stages when it is more easily suppressed. As such, knowledge of the rate of acceleration of a fire from ignition can be valuable from a fire management perspective. However, the majority of studies in wildland fire science have been dedicated to development of models for the quasi-equilibrium rate of spread attained by the fire after its acceleration phase. Comparatively little attention has been given to the development of models that specifically account for the growth phase of a fires development.

The rate of acceleration depends on many factors including variations in ambient and induced wind speed and direction, variation in moisture content of the fuel, fuel stratification and slope variation. Present models of fire growth from a point ignition are expressed as deterministic algebraic equations, thereby neglecting variability. The numerous variables involved make predictions of rate of spread from a point source very difficult.

In this paper we consider two approaches to model the acceleration phase of a fire. The first considers fitting a sigmoidal (logistic) function to experimental data using a nonlinear regression procedure. In the second approach we propose the use of stochastic differential equations to investigate the growth of a fire to quasi-equilibrium. In addition to providing a more realistic portrayal of the time series data relating to fire growth, this second approach allows for better discrimination of the mechanisms driving the growth phase of fire spread.

The models are assessed by appealing to observations of experimental fire growth. Specifically the data relate to fires growing from a point ignition under the influence of a uniform wind. The results indicate that both approaches can provide an accurate representation of the observed data, but that the approach based on stochastic differential equations yields $95 \%$ prediction bounds that are narrower than those obtained from the nonlinear regression. The difference in prediction bounds indicates that the way stochasticity is incorporated into fire growth models has implications for how models inform decisions about the likelihood of a fire selfextinguishing before it reaches quasi-equilibrium, and the magnitude of the rates of spread it is likely to exhibit during the initial stages of growth.
\end{abstract}

Keywords: Fire growth; fire acceleration; rate of spread; nonlinear curve-fitting; stochastic differential equation

\section{Introduction}

Bushfires are a significant environmental problem. In the last decade this has become increasingly apparent, with several major conflagrations causing extensive loss of life and property, and considerable environmental damage. Regardless of the ultimate size and intensity of a bushfire, all fires start small - often originating as a point ignition, or something akin to a point ignition, and growing in a manner that is governed by the ambient environmental conditions. In addition, under certain conditions a bushfire will deposit firebrands ahead of the main front, igniting spot-fires, which can also be viewed as evolving from a point source.

Landscape fires originating from a point source accelerate until they reach a quasi-equilibrium rate of spread. This behaviour has also been confirmed in laboratory experiments (McAlpine \& Wakimoto 
1991). Attacking fires while they are in their acceleration phase, before they have attained their potential quasi-steady rate of spread, provides a greater chance of suppression success (Cheney \& Gould 1997). As such, there are good reasons to understand the likely behaviour of a fire during its acceleration phase, and mathematical modelling provides a means to improve this understanding. However, in comparison with the vast literature that deals with modelling the quasi-equilibrium rate of spread, there is little that specifically considers this acceleration phase of a fires development (Cruz et al. 2015). The way a fire develops during its acceleration phase depends on the local environmental conditions, including variations in ambient and induced wind speed and direction, and variations in moisture content and structure of the fuel. Even when a fire attains quasi-equilibrium state, its rate of spread can still exhibit considerable variation about a mean value.

However, present models of fire growth from ignition are expressed as deterministic algebraic equations, thereby neglecting variability. Parameters in such models are estimated using nonlinear regression of empirical data, and once determined they are considered as fixed; separate estimates are required for different fuel types and environmental conditions. Any resulting model lacks flexibility and should not be used in circumstances where the fuel and environmental conditions are heterogeneous.

Moreover, interactions between winds, terrain, different vegetation types and structures means that the conditions affecting the initial growth of a fire (and its longer-term propagation) can never be adequately quantified in solely deterministic terms. Coupling between the fire and the atmosphere further confound this situation. The intrinsic variability of the factors affecting the initial growth of a fire means that deterministic modelling approaches are ill-suited to modelling this aspect of fire spread. Indeed, such approaches gloss over the detailed dynamics, which are inherently stochastic in nature due to the turbulent conditions under which fires develop. Recent advances in stochastic dynamical systems theory and their applications, as well as in our understanding of dynamic fire behaviour mean that we are now able to explicitly address the acceleration phase (from a point ignition) of fire growth in terms of stochastic dynamic systems.

The models we propose in this paper appear to be the first to consider the acceleration phase of a fire's growth as a stochastic dynamical system. Such an approach permits a probabilistic interpretation of fire growth, which allows for estimation of worst-case and mean-case scenarios. Also, by explicitly accounting for the intrinsic variability of fire spread and the environmental conditions that drive it, our stochastic approach has the potential to provide greater insights into the physical processes governing fire growth, particularly in its acceleration phase.

\section{Rate of spread data}

The rate of spread (ROS) data used in this study are taken from McAlpine \& Wakimoto (1991). The data were extracted using G3DATA (Frantz 2000) and are reproduced in Figure 1. The data set represents five replicates of the ROS observed in a wind tunnel experiment. In each case the fuel load was $26.3 \mathrm{~kg} / \mathrm{m}^{3}$ and burned under the influence of a wind speed of $4.8 \mathrm{~km} /$ hour. The temperature $\left(26.7^{\circ} \mathrm{C}\right)$ and humidity $(80 \%)$ were controlled for. The fuel moisture content was measured just prior to burning, and the mean of the five replicates was $8.62 \%$ (standard deviation of 0.57 ). It is of fundamental interest that even with carefully controlled conditions and a fixed wind speed, the variation in ROS can be substantial. As already mentioned, this variation may be due to differing fuel moisture content, variability in the packing of the fuel bed for each experimental run (Finney 2004), and turbulence induced by the fire itself. We treat each of the replicates in Figure 1 as independent data sets. 


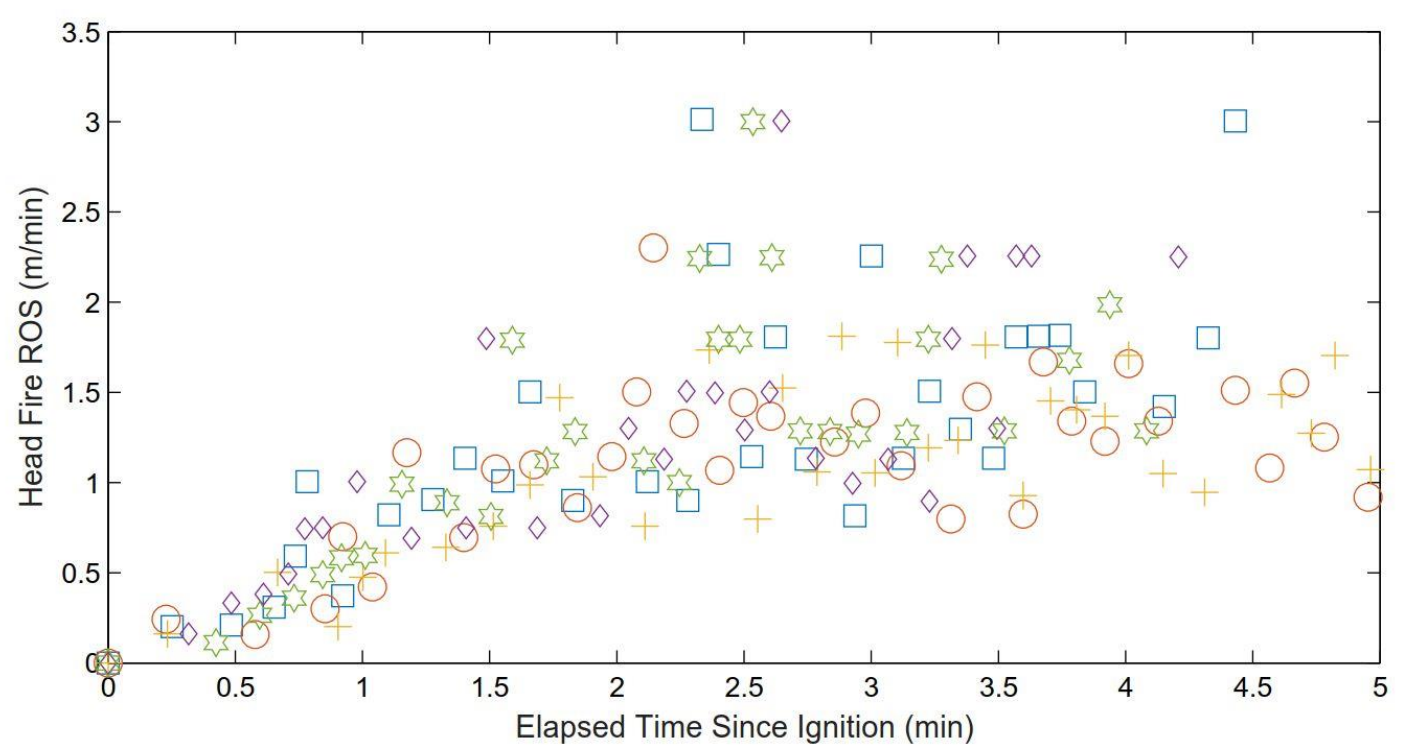

Figure 1 - Observed rate of spread for a pine needle fuel bed, with a bulk density of $26.3 \mathrm{~kg} / \mathrm{m}^{3}$ and burned at a wind speed of $4.8 \mathrm{~km} / \mathrm{hr}$. Five replications are shown, each symbol (square, circle, etc.) being a separate replication.

(Figure recreated from McAlpine \& Wakimoto (1991).)

\section{Mathematical modelling of the rate of spread}

\subsection{Nonlinear regression}

Typically, a model for the ROS, $R(t)$, is obtained by postulating a functional form with a set of unknown parameters that need to be determined. The shape of the functional form is driven by the data. McAlpine \& Wakimoto (1991) provide a summary of some well-known models that are relevant for different environmental conditions and fuel types. These include exponential-type models $R(t)=R_{e} \exp (-a / t)$ and $R(t)=R_{e}(1-\exp (-a t))$, and power-law models $R(t)=\beta t^{\alpha}$. In all cases $\alpha, \beta$ and $R_{e}$ are constants that need to be determined. In the exponential-type models, $R(t) \rightarrow R_{e}$ as $t \rightarrow \infty$, where $R_{e}$ is the asymptotic or quasi-equilibrium ROS. The power-law model never attains a quasi-equilibrium state.

Considering the data in Figure 1, it appears that there is an initial rapid increase in the ROS until reaching an inflection point, after which the acceleration begins to decrease, asymptotically approaching the quasi-equilibrium ROS. To describe this behaviour, we propose a sigmoid (logistic) function:

$$
R(t)=\frac{R_{0} R_{e}}{\left(R_{0}+\left(R_{e}-R_{0}\right) \exp (-a t)\right)}+\epsilon_{i}, \quad i=1, \ldots, n
$$

for $n$ data points $\left(t_{i}, R\left(t_{i}\right)\right)$. Here $R_{0}$ is the initial value of the ROS, the quasi-equilibrium value is $R_{e}$ and $a$ is the rate of increase of the ROS. The $\epsilon_{i}$ are independent and normally distributed errors. The basic assumptions of regression analysis are assumed except constant variance. The latter is justified since the variability in the data tends to increase with increasing ROS.

The aim is to estimate the three parameters, $R_{0}, R_{e}$ and $a$, by implementing a weighted nonlinear least-squares method to minimise the sum of the weighted square of the errors (or weighted residuals) between the observed $R\left(t_{i}\right)$ and the curve-fitted values for each $t_{i}$. The weighted sum of squares is 


$$
L_{w}\left(R_{0}, R_{e}, a\right)=\sum_{i=1}^{n} w_{i}\left(R\left(t_{i}\right)-\frac{R_{0} R_{e}}{\left(R_{0}+\left(R_{e}-R_{0}\right) \exp (-a t)\right)}\right)^{2}
$$

where $w_{i}$ are the weights. The weights determine how much each $R\left(t_{i}\right)$ value influences the final parameter estimates. Here the residuals are weighted using a bi-square weighting scheme. This method minimises a weighted sum of squares, where the weight given to each data point depends on how far the point is from the fitted line. Points near the line get full weight. Points farther from the line get reduced weight. Points that are farther from the line than would be expected by random chance get zero weight. The bi-square weights are given by

$$
w_{i}= \begin{cases}\left(1-\left(u_{i}\right)^{2}\right)^{2}, & \left|u_{i}\right|<1 \\ 0, & \left|u_{i}\right| \geq 1,\end{cases}
$$

where the $u_{i}$ are the standardised adjusted residuals. Robust fitting with bi-square weights uses an iteratively re-weighted least-squares algorithm.

The minimisation of $L_{w}$ with respect to the parameters must be carried out iteratively. The Levenberg-Marquardt method is a standard technique for solving nonlinear-least squares problems (Rowlings et al. 1998). The Levenberg-Marquardt curve-fitting method is a combination of two minimisation methods: the gradient descent method and the Gauss Newton method. In the gradient descent method, the sum of the squared errors is reduced by updating the parameters in the steepestdescent direction. In the Gauss-Newton method, the sum of the squared errors is reduced by assuming that the least squares function is locally quadratic and finding the minimum of the quadratic. The Levenberg-Marquardt method acts more like a gradient-descent method when the parameters are far from their optimal value and acts more like the Gauss-Newton method when the parameters are close to their optimal value. Both options are available in MATLAB.

\subsection{A stochastic differential equation approach}

An alternative to nonlinear regression is to reformulate the model as a differential equation (DE), specifically as a stochastic differential equation (SDE). For a dynamic process such as the spread of a fire it seems more natural to employ a DE or an SDE. Using an SDE allows for the inclusion of local environmental variability that might be due to heterogeneous fuels or changes in wind direction and strength.

With reference to Figure 1, during the early development of the fire, $R^{\prime}(t) \propto R(t)$, then as $R(t) \rightarrow R_{e}, R^{\prime}(t) \rightarrow 0$. This behaviour is captured by the logistic DE. The stochastic version of the logistic DE written in the Ito form is (Gardiner 2009),

$$
\mathrm{dR}=a R\left(1-\frac{R}{R_{e}}\right) d t+\sigma R d W, \quad R(0)=R_{0},
$$

where the parameters $R_{0}, R_{e}$ and $a$ have the same meaning as before. The first term on the righthand side is the drift and the second term is the stochastic term, often called the diffusion. The parameter $\sigma$ is the strength of the local environmental variability and $W(t)$ is a standard Wiener process with expected value $\mathbb{E}[W(t)]=0$ and variance $\mathbb{V}[W(t)]=t$. The appearance of $R(t)$ in the stochastic term assumes that the variance increases with increase in the ROS. For $\sigma=0$ the solution to the resulting (deterministic) ODE is the sigmoidal curve (1). This provides a basis for comparing the two modelling approaches.

Advances in Forest Fire Research 2018 - Page 558 
It is worth noting that equation (2) is related to the power-law nonlinear model of Dold \& Zinoviev (2009), for the modelling of fire eruptions. Under certain conditions the rate of change of the ROS satisfies

$$
\frac{\mathrm{dR}}{\mathrm{dt}}=a R\left(1-\left(\frac{R}{R_{e}}\right)^{\frac{1}{v}-1}\right)
$$

with $v<1$. Setting $v=2$, we arrive at equation (2). For this model, as with the equation (2), there exist two equilibrium points (in the absence of noise), namely $R(t)=0$ (unstable) and $R(t)=R_{e}$ (stable). This means any slight perturbation off the $R(t)=0$ equilibrium state, the ROS will accelerate towards the $R(t)=R_{e}$ state.

It should be further noted that according to equation (2), the acceleration starts off small, increasing until reaching the maximum acceleration of $R_{e} / 2$ after which the acceleration begins to decrease approaching zero as $R(t) \rightarrow R_{e}$. This is reflected in the solution curve of $R(t)$ as a concave up function during the initial phase of fire spread, followed by a change in concavity at the inflection point at $R_{e} / 2$. This is consistent with the choice of the nonlinear regression curve of equation (1).

\section{Comparing the methods}

For illustrative purposes we fit the curve to a single replicate of the ROS data (the squares) in Figure 1. The null hypothesis is tha $R_{0}, R_{e}$ and $a$ are zero, compared to the alternate hypothesis that $R_{0}, R_{e}$ and $a$ is non-zero. The estimated parameters including a $95 \%$ confidence interval (CI) is shown in Table 1.

For the ROS data, the estimated values and their CIs indicate there is statistical evidence to reject the null hypothesis, except for $R_{0}$. This is problematic as it implies the model is inadequate. To check this the integral of equation (1) was fitted to the measured distance travelled by the fire front from the time of ignition. On this ocassion all of the estimated parameters are significant (see Table 1).

Table 1 - Nonlinear curve-fitting to a single replicate (square symbol) to both the ROS and distance travelled by head of fire front

\begin{tabular}{ccccccc}
\hline \hline & \multicolumn{3}{c}{ Estimated Parameters } & & & \\
\cline { 2 - 4 } Data type & $R_{0}$ & $R_{e}$ & $a$ & RMSE & $\mathrm{R}^{2}$ & Adj $^{2}$ \\
\hline ROS & 0.203 & 1.576 & 1.622 & 0.445 & 0.648 & 0.623 \\
& $(-0.109,0.516)$ & $(1.248,1.904)$ & $(0.135,3.109)$ & & & \\
\hline Distance & 0.170 & 1.416 & 1.927 & 0.051 & 0.999 & 0.999 \\
& $(0.121,0.219)$ & $(1.366,1.466)$ & $(1.567,2.288)$ & & & \\
\hline ROS & 0.170 & 1.550 & 1.827 & 0.4263 & 0.665 & 0.654 \\
(refitted) & & $(1.305,1.809)$ & $(1.165,2.485)$ & & & \\
\hline
\end{tabular}

The reason for the difference is that the ROS, which is derived (backward differecing) from the observed distance data, is highly variable. There is an order of magnitude difference in the Root Mean Square Error (RMSE) - the sandard deviation of the weighted residuals - a measure of spread of the difference between the predicted and observed values. The estimated $\mathrm{R}^{2}$ indicates that about $65 \%$ of the variability in the ROS data is accounted for by the fitted curve and a near perfect fit for the distance data. Furthermore, plots of weighted residuals (not shown) indicate homoscedasticity of the variance and a lack of correlation in the errors.

Our aim is to compare the nonlinear regression with that of the SDE. We therefore fix the $R_{0}$ value obtained from the distance data and determined the other two parameters for the ROS data. The 
parameter estimates for the newly fitted nonlinear regression is also shown in Table 1. The CI for $R_{e}$ and $a$ are narrower, a slight reduction in RMSE and a slight increase in $\mathrm{R}^{2}$ which all amounts to an improvemnt with the refitted nonlinear curve (one less parameter to fit). A plot of the nonlinear regression curve is portrayed in Figure 2. Also shown is the 95\% prediction interval (PI) that represents an interval for a new observation of the ROS, conditioned on the observed data.

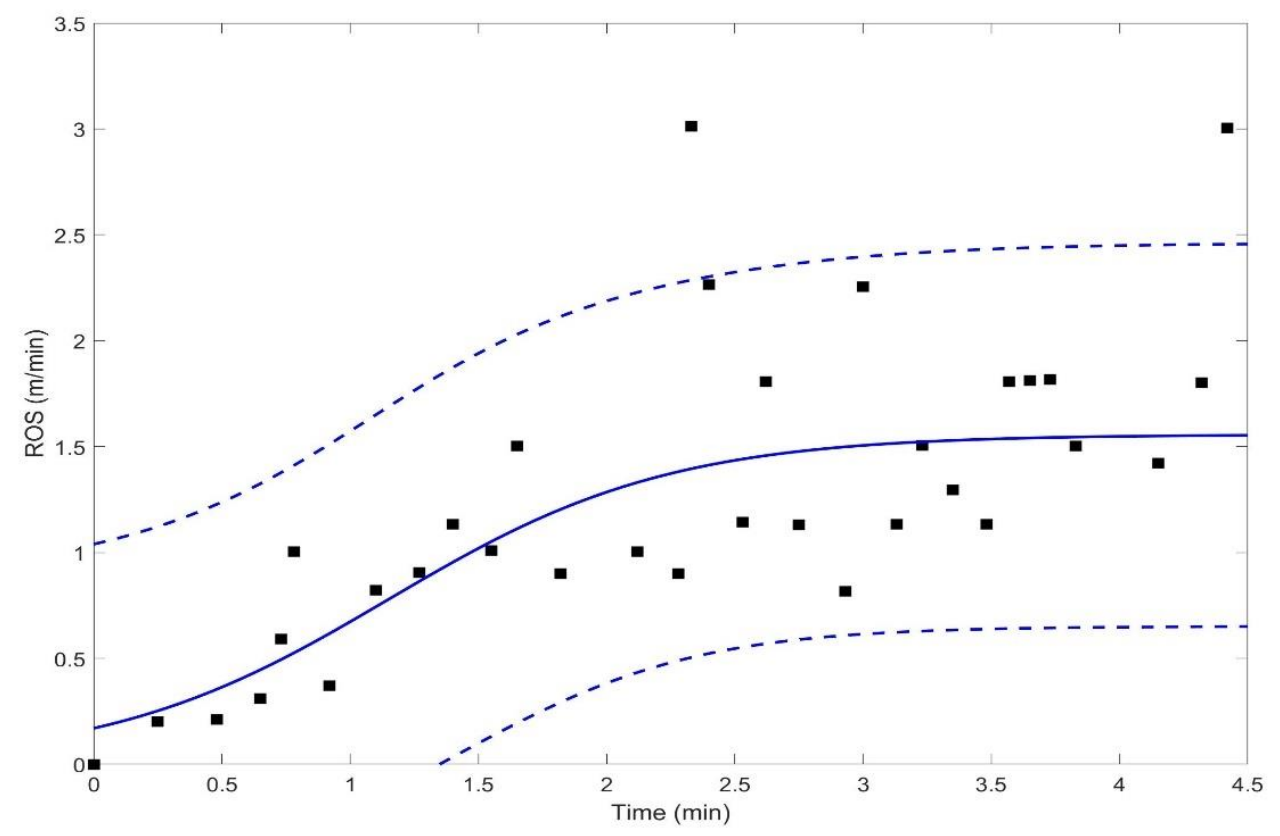

Figure 2 - Plot of the nonlinear regression curve to the ROS data including a 95\% prediction interval for a new observation of the ROS.

The solution to (2) typically requires a numerical approach, the simplest of these is the EulerMaruyama algorithm (Higham 2001). At the heart of this algorithm is the following discretisation of the SDE

$$
R_{i+1}=R_{i}+a R_{i}\left(1-\frac{R_{i}}{R_{e}}\right) \Delta t_{i}+\sigma R_{i} \Delta W_{i}
$$

where $\Delta t_{i}=t_{i+1}-t_{i}, \Delta W_{i}=W_{i+1}-W_{i}=\sqrt{\Delta t_{i}} \xi_{i}$ and $\xi_{i} \sim N(0,1)$. The numerical solution to the SDE is a single realisation, but what is of importance is to determine the expected value and variance of a large number of such realisations.

To solve equation (2) we must specify four parameters: $R_{0}, R_{e}, a$ and $\sigma$. Strictly speaking we should estimate these parameters by using maximum-likelihood methods or other related techniques directly to (2) (Kloeden et al. 1994). Here, however, we make use of the values already determined previously, and our tasks remains to find $\sigma$. This is done by searching through a range of values of $\sigma$ for which the estimated variance $\mathbb{V}[R(t)]$ (estimated at $t \approx 4.5 \mathrm{~min}$ ) is equal to the MSE (square of the RMSE). This is justifiable if we seek to make a comparison between (1) and (2).

To estimate both $\mathbb{E}[R(t)]$ and variance $\mathbb{V}[R(t)]$ we employ an Monte Carlo approach with a large number of realisations. Figure 3 depicts the results of the 100,000 realisations. The figure shows the expected ROS with a 95\% PI. Requiring $\mathbb{V}[R(t)] \approx M S E$ yields $\sigma=0.545$. It is clear that almost all of the data resides within the 95\% PI, suggesting that the proposed model provides a good description for the observed ROS. 


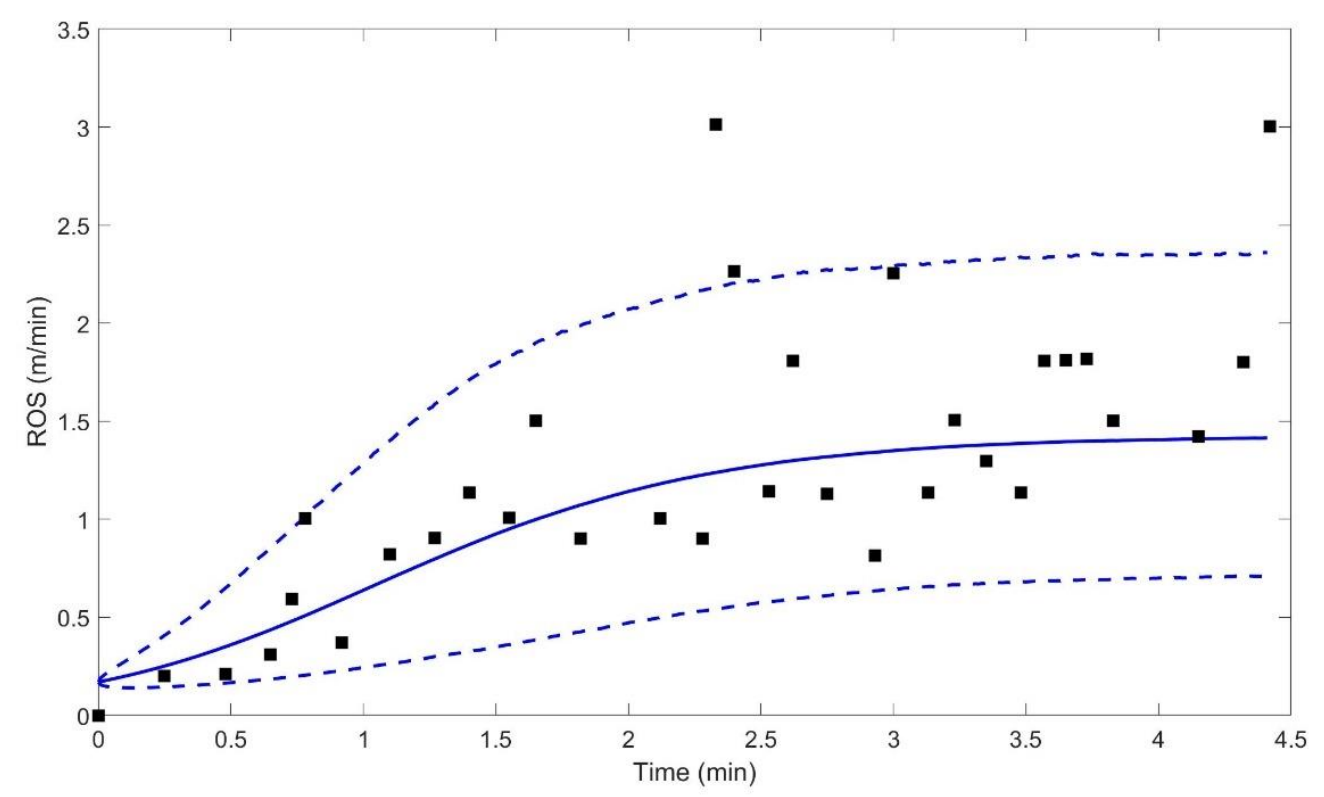

Figure 3 - Plot of the expected ROS based on 100,000 realisations of the SDE. Also shown is the 95\% prediction interval for a new realisation of the $S D E$. Here $t=0.01$.

Over the time domain relevant for the duration of the experiment, the lower of the $95 \%$ PI offers practical information about the likelihood of the fire self-extinguishing, often referred to as a 'go/nogo' threshold. The closer the lower prediction interval is to zero, the more likely the fire will selfextinguish. The upper prediction interval provides important information for fire management, as it relates to the potential for rapid initial growth of a fire in the landscape. Knowledge of the upper prediction interval can therefore give fire managers a better idea of the operational window for initial response once an ignition occurs, thus enabling more informed deployment of personnel and assets to head off a fire during its initial stages.

It is of interest to note that both of the PIs for the SDE model are roughly the same as for the nonlinear regression - the variance for both models was set to be equal once quasi-equilibrium was reached. Since they both have the same variance, any differences must be intrinsic to the way stochasticity is accounted for in each model. The major difference appears in the intial acceleration of the fire. Based on the width of the PI, the nonlinear regression predicts that self-extingishing is more likely during the first 1 min since ignition, compared to that of the SDE. The SDE on the other hand shows a rather narrower PI which increases over time. The chances of the fire to self-extinguish for the same set of parameters is greatly reduced. The result imply that a fire whose behaviour is more in accord with the SDE model is less likely to self-extinguish, and has the potential to exhibit higher rates of spread.

Computing a large number of realisations allows us to generate statistics concerning fire spread. Figure 4 shows a frequency distribution of the ROS at four different times: $1 \mathrm{~min}, 2 \mathrm{~min}, 3 \mathrm{~min}$ and 4 min. Superimposed is a continuous curve obtained via kernel smoothing to help aid in identifying the location of the distribution and the skewness that is apparent at earlier times. The distribution is narrow at earlier times, the distribution continues to evolve and by $t \approx 3 \mathrm{~min}$ the frequency distribution remains relatively unchanged, having achieved a quasi-equilibrium state. From this we can estimate the probability that the ROS is larger than a particular value, or lies within some range of values, at any time $t$ after ignition. This is in contrast to the nonlinear regression foe which the distrubution is stationary for all times. 

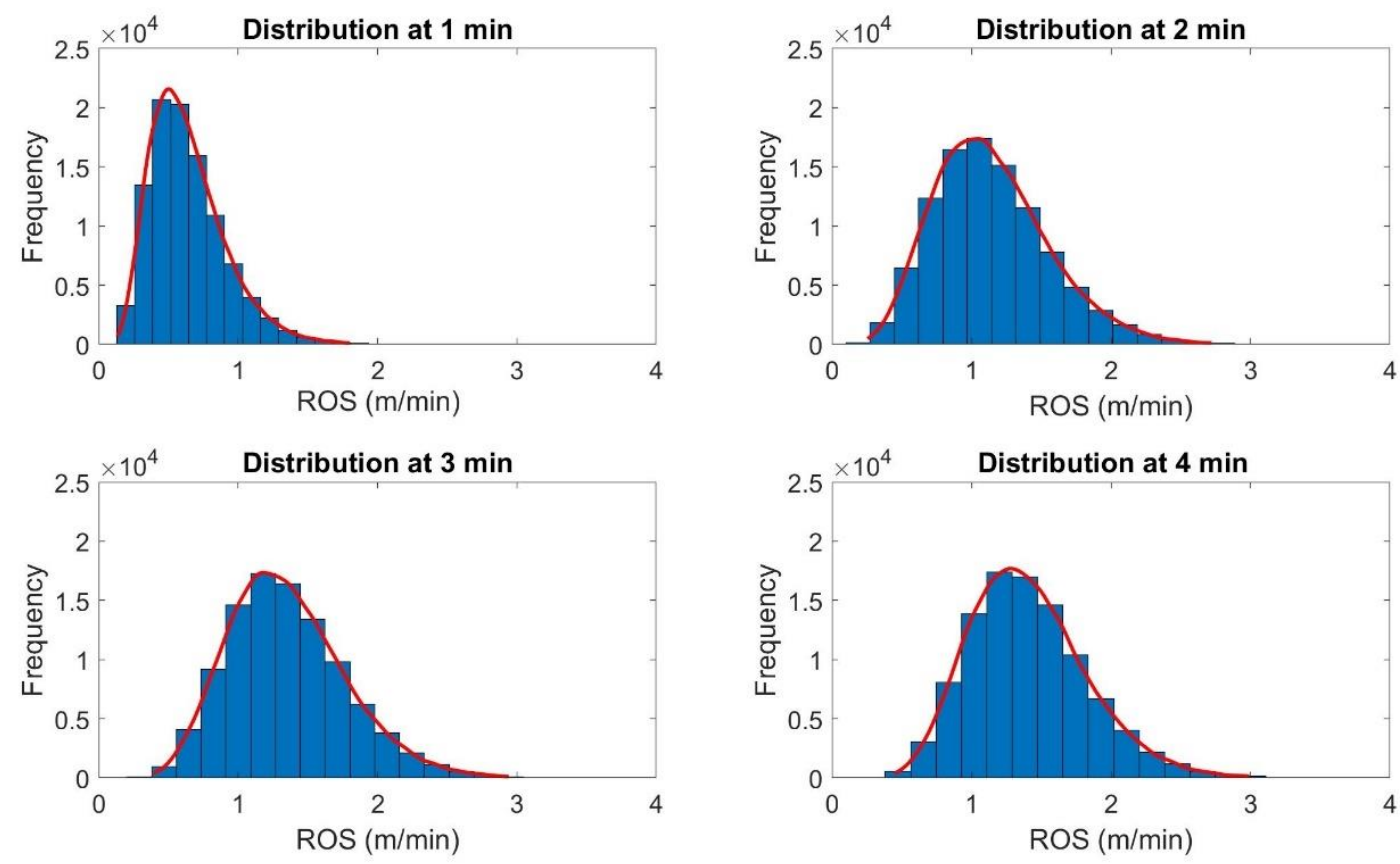

Figure 4 - The evolution of the frequency distribution for the ROS based on 100,000 realisations.

\section{Heterogeneity - preliminary analysis}

Equation (2) is a first step toward modelling the fire growth from ignition using an SDE. The manner by which stochasticity is added is a modelling issue that deserves further inquiry. Equation (2) incorporates stochasticity by aggregating the effect of growth and self-limiting of the spread, both of which are affected by local environmental variability, into a single term.

To use the nonlinear regression (1) in making predictions requires that the parameters be determined and kept fixed for a given set of environmental conditions. This means for a change in wind speed requires equation (1) be refitted, likwise if excelsior fuel is used instead of pine needles. The SDE approach (2) allows slightly more flexibility, but here too the model is inadequate as it cannot discriminate how different conditions can impact fire growth and accelaration - such as different fuel types, changes in wind strength and direction and variability in moisture content, etc. This limits the usefulness and application of both approaches.

However, some progress cna be made by treating some of the parameters as state variables. Suppose that the local quasi-equilrium rate of spread $R_{e}(t)$ is now a state variable and is a function of the wind velocity. We consider the effect of changes in wind speed and direction. The simplest of such models is described by a stochastic process

$$
\begin{gathered}
\frac{\mathrm{dR}}{\mathrm{dt}}=a R\left(1-\frac{R}{R_{e}}\right) \\
d R_{e}(t)=\sigma d W
\end{gathered}
$$

Here $d W(t)$ represents a change in the local wind direction and strength; $d W(t)>0$ helps to increase $R_{e}$, while $d W(t)<0$ to decrease $R_{e}$. The exact solution is $R_{e}(t)=R_{e}(0)+\sigma W(t)$ with $\mathbb{E}\left[R_{e}(t)\right]=R_{e}(0)$ and $\mathbb{V}\left[R_{e}(t)\right]=\sigma t$. 


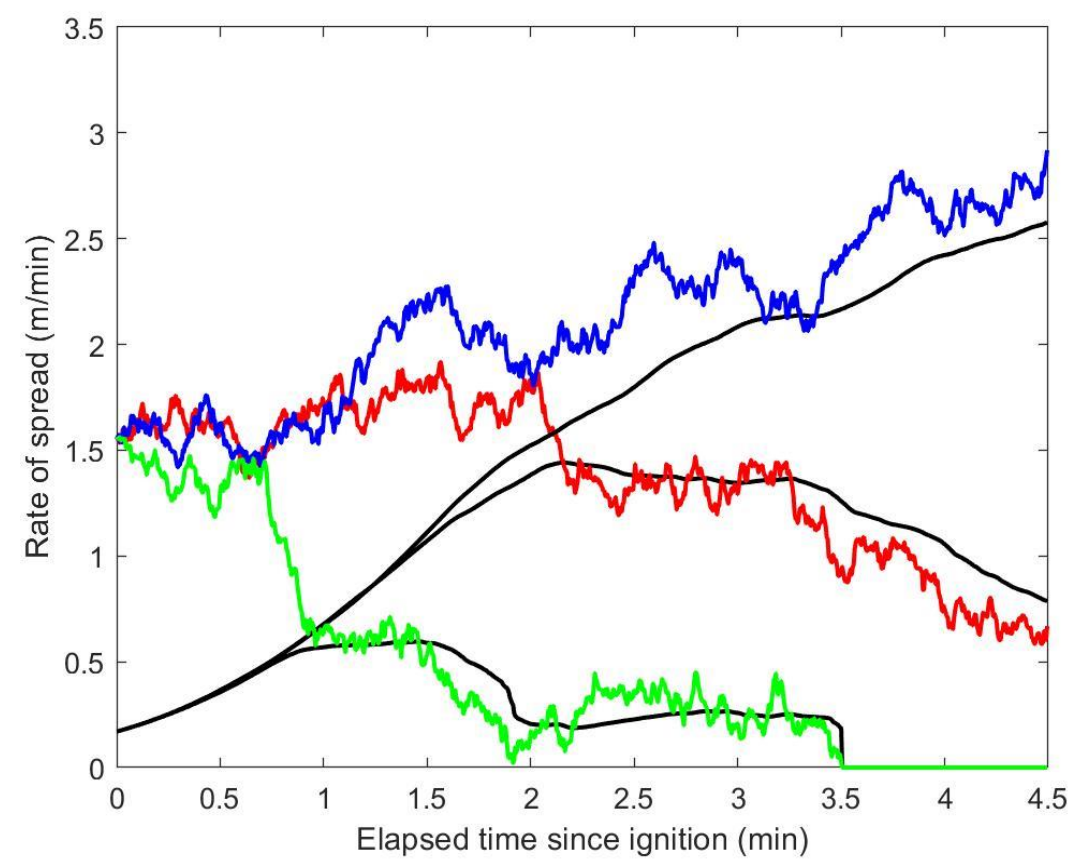

Figure 5 - Plot of three different realisations of the local quasi-equilibrium and the solution (black) for the ROS based on model (3). One realsistion is seen to self-extinguish (green).

Figure 5 depicts three realisations of equation (3) with $R_{e}(0)=1.550$. One realisation (blue) is the effect of a number of favourable wind changes that have increased the local $R_{e}(t)$ thus increasing $R(t)$. Due to a series of unfavourable wind changes in another realisation (green) the $R_{e}(t)$ is seen to have reached zero, and according to equation (3), the fire has self-extinguished.

We are now in a position to estimate the probablity that a fire may self-extinguish. Table 2 summarises the results based on 10,000 simulations of equation (3) for different levels of noise intensity. The probability increases as the ratio $R_{e}(0) / \sigma$ decreases.

Table 2 - Estimate of the probability to self-extinguish based on 10,000 realisations of equation (3)

\begin{tabular}{|c||c|c|c|c|}
\hline $\boldsymbol{\sigma}$ & 0.2 & 0.4 & 0.6 & 0.8 \\
\hline Probability & $2.6 \times 10^{-4}$ & $6.8 \times 10^{-2}$ & 0.22 & 0.36 \\
\hline
\end{tabular}

The idea that the ROS depends on the energetic ratio balance between the heat released by combustion and the energy necessary for fuel ignition is well known (Viegas, 2004). Certainly the energy required for ignition of moist fuel is greater than that of dry fuel, thus mosit fuel will have a dampening effect on $R_{e}$. Based on experiments, Rossa (2017) developed an empirical model for $R_{e}$ that is a power-law of the moisture content of the fuel. This could be incorporated into equation (3) to model the effect of a fuel with variable moisture content on the ROS as the fire develops.

Lastly, another important effect on the ROS is the curvature of the fire front. For a point-source ignition, the fire front curvature is large and as the fuel burns the radius of the fire front increases, the curvature decreases, until at such time the expanding ring of fire essentially behaves as a line fire. Point-source ignition takes a longer time than a line ignition to accelerate to $R_{e}$ (McRae 1999). However, differences can be variable and depend on the types of fuels (Finney \& McAllister 2011). Weber (1989) developed a theoretical framework for the ROS from a point-source ignition, and the conjoining of this model with that of equation (3) may be used to model the evolution of a point-source ignition to a line fire subject to variable environmental conditions. 


\section{Conclusion}

In developing models for the spread of fire it is customary to fit the data based on some prescribed functional form. Such methods provide good descriptions of the time evolution of the mean ROS from time of ignition. The aim is to obtain parameter estimates and relevant CIs of the fixed parameters for the spread rate. Alternatively, an SDE formulation is a natural way to describe the rate of change of the ROS, a quantity that can be experimentally measured. Carrying out a large number of simulations enables the construction of an empirical probability density function for the ROS, from which statistical quantities can be determined. This has practical implications for the management of fires.

The model we propose explicitly incorporates the stochastic dynamics of fire spread and environmental variability. Through our model we seek to provide a more appropriate method for assessing the likely development of fires in the landscape. It is expected that the model can be used to better inform the decisions that need to be made by fire managers through provision of a probabilistic framework that acknowledges the uncertainties inherent in fire spread.

The SDE formulation for the spread rate is particularly attractive as it provides a way to explicitly involve a fire's history (Viegas 2004). This could be accomplished by using either a distributed timedelay in the spread rate, which involves a weighted average of the spread rate taken over all previous times, or as a discrete time-lag due to the process of pyrogenesis (Dold \& Zionviev 2009). This line of research is currently being investigated.

\section{References}

Cheney, N. P. \& Gould, J. S. (1997), Fire growth and acceleration, International Journal of Wildland Fire 7, $1-5$.

Cruz, M. G., Gould, J. S., Alexander, M. E., Sullivan, A. L., McCaw, W. L. \& Matthews, S. (2015), A guide to rate of fire spread models for Australian vegetation, Technical report, CSIRO Land and Water Flagship, Canberra, ACT, and AFAC, Melbourne, Vic, 123pp.

Dold, J. W. \& Zionviev, A. (2009), Fire eruption through intensity and spread-rate interaction mediated by flow attachment, Combustion Theory and Modelling 13, 763-793.

Finney, M. A. (2004), Farsite: Fire area simulator - model development and evaluation, Technical report, USDA For. Serv., Rocky Mt. Res. Stn., Ogden, UT. Res. Pap. RMRS-RP-4 Revised. 47 p.

Finney, M. A. \& McAllister, S. S. (2011), A review of fire interactions and mass fires, Journal of Combustion 2011, article ID 548328 (14 pages).

Frantz, J. (2000), 'G3DATA', http://www.frantz.fi/software/g3data.php. [Online: accessed March 20, 2017].

Gardiner, C. (2009), Stochastic Methods: A handbook for the natural and social sciences, SpringerVerlag, Berlin.

Higham, D. J. (2001), An algorithmic introduction to numerical simulation of stochastic differential equations, SIAM. Review 43, 525-546.

Kloeden, P. E., Platen, E. \& Schurz, H. (1994), Numerical solution of SDE through computer experiments, Springer-Verlag, Berlin.

McAlpine, R. S. \& Wakimoto, R. H. (1991), The acceleration of fire from point source to equilibrium spread, Forest Science 37, 1314-1337.

McRae, D. J. (1999), Point-source fire growth in jack pine slash, International Journal of Wildland Fire 9, $65-77$.

Rossa, C. G. (2017), The effect of fuel moisture content on the spread rate offorest fires in the absence of wind or slope, International Journal of Wildland Fire 26, $24-31$. 
Rowlings, J. O., Pantula, S. G. \& Dickey, D. A. (1998), Applied regression analysis: A research tool, 2 edn, Springer-Verlag, New York.

Viegas, D. X. (2004), On the existence of a steady state regime for slope and wind driven fires, International Journal of Wildland Fire 13, 101-117.

Weber, R. O. (1989), Analytic models for fire spread due to radiation, Combustion and Flame 78, 398-408. 


\title{
Multi-scale modeling of the thermal degradation of woods
}

\author{
Guillaume Gerandi*, Virginie Tihay-Felicelli, Paul-Antoine Santoni, Valérie Leroy-Cancellieri, \\ Dominique Cancellieri \\ Université de Corse, CNRS UMR 6134 SPE, Campus Grimaldi,BP 52, 20250 Corte, France, \\ \{gerandi_g@univ-corse.fr*\}
}

\begin{abstract}
The modeling of the mass loss rate of vegetation is a key feature in models of forest fire as it allows quantifying the source term of combustible gases that supply the flames. In this work, we investigated the thermal degradation of two woods (oak and eucalyptus) using a multi-scale approach. At matter scale, experiments were carried out with a thermogravimetric analyser (TGA) under oxidizing atmosphere at several heating rates. We focussed on temperatures ranging from $150^{\circ} \mathrm{C}$ to $650^{\circ} \mathrm{C}$ in order to avoid the effect of the desication of free water and to consider only the thermal degradation of dry wood. Three kinetic mechanisms of degradation, each one consisting of four steps were developed by using the thermogravimetric results. The first one is based on an approach by constituents, the second one follows a lumped approach and the third one takes into account the formation of "active wood" which corresponds to a reduction in degree of polymerization. The kinetic parameters were calculated for each mechanism by using a genetic algorithms method. The lumped approach proved to be the mechanism that best represents the thermal decomposition of both woods. It was then tested at the material scale in the case of thermally thin wood plates heated with two radiant heat fluxes of 20 and $25 \mathrm{~kW} / \mathrm{m}^{2}$. These experiments were carried out with a cone calorimeter. The radiant heat flux was imposed at the top of the fuel sample. The mass loss rate was recorded as well as the temperature at the back surface of the wood, which was assumed equal to the temperature of the whole plate. For a radiant heat flux of $20 \mathrm{~kW} / \mathrm{m}^{2}$, the lumped approach predicted a mass loss close to the experimental results for temperatures lower than $400^{\circ} \mathrm{C}$. Above this threshold, the prediction underestimated the mass loss. Then, for a radiant heat flux of $25 \mathrm{~kW} / \mathrm{m}^{2}$, the lumped approach did not allow predicting correctly the thermal degradation of the plate. For such radiant heat fluxes, the temperature gradient within the plate has to be taken into account to test the kinetic mechanisms of degradation at material scale.
\end{abstract}

Keywords: Fuel degradation, multi-scale, fire model input

\section{Introduction}

A reliable modeling of the mass loss rate of vegetation is a key feature in detailed models of forest fires (Mell et al. 2009). The gas quantity released by the vegetal fuel corresponds to this mass loss and it supplies the flame spread during the fire. An accurate modeling of the mass loss is thus a crucial point to obtain accurate propagation models of forest fires. In detailed models of forest fire, more or less complex kinetic mechanisms are implemented to describe the thermal degradation of plant fuels. The kinetic parameters associated with these mechanisms are generally calculated from thermogravimetric experiments at different heating rates (Leroy et al. 2006, Di Blasi 2008, Poletto et al. 2012), which are much lower than those encountered in actual fires. At this scale, the experimental conditions are well-controlled but they are not representative of those encountered in actual fires. The test of these mechanisms at a larger scale with higher heating rates and where heat and mass transfers occur is essential before using them in physics-based codes of forest fires. Cone calorimeter experiments are interesting tests to assess the ability of these thermal mechanisms to model the mass loss of materials. The cone calorimeter allows reaching realistic heating rates thanks to important radiant heat fluxes and tests are performed at material scale. With this device, the thermal transfers within the material influence the degradation for thermally thick samples. For such case, the problem

Advances in Forest Fire Research 2018 - Page 566 
is complex since the degree of conversion varies within the material and its thermal parameters must be known or estimated.

There are many different approaches to modeling the thermal degradation of wood. The first approach considers that the wood decomposes in tars, chars and gases. The tars decompose then into gas and char according to two parallel reactions (referred to as secondary reactions) (Thurner and Mann 1981, Di Blasi 1993, Shafizadeh and Chin 1977). Another type of kinetic approach considers that wood consists of three biopolymers, which are hemicellulose, cellulose and lignin. Each of these polymers then decompose independently (Di Blasi 2008, Di Blasi 1993) and can be studied separately. Other approaches for the thermal degradation of wood have considered successive stages of degradation of virtual compounds. For example, Fateh et al. (Fateh et al. 2013) used a six steps successive kinetic mechanism to describe the thermal degradation of plywood whereas Benkorichi et al. (Benkorichi et al. 2017) tested a model with seven steps to represent the thermal degradation of pine needles. Finally, the last approach considers the reduction of the degree of polymerization during the degradation by introducing an "active" material. This approach was used by Shafizadeh and Bradbury (Shafizadeh and Bradbury 1979) to represent the cellulose decomposition.

In this context, we proposed to evaluate three kinetic mechanisms describing the thermal decomposition of two woods: oak and eucalyptus under oxidizing atmosphere. For this, the study was conducted at matter and material scales by using thermally thin wood plates. This kind of plates has two advantages. First of all, small pieces of this plate can be used in TGA experiments. Unlike the use of powder, the structure of the sample is not altered while avoiding temperature gradients in the material. For experiments at the material scale, using thermally thin plate avoids the temperature gradients inside the plate and the necessity of determining the thermal parameters of the material. Therefore, these experimental conditions allow to focus only on the kinetics of wood decomposition. The work presented hereafter is divided into three parts. The first section describes the experimental devices used for the experiments at both scales and the tested kinetic mechanisms. The second section presents the evaluation of the kinetic mechanisms on TGA experiments. In the last section, we present the results of the tests at material scale with regards to the kinetic mechanism, that showed the performance at matter scale.

\section{Materials and Methods}

\subsection{Experimental devices}

The multi-scale experiments were performed with two species of woods: oak (Quercus alba) and eucalyptus (Eucalyptus globulus) by using wooden sheets of $0.6 \mathrm{~mm}$ depth thick. For all experiments, the wood plates were oven-dried at $60^{\circ} \mathrm{C}$ during 24 hours to remove the moisture.

Experiments at matter scale were conducted with a PerkinElmer Pyris 1 thermogravimetric analyzer under oxidizing atmosphere. The samples were cut from the thermal thin plate in discs of $5 \mathrm{~mm}$ diameter corresponding to an initial dry mass of $4.4 \mathrm{mg}( \pm 0.6 \mathrm{mg})$ and $6.2 \mathrm{mg}( \pm 0.5 \mathrm{mg})$ for oak and eucalyptus, respectively. The discs were placed in a $33 \mu 1$ open platinum crucible. The samples were heated up to $650^{\circ} \mathrm{C}$ with five heating rates varying between 2 and $30^{\circ} \mathrm{C} / \mathrm{min}$. To focus on the thermal degradation, we only considered the mass loss recorded between 150 and $650^{\circ} \mathrm{C}$ in order to avoid the effects of the dehydration of free water. The sample temperature was controlled by a thermocouple and did not exhibit any systematic deviation from preset linear temperature programs. Three repetitions were done for each kind of wood and each heating rate.

The experiments at material scale were carried out with a cone calorimeter (Babrauskas et al. 1992). Pieces of $100 \times 100 \times 0.6 \mathrm{~mm}^{3}$ were cut in the wooden sheets for samples. Then, they were placed on ceramic wool in a sample holder mesh basket of $10 \times 10 \mathrm{~cm}^{2}$ made of stainless steel (Tihay-Felicelli et al. 2016). They were oven dried at $60^{\circ} \mathrm{C}$ during 24 hours before each experiment. The sample holder 
was then positioned on a load cell over an insulating ceramic. Before each set of experiments, the load cell was calibrated using standard weighs. Its precision was $0.1 \mathrm{~g}$ and the sampling frequency was equal to $1 \mathrm{~Hz}$. Two radiant heat fluxes $\left(20\right.$ and $\left.25 \mathrm{~kW} / \mathrm{m}^{2}\right)$ were imposed at the top of the plates. They were chosen in order to obtain fast heating rates while avoiding the flaming. The level of radiant heat flux was checked by using a flux meter before each experiment. The smoke extraction was set up with an exhaust fan at a flow rate of $24 \mathrm{l} / \mathrm{s}$. The plate temperature was recorded by two K-type thermocouples with a sampling frequency of $25 \mathrm{~Hz}$. They were placed on the back surface of the plate in order to avoid the radiation sent by the cone calorimeter. The first thermocouple was located at the center of the plate. The other one was placed at $1 \mathrm{~cm}$ from the edge of the plate. As the plates were assumed thermally thin, we considered that the temperature of the plate could be represented by the average of the values recorded with both thermocouples. The mass loss and temperature measurements were performed separately because thermocouples induce pressure on the load cell, which alter the initial mass and the mass loss recorded. At least three replicates were done for each experimental setup.

\subsection{Kinetic mechanisms}

Based on the literature (Di Blasi 1993, Shafizadeh and Chin 1977, Shafizadeh and Bradbury 1979), three kinetic mechanisms with four stages were developed for the thermal decomposition of dry wood. For all kinetic mechanisms, the degree of conversion $\alpha$ is defined as follows:

$$
\alpha=\frac{m-m_{0}}{m_{f}-m_{0}}
$$

Where $\mathrm{m}$ is the mass. The subscripts 0 and $\mathrm{f}$ correspond to the initial time and the final time respectively.

The first mechanism is based on an approach by constituents (Di Blasi 2008, Di Blasi 1993, Grønli et al. 2002) (called constituent approach). The dry wood (DW) is assumed to consist of hemicellulose, cellulose and lignin:

$$
\begin{aligned}
& D W=p_{1} \text { Hemicellulose }+p_{2} \text { Cellulose }+p_{3} \text { Lignin } \\
& \text { Hemicellulose } \rightarrow v_{1} \text { Char }+\left(1-v_{1}\right) \text { Gas } \\
& \text { Cellulose } \rightarrow v_{2} \text { Char }+\left(1-v_{2}\right) \text { Gas } \\
& \text { Lignin } \rightarrow v_{3} \text { Char }+\left(1-v_{3}\right) \text { Gas } \\
& \text { Char } \rightarrow v_{4} \text { Ash }+\left(1-v_{4}\right) \text { Gas }
\end{aligned}
$$

Where $p_{1}, p_{2}$ and $p_{3}$ represent the mass proportion of each component in the wood composition.

The reaction rates of these steps are defined as follows:

$$
\begin{aligned}
& \dot{\omega}_{i}=\left(1-\alpha_{i}\right)^{n_{i}} \cdot A_{i} \cdot \exp \left(-\frac{E_{a i}}{R \cdot T}\right) \quad \text { for } 1 \leq \mathrm{i} \leq 3 \\
& \dot{\omega}_{4}=\left(p_{1} \alpha_{1}+p_{2} \alpha_{2}+p_{3} \alpha_{3}-\alpha_{4}\right)^{n_{4}} \cdot A_{4} \cdot \exp \left(-\frac{E a_{4}}{R \cdot T}\right)
\end{aligned}
$$

The total conversion rate was then obtained with the following equation:

$$
\frac{d \alpha}{d t}=\left(1-v_{1}\right) \dot{\omega}_{1}+\left(1-v_{2}\right) \dot{\omega}_{2}+\left(1-v_{3}\right) \dot{\omega}_{3}+\left(1-v_{4}\right)\left(p_{1} v_{1}+p_{2} v_{2}+p_{3} v_{3}\right) \dot{\omega}_{4}
$$

The second mechanism (called lumped approach) (Shafizadeh and Chin 1977, Fateh et al. 2013, Benkorichi et al. 2017) considers the following steps where the dry wood is degrading in other forms of wood before leading to char and ashes: 


$$
\begin{aligned}
& D W \rightarrow v_{1} D W_{1}+\left(1-v_{1}\right) \text { Gas } \\
& D W_{1} \rightarrow v_{2} D W_{2}+\left(1-v_{2}\right) \text { Gas } \\
& D W_{2} \rightarrow v_{3} \text { Char }+\left(1-v_{3}\right) \text { Gas } \\
& \text { Char } \rightarrow v_{4} \text { Ash }+\left(1-v_{4}\right) \text { Gas }
\end{aligned}
$$

For this mechanism, the reaction rates of these steps are defined as follows:

$$
\begin{aligned}
& \dot{\omega}_{1}=\frac{d \alpha_{1}}{d t}=\left(1-\alpha_{1}\right)^{n_{1}} A_{1} \exp \left(-\frac{E_{a_{1}}}{R T}\right) \\
& \dot{\omega}_{\iota}=\frac{d \alpha_{i}}{d t}=\left(\alpha_{i-1}-\alpha_{i}\right)^{n_{i}} A_{i} \exp \left(-\frac{E_{a_{i}}}{R T}\right) \quad \text { for } 2 \leq i \leq 4
\end{aligned}
$$

The total conversion rate was then obtained as follows:

$$
\frac{d \alpha}{d t}=\left(1-v_{1}\right) \dot{\omega}_{1}+\left(1-v_{2}\right) v_{1} \dot{\omega}_{2}+\left(1-v_{3}\right) v_{1} v_{2} \dot{\omega}_{3}+\left(1-v_{4}\right) v_{1} v_{2} v_{3} \dot{\omega}_{4}
$$

In the third mechanism (called active mechanism) (Shafizadeh and Bradbury 1979, Bradbury et al. 2003), the degradation of dry wood includes a first step that accounts for the formation of "active wood", which corresponds to a reduction in degree of polymerization. Then the degradation of active wood leads to gas, tar and char. Then tars and chars undergo respectively devolatilization and oxidation:

$$
\begin{aligned}
& \text { DW } \rightarrow v_{1} \text { Active wood }+\left(1-v_{1}\right) \text { Gas } \\
& \text { Active wood } \rightarrow v_{2} \text { Char }+v_{3} \text { Tar }+\left(1-v_{2}\right) \text { Gas } \\
& \text { Tar } \rightarrow \text { Gas } \\
& \text { Char } \rightarrow v_{4} \text { Ash }+\left(1-v_{4}\right) \text { Gas }
\end{aligned}
$$

The reaction rates of this mechanism are calculated with the following relationships:

$$
\begin{aligned}
& \dot{\omega}_{1}=\frac{d \alpha_{1}}{d t}=\left(1-\alpha_{1}\right)^{n_{1}} A_{1} \exp \left(-\frac{E_{a_{1}}}{R T}\right) \\
& \dot{\omega}_{2}=\frac{d \alpha_{2}}{d t}=\left(\alpha_{1}-\alpha_{2}\right)^{n_{2}} A_{2} \exp \left(-\frac{E_{a_{2}}}{R T}\right) \\
& \dot{\omega}_{3}=\frac{d \alpha_{3}}{d t}=\left(\alpha_{2}-\alpha_{3}\right)^{n_{3}} A_{3} \exp \left(-\frac{E_{a_{3}}}{R T}\right) \\
& \dot{\omega}_{4}=\frac{d \alpha_{4}}{d t}=\left(\alpha_{2}-\alpha_{4}\right)^{n_{4}} A_{4} \exp \left(-\frac{E_{a_{4}}}{R T}\right)
\end{aligned}
$$

The total conversion rate was then obtained thanks to the following equation:

$$
\frac{d \alpha}{d t}=\left(1-v_{1}\right) \dot{\omega}_{1}+\left(1-v_{2}-v_{3}\right) v_{1} \dot{\omega}_{2}+v_{1} v_{3} \dot{\omega}_{3}+\left(1-v_{4}\right) v_{1} v_{2} \dot{\omega}_{4}
$$

The kinetic parameters ( $\mathrm{E}_{\mathrm{a}}, \mathrm{A}, \mathrm{n}$ and $\left.v\right)$ of each reaction as well as the constituent proportions were determined by using the genetic algorithms method. To determine the best set of kinetic parameter, the following objective function was minimized:

$$
\left.\left.\mathcal{F}=\sum_{i=1}^{N}\left(\alpha_{i}^{e x p}-\alpha_{i}^{c a l}\right)^{2}+\left(\frac{d \alpha}{d t}\right]_{i}^{\exp }-\frac{d \alpha}{d t}\right]_{i}^{c a l}\right)^{2}
$$

Where the exponents exp and cal represent the experimental and calculated values and $\mathrm{N}$ the number of points of the experiments. 
The performance of each set of parameters was calculated for each evaluated curve as:

$$
\text { Fit }=\sqrt{\frac{\mathcal{F}}{N}}
$$

\section{Thermal degradation at matter scale}

\subsection{Experimental results}

Figure 1 presents the evolution of the non-dimensional mass loss and the rate of change of mass (which corresponds to the mass loss rate divided by the initial mass) obtained by TGA for oak and eucalyptus with a heating rate of $10^{\circ} \mathrm{C} / \mathrm{min}$ representative of the other conditions. For both woods, the thermal degradation takes place in four steps, which is consistent with the kinetic mechanisms tested. The mass loss rate exhibits a first peak around $300^{\circ} \mathrm{C}$ followed by the most significant mass loss rate peak at about $350^{\circ} \mathrm{C}$. A shoulder is then visible around $400^{\circ} \mathrm{C}$. Finally, the last peak takes place after $500^{\circ} \mathrm{C}$ that corresponds to the char oxidation. For both woods, the non-dimensional mass loss and the rate of change of mass follow the same trends even if some differences can be observed. The different steps appear at the same temperature for both woods excepted for the char oxidation, which appears between $450^{\circ} \mathrm{C}$ and $500^{\circ} \mathrm{C}$ for oak and beyond $550^{\circ} \mathrm{C}$ for eucalyptus. This phenomenon comes from a longer duration of the shoulder observed for eucalyptus. By comparing the intensity of the rates of change of mass, we observe that the main difference occurs for the first step. The peak is nearly two times higher for oak than eucalyptus. According to literature (Grønli et al. 2002), this first reaction corresponds mainly to hemicellulose degradation, the second one to cellulose degradation whereas the shoulder is characteristic of lignin degradation. According to literature (da Silva et al. 2010, Pettersen 1984), the percentages of hemicellulose, cellulose and lignin correspond to $28 \%, 40 \%$ and $25 \%$ respectively for oak and to $17 \%, 46 \%$ and $33 \%$ for eucalyptus. Therefore, oak has more hemicellulose and less lignin than eucalyptus. This is in agreement with a higher rate of change of the first reaction for oak and with a longer duration of the shoulder for eucalyptus.
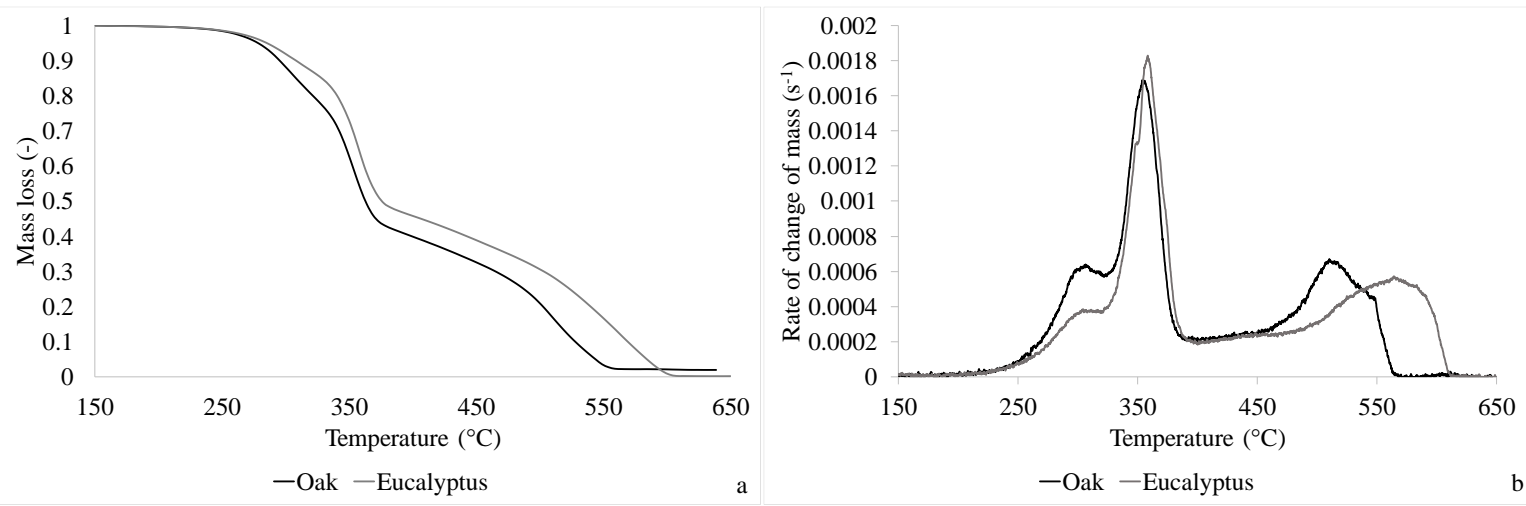

Figure 1-Evolution of a) the mass loss and b) the rate of change of mass at a heating rate of $10{ }^{\circ} \mathrm{C} / \mathrm{min}$ for oak and eucalyptus.

\subsection{Mass loss simulation}

The experimental curves obtained by TGA with the five heating rates were used to calculate the set of parameters used in the three kinetic mechanisms. Table 1, 2 and 3 present the values obtained for both woods. Considering the constituent approach, the activation energies for the degradation of hemicellulose, cellulose and lignin are around $160 \mathrm{~kJ} / \mathrm{mol}, 190 \mathrm{~kJ} / \mathrm{mol}$ and $169 \mathrm{~kJ} / \mathrm{mol}$ for both woods. These values are in agreement with literature (Chen et al. 2015, Arseneau 1971). Regarding the lumped approach, the activation energies of the three first reactions are between 161.1 and $188.9 \mathrm{~kJ} / \mathrm{mol}$ for 
oak and between 143.4 and $300.0 \mathrm{~kJ} / \mathrm{mol}$ for eucalyptus. These values are higher than those obtained for pine needles in the literature (Benkorichi et al. 2017, Fateh et al. 2017). For the active approach, the activation energies of the three first reactions are between 109 and $197 \mathrm{~kJ} / \mathrm{mol}$ and vary little for both woods. These values are higher than those found in the literature for cellulose (around $58 \mathrm{~kJ} / \mathrm{mol}$ ) (Bradbury et al. 2003). Concerning the char oxidation, there is little difference between the mechanisms. For the constituent and the lumped approaches, the activation energies are around 120 $\mathrm{kJ} / \mathrm{mol}$ whereas for the active mechanism the value is around $130 \mathrm{~kJ} / \mathrm{mol}$ for both woods. These values are close of the results of Cancellieri et al. (Cancellieri et al. 2013) but are much lower than those found by Conesa et al. (Conesa et al. 1995), for which the activation energies for char oxidation are between 179 and $219 \mathrm{~kJ} / \mathrm{mol}$. To compare the accuracy of the three mechanisms on the prediction of TGA curves, the mean value of the performance parameter calculated for the five heating rates (equation 12) was indicated in tables 1 to 3. In addition, figure 2 shows the experimental and predicted mass losses for a heating rate of $10{ }^{\circ} \mathrm{C} / \mathrm{min}$ for both woods. All kinetic mechanisms provide good predictions of the mass loss. The mass evolutions calculated with the three mechanisms by using the optimized model parameters are indeed very close to the experiments. The performance factor varies indeed between $0.96 \times 10^{-2}$ and $1.84 \times 10^{-2}$. The best agreement was obtained with the lumped approach for both woods.

Table 1 - Kinetic parameters for oak and eucalyptus with constituent approach.

\begin{tabular}{|c|c|c|c|c|c|c|c|}
\hline Species & Reactions & $\mathrm{n}_{\mathrm{i}}$ & $\begin{array}{l}\ln \left(A_{i}\right) \\
\left(s^{-1}\right)\end{array}$ & $\begin{array}{l}\mathrm{E}_{\mathrm{ai}} \\
(\mathrm{kJ} / \mathrm{mol})\end{array}$ & $v_{\mathrm{i}}$ & $\mathrm{p}_{\mathrm{i}}$ & Mean Fit (-) \\
\hline \multirow[t]{4}{*}{ Oak } & 1 & 1.43 & 29.87 & 160.99 & 0.41 & 0.26 & \multirow[t]{4}{*}{$1.37 \times 10^{-2}$} \\
\hline & 2 & 1.44 & 31.91 & 189.83 & 0.14 & 0.55 & \\
\hline & 3 & 1.46 & 21.82 & 168.39 & 0.16 & 0.19 & \\
\hline & 4 & 0.52 & 12.06 & 117.24 & 0.00 & - & \\
\hline \multirow[t]{4}{*}{ Eucalyptus } & 1 & 1.59 & 29.76 & 159.34 & 0.56 & 0.22 & \multirow[t]{4}{*}{$1.84 \times 10^{-2}$} \\
\hline & 2 & 1.47 & 31.93 & 191.06 & 0.18 & 0.55 & \\
\hline & 3 & 1.74 & 22.13 & 169.32 & 0.35 & 0.23 & \\
\hline & 4 & 0.50 & 11.63 & 121.45 & 0.00 & - & \\
\hline
\end{tabular}

Table 2 - Kinetic parameters for oak and eucalyptus with the lumped approach.

\begin{tabular}{|l|l|l|l|l|l|l|}
\hline Species & Reactions & $\mathrm{n}_{\mathrm{i}}$ & $\ln \left(\mathrm{A}_{\mathrm{i}}\right)\left(\mathrm{s}^{-1}\right)$ & $\mathrm{E}_{\mathrm{ai}}(\mathrm{kJ} / \mathrm{mol})$ & $v_{\mathrm{i}}$ & Mean Fit (-) \\
\hline \multirow{5}{*}{ Oak } & 1 & 1.17 & 29.72 & 161.06 & 0.84 & \multirow{2}{*}{$1.27 \times 10^{-2}$} \\
\cline { 2 - 6 } & 2 & 1.32 & 31.67 & 188.92 & 0.45 & \\
\cline { 2 - 6 } & 3 & 1.89 & 22.29 & 164.89 & 0.66 & \\
\cline { 2 - 6 } & 4 & 0.59 & 12.41 & 118.05 & 0.00 & \\
\hline \multirow{5}{*}{ Eucalyptus } & 1 & 1.40 & 25.97 & 143.42 & 0.88 & \multirow{2}{*}{$0.96 \times 10^{-2}$} \\
\cline { 2 - 6 } & 2 & 1.64 & 39.22 & 227.15 & 0.52 & \\
\cline { 2 - 5 } & 3 & 3.00 & 46.22 & 300.00 & 0.72 & \\
\cline { 2 - 5 } & 4 & 0.71 & 11.57 & 119.12 & 0.00 & \\
\hline
\end{tabular}


Table 4 - Kinetic parameters for oak and eucalyptus with the active approach.

\begin{tabular}{|c|c|c|c|c|c|c|}
\hline Species & Reactions & $\mathrm{n}_{\mathrm{i}}$ & $\ln \left(A_{i}\right)\left(s^{-1}\right)$ & $\begin{array}{l}\mathrm{E}_{\mathrm{ai}} \\
(\mathrm{kJ} / \mathrm{mol})\end{array}$ & $v_{i}$ & Mean Fit (-) \\
\hline \multirow[t]{4}{*}{ Oak } & 1 & 0.63 & 18.19 & 109.14 & 0.86 & \multirow[t]{4}{*}{$1.78 \times 10^{-2}$} \\
\hline & 2 & 0.82 & 24.73 & 188.81 & 0.31 & \\
\hline & 3 & 2.62 & 33.41 & 195.54 & 0.64 & \\
\hline & 4 & 0.50 & 14.58 & 128.63 & 0.04 & \\
\hline \multirow[t]{4}{*}{ Eucalyptus } & 1 & 0.65 & 19.06 & 111.51 & 0.92 & \multirow[t]{4}{*}{$1.20 \times 10^{-2}$} \\
\hline & 2 & 1.02 & 25.66 & 187.64 & 0.37 & \\
\hline & 3 & 1.82 & 33.48 & 197.68 & 0.52 & \\
\hline & 4 & 0.85 & 13.95 & 134.45 & 0.00 & \\
\hline
\end{tabular}

Oak
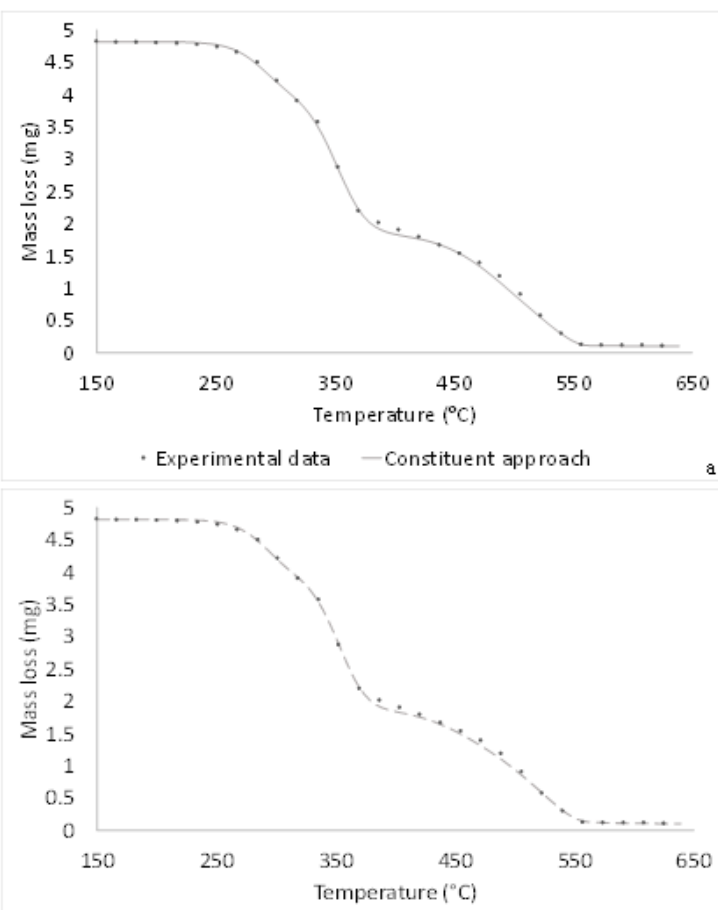

- Experimental data --Lumped approach

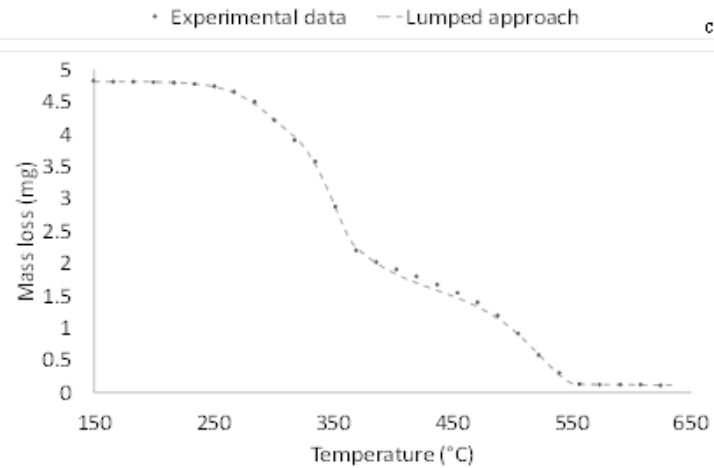

Eucalyptus

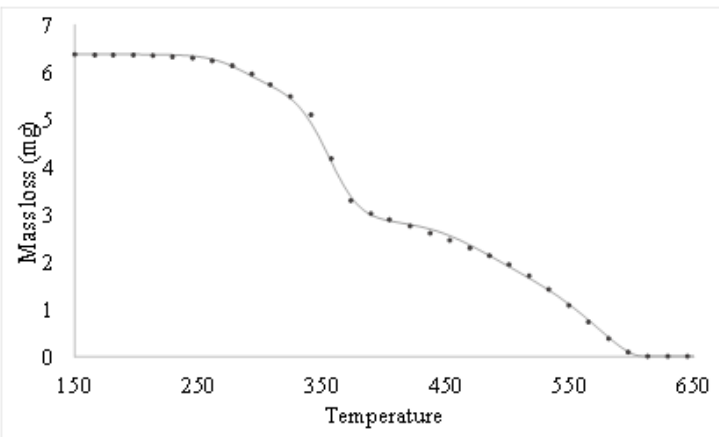

Constituent approach

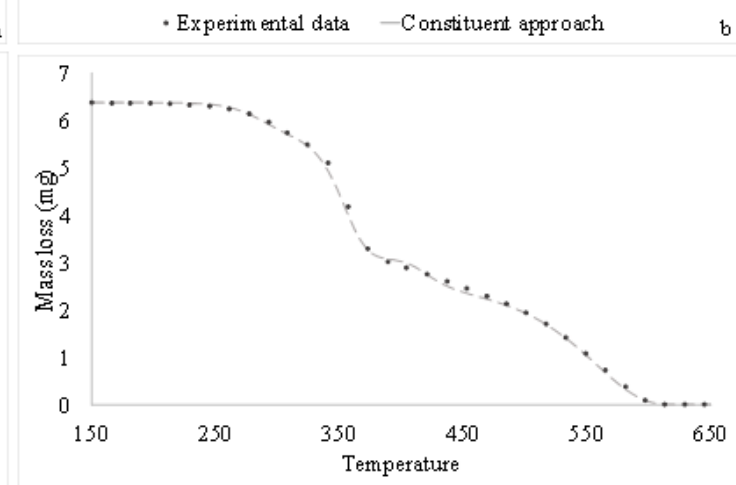

Lumped approach

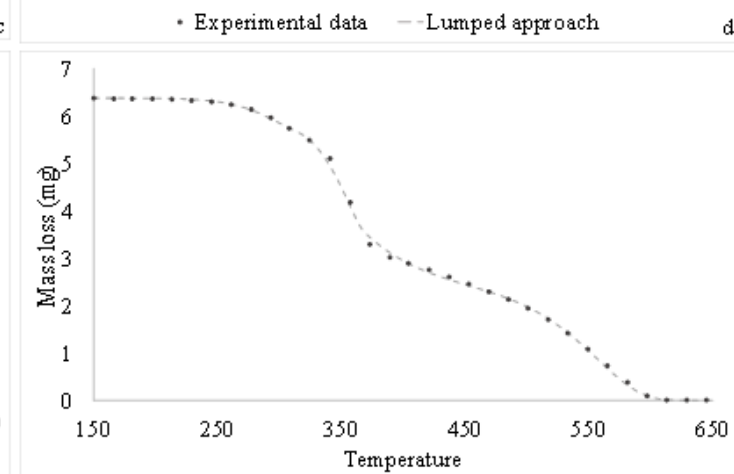

Active approach

Figure 2 - Comparison of the experimental and predicted mass losses for a heating rate of $10{ }^{\circ} \mathrm{C} / \mathrm{min}$ for oak and eucalyptus: $a$ and $b$ ) with constituent approach $c$ and d) with lumped approach $e$ and f) with active approach. 


\section{Thermal degradation at material scale}

\subsection{Experimental results}

Figure 3 presents the time evolution of the temperature and the mass loss for the plates of oak and eucalyptus exposed to a radiant heat flux of $25 \mathrm{~kW} / \mathrm{m}^{2}$. For both woods, the mean temperature recorded at the back surface of the plate follows the same trend. After the shutter opening, the plate temperature increases quasi-linearly until reaching a temperature of $615^{\circ} \mathrm{C}$. For oak, the temperature increases slightly faster than that for eucalyptus. The plates begin to degrade around $20 \mathrm{~s}$ (Fig. 3.b) which corresponds to a temperature of $230^{\circ} \mathrm{C}$ for oak and $180^{\circ} \mathrm{C}$ for eucalyptus. The mass loss is maximal near $60 \mathrm{~s}$ corresponding to a temperature around $400^{\circ} \mathrm{C}$. These results are consistent with the TGA experiments where the maximum mass loss rate occurs around $350^{\circ} \mathrm{C}$ (Fig. 1). Although no flaming occurred during the experiments, both plates lost their entire initial mass at the end of the tests. These observations are consistent with TGA experiments (Fig. 1.a).
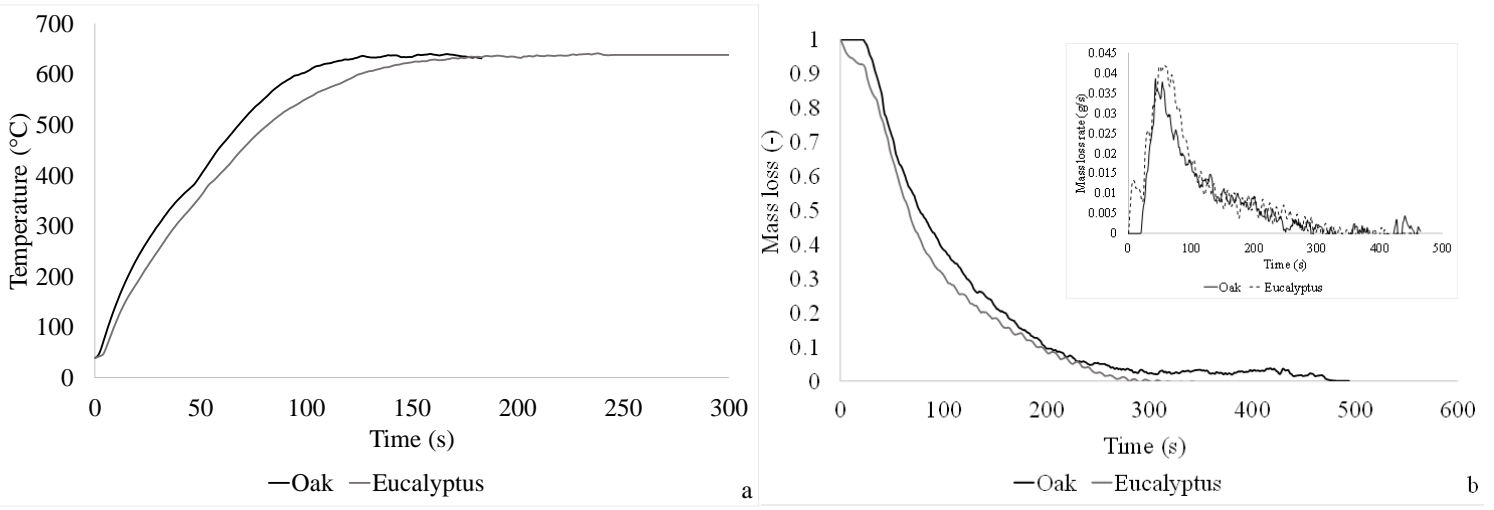

Figure 3 - Time evolution of a) the mean temperature at the back surface and b) the mass loss and mass loss rate for a radiant heat flux of $25 \mathrm{~kW} / \mathrm{m}^{2}$.

\subsection{Test of the lumped approach}

The experiments performed with the cone calorimeter were used to test the lumped approach, corresponding to the kinetic mechanism with the best performance at matter scale. Figure 4 shows the experimental and predicted mass loss obtained with the lumped approach for oak and eucalyptus for two levels of radiant heat flux $\left(20\right.$ and $\left.25 \mathrm{~kW} / \mathrm{m}^{2}\right)$. Two behaviors can be observed. For oak and eucalyptus with a radiant heat flux of $20 \mathrm{~kW} / \mathrm{m}^{2}$, until $180 \mathrm{~s}\left(400^{\circ} \mathrm{C}\right)$, the mass loss calculated with the lumped approach shows a good agreement with the experimental data. Above this threshold the model prediction overestimates the mass loss. It suggests that the model for char oxidation needs to be modified at this scale. For a radiant heat flux of $25 \mathrm{~kW} / \mathrm{m}^{2}$, a delay occurs for both woods between the simulated and experimental mass after $30 \mathrm{~s}$ (corresponding to a temperature of $200^{\circ} \mathrm{C}$ ) leading to a bad mass loss prediction. To understand why the kinetic mechanism provides a good prediction at 20 $\mathrm{kW} / \mathrm{m}^{2}$ but a bad one at $25 \mathrm{~kW} / \mathrm{m}^{2}$, the temperatures of the lower and upper faces were measured (Fig. 5). Although the wood plates are very thin $(0.6 \mathrm{~mm})$, at $25 \mathrm{~kW} / \mathrm{m}^{2}$, there is a temperature gradient inside the plate. The upper surface of the wood has indeed a higher temperature. The assumption of a thermally thin material is no more valid for this radiant heat flux. This temperature difference has strong consequences. The upper surface will indeed begin to degrade before the lower face. Taking the back face temperature to perform the mass loss prediction introduces therefore a delay. Consequently, the temperature gradient within the plate has to be taken into account to model the degradation at material scale for such radiant heat fluxes. 
Oak

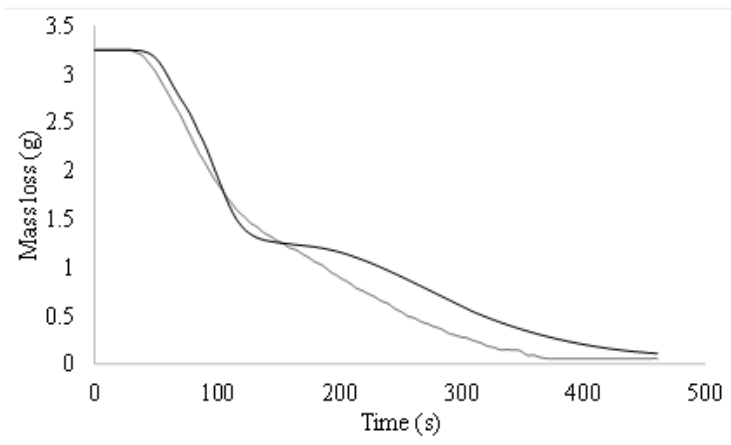

-Experimental - Simulation

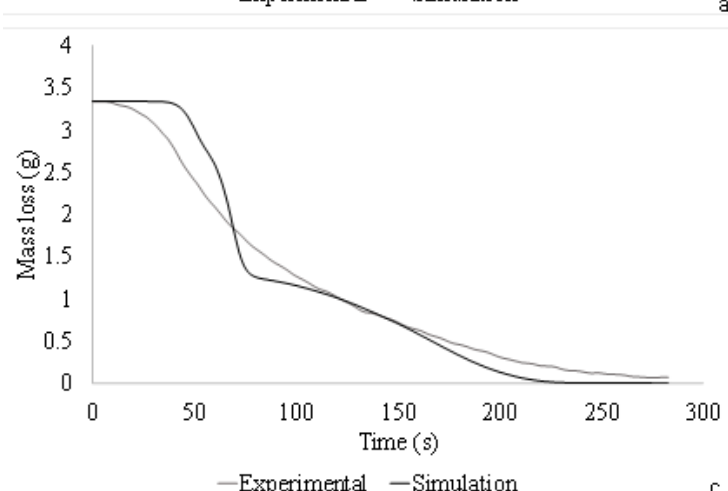

Eucalyptus
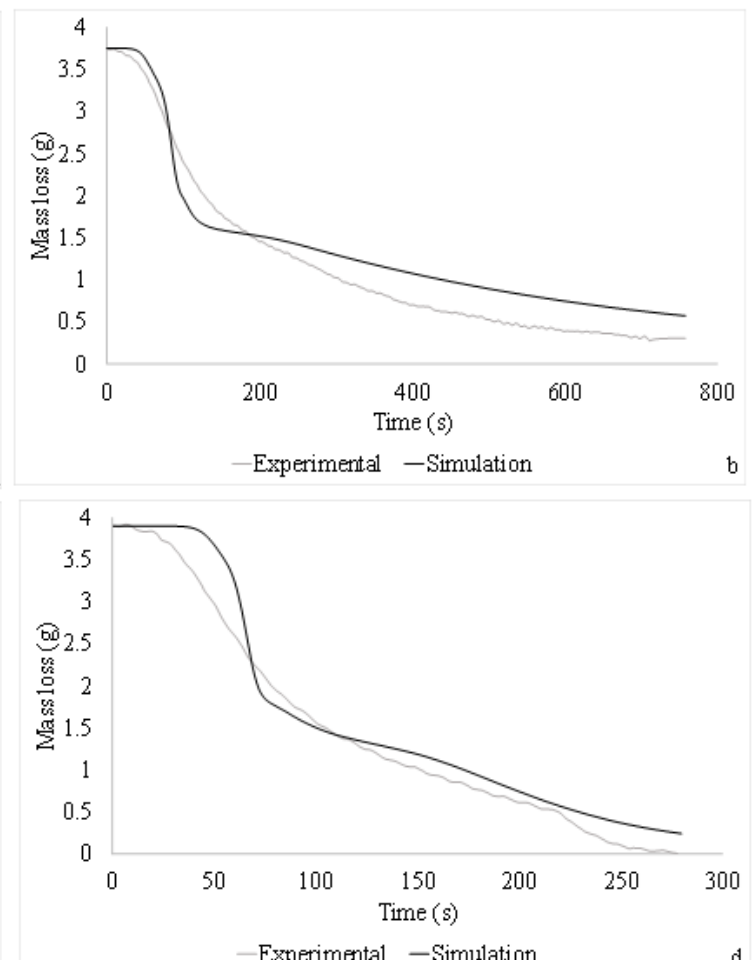

$25 \mathrm{~kW} / \mathrm{m}^{2}$

Figure 4 - Comparison between experimental and predicted mass loss for: a) oak at $20 \mathrm{~kW} / \mathrm{m}^{2}$ b) eucalyptus at 20 $\mathrm{kW} / \mathrm{m}^{2} \mathrm{c}$ ) oak at $25 \mathrm{~kW} / \mathrm{m}^{2}$ and d) eucalyptus at $25 \mathrm{~kW} / \mathrm{m}^{2}$

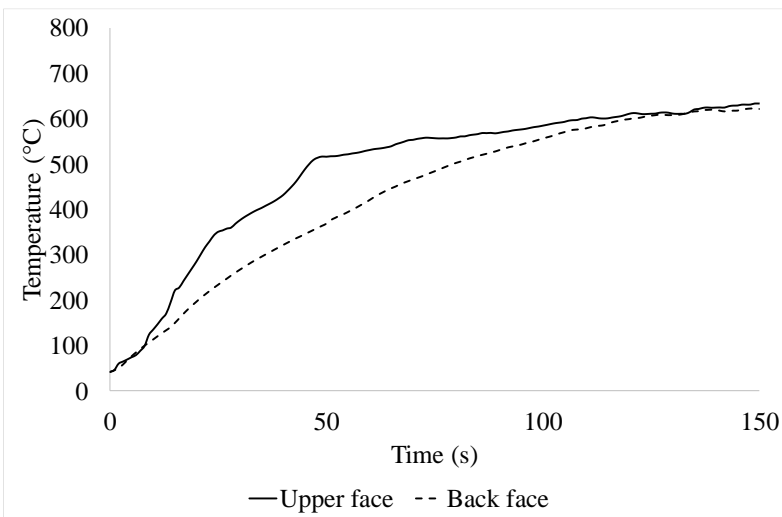

Figure 5 - Temperature recorded on the back and upper faces of the oak plate exposed to a radiant heat flux of 25 $k W / m^{2}$

\section{Conclusion}

In this article, three kinetic mechanisms were tested in order to model the thermal degradation of wood plates at matter and material scales. To focus only\{Bibliography\} on kinetics, thermally thin wood plates were used for the experiments at the different scales. The main results can be summarized as follows:

- The three models predict efficiently the thermal decomposition at matter scale for the different heating rates investigated. 
- The use of thermally thin wood plates allows testing kinetic mechanisms at $20 \mathrm{~kW} / \mathrm{m}^{2}$ without the knowledge of the thermal properties of the wood. A good concordance was observed between the experimental and predicted results except for char oxidation. For a radiant heat flux of $25 \mathrm{~kW} / \mathrm{m}^{2}$, the use of plates with $0.6 \mathrm{~mm}$ depth did not allow a thermal equilibrium within the depth of the sample. For such radiant heat flux, the temperature gradient within the plate has to be taken into account to test the kinetic mechanisms of degradation at material scale.

\section{References}

Arseneau DF (1971) Competitive Reactions in the Thermal Decomposition of Cellulose, Canadian Journal of Chemistry 49 (1971), 632-638. doi:10.1139/v71-101.

Babrauskas V, Twilley WH, Janssens M, Yusa S (1992) A cone calorimeter for controlled-atmosphere studies. Fire Materials 16, 37-43. doi:10.1002/fam.810160106.

Benkorichi S, Fateh T, Richard F, Consalvi JL, Nadjai A (2017) Investigation of thermal degradation of pine needles using multi-step reaction mechanisms, Fire Safety Journal 91, 811-819. doi:10.1016/j.firesaf.2017.03.058.

Bradbury AGW, Sakai Y, Shafizadeh F (2003) A kinetic model for pyrolysis of cellulose. Journal of Applied Polymer Science 23, 3271-3280. doi:10.1002/app.1979.070231112.

Cancellieri D, Innocenti E, Leroy-Cancellieri V (2013) WinGPYRO: A software platform for kinetic study of forest fuels. Fire Safety Journal 58, 103-111. doi:10.1016/j.firesaf.2013.01.005

Chen Z, Hu M, Zhu X, Guo D, Liu S, Hu Z, Xiao B, Wang J, Laghari M (2015) Characteristics and kinetic study on pyrolysis of five lignocellulosic biomass via thermogravimetric analysis. Bioresource Technology 192, 441-450. doi:10.1016/j.biortech.2015.05.062.

Conesa JA, Caballero J, Marcilla A, Font R (1995) Analysis of different kinetic models in the dynamic pyrolysis of cellulose. Thermochimica Acta 254, 175-192. doi:10.1016/0040-6031(94)02102-T

Da Silva MR, Machado G de O, Deiner J, Calil Junior C (2010) Permeability measurements of brazilian Eucalyptus. Materials Ressource 13, 281-286. doi:10.1590/S1516-14392010000300002.

Di Blasi C (1993) Modeling and simulation of combustion processes of charring and non-charring solid fuels. Progress in Energy and Combustion Science 19, 71-104. doi:10.1016/03601285(93)90022-7.

Di Blasi C (2008) Modeling chemical and physical processes of wood and biomass pyrolysis. Progress in Energy and Combustion Science 34, 47-90. doi:10.1016/j.pecs.2006.12.001.

Fateh T, Rogaume T, Luche J, Richard F, Jabouille F (2013) Kinetic and mechanism of the thermal degradation of a plywood by using thermogravimetry and Fourier-transformed infrared spectroscopy analysis in nitrogen and air atmosphere. Fire Safety Journal 58, 25-37.

Fateh T, Richard F, Zaida J, Rogaume T, Joseph P (2017) Multi-scale experimental investigations of the thermal degradation of pine needles. Fire and Materials 41, 654-674. doi:10.1002/fam.2407.

Grønli MG, Várhegyi G, Di Blasi (2002) Thermogravimetric analysis and devolatilization kinetics of wood. Industry and Engineering Chemistry Research 41, 4201-4208.

Leroy V, Cancellieri D, Leoni E (2006) Thermal degradation of ligno-cellulosic fuels: DSC and TGA studies. Thermochimica Acta 451, 131-138. doi:10.1016/j.tca.2006.09.017.

Mell W, Maranghides A, McDermott R, Manzello SL (2009) Numerical simulation and experiments of burning douglas fir trees. Combustion and Flame 156, 2023-2041.

Pettersen RC (1984) The Chemical Composition of Wood. The Chemistry of Solid Wood, 57-126. 
Poletto M, Zattera AJ, Santana RMC (2012) Thermal decomposition of wood: Kinetics and degradation mechanisms. Bioresource Technology 126, 7-12.

Shafizadeh F, Chin PPS (1977) Thermal Deterioration of Wood. Wood Technology: Chemical Aspects, 57-81. doi:10.1021/bk-1977-0043.ch005.

Shafizadeh F, Bradbury AGW (1979) Thermal degradation of cellulose in air and nitrogen at low temperatures. Journal of Applied Polymer Science 23, 1431-1442.

Tihay-Felicelli V, Santoni PA, Barboni T, Leonelli L (2016) Autoignition of Dead Shrub Twigs: Influence of Diameter on Ignition. Fire Technology Journal 52, 897-929. doi:10.1007/s10694-0150514-x.

Thurner F, Mann U (1981) Kinetic investigation of wood pyrolysis. Industry Engineering Chemical Process Development 20, 482-488. doi:10.1021/i200014a015. 


\title{
New Lagrangian surface forest fire propagation model
}

\author{
M. Ambroz*, K. Mikula \\ Department of Mathematics and Descriptive Geometry, Faculty of Civil Engineering, Slovak \\ University of Technology, Radlinského 11, 81005 Bratislava, Slovak Republic, \\ \{ambroz.martin.ml@gmail.com*,mikula@math.sk\}
}

\begin{abstract}
We introduce new Lagrangian surface forest fire spread model, which is based on the evolution of a three dimensional surface curve representing the fire perimeter on the topography. We split the general motion of any point of the surface curve into the normal and tangential directions. The velocity in the normal direction is given by the rate of spread, i.e. it depends on the local characteristics of fuel, terrain slope, wind speed and velocity and shape of the fire perimeter with respect to the topography (geodesic and normal curvature). The velocity in the tangential direction, which does not change the shape of the fire perimeter, is used to redistribute the curve points asymptotically uniform along the curve. This surface curve is projected into the horizontal plane as a planar curve, which evolution is numerically computed and evolved curve is mapped back to the surface. For the numerical computations we discretize the arising intrinsic partial differential equation by a semi-implicit scheme in curvature term and for the advection term we use the so-called inflowimplicit/outflow-explicit approach and implicit upwind technique which guarantee solvability of linear systems by efficient tridiagonal solver without any time step restriction and robustness with respect to singularities. Our fast and reliable treatment of topological changes (splitting and merging of the curves) with computational complexity $O(n)$ is described and presented on examples as well. We demonstrate the influence of the fire spread model parameters on a testing and real topography.
\end{abstract}

Keywords: forest fire modelling, surface curve, curve evolution, topological changes, inverse modelling

\section{Introduction}

In the mathematical literature, there exists a number of studies about the evolution of planar and surface curves with many various applications. We distinguish two main approaches to handle the curve evolution problems, the so-called Lagrangian (direct or vector-based) approach, see e.g. (Dziuk, 1999), (Mikula \& Ševčovič, 2004), (Balažovjech, et al., 2012) and the so-called Eulerian (level-set or raster-based) approach, see e.g. (Sethian, 1999), (Osher \& Fedkiw, 2002). In the Eulerian level-set approach, one solves the problem of curve evolution in a 2-D computational domain which is usually discretized by a uniform grid and the number of discrete unknowns is proportional to the number of such 2-D grid points. The evolving curve is then obtained implicitly, as the zero isoline of 2-D + time level set function. In the Lagrangian approach one evolves directly the curve discretization points, so it is spatially 1-D problem and thus computationally much simpler and faster than the level-set method. However, the Lagrangian approaches need the so-called tangential grid point redistribution (Hou, et al., 1994), (Barrett, et al., 2009), (Mikula \& Ševčovič, 2001), (Mikula \& Urbán, 2012) and efficient algorithm for the detection and treatment of topological changes during the evolution (Balažovjech, et al., 2012), (Mikula \& Urbán, 2012), (Benninghoff \& Garcke, 2014), which are on the other hand automatically handled by the level set method (Sethian, 1999), (Osher \& Fedkiw, 2002). When the Lagrangian methods are tangentially stabilized and are able to treat the topological changes fastly, they represent really efficient approaches to 2-D or surface curve evolution

We introduce a new surface forest fire spread model, which is based on the evolution of a three dimensional surface curve representing the fire perimeter on the topography. The mathematical model for curve evolution is based on the empirical laws of the fire propagation influenced by fuel, wind, 
terrain slope and the fire perimeter shape with respect to the topography (geodesic and normal curvature). Our fast treatment of topological changes (splitting and merging of the curves) is described and presented on examples as well. We demonstrate the influence of the fire spread model parameters on a testing and real topography and we reconstruct a simulated grassland fire.

\section{Surface forest fire propagation model}

In our model we use the so-called Lagrangian approach to the evolution of a surface curve, representing a fire perimeter. The surface is given as a graph of a discrete topography function $\varphi(\mathbf{x})=$ $\varphi(\mathrm{x}, \mathrm{y})$, which is given, e.g., by a digital model of terrain. The discrete surface curve points $\mathbf{X}=(\mathbf{x}$, $\varphi(\mathbf{x}))$ are evolving in the normal direction, given by outer unit normal vector $\mathcal{N}$ by following formula

$$
\mathbf{X}_{t}=\mathcal{V N},
$$

where $\mathcal{V}$ is the normal velocity of the 3-D surface curve. The outer unit normal vector $\mathcal{N}$ is given as $\mathcal{N}=\mathcal{T} \times \mathcal{N}_{\mathcal{M}}$, where $\mathcal{T}=\partial \mathbf{X} / \partial s$, with $s$ being the arc-length parameter and $\mathcal{N}_{\mathcal{M}}=(1,0, \partial \varphi / \partial x) \times(0,1, \partial \varphi / \partial y)$. For better clarity see Figure 1, where we illustrate also the curvature vector $\mathcal{K}=\partial^{2} \mathbf{X} / \partial s^{2}$ and its components, geodesic $K_{g}=\mathcal{K} \cdot \mathcal{N}$ and normal curvature $K_{n}=\mathcal{K} \cdot \mathcal{N}_{\mathcal{M}}$, which are useful in the radiation heat influence modeling.

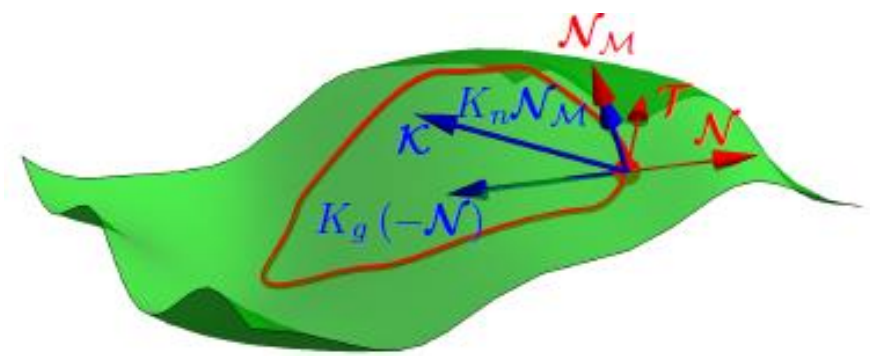

Figure 1 - Surface curve and the outer unit normal vector $\mathcal{N}$, unit tangent vector $\mathcal{T}$, normal to the surface $\mathcal{N}_{\mathcal{M}}$, curvature $\mathcal{K}$, geodesic curvature $K_{g}$ and normal curvature $K_{n}$.

Design of the normal velocity $\mathcal{V}$ is crucial for a reliable propagation model. We propose a simple formula considering fuel burnability, topography slope, wind velocity and direction and the shape of the fire perimeter with respect to the topography

$$
\mathcal{V}=\mathcal{F}\left(\delta_{\mathcal{F}}-\delta_{g} K_{g}+\delta_{n} K_{n}\right),
$$

where $\mathcal{F}$ is an external driving force, $K_{g}$ is a geodesic curvature, $K_{n}$ is a normal curvature and $\delta_{\mathcal{F}}$ , $\delta_{g}$ and $\delta_{n}$ are weights of the external force, geodesic curvature and normal curvature. This formula provides the dominant role for the external force $\mathcal{F}$ influenced by local fire perimeter shape ( $K_{g}$ and $K_{n}$ ). It also ensures that the fire perimeter will not burn into the unburnable regions due to the curvatures.

Fire perimeters curvature influences the overall rate of spread, see (Weber, 1989) and (Hilton, et al., 2017). In our approach we split the curvature $\mathcal{K}$ into geodesic $K_{g}$ and normal curvature $K_{n}$. In case of flat terrain $\left(K_{n}=0\right)$ and linear fire perimeter $\left(K_{g}=0\right)$ the fire spread depends only on the external force $\mathcal{F}$. Considering non-linear fire perimeter, i.e. the curve with convex and concave parts in the tangent plane, the geodesic curvature $K_{g} \neq 0$. In parts with $K_{g}<0$ the fire perimeter is accelerated due to radiant heat accumulation and in parts with $K_{g}>0$ the radiation heat dissipation slows down the fire perimeter propagation. If the topography is a valley or a ridge, then $K_{n} \neq 0$ and it expresses the topography influence to radiation heat transfer. In the valley $K_{n}>0$ and causes the fire spread acceleration due to the accumulated radiation heat. On the other hand, on the ridge $K_{n}<0$ slows the fire spread down, since the radiation is dissipated. 
The external driving force $\mathcal{F}$ is given by the following formula

$$
\mathcal{F}=f f_{w}(\mathbf{w} \cdot \mathcal{N}) f_{s}(\mathbf{s} \cdot \mathcal{N})
$$

where $f$ is a fuel influence, $f_{w}(\mathbf{w} \cdot \mathcal{N})$ is a wind influence and $f_{s}(\mathbf{s} \cdot \mathcal{N})$ is a slope influence on the rate of spread, with w being a 3-D wind vector, s being a 3-D slope vector and $\mathcal{N}$ being an outer unit normal vector of the surface curve.

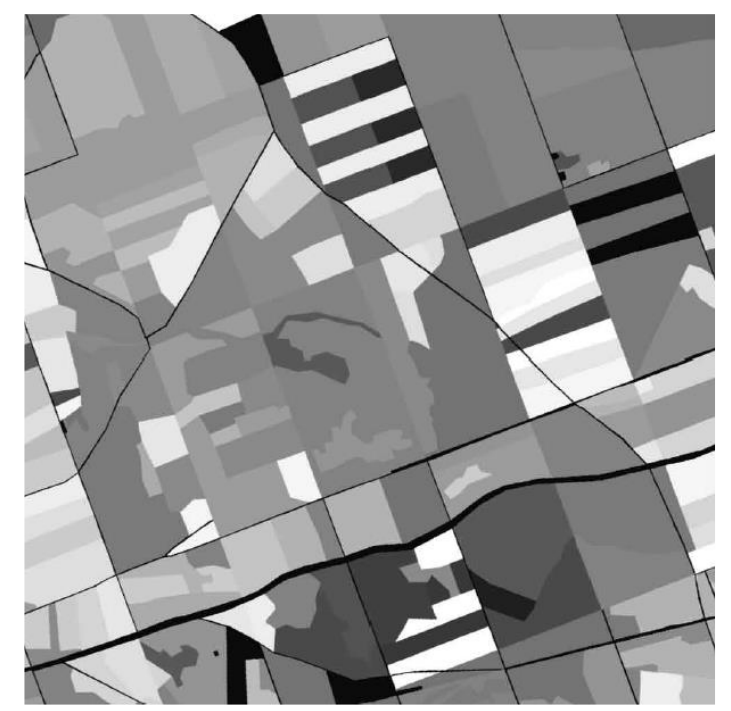

Figure 2 - The example of a 2-D scalar function $f(x)$, created as a weighted combination of selected factors, such as species, age and bulk density.

The fuel influence $f$ is in fact a scalar function $f(\mathbf{x})$ given over the whole computational domain. The values of such scalar function ranges from 0 (black color) to 255 (white color). These values express the spatial variability of rate of spread no wind and flat terrain conditions. According to (Krasnow, et al., 2009) we suppose, that the fuel influence function $f(\mathbf{x})$ is given by a weighted combination of the most important factors, such as species, age, bulk density, fuel moisture, vertical arrangement, fuel loading and compactness. Some of these factors can be determined by a typological forestry maps, like the species, age or bulk density. Their combination creates the fuel influence function $f(\mathbf{x})$, see Figure 2, where the young, dense coniferous forest is considered to be with the highest spread rate.

Wind influence on the fire spread is non-negligible. Wind increases the fuel preheating, drying and it supplies the oxygen to the fire. If the wind direction and fire spread direction are the same, the rate of spread will increase. On the other hand, in case of wind blowing against the fire spread, the rate of spread will decrease. Our model requires the 3-D wind vector $w$ which we obtain from measured 2-D wind vector $\mathbf{w}^{2 D}$ using formula

$$
\mathbf{w}=\left(\mathbf{w}^{2 D}, \nabla \varphi \cdot \mathbf{w}^{2 D}\right) \frac{\left|\mathbf{w}^{2 D}\right|}{\sqrt{\left|\mathbf{w}^{2 \mathbf{D}}\right|^{2}+\left(\nabla \varphi \cdot \mathbf{w}^{2 D}\right)^{2}}}
$$

Such vector is always parallel to the topography and $|\mathbf{w}|=\left|\mathbf{w}^{2 D}\right|$. According to (Viegas, et al., 2002), (Scott \& Burgan, 2005), the wind influences the rate of spread exponentially, so we consider the scalar product of the wind vector wand the outer normal vector $\mathcal{N}$ as an exponent of a function $f_{w}(\mathbf{w} \cdot \mathcal{N})$ in the form

$$
f_{w}(\mathbf{w} \cdot \mathcal{N})=e^{\lambda_{w}(\mathbf{w} \cdot \mathcal{N})},
$$


where $\lambda_{w}$ is a positive parameter. If those vectors are perpendicular, $\mathbf{w} \cdot \mathcal{N}=0$, the external force $\mathcal{F}$ is not influenced by wind, because $f_{w}=1$. If the vectors are parallel, $\mathbf{w} \cdot \mathcal{N}=|\mathbf{w}|, f_{w}=e^{\lambda_{w}|\mathbf{w}|}$, the influence of wind is the strongest. In all other cases, the exponent of the function $f_{w}(\mathbf{w} \cdot \mathcal{N})$ is given by a projection of the wind vector w onto the outer normal vector $\mathcal{N}$, see Figure 3.
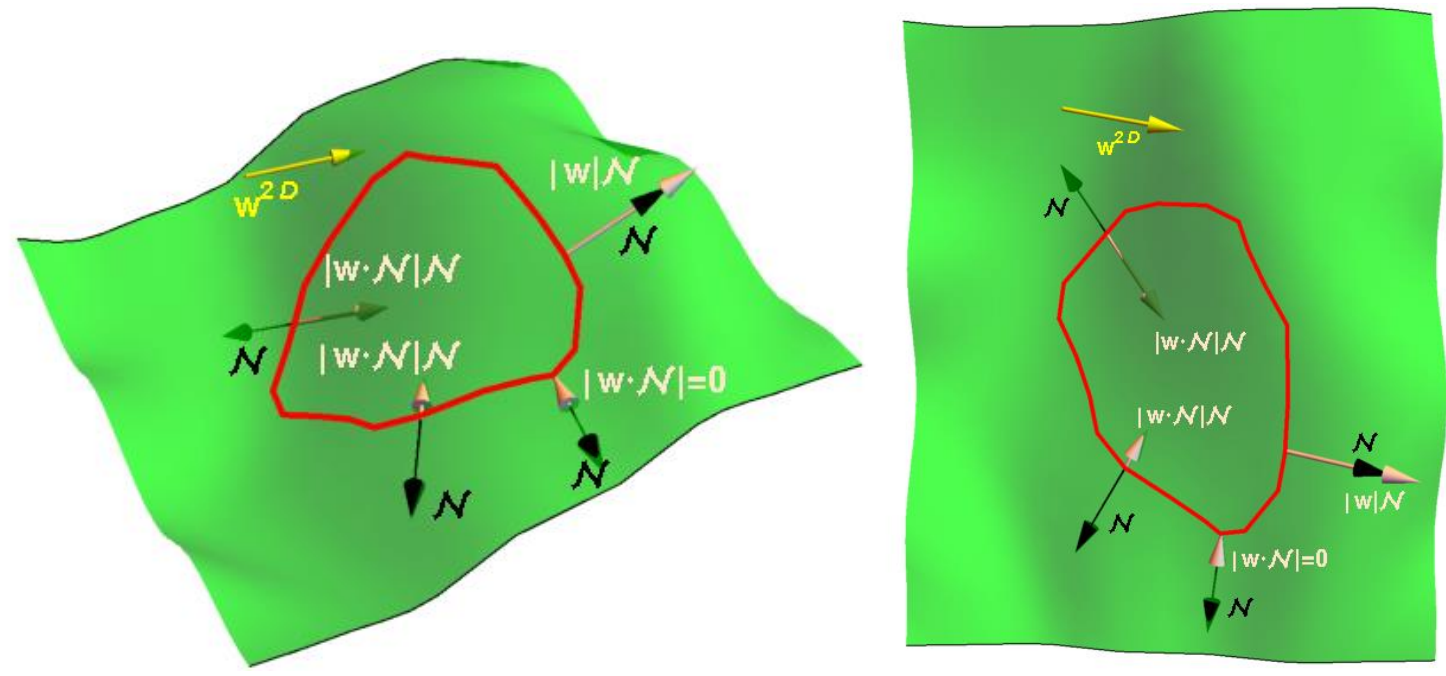

Figure 3 - The example of a projection of the wind vector $\mathrm{w}$ (white) to the normal vector $\mathcal{N}$ (black). The wind vector $\mathrm{w}$ is computed from a measured two-dimensional wind vector $\mathrm{w}^{2 D}$ (yellow). Then the dot product $\mathrm{w} \cdot \mathcal{N}$ can result in various cases, including $\mathbf{w} \cdot \mathcal{N}=0$.

The topography slope increases the radiation and convection heat transfer up the slope. Similarly to wind, slope can increase or decrease the rate of spread. The uphill fire spread rate is increased, while the downhill fire spread is slowed down. The topography slope is given by a gradient of the topography function $\nabla \varphi$. Since our model requires a 3-D vector of slope, we use formula similar to (4)

$$
\mathbf{s}=\left(\nabla \varphi,|\nabla \varphi|^{2}\right) \frac{|\nabla \varphi|}{\sqrt{|\nabla \varphi|^{2}+\left(|\nabla \varphi|^{2}\right)^{2}}}=\frac{\left(\nabla \varphi,|\nabla \varphi|^{2}\right)}{\sqrt{1+|\nabla \varphi|^{2}}} .
$$

According to (Viegas, et al., 2002), (Scott \& Burgan, 2005), (Butler, et al., 2007) the slope influence to the rate of spread is exponential and depends on the projection of the slope vector $\mathbf{s}$ onto the outer normal vector $\mathcal{N}$

$$
f_{s}(\mathbf{s} \cdot \mathcal{N})=e^{\lambda_{s}(\mathbf{s} \cdot \mathcal{N})},
$$

where $\lambda_{\mathrm{s}}$ is a positive parameter.

\section{Numerical scheme}

For numerical computation we follow (Mikula \& Ševčovič, 2006) and we numerically solve the evolution of a projected planar curve. It means, we project the 3-D surface curve into the 2-D planar curve and the evolution is computed for every discrete planar curve point $\mathbf{x}=(\mathrm{x}, \mathrm{y})$. Time evolution of the curve point $\mathbf{x}$ is given by following formula

$$
\mathbf{x}_{t}=\beta \mathbf{N}+\alpha \mathbf{T},
$$


where $\beta$ is a planar curve velocity in a direction of is outer unit normal vector $\mathbf{N}, \alpha$ is a planar curve velocity in a tangent direction, $\mathbf{T}$ is a unit tangent vector. This formula splits the general motion of any point of the 2-D projected planar curve into the normal and tangential directions. The velocity $\beta$ in the normal direction $\mathbf{N}$ changes the shape of the curve. The velocity in the tangential direction, which does not change the shape of the fire perimeter, is used to redistribute the curve points asymptotically uniformly along the curve, where we follow (Mikula \& Ševčovič, 2001), (Mikula \& Ševčovič, 2004), (Mikula \& Ševčovič, 2006), (Mikula \& Urbán, 2014).

Our evolution approach allows us to prescribe the normal velocity $\mathcal{V}$ of the 3 -D surface curve (in the previous section) and to effectively compute the evolution of the projected 2-D planar curve. To project the normal velocity $\mathcal{V}$ of the surface curve to the normal velocity $\beta$ of the planar curve we use the formula

$$
\beta=\mathcal{V} \sqrt{\frac{1+(\nabla \varphi \cdot \mathbf{T})^{2}}{1+|\nabla \varphi|^{2}}},
$$

where $\nabla \varphi$ is a gradient of a topography function $\varphi(\mathbf{x})$.

Numerical discretization is based on the flowing finite volume method. In order to get a discrete form of (8), using the Frenet equation $\mathbf{x}_{s s}=-k \mathrm{~N}$ we rewrite (8) to the form of an intrinsic partial differential equation

$$
\mathbf{x}_{t}=\varepsilon \mathbf{x}_{s s}+\alpha \mathbf{x}_{s}+w \mathbf{x}_{s}^{\perp},
$$

where

$$
\begin{aligned}
& \varepsilon=\frac{\mathcal{F} \delta_{g}}{1+(\nabla \varphi \cdot \mathbf{T})^{2}}, \\
& w=\mathcal{F}\left(\delta_{\mathcal{F}} \sqrt{\frac{1+(\nabla \varphi \cdot \mathbf{T})^{2}}{1+|\nabla \varphi|^{2}}}+\delta_{g} \frac{\mathbf{T}^{T} H(\varphi) \mathbf{T}(\nabla \varphi \cdot \mathbf{N})}{\left(1+(\nabla \varphi \cdot \mathbf{T})^{2}\right)\left(1+|\nabla \varphi|^{2}\right)}\right. \\
& \left.+\delta_{n} \frac{\mathbf{T}^{T} H(\varphi) \mathbf{T}}{\sqrt{1+(\nabla \varphi \cdot \mathbf{T})^{2}}\left(1+|\nabla \varphi|^{2}\right)}\right) \text {, } \\
& \alpha_{s}=\langle k \beta\rangle_{\Gamma}-k \beta+\omega\left(\frac{L}{g}-1\right),
\end{aligned}
$$

where $\langle k \beta\rangle_{\Gamma}$ is average value of $k \beta$ along the projected planar curve, $k$ is a curvature of planar curve, $\omega$ is parameter determining how fast the redistribution becomes uniform, $L$ is total length of planar curve and $g$ is a so-called local length of 2-D planar cuve, for details see (Ambroz, et al., Submitted).

We use a semi-implicit scheme in curvature term. For the advection term we use the so-called inflow-implicit/outflow-explicit approach (Mikula, et al., 2014) which guarantee solvability of arising linear systems by efficient tridiagonal solver without any computational time step restriction. In the end, we obtain the cyclic tridiagonal system of linear equations

$$
A_{i}^{m} \mathbf{x}_{i-1}^{m+1}+B \mathbf{x}_{i}^{m+1}+C_{i}^{m} \mathbf{x}_{i+1}^{m+1}=D_{i}^{m}
$$




$$
\begin{aligned}
& A_{i}^{m}=-\frac{\varepsilon_{i}^{m}}{h_{i}^{m}}-\frac{b_{i-\frac{1}{2}}^{i n}}{2}, \quad C_{i}^{m}=-\frac{\varepsilon_{i}^{m}}{h_{i+1}^{m}}-\frac{b_{i+\frac{1}{2}}^{\text {in }}}{2} \\
& B_{i}^{m}=\frac{h_{i+1}^{m}+h_{i}^{m}}{2 \tau}+\frac{\varepsilon_{i}^{m}}{h_{i}^{m}}+\frac{\varepsilon_{i}^{m}}{h_{i+1}^{m}}+\frac{b_{i-\frac{1}{2}}^{i n}}{2}+\frac{b_{i+\frac{1}{2}}^{i n}}{2} \\
& D_{i}^{m}=\mathbf{x}_{i}^{m} \frac{h_{i+1}^{m}+h_{i}^{m}}{2 \tau}-\frac{b_{i+\frac{1}{2}}^{\text {out }}}{2}\left(\mathbf{x}_{i}^{m}-\mathbf{x}_{i+1}^{m}\right)-\frac{b_{i-\frac{1}{2}}^{\text {out }}}{2}\left(\mathbf{x}_{i}^{m}-\mathbf{x}_{i-1}^{m}\right)+w_{i}^{m}\left(\frac{\mathbf{x}_{i+1}^{m}-\mathbf{x}_{i-1}^{m}}{2}\right)^{\perp} \text { Due to }
\end{aligned}
$$

stability reasons, e.g. in case of curve merging, this general numerical scheme is locally replaced by the implicit upwind method.

\section{Topological changes}

In the Lagrangian approach, there are some issues throughout the curve evolution left to treat additionally. The curve can be self-intersected or can be intersected by another curve. Such selfintersection can occur when the curve velocity is locally slowed down significantly (e.g. nonburnable regions) , see Figure 4. These situations require a topological change, either splitting or merging. However, the detection of such topological changes can be computationally demanding. The most often used strategy of pairwise comparison of grid points have computational complexity $O\left(n^{2}\right)$. In our propagation model we use our $O(n)$ approach, which makes the overall computations fast and reliable in fire perimeter evolution. Our main idea for the topological changes detection, see also (Balažovjech, et al., 2012), is to create an array of cells over the whole computational domain and to check a narrow strip of cells along the curves. In these cells we subsequently check whether there are two nonneighboring points of one curve, which indicates splitting. Similarly we check the cells along the curves, for the case of two points from different curves belonging to one cell. Such situation indicates merging.
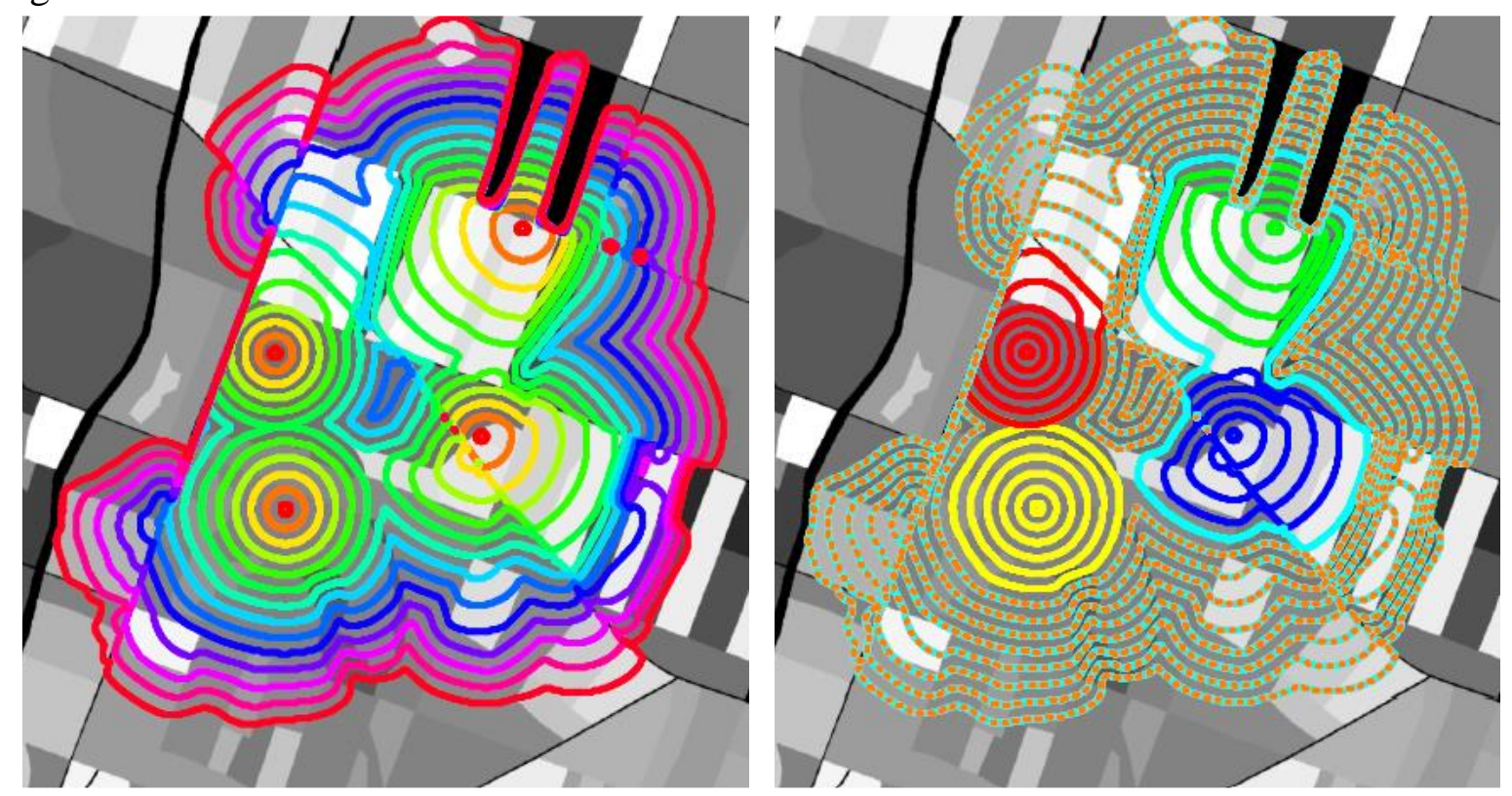

Figure 4 - Merging and splitting of the curves on a ROS map. On the left, four red circles representing the initial fire perimeters and their time evolution is plotted. Curves with the same color represent the fire perimeters position at the same time. On the right, there is the same situation, but the evolution of different fire perimeters is plotted in different color, red, yellow, green and blue, until their merging. After the merging, the color changes to a combination, e.g. green and blue curves merge as a cyan curve.

Advances in Forest Fire Research 2018 - Page 582 
During the evolution the curve length changes and thus we need to locally add or remove points in order to maintain appropriate number of grid points. Then, the asymptotically uniform redistribution ensures the desired segment length $h_{d}$. Thanks to this, we can set the dimensions of the cell along the curves to $2 h_{d} \mathrm{x} 2 h_{d}$. This ensures maximally 3 neighboring points of smooth curve in one cell.

Basic idea for the splitting detection is to traverse the curve points and mark the cells, where the points belong. First, we mark the cells where all curve points belong by 0 . Then we traverse the curve again and we mark the cells by the belonging point number. If a point belongs to a cell already marked by a non-neighboring point the curve will split, since there are more points between the point that entered the cell first time and the point entering the cell again. Those two points in one cell are not necessarily the nearest in their neighboring, thus we find the nearest by pairwise comparing. Then the curve is split in the nearest points into the enlarging (burnt area) and shrinking (unburnt area) curve. The merging detection is very similar. We traverse every curves points and mark the cells, where the points lie by 0 . Then we subsequently traverse the curve points again and we mark the cells by the curve number. When a cell is already marked by a different curve the curves will merge, since the points of two curves belong to the same cell. To merge the curve, we find the nearest points between the neighboring points of the points belonging to the same cell.

\section{Numerical experiments}

In the following experiments we present the behavior of our propagation model on a simple topography. Valley and ridge are given by two planes inclined by 30 degrees from the horizontal plane at both axis direction. The initial fire perimeter is given as a circle in the horizontal plane.

First, we present the influence of the geodesic and normal curvatures on the curve evolution velocity in the valley and on the ridge, see Figure 5. Although those experiments do not simulate the fire behavior (due to a neglected slope influence), they are suitable for the presentation of the heat accumulation or dissipation influencing the rate of spread.
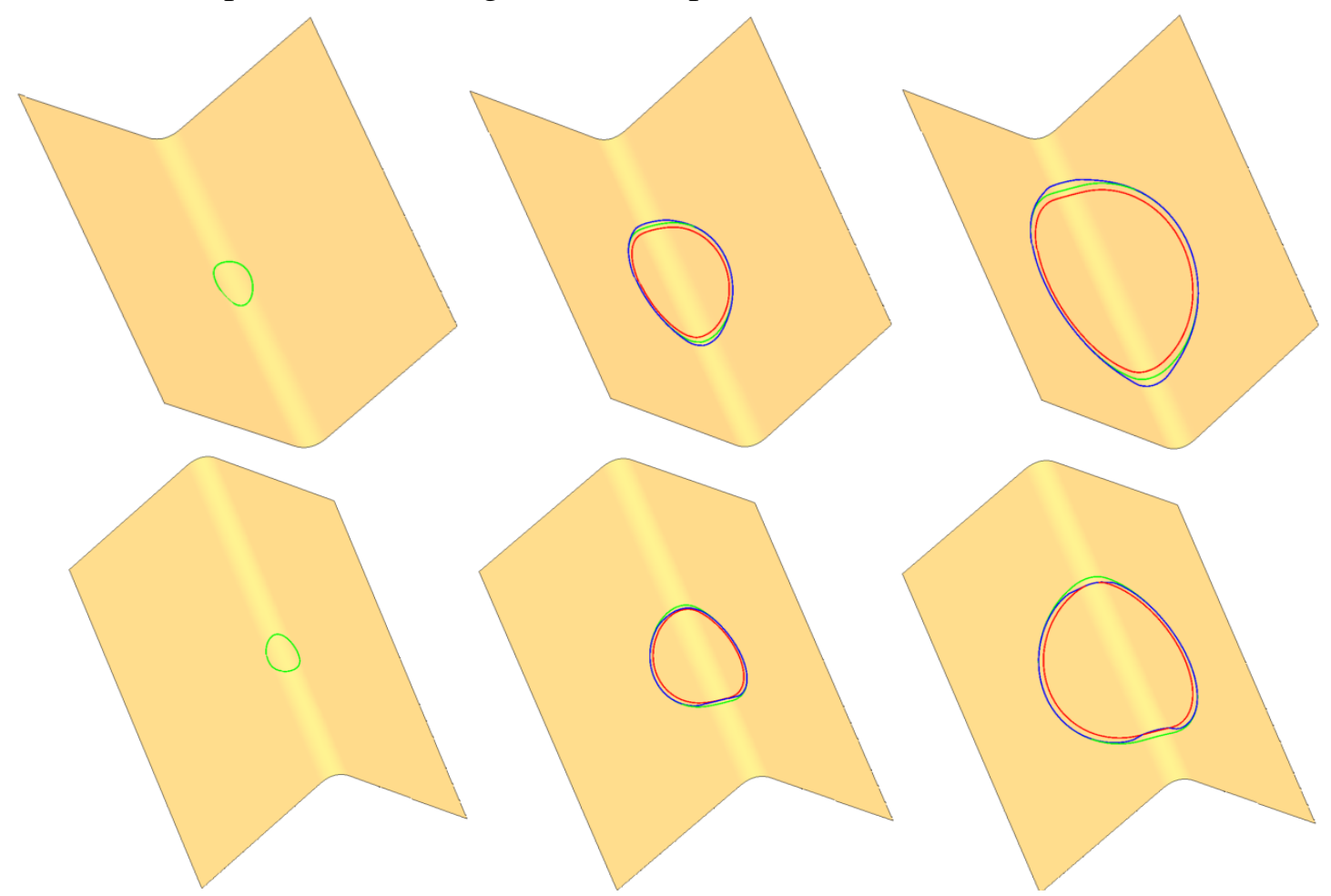

Figure 5 - The comparison of the curve evolution in the valley (top row) and on the ridge (bottom row) driven by unit external force (green) and accompanied by the geodesic curvature (red) or normal curvature (blue). Geodesic curvature slows the curve velocity locally down. The normal curvature speeds the velocity up in the bottom of the valley and slows the velocity down on the top of the ridge. 
In the next experiments we simulate the fire spread, considering all the influences in the valley, see Figure 6, and on the ridge, see Figure 7.
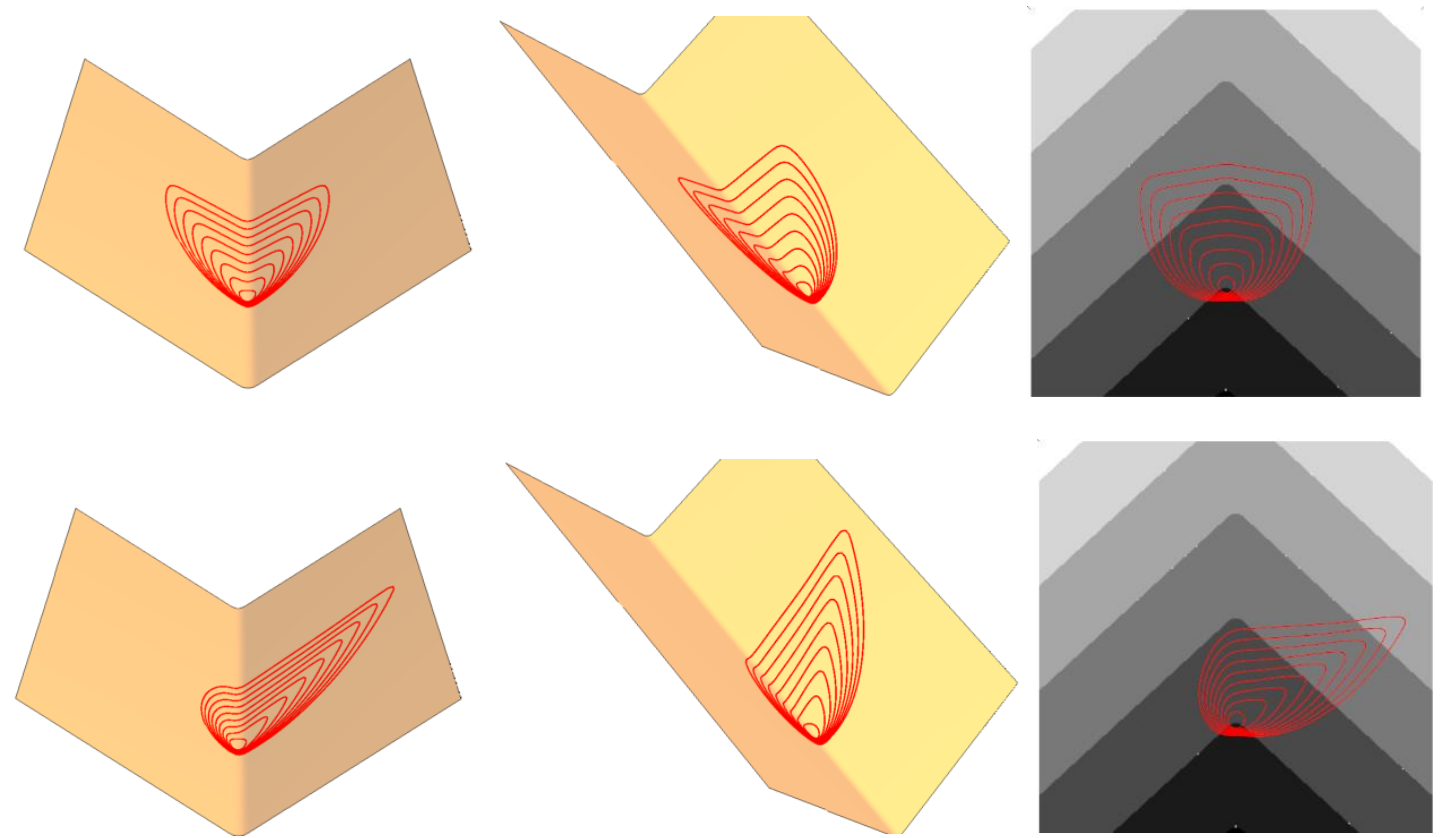

Figure 6 - Fire perimeter evolution in the valley. We consider homogenous fuel, geodesic and normal curvatures influence and the slope influence without the wind influence (top row) and considering the wind perpendicular to the valley (bottom row).
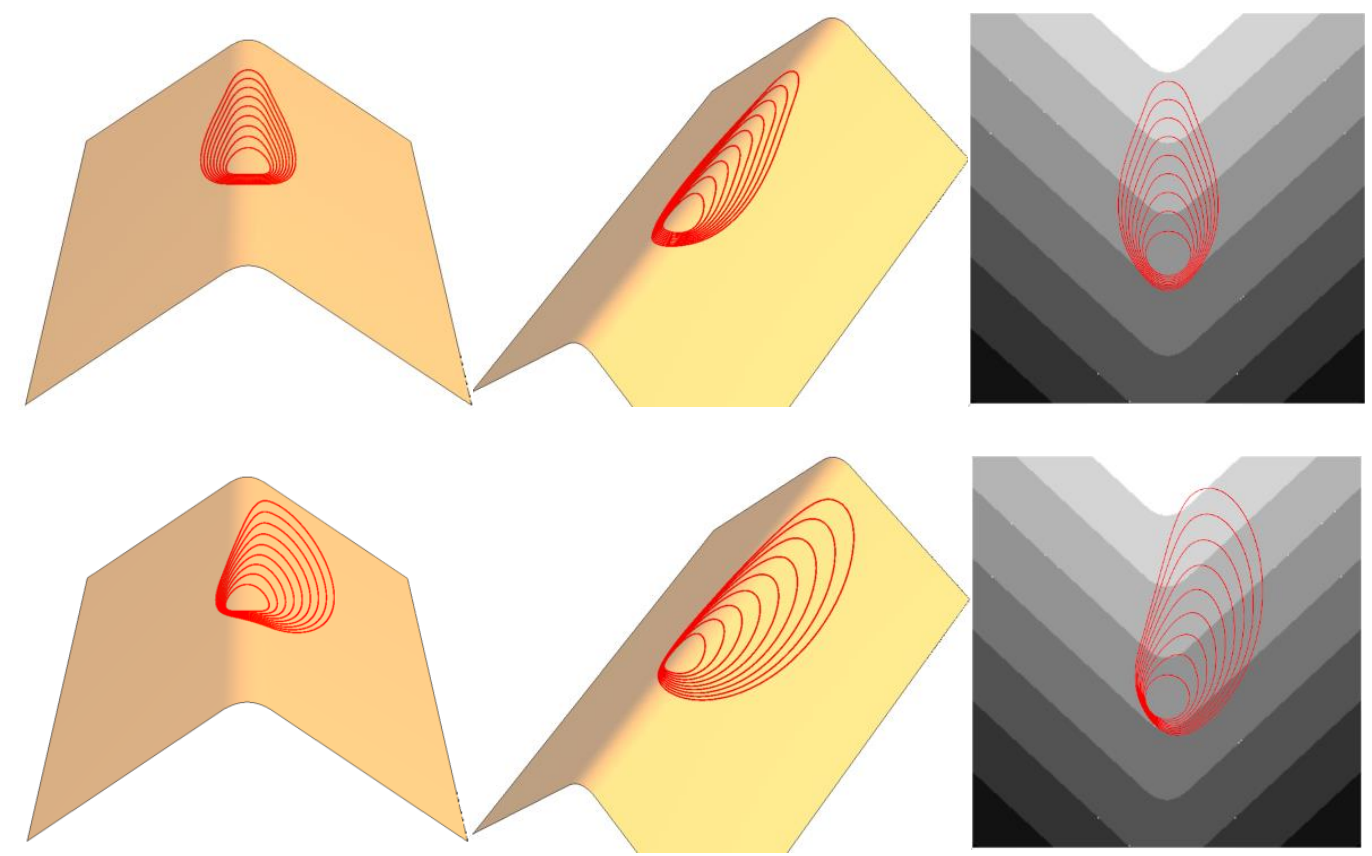

Figure 7 - Fire perimeter evolution on the ridge. We consider homogenous fuel, geodesic and normal curvatures influence and the slope influence without the wind influence (top row) and considering the wind perpendicular to the ridge (bottom row).

On the following examples, we present a simulations over a real topography of mountainous area of Staré Hory in central Slovakia. The fuel influence map was made from the forestry typological map

Advances in Forest Fire Research 2018 - Page 584 
in resolution $0.83 \mathrm{~m}$, consists of 4 colors and the rates of spread were assigned from (Prichard, et al., 2013) as follows

- black (roads, rivers) - non burnable,

- dark grey (broad-leaved forest) - $0.46 \mathrm{~m} \cdot \mathrm{min}^{-1}$,

- light grey (mixed forest) - $0.76 \mathrm{~m} \cdot \mathrm{min}^{-1}$,

- white (coniferous forest) - $1 \mathrm{~m} \cdot \mathrm{min}^{-1}$.

The other model parameters can be estimated by an inverse modeling presented in (Ambroz, et al., Submitted). Following examples show the simulations considering various wind directions and various initial conditions.
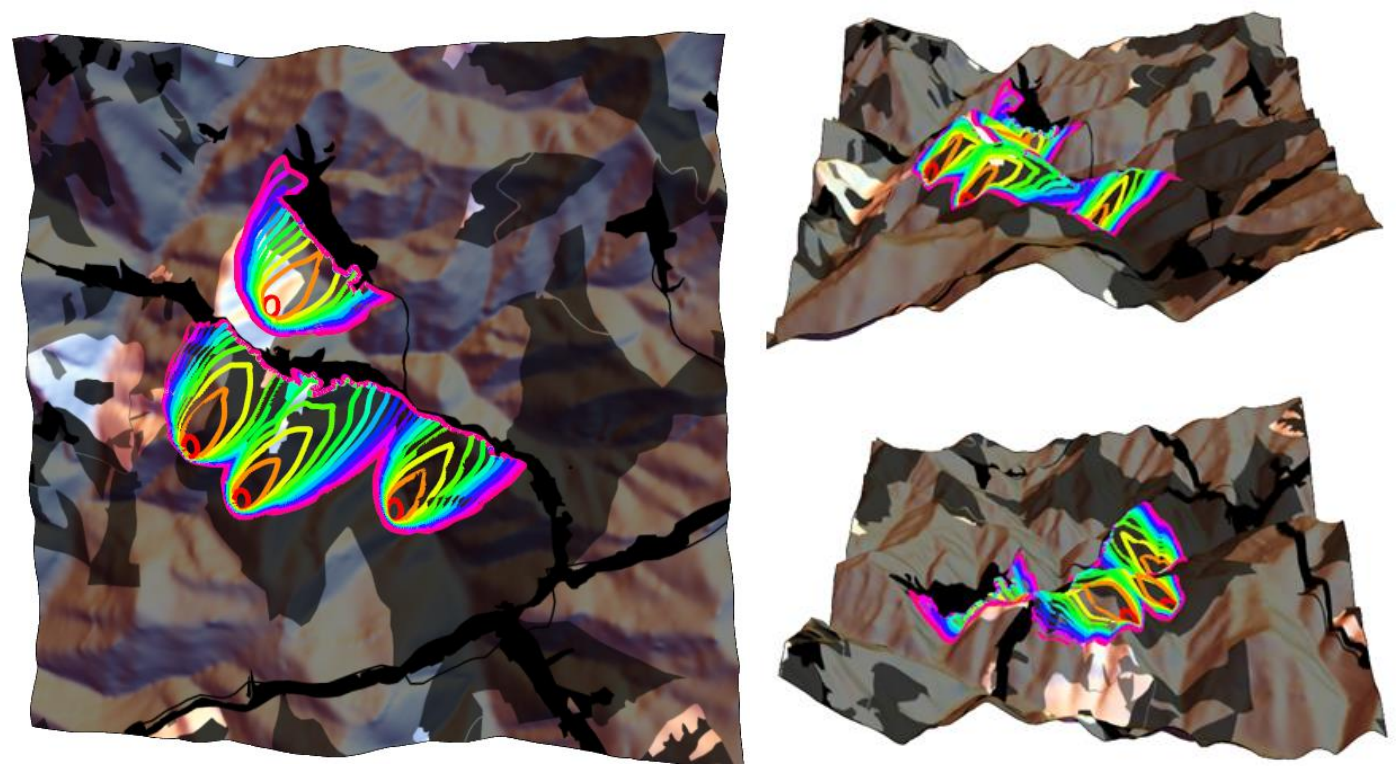

Figure 8 - Simulation with 4 initial fire perimeters (red) and their evolution in 1-hour intervals. On the left is the view from above, right top is view from south and right bottom is view from west. Wind is considered to be southwest at speed 4 m.min ${ }^{-1}$. This simulation of 10 hour spread took $45 \mathrm{~s}$ of computational time.
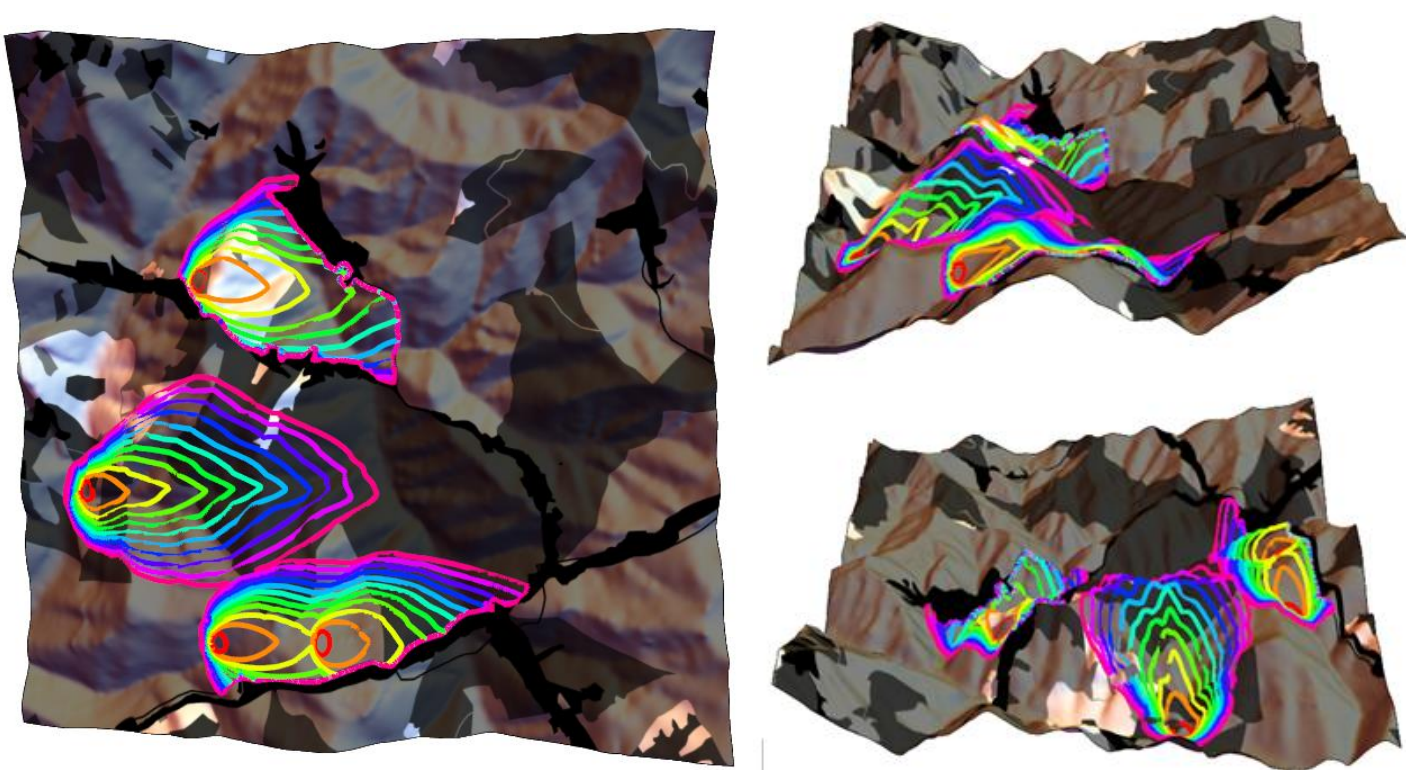

Figure 9 - Simulation with 4 initial fire perimeters (red) and their evolution in 1-hour intervals. On the left is the view from above, right top is view from south and right bottom is view from west. Wind is considered to be west at speed 4 m. $^{-1}$. This simulation of 10 hour spread took $49 \mathrm{~s}$ of computational time. 

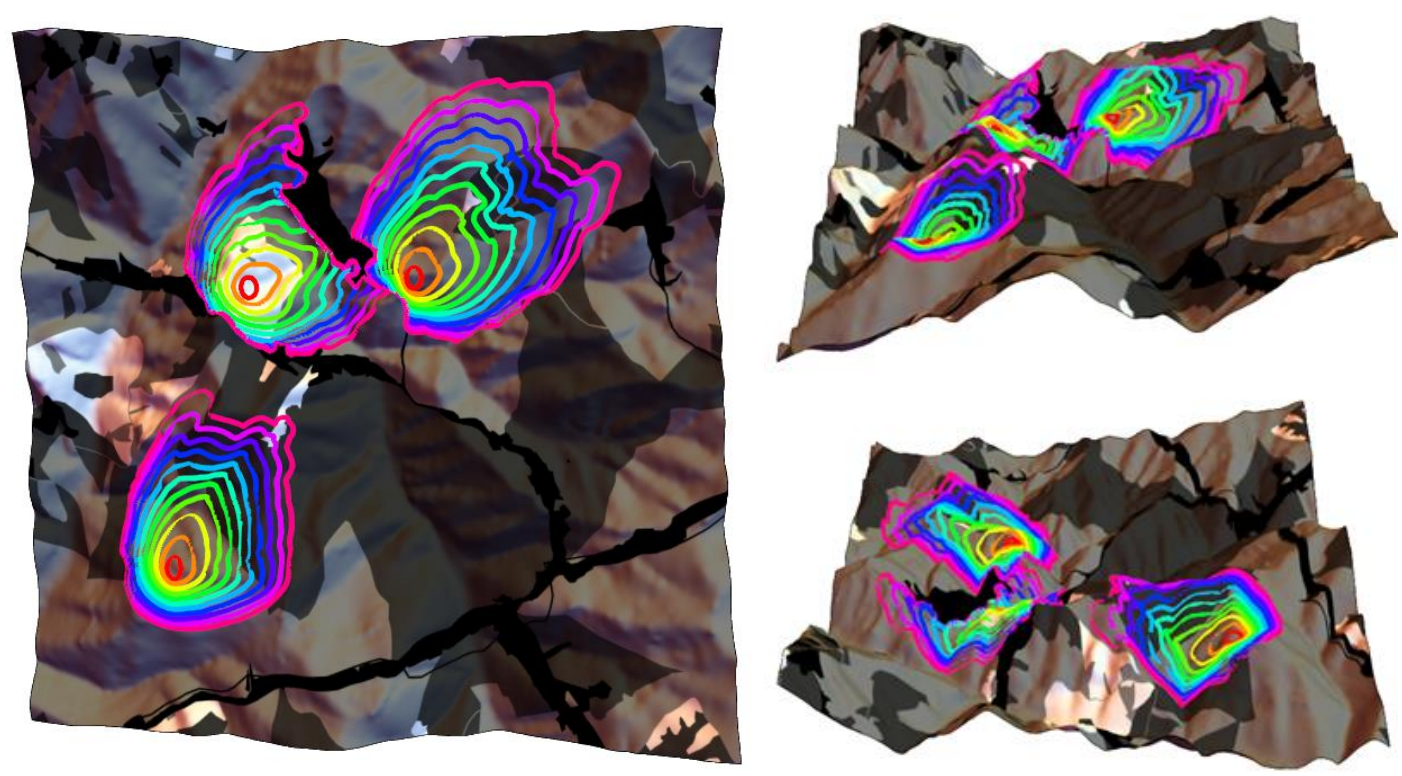

Figure 10 - Simulation with 4 initial fire perimeters (red) and their evolution in 1-hour intervals. On the left is the view from above, right top is view from south and right bottom is view from west. Wind is considered to be west at speed $1 \mathrm{~m} . \mathrm{min}^{-1}$. This simulation of 10 hour spread took $53 \mathrm{~s}$ of computational time.

\section{References}

Ambroz, M., Balažovjech, M., Medl'a, M. \& Mikula, K., Submitted. Numerical modeling of wildland surface fire propagation by evolving surface curves. Advances in Computational Mathematics.

Balažovjech, M., Mikula, K., Petrášová, M. \& Urbán, J., 2012. Lagrangean method with topological changes for numerical modelling of forest fire propagation. s.1., s.n., pp. 42-52.

Barrett, J., Garcke, H. \& Nürnberg, R., 2009. Numerical approximation of gradient flows for closed curves in $\mathrm{R}^{\mathrm{d}}$. IMA journal of numerical analysis, 30(1), pp. 4-60.

Benninghoff, H. \& Garcke, H., 2014. Efficient image segmentation and restoration using parametric curve evolution with junctions and topology changes. SIAM Journal on Imaging Sciences, Volume 7, pp. 1451-1483.

Butler, B. W., Anderson, W. R. \& Catchpole, E. A., 2007. Influence of slope on fire spread rate. s.1., s.n., pp. 75-82.

Dziuk, G., 1999. Discrete anisotropic curve shortening flow. SIAM Journal on Numerical Analysis, Volume 36, pp. 1808-1830.

Hilton, J. E., Miller, C., Sharples, J. J. \& Sullivan, A. L., 2017. Curvature effects in the dynamic propagation of wildfires. Int. J. of Wildland Fire, Volume 25, pp. 1238-1251.

Hou, T. Y., Lowengrub, J. S. \& Shelley, M. J., 1994. Removing the stiffness from interfacial flows with surface tension. J. of Comp. Physics, Volume 114, pp. 312-338.

Krasnow, K., Schoennagel, T. \& Veblen, T. T., 2009. Forest fuel mapping and evaluation of LANDFIRE fuel maps in Boulder County, Colorado, USA. Forest Ecology and Management, Volume 257, pp. 1603-1612.

Mikula, K., Ohlberger, M. \& Urbán, J., 2014. Inflow-implicit/outflow-explicit finite volume methods for solving advection equations. Applied Num. Math., Vol. 85, pp. 16-37.

Mikula, K. \& Ševčovič, D., 2001. Evolution of plane curves driven by a nonlinear function of curvature and anisotropy. SIAM J. on Applied Mathematics, Volume 61, pp. 1473-1501. 
Mikula, K. \& Ševčovič, D., 2004. A direct method for solving an anisotropic mean curvature flow of plane curves with an external force. Mathematical Methods in the Applied Sciences, Volume 27, pp. 1545-1565.

Mikula, K. \& Ševčovič, D., 2004. Computational and qualitative aspects of evolution of curves driven by curvature and external force. Computing and Visualization in Science, Volume 6, pp. 211-225.

Mikula, K. \& Ševčovič, D., 2006. Evolution of curves on a surface driven by the geodesic curvature and external force. Applicable Analysis, Volume 85, pp. 345-362.

Mikula, K. \& Urbán, J., 2012. New fast and stable Lagrangean method for image segmentation. s.1., s.n., pp. 688-696.

Osher, S. \& Fedkiw, R., 2002. Level Set Methods and Dynamic Implicit Surfaces. s.1.:Springer New York.

Prichard, S. J. et al., 2013. Fuel Characteristic Classification System Version 3.0: Technical Documentation, s.l.: s.n.

Scott, J. H. \& Burgan, R. E., 2005. Standard fire behavior fuel models: a comprehensive set for use with Rothermel's surface fire spread model. p. 66.

Sethian, J. A., 1999. Level set methods and fast marching methods. Cambridge university press.

Viegas, D., Pita, L. P., Matos, L. \& Palheiro, P., 2002. Slope and wind effects on fire spread. Roterdam, Millpress.

Weber, R. O., 1989. Analytical models for fire spread due to radiation. Combustion and flame, Volume 78, pp. 398-408. 


\title{
Observations on wildfire spotting occurrence and characteristics in Greece
}

\author{
Miltiadis Athanasiou ${ }^{1 *}$; Gavriil Xanthopoulos ${ }^{2}$ \\ ${ }^{1}$ Wildfire Management Consulting and Training. 8 Thoma Paleologou st., Acharnes, 13673, \\ Athens, Greece, \{info@m-athanasiou.gr*\} \\ ${ }^{2}$ Hellenic Agricultural Organization "Demeter". Institute of Mediterranean Forest Ecosystems. \\ Terma Alkmanos, Ilisia, 11528, Athens, Greece, \{gxnrtc@fria.gr\}
}

\begin{abstract}
This paper presents a study on the phenomenon of spotting in some of the most common forest vegetation types in Greece, during wildfires in the 2007-2017 period. Monitoring and documenting selected wildfires during this period, noting the appearance or absence of spot fires and the prevailing conditions at the time, a database of 166 field observations was developed. The database includes information on the number of observed spot fires $(\mathrm{N} \kappa)$, the in situ measured relative humidity $(\mathrm{RH}, \%)$ values, the wind speed, the forest fuel type where the firebrands had landed, namely maquis, phrygana and grasses, the maximum spotting distance $(\mathrm{D} \kappa, \mathrm{m})$ from the fire perimeter, the fire perimeter segment (head or flank) where the firebrands came from, and the fire type, namely surface, passive crown, active crown and plume dominated fire.

The database was analyzed first by examining the correlation of $\mathrm{RH}$ values with $\mathrm{N} \kappa$ for the three fuel types. An ordinal variable named $\mathrm{K} \kappa$ was created in order to represent the following four empirical classes: a) no spotting $(\mathrm{N} \kappa=0, \mathrm{~K} \kappa=0)$, b) rare spotting $(\mathrm{N} \kappa<3, \mathrm{~K} \kappa=1)$, c) limited spotting $(3 \leq \mathrm{N} \kappa \leq 9, \mathrm{~K} \kappa=2)$ and d) profuse/massive spotting $(\mathrm{N} \kappa \geq 10, \mathrm{~K} \kappa=3)$. At $\mathrm{RH}$ values higher than $46 \%$, no spotting ignition was recorded. Massive spotting that triggered extreme fire behavior, was documented for RH values lower than $17 \%$. The $\mathrm{RH}$ thresholds for spotting occurrence that were identified for the three forest fuel types on which the

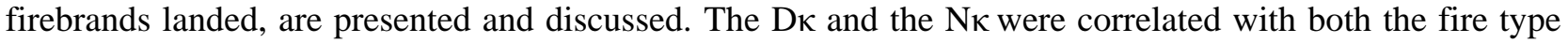
and the fire segment on which they were observed. Their descriptive statistics are also presented and discussed. The study confirmed the great spotting potential of the plume dominated wildfires, both in regard to spotting distance and the number of spot fires.
\end{abstract}

Keywords: Spotting, Spot fire, Forest fire, Wildfire behaviour, Firefighting, Greece

\section{Introduction}

Spotting ignition is one of the three significant mechanisms of wildfire spread. It can be considered as a discontinuous fire spread mechanism (Koo et al. 2010) that is synonymous with solid mass transport (Albini1979, Alexander 2009). The transport of burning fire embers outside the fire perimeter, is a cause of serious concern to firefighters because it affects fire behaviour and difficulty of control and poses a serious threat for them and for civilians.

Spotting involves the source of firebrands, how far they travel, and the probability of ignition on landing (Rothermel 1983). It is mainly caused by lofted firebrands, including burning tips of branches, cones, and pieces of bark, that fly and land beyond the main fire perimeter, but may also be caused by burning cones or pieces of wood rolling down steep slopes (Van Wagner 1988). The type of forest vegetation that is burning is important for the creation of firebrands.

The probability of ignition at the point where a firebrand lands, is a function of both firebrand size and temperature. It has been found that as firebrand size is reduced, increased temperature is required for ignition (Hadden and Scott 2011). Additionally, the probability of ignition depends on the characteristics of the dead fuels where the firebrand lands, such as fuel quantity, dimension (fineness), arrangement (compactness and continuity) and fuel moisture content (FMC, \%). Atmospheric relative

Advances in Forest Fire Research 2018 - Page 588 
humidity (RH, \%) affects directly the FMC of dead fuels, the effect being more dramatic and the response faster for the finer ones. Thus, significant differences in spotting may exist, depending on the forest vegetation properties. Firebrands in the flaming phase are more capable to ignite fuel beds with no air flow than the ones in the glowing phase with air flow (Ganteaume et al. 2009) while when fire danger is high, the ignition probability of flaming firebrands that land on fine fuels, approaches $100 \%$ (Ellis 2012).

A spotting distance of up to 200 meters $(\mathrm{m})$ corresponds to short-range spotting and is common in high intensity wildfires, while distance values between $200 \mathrm{~m}$ and 1 kilometer $(\mathrm{km})$ (Bushfire CRC 2009) or between $200 \mathrm{~m}$ and $2 \mathrm{~km}$ (Alexander 2009) can be considered as medium-range spotting. A spotting distance greater than 1 or $2 \mathrm{~km}$, which is very common in some forest types, such as the eucalypt forests of Australia, can be considered as long-range spotting.

The number of firebrands generated and the rapidity of development of the spot fires, determine the magnitude of the phenomenon and its effect on wildfire behaviour (Ellis 2012). Spotting usually exacerbates fire suppression activities and plans, is the leading cause of loss of structures in fires in Wildland Urban Interface (WUI) areas and is a major concern regarding the safety of firefighters and the public (Alexander 2009).

In Greece, spotting occurs often in all of its Mediterranean vegetation types, such as the Mediterranean pine forests, the evergreen shrublands (maquis), the low scrubland vegetation called phrygana and the grasslands (Athanasiou and Xanthopoulos 2013). As the fuel characteristics are important for the creation of firebrands and the characteristics of the fuel bed where firebrands land affect the probability of ignition, in addition to the FMC, the objective set for this study was to examine the occurrence and characteristics of spotting in three main fuel types in Greece, namely maquis, phrygana and grasses, especially in relation to the prevailing $\mathrm{RH}$, since this affects the FMC of the dead fuels. Most of the work has been carried out as part of the Ph.D. dissertation of the first author (Athanasiou 2015).

\section{Methods}

Systematic observation, recording and measurements of spotting on maquis, phryganic areas and grass during the spread of wildfires, started in 2007 in Greece and continues until today. The procedure followed has been described in Athanasiou and Xanthopoulos (2010). An initial data set of 75 cases was analysed and preliminary findings were presented in 2013 (Athanasiou and Xanthopoulos 2013). Ninety-one (91) additional spotting observations that were collected during the following fire seasons, resulted in a total of 166 cases in which the phenomenon was either present or absent, presenting an opportunity for testing and extending the initial conclusions. The length of observation for each case varied from at least five minutes to almost half an hour, depending on the conditions and the potential risk.

The database that was developed, consists of 166 spotting observations $(n=166)$ that include information about a) the number of the spot fires $(\mathrm{N \kappa}), \mathrm{b})$ the in situ measured $\mathrm{RH}$ values, c) the wind speed at the height of $10 \mathrm{~m}($ Wind $10 \mathrm{~m}, \mathrm{~km} / \mathrm{h}$ ) and at eye level (calculating one from the other, depending on which of the two was actually measured), d) the forest fuel type where the firebrands had landed, e) the maximum spotting distance from the fire perimeter $(\mathrm{D} \kappa, \mathrm{m}), \mathrm{f})$ the fire perimeter segment (head or flank) where the firebrands came from, and g) the fire type, namely surface, passive crown, active crown and plume dominated fire during the spread of which the measurements had been conducted (Figure 1). 

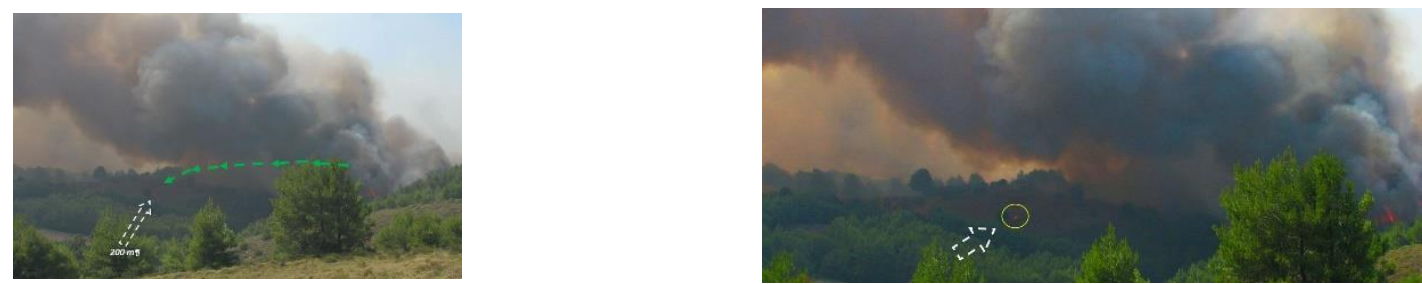

a. The first spot fire is recorded at 15:23:56 at a distance of $200 \mathrm{~m}$

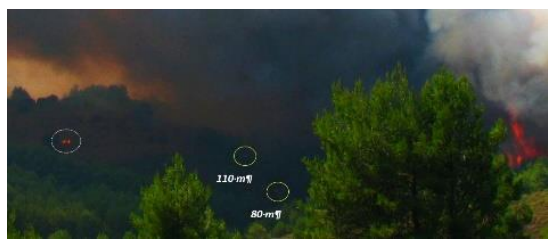

b. $\quad$ Two more spot fires at 15:24:04 at 80 and $110 \mathrm{~m}$ (while the first one at the left is growing)

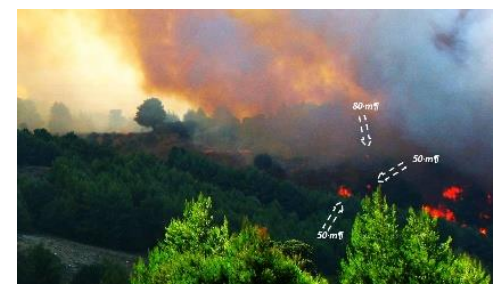

d. $\quad$ Three more spot fires at 15:26:54 at 50 and $80 \mathrm{~m}$ (in total, eleven spot fires recorded, within a period of 2 minutes and 58 seconds)

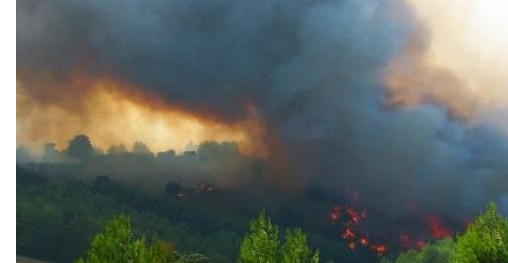

c. $\quad$ Five more spot fires have occured at 180, 200, 230 and $240 \mathrm{~m}$ and some of them have already merged, two minutes and six seconds later (at 15:26:10).

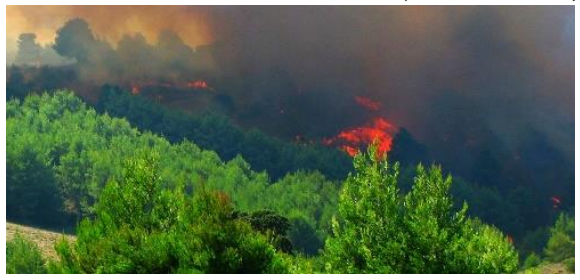

e. Wildfire evolution at 15:28:10

Figure 1 - Spot fires $\left(\mathrm{N}_{\kappa}=11\right.$ \& $\left.\mathrm{D} \kappa=240 \mathrm{~m}\right)$ recorded on a $84 \%$ slope, mainly covered by Sarcopoterium spinosum and Cistus spp. (phryganic vegetation), while a wind driven passive crown wildfire spreads through an Aleppo pine stand. Weather conditions: $T=29^{\circ} \mathrm{C}, \mathrm{RH}=43.5 \%$, Wind speed at eye level $=15 \mathrm{~km} / \mathrm{h}$

\section{Analysis}

Meteorological measurements and the relative necessary information about month and time of day, fuels and topography that had been collected on site, were utilised for calculating fine (1-h) dead Fuel Moisture Content (FDFMC, \%) values by using Rothermel's methodology (1983). The database was

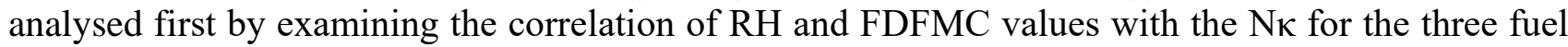
types. An ordinal variable named $\mathrm{K} \kappa$ was created in order to represent the following four empirical classes: a) no spotting $(\mathrm{N} \kappa=0, \mathrm{~K} \kappa=0)$, b) rare spotting $(\mathrm{N} \kappa<3, \mathrm{~K} \kappa=1)$, c) limited spotting $(3 \leq \mathrm{N} \kappa \leq 9$, $\mathrm{K} \kappa=2)$ and $\mathrm{d}$ ) profuse/massive spotting $(\mathrm{N} \kappa \geq 10, \mathrm{~K} \kappa=3)$. The $\mathrm{N} \kappa$ and the $\mathrm{D} \kappa$ were also examined for correlation with both the fire type and the fire perimeter segment on which they had been observed. For the observations/records where $\mathrm{N \kappa}>1$, the D $\mathrm{v}$ value was the distance of the farthest spot fire from the fire perimeter (e.g. Figure 1).

\section{Results}

At $\mathrm{RH}$ values higher than $46 \%$, no spotting ignition was recorded. Massive spotting that triggered extreme fire behaviour, was documented for $\mathrm{RH}$ values lower than 17\%. The RH and FDFMC thresholds for spotting occurrence that were found, for the three forest fuel types on which the firebrands landed, are reported in Table 1. The descriptive statistics of $\mathrm{N \kappa}$ and $\mathrm{D \kappa}$ in relation to both the fire type and the fire perimeter segment, are also presented in Table 2 and 3, respectively. Dк values were only available for 58 of the 67 cases in which spotting was observed. 
Table 1 - Ranges of values and thresholds of RH and FDFMC for spotting occurrence, on maquis, phrygana and grass

\begin{tabular}{|c|l|l|c|c|c|}
\hline \multicolumn{2}{|l|}{} & & \multicolumn{4}{|c|}{$\mathrm{n}-\mathrm{RH}-$ FDFMC } \\
\hline RH - FDFMC & $\mathrm{K} \kappa$ & $\mathrm{n}$ & Maquis & $\begin{array}{c}\text { Phrygana } \\
\text { (Sarcopoterium spinosum })\end{array}$ & Grass \\
\hline $\begin{array}{c}(15-62.5)- \\
(4-14)\end{array}$ & 0 & 99 & $43-(15-62.5)-(4-14)$ & $29-(15-50)-(4-12)$ & $\begin{array}{c}27-(23.8-55)-(4- \\
11)\end{array}$ \\
\hline $\begin{array}{c}(15-46)- \\
(3-11)\end{array}$ & 1 & 31 & $9-(20-38.5)-(4-11)$ & $15-(15-46)-(3-10)$ & $7-(16-31)-(3-6)$ \\
\hline $\begin{array}{c}(14-35)- \\
(2-9)\end{array}$ & 2 & 18 & $7-(18-33)-(4-8)$ & $4-(34.5-35)-(6-9)$ & $7-(14-30)-(2-7)$ \\
\hline $\begin{array}{c}(13-46)- \\
(3-8)\end{array}$ & 3 & 18 & $6-(16-21.4)-(3-7)$ & $1-(43.5)-(8)$ & $11-(13-46)-(3-8)$ \\
\hline Total: & 166 & 65 & 49 & 52 \\
\hline
\end{tabular}

Table 2 - Descriptive statistics of Nא, per fire type and perimeter segment for the 67 cases with spotting

\begin{tabular}{|c|c|c|c|c|c|c|}
\hline & \multicolumn{2}{|c|}{$\begin{array}{l}\text { Surface } \\
(\mathrm{n}=22)\end{array}$} & \multicolumn{2}{|c|}{$\begin{array}{l}\text { Passive crown } \\
(\mathrm{n}=21)\end{array}$} & \multirow{2}{*}{$\begin{array}{c}\begin{array}{c}\text { Active crown } \\
\text { (wind driven) }(\mathrm{n}=7)\end{array} \\
\text { Head } \\
(\mathrm{n}=7)\end{array}$} & \multirow{2}{*}{$\begin{array}{c}\text { Crown (plume) } \\
(\mathrm{n}=17)\end{array}$} \\
\hline $\mathrm{N}_{\kappa}$ & $\begin{array}{l}\text { Flank } \\
(\mathrm{n}=7)\end{array}$ & $\begin{array}{c}\text { Head } \\
(n=15)\end{array}$ & $\begin{array}{l}\text { Flank } \\
(\mathrm{n}=7)\end{array}$ & $\begin{array}{c}\text { Head } \\
(\mathrm{n}=14)\end{array}$ & & \\
\hline Mean value & 1 & 2 & 3 & 5 & 3 & 21 \\
\hline Median & 1 & 1 & 3 & 4 & 2 & 22 \\
\hline Mode & 1 & 1 & 3 & 1 & 1 & 30 \\
\hline Std. Dev. & 1 & 2 & 2 & 6 & 2 & 9 \\
\hline Minimum & 1 & 1 & 1 & 1 & 1 & 5 \\
\hline Maximum & 3 & 8 & 7 & 22 & 6 & 30 \\
\hline Wind $_{10 \mathrm{~m}}$ & & $8-30$ & & 6-111 & $10-26$ & $0-27$ \\
\hline $\mathrm{RH}$ & $16-42$ & $15-46$ & $20-35$ & $14-46$ & $20-35$ & $13-21$ \\
\hline FDFMC & $3-7$ & $3-10$ & $4-9$ & $2-11$ & $4-9$ & $3-7$ \\
\hline
\end{tabular}

Table 3 - Descriptive statistics of $D_{k}$, per fire type and perimeter segment $(n=58)$

\begin{tabular}{|c|c|c|c|c|c|c|}
\hline \multirow[b]{2}{*}{$\mathrm{D}_{\mathrm{K}}(\mathrm{m})$} & \multicolumn{2}{|c|}{ Surface $(n=22)$} & \multicolumn{2}{|c|}{ Passive crown $(n=17)$} & \multirow{2}{*}{\begin{tabular}{|c|}
$\begin{array}{c}\text { Active crown } \\
\text { (wind-driven) }(\mathrm{n}=7)\end{array}$ \\
Head \\
$(\mathrm{n}=7)$
\end{tabular}} & \multirow{2}{*}{$\begin{array}{c}\text { Crown (plume)* } \\
(\mathrm{n}=12) \\
\\
(\mathrm{n}=12)\end{array}$} \\
\hline & $\begin{array}{l}\text { Flank } \\
(\mathrm{n}=7)\end{array}$ & $\begin{array}{c}\text { Head } \\
(\mathrm{n}=15)\end{array}$ & $\begin{array}{l}\text { Flank } \\
(\mathrm{n}=6)\end{array}$ & $\begin{array}{c}\text { Head } \\
(n=11)\end{array}$ & & \\
\hline Mean value & 51 & 132 & 71 & 118 & 229 & 392 \\
\hline Median & 20 & 70 & 60 & 100 & 250 & 250 \\
\hline Mode & 15 & 10 & N/A & 100 & 250 & 200 \\
\hline Std. Dev. & 58 & 143 & 48 & 85 & 175 & 308 \\
\hline Minimum & 10 & 5 & 15 & 20 & 50 & 150 \\
\hline Maximum & 150 & 500 & 150 & 300 & 500 & 1,200 \\
\hline Wind $_{10 \mathrm{~m}}$ & & $8-30$ & & $6-36$ & $10-26$ & $0-27$ \\
\hline RH & $16-42$ & $15-46$ & $20-35$ & $15-46$ & $20-35$ & $13-18$ \\
\hline FDFMC & $3-7$ & $3-10$ & $4-9$ & $3-11$ & $4-9$ & $3-6$ \\
\hline
\end{tabular}

For the 67 cases in which spotting was observed, RH and FDFMC were also plotted versus Nא (Figure $2 \& 3$ ). 
Advances in Forest Fire Research 2018 - D. X. Viegas (Ed.)

Chapter 3 - Fire Management

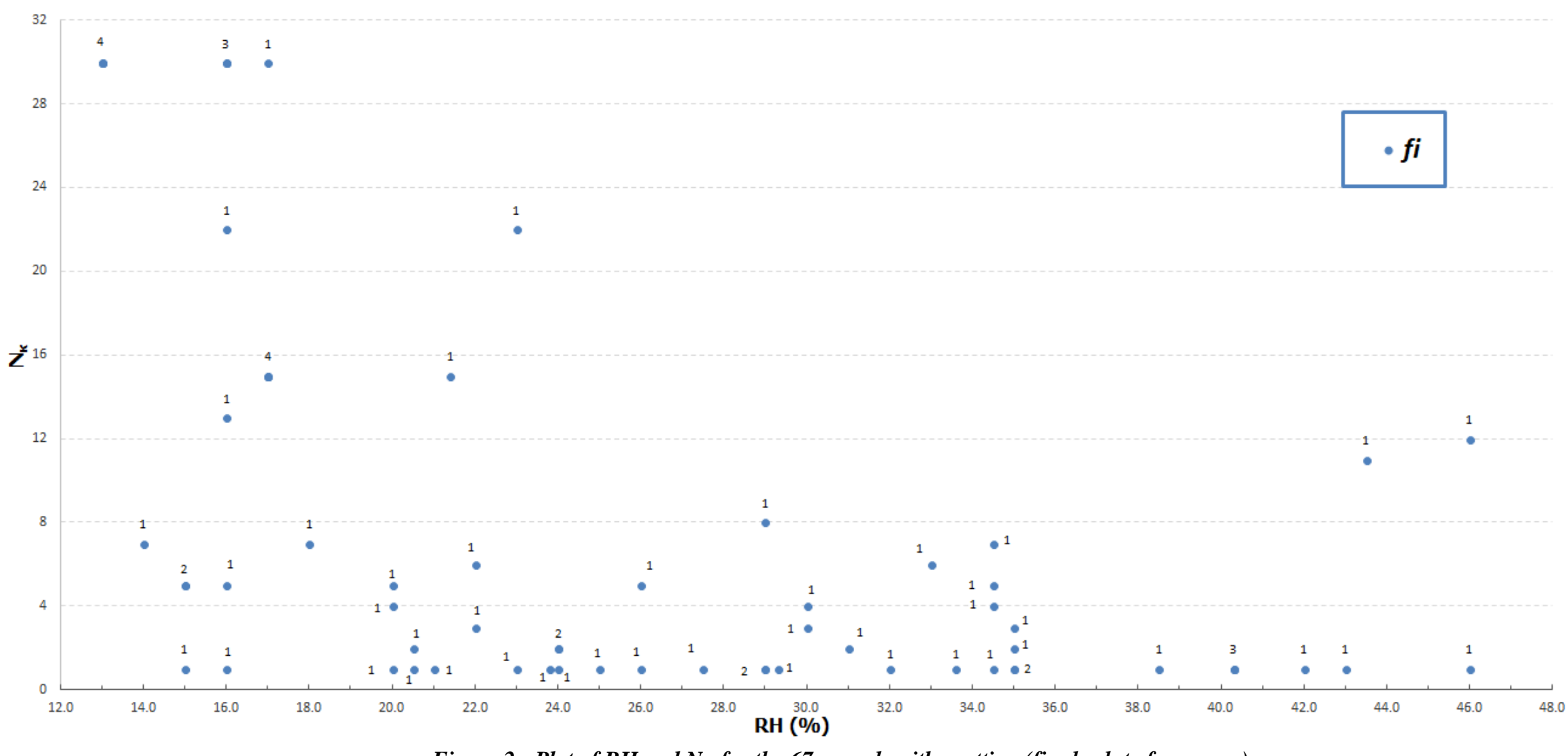

Figure 2 - Plot of $\mathrm{RH}$ and Nא for the 67 records with spotting (fi: absolute frequency) 


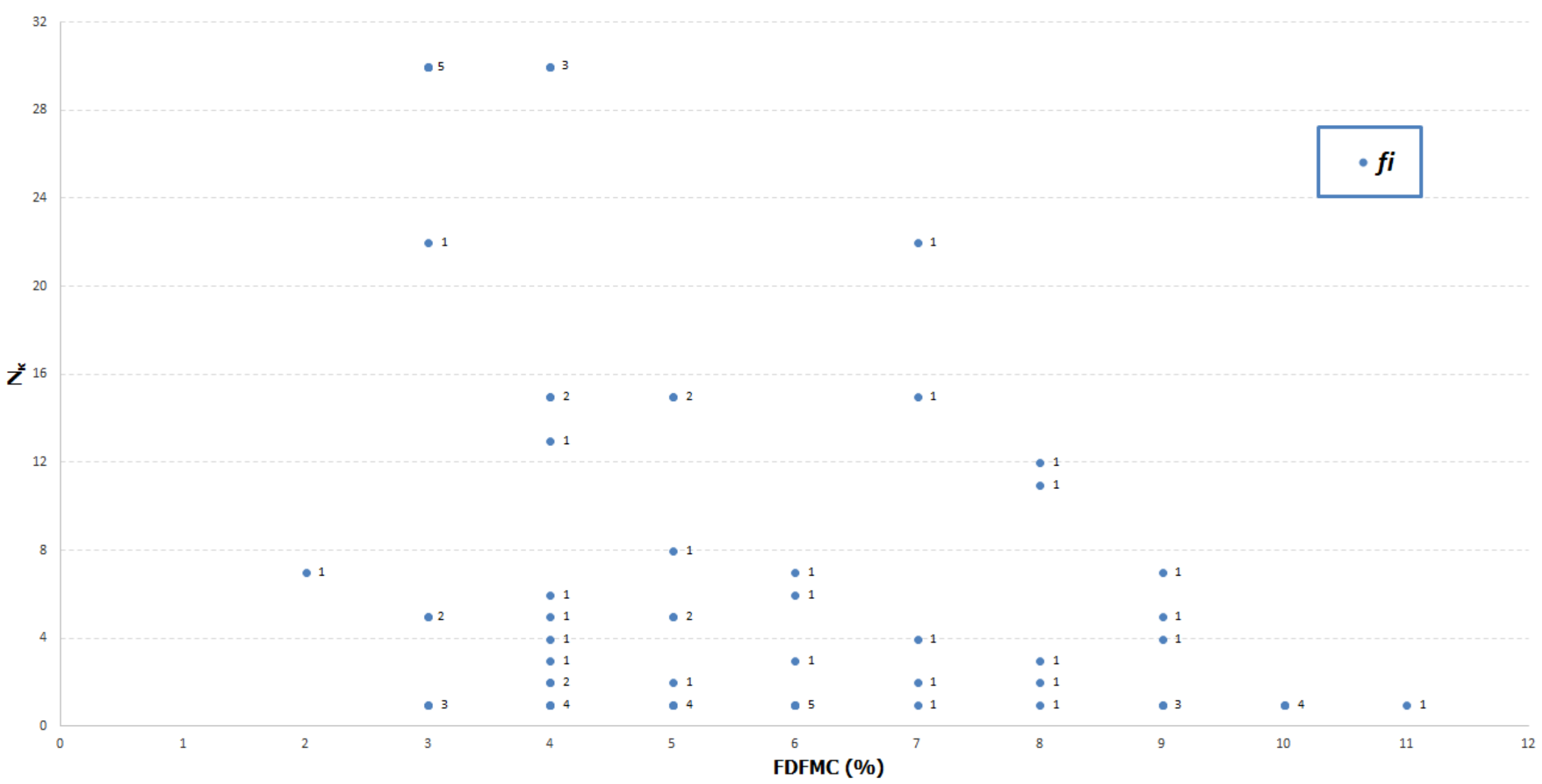

Figure 3 - Plot of FDFMC and Nא for the 67 records with spotting (fi: absolute frequency) 


\section{Discussion}

The maximum $\mathrm{RH}$ value at which spotting occurred $(\mathrm{N} \kappa=1)$ on maquis, was $38.5 \%$ (Table 1, Figure 2 ) and the corresponding calculated FDFMC value was $11 \%$ (Figure 3). The RH and FDFMC thresholds were $46 \%$ and $10 \%(\mathrm{~N} \kappa=1)$ for phrygana and $46 \%$ and $8 \%$ for grass $(\mathrm{N} \kappa=12)$. Regarding phrygana, it is worth noting that there was also one observation (Figure 1) of $K \kappa=3$ spotting class $(\mathrm{N} \kappa=11)$, at relatively high $\mathrm{RH}$ and FDFMC values, $43.5 \%$ and $8 \%$ respectively. In this case, the radiation emitted against the fuel bed, from the leaning smoke column, played a crucial role in preheating the fuels including the fine dead ones (Figure 1). Moreover, regarding the previously mentioned maximum RH and FDFMC datapoint for grass, 12 spot fires were recorded on a grassland, at $\mathrm{RH}=46 \%$ (Figure 2) and FDFMC $=8 \%$ (Figure 3), at a DK of $30 \mathrm{~m}$. The fire brands originated from a torching Pinus halepensis tree and one of them broke into smaller ones upon landing. According to Gould et al. (2007) this is a potentially important notification, and it should be included in any future analysis if available.

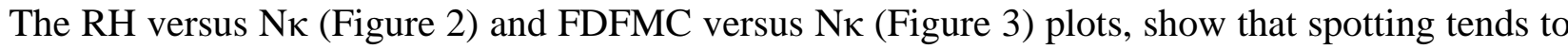
be rare when RH $>40 \%$ or when FDFMC $>10 \%$. The former trend is consistent with Weir's (2004) conclusion who examined 99 prescribed fires and found that spotting occurrence was very rare when $\mathrm{RH}$ exceeded $40 \%$ The latter one is in general agreement with the finding of Manzello et al. (2006) that embers with mass of $0.5 \mathrm{gr}$ could ignite pine needles with fuel moisture of $11 \%$ or less and with the finding of Ellis (2000) that for fine fuel moistures below 9\%, flaming embers with mass between 0.7 and $1.8 \mathrm{gr}$ had a $100 \%$ probability of igniting the Monterey pine litter while glowing ones had lower probabilities. With a light wind $(1 \mathrm{~m} / \mathrm{sec})$ the probability of ignition was found to be about $20 \%$ at fine fuel moisture content of $9 \%$ and approximately $65 \%$ at a fine fuel moisture content of $3.5 \%$ (Ellis 2000).

The great spotting potential of the plume dominated wildfires, both in regard to the $\mathrm{D} \kappa$, and the Nא, was confirmed (Table $2 \& 3$ ). Future modeling of the shed-vortex transport (Berlad and Lee 1968) and of the plumes' characteristics, may shed light into the long-distance transport aspect for this fire type. Regarding wind driven fires, Wind ${ }_{10 \mathrm{~m}}$ was not found to be a Dк predictor, not even a poor one, for the "head spread" subset. A possible reason is because a fire brand does not always originate at the fire perimeter, so the total horizontal distance it has traveled, is not known and is not necessarily equal to Dк (the distance between the fire perimeter and the farthest recorded spot fire). Furthermore, in addition to the ambient atmospheric conditions, the trajectories of fire brands are also affected by the tilted or vertical turbulence and currents of the convection column.

Additionally, the spot fires that were documented at the flanks of surface and passive crown fires, were not in a windless environment and there was a component of wind of unknown velocity and direction that temporarily drove them. Moreover, fire behavior is sometimes a poor predictor of spotting distance or number of spot fires: as found by Racher (2003), wildfires producing the most distant or numerous landing embers are not always those with the greater rate of spread, flame height or flame depth zone.

The analysis of the subset of 67 records of spotting occurrence did not show a strong correlation between Nא and RH or FDFMC. Tables 1, 2 and 3 as well as Figures 2 and 3, may offer some guidelines and relative practical advice to firefighters and fire behaviour practitioners but they are not predictive tools. Number and size of embers produced by various fuel types, is essential information, in order to estimate how many embers could be carried downwind and how far downwind they will go, to determine whether spotting ( $\mathrm{N} \kappa$ and $\mathrm{D \kappa}$ ) will affect the fire rate of spread and to assess whether the spot fires will be numerous enough to merge readily. According to the field observations, the patterns of spatial distribution of spot fires vary, depending on the fire segment the firebrands originate from, the wind field and the landscape (Figure $4 \mathrm{a} \& 4 \mathrm{~b}$ ). 


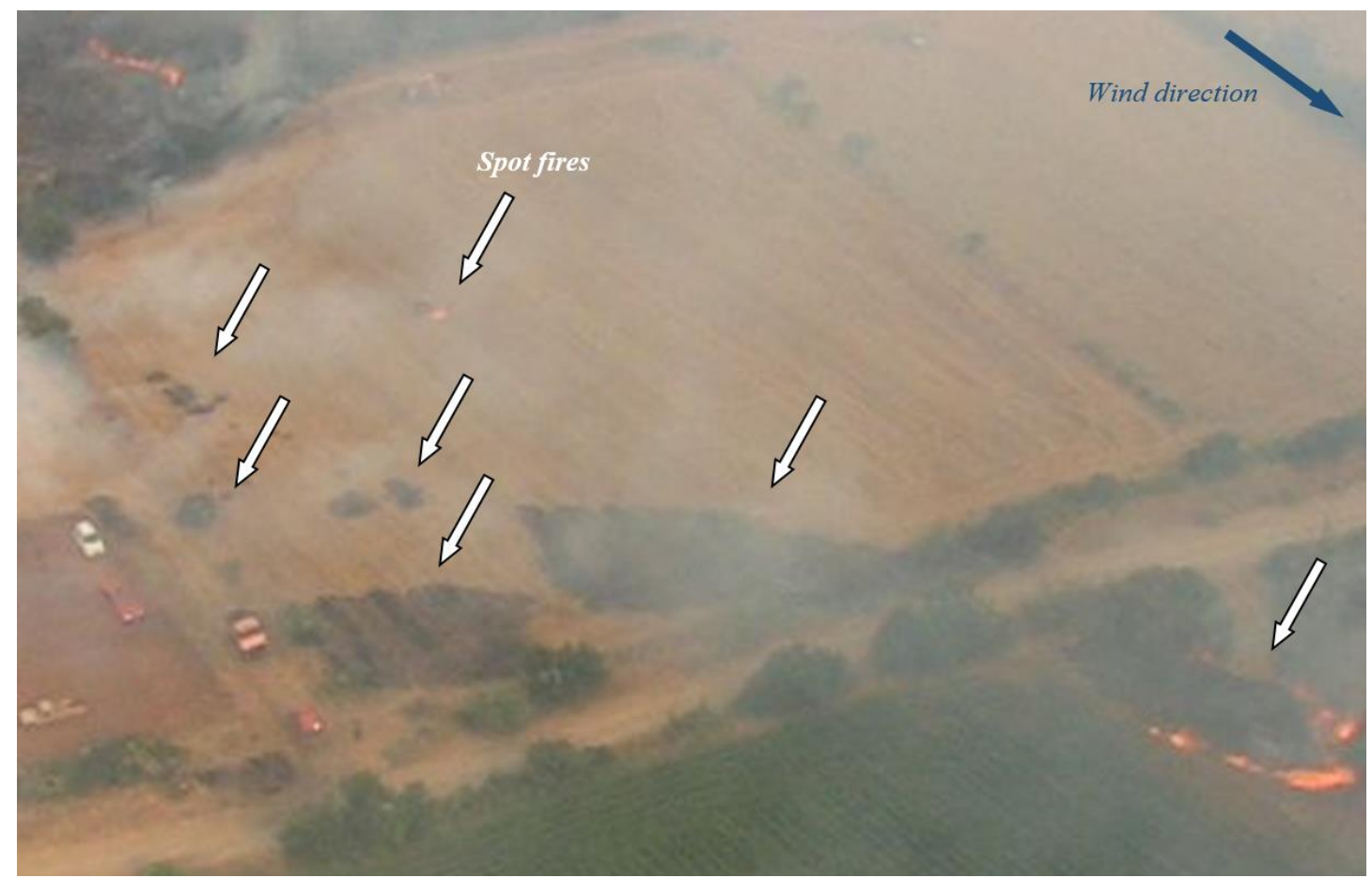

Figure 4a - An along-wind pattern of landing embers, on grass, downwind from a surface head fire

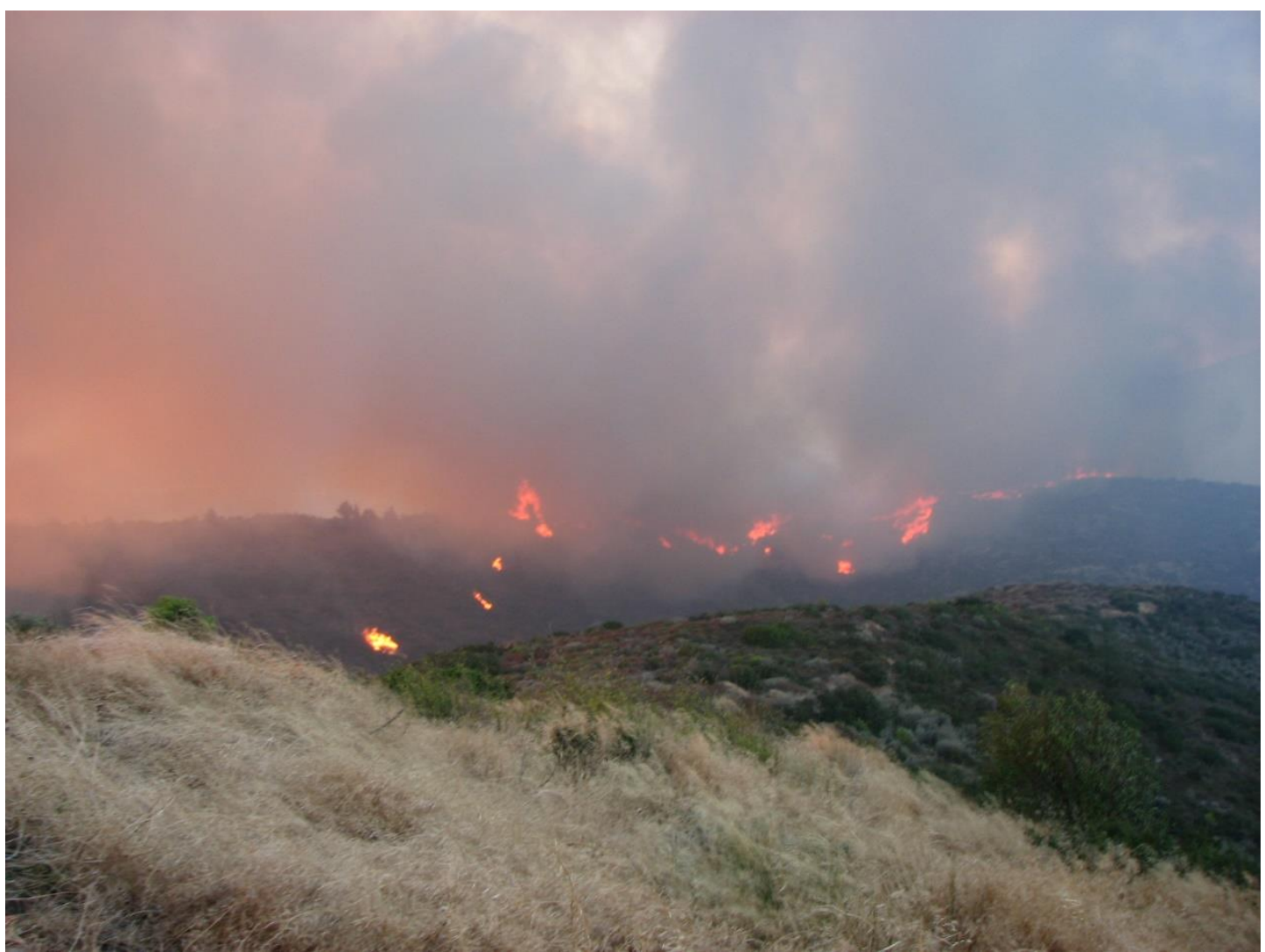

Figure $4 b$ - An across-wind pattern of landing embers, on phrygana beyond the flank of a surface wildfire that spreads through rough landscape

The along-wind pattern of groups of spot fires has been observed to form a roughly elliptical shape (Figure 4a) and seems to be more predictable, while the across-wind one (Figure $4 \mathrm{~b}$ ) is irregular. Although these patterns have not yet been thoroughly described, they may allow firefighters to get a feeling on what to expect, a practically useful information: those patterns may play a crucial role in 
the behavior as the proximity among the spot fires affects their merging rate and the overall effective rate of spread and intensity. As proposed by Gould et al. (2007), they can be studied in the field, leading to the development of empirical functions.

\section{Conclusions}

The findings of the work presented here is that no spotting ignition was recorded on maquis, phrygana and Mediterranean grasslands, at $\mathrm{RH}$ values higher than $46 \%$ and that massive spotting that triggered extreme fire behaviour, was documented for RH values lower than $17 \%$.

The RH threshold below which a spot fire is most likely to occur seems to be close to the value of $40 \%$. However, it was also observed that a significant number of spot fires may take place even if $\mathrm{RH}$ values range between $40 \%$ and $46 \%$. The finding that this is most likely to occur on fine fuels (phrygana and grass) should be taken into consideration, in operational firefighting.

The patterns of the spatial distribution of spot fires, and their basic characteristics in head and flank fires, can be included in practical guidelines about spotting

Future work is expected to shed additional light on the issues discussed in this paper, as field data continue being collected, ultimately improving fire behaviour prediction and firefighter safety in Greece.

\section{Acknowledgements}

The research reported here is part of the Ph.D. thesis of the first author. It was sponsored, in part, by the International Association of Wildland Fire (IAWF) through the Doctoral Student Scholarship Award for 2014. Participation of the second author was in the frame of the project MedWildFireLab ("Global Change Impacts on Wildland Fire Behaviour and Uses in Mediterranean Forest Ecosystems, towards a «wall less» Mediterranean Wildland Fire Laboratory") a European ERANet FORESTERRA project with funding from the General Direction for the Development and Protection of Forests and Agro-environment of the Greek Ministry of Environment and Energy.

\section{Bibliography}

Albini F.A., 1979. Spot fire distance from burning trees: a predictive model. USDA Forest Service, Intermountain Forest and Range Experiment Station, Technical Report INT-56. (Ogden, UT).

Alexander, M.E. 2009. Some pragmatic thoughts on the prediction of spotting in wildland fires. MITACS/GEOIDE Conference on Forest Fire Modelling, June 22-23, 2009 - Hinton, AB.

Athanasiou, M. and G. Xanthopoulos 2010. Fire behaviour of the large fires of 2007 in Greece. In proceedings of the 6th International Conference on Forest Fire Research, 15-18 November 2010, Coimbra, Portugal. D.G. Viegas, Editor. ADAI/CEIF, University of Coimbra, Portugal. Abstract p. 336, full text on CD.

Athanasiou M. and G. Xanthopoulos 2013. Observations of the spotting phenomenon, in wildfires in Greece. 30-40 p. In proceedings of the 16th National Forestry Conference, October 6-9, 2013, Thessaloniki, Greece. Hellenic Forestry Society. 1144 p. (in Greek).

Athanasiou, M. 2015. Development of an optimal methodology for forecasting forest fire behaviour in Greece. PhD dissertation, Department of Geology and Geoenvironment, National and Kapodistrian University of Athens, 408 p. (In Greek with English abstract).

Berlad AL, Lee SL 1968. Long-range spotting. Combustion and Flame 12, 172-174.

Bushfire CRC. 2009. Victorian Bushfires 2009 Research Taskforce. October 2009. Final Report. 
Byram, G.M. 1959. Combustion of forest fuels; Forest fire behavior. in Davis, K.P., ed. Forest fire: Control and use. New York: McGraw-Hill: 61-89, 90-123.

Ellis, P.F.M. 2000. The aerodynamic combustion characteristics of eucalypt bark - a firebrand study. A Thesis submitted to the Australian National University for the Degree of Doctor of Philosophy.

Ellis, P.F.M. 2012. A review of empirical studies of firebrand behavior. CSIRO Ecosystem Science and CSIRO Climate Adaptation Flagship.

Ganteaume A., Lampin-Maillet C., Guijarro M., Hernando C., Jappiot M., Fonturbel T., PerezGorostiaga P. and J.A. Vega. 2009. Spot fires: fuel bed flammability and capability of firebrands to ignite fuel beds. International Journal of Wildland Fire, 18, 951-969.

Gould, J.S. McCaw, W.L. Cheney N.P. Ellis, P.F. Knight, I.K. Sullivan, A.L. 2007. Project Vesta-Fire in dry eucalypt forests: fuel structure, fuel dynamics, and fire behaviour. Perth, WA: Ensis-CSIRO, Canberra ACT and Department of Environment and Conservation. 218 p.

Hadden, R., Scott, S., 2011. Ignition of Combustible Fuel Beds by Hot Particles: An Experimental and Theoretical Study. Fire Technology 47:341-355.

Koo, E., Pagni, P.J., Weise, D.R., Woycheese, J.P., 2010. Firebrands and spotting ignition in largescale fires. International Journal of Wildland Fire 19:818-843.

Manzello, S.L., Cleary, T.G., Shields, J.R., and Yang, J. C. 2006. On the ignition of fuel beds by firebrands. Fire and Materials 30: 77-87.

Racher, B. 2003. Prescription development for burning two volatile fuel types. Lubbock. TX: Texas Tech University. 72 p. Ph.D. dissertation.

Rothermel, R.C., 1983. How to predict the spread and intensity of forest and range fires. Gen. Tech. Rep. INT-143. Ogden, UT: U.S. Department of Agriculture, Forest Service, Intermountain Forest and Range Experiment Station. $161 \mathrm{p}$.

Van Wagner, C.E. 1988. Effect of slope on fires spreading downhill. Can. J. For. Res. 18:818-820.

Weir, John. 2004. Probability of Spot Fires During Prescribed Burns. Fire Management Today. 64. 24-26. 


\title{
Past fire practices and new steps towards an effective fire management approach in the Brazilian savannas
}

\author{
Eugênia Kelly Luciano Batista ${ }^{1 *}$; Jeremy Russell-Smith ${ }^{2}$; José Eugênio Côrtes Figueira ${ }^{1}$ \\ ${ }^{1}$ Department of General Biology, Federal University of Minas Gerais. 6627 Presidente Antônio \\ Carlos Ave, Belo Horizonte, MG, Brazil, \{biogenia.k@gmail.com*\} \\ ${ }^{2}$ Darwin Centre for Bushfire Research, Charles Darwin University. Ellengowan Drive, \\ Casuarina, Darwin, Australia \{Jeremy.Russell-Smith@cdu.edu.au\}
}

\begin{abstract}
Every year, widespread and severe fires cause loss of biodiversity and changes in the structure and functioning of savanna ecosystems. Currently, the Brazilian savanna (Cerrado) is threatened by the absence of a consistent fire policy. Most protected areas in Brazil still apply total fire suppression policies, instead of a more integrated fire management approach. Faced with the challenge of reconciling biodiversity conservation goals with rural livelihoods, scientists, policymakers and managers around the world have discussed potential solutions for managing fire in protected areas aimed at the sustainability of these ecosystems. Based on international experiences, an Integrated Fire Management Pilot Program was recently implemented in three Brazilian protected areas, while an adaptive and integrated approach has progressively raised the interest of other park managers. Initially, goals and actions have focused on protecting fire-sensitive vegetation and reducing the extent of areas burned, particularly at the end of the dry season. To achieve this, managers are making use of patch mosaic burning techniques to create mosaics of burnt and unburnt areas that purportedly decrease the probability of large wildfires and hence increase fire interval in fire-sensitive areas. However, despite advances in fire management policies in Brazilian savannas, an adaptive approach requires a continuous assessment of managed ecosystems, through which managers and ecologists can obtain knowledge about the responses of species and natural communities. Without a clear definition of appropriate ecological parameters, it is not possible to be sure if management actions or fire regime thresholds are actually delivering benefits for sustainable long-term biodiversity conservation. In this study, we use the example of the Canastra National Park (CNP) - Southeastern Brazil, to illustrate how widely employed suppression policies can result in inappropriate fire regimes and how these results have encouraged current changes in fire management perspectives.
\end{abstract}

Keywords: Fire regimes, Adaptive fire management, Suppression policies, Ecological monitoring, Cerrado.

\section{Suppression policies and recent changes in fire management}

Fire suppression policies have proved to be unsuitable for fire-prone environments but are still predominant in protected areas of Brazilian savannas. On the one hand, suppression policies are often associated with fire use criminalization and generally result in increased social conflicts (Batista et al. 2018). Without a dialogue with people who use fire as a management tool, it is expected that large and severe fires will occur more than would be both ecologically and socially tolerable. On the other hand, without fires, large amounts of biomass may accumulate rapidly after rains, being available for burning late in the dry season. These fires are potentially destructive because they are typically severe, less patchy (smaller and fewer unburned patches) and often grow to a large size (Oliveira et al. 2015). The homogenization of fire histories and landscape structure through large areas makes the recovery slower, especially for species with low dispersal capacity which depend on the migration of individuals from nearby areas across unsuitable habitat (Mendonça et al. 2015; Bowman and Legge 2016).

Advances in Forest Fire Research 2018 - Page 598 
It is also important to consider that slow-moving animals, those unable to fly, or with small home ranges can be easily burned during fast and hot grassland fires (Redford 1994; Koproski et al. 2006; Yates et al. 2008; Massochini Frizzo et al. 2011). Even those species that live in burrows or rock gaps can decline in abundance sometime after fire, emphasizing the relevance of indirect fire effects (especially large-scale and low-patchiness fires) on the sustainability of these species (Lawes et al. 2015). In particular, wildfires occurring at the end of dry season typically are of high intensity and with higher speed of fire fronts, which may intensify the mortality of plants and animals. These fires are of great concern in Brazilian savannas, because at the same period, animals are producing young and plants are investing in reproductive structures (Falleiro 2011). Young individuals are less able to escape or protect themselves from the flames. In plants, severe fires cause an immediate reduction in sexual reproductive success by causing abortion of flowers and fruits, interrupting the reproductive cycle and forcing vegetative resprouting (Hoffmann 1998).

From another perspective, too frequent, severe and large fires certainly result in significant economic damages. Large resource amounts are expended on fire fighting in Brazil annually. An average of $\mathrm{R} \$ 15$ million from the annual federal budget is destined to prevention and control of fires. Just in Minas Gerais, a state that encompasses many protected areas of Brazilian Cerrado, more than $\mathrm{R} \$ 20$ million is expended on fire prevention and control, mostly with airplanes and ground-based activities. Neither costs nor fire damages have reduced significantly throughout the time, which indicates that our strategies, in addition to be very expensive, are probably ineffective.

The apparent resistance in changing perspectives and actions seems to be related to a cultural heritage which considers that fire always has a negative impact on biodiversity (Bilbao et al. 2010; Durigan and Ratter 2016). Some ecologists and managers have argued that it is not possible to manage fire without scientific knowledge that could help to define ideal fire regimes for each ecosystem and support management decisions with a focus on biodiversity conservation. Meanwhile, the absence of a consistent fire management policy has resulted in inappropriate fire regimes and loss of biodiversity (Durigan and Ratter 2016).

When faced with similar challenges, Australian and South African managers concluded that fire was a natural component of savanna ecosystems that should not be excluded but properly managed to ensure the balance and sustainability of these habitats (Russell-Smith et al. 1998; Edwards et al. 2013; Van Wilgen et al. 2014). So, after years of an adaptive approach, managers and ecologists have learned by doing and currently they are able to refine their goals and actions focused on biodiversity conservation.

In Brazil, encouraged by successful international experiences and supported by federal legislation, Brazilian Environmental Agencies have implemented alternative and innovative fire management practices in some National Parks (Schmidt et al. 2018). Since 2014-2015, protected areas have been burned intentionally early in the dry season aiming to increasing the fire interval in fire-sensitive areas, producing heterogeneity and preventing high-intensity fires from reaching large sizes (Fig.1). These changes, although incipient and unrepresentative, have instigated discussions and stimulated the development of research that attempts to understand the role of fire in savanna ecosystems. 
(a)

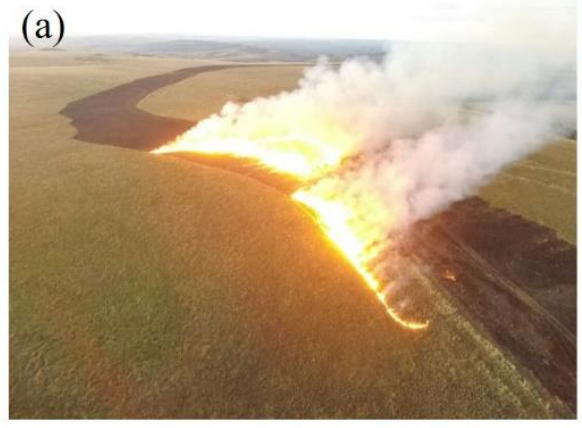

(b)
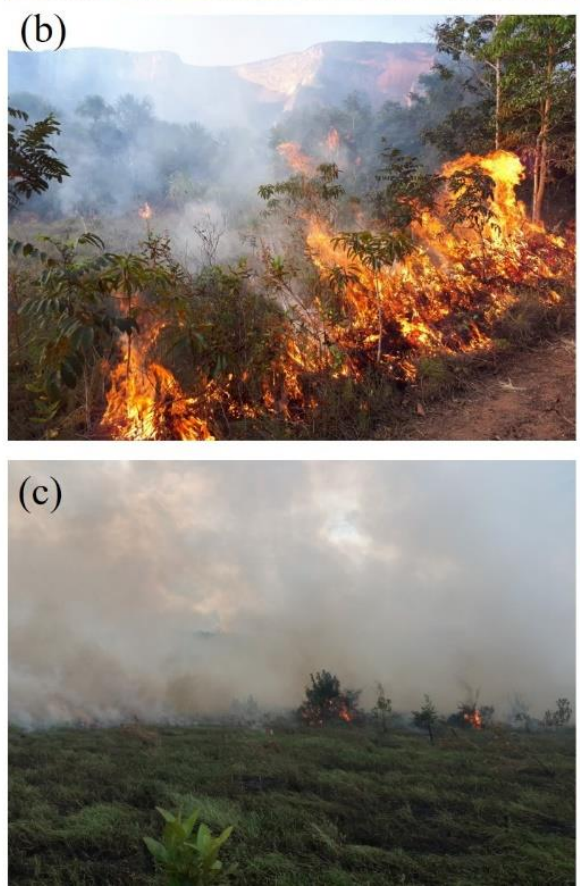
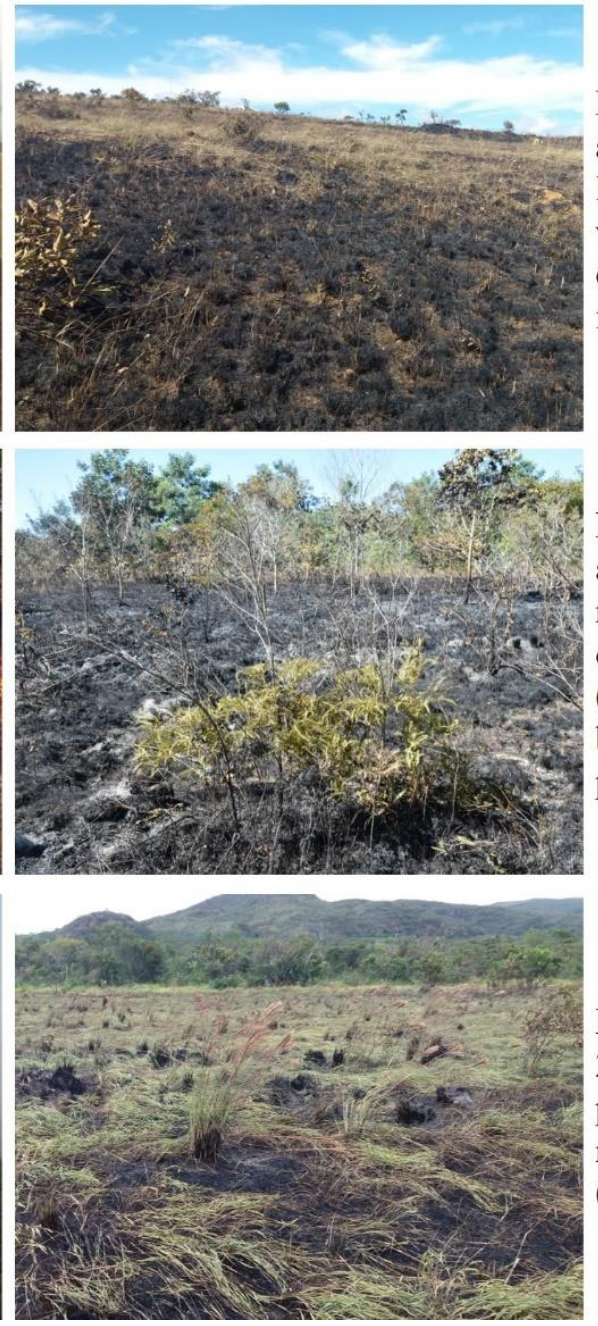

Prescribed burning in July 2016 at the CNP. Main goal: prevent large fires by increasing the width of firebreaks and establishing discontinuities in the fuel layer.

Prescribed burning in June 2018 at the CGNP. Main goal: create a mosaic of vegetation with different post-fire ages (pyrodiversity) focused on biodiversity conservation and preventing large fires.

Prescribed burning in March 2018 at the CiNP. Main goal: prevent severe fires from reaching fire-sensitive vegetation (riparian forest).

Figure 1 - Fire management in Brazilian savannas. To the left, prescribed burning. To the right, burned areas just after the fire. Note that planned fires are typically mild and patchy. At Canastra National Park (CNP) (a), the main concern has been to reduce the risks of large fires. At Chapada dos Guimarães National Park (CGNP) (b), managers have applied prescribed burnings to create a mosaic of patches in different post-fire ages. The idea is to introduce pyrodiversity expecting that the ecosystem will be able to sustain greater biodiversity. At Serra do Cipó National Park (CiNP) (c), first prescribed burnings are focused on the protection of fire-sensitive vegetation (forests).

In contrast, protected areas managed by the Brazilian States continue to support total suppression policies. Due to more restrictive legislation, the use of fire to manage landscapes and biodiversity is actually prohibited in most of these sites. As a result, large and severe fires burn extensive areas every year, causing serious damages to biodiversity and making it necessary to allocate much of the resources for firefighting rather than prevention (Fig.2). Advances in fire management policies in State protected areas would only be possible through adjustments in current legislation. 


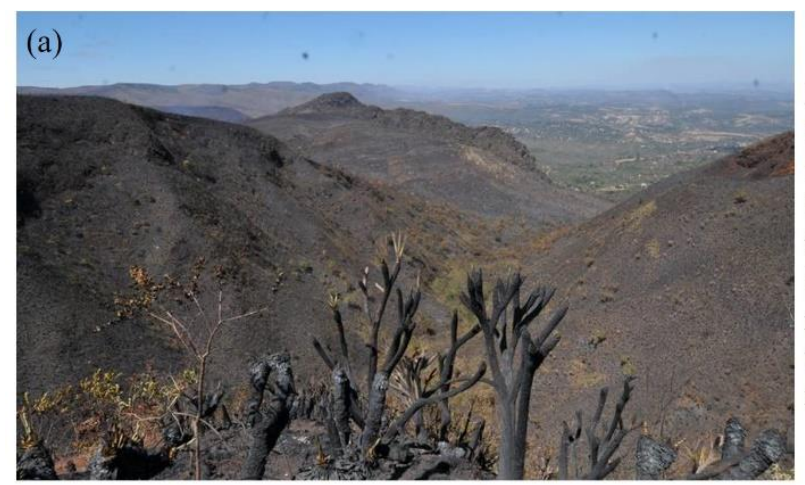

Large fires occur frequently in the Serra do Rola Moça State Park, Southeastern Brazil. In 2011, about 50\% of the Park were burned, including fire-sensitive vegetation around springs and riparian forest. In 2015, large and severe fires burned again around 55\% of the Park. Repeated fires can be seriously damaging, especially for fire-sensitive vegetation. Since inappropriate fire regimes may reduce the woody cover around springs and rivers, it is possible that the capacity of these habitats to collect and store water is also being negatively affected.

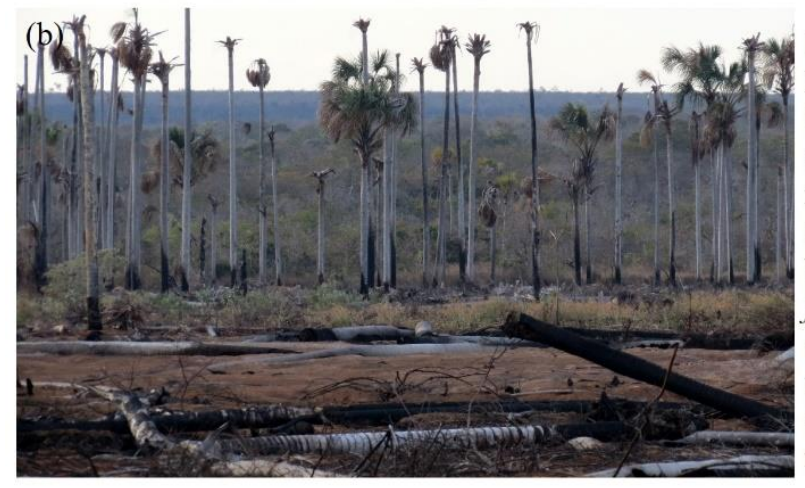

In 2017, a fire lit by a farmer in a swampy area along the Peruaçu River burned out of control and moved into the Veredas do Peruaçu State Park, Southeastern Brazil. From there, it proceeded to burn amidst grass, bushes, and palm trees (Mauritia flexuosa) for eight months. This fire smoldered underground in dried out, carbon-rich soil, spread slowly through soil and roots without any visible flames, but it was able to move up the hollow trunks of the Mauritia flexuosa and jump to the palm tree canopy. In that region, water consumption has been greater than recharge, reducing the flow of the Peruaçu River. As a result, the level of groundwater has reduced, drying up the palm swamps and making them more susceptible to new fires.

Figure 2 - Wildfires in Brazilian savannas. Large and severe fires occurring in two State Parks ((a)Serra do Rola Moça State Park and (b) Veredas do Peruaçu State Park) as a result of fire suppression policies still predominant in Brazil.

\section{Future challenges for fire management}

Firstly, fire management issues facing fire-prone ecosystems are symptomatic of ongoing conflicts between official fire exclusion policies in conservation reserves, and the livelihood aspirations and imperatives of embedded and surrounding local communities. We contend that an adaptive, inclusive approach between conservation agency authorities and local communities needs to be adopted for defining, and then collaboratively implementing, appropriate fire regimes which deliver benefits both for sustainable long-term biodiversity conservation and livelihood outcomes. Reconciling these apparently contradictory interests is the best possible solution to reduce ignitions and large, severe wildfires in protected areas.

Secondly, adaptive management provides a useful framework for integrating science and management and enables managers to work with uncertainty, incomplete knowledge and unreplicated studies. In adaptive forms of management, decisions are made as part of an ongoing process of review and evaluation of results. It involves a clear delineation of objectives, targets or thresholds of potential concern (TPC), the implementation of management actions, and the monitoring of outcomes in terms of agreed targets or thresholds (Van Wilgen et al. 2011).

If thresholds are exceeded, interventions must be done to drive the system back to within thresholds, or, alternatively, thresholds can be re-calibrated.

However, incorporating ecological thresholds into management is not a trivial task, especially in Brazilian savannas, whose landscapes are typically heterogeneous. Thus, the first issue that comes with ecological thresholds is: what is the conservation value of a given ecosystem or vegetation structure and what is the fire regime that will maintain these values? But even with clear fire regime thresholds, we can only be sure that they are actually delivering benefits for sustainable long-term 
biodiversity conservation if we monitor key ecological predictors. So, a second issue for ecologists and land managers is: what would be these predictors and how might they be assessed?

Finally, we will use the example of the Canastra National Park for making managers familiar with ecological thresholds for fire regimes and how to incorporate them into decision-making processes.

\section{Fire and management in the Canastra National Park}

The Canastra National Park was created in April 1972 and currently comprises an area of 197787 ha. In grassland areas it is mostly a short-memory system, with rapid fuel accumulation and curing of grasses and litter at the beginning of the dry season associated with low canopy cover. The uplands are characterized by an extensive matrix of grasses interspersed by small disconnected forest patches. Together, these features make the system highly vulnerable to the occurrence of large fires, which in turn creates uniformity in fire history and landscape structure, increasing the chances of subsequent large fire events. Generally, fires are initiated by farmers near, or in properties embedded within, the CNP. These fires invade the uplands and quickly become out of control (Fig.3).

$09 / 14 / 2006$

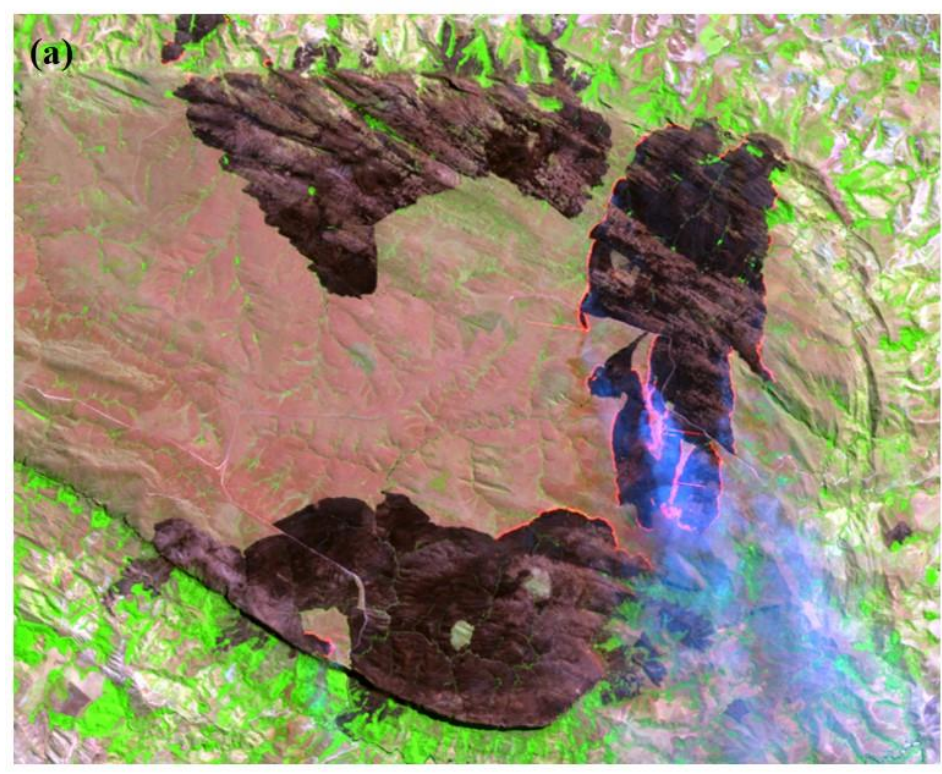

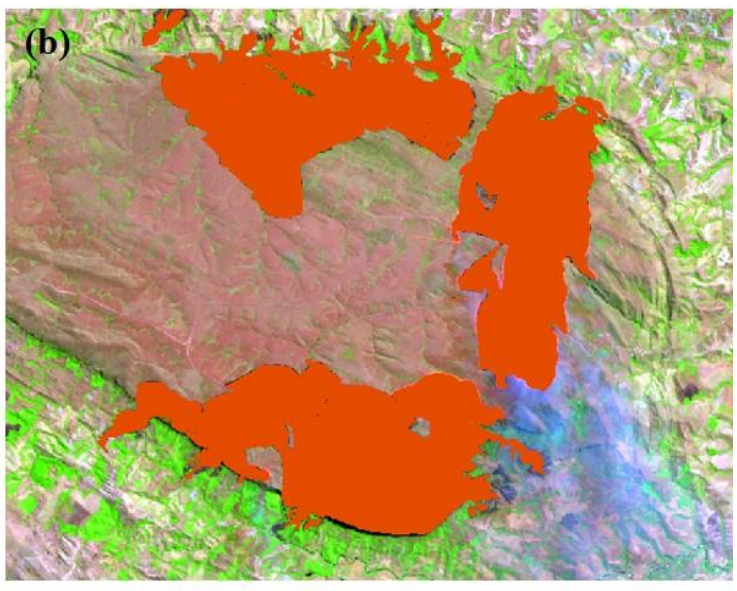

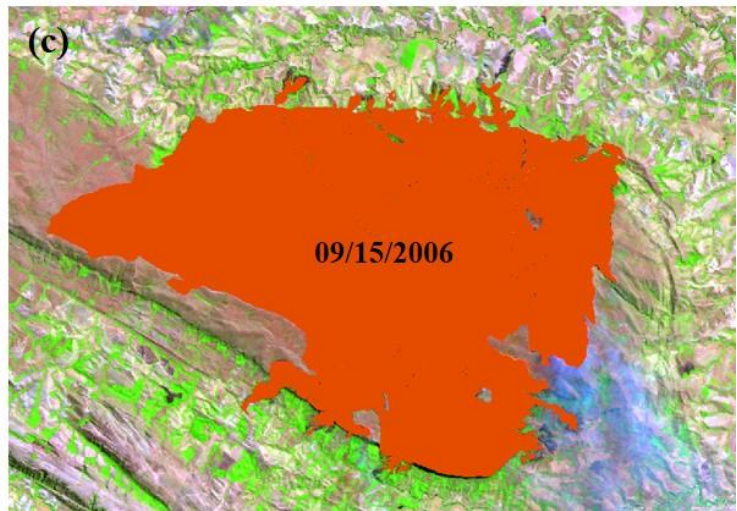

Figure 3 - Wildfires in the Canastra National Park. Fires usually are initiated by farmers near, or in properties embedded within, the CNP. These fires rapidly spread through the extensive and homogeneous grasslands and become out of control. (a) Landsat TM image (September 14th, 2006) showing different active fires invading the uplands of the Park. (b) Fire scars at the same day. (c) Fire scars extracted from subsequent images showing how quickly the fire spreads in this landscape. The date of scars was estimated through daily hotspots downloaded from the INPE website (http://www.inpe.br/queimadas/bdqueimadas).

Pastoral management undertaken by landowners is performed on patches with predominant biennial rotation, allowing the fire to occur annually and making the system entirely dependent on the fuel accumulation rates (bottom-up control). Concurrently, social conflicts intrinsic to the historical background and fire suppression policies increase the risk of unplanned fires late in the dry season. Given the high frequency of large and severe fires, especially in fire-sensitive habitats, the current fire 
regime and associated management arrangements are unlikely to deliver required sustainable biodiversity conservation management outcomes.

In an attempt to make the fire regime compatible with conservation goals, current managers have introduced alternative strategies and actions into fire management. Since 2017, prescribed burnings are being applied in grasslands to produce firebreaks perpendicular to each other. The main objective is to reduce the extent of wildfires and facilitate the fire control by creating discontinuities in the fuel layer. According to the parks' recently published Integrated Fire Management Plan, uplands will be divided into sectors that will be burnt alternately every two years. The Plan also recommends the creation of firebreaks in areas bordering the Park and around fire-sensitive vegetation, such as forests patches.

To implement thresholds appropriately, we propose to define a set of Fire Management Units (FMU) grouping physiognomies with similar fire requirements according to accumulated knowledge on the responses of species, populations and communities to fire regimes. The above delineation reveals two major FMUs: (1) Forests (fire-sensitive ecosystems) and (2) Grasslands/wooded savannas (fire-dependent ecosystems).

Forest patches in the CNP are generally small in size, which make them particularly vulnerable to fire impacts and ultimately extinction. The thin bark of forest species does not provide thermal protection to the vascular system and severe fires are usually able to kill the trees (Hoffmann and Solbrig 2003). After burning and death of trees, forest edges suffer biomass collapse and microclimate changes increasing openness making them susceptible to subsequent fire incursions. In addition, repeated topkill of saplings, sprouts and young trees prevents recruitment into adult size classes. In the course of this study, trees killed by fire were observed on the edges of forest patches of uplands, where very small patches were entirely burnt. Management actions in this FMU should focus on protecting and improving the quality of these habitats, minimizing the risk of severe fires, increasing biodiversity and controlling the edge-effects. We propose that no deliberate burning should be performed inside these units. Any late dry season fire should be extinguished. To protect the forests from fire incursions, some fuel control should be performed next to the patch borders and the maintenance of long-unburnt areas around these forest patches should be encouraged through reducing the frequency of fires. We suggest that monitoring should focus on forest -dependent taxa and specialists, sedentary species or distance-limited dispersers, and late successional species (rare and shade-tolerant). The presence of these species in viable populations would suggest that despite being a fragmented landscape, forest patches are able to support more demanding species (Broeck et al. 2017). Parameters related to the size of forest patches, their distribution through the landscape and edge effects could also be used to assess improvements in quality of these habitats after fire management (Didham and Lawton 1999; Hill and Curran 2003; Berry et al. 2015).

Grasslands and wooded savannas from the CNP are affected by large, severe and frequent wildfires. Homogenization of fire histories through large areas can delay or prevent the recovery process, especially by species with low dispersal capacity. Large fires can be especially deleterious to small, isolated populations of small mammals, slow-moving animals or those unable to fly or with small home ranges causing direct mortality, increased exposure to predators, and destruction of neighboring potential source areas. Basically, management actions in this FMU should focus on reducing the extent of wildfires (particularly at the end of the dry season), maintenance of intact unburnt vegetation and introduction of fire heterogeneity. We suggest reducing the fire frequency close to forest patches, by establishing long-unburnt areas (>2-9 years) to minimize the edge-effects on these habitats. Deliberate burning should be conducted in the grasslands before the late dry season months with the aim of increasing spatial heterogeneity, such that patches of unburnt vegetation are compartmentalized between recently burned areas and a system of strategically placed firebreaks. It would be advisable to monitor populations of small mammals, ground-dwelling birds and reptiles. These species are particularly vulnerable to increased predation after large and severe fires (Bowman and Legge 2016). 
In plants, obligate seeders and monocarpic species should be monitored for being more vulnerable to frequent fires (Noble and Slatyer 1980; Whelan 1995; Burrows et al. 2008; Figueira et al. 2016).

\section{Conclusions}

Faced with the need for a consistent fire management policy, Brazilian managers and ecologists have discussed potential solutions especially concerned with the sustainability of savanna ecosystems. The key challenge is getting park authorities and local communities to work together to devise effective fire management processes and programs. Successful integrated fire management would result in reducing the number of unplanned fires and the costs involved in firefighting. It is also important to clearly define management objectives and appropriate fire regime thresholds for each particular ecosystem. To ensure that management actions are delivering benefits for sustainable long-term biodiversity conservation, it is crucial that continuous assessment of managed ecosystems is undertaken through which managers and ecologists can actively obtain knowledge about species and natural communities ("learning by doing").

\section{References}

Batista EKL, Russell-Smith J, França H, Figueira JEC (2018) An evaluation of contemporary savanna fire regimes in the Canastra National Park, Brazil: Outcomes of fire suppression policies. Journal of Environmental Management 205, 40-49. doi:10.1016/j.jenvman.2017.09.053.

Berry LE, Lindenmayer DB, Driscoll D a. (2015) Large unburnt areas, not small unburnt patches, are needed to conserve avian diversity in fire-prone landscapes. Journal of Applied Ecology 52, 486495. doi:10.1111/1365-2664.12387.

Bilbao B a., Leal A V., Méndez CL (2010) Indigenous use of fire and forest loss in Canaima National Park, Venezuela. Assessment of and tools for alternative strategies of fire management in Pemón Indigenous Lands. Human Ecology 38, 663-673. doi:10.1007/s10745-010-9344-0.

Bowman DMJS, Legge S (2016) Pyrodiversity - why managing fire in food webs is relevant to restoration ecology. Restoration Ecology 24, 848-853. doi:10.1111/rec.12401.

Broeck A Vanden, Maes D, Kelager A, Wynhoff I, Wallisdevries MF, Nash DR, Oostermeijer JGB, Dyck H Van, Mergeay J (2017) Gene flow and effective population sizes of the butterfly Maculinea alcon in a highly fragmented, anthropogenic landscape. Biological Conservation 209, 89-97. doi:10.1016/j.biocon.2017.02.001.

Burrows ND, Wardell-Johnson G, Ward B (2008) Post-fire juvenile period of plants in south-west Australia forests and implications for fire management. Journal of the Royal Society of Western Australia 91, 163-174.

Didham RK, Lawton JH (1999) Edge Structure Determines the Magnitude of Changes in Microclimate and Vegetation Structure in Tropical Forest Fragments. Biotropica 31, 17-30. doi:10.1111/j.17447429.1999.tb00113.x.

Durigan G, Ratter JA (2016) The need for a consistent fire policy for Cerrado conservation. Journal of Applied Ecology 53, 11-15. doi:10.1111/1365-2664.12559.

Edwards AC, Maier SW, Hutley LB, Williams RJ, Russell-smith J (2013) Spectral analysis of fire severity in north Australian tropical savannas. Remote Sensing of Environment 136, 56-65. doi:10.1016/j.rse.2013.04.013.

Falleiro RDM (2011) Resgate do manejo tradicional do Cerrado com fogo para proteção das Terras Indígenas do oeste do Mato Grosso: um estudo de caso. Revista Biodiversidade Brasileira 1, 8696. 
Figueira JEC, Ribeiro KT, Ribeiro MC, Jacobi CM, França H, Neves ACO, Conceição AA, Mourão FA, Souza JM, Knegt CA de M (2016) Fire in rupestrian grasslands: plant response and management. 'Ecol. Conserv. Mt. grasslands Brazil'. (Ed GW Fernandes) pp. 1-34. (Springer International Publishing Switzerland: Belo Horizonte)

Hill JL, Curran PJ (2003) Area, shape and isolation of tropical forest fragments: effects on tree species diversity and implications for conservation. Journal of Biogeography 30, 1391-1403. doi:10.1046/j.1365-2699.2003.00930.x.

Hoffmann WA (1998) Post-burn reproduction of woody plants in a neotropical savanna: The relative importance of sexual and vegetative reproduction. Journal of Applied Ecology 35, 422-433. doi:10.1046/j.1365-2664.1998.00321.x.

Hoffmann WA, Solbrig OT (2003) The role of topkill in the differential response of savanna woody species to fire. Forest Ecology and Management 180, 273-286. doi:10.1016/S03781127(02)00566-2.

Koproski L, Mangini PR, Pachaly JR, Batista AC, Soares RV (2006) Impactos do fogo sobre serpentes (Squamata) no Parque Nacional de Ilha Grande (PR/MS), Brasil. Arquivos de Ciências Veterinárias e Zoologia da Unipar 9, 129-133. doi:10.25110/arqvet.v9i2.2006.355.

Lawes MJ, Murphy BP, Fisher A, Woinarski JCZ, Edwards AC, Russell-Smith J (2015) Small mammals decline with increasing fire extent in northern Australia: Evidence from long-Term monitoring in Kakadu National Park. International Journal of Wildland Fire 24, 712-722. doi:10.1071/WF14163.

Massochini Frizzo TL, Bonizário C, Borges MP, Vasconcelos HL (2011) Revisão dos efeitos do fogo sobre a fauna de formações savânicas do Brasil. Oecologia Australis 15, 365-379. doi:10.4257/oeco.2011.1502.13.

Mendonça AF, Armond T, Camargo ACL, Camargo NF, Ribeiro JF, Zangrandi PL, Vieira EM (2015) Effects of an extensive fire on arboreal small mammal populations in a neotropical savanna woodland. Journal of Mammalogy 96, 368-379. doi:10.1093/jmammal/gyv038.

Noble IR, Slatyer RO (1980) The use of vital attributes to predict successional changes in plant communities subject to recurrent disturbances. Vegetatio 43, 5-21. doi:10.1007/BF00121013.

Oliveira SLJ, Campagnolo ML, Price OF, Edwards AC, Russell-Smith J, Pereira JMC (2015) Ecological implications of fine-scale fire patchiness and severity in tropical savannas of Northern Australia. Fire Ecology 11, 10-31. doi:10.4996/fireecology.1101010.

Redford KH (1994) The edentates of the Cerrado. Edentata 1, 4-10.

Russell-Smith J, Ryan PG, Klessa D, Waight G, Harwood R (1998) Fire regimes, fire-sensitive vegetation and fire management of the sandstone Arnhem Plateau, monsoonal northern Australia. Journal of Applied Ecology 35, 829-846.

Schmidt IB, Moura LC, Ferreira MC, Eloy L, Sampaio AB, Dias PA, Berlinck CN (2018) Fire management in the Brazilian Savanna: first steps and the way forward. Journal of Applied Ecology. doi:10.1111/1365-2664.13118.

Whelan J. R (1995) 'The Ecology of Fire.' (Cambridge University Press: Cambridge, UK)

Van Wilgen BW, Govender N, Forsyth GG, Kraaij T (2011) Towards adaptive fire management for biodiversity conservation: Experience in South African National Parks. Koedoe 53, 1-9. doi:10.4102/koedoe.v53i2.982.

Van Wilgen BW, Govender N, Smit IPJ, MacFadyen S (2014) The ongoing development of a pragmatic and adaptive fire management policy in a large African savanna protected area. Journal of Environmental Management 132, 358-368. doi:10.1016/j.jenvman.2013.11.003. 
Advances in Forest Fire Research 2018 - D. X. Viegas (Ed.)

Chapter 3 - Fire Management

Yates CP, Edwards AC, Russell-Smith J (2008) Big fires and their ecological impacts in Australian savannas: Size and frequency matters. International Journal of Wildland Fire 17, 768-781. 


\title{
Prescribed fire experiences on crop residue removal for biomass exploitations and cost-benefits analysis, in mediterranean ecosystems.
}

\author{
Francisco Rodríguez y Silva*; Juan Ramón Molina \\ ${ }^{1}$ Forest Fire Laboratory. University of Córdoba (Spain), Edificio Leonardo da Vinci, Campus de \\ Rabanales, 14071, Córdoba, \{ir1rosif@uco.es*,jrmolina@uco.es\}
}

\begin{abstract}
The progressive increase in the use of prescribed fires, as a fire management technique for the prevention and reduction of the impact of forest fires, demands the development of new approaches. The generation of interesting utilities of this technique is not only related to the control of fuel load, but also to the creation and maintenance of operational scenarios. Biomass harvesting possibilities to rural development have led to a high woody biomass accumulation increasing potential wildfire severity. Mechanical thinning and prescribed burning practices could be jointly used to prevent large fires. The aim of this study was to assess burning treatment effectiveness in a medium-term planning (12 years) following mechanical thinning from biomass harvesting. An econometric model was designed to predict prescribed fires costs to reduce slash fuels, and as a consequence, the maximum efficiency or cost-benefit, mainly in strategic management or critical focal points.

Prescribed fires reduced available fuel in all fuel categories, mainly in fine dead fuel category. A costbenefit analysis to mitigate fire behavior was carried out using different burning severities. An efficiency burning management could reduce between $19.94 \%$ and $40.63 \%$ of the total costs in a medium-term planning (12 years). The most significant variables are related to fire severity (flame residence time) and stand characteristics, mainly canopy base height and remnant canopy cover. These variables allow us to predict costs per unit area based on different management alternatives. Managers can use this information to design technical prescription to achieve the targets while decomposed litter retention maintaining the soil properties and biodiversity under economic point of view. Understanding the most effective "burn window" should help better plan prescribed burning, in term of fire behavior, fuel consumption and the total budget that is necessary to reach the forest fires prevention and impact reduction in the forest landscapes in a medium-term planning.
\end{abstract}

Keywords: prescribed fire effectiveness, fire severity, fuel consumption, convective pruning; burning costs; presceibed fire planning

\section{Introduction}

Prescribed fires strategically allocated could be combined with traditional forest treatments, such as brush clearing, thinning and pruning, to reduce extent and severity of wildfires (Agee and Skinner, 2005). When thinning operations were made to biomass harvesting, a high load of dead fuel was appeared, independently of the skidding method. Thinned forests created mosaics of canopy gaps that provide an increment of the wind speed based on a reduction of draw coefficient (Molina et al., 2018). Prescribed fire treatments can reduce the available surface fuel, mainly 1- and 10-hr dead fuel generated by biomass harvesting, and as a consequence, the probability to transition to crown layer. Prescribed fires under these biomass harvesting stands should be achieved by a "burn window" which included tree mortality and convective heating.

There are some new sources of data on costs of burnings, prevention in costs or factor influencing the cost variability (Rodríguez y Silva, 2018). The total costs of prescribed fires includes burn preparation, burn, smoke management, general overhead and post-fire evaluation (Cleaves et al., 2000). Costs can vary depending on fire complexity, objectives, operational efficiency and unit size. 
In spite of one American study showed that burn objective was not a significant cost factor in prescribed fires (González-Cabán, 1997), the objectives of the burn could modify safety precautions based on fire severity (Rodríguez-Cabán and McKetta, 1986; Vega et al., 2001). Unit size is other of the most important factors; larger units have smaller costs. Costs also vary with the irregularity shape of the unit but it can be necessary in strategic management points. In stands areas, managers can incurs higher costs by using slower and less severe ignition techniques than head-fire.

Assessing the cost-effectiveness of fuel treatments presents many challenges, mainly in prescribed fires (Omi et al., 1999). Although prescribed fire treatments generally are lower in cost than other fuel treatments (i.e., mechanic al thinning and pruning), fire also is more variable in its effects depending on fire severity. Shaded fuelbreaks must be created in the context of the landscape within which they are placed (Agee et al., 2000). Fuel accumulation rate frequently limits prescribed fire effectiveness to a short post-treatment period (2-4 years) (Fernandes and Botelho, 2003). Prescribed fire effectiveness should be based on the mitigation or reduction of both surface and crown fire behavior. The effectiveness of a prescribed fire may be obscured by forest structure, fuel moisture, weather conditions and ignition patterns (Van Mantgem et al., 2016). Although prescribed fires reduced surface fuels, it is possible that coarse woody fuel and needles may be high from branches from convective heating (Agee and Lolley, 2006; Molina et al., 2018).

This work presents the results of combinations of biomass harvesting thinning and burning treatments. In this sense, this paper aims at developing an economic approach to identify the effects of prescribed fires on slash reduction based on a medium-term sampling (12 years). By extending prescribed fire study from the original point of fuel consumption, we have incorporated economic medium-term approach in order to identify effects of each fire severity on prescribed fire effectiveness.

\section{Material and methods}

\subsection{Study area}

The study was conducted in four study areas (Riofrio area, Sierra Alta area, Parrillas area and Velefique-Senés area) located in southern of Spain (Figure 1), occupying a sampling area about 100 ha. They are located in two regional administrative divisions (Andalusia and Castilla la Mancha) covering three 1 administrative provinces (Córdoba, Almería and Ciudad Real). All of these provinces are characterized by a Mediterranean climate with a pronounced dry period and summer temperature usually reaches $40^{\circ} \mathrm{C}$.

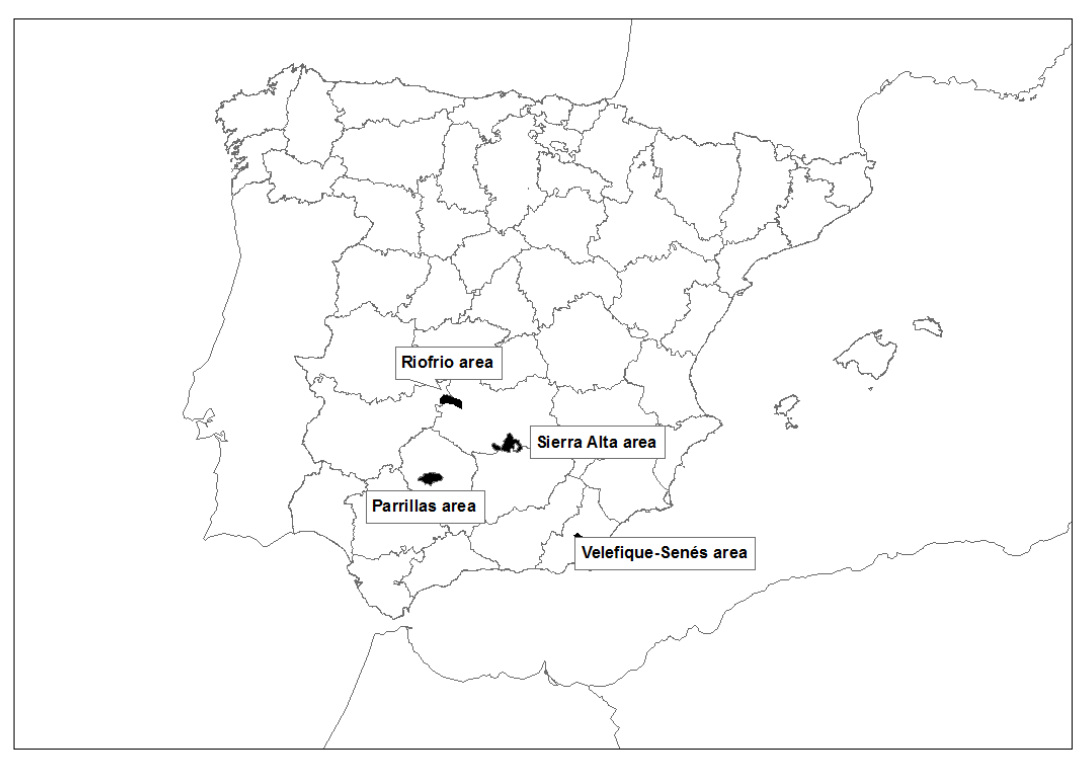

Figure 1 - Study areas location

The study was undertaken in three Pinus pinaster areas (Riofrio area, Sierra Alta area and Parrillas area) and one Pinus nigra area (Velefique-Senés area). Differences in stand characteristics (density, canopy base area, crown height,...) were shown among the different study areas.

The study area has been management from the viewpoint of forest biomass harvesting according to periodic thinning operations. Fire management agency tries to remove the important load of slash on 
different stands involved in biomass harvesting using burning. Mechanical selected thinning followed by prescribed fire was tested in combination. Stands were exploited at least two years before first burning in order to get a minimum dynamics on undergrowth fuel.

Total costs of these prescribed fires have included burn preparation (planning redaction, perimeter cleaning, pruning,...), burn (ignition, safety and liquidation resources) and post-prescribed fire evaluation (surface and crown layers). A spreadsheet was used by forest managers to include every costs. While planning redaction and post-fire evaluation was valued using number of required hours (technicians), preparation and burn resources were valued using the official cost per hour (Andalusia Government). The price of gasoil was included according to a filling-station' bill.

\subsection{Sampling design}

The data sampling collected both environmental information and fuel variables to assess prescribed fire effectiveness. A random stratified inventory was made according to proportional allocation. The forest inventory was carried out in circular plots of 1,000 $\mathrm{m} 2$ across the different stands incorporating variables diameter at breast height, tree height and basal area. The characterization of surface fuel was conducted though destructive transects using the line-intercept method (Stephens and Modhaddas, 2005). Fuel load was calculated in sampling plots with areas of $1 \times 1 \mathrm{~m}$ showing fuel load by category (live and dead) and particle size (expressed in 1-, 10-, $100 \mathrm{~h}$ time-lag). Separate live and dead fuel by particle size was needed to identify fuel moisture. Each sample underwent a 72-hours drying process in an oven set at $70^{\circ} \mathrm{C}$, at which this time, fuel weights were constant.

Thermocouples type $\mathrm{k}$ ( $50 \mu \mathrm{m}$ diameter) were placed inside the surface and crown layers. We could obtain spread rate, maximum temperature and flame residence time from these data (Table 1). Two video cameras and an infrared camera were also used to determinate flame length.

Table 1 - Equations for fire behavior modeling

\begin{tabular}{|l|l|}
\hline Variable & Equation \\
\hline Flame length & $\mathrm{L}=\mathrm{F} / \sin \mathrm{A}$ \\
\hline Fire-line intensity & $\mathrm{I}=(\mathrm{HC} * \mathrm{AF} * \mathrm{SR})$ \\
\hline Heat per unit area & $\mathrm{H}=\mathrm{HC} * \mathrm{AF}$ \\
\hline
\end{tabular}

where $\mathrm{L}$ is the flame length expressed as $\mathrm{m}, \mathrm{F}$ is the flame height measured in $\mathrm{m}, \mathrm{A}$ is the flame angle identified in ${ }^{\circ}$, I is the fire-line intensity expressed as $\mathrm{kW} / \mathrm{m}$, AF is the available fuel load measured as $\mathrm{kg} / \mathrm{m}^{2}$, SR is the spread rate expressed as $\mathrm{m} / \mathrm{s}, \mathrm{H}$ is the heat per unit area expressed as $\mathrm{kJ} / \mathrm{m}^{2}, \mathrm{HC}$ is the heat of combustion $(18,500 \mathrm{~kJ} / \mathrm{kg})$ and $\mathrm{AF}$ is the available fuel load measured as $\mathrm{kg} / \mathrm{m}^{2}$. A basic value of $18,500 \mathrm{~kJ} / \mathrm{kg}$ can be used for the heat of combustion or HC (Albini, 1976).

ANOVA analysis and Tukey HSD test were performed to identify if significant differences ( $\mathrm{p}<$ 0.05 ) existed in the dynamics of surface fuel load by categories and canopy base height according to each prescribed fire. In a similar way, a temporal horizon planning of 12 years would be used to evaluate if significant differences $(\mathrm{p}<0.05)$ existed in the economic effectiveness of each prescribed fire. Multiple regression analysis was used to develop fire prescribed cost from environmental and burning variables. In some cases, a Principal Component Analysis (PC) allowed us to reduce a smaller set of variables.

\section{Results}

Total cost of prescribed fires in Sierra Alta area ranged from $398.43 € /$ ha to $1,445.5 € /$ ha, with an average value of $911.07 € /$ ha. While dead fuel moisture ranged from 11\% (burning 2016 in Sierra Alta 
II) to $17 \%$ (burning 2011 in Sierra Alta II), flame length varied between 0.3-0.5 (burning 2016 in Sierra Alta I) to 0.8-0.9 (burning 2011 in Sierra Alta II).

Firstly, we analyzed surface fuel in Sierra Alta I during seven years: before a first moderate burning (2009), five years after the first burning and before the second prescribed fire, and one year postsecond severe burning (Figure 2). The first burning did not reduce the $1 \mathrm{~h}$ time-lag dead fuel and even it promoted the proliferation of live fuel $(0.17 \mathrm{t} / \mathrm{ha})$. The amount of $10 \mathrm{~h}$ time-lag dead fuel varied significantly after the first burning. At the second burning, all of the categories decreased in relation to the rest of the temporal monitoring.

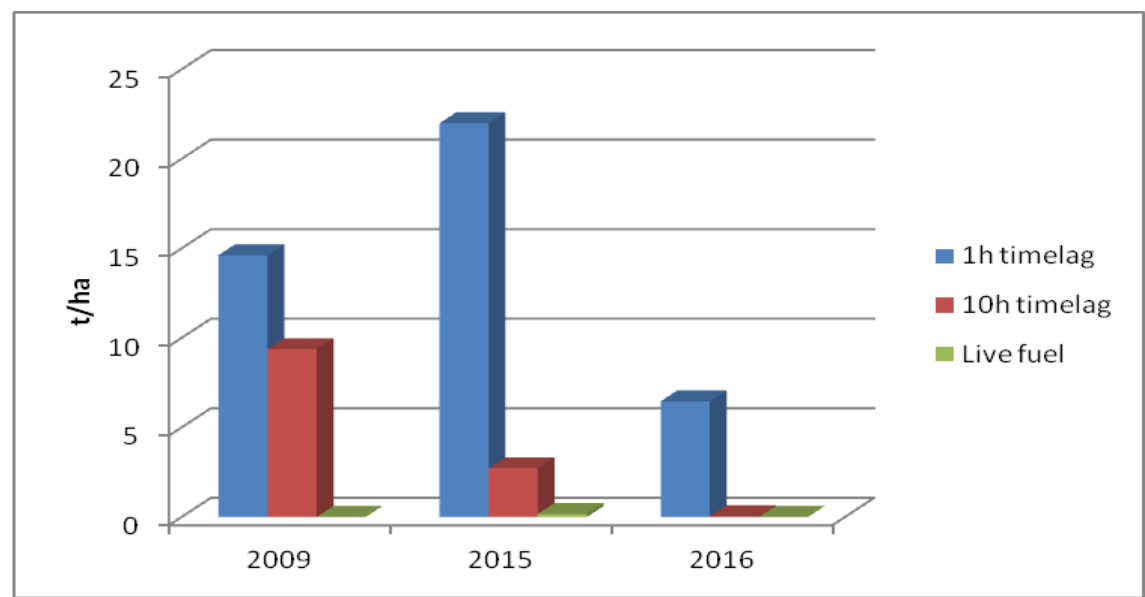

Figure 2 - Temporal evolution of surface fuel model in Sierra Alta I

We analyzed surface fuel in Sierra Alta II during six years: before first moderate burning (2010), four years post-first burning and before the second severe prescribed fire, and one year post-second burning (Figure 3). The first burning reduced dead fuel, mainly the 10h time-lag fuel. There were not significant differences between before and after the second prescribed fire in relation to $1 \mathrm{~h}$ time-lag dead fuel. In this case, live fuel appeared before the second burning $(0.08 \mathrm{t} / \mathrm{ha})$.

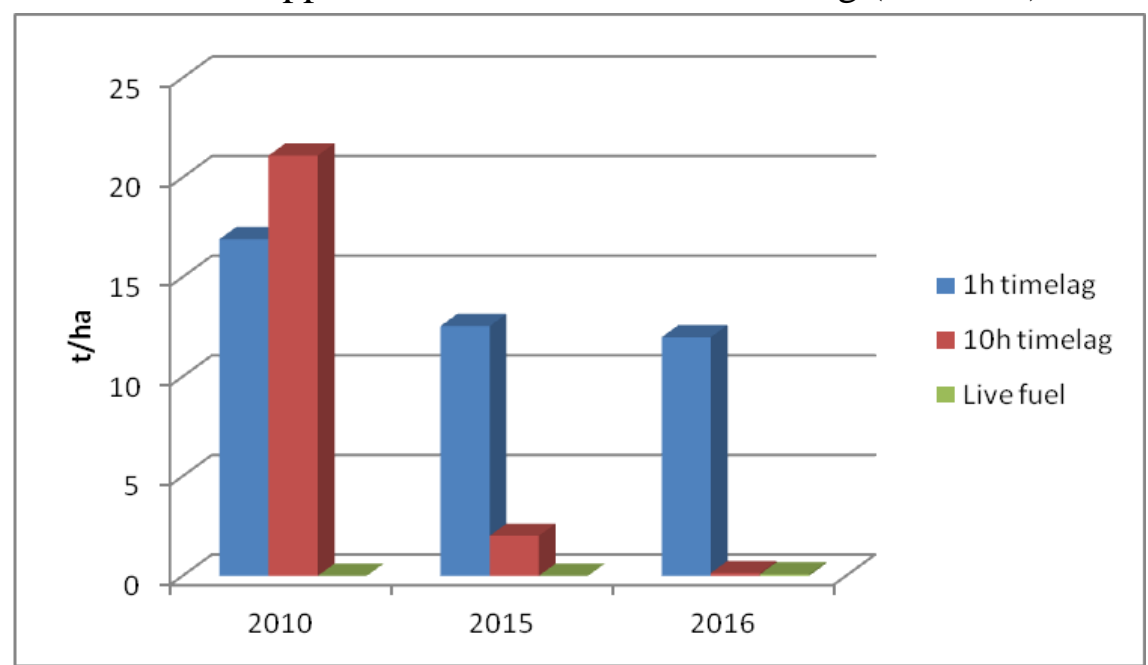

Figure 3 - Temporal evolution of surface fuel model in Sierra Alta II

The crown layer was evaluated based on canopy base height and crown scorch o crown reduction (available fuel load on canopy for crown fire). In Sierra Alta I, while a low crown height was affected 
by convective heating in the first burning, more than $50 \%$ of the crown was affected in the second burning (Figure 4). Canopy base incremented from $3 \mathrm{~m}$ to $7.12 \mathrm{~m}$ after the second burning.

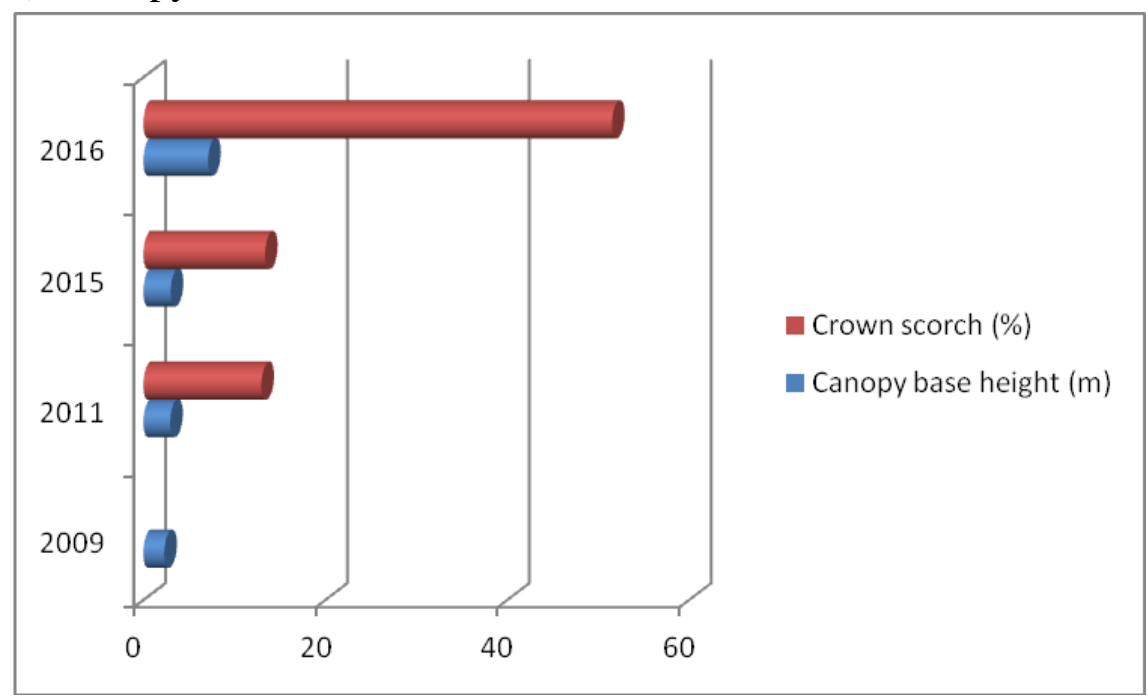

Figure 4 - Temporal evolution of crown fuel model in Sierra Alta I

In Sierra Alta II, there was a smaller affected crown height (less than $5 \%$ of the crown) than in Sierra Alta I. However, in the second burning treatment the crown scorch increased at $64.24 \%$ (Figure 5). Therefore, canopy base height was higher than in Sierra Alta I after the second burning.

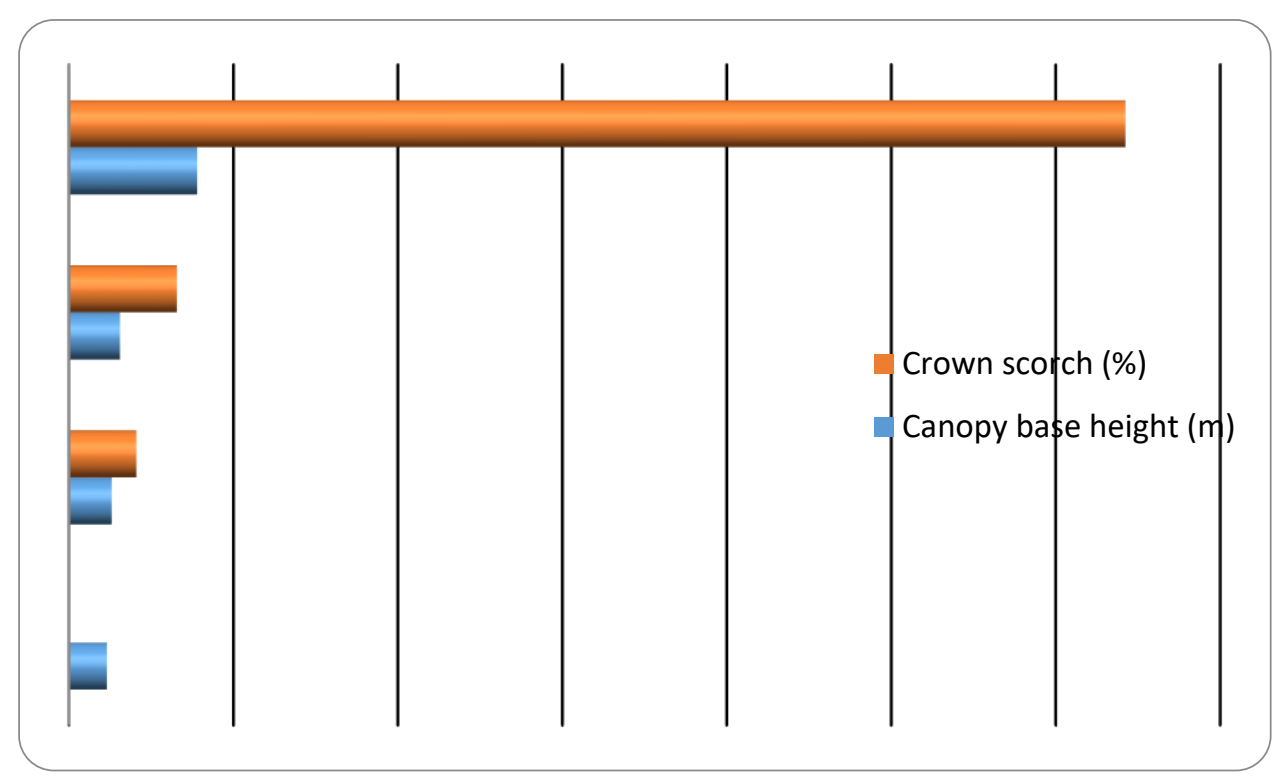

Figure 5 - Temporal evolution of crown fuel model in Sierra Alta II

In some severe convective prunings (burnings with crown scorch upper than 50\%), the reduction of canopy cover promotes the proliferation of live and very flammable fuel. In only one year, understory grew $0.08 \mathrm{t} / \mathrm{ha}$ due to the sun exposure because of the affectation of $64.24 \%$ of the tree crowns (Sierra Alta II) (Figure 6). In Parrillas area, live fuel grows exponentially (Figure 7), reaching its maximum development in a severe burning where remnant canopy cover was lower than $40 \%$. In three years, live fuel increases to $3.64 \mathrm{t} / \mathrm{ha}$ modifying the original fuel model (needles fuel model to a understory fuel model). Therefore, in Parrillas area, a higher tree mortality has been identified by more 
severe burnings (higher flame residence times). In this sense, tree mortality ranged from $6.37 \%$ (light burning) to $27.47 \%$ (severe burning). Although biomass harvesting thinning was similar, and as a consequence, the remnant canopy cover $(47.18 \%$ and $48.69 \%$, respectively), tree mortality promoted gaps where live fuel was established, modifying the original fuel model. Furthermore, it highlights the better condition of Pinus pinaster regeneration in light burning (3,392 seedlings/ha) than in severe burning (633 seedlings/ha) after three years.

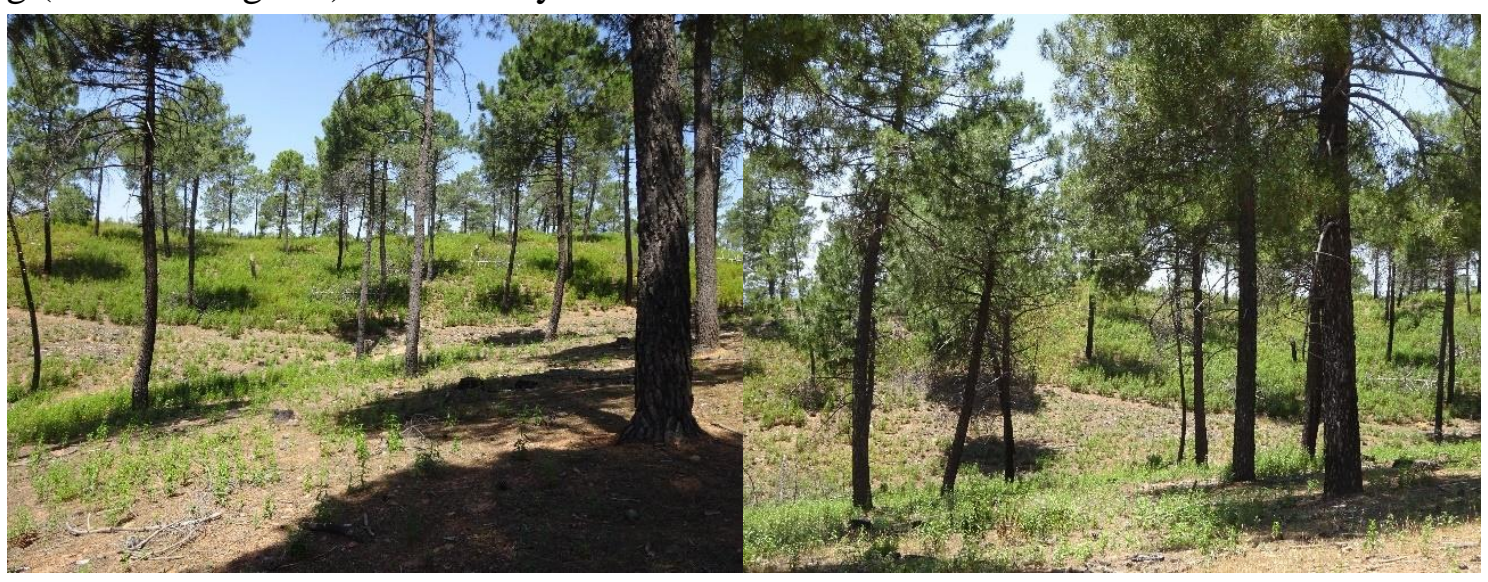

Figure 6 - Temporal evolution of two different burnings according to its fire severity

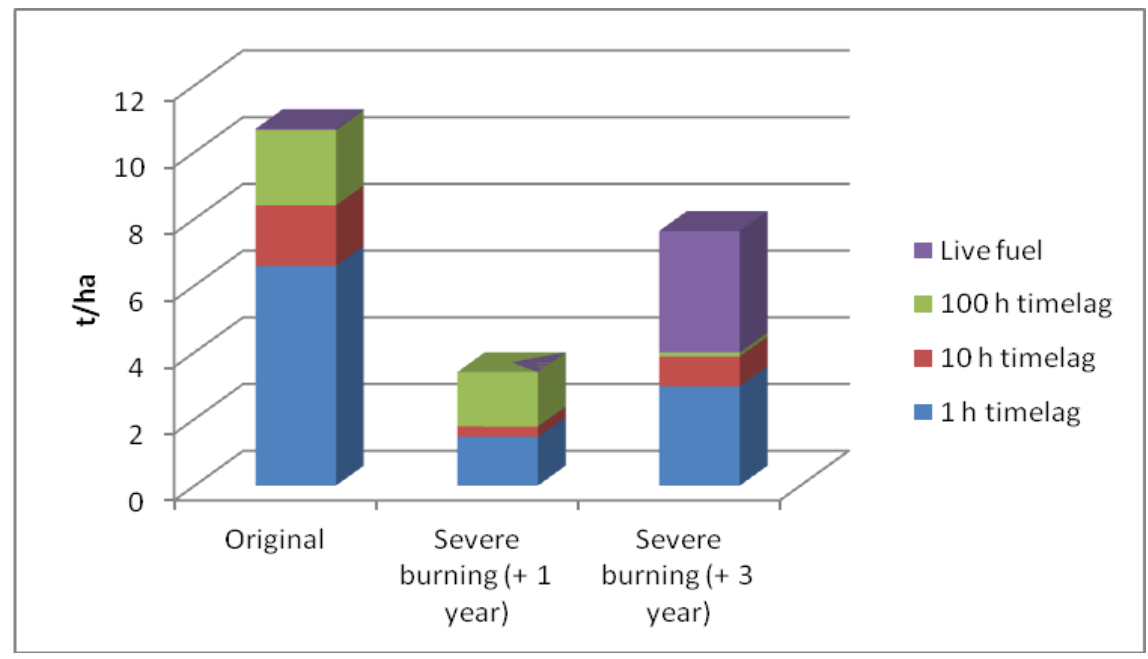

Figure 7 - Temporal evolution of severe burning in Parrillas area

Significant differences were found in Riofrio area under three different fire severities (Table 2). In this area, tree mortality ranged from $0.94 \%$ (similar to $0.63 \%$ of natural area) to $12.21 \%$ according to fire severity (Figure 8). Live and flammable fuel increased between 25 and $35 \%$ in more severe prescribed fires in only three years due to the canopy gaps.

Table 2 - Fuel load generated by three different fire severities in Riofrío area

\begin{tabular}{|c|c|c|c|}
\hline Fuel type & Severity A & Severity B & Severity C \\
\hline Needles (t/ha) & $5.37^{\mathrm{a}}$ & $4.37^{\mathrm{a}}$ & $7.46^{\mathrm{b}}$ \\
\hline 1h time-lag (t/ha) & $1.09^{\mathrm{a}}$ & $0.77^{\mathrm{b}}$ & $1.43^{\mathrm{c}}$ \\
\hline 10h time-lag (t/ha) & $0.44^{\mathrm{a}}$ & $0.70^{\mathrm{b}}$ & $1.45^{\mathrm{c}}$ \\
\hline 100h time-lag (t/ha) & $1.64^{\mathrm{a}}$ & $0.40^{\mathrm{b}}$ & $1.35^{\mathrm{a}}$ \\
\hline Live fuel & $0^{\mathrm{a}}$ & $0.32^{\mathrm{b}}$ & $0^{\mathrm{a}}$ \\
\hline Total & $8.55^{\mathrm{a}}$ & $6.57^{\mathrm{a}}$ & $11.70^{\mathrm{b}}$ \\
\hline
\end{tabular}



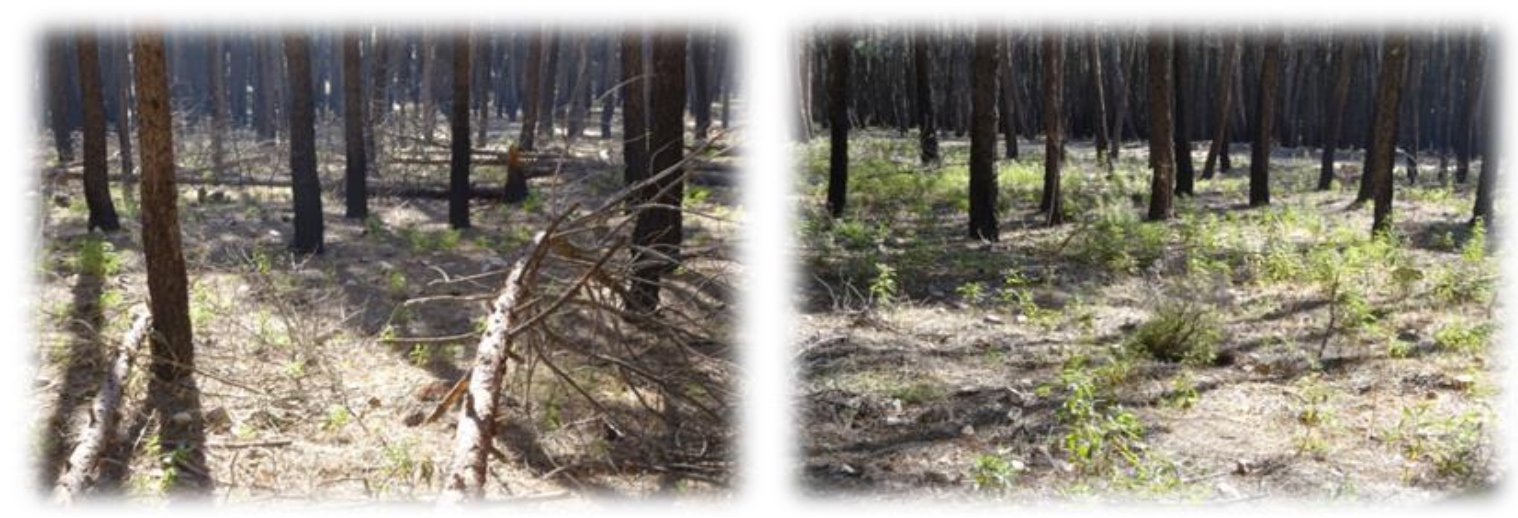

Figure 8 - Temporal evolution of severe burning in Riofrio area. Tree mortality and the proliferation of live fuel in three years can be observed

In Velefique-Senés area, most of the preliminary results were tested and validated. We can note the lower difference in the remnant condition of the surface and crown fuels due to canopy base height and the large presence of dead fuel before the burning. In this area, prescribed fire costs increases to $1,040-1,400 € /$ ha with perimeter treatment without inclusion.

\section{Discussion}

Despite the surface fuel reduction was very high in all burnings (ranging from 59.07\% to 86.18\%), needles and coarse woody fuel fell from canopy due to the convective heating (Agee and Lolley, 2006). Fire severity was one of the most important parameter in prescribed fire effectiveness (Van Mantgem et al.. 2016). When the first burning severity was moderate, a large of amount of needles fell on the ground (Sierra Alta I) showing a fuel load close to the original fuel model in six years. In these cases, it is necessary a second burning in a short-term period to mitigate crown fire probability. On the other hand, if fire severity is lower, the burning treatment may be effective six years later. Although the most efficient "burn window" was identified by a litter moisture ranged from 9\% to 15\%, (Molina et al., 2018), it only considered surface fuel consumption. If we took into account the convective heating and pruning generated to canopy trees, these conditions would be reduced between $13 \%$ and $15 \%$. Litter moisture upper than $15 \%$ did not reach the reduction targets of the burning, and as a consequence, it limits prescribed fire effectiveness.

All canopy cover changes, mainly stand density and canopy cover fraction, have provided an increment of the wind speed based on a reduction of draw coefficient. The wind speed was taken from two anemometers. located at treeless area and inside of each burn the day before burning without influence of fire and smoke. Drag coefficient ranged from 0.34 to 0.55 for the studies areas based on stand density and crown condition at the burning moment. Changes in drag coefficient have a direct influence in spread rate. and as a consequence. in fire-line intensity. Changes in dead fuel moisture were only observed by the comparison between canopy covers less than $75 \%$ and canopy covers were higher than this reference value.

Prescribed fire effectiveness was identified based on the mitigation or reduction of both surface and crown fire behavior. Prescribed fire treatments reduce the available surface fuel mainly 1- and 10-hr dead fuel generated by biomass harvesting. However, coarse woody fuel and needles were fallen from branches increasing the surface fuel load in a medium term planning. If the amount of canopy fuel on the ground is high, other burning should be made to mitigate the probability to the transition to crown fire. This fact increases considerably the costs of prescribed fires in a medium-term planning. We used Visual Behave software (Figure 9) to analyze the effects of prescribed fires on fire behavior. If the transition from surface fire to crown fire is not possible (Van Wagner, 1977), burning treatment was 
considered effective. Nevertheless, surface fire behavior was simulated taken into account that flame length should be lower than $2.5 \mathrm{~m}$ and spread rate should be lower than $7.5 \mathrm{~m} / \mathrm{min}$.

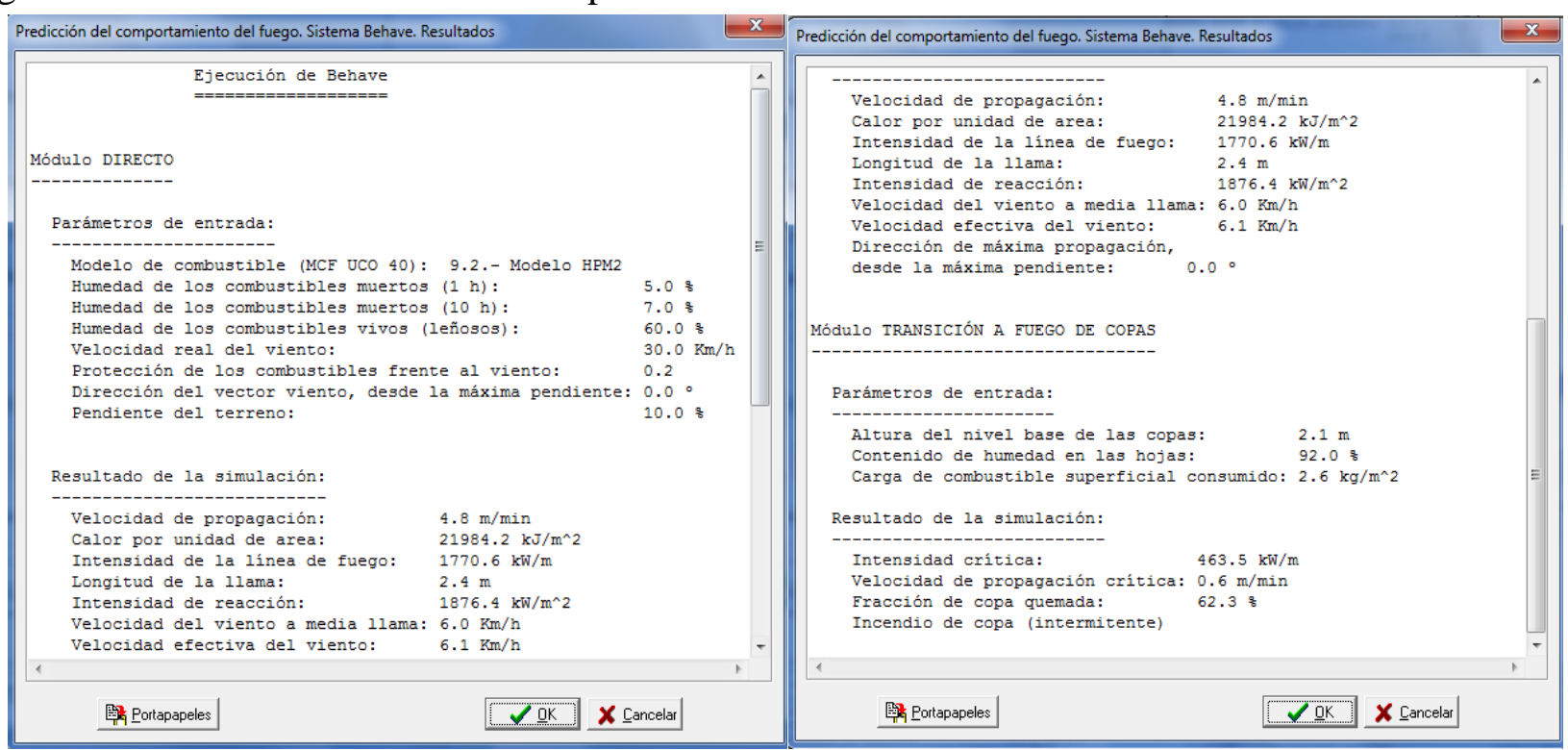

Figure 9 - Simulation of the effects of prescribed fire using Visual Behave software

A temporal planning of 12 years was used according to the context of the landscape within the duration of two periodical fire planning in Spain. Some studies limits prescribed fire effectiveness to a short post-treatment period (2-4 years) (Fernandes and Botelho. 2003). In our approach, this effectiveness varied based on burning behavior and stand characteristics. The cost-benefit of a prescribed fire planning in a medium-term (12 years) may be obscured by flame residence time, canopy base height and remnant canopy cover of the first burning. However, flame residence time is very difficult to measure "in situ" because of the smoldering process. In this case, we have modified the original model in spite to reduce its coefficient of determination and to increase the standard error including variables most easy to collect in field burnings. The final cost of prescribed fire management in a medium-term planning responds to the following econometric equation:

Cost fire planning $(€ / h a)=\alpha_{1} \mathrm{FS}_{1}+\alpha_{2} \mathrm{CBH}_{1}+\alpha_{4} \mathrm{CC}_{1}+\alpha_{5} \mathrm{FS}_{2}$

where $\mathrm{FS}_{1}$ is the fire severity classified based on 4 levels according to flame length $(\mathrm{m})$ and spread rate $(\mathrm{m} / \mathrm{min})$ in the first burning, $\mathrm{CBH}_{1}$ is the canopy base height before the first burning, $\mathrm{CC}_{1}$ is the canopy crown cover (\%) between six and nine months after the first burning, $\mathrm{FS}_{2}$ is the fire severity classified based on 4 levels according to flame length $(\mathrm{m})$ and spread rate $(\mathrm{m} / \mathrm{min})$ in the second burning.

As an example of the econometric model application, we have analyzed the cost of prescribed fire management for two real alternatives of burning of four areas based on its fire severity and according to stand characteristics (Table 3). There were significant differences in Parrillas, Riofrio and Sierra Alta areas between one and another alternative of management. In this sense, cost reduction ranged from $454.39 € /$ ha to $936.14 € /$ ha. However, costs of Velefique-Senés area were very similar based on the low canopy base height, the higher fire vulnerability of Pinus nigra and the high decomposed litter of this fuel model. 
Table 3 - Cost of prescribed fire planning (12 years) for the study areas

\begin{tabular}{|l|c|c|c|}
\hline $\begin{array}{c}\text { Cost of prescribed fire } \\
\text { planning (€/ha) }\end{array}$ & $\begin{array}{c}\text { Alternative A } \\
(\boldsymbol{\epsilon} / \mathbf{h a})\end{array}$ & $\begin{array}{c}\text { Alternative B } \\
(\boldsymbol{€} / \mathbf{h a})\end{array}$ & $\begin{array}{c}\text { Cost reduction } \\
(\boldsymbol{\%})\end{array}$ \\
\hline Parrillas Area & $2,303.89$ & $1,367.75$ & 40.63 \\
\hline Riofrio Area & $2,278.82$ & $1,824.43$ & 19.94 \\
\hline Sierra Alta Area & $2,541.87$ & $1,630.8$ & 35.84 \\
\hline Velefique-Senés Area & $2,432.82$ & $2,407,75$ & 1.03 \\
\hline
\end{tabular}

The use of Geographic Information System (GIS) and fire simulators in fire prevention planning provides flexibility when finding strategy locations to prescribed fires and increases the dynamisms of periodical planning in the case of use different alternatives. The optimal selection of prescribed fire planning increases the economic efficiency of preventive treatments under a worldwide scenario of budgetary constraints. In this sense, in some biomass harvesting areas (Parrillas, Riofrio, Sierra Alta), an efficiency management could help to facilitate an increase between $19.94 \%$ and $40.63 \%$ of the preventive treated area in a medium-term management.

\section{Conclusions}

Given the current budgetary constraints, measures for economic efficiency acquire a keystone role. In this sense, an adequate management of prescribed fires requires the medium-term monitoring of the surface and crown fuels. Prescribed fires on biomass harvesting scenarios could fulfill a meaningful fire prevention function based on fuel consumption and economic efficiency of burning treatment. This research provides a easy tool to evaluate in a medium-term period how to invest the available fire resources. It aids managers in developing prescribed fire planning in a sustainability and efficient way to mitigate potential fire impacts.

\section{References}

Agee J., Bahrob B, Finney Ma, Omi D., Skinner S., Van Wagtendonkg, Weatherspoonh P (2000), The use of shaded fuelbreaks in landscape fire management. Forest Ecology and Management 127, 5566.

Agee JK, Skinner C (2005) Basic principles of forest fuel reduction treatments. Forest Ecology and Management 211, 83-96.

Agee JK, Lolley MR (2006). Thinning and prescribed fire effects on fuels and potential fire behavior in an eastern Cascades forest. Fire Ecology 2(2), 142-158.

Cleades DA, Martínez J, Haines T (2000) Influences on Prescribed Burning Activity and Costs in the National Forest System. General Technical Report SRS-37.

Fernandes P, Botelho H (2003). A review of prescribed burning effectiveness in fire hazard reduction. International Journal of Wildland Fire 12(2), 117 - 128

González-Cabán A, McKetta C (1986) Analyzing fuel treatment costs. Western Journal of Applied Forestry 1(4), 116-121.

González-Cabán A (1997). Managerial and Institutional Factors Affect Prescribed Burning Costs. Forest Science 43, 535-543.

Molina JR, García JP, Fernández JJ, Rodríguez y Silva F (2018) Prescribed fire experiences on crop residue removal for biomass exploitations. Application to the maritime pine forests in the Mediterranean Basin. Science of the Total Environment 612, 63-70 
Omi P. Rideout D, Botti S (1999) An Analytical Approach for Assessing Cost-Effectiveness of Landscape Prescribed Fires. General Technical Report PSW- GTR-173. 237-241.

Rodríguez y Silva F, Hand M (2018) Modelling an econometric function to predict prescribed burning costs. Proceedings of Fire Continuum Conference. Missoula, Unites Stated.

Rodríguez y Silva F, Molina JR, Carmona JF (2010) Manual Técnico de Aplicaciones Informáticas para la Defensa contra Incendios Forestales. Servicio de Publicaciones Forestales. MANPAI XXI, Córdoba.

Stephens SL, Moghaddas J (2005) Experimental fuel treatment impacts on forest structure potential fire behavior and predicted tree mortality in a California mixed conifer-forest. Forest Ecology and Management 215, 21-36.

Van Mantgem PJ, Lalemand L, Keifer M, Kane J (2016) Duration of fuels reduction following prescribed fire in coniferous forests of U.S. national parks in California and the Colorado Plateau. Forest Ecology and Management 379, 265-272.

Van Wagner CE (1977) Conditions for the start and spread of crown fire. Canadian Journal of Forest Research 7, 23e34.

Vega JA, Pérez-Gorostiaga P, Cuñas P, Fontúrbel T, Fernández C (2001) Manual de Quemas Prescritas para Matogueiras de Galicia. Colección Técnica Medio Ambiente. Xunta de Galicía. Pontevedra. 


\title{
Radiative and convective heat exchanges between a fireman and the fire front and wind
}

\author{
Eusébio Z. E. Conceição ${ }^{1 *}{ }^{*}$ M $^{a}$ Manuela J. R. Lúcio ${ }^{1}$, Domingos X. Viegas ${ }^{2}$, and Maria T. Viegas ${ }^{2}$ \\ ${ }^{1}$ FCT - University of Algarve - Campus de Gambelas - 8005-139 Faro, Portugal. \\ \{econcei@ualg.pt*\} \\ ${ }^{2}$ FCT - University of Coimbra - Pinhal de Marrocos - Pólo II - 3030 Coimbra, Portugal
}

\begin{abstract}
In this work the radiative and convective heat exchanges between a fireman and the fire front and wind is made. In the radiative heat exchanges the Mean Radiant Temperature value, using the Human Thermal Response, is applied and in the convective heat exchanges the air velocity value, using the Computational Fluid Dynamics, is used.

In this work the fireman, equipped with special protective clothing, is located $5 \mathrm{~m}$ from the flame, with a height of $2 \mathrm{~m}$ and a length $10 \mathrm{~m}$, subjected to wind with air velocity of $5 \mathrm{~m} / \mathrm{s}$. The flame temperature is 1000 ${ }^{\circ} \mathrm{C}$, the air temperature is $20^{\circ} \mathrm{C}$ and the relative humidity is $50 \%$. In the paper the air velocity and the Mean Radiant Temperature around the fireman and the tissue and clothing temperature are calculated.
\end{abstract}

Keywords: Numerical Simulation, Radiative and Convective Heat Exchanges, Human Thermal Response, Mean Radiant Temperature.

\section{Introduction}

This study is a continuation of Conceição (2002), Conceição et al. (2006) and Conceição and Viegas (2010). In the previous works the radiative heat exchange was considered, using the Human Thermal Response, however in this work the convective heat exchange, using the Computational Fluid Dynamics, is also considered.

In Conceição (2002), Conceição et al. (2006) and Conceição and Viegas (2010) a numerical model, that simulates the human and clothing thermal responses, in steady-state and transient conditions, is used. This numerical model calculates not only the temperature field in the human body tissue, arterial and venous blood and clothing, but also the blood and tissue mass distribution in the human body and the water mass distribution in body skin surface and clothing. It also calculates the convective, radiative and evaporative heat exchanges, between the body and the environment and the Predicted Percentage of Dissatisfied people. This model considers the human body divided in 35 cylindrical and spherical elements, being each one sub-divided in 12 layers, which could be protected from the external environment through some clothing layers. This computational model is based on the energy balance integral equations for the human body tissue, blood and clothing as well as mass balance integral equations for the blood and transpired water in the skin surface and in the clothing. A thermoregulatory system model was implemented in order to control the human body tissue temperature.

In Conceição (2002) the present numerical model was used to study the fireman thermal sensation nearby a fire front. In the three analyzed situations, the radiant temperature, body skin and clothing temperatures and transpired sweat rate field were calculated. The more uncomfortable situation was verified when the fireman is localized in front to the fire central area and distanced $5 \mathrm{~m}$ from the flames, being the allowable exposure time, for a non-acclimatized subject with the warning criteria, of 4.5 hours. It was suggested that is important to use special protective clothing, to dispose of experimented firemen and to respect the maximum exposure time allowable. During the fire extinction was suggested that the distance between the fireman and the fire front should be the highest possible. 
It was also suggested that the fireman should avoid being located in front to the flames central area.

In Conceição et al. (2006) a numerical model, that simulates the human and clothing thermal responses, in steady-state and transient conditions, were used to study the fireman thermal sensation nearby a fire front. This numerical model was used to evaluate the thermal sensation that a fireman, equipped with special protective clothing, is subjected nearby vertical flames, with a height of $2 \mathrm{~m}$ and $10 \mathrm{~m}$ of length. In this theoretical study the fireman was placed $5 \mathrm{~m}$ distanced from the flames and localized in front to the flames central area. The body and clothing temperatures, the radiant temperature, the heat and mass fluxes fields and the comfort levels were calculated. The influence of the clothing thickness and the special protective clothing emissivity coefficient reduction, in the fireman thermal comfort sensation are analysed.

In Conceição et al. (2006) a numerical model, that simulates the human and clothing thermal responses, in steady-state and transient conditions, were used to study the fireman thermal sensation nearby a fire front. This numerical model was used to evaluate the thermal sensation that a fireman, equipped with special protective clothing, is subjected nearby vertical flames, with a height of $2 \mathrm{~m}$ and a length of $10 \mathrm{~m}$. In this theoretical study the fireman was placed $5 \mathrm{~m}$ distanced from the flames and localized in front to the flames central area. The body and clothing temperatures, the radiant temperature, the heat and mass fluxes field and the comfort levels were calculated. The influence of the clothing thickness and the special protective clothing emissivity coefficient in the fireman thermal comfort sensation was analysed. The theory used to evaluate the fireman thermal comfort is based in the extension of the PMV model. This extension, used in warm environments, combines the "static" PMV model and the adaptive model. The idea is to use the traditional PMV model, that considers the human body thermal balance, and the expectations verified in the adaptive model. It was verified that the Predicted Percentage of Dissatisfied people slightly increases when the clothing thickness increases and decreases when the special protective clothing emissivity coefficient reduces.

In Conceição et al. (2010) the radiative heat exchanges between the fire front and the firemen is made. In the radiative heat exchanges the Mean Radiant Temperature method, with correction, is applied. The Mean Radiant Temperature, the body and clothing temperature field and the Predicted Percentage of Dissatisfied people for two firemen placed perpendicularly to the fire front are calculated. In this work the radiative heat exchanges between the fire front and two firemen is evaluated. In the radiative heat exchanges the Mean Radiant Temperature method, with correction, is applied. The Mean Radiant Temperature, the skin and clothing temperature and the Predicted Percentage of Dissatisfied people are evaluated. The fireman located behind is protected to the fire front by the fireman located in front. In accordance with the obtained results the radiant protection, promoted by the fireman located in front, with a Predicted Percentage of Dissatisfied people of $25 \%$, reduces the Predicted Percentage of Dissatisfied people, that the fireman located behind is subjected, for $18.1 \%$.

\section{Physical Model}

In the Human Thermal Response and in the Computational Fluids Dynamics numerical models the human body is divided in 25 elements (see figure 1): the head, the neck, the trunk divided in three, the arms divided in four, the hands, the legs divided in four and the feet. Each element is sub-divided in 12 cylindrical or spherical layers and could be still protected of the external environment through some clothing layers. In the Human Thermal Response numerical model the elements are cylindrical or spherical, while in the Computational Fluids Dynamics numerical model the elements are boxes. 


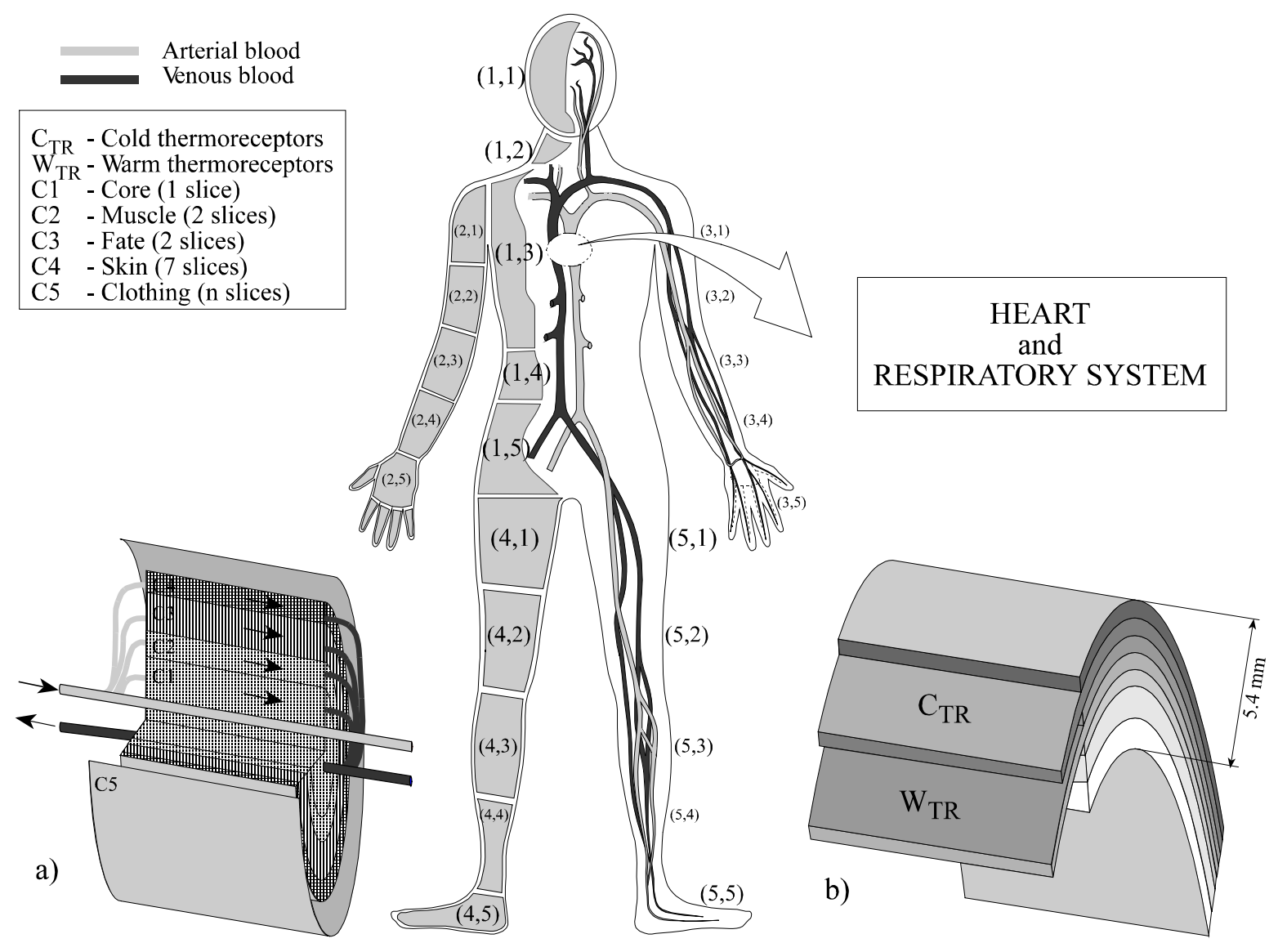

Figure 1 - Scheme of the human body divided in 35 elements. Each element divided in 12 slices and protected by clothing layers and the skin divided in 7 layers.

\section{Numerical Methodology}

This numerical model was used to evaluate the radiative and convective heat exchanges between a fireman and the fire front and wind, nearby a vertical flame, with a height of $2 \mathrm{~m}$ and $10 \mathrm{~m}$ of length, and subjected to wind boundary layer.

In this simulation the flame temperature is $1000{ }^{\circ} \mathrm{C}$ and the air mean temperature, velocity and relative humidity are, respectively, $20{ }^{\circ} \mathrm{C}, 5 \mathrm{~m} / \mathrm{s}$ and $50 \%$. In the Mean Radiant Temperature calculus the human body external surface was divided in 1256 infinitesimal areas, while the vertical fire front in 126 infinitesimal areas. In the simulation the activity level considered was $165 \mathrm{~W} / \mathrm{m}^{2}$, while the clothing level was 3 Clo.

In figure 2 the tridimentional fireman geometry used in the Human Thermal Response numerical model is presented, while in figure 3 the tridimentional localisation of the fireman in front to the flame used in the Human Thermal Response numerical model is shown. 


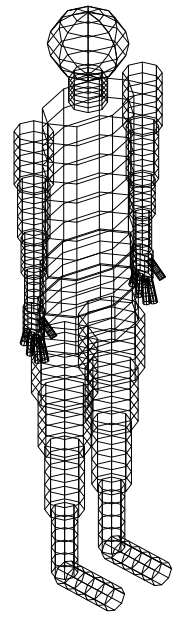

Figure 2 - Tridimentional fireman geometry used in the Human Thermal Response numerical model.

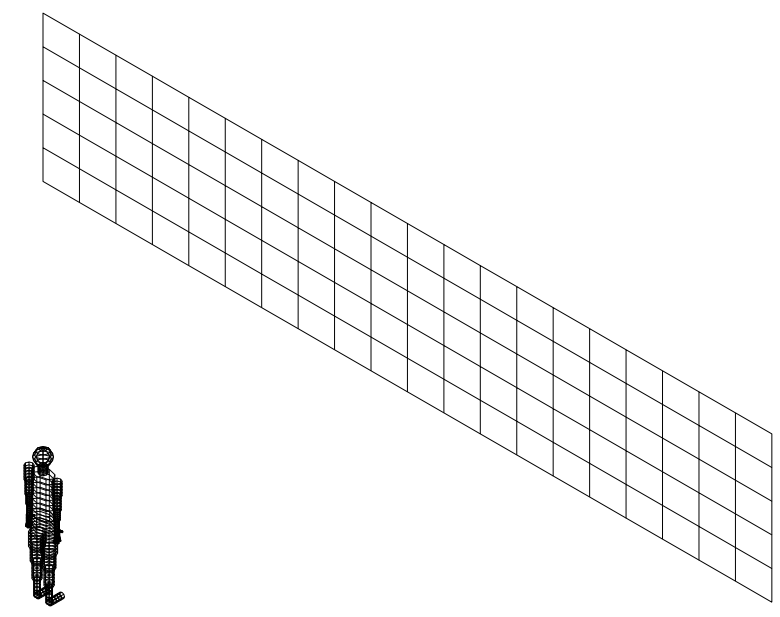

Figure 3 - Tridimentional localisation of the fireman in front to the flame used in the Human Thermal Response numerical model.

In figure 4 the tridimentional fireman geometry used in the Computational Fluids Dynamics numerical model is presented, while in figure 5 the tridimentional localisation of the fireman in front to the flame used in the Computational Fluids Dynamics numerical model is shown.

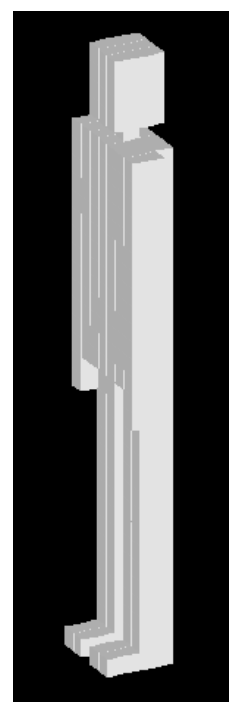

Figure 4 - Tridimentional fireman geometry used in the Computational Fluids Dynamics.

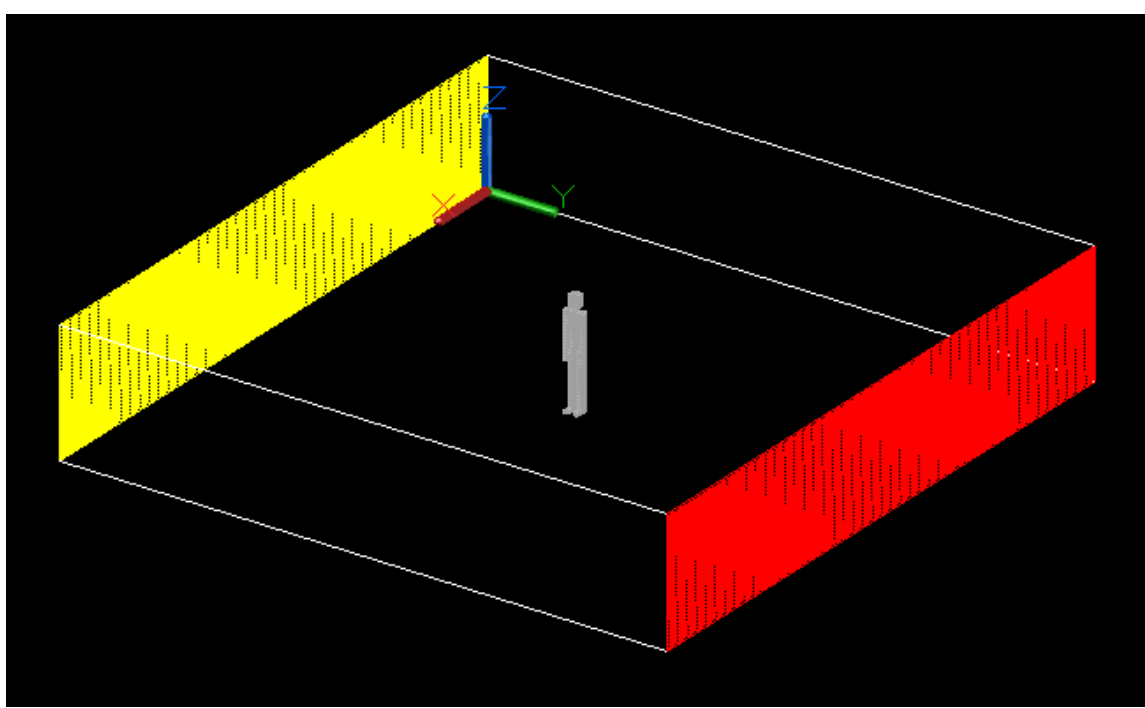

Figure 5 - Tridimentional localisation of the fireman in front to the flame used in the Computational Fluids Dynamics.

In both figures the flame front is located in front to the fireman. In figure 5 , used in the Computational Fluids Dynamics, the inlet air velocity is represented by yellow colour, in the plan XZ, while the outlet air velocity is represented by red colour, in the plan XZ.

\section{Mathematical Model}

The mathematical model is based in the Computational Fluids Dynamics and the Human Thermal Response. The two numerical models work in a coupling methodology. 


\subsection{Human Thermal Response Model}

\subsubsection{Equation System}

The human body thermal system is based on the energy and mass balance integral equations. The first one is developed for each tissue layer and for the arterial and venous blood, of each human body element. In the second one is developed, also in each element, the mass balance integral equations for the blood and for the transpired water in the skin surface.

\subsubsection{Thermoregulatory system}

The thermoregulatory system, used in the control of the human body temperature, is constituted by: heat transport from internal to external tissue, heat loss by evaporation and additional heat through shivering (see Stolwijk, 1970).

\subsubsection{Environmental variables}

The environmental variables that the model consider are the air velocity, temperature, relative humidity and Mean Radiant Temperature.

\subsubsection{Radiative Heat exchanges model}

The radiant heat exchanges by long wave phenomena are calculated, in this work, using the Mean Radiant Temperature method (Fanger, 1970), with correction. The Mean Radiant Temperature method, with correction, considers not only the heat exchanges between the human body sections and the surrounding surfaces, but also the heat exchanges between the human bodies sections of each occupant and between the human bodies sections of different occupants. The Mean Radiant Temperature method, with correction, calculates, step by step, the Mean Radiant Temperature, using the human body and surrounding surfaces temperatures, calculated in each iteration, and the precalculated view factors.

In the methodologies used in view factors determination each human body element or surrounding surfaces, with inclinations, dimensions and temperatures equal to the respective body or surrounding section, are divided in infinitesimal areas.

In the radiation by long wave phenomena calculus are also considered the shading effects that the body elements surfaces promote in each element.

\subsection{Computational Fluids Dynamics Model}

The CFD numerical model considers the three-dimensional turbulent airflows under steady-state and non-isothermal conditions. The numerical model solves the partial differential equations (using the finite volume method), namely, the mass continuity equation, moment equations (threedimensional air velocity), energy equation, turbulence kinetic energy equation and turbulence energy dissipation rate equation (using the RNG turbulence model). This numerical model is used to evaluate the airflow around the fireman (three-dimensional components of air velocity; omnidirectional air velocity, air temperature, air pressure, turbulent kinetic energy and turbulent energy dissipation rate). In the CFD numerical model each virtual fireman is based on 25 boxes elements.

\section{Results and Discussion}

In this paper the Mean Radiant Temperature and the air velocity around the fireman are calculated. In the first one is used the Human Thermal response model, while the second one is used the Computational Fluids Dynamics model.

In figure 6 is presented the Mean Radiant Temperature that the fireman are subjected in the different human body sections, while in figures 7 and 8 are presented, respectively, the air velocity that the 
fireman are subjected in a plan parallel to the airflow and the air velocity that the fireman are subjected in the different human body sections.

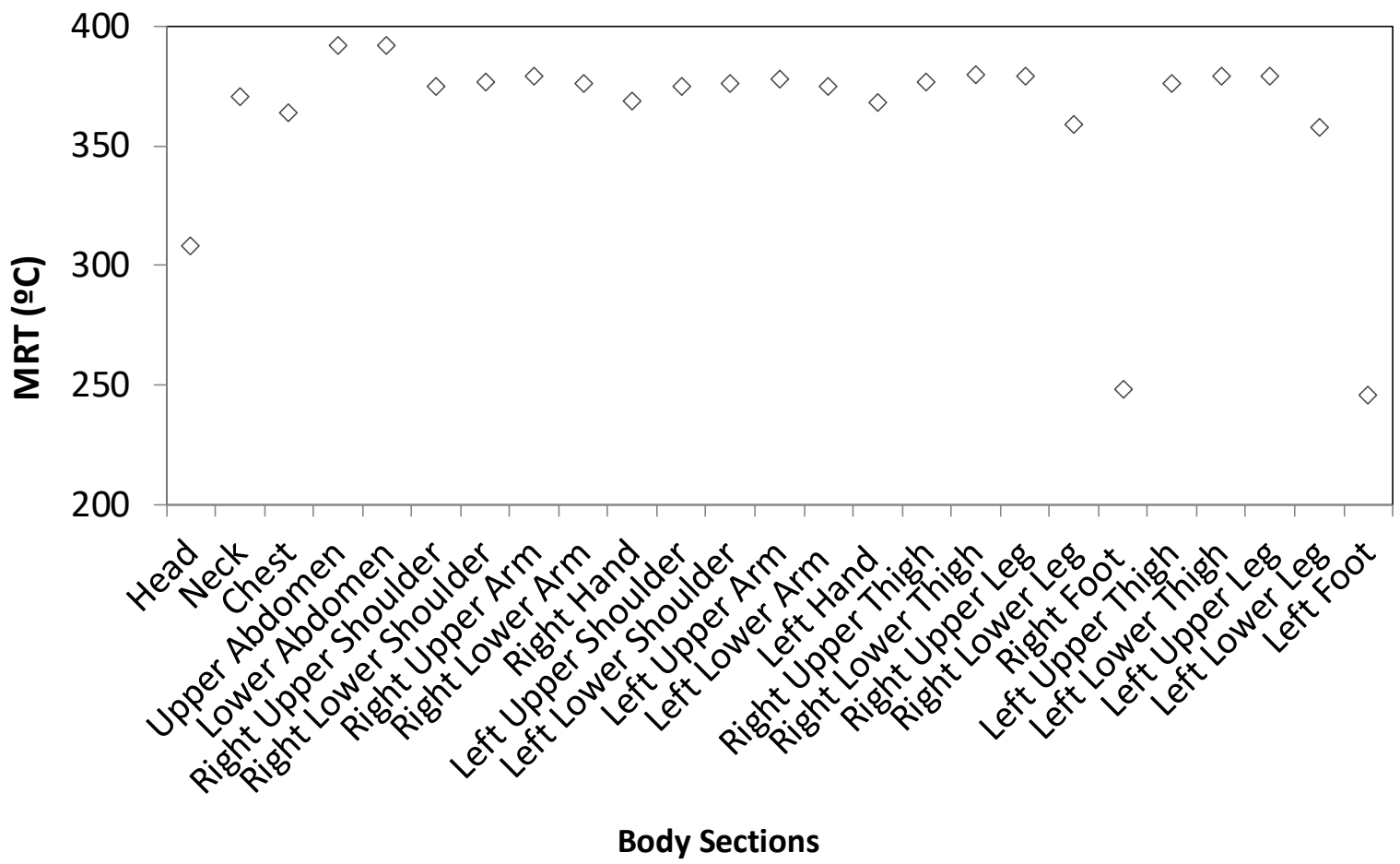

Figure 6 - Mean Radiant Temperature, MRT, that the fireman is subjected in the different human body sections.

The fire front and the surrounding environment influence the Mean Radiant Temperature. The environment influences a higher area than the fire front, while the fire front influences the higher temperature than the fire front. In accord to the obtained Mean Radiant Temperature results, is verified that:

- The Mean Radiant Temperature around the fireman is symmetrical;

- The Mean Radiant Temperature is highest in the abdomen area;

- The Mean Radiant Temperature is higher in the upper members than the lower members;

- The Mean Radiant Temperature is smallest in the feet area.

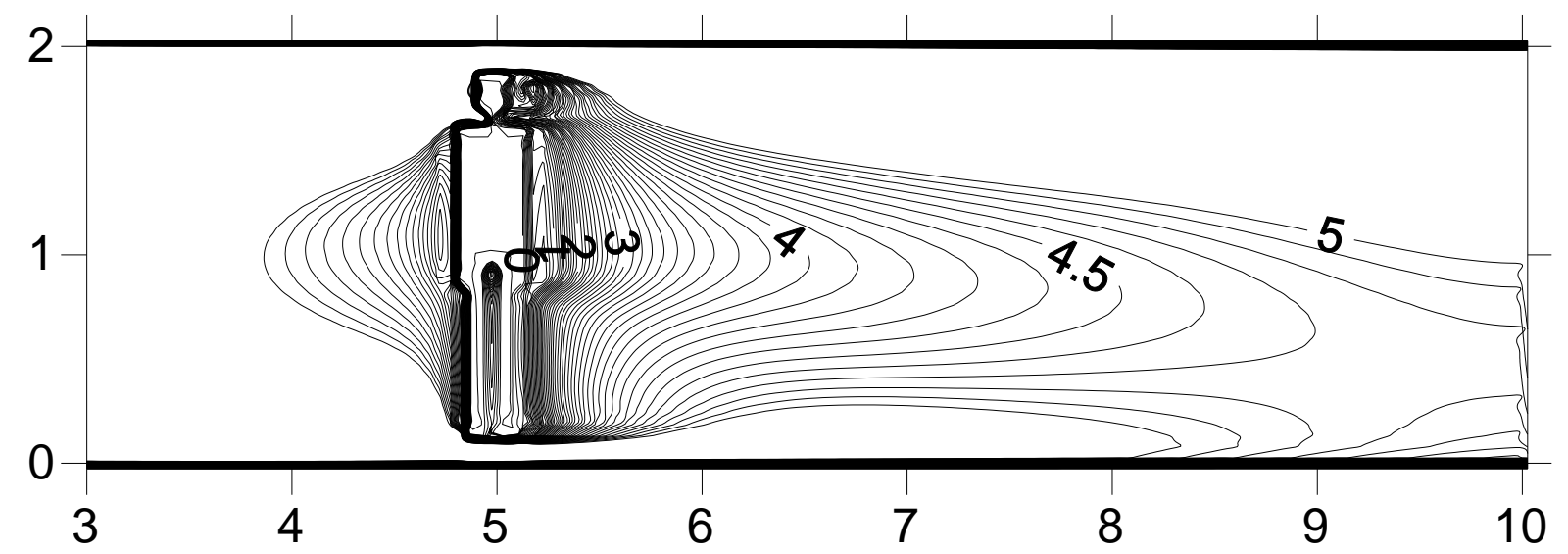

Figure 7 - Air velocity that the fireman is subjected in a plan parallel to the airflow. 


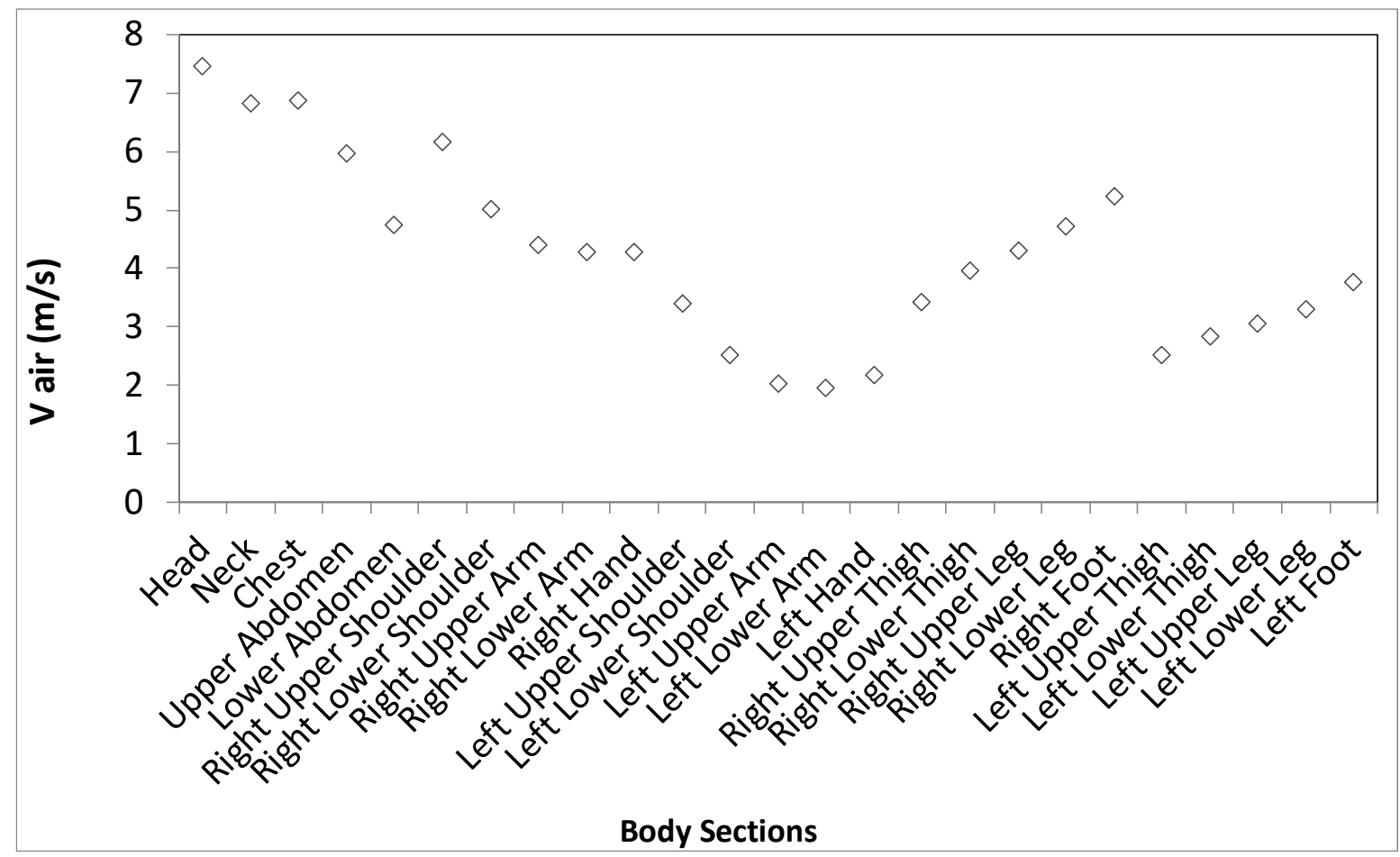

Figure 8 - Air velocity that the fireman is subjected in the different human body sections.

The airflow around the fireman guarantees a deflection in the amount area and a recirculation in downstream area. The deflection promotes a stationary area. In accord to the obtained air velocity results, is verified that:

- The air velocity is higher in the right side than in the left side;

- The air velocity is higher in the head and trunk area than in 0the members.

\section{Conclusions}

In this work the radiative and convective heat exchanges between a fireman and the fire front and wind is made. In the radiative heat exchanges the Mean Radiant Temperature is calculated, in the convective heat exchanges the air velocity is evaluated.

When the fireman is located $5 \mathrm{~m}$ from the flame, with a height of $2 \mathrm{~m}$ and a length $10 \mathrm{~m}$, the Mean Radiant Temperature is highest in the abdomen area, is smallest in the feet area and is higher in the upper members than the lower members. However, when the fireman is subjected to wind with air velocity of $5 \mathrm{~m} / \mathrm{s}$, in the right site, the air velocity is higher in the right side than in the left side and is higher in the head and trunk area than in other members.

In future work the radiative and convective heat exchanges, between a fireman and the fire front, that the fireman are subjected, will be used simultaneously in order to evaluate the human thermosphysiology and human thermal temperature distribution, for different clothing levels and activities levels.

\section{References}

Conceição E. Z. E. and Viegas D. X. 2010, Radiative Heat Exchanges Between the Fire Front and the Firemen, Proceedings of the $6^{\text {th }}$ International Conference on Forest Fire Research, Coimbra, Portugal, 15 to 18 November 2010. 
Conceição, E. Z. E. 2002. Study of Fireman Thermal Sensation Nearby a Fire Front: Evaluation of Human and Clothing Thermal Response, $4^{\text {th }}$ International Conference on Forest Fire Research, Luso, 18 to 21 November 2002.

Conceição, E. Z. E., Lúcio, Mª M. J. R. and Viegas D. X. 2006. Numerical Simulation of the Thermal Sensation of a Fireman Equipped with Special Clothing", $5^{\text {th }}$ International Conference On Forest Fire Research, Coimbra, 16 to 20 November 2006.

Fanger P.O. 1970. Thermal Comfort. Copenhagen: Danish Technical Press.

Stolwijk, J. A J. 1970. Mathematical Model of Thermoregulation", In Hardy, J. D., Gagge, A. P. and Stolwijk, J. A. J. "Physiological and Behaviour Thermoregulation", Thomas, Springfield, pp. 703721. 


\title{
RPAS and nighttime wildfire fighting: The NITROFIREX PROJECT
}

\author{
Luis M. Bordallo ${ }^{1}$, Alexander Burwitz ${ }^{2}$ \\ ${ }^{1}$ NITROFIREX S.L. C/ Baleares 4, Santiago Ribera (30720) Murcia Spain \\ \{luisbordallo@nitrofirex.com\} \\ ${ }^{2}$ NF Advanced Engineering C/Fraday, Campus Cantoblanco, 28049 Madrid \\ \{alexander.burwitz@nitrofirex.com\}
}

\begin{abstract}
The maturity of the technologies for the guidance and control of UASs allows proposing innovative operational options such as the ability to spray (a liquid) or spread (a powder) a significant amount of an agent of any sort at a pre-established point in the atmosphere. This is the case of NITROFIREX, an innovative project that integrates available technologies from the defence industry to achieve this operational capability. Of all possible applications the forest-fire fighting is where NITROFIREX places its highest priority. This is because of the ecological damage these fires bring forth, the cause social alarm they generate and the human and economic losses that take place whilst fighting them. NITROFIREX at last offers the long awaited nighttime fire fighting capability and also allows direct support to the ground crews in their relentless fight against forest fires.

The ability to transport large amount of payload in the minimum time to the area of operations is performed by heavy transport aircraft or helicopter (Launcher Aircraft L A) designed to do this at the most efficient economical way. What NITROFIREX does is to transport this payload from the transport aircraft / helicopter to the programmed release point. This is achieved by using unmanned Autonomous Gliders Containers, AGCs that are released through the rear ramp of the LAs. The AGCs fly autonomously to the programmed release point in the source of the fire realizing their contents with utmost precision and then returning to base for reuse. Once the AGG drops the extinguishing agent it performs its escape manoeuvre using its remaining speed and big weight loss to attain as much altitude as possible. Upon reaching the highest point it returns to its base of operations, empty now, so as to be reused as soon as possible. The base of operations is an airport close to the fire to which the NitroFirex system is moved when called for.

The amount of countries hit by forest fires is numerous. The heavy financial and human losses generate social unease and political concern. The impact on the environment incurs in a direct manner in harm to biodiversity and indirectly with the release of big amounts of carbon dioxide into the atmosphere. Paradoxically in the sector of aerial fire fighting no modern aeronautic technologies have been implemented up to now, especially if we compare it to all other sectors of aviation where the innovations have been really significant. Because of all that has been mentioned the NITROFIREX project raises the application of modern technology in the aviation sector with the goal to improve to improve the operative efficacy, the economical efficiency, the crews flaying safety and above all to offer night-time operation, which is the main shortcoming of current aerial means.
\end{abstract}

For further information see 3 min YOUTUBE video link: https://www.youtube.com/watch?v=MTOjTWSHR64\&feature=youtu.be

Keywords: Drones, night-time, aerial, forest, fire fighting, AGC's, launcher aircraft

\section{Introduction}

It may be the controversial climate change or perhaps human pressure on the natural environment but the fact is that, year after year, alarming reports of devastating forest fires monopolize the media. During all he last summers we saw how catastrophic fires razed through the U.S.A., Portugal, Canada, Spain Indonesia, Russia, Bolivia, Israel, India, Australia and Greece stood by helplessly before the devastation of their natural heritage. In the coming years, and this is unfortunately more than likely, we shall have to continue suffering from this modern plague that is ravaging our forests. 
In facing this bitter reality, it is necessary to ask whether political, economic and technical levels are contributing in finding solutions, or at least methods to efficiently avoid and relieve these disasters. Unfortunately, the answer is a very clear and definite negative.

According to estimates from the Intergovernmental Panel on Climate Change (IPCC), forest fires create between 14 to $20 \%$ of all $\mathrm{CO}_{2}$ projected into the atmosphere annually.

On the other hand, all the activity of commercial aviation worldwide causes only $2 \%$ of all the $\mathrm{CO}_{2}$ emission. For example, the European project CLEANSKY has a budget estimated at 1,600 millions of Euros in order to reduce this $2 \%$ to $1 \%$ by obtaining a higher efficiency of engines, the employment of biofuels, intelligent wings and the reduction of weight, etc. Paradoxically, very little technological effort is being put into research regarding the elimination or the reduction of the $14-20 \%$ emission of $\mathrm{CO}_{2}$ that results from wild fires and, above all, the lack of research into aerial fire extinction - the most versatile and effective method to fight fires.

In the face of the social alarm, political upheaval and vast economic losses engendered by these wildfires, why is it that so few technical resources are available to fight these fires with the greatest efficiency possible? Why, for example, having at our finger tips technology that literally allows us to place a guided bomb carrying 200 or 300 kilos of explosives and fired from a warplane some dozens of miles away through a window? (Figure 1) Why can't we use the same technology to drop, throughout the night, loads of 2,000 to 3,000 litres of water continuously over a burning forest?

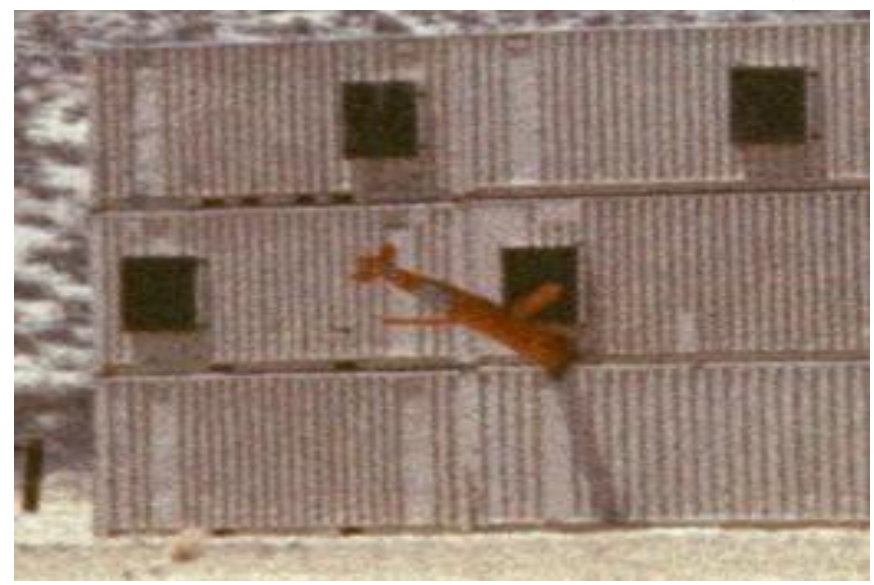

Figure 1 - Guided/guided bomb striking a target

Why is it that an aeronautical sector, which internationally moves thousands of millions of $\$ / €$, is still confined to using methods, techniques and procedures developed some 60 years ago in extreme high risk operations for their crews? Why not integrate available technology and develop the capacity to drop more fire extinguishing agents in less time, but above all, to be able to do so at night. Have we forgotten that the inability to fly at night is the greatest operative lack in present-day fire fighting?

\section{The NITROFIREX Concept}

The developing technologies in the control and guiding of RPAS allow for the development of innovative operational options, such as the possibility of 'spraying' (a liquid) or 'scattering' (a powdered solid) an important amount of extinguishing agent from a programmed point in the atmosphere.

This is the case of NITROFIREX, an innovative project that integrates the industrial technologies of the Defence Force in order to attain an operative capability that could be applied in the battle against wildfires, to combat against an atomic, chemical or biological emergency, to operate with meteorological phenomena (inducing rain, avoiding hail, dissipating fogs), to fight against plagues or to sow in remote or inaccessible regions and also the fumigation of drug plantations at night. 
Discarding all these possible applications, and due to the ecological harm caused, the social alarm generated as well as the human and economic losses that they bring forth, the project that NitroFirex is developing with maximum priority is the one to combat wildfires. For that very reason NitroFirex is concentrating on its night time operations as an indispensible and necessary complement to aerial means already in use during daytime. Heavy transport planes or helicopters will be responsible for moving great quantities of useful cargo (extinguishing agents in the case of wildfires) to the operative zone. These planes / helicopters are designed to transport at great speed big quantities of cargo at a cost per unit far lower to that of a smaller plane or helicopters.

This is the NitroFirex proposal: Launcher Aircraft (LAs), heavy cargo aircraft equipped with a rear ramp or medium/heavy helicopter (Figure 3 and 4), will be employed to transport the NitroFirex Airborne Glider Containers (AGCs) already containing around 2,500 litters of payload to the programmed drop zone (Figure 2).

The LA will then launch the AGCs from the rear ramp in a programmed sequence (Figure 3) or one by one hanged like a bucket-bamby from a helicopter (Figure 4). Once released, the AGCs will automatically homing onto their programmed target area within the drop zone and, with great accuracy, drop their load in the core of the fire. Once the drop of the fire-extinguishing agent on the fire has taken place, these containers return to base performing an "escape" manoeuvre from the danger zone by taking advantage of the great and sudden loss of weight as well as the extra speed.
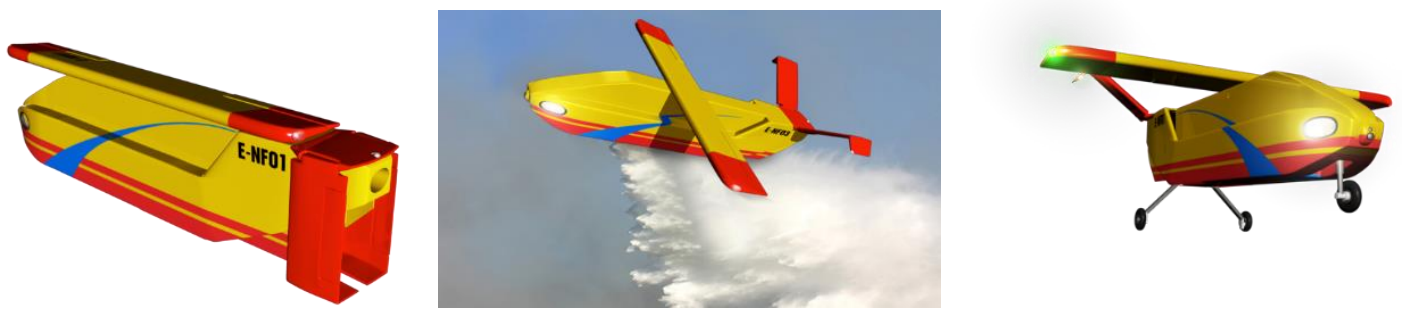

Figure 2 - NitroFirex AGC,s configurations (on board, flaying/dropping, landing or on ground)

Then the empty NitroFirex AGC begins its autonomous return to the operations base of the LA where it can be swiftly reused and loaded into the LA, thus beginning a constant turnaround operation until sunrise.

\section{The NITROFIREX Project}

Although, as we have seen in the previous paragraph, the concept has wide applications, NitroFirex is, first and foremost, a completely innovative project focused on nighttime aerial wildfire fighting. The main element used is the AGC, launched at medium altitude (six to ten thousand feet), from the rear ramp of the cargo bay of a medium/heavy transport aircraft, (Figure 3) (such as the C-295, C-27J, C-130 Hercules, AN-12, KC-390, A400M, IL-76, C-17 or even aircraft with greater cargo capacity), or from any medium/heavy helicopter hanging like a bucket (Bamby) (Figure 4) (such as Eurocopter models H-225, H-215 H-175, Bell models B-412, B-214, B-212, or Sikorsky models S61, S65, S70 or the Kamov 32 between others), which perform as the LAs.

These containers, loaded with a fire-extinguishing agent, are capable of gliding in their initial phase and are equipped with a guidance system (satellite, inertial, infrared or even laser). They fly automatically into the fire zone and drop their load, in sequence and with great accuracy, on the targeted area over the flames (Figure 5). 


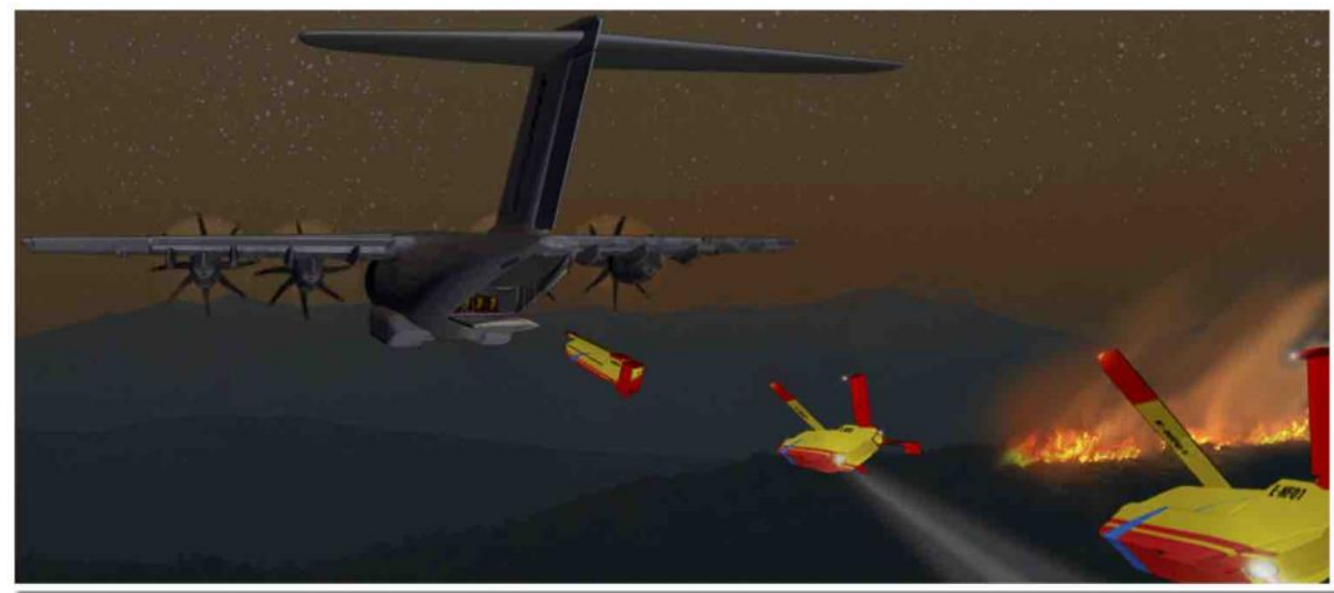

Figure 3 - Launching of AGSs from the LA (heavy cargo transport) at night

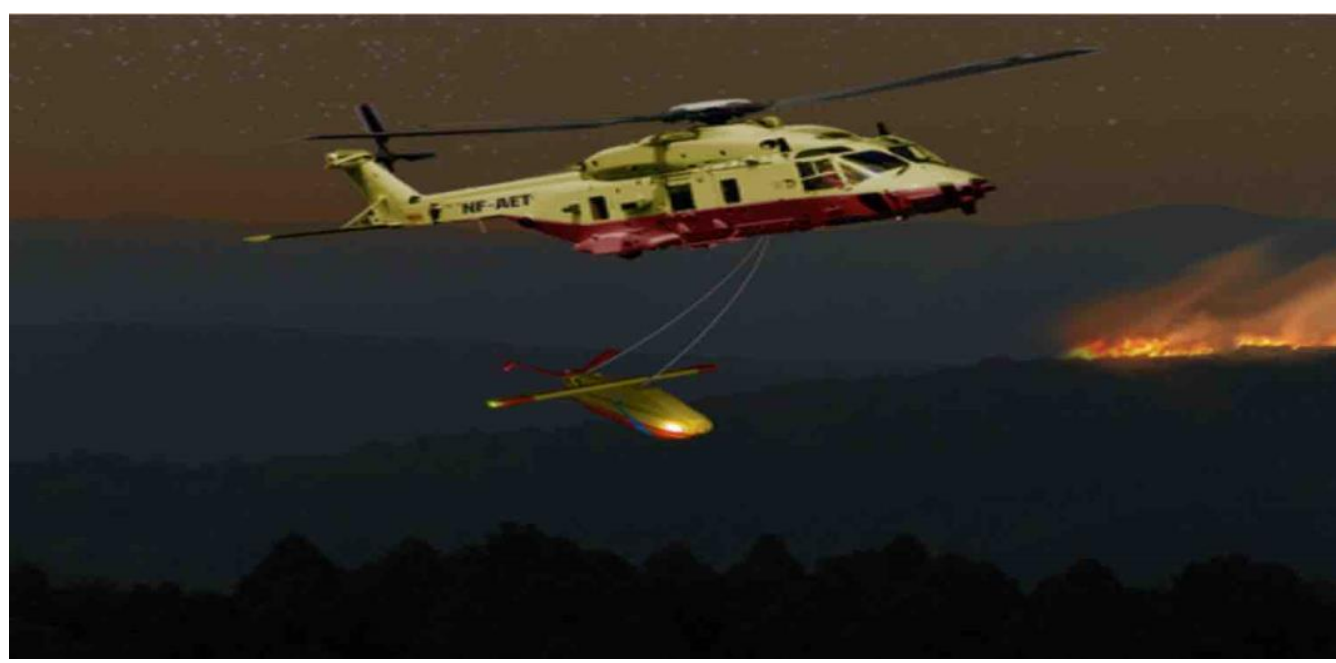

Figure 4 - Launching of AGSs from the LA (heavy cargo helicopter) at night

For safety, but also economic reasons, the AGCs are recoverable. Once the drop of fireextinguishing agent on the fire takes place, the AGCs perform an escape manoeuvre from the danger zone. Taking advantage of the great and sudden loss of weight as well as the surplus speed, the empty glider container is transformed, thanks to the previously activated small engine that propels it, into an RPAS and returns autonomously to the operation base of the LA where it can be swiftly reused. In its guided bomb role, the glider container flies no more than 90 seconds from the LA to its target over the fire.

When compared to a guided bomb, it carries a bigger payload and has more wing surface, but with an equal gliding and guide capacity. After concluding its 'escape' from the target area and until it lands at the LA.s operation base, it navigates at night as a RPAS at 500 feet above ground level and attains speeds of up to $70 \mathrm{Kts}$ (Figure 6).

By no means does NitroFirex want to substitute current aerial means whose efficiency in daytime operations has been demonstrated over decades. The truth is that NitroFirex aims to be their night-time counterpart in order to achieve H-24 aerial combat against wildfires (24 hour non-stop fighting) and to make use of, as the toiling ground crews well know, the better weather conditions that the night usually has to offer (less turbulence and wind, lower temperature and higher humidity). 


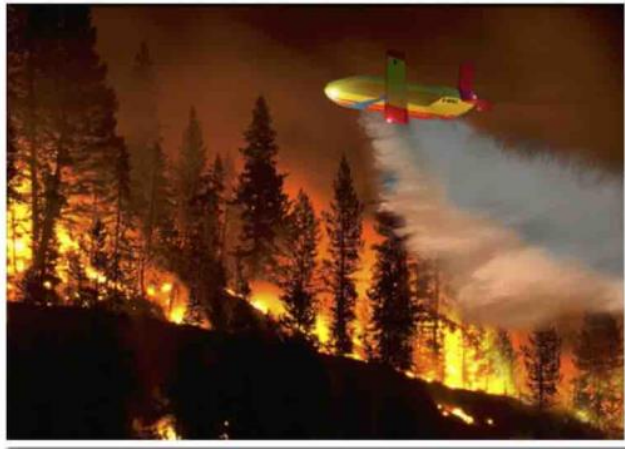

Figure 5 - AGCs dropping fire agents Extinguishing on the fire

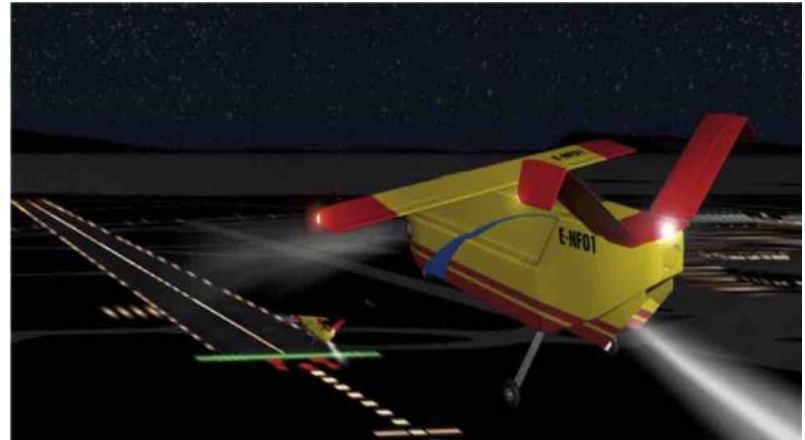

Figure6 - Coordinated autonomic return and Landing at the LA,s operation base

In the next Figures 7, 8 and 9, one can analyse these advantages from:

$1^{\circ}$ the operative point of view: litters launched per hour of the operation in function of the distance from the LA ops base to the forest fire.

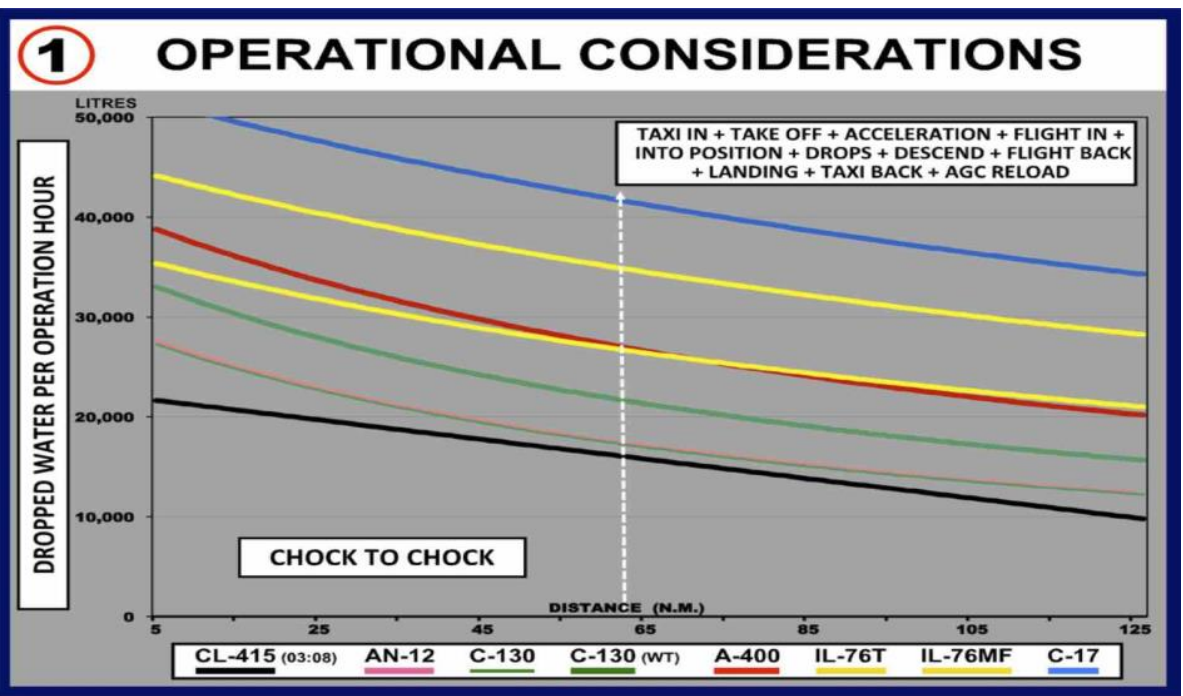

Figure 7 - Dropped water per hour of operation

$2^{\circ}$ The economic point of view: litters launched per flight hour and the cost of the dropped litter in function of the distance from the LA ops base to the forest fire.

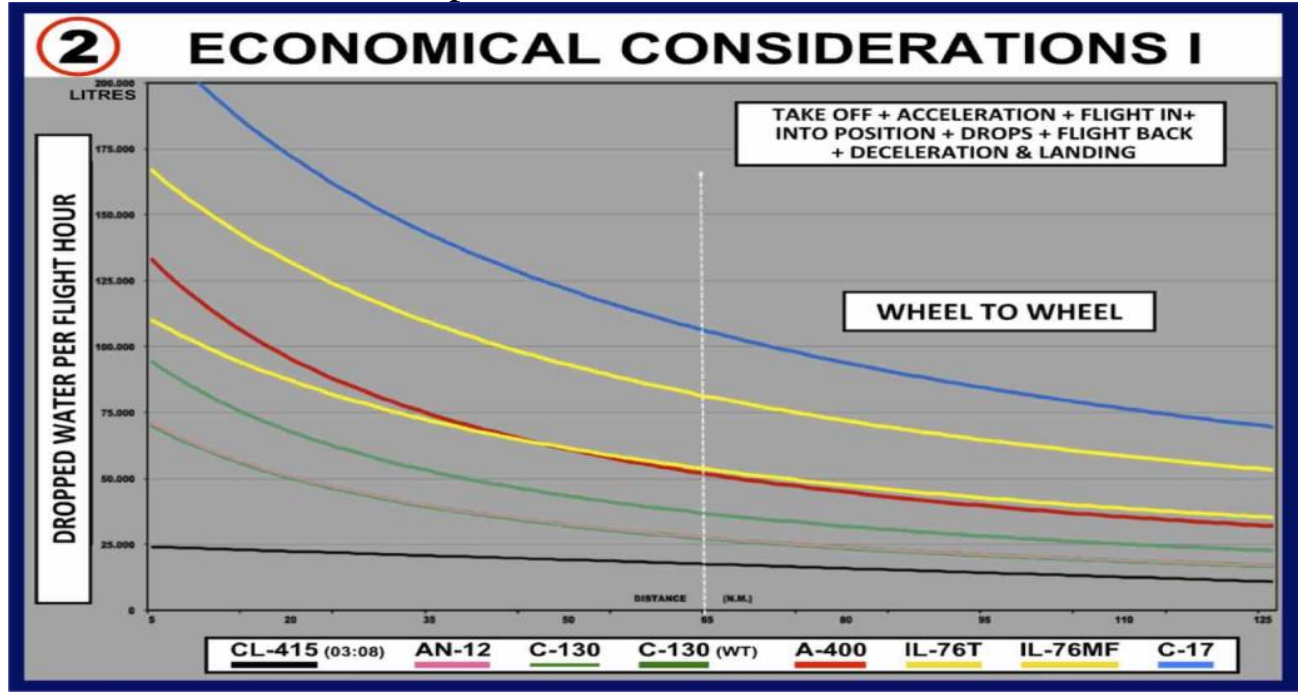

Figure 8 - Dropped water per flight hour. 


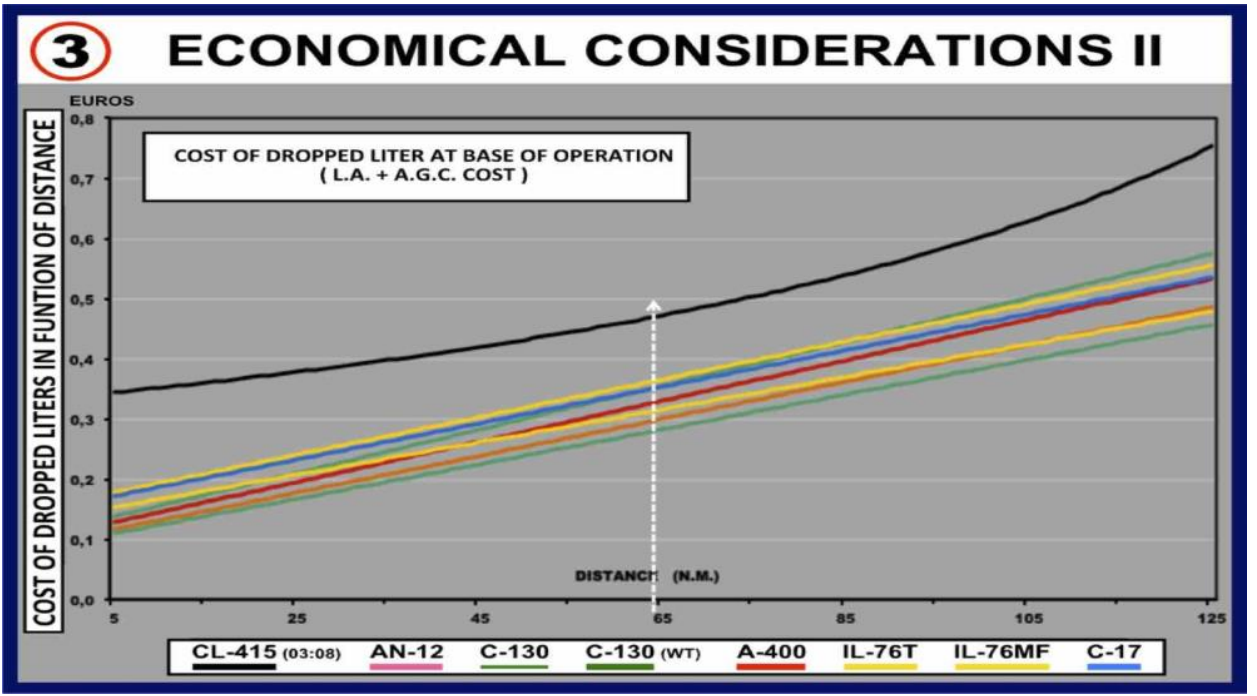

Figure 9: Cost of dropped water as a function of distance

\section{Operational Model}

Because of the great cargo capability and the increased deployment speed that the LAs offer, a new operational model will have to be put into use. This will include a regional operational base that could be situated in a strategic site in accordance to the operations area. Should a supranational operation come to be, the ideal base that would cover, for example, the Mediterranean Basin would be Marseille in France. Since the NitroFirex operation is nocturnal, we can assure that under normal conditions, at least half a day would be needed to detach the LA to any "available runway" in any country (Figure 10).

The AGCs are transported from the "regional operational base" to the "mission base" (airport, air base or aerodrome closest to the fire). Two sets of empty AGCs are stacked on two levels in a 'rack' or specifically designed shelving in the cargo hold of the LA (Figure 11).

Later, during the mission this 'rack' will be used for the fast turn-around (pre-flight check, fuelling, loading of extinguishing agent and re-stacking) of the AGCs returning from the fire zone. Once a wildfire alarm has been activated, the LA fly from their regional base to the detachment base, the airport closest to the fire, where the operation begins by downloading the rack of empty AGCs and the rest of the necessary equipment so as to proceed with the final preparation. The first set of AGCs (on the lower level of the 'rack') are filled with the extinguishing agent and are once again stacked in the LA leaving them ready for take off at dusk.

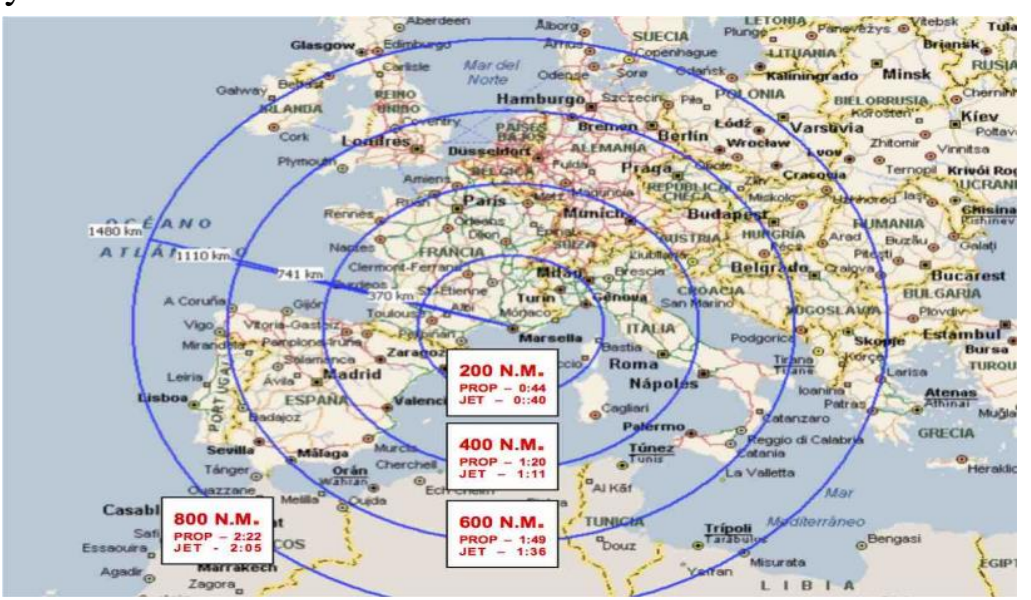

Figure 10 - Proposed base at Marseille in France in the Mediterranean Basin for a supranational operation. 


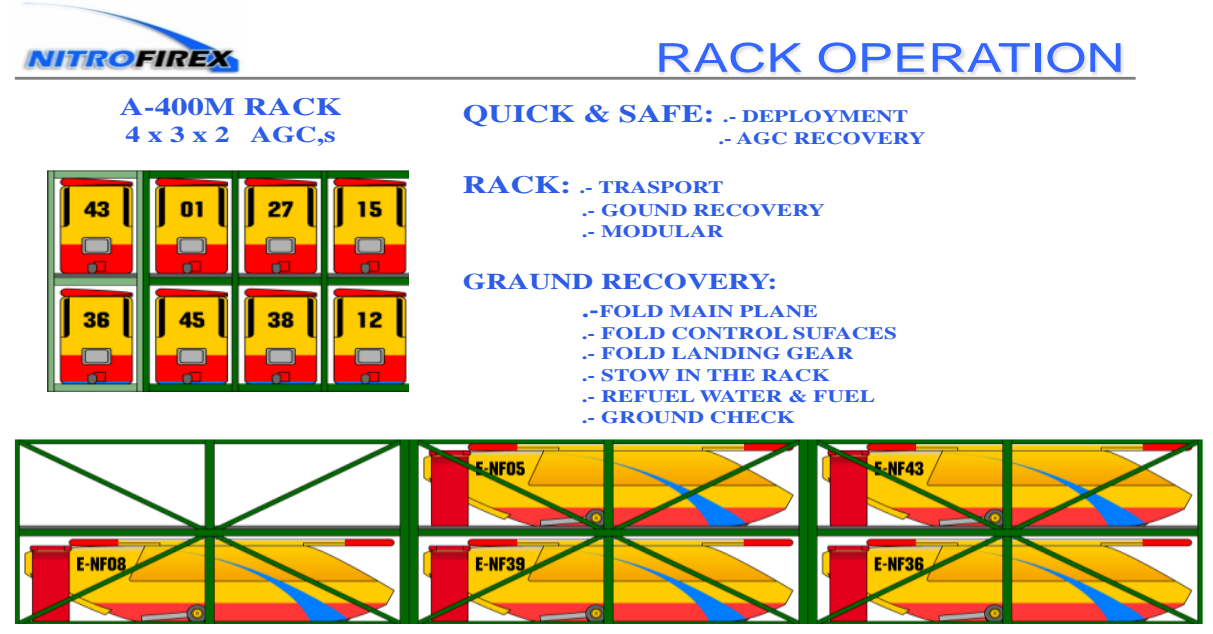

Figure 11 - Rack operations of the AGCs.

As night falls they fly to the fire zone and, coordinated by the ground teams who provide direct support, the LAs begins launching the AGCs in groups with a $90^{\circ}$ 'angle off' to the fire front, the goal being that at the moment of leaving the LA the AGCs make a $90^{\circ}$ turn, so that when several AGCs leave the LA they can form up in a "trail" formation and attack the fire.

The extinguishing agent is dropped over the area preselected by the fire management officer. In order to maximize the extinguishing effect the drops are overlapped. Once the first set of AGCs have left the LA, it returns to base to load the second set of AGCs so it can then return to the fire area in order to continue the attack on same fire or to attack more than one fire simultaneously. The AGCs autonomously return to mission-base after each drop. For example, one could consider an operation of an A-400 with a cargo capacity of 12 full AGCs.

Flying to the mission-base with a 'rack' holding 24 empty in each flight 12 containers filled with the extinguishing agent and rapidly returns to load the next 12 .

In the case of the A-400, the cargo load of each AGC would be 2,583 litres, which means that a single flight of an A-400 would be approximately equivalent to 6 drops of the Canadair CL-215/415. At a distance of $120 \mathrm{~km}(65 \mathrm{~nm})$ between the mission base and the fire zone an A-400 can perform 9 flights in one night dropping 12 AGCs with each flight.

According to operative analyses carried out by NitroFirex, and assuming the loading and stowage coefficients for the AGCs, one can establish that having one LA and its two sets of AGCs

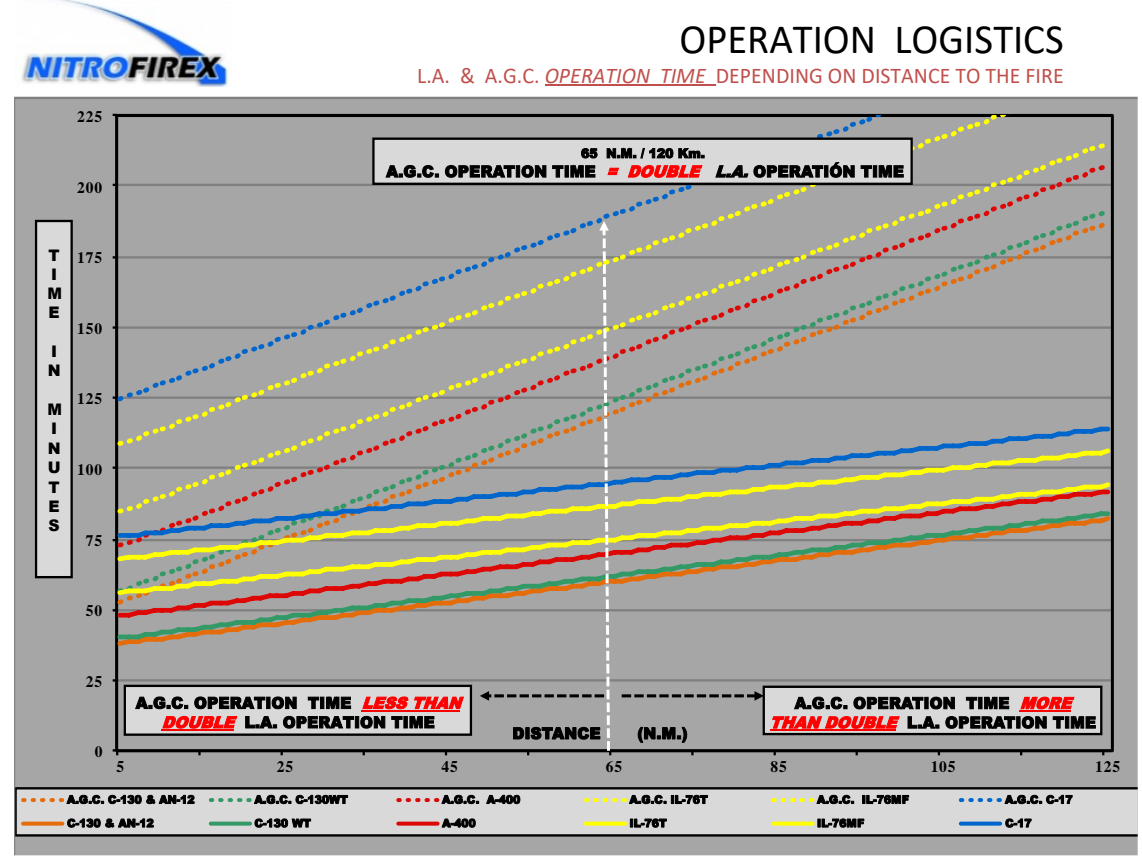

Figure 12 - NitroFirex operation logistics, Ops time LAs versus AGCs 
detached to a mission base within $120 \mathrm{~km}$ (65 N.M.), the operation over the fire zone would be nonstop.

The LA turn-around at mission base for loading the AGCs would ensure a relentless dropping of water over the intended targets (Figs. 12 and 13).

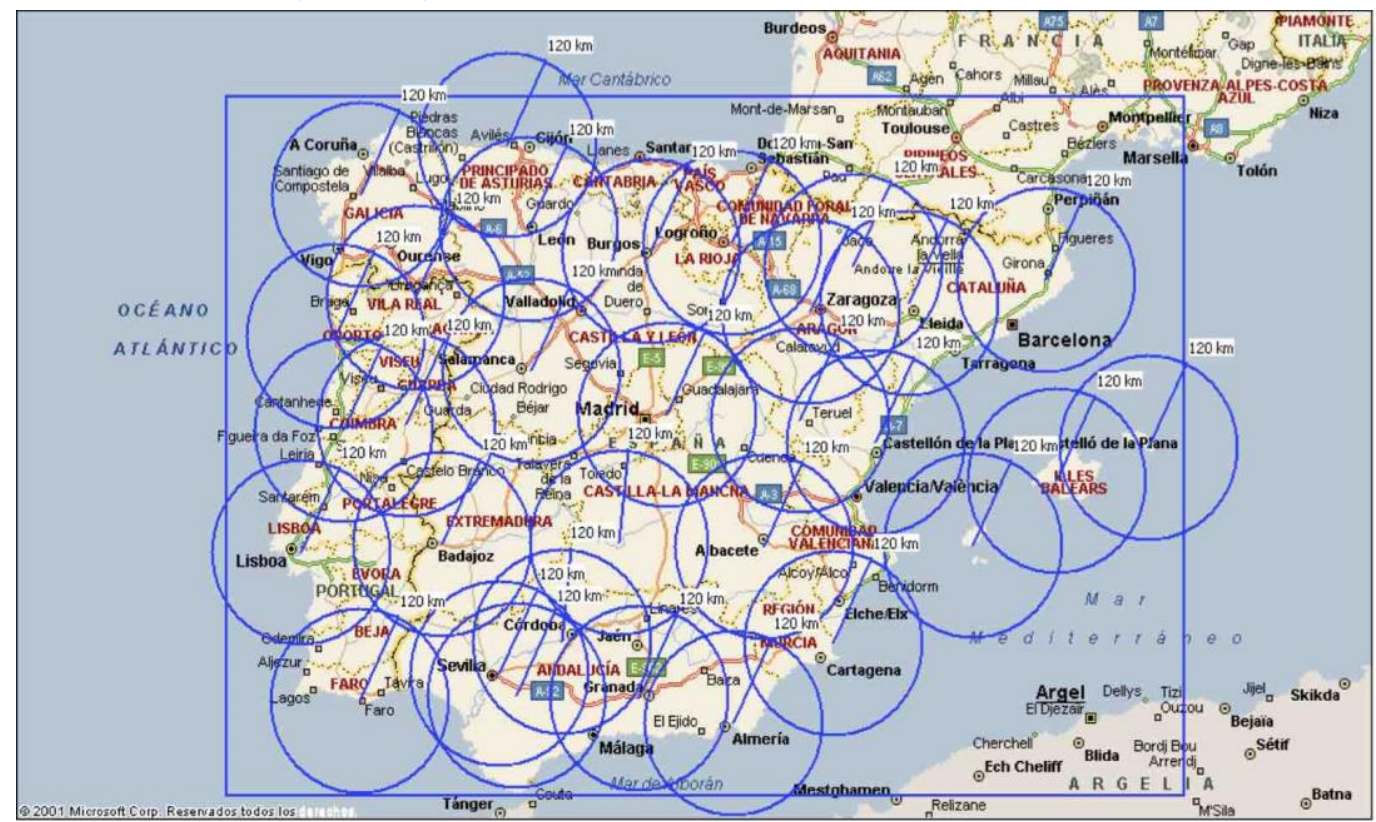

Figure 13 - NitroFirex mission analysis 65 N.M. airport radius Coordination and Safety

Those dedicated to aerial fire fighting know the importance of ground/air coordination to ensure an efficient and safe operation. With this objective in mind NitroFirex the implementation of new operative protocols and rules of communication with ground crews that are needed to adapt to the requirements of this project.

It should be the fire management officer who maintains radio contact with the LAs and the missionbase to coordinate the whole operation, principally the waiting points of the LAs as well as the approach routes and drop points of the AGCs.

At night it becomes very important that the ground crews can see the approach and the drop from the AGCs. For that reason, apart from the standard lights of all aerial vehicles (navigation, Beacon, strobe and logo lights), the NitroFirex are equipped with lights illuminating the fuselage as well as powerful headlamps. These headlamps are aimed forwards so that ground crews will be able to see them in the far distance and calculate the speed of approach to the drop point. Therefore, ground crews will be able to very clearly see the approach, the actual drop and its effect on the fire as well as the AGCs departure from the drop point.

In any type of aerial operation safety is of primordial importance at the time of planning, something to which NitroFirex is not indifferent. Consequently, the NitroFirex in their approach to the drop, as well as in the recovery phase to mission base, will have their flight parameters programmed and will accomplish them automatically and autonomously. Nonetheless, the safety concept of 'a man in a loop', in the case of having to abort the drop or reprogram the recuperation route, has been considered fundamental for the safety of the operation.

Therefore, during the approach phase, the NitroFirex will be capable of manually or automatically aborting their approach to the drop point, releasing their cargo at the abort point and then proceeding automatically into the escape and recovery phase. Should the flight trajectory deviate from the one established or an unexpected event occurs during the approach to the drop point and it becomes 
advisable not to continue, the abort can be manual, carried out by the fire management officer or by AGCs operators at LA or at mission-base. The NitroFirex AGC can automatically abort if its flight path does not match with what had been programmed or if it approaches the ground and/or a nondesired location.

In any case, the AGCs never reaches the ground filled with extinguishing agents, because if any thing deviates from what was programmed the cargo is immediately released and it then moves into the recovery phase.

On the other hand, the return route of the AGCs to mission base will be programmed for the night (they have all day to do it), at $500 \mathrm{ft}$ or less and over uninhabited areas. Should, by any chance, the AGCs engine have a flameout or any other malfunction, during the return it is equipped with a parachute and an airbag, which opens up automatically and that allows for a 'soft landing', minimizing any possible damage on ground to persons or goods.

\section{Technical Regulations for the Operation of RPAS}

Today, the great stumbling block in operating RPAS in purely civilian aerial operations is the regulations controlling the airspace where both manned and unmanned aircraft could operate safely and this regulation also has to ensure the safety of goods and persons on ground and his privacy. For promoting the development of such rules and regulations at European level the ESA (European Space Agency) established the operation of RPAS. Considering the broad range of operations and types of drones, it is proposed to establish 3 categories of operations and their associated regulatory regime: Open, Specific and Certified.

OPEN are those RPAS that basically operate in 'Visual Line of Sight' conditions, that is to say that at all moments there is visual contact between the vehicle and the operator. The operator's responsibility is to avoid any situation that could endanger any other aerial vehicle (manned or unmanned) or properties or persons on the ground and also is his responsibility to safeguard the citizen's privacy. The regulations of this category is responsibility of the different states of the European Union and the MTOW of the RPAS operating under the rules of this categories should be below $150 \mathrm{KG}$.

SPECIFIC is the category that "should be regulated in proportionate to the risk of the specific operation", this category initially has not MTOW limitations and his regulations are responsibility of UE concern agencies and at the same time of the different states of the European Union. The objective of this category is regulating for a "specific fly profile for a specific operations" ensuring that this profile does not affect fly or ground safety nor citizens privacy. Inside this category should be included what we can consider "state civil operations" like are SAR, disaster monitoring, border control, forest fire fighting, police, etc.

Finally the third category is the CERTIFIED that will be RPAS able of fly in the same operational environment (air space and endurance) that the manned aircrafts, therefore, Certified regulating for RPAS is more complex, entailing greater technical requirements that will be needed because of the new players involved. These players range from the RPAS operator to the air traffic controllers as well as the pilots of the manned aircraft.

The final operational achievements of the RPAS advancement should be logically the full development of the Certified category but to reach this objective the two other inferior categories, Open and Certified, should be fully operationally developed to demonstrate to the policy maker, regulators and the whole society that the RPAS is, in all aspects, safe enough to allows the certified RPAS operations.

Reasonably, NitroFirex stands in the second group of RPAS that operate under the rules of the Specific category. But the airspace required by the project is in fact minimal: A segregated airspace 
over of the fire would be used for an LA flying at an altitude no more of 6.000 feet over the forest fire zone and a distance of 6 to $10 \mathrm{NM}$ in respect to the intended drop zone. The LA will launch the AGCs that would need no more than 90 seconds with their filled tanks, and finally no more than an hour of navigation at an altitude of 500 feet between the fire and the mission base (the LA operating base), with the fly programmed by non populated areas to, ensure just in case any abnormal situation, a secure and soft landing.

What manned aircraft could be flying at night in the proposed zone? That is to say a zone at an altitude of 500 feet or less between a fire and the LA's mission base? It is for this very reason that a night-time operation is proposed, so that the regulations established could serve as a stepping stone for future operational regulations for RPAS in a civilian environment that need more flight time and airspace for manoeuvres.

Understanding the flight envelope proposed, and in context with the Specific regulatory authority, the profile of the AGCs recovery as proposed by NitroFirex should be the first to be regulated because the operational hours and the airspace required can not affect manned traffic. This would serve the use of RPAS in a safe and secure manner within the civilian environment of Specific category. At the same time, it may take advantage of using it as a 'launching pad' for future regular of Certified category operations in the upper layers of airspace where safety requirement and coordination are far superior owing to the altitudes and flight times needed for RPAS operations.

In one word, if in a Specific operation the low altitude profile, as proposed by NitroFirex, is not regulated and it will be extremely difficult to regulate futures Certified profiles requiring higher altitudes and longer time spans where will be flying manned aircrafts.

\section{The Aeronautical Industry and RPAS}

In the emerging and promising world of RPAS, aeronautical businesses as well as different governments and organizations are vying for good places in this race for the civil \& military market of the future that the "unmanned aviation" of air vehicles can provide. The aeronautical industry and official organizations will participate in this race with different products and business initiatives.

So, at the present moment, a differential option for the RPAS industry is to resort to ecological projects and appeal for social and political support to be able to support its participation in the development of the emergent world of unmanned aerial vehicles. The expensive, more complex and capable Certified projects must wait for better times, when the Specific category will be fully operational developed. Regarding past circumstances and now facing actual circumstances, the approach should be to find low cost projects operating in 'marginal airspace' with the purpose of keeping the regulatory bodies to a minimum.

In addition to, and taking into account present circumstances, it should be the responsibility of the organizations and businesses involved in the development of the RPAS's to refer to the economic losses, the political concern and the social fear that wild-fires generate, in order to obtain economic aid and above all political aid that a project of this nature needs for its development.

Given its relatively simple technique and minimum requirements when compared to the other RPAS projects, the NitroFirex project can and should be dealt with solely aeronautical industry, instead of having to resort to complex and problematical international alliances. In these difficult times this would allow companies to find a niche in the complex and competitive world of unmanned aerial vehicles, serving at the same time as a launching pad for the aeronautical industry to find a good position within the RPAS world by the time the civil employment of these vehicles becomes standard.

As a confirmation of the viability of the operational concept patented by NitroFirex, you can see the following video link:

https://www.dropbox.com/s/5r89j1lc9u3mwkz/VIDEO\%20COMPARTIVO-2\%20copia.mp4?dl=0 . 
This video is made with the key sequences of the NitroFirex video and the videos published, by Airbus (https://youtu.be/qCL1e1MJtSw) and by DARPA (https://www.youtube.com/watch?v=df CjHECws).

As you can see AIRBUS, with its Future Air Power program, and DARPA in its Gremlins program, assigned to be developed by Boeing and General Atomics, are both developing the operational concept patented by Nitrofirex but only for military applications.

Then what we claim is: if the NitroFirex patent concept can be developed for military purposes by Airbus, Boeing and General Atomics, why can't it be developed for this very real and very necessary civilian application as is the night aerial forest fire fighting?

Finally should be consider by the aeronautical companies that the nocturnal extinction of forest fires is a completely virgin worldwide market niche and widely demanded by experts in fire fighting as well as by policy makers all around the world so the first company that takes positions with respect to this market will be the leader of all the rest.

With the support European Space Agency (ESA) our company NF Advanced Engineering is building MicroFirex a small NitroFirex concept demonstrator able to drop around 60 litters that will be commercialized at the end of the year.

This multi-mission modular vehicle will demonstrate NitroFirex's viability. Our next step is developing a pre-commercial concept demonstrator to be in the range of one to two thousand litters dropping capability.

NitroFirex project has been presented in different national and international fairs and great interest was shown amongst the official organizations as well as private businesses in this sector, especially regarding the exclusive and original approach on the operative and technical levels of the project.

The NitroFirex concept is patented in those countries which have an economic and aeronautic capacity to face this type of project and who also have serious wildfire problems such as the USA, Canada, Australia, the Russian Federation and Europe (Spain, France, Italy, Germany and England).

\section{Conclusions}

It doesn't make sense that, well into the XXI century, at a time when ecology in all its aspects (climate change, $\mathrm{CO}_{2}$ emissions, deforestation, etc.) becomes a red hot topic on the political, social and economic levels, we've not bothered to integrate these demands in order to combat forest fires by night.

We should assume that we are using obsolete weapons against every day stronger enemy and we are losing the battle. The big fires, the destructive ones are those that last one-day, one night and at next morning are out of control, that because we believe that the priority should be to develop the first night aerial operational capability and this is the Nitrofirex's main objective.

Or we integrate modern available technologies to develop this operational capability and be able of discharge more quantity of extinguish agent in less time over de forest fire during first night and make it at safer and cheaper way than actual aerial means or our forest will burn away irretrievably.

The use of an autonomously guided glider container, such as the one proposed in the NitroFirex project, allows for nocturnal aerial fire fighting operations, eliminates risks for crews and increases the accuracy and concentration of the releases. Besides all this, the amount of water dropped both per operating hour and per flight hour is increased with respect to the aerial methods currently used.

In addition, the launcher aircraft to be used are medium/large helicopters or military transport airlifters that are not single-role and they will continue performing operations for the rest of the year once the summer forest fire season is over. This will help bring down to a great extent the final flighthour cost (considering acquisition amortization in addition to maintenance, personnel, etc.) for the part of operations performed on fire fighting, and ultimately the cost of each dispatched litter. 
The new technologies have already been developed, matured and made available. It is only necessary to integrate them towards the objective that concerns and worries us. It is therefore up to the politicians and technicians in charge of the operation to accept the seriousness of the problem and to raise a new strategy against the ecological damage, the economic loss and the social alarm that forest fires produce.

Of course we know that our approach is ambitious, but we also know that every day is more necessary and it is technically fully available. It is high time for the managerial and academic worlds to take on the challenge that an R\&D project of this nature and relevance imposes. The challenge consists in daring to develop this technological integration here and now with political decision, academicals support, managerial initiative and technological drive. 


\title{
Semi-empirical fire spread model for chamise and big sagebrush shrubs with spatially-defined fuel elements and flames
}

\author{
Chen Shen ${ }^{1}$; Thomas H. Fletcher ${ }^{1 *}$; David R. Weise ${ }^{2}$ \\ ${ }^{1}$ Chemical Engineering Dept., Brigham Young University. Provo, Utah, USA 84602, \\ \{yourjj@gmail.com,tom_fletcher@byu.edu*\} \\ ${ }^{2}$ USDA Forest Service, PSW Research Station. 4955 Canyon Crest Dr., Riverside, California, USA \\ 92507, \{dweise@fs.fed.us\}
}

\begin{abstract}
Current operational fire spread models are mostly based on experimental results from dead, low moisture fuel beds and thus do not perform well when modeling fire behavior in live shrubs. Although computational fluid dynamics (CFD) models predict more reliable combustion behavior, they have a high computational cost, large grid sizes for landscape-scale simulations, and oversimplified combustion within a shrub. There is a need for intermediate-scale shrub combustion models that describe better solid fuel combustion properties and shrub architecture, which may serve as sub-grid models for landscape fire spread simulations. A previously-developed semi-empirical shrub combustion model was improved and expanded to treat live chamise and big sagebrush. Fire spread in live chamise and big sagebrush shrubs was measured in an openroofed wind tunnel at the Pacific Southwest Research Station in Riverside, CA, which was used as the basis to develop the current model. Wind speed was held constant at $1.4 \mathrm{~m} / \mathrm{s}$. Fuel segment placement was modeled by coupling a Lindenmayer systems (L-systems) approach with LiDAR scan data. Species specific correlations for physical properties and combustion characteristics of individual fuel element were developed from individual leaf combustion experiments with a flat-flame burner system. These were incorporate in this model to simulate individual leaf flames, whose combustion history were updated every time step and further checked in flame merging sub-model to group flames and propagate flames to neighboring leaves. Fire spread in the shrub was completed when the number of burning leaves reached a minimum threshold.

Bulk density and local fuel density were found to be two major factors in shrub flame propagation. The modeling results included flame height above the shrub, fraction of shrub burned, burn time, etc. Modeling results compared well with measured time-dependent fire behavior in the wind tunnel. Fuel element placement in the semi-empirical shrub combustion model was improved when a combined L-systems and LiDAR approach was used to describe shrub architecture. This coupled architecture approach resulted in the most consistent and best predictions of combustion behavior for most experiments as compared to other random fuel element placement approaches. Variations in local fuel density affected flame spread more than the overall fuel bed density. Combustion temperature correction and species sensitive flame merging algorithm further improved the accuracy of modeling results. This model is an innovative approach to simulate shrub combustion via flame propagation at individual fuel element scale with a fairly low computational cost.
\end{abstract}

Keywords: shrub combustion, live shrubs, wildland fire, modeling

\section{Introduction}

Most current operational fire spread models (e.g. BehavePlus, FARSITE, FlamMap) (Andrews 1986; Finney 1998; Finney 2006) are based on the semi-empirical model by Rothermel (Rothermel 1972), which was developed for dead or low-moisture single-layer fuels that are contiguous to the ground. These models do not adequately describe fire spread in live shrub fuels and development of a next-generation model is hindered by the lack of fundamental understanding regarding fire behavior in live fuels (Finney et al. 2013). Computational fluid dynamics (CFD) models, including FIRETEC and WFDS (Linn 1997; Mell et al. 2007), solve the governing equations for mass and energy balances 
and thus provide insight into the physics and chemistry that influence fire spread. However, these models are computationally expensive and are generally constrained to $\sim 8 \mathrm{~m}^{3}$ grid cells for landscapescale simulations, oversimplifying the combustion process. Additionally, CFD models are restricted by inadequate knowledge regarding solid fuel physical properties and surface reactions.

This paper describes a semi-empirical, multi-leaf shrub combustion model for big sagebrush (Artemisia tridentata) and chamise (Adenostoma fasciculatum), which is developed to help fill the gap between current operational models and CFD models. This model is based on individual leaf combustion behavior measured with a flat-flame burner (Prince 2014; Gallacher et al. 2017; Shen 2018). Flames are simulated using equations based on individual leaf properties and combustion behavior; fire spread is accomplished via flame-fuel overlap. Fuel element placement is described by plant architecture sub-models developed for these two species.

\section{Methods}

\subsection{Shrub Combustion Experiment}

Multi-shrub combustion experiments were performed in an open-roofed wind tunnel at the USDA Forest Service Pacific Southwest Research Station. The fuel bed in the wind tunnel was designed to contain two shrubs arranged naturally. Geometric dimensions of the shrubs were measured manually and by pictures taken from different angles. LiDAR (Light Detection and Ranging) scans were also performed in some experiments to establish a 3-D voxel data matrix of potential fuel element locations. Excelsior placed just upwind of the first shrub was used as the shrub ignition source. The first shrub (i.e., ignition shrub) propagated the fire to the second shrub, with the goal of measuring fire behavior with minimal influence from the excelsior. Wind speed was held constant at $1.4 \mathrm{~m} / \mathrm{s}$. Combustion characteristics and time-dependent fire behavior were measured using three digital camcorders at different locations around the fuel bed. Flame angle, fire propagation path, time to burnout and flame length were then determined from video images. The effect of a surface fuel under the shrubs was also explored in some experiments. Sagebrush was collected on the San Bernardino National Forest and chamise was harvested from North Mountain Experimental Area near Riverside, CA.

\subsection{Individual Leaf Combustion Experiment}

Individual combustion experiments with live fuel particles were conducted for various plant species in a flat-flame burner (FFB) system (Prince 2014; Gallacher et al. 2017; Shen 2018). The FFB has a porous surface and produces a $1 \mathrm{~mm}$ thin premixed flame $\left(\mathrm{CH}_{4}, \mathrm{H}_{2}\right.$ and air). A glass cage surrounding the FFB prevents entrainment of ambient air. The fuel samples were placed $5 \mathrm{~cm}$ above the burner surface and ignited by the post-flame convective gases $\left(1000^{\circ} \mathrm{C}, 10 \mathrm{~mol} \% \mathrm{O}_{2}\right)$. Moisture content and geometric dimensions of each fuel sample were measured. The sample was held above the burner by a rod connected to a cantilever mass balance and were measured continuously. A K-type thermocouple was used to measure the gas temperature. Leaf sample combustion from ignition to burnout was recorded by a video camera. Combustion characteristics (e.g. flame height, time to ignition, burnout time, etc.) were determined by image analysis using MATLAB code, and then correlated vs. species, moisture content, fuel element dimensions, etc. (Shen 2017).

\subsection{Shrub Structure Modeling}

Geometrical constructions of chamise and big sagebrush were simulated by coupling an L-systems (Lindenmayer systems) approach with LiDAR data (Shen et al. in preparation). The resulting plant architecture model simulated all the branch and individual fuel segment positions, tuned for the specific shrub samples studied. Individual fuel segment physical properties and combustion characteristics were subsequently generated from statistical, species-specific correlations developed from individual leaf combustion experiments. The axiom string types were formulated for chamise 
and big sagebrush to create primary branches in the shrub. Mass measurements were used to develop correlations to predict shrub fuel mass. Individual fuel elements with physical properties populated by statistical models developed were placed onto the primary branches. The number of segments was adjusted to satisfy the targeted total dry mass of foliage and stems less than $1 / 4 \mathrm{inch}$. The ultimate direction of the L-systems strings for branches was guided by the location of the densest voxels from the LiDAR data. The resulting structure model predicted all the branch and segment positions, tuned for the specific shrub samples studied. Simulated shrubs appeared visually similar to the sampled shrubs and also generally matched the geometrical dimensions, as shown in Figure 1.
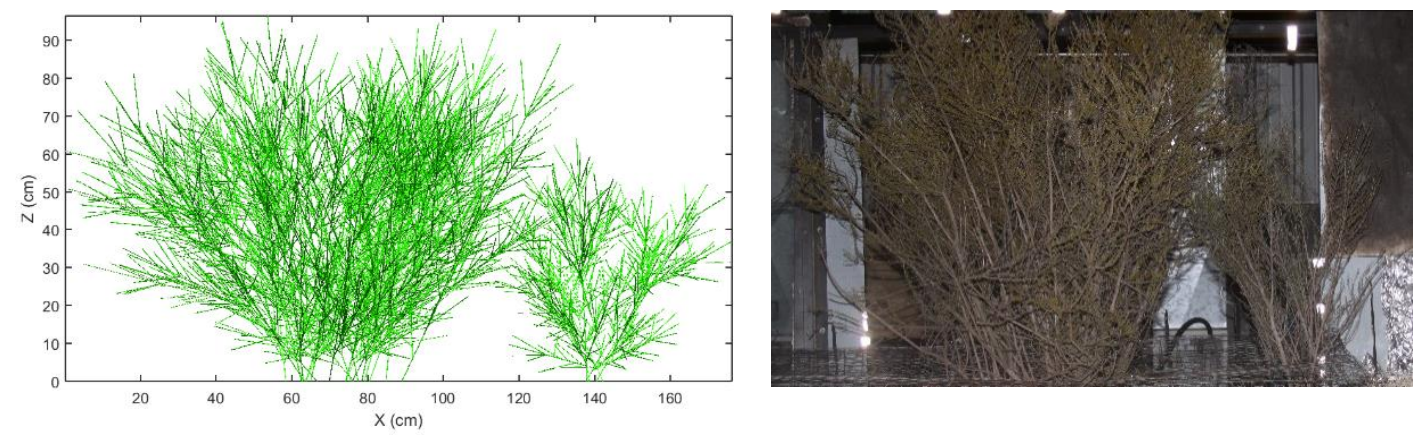

Figure 1 - A simulated chamise shrub set compared to the corresponding picture.

\subsection{Shrub Combustion Modeling}

The semi-empirical multi-leaf shrub combustion model includes the following modules: fuel element location, fuel element physical properties, fuel element combustion behavior, individual flame volume simulation, and flame merging. First, geometrical constructions of chamise and big sagebrush were simulated to establish fuel element placement. Individual segment flame volume is then simulated as a cylinder, whose dimension is determined by its combustion characteristics (including time to ignition, time to burn out, time to maximum flame height and maximum flame height) and the stage of its combustion (preheating, ignition, burning, or burn out). When an individual leaf flame contacts a neighboring leaf, the ignition sequence for that leaf commences, and the neighboring leaf may ignite. The flames then merge and contact surrounding leaves until burnout occurs. The flame merging submodel included semi-empirical correlations based on 2D flame merging experiments in the literature (Weng et al. 2004; Kamikawa et al. 2005). Both horizontal and vertical separation distances between leaf flames in three dimensions were considered to calculate the merged flame height. Those correlations were improved in the current model including:

$$
\frac{L_{f}}{L_{1}}=\frac{\alpha^{\prime}}{\alpha} \cdot\left(\rho_{\infty} c_{p} T_{\infty} \sqrt{g}\right)^{\beta-\beta^{\prime}} \cdot\left(\frac{\dot{Q}_{1}}{D_{1}^{5 / 2}}\right)^{\beta^{\prime}-\beta} \cdot\left(\frac{D_{1}}{D^{\prime}}\right)^{\frac{5}{2} \beta^{\prime}-1} \cdot\left(\frac{\dot{Q}_{D}}{\dot{Q}_{1}}\right)^{\beta^{\prime}}
$$

where $L_{f}$ is the merged flame height; $L_{1}$ is the solitary flame height; $\alpha$, $\beta$ ' and $\alpha, \beta$ are tuneable coefficients (prime designates merged flame); $D_{l}$ is the leaf segment diameter and $D^{\prime}$ is the normalized segment group characteristic diameter; $\dot{Q}_{1}$ is the heat release rate of individual leaf element combustion while $\hat{Q}_{D}$ is the segment group heat release rate. $\hat{Q}_{D} / \dot{Q}_{1}$ is currently predicted from the combustion behavior of individual leaf segments and the number of segments. This correlation is partially developed from wood crib data reported in the literature (Kamikawa et al. 2005).

A physics-based approach was established for scaling flame parameters, which included a mechanistic description of heat transfer to the leaf surface, temperature-dependent mass release, and an energy balance (Prince et al. 2017). In the current model, each leaf segment is divided into two layers, surface and interior. Species-specific multi-component one-step devolatilization models were used. Water release was tracked by a diffusion-limited model. The mass transfer was dependent on leaf temperature as well. Both convection and radiation heat fluxes were used to determine the elevated 
temperature of leaf. Finally, the heating of a leaf with the moisture evaporation was solved and the temperature history of a leaf was obtained. Based on this physics-based approach, flame parameters (end time of mass release, flame height, etc.) were scaled to match the observed fire spread conditions.

The combustion in this model was initiated by the ignition of a group of bottom corner leaf segments, which mimicked the fire front from excelsior as in the experiments. A profile of wind speed was appointed to each individual leaf segment according to its location. The combustion would terminate when there were no burning leaf segments.

\section{Results}

In total, 55 multi-shrub combustion experiments studying chamise and sagebrush were performed from 2012 to 2015. The modeling results (flame height above the shrub $(\Delta z$ f,max), fraction of shrub burned $\left(X_{s}\right)$, burn time (tburn), flame propagation speed and flame path) were compared with experimental results. Figure 2 is an example of a flame simulation of chamise shrubs compared to the corresponding experiment. Figure 3 shows that the predicted tburn by the current model for chamise was similar to the observed behavior from experiments, which is an improvement over previous predictions for most of the experimental runs. As shown in Figure 4, the prediction of the flame height $\left(\Delta z_{f, \max }\right)$ was overestimated in the previous model especially for run 3, which was a run where it was observed experimentally that the flame was not able to successfully propagate completely through the shrub. The current model is able to predict this unsuccessful propagation but underestimates the observed maximum flame height.

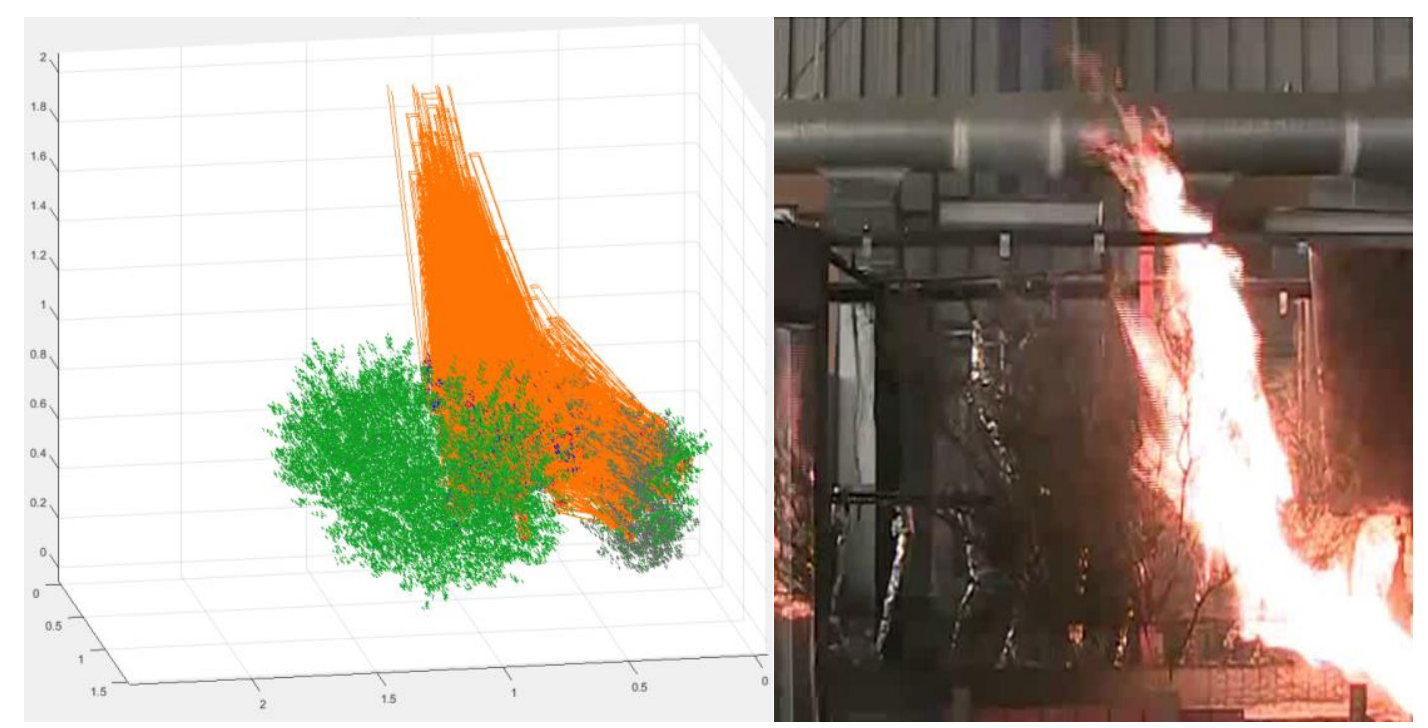

Figure 2 - Comparison of predicted flame behavior in chamise shrubs (left) using the semi-empirical shrub combustion model vs. the measured flame behavior in a wind tunnel (right). 


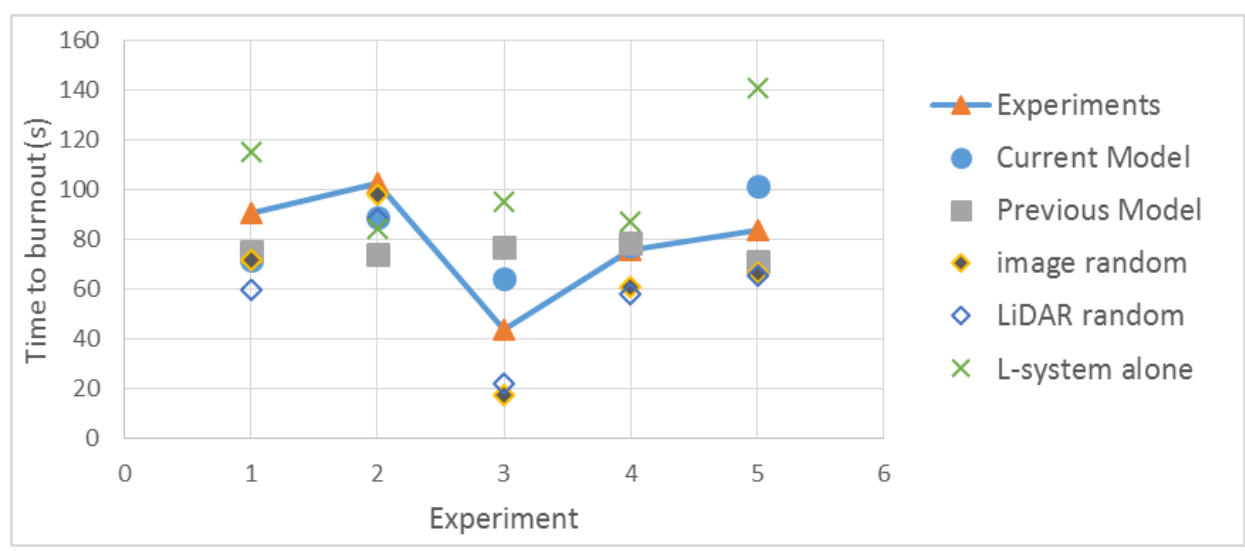

Figure 3 - Time to burnout comparison of model simulations for chamise.

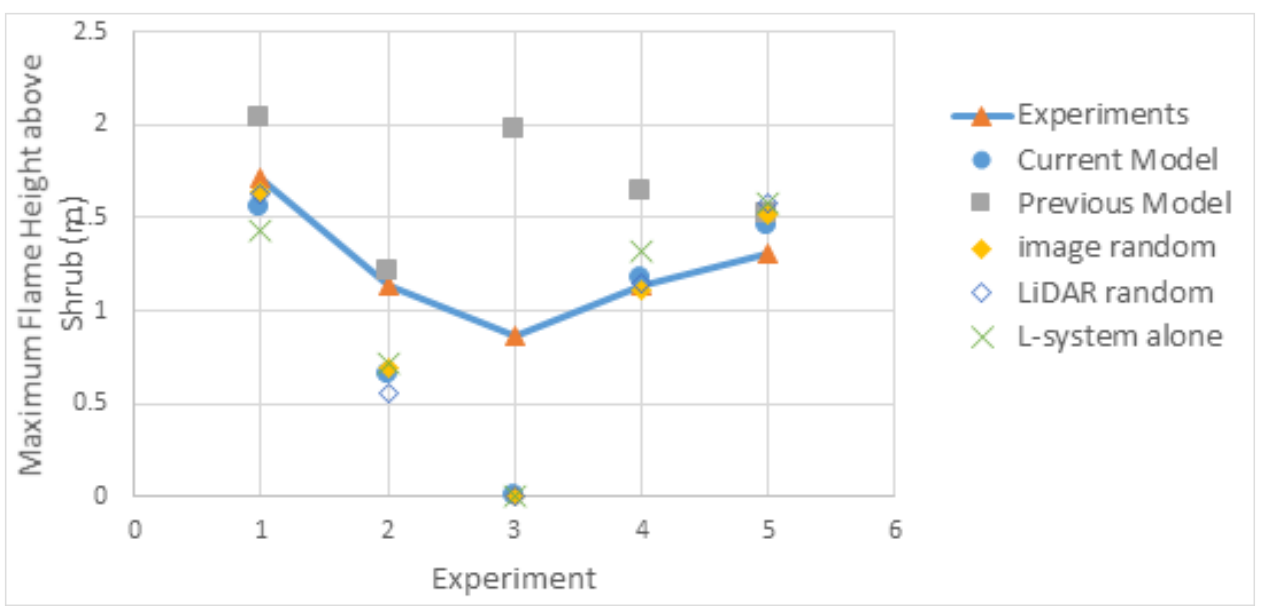

Figure 4 - Maximum flame height above the shrub comparison of model simulations for chamise.

Other less sophisticated fuel element placement approaches (random placement based on image analysis or LiDAR, and L-systems without LiDAR data) were also tested and integrated into current model. The predictions of combustion behavior with the previous fuel placement approaches are compared to the current model results (i.e., combined L-systems and LiDAR approach), which shows that the combined L-systems and LiDAR approach yielded the most consistent and best predictions of combustion behavior for most experiments.

\section{Conclusion}

Multi-shrub combustion experiments were performed in a wind tunnel facility at the Pacific Southwest Research Station in Riverside, CA. Bulk density and local fuel density were found to be two major factors in shrub flame propagation. Shrubs with high moisture content were usually observed to have a slower flame propagation rate.

Fuel element placement in the semi-empirical shrub combustion model was improved when the following were coupled: physical properties distribution, L-systems fractal theory approach, LiDAR shrub scan data and manual or image shrub measurements. More accurate flame parameters and species sensitive flame merging algorithm were developed in the semi-empirical shrub combustion model. The agreement between experimental observations and modeling results was improved. 


\section{Acknowledgements}

This work was supported by JFSP Grant 11-1-2-22 administered through USDA Forest Service PSW Research Station agreement 11-JV-11272167-054 and Brigham Young University. Special thanks to Joey Chong, Gloria Burke and Bonni Corcoran from the USDA Forest Service and Theodore Adams from the University of Montana.

\section{Reference}

Andrews PL (1986) 'BEHAVE: Fire Behavior Prediction and Fuel Modeling System- BURN Subsystem, Part 1.' USDA Forest Service, INT-194.

Finney MA (1998) 'FARSITE: Fire Area Simulator-Model Development and Evaluation.' USDA Forest Service, RMRS-RP-4.

Finney MA (2006) An overview of FlamMap fire modeling capabilities. In 'Fuels management-how to measure success: conference proceedings' pp. 28-30. (USDA Forest Service, Rocky Mountain Research Station, Fort Collins, CO)

Finney MA, Cohen JD, McAllister SS, Jolly WM (2013) On the need for a theory of wildland fire spread. International journal of wildland fire 22, 25-36.

Gallacher JR, Fletcher TH, Lansinger V, Hansen S, Ellsworth T, Weise DR (2017) Physical characteristics of shrub and conifer fuels for fire behavior models.

Kamikawa D, Weng WG, Kagiya K, Fukuda Y, Mase R, Hasemi Y (2005) Experimental study of merged flames from multifire sources in propane and wood crib burners. Combustion and Flame.

Linn RR (1997) A transport model for prediction of wildfire behavior. Ph.D. thesis, New Mexico State University.

Mell WE, Jenkins MA, Gould J, Cheney P (2007) A physics-based approach to modelling grassland fires. International Journal of Wildland Fire 16, 1-22.

Prince D, Shen C, Fletcher T (2017) Semi-empirical Model for Fire Spread in Shrubs with SpatiallyDefined Fuel Elements and Flames. Fire Technology 53, 1439-1469.

Prince DR (2014) Fire Spread in Sparse Vegetation. PhD Dissertation thesis, Brigham Young University.

Rothermel RC (1972) 'A mathematical model for predicting fire spread in wildland fuels.' USDA Forest Service, Intermountain Forest and Range Experiment Station INT-115, Ogden, Utah, USA.

Shen C (2017) Experiments and Modeling of Fire Interaction and Propagation in Big Sagebrush and Chamise Shrubs. PhD Dissertation in progress thesis, Brigham Young University.

Shen C (2018) Experiments and Modeling of Fire Interaction and Propagation in Big Sagebrush and Chamise Shrubs. PhD Dissertation in progress thesis, Brigham Young University.

Shen C, Fletcher ME, Gallacher JR, Adams T, Seielstad C, Weise D, Fletcher TH (in preparation) Geometrical Construction of Chamise and Big Sagebrush Shrubs using L-Systems Guided by LiDAR.

Weng WG, Kamikawa D, Fukuda Y, Hasemi Y, Kagiya K (2004) Study on flame height of merged flame from multiple fire sources. Combustion Science and Technology 176, 2105-2123. 


\title{
Study of growth of free-burning grass fires from point ignition
}

\author{
A.L. Sullivan ${ }^{1 *}$; M.G. Cruz ${ }^{1}$; J.E. Hilton ${ }^{2}$; M.P. Plucinski ${ }^{1}$; R. Hurley ${ }^{1}$ \\ ${ }^{1}$ CSIRO GPO Box 1700 Canberra, ACT, Australia \{Andrew.Sullivan@csiro.au*\} \\ ${ }^{2}$ CSIRO/Data61, Private Bag 10, Clayton South, Victoria, Australia
}

\begin{abstract}
Understanding wildland fire behaviour between ignition and attainment of the 'steady state' rate of spread is important for determining the speed of development of the fire and the accurate identification of the window of maximum effective first attack. As part of a larger experiment studying the behaviour of free-burning experimental grassland fires conducted in the open, the opportunity was taken to gather data on the development, behaviour and spread of fires ignited at a point, as would generally occur in a wildfire. These experimental fires, carried out in fully cured open improved pasture approximately $10 \mathrm{~km} \mathrm{NW}$ of Braidwood, NSW, Australia, were conducted on $0.1-0.2$ hectare plots and provided the most detailed insights into the behaviour of nascent fires burning in the open conducted in Australia since the work of McArthur (1966) and Cheney et al (1993).

This talk provides a general overview of this subset of experiments and the methods used to collect high spatial resolution data about the growth and development of free-burning point ignition fires in the open. As most fires in this series were very short-lived and were open to the vagaries of the prevailing wind field, traditional methods of measuring and documenting fire behaviour and spread were not practical-fires generally lasted less than two minutes. As a result, video footage taken from a quad-copter unmanned aerial vehicle (or remotely piloted aircraft or drone) hovering above the fire was used to map the progression of each fire. Details of the method by which footage is rectified and corrected, scaled and digitized will be provided as will a summary of the results obtained thus far.
\end{abstract}

Keywords: Fire behaviour, fire growth, acceleration, field experiments, wildfire, analysis, drone, remotely piloted aircraft

\section{Introduction}

Knowing precisely how long it will take a wildland fire to attain its 'steady state' rate of spread for the prevailing conditions after it breaks out is important for determining the window of opportunity when first attack or suppression will be most effective. Similarly, detailed information about the growth of an outbreak will assist with high fidelity prediction of the overall shape and extent of the fire. The behaviour of the fire during this period is generally described as the fire growth or acceleration phase (Luke and McArthur 1978, McAlpine and Wakimoto 1991). Most wildfires occur as point ignitions (e.g. from lightning, accidental causes or malicious causes). Similarly spotfires also start from points. Therefore understanding the processes involved in the transition from a point ignition to a fully developed going fire will provide insight into two very important aspects of wildland fire management (Sullivan et al. 2013).

The factors that may affect a fire's acceleration include the magnitude and variability of the speed and direction of the wind, the openness of the fuels to the wind, the moisture content, structure, availability and spatial distribution of the fuel, and local topography. A fire that has very rapid acceleration will complete its growth phase relatively quickly and achieve a behaviour and propagation rate commensurate with the prevailing conditions. A fire that has a very slow acceleration will take time to develop the flame structure and heat transfer rates associated with steady-state behaviour and thus may be more amenable to direct suppression and more readily controlled. Determining how long a fire will take to complete its acceleration phase will help enable suppression resources to respond 
most effectively to that fire-is direct attack likely to be effective when crews arrive or should they immediately implement indirect suppression tactics?

As part of a larger experiment studying the behaviour of free-burning experimental grassland fires conducted in the open, the opportunity was taken to gather data on the development, behaviour and spread of fires ignited as points for the purpose of building a dataset to enable development of an operational model of fire acceleration.

\section{Method}

\subsection{Experimental design}

The experimental fires, carried out in fully cured open improved pasture approximately $10 \mathrm{~km} \mathrm{NW}$ of Braidwood, NSW, were conducted on $0.1-0.2$ hectare plots. Plots of this size were deemed large enough to enable fire growth to be unconstrained and representative of naturally occurring fires, yet small enough to enable replication and ease of control. A preliminary analysis of seven of these fires is presented here.

Wind speed and direction was measured at $2 \mathrm{~m}$ (at two locations $<50 \mathrm{~m}$ up wind of the ignition locations) and $10 \mathrm{~m}$ above ground level (at one location within $300 \mathrm{~m}$ of each fire) at $10 \mathrm{~Hz}$. Air temperature and relative humidity were measured at $1.2 \mathrm{~m}$ at the $10 \mathrm{~m}$ measurement site at the same rate.

The experimental fires were ignited using a single match dropped at a time and location determined by the local wind gust structure and direction to maximise the potential for the fire to burn across the full length of the block with the fire allowed to burn out the entire plot. As soon as any part of the fire perimeter reached a plot boundary the experiment was considered over. Fire progression and flame characteristics were noted by a ground observer in close proximity to the fire as it developed until the fire front reached a plot boundary.

Prior to ignition a DJI Phantom 4 remotely piloted aircraft (RPA) mounted with a DJI high definition video camera was launched and positioned at an altitude and location such that its camera had as planar view of the plot as possible but which allowed for potential occlusion of the view of the head fire by smoke.

\subsection{Fire perimeter mapping and rate of spread measurement}

RPA footage was initially trimmed to the period covering fire ignition and experiment completion. The footage was then corrected for image distortion (i.e. spatially rectified to present as planar an image as was possible within the limits of the original footage, scaled and clipped to the extents of the experimental plot). This was done using purpose-built software written in the CSIRO Workspace environment (Cleary et al 2015) that utilises the OpenCV2 video analysis editing library (Bradski 2000) and the corners of the plots as control points. The fire perimeter was manually digitised using WebPlotDigitiser v3.8 ${ }^{1}$ from selected scaled images at 5 to 10 second intervals depending on fire speed. This enabled expert assessment of fire edge location which may be occluded by tall flame or smoke at a resolution of 3 and $8 \mathrm{~cm}$.

Fire behaviour metrics such as area, perimeter length, as well as rate of change in these quantities, were calculated directly from these data using an $\mathrm{R}$ script as was a fire isochrone map in which fire

${ }^{1}$ http://arohatgi.info/WebPlotDigitizer 
perimeters at each time interval were plotted. From each isochrone in the isochrones map additional fire behaviour metrics such as cumulative and interval distance and angle travelled, fire shape (length and breadth) and orientation, and headfire width (see Cheney and Gould 1995) were measured for each time step using the image analysis and processing software Image ${ }^{1}$. From these metrics, fire length to breadth ratio, cumulative and interval rate of spread, were calculated.

\section{Results}

Table 1 summarises the burning conditions of these fire experiments.

Table 1 - Summary of range of conditions for experimental point ignition fires.

\begin{tabular}{|c|c|c|c|c|c|c|c|c|}
\hline $\begin{array}{l}\text { Time of } \\
\text { day } \\
\text { (hh:mm) }\end{array}$ & $\begin{array}{l}\text { Air } \\
\text { temperature } \\
\left({ }^{\circ} \mathrm{C}\right)\end{array}$ & $\begin{array}{l}\text { Relative } \\
\text { Humidity } \\
(\%)\end{array}$ & $\begin{array}{l}\text { 10-m } \\
\text { open } \\
\text { wind } \\
\text { speed } \\
(\mathbf{k m} / \mathbf{h})\end{array}$ & $\begin{array}{l}\text { Curing } \\
\text { level } \\
(\%)\end{array}$ & $\begin{array}{l}\text { Overall } \\
\text { fuel } \\
\text { moisture } \\
\text { content } \\
(\%)\end{array}$ & $\begin{array}{l}\text { Duration } \\
\text { (s) }\end{array}$ & $\begin{array}{l}\text { Maximum } \\
\text { cumulative } \\
\text { rate of fire } \\
\text { spread }(\mathrm{m} / \mathrm{min})\end{array}$ & $\begin{array}{l}\text { Maximum } \\
\text { interval rate of } \\
\text { fire } \quad \text { spread } \\
(\mathrm{m} / \mathrm{min})\end{array}$ \\
\hline $\begin{array}{ll}11: 02 \quad- \\
16: 24\end{array}$ & $24-29$ & $21-30$ & $\begin{array}{l}14.8- \\
32.1\end{array}$ & $80-100$ & $5.9-12.9$ & $50-110$ & $15.4-66.9$ & $29.9-145.4$ \\
\hline
\end{tabular}

Figure illustrates the result of the image correction, rectification and cropping of raw RPA imagery.
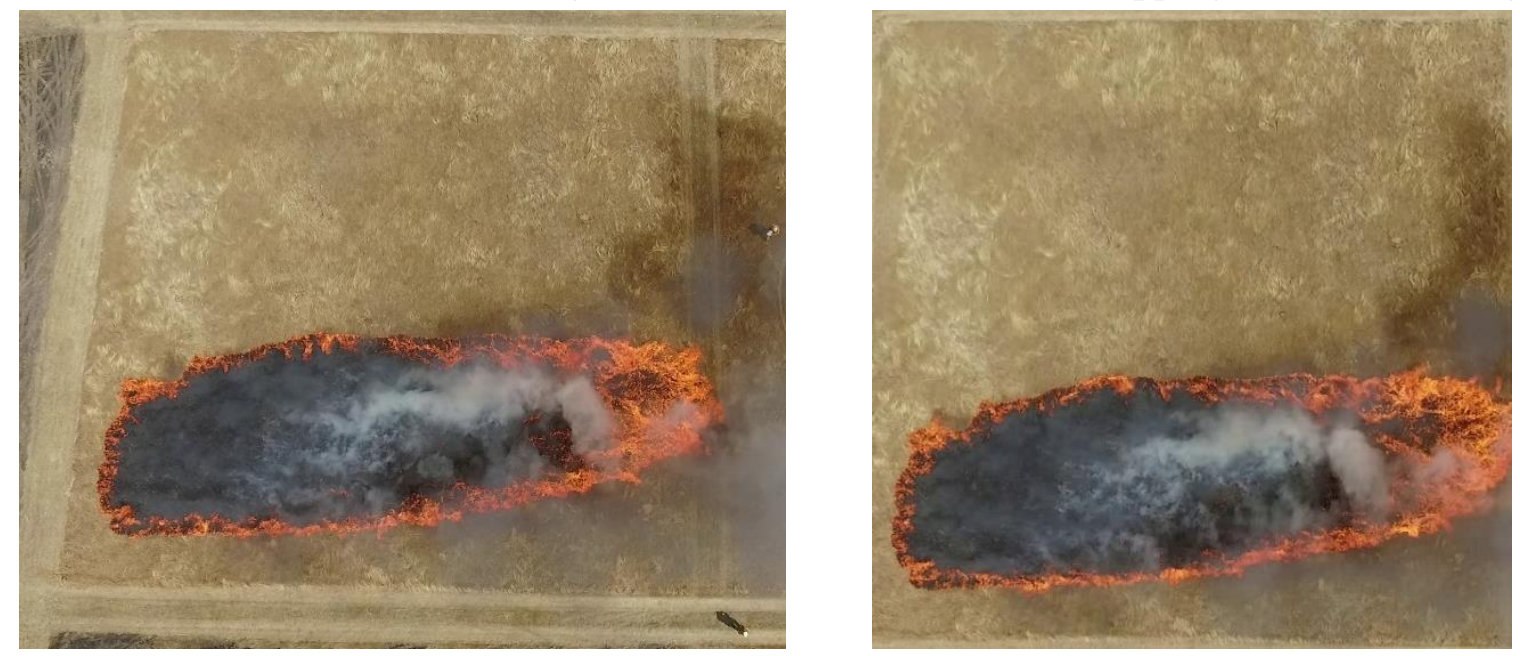

Figure 1 - (left) Example of a frame extracted from the raw RPA video of experimental fire B_S13. (right) The corrected, scaled, rectified and cropped image.

Figure illustrates an example of a series of raw images extracted from the video at 10 -second intervals showing the development of the fire and its spread across the experimental plot.

${ }^{1}$ http://imagej.nih.gov/il 

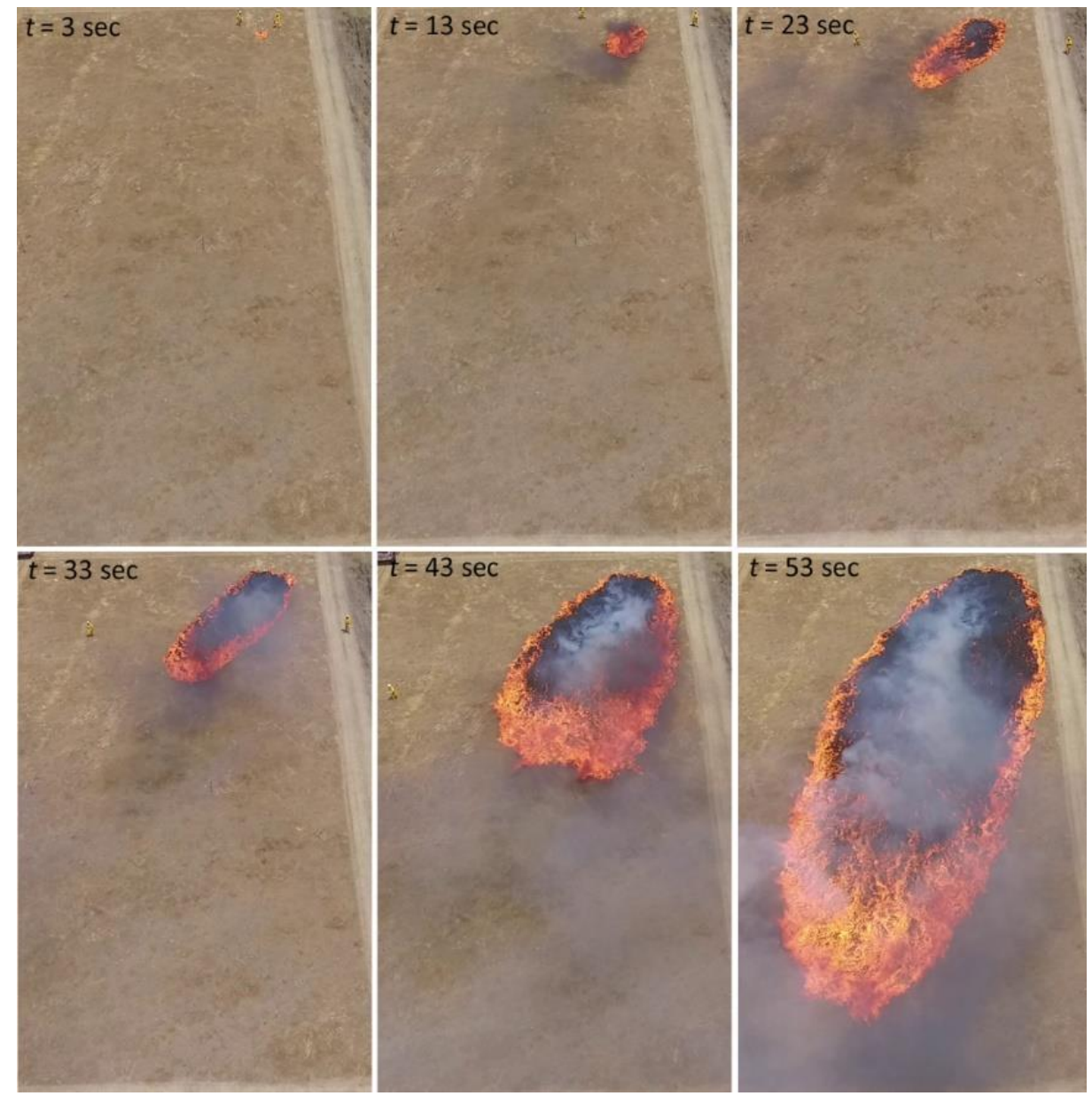

Figure 2 - Example of frames extracted from the raw RPA video of experimental fire $B \_$E28 each 10 seconds.

From the scaled and rectified imagery, the manually digitised fire perimeters enabled the construction of isochrone maps, two examples of which are shown below in Figure .
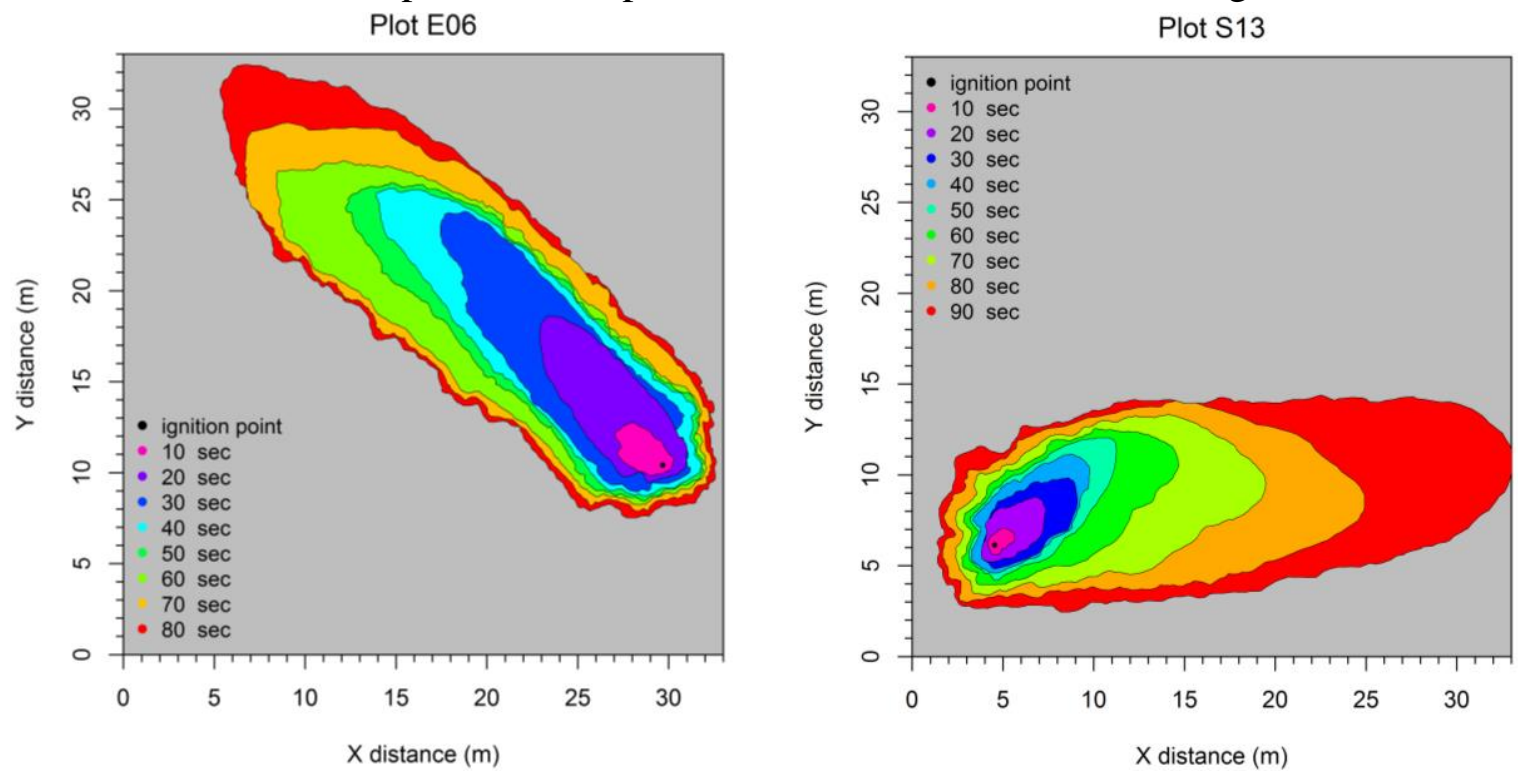

Figure 3 - Examples of two isochrones maps, one for $B \_E 06$ (left) and one for $B \_S 13$ (right). The interval and total number of isochrones depends on the speed of the fire and when it reached a plot boundary. 
Isochrone maps were then analysed for a variety of fire behaviour metrics. Figure summarises the rates of change in fire area, fire perimeter length, headfire width and length to breadth ratio over time since ignition for the subset of fires. Fire B_E28, burning on the largest experimental plot ( 0.2 ha) and under moderate fire weather conditions exhibited the greatest growth rates in terms of area increase, perimeter increase and headfire width. However fire B_E06 achieved the highest length to breadth ratio.
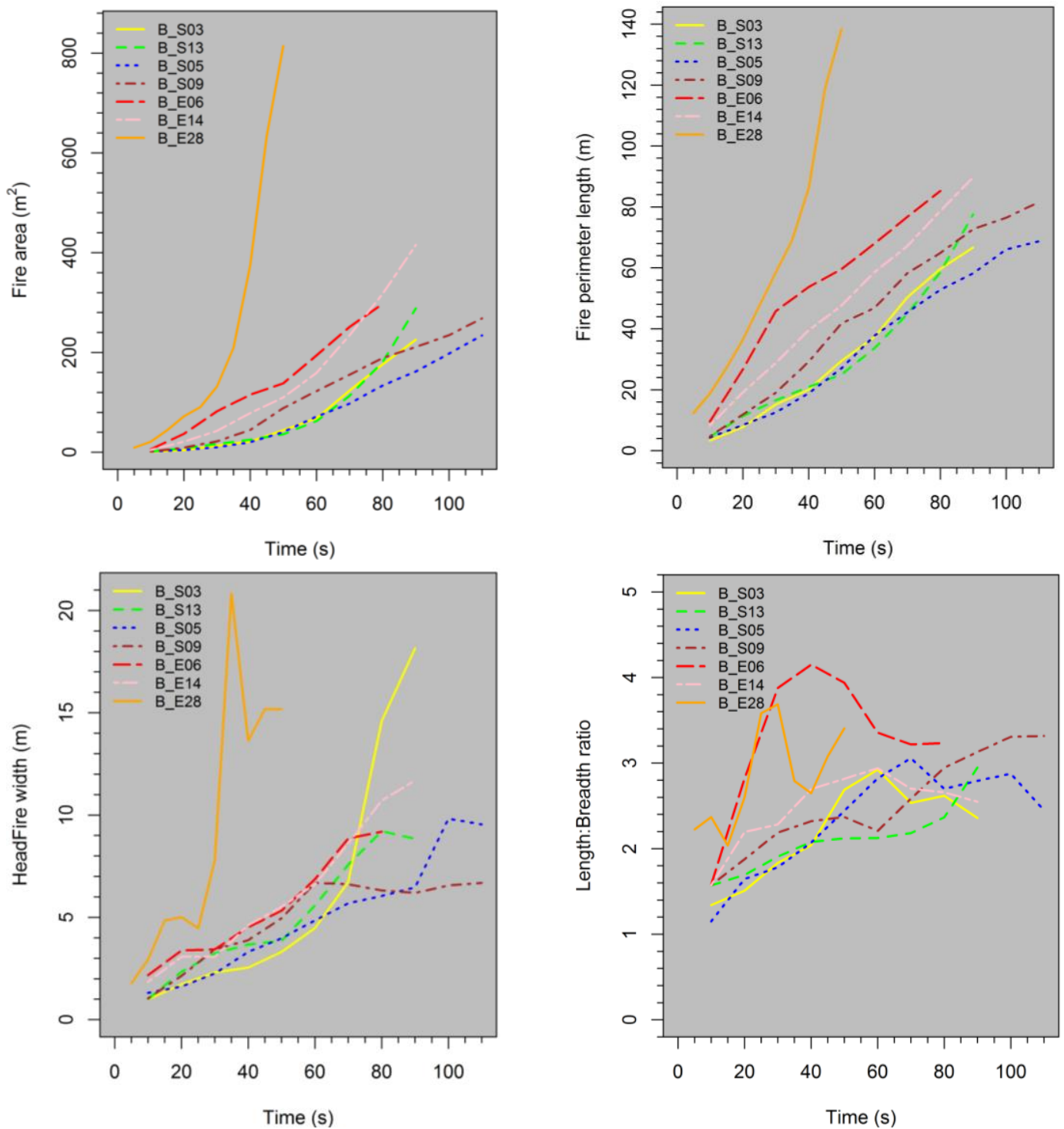

Figure 4 - Various fire behaviour and fire shape metrics for the point ignition fires. Clockwise from top left: fire area, fire perimeter length, length:breadth ratio and headfire width.

Interval spread distance and cumulative spread distance were estimated from the isochone maps as illustrated in Figure. The ignition point was determined from the location that the match was dropped and the direction of travel determined by maximum distance travelled in that interval. Graphs of interval rate of spread and interval acceleration, and cumulative rate of spread and cumulative acceleration is given in Figure and Figure, respectively. 


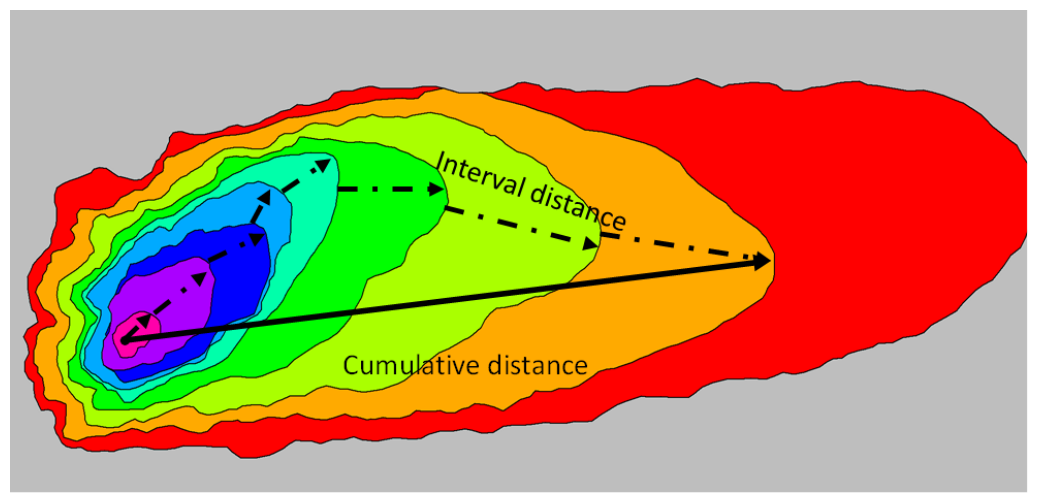

Figure 5 - Illustration of interval distance and cumulative distance in $B \_$E06. Cumulative distance is essentially the vectorial sum of the interval distances but in this case, because of the major shift in wind direction in the sixth interval, cumulative distance is much shorter than the scalar sum of the interval distances and thus will result in a lower overall rate of spread at this interval.
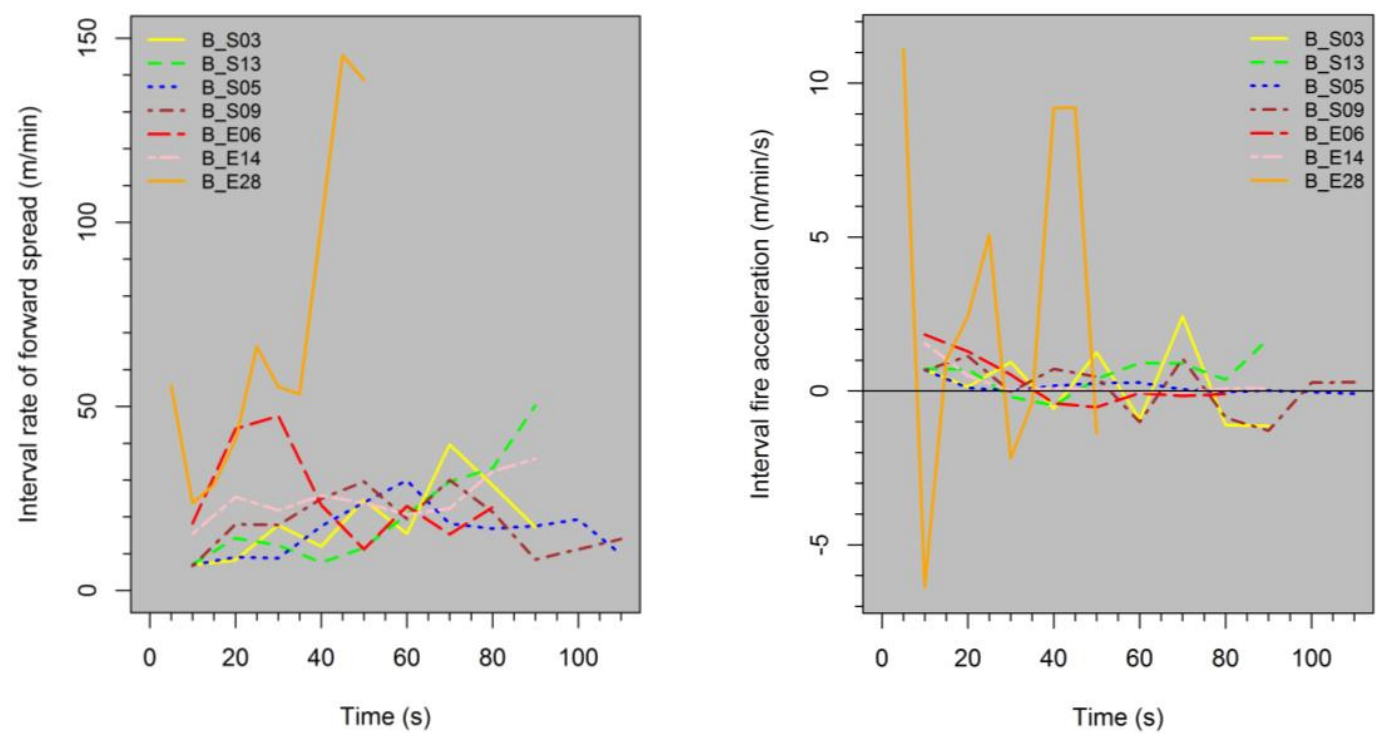

Figure 6 - Plot of interval rates of forward spread (left) and interval acceleration (right) for all fires.
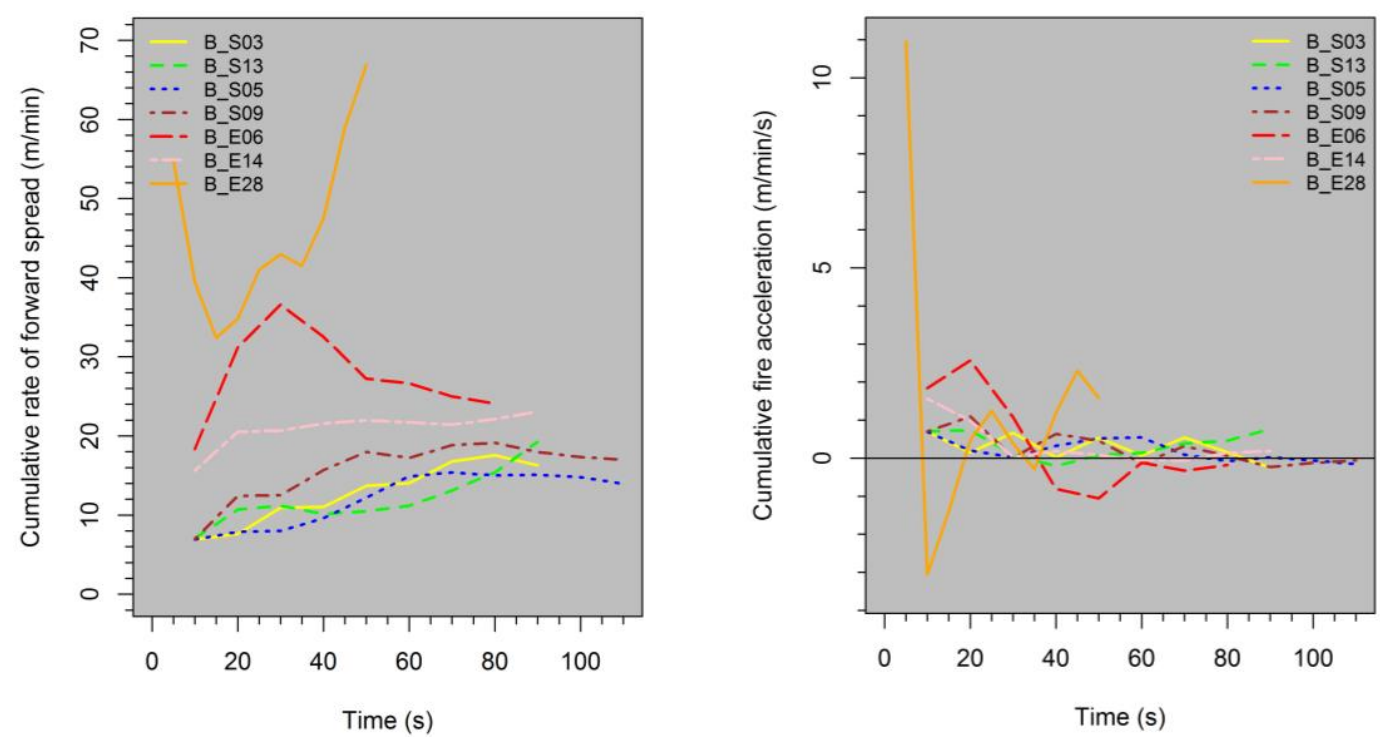

Figure 7 - Plot of cumulative rates of forward spread (left) and cumulative acceleration (right) for all fires. 


\section{Concluding remarks}

The development of a model to predict the build-up time to quasi-steady state is critical to understanding the time available to suppression crews for successful initial attack and for high fidelity landscape fire spread prediction. In the latter instance, build up time is not just applicable to new fire outbreaks or spotfires but also for break-aways from existing fire perimeters or control lines and fire spread through 'choke' points where fire width is constrained due to suppression action or changes in fuel condition.

This dataset provides a unique insight into fast developing fires, several of which very quickly attained or exceeded the predicted steady-state rate of spread for the prevailing conditions. The methodology of rectification and expert digitisation of the fire perimeters at regular but frequent intervals has produced isochrone maps of free-burning fires in the open at a previously unheard of temporal resolution.

It is envisaged that continued analysis of the dataset will enable the development of a model of grassland fire growth, particularly of wildfire outbreaks and possibly spotfires, that will benefit both fire spread simulation efforts as well as fire suppression planning and execution. This work will also be of benefit to research investigation the development of the two-dimensional shape of fires (e.g. Hilton et al 2016).

\section{Acknowledgements}

We are grateful to the Country Fire Authority of Victoria and the NSW Rural Fire Service for their support of this research. In particular we'd like to acknowledge the contributions of Alen Slijepcevic, Simon Heemstra, Laurence McCoy, Susan Kidnie, Rachel Bessell and Tim Wells for their contributions to this research. Our appreciation is also extended to numerous volunteer firefighters without whom we could not have conducted this experimental work.

\section{References}

Cheney NP, Gould JS and Catchpole WR (1993) The influence of fuel, weather and fire shape variables on fire-spread in grasslands. International Journal of Wildland Fire 3(1), 31-44.

Cheney NP and Gould JS (1995) Fire growth in grassland fuels. International Journal of Wildland Fire 5(4), 237-247.

Cleary PW, Thomas D, Bolger M, Hetherton L, Rucinski C and Watkins D (2015) Using Workspace to automate workflow processes for modelling and simulation in engineering. In: Weber T, McPhee MJ, Anderssen RS (eds), MODSIM2015, 21st International Congress on Modelling and Simulation, December 2015, pp. 669-675.

Hilton JE, Miller C and Sullivan AL (2016) A power series formulation for two-dimensional wildfire shapes. International Journal of Wildland Fire 25(9), 970-979.

Luke RH and McArthur AG (1978) Bushfires in Australia. Australian Government Publishing Service, Canberra ACT. 359 pp.

McAlpine R and Wakimoto R (1991) The acceleration of fire from point source to equilibrium spread. Forest Science 37(5), 1314-1337.

McArthur AG (1966) Weather and Grassland Fire Behaviour. Forestry and Timber Bureau Leaflet 100, Commonwealth Department of National Development. Canberra, 23 pp.

Sullivan AL, Cruz MG, Gould JS, Ellis PF, Plucinski MP, Hurley R and Koul V (2013) Fire Development, Transitions and Suppression: Final Report. CSIRO Ecosystem Sciences and CSIRO Climate Adaptation Flagship Client Report EP1312986, Canberra, ACT. 197 pp. 


\title{
The effect of interstitial flow on the burning dynamics of porous fuel beds
}

\author{
Zakary Campbell-Lochrie ${ }^{1 *}$; Carlos Walker-Rávena ${ }^{1}$; Eric V. Mueller ${ }^{1}$; Rory M. Hadden ${ }^{1}$ \\ $1 *$ University of Edinburgh. Edinburgh, \{Z.Campbell.Lochrie@ed.ac.uk\}
}

\begin{abstract}
The effect of fuel bed structure on the processes controlling flame spread in porous media is investigated. The fuel loading and bulk density of Pine needle (Pinus taeda $x$ rigida) fuel beds was varied resulting in changes in the fuel bed porosity. Experiments were conducted in no slope, quiescent conditions on a $1.5 \mathrm{~m}$ long flame spread table, which was instrumented at three locations to determine gas phase temperature and airflow. Flame height, heat release rate and burning rate were also measured.

Increases in overall fuel loading or decreases in bulk density resulted in increasing flame spread rate, flame height and HRR. At the highest fuel loading $\left(0.8 \mathrm{~kg} / \mathrm{m}^{2}\right)$ the spread rate for the lowest bulk density case $\left(10 \mathrm{~kg} / \mathrm{m}^{3}\right)$ was $180 \mathrm{~mm} / \mathrm{min}$ compared to $88 \mathrm{~mm} / \mathrm{min}$ in the highest bulk density case $\left(40 \mathrm{~kg} / \mathrm{m}^{3}\right)$, while a linear increase $\left(\mathrm{R}^{2}=0.97\right)$ in flame spread rate as a function of fuel bed porosity was also observed. The magnitude of the entrainment flow towards the approaching flame front is shown to increase with porosity, from $0.4 \mathrm{~m} / \mathrm{s}\left(94.5 \%\right.$ porosity) to $0.9 \mathrm{~m} / \mathrm{s}\left(98.6 \%\right.$ porosity), at $0.8 \mathrm{~kg} / \mathrm{m}^{2}$ fuel loading. An increase in the flow magnitude was also observed with increases in fuel loading for bulk densities of $10 \mathrm{~kg} / \mathrm{m}^{3}$, but not for bulk densities of $20 \mathrm{~kg} / \mathrm{m}^{3}$. At the higher bulk density, the effect of the increased fuel loading on the buoyancy-induced entrainment appears to be limited by porosity, resulting in reduced convective cooling ahead of the flame front, alongside the expected changes in radiation attenuation as the fuel bed depth varies.
\end{abstract}

Keywords: Flame Spread, Porous Fuel, Porosity, Flow

\section{Introduction}

The litter layer represents an important fuel stratum in wildland environments, and it has a significant role in surface flame spread. The physical phenomena involved in surface flame spread have been extensively studied (Catchpole et al. 1998; Wotton et al. 1999; Marcelli et al. 2004) however, the processes which control this require further exploration.

Existing, laboratory and field scale, flame spread studies have shown that flame spread is influenced by the weather conditions (wind, humidity), topography and fuel. The first two categories have been investigated in several previous laboratory scale flame spread experiments in natural porous fuel beds, involving a range of external flow conditions and slope angles (Mendes-Lopes et al. 2003; Liu et al. 2014; Rossa et al. 2015). To date, relatively few studies have systematically investigated the effect of the fuel bed properties on the physical processes driving flame spread (Simeoni et al. 2011; Finney et al. 2013).

Given the porous nature of natural fuel beds, flame spread is often modelled using a solid-gas multiphase approach (Grishin 1996; Sullivan 2009). Improvement of these models requires characterisation of multiple factors such as chemical, thermophysical and geometrical properties (Balbi et al. 2014) to allow development of improved sub-models.

These geometrical properties can be described at multiple scales, and encompass global fuel bed structural parameters (bulk density, porosity), local structural variations (fuel element interactions, needle orientation), and individual fuel element properties (density, surface to volume ratio).

Previous studies have shown a relationship between bulk density and fuel loading, and the flame spread rate (Morandini et al. 2013; Balbi et al. 2014). In these studies, often only the effect of fuel 
loading is studied, or, if studied, the bulk density is typically altered by changing the fuel loading. These parameters are related and changing the bulk density in this way does not allow explicit consideration of the fuel bed porosity to be independently investigated. In this study, the bulk density is controlled by altering the average height of the fuel bed, enabling fuel beds of multiple bulk densities to be investigated for any given fuel loading.

There has been little previous work to describe the effect of fuel bed characteristics on the physical mechanisms governing these variations in fire behaviour. Previous authors have used engineered materials to investigate the effect of structure within porous fuel beds (Wolff et al. 1991; Finney et al. 2013) however there remains a need to investigate the effect of structural changes within natural fuel beds on the observed variations in fire behaviour (flame spread rate, flame height, HRR) and the physical processes responsible.

\section{Experimental Methods}

\subsection{Experimental Setup}

A series of laboratory scale flame spread experiments were undertaken, involving pine needle fuel beds on a $1.5 \mathrm{~m} \times 0.67 \mathrm{~m}$ flame spread table. The table consisted of adjustable steel side walls, and a vermiculite substrate base instrumented at three measurement locations $(0.5 \mathrm{~m}, 0.8 \mathrm{~m}$ and $1.1 \mathrm{~m}$ from the ignition line) as shown in Figure .
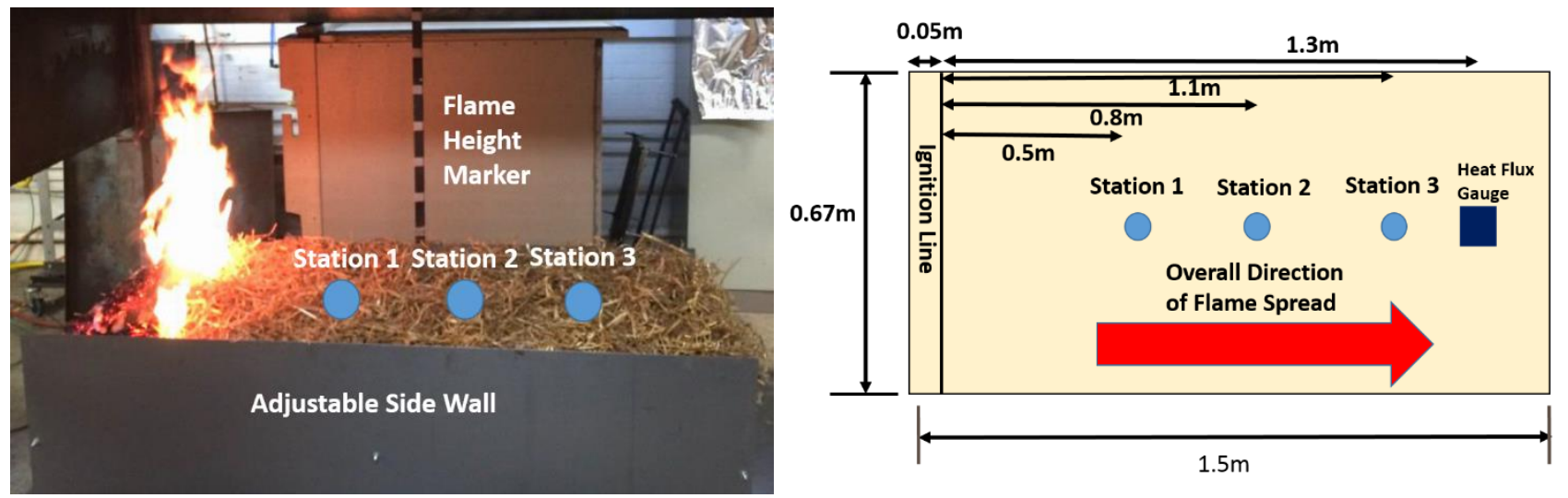

Figure 1 - Photo and diagram of experimental setup including 3 measurement station locations

Each measurement location consisted of a gas phase thermocouple $(0.25 \mathrm{~mm}$ thick, K Type $) 10 \mathrm{~mm}$ above the base; and a bi-directional pressure probe (McCaffrey and Heskestad 1976) with the centre axis located $10 \mathrm{~mm}$ above the vermiculite base. The gas phase thermocouples are used to calculate the flame spread rate, and in conjunction with the pressure probe measurements, the in-bed flow velocity.

Additionally, a water-cooled heat flux gauge was positioned flush to the upper surface of the vermiculite (bottom of fuel bed), at a distance of $1.3 \mathrm{~m}$ from the ignition line, to measure the total incident heat flux. The thermocouple, pressure probe, and heat flux gauge data were logged at $10 \mathrm{~Hz}$.

Experiments were filmed, with flame height and bed length markers allowing the flame height and spread rate to be determined through video analysis. The table was placed on a load cell to record the burning rate. The HRR was measured by oxygen consumption calorimetry (Janssens 1991).

All experiments were ignited using alumina-silica fibre $(0.67 \mathrm{~m} \times 0.05 \mathrm{~m})$ soaked in $10 \mathrm{ml}$ of acetone. The flameout time was defined as the time at which visible flaming was no longer observed within the fuel bed. 


\subsection{Fuel Properties}

\subsubsection{Pine Needle Properties}

Dead Pinus taeda x rigida (Loblolly - Pitch Pine hybrid) needles were collected in November 2017 from the Silas Little Experimental Forest in New Lisbon, New Jersey. Needles were stored indoors at ambient conditions and were otherwise unconditioned prior to use. The Fuel Moisture Content (FMC) of the pine needles was measured for each experiment, by oven drying needle samples for 24 hours at $60{ }^{\circ} \mathrm{C}$.

The geometrical properties were measured through random sampling. A needle density of $725 \mathrm{~kg} / \mathrm{m}^{3}$ [10/0.12], diameter of $1.34 \mathrm{~mm}$ [25/0.12], and surface to volume ratio of $4899 \mathrm{~m}^{-1}$ [25/446] were calculated [N/ Std. Dev.].

\subsubsection{Fuel Bed Construction}

The construction of the fuel beds followed a set procedure to ensure consistency. Unconditioned pine needles were weighed on a precision balance, and then distributed within ten equal sections of the table, to ensure uniformity of fuel loading. Within each of these sections, needles were dropped randomly on to the table with no effort to control the position or orientation of individual needles.

The height of the adjustable sidewalls was set relative to the average height of the fuel bed providing an identical edge condition in each case. The walls extended $0.03 \mathrm{~m}$ above the fuel bed preventing lateral entrainment within the fuel bed. This has been shown to result in a more linear flame front (Liu et al. 2015).

The bulk density was varied by altering the average height of the fuel bed. This allowed different bulk densities to be tested, at the same overall fuel loading. The fuel bed heights studied across the various fuel loadings, ranged from $0.01 \mathrm{~m}$ to $0.08 \mathrm{~m}$.

\subsection{Fire Behaviour Measurements}

The spread rate was calculated using the gas phase temperature measurements, which are characterised by an initial pre-heating period, followed by a large temperature spike. The flame arrival time was defined as the initial time at which the temperature exceeds $300{ }^{\circ} \mathrm{C}$, with the interval between successive arrival times used to calculate the spread rate.

The mass was measured across the duration of the experiment using a balance with accuracy of $\pm 0.001 \mathrm{~kg}$. The quasi-steady mass loss rate was calculated using a $5 \mathrm{~s}$ moving average. The HRR was calculated, through oxygen consumption calorimetry (Janssens 1991), with the peak HRR also reported for each experiment.

The flame height was determined through video analysis, with a vertical length scale $(0.05 \mathrm{~m}$ divisions) positioned at each measurement location. The flame heights were based on the upper extent of the continuous flame region.

\section{Results and Discussion}

\subsection{Flame Height, Flame Spread and Heat Release Rate Observations}

The effect of changes in global fuel bed structural characteristics is illustrated in Figure 2. This is a composite image of an experiment that involved a graduated fuel bed composed of four successive areas of both differing fuel loading and bulk density. For a change in fuel loading of $1.6 \mathrm{~kg} / \mathrm{m}^{2}$ to $0.2 \mathrm{~kg} / \mathrm{m}^{2}$ the flame height was observed to reduce from $(0.9 \pm 0.03) \mathrm{m}$ to $(0.05 \pm 0.03) \mathrm{m}$. The fire line intensity also decreased from $75 \mathrm{kWm}^{-1}$ to $9 \mathrm{kWm}^{-1}$. 


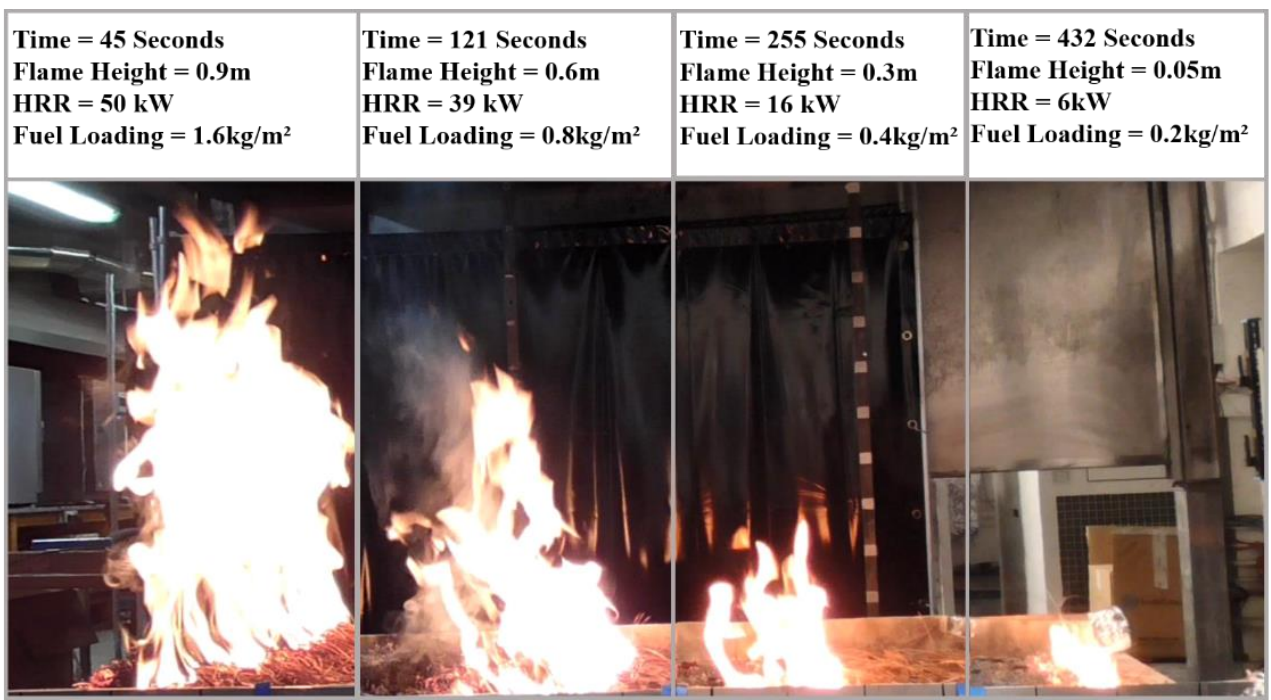

Figure 2 - Time lapsed photo of graduated fuel bed with burning characteristics at each segment centreline

These effects are in agreement with the trends observed in similar previous studies (Morandini et al. 2013) where simultaneous changes in fuel loading and bulk density led to increasing flame height, HRR and spread rate. Experiments of this nature, where both fuel loading and bulk density are simultaneously varied, are common, however previous studies (Simeoni et al. 2011; Santoni et al. 2014) have indicated that both these characteristics influence combustion dynamics and fire behaviour.

In this study, these fuel bed characteristics were therefore investigated separately through a series of experiments involving fuel beds of varying height, across a range of fuel loadings. The results from these experiments, including the average values of flame spread rate, peak HRR, mass loss rate, and flame height are summarised in Table .

Sustained flaming and flame spread were not observed at fuel loadings of $0.2 \mathrm{~kg} / \mathrm{m}^{2}$ at either bulk density investigated. This suggests that the small-scale structure of needles may be playing a significant role at low fuel loadings.

Table 1 - Effect of fuel loading and bulk density on fire behaviour for P. taeda $x$ rigida hybrid fuel beds

\begin{tabular}{|c|c|c|c|c|c|c|c|c|}
\hline $\begin{array}{c}\text { Fuel } \\
\text { Loading } \\
\text { (Wet } \\
\text { Mass) } \\
\left(\mathbf{k g} / \mathbf{m}^{2}\right)\end{array}$ & $\begin{array}{c}\text { Bulk } \\
\text { Density } \\
\left(\mathrm{kg} / \mathrm{m}^{3}\right)\end{array}$ & $\begin{array}{l}\text { Porosity } \\
\text { (\% Wet) }\end{array}$ & $\begin{array}{c}\text { Average } \\
\text { Fuel Bed } \\
\text { Height } \\
\text { (m) }\end{array}$ & $\begin{array}{c}\text { Average } \\
\text { Moisture } \\
\text { Content } \\
\text { (\% Dry } \\
\text { Basis) }\end{array}$ & $\begin{array}{c}\text { Average } \\
\text { Flame } \\
\text { Spread Rate } \\
(\mathbf{m m} / \mathbf{m i n})\end{array}$ & $\begin{array}{c}\text { Peak } \\
\text { Heat } \\
\text { Release } \\
\text { Rate } \\
(\mathbf{k W})\end{array}$ & $\begin{array}{c}\text { Quasi Steady } \\
\text { Mass Loss } \\
\text { Rate (g/s) }\end{array}$ & $\begin{array}{c}\text { Average } \\
\text { Flame } \\
\text { Height } \\
(\mathbf{m} \pm \\
\mathbf{0 . 0 2 5})\end{array}$ \\
\hline 0.2 & 20 & 97.2 & 0.01 & 16.4 & $\begin{array}{c}\text { Auto- } \\
\text { Extinguished }\end{array}$ & - & - & - \\
\hline 0.2 & 10 & 98.6 & 0.02 & 16.4 & $\begin{array}{c}\text { Auto- } \\
\text { Extinguished }\end{array}$ & - & - & - \\
\hline 0.4 & 20 & 97.2 & 0.02 & 15.5 & $96 \pm 3$ & $10 \pm 1$ & $0.4 \pm 0.2$ & 0.1 \\
\hline 0.4 & 10 & 98.6 & 0.04 & 15.2 & $129 \pm 21$ & $17 \pm 3$ & $0.4 \pm 0.2$ & 0.2 \\
\hline 0.6 & 20 & 97.2 & 0.03 & 15.2 & $132 \pm 12$ & $18 \pm 0.5$ & $0.8 \pm 0.2$ & 0.3 \\
\hline 0.6 & 10 & 98.6 & 0.06 & 15.6 & $168 \pm 3$ & $24 \pm 1$ & $1.8 \pm 0.2$ & 0.4 \\
\hline 0.8 & 40 & 94.5 & 0.02 & 16.1 & $88 \pm 7$ & $17 \pm 1$ & $0.7 \pm 0.05$ & 0.3 \\
\hline 0.8 & 20 & 97.2 & 0.04 & 15.7 & $134 \pm 12$ & $23 \pm 1$ & $1.0 \pm 0.2$ & 0.4 \\
\hline 0.8 & 11.4 & 98.4 & 0.07 & 17.4 & $177 \pm 3$ & $34 \pm 0.5$ & $1.7 \pm 0.2$ & 0.5 \\
\hline 0.8 & 10 & 98.6 & 0.08 & 15.9 & $180 \pm 3$ & $35 \pm 0.5$ & $1.4 \pm 0.2$ & 0.5 \\
\hline
\end{tabular}


For the cases of fuel loading of $0.4 \mathrm{~kg} / \mathrm{m}^{2}$ and above, sustained flame spread was observed. It was observed that an initial increase in fuel loading or a decrease in bulk density resulted in increased HRR, mass loss rate, flame height and spread rate. At greater fuel loadings $\left(0.8 \mathrm{~kg} / \mathrm{m}^{2}\right)$, the mass loss rate at $10 \mathrm{~kg} / \mathrm{m}^{3}$ reduced to $1.4 \pm 0.2 \mathrm{~g} / \mathrm{s}$, compared to $1.8 \pm 0.2 \mathrm{~g} / \mathrm{s}$, for the $0.6 \mathrm{~kg} / \mathrm{m}^{2}, 10 \mathrm{~kg} / \mathrm{m}^{3}$ case. Increasing the bulk density always resulted in a decrease in the spread rate. Increasing bulk density also resulted in increased burning rate except at the lowest fuel loading of $0.4 \mathrm{~kg} / \mathrm{m}^{2}$ where there was no variation although this may be as a result of the mass loss resolution.

\subsection{Effect of Global Structural Changes on Porosity}

It is hypothesised that these changes in fire behaviour, and the sensitivity to bulk density, arise due to changes in the processes occurring within the fuel bed. Altering the global structural characteristics of the fuel bed should result in changes in both the permeability and porosity of the fuel bed, which may then alter the in-bed flow behaviour.

The effect of fuel loading and bulk density changes can be characterised in terms of the porosity (or gaseous volume fraction, $\alpha_{\mathrm{g}}$ ), of the fuel bed which is given by,

$$
\begin{gathered}
\alpha_{g}=1-\alpha_{s} \\
\alpha_{s}=\rho^{*} / \rho
\end{gathered}
$$

Where $\alpha_{s}$ is the solid volume fraction, $\rho^{*}$ is the bulk density and, $\rho$ is the particle density of the pine needles. Figure 3 presents the flame spread rate as a function of the fuel bed porosity for the $0.8 \mathrm{~kg} / \mathrm{m}^{2}$ fuel loading. A linear increase $\left(\mathrm{R}^{2}=0.97\right)$ in flame spread rate as a function of increasing porosity is observed. This indicates that the burning dynamics are strongly influenced by porosity but does not indicate the physical mechanisms responsible for these changes.

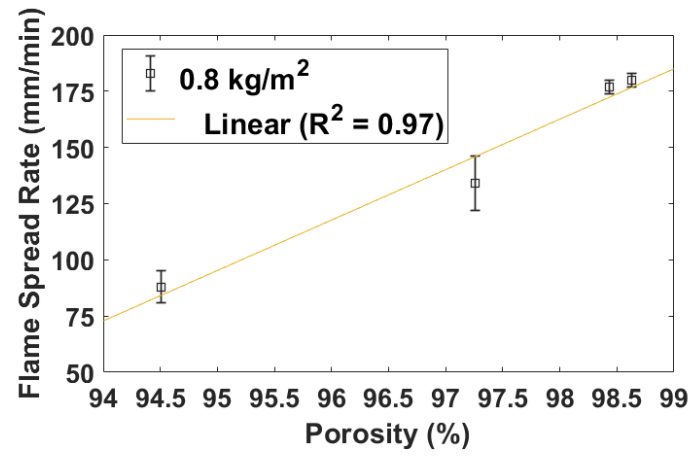

Figure 3 - Relationship between the average flame spread rate and porosity at $0.8 \mathrm{~kg} / \mathrm{m}^{2}$ fuel loading

\subsection{Heat Transfer Mechanisms}

The radiative heat transfer from the flame to the bottom of the fuel bed is influenced by the characteristics of the flame (flame length and tilt angle) (Morandini et al. 2001) and of the fuel bed (bulk density, particle size, and permeability) (Simeoni et al. 2012).

Past studies have shown both theoretically (De Mestre et al. 1989; Vaz et al. 2004a) and experimentally (Butler 1993; Vaz et al. 2004b) that increased fuel bed bulk density, and hence reduced porosity, results in increased radiation attenuation. As the porosity was changed by increasing the fuel bed height however then the depth of the absorbing media will also increase. This will reduce the effect of the extinction coefficient as the porosity is increased.

An increase in flame height (as observed to occur with increasing porosity) results in increased emittance of heat flux from the flame. As the fuel bed porosity is increased then it would therefore be expected that a greater heating period ahead of the flame front arrival would be observed. 
Measurement of the total incident heat flux to the bottom of the fuel bed however indicates a decrease in the duration of the pre-heating period, ahead of the flame front arrival, as the porosity is increased. As shown in Figure 4 (a) the time period before flame arrival in which a non-zero heat flux is observed decreases with decreasing bulk density, from $42 \mathrm{~s}$ at $40 \mathrm{~kg} / \mathrm{m}^{3}$ bulk density $(94.51 \%$ porosity), to $13 \mathrm{~s}$ at $10 \mathrm{~kg} / \mathrm{m}^{3}$ bulk density ( $98.63 \%$ porosity). The same trend is also observed (Figure $4(b))$ if the total heat flux is calculated relative to the position of the flame front from the measurement location $(\mathrm{d}=0)$, to account for the difference in spread rate.

(a)

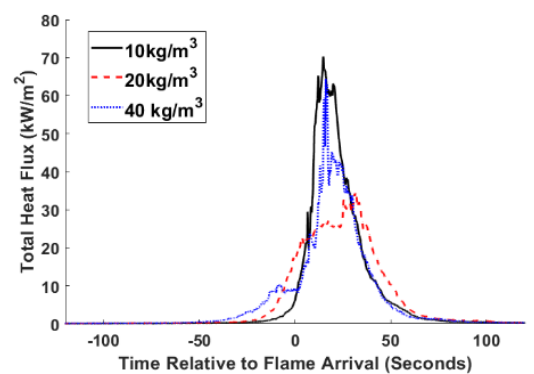

(b)

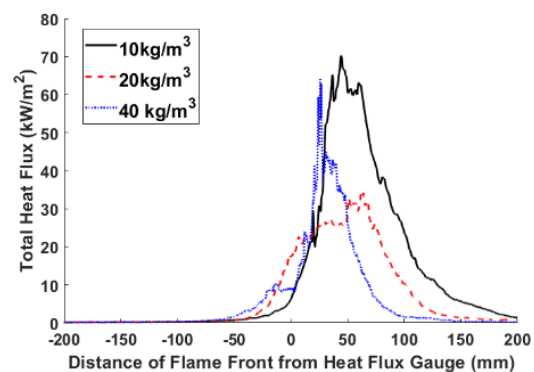

Figure 4 - Total incident heat flux to bottom surface of the fuel bed, for a range of bulk densities at a fuel load of $0.8 \mathrm{~kg} / \mathrm{m}^{2}$ relative to (a) time of flame arrival (b) flame front distance from the heat flux gauge

The total incident heat flux for fuel beds of varying fuel loading at a constant bulk density of $10 \mathrm{~kg} / \mathrm{m}^{3}$, showed an earlier initial increase in heat flux at lower fuel loadings, as shown in Figure 5. In this case the flame height increases for higher fuel loadings, but the attenuation also increases as the fuel bed depth grows with a constant extinction coefficient. For the same range of fuel loadings at a higher bulk density $\left(20 \mathrm{~kg} / \mathrm{m}^{3}\right)$ however this trend in the heat flux onset is no longer observed.
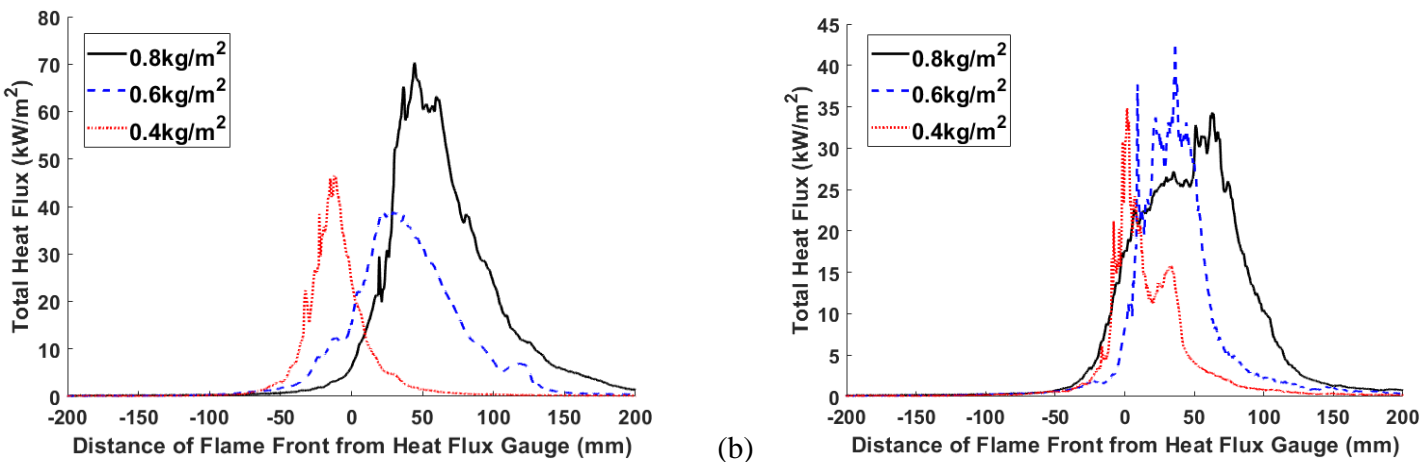

Figure 5 - Total incident heat flux to the bottom surface of the fuel bed for a range of fuel loadings at a bulk density of (a) $10 \mathrm{~kg} / \mathrm{m}^{3}$ and (b) $20 \mathrm{~kg} / \mathrm{m}^{3}$

The total heat flux to the bottom of the fuel bed also includes the effects of convective heat transfer in addition to the radiative heat transfer. This can include natural convection as well as flame-induced convection (Liu et al. 2015) driven by entrainment from the buoyant plume. Characterisation of the flow profile within the fuel bed is required to understand the influence of this buoyancy driven entrainment.

\subsection{In-Bed Flow Behaviour}

The flow data were derived from pressure measurements and processed using a Local Polynomial Regression Method (LOESS) (Cleveland 1979), with a $1 \mathrm{~s}$ window. The flow velocity is plotted relative to the distance between the flame front and the pressure probe $(d=0)$, with a negative distance indicating an approaching flame front.

The variability in flow velocity trends was assessed through comparison of the velocity data from three repeats of an identical case $\left(0.8 \mathrm{~kg} / \mathrm{m}^{2}\right.$ fuel loading, $40 \mathrm{~kg} / \mathrm{m}^{3}$ bulk density), as shown in Figure 
6. Flow data was recorded prior to ignition to provide an average value at the time of ignition, the largest standard deviation in this averaged zero value was $0.11 \mathrm{~m} / \mathrm{s}$. The greatest discrepancy in the peak entrainment towards the approaching flame front was $0.13 \mathrm{~m} / \mathrm{s}$.

(a)

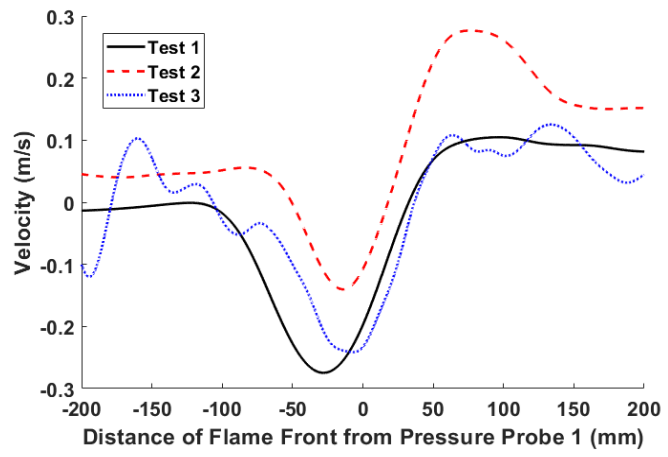

(b)

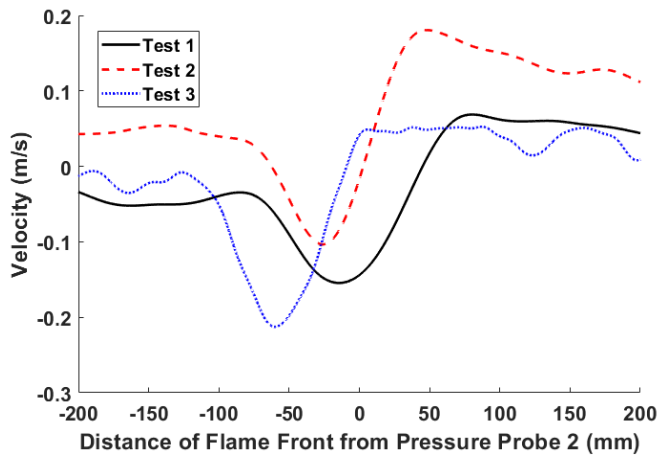

(c)

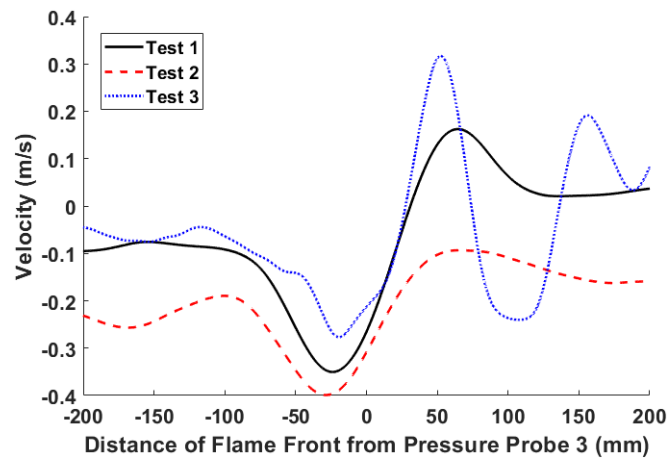

Figure 6 - In-bed flow velocity relative to flame front distance from (a) Probe 1, (b) Probe 2, (c) Probe 3, for three repeats of an identical case $\left(0.8 \mathrm{~kg} / \mathrm{m}^{2}\right.$ fuel loading, $40 \mathrm{~kg} / \mathrm{m}^{3}$ bulk density)

The in-bed flow velocity trends are generally characterised by a period of entrainment (indicated by negative velocities) prior to the arrival of the flame front, followed by a period of flow concurrent with the flame spread direction after the arrival of the flame front. In general, lower bulk densities do not follow this trend, as shown in Figure 7. It is suggested that this is due to the relatively high porosity allowing small-scale variation in needle structure to be more significant. The peak magnitude of the pre-flame arrival entrainment velocity is observed to increase, with decreasing bulk density (increasing porosity), with a peak value of $0.4 \mathrm{~m} / \mathrm{s}$ for the $40 \mathrm{~kg} / \mathrm{m}^{2}$ case, and $0.9 \mathrm{~m} / \mathrm{s}$ for the $10 \mathrm{~kg} / \mathrm{m}^{2}$ case.

(a)

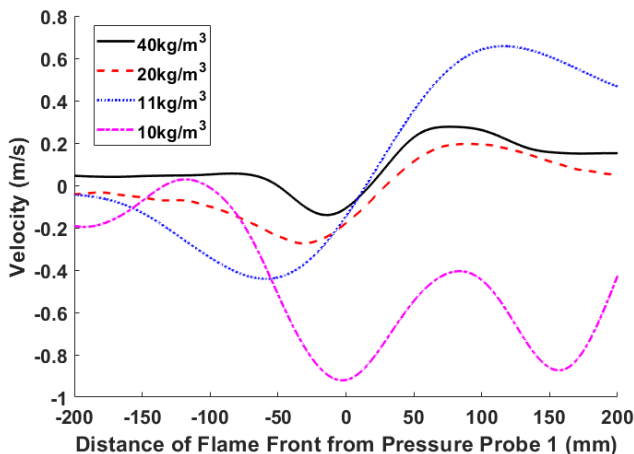

(b)

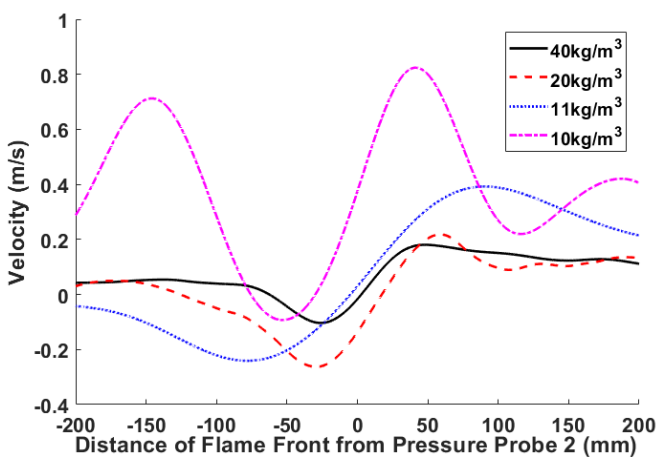


(c)

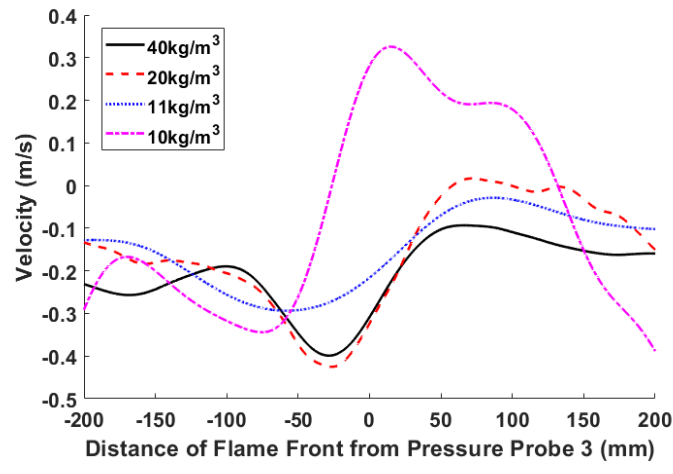

Figure 7 - In-bed flow velocity relative to flame front distance from pressure probes at (a) $0.5 \mathrm{~m}$ (b) $0.8 \mathrm{~m}$ and (c) $1.1 \mathrm{~m}$ from the ignition line, for a range of bulk density $(40,20,11,10) \mathrm{kg} / \mathrm{m}^{3}$, at $0.8 \mathrm{~kg} / \mathrm{m}^{2}$ fuel load

The increase in the pre-flame arrival entrainment velocity with reducing bulk density will result in a greater entrainment of ambient air, through the fuel bed, towards the fire front. The increased ambient airflow at the bottom of the fuel bed will result in convective cooling resulting in a reduced pre-heating period ahead of the flame front, as observed in the heat flux data.

Comparison of the in-bed flow velocity profiles for a range of fuel loadings, at a constant bulk density $\left(10 \mathrm{~kg} / \mathrm{m}^{3}\right)$, reveals a similar, but less consistent trend, as shown in Figure .

(a)

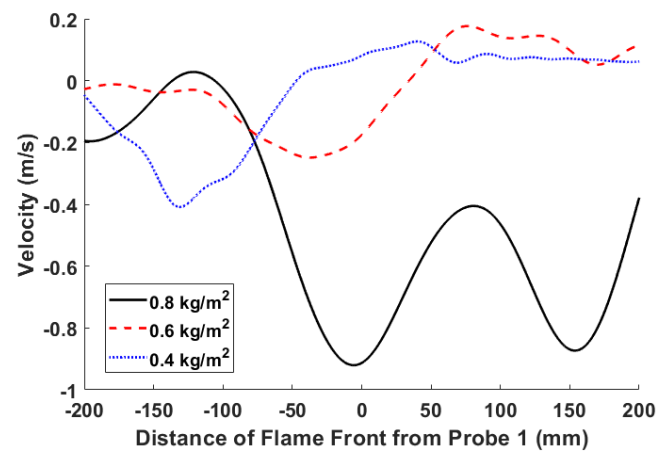

(b)

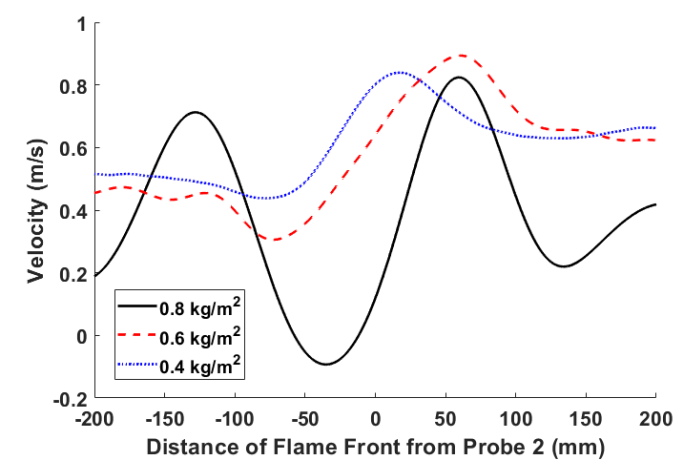

(c)

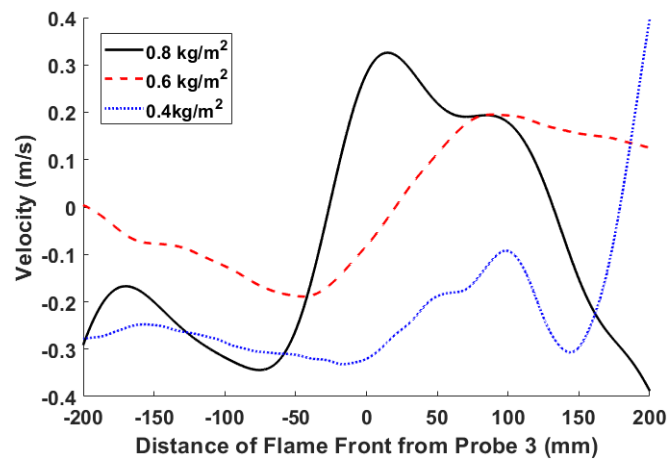

Figure 8 - In-bed flow velocity relative to flame front distance from pressure probes at (a) $0.5 \mathrm{~m}(\mathrm{~b}) 0.8 \mathrm{~m}$ and (c) $1.1 \mathrm{~m}$ from the ignition line, for a range of fuel loads $(0.8,0.6,0.4) \mathrm{kg} / \mathrm{m}^{2}$, at $10 \mathrm{~kg} / \mathrm{m}^{3} \mathrm{bulk}$ density

As shown in Figure 9 however, at a bulk density of $20 \mathrm{~kg} / \mathrm{m}^{2}$ the flow magnitude displays no clear trend with fuel loading. At the lowest fuel loading $\left(0.4 \mathrm{~kg} / \mathrm{m}^{2}\right)$, the high entrainment velocity at probe 3 may be due to the relative importance of the small-scale variations in permeability, as the fuel bed height $(0.02 \mathrm{~m})$ is equal to the height of the pressure probe. 
(a)

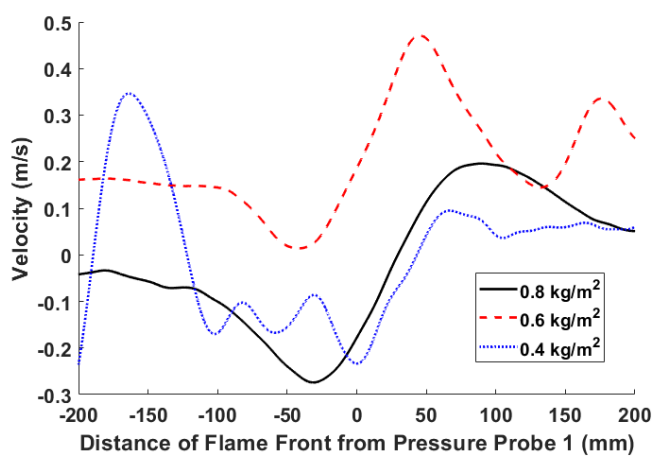

(b)

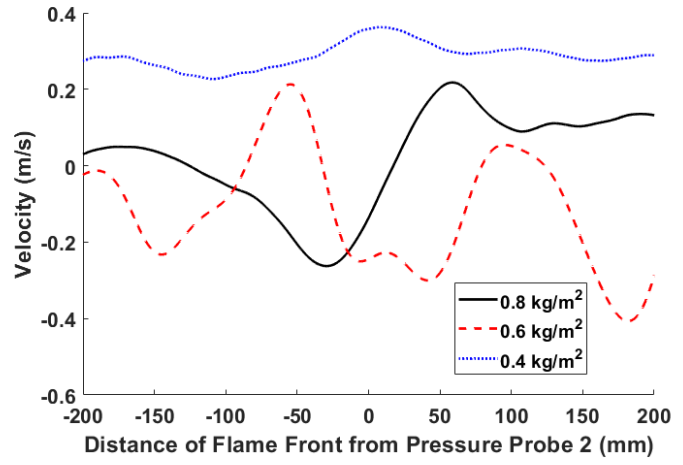

(c)

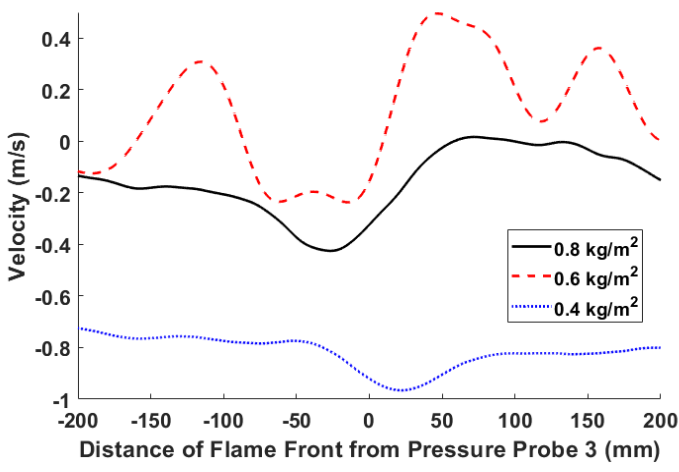

Figure 9 - In-bed flow velocity relative to flame front distance from pressure probes at (a) $0.5 \mathrm{~m}(\mathrm{~b}) 0.8 \mathrm{~m}$ and (c)

$1.1 \mathrm{~m}$ from the ignition line, for a range of fuel loads $(0.8,0.6,0.4) \mathrm{kg} / \mathrm{m}^{2}$ at $20 \mathrm{~kg} / \mathrm{m}^{3} \mathrm{bulk}$ density

The reduced effect of fuel loading on the entrainment velocity is in accordance with the lack of variation in the initial rise in total heat flux. This suggests that the fuel bed porosity in this case is limiting the effect of fuel loading increases on the entrainment flow magnitude.

\section{Conclusions}

Flame spread experiments for pine needle fuel beds of varying fuel bed height show that independent changes in either the fuel loading or the bulk density leads to observable changes in fire behaviour, with a strong coupling between burning behaviour and fuel bed porosity.

These changes in overall fuel bed porosity are observed to result in changes in the heat transfer mechanisms within the fuel bed. As the entrainment flow velocity increases with reducing bulk density, the onset of heating to the bottom of the fuel bed is delayed relative to flame arrival time. For the $0.8 \mathrm{~kg} / \mathrm{m}^{2}$ case, the initial rise in total heat incident flux at the bottom of the fuel bed was observed at $42 \mathrm{~s}$ before the flame arrival for a bulk density of $40 \mathrm{~kg} / \mathrm{m}^{3}$, compared to $13 \mathrm{~s}$ before flame arrival for a bulk density of $10 \mathrm{~kg} / \mathrm{m}^{3}$. This delay is also observed across varying fuel loadings at $10 \mathrm{~kg} / \mathrm{m}^{3}$ but not at $20 \mathrm{~kg} / \mathrm{m}^{3}$ bulk density.

The peak magnitude of the entrainment velocity, through the unburnt fuel, towards the approaching flame front, was observed to increase, with decreasing bulk density with a peak value of $0.4 \mathrm{~m} / \mathrm{s}$ for the $40 \mathrm{~kg} / \mathrm{m}^{3}$ bulk density $\left(94.5 \%\right.$ porosity) case, and $0.9 \mathrm{~m} / \mathrm{s}$ for the $10 \mathrm{~kg} / \mathrm{m}^{3}$ bulk density $(98.6 \%$ porosity) case. Increasing the fuel loading for $10 \mathrm{~kg} / \mathrm{m}^{3}$ fuel loadings led to similar increase in entrainment flow, however little trend was observed in the $20 \mathrm{~kg} / \mathrm{m}^{3}$ cases. This suggests that although the buoyancy is entrainment driven, it is limited by the porosity in the $20 \mathrm{~kg} / \mathrm{m}^{3}$ case. This highlights the importance of both the fuel loading and the structural properties of the fuel bed on the physical mechanisms within the fuel bed, and the need for a greater understanding of the effect on the overall burning characteristics. 


\section{Aknowledgements}

The authors wish to thank the Strategic Environmental Research and Development Program (SERDP) for their financial support under the project grant RC-2641. The authors would also like to thank Dr Michael Gallagher for providing the fuels used in this study, and Hugo Rouvrais for his assistance during the experiments.

\section{References}

Balbi J-H, Viegas DX, Rossa C, Rossi J-L, Chatelon F-J, Cancellieri D, Simeoni A, Marcelli T (2014) Surface Fires: No Wind, No Slope, Marginal Burning. Journal of Environmental Science and Engineering A 3, 73-86.

Butler B (1993) Experimental measurements of radiant heat fluxes from simulated wildfire flames. In 'Proc. 12th Conf. Fire For. Meteorol.', 104-111

Catchpole WR, Catchpole EA, Butler BW, Rothermel RC, Morris GA, Latham DJ (1998) Rate of spread of free-burning fires in woody fuels in a wind tunnel. Combustion Science and Technology 131, 1-37. doi:10.1080/00102209808935753.

Cleveland WS (1979) Robust locally weighted regression and smoothing scatterplots. Journal of the American Statistical Association 74, 829-836. doi:10.1080/01621459.1979.10481038.

Finney MA, Forthofer J, Grenfell IC, Adam BA, Akafuah NK, Saito K (2013) A study of flame spread in engineered cardboard fuelbeds: Part I: Correlations and observations. In 'Proc. Seventh Int. Symp. Scale Model.',

Grishin AM (1996) General mathematical model for forest fires and its applications. Combustion, Explosion and Shock Waves 32, 503-519. doi:10.1007/BF01998573.

Janssens ML (1991) Measuring rate of heat release by oxygen consumption. Fire Technology 27, 234249. doi:10.1007/BF01038449.

Liu N, Wu J, Chen H, Xie X, Zhang L, Yao B, Zhu J, Shan Y (2014) Effect of slope on spread of a linear flame front over a pine needle fuel bed: Experiments and modelling. International Journal of Wildland Fire 23, 1087-1096. doi:10.1071/WF12189.

Liu N, Wu J, Chen H, Zhang L, Deng Z, Satoh K, Viegas DX, Raposo JR (2015) Upslope spread of a linear flame front over a pine needle fuel bed: The role of convection cooling. Proceedings of the Combustion Institute 35, 2691-2698. doi:10.1016/j.proci.2014.05.100.

Marcelli T, Santoni PA, Simeoni A, Leoni E, Porterie B (2004) Fire spread across pine needle fuel beds: Characterization of temperature and velocity distributions within the fire plume. International Journal of Wildland Fire. doi:10.1071/WF02065.

McCaffrey BJ, Heskestad G (1976) A robust bidirectional low-velocity probe for flame and fire application. Combustion and Flame. doi:10.1016/0010-2180(76)90062-6.

Mendes-Lopes JMC, Ventura JMP, Amaral JMP (2003) Flame characteristics, temperature-time curves, and rate of spread in fires propagating in a bed of Pinus pinaster needles. International Journal of Wildland Fire 12, 67-84. doi:10.1071/WF02063.

De Mestre NJ, Catchpole EA, Anderson DH, Rothermel RC (1989) Uniform Propagation of a Planar Fire front Without Wind. Combustion Science and Technology 65, 231-244. doi:10.1080/00102208908924051.

Morandini F, Perez-Ramirez Y, Tihay V, Santoni PA, Barboni T (2013) Radiant, convective and heat release characterization of vegetation fire. International Journal of Thermal Sciences 70, 83-91. doi:10.1016/j.ijthermalsci.2013.03.011. 
Morandini F, Santoni PA, Balbi JH (2001) The contribution of radiant heat transfer to laboratory-scale fire spread under the influences of wind and slope. Fire Safety Journal 36, 519-543. doi:10.1016/S0379-7112(00)00064-3.

Rossa CG, Davim DA, Viegas DX (2015) Behaviour of slope and wind backing fires. International Journal of Wildland Fire 24, 1085-1097. doi:10.1071/WF14215.

Santoni PA, Bartoli P, Simeoni A, Torero JL (2014) Bulk and particle properties of pine needle fuel beds - influence on combustion. Int. J. Wildl. Fire 23, 1076-1086. doi:10.1071/WF13079.

Simeoni A, Bartoli P, Torero JL, Santoni PA (2011) On the role of bulk properties and fuel species on the burning dynamics of pine forest litters. In 'Fire Saf. Sci.', doi:10.3801/IAFSS.FSS.10-1401.

Simeoni A, Thomas JC, Bartoli P, Borowieck P, Reszka P, Colella F, Santoni PA, Torero JL (2012) Flammability studies for wildland and wildland-urban interface fires applied to pine needles and solid polymers. Fire Safety Journal 54, 203-217. doi:10.1016/j.firesaf.2012.08.005.

Sullivan AL (2009) Wildland surface fire spread modelling, 1990-2007. 1: Physical and quasiphysical models. International Journal of Wildland Fire 18, 349-368. doi:10.1071/WF06144.

Vaz GC, André JCS, Viegas DX (2004a) Fire spread model for a linear front in a horizontal solid porous fuel bed in still air. Combustion Science and Technology 176, 135-182. doi:10.1080/00102200490255343.

Vaz GC, André JCS, Viegas DX (2004b) Estimation of the radiation extinction coefficient of natural fuel beds. International Journal of Wildland Fire 13, 65-71. doi:10.1071/WF03009.

Wolff MF, Carrier GF, Fendell FE (1991) Wind-Aided Firespread Across Arrays of Discrete Fuel Elements. II. Experiment. Combustion Science and Technology 77, 261-289. doi:10.1080/00102209108951731.

Wotton BM, McAlpine RS, Hobbs MW (1999) The effect of fire front width on surface fire behaviour. International Journal of Wildland Fire 9, 247-253. doi:10.1071/WF00021. 


\title{
The fires of Mount Hymettus near Athens Greece (1996-2017): history and fire behavior characteristics
}

\author{
Gavriil Xanthopoulos ${ }^{1 *}$; Miltiadis Athanasiou ${ }^{2}$ \\ ${ }^{1}$ Hellenic Agricultural Organization “Demeter”, Institute of Mediterranean Forest Ecosystems. \\ Terma Alkmanos, Ilisia, 11528, Athens, Greece, \{gxnrtc@fria.gr*\} \\ ${ }^{2}$ Wildfire Management Consulting and Training. 8 Thoma Paleologou st., \\ Acharnes 13673, Athens, Greece, \{info@m-athanasiou.gr\}
}

\begin{abstract}
Mount Hymettus is a mountain lining the east side of Athens, the capital of Greece. Its proximity to the city, its flammable vegetation, and the variety of human activities (recreation, beekeeping, etc.) often result in problems with forest fires, which have reached a very high frequency in the last twenty years. These fires are of significant interest to firefighters, civil protection officers, foresters and the public because of their causes, their spread characteristics and associated safety risks, and their effects.

In the present paper, a brief account of the forest fires on Mt Hymettus in the 1996-2017 period is presented. Emphasis is put on the observed fire behavior which can be of interest to modeling studies of topographic influence on fire spread, and, since two of these fires have led to fatalities, may be used as case study illustrating a combination of fire environment conditions that entails great risk to firefighters. It is concluded that special care is needed regarding fire prevention and firefighting regarding fire management on Mount Hymettus. Professional and volunteer firefighters need to receive training that highlights the increased risks that fires on this mountain present. Furthermore, as the number of visitors is very high, special attention must be devoted to them, regarding both fire prevention and risk avoidance.
\end{abstract}

Keywords: Forest fire, chimney effect, canyon, fire fatality, Mount Hymettus, Greece

\section{Introduction}

Mount Hymettus is a mountain lining the east side of Athens, the capital of Greece. Its proximity to the city, its flammable vegetation, and the variety of human activities (recreation, beekeeping, etc.) often result in problems with forest fires, which have reached a very high frequency in the last twenty years or so. These fires are of significant interest to firefighters, civil protection officers, foresters and the public because of their causes, their spread characteristics and associated safety risks, and their effects.

Among Attica mountains, Mt Hymettus has been described as a "a remnant of the land that then was" in the Socratic dialogue Timaeus and Critias where the phenomenon of the erosion is described as "considerable accumulation of the soil coming down from the mountains, as in other places, but the earth has fallen away all round and sunk out of sight. The consequence is, that in comparison of what then was, there are remaining only the bones of the wasted body, as they may be called, as in the case of small islands, all the richer and softer parts of the soil having fallen away, and the mere skeleton of the land being left.". Furthermore, it can be deduced that the vegetation types covering many areas were mostly phrygana, since "mountains now only afford sustenance to bees".

Since antiquity, Mount Hymettus has been revegetated and then denuded many times, sometimes through fire but mostly through overgrazing and excessive vegetation cutting for fuelwood. At the time of the construction of the all-marble Panathenian stadium at its foothills facing Athens, for the first Modern Olympic Games in 1896, it was shown devoid of tall vegetation in all photos of that period. At the end of the World War II, tree vegetation on the mountain had again been removed 
completely because it had been used for the needs of the people of Athens. The protection the mountain received after that, and a series of reforestation efforts, led to the development of a patchy forest. This forest however, has suffered many fires in the last three decades.

In the present paper, a brief account of the forest fires on Mt Hymettus in the 1996-2017 period is presented. Emphasis is put on the observed fire behavior which can be of interest to modeling studies of topographic influence on fire spread, and, since two of these fires have led to fatalities, may be used as case study illustrating a combination of fire environment conditions that entails great risk to firefighters.

\section{Mount Hymettus and its environment as related to forest fires}

Mount Hymettus runs in a NE to SW direction defining the eastern edge of the city of Athens. It reaches a maximum elevation of $1026 \mathrm{~m}$, has a maximum length of $20 \mathrm{~km}$ and width of $6 \mathrm{~km}$, forming a perimeter of $65 \mathrm{~km}$ with a total area of $81 \mathrm{sq}$. km. Its vegetation consists of Meditereranean pine forest, Pinus halepensis and planted Pinus brutia, Mediterranean evergreen shrubs such as Quercus coccifera, Pistacia lentiscus, Genista acanthoclada, etc., and phrygana such as Cistus creticus, Cistus parviflorus, Sarcopoterium spinosum, etc. It is a Site of Community Importance (SCI) according to 92/43/EEC European Commission Habitats Directive (code GR3000006). As it belongs to the European Union's Natura 2000 network of sites, it enjoys a special environmental protection status.

Precipitation is relatively low, ranging around $400 \mathrm{~mm}$ per year. July and August, are usually completely dry. The prevailing wind during the fire season, from June to early September, is from the NE, blowing along the general direction of the mountain. This wind, known as "etisies" from the ancient Greek times, blows in the summer in all the Aegean sea, mainly from June to September, and usually runs for three to four consecutive days reaching 7 and sometimes 8 Beaufort scale force (roughly $50-70 \mathrm{~km} / \mathrm{h}$ ) in a steady direction. It is also called "meltemi" wind.

On the west side of the mountain, which faces the city and where most fires occur, the slope is perpendicular to the direction of the wind. Fire spread is in the direction of the resultant of the wind and slope vectors. This means spread in a S to SW direction with a tendency to move upslope. Further to that, and especially interesting regarding firefighter safety, is that the slopes of Mount Hymettus, especially on its western side, are characterized by a series of steep canyons.

\section{Forest fire history (1996-2017)}

In the 1996 to 2017 period at least eleven significant fires occurred on Mount Hymettus (Figure 1). They were quite large for the size of the mountain and parts of them often stopped at the fridge of the city where there was no vegetation to burn. All of them required heavy aerial support for their suppression. These larger fires are summarized in Table 1. Many other smaller fires that take place every summer are suppressed quickly through initial ground attack and help from the air.

Table 1 - Some of the most important fires of the 1996-2017 period on Mount Hymettus

\begin{tabular}{|c|l|}
\hline Wildfire & \multicolumn{1}{|c|}{ Description and Fire Behaviour } \\
\hline $\begin{array}{c}\text { July 22, } \\
\mathbf{1 9 9 8}\end{array}$ & $\begin{array}{l}\text { Wind driven through a dense and tall pine forest, explosive in a canyon at the east flank } \\
\text { close to its heel where four fatalities occurred. } \mathrm{T}=35^{\circ} \mathrm{C}, \mathrm{RH}=25 \% \text {, North wind of } 30 \\
\mathrm{~km} / \mathrm{h}, \text { gusting to } 48 \mathrm{~km} / \mathrm{h} \text {. }\end{array}$ \\
\hline $\begin{array}{c}\text { August 4, } \\
\mathbf{2 0 0 3}\end{array}$ & $\begin{array}{l}\text { Medium to low intensity surface fire in relatively sparse vegetation, that had burned in } \\
1995, \text { near the municipality of Argiroupoli }\end{array}$ \\
\hline
\end{tabular}

Advances in Forest Fire Research 2018 - Page 662 


\begin{tabular}{|c|c|}
\hline $\begin{array}{l}\text { July 9, } \\
2005\end{array}$ & $\begin{array}{l}\text { Mostly surface fire that re-burned the same canyon where the four fatalities of July } 22 \text {, } \\
1998 \text { took place. The fire moved explosively within the canyon but the intensity was } \\
\text { medium due to reduced biomass. T }=31^{\circ} \mathrm{C}, \mathrm{RH}=54 \% \text {, WSW wind of } 7 \mathrm{~km} / \mathrm{h}\end{array}$ \\
\hline $\begin{array}{l}\text { July 30, } \\
2005\end{array}$ & $\begin{array}{l}\text { Wind driven, mostly surface fire of medium intensity due to relatively sparse fuel. It } \\
\text { was suppressed quickly through strong aerial attack. }\end{array}$ \\
\hline $\begin{array}{l}\text { July 16, } \\
2007\end{array}$ & $\begin{array}{l}\text { Active wind driven crown fire spreading through a dense and tall pine forest, including } \\
\text { spotting. } \mathrm{T}=30^{\circ} \mathrm{C}, \mathrm{RH}=35 \% \text {, North wind of } 30 \mathrm{~km} / \mathrm{h} \text {. Rate of spread at the head reached } \\
2.4 \mathrm{~km} / \mathrm{h} \text {. It was controlled by strong aerial resources. }\end{array}$ \\
\hline $\begin{array}{c}\text { August } \\
25,2007\end{array}$ & $\begin{array}{l}\text { Wind driven, high intensity and fast-spreading, mostly surface fire. Started around } \\
\text { noon, fanned by a strong north wind. It started between the houses of the municipality } \\
\text { of Papagos-Cholargos and the Mount Hymettus ring road. It was controlled before dusk } \\
\text { with the intervention of strong aerial resources and the help of the ring road that } \\
\text { stopped the upslope spread of the eastern flank. }\end{array}$ \\
\hline $\begin{array}{c}\text { June } 25, \\
2008\end{array}$ & $\begin{array}{l}\text { Mostly topography and wind driven crown fire, that started at the area of Glyka Nera } \\
\text { (NE side of the mountain). It run through a dense and tall pine forest, spreading upslope } \\
\text { and westwards, until it reached the top. It was controlled on the lee side. } \mathrm{T}=38^{\circ} \mathrm{C} \text {, } \\
\mathrm{RH}=31 \% \text {, North wind of } 25 \mathrm{~km} / \mathrm{h}\end{array}$ \\
\hline $\begin{array}{l}\text { July 16, } \\
2008\end{array}$ & $\begin{array}{l}\text { Medium intensity surface and passive crown fire in a park of the municipality of } \\
\text { Kaisariani, below the ring road. Started around noon and was controlled a few hours } \\
\text { later with the help of aerial resources. }\end{array}$ \\
\hline $\begin{array}{c}\text { June 15, } \\
2009\end{array}$ & $\begin{array}{l}\text { A fire that started at 15:25 in the area of Ano Glyfada and was stopped by dusk at the } \\
\text { border of a settlement in the municipality of Voula. It exhibited high intensity and } \\
\text { became a crown fire when it burned up-canyon. }\end{array}$ \\
\hline $\begin{array}{c}\text { July 17, } \\
2015\end{array}$ & $\begin{array}{l}\text { A high intensity crown and surface fire. It burned southwards fanned by a strong } \\
\text { northern "meltemi" wind, reaching a maximum forward rate of spread of } 2 \mathrm{~km} / \mathrm{h} \text {. } \\
\text { Additionally, it backed into the perpendicular to the direction of the wind canyons, at } \\
\text { an explosive maximum up-canyon rate of spread of } 9 \mathrm{~km} / \mathrm{h} \text {. }\end{array}$ \\
\hline $\begin{array}{l}\text { August } \\
10,2017\end{array}$ & $\begin{array}{l}\text { A medium intensity surface fire between the municipalities of Kaisariani and Byron, } \\
\text { in relatively sparse vegetation that started around 14:00 and was controlled within two } \\
\text { hours with the help of aerial resources. }\end{array}$ \\
\hline
\end{tabular}




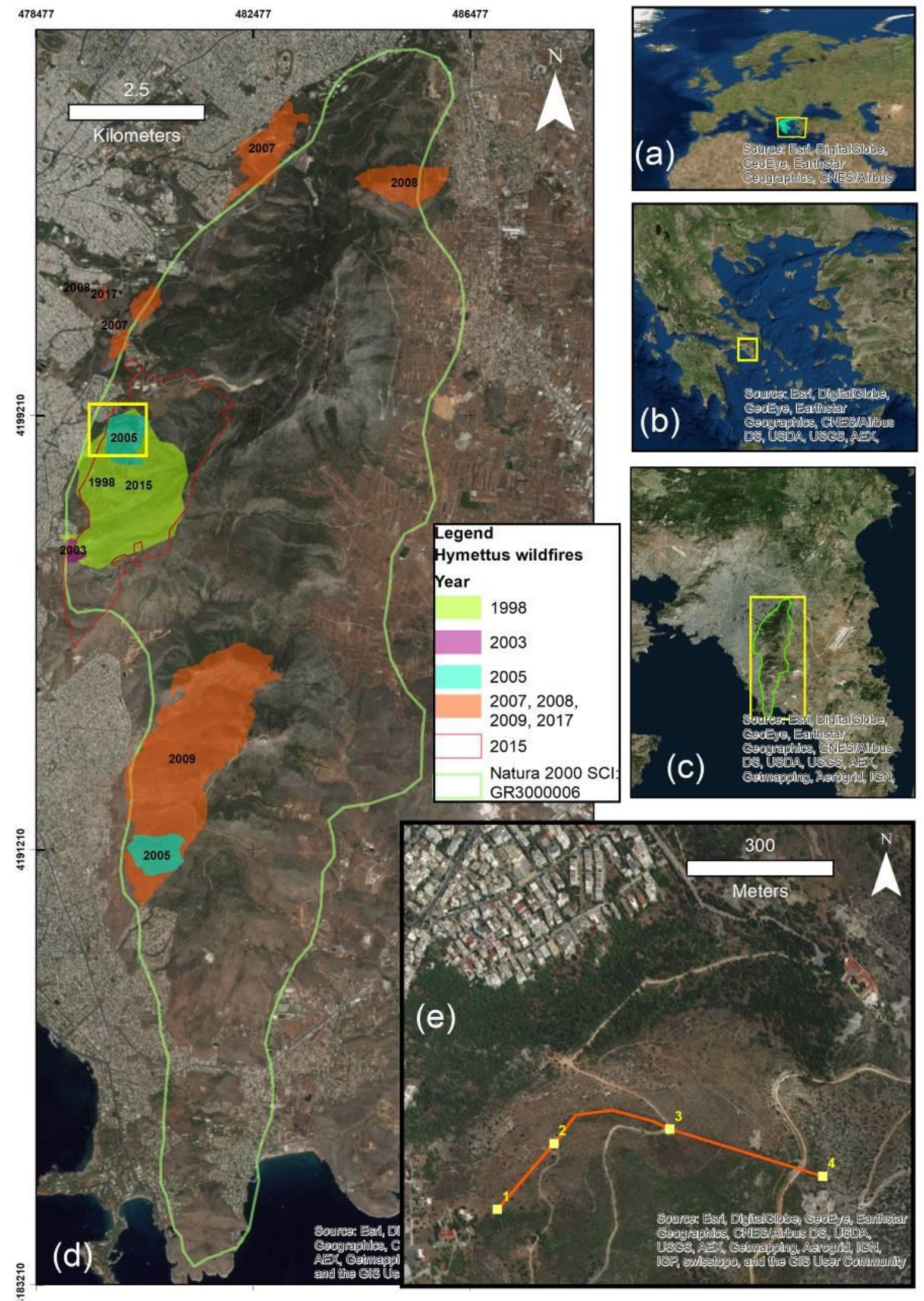

Figure 1 - The most important fires on Mount Hymettus in the 1996-2007 period. The geodetic system of the map and figure, is the Hellenic Geodetic Reference System 1987 
As the fires are clearly visible by the population of Athens, there are numerous reports, photos, opinions and comments in the Mass Media and on the Internet regarding these events. In most of these reports, the fires are attributed indiscriminately to "arsonists" who want to illegally occupy and develop forest land on Mount Hymettus. The majority of the public subscribe to this scenario or attribute the fire to political motives. In reality, there is no evidence leading to such conclusions. On the contrary, the small number of cases solved so far, include an immigrant burning old furniture (22 July 1998), a professional gun technician practicing shooting for testing ammunition (15 June 2009), a poor unemployed person with psychological problems who started multiple fires $(3,5,14$ and 22 July 2012), and two beekeepers who inadvertently started a fire while tending to their beehives using smoke (17 July 2015).

The large number of fires on Mount Hymettus and the significant damages they cause, have mobilized many people who live in the area to join volunteer groups, organized in each of the municipalities that border the mountain, offering their time for its protection. Regarding forest fires, these groups, in the summer, patrol the mountain, man lookout towers, and are immediately dispatched for initial attack when a fire is detected. Their positioning on the mountain is a major advantage because the traffic jams that Fire Service firetrucks face when coming from the Athens using the city's ring-road at the base of Mount Hymettus, often cause significant delays. On the other hand, the volunteers, having only light firetrucks, sometimes cannot cope with intense fast spreading fires.

\section{Forest fire behavior}

The majority of fires on Mount Hymettus have occurred from mid June to mid August. They usually start on days with strong wind at the base of the mountain at the fridge of the city. Fire spread under the strong wind is fast. Most importantly, as the fires run in a SW direction passing in front of the downslope "mouth" of the canyons, they are drawn-in, in a clear demonstration of the so-called "chimney effect". Fire spread within the canyon is very fast due to flame attachment (Sharples et al. 2010) and convection caused preheating of fuels mainly along its axis. As these canyons are protected to a large extent from the general wind, when there is significant amount of fuel, a strong convection column usually develops (Figure 2).

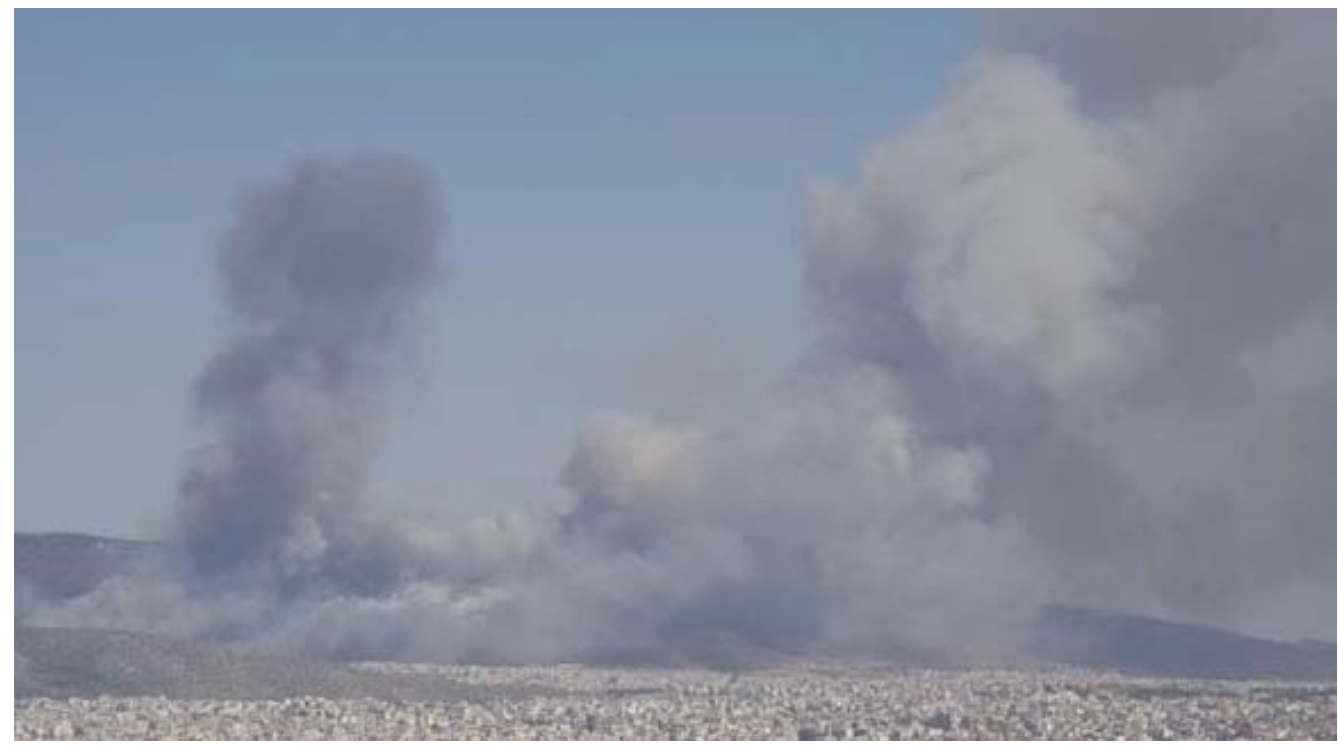

Figure 2 - An up-canyon run at the heel of a wind driven fire on Mount Hymettus, on June 15, 2009, that created a strong convection column separate from the column of the head of the fire (Source: amateur photo from www.athens.indymedia.org) 

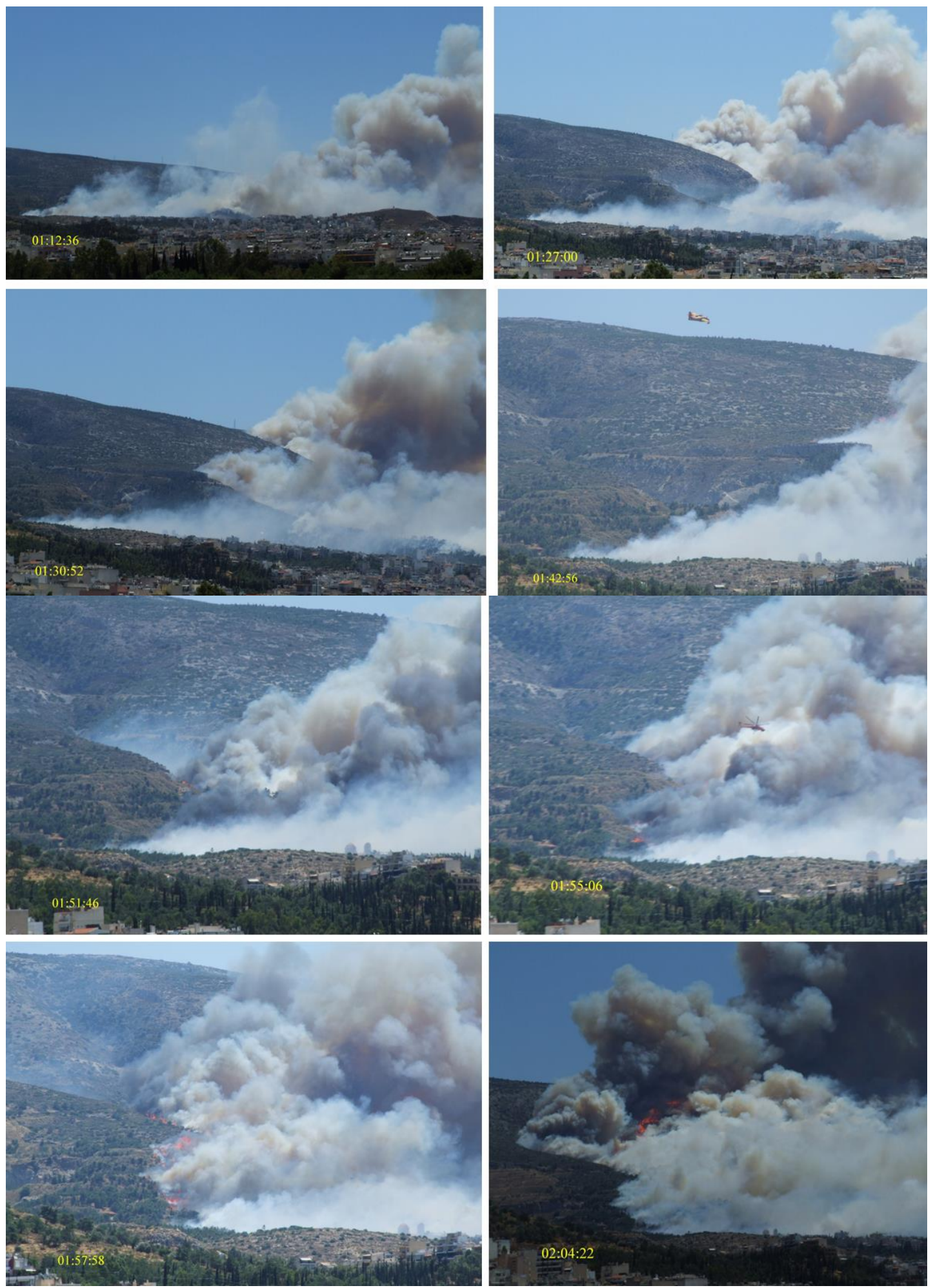

Figure 3 - The initial southwards spread of the Mount Hymettus fire of July 17, 2015, and the consecutive up-canyon runs that followed.

Especially the behaviour of the July 17, 2015 fire presented an excellent case study: the fire spread with the wind southwards and then succesively it backed-up four canyons. Recording the evolution of the fires as shown in Figure 3, and identifying the location of the fire with time, positioning it was possible to calculate the up-canyon rate of spread, in two runs, at $9 \mathrm{~km} / \mathrm{h}$ and $7.8 \mathrm{~km} / \mathrm{h}$. 


\section{Forest fire safety}

The spread within the canyons at a perpendicular direction to the prevailing wind has surprised many people and has been clearly the reason behind the deaths of three professional and one volunteer firefighters on the fire of Kareas, on July 22, 1998, in one of the canyons (Figures 4 and 5) (Xanthopoulos 2015). They stopped their fire truck at the bend of the road and tried to fight the fire that had started spreading up-canyon. They were immediately overwhelmed by the heat and the smoke and tried to run uphill in the canyon, entering a race that could not be won (Figure 6).

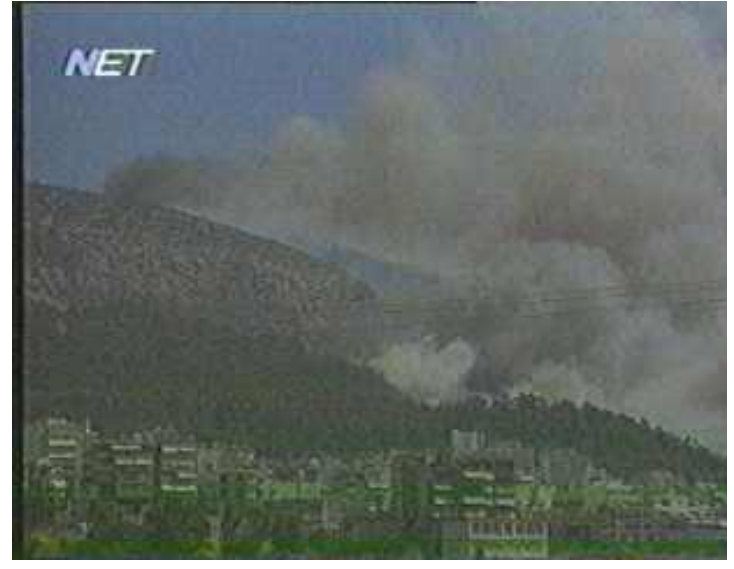

Figure 4 - The fire of Kareas of July 22, 1998, spreading within a canyon, perpendicular to the direction of the wind (Source: NET TV channel)

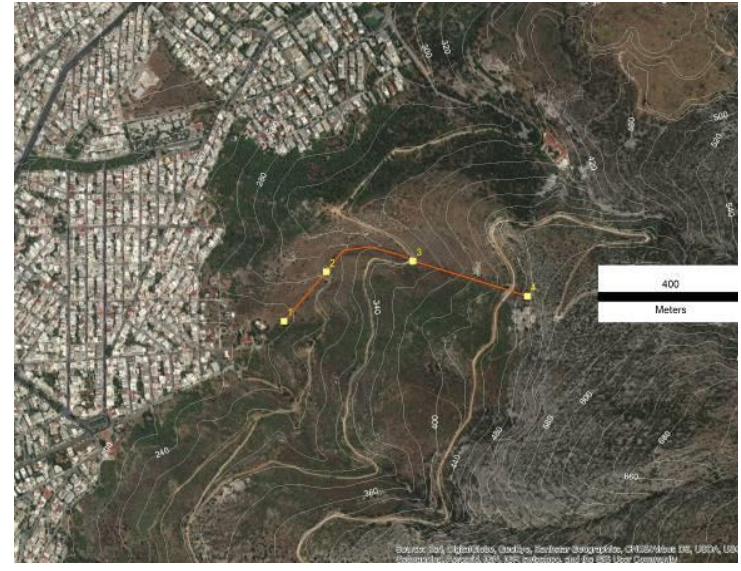

Figure 5 - The up-canyon spread of the fire of Kareas shown on a satelitte image from Google Earth. The main fire front advanced in a SW direction.

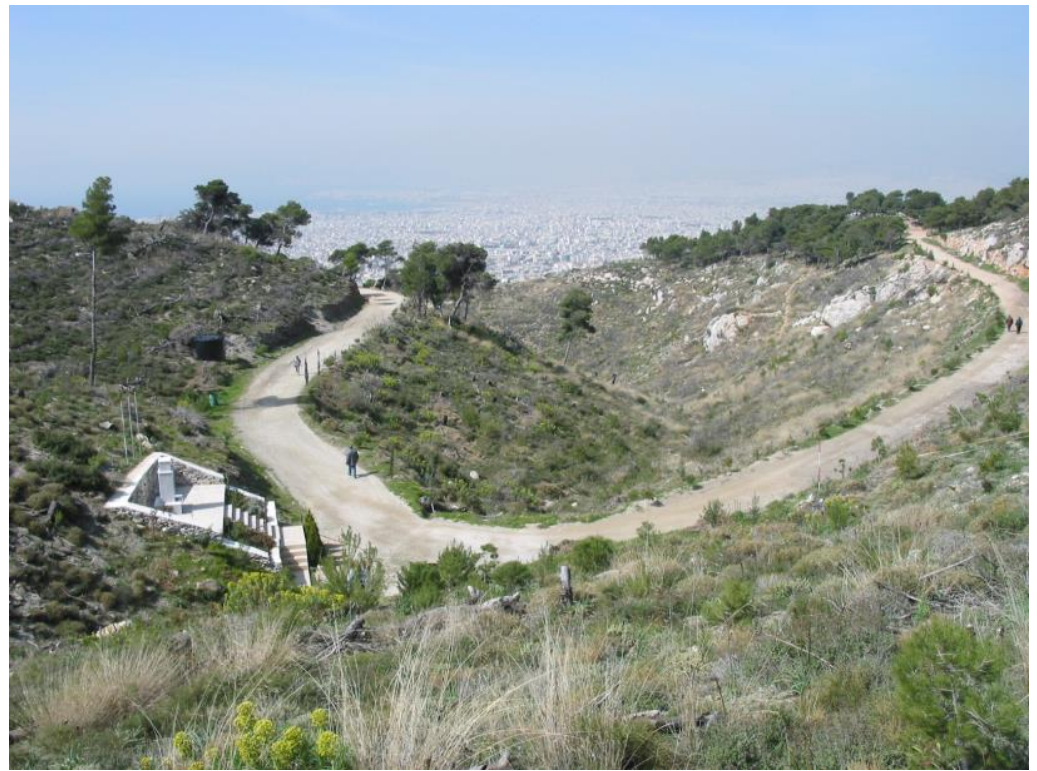

Figure 6 - The canyon within which the fire spread, photographed approximately from the locatin where the four firefighters died in 1998.

A few years later, on July 7, 2005, the same canyon burned again. This time it burned with a light up-canyon (SW) wind. In spite of reduced fuel load, the direction of the wind and the canyon topography resulted in a fire of significant intensity. Fortunately, this time the firefighters remained away from the axis of the canyon. Most of the suppression work was left to the aerial resources that included an Erickson S-64 helicopter and Canadair waterbombers (Figure 7). 


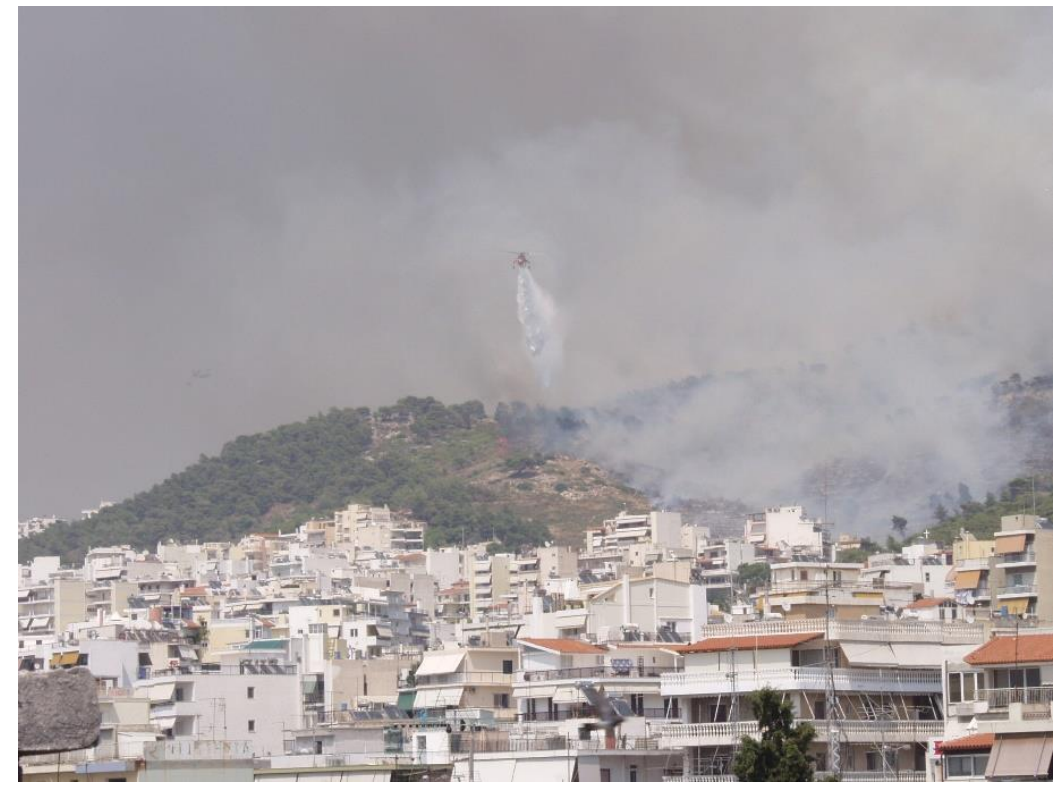

Figure 7 - Aerial firefighting during the up-canyon run of the Kareas fire on Hymettus mountain on July 7, 2005 (Photo: Foivos Theodorou, General Secretariat of Civil Protection, Greece)

The fire of July 17, 2015 also caused one fatality. This time it was a person jogging on the mountain, on a road with a north-south direction. The fire ignited and started burning below this road. The jogger called his family on his mobile to report about the fire saying that he was not worried. He was later found dead, about 100 meters higher than the road. It is likely that he was surprised by one of the upcanyon runs and tried unsuccessfully to escape to higher ground (Figure 8).

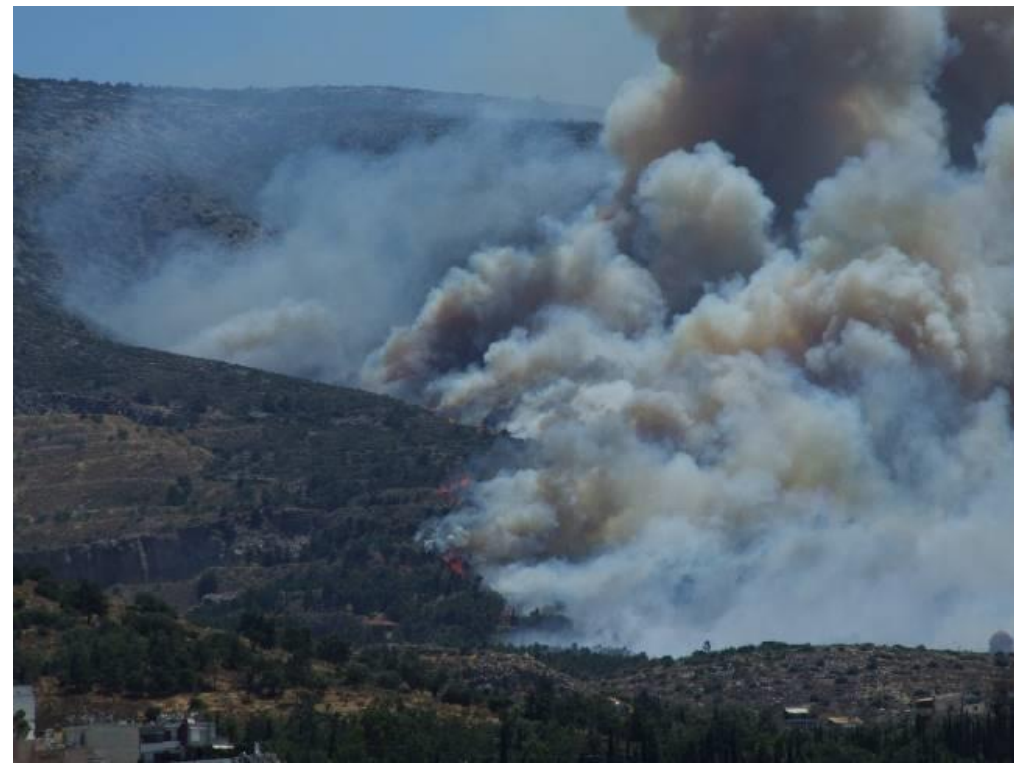

Figure 8 - The fire of July 17, 2015, on Mount Hymettus, which caused one fatality. The up-canyon fire spread in this particular canyon reached $9 \mathrm{~km} / \mathrm{h}$.

\section{Discussion and conclusions}

The fires of Mount Hymettus constitute a very good case study that encapsulates important fire prevention, behavior, firefighting and safety considerations. Regarding fire prevention the effectiveness of the measures taken for preventing fire starts, that include fire lookouts, ground patrols, and closed traffic bars on high fire danger days, can be judged as inadequate since a significant number of fires erupts every year. The response for initial attack by volunteers and professional firefighters 
with fire trucks is generally good so most of these fires remain small. However, the problem of traffic jams on the ring road at the western foothills of the mountain is clearly a central one as it results in delays in the arrival of heavier firetrucks from the city when initial attack fails. A potential measure in this direction is to condition the Athenian drivers, that often occupy the emergency lane in case of traffic jams, to refrain from such practice. This can be achieved, for example, through regular traffic police controls and special warning signs.

The fire behavior that has been observed and documented on Mount Hymettus, provides excellent material for studying, understanding and modelling the phenomenon of fire spread within "box canyons". As Viegas and Pita (2004) have shown, even in cases of quite shallow canyons a blow-up will always occur as long as space and time are available so that the fire to accelerate and create its own wind. This is related to the observation that the runs within the canyons of Mount Hymettus take place with some delay, after the general fire front has moved ahead in the direction of the wind (Figures 2 and 3$)$.

The eruptive fire behavior on Mount Hymettus must clearly be taken into consideration in firefighting plans. As stated by Viegas and Pita (2004), it is extremely dangerous to put resources at any place on the slope above the fire, especially in canyon topography, as the fire acceleration can occur at any time. This is even more important on the west-southwest facing slopes of Mount Hymettus which are mostly covered with shrub fuels, a situation that has been identified as of maximum danger by Page and Butler (2018) in a similar Mediterranean environment.

The knowledge presented here must clearly become part of the training of local firefighters, including the volunteers who have already faced a loss in 1998. Additionally, the death of the civilian in the 2015 fire shows that the public must be aware of the risk they may face in case of fires. The number of visitors on Mount Hymettus is quite large and a disastrous event including fatalities is not unlikely in case of extreme fire danger conditions. Forbidding visitor entrance to the mountain in case of such conditions, or at least providing information to those entering, may not only prevent fire starts but may also help avoid a potential disaster involving citizens.

\section{Acknowledgements}

The work presented here was carried out in the frame of the project MedWildFireLab ("Global Change Impacts on Wildland Fire Behaviour and Uses in Mediterranean Forest Ecosystems, towards a «wall less» Mediterranean Wildland Fire Laboratory") a European ERANet FORESTERRA project with funding from the General Direction for the Development and Protection of Forests and Agroenvironment of the Greek Ministry of Environment and Energy.

\section{Bibliography}

Page WG, Butler BW (2018) Fuel and topographic influences on wildland firefighter burnover fatalities in Southern California. International journal of wildland fire, 27(3), 141-154.

Sharples JJ, Gill AM, Dold JW (2010) The trench effect and eruptive wildifres: lessons from the King's Cross Underground disaster. In 'Proceedings of Australian Fire and Emergency Service Authorities Council 2010 Conference', 8-10 September 2010, Darwin, NT, Australia. (Australian Fire and Emergency Service Authorities Council: Darwin, NT, Australia) Available at http://www.maths.manchester.ac.uk/ jwd/articles/ 10-TEaEW.pdf [Verified 11 January 2018]

Viegas DX, Pita LP (2004) Fire spread in canyons. International Journal of Wildland Fire 13, 253274.

Xanthopoulos G (2015) Wildfires and safety issues in Greece. In 'Current International Perspectives on Wildland Fires, Mankind and the Environment'. (Eds B Leblon, M Alexander) (Nova Science Publishers: Huntington, NY) 


\title{
The role of forest fires in land use/land cover changes in Portugal
}

\author{
Joana Parente $^{1 *}$; Mário G. Pereira ${ }^{1,2}$; Marj Tonini ${ }^{3}$ \\ ${ }^{1}$ Centro de Investigação e Tecnologias Agroambientais e Biológicas, Universidade de Trás-os- \\ Montes e Alto Douro, Quinta de Prados, 5000-801 Vila Real, Portugal, \{joanaparente@utad.pt*; \\ gpereira@utad.pt\} \\ ${ }^{2}$ Instituto Dom Luiz, Faculdade de Ciências da Universidade de Lisboa, Lisboa, Portugal \\ ${ }^{3}$ Institute of Earth Surface Dynamics, Faculty of Geosciences and Environment, University of \\ Lausanne, Lausanne, Switzerland, \{marj.tonini@unil.ch\}
}

\begin{abstract}
In the last decades, Portugal registered substantial land use / land cover changes (LULCC) driven by the abandonment of rural areas, fast urbanization, and expansion of metropolitan areas and costal touristic centers motivated by the significant socio-economic development. Consequently, there significant transitions incurred among different land use classes as well as a homogenization of the fuel bands, increasing the extension and complexity of fire prone areas. Portugal was particularly affected by forest fires (FF) with more than 18,000 FF/year and annual burnt area (BA) of $107,000 \mathrm{ha} /$ year since 1980, while the average size of such events has increased from 4 ha to 13 ha in the last 10 years. This study analysed the FF occurred in continental Portugal between 1990 and 2013, with the main focus on: (i) assess the global and regional differences/similarities in LULCC and fire regime; (ii) evaluating the role of FF on LULCC and; (iii) to provide a better understanding (identification and characterization) of space-time cluster distribution of FF. We used the following datasets: (i) land use/land cover inventories from CORINE; (ii) BA polygons from the Portuguese Institute for the Conservation of Nature and Forests. A geospatial methodological approach, based on GIS analyses, was developed to identify LULCC and to characterize the impact of FFs on these changes; then, cluster analyses were performed to examine patterns in FFs distribution and, finally, to discover how landscape factors control FF clusters. This research provides a quantitative assessment of the relationship between LULCC and FFs in Portugal. The authors believe that the obtained results provide a valuable contribution to: (i) characterize the LULCC in Portugal; (ii) assess the role of FFs on LULCC; (iii) support a better landscape management to reduce related losses of life, property and assets; as well as, (iv) promote educational events to increase public awareness to the causes and consequences of FF.
\end{abstract}

Keywords: Land use/Land Cover changes, Forest fires, Portugal, Cluster analysis

\section{Introduction}

In the last decades, Continental Portugal registered substantial land use and land cover changes (LULCC) driven by the abandonment of rural areas, fast urbanization, expansion of metropolitan areas and costal touristic centers motivated by the significant socio-economic development (Pereira et al. 2014; Tonini et al. 2018). Consequently, there were significant changes in land-use and vegetation cover, as well as a homogenization of the fuel bands, increasing the extension and complexity of fire risk areas (Amato et al. 2018). Portugal was particularly affected by forest fires (FF) with more than $18,000 \mathrm{FF} /$ year and burnt area (BA) of 107,000 ha/year since 1980, while the average size of such events has increased from 4 ha to 13 ha in the last 10 years (San-Miguel-Ayanz et al. 2016). These fires caused a wide range of environmental, economic and human damages, including personal injury and death as well as a large amount of BA (Parente, Pereira, Amraoui, and Fischer 2018; Tedim et al.

Advances in Forest Fire Research 2018 - Page 670 
2018). The high fire incidence in Continental Portugal has been associated with several factors such as: (i) the Mediterranean climate type, characterized by conditions conducive to the development of vegetation in the cold and humid season and the dryness of life and dead fuels during the dry and hot season (Kanevski and Pereira 2017; Parente, Pereira, Amraoui, and Tedim 2018); (ii) the occurrence of weather and climatic extremes, such as heat waves (Parente, Pereira, Amraoui, and Fischer 2018) associated with anomalous atmospheric circulation patterns (Pereira et al. 2005; Amraoui et al. 2015) and episodes of drought (Bastos et al. 2011; Gouveia et al. 2012); as well as (iii) forestry, LULCC and socioeconomic characteristics and trends (Pereira et al. 2015; Parente et al. 2016). The present study analyse the FF occurrence in Continental Portugal between 1990 and 2016, with the main focus on: (i) the assessment of global and regional differences/similarities in LULCC and fire regime; (ii) the role of FF on LULCC; (iii) a better understanding (identification and characterization) of space-time cluster distribution of FF.

\section{Dataset and Methodology}

\subsection{Study area}

Portugal mainland has a surface area of $90,000 \mathrm{~km}^{2}$ and is located in the Iberian Peninsula, between Spain and Atlantic Ocean. The temperate or Mediterranean type of climate of the country provides dry and warm summers in the north and dry and hot summers in the south (Rubel and Kottek 2010). These climatic conditions promote the vegetation growth during spring and thermal and hydric stress during summer. Extreme weather favours the occurrence of FF in specific periods and other environmental characteristics (e.g., topography, land use / land cover) drive the spatial distribution of FF.

\subsection{Land use / land cover dataset}

LULCC information were gather from the 2000-2006 and 2006-2012 CORINE Land Cover Changes (CLCC) inventory (Figure 1, https://land.copernicus.eu/pan-european/corine-land-cover), which is based on image-to-image comparison of two CLC consecutive inventories which have a minimum cartographic unit of 5 ha and a thematic accuracy greater than $85 \%$.

\subsection{Forest fire dataset}

BA polygons were obtained from National Mapping Burnt Area dataset available in vector format from 1990 to 2017 and provide by the Portuguese Institute for the Conservation of Nature and Forests (http://www2.icnf.pt/portal/florestas/dfci/inc/info-geo). As this study is focused on the two most recent vector maps of CLCC inventory, we selected the BA polygons between 2000 to 2007 and 2006 to 2013. Since one of the main objectives of this study is to evaluate the influence of spatial-temporal clusters (obtained in Parente et al. (2016)), on the CLCC, the events of these two periods were split into three BA classes as in Parente et al. (2016). The final datasets for each period and for each BA classes include: (i) 3,893 (3,227) FFs for the 2000 - 2007 (2006 - 2013) period for 5 ha $\leq$ BA $<15$ ha class, which corresponds to a total BA of $35,874(28,703)$ ha; (ii) $4,518(3,534)$ FFs for the $2000-2007(2006-2013)$ period for 15 ha $\leq$ BA $<100$ ha class, which corresponds to a total BA of 173,914.3 (139,180) ha; and, (iii) 1,687 (1,139) FFs for the $2000-2007(2006-2013)$ period for $\mathrm{BA} \geq 100$ ha class, which corresponds to a total BA of $1,176,291(512,083)$ ha.

\subsection{Methodology}

A geospatial approach, based on GIS analysis, was developed to identify LULCC, and to characterize the impact of FFs on these changes; then, cluster analyses performed in Parente et al. (2016) was used to examine patterns in FFs distribution and, finally, to discover how factors as climate, soil, distributed vegetation, human activities and infrastructures control FF clusters. 

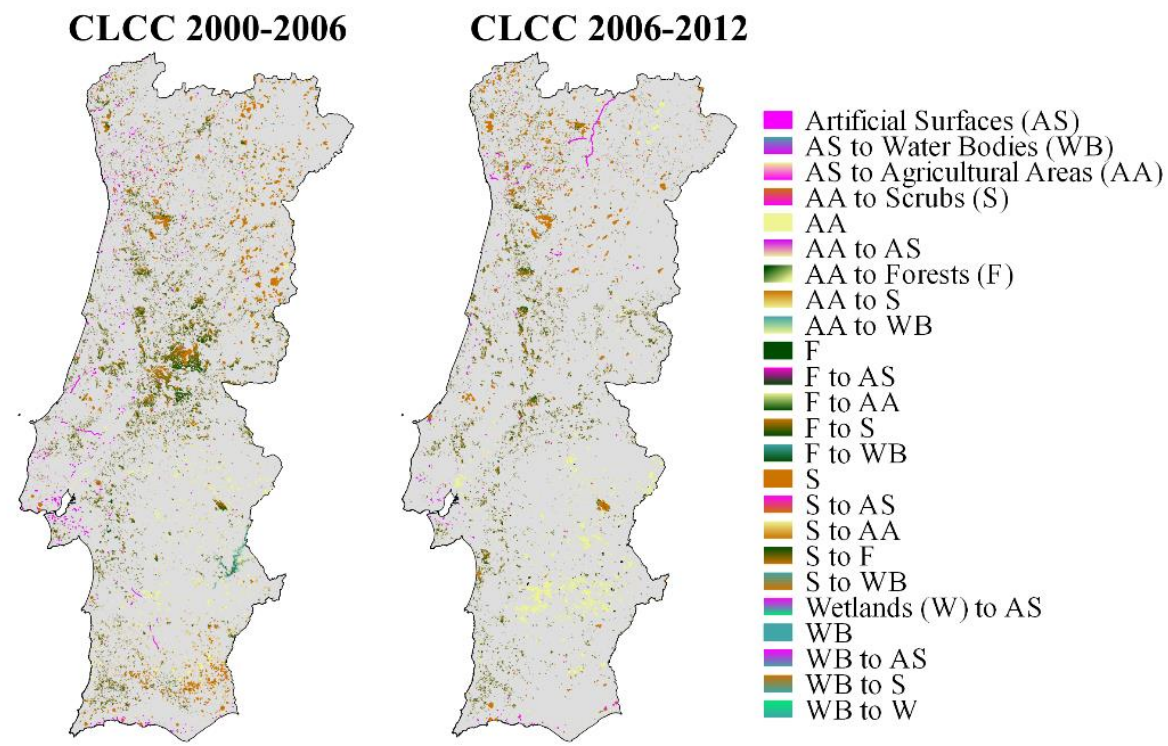

Figure 1 - CORINE land cover changes (CLCC) inventory for 2000-2006 and 2006-2012.

\section{Results}

\subsection{GIS analysis}

GIS analysis performed to CLCC inventories and BA polygons datasets for each period unveil that: (i) $9 \%$ of CLC 2000 and $6 \%$ of CLC 2006 total area have changed; (ii) the fraction of total BA within the areas of LULCC was 25\% between 2000 and 2007, with higher values in 2003, 2005 and 2006, but decrease to $13 \%$ in the $2006-2013$ period, when 2006 was the year with maximum BA in the LULCC areas. It is important to underline the role of the BA in 2006, 2007 and 2013 in the CLCC between 2000-2006 and 2006-2012. In relation to the total annual BA in each year, the BA2006 $\cap C L C C 2000-2006$ is 30\%, BA2007คCLCC2000-2006 is 8\%, BA2006 $\cap$ CLCC2006-2012 is $33 \%$ and BA2013 $\mathrm{CLCC} 2006-2012$ is $8 \%$. This suggests that BA2006 may have played an important role in both CLCC2000-2006 and CLCC2006-2012, which is consistent with the fact that CLC inventories have a minimum time consistency of plus/minus one year. On the other hand, the interception of BA2007 and BA2013, respectively, with CLCC2000-2006 and CLCC2006-2012 is only $8 \%$, which corresponds to the minimum values of BA $\cap C L C C$ in each period. These results are even more relevant if it is took into account that BA2006 and BA2007 are, respectively, the third and second lowest annual BA values, only accounting for $3.7 \%$ and $2.0 \%$ of total BA between 2000 and 2013 while, in the same period, BA2013 is the third highest annual BA value.

Obtained results also disclose that (iii) Forest and semi natural areas was the CLC class with more BA, namely 52\% in CLCC $2000-2006$ and 21\% in CLCC 2006 - 2012. The spatial distribution of the LULCC was not uniform; in fact, (iv) in the 2000 - 2006 period, most of the BA within the CLCC is concentrated in central Portugal, mainly in due to the BA of 2003, while for 2006-2012,

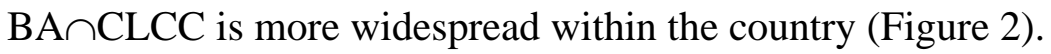



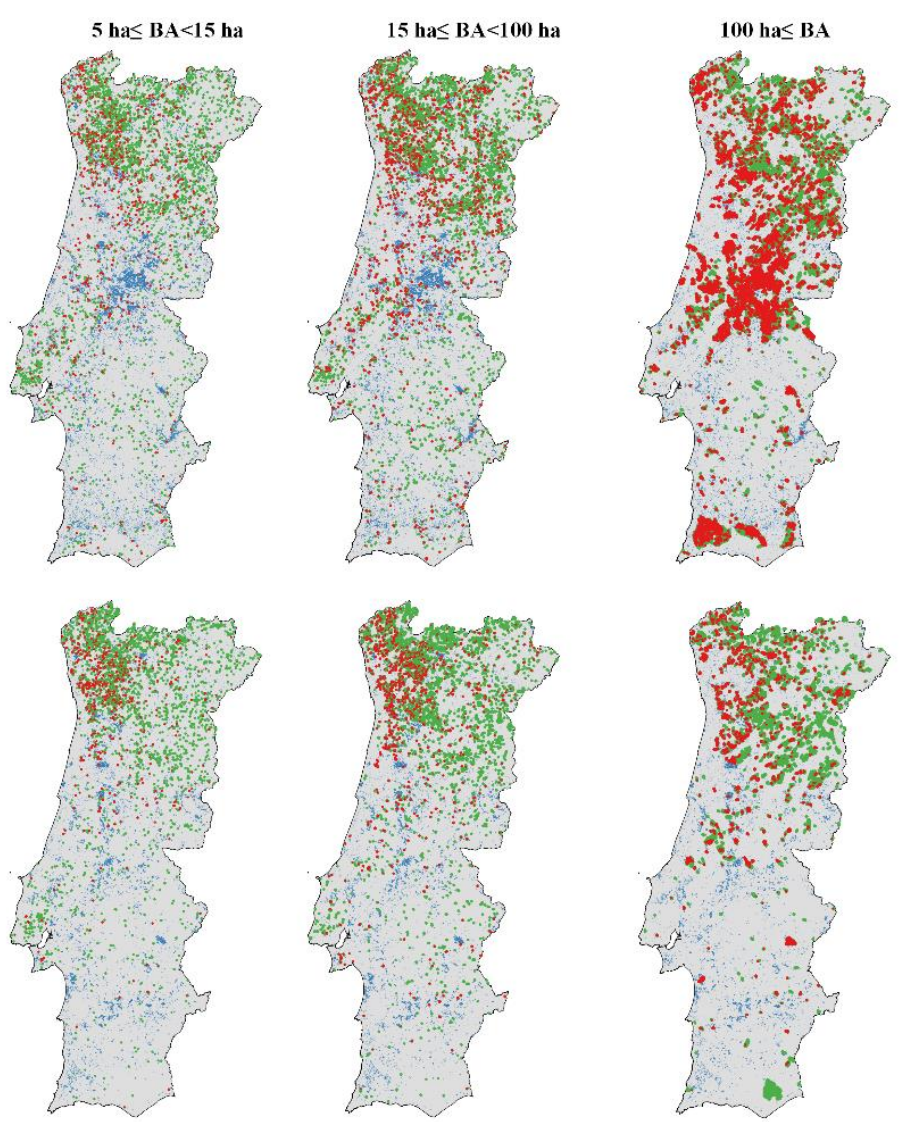
CORINE land cover changes (CLCC)
Burnt area (BA)
BA within CLCC

Figure 2 - CORINE land cover changes (CLCC) inventory, Burnt area (BA) and BA included within the CLCC for the period 2000-2006 (top panel) and 2006-2012 (bottom panel)

\subsection{Cluster analysis}

The cluster analysis performed in Parente et al. (2016) detected 16 most significant spatio-temporal clusters within the two study periods for the three different fire size classes. In the first period (Figure 3 top panel), the number of clusters is similar but their size and duration tend to be higher in the south than in the north. The largest cluster is located in the south and while its duration decreases with the increase of BA, its spatial dimension increases. In the second period and for the two first BA classes (Figure 3 bottom panel), the number of clusters increases with BA and their duration increases from north to south. Although the duration of the south clusters is almost the same, their spatial dimension decreases with the increase of BA. The largest BA class of the second period contain only 2 clusters above Tagus River on the west coast, which are very different in duration, but very similar in the spatial dimension.

LULCC statistics computed for clusters areas (CA) unveil that the fraction of total LULCC in the country inside the CA ranges between $44 \%$ and $64 \%$ in the first period $(2000-2007)$ and between $49 \%$ and $65 \%$ in the second (2006 - 2013). Apparently, statistics for smaller (BA<100 ha) FF differ from larger $\mathrm{FF}(\mathrm{BA} \geq 100 \mathrm{ha})$. For the former, LULCC in the CA ranges between $44 \%$ and $47 \%$ in the first period and between $60 \%$ and $65 \%$ in the second, while for the later class, the LULCC ranges are nearly the opposite (64\% and $49 \%$ in the first and second periods). A similar pattern is observed for $\mathrm{BA} \cap \mathrm{CLCC}$. For smaller fires, the range is $17 \%-28 \%$ in the first period and $68 \%-69 \%$ in the second while, for larger FF, BA $\cap$ CLCC is more similar (55\% and $49 \%)$ in both periods.

It should also be noted that for small fires ( 5 ha $\leq \mathrm{BA}<15$ ha) CLC the class with more LULCC

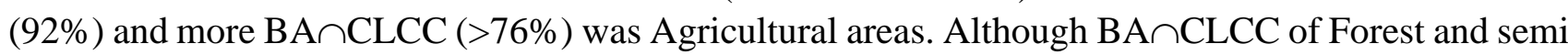
natural areas inside FFs clusters only represents $26 \%$ of total on the first period, in the second period 
it increased to $68 \%$. For this BA class, annual statistics reveal that $56 \%$ of BA $\cap$ CLCC for the period

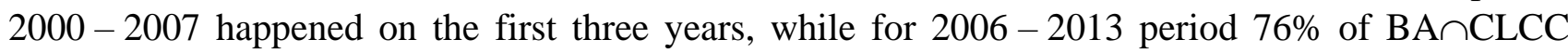
occurred between 2009 and 2012.

For the medium size class $(15 \mathrm{ha} \leq \mathrm{BA}<100 \mathrm{ha})$ Agricultural areas was also the CLC class with

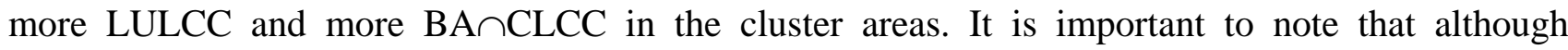
BA $\cap C L C C$ of Forest and semi natural areas inside FFs clusters only represent $16 \%$ of total on the first period, in the second period it increased to $68 \%$. Annual statistics for this BA class reveal that $52 \%$ of BA $\cap$ CLCC in the $2000-2007$ period occurred between 2003 and 2005 while, $65 \%$ of $2006-2013$ period occurred between 2009 and 2012. For the class of the largest FF (BA $\geq 100$ ha) 93\% of the

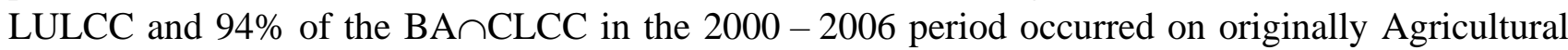
areas while these ratios change, respectively, to $4 \%$ and $19 \%$ in the second period. It is important to

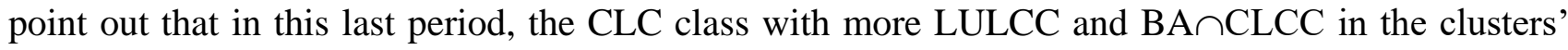
areas were Forest and semi natural areas. Annual statistics for this BA class express that $81 \%$ of

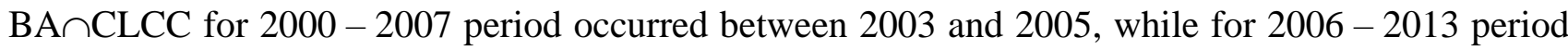
53\% were between 2009 and 2012.

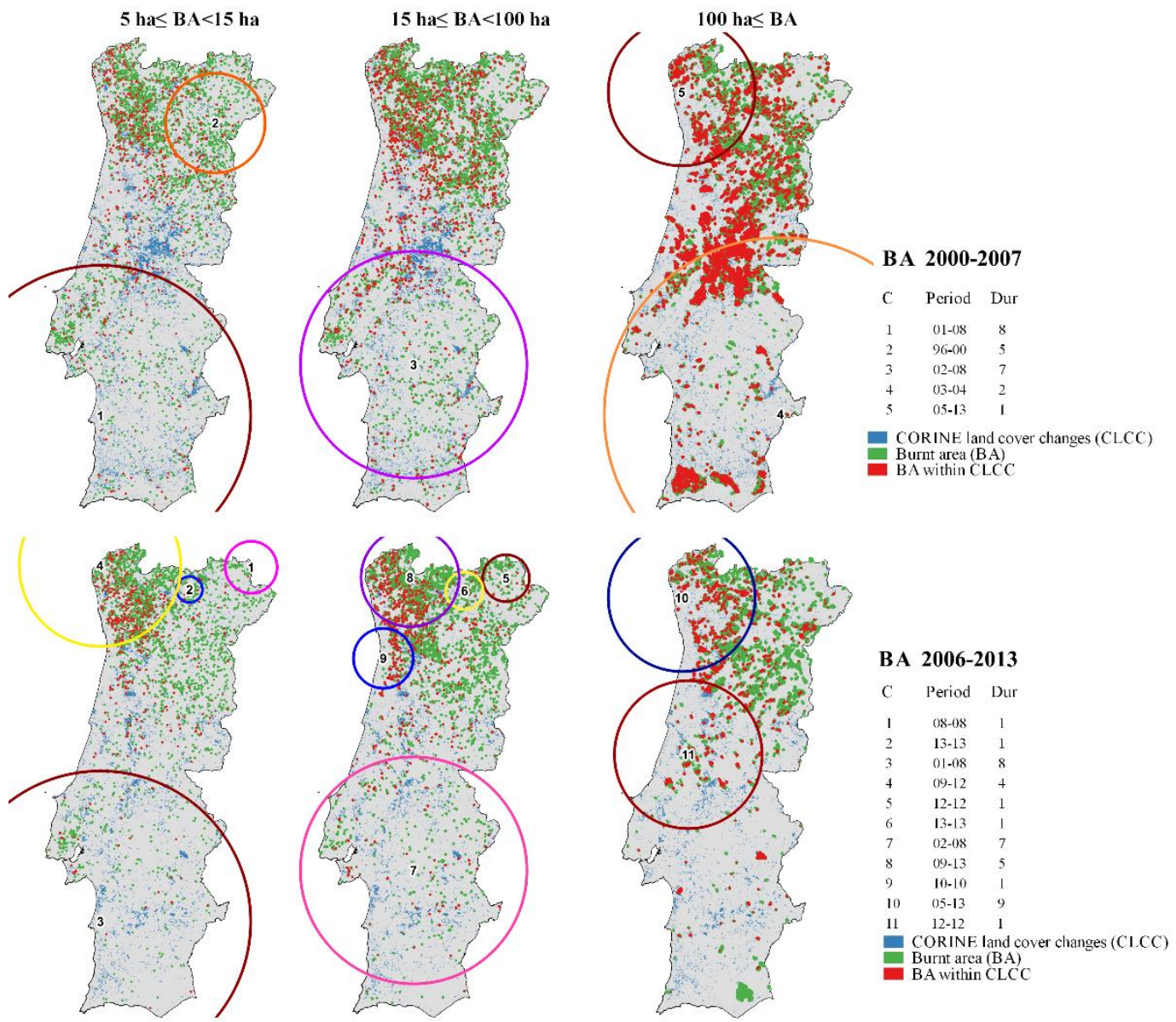

Figure 3 - CORINE land cover changes (CLCC) inventories, Burnt area (BA) and BA included within the CLCC in the periods 2000 - 2007 (top panel) and 2006 - 2013 (bottom panel). Circles $(C)$ represent the most significant spacetime clusters (p-value $<0.01 \%$ ) detected on the National Mapping Burnt Areas for forest fires within the same frame periods.

Finally, it is important to note that the CLCC from Agriculture areas were mostly to Forest and semi natural areas with the exception on the second period of the two lower FF classes, where the change to another different Agriculture areas were more noticeable. Another finding is that the CLCC of Forest and semi natural areas and Artificial surfaces were mostly to a subclass of these CLC. 


\section{Discussion and Conclusions}

This research provides a quantitative assessment of the relationship between LULCC and FFs in Portugal. Our results disclose that in $2000-2013$ period only $10 \%$ of CLC change every six years, and that the intersection of these areas with BA is decreasing in time. In the study period, Forest and semi natural areas was the CLC class with more BA (52\% and $21 \%$ of total annual BA in each period). The LULCC were uniformly distributed in the second period $(2007-2013)$ but concentrated in central areas, which should be a consequence of the impressive BA of 2003.

Cluster analysis has detected 5 and 11) significant spatio-temporal clusters of FF of different sizes in the first and second frame period, respectively. About $45 \%$ of LULCC and $63 \%$ of BA $\cap$ CLCC occurred in the cluster areas of smaller FF ( 5 ha $\leq$ BA $<100$ ha) during the first $(2000-2007)$ and second (2006-2013) periods, respectively. However, the proportions are reversed in the case of largest FF (BA $\geq 100$ ha). $\mathrm{BA} \cap \mathrm{CLCC}$ is less than $30 \%$ for small and medium FF in the first period but significantly increases to about $70 \%$ in the second period. BA $\cap$ CLCC for largest FF slightly decrease (55\% to $49 \%$ ) from the first to the second periods.

FFs events were divided into 3 BA classes of different size. For all these classes, Agricultural areas was the CLC class with more LULCC and more BA $\cap C L C C$, with the exception on the second period in largest BA class where Forest and semi natural areas prevail. Annual statistics of BA indicates that, for medium and large FFs, BA $\cap$ CLCC in the first period is highly dependent with what happened in the 2003-2005 period, increasing from 56\% to $81 \%$ from medium to large FFs. This may be due to the extremely severe years of 2003 and 2005, when a devastating sequence of medium and large FFs were observed and reached more than 440,000 ha and more than 250,000 ha of BA, respectively (Trigo et al. 2006; Oliveira et al. 2012). The duration of this period and FFs probably affected the type of the transitions of Agriculture areas. Our results reveal that most of the CLCC originally of Agriculture areas were converted in Forest and semi natural areas with the exception on the second period of the two lower classes. The transition Agriculture areas to Forest and semi natural areas may be an effect of the duration of soil heating in the soil properties, structure and its aggregation, which deteriorates with the destruction of organic matter and others proprietaries as $\mathrm{pH}$, nitrogen, sulphur, or potassium (DeBano et al. 1979).

Authors strongly believe that the obtained results contribute to characterize the recent LULCC in Portugal, the role of FFs in those LULCC, can support a better landscape management to reduce related losses of life, property and assets as well as educational events to increase public awareness to the causes and consequences of FF.

\section{Acknowledgements}

This work was prepared in the frame of project FIREXTR - Prevent and prepare society for extreme fire events: the challenge of seeing the "forest" and not just the "trees", co-financed by the European Regional Development Fund (ERDF) through the COMPETE 2020 - Operational Program Competitiveness and Internationalization (POCI Ref: 16702) and national funds by FCT-Portuguese Foundation for Science and Technology (FCT Ref: PTDC/ATPGEO/0462/2014. The study was also supported by Project Interact - Integrative Research in Environment, Agro-Chain and Technology, NORTE-01-0145-FEDER-000017, research line BEST, co-funded by FEDER/NORTE 2020.

\section{References}

Amato F, Tonini M, Murgante B, Kanevski M (2018) Fuzzy definition of Rural Urban Interface: An application based on land use change scenarios in Portugal. Environmental Modelling \& Software 104, 171-187. 
Amraoui M, Pereira MG, DaCamara CC, Calado TJ (2015) Atmospheric conditions associated with extreme fire activity in the Western Mediterranean region. Science of the Total Environment 524, 32-39.

Bastos A, Gouveia CM, DaCamara CC, Trigo RM (2011) Modelling post-fire vegetation recovery in Portugal. Biogeosciences 8, 3593.

DeBano LF, Rice RM, Eugene CC (1979) Soil heating in chaparral fires: effects on soil properties, plant nutrients, erosion, and runoff. Res Paper PSW-RP-145 Berkeley, CA: US Department of Agriculture, Forest Service, Pacific Southwest Forest and Range Experiment Station 21 p 145,.

Gouveia CM, Bastos A, Trigo RM, DaCamara CC (2012) Drought impacts on vegetation in the preand post-fire events over Iberian Peninsula. Natural Hazards and Earth System Sciences 12, 3123 3137.

Kanevski M, Pereira MG (2017) Local fractality: The case of forest fires in Portugal. Physica A: Statistical Mechanics and its Applications 479, 400-410.

Oliveira SL, Pereira JM, Carreiras JM (2012) Fire frequency analysis in Portugal (1975-2005), using Landsat-based burnt area maps. International Journal of Wildland Fire 21, 48-60.

Parente J, Pereira MG, Amraoui M, Fischer EM (2018) Heat waves in Portugal: Current regime, changes in future climate and impacts on extreme wildfires. Science of The Total Environment 631, 534-549.

Parente J, Pereira MG, Amraoui M, Tedim F (2018) Negligent and intentional fires in Portugal: spatial distribution characterization. Science of the Total Environment 624, 424-437.

Parente J, Pereira MG, Tonini M (2016) Space-time clustering analysis of wildfires: The influence of dataset characteristics, fire prevention policy decisions, weather and climate. Science of the Total Environment 559, 151-165.

Pereira MG, Aranha J, Amraoui M (2014) Land cover fire proneness in Europe. Forest Systems 23, 598-610.

Pereira MG, Caramelo L, Orozco CV, Costa R, Tonini M (2015) Space-time clustering analysis performance of an aggregated dataset: The case of wildfires in Portugal. Environmental Modelling \& Software 72, 239-249.

Pereira MG, Trigo RM, da Camara CC, Pereira JM, Leite SM (2005) Synoptic patterns associated with large summer forest fires in Portugal. Agricultural and Forest Meteorology 129, 11-25.

Rubel F, Kottek M (2010) Observed and projected climate shifts 1901-2100 depicted by world maps of the Köppen-Geiger climate classification. Meteorologische Zeitschrift 19, 135-141.

San-Miguel-Ayanz J, Durrant TH, Boca R, Libertá G, Boccacci F, Di Leo M, Pérez JL, Schulte E, Benchikha A, Abbas M (2016) Forest fires in Europe, Middle East and North Africa 2015.

Tedim F, Leone V, Amraoui M, Bouillon C, Coughlan M, Delogu G, Fernandes PM, Ferreira C, McCaffrey S, McGee T, Parente J, Paton D, Pereira MG, Ribeiro L, Viegas D, Xanthopoulos G (2018) Defining Extreme Wildfire Events: Difficulties, Challenges, and Impacts. Fire 1, 9. doi:10.3390/fire1010009.

Tonini M, Parente J, Pereira M (2018) Global assessment of land cover changes and rural-urban interface in Portugal. Natural Hazards Earth System Sciences 18, 1647-1664. doi:10.5194/nhess18-1647-2018.

Trigo RM, Pereira J, Pereira MG, Mota B, Calado TJ, Dacamara CC, Santo FE (2006) Atmospheric conditions associated with the exceptional fire season of 2003 in Portugal. International Journal of Climatology 26, 1741-1757. 


\title{
The role of the terrain-modified wind on driving the fire behaviour over hills - an Experimental and Numerical Analysis.
}

\author{
Abdelrahman Abouali*; Jorge Rafael Raposo; Domingos Xavier Viegas \\ Forest Fire Research Centre (CEIF), ADAI-LAETA, University of Coimbra, 3030 - 289 Coimbra, \\ Portugal. \{abouali@adai.pt*\}
}

\begin{abstract}
This paper analyses the fire behaviour over two hills placed in series in the direction of the wind. Laboratory-scale experiments under various slope and wind velocity conditions with changing the distance between the hills revealed two sorts of extreme fire behaviour that might take place. One is a lateral fire spread over the lee slopes (fire channelling), and the other is an eruptive fire behaviour (blow-up) happens over the windward face of the secondly ordered hill in the direction of the wind. The changes in the blowing wind velocity and the distance between the hills were found to have a significant effect on fire behaviours, where increasing the wind speed or the distance resulted in a faster rate of spread of the fire. Also, the change of the ignition point, or spreading direction of the fire relatively to the wind direction has a significant effect on the fire channelling behaviour where it was found that the fire channelling is more extreme if the fire is spreading against the main wind direction. Numerical analysis of the adiabatic flow field showed that the change the presence of these extreme fire behaviour is related directly to the terrain-modified flow topology. The interactions between the terrain-modified wind mechanisms and the fire result in accelerated flows that drive the indicated extreme fire behaviours.
\end{abstract}

Keywords: Fire channelling, lateral fire spread, fire over slopes, fire-induced wind, eruptive fire, fire blow-up

\section{Introduction}

Wildfire behaviour has caught the attention of wildfire researchers during the last decade to understand how the fire is behaving under certain conditions in order to better predict the wildfire evolution. Identifying the conditions where an extreme fire behaviour (Viegas 2004) may take place is crucial for the safety of the firefighting teams and the wildland-urban-interface (WUI) communities. The wind, complex terrain and their interactions are common conditions that may lead to extreme fire behaviour. Many numerical and experimental studies were carried out to analyse the fire behaviour over the complex terrains namely on slopes and hills. However, we still lack understanding on the behaviour of fire spreading over complex terrains, namely the effect of having other topographic obstacles around the topography of interest (i.e. the topography that the fire propagates over it). In this paper, we are investigating the fire behaviour over hills ordered in series in the direction of the wind. This configuration is closer to the existence of hills in nature, where the hills are rarely found isolated as the configuration that was investigated on the previously mentioned studies.

Previous studies (McRae 2004; Sharples et al. 2012) showed that, for a fire spreading over the leeward face of a hill with the wind blowing perpendicularly to the hill's ridgeline, a lateral enlargement of the fire front near the ridgeline occurs. McRae (2004) first noticed this phenomenon and designated it as 'fire channelling' or 'lee-slope channelling'. Sharples et al. (2012) has investigated the phenomena based on real-fire observations, where analysis of several possible mechanisms revealed that hill's lee-slope eddy plays a key role in driving the fire channelling process. The process is proposed to be a result of an interaction between the fire-induced convection (pyro-convection) and the terrain-modified winds through mechanisms that still needs to be investigated. An experimental study by (Raposo et al. 2015) was conducted on an isolated prismatic hill with horizontal ridgeline, and it demonstrated that the fire enlarges symmetrically on the two directions near the top of the crest 
due to the interaction between fire and wind. Another extreme fire behaviour is commonly found happening over slopes and in canyons, which is the eruptive fire behaviour or the fire blow-up behaviour (Viegas 2006).

In the present paper, we analyse the fire behaviour over two prismatic hills placed in series in the direction of the wind. To avoid the effect of the Reynolds number on the location of flow separation we adopted a sharp ridgeline. This configuration is important to investigate considering that terrainmodified wind field is certainly different in the existence of other hills on the landscape, which in return will affect the fire behaviour as well. We gave particular attention to the lateral fire growth behaviour (fire channelling) over the lee-slopes and the fire behaviour between the two hills where an eruptive fire behaviour may take place. The study contributes to the understanding of the conditions where this extreme behaviour occurs and its characteristics. The investigation is made by performing a set of laboratory-scale experiments under various conditions of wind, slope and distances separating the hills

As the previously mentioned studies already indicated that the terrain-modified wind is playing an essential role on driving the fire behaviour over hills, we performed an analysis for the adiabatic flow in the absence of fire using computational fluid dynamics (CFD) simulations. However, we are presenting only the results that we consider relevant to interpret the fire behaviour over the hills. We are reporting the methodology used in both experimental and numerical analysis, followed by results then conclusions.

\section{Methods and Materials}

\subsection{Hill Configuration and Terminology}

The two hills have the same size and shape which is shown in Figure . Each hill is constructed from two rectangular faces with a non-identical width between the windward and leeward faces where they are scaled 3:2 (windward face width: leeward face width). The non-identical faces allow having a steeper lee slope. Also, the larger width of the windward allows the flow to accelerate over it. Through the presentation of our study, the two angles of the windward and leeward faces are referenced as $\alpha$ and $\beta$ respectively measured from a horizontal reference. The distance between the two hills is designated as $D$. Also, we consider associating a number of 1 or 2 at the end of any parameter designation to identify that the parameter is associated to the first or second hill from the perspective of the wind direction. We designate the two lateral ends of the hill as the right end $(R)$, and the left end $(L)$, which are from a perspective of a person that stands facing the windward face of the hill and the wind is blowing from his back.

\subsection{Experimental analysis setup}

\subsubsection{Physical Simulation}

In the present study, the hills were covered by fuel and subjected to a surface fire that is originated from a single point. We, therefore, exclude spot and crown fires that may occur in a real fire.

The physical model had the dimensions shown in Erro! A origem da referência não foi encontrada.1. The hills faces are constructed from steel plates covered by a metallic grid to hold the fuel bed particles. The hill models were placed at the centre of the Combustion Wind Tunnel of the Forest Fire Research Laboratory of the University of Coimbra in Lousã (Portugal), where the midpoint of distance $\mathrm{D}$ is at the centre of the tunnel. The tunnel has an open working area of $6 \times 8 \mathrm{~m}^{2}$ with two lateral $2 \mathrm{~m}$ high walls but without ceiling (Figure ). The flow is generated by two axial fans of $72 \mathrm{~kW}$ that can produce a flow with maximum reference velocity of $7 \mathrm{~m} . \mathrm{s}-1$. The flow velocity is controlled by the rotational speed of the fans. Great care was taken to assure that the flow in the Combustion Tunnel is uniform across the test section (perpendicular to the flow). The flow over the floor of the 
tunnel is of a boundary layer type with a reference velocity $U o$ that is measured at the centre of the working section floor and $0.5 \mathrm{~m}$ above the ground in the absence of the model. A rounded end body was built for each value of $\alpha$ and attached to the model of the first hill to produce a smooth flow around the hill ends as shown in Figure. This procedure was taken to reduce the end effects on the fire propagation over the hill and as well reducing the flow turbulence that affects the area between the two hills.

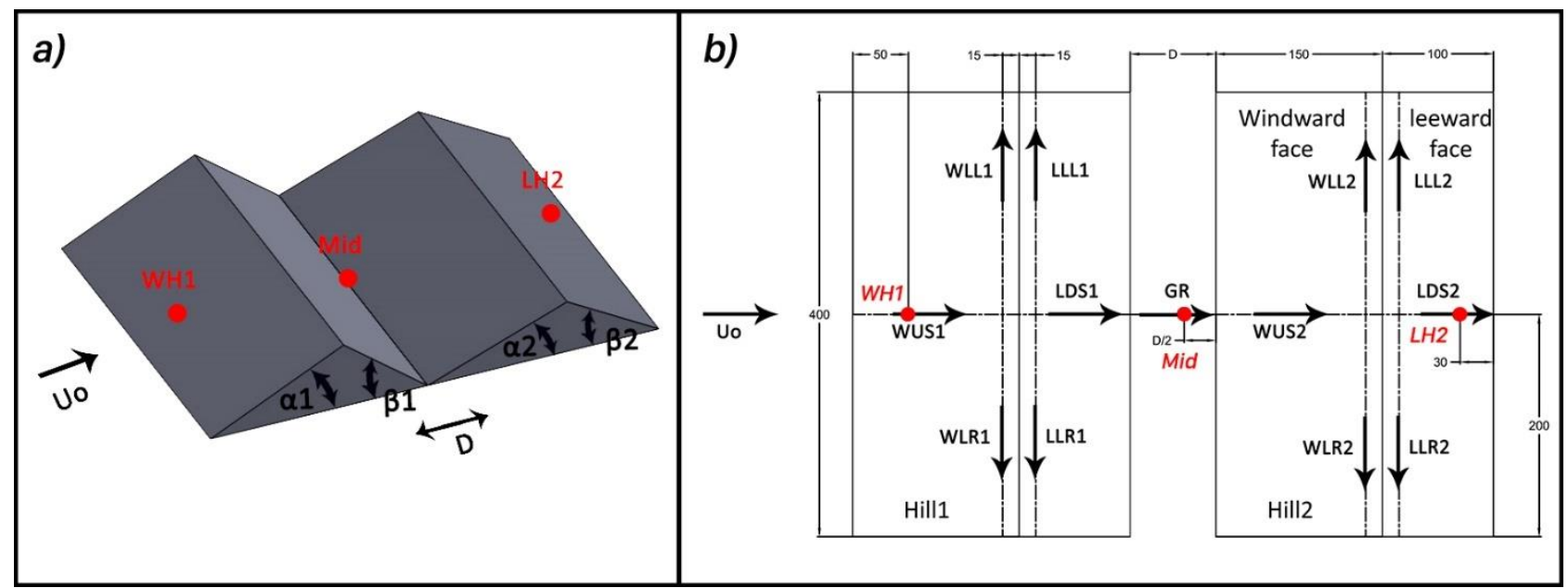

Figure 1 - a) Schematic view of the two triangular hills with the terminology configuration parameters b) Plan view of the used model size with the directions used to evaluate the ROS of the fire. The three ignition points that were used are labelled in red. The dimensions are indicated in $\mathrm{cm}$.

The used $\alpha$ values in the fire spread experiments were $20^{\circ}$ and $27^{\circ}$ (Erro! A origem da referência não foi encontrada.a). Therefore the correspondent $\beta$ values are $\sim 31^{\circ}$ and $43^{\circ}$ respectively. The reason for choosing these angle values is to limit the slope angles in all cases to be not lower than $20^{\circ}$ and not higher than $45^{\circ}$, which is the common range of hills' slope angles in the nature, also because (Sharples et al. 2012) determined that the fire channelling phenomena happens only within this range. The used $D$ values are $0.5,1,1.5$ and $2 \mathrm{~m}$; in addition to no-distance $(D=0)$, these four values correspond to about one to four times the height of the hill for $\alpha=20^{\circ}$, which is fixed inclination angle for all the tests where $D$ was changed. The considered values of $U_{o}$ are $0,1,2$ and $3 \mathrm{~m} \cdot \mathrm{s}^{-1}$. The fire was ignited at a point of three positions which are WH1, LH2 or Mid (Figure ), where WH1 corresponds to the windward face of the first hill. LH2 corresponds to the leeward face of the second hill, and Mid corresponds to the midpoint of the distance $D$. The three ignition points are at the mid-section of the hill (mid of the ridgeline) and in the cases of $W H 1$ and $L H 2$, the ignition was made at a distance of $0.5 \mathrm{~m}$ and $0.3 \mathrm{~m}$ respectively measured over the face from the ground base. A combination of all these values of the parameters $U_{o}, \alpha, D$ and ignition positions was made taking into account fixing all parameters and change only one per group of tests. In total, 17 tests are presented on this paper where their parameters and designations are reported in Table. The test designations (references) will be used from now on referring to each test without ambiguity. 


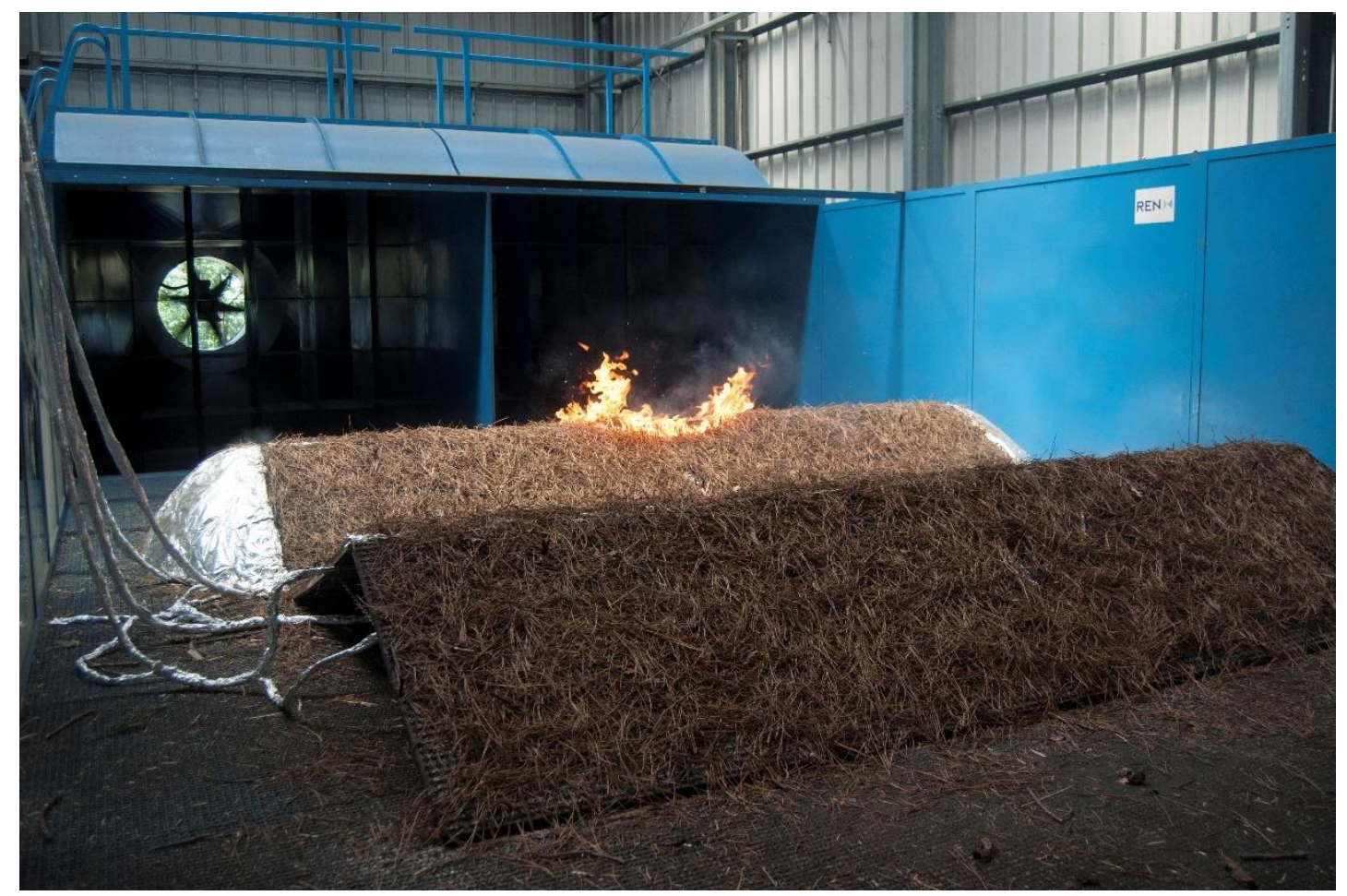

Figure 2 - Image showing the hill models placed in the wind combustion tunnel during one of the experiments, also attached the rounded ends to the left and right sides of the first hill is apparent

The hills' faces and the ground between the two hills if applicable were covered by a uniform fuel bed composed of dead pine needles (Pinus Pinaster) with a load of $0.6 \mathrm{~kg} \cdot \mathrm{m}^{-2}$ (dry basis) and an average depth of $0.05 \mathrm{~m}$. The used fuel has an average surface area to volume (SAV) ratio of $\sim 2640$ $\mathrm{m}^{-1}$. The fuel moisture content was measured for each set of experiments with an A\&D ML50 moisture analyser, and the amount of moisture was compensated to keep the fuel-loading constant on a dry basis. The range of values of the moisture content in the tests was $9 \%<\mathrm{mf}<15.4 \%$ (dry basis). We define the set of experiments as a group of experiments performed on the same day while the change in moisture content does not exceed 5\% during the testing time of the set.

For each set of experiments as well, we define a basic rate of spread (ROS) Ro which is determined by performing a reference test in no-wind and no-slope conditions. We used a flat $1 \mathrm{~m} 2$ table with strings taut over the fuel bed and spaced $10 \mathrm{~cm}$ between them to perform the reference test. The time taken by the fire to pass from a string to another is measured. The basic ROS is defined as the slope of the linear fitting between the two data sets, time versus distance, following (Viegas 2004).

After the fire is ignited, the air flow was turned on to a pre-adjusted velocity. An IR camera was used to record the fire evolution. The obtained images (frames) from the IR recording were analysed using the open source software Fire ROS Calculator (Abouali 2017), which calibrates the camera and determines the ROS values along predefined directions by the user. We will not present further details about the program as it is not the main concern here. However, the program's outputs have been verified to have an error margin on its results of $\pm 5 \%$ (Abouali 2017).

\subsubsection{Fire Spread Analysis}

Using the above methodology, we obtain the fire contour evolution for each test and the ROS. To better interpret the properties of the fire spread, we define the following directions to estimate the ROS along them (Figure b):

WUS - Upslope over the windward face. This direction is along a line that is originated at the midpoint of the ridgeline and perpendicular to it over the windward face. This direction is shifted from 
the middle of the ridgeline in some tests to follow the up-slope propagation of the fire as it didn't take place at the middle

\section{$L D S$ - Downslope over the leeward face. Along a collinear line to the WUS line but over the leeward face}

$W L$ - Lateral over the windward face. This direction is parallel to the ridgeline and at an average distance of $0.3 \mathrm{~m}$ from it. It starts from the middle of the ridgeline and ends at one of the ends. We designate the direction towards the right end as $W L R$ and towards the left end as $W L L$. Also, we designate $W L A$ to refer to the average between the two directions left and right.

$L L$ - Lateral over the leeward face. The direction has the same remarks as on the $W L$, but it is over the leeward face and an average distance of $0.15 \mathrm{~m}$ from the ridgeline.

$G R$ - Down-ridge over the windward face. This direction is collinear to the WUS and WDS but it is over the ground in the area between the two hills if $D=0$. In the case the ignition is at the Mid position, we designate $G R A$ to refer to the average between the directions towards the first and the second hills along the same collinear line.

Considering two positions $\mathrm{P}_{\mathrm{i}}$ and $\mathrm{P}_{\mathrm{i}+1}$ of the fire front along a given direction at time $t_{i}$ and $t_{i+1}$ at distances $d_{i}$ and $d_{i+1}$ measured from the same reference we can determine the instantaneous value of the ROS as:

$$
R(t)=\frac{d_{i+1}-d_{i}}{t_{i+1}-t_{i}}
$$

Assuming that the fire spreads along the reference direction is steady, knowing the distances $d_{k}$ of the fire front position at a set of times $t_{k}(\mathrm{k}=1 \ldots \mathrm{n})$, we can determine the average $\operatorname{ROS}(\bar{R})$ of the fire along that direction by calculating the slope of the linear fitting between the two data sets (Viegas 2006). The time intervals (data samples) that were used to estimate the average ROS were determined for each direction on each test separately, but they meet the criteria of having at least four data samples and the time lapse is not less than 5 seconds or greater than 30 seconds.

Although in the general case the fire spread is not steady according to (Viegas 2004), we shall consider average values of the ROS along the six above mentioned directions like for example $\bar{R}_{W U R}$, $\bar{R}_{L L A}$. Given that our method of analysis can deal with many data points, this calculation was made for several close and parallel lines which represent one of the six indicated directions, to avoid local effects and to better characterize the average value of the ROS.

To guarantee the quality of the tests' results, we replicated all tests in two experiments, where the test replication was not performed on the same day to assure different conditions. We calculated a confidence interval (CI) for the determined average ROS values from the replicates in each direction and each test. The calculated CI is expressed as a percentage of the average of the same values used to calculate the CI (the ROS values). If the percentage was lower than $20 \%$ in all directions, the result is considered valid. In the opposite case, the test was replicated until the calculated percentage from any two tests was less than $20 \%$ in all six directions. However, for the ROS values that are close to the $R_{o}$, the validity condition is increased from $20 \%$ to $50 \%$.

$$
C I=N O R M S I N V * \frac{1-\text { alpha }}{2} * \frac{\text { sigma }}{\sqrt{n}}
$$

Where NORMSINV is a Microsoft Excel (2016) iterative function that returns the inverse of the standard normal cumulative distribution; alpha is taken by 0.05 for all the tests; sigma is the standard deviation based on the entire population (n), which is taken by two always as the CI is calculated between the results of two tests.

In order to minimise the effect of small variations of fuel bed properties, namely moisture content, following (Viegas and Neto 1991), we use the non-dimensional ROS (NDROS) (R') values given by: 


$$
R^{\prime}=\frac{R}{R_{o}}
$$

In this equation, $R_{o}$ is the basic ROS measured in no-slope and no-wind conditions that were measured for each testing session.

We consider that the rate of spread is relatively low when R' is close to unity, but if it reaches values greater than 5 or if it increases rapidly then we consider that we are in the presence of an extreme behaviour (Viegas 2012).

\subsection{Numerical Simulation Analysis Setup}

\subsubsection{The Model and Domain}

A CFD simulation of the flow around the hills was performed using the open source CFD software OpenFOAM-5 (Weller et al. 1998). The adiabatic flow is simulated around a model that is similar to the experimental model but scaled down four times to not increase the size of the domain and therefore the required computational resources (The reported simulations are computed using in average 300 CPUcore and $1200 \mathrm{~Gb}$ of memory). The simulation was made for five configurations which correspond to tests 2D3, 2D14, 2D16, 2D23 and 2D24 (c.f. Table ). On these configurations, the only parameter that was changed is the distance between the hills $(D)$. We have chosen it that as this parameter is responsible for dramatic changes in the fire behaviour as we shall see on the discussion.

The dimensions of the simulation domain (Figure ) consist of a box with dimensions of $2.0 \mathrm{~m}$ width (y-direction), $1.5 \mathrm{~m}$ height and the length (x-direction) of the domain was variable according to the configuration that is being simulated, where the hill's model is placed at a distance of $0.65 \mathrm{~m}$ between the inlet section and the base windward face of the first hill, then the end of the domain was determined to be at ten times the height $(10 \mathrm{~h})$ of the simulated hill measured from the end of the leeward face of the second hill (Figure ). The choice of the end of the domain to be10h is based on the results of (Arya et al. 1981) where he estimated the reattachment point of the flow over the triangular ridge to be at 10 times the height of the hill.

The flow is assumed to be incompressible and adiabatic. The inlet flow is steady with a uniform velocity of $8.0 \mathrm{~m} . \mathrm{s}^{-1}$, which gives a Reynolds number based on the hill's height of the order of $6.5 \times 10^{4}$ assuming constant flow temperature of $25^{\circ} \mathrm{C}$. This velocity was chosen to have a flow's Reynolds number of the same order in both the simulations and experimental tests since the model sizes are different.

It is assumed that there is no flow across the lateral and the top boundaries of the domain (boundaries of a wall type). The flow near the ground is a boundary layer type developed upwind of the hill. Initially, the pressure is considered uniform and equal to 1 atmosphere in all the domain and velocity is zero. 


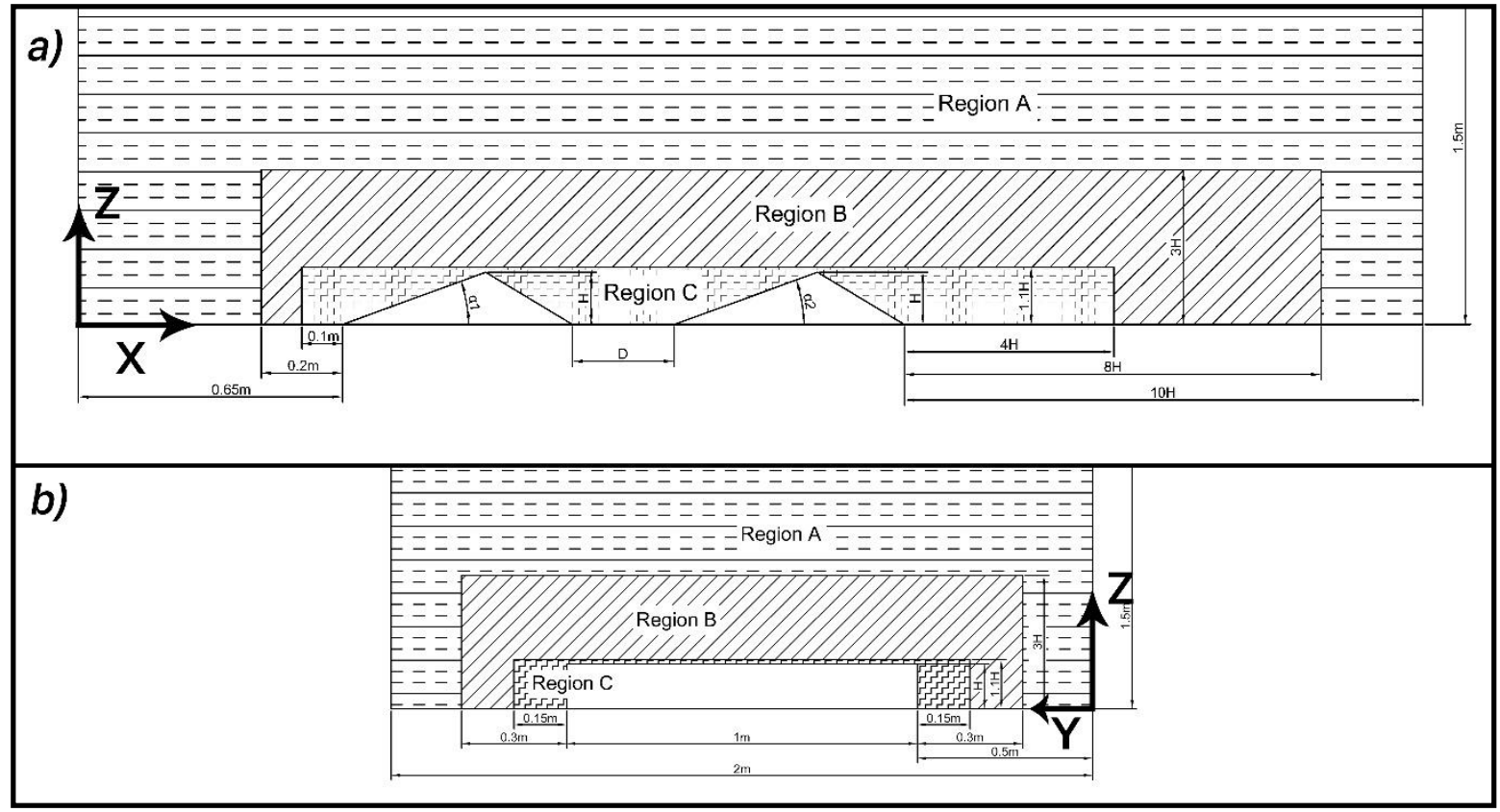

Figure 3 - Two cross sections on the calculation domain showing the axes directions, refinement regions with their dimensions and the position of the model a) parallel to the wind direction ( $x-z$ plane). $b$ ) perpendicular to the wind direction (y-z plane).

\subsubsection{The Simulation Mesh}

The mesh was created using the OpenFOAM tool SnappyHexMesh (Greenshields 2015). A course structured mesh with a cell size of $0.05 \mathrm{~m}$ was built then refined and snapped to the model. The refinement is characterised by its level where level 1 corresponds to the coarsest mesh $(0.05 \mathrm{~m})$, and then each refinement level has a cell size of the previous level divided by two (i.e. level 2 of refinement will have a cell size of $0.025 \mathrm{~m}$ ). We divided the domain into three regions A, B and C (Fig. 3) which have different levels of refinement. Region A coincides with the whole calculation domain and regions $\mathrm{B}$ and $\mathrm{C}$ are surrounding the model. Their heights (z-direction) equal to 3 and 1.1 times the height of the model respectively. Their widths (y-direction) are 1.3 and 1.15 times the width of the model respectively. The regions are centred with the model along the y-direction and their limits along the xdirection start at $0.2 \mathrm{~m}$ and $0.1 \mathrm{~m}$ measured from the base of the windward face of the first hill and ends at 8 and 4 times the height of the model measured from the base of the leeward face of the second hill. The three regions have refinement levels of 1, 3 and 4 respectively. Four transition refinement levels were configured between any two cells of different refinement levels whenever applicable to assure smooth transitions. The mesh then snapped to the model with refinement levels of 7 to 9 depending on the refinement needed for the snapping. Around the model's edges, the refinement was of level 9. Finally, 3 wall layers were added where the first layer attached to the model is of the thickness 8.5E$5 \mathrm{~m}$ and the other two layers have a growth factor of 1.1 from one to another, this results in a Y+ value of $\sim 1.9$.

\subsubsection{Turbulence model and convergence}

We tested several meshes, turbulence models and solving algorithms. The presented results were obtained using the k-omega Shear Stress Transport (SST) turbulence model (Menter et al. 2003) and the SIMPLEC algorithm. The selection of these CFD parameters along with the mesh was made based on a convergence study where we compared the simulation results, namely the pressure at 90 different pressure tabs over the surfaces of the model against experimental results obtained at the same locations from wind tunnel experiments that we performed using the same model configuration and wind velocity. The reported results achieved convergence criteria composed of two factors that are satisfied, 
which are a mean squared error less than $30 \%$ compared to the experimental measurements. The other factor is a residual error on the CFD solution less than 5E-4. However, the comparison against the experimentally obtained results was made only for the simulation of the configuration that corresponds to test 2D16 (c.f. Table), then the same setup was used to simulate the flow over the rest of the configurations.

We are not reporting the wind tunnel tests results or the setup for the sake of keeping the text focused on the most relevant results. However, we took on consideration the common procedures in performing such tests following (Tropea et al. 2007).

\subsubsection{Obtaining the results}

We focused the obtained results on the flow velocity near the surfaces of the model $(0.1 \mathrm{~m}$ above the surface for all the results) considering its importance on driving the fire behaviour. We obtained the velocity and its components at points that are redistributed along three lines, which are the middle line, quarter line and leeward line (Figure ). The middle line is a line that passes at the middle of the hills (middle of the ridgeline) and starts at the base of the windward face of the first hill and ends at the base of the second hill.

The quarter line is similar to the middle line but it passes at quarter of the ridgeline from the left side of the hill, we considered only the lift side as there is symmetry between the two sides in ideal flow conditions; the length of this line and the middle line are dependent on the configuration, namely the value of $D$. The leeward line is parallel to the ridgeline over the leeward face and spaced from it by $0.025 \mathrm{~m}(10 \%$ of the face width).

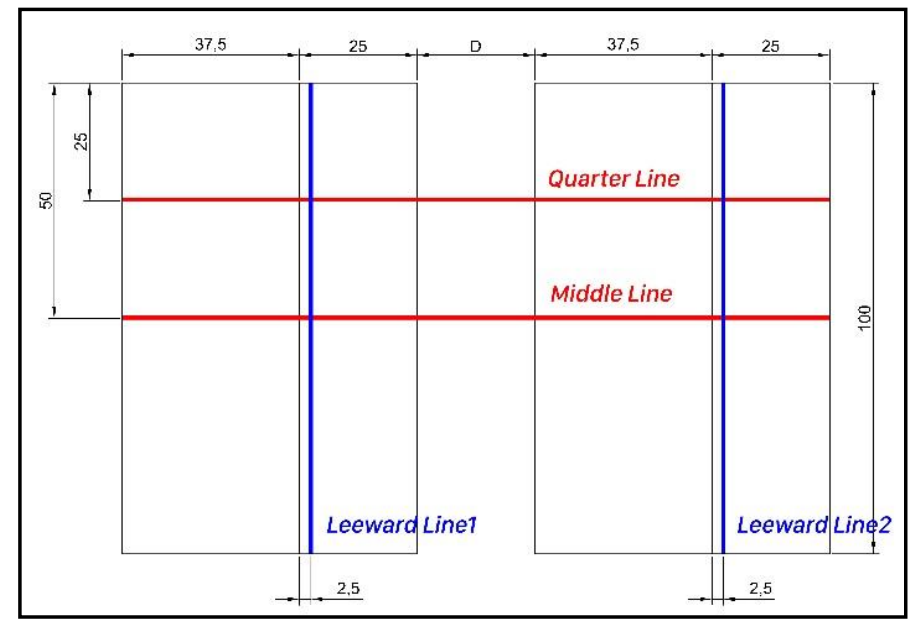

Figure 4 - a plane view showing the size of the model that was used in the simulation along with lines that the results (flow velocities) were obtained along them

\section{Results and Discussion}

\subsection{Overall Fire Behaviour}

In overall, the fire behaviour over the hills is dependent on their configuration, namely the slope; in addition to the blowing wind direction and velocity, and the approaching fire front direction and width. We performed two tests to analyse the fire behaviour in the absence of wind in order to better assess the role of the wind in driving the fire behaviour. On these two tests, 2D1 and 2D5, the two hills are configured to have equal $\alpha$ values, where we used the two values of $\alpha$ of $20^{\circ}$ and $27^{\circ}$, one for each test. The tests revealed that the fire spreads with NDROS values that are close to 1 except in the WUS1\&2 directions (Figure ), where the spread was relatively faster as expected (Viegas 2006). 
However, it's noticeable also that the spread on the WUS2 is faster than WUS1 (Table ), which is due to the fact that the fire approaches the second hill with a wider fire front.

It is well known that the fire spreads faster up-slope as we increase the slope of a surface, and in the case of having a blowing wind on the same direction of the up-slope propagation, the fire propagates even faster proportionally to the blowing wind speed. This can easily be noticed on the reported NDROS of the fire in the WUS1 direction in Table with all the tests where we had an ignition on WH1. However, over the upslope direction of the second hill (WUS2), the achieved ROS by the fire is different and it is affected by the recirculated flow between the two hills and the fire-induced flow. These flows also create the phenomena of the fire channelling. On the following two sections we discuss the fire channelling behaviour and the fire behaviour between the hills.

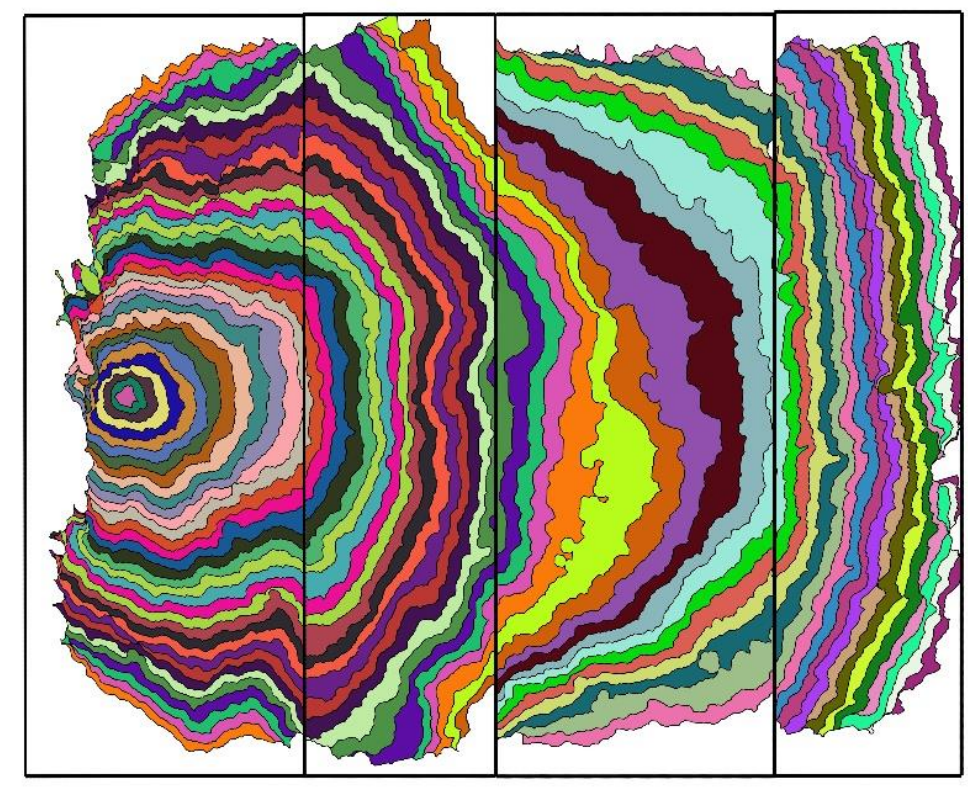

Figure 5 - Fire propagation contour map for test 2D1, showing the fire evolution over the hills in the absence of wind and with an $\alpha_{1}=\alpha_{2}=20^{\circ}$. The time lapse between frames is $30 \mathrm{~s}$.

Table 1 A list with the performed tests along with their parameters and the average NDROS of the fire along the predefined directions (Figure b) for each test

\begin{tabular}{|c|c|c|c|c|c|c|c|c|c|c|c|c|c|}
\hline Test Ref. & $U_{o}$ & $\alpha 1, \alpha 2$ & Ignition & $D$ & WUS1 & WLA1 & LDS1 & LLA1 & WUS2 & WLA2 & LDS2 & LLA2 & GRA \\
\hline 2D1 & 0 & \multirow{4}{*}{20,20} & \multirow{4}{*}{ WH1 } & \multirow{4}{*}{0} & 1.04 & 0.83 & 0.58 & 1.01 & 1.60 & 1.56 & 0.61 & 1.55 & \\
\hline 2D2 & 1 & & & & 3.01 & 0.91 & 0.74 & 2.05 & 3.46 & 2.48 & 0.87 & 2.09 & \\
\hline $2 \mathrm{D} 3$ & 2 & & & & 8.34 & 0.98 & 0.76 & 2.53 & 4.53 & 1.85 & 1.26 & 2.84 & \\
\hline 2D4 & 3 & & & & 19.12 & 2.28 & 1.56 & 5.71 & 5.06 & 2.24 & 2.28 & 7.43 & \\
\hline $2 \mathrm{D} 5$ & 0 & \multirow{4}{*}{27,27} & \multirow{4}{*}{ WH1 } & \multirow{4}{*}{0} & 1.76 & 1.25 & 0.99 & 1.32 & 3.45 & 1.78 & 0.84 & 1.70 & \\
\hline 2D6 & 1 & & & & 4.21 & 1.09 & 1.08 & 2.95 & 3.78 & 1.70 & 1.02 & 2.62 & \\
\hline $2 \mathrm{D} 7$ & 2 & & & & 8.20 & 1.28 & 1.26 & 3.58 & 7.57 & 1.55 & 1.15 & 3.23 & \\
\hline 2D8 & 3 & & & & 14.63 & 1.48 & 1.42 & 4.56 & 7.24 & 1.47 & 1.00 & 3.72 & \\
\hline $2 \mathrm{D} 17$ & 0 & \multirow{2}{*}{20,20} & \multirow{2}{*}{ Mid } & \multirow{2}{*}{0} & 0.68 & 0.91 & 1.97 & 0.91 & 1.10 & 1.11 & 0.73 & 1.19 & \multirow{2}{*}{ - } \\
\hline 2D18 & 2 & & & & 0.19 & - & 1.65 & 4.66 & 1.47 & 1.22 & 0.96 & 1.88 & \\
\hline 2D19 & 0 & \multirow{2}{*}{20,20} & \multirow{2}{*}{ LH2 } & \multirow{2}{*}{0} & 0.84 & 3.14 & 4.14 & 3.30 & 0.75 & 1.06 & 1.71 & 0.96 & \\
\hline 2D20 & 2 & & & & 0.28 & - & 3.98 & 9.64 & 0.64 & 0.47 & 1.54 & 3.73 & \\
\hline $2 \mathrm{D} 14$ & \multirow{4}{*}{2} & \multirow{4}{*}{20,20} & \multirow{4}{*}{ WH1 } & 0.5 & 10.34 & 1.16 & 0.95 & 2.80 & 2.29 & 3.07 & 1.54 & 3.62 & 0.70 \\
\hline 2D16 & & & & 1 & 6.29 & 1.14 & 0.73 & 2.56 & 6.56 & 3.85 & 0.95 & 3.11 & 0.61 \\
\hline $2 \mathrm{D} 23$ & & & & 1.5 & 8.51 & 1.23 & 0.84 & 2.82 & 18.32 & 2.46 & 1.14 & 3.23 & 1.13 \\
\hline 2D24 & & & & 2 & 7.68 & 1.35 & 8.18 & 3.10 & 14.86 & 2.83 & 1.04 & 3.47 & 1.04 \\
\hline $2 \mathrm{D} 25$ & 2 & 20,20 & Mid & 1 & - & - & 3.30 & 6.00 & 2.09 & 6.26 & 1.12 & 4.49 & 0.76 \\
\hline
\end{tabular}




\subsection{The fire channelling}

In our tests, a lateral spread of the fire or the so called fire channelling happened always if $U o>0$ over the leeward face of the first hill near the ridgeline regardless the fire front approaching direction, either from the windward face with the direction of the wind or from the leeward against the wind direction (Figure 6). Over the second hill, the channelling also happened but in some tests where the fire approached the second ridgeline with wide fire front that prevents the channelling behaviour from taking place due to the limited ridgeline length.
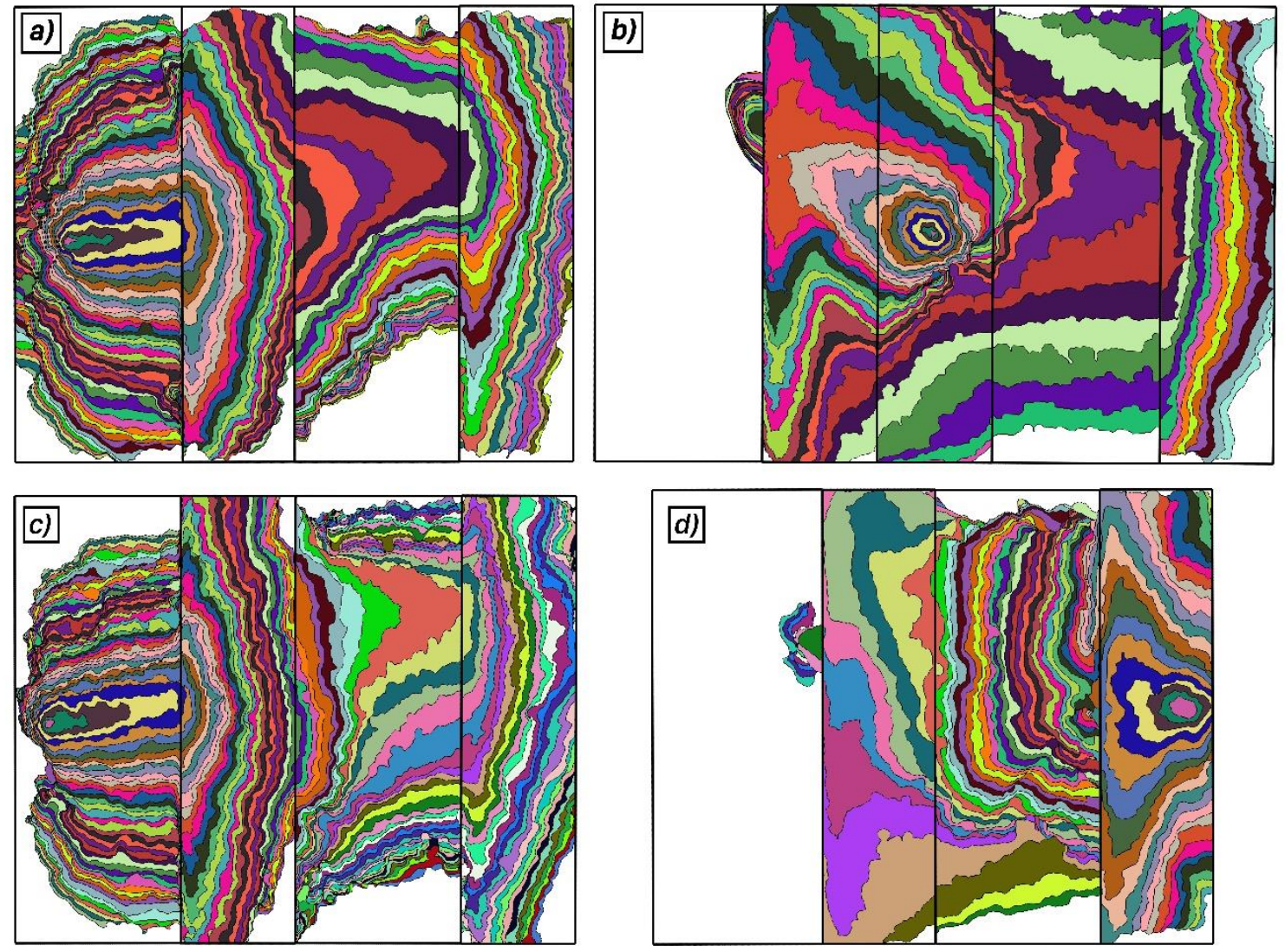

Figure 6 - Fire propagation contour maps showing the fire evolution over the hills in the presence of blowing wind with $U_{0}=2 \mathrm{m.s}-1$ a) test $2 \mathrm{D} 7$ with $\alpha 1=\alpha 2=27^{\circ}$ and $D=0 \mathrm{~m}$. The time lapse between frames is $\left.18 \mathrm{~s} . \mathrm{b}\right)$ test $2 \mathrm{D} 25$ with $\alpha 1=\alpha 2=20^{\circ}, D=1 \mathrm{~m}$ and Mid ignition. The time lapse between frames is $\left.20 \mathrm{~s} . \mathrm{c}\right)$ test $2 \mathrm{D} 3$ with $\alpha 1=\alpha 2=20^{\circ}$ and $\mathrm{D}=0 \mathrm{~m}$.

The time lapse between frames is $15 \mathrm{~s}$. d) test $2 \mathrm{D} 20$ with $\alpha 1=\alpha 2=20^{\circ}, \mathrm{D}=0 \mathrm{~m}$ and LH2 ignition. The time lapse between frames is $30 \mathrm{~s}$. The wind is blowing from left to right in all cases.

On Figure Erro! A origem da referência não foi encontrada. we plot the NDROS values of the fire on the LLA direction (the channelling direction) to illustrate the change on the behaviour with changing the distance between the hills $(D)$ on (a) and the blowing wind velocity $\left(U_{o}\right)$ on $(\mathrm{b})$. We can notice that the fire spreads on this direction with similar ROS over the first and second hills for the same test in most of the cases regardless of the configuration except for the highest tested wind velocity $U_{o}=3 \mathrm{~m} \cdot \mathrm{s}^{-1}$, where noticeably the fire spreads faster on the second hill than the first hill (Figure b). Also, we can find that the ROS of the fire is higher for higher inclination except for the case with $U_{o}$ $=3 \mathrm{~m} \cdot \mathrm{s}^{-1}$, where a sudden increase is evident in the lower inclination. By looking at the effect of changing $D$ on the channelling behaviour (Figure a), we can notice that all the values are osculating around a NDROS value of 3 with small differences when we change the value of $D$. However, the channelling has a remarkably faster spread (almost the double) (Figure a) if the fire front approaches the leeward face of the hill from its base in the opposite direction to the blowing wind (Figure b), which is the case when we did a Mid ignition while $D=1 \mathrm{~m}$. Also on another case when we had an 
ignition at LH2 (Figure d), the fire propagated even faster laterally over the first hill with a ROS that is around three times the lateral spread in case of igniting the fire at $W H 1$ (Figure a). This leads to an important remark that the fire channelling happens with faster ROS if the fire front approaches the hill from the leeward side in a contrary direction to the main stream compared to the approach from the windward side in the same direction of the wind.

a)

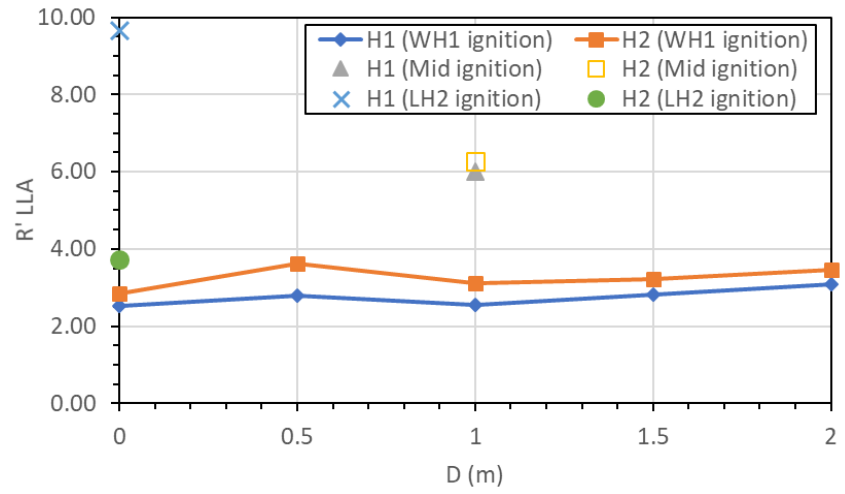

b)

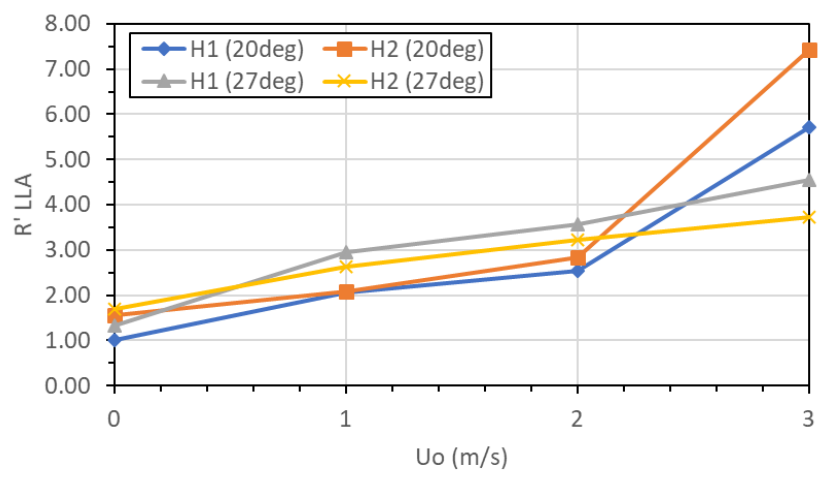

Figure 7 - The average NDROS ( $\left.R^{\prime}\right)$ values of the fire propagation on the LLA1\&2 directions plotted versus: a) the distance between the two hills $(D)$ with different ignition points. $b)$ the free stream velocity $\left(U_{o}\right)$ with the two inclination scenarios, $\alpha 1=\alpha 2=20^{\circ}$ and $\alpha 1=\alpha 2=27^{\circ}$

By looking at results of the adiabatic flow simulation (Figure ), we can see a formation of two large eddies in the wake of both hills (between the two hills and after the second hill). The two eddies that are between the two hills are rotating in opposite directions, and they spread towards the middle of the hill where they spread after that in the x-direction above the second ridgeline where the flow exits the recirculation structure. These two formed eddies cause the flow to form patterns near the surface of the leeward face (Figure a) where the flow goes in an up-slope direction at the middle of the hill then rotates to be parallel to the ridgeline and goes towards the two ends of the hill. This flow pattern is the main driver of the fire channelling behaviour.

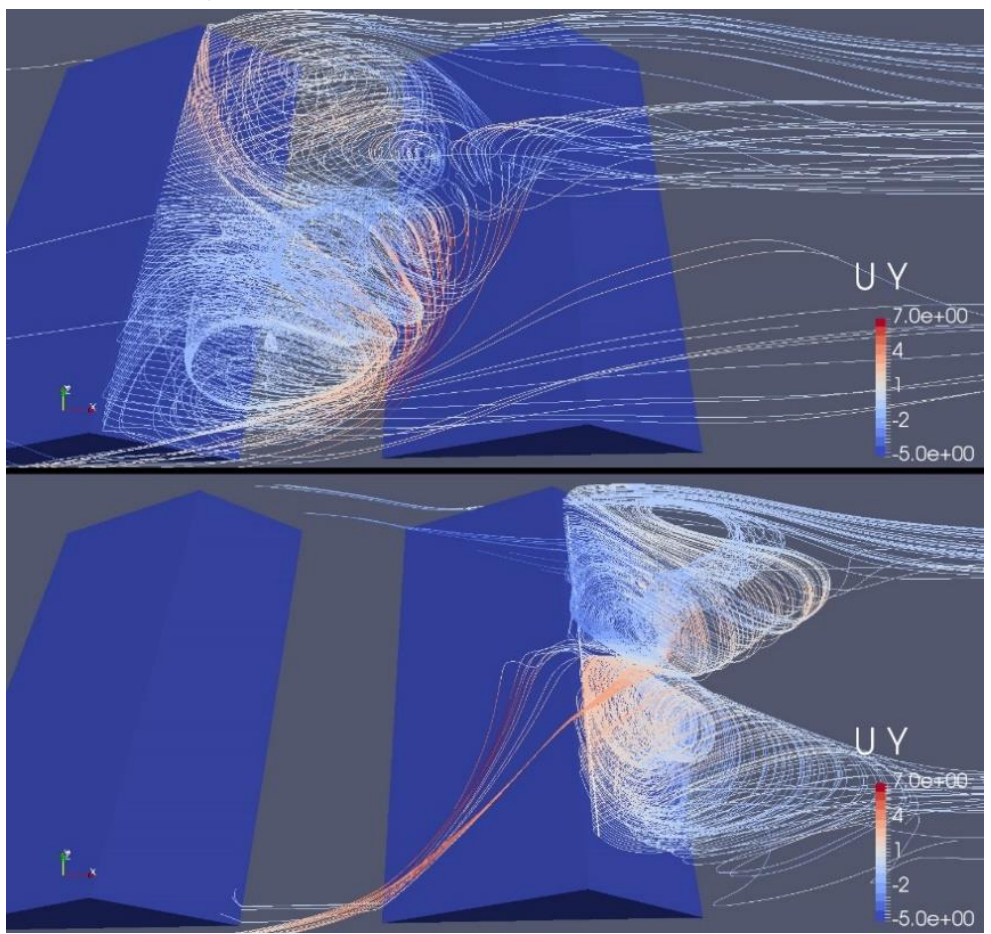

Figure 8 - Streamline of the flow in the wake of each of each of the two hills sources form a parallel line to the ridgeline (the leeward line) and coloured by the velocity y-component magnitude 


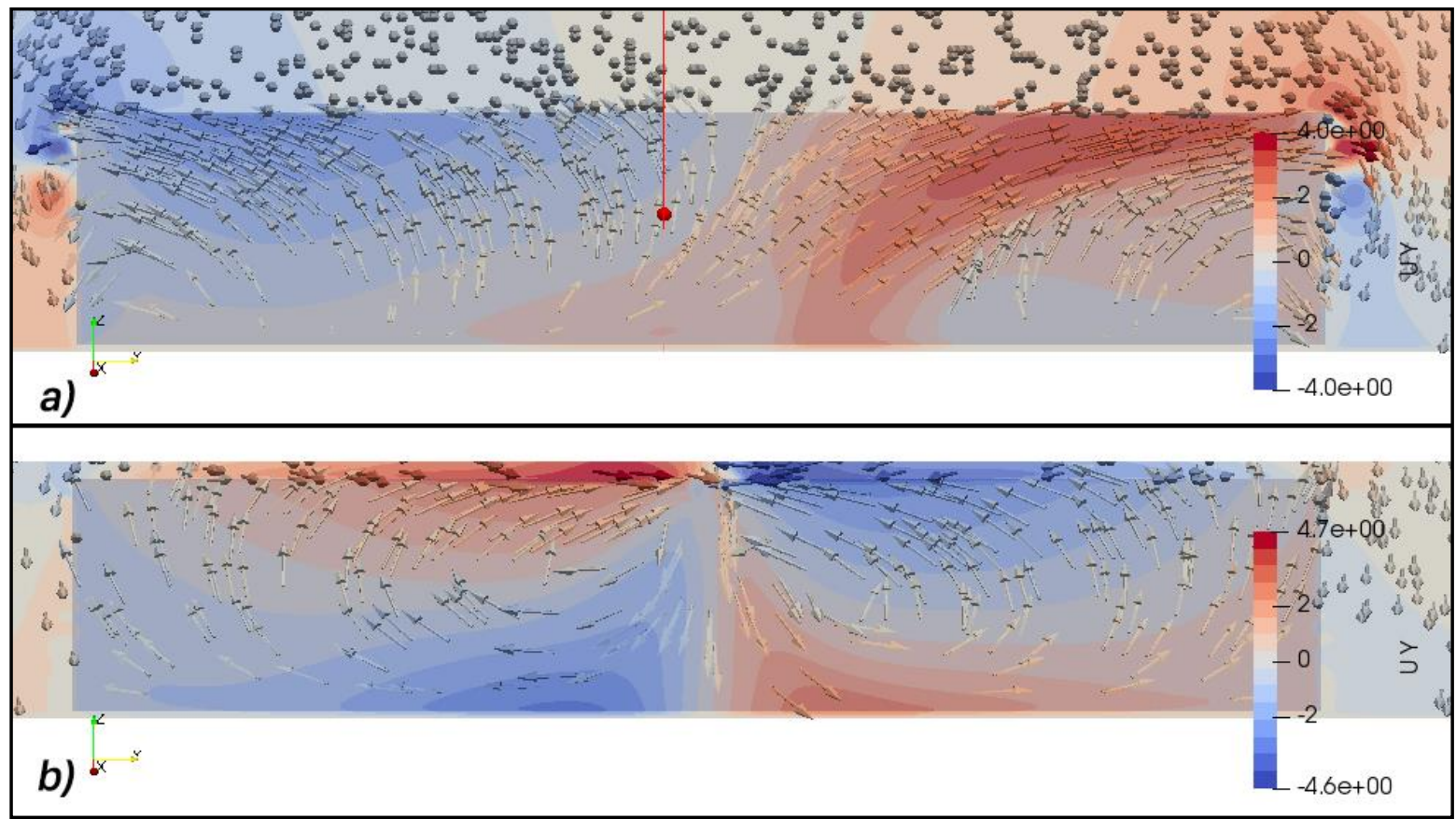

Figure 9 - Numerical results for the field of the velocity component in the $y$-direction $\left(U_{y}\right)$ on parallel planes to the hills' leeward faces and spaced from them by $0.01 \mathrm{~m}$ for the case where of $D=0.25 \mathrm{~m}$ along with the velocity vectors. a) The leeward of the first hill. b) The leeward of the second hill.

On Figure we report the values of the y-velocity component $\left(U_{y}\right)$ that was computed for the adiabatic flow along the predefined line, the leeward line. The results show that the flow near the ridgeline of the first hill (Figure a) doesn't have a y-component velocity $\left(U_{y}=0\right)$ at the middle of the hill, then a y-component starts to develop as we are heading towards the ends of the hill. However, over the second hill, the flow develops $U_{y}$ also near the ridgeline but it has an opposite direction where the flow goes towards the middle of the hill not towards the ends (Figure b), which resulted from the rotating direction of the developed eddies in the wake of the second hill (Figure b). The adiabatic flow behaviour over near the surface of the first leeward coincides with the behaviour of the fire channelling, where we can find on Figure the instantaneous ROS of the lateral spread of the fire is increasing over the course of time (accelerating). But over the second hill, the fire tends to propagate with constant ROS over the course of time or even a deceleration behaviour. These two behaviours of lateral spread over the first and second hills can be easily related to the discussed behaviour of the local adiabatic flow (Figure ). Taking into account the fire-induced flow, we can now interpret the remark that we addressed earlier about the faster lateral spread of the fire in the two ignition cases Mid and $\mathrm{LH} 2$, where the fire on this case was propagating on the same direction of the adiabatic flow towards up-slope at the middle of the hill (Figure a) then the lateral spread takes place near the ridgeline when the flow starts to develop a y-component velocity. During this scenario, the flow accelerates taking into account the fire-induced flow towards up-slope, which is not present if the fire was ignited at WHI and approached the ridgeline from the windward face (Figure a). 


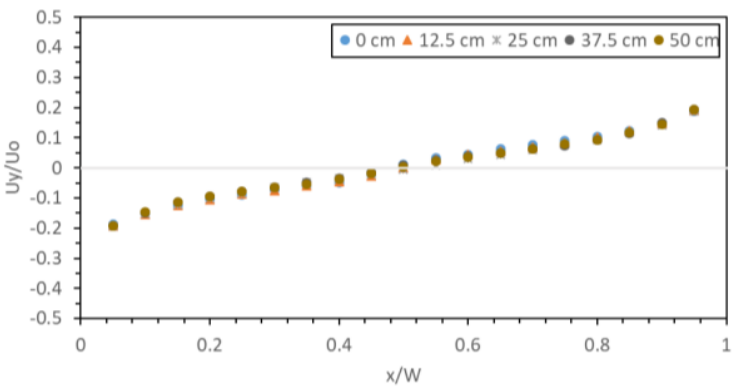

b)

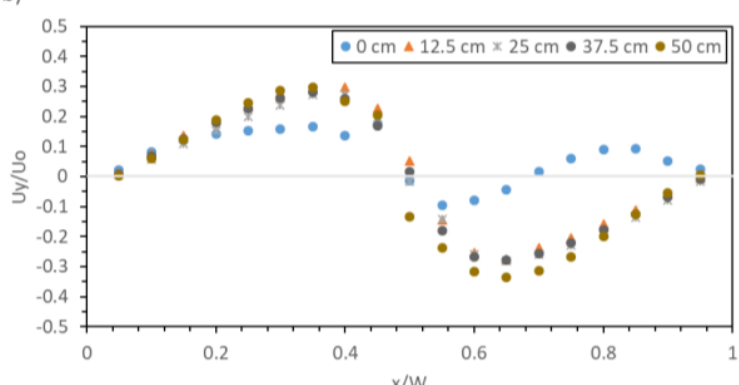

Figure 10 - Flow velocity results of the y-direction component $\left(U_{y}\right)$ computed in the adiabatic flow simulation and obtained at points along a line parallel to the ridgeline and spaced from it by $0.025 \mathrm{~m}$ (10\% of the face width) and over the leeward face surface by $0.01 \mathrm{~m}$. a) over the first hill b) over the second hill. The velocity is presented dimensionless where the values are divided by the inlet flow stream velocity $\left(U_{0}=8 \mathrm{~m} . \mathrm{s}^{-1}\right)$. The $x$-axis represents the relative distance of the point compared to the ridgeline length $(W)$, where zero corresponds to the right end of the hill and 1 is the left end.
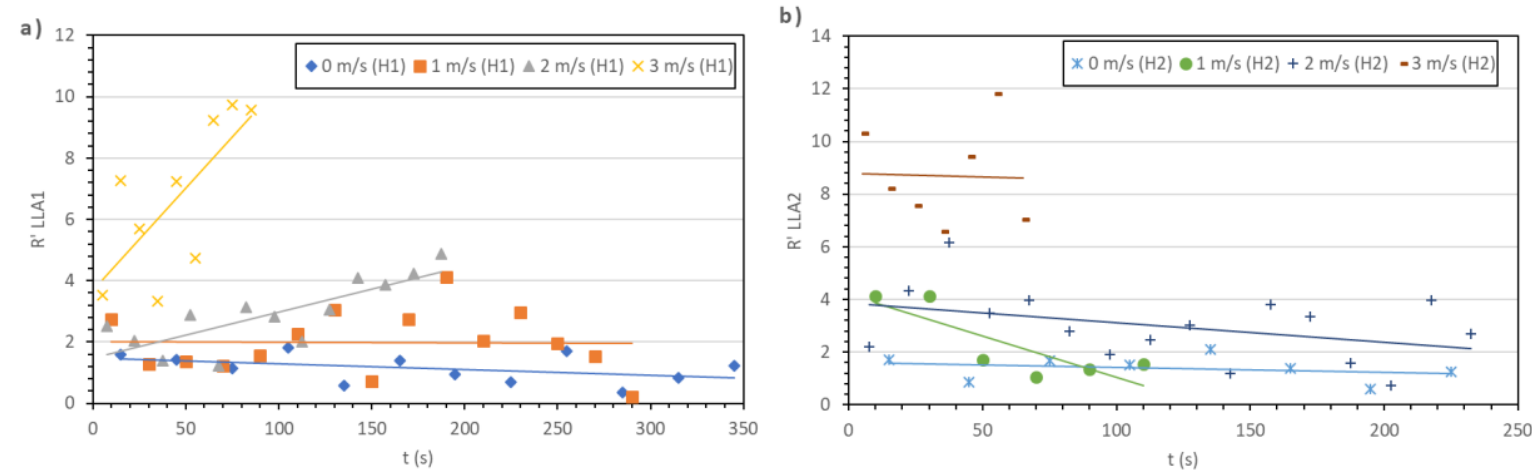

Figure 11 - The evolution of the instantaneous NDROS values over the time on LLA direction for different wind velocities $\left(U_{o}\right)$ a) over the first hill (LLA1) b) over the second hill (LLA2).

\subsection{The fire behaviour between the hills}

We are focussing this section on the fire spread on WUS2 and GRA directions, and the change of the fire behaviour along them with changing the distance between the two hills $(D)$. On Figure $\mathrm{b}$ we can find that the fire's ROS on the up-slope direction increases as we increase the velocity $\left(U_{o}\right)$, however, the increase is more evident on the WUS1 than WUS2. Also, the WUS1 is faster than WUS2 for all the tested wind velocities especially with high values of $U_{o}$ where the difference can reach up to 4 times, all while $D=0$. But when we increase the value of $D$, we can notice on Figure a that there is a slight decrease in the ROS over the WUS2 direction when $D=0.5 \mathrm{~m}$ then it increases as we increase $D$ until we reach the case of $D=1.5 \mathrm{~m}$, on these configurations we consider the eruptive fire behaviour is evident as the NDROS value is close to 18 . For $D$ values higher than $1.5 \mathrm{~m}$ we can notice a slight decrease again. It's remarkable that at $D=1 \mathrm{~m}$ the ROS over WUS1 and WUS2 are matching. However, this matching doesn't necessarily lead to the conclusion that the fire has the same behaviour on the upslope direction because the fire front is wider when approaching the second hill than it was on the first one (Figure ). 
a)

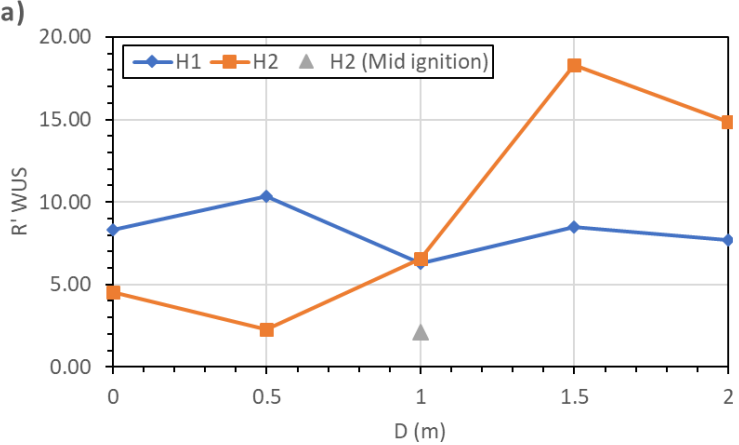

b)

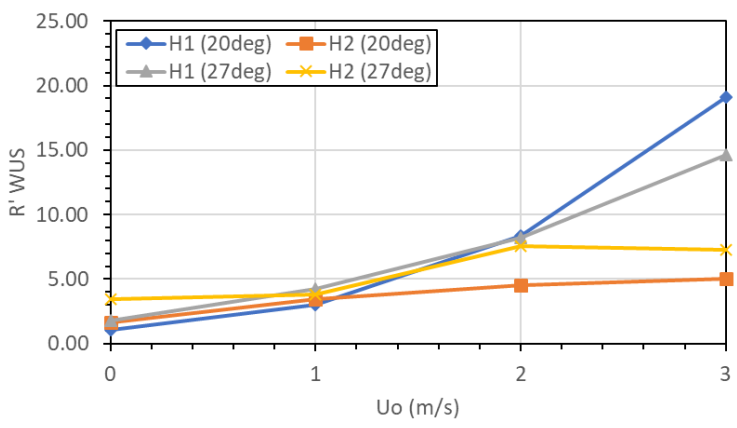

Figure 12 - The average NDROS (R') values of the fire propagation on the WUS1\&2 directions plotted versus: a) the distance between the two hills $(D)$ with different ignition points and $U_{o}=2 m \cdot s^{-1}, \alpha 1=\alpha 2=20^{\circ}$. b) the free stream velocity $\left(U_{o}\right)$ with the two inclination scenarios, $\alpha 1=\alpha 2=20^{\circ}$ and $\alpha 1=\alpha 2=27^{\circ}$, and $D=0$.
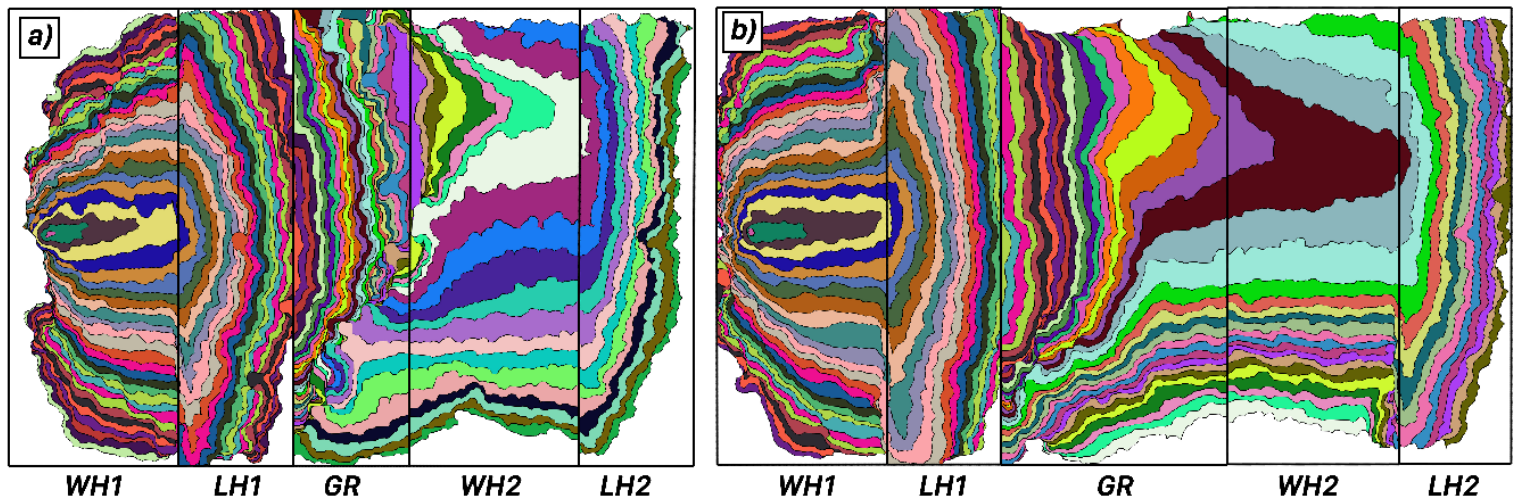

Figure 13 - Fire propagation contour maps showing the fire evolution over the hills in the presence of blowing wind with $U_{o}=2 \mathrm{~m} . \mathrm{s}-1$ and $\alpha 1=\alpha 2=20^{\circ}$ a) test $2 \mathrm{D} 16$ with $\mathrm{D}=1 \mathrm{~m} . \mathrm{b}$ ) test $2 \mathrm{D} 24$ with $\mathrm{D}=2 \mathrm{~m}$. The time lapse between frames is $30 \mathrm{~s}$ for both. The wind is blowing from left to right.

By looking at the results of the numerical solution of the adiabatic flow, we can find on Figure the flow velocity modulus values and the $U_{x}$ and $U_{z}$ components determined along the predefined middle and quarter lines (Figure ). The one may notice that the flow near the windward surface of the second hill has lower velocities than the one of the first hill. This coincides with the observation that we obtained earlier about the fire spread on the upslope direction as the ROS is higher on WUS1 compared to WUS2. Also, as we increase the distance between the hills $(D)$, the near-surface flow velocities increase, which agree as well with the fire behaviour on the WUS2 direction with increasing $D$. However, in most of our tests we noticed that the fire front approached the second windward from a point that's not at the middle but shifted towards the right or the left, as we can see on the tests that are presented on Figure and Figure, and on these cases the up-slope spread was shifted towards the left. This behaviour happens even if the fire front that is propagating on the ground reaches the middle of the hill first, as it's shown on Figure a, where the fire propagates very slowly towards up-slope until the fire reaches the left side of the windward face, then a fast up-slope (eruptive) propagation will develop. 
a)
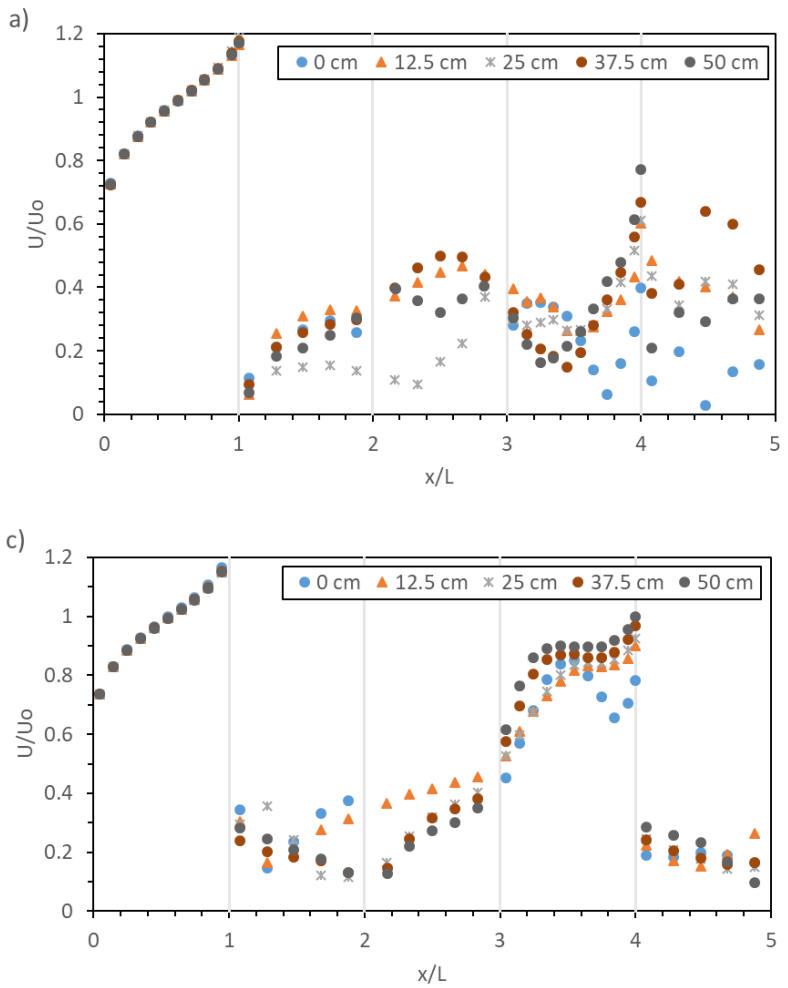
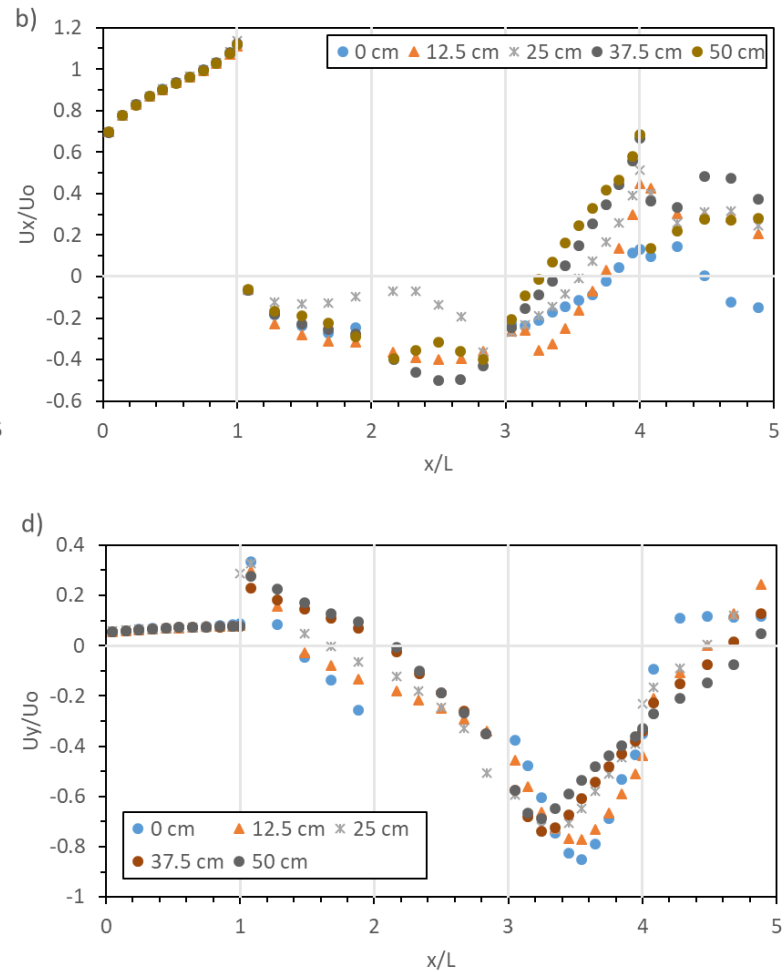

Figure 14 - Flow velocity results near the surface $(1 \mathrm{~cm})$ computed in the adiabatic flow simulation and obtained at points along two redefined lines at the middle an quarter of the hill where each line have a total length L. The velocity is presented dimensionless where the values are divided by the inlet flow stream velocity $\left(U_{o}=8\right.$ m. $\left.s^{-1}\right)$. Each unit on

the $x$-axis represents the width of each face, where from 0 to 1 is WH1 followed by LH1, GR, WH2 and LH2 respectively. a) velocity modulus along the middle line. $b$ ) velocity component in the $x$-direction $\left(U_{x}\right)$ along the middle line. $c)$ velocity modulus along the quarter line. $d)$ velocity component in the $y$-direction $\left(U_{y}\right)$ along the quarter line

By comparing the flow velocity modulus near the second windward surface along the middle line and the quarter line that are presented on Figure, we can realize that the flow has higher velocity along the quarter line than the middle line, in fact, along the middle the flow has a negative $U_{x}$ values at the beginning of the of windward surface (Figure b), which means that the flow has an opposite direction to the up-slope fire spread direction. Meanwhile, along the quarter line, the flow has high transverse flow $\left(U_{y}\right)$ with a direction towards the middle of the hill (Figure d). Furthermore, by looking at the flow topology that is presented by the streamlines on Figure, we can find that the two eddies that are formed between the hills are the ones that cause this flow pattern near the surface of the second windward face, which is presented on Figure, and we can see that there is a dead area at the middle of the hill with very low flow velocity and the velocity increases at the right and left from the middle. 


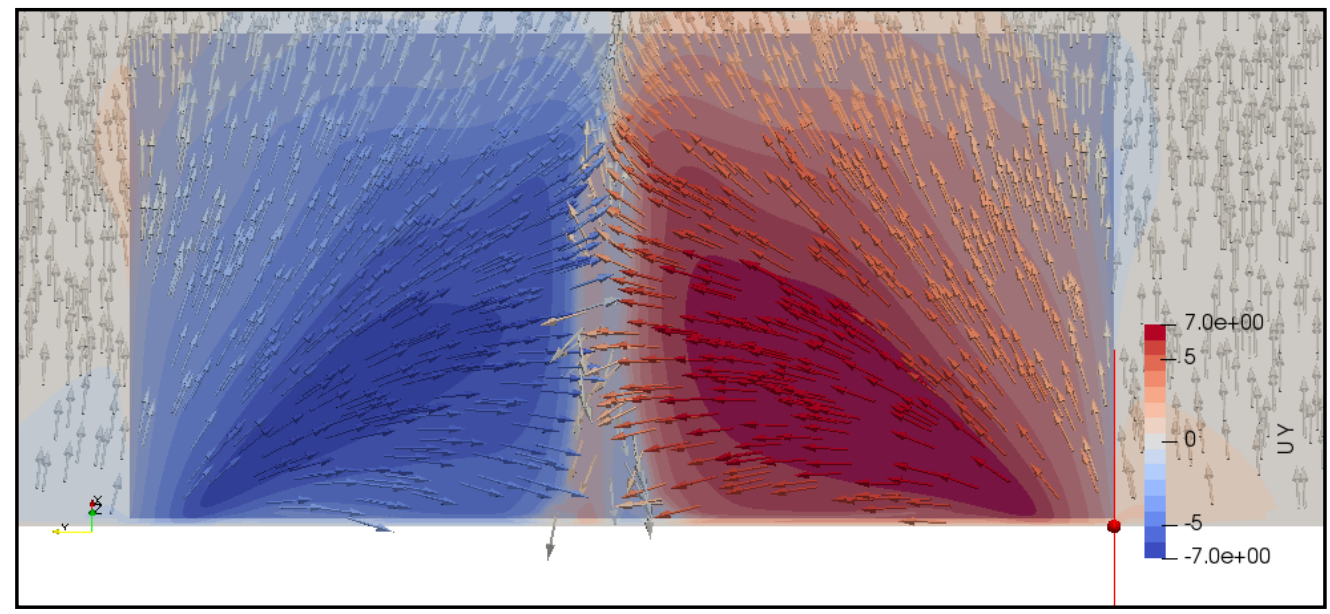

Figure 15 - Numerical results for the field of the velocity component in the $y$-direction $\left(U_{y}\right)$ on a parallel plane to the second windward face and spaced from it by $0.01 \mathrm{~m}$ for the case where of $D=0.25 \mathrm{~m}$ along with the velocity vectors.

The effect of the two circulation eddies is also evident on driving the fire behaviour on the ground $(G R)$, where the flow near the ground surface is driven on the negative $\mathrm{x}$-direction (Figure $\mathrm{b}$ ) this results in a very slow propagation of the fire as we can find the values of the NDROS are close to 1 in the GR direction (Table ). However, the negative $U_{x}$ is apparent along the middle (Figure b), but along the quarter line, we can find that the flow over the gound has higher velocities with an increasing $U_{y}$ as the flow gets closer to the second windward face (Figure d). This developed y-component of the flow drives the fire faster on the ground just before it reaches the second windward as we can see in Figure b.

From the mentioned observations about the adiabatic flow topology and patterns near the surface, we can conclude that the recirculated flow between the two hills has an apparent effect on driving the fire on the ground and the second windward face. It is noticeable also that the fire propagates in the same direction of the highest local flow velocity and enhance it by the fire-induced flow resulting in a fire propagation that is different from the typical up-slope spread (i.e. In the case that there are no obstacles before it in the direction of the wind), like the first windward in our configuration here.

\section{Conclusion}

The experiments discussed in this study explore the wildfire behaviour over two triangular hills placed on series in the direction of the wind with different configurations and applied wind velocities. We observed that the fire propagates laterally over the leeward faces of the hills near the ridgeline (fire channelling), these lateral spreads are noticeably faster if we increase the freestream velocity while the change in the fire's rate of spread with the change in the inclination of the hills or the distance between them is relatively small. Also, the lateral spread is remarkably faster on the first hill in the direction of wind than on the hill that has a topographic obstacle before it (the second hill in our study).

Based on the numerical simulations of the adiabatic flow we found that the topology of the recirculated flow in the wake of the hill is the principal cause of the lateral fire spread. The flow near the ridgeline where the fire channelling takes place has a lateral component with a value that can reach up to half of the freestream velocity value. Also, the lateral flow accelerates toward the hill sides, which coincides with the noticed accelerating fire spread behaviour from the middle of the hill towards the sides.

We also noticed that the fire channelling is faster (up to double) if the fire propagated over the leeward face towards up-slope in a contrary direction of the mainstream compared to the fire that propagates up-slope over the windward in the same direction of the mainstream. We rely this on the fire-induced flow as the up-slope propagation over the leeward face has the same direction as the local 
terrain-modified flow, therefore, the fire enhances the flow velocity locally and feeds the fire channelling

Another remarkable fire behaviour explored as well was the spread of the fire over the windward face of the second hill in the direction of the wind. The up-slope spread over this face is generally slower than the spread rate in the similar direction (up-slope) over the first hill. This is clearer with high blowing wind velocities, and without having space between the two hills, in this case, the fire's rate of spread can be up to three times slower than the spread over the first hill. However, this slower rate of spread of the fire fades if we increased the distance between the two hills, in fact, with distances higher than two times the height of the hill, the fire propagates faster over the second windward in comparison with the first one. Furthermore, we noticed that the fire's propagation towards up-slope over the second windward is shifted from the middle towards one of the sides regardless of the fire's approaching point to the windward face.

We related the behaviour of the fire in the up-slope direction over the second windward with the topology of the recirculating flow between the two hills and the local adiabatic flow velocities, where we found that the same recirculation eddies that are responsible about the fire channelling are also responsible about shifting the up slope propagation over the second windward. Additionally, this reticulated flow causes the fire to propagate on the ground between the two hills relatively slow.

From these observations and conclusions, we can summarize that the terrain-modified flow has a significant effect on driving the fire over complex topographic terrains. Our results also show the importance of simulating the flow field over these terrains with high accuracy in order to better predict the fire growth.

\section{Acknowledgements}

The authors wish to thank the Portuguese Science Foundation for the support through the project for the project "FIREWHIRL - Vorticity Effects in Forest Fires" (PTDC/EMS-ENE/2530/2014). The authors are grateful to ADAI's team, namely Nuno Luis, António Cardoso and Eduardo Melo for their help to carry on the fire investigation tests. The authors also acknowledge the Laboratory for Advanced Computing at the University of Coimbra (www.lca.uc.pt) for providing \{HPC, computing, consulting\} resources that have contributed to the research results reported within this paper and the team of the laboratory, namely Professor Pedro Vieira Alberto.

\section{References}

Abouali A (2017) Fire ROS Calculator. doi:10.5281/ZENODO.898160.

Arya SPS, Shipman MS, Courtney LY (1981) An experimental investigation of flow and diffusion in the disturbed boundary layer over a ridge-II. Diffusion from a continuous point source. Atmospheric Environment (1967) 15, 1185-1194. doi:10.1016/0004-6981(81)90309-7.

Greenshields C (2015) SnappyHexMesh. OpenFOAM v4 User Guid. https://cfd.direct/openfoam/userguide/v4-snappyHexMesh/.

McRae RHD (2004) Breath of the dragon - observations of the January 2003 ACT Bushfires. In: Proceedings of 2004 Australasian Bushfire Research Conference, May 2004, Adelaide.

Menter FR, Kuntz M, Langtry R (2003) Ten Years of Industrial Experience with the SST Turbulence Model. Turbulence Heat and Mass Transfer 4 4, 625-632. doi:10.4028/www.scientific.net/AMR.576.60.

Raposo JR, Cabiddu S, Viegas DX, Salis M, Sharples J (2015) Experimental analysis of fire spread across a two-dimensional ridge under wind conditions. International Journal of Wildland Fire 24, 1008-1022. doi:10.1071/WF14150. 
Sharples JJ, McRae RHD, Wilkes SR (2012) Wind-terrain effects on the propagation of wildfires in rugged terrain: Fire channelling. International Journal of Wildland Fire 21, 282-296. doi:10.1071/WF10055.

Tropea C, Yarin AL, Foss JF (2007) 'Springer Handbook of Experimental Fluid Mechanics.' doi:10.1007/978-3-540-30299-5.

Viegas DX (2004) Slope and wind effects on fire propagation. International Journal of Wildland Fire 13, 143-156. doi:10.1071/WF03046.

Viegas DX (2006) Parametric study of an eruptive fire behaviour model. International Journal of Wildland Fire 15, 169-177. doi:10.1071/WF05050.

Viegas DX (2012) Extreme Fire Behaviour. 'For. Manag. Technol. Pract. Impact'. (Eds ACB Cruz, REG Correa) pp. 1-56. (Nova Science Publishers, Inc.: New York)

Viegas DX, Neto LPC (1991) Wall shear-stress as a parameter to correlate the rate of spread of a wind induced forest fire. International Journal of Wildland Fire 1, 177-188. doi:10.1071/WF9910177.

Weller HG, Tabor G, Jasak H, Fureby C (1998) A tensorial approach to computational continuum mechanics using object-oriented techniques. Computers in Physics 12, 620. doi:10.1063/1.168744. 


\title{
Understanding the dynamics of inclined and wind-driven flames in wildland fires
}

\author{
M.J. Gollner ${ }^{1 *}$; W. Tang ${ }^{1}$; C.H. Miller ${ }^{1}$; S. McAllister²; M.A. Finney ${ }^{2}$ \\ ${ }^{1}$ University of Maryland, College Park, Department of Fire Protection Engineering. 3106 J.M. \\ Patterson Bldg., College Park, Maryland 20742, United States, \{mgollner@umd.edu*\} \\ ${ }^{2}$ US Forest Service, Missoula Fire Sciences Lab, Missoula, MT 59808, USA
}

\begin{abstract}
Time-dependent movements described as pulsing, puffing or swaying are among the most visible characteristics of open flames. Most existing models for wildfires, however, have assumed flames are motionless and spread at a constant rate via radiation, neglecting any clearly intermittent behaviour. Recent studies of spreading wildfires suggest that flame spread in fine fuel beds is driven by non-steady convective heating and intermittent flame contact on fuel particles. To further understand the impact and nature of these flame motions, a stationary, non-spreading fire configuration has been used as it allows for a thorough statistical analysis of the flame structure. The same intermittent heating observed in the fuel bed experiments were observed in the stationary burner, but with the ability to collect a larger data set. Scaling analyses which investigate the impact of these structures, theories on their generation and their impact to fire spread will be discussed for both wind-driven and slope-dominated flames.
\end{abstract}

Keywords: wildland fire, wind-driven, flames

\section{Overview}

Recent research (Finney et al. 2015) has emphasized the importance of convective heating in wildfire spread across fine fuel elements, indicating that an understanding of the dynamics of an intermittent flame will aid a description of the flame spread process. Although radiation was often ascribed as the dominant mechanism of flame spread in large scale fires, experimental data has shown that the fine elements present in wildland fuel beds will not ignite until they come into contact with flames due to convective cooling (Martin 1965; Rothermel and Anderson 1966; Fang and Steward 1969; Alvares et al. 1970; Baines 1990). An account of the flame dynamics responsible for this process is essential in order to develop a fundamental theory of fire spread in wildland fuels. The important mechanisms associated with flame spread in the turbulent regime of a wildland fire are very difficult to study in the field; for this reason, laboratory experiments can be employed to mimic some aspects of what is seen in the field.

Wildland fire spread is typically described as a function of fuel, weather, and topography. Therefore, an understanding of how these parameters affect the flame dynamics can help to close the gap on our understanding of the flame spread process. In particular, wind and slope are key contributors to flame spread. Steep slopes and high winds are often associated with extreme wildland behavior where flames spread faster and more erratically. As a fire spreads up a slope, the natural buoyancy of the hot gases allows flames to extend over a larger portion of unburnt fuel, accelerating the flame front in the upslope direction. Wind similarly tilts the flame forward which causes increased rates of heating and flame spread.

Although empirical models of the influence of slope and wind have been developed (Rothermel 1972), flame dynamics is still an active area of study. If fine fuels are affected by intermittent heating processes, time-dependent movements of the flame will affect heating that drives flame spread. The flames three-dimensional structure is also important to understanding this behavior, however this has yet to be fully characterized. 
This work will first review a number of studies by the authors aimed at characterizing flame structures associated with wind-driven and inclined flame spread using stationary burners. Using stationary fires, the fire's movement and behavior with respect to the wind and slope can be investigated in a consistent manner, separated from fuel ignition effects. Measurements of the flame structure and its effects in space and time are made using image analysis and heat-flux and temperature profiles of the flame under both wind and slope. While the flames used in these laboratory experiments are smaller and less turbulent than those found in a real wildland fire, their structure still retains similar features, in particular the interaction between momentum and buoyancy which governs liftoff of the flame (Quintiere 1989). Because these features are critically important to fire spread, careful study at this small scale may still help inform larger-scale work performed in the future. Results are also presented for new work focused on time-dependent movements of the flame. Previous work from the authors did not correlate these downstream frequencies which are important for intermittent heating of fine fuels, however a new relationship is presented here which provides more insight into the controlling processes of these forward movements.

\section{Review of Previous Work}

Fire science has long used stationary fires to help understand forward heating effects in concurrent flame spread (de Ris et al. 1975; Ahmad and Faeth 1979; Quintiere et al. 1986). A review of previous work on flow and geometry effects on concurrent flame spread was recently given in (Gollner et al. 2017). The focus of the review here will be on work by the authors related to stationary burners and wildland fires.

Gorham et al. studied the behavior of stationary fires using both gas burners and liquid fuel-soaked wicks under forced flows and inclined geometries (Gorham et al. 2014). A mechanism of instability was found in these flows, namely the formation of streamwise streaks, later forming spanwise "peaks and troughs" which were found to be similar to other fluid dynamic instabilities. An analysis was also made on forward movements of the flame using a level-set method on video images. A StrouhalFroude relationship was found to roughly predict the maximum frequency of forward pulsations based on fire size and wind speed. More importantly, PDF functions of the flame were found to represent the intermittent movement of the flame, which resulted in further extensions at larger fire sizes and higher wind speeds. The flame length was also found to be related to the nondimensional fire size, however no correlations were found to adequately scale inclined flames. These results were scaled and included with larger scale studies in (Finney et al. 2015)

Further study of instabilities observed in flames was carried out by (Miller, Finney, et al. 2017; Miller, Tang, et al. 2017; Miller et al. 2018). In Miller, Tang, et al. (2017) a small-scale line burner was used in crossflow to track the growth and development of a laminar streak entering a flame. It was found that a shear instability most likely induced perturbations before the flame was present and lead to the appearance of stream wise streaks in the flame. However, the growth of these structures was eventually dominated by a Rayleigh-Taylor instability. In Miller et al. $(2017,2018)$ this work was extended using hot plates and a liquid wick to track the merging and growth of flow structures. It appeared that these initial streaks grow into larger-scale structures (peaks and troughs) which then dominate some forward flame motions. In (Finney et al. 2015), these structures were found to be important in fire spread because they break up the flame front and allow for forward bursts or pulsations of flame to move forward and provide convective heating and ignition for fine fuel particles.

Tang et al. (Tang, Gorham, et al. 2017; Tang, Miller, et al. 2017) performed two detailed studies on line fires under a wind field. First, the attachment length downstream of the burner and profiles of heat fluxes were presented. These quantities were correlated using a Richardson number, comparing the buoyancy produced from the flame vs. momentum from the oncoming wind, which described liftoff of the flame from the surface. Correlations were provided that collapsed heat fluxes to the 
downstream fuel surface both within the attached region and downstream liftoff or plume region. Tang, Miller, et al. 2017 later extended this work to investigate forward pulsations of the flame following the work of Gorham et al. (2014). Correlations for the maximum frequency location were found and the work from Gorham et al. (2014) extended to different fire sizes, however a full description of the forward movement of flames was not presented.

\section{Experimental Setup}

Forced-flow experiments were performed on a specially-designed $30 \mathrm{~cm}$ cross-section wind tunnel. This laminar blower was designed and built for uniform forced-flow combustion experiments. The wind velocity from the tunnel can be as high as $6 \mathrm{~m} / \mathrm{s}$, with turbulence intensities controlled below $2 \%$ for the velocities of interest (Singh and Gollner 2015). The experimental platform was placed immediately following the outlet of the exhaust tunnel. A sand-filled gas burner was used, with a 10 $\mathrm{cm}$ deep sand-filled plenum and a $25 \times 5 \mathrm{~cm}$ surface. The top surface of the burner was mounted flush with a sheet of ceramic fiber insulation board placed at the center of the blower outlet. The insulation board, with dimensions of 90 (length) $\times 45$ (width) $\times 10$ (height) $\mathrm{cm}^{3}$, provides a quasi-adiabatic surface for downstream fire contact during experiments. Propane from a gas cylinder was passed through a programmable flow meter to provide a steady flow rate of fuel during experiments. Three different fire heat release rates, $6.3,7.9,9.5 \mathrm{~kW}$, were used during the experiments. The wind velocity is varied from about 0.8 to $2.5 \mathrm{~m} / \mathrm{s}$. High-speed videography was used to capture digital images of the flame from the side view. The camera used was high-speed Nikon DX, recording at 250 frames per second at a pixel resolution of $1000 \times 720$. The experimental setup is shown in Fig. 1.

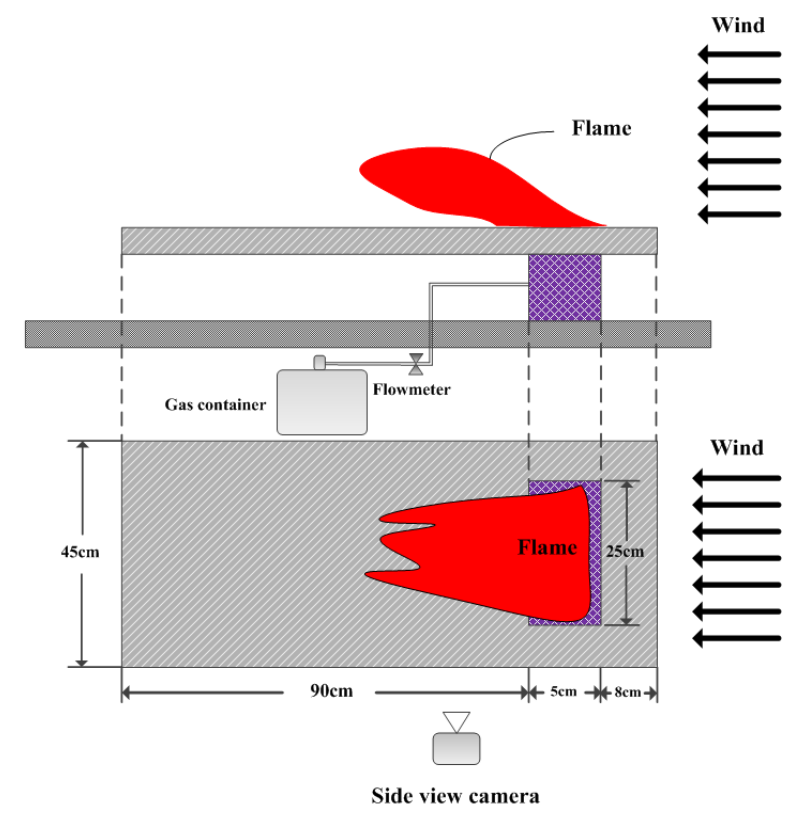

Figure 1 - Experimental setup used for forced-flow studies from (Tang, Gorham, et al. 2017).

\section{Results}

Experiments have been conducted over a variety of different wind-driven flame configurations and several inclined configurations, as reviewed above. In wind-driven studies, forward pulsation of the flame occurs as a result of the competition between the momentum provided by the ambient flow and upward buoyancy provided by the flame. A scaling analysis reveals that the forward pulsation frequency can be correlated with the Froude number (wind momentum over inertial force) and $Q^{*}$ (nondimensional buoyancy), 


$$
f=12.9\left(\frac{F r^{1 / 2}}{Q^{* 1 / 4}}\right)^{0.7}=12.9\left(\frac{F r}{Q^{* 1 / 2}}\right)^{0.35}
$$

Both the origin of structures observed on the fire line, e.g. streaks and peaks and troughs, and their role in influencing some of the macroscopic properties of the flow have also been examined. Streaks have been reproduced and characterized via experiments on stationary heated strips and liquid and gas-fueled burners in laminar boundary layer flows, providing a framework to develop theory based on both observed and measured physical phenomena. The incoming boundary layer is also established as the controlling mechanism in forming streaks, which are generated by pre-existing coherent structures, while the amplification of streaks was determined to be compatible with quadratic growth of Rayleigh-Taylor Instabilities, providing credence to the idea that the downstream growth of streaks is strongly tied to buoyancy. These local instabilities were also found to affect macroscopic properties of the flow, including heat transfer to the surface, indicating that a two-dimensional assumption may fail to adequately describe heat and mass transfer during flame spread and other reacting boundary layer flows.

Finally, new experiments on inclined surfaces with burners are been used to investigate flame attachment, where flames bend toward the surface accelerating heating and thus flame spread. The inclination also affects the formation of streaks and flame towers, suggesting these may play some role in the flame attachment process. This configuration continues to be studied, however preliminary results on attachment angle and heating will be presented.

\section{Acknowledgements}

This work was supported by the National Science Foundation (CBET-1554026) and a collaborative agreement with the USDA Forest Service (13-CS-11221637-124).

\section{References}

Ahmad T, Faeth GM (1979) Turbulent wall fires. Symposium (International) on Combustion 17, 1149 1160. doi:10.1016/S0082-0784(79)80109-5.

Alvares NJ, Blackshear PL, Kanury MA (1970) The Influence of Free Convection on the Ignition of Vertical Cellulosic Panels by Thermal Radiation. Combustion Science and Technology 1, 407-413. doi:10.1080/00102206908952220.

Baines PG (1990) Physical mechanisms for the propagation of surface fires. Mathematical and Computer Modelling 13, 83-94. doi:10.1016/0895-7177(90)90102-S.

Fang JB, Steward FR (1969) Flame spread through randomly packed fuel particles. Combustion and Flame 13, 392-398. doi:10.1016/0010-2180(69)90108-4.

Finney M a., Cohen JD, Forthofer JM, McAllister SS, Gollner MJ, Gorham DJ, Saito K, Akafuah NK, Adam B a., English JD (2015) Role of buoyant flame dynamics in wildfire spread. Proceedings of the National Academy of Sciences 112, 9833-9838. doi:10.1073/pnas.1504498112.

Gollner MJ, Miller CH, Tang W, Singh AV (2017) The effect of flow and geometry on concurrent flame spread. Fire Safety Journal. doi:10.1016/j.firesaf.2017.05.007.

Gorham DJ, Hakes R, Singh AV, Forthofer J, Cohen J, McAllister S, Finney MA, Gollner MJ (2014) Studying Wildfire Spread Using Stationary Burners. InXavier Viegas D (ed) 'VII Int. Conf. For. Fire Res.', Coimbra, Portugal.(Coimbra, Portugal)

Martin S (1965) Diffusion-controlled ignition of cellulosic materials by intense radiant energy. Symposium (International) on Combustion 10, 877-896. doi:10.1016/S0082-0784(65)80232-6. 
Miller C, Finney MA, McAllister S, Sluder E, Gollner MJ (2017) Investigating coherent streaks in wildfires via heated plates in crosswind. Fire Safety Journal 91,. doi:10.1016/j.firesaf.2017.03.035.

Miller CH, Tang W, Finney MA, McAllister SS, Forthofer JM, Gollner MJ (2017) An investigation of coherent structures in laminar boundary layer flames. Combustion and Flame 181,. doi:10.1016/j.combustflame.2017.03.007.

Miller CH, Tang W, Sluder E, Finney MA, McAllister SS, Forthofer JM, Gollner MJ (2018) Boundary layer instabilities in mixed convection and diffusion flames with an unheated starting length. International Journal of Heat and Mass Transfer 118,. doi:10.1016/j.ijheatmasstransfer.2017.11.040.

Quintiere JJG (1989) Scaling applications in fire research. Fire Safety Journal 15, 3-29. http://linkinghub.elsevier.com/retrieve/pii/0379711289900453.

Quintiere J, Harkleroad M, Hasemi Y (1986) Wall flames and implications for upward flame spread. Combustion Science and Technology 48, 191-222. http://www.bfrl.nist.gov/866/heatflux/pubs/Quintiere_Wall_Flames.pdf.

de Ris JL, Orloff L, Ris J De, Orloff L, de Ris JL, Orloff L (1975) The role of buoyancy direction and radiation in turbulent diffusion flames on surfaces. Symposium (International) on Combustion 15, 175-182. doi:10.1016/S0082-0784(75)80295-5.

Rothermel R (1972) A mathematical model for predicting fire spread in wildland fuels. (INT-115) doi:http://www.snap.uaf.edu/webshared/JenNorthway/AKFireModelingWorkshop/AKFireModeli ngWkshp/FSPro Analysis Guide References/Rothermel 1972 INT-115.pdf.

Rothermel R, Anderson H (1966) Fire spread characteristics determined in laboratory, US Forest Service Research Paper INT-30. http://www.fs.fed.us/rm/pubs_int/int_rp030.pdf.

Singh A V., Gollner MJ (2015) Estimation of local mass burning rates for steady laminar boundary layer diffusion flames. Proceedings of the Combustion Institute 35, 2527-2534. doi:10.1016/j.proci.2014.05.040.

Tang W, Gorham DJ, Finney MA, Mcallister S, Cohen J, Forthofer J, Gollner MJ (2017) An experimental study on the intermittent extension of flames in wind-driven fires. Fire Safety Journal 91,. doi:10.1016/j.firesaf.2017.03.030.

Tang W, Miller CH, Gollner MJ (2017) Local flame attachment and heat fluxes in wind-driven line fires. Proceedings of the Combustion Institute 36,. doi:10.1016/j.proci.2016.06.064. 


\title{
Understanding unburned patches patterns in extreme wildfire events: a new approach
}

\author{
Fantina Tedim ${ }^{1,2}$; Dominic Royé ${ }^{1,3}$; Christophe Bouillon ${ }^{4}$; Fernando J.M. Correia ${ }^{1}$; Vittorio \\ Leone 5 \\ ${ }^{1}$ University of Porto, Faculty of Arts, Via Panorâmica s/no 4150-564 Porto, Portugal, \\ \{ftedim@letras.up.pt*\} \\ ${ }^{2}$ Charles Darwin University, Darwin, Australia \\ ${ }^{3}$ University of Santiago de Compostela, Faculty of Geography and History, Praza da \\ Universidade, $n^{\circ}$ 1, 15782 Santiago de Compostela, Spain, \{dominic.roye@usc.es\} \\ ${ }^{4}$ Irstea, RECOVER research unit, Mediterraneans Ecosystems and risks team, Aix-en-Provence, \\ France, \{christophe.bouillon@irstea.fr\} \\ ${ }^{5}$ University of Basilicata (retired), Department of Crop Systems, Forestry and Environmental \\ Sciences, Via dell'Ateneo Lucano 10, I-85100 Potenza, Italy, \{vittorioleone40@gmail.com\}
}

\begin{abstract}
Burned area is a rather weak descriptor of wildfire activity, since it is not well correlated neither with fire severity and ecological effects, nor with socio-economic impacts. It ignores the heterogeneity in severity distribution within fire boundaries, and the existence of unburned patches (UPs). The purpose of this paper is to provide a first understanding of the trends and spatial patterns and characteristics of UPs within wildfires in Portugal as well as their explanatory variables. This research adopts a special focus on extreme wildfire events (EWEs), that represent a huge threat to society because of their high intensity, erratic behavior, and strong spot activity. Previous studies on UPs mainly followed an ecological approach, whereas our research is mainly focused on understanding how the area of wildland-urban interface (WUI) contributes to create UPs inside an EWE. This focus is of paramount importance to assist prevention and mitigation, in order to increase the safety of people and assets, in a context of more extreme fire environments. We selected as case study the Pedrógão Grande wildfire that occurred in 2017. This event is one of the most disastrous fires ever occurred worldwide, with the highest number of fatalities in a single event. We hypothesize that even in this category of fires it is possible to find UPs. Based on the wildfire perimeter dataset available, we estimated the UPs by the application of geometrical operations. The mapping of WUI was carried out by methods developed by IRSTEA and available in the Ruimap software. The WUI map was created by a combination of housing configuration and vegetation characterization or land use map. To relate the formation of UPs and the Pedrógão Grande fire intensity we used the shape file of the isochrons of fire spread provided by the Independent Technical Commission (CTI) created by the Portuguese Parliament to investigate 2017 wildfires. We created an UPs georeferenced database for Pedrógão Grande wildfire comprising several variables (e.g. size, land use characteristics, aspect, slope). Although the trend of UPs in Portugal from 1975 to 2017 is presented, the most innovative findings are related with the study case of Pedrógão Grande and the patterns and characteristics of UPs within this fire perimeter. This wildfire burned with different intensities and rate of spread but since the first thirty minutes after the ignition, fire burned above the extinction capacity (CTI 2017). In this EWE, unburned area within fire boundary were identified irrespective of fire intensity values, even in the most critical period when fire burned with intensity up to $60,000 \mathrm{kWm}^{-1}$. Our findings corroborate the previous studies but others are in contrast with what has been published so far. One of the most interesting findings is that the largest UPs were formed in the interval of maximum intensity and are of the Mix UPs type. The implications of the findings of this research in land and wildfire management are exploited.
\end{abstract}

Keywords: Extreme wildfire events (EWE), fire intensity, Portugal, unburned patches (UPs), wildland-urban interface (WUI).

Advances in Forest Fire Research 2018 - Page 700 


\section{Introduction}

The number of fire events and burned area (BA) are the most widely used metrics to characterize wildfires and evaluate their impacts. BA is a rather weak descriptor of wildfire activity, since it is not well correlated neither with fire severity and ecological effects (Keane et al. 2009; Lutz et al. 2011; Kolden et al. 2012; Birch et al. 2014; Tedim et al. 2015) nor with socio-economic impacts (Gill and Moore 1998; Tedim et al. 2018). In addition, it ignores the heterogeneity in severity distribution, and the existence of unburned patches (UPs) within a fire perimeter, also labeled unburned islands, fire islands, refugia, or inclusions; they are an important component of the burned mosaic (Kolden et al. 2012; Kolden et al. 2015; Krawchuk et al. 2016)

UPs can be considered a response of landscape matrix to a wildfire event, and can help to retrace its dynamics, because they represent a physical evidence of local changes of fire behavior with heterogeneous consumption of fuels (Meddens et al. 2018), as influenced by several biophysical and anthropic drivers and their complex interactions. UPs contribute to create landscape heterogeneity and can thereby influence the distribution and behavior of subsequent wildfires. UPs "are landscape legacies that can provide an essential environment for species sensitive to fire, and support populations that contribute to the reassembly of biotic communities after fire" (Krawchuk et al. 2016: p. 2). However, UPs size, shape, frequency, and spatial patterns are usually not considered in current fire regime descriptions (Kolden and Weisberg 2007; Kolden et al. 2012; Meddens et al. 2018).

Research on UPs has mainly followed an ecological approach, focusing on their role: as fire refugia for fire-sensitive organisms; as seed sources facilitating the regeneration of adjacent burned areas, biodiversity conservation, increase in a post fire landscape, reduction of detrimental fire impacts on hydrology and erosion (Meddens et al. 2018); and in ecological trajectories of succession, ecosystem dynamics, and wildfire risk (Kolden et al. 2012).

The pattern of UPs distribution inside fire boundaries can be very variable (Kolden et al. 2012; Tedim et al. 2013; Leonard et al. 2014). Research has shown that the number of UPs significantly decreases with increasing fire size and severity (Kolden et al. 2012). UPs size increases with the extent of fire boundary, but patches decrease in density, whereas an increase in fire intensity produces smaller size Ups (Kolden et al. 2012; Kolden et al. 2015; Meddens et al. 2018). The ecological effects of UPs are determined by size (Eberhart and Woodard 1987), and edge effect (Slik et al. 2011). Enhancing the knowledge of UPs characteristics and the factors that contribute to their formation is crucial to produce accurate modeling, predict the efficacy of localized fire prevention and mitigation actions (Corona et al. 2014; Vaillant et al. 2016; Meddens et al. 2018).

The purpose of this paper is to provide a first understanding of the spatial patterns and characteristics of UPs in extreme wildfire events (EWEs), as well as their explanatory variables in Portugal. EWEs are pyro-convective phenomena overwhelming the capacity of control (fireline intensity $\geq 10,000 \mathrm{kWm}^{-1}$ ), exhibiting erratic and unpredictable fire behavior and spread; they can likely cause relevant negative socio-economic and environmental impacts (Tedim et al. 2018). This category of wildfires represents a huge threat to society but we hypothesize that even in occasion of these events it is possible to find UPs. Although recognizing the importance of the ecological role of UPs, our research is also focused on understanding how the area of wildland-urban interface (WUI) contributes to create UPs in case of EWE, which is of paramount importance to assist prevention and mitigation, in order to increase the safety of people and assets in a context of more extreme fire environments.

In the first part of this paper, the trend of UPs distribution and size for the period from 1975 to 2017 in Portugal is analyzed. In the second part, a detailed analysis is provided for the UPs occurred in the 2017 wildfire season. In the third part, the spatial pattern and characteristics of UPs in the Pedrógão Grande wildfire are presented, which is one of the several EWEs that occurred in 2017, in Portugal. 


\section{Data and methods}

\subsection{Study area and fire history}

The 2017 wildfire season was the worst one experienced by Portugal so far, since about 465,567ha were affected, i.e. about $5 \%$ of the country surface, also provoking 112 fatalities.

In Figure 1 the location of the case study of Pedrógão Grande wildfire is presented. This event is one of the most disastrous fires ever occurred worldwide, with the highest number of fatalities (66) in a single event. Pedrógão Grande fire spread between $17^{\text {th }}$ and $20^{\text {th }}$ of June, i.e. out the usual fire season, and burned 28,624.7 ha. This wildfire reached intensities of $60,000 \mathrm{kWm}^{1}$ and during 10 minutes of its critical phase assumed a rate of spread (ROS) of $15 \mathrm{~km} / \mathrm{h}$, provoking most of the fatalities; it exhibited extreme phenomena of vorticity and of short and long-distance spotting activity (ADAI/LAETA 2017; CTI 2017). Its estimated magnitude was $568 \mathrm{GW}$, a very high value for Portugal, although it represented just one third of the potential magnitude in wildfires with higher intensity and ROS that already occurred in eucalyptus forests in Australia (CTI 2017). About 63\% of the fire perimeter burned in the first 24 hours, and 4,459 ha burned in a single hour.

In the area affected by the Pedrógão Grande fire, forests of Eucalyptus and Pinus pinaster dominate, with a high spatial continuity; the agricultural areas are small and located around the villages; grazing activity is low (1-3 livestock units per ha) (CTI 2017).
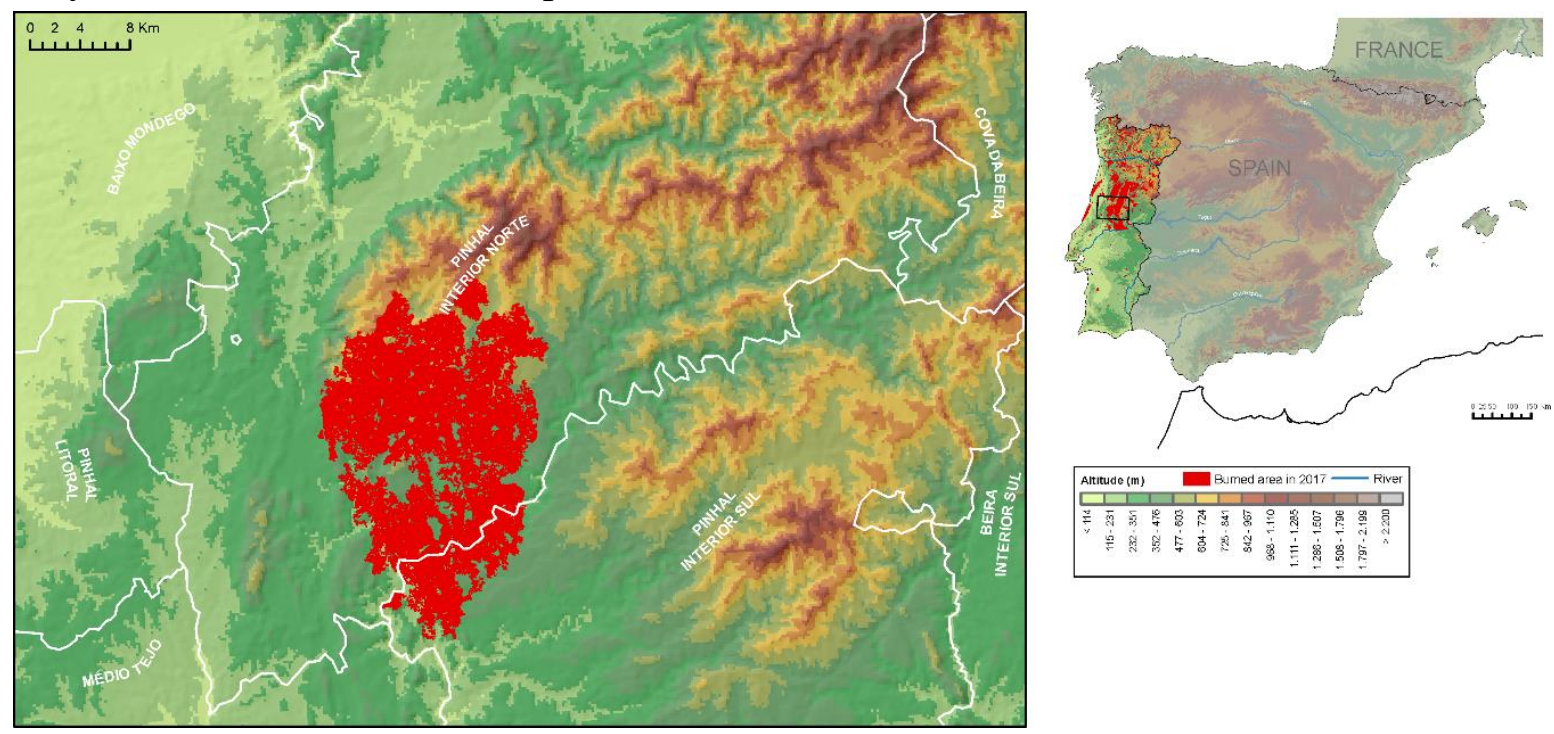

Figure 1 - Location of Portugal and the study area of Pedrógão Grande

\subsection{Data sources and processing}

\subsubsection{Wildfire dataset}

For the period 1975 to 2017, we used a dataset of wildfire perimeters polygons. To allow the comparability of our research with the findings of other studies about unburned areas (e.g., Meddens et al. 2018) we considered only the perimeters $\geq 405$ ha.

The data of burned area polygons for the period 1975-1989 were produced by the School of Agriculture (ISA - Instituto Superior de Agronomia). For the following years until 2017, we used the cartographic data available in the website of the Institute of Nature Conservation and Forests (ICNFInstituto de Conservação da Natureza e das Florestas). Furthermore, for the year 2017, we used also the dataset produced by ISA and provided by the Independent Technical Commission (CTI- Comissão Técnica Independente - created by the Portuguese Parliament to analyze the wildfire events occurred in 2017). Fire perimeters polygons considered in these datasets do not necessarily correspond to single 
wildfires, since a fire perimeter can be constituted by the coalescence of more than one fire or be a result of a single wildfire.

The two mentioned datasets used different procedures for the burned area detection and reveal different number of UPs, which shows that the mapping of fire perimeter can produce different numbers of UPs for the same event and, consequently, influences the consistency of the results. For instance, in the dataset provided by ICNF (accessed on April $3^{\text {rd }}$, 2018) 4,793 UPs were listed, of which 876 have an area $\geq 1$ ha (18.3\%). The dataset provided by the ISA, allowed the identification of 31,470 UPs, of which $8,172(25.9 \%)$ are $\geq 1$ ha. The same differences were obtained for the Pedrógão Grande wildfire (Fig. 2). While one of the datasets identifies just 45 UPs $\geq 1$ ha, from the other we obtained 522 UPs $\geq 1$ ha out of 2,039 UPs.

\subsubsection{Unburned areas database}

Unlike Meddens et al. (2016), who used random Forest and classification trees in order to separate burned from unburned locations, we estimated the unburned area by the application of geometrical operations. It means the difference between a rectangular polygon of the dimensions of each fire perimeter and the polygon of burned area, returning a new geometry only with the unburned area, excluding polygon rests which were outside the fire boundary.

a)

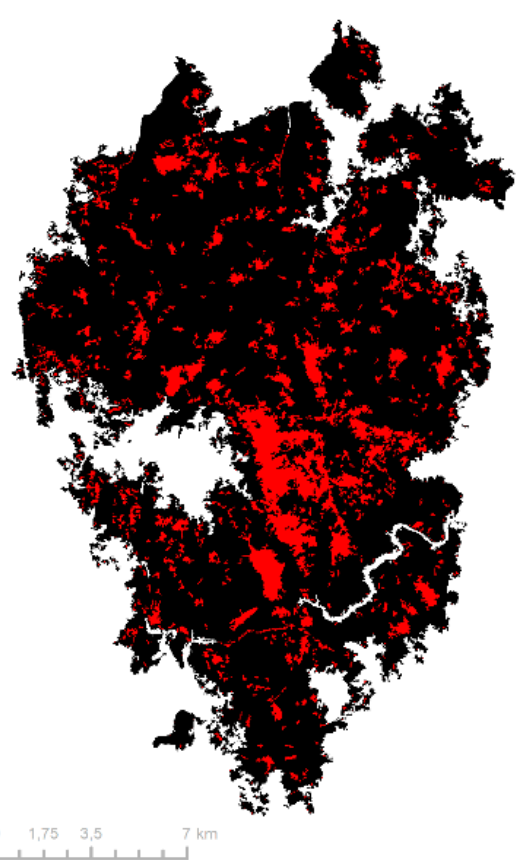

b)

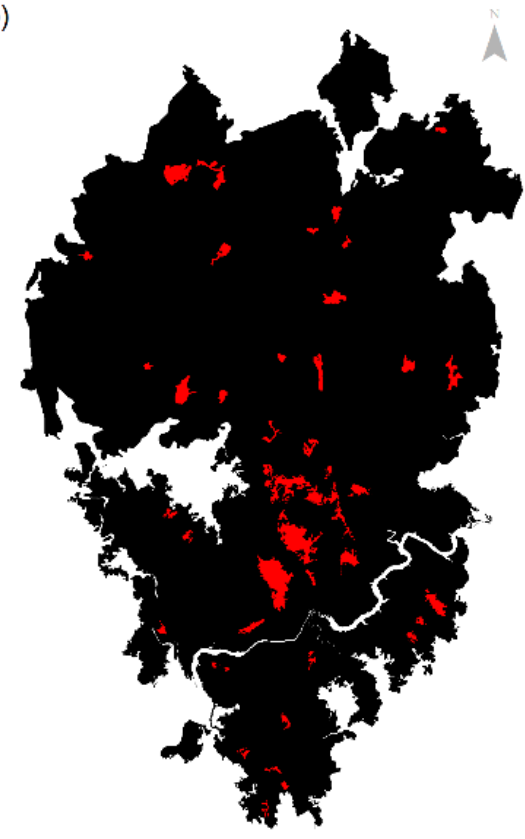

Figure 2 - The UPs in Pedrógão Grande wildfire using two different sources: a) ISA; b) ICNF

For Pedrógão Grande fire we also used the shape file of the isochrons of fire spread provided by CTI.

\subsubsection{WUI delimitation at national and local scale}

The mapping of WUI was carried out by the methods developed by the Institut National de Recherche en Sciences et Technologies pour l'Environnement et l'Agriculture (IRSTEA) and available in the Ruimap software (Sirca et al. 2017). The aim is to locate the different WUI types throughout the country in order to have an idea of WUI spatial patterns.

On the global scale, the WUI is defined by a radius of $400 \mathrm{~m}$ around houses located at less than 200 $\mathrm{m}$ from forests or shrubland. The mapping of the WUI for all Portugal using the global scale method was made on the Corine Land Cover for the year 2006, as more recent data on land use were not available. The pixel resolution is $100 \mathrm{~m}$. The WUI for the area affected by the Pedrógão Grande fire 
was also mapped using the local scale method developed in a French context by IRSTEA. In this case the WUI is defined by a radius of $100 \mathrm{~m}$ around each building located at a distance of less than $200 \mathrm{~m}$ from forests or shrublands.

Four housing configuration classes were created: isolated, scattered, dense clustered and very dense clustered housing. The WUI map was created by a combination of housing configuration and vegetation characterization or land use map; the result is a map of twelve WUI classes. For the local scale WUI mapping method applied to Pedrógão Grande fire, the input data for the buildings are from Copernicus Emergency Management Service, and the level 5 land use map of Portugal from DireçãoGeral do Território (DGT). The pixel resolution is $2.5 \mathrm{~m}$.

\subsection{UPs data analysis}

\subsubsection{National trends and spatial pattern}

The statistical analyses and illustration of the results were carried out in the $\mathrm{R}$ (3.5.) statistical environment (Team 2018), including the packages \{ggplot 2$\}$ for the graphics, the $\{s f\}$ for geometrical operations and the \{trend\} package for the Man-Kendall statistics. The linear trends in this study were estimated using the Mann-Kendall's tau test at the 95\% significance level (Libiseller and Grimvall 2002). The Man-Kendall statistic test is a rank-based nonparametric test, which has the advantage to be robust to outliers and does not assume an underlying probability distribution. For testing the correlation between different variables, the nonparametric Spearman's rank correlation coefficient was used at a 95\% significance level (Best and Roberts 1975). For the national scale, we combined the Portugal WUI with the UPs of the year 2017. The same procedure was used for Pedrógão Grande fire.

\subsubsection{UPs analysis in Pedrógão Grande fire study case}

As UPs are spatial entities, we used a pixel-based approach to investigate the factors contributing to UPs. The characterization of UPs was based on their number, size, and proportion within fire perimeter, density, and Euclidian distance using the method of nearest neighbor. Hence, we used a Digital Elevation Model (DEM) with a resolution of $25 \mathrm{~m}$, which enables us to estimate several terrain metrics: aspect, slope, elevation, Topographic Position Index (TPI) (Guisan et al. 1999) and the Terrain Ruggedness Index (Riley 1999). For the last two indices, a search radius of $1000 \mathrm{~m}$ and no distance weighting were considered. All the metric calculations were conducted in the SAGA-GIS software (version 6.3.0) by using the $\mathrm{R}$ package $\{R S A G A\}$. The morphometric indices describe different aspects of the terrain, which may play an important role in the explanation of the formation of UPs. In detail, TPI compares the elevation of each cell to the mean elevation of a specified neighborhood (identification of ridges and valleys); and TRI is the difference of elevation between a cell and the mean of an 8-cell neighborhood of surrounding cells.

The created UPs database includes several variables including the classification of UPs by their composition: Vegetation UPs constituted only by vegetation or land without any buildings; WUI UPs constituted only by WUI; and Mix UPs where WUI and vegetation areas coexist. Base map and Google Earth, both images from 2016, were used to verify the land use of each UPs, integrated by fieldwork.

\section{Results}

\subsection{National trends}

From 1975 to $2017 ; 894$ fire perimeters $\geq 405$ ha were found. There is a high interannual variability in the number, with maximum values recorded in 2005 (143 perimeters), 1998 (116), 2003 (90), and 2017 (89) (Fig. 3a). The lowest number of fire perimeters with the mentioned size occurred in 1988 and 1997. Out of the observed 894 fire perimeters, just 305 (34.1\%) contained UPs in number of 6,413, with a high interannual variability (Fig. 3b). The years with the highest number of UPs are 2017, 2013, and 2003 (Fig. 4). Since 2000, fire perimeters $\geq 405$ ha with UPs can be found every year. 
a)

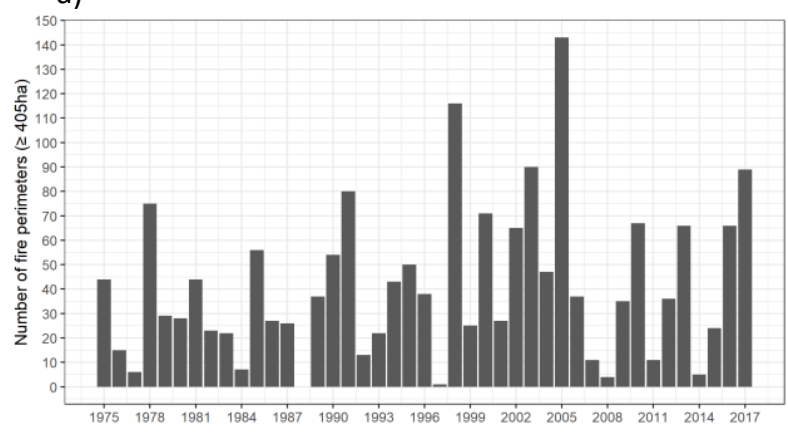

b)

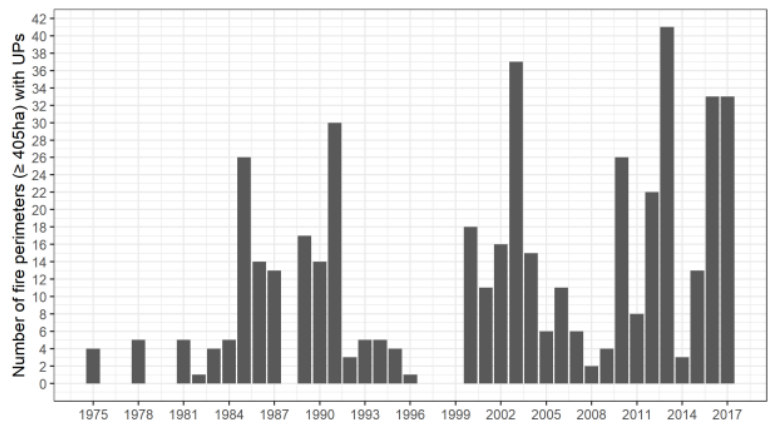

Figure 3 - a) Number of fire perimeters $\geq 405$ ha, from 1975 to 2017 , b) Number of fire perimeters $\geq 405$ ha with UPs, from 1975 to 2017 (Source: Data from ISA, ICNF)

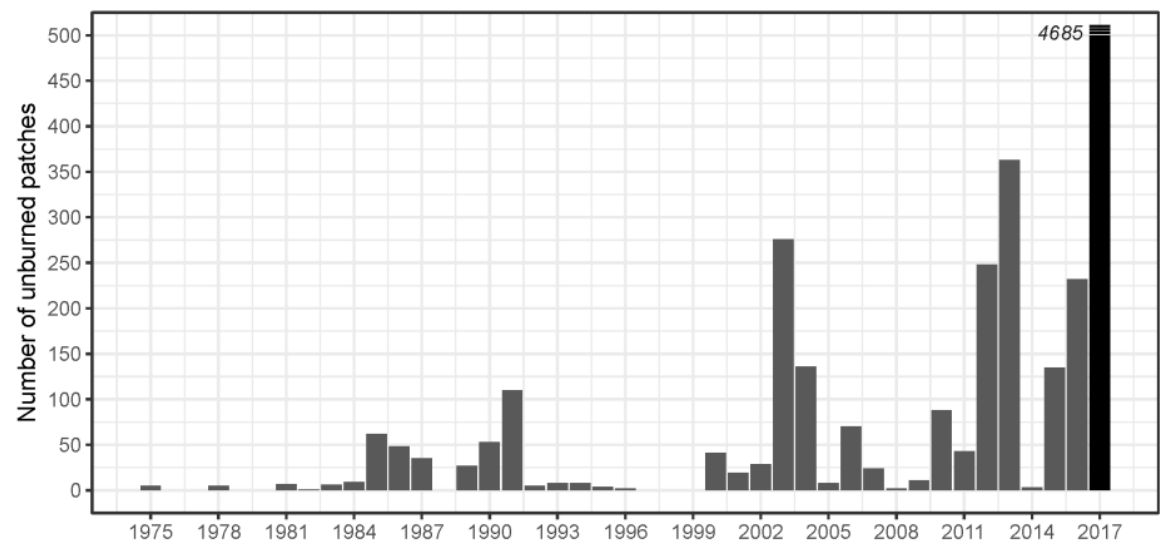

Figure 4 - Number of UPs in fire perimeters $\geq 405$ ha (Source: Data from ISA, ICNF).

For the whole period, 1975 to 2017, the area of UPs represented a proportion of 4,2\% within fire perimeters, with a range between $0.005 \%$ (for 2005) and $8.0 \%$ (for 1984, a year with very low BA in fires perimeters $\geq 405$ ha). Mann-Kendall trend test reveals positive trends for all the variables considered in the analysis: BA (tau $=0.2981 ; p$-value $=0.0056)$; UPs area (tau $=0.1901 ; p$ value $=0.0777)$; number of UPs ( $\operatorname{tau}=0.4021 ; p$-value $=0.0002)$; UPs density per 100 ha (tau=0.4354; $p$-value $\left.=5.031 \mathrm{e}^{-05}\right)$, and mean distance between UPs ( $\operatorname{tau}=0.3140 ; p$-value $\left.=0.0041\right)$. Spearman's rank correlation reveals a strong and statistically significant positive correlation between BA $\geq 405$ ha and UPs area inside those perimeters ( $r h o=0.8087 ; p$-value $=2.648 \mathrm{e}^{-07}$ ) $($ Fig. 5a). However, 2003, 2017 and 2005 are clearly highlighted as outliers: the first two exhibit a high number of BA and UPs area, whereas 2005 exhibits the third largest BA in the period, but a very low UPs area inside the perimeters $\geq 405$ ha. A strong positive and statistically significant correlation is observed between BA and the number of UPs $\left(r h o=0.8582 ; p\right.$-value $\left.=4.386 \mathrm{e}^{-11}\right)$ (Fig. $\left.5 \mathrm{~b}\right)$. The mean size of UPs ranges between 147.80 ha (1982) and 0.24 ha (2005). 
a)

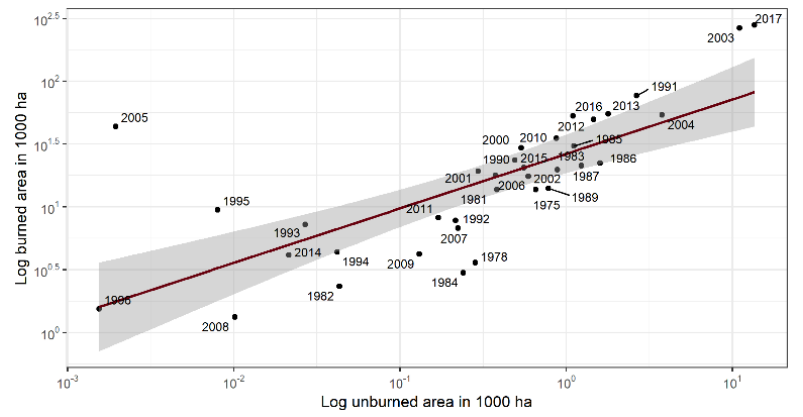

b)

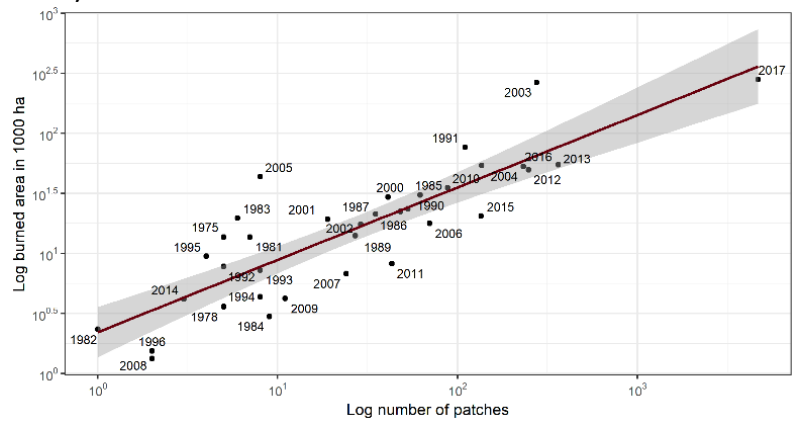

Figure 5 - Relationship between (a) BA and UPs area and (b) between BA and the number of UPs for fire perimeters $\geq 405$ ha

\subsection{The 2017 wildfire season in Portugal}

In the 2017 wildfires season in Portugal we identified 31,470 UPs occupying 55,591.4 ha within the BA. Most of the UPs had a very small size, and only 8,172 of them had a size $\geq 1$ ha. The mean size of the UPs is 1.8 ha (Q75\%: 1.0 ha; Q95\%: 4.8 ha; Q99\%: 23.7 ha), but within fire perimeters there are UPs larger than 100 ha, that is the threshold of large fires in Portugal. In fact, 63 UPs with $>100$ ha were found; the largest one reached 2,134.6 ha. The UPs density per 100 ha for all the fires considered in 2017 is 6.8 . Wildfires did not only affect forest, shrubs and agricultural areas, but also $28.2 \%$ of the WUI of Portugal. Only 5.3\% of WUI inside the 2017 fire perimeters was not affected by fires and constitutes UPs. About $44.1 \%$ (24,5 ha) of the area of UPs falls in WUI; $40.2 \%$ of it is characterized as isolated housing surrounded by forested area, followed by isolated housing surrounded by agricultural or sparse vegetation (34.2\%), and scattered housing surrounded by forested area (10.9\%) (Table A1, in Annex). UPs occurring in areas characterized as dense clustered housing, represented only 445 ha (1.8\% of UPs area within fire perimeters), a very low value, when compared with the other types of WUI.

\subsection{Pedrógão Grande wildfire}

Within Pedrógão Grande fire perimeter, there are 3,833.0 ha of WUI (Fig. 6) but just 1,705.9 ha did not burn, i.e. the $44.5 \%$ of the total affected area and about $13.5 \%(3,850.5$ ha) of the total area within fire perimeter is constituted by 2,039 UPs. The density of UPs per 100 ha in Pedrógão Grande fire is 0.4. Since 1,517 UPs (representing $74 \%$ of the number and with a surface of 231.6 ha) have $<1$ ha, we found 522 UPs $\geq 1$ ha representing an area of 3,618.9 ha. The largest UP has 751.4 ha. The mean size of the UPs is 1.9 ha, but if we consider only the UPs $\geq 1$ ha, the value increases to 6.9 ha. Using the nearest neighbor method, the mean distance between UPs is $197.77 \mathrm{~m}(\mathrm{SD}= \pm 1,459.7 \mathrm{~m}$; maximum distance $=18.3 \mathrm{~m}$; minimum distance $=15.4 \mathrm{~m}$ ).

\subsubsection{The UPs composition}

Most of the UPs (No. =1610) are vegetation UPs, but they only represent $29.1 \%$ of the unburned area. Most of area of the UPs (2,632.8 ha) belong to Mix UPs (No. =267) and 164 UPs (Area $=2.6 \%$ ) are WUI UPs. The vegetation UPs mainly have a size $>1$ ha. Only 5 of them have more than 10 ha. The largest UPs (>50 ha), with the exception of one with 61.7 ha that is a vegetation UPs, are Mix UPS. 


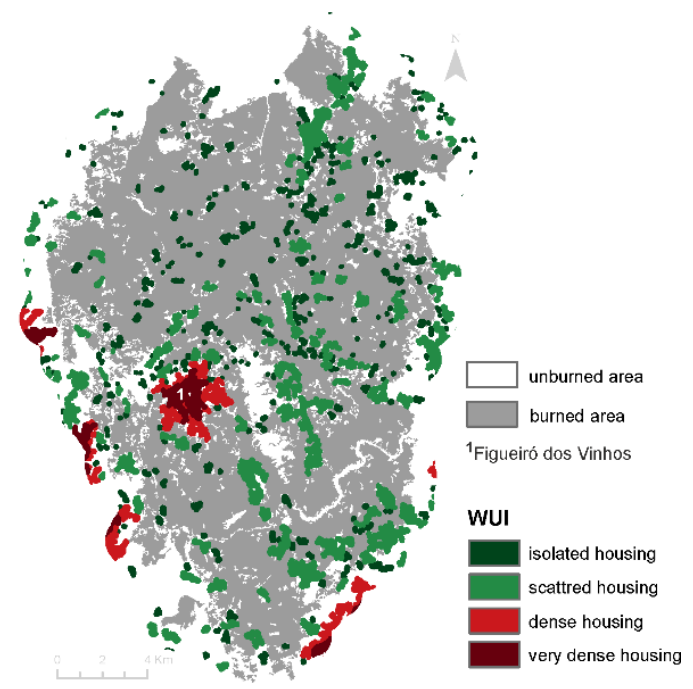

Figure 6 - The WUI within the Pedrogão Grande wildfire perimeter

\subsubsection{Terrain variables}

The terrain metrics used to explain the formation of UPs, reveal some differences between BA and UPs for all the descriptors (Table A2, in annex). Regarding the aspect, the distribution of BA and UPs seems typically bimodal, with the peaks for UPs at W and E (Fig. 7). The UPs distribution is more concentrated in some values of aspect than the one of BA. Making the analysis by the type and the size of UPs we can find that in all the situations most of the UPs are orientated to S and W (Table A3, in annex). In terms of elevation the two curves (Fig. 7) exhibit a similar mono-modal profile. The distribution of UPs seems more concentrated at lower elevation values with a lower SD $( \pm 113.20)$ than the BA. There are no significant differences in terms of size and type of UP. Regarding TRI, the BA curve presents a bimodal distribution with the two peaks with similar density but sharply right skewed. The curve of UPs is slightly different. The UPs are mostly located in lower level of TRI (level and nearly level). Although the TPI curves of BA and UPs seem almost overlapped, the latter exhibit more negative values which indicates the presence of valleys and gullies. In fact, most of the UPs $(1,038$ out of 2,039) are located in valleys. These results are corroborated by slope. It is evident that the distribution of UPs is more related with low values of slope. BA, on the contrary, are more associated with higher values of slopes and the curve exhibits some right skewness.

\subsubsection{UPs and wildfire intensity}

For the comparative analysis of the fire spread by time and the UPs location, we considered the time fire first arrived to the border of UPs. In many of the UPs fire spread around for less than 1 hour. In opposition, others faced the fire for many hours or saw it returning from another direction some hours after the first contact. The Pedrógão Grande fire burned with different intensities and ROS but since the first half an hour after the ignition, the fire burned above the extinction capacity (CTI 2017). In this EWE unburned area within fire boundary exists irrespective of fire intensity values. In the periods of higher intensity, it is possible to find a high number of UPs per hour (Fig. 8). On the $17^{\text {th }}$ of June between 19- 


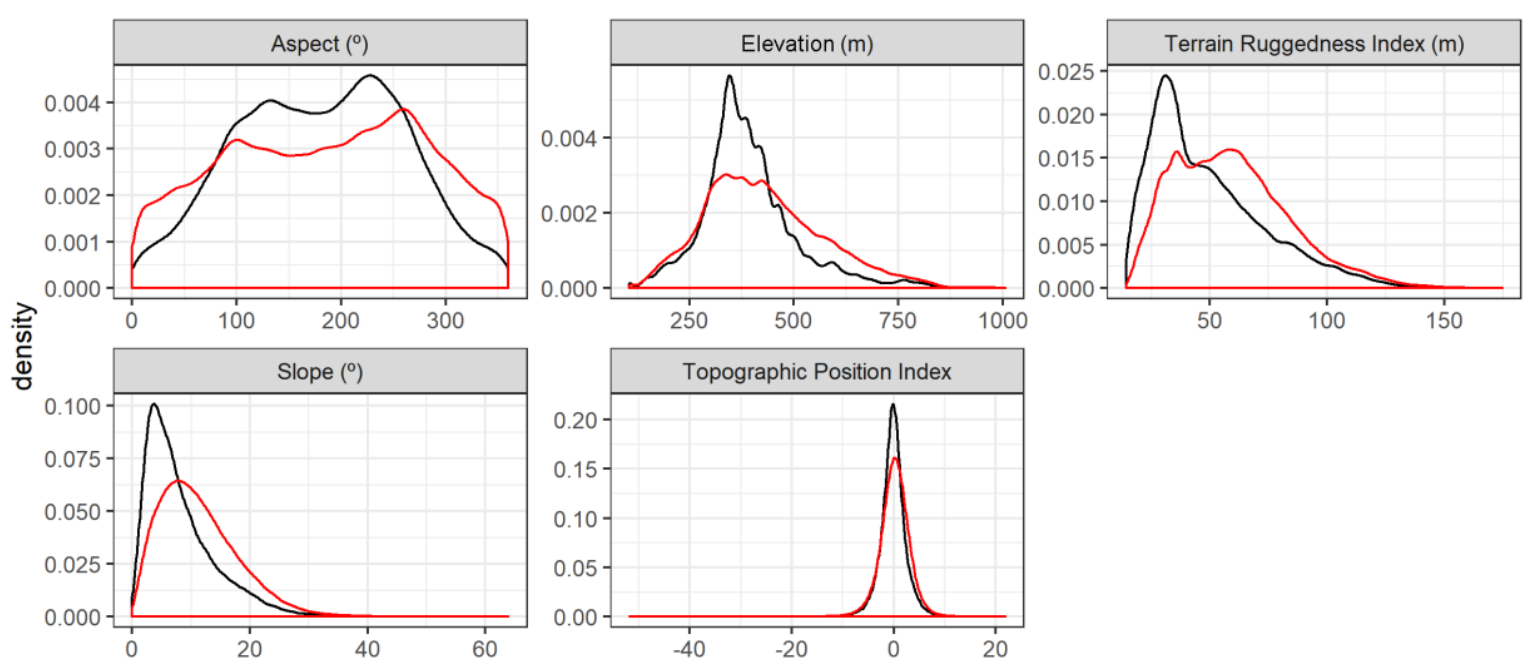

Figure 7- Probability density function of terrain metrics for BA and UPS (red line: BA; black line: UPS).

$20 \mathrm{~h}$, when fire burned with intensity up to $60,000 \mathrm{kWm}^{-1}$, we identified 82 UPs with a mean size of 6.0 ha. In the following hour, when the downburst occurred, the number of UPs reached 162 . However, in the latter period the extent of the BA was higher than in the former $(4,458.6$ ha of BA between 20 $21 \mathrm{~h}$, vs. 2,588.7 ha between 19-20 h). The density of UPs in the two mentioned intervals was respectively 31.6 and $27.5 \mathrm{UPs} / 100 \mathrm{ha}$. In the following day between 15 and $17 \mathrm{~h}$, when the fire intensity approached again to $40,000 \mathrm{kWm}^{-1}$, the number of UPs arrived to 255 (i.e. a mean of 127.5 per hour) and the UPs density assumed a value of 13.2 UPs/100 ha.

On the first day of the wildfire, in the interval between 19 and $21 \mathrm{~h}$, UPs with different sizes and characteristics occurred. The less representative was the WUI UPS (29.0 ha; mean size of the patches 0.97 ha) most of them with less than 1 ha. The maximum size was 6.7 ha. The Vegetation UPs were more numerous (137) most of them with $<1$ ha (83) and represented 117 ha (mean size of the patches 0.9 ha). The Mix UPs occupied 1,632.3 ha. They reached a larger size (mean size is 21.2 ha; maximum size 751.9 ha), as only 16 out of 77 have <1 ha; three of them have more than 100 ha. While in Vegetation and WUI UPS we could not find patches with more than 10 ha, in Mix UPs we found 21 with such size out of the 244 UPs defined for the interval between 19-21 h. Most of these UPs consist of settlements surrounded by agricultural lands, sometimes associated with small pockets of caducifolious forests, small patches of very young Eucalyptus plantations, and patches of caducifolious species close to rivers. Less frequent was to find UPs with adult Eucalyptus plantations.

\section{Discussion}

\subsection{National trends}

In Portugal the maximum size of wildfires is increasing and the overall BA reached in 2017 values higher than the previous years. WUI area represents $26,4 \%$ of the country total surface, which is a high value comparatively with other countries. For instance, South of France has only $11 \%$ of WUI (LongFournel, 2013). 


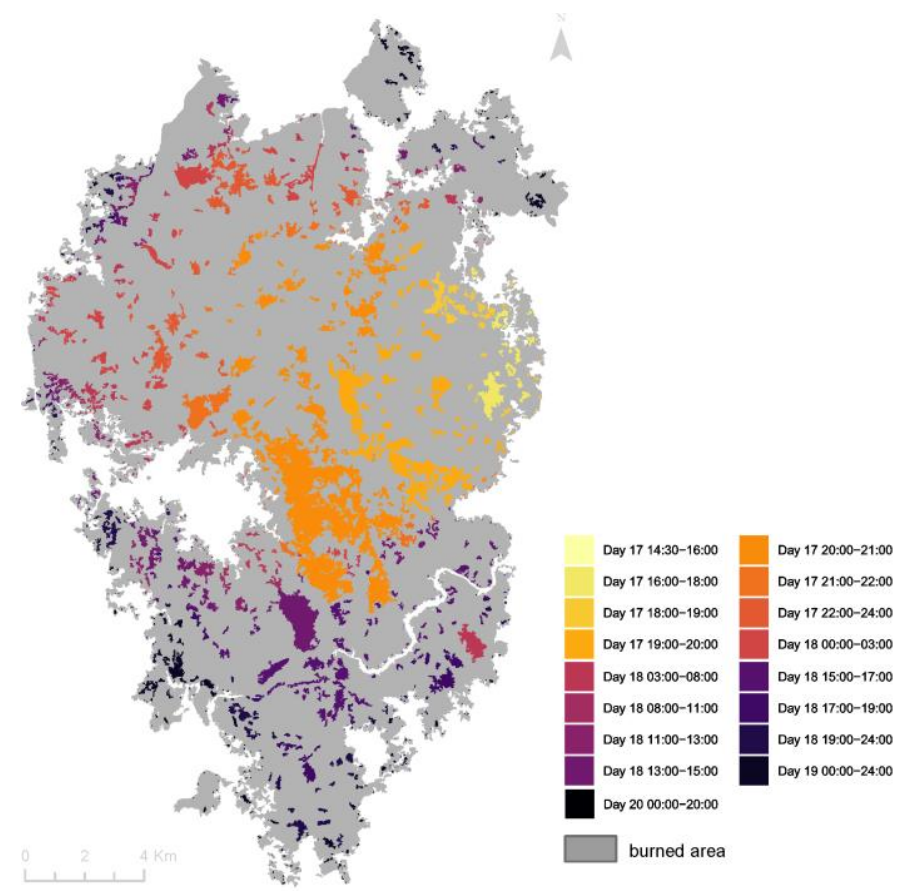

Figure 8 - Pedrógão Grande fire: Chronology of UPs appearance. The highest values of fire intensity were registered between 19 and 21 h of June $17^{\text {th }}$.

Portugal presents a high interannual variability in terms of unburned area within fire perimeters. The BA, UPs area, number of UPs are increasing in fire perimeters $\geq 405$ ha. We found a strong and positive correlation between BA and the number of Ups, as well as with the UPs area. However, we are only dealing with large fire perimeter. Nevertheless, some years presented a different behavior. For instance, 2005 is the third year with more BA but has very few UPs in fire perimeters $\geq 405$ ha. This situation deserves a deep research. In the general case of Portugal, positive trends with statistical significance were found for UPs area, number of patches, UPs density, and mean distance between patches. Our findings are in contrast with previous studies in other parts of the world where no trend was found (Meddens et al., 2018; Kolden et al. 2012, 2015).

In Portugal, the WUIs are mainly concerned by a higher fire ignition density than other areas (Bouillon et al., 2013). Isolated WUI is the most representative class, and dwellings located in the forest increase the risk for people and assets. In general, the presence of forest surrounding any type of settlement, including isolated housing, is accompanied by the increased likelihood of being affected by wildfires and suffer damage, even though the highest danger is for isolated housing. In term of proneness to be affected by wildfires, isolated housing represents the most critical situation followed by scattered housing. Dense settlements, on the contrary, seem to represent the less critical condition. But when we look at the characteristics of WUI areas that burned and the WUI UPs we found that most of the WUI UPs that did not burn were also isolated housing surrounded by forest, followed by isolated housing surrounded by agricultural lands. Of course, the presence of forest is a variable issue, as it depends on the type of forest and its condition of management, and secondarily on the distance between the edge of the forest and the buildings, as well as on fire intensity and spotting characteristics. The presence of agricultural land around the settlements does not guarantee assets protection and safety. Its efficaciousness in protecting assets mostly depends on conditions of combustibility and flammability of vegetation on the fields, in turn depending on its moisture content, its vegetative stage, and soil moisture content.

In this research we used fire perimeters produced by official organizations. We cannot guarantee that for the period of 1975 to 2017 the methodology used to map the fire perimeters has been uniform; the interpretation of satellite-derived severity can be distinctive and we are not aware if the data were 
calibrated with ground data. At the same time satellite imagery has developed a lot in that period as well as the methodologies of interpretation. If the existence of long dataset could be required to produce a better understanding of the dynamic of UPs formation and characteristics, some problems of data comparability can exist. Hence, we do not excessively valorize the results obtained for the UPs trends. The evaluation of WUI characteristic should be done with more recent land cover and land use data to validate our findings. Thus, we prefer to focalize on the findings from the case-study of Pedrógão Grande, where the mentioned potential weaknesses are not present.

\subsection{Pedrógão Grande wildfire}

Previous studies showed that in all types of wildfires (ranging from crown-fires to surface fires in grassland) it is possible to find heterogeneous patterns of fire severity including UPs (Bradstock 2008; Kolden et al. 2012). Some of UPs studies investigated the influence of fire severity in the appearance of unburned areas (Kolden et al. 2012). Our research is based on the influence of fire intensity on the UPs formation and confirms that it is possible to find UPs in case of EWEs.

In the large and high intensity Pedrógão Grande fire, UPs represented a significant percentage (11.9\%) of the area affected by the wildfire. This result is particularly interesting because Leonard et al. (2014) in the Kilmore East-Murrindindi fire complex found that UPs comprised less than one percent of the total fire area; both fires were EWEs although with different values of intensity (maximum intensity in Pedrógão Grande was $60,000 \mathrm{kWm}^{-1}$ (CTI 2017), whereas in the Australian case-study it reached up to $90,000 \mathrm{kWm}^{-1}$ (Cruz et al. 2012). The magnitude for Pedrógão Grande (568 $\mathrm{GW}$ ) was almost three times lower than the ones for the mentioned Australian fires (respectively, Kilmore East 1537 GW and Murrindindi 1642 GW; Harris et al. 2011; CTI 2017).

In the case of Pedrógão Grande, in the period of highest intensity, we found the highest number of UPs per hour and also the patches with larger size. Our study does not confirm that an increase in fire intensity produces smaller unburned areas (Kolden et al. 2012; Kolden et al. 2015; Meddens et al. 2018).

Concerning the terrain metrics influencing UPs, in our study case TPI is the one that more strongly influences their occurrence. Our results are aligned with previous studies (e.g., Leonard et al. 2014; Krawchuk et al. 2016). Slope findings corroborate previous studies (e.g., Ramon-Cuesta et al., 2009); we found UPs in very different slope conditions, including values $>50^{\circ}$, although most of them appear on slopes with very low values. On the contrary, our results contradict the recurrent opinion that unburned areas occur more frequently under the influence of $\mathrm{N}$ aspect (e.g. Ramon-Cuesta et al., 2009). In our case the most favorable aspects resulted $\mathrm{S}$ and $\mathrm{W}$.

We found UPs with very different size and characteristics. If in the smaller UPs we can identify a particular type of land use (e.g., a very young plantation of eucalyptus, a small area of caducifolious forest, a small settlement) that distinguish the unburned area from the surrounding, in the larger UPs we found heterogeneity of uses. The larger UPs found in Pedrógão Grande fire that we labelled as Mix UPS comprise different land uses.

Although the presence of forest around the settlements and mainly around isolated housing marks the riskiest condition, the relation is not so linear as we found many UPs constituted by isolated housing surrounded by forest. In parallel, the existence of an agricultural area surrounding the settlements is not a guarantee of protection. It depends on the extension, the composition, and the management of the vegetation.

The characteristics of UPs show that the big and very costly creation by the Portuguese Government of a primary network of fuel management is not necessarily the most effective in abating fire intensity. For normal fires (Tedim et al. 2018) this could have some efficacy, but in case of EWEs this kind of fuel management results absolutely useless because of the intensity and spotting activity, that can spread fire at distances of several $\mathrm{km}$ from the front of the fire. 


\section{Conclusion}

Unburned areas have been mostly analyzed using an ecological approach, but there are no studies, on our knowledge, concerning the contribution of WUI characteristics in the UPs formation. Our results must be considered absolutely innovative regarding this point, because they are quantitatively referred to categories of WUI.

The understanding of the trends and spatial patterns of UPs, mainly in the case of EWEs, is crucial to inform wildfire and land management as well as spatial planning. UPs exert important ecological benefits and influence ecosystem and communities' resilience, but they also allow to understand how to increase the efficacy of prevention and mitigation activities (e.g., to planning safer settlements location and WUI expansion).

Most of the studies relate unburned areas with severity, that is a local response of the landscape to physical characteristics of the fire; thus, we preferred to focus on the fire behavior to understand how it can affect the formation of UPs. Pedrógão Grande wildfire shows that also an EWE is able to create UPs under the complex interaction of fire behavior with fire environment, topography, vegetation, landscape characteristics, namely the WUI characteristics. The diversity of characteristics exhibited by the UPs shows that their formation is not tied to static landscape characteristics (e.g., slope, aspect, TPI) but they occur as a result of complex interactions of those factors with the dynamic factors of landscape, mainly local ones. A management goal should ensure enough landscape heterogeneity in order to maintain a consistent pattern of UPs, through the reduction of continuity of forest cover and the increase of mosaic patterns that help to provide a higher density of UPs and possibly an increase in their average size; the type and size of mosaic should be adapted to the fire regime of the different regions (e.g., low frequency, high intensity, large size; high).

Considering that fuel management is a very costly activity and exploiting the finding of our research that Mix UPs are larger than the ones with vegetation and WUI UPs, a gradual trend toward Mix UPs should be recommended as a basic model to orientate fuel management and spatial planning. This represents an advantage because inside these Mix UPs there are activities that manage the fuels, so ensuring relevant reduction of vulnerability and increase in the resilience of the territory which is one of the milestones of the new paradigm of "coexist with fire". In this direction the maintenance of productive activities (agriculture, grazing) that can break landscape continuity and connectivity can contribute to decrease vulnerability of local communities and, consequently, losses and damage. At the same time, while fuel reduction activities are very costly and difficult to implement in certain places because of the lack of work force, reducing the risk of wildfire by the introduction or maintenance of agriculture and grazing (mixing traditional with modern models of sustainable agriculture) with a high contribution to sustainable development, should be considered with priority within the measures of prevention against unwanted wildfires diffusion and spread. It is fundamental to be effective in the implementation of the United Nations Sustainable Development Goals.

\section{Acknowledgments}

This work was prepared in the frame of project FIREXTR- Prevent and prepare society for extreme fire events: the challenge of seeing the "forest" and not just the "trees" (FCT Ref: PTDC/ATPGEO/0462/2014), of which the first author is team leader, co-financed by the European Regional Development Fund (ERDF) through the COMPETE 2020 - Operational Program Competitiveness and Internationalization (POCI Ref: 16702) and national funds by FCT-Foundation for Science and Technology, Portugal. The authors would like to thank Comissão Técnica Independente for providing the shape files of Pedrógão Grande Fire perimeter and isochrons of the fire spread that were used in this study. 


\section{References}

ADAI/LAETA, 2017. O complexo de incêndios de Pedrógão grande e concelhos limítrofes, iniciado a 17 de junho de 2017. Faculdade de Ciências e Tecnologia Universidade de Coimbra.

Best, D, Roberts, D (1975) Algorithm AS 89: the upper tail probabilities of Spearman's rho. Journal of the Royal Statistical Society. Series C (Applied Statistics) 24, 377-379.

Birch, DS, Morgan, P, Kolden, CA, Hudak, AT, Smith, AM (2014) Is proportion burned severely related to daily area burned? Environmental Research Letters 9, 064011.

Bradstock, RA (2008) Effects of large fires on biodiversity in south-eastern Australia: disaster or template for diversity? International Journal of Wildland Fire 17, 809-822.

Corona, P, Ascoli, D, Barbati, A, Bovio, G, Colangelo, G, Elia, M, Garfì, V, Iovino, F, Lafortezza, R, Leone, V (2014) Integrated forest management to prevent wildfi res under mediterranean environments. Annals of Silvicultural Research 38, 24-45.

Cruz, M, Sullivan, A, Gould, J, Sims, N, Bannister, A, Hollis, J, Hurley, R (2012) Anatomy of a catastrophic wildfire: the Black Saturday Kilmore East fire in Victoria, Australia. Forest Ecology and Management 284, 269-285.

CTI, 2017. Análise e apuramento dos factos relativos aos incêndios que ocorreram em Pedrógão Grande, Castanheira de Pêra, Ansião, Alvaiázere, Figueiró dos Vinhos, Arganil, Góis, Penela, Pampilhosa da Serra, Oleiros e Sertã entre 17 e 24 de junho de 2017. Assembleia da República.

Eberhart, KE, Woodard, PM (1987) Distribution of residual vegetation associated with large fires in Alberta. Canadian Journal of Forest Research 17, 1207-1212.

Gill, AM, Moore, P (1998) Big versus small fires: the bushefires of greater Sidney, January 1994 In 'Large forest fires.' (Ed. JM Moreno.) (Backhuys Publishers).

Guisan, A, Weiss, SB, Weiss, AD (1999) GLM versus CCA spatial modeling of plant species distribution. Plant Ecology 143, 107-122.

Harris, S, Anderson, A, Kilinc, M, Fogarty, L (2011) 'Establishing a link between the power of fire and community loss: the first step towards developing a bushfire severity scale.' (Victorian Government Department of Sustainability and Environment).

Keane, RE, Agee, JK, Fulé, P, Keeley, JE, Key, C, Kitchen, SG, Miller, R, Schulte, LA (2009) Ecological effects of large fires on US landscapes: benefit or catastrophe? International Journal of Wildland Fire 17, 696-712.

Kolden, CA, Abatzoglou, JT, Lutz, JA, Cansler, CA, Kane, JT, Wagtendonk, JWV, Key, CH (2015) Climate contributors to forest mosaics: ecological persistence following wildfire. Northwest Science 89, 219-238.

Kolden, CA, Lutz, JA, Key, CH, Kane, JT, van Wagtendonk, JW (2012) Mapped versus actual burned area within wildfire perimeters: Characterizing the unburned. Forest Ecology and Management 286, 38-47.

Kolden, CA, Weisberg, PJ (2007) Assessing accuracy of manually-mapped wildfire perimeters in topographically dissected areas. Fire Ecology 3, 22-31.

Krawchuk, MA, Haire, SL, Coop, J, Parisien, MA, Whitman, E, Chong, G, Miller, C (2016) Topographic and fire weather controls of fire refugia in forested ecosystems of northwestern North America. Ecosphere 7.

Leonard, SWJ, Bennett, AF, Clarke, MF (2014) Determinants of the occurrence of unburnt forest patches: Potential biotic refuges within a large, intense wildfire in south-eastern Australia. Forest Ecology and Management 314, 85-93. 
Libiseller, C, Grimvall, A (2002) Performance of partial Mann-Kendall tests for trend detection in the presence of covariates. Environmetrics: The official journal of the International Environmetrics Society 13, 71-84.

Lutz, JA, Key, CH, Kolden, CA, Kane, JT, van Wagtendonk, JW (2011) Fire Frequency, Area Burned, and Severity: A Quantitative Approach to Defining a Normal Fire Year. Fire Ecology 7, 51-65.

Meddens, AJ, Kolden, CA, Lutz, JA, Abatzoglou, JT, Hudak, AT (2018) Spatiotemporal patterns of unburned areas within fire perimeters in the northwestern United States from 1984 to 2014. Ecosphere 9.

Riley, SJ (1999) Index that quantifies topographic heterogeneity. Intermountain Journal of sciences $\mathbf{5}$, 23-27.

Sirca, C, Casula, F, Bouillon, C, García, BF, Fernández Ramiro, MM, Molina, BV, Spano, D (2017) A wildfire risk oriented GIS tool for mapping Rural-Urban Interfaces. Environmental Modelling \& Software 94, 36-47.

Slik, JF, van Beek, M, Bernard, C, Bongers, F, Breman, FC, Cannon, CH, Sidiyasa, K (2011) Limited edge effects along a burned-unburned Bornean forest boundary seven years after disturbance. Biotropica 43, 288-298.

Team, RC, 2018. R: A Language and Environment for Statistical Computing, R Foundation for Statistical Computing, Austria, 2015. ISBN 3-900051-07-0: URL http://www. R-project. org.

Tedim, F, Leone, V, Amraoui, M, Bouillon, C, Coughlan, M, Delogu, G, Fernandes, P, Ferreira, C, McCaffrey, S, McGee, T, Parente, J, Paton, D, Pereira, M, Ribeiro, L, Viegas, D, Xanthopoulos, G (2018) Defining Extreme Wildfire Events: Difficulties, Challenges, and Impacts. Fire 1, 9.

Tedim, F, Remelgado, R, Carvalho, S, Martins, J (2015) The largest forest fires in Portugal: the constraints of burned area size on the comprehension of fire severity. Journal of Environmental Biology, 36(1):133-143.

Tedim, F, Remelgado, R, Martins, J, Carvalho, S (2013) Os grandes incêndios florestias em Portugal desafios para a gestão do risco In A. B. Gonçalves e A. Vieira (Eds.) Grandes incêndios florestais, erosão, degradação e medidas de recuperação dos solos, Guimarães, NIGP-Nucleo de Investigação em Geografia e Planeamento, Universidade do Minho 75-86.

Vaillant, NM, Kolden, CA, Smith, AM (2016) Assessing landscape vulnerability to wildfire in the USA. Current Forestry Reports 2, 201-213. 


\section{Annex}

Table A1: The WUI affected by fire in 2017, in Portugal

\begin{tabular}{|c|c|c|c|c|c|c|}
\hline \multirow{2}{*}{$\begin{array}{l}\text { Type of wildland- } \\
\text { urban interface }\end{array}$} & \multicolumn{2}{|c|}{ Burnt WUI in 2017 wildfires } & \multicolumn{2}{|c|}{$\begin{array}{c}\text { UPs in WUI in } 2017 \\
\text { wildfires }\end{array}$} & \multicolumn{2}{|c|}{ WUI Portugal } \\
\hline & Ha & $\%$ & $\mathrm{Ha}$ & $\%$ & Ha & $\%$ \\
\hline $\begin{array}{l}\text { Isolated housing - } \\
\text { mineral area }\end{array}$ & 747 & 0.6 & 223 & 0.9 & 3,450 & 0.1 \\
\hline $\begin{array}{l}\text { Isolated housing - } \\
\text { agricultural or sparse } \\
\text { vegetation }\end{array}$ & 18,869 & 14.4 & 8,384 & 34.2 & 691,831 & 28.4 \\
\hline $\begin{array}{l}\text { Isolated housing - } \\
\text { forested area }\end{array}$ & 82,653 & 63.0 & 9,867 & 40.2 & 906,432 & 37.8 \\
\hline $\begin{array}{l}\text { Scattered housing - } \\
\text { mineral area }\end{array}$ & 1,06 & 0.8 & 679 & 2.8 & 111,072 & 4.6 \\
\hline $\begin{array}{l}\text { Scattered housing - } \\
\text { agricultural or sparse } \\
\text { vegetation }\end{array}$ & 4,575 & 3.5 & 2,257 & 9.2 & 260,083 & 10.7 \\
\hline $\begin{array}{l}\text { Scattered housing - } \\
\text { forested area }\end{array}$ & 20,242 & 15.4 & 2,67 & 10.9 & 331,484 & 13.6 \\
\hline 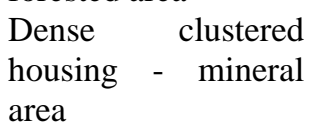 & 373 & 0.3 & 130 & 0.5 & 47,649 & 2.0 \\
\hline $\begin{array}{l}\text { Dense clustered } \\
\text { housing - agricultural } \\
\text { or sparse vegetation }\end{array}$ & 276 & 0.2 & 42 & 0.2 & 28,438 & 1.2 \\
\hline $\begin{array}{lr}\begin{array}{l}\text { Dense } \\
\text { housing }\end{array} & \text { clustered } \\
\text { area } & \text { forested }\end{array}$ & 2,424 & 1.9 & 273 & 1.1 & 57,587 & 2.4 \\
\hline Total & 131,219 & 100 & 24,525 & 100 & $2,438,026$ & 100 \\
\hline
\end{tabular}

Table A2: Summary of explanatory terrain metrics variables for UPs and BA in Pedrógão Grande wildfire

\begin{tabular}{|c|c|c|c|c|c|}
\hline Metrics & $\begin{array}{l}\text { Type of } \\
\text { area }\end{array}$ & Mean & $\pm \mathrm{SD}$ & Max & Min \\
\hline \multirow{2}{*}{ Aspect $\left(^{\circ}\right)$} & $\mathrm{BA}$ & 205.53 & \pm 2.05 & 360.00 & 0.00 \\
\hline & UPs & 182.95 & \pm 1.51 & 359.99 & 0.00 \\
\hline \multirow{2}{*}{ Elevation (m) } & BA & 426.98 & \pm 142.98 & 1006.34 & 105.36 \\
\hline & UPs & 391.77 & \pm 113.20 & 978.18 & 105.36 \\
\hline \multirow{2}{*}{ Slope $\left(^{o}\right)$} & BA & 11.28 & \pm 6.62 & 63.94 & 0.00 \\
\hline & UPs & 8.21 & \pm 0.11 & 61.29 & 0.00 \\
\hline Topographic & BA & 0.11 & \pm 3.00 & 21.84 & -51.90 \\
\hline Index & UPs & -0.23 & \pm 2.66 & 14.85 & -39.36 \\
\hline Ruggedness & BA & 11.27 & \pm 0.12 & 63.94 & 0.00 \\
\hline Index & UPs & 49.10 & \pm 24.63 & 172.60 & 14.15 \\
\hline
\end{tabular}


Table A3: Terrain metrics by UPS size and type of WUI in Pedrógão Grande wildfire

\begin{tabular}{|c|c|c|c|c|c|c|c|c|c|c|c|c|}
\hline & \multirow[b]{2}{*}{ Variables } & \multicolumn{4}{|c|}{ Vegetation UPS } & \multicolumn{3}{|c|}{ WUI UPS } & \multicolumn{3}{|c|}{ MIX UPS } & \multirow[b]{2}{*}{ Total } \\
\hline & & & $<1$ ha & 1-9.9 ha & $\geq 10 \mathrm{ha}$ & $\begin{array}{l}<1 \\
\text { ha }\end{array}$ & $\begin{array}{c}1-9.9 \\
\text { ha }\end{array}$ & $\begin{array}{c}\geq 10 \\
\text { ha }\end{array}$ & $\begin{array}{l}<1 \\
\text { ha }\end{array}$ & 1-9.9 ha & $\geq 10$ ha & \\
\hline \multirow{10}{*}{ 苞 } & \multirow{2}{*}{ North } & No. & 170 & 26 & 0 & 31 & 3 & 1 & 18 & 11 & 2 & 262 \\
\hline & & Area & 22,13 & 59,8 & 0 & 4,42 & 9,69 & 21,66 & 3,94 & 25,41 & 61,88 & 208,93 \\
\hline & \multirow{2}{*}{ East } & No. & 359 & 94 & 1 & 29 & 6 & 0 & 29 & 23 & 11 & 552 \\
\hline & & Area & 52,31 & 220,17 & 10,83 & 3,33 & 12,08 & 0 & 7,08 & 63,92 & 339,09 & 708,81 \\
\hline & \multirow{2}{*}{ South } & No. & 352 & 116 & 3 & 36 & 4 & 0 & 34 & 40 & 26 & 611 \\
\hline & & Area & 61,16 & 272,13 & 93,46 & 2,01 & 16,18 & 0 & 10,63 & 118,89 & 1703,4 & 2277,86 \\
\hline & \multirow{2}{*}{ West } & No. & 384 & 100 & 1 & 42 & 12 & 0 & 29 & 35 & 7 & 610 \\
\hline & & Area & 55,18 & 244,16 & 27,81 & 4,67 & 24,28 & 0 & 4,94 & 146,5 & 147,06 & 654,6 \\
\hline & \multirow{2}{*}{ Unclassified } & No. & 4 & 0 & 0 & 0 & 0 & 0 & 0 & 0 & 0 & 4 \\
\hline & & Area & 0 & 0 & 0 & 0 & 0 & 0 & 0 & 0 & 0 & 0 \\
\hline \multirow{12}{*}{ 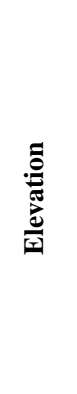 } & \multirow{2}{*}{$\geq 135<268$} & No. & 215 & 48 & 1 & 6 & 6 & 0 & 16 & 13 & 5 & 310 \\
\hline & & Area & 28,87 & 100,08 & 27,81 & 0,38 & 15,16 & 0 & 3,77 & 35,04 & 83,66 & 294,77 \\
\hline & \multirow{2}{*}{$\geq 268<362$} & No. & 306 & 97 & 2 & 39 & 6 & 1 & 24 & 22 & 12 & 509 \\
\hline & & Area & 52,12 & 203,44 & 25,94 & 4,81 & 17,41 & 21,66 & 6,3 & 80,91 & 1266,18 & 1678,77 \\
\hline & \multirow{2}{*}{$\geq 362<449$} & No. & 304 & 65 & 0 & 48 & 9 & 0 & 33 & 33 & 18 & 510 \\
\hline & & Area & 46,89 & 161,89 & 0 & 5,29 & 23,77 & 0 & 7,36 & 90,2 & 649,28 & 984,68 \\
\hline & \multirow{2}{*}{$\geq 449<549$} & No. & 215 & 60 & 1 & 38 & 3 & 0 & 33 & 36 & 9 & 395 \\
\hline & & Area & 33,09 & 144,35 & 16,66 & 3,55 & 3,92 & 0 & 8,32 & 126,87 & 227,78 & 564,54 \\
\hline & \multirow{2}{*}{$\geq 549<678$} & No. & 160 & 38 & 1 & 7 & 1 & 0 & 4 & 4 & 2 & 217 \\
\hline & & Area & 23,43 & 107,74 & 61,68 & 0,4 & 1,96 & 0 & 0,82 & 20,65 & 24,54 & 241,22 \\
\hline & $>678$ & No. & 69 & 28 & 0 & 0 & 0 & 0 & 0 & 1 & 0 & 98 \\
\hline & $=0 / 8<1000$ & Area & 6,5 & 78,76 & 0 & 0 & 0 & 0 & 0 & 1,04 & 0 & 86,3 \\
\hline & & No. & 62 & 35 & 2 & 0 & 6 & 0 & 3 & 18 & 6 & 132 \\
\hline & $\geq 10<20$ & Area & 14,66 & 80,56 & 38,64 & 0 & 18,55 & 0 & 1,61 & 46,34 & 92,23 & 292,59 \\
\hline & & No. & 180 & 84 & 1 & 19 & 9 & 1 & 24 & 29 & 12 & 359 \\
\hline & & Area & 32,99 & 203,8 & 61,68 & 3,18 & 23,61 & 21,66 & 6,24 & 88,38 & 385,29 & 826,83 \\
\hline ๕̆ & $>30<40$ & No. & 174 & 44 & 0 & 23 & 1 & 0 & 21 & 21 & 8 & 292 \\
\hline$\frac{0}{\sqrt{2}}$ & $\geq 30<40$ & Area & 27,89 & 93,51 & 0 & 2,45 & 6,1 & 0 & 5,82 & 64,4 & 996,34 & 1196,51 \\
\hline & & No. & 132 & 37 & 0 & 26 & 4 & 0 & 9 & 16 & 8 & 232 \\
\hline & $\geq 40<50$ & Area & 18,9 & 93,79 & 0 & 4,44 & 6,54 & 0 & 2,21 & 62,15 & 204,02 & 392,05 \\
\hline & & No. & 721 & 136 & 2 & 70 & 5 & 0 & 53 & 25 & 12 & 1024 \\
\hline & $\geq 50$ & Area & 96,45 & 324,59 & 31,78 & 4,36 & 7,43 & 0 & 11,11 & 93,44 & 573,56 & 1142,72 \\
\hline & & No. & 3 & 0 & 0 & 0 & 0 & 0 & 0 & 0 & 0 & 3 \\
\hline & (I) & Area & 0,18 & 0 & 0 & 0 & 0 & 0 & 0 & 0 & 0 & 0,18 \\
\hline : & $>20<-10$ & No. & 18 & 1 & 0 & 1 & 0 & 0 & 1 & 0 & 0 & 21 \\
\hline$\stackrel{8}{\&}$ & $\geq 20<-10$ & Area & 2,13 & 1,85 & 0 & 0,04 & 0 & 0 & 0,12 & 0 & 0 & 4,14 \\
\hline 气 & $<0$ & No. & 627 & 138 & 1 & 68 & 16 & 1 & 58 & 70 & 35 & 1014 \\
\hline 䒕 & z & Area & 95,8 & 295,8 & 27,81 & 6,24 & 37,25 & 21,66 & 14,29 & 229,74 & 1863 & 2591,59 \\
\hline 0 & & No. & 617 & 197 & 4 & 69 & 9 & 0 & 51 & 39 & 11 & 997 \\
\hline a & $\geq 0<10$ & Area & 92,61 & 498,6 & 104,29 & 8,15 & 24,97 & 0 & 12,18 & 124,97 & 388,43 & 1254,2 \\
\hline & & No. & 4 & 0 & 0 & 0 & 0 & 0 & 0 & 0 & 0 & 4 \\
\hline & $\geq 10$ & Area & 0,18 & 0 & 0 & 0 & 0 & 0 & 0 & 0 & 0 & 0,18 \\
\hline & & No. & 947 & 241 & 3 & 126 & 24 & 1 & 97 & 97 & 41 & 1577 \\
\hline$\stackrel{\text { S}}{=}$ & Level & Area & 146,07 & 547,46 & 42,6 & 13,1 & 60,26 & 21,66 & 24,52 & 318,22 & 2175,79 & 3349,68 \\
\hline 总 & Nearly Level & No. & 260 & 86 & 2 & 12 & 1 & 0 & 13 & 11 & 5 & 390 \\
\hline 递 & Nearly Level & Area & 38,51 & 229,62 & 89,5 & 1,33 & 1,96 & 0 & 2,07 & 32,36 & 75,65 & 471 \\
\hline$\approx$ & & No. & 59 & 9 & 0 & 0 & 0 & 0 & 0 & 1 & 0 & 69 \\
\hline 跑 & Slightly rugged & Area & 5,87 & 19,18 & 0 & 0 & 0 & 0 & 0 & 4,13 & 0 & 29,18 \\
\hline 5 & & No. & 3 & 0 & 0 & 0 & 0 & 0 & 0 & 0 & 0 & 3 \\
\hline & Intermediately rugged & Area & 0,45 & 0 & 0 & 0 & 0 & 0 & 0 & 0 & 0 & 0,45 \\
\hline
\end{tabular}




\title{
Wind measurement accuracy in fire experiments
}

\author{
François Pimont ${ }^{1 *}$; Jean-Luc Dupuy ${ }^{1}$; Rodman R. Linn ${ }^{2}$; Russ Parsons ${ }^{3}$ \\ ${ }^{1}$ URFM INRA.84914 Avignon, France, \{francois.pimont@inra.fr*, jean-luc.dupuy@inra.fr\} \\ ${ }^{2}$ EES, LANL, Los Alamos, NM 87544, USA, \{rrl@lanl.gov\} \\ ${ }^{3}$ USFS RMRS, Fire Sciences Lab.MT 5775, USA, \{rparsons@fs.fed.us\}
}

\begin{abstract}
Fire experiments generally aim to relate fire behavior to fuel and weather conditions. One of the main factors influencing the fire rate of spread is the wind speed, but its measurement in fire experiments is challenging due to the combination of wind-flow turbulence and the remote location of the sensors relative to the fire front. Differences arise because i) the feedbacks of the fire plume on the ambient wind modify the wind speed at fire location, ii) the environment at the measurement location may differ from the fire plot characteristics in non-uniform conditions (fuel, topography), which may induce a spatial bias between mean winds and iii) instantaneous fluctuations at both locations are weakly correlated, which affects the representativeness of wind measurement, because of the relatively brief duration of fire experiments.

The present work disentangles the sources of bias and variability in wind measurements (ii and iii), and provides understanding of wind measurement accuracy in fire experiments. The combination of both measurement error sources, both in representativeness and spatial bias, has to date never been studied. In the present study, we employed a modelling approach using HIGRAD/FIRETEC to compute Large-EddySimulations (LES) of wind flows over typical fire experiments. Our simulations show that the spatial bias resulting from the distance to the trailing edge of the measurement location, can be very strong in a realistic configuration and that $7 h$ (with $h$ the canopy height) is too short to get measurements representative of the ambient open wind. In this context, we recommend that the distance to the trailing edge is measured and the upwind canopy characterized (at least in terms of height), so that horizontal Wind Adjustment Factor can be applied to correct the measured wind. Another source of bias is the fact that the fire wind, defined as the average wind at $20 \mathrm{ft}$ above the fire plot, can be altered by the presence of the upwind safety break, when its size is larger than 6 to $7 h$.

The representativeness of wind measurements can be estimated by comparing values of fire and measured winds corresponding to replicates of virtual fire experiments. We confirm the finding of two pioneering studies on wind measurement representativeness, which already suggested that measurement errors resulting from both uncertainties in both measured and fire wind could be very significant in typical fire experiments, especially when the plot size was small, when the experiment duration was short, and when the number of sensors was small. For our preliminary results, obtained in more realistic configurations, we found that square fire plots larger than $200 \mathrm{~m}$ and a number of five sensors seem to provide a reasonable measurement accuracy, provided that the fire does not spread too fast (which would result in shorter experiment duration) and that the wind is not too low (as the measurement representativeness increases with ambient wind speed).
\end{abstract}

Keywords: Fire metrology, Measurement accuracy, Bias, Variance, FIRETEC, Fire behavior

\section{Introduction}

Fire experiments generally aim to relate fire behavior to fuel and weather conditions. One of the main factors influencing fire rate of spread is the wind speed. However, empirical relationships generally exhibit significant variability, which can partly be explained by wind measurement accuracy (Cruz and Alexander 2013). Indeed, measuring the wind in fire experiments is challenging, due to the combination of differences in the turbulent nature of wind flow and the location of sensor(s). In practice, the ambient wind speed is measured at some distance from the fire front, so the wind speed at the measurement location often differs from the wind speed at the fire location. 
Because no evidence was found that instantaneous measured wind relates to variations in fire spread (Cheney et al. 1993; Taylor et al. 2004), measured wind speed is generally averaged over the experiment duration and eventually, over the different sensors when exist. The rationale for that process is that the average measured wind is a good representative for the ambient wind, which drives the fire within the experimental plot. However, some differences between measured and fire wind arise because i) the feedbacks of the fire plume on the ambient wind modify the wind speed at fire location, ii) the local or upwind environment may differ (fuel, topography), which may induce differences between mean winds, and/or iii) instantaneous fluctuations at both locations are weakly or not correlated, which affects the representativeness of wind measurements.

The fire feedbacks on the fire wind are generally not included in wind measurement error (Sullivan and Knight 2001), and have to be accounted for in the fire-model formulation. As such, they are beyond the scope of the present work. The measurement error is defined as the departure between the wind speed that would have been measured at fire location during the fire experiment (without the fire), referred to as the fire-front wind $\left(\mathrm{u}_{\mathrm{f}}\right)$, and the measured wind speed at measurement location $(\mathrm{s})\left(\mathrm{u}_{\mathrm{m}}\right)$ (Sullivan and Knight 2001; Pimont et al. 2017), averaged over the same time period. The conventional approach is that $\mathrm{um}_{\mathrm{m}}$ is typically measured above bare ground, in an open area at a reference height $h_{\text {ref }}$ (20 feet or $10 \mathrm{~m}$ ), as described in Fisher and Hardy 1976). It is expected to highly depend on the surrounding obstacles (typically the canopy located in the upwind side of the open area), so that it is recommended that the measurements are done at more than $7 h$ from the trailing edge, with $h$ the height of the canopy (Fisher and Hardy 1976). In practice, this measured wind is assumed to be representative of the wind at $h_{r e f}$ above the canopy in the fire plot (Andrew 2012), which we refer later to as the fire wind $\left(\mathrm{u}_{\mathrm{f}}\right)$. This reference wind is typically converted to a wind at the height of interest through a Wind Adjustment Factor -WAF- (Andrews 2012), which depends on the fire modelling approach. A widespread assumption is to convert the fire wind to a mid-flame-height wind; in this context, calculation of WAF has important implications (e.g. Albini and Baughman 1979; Massman et al. 2017). As this height of interest potentially depends on the fire spread conditions, which determines the flame height, the scope of the present paper will be limited to the fire wind $\left(\mathrm{u}_{\mathrm{f}}\right)$, which is defined as the average wind at $h_{r e f}$ above the canopy within the fire plot, and its relationship with the measured wind $\left(\mathrm{um}_{\mathrm{m}}\right)$ in the open, which conventional approaches assume it should represent.

To a large degree, the question of wind measurement representativeness, although critical, has seldom been addressed. The measurement error can be decomposed in two subcomponents (Hastie 2009). The first one is the error due to "bias", which is caused by the difference between the expectation of the measured wind and the expectation of the fire wind (which is what we would obtain by averaging replications of the experiment under a constant weather). To the best of our knowledge, there is apparently not much scientific support to the assumption that $u_{m}$ is equal to the wind at $h_{r e f}$ above the canopy. According to Fisher and Hardy (1976), measurement in open area can be replaced by measurement at "obstacle $+h_{r e f}$ " height, "if the ground is densely covered with rocks, brush, or small trees, increase the height of the anemometer by the average height of the ground cover." There is hence a potential bias associated with the fact that the wind at $h_{r e f}$ in the open would be equal to the wind at $h_{r e f}$ above the canopy and the magnitude of this bias might varies when changing canopy properties (e.g. height, Leaf Area Index, etc).

Another bias in real-world experiments arises from safety fuel breaks which often surround shrub or forest plots. In reality, the fuel break located in the upwind side of the fire plot almost certainly affects the wind profile, which requires some distance to adjust from an "open" profile to a "fire plot" profile (e.g. in Pimont et al. 2009; Pimont et al. 2011). As a result, it is likely that the fire wind $u_{f}$, at $h_{r e f}$ above the actual fire plot, differs from the wind that would be measured at $h_{r e f}$ above a canopy, especially for the part of the fire plot located at a short distance from the plot's leading edge. From now, we will refer to $u_{t h}$ (for theoretical wind) for the wind at $h_{r e f}$ above a long homogeneous canopy. 
The second subcomponent of error is due to "variance" (or variability) in both fire and measured winds. Even if the expectations of the fire wind (at $\mathrm{z}=h+h$ ref in the fire plot, which represents the wind truly experienced by the experimental fire) and the measured wind (at $h_{\text {ref }}$ in the open) were equal (i.e. no bias), their mean values over the duration of an actual fire experiment is expected to differ because of instantaneous fluctuations of the wind. Such an impact of variance has been studied through an experimental approach by Sullivan and Knight (2001) and a numerical approach by Pimont et al. (2017). Both studies were based on comparison between wind measurements made at distant locations below homogeneous canopies, so that average winds over long periods were expected to be equal at both locations. These studies revealed that, in fact, the average winds typically differ over the duration of a fire experiment, which is typically short, causing measurement error, often on the order of $+/-$ $30 \%$. In both studies, the duration and the extent of fire experiments, the number of sensors and the measurement heights were all critical factors influencing the degree of consistency between measured and fire winds. These studies, however, focused on the variance problem in a context which differs slightly from actual fire experiments, as both fire and measured winds were estimated below a homogeneous canopy, in which variability is expected to be much larger than in the open or above a canopy. The combination of measurement error due to both bias and variance has to date never been studied in a context close to actual fire experiments. In the present study, we used Large-EddySimulations (LES) of wind flows to estimate these two subcomponents of error from wind flow simulations, typically corresponding to the ICFME experiment (Taylor et al. 2004).

\section{Method}

We employed the HIGRAD/FIRETEC model to compute Large-Eddy-Simulations (LES) of wind flows over fire plots (corresponding to virtual fire experiments), as in Pimont et al. (2017). Wind flow simulations over both homogeneous canopy and open-to-forest transitions with the HIGRAD/FIRETEC model have been validated against detailed field experiments, in terms of both average wind profiles and turbulent profiles (Pimont et al. 2009).

The forest canopy was representative of the ICFME experiment (Taylor et al. 2004) and modelled with the FuelManager software from inventory data and allometric relationships for the different species (Pimont et al. 2016). Fuel breaks of various widths were designed in the landscape (in blue, in Fig. 1), so that simulated wind flows could be used to simulate measured wind, theoretical wind and fire wind at selected heights over fire experiments.

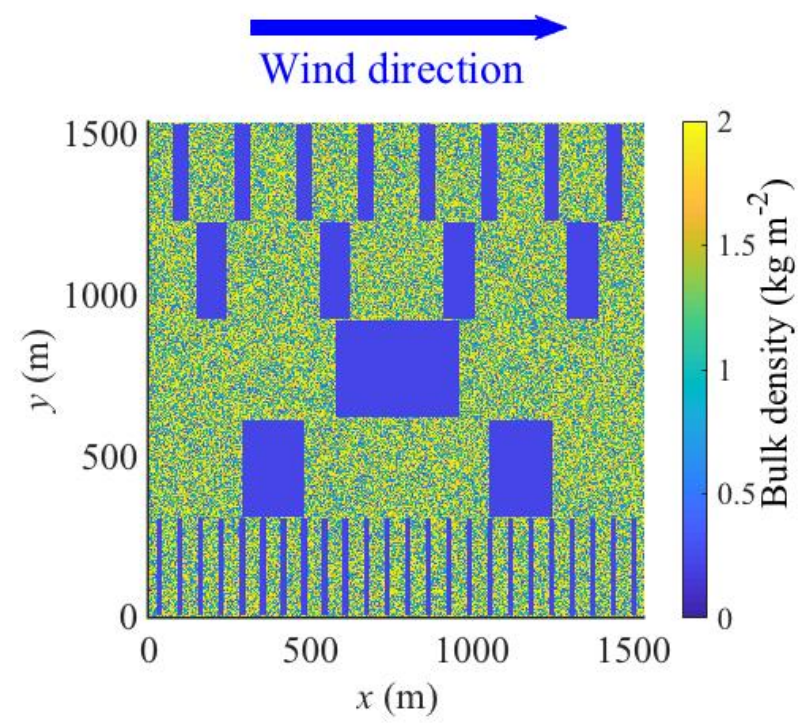

Figure 1 - Planimetric view of the fuel map (expressed in fuel bulk density), illustrating different spacing between the safety breaks (in blue, represented with very low fuel loads). This design enables comparison of simulated winds in virtual fire plots (within the canopy) and at sensor locations (within fuel breaks) to evaluate the wind measurement error in various conditions of fuel break widths, depending on the location of the fire plot in the fuel map. 
Simulated data were used to determine the sensitivity of these wind values to each configuration and to compute the resulting bias, which depends on the location of the sensor, the length of the open area and the location of the fire plot. We then compared the variance of both measured and fire wind to provide a comprehensive understanding of wind measurement error in fire experiments.

\section{Wind measurement bias}

Figure 2 shows the horizontal wind profiles in the streamwise direction, simulated at several heights in the case of a large open area $384 \mathrm{~m}$ across (shown close to the center in Fig. 1). In Figure 2, the $x$ axis is labelled with 0 at the end of the first canopy block, so that the labels correspond to the distance from this trailing edge. Close to the ground (at $z=20$ feet, in black) and at the top of the canopy (at $h=16.1 \mathrm{~m}$, in green), the wind accelerated over the open area, before slowing down when entering in next canopy block (at $d>384 \mathrm{~m}$ ). The $20 \mathrm{ft}$ curve shows that wind velocity at a distance equal to $7 \mathrm{~h}$ from the trailing edge, as recommended in Fisher and Hardy (1976) led to a measured wind of $3 \mathrm{~ms}^{-1}$, whereas the measured wind reached $4.1 \mathrm{~ms}^{-1}$ at $d=20 h$. This suggests that $7 h$, which is the distance suggested by Fisher and Hardy (1976), is too small to reach an "open" wind value, for which the canopy to forest transition requires at least $30 h$ to fully adjust to the "open" environment (e.g. Pimont et al. 2011). The red curve represents the wind at $h+20 \mathrm{ft}$, which is shown to be significantly increased above the open area with respect to the theoretical wind $u_{t h}$ at $\mathrm{z}=h+h_{\text {ref }}$ above a homogeneous forest, $u_{t h}=4.3 \mathrm{~ms}^{-1}$. Moreover, the wind at $h+20 \mathrm{ft}$ just after the open area (at $2 h$ from the canopy leading edge) further increases up to $5.4 \mathrm{~m} / \mathrm{s}$, and then gently returns to its theoretical value $u_{\text {th }}$ at only 7 to $8 h$ from the canopy leading edge. In contrast, the blue curve shows that the mean wind speed at $z=3 h$ was not much influenced by the open area. Unfortunately, such a height is too high for practical measurements with traditional anemometers.

Typically, if the measurement were made at $d=7 h$ in the open and the fire plot was within the first $100 \mathrm{~m}$ after the leading edge of the second canopy block, the fire wind would be on the order of $75 \%$ larger than the measured wind. However, if the wind was measured at $d=20 h$ and the fire was ignited at more than $200 \mathrm{~m}$ for the leading edge, the bias would be of only $5 \%$. This figure thus illustrates the importance of the location of both sensor(s) and fire plot and how this location affects the consistency of the measurement of the ambient wind.

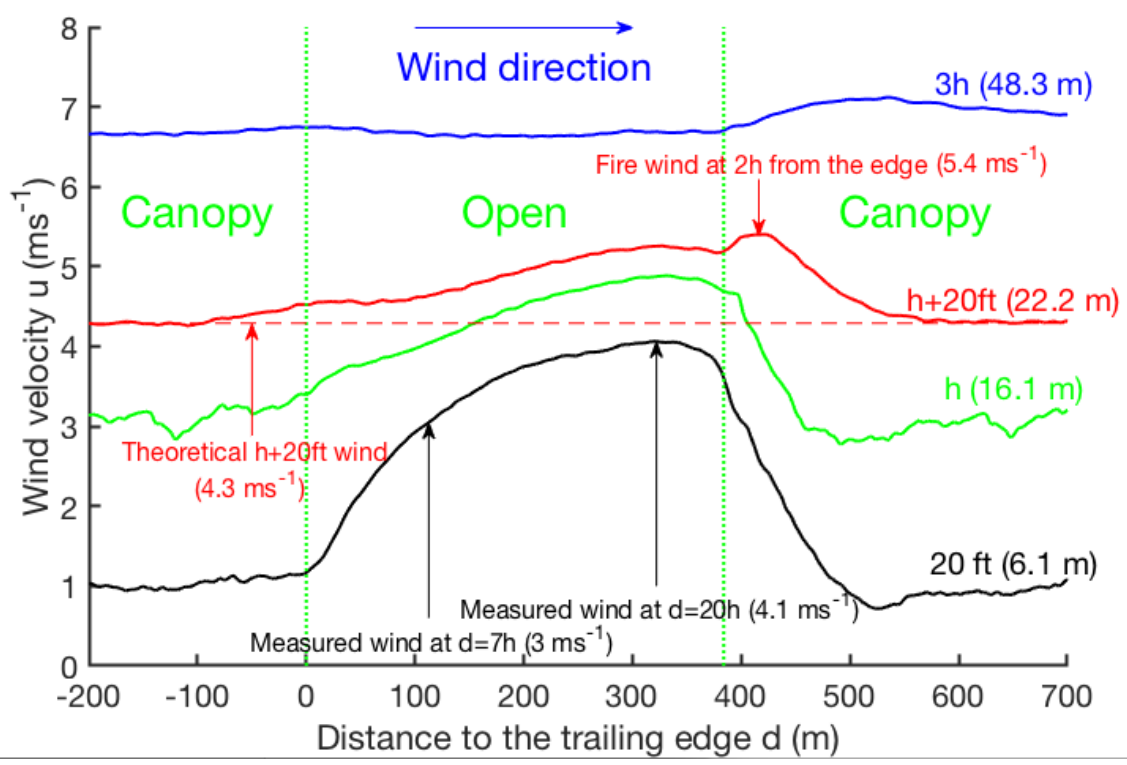

Figure 2 - Horizontal wind profiles at several heights over the $384 \mathrm{~m}$ open area ( $z=20 f$, in black; $z=h$ (canopy height), in green; $z=h+20 f t$ in red and $z=3 h$ in blue). The red dashed line corresponds to the wind speed at 20 ft above the canopy far from the open area, which is often referred to as the theoretical wind at $h+20 f t$. The wind velocity at $20 f t$ (in black) is strongly affected by the presence of the fuel break and has not converged after $d=7 \mathrm{~h}$. 
In Figure 2, the open area is very long (384 $\mathrm{m}$, which is on the order of $25 \mathrm{~h}$ ). In practice, open areas in forest are often much shorter. Figure 3 is similar to Figure 2, but for a shorter break (96 m, on the order of $6 h$, which corresponds to the four safety breaks located around $y=1100 \mathrm{~m}$, in Fig. 1). In this case the wind measured at $d=5 h$ from the edge would be $2.8 \mathrm{~ms}^{-1}$, which is much lower than the theoretical wind speed $\left(4.4 \mathrm{~ms}^{-1}\right)$. However, the shorter break induced a smaller impact on the fire wind after the break, which was much closer to the theoretical wind than after the large open area. In the present configuration, a measurement at $d=5 h$ would lead to an underestimation by $68 \%$ of the fire wind.

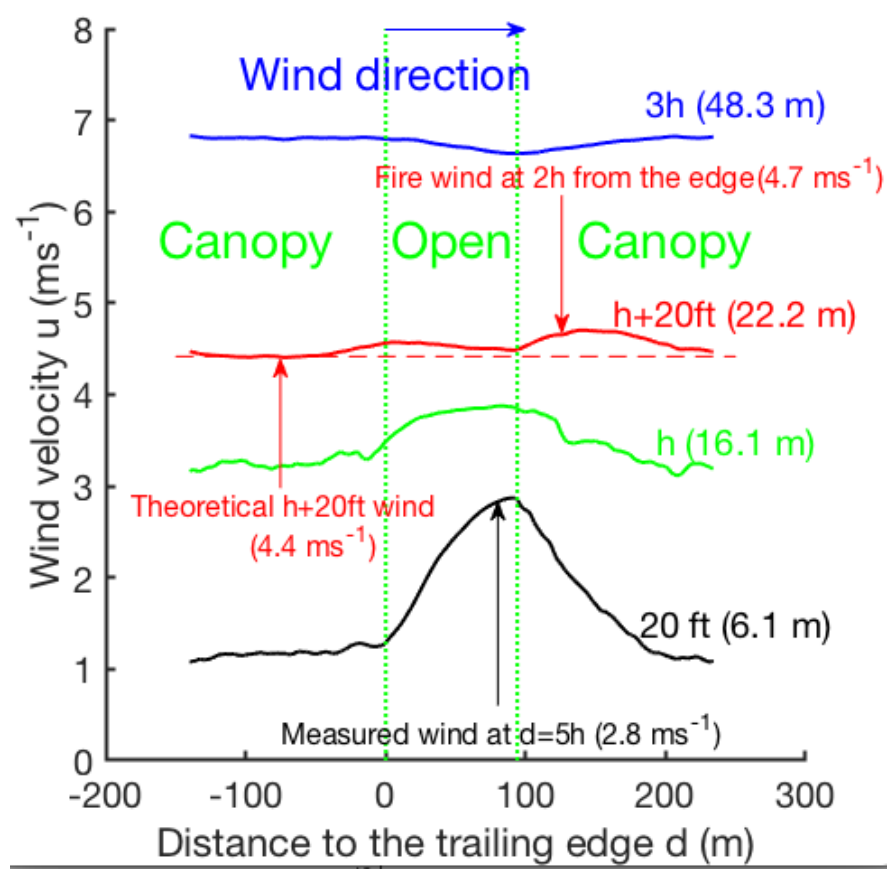

Figure 3 - Same figure as figure 2, but for the 96 m open area. ( $z=20 f t$, in black; $z=h$ (canopy height), in green; $z=h+20 f t$ in red and $z=3 h$ in blue). The red dashed line corresponds to the wind speed at 20 ft above the canopy far from the open area, which is often referred to as the theoretical wind at $h+20 f t$.

\section{Wind measurement variance}

In this subsection, we evaluated the variance in a specific configuration, in which the sensors were located at $20 \mathrm{ft}$ above the ground and at a distance $d=7 \mathrm{~h}$ from the trailing edge of the largest (centered) safety break of Fig. 1 (as recommended in Fisher and Hardy, 1976). The fire plot was in the forest area located downwind to this break (starting just after the leading edge of the second forestry block, see Fig. 1). We simulated fire winds, corresponding to mean wind at the location of a virtual fire spreading at a constant rate of spread (ROS, here $0.5 \mathrm{~ms}^{-1}$ and $1 \mathrm{~ms}^{-1}$ ) in square fire plots of a given size (50, 100 and $200 \mathrm{~m}$ ), which led to virtual experiments with durations between 50 and $400 \mathrm{~s}$. We also computed the measured wind from different numbers of virtual sensors (from one to five sensors). The design was inspired from Pimont et al. (2017), who provide more details on the method.

Figure 4 shows the comparison between fire and measured winds for the three fire plot sizes, assuming that the fire spreads at ROS $=0.5 \mathrm{~ms}^{-1}$. As expected from the previous subsection, the fire wind at 20ft above the canopy was on average much larger than the measured wind at 20ft above the ground at $d=7 h$ from the trailing edge (the no-bias line is in dashed black). 

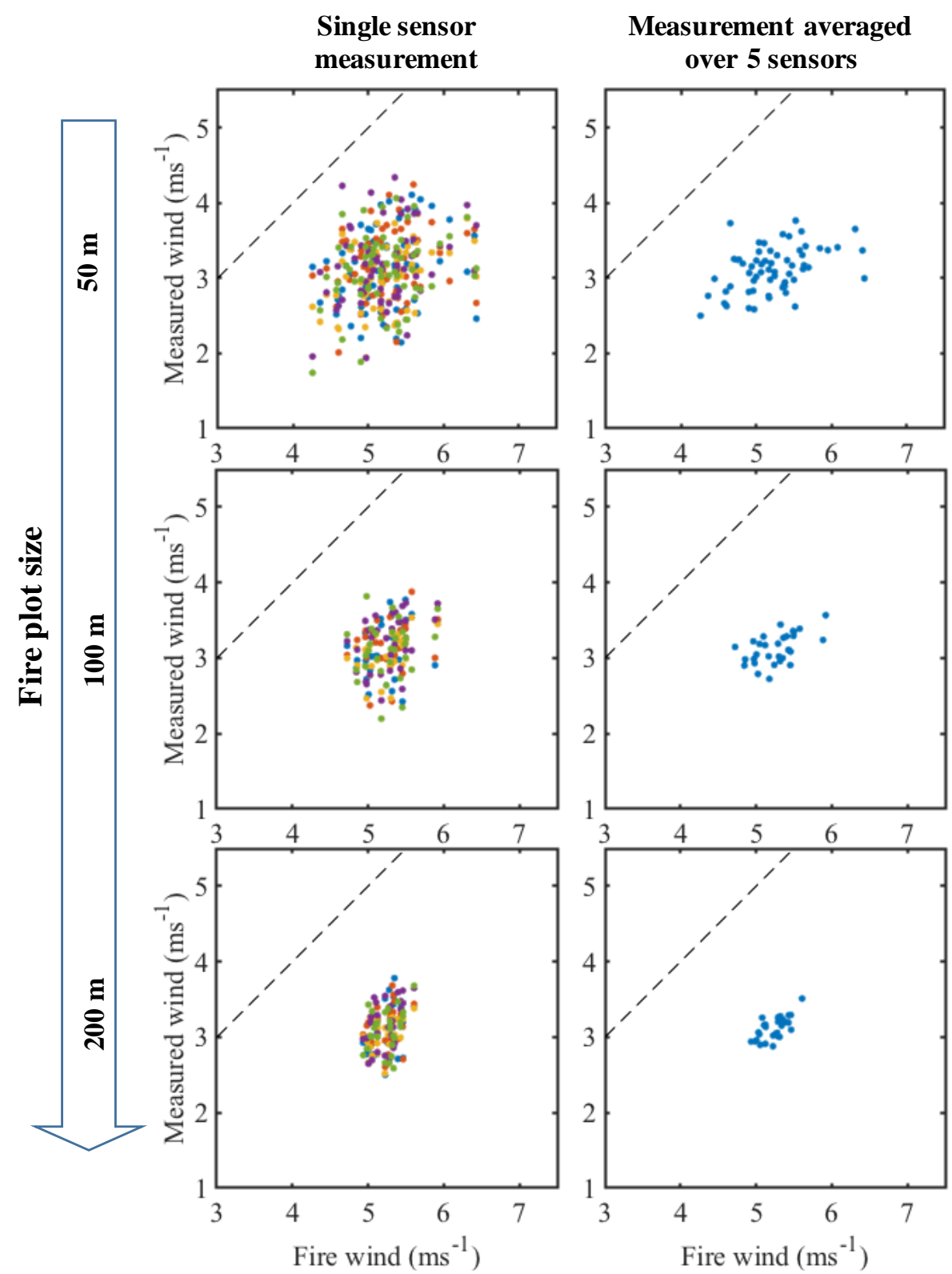

Figure 4 - Comparison between measured and fire wind speeds simulated during different virtual fire experiments in fire plots of size 50, 100 and $200 \mathrm{~m}$. As $R O S=0.5 \mathrm{~ms}^{-1}$, the durations of these experiments are respectively 100,200 and 400s. Increasing plot size (and thus experiment duration) strongly reduced the variability of both measured and fire wind speed. Also averaging over 5 sensors, rather than using only one, strongly reduced the measured wind variability.

Here, we focus on the variability of fire and measurement winds, which were obviously not much correlated, because of the short duration of the fire experiments (as already reported by Sullivan and Knight 2001 and Pimont et al. 2017). This variability was very high when measurements were made on a single sensor and with the smallest fire plot $(50 \mathrm{~m})$ which also corresponded to a short experiment duration (100 s). Increasing the size of the plot reduced the variability in both measured and fire wind speed. Measurement wind variability was reduced by the number of sensors.

Figure 5 is similar to Fig 4, but for a faster $\operatorname{ROS}\left(1 \mathrm{~ms}^{-1}\right)$, which led to shorter experiment durations. As expected, the corresponding variability was much larger than for the shorter duration. 

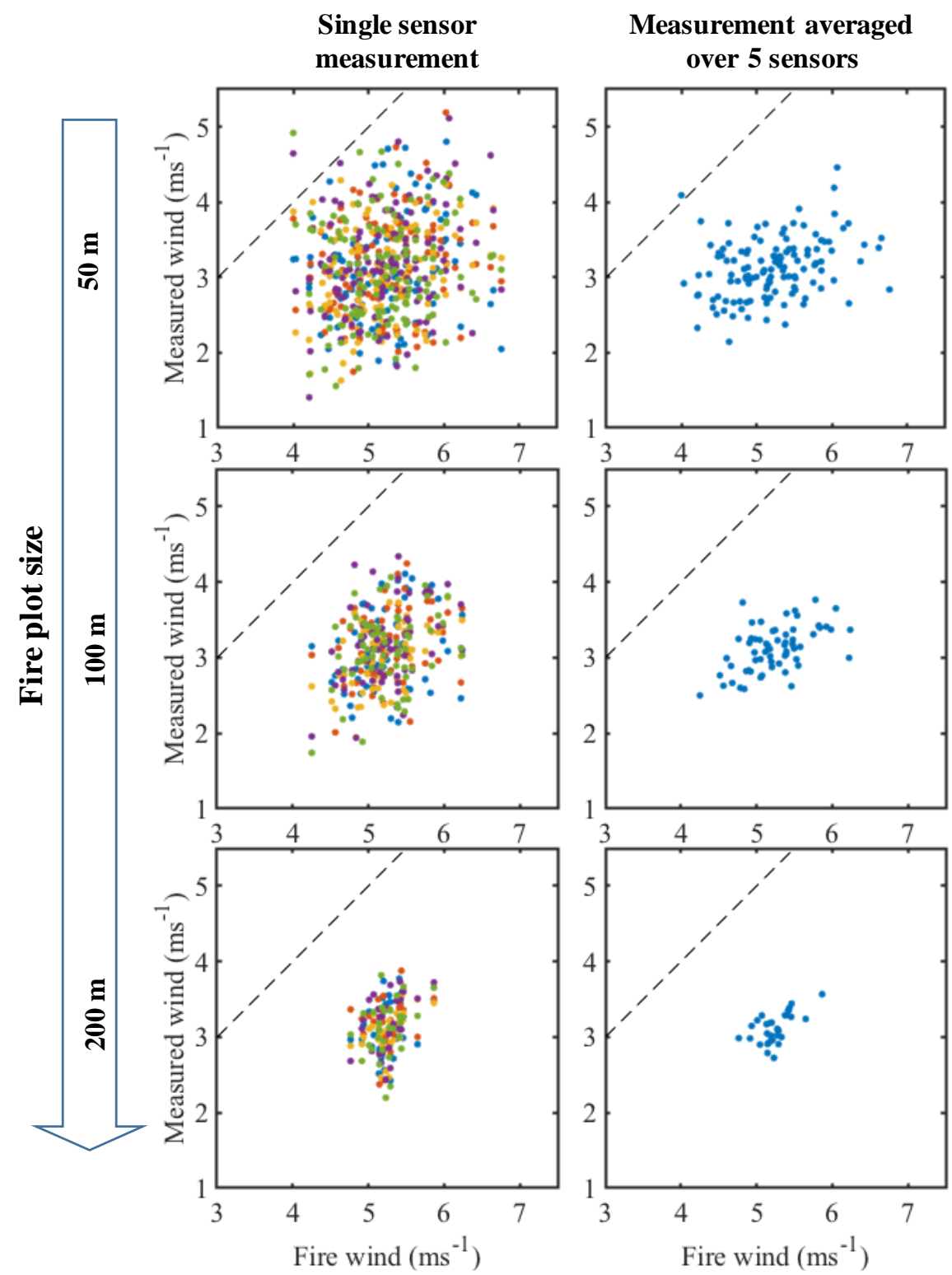

Figure 5 - Same figure as Fig. 4, but assuming a faster ROS $\left(1 \mathrm{~ms}^{-1}\right)$, and thus shorter experiment durations (50, 100 and 200s). The trends were similar as in Fig. 4, but much stronger variability was observed because of shorter averaging period.

\section{Discussion and conclusion}

The work presents an innovative analysis of the wind measurement error in fire experiments, by combining the two sources of error: the problem of wind representativeness (caused by the turbulent nature of the wind) and the heterogeneity bias, (caused by differences in physical environment between the location of wind measurements and where the fire spreads).

From both Fig. 2 and 3, we can recommend to set the fire plot after a small safety zone (6h or less), in order to limit the influence of the safety zone on the fire plot (to limit variations between the fire wind and the theoretical wind). Ideally, the fire line should be ignited at more than $2 h$ downwind to the leading edge, although this could raise practical problems. Regarding the measurement, the sensor would be placed in a very large open area (ideally larger than $20 \mathrm{~h}$ when available), on the side of the fire plot, to enable the convergence of the open wind, used to estimate the ambient wind. However, as it is often not practically doable, we recommend to measure the distance to the trailing edge and to 
describe the upwind canopy (at least the height, and eventually the Leaf Area Index and the canopy cover), so that horizontal wind adjustment factors (HWAF) can be applied, to convert the wind measured at a given distance to the actual open wind, at long distance from the edge. This is of major importance because for the same ambient wind (and thus a same expected ROS), the measured wind would be very significantly different if the measurements were made at $5 h, 7 h, 20 h$ or $30 h$ during the fire experiment. This could have been a significant source of bias in previous experiments, in which measurements were not necessarily homogeneous in terms of distance to the edge. We think that simulated or measured- HWAF could help to increase the quality of empirical fire data and to better understand the residuals in empirical models, which are in general fairly wide, as the mean absolute error is at best on the order of $+/-30 \%$ (Cruz and Alexander 2013).

Our analyses of measurement and fire wind variability confirmed the strong influence of the fire plot size, the experiment duration and the number of sensors, consistent with previous work in Sullivan and Knight (2001) and Pimont et al. (2017). The main benefit of the present work was to address this problem of wind representativeness in a context which was more realistic, as the previous studies compared measured and fire winds estimated below canopies and in horizontally homogeneous configurations. Clearly, our analyses demonstrate that the wind measurement accuracy is poor in fire plots of $50 \mathrm{~m} \mathrm{(3} \mathrm{to} 4 h)$ and limited in those of $100 \mathrm{~m}$ (6 to $7 h)$. For these specific experiments, and especially when only one sensor was available, the wind measurement variability can be reduced by averaging the measured wind on longer period than the one of the experiment (a few minutes before and after). There is no way, however, to reduce the variability of the fire wind, meaning that we basically have no idea of what the actual wind really was in the fire plot during the experiment (from 4 to $7 \mathrm{~ms}^{-1}$ ). According to our computations, a fire plot size on the order of $200 \mathrm{~m}(12 h)$ seems to be satisfactory, provided that the fire does not spread too fast. We would like to point that the magnitude of the ambient wind velocity was found to be a major factor of variation in measurement accuracy in homogeneous canopies (Pimont et al. 2017) and such a result should hold in the present more realistic context. It is thus expected that the -relative- variability of both fire and measured winds would be higher in the case of lower ambient wind.

This study also illustrates how physics-based models can be used to improve the knowledge regarding wind measurement in field experiments. More detailed analyses in a variety of fuel types and safety break design should help to provide general guidelines regarding the number of sensors and eventually to suggest some correcting factors such as the HWAF, described above. Finally, we did not investigate how the fire wind (at $h_{r e f}$ above the fire plot) relates to the mid-flame height wind, even if this vertical wind adjustment is another source of both bias and variance, which could be the scope of future investigations.

\section{References}

Albini FA, Baughman RG, Intermountain Forest and Range Experiment Station (Ogden, Utah) (1979) 'Estimating windspeeds for predicting wildland fire behavior' (Intermountain Forest and Range Experiment Station, Forest Service, U.S. Dept. of Agriculture,: Ogden, Utah:) doi:10.5962/bhl.title.68710.

Andrews PL (2012) 'Modeling wind adjustment factor and midflame wind speed for Rothermel's surface fire spread model.' (United States Department of Agriculture/Forest Service, Rocky Mountain Research Station)

Cheney NP, Gould JS (1995) Fire growth in grassland fuels. International Journal of Wildland Fire 5, 237-247. 
Taylor SW, Wotton BM, Alexander ME, Dalrymple GN (2004) Variation in wind and crown fire behaviour in a northern jack pine black spruce forest. Canadian Journal of Forest Research 34, 1561-1576.

Cruz MG, Alexander ME (2013) Uncertainty associated with model predictions of surface and crown fire rates of spread. Environmental Modelling \& Software 47, 16-28. doi:10.1016/j.envsoft.2013.04.004.

Fischer WC, Hardy, CE (1976) 'Fire-weather observers' handbook'. Agricultural handbook 494. (USDA Forest Service: Washington, DC). 152 p.

Hastie T, Tibshirani R, Friedman JH (2009) 'The elements of statistical learning: data mining, inference, and prediction.' (Springer: New York, NY)

Massman WJ, Forthofer JM, Finney MA (2017) An improved canopy wind model for predicting wind adjustment factors and wildland fire behavior. Canadian Journal of Forest Research 47, 594-603. doi:10.1139/cjfr-2016-0354.

Pimont F, Dupuy J-L, Linn RR, Dupont S (2009) Validation of FIRETEC wind-flows over a canopy and a fuel-break. International Journal of Wildland Fire 18, 775. doi:10.1071/WF07130.

Pimont F, Dupuy J-L, Linn RR, Dupont S (2011) Impacts of tree canopy structure on wind flows and fire propagation simulated with FIRETEC. Annals of Forest Science 68, 523-530. doi:10.1007/s13595-011-0061-7.

Pimont F, Parsons R, Rigolot E, de Coligny F, Dupuy J-L, Dreyfus P, Linn RR (2016) Modeling fuels and fire effects in 3D: Model description and applications. Environmental Modelling \& Software 80, 225-244. doi:10.1016/j.envsoft.2016.03.003.

Pimont F, Dupuy J-L, Linn RR, Parsons R, Martin-StPaul N (2017) Representativeness of wind measurements in fire experiments: Lessons learned from large-eddy simulations in a homogeneous forest. Agricultural and Forest Meteorology 232, 479-488. doi:10.1016/j.agrformet.2016.10.002.

Sullivan AL, Knight IK (2001) Estimating error in wind speed measurements for experimental fires. Canadian Journal of Forest Research 31, 401-409. doi:10.1139/cjfr-31-3-401. 


\section{Chapter 4}

Fire at the Wildland

Urban Interface 


\title{
Carcaixent fire impact against a self-protected WUI zone. Lessons learned
}

\author{
Ferran Dalmau Rovira*; David Caballero Valero; Francisco Quinto Peris \\ Medi XXI GSA - WUIProtect Consortium,www.wuiprotect.com, \{ferrandalmau@medixxi.com*\}
}

\begin{abstract}
In June 2016, a forest fire had a head-on impact with some misalignment, but with aggressive local topographic behavior, to the Colonia Santa Marina residential settlement. This settlement had a SelfProtection Plan approved in 2007. The Plan was based on a forest fires protection system whose acronym is WUIPROTECT@ (Wildland Urban Interface Defense System) and it included the self-projection planning for previous fire risk, the Owners Community training, fuel management and the installation defensive infrastructure. Both the Plan and the foreseen infrastructure for the nucleus were activated hours before the impact and contributed to the defense of the urbanized area.

With this methodology of operations, on one hand, the risk of affecting population and intervening professionals is reduced by lowering temperature, radiation, secondary foci ignition potential due to shortdistance flashes..., and on the other hand, environmental and infrastructures damage is reduced. Obviously, without the intervention of ground means, the result could have been different, in the same way that it would be if aerial means wouldn't join the heli-transported brigades efforts, WUIPROTECT ${ }^{\circledR}$ reduces the intensity of the fire, causing it to reach extinction capacity, emulating in this way what would be focused land-based discharges whenever they are convenient.

Due to the interest aroused by this unusual scenario, in which a developed forest fire impacts a residential area with a self-protection system, it has been considered convenient to proceed to analyze the facts to obtain some conclusions in order to identify the strengths and weaknesses of the system to improve its design, installation and operation.

This document is a summary of the technical report made based on testimonies, photographs and on-site observations of the facts and their consequences. For this purpose, an intense work of interviews with intervening professionals, eyewitnesses and owners of Colonia Santa Marina residential settlement and several expert ground visits to document the events has been carried out.
\end{abstract}

Keywords: WUI, defensive system, Fire Adapted Communities, Training, Civil Protection, WUIProtect, SIDEINFO, prevention, self-protection, risk reduction

\section{Introduction}

Colonia Santa Marina residential settlement is located between the municipalities of Carcaixent and Alzira, in Ribera Alta county (Valencia), in Barraca d'Aigües Vives valley, 500 meters from the main population nucleus on the right side of CV-50 road, towards Tavernes de la Valldigna (Figure 1).

According to the analysis carried out in the Self-Protection Plan, the vegetation surrounding in 500 meters, Santa Marina settlement is formed mainly by crops land (515 hectares) and land with forest cover (389 hectares). The forest land is covered mainly by Aleppo pine (Pinus halepensis) with close to $100 \%$ of covered fraction and plenty of Mediterranean maquis in the underwood. Colonia Santa Marina has 140 plots/properties and according to data compiled for the Self-Protection Plan, and during the summer houses a maximum estimated population of 700 to 900 people, depending on the day of the week. It should be noted that after Self-Protection Plan development, an urban development associated to Santa Marina, called Santa Marina 2, was made and meant a significant increase of the population in the area, although it is administratively segregated. The northern limit of the urbanized area matches with CV-50 road that links Lliria and Tavernes de la Valldigna and from which 
the residential area is accessed through the main entrance. A second entrance was added on the eastern boundary as part of the plan to improve the road network in the Self-Protection Plan ant it allows the access to the area from the Convent de Santa María d'Aigües Vives.

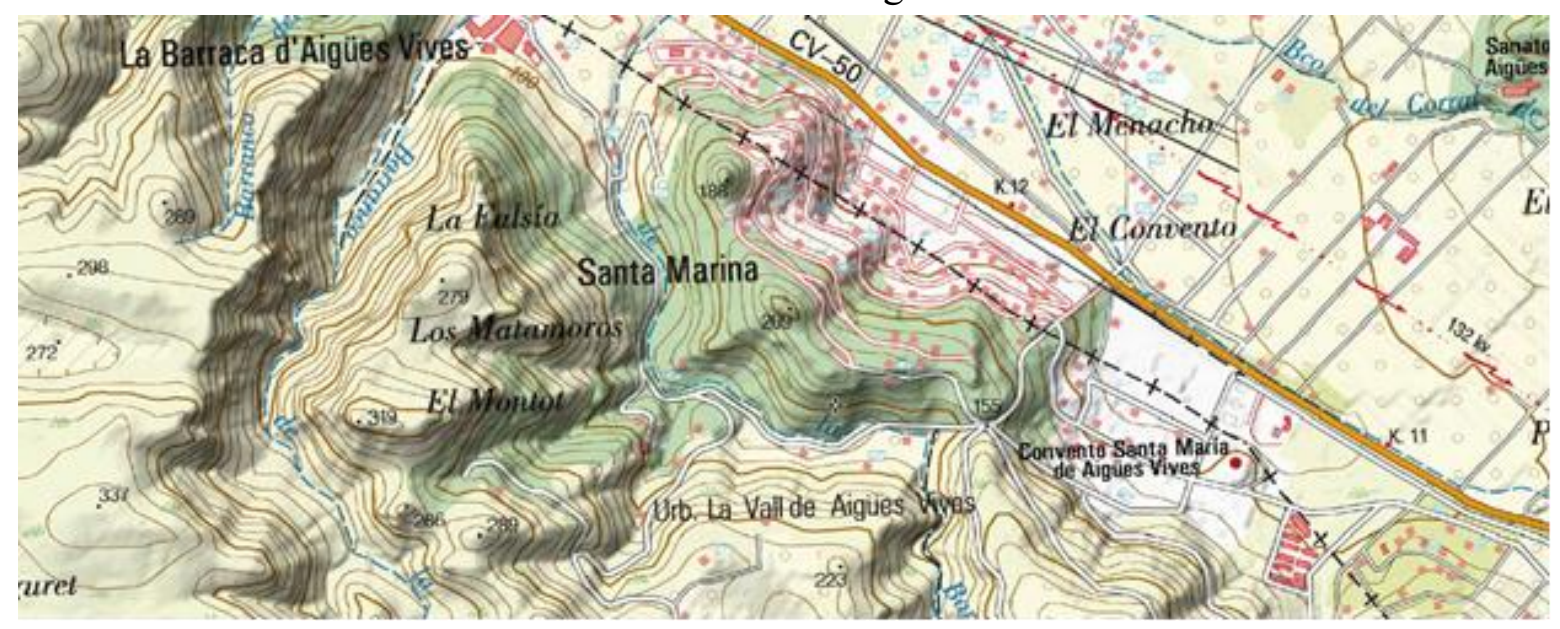

Figure 1 - General topography map of the impacted area. Fire impacts from W (left) to E (Right).

\section{Fire analysis}

\subsection{Weather conditions}

At the time and place of the fire origin, the wind predictions were higher than $20 \mathrm{~km} / \mathrm{h}$ with gusts up to $40 \mathrm{~km} / \mathrm{h}$, from general west component and getting slower during the following hours when it rolled to south-west and south component to the end of the day. The breeze at the beginning of the afternoon over the area was blocked by the effect of west component winds with intensities from moderate to strong. During the first moments of the fire, the temperature remained close to $30^{\circ} \mathrm{C}$ and the relative humidity below $30 \%$.

According to observation data from the $8300 \mathrm{X}$ station of Carcaixent, from the State Meteorogical Agency (AEMET), the wind was blowing from NNW and W component to roll to SSW at nightfall, when intensity decreased significantly. The push of the NW wind in the development of fire's left flank caused it that during the night, with SSW wind, to move towards the Colonia Santa Marina residential area, but with less intensity. On the final hillsides before the impact, the fire ran more because of topography than pushed by the wind.

The observed data by the means of extinction in the place and time the fire started on 16/6/2016 are the following: relative humidity, $25 \%$; temperature, $29,7-38,9{ }^{\circ} \mathrm{C}$; wind speed: $20,6 \mathrm{~km} / \mathrm{h}$ with gusts up to $38,9 \mathrm{~km} / \mathrm{h}$; wind direction, $280^{\circ}$ (W) to $300^{\circ}(\mathrm{NW})$. Value ranges between observations and predictions, particularly wind, could be caused by local effects and channelling by ravines and hills of the area.

\subsection{Overview of the impact zone}

The impact of the wildfire front in Santa Marina took place in different locations between 23:50h on 16/06 and 02:30h on 17/06, affecting almost all the forest perimeter of the settlement during this period. Although there was a decrease on the wind speed towards night, the fire front impacted with some misalignment in three areas where there was presence and continuity of forest fuel on both sides of the road that runs through the La Barsella ravine.

There were up to 3 high intensity wildfire fronts favoured by topography. The rest of the perimeter had a crop field (orange grove) in the lowest part that separated the forest mass. The crop field, which was farmed and maintained, acted as a firebreak at that point, giving a chance for control. In this part 
of the perimeter, two fire flanks were opened and ran with $2 / 3$ alignment by the fire relocation in the lower part of the slope. However, in all observed impact cases, the potential behavior was front/flank. The subsequent analysis evaluates the difference of the effects produced in different parts of the perimeter.

Fire managed to get through the defensive line in the southeast area (Picture 2) and in the northwest area (Picture 3). In the case of the southwest impact, it reached the interior of the residential area through the perimeter strip that had been without maintenance for 10 years. In the case of the northwest impact, it is estimated that something similar happened but the flame front could have been powered by the hill effect and the increasing intensity of local wind.

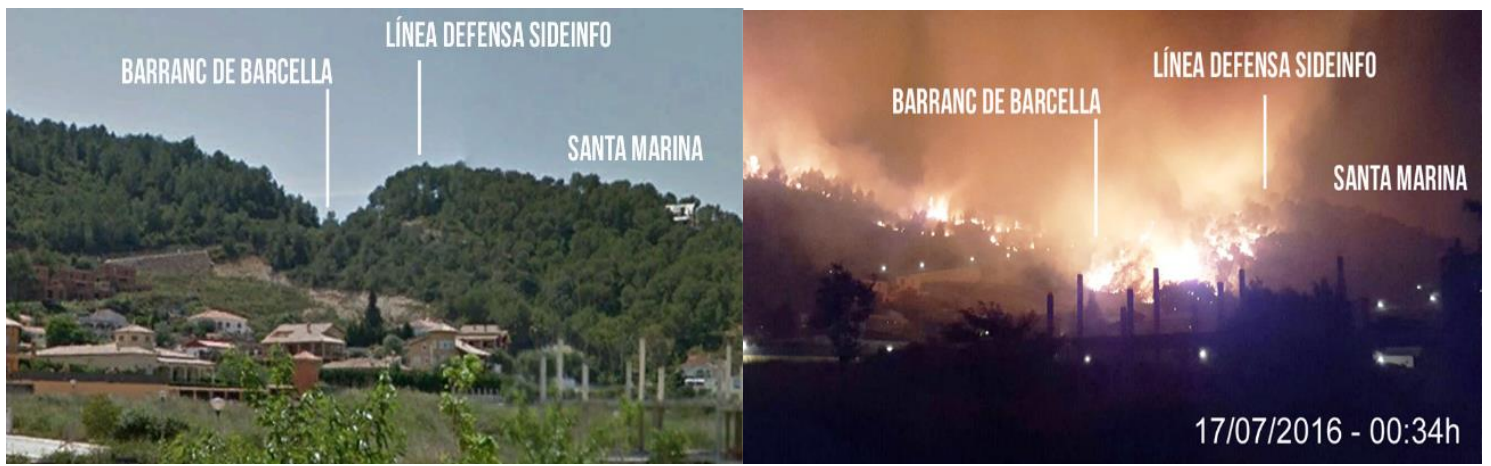

Figure 2 - Impact in area 1 (south-east). Images taken from the position of the Advanced Command Post. In the central part of the right image certain alignment is observed. When fire reaches the top of the slope, it meets the first wetted area by the WUI-PROTECT ${ }^{\circledR}$ and stops its progression. Source: Own elaboration based on Medi XXI GSA images.

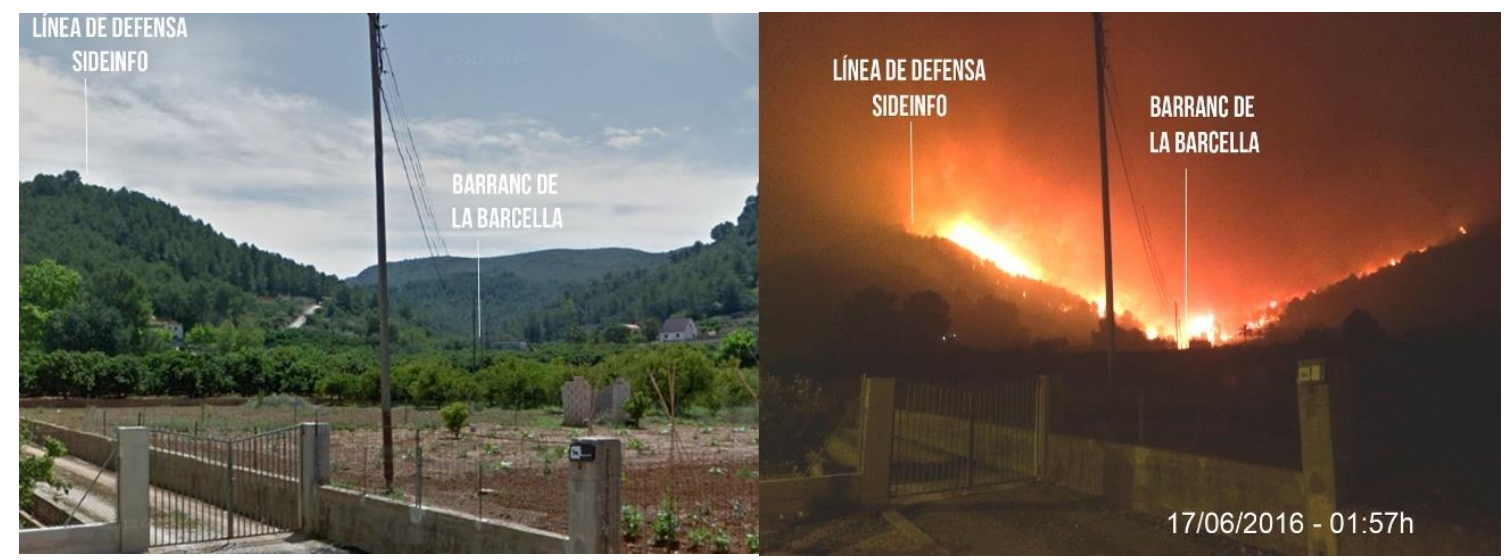

Figure 3 - Impact in area 3 (northwest). Images taken from Josep Piera Montagud Street in Barraca d'Aigïes Vives. In this area, the lack of maintenance of the perimeter fringe favoured the entry of fire inside the residential area. Source: Own elaboration based on Medi XXI GSA and J.L. Mollà pictures.

\subsection{Fire behavior at the impact site.}

The fire behaviour on the hillside through which the fire advanced and impacted on the urban settlement, based on direct observation of the forest fire and the analysis of the degree of destruction and severity in the vegetation later on (Picture 4). As mentioned before, towards the night the wind lost intensity and it rolled to a component that aligned with the slopes surrounding the urban core (SW). Both live and dead fuel was, in general, very available, due to previous drying days, due to poor recovery of the previous night, due to the west wind episode and due to the fire desiccant effect that 
entered Barsella ravine. With it, the fire that went up from the bottom of the ravine to the urban area, did it from an entry point located at the bottom of the ravine (B) and was realigned uphill pushed by the light wind but getting virulent, consuming all the available fine fuel in some points, in short runs of active crown fire that were generally driven by the undergrowth shrub. From the hillside inspection prior to impact, it can be deduced that fire developed its full potential both on the surface and on the treetops in those short distances, since there was practically no remaining fine fuel and the soil was severely burned, indicator of very high temperatures.

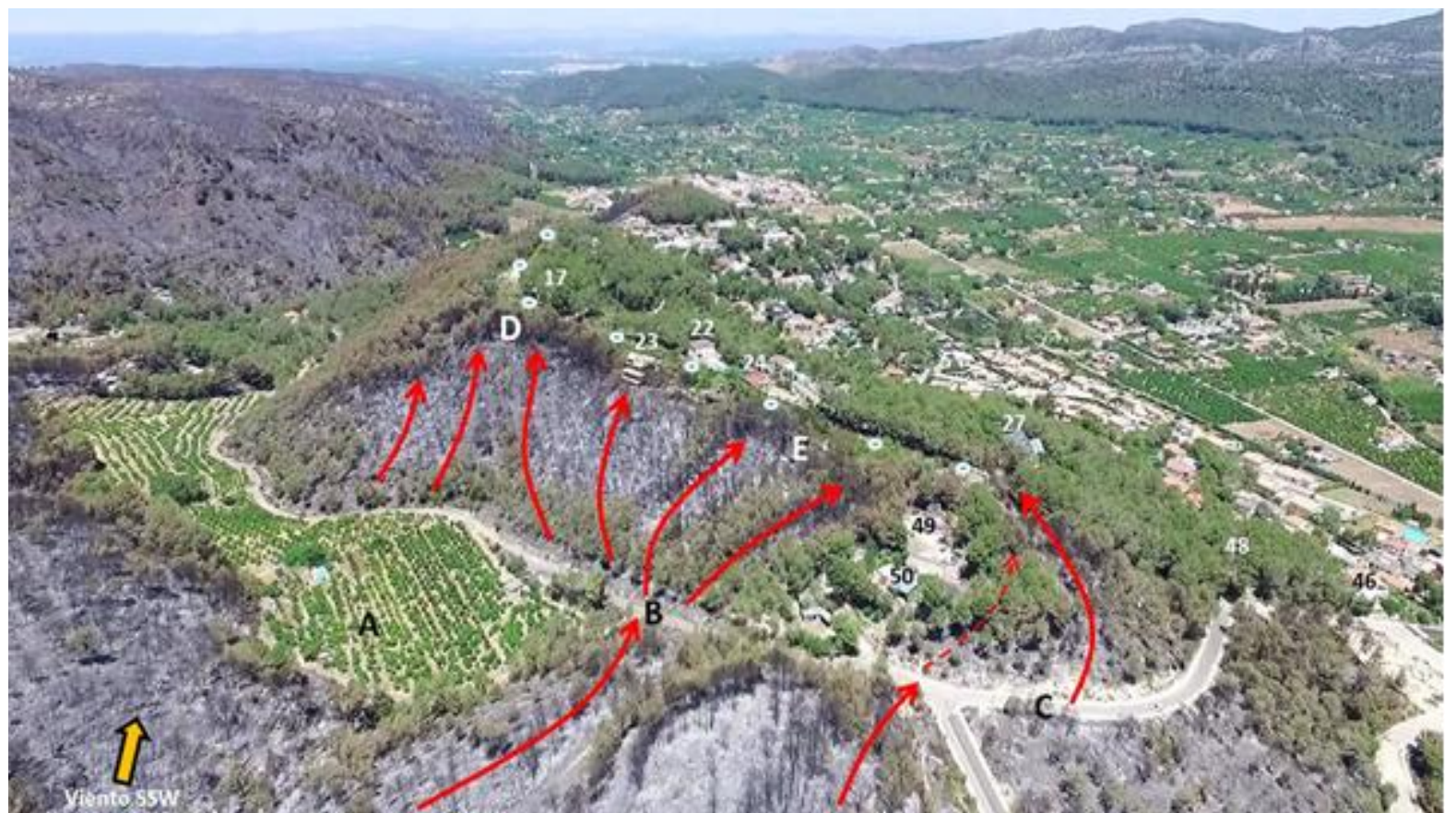

Figure 4 - Aerial view of impacted area after the fire. It shows that crops acted as a firewall (A) and also shows the possible entry point of fire to the $S W$ hill $(B)$, with wildfire front outplacement in very heavy wildfire main spreads to the top $(D$ and $E)$ and a second fire entry point $(C)$ which generated wildfire main spreads too. At the moment of the impact, $S W$ wind had a low intensity. The position of WUIPROTECT® cannons is indicated with circles. It can be appreciated that the house 23 was directly impacted by wildfire main spread head. Source: Medi XXI GSA

It is possible that fire jumped the bottom path of the ravine at some point $(\mathrm{C})$ causing new races on the southern side of the hill, some running under the undergrowth and burning pines and some other more intense climbing treetops in dependent races. It seems reasonable to think that the difference between both behaviours depend on the amount of undergrowth in each point.

\subsubsection{Flame length and intensity}

As mentioned and observed above, the flame length reached locally between 20 and 30 meters just at the pass of Barsella ravine and on the rise up the hillside. The fuel model described above, which can be seen on the right side of the picture taken before the fire, generated a high intensity flame that made it impossible for the deployed units (Military Emergency Unit, UME) to work in the area. It clearly seems that the wildfire main race through the hillside by the urban area, developed a fire whose intensity was outside capacity of extinction, forcing to defensive maneuvers to palliate the effects on affected houses.

\subsubsection{Propagation speed}

The fire spread very fast. It is estimated that during the first hours it reached propagation speeds between 50 and 75 meters per minute based on front position measures and the time elapsed since the 
beginning, burning more than 1.200 hectares between 16th and 17th of June (despite being at night) affecting finally 2.300 hectares until day19th.

\subsubsection{Smoke environment}

During all defensive operations the intervening crews were subjected to an intense smoky atmosphere and to a rain of short and long distance flashes although the fact that impacts occurred at night helped to avoid in part more secondary ignitions due to the increase relative humidity and the released water from the system in the perimeter, according on testimonies collected from the operation.

\subsubsection{Hot ashes range and secondary fires}

In the detailed post-fire inspection (made in the days immediately following the impact) a constant ash layer was observed through the streets of the residential area. This fact indicated that urban nucleus was subjected to a constant rain of ashes that arrived extinguished to the ground because by its size and mass the complete combustion happened in its flight.

Notwithstanding the foregoing, at least 3 secondary fires were documented in the first 200 meters from the main front in the impact zone. There is no record of any further jump of larger particles into the population centre that would result in secondary fires. According to testimonies gathered by the Fire Department of Valencia Provincial Consortium, the hot ashes that jumped the perimeter extinguished when they met previously wetted vegetation.

\section{WUI-PROTECT ${ }^{\circledR}$ system in Santa Marina}

As explained in annex 1, WUI-PROTECT ${ }^{\circledR}$ system consists of four components, namely: fuel management and perimeter belt, training and education, hydraulic cannon system and planning. This section focuses on the hydraulic cannon system component, specifically at the moment of the impact the installation was as follows:

Monitoring location and features: the hydraulic system of Santa Marina is composed by 15 monitors distributed on 5 sectors ( 3 monitors/sector) numbered from 1 to 5 . Picture 44 shows its association with 3 impacted areas by wildfire's front. 18 monitors were initially proposed (one more sector) but this sixth sector was not implemented for budgetary reasons. This system works at $6-7 \mathrm{Kg} / \mathrm{cm}^{2}$ with a range per monitor between 45 and 50 meters. At the base each column there is a fast-charging hydrant (15 in total). The overlap between the areas of influence of each cannon is between 15 and 20\%. Each cannon irrigates between 6.400 and $8.000 \mathrm{~m}^{2}$ with a rainfall between 7,6 and $9,31 / \mathrm{m}^{2} /$ hour.

\subsection{Sequence of events at WUI-PROTECT ${ }^{\circledR}$ operation in Santa Marina}

At 17:44h, the people in charge of the Self-Protection Plan of the Colonia Santa Marina residential area got in touch with the Advisory Committee (figure include in the Plan) specifically there was a first contact with technicians who wrote the Plan to consult on the activation of WUI-PROTECT ${ }^{\circledR}$ hydraulic system in Santa Marina. The first pictures received from the impact area were taken by SelfProtection Plan members of the urban nucleus. (Pictures 5 and 6)

After consulting municipal officers and gathering information on the weather conditions, fire situation and its expected evolution, instructions to turn on Santa Marina's WUI-PROTECT® system were given by the Advisory Committee. Immediate start-up was confirmed at 6:00 p.m. on 06/16/2016, both through the conversations held and through the graphic documentation compiled.

The recommended discharge (operation) parameters were to contribute to each sector (from 1 to 5) between 15 and 20 minutes of water and successively repeat the pattern to ensure a homogeneous contribution of wetting to the entire perimeter. 


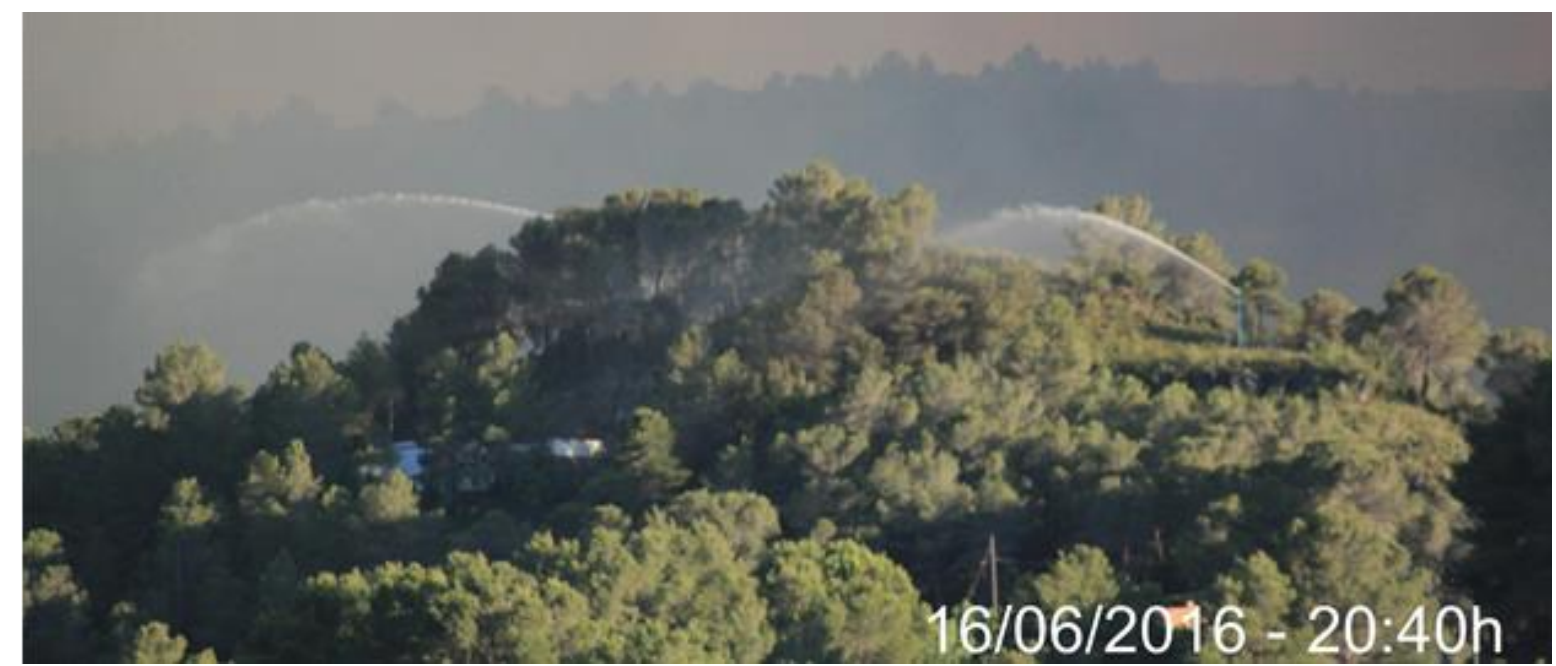

Figure 5 - Image taken on 06/16/2016 at 8:40 p.m. from Barraca d'Aigües Vives. It shows WUI-PROTECT® monitors of sector 3 providing water to the perimeter before the arrival of the flame front. Source: Vicent Vila.

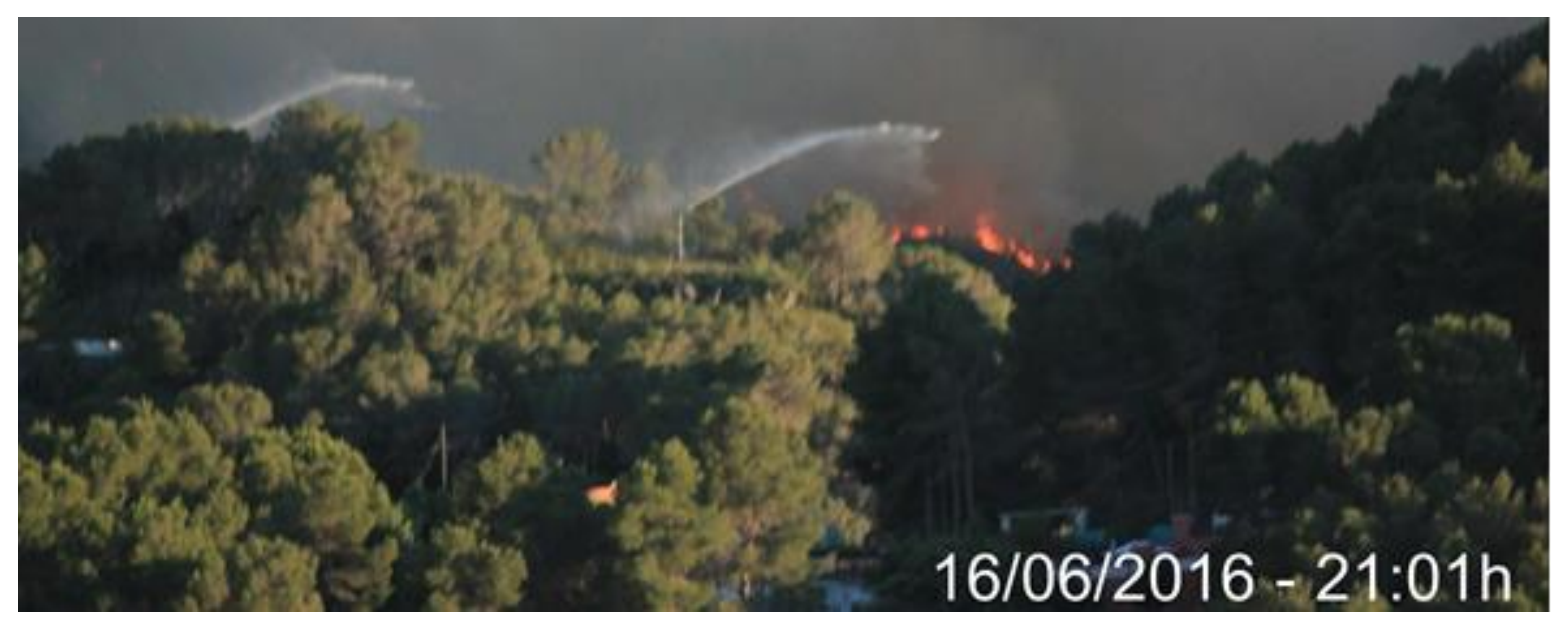

Figure 6 - Picture taken at 9:01 p.m. from Barraca d'Aigües Vives. It shows WUI-PROTECT® monitors of sector 3 as they continue supplying water, in this case outwards, before the arrival of the flame front. It shows that the water reach is less outwards due to strong upwind. However, this effect was offset with a bigger water inner scope. More water inside and less water outside. Source: Vicent Vila

\section{Analysis of the observed effect of operations on fire spread}

At the moment of the impact, the flame front locally developed its full potential, as showed by the traces observed in situ after the fire (white ashes due to its complete combustion, totally consumed treetops and so on). In the last meters of the fire run there is a significant reduction in the intensity and, therefore, in the speed of fire, especially in the areas with the greatest amount of water contributed by WUI-PROTECT ${ }^{\circledR}$. In fact, a complete stop of the fire progress is observed in some of the areas exposed to the fire front, which has come to be called "green island effect", as evidenced by the case of house 23 that, despite being on the most exposed slope, it has not suffered significant damage. In the postfire eye inspection, the authors were surprised at seeing such a remarkable gradient in the fire behaviour in the local run up the slope: from seeing a burned out ground and treetops with fine material completely consumed to see vegetation barely scorched in a narrow stripe and intact green vegetation a few meters away. Operations of deployed media were favoured by being able to work in some impacts whose flame front were in extinction capacity. With all this, it can be deduced that WUIPROTECT $^{\circledR}$ action created in part of the impacts a fire behaviour that was within the security margins 
for both flame intensity and secondary outbreaks that could end up in potential fires. WUI-PROTECT ${ }^{\circledR}$ system effect emulated aerial means unloading water during hours in the impact zone. The system had no effect on the smoke.

\section{Discussion}

Carcaixent wildfire did not badly damaged population centers due to the combination of previous actions and the sequence of events that took place between June 16th and 17 ${ }^{\text {th }}$, 2016. All affected population centers had developed a Self-Protection Plan and part of the inhabitants had received training in fire defense. Although the involvement in this kind of activities doesn't usually exceed $20 \%$ of the population, the fact of having a group of neighbours from the affected area with the necessary training on fire defense, was clue to an effective defense of Santa Marina urban nucleus. Previous training sessions were useful to have communication channels open and current. On the other hand, the mechanisms established in the Self-Protection Plan served to coordinate actions among Plan managers and external aid. Thanks to the previous work, evacuation takes place without incident and part of the neighbourhood, knowing how to act, led their own self-protection.

One of the elements that is considered necessary to improve in Santa Marina is the automation of the system. While communication technologies and automatism have been implemented on projects developed later, it has been proven that leaving the activation of the system to the Community of Owners, increases the likelihood of human error that could compromise the effectiveness of the system. It is considered that the system was activated sufficiently in advance. The problem was not the activation itself, but water consumption management. Since advisory committee members were not in operation area all the indications was given by phone and messages. By not having a complete view of the situation, Advisory Committee requested to activate the system too soon. This is not a negative fact in itself. In fact, it has always been explained that the system would be more effective the longer the advance with which it was activated. Fire Department of Valencia Provincial Consortium middle managers were the ones who determined in situ which sectors to activate at all times, correctly covering the entire perimeter.

The prescribed operations parameters consist of consuming 50\% of the water before the impact and saving the rest by the time of the impact. This parameter was not respected for various reasons related to the amount of information in this kind of events. For this reason, the idea of the need to implement automatisms (water discharge sequencer, probes for calculating water reserves) is reinforced to avoid human errors in the operation.

\section{Conclusions}

From the collection, analysis and discussion of the data and information exposed about Colonia Santa Marina forest fire, different conclusions can be deduced in order to improve protocols, methods and devices of WUI-PROTECT ${ }^{\circledR}$ wildfire defense system.

According to the data collected and the information processed throughout the preparing of this report, the combination of fire fighting crew's work and WUI-PROTECT ${ }^{\circledR}$ system contributed to significantly reduce damages inside Santa Marina urban area. As an initial conclusion, it should be noted that, although the results could have been improved, given the existing conditions, the work carried out before the fire in the four areas detailed on the methodology (developed over the ten years that the system has been installed) has been essential to provide effective self-protection against the wildfire that affected the urban nucleus.

The WUI-PROTECT ${ }^{\circledR}$ Wildfire Defense System has fulfilled its goal for which it was designed, contributing significantly to reduce damages derived from the direct impact of a high intensity wildfire, even though it was not the worst possible scenario (for example, if the fire had started 3 hours earlier 
and had arrived in full alignment and potential). However, from this report we have laid the foundations for the improvement and evolution in order to offer better solutions in the future. Some conclusions are detailed below by operational areas in order to organize the knowledge acquired throughout the process.

- Monitors, as a hydraulic part of WUI-PROTECT ${ }^{\circledR}$ defense system, have fulfilled their role of reducing intensity and speed of the flame front approaching an urban nucleus, in order to create an environment for suppression and defence operations in which fire is within extinction capacity in most of the perimeter. In the areas where it has been totally effective, working conditions have improved. (Picture 6)

- For that goal, WUI-PROTECT® monitors have served as support on fire control and extinction operations, similar to continuous water discharges from planes, in a particularly important area (an urban settlement inside the impact area) and just at the right time (especially during the night, without support from aerial means).

- According to testimonies of the operation members, WUI-PROTECT® monitors created a safer working environment for ground operations, especially by reducing the temperature. However, as a collateral effect, the soil had worst conditions in places where water accumulated, mud was formed and surface run-off was caused. WUI-PROTECT® had no effect on smoke.

- According to remains and severity analysis, everything seems to point out that WUIPROTECT $^{\circledR}$ system water posture could have contributed to reduce the intensity and the speed of propagation of a fire front that progressed over treetops in its final strech to Colonia Santa Marina. In areas where there was more water supply, it is quite clear that WUI-PROTECT ${ }^{\circledR}$ system contributed to stop the fire's progress.

- Everything seems to indicate that there is a direct correlation between the amount of water thrown and the level of damage to the vegetation. Those points in which the monitors did not operate (due to water pipes destruction), the defense and extinction operations were conditioned and also the degree of the impact on houses and vegetation presented greater severity. However, this observation must be tested in future research and development actions.

- Secondary fires did not advance inside the action range of WUI-PROTECT ${ }^{\circledR}$ monitors. This avoided internal ignitions improving the safety of the intervening units and defending buildings in the most exposed areas.

- WUI-PROTECT ${ }^{\circledR}$ water distribution on the vegetation and buildings created a "green land" effect that contributed to the fire control and to reduce building damage. Houses inside the action range of WUI-PROTECT ${ }^{\circledR}$ monitors showed no damage or damage was observed in those areas where water did not arrive. The shadow effect of vegetation and the structures themselves must be considered on future system designs.

- The total amount of water sprayed by WUI-PROTECT ${ }^{\circledR}$ monitors could have been better distributed in the different sectors; also, and according to the results on vegetation, so much water would not have been necessary to meet the objectives of reducing the intensity and speed, thus bringing the wildfire front to a scenario of extinction capacity. The sequence and the timing of water distribution was not optimal nor was it sufficiently coordinated with ground extinction operations.

- WUI-PROTECT ${ }^{\circledR}$ monitor operation is one of the four components to protect urban nucleus against wildfires. Despite the positive effect observed in controlling the progress of the fire to Santa Marina, its effectiveness could have been multiplied if the protection strip had been 
perfectly maintained, particularly at the points where the fire was able to pass into the urban nucleus. This reinforces the thesis developed by project managers. The combined action of the four lines of work (Planning, Fuel Management, Hydraulic and infrastructure, training) is fundamental. It is necessary for the future to improve the design of these defense spaces to favor the effectiveness; they must necessarily be accompanied by viable maintenance plans and assumed by the owners.

- Sections of pipe network potentially exposed to radiation and in contact with fire could cause partial or total failure of the system by the degradation or destruction of the same ones. The presence of water inside the pipes reduces or prevents such destruction as has been demonstrated. However, previous operation and hydric contribution improved conditions during the impact.

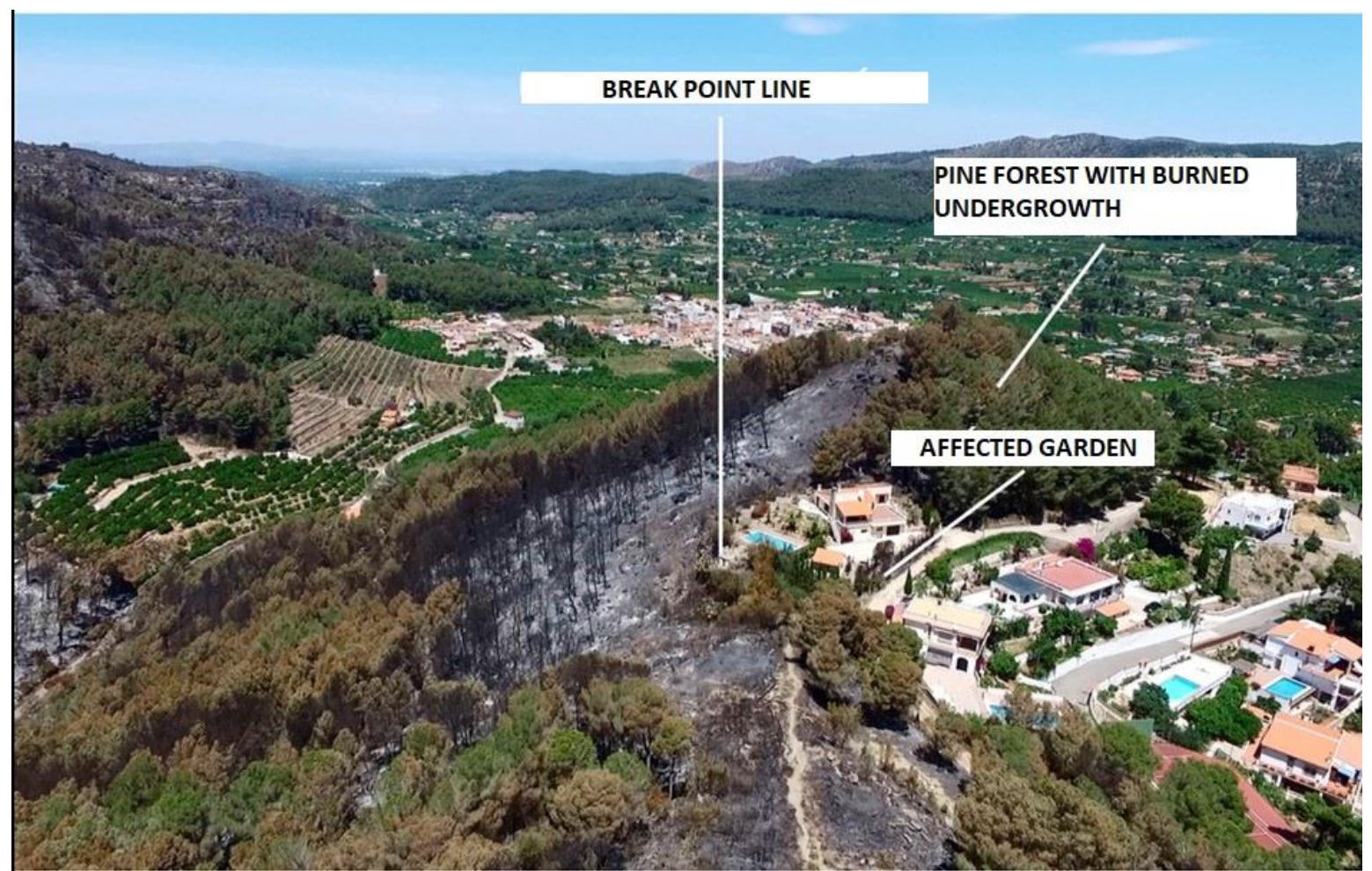

Figure 7 - Break point of the water pipe in the area of impact 3. It has been identified from the review of the entire istallation in the perimeter. Source: Medi XXI GSA

The WUI-PROTECT ${ }^{\circledR}$ design in Santa Marina is adjusted to the defensive needs of the residential area since it is not planned as an extinguishing tool, although it has a broad range of improvement on both design and execution of actions that will be based on the knowledge acquired after the Carcaixent fire, among others.

Hydraulic installation is part of a set of defensive actions (planning, fuel management, infrastructure and training) aimed at improving the self-protection capacity in urban forest interface environments with high risk of fire. The second line of protection is provided by terrestrial means. That has always been the prescribed working methodology, and in this case it has shown its effectiveness.

With this operation methodology, on one hand, the risk of harming population and interveners is reduced by lowering temperature, radiation, ignition potential of secondary fires caused by short distance spotting. On the other hand, damage is reduced at both environmental and infrastructural level. Logically, without the intervention of the terrestrial media, the result could have been different, but in the same way that the heli-transported brigades base their effort on the work combined with the 
aerial means, WUI-PROTECT ${ }^{\circledR}$ reduces the intensity so that the fire enters in extinction capacity emulating what would be focused land-based discharges where it is convenient at each moment.

The self-protection on areas at high risk of wildfire can not be a should not be a right, it must be a shared obligation betwen public administration, that must generate the necessary framework to adopt the actions, and individuals whose interests, properties and lives will be at risk at the time of the incident. It must be clearly stated that defending wildland-urban interface areas puts the lives of professionals at risk on many occasions. And the lives of the interveners are also above the defense of the goods. As long as the interveners lives are put at risk to defend private properties and the defense of environmental resources is mortgaged, the situation becomes socially, economically and environmentally unsustainable.

It has also been shown that investment in preventive / defensive measures can be much more profitable than fire extinction. And this is not just based on investment on population centers. It should also be based on the agroforestry management of their surroundings. When measures on wildlandurban areas and their immediate surroundings are prepared to receive the fire impact, control and firefighting operations can be carried out normally without consuming public resources to defend private properties. "Crews kidnapping" effect at interface areas is known among the wildfire professional sector. This way, the fact of prioritizing the defense of populated areas leads to a neglect of forest and rural areas. This ends up having as a direct consequence that public or private areas that generate benefits for the society as a whole $\left(\mathrm{CO}_{2}\right.$ and other greenhouse gases sinks, oxygen production, landscape, hydrological cycle...) are unprotected because spaces that should be self-protected (that is, protected by their own means) are using this protection.

The cost per owner for the period 2006 - 2016 was 707,46 euros. Defended urban nucleus has 140 plots. If the investment per plot had been a bit higher, protection would have been clearly more effective. Having invested 1.000 euros per owner would have reduced fire damages and it would have saved the expense that now involves recovering it to a normal state, both in gardens and in the few damaged infrastructures. This also includes damaged pipes from WUI-PROTECT ${ }^{\circledR}$ itself, obviously. As an example, Fuencaliente's wildfire (on 2009) showed that the ratio of costs of firefighting to those of subsequent recovery was 1-6. 


\title{
Coupling multi-criteria analysis and GLM for modelling houses vulnerability to forest fires at WUI
}

\author{
Eric Maillé ${ }^{1 *}$; Lilian Pugnet ${ }^{1}$; Jessica Hedan ${ }^{1}$ \\ 1*Irstea. CS40061, F-13182 Aix en Provence Cedex 5. France. \{eric.maille@irstea.fr*, \\ lilian.pugnet@gmail.com,jessica.aleys@gmail.com\}
}

\begin{abstract}
In the French Mediterranean context, a quite low number of damages are caused by the forest fires on anthropogenic values. The usual statistical correlative approaches for vulnerability modelling, aimed at explaining the observed damages with some local variables, may lack of repetitions in comparable conditions. To overcome this constraint, we coupled an expert opinion based multi-criteria analysis and modelling (MCM) approach and some generalized linear models (GLM) techniques in order to specify a vulnerability model validated in different contexts. The method consists in first defining an opinion based model of vulnerability using a multi-criteria analysis. The specified model allows calculating some vulnerability indexes in relation to the value of some vulnerability criteria. The relationship between the vulnerability indexes provided by the MCM outputs and the observed damages is then tested by comparing indexes values in the samples of comparably exposed damaged and non-damaged houses. Non-parametric tests have to be used due to the relatively low number of observations and the uncertainty of the distributions normality.

Logistic binary regressions with a logit link function are then defined with the aim to explain damaged or non-damaged comparably exposed houses to fire by the MCM vulnerability indexes. . In order to avoid autocorrelation effects, a shifted vulnerability index is integrated to the model as an additional explanatory variable, the auto-correlation having been previously assessed with the Moran index. If the binary regression is significant, a polytomic logistic regression is tested in order to assess the effect of the calculated vulnerability indexes on damage level. This latest is assessed using a six levels scale of damages on objects, the main dwelling building or the whole site.

Although the very high variance in both damages occurrence contexts and scenarios, results show the possibility to validate a general model of vulnerability by coupling the MCM approach and statistical analysis of observed damages. One of the most constraining requirement is to find some damaged and non-damaged sites that were equally exposed during the event. Physical modelling of fire behavior is often used to assess the exposure. However, the systematic data collection of damages on anthropogenic values using a normalized protocol, and its dissemination in a shared data base would be a great progress for decision support in land management and planning for WUI vulnerability mitigation.
\end{abstract}

Keywords: vulnerability, damages, multicriteria analysis, generalized linear models

\section{Introduction}

Due to global change, vulnerability more and more becomes a key concept in wild fire risk assessment together with hazard and exposure (Zscheischler \& al., 2018). Climate change impacts mainly hazard, defined as the likelyhood of occurrence of fires of a given range of intensity levels. But it may also incease vulnerability of forest ecosystems, by decreasing thier resilience and recovering potential, even if fire regimes do not change. Other components of global change, including land cover change, also increase both hazard and anthropogenic vulnerability (FUME, 2013). Large wildland urban interface resulting from the extend process of discontinous urban areas within wildlands are areas of both great ignition rate (Ganteaume 2013) and great vulnerability level (Maillé \& al. 2015). While for wildland fires the key factor for risk mitigation is hazard, vulnerability of houses and other anthropogenic stakes must also be reduce in order to limit the probability of damages in case of WUI 
fires disaster (Cohen 2003, Lampin-Maillet \& al., 2009). With this objective, vulnerability of anthropogenic stakes should first be objectively assessed with validated models (Blanchi \& al. 2011, Caballero \& al. 2003). Statistical approaches for modelling vulnerability are the only one to be able to bring the proof of model validity, based on data collection of damages on anthropogenic stakes caused by past fires. However, in the contexts of relatively low number of damages occurred in comparable conditions, the statistical approach may lack of repetitions. Alternative approaches, including multicriteria analysis of expert opinions (MCA) (Saaty 1980, Saaty 1993), bring some more information aimed at contributing to vulnerability modelling.

We coupled a multi-criteria analysis approach and some generalized linear models (GLM) techniques in order to specify a vulnerability model validated in different contexts (Hedan 2017). Given the wide variety of definition found in the litteratude, we develop de conceptuel framework chosen in the methodological section 2. Then we describe de method principles and the study fires. We present some early bird results in section 3 and discuss about the appraisal and perspective of this work in section 4 .

\section{Method}

\subsection{The conceptual framework}

A wide variety of definitions is found in the literature for the concept of vulnerability (Zscheischler \& al. 2018, Zeng \& al. 2003, D'Ercole \& Metzger 2009, Carrega 2008 ). In this work, we define vulnerability as the potential consequences in terms of damages of a hazard occurrence, i.e. a fire event with a given intensity, threatening anthropogenic stakes. A stake is defined as any valuable element of the territory that might be damaged by the fire, i.e. any valuable element with a vulnerability level different from zero. This includes anthropogenic stakes (buildings, infrastructures and also human populations themselves), and natural stakes (notably forest ecosystems themselves, and notably their biodiversity). A "value" is assigned to the stakes in order to hierarchize different classes of stakes. Econometrical methods aimed as assessing such values should be able to take into account many kinds of values, including production, patrimonial, heritage, hedonic, living environment, etc. and also to provide assessment of human being life, what is ethically unsustainable.

In this work, we consider only one class of stake, the "interface dwelling site", and we assume an equal "value" assigned to all of them. An "interface dwelling site" is a system composed of one (or exceptionally several) dwelling house(s) and its (their) immediate surrounding environment, usually including a garden and some recreational elements (swimming pool, other leisure equipment) and annexes buildings. It is generally endowed with a vegetal biomass load, composed of ornamental and semi-natural vegetation. It is often enclosed with a fence, and is managed by one unique main actor (the owner or tenant): it usually corresponds to a tenure unit. Inhabitants themselves are also considered to be part of the site. Considering that human being life (and health) always has an "overdetermining" value, we assume that attributes of persons leaving on the dwelling site (number, age, state of health...) do not change the stake value but only its vulnerability.

Three components of vulnerability are considered: $i$ ) internal vulnerability (sometimes defined as the susceptibility to damages - Carrega 2008), ii) external (or contextual) vulnerability, and iii) response capabilities. Internal vulnerability is mainly determined by building materials and building options. Contextual vulnerability is mainly determined by local potential exposure and the mechanisms of transfer of energy from the wildland fire to the stake, including notably local propagation by contact, brands projections in convective flows, and radiation possibly intercepted by opaque or semi-opaque elements. Finally, response capabilities concern the proactive behaviors of people during the event that may increase or decrease the global damages, including escape capabilities, self defense, risk The purpose of this work is to specify a model aimed at calculating a priori the vulnerability of interface dwelling sites. According to our definition, the model should be based on damages measuring, 
knowing the exposure conditions, notably the threatening fire local intensity. Local measures of fire intensity are very rare. Moreover, number of damages on anthropogenic stakes in similar conditions is very low on our case study area. In order to specify a valid model of vulnerability, tested the relationship between observed damades and the vulnerability indexes calculated by an expert oponions based vulnerability model.

\subsection{Method pinciples and study fires}

We based our modelling approach on expert opinions multi-criteria analysis and we used observations and measures of damages to check the validity of the multi-criteria model (fig 1 ).

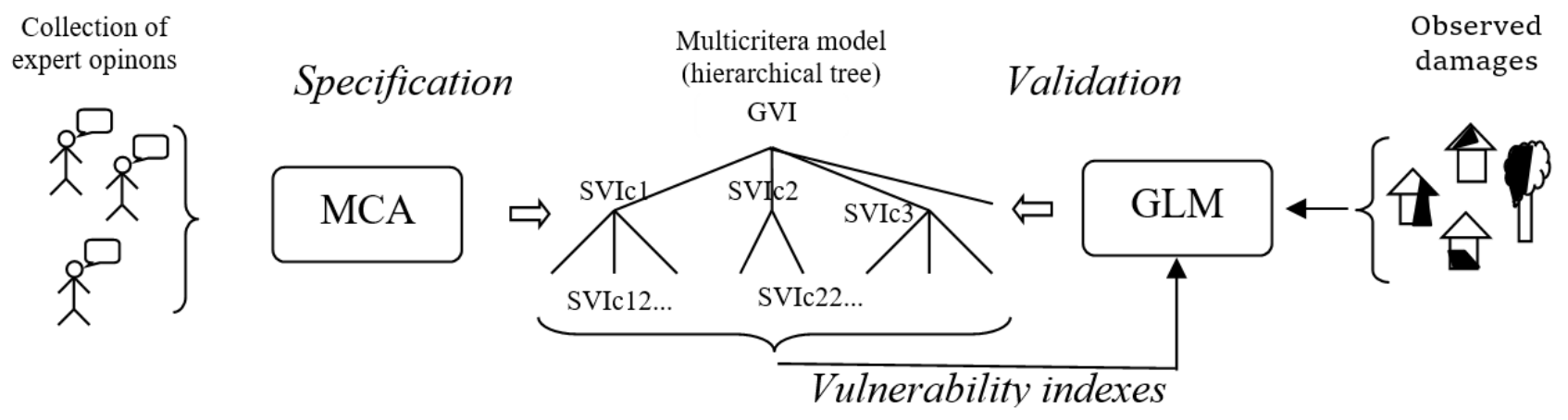

Figure 1 - The modelling approach

The first step is to specify an expert opinion based model of vulnerability using Multi-Criteria Modelling. This one allows calculating a global vulnerability index (GVI) and some indexes of vulnerability specific to criteria or to criteria families (SVIc1 to SVIc6, table 1). These latest represent the contribution of one criterion or criteria family to the global vulnerability.

The second step consists in relating the calculated indexes to some observed damages on WUI built up sites. Damages on houses in WUI of two main fires were described: the Rognac 2016 fire (10-12th of August 2016, 2663ha, 181 sites damaged, 117 buildings affected, 39 seriously and 26 totally destroyed) and the Cavaillon 2012 fire (21st of July 2012, 33ha, 20 sites affected, 13 dwelling houses damaged). The damages are described, based on three 6 levels scales of damages (0: not damaged, 5: totally destroyed/disappeared), related respectively to objects, main buildings and the whole damaged site, including usually a house and its environment. All the damaged houses could not be analyzed due to the required direct interview with the resident, who may not accept. Vulnerability indexes of non damaged but comparably exposed sites are also calculated. Given the low number of observations in the samples (20 observations for the Cavaillon fire and 36 observations for the Rognac 2016 fire, both including damaged and non damaged sites), and the uncertainty concerning the normality of the distributions, the relationship between damages and the different global and specific vulnerability indexes were tested using non parametric tests, mainly the Kruskal-Wallis test.

For significant identified relationships, an approach based on binary logistic regression, with the "logit" link function, is used to model damage occurrence (damaged/non damaged) in relation to vulnerability indexes values. Finally, in case the binary logistic regression was significant, ordinal (polytomic) logistic regression (Christensen, 2013) have been tested in order to propose a valid model able to preview damages levels in case of exposure (Cardona, 2012) in relation to vulnerability indexes values. In order to avoid autocorrelation effects, a shifted vulnerability index is integrated to the model as an additional explanatory variable, the auto-correlation having been previously assessed with the Moran index (Anselin, 1995). 


\section{Early bird results}

The following table (table 1) compares the P-value of the Kruskal-Wallis test comparing the vulnerability indexes specific to the different criteria families and the vulnerability global index of samples of damaged or not damaged houses on comparably exposed sites.

Table 1 - P-values of the Kruskal-Wallis test of the effect of different vulnerability indexes on measured damages for fires Cavaillon 2012 and Rognac 2016

\begin{tabular}{ccc}
\hline & Rognac & Cavaillon \\
& $\mathbf{2 0 1 6}$ & $\mathbf{2 0 1 2}$ \\
\hline \hline SVIc1 (local infrastructures and equipments) & 0,608 & 0,052 \\
SVIc2 (Topography) & 0,252 & 0,551 \\
\hline SVIc3 (Vegetation) & 0,1228 & 0,063 \\
\hline SVIc4 (Building arragements in WUI) & 0,061 & 0,197 \\
SVIc5 (Construction techniques and materials) & 0,529 & 0,781 \\
Global Vunerability Index (GVI) & 0,065 & 0,426 \\
\hline
\end{tabular}

The global vulnerability index separates significantly the samples of damaged sites and nondamaged for the Cavaillon fire, but not for the Rognac fire. Vulnerability indexes specific to the criteria families do not significantly differs between these two samples, although some P-values are quite close to the 5\% significance threshold. The non parametric Kruskal-Wallis test (ordinal test) is of course quite weak and some more observations of damaged building are required.

A logistic regression with the GVI and the shifted GVIs as explanatory variables of the damage occurrence (damaged/non-damaged) is then tested. The significance of the effect of each explanatory variable is tested with both the test of Wald and the likelihood ratio test (LR, Agresti 2002), while the global regression is tested by the likelihood ratio test only.

Unsurprisingly, the regression is not significant for the Rognac 2016 fire but highly significant for the Cavaillon 2012 fire (table 2).

Table 2 - Results of the binary logisitic regression on the Rognac 2016 fire and the Cavaillon 2012 fire

\begin{tabular}{ccccc}
\hline \multicolumn{2}{c}{ Rognac 2016 } & (DOM GVI + GVIs) & & \\
\hline \hline Variable & Coefficient & Test of Wald & LR test & $\begin{array}{c}\text { Global LR } \\
\text { test }\end{array}$ \\
\hline GVI & 2,724 & 0,415 & 0,396 & 0,151 \\
GVIs & $-1,296$ & 0,085 & 0,056 & Global LR \\
\hline Cavaillon 2012 (DOM GVI + GVIs) & & test \\
\hline Variable & Coefficient & Test of Wald & LR test & 0,001
\end{tabular}

\section{Conclusion/Discussion}

We proposed a contextualised explicative model of vunerability to forest fire of houses in WUI, by coupling a MCM and a GLM approaches. The obtained model is not general, and should be re-specify in different contexts. It is however explicative as it identifies the weight of the main variables (criteria) 
contributing to the global vulnerability of such anthropogenic values. Beside the operational results, this work brings some methodological lessons learned.

\subsection{The multicriteria analysis as a modelling tool}

Multi-criteria analysis (MCA) is a powerful approach of complex decision support tools implementation, but the use of expert opinion based multi-criteria analysis for a modelling purpose, known as multi-criteria modelling (MCM) is so a second level of use of MCA (Pugnet \& Maillé 2013). MCM can only model the expert knowledge about a phenomenon, and not the phenomenon itself. However MCM is a way to identify and assess the main variables (criteria) involved in vulnerability of built up sites within WUI, in situations where experimental data is missing.

Usual statistical approaches are however required in order to bring the proof of the MCM model validity. Lack of homogeneous data constrains to use non parametric tests, usually weaker than parametric ones. Generalized Linear Models, and more specifically logistic models, also require homogeneous data, but the level of significance raise quickly as soon as either the variance decrease (homogeneity of the context) or the number of observations rises.

\subsection{The need of a damages database for vulnerability modelling}

In the contexts where damages on anthropogenic values (and specially dwelling houses) remain fortunatly rare (case of Southern France), an exhautive share data collection about damages should be carried out in order to have a comprehensive approach of damage occurence. An shared protocol for this data collection should be normalize at national or international level, and a shared database implemented.

\section{References}

Agresti, A. 2002. Categorical Data Analysis. Wiley, New York.

Anselin, L.,1995. Local indicators of spatial association - LISA, Geographical Analysis, Vol. 27(2), pp. 93--115.

Blanchi, R., Leonard, J., Leicester, R., Lipkin, F., Boulaire, F., McNamara, C., 2011. Assessing vulnerability at the urban interface. The 5th International Wildland Fire Conference, Sun City, South Africa, 9-13 May.

Caballero, D., Beltran, I., 2003. Concepts and ideas of assessing settlement fire vulnerability in the WUI zone. In Proceedings (Eds Xanthopoulos, G.) of the international workshop WARM, Forest fires in the wildland-urban interface and rural areas in Europe: an integral planning and management challenge, Athens, Greece, 47-54.

Cardona, O.D., van Aalst, M.K., Birkmann, J., Fordham M., McGregor, G., R., Perez, R., Pulwarty, R.S., Schipper, E.L.F., Sinh, B.T., 2012. Determinants of risk: exposure and vulnerability. In: Managing the Risks of Extreme Events and Disasters to Advance Climate Change Adaptation. A Special Report of Working Groups I and II of the Intergovernmental Panel on Climate Change (IPCC). Cambridge University Press, Cambridge, UK, and New York, NY, USA, pp. 65-108.

Carrega, P., 2008. Forest Fire Risk in the Mediterranean Area: Understanding and Change (in french). Proceedings of the the XXIst Conference of the International Association of Climatology, September 2008, Montpellier, France. pp. 11-23.

Christensen, R. H. B., 2013. Analysis of ordinal data with cumulative link models - estimation with the ordinal package. $R$--package version 2013.09--30.

Cohen, J.D., 2003. Preventing residential fire disasters during wildfires. In Proceedings of the international workshop, Forest fires in the wildland-urban interface and rural areas in Europe: an integral planning and management challenge, Athens, Greece, 5-12. 
D'Ercole, R., \& Metzger, P., 2009. Territorial vulnerability: a new approach of risks in urban environment (in french). In. Cybergeo: European Journal of Geography, Urban vulnerabilities in Southern Regions, paper 447, on line on the 31st of March 2009, modified on the 14th of May 2009.

Hédan, J., 2017. Etude et modélisation de la vulnérabilité du bâti d'interface habitat-forêt : calibration et validation sur le feu de Rognac 2016, Rapport de Stage de Master II SET (Sciences de l'Environnement Terrestre), Université d'Aix-Marseille / Irstea.

Lampin-Maillet, C., Jappiot, M., Long M., Morge D., Ferrier, J.P., 2009. Characterization and mapping of dwelling types for forest fire prevention. Computers, Environnement and Urban Systems 33, 224-232.

Pugnet, L. et Maillé, E., 2013. Assessment of wildland-urban interface vulnerability to fire using a multi-criteria analysis. International conference on forest fire risk modelling and mapping Vulnerability to forest fire at wildland-urban interfaces, $30^{\text {th }}$ of September, $1^{\text {st }}$ and $2^{\text {nd }}$ of October 2013, Aix en Provence, France.

Saaty, T.L., 1980. The analytic hierarchy process. McGraw-Hill, New York.

Saaty, T.L., 1993. What is relative measurement? The ratio scale phantom. Mathematical and Computer Modelling, vol. 17(4/5), pp. 1-12.

Saaty, T.L., 2008. Relative Measurement and Its Generalization in Decision Making : Why Pairwise Comparisons are Central in Mathematics for the Measurement of Intangible Factors - The Analytic Hierarchy/Network Process. RACSAM, vol. 102(2), pp. 251-318.

Zeng, T., Hudsol, J., Kay, S. and Laginestra, E., 2003. A fuzzy GIS approach to fire risk assessment: a case study of Sydney Olympic Park, Australia. Spatial Sciences Conferences, 2003.

Zscheischler, J., Westra, S., van den Hurk, B.J.J.M., Seneviratne, S.I., Ward, P.J., Pitman, A., AghaKouchak, A., Bresch, D.N., Leonard, M., Wahl, T. and Zhang, X. 2018, Future climate risk from compound events, In. Nature Climate Change, May 2018, DOI: 10.1038/s41558-018-0156-3 


\title{
Defining and Mapping the Wildland-Urban Interface in Portugal
}

\author{
José M.C. Pereira*1; Patrícia M. Alexandre" ${ }^{1}$; Manuel L. Campagnolo ${ }^{1}$; Avi Bar-Massada ${ }^{2}$; Volker \\ C. Radeloff ${ }^{3}$; Pedro C. Silva ${ }^{1}$ \\ ${ }^{1}$ Centro de Estudos Florestais, Instituto Superior de Agronomia, Universidade de Lisboa. Tapada \\ da Ajuda, 1049, Lisboa, \{jmcpereira@isa.ulisboa.pt*, palexandre@isa.ulisboa.pt, \\ mlc@isa.ulisboa.pt,pcsilva@isa.ulisboa.pt\} \\ ${ }^{2}$ Department of Biology and Environment, University of Haifa. Oranim, Kiryat Tivon 36006, \\ Israel, \{barmassada@gmail.com\} \\ ${ }^{3}$ SILVIS Lab, Dep. Of Forest and Wildlife Ecology, University of Wisconsin-Madison, USA. \\ Linden Drive, \{radeloff@wis.edu\}
}

\begin{abstract}
Wildfires in Portugal often threaten urban areas, from small rural villages to the edges of large cities. Regional variations in land use and human settlement patterns originate diverse types of wildland-urban interface (WUI), which need to be mapped to aid decisions concerning landscape-scale fire prevention and suppression. We propose a novel definition of the WUI as the set of line segments where urban areas are in immediate physical contact with flammable vegetation (direct interface), or are less than a pre-specified distance away from flammable vegetation (indirect interface). We also develop a methodology to map it in detail over large areas, based on spatial analysis of the vertices of urban and wildland polygons derived from reclassifying a land cover map. Our approach to WUI delineation mapped $29977 \mathrm{~km}$ of direct WUI and 11192 $\mathrm{km}$ of indirect WUI in Portugal, and identified substantial regional variations in the length and density of both kinds of WUI, reflecting differences in degree of urbanization and extent of wildlands, and indicating different levels of urban area exposure to wildfires. Addition of fire incidence data to the results of our analysis will permit the development of a WUI typology integrating urban area exposure to wildfires with wildfire threat magnitude, useful for fire management planning at the national level.
\end{abstract}

Keywords: Direct and indirect interface, Linear WUI, Portugal, Wildfires.

\section{Introduction}

Portugal has the highest wildfire incidence of all countries in southern Europe. During the last decade, there has been an increase in the number of incident reports in which buildings and homes were directly affected by wildfires. In 2017, Portugal recorded its worst fire season on record, both in terms of total area burned, loss of human lives, and property losses, with 470.000 ha burned, over 1000 homes destroyed or damaged, and 115 fatalities. Defining and identifying the wildland-urban interface (WUI) is relevant for risk assessment, land use planning, fire prevention and suppression, but it has not been done consistently for the whole country. The main goal of our study is to delineate and characterize the WUI in Portugal.

In fire-prone countries, the WUI has been a focus of research during the last two decades. WUIspecific maps are important both as pragmatic policy tools and as powerful images, with broad appeal (Stewart et al., 2009). There are several methodologies to map the WUI, and the main differences among them relate to data availability, study area size, and, most importantly, the objective of the map. Some WUI maps focus on assets (i.e., homes, infrastructure), while others focus on vegetation (Stewart et al., 2009). The common aspect of all maps is that they highlight wildfire as a social issue, because urban areas are exposed to wildfire risk due to their proximity to flammable fuels. Existing mapping methods vary not only in purpose, but also in scale. For example, in the United States the existing mapping methods reflect limited availability of housing data, as well as a broad-policy style, which 
does not encourage fine-scale mapping. Consequently, WUI maps use relatively large minimal mapping units, at the scale of housing clusters, neighborhoods, or settlements. Therefore, these maps rely on a threshold for housing density within a pre-defined area (census blocks or other data source), as well as on quantification of the vegetation density for each area (eg. Radeloff et al., 2005; Wilmer \& Aplet, 2005). This is an example of a zonal approach for WUI mapping.

Alternatives to zonal methods of WUI mapping use actual locations of homes or buildings. These point-based methods use the spatial coordinates for each individual building and have been developed in several European countries (Lampin-Maillet et al., 2009, 2010; Bar-Massada, Stewart, Hammer, Mockrin, \& Radeloff, 2013). A second approach combines elements of zonal and point-based methods. It relies on cadastral data and assumes that each parcel centroid represents the location of a building or building cluster (Platt, 2010). Other WUI mapping methods have used building location data, but none of them consistently addresses the three aspects of WUI definition, namely vegetation density, housing density and proximity of buildings to a large vegetated area.

We present a novel, semantically accurate approach to defining and mapping the WUI. Webster's Third New International Dictionary (Gove, 1961) defines "interface: a plane or other surface forming a common boundary of two bodies or spaces." Translating this concept to the two dimensions of a map, we define the WUI as the set of line segments where urban areas are in immediate physical contact with flammable vegetation (direct interface), or are less than a pre-specified distance away from flammable vegetation (indirect interface). The linear approach to the WUI definition and mapping provides great flexibility for information storage and utilization. Each WUI segment may be tagged with information about its adjoining urban area (area, population, number of buildings), and about its surroundings (types of adjacent fuels, flammable patch area, distance to the nearest flammable patch, topographic information, distance to roads, etc). Thus, the WUI concept we propose yields useful information for fire risk assessment, fuel management, and fire suppression planning. We mapped the WUI for the whole country and provided a summary at the county level.

\section{Methods}

\subsection{Study area}

Mainland Portugal extends over $89,015 \mathrm{~km}^{2}$, and is located between $37^{\circ} \mathrm{N}$ and $42^{\circ} \mathrm{N}$ latitude, and between $6^{\circ} \mathrm{W}$ and $10^{\circ} \mathrm{W}$ longitude. Portugal has a hot-summer Mediterranean climate (Köppen class Csa) in the southern half of the country, and a warm-summer mediterranean climate (Köppen class $\mathrm{Csb}$ ) in the northern half. The natural vegetation is typically evergreen, resistant to drought and pyrophytic, and regions with rugged terrain cover a substantial part of the country. These environmental conditions render the country quite susceptible to wildland fires and socio-economic and demographic trends reinforce this susceptibility. Many rural areas have experienced substantial population decreases since the mid-20th century, leading to the abandonment of agricultural lands, decrease in the sizes of herds, and a reduction in the consumption of forest fuels by animal grazing and fuelwood collection. Areas of marginally productive agriculture were converted to forest plantations or abandoned to the natural process of old-field succession, and reverted to shrublands and woodlands. These land use / land cover changes led to increases in the connectivity of high fuel loading vegetation patches, generating a landscape prone to the spread of large fires.

\subsection{Mapping the WUI}

Our analysis relied on the national land cover map COS 2015 (Carta de Ocupação do Solo para 2015 - COS2015) created by the Portuguese Land Planning and Mapping Agency (Direcção-Geral do Território - DGT). The map has a minimum mapping unit of 1 ha and a thematic legend with nine macroclasses (figure 1a) and 48 classes, which were reclassified to the four classes shown in figure $1 \mathrm{~b}$ 
to map the WUI. The degrees of urbanization map (figure 1c), from the DEGURBA classification (Dijkstra and Poelman, 2014) are defined as: densely populated areas, or cities, where at least $50 \%$ of the population lives in high-density clusters; intermediate density areas, or towns and suburbs, where less than $50 \%$ of the population lives in rural grid cells and less than $50 \%$ live in a high-density cluster; thinly populated areas, or rural areas, where more than $50 \%$ of the population lives in rural grid cells. The fire incidence map (figure 1d) data are from the Forest Research Centre (CEF) national fire perimeter atlas, complemented with data from the Institute for Nature Conservation and Forests (ICNF).
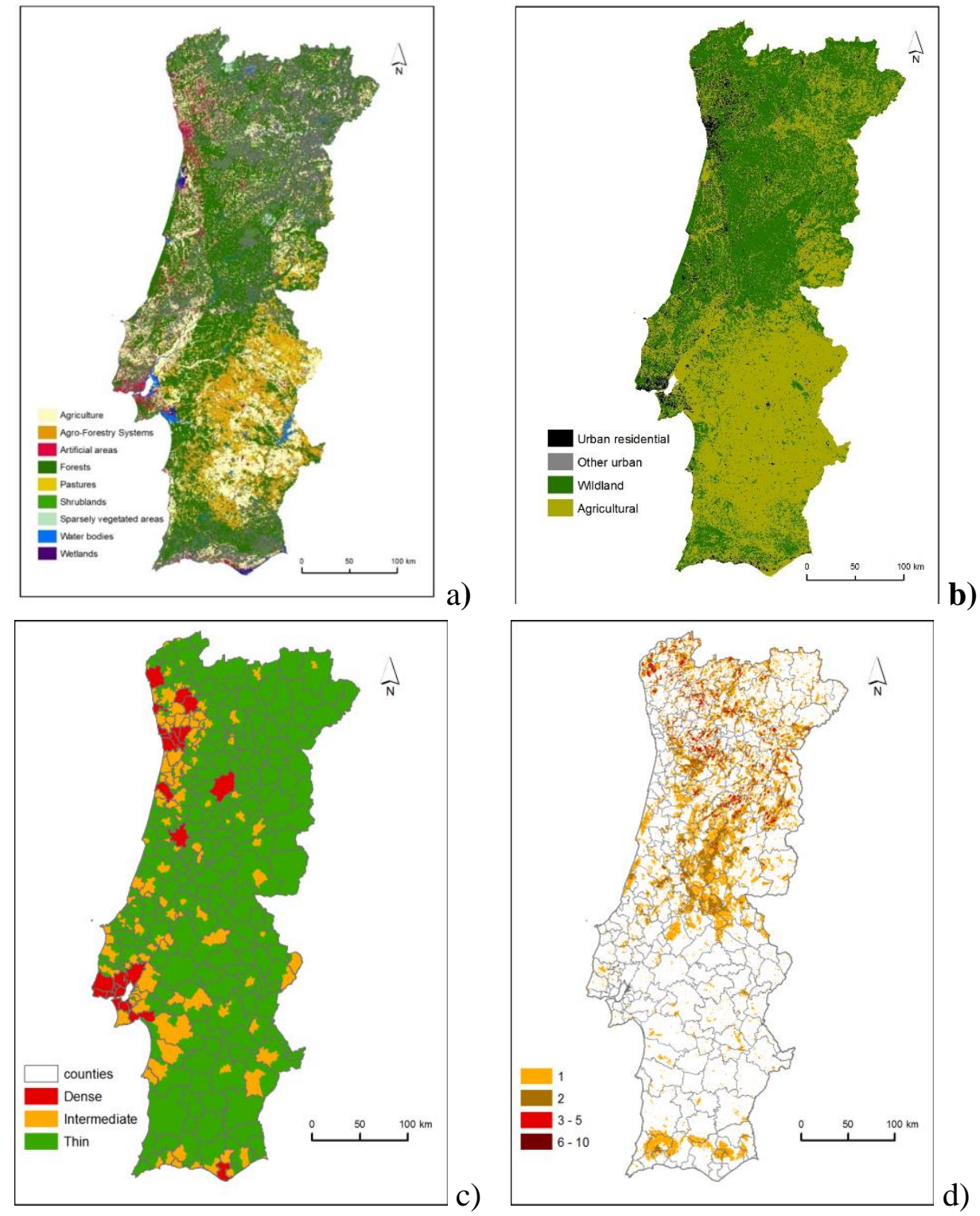

Figure 1 - a) Land cover megaclasses (COS2015); b) COS2015 simplified for WUI delineation; c) Degree of urbanization (DEGURBA) classification; d) number of times burned since 2000.

Delineation of the WUI depends primarily on the classes represented in Figure 1, "urban residential" and "Forests, shrublands, and pastures" (henceforth, wildland). We shall assume that urban area polygons and wildland area polygons share the same vertices whenever these vertices have the same location. Our definition of WUI relies on vertices rather than edges or polygons. This simplification was deemed necessary for computational efficiency reasons given the size of the data set to be analyzed. Following the legal definition of secondary fuelbreak network for the protection of settlements (Decree-Law n. ${ }^{\circ}$ 124/2006), only distances inferior to $100 \mathrm{~m}$ between urban and wildland 
patches have to be considered for the WUI delineation purpose. Therefore, we calculated, for every urban patch vertex its distance from all wildland patch vertices that lie inside a $100 \mathrm{~m}$ radius circle.

We say that an urban vertex $U$ is "protected" from its neighboring wildland vertices, if it is "protected" from all its wildland vertex neighbors within the $100 \mathrm{~m}$ range. This means that for each Wildland neighbor $\mathrm{W}$, there is an urban vertex $\mathrm{V}$ distinct from $\mathrm{U}$ that "protects" $\mathrm{U}$ from $\mathrm{W}$ in the following sense (see Figure 2):

- $\operatorname{dist}(\mathrm{U}, \mathrm{W})>0$, which guarantees that $\mathrm{U}$ cannot belong to the direct WUI (see below)

$-\operatorname{dist}(\mathrm{U}, \mathrm{V})<=\operatorname{dist}(\mathrm{U}, \mathrm{W})$ and $\operatorname{dist}(\mathrm{V}, \mathrm{W})<=\operatorname{dist}(\mathrm{U}, \mathrm{W})$, which guarantees that $\mathrm{U}$ lies "between" $\mathrm{V}$ and $\mathrm{W}$;

$-\operatorname{dist}(\mathrm{U}, \mathrm{V})+\operatorname{dist}(\mathrm{V}, \mathrm{W})<\operatorname{dist}(\mathrm{U}, \mathrm{W}) * \mathrm{~T}$, where $\mathrm{T}>1$ is an user-defined threshold $(\mathrm{T}=1.05$ provided good results).

The key condition is the last one, which states that the three vertices $\mathrm{U}, \mathrm{V}$ and $\mathrm{W}$ are nearly aligned to $5 \%$ tolerance $(\mathrm{T}=1.05)$, with $\mathrm{V}$ lying between $\mathrm{U}$ and $\mathrm{W}$. This condition is an alternative implementation, using vertices, of the intuitive idea that a vertex of a urban polygon is exposed to (i.e., not protected from) the wildland, if it can be connected by a straight line to some neighboring wildland vertex without intersecting other urban edges. In order to make this approach more effective, a previous densification of the number of vertices in each edge of the urban polygons was performed, such that no two consecutive vertices are more than $40 \mathrm{~m}$ apart. This is a good compromise between spatial accuracy and computational effort.

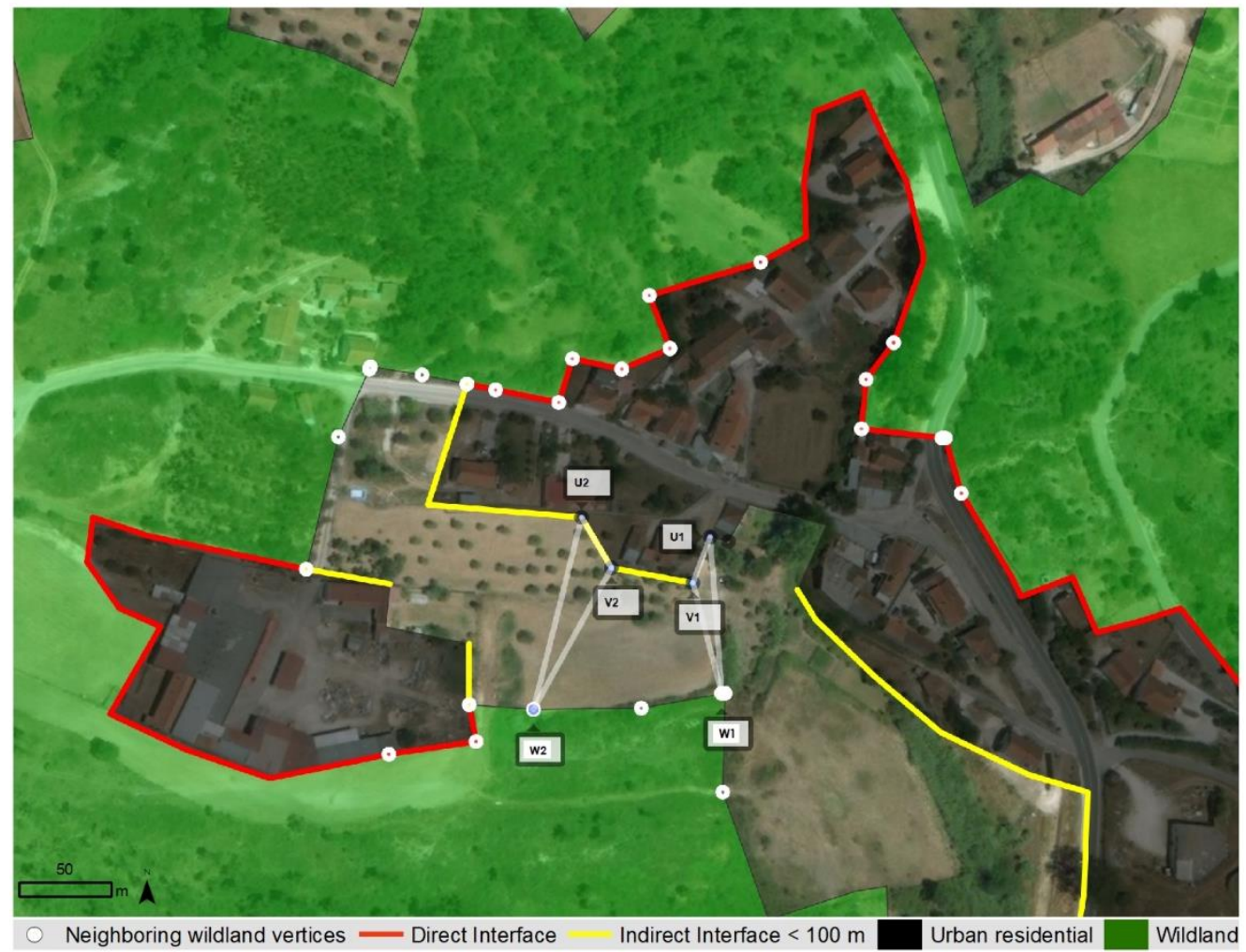

Figure 2 - WUI delineation method: the red [yellow] edges of the two urban polygons delineate the direct [indirect] WUI. Vertices $U 1, V 1$ and $W 1$ are nearly aligned within the 5\% tolerance

$(\operatorname{dist}(U 1, V 1)+\operatorname{dist}(V 1, W 1)<1.05 * \operatorname{dist}(U 1, W 1))$, and therefore urban vertex $U 1$ is protected from Wildland vertex W1by urban vertex $V 1$. On the contrary, urban vertex $U 2$ is exposed to the Wildand vertex $W 2$, since it is not protected by urban vertex V2 (dist $(U 2, V 2)+\operatorname{dist}(V 2, W 2)>=1.05 * \operatorname{dist}(U 2, W 2))$.

Using the concept described above, we define the linear WUI, as the set of edges belonging to urban polygons that have both of its vertices exposed to neighboring wildland vertices (within the $100 \mathrm{~m}$ range). In particular, two kinds of WUI can be immediately distinguished: the direct WUI (red lines in 
figure 2), where wildland and urban areas are adjacent to each other, and the indirect WUI (yellow lines in figure 2), where agroforestry, cropland, or other non-flammable land cover types, lie inbetween. Evidently, the former case implies a greater exposure of the urban area to a potential wildfire, while in the latter case agricultural areas, bare ground, or water bodies, may provide some protection to the urban area.

\section{Results}

The COS2015 land cover map contains 46480 residential urban polygons, of which 40319 correspond to some kind of WUI. Our algorithm identified $29977 \mathrm{~km}$ of direct WUI and $11192 \mathrm{~km}$ of indirect WUI. Figure 3 shows the WUI at Fafe (a), in NW Portugal, and at Vila de Rei, in the center of the country (b).
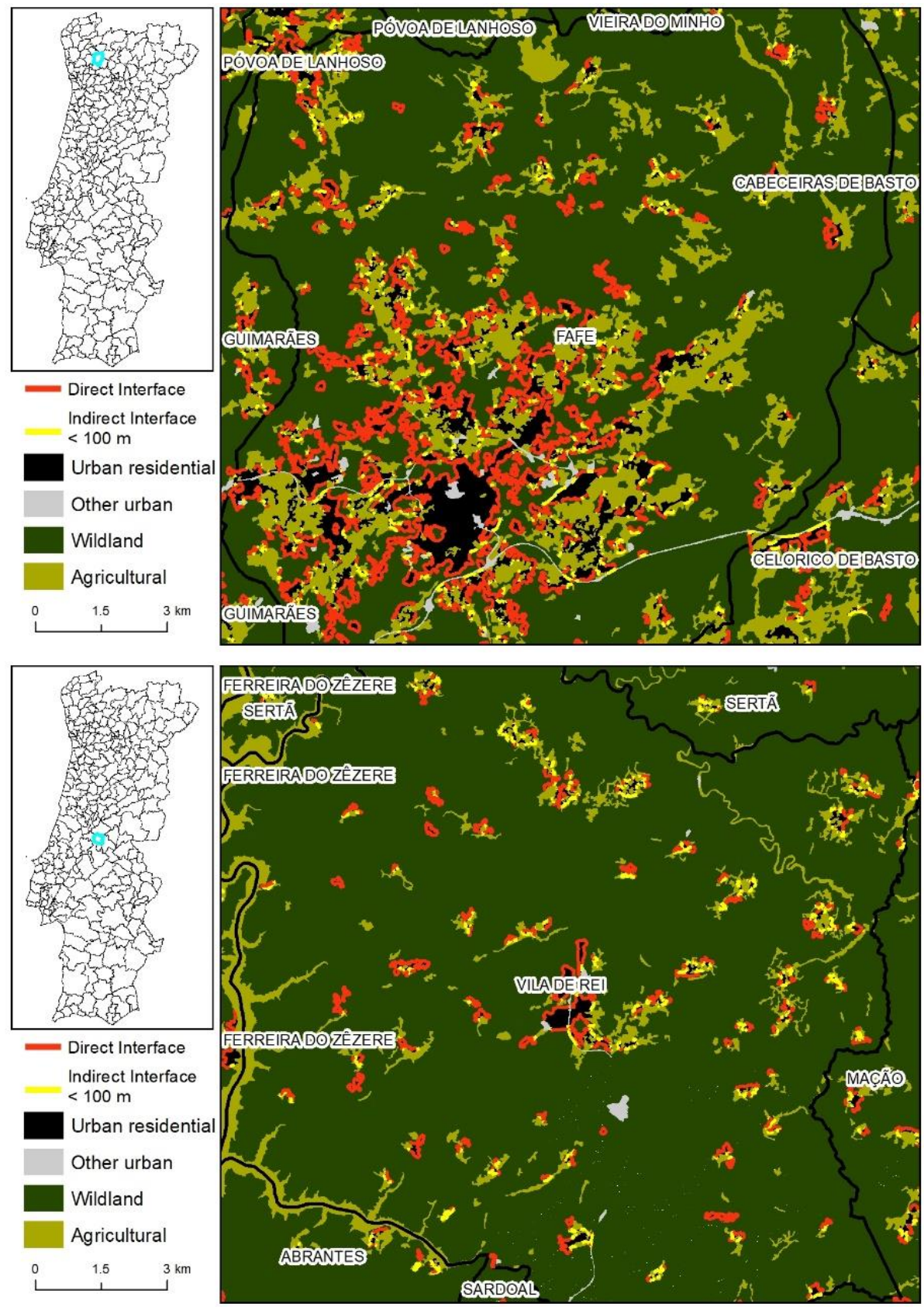

Figure 3 - Direct and indirect WUI at Fafe (a) and Vila de Rei (b), sites with contrasting land use and settlement patterns. 
The WUI length and type at the two sites is very distinct, reflecting differences in land use / land cover patterns. Fafe has a long, mostly direct WUI, while Vila de Rei has a shorter WUI, but with a higher proportion of indirect WUI than at Fafe. This landscape-level overview reveals the detailed information provided on the location, extent, and shape of the linear WUI.

Figures 4 and 5 display results of a country-wide WUI analysis of the COS2015 land cover map, aggregated at the municipal level. Figure 4 shows that the geographical patterns of direct WUI (a) and indirect WUI (b) are similar, despite quantitative differences, since the length of direct WUI are about three times higher than those of the indirect WUI.

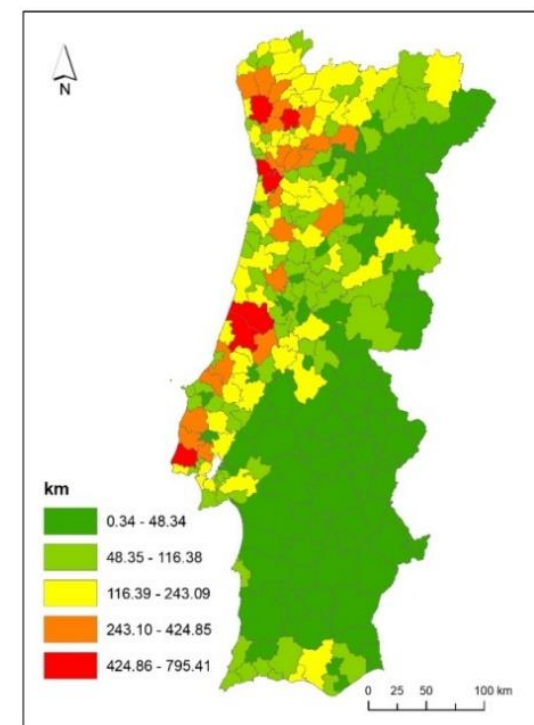

a)

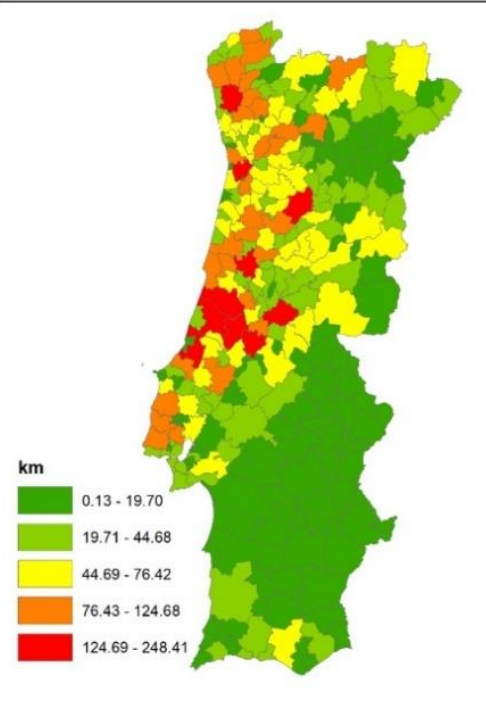

b)

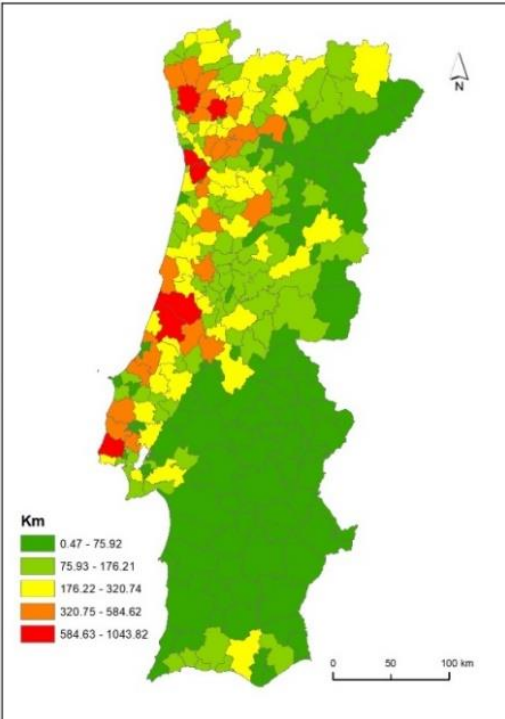

c)

Figure 4 - Length of direct (a), indirect (b) and total (direct + indirect) WUI.

Figure $4 \mathrm{c}$ shows that the largest extent of total WUI occur in regions with dense and intermediate degrees of urbanization (figure 1c), but also in a thinly urbanized coastal area in the center of the country. Rural regions in northeastern and southern Portugal display low WUI values.

WUI density (figure 5) is the ratio of WUI length to urban area. It displays substantially different geographical patterns from those shown in Figure 4, and clear distinctions between direct (a) and indirect (b) WUI.

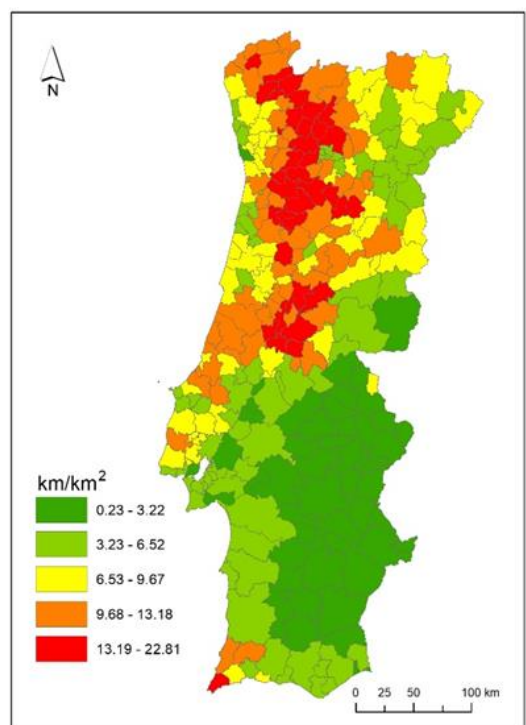

a)

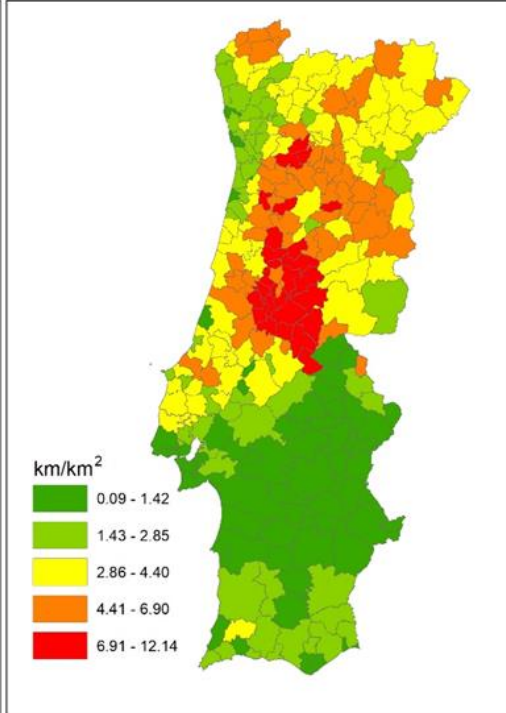

b)

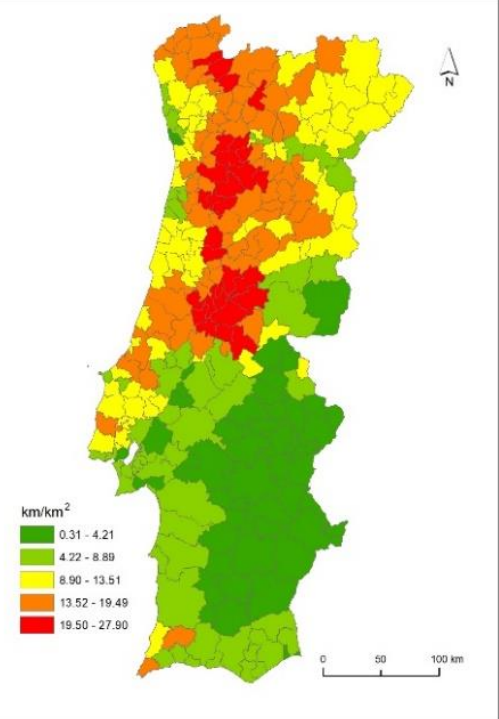

c)

Figure 5 - Direct (a), indirect (b) and total (direct + indirect) WUI density. 
Unlike WUI length, WUI density peaks in areas classified as thinly urbanized, or rural, in figure 3c. Direct WUI density (5a) is highest in NW Portugal, inland from the areas with the longest absolute WUI shown in figure $4 \mathrm{a}$, and in thinly urbanized, densely forested central region of the country. This area also has the highest values of indirect WUI density. The lowest values of direct WUI density occur in agricultural areas of rural southern Portugal. The areas with the highest total WUI density are some of the most fire-prone regions in the country (figure 1d).

\section{Discussion and Conclusions}

The linear WUI mapping algorithm we propose can be implemented over large areas, provides very detailed information on the location, extent and shape of the interface, and proved to be sensitive to variations in population density and land use. Referring back to the two test sites presented in figure 3, Fafe has 2094 inhabitants. $\mathrm{km}^{-2}$ and a landscape with relatively large areas of urban and agricultural land uses, while Vila de Rei has 18 inhabitants. $\mathrm{km}^{-2}$ and small patches of urban and agricultural areas in a forest-dominated landscape. Despite the large proportion of agricultural land in Fafe, there is extensive adjacency between urban areas and wildlands and, therefore, most of its WUI is direct interface. On the contrary, at Vila de Rei, the small patches of agricultural land surround most of the settlements, and thus a large proportion of the WUI is indirect. WUI delineation methodologies based on a coarser analysis of the relative abundance and spatial structure of the land cover might have missed this counter-intuitive result. The detailed and precise delineation of WUI segments along the perimeter of urban areas may be useful to monitor and manage the impact on WUI extent and density of processes like urban growth, cropland abandonment, and forest encroachment on rural villages.

In the densely urbanized areas of Portugal, the relative proportions and spatial distribution patterns of urban areas and wildlands produce the largest extents of linear WUI, both direct and indirect. However, city size implies that most of the built-up land is interior to the urban area, i.e. located at a relatively safe distance from the WUI. Since fire incidence typically is low in these regions, the combination of limited urban area exposure and low magnitude wildfire threat is conducive to a moderate fire risk situation. As expected, the shortest WUI values are found in predominantly agricultural rural regions, where both urban areas and wildlands are scarce. WUI density is a good metric of exposure, because landscape patches with high perimeter-to-area ratio (interface length per unit area of urban land patch) are permeable to flows from their environment, such as the energy released by a wildfire. This explains the predominant location of the highest WUI densities in thinly urbanized areas, with small rural settlements and extensive forests and shrublands. Exposed settlements in areas of high fire incidence are under severe fire risk, as tragically demonstrated in June and October, 2017. Our approach characterizes the WUI at a very detailed level as segments of the perimeter of individual settlements, with associated attributes of length, area of the adjacent urban and wildland polygons and, in the case of the indirect WUI, distance between urban area and wildland. This information concerning each WUI segment can be aggregated at the settlement level and employed to develop a WUI typology useful for setting fire prevention and suppression priorities and strategies, namely an improved delineation of the mandatory secondary fuelbreak network for the protection of rural villages and towns in fire-prone landscapes.

\section{Acknowledgments}

The research reported herein was performed under research project PTDC/AGR-FOR/2586/2014 Fire in the Rural-Urban Interface: characterisation, risk mapping, and fuel break design (RurIntFire), funded by the Foundation for Science and Technology (FCT). The Centro de Estudos Florestais (CEF) is a research unit funded by Fundação para a Ciência e a Tecnologia I.P. (FCT), Portugal (UID/AGR/00239/2013). 


\section{References}

Bar-Massada, A., Stewart, S. I., Hammer, R. B., Mockrin, M. H., \& Radeloff, V. C. (2013). Using structure locations as a basis for mapping the wildland urban interface. Journal of Environmental Management, 128, 540-547.

Dijkstra, L., \& Poelman, H. (2014). A harmonised definition of cities and rural areas: the new degree of urbanisation. Regional Working Paper 2014. DG Regional and Urban Policy, European Commission, Brussels, Belgium.

Gove, P. B. (1961). Webster's Third New International Dictionary of the English Language, unabridged. (P. B. Gove, Ed.). Springfield, Mass.: G\&C Merriam Company.

Lampin-Maillet, C., Jappiot, M., Long, M., Morge, D., Ferrier, J.P., 2009. Characterization and mapping of dwelling types for forest fire prevention. Computers, Environment and Urban Systems 33, 224-232.

Lampin-Maillet, C., Jappiot, M., Long, M., Bouillon, C., Morge, D., Ferrier, J.P., 2010. Mapping wildland-urban interfaces at large scales integrating housing density and vegetation aggregation for fire prevention in the south of France. Journal of Environmental Management 91, 732-741.

Lampin-Maillet, C., Long-Fournel, M., Ganteaume, A., Jappiot, M., \& Ferrier, J. P. (2011). Land cover analysis in wildland-urban interfaces according to wildfire risk: A case study in the South of France. Forest Ecology and Management, 261, 2200-2213.

Platt, R. (2010). The Wildland Urban Interface: Evaluating the Definition Effect. Journal of Forestry, 108, 9-15.

Radeloff, V. C., Hammer, R. B., Stewart, S. I., Fried, J. S., Holcomb, S. S., \& McKeefry, J. F. (2005). The wildland-urban interface in the United States. Ecological Applications, 15(3), 799-805.

Stewart, S. I., Wilmer, B., Hammer, R. B., Aplet, G. H., Hawbaker, T. J., Miller, C., \& Radeloff, V. C. (2009). Wildland-urban interface maps vary with purpose and context. Journal of Forestry, 107(2), 78-83.

Wilmer, B., \& Aplet, G. (2005). Targeting the community fire planning zone: Mapping matters. Washington, DC: the Wilderness Society. 


\title{
Developement of a Metodology to Assess School Bushfire Risk
}

\author{
Justin Leonard*; Raphaele Blanchi; Alessio Arena; Garth Warren; Kimberley Opie; \\ CSIRO. Australia. \{justin.leonard@csiro.au*\}
}

\begin{abstract}
This paper describes the methods used to profile the risk posed by forest fire to schools across the state of Victoria, Australia. The methods are principally spatially data driven with augmentation from local assessments and surveys. Schools are assessed in terms of their likelihood of loss from a range of fire arrival severity scenarios and their capacity to effectively provide shelter for school occupants during these events. This is achieved by considering the potential loss of school buildings. The methods also includes an estimate of the radiant heat exposure at assembly areas and egress routes.

As this is now a operational system, the paper describes both the theoretical process and the practical implementation. To address these risk asessements, the schools can implement various risk management processes such as prioritised infrastructure upgrades, vegetation management programs, pre-emptive temporary relocation, targeted education and training. These risk management strategies are considered and developed according to the risk profile of the individual school.
\end{abstract}

Keywords: Wildland, wildfire, forest, fire, bushfire, risk assessment, WUI, warnings, policy

\section{Introduction}

Wildfires or bushfires pose a danger to life and property in Australia. This risk requires active management by relevant State governments, who manage education in Australia. Hence, schools in bushfire prone areas are required to incorporate bushfire risk in their emergency management plans (EMP), which may include forced closures on days which exceed certain fire weather severity thresholds. The EMP is developed by the school with support from government and the regional fire Authority. These plans include relocation triggers, egress paths, facility management and the identification of a shelter-in-place (SIP) building. The SIP building is used as the primary emergency shelter when students and staff are on-site during a bushfire event and it is too late to safely evacuate. A SIP building may be a designated building, or one or more designated rooms within a building. EMP's also consider outside Assembly Areas (AA), as a contingency in the event that the primary shelter is compromised.

This paper presents an evidence based methodology that has been developed to identify schools at highest risk from forest fire and to quantify the risk to school assets (i.e. school buildings and grounds) and their occupants. The methodology is based on the potential for bushfire to impact school buildings and the schools' Assembly Area(s). Risk is based on modelling which uses a combination of landscape factors, including the local terrain, proximity to bushfire fuel, and the type of forest fuel. The primary output of this work is a standardised risk profile of schools, which is used to set appropriate relocation triggers and to prioritise mitigation strategies.

\section{Defining the hazard}

Bushfires can harm people and damage property through flame attack, radiant heat exposure, ember attack, wind attack, hazard and convective heat exposure (Ramsay 1987; Blanchi and Leonard 2008; Leonard and Blanchi 2012). Among these impact mechanisms, flame attack, radiant heat exposure and ember attack are the most relevant when assessing hazard to life and structures (Leonard and Blanchi 2012). The exception is when certain individuals are susceptible to smoke exposure, in which case specific mitigation measures are required.

Advances in Forest Fire Research 2018 - Page 750 
The preferred metric for estimating bushfire hazard at the landscape scale is fire line intensity (for radiant heat and flame exposure) and bark hazard (for ember attack) (Harris et al., 2012; Kilinc et al., 2014). A combination of these metrics is used to quantify hazard to buildings. Radiant heat is used as a proxy to quantify safe distance for people outside of buildings, in terms of direct radiant heat damage, as well as smoke and ember exposure.

People typically seek shelter when exposed to the radiant heat and smoke of a bushfire. Research have highlighted the importance of contingency planning, and of active sheltering and situation monitoring (Blanchi et al., 2018; Whittaker et al., 2017). Research also indicates that safe sheltering requires appropriately designed and situated shelters. Sheltering inside a building offers the most protection against the effects of fire when it is too late to evacuate safely. This raises the additional questions of when to exit the shelter, where to go, and what is the best path to take. The risks associated with egress should be properly assessed to reduce the risk to occupants. Further, any AA used as a secondary contingency situated in an open space need to be properly assessed to ensure that they provide sufficient protection.

\section{Risk Profiling methodology}

The methodology defines risk-to-life by considering the severity of a bushfire event, and assessing how vulnerable humans are to that event. Broadly, risk-to-life is considered under two circumstances:

1. When staff and students shelter within the SIP building during the bushfire event.

2. When staff and students leave the SIP building -due to loss of tenability- and move to an exernal AA.

The first circumstance requires an assessment of the likelihood of survival of the SIP building when exposed to a bushfire event. The second circumstance requires an assessment of the risks involved in moving from the SIP building to a designated AA, followed by an assessment of the risk to the AA. These circumstances are linked, given that the second contingency is only required if the SIP building ignites. Timely movement into the SIP, and then (if necessary) from the SIP to the AA is a function of school and SIP design, and the mobility and numbers of student and staff. Survival at the AA is dependent on its design and the ability of staff and students to survive in this environment until support is provided. In each case, it is assumed that suppression support is not available during the event. However, a suppression strategy and assistance through maintained hydrant systems is an important contingency at all school locations.

Factors that contribute to the risk profile of a building include the weather conditions on the day of the fire, the resulting fire exposure, and the buildings design and location (Figure ).

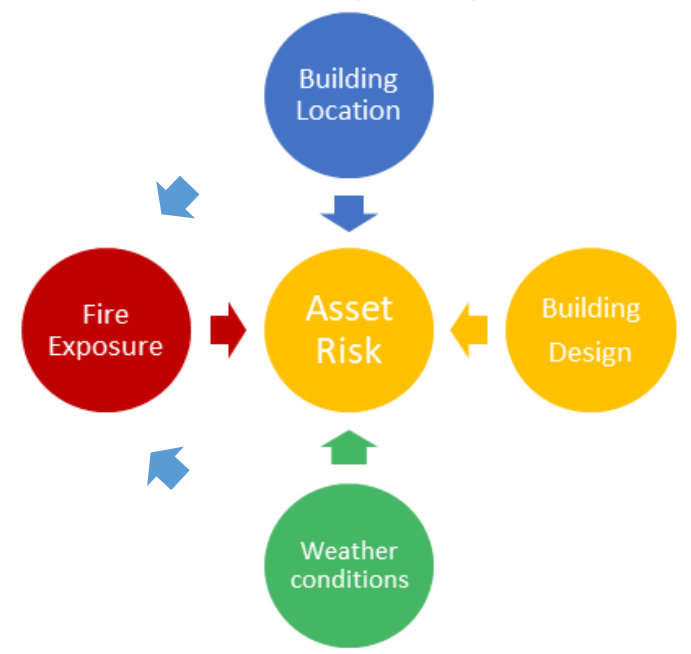

Figure 1 - Factors contributing to asset risk profiles. 
Schools and their constituent buildings are evaluated by first considering the adequacy of the SIP building based on potential building loss (Kilinc et al., 2014), including an exposure risk determined from the surrounding fire line intensity and bark hazard (Leonard et al., 2014; Newnham et al., 2015). In addition, an adjustment of loss is applied based on the buildings compliance and its exposure to other assets. The third step includes a radiant heat estimates of the AA and the egress routes between the SIP and this area.

\section{Assessing building loss potential}

The most relevant empirical analysis of building loss potential for Victoria is described by Kilinc et al. (2014). The authors found that the most important determinants of building loss were fire line intensity, bark hazard and the distance from the building to the forest fuel which supported the bushfire approach (Kilinc et al. 2014). This study was based on a large dataset of past bushfire events which impacted residential buildings with no specific bushfire mitigation measures.

\subsection{Building Loss Potential calculation}

Building Loss Potential (BLP), as described in Kilinc et al. (2014), takes into account the fire line intensity, the distance from the building to the nearest forest, and the bark hazard of any adjacent forest. Note that the definition of forest in this instance is taken from Furby (2002); where a forest is defined as any contiguous patch of vegetation that covers a minimum of 0.2 hectares, with a minimum treecrown-cover of $20 \%$ and a minimum height of 2 meters at maturity. The relationship between these parameters is described using a Generalised Linear Model with a logit link function described in equation 1:

$$
(\boldsymbol{\pi} /(\mathbf{1}-\boldsymbol{\pi}))=\boldsymbol{\eta}=\boldsymbol{\alpha}+\boldsymbol{\beta} \boldsymbol{h}+\boldsymbol{\gamma} \log (\mathrm{FLI})+\boldsymbol{\kappa} \boldsymbol{d}
$$

where $F \boldsymbol{L I}$ is Byram's fire line intensity, $\boldsymbol{d}$ is distance to forest, $\boldsymbol{h}$ denotes bark hazard level, and $\boldsymbol{\alpha}$, $\boldsymbol{\beta}$ and $\boldsymbol{\kappa}$ are the intercept and correlation coefficients respectively. Values for $\boldsymbol{\alpha}, \boldsymbol{\beta}$ and $\boldsymbol{\kappa}$ are presented in Table. These constants hold for all fire intensities excluding those below $3 \mathrm{MW} / \mathrm{m}$ or above 90 $\mathrm{MW} / \mathrm{m}$.

Table 1 - The Estimated coefficients of equation 2 (from Kilinc et al. 2014).

\begin{tabular}{|c|c|c|c|c|c|c|}
\hline $\begin{array}{l}\text { intercept } \\
(\boldsymbol{\alpha})\end{array}$ & $\begin{array}{l}\beta \mathrm{L} \\
\text { (low bark } \\
\text { hazard) }\end{array}$ & $\begin{array}{l}\beta M H \\
\text { (medium-high } \\
\text { bark hazard) }\end{array}$ & $\begin{array}{l}\beta V H \\
\text { (very high } \\
\text { bark hazard) }\end{array}$ & $\begin{array}{l}\beta E \\
\text { (extreme bark } \\
\text { hazard) }\end{array}$ & $\begin{array}{l}\gamma \\
\mathrm{FLI} \text { in } \\
\mathrm{MW} / \mathrm{m}\end{array}$ & $\begin{array}{l}\kappa \\
d \text { in meters }\end{array}$ \\
\hline-5.7295 & 0 & 2.56 & 2.87 & 3.44 & 0.87 & -0.008 \\
\hline
\end{tabular}

The prediction equation (2) for building loss is then:

$$
\hat{\boldsymbol{\pi}}=\mathbf{1} / \mathbf{1}+(-\boldsymbol{\eta})
$$

where $\hat{\boldsymbol{\pi}}$ and $\hat{\boldsymbol{\eta}}$ are estimated values from the fitted model.

\subsubsection{Fire line Intensity}

Fire line intensity is a standardised measure of the rate that an advancing head fire consumes fuel per unit timeand per unit length of fire front (Byram, 1959) and can be calculated as follows:

$$
\boldsymbol{F L I}=\boldsymbol{H} \times \boldsymbol{w} \times \boldsymbol{R}
$$

where $\boldsymbol{F L I}$ is Byram's fire line intensity (usually expressed in kilowatts per linear meter i.e. $\mathrm{kW} / \mathrm{m}$ ), $\boldsymbol{H}$ is the net latent heat of combustion $(\mathrm{kJ} / \mathrm{kg}), w$ is fuel consumed in the active flame front $\left(\mathrm{kg} / \mathrm{m}^{2}\right)$, and $\boldsymbol{R}$ is the linear rate of fire spread $(\mathrm{m} / \mathrm{sec})$. 
One of the main benefits of the fire line intensity metric is that it can be used to estimate potential flame length (Alexander et al., 2012; Byram, 1959) and thus the radiant heat expected at various distances from the flame.

A diverse range of models can be used to determine the rate of spread and fire line intensity, which are generally specific to certain fuel types (Cheney et al., 1995; Gould et al., 2007; McArthur, 1967).

\subsubsection{Bark Hazard}

Research has shown that ember attack accounts for substantially more building loss than either direct flame contact or radiant heat exposure (Leonard et al., 2009). Embers form during a bushfire due to a combination of lofted fine fuels that consist of bark, leaves and fine twigs. These fine fuels exist as deposits on the ground and on the plants themselves, different plant types and plant structures provide different levels of embers.

Bark Hazard, defined in Hines et al. (2010), can be used as a proxy for ember-attack-intensity. Kilinc et al. (2014) confirms this by empirical correlation with house loss potential. Bark hazard relates to its ease of ignition, size, shape, quantity and burning time, and is generally specific to tree species (Hines et al. 2010).

\subsection{Data requirements for BLP calculation}

\subsubsection{Potential Fuel Load}

Potential fuel load is a key driver of fire behaviour, including fire line intensity. The fuel loads used in AS3959 (Standards Australia, 2009) reflect the Australia-wide accepted level for each classification of vegetation; assuming the vegetation has reached its maximum level of fuel accumulation or 'steady state'. For this methodology, fuel loads were sourced either through local field assessments or estimated for broad Ecological Vegetation Classes $(\mathrm{EVCs})^{1}$, used for classifying native vegetation characteristics in its mature and natural state in Victoria.

\subsubsection{Topographic slope}

Topographic slope is an important variable controlling fire spread, the rate of fuel consumption and thus potential fire line intensity. The methodology developed to derive slope is based on the maximum potential slope of the landscape that could influence the rate of fire spread and fire line intensity. Slope is derived from a gridded Digital Elevation Model (DEM) using elevation values at the location and eight neighbouring pixels (Figure ).

\begin{tabular}{|c|c|c|}
\hline 1 & 2 & 3 \\
\hline 8 & $x$ & 4 \\
\hline 7 & 6 & 5 \\
\hline
\end{tabular}

Figure 2 - Calculation of landscape slope based on 8 neighbouring values.

Several digital elevation products and derived datasets exist that cover various regions of the study are. For consistence, the methodology used a national gridded DEM derived from the Shuttle Radar Topography Mission (SRTM) at 1 second $(\sim 30 \mathrm{~m})$ resolution.

${ }^{1}$ https://www.data.vic.gov.au/data/dataset (accessed May 2018). 


\subsubsection{Fire Danger Index and fire danger Rating}

Weather conditions play an important role in influencing fire behaviour, the difficulty of suppression and potential to cause damage (Luke and McArthur, 1978). Weather severity is characterised in Australia using a Forest Fire Danger Index (FFDI; McArthur, 1967) or the Grass Fire Danger Index (GFDI), depending on the dominant fuel type. The FFDI relates large-scale weather conditions to the expected fire behaviour, given common forest fuel types in eastern Australia (McArthur, 1967; Luke and McArthur, 1978). The FFDI is a useful metric for characterising the destructive potential of a fire (e.g. Bradstock and Gill, 2001; Blanchi et al., 2010). However, similar studies have not been performed to relate GFDI to similar measures of destructive potential.

In the methodology presented in this report, FFDI forms the basis of the potential fire weather severity inputs, which are used when calculating fire line intensity and bushfire hazard for school assets.

\subsubsection{Bark Hazard classification}

Bark hazard was attributed based on Ecological Vegetation Class (EVC) maps. Bark hazard can also be determined through a field assessment of vegetation if the mapped data provides insufficient information. As shown in Table , BLP considers four categories of bark hazard (taken from Hines et al., 2010). Note that the low bark hazard category poses no risk of ember-attack (bark-hazard constant of zero). Kilinc et al. (2014) found very little difference between the medium and high bark hazard levels, and that combining these categories provides a better overall estimate of BLP.

\subsubsection{Building locations}

The probability that a building will be lost to bushfire increases with fire intensity and decreases with the distance between a building and forest (Kilinc et al., 2014). Kilinc et al. (2014) used building centroids to calculate BLP.

In the proposed methodology, building footprint is defined using site plans of school. The BLP model then considers the distance from each building edge to its nearest area of unmanaged fuel that can support a significant fire line intensity $(>4 \mathrm{MW} / \mathrm{m})$. BLP is then calculated according to this potential fire line intensity. Using potential fire line intensity as a gridded dataset, we can also assess a buildings proximity to high hazard zones.

Finally, buildings that are located more than $500 \mathrm{~m}$ from any potential fire line intensity areas it will be allocated a $0 \%$ probability of loss.

\subsection{BLP workflow}

Figure presents an overview of the BLP workflow. Both the primary inputs and derived layers are shown. The assessments from these two methods can be combined in a number of ways, for example:

- A school is rated according to the better of the two methods. Hence the school's category is defined according to its most effective option out of the two methods (little redundancy in survival options).

- A school is rated according to the weaker of the two methods. Hence the school's category is defined according to its least effective option (high redundancy in survival options).

- A school is rated by the (weighted) average of the two methods. Hence the school's category is defined by averaging the effectiveness of the two methods with an approach weighting factor (some redundancy). 


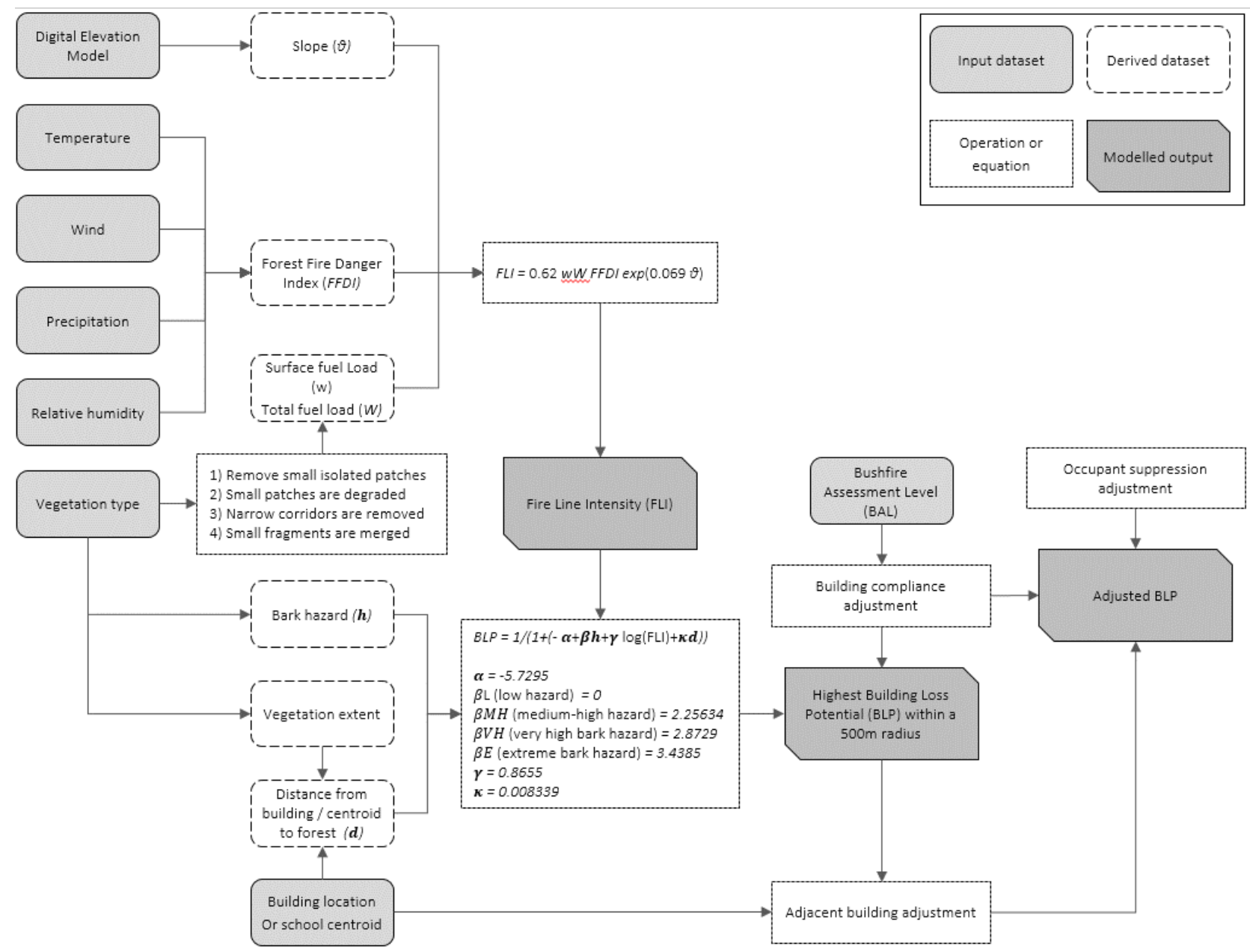

Figure 3 - Overall Building Loss Potential mapping methodology.

\section{Radiation heat exposure assessment of the Assembly Area(s)}

SIP buildings are defined as the primary location of shelter at the school. However, studies on community response have shown the need for contingency planning to account for the risk of shelter failure (Blanchi et al., 2018; Teague et al., 2010). A secondary contingency - Assembly Area (AA) such as an oval could be used as a potential secondary place of shelter either on or off site.

Radiant heat measures can be used to estimate the minimum required separation distances needed to afford safety to people and/or buildings (Cheney et al., 2001; Siggins et al., 2013; Zárate et al., 2008). In Australia, $2 \mathrm{~kW} / \mathrm{m}^{2}$ of radiant heat is often used as the maximum threshold people should sustain in open spaces ${ }^{1}$. Purser and McAllister (2016) stated that radiant heat above $2.5 \mathrm{~kW} / \mathrm{m}^{2}$ causes skin pain, followed by burns within a few seconds. A review of human exposure to radiant heat determined that a level between $4-5 \mathrm{~kW} / \mathrm{m}^{2}$ was the short term threshold beyond which significant injury may occur (Raj, 2008). 
We define the potential risk at the AA by calculating the radiant heat flux received at the location and on the egress path under different weather conditions. We develop a spatial approach to define the minimum distance from various types of vegetation to obtain a maximum tolerable exposure using 5 $\mathrm{kW} / \mathrm{m}^{2}$ as a threshold for short term exposure while moving to an alternate location, and $2.5 \mathrm{~kW} / \mathrm{m}^{2}$ for the alternate shelter location where longer exposure times may occur. The approach take into account the FLI (equation 3) to determine flame length 6 (modified for the effective slope and the vegetation and weather conditions), and then calculate the radiant heat flux based on the StefanBoltzman equation (Sullivan et al., 2003).

\section{Results and discussion}

An example of risk assessment using the methodology decscribe in this paper is presented in this section.

\subsection{BLP assessment}

We sourced information on vegetation type, fuel load, bark hazard and extent from the Ecological Vegetation Classes (EVC) map. The school in this example is located adjacent to forest herb-rich vegetation with a very high bark hazard. The SIP buiding is shown in blue in Figure . Fire line intensity (see Figure ) was then calculated using equation 1, 2 and 3 under a FFDI of 35, 50, 75 and 100, using the EVC derived maps.
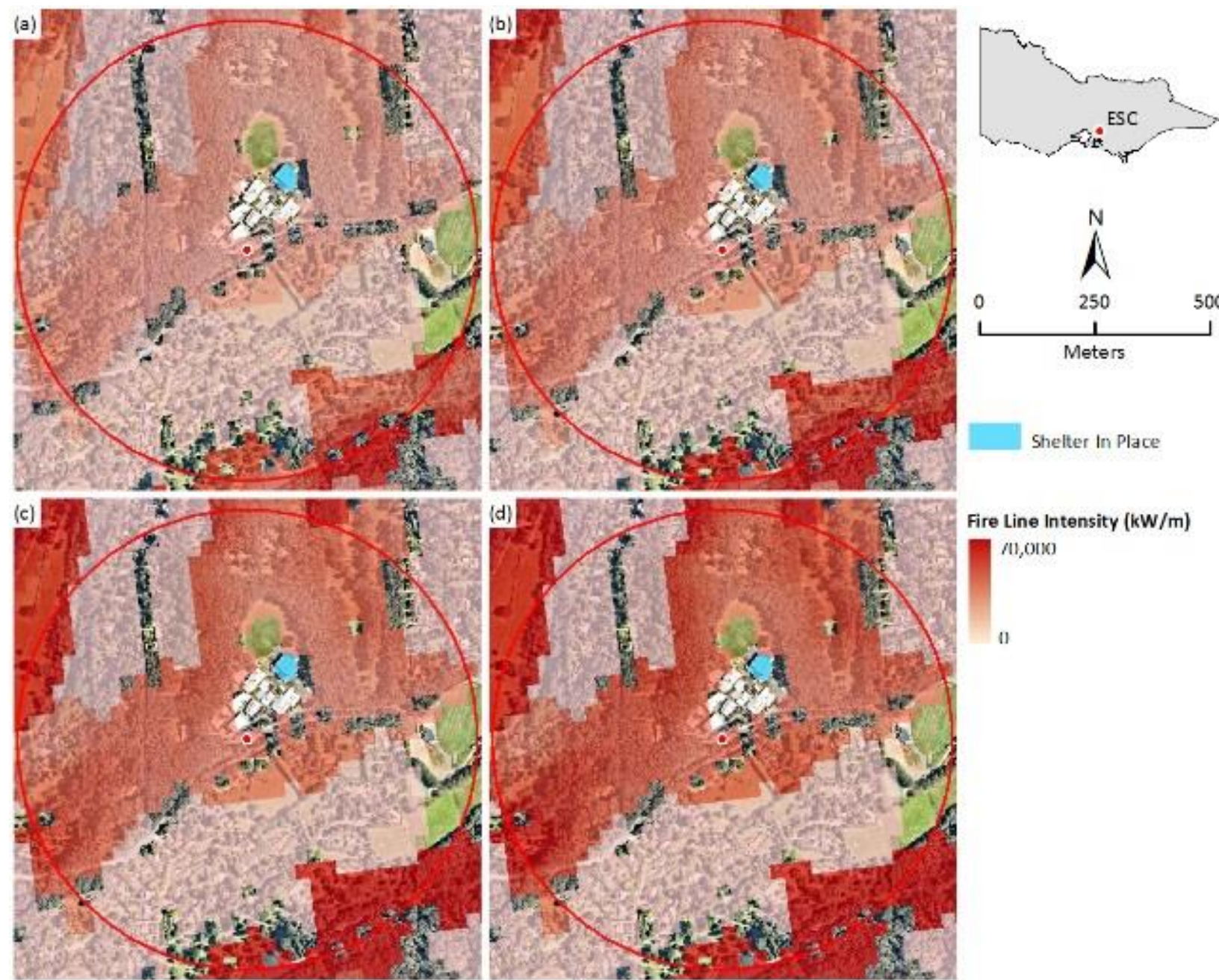

Figure 4 - Fire line intensity in the area surrounding the school SIP building (light blue). Fire Line Intensity for FFDI $35(a), 50(b), 75(c)$, and $100(d)$. 
Table 2 presents the results of BLP at different FFDI, for example BLP is $35 \%$ at a severe FFDI of 75 (Table 2).

Table 2 - Unadjusted Building loss potential at different FFDI.

\begin{tabular}{lllll}
\hline & FFDI 35 & FFDI 50 & FFDI 75 & FFDI 100 \\
\hline BLP at the SIP building & 0.216 & 0.272 & 0.347 & 0.405 \\
\hline
\end{tabular}

\subsection{Radiant Heat Flux Assessement}

The radiant heat flux received at the AA and its associated egress paths were calculated under different weather conditions (FFDI >50 and FFDI $>75$ ) to define the minimum distance from various types of vegetation needed in order to obtain the tolerable radiant heat exposure of $5 \mathrm{~kW} / \mathrm{m}^{2}$ and 2.5 $\mathrm{kW} / \mathrm{m}^{2}$.

A contour map of Radiant Heat Flux (RHF) exposures are shown in Figure . In this case, the sport oval adjacent to the SIP building will receive a radiant heat flux exceeding $2.5 \mathrm{~kW} / \mathrm{m}^{2}$ under an FFDI of 50. In order for this to qualify as an AA, fuel managment adjacent to the oval is required in order to reduce the exposure.

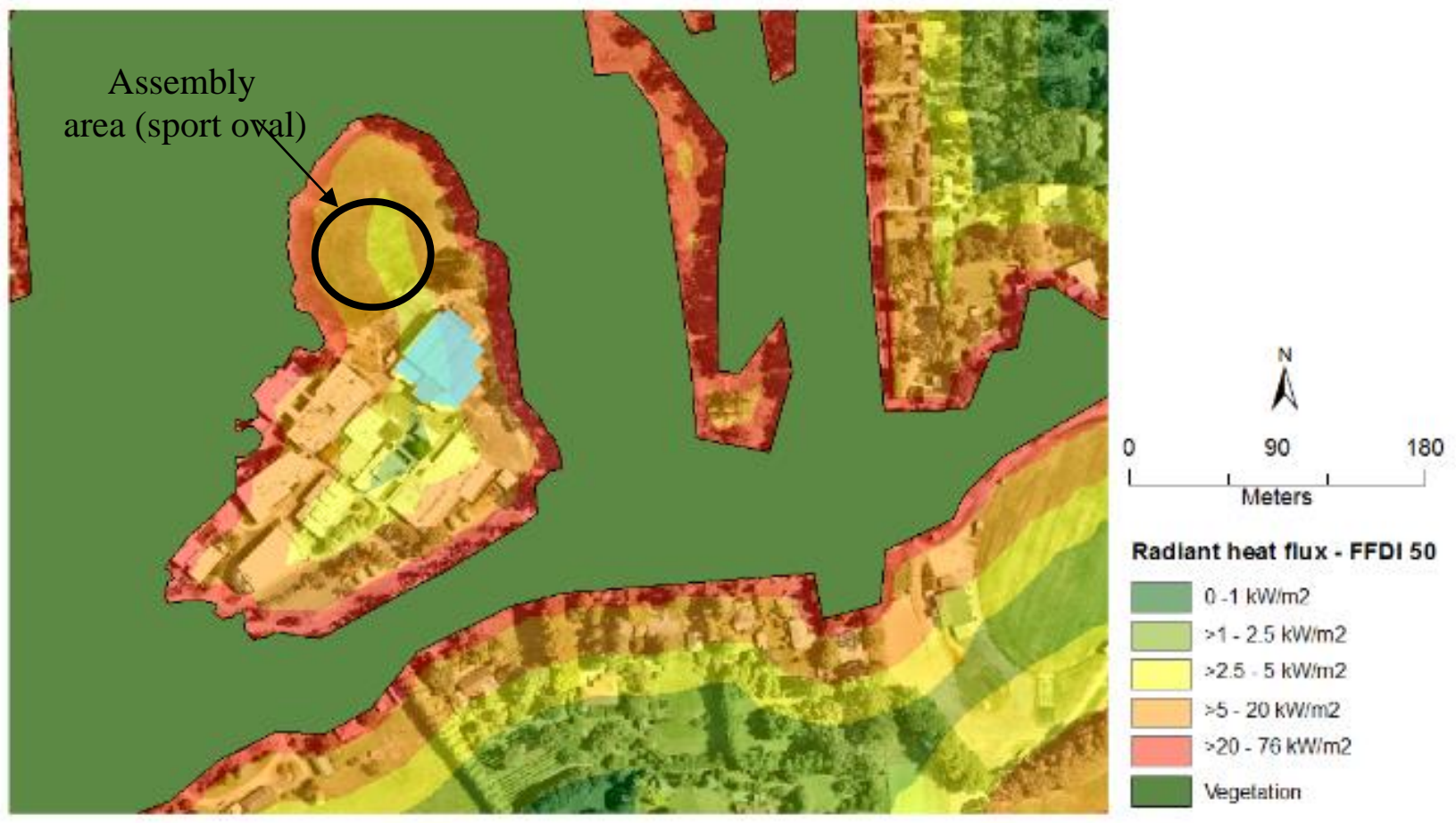

Figure 4 - Radiant heat flux exposure for FFDI 50.

At a FFDI 75 (not shown) all the oval except for a very small central area has a calculated radiant heat exposure greater than $5 \mathrm{~kW} / \mathrm{m}^{2}$. Given the level of exposurethis area is not likely to be a suitable AA for the number of staff and students that may need to utilise this area.

\subsection{Discussion and limitation}

The BLP is designed to be an indicator of hazard for school buildings with no specific bushfire design measures, as per (Kilinc et al., 2014). However, it will require an adjustment for those SIP buildings which have been upgraded using modern building design standards applicable for bushfire prone areas, such as described by AS3959 (Standards Australia, 2009).

The RHF at each location is calculated using the characteristics of the closest fuel. For the RHF calculation, 2.5 and $5 \mathrm{~kW} / \mathrm{m}^{2}$ of radiant heat are the maximum thresholds used in the method, noting 
than $2 \mathrm{~kW} / \mathrm{m}^{2}$ is a conservative medium term exposure level that is also the pain threshold for exposed non-protected skin. In adition, obscuration by buildings vegetation and terrain are not consider when determining maximum radiation levels.

An alternative and more detailed option is to model radiation exposure potential for various fire approach directions, which consider how the terrain, buildings and vegetation obscure radiation heat exposure levels at the AA. These methods can also provide time/radiation profiles which can be a more effective means of setting radiation exposure thresholds (Newnham et al., 2014, 2015).

The present method calculates risk from only forest fuel (McArthur, 1967; Noble, Bary, \& Gill, 1980). Future work will need to consider risk to schools surrounded by grassland or other vegetation types.

\section{Conclusion}

The methodology developed provides an indication of exposure for schools and their occupants. However, in specific bushfire events, risk is extremely difficult to quantify and is dependent on such factors as specific vulnerabilities and impairments of those involved, the capacity for SIP occupants to identify ignitions of the building they shelter in and to suppress them; the rate at which a SIP building may lose tenability; and the rate at which the SIP can be evacuated.

This work has been undertaken with the goal to develop a methodology to objectivly quantify a risk profile for schools. Two discrete methods have been described in this paper to qualify risk at the school level. The first is based on the potential loss of the SIP building, and the second is based on the radiant heat threshold at the AA and along its associated egress paths.

As a response to these assessments, various risk management processes will be considered. These include prioritised infrastructure upgrades, vegetation management programs, pre-empptive temporary relocation under severe weather forecasts, targeted education and training according to the risk profile of the school.

\section{Acknoledgement}

This study was co-funded by the Departement of Education and training Victoria and CSIRO. We would like to thank Wendy Grenville for her contribution and guidance.

\section{Reference}

Alexander, M. E., \& Cruz, M. G. (2012). Interdependencies between flame length and fireline intensity in predicting crown fire initiation and crown scorch height. International Journal of Wildland Fire, 21(2), 95-113. Retrieved from http://www.scopus.com/inward/record.url?eid=2-s2.084858626229\&partnerID $=40 \& m d 5=9 f c 3 d 3240 d d 6 b 7 a 0073 b a 5 c c 469 e 1 a 7 f$

Blanchi, R., Lucas, C., Leonard, J., \& Finkele, K. (2010). Meteorological conditions and wildfirerelated houseloss in Australia. International Journal of Wildland Fire, 19(7), 914-926. Retrieved from http://dx.doi.org/10.1071/WF08175

Blanchi, R., Whittaker, J., Haynes, K., Leonard, J., \& Opie, K. (2018). Surviving bushfire: the role of shelters and sheltering practices during the Black Saturday bushfires. Environmental Science and Policy, 81. Retrieved from https://doi.org/10.1016/j.envsci.2017.12.013

Bradstock, R. A., \& Gill, A. M. (2001). Living with fire and biodiversity at the urban edge: in search of a sustainable solution to the human protection problem in southern Australia. J. Mediterr. Ecol., 2,179 . 
Byram, G. M. (1959). Combustion of forest fuels. in Davis K.P. Byrma G.M. Krumm W.R. Forest fire - Control and use. McGraw-Hill Book Co., New York.

Cheney, N. P., \& Gould, J. S. (1995). Fire Growth in Grassland Fuels. International Journal of Wildland Fire, 5(4), 237-247. Retrieved from http://www.publish.csiro.au/paper/WF9950237

Cheney, P., Gould, J., \& McCaw, L. (2001). The dead-man zone - a neglected area of firefighter safety. Australian Forestry, 64(1), 45-50.

Furby, S. L. (2002). Land Cover Change: Specification for Remote Sensing Analysis. National Carbon Accounting System, Technical Report no. 9.

Gould, J. S., McCaw, W. L., Cheney, N. P., Ellis, P. F., Knight, I. K., \& Sullivan, A. L. (2007). Project Vesta-Fire in Dry Eucalypt Forest: Fuel Structure, Fuel Dynamics and Fire Behaviour. EnsisCSIRO, Canberra, ACT and Department of Environment and Conservation, Perth, WA. Retrieved from http://cbml.csiro.patron.eb20.com/Collections/ViewBookExtended/2b7558ca-863a-44259843-37390fb3c2e3

Harris, S., Anderson, W., Kilinc, M., \& Fogarty, L. (2012). The relationship between fire behaviour measures and community loss: an exploratory analysis for developing a bushfire severity scale. Natural Hazards, 63(2), 391-415. Fetrieved http://download.springer.com/static/pdf/363/art\%3A10.1007\%2Fs11069-012-0156y.pdf?auth66=1354525465_9a44c03aca914bd1d22eb076b7d76554\&ext=.pdf

Hines, F., Tolhurst, K. G., Wilson, A. A. G., \& McCarthy, G. J. (2010). Overall fuel hazard assessment guide 4th edition. Fire and adaptive management, report no. 82. Victoria: Departement of Sustainability and Environment.

Kilinc, A. M., Anderson, W., \& Anderson, D. (2014). DSE Schedule 5 : Fire Severity Rating project Technical Report 1: Project title: A methodology for state wide mapping of bushfire hazard in Victoria ,.

Leonard, J., Blanchi, R., Leicester, R., Lipkin, F., Newnham, G., Siggins, A., Opie, K., Culvenor, B., Cechet, B., Corby, N., Thomas, C., Habili, N., Jakab, M., Coghlan, R., Lorenzin, G., Campbell, D., \& Barwick, M. (2009). Building and Land use planning research after the 7th February 2009 Victorian bushfires. Preliminary findings. Melbourne: Interim report USP2008/018 - CAF122-212 .

Leonard, J., Opie, K., Newnham, G., \& Blanchi, R. (2014). A new methodology for State - wide mapping of bushfire prone areas in Queensland. Australia.

Luke, R., \& McArthur, A. (1978). Bushfires in Australia. Reprinted with corrections 1986. Canberra Publishing and Printing Co.

McArthur, A. G. (1967). Fire behaviour in eucalypt forests. Leaflet No. 107. Comm. of Australia For. \& Timber Bur.

Newnham, G., Blanchi, R., Leonard, J., Opie, K., \& Siggins, A. (2014). Bushfire Decision Support Toolbox Radiant Heat Flux Modelling: Case Study Three, 2013 Springwood Fire, New South Wales. Melbourne.

Newnham, G., Blanchi, R., Opie, K., Leonard, J., \& Siggins, A. (2015). Incorporating vegetation attenuation in radiant heat flux modelling. International Journal of Wildland Fire, 24(5), 640. Retrieved from http://www.publish.csiro.au/view/journals/dsp_journal_fulltext.cfm?nid=114\&f=WF14090

Newnham, G., Opie, K., Leonard, J., \& Flagship, W. (2015). Mapping Current Bushfire Hazard across the state of Queensland.

Noble, I. R., Bary, G. A. V, \& Gill, A. M. (1980). Mcarthur Fire-Danger Meters Expressed as Equations. Australian Journal of Ecology, 5(2), 201-203. 
Purser, D. A., \& McAllister, J. L. (2016). Assessment of Hazards to Occupants from Smoke, Toxic Gases, and Heat. In Hurley, Gottuk, Hall Jr., Harada, Kuligowski, Puchovsky, Torero, Watts Jr., \& WIECZOREK (Eds.), SFPE Handbook of Fire Protection Engineering (pp. 2308-2428). New York, NY: Springer New York. Retrieved from http://dx.doi.org/10.1007/978-1-4939-2565-0_63

Raj, P. K. (2008). A review of the criteria for people exposure to radiant heat flux from fires. Journal of Hazardous Materials, 159(1), 61-71.

Siggins, A., Newnham, G., Blanchi, R., \& Leonard, J. (2013). A 3 Dimensional ray tracing approach to modelling bushfire radiant heat flux for house using lidar derived vegetation voxel data and quadratic polygonal fire fronts. IEEE International Geoscience and Remote Sensing Symposium. Melbourne, Australia.

Standards Australia. (2009). AS3959 - Construction of buildings in bushfire prone areas. Sydney: Standards Australia. Retrieved from http://www.royalcommission.vic.gov.au/getdoc/2d486ba82cef-45a1-9f3c-6e058ec8a78a/WIT.3000.002.0108.PDF

Sullivan, A. L., Ellis, P. F., \& Knight, I. K. (2003). A review of radiant heat flux models used in bushfire applications. International Journal of Wildland Fire, 12(1), 101. Retrieved from http://www.publish.csiro.au/view/journals/dsp_journal_fulltext.cfm?nid=114\&f=WF02052

Teague, B., McLeod, R., \& Pascoe, S. (2010). 2009 Victorian Bushfires Royal Commission final report: summary. (State Government of Victoria: Melbourne).

Whittaker, J., Blanchi, R., Haynes, K., Leonard, J., \& Opie, K. (2017). Experiences of sheltering during the Black Saturday bushfires: Implications for policy and research. International Journal of Disaster Risk Reduction, 23.

Zárate, L., Arnaldos, J., \& Casal, J. (2008). Establishing safety distances for wildland fires. Fire Safety Journal, 43(8), 565-575. 


\title{
Development of a spatial analysis tool for radiant heat flux predictions
}

\author{
Alessio Arena*; Justin Leonard; Raphaele Blanchi; Glenn Newnham; Kimberley Opie \\ CSIRO Land and Water. \{alessio.arena@csiro.au*\}
}

\begin{abstract}
This paper describes a new tool developed to spatially quantify the radiant heat flux generated during a wildfire. Radiant heat generated by a wildfire can reach levels that are threatening for building structures and deadly for human life. Very dangerous situations can occur when fire shelters fail and people are required to change location. In Australia, radiant heat flux estimation has been used to model the potential exposure experienced by structures, and forms the basis for wildfire related building and planning regulations. The existing radiant heat estimation method was developed for on-site assessment, and tends to be simplistic and not scalable at a regional level. This can result in suboptimal design outcomes for builders and planners when implementing their fire risk management strategies. This paper describes a new spatial tool developed to better quantify maximum radiant heat flux at a local to regional scale. This tool builds on the estimation method already in use within the Australian legislation, improving its efficiency and expanding its spatial capabilities. The result is a comprehensive radiant heat map that can improve our understanding of conditions during wildfire as a means of understanding mechanisms for human impacts and structure loss.
\end{abstract}

Keywords: radiant heat flux, spatial, GIS, fire, risk

\section{Introduction}

Wildfires are one of the most dangerous natural hazards in Australia, impacting hundreds of properties and lives each year. Ember attack is the main cause of house and property loss (Blanchi and Leonard 2008), while radiant heat transfer poses the highest threat to firefighters and building occupants in cases of emergency shelter evacuation (Butler and Cohen 1998; Cheney et al. 2001; Whittaker et al. 2017).

The threat from radiant heat transfer needs to be accounted for at many levels of planning, including urban and emergency planning, and in building standards (Cohen 1995; Butler and Cohen 1998; Siggins et al. 2013; Newnham et al. 2014). This has lead to the development of the Australian Standards AS3959 (Standards Australia 2009) which outlines the official methodology to calculate the radiant heat flux (RHF) a person or structure could be exposed to in the 'worst case' scenario wildfire.

This standards document is currently used by all government organisations to guide many of their decisions regarding wildfires, including new development approvals and emergency planning. However, the methodology outlined in AS3959 was developed for field assessments of wildfire risk at local scale and is difficult to apply at a larger scale.

This paper outlines an adaptation of the AS3959 methodology through development of a spatial tool to allow assessment at local to regional scales. This tool was developed in Python 2.7 leveraging the ArcGIS platform to allow user friendly interaction and adaptation of relevant RHF modelling parameters.

\section{Background}

In this section we outline the state-of-the-art methods to quantify the RHF for a wildfire in Australia. This information is available in Sullivan, Ellis, \& Knight, 2003 and Standards Australia, 2009. 


\subsection{Radiant heat flux model for wildfires}

Radiation is one of the three mechanisms of heat transfer from a flame body, the others being conduction and convection. The Stefan-Boltzmann equation for a real surface defines the maximum amount of heat transferred in this way:

$$
q=\varepsilon \sigma T^{4}
$$

where $\sigma$ is the Stefan-Boltzmann constant of value $5.67 * 10^{-8} \mathrm{Wm}^{-2} \mathrm{~K}^{-4}, T$ is the absolute temperature of the radiating surface $(\mathrm{K}), \varepsilon$ is the emissive power of the surface as a ratio of total radiation emitted by the surface to that emitted by a blackbody of the same temperature, and $q$ is the emissive power per unit area $\left(\mathrm{Wm}^{-2}\right)$.

The efficiency of this mechanism is largely affected by the relative position of source and receiver, often referred to as "view factor" (Sullivan et al. 2003). This leads to the equation:

$$
E=\phi \varepsilon \sigma T^{4}
$$

where $\varphi$ is the view factor, and $E$ is the RHF received at the surface.

Additionally, the geometry of the source surface greatly affects the directional distribution of the emissions and the corresponding flux. In fact, a unit surface area radiates in all directions such that the intensity is proportional to the cosine of the angle between the direction and the normal to the surface. In the case of a wildfire front, the geometry of the radiating body is often approximated to an opaque box. Flame width, flame height and flame angle to the vertical are used to describe the geometry of the opaque box, and are important inputs for the RHF model (Sullivan et al. 2003).

\subsection{Bushfire Attack Level (BAL) assessment}

The AS3959 uses simplified radiant heat transfer estimation methods derived from Sullivan et al. 2003 and aims to capture the worst case scenario of a wildfire given information including available fuel, landscape characteristics and weather conditions. The resulting worst case RHF is then converted into a BAL using threshold values summarised in Table . Each increasing BAL increment requires increasing wildfire protection measures to meet the AS3959 requirements.

Table 1 - Summary of Bushfire Attack Levels with associated conditions and main risk factors (from Standards Australia 2009)

\begin{tabular}{|c|c|c|}
\hline $\begin{array}{c}\text { Bushfire Attack Level } \\
\text { (BAL) }\end{array}$ & Condition & Main risk factors \\
\hline BAL-LOW & Distance $>100 \mathrm{~m}$ & Insufficient risk \\
\hline BAL-12.5 & $\mathrm{RHF} \leq 12.5 \mathrm{~kW} / \mathrm{m}^{2}$ & Ember attack \\
\hline BAL-19 & $\begin{array}{c}12.5 \mathrm{~kW} / \mathrm{m}^{2}>\mathrm{RHF} \geq 19 \\
\mathrm{~kW} / \mathrm{m}^{2}\end{array}$ & $\begin{array}{c}\text { Ember attack and radiant } \\
\text { heat }\end{array}$ \\
\hline BAL-29 & $\begin{array}{c}19 \mathrm{~kW} / \mathrm{m}^{2}>\mathrm{RHF} \geq 29 \\
\mathrm{~kW} / \mathrm{m}^{2}\end{array}$ & $\begin{array}{c}\text { Ember attack and radiant } \\
\text { heat }\end{array}$ \\
\hline BAL-40 & $\begin{array}{c}29 \mathrm{~kW} / \mathrm{m}^{2}>\mathrm{RHF} \geq 40 \\
\mathrm{~kW} / \mathrm{m}^{2}\end{array}$ & $\begin{array}{c}\text { Ember attack, radiant heat } \\
\text { and direct flame interaction }\end{array}$ \\
\hline BAL-FZ & $\mathrm{RHF}>40 \mathrm{~kW} / \mathrm{m}^{2}$ & Direct flame interaction \\
\hline
\end{tabular}

The BAL assessment is performed in the field by trained personnel. Due to the complexity of the calculation, AS3959 (2009) includes a simplified 'method 1' using precomputed values in a tabulated form. This method is limited to a very small set of input values (e.g. slope increments of 5 degrees, only distances for the BAL transitions), and returns only the corresponding BAL bracket. 
The underlying 'method 2' is also provided. This is a complete calculation method, and can be generally outlined:

1. Determine the weather parameters to use as reference (typically Fire Danger Index, or FDI)

2. Determine the vegetation classification, fuel loads and characteristics

3. Determine the effective slope under the vegetation, which is the slope corresponding to the fire spread direction

4. Determine the slope of the land between the site assessed and the vegetation

5. Determine the distance between site and vegetation

6. Calculate the flame length using the rate of spread (RS) modified for the effective slope and the vegetation and weather conditions

7. Determine the flame width

8. Determine the elevation of the receiver

9. Calculate the radiant heat flux corresponding to the worst possible flame angle and resulting view factor. This is an iterative step to find the maximum value

10. Determine the BAL

Rate of fire spread for each vegetation type is calculated using empirical formulas (described in Catchpole et al., 1998; Marsdens-medley \& Catchpole, 1995; Noble, Gill, \& Bary, 1980). These formulae consider vegetation type and condition, landscape morphology and weather conditions, which are approximated into broad classifications, empirical formulas or indices. Additionally, this methodology does not consider barriers or actual visibility of the vegetation from the site. These parameters are difficult to assess during field assessment, and are therefore considered out-of-scope for

\section{Spatial calculation of radiant heat flux using AS3959 (2009) method 2}

The development of a more accurate, landscape-scale calculation of RHF requires implementation of AS3959 (2009) method 2 in a Geographic Information System (GIS) environment. Our key considerations during the development of this tool were:

- spatial scalability to address local to regional scenarios,

- performance, due to the often limited resources available using desktop GIS platforms, and

- minimal input requirement, to minimise the requirement for precomputed data, and facilitate the use of readily available and generic datasets.

\subsection{Input datasets}

GIS environments support two families of spatial datasets: raster datasets, which are comprised of discrete spatial units such as the pixels in a typical satellite image, and vector datasets which contain points, lines and features. Many publicly available large-scale datasets are rasters derived from remote sensing, including vegetation classification datasets (e.g. Ecological Vegetation Class in Victoria) that can be used to estimate fire fuel characteristics.

The typical resolution of remote-sensing derived rasters is too coarse for detailed risk analyses. To better model spatial variability, this tool requires the user to provide a more detailed vector file containing polygons of the vegetation footprint to accurately map vegetation boundaries. The input datasets required are: 
- vegetation polygons (vector), with attached vegetation type, surface fuel load and total fuel load;

- site polygons or centroids (vector), to determine the processing extent or region of interest (ROI);

- digital elevation model (raster), with its derived

$\circ$ terrain slope (raster) and

$\bigcirc \quad$ terrain aspect (raster)

- weather conditions, like fire danger index, wind speed and moisture factor.

Some of these parameters may be optional depending on the vegetation type (Standards Australia, 2009, table B1).

\subsection{Implementation}

The current implementation uses two outer loops, the first to iterate through buildings. This is used to set the Region of Interest (ROI), allowing optimisation of memory usage while being able to handle very large input datasets. At the same time, the building dataset is optional and can be omitted if a region-wise calculation is required. In this case, the processing extent will need to be set before running the tool.

The second loop iterates through vegetation polygons included within the ROI. Each of those polygons is treated as an independent source and used to calculate a RHF raster. Once all the vegetation polygons have been processed, the RHF rasters are combined to keep the maximum value for each pixel.

The calculation within the second loop is linearly structured:

1. Calculate the Euclidean distance and direction from the vegetation. The distance will be a direct input for the RHF calculation.

2. Using the Euclidean allocation algorithm, calculate the elevation, slope and aspect for the closest vegetation at any point in space (Euclidean elevation, Euclidean slope and Euclidean aspect).

3. Convert the Euclidean slope to effective slope, which is the slope a person would experience at that location by walking in the direction of the receiver. This is often referred in other disciplines as 'projected slope', 'actual slope' or 'directional slope', and is computed by:

a. Calculating the vector product between Euclidean aspect (direction where the slope underneath the vegetation is facing towards) and Euclidean direction (direction towards the receiver).

b. This product is then multiplied to the Euclidean slope.

4. Calculate the slope between source and receiver using the elevation at both points, as defined in Standards Australia, 2009.

5. Perform the RHF calculation using method 2 (Standards Australia 2009) at any point in space. Inputs are effective slope underneath the vegetation, slope between site and vegetation, distance to vegetation, vegetation and weather conditions.

A diagram of the RHF calculation steps is provided in Figure 1. 


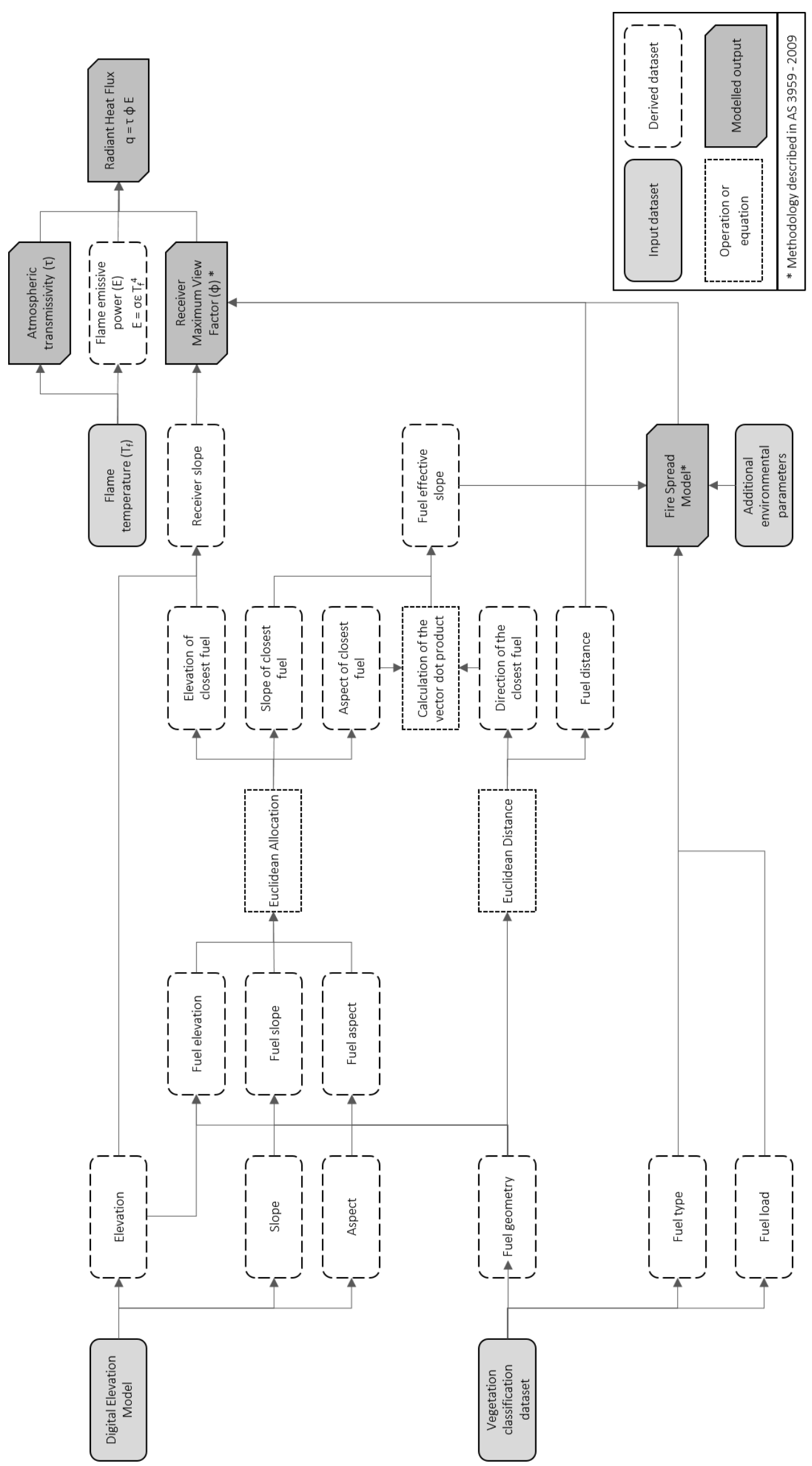

Figure 1 - Diagram representing the workflow used to calculate the radiant heat flux 


\subsection{Current limitations}

This implementation of the GIS tool calculates the RHF at any point in space using characteristics of the closest fuel. This approach allows to leverage the Euclidean distance algorithm, and efficiently calculate RHF at any location. However, the highest RHF may not be generated by the closest fuel as vegetation characteristics can have a much higher impact. Consequently, this approach will underestimate RHF values where characteristics of the closest fuel are in fact not the worst that location is exposed to. The resulting artefact will appear as a sharp change in RHF values

The use of iterations through each vegetation polygon allowed us to account for most vegetation characteristics. Each polygon is treated as an independent source and generates an intermediate RHF map. Those maps are then combined to keep only the highest RHF value given by the most dangerous vegetation polygon. However, the slope underneath vegetation can vary greatly within the same polygon, and cannot be fully accounted for by our approach.

Below is an example of the artefact we described, caused by the concave shape of the vegetation polygon that results in a large variation in effective slope.

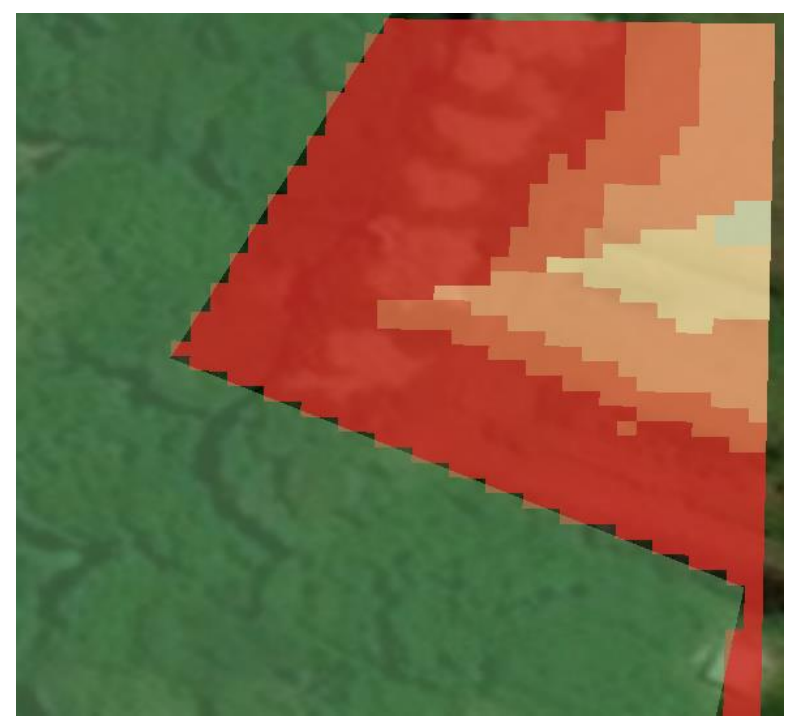

Figure 2 - Example of artefact due to the Euclidean distance based approach (top right corner). In green is the vegetation, and in red to yellow are the decreasing $B A L$ from $B A L-F Z$ to BAL12.5

\section{Results and discussion}

This tool was successfully used to produce RHF maps for 60 buildings in a case study in Victoria, to assess the relative safety of community shelters and associated offsite assembly areas in case of a wildfire. This involved assessing the likelihood of survival of the structure and the radiant heat that occupants may be exposed to during the evacuation.

This case study included vegetation information for 200 polygons over an area of 960 hectares and generating results for approximately 3.3 million locations. This analysis was completed in approximately $1.5 \mathrm{~h}$ using a moderately high specification commercially available workstation.

RHF values produced in this way were in agreement with manual calculations using both method 2 and method 1 AS3959 (2009). An example map is available in Figure . 

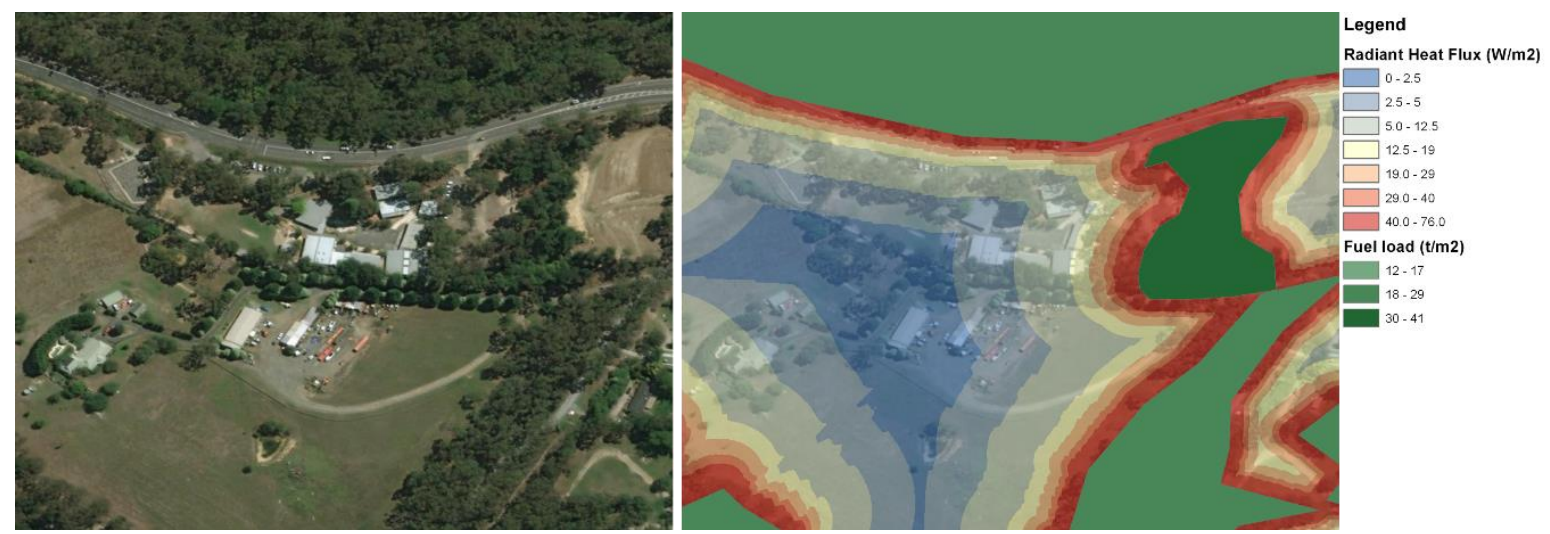

Figure 3 - Example RHF map from the case study. The area in light blue $\left(<2.5 \mathrm{~kW} / \mathrm{m}^{2}\right)$ is considered to be safe for humans without protective clothing

In comparison to a typical field assessment, the output maps derived from the tool provide a more comprehensive view of the risks that wildfire may pose to the assessed site.

This tool has the potential to be used to:

- Increase the objectivity of RHF assessments compared to the typical field assessment methods

- Assist in identifying safer emergency evacuation points that consider vegetation and terrain conditions over a larger spatial region

- Inform the definition of preferred evacuation paths for use during emergency events

This tool does not account for occlusion of the radiating source from the receiver in cases where there are opaque barriers (e.g. other buildings, fences) or complex topography. The underlying method used to perform the calculation (method 2 AS3959 (2009)) does not address this issue.

For this reason a more sophisticated approach using a ray-casting algorithm is currently under development (Leonard et al. 2014, 2015; Hilton et al. 2017). This will likely produce a more realistic radiant heat flux profile but will require a very accurate 3D scene of the site.

\section{Conclusion}

The methodology described in this paper is a valid alternative to single point-based estimates for radiant heat flux, while still conforming to the current Australian legislation. These estimates are often carried in the field at a single location and rely on the simplified 'method 1' provided by AS3959 (Standards Australia 2009). This new approach is based on the more accurate 'method 2' from AS3959 but provides a simple way to extend this method to analysis of not just a single house.

The developed tool uses readily available datasets and information to generate radiant heat maps in an objective and repeatable way. The tool proves to be computationally efficient and can be effectively used with all modern hardware configurations.

The output generated is also spatially aware, providing a more comprehensive understanding of hazard level at the landscape scale during a wildfire. This spatial context provides additional insights that can help in making more informed bushfire management decisions, such as better planning for safe sheltering and egress during fire events.

\section{References}

Blanchi R, Leonard J (2008) Property safety: judging structural safety. 'Community Bushfire Saf.' (Eds J Handmer, K Haynes) pp. 77-85 
Bradstock RA, Mccarthy G, Mccaw L (1998) Cooperative development of equations for heathland fire behaviour. https://www.researchgate.net/publication/299193502.

Butler BW, Cohen JD (1998) Firefighter Safety Zones: A Theoretical Model Based on Radiative

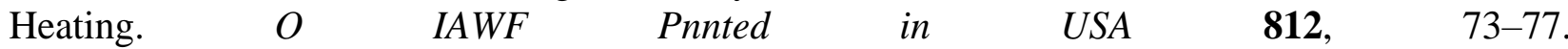
http://citeseerx.ist.psu.edu/viewdoc/download?doi=10.1.1.369.4565\&rep=rep1\&type=pdf.

Cheney P, Gould J, McCaw L (2001) The dead-man zone - a neglected area of firefighter safety. Australian Forestry 64, 45-50.

Cohen JD (1995) Structure ignition assessment model (SIAM) (DR Weise and RF Martin, Eds.). Biswell Symp. fire issues Solut. urban interface Wildl. Ecosyst. 85-92.

Hilton JE, Leonard J, Blanchi R, Newnham G, Opie K, Rucinski C, Swedosh W (2017) Dynamic modelling of radiant heat from wildfires. In 'MODSIM 2017', 1104-1110 https://www.mssanz.org.au/modsim2017/H10/hilton.pdf.

Leonard J, Newnham G, Opie K (2015) Silvan Water Treatment Plant - Fire Risk Assessment. CSIRO, https://publications.csiro.au/rpr/pub?list=SEA\&pid=csiro:EP155256\&sb=RECENT\&expert=false $\& n=2 \& \mathrm{rpp}=25 \&$ page $=1 \& \operatorname{tr}=22 \& \mathrm{q}=$ radiant heat $\& \mathrm{dr}=$ all.

Leonard J, Newnham G, Opie K, Blanchi R (2014) Millgrove Community FireRefuge - Site AssessmentJuly 2014. https://publications.csiro.au/rpr/pub?list=SEA\&pid=csiro:EP1410452\&sb=RECENT\&expert=fals $\mathrm{e} \& \mathrm{n}=5 \& \mathrm{rpp}=25 \&$ page $=1 \& \mathrm{tr}=22 \& \mathrm{q}=$ radiant heat $\& \mathrm{dr}=\mathrm{all}$.

Marsdens-medley J, Catchpole W (1995) Fire Behaviour Modelling in Tasmanian Buttongrass Moorlands .II. Fire Behaviour. International Journal of Wildland Fire 5, 215. doi:10.1071/WF9950215.

Newnham G, Blanchi R, Leonard J, Opie K, Siggins A (2014) Bushfire Decision Support Toolbox Radiant Heat Flux Modelling: Case Study Three, 2013 Springwood Fire, New South Wales. (Melbourne)

NOBLE IR, GILL AM, BARY GA V. (1980) McArthur's fire-danger meters expressed as equations. Austral Ecology 5, 201-203. doi:10.1111/j.1442-9993.1980.tb01243.x.

Siggins A, Newnham G, Blanchi R, Leonard J (2013) A 3 Dimensional ray tracing approach to modelling bushfire radiant heat flux for house using lidar derived vegetation voxel data and quadratic polygonal fire fronts. IEEE Int. Geosci. Remote Sens. Symp.

Standards Australia (2009) 'AS3959 - Construction of buildings in bushfire prone areas.' (Standards Australia: Sydney) http://www.royalcommission.vic.gov.au/getdoc/2d486ba8-2cef-45a1-9f3c6e058ec8a78a/WIT.3000.002.0108.PDF.

Sullivan AL, Ellis PF, Knight IK (2003) A review of radiant heat flux models used in bushfire applications. International Journal of Wildland Fire 12, 101. doi:10.1071/WF02052.

Whittaker J, Blanchi R, Haynes K, Leonard J, Opie K (2017) Experiences of sheltering during the Black Saturday bushfires: Implications for policy and research. International Journal of Disaster Risk Reduction 23, 119-127. doi:10.1016/j.ijdrr.2017.05.002. 


\title{
Estimating net heat flux from surrogate firebrand accumulations using an inverse heat transfer approach
}

\author{
J. Christian Thomas; Eric V. Mueller; Rory M. Hadden* \\ School of Engineering, University of Edinburgh, EH9 3BF, Edinburgh, UK. \\ \{r.hadden@ed.ac.uk*\}
}

\begin{abstract}
An experimental study is presented that investigates the heat flux from firebrand accumulations to a substrate. Surrogate firebrands produced from wood pellets were deposited on an inert solid substrate. An inverse heat transfer model is used with temperature measurements in the substrate to calculate the net heat flux into the substrate. The thermal boundary condition (heat flux) can then be used to assess the hazard from firebrand accumulations and better understand the ignition potential of combustible structural material by firebrands. Peak net heat fluxes were found to be between 4 and $9 \mathrm{~kW} \mathrm{~m}^{-2}$ and to depend primarily on the initial particle temperature. The exposure duration increases with increasing firebrand accumulation mass and decreasing deposition area. For sustained smouldering accumulations, the substrate is exposed to prolonged heating with a net heat flux between 1 and $3 \mathrm{~kW} \mathrm{~m}^{-2}$. Peak gauge heat fluxes were found between 30 and $80 \mathrm{~kW} \mathrm{~m}^{-2}$. This depends on the initial particle temperature and deposition area. The method shown herein is a robust tool to assess hazardous conditions in the wildland-urban interface, related to firebrand accumulations.
\end{abstract}

Keywords: wildfires, embers, experimentation, WUI, wood pellets

\section{Introduction}

The production, transport and deposition of firebrands represent key fire spread mechanisms in wildfires and at the wildland urban interface. This is due to the potential of firebrands to promote fire front propagation, causing long range spotting, and cause ignition of combustible structural material.

Studies available in the literature identify firebrands as a significant cause of structural ignitions in the WUI (Caton 2017). Single firebrands can ignite vegetative litter (e.g. Fernandez-Pello 2015) or porous insulation if they penetrate exterior shell of the structure (Hamann 2017). It is unlikely that a single firebrand will cause ignition of non-porous combustible solids due to the thermal mass of the substrate compared to the heating from the firebrand. However, when firebrands accumulate on solid combustible substrates, the energy transfer from an accumulation of particles may develop a hazardous thermal boundary condition sufficient to promote pyrolysis, charring and ignition (smouldering and/or flaming). A thermal boundary condition in form of a heat flux incident at the surface of combustible material is commonly used in fire safety engineering in the built environment to assess the ignition potential of that material (Torero 2016). It is the intent of this work to generate a methodology that adapts prior knowledge from the built environment to meet wildfire research needs.

A complete solution to the firebrand problem has yet to be established. Significant work has been conducted exploring firebrand production (Thomas 2017, El Houssami 2016, Manzello 2009(a)), transport and spotting (Koo 2010), ignition of porous fuel beds (Ellis 2015, Fernandez-Pello 2015, Viegas 2014, Manzello 2006), and ignition of solids (Hakes 2017(b), Santamaria 2015, Manzello 2014, Manzello 2009(b)). Hakes et al. (2017(a)) and Caton et al. (2017) produced comprehensive summaries of the state of research, which the reader is encouraged to visit.

The experimental study presented shows a methodology to assess the thermal boundary condition using direct heat flux measurements and an inverse heat transfer method. Firebrands are deposited on 
an inert substrate, which is equipped with thermocouples or heat flux gauge. The work focuses on the ignition of solid substrates, investigating the net and incident heat fluxes that are generated from firebrand accumulations. The goal is to assess what magnitude a typical flux is and if this flux causes hazardous conditions for solid combustible substrates.

\section{Methodologies}

\subsection{Fuel}

Commercially available wood pellets were used as surrogate firebrand material (Figure ). The pellets are comprised of Scottish softwood (Puffin Pellets, UK). The pellets have a quoted calorific value of $16.56 \mathrm{~kJ} \mathrm{~g}^{-1}\left(4.6 \mathrm{kWh} \mathrm{kg}^{-1}\right)$, and less than $0.5 \%$ ash content. The diameter was measured to be $6.0 \mathrm{~mm}$ and the mean length of virgin particles was $15.91 \pm 5.30 \mathrm{~mm}$ and projected area was 95.49 $\pm 31.80 \mathrm{~mm}^{2}(\mathrm{~N} \approx 600$, assuming cylindrical pellets). These particles (and resulting firebrands) are, on average, larger than firebrand sizes that has been quoted in literature (Manzello 2009(a), Thomas 2017). The moisture content of virgin wood pellets was $7.15 \pm 0.15 \%$ (dry weight).

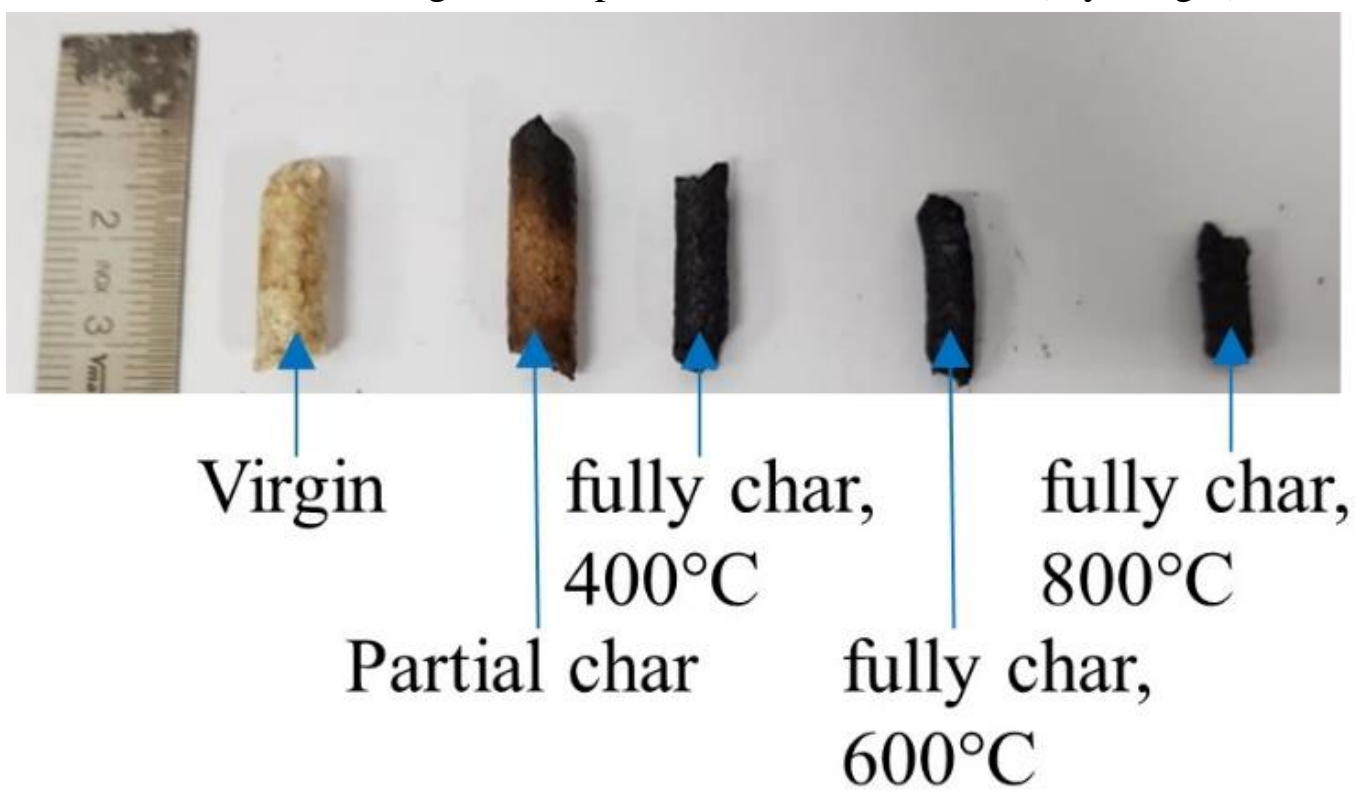

Figure 1 - Pellets used as fuel for firebrand production. At various states of generation. Temperature refers to furnace temperature (condition period: $10 \mathrm{~min}$ )

\subsection{Firebrand production}

Firebrands were produced in a muffle furnace (Nabertherm LE-14/11, Nabertherm GmbH, Germany). The furnace was set to a constant temperature and a known mass of virgin pellets was placed in the furnace for a predetermined period of time. Both the initial mass of firebrands and furnace temperature were varied in this study and the conditioning time was kept constant at $10 \mathrm{~min}$. The initial accumulation masses investigated were 50,100, and $200 \mathrm{~g}$ (of virgin pellets). Oven temperatures were varied between 400,600 and $800{ }^{\circ} \mathrm{C}$. For each initial pellet mass tested, the firebrand accumulation mass produced after conditioning was measured.

The condition of the firebrands was characterised by measuring the mass loss during conditioning at specific intervals. The resulting mass loss curves are shown in Figure . 

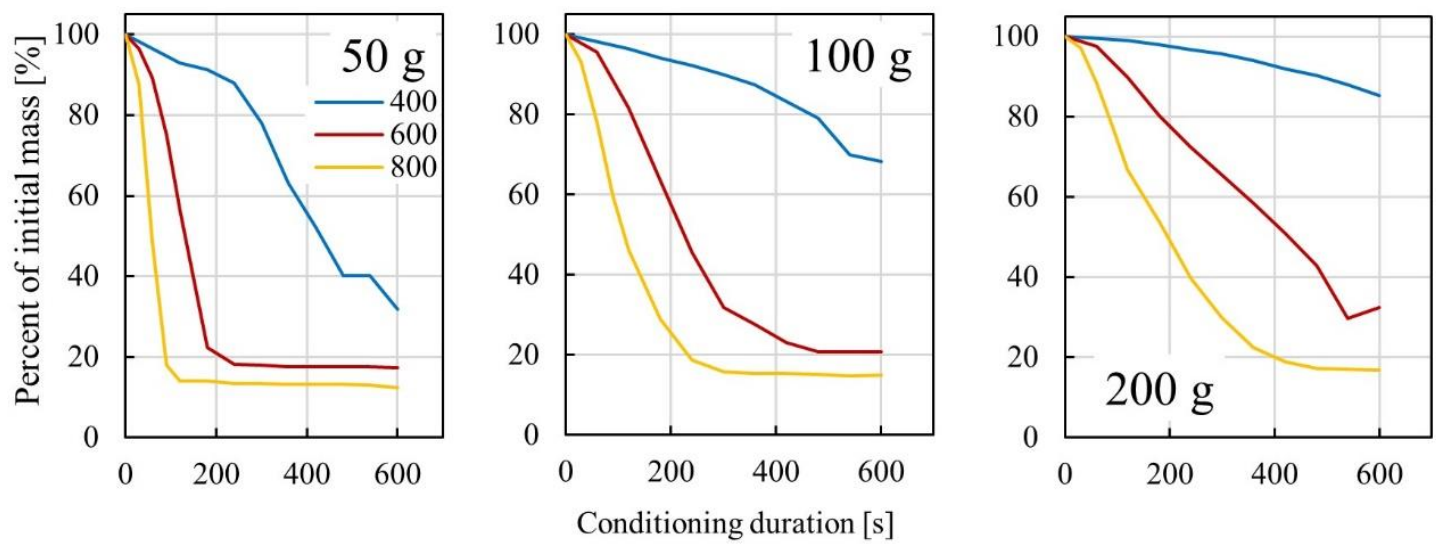

Figure 2 - Mass loss curves of firebrands generated in a muffle furnace with three different initial masses. The values in the legend refer to the furnace temperature in degree Celsius.

During the conditioning period, the virgin material undergoes dehydration, degradation and pyrolysis, and charring processes. The data shown in Figure at $600 \mathrm{~s}$ represent the firebrand mass used in experimentation discussed below. After $600 \mathrm{~s}$ at $800^{\circ} \mathrm{C}$, all samples reach fully charred state. After $600 \mathrm{~s}$ at $600{ }^{\circ} \mathrm{C}$ the $200 \mathrm{~g}$ sample still contains $32 \%$ of the initial mass. At $400{ }^{\circ} \mathrm{C}$ furnace temperature, the firebrand accumulation mass used in experimentation ranges from 32 to $85 \%$ of the initial mass, for 50 and $200 \mathrm{~g}$, respectively.

It is important to know the state of the firebrands when deposited on the substrate, i.e. whether they are partially or fully charred. During conditioning, particle properties (thermal conductivity, particle density, energy content, size, and others) change. These particle properties are important as they will affect the heat transfer between the accumulation and the substrate. The heat of combustion of the particles is also important as this characterises the total energy available in the accumulation. Figure shows pellets after various conditioning periods. For the specific pellets used here, the heat of combustion was determined using Bomb Calorimeter (IKA C1, IKA England LTD, UK) according to the principles of ISO 1716. The heat of combustion are shown in Table .

Table 1 - Heat of combustion results from Bomb Calorimeter experiments (Three repetition per condition).

\begin{tabular}{llll}
\hline & & \multicolumn{2}{c}{ Heat of comb. $\left[\mathrm{kJ} \mathrm{g}^{-1}\right]$} \\
\cline { 3 - 4 } Initial mass $[\mathrm{g}]$ & Furnace temp. $\left[{ }^{\circ} \mathrm{C}\right]$ & Avg. & St.dev. \\
\hline- & - & 18.78 & 0.06 \\
- & - & 19.92 & 0.03 \\
200 & 400 & 21.86 & 2.14 \\
100 & 400 & 22.61 & 2.34 \\
50 & 400 & 28.81 & 0.04 \\
100 & 600 & 33.54 & 0.03 \\
50 & 800 & 32.39 & 0.06 \\
\hline
\end{tabular}

Virgin pellets have the lowest heat of combustion while the fully charred particles have the highest heat of combustion. Sample accumulations with 100 and $200 \mathrm{~g}$ initial mass produced at $400{ }^{\circ} \mathrm{C}$ were a mix of virgin, partially charred and fully charred particles (see Figure ). The average heat of combustion increases, with a higher variability (see standard deviation). These values are comparable to literature values for wood and wood char (Dietenberger 2002). The temperature at which the fully charred particle are produced also has an impact on the heat of combustion although the trend is not entirely evident yet. 


\subsection{Experimental setup}

The experimental setup is shown in Figure below. After conditioning, firebrands were weighed and transported to a containment basket. This basket was made from stainless steel mesh with $63 \%$ perforation. Three different basket sizes are used with $50 \mathrm{~mm}, 75 \mathrm{~mm}$, and $100 \mathrm{~mm}$ diameter. A confined accumulation was used in order to better quantify the accumulation shape, as well as controlling the deposition area.

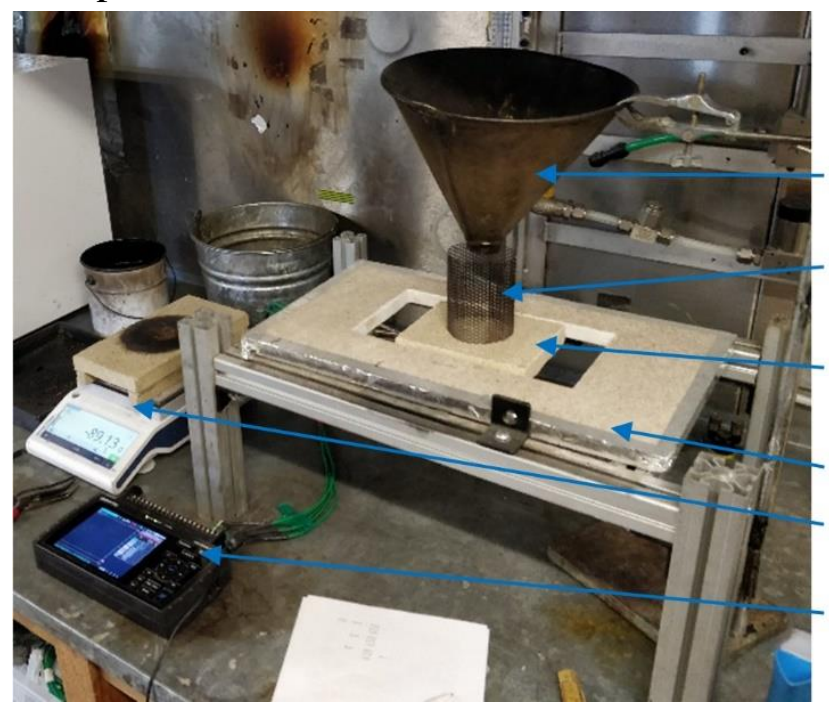

Funnel for deposition

Perforated containment basket

Substrate (vermiculite with

Embedded TCs or HFG)

Work top and frame

Load cell

Data logger

Figure 3 - Experimental setup for generation of firebrands and measuring net heat flux from firebrand accumulations.

Side view of substrate with thermocouple location:

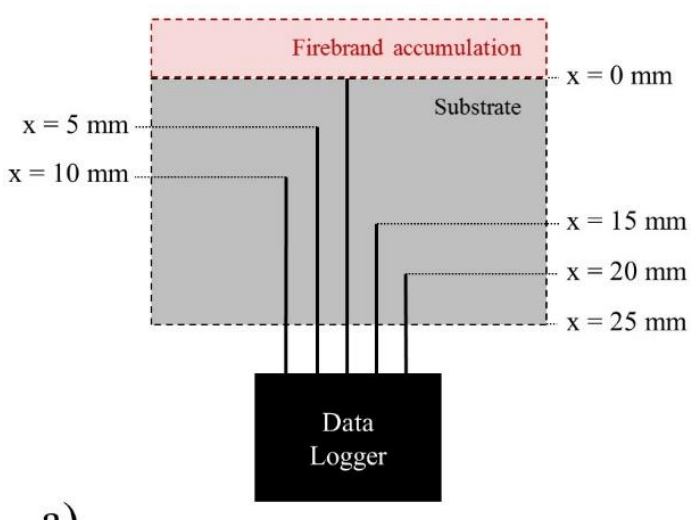

a)

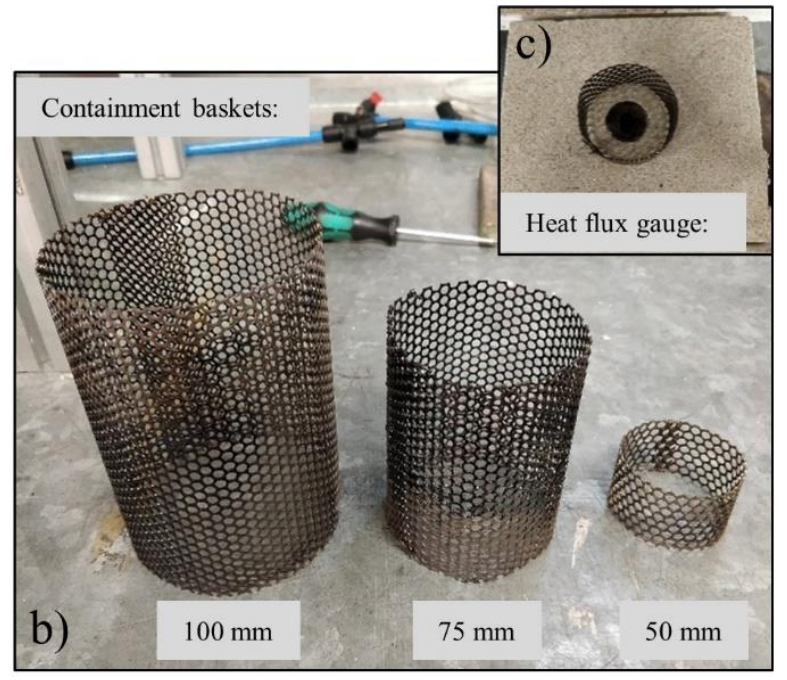

Figure 4 - (a) Schematic of thermocouple layout and (b) containment baskets and (c) heat flux gauge in substrate (with $50 \mathrm{~mm}$ basket).

The substrate used was $25 \mathrm{~mm}$ thick vermiculite insulation board (V-1100(700), Skamol, Denmark). This was chosen because it has similar properties to wood $\left(\rho=700 \mathrm{~kg} \mathrm{~m}^{-3}, k=0.19 \mathrm{~W} \mathrm{~m}^{-}\right.$ ${ }^{1} \mathrm{~K}^{-1}, C_{p}=995 \mathrm{~J} \mathrm{~kg}^{-1} \mathrm{~K}^{-1}$ ). The substrate was either instrumented with five thermocouples (K-type, 1 $\mathrm{mm}$ diameter sheath, grounded) or a water cooled heat flux gauge (Schmidt-Boelter type, Hukseflux Thermal Sensors B.V., UK, SBG01-100). Four thermocouples were located nominally 5, 10, 15, and $20 \mathrm{~mm}$ from the surface of the substrate. One thermocouple was positioned flush with the substrate surface. The heat flux gauge was flush with the surface. Temperature and heat flux data were acquired at $2 \mathrm{~Hz}$. 
In this manuscript the following notation for the description of experiments will be used: "initial accumulation mass-furnace temperature-deposition area diameter". An example is: 050-400-050. This refers to the experiment with $50 \mathrm{~g}$ initial mass, conditioned at $400{ }^{\circ} \mathrm{C}$ with $50 \mathrm{~mm}$ deposition area diameter. Note that the $50 \mathrm{~mm}$ diameter deposition area was only used with $50 \mathrm{~g}$ initial accumulation mass. Experiments with the heat flux gauge were only conducted for a small set of conditions (050400-050/075/100 and 050-800-050).

\subsection{Inverse heat transfer model}

The substrate temperature data was used as input to an inverse heat transfer model. Data from the surface thermocouple were not used in this model. This model outputs the time series of net heat flux into the substrate. Net heat flux into the vermiculite substrate was estimated using an inverse heat transfer calculation method. The 1-D conduction equation was solved numerically for the vermiculite using an implicit Crank-Nicolson scheme (Minkowycz 2006). Density, specific heat capacity and temperature dependent thermal conductivity were determined from manufacturer. Temperature dependent emissivity was obtained from Laschuetza (2017). Convective and radiative heat losses at the back of the substrate were included. The net flux condition at the upper surface was determined using Beck's method for minimizing the sum of squares between measured and estimated in-depth temperatures, using a number of future measurements for a given time step (Beck 1985). In this case, 30 future measurements were for each prediction. The calculated net heat flux is compared to the total heat flux (no losses due to radiation and convection) measured with the heat flux gauge.

\section{Results and discussion}

This section is separated into two parts. First, temperature measurements in the vermiculite substrate will be presented followed by the heat flux measurements.

\subsection{In-depth temperature measurements}

It was observed that in-depth temperature evolutions follow the same trends as the surface temperature for the duration of the experiment (Figure ).

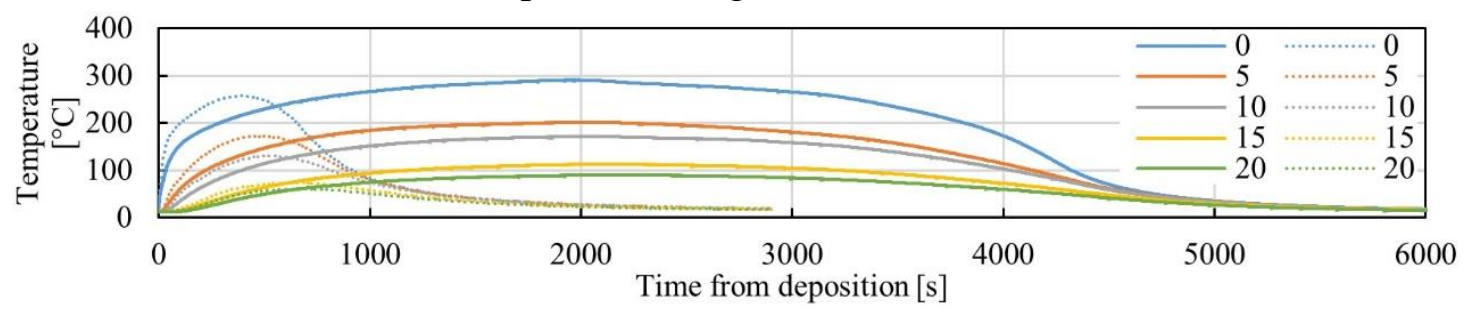

Figure 5 - Typical example of in-depth temperature evolution. Solid: 050-400-050, dotted: 050-800-050. The values in the legend refer to the thermocouple location from the surface, $0 \mathrm{~mm}$ is the surface.

The trend is characterised by a period of rapidly increasing temperature following deposition of the firebrands and a decrease in the temperature as the accumulation cools or is consumed by a smouldering reaction. The duration of the heating period is a function of the accumulation mass, temperature and deposition area.

This is expected, since the substrate is inert and no thermal degradation or combustion processes occur within the substrate matrix. Thus, for simplicity and clarity, most data presented only contain surface temperature measurements for comparison between experimental conditions. A clear distinction can be made between the two experimental conditions depicted in Figure. This distinction can also be made by just observing the temperature at the surface $(\mathrm{x}=0 \mathrm{~mm})$.

Before going into detailed analysis, a special case will be discussed: 200-400/800-075/100. Temperature at the substrate surface are shown in Figure . 


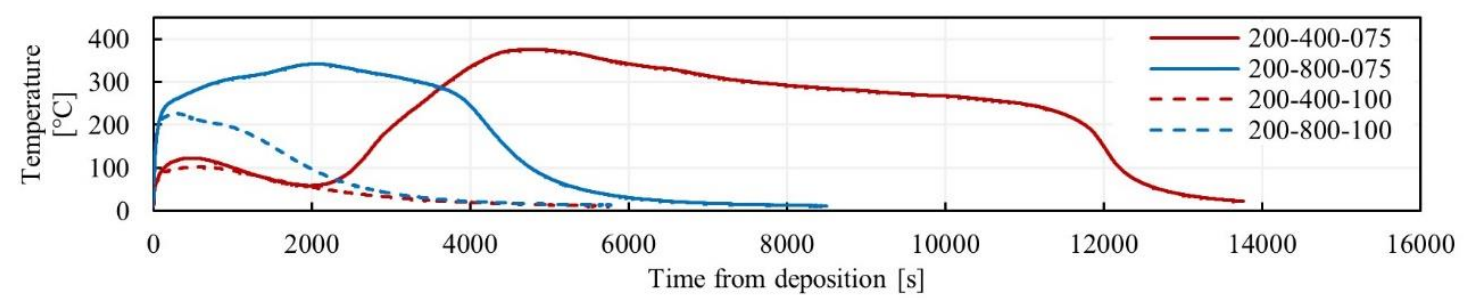

Figure 6 - Temperature evolutions at the surface for selected experiments with $200 \mathrm{~g}$ initial pellet mass.

This condition offers a good example to show importance of firebrand production, accumulation mass and deposition area. When firebrands are produced in a batch of $200 \mathrm{~g}$ at $400{ }^{\circ} \mathrm{C}$, only a small fraction of the initial mass is lost (see Figure ). Most particles are visibly indistinguishable from virgin particles, with some particles charred. When they are deposited on the substrate, they either cool down (200-400-100) or they cool down before a smouldering reaction is initiated which burns for a long duration until almost only ash is left (200-400-075). In the former case, the heat losses from the system are such that the smouldering reaction cannot be sustained. In the second case where the deposition area is smaller, the combination of heat feedback and heat losses are sufficient to drive a sustained smouldering.

When firebrands are created from $200 \mathrm{~g}$ at 600 or $800^{\circ} \mathrm{C}$, the accumulation creates a condition which promotes sustained smouldering (e.g. blue curves in Figure ; note that the firebrand mass is now lower compared to red lines). Although the behaviour of accumulations in 200-400-075/100 are interesting, they are outliers to the current data sets and represent conditions where the response of the substrate is dominated by the ignition and propagation of the smouldering fire. Therefore these two conditions will be excluded from the following discussion.

Observations of the peak surface temperatures are presented in Figure . The accumulation masses used in Figure are according to the mass loss curves shown in Figure (at 600s). The peak surface temperatures are grouped by exposure area diameters. Generally, peak surface temperature increase with increasing accumulation mass. For a given accumulation mass the peak temperature increases with decreasing deposition area. Both of these observations can be attributed to the increase in heat feedback and decrease in heat losses. A larger deposition area results in an increase in heat losses to the surrounding from the accumulation (radiative and convective). It is evident, that the range of peak temperatures measured are sufficiently high to cause pyrolysis of cellulosic material and potentially also smouldering in a combustible substrate.

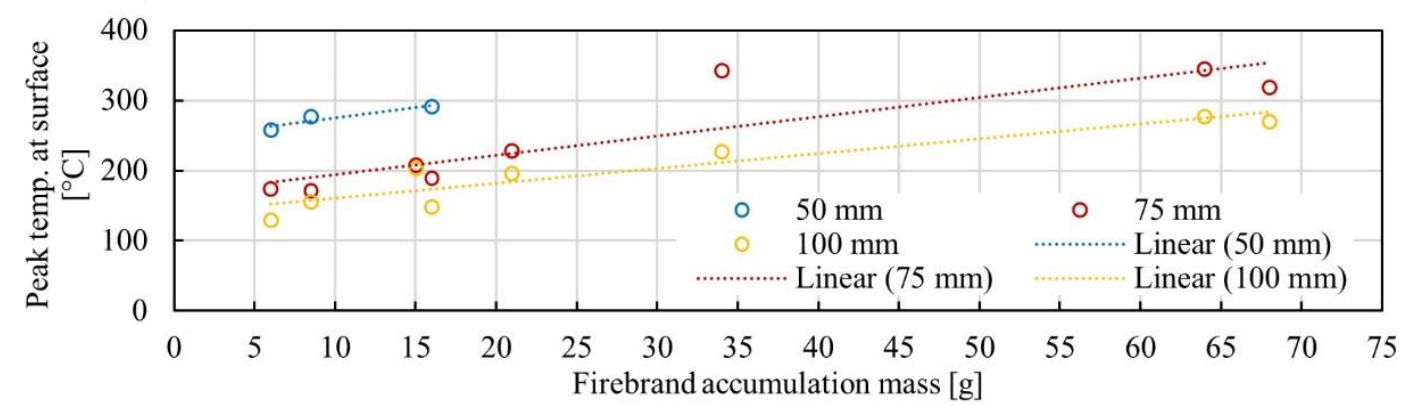

Figure 7 - Peak temperature at substrate surface plotted against firebrand accumulation mass (as deposited). The values in the legend refer to the deposition area diameter. The dotted lines are regression lines as indicated.

Increasing the furnace temperature (and keeping conditioning time constant), decreases the firebrand accumulation mass. Additionally, the initial particle temperature when deposited also increases. Observing experiments when the furnace temperature is raised shows that the peak temperature at the substrate surface only decreases slightly (Figure), the time until the substrate reaches peak temperatures is shorter (Figure ), and the initial temperature rise is faster (Figure and 
Figure ). Results in Figure show that the effect of furnace temperature is stronger for smaller deposition areas.

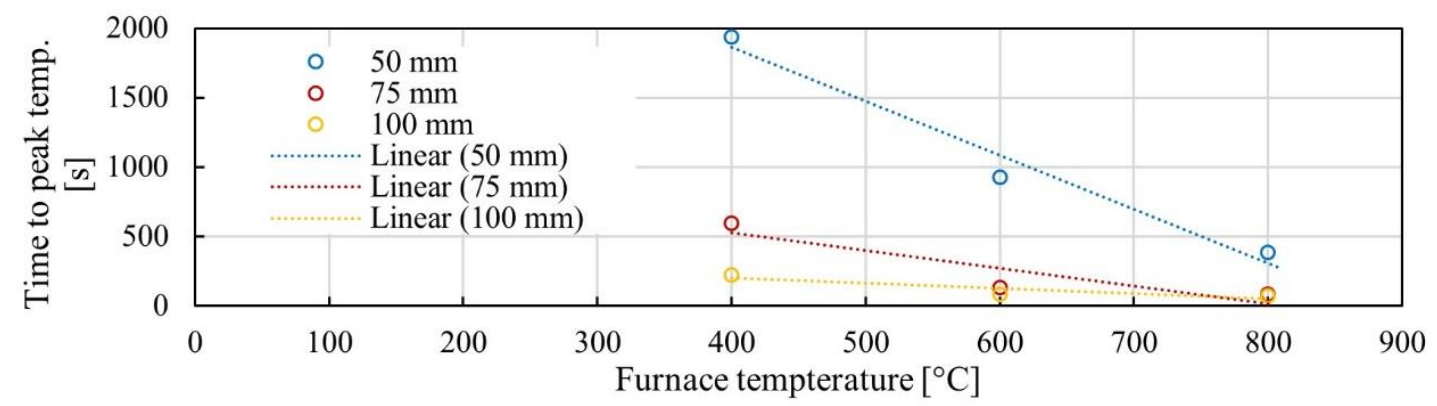

Figure 8 - Time to reach peak surface temperature plotted against furnace temperature for experiments with $50 \mathrm{~g}$ initial pellet mass.

Peak exposure conditions are not the only parameter that is important in this scenario. The duration of exposure is also a critical consideration. A low magnitude exposure applied for a long period may still present a hazardous condition. The exposure duration will be discussed further in the following section, in terms of the net heat flux absorbed by the substrate.

\subsection{Net heat flux}

The results of net heat flux for constant accumulation mass with different deposition areas and furnace temperature are shown in Figure . Deposition occurs at $t=0 \mathrm{~s}$. A positive heat flux represents the energy transferred from the accumulation into the substrate (i.e. heating) and a negative one is energy transfer from the substrate into the accumulation and surrounding (i.e. cooling). Decreasing the deposition area (Figure (a)) results in a deeper accumulation layer favouring prolonged sustained smouldering combustion (where the accumulation conditions are appropriate). The peak net heat flux occurs shortly after deposition followed by a steep drop. No significant difference between the peak net heat fluxes for different deposition areas were observed when the conditioning temperature was constant (Figure (a)) as expected from analysis of the heat transfer problem. For accumulation conditions that support sustained smouldering, for example 050-400-050, the net heat flux slowly decays from approximately $3 \mathrm{~kW} \mathrm{~m}^{-2}$ after 200 seconds to $1 \mathrm{~kW} \mathrm{~m}^{-2}$ after $3000 \mathrm{~s}$.
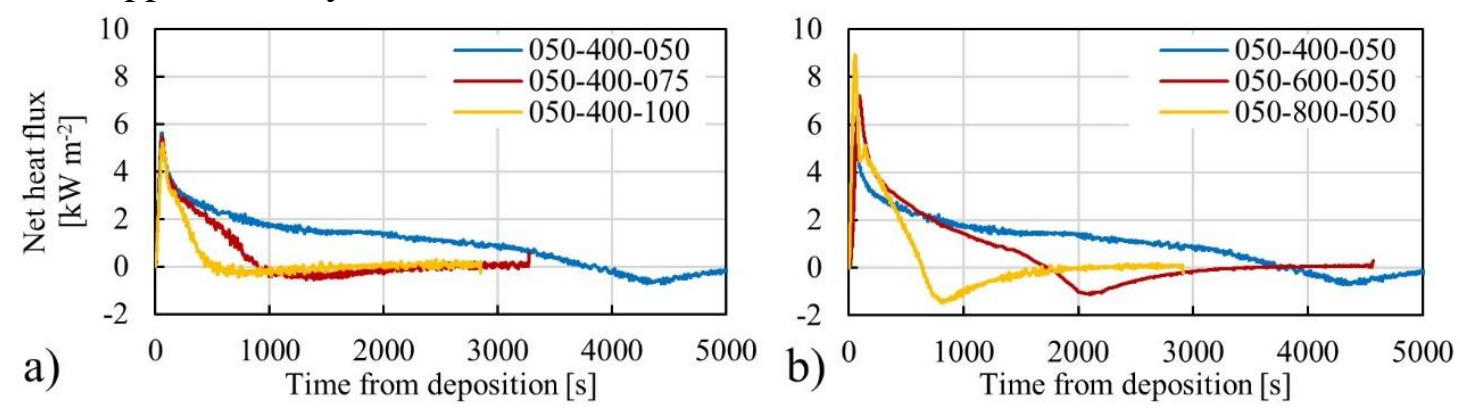

Figure 9 - Net heat flux evolution during experiments with $50 \mathrm{~g}$ initial pellet mass. (a) Varying deposition area. (b) Varying furnace temperature (firebrand accumulation mass).

Particle characteristics are critical to determining the combustion behaviour of accumulations, and firebrands from real fuel source will have properties that differ from these idealized pellets. Density, specific heat, thermal conductivity will likely different as will the particle size and shape. These determine the thermal behaviour, heat losses from the system, and hence the burning behaviour. Chemical composition and energy content are other important characteristics that are likely different. Variation in heat of combustion was already shown for different pellet firebrands (Table ). It was observed in the experimentation that, accumulations with fully charred particles do not sustain smouldering well (e.g. 050-800-050). Contrarily, accumulations with less charred or a mix of fully 
charred and near virgin pellets sustain smouldering significantly better (e.g. 050-400-050). For example, the firebrand mass of "050-400-050" is approximately two and a half times the mass of "050800-050", yet the time of heating (net heat flux $>0 \mathrm{~kW} \mathrm{~m}^{-2}$ ) is almost six times as long. This behaviour is driven in part by bulk properties but also particle properties. Both govern the heat feedback within the accumulation. This also indicates that heat of combustion alone is not sufficient to determine the risk posed by firebrands and that the physical characteristics of the particles and accumulations are also important.

A higher firebrand conditioning temperature results in a higher peak net heat flux. This can be observed in Figure (b). This tendency was observed for all accumulation masses and deposition areas tested and can be attributed to the initial particle temperature and hence a larger driving force for heat transfer. All peak heat fluxes are summarized and plotted in Figure (a) against the furnace temperature.
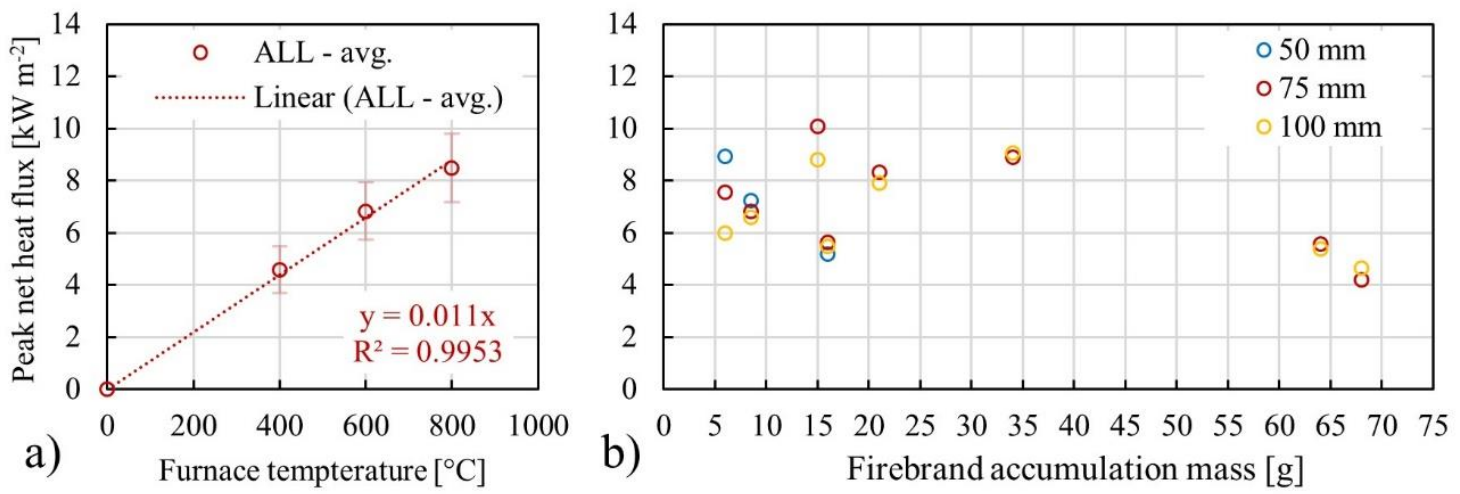

Figure 10 - (a) Averaged Peak net heat flux vs furnace temperature. Error bars: one standard deviation. (b) Peak net heat flux plotted against accumulation mass.

A linear correlation can be observed between the peak net heat flux and the range of furnace temperatures used. The error bars in Figure represent one standard deviation of the averaged values. This indicates that for the peak net heat flux, initial particle temperature is more important than accumulation mass and deposition area. No significant correlation was found between the peak net heat flux and accumulation mass (Figure (b)) again confirming that the condition (temperature/reacting or not reacting) of the particle is a key aspect of defining the risk posed by firebrand accumulations.

As discussed previously, the duration of exposure is an important consideration when defining the ignition risk posed by firebrands. To quantify the duration of exposure, Figure below shows the period of heating with a minimum net heat flux of $1 \mathrm{~kW} \mathrm{~m}^{-2}$.

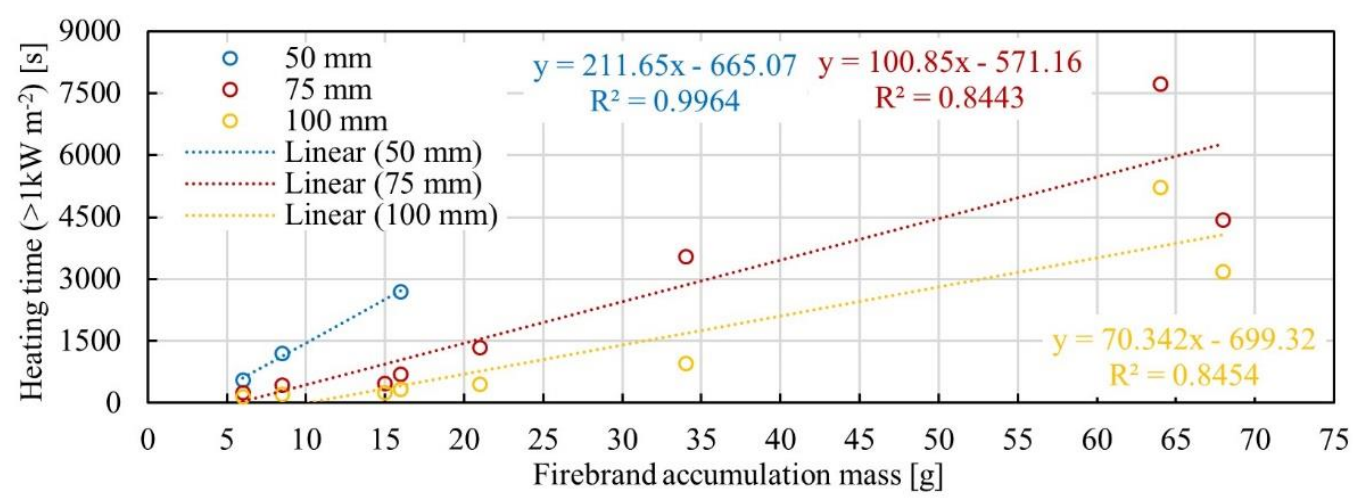

Figure 11 - Duration for which the substrate is heat with at least a net heat flux $>1 \mathrm{~kW}$ m-2 plotted against firebrand mass (as deposited). The values in the legend refer to the deposition area diameter. The dotted lines are regression lines as indicated.

The duration of heating with at least $1 \mathrm{~kW} \mathrm{~m}^{-2}$ (net) increases with increasing accumulation mass and decreasing deposition area. A higher loading on the same deposition area means there is more 
energy available in the accumulation to be transferred into the substrate, thus increasing the duration of heating. Heat feedback increases with decreases deposition area. At the same time, heat losses decrease with decreasing deposition area.

The method for calculating the net heat flux is a 1-dimensional solution. This does not account for radial conductive heat losses in the substrate. The long exposure duration means that in many cases a significant portion of the substrate is heated by conduction through the substrate. As a result, the net heat fluxes presented above are underestimations.

\subsection{Gauge heat flux}

The gauge heat flux describes the total incident heat flux for a given firebrand accumulation. This is contrasted with the net heat flux which is dependent on substrate properties and accounts for heat losses. The net heat flux is the portion of the incident heat flux that transferred into the substrate. A comparison of the two measurements is shown in Figure for experiments with $50 \mathrm{~g}$ initial mass and varying deposition area and furnace temperature.

Peak gauge heat fluxes range from 30 to $80 \mathrm{~kW} \mathrm{~m}^{-2}$. These were found to decrease with increasing deposition area, which suggests that the cooling of the particles when the accumulation is less densely packed is significant. The peak gauge heat flux is reached instantly after deposition, whereas, the time to reach peak net heat flux shows a delay. This is due to the short response time of the heat flux gauge compared to the longer response time of the vermiculite due to the diffusion of the thermal wave.
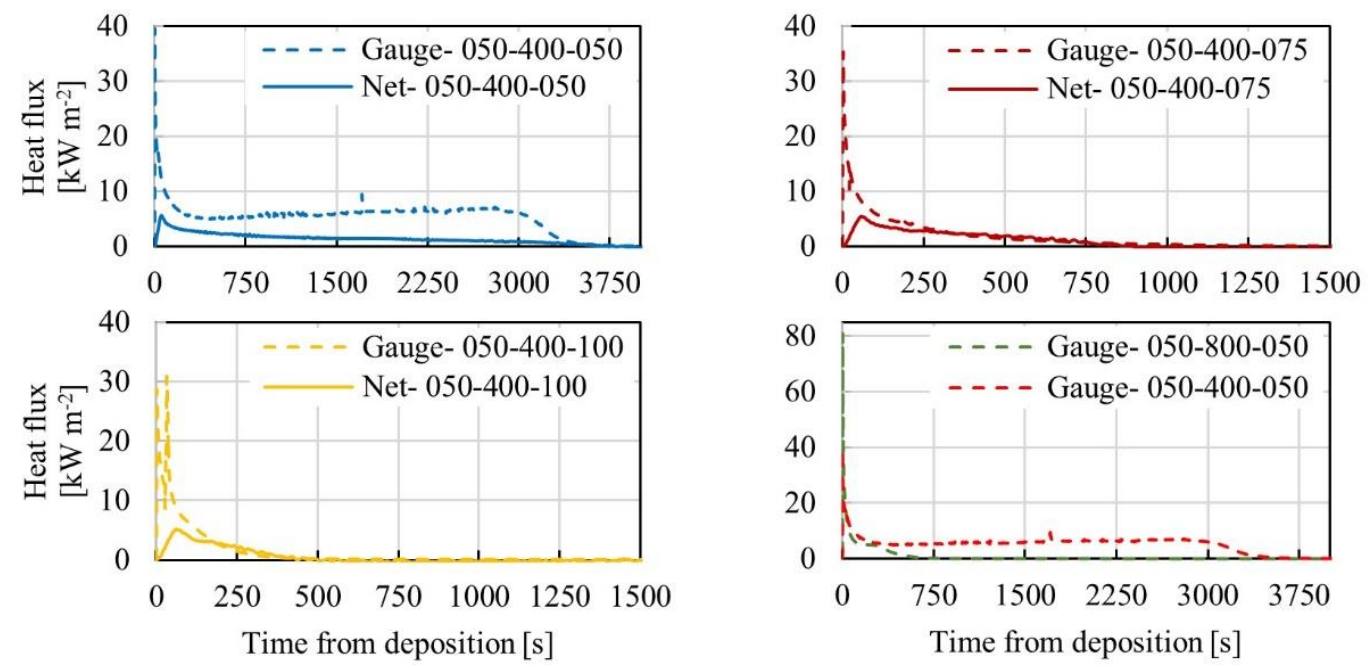

Figure 12 - Comparison between net and gauge heat flux evolution for experiments with $50 \mathrm{~g}$ initial pellet mass, varying deposition area and furnace temperature.

The measured peak gauge heat fluxes are high enough to induce flaming ignition of common materials (Torero 2016), provided they are applied for a sufficient period of time and enough oxidizer is allowed to reach the reaction zone. Peak and one minute average heat flux (net and gauge) are shown in Table. The averaged value is from the first minute after deposition.

Table 2 - Summary of peak and 1-min averaged heat fluxes. The average is the first minute after deposition.

\begin{tabular}{lllll}
\hline & \multicolumn{2}{c}{ Gauge heat flux $\left[\mathrm{kW} \mathrm{m}^{-2}\right]$} & \multicolumn{2}{c}{ Net heat flux $\left[\mathrm{kW} \mathrm{m}^{-2}\right]$} \\
\cline { 2 - 5 } Experiment ID & Peak & 1-min avg. & Peak & 1-min avg. \\
\hline $050-400-050$ & 39.6 & 17.4 & 5.63 & 3.11 \\
$050-400-075$ & 35.3 & 13.7 & 5.47 & 2.76 \\
$050-400-100$ & 30.9 & 15.2 & 5.20 & 2.52 \\
$050-800-050$ & 79.8 & 21.5 & 8.93 & 5.06 \\
\hline
\end{tabular}

The 1-min average gauge heat fluxes as well as the peak fluxes are above typical critical heat flux required to cause flaming ignition of common materials $\left(\sim 12-15 \mathrm{~kW} \mathrm{~m}^{-2}\right.$ radiative flux $)$. This illustrates 
that flaming ignition may be possible as a result of sustained heating by a firebrand accumulation. Certainly these heat fluxes are sufficient to cause pyrolysis and charring of common materials. It has been documented (Manzello 2009(b)) that flaming ignition is unlikely without forced airflow in this type of scenario (it was not observed in this study). This indicates, that under natural convection, the oxidizer flow is insufficient to support flaming combustion. and is a critical mechanism for the ignition process in this type of scenario.

Table (and the bottom right graph in Figure ) shows heat flux results for experiments with firebrands produced at higher furnace temperatures (050-800-050). These results show that peak gauge heat fluxes from accumulations produced at $800{ }^{\circ} \mathrm{C}$ are double that of accumulations produced at 400 ${ }^{\circ} \mathrm{C}$. The 1-min averaged gauge heat flux however is only a fraction larger compared to the one from accumulation produced at lower temperatures. After deposition, particles are initially hotter, but also experience much larger heat losses (convection and radiation), and therefore cool more rapidly.

Results discussed herein provide a quantification of the thermal boundary condition that develops when firebrands are deposited on a solid substrate. Although the net heat flux will change when using different substrates, vermiculite is a good benchmark to estimate what flux levels can be expected for wood and wood products due to the similar thermal properties. With more experimentation, it is desired to solidify findings and correlations, and eventually extrapolate the thermal boundary conditions to combustible substrates.

\section{Conclusions}

The heat flux from firebrand accumulations was investigated using direct heat flux measurements and an inverse heat transfer approach:

- Peak heat fluxes are mainly driven by initial particle temperature upon deposition.

- Duration of exposure is driven by firebrand accumulation mass and deposition area.

- Sustained smouldering of accumulations is a factor of deposition area, accumulation mass and particle properties.

- Peak heat fluxes range from $30-80 \mathrm{~kW} \mathrm{~m}^{-2}$ (gauge) and $4-9 \mathrm{~kW} \mathrm{~m}^{-2}$ (net).

- Sustained smouldering of accumulations produces heat fluxes of 5-7 $\mathrm{kW} \mathrm{m}^{-2}$ (gauge) and 1-3 $\mathrm{kW} \mathrm{m}{ }^{-2}$ (net).

The findings show that high temperature firebrands many not represent most dangerous condition as these particles do not retain high temperatures for long periods of time. A prolonged exposure at lower heat fluxes (arising from lower temperature firebrands) may be sufficient to cause significant pyrolysis, degradation and charring of a substrate. These accumulations are capable of sustaining smouldering compared to fully charred particle accumulations. Thus, these may be considered more hazardous to solid combustible structural material.

\section{Acknowledgements}

The Authors would like to thank the Joint Fire Science Program for their financial support (JFSP \#15-01-04-55). Furthermore, the authors thank Cameron MacLeod for his assistance with the experimental work.

\section{References}

Beck JV, Blackweel B, St Clair CR (1985) Inverse heat conduction, Ill-posed problems. (WileyInterscience Publication, New York, USA) 
Caton SE, Hakes RSP, Gorham DJ, Zhou A, Gollner MJ (2017) Review of Pathways for Building Fire Spread in the Wildland Urban Interface Part I: Exposure Conditions. Fire Technology 53, 429-473.

Dietenberger M (2002) Update for combustion properties of wood components. Fire and Materials 26, 255-267.

El Houssami M, Mueller E, Thomas JC, Simeoni A, Filkov A, Skowronski N, Gallagher MR, Clark K, Kremens R (2016) Experimental Procedures Characterising Firebrand Generation in Wildland Fires. Fire Technology 52, 731-751.

Ellis PFM (2015) The likelihood of ignition of dry-eucalypt forest litter by firebrands. International Journal of Wildland Fire 24, 225-235.

Fernandez-Pello AC, Lautenberger C, Rich D, Zak C, Urban J, Haddden R, Scott S, Fereres S (2015) Spot fire ignition of natural fuel beds by hot metal particles, embers and sparks. Combustion Science and Technology 187, 269-295.

Hakes RSP, Caton SE, Gorham DJ, Zhou A, Gollner MJ (2017a) A Review of Pathways for Building Fire Spread in the Wildland Urban Interface Part II: Response of Components and Systems and Mitigation Strategies in the United States. Fire Technology 53, 475-515.

Hakes RSP (2017b) Thermal characterization of firebrand piles. (University of Maryland, USA, MS Thesis)

Hamann E, Bahrani B, Kadel J, Stickles M, Zhou A (2017) Ignition of attic insulation foams subjected to firebrand. Proceedings of the 15th International Conference on Fire and Materials, 727-739.

Koo E, Pagni PJ, Weise DR, Woycheese JP (2010) Firebrands and spotting ignition in large-scale fires. International Journal of Wildland Fire 19, 818-843.

Laschuetza T (2017) Numerical and experimental investigation of a Thin Skin Calorimeter (TSC). University of Edinburgh, Edinburgh, UK. MSc Thesis.

Manzello SL, Cleary TG, Shields JR, Yang JC (2006) Ignition of mulch and grasses by firebrands in wildland-urban interface fires. International Journal of Wildland Fire 15, 427-431.

Manzello SL. Maranghides A, Shields JR, Mell WE, Hayashi Y, Nii D (2009a) Mass and size distribution of firebrands generated from burning Korean pine (Pinus koraiensis) trees. Fire and Materials 33, 21-31.

Manzello SL, Park SH, Cleary TG (2009b) Investigation on the ability of glowing firebrands deposited within crevices to ignite common building material. Fire Safety Journal 44 894-900.

Manzello SL (2014) Enabling the investigation of structure vulnerabilities to wind-driven firebrand showers in wildland-urban interface (WUI) fires. Fire Safety Science 11, 83-96.

Minkowycz WJ, Sparrow EM, Murthy JY (Eds) (2006) Handbook of Numerical Heat Transfer (John Wiley \& Sons, Inc., New York, USA)

Santamaria S, Kempna K, Thomas JC,El Houssami M, Mueller M, Kasimov D, Filkov D, Gallagher MR, Skowronski N, Hadden R, Simeoni A (2015) Investigation of Structural Wood Ignition by Firebrand Accumulation. Proceedings of the 1st International Conference on Structural Safety under Fire and Blast, Glasgow, UK.

Thomas JC, Mueller EV, Santamaria S, Gallagher M, El houssami M, Filkov A, Clark K, Skowronski N, Hadden RM, Mell W, Simeoni A (2017) Investigation of firebrand generation from and experimental fire: Development of a reliable data collection methodology. Fire Safety Journal 91 864-871.

Torero JL (2016) SFPE Handbook - Flaming Ignition of Solid Fuels. 5th ed. Society of Fire Protection Engineers.

Viegas DX, Alemaida M, Raposo J, Viegas CX (2014) Ignition of Mediterranean fuel beds by several types of firebrands. Fire Technology 50, 61-77. 


\title{
Fire Risk Assessment of UNESCO Historic Villages by Heat Flux from Forest Fire
}

\author{
Donghyun Kim \\ Jeonju University.303 Cheonjam-ro Jeonju Rep.of Korea, \{72donghyunkim@jj.ac.kr\} \\ International Institute for Applied Systems Analysis. Schlossplatz, 1 A-2361 Laxenburg, Austria, , \\ \{72donghyunkim@gmail.com\}
}

\begin{abstract}
Most structures in ASIA UNESCO Historic Wooden Villages are timber framed houses with thatched or shingled roofs that are combustible materials; they are thus exposed to the risk of forest fires. The study examined the effect of the heat flux on the houses in Yangdong UNESCO Historic Village as a result of forest fires to evaluate to what degree of risk they are exposed to. After distinguished the tree species of major structural components of wooden buildings. This was done by getting the precise location information on the houses in Yangdong Village with the GIS analysis, collating the information on the construction materials and conducting the numerical analysis on heat flux in case of forest fires. The results showed as follows: around $10 \%$ of the houses are directly exposed to the danger of forest fires and $5 \%$ of all the houses are expected to be subject to the indirect effects due to the proliferation of the initial forest fires. In conclusion, it was determined that appropriate and effective methods of forest fire prevention and fighting need to be sought, and they should be based on eliminating the possible fuels for forest fires that are nearby the firevulnerable houses, securing enough separation distances and installing waterproof facilities.
\end{abstract}

Keywords: UNESCO Historic Village, WUI (Wildland Urban Interface), Forest Fire, Heat Flux, Fire Risk Map.

\section{Introduction}

Many of Korea's historical and cultural properties were lost by fires including forest fires. Local cultural assets as well as national treasures were damaged by arson, forest fires, and other unidentified causes. Most of Korea's architectural cultural properties were made of wood, which is vulnerable to fires. In the case of a historical and cultural district forming a village, there will be more serious if fire occurs, in addition to danger of wood architectural cultural properties by fire. Between 2004 and 2009, a total of 15 cases of fire occurred in Seongeup Village in Jeju. The causes include short circuit and mishandling of fire arms when repairing thatched roof. Other five cases of fire occurred from 2001 to 2009 in Yangdong Village registered as World Heritage were due to short circuit and mishandling of fire arms, and negligent management of fire source in the construction site. A fire broken out on June 4, 2010 (15:39) in Andong Hahoe Village burnt Beonnam Old House to ashes while Hanju Head House was partially lost by fire occurred on January 25, 2011. In particular, as World Heritage Yangdong Village was surrounded by coniferous forests, which are vulnerable to forest fire, occurrence of fire will expose the entire village formed by wood and straw-roofed structures, to danger of fire when fire breaks out.

This study aims to assess fire risk of heat flux when fire occurs in the current forest environment and placement of residence and create a risk map based on the assessment results, thereby improving fire safety of Yangdong Village

\section{Overview of Yangdong Viliage}

Below is an example of a picture and respective legend. Use the same formatting for tables. Yangdong Village is a time-honored village formed by two clans: Wolseong Son Clan and Yeogang

Advances in Forest Fire Research 2018 - Page 780 
Yi Clan. There are Mt. Seolchangsan to the north and Soengjubong Peak of 100m in height to the south, and Yangdong Stream runs in front of the village. Beyond the west mountain unfolded Angang Plain, which was economic source of the village. The village is divided into Hachon and Sangchon as of Angye stream east and west, while Namchon and Bukchon north and south.

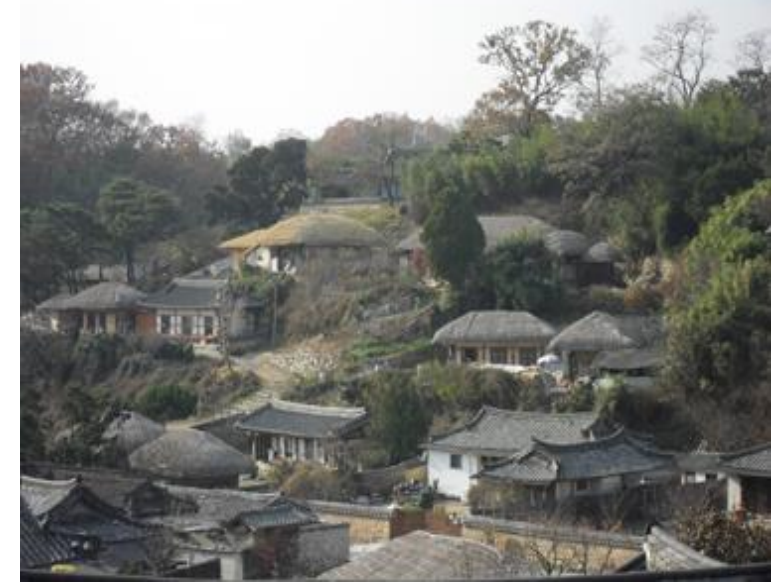

Figure 1 - A woodend and straw roof houses of Yangdong Viliage

The total number of houses in Yandong excluding non-residential structures and head houses designated as a cultural asset is 140, out of which straw-roofed houses are 78, approximately $56 \%$ of total houses. The 78 houses consist of 49 straw-roofed houses and 29 mixed (straw-roofed and tileroofed) houses, and the number of individual straw-roofed house buildings is approximately 190 (Fig. 2, Lee and Kim, 2013). Important cultural heritage properties include Tonggam sokpyeon (Supplement to the Comprehensive Mirror for Aid in Government) (National Treasure 283), Mucheomdang House in Yangdong, Gyeongju (Treasure 411), Hyangdan (Treasure 412), Hyangdan House in Yangdong, Gyeongju (Treasure 412), Gwangajeong House in Yangdong, Gyeongju (Treasure 442), Portrait of Son So (Treasure 1216), 12 important folk materials including Seobaekdang (Important Folk Material 23), and 7 cultural assets designated by the province including estate distribution documents of Son So (Gyeongbuk Tangible Cultural Heritage Property 14).

In particular, tree species of pillar, beam, and girder members of Mucheomdang House and Gwangajeong House in Yangdong designated as World Heritage was revealed to be pine tree (P. denstiflora), which was analyzed to have significant potential risk (Lee, J. H. et al., 2013).

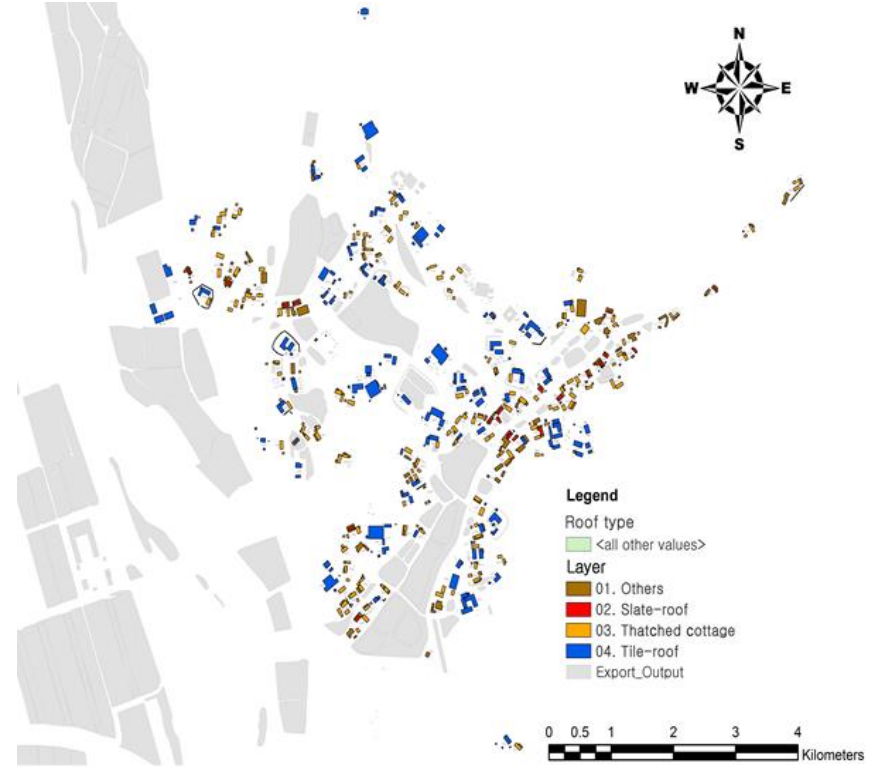

Figure 2 - Arrangement map of Yangdong Viliage 


\section{Theory Analysis of Forest Fire Flames}

\subsection{Flames of Forest Fire}

Spread of forest fire undergoes the pyrolysis process of unburned fuel by heat flux emitted through flames. Then, when the surface temperature of the burning substance reaches the igniting temperature, the process of creating flames causes the expansion of the forest fire. To assess heat flux emitted from flames, height of flames should be considered. First, to calculate the height of flames, heat release rate (HRR, $\mathrm{kW}$ ) should be estimated using Effective Heat of Combustion $(\mathrm{kJ} / \mathrm{kg}$ ) and Mass Loss Rate (MLR, $\mathrm{kg} / \mathrm{s}$ ). HRR can be calculated from Equation (1) and is proportional to MLR and Effective Heat of Combustion changed by energy equivalent, water content, combustion diameter, and density.

$$
\operatorname{HRR}(\mathrm{kW})=\Delta h_{c} \times M L R
$$

Flame height calculation models were proposed by McCaffrey (1995) and Heskestad (1998) for horizontal-plane fire of liquid flammable materials under the condition of no wind. The calculation Equation of fire flame height was presented by Albini (1981) as $H \propto I B / U$, which is an estimation Equation for one dimensional flame height, and Nelson (1986) suggested Equation (2). However, when wind velocity is 0 , it is not possible to calculate flame height with the Equation.

$$
H=\frac{a I_{B}}{U}
$$

where, $I_{B}$ is Byram' fire intensity $\left(\mathrm{kW} / \mathrm{m}^{2}\right)$ and ais $1 / 360$ and $U$ is wind velocity $(\mathrm{m} / \mathrm{s})$. Kim (2009 a) suggested a calculation Equation of flame height of solid fuel such as surface fire fuel materials as the following Equation (3):

$$
H=0.027\left(\dot{Q}^{\prime}\right)^{2 / 3}
$$

where, $\dot{Q}^{\prime}(\mathrm{kW} / \mathrm{m})$ is HRR in length, and unit length, which is an experiment setting of $Q$ calculated by Equation (1) was applied. Equations (2) and (3) are to calculate flame height under the condition of no wind and flat area, and flame height changed by wind and slope can be calculated with Equation (4).

$$
\text { H_ws }=\mathrm{H} \times \sin : f_{\mathrm{f}}:(\theta)
$$

where, $\theta\left(^{\circ}\right)$ is angle between ground surface and the flame changed by wind and slope and it is obtained by subtracting flame angle $(\varnothing)$ from $90^{\circ}$. Calculation of flame angle can be conducted using Equation (5), which represents correlation between Froude Number $(F r)$ and wind velocity under the condition of uniform wind (Kim, 2009b, 2009c).

$$
\begin{aligned}
& F r=\frac{U_{\infty}}{\sqrt{g H}} \\
& \tan \emptyset_{w s} \sin \emptyset_{w s}=1.2 \frac{U_{w s}^{2}}{g H}
\end{aligned}
$$

where, $g$ is acceleration of gravity $\left(\mathrm{m} / \mathrm{s}^{2}\right), H$ is initial flame height $(\mathrm{m})$, and $U_{w s}$ is air entrainment rate $(\mathrm{m} / \mathrm{s})$. 


\subsection{Heat Flux of Forest Fires}

Radiation heat transfer by surface fire flame is conducted through calculation of total radiant heat flux received by unburned section from individual flame for the grid defined as follows (Figure 3), where three-dimensional position values against the center point of flame obtained by Equation (3) are $x_{f}, y_{f}$, and $z f$. To calculate heat energy per unit area emitted from flames, PSM (Point Source Model) in Equation (7) was used. With the PSM can be used when calculating radiant heat flux per unit area and unit time delivered target distant by $r$ from emitting flame. Therefore, when the total heat flux reaches ignition energy, flame combustion is conducted and expanded.

$$
\dot{q}^{\prime \prime}=\frac{\dot{Q}_{r}}{4 \pi r^{2}} \cos \theta^{\prime}
$$

where, $\dot{Q}_{r}$ is radiant flux, $r$ is radius of flame depth $(\mathrm{m})$, and $\theta^{\prime}$ is angle between the center of flame and $z_{f}$ vector and expressed as Equation (8). Location coordinates of the center of flame $\left(x_{f}, y_{f}, z_{f}\right)$ needed to calculate heat flux transfer from flame can be moved by slopes and wind, which can be represented as the following Equation (9).

$$
\begin{aligned}
& \cos \theta^{\prime}=\frac{Z_{f}-Z}{r} \\
& \text { where, } \quad r=\sqrt{\left(x_{f}-x\right)^{2}+\left(y_{f}-y\right)^{2}+\left(z_{f}-z\right)^{2}}
\end{aligned}
$$

$$
\overrightarrow{P_{f}}=\left(\begin{array}{c}
x_{f} \\
y_{f} \\
z_{f}
\end{array}\right)=\left(\begin{array}{c}
x_{0}+\frac{1}{2} L_{f} \cos \emptyset \cos \alpha \\
y_{0}+\frac{1}{2} L_{f} \cos \emptyset \sin \alpha \\
z_{0}+\frac{1}{2} L_{f} \sin \emptyset
\end{array}\right)
$$

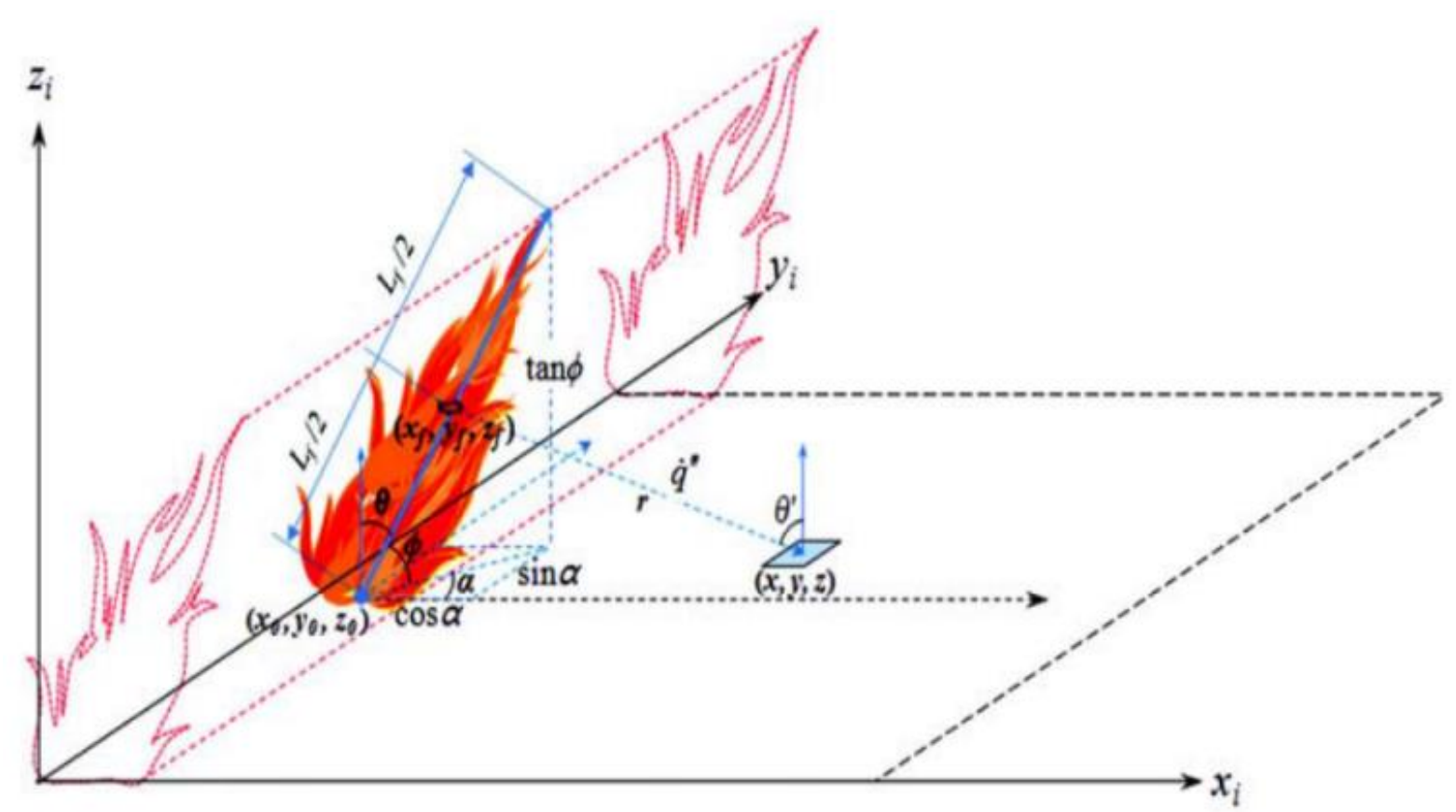

Figure 3 - The concept of heat flux transfer 


\section{Identification and Analysis of Forest Fire Risk Range}

\subsection{Digital Aerial Photo-Based Analysis}

In this study, digital aerial photograph was used to increase visibility of identified scope of risk caused by forest fires and accurately identify relevant information. As its final purpose is to provide almost real environment information, orthometric correction must be performed in displaying location information including identification of forest fire risk range. In this research, satellite image was corrected for orthometric correction by using Image Enhancement method, which improves the brightness environment of image materials and Spatial Enhancement method, which eliminates noise in the image and improves its clarity. In addition, in the case of Image Enhancement, Histogram Stretch was applied while utilizing a method that increases contrast of images by reorganizing the area of image brightness into that represented by computer (Figure 4 ).

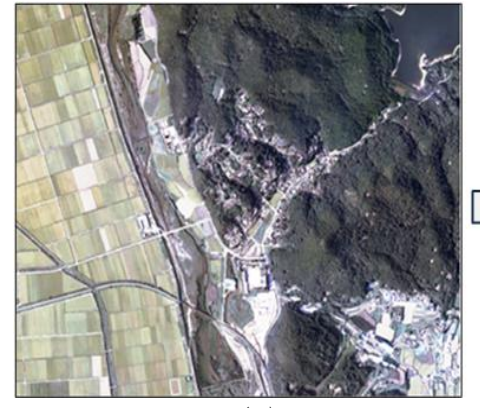

(a)

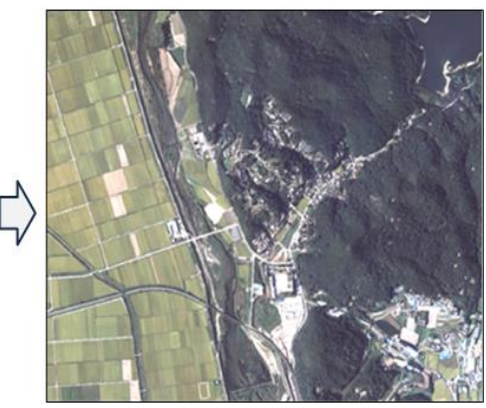

(b)

Figure 4 - Histogram stretch of a aerial photograph in Yangdong Viliage; Image Enhancement (a) to (b)

In this study, GIS (Geographic Information System) was applied to identify the scope of heat flux for Yangdong Village in case of forest fire. Risk intensity of forest fire was represented to a functional relation according to distance between forest and the structure, and GRID file was created so as to easily handle modeling work in which cumulative values of risk intensity cells should be applied to each cell of the coverage. GRID file was structured in grid pattern (width $(1 \mathrm{~m}) \mathrm{x}$ length $(1 \mathrm{~m})$ ) and in selecting the scope for GRID, the creating scope was set to Polygon with 1,230m in width and 1,000m in length so that it was possible to analyze the entire Yangdong Village in Gyeongju, and 1m-resolution GRID was created by using AutoCAD Tool to identify the scope of forest fire heat flux based on the created Polygon.

\subsection{Creating Buffer Line to Analize the Scope Identification of HRR}

To identify the scope of forest fire heat flux in Yangdong Village, GIS buffer analysis, which is an operation method to calculate certain area adjacent to a specific space object, was conducted. Buffer for buffering analysis was based on a digital stock map (1:5,000). However, as forest boundaries on the map were not accurate, digitizing was performed based on aerial photograph, and buffer line was extracted based on the accurately extracted forest boundaries. A total of 25 buffer lines were created at intervals of $1 \mathrm{~m}$ using the multiple ring buffer method. Since the acquired forest data was classified based on polygon according to tree species, it was difficult to identify the risk scope of forest fires depending on forest boundaries, so this research adopted Dissolve method, which is a GIS analysis method integrating spatial data for the same attributes based on the attributes of vector data, in order to extract forest boundaries and for the extracted boundaries, hills and pastureland in and around Yangdong Village were added and the forest was accurately partitioned off.

Since it is necessary to create a stock map or forest boundary information, which represents the scope of forest to identify risk scope of forest fire heat flux, Heads-up digitizing was performed based on aerial photograph. To input the following heat flux result values in the extracted buffer line, Split 
method was used and the buffer lines were classified per distance. The input value according to distance was entered to $25 \mathrm{~m}$ in the unit of $1 \mathrm{~m}$ (Figure 5)
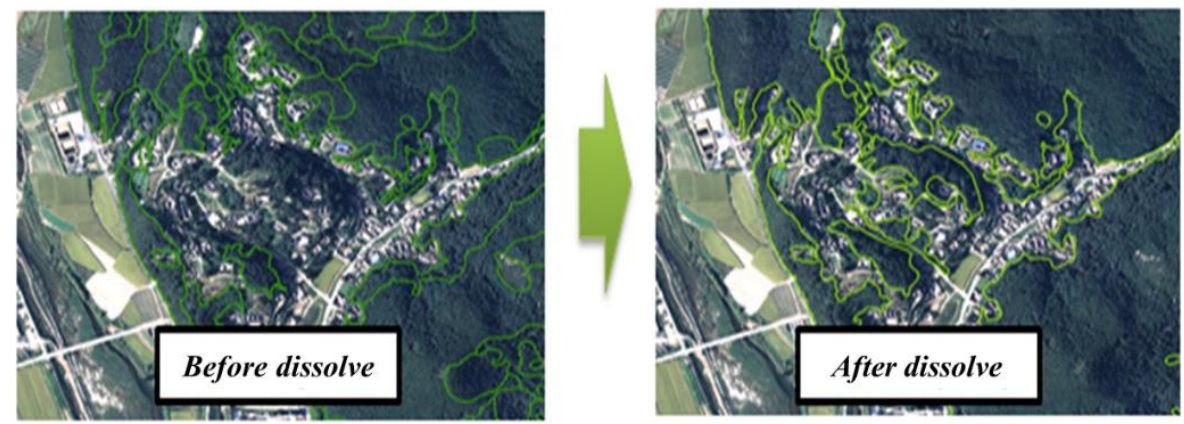

Figure 5 - Result of buffer line protection by 1 m unit

\subsection{Heat Flux Distribution and Risk Assessment}

Heat flux to ignite wood composed of cellulose is $12.7 \mathrm{~kW} / \mathrm{m}^{2}$. In other words, if heat flux is at least $12.7 \mathrm{~kW} / \mathrm{m}^{2}$, wood is ignited. Therefore, heat flux of coniferous forest fire by distance obtained from Equation 7 is shown in Figure 6 and wood house distant by approximately $4.2 \mathrm{~m}$ from the fire occurrence boundary was highly likely to ignite.

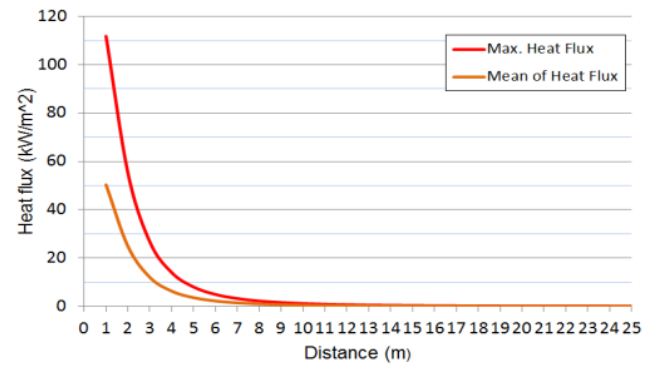

Figure 6 -- Heat flux from forest fire in Yangdong village

To identify heat flux distribution values for forest fire risk in grid pattern with the overlap method of GRID and buffer line, the buffer line should be established in the same format as the existing $1 \mathrm{~m}$ unit grid (Figure 7). For making the buffer line into grid, the format of the data was converted from vector to raster and reconverted to vector in the state where it was not simplified. The result values of the converted data were overlapped with aerial photograph that underwent orthometric correction to identify forest fire risk figures using transparency function of ARC GIS. As a result, heat flux distribution around forest-adjacent areas in Yangdong Village was revealed as the following Figure 8.
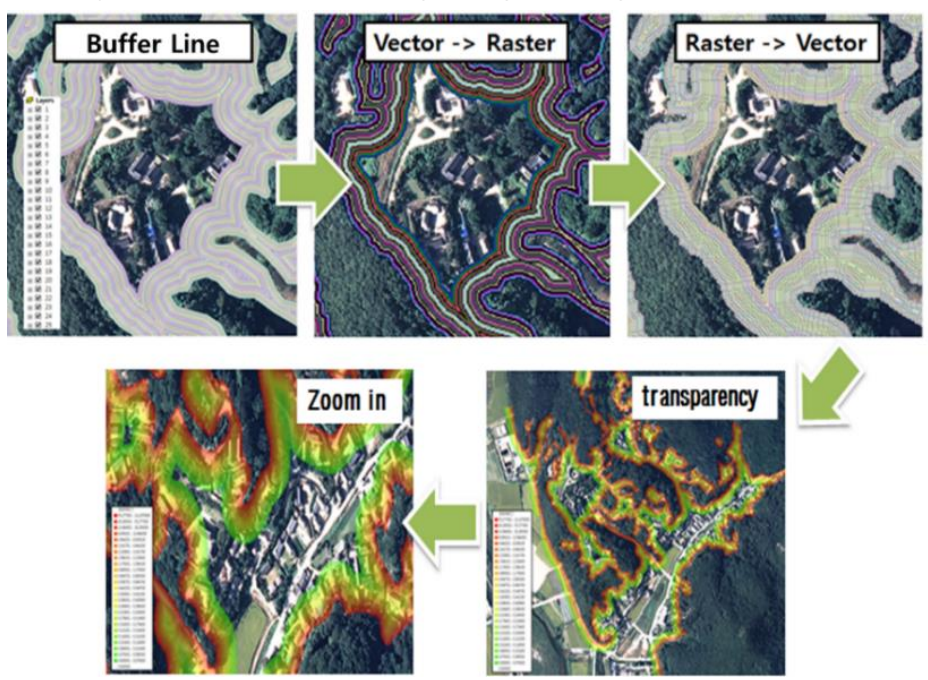

Figure 7 - The process of heat flux distribution for forest fires 


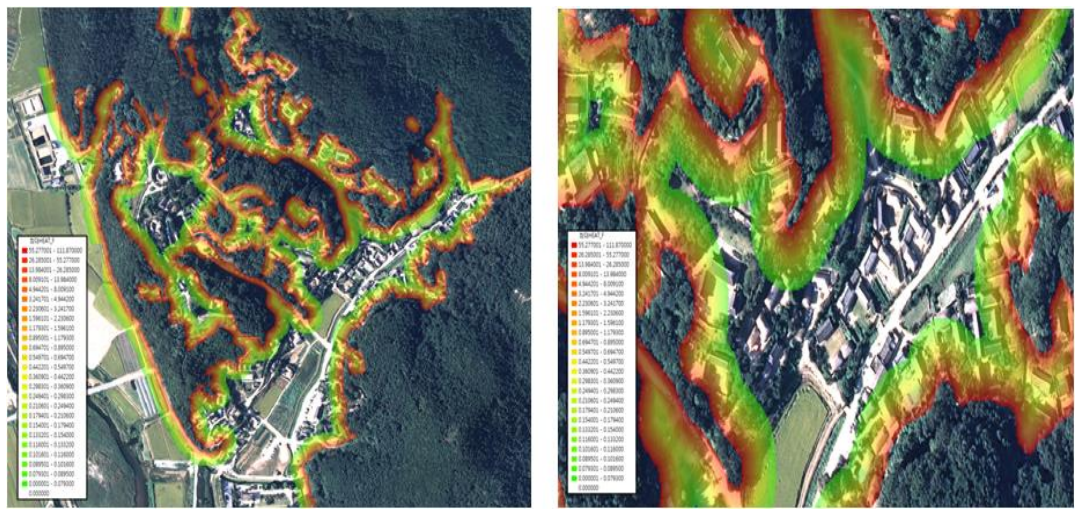

Figure 8 - Risk assessment map of Yangdong village from forest fires

\section{Considerations}

Roof types of houses in Yangdong Village registered as World Heritage include thatched roof and tile roof, and tree species of pillar, beam, and girder members of Mucheomdang House and Gwangajeong House was revealed to be pine tree ( $P$. denstiflora) and most houses in the village were wood structures. In expecting fire behavior with individual combustion property, it is highly important to identify which type of wood composing the combustible materials of the wood structure. In addition, as wood is a complex material consisting of cellulose, hemicellulose, lignin, other extracts (alcohol, ester, and resin), combined water, and compound cell structures, it undergoes a pyrolysis process when it is heated, and heat flux required for ignition energy, which can lead to flames, is known to be approximately $12.7 \mathrm{~kW} / \mathrm{m}^{2}$. Ignition energy varies by type and water content of wood. However, as experimental studies on those various variables have yet to be conducted, this study applied ignition energy measured within water content of $10 \%$ of architectural wood in the dry season where forest fire occurs, as standard value of ignition energy. As for possibility of wood structure ignition from forest fire revealed in this study, wood structures distant by $4.2 \mathrm{~m}$ from forest was highly likely to ignite as shown in Figure 6 and applicable houses were 10\% of the all houses in Yangdong Village.

In this research, expansion of forest fires by spot fires was not considered. In the case of spot fire materials, small embers become a direct cause of fire and leads to fire expansion. Spot fire distance measured in Korea is $2 \mathrm{~km}$, and since the entire Yangdong Village is within $2 \mathrm{~km}$ from forests, the village also deemed to be at danger if a forest fire occurs.

\section{Conclusion}

Assessment of fire expansion risk when a forest fire breaks out in forest adjacent to Yangdong Village, which is World Heritage, revealed that wood structures distant by about $4.2 \mathrm{~m}$ from forest fire were highly likely to ignite due to radiant heat. According to analysis conducted based on forest boundaries of the entire Yangdong Village, about $10 \%$ of the all houses in the village was evaluated that fire is transferred. In conclusion, to secure safety of village from forest fires, first, preventive measures should be used, for example replacing the coniferous trees with fire-resistance trees or eliminating fuels to secure safety zone, and second is to install water sprays or sprinklers in the houses to extinguish fire. As this research is restricted to wood structures, it is deemed that following research should be conducted including research on the risk with heat release rate of thatched roof and research on the effect of fire on adjacent houses in case of a fire breaks out in a house 


\section{References}

Albini, F.A. (1981) A Model for the Wind-blown Flame from a Line Fire, Combust. Flame. Vol. 43, 155-174.

Heskestad G. (1998), Fire Safety Journal, Vol.30, p.215.

McCaffrey B.(1995) The SFPE Handbook of Fire Protection Engineering“", 2nd ed., Society of Fire Protection Engineers and National Fire Protection Association, Quincy, MA.

Lee, J.H., Kim D.H.(2013), Combustion Properties for Tree Species of Major Structural Components of Traditional Wooden Buildings, Journal of KOSHAM, 13(3), 17-22

Kim, D.H.(2009a), A Study on Flame Height Equation for the Pinus densiflora Surface Fuel Bed, Journal of Fire Science and Engineering, 23(6), 10-15

Kim, D.H.(2009b), A Study on the Flame Tilt and Flame Spread due to Up-slope on the Surface Fuel Bed-No wind condition, Journal of KOSHAM, 9(5), 57-62.

Kim, D.H.(2009c), A Semi-numerical Study on the Flame Tilt Equation due to Wind on the Surface Fire in Forest Fire, Journal of Fire Science and Engineering, 23(5), 90-95.

Kim, M.Y., Kang, D.J.(2013), An Critical Analysis of Restoration policy about Thatched-roof House of World Cultural Heritage Yangdong Village - With focus on the Protection of Resident's Sustainable Lives , Journal of Korea Planners Association, 48(6), 57-75. 


\title{
Flammability and combustibility of potential species for use as fuel breaks
}

\author{
João Francisco Labres dos Santos*; Antonio Carlos Batista; Andressa Tres; Alexandre França \\ Tetto; Bruna Kovalsyki \\ Federal University of Paraná. Av. Lothário Meissner, 632, 80210-170, Curitiba, Paraná, Brazil, \\ \{joaolabres@ufpr.br*,batistaufpr@ufpr.br,tres.andressa@gmail.com,tetto@ufpr.br, \\ kovalsyki@ufpr.br\}
}

\begin{abstract}
In order to minimize the incidence and magnitude of fires, preventive silviculture proposes the establishment of fuel breaks, which is strips of vegetation with species of lower flammability than those of the main cultivation, with the aim of reducing or avoiding fire spread. The objective of the study was to evaluate the flammability and combustibility of eight ornamental species by means of cluster analysis, and indicate their potential for use as fuel breaks. Flammability was determined according to the methodology recommended by Valette (1990) and Petriccione (2006). For each evaluated species, 50 firing repetitions were performed in the epirradiator, each one consisting of $1 \pm 0.1 \mathrm{~g}$ of fine green combustible material $(\varnothing<$ $0.7 \mathrm{~cm}$ ). In the tests the following combustion characteristics were analyzed: Ignition Time (IT), Ignition Frequency (IF), Duration of Combustion (DC) and Flame Height (FH). The fire behavior components were measured according to the methodology presented by Batista and Biondi (2009). The experimental burns were carried out in $1 \mathrm{~m}^{2}(1 \mathrm{~m} \mathrm{x} 1 \mathrm{~m})$ plots, with a load of $1 \mathrm{~kg} \cdot \mathrm{m}^{2}$ for all treatments. The following parameters were analyzed: Flame Height (FHh), Speed of Propagation (SP) and Residual Material (RM). The Lower Calorific Value (LCV) was determined with an isoperibol calorimeter, following the standard of ABNT NBR 8633/1984 and was expressed by calories per gram (kcal.kg-1). The Flammability Value (FV) was obtained according to the methodology applied by Vallete (1990) and the fire intensity was estimated using the Byram equation (1959). The parameters IF, IT, DC and FH of flammability and FHh, SP, RM and LCV of combustibility were submitted to cluster analysis. Schinus terebinthifolius and Bougainvillea glabra were the species that presented the lowest values of flammability and combustibility being classified in the null class, as well as Rhododendron simsii in the low classification class. Jasminum mesnyi has low combustibility, but high flammability, not being indicated for composing a fuel break. Viburnum odoratissimum showed low ignition capacity, but its combustibility presented superior parameters when compared to Pinus taeda. Magnolia grandiflora, even with some inferior parameters than those of $P$. taeda, has the lowest IT, being highly flammable and not recommended for use in fuel breaks. $S$. terebinthfolius, B. glabra and $R$. simsii are the species indicated for use as fuel breaks.
\end{abstract}

Keywords: forest fires, preventive silviculture, calorimetry, combustible material.

\section{Introduction}

Natural disturbances are important for ecosystems functioning and, under normal conditions in healthy ecosystems, are an intrinsic part of nature. However, catastrophic events can seriously affect environmental functions and human activities (Montagné-Huck and Brunette 2018).

Forest fires at the Wildland-Urban Interface (WUI) are a threat to communities in many countries (Mell et al. 2010) and a growing problem with social and economic impacts (Molina et al. 2017).

The main cause of forest fires at WUI is anthropogenic (Long-Fournel et al. 2013). A lack of awareness of the risk of forest fires is observed, since residents generally tend to underestimate it (Meldrum et al. 2015). The high population density and the negligent attitude of people who inhabit or transit these regions (Castillo et al. 2011), combined with long periods of drought and accumulation 
of combustible material (Mell et al., 2010), increase the risk of ignition. In this context, it is possible to mention the recent events in Pedrógão Grande/Portugal (2017), Tathra/Australia (2018), New Mexico and Colorado/United States (2018).

Thus, land use planning should be considered as an important component of fire risk management (Syphard et al. 2013). In view of the vulnerability of WUI to forest fires, due to the risk to human life and damage to residences (Long-Fournel et al. 2013), vegetation around homes becomes a major concern as it can facilitate the reach of fire in these structures (Castillo and Correa 2012; Ganteaume et al. 2013).

One method to mitigate the probability of homes being damaged by fire is to reduce the flammability of surrounding areas (Molina et al. 2017). In this context, preventive silviculture proposes the establishment of fuel breaks, which is strips of vegetation with species of lower flammability and combustibility than those of the main cultivation, in order to reduce or prevent the fire spread (Haltenhoff 2006).

To determinate the species to compose fuel breaks, it is evaluated: i) flammability, which is the capacity of the vegetation to burn; and ii) combustibility, which characterizes the ability of the combustible material to maintain the combustion process and to propagate the fire (Soares et al. 2017). Thus, the objective of the study was to evaluate the flammability and combustibility of eight ornamental species, by means of cluster analysis, and indicate their potential for use as fuel breaks.

\section{Material and methods}

The flammability and combustibility tests were carried out in the Forest Fire Laboratory of the Federal University of Paraná, Brazil.

Nine potential species were selected for use as fuel breaks: Bougainvillea glabra Choisy., Casearia sylvestris Sw., Jasminum mesnyi Hance., Magnolia grandiflora L., Michelia champaca L., Rhododendron simsii Planch., Schinus terebinthifolius Raddi and Viburnum odoratissimum Ker Gral. Pinus taeda L. was used as control of the experiment because it is a species of high economic interest.

\subsection{Flammability test}

Flammability was determined according to the methodology recommended by Valette (1990) and Petriccione (2006). For each evaluated species, 50 firing repetitions were performed in the epirradiator, each one consisting of $1 \pm 0.1 \mathrm{~g}$ of fine green combustible material $(<0.7 \mathrm{~cm}$ in diameter) (Figure 1).
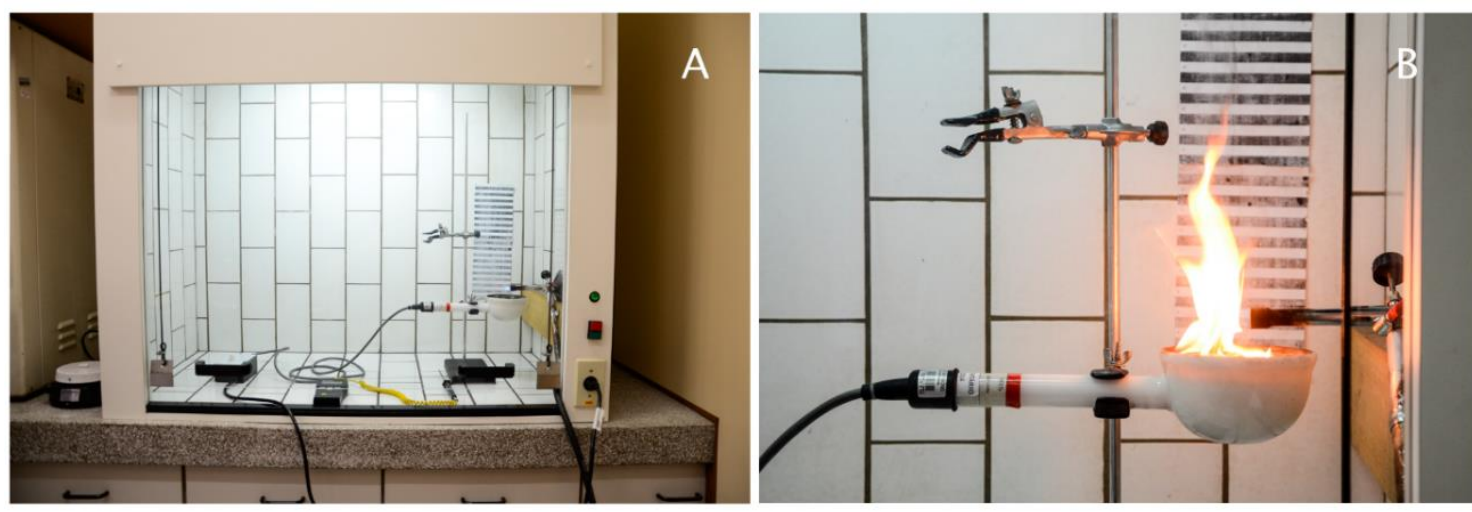

Figure 1 - Epirradiator in the fume hood (A) and epirradiador test (B)

In the tests, the following combustion characteristics were analyzed: ignition time (IT), in seconds, time of complete combustion (TC), in seconds, maximum flame height (FH), in centimeters and ignition frequency (IF), in percentage. IF is the percentage of repetitions in which ignition occurred, considering a maximum IT of 60 seconds. The burnings that exceeded this time were classified as 
"negative burning" and received standardized values: IT equal to 61 seconds; TC equal zero seconds and $\mathrm{FH}$ equal zero centimeters.

The flammability index (FI) of the selected species was obtained according to the IF and IT, varying as follow: $0=$ very low flammable; $1=$ low flammable; $2=$ moderately flammable; $3=$ flammable; 4 $=$ very flammable; and $5=$ extremely flammable $($ Table 1$)$.

Table 1 - Classification of flammability according to the ignition time (IT), in seconds, and ignition frequency (IF), in percentage

\begin{tabular}{c|c|c|c|c|c|c}
\hline \multirow{2}{*}{ IT (s) } & \multicolumn{6}{|c}{ IF (\%) } \\
\cline { 2 - 6 } & $\leq 50$ & $50-79$ & $80-84$ & $85-89$ & $90-94$ & $>94$ \\
\hline$>32.5$ & 0 & 0 & 0 & 1 & 1 & 2 \\
$27.6-32.5$ & 0 & 0 & 1 & 1 & 2 & 2 \\
$22.6-27.5$ & 0 & 0 & 1 & 2 & 2 & 2 \\
$17.6-22.5$ & 1 & 1 & 2 & 2 & 3 & 3 \\
$12.6-17.5$ & 1 & 1 & 2 & 3 & 3 & 4 \\
$<12.6$ & 1 & 2 & 3 & 3 & 4 & 5 \\
\hline
\end{tabular}

\subsection{Combustibility test}

The fire behavior was measured according to the methodology presented by Batista and Biondi (2009). For each evaluated species, 10 repetitions of experimental burnings were carried out in $1 \mathrm{~m}^{2}$ plots with a load of $1 \mathrm{~kg}$ of fine dry fuel material $(<0.7 \mathrm{~cm}$ in diameter), as presented in Figure 2 . The experimental burnings were filmed for later data collection.

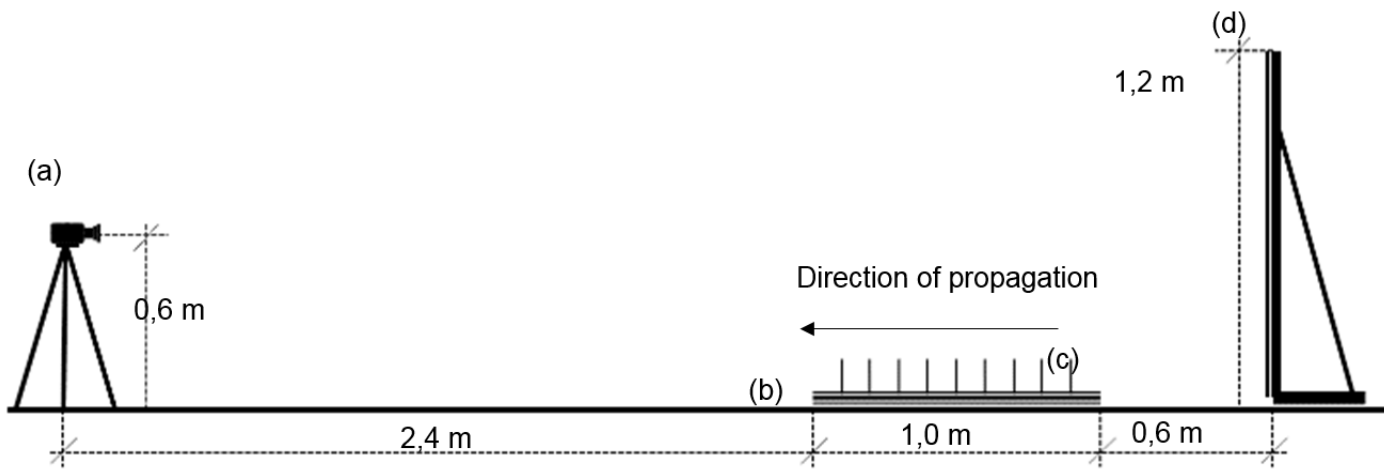

Figure 2 - Schematic illustration of components.

Note: (a) camera; (b) plot with fuel material; (c) ruler for speed of fire propagation determination; (d) panel for reading the flame height.

The following fire behavior parameters were analyzed: flame height, speed of propagation, residual material, fire intensity and lower calorific value, described below:

i. $\quad$ Flame length (FL), in centimeters: is obtained every $10 \mathrm{~cm}$ of fire propagation, having as reference a graduated rule. Eight observations are made per experimental burning. Subsequently, this variable was corrected as a function of the fire position in the plot and the flame height. For this, the following correction formulas were used:

For flame length greater than $60 \mathrm{~cm}$ (Equation 1):

$$
\mathrm{Cf}=-(\mathrm{D}+60) *\left[\frac{(\mathrm{FLv}-60)}{400}\right]
$$


For flame length less than $60 \mathrm{~cm}$ (Equation 2):

$$
\mathrm{Cf}=+(\mathrm{D}+60) *\left[\frac{(60-\mathrm{FLv})}{400}\right]
$$

where: $\mathrm{Cf}=$ correction factor, in $\mathrm{cm} ; \mathrm{D}=$ distance of the flame in relation to the beginning of the burn, in $\mathrm{cm} ; \mathrm{FLv}=$ flame length shown in the video, in $\mathrm{cm}$.

ii. Speed of propagation $(\mathrm{Sp})$, in $\mathrm{cm} \cdot \mathrm{s}^{-1}$ : determined by the relation between preestablished distances and the time spent by the fire front line to travel through them. For each experimental burning, eight observations were obtained.

iii. Residual material $(\mathrm{Rm})$, in $\mathrm{g} \cdot \mathrm{m}^{-2}$ : collected in the central area of the burning plot, using a template of $400 \mathrm{~cm}^{2}(20 \times 20 \mathrm{~cm})$.

iv. Fire intensity (I), in kcal. $\mathrm{m}^{-1} \cdot \mathrm{s}^{-1}$ : obtained by the formula of Byram (1959) (Equation 3).

$$
I=H \cdot w \cdot r
$$

where: $\mathrm{I}=$ fire intensity, in $\mathrm{kcal} \cdot \mathrm{m}^{-1} \cdot \mathrm{s}^{-1} ; \mathrm{H}=$ calorific value, in $\mathrm{kcal} \cdot \mathrm{kg}^{-1} ; \mathrm{w}=$ mass of available fuel material per area, in $\mathrm{kg} . \mathrm{m}^{-2} ; \mathrm{r}=$ speed of fire propagation, in $\mathrm{m} . \mathrm{s}^{-1}$.

v. Lower calorific value (LCV), in $\mathrm{kcal}^{\mathrm{kg}}{ }^{-1}$ : determined with an isoperibol calorimeter, following the standard of ABNT (1984) NBR 8633.

\subsection{Data analysis}

The parameters of flammability: ignition frequency (IF), ignition time (IT), time of complete combustion (TC) and flame height (FH); and combustibility: flame length (FL), speed of propagation (Sp), residual material $(\mathrm{Rm})$ and lower calorific value (LCV), were submitted to cluster analysis.

\section{Results}

In Table 2 are presented the parameters of flammability and combustibility and Figure 3 shows the cluster analysis of the studied species.

\begin{tabular}{|c|c|c|c|c|c|c|c|c|c|c|}
\hline \multirow{2}{*}{ Species } & \multicolumn{4}{|c|}{ Flammability } & \multirow{2}{*}{ FI } & \multicolumn{4}{|c|}{ Combustibility } & \multirow{2}{*}{ Intensity } \\
\hline & IF & IT & TC & FH & & FL & Sp & $\mathbf{R m}$ & LCV & \\
\hline Schinus terebinthifolius & 14 & 58.4 & 9.6 & 11.9 & $\mathbf{0}$ & 1.7 & 1.6 & 1445.9 & 4037.3 & 1.1 \\
\hline Bougainvillea glabra & 10 & 59.7 & 7.0 & 16.8 & $\mathbf{0}$ & 11.0 & 10.7 & 1643.8 & 3489.9 & 6.2 \\
\hline Michelia champaca & 86 & 19.8 & 4.5 & 9.2 & 1 & 32.6 & 70.0 & 219.0 & 3707.8 & 43.3 \\
\hline Viburnum odoratissimum & 88 & 23.3 & 5.4 & 11.9 & 1 & 32.1 & 48.8 & 200.9 & 3877.9 & 31.5 \\
\hline Rhododendron simsii & 98 & 18.8 & 26.9 & 8.6 & 2 & 24.4 & 15.6 & 290.0 & 4454.5 & 11.6 \\
\hline Casearia sylvestris & 96 & 17.5 & 5.5 & 13.7 & 3 & 23.3 & 32.4 & 395.7 & 4449.4 & 24.0 \\
\hline Pinus taeda & 100 & 16.3 & 12.5 & 12.1 & 3 & 36.6 & 55.8 & 176.6 & 4701.0 & 43.8 \\
\hline Magnolia grandifolia & 100 & 12.5 & 7.4 & 25.2 & 4 & 40.0 & 44.5 & 138.0 & 4259.3 & 31.6 \\
\hline Jasminym mesnyi & 100 & 10.9 & 18.5 & 13.9 & 4 & 14.3 & 27.1 & 440.1 & 4438.3 & 20.0 \\
\hline
\end{tabular}

Table 2 - Values of flammability and combustibility parameters of the tested species

Note: $F I=$ Flammability index; $0=$ very low flammable; $1=$ low flammable; $2=$ moderately flammable $; 3=$ flammable; 4 = very flammable; and 5 = extremely flammable.

S. terebinthifolius and B. glabra were classified as very low flammable (FI $=0)$, with an IT superior to that found by Molina et al. (2017) for species with low flammability in Spain. Kovalsyki et al. (2016), while studying the flammability of the same species, stated that both have shorter TC and $\mathrm{FH}, 1.3 \mathrm{~s}$ and $1.7 \mathrm{~cm}$ for S. terebinthifolius and $0.7 \mathrm{~s}$ and $1.2 \mathrm{~cm}$ for B. glabra, than what was found in the present study. 


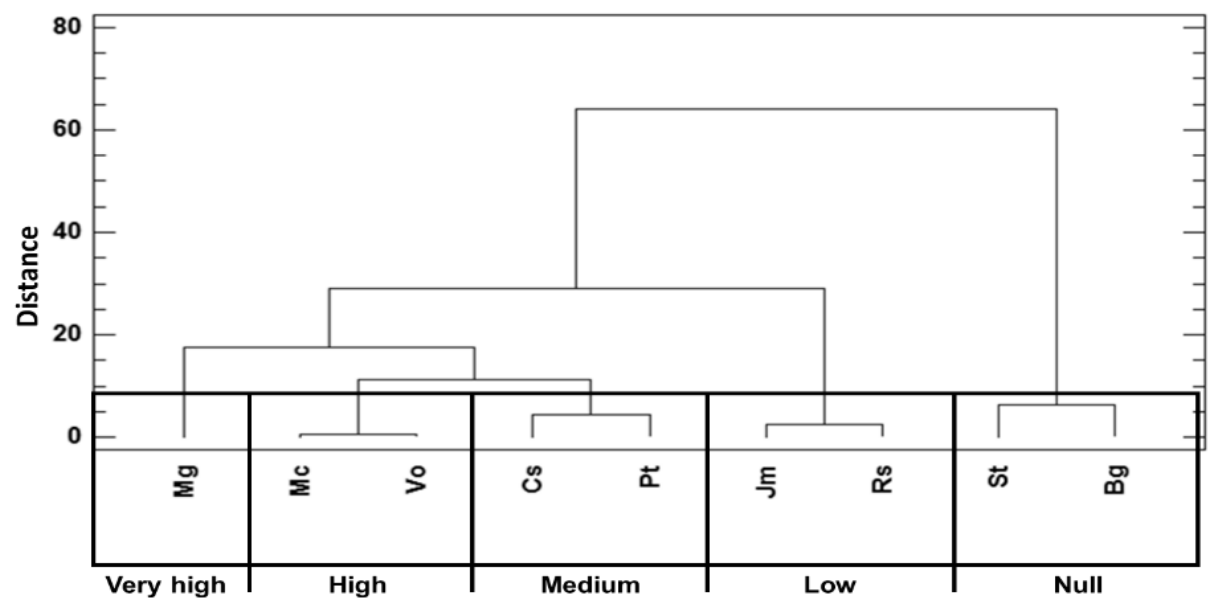

Figure 3 - Dendrogram according to the species flammability and combustibility

Another factor that favored the classification of B. glabra in class zero was a moisture content of $210.6 \%$ for this specie. The greater the moisture content, the greater the difficulty of material to ignite, where a high energy expenditure will be required to start the combustion process (White 2018).

Although S. terebinthfolius had a lower moisture content (142.4\%) and higher flammability compared to other species tested in this study, such as V. odoratissimum (163.1\%) and M. champaca (147.6\%), this species can be used as fuel breaks. Due to its low combustibility, S. terebinthfolius represents a small chance of the fire to escape control, allowing the combat in safety (Soares et al. 2017).

$J$. mesnyi has low combustibility, however is very flammable, not being indicated to act as fuel break. Even being classified as moderately flammable, $R$. simsii ignition frequency and time of complete combustion show a greater capacity to ignite and keep the flames than those species with low flammability and combustibility. Even though the heat fluxes measured during forest fires have a magnitude greater than the heat sources used in laboratory studies, it is known that ignition sources (cigarettes, bonfires, sparks, etc.) do not need to have large heat fluxes (Fernandes and Cruz. 2012).

$M$. gradiflora, even with some parameters lower than those found for $P$. taeda, has the lowest IT and combustibility, but was characterized as very flammable, not being recommended for use as fuel breaks. Results found by Mola et al. (2014) indicated that the susceptibility and severity of fires in the Apalachicola Ravines, Florida - USA were higher after the increase in the number of individuals of $M$. grandiflora.

\section{Conclusion}

The cluster analysis showed to be effective in the classification of species according to their flammability and combustibility. S. terebinthfolius, B. glabra and $R$. simsii are the species indicated for use as fuel breaks.

\section{References}

ABNT (1984) Determinação do poder calorífico - Método de ensaio. NBR 8633 (Associação Brasileira Normas Técnicas: Brasília, DF).

Batista AC, Biondi D (2009) Avaliação da inflamabilidade de Ligustrum licidum Aiton (Oleaceae) para uso potencial em cortinas de segurança na região sul do Brasil. Revista Brasileira de Ciências Agrárias 4, 435-439. 
Byram GM (1959). Combustion of forest fuels. In 'Forest Fire: Control and Use'. (Ed. KP Davis) pp. 77-84. (McGraw-Hill: New York)

Castillo MS, Correa LJ (2012) Acciones para la disminución del peligro de incendios forestales en áreas de interfaz urbanoforestal - estudio de caso. Territorium 19, 95-100.

Castillo MS, Julio GA, Quintanilla VP (2011) Vulnerabilidad y daño potencial ocasionado por incendios en áreas de interfaz urbano-forestal, provincia de Valparaíso. Chile central. Territorium 18, 247-254.

Fernandes PM, Cruz, MG (2012) Plant flammability experiments offer limited insight into vegetationfire dynamics interactions. New Phytologist 194, 606-609.

Ganteaume A, Jappiot M, Lampin C, Guijarro M, Hernando C (2013) Flammability of Some Ornamental Species in Wildland-Urban Interfaces in Southeastern France: Laboratory Assessment at Particle Level. Environmental Management 52, 467-480.

Haltenhoff, H (2006) 'Silvicultura preventiva.' (Ministerio de agricultura: Santiago)

Kovalsyki B, Takashina IK, Tres A, Tetto AF, Batista AC (2016) Inflamabilidade de espécies arbóreas para uso em cortina de segurança na prevenção de incêndios florestais. Pesquisa Florestal Brasileira 88, 387-391.

Long-Fournel M, Morge D, Bouillom C, Jappiot M (2013) La cartographie des interfaces habitat-forêt : un outil de diagnostic territorial dans la prévention du risque d'incendie de forêt dans le Sud de la France. Sciences Eaux and Territoires: la Revue du IRSTEA, 1-8.

Meldrum JR, Champ PA, Brenkert-Smith H, Warziniack T, Barth CM, Falk LC (2015) Understanding Gaps Between the Risk Perceptions of Wildland-Urban Interface (WUI) Residents and Wildfire Professionals. Risk analysis 9, 1-25.

Mell EW, Manzello SL, Maranghides A, Butry D, Rehm RG (2010) The wildland-urban interface fire problem - current approaches and research needs. International Journal of Wildland Fire 19, 238251.

Mola JM, Varner JM, Jules ES, Spector T (2014) Altered Community Flammability in Florida's Apalachicola Ravines and Implications for the Persistence of the Endangered Conifer Torreya taxifolia. Plos one 8, 1-8.

Molina JR, Martín T, Silva FR, Herrera MA (2017) The ignition index based on flammability of vegetation improves planning in the wildland-urban interface: A case study in Southern Spain. Landscape and urban planning 158, 129-138.

Montagné-Huck C, Brunette M (2018) Economic analysis of natural forest disturbances: A century of research. Journal of Forest Economics 32, 42-71.

Petriccione M (2006) 'Infiammabilità della lettiera di diverse specie vegetali di ambiente Mediterraneo.' (Università Degli Studi Di Napoli Federico II: Napoli)

Soares RV, Batista AC, Tetto AF (2017) 'Incêndios florestais: controle, efeitos e uso do fogo.' (UFPR: Curitiba)

Syphard AD, Massada AB, Butsic V, Keeley JE (2013) Land Use Planning and Wildfire: Development Policies Influence Future Probability of Housing Loss. Plos one 8, 1-12.

Valette JC (1990) lnflammabilités des espèces forestières méditerranénnes: conséquences sur la combustibilité des formations forestières (Mediterranean Forest Species Flammability, Consequences on Forest Communities Combustibility) Revue Forestière Française 42, 76-92.

White BLA (2018) Modelos matemáticos de previsão do teor de midade dos materiais combustíveis florestais finos e mortos. Ciência florestal 28, 432-445. 


\title{
Heating and Ignition from Firebrand Piles
}

\author{
R.S.P. Hakes; H. Salehizadeh; M.J. Weston-Dawkes; M.J. Gollner* \\ University of Maryland, College Park, Department of Fire Protection Engineering. 3106 J.M. \\ Patterson Bldg., College Park, Maryland 20742, United States, \{mgollner@umd.edu*\}
}

\begin{abstract}
Smoldering firebrands, also known as burning embers, cause the majority of structural ignitions in the wildland-urban interface (WUI). Firebrands can be lofted several kilometers from the main firefront and smolder for several hours before causing ignition of vegetation or structural components, such as decks or roofs. Previous studies on ignition by firebrands have focused primarily on either vegetative fuels, large scale ignition of solid fuels (e.g. wood materials), or ignition by individual firebrands. This study focuses on small-scale heating and ignition phenomena by measuring heat fluxes from piles of firebrands and connecting heating to ignition conditions. In order to capture the heating from glowing firebrand piles and understand the ignition process of WUI fuels, small-scale experiments were conducted using laboratoryfabricated cylindrical wooden firebrands. Heating by firebrand piles was de-coupled from the ignition process by initially studying heating from firebrands on an inert surface. The influences of firebrand diameter, firebrand pile mass, and wind were explored. Firebrand diameter was found to have little effect on heating, while firebrand pile mass had a moderate effect, and wind had a large effect on measured heat flux. Piles did not produce higher heat fluxes as pile mass increased. Instead, larger piles heated a larger area of the fuel for an increasingly longer duration as pile mass increased. Based on the results from heating experiments, two firebrand pile masses were studied under three wind conditions to understand the process governing the transition to ignition of a recipient fuel. Video recordings indicated a qualitative description of the transition from smoldering to flaming. Flaming ignition of the recipient fuel occurred soon after deposition of the firebrand pile for $16.2 \mathrm{~g}$ of smoldering firebrands, with a minimum critical wind speed of $1.0 \mathrm{~m} / \mathrm{s}$ required to cause transition to flaming. No smoldering of the recipient fuel occurred when only $5 \mathrm{~g}$ of firebrands were deposited.
\end{abstract}

Keywords: Firebrand ignition, Firebrands, Burning embers, Wildland-urban interface

\section{Introduction}

Over the past several decades, the area burned by wildland-urban interface (WUI) fires has increased drastically (Caton 2016). WUI fires are wildland fires that burn into developed urban areas that either abut or intermix with the wildland. The result of the increasing severity of WUI fires is that thousands of structures are lost annually to wildland fires across the globe (Grishin 2014, NICC 2017). The majority of structures are ignited in WUI fires by firebrands (Cohen 2008, Maranghides 2013, Maranghides 2015) which can travel several kilometers ahead of the main firefront (Maranghides 2013). Smoldering firebrands typically accumulate into piles (Suzuki 2017a). The smoldering piles can cause ignition of the recipient fuel on which they deposit, such as a deck or roof, which may ultimately cause full structure ignition.

Although firebrands cause the majority of structural ignitions, the heating conditions leading to ignition and causing the transition from smoldering to flaming ignition are not well understood. There is little previous work at the small-scale that explores the ignition of solid WUI fuels due to piles of smoldering firebrands. Previous work has focused primarily on ignition of vegetative fuels by individual firebrands (Ellis 2013, Ellis 2015, Manzello 2006a, Manzello 2006b, Urban 2018), or fullscale ignition of structural components (Manzello 2012, Manzello 2017). This study focuses on smallscale heating and ignition phenomena by measuring heat fluxes from piles of firebrands and connecting heating under varying wind speeds to ignition conditions. 


\section{Methods}

Two phases of experiments were conducted to quantify critical heating conditions preceding ignition and describe the transition from smoldering to flaming ignition of solid WUI fuels. For Phase I, an experimental set-up was developed to quantitatively measure the heat flux from firebrand piles under ambient conditions. For Phase II, an additional set-up was constructed to investigate the effects of wind, both on heat flux and ignition behavior. Measurements of heat flux were made on an inert surface in order to decouple the heating from the firebrand pile from ignition and to better characterize the thermal conditions critical for ignition.

For both heat flux and ignition experiments, firebrands were fabricated from cylindrical birch wooden dowels oven-dried at $103^{\circ} \mathrm{C}$ to $0 \pm 0.5 \%$ moisture content (MC) per ASTM D4442 (ASTM 2015). Firebrands were fabricated by exposing all of the wooden dowel pieces to be used for a single pile to a propane flame in a wire-mesh basket, based on a method in (Manzello 2006a). The propane flame was extinguished after the majority of the wood began flaming. Firebrands were allowed to burn freely in air until flaming combustion ceased and all firebrands were glowing. Glowing firebrands were deposited on the experimental set-up. Firebrands of length $25.4 \mathrm{~mm}$ were used at three different initial diameters, $6.35 \mathrm{~mm}, 9.52 \mathrm{~mm}$, and $12.7 \mathrm{~mm}$, for Phase I. Because diameter was found to have little effect on heating, only $12.7 \mathrm{~mm}$ firebrands were used in Phase II.

For heat flux experiments in both Phase I and II, the experimental set-up was instrumented with either an array of sixteen thin-skin calorimeters (TSCs), a single water-cooled heat flux gauge (WCHFG), or a combined array with the WC-HFG at the center of the TSC array. TSCs provided a spatial characterization of the heat flux at different points beneath the firebrand pile, while the WC-HFG provided temporally-resolved heat flux measurements at the center of the pile. Heat flux gauges of both types were placed in the center of a ceramic fiber inert insulation board, flush with the surface of the board.

Phase II ignition experiments were conducted over oriented-strand board (OSB), a common building materials in the United States. Ignition experiments and heat flux experiments with applied wind were conducted in front of a wind tunnel with a constant speed of $1.84 \mathrm{~m} / \mathrm{s}$ as that was a condition determined to repeatably cause ignition. Two firebrand pile masses, $7.9 \mathrm{~g}$ and $16.2 \mathrm{~g}$ of $12.7 \mathrm{~mm}$ firebrands, were used for Phase II tests.

\section{Results and discussion}

Heat flux tests under ambient conditions were conducted to determine the influence of firebrand diameter and firebrand pile mass on heat flux. Results from the tests at ambient conditions were used to choose a firebrand diameter and two pile sizes to compare under varied wind speeds for both heat flux tests and ignition tests. Wind was used for ignition tests because ignition of the solid fuels tested by smoldering firebrands could not be achieved without wind. In this study, TSCs were used to understand the spatial distribution of heat flux beneath a firebrand pile and to determine whether heat flux was evenly distributed through the pile.

Results from the TSC array found high variability in the heat fluxes measured in different parts of the pile. This variability is likely due to variability in individual pieces of wood and in the smoldering combustion of wood. Although the WC-HFG provides a good measure of a single point in the pile, the TSC array gives a more comprehensive view of the area heated by the firebrand pile and the extent of radiation from the pile to the rest of the inert surface. Figure shows the heat flux variability in a winddriven test at two instances: $1 \mathrm{~min}$ and $10 \mathrm{~min}$ after firebrands were deposited. Early in the test, heat flux is highest at the side of the firebrand pile facing the wind, but the areas of highest heating change later in the test. 

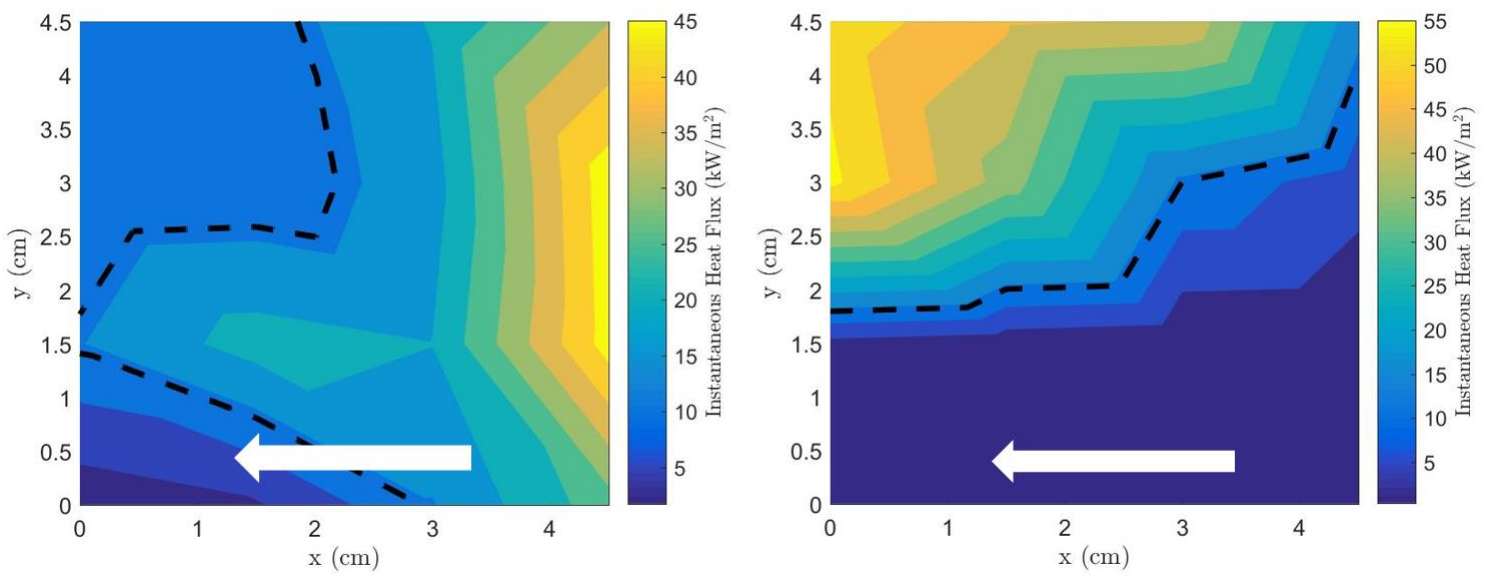

Figure 1 - Spatial distribution of heat flux under $1.84 \mathrm{~m} / \mathrm{s}$ wind for a $16.2 \mathrm{~g}$ deposited mass pile of $12.7 \mathrm{~mm}$ firebrands at (left) 1 min and (right) 10 min into test. Wind direction: right to left.

The effects of firebrand diameter and firebrand pile size (measured as the mass of glowing firebrands deposited on the test set-up) were compared using the peak heat flux and the net heat imparted by the firebrand pile to the sensors. The peak heat flux was taken to be the maximum heat flux value achieved within a given test repetition and averaged over five test repetitions. Net heat released was taken as the area under the heat flux curve over the full length of the test and averaged over five test repetitions.

Firebrand diameter had a negligible effect on both the peak heat flux and the net heat released. Previous studies using individual firebrands have found that firebrand size (measured here as the diameter of a single firebrand) made a significant difference in whether vegetative fuels ignited. Results for the firebrand pile differ as individual firebrands in the pile can interact through re-radiation. The re-radiation behavior, rather than firebrand size, is suspected to dominate the heat transfer from piles as the firebrand pile is a noncontiguous ignition source (Babrauskas 2003). Additionally, tests with a single firebrand placed on a TSC found that nearby (at a distance of $0.5-1.5 \mathrm{~cm}$ ) uncovered sensors received an average of one third of the peak heat flux received by the sensor directly in contact with the firebrand, despite the fact that uncovered sensors are subject to higher convective cooling losses. These results indicate the importance of radiation for the firebrand heating and ignition problem.

The peak heat flux for all firebrand piles was higher than the peak heat flux reached by a single firebrand; however, peak heat flux did not vary much with firebrand pile size, as shown in Figure .

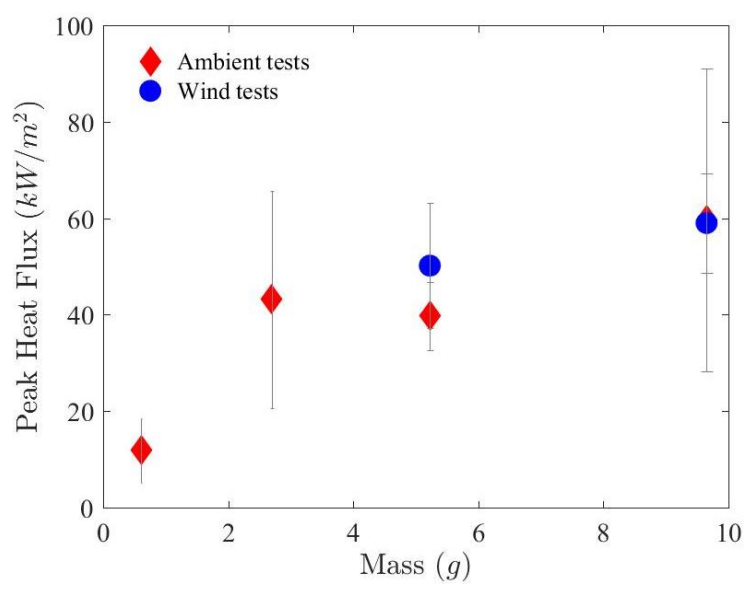

Figure 2 - Peak heat flux as a function of deposited mass for $12.7 \mathrm{~mm}$ piles under ambient and wind conditions. The smallest mass, $0.6 \mathrm{~g}$, is a single firebrand. Peak heat flux increases from an individual firebrand to a pile, but steadies with pile mass. A wind speed of $1.84 \mathrm{~m} / \mathrm{s}$ has little effect on the peak heat flux. Error bars are the standard deviation of the tests. 
The net heat imparted by the firebrand pile increased linearly with increasing pile size and with applied wind, as shown in Figure. This result is expected as a larger pile sizes produced higher sustained heat flux values throughout the tests. Heat fluxes under wind were sustained at higher heat flux values than ambient heat fluxes, but dropped off more quickly as forced flow provided increased convective cooling to the pile.

Although both peak heat flux and net heat released provide descriptions of the pile heating, neither captures the dependency on time of critical ignition conditions. Critical ignition conditions are often given as a steady heat flux or temperature value sustained over a critical exposure time.

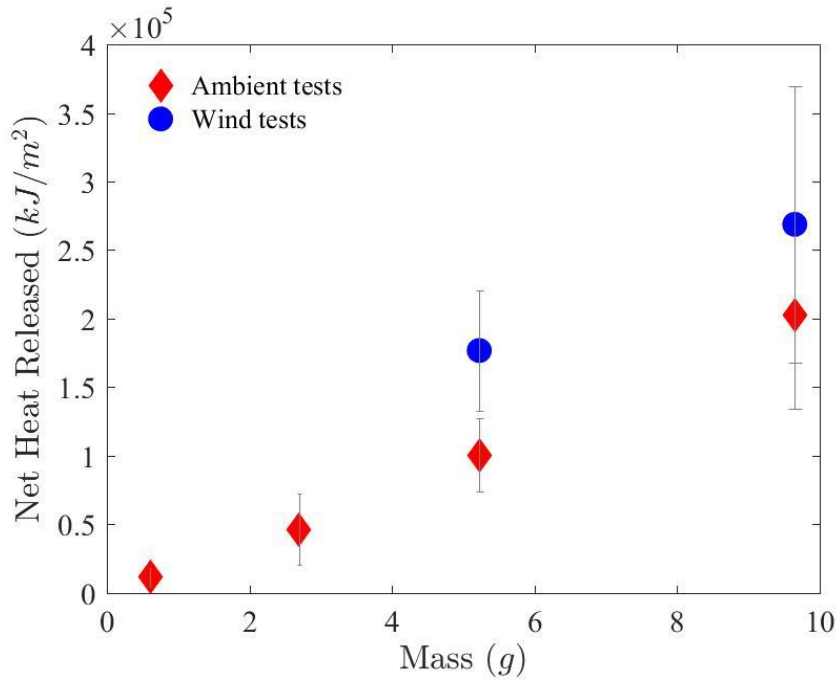

Figure 3 - Net heat released as a function of deposited mass for $12.7 \mathrm{~mm}$ diameter firebrands. The smallest mass, 0.6 $\mathrm{g}$, is a single firebrand. Net heat released increases linearly with increasing pile mass under ambient conditions. The addition of wind, $1.84 \mathrm{~m} / \mathrm{s}$, causes higher net heating values. Error bars are the standard deviation of the tests.

Ignition of the recipient fuel only occurred when it was exposed to $16.2 \mathrm{~g}$ of glowing firebrands, the larger of the two pile sizes tested. No ignition occurred under ambient conditions, and ignition by the $16.2 \mathrm{~g}$ firebrand pile required a minimum $1.0 \mathrm{~m} / \mathrm{s}$ wind speed. Figure shows ignition location for a $16.2 \mathrm{~g}$ pile of firebrands deposited on a sheet of OSB. For all tests that ignited, ignition occurred within a few minutes of the firebrand pile being deposited. The recipient fuel initially smoldered and then transitioned to flaming ignition.

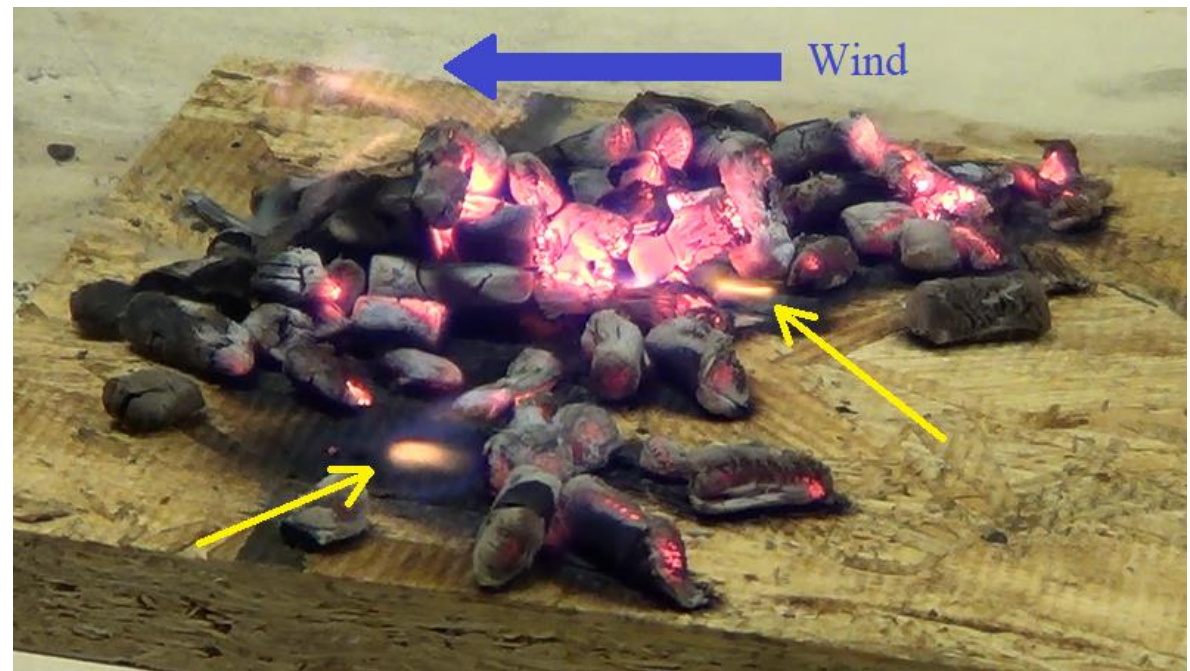

Figure 4 - Ignition of recipient OSB fuel exposed to $16.2 \mathrm{~g}$ smoldering firebrands two minutes into test under $1.84 \mathrm{~m} / \mathrm{s}$ wind. Arrows indicate areas of fuel ignition (as opposed to flaming of firebrands). 


\section{Conclusions and future work}

A methodology for measuring heat fluxes from firebrand piles has been applied under ambient and forced flow conditions. Firebrand diameter was found to have little effect on the heat flux from a firebrand pile, likely indicating that the heat transfer in firebrand piles is dominated by re-radiation and reheating rather than individual firebrand characteristics. Heat flux conditions immediately preceding ignition have been measured to indicate how conditions preceding ignition relate to the occurrence of ignition. Wind was found to play a critical role in the ignition of dense, thermally thick WUI fuels. Wind tests show that critical heat fluxes for ignition are changed when exposed to forced flow.

\section{Acknowledgements}

The authors would like to thank Alicea Fitzpatrick, Erin Griffith, Alison Davis, Jessen Oey, Evan Sluder, and Seth Lattner for their assistance with laboratory experiments, and Jiann Yang, Nelson Bryner, and the NIST WUI group for advice and support. This research was funded by the United States Department of Commerce, National Institute of Standards and Technology Fire Research Grant Program under financial assistance awards 60NANB15D358, 70NANB16H284 and 70NANB17H288.

\section{References}

American Society of Testing and Materials (2015) ASTM D4442: Standard test methods for direct moisture content measurement of wood and wood-based materials.

Babrauskas V (2003) 'Ignition Handbook.' (Fire Science Publishers: Issaquah)

Caton SE, Hakes RSP, Gorham DJ, Zhou A, Gollner MJ (2016) Review of pathways for building fire spread in the wildland urban interface part I: Exposure conditions. Fire Technology 53(2), 429-473.

Cohen JD, Stratton RD (2008) Home destruction examination: Grass Vallet Fire, Lake Arrowhead, CA. USDA Forest Service, Pacific Southwest Region Technical Paper R5-TP-026b. (Vallejo, CA)

Ellis PFM (2013) Firebread characteristics of the stringy bark of messmate (Eucalyptus obliqua) investigated using non-tethered samples. International Journal of Wildland Fire 22, 642-651.

Ellis PFM (2015) The likelihood of ignition of dry-eucalypt forest litter by firebrands. International Journal of Wildland Fire 24, 225-235.

Grishin AM, Filkov AI, Loboda EL, Reyno VV, Kozlov AV, Kuznetsov VT, Kasymov DP, Andreyuk SM, Ivanov AI, Stolyarchuk ND (2014) A field experiment on grass fire effects on wooden constructions and peat layer ignition. International Journal of Wildland Fire 23, 445-449.

Manzello SL, Cleary TG, Shields JR, Yang JC (2006a) Ignition of mulch and grasses by firebrands in wildland-urban interface fires. International Journal of Wildland Fire 15, 427-431.

Manzello SL, Cleary TG, Shields JR, Yang JC (2006b) On the ignition of fuel beds by firebrands. Fire and Materials 30, 77-87.

Manzello SL, Suzuki S, Hayashi Y (2012) Enabling the study of structure vulnerabilities to ignition from wind driven firebrand showers: A summary of experimental results. Fire Safety Journal 54, 181-196.

Manzello SL, Suzuki S (2017) Experimental investigation of wood decking assemblies exposed to firebrand showers. Fire Safety Journal 92, 122-131.

Maranghides A, McNamara D, Mell W, Trook J, Toman, B (2013) A case study of a community affected by the Witch and Guejito fires: Report \#2 - evaluating the effects of hazard mitigation 
actions on structure ignitions. National Institute of Standards and Technology Technical Note NIST TN 1796. (Gaithersburg, MD)

Maranghides A, McNamara D, Vihnanek R, Restaino J, Leland C (2015) A case study of a community affected by the Waldo Fire - Event timeline and defensive actions. National Institute of Standards and Technology Technical Note NIST TN 1910. (Gaithersburg, MD)

National Interagency Coordination Center (2017) Wildland fire summary and statistics annual report.

Suzuki S, Manzello SL (2017) Experiments to provide the scientific-basis for laboratory standard test methods for firebrand exposure. Fire Safety Journal 91, 784-790.

Urban JL, Zak CD, Fernandez-Pello C (2018) Spot fire ignition of natural fuels by hot aluminum particles. Fire Technology 54, 797-808. 


\title{
Lattice Boltzmann Method for Flow through Vegetation
}

\author{
Hayri Sezer ${ }^{1,2}$; Albert Simeoni ${ }^{1 *}$ \\ ${ }^{1}$ Worcester Polytechnic Institute, Worcester, MA, 01609, USA \{asimeoni@wpi.edu*\} \\ ${ }^{2}$ School of Engineering and Technology, Western Carolina University, USA
}

\begin{abstract}
Recently wildland/forest fires have dramatic impact on the environment and society. Therefore, it is vital to understand wildland/forest fires spread dynamics to prevent its hazardous effects on society and environment. Models based on Computational Fluid Dynamics (CFD) have been developed and improved to investigate the dynamics of the wildland/forest fires. Even for CFD based physical models, a robust understanding of the processes (i.e. drag, convective heat transfer and radiation) driving fire spread is missing for small scale. The present study focuses on obtaining drag coefficient for the complex vegetation structures in small scales by using Lattice Boltzmann method (LBM). Ultimately, the drag coefficient can be used in detailed wildland/forest fire spread models to analyze the physical mechanisms driving the fire spread in vegetation. The Lattice Boltzmann (LBM) is a relatively new numerical approach for solving Navier-Stokes equations. LBM has advantages such as simplicity, efficiency, easy treatment of boundary conditions, easy parallelization in simulating fluid flow and heat transfer. The developed model is validated with experiment for a cylinder in cross flow in the literature. The results show that LBM method can be used for the flow in complex distribution of vegetation.
\end{abstract}

Keywords: LBM, complex fuels, drag coefficient, wildland fire

\section{Introduction}

Wildland fires can be beneficial and catastrophic for the environment and society. Prescribed fires can have a positive impact on the environment including reducing grass, brushes and trees. However, wildland fires happening in the wrong place at the wrong time can have a dramatic impact on lives, property, whole communities, and natural and cultural resources (U.S. Forest Service, 2018). Therefore, it is vital to better understand the dynamics of wildfire spread to help manage forests, other ecosystems and help protect human life, property, and activities.

Fire spread in heterogenous fuels is a result of the interaction between the solid and gas phases, as well as radiative heat transfer between phases. Models based on computational fluid dynamics (CFD) have been developed to address the complicated combustion processes involved in wildland fires (Larini, M., et al., 1988; J.L. Dupuy et al., 2005; Zhou, X. et al., 2007; Mell, W. et al., 2009). However, the CFD models apply strong and unverified assumption to resolve some small-scale processes, including drag forces and convective heat transfer. For instance, the drag coefficient is an input parameter in CFD based physical models that is based on a single cylinder in a cross-flow (Mell, W., et al., 2009). In this study, an attempt has been made to resolve sub-grid scale processes involved in fire dynamics by using an in-house developed two-dimensional Navier-Stokes solver based on the Lattice Boltzmann Method (LBM).

Recently, the LBM has become an alternative numerical method for computing fluid flow and heat transfer. The LBM is particularly successful in applications including interfacial dynamics and complex boundaries because of its boundary condition implementation. The basic idea behind the LBM is to build simplified Boltzmann equations that relate the main physics of the microscopic processes (particle distribution and equilibrium distribution functions) such that the macroscopic process averaged properties including density, temperature, velocity and pressure obey the desired governing equations (i.e. Navier Stokes and scalar transport equations) (Chen, S. et al., 1998).

In the present study, the drag coefficient at grid scale is directly calculated through the LBM for different configurations of cylinders in a cross flow. 


\section{Lattice Boltzmann method}

The numerical model was developed for non-isothermal flows based on the LBM. The LBM uses a simple boundary condition scheme that can easily handle the flow in complex boundaries similar to vegetation (A.A. Mohamad, 2011). The current model is able to solve the momentum and energy equations for rigid bodies. The LBM method is based on the Boltzmann transport equation for a dynamic system. The Boltzmann equation for a system without an external force is written as (A.A. Mohamad, 2011),

$$
\frac{\partial f}{\partial t}+c \nabla f=-\frac{1}{\tau}\left(f-f^{e q}\right)
$$

Where $f, f^{e q}$ and $\tau$ are the particle distribution function, particle equilibrium function and relaxation time, respectively. Equation 1 is similar to the advection equation with a source term and it can be solved by a finite difference method. However, in LBM, Eq. 1 is solved by a streaming and a collision step (Wang, J., et al., 2007).

\subsection{Drag Coefficient}

The drag coefficient can be calculated from the estimation of the total drag force that is acting on an obstacle or from the pressure drop that is induced by the obstacle in the flow. The drag coefficient is defined as;

$$
\begin{aligned}
& \text { Drag force; } C_{D}=\frac{F_{x}}{\frac{1}{2} \rho U^{2} D} \\
& \text { Pressure drop; } C_{D}=\frac{P_{\text {in }}-P_{\text {out }}}{\frac{1}{2} \rho U^{2}}
\end{aligned}
$$

The following equation was used to estimate the total force from a fluid acting on an obstacle (Mei, R., et al., 2002):

$$
F=\sum_{x_{h}} \sum_{k \neq 0} c_{k}\left[f_{k}\left(x_{b}, t\right)+f_{k}^{-}\left(x_{b}+c_{k}^{-} \Delta t, t\right)\right] \times\left[1-\left(x_{b}+c_{k}^{-} \Delta t\right)\right]
$$

Where $x_{b}$ is the boundary nodes of the obstacle and $c_{k}^{-}$is the opposite direction of $c_{k}$.

\subsection{Verification and Validation}

A flow over an obstacle has many practical applications in buildings, bridges, heat exchangers and wildland fires. The flow, under certain conditions causes pressure vortices downstream of an obstacle and forms vortex shedding depending on the Reynolds number. To capture this dynamic fluctuation of the flow with the LBM, a square channel shown in Fig. 1 is selected, with a circular cylinder with a blockage ratio of 1 to 8 located in its center. The geometry, boundary conditions, and grid used in the simulation are shown in Fig. 1. The curvature boundary of the cylinder is zoomed out to depict the boundary nodes, solid nodes and the fluid nodes used in the simulations. On-grid bounce-back boundary conditions are used for the obstacle as well as the top and the bottom walls (Gallivan, M.A., et al., 1997). Zou-He velocity and pressure boundary conditions (Zou, Q. and He,X., 1997) are implemented at the inlet and the outlet (see Figure 1). The simulations with $\operatorname{Re}=20$ and 40 were carried out and the flow pattern is in good agreement with prior results from laboratory experiments (Bao, Y.B. and Meskas, J., 2011) (see Figure 2). 

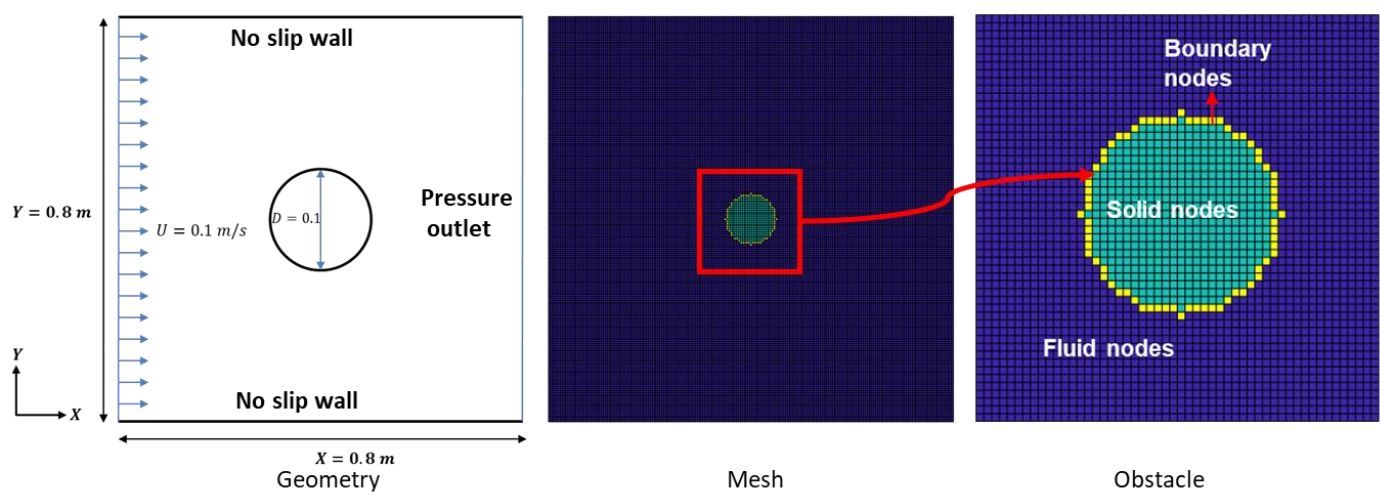

Figure 1 - Representation of geometry, mesh and obstacle used in the drag coefficient calculation

A pair of fixed symmetric vortices is generated in the wake of the cylinder in the simulation with LBM and the results match the laboratory image by Bao et al (Bao, Y.B. and Meskas, J., 2011), as seen in Fig. 2.
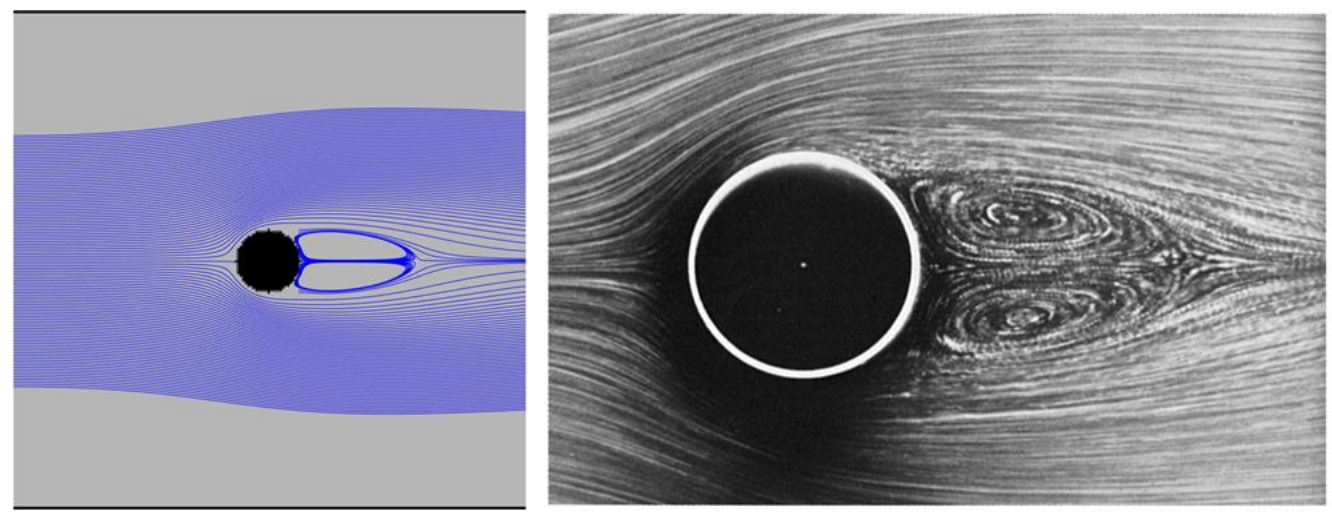

Figure 2 - A fixed pair of vortices: simulation vs experiment (Bao et al., 2011) (Bao, Y.B. and Meskas, J., 2011)

Table 1 - Drag coefficient at different Reynolds number

\begin{tabular}{|l|c|c|}
\hline & $\mathrm{Re}=20$ & $\mathrm{Re}=40$ \\
\hline Tritton experiment $^{1}$ & 2.22 & 1.48 \\
\hline Current study & 2.3 & 1.56 \\
\hline${ }^{1}$ (Tritton D., 1959) & \\
\hline
\end{tabular}

Drag coefficients with $\mathrm{Re}=20$ and 40, calculated by the present model and obtain from the literature are listed in Table 1. As it can be seen, the current model agrees reasonably well with the experimental measurements by Tritton. (Tritton D., 1959).

\section{Results and discussion}

The calculation of the vegetation drag coefficient is required for the modeling of fire spread in wildlands, but also for the flow in river beds and on flood plains (Kim, S.J. and Stoesser, T., 2011; Fischer-Antze, T., et al., 2001). The drag exerted on the flow by vegetation changes with the plant shape, rigidity, and with the spatial distribution of vegetation. In many experimental studies of flow through vegetation, the cylinder analogy is used. For example, vegetation can be represented as a bunch of rigid circular cylinders (Kim, S.J. and Stoesser, T., 2011). In the present study, the drag coefficient is directly calculated for simplified geometries that can represent a rigid vegetation such 
as, single cylinder, inline and staggered arrays of cylinders as well as randomly distributed cylinders in a cross flow.

\subsection{Single Cylinder}

The drag coefficient for a cylindrical obstacle depends on the behavior of the fluid around the cylinder. For example, depending on the Reynolds number, the flow pattern near the cylinder can vary significantly. For higher Reynolds number (i.e. Red > 40), an unsteady wake flow occurs, the characteristic of which depends on the Reynolds number. In the following simulations the Reynolds number is defined as follow:

$$
\operatorname{Re} D=\frac{U_{\infty} D}{v}
$$

Where $\boldsymbol{v}$ is the fluid kinematic viscosity, $\boldsymbol{U}_{\infty}$ is the upstream velocity, and $\boldsymbol{D}$ is the cylinder diameter.

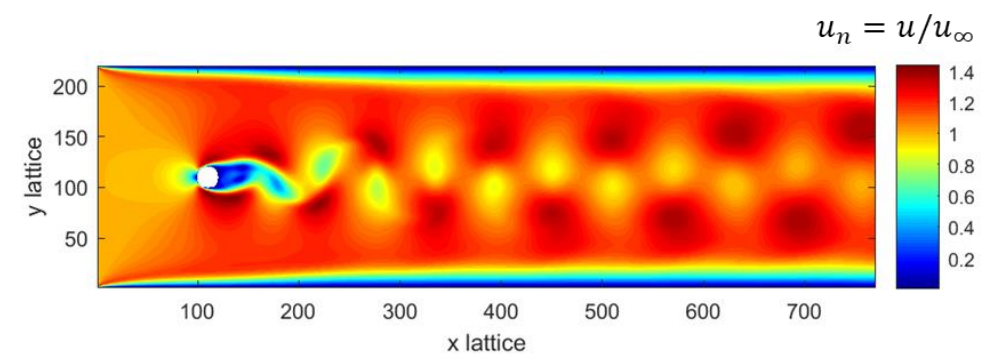

(a)

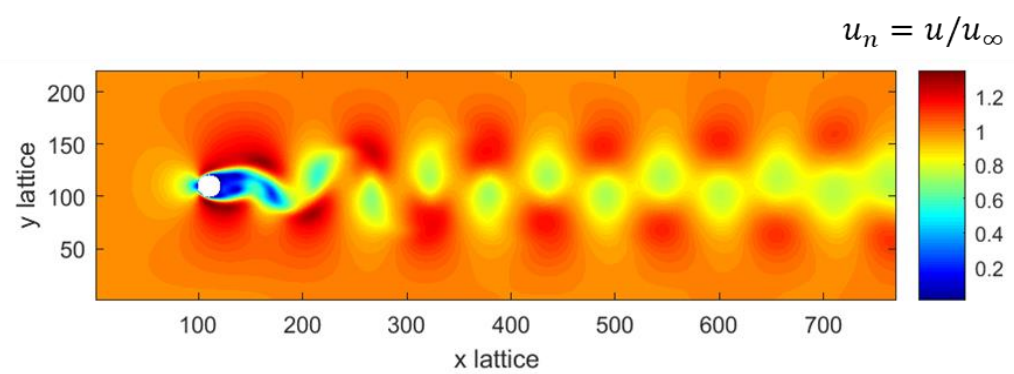

(b)

Figure 3 - Contours of velocity magnitude for flow around a cylindrical object at ReD = 100. (a) no-slip wall (b) periodic boundary conditions at top and bottom walls.

For a single cylinder, simulations have been carried out at $\mathrm{Re}_{\mathrm{D}}=100$, with no-slip and periodic boundary conditions at the walls to estimate the effect of different wall boundary condition on estimation of the drag coefficient. A uniform velocity was set at the inlet and a pressure outlet boundary condition was implemented at the outlet, as shown in Fig. 1 (geometry). Contours of the velocity magnitude shown in Fig. 4 were generated at the steady state flow for the no-slip and periodic boundary conditions. The vortex shedding can be observed downstream of the cylinders. The calculated drag coefficient for both cases and the literature values are listed in Table 2. As shown in Table 2, the current study agrees well with the literature for the no-slip wall boundary condition. However, there is a deviation when a periodic boundary condition is used. The deviation is introduced by the friction presence of a rigid wall at the boundaries. In order to remove the effect of no-slip wall condition, in the following simulations, periodic boundary condition is implemented at the walls. 
Table 2- Drag coefficient at ReD = 100 for a single cylinder .

\begin{tabular}{|l|c|}
\hline 2-D simulation & Drag coefficient at ReD $=100$ \\
\hline Braza et al. $(1986)^{1}$ & 1.34 \\
\hline Kjellgren $(1997)^{2}$ & 1.34 \\
\hline Su and Kang $(1999)^{3}$ & 1.34 \\
\hline Lam et al. $(2008)^{4}$ & 1.36 \\
\hline Current study, LBM (no-slip) & 1.34 \\
\hline Current study, LBM (periodic) & 1.25 \\
\hline${ }^{1}\left(\right.$ Braza, M., Chassaing, P., Ha Minh, H., 1986), ${ }^{2}$ (Kjellgren, P., 1997), ${ }^{3}(\mathrm{Su}$, \\
M., Kang, Q., 1999), ${ }^{4}$ (Lam, K., et al., 2008) \\
\hline
\end{tabular}

\subsection{Inline and staggered arrays of cylinders}

The drag coefficient of cylinders in a cross flow is relevant to different industrial applications, including heat exchangers, boilers, and air conditioners. The cylinders in industrial applications are usually either aligned or staggered relative to the stream-wise fluid velocity. Cylinders in a cross flow can also represent an idealized rigid vegetation. Fluid flow through circular cylinders is dictated by the boundary layer separation and wake interaction, which in-turn affect the drag coefficient. Therefore, cylinders in different configurations are used to calculate the drag coefficient. The LBM based N-S solver is used to simulate the flow through inline and staggered array of the cylinders at Reynolds number of 100. The cylinders in the inline and staggered configurations are equivalent. The top and bottom walls are 10 cylinder diameters away from the vegetation area. Figure 4 shows the contours of velocity magnitude the steady state flow for inline and staggered arrays of cylinders in a cross flow. It can be seen that the flow patterns for the inline and staggered arrays of cylinder are significantly different from each other. However, the maximum normalized velocity is similar. The drag solid fraction of the inline and staggered array of cylinders are 0.14 and 0.11 respectively, whereas, the drag coefficients are 0.57 and 0.83 . The solid fraction of the vegetation is the ratio of the solid cylinder's area to the smallest rectangular area that encompass the cylinders. As it seen in Fig. 4, the flow pattern of the inline array and staggered array are significantly different because of the cylinder's arrangement. Therefore, the drag coefficients are different for both cases even though the solid fraction is not significantly different. The LBM is able to predict the fluid flow behavior through idealized vegetation configurations such as inline and staggered arrangements. The comparison of the inline, staggered and randomly distributed cylinders are given in the subsequent sections.
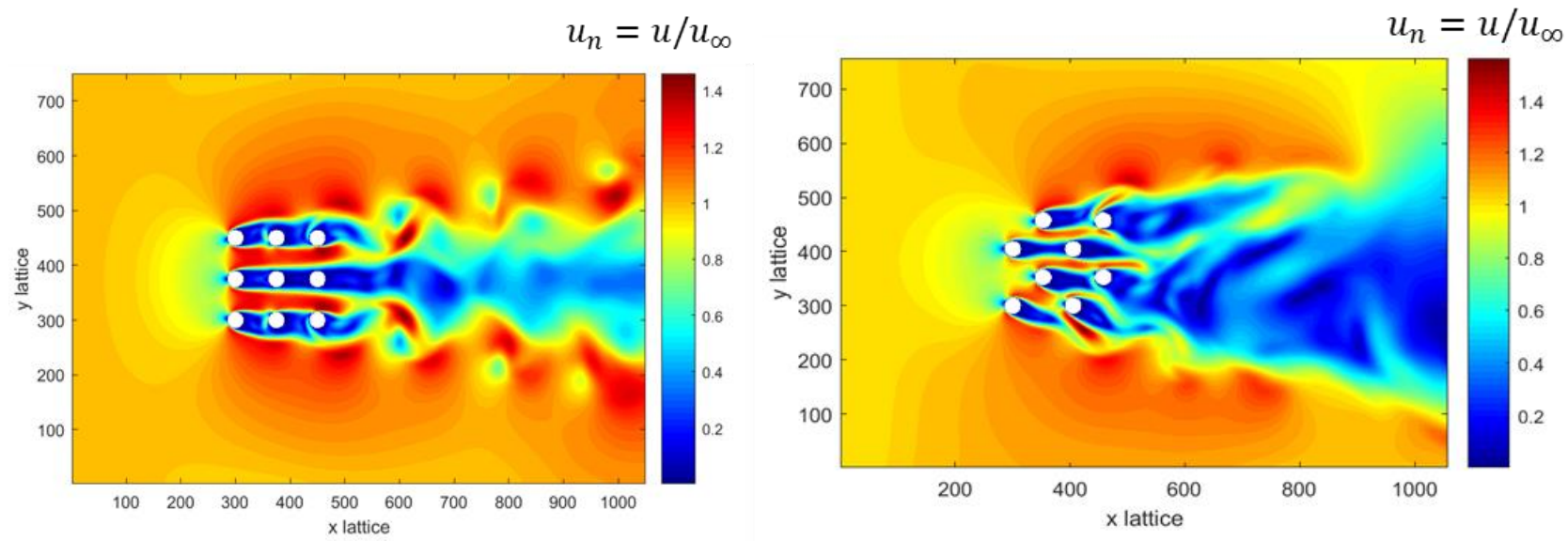

Figure 4 - Contours of velocity magnitude for a flow through an array of cylinders at $R_{D}=100 .($ top) inline, (bottom) staggered

\subsection{Randomly distributed cylinders}

A random distribution may better represent vegetation structures in their natural state than the inline and staggered arrays of cylinders. Therefore, the developed LBM code was used to calculate the drag 
coefficient for randomly distributed cylinders in a cross flow for different solid fractions and numbers of cylinders. Figure 5 shows the contours of the velocity magnitude at steady state flow for different randomly distributed cylinders in a cross flow. As it can be seen, the flow pattern is different for both cases and therefore, the drag exerted by randomly distributed rigid circular cylinders is significantly different for two densities of 0.11 and 0.093 . The corresponding drag coefficients are 1.37 and 2.7 respectively.
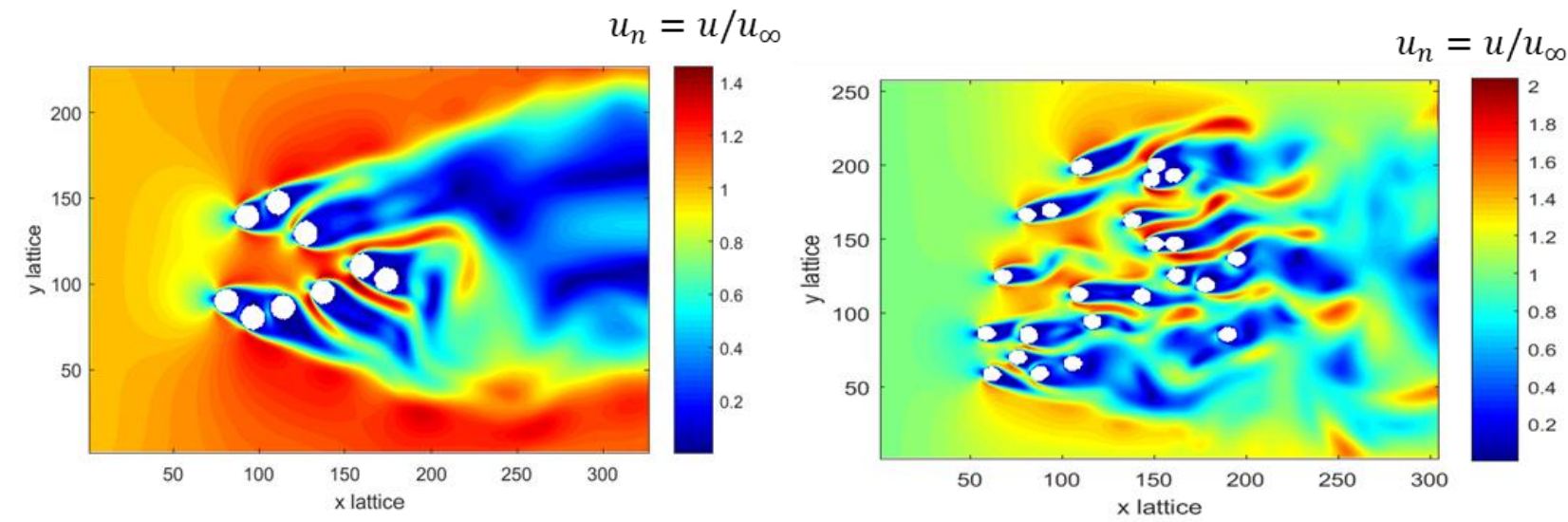

Figure 5 - Contours of velocity magnitude for a flow through randomly distributed cylinders at Re $\boldsymbol{R}_{D}=100$. (top) bulk density, $\phi=0.11$ (bottom) $\phi=0.093$

\subsection{Drag coefficient comparison}

Additional simulations are performed to analyze the drag coefficient for different configurations as well as vegetation solid fraction and compare the results with the available literature. Table 3 summarizes the numerical simulations and calculated drag coefficients. For the time being, the numerical simulations are carried out at a relatively low Reynolds number of 100 because of the current limitations of the turbulence model in the LBM.

Table 3 - Summary of the numerical simulations of drag coefficient calculation

\begin{tabular}{|c|c|c|c|c|c|c|}
\hline $\begin{array}{l}\text { solid } \\
\text { fraction }\end{array}$ & $\operatorname{Re}_{\mathrm{D}}$ & B. C. & $C_{D}$ & Shape & Configuration & study \\
\hline 0.14 & 100 & periodic & 0.57 & cylinder & inline & LBM \\
\hline 0.50 & 100 & periodic & 0.66 & cylinder & inline & LBM \\
\hline 0.26 & 100 & periodic & 0.68 & cylinder & inline & LBM \\
\hline 0.20 & 100 & periodic & 0.74 & cylinder & inline & LBM \\
\hline 0.24 & 100 & wall & 0.775 & cylinder & inline & (Lam, K., et al., 2008) \\
\hline 0.10 & 100 & periodic & 0.59 & cylinder & inline & LBM \\
\hline 0.21 & 100 & periodic & 0.5 & cylinder & inline & LBM \\
\hline 0.21 & 100 & wall & 0.64 & cylinder & inline & LBM \\
\hline 0.078 & 100 & wall & 1.5 & cylinder & inline & LBM \\
\hline 0.078 & 100 & periodic & 1.41 & cylinder & inline & LBM \\
\hline 0.11 & 100 & periodic & 0.83 & cylinder & staggered & LBM \\
\hline 0.2 & 100 & periodic & 0.44 & cylinder & staggered & LBM \\
\hline 0.087 & 695 & wall & 2.02 & cylinder & staggered & (Kim, S.J. and Stoesser, T., 2011) \\
\hline 0.11 & 100 & periodic & 1.37 & cylinder & random & LBM \\
\hline 0.065 & 100 & periodic & 1.95 & cylinder & random & LBM \\
\hline 0.093 & 100 & periodic & 2.7 & cylinder & random & LBM \\
\hline 0.04 & 100 & periodic & 2.7 & cylinder & random & LBM \\
\hline 0.11 & 100 & periodic & 2.3 & cylinder & random & LBM \\
\hline 0.16 & 100 & periodic & 2.19 & cylinder & random & LBM \\
\hline 0.15 & 193 & wall & 3.03 & cylinder & random & (Tanino, Y., and H. M. Nepf, 2008) \\
\hline 0.15 & 263 & wall & 2.86 & cylinder & random & (Tanino, Y., and H. M. Nepf, 2008) \\
\hline
\end{tabular}


As it can be seen in Table 3, the simulated results are in the range of the experimental results by Kim et al. (Kim, S.J. and Stoesser, T., 2011) and Tanino et al. (Tanino, Y., and H. M. Nepf, 2008) . However, it should be noted here that, the experimental results by Kim et al and Tanino are performed at higher Reynolds numbers. In the LBM method, fluid flow with high Reynolds number can be utilized by implementing a Multiple Relaxation Time scheme (MRT) and a Smogrosky LES-LBM model. Also, the current LBM model can be used to simulate higher Reynolds number flows with a fine grid resolution. However, a fine grid resolution simulation increases the computational cost. In future works, An MRT-LBM scheme will be developed to simulate the fluid flow at higher Reynolds numbers. In addition, a Smogrosky-LES model will be tested for drag coefficient calculations of turbulent fluid flows.

\section{Conclusion}

A computational model based on the Lattice Boltzmann method (LBM) has been developed to predict the drag coefficient for idealized vegetation structure including inline, staggered and randomly distributed configurations. The drag coefficient for a single cylinder in the cross flow has been calculated and validated with available experiments at relatively low Reynolds numbers of 20, 40 and 100. Additional simulations, with different cylinder configurations in cross flow, were performed at a Reynolds number of 100. The LBM simulations are compared with experimental and numerical studies in the literature. The LBM based simulations closely match with the numerical simulations presented in the literature. And the simulated results match with the experimental measurements reasonably well. The differences between the experimental measurements and the simulations are due to the different flow conditions i.e. higher Reynolds number flow used in the experimental work, the number of the cylinders used in the staggered and inline arrays, and the solid fractions. The model will be used to calculate the convective heat transfer and drag coefficient for complex geometries representative of vegetation structure. The prediction of this model can be used in WFDS or other CFD models to improve the modeling of vegetation, through a more accurate estimation of the drag coefficient.

\section{References}

A.A. Mohamad. (2011). Lattice Boltzmann method: fundamentals and engineering applications with computer codes. London: Springer-Verlag.

Bao, Y.B. and Meskas, J. (2011). Lattice Boltzmann method for fluid simulations. . New York: Department of Mathematics, Courant Institute of Mathematical Sciences, New York University.

Braza, M., Chassaing, P., Ha Minh, H. (1986). Numerical study and physical analysis of the pressure and velocity fields in the near wake of a circular cylinder. Journal of Fluid Mechanics , 165, 79130.

Chen, S. and Doolen, G.D. (1998). Lattice Boltzmann method for fluid flows. Annual review of fluid mechanics, 30(1), 329-364.

Fischer-Antze, T., Stoesser, T., Bates, P. and Olsen, N.R.B. (2001). 3D numerical modelling of openchannel flow with submerged vegetation. Journal of Hydraulic Research, 39(3), 303-310.

Gallivan, M.A., Noble, D.R., Georgiadis, J.G. and Buckius, R.O. (1997). An evaluation of the bounceback boundary condition for lattice Boltzmann simulations. International Journal for Numerical Methods in Fluids, 25(3), 249-263.

J.L. Dupuy, D. Morvan. (2005). Numerical study of a crown fire spreading toward a fuel break using a multiphase physical model. International Journal of Wildland Fire, 14(2), 141-151.

Kim, S.J. and Stoesser, T. (2011). Closure modeling and direct simulation of vegetation drag in flow through emergent vegetation. Water Resources Research, 47(10). 
Lam, K., Gong, W.Q. and So, R.M.C. (2008). Numerical simulation of cross-flow around four cylinders in an in-line square configuration. Journal of Fluids and Structures, 24(1), 34-57.

Larini, M., Giroud, F., Porterie, B. and Loraud, J.C.,. (1988). A multiphase formulation for fire propagation in heterogeneous combustible media. International Journal of Heat and Mass Transfer, 41(6-7), 881-897.

Mei, R., Yu, D., Shyy, W. and Luo, L.S. (2002). Force evaluation in the lattice Boltzmann method involving curved geometry. Physical Review E, 65(4), p.041203.

Mell, W., Maranghides, A., McDermott, R. and Manzello, S.L. (2009). Numerical simulation and experiments of burning douglas fir trees. Combustion and Flame, 156(10), 2023-2041.

Tanino, Y., and H. M. Nepf. (2008). Laboratory investigation of mean drag in a random array of rigid, emergent cylinders. ASCE, J. Hydraul. Eng., 134(1), 34-41.

Tritton D. (1959). Experiments on the flow past a circular cylinder at low Reynolds numbers. Journal of Fluid Mechanics, 6(4), 547-567.

U.S. Forest Service. (n.d.). U.S. Forest Service. Retrieved June 26, 2018, from https://www.fs.fed.us/managing-land/fire

Wang, J., Wang, M. and Li, Z. (2007). A lattice Boltzmann algorithm for fluid-solid conjugate heat transfer. International journal of thermal sciences, 46(3), 228-234.

Zhou, X., Mahalingam, S. and Weise, D. (2007). Proceedings of the Combustion Institute. Experimental study and large eddy simulation of effect of terrain slope on marginal burning in shrub fuel beds, 31(2), 2547-2555.

Zou, Q. and He, X. (1997). Pressure and velocity boundary conditions for the lattice Boltzmann. J. Phys. Fluids, 9, 1591-1598. 


\title{
Mathematical modeling of the forest fires initiation, spread and impact on buildings
}

\author{
Valeriy Perminov \\ Tomsk Polytechnic University. Lenin Avenue. 30, 634050. Tomsk. Russia, \{perminov@tpu.ru\}
}

\begin{abstract}
The protection of buildings and structures in a community from destruction by fire is a very important concern. At present, fire services can forecast the danger rating of, or the specific weather elements relating to, forest fire. There is need to understand and predict forest fire initiation, behavior and impact of fire on the buildings and constructions. This paper's purposes are the improvement of knowledge on the fundamental physical mechanisms that control forest fire behavior. The mathematical modeling of forest fires actions on buildings and structures has been carried out to study the effects of fire intensity and wind speed on possibility of ignition of buildings. The modeling approach is based on the use of standard non-stationary threedimensional conservation equations that are solved numerically under the input conditions specific for large crown forest fires. A multiphase mathematical model of wind-aided crown forest fires propagating through heterogeneous fuel beds has been performed. It takes into account the hydrodynamic aspects of the flow and uses Arrhenius kinetics to describe the basic physics and chemical processes of thermal decomposition heating, drying, pyrolysis, and combustion. Turbulence and radiation are considered in order to improve the physical insight. It allows to investigate the dynamics of the impact of forest fires on buildings under the influence of various external conditions: a) meteorology conditions (air temperature, wind velocity etc.), b) type (various kinds of forest combustible materials) and their state (load, moisture etc.). The calculations let to get the maximum distance from the fire to the building in which the object possible ignition. It has been found that the effect of increasing the wind speed is to increase the safety distances between forest and building. The increasing of building height is observed also led to increase the safety distances between forest and building. Specific experiments are also needed to obtain more reliable information on validation of further solution of this problem. The paper was supported from RFBR (project code: № 16-41-700022 p_a) and within the framework of Tomsk Polytechnic University Competitiveness Enhancement Program grant.
\end{abstract}

Keywords: mathematical model, forest fire, crown, ignition, spread of fire, pyrolysis, combustion

\section{Introduction}

The protection of buildings and structures in a community from destruction by forest fire is a very important concern. At present, fire services can forecast the danger rating of, or the specific weather elements relating to forest fire. A great deal of work has been done on the theoretical problem of forest fires. The first accepted method for prediction of crown fires was given by Rothermal (1972) and Van Wagner (1977). The semi-empirical models allow to obtain a quite good data of the forest fire rate of spread as a function of fuel bulk and moisture, wind velocity and the terrain slope. But these models use data for particular cases and do not give results for general fire conditions. Also crown fires initiation and hazard have been studied and modeled in detail (eg: Alexander, 1979, Xanthopoulos, 1990, Van Wagner (1999), Cruz and et al., 2002, Albini, 1995, Scott and Reinhardt, 2001). A mathematical model of forest fires was obtained by Grishin (1997) based on an analysis of known and original experimental data, and using concepts and methods from reactive media mechanics. The physical two-phase models used by Morvan (2004) may be considered as a development and extension of the formulation proposed by Grishin. There is need to understand and predict forest fire initiation, behavior and impact of fire on the buildings and constructions. This paper's purposes are the improvement of knowledge on the fundamental physical mechanisms that control forest fire behavior.

Advances in Forest Fire Research 2018 - Page 808 
The mathematical modeling of forest fires actions on buildings and structures has been carried out to study the effects of fire intensity and wind speed on possibility of ignition of buildings. The dangerous distances between forest and buildings are calculated in cases when the buildings will be ignited under the influence of forest fires.

\section{Physical and mathematical model}

It is assumed that the forest during a forest fire can be modeled as 1) a multi-phase, multistoried, spatially heterogeneous medium; 2 ) in the fire zone the forest is a porous-dispersed, two-temperature, single-velocity, reactive medium; 3) the forest canopy is supposed to be non - deformed medium (trunks, large branches, small twigs and needles), which affects only the magnitude of the force of resistance in the equation of conservation of momentum in the gas phase, i.e., the medium is assumed to be quasi-solid (almost non-deformable during wind gusts); 4) let there be a so-called "ventilated" forest massif, in which the volume of fractions of condensed forest fuel phases, consisting of dry organic matter, water in liquid state, solid pyrolysis products, and ash, can be neglected compared to the volume fraction of gas phase (components of air and gaseous pyrolysis products); 5) the flow has a developed turbulent nature and molecular transfer is neglected; 6) gaseous phase density doesn't depend on the pressure because of the low velocities of the flow in comparison with the velocity of the sound. Let the point $x_{1}, x_{2}, x_{3}=0$ is situated at the center of the surface forest fire source at the height of the roughness level, axis $0 x_{1}$ directed parallel to the Earth's surface to the right in the direction of the unperturbed wind speed, axis $O x_{2}$ directed perpendicular to $O x_{1}$ and axis $0 x_{3}$ directed upward (Fig. 1). The building is situated on the right part of the picture.

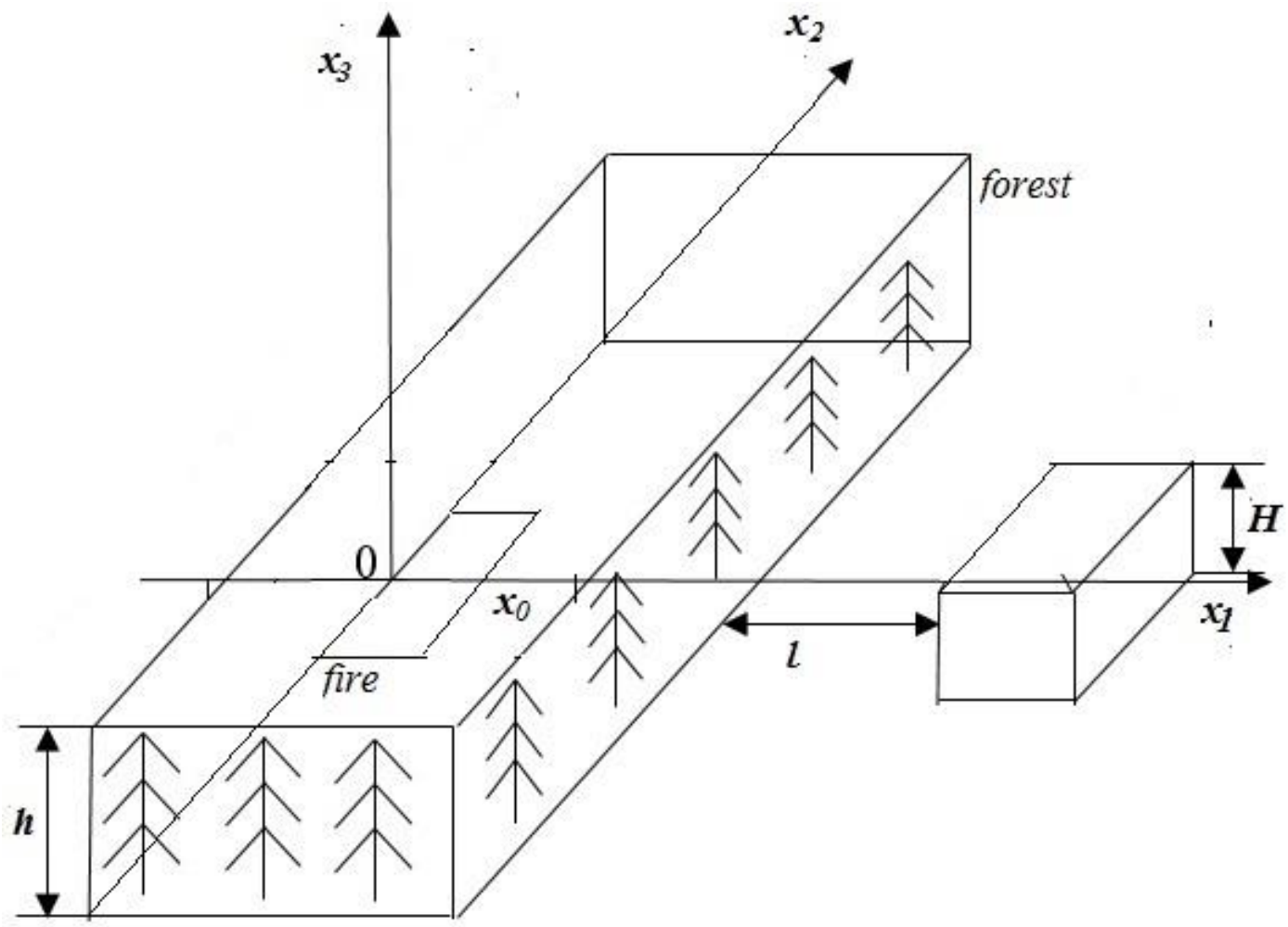

Figure 1 - The scheme of calculation domain.

Problem formulated above reduces to the solution of systems of equations (1)-(7): 
$\frac{\partial \rho}{\partial t}+\frac{\partial}{\partial x_{j}}\left(\rho v_{j}\right)=Q, j=\overline{1,3}, i=\overline{1,3}$

$\rho \frac{d v_{i}}{d t}=-\frac{\partial P}{\partial x_{i}}+\frac{\partial}{\partial x_{j}}\left(-\rho{\overrightarrow{v_{i}^{\prime}}}_{j}^{\prime}\right)-\rho s c_{d} v_{i}|\vec{v}|-\rho g_{i}-Q v_{i} ;$

$\rho c_{p} \frac{d T}{d t}=\frac{\partial}{\partial x_{j}}\left(-\rho c_{p} v_{j}^{\prime} \overline{T^{\prime}}\right)+q_{5} R_{5}-\alpha_{v}\left(T-T_{s}\right)+k_{g}\left(c U_{R}-4 \sigma T^{4}\right) ;$

$\rho \frac{d c_{\alpha}}{d t}=\frac{\partial}{\partial x_{j}}\left(-\rho \overline{v_{j}^{\prime} c_{\alpha}^{\prime}}\right)+R_{5 \alpha}-Q c_{\alpha}, \alpha=1,2 ;$

$\frac{\partial}{\partial x_{j}}\left(\frac{c}{3 k} \frac{\partial U_{R}}{\partial x_{j}}\right)-k c U_{R}+4 k_{S} \sigma T_{S}^{4}+4 k_{g} \sigma T^{4}=0$,

$k=k_{g}+k_{S}$;

$$
\begin{gathered}
\sum_{i=1}^{4} \rho_{i} c_{p i} \varphi_{i} \frac{\partial T_{S}}{\partial t}==q_{3} R_{3}-q_{2} R_{2}-k_{S}\left(c U_{R}-4 \sigma T_{S}^{4}\right)+\alpha_{v}\left(T-T_{S}\right) ; \\
\rho_{1} \frac{\partial \varphi_{1}}{\partial t}=-R_{1 s}, \rho_{2} \frac{\partial \varphi_{2}}{\partial t}=-R_{2 s}, \rho_{3} \frac{\partial \varphi_{3}}{\partial t}=\alpha_{C} R_{1 s}-\frac{M_{C}}{M_{1}} R_{3 w}, \rho_{4} \frac{\partial \varphi_{4}}{\partial t}=0 ; \\
\sum_{\alpha=1}^{3} c_{\alpha}=1, P_{e}=\rho R T \sum_{\alpha=1}^{3} \frac{c_{\alpha}}{M_{\alpha}}, \vec{v}=\left(v_{1}, v_{2}, v_{3}\right), \vec{g}=(0,0, g) .
\end{gathered}
$$

The system of equations (1)-(7) must be solved taking into account the initial and boundary conditions:

$$
\begin{gathered}
t=0: v_{1}=0, v_{2}=0, v_{3}=0, T=T_{e}, c_{\alpha}=c_{\alpha e}, T_{s}=T_{s e}, \varphi_{i}=\varphi_{i e} ; \\
x_{1}=0: v_{1}=V, v_{2}=0, v_{3}=0, T=T_{e}, c_{\alpha}=c_{\alpha e},-\frac{c}{3 k} \frac{\partial U_{R}}{\partial x_{1}}+\frac{c}{2} U_{R}=0 ; \\
x_{1}=x_{1 e}: \frac{\partial v_{1}}{\partial x_{1}}=0, \frac{\partial v_{2}}{\partial x_{1}}=0, \frac{\partial v_{3}}{\partial x_{1}}=0, \frac{\partial T}{\partial x_{1}}=0, \frac{\partial c_{\alpha}}{\partial x_{1}}=0, \frac{c}{3 k} \frac{\partial U_{R}}{\partial x_{1}}+\frac{c}{2} U_{R}=0 ; \\
x_{2}=-x_{2 e}: \frac{\partial v_{1}}{\partial x_{2}}=0, \frac{\partial v_{2}}{\partial x_{2}}=0, \frac{\partial v_{3}}{\partial x_{2}}=0, \frac{\partial T}{\partial x_{2}}=0, \frac{\partial c_{\alpha}}{\partial x_{2}}=0,-\frac{c}{3 k} \frac{\partial U_{R}}{\partial x_{2}}+\frac{c}{2} U_{R}=0 ; \\
x_{2}=x_{2 e}: \frac{\partial v_{1}}{\partial x_{2}}=0, \frac{\partial v_{2}}{\partial x_{2}}=0, \frac{\partial v_{3}}{\partial x_{2}}=0, \frac{\partial T}{\partial x_{2}}=0, \frac{\partial c_{\alpha}}{\partial x_{2}}=0, \frac{c}{3 k} \frac{\partial U_{R}}{\partial x_{2}}+\frac{c}{2} U_{R}=0 ; \\
x_{3}=0: v_{1}=0, v_{2}=0, \frac{\partial c_{\alpha}}{\partial x_{3}}=0,-\frac{c}{3 k} \frac{\partial U_{R}}{\partial x_{3}}+\frac{c}{2} U_{R}=0, \\
\rho v_{3}=\rho_{0} \omega_{0}, T=T_{0},\left|x_{1}\right| \leq x_{0},\left|x_{2}\right| \leq x_{0}, \\
\rho v_{3}=0, T=T_{e},\left|x_{1}\right|>x_{0},\left|x_{2}\right|>x_{0} ; \\
x_{3}=x_{3 e}: \frac{\partial v_{1}}{\partial x_{3}}=0, \frac{\partial v_{2}}{\partial x_{3}}=0, \frac{\partial v_{3}}{\partial x_{3}}=0, \frac{\partial T}{\partial x_{3}}=0, \frac{\partial c_{\alpha}}{\partial x_{3}}=0, \frac{c}{3 k} \frac{\partial U_{R}}{\partial x_{3}}+\frac{c}{2} U_{R}=0 .
\end{gathered}
$$


Here and above $\frac{\mathrm{d}}{\mathrm{d} t}$ is the symbol of the total (substantial) derivative; $\alpha_{v}$ is the coefficient of phase exchange; $\rho$ - density of gas - dispersed phase, $t$ is time; $v_{i}$ - the velocity components; $T, T_{S}$, temperatures of gas and solid phases, $U_{R}$ - density of radiation energy, $k$ - coefficient of radiation attenuation, $P$ - pressure; $c_{p}$ - constant pressure specific heat of the gas phase, $c_{p i}, \rho_{i}, \varphi_{i}-$ specific heat, density and volume of fraction of condensed phase (1 - dry organic substance, 2 - moisture, 3 condensed pyrolysis products, 4 - mineral part of forest fuel), $R_{i}$ - the mass rates of chemical reactions, $q_{i}$ - thermal effects of chemical reactions; $k_{g}, k_{s}$ - radiation absorption coefficients for gas and condensed phases; $T_{e}$ - the ambient temperature; $c_{\alpha}$ - mass concentrations of $\alpha$-component of gas dispersed medium, index $\alpha=1,2,3$ where 1 corresponds to the density of oxygen, 2 - to carbon monoxide $C O, 3$ - to carbon dioxide and inert components of air; $R$ - universal gas constant; $M_{\alpha}, M_{C}$, and $M$ molecular mass of $\alpha$-components of the gas phase, carbon and air mixture; $g$ is the gravity acceleration; $c_{d}$ is an empirical coefficient of the resistance of the vegetation, $s$ is the specific surface of the forest fuel in the given forest stratum. To define source terms which characterize inflow (outflow of mass) in a volume unit of the gas-dispersed phase, the following formulae were used for the rate of formulation of the gas-dispersed mixture $Q$, outflow of oxygen $R_{51}$, changing carbon monoxide $R_{52}$.

$$
\begin{aligned}
\dot{m} & =\left(1-\alpha_{c}\right) R_{1}+R_{2}+\frac{M_{c}}{M_{1}} R_{3}, \\
Q= & \left(1-\alpha_{c}\right) R_{1}+R_{2}+\frac{M_{c}}{M_{1}} R_{3}, R_{51}=-R_{3}-\frac{M_{1}}{2 M_{2}} R_{5}, R_{52}=v_{g}\left(1-\alpha_{c}\right) R_{1}-R_{5}, R_{53}=0 . \\
R_{1} & =k_{1} \rho_{1} \varphi_{1} \exp \left(-\frac{E_{1}}{R T_{S}}\right), R_{2}=k_{2} \rho_{2} \varphi_{2} T^{-0.5} \exp \left(-\frac{E_{2}}{R T_{S}}\right), R_{3}=k_{3} \rho \varphi_{3} S_{\sigma} c_{1} \exp \left(-\frac{E_{3}}{R T_{S}}\right), \\
R_{5} & =k_{5} M_{2}\left(\frac{c_{1} M}{M_{1}}\right)^{0.5}\left(\frac{c_{2} M}{M_{2}}\right) T^{-2.25} \exp \left(-\frac{E_{5}}{R T}\right) .
\end{aligned}
$$

The initial values for volume of fractions of condensed phases are determined using the expressions:

$$
\varphi_{1 e}=\frac{d\left(1-v_{z}\right)}{\rho_{1}}, \varphi_{2 e}=\frac{W d}{\rho_{2}}, \varphi_{3 e}=\frac{\alpha_{c} \varphi_{1 e} \rho_{1}}{\rho_{3}}
$$

where $d$-bulk density for surface layer, $v_{z}$ - coefficient of ashes of forest fuel, $W$ - forest fuel moisture content. It is supposed that the optical properties of a medium are independent of radiation wavelength (the assumption that the medium is "grey"), and the so-called diffusion approximation for radiation flux density were used for a mathematical description of radiation transport during forest fires. To close the system (1)-(7), the components of the tensor of turbulent stresses, and the turbulent heat and mass fluxes are determined using the local-equilibrium model of turbulence (Grishin, 1997). The system of equations (1)-(7) contains terms associated with turbulent diffusion, thermal conduction, and convection, and needs to be closed. The components of the tensor of turbulent stresses $\rho \overline{v_{i}^{\prime} v_{j}^{\prime}}$, as well as the turbulent fluxes of heat and mass $\overline{\rho v_{j}^{\prime} c_{p} T^{\prime}}, \overline{\rho v_{j}^{\prime} c_{\alpha}^{\prime}}$ are written in terms of the gradients of the average flow properties using the formulas 


$$
\begin{aligned}
& -\rho \overline{v_{i} v_{j}}=\mu_{t}\left(\frac{\partial v_{i}}{\partial x_{j}}+\frac{\partial v_{j}}{\partial x_{i}}\right)-\frac{2}{3} K \delta_{i j},-\rho \overline{v_{j} c_{p} T^{\prime}}=\lambda_{t} \frac{\partial T}{\partial x_{j}},-\rho \overline{v_{j} c_{\alpha}^{\prime}}=\rho D_{t} \frac{\partial c_{\alpha}}{\partial x_{j}} \\
& \lambda_{t}=\mu_{t} c_{p} / \operatorname{Pr}_{t}, \rho D_{t}=\mu_{t} / S c_{t}, \mu_{t}=c_{\mu} \rho K^{2} / \varepsilon
\end{aligned}
$$

where $\mu_{t}, \lambda_{t}, D_{t}$ are the coefficients of turbulent viscosity, thermal conductivity, and diffusion, respectively; $\mathrm{Pr}_{t}, \mathrm{Sc}_{\mathrm{t}}$ are the turbulent Prandtl and Schmidt numbers, which were assumed to be equal to 1 . In dimensional form, the coefficient of dynamic turbulent viscosity is determined using local equilibrium model of turbulence (Grishin, 1997). The length of the mixing path is determined using the formula $l=x_{3} k_{t} /\left(1+2.5 x_{3} \sqrt{c_{d} s / h}\right)$ taking into account the fact that the coefficient of resistance $\mathrm{c}_{\mathrm{d}}$ in the space between the ground cover and the forest canopy base is equal to zero, while the constants $\mathrm{k}_{\mathrm{t}}=0.4$ and $\mathrm{h}=\mathrm{h}_{2}-\mathrm{h}_{1}\left(\mathrm{~h}_{2}, \mathrm{~h}_{1}-\right.$ height of the tree crowns and the height of the crown base $)$. It should be noted that this system of equations describes processes of transfer within the entire region of the forest massif, which includes the space between the underlying surface and the base of the forest canopy, the forest canopy and the space above it, while the appropriate components of the data base are used to calculate the specific properties of the various forest strata and the near-ground layer of atmosphere. This approach substantially simplifies the technology of solving problems of predicting the state of the medium in the fire zone numerically. The thermodynamic, thermophysical and structural characteristics correspond to the forest fuels in the canopy of a different (for example pine) type of forest.

\section{Numerical solution and results}

The boundary-value problem (1)-(14) is solved numerically. A discrete analog was obtained by means of the control volume method using the SIMPLE like algorithm (Patankar, 1981). Difference equations that arise in the course of sampling were resolved by the method of SIP (Patankar, 1981). In order to efficiently solve this problem in a reactive flow the method of splitting according to physical processes was used. The basic idea of this method is based on the information that the physical timescale of the processes is great than chemical. In the first stage, the hydrodynamic pattern of flow and distribution of scalar functions was calculated. Then the system of ordinary differential equations of chemical kinetics obtained as a result of splitting was then integrated. The time step for integrating each function has to be smaller than the characteristic time of physical process to ensure the convergence of the numerical method. The time step was selected automatically. The accuracy of the program was checked by the method of inserted analytical solutions. Analytical expressions for the unknown functions were substituted in (1)-(14) and the closure of the equations were calculated. This was then treated as the source in each equation. Next, with the aid of the algorithm described above, the values of the functions used were inferred with an accuracy of not less than $1 \%$. The effect of the dimensions of the control volumes on the solution was studied by diminishing them. Fields of temperature, velocity, component mass fractions, and volume fractions of phases were obtained numerically. The first stage is related to increasing maximum temperature in the place of ignition with the result that a crown fire source appears. At this process stage over the fire source a thermal wind is formed a zone of heated forest fire pyrolysis products which are mixed with air, float up and penetrate into the crowns of trees. As a result, forest fuels in the tree crowns are heated, moisture evaporates and gaseous and dispersed pyrolysis products are generated. Ignition of gaseous pyrolysis products of the crown occurs at the next stage, and that of gaseous pyrolysis products in the forest canopy occurs at the last stage. As a result of heating of forest fuel elements of crown, moisture evaporates, and pyrolysis occurs accompanied by the release of gaseous products, which then ignite and burn away in the forest canopy. At the moment of ignition, the gas combustible products of pyrolysis burn away, 
and the concentration of oxygen is rapidly reduced. The temperatures of both phases reach a maximum value at the point of ignition. The ignition processes is of a gas - phase nature. At $V \neq 0$, the wind field in the forest canopy interacts with the gas-jet obstacle that forms from the forest fire source and from the ignited forest canopy and burn away in the forest canopy. The isotherms of gas phase components moved in the forest canopy by the action of wind. It is concluded that the forest fire begins to spread. The results of the calculation give an opportunity to consider forest fire spread for different wind velocity, canopy bulk densities and moisture forest fuel. It is considered the effect of forest fire front on the building which is situated near from the forest. The influences of wind velocity and distance between forest and building on ignition of building are studied numerically. The results of calculations can be used to evaluate the thermal effects on the building, located near from the forest fires. The temperature fields of crown forest fire at definite moment will be interacted with the obstacle - building (Figure 2 a) and b)) and ignited it. Fig.2. shoes temperature field at the initial moment of forest fire spread for a wind speed of $15 \mathrm{~m} / \mathrm{s}$.

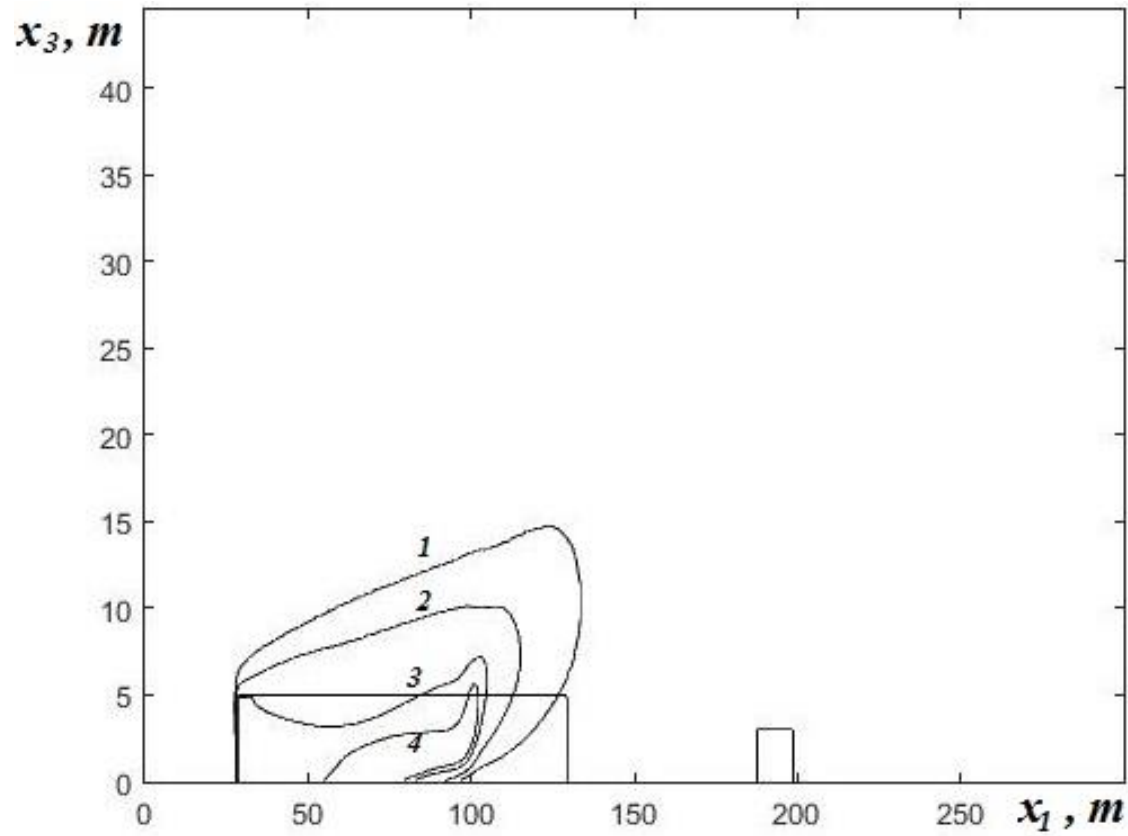

Fiure 2 - Gas temperature field at $t=15 \mathrm{~s}$ for a wind speed of $15 \mathrm{~m} / \mathrm{s} ; 1-1.5,2-2 ., 3-3 ., 4-4 ; \bar{T}=T / T_{e}, \bar{T}_{S}=T_{S} / T_{e}$, $T_{e}=300 K$.

During this process, the surface of the wall of the building heats as a result of convection and radiation heat transfer. The wood building will be ignited at definite temperature. It depends on wind velocities, distances from the forest fire to building, the height of building and others parameters. The Figures 3-5 represent the predicted distributions of temperature on the surfaces of the wall of the building as a function of vertical coordinate for the three selected wind speed values and different distances between forest and building. In paper Valendik and et al. (1979) it is showed that the wood will be ignited when its temperature exceeds $300 \mathrm{C}$. The results of calculations presented on Figures 3 5 show that the surface temperature reach these values at wind velocities more than $6 \mathrm{~m} / \mathrm{s}$. The height of building in these calculations was $H=3 \mathrm{~m}$. 
Advances in Forest Fire Research 2018 - D. X. Viegas (Ed.)

Chapter 4 - Fire at the Wildland Urban Interface

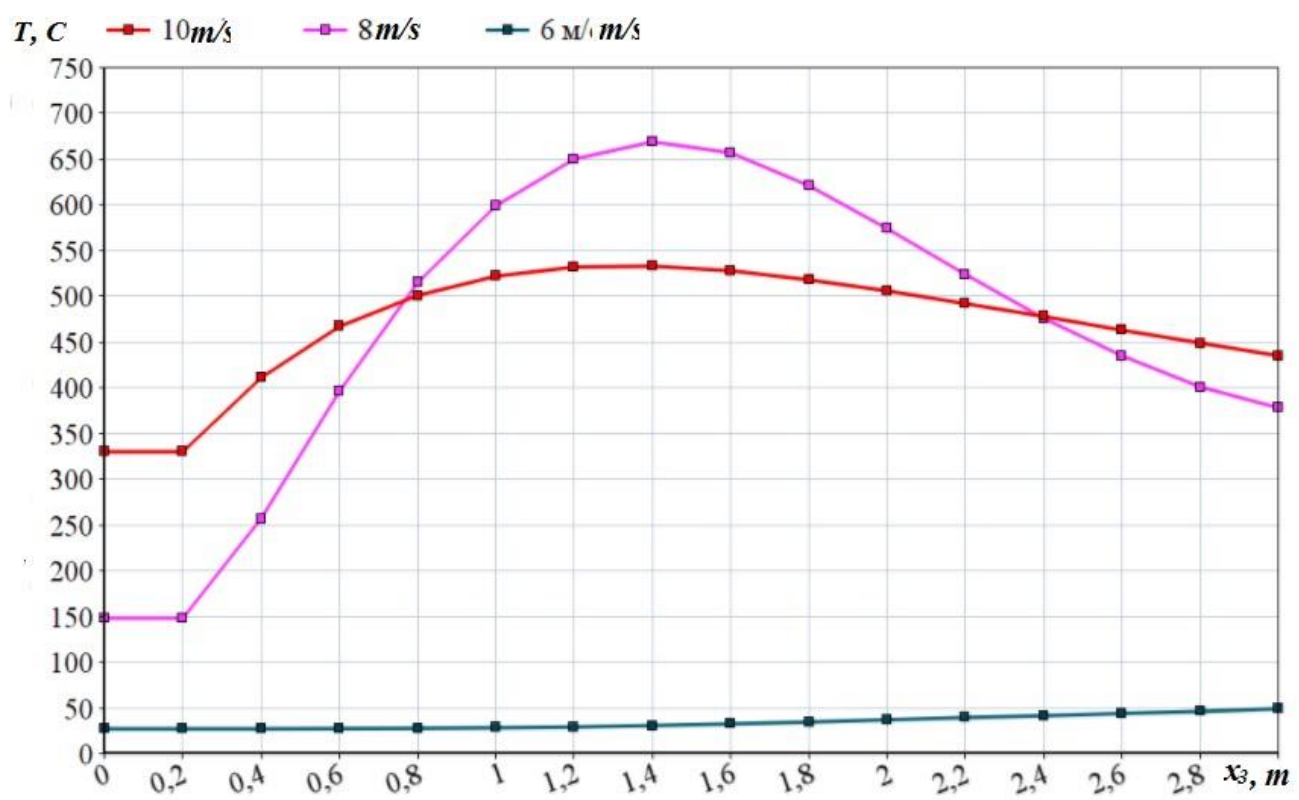

Figure 3 - The distribution of temperature on the wall of the building for three wind speed values. The distance between forest and building is $21 \mathrm{~m}$.

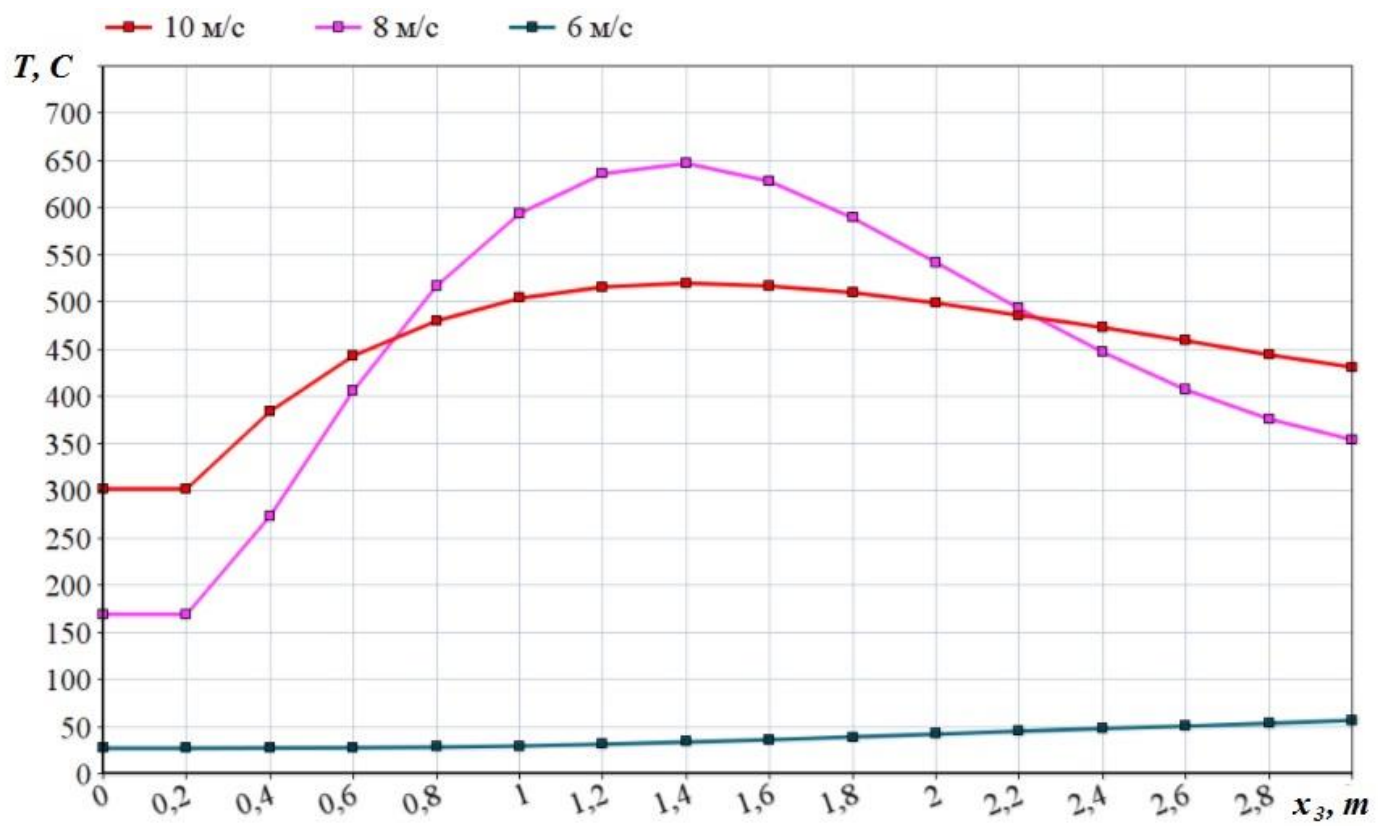

Figure 4 - The distribution of temperature on the wall of the building for three wind speed values. The distance between forest and building is $26 \mathrm{~m}$. 


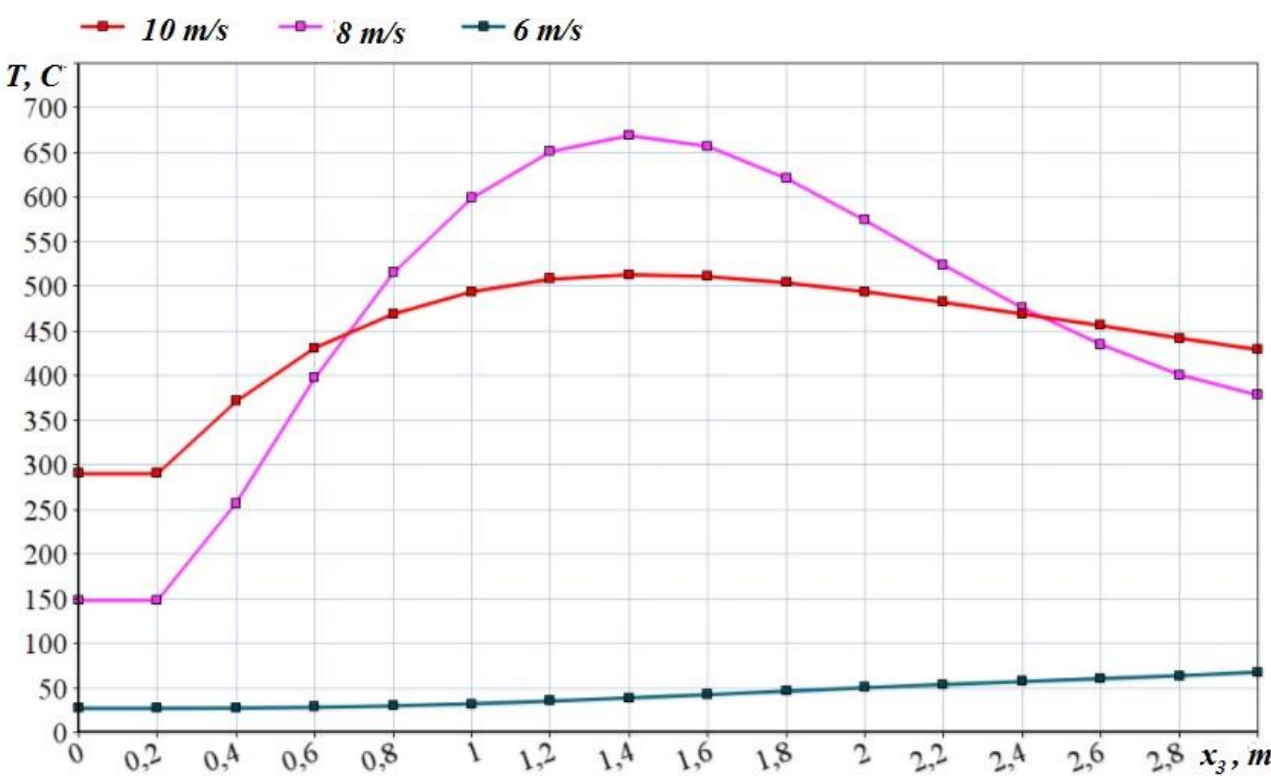

Figure 5 - The distribution of temperature on the wall of the building for three wind speed values. The distance between forest and building is $31 \mathrm{~m}$.

As a result of these calculations it was defined maximum safety distances between forest and building when the building would not have been ignited by forest fire (Fig. 6). The wind speed values increase from 6 to $14 \mathrm{~m} / \mathrm{s}$.

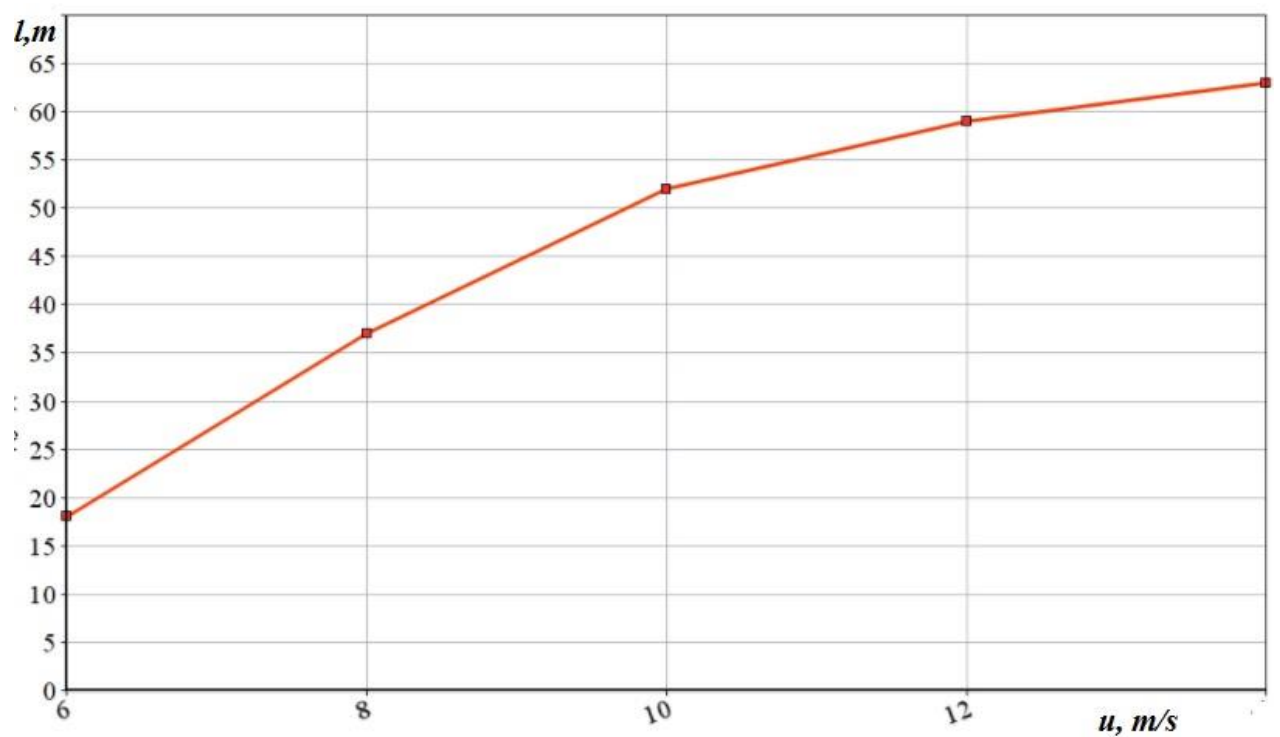

Figure 6 - The dependence of safety distances between forest and building as a function of wind speed values; $H=3 \mathrm{~m}$.

Also, it was studied the influence of the height of building on the value of safety distances. When the height of building changes from 3 to $6 \mathrm{~m}$, the safety distances $l$ also increases (Figure 7) for different values of wind speed. 


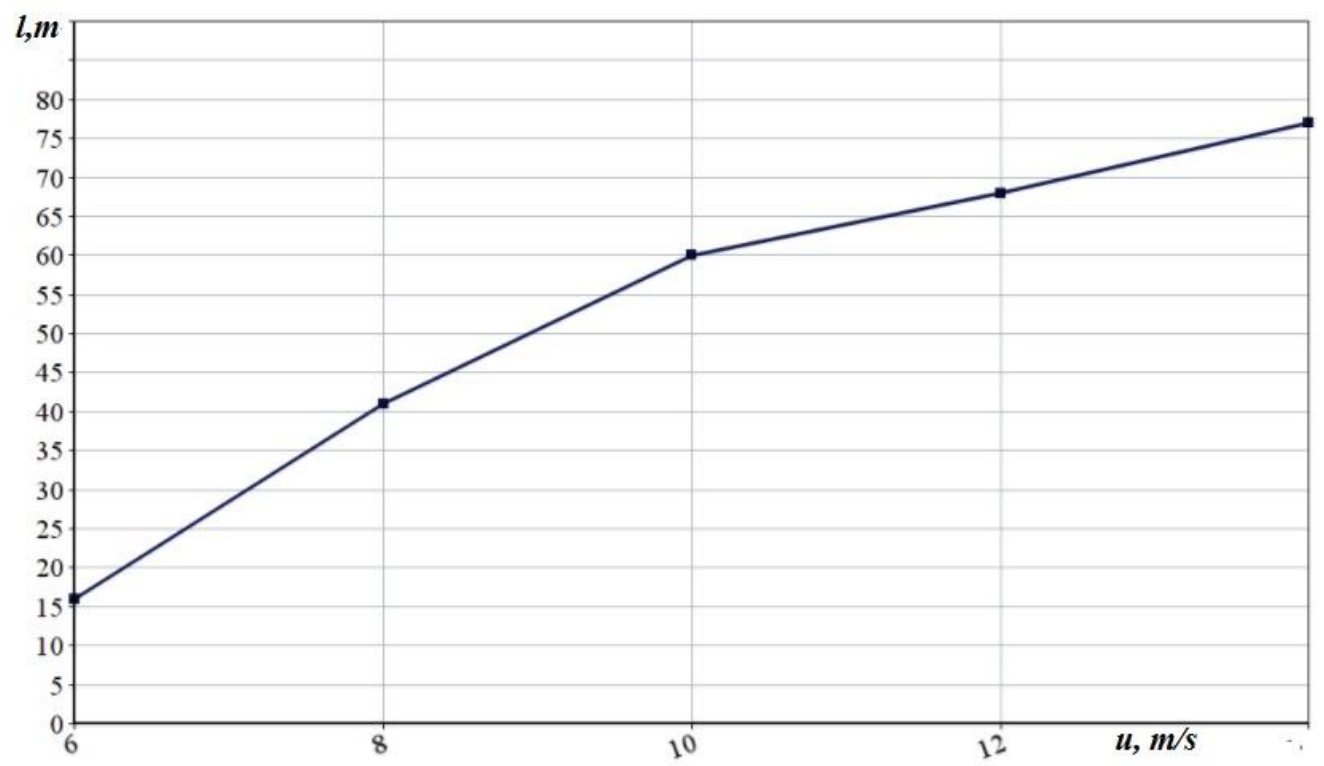

Figure 7 - The dependence of safety distances between forest and building as a function of wind speed values. $H=6 \mathrm{~m}$.

\section{Conclusion}

A mathematical model of wind-aided crown forest fires propagating through heterogeneous fuel beds has been performed. It takes into account the hydrodynamic aspects of the flow and uses Arrhenius kinetics to describe the basic physics and chemical processes of thermal decomposition heating, drying, pyrolysis, and combustion. Turbulence and radiation are considered in order to improve the physical insight. It allows to investigate the dynamics of the impact of forest fires on buildings under the influence of various external conditions: a) meteorology conditions (air temperature, wind velocity etc.), b) type (various kinds of forest combustible materials) and their state (load, moisture etc.). The calculations let to get the maximum distance from the fire to the building in which the object possible ignition. It has been found that the effect of increasing the wind speed is to increase the safety distances between forest and building. The increasing of building height is observed also led to increase the safety distances between forest and building. Specific experiments are also needed to obtain more reliable information on validation of further solution of this problem.

\section{Acknowledgments}

The paper was supported from Russian Foundation for Basic Research (project code: № 16-41700022 p_a) and within the framework of Tomsk Polytechnic University Competitiveness Enhancement Program grant.

\section{References}

Albini FA, Reinhardt ED (1995) Modeling ignition and burning rate of large woody natural fuels. International Journal of Wildland fire 5, 81-91.

Alexander ME (1979) Crown fire thresholds in exotic pine C.E. $10^{\text {th }}$ conference on fire and forest meteorology, Ottawa, Ontario. (Eds D. C. MacIver, H. Auld and R. Whitewood), 207-212.

Cruz MG., Alexander ME, Wakimoto RH (2002) Predicting crown fire behavior to support forest fire management decision-making, IV International Conference on Forest Fire Research, LusoCoimbra, Portugal. (Ed. D. X. Viegas), 11 [CD-ROM]. (Millpress). 
Grishin AM (1997) Mathematical modeling forest fire and new methods fighting them. (Publishing House of Tomsk University: Tomsk)

Morvan D, Dupuy JL. (2004) Modeling the propagation of wildfire through a mediterranean shrub using a multiphase formulation. Combustion and Flame 138, 199-210.

Patankar SV (1981) Numerical heat transfer and fluid flow. (Hemisphere Publishing Corporation: New York).

Rothermal RC (1972) Predicting behaviour and size of crown fires in the Northern Rocky Mountains, USDA Forest Service, Intermountain Forest and Range Experiment Station: Research Paper: INT438. (Ogden, UT).

Scott, JH, Reinhardt (2001) Assessing crown fire potential by linking models of surface and crown fire behavior, USDA Forest Service, Rocky Mountain Research Station. Fort Collins: Research Paper RMRS-RP-29.

Valendik EN, Mathveev PM, Safromov MA (1979) Large forest fires and fighting with them. (Science, Moscow) (in Russian)

Van Wagner CE (1977) Conditions for the start and spread of crown fire. Canadian Journal of Forest Research 7, 23-34.

Van Wagner CE (1999) Prediction of crown fire behavior in two stands of jack pine. Canadian Journal of Forest Research 23, 445-449.

Xanthopoulos G (1990) Development of a wildland crown fire initiation model, PhD thesis. (University of Montana: Montana) 


\title{
Modelling fire spread and damage in wildland-urban interfaces
}

\author{
Fabien Fernandez ${ }^{1 *}$; Bruno Guillaume ${ }^{1}$; Bernard Porterie ${ }^{2}$; Anne Ganteaume ${ }^{3}$; Fabien Guerra ${ }^{3}$ \\ ${ }^{1}$ ARIA Technologies. 8/10, rue de la Ferme - 92100 Boulogne-Billancourt, France \\ \{ffernandez@aria.fr*\} \\ ${ }^{2}$ Aix-Marseille Université, CNRS, IUSTIUMR7343, Marseille \{bernard.porterie@polytech.univ- \\ mrs.fr \} \\ ${ }^{3}$ IRSTEA Aix-en-Provence \{anne.ganteaume@irstea.fr \}
}

\begin{abstract}
Fires in wildland-urban interfaces (WUIs) are currently increasing because of the urban sprawl entailing larger contact zones with wildland areas and the on-going climate change, increasing the frequency and severity of extreme fires, especially in Mediterranean ecosystems. Heterogeneous vegetation in the WUIs, including both natural and ornamental species, allows the fire to propagate from the wildland to housing, which can be responsible for loss of life and considerable property and environmental damages. In the present study, the raster-based fire spread model SWIFFT (De Gennaro et al., 2017) is enhanced to account for fireinduced thermal damages on vegetation and building materials at the WUI. This approach is applied to two historical WUI fires. It is found that the enhanced model is particularly well suited first, to assess the changes in fire behavior, as the fire travels from the natural to the ornamental vegetation, and, second, to estimate damages on buildings. It can also be used to evaluate the effectiveness of fuel treatment strategies on wildfire exposure at the WUI scale, depending on local conditions of wind, topography and vegetation.
\end{abstract}

Keywords: Forest fire, raster-based fire spread model, wildland-urban interface, WUI, fuel treatments, building vulnerability

\section{Introduction}

Significant efforts have been made in the past to provide insights into the pattern of development and expansion of fires at the wildland urban interface (WUI) (Cohen 2000, Cohen 2008), which revealed that the wildland urban interface is a house ignitability problem and not a wildland fire control problem.

Among the many fire spread models (see Sullivan, 2009), only a few have been applied to the WUI problem. There are two main reasons for this. First, the WUI vegetation differs significantly from that of wildland areas as it is composed of a mix of wild and ornamental plant species, generally the latter surrounding buildings. Second, most fire spread models are based on the assumption of fuel continuity and homogeneity, which is somewhat questionable at the WUI. CFD physics-based models, like the wildland-urban fire dynamics simulator (WFDS, Mell et al., 2007), or raster-based models (Sullivan, 2009) are thus good candidates to model WUI fire behavior.

The approach described in this paper aims at bridging this gap, by using a raster-based model inherently designed to model fire patterns in heterogeneous landscapes. The SWIFFT model (De Gennaro et al., 2017) distributes the vegetation on a regular (here, hexagonal) network at a fine resolution. The empirical part of the model provides a description of flammability and combustibility properties of the vegetation items, including canopies. The physical part of the model includes a solid flame model for the burning sites to evaluate radiation and convection effects, as well as an energy conservation equation to describe the thermal degradation (i.e., heating, drying and ignition) of unburnt vegetative elements.

In the current work, SWIFFT is enhanced to model, at a fine scale, the fire propagation through the ornamental vegetation and the fire-induced damages to the vulnerable building materials that can be 
found at the WUI. The initial empirical fuel model in SWIFFT is also improved to fine tune the behavior of ground and canopy fuels exposed to fire (Scott and Reinhardt, 2001).

\section{Methodology}

\subsection{Basic SWIFFT model}

The starting point of the present approach is the fire spread model called SWIFFT, which considers the local conditions of wind, topography, and vegetation and combines (1) a network model to represent vegetation distribution on land with a given vegetation coverage, (2) an empirical flame model using a single burning fuel element per network site, and (3) a thermal model describing the heat transfers from burning to unburnt vegetation items, by radiation and convection processes. The reader might refer to De Gennaro et al. (2017) for more details.

In SWIFFT, the network model is composed of combustible and non-combustible cells. During a simulation, two neighborhoods are identified for each burning site: a radiation circular neighborhood, which includes all the sites that can be potentially exposed to radiation, and a convection rectangular neighborhood, which contains all the sites that can be potentially located in the wake of the burning site (Figure ). Therefore, the thermal response of unburnt fuel elements to heating depends on whether it belongs to the radiation neighborhood, the convection neighborhood, or both. The characteristic length of these neighborhoods is estimated beforehand from numerical trials.
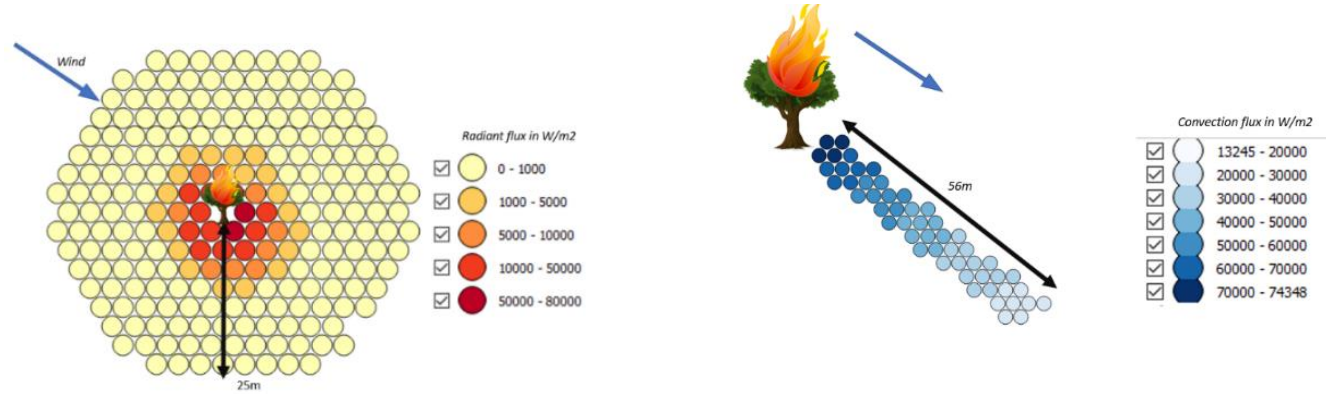

Figure 1 - Radiation neighborhood (left) and convection neighborhood (right) for a flame length of $5.6 \mathrm{~m}$ and a wind speed of 10ms-1. Here, the characteristic length of the radiation neighborhood is $25 \mathrm{~m}$, that of the convection neighborhood is ten times the flame length, thus $56 \mathrm{~m}$.

In the proposed version of the SWIFFT model, a hexagonal network of 3-m-diameter sites is used. Unlike amorphous networks (De Gennaro et al., 2017), the location of each site in a two-dimensional regular network can be easily determined using two indexes, which saves computing time.

The fire here is not simulated from its outbreak, but rather by pre-positioning the flame front slightly upstream of the WUI. This can be done empirically or using visual observations from real fires. This initial fire front is represented as a GIS polygon and the fire properties of all the network sites which belong to this polygon are calculated using the empirical flame model of SWIFFT.

\subsection{Enhancements from the SWIFFT model}

In the original SWIFFT model (De Gennaro et al., 2017), each vegetation site is composed of a single fuel type. Here, we replaced this approach with that of a multi-element fuel scene as in Rothermel et al. (1972). A fuel scene is now described with different constitutive fuel elements: dead fuels, that are referred as $1 \mathrm{hr}, 10 \mathrm{hr}, 100 \mathrm{hr}$ fuels, depending on their tendency to dry, and live fuels (herbs and shrubs). We also added the canopy fire parameterization proposed by Scott and Reinhardt (2001) to account for the increase in fire intensity and residence time due to passive and potentially active crowning, according to some average properties of canopies (height, base height, bulk density, fuel load). In addition to the default fuel map for natural forests, with a 30m-spatial resolution over the zone of interest, we used additional GIS polygons which are intersected with the site network to more 
accurately allocate the fuel types to the sites, for the wildland vegetation, as well as for the ornamental vegetation in the garden. The natural forest vegetation is modelled by a single fuel type in the case studies hereafter, whose combustion properties were kindly provided by the French Forest Service. The combustion properties of canopies (based on the Irstea's fuel database) for the ornamental vegetation of the current case study were determined tree-by-tree in the two case studies.

The weather parameters (wind, temperature, relative humidity and fuel moisture content) are inferred from the nearest weather station. The wind conditions are refined on the local topography at $100 \mathrm{~m}$ resolution from the local scale air mass-conservative model, WindNinja (Forthofer et al., 2009).

\subsection{Coupling SWIFFT with a module of fire-induced thermal degradation of building materials}

To account for the thermal degradation of materials, the vulnerable assets are not modelled as 3D structures in the model. Even if Building Intelligent Models are in growing development worldwide in cities, they are still far from being systematically applied currently, and buildings in WUI can still rarely be modelled with their full 3D structure allocations. In the model, a good compromise is found by using GIS $(2+0,5)$-D polygon objects in the zone of interest, e.g. for each polygon a description is used of (1) a single building height and (2) a list of types of building materials (e.g. glass, wooden construction elements). This nature of information is more largely spread in cities.

Horizontally, the 'vulnerable sites' through the network are identified by crossing the 2D polygon shape with the underlying site network. Vertically, the building's facades are represented as a collection of $1 \mathrm{~m}^{2}$ panels distributed every $2 \mathrm{~m}$ in height. Each facade panel can receive heat flux due to radiation from the flames and hot gas. Ignition can occur depending on the thermal properties of panel materials.

The facade height is measured relatively to the flame base height on ground. Normal vectors to the facade panels are also calculated and then used during the simulation to assess the azimuth angle, characterizing the view angle of the panel as seen by a network site from the incoming fire front.

For radiative preheating of the facade panels, as done similarly for the radiative preheating of unburnt vegetation (see De Gennaro et al., 2017), precalculated tables based on Monte Carlo simulations are used. However, for facade panels, additional parameters are introduced in the table pre-calculation: the panel azimuth angle, the output fluxes are provided on a 3D grid providing the preheating at different stock building levels. Figure illustrates the pre-calculated fluxes on two sample cases. Panels located at negative heights are also considered.

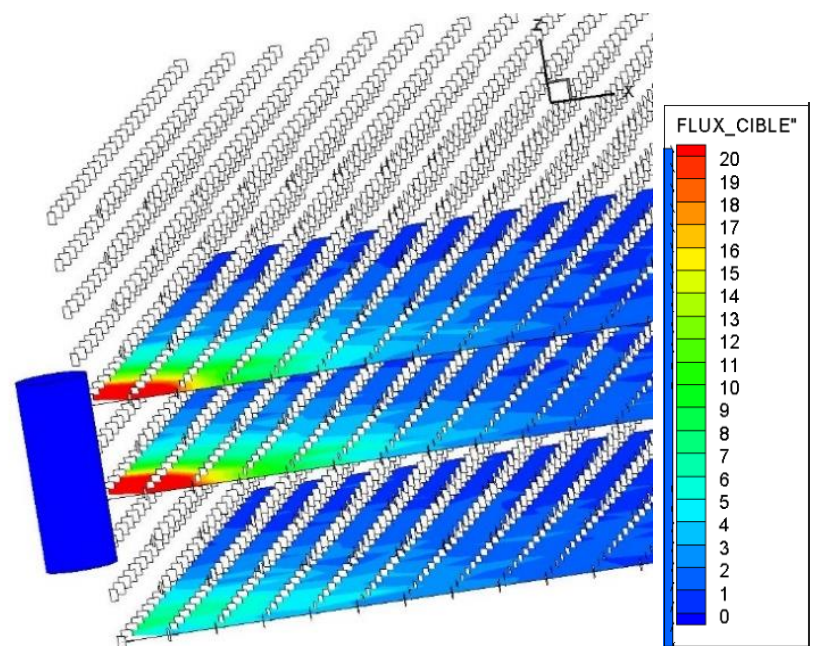

Figure 2 -Sample visualization of pre-calculated radiative fluxes (unit: $k W m-2)$ on the $3 D$ output grid (having resolutions of $d x=2 m, d y=2 m, d z=2 m$ ) with one receptor ('facade panel') positioned at each grid cell having fixed azimuth of $15^{\circ}$, for a selected flame (radius $=1.5 \mathrm{~m}$, length $=7 \mathrm{~m}$ ) for a flame angle $=0^{\circ}$ 
For reasons of symmetry, the pre-calculated fluxes are calculated only in the $\mathrm{X}+, \mathrm{Y}+$ and Z 3Dsubspace. During simulations, the vertical position of the panel height with respect to the flame base takes into account (1) the building stock height at which the panel is located, (2) the building altitude above sea-level and the (3) the flame altitude above sea-level.

For convective preheating of the facade panels, the same rectangular neighborhood is considered as for the convective preheating of the unburnt vegetation. However, the parameterization of the convective heat flux considers the relative vertical position of the panel vs. the flame base.

The case of an active crowning fire is also treated. Therefore, a facade panel can be exposed to the convective fluxes from both surface and canopy burning sites. For the latter, the reference height is taken as half the canopy height.

\subsection{SWIFFT outputs}

The first output of the model is the area burnt in the vulnerable zone, in both natural and ornamental vegetation. Moreover, the model allows the user to identify which sites exhibit surface to crown fire transition. The damage caused by fire on each material type in the vulnerable zone can be evaluated from the model. It depends on the time evolution of the total incident flux (radiation plus convection), and on the material thermal properties. Two ignition criteria are used, either based on a critical temperature or a critical total heat flux. The latter criterion is retained to model the breakage of double glazing windows. The percentages of panels damaged by fire on each building facade is an another ouput of the model. At this stage of development, fire-induced damage in the interior of houses is not considered.

\section{Validation on case studies of WUI impacted by historical fires}

This section summarizes the description of the case studies, as well as model results, in terms of fire behavior and chronology while approaching the two vulnerable zones, and damages on the vulnerable assets. In these two case studies, fire spotting risk was found to have no impact on fire spread and structures, so damage came essentially from the convective heat (hot gases) or direct contact with flames. These case studies have been selected because of their extreme behavior, with total burnt areas of 6744ha for the Vidauban fire and 2663ha for the Rognac fire, due to wind speed exceeding $30 \mathrm{kmh}^{-1}$, air relative humidity below $30 \%$ and severe drought conditions. For both cases, we detail the fire behavior and chronology, the environmental conditions (topography, fuel and weather) in the vulnerable zones during the events, as well as the precise nature of material types of the vulnerable assets exposed to fire.

\subsection{Rognac fire}

The Rognac fire occurred in the afternoon of the $10^{\text {th }}$ of August 2016, spread over 2663ha between $3 \mathrm{pm}$ and $1 \mathrm{am}$ of next day affecting seven communities, especially those of Rognac (where the fire started), Vitrolles and Les Pennes-Mirabeau. When the fire ignited, the firefighting force was limited due to an unexpected firefighting aircraft maintenance that grounded most aircrafts but also to several other fires that burned the same day in nearby locations, including the critical fire in Fos/Mer (more than 1000ha burned) that threatened the oil terminal. These concomitant fire outbreaks caused the splitting of the firefighting force onto different locations and because of this cascade of events, the fire was not contained soon enough as the massive early attack of the fire could not be achieved (usually the fire attack occurs less than 10 minutes after ignition with full force).

The average weather conditions have been reconstituted using data from the nearest meteorological station (i.e. Marseille airport, located less than $2 \mathrm{~km}$ from the vulnerable zone): wind direction of NW, wind speed of $12 \mathrm{~m} / \mathrm{s}$ in the afternoon, relative air humidity of $25 \%$, ambient air temperature of $30^{\circ} \mathrm{C}$. The fine fuel moisture was estimated to $10 \%$. 
The local vulnerable zone of the case study presented here is located on the community of Vitrolles (latitude 43.436105 and longitude 5.262842). It was also possible to piece together the local fire chronology, both from the general fire chronology of the whole fire as well as from precisions provided by the damaged house's owner. The fire arrived from the NW (see Figure ) and, after crossing a road, it restarted in the small forested groves located $100 \mathrm{~m}$ downwind the small residential district.

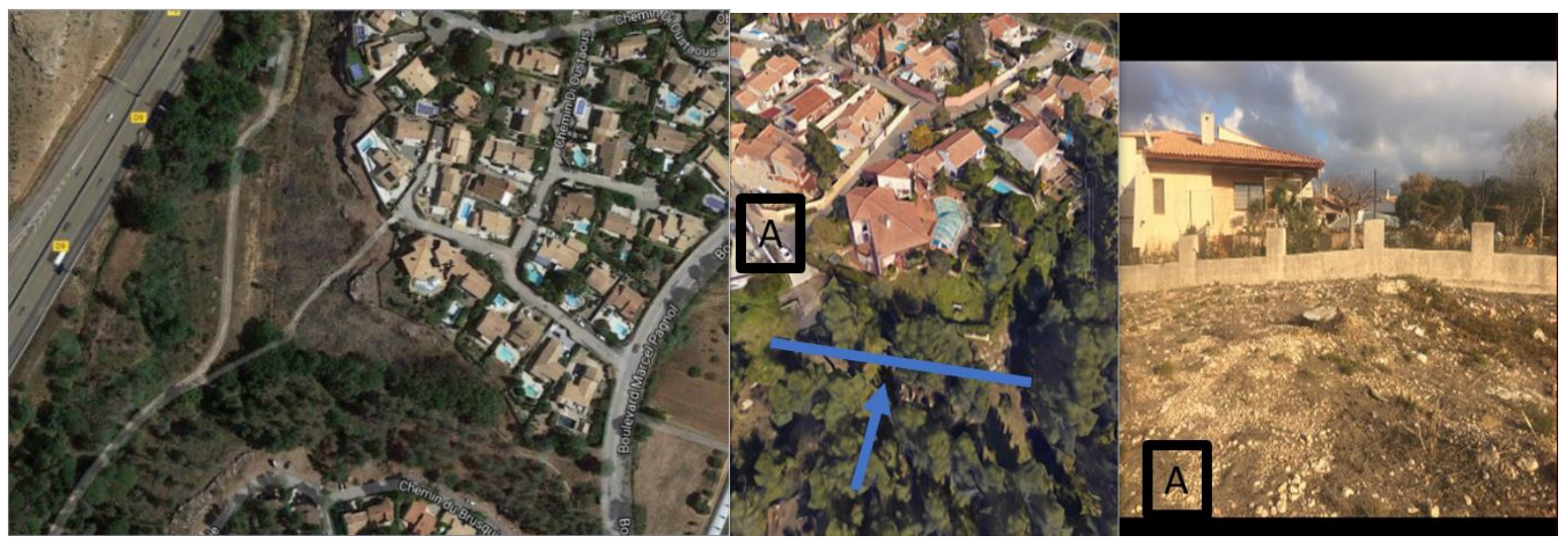

Figure 3 - left: post-fire pattern in the area of interest; middle: G-Earth view with vegetation before the fire (A left view), A right view: view of the cut trees and cleaned zone two years after the fire.

It is worth noting that the understory in the white oak stand (natural vegetation) was reported to be quite dense, since grass residues from a recent cleaning were still laying on the ground. An additional modelling assumption was to use a vegetation coverage of $91 \%$, coherent with the vegetation density as reported on Google-Earth photo before the fire.

We run a simulation of 10 min-duration, with a fire front initially positioned in the northwest corner of the WUI (Figure ). The dominant species of the wild vegetation was white oaks. A single type of material, namely the glass simple pane window, is considered. A critical heat flux for glass breakage of $10 \mathrm{~kW} / \mathrm{m}^{2}$ is used.

A general view of the vulnerable zone is given in Figure 4.
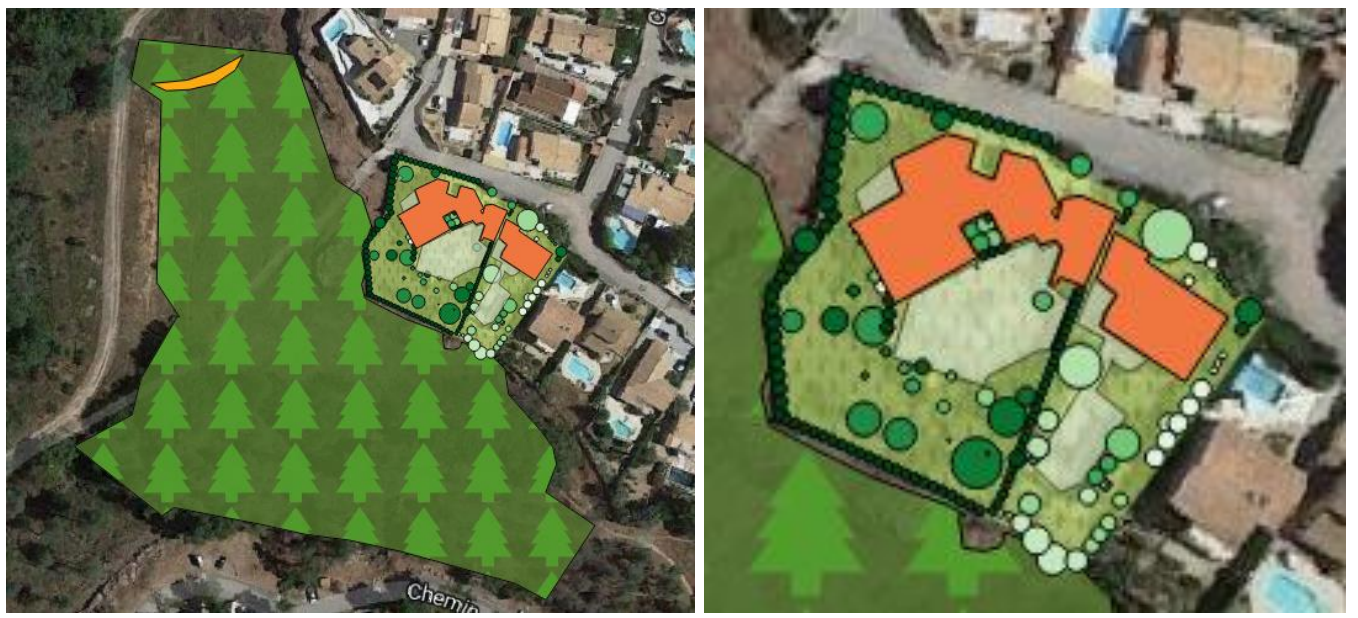

Figure 4 - Rognac fire: visualization of the initial fire front position and the different polygons used to model the natural and ornamental vegetation (in green), non-combustible materials (in grey; swimming-pool, concrete areas, etc.), and combustible material (in orange).

Figure allows the comparison between the observed and the predicted damages. Regarding observed damages, glass breakage occurred on the A1 facade, as reported by the house's owners, leading to an interior fire. Fire propagated from A1 to the ornamental hedge D, as confirmed by the house's owner. From a Google-Earth view after the fire, the ornamental hedges $\mathrm{C}$ can be considered 
as burnt. The facades of house B were kept intact, and the fire did not propagate to the garden. As shown in Figure, the simulation reproduces well the damages on $\mathrm{C}$ and A1 (only 1 on 2 facades), the damages on $\mathrm{D}$, and the no-damages on $\mathrm{B}$. The damaged house A1 was fully exposed to fire due to the continuity from the natural to the ornamental vegetation in the garden. In contrast, the house B was undamaged essentially because it was sheltered from the fire by the damaged house A1, the fire front coming from the NW direction.

The simulated fire propagated throughout the natural vegetation with average spread rate of about $1 \mathrm{kmh}^{-1}$, and average flame length of about $6 \mathrm{~m}$.
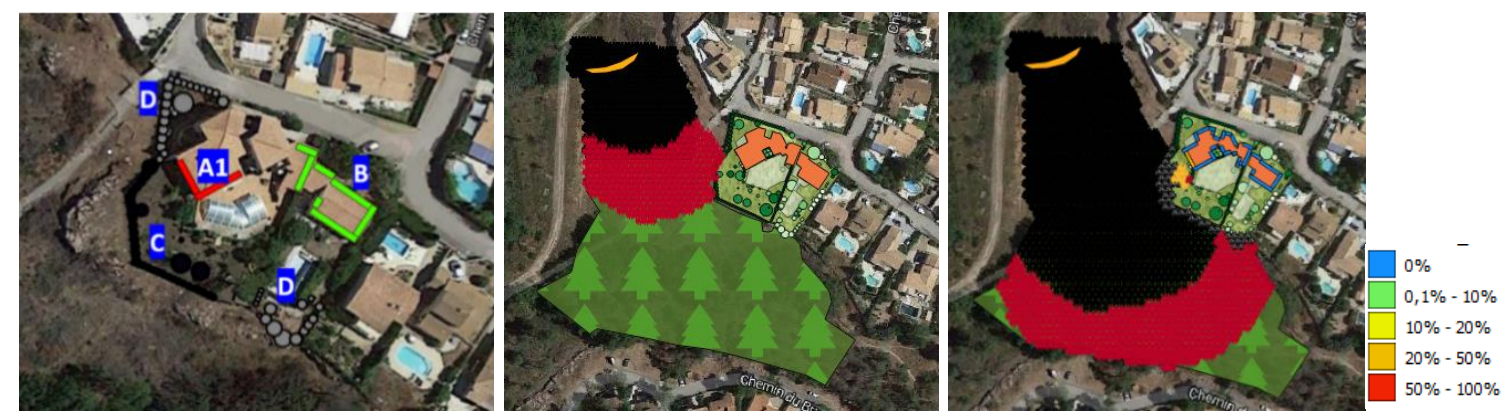

Figure 5 - Rognac fire: comparison between the observed (left) and predicted damages after $300 \mathrm{~s}$ (middle) and $600 \mathrm{~s}$ (right) of fire. For the two latter, the red areas correspond to the burning areas, the black ones to the areas burnt due to crowning, and the grey ones to the areas burnt from surface fire. Contour legend gives the percentages of panels damaged by fire on each building facade.

\subsection{Vidauban fire}

The Vidauban fire occurred on the 17th of July 2003, during a record year regarding heat wave and drought in France. It spread over 6744ha between the city of Vidauban and the Mediterranean Sea, crossing the community of Plan-de-la-Tour, where the studied vulnerable zone is located. The local vulnerable zone presented here is found approximately at location of latitude 43.368216 and longitude of 6.554530. It was possible to piece together the fire relative chronology, not in absolute time, but from its arrival in the studied garden up to when it left the property, because the house's owner had witnessed the fire from inside his house and was able to report the chronology. The fire arrived from the NW in the garden and the fire fighters were not present in this area at that time.

The exact moment of the fire in the vulnerable zone was not reconstituted, and the weather could not be precisely estimated, the nearest Meteo France weather station being too far. We took the average behavior weather conditions from $12 \mathrm{am}$ to $6 \mathrm{pm}$ as representative of the local weather: wind blowing from the $\mathrm{NW}\left(300^{\circ}\right)$ at a speed of about $10 \mathrm{~ms}^{-1}$, ambient temperature of $26^{\circ} \mathrm{C}$, and air relative humidity of $20 \%$. The fine fuel moisture was estimated at $10 \%$.
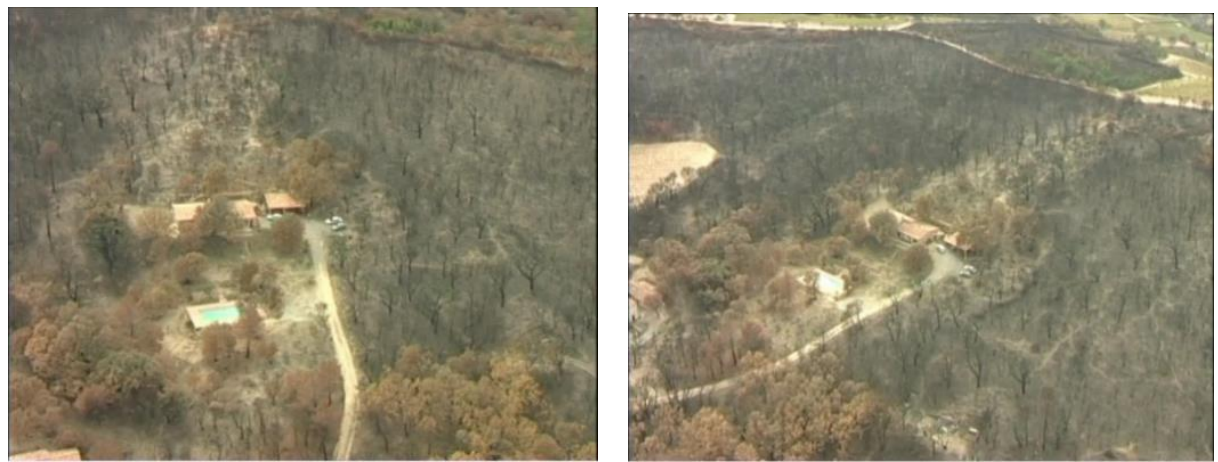

Figure 6 - Vidauban fire: two pictures taken after the fire of the WUI at two different angles of view.

The house was not damaged by the crown fire that arrived from the NW thanks to the brush-clearing of the ground vegetation around the house performed by the owner and the effective tree pruning and 
thinning, up to $1.80 \mathrm{~m}$ from the ground level. As can be seen, the vegetation is scorched but not fully burned as is the case for the vegetation outside the garden.

The owner could describe the entire vegetation present just before the fire, some of which is still in place today. He could also describe the damage that occurred to the building materials.

The natural vegetation is the same as in the previous case study, white oak with partly cleaned understory. A vegetation coverage of $91 \%$ was also used here, in accordance with owner's observations and the distribution of vegetation that was not burnt by fire in the same area. The individual trees, white oaks, have been approximately described tree-by-tree with the owner. Figure exhibits all modelled zones.

A simulation of 1000s-duration was performed, with a fire positioned according to the fire arrival seen by the owner in the natural vegetation, upstream of the two houses. The house A2 was surrounded by natural vegetation (Figure ).

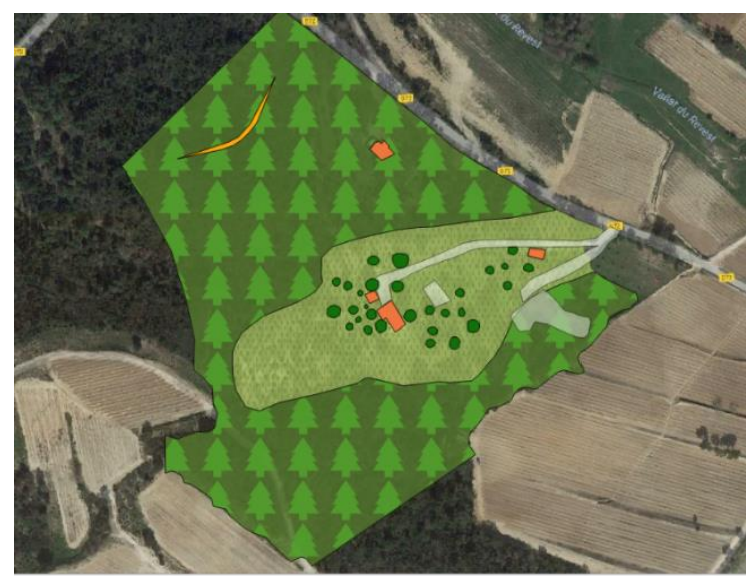

Figure 7 - Vidauban fire: visualization of the initial fire front position and the different polygons used to model the areas covered with natural and ornamental vegetation (in green), those composed of non-combustible (in grey: swimming-pool, concrete areas, etc.) and combustible (in orange) materials.

The observed and predicted damages are shown in Figure. The damages on house A2 are total: the house has been destroyed. The model simulates glass breakage on the two most exposed facades of this house which was in direct contact with the fire. However, the fact that the exterior damage was due to glass breakage is not evident.
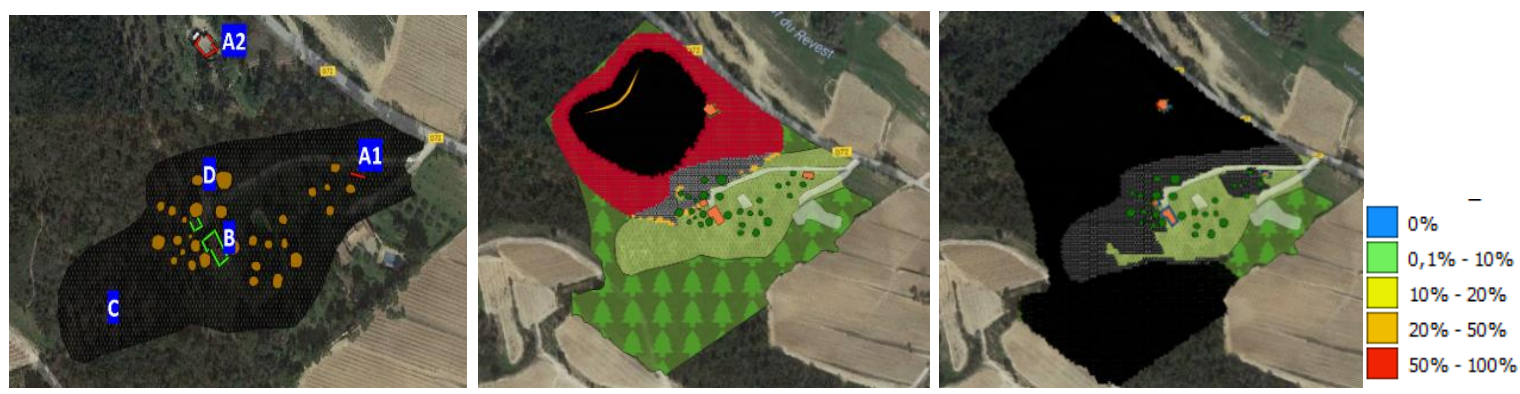

Figure 8 - Vidauban fire: comparison between the observed (left) and predicted (middle and right) damages (for color code see caption of Figure ).

The model exhibits a strong reduction in fire intensity, when it travels from the natural area to the cleared garden. The spread rate of fire is reduced by $2.5\left(2.5 \mathrm{vs} .1 \mathrm{kmh}^{-1}\right)$ and the flame length decreases from 6 to $0.8 \mathrm{~m}$. The fire intensity is so much reduced that the trees in the garden do not exhibit any crowning and that the house is undamaged.

It was observed that the house A1 was very slightly damaged, with only exterior damage. This is not reproduced by the model, which suggests that the grass fire intensity is underestimated. 


\section{Conclusions}

The SWIFFT model has been enhanced to simulate fire patterns in the heterogeneous vegetation (both natural and ornamental) and the external damages on building materials at the WUI. This approach has been tested on two WUI historical fires: the Rognac fire in 2016, and the Vidauban fire in 2003, using glass breakage as a good indicator of external damages on buildings. The observed damages and fire patterns are well reproduced by the model. The approach will be used to evaluate, on private properties or at the scale of districts, the effectiveness of fuel treatment in the reduction of fire risk.

\section{Acknowledgments}

We acknowledge the French Safe Cluster for selecting the project in its Challenge Booster Space4Earth, as well as Climate-KIC for sponsoring this work in the framework of the H2020-OASISinsurance project. We also thank the owners of the houses in the two case studies who took on their own times to provide us very useful information on the state of the houses and gardens during and after the fire.

\section{References}

Butler BW, Cohen JD (1998) Firefighter Safety Zones: A Theoretical Model Based on 494 Radiative Heating. International Journal of Wildland Fire. 8. 73-77.

Cohen J (1995), Structure Ignition Assessment Model, USDA Forest Service Gen. Tech. Rep. PSWGTR-158.

Cohen J (2000) Preventing disaster: Home ignitability in the wildland-urban interface. Journal of Forestry 98(3): 15-21.

Cohen J (2008) The wildland-urban interface fire problem: A consequence of the fire exclusion paradigm. Forest History Today. Fall: 20-26.

De Gennaro M, Billaud Y, Pizzo Y, Garivait S, Loraud J-C, El Hajj M, Porterie B (2017) Real-time wildland fire spread modeling using tabulated flame properties. Fire Safety Journal 91, 872-881. doi: 10.1016/j.firesaf.2017.03.006.

Finney MA (2004) FARSITE: Fire Area Simulator-model development and evaluation. Research Paper RMRS-RP-4 Revised. Ogden, UT: U.S. Department of Agriculture, Forest Service, Rocky Mountain Research Station.

Forthofer J, Shannon K, Butler B (2009) Simulating diurnally driven slope winds with WindNinja, in Proceedings of 8th Symposium on Fire and Forest Meteorological Society.

Mell W, Jenkins MA, Gould J, Cheney P (2007) A physics-based approach to modeling grassland fires. 2007. International Journal of Wildland fire. 16(1): 1-22.

Porterie B, Zekri N, Clerc JP, Loraud JC (2007) Modeling forest fire spread and spotting process with small world networks. Combustion and Flame - Combust. Flame, 149. 63-78. doi: 10.1016/j.combustflame.2006.12.008.

Rothermel RC (1972) A mathematical model for predicting fire spread in wildland fuels. Res. Pap. INT-115. Ogden, UT: U.S. Department of Agriculture, Intermountain Forest and Range Experiment Station. 40 p.

Sullivan A (2009) Wildland surface fire spread modelling, 1990-2007. 1: Physical and quasi-physical models. International Journal of Wildland Fire. 18. 349-368. 10.1071/WF06143. 


\title{
On the use of time-resolved three-dimensional diagnostics to characterize firebrand showers in the WUI
}

\author{
Nicolas Bouvet*; Eric D. Link; Stephen A. Fink, Erica D. Kuligowski \\ National Institute of Standards and Technology, Gaithersburg, MD, U.S.A. \\ \{nicolas.bouvet@nist.gov*\}
}

\begin{abstract}
A time-resolved, three-dimensional particle tracking and sizing diagnostic (a.k.a "emberometer") is currently being developed. It will be applied to airborne firebrands during wildland-urban interface fires; quantification of firebrand exposures would greatly improve the technical basis for WUI building codes and standards to address ignition vulnerabilities. The present paper provides an overview of the current system and reports test results demonstrating the ability of the system to characterize fluxes of artificially generated airborne firebrands.
\end{abstract}

Keywords: Firebrand; Wildland-Urban Interface (WUI); 3D Particle Tracking Velocimetry (3D-PTV); 3D Particle Shape Reconstruction (3D-PSR); firebrand flux; ember flux.

\section{Introduction}

Firebrands generated from burning wildland and structural fuels are known to be lofted into the air and transported up to thousands of meters from the active fire front (Koo et al. 2010). These firebrands can cause spot-fire ignitions of wildland fuels, as well as ignitions of structures built in the WildlandUrban Interface (WUI) (Maranghides and Mell 2009). Existing WUI-related codes and standards (e.g., NFPA 1144, California Building Code Chapter 7A) lack the research support needed to sufficiently address ignition vulnerabilities in the WUI. A detailed characterization of the exposure from firebrands impacting the WUI (e.g., number/mass flux, particle size distribution, particle temperature, etc.) will provide a more complete definition of the WUI fire hazard (Maranghides and Mell 2013), and the technical basis for further improvements to WUI standards. While conventional measurement techniques have essentially focused on time- and space-averaged post-fire data (El Houssami et al. 2016; Filkov et al. 2017; Thomas et al. 2017), very little is known during an actual firebrand assault. The present work is devoted to the development and validation of a time-resolved, three-dimensional (3D) particle tracking and sizing diagnostic applied to airborne firebrands. Among others, the diagnostic intends to resolve Lagrangian trajectories and speeds of firebrands, as well as reconstruct firebrand shapes in 3D from which characteristic dimensions are extracted.

\section{Experiment}

\subsection{Diagnostic description}

In this work, the motion and size of airborne firebrands are resolved using 3D optical techniques. Firebrand motion is studied via 3D Particle Tracking Velocimetry (3D-PTV) which allows for the time-resolved mapping of individual firebrand trajectories. 3D Particle Shape Reconstruction (3DPSR), following the principles of the visual hull concept (Laurentini 1994), is performed at each time step to yield firebrand characteristic dimensions. The following sections provide an overview of the system being developed as well as succinctly describe core steps involved in each technique.

All uncertainties are reported as expanded uncertainties, $X \pm k u_{c}$, from a combined standard uncertainty (estimated standard deviation) $u_{c}$, and a coverage factor $k=2$ (95\% confidence level, 
assuming normal distribution of the data). Results are shown uncorrected for uncertainty arising from systematic effects.

\subsubsection{Emberometer system1}

A photograph and a schematic representation of the system are provided in Figure and Figure , respectively. The measurement device, known as the "emberometer", consists of four compact, consumer-grade cameras (Sony DS-RX10 M3) aimed towards the center of a control volume $\left(\sim 2 \mathrm{~m}^{3}\right)$ located about $1.2 \mathrm{~m}$ away from the camera plane. All cameras are operated at minimum focal length $(f=8.8 \mathrm{~mm})$ and largest aperture $(f / 2.4)$. Focus is performed at the center of the control volume, being easily visualized as the intersection of four collimated lasers (CPS series, Thorlabs), carefully aligned during calibration. The cameras simultaneously record High Definition (HD) videos (1080p, $120 \mathrm{~Hz})$ of airborne firebrands traveling within the measurement volume.

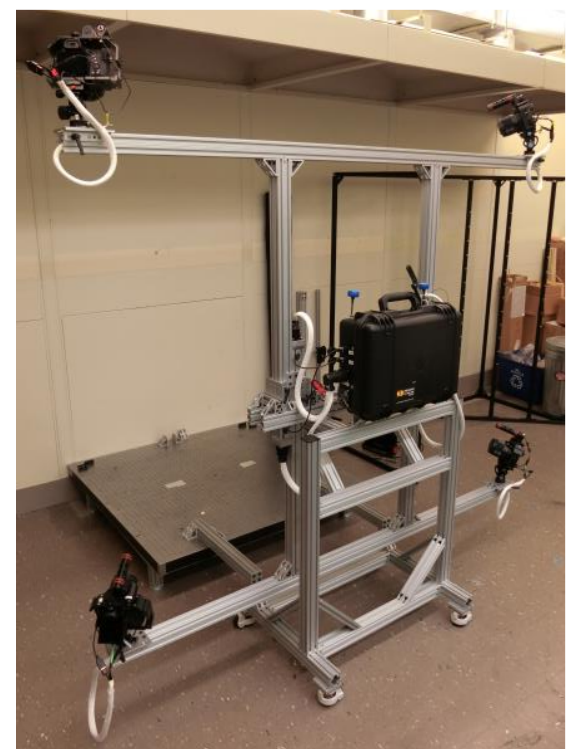

Figure 1 - Photography of the 3D-PTV/PSR system (rear view)

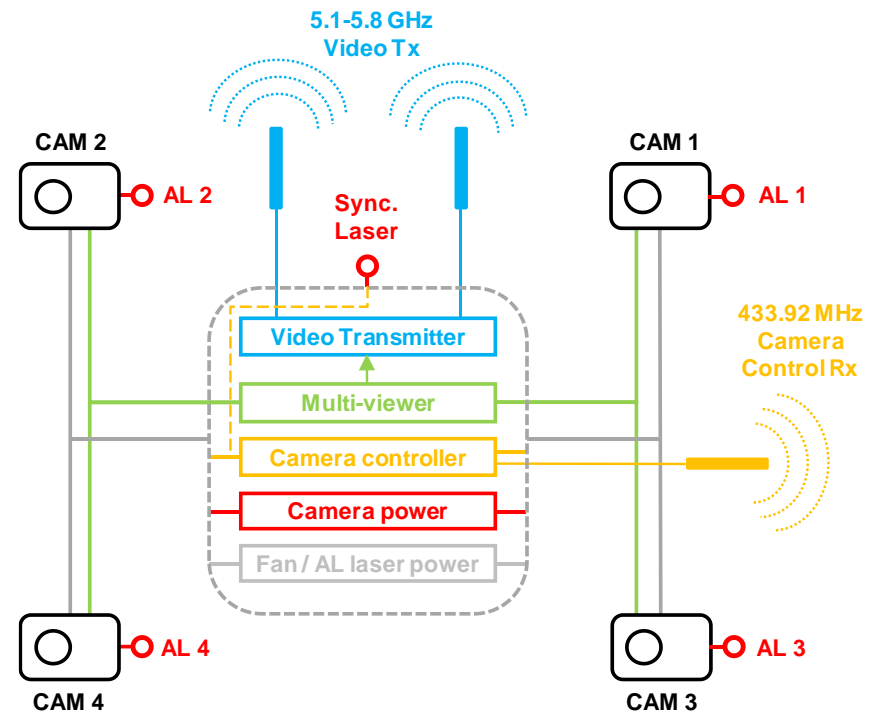

Figure 2 - Schematic representation of 3D-PTV/PSR system (AL.: Alignment Laser)

Camera triggering is achieved via a custom-developed control hub (CAMremote-4CAM, VPSystems) that can be remotely operated by users (RF $433.92 \mathrm{MHz}, \sim 2.4 \mathrm{~km}$ range). The control hub also operates a laser module set to deliver a dot visible from all camera views at the beginning of each recording sequence. The disappearance of the laser dot is used to post-synchronize the video streams across the four cameras in time. This step is essential since each camera has its own response time, even though the initial triggering event is common. Proper video synchronization has been verified by imaging a millisecond counter and is found to be within the time lapse between two consecutive frames.

${ }^{1}$ Certain commercial equipment, instruments, or materials are identified in this paper in order to specify the experimental procedure adequately. Such identification is not intended to imply recommendation or endorsement by the National Institute of Standards and Technology, nor is it intended to imply that the materials or equipment identified are necessarily the best available for the purpose. 
In addition to remote controls, the system is designed to provide a live preview for all cameras, allowing users to monitor image quality and camera settings. To this end, the four video streams are combined into a single HD stream using a quad multi-viewer (Sofly HDSW4-Q). The multi-viewer output is connected to a long range $(\sim 1 \mathrm{~km})$, low latency $(<1 \mathrm{~ms})$ HD video transmitter (Amimon Connex, RF 5.1-5.8 GHz) that delivers the video signal to a portable control rig equipped with a parent receiver. In addition to instant monitoring, users have the possibility to remotely record the four camera views at once for troubleshooting purposes. The system electronics are embedded in an air-cooled, ruggedized package to facilitate setup in case of field deployment.

\subsubsection{Emberometer system flowchart}

The simplified flow chart of the system is given in

Figure . Once acquired, video files are converted into color image sequences $(1920 \times 1080$ px $)$ using a commercial video software. Image sequences are then synchronized and their color space changed to 8-bit grayscale. 3D particle tracking is completed using the open-source software OpenPTV (www.openptv.net). Raw tracking output files are used to generate individual particle identity (PID) files, gathering a time-history of the main particle characteristics (e.g. 3D location, speed, etc.). 3D particle shape reconstruction is then performed for each particle at each time step based on color image information and previously generated PID files. In turn, the PID files are supplemented with size information extracted from the particle 3D models and further used as input for data visualization and analysis. Note that both the tracking and sizing steps require calibration using 3D targets of various nature (not discussed here). All in-house codes are developed using MATLAB and Fortran programming tools.

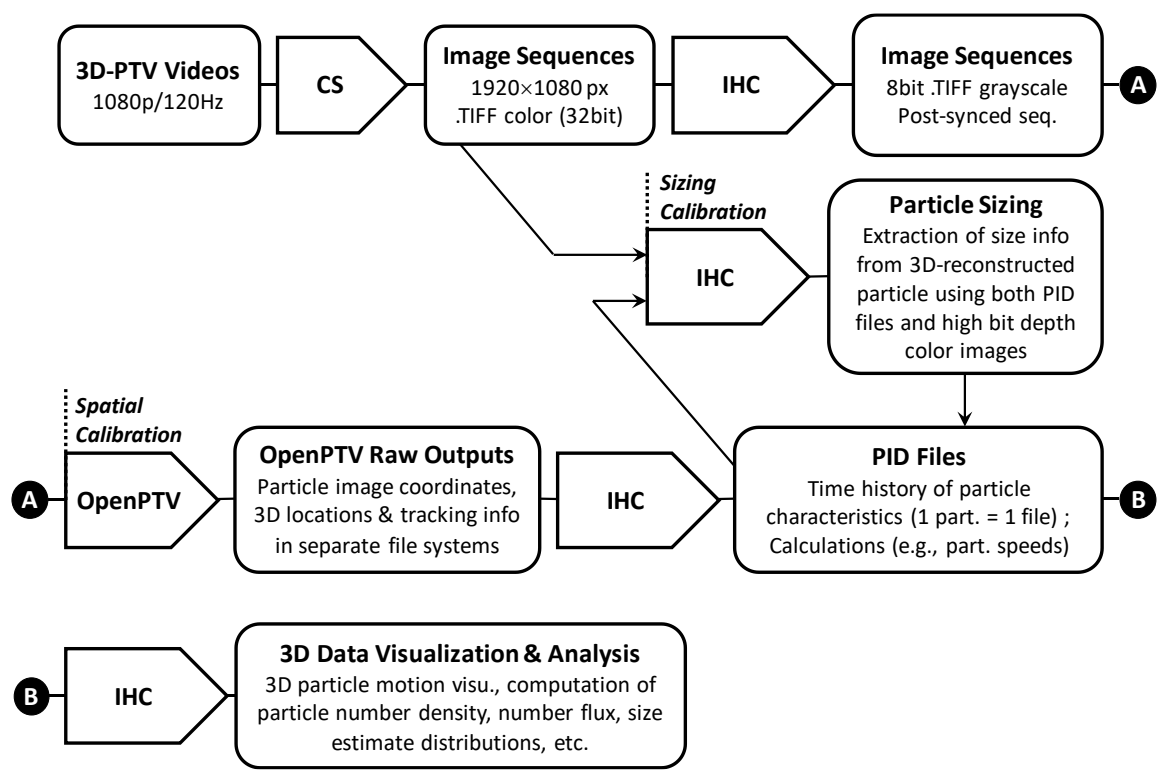

Figure 3- Simplified system flowchart (CS: Commercial Software, IHC: In-House Code)

\subsubsection{D firebrand tracking}

As indicated in the previous section, the 3D tracking of firebrands is computed via the open-source software OpenPTV (www.openptv.net). A detailed description of all operations performed by OpenPTV is clearly outside the scope of the present paper, hence only major steps are mentioned here. The reader is referred to (Willneff 2003) for theoretical insights behind most of these steps, as well as online documentation. The following set of operations are applied:

1- Calibration of the 3D-PTV system: this refers to the 3D spatial calibration of the system. A 3D target is imaged and camera orientation files are generated. These contain essential information related to the camera external parameters (positions and angles of the cameras with respect to 
a chosen referential in the measurement volume) and internal parameters (quantities related to the pinhole model used to represent the cameras, and positioning errors on the camera sensors, for instance due to optical distortions, etc.);

2- Particle detection: particles are detected using an intensity threshold operator. The particle center coordinates (found using a pixel intensity-weighted centroid operator) are listed for each 2D image;

3- Establishing particle correspondences between the different camera views: this is done applying the epipolar line intersection technique, more details can be found in (Luhmann et al. 2014);

4- Computation of $3 D$ coordinates of the particles;

5- Particle tracking: using information found both in the 2D image spaces (2D image from each camera) and 3D object space (previously computed 3D coordinates of the particles).

Steps 1 to 5 allow for the time-resolved mapping of the particle trajectories within the measurement volume. Note that in the present work, airborne firebrands are in the smoldering combustion state and therefore emit a strong orange/yellow light glow. This alleviates the need for illumination usually required in conventional particle tracking experiments to visualize tracers (Mie scattering). Step 2 applies here without any ambiguity, i.e. firebrand are well distinguished from the background elements. An example of a video frame recorded by camera 1 during firebrand generation is given in Figure .

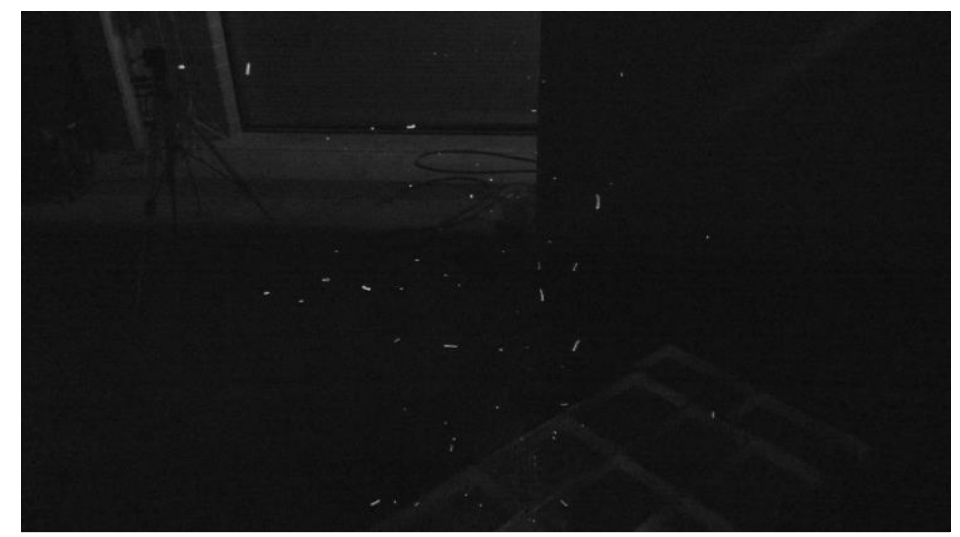

Figure 4 Example of a video frame recorded by camera 1 during firebrand generation (grayscale image).

\subsubsection{D firebrand shape reconstruction}

The 3D firebrand shape reconstruction relies on a multi-projection imaging technique, often referred to as the visual hull method (Laurentini 1994). Various practical implementations of this method have been reported in the literature; the approach adopted in the present work is outlined below. A particle silhouette obtained in a 2D image is back-projected in the measurement space using known camera orientation parameters (obtained from the tracking calibration step) and sizing calibration information. A solid projection cone, taking its origin from the camera optical center and constrained by the contour of the particle back-projected silhouette, can be formed. This process is then repeated from other viewpoints (i.e., in the present case, the 3 other camera views). The intersection of all solid cones created defines the 3D particle shape, which corresponds to the largest volumetric domain that would give identical silhouettes as the particle when observed from each of the different $2 \mathrm{D}$ views. A simplified illustration of the methodology is given in Figure for a setup involving 2 cameras. 


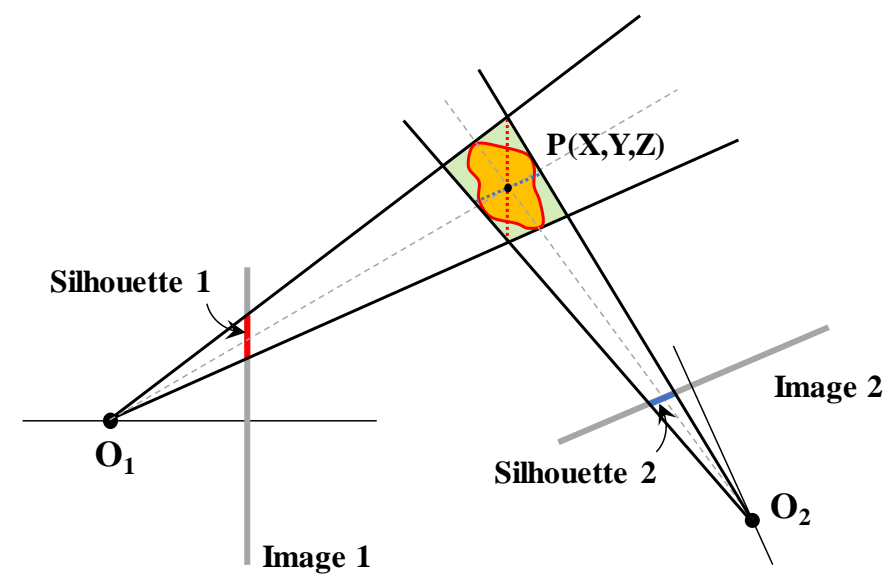

Figure 5 - Simplified 2D sketch illustrating the visual hull method for 3D particle shape reconstruction (only 2 cameras considered here; $O_{i}$ : camera perspective centers, $X, Y$ and $\mathrm{Z}: 3 D$ coord. of the particle centroid).

\subsection{Experimental layout and procedure at the National Fire Research Laboratory}

The emberometer was tested at the NIST National Fire Research Laboratory (NFRL). The experimental layout is shown in Figure. The emberometer was set downstream of the output of a firebrand generator. The generator was arranged to produce a flow of glowing firebrands that crossed the calibrated volume of the system. The firebrand generator assembly consisted of a blower (Cincinnati Fan PB-9) connected in line with cylindrical duct elements whose dimensions and arrangement are similar to those used in a previous firebrand study (Manzello et al. 2008). In the present work, the fuel was made of cylindrical birch/maple dowels (diameter: $6.6 \pm 0.3 \mathrm{~mm}$, length: $50.6 \pm 0.1 \mathrm{~mm}$ ) with chamfered edges. The fuel moisture content was measured with a handheld moisture meter (Delmhorst J-2000) and was confirmed to be less than $6 \%$.

The test with firebrands was run as follows: 1) $350 \pm 1 \mathrm{~g}$ of pristine fuel was introduced in the firebrand generator (see picture in Figure ); 2) the blower speed was set to $5 \mathrm{~Hz}$ and two propane torches (Bernzomatic TS8000) were ignited and inserted slightly below the fuel batch, on opposite sides. Ignition was maintained for $45 \mathrm{~s}$; 3) After $45 \mathrm{~s}$, the propane delivery was stopped, and a $15 \mathrm{~s}$ wait time was observed; 4) the blower speed was set to $20 \mathrm{~Hz}$. Shortly after the blower speed increase, a large flame settled at the generator output. The flame length slowly decreased until complete flame disappearance after $\sim 30 \mathrm{~s}$. The first firebrands were produced shortly after the flame onset $(\sim 3 \mathrm{~s})$, and generation continued for about $75 \mathrm{~s}$. The analysis presented in section 3 concentrates on a $33 \mathrm{~s}$ time window (beginning $27 \mathrm{~s}$ after the flame onset), when the peak production of firebrands occurred.

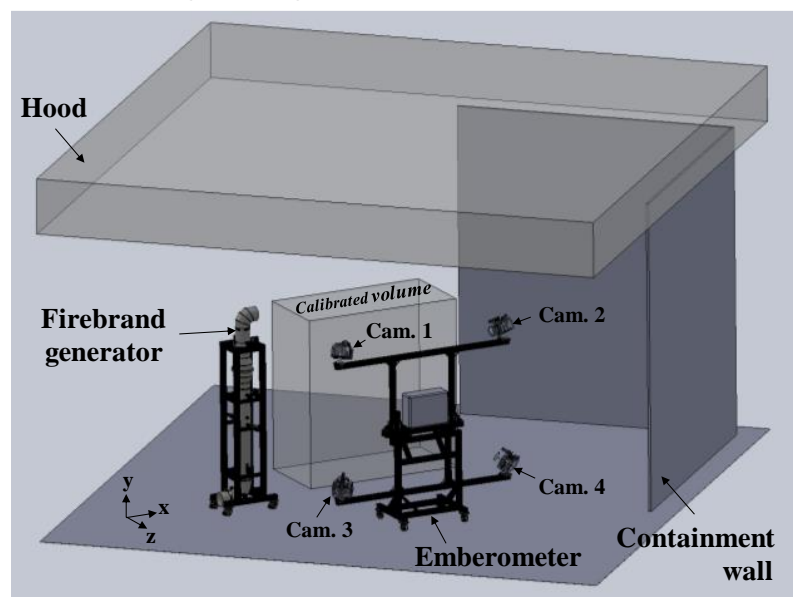

(a)

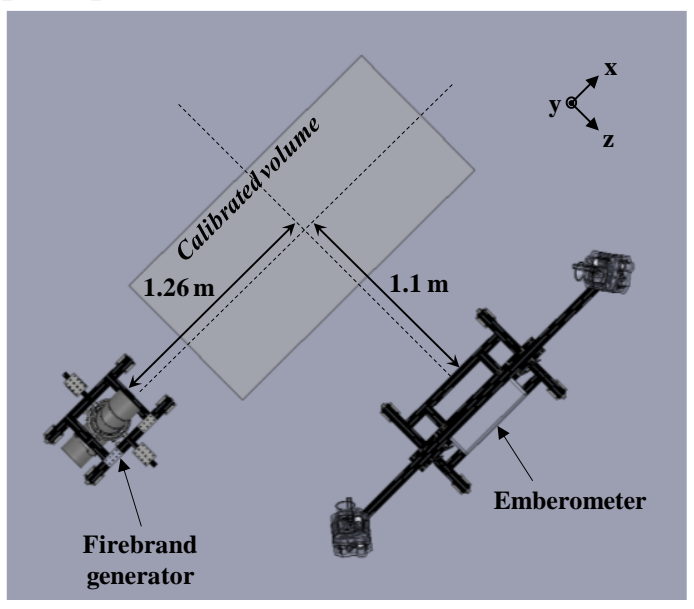

(b)

Figure 6 - Experimental layout for tests at the National Fire Research Laboratory (blower and ignition loop of the firebrand generator not shown here): (a) 3D view; (b) Top view with characteristic dimensions 
Most landing firebrands were collected in aluminum pans filled with water (see arrangement in Figure ) covering an area of about $2.4 \mathrm{~m}^{2}$ and starting $0.56 \mathrm{~m}$ downstream of the firebrand generator exit plane. Collected firebrands were dried and their sizes measured to provide an independent dataset for firebrand size distribution comparison.

Additionally, in-situ validation of the system was performed using non-combusting dowel pieces (sticks identical to those used to generate firebrands) to provide a baseline evaluation of the emberometer measurements with known particles. The sticks were ejected from the firebrand generator without ignition to provide similar trajectories and random behavior as in the combusting case. Sticks were painted white and additional illumination was provided for adequate visibility with the same camera settings as used for combusting particles.

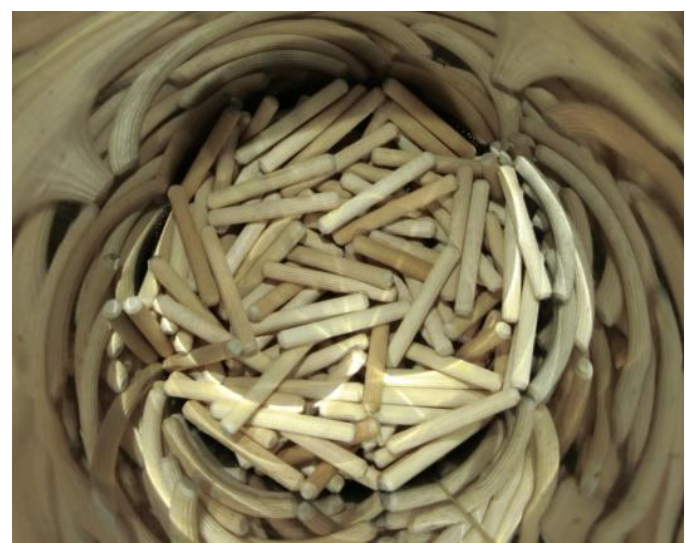

Figure 7 Fuel batch loaded in firebrand generator

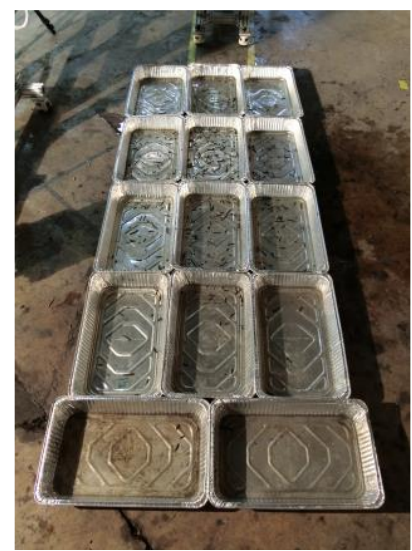

Figure 8 - Water pan arrangement for firebrand collection.

\section{Results}

\subsection{Non-burning sticks}

A total of 43 validation sticks were tracked through the control volume during a $20 \mathrm{~s}$ time period and subsequently reconstructed as described in section 2.1.4. Results of the tracking are shown in Figure . The highlighted plane, chosen to encompass all trajectories, serves as a reference surface for mass flux evaluations.

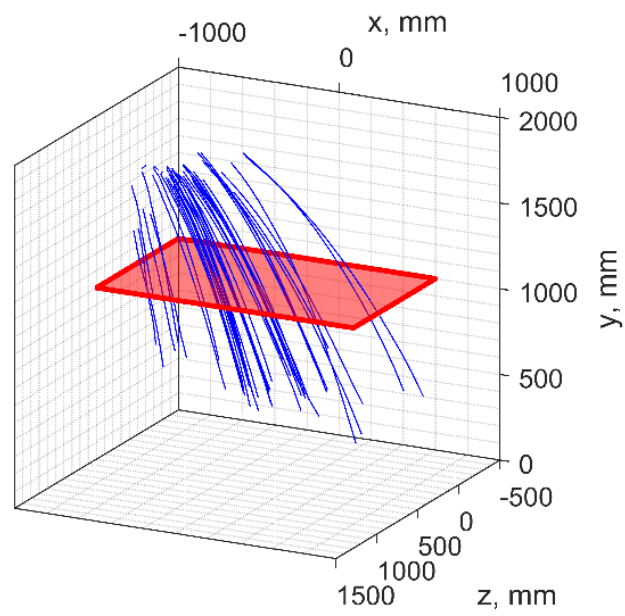

Figure 9 - Tracking results of all 43 validation particles. The highlighted plane was used to evaluate mass flux.

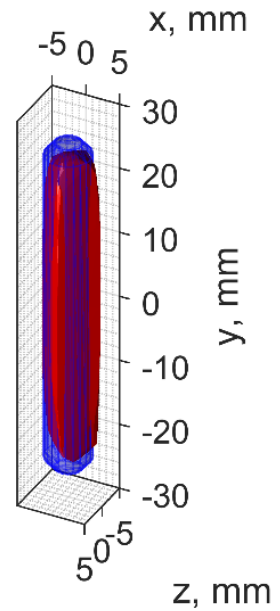

Figure 10 - Example comparison of reconstructed particle (red) with the actual known shape of the noncombusting validation particle (blue). Length $50.6 \mathrm{~mm}$, Diameter $6.6 \mathrm{~mm}$. 
Each of the 43 sticks was reconstructed using the simultaneous camera views as it passed through the control volume. Figure shows an example of a reconstructed particle (red) compared to the true shape (blue) from a single timestep in the video recording. Tracked particles are reconstructed at each timestep. Average shapes and dimensions are obtained by generating cumulative plots of particle edges using all available timesteps (after consistent particle axis re-orientation). Figure shows the resulting orthogonal views of a particle combining each individual measurement of the particle edge (black points). The average measured shape is indicated by the red outline; the blue outline, indicates the true known cylindrical shape.

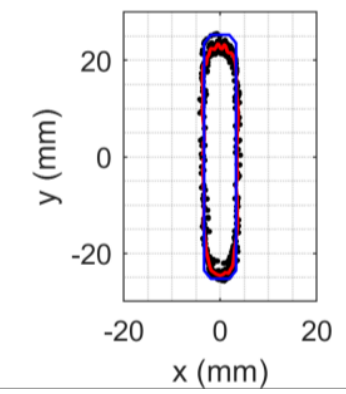

(a)

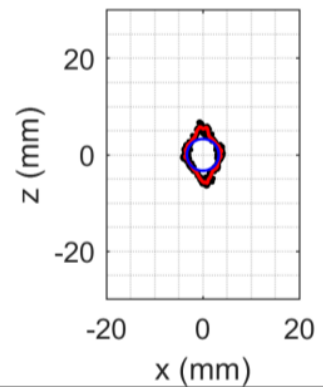

(b)

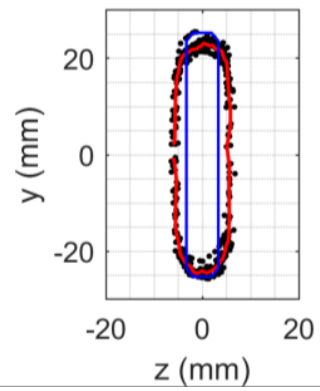

(c)

Figure 11 Orthogonal views of a reconstructed particle show good agreement, particularly in the $x$ - and $y$ directions. Added length is observed in the z-direction due to lack of visibility of the back of particles, increasing the size of the visual hull.

In general, the results are favorable and show good agreement with the true stick dimensions. Figure provides histograms displaying the distribution of dimensions measured for the 43 particles. In each plot, the red line indicates the true particle dimension and the greyed area displays an interval of length corresponding to the expanded measurement uncertainty ( $\pm k u_{c}$ ). Greatest success is shown for the $x$ and $y$ measured dimensions, where the known dimension falls within the measurement uncertainty. This is not the case, however, for dimensions along the $z$-axis. Clearly, there is a systematic error attributed to the camera configuration and the inability to view behind the particle. Because the particle reconstruction is performed using the visual hull concept, there is additional volume attributed to the particle based on the information that is visible to each camera. A simplified depiction can be seen in Figure where the intersecting cones are larger than the imaged particle. This effect can also be observed in the $z-y$ orthogonal view of the reconstructed particle in Figure c. Characterization of the systematic error is underway to define an appropriate correction method.

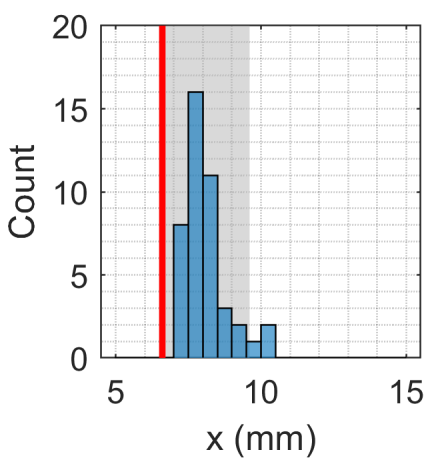

(a)

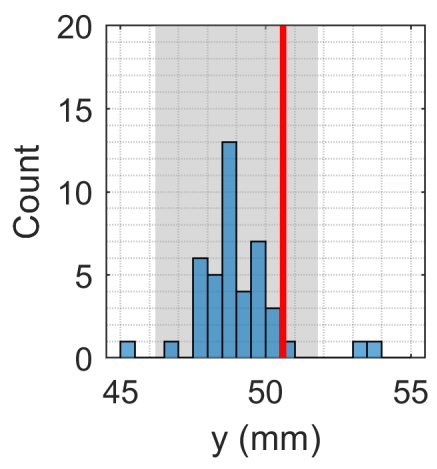

(b)

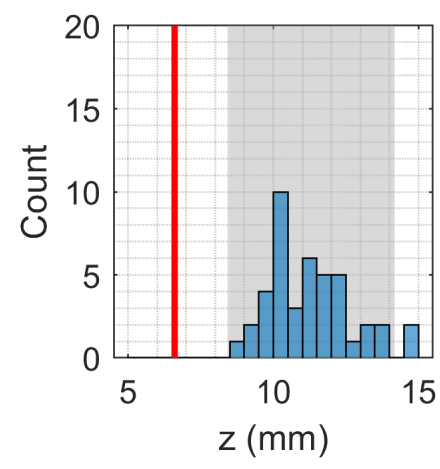

(c)

Figure 12 - Histograms of the measured dimensions of the 43 airborne sticks. Red lines: true stick dimensions; greyed areas: expanded uncertainties $( \pm 1.5, \pm 2.8$ and $\pm 2.9 \mathrm{~mm}$ for $a, b$ and $c$, respectively) 
Knowing the trajectory and volume of each particle allows for the calculation of a cumulative mass of particles (CMP) per surface area given the particle density. In the validation case, the density of the sticks is a known quantity $\left(\rho_{s}=678.7 \pm 60 \mathrm{~kg} \mathrm{~m}^{-3}\right)$, and the CMP per surface area measured by the emberometer (computed using the measured particle volumes and known density) can be compared to its "true" value (using the cumulative number of particles and known average particle mass). Figure shows results for both calculations using the reference surface specified in Figure .

The blue line plots the cumulative number of particles per unit area during the analyzed time period and is read on the left axis. The other three lines relate to the CMP, and are read to the right axis. The actual CMP is known based on the true mass of the particles (green line). The solid red line plots the emberometer measured CMP based on the reconstructed particle volume and density. As previously noted, the volume measurement is overestimated, resulting in a corresponding overestimate of the CMP. It is observed that correcting the emberometer CMP by the ratio between the actual and average measured particle volumes (i.e., a constant factor equal to 0.751) is sufficient to collapse the measured CMP to the actual CMP. Assuming CMP linearity vs. time, the mean measured mass flux, i.e. $2.05 \pm 0.66 \mathrm{~g} \mathrm{~m}^{-2} \mathrm{~s}^{-1}$, closely collapses onto the actual mass flux, $1.55 \pm 0.19 \mathrm{~g} \mathrm{~m}^{-2} \mathrm{~s}^{-1}$. The constant correction factor result, while remarkable, is not intuitive, as it is independent of individual particle position or orientation within the control volume. Additional work is planned to better characterize the volume errors.

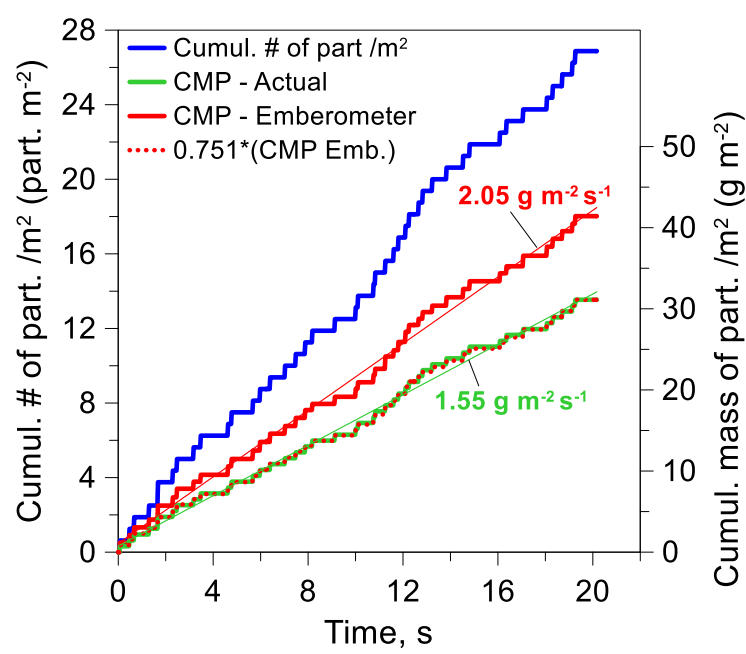

Figure 13 Cumulative number and mass of particles (non-burning stick case). CMP plot labels indicate the emberometer (red) and actual (green) mass fluxes, respectively.

\subsection{Firebrands (Burning sticks)}

Actual combusting firebrands were generated using dowel pieces with the same dimensions as the non-combusting validation case. Glowing/smoldering firebrands were directed into the control volume following the procedure highlighted in section 2.2. The complete tracking results are shown in Figure , along with the designated area used for flux calculations. A total of 1032 particles were tracked and measured during the test. 


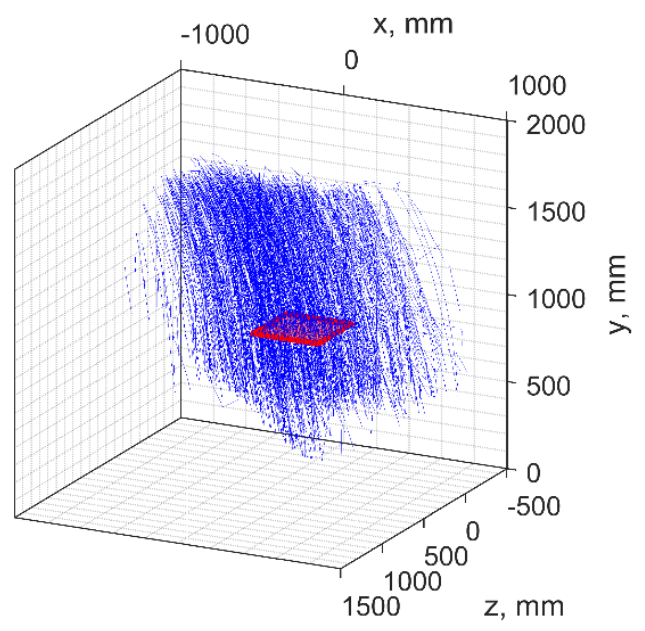

Figure 14 - Firebrands tracked through the control volume $(N=1032)$.

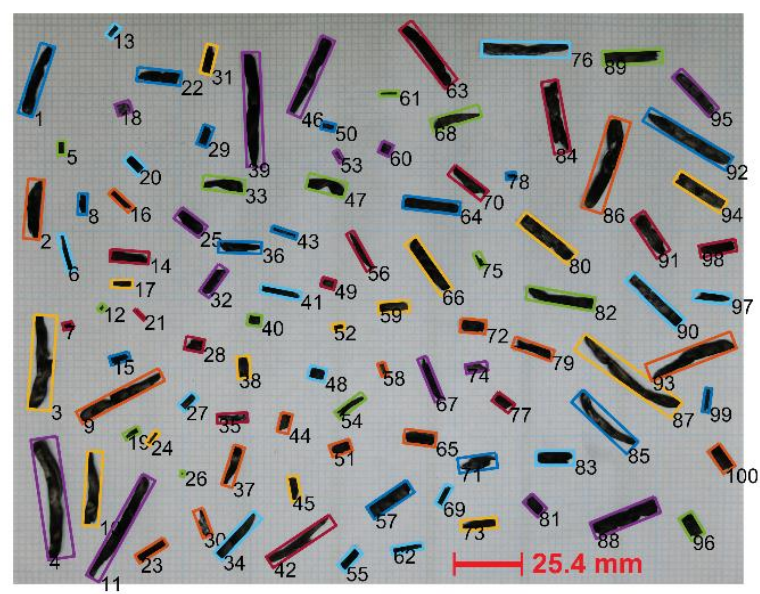

Figure 15 - Burning sticks were captured and quenched in pans of water. Dimensions of each captured firebrand $(N=$ 1155) were measured using a calibrated image.

Additionally, the firebrands were captured and quenched in pans of water arranged on the floor for subsequent counting and measuring. After the test, accumulated firebrands were dried and positioned for photographs. Calibrated images were used to measure 1155 recovered particles using an in-house analysis script. A selection of recovered firebrands is seen in Figure, along with the bounding boxes used to automatically evaluate the dimensions of each particle. Although care was taken during firebrand collection, transport, drying, and photographing, it is acknowledged that some pieces may have broken/spalled into multiple smaller pieces, and that some pieces may have been discarded. It is not possible to account for these effects; however, the authors believe the sample to be representative of the firebrands that landed in the water pans. Limitations of this image-based measurement method include the characterization of particle width, particularly for the longer curved particles (e.g. particle \#4 of Figure ). The bounding box was aligned with the longest axis, and was determined as the smallest bounding rectangle. This method is comparable to the sizing method based on the orthogonal views of the emberometer reconstructed particles, as presented in Figure .

Histograms of the firebrand size distributions are shown in Figure . Distributions for both the collected firebrands and airborne emberometer measurements are compared, normalized by the total number of firebrands collected by each method. Blue bars correspond to the collected firebrands while the orange bars correspond to emberometer measurements of airborne firebrands. Figure a shows the distribution of the long dimension. It is observed that the combustion and forced ejection processes of the firebrand generator significantly alter the dimensions of the particles, and there is a significant number of shorter particles compared to the initial length. Very good agreement is observed between the two measurement methods, as the distributions overlap. In both cases, there is the potential limitation of undercounting the smallest firebrands, particularly as the tiny brands may be buoyant enough to escape the emberometer control volume, as well as the collection pans, as they are transported out through the exhaust hood. 


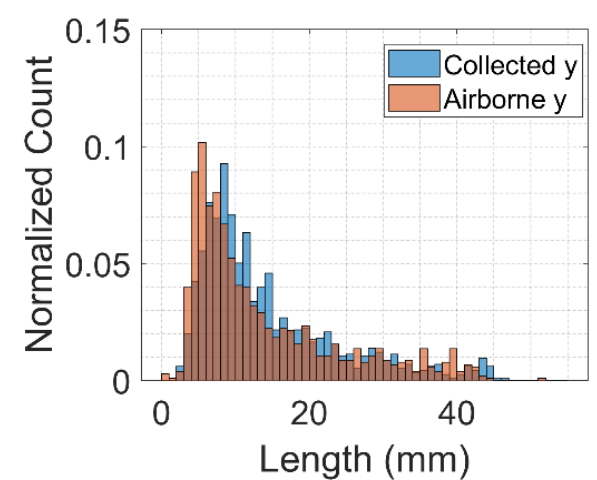

(a)

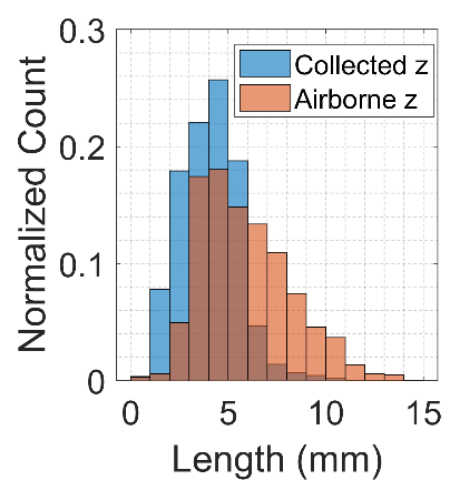

(b)

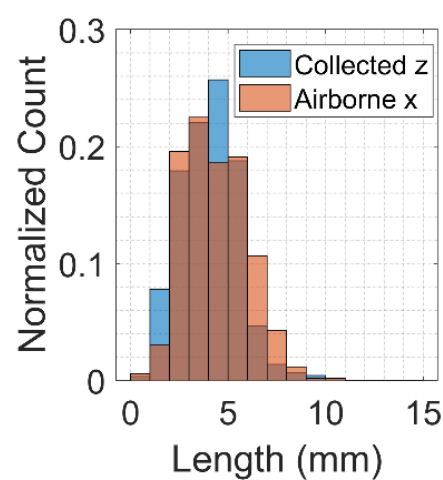

(c)

Figure 16 Distribution of firebrand dimensions as measured by the emberometer compared to those physically collected in the water pans. Bars are normalized by number of brands in each sample, bins are increments of 1 mm. Uncertainties are expected to be on the order of those presented in Fig. 12

An additional limitation is that sizing the quenched firebrands using the imaging technique yields only two dimensions. For comparison to the emberometer measurements, the appropriate dimension should be selected. Based on the orientation of the collected particles in the photographs, the corresponding view is of the $y-z$ plane, along the $\mathrm{x}$-axis (i.e. the two longest dimensions are visible). This comparison is presented in Figure 16b. It is noted that the error in the emberometer measurement systematically overpredicts the particle size in a similar manner as to the error observed in Figure 11c. In the current case, the collected particles can be assumed to have comparable dimensions along the $\mathrm{x}$ and $\mathrm{y}$ axes (based on their original shapes), thus the $\mathrm{x}$-dimension is likely to provide a better measurement of the short dimension of the firebrands. The $\mathrm{x}$-dimension of the particle is plotted in Figure 16c, and shows better agreement with the collected firebrand size distribution.

As was done for the validation case, the flux of firebrands was also considered in the combusting case. Due to the current limitations regarding the reconstructed volume accuracy, only the number flux of firebrands is presented here. In future work, improved characterization of particle volume errors will reduce the uncertainty in mass flux measurement. However, the number flux of particles is measurable now, and is presented in Figure 17. The black points correspond to the cumulative number of particles passing through the designated area, and is read to the left axis. The red line corresponds to the number flux as a function of time, and is read to the right axis. The fluctuations in number flux are confirmed to correlate with visually observed fluctuations in firebrand generation during the test.

\section{Conclusion}

A unique methodology for the measurement and characterization of firebrand showers was tested using both non-combusting validation particles as well as glowing firebrands. Overall, measurements of airborne firebrand dimensions agree well with validation particles and physically collected quenched firebrands. The true dimensions of validation particles are within the uncertainty of the measurement of the longest ( $y$-axis) and shortest ( $x$-axis) dimensions of particles. Due to the configuration of the cameras, the third dimension $(z-$ axis), and consequently the particle volume, shows higher deviation from the known value.

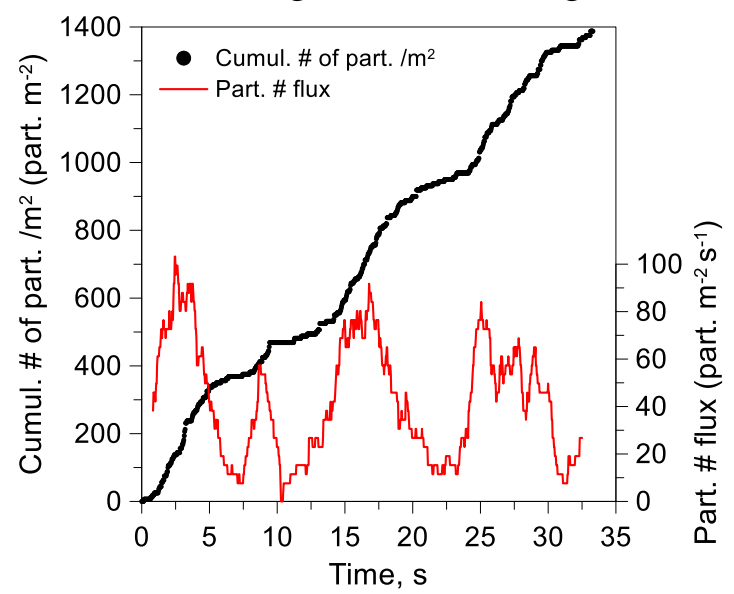

Figure 17 - Cumulative number of firebrands (per $m 2$ ) and the number flux of firebrands during the test period. 
More analysis is required to improve the particle volume measurement of airborne firebrands, which will more accurately measure mass flux. However, in the validation case, where mass flux was independently measured, a constant volume correction factor adequately recovers the true mass flux.

Future work regarding the emberometer includes improved characterization of measurement errors and camera configurations, as well as deployment considerations for measurement of firebrands from actual wildland urban-interface fires. These future measurements may be used to better understand the true exposure conditions experienced from WUI fires and can be related to structure ignition mitigation as well as fire spread and spotting predictions.

\section{Acknowledgments}

The authors would like to thank Ed. Hnetkovsky for machining various parts for the system. Technical support by the NFRL personnel is also gratefully acknowledged.

\section{References}

El Houssami, M, Mueller, E, Filkov, A, Thomas, JC, Skowronski, N, Gallagher, MR, Clark, K, Kremens, R, Simeoni, A (2016) Experimental Procedures Characterising Firebrand Generation in Wildland Fires. Fire Technology 52, 731-751.

Filkov, A, Prohanov, S, Mueller, E, Kasymov, D, Martynov, P, Houssami, ME, Thomas, J, Skowronski, N, Butler, B, Gallagher, M, Clark, K, Mell, W, Kremens, R, Hadden, RM, Simeoni, A (2017) Investigation of firebrand production during prescribed fires conducted in a pine forest. Proceedings of the Combustion Institute 36, 3263-3270.

Koo, E, Pagni, PJ, Weise, DR, Woycheese, JP (2010) Firebrands and spotting ignition in large-scale fires. International Journal of Wildland Fire 19, 818-843.

Laurentini, A (1994) Visual hull concept for silhouette-based image understanding. IEEE Transactions on Pattern Analysis and Machine Intelligence 16, 150-162.

Luhmann, T, Robson, S, Kyle, S, Boehm, J (2014) 'Close Range Photogrammetry and 3D Imaging.' (Walter De Gruyter GmbH: Göttingen)

Manzello, SL, Shields, JR, Cleary, TG, Maranghides, A, Mell, WE, Yang, JC, Hayashi, Y, Nii, D, Kurita, T (2008) On the development and characterization of a firebrand generator. Fire Safety Journal 43, 258-268.

Maranghides, A, Mell, W (2009) A Case Study of a Community Affected by the Witch and Guejito Fires. National Institute of Standards and Technology, NIST Technical Note 1635.

Maranghides, A, Mell, W (2013) Framework for Addressing the National Wildland Urban Interface Fire Problem - Determining Fire and Ember Exposure Zones using a WUI Hazard Scale. National Institute of Standards and Technology, NIST Technical Note 1748.

Thomas, JC, Mueller, EV, Santamaria, S, Gallagher, M, El Houssami, M, Filkov, A, Clark, K, Skowronski, N, Hadden, RM, Mell, W, Simeoni, A (2017) Investigation of firebrand generation from an experimental fire: Development of a reliable data collection methodology. Fire Safety Journal 91, 864-871.

Willneff, J (2003) A spatio-temporal matching algorithm for 3D particle tracking velocimetry. Ph.D. thesis, Swiss Federal Institute of Technology Zurich. 


\title{
Proper Width Calculation to a firebreak line to protect flame spread from forest fire
}

\author{
Donghyun Kim \\ Jeonju University. 303 Cheonjam-ro Jeonju Rep. of Korea, \{72donghyunkim@jj.ac.kr\} \\ International Institute for Applied Systems Analysis. Schlossplatz 1 A-2361 Laxenburg, Austria, \\ \{72donghyunkim@gmail.com\}
}

\begin{abstract}
Building a firebreak line against forest fire spread is a typical indirect suppression method that stops spread of flame by removing fuel, such as trees and bushes. In the view of fire dynamic, building a firebreak line is to set a section which will block thermal energy from igniting on virgin fuel. This study suggests and evaluates a calculation method for width of firebreak against forest fires for variant wind and slope conditions by applying the Point Source Model (PSM) to Korean fuel types. Width of firebreak was measured based on the distance the threshold radiant heat igniting forest surface fuels wat the heat flux of $4.9 \mathrm{~kW} / \mathrm{m}^{2}$ from surface and crown fires in forest. This study applied the condition of wind and slope as the wind velocity of $0 \sim 5 \mathrm{~m} / \mathrm{s}$ and the slope of $0 \sim 50^{\circ}$. As a result, Proper width of a firebreak line was calculated $1.1 \mathrm{~m}$ in surface fires and $14.6 \mathrm{~m}$ in crown fires. Additional comparative analyses through experiments and field surveys are deemed necessary to determine appropriate widths of firebreak for different types of surface fuel.
\end{abstract}

Keywords: Forest Fires, Firebreak Line, Heat Transfer, Flame Spread, Point Source Model (PSM)

\section{Introduction}

Fire forests spread to surface fire and even crown fire due to radiant heat flux generated from flames. In a case where materials are burned in open space as forest fires, most of heat transfer is conducted by radiant heat transfer (McCaffery, 1995). Fire line has a function to prevent flame expansion from forest fire and protect main facilities adjacent to the forest while securing safety zones for fire fighters and ordinary people. In establishing a fire line, there are several methods including eliminating combustible materials and spaying fire extinguishing agents such as Foam Chemical and Retardant toward incombustible materials with a specific amount to prevent flame expansion. Therefore, to prevent spread of forest fire, appropriate width and promptness of the establishment of fire line. Figure 1 is a conceptual diagram on the establishment of fire line to prevent spread of surface fire and fire line establishment can be explained as securing Section (A) as shown in Figure 1. It can be said that appropriate width of fire line is key to success of indirect extinguishment of forest fires. For example, if fire line width is less than the distance to prevent ignition of unburned materials by heat transfer, flames will continue to spread, while if fire line width is too large, it will be waste of resources and it will take a long time to establish fire line, and accordingly a problem may occur in extinguishing forest fires effectively. In this regard, this research aims to provide methods and results to calculate appropriate width of fire line in order to prevent spread of surface fire flames.

\section{Background}

In Korea, width of fire line shall be about $1.5 \mathrm{~m}$ according to guidelines prepared by Korea Forest Service in 2005), while in the USA and Canada, ground surface fuels including standing trees shall be eliminated in width of 6 9m in accordance with the applicable guidelines (NWCF, 2004), and in particular, it is specified to set width of fire line to four times the flame height to prevent spread of crown fire (NWCG, 2004) and it is reported that fire line width should be set considering fire intensity 
depending on fuel load per unit area. In the case of studies conducted by overseas countries, there was a study using a mathematical model on fire line width and flame height, but in Korea, there is lack of research on the calculation of fire line width to prevent spread of forest fires. Although fire line should be established by calculating fire intensity $\left(\mathrm{kW} / \mathrm{m}\right.$ or $\left.\mathrm{kW} / \mathrm{m}^{2}\right)$ or flame length, it has limitations to consider all of the conditions changed by fuel load per unit area, slope, and wind velocity.

\subsection{Fire Break Building Work}

Building of forest fire break line on the ground is conducted by forest fire extinguishers in groups of more than one person or two persons by using fire rakes or air pumps. The progress of fire line building work varies by skills of fire extinguishers, the number of workers, and fire line establishment method. Figure 1 shows fire line building methods for the group of one, group of two and group of three.
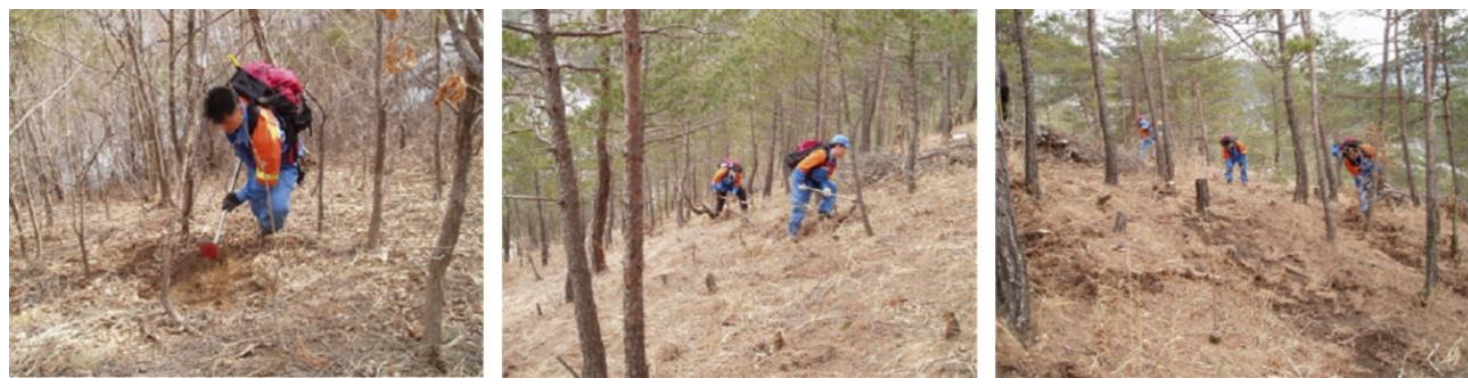

Figure 1 - Fire break line establishement methods (a: group of one. b: group of two, c: group of three)

As the result of measuring working hours for each fire extinguisher to build a fire line (1m (width) $\mathrm{x} 100 \mathrm{~m}$ (length)) in the forest with 23-degree slope, working hours in deciduous were shorter than that in forest coniferous forest by $22 \%$, which indicated that working in coniferous forest is more difficult. It was reported that the difference was caused by forest density and securing of moving passages. In terms of working speed, smoke jumpers were two times faster than normal fire fighters, and in relation to the number of workers, the group of three was 1.85 and 1.2 times faster than the group of one and group of two, respectively. Likewise, previous studies suggested that work efficiency varied by operation and methods of limited fire extinguishing resources. Therefore, as the calculation of effective fire line width reduces risk of fire expansion as well as suggesting the most effective fire line establishment work, it can suggest standards to extinguish forest fires efficiently with limited manpower.

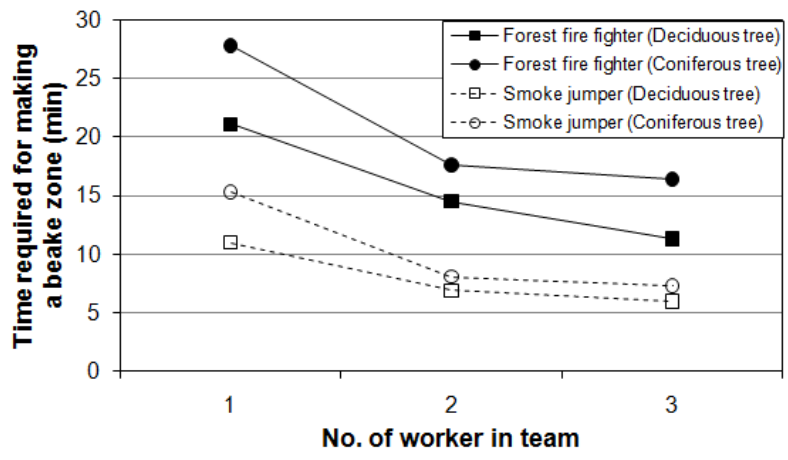

Figure 2 - Work amount for fire line establishment

\subsection{Specifications of Surface Fire}

\subsubsection{Flame Height}

Spread of forest fire undergoes the pyrolysis process of unburned fuel by heat flux emitted through flames. Then, when the surface temperature of the burning substance reaches the igniting temperature, 
the process of creating flames causes the expansion of the forest fire. To assess heat flux emitted from flames, height of flames should be considered. First, to calculate the height of flames, heat release rate (HRR, kW) should be estimated using Effective Heat of Combustion (kJ/kg) and Mass Loss Rate (MLR, $\mathrm{kg} / \mathrm{s}$ ). Heat release rate can be calculated from Equation (1) and is proportional to MLR and Effective Heat of Combustion changed by energy equivalent, water content, combustion diameter, and density.

$$
\operatorname{HRR}(\mathrm{kW})=\Delta h_{c} \times M L R
$$

Flame height calculation models were proposed by McCaffrey (1995) and Heskestad (1998) for horizontal-plane fire of liquid flammable materials under the condition of no wind. The calculation Equation of fire flame height was presented by Albini (1981) as $H \propto I B / U$, which is an estimation Equation for one dimensional flame height, and Nelson (1986) suggested Equation (2). However, when wind velocity is 0 , it is not possible to calculate flame height with the Equation.

$$
H=\frac{a I_{B}}{U}
$$

where, $I_{B}$ is Byram' fire intensity $\left(\mathrm{kW} / \mathrm{m}^{2}\right)$ and ais $1 / 360$ and $U$ is wind velocity $(\mathrm{m} / \mathrm{s})$.

$\operatorname{Kim}(2009$ a) suggested a calculation Equation of flame height of solid fuel such as surface fire fuel materials as the following Equation (3):

$$
H=0.027\left(\dot{Q}^{\prime}\right)^{2 / 3}
$$

where, $\dot{Q}^{\prime}(\mathrm{kW} / \mathrm{m})$ is heat release rate in length, and unit length, which is an experiment setting of $Q$ calculated by Equation (1) was applied. Equations (2) and (3) are to calculate flame height under the condition of no wind and flat area, and flame height changed by wind and slope can be calculated with Equation (4).

$$
H_{w s}=H \times \sin (\theta)
$$

where, $\theta\left(^{\circ}\right)$ is angle between ground surface and the flame changed by wind and slope and it is obtained by subtracting flame angle $(\varnothing)$ from $90^{\circ}$. Calculation of flame angle can be conducted using Equation (5), which represents correlation between Froude Number $(\mathrm{Fr})$ and wind velocity under the condition of uniform wind (Kim, 2009b, 2009c).

$$
\begin{aligned}
& F r=\frac{U_{\infty}}{\sqrt{g H}} \\
& \tan \emptyset_{w s} \sin \emptyset_{w s}=1.2 \frac{U_{w s}^{2}}{g H}
\end{aligned}
$$

where, $g$ is acceleration of gravity $\left(\mathrm{m} / \mathrm{s}^{2}\right), H$ is initial flame height $(\mathrm{m})$, and $U_{w s}$ is air entrainment rate $(\mathrm{m} / \mathrm{s})$.

\section{Methods}

\subsection{Application Conditions}

As shown in Table 1, to calculate appropriate fire line width, there were a total of 12 conditions set: 6 conditions at $1 \mathrm{~m} / \mathrm{s}$ intervals within $0 \sim 5 \mathrm{~m} / \mathrm{s}$ of wind velocity for the dried pine tree litter layer in the spring season of Korea and the other 6 conditions at intervals of $10^{\circ}$ within gradient $0 \sim 50^{\circ}$. Properties of the pine tree litter layer are factors to calculate flame height and heat release rate and conditional variables applied to Formulas 1 and 4. From Formula 4, flame height of the pine tree liter layer was $0.71 \mathrm{~m}$ on average and $1.46 \mathrm{~m}$ at maximum (Kim, 2009b). 
Table 1 - Conditions for wind speeds, slopes and surface fuels

\begin{tabular}{|c|c|c|c|c|}
\hline \multicolumn{3}{|c|}{ Surface fuels } & \multirow{2}{*}{$\begin{array}{l}\text { Wind speed } \\
(\mathrm{m} / \mathrm{s}, 6 \\
\text { conditions })\end{array}$} & \multirow{2}{*}{$\begin{array}{c}\text { Slope } \\
(\circ, 6 \text { conditions })\end{array}$} \\
\hline $\begin{array}{l}\text { Fuel density } \\
\qquad\left(\mathrm{kg} / \mathrm{m}^{3}\right)\end{array}$ & $\begin{array}{c}\text { FMC } \\
(\%)\end{array}$ & $\begin{array}{l}\text { Fuel depth } \\
\text { (m) }\end{array}$ & & \\
\hline 20 & $13 \pm 2$ & 0.1 & $\begin{array}{c}0 \sim 5 \\
(\text { each } 1 \mathrm{~m} / \mathrm{s})\end{array}$ & $\begin{array}{c}0 \sim 50 \\
\left(\text { each } 10^{\circ}\right)\end{array}$ \\
\hline
\end{tabular}

\subsection{Heat Flux Calculation}

\subsubsection{Point Source Model Flame Height}

Spread of forest fire undergoes the pyrolysis process of unburned fuel by heat flux emitted through flames. Then, when the surface temperature of the burning substance reaches the igniting temperature, the process of creating flames causes the expansion of the forest fire. To assess heat flux emitted from flames, height of flames should be considered.

As shown in Table 1, to calculate appropriate fire line width, there were a total of 12 conditions set: 6 conditions at $1 \mathrm{~m} / \mathrm{s}$ intervals within $0-5 \mathrm{~m} / \mathrm{s}$ of wind velocity for the dried pine tree litter layer in the spring season of Korea and the other 6 conditions at intervals of $10^{\circ}$ within gradient $0-50^{\circ}$. Properties of the pine tree litter layer are factors to calculate flame height and heat release rate and conditional variables applied to Formulas 1 and 4. From Formula 4, flame height of the pine tree liter layer was $0.71 \mathrm{~m}$ on average and $1.46 \mathrm{~m}$ at maximum (Kim, 2009b).

First, to calculate the height of flames, heat release rate (HRR, $\mathrm{kW}$ ) should be estimated using Effective Heat of Combustion ( $\mathrm{kJ} / \mathrm{kg}$ ) and Mass Loss Rate (MLR, $\mathrm{kg} / \mathrm{s}$ ). Heat release rate can be calculated from Equation (1) and is proportional to MLR and Effective Heat of Combustion changed by energy equivalent, water content, combustion diameter, and density.

$$
\operatorname{HRR}(\mathrm{kW})=\Delta h_{c} \times M L R
$$

Flame height calculation models were proposed by McCaffrey (1995) and Heskestad (1998) for horizontal-plane fire of liquid flammable materials under the condition of no wind. The calculation Equation of fire flame height was presented by Albini (1981) as $H \propto I B / U$, which is an estimation Equation for one dimensional flame height, and Nelson (1986) suggested Equation (2). However, when wind velocity is 0 , it is not possible to calculate flame height with the Equation.

$$
H=\frac{a I_{B}}{U}
$$

where, $I_{\text {Eis }}$ Byram' fire intensity $\left(\mathrm{kW} / \mathrm{m}^{2}\right)$ and ais $1 / 360$ and $\mathrm{U}$ is wind velocity $(\mathrm{m} / \mathrm{s})$.

Kim (2009 a) suggested a calculation Equation of flame height of solid fuel such as surface fire fuel materials as the following Equation (3):

$$
H=0.027\left(\dot{Q}^{\prime}\right)^{2 / 3}
$$

where, $Q^{\prime}(\mathrm{kW} / \mathrm{m})$ is heat release rate in length, and unit length, which is an experiment setting of $Q$ calculated by Equation (1) was applied.

Equations (2) and (3) are to calculate flame height under the condition of no wind and flat area, and flame height changed by wind and slope can be calculated with Equation (4).

$$
H_{w s}=H \times \sin (\theta)
$$

where, $\theta\left(^{\circ}\right)$ is angle between ground surface and the flame changed by wind and slope and it is obtained by subtracting flame angle $(\varnothing)$ from $90^{\circ}$. Calculation of flame angle can be conducted using 
Equation (5), which represents correlation between Froude Number $(F r)$ and wind velocity under the condition of uniform wind (Kim, 2009b, 2009c).

$$
\begin{aligned}
& F r=\frac{U_{\infty}}{\sqrt{g H}} \\
& \tan \emptyset_{w s} \sin \emptyset_{w s}=1.2 \frac{U_{w s}^{2}}{g H}
\end{aligned}
$$

where, $g$ is acceleration of gravity $\left(\mathrm{m} / \mathrm{s}^{2}\right), H$ is initial flame height $(\mathrm{m})$, and $U_{w s}$ is air entrainment rate $(\mathrm{m} / \mathrm{s})$.

\subsection{Heat Flux of Forest Fires}

Radiation heat transfer by surface fire flame is conducted through calculation of total radiant heat flux received by unburned section from individual flame for the grid defined as follows (Figure 3), where three-dimensional position values against the center point of flame obtained by Equation (3) are $x f, y f$, and $z f$. To calculate heat energy per unit area emitted from flames, Point Source Model in Equation (7) was used. With the Point Source Model can be used when calculating radiant heat flux per unit area and unit time delivered target distant by $r$ from emitting flame. Therefore, when the total heat flux reaches ignition energy, flame combustion is conducted and expanded.

$$
\dot{q}^{\prime \prime}=\frac{\dot{Q}_{r}}{4 \pi r^{2}} \cos \theta^{\prime}
$$

Where, $\dot{Q}_{r}$ is radiant flux, $r$ is radius of flame depth (m), and $\theta^{\prime}$ is angle between the center of flame and $z_{f}$ vector and expressed as Equation 7. Location coordinates of the center of flame $\left(x_{f}, y_{f}, z_{f}\right)$ needed to calculate heat flux transfer from flame can be moved by slopes and wind, which can be represented as the following Equation (9).

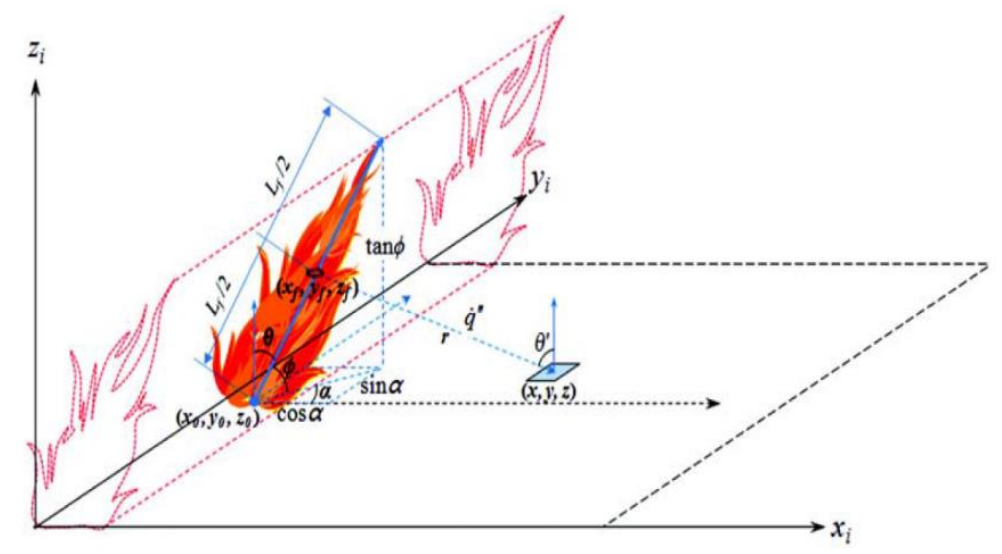

Figure 3 - The concept of heat flux transfer

$$
\begin{gathered}
\cos \theta^{\prime}=\frac{z_{f}-Z}{r} \\
\text { where, } \quad r=\sqrt{\left(x_{f}-x\right)^{2}+\left(y_{f}-y\right)^{2}+\left(z_{f}-z\right)^{2}} \\
\overrightarrow{P_{f}}=\left(\begin{array}{c}
x_{f} \\
y_{f} \\
z_{f}
\end{array}\right)=\left(\begin{array}{c}
x_{0}+\frac{1}{2} L_{f} \cos \emptyset \cos \alpha \\
y_{0}+\frac{1}{2} L_{f} \cos \emptyset \sin \alpha \\
z_{0}+\frac{1}{2} L_{f} \sin \emptyset
\end{array}\right)
\end{gathered}
$$


In this case, the cell condition was set to $0.3 \mathrm{~m} \times 0.3 \mathrm{~m}$ for numerical analysis of radiative heat transfer in Figure 4, which is the experimental size of the existing P. densiflora litter layer basket (Kim, 2009b). In this study, a y-axis flame grating is to 9 grids, total 2.7 meters in order to analyze the heat transfer of individual flames for each surface fuels. Therefore, the radiant heat flux of the surface fuels for each unit flame can be calculated from Equation 7 to Equation 10. Where $F$ is the individual flame and $\mathrm{S}$ is the target cell.

$$
\dot{q}^{\prime \prime}=\int_{F} \int_{S} \frac{\dot{Q}_{r}}{4 \pi r} d F d S
$$

\section{Results}

\subsection{Characteristics of Heat Flux Distribution}

Analysis of flame heat flux distribution using PSM revealed that under the condition of no wind and flat area as shown Figure 4, 5, heat flux decreased as distance from flames were longer and horizontal heat influx for fire line is distributed the highest at the center of fire line. Based on the characteristics of heat flux, maximum heat flux should be calculated focusing on the main axis of fire line (y5).

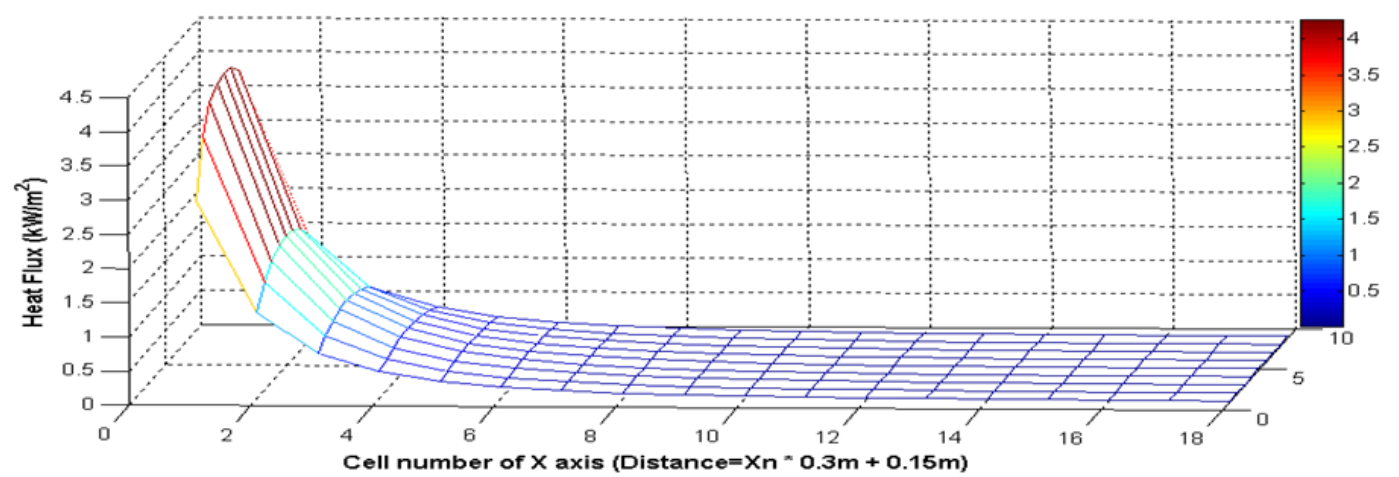

Figure 4 - Heat flux under the condition of no wind -flat area

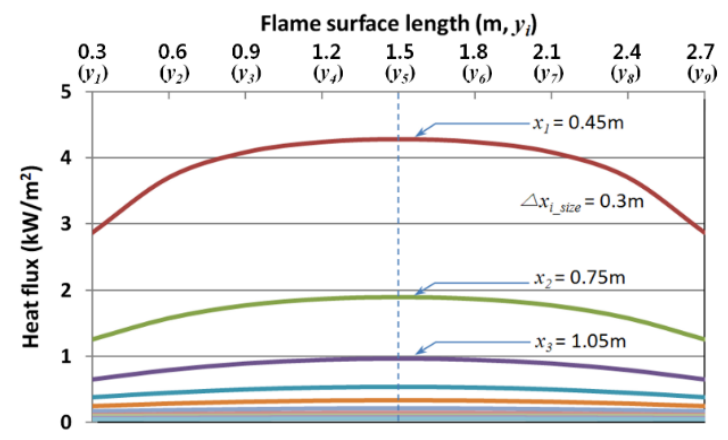

Figure 5 - Distribution of heat flux per distance according to average flame height

\subsection{Distribution of Heat Flux According to Distance}

\subsubsection{Average Flame Height}

As shown in Figure 6, heat flux distribution under the condition of wind velocity and slop for about $0.71 \mathrm{~m}$ of average flame height of the pine tree litter layer was in inverse proportion by distance and as wind velocity and slope increased, heat flux raised. Distribution of heat flux per distance from flames was calculated based on about $0.35 \mathrm{~m}$, which is center height of the flames. At that time, heat flux at 
$0.35 \mathrm{~m}$ was measured to $4.28 \sim 106.98 \mathrm{~kW} / \mathrm{m}^{2}$ for $0-5 \mathrm{~m} / \mathrm{s}$ of velocity and $4.28 \sim 21.81 \mathrm{kw} / \mathrm{m}^{2}$ for $0 \sim 50^{\circ}$ of slope. In addition, heat flux at $1.2 \mathrm{~m}$ was $1 \mathrm{~kW} / \mathrm{m}^{2}$ or less in both conditions.

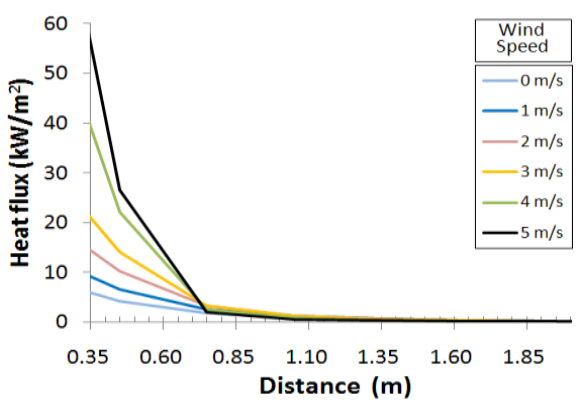

(a)

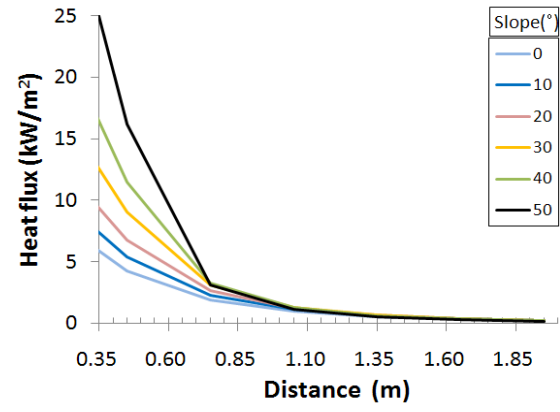

(b)

Figure 6 - Distribution of heat flux per distance according to average flame height; (a) Wind velocity condition, (b) Slope condition

\subsubsection{Maximum Flame Height}

As shown in Figure 7, heat flux distribution under the condition of wind velocity and slop for about $1.46 \mathrm{~m}$ of maximum flame height of the pine tree litter layer was in inverse proportion by distance and as wind velocity and slope increased, heat flux raised. Distribution of heat flux per distance from flames was calculated based on about $0.75 \mathrm{~m}$, which is center height of the flames. At that time, heat flux at $0.75 \mathrm{~m}$ was measured to $4.28 \sim 106.98 \mathrm{~kW} / \mathrm{m}^{2}$ for $0-5 \mathrm{~m} / \mathrm{s}$ of velocity and $4.28-21.81 \mathrm{kw} / \mathrm{m}^{2}$ for $0 \sim 50^{\circ}$ of slope. In addition, heat flux at $1.2 \mathrm{~m}$ was $1 \mathrm{~kW} / \mathrm{m}^{2}$ or less in both conditions.

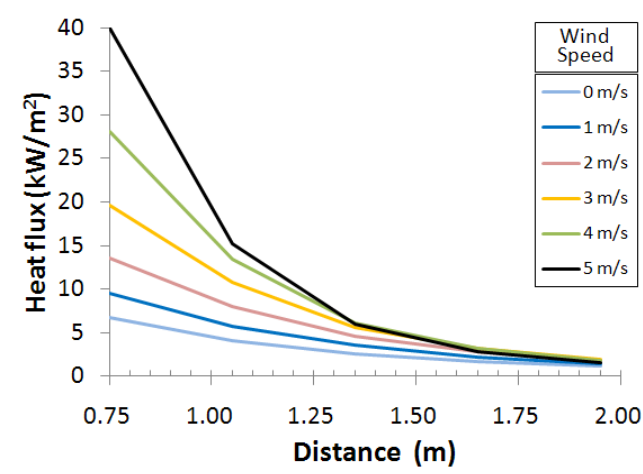

(a)

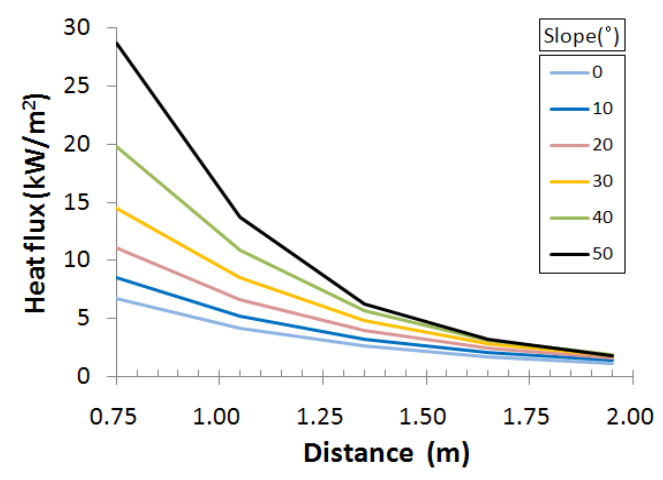

(b)

Figure 7 - Distribution of heat flux per distance according to maximum flame height; (a) Wind velocity condition, (b) Slope condition

\subsubsection{Width of Fire Break Line}

Radiant heat flux required for ignition of fallen leaves of pine trees is $7.9 \mathrm{~kW} / \mathrm{m}^{2}$ and $4.9 \mathrm{~kW} / \mathrm{m}^{2}$ in case of self-ignition and pilot ignition, respectively (Kim, 2010). Considering characteristics of surface fire flame spread and safety rate, it is desirable to apply $4.9 \mathrm{~kW} / \mathrm{m}^{2}$ of heat flux for pilot ignition as critical radiant heat flux for ignition of pine tree fallen leaves. Influential distances at $4.9 \mathrm{~kW} / \mathrm{m}^{2}$ of critical radiant heat flux for flame angle ( ) at $0 \sim 5 \mathrm{~m} / \mathrm{s}$ of velocity and $0 \sim 50^{\circ}$ of slope were calculated to be $0.35 \sim 0.65 \mathrm{~m}$ for the average flame height and $0.75 \sim 1.05 \mathrm{~m}$ for maximum flame height as shown in Figure 8. Therefore, optimal fire line width to prevent flame spread was appeared to be about $1.05 \mathrm{~m}$ based on maximum flame height applied 


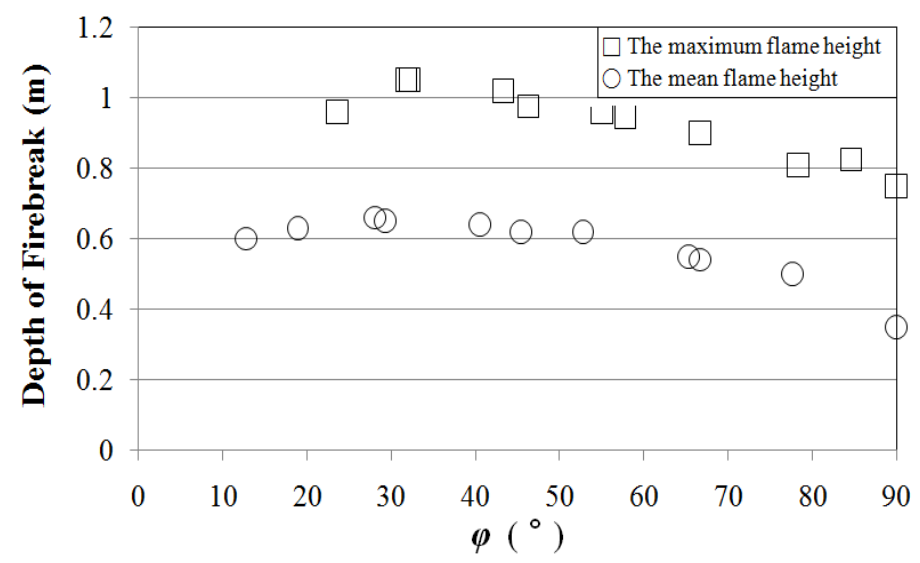

Figure 8 - Results of calculating fire line establishment width for P. densiflora tree litter layer

\section{Conclusion}

To prevent spread of surface fire flame in the pine tree litter layer, optimal fire line establishment widths were calculated. For the calculation, numerical analysis of two-dimensional radiant heat transfer was conducted using a heating point model. As a result, the following conclusion was derived.

First, calculating optimal width of fire line for surface fire can be an approach to increase work efficiency of forest fire fighters as well as minimizing spread of flames according to errors in working on appropriate fire line width.

Second, as of $4.9 \mathrm{~kW} / \mathrm{m}^{2}$ of critical radiant heat flux for ignition of pine tree fallen leaves, fire line establishment width at $0 \sim 5 \mathrm{~m} / \mathrm{s}$ of velocity and $0 \sim 50^{\circ}$ of slope were calculated to be $0.35 \sim 0.65 \mathrm{~m}$ for the average flame height and $0.75 \sim 1.05 \mathrm{~m}$ for maximum flame height.

Third, optimal fire line considering safety rate is deemed to be about $1.05 \mathrm{~m}$ based on maximum flame height applied.

Fourth, assessment for rolling fire and spotting fire by strong wind has yet to be conducted, and therefore, when establishing fire line on the site, it should be considered.

In the near future, it is deemed to need to conduct follow-up studies including research on the comparative analysis of appropriate fire line establishment width per ground surface-layer fuel and research on the methods to calculate fire line establishment width to prevent spread of crown fire, through experiments and field case studies.

\section{References}

Kim, D.H.(2009a), A Study on the Flame Tilt and Flame Spread due to Up-slope on the Surface Fuel Bed-No wind condition, Journal of KOSHAM, 9(5), 57-62.

Kim, D.H.(2009b), A Study on Flame Height Equation for the Pinus densiflora Surface Fuel Bed, Journal of Fire Science and Engineering 23(6), 10-15

Kim, D.H.(2009c), A Semi-numerical Study on the Flame Tilt Equation due to Wind on the Surface Fire in Forest Fire, Journal of Fire Science and Engineering 23(5), 90-95.

Albini, F.A. (1981) A model for the wind-blown flame from a line fire. Combust. Flame 43, 155-174. Albini F.A (1985) A model for fire spread in wildland fuels by radiation. Combustion Sci. Technology 42, 229-58.

Andrew A. G. Wilson (1988), Width of firebreak that is necessary to stop grass fires: some field experiments. Can. J. For. Res. 18(6), 682-687. 
B. McCaffrey (1995), The SFPE Handbook of Fire Protection Engineering, $2^{\text {nd }}$ ed. Society of Fire Protection Engineers and National Fire Protection Association, Quincy, MA.

KIM Donghyun (2010), A study for surface fire behavior and flame spread model in forest fire. Ph.D Dissertation, Kyoto University.

F. Morandini, P.A. Santono, J.H. Balbi (2001), The contribution of radiant heat transfer to laboratoryscale fire spread under the influences of wind and slope. Fire Safety Journal 36, 519-543.

Guy R. McPherson, G. Allen Rasmussen, Henry A. Wright, Carltonm M. Britton (1986), Management notes "Getting Started in Prescribed Burning". Texas Tech University, 1-6.

NWCG (2004), Fireline Handbook, A publication of the National Wildfire Coordinating Group PMS 410-1 NFES 0065, 437.

P.A. Santoni, J.H. Balbi (1998), Modelling of two-dimensional flame spread across a sloping fuel bed. Fire Safety Journal 31, 201-225.

R Mees, D Strauss and R Chase (1993), Modeling Wildland Fire Containment With Uncertain Flame Length and Fireline Width. International Journal of Wildland Fire 3(3), 179-185.

TL Clark, MA Jenkins, JL Coen, DR Packham (1996), A Coupled Atmosphere-Fire Model: Role of the Convective Froude Number and Dynamic Fingering at the Fireline. International Journal of Wildland Fire, 10, 177-190. 


\title{
Terpene-containing species vs terpeneless species: what best drives flammability of ornamental species used in WUI?
}

\author{
Anne Ganteaume*; Bastien Romero \\ IRSTEA, RECOVER-EMR Aix-en-Provence, France \{anne.ganteaume@irstea.fr*, \\ bastien.romero@irstea.fr\}
}

\begin{abstract}
In wildland-urban interfaces (WUI), the vegetation surrounding housing can be an efficient vector of fire propagation towards buildings possibly resulting in extensive damage. The current work aimed at highlighting the most flammable species in WUI and the driving factors of their leaf flammability among a set of leaf characteristics including terpene content supposed to enhance plant flammability. This hypothesis needed to be clarified and the role of the three main classes (mono-, sesqui-, and diterpenes) determined in the species that contained such molecules.

After the screening of the live leaf terpene contents in 16 of the most common species in WUI of SE France, only five presented terpenes: Pinus halepensis, Cupressus arizonica, C. sempervirens, Cupressocyparis leylandii, and Thuja occidentalis. Burning experiments provided data allowing the species to be ranked from the most to the least flammable. P. halepensis belonged to the most flammable species contrary to the four others. Relationships between flammability and leaf characteristics (terpene contents, leaf thickness, surface-to-volume ratio, mass-to-volume ratio, and FMC) were tested to highlight the most significant flammability drivers. Except for ignition frequency hindered by diterpene content, the flammability of species containing terpenes was mostly driven by sesquiterpene content (positive effect), sometimes combined to other leaf characteristics; P. halepensis best characterized these relationships. Leaf thickness best predicted flammability of the terpeneless species (negative effect) but when all species were pooled, sesquiterpene content was still one of the best predictors.

$P$. halepensis containing the highest amount of sesquiterpenes and being among the most flammable species should be avoided in WUI, along with species with low FMC and leaf density. The other species presenting high monoterpene and diterpene contents were characterized by less flammable thick and dense leaves; however, the amount of dead fuel trapped in the canopy of Cupressus sempervirens strongly increases its flammability and should also be avoided near housing.
\end{abstract}

Keywords: WUI, leaf flammability, terpenes, ornamental vegetation, Pinus halepensis

\section{Introduction}

In wildland-urban interfaces (WUI), where the fire risk is high, the vegetation surrounding housing (i.e. WUI vegetation also called ornamental vegetation composed of both native and strictly ornamental species) can be an efficient vector of fire propagation towards structures, possibly resulting in damaging or destroying these buildings. Consequently, to better understand the role of the ornamental vegetation in this propagation, either due to spot fires or to the radiant heat emitted by the flame front, previous studies have focused on the assessment of this vegetation's flammability (Ganteaume et al. 2013a, 2013b), targeting live and dead surface fuels but without taking into account chemical characteristics. Others works showed that some of these characteristics (i.e. nitrogen, phosphorus, and tannin contents) could affect plant flammabilty (Grootemat et al. 2015, 2017). Terpenes are among the most frequent chemical compounds hypothesized or even assumed to promote flammability but results were not always significant or differed between studies (Dimitrakopoulos 2001; Alessio et al. 2008; Ormeño et al. 2009; Pausas et al. 2016). To increase knowledge on the WUI vegetation's flammability and to be able to select "firewise species" for landscaping in these areas, the

Advances in Forest Fire Research 2018 - Page 846 
current work aimed at highlighting the most flammable species that can be deleterious in WUI and the driving factors of their flammability among a set of leaf characteristics including leaf terpene content characterized by three classes (monoterpenes, sesquiterpenes, and diterpenes). The terpene content is supposed to enhance plant flammability (Pausas et al. 2016; Alessio et al. 2008) but this hypothesis needed to be clarified and the role of the different classes determined in species that contained such molecules.

\section{Methodology}

\subsection{Study Species and Sampling}

The 16 species studied in the current work (Table 1) are common in WUI of SE France and this WUI vegetation is composed of both native (such as Viburnum tinus, Pinus halepensis) and exotic species (such as Thuja occidentalis), some being created for strictly ornamental purposes (i.e. C. Leylandii). Depending on their location, these species can be involved in fire propagation to the nearby buildings, especially those used in ornamental hedges characterized by a strong horizontal fuel continuity. During a fire, some of these species (e.g. Pinus halepensis, Cupressus sempervirens) can also be responsible for damage and even for the destruction of buildings when the plants are too close to buildings, as often witnessed by the firefighters.

The live leaf sampling was carried out on five individuals per species, in summer (July 2016), when the climate conditions were the most severe in SE France and avoiding $48 \mathrm{~h}$ following precipitations to avoid any impact of the recent rain on FMC.

Table 1 - List of the 16 species studied: abbreviations (Abb.), terpene content (yes or no), mean leaf thickness and mean $( \pm S D)$ leaf moisture content (FMC)

\begin{tabular}{lcc}
\hline Latin name & Abb. & Terpene content \\
\hline Viburnum tinus & $V t$ & No \\
Cupressus sempervirens & $\mathrm{CuS}$ & Yes \\
Cotoneaster franchetii & $\mathrm{Co}$ & No \\
Prunus laurocerasus & $\mathrm{Pr}$ & No \\
Elaeagnus ebbingei & $E l$ & No \\
Cupressus arizonica & $\mathrm{CuA}$ & $Y e s$ \\
Phyllostachys sp. & $\mathrm{Phy}$ & No \\
Pyracantha coccinea & $P y$ & No \\
Cupressocyparis leylandii & $\mathrm{CuL}$ & $Y e s$ \\
Nerium oleander & $\mathrm{Ne}$ & No \\
Pinus halepensis & $P h$ & $Y e s$ \\
Photinia fraseri & $P h o$ & No \\
Euonymus japonicus & $E u$ & No \\
Ligustrum japonicum & $\mathrm{Lj}$ & No \\
Pittosporum tobira & $P i$ & No \\
Thuja occidentalis & $T o$ & $Y e s$ \\
\hline
\end{tabular}




\subsection{Terpene analyses}

The content of the three main terpene classes (monoterpenes, sesquiterpenes, diterpenes) was screened in the leaves of the 16 species studied. The analyses were conducted on $10 \mathrm{~g}$ leaf samples using a GC-MS (GC System 7890B - Agilent Technologies $\left.{ }^{\circledR}\right)$.

\subsection{Burning experiments}

Just after sampling, the flammability of the 16 species studied was assessed burning thirty $1 \mathrm{~g}$ leaf samples per species using a 500W epiradiator. Ignition frequency (IF, \%), time-to-ignition (TTI, s), and flaming duration (FD, s) were recorded during the burning experiments.

Just before the experiments, $5 \mathrm{~g}$ of leaves of each individual were oven-dried for $48 \mathrm{~h}$ at $60{ }^{\circ} \mathrm{C}$ in order to measure their moisture content at the time of burning. Along with the terpene content and FMC, leaf characteristics - thickness (Thi), surface-to-volume ratio (SVR), density or mass-to-volume ratio (D) were also taken into account as flammability explanatory factors.

\subsection{Data analyses}

In order to check if flammability and terpene contents varied according to species, intra- and interspecific variations in leaf flammability were tested with variance analysis (one-way ANOVA, Fisher test). According to the flammability variables recorded during the burning experiments, species were ranked from the most flammable to the least flammable performing Hierarchical Cluster Analysis. Then relationships between flammability and leaf characteristics (monoterpene, sesquiterpene, and diterpene contents, leaf structural characteristics, and FMC) were tested to highlight the most significant drivers of each flammability variable performing regression and co-inertia analyses.

\section{Results and discussion}

\subsection{Terpene Contents}

Out of the 16 species studied, only five presented terpenes: Pinus halepensis, Cupressus arizonica, C. sempervirens, Cupressocyparis leylandii, and Thuja occidentalis. These species present storage structures for volatile organic compounds, such as resin ducts (Yani et al. 1993; Ormeño et al. 2008)

Regarding terpene diversity, a total of 54 different compounds were identified in the leaves and litter of the species studied. Sesquiterpenes presented the higher diversity in terpenes ( 24 compounds) mostly in Cupressocyparis leylandii and Cupressus arizonica, compared to diterpenes (19 compounds) mainly found in C. leylandii and in Cupressus sempervirens, and to monoterpenes (11 compounds), mostly in $C$. Leylandii. This latter species presented the highest terpene diversity with 34 different compounds.

Regarding terpene contents, C. leylandii presented the highest monoterpene content $\left(2.54 \mathrm{mg} \mathrm{g}^{-1}\right)$, $P$. halepensis presented the highest sesquiterpene content $\left(1.2 \mathrm{mg} \mathrm{g}^{-1}\right)$, mostly due to caryophyllene, and diterpene content was the highest in Thuja occidentalis $\left(1.04 \mathrm{mg} \mathrm{g}^{-1}\right)$, mostly due to nezukol.

The intra-specific variation in the terpene content $(31 \%)$ was not considered as significant. The inter-specific variations in the terpene content were significant regardless of the class of terpene (One way ANOVA, p<0.0001).

\subsection{Species Flammability}

Within the same species, flammability variables varied only slightly between individuals (the coefficient of variation was, on average, 25.6\%). Inter-specific differences were tested for each flammability variable and all the flammability variables significantly differed between species (Oneway Anova, $\mathrm{p}<0.001)$. When the flammability of species containing terpenes was compared to that of terpeneless species, results were contrasted; the former presenting longer flaming duration and timeto-ignition. The ranking of species according to their flammability revealed that the terpene-containing 
species were split into two groups of different flammability (Fig. 1). Agreeing with previous works (Trabaud, 2000; Dimitrakopoulos, 2001), P. halepensis belonged to the most flammable species, along with two terpeneless species Cotoneaster franchetti and Elaeagnus ebbingei (mostly due to high ignition frequency and long flaming duration) while the four others were ranked as not very flammable (mostly due to long time-to-ignition).

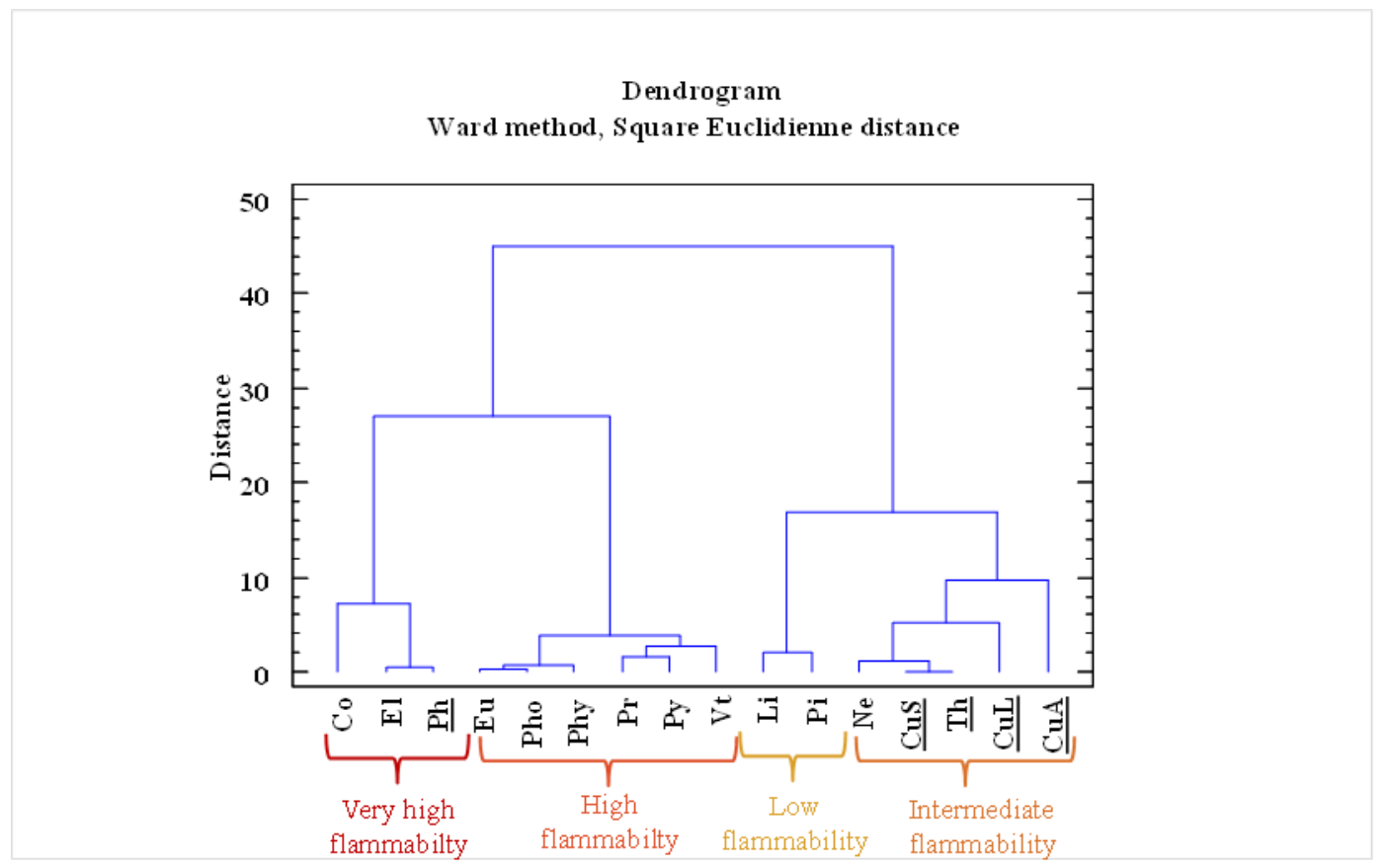

Figure 1 - Ranking of the 16 ornamental species from the most flammable to the least flammable species according to leaf flammability (underlined: species containing terpenes, Co: Cotoneaster franchetii, CuA: Cupressus arizonica, CuL: Cupressocyparis leylandii, CuS: Cupressus sempervirens, El: Elaeagnus ebbingei, Eu: Euonymus japonicus, Li: Ligustrum japonicum, Ne: Nerium oleander, Ph: Pinus halepensis, Pho: Photinia fraseri, Phy: Phyllostachys sp., Pi: Pittosporum tobira, Pr: Prunus laurocerasus, Py: Pyracantha coccinea, Th: Thuja occidentalis, Vt: Viburnum tinus).

Along with the high sesquiterpene, $P$. halepensis' leaves also presented high SVR as well as low density and FMC in contrast to less flammable species containing terpenes whose leaves were thicker and denser and presented higher monoterpene and diterpene contents. The least flammable species (Pittosporum tobira and Ligustrum japonicum) did not contain terpenes and presented low ignitability and sustainability, mostly due to higher leaf moisture content along with high density and low SVR (Fig. 2).

Except for ignition frequency significantly predicted by diterpene content (negative effect, logistic regression, $\mathrm{p}=0.002$ ), the flammability of species containing terpenes was mostly driven by sesquiterpene content (positive effect), combined to leaf density (negative effect) regarding flaming duration (multiple regression, $\mathrm{p}=0.002$ ) and to $\mathrm{FMC}$ and thickness (positive effect) regarding time-toignition (multiple regression, $\mathrm{p}<0.0001$ ). P. halepensis was the species best characterizing these relationships, this species presenting the highest amount of sesquiterpenes (mostly due to caryophyllene: $0.905 \mathrm{mg} \mathrm{g}^{-1}$ which was the most concentrated molecule among the entire set of compounds identified). When this latter species was removed from the analyses, sesquiterpene content was not a significant driver of flammability anymore, replaced by diterpene content (negative effect on TTI). Several other fire-prone species also presented high sesquiterpene contents, such as the Australian Malaleuca quinquenervia (Ireland et al. 2002) or several Mediterranean species of Cistus (especially caryophyllene for C. monspeliensis; Llusià and Peñuelas 1998) whose germination is triggered by fire. In the current study, monoterpene content was not a significant driver of flammability contrary to the results of Pausas et al. (2016) obtained on Rosmarinus officinalis. This latter species 
produces monoterpenes (some presenting high contents such as camphrene) differing from those found in the species we studied; this could be an explaination to this difference. Other works showed, however, that monoterpenes were poorly related to flammability or were overridden by FMC (Alessio et al. 2008a, 2008b).

For the terpeneless species, leaf thickness best predicted flammability (negative effect) combined to surface-to-volume ratio and FMC (negative effect) regarding flaming duration, to leaf density (positive effect) regarding time-to-ignition, and to leaf density and surface-to-volume ratio (negative effect) regarding ignition frequency (for all relationships, $\mathrm{p}<0.0001$ ). When all the species were pooled in the multiple regression analyses, sesquiterpene content was still one of the best predictors of timeto-ignition and flaming duration, highlighting the strong impact of Pinus halepensis in the relationships.

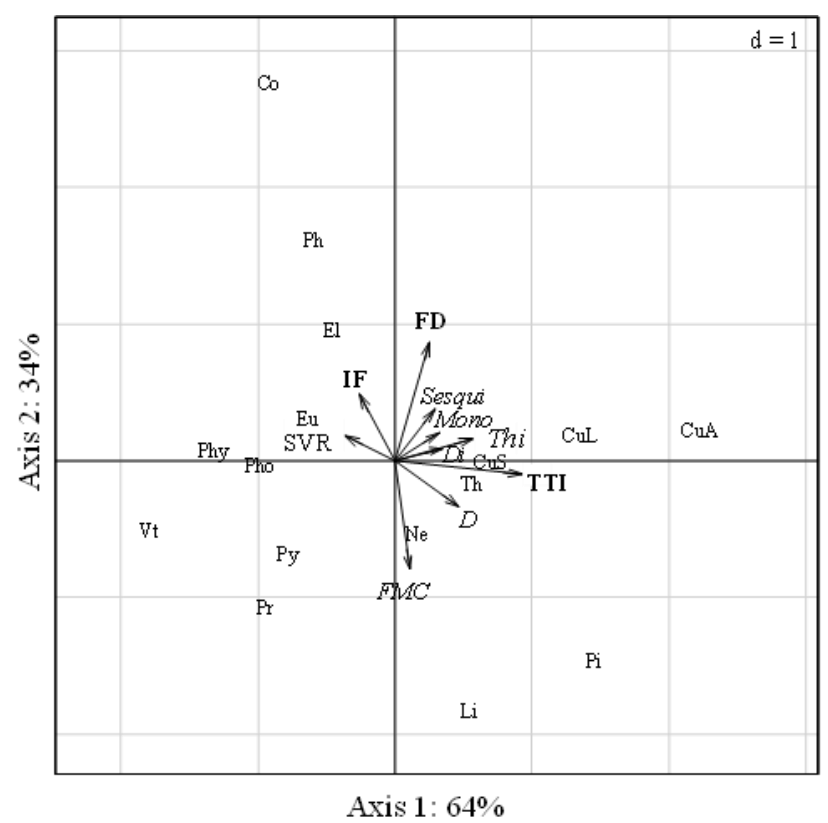

Figure 2 - Co-inertia analysis highlighting the relationships between the leaf characteristics of the 16 ornamental species and their flammability variables. The two first components together explain $98 \%$ of the total variance (D: leaf density, SVR: surface-to-volume ratio, FMC: fuel moisture content, Thi: leaf thickness, Mono: monoterpene content, Sesqui: sesquiterpene content, Di: diterpene content, Co: Cotoneaster franchetii, CuA: Cupressus arizonica, CuL: Cupressocyparis leylandii, CuS: Cupressus sempervirens, El: Elaeagnus ebbingei, Eu: Euonymus japonicus, Li: ligustrum japonicum, Ne: Nerium oleander, Ph: Pinus halepensis, Pho: Photinia fraseri, Phy: Phyllostachys sp., Pi: Pittosporum tobira, Pr: Prunus laurocerasus, Py: Pyracantha coccinea, Th: Thuja occidentalis, Vt: Viburnum tinus).

\section{Conclusion}

The present work showed that the sesquiterpene content (especially the caryophyllene content) was one of the strongest predictor of flammability highlighting that species containing high amount of this terpene (e.g. P. halepensis) would be among the most flammable and thus should be avoided in WUI, along with terpeneless species with low FMC and leaf density (e.g. C. franchetti and E. ebbingei contrary to P. tobira and L. japonicum). The other species that presented high monoterpene and diterpene contents were also characterized by thicker and denser leaves that were less flammable. However, the amount of dead fuel trapped in the canopy of some of these species (e.g. Cupressus sempervirens) strongly increases their flammability (Ganteaume et al. 2013a) and should also be avoided close to housing, especially when they form horizontal fuel continuity (as in ornamental hedge) that can easily propagate the fire. Moreover, as flammability can differ between live leaf and dead surface fuel within a given species (Ganteaume 2018), it will be important to also assess the role of terpene contents on litter flammability. 


\section{References}

Alessio GA, Peñuelas J, Llusià J, Ogaya R, Estiarte M, De Lillis M (2008a) Influence of water and terpenes on flammability in some dominant Mediterranean species. Int. J. Wildland Fire 17, 274286.

Alessio GA, Penuelas J, De Lillis M, Llusia J (2008b) Implications of foliar terpene content and hydration on leaf flammability of Quercus ilex and Pinus halepensis. Plant Biology 10, 123-128.

Dimitrakopoulos A (2001) A statistical classification of Mediterranean species based on their flammability components. Int. J. Wildland Fire 10, 113-118.

Ganteaume A (2018) Does plant flammability differ between leaf and litter bad scale? Role of fuel characteristics and consequences for flammability assessment. Int. J. Wildland Fire. https://doi.org/10.1071/WF17001.

Ganteaume A, Jappiot M, Lampin C, Guijarro M, Hernando C (2013a) Flammability of some ornamental species in wildland-urban interfaces in southeastern France: laboratory assessment at particle level. Environ manag. 52, 467-480.

Ganteaume A, Jappiot M, Lampin C (2013b) Assessing the flammability of surface fuels beneath ornamental vegetation in wildland-urban interfaces in Provence (southeastern France). Int. J. Wildland Fire 22, 333-342.

Grootemaat S, Wright IJ, Bodegom PM, Cornelissen JH, Cornwell WK (2015) Burn or rot: leaf traits explain why flammability and decomposability are decoupled across species. Functional Ecology 29, 1486-1497.

Grootemaat S, Wright IJ, van Bodegom PM, Cornelissen JH. 2017. Scaling up flammability from individual leaves to fuel beds. Oikos 126, 1428-1438.

Ireland B, Hibbert D, Goldsack R, Doran J, Brophy J (2002) Chemical variation in the leaf essential oil of Melaleuca quinquenervia (Cav.) ST Blake. Biochemical Systematics and Ecology 30, 457470.

Llusià J, Peñuelas J (1998) Changes in terpene content and emission in potted Mediterranean woody plants under severe drought. Canadian Journal of Botany 76, 1366-1373.

Ormeno E, Cespedes B, Sanchez IA, Velasco-García A, Moreno JM, Fernandez C, Baldy V. (2009) The relationship between terpenes and flammability of leaf litter. For. Ecol. Manag. 257, 471-482.

Pausas JG, Alessio GA, Moreira B, Segarra-Moragues JG (2016) Secondary compounds enhance flammability in a Mediterranean plant. Oecol. 180,103-110.

Trabaud L (2000) Post-fire regeneration of Pinus halepensis forests in the West Mediterranean. Ecology, Biogeography and Management of Pinus halepensis and P. brutia Forest Ecosystems in the Mediterranean Basin. Backhuys Publishers, Leiden, The Netherlands, 257-268.

Yani A, Pauly G, Faye M, Salin F, Gleizes M. (1993) The effect of a long-term water stress on the metabolism and emission of terpenes of the foliage of Cupressus sempervirens. Plant, Cell \& Environment 16, 975-981. 


\title{
The large fire of Pedrógão Grande (Portugal) and its impact on structures
}

\author{
Luís Mário Ribeiro*; André Rodrigues; Davi Lucas; Domingos Xavier Viegas \\ Forest Fire Research Centre (CEIF), ADAI-LAETA, University of Coimbra, 3030 - 289 Coimbra, \\ Portugal. \{luis.mario@adai.pt*\}
}

\begin{abstract}
The fire complex that began on 17/6/2017, in Central Portugal, will be for always remembered, not only because of the extensive burned area, but mainly because it caused 65 deaths, more than 200 injured and destroyed hundreds of structures, making it one of the most serious accidents in the country, and one of the worst in Europe. The Forest Fire Research Centre of ADAI (University of Coimbra, PT) performed a detailed analysis and report on this fire complex focused on aspects related to the fire spread and behaviour, the mortal accidents and the fire impact on local communities. The work presented here is related to the latter, i.e., the destruction of property. The greatest impact was observed in the municipalities of Pedrógão Grande, Castanheira de Pera and Figueiró dos Vinhos, where the deaths occurred and where most of the damaged structures were located. This component of structural damage was the subject of an intensive fieldwork, during which we inventoried several aspects related to fire behavior, the structures and their surroundings. During the field work, 1043 structures damaged by fire were identified. They are mostly support structures, such as barracks or storage (38.6\%), but there is a considerable number of damaged dwellings, either permanent $(13.3 \%)$ or temporary $(11.9 \%)$. One of the most important aspects of the study relates to the type and location of structural ignition. More than $60 \%$ of the structures have been ignited due to the deposition of incandescent particles (firebrands) in different weak points. Likewise, more than $60 \%$ of these ignitions occurred on the roofs, mainly because of the vulnerability associated to the structures and materials supporting them. Another relevant factor is the observation of the advanced age of most buildings affected (over 30 years), which may indicate less resistance to the passage of a fire. Nevertheless, and for the Portuguese reality, we can say that buildings are generally safe during the passage of a fire, if themselves and their surroundings are kept in good conditions. The fact that there are people inside can be essential in their defense and resistance to the passage of fire.
\end{abstract}

This paper presents the main results of the assessment of the fire impact on structures, part of a larger work on the Fire Complex of Pedrógão Grande and neighboring municipalities.

Keywords: WUI; impact on structures; large fire

\section{Introduction}

The analysis of the impact of a fire on a given community has inherent the concept of Wildland Urban Interface (WUI), or simply interface. WUI can be simply defined as the space where structures and vegetation coexist in a fire prone environment (BRP, 2008). We must also add the human component (Ribeiro, 2016), because it is mainly people who are affected. This impact can be hard to evaluate, given the difficulty in obtaining measurable data that allows the establishment of gradations of social, economic or even emotional and familiar impact. From a purely structural impact perspective, it becomes more feasible to gather a set of parameters that allow us to estimate how the fire has impacted the community affected.

The way in which the structures in the WUI are damaged by wildfires has received special attention all over the world (e.g. Cohen, 2003; Cohen \& Saveland, 1997; Gollner et al., 2015; Graham et al., 2012; Westhaver, 2016) and efforts have been made by the scientific community, but also the operational and technical ones, in order to understand the ignition mechanisms of the structures and

Advances in Forest Fire Research 2018 - Page 852 
the weaknesses they present to the passage of a wildfire. For instance, Blanchi et al., (2012) carried out an historical analysis on the impact of fire on people and structures across Australia between 1901 and 2011. During this work specific databases were created to allow data harmonization and collection. Inspired by this report, we designed customized geodatabases to allow us the systematic collection of data related to the impacts of the wildfire complex of Pedrógão Grande and neighbouring municipalities, namely on personal accidents resulting in deaths, and on structures damaged. The geodatabases where simple but were designed to collect the maximum amount of detail in the field work, considering the time available to execute the field campaign.

After the extensive field campaign, a detailed analysis of all the collected data was performed, and published in the respective fire report (Viegas et al., 2017). We present here what we believe to be some of the most important results obtained.

\section{Methods}

The analysis of the impact of fire on structures was based on field verification of all buildings damaged by fire, regardless of their use or type. For this purpose, a geodatabase and a geospatial form of simple interpretation and completion were constructed. The database comprised 3 sections: i) description of the structures, for example the type of structure, age, fuel management in the surroundings, condition before and after the fire and usage, among others. The second section was related to describing how the fire affected the structure, namely, the ignition point and the way the structure ignited. The last section was related to fire details (time of arrival, impact on electricity, water, communications) and human behavior (escape/stay and defend, injuries, deaths).

Given the size of the affected area and the time available to carry out the survey and analysis, visiting the entire burned area searching for affected structures would be extremelly hard to accomplish. Therefore, the initial planning comprised the search for initial data sources related to the location of damaged structures. For this, we asked the affected municipalities for information on the structures impacted by the fire in each of them. From the municipalities of Castanheira de Pera, Figueiró dos Vinhos, Penela and Sertã we obtained georeferenced data of the structures that they identified as having been damaged in some way by the fire. Together with Pedrogão Grande, these were the municipalities selected to carry out the fieldwork and the respective analysis. To our knowledge, these surveys were carried out in all municipalities in order to allow applications for reconstruction or rehabilitation support. Later we obtained this data also from the municipality of Góis (27 houses damaged by fire), Pampilhosa da Serra ( 8 dwellings and 20 agricultural wharehouses) and Alvaiázere (10 structures but only a secondary housing), but they were not included in the fieldwork and therefore the analysis. From the municipality of Pedrógão Grande we were not able to obtain this information. However, at the beginning of this work we were invited to join an initiative of Esri Portugal - Sistemas e Informação Geográfica, SA (the official distributor of the North American Esri - Environmental Systems Research Institute, world leader in the technology of Geographic Information Systems), to support the decision in the wildfire of Pedrógão. This initiative was titled "FireHub 2017" and consisted in the creation of a collaborative open data platform (available in June 2017 at http://arcg.is/2rMwc0B). Using this platform, we had access to a set of georeferenced points representing structures allegedly damaged by fire, especially in the municipality of Pedrógão Grande, but with some cases in Castanheira de Pera and Penela. These points were not validated by any entity, so we proceeded with some care to include them in our work.

The impact of the fire was especially noted in the municipalities of Pedrógão Grande, Figueiró dos Vinhos and Castanheira de Pera, so the analysis presented focuses mainly on that region. We also decided to include the neighboring municipalities of Penela and Sertã, since they have suffered considerable damage, albeit in a smaller area. In the municipalities of Góis and Pampilhosa da Serra 
the impact of the fire was lower. It was not possible to extend the study to those two municipalities, due to difficulties related to the execution time of this work.

We were able to gather a substantial set of georeferenced points, which allowed us to schedule the field visits. These points, for a total of 704, represented, for the most part, permanent housings. During the fieldwork, 289 of these points proved to be false. In some cases, there were not even structures in the place indicated by the points, in others the structures had not been damaged by the fire. As we said before, we were interested in all structures damaged by the fire, not only the housings, so during the field work we inventoried another 684 points that were not listed initially. In total, 1388 points were visited, of which 1099 points were initially considered valid. Figure 1 shows the location of all points visited (valid and false) as well as the points not visited, referring to Góis (27), Pampilhosa da Serra (28) and Alvaiázere (10).

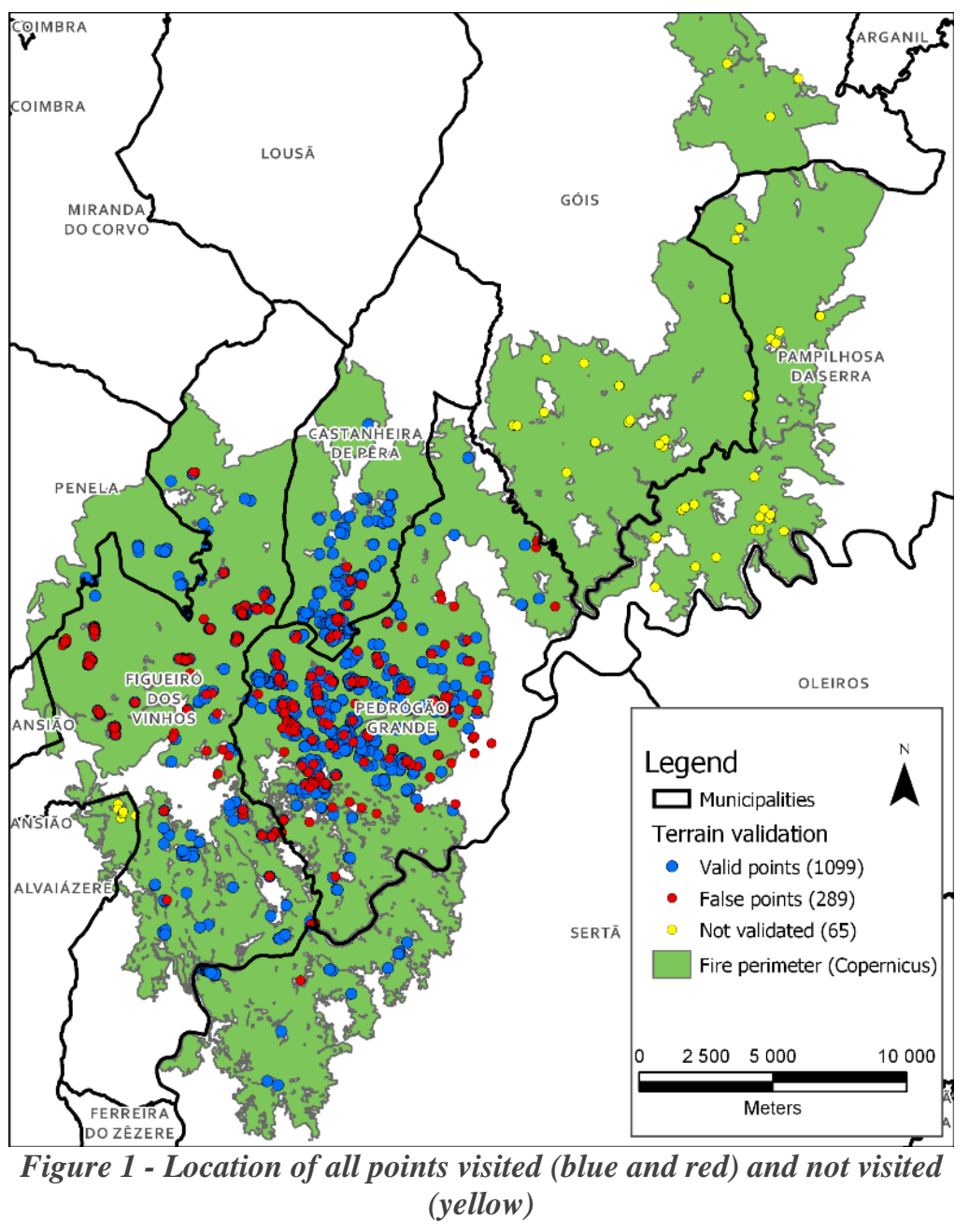

During the field work we encountered several structures in ruins that were already in that state before the fire. Although we started registering them, the number we encountered was so large (and the data gathered not that meaningful) that we decided to leave them out of the analysis. In total, discounting these structures, we finished with 1043 valid points that fulfilled the requirements for analysis.

\section{Results and discussion}

In total, 1043 points (representing affected structures) were considered valid for analysis, from approximately 1400 points visited. The data collected in the field work was treated and analyzed in ArcMap and IBM SPSS Software. Figure 2 shows a density map of damaged structures, or "heatmap". It can be clearly seen the area where the impact was greatest. This area, comprised of five municipalities, also coincides with the region of more extreme fire behavior and to where the 65 victims were found. 


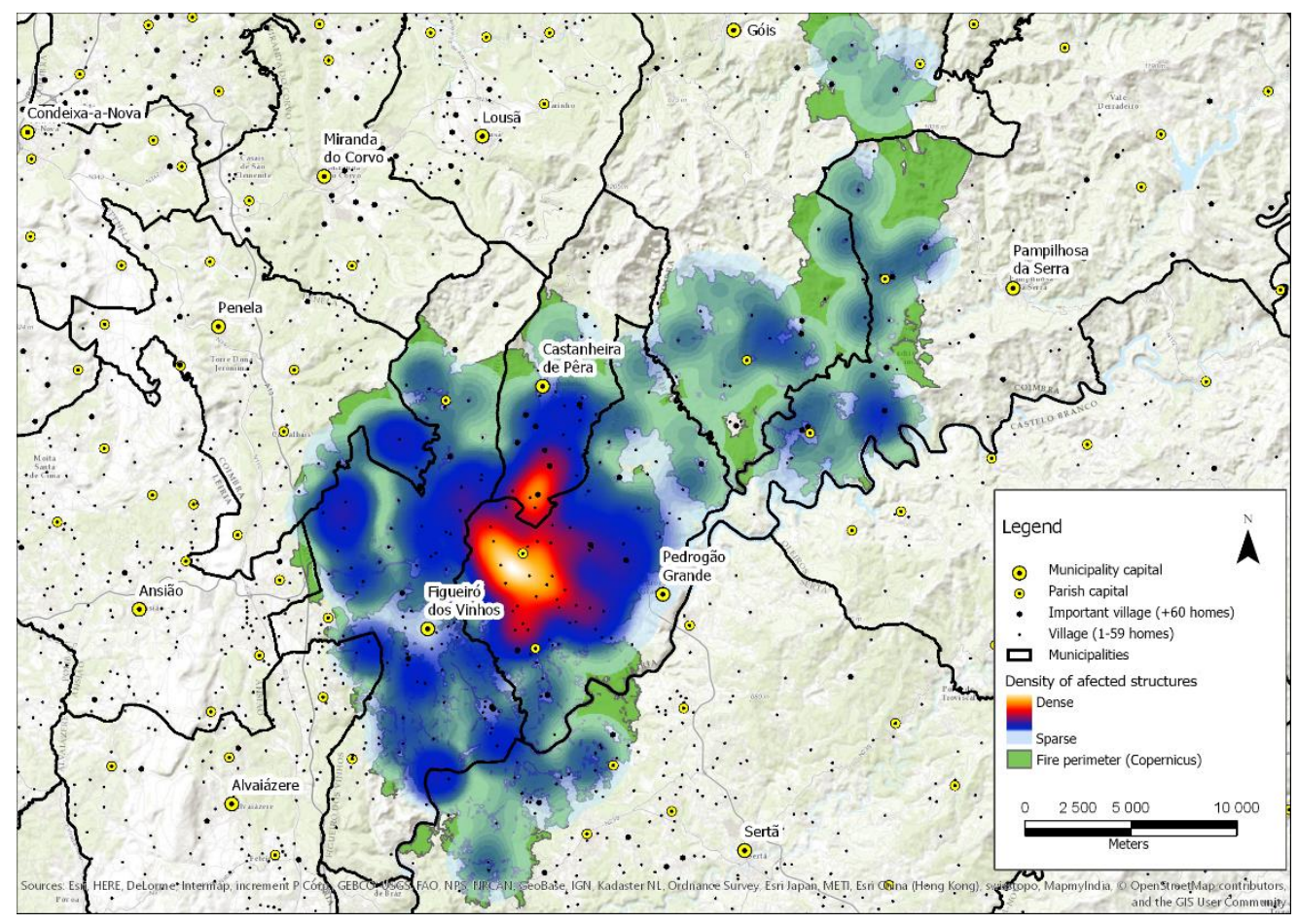

Figure 2 - Heatmap of damaged structures

This was the region chosen for the fieldwork and subsequent analysis. The data collected was treated and analyzed in ArcMap and IBM SPSS Software. Results were grouped in three categories: i) relating to the structure, ii) relating to the surroundings of the structure and iii) relating to the arrival and impact of fire. In the next tables (Tables 1 through 3) we present some of the most relevant results from each of these categories. A most complete set of results can be consulted in Viegas et al., 2017.

Table 1 - Results from the field work related to $i)$ the structure

\begin{tabular}{|c|c|c|c|c|c|c|}
\hline \multirow[b]{2}{*}{ Variable } & & \multicolumn{5}{|c|}{ Structure condition after the fire } \\
\hline & & $\begin{array}{c}\text { Slightly } \\
\text { damaged }\end{array}$ & $\begin{array}{c}\text { Moderately } \\
\text { damaged }\end{array}$ & $\begin{array}{c}\text { Highly } \\
\text { damaged }\end{array}$ & $\begin{array}{c}\text { Totally } \\
\text { destroyed }\end{array}$ & Total \\
\hline \multirow{12}{*}{$\begin{array}{c}\text { Type of } \\
\text { structure }\end{array}$} & Permanent housing & $35^{\circ}$ & 17 & $46^{\circ}$ & 41 & 139 \\
\hline & Secondary housing & 19 & 9 & 46 & 50 & 124 \\
\hline & Agricultural Warehouse & 1 & 5 & 28 & 40 & 74 \\
\hline & Shed / Storage & 12 & 20 & 179 & 192 & 403 \\
\hline & Garage & 5 & 9 & 22 & 24 & 60 \\
\hline & Comerce facility & 0 & 1 & 0 & 0 & 1 \\
\hline & Industry & 0 & 2 & 5 & 8 & 15 \\
\hline & Uninhabited house & 2 & 4 & 36 & 16 & 58 \\
\hline & Devolute structure & 1 & 3 & 56 & 72 & 132 \\
\hline & Stable & 2 & 1 & 8 & 9 & 20 \\
\hline & Outdoor kitchen & 0 & 2 & 2 & 2 & 6 \\
\hline & Other & 2 & 1 & 4 & 4 & 11 \\
\hline \multirow{3}{*}{$\begin{array}{l}\text { Age of the } \\
\text { structure }\end{array}$} & $<10$ years & 6 & 5 & 2 & 13 & 26 \\
\hline & between 10 and 30 years & 17 & 11 & 26 & 64 & 118 \\
\hline & $>30$ years & 56 & 58 & 399 & 380 & 893 \\
\hline \multirow{5}{*}{$\begin{array}{c}\text { Type of } \\
\text { construction }\end{array}$} & Concrete & 71 & 57 & 195 & 207 & 530 \\
\hline & Stone & 7 & 14 & 222 & 177 & 420 \\
\hline & Wood & 0 & 0 & 0 & 29 & 29 \\
\hline & Metal & 1 & 2 & 14 & 43 & 60 \\
\hline & Other & 0 & 1 & 1 & 1 & 3 \\
\hline
\end{tabular}


Most of the structures affected by the fire are support structures, such as barracks or storage $(38.6 \%)$, but there is a considerable number of damaged dwellings, either permanent $(13.3 \%)$ or temporary $(11.9 \%)$. The inventoried structures are in general aged, with $86 \%$ being older than 30 years, but they are mainly support structures (only 23\% of affected houses have more than 30 years). Construction in Portugal is mainly of concrete, but in this area we found many old stone structures. Age and building materials are important factors to be taken into account in the structural strength analysis but are themselves dependent on the degree of conservation or maintenance in which the owners maintain them. During the visits it was possible to observe cases of old houses, mainly of schist, recovered and maintained in excellent conditions (mainly by foreigners), while at the same time relatively recent structures were seen, but with a very low degree of maintenance.

Table 2. Results from the field work related to Results from the field work related to ii) the surroundings of the structure

\begin{tabular}{|c|c|c|c|c|c|c|}
\hline \multirow{2}{*}{ Variable } & & \multicolumn{5}{|c|}{ Structure condition after the fire } \\
\hline & & $\begin{array}{c}\text { Slightly } \\
\text { damaged }\end{array}$ & $\begin{array}{l}\text { Moderately } \\
\text { damaged }\end{array}$ & $\begin{array}{c}\text { Highly } \\
\text { damaged }\end{array}$ & $\begin{array}{c}\text { Totally } \\
\text { destroyed }\end{array}$ & Total \\
\hline \multirow{9}{*}{$\begin{array}{l}\text { Land use (COS } \\
\text { 2010, level 2) }\end{array}$} & 1.1 Urban Tissue & 48 & 39 & 181 & 235 & 503 \\
\hline & $\begin{array}{l}1.2 \text { Industry, commerce and } \\
\text { transportation }\end{array}$ & 0 & 1 & 3 & 4 & 8 \\
\hline & $\begin{array}{l}\text { 1.3 Areas of inert extraction, waste } \\
\text { disposal sites and construction sites }\end{array}$ & 0 & 1 & 3 & 1 & 5 \\
\hline & $\begin{array}{l}\text { 1.4 Urban green spaces, sports, } \\
\text { cultural and leisure facilities, and } \\
\text { historical areas }\end{array}$ & 0 & 0 & 1 & 1 & 2 \\
\hline & 2.1 Temporary crops & 1 & 2 & 4 & 3 & 10 \\
\hline & 2.2 Permanent crops & 4 & 4 & 19 & 18 & 45 \\
\hline & 2.4 Heterogeneous agricultural areas & 20 & 21 & 152 & 126 & 319 \\
\hline & 3.1 Forests & 4 & 6 & 55 & 58 & 123 \\
\hline & $\begin{array}{l}\text { 3.2 Open forests and shrub and } \\
\text { herbaceous vegetation }\end{array}$ & 2 & 0 & 14 & 12 & 28 \\
\hline \multirow{3}{*}{$\begin{array}{c}\text { Fuel } \\
\text { Management }\end{array}$} & Absent & 17 & 20 & 183 & 203 & 423 \\
\hline & Partial & 37 & 40 & 198 & 211 & 486 \\
\hline & Complete & 7 & 8 & 19 & 20 & 54 \\
\hline
\end{tabular}

Analyzing the land use obtained with the level 2 of the official Land Use Map (COS 2010), produced by the Portuguese Direção Geral do Território (General Directorate of Territory), it is possible to verify that most of the impact was on the urban areas (50\%), specially the urban tissue (48\%). The most affected area after this was the one classified as "heterogeneous agricultural areas" (30\% of the total). The General Directorate of the Territory (Direção-Geral do Território, 2016) describes these areas as "agricultural areas with various types of associations between temporary crops, pastures, permanent crops and natural areas. Includes temporary crops and/or pastures associated with permanent crops, temporary or permanent crops grown under forest cover, areas of temporary crop mosaics, pastures and permanent crops, and landscapes in which crops and pastures are mixed with natural or semi-natural areas. Only about $11 \%$ of damaged structures were within forest areas.

Fuel management is a difficult parameter to assess after the passage of the fire. Nevertheless, and with the help of the local inhabitants, we were able to inventory this aspect in 963 structures. The criteria we used was that, in order to have a "partial" fuel management, at least one of the sides of the structure should have a discontinuity of no less than 3 meters. A "full" fuel management would mean that all 4 sides of the structure had it. Although the results may be statistically not representative, as we did not inventory non-affected houses, they suggest the idea that having "partial" or "absent" fuel 
management may be indifferent to the structure survival. In other words, either fuel management is active on all the surroundings of the structure or its effectiveness may be compromised.

Table 3. Results from the field work related to iii) the arrival and impact of the fire

\begin{tabular}{|c|c|c|c|c|c|c|}
\hline \multirow[b]{2}{*}{ Variable } & & \multicolumn{5}{|c|}{ Structure condition after the fire } \\
\hline & & $\begin{array}{c}\text { Slightly } \\
\text { damaged }\end{array}$ & $\begin{array}{c}\text { Moderately } \\
\text { damaged }\end{array}$ & $\begin{array}{c}\text { Highly } \\
\text { damaged }\end{array}$ & $\begin{array}{c}\text { Totally } \\
\text { destroyed }\end{array}$ & Total \\
\hline \multirow[t]{5}{*}{ Type of ignition } & Embers (spotting) & 27 & 54 & 294 & 261 & 636 \\
\hline & Direct fire impact & 7 & 8 & 91 & 116 & 222 \\
\hline & Materials burning on the vicinity & 7 & 9 & 43 & 74 & 133 \\
\hline & Neighbour structure & 2 & 1 & 3 & 6 & 12 \\
\hline & Damaged but no ignition & 35 & 2 & 1 & 0 & 38 \\
\hline \multirow{8}{*}{$\begin{array}{l}\text { Location of the } \\
\text { ignition }\end{array}$} & Roof & 16 & 36 & 299 & 293 & 644 \\
\hline & Window & 14 & 17 & 70 & 68 & 169 \\
\hline & Door & 4 & 7 & 36 & 28 & 75 \\
\hline & Open structure & 2 & 6 & 13 & 47 & 68 \\
\hline & Wall & 5 & 4 & 0 & 14 & 23 \\
\hline & Ventilation & 0 & 1 & 12 & 6 & 19 \\
\hline & Other & 3 & 1 & 1 & 0 & 5 \\
\hline & Damaged but without ignition & 35 & 2 & 1 & 0 & 38 \\
\hline
\end{tabular}

One of the most important aspects of the study relates to the type and location of structural ignition. More than $60 \%$ of the structures have been ignited due to the deposition of incandescent particles (firebrands) in different weak points. This percentage could even be higher, as 133 ignitions (12\%) were identified as being related to different materials burning in the vicinity of the structure. These were most probably also ignited by embers. More than $60 \%$ of these ignitions occurred on the roofs, mainly because of the vulnerability associated to the structures and materials supporting them.

\section{Conclusion}

The largest impact of this fire complex occurred in the Pedrogão Grande, Castanheira de Pera and Figueiró dos Vinhos municipalities, including small areas in the neighboring municipalities of Penela, Alvaiázere and Sertã. Most damaged structures occurred in this area. These are mainly structures of advanced age (over 30 years), but the majority without being housing. In the fieldwork carried out in the six mentioned counties, we inventoried 263 damaged dwellings (primary and secundary), 91 of which were destroyed. Most ignitions were registered as being originated by firebrands, and mainly affecting roofs and windows. The partial or full absence of fuel management in the surroundings of the houses is a constant in all the burned area. That may explain, in part, the destructive outcome of the Pedrógão fire. Another relevant factor is the observation of the advanced age of most buildings affected (over 30 years), which may indicate less resistance to the passage of a fire. Nevertheless, and for the Portuguese reality, we can say that buildings are generally safe during the passage of a fire, if themselves and their surroundings are kept in good conditions. The fact that there are people inside can even be essential in their defense and resistance to the passage of a wildfire.

\section{Acknowledgements}

The authors wish to acknowledge the support of the Portuguese Government in funding this work and the partial support of project FIREXTR (Prevent and prepare society for extreme fire events: the challenge of seeing the "forest" and not just the "trees"), co-financed by the European Regional 
Development Fund (ERDF) through the COMPETE 2020 - Operational Program Competitiveness and Internationalization (POCI Ref: 16702) ) and national funds by FCT-Foundation for Science and Technology (Proj Ref: PTDC/ATPGEO/0462/2014).

\section{References}

Blanchi, R., Leonard, J., Haynes, K., Opie, K., James, M., Kilinc, M., Dimer de Oliveira, F., \& Van den Honert, R. (2012). Life and house loss database description and analysis Final report. CSIRO, Bushfire CRC report to the Attorney-General's Department. CSIRO EP-129645.

BRP. (2008). The Blue Ribbon Panel on Wildland/Urban Interface Fire. Blue Ribbon Panel. International Code Counci (ICC), USA.

Cohen, J. D. (2003). Preventing Residential Fire Disasters During Wildfires. Em G. Xanthopoulos (Ed.), Proceedings of the International Workshop"Forest Fires in the Wildland-Urban Interface and Rural Areas in Europe: An integral planning and management challenge", May 15-16. Athens, Greece.

Cohen, J. D., \& Saveland, J. (1997). Structure Ignition Assessment Can Help Reduce Fire Damages in the W-UI. Fire Management Notes, 57(4), 19-23. http://doi.org/http://www.firewise.org/wildfirepreparedness/wui-home-ignition-research/the-jack-cohen-files.aspx?sso=d1d65917-4f03-46dabb5f-0b815f0784ea

Direção-Geral do Território. (2016). Especificações Técnicas da Carta de Uso e Ocupação do Solo (COS) de Portugal Continental para 1995, 2007 e 2010. Relatório Técnico. Direção-Geral do Território. Coordenação: Mário Caetano, Cristina Igreja e Filipe Marcelino.

Gollner, M. J., Hakes, R., Caton, S., \& Kohler, K. (2015). Pathways for Building Fire Spread at the Wildland Urban Interface. Fire Protection Research Foundation.

Graham, R., Finney, M., McHugh, C., Cohen, J., Calkin, D., Stratton, R., Bradshaw, L., \& Nikolov, N. (2012). Fourmile canyon fire findings. USDA Forest Service - General Technical Report RMRSGTR.

Ribeiro, L. M. (2016). Os incêndios na interface urbano-florestal em Portugal: uma análise de diagnóstico. Dissertação para a obtenção do grau de Mestre em Dinâmicas sociais, riscos naturais e tecnológicos. Faculdade de Ciências e Tecnologia da Universidade de Coimbra.

Viegas, D. X., Almeida, M. F., Ribeiro, L. M., Raposo, J., Viegas, M. T., Oliveira, R., Alves, D., Pinto, C., Jorge, H., Rodrigues, A., Lucas, D., Lopes, S., \& Silva, L. F. (2017). O complexo de incêndios de Pedrógão Grande e concelhos limítrofes, iniciado a 17 de junho de 2017. Centro de Estudos sobre Incêndios Florestais (CEI/ADAI/LAETA).

Westhaver, A. (2016). Why some homes survived: Learning from the Fort McMurray wildland/urban interface fire disaster. Toronto, Ontario (Canada): Institute for Catastrophic Loss Reduction Research paper series (56). 


\title{
The urban side of the Wildland-urban interface - a new fire audience identified following an extreme wildfire event in Aotearoa/New Zealand
}

\author{
E.R. (Lisa) Langer ${ }^{1 *}$; H. Grant Pearce ${ }^{1}$; Simon Wegner $^{3}$ \\ ${ }^{1}$ Scion. PO Box 29 237, Christchurch 8440, New Zealand, \{lisa.langer@ scionresearch.com*, \\ grant.pearce@scionresearch.com\} \\ ${ }^{3}$ Scion, Private Bag 3020, Rotorua 3046, New Zealand, \{simon.wegner@scionresearch.com\}
}

\begin{abstract}
Wildland-urban interface wildfires are increasingly impacting communities worldwide. In New Zealand this has been demonstrated by the destruction or damage of over 25 homes during the 2016-2017 fire season, the greatest number lost in 100 years. The wildfire which most impacted these statistics is the Port Hills fire, which resulted in the loss of nine houses and the evacuation of over 1400 residents on the margin of the city of Christchurch in February 2017. This wildfire opens the door to a new focus of attention required to address wildfire mitigation needs of suburban properties in the urban fringe in wildfire prone areas.

Although mitigation measures for large and small rural properties are well understood by fire officials and conveyed to property owners, uptake has been minimal in the dense suburban communities which extend into the WUI. Suburban property owners in the urban fringe will require greater focus by fire managers to ensure residents are aware of the risk of wildfires, and adequately prepared to face the risk of potentially devastating wildfires in the future. However, these communities are not merely denser versions of their rural neighbours and lessons from other parts of the WUI may not apply. Engaging with them will necessitate understanding their differences and developing new messages appropriate to their context.
\end{abstract}

Keywords: urban fringe, risk awareness, mitigation, preparedness

\section{Background}

Frequent small, and occasional large, wildfires occur in New Zealand (about 4100 wildfires were recorded burning approximately 4170 ha per year from 2005-06 to 2014-15; National Rural Fire Authority data 2015). The majority of these wildfires are caused by human activity (Anderson et al. 2008). However, the impact of wildfire events on communities in the wildland-urban interface (WUI, or rural-urban interface (RUI) as it is more commonly referred to in New Zealand) has grown in recent years with over 25 homes destroyed throughout country during the 2016-2017 fire season, the greatest number lost in 100 years (AFAC 2017).

The WUI generally is defined as areas where houses mix or intermingle with potentially flammable vegetation, such as where buildings are in close proximity to large contiguous patches of flammable vegetation (interface) and where buildings are interspersed within flammable vegetation (intermix) (Radeloff et al. 2005). However, properties impacted by WUI wildfires are not just those surrounded by rural land, but include those that lie within suburbs, with either one boundary bordered by rural land or completely within the city limits, on the margins of urban areas or what is often referred to as the urban fringe.

There is an increasing worldwide trend of wildfires impinging on the margins of suburban areas of cities with an associated increasing risk to lives and property (for example, Hobart, Australia, 1967; Canberra, Australia, 2003; and Fort McMurray, Canada, 2016). Similarly wildfire events on the margins of urban areas are not new to New Zealand (Fogarty, 1997; Graham and Langer 2009; Hart and Langer 2014), but up until 2017 they were relatively infrequent and few properties were destroyed 
or fatalities experienced. This changed in February 2017, however, when a large wildfire occurred on the Port Hills, adjoining the city of Christchurch in the South Island, significantly adding to the tally of destruction experienced during the 2016/17 fire season. Although regarded as a moderately small wildfire in international terms, the Port Hills wildfire is considered one of the biggest and most severe in recent New Zealand history, bringing with it new wildfire management and community preparedness concerns.

Research in New Zealand to date has focused on wildfire-affected rural and WUI communities, including lifestyle ${ }^{1}$ block owners, from a rural perspective. That is, the WUI has largely been characterised as areas where formerly urban residents' have relocated into rural spaces. These new lifestyle block properties may be clustered together, but generally retain the low density and character of an otherwise rural landscape. Effectively, a wildfire impacts a single property and then may spread to neighbouring lifestyle properties. Therefore, while their owners represent a different audience, the fire landscape and the messages fire officials wish to convey are largely unchanged.

Fires on the margins of cities, however, bring residents from suburban properties into the spot light. Although they may be adjacent to areas with lifestyle blocks, denser suburban developments on previously undeveloped land have an entirely different character, essentially transforming pockets of space from rural to urban. A single wildfire can impact on multiple properties at once, and even spread from structure to structure within the suburban area.

Suburban residents represent an entirely different audience as well as a different landscape, changing the nature of their threat and range of preparation options at hand. This will require a shift in fire management, supported by research to ensure that they too have adequate awareness of the risk of future wildfires that could impact them and prepare their properties accordingly.

\section{Methodology}

This paper considers the Port Hills wildfire event on the margin of Christchurch in February 2017 and highlights impacts on local residents living in the denser suburban areas of the WUI. Drawing on ongoing research into the fire and its aftermath, it discusses the changing nature of the wildfire threat in New Zealand and the implications these changes have for communities, for fire and land managers and, consequently, for researchers.

International and national reporting of wildfires that have affected communities on the fringe of urban areas, community audiences and their risk awareness and preparedness have been evaluated to set the context for this think piece.

\section{Port Hills fire event}

The Port Hills wildfire occurred on the fringe of urban areas of Christchurch city and burned 1660 ha of largely ungrazed grass, conifer plantations, pockets of native forest, and regenerating native and exotic scrub vegetation, within a perimeter of $60 \mathrm{~km}$ (see Figure 1). The wildfire began as two separate fires on 13 February 2017, starting within two hours of each other in different areas of the Port Hills and then joining two days later to become an extreme wildfire event. A state of emergency was

\footnotetext{
${ }^{1}$ A lifestyle property is a New Zealand term introduced by real estate agents in the 1980's to describe rural small holdings purchased by people who want to live a rural lifestyle, often with a small number of farm animals or other agricultural activities, but who derive their principal income from non-farming activities (Paterson, 2005).
} 
declared to support emergency services, and the fire was not officially considered to have been extinguished for 66 days (AFAC 2017).

The fire resulted in the loss of nine homes and fire damage to five others on small lifestyle properties surrounded by rural land. The wildfire caused the evacuation of about 450 households (estimated over 1400 residents) for 3-9 days. Significant loss or threat also occurred to infrastructure, including major power lines, airport radar, communications networks, radio and television transmission towers, and a mountain-bike and recreational facility. Tragically, a helicopter pilot was killed in an accident while fighting the fire. Although most devastation was experienced by those living on lifestyle properties, the majority of threatened households lay on small properties of less than $1000 \mathrm{~m}^{2}$ within suburban areas and had either one boundary bordered by rural land or were completely within the city limits.

The Port Hills, with moderate to steep ridges and gullies and slopes up to 30 degrees, and a range of flammable fuel types including particularly sprayed gorse (Ulex europaeus) and cured rank grass, had fire behaviour potential which ranged between high and extreme. Thus, the area exhibited a particular susceptibility to wildfire events at this time of the summer and a total fire ban was in place.

At the time of this wildfire event, rural fire control was the responsibility of Rural Fire Authorities, comprising local territorial authorities (district and city councils), the Department of Conservation, and Defence and forestry-based Rural Fire Districts. Four months later, on 1 July 2017, the urban and rural fire agencies were merged into the single Fire and Emergency New Zealand organisation.

The cause of both wildfires ignitions remains undetermined, but the post-fire investigation believed them to have been suspicious. Based on the information available, Fire and Emergency New Zealand believe the first ignition to have been deliberately lit, and the second either accidental or deliberately lit (FENZ, 2017).

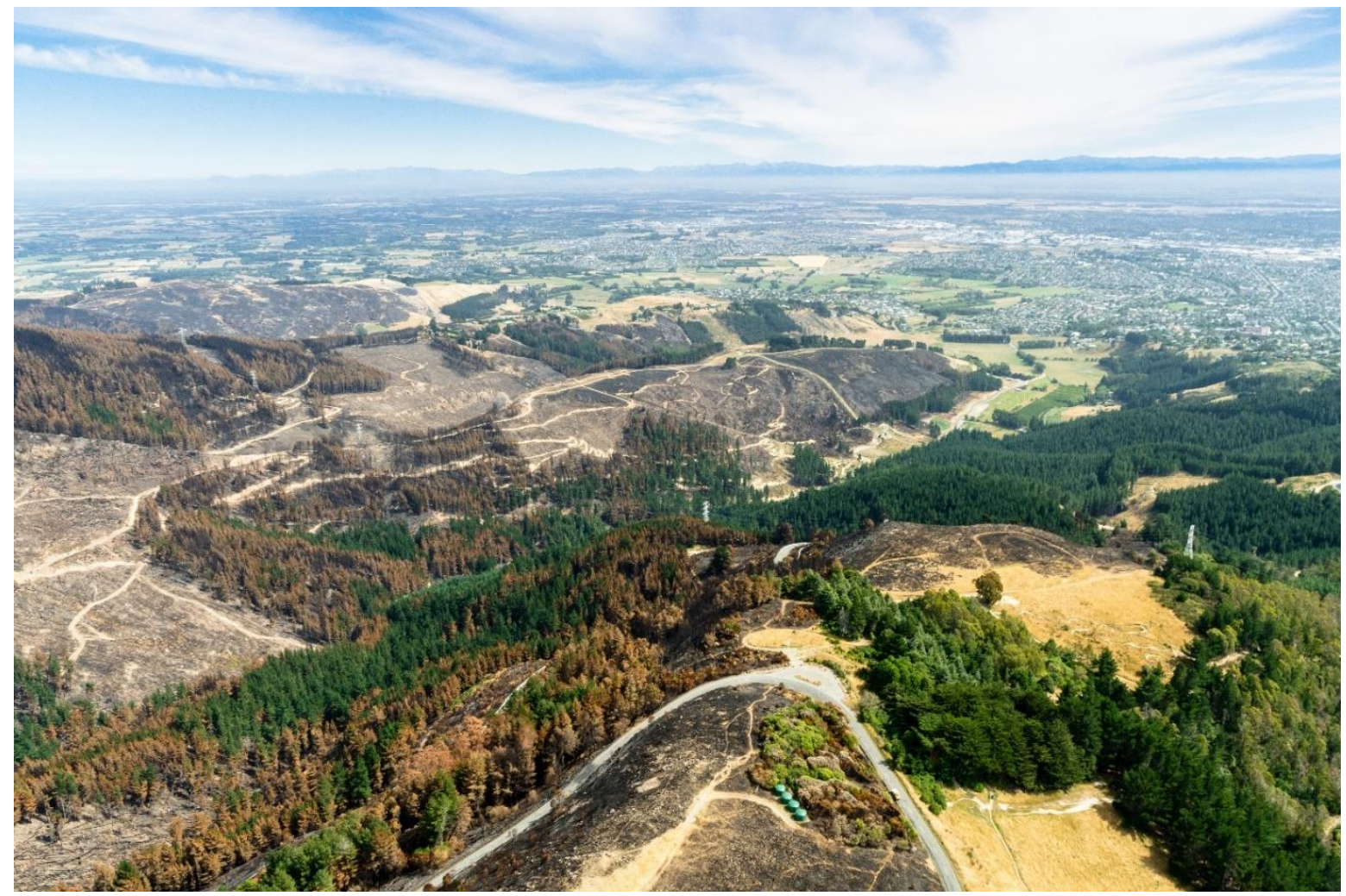

Figure 1 - Aerial view of the wildfire which occurred on the Port Hills, adjoining Christchurch, burning 1660 ha of farmland, plantation and native forest and recreational areas, destroying nine homes and damaging a further five, and causing the evacuation of about 450 households within a perimeter of $60 \mathrm{~km}$ in February 2017 (V. Clifford, Scion). 


\section{Drivers of change leading to increased wildfire risk}

Though fires on the margins of urban areas in New Zealand have been relatively rare in the past, they are likely to become more common. Climate change modelling predicts that New Zealand will become hotter and drier, creating conditions that increase both the frequency and severity of wildfire events (Pearce et al. 2005; Pearce and Clifford 2008; Pearce et al. 2011). With the overall risk of wildfire expected to increase, so too is the likelihood that these fires will occur within the WUI (Reisinger et al. 2014).

The increased risk associated with changing climate is compounded by a changing landscape. As is common worldwide, the area of New Zealand encompassed by the WUI and the number of people living and recreating within that zone are growing (Bayley and Goodyear 2004). Andrew and Dymond (2012) estimated that 5800 new lifestyle blocks have been added annually since 1998. Not only does this mean that more people are exposed to wildfire risk, the growing number of people and homes also increases the costs of fire suppression (Gude et al. 2013) and introduces more opportunities for humancaused wildfires to occur (Radeloff et al. 2018), through accidental ignition sources such as mower blade strikes, recreational fires and fireworks, escaped rubbish burns or electrical infrastructure faults as well as through intentional arson. Additionally, many new WUI residents will have little knowledge about fire risks or experience with fire use, so will pose greater individual risk of starting accidental wildfires than previous rural residents (Jakes et al. 2010; Hart and Langer 2014).

At the same time, in some parts of New Zealand, land near the WUI that was formerly grazed pasture is being retired from production and used instead for conservation or recreation purposes. Often, this results in rising fuel loads as grasses are slowly replaced by woody shrubs and native bush. The combined impacts of these changes are already becoming apparent in a recent increase in WUI fire incidents and increased number of homes threatened, damaged or lost (Pearce unpubl. data).

\section{WUI in New Zealand}

\subsection{Audiences, risk awareness and preparedness}

Turning to the literature, we are informed that not all residents have the same level of fire experience, fire risk awareness or preparedness. Internationally, research has demonstrated that residents living in high-risk areas (McCaffrey et al. 2013,2015) or where a recent wildfire has occurred (McGee et al. 2009; Champ and Brenkert-Smith 2016) are more likely to be aware of wildfire risk. Research in New Zealand has identified similar differences in awareness between and within communities. Those with greater experience of rural living, or of wildfire, have been found to be more aware of wildfire risk and more likely to take preventative action (Jakes and Langer 2012; Langer and Hart 2014; Langer and McGee 2017; Nicholas and Hepi 2017). For example, in a study of a wildfireaffected peri-urban community in Canterbury, newcomers with shorter residency were found to have less awareness of wildfire risk and preparedness than longer-term residents (Jakes et al. 2010). Likewise, drawing on three case studies in wildfire-prone areas across New Zealand, Hart and Langer (2014) found varying knowledge of wildfire risk, fire restrictions and preparedness measures between long-term rural or semi-rural fire users and non-resident land owners or suburban dwellers who had less fire experience and did not use fire.

It is becoming increasingly clear, however, that there can also be significant variations in how people perceive and act upon wildfire risks. While experience with wildfire is associated with increased risk awareness in the short term, the connection is not universal and does not always lead to preventative or mitigative action. In an American study, for example, Olsen et al. (2017) found that WUI residents' perceptions of wildfire risk had little impact on levels of preparedness.

Much of the variation may be because of differences in how people interpret and act upon their perceptions of wildfire risk (Paveglio and Edgeley, 2017; Sword-Daniels et al. 2018). Though the

Advances in Forest Fire Research 2018 - Page 862 
literature typically treats geographic communities as homogenous units with shared perspectives and experiences, differences between socio-economic, cultural and demographic sub-communities can have profound impacts on how they interpret fire experience and risk (Paveglio and Edgeley 2017; Hart and Langer unpubl. data). For example, Langer and Hart (2014) described how, in a community that had recently been affected by wildfires, town dwellers who had witnessed wildfire nearby tended to have seen it as a spectacle rather than a threat and, therefore, showed little appreciation of risk to their home or person and tended not to take mitigative action. On an individual level, values, identities, lived experiences and other psycho-social factors shape how people interpret risks and decide how to act (Sword-Daniels et al. 2018). An exploratory study of media and social media during and after the Port Hills fire, has already identified significant differences in how people made sense of the fires in the context of underlying socio-political debates and where people believe responsibility for action lies (Langer and Wegner unpubl. data). Further research will be necessary to fully understand the impact that these fires have had.

However, the expanding WUI brings added social challenges beyond simply introducing another, different audience. As WUI communities grow in population, the potential complexities within them become even greater. Moreover, because a high proportion of the growth is in developments on previously undeveloped land, they may lack any form of community per se. There is also potential for conflict between new WUI residents and longer-term, previously rural residents who have experienced the landscape and community around them changing (Curran-Cournane et al. 2016). These considerations have been missing from the New Zealand literature and is underdeveloped internationally. Thus, research will be needed to better understand the more urban populations and communities living within the WUI and how they might differ from previous conceptions of communities at risk from wildfire.

\subsection{Mitigation}

Although there is well-established literature discussing mitigation options for landowners to reduce the likelihood and impact of wildfires (e.g. Cohen, 2000; Mell et al. 2010), this has not translated into significant mitigation in developing WUI areas of New Zealand.

Fire and land management agencies throughout the world recognise both wildfire risk and appropriate mitigation options (e.g. Cohen, 2000; Calkin et al. 2014). The necessity of having planning restrictions in place to ensure appropriate house siting and the use of fire-resistant construction materials for buildings, and increased homeowner awareness and education to encourage fire risk mitigation activities properties, such the management of fuels and maintenance of 'defensible (or defendable) space'1 (Syphard et al. 2014; Kornakova and March, 2017) are well recognised by fire managers.

Worldwide, people choose to live in wildfire prone areas and often do not fully understand the severity of wildfire risk (relative to other risks). The lack of awareness is demonstrated by the fact that limited mitigation measures are put in place by residents on their individual properties, collectively with their neighbourhoods, or in partnership with fire and other land management agencies (e.g.

${ }^{1}$ The concept of a defensible space is to remove or reduce the amount of flammable vegetation within a zone of at least 10-30 m wide (and preferably greater, where space permits) around the home which can limit fire spread and flame contact, and reduce radiated heat, and thereby increasing the chances of house survival (Wilson and Ferguson 1986; Cohen 2000). Regular maintenance to remove the build-up of dead material, including in roof gutters where leaf litter can provide a potential ignition point for windblown embers is the key to a successful defensible space. Low flammability species also can significantly reduce fire spread and intensity. 
Toman et al. 2013; McCaffrey 2015) and homes and lives continue to be lost in WUI fires. Successful community programmes have been adopted in North America and South Africa (such as the US FireWise and the Canadian FireSmart programmes (Haynes and Madsen 2017; Partners in Protection 1999) and, more broadly, the Fire Adapted Communities Learning Network (Anon. 2014)). However, in New Zealand, the National Rural Fire Authority's FireSmart programme (NRFA 2009) has struggled to get traction (Hart and Langer 2014; Pearce et al. 2014).

To date planning and wildfire messages to encourage mitigation by residents in the WUI has focused on rural and lifestyle properties. However, the Port Hills fire, and those that have preceded it that have either damaged or threated suburban properties in the urban fringe, identifies a new audience that needs to be considered. It also raises the question of whether instructions and target audiences need to be broadened and modified to suit different properties. For example, a defensible space of 10$30 \mathrm{~m}$ may be hard to implement on smaller suburban properties.

\section{Discussion}

The devastating 2017 Port Hills wildfire, and other similar WUI fire incidents from the past fire season, must serve as a major impetus to New Zealand fire agencies and property owners alike to raise awareness of the WUI fire issue, and increase education and guidance to at-risk communities on options for mitigating wildfire risk. Just as importantly, they should also prompt a significant review of the treatment of wildfire risk in local planning processes across the country, including improved identification of wildfire prone areas, and development of stronger requirements around development, construction and provision of defensible space in these high wildfire risk areas.

The risk of WUI wildfires like the Port Hills wildfire is not new. Similar wildfires have been reported widely over the years, both internationally (Butler 1974; Gale and Cortner 1987) and in New Zealand (Anon. 1982, FRFANZ 1994). The Port Hills wildfire, which resulted in the greatest reported property loss in an individual fire in almost 100 years, and followed a number of other WUI fires during the same fire season, serves as a wakeup call to fire and land managers. It provides a window into the future for New Zealand, with the likelihood of many similar wildfire events in the WUI impacting on communities, intensified by climate change, changing land use and the development of further subdivisions in the urban fringe of cities. Although affected communities are likely to have heightened awareness for the following wildfire season if dry conditions prevail, this is unlikely to be translated into increased mitigation by households, neighbourhoods and communities to reduce the longer term prospect of adverse impacts in the future. This step will not occur without considerable guidance and continued input from agencies - guidance which has been adapted to suit the specific audience.

The Port Hills wildfire has identified a new audience that will require special consideration by fire and land managers to ensure suburban residents in the urban fringe are aware of the risk wildfires could pose and adequately prepared for likely future disasters.

Fire and land management agencies have the responsibility to reduce the probability of home exposure to wildfire, through fire prevention measures (such as fire season restrictions and activity controls) that reduce the prospect of future wildfires events, and also readiness and response activities to suppress wildfires when they do occur. The management of fuels can also help reduce the chances of fire spread, decrease potential fire intensity, and hence increase the likelihood of successful fire suppression. Significant WUI fires such as the recent Port Hills wildfire clearly show that not all fires can be prevented, or controlled before they adversely impact people and property. This highlights the necessity for local government to ensure appropriate local planning and land use zoning controls to reduce exposure to wildfires.

In New Zealand, FireSmart community fire protection initiatives led by the National Rural Fire Authority/NZ Fire Service (NRFA 2004, 2009) have struggled to gain traction (Hart and Langer 2014)

Advances in Forest Fire Research 2018 - Page 864 
and perceived wildfire risk is considered to be a low priority compared to other hazards, such as flooding or earthquakes. It is essential that planners give greater recognition to wildfire risk, particularly in wildfire prone regions, and convert this into local planning processes. Glavovic (2010) and others (e.g. Saunders and Kilvington 2016, Crawford et al. 2018) have clearly articulated the benefits of natural hazards planning in New Zealand, and identified the barriers and priority actions required to realise the potential for disaster risk reduction. Key to achieving this is the strengthening of links between planners and emergency managers (Saunders et al. 2007), and also engaging and involving communities in the planning process. The latter is by no means an easy task, but initiatives such as Community Wildfire Protection Plans (CWPPs) have been found to be very successful in the U.S. (Jakes and Sturtevant 2013). However, this requires an understanding of the make-up of communities (Carroll and Paveglio 2016; Hart and Langer, unpubl. data) and what they value (Beilin and Reid 2015; Rawluk et al. 2017).

An onus remains on homeowners to take some responsibility for protecting their property from wildfires (Calkin et al. 2014) with or without specific local government wildfire risk planning requirements or community-led fire risk reduction initiatives, However, observance of planning requirements will not prevent all homes from being destroyed. Similarly, fire agencies cannot protect every property during a major event.

Research to date highlights clear differences in wildfire risk awareness and preparedness actions by urban and rural residents. In tandem with this, it illustrates complexities and divisions within communities that influence how people make sense of wildfire experiences (McCaffery 2015; Jakes and Langer 2012; Langer and McGee 2017). Opposing understandings of responsibility may help to explain the difficulty that fire experts face in promoting community action and, in particular, the poor correlation between people being aware of risks from wildfire and taking actions to mitigate those risks. The connection between awareness and taking actions to mitigate risk may well depend on where the ownership of risk and responsibility is seen to lie (Hart and Langer 2014; Langer and Wegner unpubl. Data). Hart and Langer (unpubl. data) have demonstrated the need for fire managers to proactively work with sectors of communities on an individual basis to build the community preparedness for future wildfire events.

This discussion paper paves the way for an in-depth study of the community affected by the 2017 Port Hills fire to gain a better understanding of wildfire awareness and preparedness of residents living in suburban properties in the urban fringe. This identified new audience will require special consideration by fire and land managers to ensure residents are aware of the risks wildfires could pose and adequately prepared for likely future disasters. Proposed research by the authors will advance knowledge to determine whether the recent experience of those living in the urban fringe has changed their awareness of wildfire risk, and whether the ways that residents interpret their experiences and the social norms surrounding loss may inhibit change.

\section{Conclusion}

With the growing number of people living in the WUI, and the number and severity of wildfires in the WUI expected to increase, the Port Hills fire of February 2017 serves as a clear indication to New Zealand communities of the likely wildfire threats to come. The fire signals that the changes ahead are not merely an increase in the risk to people at the fringe, but represent a change in the nature of the audience and landscape at risk. Previous work has focussed on the rural side of the WUI, with management efforts targeted at that context. However, denser pockets of the urban fringe, often recently developed and populated by urban residents, have not been well incorporated into the existing New Zealand wildfire literature or into fire managers' risk mitigation messaging. These areas will require special consideration in terms of the different audiences found within, and the different awareness and preparation messages they will require. 


\section{Acknowledgments}

Funding for this work was provided by New Zealand's Ministry of Business Innovation and Employment through Resilience to Nature's Challenges National Science Challenge and the Endeavour fund via contract C04X1602, 'Preparing New Zealand for Extreme Fire'.

\section{References}

AFAC (2017) Independent Operational Review: A review of the management of the Port Hills fires in February 2017. Report Prepared for Fire and Emergency New Zealand. https://fireandemergency.nz/assets/Documents/Files/AFAC-Port-Hills-Review.pdf

Anderson SAJ, Doherty J, Pearce HG (2008) Wildfires in New Zealand from 1991 to 2007. New Zealand Journal of Forestry 53, 19-22.

Andrew, R, Dymond, JR (2012) Expansion of lifestyle blocks and urban areas into high-class land: an update for planning and policy. Journal of the Royal Society of New Zealand 43(3), 128-140.

Anon. (2014) The National Strategy: The Final Phase in the Development of the National Cohesive Wildland Fire Management Strategy. Available at https://www.forestsandrangelands.gov/strategy/documents/strategy/CSPhaseIIINationalStrategyA pr2014.pdf

Bayley A, Goodyear R (2004) 'New Zealand: an Urban/Rural Profile.' (Statistics New Zealand: Wellington, NZ)

Butler, CP (1974) The urban/wildland fire interface. In 'Proceedings of Western States Section, 6-7 May 1974, Spokane, Washington' (Washington State University: Pullman, WA) Combustion Institute Papers, 74(15), 1-17.

Calkin DE, Cohen JD, Finney MA, Thompson MP (2014) How risk management can prevent future wildfire disasters in the wildland-urban interface. Proceedings of the National Academy of Sciences of the United States of America 111, 746-751.

Calkin DE, Rieck JD, Hyde KD, Kaiden JD (2011) Built structure identification in wildland fire decision support. International Journal of Wildland Fire 20, 78-90.

Carroll M, Paveglio T (2016) Using community archetypes to better understand differential community adaptation to wildfire risk. Philosophical Transactions of the Royal Society B 371, 20150344.

Champ PA, Brenkert-Smith H (2016) Is seeing believing? Perceptions of wildfire risk over time. Risk Analysis 36, 816-830.

Cohen JD (2000) Preventing disaster: home ignitability in the wildland-urban interface. Journal of Forestry 98, 15-21.

Curran-Cournane F, Cain T, Greenhalgh S, Samarsinghe O (2016) Attitudes of a farming community towards urban growth and rural fragmentation-An Auckland case study. Land Use Policy 58, 241250.

Fire and Emergency New Zealand (2017) Fire and Emergency New Zealand releases findings of the Cause and Origin of Port Hills fires. Fire and Emergency New Zealand press release, 3 November 2017. Available at https://fireandemergency.nz/assets/Documents/Files/Port-Hills-Cause-andOrigin.pdf

Fogarty L (1995) Creating less fire prone environments - an example from the gorse covered hills of Wellington and the Hutt Valley. In 'Proceedings, Forest and Rural Fire Association of New Zealand (FRFANZ) 5th Annual Conference, 2-4 August 1994, Nelson’ pp 135-151 (FRFANZ: Rotorua, $\mathrm{NZ)}$ 
Gale RD, Cortner HJ (Eds) (1987) 'People and Fire at the Wildland/Urban Interface: A Sourcebook' . (USDA Forest Service: Washington, DC)

Graham R, Langer ER (2009) Lessons Learned: Wither Hills Fire, Blenheim, December 2000. Scion Client Report No. 16183 (Christchurch, NZ)

Gude PH, Jones K, Rasker R, Greenwood MC (2013) Evidence for the effect of homes on wildfire suppression costs. International Journal of Wildland Fire 22, 537-548.

Hart M, Langer ER (2014) Effective communication: communities and wildfire in New Zealand. Contract report to Bushfire Cooperative Research Centre. Scion Client Report No. 21017. (Christchurch, NZ)

Haynes H, Madsen R (2017) Wildland/Urban Interface: Fire Department Wildfire Preparedness and Readiness Capabilities - Final Report, National Fire Protection Association, January 2017. Available at http://www.nfpa.org//-/media/Files/News-and-Research/Fire-statistics/Fireservice/osWUI2017.pdf

Jakes PJ, Kelly L, Langer ER (2010) An exploration of a fire-affected community undergoing change in New Zealand. The Australian Journal of Emergency Management 25, 48-53.

Jakes P, Langer ER (2012) The adaptive capacity of New Zealand communities to wildfire. International Journal of Wildland Fire 21, 764-772. doi:10.1071/WF11086

Jakes PJ, Sturtevant V (2013) Trial by fire: community wildfire protection plans put to the test. International Journal of Wildland Fire 22, 1134-1143.

Langer ER, Hart M (2014) Effective communication of wildfire messages for New Zealand communities. Fire Technology Transfer Note 43 (Scion: Christchurch, NZ)

Langer ER, McGee TK (2017) Indigenous and non-Indigenous rural residents' wildfire risk awareness and safe use of fire on the Karikari Peninsula, Aotearoa New Zealand. International Journal Wildland Fire 26(9), 820-828. doi:10.1071/WF16133

McCaffrey S (2004) "Thinking of wildfire as a natural hazard." Society \& Natural Resources 17 (6):509-516. doi: 10.1080/08941920490452445

McCaffrey S (2015) Community Wildfire Preparedness: a Global State-of-the-Knowledge Summary of Social Science Research. Current Forestry Report 1: 81. https://doi.org/10.1007/s40725-0150015-7

McCaffrey S, Toman E, Stidham M, Shindler B (2013) Social science research related to wildfire management: an overview of recent findings and future research needs. International Journal of Wildland Fire 22, 15-24. doi:10.1071/WF11115

McCaffrey S, Toman E, Stidham M, Shindler B (2015) Social science findings in the United States. In 'Wildfire hazards, risks, and disasters'. (Eds D. Paton, J. F. Shroder) pp. 15-34. (Elsevier: Amsterdam, Netherlands)

McGee TK, McFarlane BL, Varghese J (2009) An examination of the influence of hazard experience on wildfire risk perceptions and adoption of mitigation measures. Society \& Natural Resources 22, 308-323. doi:10.1080/08941920801910765

Mell WE, Manzello SL, Maranghides A, Butry D, Rehm RG (2010) The wildland-urban interface fire problem - current approaches and research needs. International Journal of Wildland Fire 19, 238251.

NRFA (2004) 'FireSmart: Partners in Protection - Protecting our Communities from Interface Fire.' (National Rural Fire Authority: Wellington, NZ)

NRFA (2009) 'FireSmart: Home Owners Manual - Protecting Your Home from Interface Fire', 2nd edn. (National Rural Fire Authority: Wellington, NZ) 
Nicholas G, Hepi M (2017) Engaging Owners of Lifestyle Blocks in Understanding and Mitigating Wildfire Risks. Fire and Emergency New Zealand Research Report No.157. (Wellington, New Zealand)

Officials' Committee for Domestic and External Security Coordination (2007) National Hazardscape Report. (Wellington, NZ)

Oliver C (1994) Wildland Urban Interface: other perspectives. In 'Proceedings, Forest and Rural Fire Association of New Zealand (FRFANZ) 4th Annual Conference, August 3-5, 1994, Rotorua' (FRFANZ: Rotorua, NZ)

Olsen CS, Kline JD, Ager AA, Olsen, KA, Short KC (2017) Examining the influence of biophysical conditions on wildland-urban interface homeowners' wildfire risk mitigation activities in fire-prone landscapes. Ecology and Society 22(1), 21.

Partners in Protection (1999) 'FireSmart: protecting your community from wildfire' (Partners in Protection: Edmonton, Canada)

Paterson J (2005) What is a lifestyle block and is it a form of rural gentrification? A paper presented to the Waikato Branch of the Geographical Society, The University of Waikato. (Hamilton, NZ)

Paveglio TB, Edgeley C (2017) Community diversity and hazard events: understanding the evolution of local approaches to wildfire. Natural Hazards 87(2), 1083-1108. doi:10.1007/s11069-017-2810$\underline{\mathrm{x}}$

Pearce HG, Clifford V (2008) Fire weather and climate of New Zealand. New Zealand Journal of Forestry 53(3), 13-18.

Pearce HG, Kerr J, Clark A, Mullan B, Ackerley D, Carey-Smith T, Yang E (2011) Improved estimates of the effect of climate change on NZ fire danger. MAF Technical Paper No. 2011/13.

Pearce HG, Mullan AB, Salinger MJ, Opperman TW, Woods D, Moore JR (2005) Impact of climate change on long-term fire danger. New Zealand Fire Service Commission Research Report 50, National Institute of Water \& Atmospheric Research Limited.

Radeloff VC, Hammer RB, Stewart SI, Fried JS, Holcomb SS, McKeefry JF (2005) The wildlandurban interface in the United States. Ecological Applications 15(3), 799-805.

Radeloff V, Helmers DP, Kramer HA, Mockrin MH, Alexandre PM, Bar-Massada A, Butsic V, Hawbaker T, Martinuzzi S, Syphard AD, Stewart S (2018) Rapid growth of the US wildland-urban interface increases wildfire risk. Proceedings of the National Academy of Sciences of the United States of America 115, 3314-3319.

Reisinger A, Kitching R, Chiew F, Hughes L, Newton P, Schuster SS, Tait A, Whetton P (2014) Chapter 25: Australasia. In Climate Change 2014: Impacts, Adaptation and Vulnerability Contribution of Working Group II to the Fifth Assessment Report of the Intergovernmental Panel on Climate Change. (Eds VR Barros, DJ Dokken, CB Field, MD Mastrandrea, KJ Mach, TE Bilir, M Chetterjee, KL Ebi, YO Estrada, RC Genova, B Girma, ES Kissel, AN Levy, S MacCracken, PR Mastrandrea, LL White), pp. 1371-1438. (Cambridge University Press: Cambridge and New York)

Sword-Daniels V, Eriksen C, Hudson-Doyle EE, Alaniz R, Adler C, Schenk T, Vallance S (2016) Embodied uncertainty: living with complexity and natural hazards. Journal of Risk Research 21(3), 290-307.

Syphard AD, Brennan TJ, Keeley JE (2014) The role of defensible space for residential structure protection during wildfires. International Journal of Wildland Fire 23(8), 1165-1175.

Toman E, Stidham M, McCaffrey S, Shindler B (2013) Social science at the wildland-urban interface: a compendium of research results to create fire-adapted communities. General Technical Report NRS-111 (USDA, Forest Service, Northern Research Station: Newtown Square, PA) 
Twigg SM (1994) Wildland Urban Interface: The Planners perspective. In 'Proceedings, Forest and Rural Fire Association of New Zealand (FRFANZ) 4th Annual Conference, August 3-5, 1994, Rotorua' (FRFANZ: Rotorua, NZ)

VBRC (2010) 2009 Victorian Bushfires Royal Commission Final Report: Summary. Parliament of Victoria, 2009 Victorian Bushfires Royal Commission (Melbourne, AUS)

Wilson AAG, Ferguson IS (1986) Predicting the probability of house survival during bushfires. Journal of Environmental Management 23, 259-270. 


\title{
Wildland Urban Interface assessment and prediction in relation to land use and land cover changes. The Portuguese case study
}

\author{
Marj Tonini $^{1 *}$; Federico Amato ${ }^{1}$; Joana Parente ${ }^{3}$; Mário G. Pereira ${ }^{2,3}$ \\ ${ }^{1}$ Institute of Earth Surface Dynamics, Faculty of Geosciences and Environment, University of \\ Lausanne, Géopolis Building, UNIL Mouline - 1015 Lausanne, Switzerland, \\ \{marj.tonini@unil.ch*,federico.amato@unibas.it\} \\ ${ }^{2}$ Centro de Investigação e Tecnologias Agroambientais e Biológicas, Universidade de Trás-os- \\ Montes e Alto Douro, Quinta de Prados, 5000-801 Vila Real, Portugal, \{joanaparente@utad.pt; \\ gpereira@utad.pt\} \\ ${ }^{3}$ Instituto Dom Luiz, Faculdade de Ciências da Universidade de Lisboa, Lisboa, Portugal, \\ \{gpereira@utad.pt\}
}

\begin{abstract}
The Wildland-Urban Interface (WUI) refers to the zone of transition between wildlands and human development. Its spatio-temporal evolution is strongly influenced by land use/land cover changes (LULCC). In Mediterranean countries, WUI represents the most prone area for human-caused wildfires, and is, at present, a central focus of wildfire policy. Traditionally, WUI maps rest on the measurement of the distances among human settlements and wildlands. This methodology is effective to produce detailed maps and to give precise indications, but it is rigid and needs to pre-define fixed parameters. To overcome this uncertainty, we introduce a LULCC simulation model based on Machine Learning approach, allowing to produce prediction maps for the land cover classes related to the WUI. Finally, Fuzzy Set Theory is used to develop continuous non-categorical maps expressing the possibility of being part of this interface. The proposed methodology has been applied to the case study of Portugal, and considers the land covers referred to different epochs $(1990,2000$, and 2012). Both a classical geospatial analyses and an innovative simulation approach were adopted: as result we obtained traditional maps of the WUI for the different investigated periods ("hard" boundaries), and a fuzzy WUI map ("soft" prediction) for the year 2030. The framework proposed in this paper correctly identifies the areas belonging to this interface, providing useful information for wildfiresprevention policies.
\end{abstract}

Keywords: WUI, land use / land cover changes, simulation model, GIS, Machine Learning

\section{Introduction}

The Wildland-Urban Interface (WUI) is known as the area where structures and other human development meet or intermingle with wildland and rural area (USDI and USDA 2001). Humancaused wildfires have a very high probability of starting and spreading within the WUI, representing a main hazard for people, houses and other infrastructures. For these reasons, WUI mapping became a key tool to identify areas where to concentrate financial and technical resources for fire protection and prevention actions. Anthropogenic and environmental features, such as urban growth, fragmentation of rural areas, deforestation and land use / land cover changes (LULCC), shaped the WUI's extension. In Portugal, significant LULCC occurred in the last decades in response to trends associated with the socioeconomic development, such as rural abandonment, migration, ageing of population (Van Doorn and Bakker 2007; Diogo and Koomen 2012; Pereira et al. 2014). In the present study, authors consider LULCC for the period 1990 - 2012 as base data to elaborate WUI maps of Continental Portugal and to assess the evolution of its extension. Afterwards, an innovative approach resting on LULCC simulation model is introduced; this allowed to make prediction on the future extension of the land cover classes related to the WUI in the following two decades (up to 2030). 
Finally, the fuzzy set theory has been used both to overly urban with rural scenarios and to elaborate a fuzzy WUI possibility map for the year 2030. The main novelty of this method is that it escapes from the definition of rigid boundaries with their dependence on predefined parameters.

\section{Materials and methods}

\subsection{Study area: Portugal}

Mainland Portugal (Fig.1) covers the western coast of the Iberian Peninsula with a land surface of $89,000 \mathrm{~km}^{2}$ and an altitude ranging from sea level to about $2000 \mathrm{~m}$ in the north-central region.

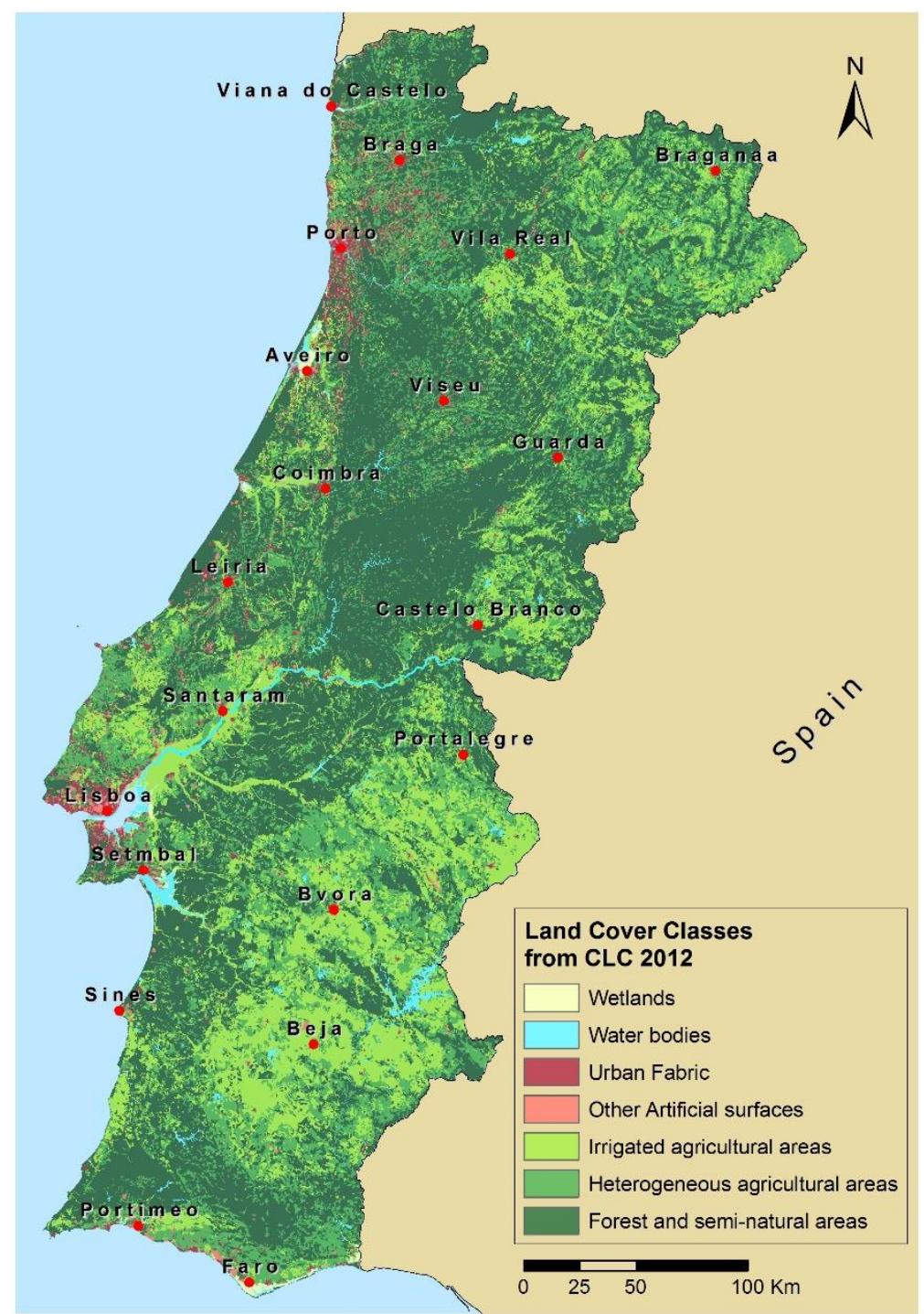

Figure 1 - Continental Portugal with main cities and land cover based on the second level of CORINE land cover (CLC) inventory 2012

The climate is temperate and typical Mediterranean. Vegetation grows in the spring season, while it experiences hydric and thermal stress during the summer months (Amraoui et al. 2015), when most burnt areas (about 90\%) are reported. Forests are predominant in the northern half of the country, while in the southern part prevail agricultural lands and scrub vegetation. Weather and climate are responsible for about two-thirds of the total annual burnt area inter-annual variability, which results unevenly distributed in space and in time within the country (Tonini et al., 2018). 


\subsection{Data acquisition and processing}

Information on land use / land cover came from the CORINE Land Cover (CLC) inventory, provided by European Environment Agency (https://land.copernicus.eu/pan-european/corine-landcover). CLC inventory is organised in different classes articulated over three levels and are currently available for four periods $(1990,2000,2006$, and 2012) with a minimum time consistency of plus/minus one year and a minimum mapping unit of 25 hectares. For the present purpose, CLC maps have been reclassified with reference to the second level as reported in Table 1.

Table 1 - Land cover classification based on the second level of the CORINE Land Cover (CLC) nomenclature.

\begin{tabular}{|l|l|}
\hline Proposed Classification & CLC Nomenclature (Level 2) \\
\hline Urban fabric & Urban fabric \\
\hline \multirow{4}{*}{ Other artificial surfaces } & Industrial, commercial and transport units \\
\cline { 2 - 2 } & Mine, dump and construction sites \\
\cline { 2 - 2 } & Artificial, non-agricultural vegetated areas \\
\hline \multirow{3}{*}{ Irrigated agricultural areas } & Arable land \\
\cline { 2 - 2 } & Permanent crops \\
\cline { 2 - 2 } & Pastures \\
\hline Heterogeneous agricultural areas & Heterogeneous agricultural areas \\
\hline \multirow{3}{*}{ Forest and semi-natural areas } & Forest \\
\cline { 2 - 2 } & Shrub and/or herbaceous vegetation associations \\
\cline { 2 - 2 } & Open spaces with little or no vegetation \\
\hline \multirow{2}{*}{ Wetlands } & Inland wetlands \\
\cline { 2 - 2 } & Coastal wetlands \\
\hline \multirow{2}{*}{ Water bodies } & Inland waters \\
\cline { 2 - 2 } & Marine waters \\
\hline
\end{tabular}

A number of driving variables were selected to calibrate the simulation model. Driving forces are defined as those factors that cause LULCC and having a marked association with the nature of the change (Meyfroidt, 2016). In the present study, we considered the following 17 driving variables: digital elevation model (DEM) and derivatives (slope, aspect), provided by the European Environment Agency (EEA) under the framework of the Copernicus programme; agricultural and population census data, derived from the Portuguese National Institute of Statistics (Portal do Instituto Nacional de Estatística n.d.); distance from primary roads and from the cities with more than 50.000 inhabitants coming from the Open Street Map Database (https://www.openstreetmap.org); soil thickness, ph, texture and ecological values were made available from EPIC WebGIS, an interactive spatial data infrastructure which provides georeferenced cartography at Portuguese national scale. Supplementary variables were finally derived from a change detection analysis between the inputs of CLC maps. All data were reprocessed on a regular grid (i.e. raster format) with a spatial resolution of 500 by 500 meters.

\subsection{WUI mapping}

A geoprocessing workflow based on a GIS analysis was implemented to produce WUI's maps for different periods, corresponding the available CLC inventories. Subsequently, an approach based on LULCC simulation models was designed to elaborate scenarios allowing to predict WUI extension for 2030.

\subsubsection{Geoprocessing workflow}

A geoprocessing workflow was developed and implemented into a mode builder, a specific application available in ArcGIS ${ }^{\mathrm{TM}}$ software environment and allowing to string together sequences of

Advances in Forest Fire Research 2018 - Page 872 
geoprocessing tools. This approach have the advantage of automating the operations and making them reproducible. The specific objective is to extract the intersection areas between a buffer around the urban fabric and the area resulting from the sum of the forest and semi-natural areas plus the heterogeneous agricultural areas, which denote the wildlands. A buffer width of $1 \mathrm{~km}$, corresponding to two times the spatial resolution of CLC inventories, was retained. Different studies in other countries applied similar distance-values for WUI mapping (Radeloff et al. 2005; Vilar et al. 2016) and, at the same time, we estimated that this buffer is sufficiently large to avoid bias in the results due to the base cartographic map unit. As regards the methodology and the choice of the CLC classes to define the WUI, we refer to the work of Tonini et al. (2018), with the difference that in the present study authors include only the continuous and discontinuous urban fabric in the definition of the urban area.

\subsubsection{Simulation model}

A future scenario for LULCC was computed for the year 2030 using a spatially explicit inductive method, based on the transitions between two observed land covers (CLC1990 - CLC2000 and CLC2000 - CLC2012) (Fig. 2). The transition potential for the subsequent period (i.e. 2012 for the calibration phase and 2030 for the simulation phase) was computed using Multilayer Perceptron (MLP), a popular Machine Learning algorithm resting on feedforward artificial neural network, given as input the described driving variables. The real quantities of changes, measured through a change detection, were then spatially allocated based on MLP outputs. The model was validated via Kappa statistics, by comparing the resulting 2012-simulation map with the reference CLC map for the same year. Once calibrated the model, the resulting best parameters were retained to elaborate the future land cover scenarios for the year 2030, allocating the expected changes via a Markov Chain procedure. Hence, the resulting scenarios toward the wildlands and the urban areas were used to predict the future WUI extension. To this end, fuzzy set theory was applied to produce fuzzy maps expressing the possibility of each pixel of experiencing a transition toward wildland coverages and of being in a distance from urban areas. These maps were then overlapped through joint membership fuzzy functions, resulting in a map expressing the possibility of an area to belong to the WUI in 2030. This procedure, although quite complex, is extremely robust and has the advantage of not relying on expert knowledge inputs. An exhaustive explanation of the methodology and obtained results can be found on Amato et al. (2018).

\subsubsection{Model validation}

To validate the ability of the MLP to correctly predict the land cover changes, the real transition matrix measured for the period 2000-2012 was used to model change demand. The result was a land cover simulation for the year 2012 which was compared with the reference map (i.e. CLC2012) obtaining an overall Kappa of 0.8227. This value expresses a high capacity of the model to simulate the real-occurred land cover transitions. The validation of the obtained possibility maps for WUI was done via the relative operating characteristic (ROC). To this end, we selected a dataset of point falling inside the WUI2012 map ("presence" observation), than divided in training/testing subset. Furthermore, randomly chosen pseudo-absence points were added to both the training and testing sets. 
Calibration

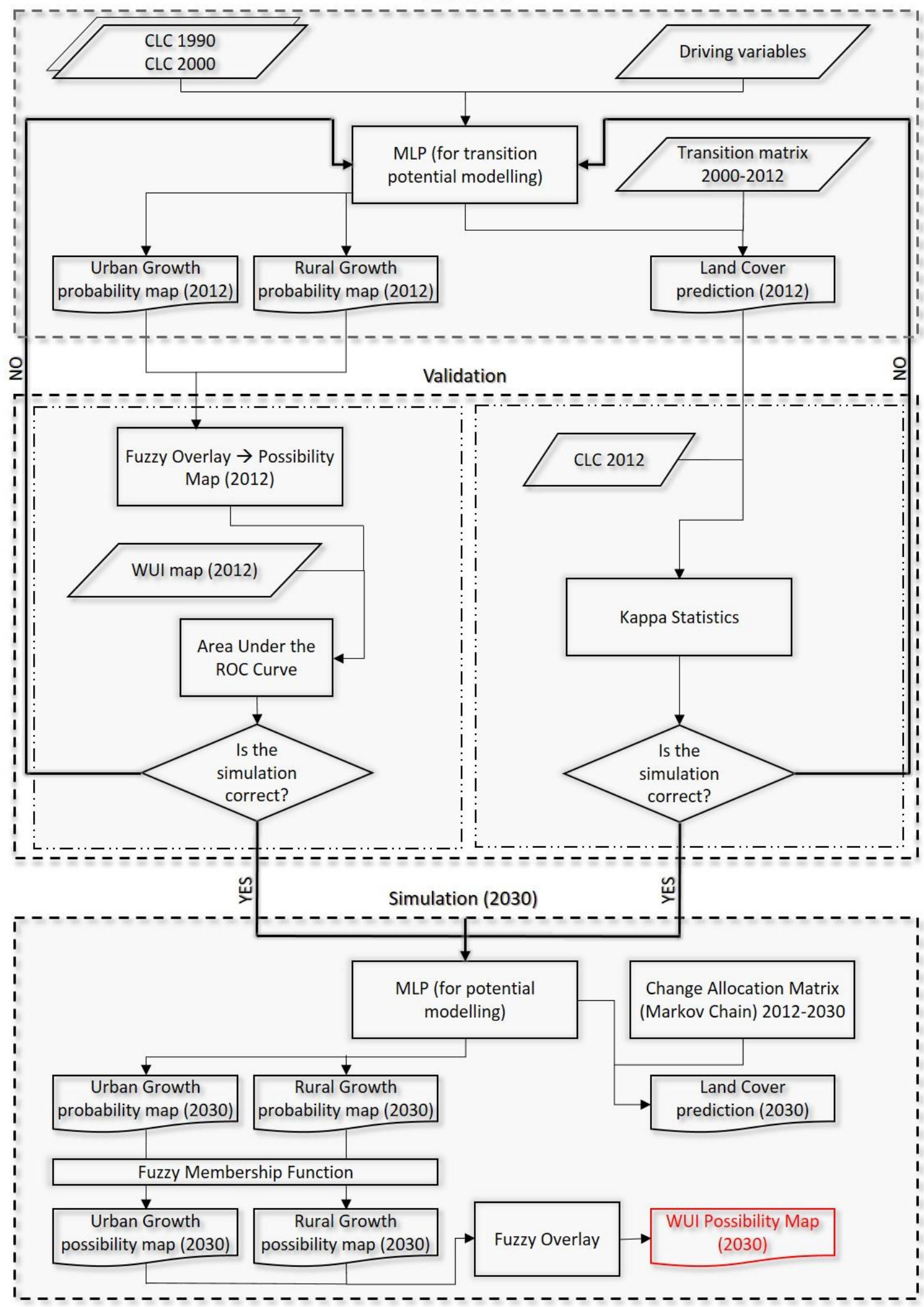

Figure 2 - Flowchart of the entire procedure including the land use / land cover changes simulation model and the fuzzy set theory allowing to produce the Wildland-Urban Interface (WUI) possibility map for 2030 (CLC $=$ CORINE Land Cover; MLP= Multilayer Perceptron) 


\section{Results}

The proposed methodology allowed to produce maps of the WUI for different periods (1990, 2000, and 2012) and to predict both the "hard" boundaries and the "fuzzy" WUI map (i.e. "soft prediction") for the year 2030 (Fig. 3 and Fig. 4).

Globally, WUI increased from about $690 \times 10^{3}$ ha in 1990 up to about $1050 \times 10^{3}$ ha in 2012 (Table 2 ). This growth is more pronounced in the north-west and along the coast, mainly due to the urban growth. From our analyses, WUI is not expected to increase further in the next 20 years.

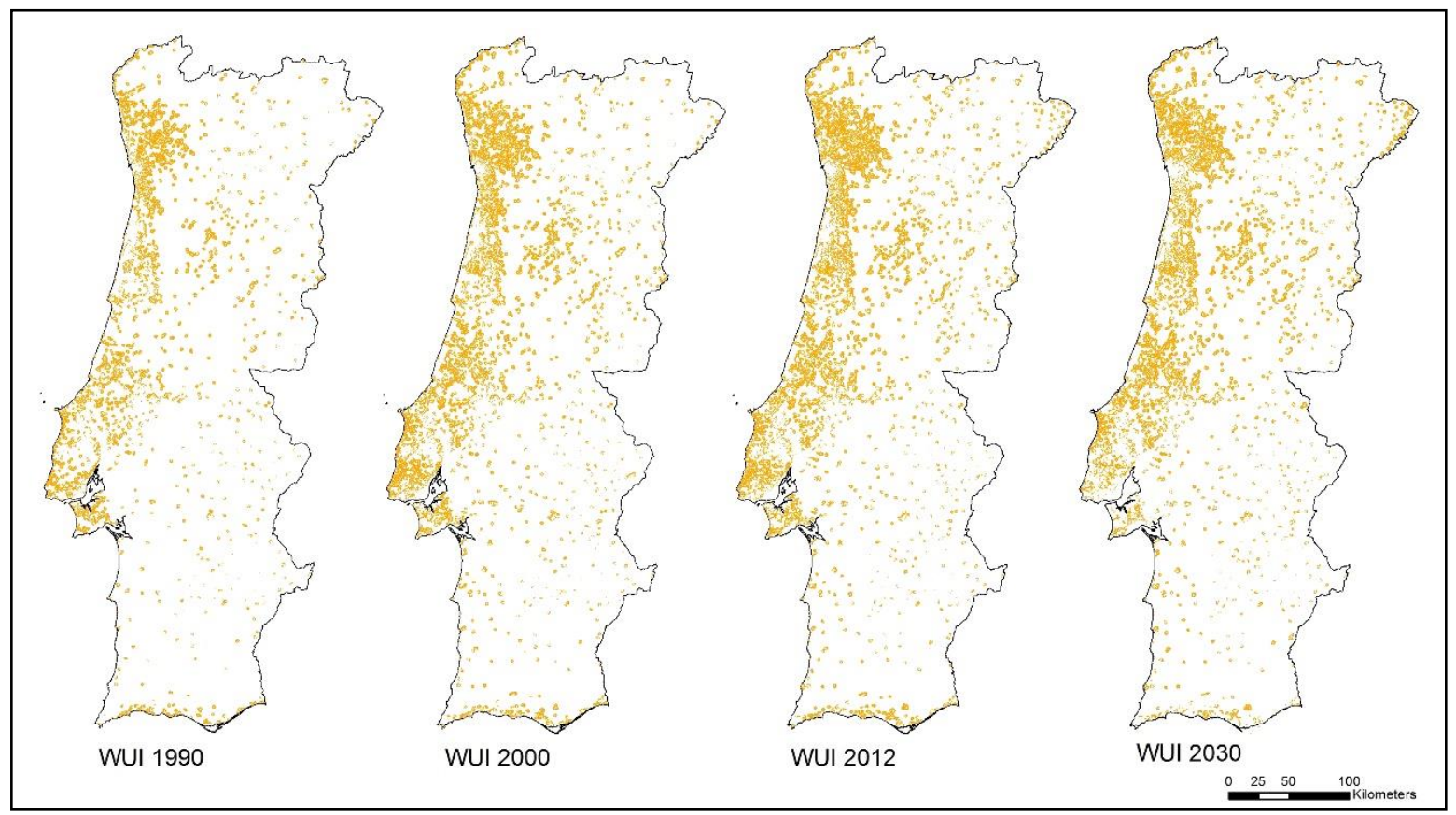

Figure 3 - Maps of the Wildland-Urban Interface (WUI) in Portugal estimated for the different periods of investigation $(1990,2000,2012,2030)$

From 1990 to 2012 the urban areas and other artificial surfaces registered an increase in each frame period in comparison to the previous one, face of a decrease of the irrigated agricultural areas. This behaviour is more noticeable from 1990 to 2000 and less in the last frame period. The model predicts a slower growth of the urban area from 2012 to 2030 than it was during the two previous frame periods (1990 - 2000 and, 2000 - 2012). As regards the wildlands, both the heterogeneous agricultural and the forest and semi natural areas stay almost unchanged during the entire simulation period.

Table 2 - Area (in hectares) of Wildland-Urban Interface (WUI) and different land cover classes affecting the WUI for each investigated period (on the top). For each frame period, net percentage changes are computed relative to the total area of each classes in the later period (on the bottom).

\begin{tabular}{|c|c|c|c|c|c|c|}
\hline Year & WUI & $\begin{array}{c}\text { Urban } \\
\text { area }\end{array}$ & $\begin{array}{c}\text { Other } \\
\text { artificial } \\
\text { surfaces }\end{array}$ & $\begin{array}{c}\text { Irrigated } \\
\text { agricultural } \\
\text { areas }\end{array}$ & $\begin{array}{c}\text { Heterogeneous } \\
\text { agricultural } \\
\text { areas }\end{array}$ & $\begin{array}{c}\text { Forest and } \\
\text { semi natural } \\
\text { areas }\end{array}$ \\
\hline 1990 & 688989 & 133704 & 35630 & 1975871 & 2371018 & 4274600 \\
\hline 2000 & 966972 & 214379 & 67432 & 1905894 & 2344481 & 4252424 \\
\hline 2012 & 1049825 & 241822 & 92749 & 1811715 & 2349977 & 4264167 \\
\hline 2030 & 1013235 & 283900 & 133543 & 1699367 & 2355895 & 4287690 \\
\hline $\begin{array}{l}\text { Frame } \\
\text { period }\end{array}$ & \multicolumn{7}{|c|}{ Net \% of changes } \\
\hline $1990-2000$ & $28.75 \%$ & $37.63 \%$ & $47.16 \%$ & $-3.67 \%$ & $-1.13 \%$ & $-0.52 \%$ \\
\hline $2000-2012$ & $7.89 \%$ & $11.35 \%$ & $27.30 \%$ & $-5.20 \%$ & $0.23 \%$ & $0.28 \%$ \\
\hline $2012-2030$ & $-3.61 \%$ & $14.82 \%$ & $30.55 \%$ & $-6.61 \%$ & $0.25 \%$ & $0.55 \%$ \\
\hline
\end{tabular}


The WUI fuzzy map (Fig. 4) predicts a scenario for the year 2030, expressed in terms of possibility for an area of belonging to the WUI. The tests performed for validation (i.e. Kappa statistics and ROC curves) demonstrate the correctness and good performance of the proposed model. It results that the highest WUI extension will take place in the areas enclosed by Braga, Porto, Aveiro and Vila Real, in the north-western part of Portugal, with a spatial contiguity along the coast up to Lisbon and crossing the city of Coimbra. In the Centro Region, nearby the city of Viseu, we can observe another highly predisposed WUI area. The growing peri-urban fringes probably drove this trend. In the southern part of the country, we can observe scattered hotspot for WUI areas, extending in the mountainous region towards the interior, most likely caused by the progressive abandonment of agricultural activities and the consequent spreading of the forest in these areas. WUI will probably also extend along the coastline in the Faro Region, caused by the expansion here of tourism-led developments.

\section{Conclusions}

Obtained results highlight the effectiveness of the proposed framework in identifying the WUI through a flexible approach, which combine a LULCC simulation model with the fuzzy set theory. The main objective is to avoid the rigid definition of boundaries rising from the classical binary classification methods. In this sense, fuzzy set theory offers a solid mathematical background on which solve the issue of labelling each pixel as belonging or not to the WUI through the assignation to each pixel of a degree of possibility of being part of this interface.

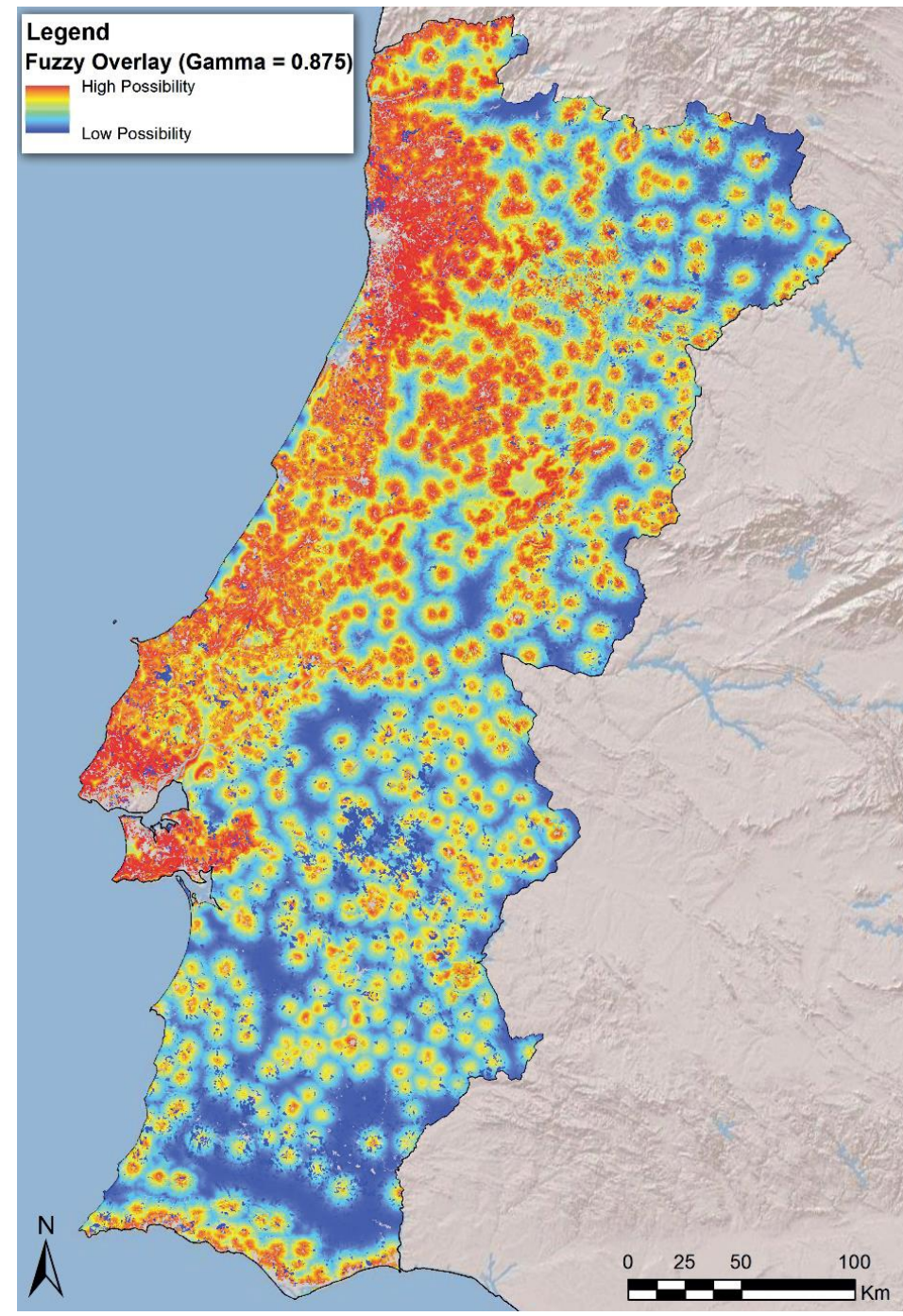

Fig. 4 - Wildland-urban interface (WUI) fuzzy map for the year 2030 


\section{References}

Amato F, Tonini M, Murgante B, Kanevski M (2018) Fuzzy definition of Rural Urban Interface: An application based on land use change scenarios in Portugal. Environmental Modelling \& Software 104, 171-187.

Amraoui M, Pereira MG, DaCamara CC, Calado TJ (2015) Atmospheric conditions associated with extreme fire activity in the Western Mediterranean region. Science of the Total Environment 524, $32-39$.

Diogo V, Koomen E (2012) Land-Use Change in Portugal, 1990-2006: Main Processes and Underlying Factors. Cartographica: The International Journal for Geographic Information and Geovisualization 47, 237-249.

Pereira MG, Aranha J, Amraoui M (2014) Land cover fire proneness in Europe. Forest Systems 23, 598-610.

Radeloff VC, Hammer RB, Stewart SI, Fried JS, Holcomb SS, McKeefry JF (2005) The wildlandurban interface in the United States. Ecological applications 15, 799-805.

Tonini M, Pereira MG, Parente J, Orozco CV, (2017). Evolution of forest fires in Portugal: from spatiotemporal point events to smoothed density maps, Natural Hazards 85, 1489-1510

Tonini M, Parente J, Pereira M (2018) Global assessment of land cover changes and rural-urban interface in Portugal. Natural Hazards Earth System Sciences 18, 1647-1664. doi:10.5194/nhess18-1647-2018.

USDI UD of the I, USDA UD of A (2001) Urban wildland interface communities within the vicinity of federal lands that are at high risk from wildfire. Federal Register 66, 751-777.

Van Doorn AM, Bakker MM (2007) The destination of arable land in a marginal agricultural landscape in South Portugal: an exploration of land use change determinants. Landscape ecology 22, 10731087.

Vilar L, Camia A, San-Miguel-Ayanz J, Martín MP (2016) Modeling temporal changes in humancaused wildfires in Mediterranean Europe based on Land Use-Land Cover interfaces. Forest Ecology and Management 378, 68-78. 


\title{
WUI, Planning and fires in South of France
}

\author{
Christophe Bouillon ${ }^{1 *}$; Anne Ganteaume ${ }^{1}$; Khalil Djazouli ${ }^{2}$, Chaimaa Ennesr ${ }^{3}$ \\ ${ }^{1}$ IRSTEA-RECOVER Mediterranean ecosystems and risks, Aix-en-Provence France, \\ \{christophe.bouillon@irstea.fr*,anne.ganteaume@irstea.fr\} \\ ${ }^{2}$ Université de Nice Sophia-Antipolis, nice, France, \{djazouli.khalil@gmail.com\} \\ ${ }^{3}$ Université de Lille 1, France, \{chaimaa.ennesr@gmail.com\}
}

\begin{abstract}
In France, after a slowdown following the 2008 crisis, there has been an increase in urbanization. Since 2015, in the French Southern region, there has been an increase of urbanization in risk areas. Decision-makers and especially city councilors are greatly involved in the land management because of their capacity in decision-making on diverse topics such as planning and naturals risks. Concerning fire risk, they need to get a correct knowledge of the fire ignitions occurring within their community in order to be able to secure a place, inform the population and ensure fire prevention

Urban sprawl has progressively gained land classified in "NB" (large building plots in natural or agricultural areas) or abandoned by agricultural activities, by creating an interface zone where habitats and natural spaces are intertwined. The coexistence of spaces and continuities often unkempt, with dense vegetation, creates the conditions for the outbreak and the spread of fires in a complex area, difficult to defend. (In France, 25000 ha / year are urbanized mainly at the expense of agricultural, natural and forest areas).

In this context, this study aims at providing decision-makers with all useful information about fire ignitions and their specialization in the community, especially in the wildland-urban interfaces taking into account the planning and the mandatory brush-clearing area and taking the département de l'Hérault as the study area.

The studies and results proposed in the paper are a part of a Natural Risk program founded by the French Ministry of Environment. It will give an analysis and information to stakeholders concerning relationships between land planning, WUI and wildfires in their municipality. The aim is to provide knowledge of habitat vulnerability to wildfire and the opportunity of a careful consideration of new urbanization zones. The high demands of land along the Mediterranean coast and hinterland have issued a large extension of urban areas near natural or rural land, creating new WUI at risk.

A spatial analysis of the points of departure of fire was carried out with the taking into account of various aspects: fire ignitions and interfaces types, fire ignitions and land use, fire ignitions zoning in planning documents.

We try to find relationships between fire ignition location and planning document according to the LULC in the study area in the context of WUI.

The results confirm a strong relationship between WUI and fire ignition. In the WUI the fire density is three times higher than outside.

Considering this, a decision support tool was developed to help the stakeholders to simulate the new WUI configuration for new buildings and development zone in connection with the existing WUI.
\end{abstract}

Keywords: Wildfire, wildland urban interface, planning, fire risk, decision making

\section{Introduction}

The studies and results proposed in this paper are a part of a Natural risk program founded by the French Ministry of Environment. It will give an analysis and information to stakeholders concerning relationships between land planning, WUI and wildfires in their municipality. The aim is to provide knowledge of habitat vulnerability to wildfire and a careful consideration of the proposed extension of urbanization zones. The high demands of land along the Mediterranean coast and hinterland have 
created a large extension of urban areas near natural or rural land creating new WUI at risk.

In France, after a slowdown following the 2008 crisis, there has been an increase in urbanization. Since 2015, in the French Southern region, there has been an increase in urbanized zones in risk areas with a significant increase in population and an uncontrolled consumption of space. This is the case for peri-urban areas in the conurbations and in the Atlantic and Mediterranean littoral zones. On the Mediterranean coast, we can perceive a saturation effect: The consumption of space moves in the municipalities of the hinterland. (25000 ha / year are urbanized mainly at the expense of agricultural, natural and forest areas.)

Decision-makers and especially the city councilors are greatly involved in the land management because of their capacity in decision-making on different topics such as planning and naturals risks. Concerning fire risk, they need to get a correct knowledge of the fire ignitions occurring within their community in order to be able to secure a place, to inform people and ensure fire-prevention.

In this context, this study aims at providing the decision-makers with all useful information about the fire ignitions and their spatialization in the community, especially in the wildland-urban interfaces but also about the land-cover and the mandatory brush-clearing area, taking the département de l'Hérault as the study area.

The ancient planning document - the land use plan (POS) in force between 1967 and 2017 included areas allowing the construction on large plots, coded "NB" (large building plots in natural or agricultural areas) which led to a dissemination of houses located in the forest or in their approach. The law Alur (Loi no 2014-366 du 24 mars 2014) has planned the end of the POS and NB areas on March 27, 2017. It has been replaced by the local urban plan (PLU), this is the major planning document in use in most of the municipalities. It includes rules on the prevention of forest fires, clearing and in the case of a fire risk prevention plan, the specific urban planning rules applicable to the concerned areas. Changes in land use, especially in agricultural abandonment, create an interface zone where habitats and natural spaces are intertwined. The coexistence of spaces and continuities often unkempt and with dense vegetation creates the conditions for the outbreak and the spread of fires, in a complex area difficult to defend. The final purpose of this study consists in providing them with a decision-making support tool when they come to produce or review the documents. This tool will take into account all the parameters inherent in the fire-risk that are pointed out in this work. It will also help the decision-makers to understand better the impact of forest fires in terms of vulnerability and gauge the greater risk in urban development zones.

\section{Materials}

\subsection{Study zone}

The chosen study area (Figure 1) is the département de l'Hérault, an administrative territory, in the Occitanie Region. This zone is located in the south of France near the Mediterranean Sea and with Montpellier, a major city and urban area of 600000 people.

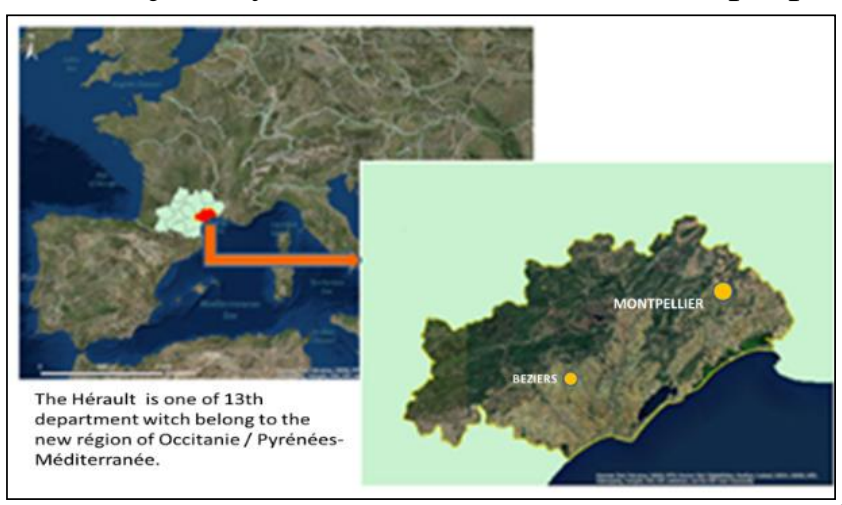

Figure 1 - Study zone location- France

Advances in Forest Fire Research 2018 - Page 879 
This département is facing a profound change in its environment. A strong dynamics of urbanization is developing in this area. The new urbanized areas are progressing in areas that are changing and sensitive to forest fires. The areas concerned are in particular areas abandoned by agriculture, especially old vineyards.

"In addition these areas belong to the top most average of fire prone areas located in the South of France and among the highest fire ignition numbers (Figure 2).

A special feature is the increase of so called "AFERPU - autres feux de l'espace rural et péri urbain", also named peri-urban fires. If generally this kind of fire is smaller than forest fire it can be very dangerous for the people and belongings as it spreads in the WUI areas. Sometimes this type of fire can threaten larger natural or forested areas and become a forest fire.

\subsection{Data and softwares}

The choice of this study zone was directed by the availability of updated data. The stakeholders of Direction départementale des Territoires et de la Mer du département de l'Hérault (DDTM 34) concerned by forest fire, managed a fire database with detailed facts and linked to the official forest fire database for the French Mediterranean region "Promethée". It contains detailed information on all fires between 2005 and 2014, including the geographical location of fire points and is an essential source of information for this study

The advantage of the choosen study area is the availabililty of up-to-date databases, especially concerning fires. Fot his study, we use :

- Land cover - SIG L-R, 2006 from OpenIG Occitanie Pyrénées

- Buildings BD TOPO® v2.1 2011 révised 2013, 1m precision from Institut National de l'Information Géographique et Forestière

- Clearing law zone from DDTM 34

- Planning documents géographiques from Adélie database, 2015

- Ignition points, burnt areas and causes from Adélie and Prométhée databases, 2015

All the data were georeferenced and processed with ArcGis, the statistical calculations with Statgraphics.

For the ignition points, 5 main classes of causes were used : (Table 1) : natural, accidental from installations, volontary, unintentionnal professionnal works and unintentionnal private owners works.

Table 1 - Causes of fire

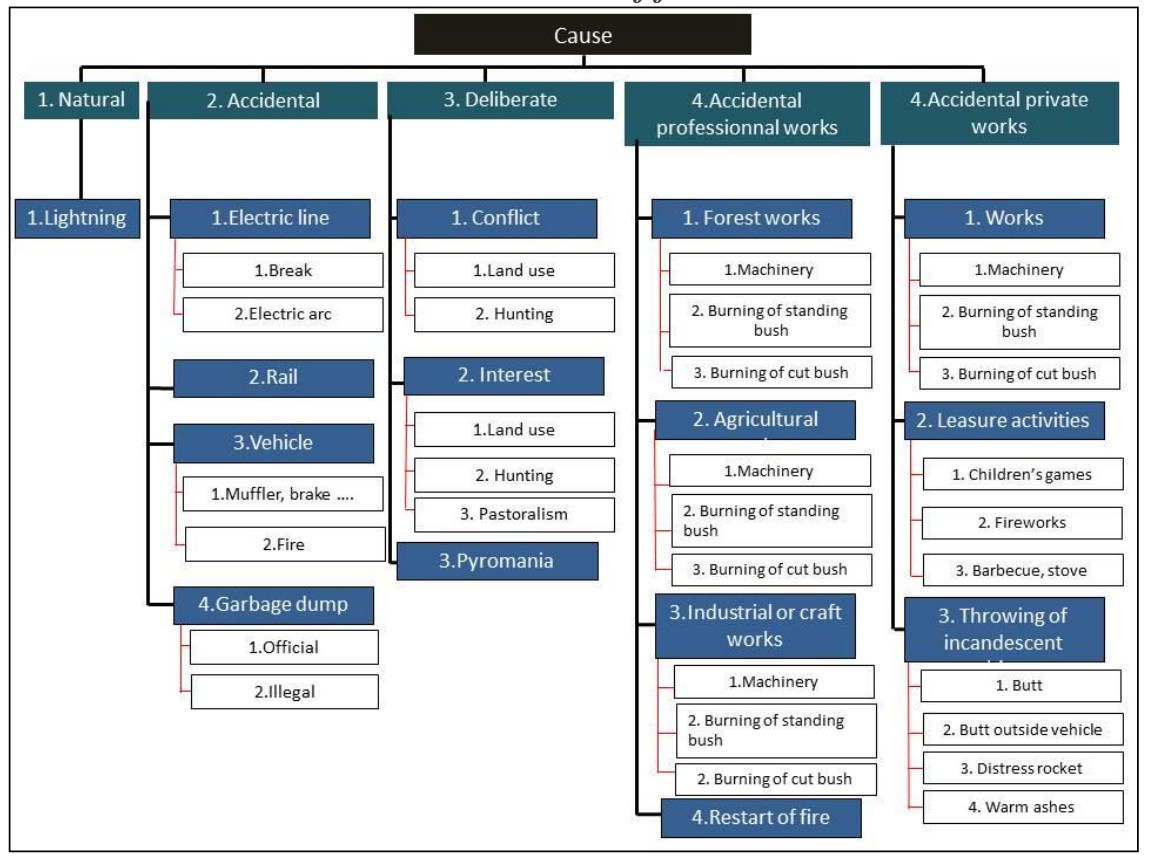

Advances in Forest Fire Research 2018 - Page 880 


\section{Methods}

\subsection{Fire definitions}

In the French fire databases, these main types of fires are recorded.

Forest fire, scrubland, maquis and moors : Fire that has reached forests, moors, scrubland or maquis with an area of at least one hectare in one piece (and regardless of the area covered). Nota Bene : The term "reaches" implies that at least a part of the shrub or tree stage has been destroyed.

Other fires (AFERPU): fires that concern peri-urban and rural areas and that do not threaten the forest - Fires of the rural and peri-urban space, or fire of vegetation not belonging to the preceding category. Six types are identified: fires in beds of less than 1 ha, linear afforestation fires, grass fires, other agricultural fires, garbage dumps, other.

\subsection{WUI mapping}

The mapping of WUI uses the method developed by Irstea (Lampin 2009) and the software developed for an automated calculation of maps (Lampin \& Bouillon 2011). Based on housing configuration (4 classes) and vegetation aggregation (3 classes), this method creates a 12 class WUI. The input data are the buildings and the land use or vegetation aggregation index from aerial or satellital image when available.

This method is used in different studies across Europe (FUME European program). The land use land cover (LULC) study was made with the SIG LR LULC data from the Occitanie Region.

\subsection{Analysis process}

The digital planning documents (from DDTM 34), were harmonized in 5 classes representing the major groups of zoning (Natural, Agricultural, Urban, to be Urbanized.)

Then a Spatial analysis of ignition fire was carried out with the taking into account of various aspects: fire ignitions and types of interfaces, fire ignitions and types of land use, fire ignitions and types of zoning in planning documents.

\section{Results}

The study area is part of a the former Languedoc-Roussillon region concerned with forest fires in France. The higher risk is located in the southerm part of the country along the Mediterranean coast and with hinterland areas and the Landes forest in the South-West of the country. (Figure 2)

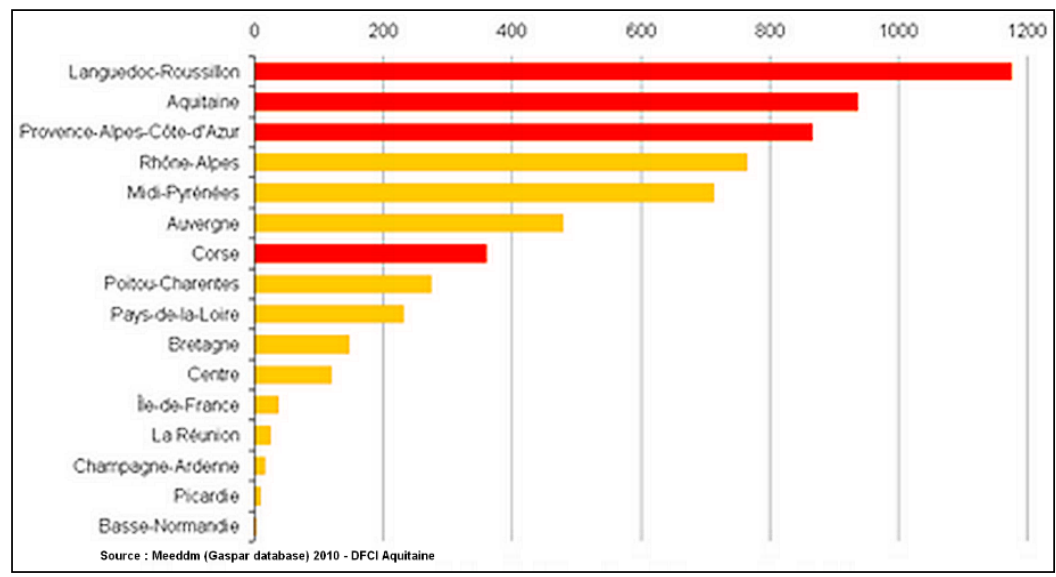

Figure 2 - Municipalities by regions exposed to forest fire risf - France

Regarding the forest fire risks, the main concerned public policies are : the planning policies, the prevention policy and other national policies like agricultural ones. 
The main rules of urban planning in France have been based since 2000 upon the plan local d'urbanisme (PLU). It is the main planning document in use in municipalities. In some other cases, the old plan d'occupation des sols (POS) or carte communale (CC) remain. If no local plan or document exists, the national rules of planning (RNU) are applied.

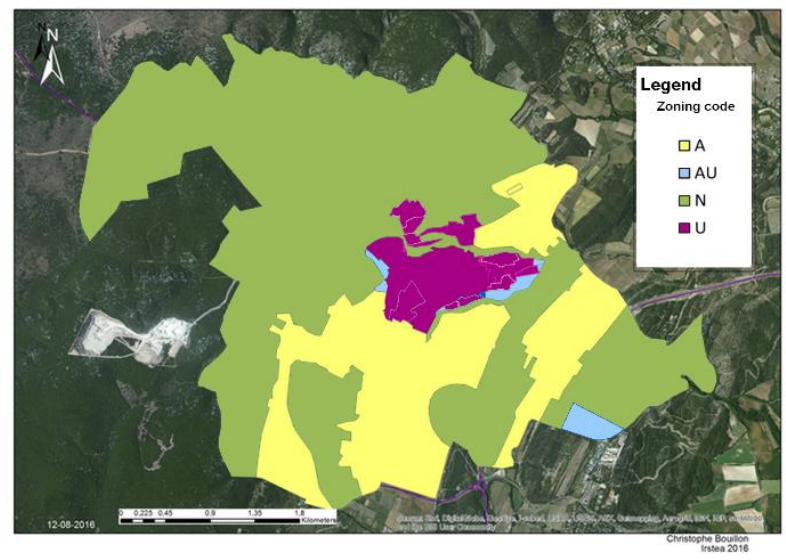

Figure 3 - Planning zones- Les Matelles municipality

The zoning is composed of 4 major categories (Figure 1)

- $\quad \mathrm{U}$ : urban area

- $\quad \mathrm{N}$ : natural area

- A : agricultural area

- $\quad$ AU : to be constructed area

For each zone, some rules were set up such as : construction distances, height, window types and the constructible area for each lot with a soil occupation coefficient (COS)

\subsection{Ignition points and types of interfaces}

The study is based upon the localization of the starting points of fire in the WUI.

The WUI covers $23.52 \%$ of the total area of the department of Hérault. The majority of the interfaces belongs to the isolated housing type with $35 \%$, followed by very dense with $30 \%$. housing type (Table 2)

Table 2 - Types of interfaces in the study area

\begin{tabular}{|l|c|l|}
\hline Housing type & Area (ha) & $\mathbf{( \% )}$ \\
\hline Isolated & 51729 & $35 \%$ \\
\hline Scattered & 36144 & $25 \%$ \\
\hline Dense & 14672 & $10 \%$ \\
\hline Very dense & 44061 & $30 \%$ \\
\hline Total & 146606 & $100 \%$ \\
\hline
\end{tabular}

As a matter of fact, the spatial organization of buildings in the Hérault is very dense along the coastline, particularly in the municipalities of Montpellier and Béziers which are the most populated area. The housing types mentioned bove are also observed near the urban core of other most important municipalities. The further we get off the coast, the more the urban fabric becomes loose, and we find more isolated habitat. The scattered habitat represents $25 \%$ of the interfaces and it is localized on the periphery of the aforementioned urban cores and sometimes operates the junction between the two areas of dense housing. It should be noted that the dense structure covers only $10 \%$ of the surface, and is mostly an extension of very dense areas. 
The ratio between the surface of the WUI and the number of ignition points is very significant as $30 \%$ of fires starts in these areas.(Figure 5)

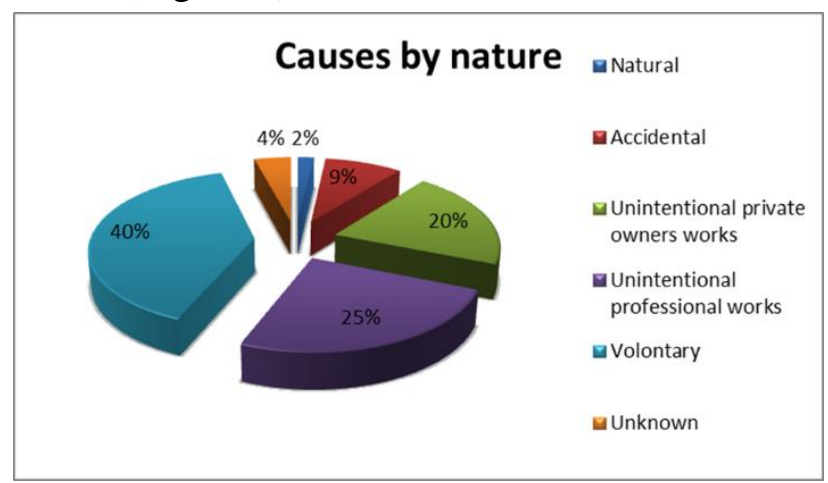

Figure 5 - Fire causes in the study area

The WUI with isolated housing classes are most at risk of fire. It is due to the layout of space with large areas which increases the degree of fire risk because of vegetation not managed.

Table 3 - Causes of fire with simplified classification (K Djazouli, source Adelie 2015)

\begin{tabular}{|l|l|l|}
\hline Nature of cause & Nb & Burnt area (ha) \\
\hline Natural & 31 & 4 \\
\hline Accidental & 148 & 890 \\
\hline Unintenional private owners works & 346 & 1305 \\
\hline Unintentional professional works & 424 & 2232 \\
\hline Volontary & 669 & 6131 \\
\hline Unknown & 70 & 240 \\
\hline Total & 1688 & 10802 \\
\hline
\end{tabular}

Dense and very dense interfaces are very rarely affected by fires. Groupings of structures act as fuel cuts in case of a fire starting. The results, presented in the tables, show that out of the 1688 ignition points, $40 \%$ of fire causes are due to arsonism, followed by unintended causes related to professional work $(25 \%)$, as well as unintentional causes related to specific work at $20 \%$. To a lesser degree, the accidental and natural causes also represent respectively $9 \%$ and $2 \%$. To specify that, only a minimal part of the causes unidentified is $4 \%$ of the total data.

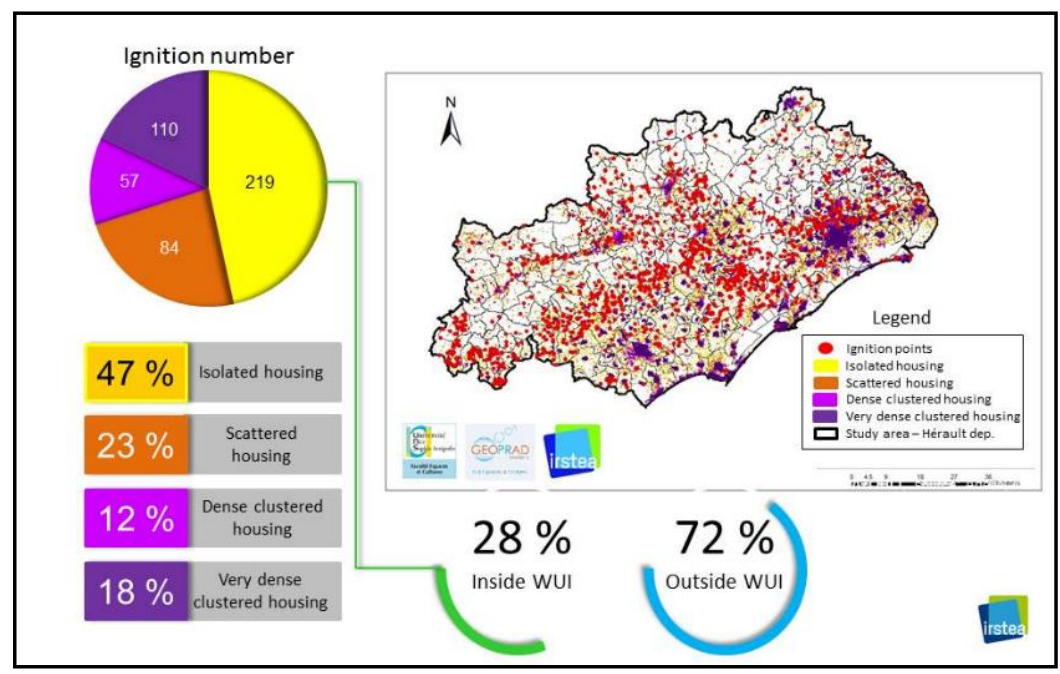

Figure 4 - Causes of fire starting in the interfaces 
Table 4 - Fire starting number in interface zone surface (communal data)

\begin{tabular}{|l|l|l|}
\hline \multicolumn{1}{|c|}{ Housing type } & $\%$ & Ignition points \\
\hline Isolated & $46 \%$ & 219 \\
\hline Scattered & $24 \%$ & 110 \\
\hline Dense & $12 \%$ & 57 \\
\hline Very dense & $18 \%$ & 84 \\
\hline Ignitions points in WUI & 470 \\
\hline Outside WUI & 1218 \\
\hline
\end{tabular}

\subsection{Ignition points and types of land use}

The following analysis was made by crossing the ignition point locations according to the land use with WUI types. We can see that within artificialized territories, outbreaks are essentially born in discontinuous urban fabric with 41 out of 64 fire ignitions across the artificialised territories. It should also be noted that within the scattered housing buildings, there are also 18 fire outbreaks.

Table 5 - Ignition points and Land Cover (Khakil Djazouli, DDTM 34)

\begin{tabular}{|c|c|c|c|}
\hline \multicolumn{2}{|r|}{ Land cover } & Ignitions & $\%$ \\
\hline \multirow{5}{*}{$\begin{array}{l}\text { Territoires } \\
\text { Artificialisés }\end{array}$} & Discontinuous urban fabric & 41 & $7 \%$ \\
\hline & Scattered housings & 18 & $4 \%$ \\
\hline & Industrial & 2 & $0,5 \%$ \\
\hline & Consruction zone & 1 & $0,5 \%$ \\
\hline & Leasure and sports facilities & 2 & $0,5 \%$ \\
\hline \multirow{7}{*}{$\begin{array}{l}\text { Agriculture et } \\
\text { végétation éparse }\end{array}$} & Arable lands & 10 & $2 \%$ \\
\hline & Vineyards & 120 & $25 \%$ \\
\hline & Olive trees & 2 & $0,5 \%$ \\
\hline & Complex agricultural plots systems & 17 & $4 \%$ \\
\hline & Agriculture and sparse natural vegetation & 1 & $0,5 \%$ \\
\hline & Moors & 4 & $1 \%$ \\
\hline & Sparse vegetation & 14 & $3 \%$ \\
\hline \multirow{6}{*}{$\begin{array}{l}\text { Forêts et } \\
\text { Végétation dense }\end{array}$} & Deciduous forests & 36 & $8 \%$ \\
\hline & Coniferous forests & 59 & $13 \%$ \\
\hline & Mixed forests & 1 & $0,5 \%$ \\
\hline & Natural pastures & 8 & $2 \%$ \\
\hline & Shrublands & 50 & $10,5 \%$ \\
\hline & Forest and evoluting shrubland & 81 & $16 \%$ \\
\hline Divers & & 3 & $1 \%$ \\
\hline TOTAL & & 470 & $100 \%$ \\
\hline
\end{tabular}

Within farmland, with sparse vegetation, nearly three quarters of fire ignition start in wineyards. Finally, at the level of dense vegetation types, there are 81 fire ignitions in the forest and shrubby vegetation, followed by 59 fire outbreaks in coniferous forests and 50 fire ignitions in shrublands (Table 5)

\subsection{Ignition points and planning documents}

In Hérault (343 municipalities), 129 communes, nearly $40 \%$ of the municipalities in the department, adopted the Local Urban Plan as a document. However, 123 municipalities have retained 
the Land Use Plan.19 municipalities, nearly 5\% of municipalities, have the Communal Map and 20\% refer to the National Urban Planning Regulation. Finally, 4 communes turned to the Local Urban Plan.

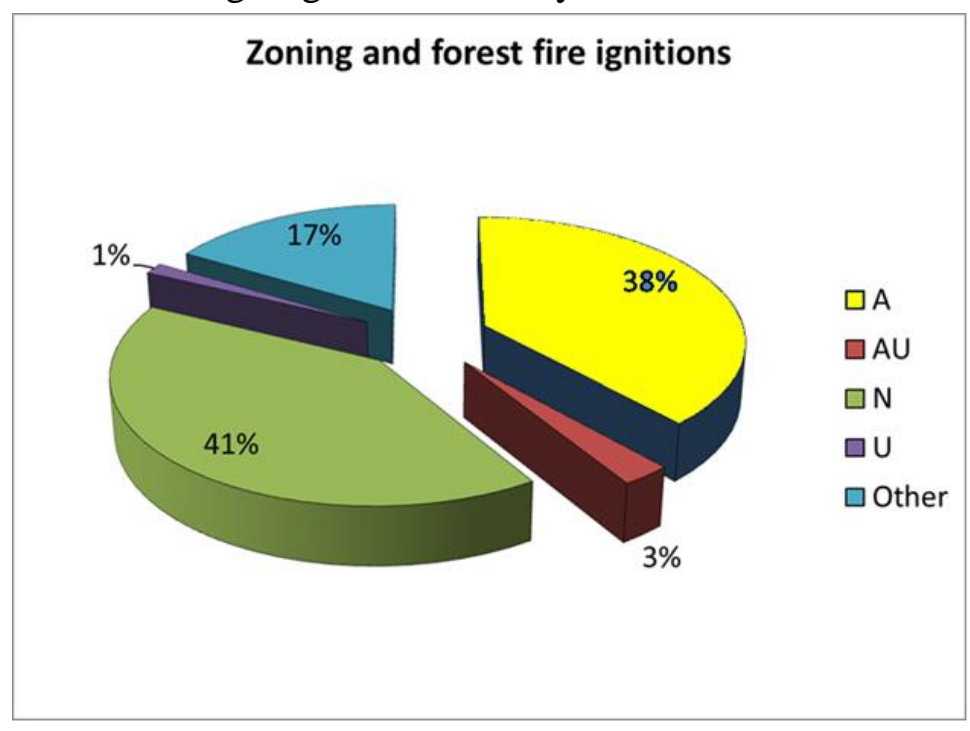

Figure 6 - Fire ignition and planning zones

We can see that the majority of ignition points are located in natural areas (Figure 6) with 693 points, followed by agricultural areas which were affected by 647 outbreaks. Thus, the areas to be urbanized and the urban areas, recorded respectively only 46 and 24 fire ignition points. It should be noted that 278 points are not included in the new zoning, because the zoning types were not available for certain municipalities with RNU.

In a part of the research, we try to find relationships between fire ignition location and planning document according to the LULC, in the study área, in the context of WUI.

The results confirm the relationship between WUI and fire ignition. In the WUI, the fire density is three times higher than outside.

Other types of fire summed up by municipalities (Figure 7)show a correlation between the WUI area and the number of peri-urban and rural fires and between AFERPU fires and AU zones (to be constructed area).

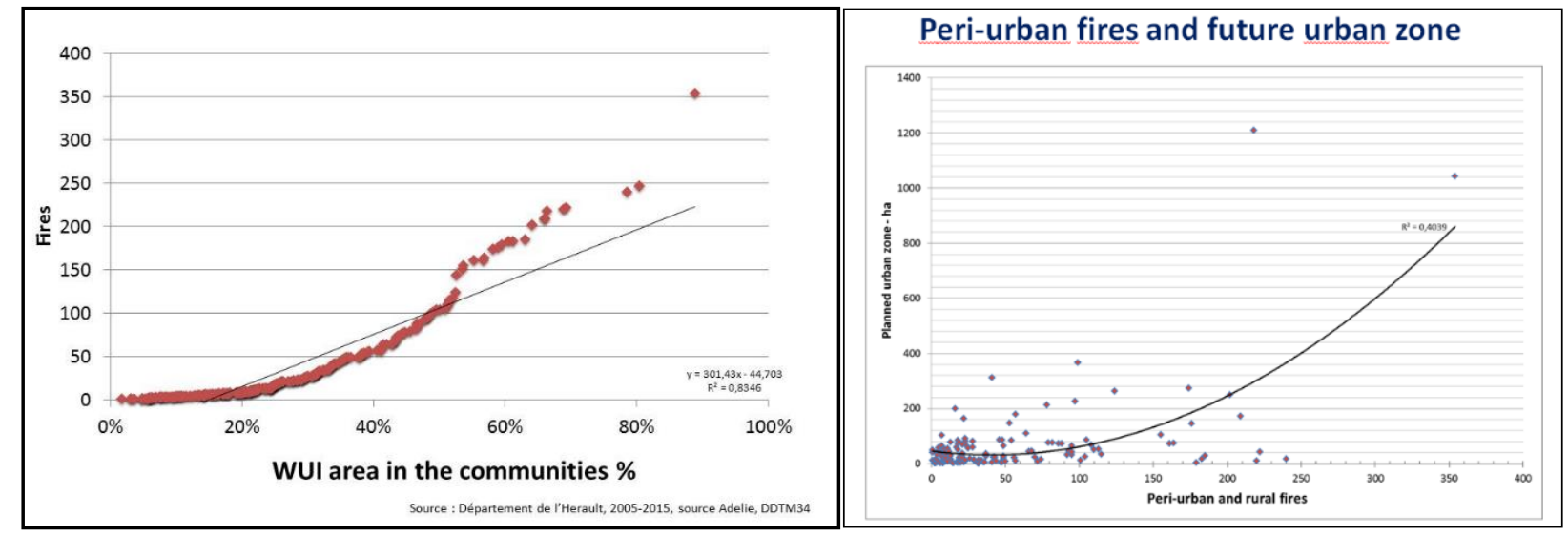

Figure 7 - Fire ignition, WUI and planning zones 


\section{Discussion}

\subsection{Planning and fire risk: the spreading of urbanization and WUIs}

The ancient planning document - the land use plan (POS) in force between 1967 and 2017- included areas allowing the construction on large plots, coded "NB" which led to a dissemination of houses located in the forest or in their approach. The law Alur (Loi no 2014-366 du 24 mars 2014) has planned the end of the POS and NB areas on March 27, 2017.

The consequences of old « NB » zoning are that they allowed constructions in agricultural and forested area, with large construction plots 2000 to $30000 \mathrm{~m}^{2}$. These areas have been difficult to manage and comply with forest fire prevention. Their locations have increased fire ignition risk because of human activities within natural areas or near them. The « NB » zoning also contributes to the creation of WUI zones with complex organizations difficult to defend in case of fire. The possibility of dividing these properties into several lots has reinforced the phenomenon. This type of zoning was cancelled by law in 2017, but these areas often keep a lot of burnable fuel.

The numerous fire ignitions in vineyards are surprising and an explanation is to be found. We found it thanks to the many readings. The results of these facts were confirmed by other studies concerning the same areas and topics. The CEREMA- (Centre d'études et d'expertises sur les risques, l'environnement, la mobilité et l'aménagement) observatory reports some statistics about soil consumption in France and FranceAgriMer studies the consequences of the uprooting program in Languedoc-Roussillon former region. We found facts and figures which confirm our results and give us some clues about the WUI and fire issues.

The extension of urbanization has been a continuous process for many years. Despite the SRULaw (Solidarity and Urban Renewal) of 13 December 2000, and despite a careful and thrifty management of the territory, most of the country has been affected by a consumption of land for urbanization.

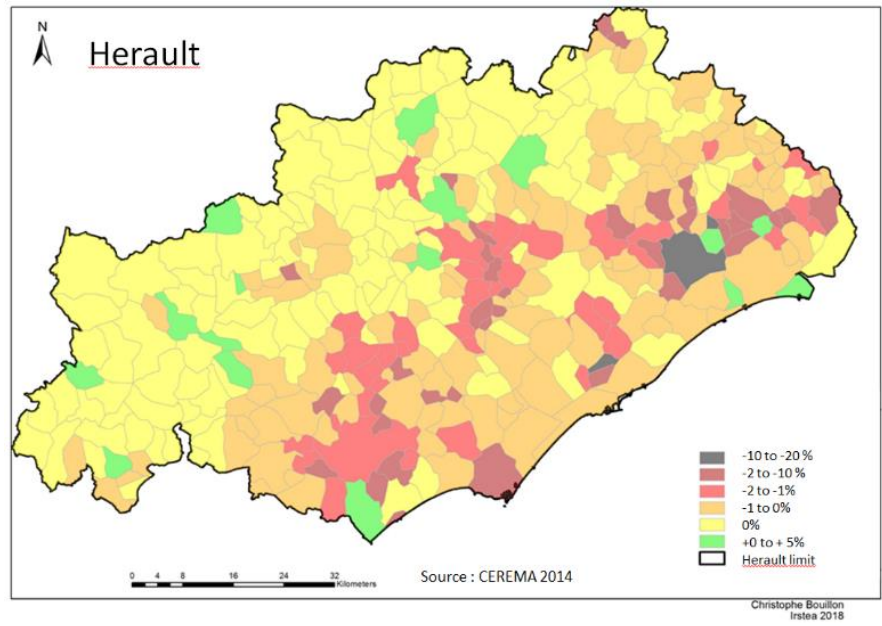

Figure 8 - Evolution of natural, agricultural or forested land in Hérault - 2006-2013 - Source CEREMA

The main land consumption is for individual housings and infrastructures. These extensions were made on agricultural, natural or forested lands. (Agreste, 2004)

\subsection{Impact of State agricultural policies}

Regarding agricultural policies and the impacts in the study area, new fire prone areas were created. The aim of this policy was to increase the vineyard quality, to improve the wine European market and replace low quality wines with better ones. 


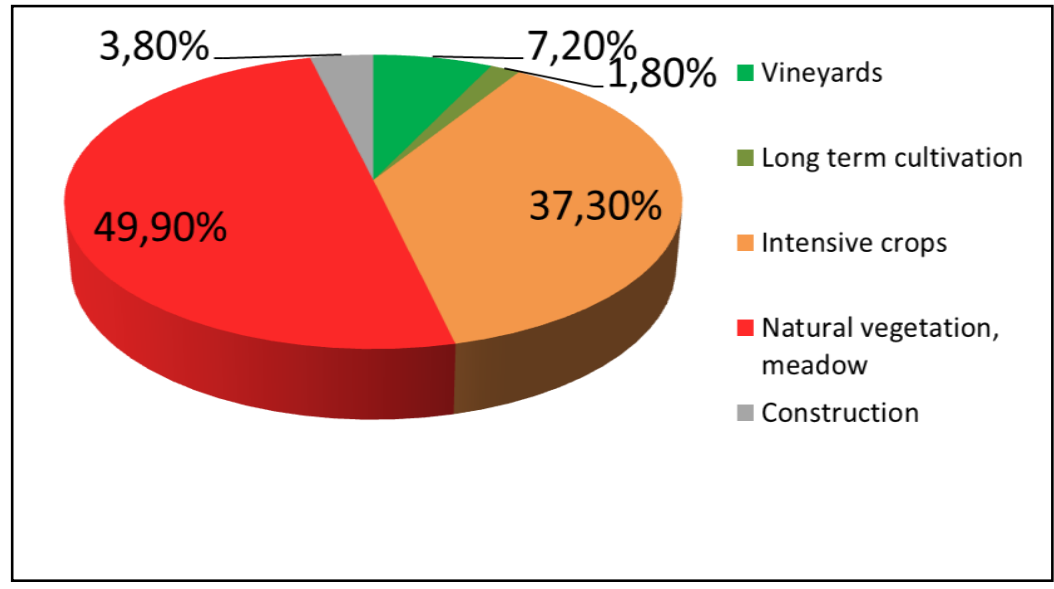

Figure 3 - Land use of uprooted wineyards in th Hérault in 2014- Source FranceAgriMer

From 1980 to 2011, the Hérault department faced the uprooting of half of its vineyards, within the framework of the Permanent Abandonment Premiums, an European structural measure aimed at cleaning up the European wine market. This led to profound changes in the landscapes of the Hérault territories, with a change in destination of the uprooted plots and a disruption of the balance between production and vineyard landscapes. The question of the future of the uprooted vine lands is acute, resulting in the mobilization of local actors on different scales. They take public policies intended to overcome land abandonment and to accompany changes in rural landscapes affected by the uprooting.

\section{Conclusion}

The risk of fire is greater in the WUI and additional observations can be made on the current LULC in these fire-sensitive areas.

In the département de l'Hérault prone to important changes, the zoning of urban areas (U) are still occupied at $21 \%$ by areas with agricultural land use; these areas are sometimes abandoned with brush vegetation and may change into forested areas.

Areas to be urbanized (coded AU) have a land-use composition with potentially significant combustible surfaces. The study shows that $45 \%$ are classified as natural areas while $41 \%$ are classified as agricultural areas, sometimes abandoned.

These unkempt "transitional" areas are conducive to fire-outbreaks and spreading. This state of awaiting projects, construction, infrastructure or other activities, can last for a long time.

The zoning-modification (local urban plan, etc.) has sometimes consequences on the vulnerability of the buildings, infrastructures, networks which are at stake. This is particularly true when new building zones are created. The addition of new buildings leads to the creation of new areas of interfaces and therefore increases the vulnerability of the facilities.

Sometimes an "AU" zone at the edge of an interface already present, and in contact with natural vegetation increases risk. The future area to be protected will increase the interface area subject to legal clearing requirements too. The potential risk is twofold: the natural area may be affected by a fire-outbreak in this new interface zone, and conversely a fire propagating in the natural area may threaten the future buildings of the new zone.

In other cases the AU zones are under development, which can last several years.

The formerly visible agricultural areas are sometimes abandoned pending projects (building plots). Therefore some lands abandoned by agriculture are particularly sensitive to fire.

On the outskirts of larger towns, the colonization of agricultural lands by the progression of the AU zones, creates unkempt islands near existing buildings and is the source of many fires. 
A recent study of abandonned agriculture land reinforces our study, as the sampling of ancien agricultural zones show the state of abandonment in the study area.

The different public policies, from the municipal level to a national one, could have consequences on the fire risk and not take them into account.

\section{References}

Arnal, C., Laurens, L., Soulard, C.,2013, The landscape mutations generated by the uprooting of vineyard, a vector for the mobilization of local actors in the Herault department [Article @ Les mutations paysagères engendrées par l'arrachage viticole, un vecteur de mobilisation des acteurs territoriaux dans l'Hérault] (2013) Mediterranee, 120 (1), pp. 49-58.

Bouillon, C., Fernández Ramiro M.M. Fierro García B, Sirca C.,Long-Fournel M, Casula F., Pellizaro, G., Arca, B., Maillé, E., Bellet, A., Pugnet, L., Vila-Molina, B., Ferrara, R., Pintus, G.V. - 2013. FUME (Forest fires under climate, social and economic changes in Europe, the Mediterranean and other fire-affected areas of the world ) European Program Déliverable D 1.1.5. The RUI and fire risk Analysis and mapping of the spatial and temporal changes of the RUI on local and regional scales and of fire occurrence Aix-en-Provence 148

CEREMA, 2016, La consommation d'espaces et ses déterminants d'après les Fichiers fonciers dela DGFiP, Analyse et état des lieux au 1er janvier 2015 Direction territoriale Nord-Picardie : 2, rue de Bruxelles - CS 20275 - 59019 Lille Cedex, 41p.

Costantino Sirca, Franco Casula, Christophe Bouillon, Belen Fierro García , Monica Maria Fernandez Ramiro , Beatriz Vila Molina, Donatella Spano , 2017, A wildfire risk oriented GIS tool for mapping Rural-Urban Interfaces, Environmental Modelling \& Software 94 (2017) 36-47 https://doi.org/10.1016/j.envsoft.2017.03.024

FranceAgriMer-LR, 2012, Enquête sur le devenir de 500 parcelles arrachées primés PAD entre $2005 / 2006$ et $2010 / 2011$

Lampin-Maillet \& C., Bouillon C. , 2011, WUImap: a software tool for mapping rural-urban interfaces at a large scale over a large area in Mediterranean European context. Journal of Environmental Science and Engineering, 5, 2011-4

Lampin-Maillet, 2009, Caractérisation de la relation entre orgzniqtion spatiale d'un territoire et risque d'incendie : le cas des interfaces habitat-foret du sud de la fgrance, thèse en géographie, Université de Provence, Aix-Marseille, 396p.

Ministère de l'agriculture et de la pêche, Agreste, 2009, "La maison individuelle grignote dles espaces naturels, Agrest Primeur n]219, janvier 2009,

Ministère de l'Agriculture, L'utilisation du territoire en 2014, Agreste chiffres et donnes Agriculture $\mathrm{n}^{\circ} 229$

Ministere de l'agriculture de l'alimentation, de la pêche, de la ruralité et de l'aménagement du territoire; Ministère de l'intérieur de l'outre-mer, des collectivités territoriales et de l'immigation, 1973-2018, Prométhée (C), base de données officielle pour les incendies de forêt dans la zone méditerranéenne française, v1.0.39 


\section{Chapter 5}

Decision Support

Systems and Tools 


\title{
AdriaFirePropagator and AdriaFireRisk - user friendly Web based wildfire propagation and wildfire risk prediction software
}

\author{
Marin Bugarić*; Darko Stipaničev; Toni Jakovčević \\ ${ }^{1}$ University of Split Faculty of Electrical Engineering, Mechanical Engineering and Naval \\ Architecture in Split, Ruđera Boškovića 32, 21000 Split, Croatia, \{marin.bugaric@fesb.hr*, \\ darko.stipanicev@fesb.hr,toni.jakovcevic@fesb.hr\}
}

\begin{abstract}
The HOLISTIC Project with full name Adriatic Holistic Forest Fire Protection was a project funded by Adriatic IPA funds that has gathered together almost all regions around Adriatic Sea in order to improve wildfire prevention, protection, fighting and post-fire recuperation. It has started in December 2013 and ended in December 2016. A part of this project was to establish user friendly and simple regional wildfire propagation and wildfire risk prediction simulation tools that could be easily used by fire fighters in everyday fire fighting operative work, but also as a tool for fire season preparation. The final results were AdriaFirePropagator and AdriaFireRisk and additionally AdriaFireRisk sub-module named AdriaFireRiskPanels, and they are the main topics of this paper. AdriaFirePropagator is a Web based system for wildfire behaviour modelling and wildfire spread simulation. Web based means that all the data are stored on the server, and all the applications in the fire simulation software are server based programs that run when requested by the users and the only user interface is a standard web browser. AdriaFireRisk is additional module, running parallel on the same server using the same GIS calculation engine. AdriaFireRisk calculates site-specific wildfire risk index based on experimental system for micro location wildfire risk index calculation developed for Split - Dalmatia County. AdriaFireRisk is also a support system for additional HOLISTIC project result - AdriaFireRiskPanels designed to raise the awareness about the wildfire risk in order to increase caution and reduce the number of accidental wildfires. The paper describes technical details of these systems, as well as their practical implementation.
\end{abstract}

Keywords: wildfire propagation simulation, wildfire risk prediction, wildfires, forest fires, EU project

\section{Introduction}

The regions around Adriatic Sea have high risk of wildfires, especially during the summer due to very warm climate with low level of humidity, densely spaced Mediterranean vegetation, particularly conifer forests and lot of tourists. During fire season that is usually between July 1 and October 31, there are lot of wildfires in this region. For example in Republic Croatia located on the east side of Adriatic Sea in the last 10 years 2.320 wildfires have been recoded that affected 84.250 hectares of forests and forest land.

Therefore, the biggest Croatian county Split-Dalmatia County has initiated in 2007 preparation of strategic project target to Adriatic IPA funds, based on Split-Dalmatia County holistic approach to forest fires protection and prevention. The HOLISTIC Project has been finally accepted in 2013 as Adriatic IPA project under priority 2 Natural and Cultural Resources and Risk Prevention, measure 2.2 Natural and cultural resource management and prevention of natural and technological risks. HOLISTIC project, with full name Adriatic Holistic Forest Fire Protection, ended in December 2016 (Stipanicev et al, 2018). A part of this project was to establish user friendly and simple regional wildfire propagation and wildfire risk prediction simulation tool that could be easily used by fire fighters in everyday fire fighting operative work, but also as a tool for fire season preparation. The final results were AdriaFirePropagator and AdriaFireRisk and additionally AdriaFireRisk sub-module named AdriaFireRiskPanels. These three operative tools are the main topics of this paper. 


\section{Simulator description and architecture}

Wildfires can have severe consequences to the environment, therefore many efforts have been put into investigating events related to fire ignition and fire spread. Several models have been developed throughout the years: "fire ignition modelling", "fire intensity modelling" and "fire spread modelling". "Fire spread modelling" is useful not only during the actual wildfire for predicting how wildfires will spread, but also in pre- and post-fire activities. In the pre-fire, preventive phase, it can be used for organizing the fire fighting units, planning the position of ground fire fighting equipment, or simply for training the fire fighting personnel. In the case of post-fire activities, previous wildfires can be investigated to improve efficiency in the future.

Along with development of fire spread models, fire risk models have improved as well. Fire risk index is a numerical indicator that describes the level of fire risk at certain geographical area. Identifying the fire danger is important for both wildfire prevention and protection. It can be useful for improving automatic smoke and fire detection systems, as well as for other fire-fighting activities that happen before the actual wildfire.

Both fire spread models and fire risk models strongly rely on correct input parameters. Speaking of fire propagation, most of the existing models that have been developed in the last 50 years are mostly based on the following parameters: meteorological conditions (wind speed and direction, temperature, humidity), vegetation (fuel) models, fuel moisture and topography, primarily aspect and slope of the terrain. Similarly, most of the existing fire risk models rely on the very same parameters, although they often consider at least a few other parameters as well. Therefore, a system with a robust and timely access to current meteorological conditions along with an up-to-date GIS database is necessary to develop either a fire spread simulation or fire risk calculation system.

Fire spread systems are usually grouped into three categories:

- Physical and quasi-physical models,

- Empirical and quasi-empirical models,

- Models based on mathematical analogies and simulation models.

Physical models are based on physical and chemical laws related to fire propagation, while quasiphysical models are based solely on physical description of fire propagation. Empirical models are predicting more probable fire behaviour from average conditions and accumulating acknowledges obtained from laboratory and outdoor experimental fire, or historical fires. Semi-empirical models combine analytic formulation of physical phenomena with statistical information. Models based on mathematical analogies use mathematical laws instead of physical, whereas simulation models use any of the aforementioned models with the main aim of simulation of fire spread in two-dimensional space. One of the most famous fire spread models is Rothermel's surface fire spread model. It is a semiphysical model, meaning that it has components that rely on the physics of combustion and components that rely on laboratory experimental results.

The overall system architecture of AdriaFirePropagator is based on two basic modules:

- "Before wildfire" module that includes fire spread simulation based on archived meteorological data (education and investigation mode suitable for pre-fire and post-fire activities), and

- "During wildfire" module that includes fire spread simulation based on real-time meteorological data

AdriaFirePropagator has two main data sources that ensure the correct operation of the system. First, and most important one is a GIS database. GIS database stores not only standard GIS data (such as terrain map, satellite map, road map, settlements, water sources...), but also GIS data specific for 
both fire spread simulation and fire risk index calculation systems. Some of GIS layers are used solely for visualisation and are not required for actual calculations, however, without those layers it would be difficult to visualize the simulation results to the client.

The second data source is a meteorological database. Meteorological data is downloaded automatically several times a day from official server of Croatian Meteorological and Hydrological Service (DHMZ, 2018), with hourly forecast based on ALADIN model, allowing the "during wildfire" module to run simulations throughout the day. Simultaneously, meteorological data is stored in the database for future use when "before wildfire" module is executed.

Fire propagation module is responsible for the actual wildfire simulation and provides simulation results. It is initiated once the user starts a simulation from a web-based user interface. If all input data is provided, the fire propagation module produces simulation results that are then displayed back to the user in the same web-based user interface.

The main tool behind the fire propagation module is a Geographical Information System (GIS), more specifically GRASS GIS, a free, open source system capable of handling raster, topological vector, image processing, and graphic data. We have improved the original fire spread module, developed for GRASS GIS, based on Rothermel's surface fire spread model and cellular automata approach, to allow more customisation of all parameters, with special emphasis on customisation of fuel parameters. This allowed us to better adapt the fire simulation module to Mediterranean climate and vegetation.

Along with fire propagation module, the same GIS is used for two additional modules responsible for calculation of AdriaFireRisk - overall wildfire risk index and AdriaFireEruptiveRisk - eruptive wildfire risk index. AdriaFireRisk module is used to calculate fire risk that is adapted to the microlocation (site-specific wildfire risk index), while AdriaFireEruptiveRisk module is used to detect zones where eruptive fire behaviour could happen.

Both risk indexes are calculated automatically in the background every three hours. Resulting risk maps are served through a WMS service and displayed as layers within AdriaFirePropagator system.

AdriaFirePropagator is a Web based application so users can access the system from any location, using any available wired or wireless ways of accessing the Internet. It is also designed as a multi-user environment, meaning that multiple users can use the system simultaneously and independently. After authenticating with the system, all users are presented with their own user interface that they can customize to their needs and from which they control all their simulation actions, independently of other users.

An additional API was also developed that allows other (authorized) systems to communicate with AdriaFirePropagator. API calls can easily be used to initiate fire propagation module, either in "before wildfire" or "during wildfire" mode, but also to obtain the results of such simulations. Similarly, AdriaFirePropagator allows other systems to display results of AdriaFireRisk and AdriaFireEruptiveRisk calculations using WMS services. This allows other systems to use AdriaFirePropagator as a "single-click" operation to simplify usage in case of emergency. However, it also uses a high degree of customization for more advanced users.

\section{AdriaFirePropagator}

AdriaFirePropagator is based on our previous experience with experimental wildfire spread simulation for Split-Dalmatia County and the following:

- Generic model of Web based wildfire behaviour modelling and wildfire spread simulation adapted for Adriatic region characteristics in HOLISTIC project,

- $\quad$ MOPP - experimental system for wildfire spread simulation developed at University of Split for Split-Dalmatia County (CIPOP, 2015), 
- Wildfire spread simulation modules of Open Source GIS system GRASS GISS developed by various authors under MIT license (GRASS GIS ROS, 2015), but further modified through MOPP and HOLISTIC project.

AdriaFirePropagator user interface is shown in Figure 1, together with an example of a typical wildfire simulation result in AdriaFirePropagator. AdriaFirePropagator was designed primarily as a fire fighters software tool that could be used in pre-fire or post-fire activities, as well as during-fire activities. To support this, three main groups of users were introduced:

1. System administrator,

2. Fire-fighting activities planner, and

3. Sentry in the fire-fighting centre.

System administrator has a full access to all the programs, modules and data in the system. Fire fighting activities planer is a fire-fighting operator who uses fire propagation module for simulating wildfire behaviour based on custom weather conditions or conditions extracted from historical data. Sentry in the fire-fighting centre is a fire-fighting operator who uses the fire propagation module in a case of a real wildfire. In this case, the simulation is performed on the actual (current) meteorological conditions and data.

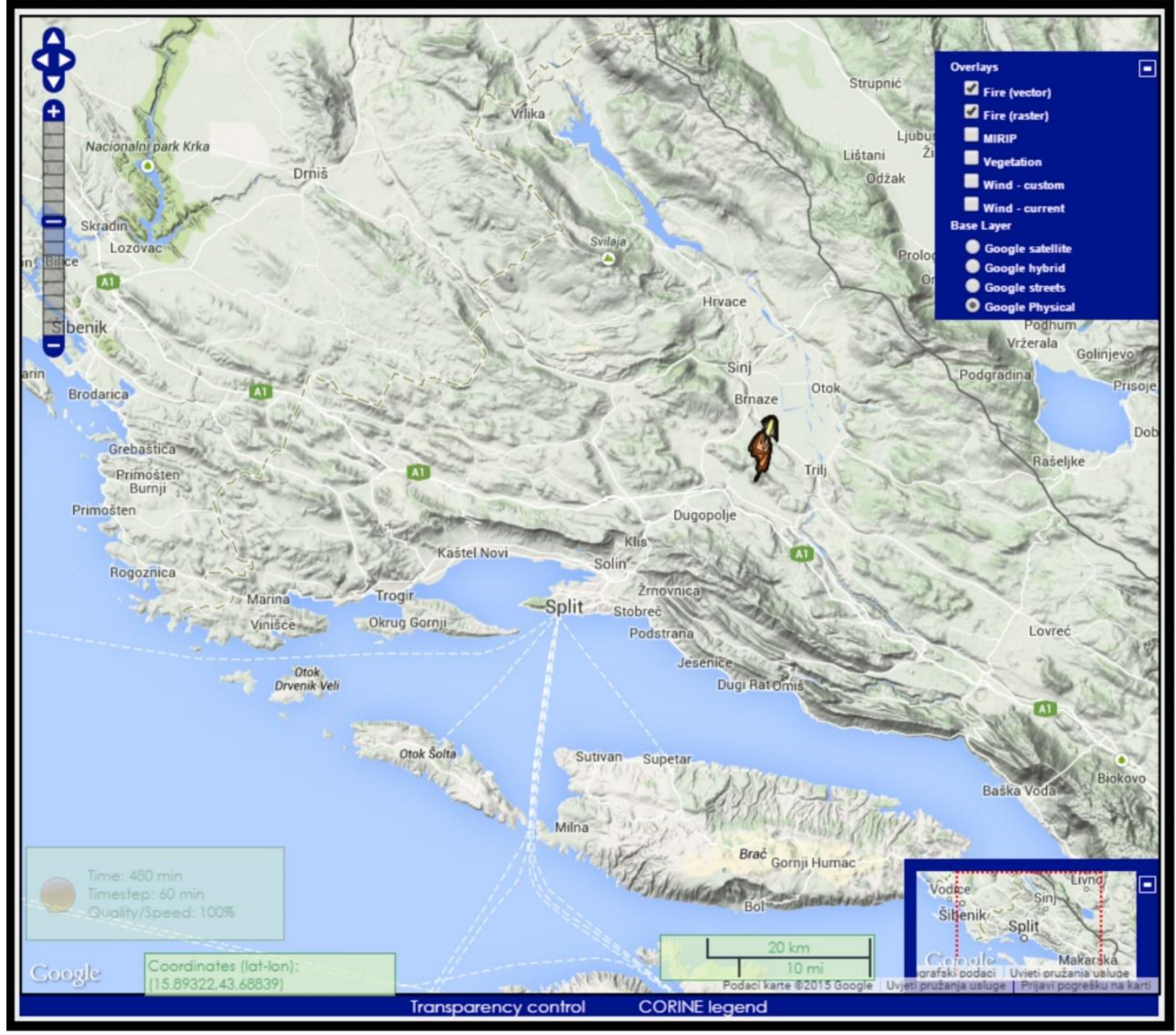

Figure 1 - AdriaFirePropagator screen 
AdriaFirePropagator support two different wildfire simulation scenarios: simulations based on current meteorological conditions and simulations based on custom meteorological conditions, demonstrating two modes of the system: active and simulation mode, respectively.

Before initiating the actual simulation, the user must first specify various simulation settings such as the duration of the simulated fire, time step that will be used to mark the fire front at certain time instants, spatial resolution of the simulation, etc. After choosing the initial point or initial area of the wildfire the user decides whether to run the simulation based on current or custom meteorological conditions. If the user initiates the simulation based on custom meteorological conditions, the system first checks if all the data is prepared. If so, the simulation proceeds with execution. Similarly, if the user initiates the simulation based on current meteorological conditions, the system first checks whether the most recent data is obtained from meteorological server. After preparing all the required data, the simulation proceeds with execution. Finally, all the results are saved in the right format and published in a form of a WMS layer. Fire propagation module then sends a signal to the user interface that automatically refreshes the view to display the latest result.

AdriaFirePropagator checks whether current meteorological data is up to date at the moment when the simulation is run in active mode, other mechanisms exist that do this task on a frequent basis. This is so to avoid unnecessary delay in execution time of the simulation. Time-based job scheduler is set to automatically download meteorological data every three hours from Croatian Hydro Meteorological Service. Current meteorological data is automatically stored into meteorological database (allowing these data for the future use). Simultaneously, all GIS layers that rely on meteorological data are automatically prepared, as explained in the rest of this section. Meteorological data consist of: wind (speed and direction), relative humidity, rainfall and temperature. Most of these data require adjustment before use in propagator. Wind data is first corrected to obtain mid-flame wind speed from standard $10 \mathrm{~m}$ high, whereas relative humidity, rainfall and temperature are used to calculate Canadian Fire Weather Index (FWI) and live, 1-hour, 10-hour and 100-hour fuel moistures. Fuel moisture, both live and dead is a very sensitive parameter in wildfire spread simulation.

Vegetation (fuel) model used in AdriaFirePropagator depends on two inputs: fuel maps and fuel parameters. AdriaFirePropagator supports Albini-Anderson and Scott-Burgan's fuel models as well as fine adjustment of fuel parameters for both, as shown in Figure 2.

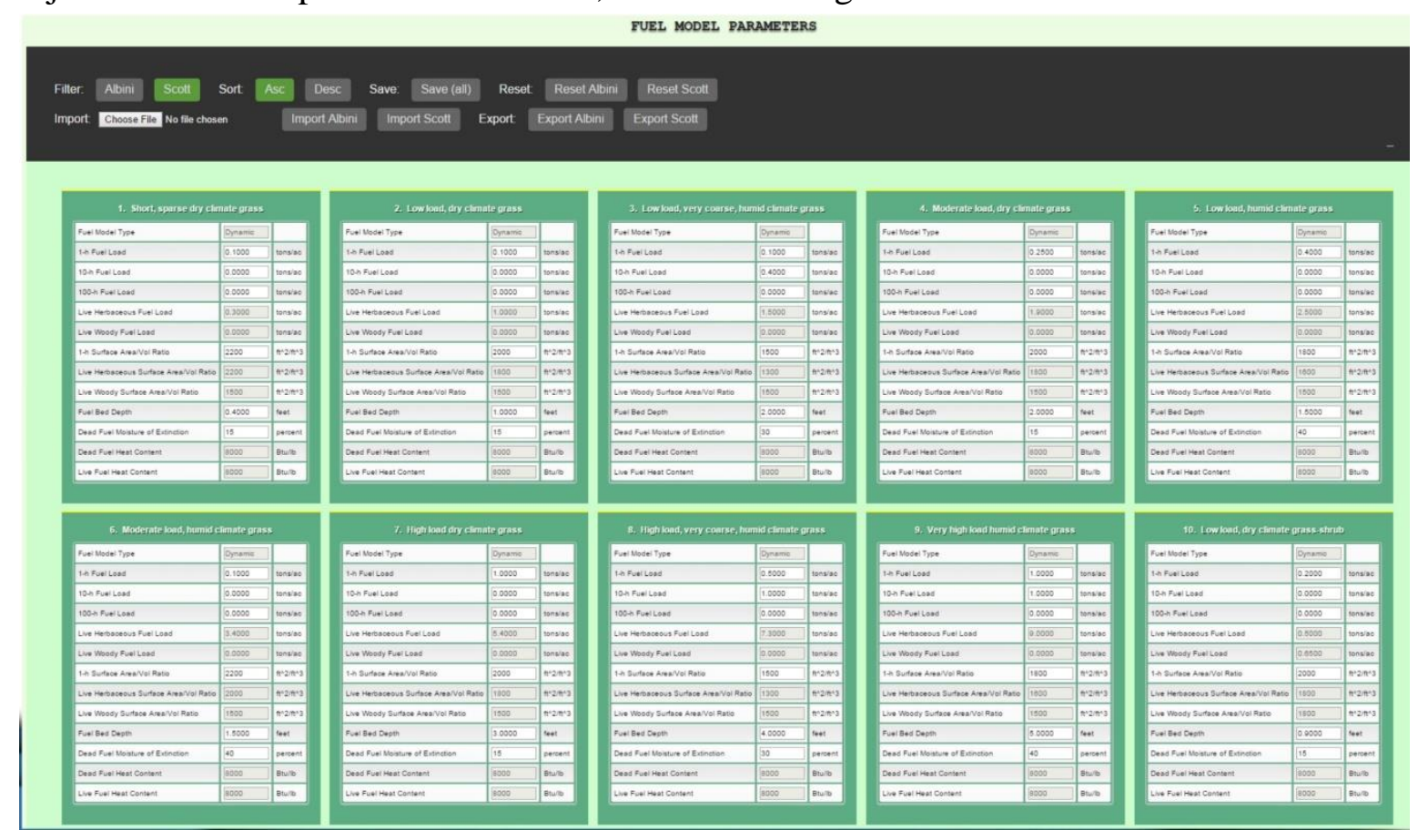

Figure 2 - Adjustment of fuel parameters of applied fuel models. 
Finally, meteorological data, fuel data and topography are used to calculate Rate of spread, a layer that is later used along with ignition point (or area) to calculate the actual simulation result.

Additional possibilities of AdriaFirePropagator is simple adding of fire barriers to simulate unburnable areas and specify the ignition location as a single point or as arbitrary polygon, so that various initial ignition situations could be simulate.

Simultaneously with GIS layers preparation, eruptive wildfire risk index (AdriaFireEruptiveRisk) is calculated. Eruptive fire risk considers the topography of the terrain and current wind data to estimate areas where possible eruptive fire behaviour could happen. Calculations are mostly based on Viegas recommendations (Viegas, 2005). Eruptive wildfire risk is divided in 4 risk categories coded with different shades of red - purple: Category I - Possibility of Landscape caused eruptive fire, Category II - Possibility od Wind \& Landscape caused eruptive fire, Category III - High possibility od Wind \& Landscape caused eruptive fire and Category IV - Very high possibility od Wind \& Landscape caused eruptive fire. Figure 3 shows a typical map where eruptive fire behaviour could happen for part of Split - Dalmatia County on June 30, 2015.

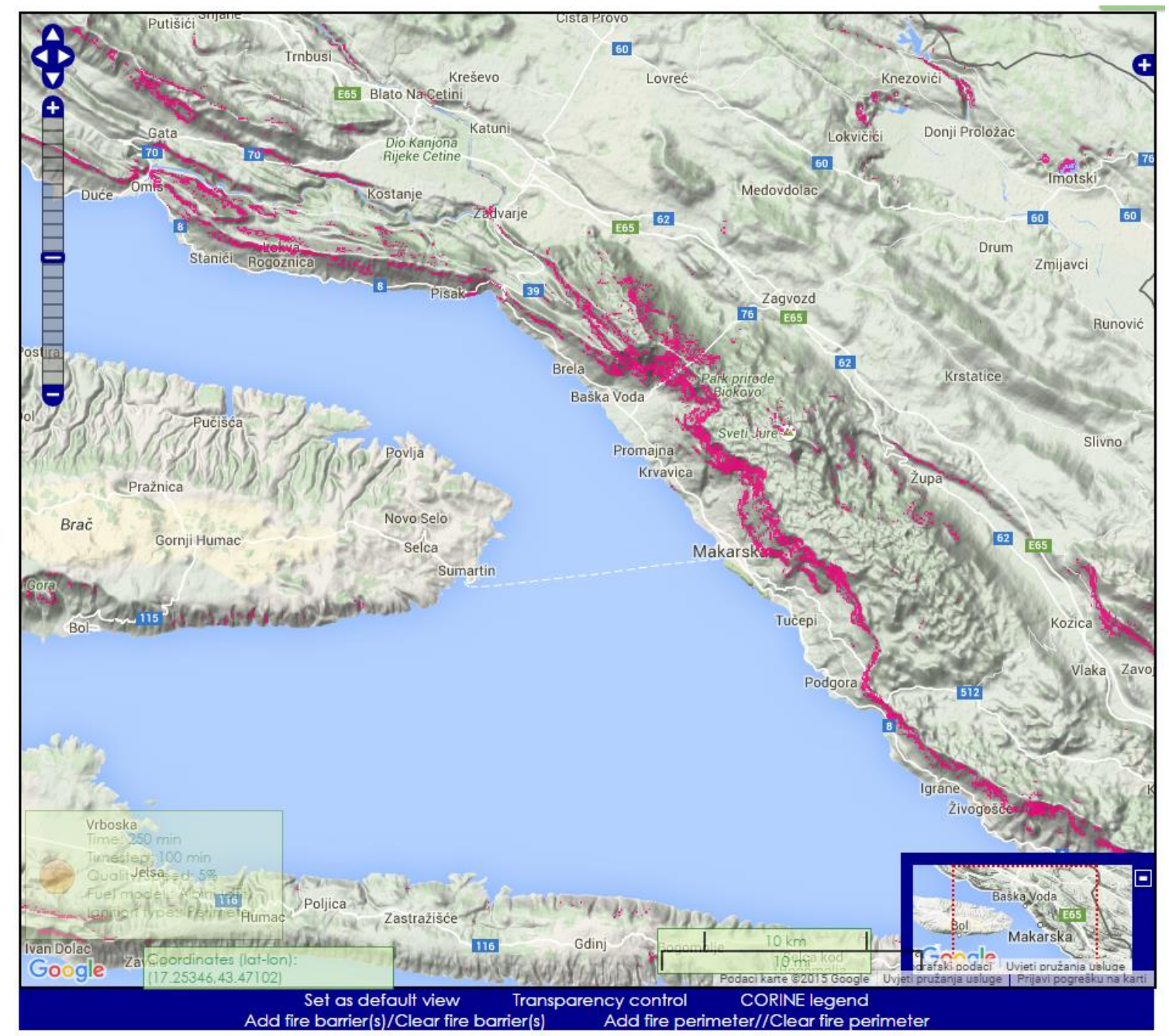

Figure 3 - Map of AdriaFireEruptiveRisk for Split - Dalmatia Counties on June 30, 2015

\section{AdriaFireRisk and AdriaFireRiskPanels}

AdriaFireRisk is an additional module, running parallel on the same server as AdriaFirePropagator, using the same GIS engine based on GRASS GIS application. AdriaFireRisk module calculates sitespecific wildfire risk index based on MIRIP - experimental system for micro location wildfire risk 
index calculation developed at University of Split for Split - Dalmatia County (CIPOP, 2015), (Bugarić et al., 2014).

AdriaFireRisk index is Site-specific Wildfire Risk Index (SWRI) with quite satisfactory spatial resolution. It is not based solely on meteorological parameters, like most existing wildfire risk indexes. It also takes into account other parameters for which we have, after carrying out a detailed statistical analysis, proven that they have a significant influence on the risk of wildfires in the Adriatic region, particularly central part of Adriatic (Bugarić et al., 2014). More specifically, AdriaFireRisk index is based on the following parameters: climatological and meteorological parameters, vegetation, terrain configuration and anthropogenic parameters.

AdriaFireRisk index could be seen as an additional layer of AdriaFirePropagator, but it could be included in any GIS interface as an external WMS layer. An example of the AdriaFireRisk index map for part of Split and Dalmatia County in Croatia, during a small and high-risk index is shown in Fig. 4.
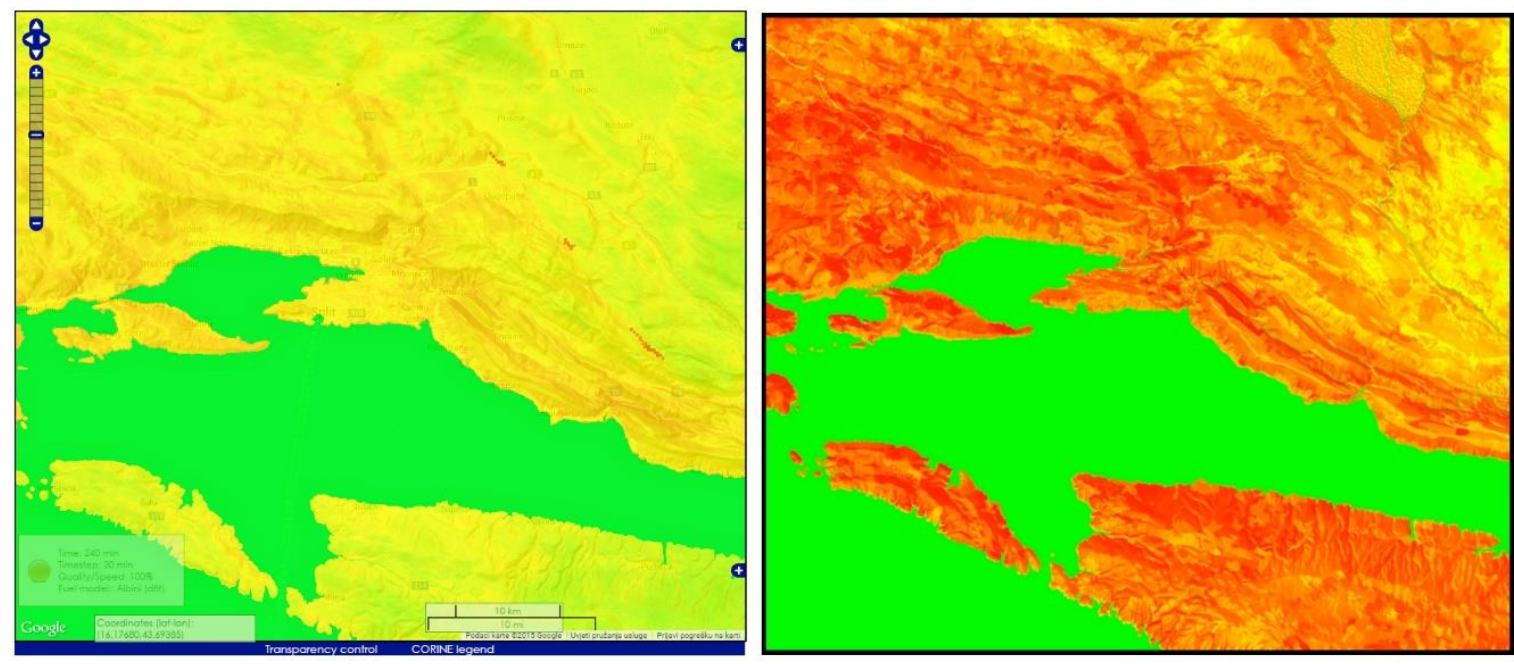

Figure 4 - Site-specific Wildfire Risk Index for part of Split-Dalmatia County during low and high risk.

Climatological and meteorological parameters are dynamic parameters, based on actual meteorological measurements, as well as on prediction of meteorological data calculated by ALADIN numerical weather prediction system of Croatian Meteorological and Hydrological Service (DHMZ). Meteorological data are automatically collected from DHMZ server.

The most important climatological and meteorological parameter within AdriaFireRisk is Canadian Fire Weather Index (FWI). Throughout the years, it has proven to be a good indicator of fire risk, however, it is not well adapted to the micro-location. AdriaFireRisk system calculates FWI every day based on temperature, relative humidity, rainfall and wind data. Meteorological data is also used in combination with other GIS layers. Our study has shown that the slope of the terrain in combination with wind speed or aspect of the terrain in combination with wind direction have greater influence on wildfire risk than wind speed and wind direction alone.

Vegetation is one of the most important parameters that build up fire risk index. FBPS (Fire Behaviour Prediction System) is a fuel model that is used to describe burning characteristics of different vegetation categories. Unfortunately, there is no map that classifies Croatian territory into these fuel categories based on type of vegetation, therefore we decided to use Corine Land Cover map. We have created conversion guidelines that can be used to convert from CLC model to FBPS model.

As already mentioned, topography is used together with meteorological data as an input for AdriaFireRisk calculation. However, terrain configuration has even a more significant influence on fire risk. With higher altitudes the temperatures are lower. Also, there is a direct relation between vegetation types and altitude. 
Finally, proximity to people and infrastructure is of great importance when talking about wildfire risk. Fire risk is higher in the locations inhabited with more people. Historic wildfires have shown high correlation to anthropogenic parameters, more specifically distance to buildings, distance to roads and distance to transmission lines. Therefore, those were considered as additional input for AdriaFireRisk.

AdriaFireRisk is also a support system for additional HOLISTIC project result AdriaFireRiskPanels designed to raise the awareness about the wildfire risk in order to increase caution and reduce the number of accidental wildfires. The panels have been placed on spots of important public interest sensitive to wildfires, such as national parks, city forests, protected islands etc.

Appropriate information about current fire risk for a given area plays an important role in fire prevention. AdriaFireRiskPanels serve as an instrument for disseminating this information to the general public. The main role of these panels is to raise the awareness about wildfires and subsequently influence the behaviour of people in the high-risk areas.

The panels are a visual tool for spreading this information, so they should be easy to interpret, and also legible from larger distances. The panel is divided into five areas of different colours, each representing a certain fire risk index. Figure 5 shows one typical installation at the entrance of protected Park Forest Marjan in Split, Croatia.

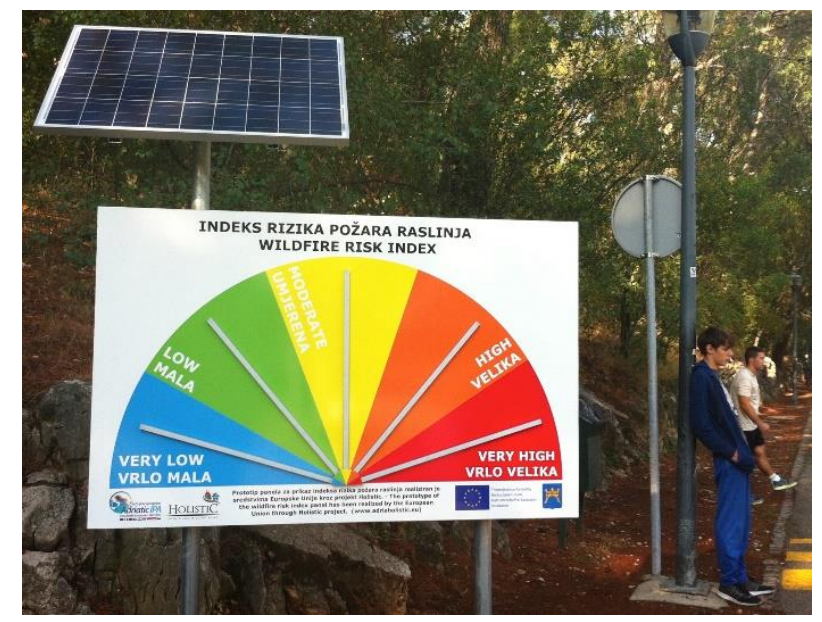

Figure 5 - Wildfire risk index panel installed in Park Forest Marjan in Split, Croatia

LED (Light Emitting Diode) strips are used to signal which fire risk index is currently predicted for the given location. This is indicating by blinking of the strip. Blinking lights are better at drawing attention of the passing by pedestrians than continuous light, and additionally serves as energy conservation mechanism. It is important that the lights are visible even in the midday sunlight, so in the design phase testing was performed to determine which kind of stripes regarding LED type and power categories are most suitable for this kind of application. Figure 5 show the setup used for testing visibility of different LED stripes on different background colours.

Many of potential installation location for fire risk panels are in natural environment, often without access to electrical power source; therefore it is important that these panels are self-sufficient in terms of energy needed for their operation. In such cases panels are equipped with solar panels and deep cycle batteries.

The fire risk index for a given location is calculated on fire risk server and the task of the panel is to retrieve this information and display it properly. The communication between panels and the server is performed using an embedded networking device that has an integrated GPRS modem. This means that the panels must be in range of cellular networks in order to have a means of communication with the server.

The information about the fire index for a given location is served by the server in the form of an XML document that is parsed by the embedded device in the panel. 
The embedded device also operates the relays controlling the LED stripes. It is important that the system is robust when it comes to dealing with communication problems with the servers. The risk index on the panel is refreshed every hour with the information from the server. In case the panel cannot obtain fresh information from the server in the period of 24 hours, the LED stripes are automatically switch off until communication is re-established.

One central server calculates fire risk index for all the locations where panels are installed. Panels do not communicate between themselves, but only with the central server.

Several panels can be deployed for different points in the same location and can use the same fire risk calculation, but generally the deployment is such that panels are deployed for different location and have separate risk calculations. Figure 6 show a diagram for multiple panel system.

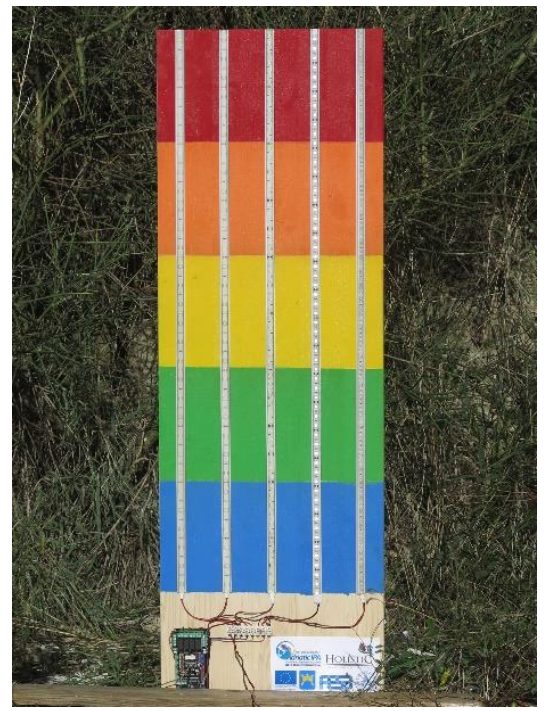

Figure 6 - Setup for LED visibility testing

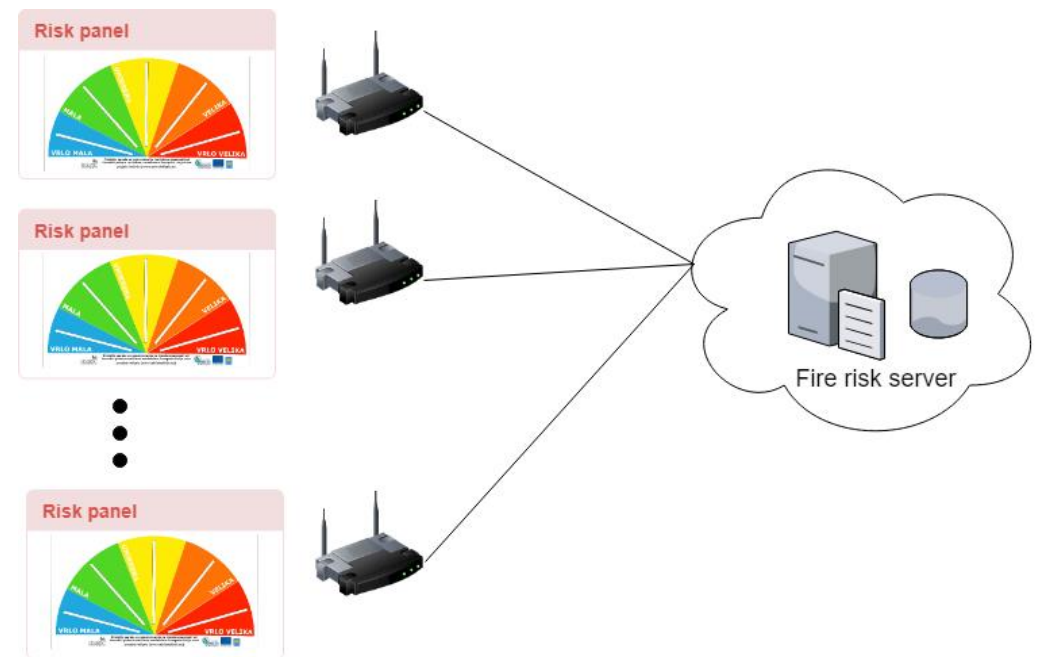

Figure 7 - System with multiple panels

AdriaFireRiskPanels were designed at University of Split Faculty of Electrical Engineering, Mechanical Engineering and Naval Architecture as Open Hardware and Open Software project, so anyone who want to deploy panels could get all project details.

\section{Conclusion}

A part of IPA ADRIATIC HOLISTIC project was to establish user friendly and simple regional wildfire propagation and wildfire risk prediction simulation tool that could be easily used by fire fighters in everyday fire fighting operative work, but also as a tool for fire season preparation. The final results were AdriaFirePropagator and AdriaFireRisk and additionally AdriaFireRisk sub-module named AdriaFireRiskPanels. These three operative tools are the main topics of this paper.

AdriaFirePropagator is a Web based system for wildfire behaviour modelling and wildfire spread simulation. Web based means that all the data are stored on the server, and all the applications in the fire simulation software are server-based programs that run when requested by the users and the only user interface is a standard web browser. 
Based on our previous experience with experimental wildfire spread simulation for Split - Dalmatia County, AdriaFirePropagator was proposed as a software tool for fire fighters that could be used in pre-fire (before-fire) or post-fire (after-fire) activities, as well as for fire (during-fire) activities. In prefire and post-fire activities AdriaFirePropagator could be used for fire behaviour modelling and fire spread simulation based on archived meteorological data (education mode for training and preparing fire fighters for possible future wildfire scenarios, as well as for investigation of some past fires. AdriaFirePropagator could be also a quite useful tool for during-fire activities. In this phase its main task is to predict possible wildfire spread for existing fire, and therefore it is based on real time meteorological data. Knowing a potential fire spread pattern is particularly important for fire fighters safety issues.

AdriaFirePropagator could be used as 'one-click' application, but for more serious fire propagation simulation, a lot of features and parameters can additionally be modified and tuned.

AdriaFireRisk and AdriaFireEruptiveRisk are two additional modules, running in parallel on the same server as AdriaFirePropagator, using the same input data. AdriaFireRisk module calculates sitespecific wildfire risk index. AdriaFireEruptiveRisk module predicts potential areas where there is enhanced risk of eruptive fire behaviour. AdriaFireRisk index is based on several parameters that can be classified into two categories: static and dynamic. Static parameters change slowly over time; therefore they can be computed once before each fire season, while dynamic parameters depend upon current conditions and must be calculated on a daily basis. Those parameters are: climatological and meteorological parameters, vegetation, terrain configuration and anthropogenic parameters. Climatological and meteorological parameters are dynamic parameters, based on actual meteorological measurements, as well as on prediction of meteorological data calculated by ALADIN numerical weather prediction system of Croatian Meteorological and Hydrological Service (DHMZ). Meteorological data are automatically collected from DHMZ server. Other parameters belong to the set of static indicators and they were collected for the all partner participating in HOLISTIC project. AdriaFireRisk index could be seen as an additional layer of AdriaFirePropagator, but it could be included in any GIS interface as an external WMS layer.

AdriaFireRisk is also a support system for additional HOLISTIC project result AdriaFireRiskPanels designed to raise the awareness about the wildfire risk in order to increase caution and reduce the number of accidental wildfires. The panels have been placed on spots of important public interest sensitive to wildfires, such as national parks, city forests, protected islands etc. The paper describes technical details, particularly panels automatic synchronization with AdriaFireRisk system.

\section{References}

Bugarić et al., 2014 Bugarić, M., Braović, M., Stipaničev, D. Statistical evaluation of site-specific wildfire risk index calculation for Adriatic regions // Proceedings of VII International Conference on Forest Fire Research / D.X.Viegas (ur.). Coimbra, Portugal, 17-20.11.2014.

CiPOP, 2015 Center for Wildfire Research, http://cipop.fesb.hr

DHMZ, 2018) Croatian Meteorological and Hydrological Service,

http://www.dhmz.htnet.hr/index_en.php

GRASS GIS ROS, 2015 GRASS GIS SOFTWARE - http://grass.osgeo.org

Stipaničev et al., 2018 Stipaničev, D., Šeric, Lj., Krstinić D., Bugarić, M., Vuković A. "Adriatic Holistic Forest Fire Protection Project - results and experiences a year after" VIII Int. Conference on Forest Fire Research. Coimbra, 2018. (this conference)

Viegas, 2005 Viegas D.X. Forest Fire Meteorology Reaearch and Application, Presentation at WWRP Wildfire Workshop, Melburn, Australia, 6-10 June 2005 


\title{
An upper non-reflecting boundary condition for atmospheric compressible flow
}

\author{
Aurélien Costes* ${ }^{1}$; Christine Lac ${ }^{2}$; Valéry Masson ${ }^{2}$; Mélanie Rochoux ${ }^{3}$ \\ ${ }^{1}$ CERFACS, CNRM, Toulouse, France. \{aurelien.costes@meteo.fr*\} \\ ${ }^{2}$ CNRM,Toulouse,France. \{christine.lac@meteo.fr,valery.masson@meteo.fr\} \\ ${ }^{3}$ CERFACS, Toulouse, France. \{melanie.rochoux@cerfacs.fr
}

\begin{abstract}
So as to predict fire spread after its ignition, the Meso-NH atmospheric model has been coupled to the forest fire model ForeFire. Meso-NH uses the anelastic hypothesis that provides an intrinsic acoustic filter. Nevertheless, this approximation does not allow horizontal density variations which leads to problems close to the fire front because of the high heat release. That is why the full Euler compressible system shall be used to take into account air expansion at the fire level. This governing equations system transition comes with acoustic waves propagation. Although those waves are uninteresting for meteorology, they bounce on the ground and on the roof of the domain and disturb the inner solution. Indeed, the actual rigid lid as upper boundary condition does not allow acoustic waves radiation. The Rayleigh top damping layer already implemented in Meso-NH provides high frequency filtering, meaning that the new upper boundary shall only evacuate the remaining low frequency waves.

In order to build a reliable and precise upper boundary condition for a reasonable computational cost, we shall verify that the new boundary (1) lets low frequency waves pass through and (2) does not force the interior domain state. So as to preserve the scalability of the code, the boundary shall also (3) be localized in space and (4) as localized as possible in time to limit memory storage.

Several methods could be used to evacuate acoustic waves from the domain. Our requirements have conducted us to base our new upper boundary condition for Meso-NH on Characteristic-based Boundary Conditions, known as NSCBC, proposed by Poinsot and Lele. This boundary condition (BC) is known to be "non-reflecting" and also local in space and time. Nevertheless, the NSCBC formulation based on the characteristic theory of Thompson, comes out as "partially non-reflecting" as its behavior is more a low pass filter. An extended method called Plane Wave Masking, PWM, has been proposed by Polifke et al. to get a near zero reflexion coefficient for low frequency waves.

In PWM theory, the acoustic waves are assumed plane and incoming with normal incidence at the boundary. Nevertheless, the normal incidence of wave represents a strong limitation of the formulation. Lodato et al. have built a 3D formulation of NSCBC that computes transverse gradient to take into account oblique waves.

In purpose of testing the PWM formulation for its requirements, an offline model has been created in Python. This simplified model has been used to check the PWM boundary behavior facing plane waves and spherical waves. Once the PWM method has been proved as compliant to each requirement (1-4), it is implemented in the Meso-NH compressible code. Then, different academic and classic Meso-NH cases are run to ensure the correct behavior of the new boundary as orographic waves as well as fire experiments. The compressible assumption will be evaluated with the FireFlux experience.
\end{abstract}

Keywords: Acoustics, Boundary conditions, Non-reflecting, Meso-NH, Compressible, Computational Fluid Dynamics

\section{Introduction}

So as to predict fire spread after its ignition, the Meso-NH atmospheric model (Lafore et al. 1998, Lac et al. 2018) has been coupled to the forest fire model ForeFire (Filippi et al. 2009). Meso-NH uses the anelastic hypothesis that provides an intrinsic acoustic filter. Nevertheless, this approximation does 
not allow horizontal density variations which leads to problems close to the fire front because of the high heat release. That is why the full Euler compressible system shall be used to take into account air expansion at the fire level. This governing equations system transition comes with acoustic waves propagation. Although those waves are uninteresting for meteorology, they bounce on the ground and on the roof of the domain and disturb the inner solution. Indeed, the actual rigid lid as upper boundary condition does not allow acoustic waves radiation. The Rayleigh top damping layer already implemented in Meso-NH provides high frequency filtering, meaning that the new upper boundary shall only evacuate the remaining low frequency waves.

In order to build a reliable and precise upper boundary condition for a reasonable computational cost, we shall verify that the new boundary (a) lets low frequency waves pass through and (b) does not force the interior domain state. So as to preserve the scalability of the code, the boundary shall (c) be localized in space and (d) also localized in time to limit memory storage.

Several methods could be used to evacuate acoustic waves from the domain as mentioned by Colonius (2004). Our requirements have conducted us to base our new upper boundary condition for Meso-NH on Characteristic-based Boundary Conditions, known as NSCBC, proposed by Poinsot and Lele (1992). This boundary condition (BC) is known to be "non-reflecting" and also local in space and time. Nevertheless, the NSCBC formulation based on the characteristic theory of Thompson (1987), comes out as "partially non-reflecting" as its behavior is more a low pass filter as shown in Selle et al. (2004). An extended method called Plane Wave Masking, PWM, has been proposed by Polifke et al. (2006), to get a near zero reflexion coefficient for low frequency waves.

\section{Boundary formulation based on characteristic analysis}

The new boundary is built on the full Euler compressible system ignoring Coriolis and subgrid effects as well as the atmosphere is considered dry, i.e. the mixing ratio of total water substance is null. Those equations used in the compressible version of MesoNH are given by Eq. (2-4). The characteristic study of this system is based on Thomson 1987 analysis which require to write the system of equations as

$$
\begin{aligned}
\frac{\partial \underline{\tilde{U}}}{\partial t}+\frac{\partial \underline{F}_{x}}{\partial x}+\frac{\partial \underline{F}_{y}}{\partial y}+\frac{\partial \underline{F}_{z}}{\partial z}+\underline{D}^{\prime} & =\underline{0} . \\
\frac{\partial \rho_{d}}{\partial t}+\frac{\partial \rho_{d} u_{i}}{\partial x_{i}} & =0 \\
\frac{\partial \rho_{d} u_{i}}{\partial t}+\frac{\partial \rho_{d} u_{i} u_{j}}{\partial x_{j}}+\frac{\partial P}{\partial x_{i}}+\rho_{d} g \delta_{i 3} & =0 \\
\frac{\partial \rho_{d} \theta}{\partial t}+\frac{\partial \rho_{d} \theta u_{i}}{\partial x_{i}} & =0
\end{aligned}
$$

In Eq. (1), $\underline{U}$ is the vector of conservative variables, $\underline{F}$ the flux vectors and $D^{\prime}$ an inhomogeneous term not containing derivatives. Another form of the system uses the vector of primitive variables $\underline{U}$ chosen as

$$
\underline{U}=\left[\begin{array}{lllll}
\rho_{d} & u & v & w & \theta
\end{array}\right]^{T} .
$$

One can rewrite the Eq. (1) with the new vector of variables.

$$
\frac{\partial \underline{U}}{\partial t}+\underline{\underline{A}} \frac{\partial \underline{U}}{\partial x}+\underline{\underline{B}} \frac{\partial \underline{U}}{\partial y}+\underline{\underline{C}} \frac{\partial \underline{U}}{\partial z}+\underline{D}=0
$$


Then, matrix $A, B$ and $C$ can be diagonalized and lead to

$\frac{\partial \underline{U}}{\partial t}+\underline{\underline{R}} \underline{\underline{L}} \underline{\underline{R}}^{-1} \frac{\partial \underline{U}}{\partial x}+\underline{\underline{S}} \underline{\underline{M}} \underline{\underline{S}}^{-1} \frac{\partial \underline{U}}{\partial y}+\underline{\underline{T}} \underline{\underline{N}} \underline{\underline{T}}^{-1} \frac{\partial \underline{U}}{\partial z}+\underline{D}=\underline{0}$,

with $L, M$ and $N$ diagonal matrix. Let us identify waves as the following terms

$\underline{\mathcal{L}}=\underline{\underline{L}} \underline{\underline{R}}^{-1} \frac{\partial \underline{U}}{\partial x}, \quad \underline{\mathcal{M}}=\underline{\underline{M}} \underline{\underline{S}}^{-1} \frac{\partial \underline{U}}{\partial y}, \quad \underline{\mathcal{N}}=\underline{\underline{N}} \underline{\underline{T}}^{-1} \frac{\partial \underline{U}}{\partial z}$.

For the following, we will focus only on $z$ direction as this is the normal direction of the upper boundary. The characteristic study gives the waves amplitude as a function of normal spacial derivatives.

$$
\begin{aligned}
& \mathcal{N}_{1}=\frac{w-c}{2}\left[\frac{\partial \rho_{d}}{\partial z}-\frac{\rho_{d}}{c} \frac{\partial w}{\partial z}+\varphi \frac{\partial \theta}{\partial z}\right] \\
& \mathcal{N}_{2}=\frac{w+c}{2}\left[\frac{\partial \rho_{d}}{\partial z}+\frac{\rho_{d}}{c} \frac{\partial w}{\partial z}+\varphi \frac{\partial \theta}{\partial z}\right] \\
& \mathcal{N}_{3}=-w \varphi \frac{\partial \theta}{\partial z} \\
& \mathcal{N}_{4}=\frac{w}{2} \frac{\partial u}{\partial z} \\
& \mathcal{N}_{5}=\frac{w}{2} \frac{\partial v}{\partial z}
\end{aligned}
$$

with $\varphi=\frac{\rho_{d}}{\gamma \theta}, u, v, w$ the three components of the wind, $c$ the sound speed defined as $c^{2}=\frac{\gamma P}{\rho_{d}}, \gamma$ the adiabatic index, $P$ the pressure and $\theta$ the potential temperature. The terms described above are the amplitudes of characteristic waves associated with each characteristic velocity as eigenvalues of the matrix $A, B$ and $C$. The first wave moves at the speed of sound $(w-c)$ in the negative $z$ direction it "comes in" the domain at the boundary (Fig. 1). The second one travels also at the speed of sound $(w+c)$ but in the positive $z$ direction. Then, the third characteristic wave is associated to the convective velocity which is the speed of the entropy waves and finally, the last waves correspond to the advection by the vertical wind $w$. At the upper boundary the spacial derivatives can be computed by uncentered schemes and the other variables are available locally which is compliant with our constraints (c).

The time integration used is a simple Forward In Time (FIT) scheme which leads to the following system.

$$
\begin{aligned}
& \underline{U}^{n+1}=\underline{U}^{n}-\Delta t\left[\underline{\underline{T}}^{n} \underline{\mathcal{N}}^{n}+\underline{D}^{n}\right], \text { Poinsot and Lele (1992) } \\
& \underline{U}^{n+1}=\underline{U}^{n}-\Delta t\left[\underline{\underline{R}}^{n} \underline{\mathcal{L}}^{n}+\underline{\underline{S}}^{n} \underline{\mathcal{M}}^{n}+\underline{\underline{T}}^{n} \underline{\mathcal{N}}^{n}+\underline{D}^{n}\right], \text { Lodato et al. (2008) }
\end{aligned}
$$

The exponent $n$ means at time $t^{n}=n \Delta t$ and so $n+1$ is the next time step $\Delta t$. The method used by Poinsot and Lele (1992) is one-dimensional and considers only the normal direction informations to compute the boundary condition. Like most of the non-reflective boundary conditions, Poinsot and Lele (1992) assume that the flow at the boundary can be viewed as locally one-dimensional and aligned with the boundary normal direction. The incoming waves are then quantified by the LODI (Locally One Dimensional Inviscid) system which is directly derived from the Navier-Stokes equations written for primitives variables in characteristic form. Lodato et al. (2008) proposed to use the three directions of space to better describe oblique waves. Each method requires a special treatment for the first wave which comes from the outer to inner of the computational domain. 


\section{Entering wave amplitude term}

\subsection{Classic NSCBC}

First, let us focus on the LODI formulation for the "entering wave" $\mathcal{N}_{1}$. The wave amplitude is modified in order to make the boundary non-reflective. One can impose the amplitude as 0 but this will lead to an unconstraint density which is most generally unstable or conduct to drift in pressure at the boundary. Then the "perfectly non-reflecting" boundary condition would lead to stability issues due to the lack of informations on the mean density $\rho_{\infty}$ far from the computational domain. Therefore, this mean density can be imposed at the boundary but this treatment would generate reflected waves.

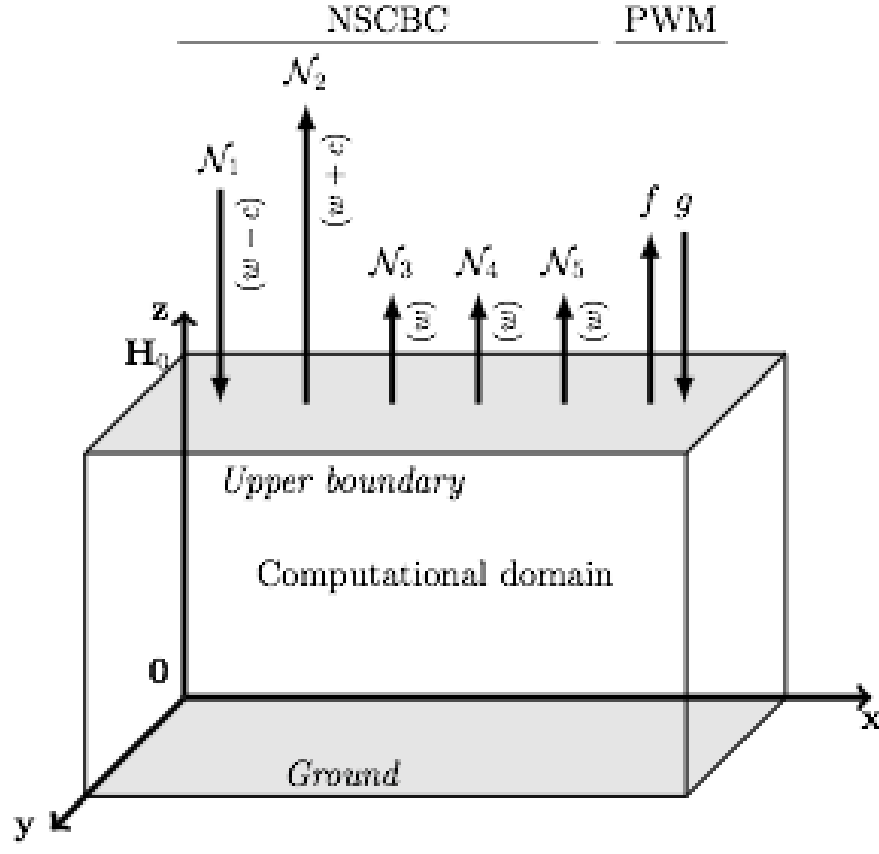

Figure 1 - Outgoing and entering waves through the upper boundary $\left(z=H_{0}\right)$ of the computational domain for classic NSCBC and PWM theories
In that case, we should add some physical informations on $\rho_{\infty}$ to the boundary in a "soft" way to avoid strong reflection. A simple way to do it is to set the entering wave as the linear relaxation term of Rudy and Strikwerda (1980)

$$
\mathcal{N}_{1}=K\left(\rho-\rho_{\infty}\right)
$$

where $K$ is a constant $\left[s^{-1}\right]$. When $K$ is set to 0 , we get back to the unstable "perfectly nonreflecting" boundary and when $K$ is big enough, the boundary is closer to the fully reflecting behavior. Selle et al. (2004) have studied the impact of the coefficient $K$ on the boundary reflection and have shown that the boundary is equivalent to a low-pass filter with $K / 4 \pi$ as cut off frequency. Nevertheless, in our problem, the Rayleigh top damping layer provides high frequency filtering, meaning that the new upper boundary shall only evacuate the remaining low frequency waves which can not be done with this theory.

\subsection{Plane Wave Masking}

An extension of the "classic" NSCBC presented above called PWM (Plane Wave Masking) is built to filter the low frequency waves. There, it is favorable to distinguish turbulent (" ' ") and acoustic (" $\sim$ ") perturbations of the flow variables $e . g$.

$$
\begin{aligned}
\rho_{d}(x, y, z, t) & =\overline{\rho_{d}}(x, y, z)+\rho_{d}^{\prime}(x, y, z, t)+\tilde{\rho}_{d}(z, t) \\
w(x, y, z, t) & =\bar{w}(x, y, z)+w^{\prime}(x, y, z, t)+\tilde{w}(z, t)
\end{aligned}
$$

Here again, the acoustic perturbations are assumed one-dimensional and normal to the boundary. For sufficiently small fluctuation amplitude, the linearized characteristics wave amplitudes are given by $f=f(z-(\bar{w}+\bar{c}) t)$ and $g=g(z-(\bar{w}-\bar{c}) t)$, travelling respectively in the positive and negative vertical direction. Using the acoustic fluctuation, one can write

$$
f=\frac{1}{2}\left[\frac{\tilde{P}}{\bar{\rho} \bar{c}}+\tilde{w}\right], \quad g=\frac{1}{2}\left[\frac{\tilde{P}}{\bar{\rho} \bar{c}}-\tilde{w}\right]
$$


It is then possible to adapt the classic NSCBC to get a non-reflecting boundary for low frequency waves. The idea of PWM is to identify outgoing waves at the boundary using the above equation and then explicitly eliminate outgoing wave contribution from the linear relaxation term.

$$
\mathcal{N}_{1}=K\left[\rho-\rho_{\infty}-\frac{\bar{\rho}}{\bar{c}} f\right]
$$

The "masked" outgoing wave no longer contributes to the "entering" wave $\mathcal{N}_{1}$ ans should leave the domain without any reflection.

\subsection{Reflection coefficient}

In order to evaluate the boundaries efficiency, it is important to properly define the reflection of the incoming wave. The reflection coefficient $r(\omega)$ is typically dependent of the acoustic incoming wave frequency (or pulsation). Basically, the reflection is the ratio between the reflected wave, seen as the "entering wave", amplitude ans the incoming wave amplitude. The incoming wave can be seen either as $\mathcal{N}_{2}$ or $f$. Therefore, the reflected wave becomes respectively $\mathcal{N}_{1}$ or $g$. Selle et al. (2004) define the reflection coefficient as

$$
r(w)=\frac{\mathcal{N}_{1}}{\mathcal{N}_{2}}
$$

whereas Polifke et al. (2006) use

$$
r(w)=\frac{\hat{g}}{\hat{f}} .
$$

The ".." denotes the Fourier transform of the quantity ". ". This last definition only stands if the coefficient $K$ is large enough so that the drift of density or velocity is effectively eliminated. Thus, deviations from the target density at the boundary will be controlled by the acoustic signal. This coefficient gives a first comparison point between the classic NSCBC and the PWM extension. According to Polifke et al. (2006), with $\tau \equiv 2 / K$,

The classic formulation is a low-pass whereas the plane wave masking extension allows, by construction, the radiation of the low-frequency waves. This study is only valid for harmonic plane waves of arbitrary non-zero frequency. This post-processing coefficient is useful to measure the efficiency of the boundary and this shows that the PWM should be the best solution as it is compliant at least with (a) and (c).

\section{MesoNH implementation}

The two solutions have been implemented in an off-line Python code to investigate the performances of the new boundary. This implementation has required several changes at the term computation method level but not on the philosophy of the process. Indeed, the characteristic boundary conditions have not been written in a stratified atmosphere and this hypothesis requires some adjustments. In this section, we will focus on 1D formulation, i.e. Eq. (14), with plane wave masking. Let us write the equations system really solved by MesoNH at the new boundary for the density $\rho$, the potential temperature $\theta$ and the vertical wind $w$. 


$$
\begin{array}{r}
\frac{\partial \rho}{\partial t}+\mathcal{N}_{1}+\mathcal{N}_{2}+\mathcal{N}_{3}=0 \\
\frac{\partial w}{\partial t}+\frac{c}{\rho}\left(\mathcal{N}_{2}-\mathcal{N}_{1}\right)+K_{3} \Delta_{z} w \\
\hdashline \frac{\partial \theta}{\partial t}-\frac{1}{\varphi} \mathcal{N}_{3}+\frac{\partial^{L} \theta}{\partial z}+K_{4} \Delta_{z} \theta
\end{array}
$$

The framed terms are added to the original system and will be detailed further. The wave amplitudes have also been modified from the original method.

$$
\begin{aligned}
& \mathcal{N}_{1}=K_{1}\left(\rho-\rho_{L S}\right)-K_{2} \frac{\rho_{L S}}{c_{L S}} f \\
& \mathcal{N}_{2}=\frac{w+c}{2}\left[\frac{\partial^{*} \rho}{\partial z}+\frac{\rho}{c} \frac{\partial^{*} w}{\partial z}+\varphi \frac{\partial^{*} \theta}{\partial z}\right] \\
& \mathcal{N}_{3}=-w \varphi \frac{\partial^{*} \theta}{\partial z}
\end{aligned}
$$

Several main terms have been changed so let us have a look on each modification and theirs consequences.

\subsection{Background stratification and hydrostatic equilibrium}

The atmosphere is a vertically stratified domaine due to the hydrostatic equilibrium which can be described by

$$
\frac{\partial P}{\partial z}+\rho g=0 .
$$

The stratification directly implies that the spacial derivative computed at the boundary for the NSCBC, with or without PWM extension, are non-zero even in equilibrium state. If the original system was implemented without any modifications, the hydrostatic state would have been treated as a fluctuation leading to an unstable boundary. Then, the first step is to split the hydrostatic part and the perturbational part of the signal. For this purpose, we uses the large scale fields for the density, the wind and the potential temperature. They are provided by another simulation from a larger scale model as AROME. This previous simulation has already computed fields at the boundary taking into account the hydrostatic equilibrium. Thus, the main variables are written using the large scale fields denoted by the subscript "LS" and a perturbation term denoted by " $\sim$ ".

$$
\begin{array}{ll}
\rho=\rho_{L S}+\tilde{\rho}, & w=w_{L S}+\tilde{w} \\
\theta=\theta_{L S}+\tilde{\theta}, & P=P_{\infty}+\tilde{P}
\end{array}
$$

The pressure $P$ is not provided by the large scale model and its reference state should be computed using other variables which have a large scale field available. As the fluctuations of pressure are an important part of the PWM extension, a specific attention should be taken to define its reference state (see section 4.4). Therefore, this notation allows to create two new derivative operators related to each term.

$$
\begin{aligned}
\frac{\partial^{L S} \star}{\partial z} & \equiv \frac{\partial \star_{L S}}{\partial z} \\
\frac{\partial^{*} \star}{\partial z} & \equiv \frac{\partial \star}{\partial z}-\frac{\partial^{L S} \star}{\partial z} \\
\star & =\{\rho, w, \theta\}
\end{aligned}
$$


The new derivative operators takes into account the whole hydrostatic equilibrium and then the gravity term which can be found in $D$ in Eq. (14) is no longer relevant and does not appear in Eq. (26).

\subsection{Potential temperature transport}

The potential temperature should be advected at the wind velocity $w$ in the Eq. (27). Without any compensation, framed terms in Eq. (27), the boundary would have advected the perturbation state only as it is reduced to

$$
\frac{\partial \theta}{\partial t}+w \frac{\partial^{*} \theta}{\partial z}=0
$$

By adding the large scale field transport, $w \frac{\partial^{L S} \theta}{\partial z}$, to Eq. (36) and using the definition of operators in Eq. (35), it becomes

$$
\begin{aligned}
& \frac{\partial \theta}{\partial t}+ w \frac{\partial^{*} \theta}{\partial z}+w \frac{\partial^{L S} \theta}{\partial z}=0 \\
& \Longleftrightarrow \frac{\partial \theta}{\partial t}+w \frac{\partial \theta}{\partial z}=0
\end{aligned}
$$

This new term balance the advection of the total potential temperature which is coherent with the overall transport equation - Eq. (4).

The last two terms in Eq. (26) and Eq. (27) are designed to stabilize the overall boundary behavior, i.e. $\frac{\partial^{*} \theta}{\partial z} \underset{t \rightarrow \infty}{\rightarrow} 0$ and $\frac{\partial^{*} w}{\partial z} \underset{t \rightarrow \infty}{\rightarrow} 0$. They will not going to be detailed further in this study but it is shown that they do not affect the dynamic behavior of the boundary which is the most important when radiating waves.

\subsection{PWM extension formulation}

In this section, we will focus on the formalism used to compute the "entering wave" $\mathcal{N}_{1}$. There is two main parts in this term as shown Eq. (28). The first one corresponds to the classic linear relaxation term of (Rudy and Strikwerda 1980) but the reference state used is the large scale density. The second one is the plane wave masking expression. Using Eq. (19) and Eq. (32-33), we set

$$
f=\frac{1}{2}\left[\frac{\tilde{P}}{\rho_{L S} c_{L S}}+\tilde{w}\right]
$$

Here, the definition of $P_{\infty}$ is very important and it can lead to a significant error of $\rho(t \rightarrow \infty)$. The pressure is a diagnostic variable computed by

$$
P=P_{00}^{1-\gamma}\left[\rho R_{d} \theta\right]^{\gamma},
$$

where $P_{00}$ is a reference value of the pressure and $R_{d}$ the gas constant for dry air. A natural way to define $P_{\infty}$ with large scale fields is

$$
P_{\infty}=P_{00}^{1-\gamma}\left[\rho_{L S} R_{d} \theta_{L S}\right]^{\gamma} \text {. }
$$

Therefore, the used definition of the reference pressure is

$$
P_{\infty}=P_{00}^{1-\gamma}\left[\rho_{L S} R_{d} \theta\right]^{\gamma} \text {. }
$$

The onl y difference between the expression is the use of the total potential temperature instead of the large scale one. 


\subsection{Drift in mean density depending on the reference pressure definition}

In order to show the consequences of such a choice, let us consider the steady state reached after a wave radiation. Each temporal derivative is null in Eq. (25-27). For the sake of clarity, $K_{3}=K_{4}=0$ which means that no stability correction is applied. Moreover, in general case, the large scale potential temperature profile is not constant, i.e. $\frac{\partial^{L S} \theta}{\partial z} \neq 0$. On the another hand, we assume that the wave had changed the local equilibrium of the potential temperature as $\tilde{\theta} \neq 0, \frac{\partial^{*} \theta}{\partial z}=0$. The last hypothesis is that the large scale vertical wind is null, i.e $w_{L S}=0$. Under those hypothesis, we have necessarily $w=$ $w=0$ in order to satisfy Eq. (27). The wave amplitude $\mathcal{N}_{3}$ is also null due to the local constant vertical

profile of the potential temperature fluctuation. Finally, the system is reduced to two equations

$$
\begin{aligned}
& \mathcal{N}_{1}+\mathcal{N}_{2}=0, \\
& \mathcal{N}_{2}-\mathcal{N}_{1}=0 .
\end{aligned}
$$

This system is equivalent to

$$
\mathcal{N}_{1}=\mathcal{N}_{2}=0 \text {. }
$$

For $\mathcal{N}_{2}$, it can be seen as each perturbation field is locally, at least, constant over $z$ which is quite good for a steady state. Thus, the only tricky equation is

$$
\mathcal{N}_{1}=K_{1} \tilde{\rho}-K_{2} \frac{\rho_{L S}}{c_{L S}} f=0
$$

With $c_{L S}=\frac{\gamma P_{L S}}{\rho_{L S}}$, we have

$$
K_{1}\left[\frac{\rho}{\rho_{L S}}-1\right]=\frac{K_{2}}{2 \gamma}\left[\frac{P}{P_{L S}}-1\right] .
$$

At this stage, the definition of the reference pressure becomes important. If the Eq. (40) is used, the ratio $\alpha=\left[\frac{\theta}{\theta_{L S}}\right]^{\gamma}$ will appear and we can show that the deviation on density at the steady state is

$$
\frac{\tilde{\rho}}{\rho_{L S}}=\frac{1}{\gamma} \frac{K_{2}(1-\alpha)}{\alpha K_{2}-2 K_{1}} .
$$

This ration is zero if $K_{2}=0$ or $\alpha=1$. The first solution gives a exact steady state if the used method is NSCBC without plane wave masking. This solution is not satisfying because PWM is necessary to ensure low-frequency waves radiation. In the other solution, the ratio $\alpha$ should be unity which is in contradiction with the baseline assumption of $\theta \neq 0$. Therefore, this definition of $P_{\infty}$ leads to inaccuracy of the density steady state.

On the other hand, if the Eq. (41) is used, the ratio $\alpha$ does not appear - equivalent to always unity - which leads to an accurate boundary. Those behavior have been verified numerically but the results are not presented here and for following simulations, the Eq. (41) will always be used as the reference pressure.

\section{Simulation results}

Full compressible simulation of a gaussian density perturbation has been performed to validate the non-reflective character of the new boundary. The initial condition is the hydrostatic state, here equivalent to the large scale state, to which a density perturbation is added as 


$$
\rho(z, t=0)=\rho_{L S}(z)+\rho_{a} \exp \left[-\frac{\left(z-\frac{H_{0}}{2}\right)^{2}}{d_{a}^{2}}\right],
$$

where $\rho_{a}$ is the perturbation amplitude $\left[\mathrm{kg} \cdot \mathrm{m}^{-3}\right], d_{a}$ the characteristic spreading $[\mathrm{m}]$ of the initial perturbation and $H_{0}$ the height of the domain. The ground is set as slip wall and other boundaries are cyclic. The cells are $50 \mathrm{~m}$ long in each direction and the grid is $7 \times 3 \times 102$ in $x, y, z$ direction respectively. The problem is actually quasi $1 \mathrm{D}$ at this point. This time step used is $0.05 \mathrm{~s}$. No turbulence, relaxation and Rayleigh damping layer was added to look a the boundary efficiency without any help of other methods.

The wind advection scheme used is $4^{\text {th }}$ order centered scheme used with RKC4 scheme, the scalar advection scheme is PPM in its monotonic and positive definite version. The numerical viscosity added to the momentum equation is set to $3 \cdot 10^{3}[\mathrm{~Pa} \cdot \mathrm{s}]$. The Brunt-Väisälä frequency, defined as $N=\sqrt{\frac{g}{\theta} \frac{d \theta}{d z}}$, is set to $0.02\left[s^{-1}\right]$.

\subsection{Results for one-dimensional wave}

For the simulations discussed in this section, the density perturbation is set to $\rho_{a}=0.01\left[\mathrm{~kg} \cdot \mathrm{m}^{-3}\right]$ and $d_{a}=300[\mathrm{~m}]$. The wind is set to zero everywhere so only the acoustic signal will generate wind. The stability coefficient are set to $K_{3}=0\left[\mathrm{~s}^{-1}\right], K_{4}=0.1\left[\mathrm{~s}^{-1}\right]$. The relaxation coefficient $K_{1}$ is set to $0.1\left[\mathrm{~s}^{-1}\right]$ which allows no drift from the large scale density. For the NSCBC simulation, $K_{2}=0\left[\mathrm{~s}^{-1}\right]$ and for the plane wave masking simulation, it is equal to $K_{1}$. Both simulation are compared with the old boundary condition, denoted $\mathrm{MNH}$, which forces $w=0$ at the top and computes othe $\mathrm{r}$ quantities with hydrostatic equilibrium equation.

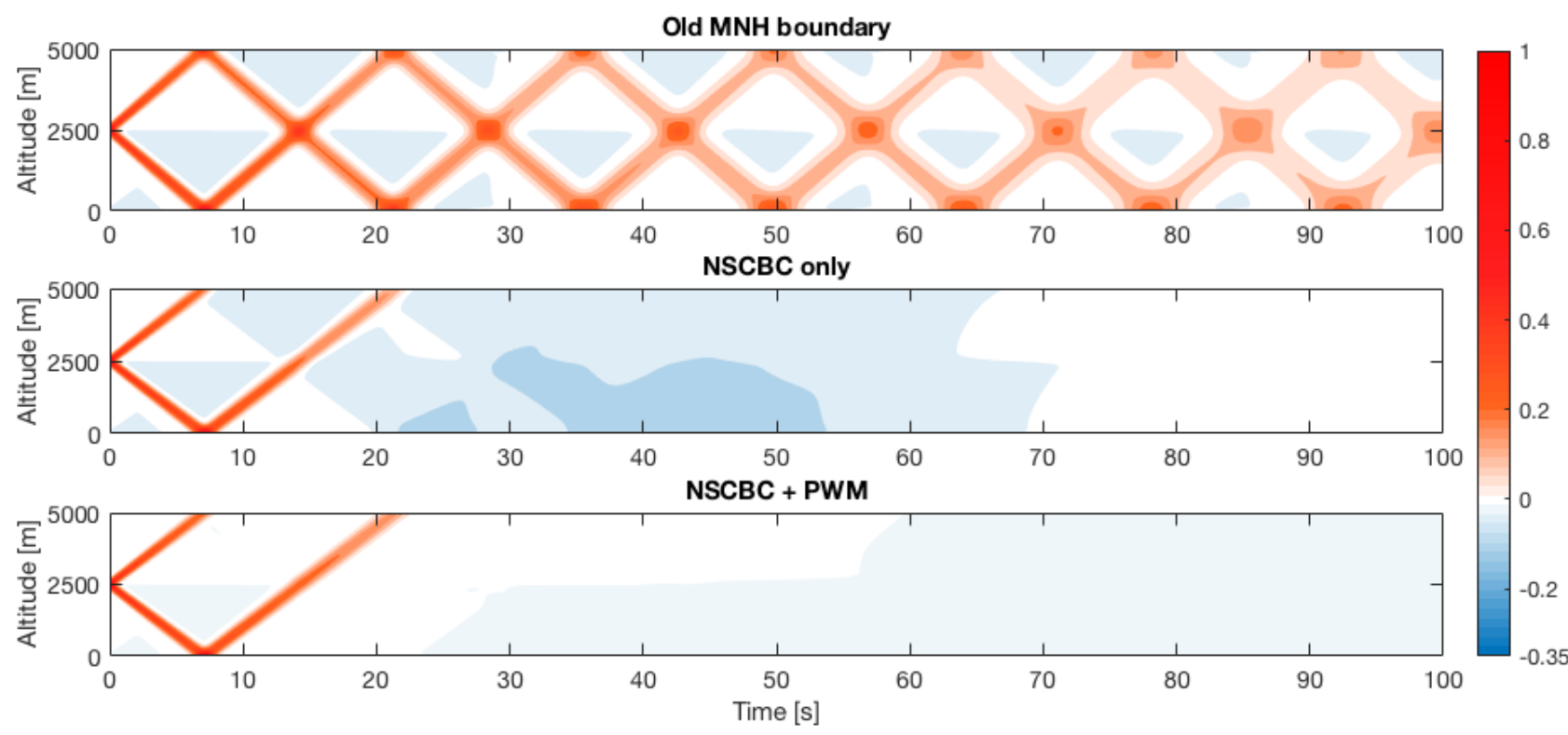

Figure 2 - Relative density anomaly [-] for the different boundaries. CI : 0.05

The relative density anomaly is defined by

$$
\tilde{\rho}^{*}=\frac{\tilde{\rho}}{\rho_{a}}=\frac{\rho-\rho_{L S}}{\rho_{a}} .
$$




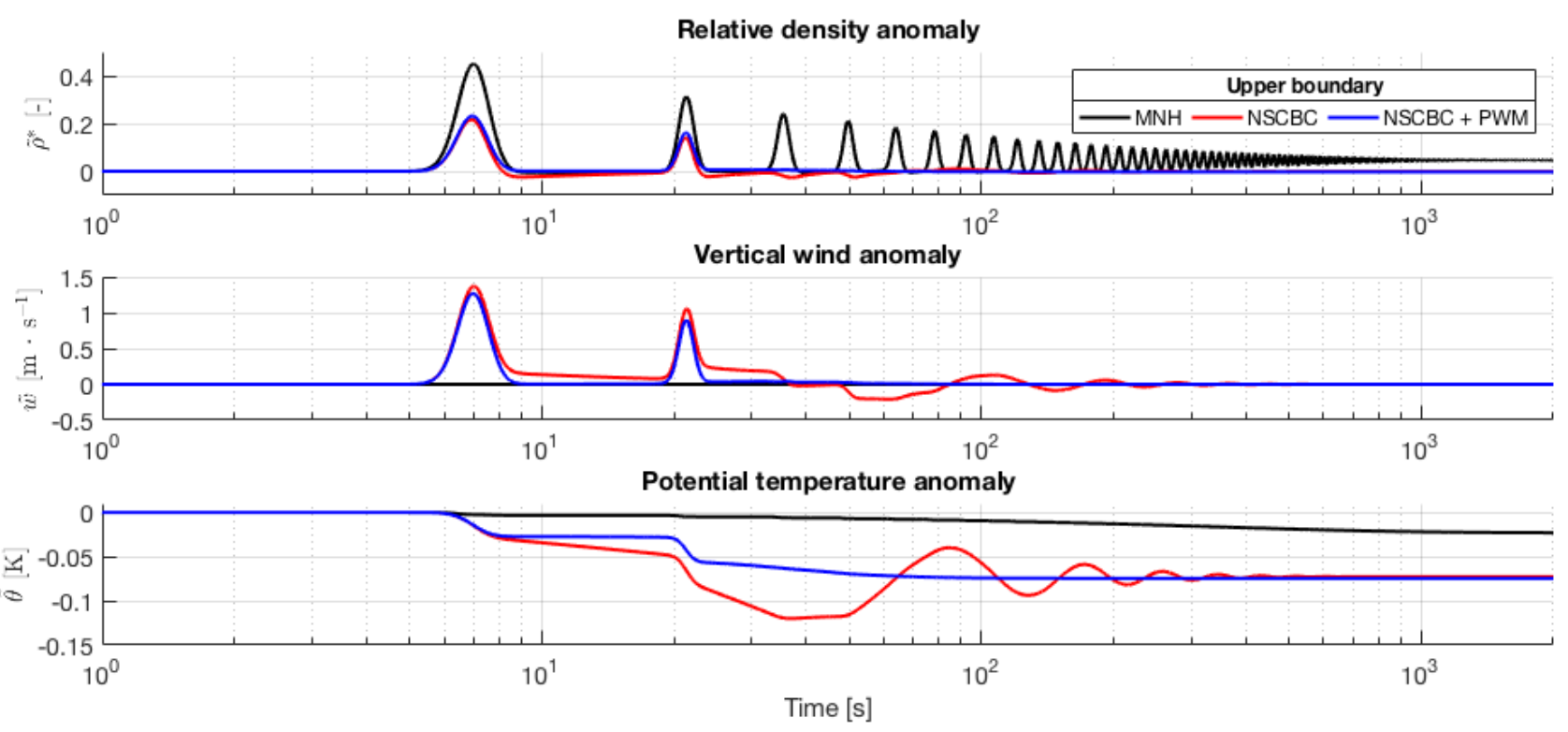

Figure 3 - Main fields anomaly over time at the upper boundary for the different methods for 1D gaussian wave perturbation

As shown Fig. 2, the old boundary reflects the whole signal and the numerical viscosity diffuses it through time, that is why the amplitude of the relative density anomaly decreases and its spreading increases. The behavior is completely non satisfying because of the perturbation near the ground that were reflected on the upper boundary. The NSCBC does a quite good job with this coefficient but their is still an important reflection which is not compliant enough with the first criterion (a). The plane wave masking extension does a great improvement in radiating the waves as no reflection occurs. In order to better understand and quantify the dynamic and static behavior of each boundaries, the difference fields are studied at the boundary.

In Fig. 3, the static behavior is reached around 1000s for both MNH and NSCBC boundaries whereas the PWM extension strikes the balance in 400s. Moreover, the final state is the same for NSCBC, with and without PWM, which is consistant with the study in section 4.4. The new boundary converges to zero anomaly for both wind and density and advects the potential temperature in the same way that the inner domain, i.e. the vertical gradient of $\theta$ is zero. On the other hand, the dynamic behavior is the capability to radiate efficiently an incoming wave. The MNH boundary is not good at this exercice as the wind is strictly zero at the boundary, which explain the low advection of the potential temperature. The waves are constantly bouncing on the boundaries while the numerical diffusion weakens the wave amplitude. The NSCBC boundary is more efficient but is not perfect as some residual wind and density anomaly are still existing after the wave passage across the domain limit. Therefore, some oscillations of the wind and consequently on potential temperature are induced in the domain. The plane wave masking extension fixes those problems in changing the dynamic behavior of the boundary which leads to zero residual density and wind anomalies after the wave crossing.

The overall performance of the boundaries can also be shown on the reflection coefficient. On Fig. 4 , the plane wave masking gets near zero reflection for low-frequencies as predicted. The classic NSCBC is partially non-reflecting and is partially compliant with the requirements for the new boundary. 


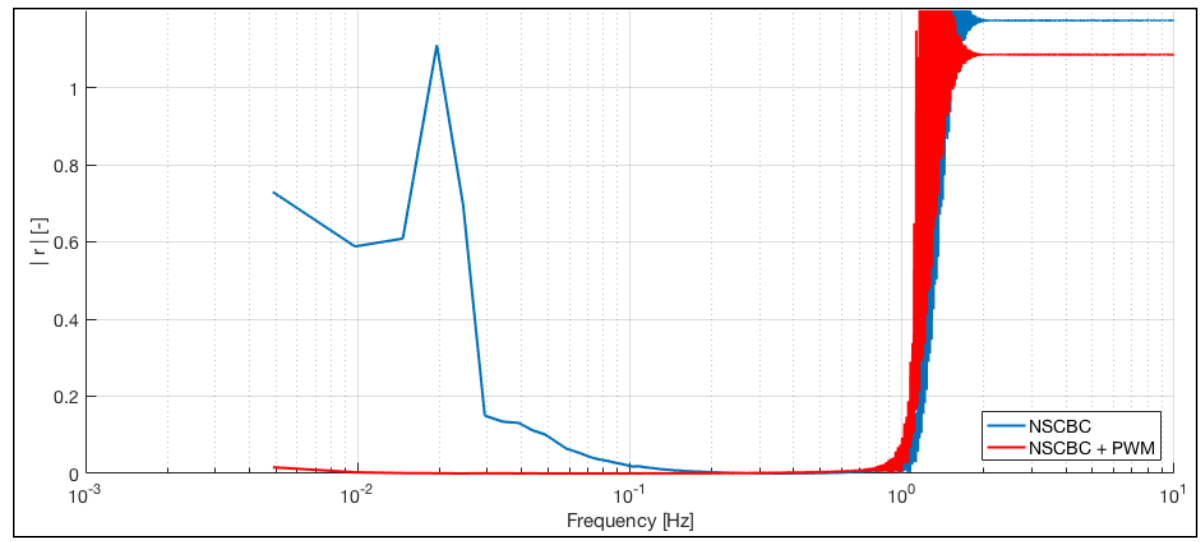

Figure 4 - Reflection coefficient for one-dimensional wave without and with plane wave masking

\subsection{Results for spherical wave}

The gris used is now $326 \times 3 \times 102$ in $x, y, z$ directions respectively. Cell size, time step, temporal and spacial schemes are the same that the ones used in section 5.1. The initial perturbation formulation is now two dimensional in order to test the boundary for non-normal incoming waves.

$$
\rho(z, t=0)=\rho_{L S}+\rho_{a} \exp \left[-\frac{\left(z-\frac{H_{0}}{2}\right)^{2}+\left(x-\frac{L_{0}}{2}\right)^{2}}{d_{a}^{2}}\right]
$$

Fig. 5 shows that the new boundary radiates the spherical wave as well as the normal wave. The two top images show the wave expansion before the boundary interaction. The bottom left image points the reflection on the left part and the wave passing through on the right side. At that moment, the incoming wave reaches the boundary with normal direction, so the behavior is consistant with the one dimensional study. The bottom right image show the strong reflection for the old boundary condition and the correct radiation for the new boundary even if the wave comes with an oblique direction. The sphericity is not affect by the local 1D hypothesis at the boundary.

\section{Conclusion and prospects}

The new boundary implemented in the compressible version of Meso-NH has shown great interests in evacuating acoustic waves in order to get a better precision near of the ground. To allow waves to go out of the domain, the NSCBC theory with the plane wave masking layer has been implemented and tested for planar, oblique and spherical waves. Its behavior has also been checked for various academic and classic Meso-NH cases as hydrostatic and non-hydrostatic orographic waves as well as fire experiments. Then, the new boundary is compliant with all of the criterions given in section $\mathbf{1}$. The new boundary should lead to a better accuracy near of the fire front for futur simulations. The compressible assumption will be evaluated with the FireFlux experiment (Filippi et al. 2013) and compared to results already gathered by the anelastic version of Meso- $\mathrm{NH}$ on Aullene fire in Corsica (Filippi et al. 2018). 

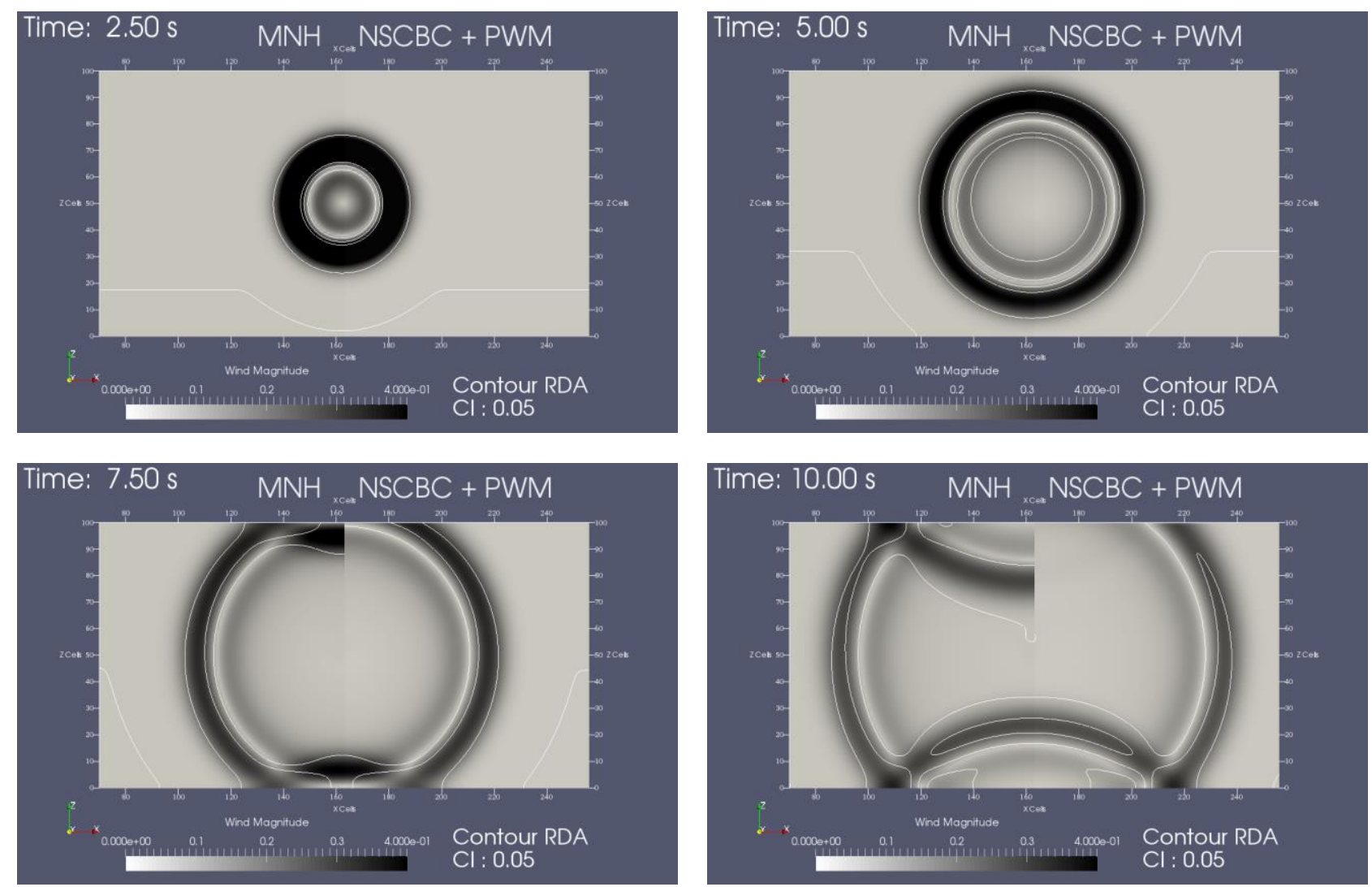

Figure 5 - Comparison of spherical wave radiation for MNH (left part of each image) and NSCBC + PWM (right part of each image). Wind magnitude is presented in colored contour and Relative Density Anomaly (RDA) in white line contour for $-0.05,0$ and 0.05 [-].

\section{References}

Colonius, T. (2004). Modeling Artificial Boundary Conditions for Compressible Flow. Annual Review of Fluid Mechanics, 36(1), 315-345.

Filippi, J.-B., F. Bosseur, C. Mari, C. Lac, P. Le Moigne, B. Cuenot, D. Veynante, D. Cariolle, and J. H. Balbi, (2009). Coupled atmosphere-wildland fire modelling, J. Adv. Model Earth Syst., 1, \#11.

Filippi, J.-B., X. Pialat, and C. G. Clements, (2013). Assessment of ForeFire/Meso-NH for wildland fire/atmosphere coupled simulation of the FireFlux experiment, Proc. Combustion Inst., 34, 26332640.

Filippi, J.-B., Bosseur, F., Mari, C., \& Lac, C. (2018). Simulation of a large wildfire in a coupled FireAtmosphere model, Atmosphere, 9(6), 218.

Lac, C., J.-P. Chaboureau, V. Masson, J.-P. Pinty, P. Tulet, J. Escobar, M. Leriche, and co-authors (2018). Overview of the Meso-NH model version 5.4 and its applications, Geosci. Model Dev., p. in discussion.

Lafore, J. P., J. Stein, N. Asencio, P. Bougeault, V. Ducrocq, J. Duron, C. Fischer, P. Hereil, P. Mascart, J. P. Pinty, J. L. Redelsperger, E. Richard, and J. Vila-Guerau de Arellano, (1998). The Meso-NH Atmospheric Simulation System. Part I: Adiabatic formulation and control simulations. Annales Geophysicae, 16, 90-109.

Lodato, G., Domingo, P., \& Vervisch, L. (2008). Three-dimensional boundary conditions for direct and large-eddy simulation of compressible viscous flows. Journal of Computational Physics, 227(10), 5105-5143. 
Polifke, W., Wall, C., \& Moin, P. (2006). Partially reflecting and non-reflecting boundary conditions for simulation of compressible viscous flow. Journal of Computational Physics.

Poinsot, T. J., \& Lele, S. K. (1992). Boundary conditions for direct simulations of compressible viscous flows. Journal of Computational Physics, 101(1), 104-129.

Rudy, D. H., \& Strikwerda, J. C. (1980). A nonreflecting outflow boundary condition for subsonic navier-stokes calculations. Journal of Computational Physics, 36(1), 55-70.

Selle, L., Nicoud, F., \& Poinsot, T. (2004). Actual Impedance of Nonreflecting Boundary Conditions: Implications for Computation of Resonators. AIAA Journal, 42(5), 958-964.

Thompson, K. W. (1987). Time dependent boundary conditions for hyperbolic systems. Journal of Computational Physics, 68(1), 1-24. 


\title{
Applying GPU Parallel Technology to Accelerate FARSITE Forest Fire Simulator
}

\author{
Carlos Carrillo*; Ana Cortés; Andrés Cencerrado; Antonio Espinosa; Tomàs Margalef \\ Computer Architecture and Operating Systems Department. Universitat Autònoma de Barcelona, \\ Spain.\{carles.carrillo@uab.cat*,ana.cortes@uab.cat,andres.cencerrado@uab.cat, \\ antoniomiguel.espinosa@uab.cat,tomas.margalef@uab.cat\}
}

\begin{abstract}
Forest fires are one of the most common natural hazards in the countries of the Mediterranean region. The forest fires spread simulators have proven to be very effective tools in the fight against these disasters. But to be able to use these simulators in an effective way it is necessary to be able to obtain realistic predictions of the behavior of the fire in a relatively short time. In the last decades, the advances in the field of computing have allowed different strategies to improve the effectiveness of simulators. With this objective, it has been tried to take advantage of the computing power of the GPUs (Graphic Processors Units) to accelerate the simulation of the propagation of fires. The problem with the use of GPUs is that the data transfer between the memory of the system and the GPU memory increases the execution time. For this reason, the computation operations have to compensate the transfer time of the data from the CPU (Central Processor Unit) to the memory of the GPU; which means, that the data volume must be sufficient to compensate this effect. The main objective of this work is to analyze the execution time of the forest fire spread simulation in a CPU and in a GPU, and to see from what data volume the execution in GPU is more efficient than in the CPU. For this study, a fire simulator has been designed based on the basic model for one point evolution in the simulator FARSITE. As study case, a synthetic fire was used where the fire progresses in a linear front and in a single direction, maintaining constant wind, terrain and vegetation conditions for all points of the fire front and throughout the simulation.
\end{abstract}

Keywords: Forest Fire Simulation, Parallel computing, GPU

\section{Introduction}

Forest fires are significant hazards that every year cause important damages around the world and have a deep impact on the natural environment, people and communities. This type of catastrophes is especially important in warm countries, like the countries of the Mediterranean zone, where thousands of hectares of forest burn every year, causing significant damages to the environment and economic losses. Proper prediction of the fire evolution allows to manage the firefighting equipment properly in order to mitigate the fire effects. Fire spread models have been developed and implemented in computing simulators to help command and control centers in taking the adequate decisions. To use these models and simulators effectively, it is of paramount importance to establish an appropriate measure of confidence in their performance with an acceptable execution time.

In order to improve the effectiveness of fire simulators, in the past decade different strategies have been developed to improve the accuracy and reduce the execution time using multicore architectures. In this context, most studies focus on multicore architectures based on CPU (Cencerrado et al., 2015; Brun et al., 2014). However, in the last years, the computational capacity of Graphics Processing Units (GPUs) has increased enormously (see Fig. 1). Due to their computational power, GPUs have become increasingly attractive for complex systems modelling, (like forest fire spread). The GPU architecture is ideally suited for high volume data processing applications because can process millions of operations simultaneously. 


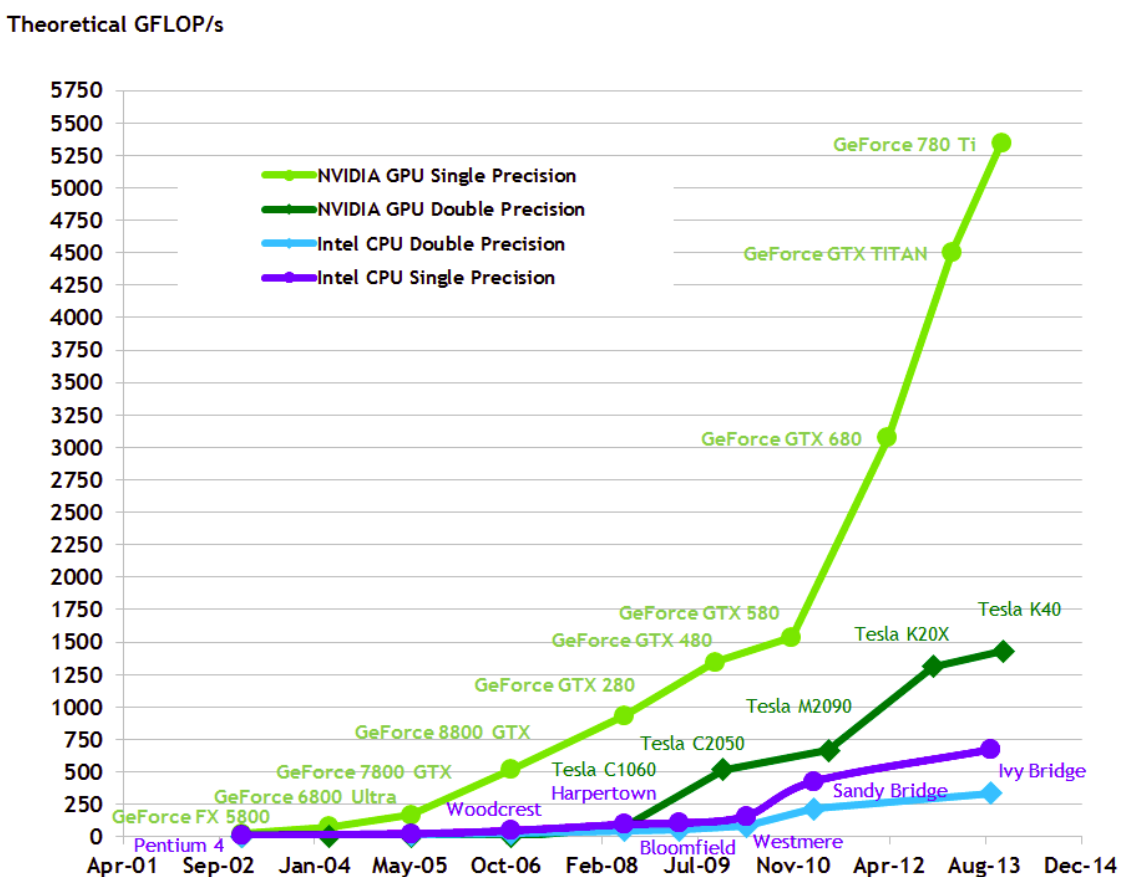

Figure 1 - Computation capacity of GPUs in the last few years in Gigaflops per second.

In recent years, different works have been carried out to apply the computational capacity of GPUs to the simulation of forest fires behavior and to accelerate these simulations (Gregoria et al., 2013; Ntinas et al., 2017; Sousa et al., 2012). These works have been focused on the application in simulators based on cellular automata (CA) that are relatively easy to parallelize. These studies have demonstrated that the use of GPU can reduce the execution time significantly without losing resolution. However, the main limitation is the intrinsic low accuracy of the simulators based on CA approach.

In order to effectively use a GPU, programmers need to build scalable programs, typically in CUDA programming model, that are able to use all GPU resources. For that, they have to deal with memory management, load balance of the execution threads, data races and data transfer cost. A GPU program workflow follows the general idea shown in Fig. 2. The necessary data is downloaded to the GPU (Device) from the CPU (Host). The operations are executed in parallel in the GPU and the result obtained is returned to the CPU.

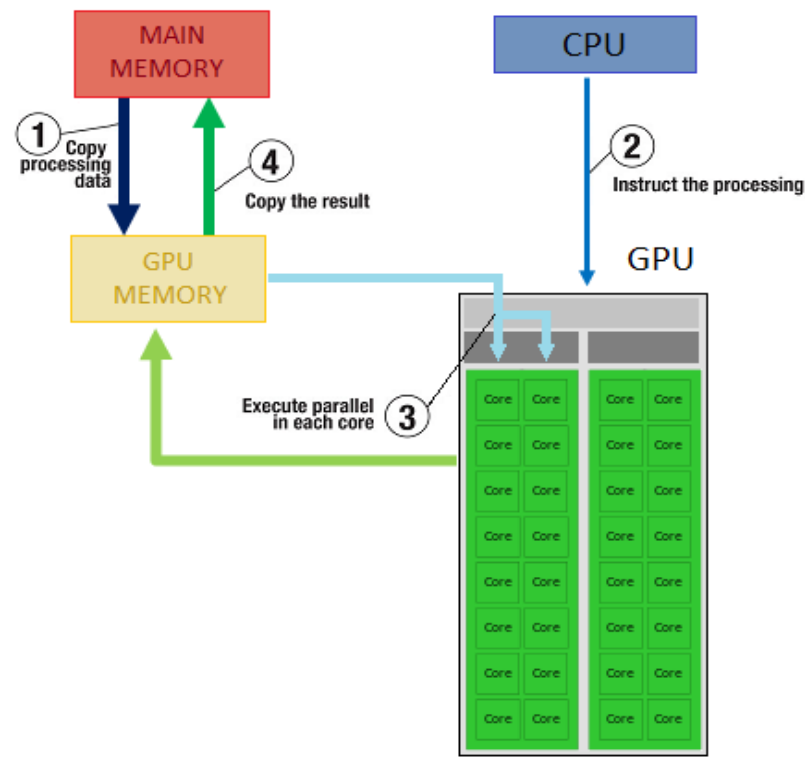

Figure 2 - General workflow of a GPU program 
This data flow between the CPU and the GPU means that part of the program's execution time is wasted in the data copy, which implies that the GPU utilization only makes sense if the size of our problem is large enough, so that the data copying time is compensated by the computation time gain.

The main objective of this work is to analyze both the CPU and GPU execution time of a given forest fire spread simulator in order to determine the data volume requirements that implies higher efficiency using GPU than CPU.

In particular, in this work, we apply the GPUs parallel technology to FARSITE (Fire Area Simulator) (Finney, 2004), which is a forest fire simulator based on the Huygens principle and on the Rothermel's model as forest fire propagation (Rothermel, 1972). Simulators based on the Huygens principle, or elliptical wave propagation, have a higher precision than those based on cellular automata, however their execution time is higher. To carry out our study, we have extracted the FARSITE simulation kernel and we have implemented it in CUDA (Compute Unified Device Architecture) to reincorporate it into the FARSITE body, and thus compare the two implementations. As first approach to the problem, a synthetic fire is used, which consists of a linear front in a flat terrain, maintaining constant wind speed, wind direction and the vegetation conditions throughout the simulation. To be able to compare the two executions (GPU and CPU) the simulations have been limited to one hour propagation, consequently, the execution time will be mainly linked to the volume of data to be treated and not to the propagation time of the fire to be simulated.

This paper is organized as follows. Section 2 describes different types of forest fires spread simulators. Section 3 includes a brief introduction to the GPU architecture and the methodology used to perform the spread simulation of the fire in this device. Section 4 presents the experimental procedure and the obtained results and finally, Section 5 summarizes the main conclusions and future work.

\section{Forest Fires Spread Simulators}

In the last decades, a large number of forest fires spread simulators have been developed. Most of these simulators are based on the Rothermel's fire spread model (Rothermel, 1972), which is formulated in the following way:

$$
\boldsymbol{R}=\frac{I_{R} \xi \cdot\left(\vec{n}+\vec{\phi}_{w}+\vec{\phi}_{s}\right)}{\rho_{b} \varepsilon Q_{i g}}
$$

where $R$ represents the rate of spread in a particular point, $I_{R}$ is the reaction intensity, $\xi$ is the propagation flux ratio, $\rho_{b}$ is the ovendry bulk density, $\varepsilon$ is the effective heating number, Qig is the heat of pre-ignition, $\overrightarrow{\boldsymbol{n}}$ is the normal direction to the fire perimeter on that particular point $\overrightarrow{\boldsymbol{\phi}}_{W}$, is the wind factor and $\vec{\phi}_{S}$ is the slope factor. Except, $\vec{\phi}_{W}$ and $\vec{\phi}_{S}$, all the factors only depend of the vegetation of the cell. Equation 1 can be rewritten in the following way:

$$
R=R_{0} \cdot\left(\vec{n}+\vec{\phi}_{W}+\vec{\phi}_{s}\right)
$$

where $\mathrm{R}_{0}$ represents the rate of spread in a particular point with no wind and no slope.

If we make a classification of the forest fire spread simulators according to the method used to propagate the fire front, three large groups can be defined:

- Raster-based Approaches or Cellular Automata: In the Raster-based or Cellular Automata simulators, the area where the fire takes place is divided into cells forming a network of cells (Alexandridis et al., 2008). The fire advances from one cell to the next through a set of rules that describe the spread of fire (Fig. 3). These rules are determined by the fire model used by the simulator. While faster and simpler to implement, they lack 
precision when compared to other approaches (Pastor et al., 2003), but its parallelization is simpler than the Vector-based Approaches parallelization which is subsequently described. Fire simulators that use this approach are FireLib, FireMap and Cardin.

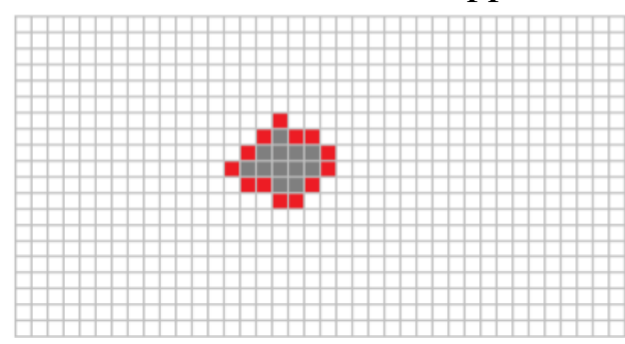

(a) Initial perimeter at $t=0$

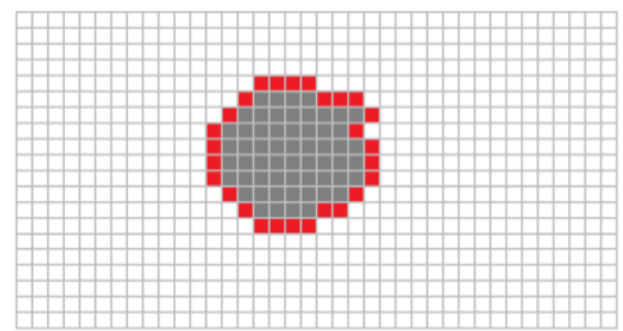

(c) Fire perimeter at $t=t_{2}$.

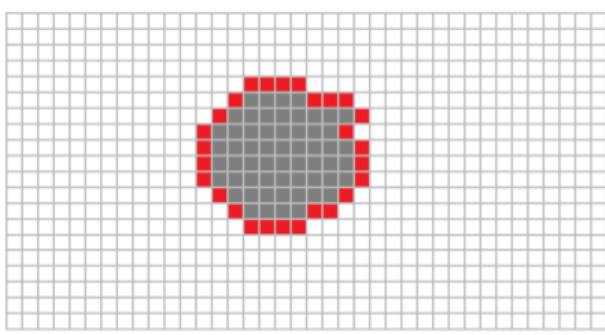

(b) Fire perimeter at $t=t_{1}$.

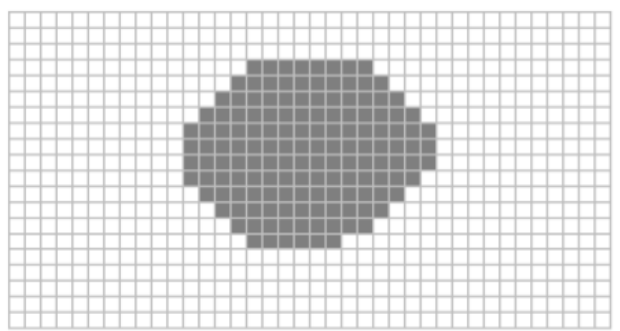

(d) Final burned area.

Figure 3 - Simulators based on cellular automata. In this case, the white color indicates unburned cells, the red indicates the cells burning at that instant and the gray cells are those completely burned.

- Vector-based Approaches or Elliptical wave propagation: In this type of simulators the perimeter of the fire is divided into series of points, where the distance between the different points will be determined by the resolution of the simulator (Knight et al., 2003). To obtain the evolution of the perimeter of the fire, for each point a ellipse is generated. The shape of the ellipses is determined by the local characteristics at each point. In this way, the new perimeter is obtained by joining the obtained ellipses (Fig. 4). In this type of simulators, the propagation of each point of the perimeter is done in a continuous space. The accuracy of this type of simulators is greater than the CA approaches; however, their complexity and time requirements are higher. SiroFire, Prometheus, and FARSITE are relevant examples of this kind of forest fire spread simulators.

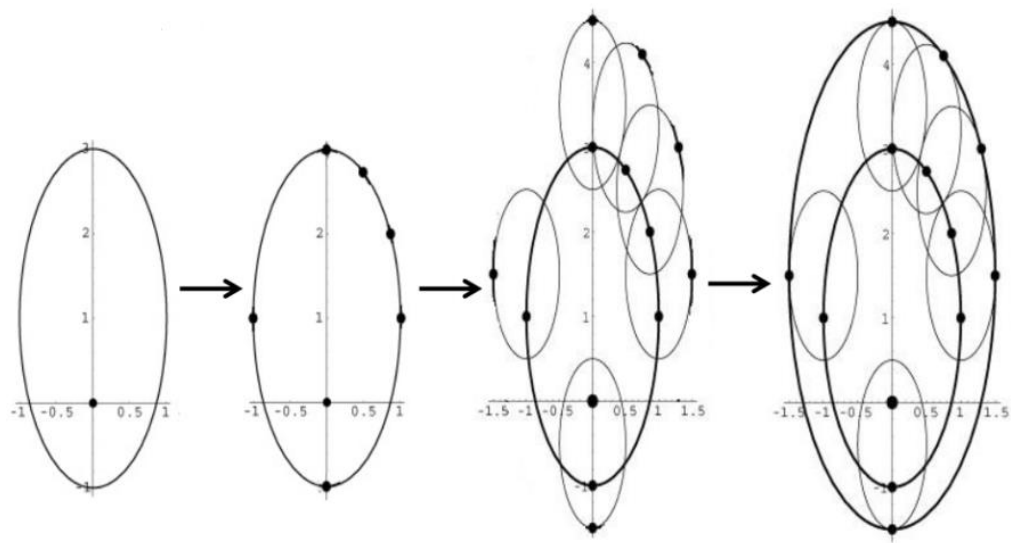

Figure 4 - Elliptical wave propagation from 11 to $t 2$ 
- Level Set Method: In this kind of fire simulators, the burned region is defined as a level set function $\psi=\psi(\vec{x}, t)$ whose values are:

$$
\left\{\begin{array}{l}
\psi(\vec{x}, t) \leq 0 \text { Burned Area } \\
\psi(\vec{x}, t)=0 \text { Fire Front } \\
\psi(\vec{x}, t)>0 \text { Saved Area }
\end{array}\right.
$$

and it evolves through the partial differential equation

$$
\partial \psi / \partial t=R\|\nabla \psi\|
$$

where $\mathrm{R}$ is the rate of spread (Farguell et al., 2017). Equation 4 can be solved numerically given initial and boundary values for $\psi$, as well as, a model to compute the rate of speed. An advantage of this method is that the behavior of fire fronts arises naturally from the underlying mathematics and thus does not require the special handling that is needed when an Elliptical wave propagation method is used. WRF-SFIRE uses this approach (Fig. 5).

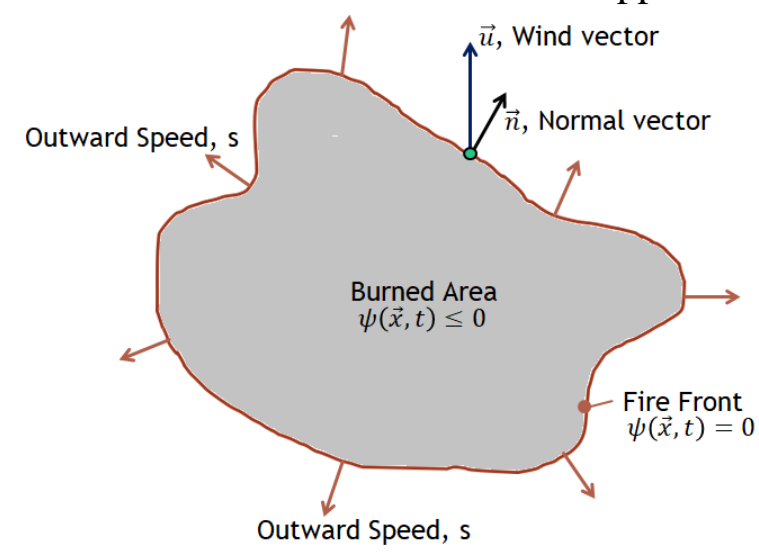

Figure 5 - Simulator based on Level Set Method.

\section{Forest Fires Simulator Based on GPUs}

As previously mentioned, in this study we focused on FARSITE simulator, which is based on elliptical wave propagation. As we have seen, in the simulators based on the Huygens principle, the expansion of a point only depends on the local conditions of this point and, therefore, it does not depend on the expansion of the other points. When a simulation in FARSITE is started, the resolution of the perimeter must be indicated. This perimeter resolution defines the distance between points of the perimeter of the fire. Higher perimeter resolution provides more accurate simulations. But, on the other hand, a higher resolution implies a greater number of points to compute, and therefore, a longer execution time. 


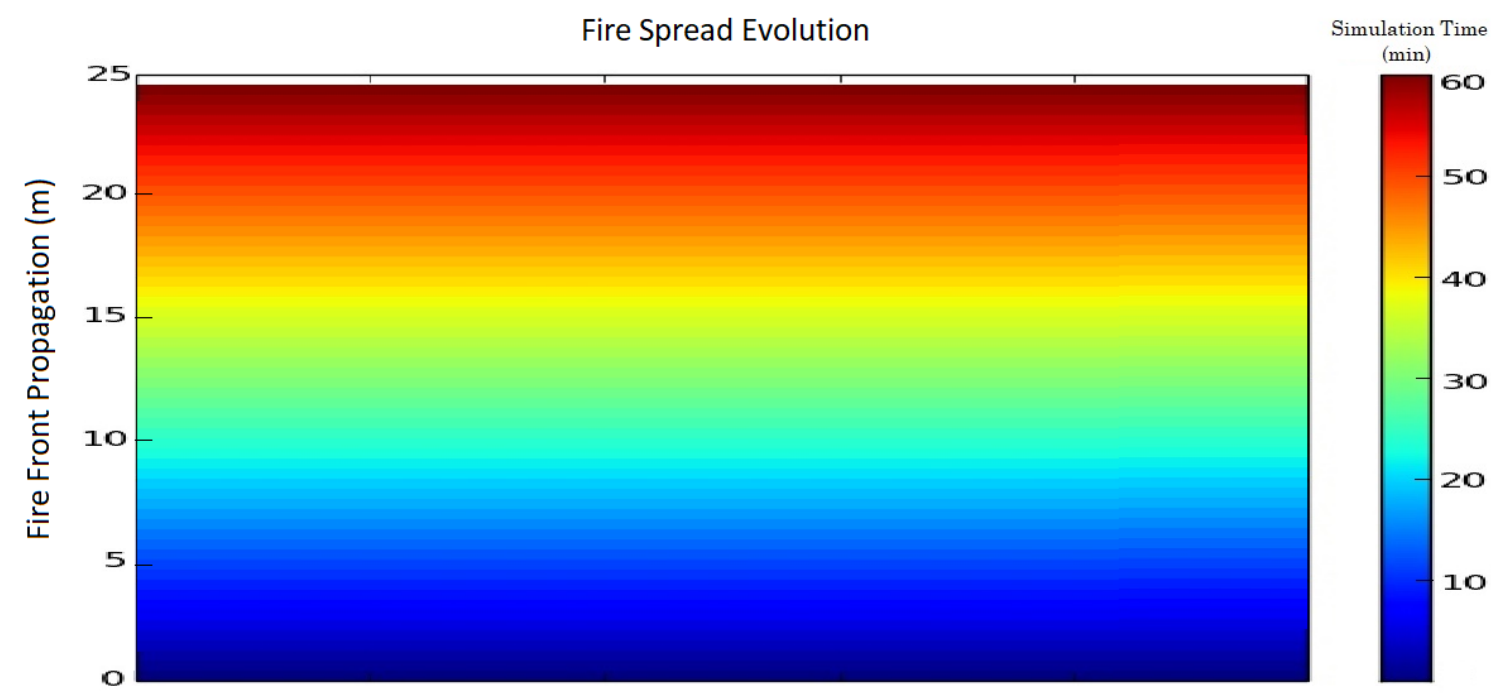

Fire Front

Figure 5 - Evolution of the fire front. The y-axis shows the propagation distance of the fire in meters. The color scale indicates the time step of the simulation in minutes.

As a first approximation, a synthetic fire that advances on a linear front has been used. As a simplification, the boundary problems at the front ends are ignored. In this particular case, it has been considered a flat terrain, with a homogeneous vegetation and constant wind speed and wind direction during the whole simulation. Under these conditions the propagation will be the same for all points of the fire front. Fig. 5 shows the evolution of the fire front, the color scale indicates the evolution time of the fire, while the ordinates axis shows the distance advanced by the front.

When executing the simulation on CPU, the point's evolution is computed sequentially, see Fig. 6 . Therefore, if the number of points to expand is increased, the execution time will proportionally increase. For that reason, simulations with high resolutions provide high execution time, which limits their use in real situations. On the other hand, reducing the resolution implies that the execution time will be reduced, but the accuracy of the simulation will be penalized and therefore, the simulation will not properly describe the behavior of the fire.

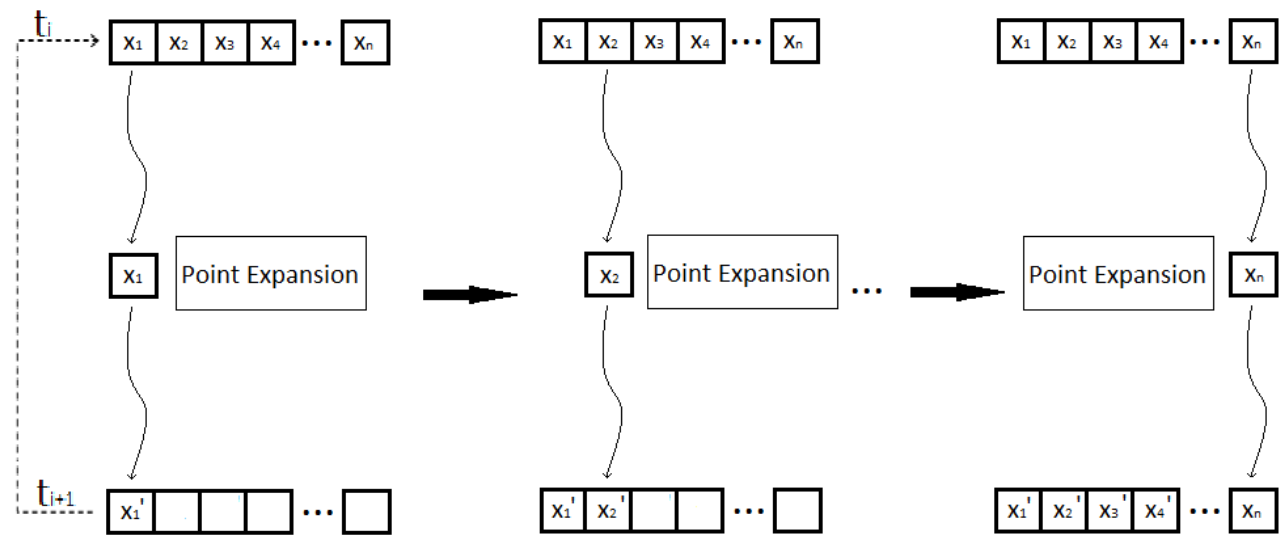

Figure 6 - Workflow execution of point's expansion in the CPU. 


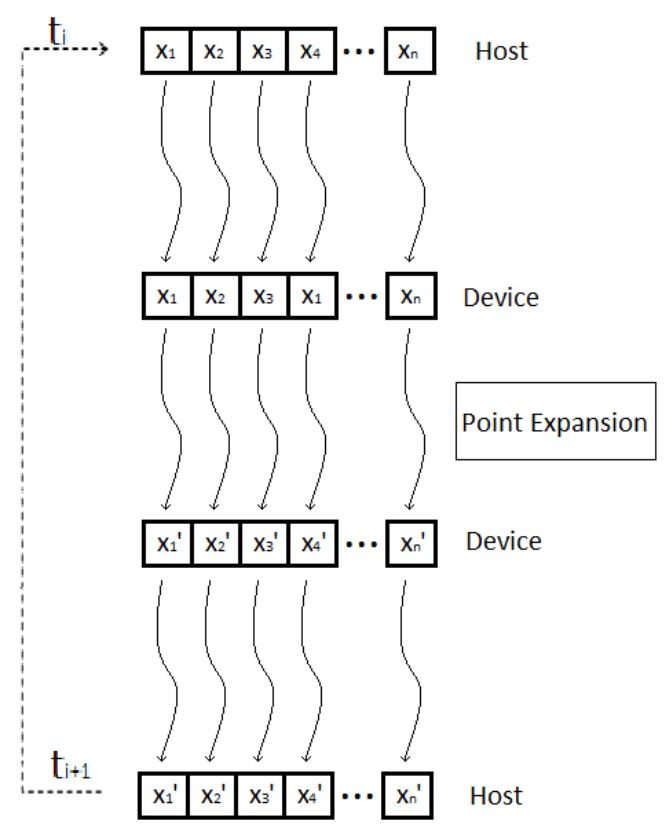

Figure 7- Workflow execution of point's expansion in the GPU.

One way to manage a simulation with high resolution is to be able to compute as much points of the fire front as possible in parallel. Computational accelerators like GPUs, provide the computer characteristics that allow to exploit this parallelization requirement. Therefore, the previous described point propagation scheme has been modified to fit the parallel scheme required by a GPU (Fig. 7). In this implementation, the number of fire points can be increased without increasing the execution time excessively.

To evaluate the performance of the proposed parallelization, five different simulations have been performed on CPU and GPU for each number of points between 1,000 and 50,000, with intervals of 1,000 points. The obtained results are presented in the next section.

\section{Experimental Study and Results}

As execution platform, we have used an Intel (R) Xeon (R) CPU E5-2603 v2 @ 1.80GHz, with 4 cores and for the GPU simulations, a GeForce GTX TITAN X with 3072 CUDA cores was used. As it was previously mentioned, the fire propagation time has been limited to one hour, so the execution time of each simulation will be mainly determined by the volume of data to be treated, not by the fire evolution time.

Fig. 8 shows the execution time when the simulation is done in CPU (Red) and in GPU (Blue) depending on the number of points of the fire front. As it can be seen, the execution time in the CPU increases significantly when the number of points increases. We can observe that for a small number of points, the execution in GPU is slower than executing the same number of points in CPU. This is because data transfer from CPU memory to GPU memory consumes most of the execution time. From 15,000 points the execution in GPU is faster than the execution in CPU. 


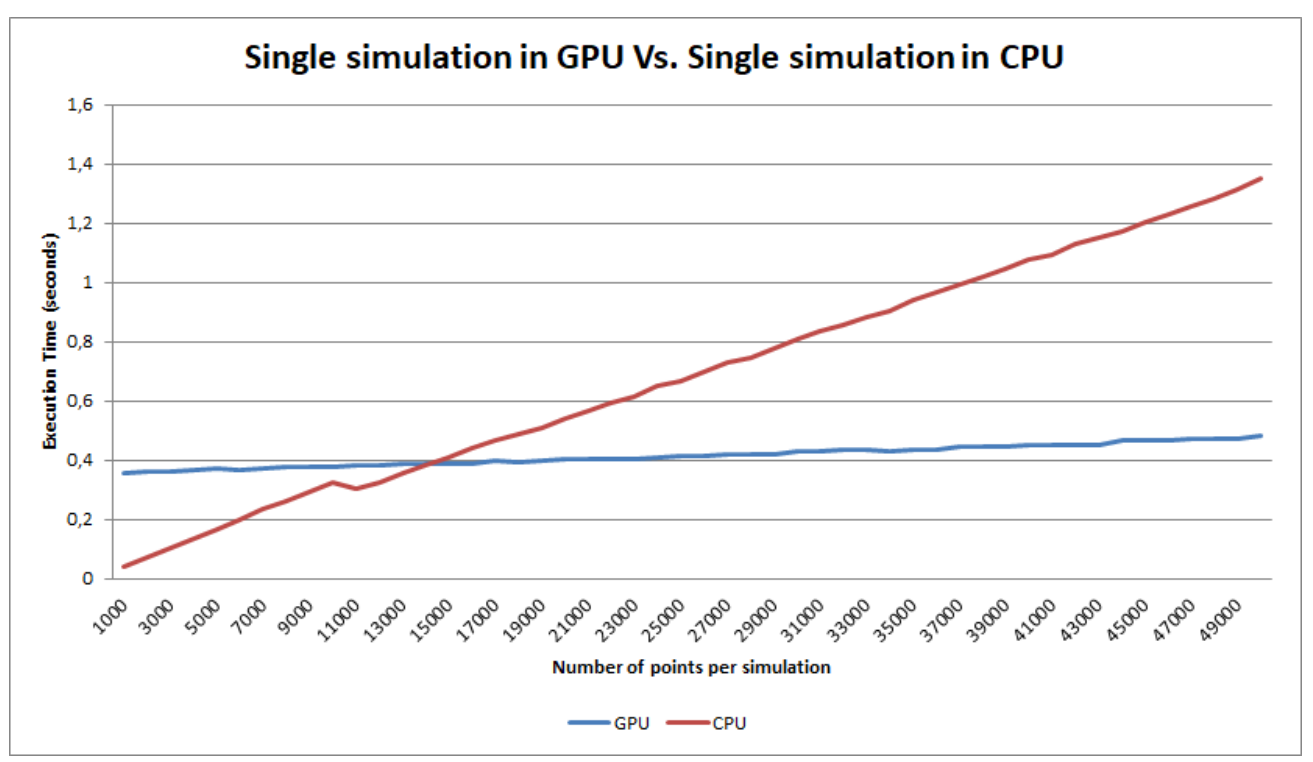

Figure 8 - Average execution time in CPU (Red) and GPU (blue) depending on the number of simulated points.

The Fig. 9 shows the speed-up of the simulator when it is running on the GPU. As it can be seen, when the number of points are above 15,000 , the speed-up is higher than 1 , which indicates that the execution in the GPU is more efficient than on the CPU.

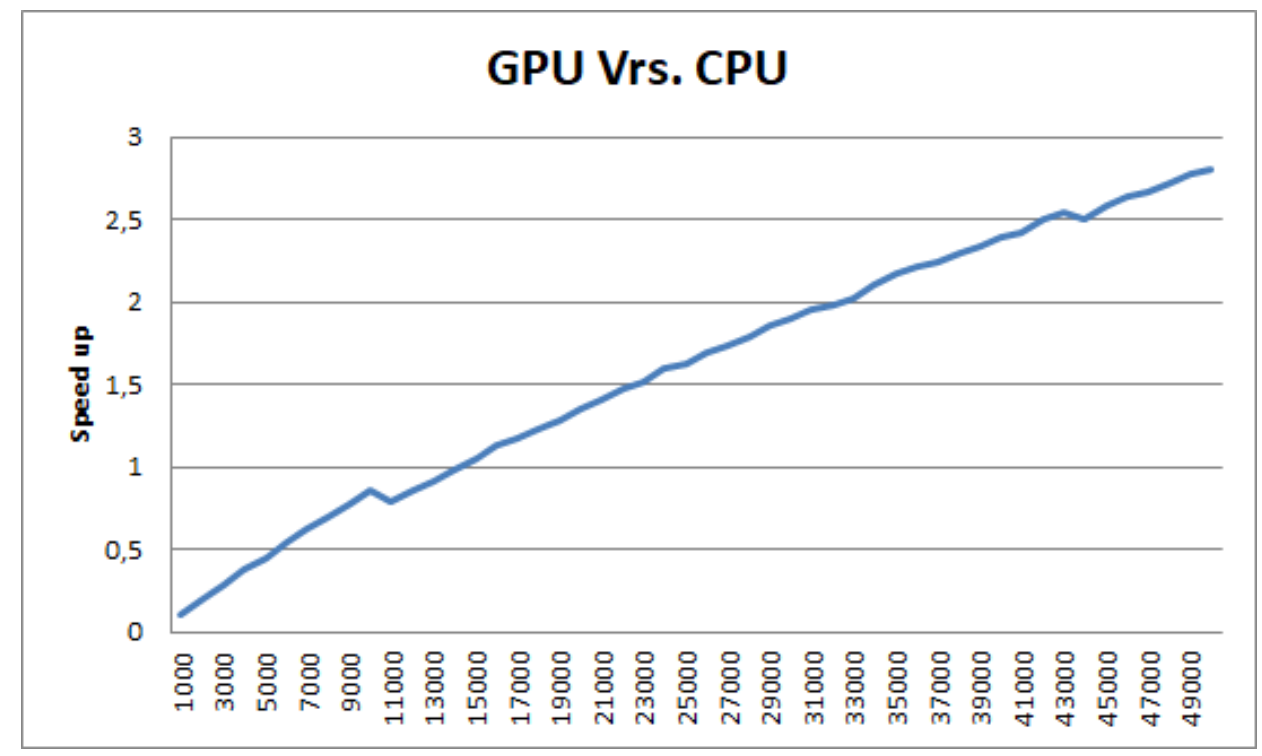

Figure 9: Average speed-up of the simulator in GPU depending on the number of front points.

\section{Conclusions and Future work}

In recent years, we have witnessed a significant increase in the computational capabilities of GPUs. This computing power makes GPUs ideal for those problems that require the processing of a large amount of data. In our case, we have focused on the study of forest fire propagation simulators based on the Huygens principle, in particular FARSITE. In this work, a synthetic fire has been used, with a linear fire front and constant wind and vegetation conditions throughout the simulation.

According to the study carried out, when the simulator is executed in the GPU most of the execution time is invested in the data copy between memories. For this reason, the volume of data to be computed must be large enough to be able to justify this copying time. It has been shown that, with a number of points greater than 15,000 points, the copying time is compensated by the computation time, so it is advisable to perform the execution on the GPU. On the other hand, when the number of 
points is lower, the copying time is not compensated by the execution time, so the execution in CPU is more efficient than in GPU.

That preliminary work open a new way of approaching forest fire spread simulation in the sense that we expect to obtain more accurate results and, at the same time, faster and, therefore operationally simulation time

\section{Acknowledgments}

This research has been supported by MINECO-Spain under contract TIN2014-53234-C2-1-R and TIN2017-84553-C2-1-R, and by the Catalan government under grant 2017-SGR-313.

\section{References}

A. Cencerrado, T. Artés, A. Cortés, and T. Margalef, "Relieving uncertainty in forest fire spread prediction by exploiting multicore architectures," in Proceedings of the International Conference on Computational Science, ICCS 2015, Computational Science at the Gates of Nature, Reykjavík, Iceland, 1-3, June, 2015, 2014, 2015, pp. 1752-1761.

C. Brun, T. Margalef, A. Cortes, and A.a Sikora, "Enhancing multi-model forest fire spread prediction by exploiting multi-core parallelism," The Journal of Supercomputing, vol. 70, no. 2, pp. 721-732, 2014.

Finney, M.A. "FARSITE: Fire Area Simulator-model development and evaluation". Research Paper RMRS-RP-4 Revised. Ogden, UT: U.S. Department of Agriculture, Forest Service, Rocky Mountain Research Station, 2004.

Gregorio S.D., Filippone G., Spataro W., Trunfio G.A. “Accelerating wildfire susceptibility mapping through GPU”. Journal of Parallel and Distributed Computing 2013; 73(8):1183-1194.

Ntinas V.G., Moutafis B.E., Trunfio G.A., Sirakoulis G.C. "Parallel fuzzy cellular automata for datadriven simulation of wildfire spreading". Journal of Computational Science 2017; 21:469 - 485.

Sousa F.A., dos Reis R.J.N., Pereira J.C.F." Simulation of surface fire fronts using firelib and GPUs". Environmental Modelling and Software 2012; 38:167-77.

Rothermel R. "A mathematical model for predicting fire spread in wildland fuels"; 1972.

A. Alexandridis, D. Vakalis, Constantinos I. Siettos, and George V. Bafas, "A cellular automata model for forest fire spread prediction: The case of the wildfire that swept through spetses island in 1990," Applied Mathematics and Computation, vol. 204, no. 1, pp. 191-201, 2008.

E. Pastor, L. Zárate, E. Planas, J. Arnaldos. "Mathematical models and calculation systems for the study of wildland fire behaviour". Progress in Energy and Combustion Science, 29(2):139-153, 2003.

I. Knight and J. Coleman, "A fire perimeter expansion algorithm-based on Huygens wavelet propagation," International Journal of Wildland Fire 3, 73-84. 1993.

À. Farguell, A. Cortés, T. Margalef, J.R. Miro, and J. Mercader. Data resolution effects on a coupled data driven system for forest fire propagation prediction. Procedia Computer Science, 108:1562 1571, 2017. International Conference on Computational Science, ICCS 2017, 12-14 June 2017, Zurich, Switzerland. 


\title{
Atmospheric flow and a Large fire interaction: the unusual case of Pedrogão Grande, Portugal (17 June 2017)
}

\author{
Paulo Pinto*; Álvaro Silva \\ Instituto Português do Mar e da Atmosfera (IPMA), Lisbon, 1749-077, Portugal. \\ \{Paulo.Pinto@ipma.pt*\}
}

\begin{abstract}
On the afternoon of 17th June 2017 the fire was progressing in a hilly region of central mainland Portugal. A hot and dry atmosphere, characterized by a classic inverted $\mathrm{V}$ profile, typical from dry downburst environments (Weisman and Klemp, 1986), was in place, thus favoring high level based convection. In fact, an MCS that was observed both on satellite imagery and weather radar, has developed southeast of the fire area and, during the afternoon of this day, produced several downbursts that were recorded in several automatic weather stations (AWS). It was found that several shear lines, identified on Doppler radar, were considered to be consistent with convective outflow gust front surges that emanated from a downburst, or downbursts, generated by the MCS, and propagated over the fire area. It was verified that as this happened, the fire suffered an outstanding intensification, having produced a very violent and widespread firestorm that materialized an Extreme Fire Behavior (EFB), confirmed by ground survey (Viegas et al, 2017). As an outcome of this first interaction between the atmosphere and the fire, it was observed that a vertically developed dry pyroconvective column formed and made its way through the MCS anvil, placed overhead the fire by that time. Soon after, this smoke column top altitude decreased more than $5000 \mathrm{~m}$ in just 10 minutes. This remarkable behavior, resembling a downburst top collapse, was accompanied by a rapid low level plume displacement, interpreted as a result of the downburst induced circulation close to the ground. Just 10 minutes later, another fire blowup occurred, as the plume started to grow vertically. This second outstanding pyroconvective column growth was seen as a direct result of the stated downburst induced circulation at low levels that, as it was manifesting, induced another EFB episode.
\end{abstract}

Keywords: mesoscale convective system; outflows; shear lines; fire blowup; downburst; weather radar

\section{Introduction}

As recognized in specialized literature (Werth, $\mathrm{P}$, et al, 2016), prolonged periods of drought are positively correlated with the occurrence of intense hot fires, although several studies (e.g. Viegas and Simeoni, 2011) have also clearly linked extreme fire behavior (EFB) with fuel properties and topography. The Pedrogão Grande (PG) large fire occurred in central mainland Portugal, after several months of under average rainfall records (April was the driest since 1931) and above average temperatures. By late May, more than two thirds of the country were in a severe drought level, according to Palmer Drought Severity Index (IPMA, 2017, in portuguese).

The PG large fire started during the early afternoon of the $17^{\text {th }}$ June 2017 . Less than one hour later, a second ignition originated a separate fire, a few kilometers far from the first location. By the same time, a multicell convective convective system (MCS) formed and started to propagate to the eastsoutheast of the fire area, in a very hot and dry low troposphere environment. It was observed, both on radar and ground stations, that several convective outflows were produced by the MCS. As the meteorological system was propagating closer the fire, some gust front surges started to interact with it and, on a certain occasion, a first outstanding fire intensification was observed. It materialized an $\mathrm{EFB}$, as confirmed by ground survey. As it happened, the fire went out of control and more than 50 people were killed. 


\section{Data}

For the study of this interaction between the atmospheric flow and the fire, there were used ground, weather radar and lightning detection network observations. Numerical model data were also considered.

The area where the EFB occurred, referred to as PG fire area, is located in central mainland Portugal, as highlighted in Figure 1. Meteorological parameters from IPMA automatic weather stations (AWS) located in the fire area and surroundings (Figure 1) were analyzed, regarding air temperature, relative humidity and wind. The Tomar/Valdonas AWS was particularly important to validate a radar product that was used to diagnose the interaction. On the other hand, two C-band Doppler weather radars of the IPMA network were used (Figure 1). These were about $100 \mathrm{~km}$ far from the study area. The system installed to the north is a dual polarization (DP) radar, located at around $1100 \mathrm{~m}$ altitude (Porto radar, Figure 1). The system installed to the south is a single polarization system, located at around $200 \mathrm{~m}$ altitude (Lisboa radar, Figure 1). While the Porto radar did not perform observations below $1400 \mathrm{~m}$ altitude over the fire area, due to its large installation height, it was crucial in order to get a microphysical insight of the fire plumes, enabled by its DP capability. The Lisboa radar performed observations over the fire site, as low as $900 \mathrm{~m}$ altitude, although limited to single polarization moments. The real resolution of radar observations was about $1 \mathrm{~km}$ over the fire area, both in azimuth and range, matching a nominal cartesian resolution of the same magnitude. Reflectivity, Doppler velocity and several DP moments were used in this study. The analysis of the fire-atmosphere interaction evolution was mainly based on the use of single PPI's and vertical sections of radar observations. The vertical sections were performed over a segment that is referenced to in Figure 1. Nine lightning detection sensors were accessed, 3 of them operated by IPMA and the remaining by AEMET (Spanish Meteo Service), installed close to the Portuguese border. The nearest 4 of these 9 sensors are represented in Figure 1.

The vertical thermodynamic structure of the atmosphere was analysed, both using observed atmospheric profiles (rawinsonde data from 08579 Lisboa station, $17^{\text {th }}$ June, 12 UTC, not shown) and vertical profiles obtained from numerical model processing. From these, a vertical profile from 18 UTC (19:00h, local time) from the Pedrogão Grande location (Figure 1) was extracted and discussed.

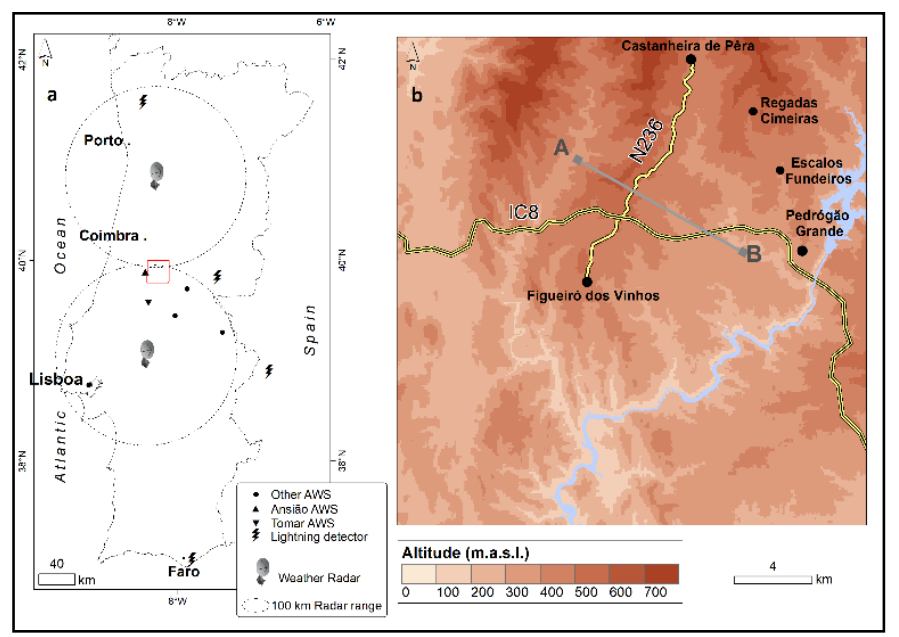

Figure 1 - Parts of western Iberia a) and zoomed Pedrogão Grande Fire area b). a) Fire area (red square) zoomed in b), relevant automatic weather radar stations, 4 of the 9 nearest lightning used sensors and weather radar stations. b) terrain, important locations and roads, numerical model profile point (Pedrogão Grande), relevant fire ignitions at Escalos Fundeiros and Regadas; radar transect (A-B) segment referred to in the text. $100 \mathrm{~km}$ radar ranges are marked for reference. 


\section{Meteorological environment}

By 13:00h local time, $17^{\text {th }}$ June 2017, the ECMWF mean sea level pressure analysis was showing a high pressure system centered over southern England (Figure 2) and, to the south of it, a thermal trough pattern over western Iberia, in association with the typical Iberian thermal low. This was promoting a weak easterly flow over mainland Portugal. Offshore northern Morocco there was an upper level low, significant just above $700 \mathrm{hPa}$ (not shown). During early afternoon, a cool air advection in the 700-500 hPa layer (not shown) that was driven by the stated high pressure system, and the strong low level heating, increased the atmospheric instability. By 16:00h local time, as shown by ECMWF data, a short forecast was showing some humidity convergence at mid levels over central mainland Portugal (Figure 3).

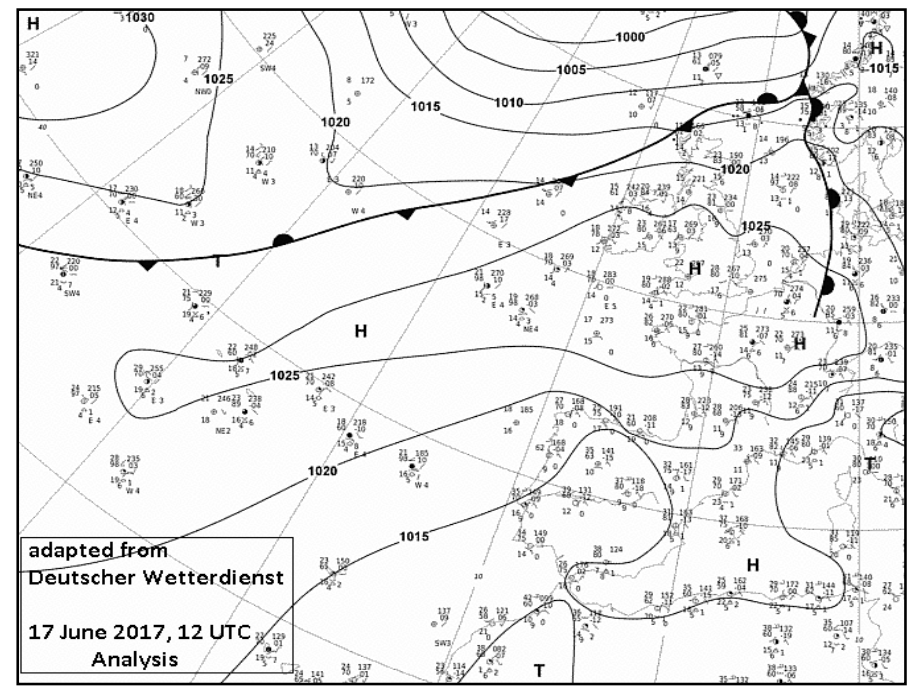

Figure 2 - Mean sea level pressure (solid contours, 5hPa intervals) over northeast Atlantic; analysis adapted from Deutscher Wetterdienst, 13:00h, local time, $17^{\text {th }}$ June 2017.

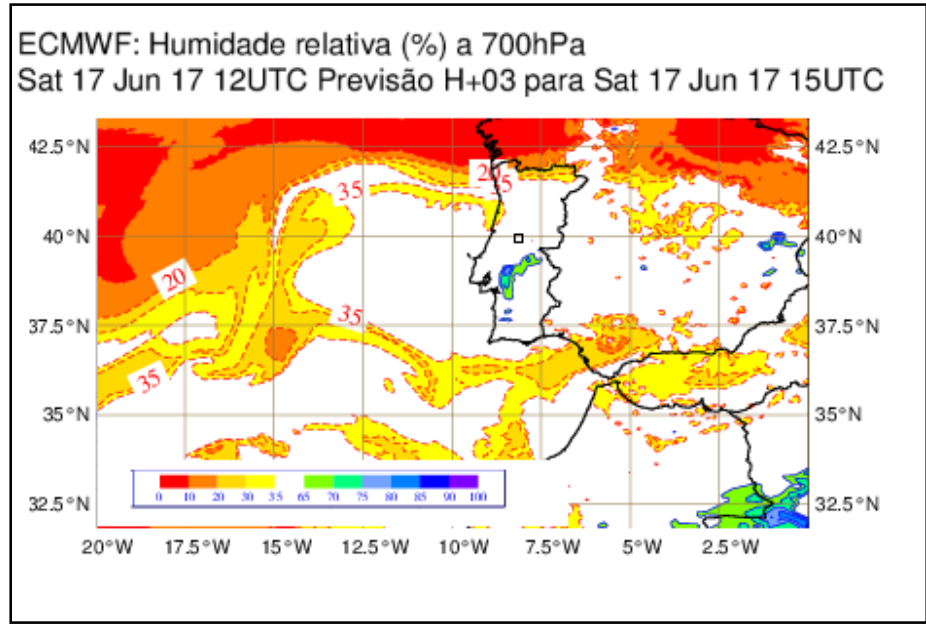

Figure 3 - Relative humidity (color shaded, \%) at 700 hPa over Iberia and northern Morocco, ECMWF forecast for 16:00h, local time. $17^{\text {th }}$ June 2017.

A vertical atmospheric profile valid at 19:00h was derived from ECMWF short term forecasts (Figure 4), for a point located a few kilometers east of the fire perimeter. This profile showed a hot and dry low troposphere, resembling the classic "inverted V" profile, typical from dry downburst environments (Weisman and Klemp, 1986). Above $700 \mathrm{hPa}$ the atmosphere was unstable. Total precipitable water was around $30 \mathrm{~mm}$. This was consistent with an environment prone to high level based convection to the southeast of the fire area. 


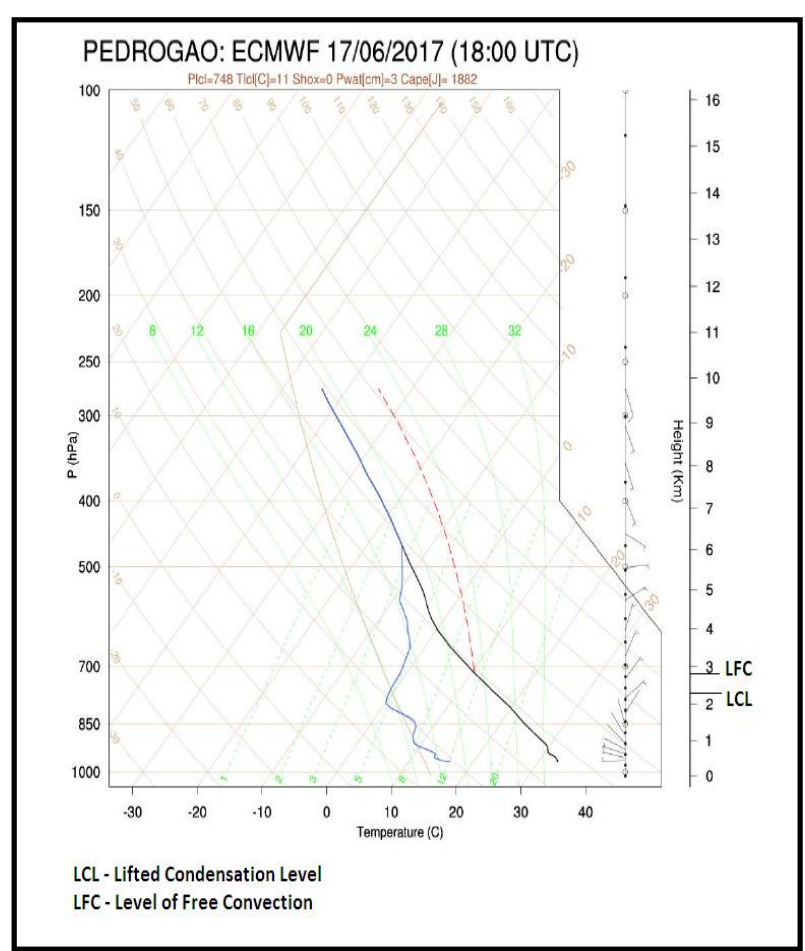

Figure 4 - SkewT chart (ECMWF profile) for the Pedrogão Grande area at 19:00h, local time, $17^{\text {th }}$ June 2017. Dry bulb air temperature (black curve, to the right), dew point temperature (blue curve, to the left) and parcel trajectory (dashed orange curve) are represented. Wind barbs at different levels also represented. LCL and LFC are depicted over the height scale.

As revealed both by observations derived from the Lisboa radar scans and satellite imagery, a multicell convective system was propagating over central-southern mainland Portugal (Figure 5) during the afternoon of the $17^{\text {th }}$ June. By $18: 30 \mathrm{~h}$, the system was already entering a dissipating stage, clearly visible by a developed and extensive anvil, spawned for many thousands of squared kilometers. Its signature was remarkably visible on satellite, over the area where the fire was progressing (Figure $5 b)$. Nevertheless, by this time, the radar was showing several convective core signatures, consistent with the multicell nature of the convective system (Figure 5a).
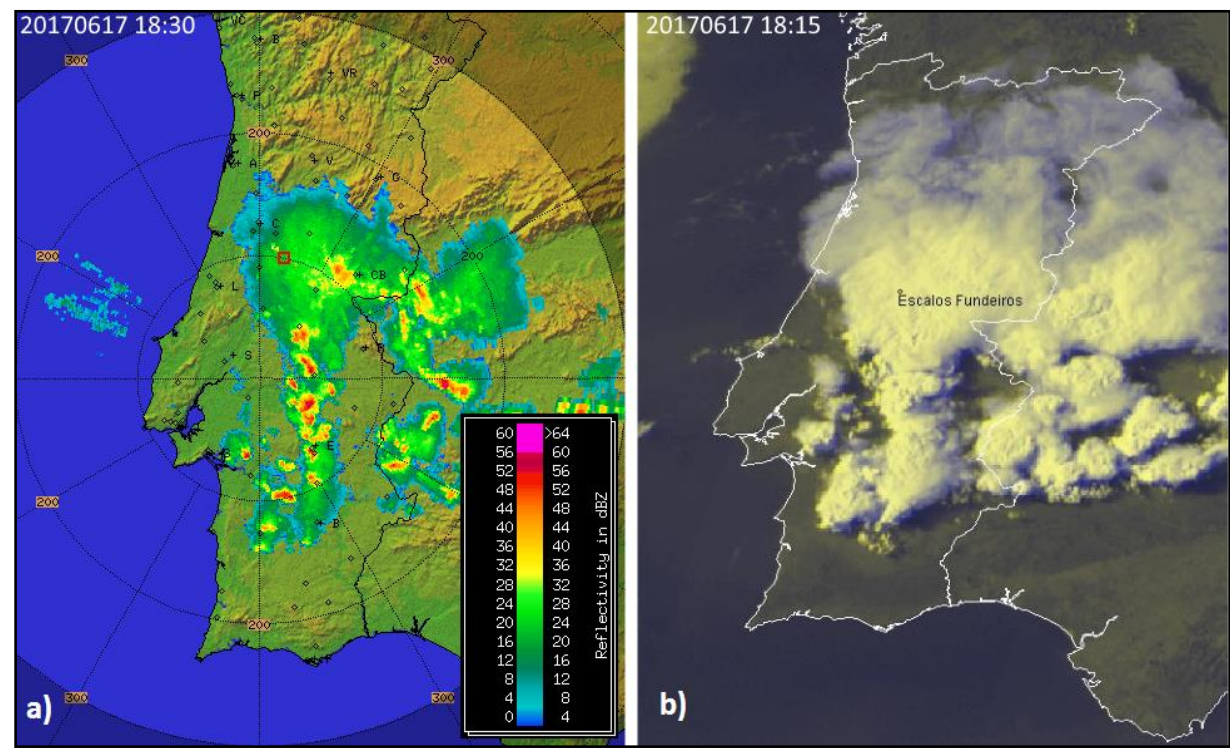

Figure 5 - a) Maximum returns of reflectivity (dBZ) for 18:30h, local time, Lisboa radar. Fire area marked with a red square (over the northern end of $100 \mathrm{~km}$ range). A large circular reflectivity pattern corresponds to an extensive anvil signature; b) High resolution visible MSG image for 18:15h, local time. Location Escalos Fundeiros (Fire area) is marked. $17^{\text {th }}$ June 2017.

As frequently observed, some atmospheric environments can be supportive of both wet and dry downbursts. On the $17^{\text {th }}$ June the situation was of this type, in accordance with several reports and 
records on film (not shown). This atmospheric environment, prone to downbursts did, in fact, develop several of these phenomena that were observed in a number of IPMA AWS and on Doppler radar.

\section{Convective outflows}

More frequently than recognized during the past decades, convectively-cooled air from small scale convective perturbations may impact the ground levels. Usually, the surface boundary (marked by a gust front) formed by the horizontal spread of such cooled air, does not play a significant role. Nevertheless, provided the required ingredients are in place, convective outflow boundaries may act as sources of secondary convection and even present hazardous conditions to aircrafts takeoff and landing. In extreme cases these outflows may generate large turbulent regime flows, especially in the area of the gust front, where sudden wind direction and intensity may be expected. These conditions may persist even at far ranges of the originating convective system.

\subsection{Gust front detection in favorable conditions}

The atmospheric volumes where a convective outflow leading edge is propagating may, or may not, be favorable to the detection on a weather radar perspective. When scatterers, such as insects, are well distributed throughout the observational volumes, thin reflectivity lines and Doppler signatures (Klingle and Smith, 1987) may be detectable and allow the diagnostic of low level convergence lines (or shear lines) associated with the gust fronts. This was the case in the region located to the south and southwest of the multicell system (not shown), in an early stage, as the system was developing, still to the southeast of the fire area. However, radar reflectivity analysis may render useless if scatterers are poorly distributed in the observed volumes. Furthermore, Doppler velocity signatures of these gust fronts may also be very hard or impossible to identify, due to the lack of scatterers and the complexity of signatures, themselves. This unfavorable detection environment was found in the area located between the convective system cores and the fire location, just before the largest interaction took place. This fact required a non-standard approach.

\subsection{Gust Front detection in unfavorable conditions}

A fine tune calibration of a low level PPI of horizontal shear (azimuthal and radial shear) was performed, so that any shear lines propagating, eventually, over the fire area could be identified even in unfavorable conditions. This calibration was achieved using several AWS data located in the favorable detection area. If the validity of this non-standard radar product would be confirmed in the favorable detection area, its use during the afternoon of this day would be extended to the required unfavorable detection area.

It was found an optimal fine tune situation by directly comparing a PPI of shear with a simultaneous PPI of reflectivity (Figure 6). In the example of Figure 6a the subjectively detected fine line of reflectivity, using space and time coherence criteria, is represented. In Figure 6b, over the horizontal shear field, a line showing space and time coherence on radar, matching the one already identified in Figure $6 \mathrm{a}$, was represented. In this example, by 18:06h, the identified line was propagating westerly and very close, but still to the east, of the Tomar AWS (Figure 6). Meanwhile, just before 18:20h, the AWS observations, 10 minutes average, were showing a $2 \mathrm{~m}$ air temperature over $40^{\circ} \mathrm{C}$ and a relative humidity close to $20 \%$, typical of the low level atmospheric environment of that day (Figure 7). Then, by $18: 20 \mathrm{~h}$, a sudden temperature decrease and relative humidity increase started, followed by an easterly gust of $15 \mathrm{~m} / \mathrm{s}$, shortly after. This evolution was absolutely consistent with the propagation of a density current over the station. This calibrated horizontal shear field, tested against other ground observations (not shown) was considered as a reliable tool for the identification of the shear lines in hard observational conditions. 
The diagnose of shear lines was especially difficult in the vicinity of the fire area, due to the stated lack of scatterers and to an unfavorable beam orientation of the Lisbon radar over there. In fact, to the southeast of the fire area, the beam was facing the main flow (from the east-southeast) at an unfavorable right angle.

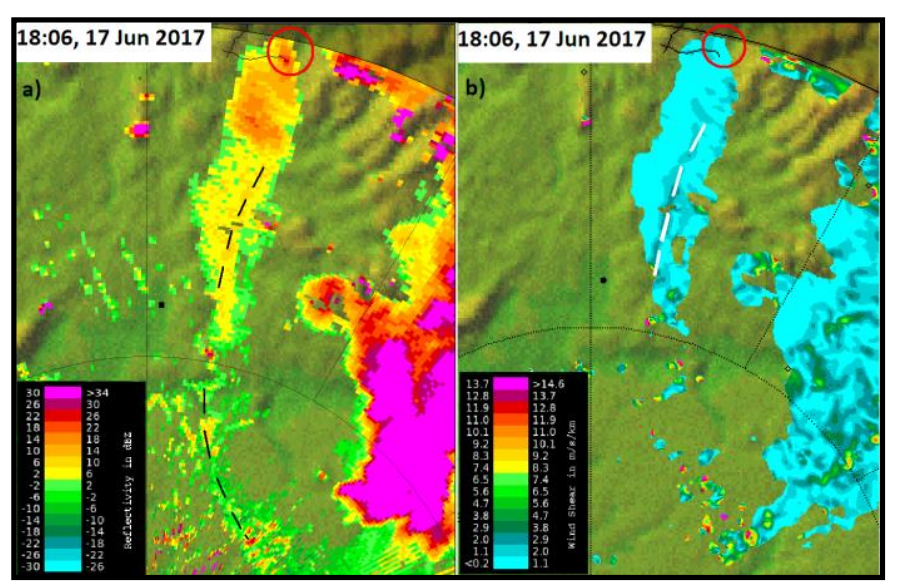

Figure 6 - Plane position indicator, $0.1^{\circ}$ elevation, 18:06, $17^{\text {th }}$ June 2017, Lisboa radar for a) reflectivity (-30 to $>30 \mathrm{dBZ}), \mathrm{b})$ horizontal shear (radial and azimuthal) $(0$ to $>14,6 \mathrm{~m} / \mathrm{s} / \mathrm{km})$. A shear line signature is marked with a dashed black line in a) and a dashed white line in c). Tomar AWS location is marked with a black dot. Fire plume area (low level) by 18:06, is marked with a red circle. Radar observations over the fire area are performed at $900 \mathrm{~m}$ altitude (radar site to the bottom of the image).

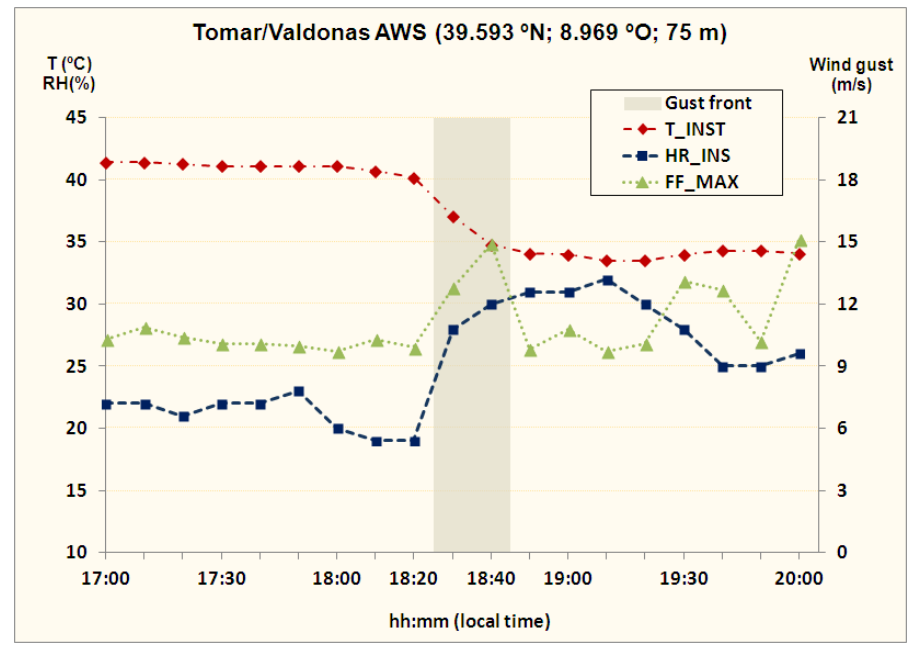

Figure 7 - Tomar/Valdonas AWS observations: $2 m$ air temperature (red), $2 m$ relative humidity (dark blue), $10 \mathrm{~m}$ wind gust (green), 17:00-20:00h, local time, $17^{\text {th }}$ June 2017. The vertical shaded bar (18:25-18:45) signs the onset of the convective outflow over the AWS.

\section{Fire-atmosphere interaction}

The Lisboa radar was very helpful to follow up the fire-atmosphere interaction, at low levels. It performed observations as low as $900 \mathrm{~m}$ altitude over the study area, where the average ground was about $400 \mathrm{~m}$. The lowest level PPI of reflectivity enabled the identification of relevant maximum reflectivity patterns. These, if showing vertical consistency with upper level PPI's, were considered as fine identifiers of the approximate low level pyroconvective plume position ( $900 \mathrm{~m}$ altitude). So, the fire-atmosphere interaction was, in fact, studied from a pyroconvective plume-atmosphere interaction perspective. Radar reflectivity is proportional to the first power of the number of scatterers in an observation volume and to the sixth power of their diameter. Thus, larger reflectivity values at low 
levels are positively correlated with more intense burning processes at ground levels. Vertical sections using several PPI's were also used.

On the other hand, the Porto radar was used to diagnose the microphysics of the observed atmospheric volumes. During the more relevant pyroconvective plume-atmosphere interaction stage, as it will be shown, the plume made its way through the anvil of a convective system that was, then, in place above the fire. A distinction between the approximate boundaries of the smoke plume and the ones of the intervening meteorological system was crucial. A useful DP parameter, the correlation coefficient $(\rho \mathrm{hv})$, was used, as it is considered a fine particle type discriminator (Balakrishnan and Zrnic, 1990). It corresponds to a correlation coefficient, in time series, between horizontally and vertically polarized echoes. Recently (Lang et al, 2014; LaRoche and Lang, 2017) it was found that a

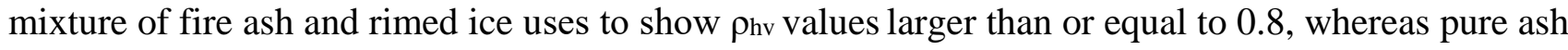
uses to feature $\rho_{\mathrm{hv}}$ values lower than 0.8 . So, it was roughly considered that volumes presenting $\rho_{\mathrm{hv}}$ values less than 0.8 were typical from the dry pyroconvective plume.

\subsection{Stage 1: 14:30h-19:15h}

The PG fire started by 14:30h, near Escalos Fundeiros (Figure 1). A second ignition occurred close to Regadas Cimeiras (Figure 1), reported by 15:40h and originating a separate fire, less than $3 \mathrm{~km}$ apart from the first location. By this time the low level wind was weak and blowing from the northwest, as revealed by video camera recordings and AWS stations (not shown). As mentioned before, a multicell convective system started to propagate over central-southern mainland Portugal by this time.

During the first few hours of this stage, the fire-atmosphere interaction did not produce remarkable effects. After 18:00h, though, the approaching convective system started to increase its influence, as a stronger southeast flow, at low levels, became predominating. Several fire intensifications were, then, observed on radar, as the fire plume pattern showed transient, although not very spectacular, increase on its reflectivity and vertical extension (not shown). These intensifications were also confirmed by ground survey (Viegas et al., 2017, in portuguese) and video camera recordings.

\subsection{Stage 2: 19:15h-20:00h}

Radar observations have shown that the pyroconvective column, inspected along the transect segment marked in Figure 1, started to suffer a huge growth by 19:20h (Figure 8) having peaked by 19:50h (Figure 9), as it topped at $12500 \mathrm{~m}$ altitude. During this outstanding development, it is perfectly illustrated in Figure 10 that the pyroconvective column ( $\rho$ hv values $<0.8$, in greenish color), made its way through the convective system deep anvil ( $\rho_{\text {hv }}$ values $>0.8$, in yellowish color). The anvil was more than $3000 \mathrm{~m}$ below the one of the column top (Figure 8).

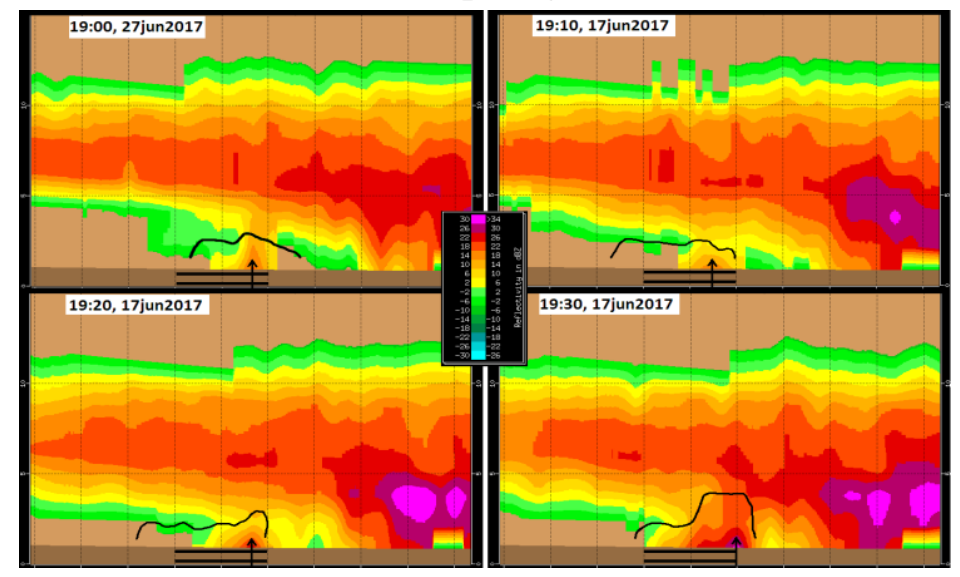

Figure 8 - Vertical cuts on reflectivity (-30 to $>34 \mathrm{dBZ})$, Lisboa radar, June $17^{\text {th }} 2017$ (19:00h-19:30h). Section is along the segment marked in Figure 1, observer looking from the south (horizontal double lines mark the transect itself). Black arrow represents the plume location at $900 \mathrm{~m}$ altitude. Black contour represents $0.8 \rho_{h v}$ value (dry smoke threshold). Vertica/horizontal scales, $5 \mathrm{~km}$ discretization. 


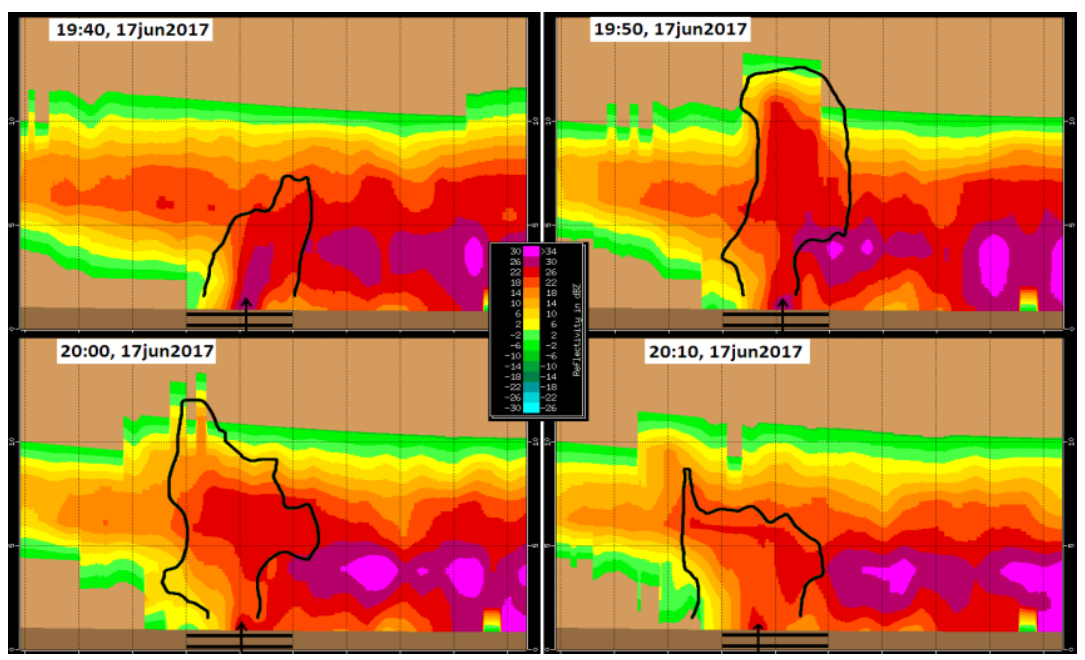

Figure 9 - Vertical cuts on reflectivity (-30 to $>34 \mathrm{dBZ})$, Lisboa radar, June $17^{\text {th }} 2017$ (19:40h-20:10h). Section is along the segment marked in Figure 1, observer looking from the south (horizontal double lines mark the transect itself). Black arrow represents the plume location at $900 \mathrm{~m}$ altitude. Black contour represents $0.8 \rho \mathrm{hv}$ value (dry smoke threshold). Vertical/horizontal scales, $5 \mathrm{~km}$ discretization.

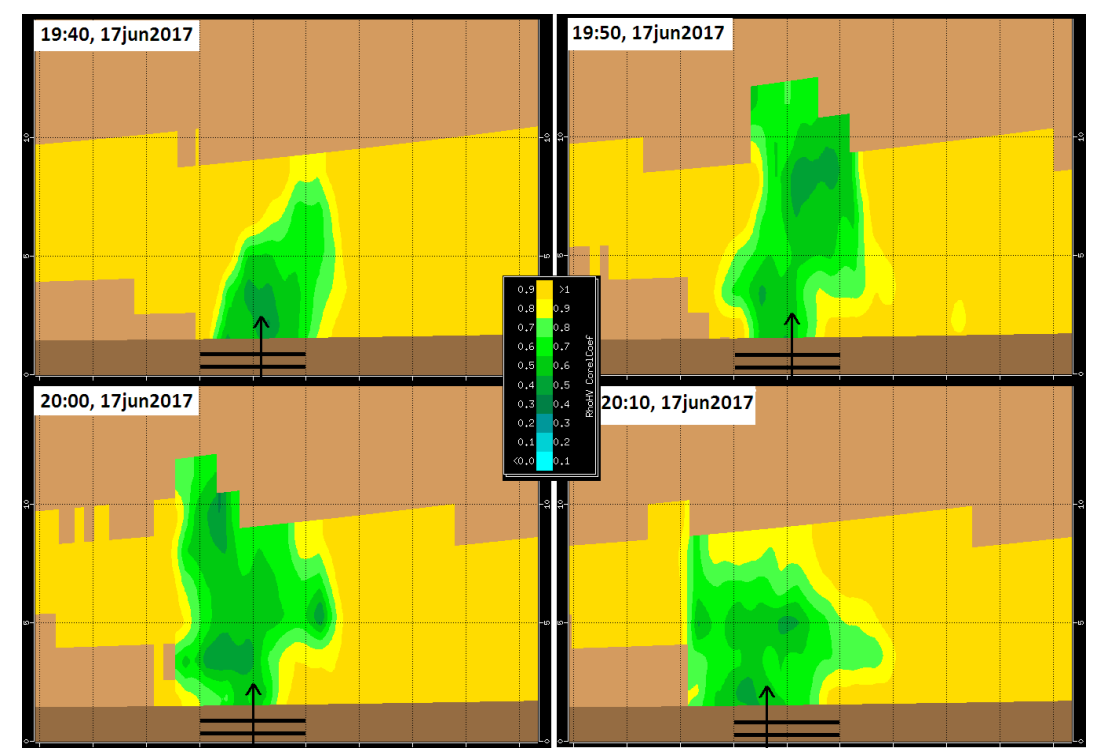

Figure 10 - Vertical cuts over the correlation coefficient field ( 0 to1), Porto radar, June 17 ${ }^{\text {th }}$ 2017 (19:40h-20:10h). Section is along the segment marked in Figure 1, observer looking from the south (double lines mark the transect itself). Black arrow represents the plume location at $1100 \mathrm{~m}$ altitude. Greenish colors represent $\rho_{h v}<0.8$ (dry smoke threshold). Vertica/horizontal scales, $5 \mathrm{~km}$ discretization.

The atmospheric volumes upwind of the fire area were inspected using radar observations, starting at a considerable time before the observed fire behavior modification. These observations corresponded to the calibrated horizontal shear product (see 4.2). Using this product, no coherent shear lines were identified on radar between $18: 10 \mathrm{~h}$ and 18:50h. However, by 18:56h, a shear line was detected, to the southeast of the fire (Figure 11a; the fire area for reference is the one of the low level fire plume area observed on radar by 19:16h). By 19:06h and 19:16h this line and another one, to the northwest of it, could be identified, while propagating towards the fire area (Figures 11b, c). By 19:26h the first detected shear line became more visible (Figure 11d) and by 19:36h could be clearly identified over a larger area (Figure 11e). More shear lines were detected to the southeast of the fire area, especially by 19:36h, although less defined (not marked).

All these shear lines resemble the ones previously mentioned (section 4.2) and propagated to the west/northwest, in a direction coherent with a divergent outflow, or outflows, emanated from a 
downburst, or downbursts, that were generated on the multicell system that was propagating from the southeast, towards the fire area. It is considered that these lines were directly associated with gust front surges that interacted with the fire.

While these shear lines were propagating at low levels, the fire was evolving on the terrain. As revealed by a detailed study (Viegas et al., 2017, in portuguese) it was verified that by 19:30h and slightly before, the two fires (Escalos and Regadas) were still separated, but with the corresponding fronts very closely organized. Two separate fire columns existed at that time, according to available photos (Viegas et al., 2017, in portuguese). According to the same study, the fires merged shortly after 19:30h and an EFB has started.

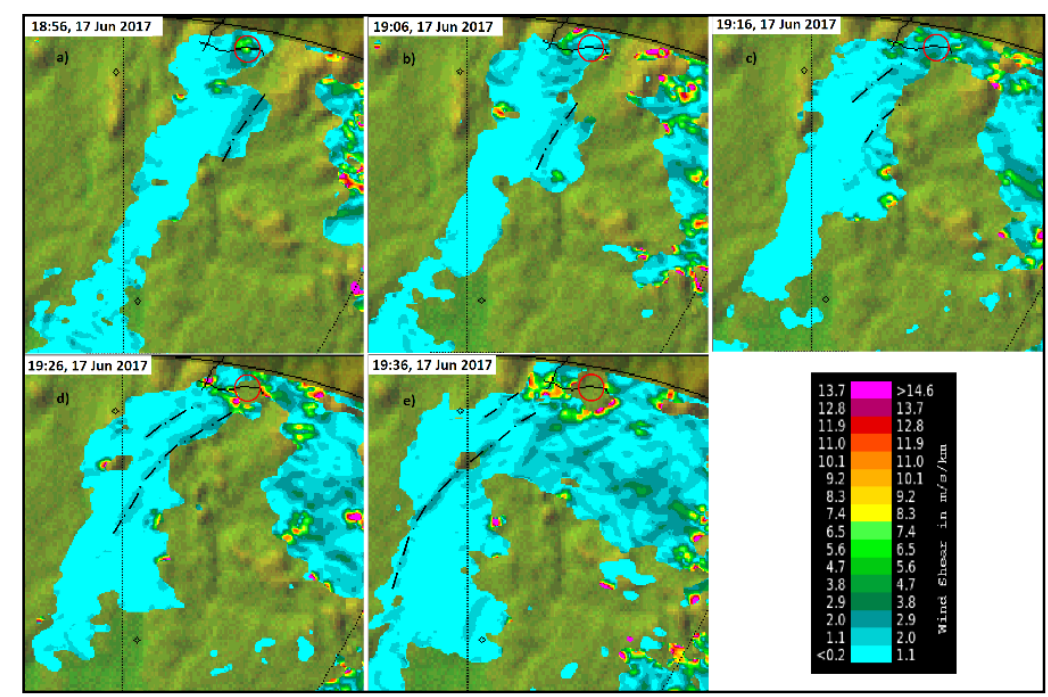

Figure 11 - Plane position indicator, $0.1^{\circ}$ elevation, 18:56h-19:36h, local time, $17^{\text {th }}$ June 2017, Lisboa radar, for horizontal shear (radial and azimuthal, $\mathrm{m} / \mathrm{s} / \mathrm{km}$ ). Shear line signatures are marked with black dot dash lines. Fire plume area (low level) by 19:16h reference, is marked with a red circle. Radar observations over the fire area are performed at $900 \mathrm{~m}$ altitude (radar site to the bottom of the images).

The pyroconvective plume position, at $900 \mathrm{~m}$ altitude, was identified by its radar reflectivity maximum values (section 5.). Since these are positively correlated with the corresponding fire intensity, the time evolution of those maximum was followed, between 17:00h and 19:50h. Figure 12 shows that there were several fluctuations during the considered period. But the fact is that from 17:00h to 19:06h the fluctuations were not very impressive. They were consistent with the already mentioned fire intensifications, confirmed by ground survey and video camera recordings, during the same period (Viegas et al., 2017, in portuguese). However, between 19:06h and 19:16h, it was observed a huge increase in the maximum reflectivity values, sustained afterwards and consistent with the onset of an EFB. In fact, taking into account that the average value of the plume maximum reflectivity increased from $21 \mathrm{dBZ}$, before the interaction, to $34 \mathrm{dBZ}$, after it (Figure 12) and that this unit is logarithmic, this increase rises to several orders of magnitude if linear units are considered. This 10 minute period was the first in which two shear lines (representing gust front surges) were detected very close to the fire area (Figure 11c). This interaction, by 19:16h, was in place 15 minutes before the reported fires merging and was seen as a cause for the two fires intensification, a forcing element that drove an increase in their rate of spread and, ultimately, to their merging, slightly after 19:30h. Afterwards, an EFB occurred, and an enormous area was burned in a few minutes. 


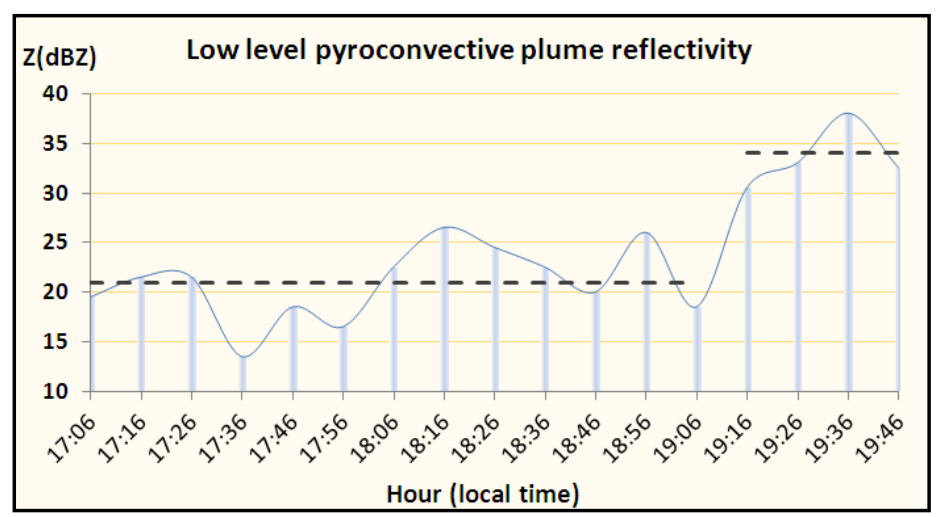

Figure 12 - Pyroconvective plume maximum reflectivity values (dBZ) at 900m altitude, 17:00h-19:50h, 17 June 2017, Lisboa radar observations. The horizontal dashed segments represent average values during 17:00h-19:10h $-21 \mathrm{dBZ}$ - and 19:15h-19:50h - $34 \mathrm{dBZ}$. The difference between 21dBZ and $34 \mathrm{dBZ}$, logarithmic units, are several orders of magnitude in original (not represented) linear units.

\section{Final remarks}

This study has treated a case in which a multicell convective system, propagating in a very hot and dry low troposphere environment, revealed to be a prolific downburst producer. This system propagated from the southeast, towards a forest fire, that was in place over central mainland Portugal. The fire initiated by 14:30h, local time, of $17^{\text {th }}$ June 2017. A second ignition produced another fire, a few minutes later, less than 3 kilometers far from the first ignition. The fire area was, initially, under a weak low level flow but, as the convective system approached, this flow intensified and several interactions with the fire were observed. No significant interactions occurred before 19:10h according to radar analysis. However, from 18:50h onwards, several shear lines were detected at low levels, propagating upwind of the fire and towards it (Figure 13). These lines were coherent with divergent outflows emanated from a downburst, or downbursts, generated on the convective system. This study showed that the lines corresponded to gust front surges that started to strongly interact with the fire, about 15 minutes before the observed fire behavior modification, so around 19:15h. These first gust front surges were considered as an important factor for the fires intensification and, in the end, for their merging, after $19 \mathrm{~h} 30$.

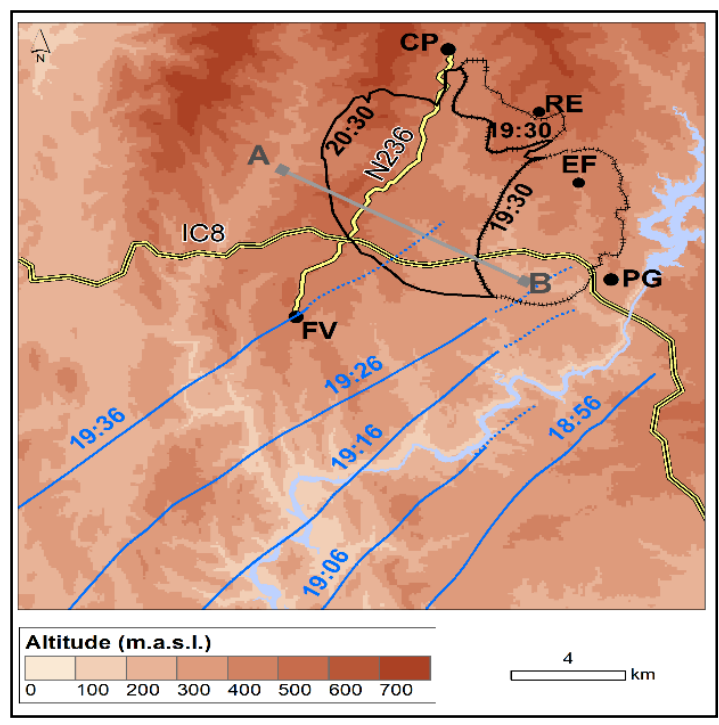

Figure 13 - Pedrogão Grande Fire area (zoomed in, Figure 1). Terrain, important locations (CPCastanheira de Pera, RE - Regadas, EF-Escalos Fundeiros, PG-Pedrogão Grande and FV-Figueiró dos Vinhos) and roads, relevant fire ignitions ( $\mathrm{EF}$ and $R E)$; radar transect $(A-B)$ referred to in the text. Fire fronts position estimated by 19:30h and 20:30h (adapted from Viegas et al, 2017) marked in black contour. Shear line, as detected on radar, time stamped in blue color. 
As the fires merged, an EFB started and a large area was quickly burned. This EFB was conceptually explained on another study (Viegas et al., 2017, in portuguese). The topography of the area in between the fire front position estimated at 19:30h, and the N236 road, shows two canyons, clearly visible (Figure 13). The relative orientation of the gust fronts in respect to these canyons, suggests a small angle between the predominant gusty winds of the outflow (from the southeast, roughly at right angle to the gust fronts) and the canyons orientation. This may have contributed to locally intensify the flow and, thus, foster the interaction with the fire.

The complex interaction phenomena between the flow and the fire, was not limited to this first fire blowup. By 20:00h-20:10h a sudden decrease of the pyroconvective plume top was noticed on radar. This decrease, resembling a downburst of the smoke plume itself, is still of undetermined origin. According to weather radar data analysis, this phenomenon produced another activation of the fire and, as a consequence, a $13000 \mathrm{~m}$ altitude convective plume was produced in a very short time period. This convective plume collapse and consequent pyroconvection activation will be object of another study.

\section{References and bibliography}

Balakrishnan, N., and Zrnic, D., 1990: Use of Polarization to Characterize Precipitation and Discriminate Large Hail, Journal of the Atmospheric Sciences, Volume 47, No. 13, 1525-1540.

IPMA, 2017: Boletim Climatológico junho 2017, Portugal Continental, ISSN 2183-1076 (in portuguese).

Klingle, D., and Smith, D., 1987: Gust Front Characteristics as Detected by Doppler Radar, Monthly Weather Review, Volume 115, 905-918.

Lang, T., B. Dolan, P. Krehbiel, W. Rison, and D. T. Lindsey, 2014: Lightning in wildfire smoke plumes observed in Colorado during summer 2012. Monthly Weather Review, 142, 489-507.

LaRoche, K., and Lang, T., 2017: Observations of Ash, Ice, and Lightning within Pyrocumulus Clouds Using Polarimetric NEXRAD Radars and the National Lightning Detection Network, Monthly Weather Review, Volume 145, 4899-4910.

Viegas, D. X., M. F. Almeida, L. M. Ribeiro, J. Raposo, M. Viegas, R. Oliveira, D. Alves, C. Pinto, H. Jorge, A. Rodrigues, D. Lucas, S. Lopes and L. F. Silva, 2017: O complexo de incêndios de Pedrogão Grande e Concelhos Limítrofes, iniciado a 17 de Junho de 2017. ADAI/LAETA, Dep. Eng. Mecânica, F. C. T., Universidade de Coimbra (in portuguese).

Viegas, D. X., and Simeoni, A., 2010: Eruptive behaviour of forest fires. Fire Technology. 47(2): 303.

Weisman, M. L., and Klemp, J. B., 1986: Characteristics of isolated convective storms. Mesoscale Meteorology and Forecasting, P. S. Ray, Ed., Amer. Meteor. Soc., 331-358.

Werth, P, et al., 2016: Synthesis of Knowledge of Extreme Fire Behavior, Volume 2 for Fire Behavior Specialists, Researchers, and Meteorologists, USDA, 260 pp. 


\title{
Biomass burning emissions using the Sentinel 2 Small Fire Database
}

\author{
Rubén Ramo ${ }^{1 *}$; Ekhi Roteta2 ${ }^{2}$ Ioannis Bistinas ${ }^{3}$; Aitor Bastarrika²; Emilio \\ Chuvieco ${ }^{1}$; Guido van der Werf ${ }^{3}$ \\ ${ }^{1}$ Enviromental Remote Sensing Research Group, Department of Geology, Geography and the \\ Environment, University of Alcalá. Colegios 2, 28801 Alcalá de Henares, Spain. \\ \{ruben.ramo@uah.es*,emilio.chuvieco@uah.es\} \\ ${ }^{2}$ Department of Mining and Metallurgical Engineering and Materials Science, School of \\ Engineering of Vitoria-Gasteiz. University of the Basque Country UPV/EHU, Nieves Cano 12, 7 \\ 01006 Vitoria-Gasteiz, Spain. \{ekhi.roteta@gmail.com, aitor.bastarrika@ehu.es\} \\ ${ }^{3}$ VU University, Faculty of Sciences, Department of Earth Sciences, $1081 \mathrm{HV}$, Amsterdam, \\ Netherlands.\{i.bistinas@vu.nl,guido.vander.werf@vu.nl\}
}

\begin{abstract}
Burned area (BA) estimates based on moderate resolution (500 meters) MODIS instruments show that globally, 3,500,000 hectares burns each year. MODIS has difficulty capturing fires smaller than 25 ha and studies estimate this lost variability up to $24 \%$. These estimates are highly uncertain.

We use burnt area from the Sentinel 2 Small Fire Database (SFD) at 20m spatial resolution for six tiles $\left(5 \times 5^{\circ}\right)$ in northern hemisphere Africa in areas that burn frequently. We find that in large fires $(>100 \mathrm{ha})$, MODIS (MCD64) and SFD show a reasonable agreement (92\%). However, MODIS misses most small fires ( $<25 \mathrm{ha}$ ) that add an additional $37 \%$ area burnt. SFD reveals a total burnt area that is $50 \%$ higher than MODIS for the studied areas.

We then calculate fire emissions from the SFD and we compare to the Global Fire Emissions Database GFED4 and GFED4s. The contribution of small fires in terms of total burned area and emissions has been significantly underestimated and we find emissions up to 56\% higher than GFED4 and 37\% higher than GFED4s for this regions. This analysis highlights the importance of accounting for small fires for both burned area and fire emissions.
\end{abstract}

Keywords: Sentinel 2, MODIS, small fires, burned area, emissions

\section{Introduction}

Fire is one of the most important factors in the ecosystem disturbance. It affects to the landscape, vegetation structure, biodiversity and also to humans (Kloster et al. 2012; Mouillot et al. 2014). As a result of the combustion, greenhouse gasses are emitted to the atmosphere being an important part of the carbon cycle making alterations to the atmospheric composition and hence affecting to the climate (van der Werf et al. 2010).

Because of these reasons fire disturbance is considered one of the Essential Climate Variables (ECVs) by the Global Climate Observing System (GCOS) (Bojinski et al. 2014). In this context, there are multiple agencies, projects and studies that focus on the cartography of burned areas in order to assess the impact of fires and to provide reliable information to the climate modelling community based on remote sensing data. One of them is the Fire_cci project, carried out by the European Spatial Agency in the frame of the Climate Change Initiative (CCI) program (Hollmann et al. 2013). The Fire_cci main objective is to elaborate global burned area maps in long time series.

There are a wide variety of sensors and methods to estimate the burned area. The traditional approach to provide global burned area mapping is by the use of moderate resolution images such as 
Advance very high resolution radiometer (AVHRR) (Riaño et al. 2007), VEGETATION (Tansey et al. 2008), Moderate Resolution Imaging Spectrometer (MERIS) (Alonso-Canas and Chuvieco 2015) or Moderate Resolution Image Spectroradiometer (MODIS) (Chuvieco et al. 2018; Giglio et al. 2006; Roy et al. 2008). The products developed by these sensors have been widely used to make emissions estimations (Chuvieco et al. 2016; Giglio et al. 2013; Van Der Werf et al. 2017), analyze the human impact on fire (Hantson et al. 2015) or to study the fire regimes (Archibald et al. 2010).

One of the drawbacks of these products is the high omission mainly related to the spatial resolution of these products $(1 \mathrm{~km}$ to $300 \mathrm{~m}$ ). The influence of the small fires have been studied (Randerson et al. 2012) in terms of the total burned and carbon emitted, concluding that it represents a significant part of the total burned, but there is uncertainty around these estimations. Nowadays, thanks to the advances in computing science and the new generation of high-resolution satellite images such as Landsat or Sentinel 2 (10-30 meters resolution) is possible to provide better estimations of fire impact.

There are several studies that perform burned area based on Landsat (Bastarrika et al. 2014) or Sentinel (Fernández-Manso et al. 2016; Huang et al. 2016), but none of them have been applied to a continental scales or to a long time period. In this frame, the Fire_cci project has developed a new product named Small Fire Database (SFD) using Sentinel 2 images. The database extent covers the African continent in 2016 at 20 meter resolution and is planned to be released in the next months.

The objective of this paper is to analyze the potential of the SFD in six different $5 \times 5^{\circ}$ regions of the north of Africa using the first version of the database that only comprises the first nine months of the year. A comparison with the MCD64 product was performed in terms of total burned area, fire size distribution, and emissions performed by the Global Fire Emissions Dataset (GFED) (Van Der Werf et al. 2017).

\section{Methods}

\subsection{Burned area databases}

The MCD64 product provides the burned information extracted from MODIS reflectances from Terra and Aqua at 500 meter resolution (Giglio et al. 2009). This product is available from November of 2000 until now in monthly layers and encodes the day of burned together with the uncertainty for the burned pixels. In general terms, the product detects the burned area using a temporal composites of vegetation indices based on the shortwave infrared (SWIR) wavelength applied to a temporal series. The algorithm also uses the active fire information from MODIS to characterize the burned signal and to extend the segmentation to the whole image, making the thresholds able to regional adaptation and hence to multiple fire conditions.

One of the most widely used emission product is GFED (Van Der Werf et al. 2017) that is extensively used for climate, atmospheric and biochemical modelling (Aouizerats et al. 2015; Schwietzke et al. 2016). In this frame, the MCD64 product is one of his main source of information used to perform the emissions estimations from the forest fires. GFED use the CASA (CarnegieAmes-Stanford Approach) to derive the ecosystem productivity through the use of remote sensing data for finally produce a global estimation of carbon emissions and other trace gases. There are different versions of this database, the last versions are GFED4 and GFED4s. Both use MCD64 as burned area input, but GFED4s also include an estimation of the burned fraction caused by the small fires. This estimation comes from the influence of the active fires that have enough sensitivity to detect fires below of their original resolution.

The SFD uses Sentinel 2-MSI data at 20 meter resolution to estimate the burned area in Africa (Roteta et al. 2018). It has been applied in the north of Africa for the nine first months of 2016 in a first phase, but the processing chain has been extended for the entire year and continent. Here a selection of six different study areas (Figure 1) were selected to test the potential of the database. 


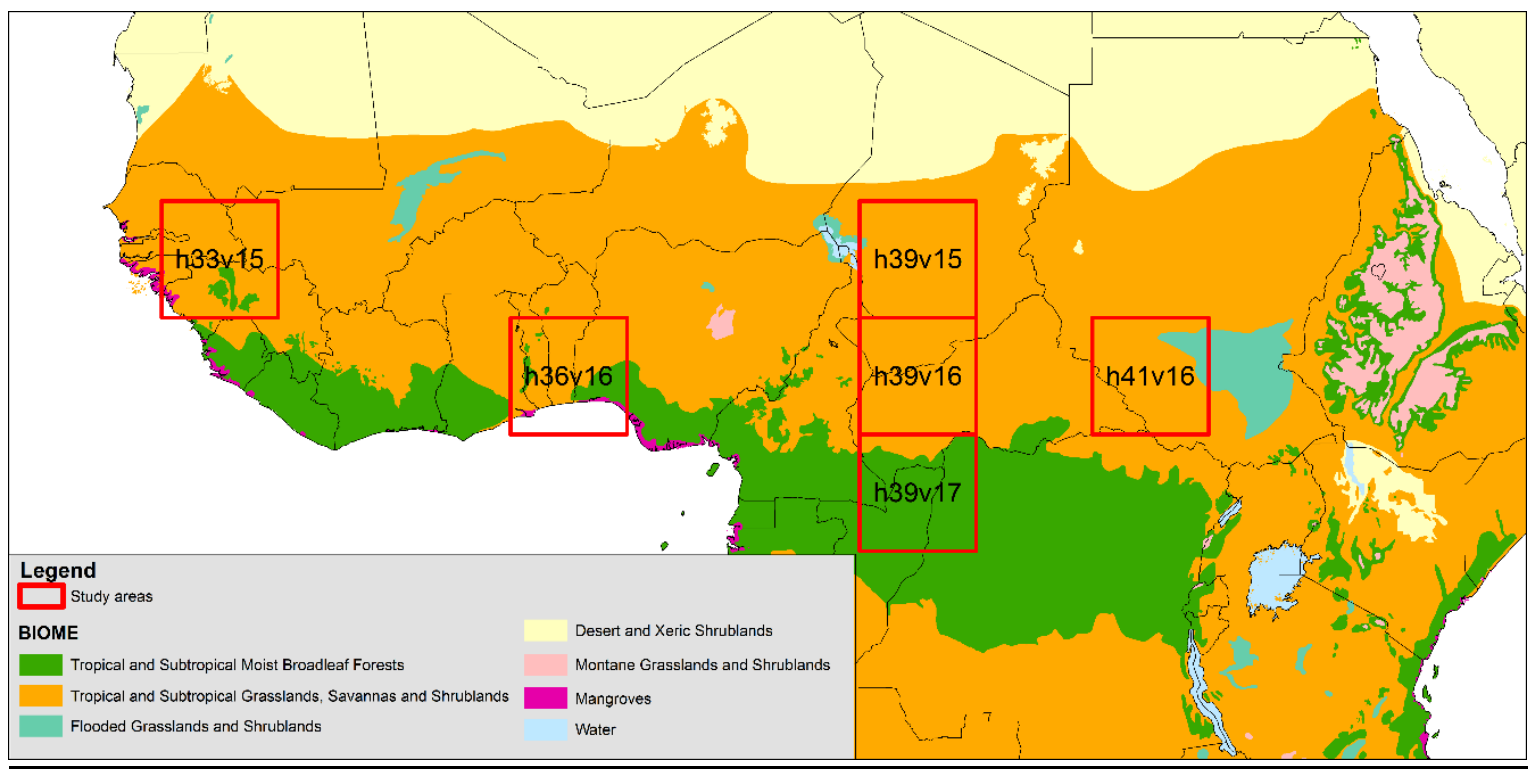

Figure 1 - Locations of the study area

The algorithm derives the burned area using a hybrid multitemporal approach classifying pairs of images from the available images. The structure of the algorithm has three different steps. First, a group of burned area indices were performed. After that an initial segmentation of the image using those indices to derive an early burned area. This result is crossed with the active fire observations in order to extract some high probability burned pixels or seeds. The seed is introduced in a probability distribution function of two different indices normalized burned ratio (NBR) and the mid-infrared burned index (MIRBI). Finally following the distribution function the pixels are classified using a certain threshold.

This version SFD have been validated following a systematic sampling scheme using 27 test areas. The perimeters were generated in a first step using a semiautomatic burned area mapping tool (Bastarrika et al. 2014) to finally perform a visual analysis to guarantee the quality of the data. The way to obtain the burned area from the test perimeters was de same used in the Fire_cci project (Padilla and Chuvieco 2014; Padilla et al. 2015) and follows the CEOS-CalVal protocol. The results obtained in the validation shows a commission error of $7.3 \%$ while omission is $18.4 \%$, further details are shown in Roteta et al (2018).

\subsection{Data processing}

To carry out the comparisons at pixel level the MCD64 collection 6 were used. MODIS products are developed under the observations of two different satellites (Terra and Aqua), thus the revisited period is significantly higher (twice per day) than the one used by the SFD (once per ten days). Because the temporal resolution, pixels that have a contiguous day of burned can be considered a single fire event and the changes in the day of burned are due to the fire spread (Archibald and Roy 2009). To separate individual fires for MODIS a flood algorithm was used to connect fires that have a spatial connectivity in a $3 \times 3$ window within a temporal frame of four days. Once this process is over, the next step is to individualize the fires labelling each one with a unique value performing at the same time the fire size. To do so pixels with a similar day of burned connected by $3 \times 3$ window are considered one single event. The fire size was divided in 7 different ranks: 0.04 ha, 0.04-1 ha, 1-5 ha, 5-25 ha, $25-$ 50 ha, 50-100 ha, >100 ha.

For the emissions estimation, the SFD was converted to $0.25^{\circ}$ summarizing the total amount of burned area for each grid cell. This information was used to replace the source of burned of the GFED model, which used to be MCD64 collection 5. The emission was performed for three databases SFD, GFED4, and GFED4s using the same parameters to make able the comparison. 


\section{Results}

The next figure shows the amount of burned area observed in the six test areas ordered by fire size.

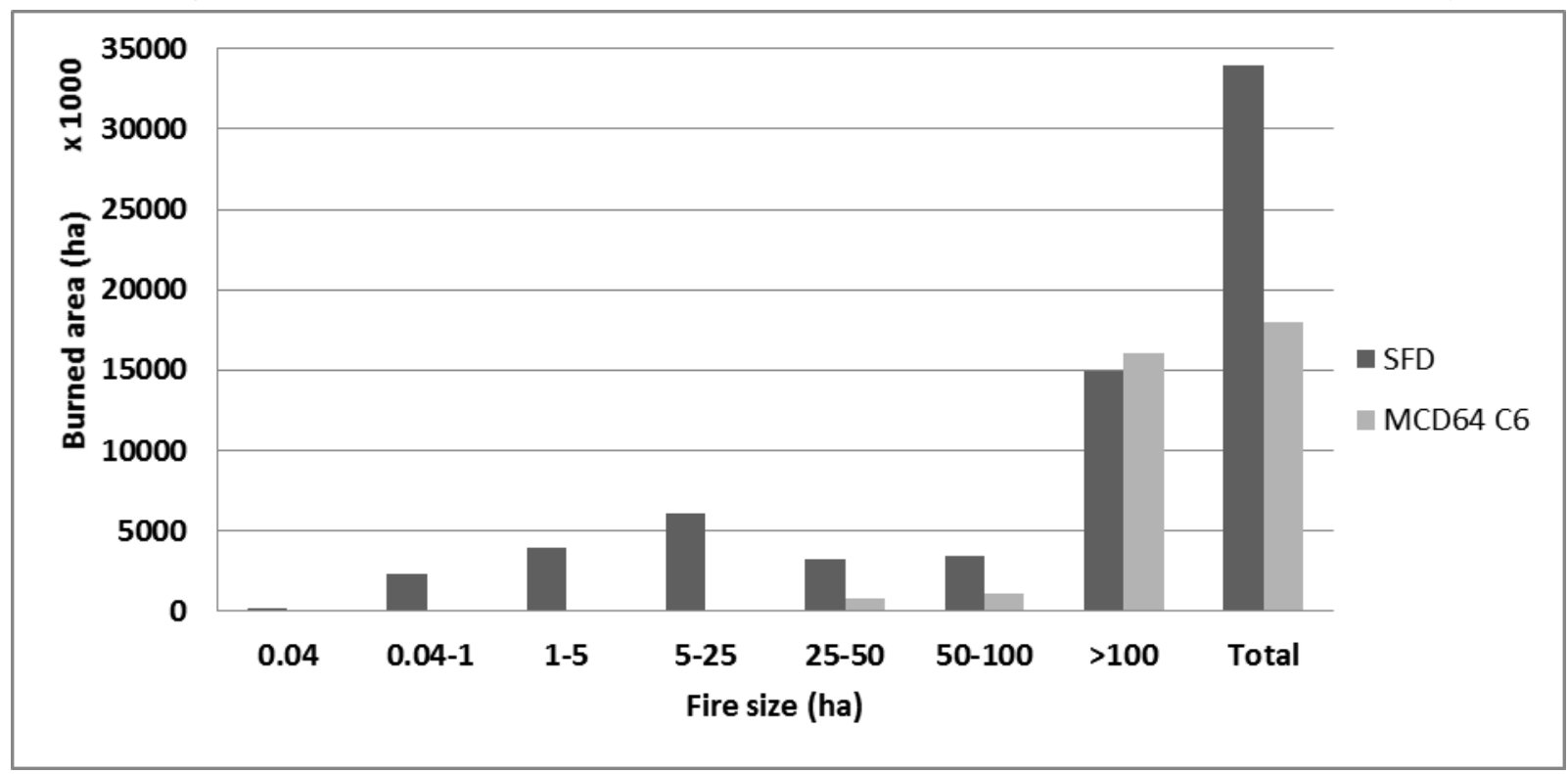

Figure 2 - Total burned by size for the six test areas.

The total burned observed for MCD64 is $47 \%$ lower than the estimations through SFD. The fires below the MODIS resolution represent the $37 \%$ of the total burned. SFD also detect more burned area between 25-100 ha, while for fires bigger than 100 ha MODIS detect slightly more burned area.

Figure 3 shows the fire counts for single fire events for both products. SFD shows a higher number of fires in all the range size including the $>100$ fire size. The number of fire counts shows an exponential trend where the maximum is reached with fires between $0.04-1$ ha.

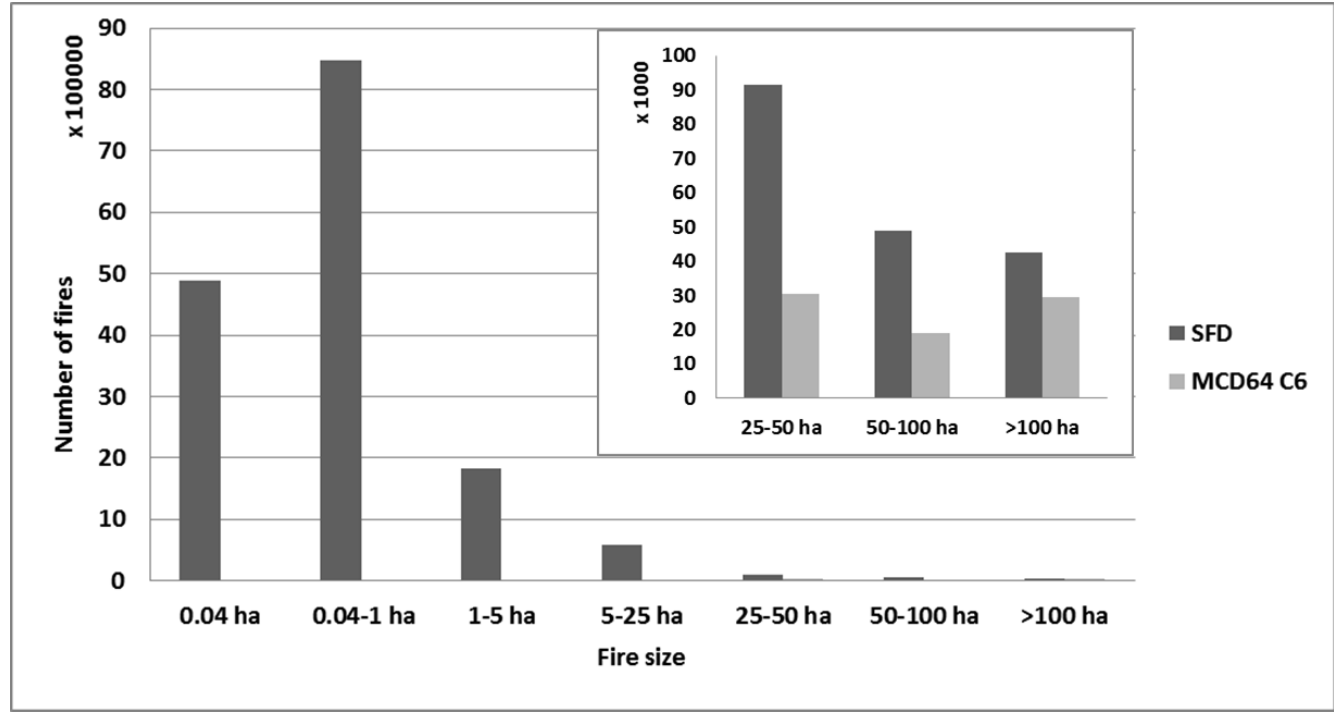

Figure 3 - Number of individual fires for MCD64 and SFD

The next figure shows the total burned observed for the three fire databases along the year. The observed trends are similar for all products but the amount of burned area not, being SFD the database that shows more followed by GFED4s and GFED4. 


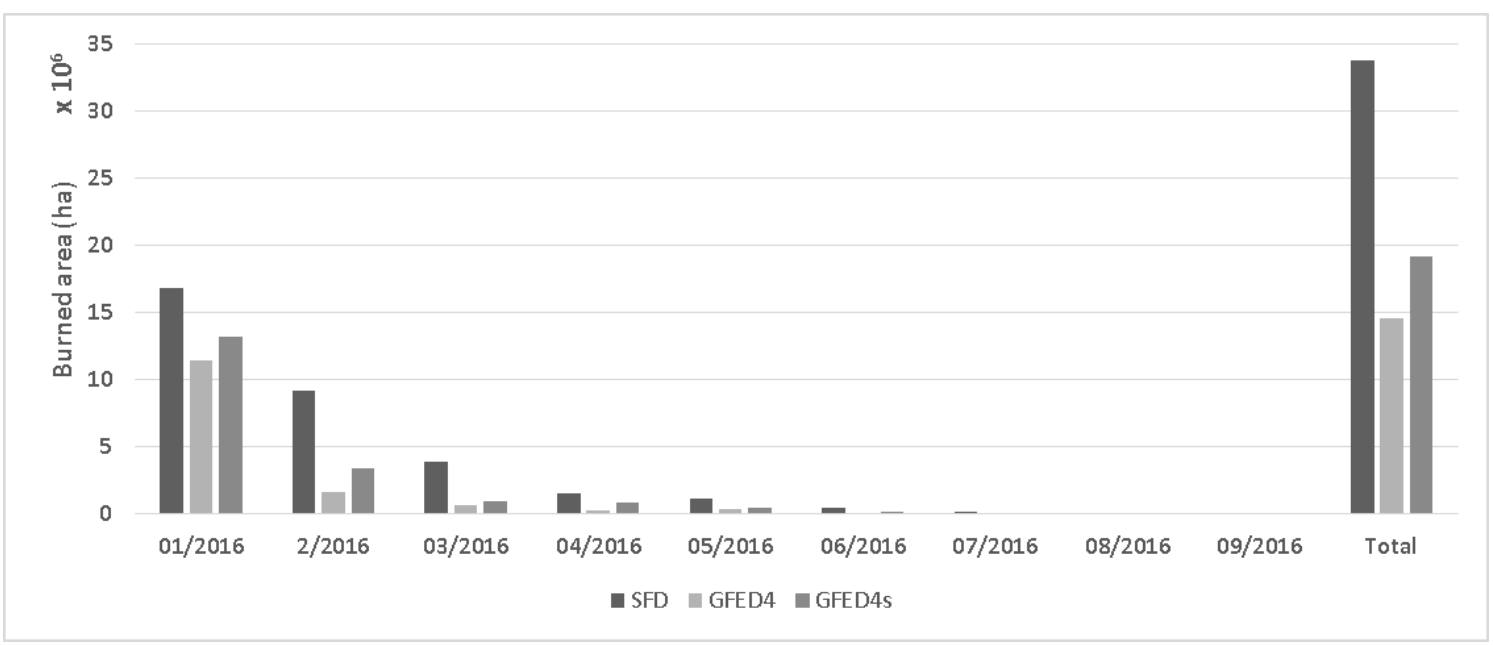

Figure 4 - Burned area distribution for SFD, GFED4 and GFED4s.

The total burned area founded by SFD is 57\% higher than for GFED4 and 43\% more than GFED4s. The higher differences are observed after the fire season in January, especially in February and March.

The emissions derived from these burned area databases are shown in figure 5. The observed trends are similar to those observed in burned area. The amount of carbon emitted by SFD is $56 \%$ higher than GFED4 and 37\% in comparison with GFED4s.

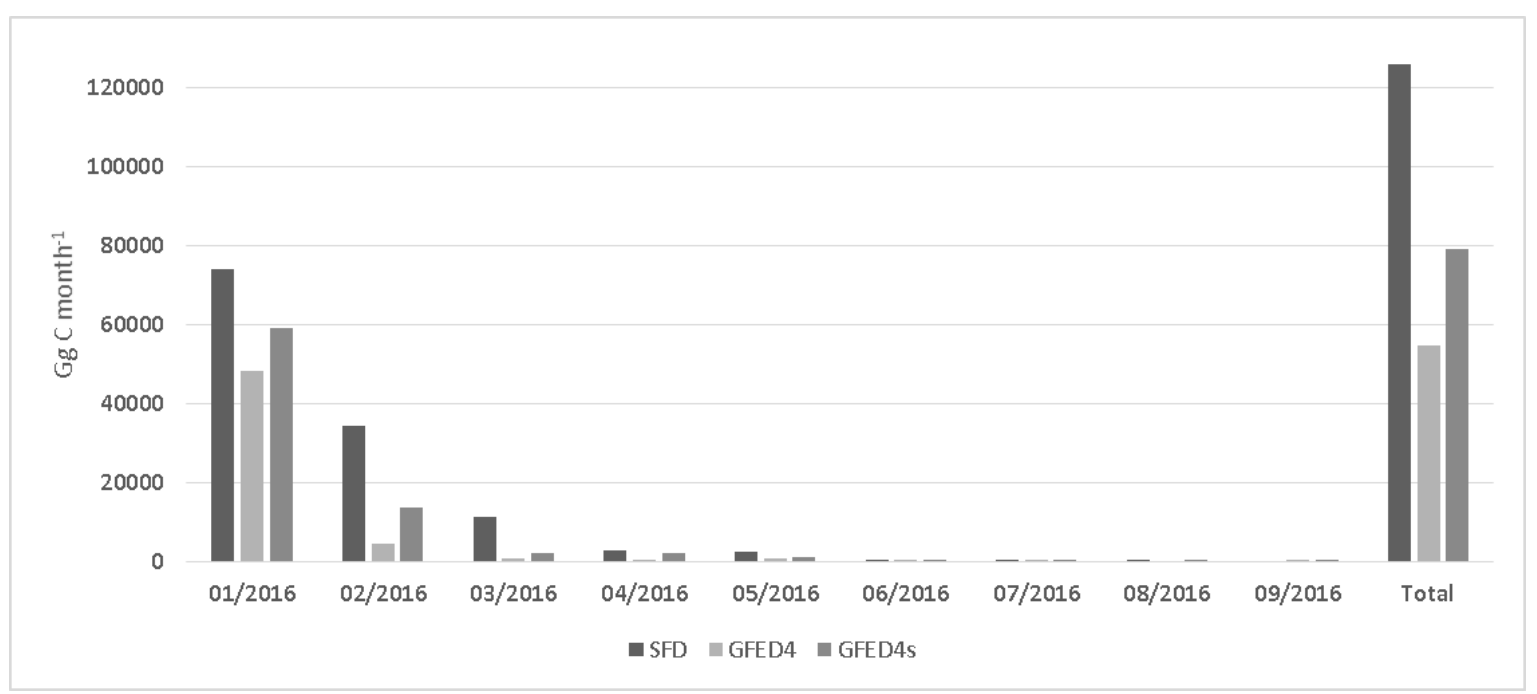

Figure 5 - Emissions for SFD, GFED4 and GFED4s

\section{Discussion and conclusions}

This study analyzes six different tiles of the new SFD produced by the Fire_cci project. We compared the SFD at pixel level with the standard product MCD64 collection 6 in terms of total burned, number of fires and fire size distribution. We derive emissions at $0.25^{\circ}$ grid using the GFED model and we show a comparison between SFD, GFED4 and GFED4s.

The results obtained in the comparison at pixel level reveal that there is a significant difference between the SFD and MCD64. Those differences represent almost the 50\% of the total burned. Between both products, there is an agreement solely in the big fires (>100 ha) with only 8\% difference. For the rest of the fire sizes, MODIS detects less burned area even above the minimum resolution that the sensor can detect (25-100 ha). The total burned area is $29 \%$ less than for the Sentinel product for 
these fire intervals. The underestimation of the MCD64 product is related in part to the influence of the small fires but also with the mixed signatures that comes from other surface reflectances.

In terms of number of fires the SFD detect more in all the observed intervals, even in the biggest fire range where MCD64 shows more burned area. This indicates that the SFD shows smaller and more fragmented fires than MODIS because of the resolution effect. The smallest fire categories $(0.04-$ $1 \mathrm{ha}$ ) represent less than the $10 \%$ of the total burned, but in number of fires are the $84 \%$ of the total fire events. In this context, small fires ( $<25 \mathrm{ha}$ ) are $98.8 \%$ of the total ignitions and group $37 \%$ of the total burned area with events of 0.04-1 ha being the most common fire size (53\% of the total).

The differences in amount of burned area between GFED4 and SFD is 57\% while for GFED4s is $43 \%$. For GFED4 the differences are higher than the observed at pixel level (50\%) because this model uses the data provided by the collection 5 instead of the sixth from the MCD64. Furthermore, when including small fires in GFED (GFED4s), the emissions are still lower than from the SFD. Those differences, located mainly between February and May, are related with the sensitivity of the active fire observations. GFED4s estimates the contribution of the small fires using the fraction of hotspots that are located out of the burned area from the MCD64. The basic principle is that the active fires observations are more sensitive to fires than their native resolution. That approximation is a result of a statistical analysis, while SFD is purely based on higher resolution observation. The differences in terms of emissions between the analyzed products are slightly lower than the one founded in burned area. Emissions are dependent on other factors like the biomass or land cover. In this case, the estimations provided by GFED4s are 6\% closer to the SFD than the burned area approximation.

Despite this study is limited in temporal and spatial extent, the results confirm the evidences of other researches (Randerson et al. 2012) that small fires have a significant impact in terms of burned area and emissions. To better characterize the role of the small fires and their influence on climate, further efforts to develop long time series of high resolution burned area maps are vital.

\section{References}

Alonso-Canas, I., \& Chuvieco, E. (2015). Global Burned Area Mapping from ENVISAT-MERIS and MODIS active fire data. Remote Sensing of Environment, 163, 140-152

Aouizerats, B., Van Der Werf, G.R., Balasubramanian, R., \& Betha, R. (2015). Importance of transboundary transport of biomass burning emissions to regional air quality in Southeast Asia during a high fire event. Atmospheric Chemistry and Physics, 15, 363-373

Archibald, S., Nickless, A., Govender, N., Scholes, R.J., \& Lehsten, V. (2010). Climate and the interannual variability of fire in southern Africa: a meta-analysis using long-term field data and satellitederived burnt area data. Global Ecology and Biogeography, 19, 794-809

Archibald, S., \& Roy, D.P. (2009). Identifying individual fires from satellite-derived burned area data. In, Geoscience and Remote Sensing Symposium,2009 IEEE International,IGARSS 2009 (pp. III160-III-163)

Bastarrika, A., Alvarado, M., Artano, K., Martinez, M.P., Mesanza, A., Torre, L., Ramo, R., \& Chuvieco, E. (2014). BAMS: A Tool for Supervised Burned Area Mapping Using Landsat Data. Remote Sensing, 6, 12360-12380

Bojinski, S., Verstraete, M., Peterson, T.C., Richter, C., Simmons, A., \& Zemp, M. (2014). The concept of essential climate variables in support of climate research, applications, and policy. Bulletin of the American Meteorological Society, 95, 1431-1443

Chuvieco, E., Lizundia-Loiola, J., Pettinari, M.L., Ramo, R., Padilla, M., Tansey, K., Mouillot, F., Laurent, P., Storm, T., Heil, A., \& Plummer, S. (2018). Generation and analysis of a new global burned area product based on MODIS $250 \mathrm{~m}$ reflectance bands and thermal anomalies. Earth System Science Data, 1-24 
Chuvieco, E., Yue, C., Heil, A., Mouillot, F., Alonso-Canas, I., Padilla, M., Pereira, J.M., Oom, D., \& Tansey, K. (2016). A new global burned area product for climate assessment of fire impacts. Global Ecology and Biogeography, 25, 619-629

Fernández-Manso, A., Fernández-Manso, O., \& Quintano, C. (2016). SENTINEL-2A red-edge spectral indices suitability for discriminating burn severity. International Journal of Applied Earth Observation and Geoinformation, 50, 170-175

Giglio, L., Loboda, T., Roy, D.P., Quayle, B., \& Justice, C.O. (2009). An active-fire based burned area mapping algorithm for the MODIS sensor. Remote Sensing of Environment, 113, 408-420

Giglio, L., Randerson, J.T., \& Werf, G.R. (2013). Analysis of daily, monthly, and annual burned area using the fourth-generation global fire emissions database (GFED4). Journal of Geophysical Research: Biogeosciences, 118, 317-328

Giglio, L., Van der Werf, G., Randerson, J., Collatz, G., \& Kasibhatla, P. (2006). Global estimation of burned area using MODIS active fire observations. Atmospheric Chemistry and Physics, 6, 957974

Hantson, S., Lasslop, G., Kloster, S., \& Chuvieco, E. (2015). Anthropogenic effects on global mean fire size. International Journal of Wildland Fire, 24, 589-596

Hollmann, R., Merchant, C., Saunders, R., Downy, C., Buchwitz, M., Cazenave, A., Chuvieco, E., Defourny, P., De Leeuw, G., \& Forsberg, R. (2013). The ESA climate change initiative: Satellite data records for essential climate variables. Bulletin of the American Meteorological Society, 94, $1541-1552$

Huang, H., Roy, D.P., Boschetti, L., Zhang, H.K., Yan, L., Kumar, S.S., Gomez-Dans, J., \& Li, J. (2016). Separability analysis of Sentinel-2A multi-spectral instrument (MSI) data for burned area discrimination. Remote Sensing, 8, 873

Kloster, S., Mahowald, N., Randerson, J., \& Lawrence, P. (2012). The impacts of climate, land use, and demography on fires during the 21st century simulated by CLM-CN. Biogeosciences, 9

Mouillot, F., Schultz, M.G., Yue, C., Cadule, P., Tansey, K., Ciais, P., \& Chuvieco, E. (2014). Ten years of global burned area products from spaceborne remote sensing - A review: Analysis of user needs and recommendations for future developments. International Journal of Applied Earth Observation and Geoinformation, 26, 64-79

Padilla, M., \& Chuvieco, E. (2014). ESA CCI ECV Fire Disturbance - Product Validation Report II Results from the Global Sample. In. http://www.esa-fire-cci.org/documents

Padilla, M., Stehman, S.V., Ramo, R., Corti, D., Hantson, S., Oliva, P., Alonso-Canas, I., Bradley, A.V., Tansey, K., \& Mota, B. (2015). Comparing the accuracies of remote sensing global burned area products using stratified random sampling and estimation. Remote Sensing of Environment, $160,114-121$

Randerson, J., Chen, Y., Werf, G., Rogers, B., \& Morton, D. (2012). Global burned area and biomass burning emissions from small fires. Journal of Geophysical Research: Biogeosciences, 117

Riaño, D., Moreno Ruiz, J., Isidoro, D., \& Ustin, S. (2007). Global spatial patterns and temporal trends of burned area between 1981 and 2000 using NOAA-NASA Pathfinder. Global Change Biology, $13,40-50$

Roteta, E., Bastarrika, A., Storm, T., \& Chuvieco, E. (2018). Development of a Sentinel-2 Burned Area algorithm: generation of a Small Fire Database for Northern Hemisphere Tropical Africa (Under review). Remote Sensing of Environment

Roy, D., Boschetti, L., Justice, C., \& Ju, J. (2008). The collection 5 MODIS burned area productGlobal evaluation by comparison with the MODIS active fire product. Remote Sensing of Environment, 112, 3690-3707 
Schwietzke, S., Sherwood, O.A., Bruhwiler, L.M., Miller, J.B., Etiope, G., Dlugokencky, E.J., Michel, S.E., Arling, V.A., Vaughn, B.H., \& White, J.W. (2016). Upward revision of global fossil fuel methane emissions based on isotope database. Nature, 538, 88

Tansey, K., Grégoire, J.M., Defourny, P., Leigh, R., Pekel, J.F., Van Bogaert, E., \& Bartholomé, E. (2008). A new, global, multi-annual (2000-2007) burnt area product at $1 \mathrm{~km}$ resolution. Geophysical Research Letters, 35

van der Werf, G.R., Randerson, J.T., Giglio, L., Collatz, G., Mu, M., Kasibhatla, P.S., Morton, D.C., DeFries, R., Jin, Y.v., \& van Leeuwen, T.T. (2010). Global fire emissions and the contribution of deforestation, savanna, forest, agricultural, and peat fires (1997-2009). Atmospheric Chemistry and Physics, 10, 11707-11735

Van Der Werf, G.R., Randerson, J.T., Giglio, L., Van Leeuwen, T.T., Chen, Y., Rogers, B.M., Mu, M., Van Marle, M.J., Morton, D.C., \& Collatz, G.J. (2017). Global fire emissions estimates during 1997-2016. Earth System Science Data, 9, 697 


\title{
CeaseFire: a website to assist fire managers in Portugal
}

\author{
Carlos C. DaCamara*1; Ricardo M. Trigo ${ }^{1}$; Miguel M. Pinto ${ }^{1}$; Sílvia A. Nunes ${ }^{1}$; Isabel F. Trigo ${ }^{2}$; \\ Célia M. Gouveia ${ }^{2}$; Manuel Rainha ${ }^{3}$ \\ ${ }^{1}$ Instituto Dom Luiz (IDL), Faculdade de Ciências, Universidade de Lisboa, Lisbon, 1749-016, \\ Portugal. \{cdcamara@fc.ul.pt*,rmtrigo@fc.ul.pt,mnpinto@fc.ul.pt,sanunes@fc.ul.pt\} \\ ${ }^{2}$ Instituto Português do Mar e da Atmosfera (IPMA), Lisbon, 1749-077, Portugal. \\ \{isabel.trigo@ipma.pt,celia.gouveia@ipma.pt\} \\ ${ }^{3}$ The Navigator Company, Cacia, 3800-536, Portugal. \\ \{manuel.rainha@thenavigatorcompany.com\}
}

\begin{abstract}
Climate and weather are major drivers of fire activity in Portugal. We present CeaseFire, a new website designed to provide the user community in Portugal with relevant real-time information on fire activity and meteorological fire danger.

CeaseFire aims at linking academia with practice within the fire community at large by providing easily accessible and simple to use products that are customized to specific user needs. Products supplied include the set of indices that integrate the Canadian Fire Weather Index (FWI) System, classes of fire danger, seasonal outlooks, hourly forecasts and information for prescribed burning. The number of registered users of the platform is currently up to 650 users that include forest managers, firemen and civil protection officers, personnel from municipalities, academic researchers and private owners. Received positive feedbacks and suggestions for other products reflect the success of the project.

Since its beginning, in March 2016, CeaseFire has been sponsored by The Navigator Company and has the institutional support of the Portuguese Authority for Forests (ICNF).
\end{abstract}

Keywords: fire danger, megafires, fire management, fire meteorology, web platform

\section{Introduction}

Severe wildfires in Mediterranean Europe have strong impacts and adverse effects at the social, economic, ecologic and environmental levels that include the destruction of property, the loss of human lives, the degradation of the landscape and the emission of greenhouse gases (Amraoui et al., 2015). The last fire season of 2017 is a tragic example with about 500 thousand hectares of burned area and 116 deaths in the fire events. Moreover, according to the different scenarios of future climate in the Mediterranean, there is a high probability of such extreme events to become more frequent (Flannigan et al., 2013; Mori and Johnson, 2013) embedded in a clear increment of the expected average burned area in all western Iberia (Sousa et al, 2015).

The Mediterranean region is responsible for up to 85\% of burned area in Europe (San-MiguelAyanaz et al., 2013) and Portugal, in particular, presents the highest score of fire occurrences in the Mediterranean Basin (Pereira et al., 2005). According to the official records of Instituto de Conservação da Natureza e das Florestas (ICNF), the Portuguese Authority for Forests, 4,416,981 hectares have burned in Continental Portugal during the period 1980-2017.

Like in Mediterranean Europe, fire activity in Portugal is a natural phenomenon linking climate, humans and vegetation (Lavorel et al., 2007, Costa et al., 2010). The rainy and mild winters that are followed by dry and warm summers tend to make the vegetation stressed and prone to the occurrence of severe fire events, their spread and ignition being enhanced by the high temperature, strong wind and low relative humidity during summer (DaCamara et al., 2014, Ruffault et al. 2016). The two 
most important factors for fire occurrence in Mediterranean Europe are of anthropogenic and environmental origins. The first one is the main cause of ignition, reaching 95\% of all cases (SanMiguel-Ayanz et al., 2013) whereas the most significant environmental factors are weather, fuel availability and topography (Costa et al., 2010).

There is therefore a growing demand for the operational availability of a suite of products related to fire danger and specifically tailored to the needs of the fire community that will allow decision makers taking better decisions and adopting more adequate measures to mitigate fire damage. We present CeaseFire, a new web platform designed to provide the user community in Portugal with relevant real-time information on fire activity and meteorological fire danger.

\section{The website}

The CeaseFire website (http://idlcc.fc.ul.pt/CeaseFire/index.php) was created in March 2016 with the aim of providing an area where researchers at Instituto Dom Luiz (IDL) could exchange knowledge, data and scientific work with other universities and specialized entities. Since its beginning, the platform has relied on information provided by EUMETSAT Satellite Application Facility on Land Surface Analysis (LSA SAF), based at the Portuguese Weather Service (IPMA), that operationally disseminates a suite of products related to fire activity and meteorological fire danger (Trigo et al., 2011).

\subsection{Structure}

As shown in Figure 1, CeaseFire is organized into four main areas: 1) User; 2) Danger; 3) Prescribed Fire; and 4) Data Download. Currently users have access to values of meteorological parameters and to indices of fire danger, to an interactive display of weather and fire danger maps, to an historical archive of data and maps and to information related to fire management activities such as prescribed burning in eucalyptus, pine and bush areas. Available information at the website covers mainland Portugal and ranges from five days prior to the current day up to the next 72 hours.

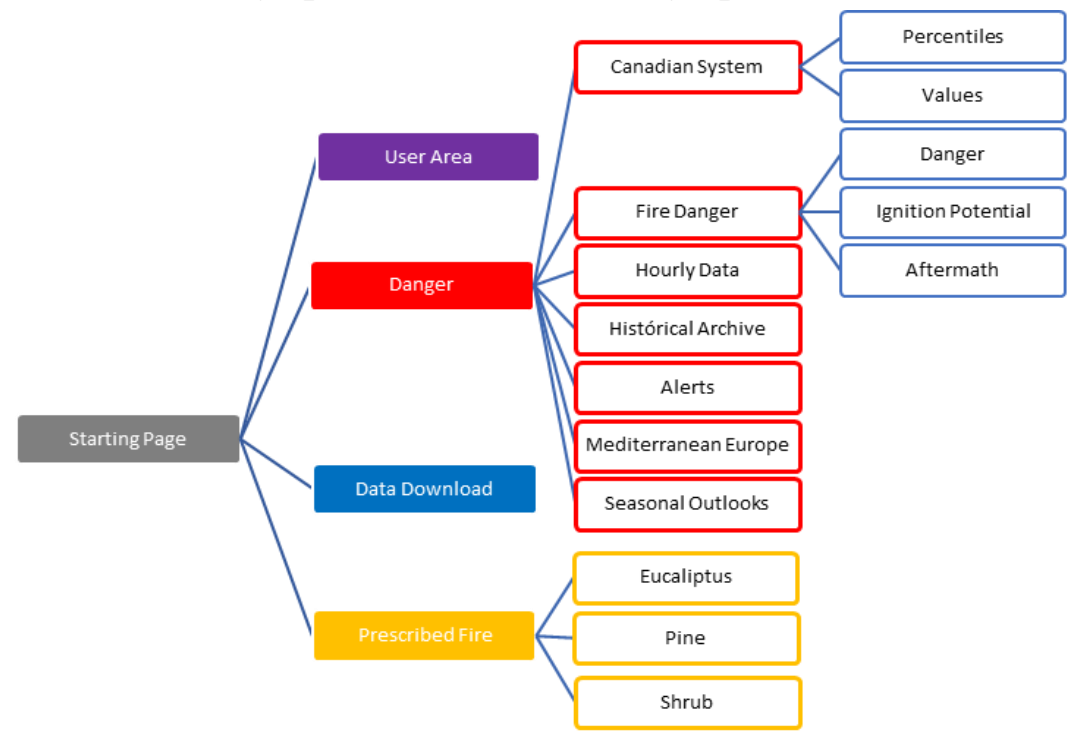

Figure 1 - Overall structure of the CeaseFire webpage

\subsubsection{User Area}

This area is devoted to the customization to specific needs of each user, from changing password for access to the website up to specifying types of pop-up alerts (e.g. an active fire close to his property). 


\subsubsection{Danger Area}

This area is the core of the CeaseFire platform and provides information about fire danger to assist the user on fire prevention and planning and on firefighting actions during a fire event. Information is available both as numeric data and as maps with customized information for different types of users. Active fires may be also displayed on maps in real time as identified by Meteosat, a geostationary satellite operated by EUMETSAT that provides information about location and energy released every 15 minutes (Trigo et al., 2011; Wooster et al., 2015) or as retrieved from the database of the Portuguese Civil Protection Authority (ANPC).

The Danger Area is organized into seven subareas:

\section{a) Canadian System}

This subarea provides data and interactive displays of maps of the six components of the Canadian Fire Weather Information (FWI) System (Van Wagner, 1974) as supplied by the LSA SAF based on forecasts provided by the European Centre for Medium-Range Weather Forecasts (ECMWF). As an option, contour lines of indices may be superimposed on a coloured background (Figure 2, left panel) providing information about the spatial distribution of percentiles of the records for the chosen day in the last 38 years (1979-2017). When a pixel is selected on the map, information about the chosen location is provided together with numeric information for all indices and a time series is displayed of the chosen index and respective percentiles for the past five days and the next two (Figure 2, right panel).
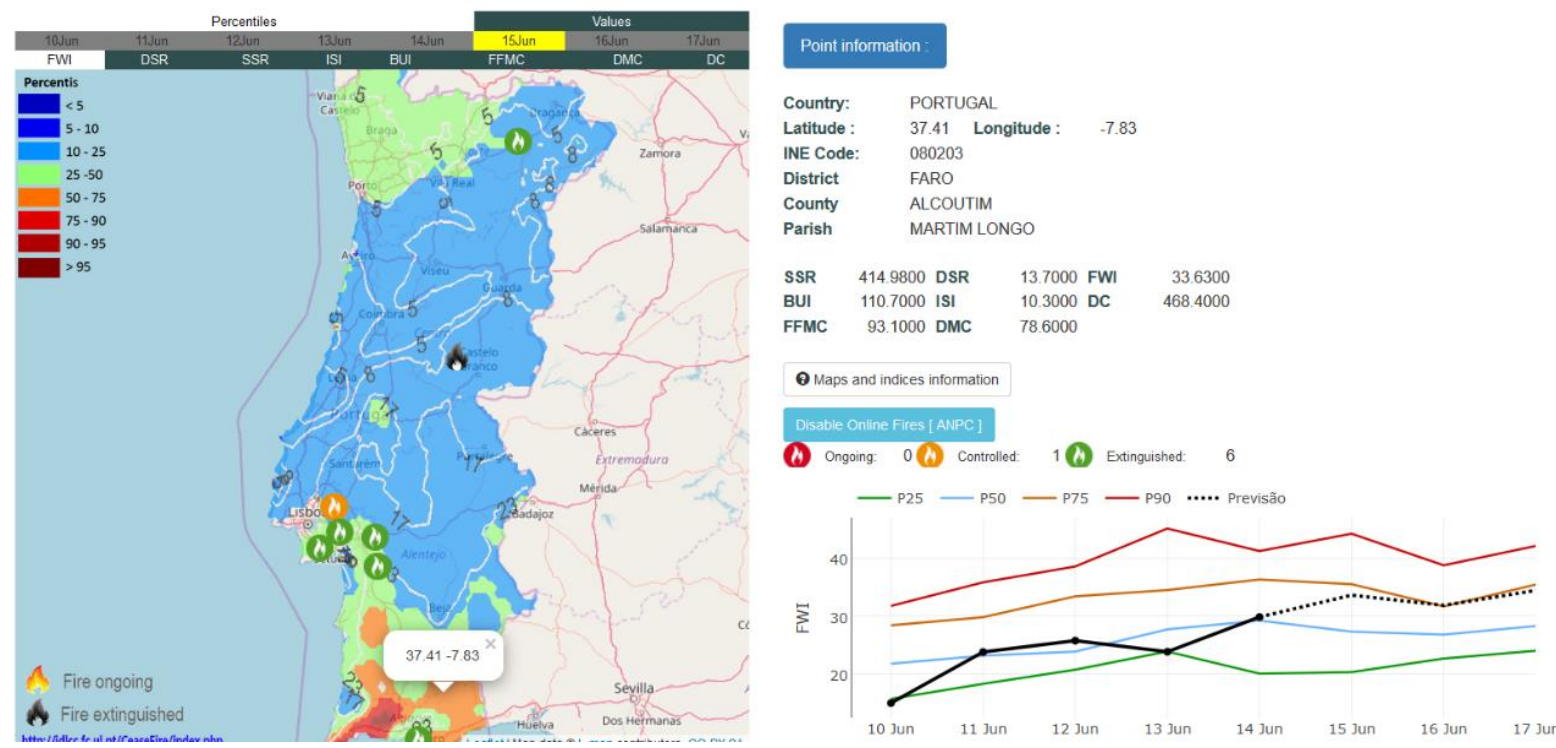

Figure 2 - FWI on 15 June 2018 over the climatological background (left panel) and information about fire danger at a chosen location (right panel).

\section{b) Fire Danger}

This subarea provides interactive displays of maps of classes of fire danger (Figure 3) as obtained from a statistical model that uses historical information about fire events together with current meteorological conditions (Pinto et al., 2018) as well as displays of maps of classes of potential of ignition and aftermath that were empirically derived from systematic analyses of past fire events (Fernandes et al., 2015). 

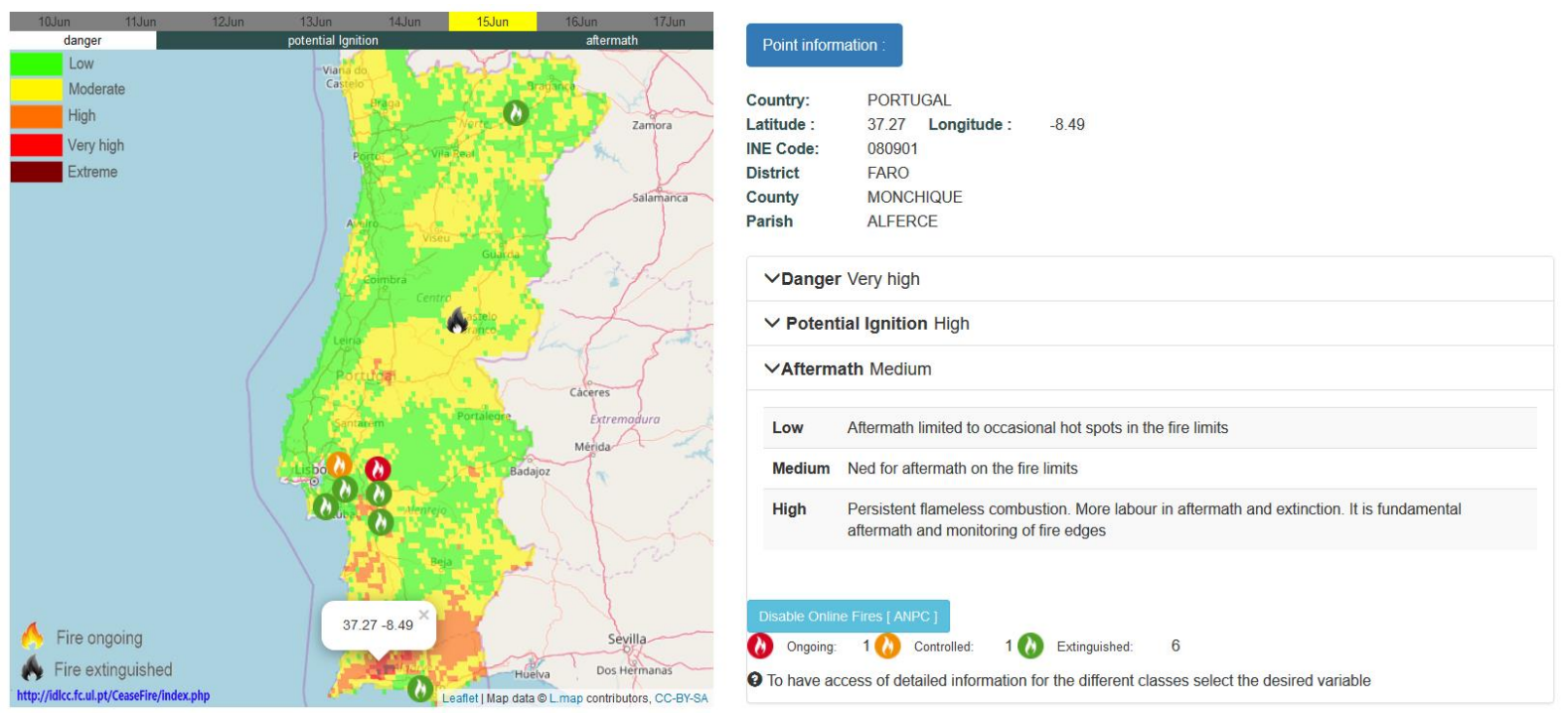

Figure 3 - As in Figure 2 but for classes of fire danger and active fires from satellite and ANPC (left panel) and information at a chosen location about fire danger, potential ignition and aftermath (right panel).

c) Hourly Data

This subarea provides animated displays of maps with hourly forecasts of meteorological parameters as obtained from the WRF mesoscale model (Skamarock et al., 2008) as well as forecasts of hourly FWI that resulted from a novel procedure that integrates hourly meteorological data with meteorological conditions at 12 UTC from the two previous days (Figure 4).
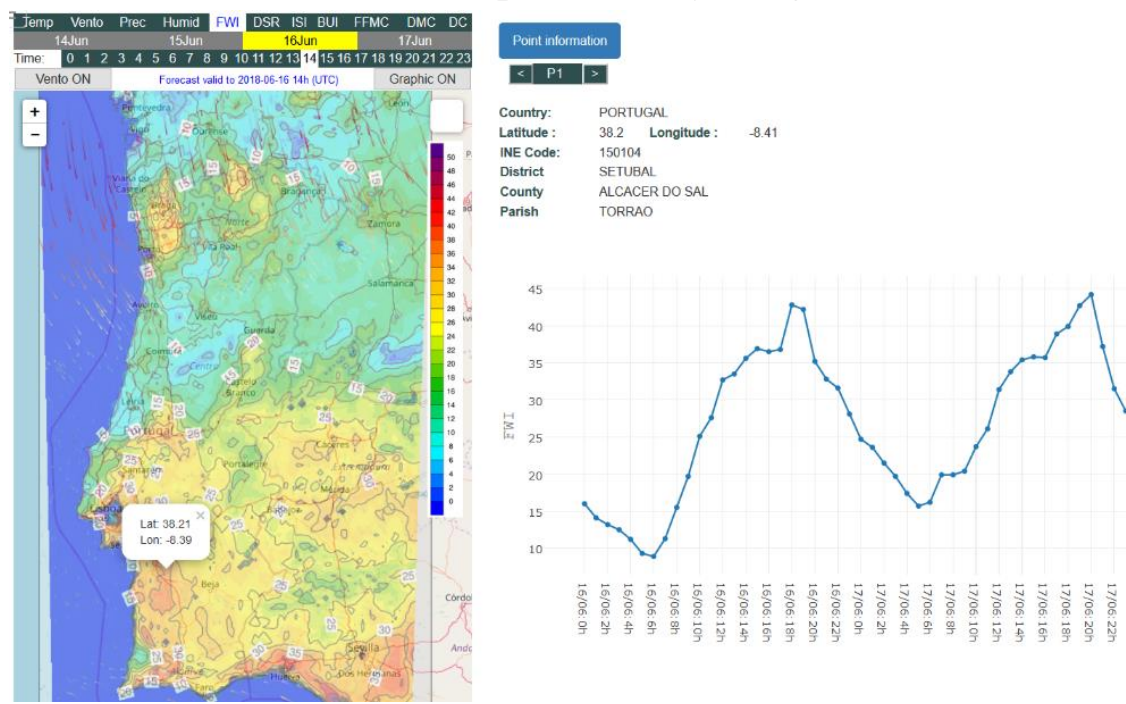

Figure 4 - Hourly FWI on 16 June 2018 (left panel) and daily cycles of hourly FWI at a chosen location (right panel).

\section{d) Alerts}

This subarea contains customized levels of alerts where the user can define the area of interest, the ranges of meteorological variables, indices and classes, and the number of levels and their meaning. Currently this area is restricted to the staff of The Navigator Company and information provided consist of maps with four degrees of alerts at the level of municipality and/or property. Besides the availability on the platform, this information is sent by e-mail on a daily basis to the supervisors in order to assist them in defining what kind of tasks are to be allowed in order to minimize the danger of originating an uncontrolled fire (Figure 5). 

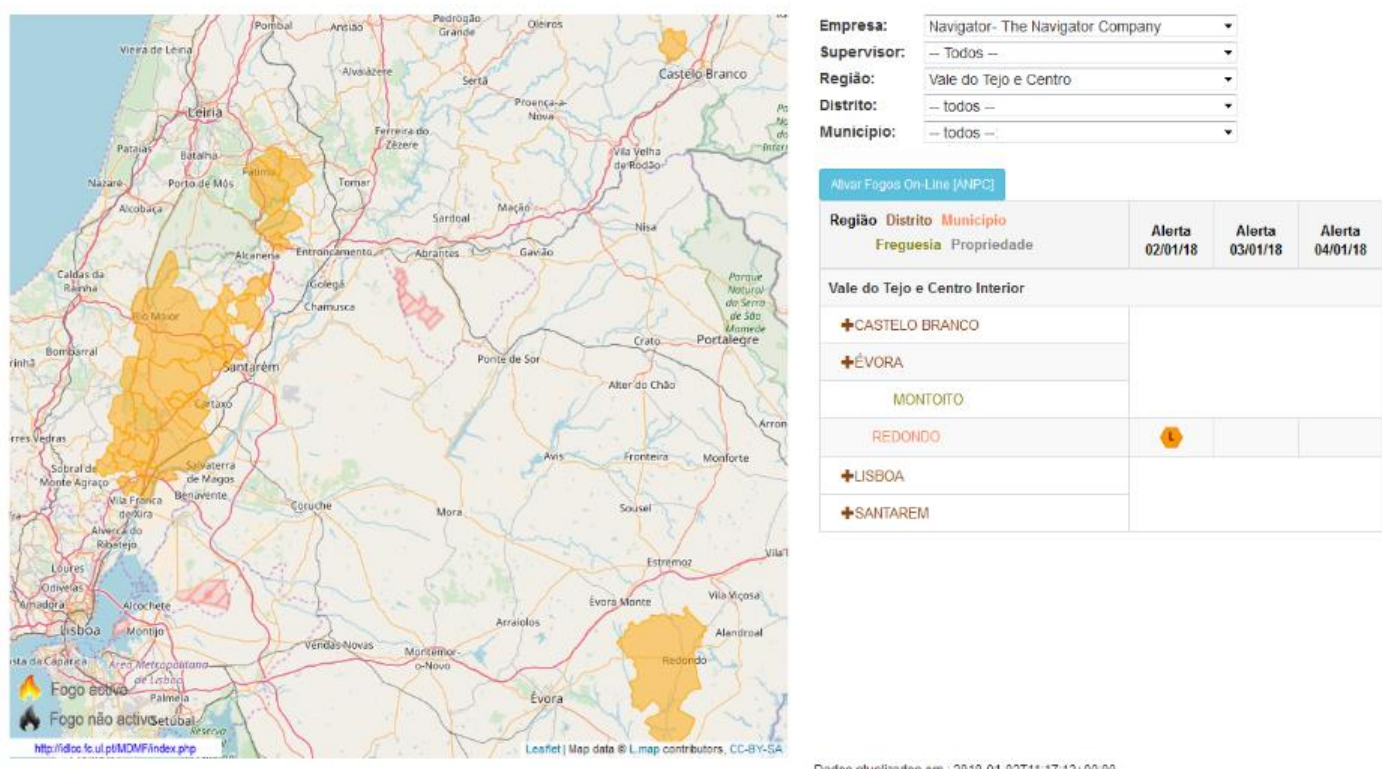

Figure 5 - Alerts at the level of the properties of The Navigator Company on 15 June 2018.

\section{e) Seasonal Outlooks}

This subarea provides graphical information about the likelihood of having a severe or weak fire season (in terms of expected burned area) for different regions of Portugal (Figure 6), as derived from a statistical model based on long-term indicators of fire activity (Nunes et al., 2014). Seasonal outlooks (for July-August) are computed starting on April 1 of each year and updated on a daily basis. For each region, the daily energy released by active fires as detected by satellite is also provided.

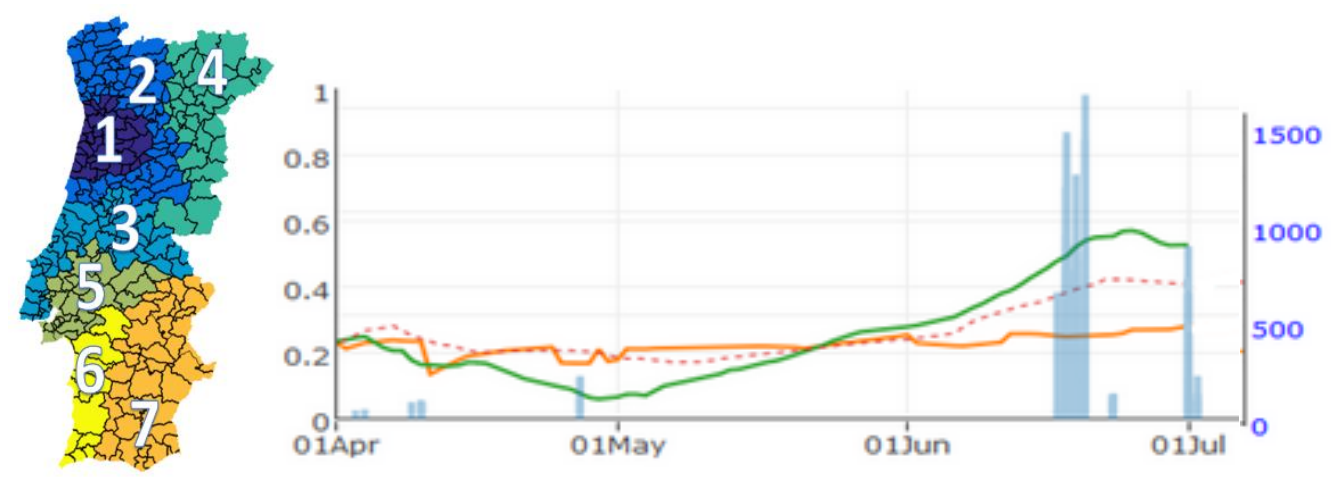

Figure 6 - Seasonal outlook for region 3 and for the year of 2017 (green line) and for the severe year of 2005 (red dotted line), reference threshold (orange line) and daily energy released in GJ by active fires detected from satellite (blue columns).

\section{f) Historical Archive}

The historical archive contains daily maps of all indices of the Canadian FWI System and of classes of fire danger since July 15, 2015, together with information of location and energy released by active fires detected from satellite. (Figure 7) 


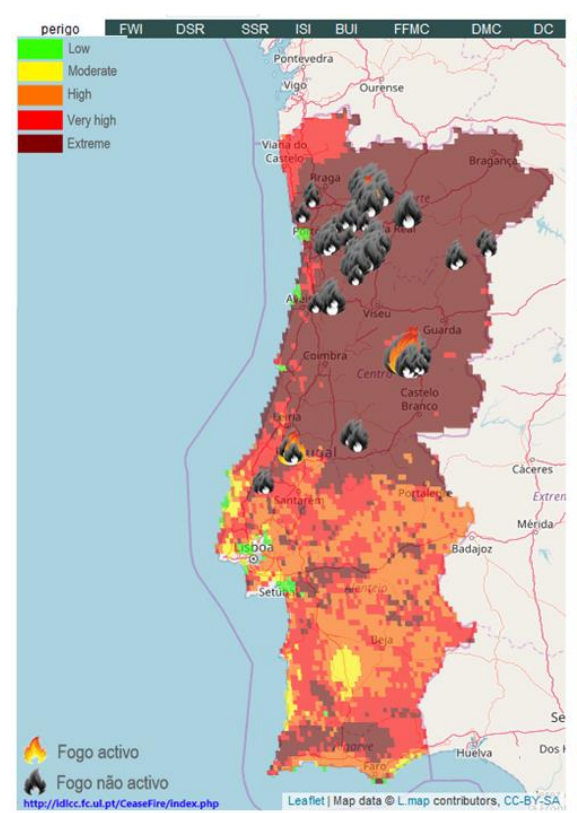

Figure 7 - Historical information about classes of fire
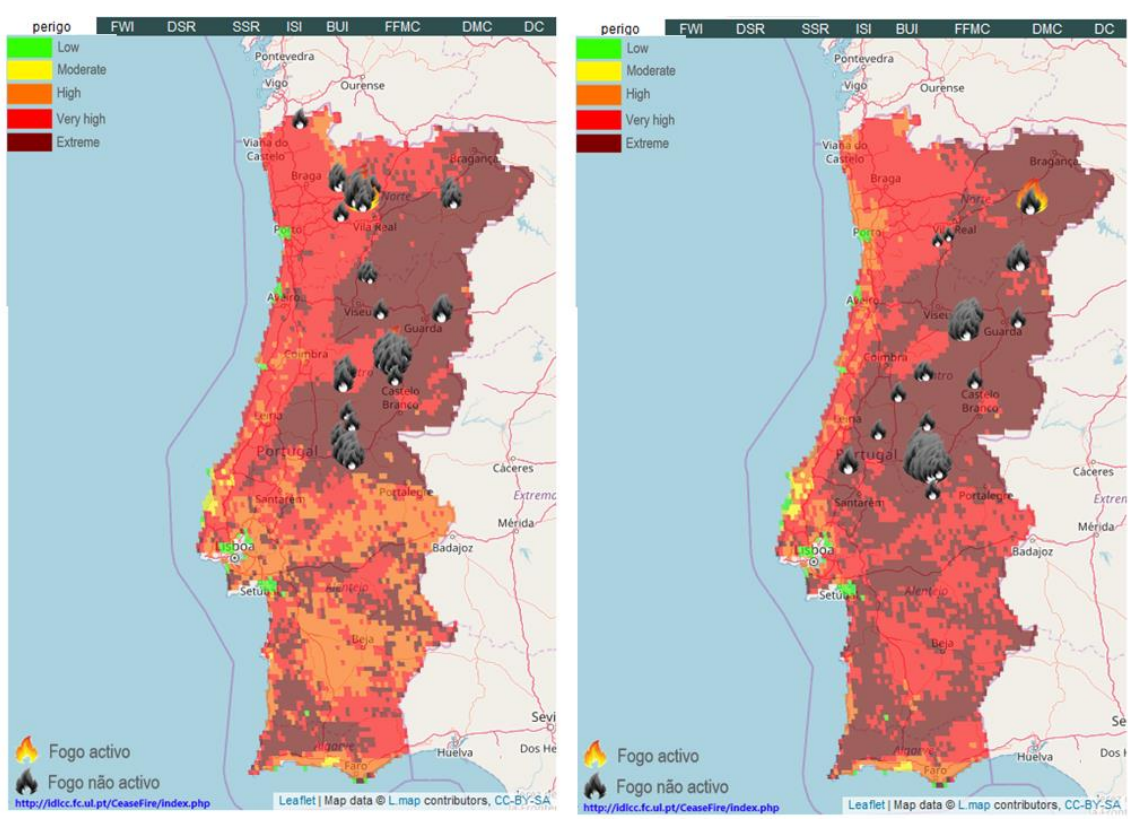
August 2017.

This subarea provides regional maps with forecasts of meteorological fire danger for the current day and the next two days over Mediterranean Europe as derived from the LSA SAF fire products (Figure 8). A similar product is also available at the LSA SAF website (http://lsa-saf.eumetsat.int/).

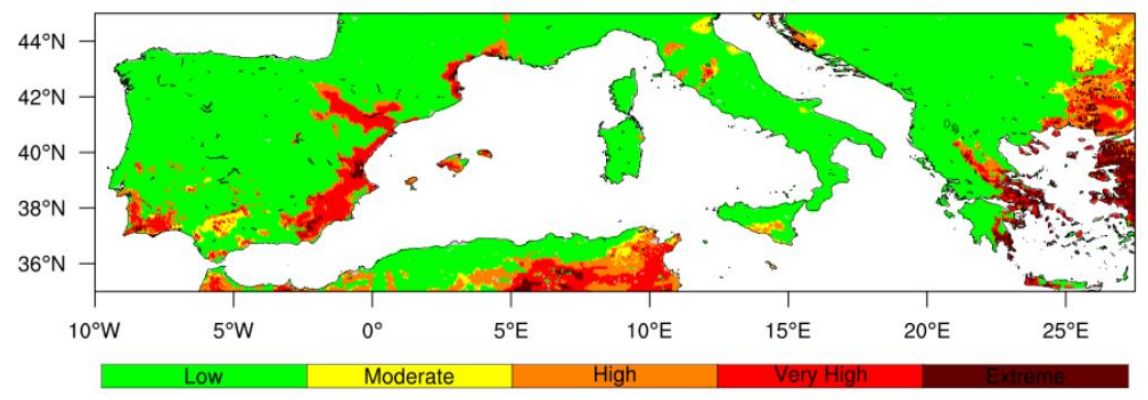

Figure 8 - Classes of fire danger in Mediterranean Europe on 15 June 2018.

\subsubsection{Prescribed Fire Area}

This area contains interactive displays of maps (Figure 9) with information allowing to define optimal windows of opportunity to perform activities related to prescribed burning in areas of eucalyptus, pine and shrub. Information is also provided, at any location selected by the user, about values of the Canadian FWI System and their role on the choice of the class of prescribed burning (Fernandes, 2018).

A special area for accredited technicians is now being developed providing hourly data for the day, time and location of the burning in return of real hourly data measured in the location. In the end, the technician receives an automatic report of the activity, a mandatory requirement to grant these users the possibility to proceed doing these activities. 


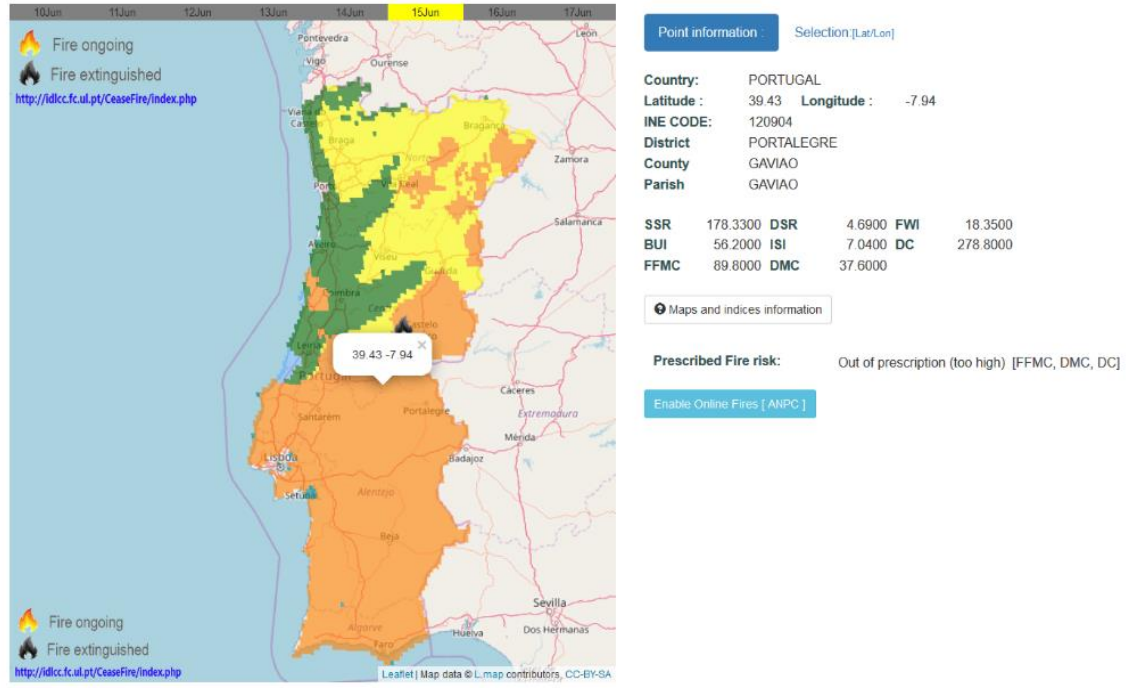

Figure 9 - Classes of prescribed fire on 15 June 2018 (left panel) and indices of fire danger at a selected location (right panel).

\subsubsection{Data Download Area}

This is a restricted area for download of information about meteorological parameters and all indices of the Canadian FWI System.

\section{The APP}

A first version of a mobile application for Android has been released to restricted users and is currently being tested. This APP aims at providing in a more efficient way information assisting the users in making decisions on fire management and combat. Information released includes daily alerts, maps of fire danger, ignition potential and aftermath for the day and the two following days, and location of active fires in real time provided by ANPC and satellite (Figure 10).

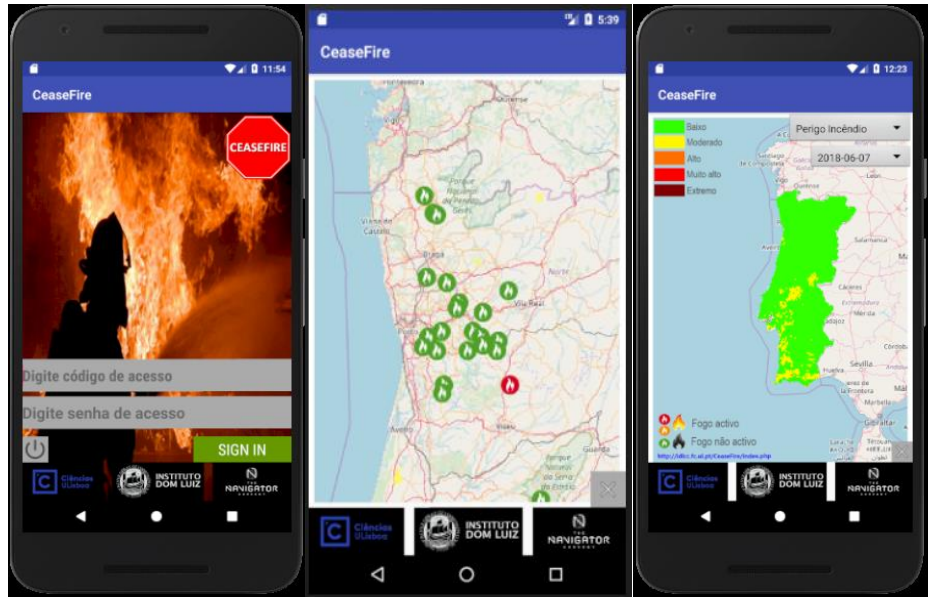

Figure 10 - Screenshots of the recently released APP: login area (left panel), active fires (central panel) and classes of fire danger (right panel).

Several improvements are currently being introduced to the APP and, in the next release, the location of the mobile phone will be identified via GPS and information will be provided about the location of active fires within a radius of $50 \mathrm{~km}$ (Figure 11). Hourly information about meteorological variables (temperature, wind speed and direction, humidity) and components of the Canadian FWI System will also be provided for each of the identified active fires. 


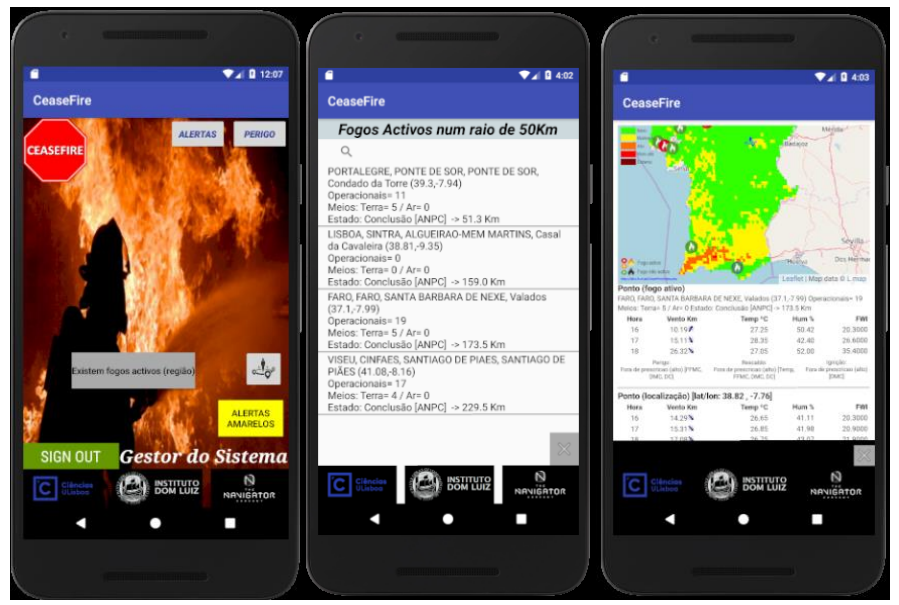

Figure 11 - As in Figure 9 but for the new features of the next release of the APP.

\section{Final Remarks}

Since its starting, in March 2016, the CeaseFire website has been sponsored by The Navigator Company, a leading force in the global pulp and paper industry and has received institutional support from the Portuguese Authority for Forests (ICNF). The number of registered users has been steadily increasing and is currently up to about 650 users from a wide range of backgrounds that include forest managers, firemen and civil protection officers, personnel from municipalities, academic researchers and private owners.

The feedback from the users about the currently available products and the interest shown in the development of new ones is a measure of the usefulness of the CeaseFire platform, filling the gap between the scientific community and the fire community at large. This link between academia and practical users has shown to be beneficial for both sides. On the one hand, the user community has access to products derived using state of the art procedures and tailored to their specific needs; on the other hand, product developers are challenged by users to deliver new products, improve the existent ones and even extend applications to other fields like plague and pest control.

\section{Acknowledgments}

Main research is in the framework of Brazilian Fire-Land-Atmosphere System (BrFLAS) Project financed by the Portuguese and Brazilian science foundations, FCT and FAPESP (project references FAPESP/1389/2014 and 2014/20042-2) and of EUMETSAT Satellite Application Facility for Land Surface Analysis (LSA SAF) Project. Part of research was supported by The Navigator Company in the framework of the CeaseFire platform. Research by Sílvia A. Nunes was supported by a grant in the framework of Project "Fire Early Warning System in Mozambique" (World Bank Group Contract 7184114). Research by Miguel M. Pinto was supported by the Portuguese Science Foundation (FCT) through PhD grant PD/BD/142779/2018.

\section{References}

Amraoui M, Pereira MG, DaCamara CC, Calado TJ (2015) Atmospheric conditions associated with extreme fire activity in the Western Mediterranean region. Sci. Total Environ. 524-525, 32-39. https://doi.org/10.1016/j.scitotenv.2015.04.032

Costa L, Thonicke K, Poulter B, Badeck F (2010) Sensitivity of Portuguese forest fires to climatic, human, and landscape variables: subnational differences between fire drivers in extreme fire years and decadal averages. Reg. Environ. Change 11, 543-551. https://doi.org/10.1007/s10113-0100169-6 
DaCamara CC, Calado TJ, Ermida SL, Trigo IF, Amraoui M, Turkman KF (2014) Calibration of the Fire Weather Index over Mediterranean Europe based on fire activity retrieved from MSG satellite imagery. International Journal of Wildland Fire 23, 945-958. https://doi.org/10.1071/WF13157.

Flannigan M, Cantin AS, de Groot WJ, Wotton M, Newbery A, Gowman LM (2013) Global wildland fire season severity in the 21st century. Forest Ecology and Management 294, 54-61. https://doi.org/10.1016/j.foreco.2012.10.022.

Fernandes, P, Palheiro, P (2015) Interpretação dos índices do Sistema Canadiano de Indexação do Perigo de Incêndio Florestal. https://doi.org/10.13140/RG.2.1.3683.8568. (in portuguese)

Fernandes, P (2018) Scientific support to prescribed underburning in southern Europe: What do we $\begin{array}{llllll}\text { know?, } & \text { Science of } & \text { The } & \text { Total } & \text { Environment, } & 630,\end{array}$ https://doi.org/10.1016/j.scitotenv.2018.02.214.

Lavorel S, Flannigan MD, Lambin EF, Scholes MC (2007) Vulnerability of land systems to fire: Interactions among human, climate, the atmosphere, and ecosystems. Mitigation and Adaptation Strategies for Global Change, 12, 33-53. https://doi.org/10.1007/s11027-006-9046-5.

Mori AS, Johnson EA (2013) Assessing possible shifts in wildfire regimes under a changing climate in mountains landscapes. Forest Ecology and Management 310, 875-886. https://doi.org/10.1016/j.foreco.2013.09.036.

Nunes SA, DaCamara CC, Turkman KF, Ermida SL, Calado T.J (2014) Anticipating the severity of the fire season in Northern Portugal using statistical models based on meteorological indices of fire danger. Advances in forest fire research 1032. https://doi.org/10.14195/978-989-26-0884-6_180.

Pereira MG, Trigo RM, DaCamara CC, Pereira JMC, Leire SM (2005) Synoptic patterns associated with large summer forest fires in Portugal. Agricultural and Forest Meteorology 129, 11-25. https://doi.org/10.1016/j.agrformet.2004.12.007.

Pinto MM, DaCamara CC, Trigo IF, Trigo RM, Turkman KF (2018) Fire danger rating over Mediterranean Europe based on fire radiative power derived from Meteosat. Natural Hazards Earth System Science 18, 515-529, https://doi.org/10.5194/nhess-18-515-2018.

Ruffault J, Moron V, Trigo RM, Curt T (2016) Daily synoptic conditions associated with large fire occurrence in Mediterranean France: evidence for a wind-driven fire regime. Int. J. Climatol. 37, 524-533. https://doi.org/10.1002/joc.4680

San-Miguel-Ayanz J, Moreno JM, Camia A (2013) Analysis of large fires in Mediterranean landscapes: Lessons learned and perspectives. Forest Ecology and Management 294, 11-22. https://doi.org/10.1016/j.foreco.2012.10.050.

Skamarock WC, Klemp JB, Dudhia J, Gill DO, Barker DM, Duda MG, Huang X, Wang W, Powers JG (2008) A Description of the Advanced Research WRF Version 3. NCAR Technical Note NCAR/TN-475+STR. https://doi.org/10.5065/D68S4MVH.

Sousa PM, Trigo RM, Pereira MG, Bedia J, Gutiérre JM (2015) Agricultural and Forest Meteorology 202 11-25. doi: http://dx.doi.org/10.1016/j.agrformet.2014.11.018

Trigo IF, DaCamara CC, Viterbo P, Roujean J-L, Olesen F, Barroso C, Camacho de Coca F , Freitas SC , García-Haro J, Geiger B , Gellens-Meulenberghs F, Meliá J, Pessanha L, Siljamo N (2011) The Satellite Application Facility for Land Surface Analysis. International Journal of Remote Sensing 32, 2725-2744. https://doi.org/10.1080/01431161003743199.

Van Wagner, CE (1974) Structure of the Canadian Forest Fire Weather Index. Can. Forestry Serv., Publication 1333, Ottawa, Ontari, pp. 49.

Wooster MJ, Roberts G, Freeborn PH, Xu W, Govaerts Y, Beeby R, He J, Lattanzio A, Fisher D, Mullen R (2015) LSA SAF Meteosat FRP products - Part 1: Algorithms, product contents, and analysis. Atmos. Chem. Phys. 15, 13217-13239. https://doi.org/10.5194/acp-15-13217-2015. 


\title{
Coupled fire-atmosphere-smoke forecasting: current capabilities and plans for the future
}

\author{
Adam K. Kochanski ${ }^{1 *}$; Jan Mandel²; Martin Vejmelka³ ${ }^{3}$ Dalton Burke²; Lauren Hearn ${ }^{2}$; James \\ Haley $^{2}$; Angel Farguell Caus ${ }^{4}$; Sher Schranz ${ }^{5}$ \\ ${ }^{1}$ University of Utah. Salt Lake City, Utah, \{adam.kochanski@utah.edu*\} \\ ${ }^{2}$ University of Colorado Denver. Denver, Colorado, \{jan.mandel@ucdenver.edu, \\ dalton.burke@ucdenver.edu,lauren.hearn@ucdenver.edu,james.haley@ucdenver.edu\} \\ ${ }^{3}$ CEAi, Inc., Prague, Czech Republic,0000-0003-2309-5869, \{vejmelkam@gmail.com\} \\ ${ }^{4}$ University of Colorado Denver and Autonomous University of Barcelona. Denver, Colorado, \\ \{angel.farguell@gmail.com\} \\ ${ }^{5}$ Colorado State University, Ft. Collins, Colorado \{sher@rams.colostate.edu\}
}

\begin{abstract}
In this paper, we present an integrated wildland fire forecasting system based on combining a high resolution, multi-scale weather forecasting model, with a semi-empirical fire spread model and a prognostic dead fuel moisture model. The fire-released heat and moisture impact local meteorology which in turn drives the fire propagation and the dead fuel moisture. The prognostic dead fuel moisture model renders the diurnal and spatial fuel moisture variability. The local wind and the fuel moisture variation drive the fire propagation over the landscape.

The sub-kilometer model resolution enables detailed representation of complex terrain and small-scale variability in surface properties. The fuel moisture model assimilates surface observations of the $10 \mathrm{~h}$ fuel moisture from Remote Automated Weather Stations (RAWS) and generates spatial fuel moisture maps used for the fire spread computations. The dead fuel moisture is traced in three different fuel classes $(1 \mathrm{~h}, 10 \mathrm{~h}$ and $100 \mathrm{~h}$ fuel), which are integrated at any given location based on the local fuel description, to provide the total dead fuel moisture content at the fire-model grid, of a typical resolution of tens of meters.

The fire simulations are initialized by a web-based control system allowing a user to define the fire anywhere in CONUS as well as basic simulation properties, such as simulation length, resolution, and type of meteorological forcing for any time meteorological products are available to initialize the weather model. The data is downloaded automatically, and the system monitors execution on a cluster. The simulation results are processed while the model is running and displayed as animations on a dedicated visualization portal.
\end{abstract}

Keywords: Fire forecasting, Plume rise, WRF-SFIRE, WRFX, wrfxpy, Coupled fire-atmosphere modeling, Web portal, Automatic setup

\section{Introduction}

As the resolution of the weather forecasting models increases, the fire-atmosphere interactions and smoke plumes that had to be treated as sub-grid-scale processes requiring a simplified treatment through external parameterizations become explicitly resolvable on fine model grids. Thanks to improved computational capabilities, operational applications of coupled-fire atmosphere models have become increasingly feasible. The rapid increase in the resolution of numerical weather prediction products observed over recent years has opened new avenues for development and implementations of integrated systems (e.g. WRF-SFIRE-CHEM; Kochanski et. al., 2016) that resolve in a fully coupled way fire progression, plume rise, smoke dispersion, and chemical transformations. In this paper, we present an integrated wildland fire forecasting system based on a high-resolution, multi-

Advances in Forest Fire Research 2018 - Page 950 
scale weather forecasting model coupled with a semi-empirical fire spread model and a prognostic dead fuel moisture model.

\section{Description of the simulation system}

The core of this forecasting framework is WRF-SFIRE (Mandel et al., 2009, 2011, 2014a), which is a coupled fire-atmosphere model based on the Weather Research and Forecasting model (WRF; Skamarock et al., 2008) and the Rothermel (1972) fire-spread model implemented using a level set method to evolve the fire front on a Eulerian grid in time. WRF-SFIRE evolved from CAWFE (Clark et al. 2004) and it has been a part of WRF release as WRF-Fire since 2011 (Mandel et al. 2011; Coen et al 2013). WRF-Fire was recently selected as foundation of the Colorado Fire Prediction System (CO-FPS) and the level set method in WRF release was improved (Muñoz-Esparza et al. 2018).

Fire-emitted heat and moisture fluxes computed at the fire mesh are integrated into WRF's coarser mesh. The fluxes affect the atmospheric state, thereby changing local winds that drive fire propagation (Mandel et al. 2011).

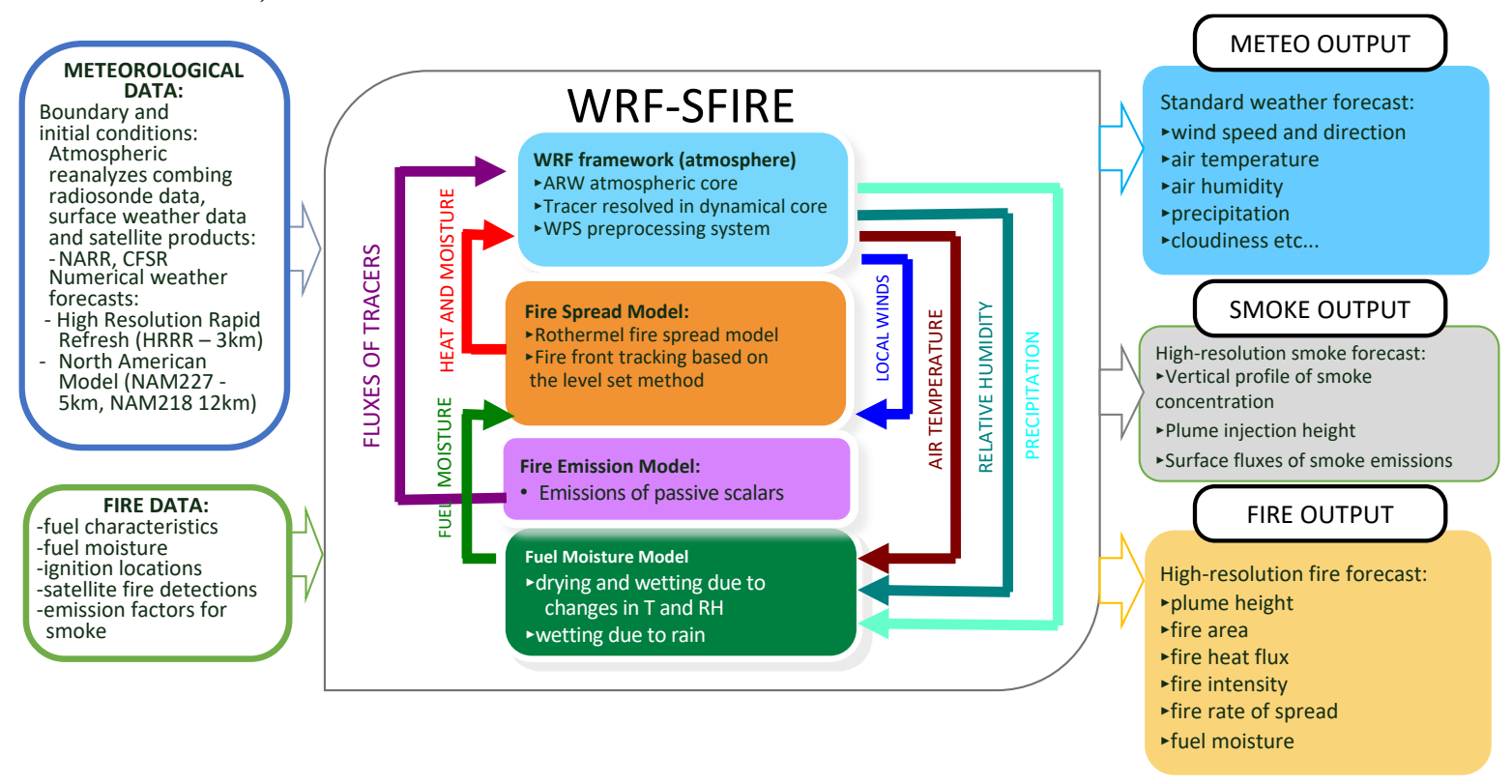

Figure 1 - Schematic of the WRF-SFIRE modeling system.

WRF-SFIRE is also coupled to a prognostic fuel moisture model, which assesses the moisture based on local WRF meteorology (air humidity, temperature, and precipitation (Mandel et al. 2014a). WRFSFIRE is designed to simulate the landscape-scale physics of the coupled fire-atmosphere phenomena and focuses on the importance of rapidly changing meteorological conditions at the fire line by taking into account local feedbacks between the fire, fuel, terrain, and the evolving atmospheric boundary layer. It can be driven by operational numerical weather prediction products to generate downscaled weather forecasts at a resolution of several hundred meters that resolve small-scale interactions between, for example, thunderstorm outflows, terrain, and fire. WRF-SFIRE is capable of simulating large-scale, high-intensity fires, under various topographical, meteorological, and vegetation conditions (Kochanski et al., 2013). Through the two-way coupling, WRF-SFIRE resolves basic fireatmosphere feedbacks, pyro-convection, and plume rise without relying on an external plume parameterization. A schematic of the WRF-SFIRE modeling system is presented in Figure 1. 


\section{The WRFX System}

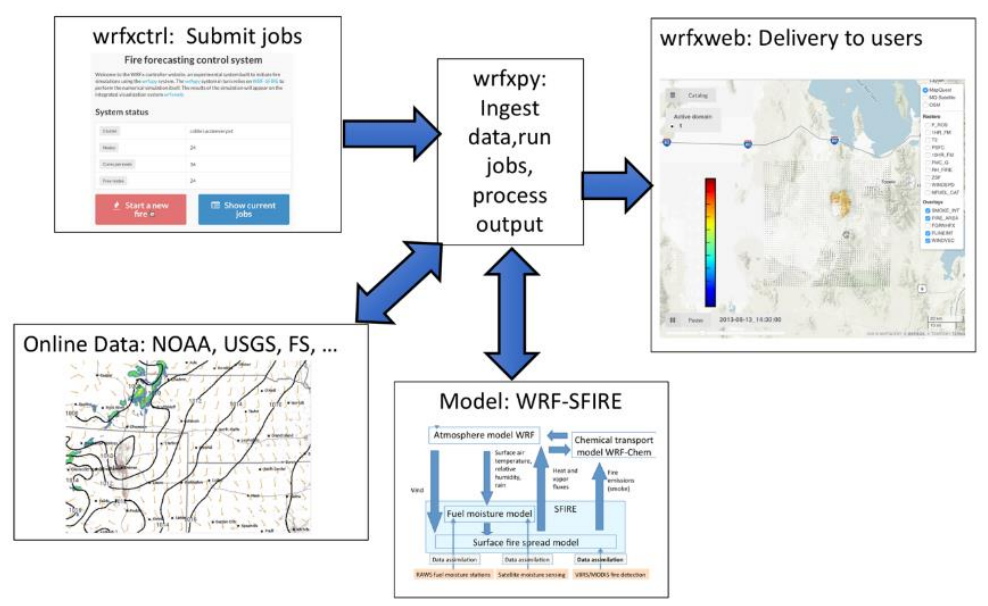

Figure 2 - The WRFX simulation management system.

\section{Description of the management system and web portal}

The WRFX system built around WRF-SFIRE enables automated real-time fire simulations and visualization of the model output. WRFX is a successor of an earlier prototype integrated system (Beezley et al. 2012). WRFX is comprised of three main modules: wrfctrl which allows the user to define simulation parameters and launch the forecast through the web interface, wrfxpy responsible for data collection, preprocessing, job submission, and visualization, and wrfxweb which displays the forecast on the web (Figure 2).

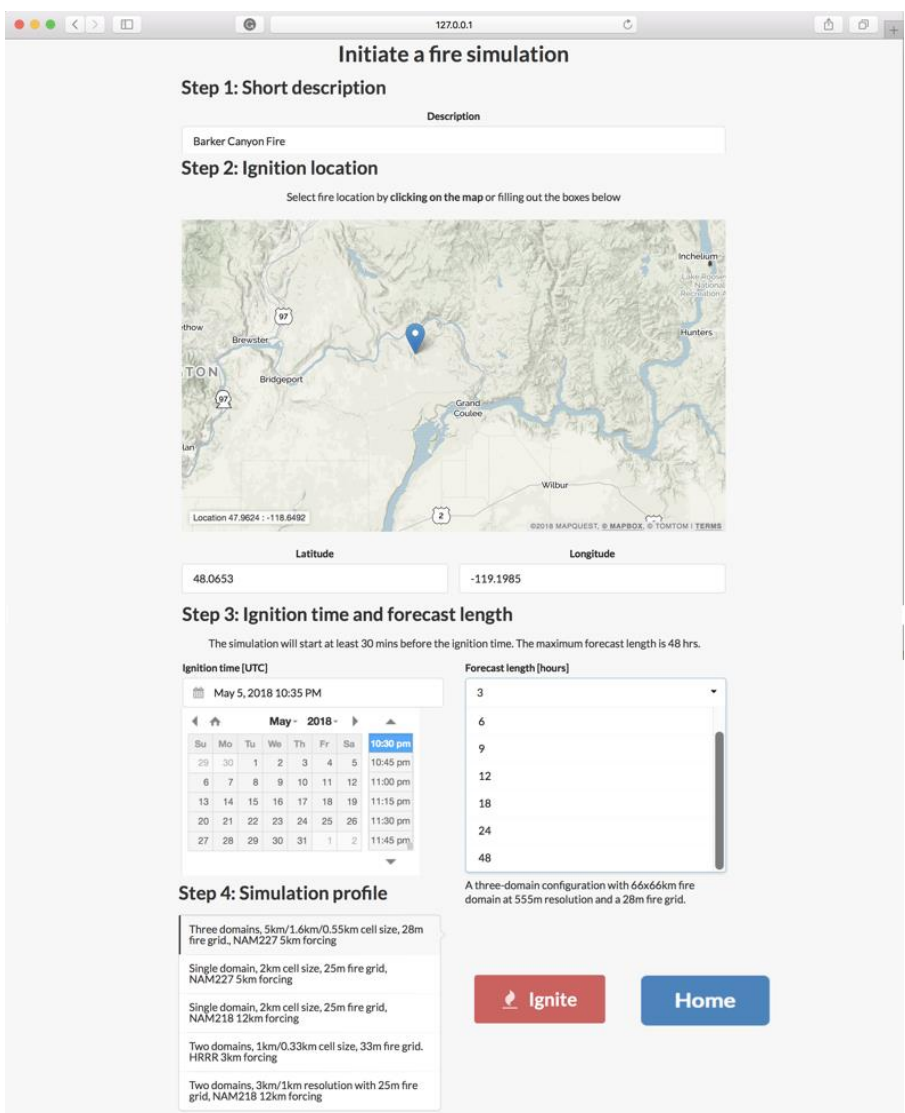

Figure 3. The wrfxctrl module used to define simulation parameters and initiate a forecast. 
Table 1 - Weather products integrated with the system.

\begin{tabular}{|l|l|l|}
\hline Weather product & Resolution & Forecast length \\
\hline North American Model NAM 218 & $12 \mathrm{~km}$ & 84 hours \\
\hline North American Model NAM 227 & $5 \mathrm{~km}$ & 60 hours \\
\hline High Resolution Rapid Refresh (HRRR) & $3 \mathrm{~km}$ & 36 hours \\
\hline North American Regional Analysis (NARR) & $32 \mathrm{~km}$ & Hindcast only \\
\hline Climate Forecast System Reanalysis (CFSR) & $38 \mathrm{~km}$ & Hindcast only \\
\hline
\end{tabular}

Fire simulation monitoring

\begin{tabular}{|c|c|c|c|}
\hline nformation & & Home & List jobs \\
\hline Current time & Fri, July 6th 2018, 14:52:56 & & \\
\hline WRF state & waiting & & \\
\hline Simulation started & 3 minutes ago & & \\
\hline Ignition coordinates & $40.3883,-112.6748$ & & \\
\hline Ignition time & 2018-07-06_20:47:00 UTC & & \\
\hline Forecast length & 24 hours & & \\
\hline Short description & test patch & & \\
\hline Simulation start time & 2018-07-06_20:00:00 UTC & & \\
\hline Simulation end time & 2018-07-07_20:00:00 UTC & & \\
\hline System identifier & from-web-2018-07-06_20-49-57 & & \\
\hline Visualization & no visualization & & \\
\hline
\end{tabular}

Detailed status

\begin{tabular}{|l|l|}
\hline GEOGRID & Running \\
\hline Ingest & Success \\
\hline UNGRIB & Success \\
\hline METGRID & Waiting \\
\hline REAL & Waiting \\
\hline WRF & Wa \\
\hline Output & Waiting \\
\hline
\end{tabular}

Event log

Figure 4 - Monitoring of wrfpy job execution by wrfxtrl.

The wrfctrl module enables the user to define the fire location, the start date of the forecast, its length, as well as domain configuration and the source of weather data to be used. A sample screenshot of the wrfctrl portal is presented in Figure 3. The forecast information provided by the user is fed to the wrfxpy module that performs additional steps needed to prepare the forecast. Once the fire is 'ignited', wrfxpy automatically generates a set of nested domains around the defined ignition location and starts data preprocessing tasks and then WRF execution, which are all monitored by wrfxctrl (Figure 4). 
As a first step, static data such as land use classification, soil type, vegetation type, fuel type, and elevation are interpolated to the model grids. The fuel and moisture data at $30 \mathrm{~m}$ resolution are stored locally as GeoTIFF files covering the whole contiguous U.S., so they can be accessed quickly without any delays associated with the data download from external sources. In parallel to the static data processing, wrfxpy acquires large-scale meteorological data, decompresses it and interpolates horizontally to the model grid. The currently supported meteorological sources are listed in Table 1.

Once the data are acquired and processed, wrfxpy generates WRF input and boundary condition files and submits a job to the queuing system on a computing cluster whch runs the forecast. The forecast length is limited by the length of the weather product used to generate boundary conditions. The wrfxctrl module contains a catalog of previous simulations initiated in wrfxctrl, so the user can monitor multiple simulations and manage currently running as well as past simulations (Figure 5).

\section{List of fire simulations}

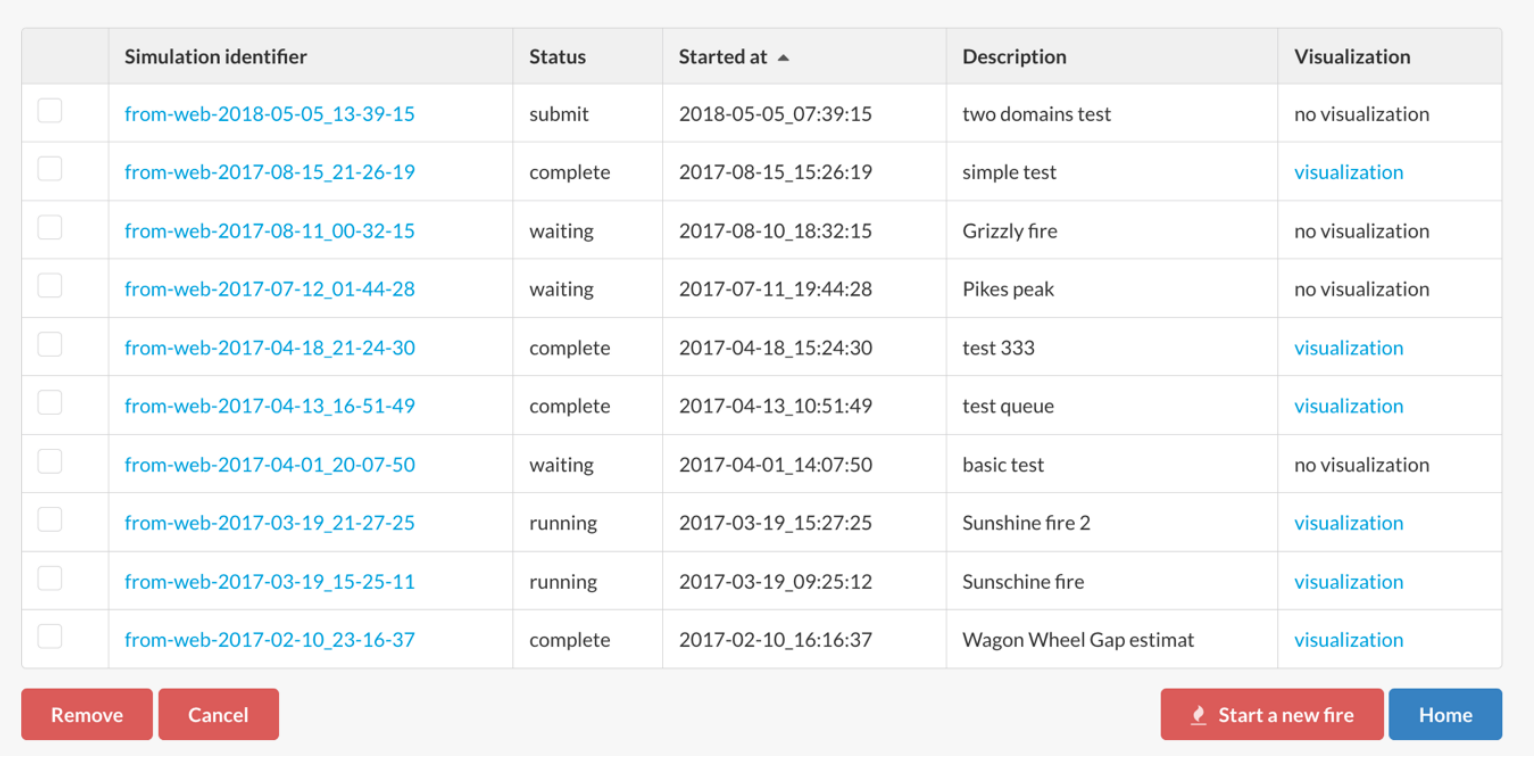

Figure 5 - Managing the simulations from the webcontrol interface wrfxctrl.

After the simulation job is submitted, the system controls the progression of the job, generates visualizations, and uploads them to wrfxweb. The portal enables easy access to the forecast, and progressively displays additional time frames as the simulation progresses, so the users have access to the partial forecast even before the full simulation is completed (Figure 6). The forecast includes variables such as $10 \mathrm{~m}$ wind speed and direction, $2 \mathrm{~m}$ air temperature, fire area, fireline intensity, fire heat flux, surface pressure, smoke intensity, plume top height, and fuel moisture. Any of these variables can be displayed or hidden in the portal by turning the particular forecast layer on or off, and animated using the time slider. Custom postprocessing and new layers, such as the plume height, used in Kochanski et al. (2018) can be easily added. The simulation to be displayed is selected from a catalog, which provides access any prior simulation stored in the wrfxweb subsystem.

The visualizations are also available for download as animated Google Earth files (Figure 7), where the simulation can be easily integrated with other information available in Google Earth format, such as satellite data. 


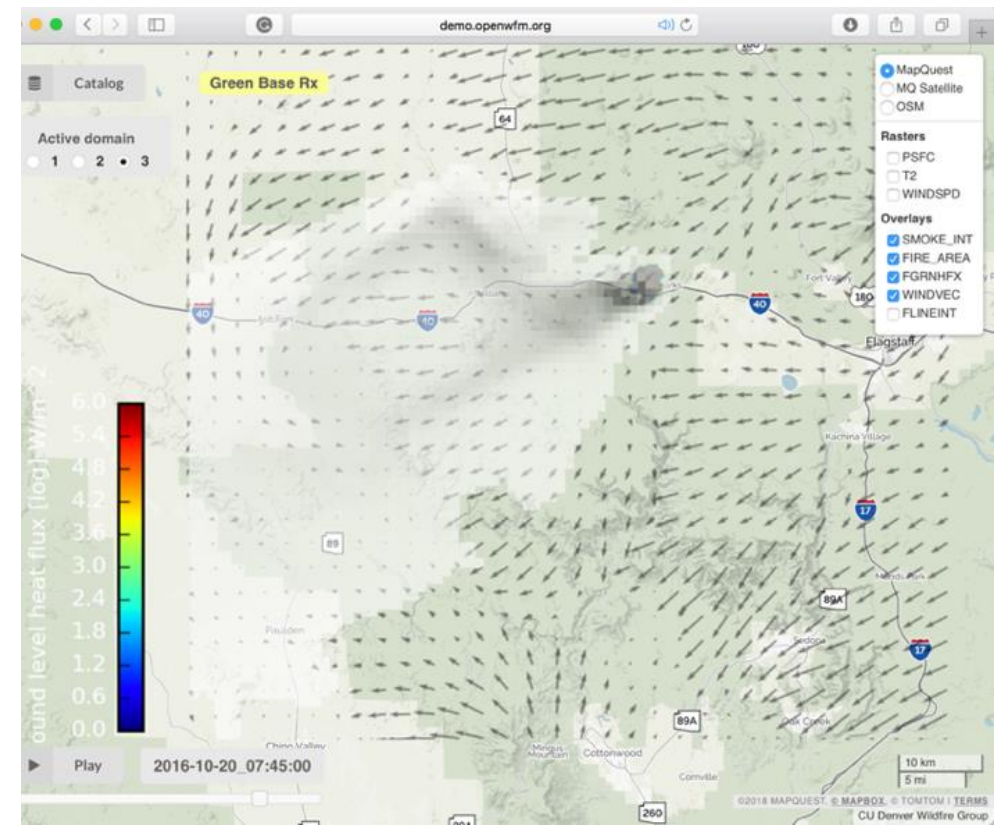

Figure 6 - WRFX web portal (wrfxweb) showing a sample visualization of a coupled fire atmosphere forecast.

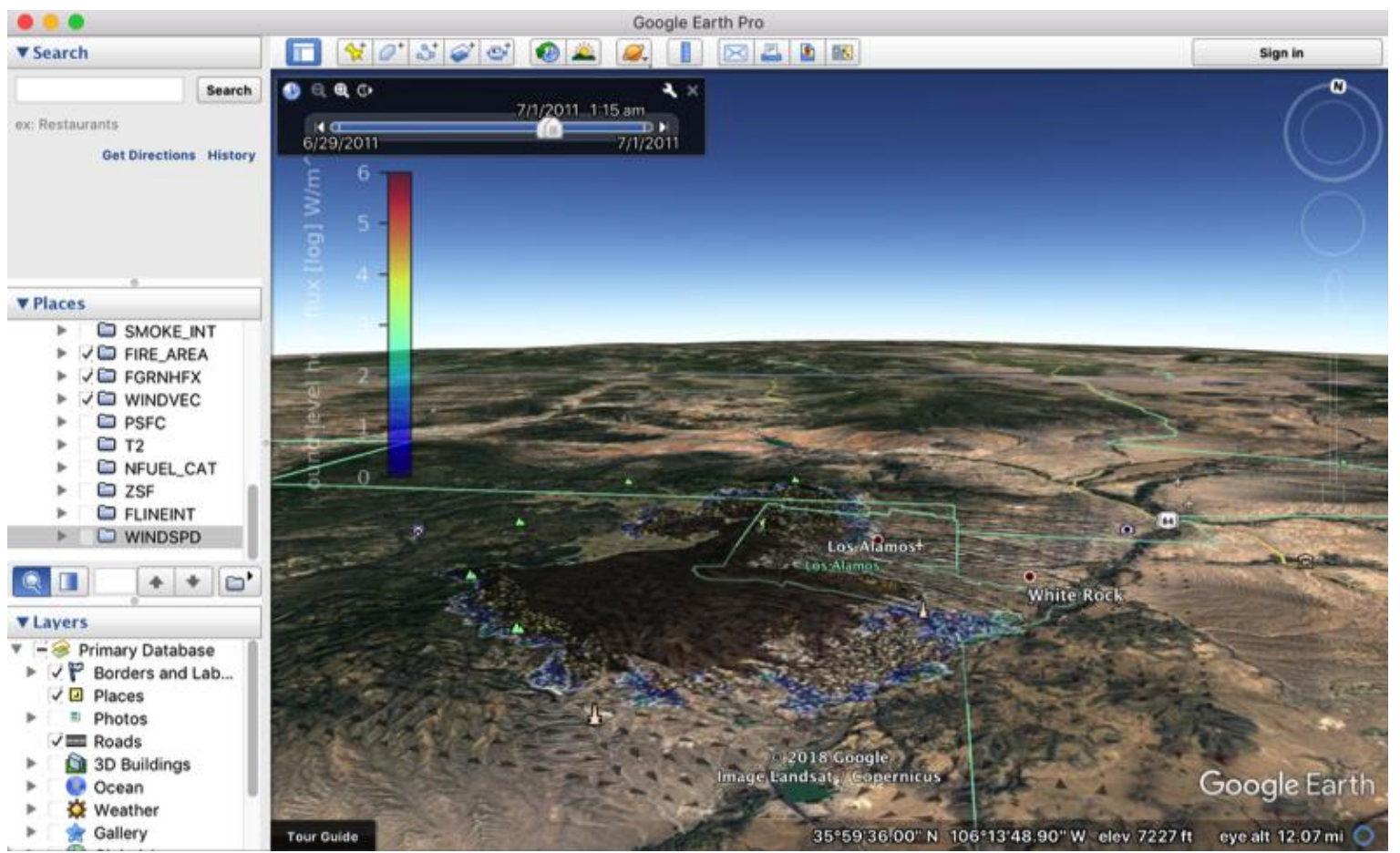

Figure 7 - An animated visualization in Google Earth (2011 Las Conchas fire).

The same wrfxweb portal also displays nowcast maps of the dead fuel moisture (Figure 8). They are computed for predefined geographical regions using the fuel moisture data assimilation system (Vejmelka et al., 2015), which automatically acquires and assimilates observations of dead fuel moisture by Remote Automated Weather Station (RAWS). 


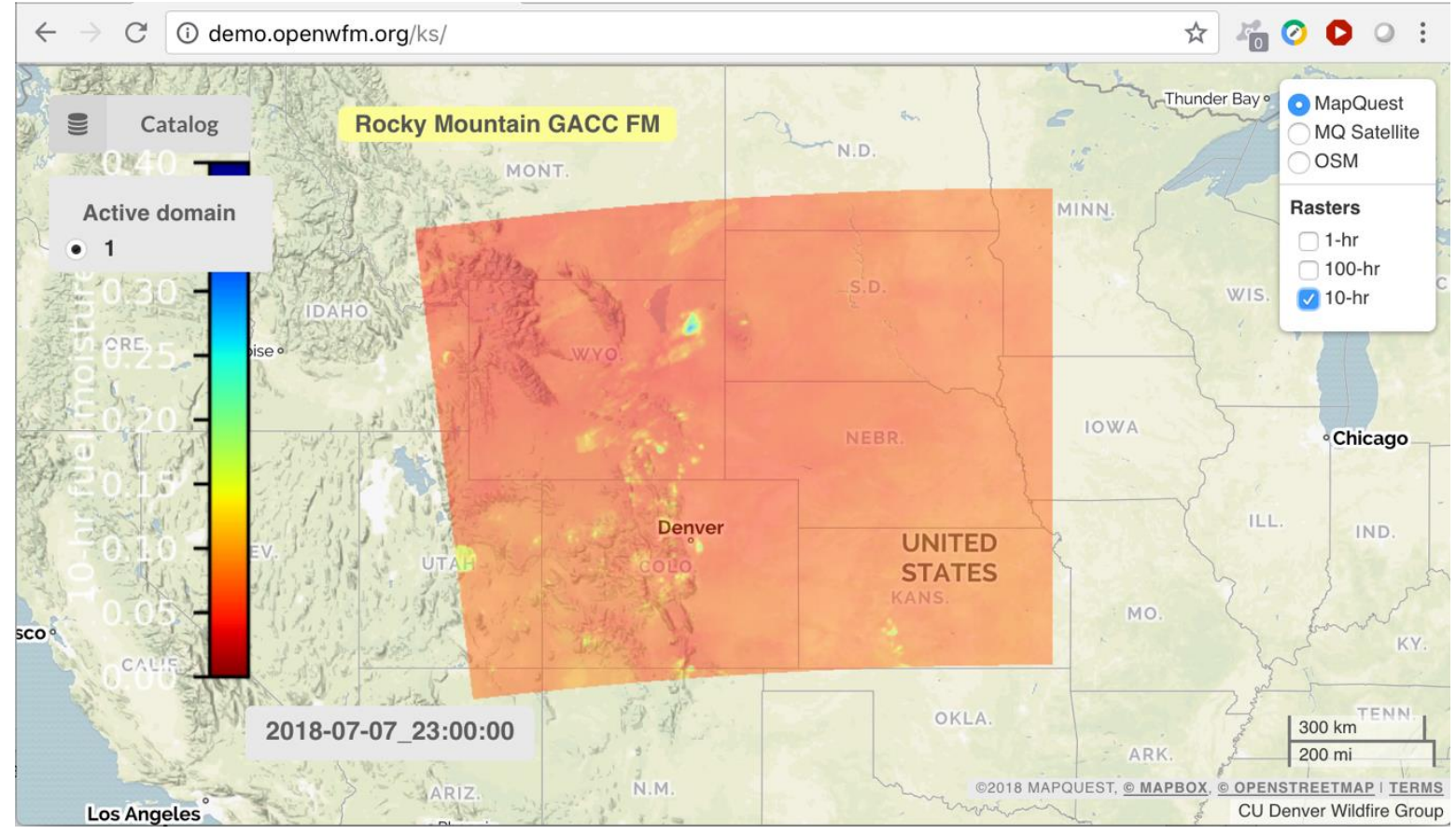

Figure 8 - Fuel moisture nowcasting from weather analysis and RAWS fuel moisture data assimilation.

\section{Conclusion and future plans}

In order to improve models like WRF-SFIRE, which utilize large-scale meteorology for the purpose of operational wildfire prediction, novel solutions must be applied to faithfully represent smaller-scale processes. The radiation and convective heat transfer from the fire to the atmosphere, the buoyancy effects on the fire-line winds, flow over complex terrain, changing fuel types, and structures (including canopies or individual trees and buildings) must be accounted for while remaining computationally viable. The ongoing work focuses on possible solutions in the form of coupling with complementary, reduced-physics diagnostic models such as QUIC-EnvSim (Overby et al., 2016). In this approach, the WRF model would be a source of coarser-scale weather data used to drive the prognostic QUIC model similarly as it was done for urban simulations presented in Kochanski et al. (2015). This coupling would enable better representation of the small-scale flow features driven by the terrain and the floe under a tree canopy. Additional endeavor aims at improvement of the fire-spread component of the system, such as replacing the Rothermel model with the Balbi (2009) model, improving the fuel consumption (e.g., fuel loads) and emissiona, through the integration with fuel consumption models using models that account for smoldering e.g., CONSUME 3.0 (Ottmar, 2009).As a flexible system of transparent Python scripts, WRFX is uniquely suited for extensions and integration of new functions.

The wrfxpy module can already acquire all relevant MODIS and VIIRS fire detection data for data assimilation. This module is currently in a transition to the new NASA https API access. The satellite products will be accessed by a uniform internal API, which will integrate additional data products, such as from GOES satellites (Koltunov et al., 2016). The visualization engine in WRFX enables an integration of the fire detections from various sources as additional layers.

Thus, WRFX can serve as a hub for research and implementation of data assimilation algorithms for coupled fire-atmosphere modeling, such as those in Mandel et al. (2014b, 2016). WRFX already includes a subsystem for initializing and monitoring of coupled fire-atmosphere simulations, which can be extended to manage analysis cycles, and spin-up for data assimilation. The ignition data from dispatch systems, such as IRWIN, and in principle even the effect of fire suppression actions can be 
likewise integrated into WRFX. The effect of fire suppression can be already modeled by hand modification of fuel maps, and WRFX supports restarting of hand-modified problems. Such modification can be automated and integrated into WRFX data preprocessing workflow.

The fuel moisture model with RAWS data assimilation is currently running in a standalone mode using Real Time Mesoscale Analysis (RTMA), which is available only for the present as a nowcast. Thus, the spin-up of the assimilated state has to happen in real time. In addition, the fuel moisture maps driven by the RTMA data can show artifacts, such as radar circles. We plan to switch to other data sources including the HRRR surface layer (HRRR is a forecast only product, but 3rd party archives exist) in order to support historical fuel moisture analyses and integrate the assimilated fuel moisture with the online fuel moisture model within WRF-SFIRE.

Both WRF-SFIRE and WRFX are publicly available at https://github.com.openwfm. The wrfxweb software is running at http://demo.openwfm.org provides visualization of test simulations as well as the near-real-time nowcasts of the dead fuel moisture.

\section{Acknowledgements}

This research was partially supported by NSF ICER-1664175 and NASA NNX13AH59G. The high-performance computing support from the Center for High Performance Computing at the University of Utah and Cheyenne (doi:10.5065/D6RX99HX) at NCAR Computational and Information Systems Laboratory, sponsored by the NSF, are gratefully acknowledged.

\section{References}

Balbi JH, Morandini F, Silvani X, Filippi JB, Rinieri F (2009), A physical model for wildland fires, Combustion and Flame 156, 2217-2230. doi:10.1016/j.combustflame.2009.07.010

Beezley JD, Martin M, Rosen P, Mandel J, Kochanski AK (2012) Data management and analysis with WRF and SFIRE. In 2012 IEEE Interntional Geoscience and Remote Sensing Symposium (IGARSS), 5274-5277, IEEE. doi: 10.1109/IGARSS.2012.6352419

Clark TL, Coen J, Latham D (2004) Description of a coupled atmosphere-fire model. International Journal of Wildland Fire 13, 49-63. doi:10.1071/WF03043

Coen JL, Cameron M, Michalakes J, Patton EG, Riggan PJ, Yedinak KM (2013) WRF-Fire: Coupled weather-wildland fire modeling with the Weather Research and Forecasting model. Journal of Applied Meteorology and Climatology 52, 16-38. doi:10.1175/JAMC-D-12-023.1

Kochanski AK, Jenkins MA, Krueger SK, Mandel J, Beezley JD (2013) Real time simulation of 2007 Santa Ana fires. Forest Ecology and Management 15, 136-149. doi: 10.1016/j.foreco.2012.12.014

Kochanski AK, Pardyjak ER. , Stoll R, Gowardhan A. , Brown MJ., and Steenburgh WJ., (2015) Oneway coupling of the WRF-QUIC urban dispersion modeling system. $J$. Appl. Meteorol. Climat., 52, 2119-2139, 2015. doi: 10.1175/JAMC-D-15-0020.1

Kochanski AK, Jenkins MA, Yedinak K, Mandel J, Beezley J, Lamb B (2016) Toward an integrated system for fire, smoke, and air quality simulations. International Journal of Wildland Fire 25, 534546. doi: 10.1071/WF14074

Kochanski AK, Fournier A, Mandel J (2018) Experimental Design of a Prescribed Burn Instrumentation, arXiv:1806.06460. Submitted to Atmosphere.

Koltunov A, Ustin SL, Quayle B, Schwind B, Ambrosia VG, Li W (2016) The development and first validation of the GOES Early Fire Dectection (GOES-EFD) algorithm. Remote Sensing Environment, 184, 436-453, doi: 10.1016/j.rse.2016.07.021 
Mandel J, Beezley JD, Coen JL, Kim M (2009) Data assimilation for wildland fires: Ensemble Kalman filters in coupled atmosphere-surface models. IEEE Control Systems Magazine 29, 47-65. doi:10.1109/MCS.2009.932224

Mandel J, Beezley JD, and Kochanski AK (2011) Coupled atmosphere-wildland fire modeling with WRF 3.3 and SFIRE 2011. Geoscientific Model Development 4, 591-610. doi: 10.5194/gmd-4591-2011

Mandel J, Amram S, Beezley JD, Kelman G, Kochanski AK, Kondratenko VY, Lynn BH, Regev B, Vejmelka M (2014a) Recent advances and applications of WRF-SFIRE. Natural Hazards and Earth System Science 14, 2829-2845. doi:10.5194/nhess-14-2829-2014

Mandel J, Kochanski AK, Vejmelka M, Beezley JD (2014b) Data assimilation of satellite fire detection in coupled atmosphere-fire simulations by WRF-SFIRE. Advances in Forest Fire Research, D. X. Viegas, ed., Coimbra University Press, 716-724. doi:10.14195/978-989-26-0884-6_80

Mandel J, Fournier A, Jenkins MA, Kochanski AK, Schranz S, Vejmelka M (2016) Assimilation of satellite active fires detection into a coupled weather-fire model. Proceedings for the 5th International Fire Behavior and Fuels Conference April 11-15, 2016, Portland, Oregon, USA, International Association of Wildland Fire, Missoula, Montana, USA, 17-22, http://www.iawfonline.org/proceedings.php.

Muñoz-Esparza D, Kosović B, Jiménez PA, Coen JL (2018) An accurate fire-spread algorithm in the Weather Research and Forecasting model using the level-set method. Journal of Advances in Modeling Earth Systems 10, 908-926. doi: 10.1002/2017MS001108

Overby M, Willemsen P, Bailey BN, Halverson S, and Pardyjak ER (2016) A rapid and scalable radiation transfer model for complex urban domains. Urban Climate 15, 25-44. doi: 10.1016/j.uclim.2015.11.004

Ottmar R (2009) Consume 3.0 - a software tool for computing fuel consumption. Fire Science Brief 55, 1-6, June 2009. Available at http://www.firescience.gov/projects/98-1-9-06/supdocs/98-1-906_FSBrief55-Final.pdf

Rothermel RC (1972) A mathematical model for predicting fire spread in wildland fires. USDA Forest Service Research Paper INT-115.

Skamarock WC, Klemp JB, Dudhia J, Gill DO, Barker DM, Duda MG, Huang X-Y, Wang W, and Powers JG (2008) A description of the Advanced Research WRF version 3. NCAR Technical Note 475. doi:10.5065/D68S4MVH

Vejmelka M, Kochanski AK, Mandel J (2016) Data assimilation of dead fuel moisture observations from remote automatic weather stations. International Journal of Wildland Fire 25, 558-568. doi:10.1071/WF14085 


\title{
Data Likelihood of Active Fires Satellite Detection and Applications to Ignition Estimation and Data Assimilation
}

\author{
James Haley ${ }^{1}$; Angel Farguell Caus ${ }^{2}$; Adam K. Kochanski ${ }^{3}$; Sher Schranz ${ }^{4}$; Jan Mandel ${ }^{1 *}$ \\ ${ }^{1}$ University of Colorado Denver, Denver, Colorado, \{james.haley@ucdenver.edu, \\ jan.mandel@ucdenver.edu*\} \\ ${ }^{2}$ University of Colorado Denver, Denver, Colorado, and Autonomous University of Barcelona, \\ Spain,0000-0003-2986-3857, \{angel.farguell@gmail.com\} \\ ${ }^{3}$ University of Utah, Salt Lake City, Utah, \{adam.kochanski@utah.edu\} \\ ${ }^{4}$ CIRA, Colorado State University, Ft. Collins, Colorado \{sher@rams.colostate.edu\}
}

\begin{abstract}
Data likelihood of fire detection is the probability of the observed detection outcome given the state of the fire spread model. We derive fire detection likelihood of satellite data as a function of the fire arrival time on the model grid. The data likelihood is constructed by a combination of the burn model, the logistic regression of the active fires detections, and the Gaussian distribution of the geolocation error. The use of the data likelihood is then demonstrated by an estimation of the ignition point of a wildland fire by the maximization of the likelihood of MODIS and VIIRS data over multiple possible ignition points.
\end{abstract}

Keywords: Active Fires, MODIS, VIIRS, Coupled Fire-Atmosphere Modeling, Remote Sensing, Maximum Likelihood, Data Assimilation, Data-Driven Simulation

\section{Introduction}

Satellite-based sensors are a commonly used data source because of their large spatial coverage, but the mismatch of scales, geolocation errors, the probabilistic character of the fire detection, and missing data present a challenge. A practical resolution for fire behavior models is given by fuel data availability, typically about $30 \mathrm{~m}$, while the resolution of satellite-based fire detections is from $375 \mathrm{~m}$ to $2 \mathrm{~km}$ and up. Therefore, initializing a model directly by igniting entire detection squares results in blocky, fragmented fire shape. Furthermore, in coupled fire-atmosphere models, such ignitions generally result in numerical instabilities induced by the sudden heat release from the ignited pixel, as well fire state inconsistent with the atmospheric state at the ignition time. In the presented method, we use satellite data to improve the fire modelling in a statistical sense. The basic tool we use is data likelihood, which is defined as the probability of the fire detection outcome given the state of the firespread model.

Data likelihood is one of the techniques to evaluate a model state relative to data, and a basic ingredient of many data-driven simulation methods. Data likelihood is used e.g., in data assimilation to update importance weights in particle filters, or as a term in an objective function of an optimization method in maximum likelihood or maximum aposteriori probability (MAP) estimates.

Other, less formal pragmatic fitness functions are often used for model evaluation, see a survey in Filippi et al. (2013). Approaches used in wildland fire spread include evaluation of a model solution by the difference of burned area e.g., Brun et al. (2017), who update the solution by genetic algorithms; least squares, or, equivalently, Gaussian data likelihood of perimeter position with update by sequential Monte Carlo (e.g., Srivas et al. 2017), or by the ensemble Kalman filter (EnKF) in Rochoux et al. (2014); Gaussian likelihood of sensor data (e.g., Gu 2018; Xue et al. 2012); and the size of a spatial deformation needed to match two fires, with the spatial deformation mapping extending the state in 
the EnKF (Beezley and Mandel, 2008; Mandel et al. 2009). See also a survey of data assimilation for wildland fire spread modeling in Gollner et al. (2015, Sect. 4).

Approaches to wildland fire modeling using satellite data include periodic reinitialization from new detections (Coen and Schroeder 2013; Sá et al. 2014) and identification of ignition as the first active fires detection within a reported perimeter (Benali et al. 2016). However, because of the missing data, the statistical uncertainty of detection, the geolocation uncertainty, and the mismatch of scales between the fire model and the satellite sensor, direct use of satellite data at fuel map scale is of limited value and the data is better suitable to improve models in a statistical sense. Fire behavior models run on a mesh given by fuel data availability, typically with about $30 \mathrm{~m}$ resolution and aligned with geographic coordinates and with time step of the order of seconds. The satellite fire detections are commonly used due the large spatial coverage, but the gap of scales and data errors present a challenge. The resolution of satellite-based sensors is much coarser, from $375 \mathrm{~m}$ once a day for VIIRS (Schroeder et al. 2014), $1.1 \mathrm{~km}$ twice a day for MODIS (Giglio et al. 2016), aligned with flight coordinates, to $2 \mathrm{~km}$ every 5 minutes for GOES-16 (Koltunov et al. 2016). Geolocation error can be significant, e.g., $1.5 \mathrm{~km}$ at $3 \sigma$ for VIIRS (Sei, 2011). Pixels may be missing for various reasons such as clouds. While consumergrade data provide only fire detection pixels, science-grade data make an important distinction between missing data and absence of fire detections.

Spatial statistical interpolation of the first detection time by kriging to obtain a continuous fire arrival time field was proposed by Veraverbeke et al. (2014). Sá et al. (2017) proposed a measure of spatial discrepancy between fire spread simulation and satellite data. The probability of fire detection in a sensor pixel, given the state of the fire and the properties of the surface in the pixel, was estimated in a validation study (Schroeder et al., 2008) by logistic regression. Mandel et al. (2014b) suggested a form of data likelihood for satellite active fires detection in a pixel and used it for data assimilation by a MAP estimate. The form of the data likelihood from Mandel et al. (2014b) was further motivated in Mandel et al. (2016b) by substituting the fire heat flux into the logistic regression from Schroeder et al. (2008), which explained the behavior of the likelihood function after the fire arrival time. This likelihood function was used to find a maximum likelihood estimate of an ignition point in Mandel et al. (2016a).

In this paper, we build a data likelihood function by adding a geolocation error to the construction in Mandel et al. (2016b), which combines a heat release model with logistic regression for the active fires detections. For a single pixel, we recover and justify the form of data likelihood function proposed in Mandel et al. (2014b, 2016b). We then demonstrate use of the data likelihood on identifying the ignition point on a realistic example.

We have used WRF-SFIRE (Mandel et al., 2009, 2011, 2014a) in the examples in this paper. WRFSFIRE evolved from CAWFE (Clark et al. 2004) and it has been a part of WRF release as WRF-Fire since 2011 (Mandel et al. 2011; Coen et al 2013).

\section{Data Likelihood of Active Fires Satellite Detection}

The state of the fire-spread model is encoded as fire arrival time on a grid of locations of Earth surface. The data likelihood is obtained from the fire arrival time by substituting the heat release into the logistic sensitivity function and convolution of the result with a Gaussian kernel to account for the geolocation error (Figure 1). See Appendix A for mathematical details. 


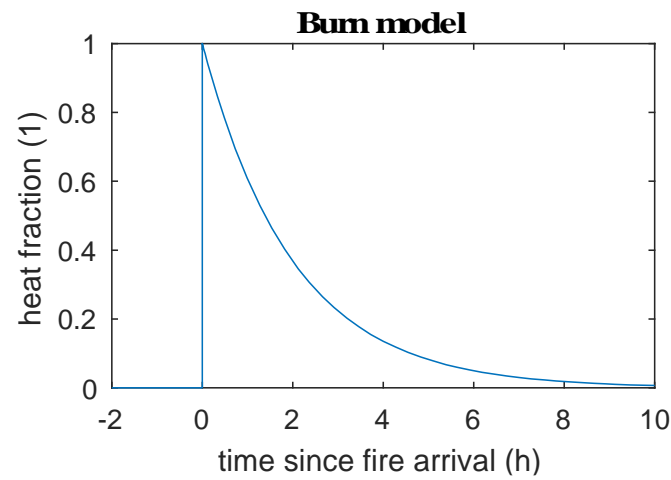

(a)

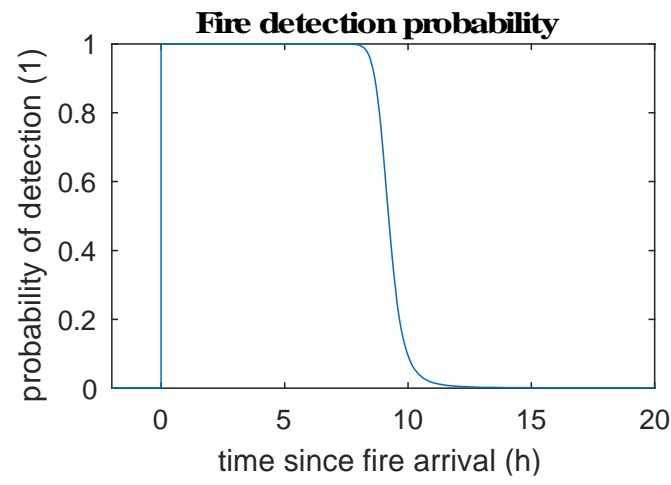

(c)

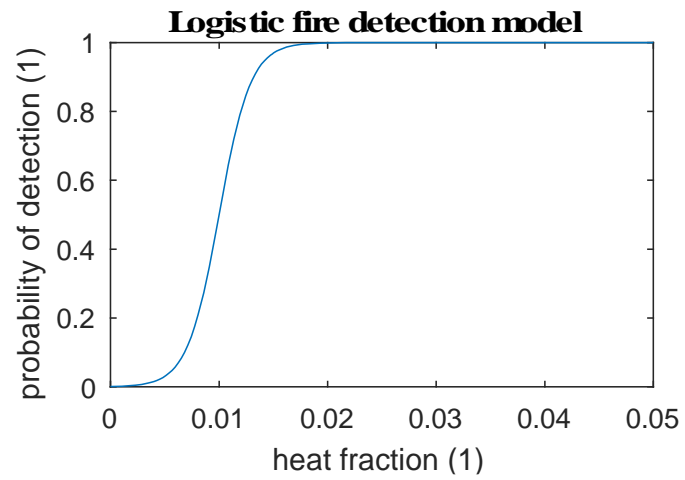

(b)

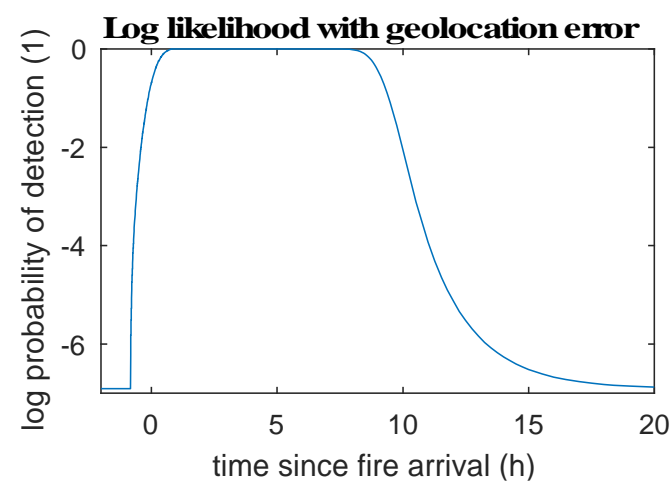

(d)

Figure 1 - (a) Heat release model with exponential decay. The maximum heat fraction of 1 drops to 1/e in the characteristic time of $2 \mathrm{~h}$. (b) Probability of fire detection as a logistic function of the fraction of maximum heat release, with $50 \%$ probability of detection at $1 \%$ heat fraction, and $0.1 \%$ false detection rate. (c) Probability of detection as a function of time elapsed since fire arrival, obtained by substituting the heat release fraction from (a) to the logistic curve (b). (d) Final log probability of detection from Eq. (7), obtained by convolution of (c) with a Gaussian kernel. The fire rate of spread was $1 \mathrm{~m} / \mathrm{s}$ and Gaussian geolocation error was $\sigma=2000 \mathrm{~m}$, equal to a rounded sum of the diagonal of a $1.1 \mathrm{~km}$ pixel plus the standard deviation of $0.5 \mathrm{~km}$ of the geolocation error from VIIRS specifications (Sei, 2011).

The log likelihood suggested in Mandel et al. (2014b, 2016b) was based on the pragmatic consideration that the probability of detection is close to one for some time after the fire arrival, with quadratic tails, and the leading edge is steeper than the trailing edge. Note that the log likelihood curve in Figure 1(d) has exactly this type of shape above the transition to the constant nonzero false detection rate.

\section{Retrieving the Ignition Location and Time}

\subsection{A Simple Test}

As a first test of whether the likelihood function can be used to retrieve the ignition location and time of a wildfire we made a simple experiment simulating an idealized fire over flat terrain with homogenous fuels and no winds. We picture the progression of the perimeter of such a fire to be originating from a point and growing outward like the wave caused by a stone dropped into a pool of still water. With $T$ representing the fire arrival time at some particular spatial point $(x, y)$, we model the progression of the fire as cone

$$
T(x, y)=\sqrt{x^{2}+y^{2}} .
$$

Working with a spatial domain of 1000 units square, we simulate a fire with ignition point $(x, y)=(500,500)$ and ignition time $t=30$ with the function 


$$
T(x, y)=\sqrt{(x-500)^{2}+(y-500)^{2}}+30 .
$$

We then simulated satellite fire detections at several points along the simulated fire perimeter corresponding to the level-curves of the function at time $t=300$. In a real-world setting, these detections may be the first information we have about a fire and in order to achieve the best simulation of it we need to have a good estimate of the ignition point. We find this estimate by running many simulations of the fire starting at locations and times near those of the satellite detections and then computing the log-likelihood of the simulated satellite detections according to equation (6). The largest log-likelihood obtained from the set of simulations gives us the best estimate of the location and time of the fire ignition.

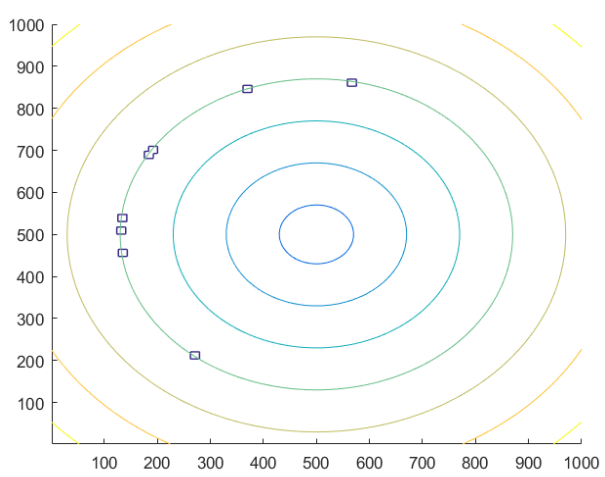

(a)

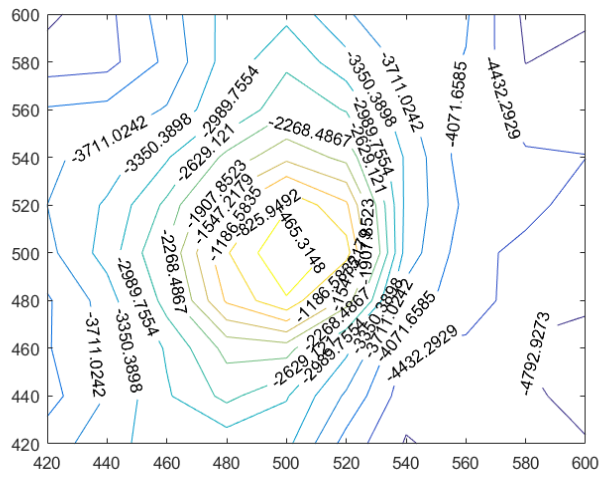

(b)

Figure 2 - (a) Simulated fire with no wind and homogenous fuel. The concentric circles represent the fire perimeter at various times, increasing from the center outward. The small squares represent simulated satellite fire detections at a time concurrent with the time of the fire perimeter which they overlay. (b) Contour map of data log-likelihood for simulated fire with ignition time $t=20$. The actual ignition point of the simulated fire was at the point (500,500).

For this first test, a collection of 500 simulations was made over a 10-by-10 spatial grid at five separate times. The data likelihood of each simulation was then computed and the best estimate of the time and place of ignition was determined using the likelihood function in equation (5). For the set of simulated detections used, the maximum likelihood of all simulations occurred at the correct "true ignition point" but at an incorrect ignition time. The "true ignition time" was $t=30$, but the estimation procedure gave an earlier time $t=20$. This is not a surprising result, as an earlier ignition time results in fire perimeter containing more of the area encompassed by the simulated fire detection pixels. As seen by the shape of the likelihood curve in Figure (d), areas within and close to the fire perimeter have a high probability of detection but areas outside of the perimeter at a similar distance may have a very low probability of detection. The earlier ignition time makes for a larger fire, which covers more of the detection squares, which leads to a greater data likelihood.

\subsection{Test Using the WRF-SFIRE Hill Ideal Experiment}

A second test of the data likelihood function to estimate time and place of ignition was made using the WRF-SFIRE coupled atmosphere-wildfire model. This system will be used to work with real fire data but for this second test, simulated fire data created by the "Hill Experiment" within WRF-SFIRE was used. The "Hill Experiment" simulates a fire in a square region with sides of length $2 \mathrm{~km}$ and contains a small, dome-shaped hill 100 meters tall situated in its center. A simple atmosphere state, with winds blowing from the northeast was used to create the initial conditions of the weather. Like the first simple test, a simulated fire was created, this time using the WRF-SFIRE model, and then artificial satellite fire detection pixels were created by hand by placing the centers of fire detection pixels on top of a particular fire perimeter. For this simulated fire, the ignition point was chosen to be 
$(x, y)=(1400 \mathrm{~m}, 1400 \mathrm{~m})$ and the ignition time was chosen to be $t=60 \mathrm{~s}$. Fire detection pixels were placed over the fire perimeter line corresponding to the time $t=400 \mathrm{~s}$.

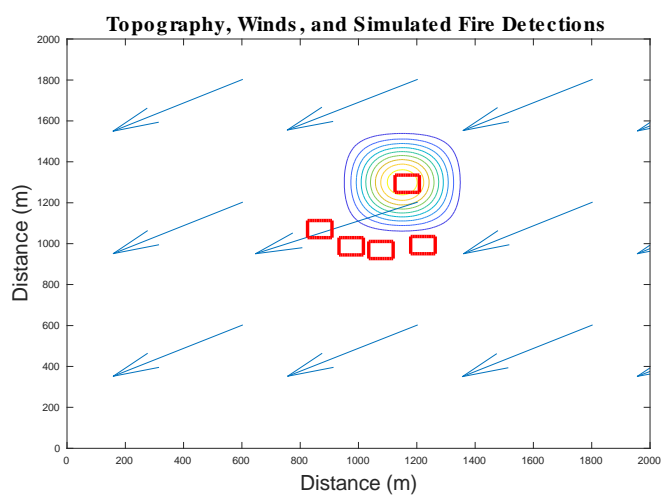

(a)

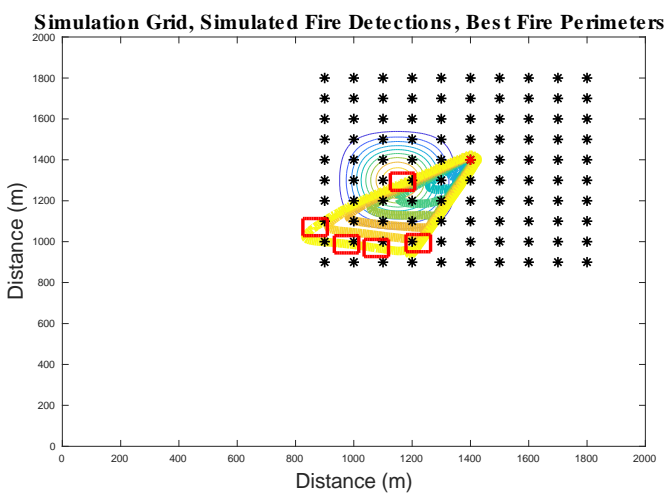

(b)

Figure 3 - (a) Topography, winds, and simulated fire detection pixels of the "Hill Experiment." The contour lines represent a small hill in the center of a simulated fire domain with winds initially blowing from the upper right corner. The rectangles within the figure are simulated fire detection pixels. (b) Fire simulation grid and fire perimeters of the simulation with largest data likelihood. Fire simulations were started at each of the points on the grid in the figure and the data likelihood of each simulation was then calculated. The colored contour lines represent the fire perimeters of the simulation with the maximum data likelihood.

Again, a large number of fire simulations were then run at various locations and times and the data likelihood of each was computed to give an estimate of the time and place of the true fire ignition. We ran 300 simulations of the fire on with trial ignition points selected on a 10-by-10 spatial grid, at three distinct ignition times. The true time and place of ignition was in the exact center of this threedimensional grid of the trial ignition points. The likelihood of the data associated with each simulation was computed and the maximum value found corresponded to the true ignition time and place.

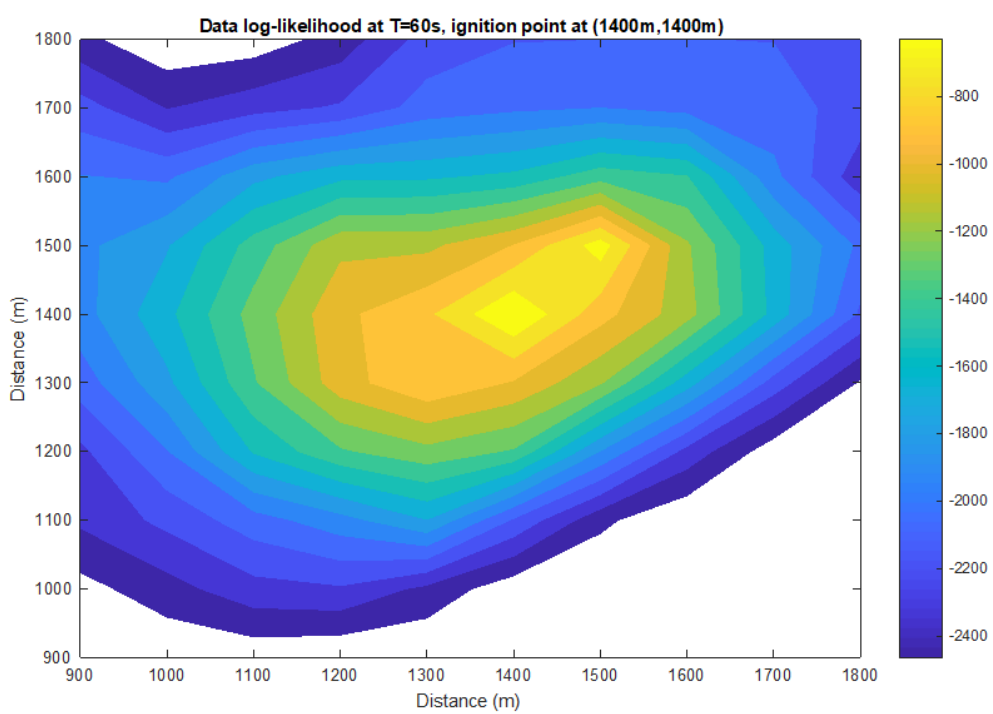

Figure 4 - Contour map of data log-likelihood for ignition points of the WRF_SFIRE Hill Experiment. In this case, the maximum likelihood gives the correct time and location of the fire ignition.

\subsection{The 2013 Patch Fire Experiment}

A third experiment was completed using satellite data from both MODIS and VIIRS to estimate the time and place of ignition of a real fire. The fire modeled is known as the "Patch Springs Fire" and occurred southwest of Salt Lake City, Utah in August, 2013. As with the other cases previously 
detailed, the time and place of the fire's ignition was estimated by running an ensemble of WRFSFIRE simulations at various times and places with the intent to estimate the ignition point by evaluating the data likelihood for each simulation. In this case, 1000 fire simulations were run on a 10by-10 spatial grid (Figure 5) at ten different ignition times. The spatial grid resolution was approximately 500 meters and the ignition times were spaced two hours apart, with the first ignition time occurring at 21:00 UTC on August 10. The data likelihood of each simulation was evaluated using satellite data from the first two days of the fire simulation period.

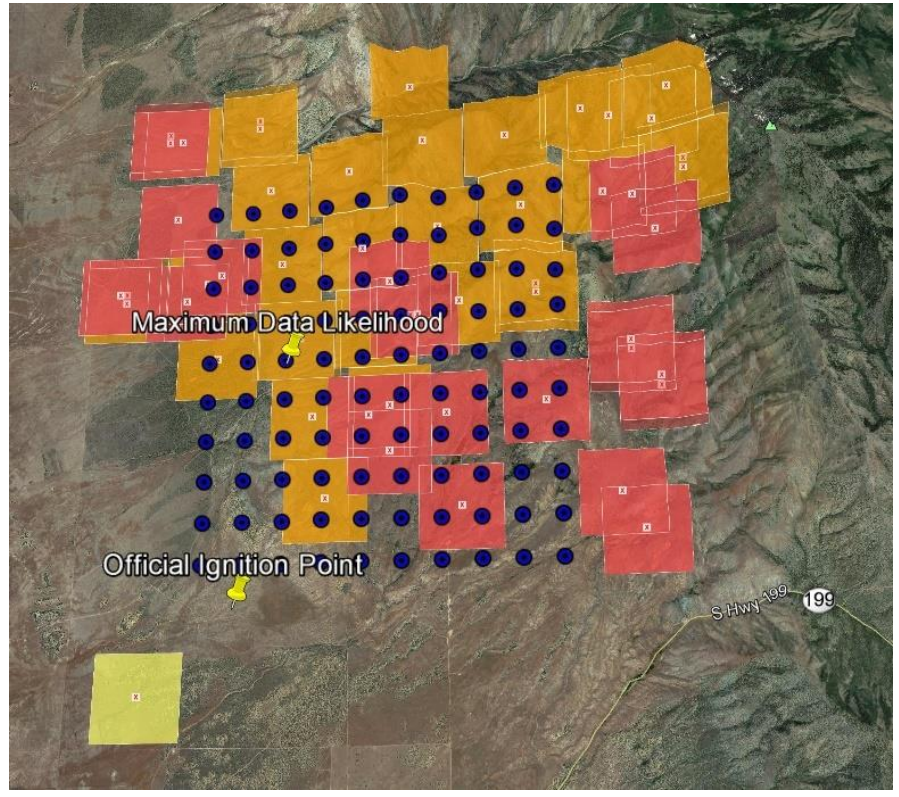

Contours of Data Likelihood

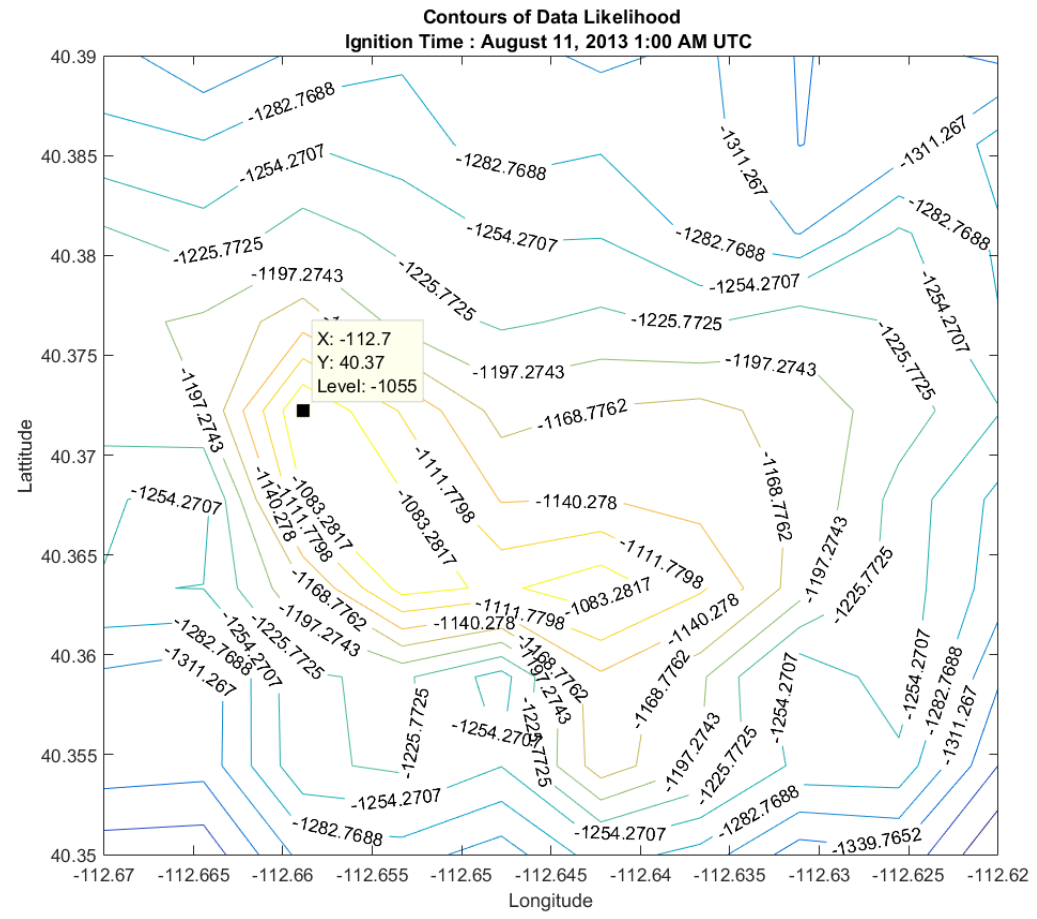

Figure 5 - Satellite fire detections and WRFSFIRE simulation locations for the Patch Springs Fire of August 2013. The large colored squares represent $1 \mathrm{~km}$ active fire locations from the MODIS satellites during the first two days of the fire. The evenly spaced blue points are locations at which fire simulations were run in order to estimate the time and place of the fire ignition. The official estimate of the ignition point lies just outside of the grid of simulations and the estimate of this location obtained by maximum data likelihood lies nearly $3 \mathrm{~km}$ to the north.

With all simulations run and the data likelihood of each evaluated, the estimated time and place of ignition was determined to be $40.372^{\circ} \mathrm{N},-112.659^{\circ} \mathrm{W}$ at $1: 00 \mathrm{UTC}$ on August 11, 2013 (Figure 6). Spatially, this estimate differs by nearly $3 \mathrm{~km}$ from the official ignition point determined by investigators. The estimated time of ignition is within an hour of the official ignition time of 2:00 UTC. This fire initially progressed to the northeast but eventually spread southward. It is probable that if that

Figure 6 - Contour map of data likelihood for simulations of the Patch Springs Fire on August 11, 2013. The contour lines are drawn from the data likelihood of all fires with simulated ignition times of 1:00 UTC. The best estimate of the fire's ignition point lies just left of center at approximately $40.37^{\circ} \mathrm{N},-112.7 \mathrm{~W}$. 
if longer simulations were run and more satellite fire detections showing this southward progression of the fire were used then our estimate would be closer to the official fire ignition location.

\section{Conclusion}

We have derived a physics-motivated likelihood function for active fires satellite detection data, and demonstrated its utility on identifying the ignition point of a wildland fire in time and space. The new data likelihood can be also useful in data assimilation for fire spread models, e.g., as a part of the objective function in an optimization approach (Farguel Caus et al. 2018).

\section{Acknowledgements}

This research was partially supported by grants NSF ICER-1664175 and NASA NNX13AH59G. High-performance computing support at CHPC at the University of Utah and Cheyenne (doi:10.5065/D6RX99HX) at NCAR CISL, sponsored by the NSF, are gratefully acknowledged. We would like to thank Brad Quayle and David Hayes from U.S. Forest Service for supplying the satellite data used in the example in Section 3.3.

\section{References}

Beezley JD, Mandel J (2008) Morphing ensemble Kalman filters. Tellus 60A, 131-140. doi:10.1111/j.1600-0870.2007.00275.x

Benali A, Russo A, Sá ACL, Pinto RMS, Price O, Koutsias N, Pereira JMC (2016) Determining fire dates and locating ignition points with satellite data. Remote Sensing 8, Article number 326. doi:10.3390/rs8040326

Brun C, Artes T, Cencerrado A, Margalef T, Cortés A (2017) A high performance computing framework for continental-scale forest fire spread prediction. Procedia Computer Science 108, 1712-1721. doi:10.1016/j.procs.2017.05.258

Clark TL, Coen J, Latham D (2004) Description of a coupled atmosphere-fire model. International Journal of Wildland Fire 13, 49-63. doi:10.1071/WF03043

Coen JL, Cameron M, Michalakes J, Patton EG, Riggan PJ, Yedinak KM (2013) WRF-Fire: Coupled weather-wildland fire modeling with the Weather Research and Forecasting model. Journal of Applied Meteorology and Climatology 52, 16-38. doi:10.1175/JAMC-D-12-023.1

Coen JL, Schroeder W (2013) Use of spatially refined satellite remote sensing fire detection data to initialize and evaluate coupled weather-wildfire growth model simulations. Geophysical Research Letters 40, 5536-5541. doi:10.1002/2013GL057868

Farguell Caus A, Haley J, Kochanski AK, Cortés Fité A, Mandel J (2018) Assimilation of fire perimeters and satellite detections by minimization of the residual in a fire spread model. Lecture Notes in Computer Science 10861, 711-723. doi: 10.1007/978-3-319-93701-4_56

Filippi J-B, Mallet V, Nader B (2013) Representation and evaluation of wildfire propagation simulations. International Journal of Wildland Fire 23, 46-57. doi:10.1071/WF12202

Giglio L, Schroeder W, Justice CO (2016) The collection 6 MODIS active fire detection algorithm and fire products. Remote Sensing of Environment 178, 31-41. doi:10.1016/j.rse.2016.02.054

Gollner M, Trouvé A, Altintas I, Block J, de Callafon R, Clements C, Cortes A, Ellicott E, Filippi JB, Finney M, Ide K, Jenkins MA, Jimenez D, Lautenberger C, Mandel J, Rochoux M, Simeoni A (2015) Towards data-driven operational wildfire spread modeling: A report of the NSF-funded WIFIRE workshop. https://wifire.ucsd.edu/files/pdf/WIFIRE_Wks_Report_FINAL.pdf 
Gu F (2018) On-demand data assimilation of large-scale spatial temporal systems using sequential Monte Carlo methods. Simulation Modelling Practice and Theory 85, 1-14. doi:10.1016/j.simpat.2018.03.007

Hawbaker TJ, Radeloff VC, Syphard AD, Zhu Z, Stewart SI (2008) Detection rates of the MODIS active fire product in the United States. Remote Sensing of Environment 112, 2656-2664. doi:10.1016/j.rse.2007.12.008

Koltunov A, Ustin SL, Quayle B, Schwind B, Ambrosia VG, Li W (2016) The development and first validation of the GOES early fire detection (GOES-EFD) algorithm. Remote Sensing of Environment 184, 436-453. doi:10.1016/j.rse.2016.07.021

Mandel J, Beezley JD, Coen JL, Kim M (2009) Data assimilation for wildland fires: Ensemble Kalman filters in coupled atmosphere-surface models. IEEE Control Systems Magazine 29, 47-65. doi:10.1109/MCS.2009.932224

Mandel J, Beezley JD, Kochanski AK (2011) Coupled atmosphere-wildland fire modeling with WRF 3.3 and SFIRE 2011. Geoscientific Model Development 4, 591-610. doi:10.5194/gmd-4-591-2011

Mandel J, Amram S, Beezley JD, Kelman G, Kochanski AK, Kondratenko VY, Lynn BH, Regev B, Vejmelka M (2014a) Recent advances and applications of WRF-SFIRE. Natural Hazards and Earth System Science 14, 2829-2845. doi:10.5194/nhess-14-2829-2014.

Mandel J, Kochanski AK, Vejmelka M, Beezley JD (2014b) Data assimilation of satellite fire detection in coupled atmosphere-fire simulations by WRF-SFIRE. Advances in Forest Fire Research, D. X. Viegas, ed., Coimbra University Press, 716-724. doi:10.14195/978-989-26-0884-6_80

Mandel J, Fournier A, Haley JD, Jenkins MA, Kochanski AK, Schranz S, Vejmelka M, Yen TY (2016a) Assimilation of MODIS and VIIRS satellite active fires detection in a coupled atmospherefire spread model. Poster, 5th Annual International Symposium on Data Assimilation, 18-22 July 2016, University of Reading, UK, http://www.isda2016.net/abstracts/ posters/MandelAssimilationof.html, retrieved December 2016.

Mandel J, Fournier A, Jenkins MA, Kochanski AK, Schranz S, Vejmelka M (2016b) Assimilation of satellite active fires detection into a coupled weather-fire model. Proceedings for the 5th International Fire Behavior and Fuels Conference April 11-15, 2016, Portland, Oregon, USA, International Association of Wildland Fire, Missoula, Montana, USA, 17-22, http://www.iawfonline.org/proceedings.php.

Rochoux MC, Emery C, Ricci S, Cuenot B, Trouve A (2014) Towards predictive simulation of wildfire spread at regional scale using ensemble-based data assimilation to correct the fire front position. Fire Safety Science 11, 1443-1456. doi:10.3801/IAFSS.FSS.11-1443

Sá A, Benali A, Pinto R, Fernandes P, Russo A, Santos F, Trigo R, Pereira J, Jerez S, Camara Cd (2014) Improving wildfire spread simulations using MODIS active fires: the FIRE- MODSAT project. Advances in Forest Fire Research, D. X. Viegas, ed., Coimbra University Pres, Coimbra, 811-822. doi:10.14195/978-989-26-0884-6_90

Sá ACL, Benali A, Fernandes PM, Renata P, Trigo RM, Salis M, Russo A, Jerez S, Soares PMM, Schroeder W, Pereira JMC (2017) Evaluating fire growth simulations using satellite active fire data. Remote Sensing of Environment 190, 302-317. doi:10.1016/j.rse.2016.12.023

Schroeder W, Oliva P, Giglio L, Csiszar IA (2014) The New VIIRS 375 m active fire detection data product: Algorithm description and initial assessment. Remote Sensing of Environment 143, 8596. doi:10.1016/j.rse.2013.12.008

Schroeder W, Prins E, Giglio L, Csiszar I, Schmidt C, J. Morisette, and D. Morton, 2008: Validation of GOES and MODIS active fire detection products using ASTER and ETM+ data. Remote Sensing of Environment 112, 2711-2726. doi:10.1016/j.rse.2008.01.005 
Sei A (2011) VIIRS active fires: Fire mask algorithm theoretical basis document. Available at https://www.star.nesdis.noaa.gov/jpss/documents/ATBD/D0001-M01-S01021_JPSS_ATBD_VIIRS-Active-Fires.pdf, Accessed May 2018.

Srivas T, de Callafon RA, Crawl D, Altintas I (2017) Data assimilation of wildfires with fuel adjustment factors in FARSITE using ensemble Kalman filtering. Procedia Computer Science 108, 1572-1581. doi:10.1016/j.procs.2017.05.197

Veraverbeke S, Sedano F, Hook SJ, Randerson JT, Jin Y, Rogers BM (2014) Mapping the daily progression of large wildland fires using MODIS active fire data. International Journal of Wildland Fire 23, 655-667. doi:10.1071/WF13015

Xue H, Gu F, Hu X (2012) Data assimilation using sequential Monte Carlo methods in wildfire spread simulation. ACM Transactions on Modeling and Computer Simulation 22, Article No. 23. doi:10.1145/2379810.2379816

\section{Appendix A}

The probability of MODIS fire detection is estimated in validation studies (Hawbaker et al. 2008; Schroeder et al. 2008) by logistic regression as a function of the fire size, or the fraction of the pixel actively burning, as

$$
P(d=1)=\frac{1}{1+\exp (-a F+b)},
$$

where $d=1$ means that a fire was detected and $d=0$ that it was not. The constants $a$ and $b$ depend on the properties of the fuel and its loading. They are commonly specified in terms of the false detection rate $1 /(1+\exp (b))$ for $F=0$, and the quantity $F_{0.5}$, defined by $1 /\left(1+\exp \left(-a F_{0.5}+b\right)\right)=0.5$, which determines $a$ as $a=b / F_{0.5}$. We consider the quantity $F$ in the logistic regression (1) as a proxy for the heat flux $h(x)$ over the pixel at $x$, and thus use (1) with $h(x)$ in place of $F$. This view is supported by the facts that the quantity the hardware sensor actually measures is the radiative power (in its frequency bands); a more accurate regression was obtained in Schroeder et al. (2008) by adding as another regressor the maximum contiguous area burning, which is essentially a proxy for the fire intensity and thus radiative power, which the fine-resolution sensor used for the validation could not measure; and 50\% probability of VIIRS detection occurs at nearly constant product of fire size and black body radiation intensity over a range of fire sizes and fire temperatures, as one can compute from Figure 4 in Schroeder et al. (2014).

The heat flux is modelled from the burn model in WRF-SFIRE (Mandel et al., 2011) as identically zero before the fire arrival time $T$ and by a decaying exponential function afterwards,

$$
h(T)=\left\{\begin{array}{lll}
e^{-\left(T_{\text {now }}-T\right) / c} & \text { if } & T_{\text {now }} \geq T \\
0 & \text { if } & T_{\text {now }}<T
\end{array}\right.
$$

where $T_{\text {now }}$ is the time of the satellite imaging (Figure 1(a)). However, because of the geolocation error, fire detection in a pixel with nominal coordinates $x=\left(x_{1}, x_{2}\right)$ is a two-step random process. Assume that the geolocation error is a Gaussian random variable in two dimensions with zero mean and isotropic standard deviation $\sigma$. Then, the location $y=\left(y_{1}, y_{2}\right)$ that the sensor is actually looking at is a random variable with the probability density

$$
P(y \mid x)=\frac{1}{2 \pi \sigma^{2}} \exp \left(-\frac{\|x-y\|^{2}}{2 \sigma^{2}}\right),
$$


and the probability of detection is given by the logistic formula (1) with $F=h(T(y))$, the fire heat flux at $y$, with $y$ sampled following (3). Assuming further that the geolocation error and the fire detection outcome are independent random variables, the probability of the detection is then given by the probability mixture

$$
P(d=1 \text { at } x \mid T)=\iint \frac{1}{2 \pi \sigma^{2}} \exp \left(-\frac{\|x-y\|^{2}}{2 \sigma^{2}}\right) \frac{1}{1+\exp (-a h(T(y))+b)} d y_{1} d y_{2} .
$$

The probability of no fire detection (i.e., ground without fire) is the complementary probability,

$$
\begin{aligned}
P(d=0 \text { at } x \mid T) & =1-P(d=1 \text { at } x \mid T) \\
& =\iint \frac{1}{2 \pi \sigma^{2}} \exp \left(-\frac{\|x-y\|^{2}}{2 \sigma^{2}}\right)\left(1-\frac{1}{1+\exp (-a h(T(y))+b)}\right) d y_{1} d y_{2}
\end{aligned}
$$

since

$$
\iint \frac{1}{2 \pi \sigma^{2}} \exp \left(-\frac{\|x-y\|^{2}}{2 \sigma^{2}}\right) d y_{1} d y_{2}=1
$$

To recover a form of data likelihood similar to that in Mandel et al. (2014b), consider the case of a straight fire line propagating in unit normal direction $n$ with rate of spread $R$. Then,

$$
T(y)=T(x)+\frac{\langle y-x, n\rangle}{R} .
$$

Substituting $T(y)$ into (4), we see that, in particular, the probability of fire detection at $x$ depends on $T(x)$ only. Now take $n=(0,1)$ and $x=(0,0)$, then (6) becomes $T(y)=T(0,0)+y_{1} / R$, and we have that

$$
P(d=1 \text { at } x \mid T(x))=\iint \frac{1}{2 \pi \sigma^{2}} \exp \left(-\frac{\left(x_{1}-y_{1}\right)^{2}+y_{2}^{2}}{2 \sigma^{2}}\right) \frac{1}{1+\exp \left(-a h\left(T(x)+\frac{y_{1}}{R}\right)+b\right)} d y_{1} d y_{2} \text {. }
$$

The graph of an example of this likelihood function is shown in Figure 1(d), which is indeed similar to the likelihood from Mandel et al. (2014b, 2016b).

For data assimilation and identification of the ignition point, however, we need likelihood computed for the entire satellite image, not just one pixel. First, from (5), we have for both $d=1$ and $d=0$ that

$$
P(d \text { at } x \mid T)=\iint \frac{1}{2 \pi \sigma^{2}} \exp \left(-\frac{\|x-y\|^{2}}{2 \sigma^{2}}\right)\left(1-d+\frac{2 d-1}{1+\exp (-a h(T(y))+b)}\right) d y_{1} d y_{2} .
$$

So suppose we are given a mesh of pixels at nominal locations $x^{i}=\left(x_{1}{ }^{i}, x_{2}{ }^{i}\right)$ and corresponding detection data $d_{i}=0$ or 1 , with confidence levels $c_{i}$ between 0 and 1 . Confidence level 0 means missing data. Assume for simplicity that the detections are independent random variables, thus the detection probabilities at individual pixels multiply (and their logarithms add), and discretize the integral in (4) by summation over the same mesh. Then, we can approximate the log likelihood of the detection array $d=\left(d_{i}\right)$ given the array of fire arrival times $T=\left(T\left(x^{j}\right)\right)$ by

$$
\log P(d \mid T)=\sum_{i} c_{i} \log \sum_{j} w_{i} \exp \left(-\frac{\left\|x^{i}-x^{j}\right\|^{2}}{2 \sigma^{2}}\right)\left(1-d_{i}+\frac{2 d_{i}-1}{1+\exp \left(-a h\left(T\left(x^{j}\right)\right)+b\right)}\right),
$$

where $w_{i}$ are normalization constants such that

$$
w_{i} \sum_{j} \exp \left(-\frac{\left\|x^{i}-x^{j}\right\|^{2}}{2 \sigma^{2}}\right)=1 .
$$

The data likelihood (9) is used in the examples in Section 3. 


\title{
Detecting Peatland Combustion using Shortwave and Thermal Infrared Landsat-8 data
}

\author{
Parwati Sofan ${ }^{1 *}$; David Bruce ${ }^{2}$; Eriita Jones ${ }^{3}$, Jackie Marsden ${ }^{4}$ \\ ${ }^{1}$ PhD candidate, the University of South Australia (UniSA); Remote Sensing Application Center of \\ Indonesian Institute of Aeronautics and Space (LAPAN), Adelaide South Australia, \\ \{parwati.sofan@mymail.unisa.edu.au*\} \\ ${ }^{2}$ Natural and Built Environments Research Centre, UniSA, Adelaide, South Australia, \\ \{david.bruce@unisa.edu.au\} \\ ${ }^{3}$ School of IT and Mathematical Sciences, UniSA. Adelaide, South Australia, \\ \{eriita.jones@unisa.edu.au\} \\ ${ }^{4}$ Imagery, Landgate. Midland, West Australia, \{Jackie.Marsden@landgate.wa.gov.au\}
}

\begin{abstract}
Peatland fires occur every year in Indonesia. These fires usually occur in the dry season due to agricultural and plantation expansion practices, which include slash and burn activities. Peatland combustion fires can be classified into smouldering and flaming. Smouldering is described by lower temperatures, spread rates and combustion power compared with flaming. Most of the standard fire products from remote sensing satellites use an apparent fire temperature at $4 \mu \mathrm{m}$ in the Middle Infrared (MIR) and background temperature at $11 \mu \mathrm{m}$ in Thermal Infrared (TIR). These products only report combustion in a single term as an "active fire" or "fire hotspot", and do not discriminate between smouldering and flaming states. In this research, the Shortwave Infrared (SWIR) and TIR bands of day-time Landsat-8 were explored to discriminate smouldering and flaming states of peatland combustion. Initial identification of possible combustion areas was from visual observation of false colour composite images (bands 764 to RGB) of Landsat-8, which presented smoke plumes in pale bluish tones emanating from a defined ground location. The potential combustion areas were further investigated using an implementation of the Hyperspectral Fire Detection Index based on SWIR of Landsat-8 and named here as the Shortwave Infrared Combustion Index (SICI). Potential combustion areas were also analysed using radiances of the SWIR (band 7) and TIR (band 10), which exhibited values close to their saturation. From these radiances, a radiative transfer model using MODTRAN4 and Planck's law, the fire temperatures were estimated. Preliminary results showed that the flaming states, with higher temperatures than smouldering states, are coincident with SWIR radiances near saturation $\left(24-29 \mathrm{Wm}^{-2} \mathrm{sr}^{-1} \mu \mathrm{m}^{-1}\right.$ for band 7) and TIR (band 10) $>12 \mathrm{Wm}^{-2} \mathrm{sr}^{-1} \mu \mathrm{m}^{-1}$, while smouldering states, with lower temperatures, occur near flaming areas with lower SWIR radiance and TIR radiance above $11 \mathrm{Wm}^{-2} \mathrm{sr}^{-1} \mu \mathrm{m}^{-1}$. Based on Landsat-8 imagery on $3^{\text {rd }}$ and $19^{\text {th }}$ August 2015, fire temperatures of flaming areas were estimated at $700-758 \mathrm{~K}$, mixtures of flaming and smouldering at $500-665 \mathrm{~K}$, and smouldering at $320-499 \mathrm{~K}$. This result aligned with prior research that sampled in situ smouldering, smouldering close to flaming, and flaming temperatures taken from Indonesian peatland. The algorithm developed in the research reported here was able to map some combustion areas that could not be mapped using lower spatial resolution imagery from MODIS. However, the results will undergo further investigation for atmospheric influence such as haze and thin cloud. Results also will be validated with other data such as field measurements. The algorithm developed in this research has potential to be used on a routine basis due to the availability of day-time Landsat- 8 data which, at best, has a temporal resolution of 16 days. It can also be implemented for forest fire detection at a detailed scale to complement other fire information, but again temporal resolution is an issue. This research is part of a larger project which is utilising automated smoke detection and soil moisture measurement in the mapping of peatland combustion from space imagery.
\end{abstract}

Keywords: Flaming, smouldering, peatland fires, Landsat-8, remote sensing, SWIR, TIR 


\section{Introduction}

In Indonesia, peatland combustion usually has an anthropogenic origin due to agricultural and plantation expansions, which are mostly accomplished through slash and burn activities (Miettinen et al. 2012; Rieley and Page 2016). Most peatland fires occur during the dry season (July-September) when the peatland is drier than in other times of the year and more susceptible to combustion (Usup et al. 2014, Hayasaka et al. 2016). The devastating forest and peatland fires in Indonesia in 2015 caused a total economic loss of about USD 16 billion (Worldbank 2016), released approximately $1.75 \mathrm{gt}$ of atmospheric CO2 (Worldbank 2016) and contributed to 100,000 premature deaths (Burrows 2016).

In general, peatland combustion consists of flaming and smouldering. Smouldering occurs with lower temperatures, spread rates and combustion power compared with flaming. Once ignition has occurred, smouldering can exist for long periods and extinguishment is dependent on heavy rainfall or fire-fighting attempts (Rein 2016; Zaccone et al. 2014). Determination of flaming and smouldering phases of peatland combustion from remote sensing data is important for peat fire management before the fire extends into a large area. However, routine satellite-based monitoring to supply smouldering and flaming information has not yet been effectively established. MODIS and VIIRS sensors, generate routine global active fire monitoring products with a low spatial resolution of $1000 \mathrm{~m}$ (MODIS), 375 $\mathrm{m}$ and $750 \mathrm{~m}$ (VIIRS). These sensors utilise the Middle Infrared (MIR) and Thermal Infrared (TIR) to detect active fires with algorithms utilising at a contextual approach, aimed at identifying a combustion pixel relative to a local background and consist of pathways to establish potential fire pixels (Justice et al. 2002; Giglio et al. 2016; Schroeder et al. 2014; Csiszar et al. 2014).

Research on detection of peatland combustion has been conducted with medium spatial resolution at 30-100 m, Landsat-8 night-time data, using an implementation of Planck's Law on radiant emission and temperature from Shortwave Infrared (SWIR) and TIR in Indonesia's Kalimantan peatland (Elvidge et al. 2015). At night, no sunlight interferes with the SWIR and TIR radiances over fire areas with sensors only recording emitted radiation. However, the availability of Landsat- 8 night-time data is limited with provision only for special requests. There is a possibility to utilise day-time Landsat- 8 data, which is freely available every 16 days (atmosphere permitting) to produce flaming and smouldering information. Flaming detection has been explored by Schroeder et al. (2016) who measured SWIR radiance (band 7) of Landsat-8, which reached saturation from $30 \mathrm{~m} 2$ areas of woody burning material in Germany and Brazil, where ground fire temperatures were measured between 870 and $970 \mathrm{~K}$ as obtained from a thermal handheld sensor. Dennison and Roberts (2009) found that SWIR bands at 2.061 and $2.429 \mu \mathrm{m}$ could be utilised in the Hyperspectral Fire Detection Index (HFDI) for flaming detection. Meanwhile, the detection of peatland smouldering based on satellite remote sensing in day-time data remains a challenge, due to its lower radiant emission than that from flaming. The lower temperature of smouldering combustion may be more accurately captured through the use of the longer wavelength infrared or TIR.

In this research, investigation of day-time Landsat- 8 data was conducted to distinguish peatland flaming and smouldering states. Landsat- 8 has the spectral capability to detect combustion from SWIR and TIR, and also with its medium spatial resolution of $30 \mathrm{~m}$ and $100 \mathrm{~m}$, potentially able to detect small burning areas that are often found in the field especially in the initial stages of combustion. This information will be useful for early fire suppression before it extends into a larger area.

\section{Materials and Methods}

The study area for this research is located in Central Kalimantan, one of the severe peat fire areas in Indonesia. Level 1T (Terrain Corrected) Landsat 8 data (path/row 118/062; August 3rd and 19th, 2015) of the study area were accessed through the U.S. Geological Survey (USGS) Earth Explorer website. The MODIS fire-mask product-collection 6 for both days 3rd and 19th August 2015 were used in order to compare with the Landsat-8's results. 
The first identification of possible combustion was from visual inspection of the false colour image composite: bands 764 to Red Green Blue (RGB). Potential combustion areas were further investigated using a SWIR index which is here named as the Shortwave Infrared Combustion Index (SICI), the ratio of radiance of bands 6 and 7 of Landsat-8, combined with the SWIR and TIR radiances. Based on Planck's Law (relating temperature and wavelength), smouldering fire states will have lower SWIR radiance than flaming, and higher TIR radiance. Subsequently the combustion temperature was estimated using a radiative transfer model (MODTRAN4) combined with Planck's equation for SWIR and TIR (Barduci et al. 2004; Qian and Kong 2012). To evaluate the precision of the combustion detection, the results were overlaid with the same data of RGB images of Landsat- 8 . In addition, the results were also compared with the MODIS fire-mask product.

\section{Results and Discussion}

\subsection{Peat Combustion Identification}

Since the ground validation of peatland combustion was not available at the date of Landsat-8 observations of the study area, the potential combustion areas were visually interpreted from the RGB764 of Landsat-8. There were 9 and 22 samples of potential combustion areas visible in these images in August 3rd and 19th, 2015 respectively in Central Kalimantan's peatland, as shown in Figure 1a and $1 \mathrm{~b}$. The enlarged picture of sample number 9 in Figure 1a shows bluish smoke emanating from combustion areas surrounding the burnt area in magenta tones. Sixteen days after that, Figure $1 b$ showed an extended burnt area in and around and south-west of sample number 9 with some active combustion areas showing bluish smoke in the lower-left part of burnt area. The combustion pixels exhibit in light yellow tones surrounded with orange and pink tones. All the potential combustion areas and non-combustion areas were then analysed statistically to obtain the radiance value of each SWIR and TIR band of Landsat-8. The potential combustion areas and non-combustion areas were chosen by selecting the area which has SWIR and TIR radiances less than and equal to $33 \%$ of coefficient of variation (the ratio of the standard deviation to the mean) from each landcover. In the study area, the landcover types which represented the non-combustion areas were burnt area, forest, and urban. Most combustion areas were located around burnt areas or vegetated areas (forest) as extensions of plantation areas. Urban/city areas are typically bright (high radiance) and therefore could be a potential source of commission errors in the calculation of combustion area. The selected areas are shown in Figure 1a. There were 563 potential combustion pixels. Non-combustion areas consisted of 1,594 pixels of burnt area, 84,049 pixels of forest, and 8,424 pixels of urban that were visually interpreted from the RGB images of Landsat-8. In Figure 1c box plots contain the minimum, quartile-1, median, quartile-3, and maximum values for combustion and non-combustion areas for every SWIR's radiance (band 6 and 7) and TIR's radiance (band 10). These show, not unsurprisingly, that combustion areas had highest radiance in bands 6 and 7 compared with other objects, while in band 10 the radiances of combustion areas were close to those of burnt areas. Some of the pixels in the combustion areas were close to saturation at radiances of $89.8,28.5$, and $20.9 \mathrm{Wm}-2 \mathrm{sr}-1 \mu \mathrm{m}-1$ at band 6 , band 7 , and band 10, respectively. Even though the median values of combustion and burnt areas were about $11 \mathrm{Wm}-2 \mathrm{sr}-$ $1 \mu \mathrm{m}-1$, none of the burnt area radiances were close to saturation in band 10. In Figure 1d, the SICI box plot of combustion areas shows about $75 \%$ of SICI were different to burnt area SICI values. About $25 \%$ of the SICI values in combustion areas exhibited the same values as that of burnt and urban areas. In order to have the maximum identification of potential combustion areas, and minimum mixture with burnt and urban areas, SICI values less than the 1st quartile of burnt areas (3.0) were adopted. The SICI value at 3.0 is shown with a blue line in Figure 1d. The potential combustion areas were then investigated further using radiance values of bands 7 and 10 as presented in Figure 1c to separate the combustion areas from non-combustion areas (burnt area, urban, and forest). 
a)
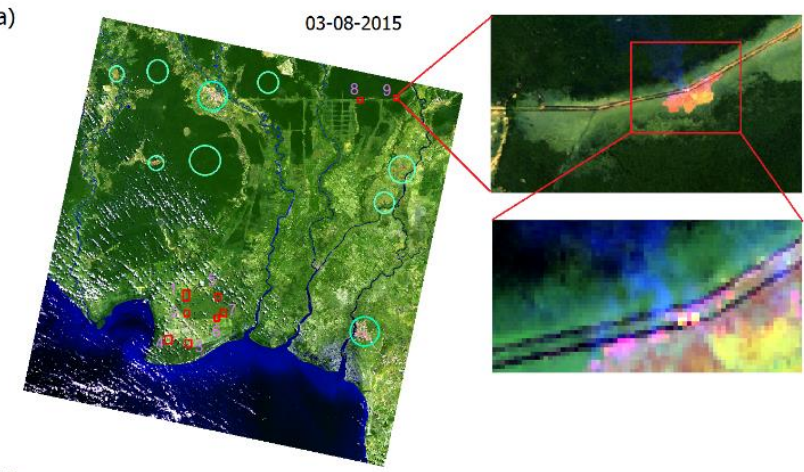

b)

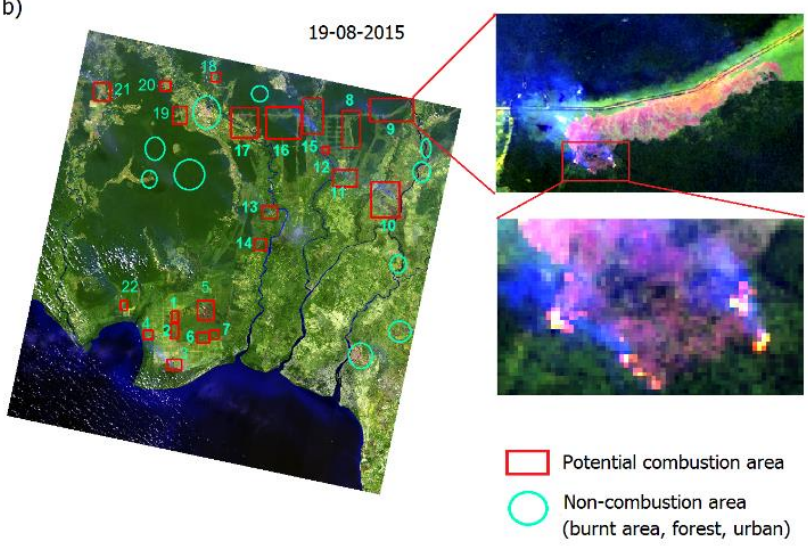

c)
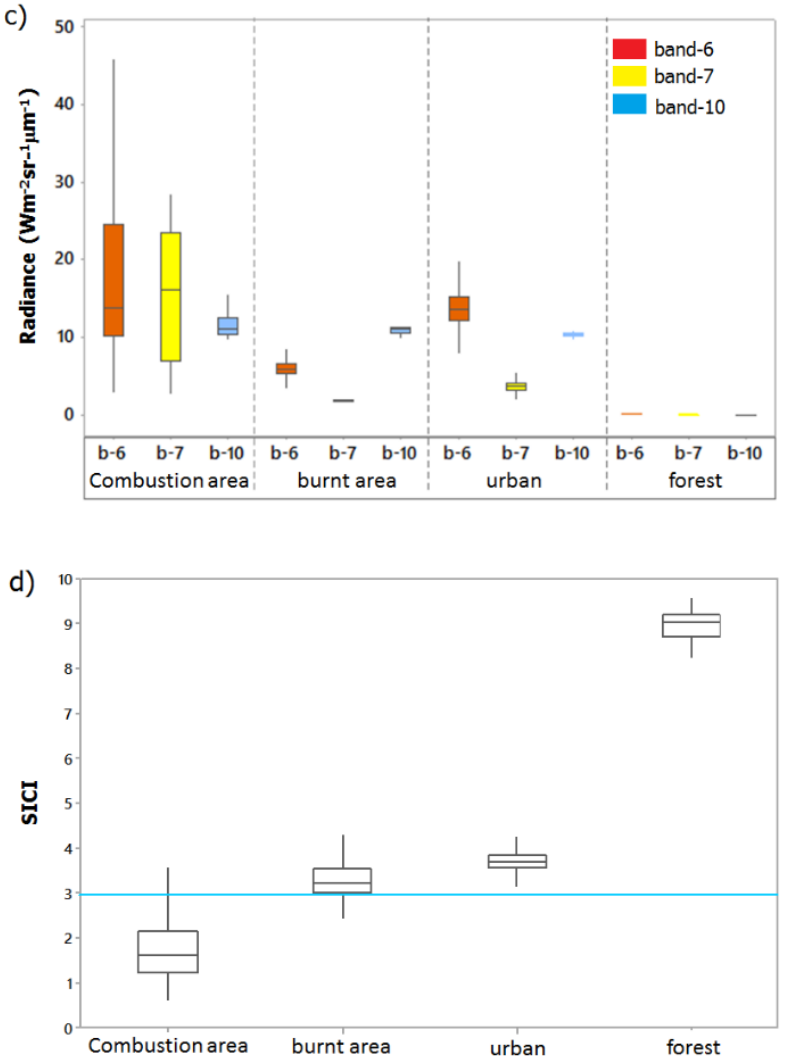

Figure 1 - $(a, b)$ The 764-RGB image of Landsat-8 on August $3^{\text {rd }}$ and August 19 ${ }^{\text {th }}, 2015$ with 9 samples and 21 samples respectively of potential combustion areas shown inside red squares and non-combustion areas inside cyan circles, respectively. An enlargement of one potential combustion sample area is presented on the right side of each Landsat image, (c) Box plots of radiance values of SWIR and TIR bands over combustion, burnt, forest, and urban areas, (d) SICI's box plots in combustion and non-combustion areas with the blue line indicating a threshold of 3.0 (the $1^{\text {rd }}$ quartile value of burnt area) which was used to separate combustion from other areas. 
An example of identification of peatland combustion using the SICI, SWIR radiance, and TIR radiance is shown in Figure 2. The combustion area in the 764-RGB image, shown in orange to yellow tones (Fig. 2a), had radiance values that were close to SWIR (band 7) saturation at $24-29 \mathrm{Wm}-2 \mathrm{sr}-$ $1 \mu \mathrm{m}-1$ (Fig. 2b). This agreed with findings by Schroeder et al. (2016). These areas are observed with TIR radiance greater than $12 \mathrm{Wm}-2 \mathrm{sr}-1 \mu \mathrm{m}-1$ (saturation occurs at $20.9 \mathrm{Wm}-2 \mathrm{sr}-1 \mu \mathrm{m}-1$ for band 10) (Fig. 2c). SICI values less than 3 represented the potential combustion area shown in Fig. 2d. Pixels with SWIR and TIR's radiance close to saturation were assumed in a flaming stage (Fig. 2e), while the other neighbouring pixels located inside the SICI $<3$ area with lower radiance values than flaming pixel, i.e. $11-20 \mathrm{Wm}-2 \mathrm{sr}-1 \mu \mathrm{m}-1$ for band 7 and $11-20.9 \mathrm{Wm}-2 \mathrm{sr}-1 \mu \mathrm{m}-1$ for band 10 , were assumed to be in a smouldering stage (Fig. 2f); these corresponded to the orange tones in the 764-RGB image (Fig 2a). The classified flaming and smouldering areas were merged in Fig. 2g. However, some of combustion pixels were not identified as flaming or smouldering pixels in Fig. $2 \mathrm{~g}$. These were then classified as mixed pixels, exhibiting both flaming and smouldering states. The mixed pixels had lower TIR (band 10) radiances compared with flaming and smouldering pixels. In Figure $2 \mathrm{~h}$, the mixed flaming and smouldering pixels are mapped in a cyan tone. The median values of SICI of flaming, smouldering, and mixture of flaming and smouldering pixels were 1.4, 2.2, and 2.6, respectively (Fig. 2i).
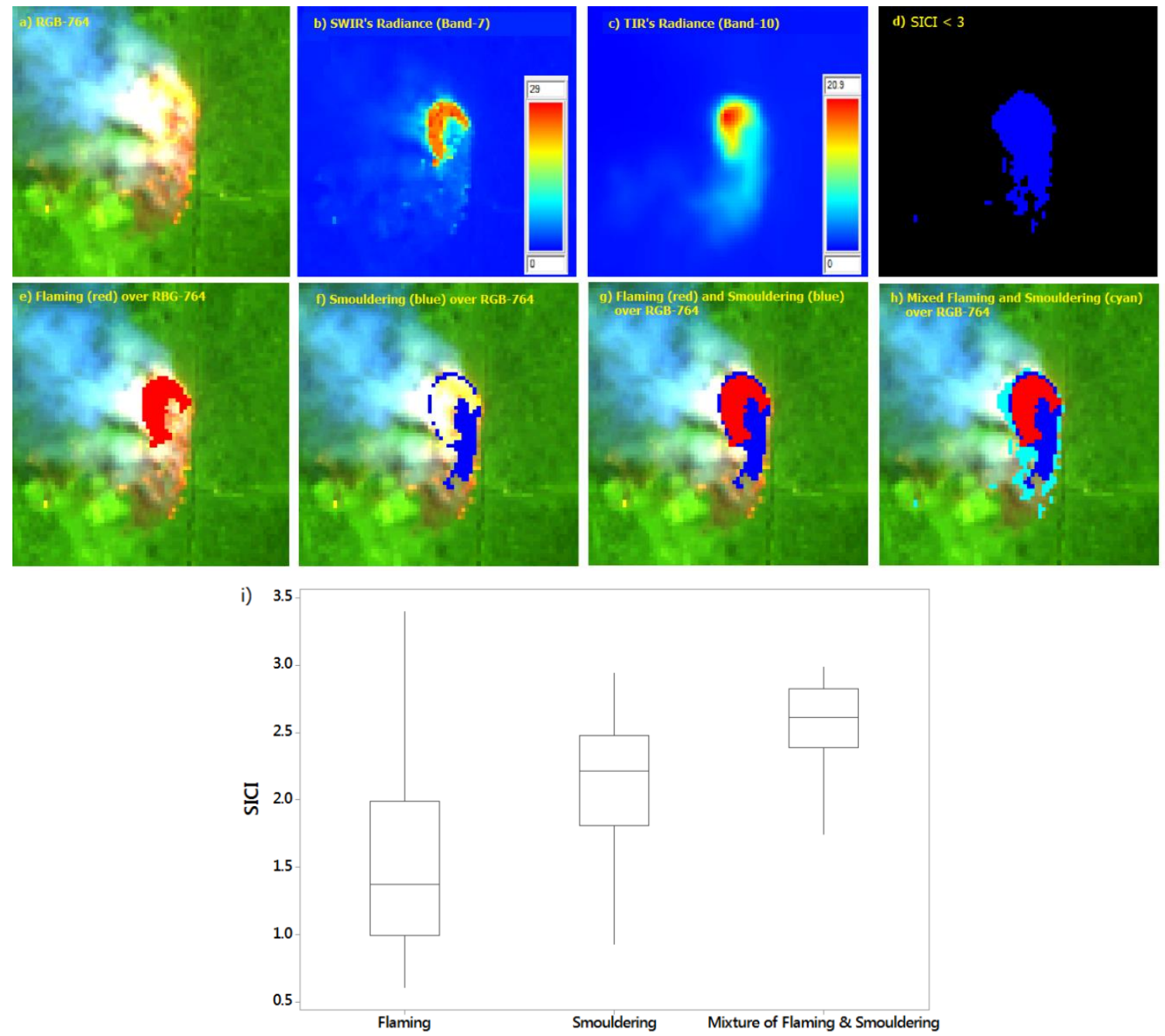

Figure 2 -Example of peatland combustion identification (a) Smoke in pale bluish tones showed in 764-RGB with the potential combustion area in orange to yellow tones, $(b \& c)$ Pseudo colour of the radiance of band-7 and band-10 respectively were just under / at saturation, (d) The SICI <3 in blue as the potential combustion area, $(e, f, g)$ The identified flaming (red) and smouldering (blue) areas, (h) Mixture of flaming and smouldering areas shown in cyan over the 764-RGB image, (i) Box plots of SICI over flaming, smouldering, and mixture of flaming and smouldering areas 


\subsection{Fire Temperature Estimation}

The estimated fire temperature based on SWIR (bands 6-7) and TIR (band 10) over identified combustion pixels in Landsat-8 images on $3^{\text {rd }}$ and $19^{\text {th }}$ August 2015 is plotted in Figure 3. Figure $3 \mathrm{a}$ shows a boxplot representing the minimum, quartile $1^{\text {st }}$, median, quartile $3^{\text {rd }}$ and maximum values of smouldering (S), mixture of flaming and smouldering (FS), and flaming (F). There were $402 \mathrm{~S}$ pixels in range of $320 \mathrm{~K}$ to $499 \mathrm{~K}, 8,587 \mathrm{FS}$ pixels in range of $500 \mathrm{~K}$ to $665 \mathrm{~K}$, and $103 \mathrm{~F}$ pixels with temperature of $700 \mathrm{~K}$ to $758 \mathrm{~K}$. The median values of $\mathrm{S}, \mathrm{FS}$, and $\mathrm{F}$ temperatures were about $476 \mathrm{~K}$, $585 \mathrm{~K}$, and $722 \mathrm{~K}$, respectively. According to Usup et al (2014), smouldering temperatures observed from ground measurements were about 320 - $500 \mathrm{~K}$, and Elvidge et al (2015) found smouldering close to flaming at about $500-700 \mathrm{~K}$, and flaming at about $700-1000 \mathrm{~K}$. Thus, the estimated peatland fire temperatures from Landsat- 8 in all the peatland combustion stages were in the range of those provided by Usup et al (2014) and Elvidge et al (2015) but estimated flaming temperatures could not be higher than $758 \mathrm{~K}$ due to the saturation of band-6 in Landsat-8. Figure $3 \mathrm{~b}$ shows a dotplot of $\mathrm{S}$, FS, and F temperatures with each dot representing the average of up to 32 observations. The dotplot shows the distribution of the estimated fire temperature from SWIR and TIR bands. Figure $3 \mathrm{~b}$ also shows the distribution of FS temperature with two peaks of temperature of $505 \mathrm{~K}$ and $595 \mathrm{~K}$ which represented the most common temperatures in FS. However, the dotplot of FS shows a left skewed distribution which possibly means a higher contribution of flaming than smouldering temperatures.

a)

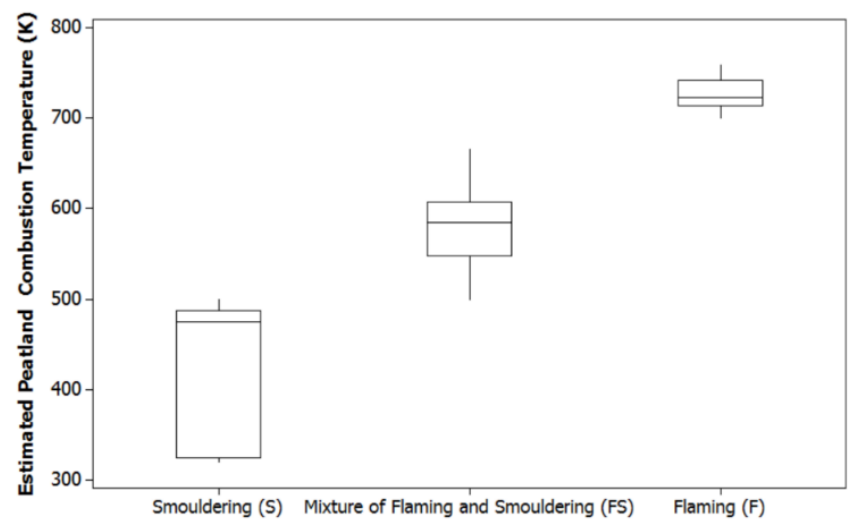

b)

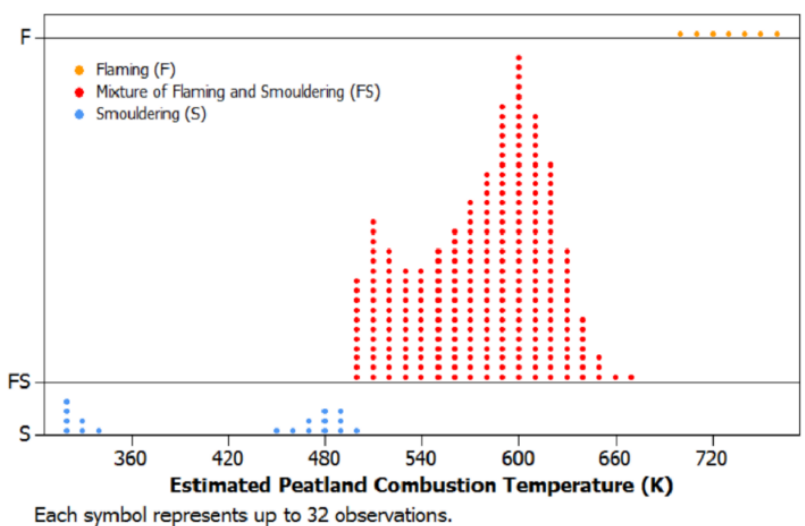

Figure 3 - (a) Boxplot represents the minimum, quartile $1^{\text {st }}$, median, quartile $3^{\text {rd }}$ and maximum values of smouldering

$(S)$, mixture of flaming and smouldering $(F S)$, and flaming $(F),(b)$ The dotplot of estimated peatland combustion temperature on F, FS, and $S$ with two peaks of temperature and left-skewed distribution indicating a higher contribution of flaming than smouldering to FS temperature. 


\subsection{Precision and Comparison}

The methodology outlined in section 3.1 was implemented over 2 images including all 31 of potential combustion areas and non-combustion areas. Table 1 shows the 31 of potential combustion areas identified as flaming $(\mathrm{F})$, smouldering $(\mathrm{S})$ or mixture of flaming and smouldering (FS). Overall, the result of combustion identification over all the 31 samples shows good mapping precision (Table 1). The mixture of flaming and smouldering pixels was identified in all the samples, while flaming or smouldering did not exist in every sample. The spatial resolution $(100 \mathrm{~m})$ of TIR may lead to the apparent mixture of flaming and smouldering stages. In one case, the mixture of flaming and smouldering was also found in a commission error in a burnt area separate from a combustion area. In one combustion area experiencing smoky conditions, the flaming and smouldering detection had an omission error leading to less combustion pixels being detected. The mixed flaming and smouldering pixels were also over mapped, producing commission errors over urban areas. The total hit detection was 31 samples with 1 sample of omission error and 6 samples of commission error. Overall, the precision of combustion detection was about $77 \%$ compared to the visual interpretation of 764-RGB image. This is an early estimation of mapping precision and is not an estimate of accuracy which can only be achieved with independent data such as ground data. To reduce omission and commission errors issues associated with smoke, cloud, urban land cover, and bright objects will be dealt with in the next step in the research.

Table 1 - Detection of flaming (F), smouldering (S), and mixture of flaming and smouldering (FS) over 2 Landsat-8 images (03-082015 and 19-08-2015). The hit, Commission $(C)$ and Omission $(O)$ Errors were derived from comparison with the 764-RGB of Landsat-8.

\begin{tabular}{|c|c|c|c|c|c|c|c|c|c|c|c|c|c|}
\hline \multicolumn{7}{|c|}{ Landsat-8 (03-08-2015) } & \multicolumn{7}{|c|}{ Landsat-8 (19-08-2015) } \\
\hline \multirow{2}{*}{ No } & \multicolumn{3}{|c|}{ Hit } & \multirow{2}{*}{$\mathbf{O}$} & \multirow{2}{*}{$\mathbf{C}$} & \multirow{2}{*}{ Note } & \multirow{2}{*}{ No } & \multicolumn{3}{|c|}{ Hit } & \multirow{2}{*}{$\mathbf{O}$} & \multirow{2}{*}{$\mathbf{C}$} & \multirow{2}{*}{ Note } \\
\hline & $\mathbf{F}$ & $\mathbf{S}$ & FS & & & & & $\mathbf{F}$ & $\mathbf{S}$ & FS & & & \\
\hline 1 & $\checkmark$ & - & $\checkmark$ & - & - & with smoke & 1 & $\checkmark$ & - & $\checkmark$ & - & - & with smoke \\
\hline 2 & $\checkmark$ & - & $\checkmark$ & - & - & with smoke & 2 & $\checkmark$ & & $\checkmark$ & - & - & $\begin{array}{l}\text { with smoke; Extended burning area from 03- } \\
08-2018\end{array}$ \\
\hline 3 & $\checkmark$ & - & $\checkmark$ & - & $\checkmark$ & $\begin{array}{l}\text { Commission on FS } \\
\text { over burnt area }\end{array}$ & 3 & $\checkmark$ & $\checkmark$ & $\checkmark$ & - & - & with smoke \\
\hline 4 & - & - & $\checkmark$ & - & $\checkmark$ & No smoke & 4 & $\checkmark$ & - & $\checkmark$ & - & $\checkmark$ & No smoke \\
\hline 5 & $\checkmark$ & - & $\checkmark$ & - & - & with smoke & 5 & $\checkmark$ & - & $\checkmark$ & - & - & $\begin{array}{l}\text { Extended burning area from 03-08-2018 with } \\
\text { smoke }\end{array}$ \\
\hline 6 & $\checkmark$ & - & $\checkmark$ & - & - & with smoke & 6 & $\checkmark$ & $\checkmark$ & $\checkmark$ & - & - & $\begin{array}{l}\text { Extended burning area from 03-08-2018 with } \\
\text { smoke }\end{array}$ \\
\hline 7 & - & - & $\checkmark$ & - & - & No smoke & 7 & $\checkmark$ & - & $\checkmark$ & - & - & No smoke \\
\hline 8 & - & - & $\checkmark$ & - & - & with smoke & 8 & $\checkmark$ & - & $\checkmark$ & - & - & with smoke \\
\hline 9 & $\checkmark$ & - & $\checkmark$ & - & - & with smoke & 9 & $\checkmark$ & $\checkmark$ & $\checkmark$ & - & - & with smoke \\
\hline \multirow{14}{*}{$*$} & \multirow{14}{*}{-} & \multirow{14}{*}{-} & \multirow{14}{*}{-} & \multirow{14}{*}{-} & \multirow{14}{*}{$\checkmark$} & \multirow{14}{*}{$\begin{array}{l}\text { Non-combustion } \\
\text { area, commission } \\
\text { error of FS over } \\
\text { urban and cloud }\end{array}$} & 10 & $\checkmark$ & $\checkmark$ & $\checkmark$ & - & $\checkmark$ & $\begin{array}{l}\text { with smoke; commission of FS over burnt } \\
\text { area }\end{array}$ \\
\hline & & & & & & & 11 & $\checkmark$ & - & $\checkmark$ & - & - & with smoke \\
\hline & & & & & & & 12 & - & - & $\checkmark$ & - & - & with smoke \\
\hline & & & & & & & 13 & $\checkmark$ & $\checkmark$ & $\checkmark$ & $\checkmark$ & - & less detection over smoky condition \\
\hline & & & & & & & 14 & - & $\checkmark$ & $\checkmark$ & - & - & with smoke \\
\hline & & & & & & & 15 & $\checkmark$ & $\checkmark$ & $\checkmark$ & - & - & with smoke \\
\hline & & & & & & & 16 & $\checkmark$ & $\checkmark$ & $\checkmark$ & - & - & with smoke \\
\hline & & & & & & & 17 & $\checkmark$ & - & $\checkmark$ & - & - & with smoke \\
\hline & & & & & & & 18 & - & - & $\checkmark$ & - & - & with smoke \\
\hline & & & & & & & 19 & $\checkmark$ & $\checkmark$ & $\checkmark$ & - & - & with smoke \\
\hline & & & & & & & 20 & $\checkmark$ & - & $\checkmark$ & - & - & with smoke \\
\hline & & & & & & & 21 & - & $\checkmark$ & $\checkmark$ & - & - & with smoke \\
\hline & & & & & & & 22 & $\checkmark$ & & & - & - & with smoke \\
\hline & & & & & & & $*$ & - & - & - & - & $\checkmark$ & $\begin{array}{l}\text { Non-combustion area, commission error of FS } \\
\text { over urban }\end{array}$ \\
\hline
\end{tabular}


In addition to precision analysis, the results were also compared with the MODIS fire-mask product at the same date and the same location, but slightly different in time. In the first image acquired on August $3^{\text {rd }}, 2015$, there was only one MODIS acquisition at the location with a time about 4 hours later than Landsat-8 (Landsat-8 acquired at 09.34 am while MODIS at $01.35 \mathrm{pm}$ ). According to Usup et al. (2004) the maximum spreading time of peatland fire is $6.5 \mathrm{~cm} /$ hour at the peat surface $(0-20 \mathrm{~cm})$; thus within 4 hours of the Landsat- 8 observation the MODIS observations of the peatland fires potentially corresponded to combustion $26 \mathrm{~cm}$ different in position to that observed by Landsat 8 . However, of all nine samples of combustion areas found in Landsat-8, the MODIS fire-mask only detected one at location number 2 (Figure 4). The MODIS fire mask did not detect the other eight combustion areas. Of the eight combustion areas not detected by the MODIS fire-mask product, two combustion areas were cloud covered, which possibly caused the non-detection of fire, while six combustion areas with clear sky were undetected by MODIS.

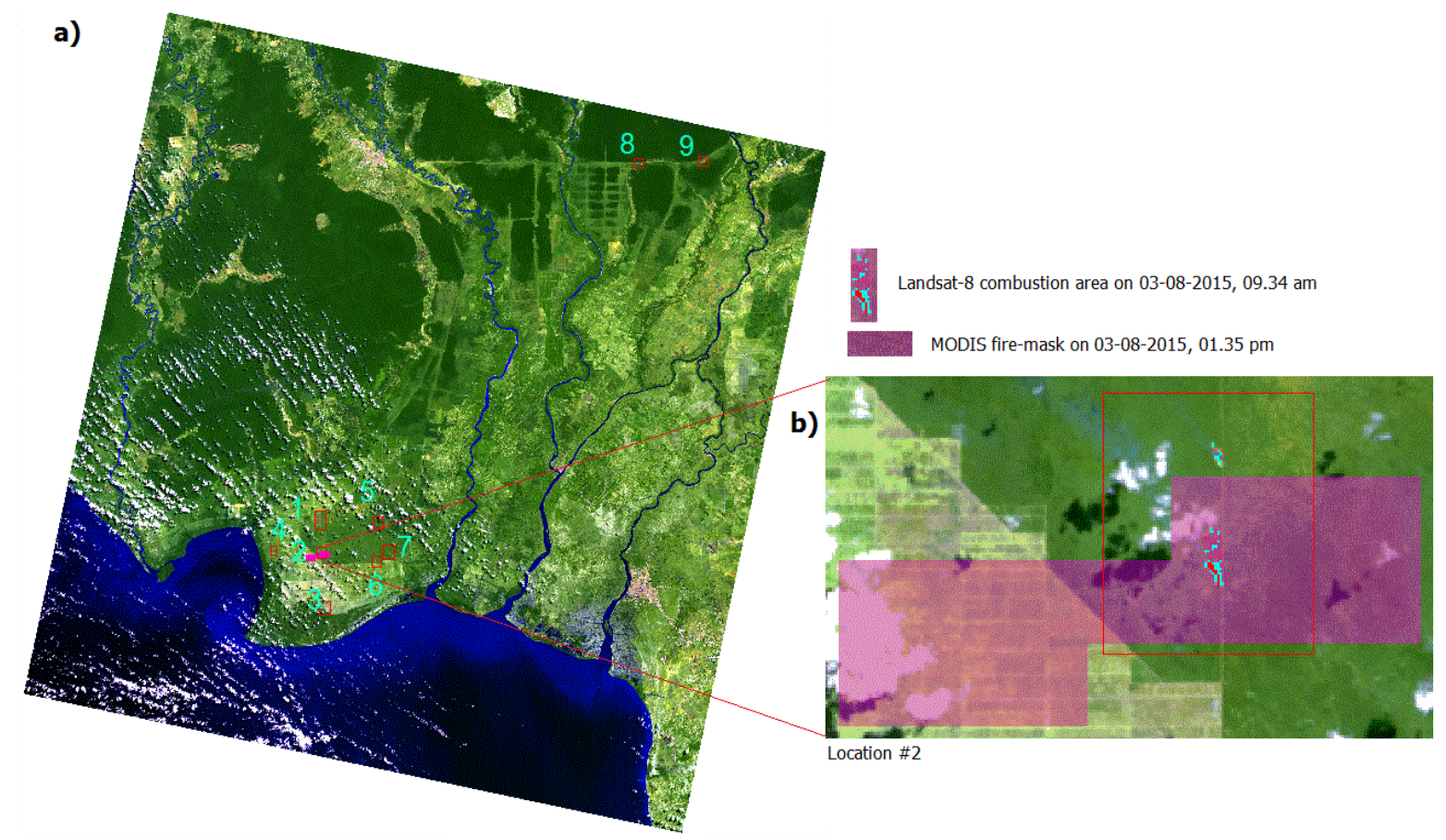

Figure 4 - (a) The 9 combustion areas shown in red square and MODIS fire-mask (pink) overlaid on the Landsat-8 764-RGB image of August $3^{\text {rd }}$, 2015. The MODIS fire-mask only appeared in location \#2, (b) Enlargement of location \#2 showing the MODIS fire-mask (pink) transparency overlaid on the Landsat-8 image which is showing a combustion area in red and cyan (centre right).

The second Landsat- 8 image in study area was acquired on $19^{\text {th }}$ August 2015 at 09.35 am, while on the same date and location MODIS fire-masks were available at $01.35 \mathrm{pm}$ and $10.20 \mathrm{pm}$, thus generating a difference in time with the Landsat acquisition of about 4 hours and 12 hours respectively. These time differences translate to maximum peat fire spread of about $26 \mathrm{~cm}$ and $78 \mathrm{~cm}$ (Usup et al 2004), respectively. Figure 5a shows the 22 combustion areas (red squares) on the 764-RGB image of Landsat- 8 overlaid with MODIS fire-mask data at $01.35 \mathrm{pm}$ (magenta) and $10.20 \mathrm{pm}$ (yellow). There were only six combustion areas detected by the MODIS fire-mask at $01.35 \mathrm{pm}$, with only two of them remaining detected at $10.20 \mathrm{pm}$, and two other combustion areas additionally detected at $10.20 \mathrm{pm}$; thus, a total of 8 combustion areas detected by MODIS fire-mask. Of the other 14 combustion areas not detected by MODIS fire-mask, five of them were due to cloud cover, while the nine remaining combustion areas were undetected even with a clear sky condition. Figure $5 \mathrm{~b}$ is the enlargement of combustion areas 15 and 16, which matched with the MODIS fire-mask, while in Figure 5c there was one commission error of MODIS fire-mask at 01.35 pm over a bare plantation area. 
Overall, the comparison of data from the MODIS fire-mask for peat combustion areas with combustion identified from Landsat- 8 resulted in 9 agreements ( $29 \%$ ) out of 31 for combustion, while 22 areas were undetected (71 \%). The undetected combustion areas in the MODIS fire-mask were due to cloud cover ( 7 areas), low fire temperature and MODIS pixel size, which at $1 \mathrm{~km}$ would reduce detectability of small fires. One commission error in the MODIS fire mask was also found over a bare plantation area.

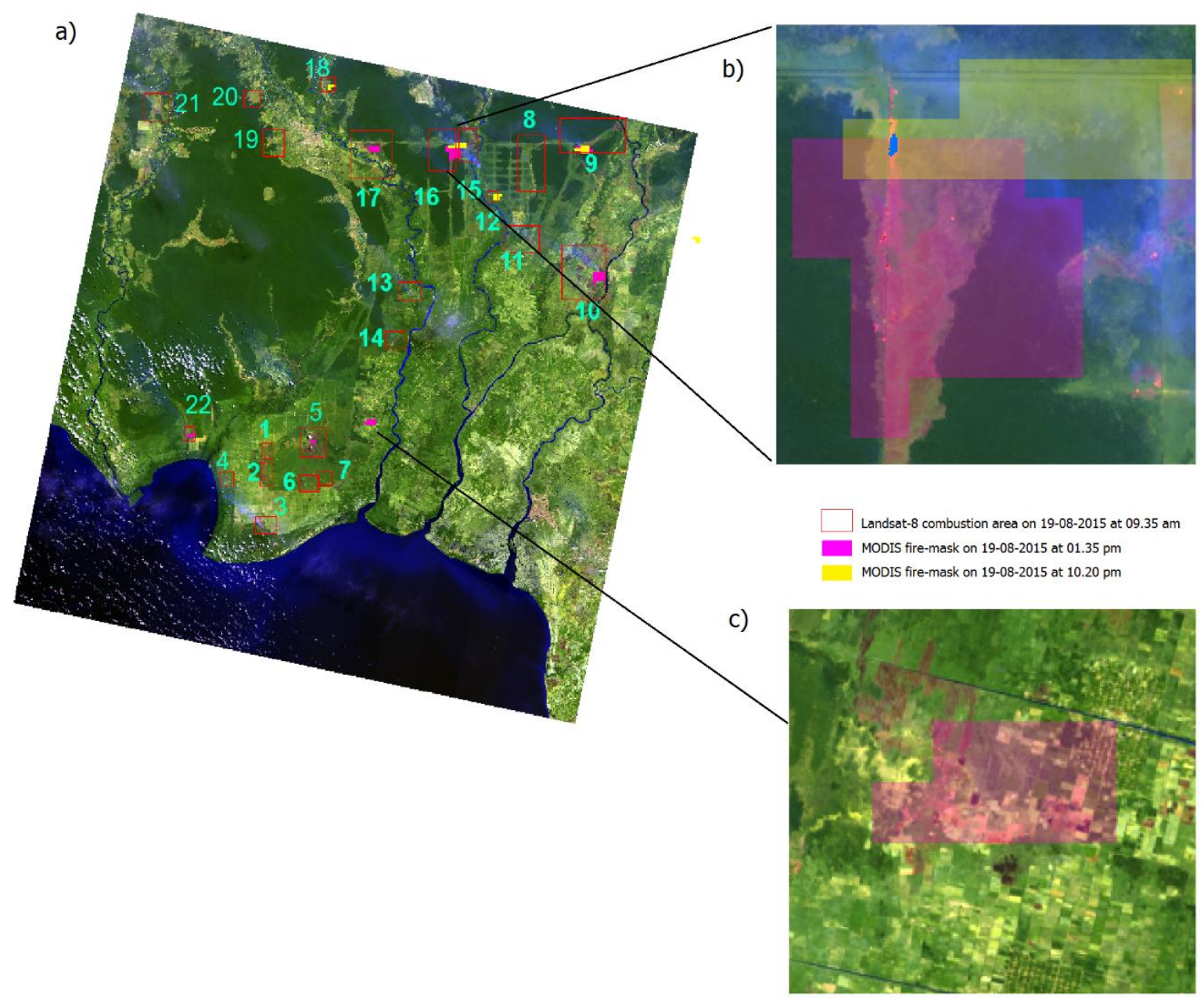

Figure 5 - a) The 22 combustion areas shown in red squares and MODIS fire-mask (pink and yellow) overlaid on the Landsat-8 764-RGB image of August 19 ${ }^{\text {th }}, 2015$, b) Enlargement of locations of \#15 and \#16 showing the MODIS fire-mask (pink and yellow) transparency overlaid on Landsat-8's combustion area; the blue polygon represents smouldering area generated from Landsat-8, c) Enlargement of commission error of MODIS fire-mask at 01.35 pm over bare plantation area.

\section{Conclusion}

Analysis of the SWIR and TIR bands of day-time Landsat- 8 data indicates that it is potentially possible to discriminate flaming and smouldering stages in peatland combustion. The SICI integrated with the radiances of band- 7 and band-10 of Landsat- 8 can be used for separating combustion and non-combustion areas. The detected peatland combustion showed about $77 \%$ precision compared with visual observations of the 764-RGB images of Landsat-8. Whilst there is room for further improvement, this is significantly better than the $71 \%$ of MODIS fire-mask which is widely used for global fire monitoring. The algorithm when applied to Landsat 8 imagery was able to detect combustion areas that could not be mapped using the lower spatial resolution MODIS fire-mask, especially in small burning areas as usually occur in peatland fires. The estimated peat fire 
temperatures from Landsat-8 aligned with prior research, but the estimated flaming temperatures can not be higher than $758 \mathrm{~K}$ due to the saturation temperature of Landsat-8. However, further analysis is required for verification with a simultaneous field measurements. This algorithm has the potential to be used on a routine basis due to the availability of Landsat- 8 data during day-time every 16 days, provided cloud cover permits. It is possible that some merging of information from Landsat and higher temporal but lower spatial resolution satellites (e.g. Sentinel-2 and Sentinel-3, and maybe Himawari) can assist in interpolating the temporal gaps in Landsat data and when cloud cover inhibits use of Landsat. The algorithm presented in this research can also be implemented for forest fire mapping at a detailed scale, as its spatial resolution $(30 \mathrm{~m})$ is higher than the standard fire products $(1 \mathrm{~km})$. We are currently trialling and validating other algorithms with further research ongoing, as this work is part of a larger project, which is investigating automated smoke detection and soil moisture measurements in the mapping of peatland combustion.

\section{Acknowledgements}

This research was funded by the Research and Innovation in Science and Technology Project (RISET-Pro), Ministry of Research, Technology and Higher Education of the Republic of Indonesia (Kemenristekdikti) and the University of South Australia.

\section{References}

\%Barducci A, Guzzi D, Marcoionni P, Pippi, I (2004) Comparison of fire temperature retrieved from SWIR and TIR hyperspectral data. Infrared Physics \& Technology 46, 1-9. doi:10.1016/j.infrared.2004.03.001

Burrows L (2016) Smoke from 2015 Indonesian fires may have caused 100,000 premature deaths. Harvard John A. Paulson School of Engineering and Applied Sciences. updated 19 September 2016, viewed 10 February 2018, <https://www.seas.harvard.edu/news/2016/09/smoke-from-2015Indonesian-fires-may-have-caused-100000-premature-deaths >

Csiszar I, Schroeder W, Giglio L, Ellicott E, Vadrevu KP, Justice CO, Wind B (2014) Active fires from the Suomi NPP Visible Infrared Imaging Radiometer Suite: Product status and first evaluation results. Journal of Geophysical Research: Atmospheres 119, 803-816. doi:10.1002/

\section{JD020453}

Dennison PE, Roberts DA (2009) Daytime fire detection using airborne hyperspectral data. Remote Sensing of Environment 113, 1646-1657. doi: 10.1016/j.rse.2009.03.010

Elvidge CD, Zhizhin M, Hsu F, Baugh K, Khomarudin M, Vetrita Y, Sofan P, Suwarsono, Hilman D (2015) Long-wave infrared identification of smouldering peat fires in indonesia with nighttime landsat data. Environmental Research Letters 10, 065002. doi:10.1088/1748-9326/10/6/065002

Giglio L, Schroeder W, Justice C (2016) The collection 6 MODIS active fire detection algorithm and fire products. Remote Sensing of Environment 178, 31-41. doi:10.1016/j.rse.2016.02.054

Hayasaka H, Takahashi H, Limin SH, Yulianti N, Usup A (2016) Peat Fire Occurrence. In 'Tropical Peatland Ecosystems'. (Eds M Osaki, N Tsuji) pp. 377-395. (Springer: Tokyo). doi: 10.1007/9784-431-55681-7_25

Justice C, Giglio L, Korontzi S, Owens J, Morisette J, Roy D, Descloitres J, Alleaume S, Petitcolin F, Kaufman Y (2002) The MODIS fire products. Remote Sensing of Environment 83, 244-262.

Miettinen J, Hooijer A, Wang J, Shi C, Liew SC (2012) Peatland degradation and conversion sequences and interrelations in Sumatra. Regional Environmental Change. 12, 729-737. doi: $10.1007 / \mathrm{s} 10113-012-0290-9$ 
Qian, YG, Kong XS (2012) A method to retrieve subpixel fire temperature and fire area using MODIS data. International Journal of Remote Sensing 33, 5009-5025. doi: 10.1080/01431161.2012.657365

Rein G (2016) Smouldering Combustion. In 'SFPE Handbook of Fire Protection Engineering'. (Eds MJ Hurley) pp. 581-603. (Springer: New York). doi: 10.1007/978-1-4939-2565-0_19

Rieley J, Page S (2016) Tropical Peatland of the World. In 'Tropical Peatland Ecosystems'. (Eds M Osaki, N Tsuji) pp. 3-32. (Springer, Tokyo). doi: 10.1007/978-4-431-55681-7_1

Schroeder W, Oliva P, Giglio L, Csiszar I (2014) The New VIIRS 375 m active fire detection data product: Algorithm description and initial assessment. Remote Sensing of Environment 143, 85-96. doi: 10.1016/j.rse.2013.12.008

Usup A, Hashimoto Y, Takashi H, Hayasaka H (2004) Combustion and thermal characteristics of peat fire in tropical peatland in Central Kalimantan, Indonesia. Tropics 14, 1-19.

World Bank (2016) The Cost of Fire: An Economic Analysis of Indonesia's 2015 Fire Crisis. Indonesia Sustainable Landscape Knowledge Note: 1 (World Bank Group)

Zaccone C, Rein G, D’Orazio V, Hadden RM, Belcher CM, Miano TM (2014) Smouldering fire signatures in peat and their implications for palaeoenvironmental reconstructions. Geochimica et Cosmochimica Acta. 137, 134-146. doi:10.1016/j.gca.2014.04.018 


\title{
Development of multilingual network resource for comparative- contrastive dictionary of fire science terminology
}

\author{
Tatiana M. Sofronova ${ }^{1 *}$; Alexandra V. Volokitina ${ }^{2}$ \\ $1 *$ Astafiev Krasnoyarsk State Pedagogical University, ul. A. Lebedevoy,89, Krasnoyarsk 660049, \\ Russia, \{tmsofronova@gmail.com*\} \\ ${ }^{2}$ Sukachev Institute of Forest SB RAS, ul.Akademgorodok,50/28, Krasnoyarsk 660036, Russia, \\ \{volokit@ksc.krasn.ru\}
}

\begin{abstract}
Our ambition is to obtain experience in terminology management of large international lexicographic projects through cooperation with the international terminology and lexicography experts and to improve the software for implementation of the concept of the multilingual network resource for a comparativecontrastive dictionary of fire science terminology (on the example of English and Russian). Since the project implementation requires international team efforts for its logical completion, Tatiana M. Sofronova (being both a terminologist and a fire scientist) would like to become a uniting, organizing and conceptual link in promotion of this international project and to earn the support of recognized international experts in Lexicography and Terminology Management.

The project goal is development of a multilingual network resource for a comparative-contrastive dictionary of fire science terminology (FST) at the high modern technological level. This dictionary is meant to: 1) combine features of translation, explanatory, encyclopedic, ideographic and student's dictionaries; 2) help to treat each national FST with care preserving the uniqueness of each system of knowledge; 3) enable comparison of different countries' scientific terminologies for their harmonization (at the level of systems through terminology comparison and at the level of individual terms through terminology contrasting); 4) suggest joint ways of solving translation issues by terminology translators and fire science experts; 5) reflect diachronic development of a scientific notion behind a term and existing approaches to its explanation; 6) be open for updating and editing; 7) provide potential involvement of FSTs from different languages and countries to this project.

The main project objectives include: 1. Obtaining theoretical experience in management of large international terminological projects through collaboration with leading international experts in the field of terminology and lexicography. 2. Improving the software for the multilingual network resource aimed at development of a comparative-contrastive fire science dictionary on the basis of the elaborated prototype of the bilingual fire science glossary. 3. Preparing relevant project documents for the implementation of the multilingual network resource for the comparative-contrastive fire science dictionary that could clearly guide efforts of fire scientists and translators/terminologists in different countries.
\end{abstract}

Keywords: network resource, terminological lexicography, multilingual modeling, fire science terminology

\section{Background}

It is known that the issues of lexicographical modeling of bilingual and multilingual terminological dictionaries occupy a special place in the wide range of terminological and lexicographical works (Tabanakova et al., 2009). However, none of the studies individually, or their combination can claim to be a complete and universal scheme of stages of work on a dictionary, in each case, project development should take into account special features of a terminology, requirements of potential users, and working conditions (Kudashev, 2007).

The theory and practice of modern terminological lexicography attaches a great importance to contrastive descriptions of the meanings of words from different semantic categories and different 
languages (see: Sternin, 2007). Among recent works in this direction is A.M. Bobunov's contrastive dictionary of Russian and English language folk songs (2012). Such dictionaries are valuable for contrastive presenting of non-terminological words, since creation of a contrastive dictionary of scientific terminology requires systematic analysis of interlingual terms and scientific concepts behind them both at the level of comparable subsystems (comparison), and at the level of parallel pairs of terms (contrasting). This dictionary should be focused not only on the linguistic, but also on the extralinguistic information, on how scientists perceive the world through the prism of scientific concepts.

Of paramount importance is lexicographic description of young terminologies, which have developed their distinctive features in different countries and scientific schools and have never been subjected to linguistic research. Among them is fire science terminology (FST). Having originated from the depths of the forest management terminology in the middle of the XX century. To date, the basic research in the field of FST is carried out and summarized in the monograph Lexicographical modeling of Russian and English fire science terminology (Felde, Sofronova, 2017), and is published in eight peer-reviewed papers.

As a result of Sofronova's training in Environmental Sciences at the University of Montana and the Missoula Fie Lab within the Fulbright Faculty Development Program (2008-2009), she developed an electronic prototype of a comparative-contrastive bilingual fire science glossary (Russian state registration number 0321201925), established contacts with the leading specialists in the US and Russian fire science, and defended her PhD thesis on the fundamentals of lexicographic modeling of Russian and English fire science terminology at the Tyumen State University (Russia, 2013).

\section{Problematic}

When considering the history of the formation of Russian and English FST we distinguished three similar stages in its development (Sofronova, 2008) and concluded that the modern fire science is at an extraordinary stage of development (Kuhn, 1962). The latter implies unwanted terminological confusion as a result of distinctive terminology development in different countries, in different scientific schools, thus preventing mutual understanding of scientists from different scientific schools and countries. Therefore, coordination and harmonization of terms and notions is a long overdue problem in the modern fire science.

When disclosing the degree of order in modern FST, we have found that this terminology is poorly ordered and poorly harmonized (Sofronova, 2010). The Russian terminology faces the following issues: (a) absence of fire science dictionaries; (b) low quality of state standards on fire management terminology (e.g., lack of coordination in the extraction of generic terms, variability in definitions, erroneous definitions). The English terminology is characterized by: (a) absence of formal ideographic dictionaries in Fire Science; (b) national specific features of the English FST in the United States, Canada and Australia. Harmonization of the Russian and English FST is interfered by poor quality of translation dictionaries, which is proved by: a) partial cover of fire science terms in forestry dictionaries (Filipchuk, 2002; Rodin, Filipchuk, 2006; Helms, 1998); b) presence of artificial translation equivalents (International multilingual fire management terminology, www.fire.unifreiburg.de); c) presence of several translations of a term without disclosing their distinguishing features and presence of terms with erroneous translations (Mozhaev et al., 1998). This situation is due to disparity of the Russian and English terminological fields, which is caused by unique national term formation, variability in understanding of terms by different scientists and scientific schools and misleading terms. Mechanical comparison of terminological systems with unique segmentation of the area of knowledge only increases terminological confusion (as an example, see FAO/ GFMC ..., 2003). In 2006-2007, scientists of the St. Petersburg Research Institute of Forestry in collaboration with Canadian colleagues attempted to harmonize the Russian and English FST, but the results of their 
work remained unpublished. In 2012, European scientists created an English-language European Wildfire Glossary, which is a mixture of different terminologies of European countries and is suitable mainly for practitioners working at the international level. For scientists, every scientific term is associated with a specific terminological system, and scientific concepts do not remain static over time. Therefore, this confusion of terminologies can only hinder the development of the scientific thought.

\section{Specific aim and Objectives}

The project specific aim is development of a multilingual network resource for a comparativecontrastive dictionary of fire science terminology (on the example of English and Russian) at the high modern technological level. This dictionary is meant to: 1) combine features of translation, explanatory, encyclopedic, ideographic and student's dictionaries; 2) help to treat each national fire science terminology with care preserving the uniqueness of each system of knowledge; 3) enable comparison of different countries' scientific terminologies for their harmonization (at the level of systems through terminology comparison and at the level of individual terms through terminology contrasting); 4) suggest joint ways of solving translation issues by terminology translators and fire science experts; 5) reflect diachronic development of a scientific notion behind a term and existing approaches to its explanation; 6) be open for updating and editing; 7) provide potential involvement of fire science terminologies from different languages and countries to this project.

The main project objectives include: 1. Obtaining theoretical experience in management of large international terminological projects through collaboration with leading international experts in the field of terminology and lexicography. 2. Improving the software for the multilingual network resource aimed at development of a comparative-contrastive fire science dictionary on the basis of the elaborated prototype of the bilingual fire science glossary. 3. Preparing relevant project documents for the implementation of the multilingual network resource for the comparative-contrastive fire science dictionary that could clearly guide efforts of fire scientists and translators/terminologists in different countries.

\section{Significance}

Prior to our research, the terminology of forest fire science had never been involved in the linguistic studies and had never been subjected to systematic comparative and lexicographical analysis. Russia has no special dictionaries in this field of knowledge yet. There are only general reference materials on forestry and forest management covering the FST only partially. The first brief glossary of fire science terms was published by Prof. N.P. Kurbatskiy in the 1972 collection of articles. The US, on the contrary, have official glossaries of fire management terminology, which, however, do not always reflect the modern development of the scientific thought in the field of fire science (Glossary ..., 1990; Glossary ..., 2007). In 2007, a US electronic fire science glossary FireWords (Scott, Reinhardt, 2007) was created. It was aimed at clarifying understanding of 300 fire science terms. Unfortunately, the work on the project was terminated for financial reasons, and about $25 \%$ of the terms were not provided with glossary entries, but the results of this work were published on the website: http://www.firewords.net/. During my Fulbright training, the authors of the FireWords kindly provided the accumulated material as a legacy and software for use in the prototype of the bilingual glossary of the Russian and US FST.

The theoretical significance of our study lies in realization of the strategy for comparativecontrastive lexicographic modeling of the dynamically evolving terminology of a relatively young fire science that is a definite contribution to the theory of bilingual (and potentially, multilingual) terminological lexicography. The strategy of the proposed network resource modeling makes it possible to disclose the variability in understanding of terms by different scientists and scientific 
schools, to correct or logically synthesize definitions, leaving a user the right to their own conclusions in the analysis of the discussion section in the dictionary entry.

The practical importance of the project lies in the versatility of the lexicographical product being developed that may be addressed to fire scientists, translators/interpreters, and students of forestry departments majoring in forest fire protection. Development of the multilingual dictionary of fire science terms and concepts systematized within a language / country and harmonized among languages /countries should improve mutual understanding and exchange of information and experience among scientists of different countries, and also should help involve them in the process of systematization of their national terminology and in the discussion of problematic aspects of its harmonization with the terminology of other countries. As a result, such an electronic resource may become the basis for a large international project on development of a multilingual network resource on similar informational and technological but more rigorous scientific principles as Wikipedia or Wiktionary. Creation of such a dictionary should facilitate (and, possibly, automate, to some extent) translation of scientific works, which remain untranslated or poorly translated.

\section{Methodology}

Creation of any bi- or multilingual lexicographical product involves comparative and contrastive studies. They are based on the general principles of terminology analysis such as the principles of comparability, consistency, sequence of analysis of a linguistic material. Their main difference lies in the level of description of the object of study and the sequence of comparison: comparative analysis is conducted at the level of subsystems, fields, groups, independently with the following comparison, and contrastive analysis is conducted at the level of individual terminological units in the direction from a unit of one language to its possible correspondences in other languages (Sternin, 1992).

Effective semantization of scientific terms in the dictionary and transfer of the accumulated scientific experience with the preservation of national terminological peculiarities is possible through realization of the algorithm, the key elements of which are: a) terminological system modeling through comparable logical-conceptual schemes; b) diachronic analysis of the development of scientific concepts in dictionary entries and synthesis of definitions based on the accumulated scientific expertise; c) translation commentary which takes into account the current scope of the term concept in multilingual terminological systems and offers a contrastive analysis of relative term equivalents or suggests artificial equivalents in case of non-equivalent terminology.

The prototype of the comparative-contrastive bilingual electronic glossary of the Russian and US FST (Fig. 1 and 2) developed according to the algorithm will serve as a stepping stone for the realization of a multilingual network resource for fire science dictionary. This dictionary will combine the parameters of translation, explanatory, encyclopedic, ideographic and student's dictionaries and should be a means of harmonization of the FST among different languages and countries.

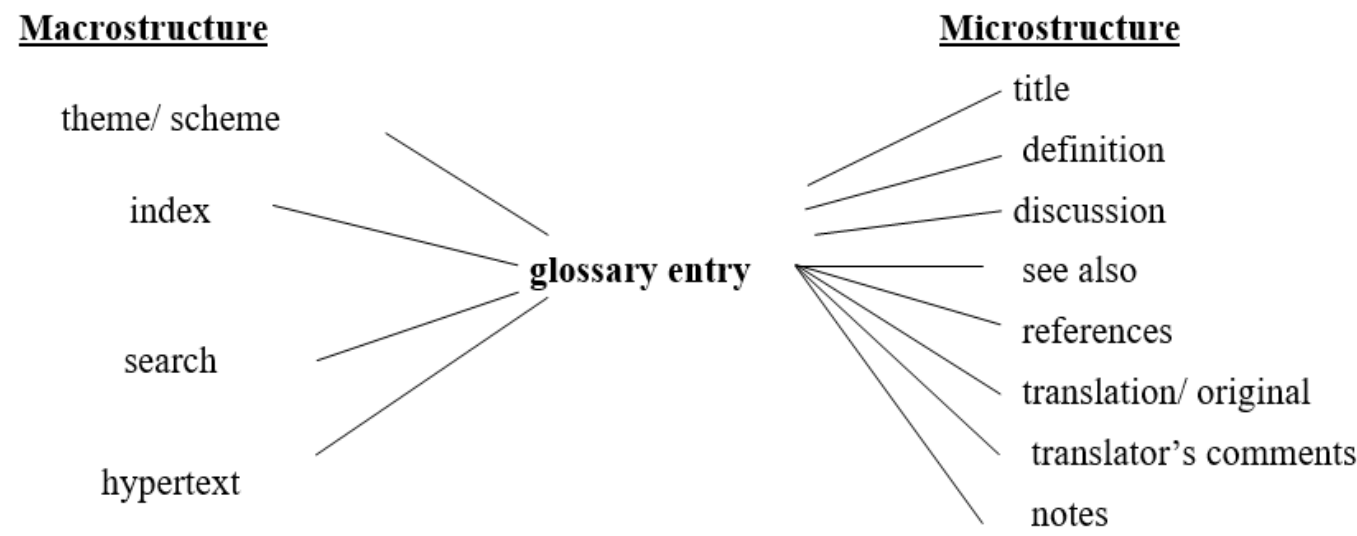

Figure 1 - Model of the bilingual fire science glossary 

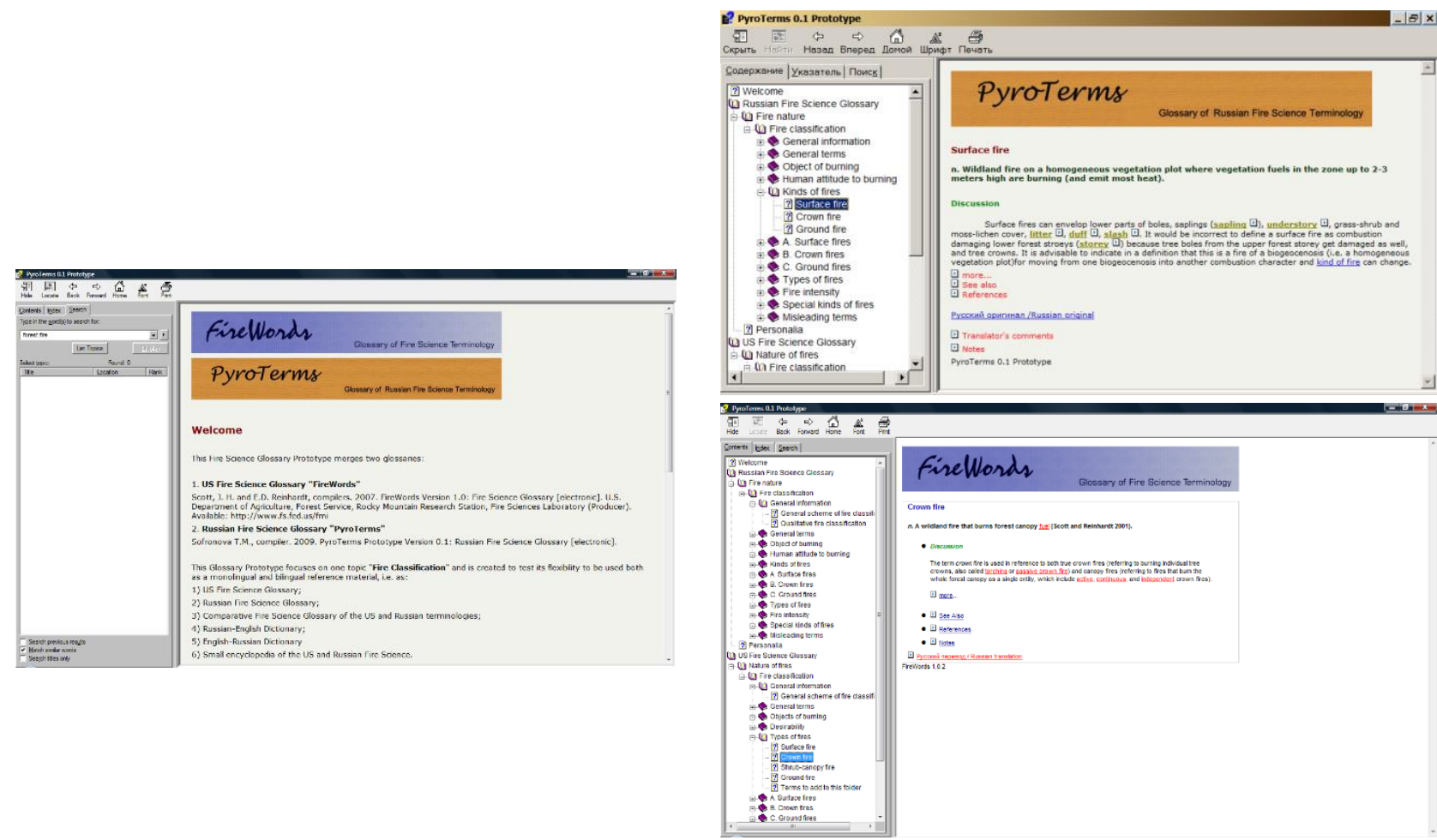

Figure 2 - Interface of the bilingual fire science glossary prototypey

\section{Research Programme}

The proposed project of qualitative nature involves: 1) consultations and workshops with terminology experts from the ATILF Laboratory (Nancy Cedex) and INALCO (Paris) on issues of modern bilingual and multilingual terminological lexicography as well as business trips (funds permitting) or video conferencing with terminology experts from other countries (Finland, the USA); 2) analysis of published works on modern foreign research and technology in this field; 3) creation of fire science corpora on the basis of the Fire Research Institute Library (on the example of English and Russian languages) for extraction of scientific terms and their contextual meanings that have emerged over the past 40-50 years, i.e. during the period of existence of the fire science as an independent field of scientific knowledge; distribution of the terms among the main fire science subfields and in the logical-conceptual schemes: 4) learning the basics of a network approach and programming of network lexicographical products with the specified parameters for the development of an updated software for a multilingual network resource on fire science terminology; 5) interaction with international organizations and foundations potentially interested in the proposed project on development of a multilingual network for a fire science dictionary (e.g., Global Fire Monitoring Center, Germany; FAO, Italy; International Association of Wildland Fires, USA; World Bank, USA); 6) preparation of the necessary documents for implementation of an international project on creation of this multilingual network resource; 7) development of a demo version of a specialized website on the dictionary project with information about the status of work, the latest updates and lexicographical products available for download.

Our terminology institutions and contacts include:

Hosting institution: ATILF CNRS Laboratory (Nancy, France), Prof. Alain Polguère, alain.polguere@univ-lorraine.fr

Partnership Institution: INALCO (Paris, France), Prof. Svetlana G. Krylosova, svetlana.krylosova@inalco.fr 
International contacts:

University of Tampere / University of Helsinki (Finland), Prof. Igor. S. Kudashev, Igor.Kudashev@staff.uta.fi

Kent State University (Ohio, USA), the Institute of Applied Linguistics at the Department of Studies of Modern and Classical Languages, Professors Sue Ellen Wright, swright@kent.edu.

\section{References}

Bobunov AM (2012) Contrastive dictionary of the English language and Russian folk songs as the basis of cross-cultural studies. (Voronezh)

European Glossary for Wildfires and Forest Fires (2012). URL: http://www.fire.unifreiburg.de/literature/EUFOFINET-Fire-Glossary.pdf

FAO / GFMC Wildland Fire Management Terminology (2003) URL: http://www.fire.unifreiburg.de/literature/glossary.htm.

Filipchuk AN (Ed) (2002) 'Forestry: Terminological dictionary.' (VNIILM: Moscow)

Glossary of wildland fire management terms used in the United States (1990) (Society of American Foresters)

Glossary of wildland fire terminology (2007) (National Wildfire Coordinating Group Incident Operations Standards Working Team). URL: http://wwwnwcg.gov.

Helms A (Ed.) (1998) 'The Dictionary of Forestry.' (The Society of American Foresters: Bethesda, MD)

International multilingual fire management terminology (English - Russian - Mongolian - German). Center for Global Fire Monitoring. URL: http://www.fire.uni-freiburg.de/literature/RUS-MONGER-ENG-Glossary-Web.pdf

Kudashev IS (2007) 'Development of translation dictionaries for specialized vocabulary.' (Helsinki University Print: Helsinki)

Kuhn TS (1962) 'The Structure of Scientific Revolutions.' (Univ. of Chicago Pr.: Chicago)

Kurbatsky NP (1972) Terminology of forest fire science. In 'Forest fire science issues'. pp. 171-231. (ILiD SB RAS: Krasnoyarsk)

Mozhaev DV, Novikov B, Rybakov DM (Eds) (1998) 'English-Russian and Russian-English Dictionary of Forestry.' (Moscow: Rousseau, 1998. 857 p.

Rodin SA, Filipchuk AN (2006) 'Encyclopedia of Forestry.' (VNIILM: Moscow)

Scott JH, Reinhardt ED (compilers) (2007) FireWords Version 1.0: Fire Science Glossary [electronic]. U.S. Department of Agriculture, Forest Service, Rocky Mountain Research Station, Fire Sciences Laboratory (Producer). URL: http://www.firewords.net/.

Sofronova TM (2008) Formation of fire science sub-language in the aspect of its history. In: Proceedings of the VI International Scientific Conference on comparative historical linguistics on Language contacts in the aspect of history, 29-31 January 2008 Moscow. pp. 101-102. (Lomonosov Moscow State University: Moscow)

Sofronova TM (2010) Harmonisation of fire science terminology in Russian and English languages. In: Proceedings of the II International Symposium on Terminology and knowledge (Moscow, May, 21 - 22, 2010). pp. 343-351. (Azbukovnik: Moscow)

Sofronova TM (2012) Prototype of bilingual fire science glossary: Version 1.0. Krasnoyarsk: Siberian Federal University (CD). State registration number 0321201925. - ISBN 978-5-7638-2578-7.

Sofronova TM, Felde OV (2017) 'Lexicographical modeling of Russian and English fire science terminology.' (Siberian Federal University: Krasnoyarsk) 
Sternin IA (1992) National specificity of the word notion and lexicography. In 'Contemporary problems of lexicography’ (Ed. Dubichinskiy VV, Vakulenko SV, Danilenko AI). pp. 214-216. (Kharkov University: Kharkov)

Sternin IA (2007) 'Contrastive studies and contrastive dictionaries.' (Voronezh State University: Voronezh)

Tabanakova VD, Sotnikova TV, Antonova SN, et al. (Eds) (2009) 'Linguistic modeling.' (Vector Book: Tyumen) 


\title{
Do we need weather prediction models to account for local weather modifications by wildland fires?
}

\author{
Adam Kochanski ${ }^{1}$; Derek V. Mallia ${ }^{1}$, Matthew G. Fearon ${ }^{2}$, Tim Brown ${ }^{2}$, Jan Mandel ${ }^{3}$, Joseph K. \\ Vaughan ${ }^{4}$ \\ ${ }^{1}$ University of Utah. Salt Lake City,Utah,USA. \{adam.kochanski@utah.edu*, \\ derek.mallia@utah.edu\} \\ ${ }^{2}$ Desert Research Institute, Reno, Nevada, USA. \{matthew.fearon@dri.edu,tim.brown@dri.edu\} \\ ${ }^{3}$ University of Colorado Denver, Denver, Colorado,USA. \{jan.mandel@ucdenver.edu\} \\ ${ }^{4}$ Washingon State University, Pullman,WA, USA. \{jvaughan@wsu.edu\}
}

\begin{abstract}
During the summer of 2015, a number of wildfires fires burned across northern California, which produced significant smoke across the region. Smoke from these wildfires hindered fire-fighting efforts by delaying helicopter operations and exposed communities to high concentrations of atmospheric pollutants. Nighttime inversions are common across the western U.S. and usually mix out during the early afternoon as a result of convective mixing from daytime heating. However, atmospheric conditions in valleys adjacent to the aforementioned wildfires remained stable throughout the afternoon. It is hypothesized that the smoke from nearby wildfires enhanced atmospheric stability due to surface cooling caused by reduced incoming solar radiation, and possibly by warming aloft due to absorption of the incoming solar radiation in the smoke layer. At the same time, mid-level heating from the wildfire could have increased atmospheric stability and extended the duration of the inversion.

In this study, we utilize the WRF-SFIRE-CHEM modeling framework, which couples an atmospheric, chemical, and fire spread model in an effort the model the impacts of smoke on local inversions and to improve the physical understanding behind these smoke-induced inversion episodes. This modeling framework was used to simulate the Route and South Complex fires between August 10 - August $26^{\text {th }}, 2015$. Preliminary results indicate that wildfire smoke may have significantly reduced incoming solar radiation, leading to local surface cooling by up to 2-3 degrees. Direct heating from the fire itself does not significantly enhance atmospheric stability. However, mid-level warming was observed in the smoke layer suggesting that absorption in this layer may have enhanced the inversion. This study suggests the including the fire-smokeatmosphere feedbacks in a coupled modeling framework such as WRF-SFIRE-CHEM may help in capturing the impacts of wildfire smoke on near-surface stability and local inversions.
\end{abstract}

Keywords: smoke, air quality, inversion

\section{Introduction}

It has long been recognized that weather can have large impacts on fire behavior and that sudden changes in winds at the fire line can have significant influences on fire behavior and other hazards (Albini 1982). Since wind at the fire line can impact fire spread and other fire phenomena such as plume rises, and smoke generation and transport, the prediction of the meteorology driving the fire must accurately account for all scales of motion ranging from synoptic-scale down to the fire line scale. This requirement is difficult to satisfy, as fire is dynamically coupled with the atmosphere. Recent studies by Clements et al. (2007), Lareau and Clements (2015), and Lareau and Clements 
(2017) provide observational evidence of fire-driven modifications of local weather, including the development of the pyro-convective plumes, in addition to the modification of local-scale winds that can extend several kilometers from the fire.

Weather data used to drive operational fire propagation models like BehavePlus (Andrews 2009) typically come from nearby weather stations or numerical weather forecasts. In order to refine relatively coarse resolution numerical weather prediction product and improve the accuracy of wind forecasts in complex terrain, diagnostic models such as WindNinja (Forthofer et al. 2014) are used to refine winds to finer scales. These models typically downscale wind forecasts at coarser resolutions ( 1-12 km in order to account for local terrain-induced circulations. However, downscaling is applied to the larger scale weather forecasting model, that does not account for the dynamical feedbacks between fire and the atmosphere. From that standpoint, it is important to assess if the local weather modifications due to the fire are significant enough to motivate coupling between the numerical weather prediction models, and the fire models.

Dynamical impacts of fire on the atmosphere exist at a variety of different scales ranging from meters to kilometers. Physically, fire-induced weather modifications may include:

1. modification of horizontal winds near the fire line as well as the vertical wind profile

2. formation of pyro-convective columns, and upper-level warming due to the condensation of water vapor in the smoke column

3. change of the atmospheric chemical composition due to the injection of smoke

4. atmospheric warming due to the heat release from the fire, and

5. radiative effects of smoke aerosols

In this paper, we focus on the last two aspects of fire-induced weather modifications, the direct warming due to the fire heat-release and the radiative impacts of smoke through atmospheric absorption and scattering.

\section{Motivation}

During the summer of 2015, a number of large wildfires burned across northern California in areas of localized topographic relief (Figure a). Persistent valley smoke hindered fire-fighting efforts, delayed helicopter operations, and exposed communities to high concentrations of atmospheric pollutants.

a)

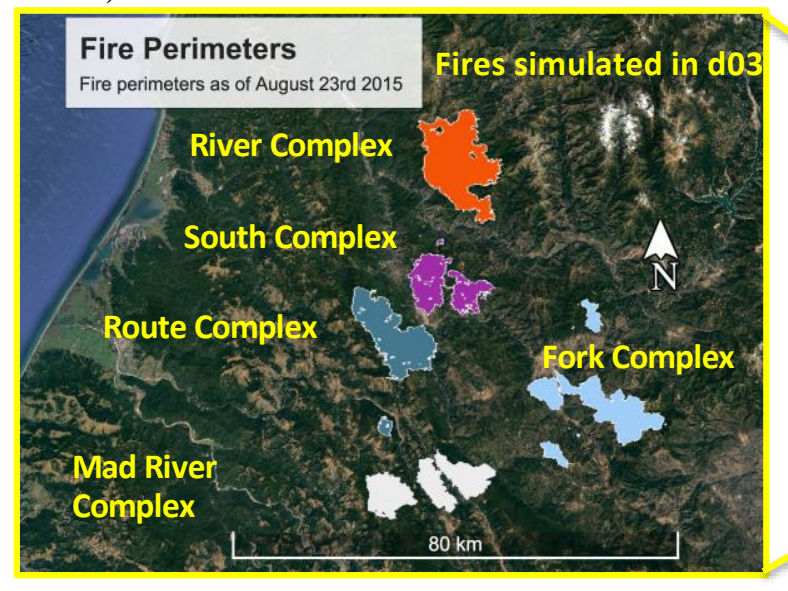

b)

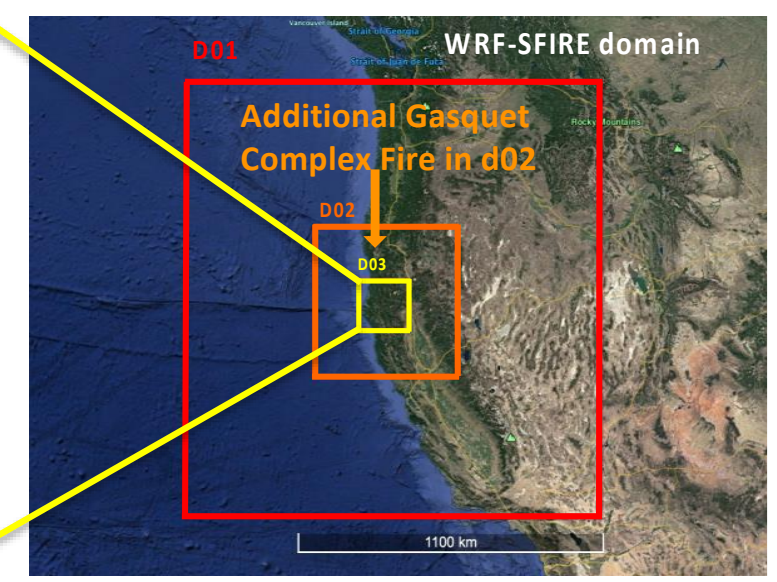

Figure 1 - Active fires across northern California on August $23^{r d}$, 2015 within the innermost domain d03 (a), and WRF-SFIRE-CHEM domain setup with the indication of the additional fire within domain d02 (b).

While nighttime inversion conditions are common in valleys across the West, the inversion during this event lasted longer than typical, as it persisted through the late afternoon. Pronounced cooling was 
observed in valleys across northern California, while upper-elevation locations above the smoke layer saw minimal decreases in surface temperatures. It was hypothesized that the combination of increased scattering and absorption within the smoke layer and mid-level atmospheric heating from the fire helped maintain a feedback process that extended the inversion period. This project utilized the coupled WRF-SFIRE-CHEM system to examine this hypothesis and to determine whether this modeling framework could be used to accurately forecast smoke-induced inversions in the future.

\section{Methodology}

Here we used WRF-SFIRE-CHEM, which is an extension of WRF-SFIRE modeling framework (Mandel et al. 2011). This model framework couples the SFIRE propagation model with a chemical transport model (WRF-CHEM; Grell et al. 2011). The integration of the atmospheric, fire and chemical transport models enables direct simulations of the fire propagation, plume rise, as well as smoke dispersion and chemical transformations (Kochanski et al. 2016). For the purpose of this study, the existing coupling between the fire and the chemistry model has been extended by implementing the Goddard Chemistry Aerosol Radiation and Transport (GOCART) scheme (Chin et al. 2000) to account for aerosol impacts on incoming solar radiation. A schematic diagram showing the coupling mechanisms between WRF-SFIRE-CHEM components is presented in Figure .

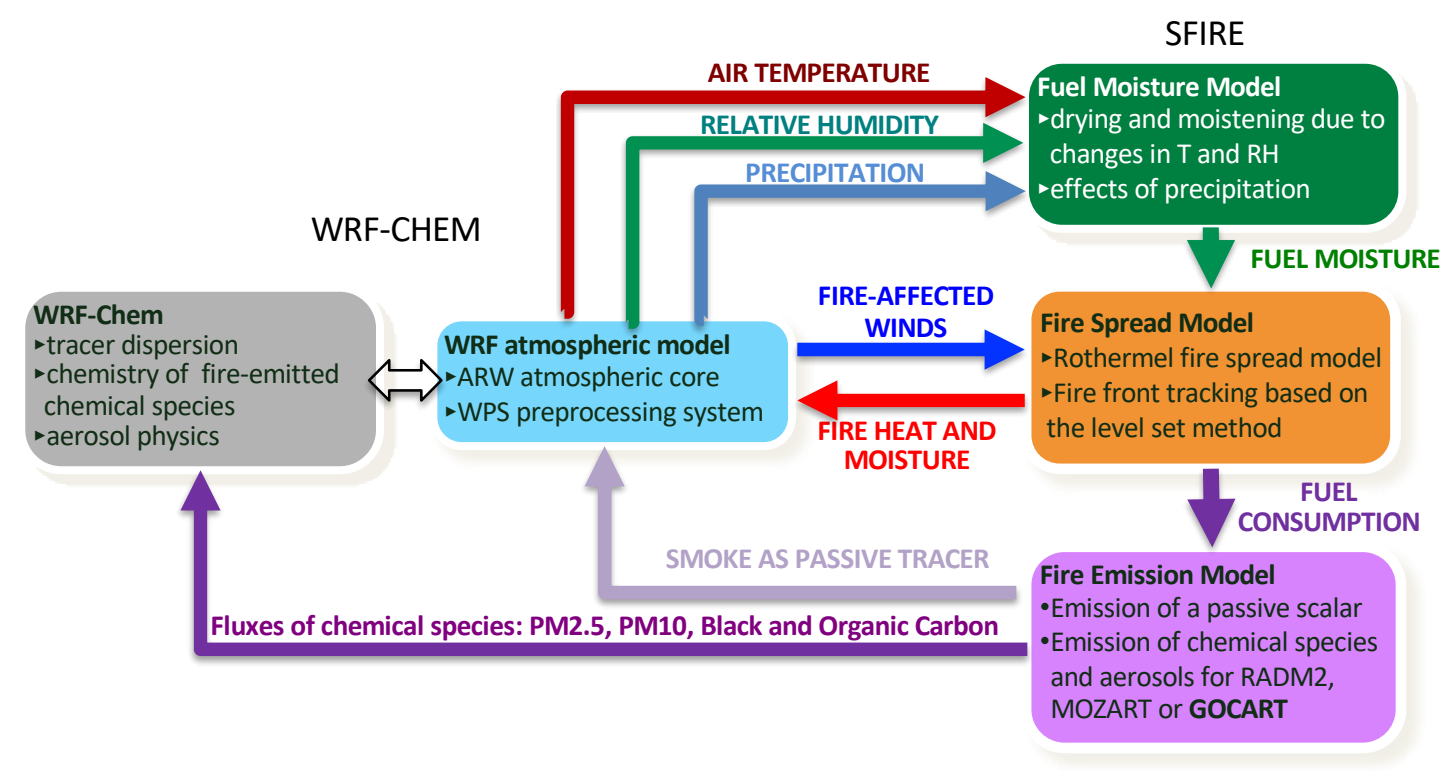

Figure 2 - Diagram of the WRF-SFIRE-CHEM model.

WRF-SFIRE-CHEM has been configured with three nested domains with horizontal resolutions of $12 \mathrm{~km}, 4 \mathrm{~km}$, and $1.33 \mathrm{~km}$, respectively. A total of 6 fires were simulated, with 5 of them being located within the innermost domain $\mathrm{d} 03$ and one within domain d02 (see Figure b). The simulation was initialized on August $16^{\text {th }} 0000$ UTC and ran for seven days, with boundary conditions being derived from the Climate Forecast System Reanalysis (CFSR) data (Saha et al. 2014). The fire model was executed on a 1:20 refined mesh, with the horizontal resolution of $67 \mathrm{~m}$. The fuel description came from the LANDFIRE dataset available at https://www.landfire.gov/.

Two numerical simulations were carried out in order to quantify the impacts of smoke on surface temperatures across northern California. The first one was defined as the "baseline simulation", where WRF-SFIRE was ran with no radiative smoke impacts. The second model configuration used WRFSFIRE-CHEM, where smoke emissions computed from fuel consumption were linked to the GOCART aerosol scheme, which interacts with the atmospheric radiation scheme. As a result, this WRF-SFIRE-CHEM configuration is able to account for the impacts of smoke aerosols on incoming 
solar radiation. Both model simulations had fire growth prescribed using the fire arrival time derived as a spatial interpolation between observed fire perimeters. An example of the encoded fire progression is shown in Figure .

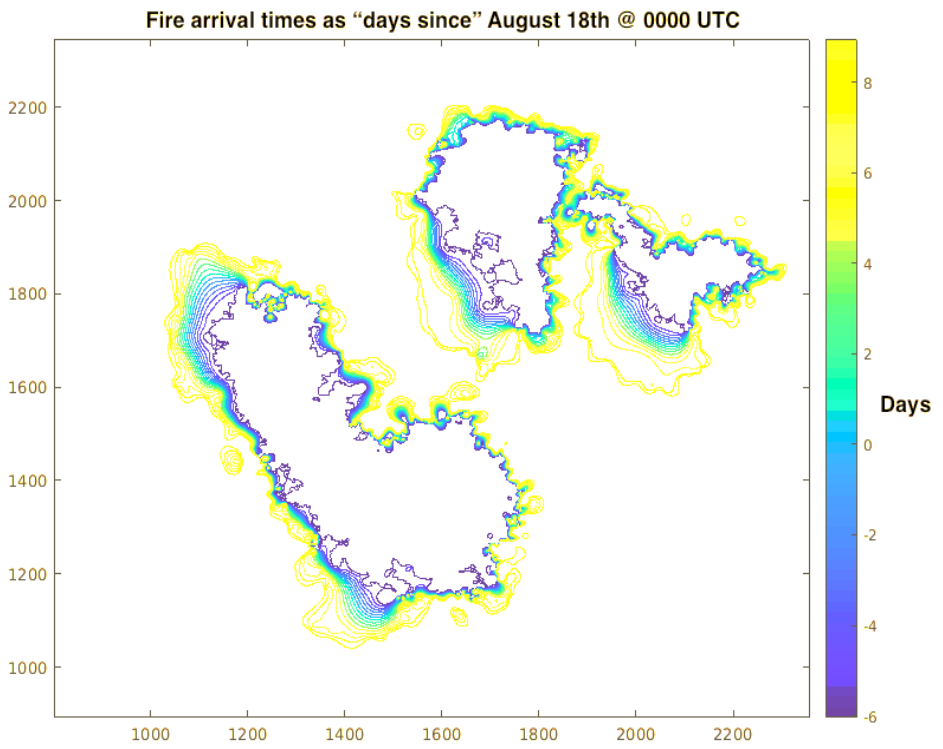

Figure 3 - An example of the fire arrival time derived from observed fire perimeters for the Route and South Complex fires.

\section{Results}

Temperature and incoming solar radiation from both WRF-SFIRE model configurations were compared to observations at two stations that were adjacent to the River Complex and South Complex fires. The first station (Big Bar; BGBC1) was located within the Trinity River valley, with an elevation of 524-m, while the second site was located at the top of the Trinity River valley and had an elevation of 1005-m (Trinity Camp - TCAC1). The elevation map of the innermost model domain and the location of these two stations are shown in Figure.

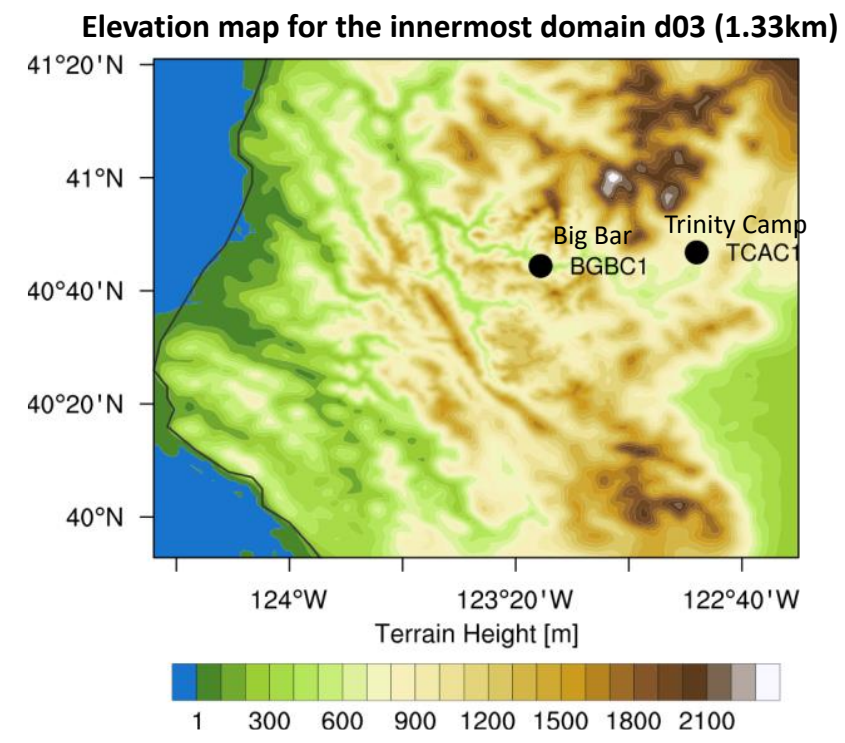

Figure 4 - Elevation map of the innermost domain 103 and the location of measurement stations used in the analysis 
Observational data from these stations indicated that the Big Bar station, located deeper in the valley, observed a more significant reduction in the incoming solar radiation, than the Trinity Camp, located higher in the mountains. It was hypothesized, that these differences were a consequence of each site's geographic location resulting in different aerosol optical depths in the smoke layers above each site. Model simulations performed with, and without the radiative impact of smoke confirmed that the observed deficit in the incoming solar radiation was associated with the presence of the smoke layer.

The time series of the simulated and observed incoming solar radiation at these two stations presented in Figure illustrate that during the first three days when the stations were mostly smokefree, the simulations with and without radiative impacts of smoke showed very similar results. However, at the end of August 19 ${ }^{\text {th }}$, and the beginning of August $20^{\text {th }}$, (Figure ), a dramatic decrease in the incoming solar radiation was observed at the Big Bar station in the WRF-SFIRE-CHEM run accounting for the radiative impact of smoke. The Trinity Camp also experiences a decrease in the insolation but not as significant as the Big Bar.

These results suggest that the smoke covering the Trinity River Valley could have been a reason for the observed discrepancies between the Big Bar station located deeper in the valley and consequently covered by a thick layer of smoke, versus the Trinity Camp station, located higher and covered by a shallower smoke layer.

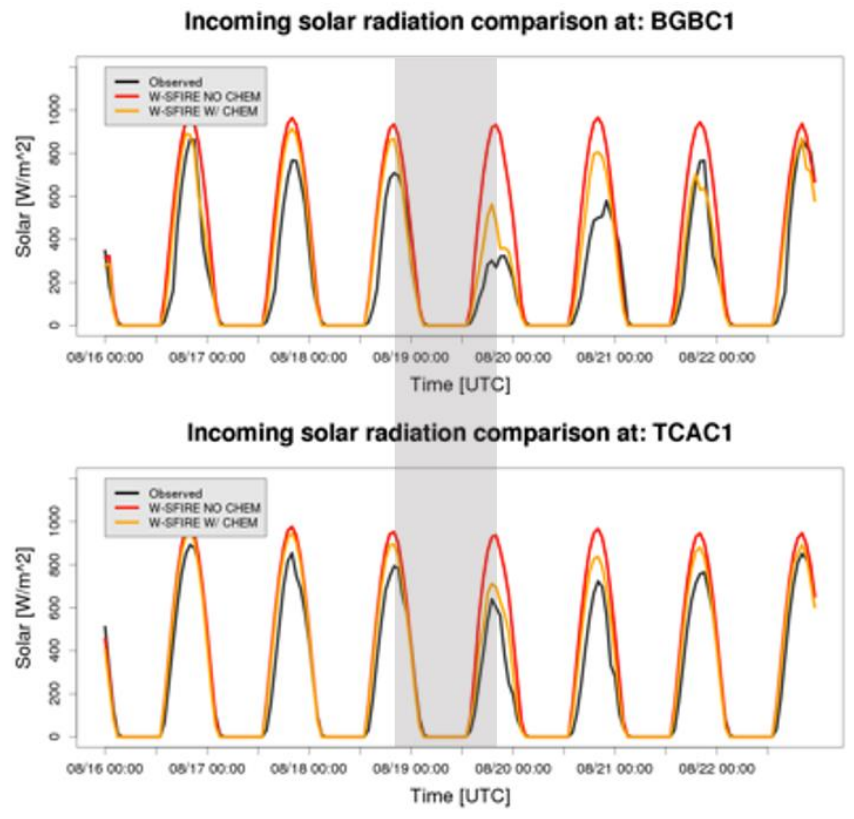

Figure 5 - Time series of the simulated and observed incoming solar radiation at the Big Bar station (BGBC1) - top panel, and the Trinity Camp station (TCAC1) - bottom panel. Observed temperatures plotted as the black line, the baseline simulation is plotted in the red line, and the orange line represents the WRF-SFIRE simulation with aerosol impacts. Gray shading denotes period where smoke-inversion conditions were present.

The impact of the fire smoke is also evident in the $2 \mathrm{~m}$ air temperature time series presented in Figure . Prior to the smoke inversion conditions on the 19 and $20^{\text {th }}$ of August, the simulations with and without radiative impacts of smoke are nearly identical. However, on August $19^{\text {th }}$ and $20^{\text {th }}$ the temperatures for these simulations diverge. The simulation accounting for the smoke shading showed a temperature that was $3^{\circ} \mathrm{C}$ cooler at Big Bar relative to the baseline run. The timeseries for the Trinity Camp station also showed some cooling due to the smoke, but as expected, it was not as pronounced as the cooling at the Big Bar, which is located deeper in the valley. Model comparisons across northern California on August $19^{\text {th }}$ at 2100 UTC indicates that much of the area was infiltrated by smokeemitted $\mathrm{PM}_{2.5}$, with concentrations exceeding $100 \mu \mathrm{g} \mathrm{m}^{-3}$ (Figure a). 


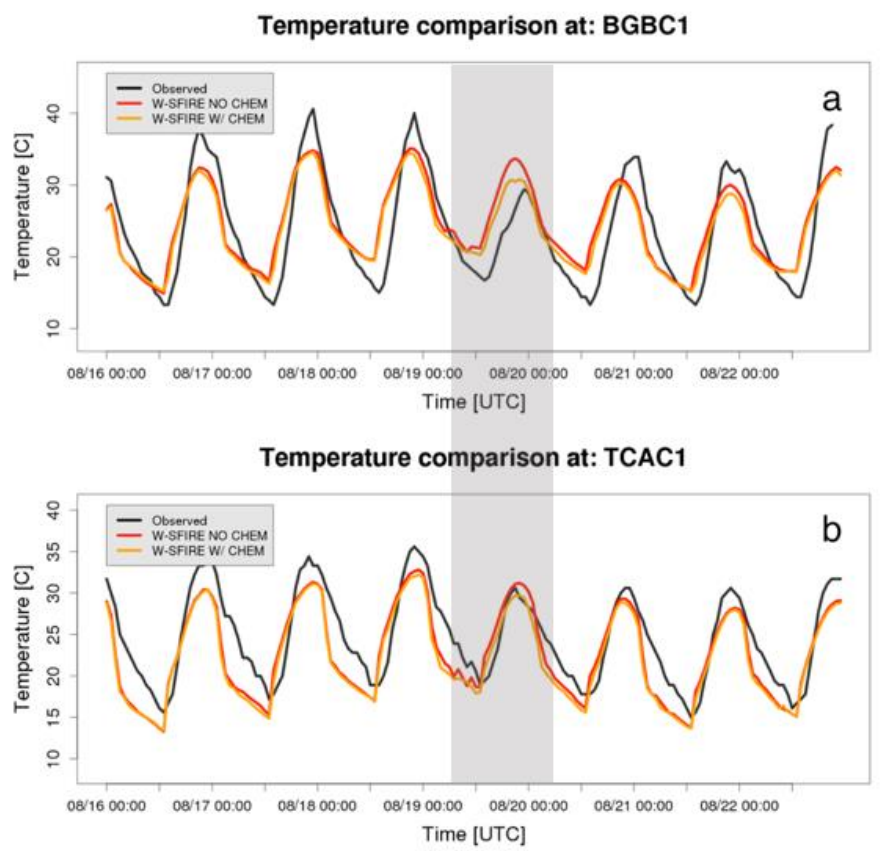

Figure 7 - Same as Figure 5, but for 2-m temperature..

Incoming solar was significantly reduced in the WRF-SFIRE simulation that included aerosol impacts, which saw deceases approaching $600 \mathrm{Wm}^{-2}$ at some locations (Figure b). Similar decreases were also seen in 2-m temperatures, which were mostly confined to river valleys across northern California (Figure c). Finally, wind speed decreases were also observed across the study region (Figure d), which was likely the result of smoke-induced inversions decoupling surface winds from the free troposphere.
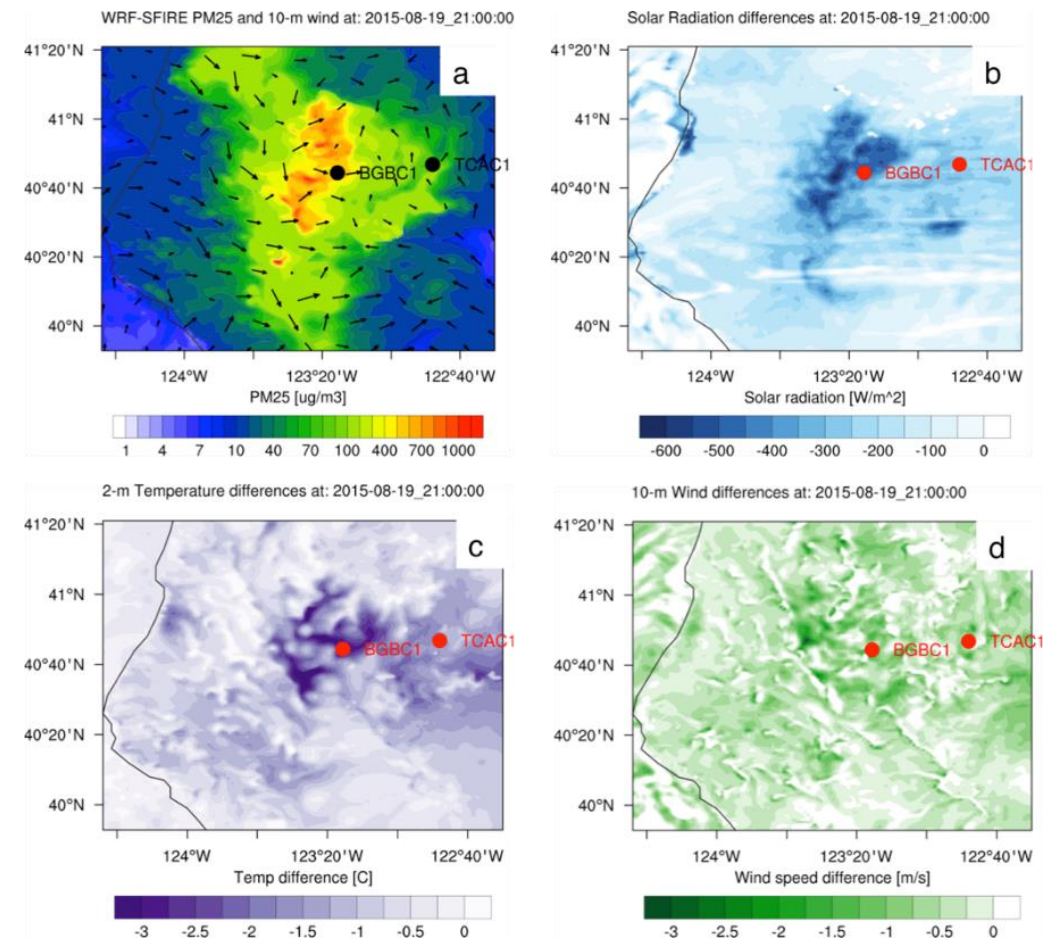

Figure 7 - (a) Modeled PM2.5 concentrations and 10-m winds, differences between the WRF-SFIRE configuration that included aerosol impacts and the baseline simulations for (b) solar radiation, (c) 2-m temperature differences, and (d) wind speed. Panels are for August $19^{\text {th }}$, 2015 at 2100 UTC. 
The visible correlation between the patterns of $\mathrm{PM}_{2.5}$ concentration, radiation reduction, and surface cooling indicates that the observed dampening of the diurnal temperature cycle at the Big Bar station was related to the local impacts of the fire smoke that strengthened inversions by decreasing incoming solar radiation and surface temperatures.

In order to assess the fire impact on the thermal structure of the atmosphere, the vertical temperature profiles over the Trinity Valley was analyzed. The comparison between the baseline simulation and the pure atmospheric run without fire didn't reveal any measurable differences in the temperature profiles. This suggests that in the case of low-intensity fires, the direct thermal effect of the fire is negligible and didn't significantly contribute to the observed inversions. However, the differences between the baseline simulation and the WRF-SFIRE-CHEM run indicate that smoke layer had a significant impact on the thermal structure of the atmosphere (Figure ). The presence of the smoke layer has a dual effect. The smoke shading, (radiation blocking) results in surface cooling, while the absorption of radiation at higher elevations results in slight warming. This double effect tends to stabilize the atmosphere and promote persistent smoke-enhanced inversions.

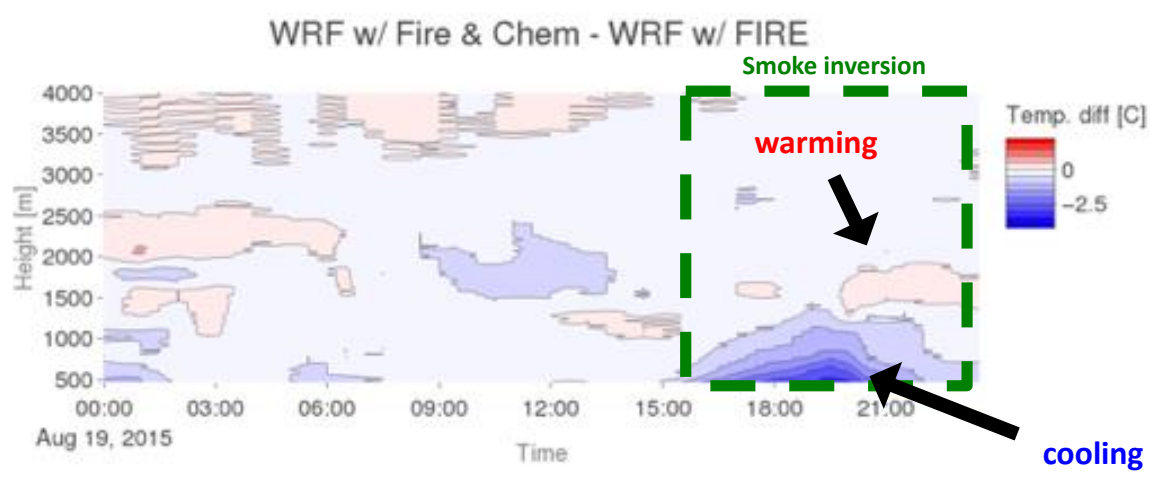

Figure 8 - Difference between the temperature from the baseline WRF-SFIRE run and the WRF-SFIRE-CHEM run with radiatively active smoke, showing time evolution of the vertical temperature modification due to the smoke.

\section{Conclusions}

Preliminary results indicate that local weather modifications due to the wildfire smoke are significant enough to motivate the inclusion of fire smoke impacts on local weather conditions within the numerical models. In the analyzed case the wildfire smoke reduced incoming solar radiation by up to $600 \mathrm{~W} / \mathrm{m}^{2}$, which lead to local surface cooling reaching $3{ }^{\circ} \mathrm{C}$. This work suggests that the inclusion of fire-smoke-atmosphere feedbacks in a coupled modeling framework such as WRF-SFIRE-CHEM may help capture the impacts of wildfire smoke on near-surface stability and local inversions. Furthermore, results here suggest that smoke may have impacts beyond increasing atmospheric stability and decreasing temperatures in smoke infiltrated valleys. The smoke-enhanced inversions tend to inhibit the vertical mixing and consequently reduce near-surface winds. This interaction generates positive feedback, in a sense that the smoke layer cools down the surface, stabilizes the atmosphere, and reduces surface winds which in turn limits the smoke ventilation and promote persistent inversions. Also, as the smoke-induced inversions inhibit the surface flow, the inversion break-ups, or the upslope fire propagation protruding through the inversion layer may lead to significant fire acceleration.

\section{Acknowledgements}

This research was sponsored by the USDA grant 16-CS-11132543-075. The authors would also like to acknowledge the support of the National Science Foundation grants DMS-1216481, ICER1664175, the NASA grant NNX13AH59G to Colorado State University, Sher Schranz, PI, CIRA in 
affiliation with NOAA/ESRL, as well as the JFSP grant 15-1-02-2 to the Washington State University. The authors would like to acknowledge high-performance computing support from Cheyenne (doi:10.5065/D6RX99HX) provided by NCAR's Computational and Information Systems Laboratory, sponsored by the National Science Foundation. The computing support from the University of Utah Center for High Performance Computing is greatly appreciated.

\section{References}

Albini, F. A., 1982: Response of free-burning fires to nonsteady wind. Combustion Science and Technology, 29(3-6), 225-241.

Andrews, Patricia L. 2009. BehavePlus fire modeling system, version 5.0: General Technical Report RMRS-GTR-213WWW. Revised. Fort Collins, CO: U.S. Department of Agriculture, Forest Service, Rocky Mountain Research Station. 111 p.

Chin, M., R. B. Rood, S.-J. Lin, J. F. Muller, and A. M. Thomspon, Atmosphericsulfur cycle in the global model GOCART: Model description and global properties, J. Geophys. Res., 105, 24,67124,687, 2000.

Clements, C.B., Perna, R., Jang, M., Lee, D., Patel, M., Street, S., Zhong, S., Goodrick, S., Li, J., Potter, B.E. and Bian, X., 2007: Observing the dynamics of wildland grass fires: Fireflux - a field validation experiment. Bull. Amer. Meteor. Soc., 88,1369-1382.

Forthofer, J.M., Butler, B.W., and N.S. Wagenbrenner, 2014a: A comparison of three approaches for simulating fine-scale winds in support of wildland fire management: Part I. Model formulation and accuracy. Int. J. Wildland Fire, 23, 969-981.

Grell, G., and A. Baklanov, 2011: Integrated modeling for forecasting weather and air quality: a call for fully coupled approaches. Atmos. Environ., 45, 6845-6851.

Kochanski, A. K., M. A. Jenkins, K. Yedinak, J. Mandel, J. Beezley, and B. Lamb, 2016: Toward an integrated system for fire, smoke, and air quality simulations. Int. J. Wildland Fire 25, 558-568. doi:10.1071/WF14074

Lareau, N.P., and C. B. Clements, 2015: Cold Smoke: smoke-induced density currents cause unexpected smoke transport near large wildfires. Atmos. Chem. Phys., 15, 11513-11520.

Lareau, N.P., and C. B. Clements, 2017: The mean and turbulent properties of a wildfire convective plume. J. Appl. Meteorol. and Climatol., 56, 2289-2299.

Mandel, J., S. Amram, J. D. Beezley, G. Kelman, A. K. Kochanski, V. Y. Kondratenko, B. H. Lynn, B. Regev, and M. Vejmelka, 2014: Recent advances and applications of WRF-SFIRE. Natural Hazards and Earth System Sciences, 14, 2829.

Saha, Suranjana, and Coauthors, 2014: The NCEP Climate Forecast System Version 2. J. Climate, 27, 2185-2208 


\title{
Examination of the forest fires detection - The relationship between the fire and the detection
}

\author{
László Bodnár *; Ágoston Restás \\ 1* National University of Public Service, Hungary. 1101, Budapest, Hungária krt. 9-11, \\ \{bodnar.laszlo@uni-nke.hu*,restas.agoston@uni-nke.hu\}
}

\begin{abstract}
Introduction: Forest fires are one of the most typical natural disasters in the world. As a result of the global climate change a continuous average temperature increase can be determined. Consequently, the risks of the forest and the vegetation fires are increasing. Because of the global climate change, the dry and rainless periods of the weather conditions can be extended. It gives bigger opportunity to ignite the biomass. For the effective firefighting it is necessary to make a quick detection. The detection of the forest and vegetation fires is carrying out mostly on a passive way, mainly based on public announcement. If there is no announcement of a fire, it does not mean a fire for the fire departments. So that is why the authors make an examination of the forest fire detection, focusing on the relationship between the fire and the detection.

Methods: When writing the paper, it was important to analyze and study the relevant literatures of the topic, and the related data collection. In addition, it is important to mention the personal consultations with various experts, the logical conclusions and the personal experiences of the authors on the subject. The authors expressed the effectiveness of the observation with an simple mathematical formula.

Results: The authors set out to examine the detection of the forest and vegetation fires as a factor influencing on the effective firefighting. The paper shows the relationship between the observer and the fire. The authors were analyzing the operation methods of the human eyes. Including the importance of the angle of view, the whole field of view of the human eyes. In the paper appear the obstructive factors of the detection, such as the distance and the terrain. As a result of the paper, the authors determined a formula, which shows the efficiency of the observation and its most important circumstances.
\end{abstract}

Keywords: forest fire, vegetation fire, observer, detection, efficiency, angle of view

\section{Introduction}

The global climate change is a real threat in the past decades, (Westerling ALR. 2016) but it has begun in the 20th century. (Morgan et al. 2008) Global warming affects all elements of firefighting. The in time observation of the forest fires is very important, as it has an indirect impact on the size of burned areas and on the effectiveness of firefighting. Firefighting is a very long and complicated process that requires proper firefighting tactics and well - considered decisions from the leader of the firefighting. (Restás 2015) When doing researches in connection with the detection of the forest fires, it is very important to mention some results from other authors. According to Ahmad A. A. Alkhatib 2014 there are a lot of methods and systems, which can help for the early detection. Each technique has its own advantages and disadvantages. For example, a well-functioning camera system can be a big help in the early detection. Authors like other experts prefer unnamed aerial vehicles for the effective detection. Drones can monitor forest fires and make measurements (L. Merino et al. 2012) or software's can provide functions on processing UAV aerial image data, according to the requirements of forestry area application on UAV platform. It gives a real-time and remote watch on fires. (Lan Zhang et al. 2015) But there are some other researchers, who suggest wireless sensor networks for the reliable detection. It promises green technology for the future in detecting efficiently the forest and the vegetation fires. (Bouabdellah et al. 2013) In case of a fire, in many cases the innovative devices are not available. Fire detection is a civic duty in which the efficiency of the fire detection depends on the observer. Authors will examine this problem and its circumstances. 
A firefighting process is a result of many complex steps. However, these steps can be called effective only when the first steps are time-consuming. These first steps will take place even before the marching of the professional fire departments. The most important of these steps are the generation of the fire, the fire detection, and the assessments of fire detection, the determination of alert state and the alarm. This is illustrated in Figure 1 below.

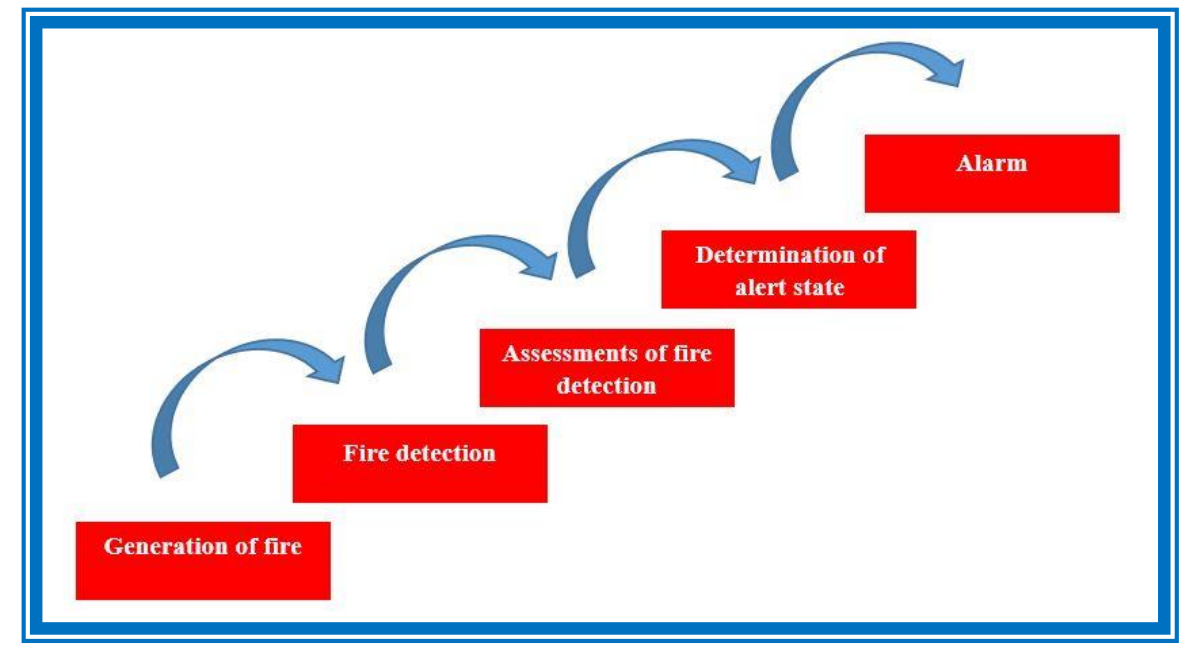

Figure 1 - The first 5 steps in the firefighting process

It is a fact that most of forest fires are the result of human negligence or intent, therefore, the authors state that the first two steps of the firefighting process are related to the citizens. The generation of fires can be prevented by laws and modern innovative tools. (Blum 2012; Condera et al. 2012) However, in many cases they do not provide the opportunity to prevent the fires. The generation of a fire causes heat and fumes. The colour of smoke can be detected within a short time by the citizens. The quickness of the observation and the signalling is very important, as the later the fire alarm is realized, the later the professional fire departments will be alerted, and it will result in more burnt areas. It means significant costs for the national economy. (Restás 2014; Bodnár 2017)

In connection with the firefighting it is important to mention that according to common law, who detects a fire has an obligation to signal it to the professional fire departments. If it is not possible, the observer has to make a signal for the police, the ambulance service or the mayor's office of the competent municipality.

The fire detection can be done by personal communication, by telephone or by voice and light signalling systems. In this case the most important facts are the quickness and the statement. According to the experiences of experts the observer must always report:

- The exact location of the fire

- What is burning?

- What kind of damage has happened

- What is in danger (gas tanks, dangerous goods, vehicles)

- Is there a life threat?

- The name and phone number of the observer

The statement and the speed of these data are very important for the effective firefighting. However, detection of fire is not always easy. There are some cases when detection is affected by various circumstances. In addition, it is important to examine and analyse the angle of view and the function of the human eye. With this function, the authors make considerations in the following chapter. 


\section{Examining the function of the human eye and the angle of view}

A basic condition for remote detection is that it has to reach the minimum level of the sensitivity threshold of the detection. The typical parameter of the fire is the smoke, which can be detected visually even from a long distance. The smoke is a complex flue-gas system. Its composition depends on the combustible material and the conditions of combustion. The most important factor in the burning of vegetation is the water content of the vegetation. Depending on the circumstances, the location of the burning vegetation can be observed from long distance, because of fume. The observation is basically influenced by the colour and intensity of the smoke, the water content of the given vegetation and the atmospheric conditions. The released aqua vapour during the combustion dyes the fumes grey-white. The opportunity of the long distance detection is determined by the distance between the observer and the fire, the obstacles between them and their relative positions. But it is also important to investigate field of view and the operation of the photoreceptors.

Eye is one of the most important senses of people. By using our eyes, we are able to detect and find out everything. The field of view is the space that our eye sees in straight forward look. During a perimetry, the two eyes must be examined individually. Looking straight forward, the eye fixes a point, in the visual field hemispheres, but whenever a spotlight is displayed, it is indicated by a button push. During the perimetry, the testing hemisphere and the intensity of the test light are accurately calibrated. Based on the test signal, two types of perimetry can be distinguished.

During the kinetic perimetry, the light point is moved by the investigator in the perimeter sphere from the periphery to the centre (hence kinetic). The circle or oval line within which the test point of a given size and brightness is detected by the test is a so-called isopter. The ability of the light sensor retina is centrally the largest, and to the edge is gradually more imperceptable. Therefore, the smaller and weaker is the test point, the smaller is the associated isopter, so the field of view is narrower.

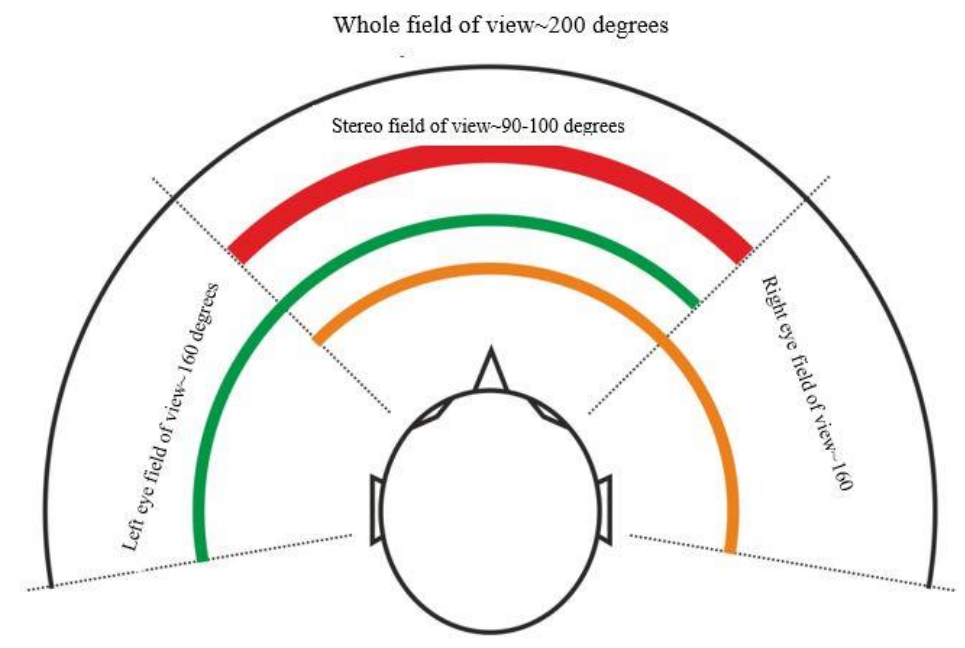

Figure 2 - The whole field of view of the human eyes. Source (Süveges 2010)

During the static perimetry, the test signal does not move, but it is displayed at different locations with different light intensity. The purpose of the test is to determine the light detection threshold value at the retinal sites under examination. (Steven 2016)

According to the Figure 2, the human eye is generally capable of measuring a 200-degree angle. Within the field of sharp eyesight, in the centre of the field of vision, the eyes are able to see angle of view about 90-100 degrees. Within this area, a healthy person's eyes can detect anything and capture it in brain in a good quality. (red line) If the two eyes are examined separately, it can be stated that the angle of view of each eyes has approximately an angle of 160 degrees. (green and orange lines) 
Separately, they do not cover the entire field of view but the full visibility is achieved only with the operation of both eyes.

The importance of the eyes and the field of view also plays a significant role in the detection of fire. Detection is effective if the observers perform their activity at the widest possible angle and in the best possible quality. If these two conditions are not fulfilled, the detector may receive a false image of the fire and the fire spread. As a result of this, false data can be sent to the professional fire departments, which also has an influence on the number of the alert forces and on determination of the alert state. Almost everywhere, a large number of forest fires were generated due to bad detections data due to classifications of the alert state. Later this problem was solved by the leader of the firefighting. (Bodnár 2017)

As a result of this chapter, the authors stated that the detection may have a significant effect on firefighting. Because the detection of a fire is still made by the citizens in most cases, it is very important to examine the function and the operation of the human eye. If a person who detects a fire does not perform their obligation well, it will result in a more complicated intervention for the firefighters. The incorrect detection gives a bad signal to the interveners, so it will lead to problems during the firefighting. In addition, it means extra costs for the national economy

\section{Factors hindering the detection}

The early detection of the generation of fire is very important. In some cases, observation is hampered by various obstacles. In this chapter, the authors examine the obstacles of the fire detection and its methodological background. If the fire is detected from a long distance it is important to have a feature which reaches the minimum level of sensitivity threshold for the detection. The characteristic parameter of the fire is smoke. It can be perceived visually from even longer distance.

\subsection{Examining the role of the distance in connection with the detection}

One of the most typical difficulties of detecting a fire is distance, because this is a factor that basically has a major influence on the effectiveness of the observation. In this connection, the authors still consider that it is important to examine the function of the human eyes and its adaptation to the distance. The eyes must also give a sharp picture from various distances in order to keep the image distance. it is facilitated by the long-distance adaptation and accommodation of the eye.

The eyepieces are fixed to the ciliary body in the surroundings with a help of so-called elastic and inflexible lenses. If the ciliary muscle relaxes in the ciliary body (resting position), then the hanging fibres are strained, so the eyepiece will flatter and the focal length will be greater. It is called far-reaching adaptation. If the ciliary muscle will contract, (the diameter of the radial body decreases) the hanging fibres will slack, so the flexible lens will curle, and its focal length will decrease. This is the close adapted eye. Without any exertion the normal eye can adapt to the distance of so-called perspicacity. For a healthy eye, the clearity distance is approximately $25 \mathrm{~cm}$. (Goldsmith 1990) Of course, detecting forest fires, the observer cannot detect from a $25 \mathrm{~cm}$ of distance. Its distance is significantly higher. (Dancy and Christian 2014) Of course, the eye can adapt to longer distances. Its operation is illustrated in Figure 3. While increasing the distance, the possibility of the observation decreases proportionately. The reason for this is that on one

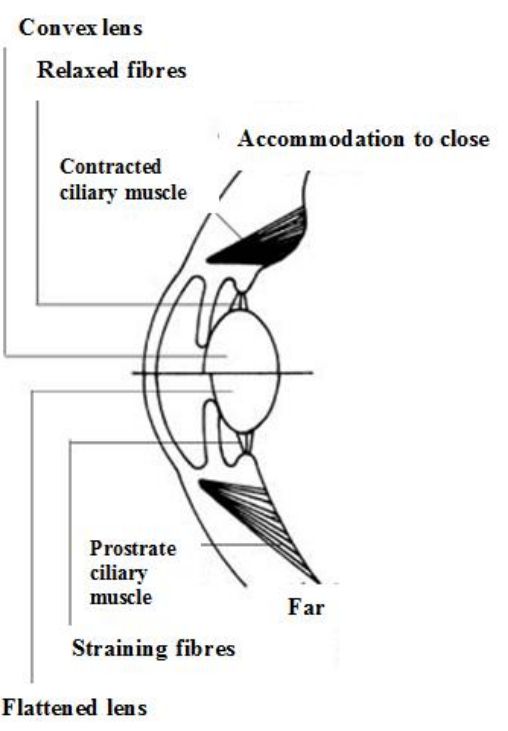

Figure 3 - The long distance adaptation of the human eye. Source: (Sulinet 2018) 
hand the visible surface decreases in proportion to the square of the distance and, on the other hand, the humidity of the air reduces the visibility of the contours. If the smoke does not reach the threshold of sensitivity of the observer, it remains invisible.

When examining the operation of the eye it can be stated that our eyes can adapt to various distances. However, in some cases, because of the distance between the eye and the detected area, the observer can get distorted view. It can also have an affect to the fire alarm. But when the observer does not get a real picture of the circumstances of the fire, the signal can be false. In the case of a false fire alarm, the marching will be unnecessary. It means further costs for the economy system.

\subsection{Other obstacles during a detection}

After investigating the detection and the distance, the authors consider to analyse other obstacle factors during the observation.

There may be many obstacles between the location of the observer and the burning area. It can reduce or exclude the possibility of detection. It could be for example the terrain and the vegetation of the detected area. The perceptibility of various fires in valleys can only be observed by the smoke, when it rises above the top of the vegetation. In this case, the flames can not be detected. In many cases, it has led to major problems, because basically, smoke does not always mean a fire in the forest. Ignition is allowed at designated fireplaces in the forest, but it has also smoke phenomenon. In many cases the observer thinks of this and not of a real forest fire. In addition, it is considered by the authors to mention that the content of the air humidity and the relative humidity reduces the option of the detection in proportion with the distance. In addition, it should be noted that the low humidity increases to both the propagation of fire and the possibility of the detection.

The detection of forest fire is basically influenced by the relationship between the observer and the fire. It can be an advantage or even a disadvantage in the detecting activity. The lower level you can imagine it in a hilly, pointed area when the observer is in the valley and the fire on a hillside or on a hilltop. In this case, the angle of view allows not only the detection of the smoke, but also the visibility of the frontline of the fire. This is a clear sign of a forest fire, so the phenomenon of smoke does not deceive the observer, so he can warn quickly. The same level of the location allows the detection of smoke, the visibility of the frontline of the fire depends on the height of the vegetation or the parameters of the fire. In this case, there can be some doubts from the observer, because he cannot see the flames, only the smoke. The higher detection level of the observer allows the detection of both the smoke and the frontline of the fire due to the better angle of view. The smoke can reduce the detection of the frontline fire. Detection has a psychological background as well. It has been a concern for a long time. (Wade and Rovira 1981)

Table 1 - The observer's relationship to the fire.

\begin{tabular}{|c|c|c|c|}
\hline $\begin{array}{c}\text { observer's } \\
\text { relationship with } \\
\text { the fire }\end{array}$ & $\begin{array}{c}\text { only smoke } \\
\text { detection }\end{array}$ & $\begin{array}{c}\text { flame and } \\
\text { smoke } \\
\text { detection }\end{array}$ & $\begin{array}{c}\text { only flame } \\
\text { detection }\end{array}$ \\
\hline lower & & $\mathrm{X}$ & \\
\hline same & $\mathrm{X}$ & & \\
\hline higher & & $\mathrm{X}$ & \\
\hline
\end{tabular}

As a result of this chapter, the authors define a formula for the effective human detection. The efficiency of the detection is influenced by the distance between the fire and the observer, the time of the detection (response time), the terrain of the area and the phenomenon of the flames and smoke. In this context, these factors will be reflected in the formula defined by the authors.

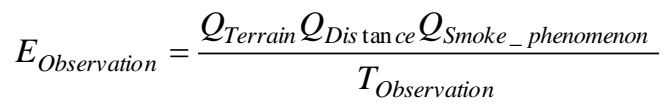

- Eobservation: effectiveness of the observation 
- QTerrain: characteristics of the territory

- QDistance: distance between the fire and the observer

- Qsmoke_phenomenon: characteristics of the smoke (fire)

- Tobservation: time between fire ignition and observation

$$
\begin{aligned}
& O=\frac{\mathrm{S}+\mathrm{Te}+\mathrm{Ph}}{\mathrm{To}} \\
& \text { O: Observation } \\
& \mathrm{Te}: \text { Terrain } \\
& \mathrm{S}: \text { Distance between the fire and the observer } \\
& \text { To: Time of the Observation } \\
& \mathrm{Ph} \text { : Phenomenon } \\
& \text { Figure } 4 \text { - Formula of the efficient observation. }
\end{aligned}
$$

According to the formula the authors determined the efficient detection. The efficient observation is realised, when the observer in a certain period of time (To), from a certain distance (S), under certain terrain conditions $(\mathrm{Te})$ detects a phenomenon of fire $(\mathrm{Ph})$ and warns the disaster management. The less time it is realized the fire alarm will be more efficient. A quick fire detection is a key for the effective firefighting. In connection with the early detection it is important to mention that beside the human observation, there are other technical solutions (for example drones) to detect the wildfires. Many suggestions have been made to this in the international publications in connection with forest fires (Restás 2015; Bodnár et al. 2018) and in other fields of science (Muyambo et al. 2017)

\section{Summary}

In this article, the authors set out to examine the detection of the forest and vegetation fires. The paper also examines and analyse the operation and methodology of the human eye, as well as the conclusions and actualities to the wildfires. In addition, the authors performed a research in connection with the detection factors and the difficulties of the observation. In this context, the potential connections of the observer were presented to the fire, thus examining the circumstances of the detection.

The result of the paper is a formula defined by the authors that determines the effectiveness of the fire detection. It is important because the first step in any firefighting activity is the fire detection of the citizens. If it does not happen quickly and efficiently, it significantly determines the effectiveness of firefighting. The number of the intervening forces depends on the fire detection and on the previous experiences. If less fire forces will be alerted, the leader of the firefighting has to alarm more fire forces. It takes time. During this period of time, the size of burned areas will grow continuously, which means significant expense to the national economy system.

In addition, the authors drew attention to the importance of false fire detection. Even in this case, they are alerted by professional fire brigades and they are on the scene due to the signal. Different alarms of a fire have their own costs, even if it is a false alarm; it means more costs for the economy system. Overall, it can be stated that early detection of fire is extremely important, so it requires further researches and analysis.

\section{References}

Ahmad A. A. Alkhatib (2014) A Review on Forest Fire Detection Techniques. International Journal of Distributed Sensor Networks 10, 1-12.

$$
\text { http://journals.sagepub.com/doi/pdf/10.1155/2014/597368 }
$$


Blum, S. (2012) Eine neue Methode warnt vor Waldbrand. Tierwelt 31, 22-23.

Bodnár L (2017) Case study of "Hortobagy" and "Kunfeherto" fires, Hungary: disaster in costs of their elimination's view. Ecoterra: Journal of environmental research and protection 14, 40-46.

Bodnár L, Restás Á, Qiang X (2018) Conceptual Approach of Measuring the Professional and Economic Effectiveness of Drone Applications Supporting Forest fire Management. Procedia Engineering 211, 8-17.

Bouabdellah K, Noureddine H, Larbi S, (2013): Using Wireless Sensor Networks for Reliable Forest Fires Detection. Procedia Computer Science 19, 794-801.

Condera, $\mathrm{M}$ et al. (2012): Verifica sperimentale del sistema FireLess2: stima dell'umidità dei combustibili forestali e del pericolo di incen- dio. Sherwood 18, 185, 25-31.

Dancy M, Christian W (2014) The applet of my eyes. (Translate: Nagy S) https://nagysandor.eu/ChristianBelloni/optics/applications/illustration36_1.html (download 22 $2^{\text {th }}$ of June 2018)

Goldsmith T. H (1990) Optimization, Constraint, and History in the Evolution of Eyes. The Quarterly Review of Biology 65, 281-322

Lan Z, Bing W, Weilong P, Chao L, Zeping L, Yan G (2015): A Method for Forest Fire Detection Using UAV. Advanced Science and Technology Letter 81 69-74. https://pdfs.semanticscholar.org/c353/eec25fc3a25675e57e129817a94ac7e1c136.pdf

Merino L, Caballero F, Martínez-de-Dios J. R, Maza I, Ollero A, (2012) An unmanned aircraft system for automatic forest fire monitoring and measurement. Journal of Intelligent \& Robotic Systems $\mathbf{6 5}$ 533-548

Muyambo F, Restás A, Jordaan A, Bodnar L, (2017) A life-saving technology supporting crisis management unnamed aerial vehicle (UAV) application in developing countries. Delta XI, 20-27.

Morgan P, Heyerdahl EK, Gibson CE. 2008 Multi-season climate synchronized forest fires throughout the 20th century, northern Rockies, USA. Ecology, 89 717-728.

Restás Á (2014) Theoretical approaches for evaluating the economic efficiency of the aerial firefighting helping strategic planning In: Domingos Xavier Viegas Luis Mário Riberio, Abstracts of the VII International Conference on Forest Fire Research.Coimbra, Portugal, 2014.11.142014.11.21. Coimbra: University of Coimbra, 2014. p. 17. 1 p.

Restás Á (2015) Drone Applications for Supporting Disaster Management. World Journal of Engineering and technology 3, 316-321

Restás Á (2015) How fire managers make decision in case of time pressure. In: W J de Groot The 6th International Wildland Fire Conference. Seoul, South Korea, 12.10.2015-16.10.2015. Seoul: Institute for Environmental Technology and Sustainable Development, Korea University, 2015. Paper 0508.

Steven M. LaValle (2016) Virtual Reality. Cambridge University Press. Cambridge http://msl.cs.uiuc.edu/vr/vrch5.pdf

Sulinet (2018) http://tudasbazis.sulinet.hu/hu/termeszettudomanyok/fizika/fizika-11-evfolyam/azemberi-szem/a-szem-alkalmazkodasa-a-tavolsaghoz (download $20^{\text {th }}$ of June 2018)

Süveges I (2010) Szemészet. (Medicina Publishing: Budapest)

Wade M, Rovira M (1981) Signal Detection Theory - Its Implications for Social Psychology. Personality and Social Psychology Bulletin 7, 232-239.

Westerling ALR (2016) Increasing western US forest wildfire activity: sensitivity to changes in the timing of spring. Philosophical Transactions Biological Sciences 371, 1-10. 


\title{
Factors Contributing to High and Low Risk Fires in the United States: An Analysis of the Relative Risk Assessment
}

\author{
Erin Noonan-Wright ${ }^{1 *}$; Carl Seielstad ${ }^{2}$ \\ ${ }^{1} 5775$ W. Broadway, Missoula, MT 59808, USA. \{enoonan02@fs.fed.us*\} \\ ${ }^{2}$ University of Montana, CHCB 441, 32 Campus Drive, Missoula, MT 59812. USA. \\ \{carl@firecenter.umt.edu\}
}

\begin{abstract}
Discussion of risk permeates every U.S. federal wildland fire agency directive before, during and after fire season. In this study, we explore patterns of wildfire risk across the U.S. from the systematic operational risk assessments conducted by land managers on 5,087 wildfire incidents from 2010 - 2017. The Relative Risk Assessment (RRA) is a systematic, semi-quantitative assessment of risk which integrates ratings of high, moderate, and low 'Values', 'Hazard', and 'Probability' for each wildland fire. Each entry also includes notes, where decision makers describe qualitatively their reasons for their rating. The RRA is a mandated component of the Wildland Fire Decision Support System - WFDSS, decision making system for U.S. federal wildland fires. We know that perceptions of risk are different in geographic areas of the United States. The Southwest region perceives low risk while the Northwest perceives high risk more frequently when compared to the U.S. as a whole. The reasons for differences in risk perceptions are complex, but previous analysis of the relative risk data suggest the Southwest has a greater frequency of low ratings for Values in comparison to the Northwest. Other geographic areas follow similar trends. Here, we strive to define the attributes of the RRA that are most prevalent for high versus low risk fires by evaluating qualitative content associated with the RRA. "Private" values in the Northwest are documented with greater frequency for the Values element compared to the Southwest, where "cultural" values occur with greater frequency. Qualitative analysis illuminated specific geographic trends previously analyzed quantitatively. As we strive to make a better connection between perceived and actual risk, we hope the results of this analysis demonstrates that there are components of perceived risk that should be addressed with greater emphasis before and during wildland fire planning efforts.
\end{abstract}

Keywords: wildland fire, United States, WFDSS, risk

\section{Introduction}

The topic of risk permeates every facet of U.S. federal wildland fire decision-making. Attempts have been made to identify how individual decision makers address risk. Maguire and Albright (2005) contribute excessive risk aversion in wildland fire management decision making to mental shortcuts developed during uncertain and conflicting decision environments. The flexibility in fire management policy has inadvertedly resulted in more suppression strategies to manage wildland fire due to the lack of planning (Seielstad 2014). Yet the need to embrace the uncertainty of wildland fire is necessary to adequately achieve the missions and goals of long-term land management. Solutions to address limitations in risk perception have manifested in solutions such as those proposed by Marcot et al. 2012, with formal procedures encompassing the four stages of structured decision making: problem structuring; analysis; decision point; and monitoring; addressing uncertainty with decision making in planning environments. Taber et al. (2013) specified a decision making process specific to incident level wildland fires that alluded to the same principles as (Marcot et al. 2012). A step by step case study of the incident level risk management process was demonstrated for the Gold Pan Fire 2013 using the tools and information available in Wildland Fire Decision Support System - WFDSS (Noonan-Wright and Opperman 2014). 
We can learn something about how real/actual risk is conceived and what factors govern its formulation by examining spatial patterns of risk where the rubber meets the road- in the systematic operational risk assessments conducted by land managers on thousands of wildfire incidents. By illuminating these patterns, we hope to stimulate examination of the social, cultural, and physiographic factors that drive risk-informed decision-making and expect that improved understanding of the geographic diversity of risk will ultimately lead to improvements in decision making. We illustrate differences between two geographic areas in the United States: the Southwest and Northwest by evaluating their selection preferences towards Values and the notes that support these preferences.

\subsection{The Wildland Fire Decision Support System and The Relative Risk Assessment}

The Wildland Fire Decision Support System - WFDSS was created to facilitate a deliberate risk assessment process on wildland fires by establishing a web-enabled collection of tools and information to support decision making (Calkin et al. 2011; Noonan-Wright et al. 2011). WFDSS was chartered in 2005 to capitalize on the availability of existing spatial fire behavior models to predict fire behavior spread, co-incident with the availability of a plethora of national-scale GIS cadastral and critical infrastructure data, ultimately leading to the ability to better quantify the threat and hazard to values at risk. This was an important step in the advancement of quantifying risk on wildland fires (Finney 2005).

The Relative Risk Assessment (RRA) is not a quantitative risk assessment; however, the process of evaluating risk could include elements of quantitative risk. Alternatively, the RRA capitalizes on the knowledge of the decisionmaker to qualitatively assign ratings of high, moderate or low to values, hazard and probability elements. Each main element (values, hazard or probability) is composed of subelements that are rated to determine a final rating for each main element and the relative risk (Figure 1). Each land manager must evaluate their perspective risk using the RRA for every wildland fire in WFDSS.

The RRA was initially composed of a square box to support a decision of whether to aggressively suppress a wildland fire or allow it to burn to achieve some natural resource benefit. When the fire policy was revised in 1995, there was a need to create a quick justification for this 'Go/No Go' decision to allow a wildland fire to burn to achieve resouce objectives without aggressive suppression tactics and

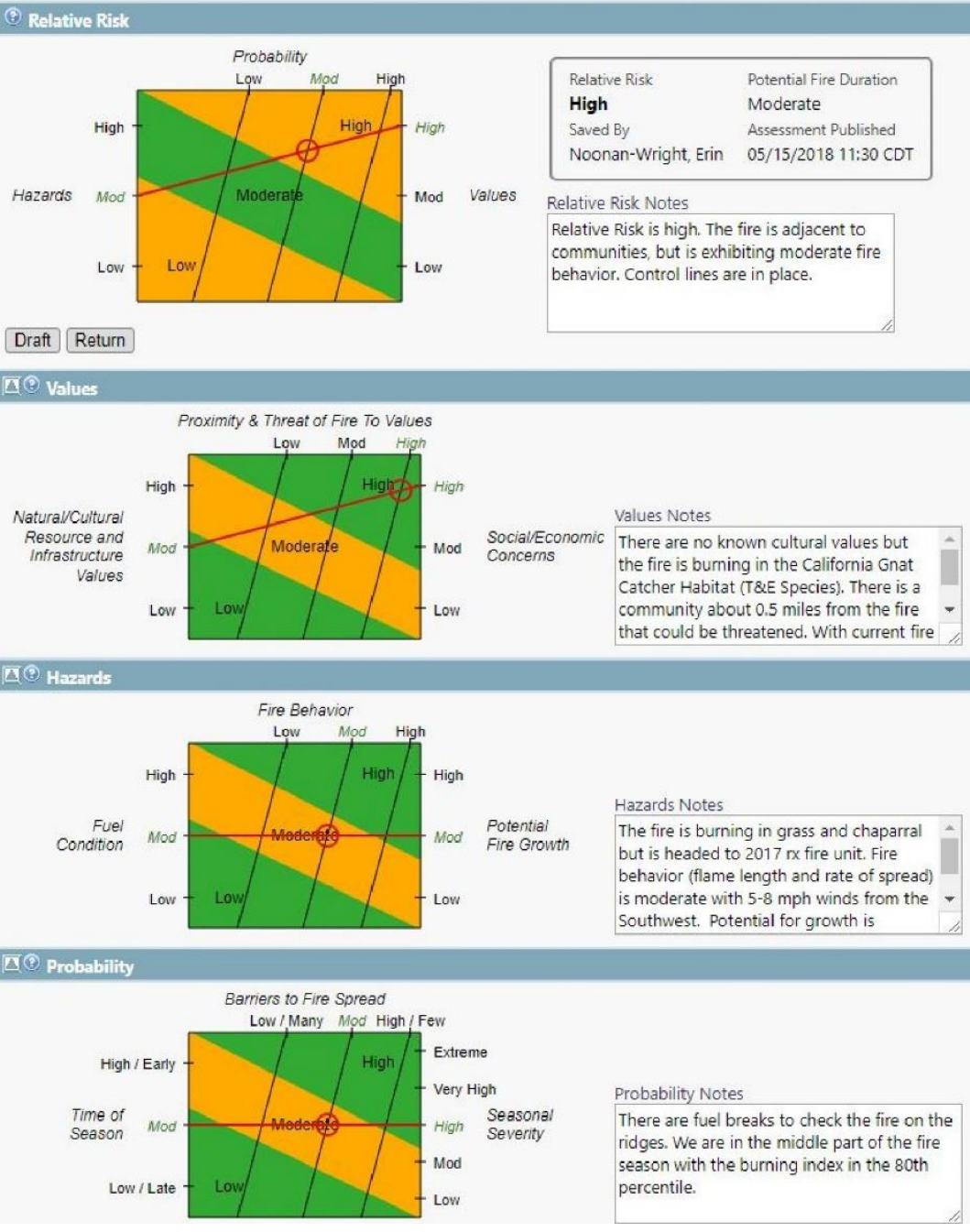

Figure 1 - An example of the Relative Risk Assessment process in the Wildland Fire Decision Support System - WFDSS 
strategy. Initially the preliminary relative risk assessment was purely subjective and qualitative and intended to be done in about ten minutes given that few climatological/fire behavior tools existed to quantify this first assessment. However, the process to compute an initial assessment of risk resulted in some confusion and was revised in 2005. The revision was based, in part, on the incorporation of the hauling chart (Andrews and Rothermel 1982) and the National Fire Danger Rating System (Deeming, Burgan, Cohen 1978). Because the assessment documented the initial, qualitative risk, it was termed a "relative" risk assessment (Zimmerman 2017).

\section{Methods}

WFDSS is a J2EE, java server faces (JSF) web application using a service-orientated architecture (SOA) which integrates a number of other technologies in order to store, create, query, and display geospatial and tabular data through the application server as well as other services (Noonan-Wright et al. 2011). Data are stored in a relational data stream management system (RDSMS) and are queried though the use of Structured Query Language (SQL) to link data tables and extract all the fire records in WFDSS from 2010 through 2017. Duplicates and other anomalies with the data were remedied using CRAN - R (R Core Team 2013) and various packages to compute time (Grolemund, Wickham 2011), create and append data tables (Wickham 2011, Wickham et al. 2017) and expedite processes (Bache and Wickham 2014). While numerous relative risk assessments are done for long-duration fires as conditions change, we chose the most frequently used relative risk rating to represent the wildland fire. Consequently, each fire record has one relative risk rating to represent its relative risk. The two most different risk profiles by geographic area (G.A.), the Southwest and the Northwest, were compared for their rating selections of the sub-elements (Noonan-Wright and Seielstad, in prep). The Southwest tends to prefer 'low' ratings while the Northwest prefers 'moderate' and 'high' ratings for sub elements, main elements and relative risk. When these G.A.s are compared to the U.S. frequencies, the Northwest more clearly prefers 'high' ratings while the Southwest prefers 'low' ratings.

Individual responses to each of the sub-elements (time of season - tos, barriers to fire spread - bar, seasonal severity - ss, fuel condition - fuel, fire behavior -fbeh, potential fire growth - pot, natural/cultural resource and infrastructure values - res, proximity and threat of fire to values - threat, and social and economic concerns - conc) were tallied by geographic area (Southwest and Northwest) and the rating (high, moderate, or low). Extreme and very high ratings are only applicable to seasonal severity. A preference metric was computed to show preference or aversion to specific ratings:

\section{Preference metric $=(\%$ observed $\div \%$ expected $)-1$}

Where percent expected was based on expectation values from the United States for a particular rating. Percent observed were tallied ratings for a specific sub-element normalized by the totals for specific geographic area.

In addition, CRAN - R, the text mining package, 'tm' (Feinerer et al. 2008) was used to evaluate word frequencies and associations from the 'Values' notes from the relative risk assessment (Figure 1). Word frequencies identified the words of 'private' being one of the most frequently used for the Northwest, while the word 'cultural' was more frequently used in the Southwest. Correlations with those words by geographic area more clearly identify the specific 'values' each geographic area is most concerned with.

\section{Results}

The Northwest (NW) geographic area prefers 'high' then 'moderate' ratings for the Values subelements: conc - social, economic and political concerns; threat - proximity and threat of the fire to values at risk; and res - natural/cultural resources or infrastructure values. Comparatively, the Southwest (SW) prefers mostly 'low' or 'moderate' ratings for those same elements, when compared 
to the United States frequencies. In general, the Southwest does not prefer 'high' ratings and the Northwest does not prefer 'low' ratings.

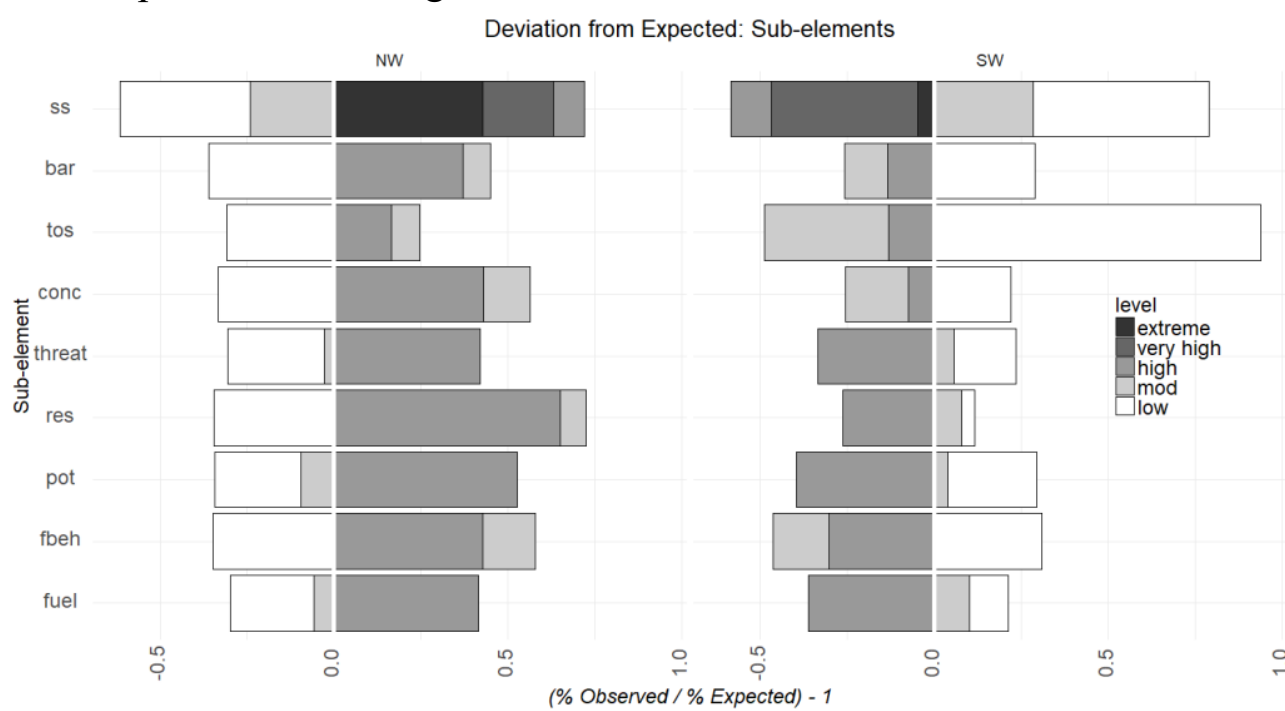

Figure 2 - Percent frequencies for each sub-element of the Relative Risk Assessment divided by the percent frequencies of the U.S. subtracted by one to show preference (positive) or aversion to (negative) a specific rating. The Values main element is composed of three sub-elements (conc-Social, Political, and Economic concerns; threat proximity and threat of fire to values; and res - Natural/Cultural Resources or Infrastructure values). $N W$ represents the sub-elements for the Northwest Geographic Area; while SW represents the Southwest Geographic Area.

To better identify specific values of concern described by each geographic area, we examined the 'Values' notes (Figure 1). The Northwest identified the word, "private" as one of the most frequently used in the Values notes; while the Southwest identified the word, "cultural" as one of the most frequently used. Correlations with those words further describe associations with those values (Figures 3, 4). The G.A.s share correlations with some words related to "private' which include: "property", "land", and "blm". The Northwest also documents words related to values with discrete economic value such as, "structures", "property", "hut", and "snowmobile" that are not included in the Southwest notes. The Southwest appears to document commercial values associated with grazing. Words such as "lands", "property", "permittees", "holdings", and "allotments" are more commonly associated with the word "private". The influence of multiple jurisdictions seems evident. Both G.A.s use the word "blm" or Bureau of Land Management, while only the Northwest correlates "airforce" to the word "private".

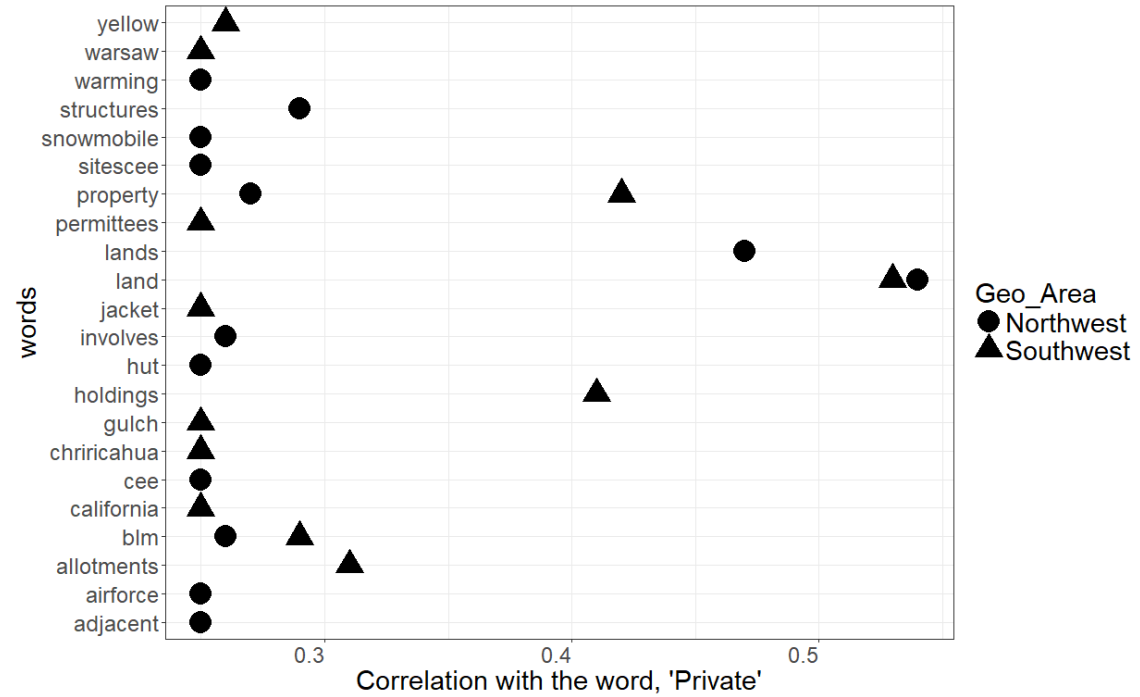

Figure 3 - Correlations with the word, "Private", for the Values notes in the Relative Risk Assessment. 
Cultural values are included in the 'res' or 'Natural/Cultural Resources and Infrastructure Values" sub-element. The word, 'cultural' is frequently used for both G.A.s but more commonly in the Southwest. Both G.A.s associate the words "social", "resources", "proximity", "natural", "fire" and "economic" with the word, "cultural". The word, "natural" appears to be the most highly correlated word with "cultural" for the Southwest while the words "resources" and "natural" are the most highly correlated for the Northwest, likely in reference to the name of the sub-element. The Southwest also correlates "infrastructure" and "sites" with "cultural", suggesting that cultural resources may also have adjacency to infrastructure. The Northwest correlates the words "tribal" with "cultural" suggesting a link to cultural values associated with Northwest Native American tribes.

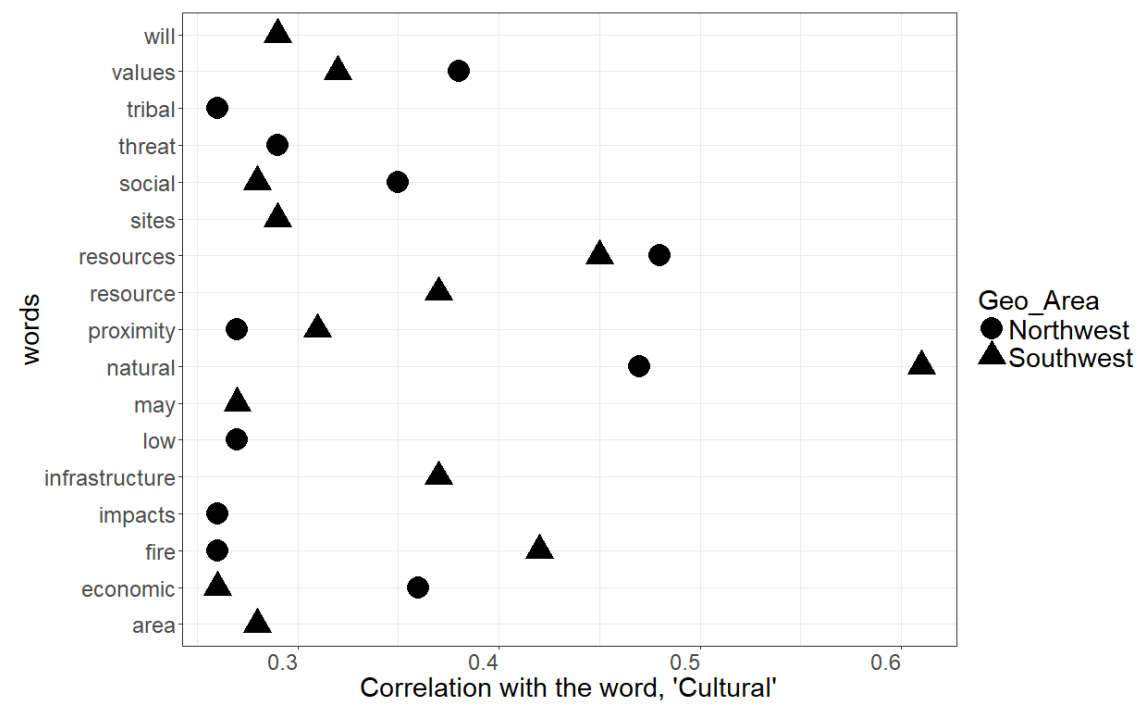

Figure 4 - Correlations with the word, "cultural", for the Values notes in the Relative Risk Assessment.

\section{Discussion}

While the Northwest perceives their values as higher compared to the U.S. and the Southwest perceives their values as lower compared to the U.S., one cannot conclude that these perceptions are real. Specific sub-elements are rated 'high', 'moderate' or 'low" by land managers for a specific wildland fire. These ratings compose the Relative Risk Assessment that becomes one part of strategic decision documented in WFDSS, ultimately signed by an authorative land manager such as a district ranger or forest supervisor. However, perceptions of risk can influence strategic decision making and how wildland fire is managed. Fire management strategies that were costly and less risk averse were more favored by fire managers given social and political constraints simulated during hypothetical scenarios (Calkin, Wibbenmeyer, Thompson 2012).

Contextual analysis of qualitative information can help identify meaningful trends. When discussing Values, the Southwest uses words related to natural and cultural resources. The Southwest also prefers 'low' and 'moderate' ratings for the Values sub-elements, suggesting that the lack of infrastructure values threatened by wildland fires may result in lower perceptions of risk to values and a general acceptance of the role of fire interacting with cultural and natural resources. The Northwest uses the word "private" with the most frequency when documenting values, suggesting that non federal government lands and associated infrastructure are influential in determining high perceptions of risk related to values.

"Cultural" is a commonly used word for all geographic areas, regardless of the specfic rating. Perhaps the difficulty in identifying these values during wildland fires plays a role in why they are discussed so frequently. During wildland fires, it is incumbent on the land manger and her staff to work with the local community to identify cultural values and make their general vicinity known to 
limit the negative effects from fire fighting efforts. The general vagueness of what these values consist of and their exact location may be one reason they are discussed so frequently in the 'Values' notes.

WFDSS became the decision documentation support system in 2009 used by federal land management agencies to document wildland fires that exceeded initial attack or were to be managed as long duration events. Eight years of wildland fires $(2010$ - 2017) were used for this study to summarize perceptions of risk in an attempt to highlight decision making patterns during wildland fires. As we identify trends through this study, we hope the results will be a starting point for more thoughtful pre-season fire preparation and the evaluation of fire management strategies.

\section{References}

Andrews PL, Rothermel RC (1982) Charts for interpreting wildland fire behavior characteristics. USDA Forest Service, Intermountain Forest and Range Experiment Station Gen. Tech. Rep. INT131. (Ogden, UT)

Bache SM, Wickham H (2014). magrittr: A Forward-Pipe Operator for R. R package version 1.5. https://CRAN.R-project.org/package=magrittr

Calkin DE, Thompson MP, Finney MA, Hyde KD (2011) A real-time risk assessment tool supporting wildland fire decision-making. Journal of Forestry 109(5), 274-280.

Calkin DE, Venn T, Wibbenmeyer M, Thompson MP (2012) Estimating US federal wildland fire managers' preferences toward competing strategic suppression objectives. International Journal of Wildland Fire 22(2) 212-222.

Deeming JE, Burgan RE, and Cohen JD (1978) The national fire danger rating system - 1978. USDA Forest Service, Intermountain Forest and Range Experiment Station Gen. Tech. Rep. INT-39. (Ogden, UT)

Feinerer I, Hornik K, Meyer D (2008) Text Mining Infrastructure in R. Journal of Statistical Software 25(5): 1-54. URL: http://www.jstatsoft.org/v25/i05/.

Grolemund G, Wickham H (2011) Dates and Times Made Easy with 'lubridate'. Journal of Statistical Software, 40(3), 1-25. URL http://www.jstatsoft.org/v40/i03/.

Maguire LA, Albright EA (2005) Can behavioral decision theory explain risk-averse fire management decisions? Forest Ecology and Management 211, 47-58.

Marcot BG, Thompson MP, Runge MC, Thompson FR, McNulty S, Cleaves D, Tomosy M, Fisher LA, Bliss A (2012) Recent advances in applying decision science to managing national forests. Forest Ecology and Management 285, 123-132.

Noonan-Wright EK, Opperman TS (2014) Applying the Wildland Fire Decision Support System (WFDSS) to Support Risk-informed Decision Making: The Gold Pan Fire, Bitterroot National Forest, Montana, USA, Proceedings of the large wildland fires conference; May 19-23, 2014; Missoula, MT. Proc. RMRS-P-73. Fort Collins, CO: U.S. Department of Agriculture, Forest Service, Rocky Mountain Research Station. 345 p.

Noonan-Wright EK, Opperman TS, Finney MA, Zimmerman TG, Seli RC, Elenz LM, Calkin DE, Fiedler JR (2011) Developing the U.S. Wildland Fire Decision Support System (WFDSS). Journal of Combustion. Article ID 168473: doi: 10.1155/2011/168473. 14 p.

R Core Team (2013) R: A language and environment for statistical computing. R Foundation for Statistical Computing, Vienna, Austria. URL http://www.R-project.org/.

Seielstad, C (2014) Reconsidering Wildland Fire Use: Perspectives From the Northern Rockies, Proceedings of the large wildland fires conference; May 19-23, 2014; Missoula, MT. Proc. RMRSP-73. USDA Forest Service, Rocky Mountain Research Station (Fort Collins, CO) 
Taber MA, Elenz LM, Langowski PG (2013) Decision Making for Wildfires: A Guide for Applying a Risk Management Process at the Incident Level. Gen. Tech. Rep. RMRS-GTR-298WWW. USDA Forest Service, Rocky Mountain Research Station (Fort Collins, CO)

Wickham H (2011) The Split-Apply-Combine Strategy for Data Analysis. Journal of Statistical Software, 40(1), 1-29. URL http://www.jstatsoft.org/v40/i01/.

Wickham H, Francois R, Henry L, Müller K (2017). dplyr: A Grammar of Data Manipulation. R package version 0.7.4. https://CRAN.R-project.org/package=dplyr

Zimmerman T (2017 December 17) Phone Interview. 


\title{
Fast computational models of dynamic fire propagation using near-field techniques
}

\author{
J. E. Hilton ${ }^{1 *}$; J. J. Sharples ${ }^{2}$; A. L. Sullivan ${ }^{3}$; W. Swedosh ${ }^{1}$ \\ ${ }^{1}$ CSIRO, Clayton, VIC 3168, Australia, \{james.hilton@csiro.au*\} \\ ${ }^{2}$ UNSW, Canberra, ACT 2610, Australia \\ ${ }^{3}$ CSIRO, Canberra, ACT 2601, Australia
}

\begin{abstract}
Modelling of wildfires is required for risk assessment and predicting the behavior of a potential or ongoing wildfire. Computational physics-based models can calculate the full combustion dynamics of a wildfire, but these are currently too computationally costly to be used for rapid operational predictions. As a result, many operational prediction systems for wildfires are based on rapid two-dimensional propagation algorithms which model only the outward growth of the fire perimeter. These take a fraction of the calculation time of physics-based models as factors such as the rate-of-spread of the fire are generally implemented using empirical expressions. The predictive ability of these perimeter propagation models is, however, governed by the limitations of the algorithm used. We introduce a two-dimensional perimeter propagation model that incorporates aspects of a full three-dimensional physics-based model using near-field approximations to fireinduced air flows. The model comprises a two-dimensional perimeter propagation approach with an additional near-field physics-based component allowing more complex fire behavior to be predicted rapidly enough for operational usage. We show examples of various aspects of fire behavior which can be modelled using a near-field approach that were previously difficult, or impossible, to simulate in two-dimensional perimeter propagation approaches. These include fire line interaction, wind and terrain effects, fire shape development and vortex sources. The method allows all of these aspects to be incorporated in perimeter propagation using a single computational approach. Comparison to experimental results are provided, showing an excellent fit between the model and experimental fires. The near-field approach may improve the accuracy of rapid computational models with low additional overhead, suitable for operation usage.
\end{abstract}

Keywords: Wildfire modelling, wildfire behaviour

\section{Introduction}

Computer modelling of wildfire behaviour ranges from risk management and operational predictions of a potential or actual wildfire in progress, to physics-based models investigating the complex processes and dynamics of wildfires. Currently, many operational prediction systems use rapid two-dimensional perimeter propagation models, which are based on empirical rate-of-spread models for specific fuel types. Perimeter propagation models use a range of different computational algorithms such as cellular automata, front-tracking techniques and level set methods (Sullivan 2009a). Generally, these methods track or model the fire perimeter and advance the perimeter based on local fuel, weather and topographic information to provide a prediction of the wildfire's extent at future times. Although very fast (taking on the order of seconds to minutes to complete a prediction of several simulated hours) they are limited by the analytical nature of the perimeter propagation algorithm. In contrast, three-dimensional physics-based wildfire models can model the entire combustion and air flow dynamics around a wildfire to a high degree of accuracy as they are based on discretisation and solution of the fluid and thermo-dynamic equations (Sullivan 2009b). Currently, however, these models are too computationally costly to be used in operational predictions, taking on the order of hours to days for a simulation to complete on a supercomputer. 


\section{Near-field techniques}

We have developed a two-dimensional perimeter propagation model that incorporates aspects of a full three-dimensional physics-based model using near-field approximations to fire-induced flows. The model is fully detailed and compared to a number of experimental results in Hilton et al. (2018). Specifically, the model comprises a two-dimensional perimeter propagation approach with an additional physics-based component allowing new types of fire behaviour to be predicted rapidly enough for operational usage. The additional component is a near-field approximation to the groundlevel fire-induced flow $\mathbf{u}$, which is represented using a Helmholtz decomposition in terms of a local scalar potential, $\psi$, and a vector potential, $\chi$.

$$
\mathbf{u}=\nabla \psi+\nabla \times \chi
$$

The scalar 'pyrogenic' potential is, essentially, the ground-level pressure field in the presence of any fires and the vector potential arises from any large-scale sources of vorticity present around the fire.

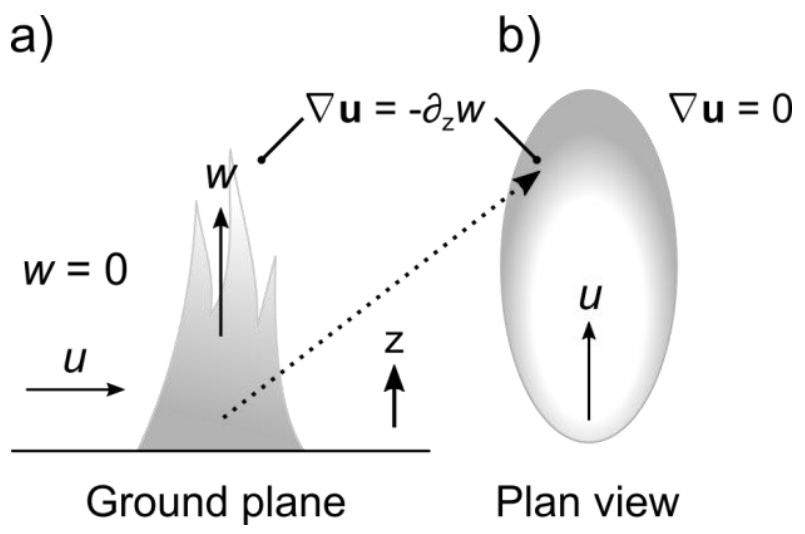

Figure 1 - Schematic overview of near-field model, a) flaming region on ground plane and b) fire perimeter from above. The forcing in Eq. (2) is assumed to be non-zero only within the flaming regions.

The near-field model requires two extra computational steps in addition to a standard perimeter propagation model. The first is the calculation of the source terms for the near-field and the second is the calculation of the field itself requiring the solution of a two-dimensional Poisson equation for the scalar potential and a solution of set of two-dimensional Poisson equations for the vector potential:

$$
\nabla^{2} \psi=-\partial_{z} w \quad \nabla^{2} \boldsymbol{\chi}=\boldsymbol{\omega}
$$

where $w$ is the vertical air flow and $\omega$ is a specified vorticity. Once the set of Poisson equations, Eq. (2), are solved, the local wind field due to near-field effects can be calculated using Eq. (1) and added to the global (ambient) wind field. In this work, all simulations were carried out in the Spark framework (Miller et al., 2015), a level set based perimeter propagation solver. The Poisson equations, Eq. (2), were solved using a multigrid technique. Although the near-field model has been implemented here using a level set solver, the process could be applied to other perimeter propagation methods such as front tracking or cellular automata methods. A related method using cellular automata has previously been developed (Sullivan and Knight, 2004), which used the geometric centre of all burning cells to model a central convective column to provide an additional in-drawn component of wind. This previous model replicated the key effects of the model presented here using a semi-physical approach in which the forcing was essentially reduced to a single point at the centre of the fire.

From investigation and comparison to experimental fires the vector potential appears to be negligible in most cases, although may be important in certain situations where wind interacts with topography to form lateral vortices (Simpson et al., 2013). A key component of the scalar potential is the upward forcing of air within flaming regions of the fire. A schematic overview of this forcing is 
shown in Figure. The model uses the simple approximation of the vertical wind component, $w$, being zero outside the fire and non-zero within any flaming regions. Similarly, the vertical gradient of this component, $-\partial_{z} w$, required for Eq. (2) is similarly assumed to be non-zero only within the flaming regions.
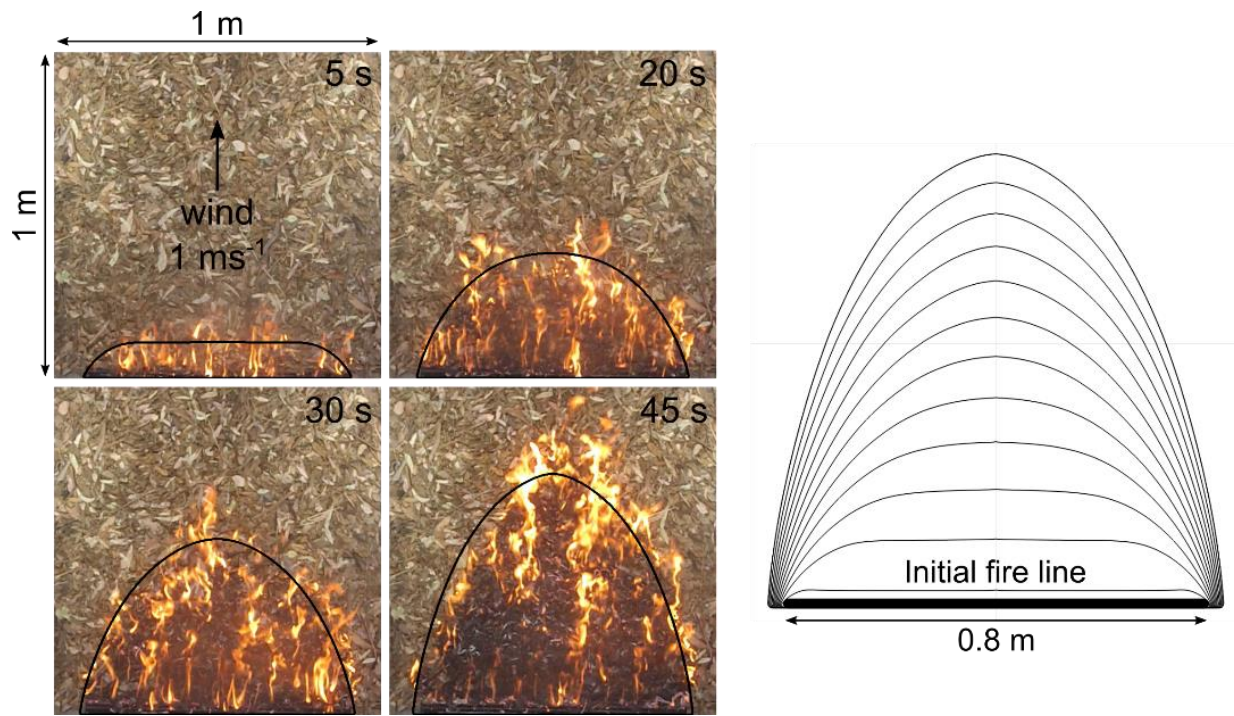

Figure 2 - Comparison of near-field model to experimental results (left) and simulation isochrones showing parabolic rounding arising naturally from the model (right).

The exact form and relationship between this vertical component and the parameters of the fire are currently being investigated and compared to experiments, the aim of which will be to characterise this vertical gradient for real-world conditions. However, the forcing will clearly be dependent of some function of heat release or flame temperature driving buoyant convection. Heat release can be modelled using a combustion curve of the form:

$$
I(t)=\frac{H M_{0}}{\tau} e^{-\frac{t}{\tau}}
$$

where $I$ is the heat intensity $\left(\mathrm{W} \mathrm{m}{ }^{-2}\right)$ at time $t(\mathrm{~s}), H$ is the heat of combustion $\left(\mathrm{J} \mathrm{kg}^{-1}\right), M_{0}$ is the initial fuel load $\left(\mathrm{kg} \mathrm{m}^{-2}\right)$ and $\tau$ is the mean lifetime of the burning fuel. Results using Eq. (3) have shown a close match to experimental ring fires simulated using fully physical models (Thomas, 2018). However, a simple forcing of the form $I \propto s$, where $s$ is the local speed of the fire, has been found to give surprisingly good results in comparison to small and medium-scale experiments. Simulations using this simple forcing are shown in Figure , with $-\partial_{\mathrm{z}} w=k s$ and the rate-of-spread in the normal direction $\mathbf{n}$ is a 'first-order' model (Hilton et al. 2016) given by:

$$
s=u_{0}+u_{1} \max (\hat{\mathbf{u}} \cdot \hat{\mathbf{n}}, \mathbf{0})
$$

with $\mathrm{u}_{0}=0.5 \mathrm{~mm} \mathrm{~s}^{-1}, \mathrm{u}_{1}=0.04$ and $k=80$, where these values were manually matched to the experimental data. The predicted fire perimeter is shown as a black line superimposed over images of experiments performed in the CSIRO Pyrotron (Sullivan et al., 2013).

The near-field model has also been shown to match results from larger grass fire experiments on open plots of 33 meters per side (Hilton et al., 2018). We are currently investigating whether the model is applicable to larger or more intense wildfires as the experimental data sets used so far cover only a limited range of the possible parameter space for fires and, of course, cannot model complex transitions in fire behaviour such as flame attachment (Apte et al., 1991, Tang et al., 2017). Despite this, the model appears to provide a close match to the experimental fires under the range of parameters considered and has the potential to include more complex modes of fire behaviour at low cost. 


\section{Inclusion of additional fire behaviour effects}

The use of near-field techniques permits modelling of aspects of fire behaviour that were previously difficult, or impossible, to simulate in two-dimensional perimeter propagation approaches. This includes the attraction between nearby fires, an example of which is shown in Figure for two fires lit in close proximity to each other. The results on the left show isochrones from a standard simulation, and the results on the right show the isochrones using the near-field model. For comparison these simulations use the same rate-of-spread model given in Eq. (4). However, the attractive behaviour is independent of the underlying rate-of-spread model used as it is a consequence of coupling though the near-field interaction.
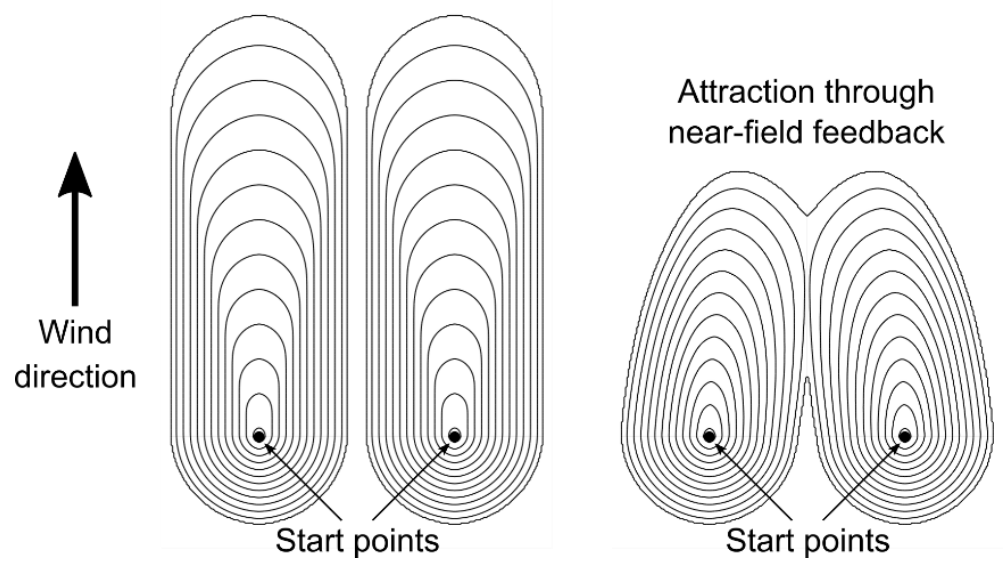

Figure 3 - Isochrones from two fires side-by-side without near-field coupling (left) and with coupling (right). The near-field coupling predicts the fires attract, a phenomenon observed in experiments.

This attraction has been observed in experimental ' $\mathrm{V}$ '-line or junction fires (Sullivan et al., in preparation) and may play a major role in fires ignited by ember storms as the coalescence rate of multiple small spot fires is significantly higher when this attractive effect is taken into account (Hilton et al., 2018).

A second behaviour is the parabolic rounding exhibited by a wind-driven fire line, as shown in Figure for a fire line lit in uniform wind conditions. The parabolic rounding arises naturally when using the near-field approach and is not required to be added as an additional phenomenological component to a two-dimensional rate-of-spread model. The close match to observed fires also suggests that a pressure, rather than radiative, effect may be responsive for such parabolic shapes, at least for the limited set of fire conditions investigated for this and previous studies.
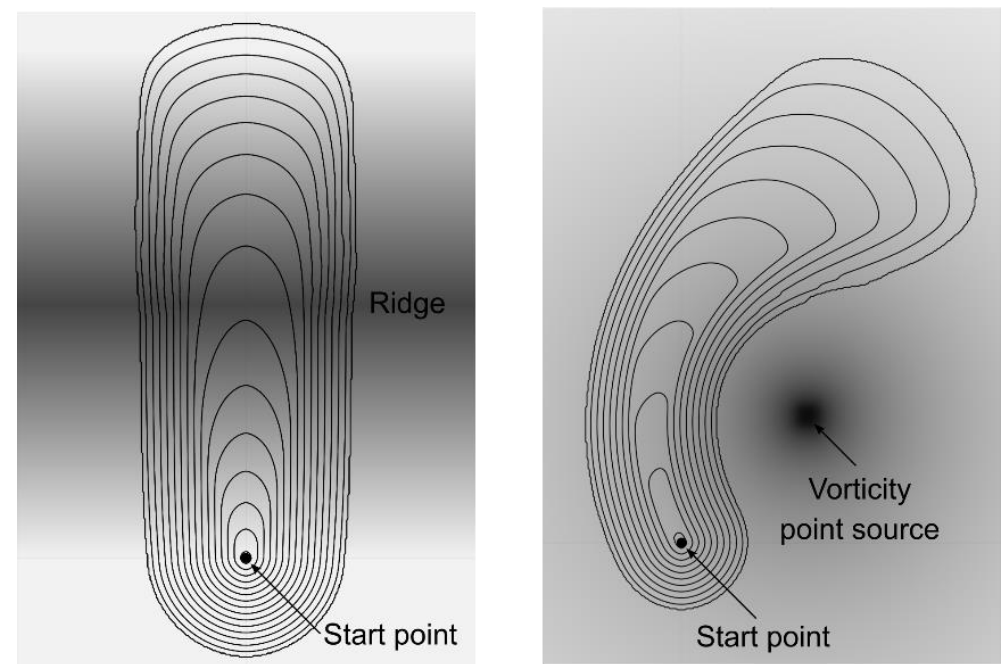

Figure 4 - Incorporation of terrain into the near-field model as a source term for the scalar potential (left). Example of fire propagation in the presence of a vortex point source (right). 
The expression for the source term in Eq. (2), $\partial_{z} w$, is a vertical displacement of air from the fire. This is mathematically identical to a forcing term from lifting of air over terrain which easily be incorporated into the model using the local terrain gradient, an example of which is shown on the left of Figure. The simulated fire in this example is started downwind of a ridge (elevation is shown as shaded grey in the image, with dark grey high elevation) and can be seen to accelerate on the windward slope and decelerate on the lee slope of the ridge, in general agreement with empirical models of fire behaviour on slopes (McArthur, 1967). The applicability of the model to provide a topological correction in this manner is currently under investigation. The use of the near-field model in this manner is, essentially, a single-level approximation to a diagnostic wind field mass correction (Ross et al., 1988) which can be implemented at a substantially reduced computational cost as a threedimensional terrain-following discretisation does not have to be used.

Fully three-dimensional vorticity fields can be applied using the model if required, but for circulation in the ground plane the vector potential reduces to a single component $\omega_{z}$ requiring the solution of only one additional Poisson equation. An example of incorporating circulation in the ground plane is shown on the right of Figure, where a single point source of vorticity has been applied. The resulting isochrones from a fire starting at a point nearby are shown, superimposed over vorticity potential $\chi_{z}$ (shaded by magnitude in greyscale). The simulation isochrones can be seen to follow the circulation, the strength of which is given by the magnitude of the original point source.

Notably, as the near-field model is linear terrain correction and near-field pressure effects from buoyant convection can simply be added in the model and require no additional computational effort as only one scalar Poisson equation needs to be solved.

\section{Conclusions}

The near-field modelling technique presented here potentially allows fire line interaction, wind and terrain effects, fire shape development and vortex sources to be incorporated in perimeter propagation using a unified computational approach. Development of the model is currently ongoing with research into an appropriate and representative forcing for large-scale wildfires. This near-field approach may improve the accuracy of rapid computational models with low additional overhead suitable for operation usage.

\section{References}

Apte V, Bilger R, Green A, Quintiere J (1991) Wind-aided turbulent flame spread and burning over large-scale horizontal PMMA surfaces. Combustion and Flame 85, 169-184.

Hilton JE, Sullivan AL, Swedosh W, Sharples JJ, Thomas C (2018) Incorporating convective feedback in wildfire simulations using pyrogenic potential, Environmental Modelling \& Software, 107, 12 24.

Hilton JE, Sharples JJ, Sullivan AL, Swedosh W (2017) Simulation of spot fire coalescence with dynamic feedback, In Syme, G., Hatton MacDonald, D., Fulton, B. and Piantadosi, J. (eds) MODSIM2017, 22nd International Congress on Modelling and Simulation. Modelling and Simulation Society of Australia and New Zealand, 1111-1117.

JE Hilton, C Miller, AL Sullivan (2016) A power series formulation for two-dimensional wildfire shapes, International Journal of Wildland Fire, 25, 970-979.

McArthur AG (1967) Fire behaviour in eucalypt forest. Commonwealth of Australia Forestry and Timber Bureau, Canberra, ACT. Leaflet No. 107.

Miller C, Hilton JE, Sullivan AL, Prakash M (2015) Spark - A Bushfire Spread Prediction Tool. In: Denzer R., Argent R.M., Schimak G., Hřebíček J. (eds) Environmental Software Systems. 
Infrastructures, Services and Applications. ISESS 2015. IFIP Advances in Information and Communication Technology, 448. Springer, Cham.

Ross DG, Smith IN, Manins PC, Fox DG (1988) Diagnostic wind field modeling for complex terrain: model development and testing, Journal of Applied Meteorology, 27, 785-796.

Simpson CC, Sharples JJ, Evans JP, McCabe MF, (2013) Large eddy simulation of atypical wildland fire spread on leeward slopes. International Journal of Wildland Fire, 22 599-614.

Sullivan AL, Knight IK (2004) A hybrid cellular automata/semi-physical model of fire growth. AsiaPacific Conference on Complex Systems, Complex 09, Cairns, Australia.

Sullivan AL (2009a) Wildland surface fire spread modelling, 1990-2007. 3: Simulation and mathematical analogue models. International Journal of Wildland Fire, 18, 387-403.

Sullivan AL (2009b) Wildland surface fire spread modelling, 1990-2007. 1: Physical and quasiphysical models. International Journal of Wildland Fire, 18, 349-368.

Sullivan AL, Knight IK, Hurley R, Webber C (2013) A contractionless, low-turbulence wind tunnel for the study of free-burning fires. Experimental Thermal and Fluid Science 44, 264-274.

Sullivan AL, Swedosh W, Hurley R, Sharples JJ, Hilton J E, (In Preparation) Investigation of the effects of interactions of intersecting oblique fire lines, with and without wind.

Tang W, Miller CH, Gollner MJ, (2017) Local flame attachment and heat fluxes in wind-driven line fires. Proceedings of the Combustion Institute 36, 3253-3261.

Thomas C.M., Sharples J.J., Hilton J., Evans JP, (2018) Rate of spread in coupled models: fireline curvature and pyrogenic potential, In D. X. Viegas (Ed.), Advances in Forest Fire Research. 


\title{
Feature Selection for Burned Area Classification in the Castelo de Paiva Region
}

\author{
Carlos Viegas Damásio*² ${ }^{1}$, Lourdes Bugalho ${ }^{2}$; Pedro Medeiros ${ }^{1}$; Susana Nascimento ${ }^{1}$; Luís Duque \\ Santos ${ }^{1}$ \\ ${ }^{1}$ NOVA LINCS, Departamento de Informática, FCT/UNL, Portugal, \{cd@fct.unl.p*t\} \\ ${ }^{2}$ Instituto Português do Mar e da Atmosfera, Portugal
}

\begin{abstract}
Burned area mapping is a fundamental activity for the study of wildfires, but good quality data requires long in situ campaigns performed by specialized personnel. The use of machine learning algorithms and the availability of high-quality remote sensing data bring new opportunities to make the process more precise and expedite. This paper elicits the best combinations of features to be used in machine learning pixel-based burned area mapping, obtained by LASSO regression. The feature selection is applied to ground-truth obtained in the region of Castelo de Paiva, Portugal, in 2016.

The selected number of features is 9 , from an initial set of 51 features, and includes besides a new index a mix of prefire, postfire as well delta differences of spectral indices (namely dNBR). The selected features can be used with Sentinel 2, Landsat, and MODIS imagery.

In parallel, the identified features have been fed to several classifiers, namely a multilayer neural network, gradient boost and extreme gradient boosting, support vector machines and K-nearest neighbour classifiers to validate the choice performed by the LASSO regression. Accuracies of $96 \%$ and kappa of 0.86 , are consistently obtained for Sentinel 2 imagery, and Landsat 8 also scores very well; as expected, MODIS results in score reduction to the coarser spatial resolution. Confusion maps are presented to visualise the quality of the obtained results, as well as to pinpoint existing problems with the ground-truth.

Extreme Gradient Boosting shows to combine very high classification metrics with very efficient processing, possible via the Graphics Processing Units (GPU) implementation of XGBoost. A simple processing architecture is proposed to support an automatic classification system based on the publicly available satellite imagery, supported by the benchmarks obtained in this paper. A set of Sentinel 2 granules covering Portugal can be processed in less than 10 minutes in consumer hardware, for each satellite passage.
\end{abstract}

Keywords: Remote Sensing, Burned Area Indices, Machine Learning, Sentinel 2, Landsat 8, MODIS

\section{Introduction}

Portugal is a country with a recurrent history of wildfires. Every year thousands of hectares are burned as result of wildfires running rampant. These events have greater intensity in the center and northern regions of the country. A timely and accurate assessment of burned areas is important to have reliable data that can be used at the planning stage, and even at the operational level. The existence of ground-truth for the entire country provided by the ICNF (Instituto da Conservação da Natureza e das Florestas), as well as the availability of public remote sensing date fosters the study of machine learning classifiers/techniques to provide better identification of burned areas for the Portuguese territory. However, the use of pixel-based techniques requires the identification of the appropriate set of features both to reduce time as well as overfitting, and this the main concern of this paper.

Presently there are also other repositories focussed on fire related data as for examples the European Forest Fire Information System (EFFIS), which consists on web geographic information system that provides near-real time historical information on forest fires and their regimes. This service encompasses the European, Middle Eastern and North African regions. EFFIS is composed of five 
modules, Fire Danger Assessment, Rapid Damage Assessment (these last two have near real time availability), Emissions Assessment and Smoke Dispersion, Potential Soil Loss Assessment, and Vegetation Regeneration (COPERNICUS 2018).

For the present work we defined as study area the Castelo de Paiva region that had significant fire activity during August 2016. The area of study is situated in northern Portugal where the Douro and Tâmega rivers intersect. The area selected has $559 \mathrm{Km}^{2}$ of which by the end of the year nearly 118 $\mathrm{Km}^{2}$ had burned (circa $20 \%$ of the area). Using burned area shapefiles provided by the ICNF (Instituto da Conservação da Natureza e das Florestas) as ground-truth, and feeding a variety of spectral indices over MODIS, LANDSAT 8, and Sentinel 2 imagery to machine learning classifiers, we evaluate and obtain a very accurate burned area classifier over a selected set of those indices. In this process, we have defined a new burned area index that provides high-quality discrimination between burned and non-burned areas.

The paper is organized as follows. First, in Section 2, we present the general methodology used for this work. Section 3, presents the results regarding the best combination of features to be used in the classification approach; these features are then used to determine the best performing classifier, both in terms of classification scores and execution times. Section 4 presents the classification results and analyses them, contrasting the use of standard burned area indices with the classification approach based on the ensemble of classifiers. Section 5 describes a proposed automatic system, and discusses its feasibility supported by the results previously obtained. Section 6 , closes the papers with the main conclusions.

\section{Methodology}

The difference between pre and post-fire imagery displays a difference between near and mid infrared values, namely, the near values are very high and the mid values are very low in pre-fire, the opposite is verified in a post-fire scenario. It is the relationship between these two bands that the several Burned Area Reflectance Classification (BARC) methods exploit. The areas with the largest differences are the best candidates for being severely burnt while remaining areas are likely to be unburned or lightly burned (USFS 2017). Indices are used to capture specific changes of the earth's surface through the use of spectral bands. The indices chosen for burned area identification in our experiments are known vegetation indices, namely Normalized Difference Vegetation Index (NDVI) (Pettorelli et al. 2005), Soil Adjusted Vegetation Index (SAVI) (Rondeaux et al. 1996), Moisture Stress Index (MSI) (Welikhe et al. 2017), and the BARC specific indices, like the Mid Infrared Burn Index (MIRBI) (Trigg et al. 2001), Burn Ratio (BR) and Normalized Burn Ratio (NBR) (Key et al. 2006), two variations (NBR2, NBR3) and a new proposed index (NBR4), as well as the differences (deltas) of these indices from the post-fire scenario to the pre-fire one. The rationale behind NBR4, is based on the fact that the green spectrum is greatly influence by fire activity, thus being able to contribute in separation between burned and unburned areas, since the areas with greater amounts of green will trend toward the lower end of the index's range and vice versa. The equations for determining these indices can be found in Figure, where Green and Red are the visible light satellite bands, NIR, LSWIR, and SSWIR are respectively the Near Infrared, Longer short wave infrared and Shorter short wave infrared spectral ranges. The exact band ranges are slightly distinct from satellite to satellite, but here those differences are ignored. 


$$
\begin{gathered}
N D V I=\frac{(N I R-R e d)}{(N I R+R e d)} \\
S A V I=(1-L) * \frac{(\text { NIR-Red })}{(\text { IIR }+ \text { Red }+L)}, \text { with } \mathbf{L}=\mathbf{0 . 5} \\
M S I=\frac{S S W I R}{N I R} \\
M I R B I=10 * S S W I R-9.8 * L S W I R \\
B R=\frac{N I R}{L S W I R} \\
N B R=\frac{(N I R-L S W I R)}{(N I R+L S W I R)} \\
N B R 2=\frac{(2 * N I R)}{(S S W I R+L S W I R)} \\
N B R 3=\frac{(S S W I R-\text { LSWIR })}{(S S W I R+\text { LSWIR })} \\
\text { NBR4 }=\frac{(\text { NIR }- \text { LSWIR })}{(((N I R+\text { LSWIR } * \text { Green })+1)}
\end{gathered}
$$

Figure 1 - Remote sensing indices used for burned-area classification

In this study Sentinel 2, Landsat 8 and MODIS satellite imagery were used. Sentinel 2 is characterized by its great resolution $(10 \mathrm{~m} \times 10 \mathrm{~m})$ generating the same granule every 5 days. While Landsat 8 presents a three-fold reduction in resolution $(30 \mathrm{~m} \times 30 \mathrm{~m})$ in comparison with Sentinel 2 and generates the same granule every 16 days. Finally, MODIS, with a $250 \mathrm{~m}$ x 250m, yet, a daily granule generation.

The Sentinel 2 raster images from July 29th (pre-fire) and September 27th were processed with a DOS1 (Dark Object Subtraction 1) correction and then clipped to the size of the study area. Sentinel 2 was chosen due to the fact of it possessing largest quantity of samples, providing a larger amount of information about the land cover. The dataset was then compiled using all the pre and post-fire Sentinel 2 bands, indices and deltas.

This dataset was then subjected to a LASSO (Least Absolute Shrinkage Selection Operator) regression: "The LASSO is a shrinkage method like ridge, with subtle but important differences" (Hastie et al. 2008). LASSO is a regression method used in both variable selection and regularization in order to enhance the prediction accuracy of the classification process. As such, the LASSO method was used to select a small subset out of a considerably larger set of the provided features with the intent of reducing the dimension of the dataset and improving their prediction capabilities. The LASSO regression method constrains the regression coefficients sizes, encouraging the simplification of the models. This method is useful for automating feature selection and to better deal with models where the correlation levels between variables are high. Weights are the normalization factor associated with this regression. These weights, define the amount of severity applied in the "punishment" of the outliers. By using various weights to select the best features and subsequently averaging the importance attributed to each feature across all weight, a simple heuristic created. This heuristic not only makes our choice of the amount of features less biased, since the number of features is not being directly chosen, but ensures that the most useful features are used in the classification process in addition to making the model generation more efficient.

Using the selected indices as features, SVM (Support Vector Machine), Gradient Boosting (GB), Artificial Neural Networks (ANN) and K Nearest Neighbours (KNN) classifiers were trained with 
using different training set percentages in a pixel-based approach, and confusion maps were calculated as well as overall accuracy and kappa statistics for accuracy assessment. We will take special attention in the use of GPU-accelerated classifiers, mostly due to the magnitude of data that needs to be processed. This is the precise scenario GPUs excel, since they are optimized for mass parallel execution of operations, since the major caveat of traditional approach is that some operations take too long to execute when the dataset exceeds a certain dimension. All tests of this study were run on a laptop machine with an Intel Core i7 6700HQ, 16GB of RAM and an NVIDIA GTX950M with 4GB, running on Windows 10 version 1709.

\section{Feature selection and classifier evaluation}

The objective of the work reported in this section is the selection of remote sensing based features, and the corresponding most appropriate classifier to be used for burned area classification. The result is the definition of the classification setting to be used.

\subsection{Feature selection}

In order to perform feature selection, we applied a LASSO regression to the dataset containing all Sentinel 2 pre and post-fire scenario band data and indices, as well as the indices' variation between the sensing dates of July 29th and September 27 $7^{\text {th }}, 2016$ (deltas), and all the original satellite bands with a total of 51 features per pixel. The LASSO regression was run several times with different values for the weight parameter, as choosing a single weight value and opting for the resulting feature selection would turn our choice biased. A solution found for the problem was to define a heuristic to help us choose the most relevant features, in this case an average. The mean value for each feature across all the different weight values being greater than zero equates to that particular feature having some relevance. As a result of the LASSO regression, the pre-fire NDVI and NBR4, the post-fire MIRBI and NBR as well as the delta values of the MSI, MIRBI, BR, NBR and NBR4 indices, were considered to be the features that better help in solving the presented classification problem. The remaining features had a mean score of zero, meaning that they are not as relevant as the ones with non-zero values. We used the Sentinel 2 imagery due to it having the best spatial resolution of the three satellites considered, which equates to a greater amount of data points to assist in finding the best combination of features for our models.

\subsection{Classifier evaluation}

Classification processes were run using five different classifiers for Sentinel 2 data, and for each the best training set size combinations for this dataset were collected. The criteria chosen for ranking the classifiers, by order, were Accuracy, followed by Kappa score and lastly its total execution time.

The used classifiers were off the shelf MLP, GB, and KNN, from Python's scykit-learn library, as well as XGBoost and LiquidSVM. XGBoost. XGBoost is short for "Extreme Gradient Boosting", where the term "Gradient Boosting" is proposed in the paper Greedy Function Approximation: A Gradient Boosting Machine, by Friedman 1999, and is a variant of the original Gradient Boosting algorithm implemented in scykit-learn. It has a plugin that adds the option for GPU accelerated tree construction and prediction algorithms. LiquidSVM is an implementation of SVMs whose key features are fully integrated hyperparameter selection and extreme speed on both small and large data sets. XGBoost, MLP and LiquidSVM are GPU accelerated, while GB and KNN are not. 
Table 1. The top 5 classifiers for Sentinel 2 data

\begin{tabular}{|l|c|c|c|c|}
\hline Classifier & $\begin{array}{c}\text { Training Set } \\
\text { (percentage) }\end{array}$ & Accuracy & Kappa & $\begin{array}{c}\text { Total Time } \\
\text { (s) }\end{array}$ \\
\hline XGBoost & $5 \%$ & 0.96 & 0.86 & 6.68 \\
\hline MLP & $20 \%$ & 0.96 & 0.86 & 77.42 \\
\hline GB & $10 \%$ & 0.96 & 0.86 & 215.78 \\
\hline LiquidSVM & $5 \%$ & 0.96 & 0.86 & 898.94 \\
\hline KNN & $10 \%$ & 0.95 & 0.85 & 126.98 \\
\hline
\end{tabular}

The obtained experimental results are present in Table 1 and it is clear that very high classification accuracy and kappa values are obtained, independently of the classifier. The best results for the classification process were obtained with the GPU XGBoost library (version 0.6), with 10\% training data (559311 pixels) using a stratified split on the dataset in order to maintain the ratio between labels. This combination of parameters obtained an accuracy score of 0.96, a kappa score of 0.86 and a total execution time of 6.68 seconds.

The classification processes were subsequently repeated using different combinations of training set size and satellite data for the XGBoost classifier, obtaining:

- Sentinel 2 with 0.96 Accuracy and 0.86 Kappa in 6.68 seconds with 5\% of sample as training data;

- Landsat 8 with 0.95 Accuracy and 0.83 Kappa in 1.41 seconds with 5\% sample;

- MODIS with 0.89 Accuracy and 0.61 Kappa in 0.98 seconds with 20\% sample.

Mark again that these timings were obtained with a standard consumer laptop, and therefore for professional hardware better results are expected. These findings support the use of XGBoost as a top choice classifier for burned area identification classification problems.

\section{Classification Results}

In order to visually analyse the resulting classifications, confusion maps were created in order to provide a visual, spatial representation of a regular confusion matrix. An example of the map for XGBoost is depicted in Figure .

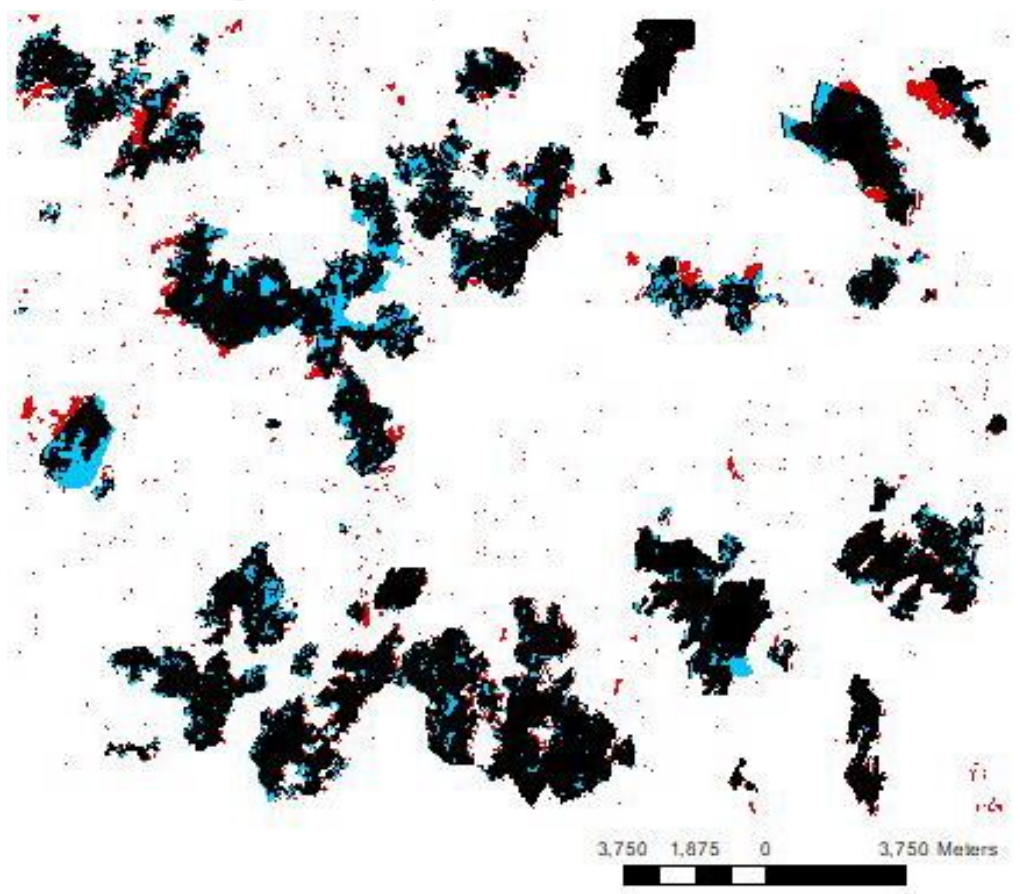

Figure 2 - Confusion map generated for the XGBoost classification with Sentinel2 imagery. Black and red areas are the burned areas identified by the classifier, and black plus blue areas form the ground-truth 
There is a very good agreement of the ground-truth with the classifier results, however some differences are clear. Blue areas correspond to burned areas marked in the ground-truth but not identified as burned by the classifier (false negatives); by visual inspection of the images these correspond mostly to villages or rock formations inside the perimeter of fire. Reversely, red areas are classified as burned according to our classifier and may correspond to errors in the ground-truth (false positives), namely due to the fact that till recently ICNF did not record rural fires. This shows that using a pixel-based machine learning approach might obtain finer burned area maps inside the perimeter of fires and show that the methods are robust to noise that may exist in the ground-truth.

To assess the behaviour of the machine learning based approach, we compared the XGBoost results to the differences (or delta) of the selected indices. For instance, the dNBR is one of the standards for burn severity classification but can be simplified in order to map burned areas, according to Lutes et al. 2006, burned areas correspond to values of dNBR $\geq 100$. However, a threshold of 200 is used since it can vary \pm 100 and should be chosen according to the existing data (USGS 2004). Our proposed index NBR4 marks burned areas when its value is greater than zero. By using these thresholds burned area confusion maps were generated (see Figure ).

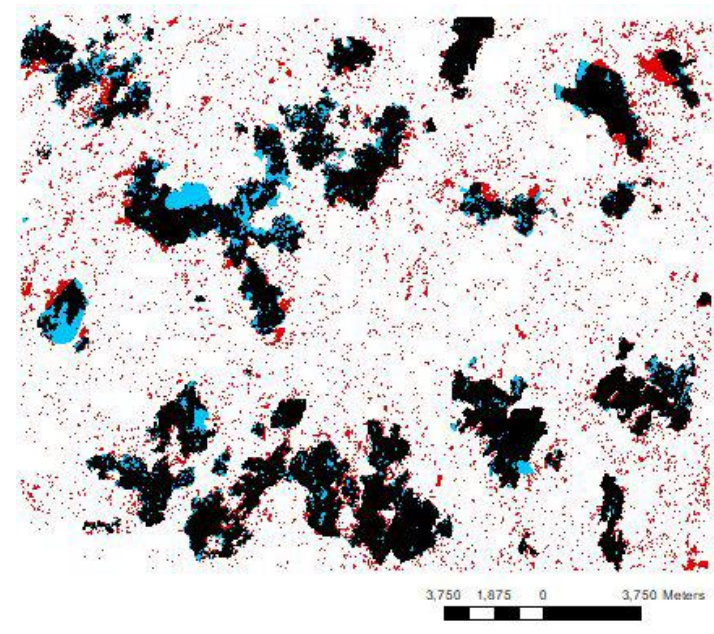

a) $\mathrm{dNBR} \geq 200$

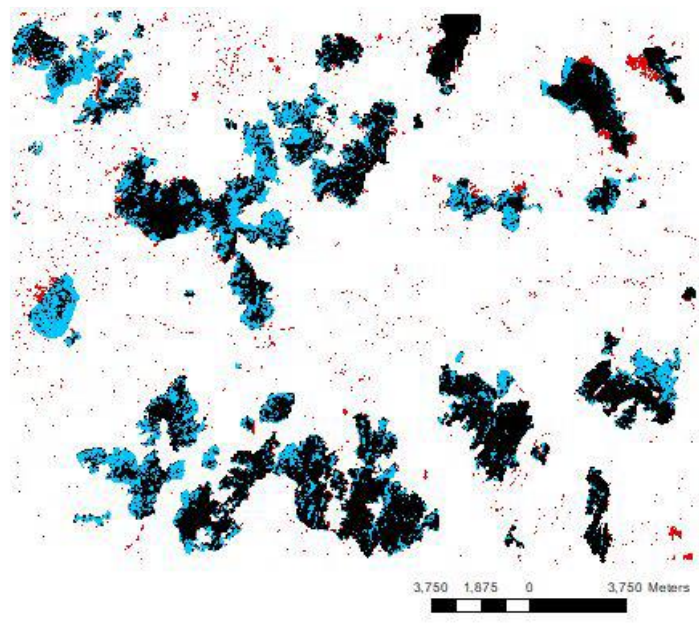

b) $\mathrm{dNBR} 4 \geq 0$

Figure 3 - burned area classification confusion maps for delta indices over a threshold

The dNBR presents a moderate amount of false positives in its classification. Our dNBR4 index has far less false positives, but more false negatives concentrated in some regions. By contrasting with Figure, it can be easily concluded the superior performance of the classification-based approach.

To be able to quantify the above empiric result, the delta indices are compared to the result with the XGBoost classification, as well as the majority vote of the top 5 distinct classifiers described in Section 3. A weighted confusion map was determined, in order to observe the overall opinion of an ensemble composed by the different classifiers, voting on which category should be attributed to a specific pixel. For this intent, the Sentinel 2 dataset was chosen due its greater resolution, as well as its overall results in previous tests. The results are summarised on Table .

Table 2. Comparison of classification scores

\begin{tabular}{|l|l|l|}
\hline Index & Accuracy & Kappa \\
\hline dNBR & 0.91 & 0.73 \\
\hline dNBR4 & 0.92 & 0.72 \\
\hline XGBoost & 0.96 & 0.86 \\
\hline Majority & 0.96 & 0.86 \\
\hline
\end{tabular}

When comparing the dNBR with the dNBR4, one can verify an increase in accuracy, but kappa reduces; the exact determination of the most appropriate discriminating threshold is ongoing work. 
This was expected and can be associated in the reduction of the "salt and pepper" effect present in dNBR4. The classification approaches are better, with no quantitative clear distinction between XGBoost and Majority. However, the generated confusion map based on from the ratios of the majority vote, enables us to visualize how the overall classifier ensemble consensus on the label outcome and shows the benefits of using an ensemble of classifiers. It is clear the reduction of the salt-and-pepper effect with respect to Figure and Figure .

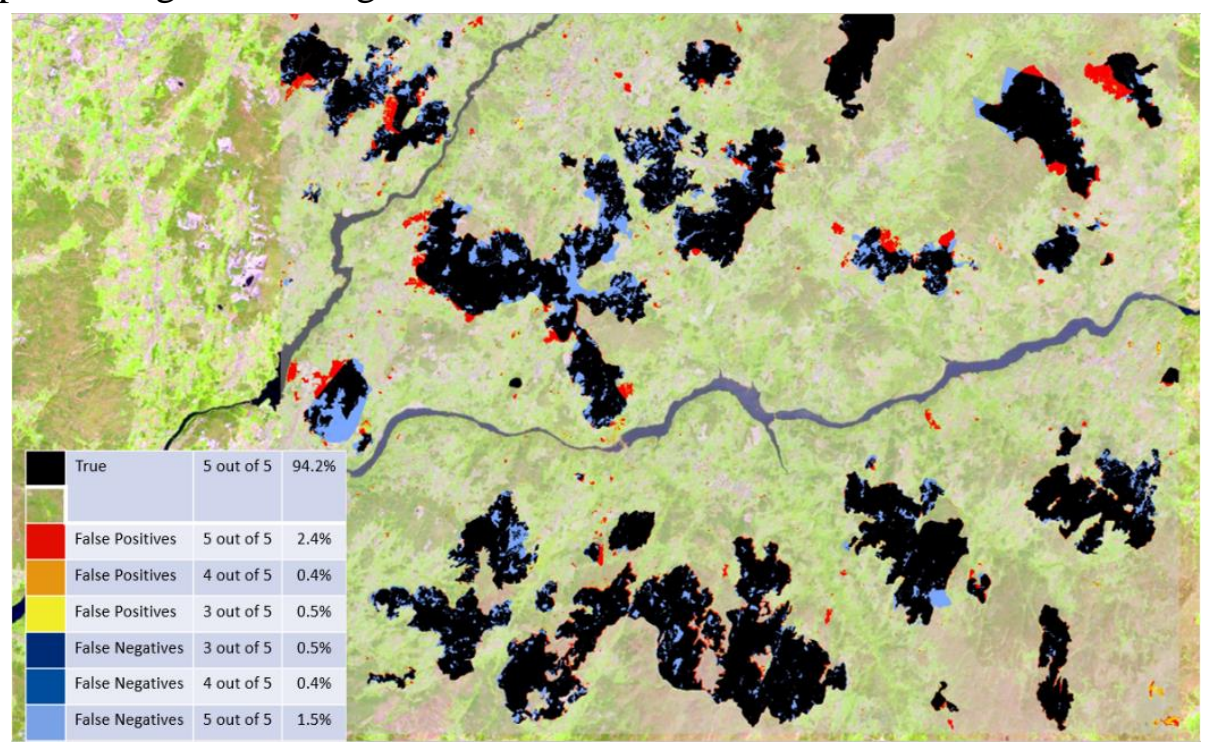

Figure 4 - Confusion map for the majority vote ensemble of classifiers

When analysing the images it became evident which areas where or where not controversial as well as some previously "hidden" terrain features became evident such as roads for example.

Figure depicts some aspects of the generated map.

\section{Fire Season Classification}

The purpose of this experiment is to assess the feasibility to perform burned area identification for all the days in the fire season (May 1st to October 31st), using the selected features. For this intent we defined our "pre-fire scenario" to the day before the official start of the season (April 30th). The model chosen was a XGBoost classifier trained with $30 \%$ of the MODIS dataset, referring to the $1^{\text {st }}$ of August, and the following parameters: max_depth $=4$, min_samples_split $=2$ and n_estimators $=100$. The choice of MODIS is mainly based on its daily availability of products, The differences between days were calculated using the the August $1^{\text {st }}$ date and the day we intended to differentiate. In order to minimize the overall variation of the classification, this process was executed 5 times and the classification for each day was submitted to a majority vote. The metrics obtained from this process are present in Table.

Table 3 - XGBoost burned-area classification timings for seasonal MODIS data

\begin{tabular}{|lr|}
\hline Operation & Time (s) \\
\hline Load train data & 0.06 \\
\hline Load Fire Season Data & 340.33 \\
\hline Total Classification Time & 4119.27 \\
\hline Write Classifications & 42.90 \\
\hline Total Time & 4539.00 \\
\hline
\end{tabular}

\subsection{Granule Classification}

Granules from Sentinel 2, Landsat 8 and MODIS were submitted to a classification process in order to obtain performance metrics. These granules where trained with a small portion of the granule and 
then the training classifier was used to classify the entire granule, and the obtained timings are present in Table .

Table 4 - Train and Classification times for each satellite tile/granule

\begin{tabular}{|lrr|}
\hline Granule & Train (s) & Classification (s) \\
\hline Sentinel 2 & 3.02 & 37.52 \\
\hline Landsat 8 & 1.04 & 20.06 \\
\hline MODIS & 0.92 & 2.89 \\
\hline
\end{tabular}

The classification time for the entire fire season can be approximately extrapolated from the classification time for each granule and the fact that 16 Sentinel 2 granules, 8 Landsat 8 tiles, and 2 MODIS tiles cover Portugal. This would equate to a total time of about 603 seconds with Sentinel 2 data, 162 seconds with Landsat 8 and 5.78 seconds with MODIS, for each satellite's passage. For half year of data the computation time required would be circa 6 hours for Sentinel 2, 32 minutes for Landsat 8, and 18 minutes for MODIS data. Note again that Table 1 timings for MODIS were obtained from 5 executions and some dates were discarded because of cloud contamination, justifying the differences of our estimation of MODIS data to the data in Table .

\subsection{Architecture}

The processing architecture is supported from publicly data available in the Web, and is depicted in Figure .

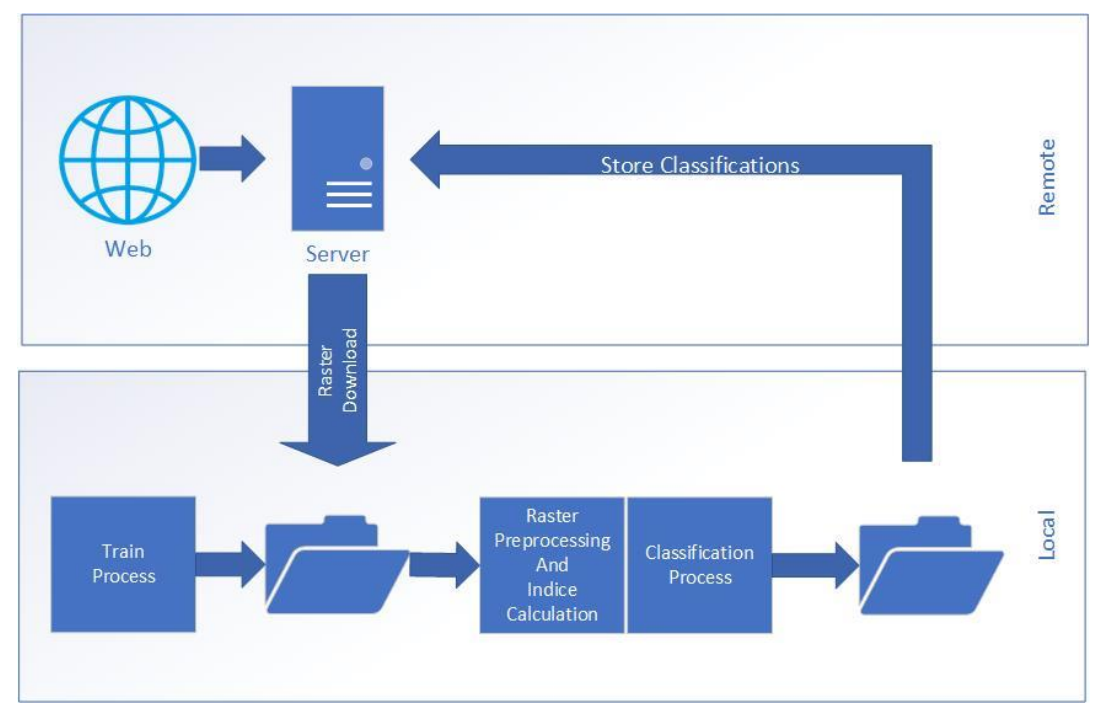

Figure 5 - Processing architecture

The system daily checks if new satellite products are available, downloads them and calculates the input indices using a bash/python script using the numpy library. The classifiers are previously trained with ICNF ground-truth over a small predefined area. This trained classifier can then be repeatedly used in the classification of the entire satellite granule. For the classification of imagery with substantial size, like Sentinel 2, the granule can be too big to fit in GPU's memory. This fact requires the granule to be split into smaller sized regions that are then classified independently since the approach is pixel-based. The classification results are then updated in a summary raster, containing the burned area since the start of the fire season period. From the results of this work, this system can be constructed with consumer hardware.

\section{Conclusions}

The reported experiments, show that very high classification scores can be obtained for artificial neural networks, support vector machine, gradient boosting, and k-nearest neighbour classifiers, using 
selected features by the LASSO regression. The gradient boosting algorithm, XGBoost, obtained excellent results for burned area classification with Sentinel 2 imagery taken in two dates, by combining pre-fire NDVI and our proposed NBR4, the post-fire MIRBI and NBR as well as the delta values of the MSI, MIRBI, BR, NBR and NBR4 indices. By employing GPU accelerated algorithms, a single Sentinel 2 granule can be classified in less than 40 seconds in a consumer laptop. This shows the appropriateness of the selected algorithm for burned area identification at the national and continental levels.

Based on the analysis of the collected metrics and scores, visual comparison between the dNBR, one of the standards in burned area assessment and the proposed index (dNBR4) its selection by the LASSO regression, makes it is clear that the reduction in the amount of misclassifications present in dNBR4 may result in more efficient burned area classifications. Yet, it still falls behind both the Majority Votes and XGBoosts in terms accuracy. More work is required to compare the proposed index with the other existing indices, for larger areas and different periods of time. This is possible, due to the existence of Landsat imagery and ICNF ground-truth, as well as the efficient classifiers identified.

An interesting outcome of the work, is the fact that the classifier are shown to be robust regarding noise in the ground-truth, and in practice almost perfect classification results may be obtained with the discussed techniques and algorithms, for the cases of burn scars visible from space. Therefore, obtaining very accurate ground-truth will be very important to validate these findings.

Finally, the proposed architecture will be made available to the community in a public web site.

\section{References}

Copernicus and European Committee, emergency management services, 2018

D. Lutes, R. Keane, J. Caratti, C. Key, N. Benson, S. Sutherland, L. Gangi, Integration of Standardized Field Data Collection Techniques and Sampling Design With Remote. Sensing to Assess Fire Effects. USDA Forest Service Gen Tech. Rep RMRS-GTR-164-CD. 2006

Friedman J. H., Greedy Function Approximation: A Gradient Boosting Machine, Stanford university, February 1999

Key, C., Benson, N.: Landscape Assessment (LA) Sampling and Analysis Methods, USDA Forest Service, Gen Tech. Rep, 2006

Pettorelli, N., Vik, J., Mysterud, A., Gaillard, J., Tucker, C., Stenseth, N. , Using the satellite-derived NDVI to assess ecological responses to environmental change. TRENDS in ecology and Evolution, Vol.20 No.9 September 2005

Rondeaux, G., Steven, M., Baret, F.: Optimization of Soil-Adjusted Vegetation Indices. REMOTE SENS. ENVIRON. 55:95-107 (1996)

S. Trigg and S. Flasse (2001) An evaluation of different bi-spectral spaces for discriminating burned shrub-savannah, International Journal of Remote Sensing, 22:13, 2641-2647

T. Hastie, R. Tibshirani, J. Friedman, The Elements of Statistical Learning, Data Mining, Inference and Prediction, August 2009.

US Forest Service: Burned Area Reflectance Classification

USGS, The Normalized Burn Ratio (NBR) - Brief Outline of Processing Steps. FIREMON BR Cheat Sheet V4, June 2004

Welikhe, P., Essamuah, Quansah, J., Fall, S., McElhenney, W.: Estimation of Soil Moisture Percentage using LANDSAT-based Moisture Stress Index. Journal of Remote Sensing and GIS 2017, 6:2 


\title{
Front's dynamics of quasi-infinite grassland fires
}

\author{
Nicolas Frangieh $^{* 1}$; Gilbert Accary ${ }^{2}$; Sofiane Méradji ${ }^{3}$; Dominique Morvan ${ }^{1}$ \\ ${ }^{1}$ Aix-Marseille Univ, CNRS, Centrale Marseille, M2P2, Marseille, France, \\ \{nicolas.frangieh1@hotmail.com*,dominique.morvan@univ-amu.fr\} \\ ${ }^{2}$ Scientific Research Center in Engineering, Lebanese University, Lebanon, \{gaccary@ul.edu.lb\} \\ ${ }^{3}$ IMATH, EA 2134, Toulon University, France, \{meradji@univ-tln.fr\}
}

\begin{abstract}
Numerical simulations of grassland fires were carried out to study the effects of the wind speed on the dynamics of the fire front. This study was performed using a detailed physical model based on a multiphase formulation, consisting in solving the balance equations (mass, momentum, energy ...) governing the behavior of the coupled system formed by the vegetation layer and the surrounding atmosphere. The objective of this study is to focus on the 3D interactions between a quasi-infinite fire front propagating through a homogeneous vegetation layer (a grassland) and an atmospheric boundary layer flow representing an idealized wind flow. The behavior of a surface fire results from the competition between the buoyant force of the thermal plume and the inertial force of the wind. In 3D, the interaction between these two forces is at the origin of the structuration of the fire front as a succession of peaks and troughs that modify the flames dynamics in a manner different to what can be observed in a simplified 2D assumption. To reproduce a quasi-infinite fire front, the numerical simulations were carried out using periodic boundary conditions along the two lateral sides of the computational domain. The numerical results highlights the 3D structuration of the fire both vertically and horizontally, and the significant effect of an increase of the wind velocity on the rate of spread (ROS) and on the fire intensity. Results also show that the correlation between Froude's number (based on the fire-front structures characteristic-wavelength) and Byram's number is the same at small scale (litter fires) and at large scale (grassland fires).
\end{abstract}

Keywords: Fire dynamics, Physical model, Numerical simulation, Grassland fire

\section{Introduction}

From a physical and mathematical point of view, a wildfire is a multiscale and complex problem; for this reason, most of operational tools predicting wildfires behavior, such as PHOENIX (Tolhurst et al, 2008) in Australia and FARSITE in the US (Finney, 1998), are based on statistical or semiempirical approaches, namely the MK5 model (McArthur, 1967) in Australia and the BEHAVE model in the US (Rothermel, 1972). However, in many situations, the quality of the predictions compared to real observations on well documented fire can be qualified at least as poor (Hanson et al, 2000). The limitations highlighted in empirical or semi-empirical models are mainly due to the fact that the predictions outside the data-set range used for the model statistics are generally subjected to great uncertainties and generally not suitable. Consequently, other approaches have been proposed to improve the understanding of the physical processes governing the behavior of fires. This alternative class of fire models is based on more or less realistic description of the physical phenomena governing the heat transfer between the fire front and the vegetation (Sullivan, 2009). A fully physical model addresses the problem of fire spread by analyzing its behavior through its physicochemical aspects (Grishin and Albini, 1997, Mell et al, 2007). This approach minimizes the need of using an empirical parameterization, even if, compared to empirical and semi-empirical models, it needs bigger computational resources, it is also more promising in the understanding of the physics of fires (Mell et al, 2007, Linn and Cunningham, 2005, Morvan et al, 2009). The fully physical approach has also a great potential in the management of fire hazard in wildland-urban interfaces, such as the dimensioning of a fuel break, the evaluation of heat flux on a target located inside the WUI, the interaction between two fire fronts (Morvan, 2015, Mell et al, 2010) 
The 3D model used in this study (FireStar3D) is based on a multiphase formulation and solves the conservation equations of the coupled system formed by the vegetation and the surrounding gaseous medium (Grishin and Albini, 1997, Morvan et al, 2018). The predictive potential of FireStar3D model was tested at a small scale in the case of litter fires (Morvan et al, 2018), as well as at a larger scale in the case of grassland fires (Frangieh et al, 2018) that have been extensively studied experimentally (Cheney et al, 1998). In this study, the fire-front dynamics and spread through a homogeneous grassland is analyzed, for different wind speeds, in terms of rate of fire spread, fire intensity, and characteristic-wavelength of the fire-front structures. The results are compared with data collected during experimental campaigns and predictions from semi-empirical and physical models.

\section{Modeling and Numerical Method}

The mathematical model is based on a multiphase formulation, it consists in a first step of averaging the conservation equations (mass, momentum, energy ...) governing the behavior of the coupled system formed by the vegetation and the surrounding atmosphere inside elementary control volumes including both the solid phase (the vegetation) and the gaseous phase. This results in the introduction of source/sink terms on the right hand side of the equations, representing the contributions of the interaction terms (exchanges of mass, drag, heat flux ...) between the gaseous phase and the vegetation. The details of the model have been widely presented in previous publications, we invite the reader to consult Grishin and Albini, 1997, and Morvan et al, 2009, for more information.

The model consists of two parts that are solved on two distinct grids. The first part consists of the equations of a reacting turbulent flow in the gaseous phase composed as a mixture of fresh air with the gaseous products resulting from pyrolysis and combustion. The second part consists of the equations governing the state and the composition of the solid phase.

The gaseous phase model consists in the conservation equations of mass, momentum, energy, and chemical species $\left(\mathrm{O}_{2}, \mathrm{~N}_{2}, \mathrm{CO}, \mathrm{CO}_{2}\right.$, and $\left.\mathrm{H}_{2} \mathrm{O}\right)$ filtered using either a LES approach (Sagaut, 2006) or an Unsteady RANS approach (URANS) with Favre average formulation (Cox, 1995). Both approaches are available, but the LES model was used in this study. The closure of the averaged conservation equations is based on the concept of eddy viscosity (Sagaut, 2006) obtained from an evaluation of the turbulent kinetic energy. The temperature dependence of the gas-mixture enthalpy is based on CHEMKIN thermodynamic tables (Kee et al, 1992). A combustion model based on Eddy Dissipation Concept (EDC) (Cox, 1995) is used to evaluate the combustion rate occurring in the gaseous phase. Finally, the field of soot volume-fraction in the gas mixture is calculated by solving a transport equation including a thermophoretic contribution in the convective term and taking into consideration soot oxidation (Syed et al, 1991).

During the thermal degradation of the solid phase, the composition of the fuel particles representing the vegetation is represented as a mixture of dry material (generic term for a mixing of cellulose, hemicellulose, and lignin), charcoal, moisture, and residual ashes. For each solid particle, the model consists in the conservation equations of the mass fractions of water, of dry material, of charcoal, as well as of the total mass of the solid particle, its volume fraction and its temperature (Grishin and Albini, 1997, Morvan, 2015, Morvan et al, 2018). The degradation of the vegetation is governed by three temperature-dependent mechanisms: drying, pyrolysis, and charcoal combustion. The pyrolysis process starts once the drying process is completed and charcoal combustion starts once the pyrolysis process is achieved.

The interaction between the gaseous phase and the solid one obtained through coupling terms that appear in both parts of the model. The coupling in the momentum and turbulence equations is obtained by adding aerodynamic drag terms (Morvan et al, 2018) that include a drag coefficient (evaluated empirically, equal to 0.15 in the present study) multiplied by a reference surface, defined as the Leaf Area Density (LAD). Heat transfer between the gas mixture and the solid fuel is based on empirical 
correlations for convective transfer (Incropera and DeWitt, 1996), and on the resolution of the radiative transfer equation (Modest, 2003) that accounts for the presence of soot in the flaming zone and for the presence of hot particles in the vegetation layer (embers) (Grishin and Albini, 1997). Finally, mass transfer from the solid phase to the gaseous phase is obtained by adding source/sink terms in the mass conservation equations of both phases.

The Radiative Transport Equation (RTE) is solved using a Discrete Ordinate Method (DOM), consisting in solving the radiation-intensity equation in a finite number of directions (Modest, 2003). This set of discrete contributions is then integrated using a numerical Gaussian quadrature rule (a S8 method is used here) for the calculation of the total irradiance. The radiative transfer equation accounts for gas-soot mixture absorption of radiative intensity depending on the amounts of combustion products $\left(\mathrm{CO}_{2}\right.$ and $\left.\mathrm{H}_{2} \mathrm{O}\right)$, on the gas mixture temperature, and of the soot volume fraction (Kaplan et al, 1994).

\section{Grassland Fire Configuration}

To reproduce the conditions similar to those encountered in the case of a quasi-infinite fire front and to avoid the border effects induced by a finite-length ignition line on the fire behavior (Cheney and Gould, 1995, Linn et al, 2012), the numerical simulations were carried out using periodic boundary conditions along the two lateral sides of the computational domain (Canfield et al, 2014), as shown in Fig. 1. The length $L$ of the computational domain was increased with the wind speed from $120 \mathrm{~m}$ up to $600 \mathrm{~m}$ in order to reach a steady rate of spread, while the fire front is still far enough from the end of the domain. The homogeneous vegetation layer is located at $20 \mathrm{~m}$ from the domain inlet and has the following main properties (Linn and Cunningham, 2005, Morvan et al, 2009): vegetation height $\delta=0.7 \mathrm{~m}$, volume-fraction $\alpha=0.002$, surface-to-volume ratio $\sigma=4000 \mathrm{~m}^{-1}$, moisture content $M=$ $5 \%$, dry material density $\rho=500 \mathrm{~kg} \cdot \mathrm{m}^{-3}$, solid-fuel particles are assumed to have cylindrical shape and to behave as a black body. The simulations were carried out for seven values of the $10-\mathrm{m}$ open wind speed: $1,3,5,8,10,12$, and $16 \mathrm{~m} / \mathrm{s}$. These velocities were measured at the domain inlet, $10 \mathrm{~m}$ above ground.

For all wind speeds, the same mesh size was used in the $y$ - $z$ plane: for the solid phase a uniform grid with $(\Delta y, \Delta z)=(0.25 \mathrm{~m}, 0.035 \mathrm{~m})$ was used, while for the fluid phase a uniform grid with $(\Delta y$, $\Delta z)=(0.5 \mathrm{~m}, 0.07 \mathrm{~m})$ was used within the vegetation before being gradually coarsened in the vertical direction. Both the solid-phase grid and the fluid-phase one were characterized by cells sizes below the extinction length scale (Morvan et al, 2009) within the vegetation given by $4 / \alpha \sigma$ and equal to 0.5 $\mathrm{m}$ in our case. This value is not to be exceeded in order to avoid fire extinction, especially in the case radiation-dominated fire propagation (i.e. when the wind speed is low). A uniform mesh was used in the $x$ direction but its size was increased with the wind speed: for the solid phase, the mesh size varied from $\Delta x=0.25 \mathrm{~m}$ for $U=1 \mathrm{~m} . \mathrm{s}^{-1}$ up to $\Delta x=0.5 \mathrm{~m}$ for $U=16 \mathrm{~m} \cdot \mathrm{s}^{-1}$, the mesh size of the fluid phase was twice that of the solid one.

Before activating the burner, the hydrodynamic module of the code was run long enough until reaching a statistically quasi-steady state. During this purely dynamic phase, homogeneous Neumann boundary conditions were imposed at the top, inlet, and outlet boundaries of the computational domain for all primary variables of the problem except for $y$ and $z$-velocity components that were set to zero. In addition, a negative pressure gradient is applied in the wind direction $(O x)$; this pressure gradient was automatically adjusted during this dynamic phase to obtain the desired 10-m open wind speed. This procedure allowed collecting the turbulent fields at the open boundaries, and these fields were then used during the burning phase, in particular for the management of the entering turbulent fluxes. Once the flow had reached a statistically-steady state, the burner was activated along an ignition line by injecting $\mathrm{CO}$ gas at $1600 \mathrm{~K}$ in the burning zone from the bottom boundary of the domain. The burner was activated during $25 \mathrm{~s}$ (at most) or until the consumption of a solid-fuel mass equal to that 
available above the burning area (i.e. contained in a volume of $2 \times 100 \times \delta \mathrm{m}^{3}$ ). More details about the ignition procedure are given in (Frangieh et al, 2018).

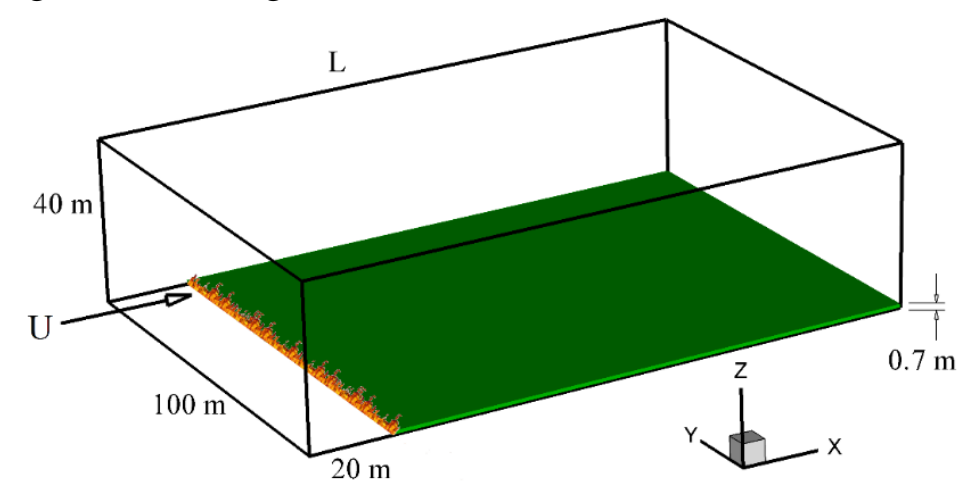

Figure 1 - Perspective view showing the dimensions of the computational domain and of the vegetation cover, the ignition line is $2 \mathrm{~m}$ wide.

The results presented in this study were obtained using a variable time step strategy based on the truncation-error control, with time step values varying between $0.001 \mathrm{~s}$ and $0.01 \mathrm{~s}$. At each time step, the solution is assumed to be obtained when the residuals of all conservation equations had reached $10^{-4}$ in normalized form. As a rough estimation of the computational cost, the simulation of $1 \mathrm{~s}$ of fire propagation required about $7 \mathrm{~h}$ of CPU time on a 24 -cores node.

\section{Numerical Results}

The description of the results is limited to local three-dimensionality of quasi-infinite grassland fire and its effect on fire line propagation with a specific focus on rate of spread, fire intensity, fire regime, and characteristic-wavelength of the fire-front structures for different wind speeds.

To understand the effect of the wind speed on the flame structure and more generally on the dynamics of fire, 3D views of the fire propagation obtained for $U_{10}=1 \mathrm{~m} / \mathrm{s}$ and $10 \mathrm{~m} / \mathrm{s}$ are shown in Fig. 2. For a small value of the wind speed $\left(U_{10}=1 \mathrm{~m} / \mathrm{s}\right)$, the flame plumes rise is not noticeably affected by the action of the cross wind. In this case the fire front can be assimilated to an obstacle, and the air flow is deflected vertically by the plume. On the other hand, figure 2 shows how larger value of wind speed $\left(\mathrm{U}_{10}=10 \mathrm{~m} / \mathrm{s}\right)$ affects more significantly the rise of the flame plumes by crossing the fire front and pushing the hot gases toward the unburned vegetation. We can also notice that the wind speed increases significantly the depth of the fire front.

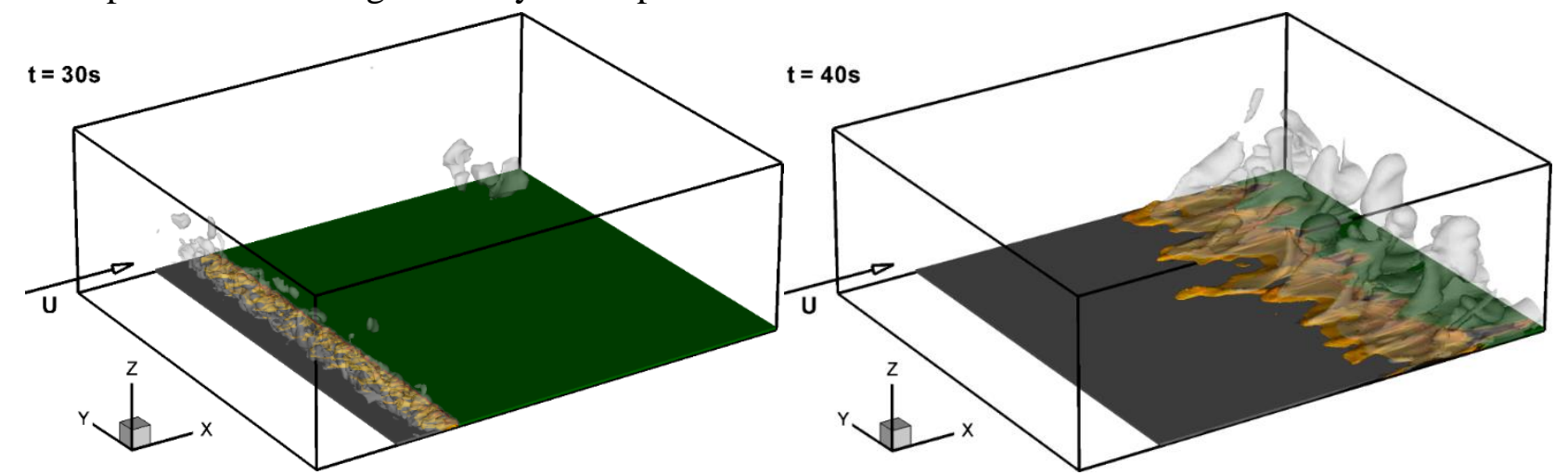

Figure 2 - 3D view of an isovalue surface of the soot volume fraction $\left(10^{-6}\right)$ colored by the gas temperature (in yellow) and an isovalue surface of the water mass fraction $\left(10^{-3}\right)$ (in grey with $50 \%$ of transparency) for $U_{10}=1 \mathrm{~m} / \mathrm{s}$ (top) and $U_{10}=10 \mathrm{~m} / \mathrm{s}$ (bottom), showing the effect of wind speed on the fire dynamics.

These phenomena are further illustrated by Fig. 3, where we notice that for a low wind speed, fresh air is aspirated from the vicinity of the fire front supplying the thermal plume; this regime is often 
referred to as "plume dominated fires". These results highlight the capability of the code to reproduce the backflow generated by the fire front on the leeward direction. For stronger wind conditions, the structure of the air flow is less affected by the fire front as shown in Fig. 3 (bottom), and this effect is limited to the local acceleration of the flow (resulting from a local expansion of the gas) in the plume; this regime is often referred to as "wind-driven fires". The streamlines show clearly the possibility for the inlet air flow to cross the fire front that must be structured vertically in peaks and troughs (Beer, 1991). The reproduction of this configuration is not possible in $2 \mathrm{D}$, because in $2 \mathrm{D}$ the fire front represents a uniform thermal barrier.

The effect of the wind speed on the flow structures is shown in Fig. 4 through the $Q$-criterion. This invariant of velocity gradient tensor represents the balance between the rotation and strain rates and is an appropriate tool for the visualization of coherent structures in a turbulent flow (Hunt et al, 1988). Figure 4 clearly shows that the assumption of a homogeneous plane made for the fire front in the radiant panel theory is not valid, the fire front is structured as a succession of peaks and troughs allowing for the air flow to find a way across it (Beer, 1991). This heterogeneity of the flame and the flow structure along the transverse direction, which is a great demonstration of 3D effects in a fire, affects a lot the propagation of the fire, as it has been clearly demonstrated experimentally at small scale (Finney et al, 2015) and numerically at larger scale (Linn et al, 2012).
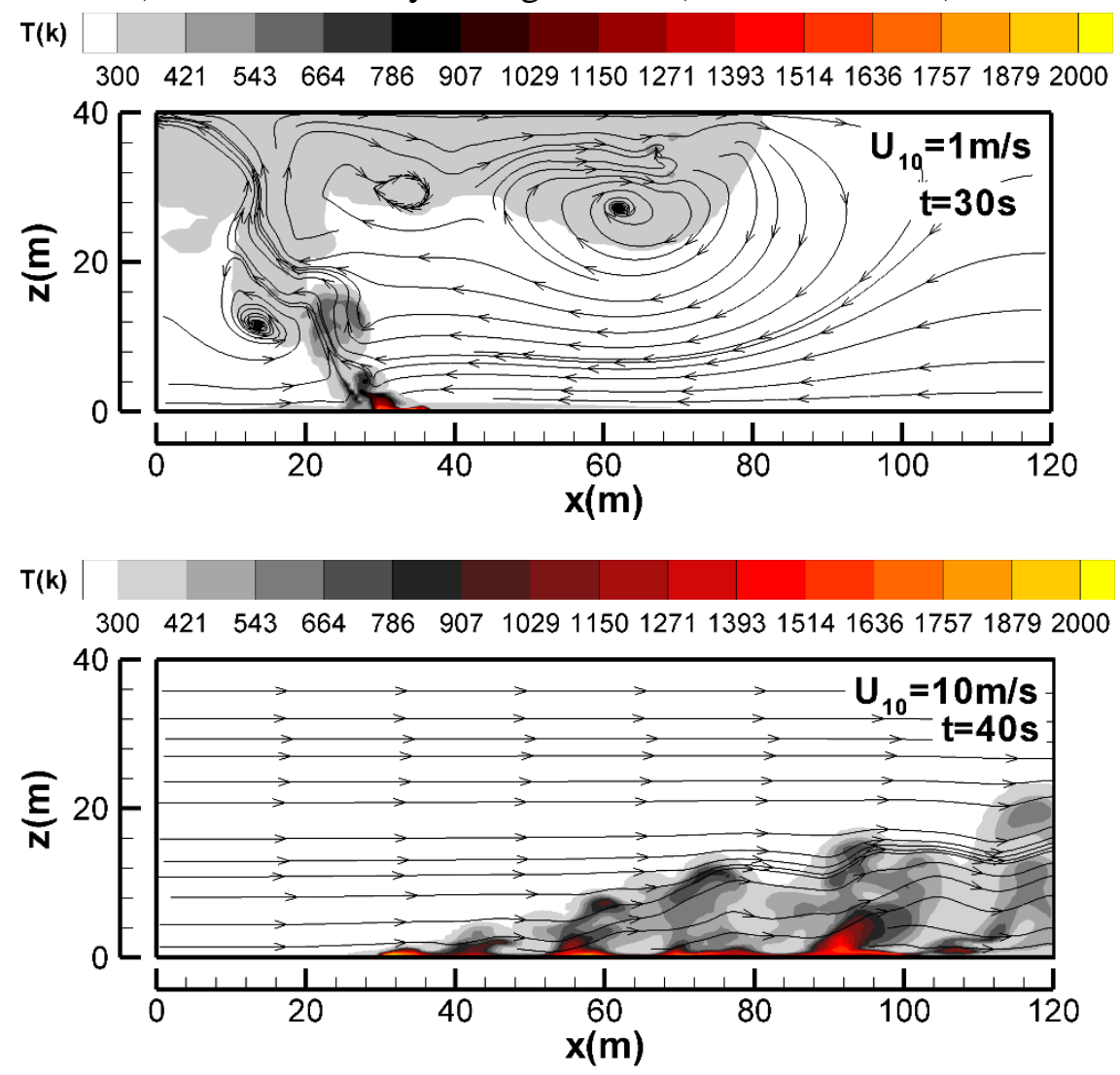

Figure 3 - Temperature field and streamlines of the gaseous phase, obtained in the vertical median plane $(y=$ $70 \mathrm{~m}$ ) for $U_{10}=1 \mathrm{~m} / \mathrm{s}($ top $)$ and $U_{10}=10 \mathrm{~m} / \mathrm{s}$ (bottom), corresponding to the 3D fields shown in Fig. 2. 

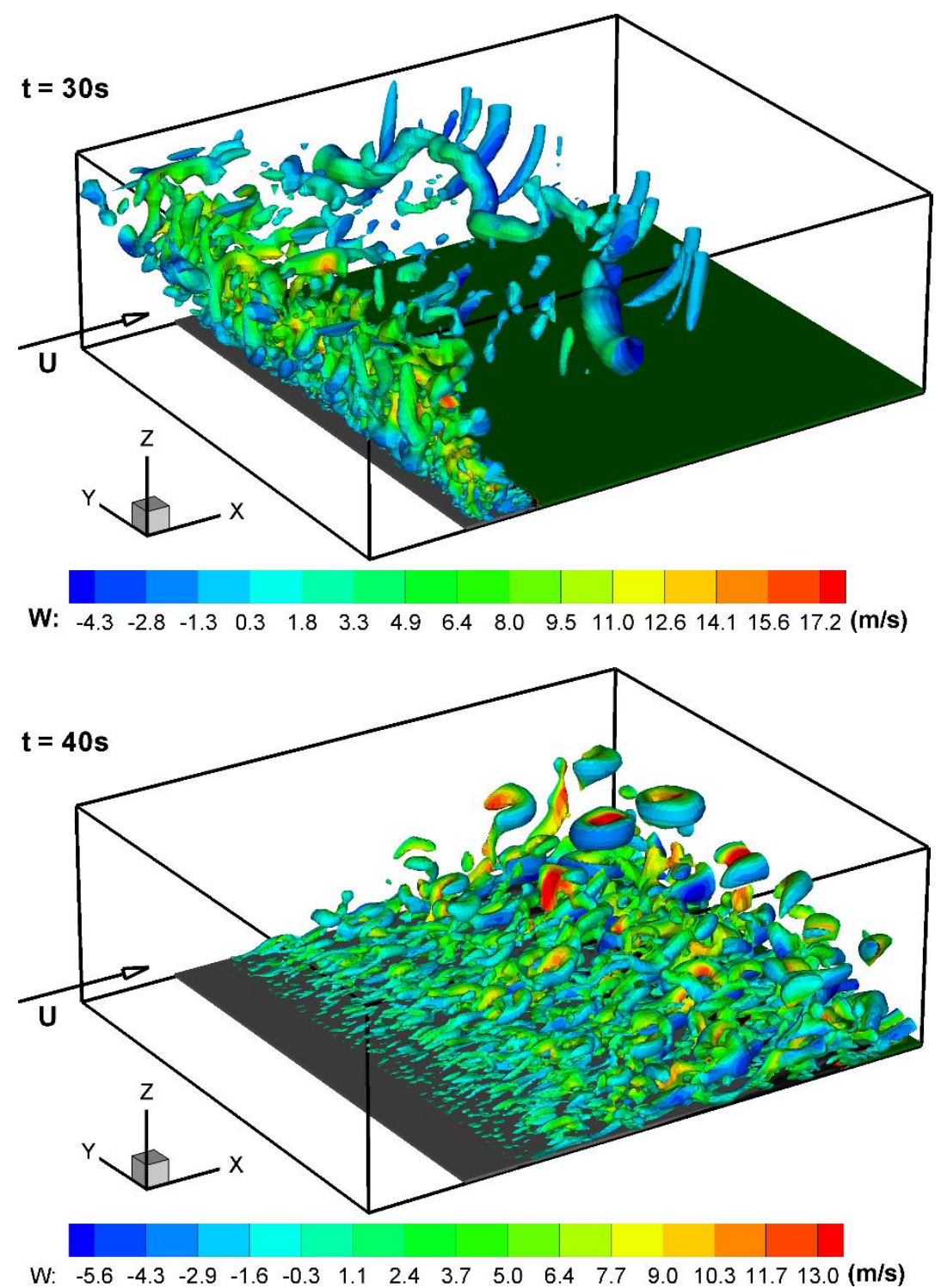

Figure 4 - Flow structure in a grassland fire for $U_{10}=1 \mathrm{~m} / \mathrm{s}$ (top) and $U_{10}=10 \mathrm{~m} / \mathrm{s}$ (bottom), shown using an isovalue surface of the $Q$ criterion $\left(Q=1 \mathrm{~s}^{-2}\right)$ colored by the vertical component $w$ of the velocity field, corresponding to the results shown in Figs. 2 and 3.

The Rate of Spread (ROS) of fire was estimated from the time derivative of the average position of the pyrolysis front at the surface of the vegetation cover, and shown in Fig. 5 versus with the 10-m open wind speed. The experimental data shown in Fig. 5 were obtained from Cheney and Gould, 1995, and Cheney et al, 1998, for different lengths $w$ of the ignition line. These experimental studies show that the ROS increases with $w$, as found by FIRETEC model predictions (16 m and $100 \mathrm{~m}$ ) (Linn and Cunningham, 2005), before reaching an asymptotic value for $w>200 \mathrm{~m}$. The relatively large dispersion of the experimental measurements can result from the unsteadiness nature of the wind flow (Morvan, 2014). On the other hand, the reported experimental data for $\mathrm{U}_{10} \geq 8 \mathrm{~m} / \mathrm{s}$ (Cheney et al, 1998) were estimated from measurements recorded during real wildfires with significantly large fire front, but for which wind speed and vegetation characteristics are not under control as in experimental fires. For low to moderate $U_{10}$ values (up to $6 \mathrm{~m} / \mathrm{s}$ ), FireStar3D results compare well with experimental data and with other predictions, and we can observe a quasi-linear evolution of the ROS. For $\mathrm{U}_{10} \geq 8 \mathrm{~m} / \mathrm{s}$, the results are consistent with the predictions of other models (FIRETEC and WFDS) and with the experiments, despite the relative dispersion of the experimental measurements which prevents a significant comparison. 


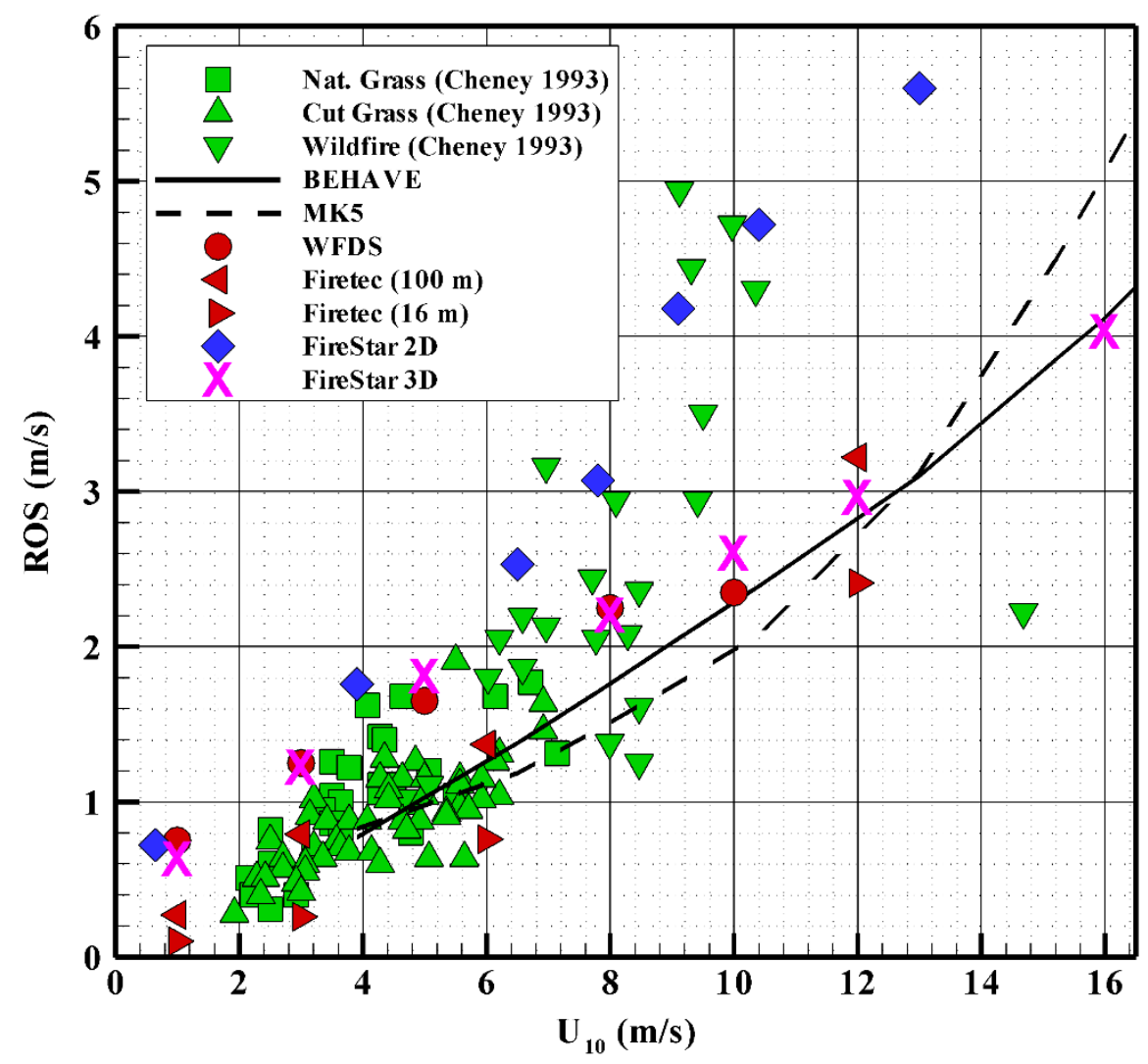

Figure 5 - Rate of fire spread (ROS) through a uniform grassland obtained for different 10-m open wind speeds. The results of FireStar3D are compared to those obtained experimentally by Cheney and Gould, 1995, and by Cheney et al, 1998, using the empirical model MK5 (McArthur, 1967), the semi-empirical model BEHAVE (Rothermel, 1972), 3D numerical models FIRETEC (Linn and Cunningham, 2005) and WFDS (Mell et al, 2007), and the 2D numerical model FireStar2D (Morvan et al, 2009).

The fireline intensity $I_{B Y R}$ (Byram's intensity) can be evaluated from $I_{B Y R}=m \times \Delta H \times R O S$ (Byram, 1959), where $m=\alpha \rho \delta$ is the fuel load (equal to $0.7 \mathrm{~kg} / \mathrm{m}^{2}$ for the present study), and $\Delta H$ is the heat yield of the fuel. To avoid the uncertainty on the value of $\Delta H$, the heat release rate $H R R$ given by Eq. 1 was computed, where $\omega_{v a p}, \omega_{p y r}, \omega_{c h a r}, \omega_{C O}$ and $\omega_{\text {soot }}$ are respectivelly the total mass rates of water evaporation, pyrolysis, char combustion, combustion of $\mathrm{CO}$ in the gas mixture, and soot combustion, and $\Delta H_{\text {vap }}, \Delta H_{p y r}, \Delta H_{\text {char }}, \Delta H_{C O}$ and $\Delta H_{\text {soot }}$ are the corresponding specific heats.

$$
H R R=-\omega_{\text {vap }} \cdot \Delta H_{\text {vap }}-\omega_{\mathrm{pyr}} \cdot \Delta H_{p y r}+\omega_{\text {char }} \cdot \Delta H_{\text {char }}+\omega_{C O} \cdot \Delta H_{C O}+\omega_{\text {soot }} \cdot \Delta H_{\text {soot }}
$$

Note that $\Delta H_{\text {char }}$ is not constant, it depends on the proportion of $\mathrm{CO}$ to $\mathrm{CO}_{2}$ produced during charcoal combustion (Morvan et al, 2018), it varies between $9 \mathrm{MJ} / \mathrm{kg}$ (for an incomplete combustion) and $30 \mathrm{MJ} / \mathrm{kg}$ (for a complete combustion).

The fireline intensity is then obtained from $I=H R R_{s s} / w$, where $H R R_{s s}$ is the average value of the heat release rate obtained at steady state and $w=100 \mathrm{~m}$ is the fireline width. As shown by Fig. 6, the present numerical results are compared with the predictions obtained using the operational models MK5 and BEHAVE and the predictions of the 2D numerical model FireStar2D; we have also reported some evaluations extracted from direct observations of prescribed and wildland fires on the field (Alexander and Fogarty, 2002). The transition between a high and a very high fire danger index corresponds to a fire intensity of $6 \mathrm{MW} / \mathrm{m}$. Almost all the results obtained using the present model (except $\mathrm{U}_{10}=1 \mathrm{~m} / \mathrm{s}$ ) are located above this threshold value, which means that, even if the wind speed remains quite moderate, the fire can exhibit a very dangerous behavior because the fuel moisture content is very low $(5 \%)$. 


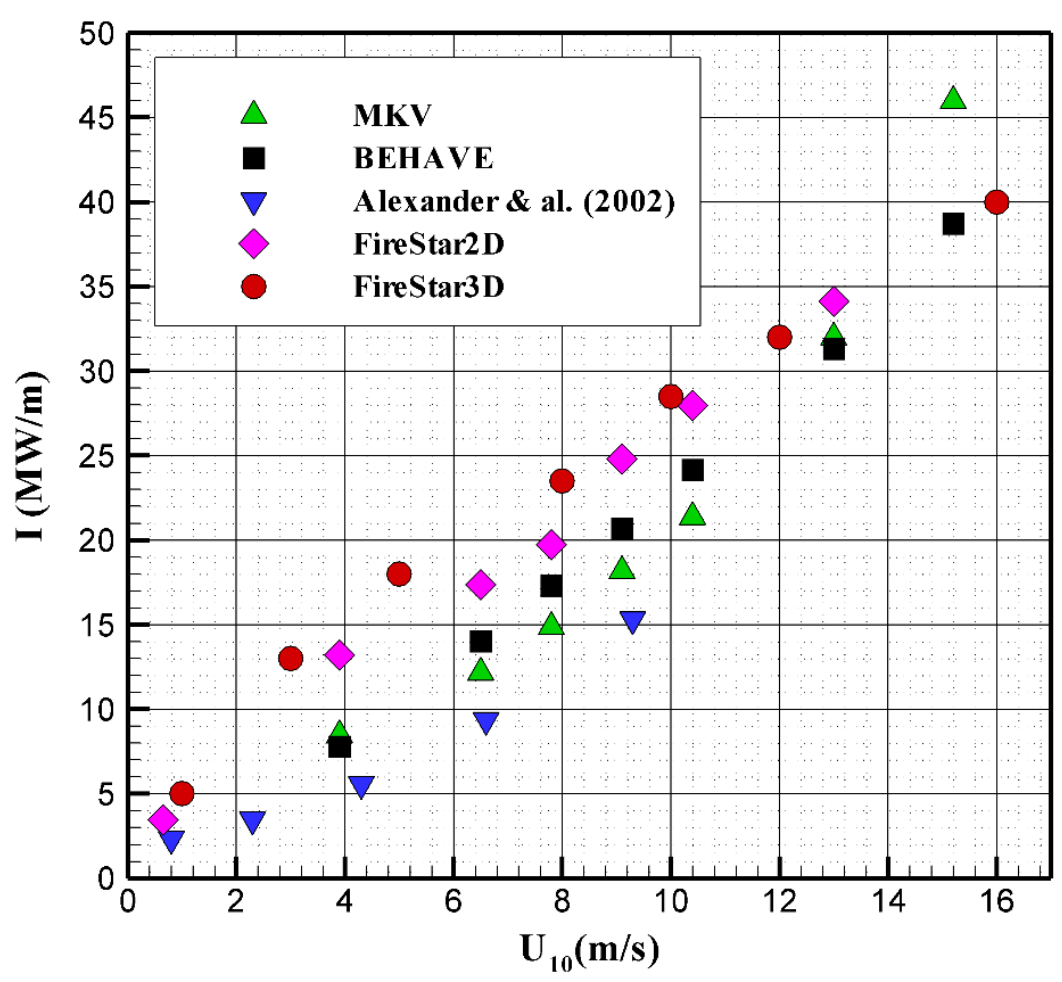

Figure 6 - Fireline intensity for fire propagation through a uniform grassland obtained for different 10-m open wind speeds. The results of FireStar3D are compared to those obtained experimentally by Alexander and Fogarty, 2002, using the empirical model MK5 (McArthur, 1967), the semi-empirical model BEHAVE (Rothermel, 1972), and the 2D numerical model FireStar2D (Morvan et al, 2009).

Finally, the heterogeneity of the fire front and its structuration as a succession of peaks and troughs is characterized by determining (using direct Fourier transform) the dominant wavelength of the temperature profile obtained along the transverse direction, $2 \mathrm{~m}$ above the fire front. This operation is performed during steady-state fire propagation and with a time interval of $1 \mathrm{~s}$. The wavelength $\lambda$ characterizing the fire front heterogeneity is then determined as the average value of the wavelengths obtained at different times. Froude's number based on the characteristic wave length $\lambda$, given by Eqs. 2 where $g$ is Earth gravity, is plotted in Fig. 7 versus Byram's convective number $N_{C}$ defined as the ratio between the buoyancy force and the inertial force due to the wind (Nelson, 2015) and given also by Eqs. 2, where $\rho_{0}$ and $C_{P O}$ are the density and the specific heat of ambient air at temperature $T_{0}=300$ $\mathrm{K}$.

$$
F r=\frac{g \lambda}{\left(U_{10}-R O S\right)^{2}} \quad N c=\frac{\rho_{0} C_{P 0} T_{0}\left(U_{10}-R O S\right)^{3}}{2 g I}
$$

Large values of Byram's number are normally obtained in plume dominated fires, whereas small values of Byram's number are obtained in wind driven fires (Nelson, 2015). Figure 7 also shows the experimental results obtained by Finney et al, 2013, at laboratory scale in the case of flame spread in engineered cardboard fuelbeds; to our knowledge, these are the only available experimental data about the 3D structuration of the fire front. It was foreseeable to find that Froude's and Byram's numbers were well correlated with a certain exponent (equal here to 0.633 for the experimental data), since they both compare the ratio between the buoyant forces and the inertial forces. It was however interesting is to find that the correlations are almost the same at a small scale (experimental data) and at large scale (numerical results). 


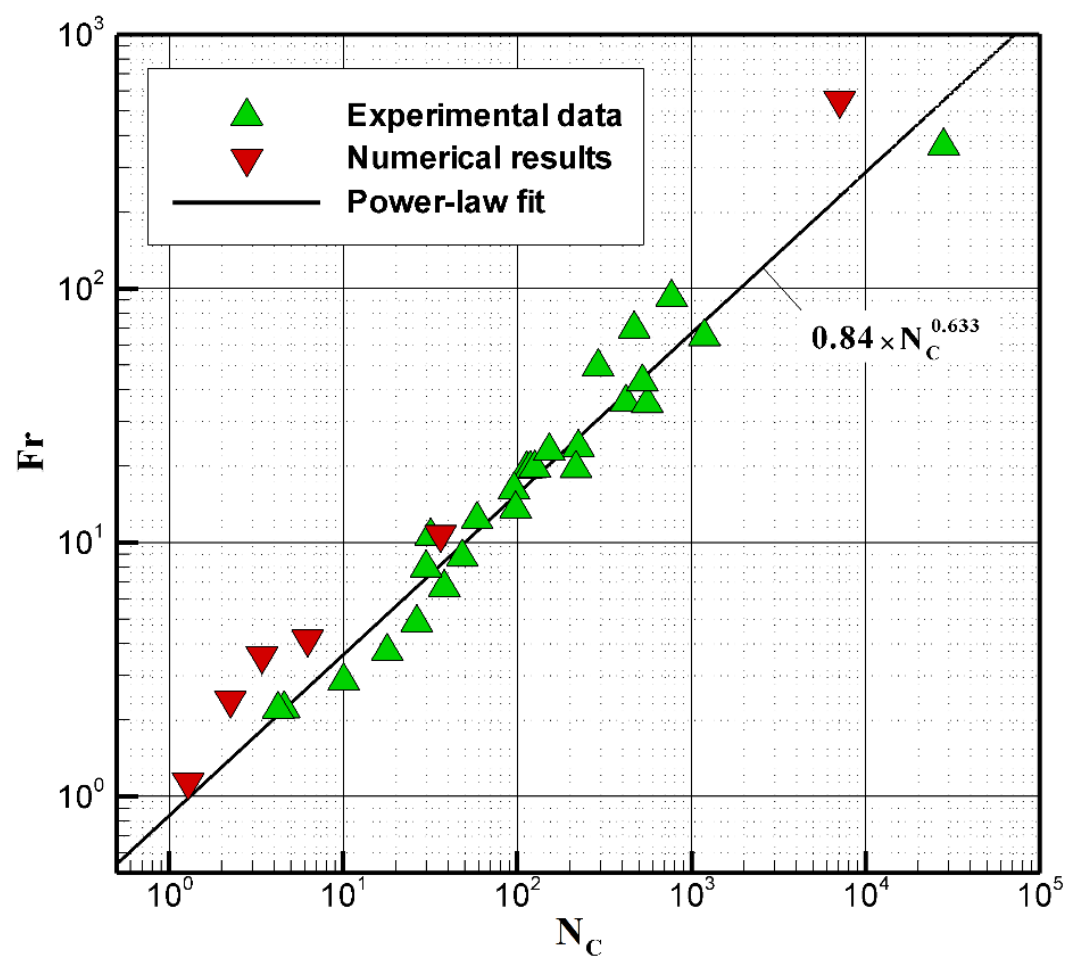

Figure 7 - Froude number based on the wavelength characterizing the fire front structure versus Byram's convective number. The results of FireStar3D obtained in the case of grassland fire are compared to those obtained experimentally at laboratory scale by Finney et al, 2013.

\section{Conclusions}

This study reported LES of fire spread of a quasi-infinite width fireline (obtained using periodic conditions at the lateral boundaries) through a homogeneous grassland. The results were obtained using a new 3D Computational Fluid Dynamic code based on a fully-physical multiphase model FireStar3D. The simulated Rate Of Spread of fire (ROS) and fire intensity were in good agreement with the data of the experimental campaign, with the predictions of operational empirical models (such as MK5 and BEHAVE), and with the numerical results of other 3D physical models (FIRETEC and WFDS). The simulations highlighted the effects of the wind speed on the ROS and on the intensity of fire, as well as the competition between the buoyant forces of the thermal plumes and the inertial forces of the wind. This competition is at the origin of the structuration of the fire front as a succession of peaks and troughs. Results also showed that the correlation between Froude's number (based on the fire-front structures characteristic-wavelength) and Byram's number is the same at small scale (litter fires) and at large scale (grassland fires). Many other fundamental aspects of the wildfire dynamic are not well understood, such as the role played by the field slope (and the competition between the wind and the slope when their directions are not aligned), the impact of the fuel moisture content (its threshold effect in the burn/no burn process, the linear or exponential decay of the rate of spread) ... In complement to experimental investigations, detailed physical models (such as FireStar3D) could be good tools for the understanding of the basic physical processes governing the behavior of wildfires.

\section{Acknowledgment}

The authors thank Aix-Marseille University for granting access to the HPC resources funded by Equip@Meso project (ANR-10-EQPX-29-01) of "Investissements d'Avenir" program, as well as the Lebanese University for the financial support in the framework of the research-projects funding program. 


\section{References}

Alexander ME, Fogarty LG (2002) A pocket card for predicting fire behaviour in grasslands under severe burning conditions. Fire Technology Transfer Note 25.

Beer T (1991) The interaction of wind and fire. Boundary-Layer Meteorology 54, 287-308.

Byram GM (1959) Combustion of Forest Fuels. In K. P. Davis (Ed.), Forest Fire: Control and Use. (New York: McGraw Hill)

Canfield JM, Linn RR, Finney M, Forthhofer J (2014) A numerical investigation of the interplay fireline length geometry, and rate of spread. Agricultural and Forest Meteorology 189-190, 48-59.

Cheney NP, Gould JS, Catchpole WR (1998) Prediction of fire spread in grasslands, International Journal Wildland Fire 8, 1-13.

Cheney NP, Gould JS (1995) Fire growth in grassland fuels. International Journal of Wildland Fire 5(4), 237-247.

Cox G (1995) Combustion fundamentals of fire. (Academic Press: UK)

Finney MA (1998) FIRESITE: Fire Area Simulator-Model Development and Evaluation, Rocky Mountain Research Station Research Paper RMRS-RP-4. (Ogden, UT)

Finney MA, Cohen JD, Forthofer JM, McAllister SS, Gollner MJ, Gorham DJ, Saito K, Akafuah NK, Adam BA, English JD (2015) Role of buoyant flame dynamics in wildfire spread. Proceedings of the National Academy of Sciences 112, 9833-9838.

Finney MA, Forthhofer J, Grenfell IC, Adam BA, Akafuah NK, Saito K (2013) A study of flame spread in engineered cardboard fuelbeds, Part I: correlation and observations. $7^{\text {th }}$ International Symposium on Scale Modeling (ISSM-7), Hirosaki, Japan.

Frangieh N, Morvan D, Accary G, Méradji S, Bessonov O (2018) Numerical simulation of grassland fires behavior using an implicit physical multiphase model. Fire Safety Journal (available online 20 June 2018).

Grishin AM, Albini FA (Eds) (1997) Mathematical modeling of forest fires and new methods of fighting them. (Publishing House of the Tomsk University: Russia)

Hanson HP, Bradley MM, Bossert JE, Linn RR, Younker LW (2000) The potential and promise of physics-based wildfire simulation, Environmental Science \& Policy 3, 161-172

Hunt JC, Wray AA, Moin P (1988) Eddies, streams, and convergence zones in turbulent flows, In its Studying Turbulence Using Numerical Simulation Databases $2^{\text {nd }}$ Proceedings of the Summer Program, 193-208.

Incropera FP, DeWitt DP (1996) Fundamentals of Heat and Mass Transfer. (John Wiley and Sons: UK).

Kaplan CR, Bek SW, Oran ES, Ellzey JL (1994) Dynamics of a Strongly Radiating Insteady Ethylene Jet Diffusion Flame Combustion and Flame 96, 1-21.

Kee RJ, Rupley FM, Miller JA (1992) The Chemkin Thermodynamic Data Based. (Sandia National Laboratory)

Linn RR, Cunningham P (2005) Numerical simulations of grass fires using a coupled atmosphere-fire model: Basic fire behavior and dependence on wind speed. Journal of Geophysical Research 110, D13107.

Linn RR, Canfield JM, Cunningham P, Edminter C, Dupuy JL, Pimont F (2012) Using periodic line fires to gain a new perspective on multi-dimensional aspects of forward fire spread. Agricultural and Forest Meteorology 157, 60-76.

McArthur AG (1967) Fire behaviour in eucalypt forests, Forest Research Institute, Leaflet No. 107 (Canberra, Australia) 
Mell W, Jenkins MA, Gould J, Cheney P (2007) A physics-based approach to modelling grassland fires. International Journal Wildland Fire 16, 1-22.

Mell W, Manzello S, Maranghides A (2010) The Wildland-Urban Interface Problem - Current Approaches and Research Needs, International Journal Wildland Fire 19, 238.

Modest MF (2003) Radiative Heat Transfer. (Academic Press: UK)

Morvan D, Méradji S, Accary G (2009) Physical modelling of fire spread in Grasslands. Fire Safety Journal 44, 50-61.

Morvan D (2015) Numerical study of the behaviour of a surface fire propagating through a firebreak built in a Mediterranean shrub layer, Fire Safety Journal 71, 34-48.

Morvan D, Accary G, Meradji S, Frangieh N, Bessonov O (2018) A 3D physical model to study the behavior of vegetation fires at laboratory scale. Fire Safety Journal 101, 39-52.

Morvan D (2014) Wind Effects, Unsteady Behaviors, and Regimes of Propagation of Surface Fires in Open Field. Combustion Science and Technology 186, 869-888.

Nelson RM (2015) Re-analysis of wind and slope effects on flame characteristics of Mediterranean shrub fires. International Journal of Wildland Fire 24, 1001-1007.

Rothermel RC (1972) A mathematical model for predicting fire spread in wildland fuels, USDA Forest Service Research Paper 40 (INT, USA)

Sagaut P (2006) Large eddy simulation for incompressible flows: An introduction. (Springer-Verlag Berlin Heidelberg)

Sullivan AL (2009) Wildland surface fire spread modelling, 1990-2007. 1: Physical and quasiphysical models, International Journal of Wildland Fire 18, 349-368.

Syed KJ, Stewart CD, Moss JB (1991) Modelling soot formation and thermal radiation in buoyant turbulent diffusion flames. In $23^{\text {rd }}$ International Symposium on combustion (Pittsburgh), The Combustion Institute 23, 1533-1541.

Tolhurst KG, Shields B, Chong DM (2008) Phoenix: Development and Application of a Bushfire Risk Management Tool. Australian Journal of Emergency Management. 23, 47. 


\title{
Generation of a global burned area product from satellite data: the ESA Fire_cci project
}

Emilio Chuvieco $^{1 *}$; M. Lucrecia Pettinari ${ }^{1}$; Joshua Lizundia-Loiola ${ }^{1}$; Ekhi Roteta ${ }^{2}$; Marc Padilla Parellada $^{3}$; Duarte Oom ${ }^{4}$; Philip Lewis ${ }^{5}$; Thomas Storm ${ }^{6}$; Johannes Kaiser ${ }^{7}$; Florent Mouillot ${ }^{8}$; Pierre Laurent ${ }^{9}$; Ioanis Bistinas ${ }^{10}$

${ }^{1}$ Grupo de Investigación de Teledetección Ambiental, Universidad de Alcalá, Calle Colegios 2, 28803 - Alcalá de Henares, Spain. \{emilio.chuvieco@uah.es* ${ }^{*}$ mlucrecia.pettinari@uah.es, joshua.lizundia@uah.es\}

${ }^{2}$ Universidad del País Vasco, Barrio Sarriena s/n, 48940 - Leioa, Spain. \{ekhi.roteta@ehu.eus\}

${ }^{3}$ Department of Geography, University of Leicester, University Road, Leicester, LE1 7RH, United Kingdom. \{mp489@leicester.ac.uk\}

${ }^{4}$ Instituto Superior de Agronomia, Universidade de Lisboa, Tapada da Ajuda

1349-017 Lisboa Portugal. \{duarte.oom@gmail.com\}

${ }^{5}$ Department of Geography, University College London, Gower St., London WC1H 6BT, United Kingdom. \{ucfalew@ucl.ac.uk\}

${ }^{6}$ Brockmann Consult GmbH, Max-Planck-Str. 2, 21502 - Geesthacht, Germany. \{thomas.storm@brockmann-consult.de\}

${ }^{7}$ Max Planck Institute for Chemistry, Hahn-Meitner-Weg 1, A.3.53, 55128 - Mainz, Germany. \{j.kaiser@mpic.de\}

${ }^{8}$ Institut de Recherche pour le développement, CEFE/CNRS, Campus du CNRS, 1919 route de Mende, 34293 - Montpellier 5, France. \{florent.mouillot@cefe.cnrs.fr\}

${ }^{9}$ Laboratoire des Sciences du Climat et de l'Environnement, Bât. 12, Avenue de la Terrasse,F91198 GIF-SUR-YVETTE, France. \{pierre.laurent@lsce.ipsl.fr\}

${ }^{10}$ Faculty of Earth and Life Sciences, Vrije Universiteit Amsterdam, De Boelelaan 1105, 1081 HV Amsterdam,The Netherlands. \{i.bistinas@vu.nl\}

\begin{abstract}
Biomass burnings (including forest, grassland, peatland and agricultural fires) have important impacts on global terrestrial and atmospheric systems, affecting land cover, surface albedo, and the atmospheric concentration of greenhouse gases, chemically reactive species and aerosols. Several products have been generated in the last years to estimate total burned area, but uncertainties remain, particularly those associated to small and low intensity fires. Impact of climate and societal changes modify traditional fire regimes, extending fire seasons, increasing fire severity or introducing fire in sensitive areas.

The Fire_cci project of the European Space Agency Climate Change Initiative aims to generate consistent time series of burned area products to assess the extent of biomass burnings, as well as their spatial and temporal characteristics. Fire impacts on atmospheric and terrestrial processes are assessed, including modifications of vegetation patterns and biomass availability.

The global burned area products of the Fire_cci program currently available are derived from MERIS FRS and MODIS 250m sensors and cover the period from 2001 to 2016. These global burned area products are complemented with a small-fire database generated from medium resolution sensors (Sentinel-2 and Sentinel1) for the African continent. BA algorithms for new Sentinel-3 sensors (OLCI and SLSTR) are also being developed. Validation of the global products is based on a statistical sampling design of 1200 Landsat scenes.
\end{abstract}

Keywords: Burned area, Remote Sensing, Earth Observation, Global, Fire impacts, ESA, CCI 


\section{Introduction}

Fire has shaped human history from the very beginning. It has been used to cook, change vegetation composition, hunting and creating harder tools. Nowadays, fire is still used widely to transform land use, improve soil nutrients, help hunting, mining and other human activities (Bowman et al., 2013; Hantson et al., 2015). Current estimations show that 3.5-4 $\mathrm{Mkm}^{2}$ are burned every year (Alonso-Canas and Chuvieco, 2015; Giglio et al., 2013). Fire mainly affects tropical forests and savannas in Africa, Brazil and Indonesia, temperate forest and grasslands in USA, South America and Eurasia, and boreal forest in Siberia and N. America. Fire effects include impacts on atmospheric composition, carbon budgets and vegetation dynamics. Global estimates of fire emissions are between 1 and $3 \mathrm{Pg} \mathrm{C}$ year-1 (van der Werf et al., 2010). Fire reduces tree cover and in consequence the ability of terrestrial ecosystems to sequester carbon (Yue et al., 2014).

Even though the current understanding of climate-fire interactions is limited, there is also increasing evidence on their mutual influence (Hantson et al., 2015; Kloster et al., 2012; Yue et al., 2015). Climate has a strong influence on all aspects of the fire regime. It is related to seasonal timing temperature and humidity, as well as wind (that helps spreading the fire). Climate also influences the nature and availability of fuel, through its influence on the productivity and type of vegetation. Expected temperature (dryer vegetation conditions) and rainfall changes are likely to increase wildfire frequency in some regions, while others may experience decrease in fire activity as a result of decreased biomass production (Krawchuk et al., 2009). On the other hand, fires also affect climate. GHG and aerosol emissions, as well as albedo changes (from vegetation to soil) affect overall radiation budget, although their impacts are not yet well defined. For instance, the impact of increasing boreal forest fires on climate warming may be limited or even result in regional cooling because of the negative forcing from increased surface albedo following a fire (snow albedo is much higher than vegetation's). Climate modelling studies suggest that aerosols derived from burning may lengthen or intensify periods of drought in the Equatorial regions. The relevance of fires in the global terrestrial system has pushed the development of fire modules within Dynamic Global Vegetation Models (DGVM), such as the SPITFIRE (Yue et al., 2015).

The global impacts of fire justify a growing interest of deriving global burned area products. This is the basis of selecting Fire disturbance as one of the Essential Climate Variables (ECV) defined by the Global Climate Observing System (GCOS). These ECVs are intended to have a systematic and long-term record of observations that are relevant for climate modelling. Many of these variables can be systematically retrieved using different satellite Earth observation systems, and therefore GCOS has provided different guidelines for creating international programmes to generate those ECVs (GCOS, 2016).The European Space Agency (ESA) responded to this petition by creating the Climate Change Initiative (CCI) programme in 2010. The ESA CCI programme includes 13 variables, covering atmospheric, oceanic and land ECVs, which are systematically derived from up to date satellite sensors (Hollmann et al., 2013).

Fire disturbance (defined as the extent and characteristics of burned areas) was one of those variables included in the ESA CCI programme in 2010. Since then, the project teams have generated global databases of burned areas (BA), with the adequate auxiliary information to fit climate modellers' needs. This paper presents the main outputs of the Fire_cci project, including review of user needs, data generation, validation and assessment. In addition to global products, a small fire database (SFD) has been generated for Sub-Saharan Africa, which will not be covered in this manuscript. 


\section{Methods}

\subsection{User requirement analysis}

Since the main target community of the CCI programme are climate modellers, a survey was conducted to better understand user needs and prepare product specifications accordingly. The review of Mouillot et al. (2014) identified the main uses of BA products and the specific demands of modellers. This work was extended by dedicated questionnaires to different users of BA products, including carbon and atmospheric chemistry modellers, fire ecologists and civil protection services. As a result of the literature review and user interviews, several priorities for global BA products were identified:

(i) Long-term series data for understanding the long-term fire, climate and vegetation interaction. Temporal consistence should be assured within a 5\% threshold.

(ii) Temporal resolution should be daily data at the original spatial resolution of the sensor, weekly, 10-day or monthly basis on global scale.

(iii)Spatial resolution of the product should be 5-25 hectares for regional analysis and 0.25 to 0.5 degrees at global scale.

(iv)Maximum omission and commission errors should be below 15\%, with demonstrated systematic and adequate validation using internationally agreed validation protocols.

(v) Error traceability and uncertainty characterization should be included in both the pixel and grid products, in a manner that can be easily understood and utilized by users in various applications.

(vi)Easy access to data, including auxiliary information and metadata on burned cover, burn patch distribution and burn severity/efficiency.

\subsection{Product specifications}

Following recommendations from users, two sets of Fire_cci BA products were defined. They include pixel outputs (at the level of spatial detail of each sensor) and grid products (global summaries at coarser spatial resolution, which better fits climate models' resolution). Each product included a set of auxiliary variables to help the main user communities. They are:

- Pixel product: continental tiles of monthly BA, including three variables: date of burned, confidence level and land cover burned (extracted from the Land Cover CCI project, Kirches et al., 2013). The product is delivered in GeoTIFF format.

- Grid product at $0.25 \times 0.25$ degree resolution cells accumulating data every 15 days. It includes 23 layers: total burned area in the period, standard error, fraction of burnable area, fraction of observed area, number of fire patches, and total BA in each of 18 different land covers. These files are distributed in NetCDF-CF format.

Both data sets are supported by metadata provided as XML in the case of the pixel product and embedded in the NetCDF format for the grid product.

\subsection{Burned Area algorithm}

A set of BA algorithms have been developed within the Fire_cci project adapted to the different input sensors:

- Continental scale for the small fire database. They are based on Sentinel-1 radar (SAR), and Sentinel-2 MSI (optical) data.

- Global scale: MERIS (300 m) on board ENVISAT, MODIS (250m) on board TERRA and OLCI and SLSTR on board the Sentinel-3 satellite. This paper focuses on these datasets.

The MERIS BA algorithm combined temporal changes in near infrared (NIR) reflectance with location of active fires (Alonso-Canas y Chuvieco, 2015). A similar approach was followed for the 
MODIS sensor, in this case including only information from the highest resolution channels (250 $\mathrm{m}$ ), to complement current NASA BA products based on $500 \mathrm{~m}$ resolution data (Giglio et al., 2013). Both algorithms detect burned pixels in two phases. The first one aims to detect seed pixels (those more clearly burned), thus minimizing commission errors, while the second one applies a contextual criterion to discriminate the complete BA patch from the seed pixels, aiming to reduce omission errors.

Before the seed phase was carried out, a monthly composite of images was generated. The compositing criterion was based on selecting the nearest day to the closest hotspot of each pixel. This criterion tried to identify those days when the fire signal had the highest contrast from the background, avoiding clouds, cloud shadows and other artefacts.

Once the composite was created, the seed phase tried to identify the most clearly burned pixels. Since burned areas are very diverse worldwide and therefore the changes in reflectance may greatly vary among different ecosystems, the selection of seeds was guided by a regional-adapted statistical approach. Detection thresholds were established for each 10x10 degree tile and each monthly period based on the probability distribution function of the NIR reflectances of the pixels detected as active fires in that region and period. A criterion to maximize separability between the distribution functions of burned and unburned pixels was applied to establish a spatio-temporal threshold. This was complemented with other temporal change thresholds in NIR reflectances to further assure that pixels selected were actually burned. Once the seeds were detected, a contextual criterion was applied to extend the seeds to their neighbours that were likely to be burned as well. This region-growing process was based on the NIR values, and the change in red and NIR reflectance between the seed pixels and the surrounding ones. A similar approach was applied to the MODIS R and NIR channels, adapting several thresholds to better suit the MODIS sensor characteristics.

The BA algorithm was processed globally for both sensors. For MERIS full resolution images the time series made public includes the period from 2005 to 2011 (Chuvieco et al., 2016), while for MODIS images, the processed period spans from 2001 to 2016 (Chuvieco et al., 2018). Both BA products are made publicly available and freely downloadable (https://geogra.uah.es/fire_cci/ last accessed June, $4^{\text {th }}$ 2018).

\subsection{Validation}

The reference data used for the validation was derived from pairs of Landsat images consecutive in time, following CEOS Cal-Val guidelines (Boschetti et al., 2009). Reference fire perimeters were derived from a semi-automatic algorithm and revised visually by two interpreters. Final sampling included 1200 Landsat pairs following a stratified random sampling design (Padilla et al., 2017). Accuracy measurements were derived from the error matrix, obtained from the cross tabulation between product and reference maps: overall accuracy (OA), commission error (CE) ratio, omission error (OE) ratio, Dice coefficient (DC), bias (B) and the relative bias (rel B), the last two referring to the trend towards under or overestimation of BA.

\section{Results}

\subsection{Spatial trends}

Figure 1 includes an example of the global BA datasets for 2016 based on the MODIS Fire_cci v5.0 product (called FireCCI50 for short). The most extensive burnings occurred in the Tropical regions, particularly in the African continent, followed by the Northern regions of Australia, Central Brazil, Venezuelan and Colombian Llanos, and Southeast Asia. A second belt of burned regions is noticeable in the temperate grasslands and croplands of central Asia, and Southeast USA. The boreal forests of Russia and Canada have also an important role in global biomass burnings. 


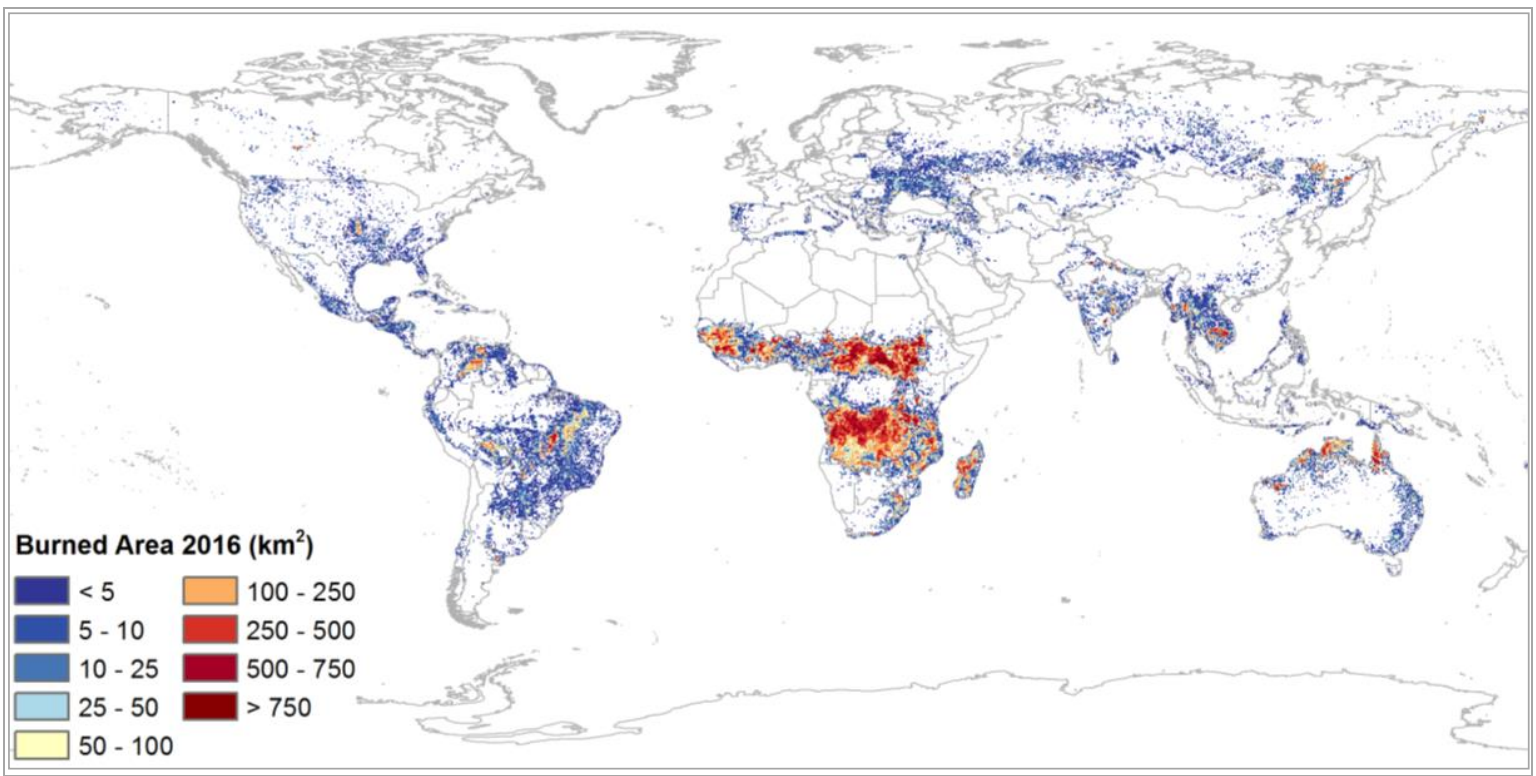

Figure 1 - Global Burned area of 2016 from FireCCI50

\subsection{Temporal trends}

Average yearly BA was $3.81 \mathrm{Mkm}^{2}$, approximately the size of India and France combined. Years with largest BA were 2004 and 2011 with more than $4.16 \mathrm{Mkm}^{2}$, while only 2013 and 2016 had less than $4 \mathrm{Mkm}^{2}$. These global trends are very similar to the GFED4, the most widely used BA database, which accounts for the same years an average total value of $3.38 \mathrm{Mkm}^{2}$, with yearly variations between 2.87 and $3.77 \mathrm{Mkm}^{2}$. Recent estimations from the MODIS BA product MCD64 c6 increase the estimations of our product by $15 \%$. Figure 2 shows the seasonal and multiyear cycle of burn activity comparing our results with the GFED4, MCD64 c6 and the MERIS Fire_cci v4.1 (FireCCI41) BA databases.

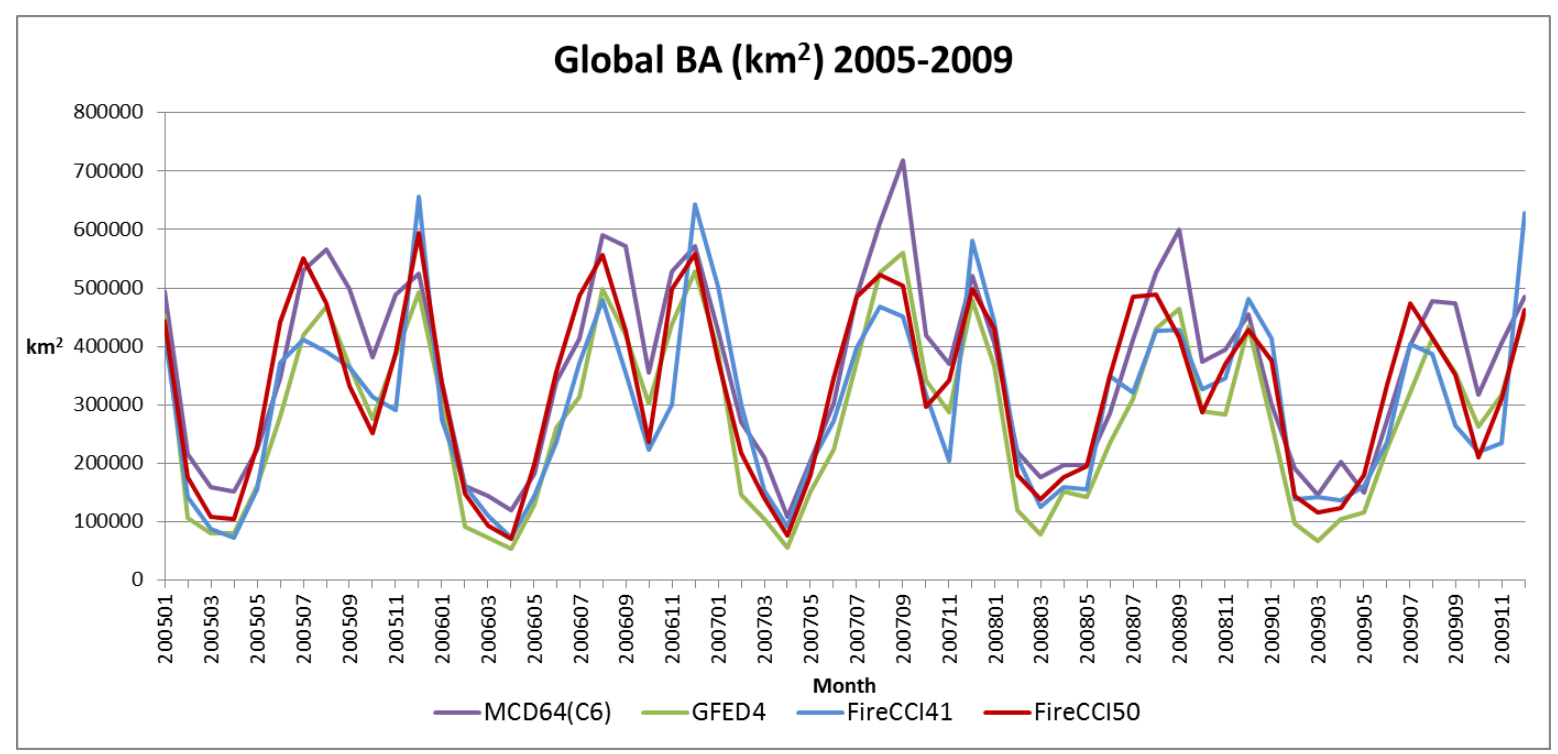

Figure 2 - Temporal trends of burn area in different global BA products

A clear seasonal pattern is observed throughout the years, with two peaks. The first one is located in December-January, which corresponded mainly to the dry season of the Northern Hemisphere (tropical burnings of Sub-Saharan Africa, Northern Australia, SE Asia, Central America and Northern fringe of South America). The second occurred in July-September and referred to the burnings of the Southern Hemisphere tropical regions of Africa and South America, the grasslands and agricultural 
burnings of South Asia, the temperate forest burnings of the Mediterranean, USA and Australia, and the Boreal regions of Canada, Russia and Alaska. The four BA databases agreed quite well, although MCD64 provided generally higher values. The GFED4 product, based on MODIS images, and the Fire_cci products showed similar trends, with a tendency to overestimate BA in the peak of DecemberFebruary in the case of the FireCCI41 product.

\subsection{Spatio-temporal patterns of the pixel product}

Figure 3 illustrates an example of the pixel products generated from the FireCCI50 dataset. It includes the date of detection and provides a much higher resolution than the grid product to analyse spatial and temporal patterns.

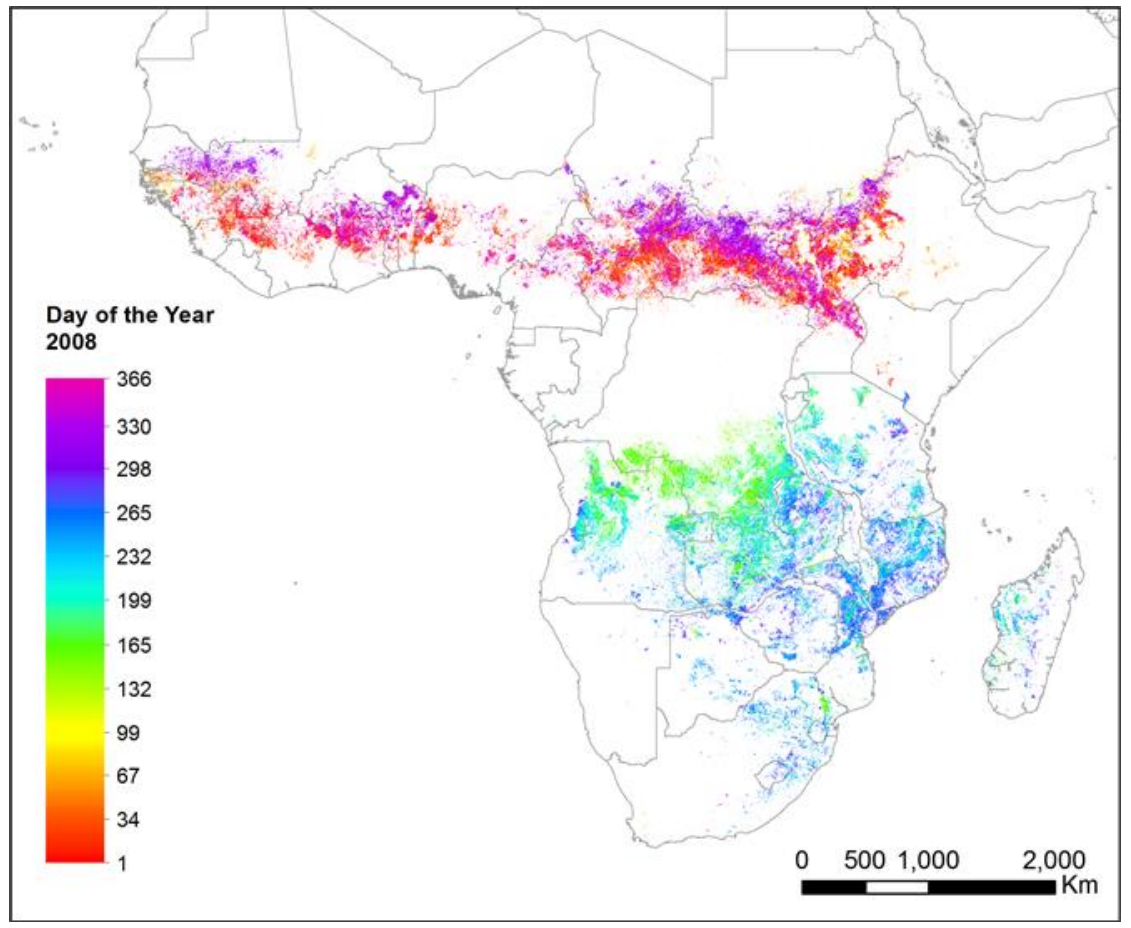

Figure 3 - Day of detection for Sub-Saharan Africa for the year 2008.

As it may be expected, two parallel fringes are observed, one in the Northern Hemisphere Tropical belt (Senegal, Ivory Coast, Ghana, Nigeria, Cameroun, Central African Republic, Chad and Sudan), mostly burned at the beginning or end of the year, in coincidence with the dry season of the Northern Hemisphere Tropics, and another one to the South of Equator, where a dry season occurs between June and September. Angola, Zambia, Zimbabwe, South Africa, Mozambique, Madagascar and Tanzania are the most affected countries in this latter region. Pixel products can be used for different objectives, both at global and regional scales. The Fire_cci pixel product has been used to analyse burn shapes (Chuvieco et al., 2018; Chuvieco et al., 2016), which is an interesting variable to better understand fire propagation patterns.

\subsection{Validation}

At global scale and for the time period with available data, the accuracy of FireCCI50 expressed by the Dice coefficient $(\mathrm{DC})$ was 0.365 (standard error $=0.026$ ). This value is significantly higher than for the FireCCI41 product ( $\mathrm{DC}=0.248$ with $\mathrm{SE}=0.030$ ), but lower than for the MCD64A1 c6 product ( $\mathrm{DC}=0.478$ with $\mathrm{SE}=0.031$ ). Similarly this is expressed by the commission and omission error ratios (Ce and Oe), $0.512(0.020)$ and $0.708(0.30)$ for FireCCI50, $0.643(0.045)$ and 0.810 (0.030) for FireCCI41, and $0.353(0.016)$ and $0.622(0.038)$ for MCD64A1 c6 respectively. FireCCI50 underestimates a fraction of $0.402(0.058)$ of the BA reported in the reference data, as expressed by 
the relative bias (relB), slightly less than MCD64A1 c6, 0.415 (0.056), and lower than FireCCI41, with a vaule of $0.468(0.094)$.

We should point out that these errors are most likely inflated, as they do not take into account reporting accuracy inaccuracies. This means that pixels correctly classified as burned may be labelled as omission errors when detected after the reference date of the Landsat multitemporal pairs used for generating reference files. Similarly, pixels correctly detected as burned but labelled a few days after the beginning of the Landsat multitemporal pair may be considered as commission errors. To analyse these impacts, three large areas (around 250,000 $\mathrm{km}^{2}$ ) were selected in Australia, Canada and California from where national fire perimeters were available. The omission and commission errors for these sites were much lower than those observed for the global sample (commission 0.22 to 0.4 and omission 0.35 to 0.5 ) for the FireCCI50 product.

\section{Conclusions}

This paper has shown results provided by the Fire_cci project, included in the Climate Change Initiative programme of the European Space Agency. The first assessment of these results provides coherent spatial and temporal trends, with similar patterns as the most widely used global BA products. Preliminary validation shows a high overall accuracy, but also high omission and commission errors of the burned class, which should partially be related to the temporal reporting accuracy and the impact of small fires in some regions.

\section{Acknowledgments}

The Fire_cci project is funded by the European Space Agency (ESA) as part of the Climate Change Initiative Programme.

\section{References}

Alonso-Canas I, Chuvieco E (2015) Global Burned Area Mapping from ENVISAT-MERIS data. Remote Sensing of Environment 163, 140-152.

Boschetti L, Roy DP, Justice CO (2009) International Global Burned Area Satellite Product Validation Protocol. Part I - production and standardization of validation reference data. http://lpvs.gsfc.nasa.gov/DOC/protocol_revised_Apr09.doc.

Bowman DM, O'Brien JA, Goldammer JG (2013) Pyrogeography and the global quest for sustainable fire management. Annual Review of Environment and Resources 38, 57-80.

Chuvieco E, Lizundia-Loiola J, Pettinari ML, Ramo R, Padilla M, Tansey K, Mouillot F, Laurent P, Storm T, Heil A, Plummer S (2018) Generation and analysis of a new global burned area product based on MODIS 250m reflectance bands and thermal anomalies. Earth System Science Data Discussion, https://doi.org/10.5194/essd-2018-46.

Chuvieco E, Yue C, Heil A, Mouillot F, Alonso-Canas I, Padilla M, Pereira JMC, Oom D, Tansey K. (2016) A new global burned area product for climate assessment of fire impacts. Global Ecology and Biogeography 25, 619-629.

GCOS (2016) The Global Observing System for Climate: Implementation Needs, GCOS-200. World Meteorological Organization. (Geneva, Switzerland)

Giglio L, Randerson JT, van der Werf GR (2013) Analysis of daily, monthly, and annual burned area using the fourth generation global fire emissions database (GFED4). Journal of Geophysical Research: Biogeosciences 118, 317-328. 
Hantson S, Pueyo S, Chuvieco E (2015) Global fire size distribution is driven by human impact and climate. Global Ecology and Biogeography 24, 77-86.

Hollmann R, Merchant CJ, Saunders R, Downy C, Buchwitz M, Cazenave A, Chuvieco E, Defourny P, de Leeuw G, Forsberg R, Holzer-Popp T, Paul F, Sandven S, Sathyendranath S, van Roozendael M, Wagner W (2013) The ESA Climate Change Initiative: satellite data records for essential climate variables. Bulletin of the American Meteorological Society 94, 1541-1552.

Kirches G, Krueger O, Boettcher M, Bontemps S, Lamarche C, Verheggen A, Lembrée C, Radoux J, Defourny P (2013) Land Cover CCI: Algorithm Theoretical Basis Document Version 2. Land_Cover_CCI_ATBDv2_2.3. (Louvain, Belgium).

Kloster S, Mahowald N, Randerson J, Lawrence P (2012) The impacts of climate, land use, and demography on fires during the 21st century simulated by CLM-CN. Biogeosciences 9, 509-525.

Krawchuk MA, Moritz MA, Parisien M-A, Van Dorn J, Hayhoe K (2009) Global Pyrogeography: the Current and Future Distribution of Wildfire. PLOS ONE 4, e5102.

Mouillot F, Schultz MG, Yue C, Cadule P, Tansey K, Ciais P, Chuvieco E (2014) Ten years of global burned area products from spaceborne remote sensing - A review: Analysis of user needs and recommendations for future developments. International Journal of Applied Earth Observation and Geoinformation 26, 64-79.

Padilla M, Olofsson P, Stehman SV, Tansey K, Chuvieco E (2017) Stratification and sample allocation for reference burned area data. Remote Sensing of Environment 203, 240-255.

van der Werf GR, Randerson JT, Giglio L, Collatz GJ, Mu M, Kasibhatla PS, Morton DC, DeFries RS, Jin Y, van Leeuwen TT (2010) Global fire emissions and the contribution of deforestation, savanna, forest, agricultural, and peat fires (1997-2009). Atmospheric Chemistry and Physics 10, 11707-11735.

Yue C, Ciais P, Cadule P, Thonicke K, Archibald S, Poulter B, Hao WM, Hantson S, Mouillot F, Friedlingstein P, Maignan F, Viovy N (2014) Modelling the role of fires in the terrestrial carbon balance by incorporating SPITFIRE into the global vegetation model ORCHIDEE-Part 1: simulating historical global burned area and fire regimes. Geoscientific Model Development 7, 2747-2767.

Yue C, Ciais P, Cadule P, Thonicke K, van Leeuwen TT (2015) Modelling the role of fires in the terrestrial carbon balance by incorporating SPITFIRE into the global vegetation model ORCHIDEE - Part 2: Carbon emissions and the role of fires in the global carbon balance. Geoscientific Model Development 8, 1321-1338. 


\title{
Integration of the emissions and smoke dispersion models in the European Forest Fire Information System
}

\author{
Pieralberto Maianti ${ }^{1,2 *}$; Tomàs Artés Vivancos ${ }^{2}$; Bruno Guillaume ${ }^{3}$; Ana Isabel Miranda ${ }^{4}$; \\ Alexandra Monteiro ${ }^{4}$; Carla Gama ${ }^{4}$; Tracy Houston Durrant ${ }^{2,5}$; Giorgio Libertà ${ }^{2}$; Roberto Boca; \\ Alfredo Branco ${ }^{1}$; Daniele de Rigo ${ }^{1}$; Davide Ferrari ${ }^{2,6}$; Fabio Lana ${ }^{1}$; Hugo Costa ${ }^{2}$; Jesús San- \\ Miguel-Ayanz ${ }^{2 *}$ \\ ${ }^{1}$ External consultant for the European Commission (ARCADIA SIT s.r.l). ARCADIA SIT s.r.l, \\ Vigevano $(P V)$, Italy. \\ ${ }^{2}$ European Commission, Joint Research Centre (JRC), Directorate for Space, Security and \\ Migration, Disaster Risk Management Unit, Ispra(VA), Italy. \{JRC-EFFIS@ec.europa.eu*\} \\ ${ }^{3}$ ARIA Technologies. 8/10, rue de la Ferme - 92100 Boulogne-Billancourt, France, \\ \{bguillaume@aria.fr\} \\ ${ }^{4}$ University of Aveiro. Campus Universitário de Santiago, 3810-193 Aveiro, Portugal, \\ \{miranda@ua.pt; alexandra.monteiro@ua.pt; carlagama@ua.pt\} \\ ${ }^{5}$ External consultant for the European Commission (GFT Italia s.r.l). GFT Italia s.r.l., Milan (MI), \\ Italy. \\ ${ }^{6}$ External consultant for the European Commission, European Dynamics, Luxembourg, \\ Luxembourg
}

\begin{abstract}
Since 2000, the European Forest Fire Information System (EFFIS) has provided continuous support and statistics on wildland fire events. The monitoring system covers all the European Countries, North Africa and the Mediterranean Countries of the Middle East, and provides information on the various aspects involved in the fire events, from fire danger forecasting to post-event analysis. One of the major impacts on the environment produced by the wildland fires is the atmospheric pollution. Currently, in EFFIS the only available information on this impact is the amount of atmospheric emissions for each fire event. The current emissions model is static and does not take into account the fire behavior during the event; moreover, it cannot distinguish the contribution of flaming and smoldering fire to the emissions. The workgroup in EFFIS is working on the current atmospheric pollution assessment system to provide a new and improved emissions model integrated with a smoke dispersion model that simulates the distribution of the pollutants in the impacted area. The new emissions estimation model will take as input the evolution of the fire events, a map of the fuel classes and forest types, a digital elevation model and the meteorological data covering the whole Europe. Based on these input data, the new emissions model simulates the behaviour of the fire using the NEXUS, FOFEM5 and CONSUME3 models, calculates the impact of the fire on the fuels and provides an assessment of the ensuing emissions. The new emissions module provides an assessment of the major pollutants emitted by fires, such as $\mathrm{CO}_{2}, \mathrm{CO}, \mathrm{CH}_{4}, \mathrm{PM}_{2.5}, \mathrm{PM}_{10}$. Large wildfires, although not very frequent, are responsible for a remarkably high percentage of the overall annual burnt area in Europe, with the highest emissions per fire event. In this paper, we present the results of the emissions estimations on the study case of a representative large wildfire event that took place in Zamora (Spain) during the 2017 fire season. The dispersion model, developed along with the new emissions module, takes as an input the output of the emissions model and provides a simulation of the dynamic of the smoke plume for each of the aforementioned pollutants. The dispersion module is a framework of integrated models able to estimate the dispersion of fire plumes in the atmosphere in 3D grid format. There are four main modules: WRF is dedicated for the computation of the meteorological background data, SWIFT for the higher resolution focus on a specific study area, CHIMERE for the assessment of the concentration of the background pollutants at regional scale,
\end{abstract}


and SCICHEM for the simulation at local scale of the smoke plume of a selected event. The output of the model is a series of NetCDF files (one for each of the pollutants taken into account) that simulate the 3D spatial and temporal development of the smoke plume. The application of the dispersion model on the Zamora study case shows an example of the output product.

Keywords: Forest fires; Wildfires; Atmospheric emissions modelling; Pollutant dispersion; EFFIS.

\section{Introduction}

Since 2000, the European Forest Fire Information System (EFFIS, http://effis.jrc.ec.europa.eu/) of the European Commission, Joint Research Centre (JRC) has systematically delivered information and statistics of burnt areas mapped from satellite imagery (San-Miguel-Ayanz et al., 2013). It is a complex monitoring system covering all the European Countries, North Africa and the Mediterranean Countries of the Middle East. EFFIS is able to collect and disseminate information on the many aspects involved in the fire events, from fire danger forecasting to post-event analysis.

The impact of forest fires on air quality is progressively concerning the population of the main affected areas (Cornwall, 2018; Finlay et al., 2012; Carvalho et al., 2011; Stefanidou et al., 2008). Forest fire smoke can be synonymous of severe crisis, which in recent years increased the number of hospitalizations for respiratory diseases (Wegesser et al., 2009; Ignotti et al. 2010). The integration of the new fire emissions and smoke dispersion modules in EFFIS will provide improved tools for air quality assessment both at local and European scale. The new emissions module will take into account the evolution of the burned areas mapped on a daily basis, and provides an assessment of the major pollutants emitted by fires, such as $\mathrm{CO}_{2}, \mathrm{CO}, \mathrm{CH}_{4}, \mathrm{PM}_{2.5}$, and $\mathrm{PM}_{10}$. The output of the new emissions module will be the input of the dispersion module, which provides the dynamic of the smoke plume for each of the aforementioned pollutants on selected severe fire events. The objective of this paper is to describe the components and the workflow of the new emissions and dispersion models, and to present their application on a study case.

\section{Emissions module}

\subsection{Module description}

The current version of the emissions module in EFFIS estimates the concentration of pollutants (Barbosa et al., 2008; Monteiro et al., 2014) relying on classical methodologies of fuel map-based computation and constant burning efficiencies given by fuel types (San Miguel et al., 2011). In order to better reproduce the complex behaviour of the phenomenon, this model has been restyled, updated and integrated in recent years.

In the new version of the model, a richer set of features is considered:

- Burnt areas and their evolutions provided by EFFIS daily mapping (in the current model only the final burnt area is considered);

- Digital Terrain Model, fuel composition (JRC forest types static maps) and meteorological data from the European Centre for Medium-Range Weather Forecast (ECMWF);

- Evaluation of the key variables fuel moisture (provided by the Live Fuel Moisture Content, LFMC model), fireline intensity and fire rate of spread (NEXUS model, Scott, 1999, Scott and Reinhardt, 2001);

- Assessment of burning efficiencies and amounts of fuel burned or consumed during the different combustion phases (provided by the models CONSUME3, Prichard et al., 2005, Ottmar et al., 1993; and FOFEM5, Keane et al., 1995, Reinhardt and Dickinson, 2010, Prichard et al., 2014); 
- Emissions estimations from burned fuel loadings applying emission factors taken from the "European biomass burning" (Miranda et al., 2005).

The workflow of the module is represented in Figure 1. The main application of the Emissions module is the daily estimation of the pollutants, which is based on the preliminary mapping of the burnt areas. It is scheduled at 1:00 AM of the day following a burnt area detection, and takes into account the perimeters detected during the previous day. As the definition of the perimeters can be reviewed and corrected, a second typology of the module usage is the reanalysis mode, which consists of a simulation run usually performed at the end of a fire season. The reanalysis mode takes as an input the final perimeters in order to obtain the definitive assessment of the emissions.

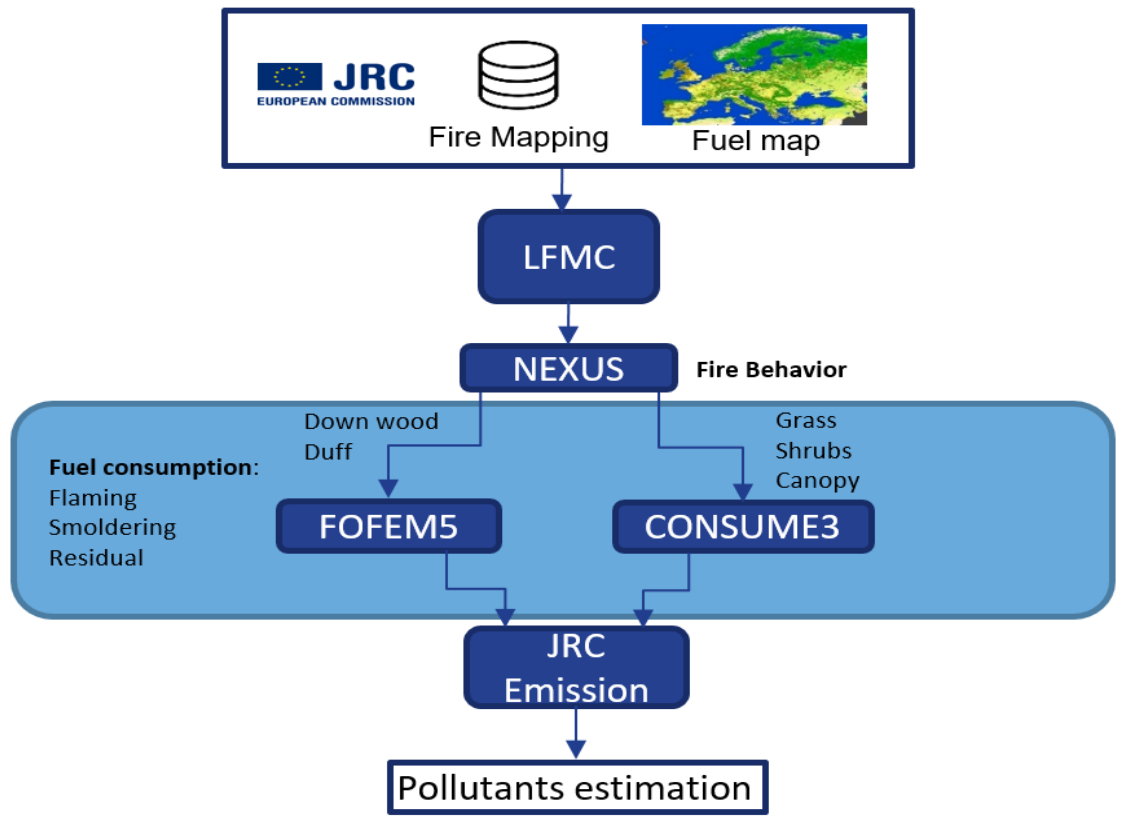

Figure 1 - Emissions module workflow.

When applied for the evaluation of the emissions produced within a burnt area, the new emissions module provides a shapefile with the geometry of the input burnt areas and lists in the associated attribute table the estimated concentrations of the pollutants. For each of these polluting components (Table 1), the module simulates the evolution of the concentrations produced (measured in $10^{3} \mathrm{~kg}$ ) over the day of fire activity with a time step of three hours (from 0 to 24). Compared to the current model, the new version expands the set of output variables and provides an assessment for the $\mathrm{SO}_{2}$, $\mathrm{NH}_{3}, \mathrm{BaP}, \mathrm{LVGC}$ pollutants (see list on Table 1).

Table 1 - List of the variables (aggregated) reported in the emissions simulation module.

\begin{tabular}{|c|c|c|c|c|c|c|c|}
\hline Variable & $\mathrm{CO}_{2}$ & $\mathrm{CO}$ & $\mathbf{C H}_{4}$ & $\mathbf{P M}_{2.5}$ & $\mathbf{P M}_{10}$ & NMHC & VOC \\
\hline $\begin{array}{l}\text { Extended } \\
\text { name }\end{array}$ & Dioxide carbon & $\begin{array}{l}\text { Monoxide } \\
\text { carbon }\end{array}$ & Methane & $\begin{array}{l}\text { Particulate } \\
\text { matter } \\
\leq 2.5 \mu \mathrm{m}\end{array}$ & $\begin{array}{l}\text { Particulate } \\
\text { matter } \leq 10 \mu \mathrm{m}\end{array}$ & $\begin{array}{l}\text { Non-methane } \\
\text { hydrocarbons }\end{array}$ & $\begin{array}{l}\text { Volatile } \\
\text { Organic } \\
\text { Compounds }\end{array}$ \\
\hline Variable & $\mathbf{N O}_{\mathrm{x}}$ & OC & BC & $\mathbf{S O}_{2}$ & $\mathbf{N H}_{3}$ & $\mathrm{BaP}$ & LVGC \\
\hline $\begin{array}{l}\text { Extended } \\
\text { name }\end{array}$ & $\begin{array}{l}\text { Nitrogen } \\
\text { Oxides }\end{array}$ & Organic carbon & Black Carbon & Sulphur dioxide & Ammonia & $\begin{array}{l}\text { Benzo(a)- } \\
\text { pyrene }\end{array}$ & Levo- glucosan \\
\hline
\end{tabular}

As ancillary information, the module also reports the following variables:

- Fire intensity (in $\mathrm{kW} \mathrm{m}^{-2}$ ), which is used as input in the dispersion module to estimate the maximum height of injection of the emissions in the atmosphere; 
- Fuel consumption in the fire phases of flaming (fire front) and smouldering (after the passage of the fire front), expressed in $\mathrm{kg}$ of fuel / 3hours;

- Area increase over each 3 hours time step, assumed proportional to the 3 hours average rate of spread of the fire, expressed in $\mathrm{m}^{2} / 3$ hours.

\subsection{Study case results}

In cases where the fire event takes two or more days of burning activity, and as long as the clouds conditions and smoke presence allow constant monitoring, the mapping activity can theoretically detect the evolution of the event on a daily basis. When these conditions are present, the emissions module estimates the amount of emissions for each day of the mapping.

As an example of the emissions and dispersion modules application, in this paper we report the test case of the fire that took place in Zamora province (Spain) during the day 29/07/2017 and concluded on 30/07/2017 (Figure 2). This study case is representative of a large wildland fire in Europe. Although the number of large wildfires represent a small proportion of the total fire events in Europe, their impact is disproportionally high. Large wildland fires are also the most endangering and impactful for the population. In the Fire Database of EFFIS (a repository of over 2 million individual fire records from 24 countries in Europe and North Africa), the wildfires larger than 1000 ha represent only $0.1 \%$ of the recorded fire events, but are responsible for $30 \%$ of the total burnt area. Only less than $0.02 \%$ of the wildfires spread over 3000 ha: however, this small percentage contributes $16 \%$ of the total damaged area in Europe (Camia et al., 2014; de Rigo et al., in prep.).

The wildfire in Zamora spread over a total burnt area of 3087 ha (EFFIS source). In this case, it was not possible to follow the evolution of the fire during the development of the event due to smoke plume coverage on the area, which impeded the constant monitoring during the days of activity, and the speed of the fire spread and consumption.

Table 2 reports the output of the new fire emissions model, along with those provided by the static model currently used by EFFIS for the emissions assessment. For the variables $\mathrm{SO}_{2}, \mathrm{NH}_{3}, \mathrm{BaP}, \mathrm{LVGC}$ there is no information provided by the current model and no comparison was therefore possible.

Table 2 - Emissions estimations for the test case of wildfire in Zamora province (Spain).

\begin{tabular}{|c|c|c|c|c|c|c|c|c|c|c|c|c|c|c|}
\hline Variable & $\mathbf{C O}_{2}$ & CO & $\mathrm{CH}_{4}$ & $\mathbf{P M}_{2.5}$ & $\mathbf{P M}_{10}$ & NMHC & VOC & $\mathbf{N O}_{\mathbf{x}}$ & OC & $\mathbf{B C}$ & $\mathbf{S O}_{2}$ & $\mathbf{N H}_{3}$ & $\mathrm{BaP}$ & LVGC \\
\hline $\begin{array}{l}\text { New emission } \\
\text { model }\left(\mathrm{kg} \cdot 10^{3}\right)\end{array}$ & 69180 & 3450 & 139 & 335 & 374 & 387 & 263 & 233 & 228 & 37 & 49 & 25 & 1 & 15 \\
\hline $\begin{array}{l}\text { Static model } \\
\left(\mathrm{kg} \cdot 10^{3}\right)\end{array}$ & 54875 & 2466 & 126 & 238 & 281 & 102 & 123 & 172 & 140 & 16 & - & - & - & - \\
\hline $\begin{array}{l}\text { Variation } \\
\text { Static } \rightarrow \text { New }(\%)\end{array}$ & 26 & 40 & 10 & 41 & 33 & 279 & 114 & 36 & 63 & 128 & - & - & - & - \\
\hline
\end{tabular}

In the described study case, the new emissions model provides values of pollutant emissions greater than those estimated by the current one. The most remarkable increases are those recorded on NMHC, VOC and BC, for which the estimations result higher than $100 \%$ compared to the ones of the current model. The consistency of the increase may be due to the contribution brought by the smouldering fire, which is taken into account with the new model. 


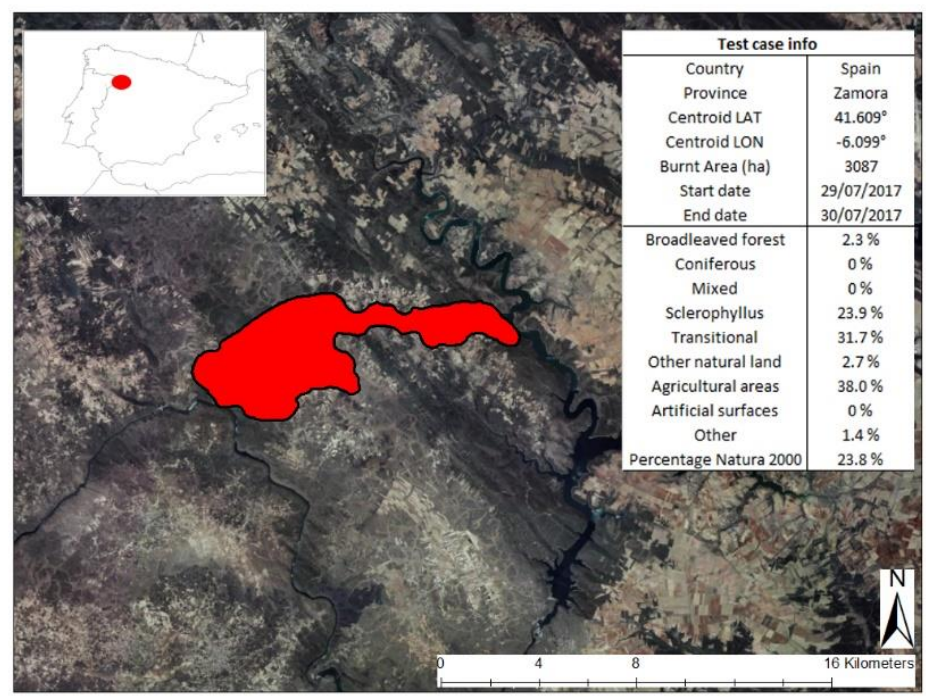

Figure 2 - Localization, shape and ancillary information on the test case in Zamora (Spain).

\section{Dispersion module}

\subsection{Module description}

The smoke plume dispersion module is a new feature developed along and integrated with the new emissions module. It consists of a framework of integrated models that take as input the emissions generated by the new emissions module, and simulates over time in 3D grid format their dispersion plumes in the atmosphere. The processing is applied only on selected severe wildland fire events, for which the smoke plume modelling or post-event analysis is of particular interest. The four main modules of the framework are:

- WRF: Weather Research and Forecasting model;

- CHIMERE: Chemistry-transport model;

- SWIFT: Stationary Wind Field and Turbulence;

- SCICHEM: Second-order Closure Integrated puff model with CHEMistry.

The WRF model (Michalakes et al., 2005) is assigned to the computation of the meteorological background data at regional scale (European continent), and SWIFT to the local focus on a specific selected study area. The concentration of the pollutants is then assessed by CHIMERE (Bessagnet $e t$ al., 2004) (for the regional pollutant background assessment), while the evolution of the plume at local scale by SCICHEM (Chowdhury et al., 2015) (Figure 3). The final output of the dispersion module is a series of Network Common Data Form (NetCDF) files (one for each of the pollutant emissions generated by the emissions module) that simulate the 3D spatial and temporal development of the smoke plume with a time step of 3 hours. 


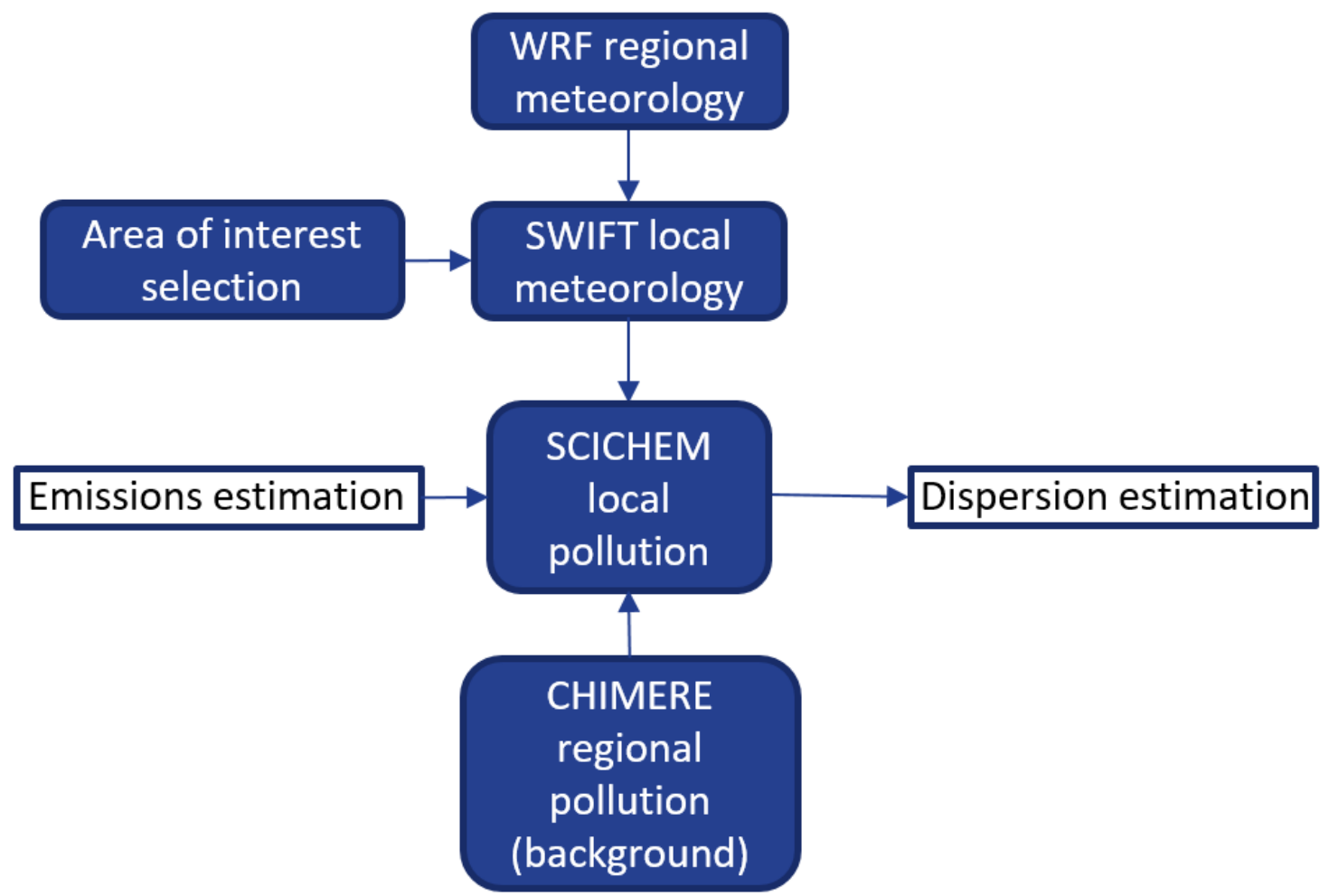

Figure 3 - Dispersion module workflow

\subsection{Study case results}

The Zamora (Spain) fire event was taken into account for the representation of the dispersion of pollutants on an area of approximately $70 \times 70 \mathrm{~km}^{2}$ surrounding the centroid of the burnt area. The simulation was performed setting a grid sampling resolution to $1 \mathrm{~km}$ in $\mathrm{X}$ and $\mathrm{Y}$ directions, and $0.5 \mathrm{~km}$ in $\mathrm{Z}$ from the ground level. Figure 4 shows the spatial representation of the concentration of the $\mathrm{CO}$ emissions produced by the fire in Zamora, values of which were estimated and reported in Table 2. The maps shown are centered in the centroid of the burnt area, and the plots show the evolution of the smoke plume dispersions during the 24 hours of the day 29/07. The CO dispersions in Figure 4 are related to the ground level, which is the one of greatest interest due to the impacts of the pollution on the population.

The following Figure 5 represents the progression of $\mathrm{CO}$ concentrations in time steps (top to bottom in columns) and height (left to right in the rows; ground level, 500 and $1000 \mathrm{~m}$ ). 


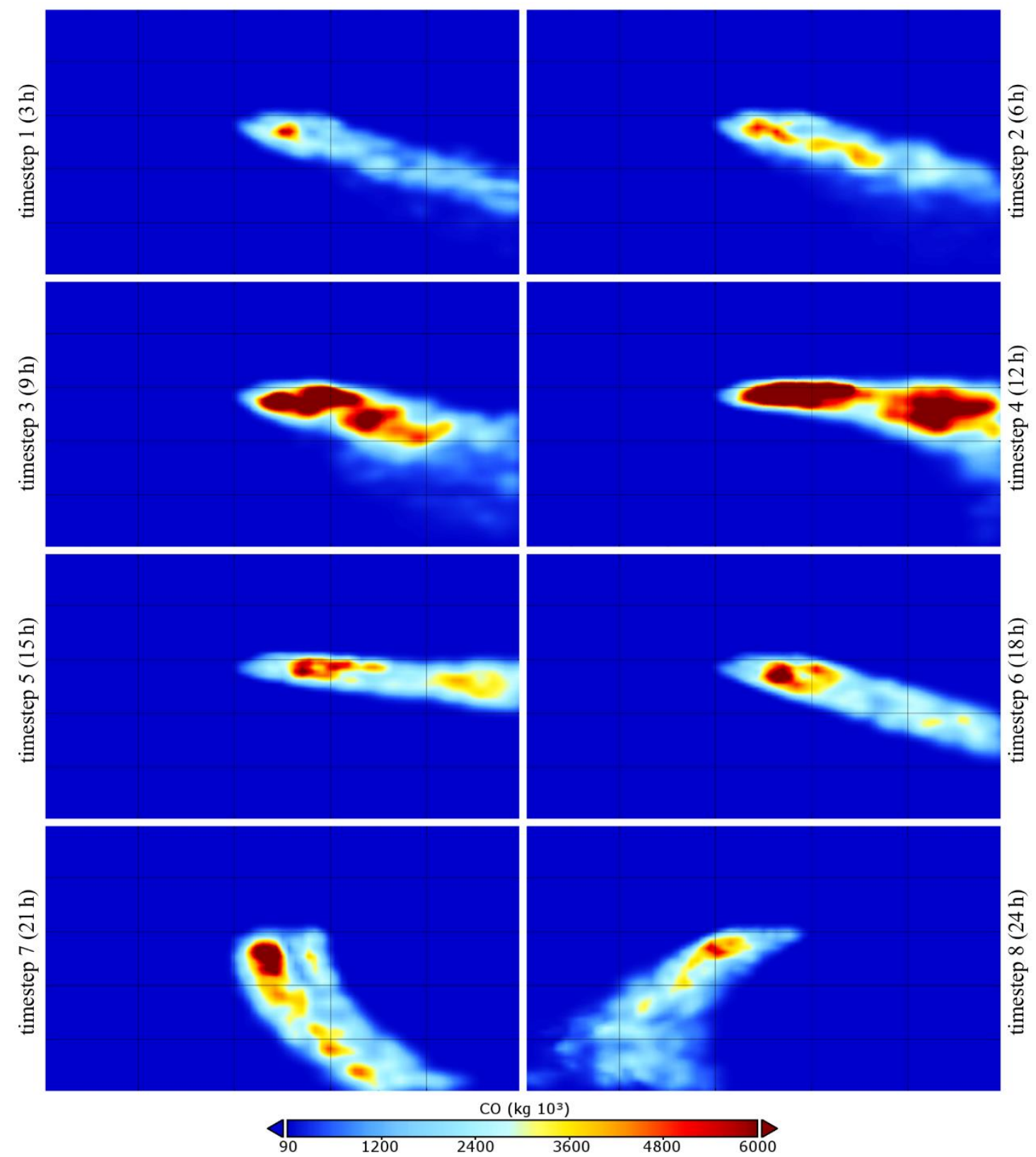

Figure 4 - Progression of the concentration of $\mathrm{CO}$ at ground level produced by the wildfire in Zamora (2930/07/2017). 


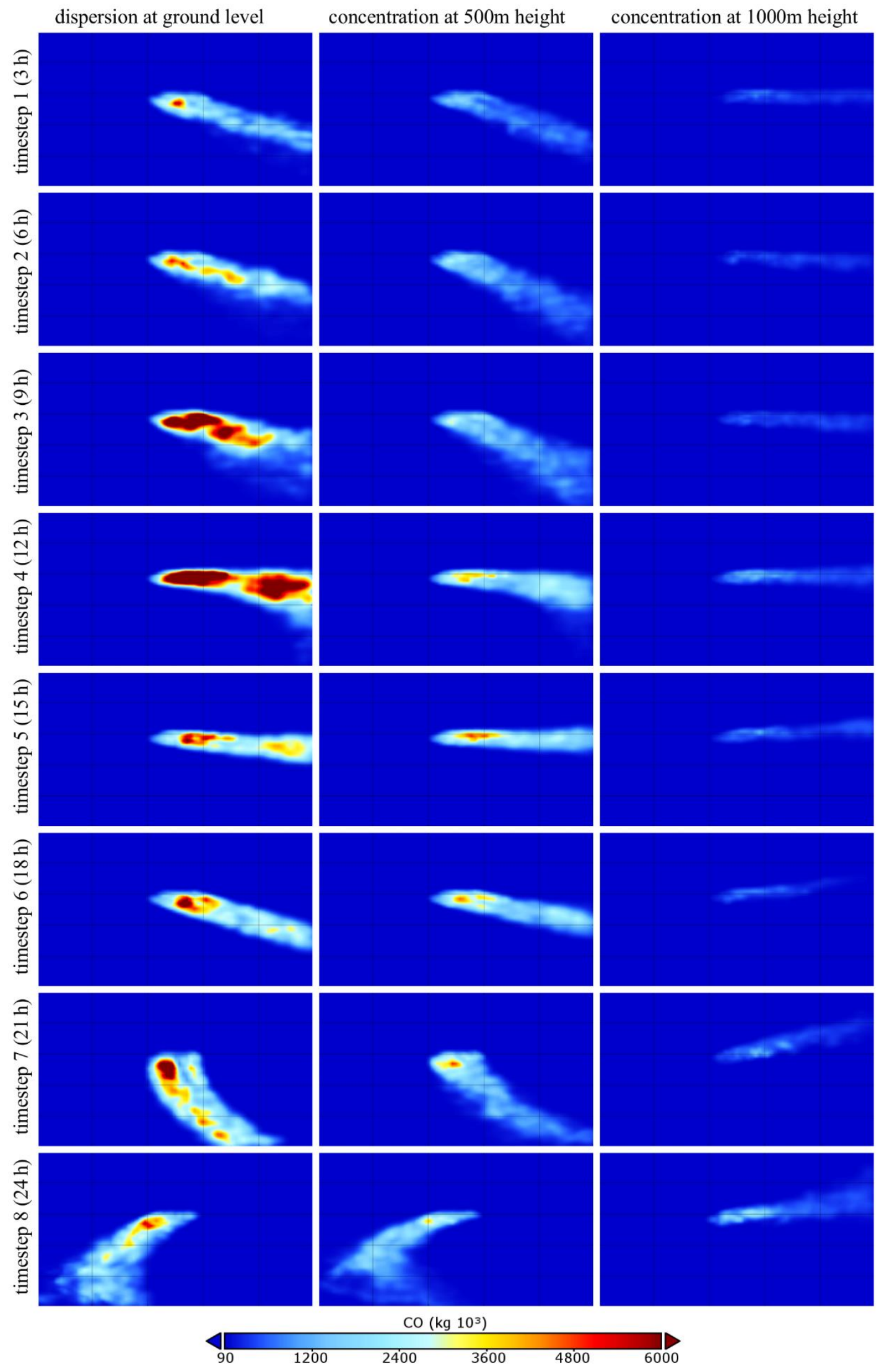

Figure 5 - Dynamics of $\mathrm{CO}$ concentrations in timesteps (top to bottom in columns) and height (left to right in the rows) simulated for the Zamora fire episode (29-30/07/2017).

The last step of the processing is the conversion of the resulting concentrations in GeoJSON format isolines for easier visualization on webmaps.

Advances in Forest Fire Research 2018 - Page 1050 


\section{Conclusions and way forward}

The integration of the new fire emissions and dispersion modules in EFFIS will provide a unique tool for the assessment of the air pollution caused by forest fires in Europe. The resulting information at regional and local scale will be extremely valuable for integrated-assessment applications both during the fire season and in post-event analysis (Lentile et al., 2006; de Rigo et al., 2013; Di Leo et $a l ., 2013$ ). On the study case fire event of Zamora (Spain), the comparison of the new with the current emissions model used in EFFIS shows a consistent increase in the estimation of the concentration for each one of the pollutants, with three peaks in NMHC, VOC and BC. The application of the dispersion model on the outputs of the emissions model will also provide a 3D assessment of the evolution of the smoke plume trajectory. The spatial distribution of the concentrations at ground level represents valuable information on the impact of the smoke plume pollutant on the population resident in the surrounding area. The next steps of the project will consist of the comparison with field data on various test cases and the Sentinel-5P atmospheric pollution estimations. This will be the basis for a biasadjustment module to be added, so as for the field information to improve the physically based estimations of the fire emission and dispersion modules in EFFIS. At last, the dissemination of the results for each fire season in the EFFIS web application will be available.

\section{References}

Barbosa P, Camia A, Kucera J, Libertà G, Palumbo I, San-Miguel-Ayanz J, Schmuck G (2008). Assessment of forest fire impacts and emissions in the European Union based on the European Forest Fire Information System. Vol. 8 of Developments in Environmental Science. Elsevier, pp. 197-208.

Bessagnet B, Hodzic A, Vautard R, Beekmann M, Cheinet S, Honoré C, Liousse C, Rouil L (2004). Aerosol modelling with CHIMERE: preliminary evaluation at the continental scale. Atmospheric Environment 38, 2803-2817.

Camia A, Houston Durrant T, San-Miguel-Ayanz J (2014). The European Fire Database: technical specifications and data submission. Publications Office of the European Union, Luxembourg.

Carvalho A, Monteiro A, Flannigan M, Solman S, Miranda AI, Borrego C (2011). Forest fires in a changing climate and their impacts on air quality. Atmospheric Environment 45 (31), 5545-5553.

Chowdhury B, Karamchandani PK, Sykes RI, Henn DS, Knipping E (2015). Reactive puff model SCICHEM: Model enhancements and performance studies. Atmospheric Environment 117, 242258.

Cornwall W (2018). Scientists aim to smoke out wildfire impacts. Science 360 (6392), 948-949.

de Rigo D, Costa H, Libertà G, Houston Durrant T, San-Miguel-Ayanz J (2018). European wildfire danger and damage in a changing climate: towards integrating risk dimensions. Publication Office of the European Union, Luxembourg, (in preparation).

de Rigo D, Rodriguez-Aseretto D, Bosco C, Di Leo M, San-Miguel-Ayanz J (2013). An architecture for adaptive robust modelling of wildfire behaviour under deep uncertainty. IFIP Advances in Information and Communication Technology 413, 367-380.

Di Leo M, de Rigo D, Rodriguez-Aseretto D, Bosco C, Petroliagkis T, Camia A, San-Miguel-Ayanz J (2013). Dynamic data driven ensemble for wildfire behaviour assessment: a case study. IFIP Advances in Information and Communication Technology 413, 11-22.

Finlay SE, Moffat A, Gazzard R, Baker D, Murray V (2012). Health impacts of wildfires. PLoS Currents Disasters, 1881+. 
Ignotti E, Hacon SS, Longo K, Freitas S, Mourao DS, Junger WL, Artaxo P (2010). Air Pollution and Hospital Admissions for Respiratory Diseases in the Subequatorial Amazon: A Time Series Approach. Cadernos de Saúde Pública (ENSP. Impresso), 26, 747-761.

Keane, RE, Reinhardt, ED, \& Brown, JK (1995). FOFEM: a first order fire effects model. General technical report INT (USA).

Lentile LB, Holden ZA, Smith AMS, Falkowski MJ, Hudak AT, Morgan P, Lewis SA, Gessler PE, Benson NC (2006). Remote sensing techniques to assess active fire characteristics and post-fire effects. International Journal of Wildland Fire 15 (3), 319+.

Michalakes J, Dudhia J, Gill D, Henderson T, Klemp J, Skamarock W, Wang W (2005). The Weather Research and Forecasting Model: software architecture and performance. Proceedings of the Eleventh ECMWF Workshop on the Use of High Performance Computing in Meteorology, 156168.

Miranda AI, Ferreira J, Valente J, Santos P, Amorim JH, Borrego C (2005). Smoke measurements during Gestosa 2002 experimental field fires. International Journal of Wildland Fire 14, 107-116.

Monteiro A., Corti P., San-Miguel-Ayanz J., Miranda A. I., Borrego C. (2014). The EFFIS forest fire atmospheric emission model: application to a major fire event in Portugal. Atmospheric Environment 84, 355-362.

Ottmar, RD, Burns, MF, Hall, JN, Hanson, AD (1993). CONSUME: users guide. Gen. Tech. Rep. PNW-GTR-304. Portland, OR: U.S. Department of Agriculture, Forest Service, Pacific Northwest Research Station.

Prichard SJ, Ottmar RD, Anderson GK (2005). Consume 3.0 User's Guide, United States Department of Agriculture Forest Service, Pacific Northwest Research Station, Seattle. WA.

Prichard, SJ, Karau, EC, Ottmar, RD, Kennedy, MC, Cronan, JB, Wright, CS, \& Keane, RE (2014). Evaluation of the CONSUME and FOFEM fuel consumption models in pine and mixed hardwood forests of the eastern United States. Canadian journal of forest research, 44(7), 784-795.

Reinhardt, ED, \& Dickinson, MB (2010). First-order fire effects models for land management: overview and issues. Fire Ecology. 6(1), 131-142.

San Miguel-Ayanz J, Schulte E, Schmuck G, Camia A, Strobl P, Liberta G, Giovando C, Boca R, Sedano F, Kempeneers P, McInerney D, Withmore C, Santos de Oliveira S, Rodrigues M, Durrant T, Corti P, Oehler F, Vilar L, Amatulli G (2011). Comprehensive monitoring of wildfires in Europe: The European Forest Fire Information System (EFFIS), in Tiefenbacher, J. (Ed.) Disaster Management, ISBN 978-953-307-651-5.

San-Miguel-Ayanz J, Schulte E, Schmuck G, Camia A (2013). The European Forest Fire Information System in the context of environmental policies of the European Union. Forest Policy and Economics 29, 19-25.

Scott, JH (1999). NEXUS: a system for assessing crown fire hazard. Fire Management Notes.

Scott JH, Reinhardt ED (2001). Assessing crown fire potential by linking models of surface and crown fire behavior. Res. Pap. RMRS-RP-29. Fort Collins, CO: Introduction to Wildfire Behavior Modeling Appendix A 143 U.S. Department of Agriculture, Forest Service, Rocky Mountain Research Station.

Stefanidou M, Athanaselis S, Spiliopoulou C (2008). Health impacts of fire smoke inhalation. Inhalation Toxicology 20 (8), 761-766.

Wegesser TC, Pinkerton KE, Last JA (2009). California Wildfires of 2008: Coarse and Fine Particulate Matter Toxicity. Environmental health perspectives 117 (6), 893-897. 


\title{
Mapping fire severity levels of burned areas in Galicia (NW Spain) by Landsat images and the dNBR index: Preliminary results about the influence of topographical, meteorological and fuel factors on the highest severity level
}

\author{
Stéfano Arellano-Pérez ${ }^{1 *}$; Ana Daría Ruiz-González ; Juan Gabriel Álvarez-González ${ }^{1}$; Jose \\ Antonio Vega-Hidalgo ${ }^{2}$; Ramón Díaz-Varela ${ }^{1}$; Cecilia Alonso-Rego ${ }^{1}$ \\ ${ }^{1}$ Department of Agricultural and Forestry Engineering. University of Santiago de Compostela. \\ Campus universitario s/n 27002,Lugo,Spain.\{stefano.arellano@usc.es*,anadaria.ruiz@usc.es, \\ juangabriel.alvarez@usc.es,ramon.diaz@usc.es,c.alonso.rego@usc.es\} \\ ${ }^{2}$ Centro de Investigación Forestal de Lourizán, PO Box 127, 36080, Pontevedra, Spain. \\ \{jose.antonio.vega.hidalgo@xunta.gal\}
}

\begin{abstract}
Fire severity assessment and mapping are essential for prioritizing post-fire emergency rehabilitation actions. The present study, carried out in Galicia (one of the regions most severely impacted by wildfire in the Iberian Peninsula), focused on 36 wildfires affecting areas of more than 200 ha during the period 20062016. The aims of the study were i) to map the severity in these wildfires by using the differenced Normalized Burn Ratio (dNBR) index, and ii) to explore how the highest levels of fire severity and canopy damage are related to topographical, meteorological and forest fuel variables. Pre and post-fire Landsat images (Path 204 and Row 31), provided by the European Space Agency (ESA), were radiometrically and topographically corrected. The dNBR index was calculated for each wildfire, and the values obtained were used to establish four classes of fire severity and four levels of canopy damage. A total of 23 topographical, meteorological and fuel variables were also determined in order to characterize each wildfire. The non-parametric Random Forests method was used to relate the burned area (\%) corresponding to the highest levels of fire severity and crown fire damage to the various environmental variables. The variables that best differentiated the most severely burned areas were in both cases wind speed and percentage of burned area with slope ranging from 30 to $45 \%$.
\end{abstract}

Keywords: Fire severity, Landsat, dNBR, fire mapping, crown damage, EGIF

\section{Introduction}

The total number of wildfires, number of large wildfires (>500 ha) and the total area burned are higher in Galicia than in other regions of Spain, representing respectively $42 \%, 24 \%$ and $25 \%$ of the overall values for the whole of the country (MAPAMA 2012). Moreover, current forecasts for the fire regime indicate that the wildfire problem will be exacerbated by climate change (Moreno 2005, Arellano 2008, Vega et al. 2009), with increased frequency, extent, intensity and severity of wildfires. Fire severity has been broadly defined as the level of damage or disturbance caused by fire in an ecosystem or any of its components (Key and Benson 2006).

Fire severity assessment and mapping are essential tasks in post-fire emergency rehabilitation planning. These processes enable prioritization of the responses by focusing on the areas most affected by fire with the aim of minimizing the impacts and shortening the recovery time of the ecosystems affected (Robichaud et al. 2000, Miller and Yool 2002, Chuvieco et al. 2006).

Satellite spectral indices are useful for obtaining information on fire severity at landscape scale (Hudak et al. 2007; Parsons et al. 2010). These indexes also enable analysis of spatial patterns of 
different levels of damage caused by fire and identification of the most important factors influencing these patterns. Advantages of the use of remote sensing indices include the ability to assess fire severity in inaccessible sites or sites where field work is very expensive, because of the topographical conditions or the presence of very dense vegetation. However, the indexes can be limited by cloudy conditions, low spatial resolution and their poor representation of lower strata of the tree canopy and the soil (Hudak et al. 2007, Meng and Meentemeyer 2011). The differenced Normalized Burn Ratio or dNBR (Key and Benson 2006), obtained from Landsat images, is the index most widely used in many countries to estimate fire severity. Specifically, in the region of Galicia, fire severity evaluation and annual mapping of the most important fires is carried out using the dNBR index. This task is the first step in designing emergency actions aimed at reducing the hydrological and erosive risks associated with wildfire (Vega et al. 2013) and that are included in the regional Plan for Preventing and Fighting Forest fires (PLADIGA; Xunta de Galicia, 2017).

In a study relating values of the dNBR index to the fire severity and the levels of canopy damage observed in wildfires that occurred in Galicia in the 2013 fire season, Arellano (2014) proposed four specific classes of fire severity and four classes of canopy damage for the region. The present study used these classifications to characterize the wildfires that occurred during the period 2006-2016 in one of the areas most affected by wildfire in Galicia. The aims of the study were i) to map the levels of fire severity associated with the wildfires by using the dNBR index; and ii) to explore the most important variables (topographical, meteorological and forest fuels) influencing the highest levels of fire severity and canopy damage.

\section{Material and methods}

The study area is the XIV Forest District (Verín-Viana), a mountain region covering an area of 175,296 hectares in the south of the province of Ourense (Galicia, NW Spain). The region borders to the north and west with respectively the XIII Forest District (Valdeorras - Trives) and the XV Forest District (A Limia), to the east with the region of Castilla-León (Spain) and to the south with the region of Tras-Os-Montes Alto Douro (Portugal).

The study considered wildfires affecting an area of more than 200 hectares that occurred during the 11-year period between 2006 and 2016 (a total of 36 wildfires). The burned areas were selected from the database of the Spanish General Statistics on Forest Fires (EGIF), provided by the Ministry of Agriculture and Fisheries, Food and Environment (MAPAMA).

The perimeters and severity classes were mapped using a collection of pre and post-fire Landsat images (Path 204 and Row 31) that covered the entire area and study period. Some of the Landsat 5 TM, 7 ETM and 8 OLI images were downloaded from the European Space Agency website (ESA, https://earth.esa.int/web/guest/eoli), while others were provided by the Galician Institute of Territorial studies (IET). Scenes were radiometrically corrected by digital levels (ND), according to Chander et al. (2009), into top of atmosphere (TOA) reflectance. The topographic correction was also applied according to the Minnaert Correction with slope method (Riaño et al. 2003).

The fire severity class was assigned for each pixel by calculating the dNBR index (Key and Benson 2006) and applying the classification thresholds previously established by Arellano (2014). These thresholds are based on the existing relationship between the Composite Burning Index (CBI, Key and Benson 2006) and the dNBR for several wildfires that occurred in Galicia in 2013: unburned $(\mathrm{dNBR}<12.3)$; low $(12.3 \leq \mathrm{dNBR}<262.4)$; moderate $(262.4 \leq \mathrm{dNBR}<577.2)$ and high $(577.2 \leq \mathrm{dNBR})$. Likewise, a level of canopy damage was assigned to each tree-covered pixel according to Arellano (2014) and Arellano et al. (2017). The four levels of canopy damage considered are shown in Table 1.

Advances in Forest Fire Research 2018 - Page 1054 
Table 1 - Description of canopy damage levels and dNBR thresholds

\begin{tabular}{llc}
\hline $\begin{array}{l}\text { Level of canopy } \\
\text { damage }\end{array}$ & Description & dNBR \\
\hline Green & $\begin{array}{l}\text { Area covered by trees in which the crown remained } \\
\text { green after fire }>50 \%\end{array}$ & 282.9 \\
Green-scorched & $\begin{array}{l}\text { Area covered by trees with scorched crown after fire: } \\
50-90 \% \text {. The rest of the area, up to 100\%, occupied } \\
\text { by trees in which the crown remained green. }\end{array}$ & \\
& $\begin{array}{l}\text { Area covered by trees with scorched crown after fire: } \\
50-100 \% \text {. The rest of the area, up to 100\%, occupied }\end{array}$ & $443.2-626.6$ \\
Scorched-consumed & $\begin{array}{l}\text { by trees with the crown consumed. } \\
\text { Area covered by trees with the crown consumed }\end{array}$ & $\geq 626.6$ \\
\hline Consumed & $>50 \%$ & \\
\hline
\end{tabular}

The values of the dNBR index statistics (mean, standard deviation, minimum, median and maximum) were calculated for each wildfire. The proportion of burned area was also calculated for each fire severity class and each level of canopy damage in each wildfire.

Topographical, meteorological and fuel-related data were compiled for each wildfire. The digital elevation model (DEM), with a spatial resolution of $25 \mathrm{~m}$, was downloaded from the Spanish Geographic Institute (IGN) website (http://www.ign.es/web/ign/portal) and used to generate slope and aspect cartography for each burned area. Five levels of slope (0-10\%, 10-20\%, 20-30\%, 30-45\%, $>45 \%)$ and the four cardinal directions (N, S, E and W) were considered. Descriptive statistics on altitude and slope (mean, maximum, minimum, median and standard deviation) were computed. Meteorological databases were downloaded from the Meteogalicia website (http://www.meteogalicia.gal) and used to determine the following variables at the start of each wildfire: temperature $\left({ }^{\circ} \mathrm{C}\right)$, relative humidity $(\%)$, wind speed $(\mathrm{km} / \mathrm{h})$, wind direction $\left({ }^{\circ}\right)$, wind gust speed $(\mathrm{km} / \mathrm{h})$ and wind gust direction $\left(^{\circ}\right)$. Data from seven different meteorological stations in the Xunta de Galicia network that are close to the burned areas were used. Finally, the Spanish Forest Map (MARM 2011) was used to classify the burned areas into four different categories according to the fuel type (tree-covered land, scrubland, agricultural land and riparian forest land) and to estimate the proportion of each class in each area affected by wildfire.

A total of 23 variables were compiled for each wildfire. The non-parametric Random Forests method was then used to relate the proportion of the total burned area affected by the high fire severity class and the proportion of the total burned area affected by the most severe level of canopy damage, with the respective topographical, meteorological and fuel characteristics.

The study only considered the highest levels of both fire severity and canopy damage as these are the variables of most interest in relation to making decisions about the areas where recovery activities should be carried out.

The randomForest package developed for R (R Core Team 2013) by Liaw and Wiener (2012) was used to fit 500 regression trees for each variable analysed. For fitting each regression tree, 2/3 of the total fires were selected at random and 8 of the total descriptor variables were selected at random.

Finally, the relative importance of each descriptive variable was estimated by normalizing the values of the Increment of Node Purity statistic to a maximum value of 100 . The importance of a variable in the classification process increases with the value of this statistic.

\section{Results and discussion}

The locations and perimeters of the wildfires are shown in Figure 1. 
The average values of elevation, slope, temperature, relative humidity, wind and gust speed of the 36 wildfires were respectively $957 \mathrm{~m}, 22 \%, 23{ }^{\circ} \mathrm{C}, 34 \%, 13 \mathrm{~km} / \mathrm{h}$ and $24 \mathrm{~km} / \mathrm{h}$. The combination of the most frequent wind direction (SE) with the predominant orientation (E) promoted wildfire spread in the study area. In general, the percentage of burned shrub area exceeded that of tree-covered burned area. Considering all of the wildfires, the average percentage values of the different dNBR severity classes were as follows: unburned $=3 \%$; low $=12 \%$; moderate $=39 \%$ and high $=46 \%$. For the levels of canopy damage, the average percentages were as follows: green $=25 \%$, green-scorched $=17 \%$, scorched-consumed $=21 \%$ and consumed $=37 \%$. These values indicate the high level of impact of the wildfires in the study area.

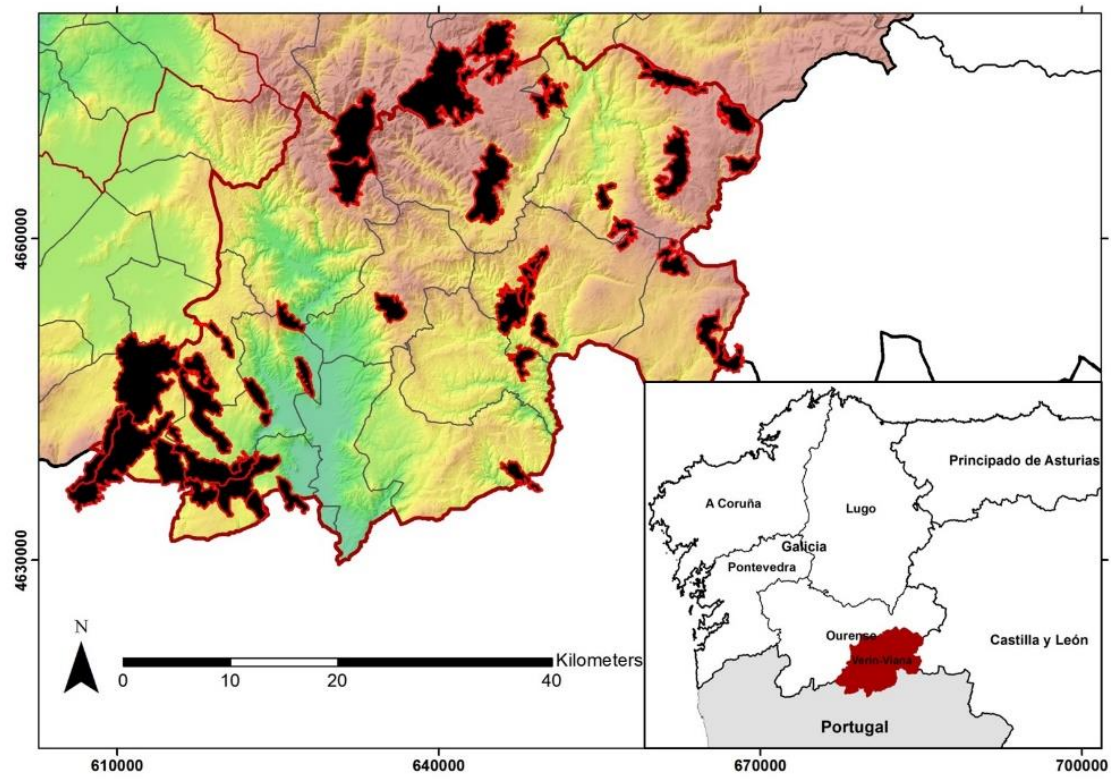

Figure 1 - Location and perimeter of the wildfires studied

The spatial distribution of dNBR severity classes in two of the wildfires (by way of example) is shown below (Figure 2).
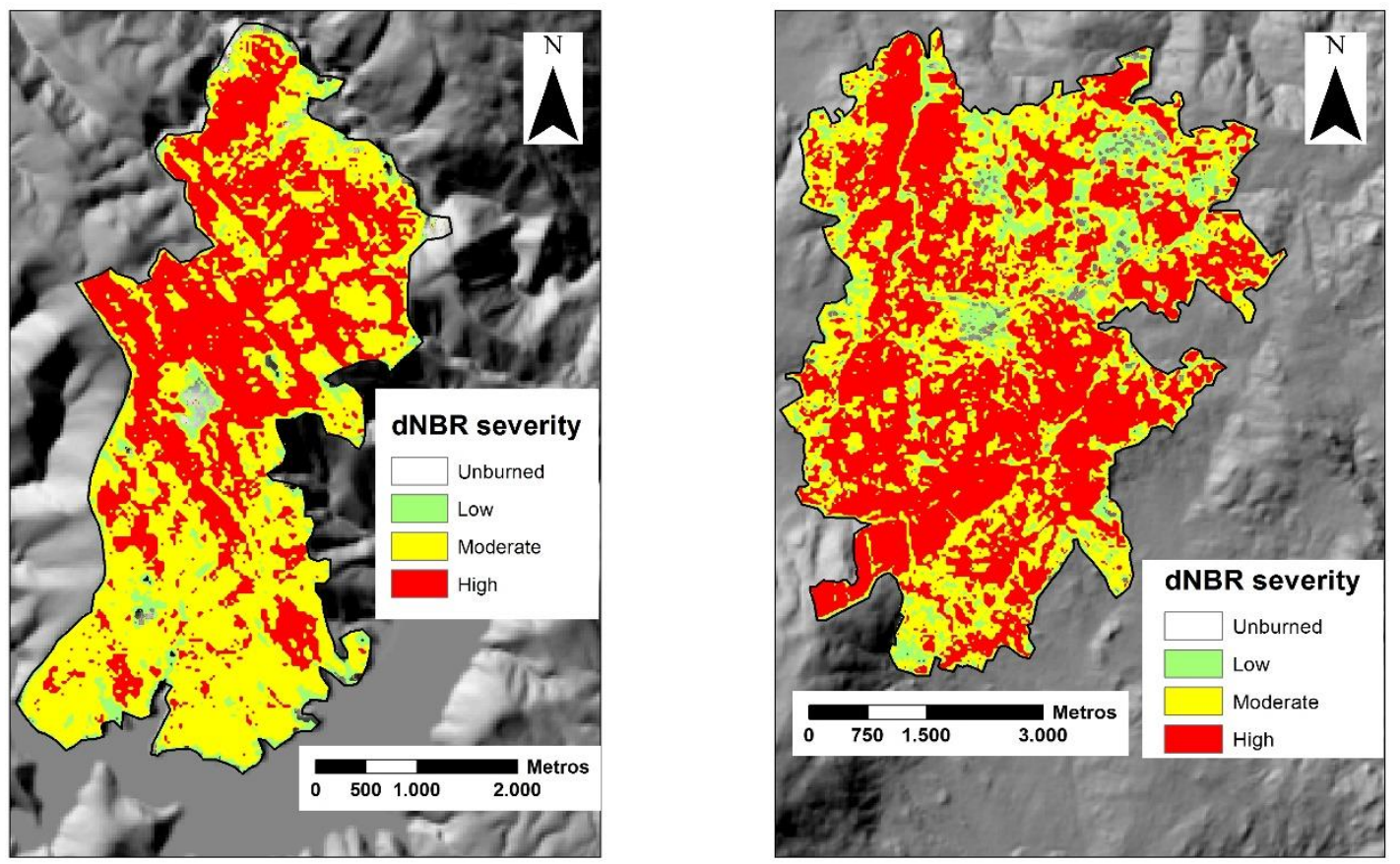

Figure 2 - dNBR Fire severity map: San Mamede de Hedrada Fire 2011 (left) and Lucenza Fire 2015 (right) 
Box plots were constructed of the distribution of the mean values of dNBR obtained for each fire, grouped by year (Figure 3). The mean values exceeded the threshold of dNBR of 577.2, corresponding to the highest level of fire severity, in only three years (2008, 2015 and 2016). Interestingly, the average dNBR values were highest in shrubby habitats, followed by tree-covered land and were lowest in riparian forest and agricultural land.

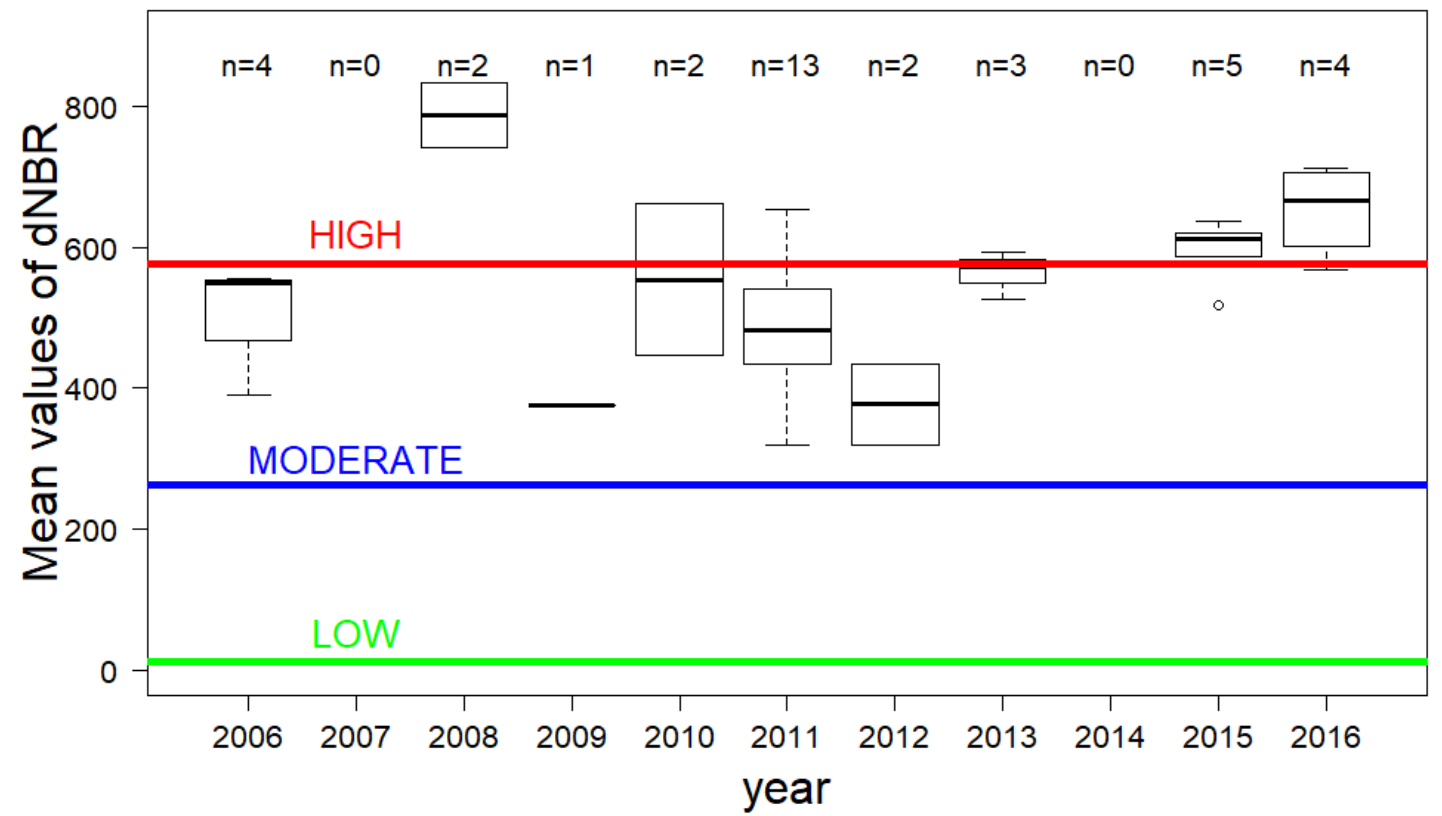

Figure 3 - Box plots of the distribution of averages values of $\mathrm{dNBR}$ throughout the study period

The relative importance of the main features explaining the percentage of burned area classified as high fire severity class and the classified with the highest level of canopy damage ("crown consumed", equivalent to crown fire) are shown in Figures 4 and 5. The two features that best differentiated the areas affected by high fire severity class and the level of crown fire were the wind speed and the burned area percentage with slope, ranging from 30 to $45 \%$, coinciding for both random forests.

These results are consistent with those of Fernández Alonso et al. (2017), who reported that wind speed was also the most important variable explaining the spatial pattern of crown fire in several wildfires in Galicia. Other studies (Lentile et al. 2006, Holden et al. 2009, Lecina-Diaz et al. 2014) have also demonstrated the relationship between slope and -extreme fire severity. Overall, the results confirm the well-known overwhelming importance of wind and slope angle in wildland fire behaviour (Rothermel 1983) and particularly in high intensity fires (Werth et al. 2016). Other variables that proved important in explaining high levels of fire severity were the southern orientation and the wind direction, underlying the probable influence of lower dead fuel moisture content and the alignment of wind and slope as factors contributing to strengthening the intensity of fire. Finally, the maximum slope and the temperature at at the start of the wildfire were also important for predicting the crown fire. The latter is probably related to dead fuel moisture. 


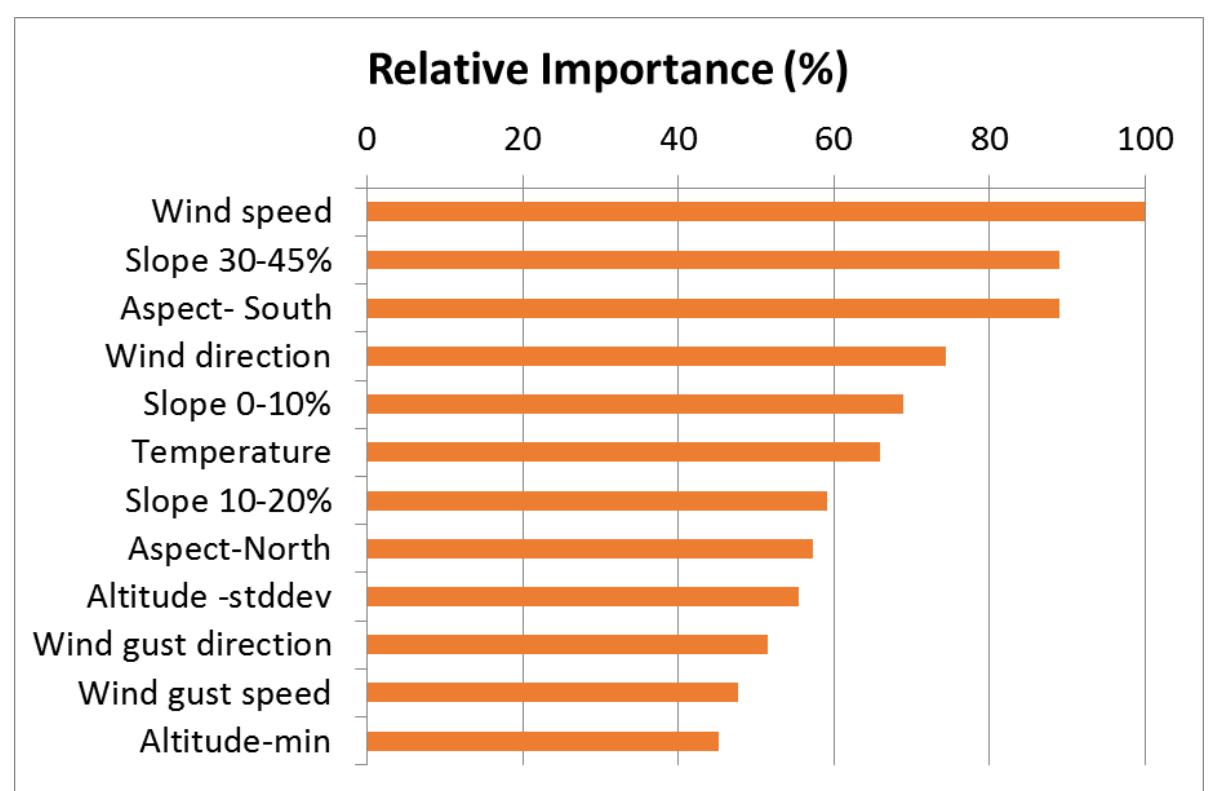

Figure 4 - Relative importance of each variable as determined by the Random Forests method used to explain the proportion of the highest level of fire severity

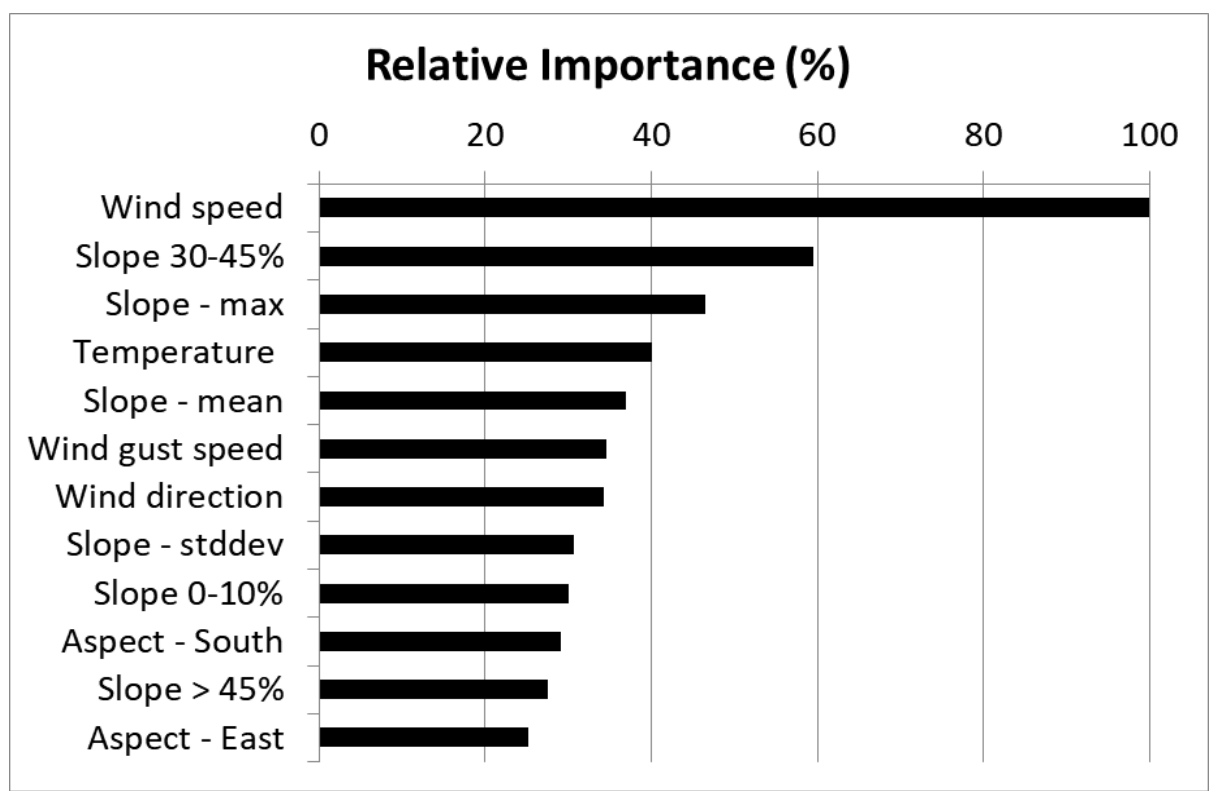

Figure 5 - Relative importance of each variable as determined by the Random Forests method used to explain the proportions of crown consumed

\section{Conclusions}

Mapping burned areas and fire severity in all wildfires of more than 200 hectares that occurred in the Verín-Viana Forest District over a period of 11 years is a fundamental starting point for new studies such as the analysis of the fire recurrence in the area, the location of strategic management areas and characterization of wildfires according to the main variables involved in their spread.

The dNBR index is capable of discriminating different levels of canopy damage caused by fire. These levels can be used as the basis for proposing rehabilitation actions in burned areas and for prioritizing these actions in areas affected by crown fires. However, dNBR severity thresholds must be calibrated for a longer period than used in this study. Identification of levels of fire damage in scrubland, in addition to wooded areas, would also be of interest. 
The meteorological information used could also be improved. Including average variables, at least during the first hours of wildfire development, instead of specific information on the start time, would be useful. Use of the WindNinja (Forthofer 2007) software, which simulates the spatial distribution of winds by taking into account the physiography would enable studies to be carried out at much more detailed (pixel) level.

Future studies should also aim to characterize the fuel more comprehensively. The use of historical orthophotos and LIDAR data, when possible, would enable estimation of fuel related variables that may be important for predicting fire severity.

\section{Acknowledgements}

This study was funded by the Spanish Ministry of Economy, Industry and Competitiveness through the GEPRIF research project (RTA2014-00011-C06-04). We are also grateful to ADCIF (MAPAMA) for providing the EGIF database, METEOGALICIA for providing free weather data on its website and IET for providing Landsat imagery.

\section{References}

Arellano S (2008) Índices meteorológicos de peligro de incendios forestales en Galicia: Evidencias de cambio climático y su relación con la frecuencia de fuegos y superficie afectada- Proyecto fin de carrera. Escuela Técnica de Ingeniería Forestal. Universidad de Vigo. Pp 409.

Arellano S (2014) Comparación de la capacidad de los índices dNBR y RdNBR para evaluar la severidad del fuego en incendios forestales de Galicia. Trabajo Fin de Máster. Master Fuego. Universidades de Lérida, Córdoba y León.

Arellano S, Vega JA, Rodríguez y Silva F, Fernández C, Vega-Nieva D, Álvarez JG, Ruiz AD (2017) Validación de los índices de teledetección dNBR y RdNBR para determinar la severidad del fuego en el incendio forestal de Oia-O Rosal (Pontevedra) en 2013. Official Journal of the Spanish Association of Remote Sensing. No 49 Special issue: Avances en el análisis de la severidad y la dinámica ambiental post-fuego mediante teledetección.

Chander G, Markham ML, Helder DL (2009) Summary of current radiometric calibration coefficients for Landsat MSS, TM ETM+, and EO-1 ALI sensors. Remote Sensing of Environment 113, 893903.

Chuvieco E, Riaño D, Danson FM, Martín MP (2006) Use of radiative transfer model to simulate the post-fire spectral response to burn severity. Journal of Geophysical Research 111, G04S09.

ESA (2017) European Space Agency. https://www.esa.int/esl/ESA_in_your_country/Spain. Accessed 29 June 2017.

Fernández Alonso JM, Vega JA, Jiménez E, Ruiz González AD, Álvarez González JG (2017) Spatially modeling wildland fire severity in pine forests of Galicia, Spain. Eur J. Forest Res 136,105-121.

Forthofer J (2007) Modeling wind in complex terrain for use in fire spread prediction. Thesis Colorado State University. Fort Collins, Colorado.

Holden ZA, Morgan P, Evans JS (2009) A predictive model of burn severity based on 20-year satelliteinferred burn severity data in a large southwestern US wilderness area. For. Ecol. Manage. 258, 2399-2406.

Hudak AT, Morgan P, Bobbitt MJ, Smith AMS, Lewis SA, Lentile LB (2007) The relationship of multispectral satellite imagery to immediate fire effects. Fire Ecology 3, 64-90.

IGN (2017) Instituto Geográfico Nacional. http://www.ign.es/web/ign/portal. Accessed 3 June 2017. 
Key CH, Benson NC (2006) Landscape assessment: Ground measure of severity the Composite burn index, and remote sensing of severity, the Normalized Burn index. USDA Forest Service. RMRSGTR-164-CD: LA1-51(Ogden, UT).

Lentile LB, Smith FW, Shepperd WD (2006) Influence of topography and forest structure on patterns of mixed severity fire in ponderosa pine forests of the South Dakota Black Hills, USA. Int $J$ Wildland Fire 15, 557-566.

Liaw A, Wiener M (2002) Classification and regression by randomForest. $R$ News 2, 18-22.

Lecina-Diaz J, Alvarez A, Retana J (2014) Extreme Fire Severity Patterns in Topographic, Convective and Wind-Driven Historical Wildfires of Mediterranean Pine Forests. PLOS ONE 9 (1): e85127.

MAPAMA (2012) Los incendios Forestales en España (decenio 2001-2010). Enríquez Alcalde E, del Moral Vargas L Coor. ADIF. Madrid.

MAPAMA (2017) Mapa Forestal Español (MFE25). http://www.mapama.gob.es/es/. Accessed 19 June 2017.

Meng Q, Meentemeyer RK (2011) Modeling of multi-strata forest fire severity using Landsat TM Data. International Journal of Applied Earth Observation and Geoinformation 13, 120-126.

METEOGALICIA (2017). http://www.meteogalicia.gal/web/index.action. Accessed 1 June 2017.

Miller JD, Yool SR (2002) Mapping forest post-fire canopy consumption in several over story types using multi-temporal Landsat TM and ETM data. Remote Sensing of Environment 82, 481-496.

Moreno JM (2005) Riesgos de Origen Climático: Impactos sobre los Incendios Forestales. En: Evaluación Preliminar de los Impactos en España por Efecto del Cambio Climático (581-615). Ministerio de Medio Ambiente.

Parsons A, Robichaud PR, Lewis SA, Napper C, Clark JT (2010): Field Guide for Mapping Post-Fire Soil Burn Severity. USDA Forest Service. Rocky Mountain Research Stattion. General Technical Report RMRS-GTR-243 pp. 56.

Riano D, Chuvieco E, Salas J, Aguado I (2003) Assessment of different topographic corrections in Landsat- TM data for mapping vegetation types. IEEE Transactions on Geoscience and Remote Sensing 41, 5.

Robichaud PR, Beyers JL, Neary DG (2000): Evaluating the effectiveness of postfire rehabilitation treatments. USDA Forest Service. General Technical Report. RMRS-GTR.-63.

Rothermel RC (1983) How to predict the spread and intensity of forest and range fires. USDA For. Serv. Gen. Tech. Rep. INT-143.

Vega JA, Fonturbel T, Fernández C, Arellano A, Díaz-Raviña M, Carballas MT, Martín A, González-Prieto S, Merino A, Benito E (2013) Acciones urgentes contra la erosión en áreas forestales quemadas: Guía para su planificación en Galicia. Santiago de Compostela.

Vega JA, Fernández C, Jiménez E, Ruiz AD (2009) Evidencias de cambio climático en Galicia a través de la tendencia de los índices de peligro de incendios forestales. En: Evidencias e Impactos do Cambio Climático en Galicia (Cap.8, 173-194). XUNTA DE GALICIA, Consellería de Medio Ambiente e Desenvolvemento Sostible, Santiago de Compostela.

Werth PA, Potter BE, Alexander ME, Clements CB, Cruz MG, Finney MA, Forthofer JM, Goodrick SL, Hoffman C, Jolly WM, McAllister SS, Ottmar RD, Parsons RA (2016) Synthesis of knowledge of extreme fire behavior: volume 2 for fire behaviour specialists researchers, and meteorologists. Gen. Tech. Rep. PNW-GTR-891. Portland, OR: USDA Forest Service, Pacific Northwest Research Station. 258 p.

XUNTA DE GALICIA PLADIGA. http://mediorural.xunta.gal/es/areas/forestal/incendios_forestales/pladiga_2017/. Accessed 15 June 2017. 


\title{
Mean atmospheric flow pattern and forest fire risk on the Adriatic coast of Croatia
}

\author{
Izidor Pelajić*; Marija Mokorić; Krunoslav Mikec; Tomislav Kozarić \\ Meteorological and Hydrological Service, Croatia, Zagreb, \{pelajic@cirus.dhz.hr*\}
}

\begin{abstract}
Forest fires are the most common natural disaster during the warm part of the year on the Adriatic coast of Croatia causing extensive damage and sometimes even an ecological catastrophe. It is therefore important to understand all weather and climate conditions leading to an increased risk of forest fire ignition and spread. In this paper the relationship between mean monthly atmospheric flow pattern (monthly weather type) and mean monthly forest fire risk (monthly severity rating) has been investigated. The analysis was done for the Adriatic coastal areas in the period from 1981 to 2015 during the fire season, between May and October. The results show that in the fire seasons of the observed 35-year period the most common monthly weather types were non-gradient field, northwesterly flow and westerly flow $(20 \%, 16 \%$ and $20 \%$ of the cases, respectively). The most frequent monthly severity rating was "high" with $44 \%$ of all cases, followed by "moderate". On the other hand, the highest monthly severity rating ("very high") was present in just 5\% of cases. Dry monthly weather types (ridge and front side of the ridge, $8 \%$ and $7 \%$ of the cases, respectively) showed stronger relationship with higher monthly severity ratings, while wet types (southwesterly flow and trough) appeared very rare in case of higher monthly severity ratings. The aforementioned most common monthly weather types, denoted as mixed types, were associated with "moderate" to "high" monthly severity ratings in less than $30 \%$ of the cases, making those types difficult to connect with monthly severity ratings. The "Consecutive Month Diagrams" (CMDs) concept was introduced in order to inspect the time correlations between monthly weather types and monthly severity ratings. In general, dry weather types supported fast transition to weather conditions favoring higher severity ratings, almost regardless of the previous month conditions, with two exceptions noted. If weather type time series were expressed as "cumulative fractions in total", the trends and ratios between weather types could be easily observed in the 35-year period. Since 1990s differences between fractions of dry and wet weather types have been increasing, suggesting more months with atmospheric flow patterns connected with higher forest fire risk. The results and concepts introduced in this work, as well as further analysis of atmospheric flow patterns and forest fire risk, can help to improve the short-term and especially long-term forecasts of forest fire risk.
\end{abstract}

Keywords Adriatic, forest fire risk, severity rating, weather type

\section{Introduction}

On the eastern Adriatic coast under climate forcing forest fires are most frequent in warm part of the year (Republic of Croatia, 2014). Fire season and related activities are conducted from spring to autumn, usually from 1st of May until the end of October. On the national level Meteorological and Hydrological Service of Croatia (DHMZ) is acting as advisory body in fire suppression system providing specialized meteorological information to the stakeholders, Civil Protection Service and fire departments with focus on meteorological condition favoring fire ignition, rapid fire spread potential and fire severity rate (Republic of Croatia, 2015).

In last decades several high temperature and drought extremes have been recorded in Croatia, leading to higher forest fire risks $(2003,2011,2012,2015$.) and climate analysis revealed mean temperature increase with prominent positive trend since year 2000 (Republic of Croatia, 2014). Climate is average state of the atmosphere comprised of an average from a number of weather conditions (usually 30 years), both near the surface as well as in the upper levels. In extremely dry years, in the warm part of the year, surface weather in Croatia is associated with higher temperatures 
and lower precipitation (Plačko Vršnak et al., 2014).

Transports of heat and moisture are influenced by surface and atmospheric flow in upper levels, especially around $5500 \mathrm{~m}$ that can be analyzed from geopotential height at AT500 $\mathrm{hPa}$ (Absolute Topography of isobaric plane at $500 \mathrm{hPa}$ ). Atmospheric flow at AT500 and surface weather are related. In forest fire assessments (outbreaks potential, spread, severity) daily surface weather realizations and atmospheric circulation plays as important role as the monthly means of upper level atmospheric flows.

\section{Flow pattern and forest fire risk}

Atmospheric flow patterns (weather types) over Southern Europe can be categorized in 8 groups (Poje, 1965). According to AT500 monthly mean fields, upper level atmospheric flow was identified as one of the following: ridge (R); front side of the ridge (R-NW); non-gradient field (NG); northwesterly flow (NWF); trough (T); westerly flow (WF); southwesterly flow (SWF) and upper level low (ULL). Each weather type is associated with prevailing surface conditions (Lončar and Bajić, 1994), monthly means of temperature and precipitation on the Adriatic (Lončar and Vučetić, 2003) and Croatia (Lončar and Bajić, 1994), so consequently monthly weather type can be associated to the mean monthly forest fire risk.

Since the 1980s DHMZ has been using a Fire Weather Index (FWI), meteorological index for forest fire risk assessment based on the methodology proposed and developed by Meteorological Service of Canada in 1960s. FWI is daily computed and contains information on vegetation, wind speed, daily precipitation, temperature and humidity. Based on the FWI as a predictor, calibrated index of daily forest fire severity rating (DSR) is calculated. Monthly severity rating (MSR) index is monthly mean of daily values (DSR). Severity rating (DSR or MSR) is numerical estimator that reflects the expected efforts required for fire suppression activities expressed in five classes: very low (1), low (2), moderate (3), high (4) and very high (5) being the most difficult. Monthly severity rating represents mean monthly forest fire risk.

In recent decades significant improvements were achieved in numerical weather prediction systems (NWP). Successful prediction of upper level atmospheric state is extended from about 3 days in 1980s to somewhere from 7 to 10 days (Haiden et al., 2015), and while seasonal forecasts in extratropics still need improvements, useful results are already achieved at least for the first month (Molteni et al., 2011). It is reasonable to assume that next progress in extended and long range NWP will first be achieved in the upper levels. To explore the possible benefits of longer lead times (up to a month), relationship between mean monthly atmospheric flow pattern (monthly weather type, MWT) and mean monthly forest fire risk (monthly severity rating; MSR) has been investigated.

\section{Data analysis}

In this work monthly means of the upper level atmospheric flows (AT500) in the warm part of the year (May to October) for the period of 35 years, from 1981 to 2015, were analysed for the Adriatic coast of Croatia. Relation of monthly means of the atmospheric flows (weather types) and forest fire risks (severity ratings) was investigated.

Weather type for each month was categorized based on subjective analysis of monthly mean atmospheric flows at AT500 fields that were retrieved from European Centre for Medium Range Forecast (ECMWF) reanalysis and Deutscher Wetterdienst (DWD) upper level analysis for Northern hemisphere.

Daily severity rating index was calculated for 30 stations on the Croatian coast and islands (dominant vegetation of macchia shrubland) based on the FWI methodology using measured meteorological elements. Monthly severity rating was calculated for each station to build up a contingency table of MSR and MWT. 
Based on the DSR, analysis of variance was conducted for 30 stations to investigate the differences between WTs according to SRs, as a requirement for any further analysis. Analysis of variances of unequal sample sizes at the significance level of 0.05 followed by Tukey-Kramer test provided statistically significant evidences for connection between at least some MWTs and MSR index for most of the stations (<30), based on the 35 year dataset (1980-2015, summer seasons, MJJASO). Exceptions in a-posteriori analysis ( 2 island stations, Komiža and Goveđari) were attributed to gaps in stations time series.

Local atmospheres' potential for memory of MSR index was investigated, autocorrelation function was introduced to MSR time series.

\section{Results}

The most frequent MSR index in 35-year period (season MJJASO) was "high" (44\%) indicating overall high forest fire risks on the Adriatic coast of Croatia (Figure 1). The most frequently observed MWTs in the same period were westerly flow (20\%) and non-gradient field (20\%), followed by northwesterly flow (16\%) while there has been only one month in 35 years with upper level low identified as dominant monthly weather type.

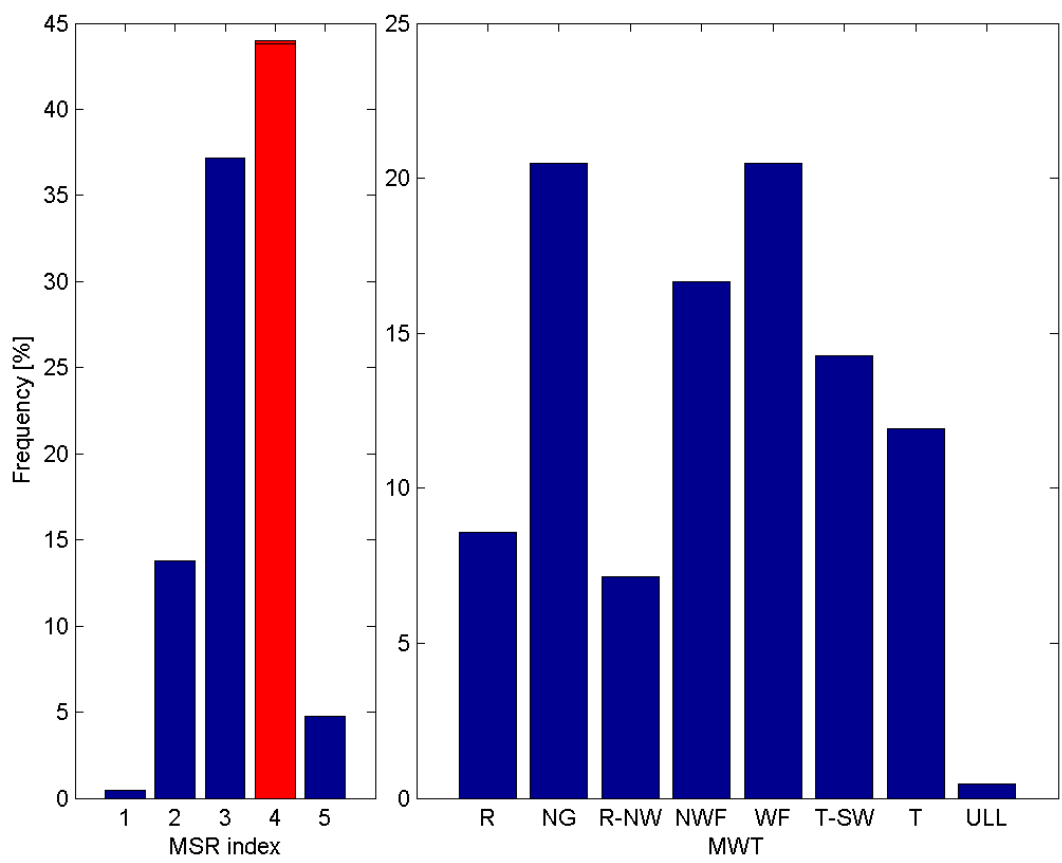

Figure 1 - Occurance of monthly severity rating (MSR index at left) and monthly weather type (MWT at right) in the warm part of the year for the Adriatic coast of Croatia from 1981 to 2015

Out of eight main atmospheric circulation patterns in upper atmosphere, two are associated with relatively dry and warm weather (R, R-NW), which could be connected with higher MSR and increased number of forest fires (Mokorić and Kalin, 2006). Northwest flow brings cold air with local showers or thunderstorms, west flow is characterized by moderate temperatures and variable precipitation amount (Mokorić and Kalin, 2006), while nongradient field also permits local convection, making those types (NWF, WF, NG) difficul to connect to MSR. Southwest flow and trough cause in average more precipitation which can be translated to lower forest fire risks and smaller MSR values. Alternative weather typing is proposed in this work, based on above mentioned discrimination, on Dry, Mixed and Wet monthly weather type. Analysis reveald only slight differences within eight weather types in respect to MSR (Figure 2, left), while larger differences between Wet and Dry weather type has been observed (Figure 2, right). Spatial differences between stations are sources of diversity for MSR and therefore higher spread of the MSRs within WT, this in turn has 
negative effect on basic assumption of connection between MSR and MWT. When Mixed types (NWF, WF, NG, ULL) are excluded, stronger relationship between Dry (R, R-NW) and Wet (T, T$\mathrm{SW})$ types with respect to their average severity rate is revealed and tested by t-test statistics using 95\% confidence interval. Within proposed alternative weather type clasification only about $45 \%$ of the months fall into categories Dry and Wet (Figures 1 and 3).

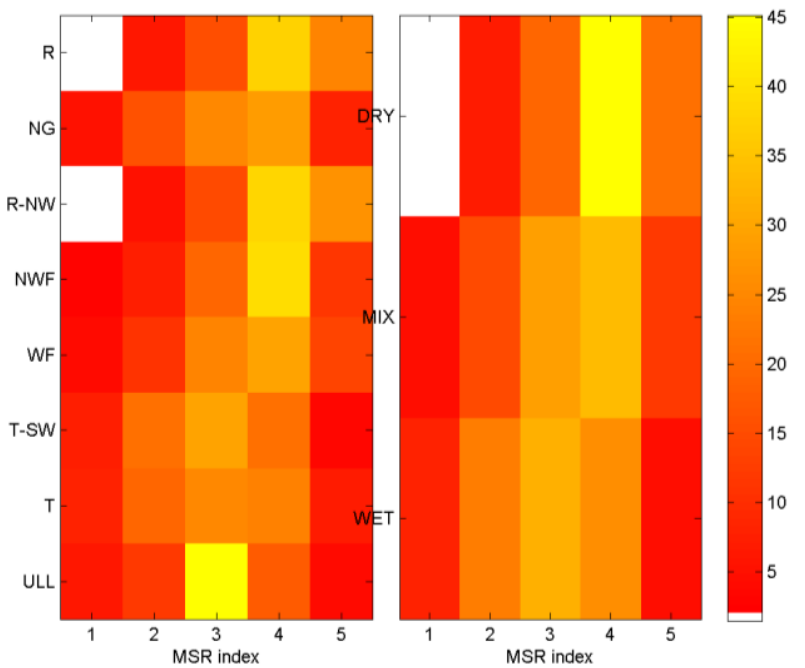

Figure 2 - Percentage (color coded) of MSR within the MWT (left) and MSR within dry, mixed and wet MWT groups (right) in the warm part of the year for the Adriatic coast of Croatia from 1981 to 2015.

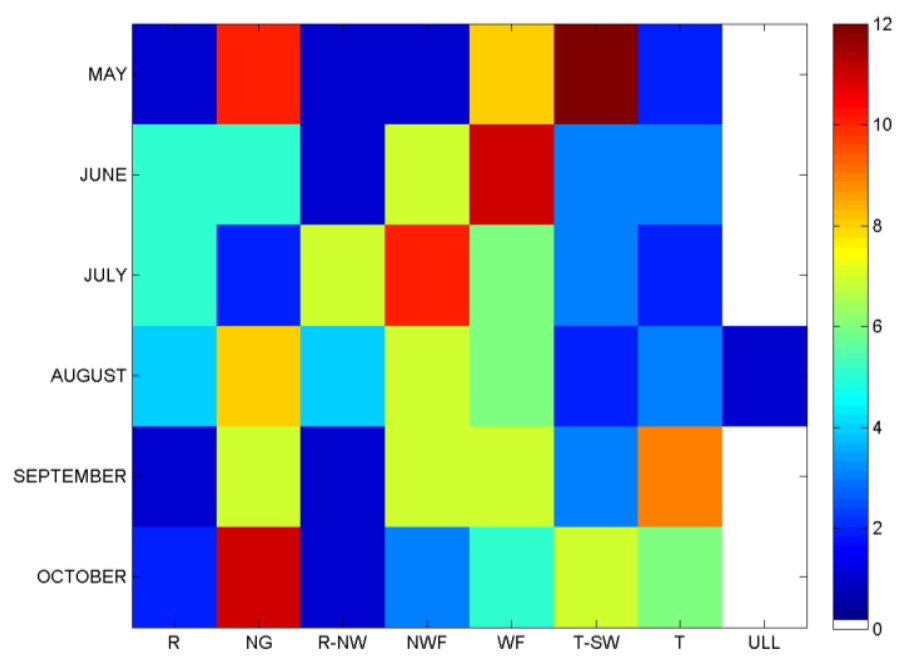

Figure 3 - Occurrence in number of weather type per month in warm part of the year from 1981 to 2015. Upper level low was observed once.

Consecutive months diagrams" (CMDs) were constructed from 35 years of monthly data for MSR (average value) and MWT (number of months). Previous month is on X-axis and current month is on Y-axis. In the diagrams average value of all recorded pairs is plotted for MSR (Figure 4) and total number of months for MWT (Figure 5). 


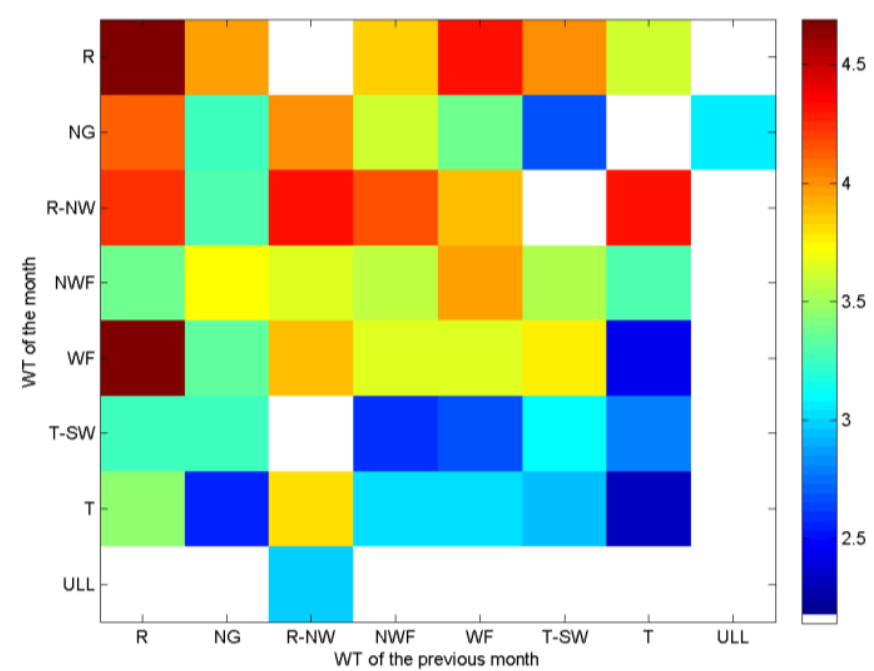

Figure 4 - "Consecutive Month Diagram" for MSR (color coded is MSR of MWT transition pair) for the Adriatic coast of Croatia based on the 35 years of data (1981-2015) for warm part of the year.

From Figure 4 can be seen that very high monthly severity rating (MSR) was recorded in months with main atmospheric circulation patterns in upper atmosphere identified as ridge $(\mathrm{R})$ or westerly flow (WF) if in a preceding month MWT was ridge (R). This combinations however are not often recorded, as it can be seen from low values in CMD on Figure 5. The most frequent pairs are west flow followed by westerly flow (WF $\rightarrow \mathrm{WF}$ ), and northwesterly flow followed by northwesterly flow $(\mathrm{NWF} \rightarrow \mathrm{NWF}$ ), both combination yielding on average moderate to high values of monthly severity rating.

When southwesterly flow associated with the trough (T-SW) was followd by nongradient field (NG) low and very low average MSR index was observed. Transition pair (T-SW $\rightarrow \mathrm{NG}$ ) is quite frequent on the Adriatic coast of Croatia. In general, T-SW in a previous month is a good indicator of low and medium MSR with an exception of month when ridge $(\mathrm{R})$ was observed. Another Wet class member, trough (T), in the previous month can also be considered as good indicator for low and medium classes with noticable exception in month when northwesterly flow associated with the ridge (R-NW) was observed. It should be noted (Figure 4) that R-NW and R types are supporting fast transitions to weather conditions favorable to higher severity rating, almost regardless of the previous month conditions. Exceptions are NG in previous month for $\mathrm{R}-\mathrm{NW}(\mathrm{NG} \rightarrow \mathrm{R}-\mathrm{NW})$ and trough preceding ridge $(\mathrm{T} \rightarrow \mathrm{R})$, which was quite common transition pair.

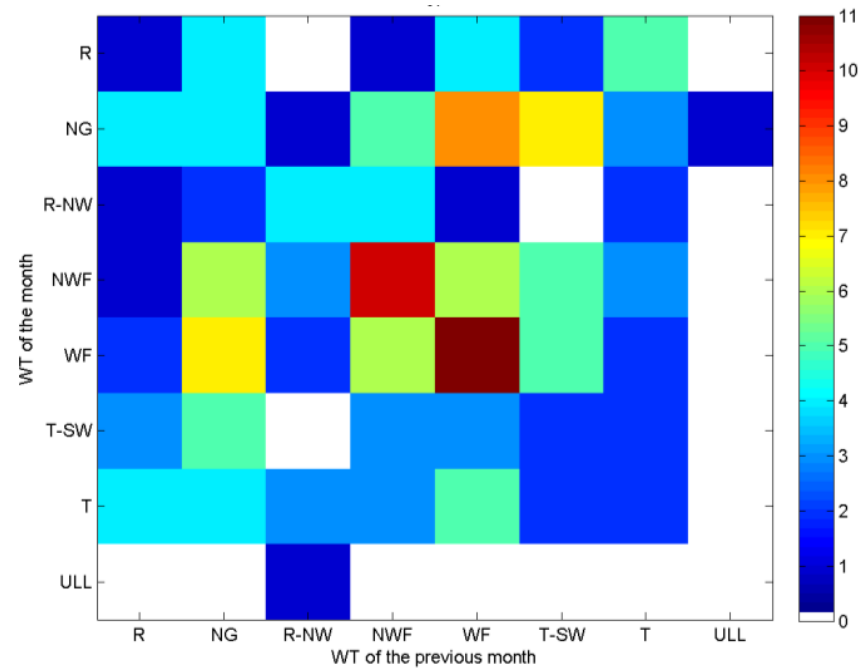

Figure 5 - "Consecutive Month Diagram" for MWT occurance (color coded is number of cases of the MWT transition pair) for the Adriatic coast of Croatia based on the 35 years of data (1981-2015) for warm part of the year. 
Time series of weather types and alternative weather types were constructed. Due to low number of samples within each weather type Mann-Kendall test was not applicable to test for the trends in weather types. In order to assess, at least to some degree, the temporal changes in weather types, cumulative fraction functions were introduced to the data. At each time step (month) cumulative number of occurrence of each weather type from the start of the period (May, 1981) was normalized by a total number of months within the whole period. In the end, sum of the weather type's cumulative fractions on the right-hand side equals 1 on Figures 6 and 7.

Jumps in the weather type's and alternative weather type's cumulative fraction functions are indicators of higher than normal number of particular weather type, while flat intervals indicate lack of particular weather type in some period.

Since 1995 Mixed weather types are dominant in the Adriatic coast of Croatia. Mixed weather types can be associated both with low and high MSR. Forecasting difficulty of MSR is increasing with the increase of Mixed Types.

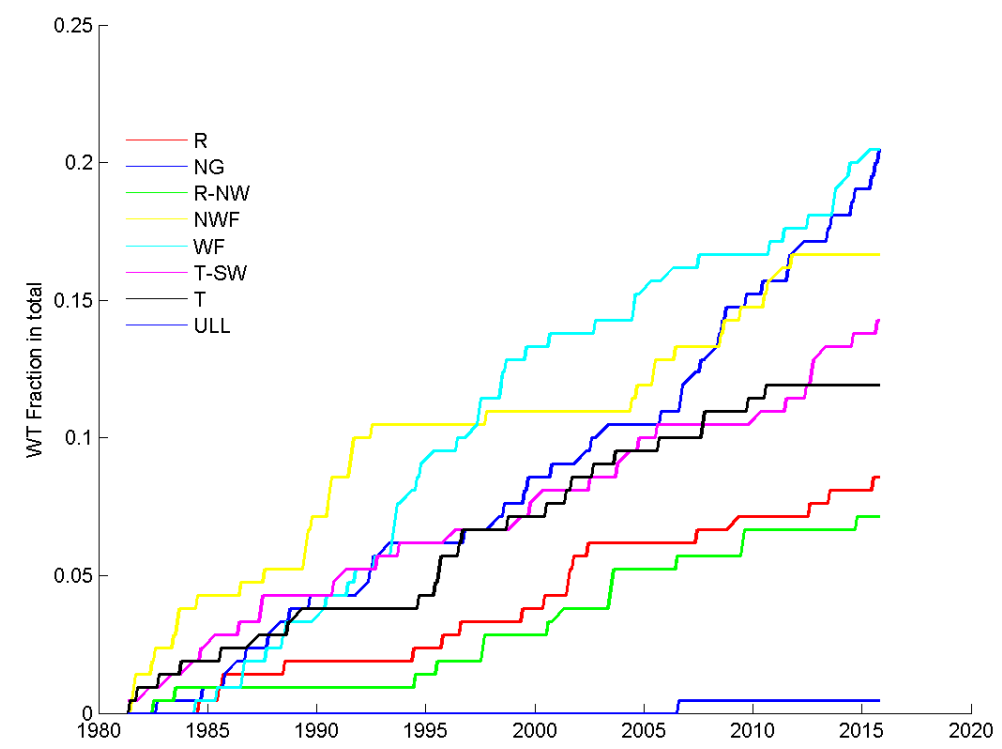

Figure 6 - Weather type (WT) cumulative fraction in total of 35 (1981-2015) warm seasons (MJJASO) in the Adriaitic coast of Croatia.

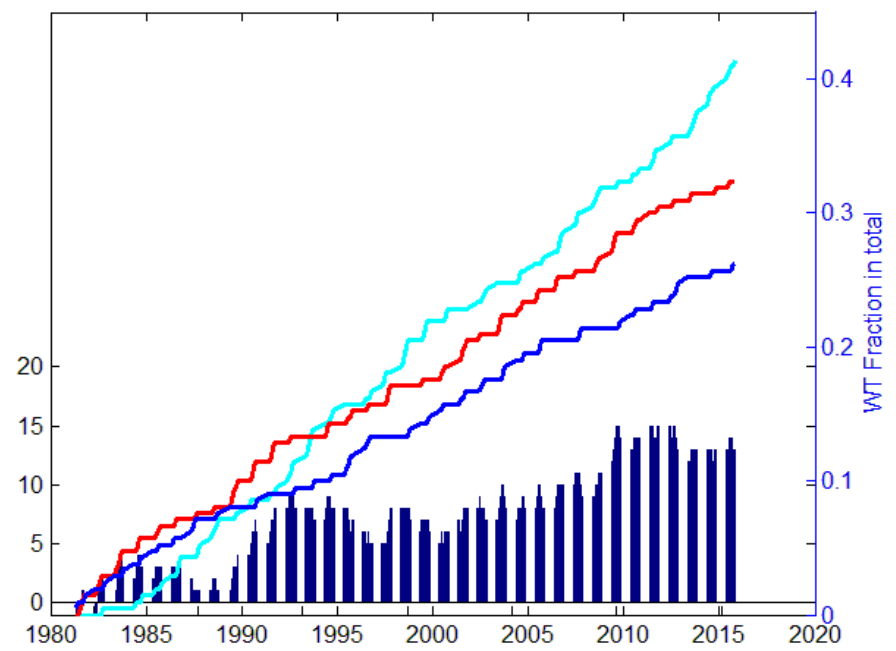

Figure 7 - Alternative weather type (WT) cumulative fraction in total. Mixed (cyan), Dry (red) and Wet (blue) as lines (right), with Dry-Wet differences in bars (left). 


\section{Discussion and conclusions}

Manual typing of monthly means of upper level atmospheric flow provided the basis for weather type analysis in warm part of the years between 1981 and 2015 with total of 210 month analyzed. Weather type analysis results were in line with expectations and work by other authors (Mokorić and Kalin, 2010).

Analysis of variances provided statistical confidence in at least some relation between MWT and MSR index, and although the nature of their interconnection was not in the focus of the work presented in this paper, it provided the basis for the inspection of time correlations. Autocorrelation of MSR time series was found to be above $95 \%$ confidence interval for time lag of 1 month indicating atmospheric potential for 1 month "memory" and providing grounds for construction of 2 consecutive months MSR and MWT histograms, "Consecutive Month Diagrams" or CMDs.

Shortcoming of the methodology is subjective weather typing sensitive to individual experience. There is a potential to overcome it using Principle Component Analysis of mean upper level atmospheric circulation fields and Clustering, however it is beyond the scope of this work. Although PCA would yield higher degree of generalization, original weather typing with 8 classes is widely used in synoptic practice in Croatia and justification of this methodology could be seen on national level where experts can easily attribute weather conditions to familiar upper level atmospheric flow patterns. Proposed alternative weather typing provided stronger statistical discrimination between mean Severity Rating but is should be noted that less than a half of all months in 35 year period (MJJASO) fall in Wet or Dry category which is limiting factor for this approach.

Severity rating based on the FWI seems to produce good results for Adriatic coast of Croatia (Vučetić and Vučetić, 2011), however, from Figure 1 with average MSR class "high" it is clear that calibration of classes needs to be updated in order to accommodate for the warming trend and its effects, observed in Mediterranean region (and Croatia) in last decades (Republic of Croatia, 2014).

Weather types and alternative weather types time series, expressed as cumulative fractions in total, revealed relatively stable ratios of upper level atmospheric flows with increase of the NG type and decrease in NWF in recent years. Interestingly, WF had noticeable increase in 1990s and especially in 2000s, but although still dominant in warm part of the year, in recent years there was a decrease of WF. Westerly flow cannot be connected to MSR due to high spatial variability of precipitation across the Eastern Adriatic Coast associated with this weather type. Decrease in WF fraction is not followed by increased confidence in alternative weather type association to MSR, since in the whole period steady increase of Mixed weather types was observed (Figure 7). However, differences between fractions of Dry and Wet weather types have been increasing since 1990 suggesting more months with Dry weather types that can be connected to higher classes of MSR.

Constructed CMDs contain enough data for justification of the conclusions drawn from it. MSR and MWT CMDs are coupled and should be interpreted in parallel. Higher numbers in MWT CMD per transition pair (WT0 $\rightarrow$ WT1) imply more significant value of MSR for that pair. MWT and MSR CMDs may have a potential in forecasting environment on extended time scales where present day NWP systems are managing to reproduce upper level fields but struggle to adequately reproduce surface weather. In such circumstances, for the Adriatic coast of Croatia, CMDs statistically constructed in this work could serve as mapping functions between forecasted upper level atmospheric conditions and possible monthly severity rating. This concept however, needs to be developed further and tested.

\section{Acknowledgement}

Authors would like to express gratitude to Nevenka Kadić Vlahović from Meteorological and Hydrological Service of Croatia for her professionalism and work in raw data retrieval. 


\section{References}

Haiden T, Janousek M, Bauer P, Bidlot J, Dahoui M, Ferranti L, Prates F, Richardson DS, Vitart F (2015) Evaluation of ECMWF forecasts, including 2014-2015 upgrades. ECMWF, Technical memorandum 765. (Reading, UK)

Lončar E, Bajić A (1994) Tipovi vremena u Hrvatskoj. Hrvatski meteorološki časopis. 29, 31-41. http://hrcak.srce.hr/68042

Lončar E, Vučetić V (2003) Tipovi vremena i njihova primjena na sjeverni Jadran. Hrvatski meteorološki časopis 38, 57-81. http://hrcak.srce.hr/67265

Mokorić M, Kalin L (2006) Evaluation of meteorological index for forest fire protection in Croatia. Forest Ecology and Management 234. doi:10.1016/j.foreco.2006.08.102.

Mokorić M, Kalin L (2014) The weather circulation analysis over Adriatic region of Croatia in warm period 1981-2013, In 'Advances in forest fire research'. (Ed Viegas DX Imprensa da Universidade de Coimbra: Coimbra). http://dx.doi.org/10.14195/978-989-26-0884-6_140.

Molteni F, Stockdale T, Balmaseda M, Balsamo G, Buizza R, Ferranti L, Magnusson L, Mogensen K, Plamer T, Vitart F (2011) The new ECMWF seasonal forecast system (System 4). ECMWF, Technical memorandum 656. (Reading, UK)

Plačko Vršnak D, Mokorić M, Mikec K (2014) The comparation of weather types in the years of extreme drought over Croatia (Poster). 14th EMS Annual Meeting and 10th European Conference on Applied Climatology (Prague, CZ). http://www.ems2014.eu/ems2014_programme_book.pdf

Poje D (1965) Glavni tipovi vremena u Jugoslaviji i njihova zavisnost o visinskim strujanjima (Doctoral dissertation). University of Zagreb. (Zagreb, HR)

Republic of Croatia, Government of the Republic of Croatia (2015) Program aktivnosti u provedbi posebnih mjera zaštite od požara od interesa za Republiku Hrvatsku u 2015. godini. http://narodnenovine.nn.hr/clanci/sluzbeni/2015_03_36_743.html

Republic of Croatia, Ministry of Environmental and Nature Protection (2014) 6st National Communication and 1st Biennial Report of the Republic of Croatia Under the United Nations Framework Convection on Climate Change. http://unfccc.int/files/national_reports/annex_i_natcom_/application/pdf/hrv_nc6.pdf

Vučetić M, Vučetić V (2011) Analiza opasnosti od požara za vrijeme kornatskog požara 30. kolovoza 2007. godine. Vatrogastvo i upravljanje požarima I. 12-25. 


\title{
Non-Rigid Feature Extraction Methods in Real Time Forest Fire Detection Algorithms
}

\author{
Azarm Nowzad*1,2; Andreas Jock ${ }^{1}$;Ralf Reulke ${ }^{2}$ \\ ${ }^{1} I Q$ wireless GmbH, Berlin, Germany, \{azarm.nowzad@iq-wireless.com, andreas.jock@iq- \\ wireless.com\} \\ ${ }^{2}$ Department of computer science, Humboldt-Universität zu Berlin, Berlin, Germany, \\ \{reulke@informatik.hu-berlin.de\}
}

\begin{abstract}
In this paper a smoke detection algorithm for real time forest fire detection is proposed. The scene complexity in open-air environment and the non-rigid nature of the smoke cause high false positive alarms in many detection algorithms. To increase the efficiency of the algorithm, a multi features smoke approach is presented in this work. To segment the possible smoke regions, a change detection method is applied to the image. Afterwards, static and dynamic features of smoke are analyzed. Merging the extracted smoke features and applying morphological processes, region(s) with the highest probability of having smoke pixels are extracted. Two complementary texture features, Gabor filter and Local Binary Pattern (LBP), are applied to the input images. The input image sequence are first characterized by bank of Gabor filters covering the spatial- frequency domain. As multichannel filtering approach, Gabor filters extract features at different orientations and scales. By segmenting the energy image, smoke candidates are extracted and examined using An eXtended Center-Symmetric Local Binary Pattern (XCS-LBP). The image is converted to an array of integer labels as feature vectors for further analysis on smoke and non-smoke classification. The smoke area shows a blurred and smooth texture characteristic compared to the non-smoke areas. This criterion is examined using the histogram of LBP. A number of 5000 labelled smoke blocks are applied to the XCS-LBP operator and the average histogram is calculated and normalized as a priori variable. Applying spectral analysis, a fuzzy logic decision process is implemented on a chromatic analysis enhanced in the HSI (HueSaturation-Intensity) color mode. To define the fuzzy rules, empirical analysis is applied on a set of image data. A trial and error method is then used to reduce the failures. The algorithm is tested on a natural scene forest fire data set, collected from three different sits in Germany. Experimental results show high performance accuracy in smoke classification.
\end{abstract}

Keywords: Non-Rigid object detection, Forest fire detection, smoke features, smoke classification, fuzzy logic smoke detection, smoke texture analysis

\section{Introduction}

Imaging techniques are the main processing element of fire detection technologies and have been developed to extract information from images and, ultimately, to detect fires. Since the effectiveness of any fire detection system is measured by its speed in reporting a fire to the authorities, image-based fire detection systems prioritize the reliability of early stage fire detection and real-time performance.

The majority of image-based techniques search the images for traces of smoke as pre-fire indicators, therefore allowing the discovery of a fire as soon as its smoke signature is visible, enabling a prompt intervention and an easy extinction.

Several solutions have been proposed to handle the early detection of forest fires. Detecting real smoke with low false alarm occurrences and a high detection rate is still a challenging problem, especially in complex natural environments, like forests. Smoke is semi-transparent at the beginning of a fire. The background manifold, natural scene illumination conditions, cloud shadows, wind, and various natural phenomena can drastically degrade the reliability of the detection systems. 
Therefore, improvements in the detection rate as well as in the classification of the smoke features are still a topic of research.

The reliability and efficiency of a detection algorithm is strongly connected to the number of smoke features handled by the algorithm. Many image techniques focus on vision capabilities and have been developed to combine visualization fire properties with some extra features such as motion, flicker, heat and chemical characteristic of aerosols.

Camera-based automated smoke detection systems use image processing algorithms to detect smoke by analyzing typical features of smoke signatures, such as local changes of brightness, contrast, transparency and direction of smoke movement. Each of these smoke features is addressed by a specific algorithm individually.

In this study different smoke feature extraction methods and classification approaches are presented by using the data from a ground-based forest fire surveillance system. Change detection is applied to segment the possible smoke regions in the image, followed by static and dynamic analysis of the smoke features. Combining the extracted smoke features and applying morphological processes, Regions of Interest (ROIs) are extracted as region(s) with the highest probability of having smoke pixels.

\section{Non-uniform illumination}

Non-uniform illumination is an indivisible part of the natural scene environments. Barrow \& Tenenbaum (1978) present recovering intrinsic scene characteristic from images as reflectance and illumination components. To do so, assumptions about the nature of the scene and the physics of image processing need to be taken. The physics consists of many unknown parameters such as camera parameters and lighting characteristic of the scene (Stainvas and Lowe 2003). In image processing, a simplification of physics is applied, which represents the intensity values of the image as the product of the illumination of the scene and the reflectance of the objects.

$$
I(x, y)=L(x, y) R(x, y)
$$

where $L(x, y)$ is the illumination and $R(x, y)$ is the reflectance.

The illumination of a scene is characterized by low frequencies and the lightening conditions, whereupon the reflectance arises by high spatial variations, especially at object edges. The main idea is to remove the illumination component as multiplicative noise signal, to achieve a uniform illumination scene.

Homomorphic filtering, an image enhancement technique, is applied to remove the multiplicative noise signal from the input images. The procedure is shown in Figure .

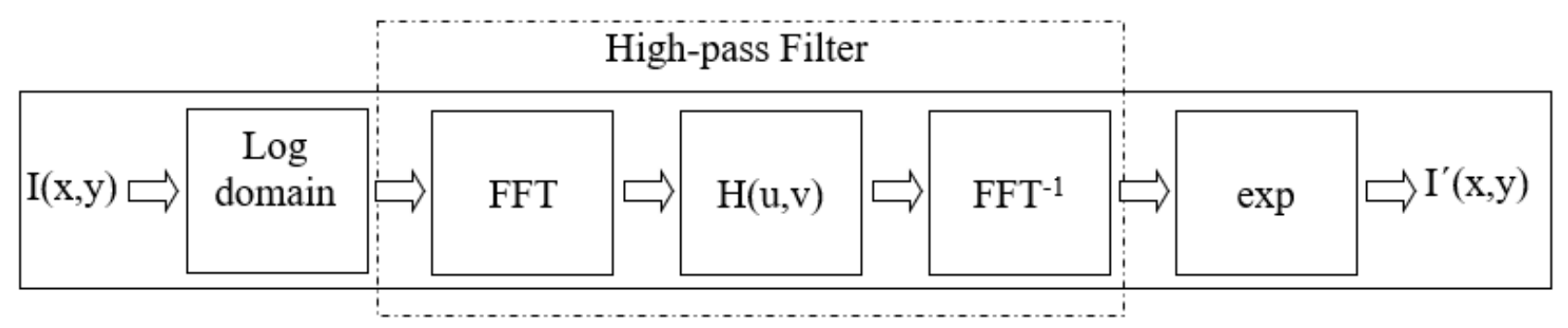

Figure 1 - Homomorphic filtering process in frequency domain

The illumination component is considered as additive component in log-transformed image and is filtered out using a high-pass filter. A frequency-domain high pass filter is constructed to amplify high frequency components. The exponential function is then applied to invert the log-transformed image. The result is a homomorphic filtered image including reflectance components. 


\section{Change detection}

Change detection is one of the main steps in computer vision applications such as object detection, video surveillance and remote sensing systems. A common approach in detecting foreground pixels in an image is the inter-frame subtraction method, which is affected by environmental conditions and is sensitive to noise.

As the first viable step, background subtraction algorithm must be in the position to extract all possible changes, including semi-transparent smoke pixels.

By applying inter-frame subtraction and combining the result with a binary AND operation, pixels of moving regions are extracted. Considering smoke characteristics, in particular the semitransparency, high frequency signals of the background are affected. Difference of Gaussian (DoG) is computed for background frame $B(x, y)$, by convolving image with a Gaussian kernel (Hsu et al. 2016) as

$$
B_{D O G}(x, y)=\left(G_{\sigma 1}(x, y)-G_{\sigma 2}(x, y)\right) * B(x, y)
$$

where $G_{\sigma}(x, y)$ is a Gaussian kernel with variance $\sigma^{2}$ and zero mean.

The DoG image of the background includes high frequency information such as edges and textures. A local entropy image is calculated on a 9x9 neighborhood. By thresholding the entropy image, the result of the background subtraction is examined on high frequency areas.

The segmented ROIs contain false positive incidences, which is filtered out in further analysis.

\section{ROI classification}

Understanding the natural scene environment helps to extract extra information to segment the target. The classification is observed as a non-trivial problem in natural scene due to the dynamic changes and topographical variations. Methods based on spatial relationships besides intensity changes are preferred for complex scene analysis. To analyze complex scene scenarios, more than one feature is required to extract classification information of the scene.

\subsection{Texture descriptors}

Texture descriptors are visual features for characterizing image texture and are used for classification purposes .Gabor filters and Local Binary Pattern (LBP) are two rich texture features with complementary characteristics. While LBP extracts small texture details, Gabor filters encodes texture analysis in a broader range (Tan X and Triggs B 2007).

For subsequent classification purposes, Gabor filters are widely used with the advantage of being invariant to illumination, translation and orientation changes to some extent (Sun $\mathrm{Z}$ et al. 2002).

Local Binary Pattern operator, introduced by Ojiala et al.1996, is a mean of summarizing local graylevel structure as feature vectors and converting an image into an array of integer labels. The statistics of the labels, mostly histograms, are used for image analysis.

\subsubsection{Gabor filter bank}

Gabor filters are a multichannel filtering approach that extracts features at different orientations and scales. As a matter of fact, dominant spatial frequency components of different textures are unequal. Image texture is defined as a Gaussian modulated sinusoid, which can model the texture orientation and the spatial frequency. The filter characteristic must be set in a proper set up to best capture texture information. To cover the spatial-frequency domain, multiple filters can be generated by tuning the frequency and angle. The filter position $\left(F_{x}, F_{y}\right.$ :center frequency, $\theta$ : orientation) and the standard deviations $\left(\sigma_{x}, \sigma_{y}\right)$ of the Gaussian envelope needs to be assigned correctly to extract texture information (Al-Kadi 2017). 

1999)

A two dimensional spatial Gabor function along the $\mathrm{x}-\mathrm{y}$ axis is given by (Weldon and Heggins

$$
g(x, y)=a(x, y) c(x, y)
$$

where

$$
a(x, y)=\frac{1}{2 \pi \sigma_{x} \sigma_{y}} \exp \left\{\frac{1}{2}\left[\frac{x^{2}}{\sigma_{x}^{2}}+\frac{y^{2}}{\sigma_{y}^{2}}\right]\right\}
$$

is the gaussian component and

$$
c(x, y)=\cos \left(2 \pi\left(F_{x} x+F_{y} Y\right)\right)
$$

is the sinusoidal component.

The radial frequency of the filter, with a width of $\mathrm{N}_{\mathrm{c}}$ pixels, is assigned as follows

$$
1 \sqrt{2}, 2 \sqrt{2}, 4 \sqrt{2}, \ldots,\left(\frac{N_{c}}{4} \sqrt{2}\right) \frac{\text { cycle }}{\text { image width }}
$$

which ensures that the passband of the highest frequency lies inside the image. The sampling orientation is in steps of 30 degrees, leading to 6 different orientations. Figure shows an example of applying Gabor filter on a real smoke image.
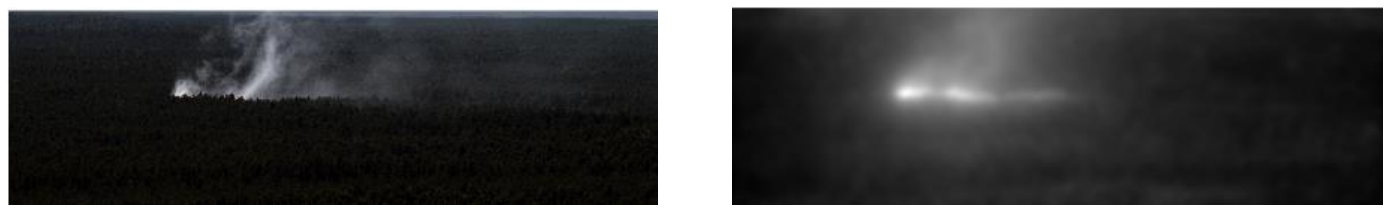

Figure 2 - Real smoke image (left), resulted energy image (right)

\subsubsection{Local Binary Pattern}

Local Binary Pattern (LBP) is a local texture descriptor, which is gray scale invariance. A binary number is formed by thresholding the neighborhood of each center pixel in a cell which is created by dividing the examined window. A normalized histogram is then computed for each cell. The LBP for texture analysis, proposed in by Ojala et al.1996 can be defined as

$$
L B P_{P, R}=\sum_{p=0}^{P-1} s\left(g_{p}-g_{c}\right) 2^{p}, \quad s(x)=\left\{\begin{array}{l}
1, x \geq 0 \\
0, x<0
\end{array},\right.
$$

where $g_{p}$ and $g_{c}$ represent the gray values of center pixel and neighbouring pixels respectively. $\mathrm{R}$ is the circle radius and $\mathrm{P}$ is the total number of sample points in the neighborhood.

Figure shows a $3 \times 3$ neighborhood sample and the computation of $\mathrm{LBP}$ with $\mathrm{P}=8$ and $\mathrm{R}=1$.

\begin{tabular}{|c|c|c|}
\hline 6 & 3 & 1 \\
\hline 8 & 5 & 4 \\
\hline 9 & 2 & 7 \\
\hline
\end{tabular}

Sample

\begin{tabular}{|c|c|c|}
\hline 1 & 0 & 0 \\
\hline 1 & & 0 \\
\hline 1 & 0 & 1 \\
\hline
\end{tabular}

Threshold

\begin{tabular}{|c|c|c|}
\hline 1 & 2 & 4 \\
\hline 128 & & 8 \\
\hline 64 & 32 & 16 \\
\hline
\end{tabular}

Weight

$$
L B P=1+16+64+128=209
$$

Figure 3 - Calculation of LBP 
The neighboring pixels are multiplied by the binomial weights after thresholding. The LBP for each cell is then calculated by summing up the values. An input image with LBP result and the corresponding histogram is illustrated in Figure .

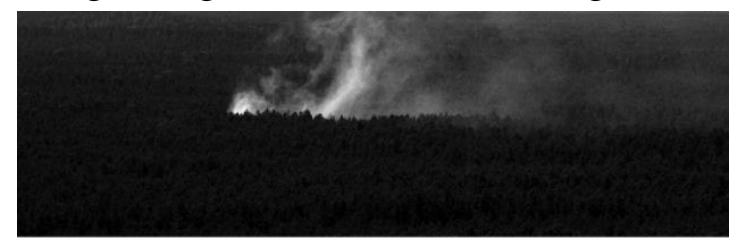

Input Image

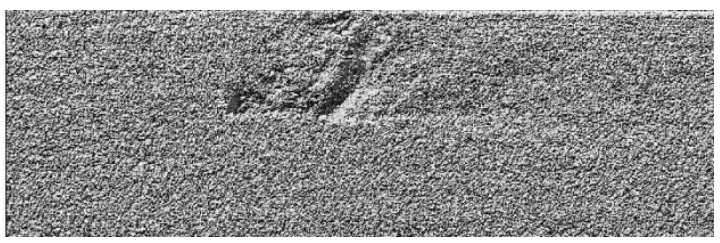

LBP Image

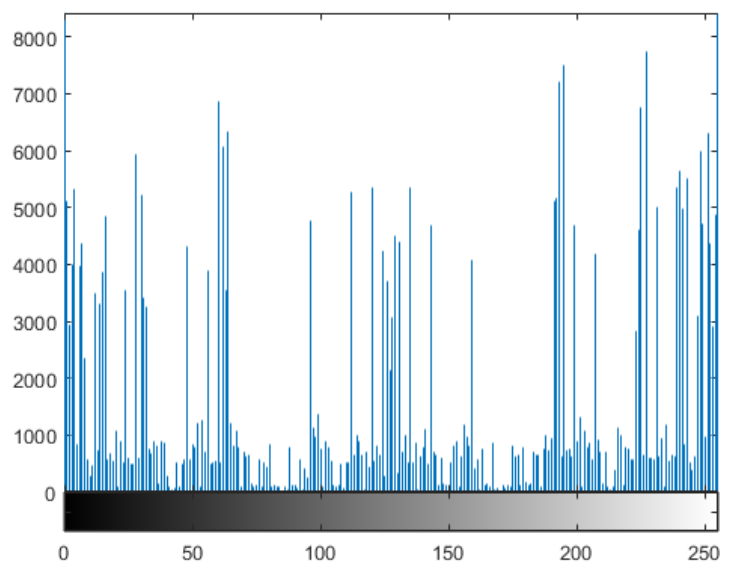

Figure 4 - Example of an input image, the corresponding LBP image and Histogram

As a local operator, LBP converts an image in to an array of integer labels which can be uses as feature vectors for further analysis of smoke and non-smoke classes.

\subsection{Spectral analysis}

Monochrome detectors are widely used in many video based detection systems. In comparison to color sensors, the light sensitivity, the spatial and optical resolutions and the dynamic range of these detectors exceed the color sensor function. However, the lack of color information increases the likelihood of falsely detected smoke and may degrade the system efficiency.

Input images, in Red, Green and Blue channels are converted into HSI color map, describing color in hue, saturation and brightness, which decouples brightness from the chroma components.

The color of the smoke is categorized in a wide range of dark gray $\left(\mathrm{D}_{1}, \mathrm{D}_{2}\right)$ and light gray $\left(\mathrm{L}_{1}, \mathrm{~L}_{2}\right)$ based on many variables such as combustion material and burning temperature. The brightness component is used to describe these two different gray level regions. By assigning a fix threshold for the gray color of smoke, the range of the dark and light gray are defined based on statistical data.

Barring the variation given by a constant $\alpha$, the intensity (I) of a gray colored pixel in RGB channels is almost the same. The decision function rules display as:

$$
\begin{aligned}
& R \pm \alpha=G \pm \alpha=B \pm \alpha \\
& L_{1} \leq I \leq L_{2} ; D_{1} \leq I \leq D_{2}
\end{aligned}
$$

The values of $\alpha, D_{1}, D_{2}, L_{1}$ and $L_{2}$ are assigned by empirical analysis of test frames. The main drawback of this method is the failure in smoke recognition, whenever the smoke intensity range exceeds the predefined range.

A reliable "Yes" and "No" decision for the smoke is replaced with a fuzzy logic based process in the proposed method to deal with uncertain and vague information.

As presented in Nowzad A et.al 2014, by evaluating the saturation and the intensity value ranges of the smoke and non-smoke pixels in natural environment, a fuzzy set is built to show how likely a given pixel belongs to a smoke class.

In fuzzy set theory, image $\mathrm{A}$ is defined as

$$
\sum_{i=0}^{M-1} \sum_{j=0}^{N-1}\left[x_{i j}, \mu\left(x_{i j}\right)\right], \forall x \in A, i=0,1, \ldots, M-1, \quad j=0,1, \ldots, N-1
$$


Where $x_{i j}$ is the pixel value at $(\mathrm{i}, \mathrm{j})$ and $\mu\left(x_{i j}\right)$ donates as membership value in the range of $[0,1]$.

To specify the likelihood of an input pixel to the smoke class, $\left(\operatorname{P}_{f}(i, j)\right)$ is defined as fuzzy output. The higher the value of $\left(\mathrm{P}_{\mathrm{f}}(\mathrm{i}, \mathrm{j})\right)$, the more likely belongs the pixel to the smoke class.

Input (Saturation $(i, j)$, Intensity $(i, j))$ and output $\left(\mathrm{P}_{\mathrm{f}}(\mathrm{i}, \mathrm{j})\right)$ variables are defined by membership functions. To build an interface between input and output variables, a series of IF-THEN rules are defined by analyzing the image dataset.

Table shows the rule table for the fuzzy inference system, which represents the final decision in a linguistic term.

Table 1 - Rule table for fuzzy inference system

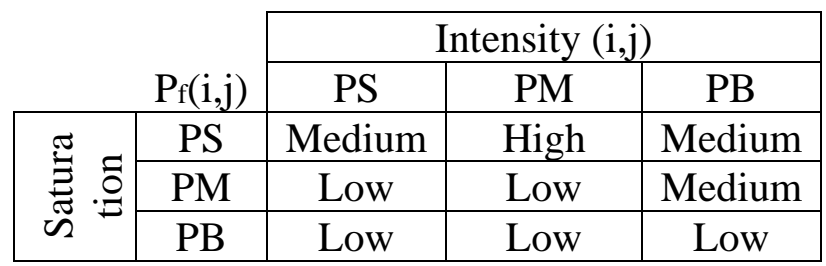

PS, PM and PB stand for "Positive Small", "Positive Medium" and "Positive Big" respectively, which are the membership functions of the input channels.

Depending on the weight of each rule, qualified consequents are generated and aggregated. The output is then assigned by means of the "Defuzzification" interface.

\section{Proposed algorithm}

Consecutive frames in a rate of 1 frame per second are extracted from the stationary video surveillance camera. Preprocessing steps are applied on the input images to increase the performance of the detection algorithm.

A Gabor filter bank is designed using six different orientations $(\theta=0,30,60,90,120,150)$ and the radial frequencies of $\left(2^{2} \sqrt{2}, 2^{3} \sqrt{2}, 2^{4} \sqrt{2}, 2^{5} \sqrt{2}, 2^{6} \sqrt{2}, 2^{7} \sqrt{2}, 2^{8} \sqrt{2}\right)$, with orientation and frequency bandwidths of each filter be $30^{\circ}$ and 1 octave, respectively. The bandwidths are assigned based on the ability of the human visual system and computational costs (Jain and Farrokhnia 1991). The Gabor magnitude features is then extracted and went through some postprocessing steps. First, a low pass Gaussian filter is applied to the energy image, to compensate local variations in constant texture regions. A map of spatial location is added to the Gabor features for classification purposes. For each pixel in the image, there are 42 Gabor features and two additional spatial locations. Principle Component Analysis (PCA) is applied to visualize the energy image from $44 \mathrm{D}$ to $1 \mathrm{D}$ intensity value per pixel. The magnitude features of the candidate areas are then extracted by segmenting the result image.

Applying the proposed change detection method on energy images, ROIs are extracted. The small objects in the foreground and the holes are removed, while the small islands in the image in close distance are combined together using morphological filters. The extracted ROIs are further analyzed by applying LBP descriptor.

The texture characteristic of the smoke area is blurred and smooth compared to the non-smoke areas. This can be checked using the histogram of LBP.

An eXtended Center-Symmetric LBP (XCS-LBP), presented in by Silva C et.al 2015, is computed for each image block is as a 16 dimensional feature vector. A number of 5000 labelled smoke blocks are applied to the XCS-LBP operator and the average histogram is calculated and normalized as a priori variable. The similarity of the histogram is defined by measuring the distance from a suspected ROI to smoke class. If the similarity measurement meets the predefined condition, the block will be conducted for spectral analysis. 
The spectral analysis is implemented by converting the RGB image to HSI color map and applying fuzzy-based rules. A total number of 9 rules are defined to link the possible combinations of the input variables as shown in Table . To define the fuzzy rules, empirical analysis is applied on a set of image data. A trial and error method is then used to reduce the failures and makes adaptive changes to achieve best results.

\section{Experimental results and discussion}

The proposed method is evaluated on real set smoke image sequences ( 8 frames/ sequence) with 32 sequences of smoke and 20 sequences of non-smoke events. The images are taken from three different sites in Germany and a resolution of $512 \times 1360$ pixels.

Figure and Figure show some examples of the smoke and non-smoke forest images, under various illumination conditions and the detection results.
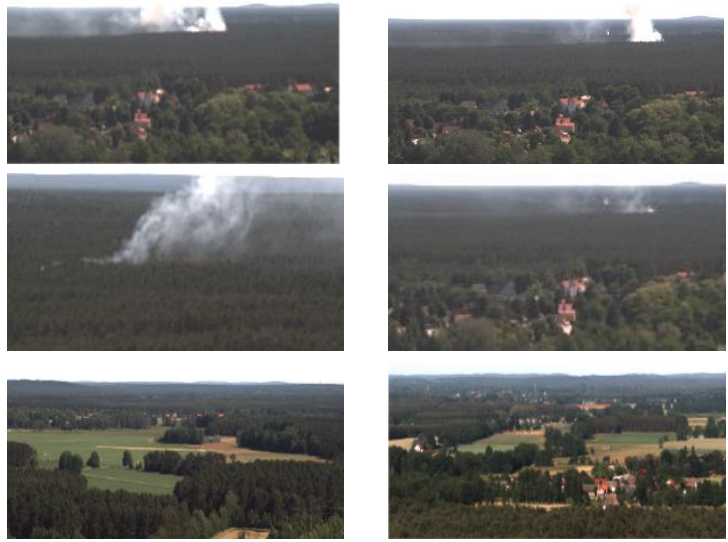

Figure 5 - Examples of image data set. The first two rows are smoke images
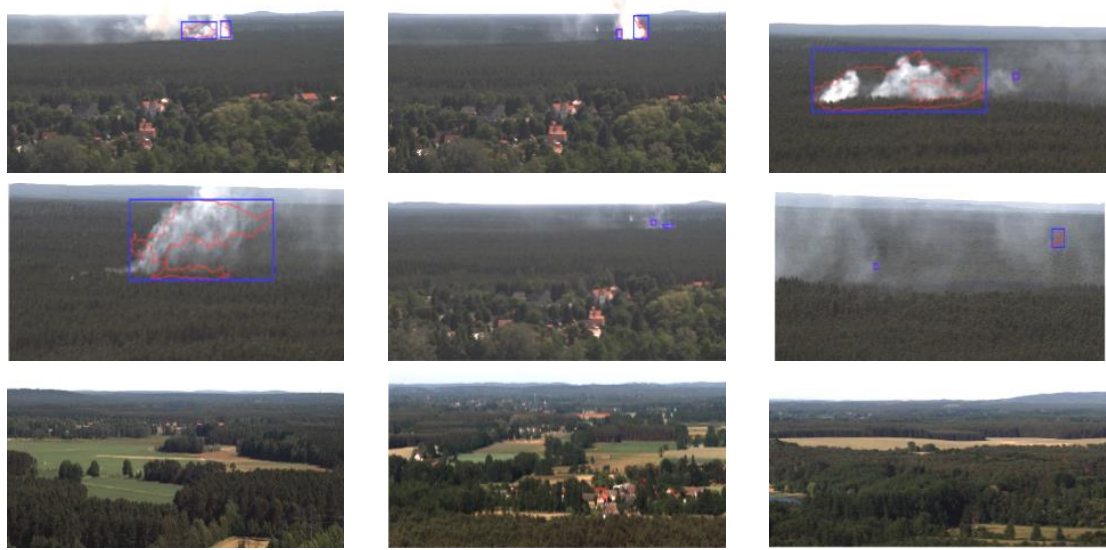

Figure 6 - Detection results on real scene images
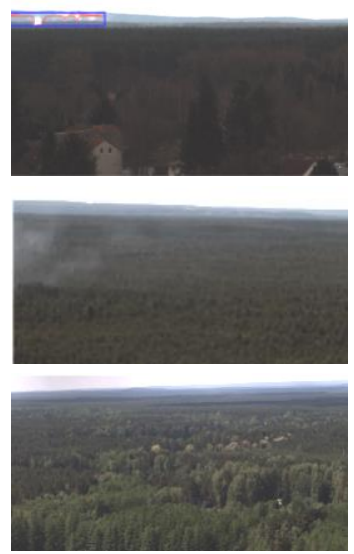
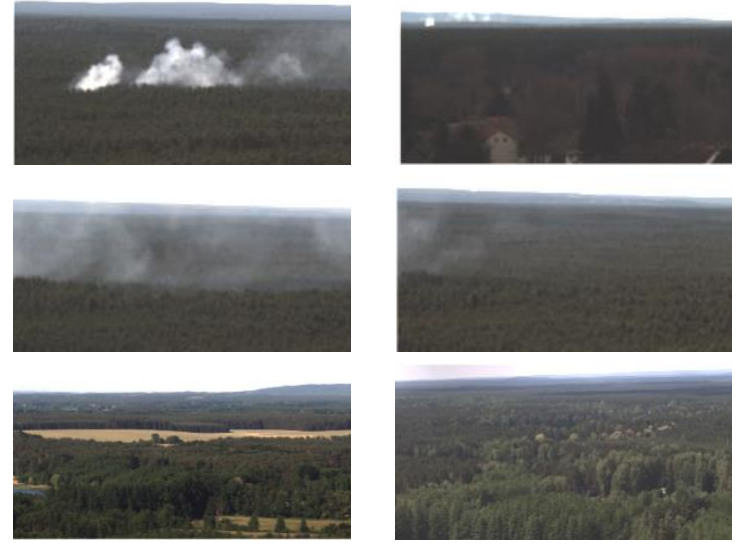

As illustrated in Figure, the smoke detection algorithm detects and locates smoke regions within the frame. The last example in the second row shows a low density smoke event, which is not detected. The number of non-detected smoke events is only three, with two events of low transparency and a small smoke cloud at long distance. 


\section{Conclusion}

In this paper, a texture and color-based smoke detection algorithm is presented. The efficiency of the algorithm is increased by implementing a multi features smoke approach. To segment the possible smoke regions, a change detection method is applied to the homomorphic filtered image sequence, including reflectance components.

Merging the extracted smoke features generated by static and dynamic analysis, morphological processes are applied to retrieve region(s) with the highest probability of smoke pixels.

A Gabor filter bank is designed covering the spatial-frequency domain. Texture information are segmented from the calculated energy image. Applying change detection, further analyses on fine texture changes, caused by smoke pixels, are done using an eXtended Center-Symmetric Local Binary Pattern (XCS-LBP). Finally, a spectral examination is performed on the suspected regions based on fuzzy-rules to classify smoke and non-smoke events. Experimental results show high performance accuracy in smoke classification. Most of the non-detected smoke events are caused by smoke transparency, which is a vague decision criterion. The sensitivity of the proposed smoke detection algorithm can be tuned to detect transparent smoke up to a defined level. However, higher sensitivity is directly connected to false positive misclassification.

\section{References}

Al-Kadi O.S (2017) Gabor Filter Texture Analysis Approach for Histopathol brain Tumour Subtype Discrimination. ISESCO JST Journal

Barrow H.G, Tenenbaum J.M (1978) Recovering intrinsic scene characteristics from images. In 'Computer Vision Systems'. (Eds A Hanson, E Riseman) pp.3-26. (Academic Press: New York)

Hsu Y.C, Dille P.S, Sargent R, Nourbakhsh I (2016) Industrial Smoke Detection and Visualization, Robotics Institute, Carnegie Mellon University Tech. Report, CMU-RI-TR-16-55

Jain A.K, Farrokhnia F (1991) Unsupervised texture segmentation using Gabor filters. Pattern Recognition 1167-1186.

Nowzad A, Jock A, Krane U, Jaeckel K, Vogel H (2014) Development of an automatic smoke detection algorithm using color images and a fuzzy logic approach for real-time forest fire detection applications. Proceedings of the 15th International Conference on Automatic Fire Detection 313320.

Ojala T, Pietikainen M, Harwood D (1996) A comparative study of texture measures with classification based on feature distributions. Pattern Recognition 51-59.

Silva C, Bouwmans T, Frélico C (2015) An eXtended Center-Symmetric Local Binary Pattern for Background Modeling and Subtraction in Videos. Proceedings of the 10th International Conference on Computer Vision Theory and Applications 395-402.

Stainvas I, Lowe D (2003) A generative model for separating lllumination and reflectance from images. Journal of Machine Learning Research 1499-1519.

Sun Z, Bebis G, Miller R (2002) On-road vehicle detection using Gabor filters and support vector machines. 14th International Conference on Digital Signal Processing Proceedings 1019-1022.

Tan X, Triggs B (2007) Fusing Gabor and LBP Feature Sets for Kernel-Based Face Recognition. In ‘ Analysis and Modeling of Faces and Gestures'. ( Eds S.K Zhou, W Zhao, X Tang, S Gong) pp. 235-249. (Springer: Berlin Heidelberg)

Weldon T, Higgins W.E (1999) Designing multiple Gabor filters for multitexture image segmentation. Optical Engineering 1478-1489. 


\title{
On the use of compact thermal cameras for quantitative wildfire monitoring
}

\author{
Mario M. Valero ${ }^{1 *}$; Dan Jimenez ${ }^{2}$; Bret Butler ${ }^{2}$; Christian Mata ${ }^{1}$; Oriol Rios ${ }^{1}$; Elsa Pastor ${ }^{1}$; Eulàlia \\ Planas $^{1}$ \\ ${ }^{1}$ Centre for Technological Risk Studies, Universitat Politècnica de Catalunya-BarcelonaTech. \\ Barcelona, Spain. \{mario.miguel.valero@upc.edu*\} \\ ${ }^{2}$ Fire Sciences Laboratory, Rocky Mountain Research Station, USDA Forest Service, Missoula, \\ $M T, U S A$
}

\begin{abstract}
In recent times, there have been significant developments in remote sensing techniques applied to forest fire research. In particular, infrared (IR) imagery has proved effective to measure variables such as rate of spread, fire radiative power and Byram's intensity. Remote sensing methodologies provide spatially explicit data in a cost-effective manner and reduce the need for intensive surveying campaigns. Concurrently, IR technology has seen remarkable maturing and thermal cameras have become increasingly light, compact and affordable. New models weigh only a few hundreds of grams, they have the size of a phone and they can be easily installed aboard remotely piloted aircraft (RPAS). Whereas these advances may be the base for powerful quantitative fire monitoring systems, there are some practical issues that need to be addressed before the full potential of this technology can be achieved. In this paper, we present our experience with two modern compact thermal cameras, and we explain how we overcame the difficulties we found. Two medium-scale experimental burns were recorded and computer vision algorithms were used to track the fire perimeter and measure its rate of spread (ROS). For performance reasons, one of the cameras encapsulated raw radiometric information in binary files with a non-standard proprietary video format. Adequate processing of this format was required to obtain brightness temperature distributions with a high temporal resolution (27 frames per second). On the other hand, the second camera stored IR frames as JPEG images with no radiometric information and with a low temporal resolution (approximately 1 frame per second, not constant). Additionally, this camera was operated from a small drone and significant jitter was present in the recorded video. To make data from the second camera usable, we applied video stabilization algorithms and we retrieved the exact time of acquisition of each frame from the metadata saved with each image. Ultimately, pre-processed IR data from both cameras was successfully used to detect the fire front, track the fire perimeter and compute rates of spread. All these tasks were performed automatically. Still, human interaction was required to georeference the imagery using ground control points. Georeferencing aerial imagery automatically remains one of the main challenges for the achievement of a fully automated fire monitoring system. Nevertheless, results obtained here were satisfactory. The algorithms for fire front detection, fire perimeter tracking and ROS estimation worked successfully, even when available data had significant limitations. The information retrieved from IR imagery through these algorithms can be further combined with other remote sensing products that provide details about fuel, weather and terrain. Moreover, observed data may be used to adjust data-driven fire spread simulators in real time so that they can produce accurate forecasts about the fire evolution, at least in the short term. These technological developments make important contributions towards the accomplishment of affordable, yet reliable, operational decision-support systems (DSS) that can be deployed during an emergency.
\end{abstract}

Keywords: Decision Support Systems, Fire Monitoring, Fire Segmentation, Remote Sensing, Thermal Infrared Cameras, Unmanned Aerial Vehicles

\section{Introduction}

Quantitative wildfire monitoring is essential for two reasons. On the one hand, to provide emergency response teams with meaningful, accurate information about the state of the fire during an incident. On the other, to study a number of aspects related to fire behavior. Extensive experimental 
campaigns have been conducted with the aim of improving scientific knowledge of wildfire dynamics, e.g. Clements et al. (2007, 2014), Cruz et al. (2010), Ottmar et al. (2016). Within the acquired datasets, spatially and temporally explicit measurements of fire radiant emission and fire rate of spread have been considered to be critical for the investigation of fire behavior and effects (O'Brien et al. 2016). Similarly, continuous surveillance of the fire perimeter and remote measurement of its rate of spread during a real emergency would greatly assist decision making. Current telecommunication systems and information management technologies support the combination of such measurements with other relevant data such as fuel properties, fuel spatial distribution, digital elevation models and updated weather variables. Furthermore, there exist fire spread simulators that can forecast the fire evolution in the short term. The availability of all this information in real time would notably increase the level of situational awareness in a scenario that can quickly become dangerous.

However, this technology-assisted decision-support context is incomplete at present. Real-time information available during a wildfire incident is, in the best case, limited to crew positions, weather data and discrete observations of the fire state. There is no continuous monitoring of the fire perimeter location, the active fire lines, their rate of spread or their burning intensity. Furthermore, operational fire spread simulators have insufficient accuracy to be relied on in a potentially dangerous scenario (Cruz and Alexander 2013). A proposed solution to the limited accuracy of operational fire spread simulators is the use of data assimilation techniques (Mandel et al. 2008; Rios et al. 2014, 2016; Rochoux et al. 2014, 2015), but this approach needs frequent, updated and spatially-explicit information about the fire evolution.

Thermal Infrared (TIR) imagery has proved to be a suitable technology to obtain continuous information about an active fire. Some variables that can be estimated remotely using IR cameras are the fire perimeter location, the rate of spread, the Fire Radiative Power (FRP) and Byram's fire intensity (Pérez et al. 2011; Paugam et al. 2013; Stow et al. 2014; Butler et al. 2016; Dickinson et al. 2016; Johnston et al. 2017). In this regard, our previous work contributed to automate the analysis of aerial TIR imagery through computer vision techniques (Valero et al. 2017, 2018). Algorithms of this type allow the automated acquisition of spatially explicit, high-frequency fire information, and they may be the base of a quantitative monitoring system with applicability to wildfire incident management and wildfire research. Furthermore, thermal infrared cameras, which had historically been expensive, complex and heavy, have experienced a notable decrease in size, weight and price, until the point of being suitable for installation aboard Remotely Piloted Aerial Systems (RPAS) and Unmanned Aerial Systems (UAS). RPAS and UAS have numerous advantages and a promising potential in forest fire management and research. Being able to use IR imagery acquired by these small aircraft would be a remarkable step forward towards the accomplishment of powerful, yet affordable, fire monitoring systems.

Nonetheless, to the best of our knowledge, all previous studies that successfully used infrared imagery to derive fire behavior information employed conventional thermal cameras (Plucinski and Pastor 2013; Paugam et al. 2013; Butler et al. 2016; O'Brien et al. 2016; Johnston et al. 2017; Valero et al. 2018). There have been very few attempts to use modern compact IR cameras to sense fire behavior, and there is a general concern in the scientific community about the image quality achievable by low-end IR cameras. Furthermore, some unsuccessful experiences have been reported when using RPAS, mainly due to difficulties in georeferencing the imagery and saturation of the IR sensors (Dickinson et al. 2016; Zajkowski et al. 2016).

In this work, we present a successful coupling of quantitative fire monitoring algorithms with compact, affordable IR cameras. Two camera models, from two different manufacturers, were tested in medium-scale experimental burns using dissimilar setups. An RPAS was successfully used in one of the experiments, and collected imagery was used to follow the fire perimeter evolution and to measure its rate of spread. Existing computer vision algorithms were adapted and additional preprocessing modules were developed to deal with the limitations of each camera. 


\section{Compact IR cameras}

Thermal infrared cameras have seen an outstanding development in the last few years, on both market ends. On the one hand, high-end devices that work at high speed and with high resolution are allowing unprecedented studies of fire behavior and characteristic fire structures at different scales (McAllister and Finney 2017; Miller et al. 2017). On the other, cameras with basic capabilities have become smaller, lighter and cheaper than ever.

Fire monitoring entails specific requirements such as a wide temperature measurement range to avoid sensor saturation and, at least, medium resolution so that fire behavior can be sensed from a distance that might go from a few meters to a few hundreds of meters. Until recently, these requisites prevented fire researchers from relying on low-end IR camera models. However, camera manufacturers have started to produce compact cameras that meet the needs of many wildfire studies. We had the opportunity to test two models from two known international manufacturers. The main specifications of the tested models are summarized in table 1.

Table 1 - Technical specifications of the two tested cameras

\begin{tabular}{c|ccccccc} 
Camera & $\begin{array}{c}\text { Commercial } \\
\text { Name }\end{array}$ & $\begin{array}{c}\text { Dimensions } \\
(\mathrm{mm})\end{array}$ & $\begin{array}{c}\text { Weight } \\
(\mathrm{g})\end{array}$ & $\begin{array}{c}\text { Image } \\
\text { Resolution } \\
(\text { pixels })\end{array}$ & $\begin{array}{c}\text { Spectral } \\
\text { range }(\mu \mathrm{m})\end{array}$ & $\begin{array}{c}\text { Temperature } \\
\text { measurement } \\
\text { range }{ }^{*}\left({ }^{\circ} \mathrm{C}\right)\end{array}$ & Output \\
\hline 1 & Optris PI 400 & $46 \times 56 \times 90$ & 320 & $382 \times 288$ & {$[7.5,13]$} & {$[200,1500]$} & $\begin{array}{c}\text { Radiometric video, } \\
\text { proprietary format }\end{array}$ \\
2 & $\begin{array}{c}\text { DJI Zenmuse } \\
\text { XT (developed } \\
\text { by FLIR) }\end{array}$ & $\begin{array}{c}103 \times 74 \times 102 \\
(\text { Gimbal } \\
\text { included })\end{array}$ & 270 & $640 \times 512$ & {$[7.5,13.5]$} & {$[-40,550]$} & $\begin{array}{c}\text { Individual JPEG } \\
\text { images, no radiometric } \\
\text { information }\end{array}$
\end{tabular}

*Manufacturers usually offer several calibration options for different temperature ranges. The values shown in this table correspond to the measurement ranges used during the tests described in this article.

The two IR cameras listed in table 1 have some characteristics in common. Both are compact and based on uncooled microbolometers. They both need a reduced amount of power to work and they cannot operate on their own but need to be connected to some kind of controlling hardware. The controller might be a laptop, a tablet or a single-board microcomputer. These characteristics make them especially convenient for portable field experimental setups and for installation aboard RPAS. In this study, camera 1 was connected to a laptop whereas camera 2 was operated from a drone through an on-board microcomputer.

Despite meeting the basic technical needs for large-scale fire monitoring (i.e. suitable temperature measurement range and sufficient resolution), these cameras entail significant limitations in the data processing workflow. Each manufacturer normally uses its own private software to operate their cameras and their own private format to store data. Raw IR data can afterwards be inspected only with their own software. Manufacturers' programs, provided with easy-to-use graphical user interfaces, are convenient for most industry clients. However, the use of non-standard formats and the lack of transparency in the camera control software constitute serious drawbacks for fire researchers, who usually need full access to the raw thermal data. Furthermore, there are other practical constraints when using these cameras in situations with limited resources, such as a drone or in the field. For instance, IR video files can easily become too large for modest systems, which usually implies restrictions in the recording frame rate. In the next sections, we discuss how these limitations affect quantitative fire monitoring algorithms and how they can be worked around.

\section{Experimental sites}

We tested our cameras in two medium-scale experimental burns. Test 1 was performed at the Tall Timbers Research Station in Tallahassee, FL, USA, in April 2017. Test 2 was conducted on The Nature Conservancy land next to Baxley, GA, USA, in January 2017. The cameras were used differently in 
each occasion. Test 1 was recorded with camera 1 from a fixed overhead position that provided an oblique view of the plot. In test 2, camera 2 was operated from a small RPAS hovering above the plot.

\subsection{Test 1: Fixed vantage point, off-nadir}

Test 1 was conducted in plot $\mathrm{C} 1$ of the Pebble Hill sites at Tall Timbers, which consisted of mixed southern rough/long leaf pine fuels. This plot was equipped with two highly-instrumented plots (HIPs) consisting of several radiometers, visual and thermal cameras. These items were geolocated and used as ground control points (GCPs) for imagery geocorrection. Furthermore, a thermal camera (camera 1 of this study) was installed on top of a boom lift situated at the south-east corner of the plot. Figure 1 shows the layout and some representative distances. Most of the GCPs were distinguishable in the IR video, but in different frames. Consequently, a composite figure was created from frames at different times in order to facilitate the annotation of GCPs.

\subsection{Test 2: Drone, nadir view}

In the case of test 2 , the fact of having a clear nadir view of the complete plot considerably simplified image georegistration. Measurement of one ground distance between two GCPs sufficed to estimate the conversion between image and ground coordinates. No projective transformation was needed. A sample IR frame in which these GCPs can be observed is displayed in figure 2. The main difficulty during this test arose from the drone instability. The footage had some jitter that needed to be cancelled through image processing techniques. Furthermore, exportable data from this video sequence was not radiometric. Available pixel values did not correspond to brightness temperatures. This type of representation is common in industry.

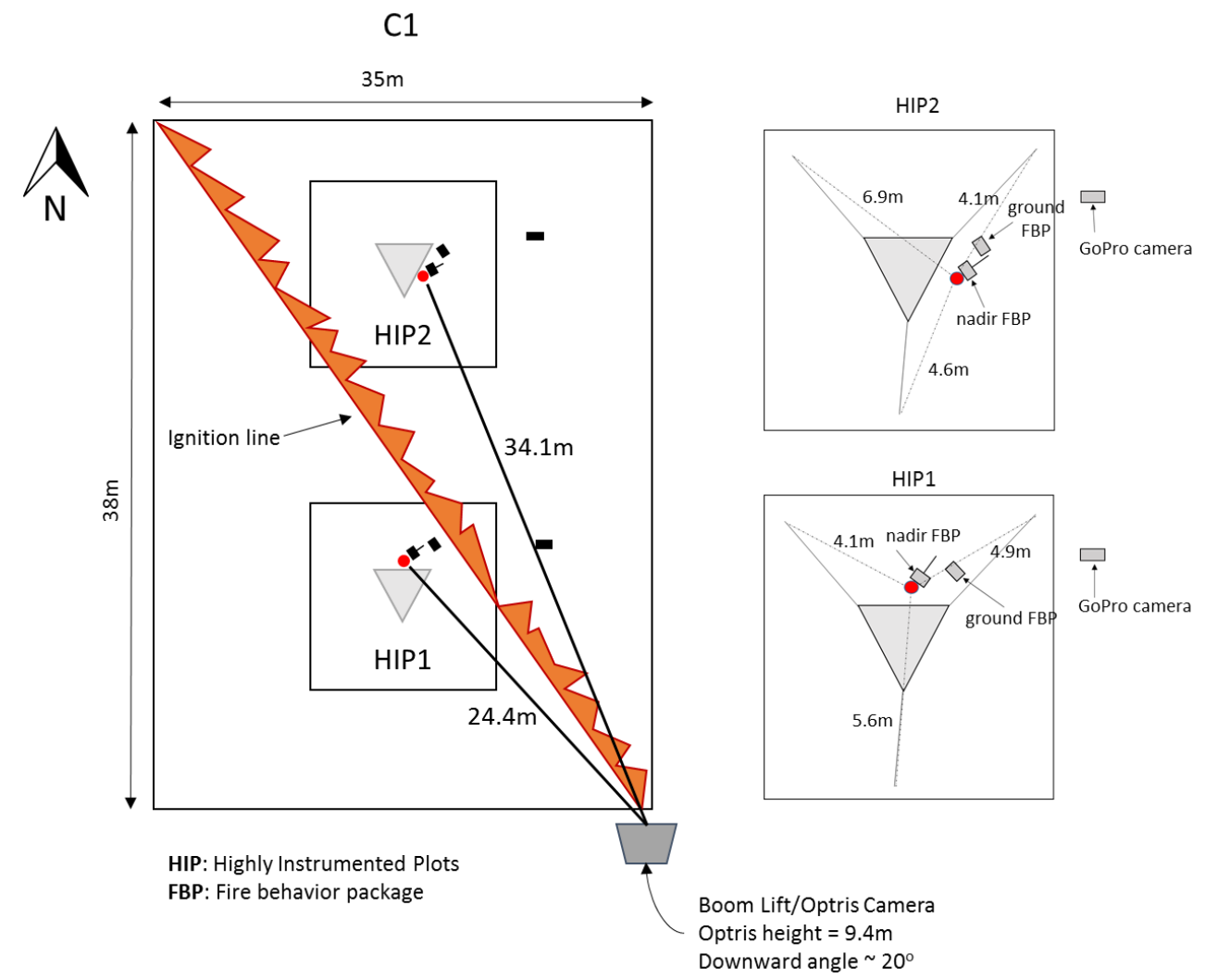

Figure 1 - Diagram of the item layout existing in test 1. All indicated distances are horizontal at ground level. 


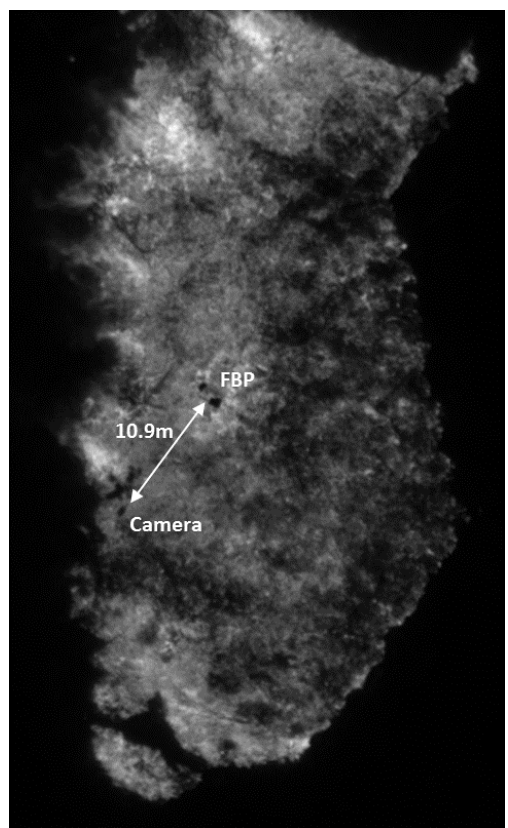

Figure 2 - Sample IR frame corresponding to test 2, with GCPS used for imagery geocorrection. FBP means Fire Behavior Package.

\section{Quantitative fire monitoring}

\subsection{Pre-processing modules}

By default, software provided by the manufacturer of camera 1 always stores acquired IR video in a private binary format. This format can only be read using the same manufacturer's software. IR video encapsulated this way can neither be exported to other formats. Moreover, data analysis tools officially provided by Optris are rather limited. To overcome these issues, we used the $\mathrm{C}++$ libraries provided by the manufacturer to build two small applications: one that directly operates the camera and stores radiometric data in a readable format, and another that retrieves thermal data from existing IR video. By using these applications, we could read and process high-frequency video containing actual brightness temperatures.

In the case of camera 2, manufacturer's software did allow exporting IR video in a standard format, but such video did not contain actual brightness temperatures. Frames could only be exported as JPEG images, valid for visualization but without radiometric information. Furthermore, this information was available with a low temporal resolution. Frame rate was close to $1 \mathrm{~Hz}$, but it was not kept constant. Greyscale frames could be used successfully to detect the fire perimeter evolution because our computer vision methods do not rely on precise radiometric data. However, timing was important to estimate rates of spread. Fortunately, the exact time at which each frame was originally acquired could be retrieved from each file's metadata. We used the open-source program Exiftool ${ }^{1}$ for this purpose.

In addition to format limitations, footage taken during both tests needed some degree of geometric correction. Imagery from test 1 was oblique and the corresponding $2 \mathrm{D}$ projective transformation between the camera plane and the ground was estimated using a set of Ground Control Points (GCPs). A composite image was created using different frames from the video sequence to simplify this task. Available GCPs were identified in this composite image and their location was used to estimate the

\footnotetext{
${ }^{1}$ Originally developed by Phil Harvey. Website: https://www.sno.phy.queensu.ca/ phil/exiftool/
} 
transformation between image and ground coordinates. Afterwards, this transformation was applied to all video frames.

Footage 2 did not need the application of a projective transformation because it was acquired from a nadir viewpoint. However, instabilities in the drone position translated into slight jitter in the video sequence. Light aircraft are usually susceptible to wind gusts, especially when they fly over an active fire. Although the DJI Zenmuse camera is specifically equipped with a mechanical stabilization system to minimize the impact of this, significant vibrations were present in the output video. This issue was resolved through additional video stabilization software, based on image registration and feature matching. Features are regions of pixels that can be described in terms of certain parameters. If those descriptors are invariant to translations, rotations and changes in scale, the same points can be detected in two consecutive video frames and their corresponding locations can be matched. This methodology was followed here to estimate the relative movement between each consecutive pair of frames. Once these movements were known, each frame was corrected and registered to the previous one. The final result was a stable footage.

\subsection{Computer vision algorithms for perimeter tracking}

Pre-processed IR imagery was used to follow the fire perimeter evolution and to measure its rate of spread. Both tasks were accomplished automatically using an adaptation of the algorithms described in Valero et al. (2018). The basis of these algorithms lie on edge detectors that search the highest brightness gradients within each image. Pixels are classified as edges or non-edges using two thresholds for the gradient magnitude. The correct thresholds are automatically estimated for each frame by the algorithm itself. Finding edges in the image allows the software to estimate the location of the fuel burning interface. If a time series is available, the evolution of the burning line can be reconstructed. Morphological operations are applied to derive an estimation of the burned perimeter location at each instant in time. Finally, the difference in fire perimeter positions between consecutive frames allows the computation of spatially-explicit rates of spread.

This methodology is not exempt of difficulties. The most significant detected so far consists in the rejection of ROS outliers. Actively burning lines usually exhibit flames whose length is not negligible. These flames must be avoided when detecting the fuel burning interface and computing ROS. Our edge detection algorithms are insensitive to flames up to a certain size, but the system becomes biased in the presence of regions with a high concentration of flames, especially if the same region keeps flaming for a long period of time. In these cases, unrealistic ROS values may be produced and they need to be detected and rejected. There are image processing techniques to achieve this by directly filtering flames out (Valero et al. 2017), but such methods are complex to implement and need considerable computational resources. Sometimes it is enough with the application of common statistical rules for outlier rejection. In this case, we applied the generalized extreme studentized deviate (GESD) test (Rosner 1983) to the ROS values obtained along each isochrone. This test is especially suitable for datasets that have multiple outliers but where the number of outliers is not known a priori.

\section{Results}

Once converted to a readable format, footage 1 provided reach radiometric data about test 1 , with a high temporal resolution $(27 \mathrm{~Hz}$ ). For its part, footage 2 had to be stabilized before becoming useful. Figure 3 exemplifies the video stabilization methodology based on feature matching. 


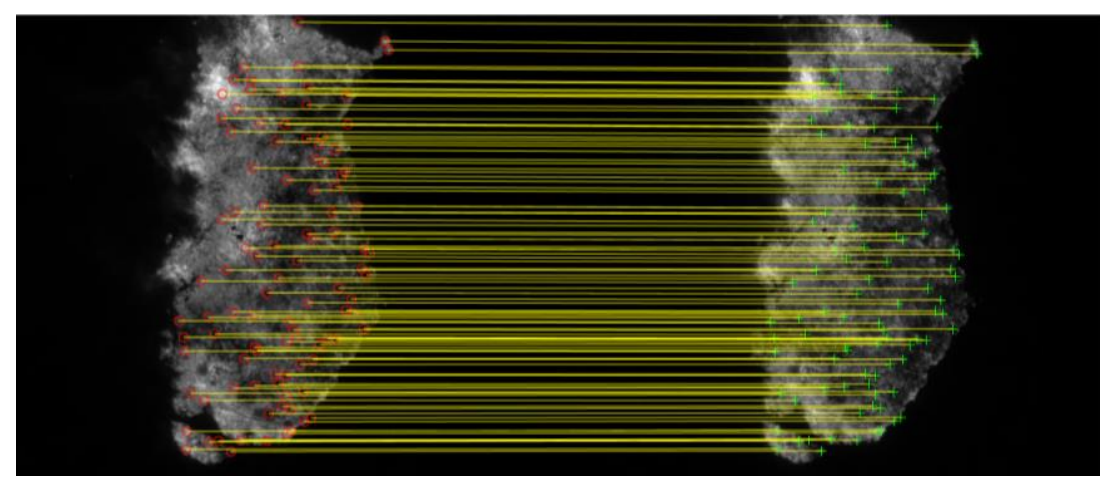

Figure 3 - Subset of matching features detected in two sample consecutive frames during the stabilization of the drone IR footage (test 2). Red and green points represent features. Yellow lines connect matched features. The relative movement between these two frames consisted of a translation of 13.74 pixel units and a scale change of $1.2 \%$.

After pre-processing, the perimeter tracking algorithms were applied to both video sequences at an approximate framerate of $1 \mathrm{~Hz}$. A subset of the obtained results is shown in figure 4. Complete animated video with the detected perimeter evolution is available at the following links: https://goo.gl/bKb4Cu; https://goo.gl/47CxcU.
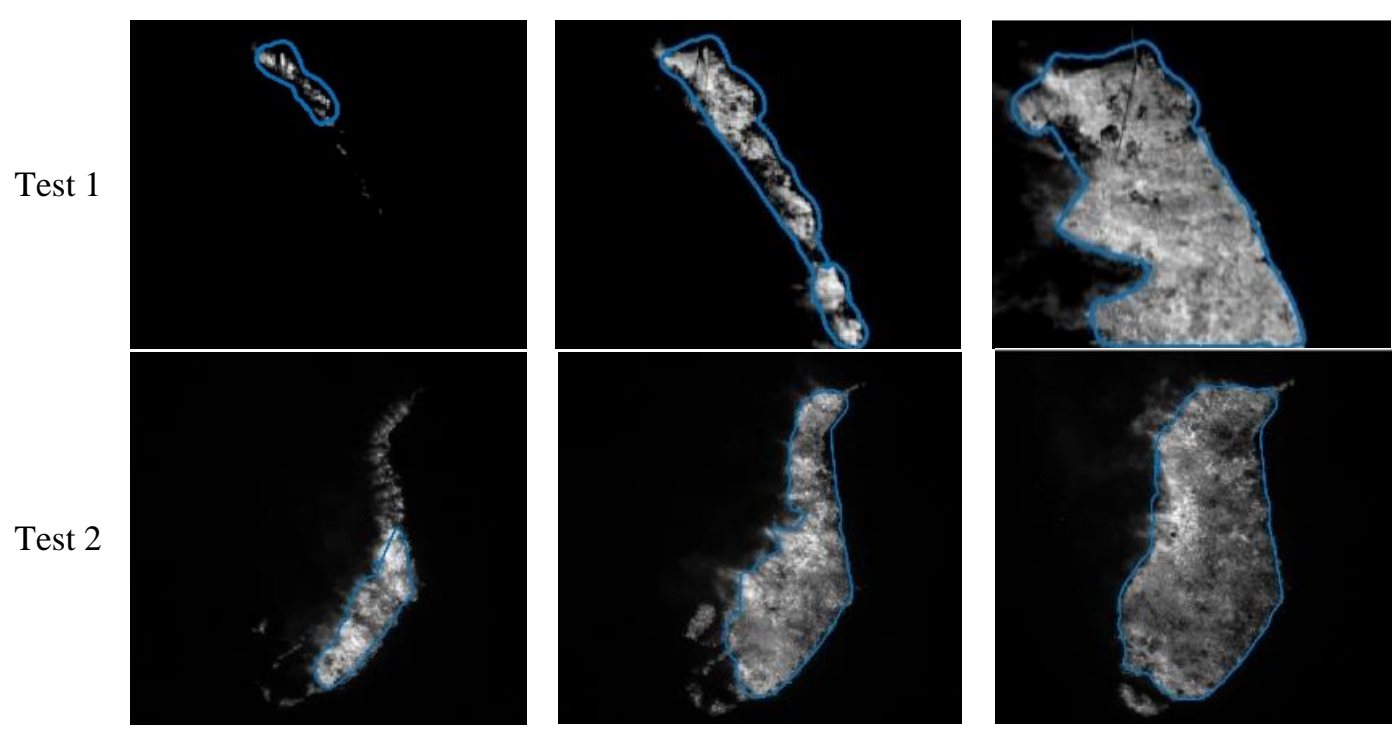

Figure 4 - Burning area automatically detected in TIR frames acquired by compact cameras. Displayed frames approximately correspond to footage beginning (left), midway (center) and end (right).

Finally, geocorrection information allowed the computation of ROS spatio-temporal distributions. Figure 5 summarizes this information. 

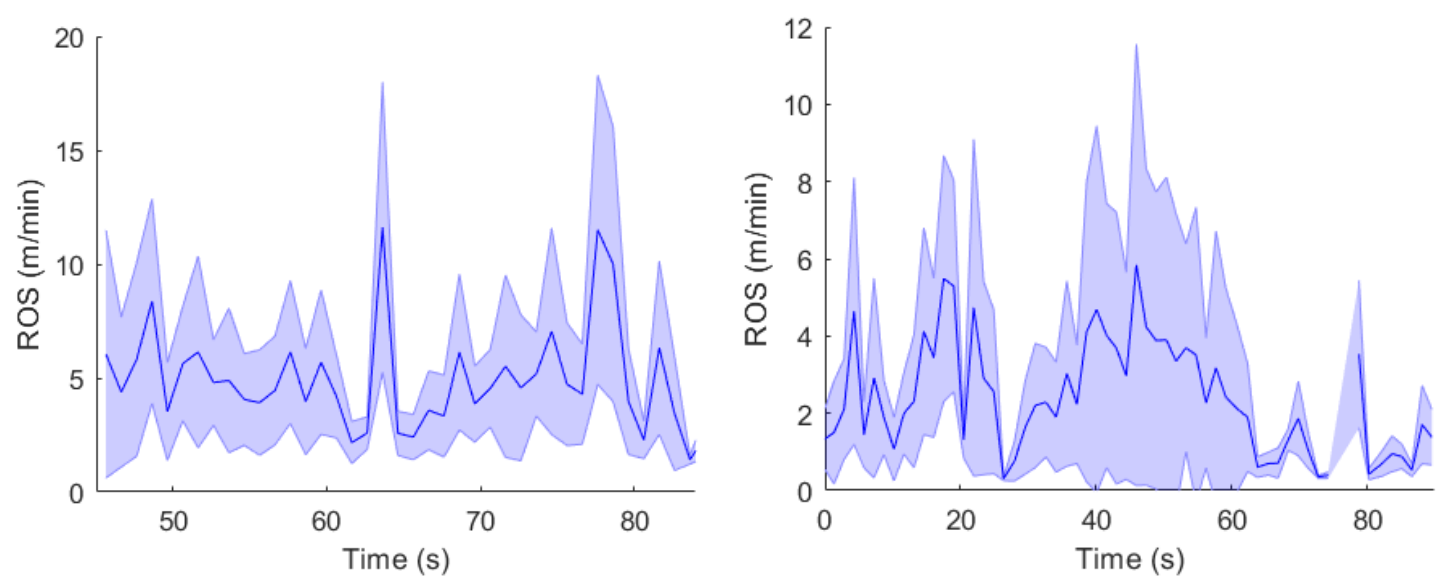

Figure 5 - Temporal evolution of remotely sensed rates of spread. Central solid line represents average values within each isochrone. Shaded area represents standard deviation within each isochrone. Left: test 1; right: test 2.

Rates of spread were not measured in situ, so values obtained remotely could not be compared with other experimental information. Nonetheless, results are in agreement with visual estimations. Moreover, the evolution of the detected fire perimeter represented in figure 4 and in the associated animated video is very similar to what a human operator would annotate manually.

\section{Discussion and conclusions}

The main goal of this paper was to assess the suitability of compact IR cameras for wildland fire monitoring. The camera models tested in this study as well as others have significant strengths for field deployment: they are light and easy to use, they consume little power and they are relatively affordable. However, they have certain limitations that need to be resolved. Some manufacturers enclose radiometric data into private formats, while their official software does not provide full processing capabilities. Furthermore, some recording configurations do not store actual brightness temperatures but just a visual representation of 'what is hot and what is cold'. Finally, one of the star applications of these cameras is their installation aboard RPAS, but small aircraft is highly susceptible to aerodynamic instabilities and video recorded from these platforms is usually shaky. A video stabilization step is often necessary before such imagery can be further processed.

This work proposes solutions for some of these issues, and the obtained results prove that data acquired with compact IR cameras has sufficient quality for a variety of wildfire applications: firstly, if an active fire is recorded from an overhead perspective, be it nadir or off-nadir, IR imagery can be processed to detect the location of the fuel burning interface and the fire perimeter at a certain time. Furthermore, if time series information is also available with sufficient frequency, rates of spread can be estimated remotely. This type of data may be useful for fire behavior research, but it may also have a relevant utility at operational level. Emergency responders could benefit from updated quantitative information about the state of the fire during an incident. Moreover, wildfire monitoring software can be integrated into broader decision support systems that combine fire information with fuel, terrain and weather data. Such systems may even include data-driven fire spread simulators which, if timely fed accurate fire observations, are able to deliver reliable forecasts of the fire evolution in the short term.

Key aspects to be addressed in the future relate to imagery geocorrection and the communication infrastructure. Georeferencing IR aerial imagery is the only step that remains manual in the image processing workflow. Its automation would allow the achievement of a totally autonomous fire monitoring system. On the other hand, extracted information will only be useful at operational level if it can be reliably transmitted from the aircraft to a command center in near real time. This requires 
important hardware developments such as on-board data processing architectures and deployable communication networks.

\section{Acknowledgements}

This research was partially funded by the Spanish Ministry of Economy and Competitiveness (projects CTM2014-57448-R and CTQ2017-85990-R, co-financed with FEDER funds) and the Spanish Ministry of Education, Culture and Sport (FPU Programme). The IR data was acquired using hardware from the University of Montana, National Center for Landscape Fire Analysis.

\section{References}

Butler B, Teske C, Jimenez D, O’Brien J, Sopko P, Wold C, Vosburgh M, Hornsby B, Loudermilk E (2016) Observations of energy transport and rate of spreads from low-intensity fires in longleaf pine habitat - RxCADRE 2012. International Journal of Wildland Fire 25, 76-89. doi:10.1071/WF14154.

Clements CB, Davis B, Seto D, Contezac J, Kochanski A, Fillipi J-B, Lareau N, Barboni B, Butler B, Krueger S, Ottmar R, Vihnanek R, Heilman E, Flynn J, Jenkins M, Mandel J, Teske C, Jimenez D, O'Brien J, Lefer B (2014) Overview of the 2013 FireFlux II grass fire field experiment. 'Adv. For. fire Res. - Proc. 7th Int. Conf. For. Fire Res.’ pp. 392-400 doi:10.14195/978-989-26-0884-6_43.

Clements CB, Zhong S, Goodrick S, Li J, Potter BE, Bian X, Heilman WE, Charney JJ, Perna R, Jang M, Lee D, Patel M, Street S, Aumann G (2007) Observing the Dynamics of Wildland Grass Fires: FireFlux - A Field Validation Experiment. Bulletin of the American Meteorological Society 88, 1369-1382. doi:10.1175/BAMS-88-9-1369.

Cruz MG, Alexander ME (2013) Uncertainty associated with model predictions of surface and crown fire rates of spread. Environmental Modelling and Software 47, 16-28. doi:10.1016/j.envsoft.2013.04.004.

Cruz MG, Matthews S, Gould J, Ellis P (2010) Fire dynamics in mallee-heath: fuel, weather and fire behaviour prediction in south Australian semi-arid shrublands. Bushfire Cooperative Research Centre, Technical Report A1001. http://www.bushfirecrc.com/sites/default/files/firedynamicsinmalleeheathreport.pdf.

Dickinson MB, Hudak AT, Zajkowski T, Loudermilk EL, Schroeder W, Ellison L, Kremens RL, Holley W, Martinez O, Paxton A, Bright BC, O’Brien JJ, Hornsby B, Ichoku C, Faulring J, Gerace A, Peterson D, Mauceri J (2016) Measuring radiant emissions from entire prescribed fires with ground, airborne and satellite sensors - RxCADRE 2012. International Journal of Wildland Fire 25, 48-61. doi:10.1071/WF15090.

Johnston JM, Wooster MJ, Paugam R, Wang X, Lynham TJ, Johnston LM (2017) Direct estimation of Byram's fire intensity from infrared remote sensing imagery. International Journal of Wildland Fire 26, 668-684. doi:http://dx.doi.org/10.1071/WF16178.

Mandel J, Bennethum LS, Beezley JD, Coen JL, Douglas CC, Kim M, Vodacek A (2008) A wildland fire model with data assimilation. Mathematics and Computers in Simulation 79, 584-606. doi:10.1016/j.matcom.2008.03.015.

McAllister S, Finney M (2017) Autoignition of wood under combined convective and radiative heating. Proceedings of the Combustion Institute 36, 3073-3080. doi:10.1016/j.proci.2016.06.110.

Miller C, Finney MA, McAllister S, Sluder E, Gollner MJ (2017) Investigating coherent streaks in wildfires via heated plates in crosswind. Fire Safety Journal 91, 735-741. doi:10.1016/j.firesaf.2017.03.035. 
O’Brien JJ, Loudermilk EL, Hornsby B, Hudak AT, Bright BC, Dickinson MB, Hiers JK, Teske C, Ottmar RD (2016) High-resolution infrared thermography for capturing wildland fire behaviour: RxCADRE 2012. International Journal of Wildland Fire 25, 62-75. doi:10.1071/WF14165.

Ottmar RD, Hiers JK, Butler BW, Clements CB, Dickinson MB, Hudak AT, O’Brien JJ, Potter BE, Rowell EM, Strand TM (2016) Measurements, datasets and preliminary results from the RxCADRE project-2008, 2011 and 2012. International Journal of Wildland Fire 25, 1-9.

Paugam R, Wooster MJ, Roberts G (2013) Use of Handheld Thermal Imager Data for Airborne Mapping of Fire Radiative Power and Energy and Flame Front Rate of Spread. IEEE Transactions on Geoscience and Remote Sensing 51, 3385-3399. doi:10.1109/TGRS.2012.2220368.

Pérez Y, Pastor E, Planas E, Plucinski M, Gould J (2011) Computing forest fires aerial suppression effectiveness by IR monitoring. Fire Safety Journal 46, 2-8. doi:10.1016/j.firesaf.2010.06.004.

Plucinski M, Pastor E (2013) Criteria and methodology for evaluating aerial wildfire suppression. International Journal of Wildland Fire 22, 1144-1154. doi:10.1071/WF13040.

Rios O, Jahn W, Rein G (2014) Forecasting wind-driven wildfires using an inverse modelling approach. Natural Hazards and Earth System Sciences 14, 1491-1503. doi:10.5194/nhess-141491-2014.

Rios O, Pastor E, Valero MM, Planas E (2016) Short-term fire front spread prediction using inverse modelling and airborne infrared images. International Journal of Wildland Fire 25, 1033-1047. doi:10.1071/WF16031.

Rochoux MC, Emery C, Ricci S, Cuenot B, Trouvé A (2015) Towards predictive data-driven simulations of wildfire spread - Part II: Ensemble Kalman Filter for the state estimation of a fronttracking simulator of wildfire spread. Natural Hazards and Earth System Sciences 15, 1721-1739. doi:doi:10.5194/nhess-15-1721-2015.

Rochoux MC, Ricci S, Lucor D, Cuenot B, Trouvé A (2014) Towards predictive data-driven simulations of wildfire spread - Part I: Reduced-cost ensemble Kalman filter based on a polynomial chaos surrogate model for parameter estimation. Natural Hazards and Earth System Sciences 14, 2951-2973. doi:10.5194/nhess-14-2951-2014.

Rosner B (1983) Percentage Points for a Generalized ESD Many-Outlier Procedure. Technometrics 25, 165-172. doi:10.1080/00401706.1983.10487848.

Stow DA, Riggan PJ, Storey EJ, Coulter LL (2014) Measuring fire spread rates from repeat pass airborne thermal infrared imagery. Remote Sensing Letters 5, 803-812. doi:10.1080/2150704X.2014.967882.

Valero MM, Rios O, Pastor E, Planas E (2018) Automated location of active fire perimeters in aerial infrared imaging using unsupervised edge detectors. International Journal of Wildland Fire 27, 241-256. doi:10.1071/WF17093.

Valero MM, Verstockt S, Rios O, Pastor E, Vandecasteele F, Planas E (2017) Flame filtering and perimeter localization of wildfires using aerial thermal imagery. In 'Proc. SPIE 10214. Thermosense Therm. Infrared Appl. XXXIX', doi:10.1117/12.2262100.

Zajkowski TJ, Dickinson MB, Hiers JK, Holley W, Williams BW, Paxton A, Martinez O, Walker GW (2016) Evaluation and use of remotely piloted aircraft systems for operations and research RxCADRE 2012. International Journal of Wildland Fire 25, 114-128. doi:10.1071/WF14176. 


\title{
Predictive distribution modeling of forest fire in pine zone of Uttarakhand Himalayas of India
}

\author{
Amit Kumar Verma ${ }^{1 *}$; Namitha Nhandadiyil Kaliyathan ${ }^{1}$; N S Bisht ${ }^{2}$, Raman Nautiyal ${ }^{2}$, S D \\ Sharma ${ }^{2}$ \\ ${ }^{1}$ Forest Research Institute, P.O. New Forest, Dehradun, India. \{amitvermafri@gmail.com*, \\ namithafri@gmail.com\} \\ ${ }^{2}$ Indian Council of Forestry Research \& Education, P.O. New Forest, Dehradun, India, \\ \{bishtnsifs@yahoo.com,nautiyal.raman@gmail.com,sharmasatinder95@gmail.com\}
}

\begin{abstract}
Periodic forest fire in the Pine zone of Indian Himalayas has been portrayed as the great destroyer of forests. The coniferous forests in the Himalayan region, notably Pinus roxburghii stands are highly prone to fire. The lack of proper forest fire monitoring system in Pine zone is a major management issue needs attention for the long term viability. Hence the present study was attempted using Maximum Entropy (MaxEnt) method to predict the potential areas under fire across the Pine zone and to identify key variables associated with fire occurrence. A total of $n=529$ spatiotemporally independent fire incidence locations were used after auto-correlation testing. Bioclimatic environmental variables were used along with other anthropogenic, topographic, Forest type/canopy density variables. Spatial multi-collinearity of variables was tested where the variables with $r>0.7$ were dropped from the analysis. The accuracy of the model was assessed using the area under the curve (AUC) and to assess the variables importance jackknife procedure was adopted. Hence, 100 model predictions were averaged to produce a probability map. The map was classified into four categories based on the probability value viz., Very highly, highly, moderately and least susceptible to fire. The results showed that the forest areas having moderate canopy density and near to village is highly affected by fire. The areas identified as very highly and highly susceptible to fire can be used for preparatory planning for management of forest fire in Pine Zone of Uttarakhand Himalayas.
\end{abstract}

Keywords: Pine Zone, MaxEnt, Forest Fire, MODIS, Modeling, Himalayas

\section{Introduction}

Pines are natives of tropics as well as the temperate climates. They are found abundantly in the Himalayas (Gamble, 1875; Craw, 1875). The major problem associated with the pine forest is that the area becomes highly vulnerable to fire due to the presence of pine needles in very large amounts. Needles are fine fuels that ignite and spread flames faster than coarse woody fuels and represent an important portion of the total fuel consumption in forest fires. It is not the thick bark but exposed resin ducts that make the pine trees prone to fire. In the Chir forests, the resin channels increase the height of the flames to about $4 \mathrm{~m}$ from where the crowns of the small poles, bigger poles and then bigger trees, in turn, catch fire thus enabling the fire to rise vertically upwards. In the Central Himalayas, particularly Uttarakhand, frequent man made fires are an integral part of the pine forest especially in P. roxburghii (Chir pine) forest between $800 \mathrm{~m}$ and $2000 \mathrm{~m}$ altitude. P. roxburghii forests encompass approximately $39,416.38$ ha or $16.15 \%$ of the total forest area of Uttarakhand. A massive forest fire in 1995 engulfed 5,948.88 ha of valuable forest area through 2,272 forest fire incidents in the state, which resulted in the loss of crores of rupees and created various long-lasting ecological consequences (Dhaundiyal and Gupta, 2014).

The major constraint in forest fire management is the lack of scientific study on identification of fire hazard areas across the country. The information about peak fire season, factors associated with fire occurrence and the areas where forest fire are likely to occur is lacking. Further, the comprehensive 
information needed to relate the fire susceptibility with habitation, road network, drainage network, distance to fire station, forest composition and density, slope, aspect, altitude, fuel type, temperature, precipitation etc. is also lacking. In Uttarakhand, the topography is highly undulating and it is difficult to control the forest fire in time. During summer, forest fires are common in these areas as pine needles, which keep falling off the trees from the middle of March till the onset of the rains in July, are highly inflammable. Fire prediction modeling and risk assessment are important tools to predict, and design measures to minimize, both direct and indirect losses due to fire. Verma et al., (2013) developed a forest fire risk zone map of Raipur Range of Mussoorie Forest Division in Uttarakhand using remote sensing and GIS. Verma and Kumar (2015) developed a Fire Hazard Map of Rajaji National Park, Uttarakhand by using multi source data comprising of cartographic documents, satellite imageries and statistical information with a combination of remote sensing and GIS data. Verma (2016) used the Maximum Entropy (MaxEnt) ecological niche modeling framework to predict the potential areas under fire across the Lansdowne Corridor of Lesser Himalayas and identified key environmental variables associated with fire occurrence. Hence similar model i.e. MaxEnt ecological niche modeling framework was used to predict the potential areas under fire across the Pine Zone and to identify the key environmental variables associated with fire occurrence.

\section{Materials and Methods}

\subsection{Study area}

The present study was conducted in Uttarakhand Himalayas of India (Figure 1). It lies on the southern slope of the Himalayan range, located between $28^{\circ} 44^{\prime}$ to $31^{\circ} 28^{\prime} \mathrm{N}$ Latitude and $77^{\circ} 35^{\prime}$ to $81^{\circ} 01^{\prime}$ E Longitude and spread over an area of $53,483 \mathrm{~km}^{2}$. Topographically, Uttarakhand can be divided into three zones namely the Himalayas, the Siwaliks and the Terai region. The terrain and topography of the State is largely hilly with large areas under snow cover and steep slopes. Major portion of the State is mountainous and these mountains (Himalayas) are one of the youngest mountain systems of the World, hence ecologically very fragile and relatively much more susceptible to earthquakes and landslides (http://sbb.uk.gov.in/pages/display/93-about-uttarakhand).

In the present study two Pine species viz., Pinus roxburghii (Chir pine) and Pinus wallichiana (blue pine) have been considered for Pine zone in Uttarakhand. According to Troup (1921), Chir pine occurs from $457 \mathrm{~m}$ to 2,286 $\mathrm{m}$ and blue pine occurs from 1,219 $\mathrm{m}$ to 3,658 $\mathrm{m}$ in Himalayas.

\subsection{Fire occurrence data}

The fire data for the period of year 2001-2016 were downloaded from https://firms.modaps.eosdis.nasa.gov in the form of shape file as point feature. However for the present study only 529 spatiotemporally independent fire incidences were used for model building. The spatiotemporally auto-correlated locations were removed after testing using Software Program DivaGIS (Hijmans et al., 2002; DIVA GIS version 2). Only one location per $1 \mathrm{~km}$ grid cell if $>1$ observation was used by clustered in a grid (i.e., to avoid autocorrelation with low sample size; Phillips et al., 2006; Pearson et al., 2007).

\subsection{Environmental variables}

The environmental and bioclimatic environmental variables were obtained from WorldClim database (Version 1.4, http://www.worldclim.org/bioclim.htm; Hijmans et al. 2005). These metrics are derived from monthly temperature and rainfall climatology and represent biologically meaningful variables for characterizing a species range. We used 19 environmental variables (11 variables for temperature, 8 for precipitation variables, expressing spatial variations in annual means, seasonality and extreme or limiting climatic factors). Forest Type layer was generated from Landsat- 8 satellite 
data by using supervised classification approach in which 30 training sets were taken from Google earth as ground control points.

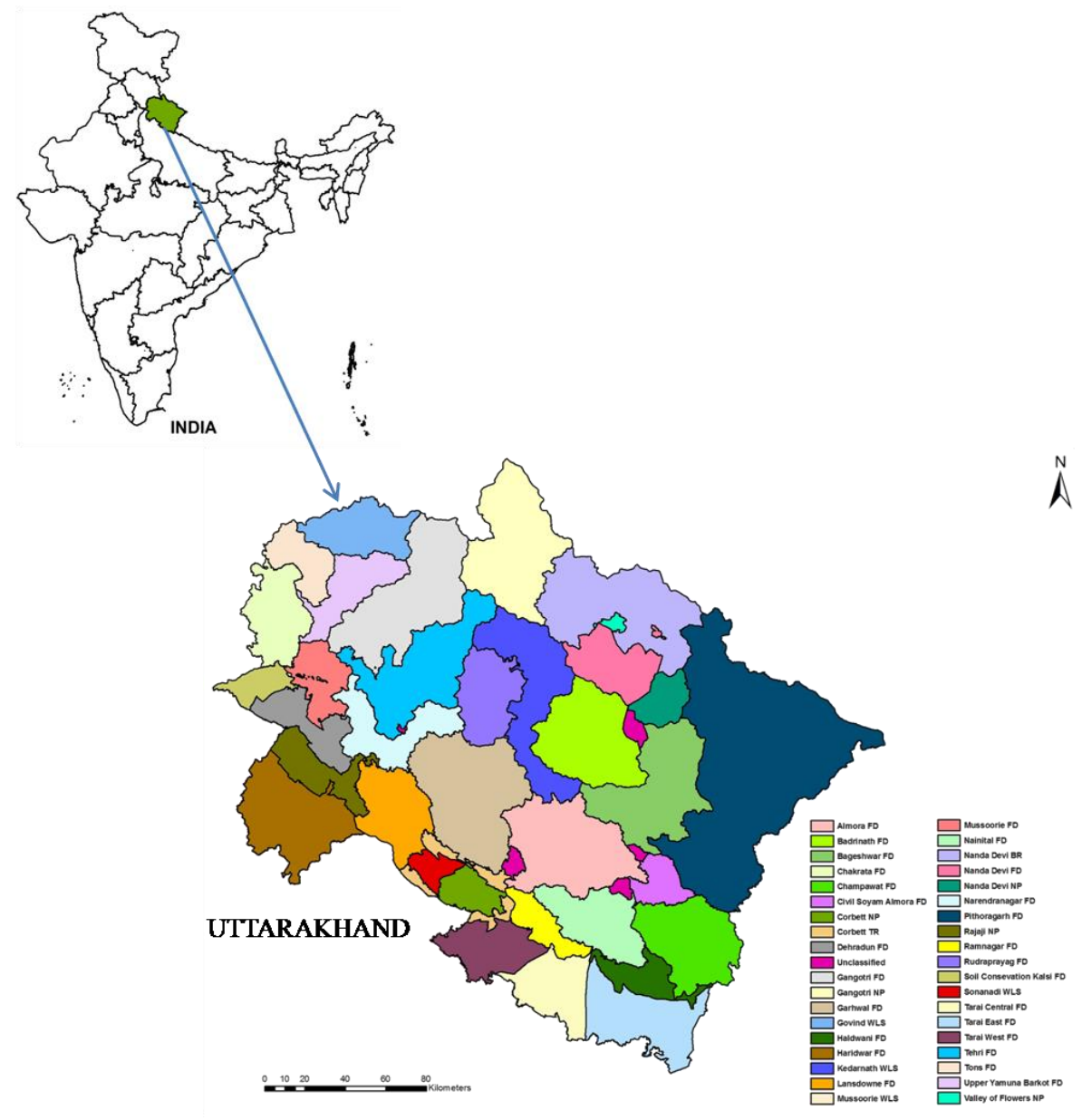

Figure 1 - Location map of the study area

Based on the field visit the study area was classified into 4 classes (table 1). Forest cover map was generated using unsupervised classification approach was used for Vegetation Density Mapping using ERDAS imagine 2013 software. The study area was classified into 50 spectral classes using unsupervised image classification approach. Eventually the Vegetation of study area was stratified into four major types on the basis of density (table 1) as per the fundamental criteria of Forest Survey of India (FSI, 2013). Slope, Aspect and Elevation map was generated from CARTOSAT 30m DEM using boundary vector layer with the help of Arc map 10.1. Road Network, Drainage Network were digitized from Survey of India Toposheet at 1:50,000 scale with the help of Arc map 10.1 software. GPS location of Habitation and Forest watch station was collected from field visits. Initially 19 'bioclimatic' variables were considered from WorldClim database. A subset of Bioclim 30m resolution of study area was clipped using boundary vector layer in Arc map 10.1.

The ENM Tools version 1.3 (Warren et al. 2010) was used to test multi co-linearity between 31 predictor variables. The criteria adopted to predict multi-collinearity between the variables was Pearson Correlation Coefficients ( $r$ ). On the basis of $r$ value we can determine whether two different variables will have distinct spatial effect on the distribution of the species or not. Henceforth the variables with $r>0.7$ i.e. variable showing more than $70 \%$ correlation were not used in analysis and model building. These variables were found to be correlated and hence only those variable were used that were found to be spatially distinct in this study. 
Table 1 - Predictor variables tested for prediction modeling of fire in Pine zone

\begin{tabular}{|c|c|c|c|c|c|}
\hline \multicolumn{3}{|c|}{ Variable } & Code & source & Type \\
\hline \multirow{19}{*}{ Climate } & \multicolumn{2}{|c|}{ Bio 1 = Annual Mean Temperature } & Biol & \multirow{19}{*}{ Worldclim } & \multirow{19}{*}{ continuous } \\
\hline & \multicolumn{2}{|r|}{$\begin{array}{l}\text { Bio } 2=\text { Mean Diurnal Range (Mean of monthly (max temp } \\
- \text { min temp) }\end{array}$} & Bio2 & & \\
\hline & \multicolumn{2}{|r|}{ Bio 3 = Isothermality (P2/P7)*(100) } & Bio3 & & \\
\hline & \multicolumn{2}{|r|}{ Bio 4 =Temperature Seasonality (standard deviation*100) } & Bio4 & & \\
\hline & \multicolumn{2}{|c|}{ Bio 5 = Max Temperature of Warmest Month } & Bio5 & & \\
\hline & \multicolumn{2}{|c|}{ Bio $6=$ Min Temperature of Coldest Month } & Bio6 & & \\
\hline & \multicolumn{2}{|c|}{ Bio 7 =temperature Annual Range (P5-P6) } & Bio7 & & \\
\hline & \multicolumn{2}{|r|}{ Bio $8=$ Mean Temperature of Wettest Quarter } & Bio8 & & \\
\hline & \multirow{2}{*}{\multicolumn{2}{|c|}{ Bio 9=Mean Temperature of Driest Quarter }} & Bio9 & & \\
\hline & & & Biol0 & & \\
\hline & \multicolumn{2}{|r|}{ Bio 11 =Mean Temperature of Coldest Quarter } & Bioll & & \\
\hline & \multicolumn{2}{|c|}{ Bio $12=$ Annual Precipitation } & Biol2 & & \\
\hline & \multicolumn{2}{|c|}{ Bio $13=$ =Precipitation of Wettest Month } & Biol3 & & \\
\hline & \multicolumn{2}{|c|}{ Bio $14=$ Precipitation of Driest Month } & Biol4 & & \\
\hline & \multicolumn{2}{|r|}{$\begin{array}{l}\text { Bio } 15=\text { Precipitation of Seasonality (Coefficient of } \\
\text { Variation) }\end{array}$} & Biol5 & & \\
\hline & \multicolumn{2}{|c|}{ Bio 16 =Precipitation of Wettest Quarter } & Bio16 & & \\
\hline & \multicolumn{2}{|c|}{ Bio $17=$ Precipitation of Driest Quarter } & Biol7 & & \\
\hline & \multicolumn{2}{|c|}{ Bio $18=$ Precipitation of Warmest Quarter } & Biol8 & & \\
\hline & \multicolumn{2}{|c|}{ Bio $19=$ =Precipitation of Coldest Quarter } & Bio19 & & \\
\hline \multicolumn{3}{|c|}{ Elevation (m) } & elevation & $\begin{array}{l}\text { CARTOSAT } \\
\text { DEM }\end{array}$ & continuous \\
\hline \multicolumn{3}{|c|}{$\begin{array}{l}\text { Slope }\left({ }^{0}\right) \\
\text { Aspect }\left({ }^{0}\right)\end{array}$} & $\begin{array}{l}\text { Slope } \\
\text { Aspect }\end{array}$ & $\begin{array}{l}\text { Calculated } \\
\text { from } \\
\text { CARTOSAT } \\
\text { DEM }\end{array}$ & continuous \\
\hline \multicolumn{3}{|c|}{$\begin{array}{l}\text { Distance to the nearest village/ } \\
\text { tribal settlement }(\mathrm{m})\end{array}$} & $D 2 v$ & $\begin{array}{l}\text { Field data GPS } \\
\text { location }\end{array}$ & continuous \\
\hline \multicolumn{3}{|c|}{ Distance to the nearest water source (m) } & $D 2 d$ & SOI-toposheet & continuous \\
\hline Distance & 0 the & nearest watch station (m) & $D 2 w t$ & $\begin{array}{l}\text { Field data GPS } \\
\text { location }\end{array}$ & continuous \\
\hline Distance & o the & nearest road (m) & $D 2 r$ & SOI-toposheet & continuous \\
\hline Actual E & apo-t & anspiration & AET & CGIR & continuous \\
\hline Aridity i & & & AI Index & CGIR & continuous \\
\hline Populati & n den & & $\begin{array}{l}\text { Population } \\
\text { density }\end{array}$ & Diva GIS & continuous \\
\hline & & $1=$ Very Dense Forest & & & \\
\hline & & $2=$ Moderately Dense Forest & & & \\
\hline Forest $\mathrm{C}$ & ver & $3=$ Open Forest & $f_{c m}>$ & Satellite data & categorical \\
\hline Тур & & $4=$ Scrub & $J c m$ & Satemine data & \\
\hline & & $5=$ Water & & & \\
\hline & & $6=$ Non Forest & & & \\
\hline & & 1=Tropical Moist Deciduous & & & \\
\hline & & 2=Dry Deciduous & $f t m$ & Satellite data & \\
\hline Forest $\mathbf{T}$ & & $3=$ Northern Sub-Tropical Broadleaved & Jim & satemite data & categorical \\
\hline & & 4=Himalayan Temperate Forest & & & \\
\hline
\end{tabular}

\subsection{Fire modelling}

MaxEnt software package (version 3.3.3.e; Phillips et al., 2004; http:// www.cs.princeton.edu/ schapire/maxent/) was used for fire modeling that implements a maximum entropy algorithm, which generates a probability distribution map of similar conditions across the landscape considering the characteristics of the occurrence GPS locations (Phillips et al., 2006; Elith et al., 2011). The Maxent is a machine learning algorithm used to predict robust ecological niches of a species, with presence 
records, even when only a few are available (Elith et al., 2006; Phillips et al., 2006; Papeş and Gaubert, 2007; Kumar and Stohlgren, 2009). This model has an advantage where presence/available data are limited (Elith et al., 2006; Phillips and Dudik, 2008). In the present study based on the assumptions of the MaxEnt model a fire prediction modeling was attempted (Phillips et al., 2006).

\subsection{Model parameter settings}

The maximum number of background points was 5,000 and implementing linear, quadratic and hinge features was used (Phillips and Dudik 2008). 100 replicates were kept for model building (Flory et al., 2012) and the occurrence locations were partitioned randomly into two sub samples, using $75 \%$ of the locations as the training dataset and the remaining $25 \%$ for testing the resulting (partitioned) models. The accuracy of the model was using the area under the curve (AUC) of a receiver operating characteristic $(\mathrm{ROC})$ plot (ranging from $0.5=$ random to $1=$ perfect discrimination). Jackknife procedure was adopted to assess the variables importance (Yang et al., 2013). Average of 100 model predictions were used to produce a probability map of fire occurrence. However the other settings for the model parameters and values were kept as default.

Additionally, for demonstration of very highly, highly, moderately and least susceptible fire areas, all values above 0.8 were categorized as very highly susceptible to fire, the values between 0.6 and 0.8 as highly susceptible fire area and those between 0.2 and 0.4 as moderately susceptible fire areas. Based on a $10 \%$ training presence logistic threshold, values below 0.2 were selected as least susceptible fire area.

\section{Results and discussion}

A total of 529 fire incidences were found to be saptio-temporally independent. Multi-collinearity test showed that out of 31 variables only 22 variables were not correlated wth each other. Jackknife test results showed that based on the percentage contribution, "Forest canopy density" (18.2\%), "distance from nearest village" (d2v) (15\%), "Isothermality" (bio3) (12.4\%) and "Forest Type" $(10.1 \%)$ were the highest contributors. Based on permutation importance, "Precipitation of seasonality" (bio15) was the most significant variable (16.6\%) followed by "Isothermality" (bio3) (15.5\%) (figure 2).

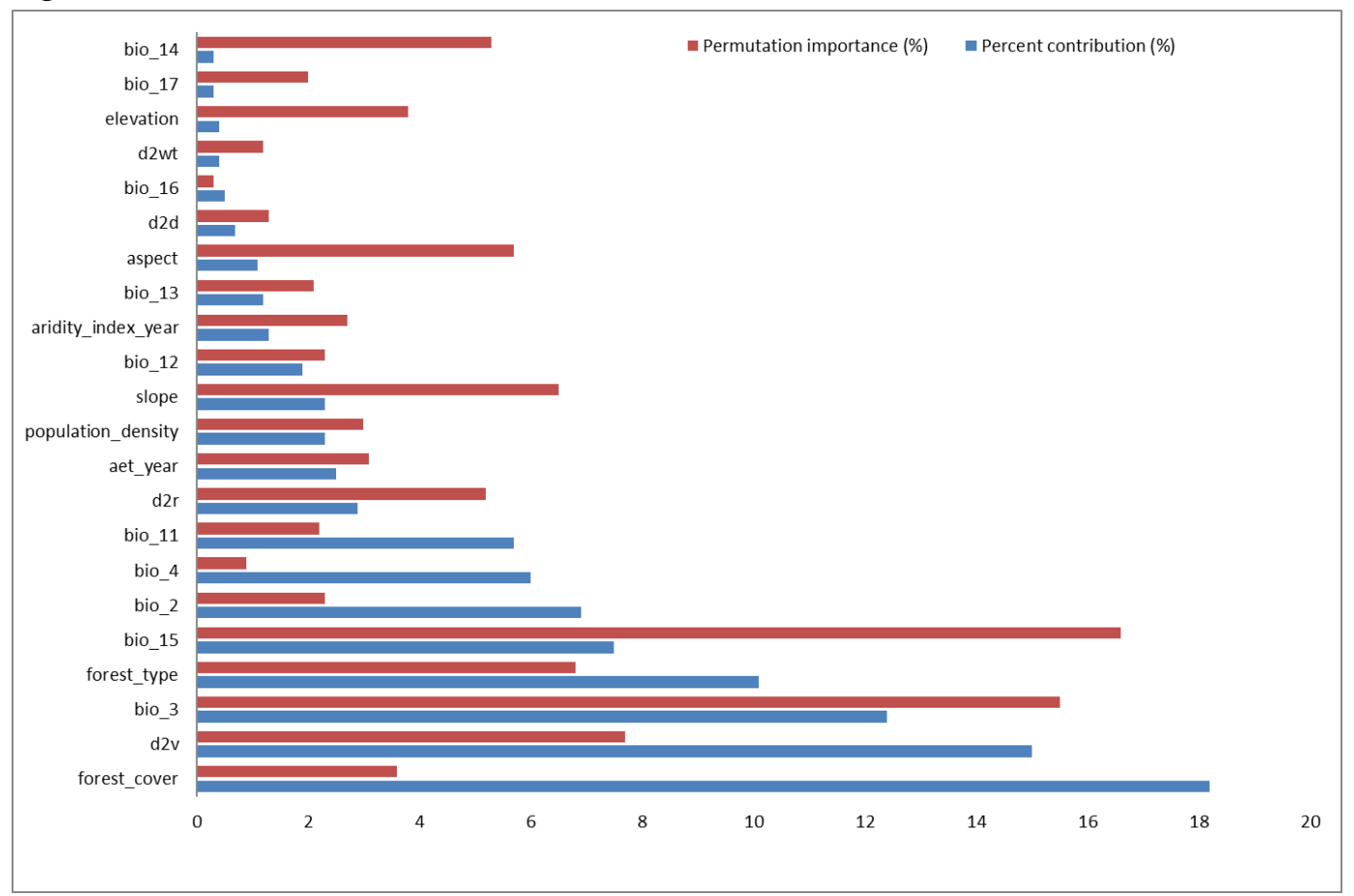

Figure 2 - Percent contribution and permutation importance of variables 
The area under the curve (AUC) score was 0.896 for the training data from our model, which indicates moderate to excellent predictive ability of the model (figure 3 ).

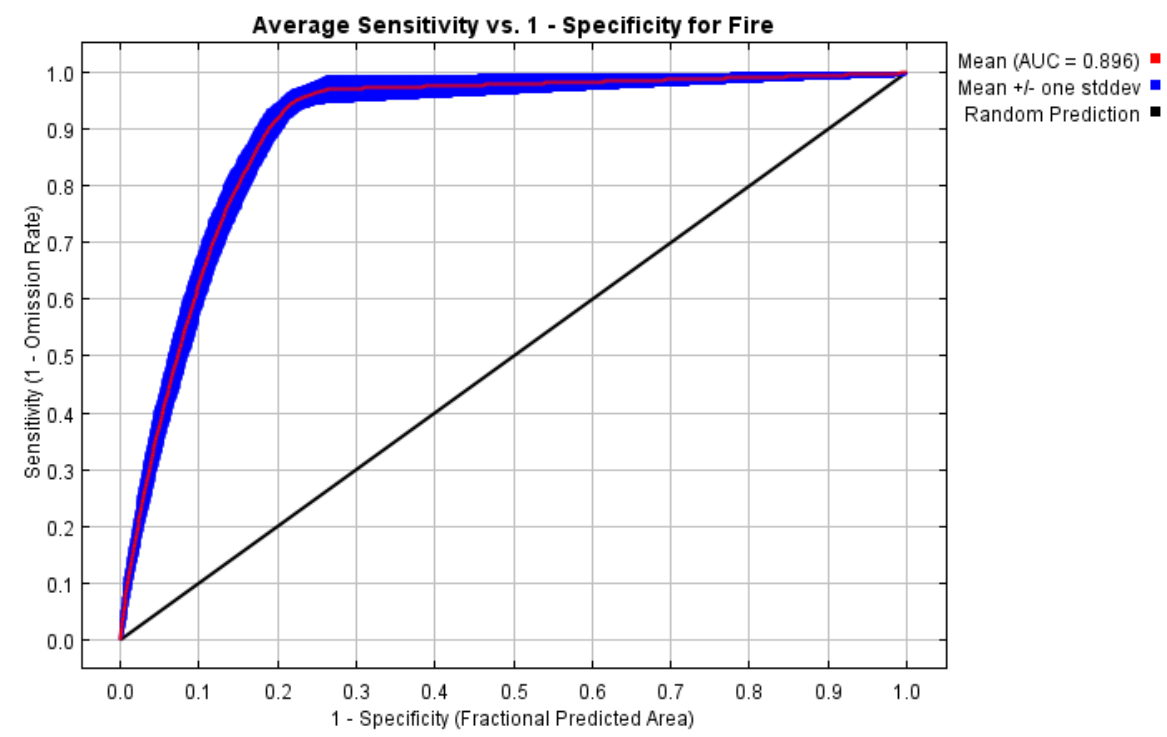

Figure 3 - Area under Curve of Fire (AUC)

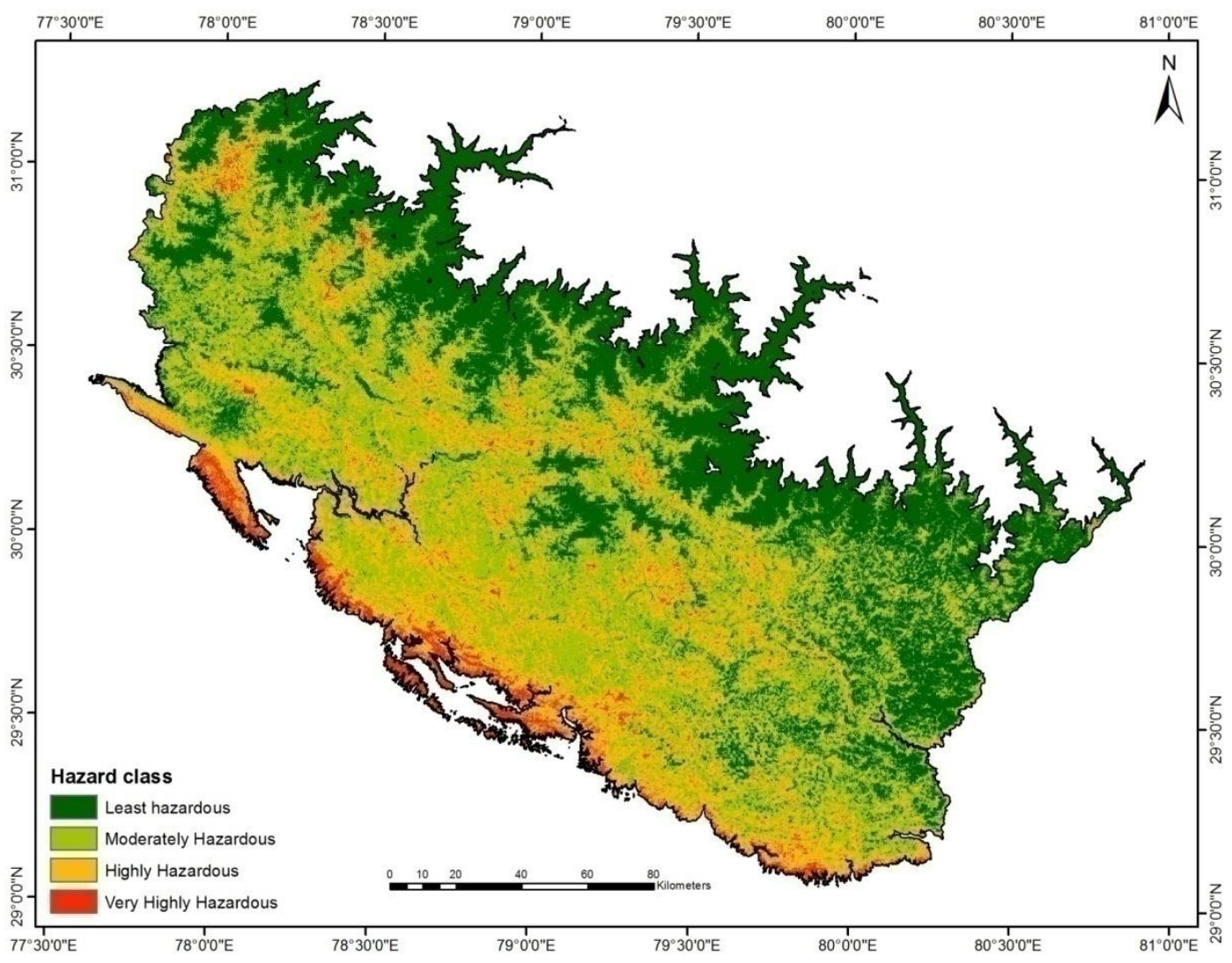

Figure 4 - Classified fire hazard map

The classified fire prediction map showed good discrimination between very high, high, moderate and least susceptible categories. The result demonstrated that out of the total geographical area of pine zone 120933.16 ha area (3.64\%) was under very high susceptible category, 708722.55 ha area $(21.39 \%)$ under high susceptible category, 1318959.84 ha area $(39.81 \%)$ under moderately susceptible 
category and 1164751.26 ha area (35.16\%) under least susceptible category (figure 4$)$. The areas near to village having moderate canopy density were highly affected by fire.

\section{Conclusion}

Pine forests at lower elevation and near to settlement are badly impacted by fire. The present methodology provides substantial improvement over frequency analysis method as used by earlier workers for developing fire hazard models in pine zone. Such types of models if recalibrated using future data can lead to higher degree of accuracy of fire prediction. Validation of fire hazard map shows that it resembles the ground situation, hence it can act as a baseline map for planning fire control in pine zone and can be useful for finalising best locations for fire lines, establishment of new Fire Crew Stations, Forest Guard Chowkis etc. GIS based modelling is helpful in identifying, mapping, quantification of biophysical parameters at spatio-temporal scale and also for the future predictions of forest fire. Geospatial modelling can potentially enhance forest fire management by providing spatially mapped fire hotspots. The forest fire hazard model as generated under this study would be helpful in detecting and mitigating the fire in pine zone of Himalayas and can be used for preparatory planning to minimize the fire frequency and losses thereof. Our results showed that MaxEnt may become an important tool to guide on-the-ground decisions on fire prevention actions and planning more effectively for management of forest fire with respect to conservation of forest and biodiversity in Pine Zone.

\section{References}

Craw. (1875). A few notes on the nursery treatment of Chil, Chir. Indian Forester, (1), 305.

Dhaundiyal, A. and Gupta, V. K. (2014). The analysis of pine needles as a substrate for gasification. Hydro Nepal: Journal of Water, Energy and Environment; 15, 73-81.

Elith, J, Phillips, S.J, Hastie, T, Dudík, M, Chee, Y.E., Yates, C.J. (2011). A Statistical Explanation of MaxEnt for Ecologists. Diver. and Distri. 17, 43-57

Elith, J, Graham, C.H., Anderson, R.P., Dudík, M., Ferrier, S., Guisan, A., Hijmans, R.J., Huettmann, F., Leathwick, J.R., Lehmann, A., Li J., Lohmann, L.G., Loiselle, B.A., Manion, G., Moritz, C., Nakamura, M., Nakazawa, Y., Overton, J.M., Peterson, A.T., Phillips, S.J., Richardson, K.S., Scachetti-Pereira, R., Schapire, R.E., Soberón, J., Williams, S., Wisz, M.S., Zimmermann, N.E., (2006). Novel methods improve prediction of species' distributions from occurrence data. Ecography, 29, 129-151.

Flory, A.R., Kumar, S., Stohlgren, T.J., Cryan, P.M. (2012). Environmental conditions associated with bat white nose syndrome mortality in the north-eastern United States. J Appl Ecol, 49: 680-689.

Forest Survey of India. (2013). India State of Forest Report. MOEF, Govt. of India.

Gamble, J. S. (1875). The Darjeeling Forests. Indian Forester (1) 81.

Hijmans, R. J. et al; (2002) DIVA-GIS Version 2, A geographic information system for the analysis of biodiversity data Manual.

Hijmans, R. J., Cameron SE, Parra JL, Jones PG, Jarvis, A. (2005). Very high resolution interpolated climate surfaces for global land areas. Int. J. Clim. 25, 1965-1978.

Kumar, S, Stohlgren, T.J. (2009).Maxent modeling for predicting suitable habitat for threatened and endangered tree Canacomyrica monticola in New Caledonia. J. Ecol. and Nat. Envir. 1(4), 94-98

Phillips, S. J. et al., (2006).Maximum entropy modelling of species geographic distributions.

Phillips, S. J., Miroslav, D, Schapire, R.E. (2004). Maxent Software for Species Distribution Modeling. http://cs.princeton.edu/ schapire/Maxent/ 
Phillips, S. J., Anderson, R.P, Schapire, R.E. (2006). Maximum entropy modeling of species geographic distributions. Ecol Mod, 190: 231-259

Pearson, R., Raxworthy C, Nakamura M, Peterson, A. (2007). Predicting species distributions from small numbers of occurrence records: a test case using cryptic geckos in Madagascar. J Biogeo, 34:102-117.

Phillips, S. J., Dudík, M (2008). Modeling of species distributions with Maxent: new extensions and a comprehensive evaluation. Eco gra, 31: 161-175

Troup, R.S. (1921). The Silviculture of Indian Trees. Vol. III. Clarendon Press, Oxford, UK.

Verma, A. K., and Kumar,S. (2015). Mapping Fire Hazard in Rajaji National Park, Future Perspective of Wildlife Habitat Conservation by Using Remote Sensing and GIS. International Journal of Technical Research and Applications. 5(3), 81-88. ISSN: 2320-8163.

Verma, A.K., Singh, D., Sharma, S.D. and Khanduri, K. (2013). Forest Fire Risk Zonation in Raipur Range, Mussoorie Forest Division using: GIS and Remote Sensing Technology. International Journal of Advanced Scientific and Technical Research, 6(3), 141-150. ISSN 2249-9954

Verma, A.K. (2016). Predictive modeling and mapping of wildland fire in Lansdowne corridor of Lesser Himalayas: A future perspective for Tiger conservation. In: Proceedings for the $5^{\text {th }}$ International Fire Behavior and Fuels Conference, International Association of Wildland fire, Missoula, Montana, USA.

Warren, D.L., R.E., Glor, R.E., Turelli, M (2010). ENM Tools: a toolbox for comparative studies of environmental niche models. Ecogr, 33:607-611

Yang, X., Kushwaha, S. P. S., Saran, S., Xu, J., Roy, P.S. (2013). MaxEnt modeling for predicting the potential distribution of medicinal plant, Justicia adhatoda L. in Lesser Himalayan foothills, Ecological Eng, 51, 83- 87.

\section{Websites:}

http://sbb.uk.gov.in/pages/display/93-about-uttarakhand http://www.worldclim.org/bioclim https://firms.modaps.eosdis.nasa.gov 


\title{
System for personnel training in decision making of wildfires fighting
}

\author{
Georgy Dorrer*; Alexandra Dorrer; Igor Buslov; Sergey Yarovoy \\ Reshetnev Siberian State University of Science and Technology, Krasnoyarsk, Russia \\ \{g_a_dorrer@mail.ru*\}
}

\begin{abstract}
Wildfires (forest, peat, steppe, etc.) fighting is a complex and often dangerous process, connected with cooperation of many organizations and crews. Thus, suppression of a wildfire is a complicated process that requires a good organization. An important role in this process plays the qualitative training of personnel engaged in fighting the fire. In the process of extinguishing the fire crew of various organizations are involved: employees of Russian Aviation Forest Protection agency Avialesoohrana, crews of the Russian Ministry of Emergencies, members of voluntary fire crews created by municipalities. In accordance with the standard issued in Russia in 2018, a new working specialty - forest firefighter - has appeared. To such specialists are put quite high demands. They should not only be able to extinguish fires, but also have the skills of firefighting tactics, search engines, rescuers, psychologists, organizers and communication specialists.

Training of personnel in the techniques and tactics of combating wildfires is carried out in educational systems at various levels - from special education in the university to short-term courses for recruited employees of outside organizations. However, regardless of the educational institution level in which the training is conducted, members of firefighting crews should have basic knowledge of fire safety techniques, methods and tactics for wildfires combating.

As it known, one of the currently used effective forms of personnel training and professional development is interactive computer training systems that allow individual approach to the trainees both in material mastering and testing. A separate class of training systems are computer simulators widely used in various fields. Given the dynamic nature of the wildfire spread and the need to make operational decisions, training system should include the elements of a simulator - an imitation subsystem, which helps the trainee to observe the development of fire situation on the map, make decisions and evaluate their effectiveness.

In the work presented, an interactive simulation system FIREMAN, developed by the authors, is discussed. The system was designed to train the personnel on the basics of knowledge of wildfires and decision making to combat them. The structure of the system is described and an example of working system is given.
\end{abstract}

Keywords: wildfires simulation; personnel training; decision making, learning management system MOODLE

\section{Introduction}

Wildfires fighting is a complex and often dangerous process, connected with cooperation of many organizations and crews. Thus, suppression of a wildfire is a complicated process that requires a good organization (Davis, 1959, Deeming, 1972, Middlebon, 1977, Schetinski, 2002).

Wildfires cause huge economic and environmental damage to the countries and regions in which they occur, leading to the death of people. To support effective control of wildfires, a large number of information systems have been developed for simulation and prediction fire behavior (BehavePlus (Andrews, 2003), FARSITE (Finney, 1998), WFDS (Mell, 2007), etc.).

However, in addition to modeling directly the edge of the natural fire, there is a need to simulate various external influences on the fire for its localization and elimination. Such models can be actively used in the management of the fire situation, in particular, to solve the problem of effective 
management of firefighting forces and facilities (European Forest Fire Information System, 2009, Russian information system of remote monitoring ISDM-Rosleskhoz , 2010), etc.

An important role in this process plays the qualitative training of personnel engaged in fighting the fire. In the process of extinguishing the fire crews of various organizations are involved: employees of Russian Aviation Forest Protection agency Avialesoohrana, crews of the Russian Ministry of Emergency Situations, members of voluntary fire crews created by municipalities.

In accordance with the standard issued in Russia in 2018, a new working specialty - forest firefighter - has appeared. To such specialists are put quite high demands. They should not only be able to extinguish fires, but also have the skills of firefighting tactics, search engines, rescuers, psychologists, organizers and communication specialists.

In fire extinguishing are involved, for example, fire jumpers, paratroopers, personnel of the fire and chemical stations. Forest firefighters can work in land groups, and in aviation units. These structures are under the jurisdiction of Avialesoohrana and are subordinated to Rosleskhoz. Professional standard unites these two spheres into one working profession.

According to the professional standard, forest fire extinguishers are divided into three classes. Firemen of the third class should be able to extinguish fires with the help of hand-held technical means (for example, a shovel or a fire extinguisher), the second - with the use of special equipment (including tractors for extinguishing forest fires). Firefighters of the first class, in addition, must have skills of organizers and be able to conduct search and rescue operations. Forest firefighters in special cases should be able to perform the functions of managers. It is impossible to find so many firefighters to provide each group with a leader.

According to the professional standard, forest firefighters should be able to correctly apply various means and methods of extinguishing, taking into account the characteristics of the terrain and fires, to choose safe places for creating strong points, to navigate in the forest. They should be able to act in extreme situations, adverse weather conditions, under the influence of stress factors.

The personnel training in the techniques and tactics of combating wildfires is carried out in educational systems at various levels - from special education in the universities (Matveev, 2002), where the course of forest pyrology is taught, to short-term courses for recruited employees of outside organizations. However, regardless of the level of the educational institution in which the training is conducted, members of firefighting crews should have basic knowledge of fire safety techniques, methods and tactics for wildfires combating.

As it known, one of the currently used effective forms of personnel training and professional development is interactive computer training systems that allow individual approach to the trainees both in material mastering and testing. A separate class of training systems are computer simulators widely used in various fields. Given the dynamic nature of the wildfire spread and the need to make operational decisions, training system should include the elements of a simulator - an imitation subsystem, which helps the trainee to observe the development of fire situation on the map, make decisions and evaluate their effectiveness.

In the work presented, the interactive simulation system FIREMAN, developed by the authors, is discussed. The system was designed to train the personnel in the basics of knowledge of wildfires and decision making to combat them.

\section{Structure and functions of the fireman system}

The system is a web-oriented software package based on learning management system (LMS) (MOODLE, 2010) and the TAIGA simulation program. Structure and components of the FIREMAN system are shown in Figure. 1. 


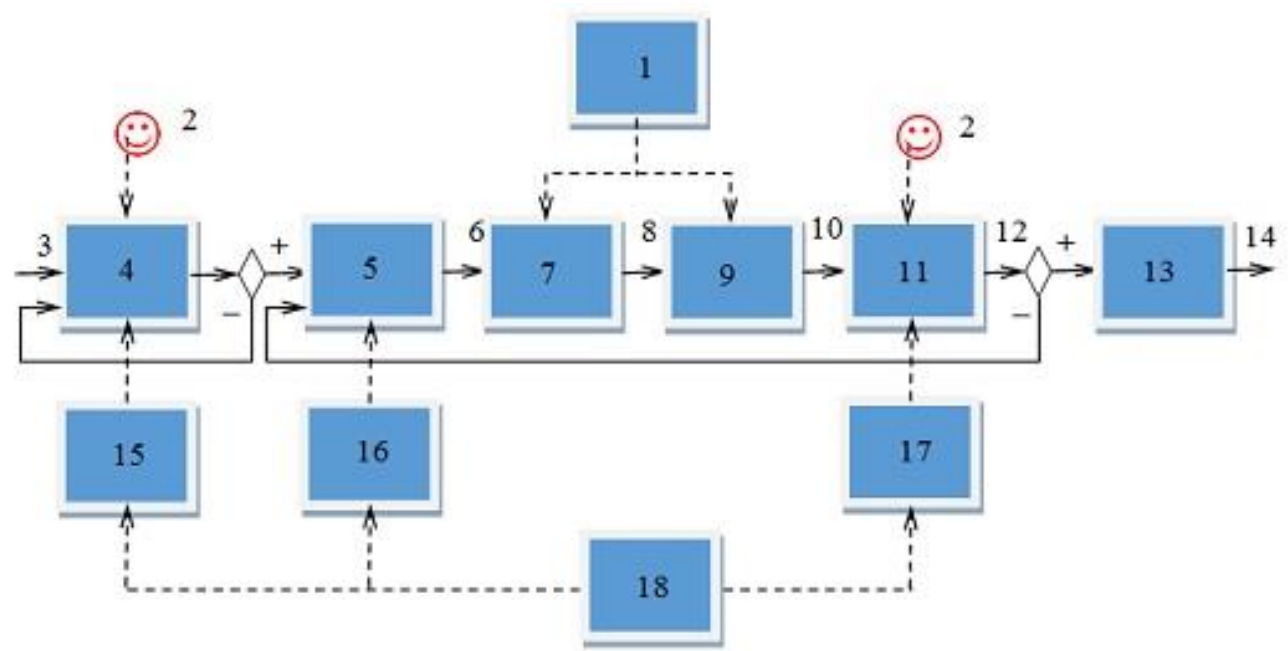

Figure 1 - The structure of the imitation training system FIREMAN. Legend: 1 - simulation program TAIGA; 2 tutor; 3 - login to the system; 4 - preliminary training and testing; 5 - game scenario selection; 6 - scenario data; 7 firefighting process simulation; 8 - system events; 9 - events protocol formation; 10 - events protocol; 11 - trainee's actions appraisal; 12 - trainee's actions assessment; 13 - training passage report formation; 14 - end of training; 15 e-learning course on basics of firefighting and safety techniques; 16 - scenarios base; 17 - trainee's work appraisal rules; 18 - LMS Moodle.

\subsection{Functions of LMS MOODLE:}

- preliminary training and testing of trainees,

- assignment to a trainee the game scenario variant for the TAIGA simulator,

- receiving game events protocol from TAIGA,

- appraisal of trainee's decisions by tutor,

- formation an overall assessment of the trainee's work,

- formation the report with final grades.

\subsection{Functions of the TAIGA program:}

- demonstration of various scenarios for wildfire development and elimination;

- creation a game situation based on the initial data of the scenario: a map of the terrain with the indication of possible protection objects, characteristics of vegetable fuel materials, fire danger class, wind speed and direction, initial configuration of the fire, list of available firefighting forces and facilities;

- modeling the fire dynamics on basis of agent approach and displaying fire situation on the map of the terrain: fire spreading, action of fire-fighting forces and facilities (Dorrer, 2008, Yarovoy, 2016);

- messages generation on the fire dynamics: area passed by fire, length of the burning edge, distance to the object of protection - after a predetermined time interval;

- messages generation about the decisions made by a trainee: calling personnel and technical means, instructions for their movement, placing firemen along the edge of the fire, building fire protection bands, etc., time-bound;

- detection of catastrophic situations: objects of protection captured by fire, firefighters or equipment affected by fire, uncontrolled growth of fire area and length of a burning edge, etc.;

- events data transfer to MOODLE.

\subsection{Functions of the tutor (or moderator):}

- consultations and assistance to a trainee at the stage of preliminary training and supervision; 
- appraisal of a trainee's actions when his work with the imitation program is over, formation of a report on the training completion.

Let us consider in more detail the components of the system.

\section{Learning subsystem on the base of MOODLE}

Learning Management System (LMS) MOODLE is today one of the most popular in the world and is used both in traditional educational process, and in personnel training. The acronym MOODLE is formed from the initial letters of the name Modular Object-Oriented Dynamic Learning Environment.

In the MOODLE system, you can create and store electronic learning materials and specify the sequence of their study. Electronic format allows you to use not only text, but also interactive resources of any format from an article in Wikipedia to a video on YouTube. To do this, the system provides a lot of tools: wiki, glossary, blogs, workshops, hyperlinks, files and much more. Thus, Moodle is designed to create quality distance courses and to organize an effective educational process.

Moodle can also be useful for automating of personnel training in basis of combating wildfires tactics. To solve such a problem, a distance course integrated with a computer simulator TAIGA was created.

The course consists of three main sections. The first section is called "Testing" and contains a block with input tests (Figure 2). Choice of the input test for a trainee is determined by tutor. An example of the input test is shown in the Figure 3. If the trainee correctly answered more than $90 \%$ of questions, a link to the computer simulator TAIGA, which is located in the second section of the course, becomes available to him. The second section is called "Interactive imitation system FIREMAN" (Figure 4).

\section{Educational and training system Taiga}

Educational and training system TAIGA developed by the authors can be used for training of various categories of trainees in educational and playful way in the basics of tactics to combat wildfires. TAIGA is a network geographic information system (GIS) with reference to the real terrain. The system has client-server architecture. To reduce the load on the network and better scalability, most calculations are performed on the client side that allows a user to distribute the load evenly between all devices. Server part is developed on PHP. The server contains a database that stores information about the simulation environment (real terrain). Client part is developed on JavaScript using a library of open source OpenLayers. This library is used to create maps based programming interface (API) and allows a user to create web-based interface for the maps display. This library can work with data from different map services, such as OpenStreetMap, Google, Yandex and others. 


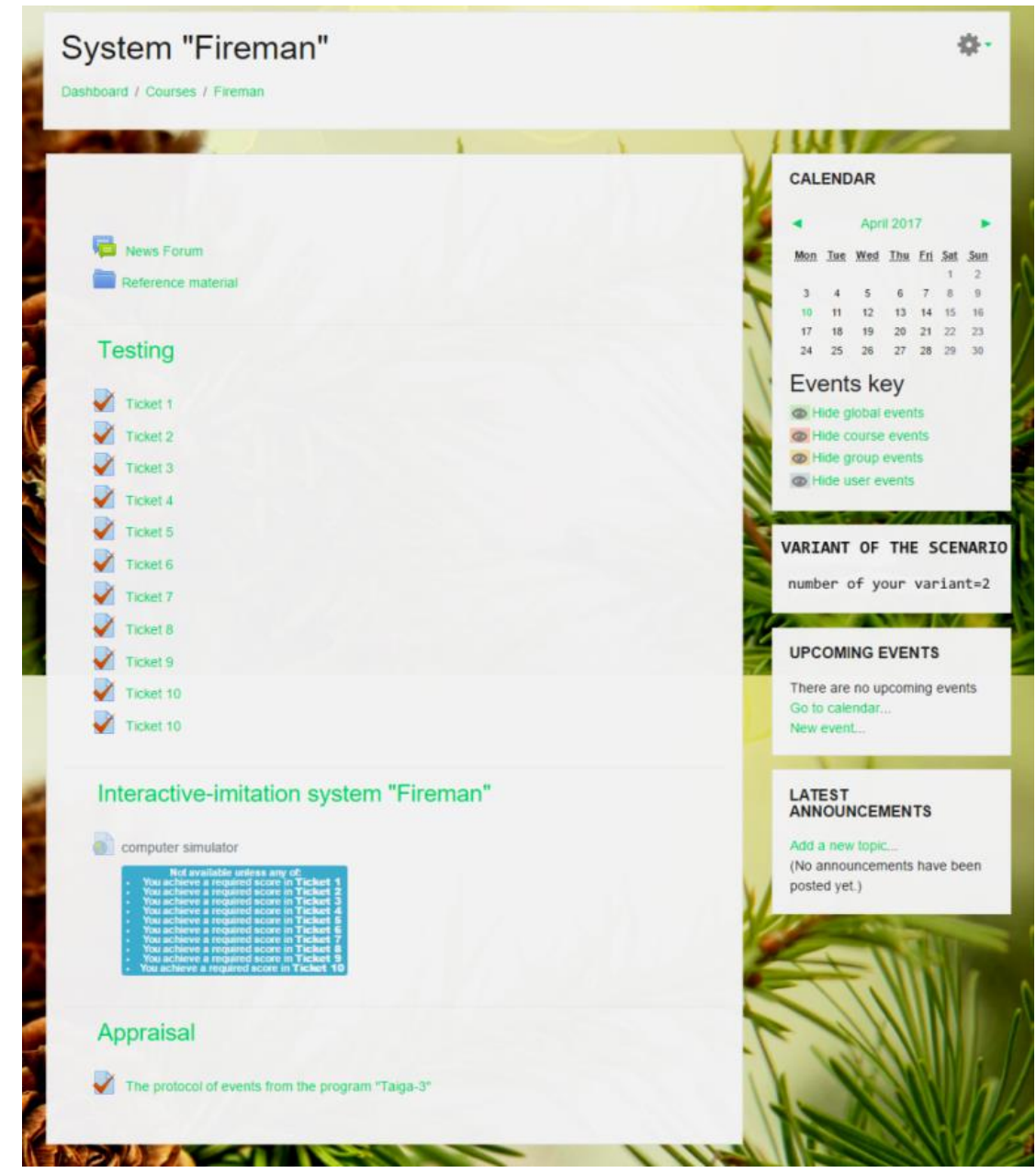

Figure 2 - Appearance of the Fireman Course Page

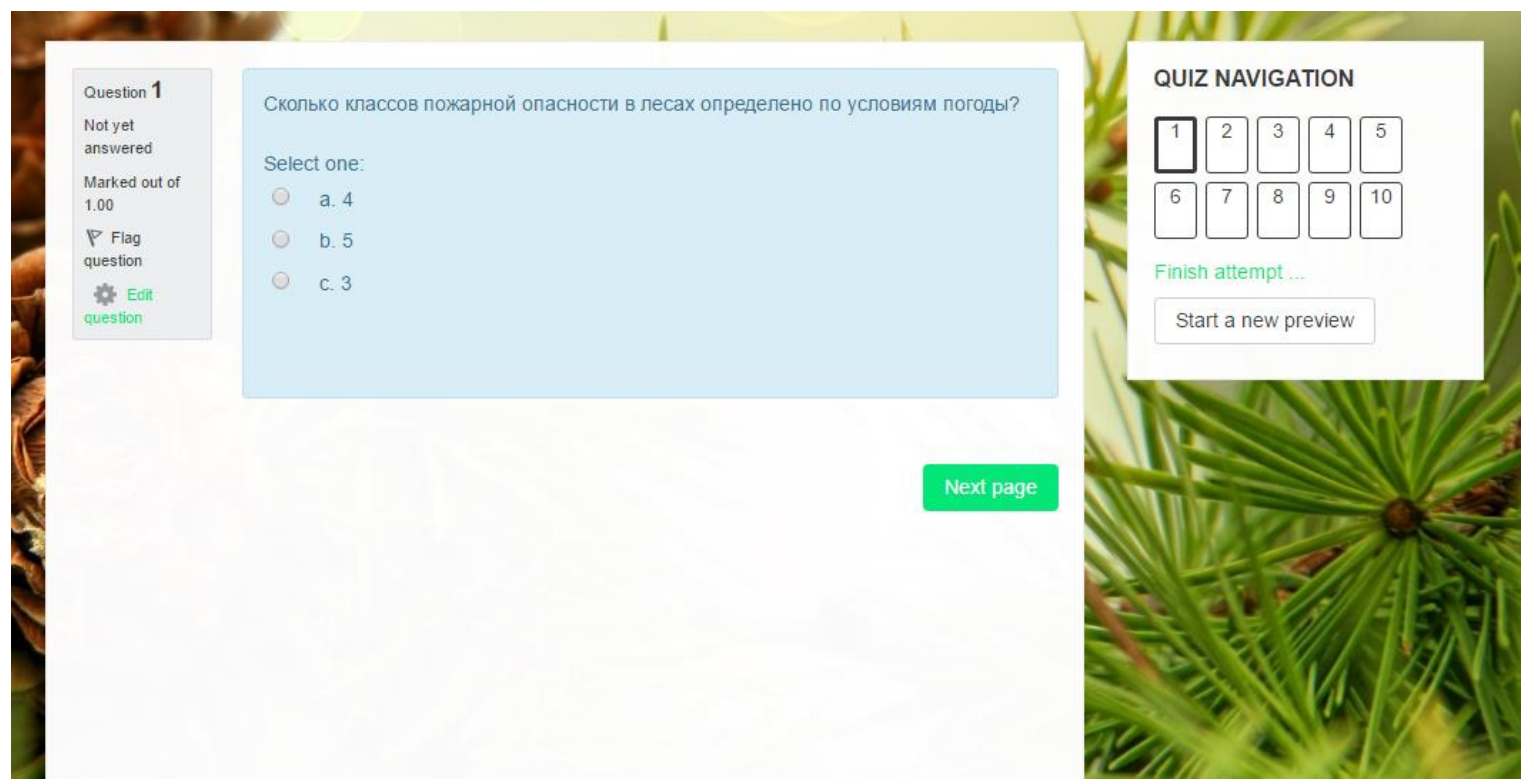

Figure 3 - Preliminary Test Example

The authors have developed an agent-based system containing two types of agents, designated A and B. Type A agents simulate the movement of the wave front. They can be in active and passive states. A-agent, which is in the active state, determines the motion of the process front. Agents of type 
B (B agents) simulate the impact on agents of type A. They pursue a single goal - to translate all Aagents into a passive state. To do this, the B-agent moves through the simulation environment to the nearest A-agent and, approaching it, carries out a control action aimed at destroying it.

Figure 4 shows the localization menu, which is located on the right side of the system interface. This menu consists buttons, switches and text fields. On the left side of the system interface there is a map of the real terrain.

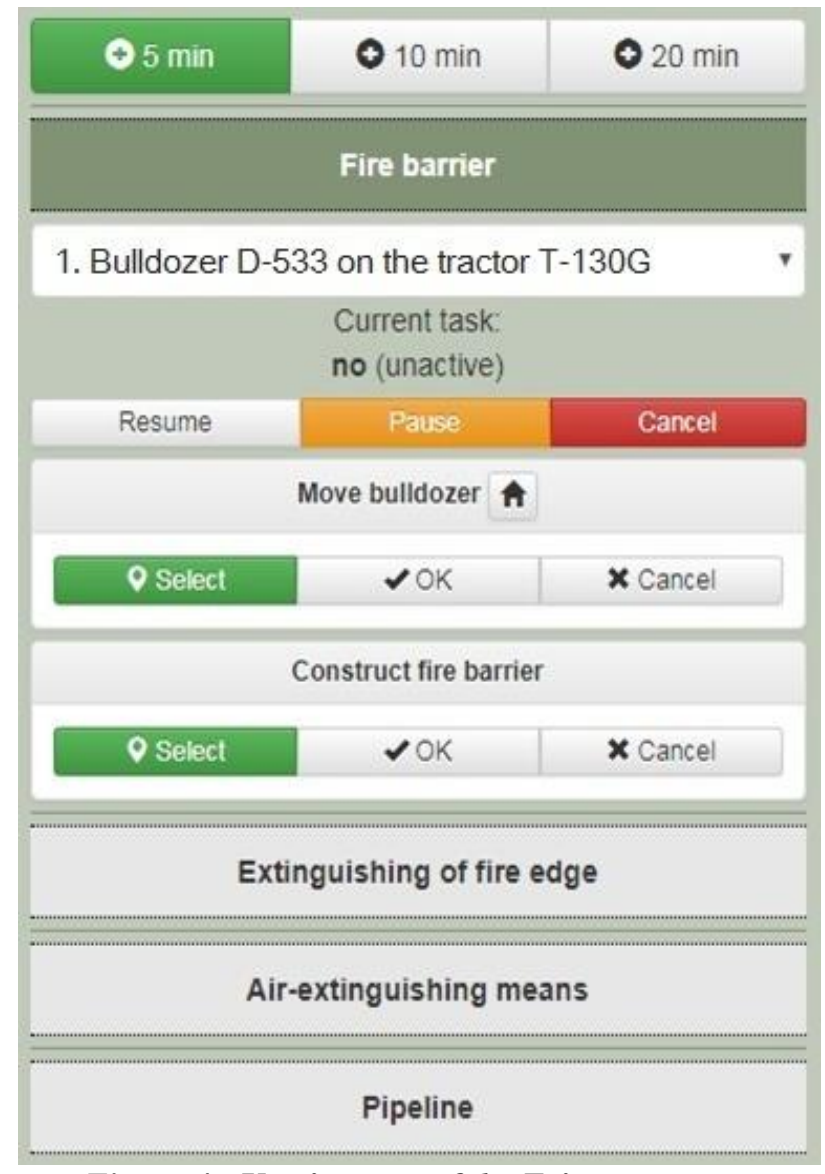

Figure 4 - User's menu of the Taiga system

\section{An example of decision making}

A simple example of wildfire fighting imitation is given below.

The environment settings of the fire simulation are the next.

- Polygon on the right-hand side of the figure, circled by a dashed line, represents a locality (protected object);

- area of the wildfire at the start is 0.22 ha;

- wind speed under the canopy is $2 \mathrm{~m} / \mathrm{s}$;

- wind direction from the north.

- fire danger class is four.

- aim of the game - to protect object from wildfire.

The place where the fire starts is appointed by the tutor. Initial fire contour is constructed automatically. In this example, the trainee decided to construct fire barriers to exclude the possibility of reaching by wildfire a protected area. Figures $5 \mathrm{~A}-5 \mathrm{C}$ shows the fire propagation dynamics. A 
reader can see that the thickness of the fire barrier was not enough. The wildfire continued to spread and reached the settlement. Thus, simulation is over with the negative result.
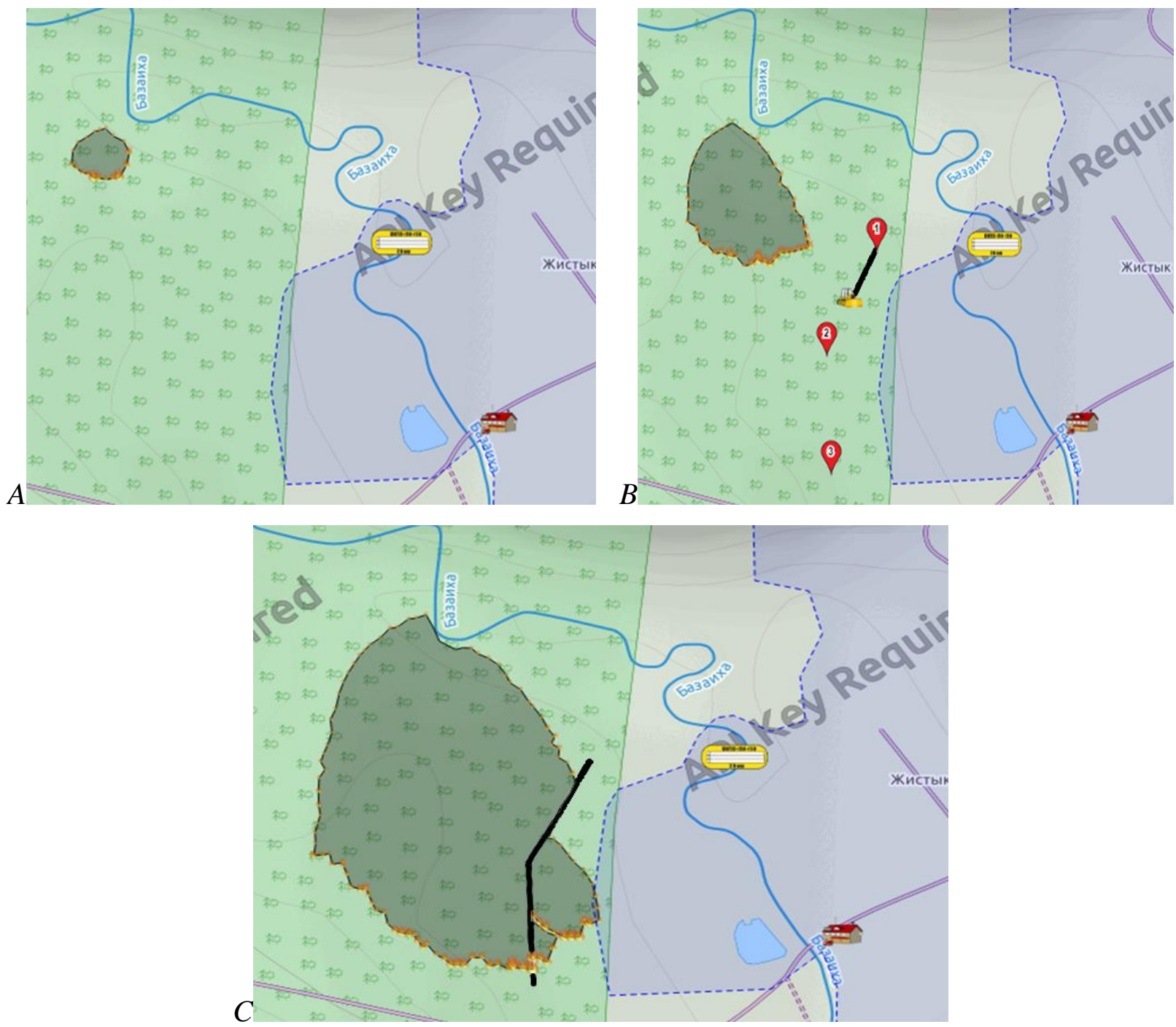

Figure 5 - Illustration of simulation process. Legend: A - start of the game, B - trainee desides to create a fire barrier and call a bulldoser, $C$ - end of the simulation process, object was not protected

During the simulation process, an event log of the trainee's actions was generated. The fire situation protocol is presented below.

\section{Time: 0 h, 0 m}

Detected wildfire with area $0,22 \mathrm{ha}$;

Distance to the protection object $393 \mathrm{~m}$;

Fire spread is $2,8 \mathrm{~m} / \mathrm{min}$;

Time: 0 h, $\mathbf{4 5}$ m

Trainee calls bulldozer D-533 for piercing the fire barrier;

\section{Time: 1 h, 0 m}

Area of wildfire 0,95 ha;

Distance to the protection object $281 \mathrm{~m}$;

Fire spread is $3,19 \mathrm{~m} / \mathrm{min}$;

Time: 1 h, 25 m

Bulldozer D-533 began piercing the fire barrier width 0,5 m; 


\section{Time: 2 h, $\mathbf{0} \mathbf{~ m}$}

Area of wildfire 1,995 ha;

Distance to the protection object $183 \mathrm{~m}$;

Fire spread is $2,08 \mathrm{~m} / \mathrm{min}$;

\section{Time: $\mathbf{2}$ h, 10 m}

Bulldozer D-533 ended construction the fire barrier;

\section{Time: $3 \mathbf{~ h , ~} \mathbf{0}$ m}

Area of wildfire 3,413 ha;

Distance to the protection object $110 \mathrm{~m}$;

Fire spread is $3,34 \mathrm{~m} / \mathrm{min}$;

\section{Time: $4 \mathbf{~ h , ~} 0$ m}

Area of wildfire 5,435 ha;

Distance to the protection object $50 \mathrm{~m}$;

Fire spread is $2,42 \mathrm{~m} / \mathrm{min}$;

\section{Time: $\mathbf{4} \mathbf{h}, \mathbf{4 0} \mathrm{m}$}

Area of wildfire 6,995 ha;

Distance to the protection object $0 \mathrm{~m}$;

Fire spread is $3,36 \mathrm{~m} / \mathrm{min}$;

Wildfire reached the protected object (see Figure 5C)

\section{Conclusion}

In the work presented the interactive imitation computer system FIREMAN is described. This system is intended for firefighting personnel training in basics of the wildfires struggle rules and tactics.

The system is a combine of popular e-learning system MOODLE and wildfire imitation system TAIGA developed by the authors. Such combination permits to obtain effective means for firefighters of different qualification levels training.

At the moment FIREMAN is tested in Reshetnev Siberian University of Science and Technologiy and in Siberian Fire and Rescue Academy.

\section{References}

Andrews PL et al. (2003). BehavePlus fire modeling system, version 2.0: User's Guide. USDA Forest Service Gen. Techn. Rep. RMRS-GTR-106WWW. (Ogden, UT).

Finney MA (1998) FARSITE: Fire Area Simulator-Model development and evaluation. USDA Forest Service, Rocky Mountain Research Station Research Paper RMRS-RP-4. (Ogden, UT).

Mell W et al. (2007). A physics-based approach to modeling grassland fires. International Journal of Wildland Fire. 16, 1-22.

Davis KP (1959) 'Forest fire: control and use' (McGraw-Hill Book Co. Inc. New York -Toronto London).

Schetinski EA (2002). 'Wildfires extinguishing: instruction for forest firefighters' (Moscow) (in Russian).

European Forest Fire Information System (2009) (European Commission Joint Research Center, Institute of Environment and Sustainability) URL: http://effisviewer.jrc.ec.europa.eu/wmi/viewer.html.

Information system of remote monitoring of the Federal forestry agency (2010) (Pushkino: Federal agency Avialesookhrana): URL: http://www.pushkino.aviales.ru/rus/main.sht. 
Matveev PM (2002). 'Forest pyrology: Textbook for students' (Krasnoyarsk: Siberian State Technological University) (in Russian).

Middlebon LJ (1977) 'User's manual for forest fire control equipment inventory and personnel programs'. (Canadian Forestry Service Inf. Rep. FF-X-63, Ottawa).

Deeming JE (1972). 'The National Fire-Danger Rating System' ( USDA Forest Service Research Paper RM-84) ( Fort Collins).

Learning management system MOODLE 3.5 (2018) URL: https://docs.moodle.org/35/en/Main_page.

Yarovoy SV (2016) 'Simulation of forest fire situations using an agent-based model'. Electronic scientific journal Software products, systems and algorithms. 2 (in Russian)

Dorrer GA, Yarovoy SV (2017) Describing the process of propagation and eliminating wildfires with the use of agent models. Siberian journal of forest science, 5, 105-113. (in Russian). 


\title{
The Spark Wildfire Prediction System
}

\author{
J. E. Hilton ${ }^{1 *}$; A. L. Sullivan*2; W. Swedosh ${ }^{1} ;$ M. G. Cruz ${ }^{2}$; M. P. Plucinski²; R. J. Hurley ${ }^{2}$; C. \\ Huston $^{1} ;$ M. Prakash ${ }^{1}$ \\ ${ }^{1}$ CSIRO, Clayton, VIC 3168, Australia, \{james.hilton@csiro.au*\} \\ ${ }^{2}$ CSIRO, Canberra, ACT 2601, Australia, \{andrew.sullivan@csiro.au\}
}

\begin{abstract}
Spark is a computational platform for modelling wildfire behavior, developed for research, risk assessment and operational use. The platform has been designed for flexibility, allowing users to define wildfire models, process weather streams and spatial data, and visualize and analyze the resulting simulations. The core fire propagation solver is a raster-based level set method that calculates the progression of a fire perimeter over given terrain layers and allows the creation, merging and interaction of any number of individual fire perimeters. Propagation in different fuel types is implemented using text-based scripts that define the fire behavior and rate-of-spread, rather than having pre-determined models built into the system. This gives the system flexibility to implement any available fire behavior models. The system is currently being used to research and implement many aspects of fire behavior including the development of new rate-of-spread models, integration with meteorological and remote sensing data and the modelling of firebrand dynamics and fire-atmosphere interaction. The system is freely available to researchers, practitioners and managers in the wildfire community.
\end{abstract}

Keywords: Wildfire modelling, bushfire, simulation, software

\section{Introduction}

Wildfires are a worldwide threat, causing major environmental damage and posing significant danger to lives and infrastructure when impacting on urban interfaces. Spark is a computational platform that can rapidly model two-dimensional wildfire spread for research, risk assessment and operational use (Miller et al., 2015). The platform has been developed to be entirely user-configurable at all levels, from the behaviour of the fire down to the central workflow for running and processing wildfire simulations. This flexibility allows users complete control in processing input layers and data, implementing wildfire models, post-processing the results of simulation and visualising data. The system leverages the power of new graphical processing units (GPUs) for rapid geoprocessing allowing complex fire behaviour models to be implemented for multiple fuel types. Spark can also spatially ensemble the results of multiple predictions to build statistical maps for risk assessment and fire management.

\section{Architecture and Configurability}

The core of Spark is a fire propagation solver, based on a computational level set method (Hilton et al., 2015). This raster-based method manages the creation, movement and merging of fire perimeters over a set of given terrain layers according to user-defined empirical fire spread models. The level set method is particularly well suited to modelling fire spread as it can handle irregular boundary conditions, run at any required resolution and handle the interaction of any number of individual fire perimeters, for example, multiple spot fire ignitions ahead of a fire front. 


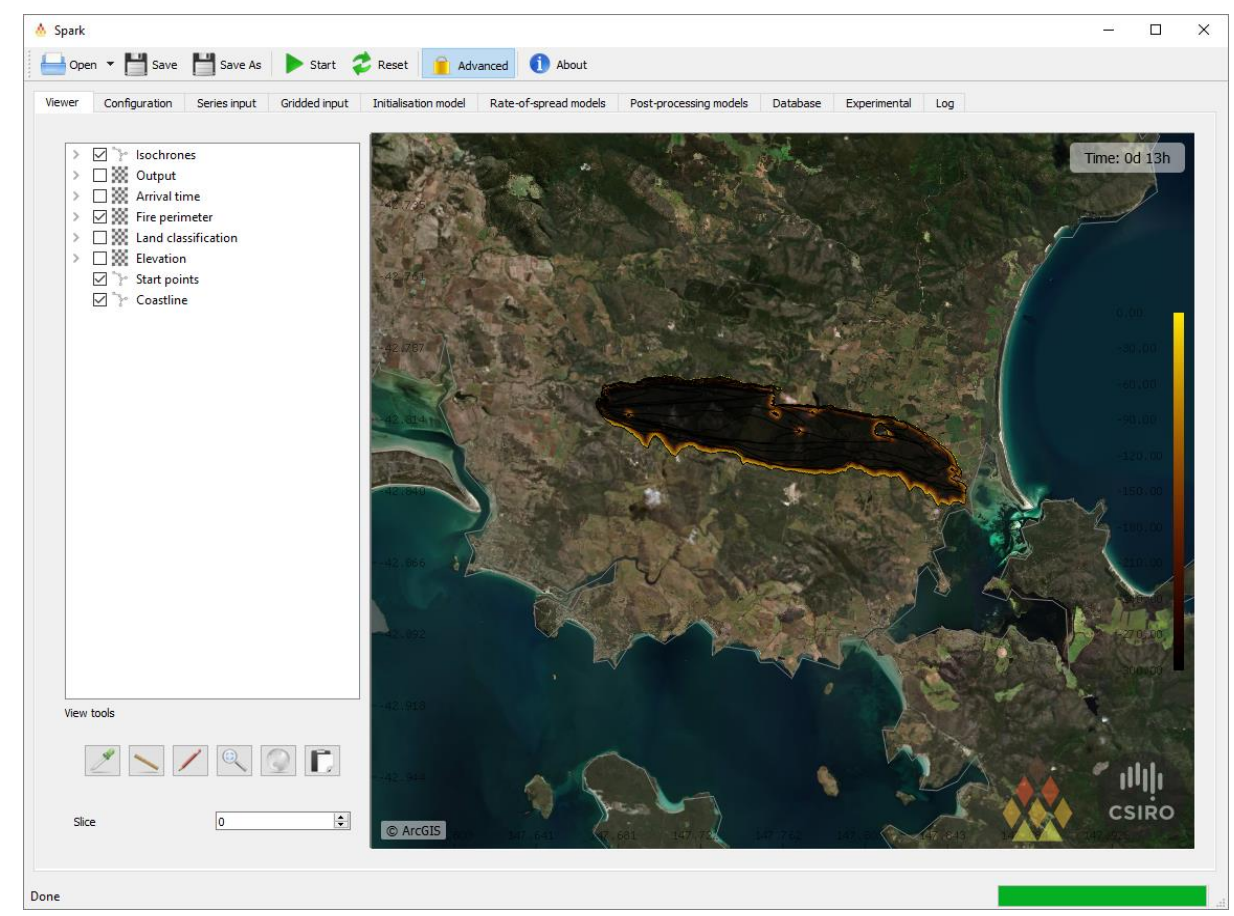

Figure 1 - Spark configured as a graphical-user-interface suitable for operational usage showing a fire in Tasmania, Australia.

The fire propagation solver can be configured in various user-dependent ways, such as within an interactive graphical-user interface (Figure 1), a workflow-based system for research (Figure 2) or a headless command line version for servers and cloud computation. The system is supported on both the Linux and Windows platforms. Fire front propagation in different fuel types is implemented using text-based scripts to define the fire behaviour and rate-of-spread, rather than having pre-determined models built into the system. This gives the system flexibility to implement any available fire behaviour models and fire templates, such as elliptical or more complex shapes, and to enable testing of new models (Hilton et al. 2016). We have currently implemented most fire rate-of-spread models used operationally in Australia (Cruz et al. 2015), examples of which are available on our research website (Spark, 2018) as well as version of the software, documentation and modelling guides.

The data layers required for these fire rate-of-spread models can be loaded into the system in any common geospatial formats. The layers are transparently re-projected and interpolated to a required resolution within Spark. The system can also pre and post-process any combination of these layers using user defined scripts. For example, a pre-processing implementation has been developed which directly reads remote sensing data tiles from Landsat imagery and converts these into fuel condition data suitable for fire simulations in Eucalypt forest (Massetti et al. 2018). Post-processing can include models for combustion, radiant heat flux and flame height, or models for impact assessment with asset layers. 


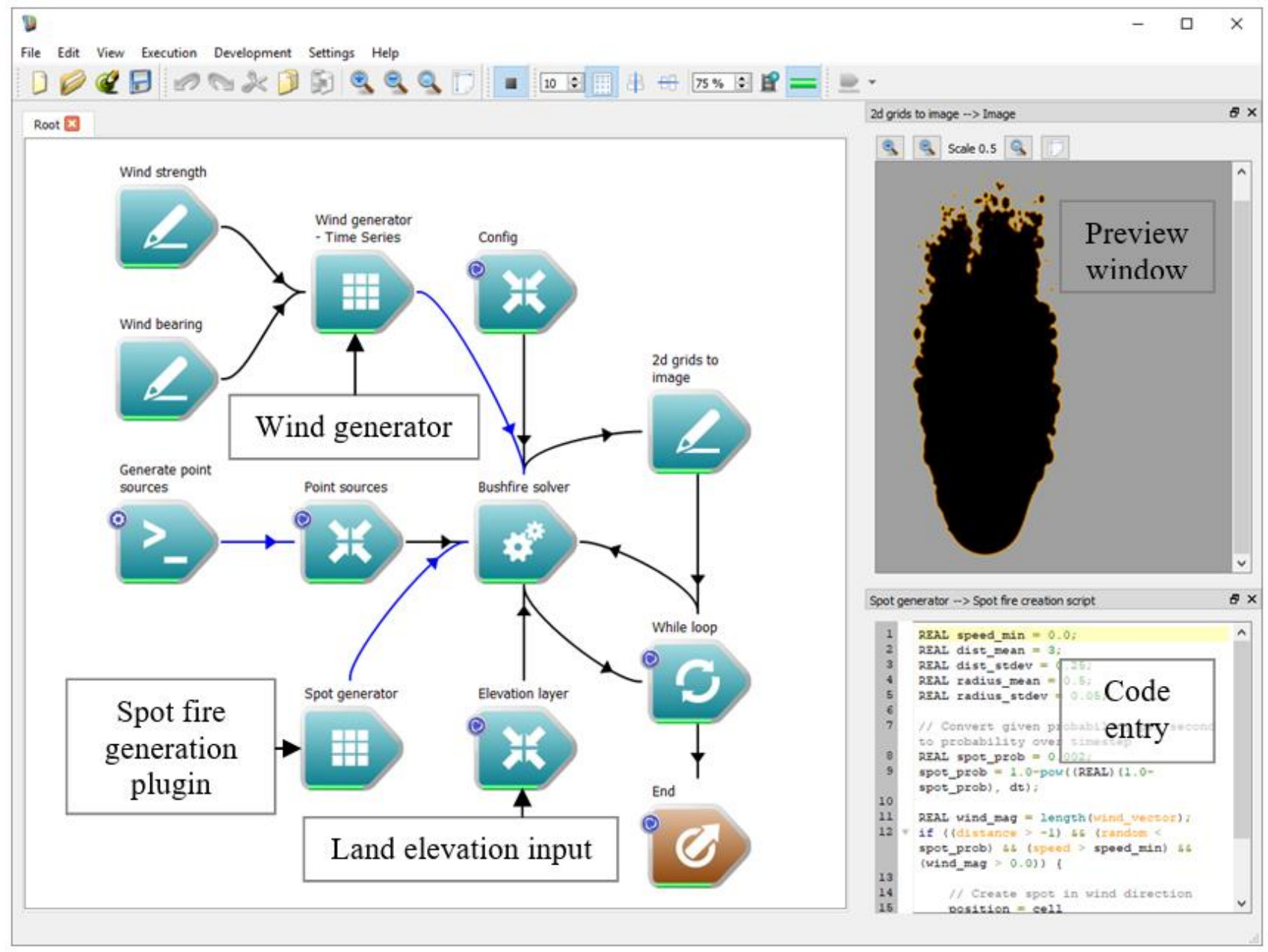

Figure 2 - Spark workflow in the Workspace editor.

Surrounding the core propagation algorithm is a modular geospatial processing architecture based on a workflow technology called Workspace developed by CSIRO (Watkins et al., 2017). This workflow framework allows Spark to be easily configured in multiple ways for different purposes, for example, development and research of new fire behaviour models. An example workflow canvas is shown in Figure 2 where the individual elements of the workflow are blue boxes. These elements include modules to create the input starting conditions, generate the wind field and input data layers. The core fire propagation solver supports modular plug-ins for various aspects of fire behaviour, such as the firebrand generation model shown in Figure 2 A preview of the simulation results is shown in a window on the upper right-hand side and the user-defined script for configuring the firebrand model is shown on the lower right-hand side.

The system can also be used to quantify the risks from wildfires in hypothetical scenarios by running ensembles of input or weather conditions. These ensembles can be configured and composited in various ways, for example, varying the starting location of a fire and computing the resulting fire footprints into a probability map, as shown in Figure 3. The heat-map shown in the left-hand figure is shaded by frequency of fire impact. As with all models in Spark the composition and processing of the ensembles is user-defined, with Spark providing the ability to calculate global metrics over each ensemble, or to calculate a running spatial ensemble over all ensemble members. The results can be written to a PostGIS database, if required, for further downstream processing. 


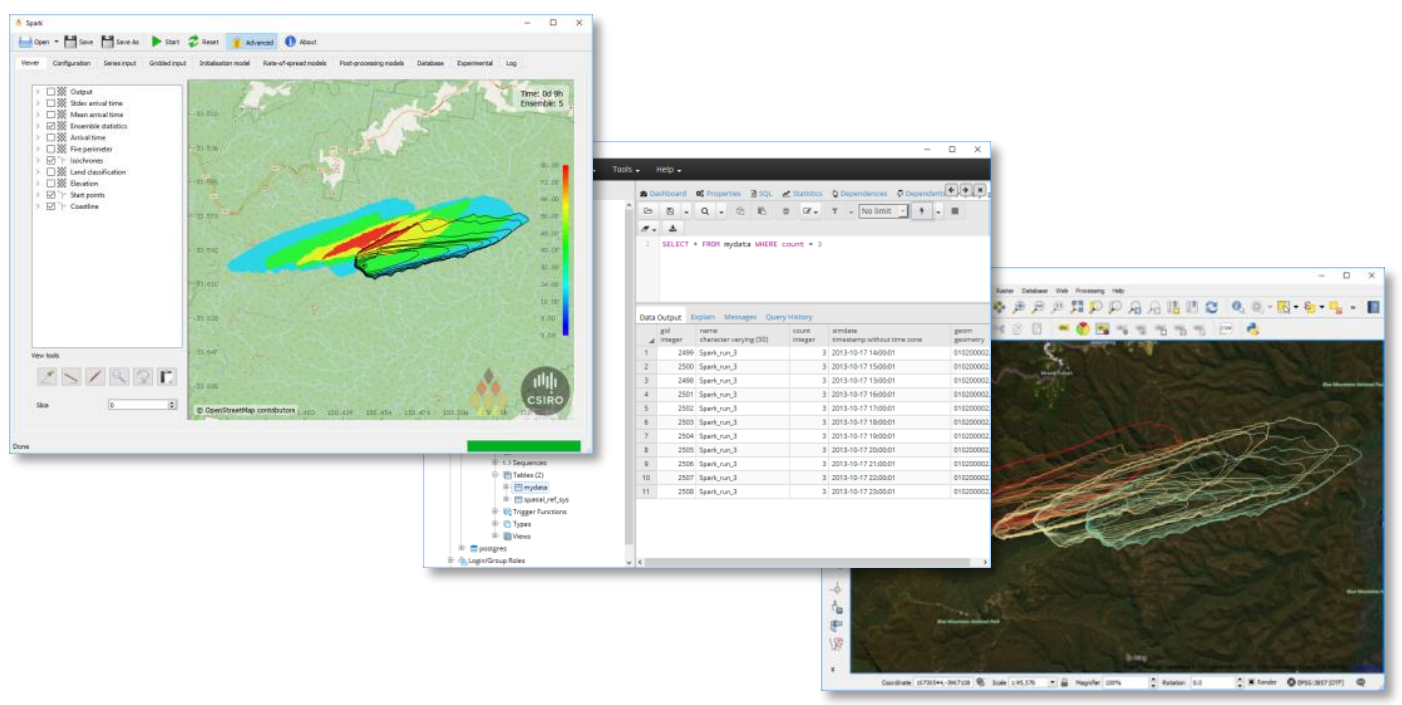

Figure 3 - Spark ensemble analysis. The Spark graphical-user-interface (left) showing impact frequency from several possible fires as a coloured heat-map. The fire isochrones can be directly written to PostGIS (centre) and visualised in and external GIS tool (right).

Sub-models currently available within the framework include a mass-conservation wind correction model, a firebrand transport and ignition model and a fuel break model for firebreaks, roads and waterways, an example of which is shown in Figure 4. The fuel break model uses a set of line features of inputs which stops the spread of fires at these lines until a user-defined condition is met. In the example shown in Figure 4, a set of roads is used as input and the road has a probability of being breached based on a constant hazard function. As the rule for breaching is probabilistic an ensemble of simulations has been run to find the probability of the fire arriving at each point on the map. This is shown as a heat-map of impact probability.

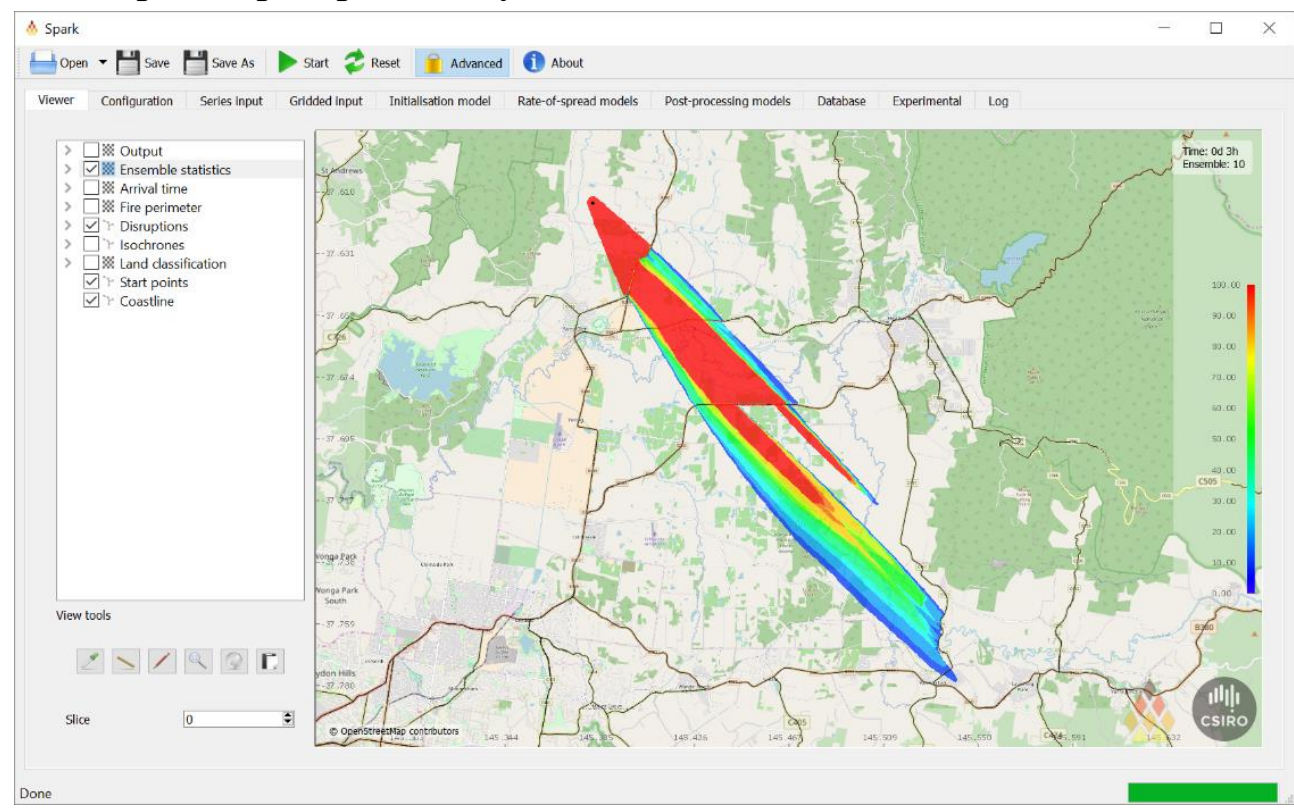

Figure 4 - Ensemble of simualtions from a set of fires with a random probability of crossing a road. The shaded heat map shows the impact probability of a fire arriving at a given point.

Spark harnesses the power of new graphical processing unit technology (GPUs). Almost all current computers have graphical processing units which, if used for computation, can carry out multiple calculations in parallel. This can result in speed increases hundreds of times greater than standard 
computations on the central processing unit (CPU) of a computer. This translates into the ability to run complex fire behaviour algorithms at high spatial resolutions in a very short computational time. Additionally, such GPUs are available as a cheap, reliable and scalable resource on several cloudbased systems such as Amazon Web Services (AWS).

\section{Research and Operation}

An aim of developing the Spark system was to connect the research community with operational users by creating a common platform through which new research could be easily tested and deployed. We are currently using Spark for internal research as well as collaborating with several university groups to develop new methods and models for predicting fire behaviour. This includes research into the sensitivity to meteorological data, direct integration of remotely sensed data products into fire simulations, firebrand dynamics, radiant heat flux modelling for urban interfaces (Hilton et al. 2017), new methods for modelling firebreaks (Swedosh et al. 2017) and fire-atmosphere interaction such feedback effects from the fire (Hilton et al., 2018). We are also researching more efficient methods for ensemble modelling and web-based deployment on cloud infrastructure for large sets of ensembles (Garg et al., 2018).

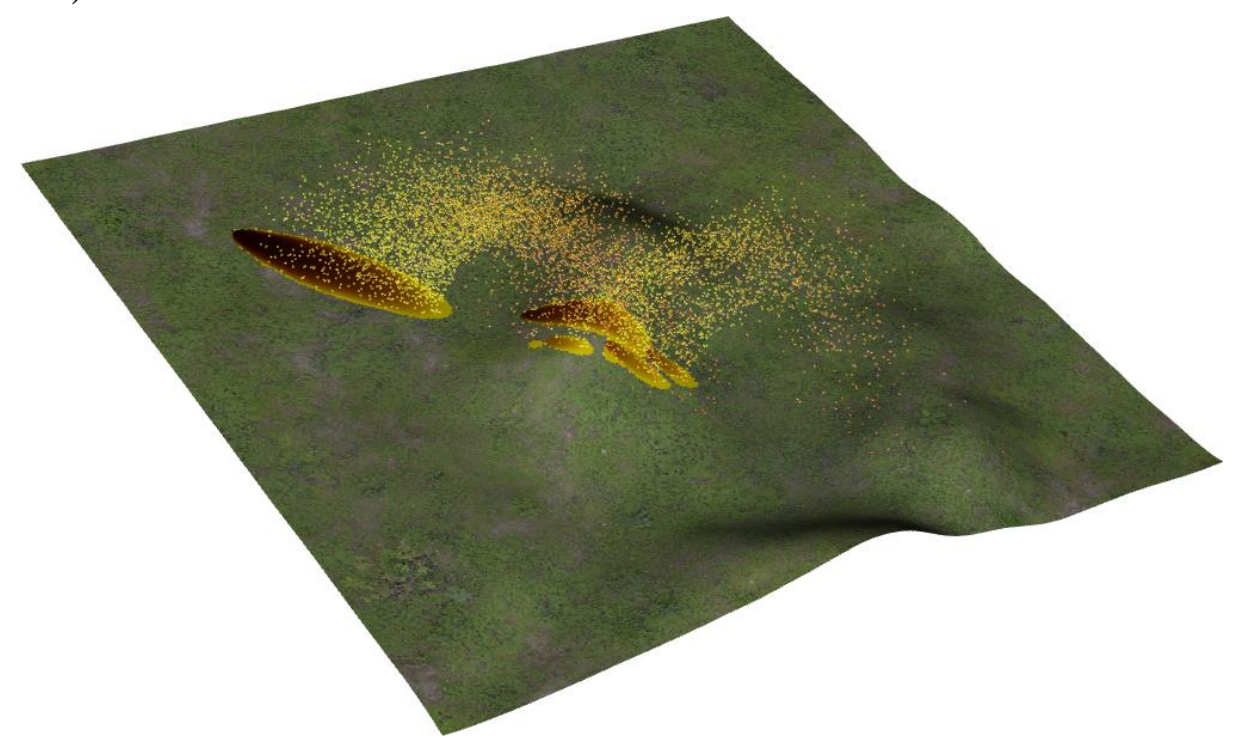

Figure 5 - Three-dimensional visualisation of Spark firebrand modelling system. Each firebrand is individually controlled in the model using a user-defined script.

An example of a research module is the Spark firebrand processor, a visualisation of which shown in Figure 5. This module allows the user to define models for the creation and transport of firebrands as well as the impact with the ground and subsequent chance of spot-fire creation. The models are defined in a similar way to the rate-of-spread models, for example, the user specifies the acceleration of the firebrand in a script based on properties of the firebrand, such as time since ignition, and local fields, such as the wind vector at the location of the firebrand. We are currently collaborating with university groups to research and develop models for firebrand distribution and spot formation using the Spark platform.

\section{Conclusion}

Spark has been designed as a platform for rapid and configurable modelling of two-dimensional fire spread. The system can be used for research or to interactively provide a visual awareness of the potential impact of a wildfire, enabling early warnings, ability to manage resources and guide mitigation strategies. Alternatively, the system can be configured as a risk modelling system capable 
of generating spatial risk metrics from large ensembles of potential wildfires. The modular nature of the system allows configurability and customisation for the end user, or the ability for developers to add new types of more complex fire behaviour models. The system is freely available to the research and operational community (Spark, 2018).

\section{References}

Cruz MG, Gould JS, Alexander ME, Sullivan AL, McCaw WL, Matthews S (2015) Empirical-based models for predicting head-fire rate of spread in Australian fuel types. Australian Forestry 78, 118 158.

Garg S, Forbes-Smith N, Hilton JE, Prakash M, (2018) SparkCloud: A Cloud-Based Elastic Bushfire Simulation Service, Remote Sensing, 10, 74

Hilton JE, Leonard J, Blanchi R, Newnham G, Opie K, Rucinski C, Swedosh W (2017) Simulation of spot fire coalescence with dynamic feedback, In Syme, G., Hatton MacDonald, D., Fulton, B. and Piantadosi, J. (eds) MODSIM2017, 22nd International Congress on Modelling and Simulation. Modelling and Simulation Society of Australia and New Zealand, 1104-1110.

Hilton JE, Miller C, Sullivan AL, Rucinski C (2015) Effects of spatial and temporal variation in environmental conditions on simulation of wildfire spread, Environmental Modelling and Software, 67, 118-127

Hilton JE, Miller C, Sullivan AL (2016) A power series formulation for two-dimensional wildfire shapes. International Journal of Wildland Fire, 25, 970-979

Hilton JE, Sullivan AL, Swedosh W, Sharples J, Thomas C (2018) Incorporating convective feedback in wildfire simulations using pyrogenic potential, Environmental Modelling \& Software, 107, 1224

Massetti A, Yebra M, Hilton J, Rüdiger C, (2018) Determining Spatio-Temporal Fuel Accumulation Using the Vegetation Structure Perpendicular Index from Optical Remote Sensing: Application to Dynamic Fire Spread Simulations, In D. X. Viegas (Ed.), Advances in Forest Fire Research

Miller C, Hilton JE, Sullivan AL, Prakash M (2015) Spark - A Bushfire Spread Prediction Tool. In: Denzer R., Argent R.M., Schimak G., Hřebíček J. (eds) Environmental Software Systems. Infrastructures, Services and Applications. ISESS 2015. IFIP Advances in Information and Communication Technology, 448. Springer, Cham

Spark research website (2018), https://research.csiro.au/spark/

Swedosh W, Hilton JE, Prakash M (2017) S Modelling Firebreaks in a Two-Dimensional Dynamic Fire Spread Simulator, In Syme, G., Hatton MacDonald, D., Fulton, B. and Piantadosi, J. (eds) MODSIM2017, 22nd International Congress on Modelling and Simulation. Modelling and Simulation Society of Australia and New Zealand, 1104-1110.

Watkins D, Thomas D, Hetherton L, Bolger M, Cleary PW (2017) Workspace - a Scientific Workflow System for enabling Research Impact, In Syme, G., Hatton MacDonald, D., Fulton, B. and Piantadosi, J. (eds) MODSIM2017, 22nd International Congress on Modelling and Simulation. Modelling and Simulation Society of Australia and New Zealand, 1-7 


\title{
Tracking of uncertainty when aggregating from fine to coarse spatial resolution
}

\author{
J. Brennan ${ }^{1,2}$; P. Lewis ${ }^{1,2} ;$ J. Gómez-Dans ${ }^{1,2}$; M. Chernetskiy1 ${ }^{1,2 *}$; E. Chuvieco ${ }^{3}$;. Lizundia ${ }^{3}$; M. \\ Campagnolo $^{4}$; J Pereira ${ }^{4}$; D. Oom ${ }^{4}$ \\ ${ }^{1}$ Department of Geography, University College London, Gower Street, London, WC1E 6BT, UK, \\ \{m.chernetskiy@ucl.ac.uk*\} \\ ${ }^{2}$ National Centre for Earth Observation (NCEO), Department of Geography, University College \\ London, Gower Street, London, WC1E 6BT, UK \\ ${ }^{3}$ Env. Rem. Sens. Group, University of Alcala, Alcal'a, Spain \\ ${ }^{4}$ Dept. of Forestry, ISA, Technical University of Lisbon, Portugal
}

\begin{abstract}
Uncertainty characterisation and validation are critical phases to generate any Essential Climate Variable (ECV), and therefore both have been included as key deliverables of the ESA CCI programme (Rainer et al 2013). All products generated by the CCI are required to have an associated per pixel uncertainty characterisation. This paper describes both the uncertainty characterisation framework and the related uncertainty validation exercise of the Fire-CCI project.
\end{abstract}

Keywords: Burnt Area, Uncertainty, Aggregaion, Fire CCI.

\section{Uncertainty definition within fire-cci}

Burnt Area algorithms broadly consist of a classification into a binary Burnt/Unburnt product. As with all remote sensing retrievals these algorithms can be phrased within inverse theory:

$$
B=\boldsymbol{G}(d)
$$

Where the outputted burnt area product $\mathrm{B}$ is determined by the data $\mathrm{d}$ and the burnt area algorithm G. Remote sensing measurements contain uncertainty (Poveu and Grainger, 2015). As such the relationship above is better described by:

$$
B=\boldsymbol{G}(d)+v
$$

Where $v$ represents uncertainty in the value of the measurement $d$. Often the probability distribution function of $\mathrm{v}$ is assumed normal such that the observation arrives as:

$$
d_{\text {observed }}=d_{\text {true }}+N(0, \delta)
$$

where $\delta$ is an estimate of the observation uncertainty. Naturally the uncertainty in the observations leads to uncertainty in the retrieval of burnt area. To fully describe this uncertainty requires definitions of uncertainty at the relevant scales of the products. Within the ESA CCI programme there is an expectation of pixel-level uncertainty information (Rainer et al 2013). Further, the Fire-CCI products are also to be provided at a lower resolution Climate Model Grid (CMG) $\left(0.25^{\circ}\right)$.

\subsection{Pixel resolution}

At the pixel scale we define an appropriate uncertainty characterisation to be the probability that the pixel is burnt $\mathrm{Pb} . \mathrm{Pb}$ then provides a probabilistic confidence that the pixel is burnt given the observations. $\mathrm{Pb}$ must follow the axiomatic laws of probability, e.g the probability that the pixel is not burnt $\mathrm{P}_{\mathrm{u}}$ is:

$$
P_{u}=1-P_{b}
$$

Advances in Forest Fire Research 2018 - Page 1110 
The full pixel level uncertainty is then described by a Bernoulli distribution B:

$$
B= \begin{cases}P_{u}=1-P_{b}, \text { if } B=\text { Unburnt } \\ P_{b}, & \text { if } B=\text { Burnt }\end{cases}
$$

\subsection{CMG resolution}

At the CMG scale the definition of uncertainty is different. Burnt area at the CMG is classically defined as the summation of the number of burnt pixels $\mathrm{N}_{\mathrm{b}}$. However to propagate the uncertainty to the $\mathrm{CMG}$ we suggest that the CMG burnt area BCMG is better described by a Poisson binomial distribution. The Poisson binomial distribution describes the probability of $\mathrm{N}$ independent Bernoulli distributions. As $\mathrm{N}$ grows large the distribution is well described by a normal distribution with mean $\mu$ and standard deviation $\delta$ :

$$
\mu=\sum_{i}^{N} p_{i} \quad \delta=\sum_{i}^{N}\left(1-p_{i}\right) P_{\bar{i}}
$$

This means that at the CMG scale each grid cell is approximated as a normal random variable which encodes the uncertainty in the burnt area. Crucially, all pixels are included in the calculation even if they have a low $\mathrm{Pb}$.

\section{Uncertainty characterisation exercise}

An uncertainty validation methodology was developed for testing the uncertainty characterisation of algorithms within Fire-CCI. The purpose of the validation exercise was to assess the presently developed uncertainty characterisations within a framework which provides realistic estimates of the true product uncertainties. In this paper we address the validation of uncertainties for two algorithms within Fire-CCI.

\subsection{Algorithm uncertainty estimates $\widehat{P}_{b}$}

The two algorithms make an estimate of the per-pixel uncertainty $\widehat{P}_{b}$ in different manners. Algorithm A estimates $\widehat{P}_{b}$ based primarily on a pixel's distance to the nearest active fire observation. While algorithm B defines $\widehat{P}_{b}$ based on the distance to the classification boundary between the burnt and unburnt classes within the algorithm.

\subsection{Algorithm true uncertainty $P_{b}$}

Evidently the true uncertainty $P_{b}$ is determined by the confidence the algorithm places in the detection given the uncertainty in the data. To take account of the uncertainty in the observations, algorithms must marginalise over the probability distribution of the observation:

$$
P_{b}=\int_{d} P(d) G(d) \delta d
$$

\subsection{Sampling Framework}

However the algorithms presently are unable to perform this integral. Instead a monte-carlo framework was developed to approximate this integral. We can derive an estimate of the true $P_{b}$ for an algorithm based on sampling from the data distribution $P(d)$.

Three test sites were selected, representing three significant pyromes for burnt area: savanna, boreal forest and tropical forest (which are shown in figure 1). An estimate of daily surface reflectance for each site was provided from the MODIS Collection 6 surface reflectance products (MOD/MYD09).

To generate each realisation of the reflectance $\mathrm{R}_{\mathrm{i}}(\lambda)$, each recorded measurement from MODIS $R_{M O D I S}(\lambda)$ was described by a multivariate normal distribution: 


$$
R_{i}(\lambda)=R_{\text {MODIS }}(\lambda)+N\left(0, C_{\lambda}\right)
$$

where the observation uncertainties are represented by the diagonal covariance matrix $C_{\lambda}=\operatorname{diag}\left(\delta_{i}^{2} \ldots \delta_{\lambda}^{2}\right)$. Per channel uncertainties $\delta_{\lambda}^{2}$ where provided from the MODIS uncertainty characterisation (see table 1) (Vermote et al, 2002).
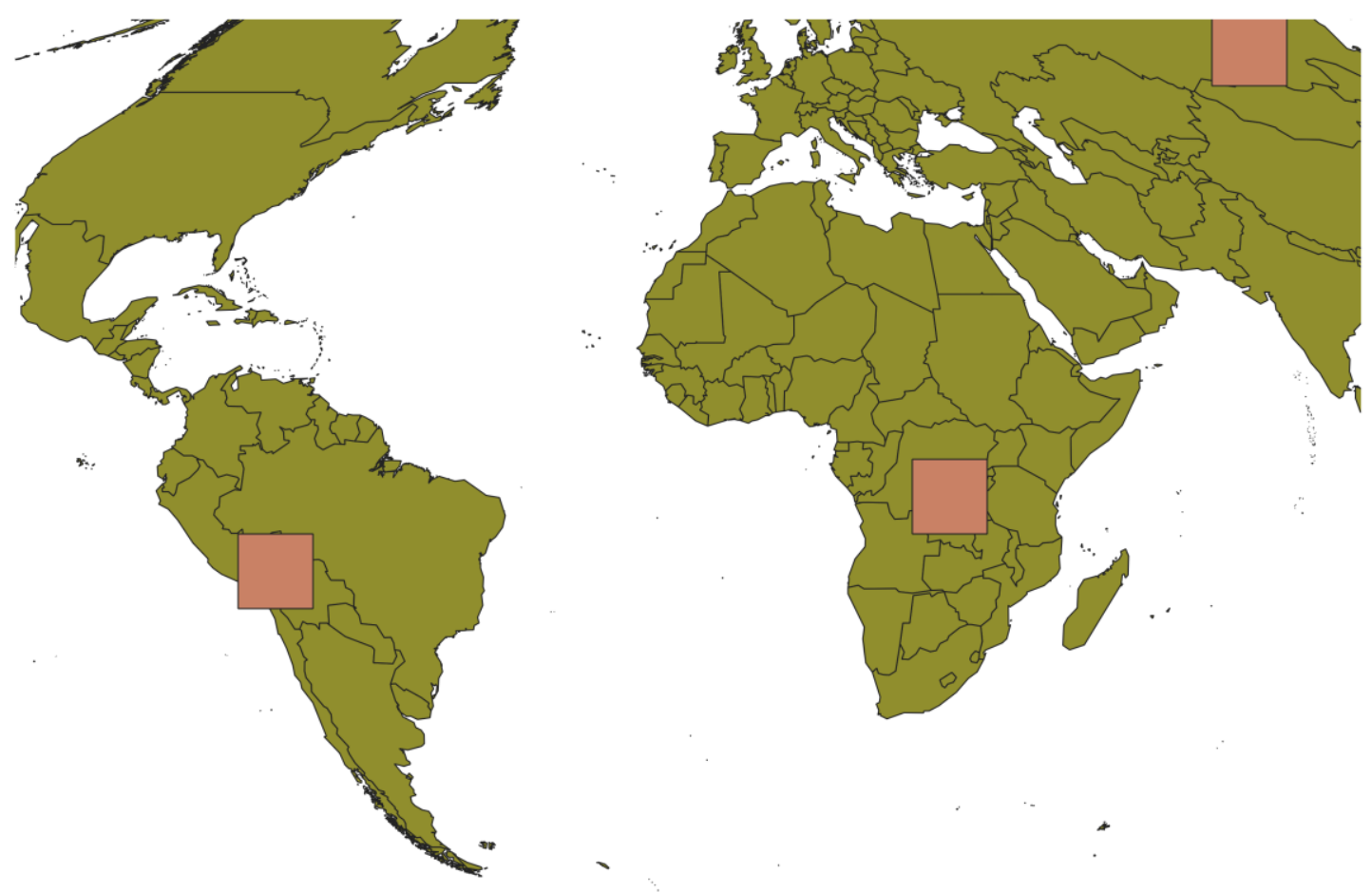

Figure 1 - Location of the selected test sites

To consider that algorithms were properly representing the uncertainty in $P(d)$, three sets of realisations were computed for each site with three different levels of observational noise. To do this, the MODIS covariance matrix was scaled by a noise factor $c$, with the values of $0.5,1.0,1.5$. As $c$ increases, it would be expected that $P_{b}$ would tend more towards ignorance, as described by $P_{b}$ values approaching 0.5 . Both algorithms were run on the $N$ realisations of a remote sensing dataset sampled from $P(d)$ for each site. As a result, for a pixel $p$ we have $N$ realisations of the product:

$$
P=[b, u, u, b, b, u, b, u, u, u, . .]
$$

where $b$ corresponds to a burnt detection and $u$ and unburnt detection. A good approximation to the true algorithm $P_{b}$ is then a function of the number of burnt draws to unburnt draws. When $B$ is defined as a Bernoulli variable, we can estimate the maximum likelihood estimate of $P_{b}$ from the function:

$$
\max P_{b}^{B}\left(1-P_{b}\right)^{U}
$$

where $B$ and $U$ is the number of burnt and unburnt outputs respectively. $P_{b}$ is then simply:

$$
P_{B}=\frac{B}{N}
$$

Given the limited number of sample runs, this estimate may be poor (Megill and Pavicic, 2011). Instead an adjusted estimate is provided by:

$$
P_{B}=\frac{B(B+2, N-N+2)}{B(N+1, n-B+1)}
$$


where $B$ is the Beta function. Each realisation of $\widehat{P}_{b}$ was then aggregated to the grid-scale along with the algorithm true $P_{b}$ as outlined in section 1.2.

Table 1 - Theoretical per-pixel uncertainty for MODIS channels. From: [3]

\begin{tabular}{rccccccc}
\hline MODIS channel & 1 & 2 & 3 & 4 & 5 & 6 & 7 \\
\hline central $\lambda$ & $645 \mathrm{~nm}$ & $858 \mathrm{~nm}$ & $469 \mathrm{~nm}$ & $555 \mathrm{~nm}$ & $1240 \mathrm{~nm}$ & $1640 \mathrm{~nm}$ & $2130 \mathrm{~nm}$ \\
\hline$\delta_{\lambda}^{2}$ & 0.004 & 0.015 & 0.003 & 0.004 & 0.013 & 0.010 & 0.006 \\
\hline
\end{tabular}

\section{Results}

Figure 2 shows an example of the pixel level estimates of $P_{b}$ from two Fire-CCI algorithms compared to the true uncertainty characterisation. Primarily we see that the two algorithms show considerably different uncertainty estimates while having similar true uncertainties. Algorithm A shows high $\widehat{P}_{b}$ for unburnt areas (0.2-0.4) (with a low true $P_{b}$ ). Within the burn scars $\widehat{P}_{b}$ is highest (typically 0.7-0.9) but still below the true uncertainty for these areas. Algorithm B matches more closely to the true uncertainty, especially in areas of low probability. However in the burn scars the estimated probabilities are considerably lower than the truth.
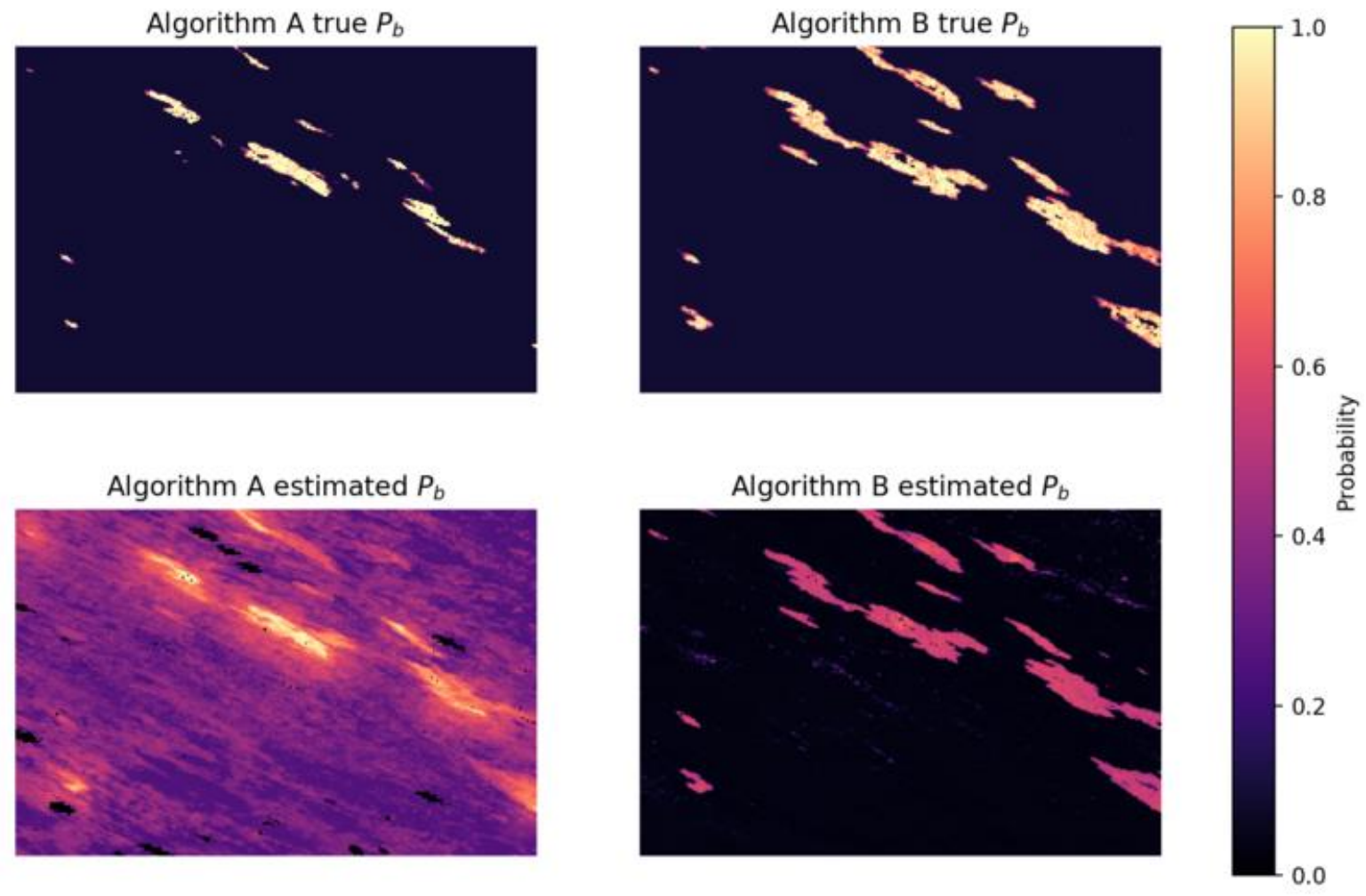

Figure 2 - Pixel level estimates of Pb for two Fire-CCI algorithms over the boreal test site. Top) True Pb derived from the sampling framework. Bottom) Algorithm estimate of $\mathrm{Pb}$

Figure 3 shows the resulting CMG estimates of burnt area $\mathrm{km}^{2}$ from two Fire-CCI algorithms. The differences in the pixel level uncertainties clearly propagates to the CMG distributions. The over estimation of uncertainties in algorithm A leads to a large over estimation in the burnt area. Algorithm A also has an increased standard uncertainty relative to the true standard uncertainty given the data. Overall algorithm A estimate of burnt area does not match the true distribution well. Estimated distribution: $\mu: 23267 \mathrm{~km}^{2} \delta: 13306 \mathrm{~km}^{2}$ vs the sampling-based distribution: $\mu: 6395 \mathrm{~km} 2 \delta: 4951 \mathrm{~km} 2$. Algorithm B performs more favourably with a closer estimate to the true distribution as well as the true burnt area. However it slightly underestimates the mean due to the under-estimation of $\widehat{P}_{b}$ within 
the burn scars. Algorithm B shows a slight over-estimation in the standard uncertainty but is closer to the true standard uncertainty. Estimated distribution: $\mu: 4050 \mathrm{~km}^{2} \delta: 2838 \mathrm{~km}^{2}$ vs the sampling-based distribution: $\mu: 8195.5 \mathrm{~km}^{2} \delta: 5077 \mathrm{~km}^{2}$.

Figure 4 shows the error in the algorithm uncertainty estimates for the three test sites. Here, the error in the uncertainty characterisation is encoded by the difference between the algorithm $P_{b}$ estimate and the true $P_{b}$ from the sampling. Generally therefore, an accurate uncertainty characterisation occurs when this error is near zero. Further, we would expect no biases in this error as a function of the noise factor $c$. Such an outcome indicates that the uncertainty method correctly identifies the magnitude of the uncertainty in the observations. A general feature of note is the overall bias in algorithm B towards an underestimate of $P_{b}$ as was seen for the boreal site in figure 2. An encouraging feature for algorithm B is that the error in $P_{b}$ is not a feature of the degree of noise imposed by $c$. We can see that both the bias and distribution of errors in $P_{b}$ remain consistent across noise levels. This indicates that the algorithm provides an estimate of the uncertainty which is not sensitive to the noise sampling of the input datasets. Algorithm A shows a larger sensitivity to the noise factor $c$. In each test site, the distribution of errors in $P_{b}$ is sensitive to the level of noise $c$. This indicates that the present uncertainty characterisation needs to be refined to remove such sensitivity. Further, for algorithm A the biases in $P_{b}$ are inconsistent across the three sites unlike for algorithm B. In the boreal site, the algorithm provided $P_{b}$ is less than the true $P_{b}$. However in the tropical and savanna sites, the algorithm $P_{b}$ is larger than the true $P_{b}$. This feature indicates that the present uncertainty method needs to be refined to be suitable for each biome.
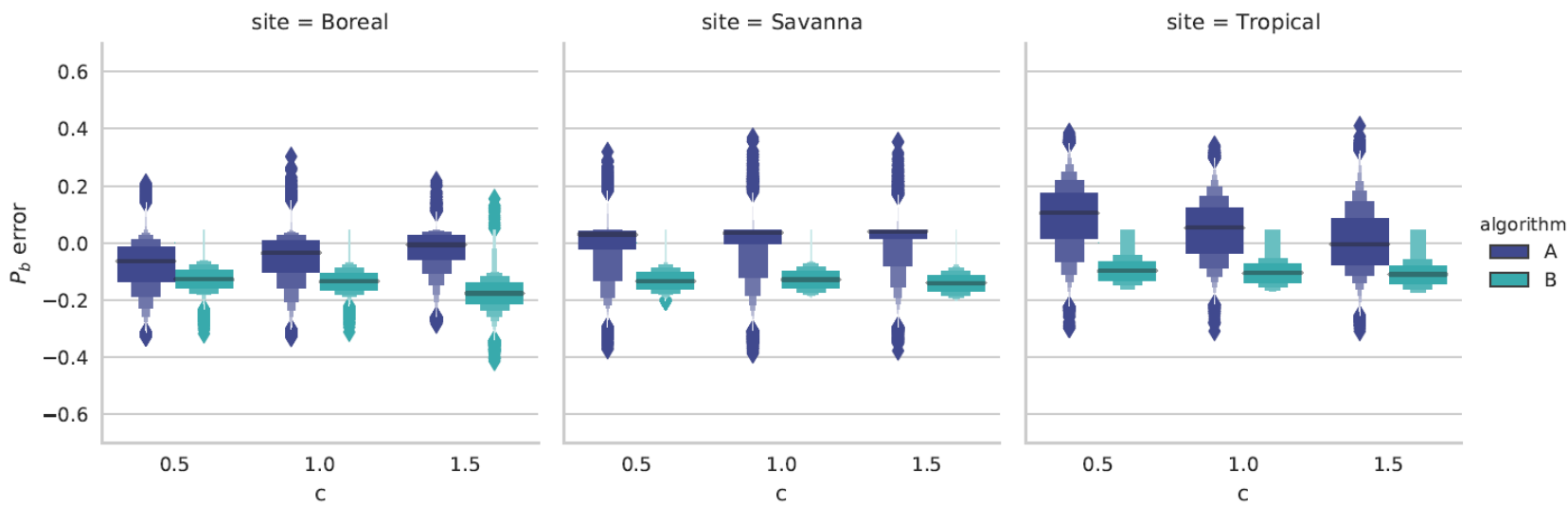

Figure 3 - Error in algorithm estimates of $\boldsymbol{P}_{b}$ for the two FireCCI algorithms. $P_{b}$ error is the difference between the algorithm $\boldsymbol{P}_{b}^{a}$ and the estimated true $\boldsymbol{P}_{b}^{t}$.

\section{Final remarks}

The described framework represents an initial attempt at producing uncertainty quantified BA products at both the pixel and CMG scales. Further a methodology for validating uncertainty estimates based on sampling from the data distribution is presented. Results indicate that present uncertainty estimates need to agree more closely with the present binary estimates of products. Going forward this can be achieved by full uncertainty propagation within algorithms following metrological principles. An appropriate best practice framework for uncertainty characterisation is provided by the Guidelines for Uncertainty in Measurement (GUM) (IUPAC 2008). Secondly there is a need to consider uncertainty within the retrieval algorithm itself. The presented framework details the implications of the uncertainty in remote sensing observations and the effect these have on the algorithm performance, but the algorithm is here assumed to introduce no uncertainty. 


\section{References}

BIECIIIS IUPAC and IUPAP OIML, "Evaluation of measurement dataguide to the expression of uncertainty in measurement," 2008.

Hollmann. Rainer, Chris J Merchant, Roger Saunders, Catherine Downy, Michael Buchwitz, Anny Cazenave, Emilio Chuvieco, Pierre Defourny, Gerrit de Leeuw, Ren'e Forsberg, et al., "The esa climate change initiative: Satellite data records for essential climate variables," Bulletin of the American Meteorological Society, vol. 94, no. 10, pp. 1541-1552, 2013.

Megill Norman D and Mladen Pavicic, "Estimating bernoulli trial probability from a small sample," arXiv preprint arXiv:1105.1486, 2011.

Povey AC and RG Grainger, "Known and unknown unknowns: uncertainty estimation in satellite remote sensing," Atmospheric Measurement Techniques, vol. 8, no. 11, pp. 4699-4718, 2015.

Vermote Eric F, Nazmi Z El Saleous, and Christopher O Justice, "Atmospheric correction of modis data in the visible to middle infrared: first results," Remote Sensing of Environment, vol. 83, no. 12, pp. 97-111, 2002. 


\title{
Wildfires - web application concept and prototype
}

\author{
Sandra Nieves ${ }^{1 *}$; Olga Mordvinova ${ }^{2}$ \\ ${ }^{1}$ Data and You. Mathy Straße 31, 76133 Karlsruhe, \{sandra@data-and-you.eu*\} \\ ${ }^{2}$ incontext.technology GmbH, Friedrich-Ebert-Anlage 27, 69117 Heidelberg, \{olga@inctec.de\}
}

\begin{abstract}
In 2016 there were 50,653 wildfires in Europe, which destroyed 352,729 hectares of land - about the size of 490,000 soccer fields. $46 \%$ of all land destroyed was in Portugal (European Commission, 2016). That amounts to $161,522 \mathrm{ha}$ (Figure 1).

According to the news, the fire around Pedrógão Grande, Portugal in June 2017 caused losses that are estimated to be $€ 497$ mil., plus $€ 300$ mil. for future prevention. In this one fire, 481 houses burnt, 374 people lost their jobs, 45ha of land were destroyed and 64 people died (ECO News, 2017). By October 2017 fires in Portugal have already burnt 520,000ha (abc Net, 2017).

Fires have had a tremendous impact on people, especially in Southern Europe. People living in rural areas, are often on their own to protect themselves. Unfortunately, following one's gut feeling might not be the best choice. Last year in Portugal, most people died on the roads, when trying to escape the flames.

We conducted qualitative research which revealed, that there is a lack of reliable, current and contextual information available to people, that can support good decisions during fire emergencies.

There is an abundance of quantitative research coming out of the fire science and geo science community, that has explored the topic, and scientists have discussed and evaluated data driven- and artificial intelligence approaches to predict fire behavior and fire danger.

However, we did not find a solution that applies insights from qualitative research and knowledge from quantitative studies in form of a practical web application, that can be intuitively used by people in affected areas.

In this paper, we illustrate how design thinking - a process for creative problem solving, can bring together technology and science with needs of people, and to create a solution that enables them to make better decisions related to forest fires.
\end{abstract}

Keywords: forest fires, fire science, geo science, design thinking, data visualization, user experience design, artificial intelligence, data science, geo informatics, software engineering

\section{Introduction}

On October 15, 2017 there were 344 active recorded fires that were "on-going" or "in resolution" based on data from a Portuguese website (fogos.pt) that provides information regarding forest fires.

Figure 2 shows that fires, between September 19 and October 30th, were concentrated mostly in the Central and Northern regions - which is where we conducted our user research.

Figure 3 shows the exceptionally high number of active fires on October 15, 2017, which put the Portuguese firefighting capacity beyond their limit - judged by the local observations, where no firefighting means were available to protect people. Civilians had no choice, but to take it upon themselves to fight the flames and to figure out how to stay safe. 


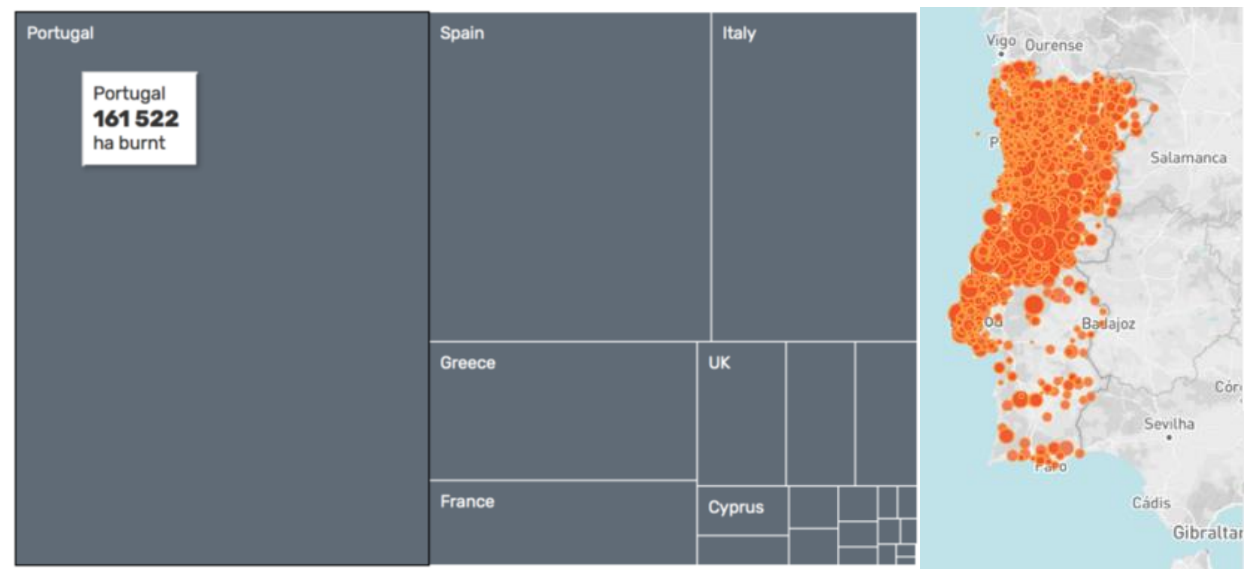

Figure 1 - (left): 2016 burnt area in ha (based on the European Commission, JRC science for policy report, 2016) Figure 2 - (right): Fires with status "on-going” or "in resolution", in Portugal between September 19 and December 28, 2017. It shows that Central and North Portugal were most affected. The size of the bubbles represents the number of fire fighters on site. (The data was derived from fogos.pt.)

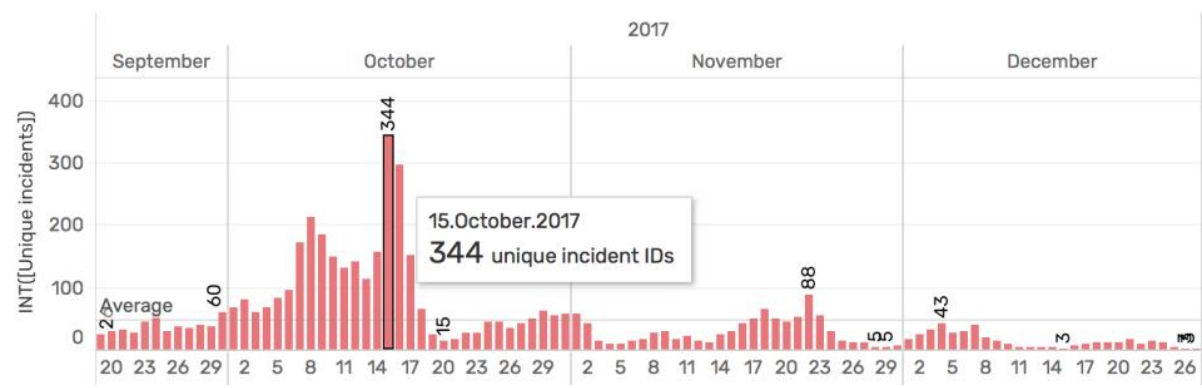

Figure 3 - Number of unique fire IDs with status "on-going” or "in resolution", in Portugal between September 19 and December 28, 2017. (The data was derived from fogos.pt.)

Our user research revealed that available information offerings do not consolidate enough context information - in form of an intuitive user experience, to paint a coherent picture, so that people can quickly gain a $360^{\circ}$ view of the situation, prior to making decisions. We observed people using multiple information services concurrently to try to understand what is going on - which is cumbersome and time consuming.

On other hand, we found quite a lot of research, that approaches the topic from a scientific angle.

Described below are just a few examples of scientific research which addresses fuel moisture determination, how to work with data captured by satellites, and fire behavior predictions, that are relevant to the solution we propose. New papers around the topic are surfacing every day, which demonstrates that science already holds answers to difficult questions in this context.

The NDVI is a remote sensing technique to identify a vegetation's vitality. It is based on the spectral signatures of plant species and is a comparatively simple method to estimate the moisture content of plants. It is based on red and near infrared reflectance (Joyce, 2016).

Our solution proposes to use the NDVI, to discriminate plant species and to visualize land cover types that play a significant role in determining how fire spreads.

A worldwide compilation of the available fire behavior data was collected in the BONFIRE project (Fernandes at al. 2018). The data variables used in this research are similar to the ones proposed in Wildfires.

The challenges BONFIRE faces, relating to data quality, are pretty much universal, when it comes to big data applications: 1) availability, 2) completeness and 3) reliability of the data. How Wildfires handles these issues is addressed later in this paper (see chapter 2.2. Data Technology). 
Different models to predict the rate of fire spread for different fuel types have been evaluated (Cruz et al., 2018). While the results showed a consistent improvement in prediction accuracy of newer models in comparison to older ones, Cruz estimates that fire behavior predictions could be improved, e.g. by improving classifications of plant species and better mapping of fuel moisture.

It is suggested, that quantifying the uncertainty of predictions for fire spread in an operational setting, should be favored over deterministic approaches (Pinto et al. 2016)

From our experience in developing predictive applications, uncertainty distributions require data science skills to draw sensible conclusions and to trust the outcome. People living in rural areas most likely do not possess these skills, therefore we propose to visualize the prediction model's input data, so that they can draw their own conclusions. Probabilistic fire behavior predictions, in our view, should only be exposed to trained fire management professionals - if qualitative research with these potential users can positively validate its usefulness.

The following chapter describes, how design thinking, a process where interdisciplinary teams work together to solve complex problems in an iterative way, can bring together available research with data technology, and to create an intuitive user experience that delivers value for people.

\section{Design thinking, data technology and user experience to improve decision making}

\subsection{Design Thinking}

Design thinking is a human-centered approach to innovation that draws from the designer's toolkit to balance the needs of people, the possibilities of technology and business requirements, in order to come up with successful solutions (Brown, 2018). It is a generic method and therefore can be applied to design anything, from a simple toothbrush to complex software.

In software development, design thinking has a proven track record of success. For example, Hasso Plattner, who is one of the founders of SAP - a German-based European multinational software corporation, infused the company with design thinking, to put users in the center to design business software, in order to not overwhelm them, and instead help them to reach their goal.

Other examples show how design thinking was applied to social innovation, e.g. to find ways to provide low-cost healthcare throughout the world (Brown and Wyatt, 2010).

Recently, IDEO, one of the most successful design consultancies, acquired Datascope, a data science company, to help merge machine learning and human-centered design (Budds, 2017), much like Wildfires is proposing.

In summary, design thinking will help to achieve a balance of goals of the different stakeholders of our web application Wildfires.

Figure 4 shows the design thinking process applied to user experiences involving data technology.

research + synthesis

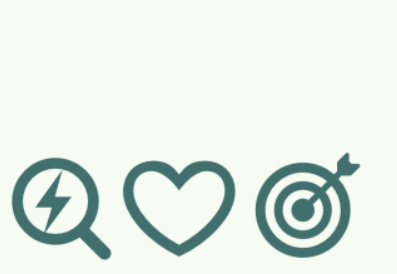

terative prototyping

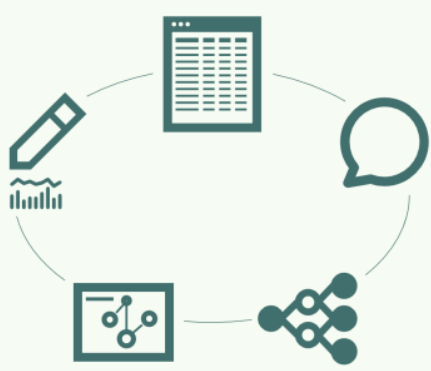

explore discover define

problem user goals

space needs sketch out ideas - explore data - collect user feedback - develop models - iterate the solution value creation

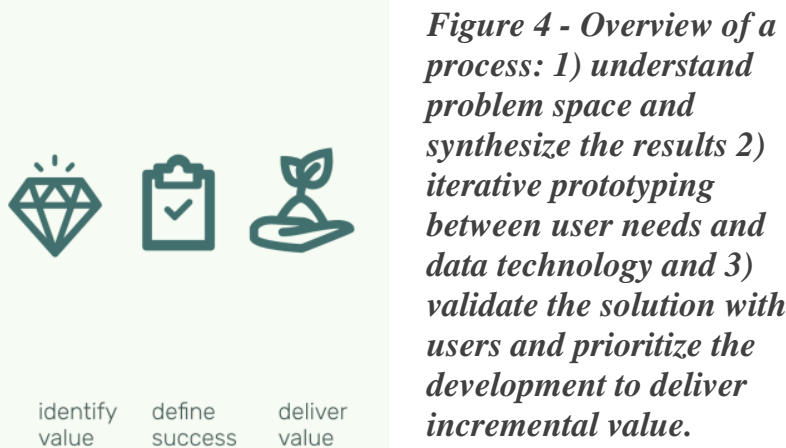

success value criteria 


\subsubsection{Design Thinking process used for the Wildfires solution concept}

Design thinking begins with qualitative research - also called design research, which aims at discovering user needs, pain points, process steps and usage context. Design research data is not evaluated using statistical methods. It serves as a means to develop empathy for the target users, by unmasking underlying motivations.

The results are qualitative statements, that enable the team to make design choices. When doing qualitative research, you start to discover patterns in users' statements after 5-7 interviews.

For Wildfires, we conducted 10 interviews, in October 2017 - prior to, and after the fire on October 15th has affected the region around Benfeita in central Portugal. The participants were Englishspeaking residents. We recorded and transcribed the interviews, and then captured individual data points on post-it notes. During the so-called synthesis phase, the following themes emerged:

1. There is a lack of clear and reliable information available related to forest fires, so it is difficult for people to gage what is going on.

2. Some people panic, they spread misinformation, or sometimes they are not capable of providing accurate location information.

3. People feel, that they are left to themselves, to defend their lives and livelihoods.

4. Trying to escape is dangerous, because the location of the fire changes quickly and information whether or not the roads were safe, was not available.

People organize civilian firefighting that relies on a functioning communication infrastructure, without which, everything breaks down.

\subsection{Data technology}

\subsubsection{Data that supports the solution}

To support the needs discovered during research and to enable better prevention planning and decision making during forest fires, data from different sources can be collected. The source spectrum begins with a) data from official authorities, who provide information about fire locations, firefighting activities, and publicly available mission reports; b) basic environmental data, such as satellite data - that can be used to derive land cover and fuel moisture information c) weather data from service providers d) end user data provided by people in the affected areas, such as current fire locations, road blocks, shelter locations or messages. Users' input might, on the one hand, offer more recent updates than authorities, but at the same time might be biased by subjective perception (in chapter 2.3 The underlying platform, we show how we apply data technology to mitigate risks of mis-information); e) data collected from users' mobile devices, i.e. location information and user IDs. Figure 5 provides an overview of expected data sources.

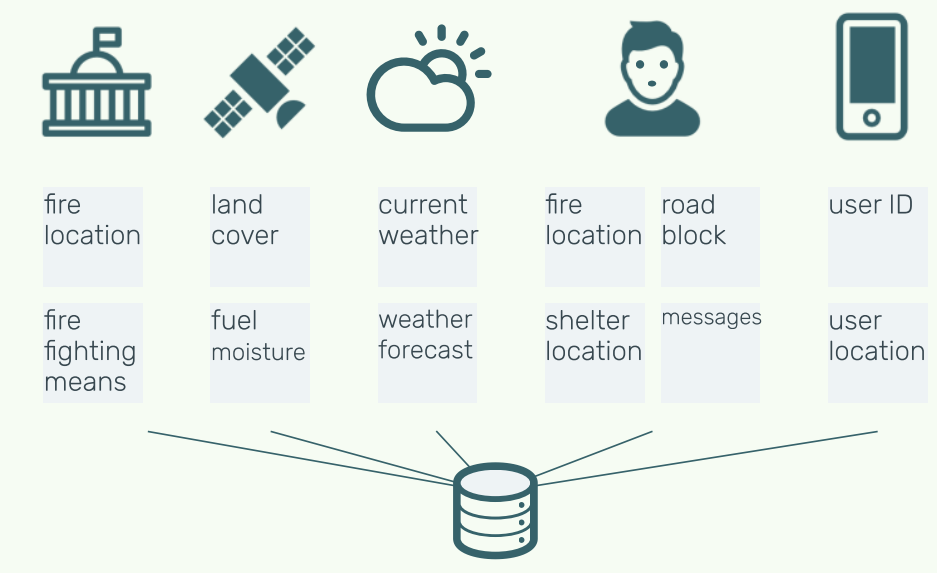

Figure 5 - Overview of possible data sources and data types. 
According to its nature, processed data is classified into 3 categories a) structured data, i.e. geo or weather data; b) semi-structured, i.e. meta data about user input and c) non-structured data, i.e. enduser messages. In the next chapter, we talk about how these different data classifications impact the design of the underlying platform to store and process the data.

As for its size, we expect to handle large data volumes, in particular due to targeted long-term objective, to create fire spread predictions, which require sufficient data history.

\subsubsection{The underlying platform}

The main requirement for the underlying data platform architecture is its ability to efficiently collect, store, process and expose large amounts of heterogeneous data, needed to paint a coherent picture for end users. Besides this, the technological foundation should be openly accessible, so that it can be extended by a broad community which is, motivated to contribute and improve the platform. This imperative led to the selection of mature, open source components as the application foundation. As shown in figure 6, we split the platform into three layers: a) data collection and storage, b) data modeling and analytics, incl. methods of artificial intelligence and c) the data visualization and user experience.

For the collection and consumption of the heterogeneous mass data, we use different types of data stores, that are tightly bound together: 1) Classical databases - for structured data. 2) Search engine technologies, that let you perform searches for structured, unstructured, geo, and metric data (Elastic Search). 3) Unstructured data and NoSQL for large, scalable data storage - using a framework that allows for distributed processing across clusters of computers using simple programming models (Hadoop).

We also integrate common application interfaces, for the consumption of constantly changing resources i.e. during peak times of fire reports and access to weather information, in JSON, CSV or other formats, as well as streaming - using a distributed streaming platform (Kafka).

The objective of the modeling layer is the combination and transformation of data into advanced analytical and decision-making capabilities.

The provided logical models work with distributed SQL, Python, and Scala programming languages and leverage the data processing capabilities of a leading open source data science and machine learning platform (H2O) with its set statistical algorithms like K-means clustering, naive bayes, principal component analysis and more.

By assigning data to particular models, the data can be checked for its plausibility, for example by comparing fire locations reported by civilians with data from official authorities, weather information, satellite data, as well as fire spread predictions.Finally, the user interaction layer supports an effective and scalable visualization technology by using latest web trends in progressive web applications (PWA) and mobile cross-platform development approaches (Apache Cordova).

\begin{tabular}{|c|c|c|}
\hline \multirow{2}{*}{$\begin{array}{l}\text { User Interaction } \\
\text { Visualization } \\
\text { Collaboration Services } \\
\text { Responsive Design }\end{array}$} & Vue.js & PWA \\
\hline & D3.js & Cordova \\
\hline \multirow{3}{*}{$\begin{array}{l}\text { Analytical Modelling } \\
\text { \& Data Science } \\
\text { Distributed SQL } \\
\text { Machine Learing } \\
\text { Artificial Intelligence }\end{array}$} & Spark & $\mathrm{H} 2 \mathrm{O}$ \\
\hline & Python & Scala \\
\hline & Drill & SQL \\
\hline \multirow{3}{*}{$\begin{array}{l}\text { Data } \\
\text { SQL / NOSQL } \\
\text { Indexes } \\
\text { Files } \\
\text { Streaming }\end{array}$} & Open Data & Kafka \\
\hline & SQL DB & ElasticSearch \\
\hline & JSON / CSV & Hadoop \\
\hline
\end{tabular}

Figure 6 - Overview of proposed freely available technology components suitable for collection, processing and visualization of fire-related data. 


\subsection{User experience}

\subsubsection{Design principles}

The Wildfires user experience is based on design principles that were developed during the synthesis phase of the design process.

There are two main factors that drive them. 1) Wildfires is intended for use during emergencies, where people are under immense pressure to make good decision. 2) Wildfires uses large amounts of divers data types and technology that only creates value, if it is adopted by users.

Design Principles:

1. simple, and intuitive visual information and interaction

2. information related to forest fires needs to be reliable, current and coherent

3. provide transparency, into data sources, system status and validity of the data

4. people are in control, visual information should support them

5. availability at all times, particularly during emergencies

6. minimal technical requirements for end user devices

\subsubsection{Solution concept}

The following section describes how our solution concept addresses the needs we heard during user research.

\subsubsection{1. "We want to be prepared, or prevent fires in the first place"}

Visualizing geographic information, such as current land cover types, fuel moisture or topography, in the context of a fire map, which includes the person's current location, enables people to quickly assess, how relevant on-going fires are to her.

Wildfires translates information from multiple sources into visualizations, so that users are not overwhelmed by data and instead are able to consider the most critical factors influencing fire spread in their personal decision-making process. Having data that includes e.g., 12-hour weather forecasts supports people in being proactive. Land cover and fuel moisture visualizations can unmask high risk areas, that require action and fire prevention measures.

\subsubsection{2. “We need reliable information”}

During user research we heard, that people are posting fire locations on Facebook. However, there were situations, when people were not capable of providing accurate location information - which misguided fire fighters to the wrong location in one instance - which is a problem.

In the analytical modeling and data science layer of our data platform, relationships between data types are created and plausibility checks can be performed using machine learning algorithms. These data quality checks, along with a user's automatically captured location information, can significantly reduce the spread of misinformation

\subsubsection{3. "Fire is not static - we need to see how the fire moves"}

Not knowing where exactly a fire was at any given time, was the biggest pain point we heard. With Wildfires, people can easily report fires and provide an up to date picture of the situation - along with other helpful information, such as roadblocks or shelter locations. 

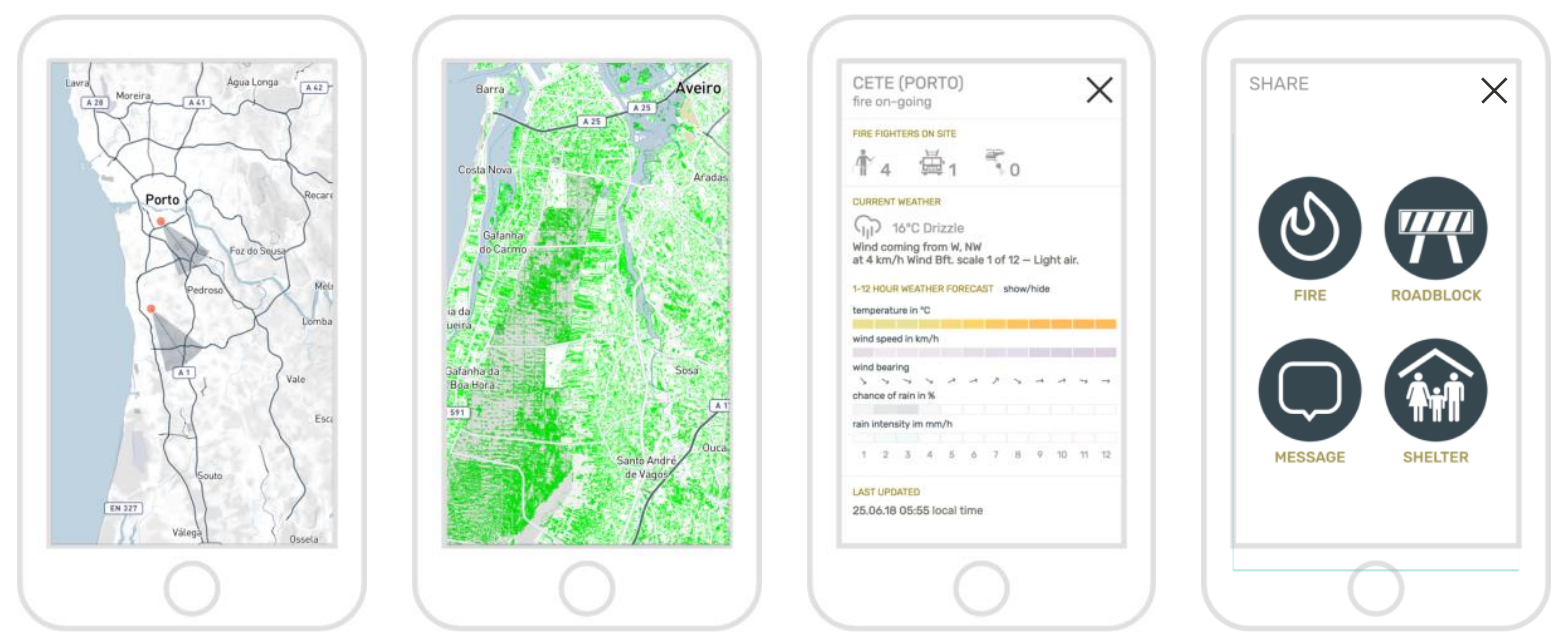

Figure 6 (left to right): Current, functional prototype of a minimal viable solution scope visualizes 1) active fires and wind speed and direction at the location. 2) "forest" areas, based on the NDVI. 3) further information, such as a 12hour weather forecast and data sources. 4) a conceptual prototype to share fire locations and more

\section{Summary and conclusion}

Our data platform collects, stores, processes and makes available large amounts of divers data. Data that is accessible for the analytical modeling layer, where artificial intelligence can deliver answers to complicated questions, such as "Where will the fire go?" or "How reliable is the input data?".

Design thinking ensures that the solution focusses on those questions, that are most relevant for the target users, and that the great scientific achievements become available to create value for people.

Data visualization and user experience design ensure that people can see clearly and draw the right conclusions in times of emergencies. In addition, by seeing areas that pose high fire risks Wildfires can deliver evidence to support prevention measures.

\section{References}

abc Net (2017). Portugal fires: Interior Minister resigns as death toll passes 100, PM under fire to stand down. (See www.abc.net.au/news/2017-10-19/portuguese-minister-resigns-as-wildfire-tollpasses-100/9065714, visited on October 19th, 2017)

Apache Cordova. Explanation: Apache Cordova is a mobile application development framework to build mobile apps with HTML, CSS \& JS that lets you target multiple platforms with one code base. (See https://cordova.apache.org/ visited in June 2018)

Diana Budds (2017). Exclusive: Ideo's Plan To Stage An AI Revolution. Co-Design. (See https://www.fastcodesign.com/90147010/exclusive-ideos-plan-to-stage-an-ai-revolution, visited in May 2017)

ECO News (2017). Pedrógão fires: Government estimates losses of 497 million euros. (See https://econews.pt/FSRJV, visited on July 3rd, 2017)

Elastic search. Explanation: Elastic search is a distributed, RESTful search and analytics engine capable of solving a growing number of use cases. As the heart of the Elastic Stack, it centrally stores your data so you can discover the expected and uncover the unexpected. (See https://www.elastic.co/ visited inJune 2018)

European Commission. Joint Research Centre in collaboration with other Directorate Generals of the European Commission, including DG ENV, DG GROW and DG ECHO, and the national wildfire administrations of the countries in the Expert Group on Forest Fires (2016); JRC science for policy report. Forest Fires in Europe, Middle East and North Africa 2016. European Commission (Luxembourg) 
http://effis.jrc.ec.europa.eu/media/cms_page_media/40/Forest_fires_in_Europe_Middle_east_and _North_Africa_2016_final_pdf_JZU7HeL.pdf)

$\mathrm{H} 2 \mathrm{O}$. Explanation: $\mathrm{H} 2 \mathrm{O}$ combines the power of highly advanced algorithms, the freedom of open source, and truly scalable in-memory processing for big data on one or many nodes. (see https://www.h2o.ai/h2o/ visited in June 2018)

Hadoop. Explanation: The Apache ${ }^{\mathrm{TM}}$ Hadoop ${ }^{\circledR}$ project develops open-source software for reliable, scalable, distributed computing. (See http://hadoop.apache.org/ visited in June 2018)

Kafka. Explanation: Kafka is used for building real-time data pipelines and streaming apps. (See https://kafka.apache.org/ visited in June 2018)

Karen Joyce (2013). Vitality of plants based on the NDVI - Normalized Difference Vegetation Index. (See https://youtu.be/rxOMhQwApMc Channel for remote sensing educational resources, visited in May 2017)

Miguel G Cruz, Martin Edward Alexander, Andrew Sullivan, James S. Gould and Musa Kilinc (2018). Assessing improvements in models used to operationally predict wildland fire rate of spread (page 1). Part of the project: Wildland fire behaviour and spread prediction systems (DOI: 10.1016/j.envsoft.2018.03.027) (See https://www.researchgate.net/publication/324845113_Assessing_improvements_in_models_used _to_operationally_predict_wildland_fire_rate_of_spread)

Paulo Fernandes, Davide Ascoli, Carlos G. Rossa, Mário G. Pereira, Miguel G Cruz, Martin Edward Alexander, Ângelo Sil and Malik Amraoui (2018). BONFIRE - gloBal-scale analysis and mOdelliNg of FIRE behaviour potential (PTDC/AAGMAA/2656/2014) (page 5, 6). Paulo Fernandes's Lab, Universidade de Trás-os-Montes e Alto Douro. (Vila Real, Portugal) (See https://www.researchgate.net/project/BONFIRE-gloBal-scale-analysis-and-mOdelliNg-of-FIREbehaviour-potential-PTDC-AAG-MAA-2656-2014)

PWA. Explanation: Progressive Web App (PWA) is a term used to denote a new software development methodology. Unlike traditional applications, progressive web apps are a hybrid of regular web pages (or websites) and a mobile application. This new application model attempts to combine features offered by most modern browsers with the benefits of mobile experience. (See https://medium.com/@deepusnath/4-points-to-keep-in-mind-before-introducing-progressive-webapps-pwa-to-your-team-8dc66bcf6011 visited inJune 2018)

Renata Pinto, Akli Benali, Ana C. L. Sá, Paulo Fernandes, P.M.M. Soares, Rita M Cardoso, Ricardo M Trigo and José M.C. Pereira (2016). Probabilistic fire spread forecast as a management tool in an operational setting (page 9, 10). SpringerPlus 5(1):1205. (DOI: 10.1186/s40064-016-2842-9). (See

https://www.researchgate.net/publication/305401391_Probabilistic_fire_spread_forecast_as_a_m anagement_tool_in_an_operational_setting)

Tim Brown (2018). CEO of IDEO. (See https://www.ideou.com/pages/design-thinking, visited in May 2017)

Tim Brown and Jocelyn Wyatt (2010). Design Thinking for Social Innovation. Stanford Social Innovation Review. (See https://ssir.org/articles/entry/design_thinking_for_social_innovation, visited in May 2017) 


\section{Chapter 6}

Socio Economic Issues 


\title{
Effects of law enforcement efforts on intentional wildfires
}

\author{
J. Prestemon ${ }^{1}$; D. Butry ${ }^{2}$; M.L. Chas-Amil*3; ${ }^{*}$ J. Touza ${ }^{4}$ \\ ${ }^{1}$ Southern Research Station, USDA Forest Service, Research Triangle Park, NC 27709, USA, \\ \{jprestemon@fs.fed.us\} \\ ${ }^{2}$ National Institute of Standards and Technology, 100 Bureau Drive, Mailstop 8603. Gaithersburg, \\ MD 20899-8603, \{david.butry@nist.gov\} \\ ${ }^{3}$ Universidade de Santiago de Compostela, Baixada Burgo das Nacións s/n, 15782 Santiago de \\ Compostela, Spain, \{marisa.chas@usc.es*\} \\ ${ }^{4}$ University of York, Environment Department, Heslington, YO10 5NG, UK, \\ \{julia.touza@york.ac.uk\}
}

\begin{abstract}
Wildland managers and law enforcement organizations devote significant resources attending to the consequences of illegal firesetting across many parts of the world. Intentional wildfires may be set for reasons of malice, as acts of protest, or as pure vandalism. Incendiary wildfires result in deaths, property, and resource damages that exceed rates found for fires of accidental or natural origin. In fact, there is a great concern with how such fires tend to occur nearer populations and values at risk. Extensive evidence suggests that arsonists were behind many of the deadly wildfires that have occurred in many countries, particularly in 2017 in the United States (California), Chile, Portugal, and Spain (Galicia). Apart from firefighting resources, prevention measures may reduce wildfire occurrence. Prevention measures may include fuel reductions (thinnings, fuel breaks, prescribed fire). Research has also shown that education programs and information campaigns, fire investigations, surveillance efforts, as well as law enforcement efforts could reduce unwanted ignitions. Studies of the effects of law enforcement or other wildfire prevention measures have shown that these interventions can reduce wildland arson occurrence overall. Based on Becker's economic theory of crime, law enforcement affects crime in a direct way, through fines and incarceration, and in an indirect way, through deterrence. Criminologist have also recognized the possibility that efforts by law enforcement intended to reduce crime in one time or place or of one type could lead to displacement of crime in a variety of ways (temporal, spatial, target, tactical, and offense), but some research has shown that the opposite could happen - diffusion. In the spatial sense, one kind of diffusion is where law enforcement efforts in one defined location lead to reduced crime across many neighboring locations. The objective of this paper is to describe our efforts to analyze the effect of law enforcement measures — namely, arrests for intentional firesetting on intentionally-set illegal wildfires. We have conducted two studies (Prestemon et al. 2012, forthcoming) showing evidence for Galicia (NW of Spain). Firstly, we investigated, the effect of an arrest for intentional firesetting on future intentional wildfires in the same location and in neighboring locations. Secondly, we evaluated whether arrests have displacement or diffusion effects, as they may increase or decrease intentional wildfires in nearby locations or later periods, due to both a risk-perception adjusting effect and an incapacitation effect. Therefore, by relating intentional wildfire occurrence to arrest of suspected firesetters, we identify a likely principal mechanism for achieving law-enforcement success. Additionally, we separately quantify the effects of an arrest on agriculturally-based versus non-agriculturally-based intentional firesetting, leaving open the prospect that these two subcategories have different behavioral responses to law enforcement actions and other variables. Such differences could be key to the design of effective policing strategies in response to firesetting in the region.
\end{abstract}

Keywords: arson, arrest, crime, Galicia, incendiary, Spain,

\section{Introduction}

Wildland managers and law enforcement organizations devote significant resources attending to the consequences of illegal firesetting across many parts of the world. Intentional wildfires may be set out of of malice, as acts of protest, or as pure vandalism, among other reasons. Incendiary wildfires result in deaths, property, and resource damages that exceed rates found for fires of accidental or 
natural origin. In fact, there is great concern with how such fires tend to occur nearer populations and values at risk. Extensive evidence suggests that arsonists were behind many of the deadly wildfires that have occurred in many countries, particularly in 2017 in the United States (California), Chile, Portugal, and Spain (Galicia). Apart from firefighting resources, prevention measures, such as fuel reductions (thinnings, fuel breaks, prescribed fire), may reduce wildfire occurrence. Research has also shown that education programs and information campaigns, fire investigations, surveillance efforts, as well as law enforcement efforts could reduce unwanted ignitions (e.g., Donoghue and Main 1985, Prestemon and Butry 2005, Butry and Prestemon 2005, Prestemon and Butry 2010, Abt et al. 2015). Based on Becker's (1968) economic theory of crime, law enforcement affects crime directly, through fines and incarceration, and indirectly, through deterrence. Criminologist have also recognized the possibility that efforts by law enforcement intended to reduce crime in one time or place or of one type could lead to displacement of crime in a variety of dimensions (temporal, spatial, target, tactical, and offense), but some research has shown that the opposite could happen-diffusion. In the spatial sense, one kind of diffusion is where law enforcement efforts in one defined location lead to reduced crime across many neighboring locations.

The objective of this paper is to describe our efforts to analyze the effects of law enforcement measures — namely, arrests for intentional firesetting — on intentionally-set illegal wildfires. We have conducted two studies (Prestemon et al. 2012, forthcoming) where we describe intentional wildfire in a mixed Routine Activity and Rational Choice (in the Becker [1968] context) theoretical framework, showing evidence for Galicia (NW of Spain). The choice of our study area is relevant because the region of Galicia has the highest wildfire incidences of Spain, and nearly all the fires are human-caused $(88 \%)$, which have traditionally been associated with illegal behaviour through intentional ignitions (82\%). In addition, Galicia is among the most heavily forested regions of Spain, with nearly $70 \%$ of the territory classified as forestland, 2 million hectares (ha), which produce $59 \%$ of Spain's timber. The primary objective of Prestemon et al. (2012) was to develop and test the forecast performance of forward-looking intentional fire hotspotting models that could be used by managers and law enforcement. A secondary objective was to evaluate whether spatio-temporal lags of intentional wildfire occurrence could be used to aid in these forecasts. Thus, we investigate the effect of an arrest for intentional firesetting on future intentional wildfires in the same location and in neighboring locations. The primary innovation of this work is that we compare competing alternative forwardlooking models and identify a superior forecasting empirical model. Our testing framework is rigorous, in the sense that the fit of a forecast is compared against the fit of plausible alternatives, with parameters estimated over a training dataset which pre-dates the long forecast sample. A secondary innovation is that we successfully identify the form of spatio-temporal relationships of intentional wildfire occurrence. Third, we confirm the validity of existing crime theories in the context of intentional fires, particularly in Galicia, Spain, where such tests have not been made. Fourth, and unlike previous research, our models relate intentional wildfire occurrence to a more proximate measure of law enforcement effort: previous arrests of suspected firesetters. The implication of the joint findings of significant spatio-temporal relationships in fire occurrence and of arrests is that law enforcement has broad-scale, long-run impacts that reach far beyond the immediate landscape or short time horizons following arrest. Prestemon et al. (forthcoming) evaluate whether arrests have displacement or diffusion effects, as they may increase or decrease intentional wildfires in nearby locations or later periods, due to both a risk-perception adjusting effect and an incapacitation effect. Therefore, by relating intentional wildfire occurrence to arrest of suspected firesetters, we identify a likely principal mechanism for achieving law-enforcement success. Additionally, accurate quantification of the spatiotemporal displacement of arrests on intentional firesetting might depend on the motivations of firesetters. Thus, we separately quantify the effects of an arrest on agriculturally-based versus nonagriculturally-based intentional firesetting, leaving open the prospect that these two subcategories have different behavioral responses to law enforcement actions and other variables. Such differences could be key to the design of effective policing strategies in response to firesetting in the region. The 
remainder of this work describes data, methods and main results of these two papers, and, finally, the main conclusions obtained.

\section{Forecasting intentional wildfires using temporal and spatiotemporal autocorrelation (Prestemon et al. 2012)}

\subsection{Data}

We relate reported daily ignitions of intentional wildfires in Galicia, covering January 1, 1999, to December 31, 2006, to intentional wildfire arrests in the forest districts and spatial lags of these arrests at different distances. Variables hypothesized to relate to intentional wildfire occurrences (counts) include: day dummies indicating leisure (Friday, Saturday, Sunday, and non-weekend Holiday), which index potentially lower opportunity costs of crime commission through foregone wages; month dummy variables, which may index the direct costs of successful crime commission, related to fuel conditions and average weather; the Modified Fire Weather Index (MFWI) (Goodrick 2002), which also measures fuel conditions and direct costs; daily minimum relative humidity and maximum onehour wind speed, capturing direct costs; aggregate income in the spatial unit of inference, and income per capita in the spatial unit of inference, both of which index opportunity costs of crime commission and being caught; and the number of arrests of individuals for intentionally and illegally igniting wildfires in the previous year in the region of Galicia.

\subsection{Methods}

We develop daily spatio-temporal ignition count models for intentional wildfires as a function of arrests, weather and climate, and socio-economic factors. We specify statistical intentional wildfire forecast models that could be used by managers and law enforcement as tactical and strategic tools. We estimated Poisson autoregressive models of order p--PAR(p) models--and three competing alternative models (a static Poisson, a constant rate, and a random walk) at the daily time step. Models are estimated independently for each of the 19 forest districts in Galicia, Spain, over a 1999-2003 training dataset and then evaluated out-of-sample with a 2004-2006 dataset. PAR(p) and static Poisson models included covariates deriving from crime theory and an understanding of the drivers of wildfire occurrence. Among the covariates were dummy variables indicating the occurrence of intentional wildfires in nearby and distant forest districts in days $\mathrm{t}-1$ and $\mathrm{t}-2$.

\subsection{Results}

Models show highly significant autoregressive components, lasting up to four days, in intentional firesetting across all of Galicia's forest districts. This autoregressivity was the primary source of superior forecast performance of the PAR(p) models over all competing models, reducing out-ofsample forecast root mean squared error of forecast counts for most forest districts by an average of 15 percent compared to the static Poisson and larger amounts for the constant rate and random walk alternatives. Most additional covariates were statistically significantly different from zero and signed consistent with theory. Arrests of individuals for illegal firesetting in Galicia and spatio-temporal lagged occurrences of wildfires in other districts were found to be predictive of wildfire occurrences. This suggests the existence of serial and copycat behavior, and the potential spatial and temporal broad scale impact of law enforcement. We find that the combined temporal and spatio-temporal long-run marginal effect of an additional arrest, summed across forest districts in Galicia, is -139.6 intentional wildfires, equivalent to a long-run elasticity of -0.94 .

\section{Displacement of Wildland Arson by Law Enforcement Efforts (Prestemon et al. forthcoming)}

\subsection{Data}


Using daily wildfire count data, from January 1, 1999, to December 31, 2014, we develop daily spatio-temporal ignition count models for municipalities. These intentional wildfires are further subdivided into those related to the (illegal) firesetting associated with agricultural activities (fires started by farmers to eliminate shrubs and agricultural debris) and those that are not. Separate model estimates were generated for agricultural intentional, non-agricultural intentional, and their sum. As covariates, we use arrests, the election cycle, day of week, weather and climate, and socio-economic factors.

\subsection{Methods}

Estimation of the autoregressive count models was done using various assumptions about the spatio-temporal firesetting process. We estimated fixed effects negative binomial models (FENB), random effects negative binomial (RENB) models, and zero-inflated negative binomial (ZINB) models with intercept-shifting municipality dummy variables. The ZINB modeling approach was done because over 100 municipalities had very low counts (fewer than ten per year, from 1999 through 2014) of intentionally ignited wildfires and because for these and many other municipalities, at least half of the year, there were virtually zero intentionally ignited wildfires. This inflated count of zero fires therefore was captured by the first-stage logit equation relating the zero count for a day to municipality dummy variable shifters and other factors. In all models, we attempted to specify multiple autoregressive parameters, but it was clear upon estimation that this was preventing many specifications from converging in maximum likelihood. In the end, we opted for a single autoregressive term to control for the autocorrelation in firesetting that is common in intentional fires.

Effects of arrests on wildfire occurrences in the municipality and in neighboring radii were computed using arrests in two temporal running time blocks: the previous 366 to 545 days, and the previous 1 to 365 days. Buffer radii were 10, 15, and 25 kilometers, justified by the fact that the most firesetters arrested in Spain set a fire close to their place of residence (Sotoca 2016). Effects are reported for the short-run, which ignores the autoregressive effect of wildfires within a municipality, and the long-run, which incorporates the autoregressive effect. When estimating separate agricultural and non-agricultural intentional fire models, we included the lagged count and lagged zero count indicators of both categories. When calculating the long-run effects of elections and arrests, we used only the lagged count of the own type (i.e., the coefficient on agricultural intentional lagged fires was used to make the long-run adjustment, and the same for the non-agricultural category of intentional fires).

\subsection{Results}

Across all specifications and model versions, arrests reduced the counts of intentional wildfires. This suppressive effect was also identified for progressively farther "donuts" around municipalities, with each spatio-temporal lag negatively related to the count of intentional fires. For agricultural models, the effects of arrests were substantially larger. Non-agricultural intentional fires were more inelastically related to arrests but still demonstrated more elastic responses to arrests compared to total intentional fires. The overall more elastic responses for each of agricultural and non-agricultural wildfires reveal an aggregation bias, attenuating the size of the coefficients on arrests, existing in the total intentional fire model estimates.

An examination of the temporally and spatio-temporally lagged effects of arrests in the different models nearly universally reveal a statistically significant effect of own municipality and neighborhood municipality arrests on the count of intentional fires of both agricultural and nonagricultural fires and their sum. We can conclude that arrests do not tend to displace future firesetting to nearby locations in Galicia; instead, arrests tend to dampen firesetting for at least 1.5 years within and across nearby municipalities. In other words, our models support a diffusion effect of arrests. 


\section{Conclusions}

Our work revealed that an arrest for intentional firesetting could reduce future intentional wildfires for at least one year. These effects of arrests are both direct, stemming from successes within forest districts, and indirect, stemming from successes in other forest districts. This spatio-temporal impact, whose effects until now have never before been quantified in the refereed literature on intentional wildfires, are important and consistent with expectations of a process that contains copycat or even moving serial elements in the firesetting population. Arrests, we find, have wide-ranging and large impacts because of this spatio-temporal element of intentional wildfire patterns in Galicia. Moreover, we also detect that arrests may decrease intentional wildfires in nearby locations or later periods, consistent with a pattern associated with diffusion effects. Thus, we find evidence that arrests diffuse (reduce) future intentional fires across space but do not displace them. This research offers a tool that is needed by policy makers and forest managers to assess the overall impacts of enhanced efforts to reduce the occurrence of intentional wildfires,

\section{Acknowledgements}

We are thankful to the Spanish Ministry of Agriculture and Fisheries, Food and Environment (MAPAMA), and AEMet (Agencia Estatal de Meteorología) for providing the forest fires and climatic databases, respectively. We are also grateful to Laboratorio de Criminalística-Zona Guardia Civil de Galicia for facilitating arrest information. This work was partly funded by Project ECO2017-89274-R MINECO/AEI/FEDER,UE.

\section{References}

Abt, K.L., D.T. Butry, J.P. Prestemon, and S. Scranton. 2015. Effect of fire prevention programs on accidental and incendiary wildfires on tribal lands in the United States. International Journal of Wildland Fire 24(6):749-762.

Becker, G.S. 1968. Crime and punishment: An economic approach. Journal of Political Economy 76:169-217.

Butry, D.T., and J.P. Prestemon. 2005. Spatio-temporal wildland arson crime functions. Paper presented at the Annual Meeting of the American Agricultural Economics Association, July 26-29, 2005, Providence, Rhode Island. 18 p. Published on the Internet, http://purl.umn.edu/19197

Donoghue, Linda R.; Main, William A. 1985. Some factors influencing wildfire occurrence and measurement of fire prevention effectiveness. Journal of Environmental Management 20(1):87-96.

Goodrick S.L. 2002. Modification of the Fosberg fire weather index to include drought. International Journal of Wildland Fire 11:205-221.

Prestemon, J.P., and D.T. Butry. 2005. Time to burn: modeling wildland arson as an autoregressive crime function. American Journal of Agricultural Economics 87(3):756-770.

Prestemon, J.P., and D.T. Butry. 2010. Wildland arson: a research assessment. P. 271-283 In Pye, J.M., H.M. Rauscher, Y. Sands, D.C. Lee, and J.S. Beatty (eds.), Advances in Threat Assessment and their Application to Forest and Rangeland Management. Gen. Tech. Rep. PNW-802. Portland, OR: U.S. Department of Agriculture, Forest Service, Pacific Northwest Research Station. 708 p.

Prestemon, J.P., M.L. Chas-Amil, J. Touza Montero, and S.J. Goodrick. 2012. Forecasting intentional wildfires using temporal and spatiotemporal autocorrelations. International Journal of Wildland Fire 21(6):43-54.

Prestemon, J.P., D.T. Butry, M.L. Chas-Amil, and J. Touza Montero. Forthcoming. Displacement or Diffusion of Intentional Wildfires by Law Enforcement Efforts? Evidence from Spain.

Sotoca Plaza, A. 2016. Perfil criminológico del incendiario forestal: estudio empírico basado en la evidencia. PhD Dissertation. Universidad Complutense de Madrid. http://eprints.ucm.es/37600/1/T37195.pdf (Accessed 11/10/2017). 


\title{
Human perception of fire hazard in wildland urban interface areas - a Portuguese survey analysis of spot fires
}

\author{
Ricardo Oliveira*²; Sandra Oliveira ${ }^{2}$; José Zêzere ${ }^{2}$; Domingos Viegas ${ }^{1,3}$ \\ ${ }^{1}$ Forest Fire Research Centre (CEIF), ADAI-LAETA, University of Coimbra, 3030 - 289 Coimbra, \\ Portugal, \{ricardo@adai.pt*\} \\ ${ }^{2}$ Instituto de Geografia e Ordenamento do Território; Universidade de Lisboa - Lisboa, Portugal, \\ ${ }^{3}$ FCTUC; Departamento de Engenharia Mecânica - Coimbra, Portugal.
}

\begin{abstract}
Nowadays, wildfires are the most devastating natural hazard in Portugal with the highest number of victims and mortality of both operational agents and civilians. During the yearly Wildfire Season, which is determined by law from 15th of May until 15th of October, extra efforts are carried out by the civil protection through dedicated financial and human resources. In the recent years, most of the available resources were employed with their highest capacities in Portugal to control wildland fires. However, still, a high number of fires escape first attach and results in large fires (>100 ha). On the years 2012, 2013, 2016 and 2017 there is temporal variances appeared in the recorded ignitions, where high ignitions recorded during the spring and some of them developed in large and deadly wildfires like the fires ignited in 17th of June 2017 in the Centre of Portugal with 65 mortalities. In the autumn of the same year as well, 2017, large fires were ignited in 15th of October 2017 with 47 mortalities. In 2015, the Institute for Conservation of Nature and Forests (ICNF) recorded a total of 15851 occurrences, the National Republican Guard investigated $76 \%$ of those and identified $47 \%$ of them occurred due to negligent human behaviour. $89 \%$ of these occurrences are due to leftover burnings. With such a high number of anthropogenic causes, there is a need arises to evaluate the human perception of wildfires hazards, particularly in Wildland-Urban Interfaces (WUI). The evaluation will assist in reducing the occurrences due to negligent behaviours, which is the aim of this study in addition to highlighting the needed awareness actions based on that perception. As a methodology, we defined territories in Mainland Portugal recently affected by wildfires; then we characterised them based on human and physical aspects. A survey with 40 questions was generated. We divided the survey into four sections according to the purpose of the questions, which are sociographic characterisation; perception of the participant regarding the wildfire hazard; the impact of wildfire in the area of the participant; and finally, the participant suggestion of mitigation and prevention measures. The query was pre-tested first by selecting a small population group formed of 30 residents, which is in Algeriz, located in the district of Aveiro. As some preliminary results, a pre-test showed that $85.2 \%$ of the sample addressed wildfires as the most significant natural hazard impacting his/her life, followed by floods. $3.7 \%$ of the participants reported that they suffered from housing damage due to wildfires To complete our understanding of the people perception of wildfire hazards, this survey will be extended to several other territories of Portugal Mainland, and analyse the patterns of answers.
\end{abstract}

Keywords: wildfire, impact, perception, survey, spot fire

\section{Introduction}

Wildfires are the most devastating environmental hazard in Portugal, with the highest number of victims and mortality of both operational agents and civilians, and causing severe economic and environmental consequences (Oliveira et al., 2017) (Nunes et al., 2016) (San-Miguel-Ayanz et al., 2013) (Tedim et al., 2015) (Turco et al., 2016). Most fires in Portugal result from human activities, with negligence associated with leftover burning as the primary cause (ICNF, 2016a). The year of 2017 was particularly severe, with 112 fatal victims and a total burned area in the country of around 500.000 ha (ICNF, 2017). The temporal trends of wildfire occurrence during this year indicate that the larger fires, that caused higher losses, occurred in late spring (June) and autumn (October). In Portugal,

Advances in Forest Fire Research 2018 - Page 1130 
the main wildfire season is established by Law and extends from $15^{\text {th }}$ May to $15^{\text {th }}$ October; during this period, an extra effort is carried out by the civil protection services through additional financial and human resources, and most fires are controlled in the early stages of development (Diário da República, 2006). However, a certain number of fires still escape control during the early attack by suppression resources and may result into fires above 500 ha in size. When driven by favorable weather conditions, in places with irregular topography and higher slopes, these fires exhibit extreme and unexpected behaviour and escape the control of suppression resources (Viegas et al., 2017).

The tragic situation verified in Central Portugal in 2017 also derived from other characteristics of the territory, in particular the dispersion of small settlements interspersed in wildland areas, where fuels accumulated due to the abandonment of agricultural activities in the last decades (Nunes et al., 2016) (Moreira et al., 2011)(San-Miguel-Ayanz et al., 2012). These areas, generally defined as Wildland-Urban Interface (WUI), have been the focus of multiple studies regarding wildfire mitigation, since the coexistence of vegetated areas with human structures and potential ignition agents increase risk levels (Modugno et al., 2016) (Badia et al., 2011).

\subsection{Spot fires}

According to the conditions of propagation, fires are divided in four types: surface fires, crown fires, ground fires and spot fires (Almeida, 2011). Spot fires are caused by the projection of sparks or embers at a distance from the main fire and are a characteristic of extreme wildfire behavior (Viegas, 2012) (Almeida et al., 2014). Previous studies indicate a direct relation between damages in buildings and spot fires, with damages resulting from the direct contact with a fire front being rarer (Cohen, 2010) (Blanchi \& Leonard, 2008) (Caballero, 2017).

Research on spot fires and their relation with wildfire behavior dates back from the fifties of the last century (Byram, 1954) (McArthur, 1967) (Lee \& Hellman, 1969); however, several knowledge gaps still remain, mainly regarding the difficulty in determining with high accuracy the amount, size, shape and landing spot of burning particles, as well as their ability to ignite a new fire. As such, the results of this thematic research are usually reflected in guidelines and good practices to help mitigating the effects of spot fires in human structures, through fuel management options and in the involvement of communities in decision-making.

Considering the strong human dimension of fire causes, the characteristics of the WUI, the relevance of spot fires in wildfire behavior and the severe impacts of wildfires in the country, it is crucial to understand how people perceive fire occurrence in their area of residence and their knowledge on spot fires as a means of fire propagation in extreme conditions. This study aimed to evaluate people's perception of spot fires in wildland-urban interface areas, in order to identify potential mitigation and prevention (awareness) actions based on their knowledge, experience and needs.

\section{Materials and methods}

\subsection{Study area}

This study was carried out in two test areas of mainland Portugal that were recently affected by wildfires: Algeriz (Vila Nova (VN) de Monsarros - Anadia) in 2016 and Pedrógão Grande in 2017 (Figure ).

Area 1 - Algeriz. Village located in the countryside of the civil parish of Vila Nova de Monsarros, in the municipality of Anadia, Aveiro district (Figure ). According to the latest Census Survey 2011 (INE, 2012), the municipality of Anadia has 29150 residents, distributed by 10 civil parishes and a population density of 134,58 people/ $\mathrm{km}^{2}$. The civil parish of VN Monsarros occupies an area of 23,72 $\mathrm{km}^{2}$ and has 1713 inhabitants, with a population density of 72,2 people/ $\mathrm{km}^{2}$, lower than the municipal average. In Algeriz village, currently live 30 people. 
This municipality was affected by a wildfire that started on 10 August 2016, with a burned area extending for 2538,9 ha, which corresponds to $11.7 \%$ of the municipality area. The civil parish of VN Monsarros by itself contributed with 1691 ha, corresponding to $71.29 \%$ of its total area.

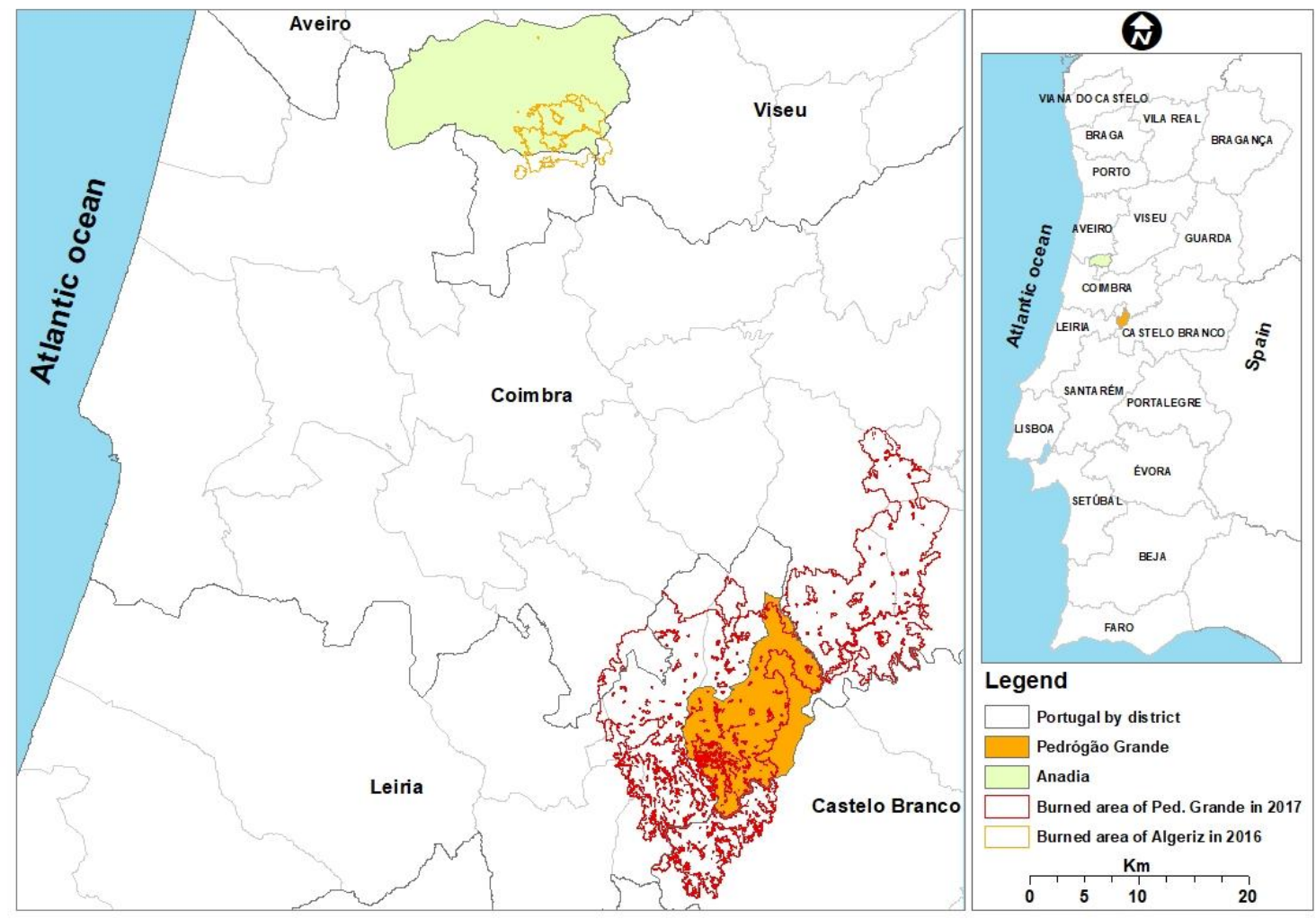

Figure 1 - Location of the two test areas

The cause of the fire has supposedly been the contact between a fallen electric line in a steep slope and the vegetation, mainly composed of eucalyptus, under windy conditions (ICNF, 2016b).

Area 2 - Pedrógão Grande is a municipality located in the district of Leiria, in the Central Region. It has an area of 128,75 $\mathrm{km}^{2}$ and 3915 residents (INE, 2012), divided in 3 civil parishes. The population density corresponds to 30,4 people $/ \mathrm{km}^{2}$, much lower than the national average of 114,5 people $/ \mathrm{km}^{2}$ and less than half of the population density of VN Monsarros.

This municipality was severely affected by a wildfire that started on 17 June 2017, with a burned area extending for $9407,6 \mathrm{ha}$, corresponding to $73.06 \%$ of the municipality area (Viegas et al., 2017). The probable cause of the fire has been identified as the contact between an electric line and the canopy of a cork oak tree, in dry and windy weather conditions (Viegas et al., 2017).

\subsection{Data collection - survey questionnaire}

Data on prior experience and perception of wildfire occurrence by residents was obtained through the implementation of a survey questionnaire in the test areas. In total, 47 questionnaires were made face-to-face.

The questionnaire was composed of 40 questions, divided into 4 sections:

i. The first section included questions regarding the individual characteristics of people (age, gender, nationality, place of origin, education level and occupation). Additionally, the relation of people with forest activities and prior experience with wildfires were also included;

ii. The second section comprised essentially the conceptual understanding of wildfire risk, spot fires and wildland-urban interface areas; 
iii. The third section focused on the prior experience of people with different wildfire impacts and their knowledge on propagation conditions related to construction and property;

iv. The fourth section included questions about the knowledge and preferences of people regarding wildfire prevention and mitigation measures.

This paper focuses mainly on the results obtained with sections 1 and 2 of the questionnaire.

\subsection{Data analysis}

The personal characteristics and wildfire experience of the interviewees, compiled in section 1 of the questionnaire, were first analyzed with descriptive statistics, to uncover the overall sociodemographic context of the respondents. These characteristics were subsequently tested for association with the responses given in the second section, regarding the understanding of several wildfire-related concepts. To attain this goal, the Pearson $\chi^{2}$ (chi-square) test was applied to multiple pairs of questions. This test measures the difference between the observed values in each category and the expected values, which should be similar among categories if no significant association exists (Scheffe, 1959). For open-ended questions, to which no prior option was provided and where the responses were based on the individual's own knowledge or experience, a data-driven analysis based on text mining techniques was applied. These techniques allow to explore the frequency of specific terms, the relation between different words and to visualize graphically the most common words that people associate with the question posed (with word clouds, for example). These results were used to define particular categories of responses retrieved from the open-ended questions, for further analysis regarding their association with personal characteristics and prior wildfire experience. The statistical analysis of the questionnaire responses was made with the R software (R Project for Statistical Computing) (Bunn \& Korpela, 2016), to create automatic routines and to enable the replication of the analysis procedure in other areas and with new data.

\section{Results and discussion}

Initial results showed that $64 \%$ of the participants were women, $79 \%$ were above 50 years old and $52 \%$ had no education level or only the elementary school (Erro! A origem da referência não foi encontrada.). About $96 \%$ of the interviewed people were previously affected by wildfires and only $11 \%$ have a job related to forest activities (Table 1 ).
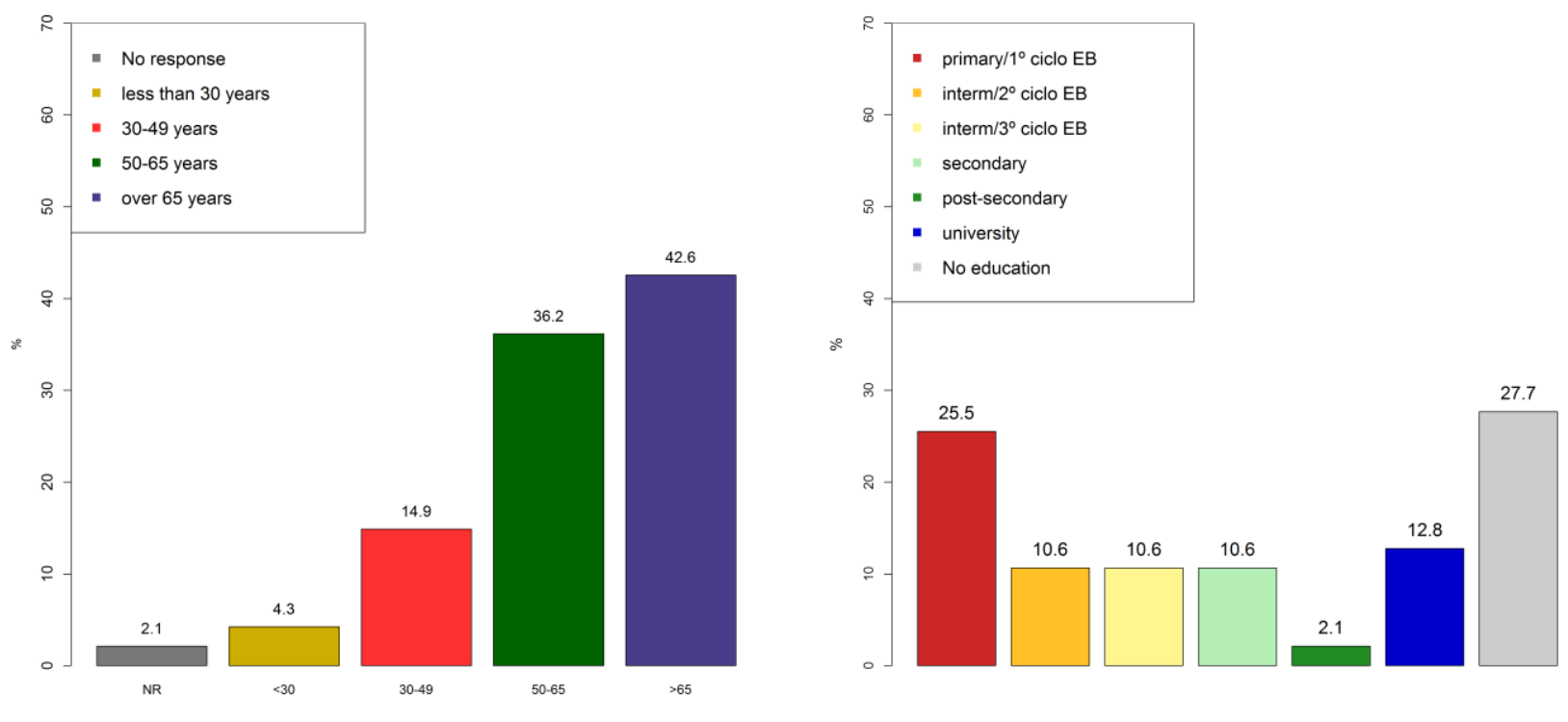

Figure 2 - Proportion of people by age groups (left) and by education level (right) 
Table 1-Percentage of people with regards to residency, professional status and job related to forest

\begin{tabular}{|l|c|l|c|l|c|}
\hline $\begin{array}{c}\text { Resident in the parish } \\
\text { of origin }\end{array}$ & \multicolumn{2}{c|}{ Professional status } & \multicolumn{2}{c|}{ Job related to forest } \\
\hline Categories & $\%$ & Categories & $\%$ & Categories & $\%$ \\
\hline Yes & 85.1 & Employed & 46.8 & Yes & 10.7 \\
No & 14.8 & Unemployed & 8.5 & No & 89.3 \\
& & Retired & 44.7 & & \\
\hline
\end{tabular}

These personal characteristics and experience showed association with the responses given in section 2. The concept of wildfire risk was different depending on age groups, education level and professional status (Table ). Regarding age groups, people above 65 years old answered mostly that they didn't know, or didn't want to answer ( $80 \%$ ), whereas only $14 \%$ of people below 50 years old didn't give an answer. The majority of people who responded mentioned that wildfire risk is not probability, weather conditions nor fuel management, giving a wide diversity of opinions that are difficult to classify ("Others"). Regarding education levels, the proportion of people that responded didn't know/respond was higher for people with no education $(85 \%)$ or people with elementary school level $(58 \%)$. The relation of wildfire risk with Probability was mostly selected by people with university level (33\%) and by people with secondary education (20\% of the total people in this level). In relation to the professional status, for the people who are currently employed (47\%), $18 \%$ relate wildfire with Probability, $14 \%$ with Risk, $5 \%$ with weather conditions, $5 \%$ with lack of fuel management and $45 \%$ relate it to other conditions. Retired people correspond to $45 \%$ of the interviewees and the majority answered, "didn't know/respond" (76 \%).

Table 2 - Association between personal characteristics and the answers given in section 2 of the survey

Q10 - What is wildfire risk?

\begin{tabular}{|l|c|c|c|}
\hline Characteristics \& Experience & $\chi 2$ & $p$-value & df \\
\hline Age groups & 36,39 & 0,0138 & 20 \\
\hline Education level & 46,03 & 0,0308 & 30 \\
\hline Professional status & 22,14 & 0,0144 & 10 \\
\hline
\end{tabular}

Q11 - How do you classify your knowledge on wildfire risk?

\begin{tabular}{|l|c|c|c|}
\hline Characteristics \& Experience & $\chi 2$ & $p$-value & $\mathrm{df}$ \\
\hline Job related to forest & 18,16 & 0,0004 & 3 \\
\hline
\end{tabular}

Q12 - Do you know what are spot

fires?

\begin{tabular}{|l|c|c|c|}
\hline Characteristics \& Experience & $\chi 2$ & $p$-value & $\mathrm{df}$ \\
\hline Job related to forest & 8,66 & 0,0131 & 2 \\
\hline
\end{tabular}

Q20 - Do you think you can defend yourself, your family and your property against wildfires?

\begin{tabular}{|l|c|c|c|}
\hline Characteristics \& Experience & $\chi 2$ & $p$-value & df \\
\hline Education level & 27,60 & 0,0063 & 12 \\
\hline Previously affected by fires & 6,46 & 0,0393 & 2 \\
\hline
\end{tabular}

$\chi^{2}$ - value of the Pearson $\chi^{2}$ test; $p$-value indicates significance of the association; if the p-value is less than 0.05, the difference in the responses related to the personal characteristic tested is significant at $95 \%$ level. $d f$-degrees of freedom 
The level of knowledge on wildfire risk and spot fires activity (Question 11) is associated with having a job related to forest or not; from those people who don't work in forest activities (90\%), the majority says their knowledge is low $(64 \%)$, whereas the people with forest-related activities (10\%) mostly gave the answer "Doesn't know/respond" (66\%) and none said their knowledge was high. This may be related to their age and education level, since most of the people with forest-related activities are above 60 years old. Regarding spot fires (Question 12), about $56 \%$ of the people who don't work in forest activities said they know what they represent, which means that this knowledge may be disseminated through other sources, eventually by past experiences.

Regarding the possibility of self-defense (Question 20), $92 \%$ of the people with no education mention they can't protect themselves properly; on the other hand, most of the people with secondary and university education levels also said they had no conditions for self-protection $(80 \%$ and $83 \%$, respectively). These results can be related to the lack of fire experience of the younger and more educated population, whose relation with fire is different from older generations.

\section{Further work}

To deepen our understanding on the perception of wildfire occurrence in the country, this survey is being extended to several other areas in the Portuguese mainland. The responses will be analyzed with the same procedure to further explore the relationship between personal characteristics and prior experience with wildfire knowledge of wildfires. These results can contribute to improve educational programmes dedicated to students in schools; furthermore, prevention and mitigation options can be adapted to the needs and experience of people who live in wildland-urban interface areas, in order to improve wildfire awareness.

\section{References}

Almeida, M. A. de F. B. de. (2011). Propagação de incêndios florestais por focos secundários. Retrieved from https://estudogeral.sib.uc.pt/handle/10316/16441

Almeida M. Viegas D. X. Raposo J. (2014). Analysis of firebrand release on the spot fire mechanism. In AdvAnces in Forest Fire reseArch (pp. 4-6). Coimbra.

Badia, A., Serra, P., \& Modugno, S. (2011). Identifying dynamics of fire ignition probabilities in two representative Mediterranean wildland-urban interface areas. Applied Geography, 31(3), 930-940. https://doi.org/10.1016/j.apgeog.2011.01.016

Blanchi, R., \& Leonard, J. (2008). The influence of human behaviour on house loss, 2008.

Bunn, A., \& Korpela, M. (2016). Crossdating in dplR, 2, 1-12. https://doi.org/10.1016/j.dendro.2009.12.001

Byram, G. M. (1954). Atmospheric conditions related to blowup fires. Station Paper SE-SP-35. Asheville, NC: USDA-Forest Service. Southeastern Forest Experiment Station., 36.

Caballero, D. (2017). Wildland-Urban Interface Forest Fire Risk Observatory and Interest Group in Europe WUIWATCH Agreement № ECHO/SUB/2014/694556.

Cohen, J. (2010). The wildland-urban interface fire problem. Fremontia, 38(2), 16-22. https://doi.org/10.1071/WF07131

Diário da República. (2006). Decreto-Lei 124/2006 de 28 de Junho. Diário Da República, $1^{a}$ Série $A\left(\mathrm{~N}^{\circ} \quad 123\right), \quad 4586-4599 . \quad$ Retrieved from https://dre.pt/application/dir/pdf1sdip/2006/06/123A00/45864599.pdf

Domingos Xavier Viegas, Miguel Figueiredo Almeida, Luís Mário Ribeiro, Jorge Raposo, Maria Teresa Viegas, Ricardo Oliveira, Daniela Alves, Cláudia Pinto, Jorge Humberto, André Rodrigues, Davi Lucas, Sérgio Lopes, Luís Filipe Silva. (2017). O complexo de incêndios de Pedrógão Grande $e$ concelhos limítrofes, iniciado a 17 de junho de 2017. Retrieved from 
https://www.portugal.gov.pt/download-ficheiros/ficheiro.aspx?v=3bb9773b-59fb-4099-9de5a22fdcad1e3b

ICNF. (2016a). RELATÓRIO ANUAL DE ÁREAS ARDIDAS E INCÊNDIOS FLORESTAIS EM PORTUGAL CONTINENTAL.

ICNF. (2016b). Relatório de estabilização de emergência - INCÊNDIO FLORESTAL DE ALGERIZ.

ICNF. (2017). 10. ${ }^{\circ}$ RELATÓRIO PROVISÓRIO DE INCÊNDIOS FLORESTAIS - 201701 DE JANEIRO A 31 DE OUTUBRO. Lisboa.

Instituto Nacional de Estatística. (2012). Censos 2011 Resultados Definitivos. Censos 2011, 26, 1-41. https://doi.org/ISBN 978-989-25-0148-2

Lee, S.L., \& Hellman, J. M. (1969). Study of firebrand trajectories in a turbulent swirling natural convection plume. Combustion and Flame, 13(6), 645-655. https://doi.org/10.1016/00102180(69)90072-8

McArthur A. (1967). Fire behaviour in eucalypt forests., (107), 144.

Modugno, S., Balzter, H., Cole, B., \& Borrelli, P. (2016). Mapping regional patterns of large forest fires in Wildland-Urban Interface areas in Europe. Journal of Environmental Management, 172(July), 112-126. https://doi.org/10.1016/j.jenvman.2016.02.013

Moreira, F., Rego, F. C., \& Ferreira, P. G. (2001). Temporal (1958 - 1995) pattern of change in a cultural landscape of northwestern Portugal : implications for fire occurrence. Landscape Ecology, 2(Ccrn 1995), 557-567. https://doi.org/10.1023/a:1011169614489

Moreira, F., Viedma, O., Arianoutsou, M., Curt, T., Koutsias, N., Rigolot, E., Bilgili, E. (2011). Landscape - wildfire interactions in southern Europe: Implications for landscape management. Journal of Environmental Management, 92(10), 2389-2402. https://doi.org/10.1016/J.JENVMAN.2011.06.028

Nunes, A. N., Lourenço, L., \& Meira, A. C. C. (2016). Exploring spatial patterns and drivers of forest fires in Portugal (1980-2014). Science of The Total Environment, 573, 1190-1202. https://doi.org/10.1016/J.SCITOTENV.2016.03.121

Oliveira, S., Zêzere, J. L., Queirós, M., \& Pereira, J. M. (2017). Assessing the social context of wildfire-affected areas. The case of mainland Portugal. Applied Geography, 88, 104-117. https://doi.org/10.1016/j.apgeog.2017.09.004

San-Miguel-Ayanz, J., Marcos Rodrigues Q., Oliveira S.,Pacheco C.K., Moreira F., Duguy B., C. A. (2012). Land Cover Change and Fire Regime in the European Mediterranean Region. Chapter 2, In: Post-Fire Management and Restoration of Southern European Forests, 2012 Edited by Moreira F., Arianoutsou M., Corona P. De Las Heleras J., 02/2012: Chapter Land Cover Change and Fire Regime in the European Mediterranean Region: Pages 21-43;, 24(102162), 2012.

San-Miguel-Ayanz, J., Moreno, J. M., \& Camia, A. (2013). Analysis of large fires in European Mediterranean landscapes: Lessons learned and perspectives. Forest Ecology and Management, 294, 11-22. https://doi.org/10.1016/J.FORECO.2012.10.050

Scheffe, H. Y. (1959). The Analysis of Variance.

Tedim, F., Xanthopoulos, G., \& Leone, V. (2015). Forest Fires in Europe: Facts and Challenges. Wildfire Hazards, Risks and Disasters, 77-99. https://doi.org/10.1016/B978-0-12-4104341.00005-1

Turco, M., Bedia, J., Di Liberto, F., Fiorucci, P., Von Hardenberg, J., Koutsias, N., ... Provenzale, A. (2016). Decreasing fires in mediterranean Europe. PLoS ONE, 11(3). https://doi.org/10.1371/journal.pone.0150663

Viegas, D. X. (2012). EXTREME FIRE BEHAVIOUR. LAETA-ADAI Departamento de Engenharia MecâNica Da Universidade de Coimbra, 56. 


\title{
Integrating paleoecology into landscape management
}

\author{
Kendrick J. Brown ${ }^{1,2, *}$, Mitchell J. Power ${ }^{3}$, and Nicholas J.R. Hebda ${ }^{2}$ \\ ${ }^{1}$ Natural Resources Canada. 506 West Burnside Road, Victoria, BC, V8Z 1M5, \\ \{kendrick.brown@canada.ca*\} \\ ${ }^{2}$ University of British Columbia, Okanagan. FIP-339, Fipke Building, 3247 University Way, \\ Kelowna, British Columbia, Canada, VIV1V7, \{nhebda@alumni.ubc.ca\} \\ ${ }^{3}$ Department of Geography, Natural History Museum of Utah, University of Utah. 301 Wakara \\ Way, Salt Lake City, UT, USA, \{mitchell.power@geog.utah.edu\}
}

\begin{abstract}
Paleoecology has traditionally been used to examine how ecosystems evolve through time. Focusing on fossil plant reconstructions, conventional approaches examine the origin, expansion, contraction, and dynamic processes influencing plant communities over time. Recognizing that vegetation types are predominately influenced by climate, paleoecological records are frequently used to reconstruct climate through time, using both qualitative and quantitative approaches. In addition to examining vegetation and climate, evidence from natural disturbances, including charred plant remains from wilfires deposited in lake sediments, has increased in recent decades, enabling researchers to examine fire dynamics. Increasingly, there is growing urgency to integrate paleoecological studies and landscape management, rendering unique spatial (local to global) and temporal (years to millennia) perspectives that offer valuable insights for land managers today. This manuscript presents a suggested framework to acheive this goal, with emphasis on integrating paleofire data and managment applications. The framework illustrates several potential applications by scale, including direct local-scale applications within a managed municipal watershed, and medium-to-long-term perspectives on the causes and consequences of large fire events in forested ecosystems.
\end{abstract}

Keywords: paleoecology, fire, charcoal, Holocene, climate, management

\section{Introduction}

Traditionally, paleoecological studies aim to examine how ecosystems change through time and in response to various forcing mechanisms. Plant remains preserved in sedimentary sequences provide insights into the origin, development, and dynamics of plant communities. Recognizing that climate influences plant distribution, the utility of paleoecological records expanded to include reconstructing climate, using both qualitative and quantitative approaches (Birks et al., 2010). In addition to examining vegetation and climate, analyses of charcoal fragments and other fire-derived indicators preserved in stratigraphic records have increased in recent decades (Brown and Power, 2013), enabling researchers to examine fire disturbance dynamics through time, including fire regime change and drivers.

Increasingly, there is a desire within the paleoecological community to integrate results into landscape management practices. For example, there is a growing awareness that perspectives on wildfire regimes, drivers, and effects at different scales are critical to understanding current and future fire occurrence and effects. Information about wildfire can be derived from many different sources, including administrative records, case studies, foret stand age-classes, dendrochronology, laboratory and field experiments, remote sensing, and modelling. However, fire history information extending beyond the comparatively short instrumental and observational interval is primarily derived from natural paleoenvironmental archives (Smol, 2008) and, in some cases, traditional knowledge (Lake et al., 2017). 
Paleofire proxies are natural materials (e.g., charcoal, pollen grains, biomarkers, minerals) or specific metrics of these materials (e.g., particle size, magnetic susceptibility, charred plant morphotypes) that have been directly generated or indirectly influenced by past fires or fire-related processes (Simoneit, 2002; Conedera et al., 2009; Brown and Power, 2013). These materials accumulate in lake sediments during and/or after wildfire via various pathways and linkages, creating a sequential record of physical, chemical, and biological responses to disturbance events (Birks, 1997; Conedera et al., 2009). These proxies can be extracted from sediment cores and systematically analyzed to enable qualitative interpretations or quantitative reconstructions of past fire dynamics based on modern fire-environment relationships.

\section{Framework}

A tentative framework for integrating paleoecological data, specifically paleofire data, into management is herein presented (Fig. 1), together with examples. While select examples are presented in this discussion, potenial opportunities for paleofire data within management contexts are more expansive, spanning multiple temporal and spatial scales. The framework presented here includes direct applications coupled with insights across scales, illustrating the robustness of the approach. Beyond management applications, paleo records documenting change through time are also valuable teaching tools for public education that resonate with wide-ranging audiences. For example, paleo studies can be used to illustrate the dynamic behavior of the earth's climate system through time, document rates and magnitudes of change in vegetation communities, and uncover the role of wildfires in shaping our modern landscape. Moreover, given that sedimentary records contain evidence of past fire, they can, if sampled at appropriate temporal resolution, provide insight into fire-induced terrestrial and aquatic effects and recovery times. The recent creation of the Global Modern Charcoal Database further aims to better calibrate recent charcoal depostion events with various $21^{\text {st }}$ century fire metrics (Hawthorne et al., 2017). Finally, paleoecology has much to offer conservation, providing baselines as well as measures of habitat, biodiversity, and processes, which in turn can aid the development of restoration goals (Hebda, 1998; Brown and Pasternack, 2004; 2005).

Paleoecological records can have relevance at the event-scale, where some of the largest areas burned can occur within days, by providing insights into the climate mode linked to the event, as well as other important controls (e.g., fuel types and density) and responses (e.g., geochemical and mineralogical changes). For example, the time between large charcoal peaks within a sediment archive may capture the frequency distribution of higher severity fire events (Dunnette et al., 2014). At annualscales, another potential application relates to cumulative impacts. Given that paleoecological records contain biological, chemical, and physical indicators of pre- and post-disturbance conditions, they capture cumulative impacts or trends, as well as short-term responses. For example, the flammability of fuel loads in boreal or sub-boreal forests could be enhanced through insect-induced forest mortality, evidenced through changes in pollen and insect macros, increasing fire potential, assessed through changes in stratigraphic charcoal. The subsequent loss of vegetation following a fire increases overland flow and nutrient input into downstream basins, captured in sediment records by changes in grain size and diatom communities, respectively. 


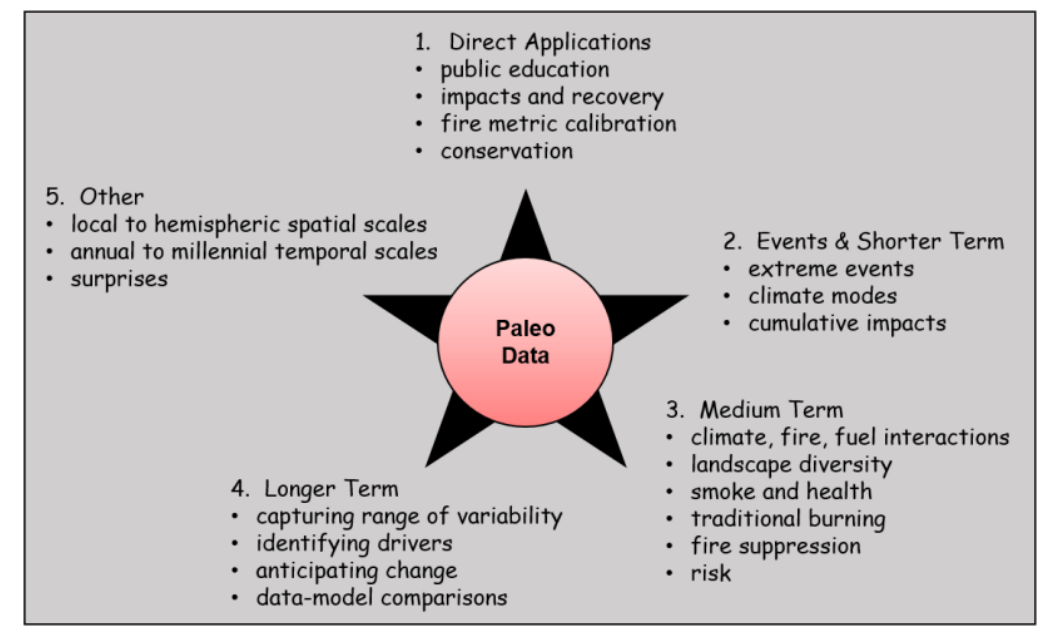

Figure 1 - Suggested framework for integration of paleoecological research into landscape management, with an emphasis on paleofire data.

Over the medium term (decades to centuries), paleoecology informs about the interaction between climate, vegetation, and fire. With each of these parameters interacting with the others, creating feedbacks within the system. For example, prolonged droughts may initially increase fire frequency and reduce fuel loads, but fire will utlimately become limited through an overall reduction in primary productivity in the absence of precipitation under severe drought conditions. Pollen records capture the integrated signal of the resulting landscape mosaic. Fire disturbance also creates a range of chemical products through combustion products, including slightly charred particles, residues like charcoal, condensates like soot, and a variety of other chemical markers, including fire-derived compounds (Simoneit, 2002). During a wildfire, these compounds become atmospherically lofted, transported, and later removed by wet and dry deposition. During this latter stage, some of the compounds accumulate in depositional environments, preserving a record of fire and serving as tracers of smoke. Likewise, the records are also suitable for discerning and characterizing the effects of traditional burning and fire suppression. Finally, medium- to long-term tracking of natural disturbances through time also provides insight into risk. For example, the frequency of megathrust earthquake events has been ascertained and refined using turbidites preserved in marine cores (Goldfinger et al., 2003), informing the public about seismic risk. Adopting a similar approach targeting lake sedimentary records could inform communities about wildfire risk in terms of the average time between large-scale wildfires, with the caveat that non-analog climate will change fuel charateristics and disturbance regimes

On yet longer timescales (centuries to millennia), paleoecological studies can reveal natural ranges of variation and identify drivers of change. Paleo data can aid model calibration and validation, and facilitate data-model comparisons (Flannigan et al., 2001; Marlon et al., 2012). Paleoecological data are used to understand landscape evolution and processes occurring at spatial and temporal scales beyond the recent establishment of geo-political boundaries and human-built systems located in regions where disturbances may skip several human generation times (e.g., fires occuring on average every 300 years). Finally, paleodata can inform about surprises in the earth system. For example, several paleoecological studies have shown that during the northern hemisphere warm mid-Holocene interval about 4000-8000 years ago, conditions were favorable for widespread burning. However, spatially explicit paleofire data show that fire actually decreased in certain parts of the circumboreal, including Quebec (Girardin et al., 2013) and Fennoscandia (Brown and Giesecke, 2014). This nonlinear response was possibly a result of the expansion of broadleaf vegetation, which altered microclimatic conditions and fuel type, arrangement, moisture content and flammability, and lowered mid-Holocene fire activity in certain regions. In another example from boreal Alaska, the expansion 
of flammable black spruce resulted in an increase in fire disturbance even though climate was cooling and moistening (Lynch et al., 2003).

\section{Example from a managed municipal watershed}

A specific example of integrating paleoecological data into landscape management heralds from a study in the Greater Victoria Water Supply Area (GVWSA), British Columbia, Canada (Fig. 2). The Sooke Lake Reservoir (SLR) is a dammed natural lake that supplies high quality drinking water to $>350,000$ people at comparatively low cost. However, because the watershed is densely forested, it is susceptible to fire disturbance. Elevated post-fire erosion can contaminate water supplies by increasing delivery of suspended sediment, nutrients, and other pollutants to reservoirs for periods of days to years (Emelko et al., 2011; Shakesby and Doerr, 2006; Smith et al., 2011). Resultant changes in water turbidity, chemistry, and biology may leave it unsuitable for drinking (Smith et al., 2011; White et al., 2006), and treatment may be costly or unfeasible (Emelko et al., 2011). This problem is exacerbated by climate change, which is projected to drive a regional increase in wildfire severity and area burned (Haughian et al., 2012).

In response, GVWSA managers have identified wildfire as a major threat to drinking water supply and seek to understand how fire activity has changed within the watershed through time and in response to various forcing mechanisms. Of particular interest is the early Holocene interval, 11,7007,000 years ago, since it represents a time when climate was warmer and drier than present, potentially providing an window into current and future fire conditions. A paleoecological investigation was initiated to characterize the long-term relationships among climate, vegetation and fire disturbance in the watershed (Brown et al., submitted). Lake sediment cores were collected and analysed for a suite of paleoenvironmental proxies, including pollen, charcoal, and magnetic susceptibility. The results indicate that a longer fire season and mixed-severity fire regime occurred in the warmer, drier early Holocene (Fig. 3). Futhermore, increased fire disturbance in the mid-Holocene was likely due to increased human activity and/or climate variability. Combining the results from this investigation with paleoclimate modeling, modern management practices and forecast simulations can aid the development of strategies that account for these types of ecosystem change within the watershed (sensu a past-present-future data continuum), leading to a more robust and informed climate adaptation strategy.

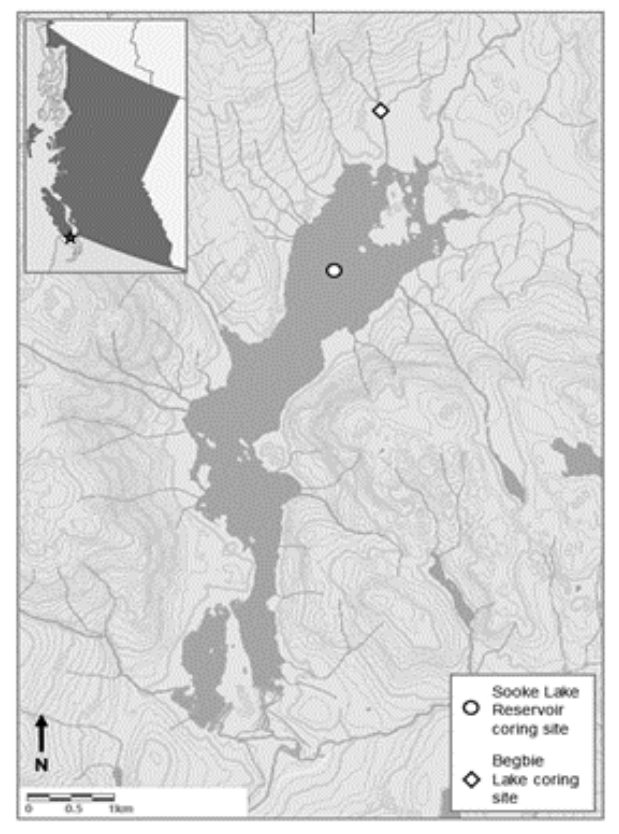

Figure 2 - Sediment coring locations within the Greater Victoria Water Supply Area, BC, Canada. 


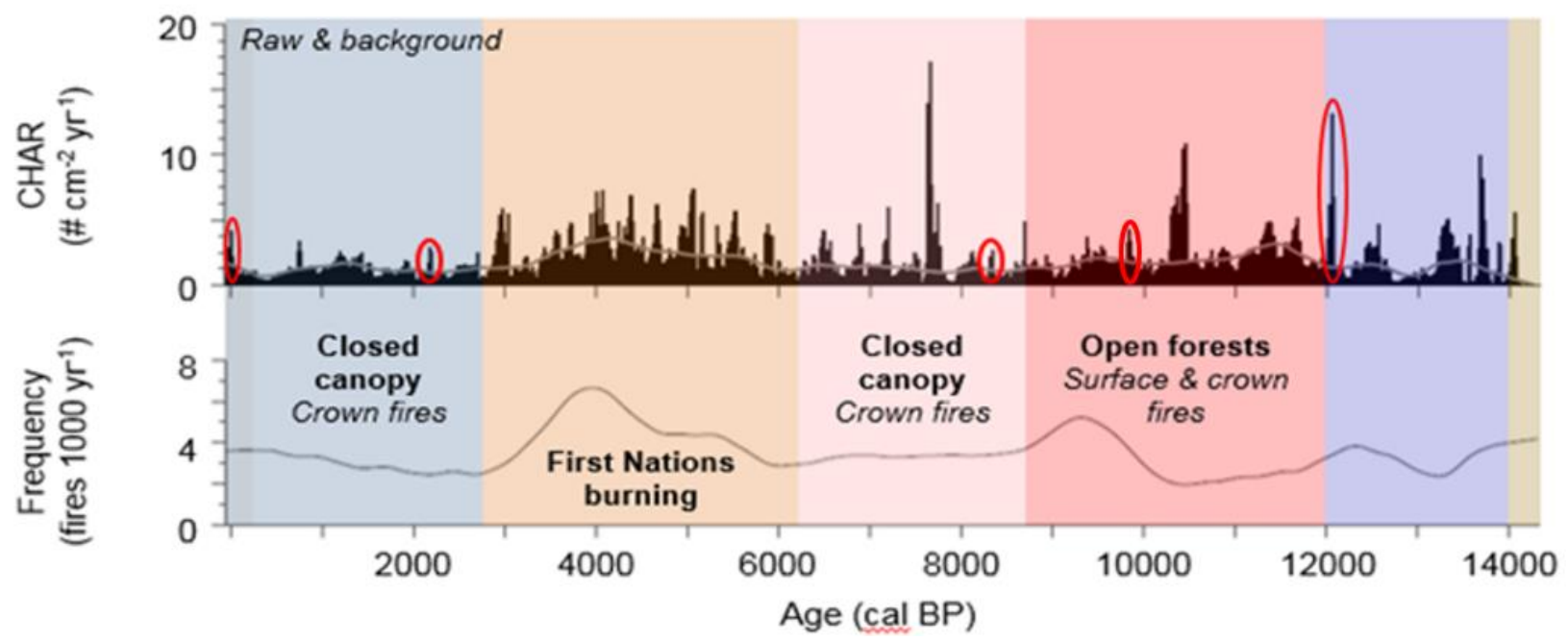

Figure 3 - GVWSA Holocene charcoal time series showing fire regime change through time. Red ovals indicate the fire episodes being examined at high resolution.

Furthermore, the investigation includes high-resolution analyses of select individual fire episodes recorded in the sediment to examine short-term fire effects and recovery in the terrestrial and aquatic environments (Fig. 3). Several fire events, each occurring under different past climatic conditions, are being examined in detail at high temporal resolution. The ecological and water quality effects of these fires are being evaluated using a variety of terrestrial and aquatic paleoenvironmental indicators including charcoal, pollen, diatoms, total carbon (C) and nitrogen (N), grain size, magnetic susceptibility, and trace elements. Initial results show strong evidence of fire-induced effects (Fig. 4Figure ). Charcoal, magnetic susceptibility, and total $\mathrm{C}$ and $\mathrm{N}$ exhibit clear fire responses. Coincident changes observed in the $\mathrm{C}: \mathrm{N}$ ratio provide insight into post-fire changes in carbon as well as changes in aquatic productivity. Preliminary grain size results reveal apparent post-fire fining of sediment deposits. Pollen analyses show variable vegetation response, including post-fire increases in fireadapted species abundance and concurrent decreases in fire-sensitive species. Diatom community composition likewise exhibits marked changes coincident with charcoal and magnetic susceptibility peaks. A wide variety of trace elements increase in the sediment during the post fire intervals. Although the investigation is ongoing, the targeted paleoenvironmental indicators variously exhibit compelling evidence of fire-related ecosystem impacts. As further data become available, statistical analysis and interpretation of this multi-proxy tool kit will facilitate both qualitative and quantitative reconstructions of past fire effects in the GVWSA. Viewing these data under different climate scenarios in the past will inform watershed management recommendations for mitigating future wildfire risk to the supply of drinking water. 


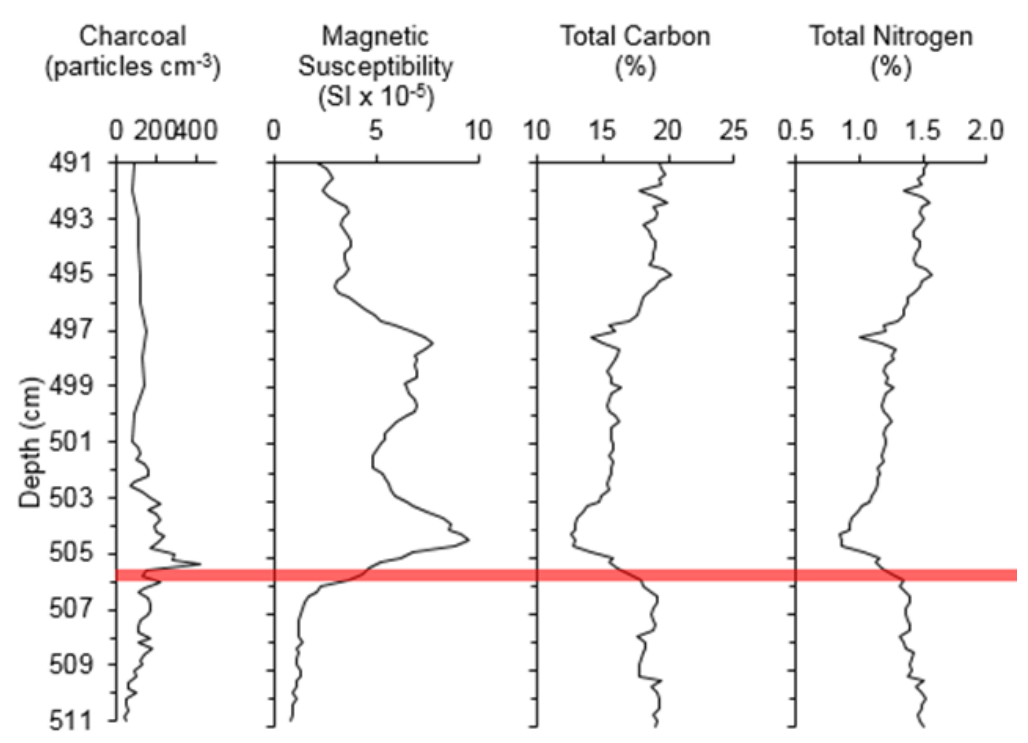

Figure 4 - Responses of select proxies to fire disturbance. Red line indicates timing of fire event.

\section{Example from western USA}

An example of a 13,000 year-long high-resolution pollen and charcoal record from a low-elevation record, Foy Lake, in western Montana (Fig. 5) provide novel perspectives on the evolution of fire and the relative importance of mega-fire events, like the fires of AD 1910. Following the last Ice Age approximately 20,000 years ago, low elevations in western Montana comprised subalpine parklands that supported a low incidence of fire. As the climate warmed during the late glacial and early Holocene, the region became effectively drier and more fire-prone as lodgepole pine and Douglas-fir and western larch forests developed at low elevations (Fig. 6; Power et al., 2011). In high-elevation forests of white pine and spruce, fires increased abruptly after 12,500 years ago in response to higherthan-present early summer temperatures and an increasing abundance of vegetation on the landscape. As fire activity increased during the early and mid Holocene, fire-adapted Douglas-fir and western larch forests developed at both low- and mid-elevations, with evidence that burning generally increased across all elevations at least until $\sim 6000$ years ago. The modern vegetation communities developed in this region during the last several millennia as the regional climate generally became cooler and wetter. The exception to this long-term trend of reduced fire occurred around 2000 years ago, when a mixed-severity fire regime developed. A synthesis of dozens of fire records from the region show several centuries of high fire activity (Power et al., 2011). This time of high-fire was also when prehistoric populations expanded in the region. This abrupt increase in fire 2000 years ago was embedded within a long-term trend of climate cooling and was likely linked to short-term climate varibility and potentially amplified by human ignitions. 


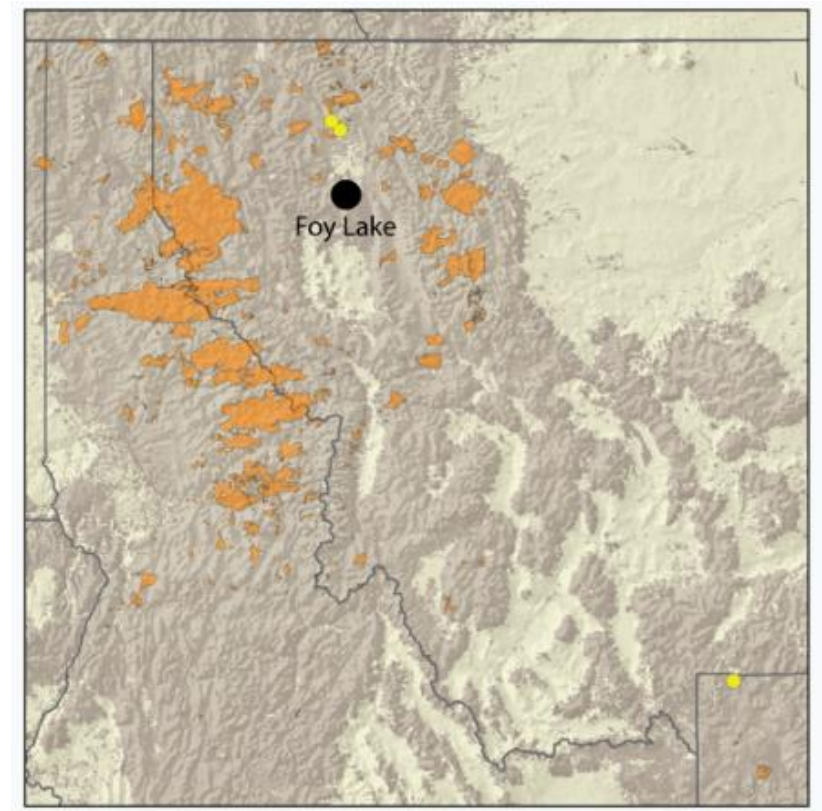

Figure 5 - Location of study site in western Montana (black circle) and the area burn maps of the fires of 1910 (orange polygons).

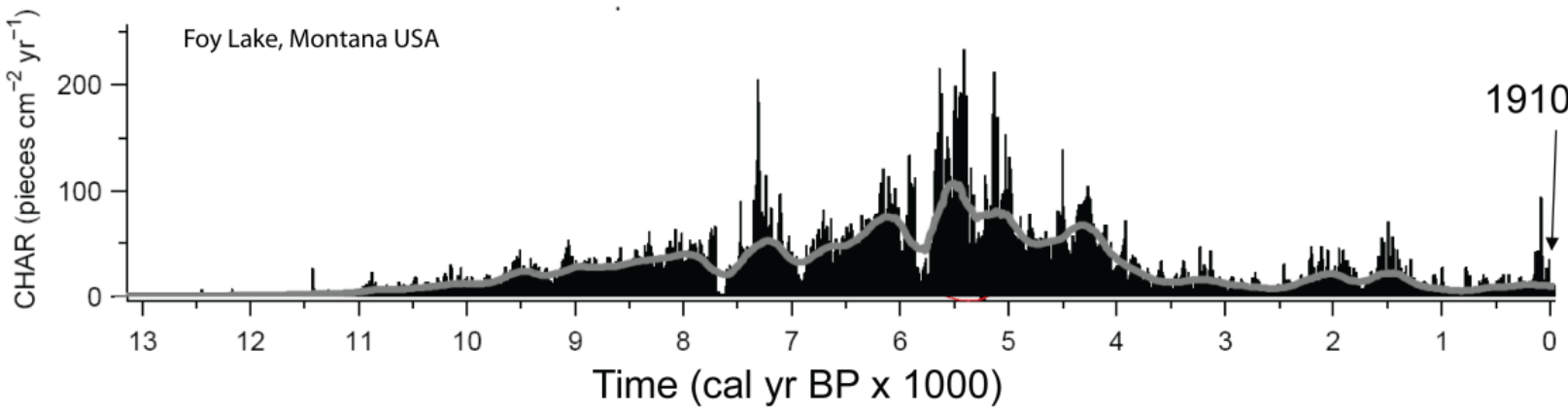

Figure 6 - A 13,000-year long fire history reconstruction from Foy Lake, Montana, showing the relative scale of the AD 1910 fire when compared to the mid-Holocene period of extreme fire conditions.

This research also provides a context to the AD 1910 fires that were responsible for numerous deaths and policy changes within the U.S. Department of Agriculture. When the 1910 fires are placed in the context of the last 13,000 years (Fig. 6), the significance of the disturbance event is obscured by the magnitude and frequency of fire events throughout the middle Holocene. In recent years, fires have increased across the region at both high and low elevations, suggesting the interplay among climate conditions (e.g., seasonal temperature, moisture, and snowpack), a century of fire-supression management practices, and an increasing abundance of ignition sources, both human and natural. Understanding the range of variability in western forests will ultimately help forest managers prepare and potentially mitigate worse-case scenarios under future climate conditions.

\section{Conclusion}

Paleoecological records, including paleofire proxies, provide a rich and varied archive of ecosystem and fire history information reaching back millennia, integrating information from local to global spatial scales. A more thorough understanding of current and future fire incidence and impacts can be gained by studying past natural fire variability, patterns, drivers, and consequences. By combing insights from paleodata with present-day monitoring efforts, management practices and future 
simulations, it is possible to generate a past-present-future data continuum (Brown et al., 2017), each rendering unique spatial and temporal insights. The combined information can be consulted when developing best practices and policies, especially during times of rapid change.

\section{References}

Birks HJB (1997) Reconstructing environmental impacts of fire from the Holocene sedimentary record. In 'Sediment Records of Biomass Burning and Global Change'. (Eds JS Clark, H Cachier, JG Goldammer, B Stocks) pp. 295-311. (Springer: New York)

Birks HJB, Heiri O, Seppa H, Bjune, AE (2010) Strengths and weaknesses of quantitative climate reconstructions based on Late-Quaternary biological proxies. Open Ecology Journal 3, 68-110.

Brown KJ, Power MJ (2013) Charred particle analyses. In 'Encyclopedia of Quaternary Science, $2^{\text {nd }}$ Edition'. (Ed. SA Elias) pp. 716-729. (Elsevier: Amsterdam)

Brown KJ, Hebda NJ, Conder N, Golinski KG, Hawkes B, Schoups G, Hebda RJ (2017) Changing climate, vegetation, and fire disturbance in a sub-boreal pine-dominated forest, British Columbia, Canada. Canadian Journal of Forest Research 47, 615-627.

Brown KJ, Giesecke T (2014) Holocene fire disturbance in the boreal forest of central Sweden. Boreas 43, 639-651.

Brown KJ, Pasternack GB (2005) A paleoenvironmental reconstruction to aid in the restoration of floodplain and wetland habitat on an upper deltaic plain, California, USA. Environmental Conservation 32, 103-116.

Brown KJ, Pasternack GB (2004) The geomorphic dynamics and environmental history of an upper deltaic floodplain tract in the Sacramento-San Joaquin Delta, California, USA. Earth Surface Processes and Landforms 29, 1235-1258.

Brown KJ, Hebda NJR, Schoups G, Conder N, Smith KAP, Trofymow JA (submitted) Long-term climate, vegetation and fire regime change in a managed municipal water supply area, British Columbia, Canada. Quaternary Science Reviews.

Conedera M, Tinner W, Neff C, Meurer M, Dickens AF, Krebs P (2009) Reconstructing past fire regimes: Methods, applications, and relevance to fire management and conservation. Quaternary Science Reviews 28, 555-576.

Dunnette PV, Higuera PE, McLauchlan, KK, Derr KM, Briles CE, Keefe, MH (2014) Biogeochemical impacts of wildfires over four millennia in a Rocky Mountain subalpine watershed. New Phytologist 203, 900-912.

Emelko MB, Silins U, Bladon KD, Stone M (2011) Implications of land disturbance on drinking water treatability in a changing climate: Demonstrating the need for "source water supply and protection" strategies. Water Research 45, 461-472.

Flannigan M, Campbell I, Wotton M, Carcaillet C, Richard P, Bergeron Y (2001) Future fire in Canada's boreal forest: Paleoecology results and general circulation model-regional climate model simulations. Canadian Journal of Forest Research 31, 854-864.

Girardin MP, Ali AA, Carcaillet C, Blarquez O, Hely C, Terrier A, Genries A, Bergeron Y (2013) Vegetation limits the impact of a warm climate on boreal wildfires. New Phytologist 199, 10011011.

Goldfinger C, Nelson CH, Johnson, JE (2003) Holocene earthquake records from the Cascadia subduction zone and northern San Andreas fault based on precise dating of offshore turbidites. Annual Review of Earth and Planetary Sciences 31, 555-577.

Haughian SR, Burton PJ, Taylor SW, Curry C (2012) Expected effects of climate change on forest disturbance regimes in British Columbia. BC Journal of Ecosystems and Management 13, 1-24. 
Hawthorne D, Courtney Mustaphi CJ, Aleman JC, Blarquez O, Colombaroli D, Daniau AL, Marlon JR, Power M, Vannière B, Han Y, Hantson S, Kehrwald N, Magi B, Yue X, Carcaillet C, Marchant R, Ogunkoya A, Githumbi EN, Muriuki RM (2017) Global Modern Charcoal Dataset (GMCD): A tool for exploring proxy-fire linkages and spatial patterns of biomass burning. Quaternary International, 1-15.

Hebda RJ (1998) Atmospheric change, forests and biodiversity. Environmental Monitoring and Assessment 49, 195-212.

Lake FK, Wright V, Morgan P, McFadzen M, McWethy D, Stevens-Rumann C (2017) Returning fire to the land: celebrating traditional knowledge and fire. Journal of Forestry 115, 343-353.

Lynch, JA, Clark, JS, Bigelow, NH, Edwards, ME, Finney, BP (2003) Geographic and temporal variations in fire history in boreal ecosystems of Alaska. Journal of Geophysical Research 107, 8152.

Marlon JR, Bartlein PJ, Gavin DG, Long C, Anderson RS, Briles C, Brown KJ, Colombaroli C, Hallett DJ, Power MJ, Scharf EA, Walsh MK (2012) Long-term perspective on wildfires in the western USA. Proceedings of the National Academy of Sciences 109, E535-E543.

Power MJ, Whitlock C, Bartlein PJ (2011) Postglacial fire, vegetation, and climate history across an elevational gradient in the Northern Rocky Mountains, USA. Quaternary Science Reviews 30, 2520-2533.

Shakesby RA, Doerr SH (2006) Wildfire as a hydrological and geomorphological agent. Earth Science Reviews 74, 269-307.

Simoneit, BRT (2002) Biomass burning - a review of organic tracers for smoke from incomplete combustion. Applied Geochemistry 17, 129-162.

Smith HG, Sheridan GJ, Lane PNJ, Nyman P, Haydon S (2011) Wildfire effects on water quality in forest catchments: A review with implications for water supply. Journal of Hydrology 396, 170 192.

Smol JP (2008) 'Pollution of lakes and rivers: A paleolimnological perspective, $2^{\text {nd }}$ edition'. (John Wiley \& Sons: Oxford).

White I, Wade A, Worthy M, Mueller N, Daniell T, Wasson, R (2006) The vulnerability of water supply catchments wildfires on the Australian Capital Territory. Australian Journal of Water Resources 10, 179-194. 


\title{
Modeling the productivity of forest fire suppression operations using production functions. A methodological approach
}

\author{
Francisco Rodríguez y Silva*1; ${ }^{\text {; }}$ Michael Hand ${ }^{2}$ \\ ${ }^{1}$ Forest Fire Laboratory. University of Córdoba (Spain), Edificio Leonardo da Vinci, Campus de \\ Rabanales, 14071, Córdoba, Spain. \{irlrosif@uco.es*\} \\ ${ }^{2}$ USDA Forest Service, Rocky Mountain Research Station. 201 14th St., SW. Washington, DC \\ 20024,USA. \{mshand@fs.fed.us\}
}

\begin{abstract}
Society, forest landscapes and fires constitute a triangle of interaction with important requirements and consequences. These interdependencies offer a field for the application and development of research with a clear focus on social sciences, in which the fire prevetion, risk analysis and socio-economic conditions are shaped as basic pillars.

Environmental dryness, climate and the consequences of fire exclusion, aggravated by urban growth in forest areas, with absence of preventive norms and under a scenario of high forest fires risk creates an environment susceptible to large forest fires that can affect the well-being of citizens. In this sense, the authorities and goverment are commiting large budgetary investments for the prevention and suppression of wildfires.

The knowledge advances of the dynamics of fire behavior have allowed to dimension the expansive capacity of fire and, as a function of it, to identify a priori the potential impacts. In this sense, the integration of this knowledge together with social and economic aspects, have allowed us to advance in the studies of costs and benefits, spatial-temporal determination of risks in probabilistic terms and others such as the evaluation of the fire severity, the operational strategy, the reduction of uncertainty and the help in the decision making process.

The present work explores the development of a methodology to incorporate fire suppression experience, detailed historical data on forest fire management from Mediterranean ecosystems, and empirical models to analyze efficiency and production. The resulting models of wildfire suppression production provides a basis for generating a conceptual framwork to study suppression operations.

The development of decision support tools based on econometric models of fire suppression production functions requires the selection of variables that define and determine the problem. The present work includes the main methodological considerations for the definition and development of an econometric model based on the study of efficiency and productivity through the development of production functions that can predict the operational suppression yields in different scenarios of forest fires (meters per hour of fire spreading containment and suppression). The resulting model may represent an opportunity to aid in the development of suppression operations plans, helping the fire managers determine the production rates of the resources dispatched to fire suppression.
\end{abstract}

Keywords: Suppressions operations, production function, econometric models, fire weather danger, production rates, suppression costs, ground resources, aerial resources, suppression difficulty index

The views expressed in this paper are the authors' and do not necessarily reflect those of the U.S. Department of Agriculture or the USDA Forest Service. Results are preliminary and subject to revision.

\section{Introduction}

The main lines of research developed from the middle of the first quarter of the twentieth century to the present, have allowed us to conceptualize the problem of the occurrence of forest fires, not only in physical, ecological, landscape management or operations suppression terms, but also the knowledge acquired due to the science associated with the study of forest fires, they define a new 
dimension with a very clear socio-economic perspective. On the other hand, the envelope of the researches takes on a globalizing way a vision of strong social and economic interaction, due to its transcendence and implications in the role played by the population and the landscape in the wildfire problems.

The current possibilities of the economic analysis supporting studies on the potential danger of forest ecosystems and the suppression difficulty operations based on fire behavior, represents an important advance in the decision making tools for fire management. Undoubtedly the incorporation of modern econometric tools based on efficiency and productivity concepts, as well as in the developing of utility functions, can help to carried out guidelines for the budgetary definition to support protection of ecosystems, considering the value of natural resources and the suppression costs.

The object of the present work is to carry out an methodological approach to modeling the productivity of forest fire extinction (or, suppression) operations. For this purpose it has been considered important to determine the structure and composition of the database made with the report of the forest fires occurred in a certain region, allow us to identify the variables that can provide consistent information in the econometric modeling of forest fires suppression productivity.

\section{Material and methods}

In the methodological approach, the deterministic determination of the suppression operational productivity evaluation option have been considered. For the development of this work, a total number of 20 fires were selected from the statistical forest fires database of the Andalucía Region (Spain) (table 1).

An updateable database of operations plans to fires extinction with the identification of behavior and influences of human factors, environmental conditions (scenarios of the events) and strategies suppression adopted, provides the information that gives access to past experiences, facilitating the sharing of knowledge at the level of capitalization and subsequent use of information in terms of the lessons learned, making possible the establishment of a "network of experience transmission " between the different people in charge of the forest fires management.

Table 1 - Relation and data base of the forest fires selected for this study

\begin{tabular}{|c|c|c|c|c|c|c|c|}
\hline $\begin{array}{c}\text { No } \\
\text { Wildfire }\end{array}$ & Date of ignition & Municiplity & $\begin{array}{c}\text { Total fire } \\
\text { perimeter } \\
(\mathrm{m})\end{array}$ & $\begin{array}{c}\text { Total } \\
\text { surface } \\
(\mathrm{ha})\end{array}$ & $\begin{array}{c}\text { Surface } \\
\text { contraction } \\
\text { factor FCS\% }\end{array}$ & $\begin{array}{c}\text { Time to } \\
\text { control } \\
\text { (min) }\end{array}$ & $\begin{array}{c}\text { Production } \\
\text { rate } \\
(\mathrm{m} / \mathrm{min})\end{array}$ \\
\hline 1 & 9 -ago-02 & MALAGA & 7355 & 120,5 & 11,14 & 1089 & 6,8 \\
\hline 2 & 22 -jul-06 & EL BURGO & 6456 & 110,54 & 0,56 & 1307 & 4,9 \\
\hline 3 & 9 -sept-03 & OJEN & 1467 & 14 & 13,79 & 1280 & 1,1 \\
\hline 4 & 9 -ago-05 & MALAGA & 3956 & 60,3 & 5,31 & 1010 & 3,9 \\
\hline 5 & 22 -ago-04 & FARAJAN & 3425 & 21 & 18,22 & 350 & 9,8 \\
\hline 6 & 26 -jul-02 & BENALMADENA & 1879 & 7 & 46,65 & 840 & 2,2 \\
\hline 7 & 20 -jun-02 & MALAGA & 1673 & 6,4 & 90,14 & 645 & 2,6 \\
\hline 8 & 2 -ago-04 & CORTES DE LA F. & 2819 & 17 & 24,91 & 750 & 3,8 \\
\hline 9 & 10 -oct-05 & ESTEPONA & 7349 & 69,9 & 14,34 & 1190 & 6,2 \\
\hline 10 & 9 -jul-06 & CASARES & 7768 & 99,9 & 12,46 & 200 & 38,8 \\
\hline 11 & 12 -ago-04 & COMPETA & 2965 & 17,9 & 87,71 & 215 & 13,8 \\
\hline 12 & 21 -ago-04 & GAUCIN & 3245 & 20 & 98,47 & 515 & 6,3 \\
\hline 13 & 14 -ago-04 & MIJAS & 3128 & 18,45 & 70,38 & 1418 & 2,2 \\
\hline 14 & 18 -ago-05 & CASARES & 5183 & 61,69 & 35,83 & 930 & 5,6 \\
\hline 15 & 4 -ago-06 & FRIGILIANA & 11234 & 210 & 77,35 & 1385 & 8,1 \\
\hline 16 & 3-ago-06 & CORTES DE LA F. & 7956 & 158,6 & 87,31 & 861 & 9,2 \\
\hline
\end{tabular}




\begin{tabular}{|c|c|c|c|c|c|c|c|}
17 & 7 -oct-06 & MARBELLA & 13489 & 457,33 & 45,14 & 780 & 17,3 \\
\hline 18 & 8-oct-06 & CORTES DE LA F. & 16781 & 602,1 & 74,85 & 1260 & 13,3 \\
\hline 19 & 16 -sept-06 & CASARES & 12689 & 320,66 & 55,72 & 774 & 16,4 \\
\hline 20 & 21 -sept-06 & CASARES & 14987 & 455 & 48,52 & 795 & 18,9 \\
\hline
\end{tabular}

The definition of the experience need previously of the investigation and reconstruction of the events (the wildfires), the analysis and the comprehension of the same, it allows to later extend the learned and recognized towards the capitalization of the experiences lived (Rodríguez y Silva,1999), (Rodríguez y Silva et al., 2007). From the generation of the database obtained through the capitalization, it is necessary to select the variables and parameters that will allow analyzing and evaluating the extinction operations productivity. The following should be considered:

- Fire perimeter controled by time unit (production rate) $(\mathrm{m} / \mathrm{min})$

- Surface contraction factor ACF, (understood as the rate calculated how the difference between one and the quotient between the real surface affected by the propagation of the fire and the surface obtained in free evolution, that is without extinction operations, subject to the time elapsed since the detection until the fire is controlled) (Rodríguez y Silva, 2007), (Rodríguez y Silva, and González-Cabán, 2010), (Rodríguez y Silva, and González Cabán, 2016)

- Average surface affected by each forest fire registered (ha / fire)

- Average extinction costs per affected area, $(€ /$ ha)

- Unitary costs of the differents resources to fires suppression

- Production rates of each differents resources

- Quotient between suppression cost and net change value of the natural resources

- List of the total resources despached to the fires, with special detail in the proportion of individual operations times in relation to the total sum of operational times of all resources involved in a fire.

- Factors related to suppression inefficiency causes, such as the difficulty of the extinction activities, adverse meteorological effects, simultaneity of fires ocurrence and WUI operational difficulties.

The database prepared for the development of the suppression production function, includes for each each of the 20 forest fires, the "Surface Contraction Factor (SFC)", (Rodríguez y Silva, 2007), (Rodríguez y Silva, and González-Cabán, 2010, 2016). This parameter is useful because it includes the control operational effects in the perimeter of the fire, as opposed to what would be the final result without carrying out extinction activity. The database was also expanded, incorporating how independent variables the meteorological danger indexes of the occurrence of forest fires (Rodríguez y Silva, 2002, 2004) and the suppression difficulty index (Rodríguez y Silva, et al., 2014), as well as the weighted extinction means mobilized for each fire disaggregated into ground resources and aerial resources.

\section{Deterministic model of suppression operational productivity}

The analysis of the productivity has been widely used in efficiency studies in many fields related to economics ((Aigner et al., 1977; Battese and Coelli, 1992, 1995; Greene, 2004; 2003; Hadri et al., 2003; Meeusen and Den Broeck, 1977). Likewise, functions have been modeled to provide information on the efficiency that can be achieved, depending on the effort applied in the resolution of emergencies or damage reduction., effects related to disturbances in natural resources (Huang et al., 2013; Penman et al., 2013; Grimsrud et al., 2008; Kovacs et al., 2014).

The background in the application of production functions in the modeling of actions aimed at defending against forest fires is reduced, among others, Holmes and Calkin of the analysis (2013), Katuwal et al. (2016). The research on efficiency under the approach of comparative analysis between the suppression costs and the economic value of natural resources saved (Molina et al. 2009; Rodríguez 
y Silva et al. 2009), provide important results that help qualify the utility of budgetary investments in defense against forest fires (Rodríguez y Silva and González-Cabán, 2016).

In this way the analytical process of the perimeter control fire productivity rate evaluation, under a deterministic model of the production function that can best predict operational performance is included below. The method used is to compare the results provided by two widely applied models in the econometric production function modeling, in the framework of the microeconomic analysis of business activities. The model functions selected are the Cobb-Douglas function and the CES function. The Cobb-Douglas production function (CD, hereafter (1)) can be written as:

$$
Y=A \cdot x_{1}^{\alpha_{1}} \cdot x_{2}^{\alpha_{2}} \cdot \ldots \ldots \cdot x_{n}^{\alpha_{n}}
$$

The parameter A can be interpreted as a measure of technical efficiency of the productivity (that is, $\mathrm{A}=\mathrm{Ai}$ where the subscript $\mathrm{i}$ denotes the ith fire) since for any combination of inputs, the output increases with A. If the observations on which the function is defined are of time series and correspond to a certain sector, then A can be interpreted as an indicator of the state of the technology (in this case, A can be written as A ( $\mathrm{t}$ ) where $\mathrm{t}$ is the time variable) and its temporal variation as the effect of technical change about production (Álvarez Pinilla et al. 2003).

The Cobb-Douglas function is transformed into a linear multivariate function by taking logs, an extended form for the econometric resolution of the determination of the corresponding explanatory parameters of each of the independent variables, the transformed expression being the following (2):

$$
L n Y=\alpha_{0}+\alpha_{i} \sum_{1}^{i} \operatorname{Ln} x_{i}
$$

As an alternative and in order to overcome the rigidities of the Cobb-Douglas function, and in particular, that the elasticity of substitution is always equal to 1, Arrow, Chenery, Minhas and Solow (1961) introduced the CES function (Constant Elasticity of Substitution, that is, Constant Replacement Elasticity (3)) . This function for the case of two explanatory variables, can be expressed as follows:

$$
Y=A\left(\alpha \cdot x_{1}^{-\rho}+(1-\alpha) \cdot x_{2}^{-\rho}\right)^{-\eta / \rho}
$$

For the direct estimation of the production function, it is necessary to use non-linear regression analysis techniques. As a mathematical solution we can use a Taylor expansion around the value of $\rho$ $=0$, which gives us a functional approximation in neperian logarithms that adopts the following expression (4):

$$
\operatorname{Ln} Y=\operatorname{Ln}(A)+\eta \alpha \operatorname{Ln}\left(x_{1}\right)+\eta(1-\alpha) \operatorname{Ln}\left(x_{2}\right)-\rho \alpha \eta(1-\alpha)\left(\operatorname{Ln}\left(x_{1}\right)-\operatorname{Ln}\left(x_{2}\right)\right)^{2}
$$

Generalizing for (n) explanatory variables and making a change of parametric coefficients, the following expression is obtained (5):

$$
\operatorname{Ln} Y=\beta_{0}+\sum \beta_{i} \operatorname{Ln} x_{i}+\beta_{1+i}\left(\operatorname{Ln}\left(x_{1}\right)-\left(\sum \ln x_{1+i}\right)\right)^{2}
$$




\section{Results}

As indicated in the methodological section and in terms of the productivity analysis, we have been considered in addition, the surface contraction factor (SCF), the suppression difficulty index, and fire weather index. The incorporation of these variables of each fire, allowed to collect the meteorology effects in the different production functions developed in the framework of efficiency.

The Idex, represents the suppression difficulty index (simplified), calculated for the fire behavior average conditions of each wildfire. In its determination, the effects of heat per unit area caused by fire and flame length have been considered (Rodríguez y Silva et al., 2014). The IPMIF, represents the fire weather index of forest fire ocurrence occurrence (Rodríguez y Silva, 2002), (Rodríguez y Silva and Aguado, 2004).

RpMAR, represents the accounting of aerial resources number and type dispached to each wildfire, obtained through the weighting of the number of units and the factor of proportionality of the operation time for each one type in relation to the total suppression time spent of all aerial ressources. RpMT, represents the accounting of ground resources number and type dispached to each wildfire, obtained through the weighting of the number of units and the factor of proportionality of the operation time for each one type in relation to the total suppression time spent of all ground ressources. Fcs\%, represents the surface contraction factor $(\mathrm{FCs} \%=\mathrm{SCF})$.

The independent variables considered to evaluate the deterministic function of suppression operational productivity, such as the perimeter length controlled per unit of time $(\mathrm{m} / \mathrm{min})$, are the following (table 2) :

Table 2 - Variables considered to develop the deterministic models

$\begin{array}{cccc}\text { Aerial resources } & \text { Ground resources } & \text { Suppression } & \text { Fire weather } \\ \text { suppression effect } & \text { suppression effect } & \text { Difficulty } & \text { index } \\ \text { Rp MAR } & \text { Rp MT } & \text { Index (Idex) } & \text { IPMIF }\end{array}$

The table elaborated for the modeling of the suppression operations productions, allows to compare the results of each one of the variables considered for the twenty fires analyzed (table 3 ).

Table 3 - Values of the variables considered to develop the deterministic models

\begin{tabular}{|c|c|c|c|c|c|}
\hline $\begin{array}{c}\text { № } \\
\text { wildfire }\end{array}$ & $\begin{array}{l}\text { Control fire } \\
\text { perimeter } \\
\text { production } \\
\text { rate } \mathrm{m} / \mathrm{min}(\mathrm{Y})\end{array}$ & Rp MAR & Rp MT & Idex & IPMIF \\
\hline 1 & 6,75 & 11,51 & 37,28 & 6,49 & 228,31 \\
\hline 2 & 4,93 & 8,34 & 37,38 & 6,46 & 188,31 \\
\hline 3 & 1,14 & 15,55 & 59,93 & 5,25 & 131,52 \\
\hline 4 & 3,91 & 19,32 & 48,23 & 6,28 & 171,07 \\
\hline 5 & 9,78 & 31,65 & 39,35 & 5,59 & 149,13 \\
\hline 6 & 2,23 & 17,83 & 34,92 & 5,38 & 133,42 \\
\hline 7 & 2,59 & 18,91 & 33,53 & 4,87 & 128,86 \\
\hline 8 & 3,75 & 14,13 & 29,64 & 6,05 & 158,8 \\
\hline 9 & 6,17 & 29,16 & 45,77 & 6,45 & 187,84 \\
\hline 10 & 38,84 & 62,98 & 79,72 & 6,58 & 209,83 \\
\hline 11 & 13,79 & 52,25 & 84,65 & 6,25 & 160,1 \\
\hline 12 & 6,30 & 34,00 & 73,07 & 6,28 & 169,1 \\
\hline 13 & 2,20 & 10,48 & 55,02 & 6,31 & 178,56 \\
\hline 14 & 5,57 & 14,20 & 45,71 & 6,63 & 209,83 \\
\hline 15 & 8,11 & 36,78 & 39,7 & 6,78 & 295,08 \\
\hline 16 & 9,24 & 32,55 & 43,74 & 6,59 & 252,48 \\
\hline
\end{tabular}




$\begin{array}{llllll}17 & 17,29 & 36,77 & 43,79 & 7,35 & 380,73 \\ 18 & 13,31 & 35,05 & 52,71 & 8,12 & 420,89 \\ 19 & 16,39 & 10,22 & 41,79 & 7,86 & 318,41 \\ 20 & 18,85 & 29,80 & 48,62 & 7,14 & 361,89\end{array}$

The production function of the suppression activities determined with the structure and econometric composition of the Cobb-Douglas function, has been estimated as follows (table 4):

$$
\begin{aligned}
& \ln y=-3.15+0.881 * \ln x_{1}-0.360 * \ln x_{2}+5.18 * \ln x_{3}-0.336 * \ln x_{4} \\
& \left.\begin{array}{llll}
(1.06) & (0.299) & (0.562) & (2.48)
\end{array}\right] \\
& \mathrm{n}=20, \mathrm{R}^{2}=0.739
\end{aligned}
$$

Table 4. Model 1 (Cobb-Douglas): MCO, using observations 1-20

$\begin{array}{lccccc} & \text { Coeficients } & \text { Desv. stand. } & t & p \text { value } & \\ \text { const } & -3.1524 & 1.05775 & -2.9803 & 0.0093 & * * * \\ \ln (\mathrm{Rp} & 0.880821 & 0.298649 & 2.9494 & 0.0099 & * * * \\ \text { MAR }) & & & & & \\ \ln (\mathrm{Rp} \text { MT }) & -0.359546 & 0.561508 & -0.6403 & 0.5316 & \\ \ln (\mathrm{Idex}) & 5.18219 & 2.47994 & 2.0896 & 0.0541 & * \\ \ln (\mathrm{IPMIF}) & -0.33553 & 0.817756 & -0.4103 & 0.6874 & \end{array}$

$\begin{array}{lllr}\text { Mean of vble. dep. } & 0.832020 & \text { D.T. vble. dep. } & 0.377385 \\ \text { Sum squard w. } & 0.706283 & \text { D.T. of the regresion } & 0.216992 \\ \mathrm{R}^{2} & 0.738990 & \mathrm{R}^{2} \text { adjusted } & 0.669388 \\ \mathrm{~F}(4,15) & 8.503972 & \text { P value (of F) } & 0.000863 \\ \text { Log- likelihood } & 5.055939 & \text { Akaike criteria } & -0.111879 \\ \text { Schwarz criteria } & 4.866783 & \text { Hannan-Quinn criteria } & 0.860008\end{array}$

The production function of the suppression activities determined with the structure and econometric composition of the CES function, has been estimated as follows (table 5):

$$
\begin{gathered}
\ln y=13.3+10.8 * \ln x_{1}-9.99 * \ln x_{2}-5.17 * \ln x_{3}-9.95 * \ln x_{4}+1.42^{*} x_{5} \\
(3.30)(2.18)
\end{gathered}
$$

The variable $\mathrm{x}_{5}$, is following the equation $(5)=\left(\ln \mathrm{x}_{1}-\left(\ln \mathrm{x}_{2}+\ln \mathrm{x}_{3}+\ln \mathrm{x}_{4}\right)\right)^{2}$

$$
\mathrm{n}=20, \mathrm{R}^{2}=0.813
$$


Table 5 - Model 2 (CES): MCO, using observations 1-20

\begin{tabular}{|c|c|c|c|c|}
\hline & Coeficients & Desv. Stan. & $t$ & p-value \\
\hline const & 13.3182 & 3.29606 & 4.0407 & 0.0012 \\
\hline $\ln (\mathrm{Rp} \mathrm{MAR})$ & 10.7661 & 2.18035 & 4.9378 & 0.0002 \\
\hline $\ln (\mathrm{Rp} \mathrm{MT})$ & -9.98696 & 2.00213 & -4.9882 & 0.0002 \\
\hline $\ln (\operatorname{Idex})$ & -5.16911 & 2.32676 & -2.2216 & 0.0433 \\
\hline $\ln (\mathrm{IPMIF})$ & -9.95401 & 2.27882 & -4.3681 & 0.0006 \\
\hline $\begin{array}{c}(\ln (\text { RpMAR })- \\
(\operatorname{lnRpMT}+\ln (\text { Idex })+\ln (\text { IPMIF }))^{2}\end{array}$ & 1.41696 & 0.293173 & 4.8332 & 0.0003 \\
\hline Mean of vble. dep. & 0.832020 & \multicolumn{2}{|c|}{ D.T. of vable. dep. } & 0.377385 \\
\hline Sum squard w. & 0.505963 & \multicolumn{2}{|c|}{ D.T. of regresion } & 0.190106 \\
\hline $\mathrm{R}^{2}$ & 0.813019 & \multicolumn{2}{|c|}{$\mathrm{R}^{2}$ adjusted } & 0.746241 \\
\hline $\mathrm{F}(5,14)$ & 10.12371 & \multicolumn{2}{|c|}{$\mathrm{p}$ value $(\mathrm{F})$} & 0.000291 \\
\hline Log- likelihood & 8.391472 & \multicolumn{2}{|c|}{ Akaike criteria } & -4.782945 \\
\hline Schwarz criteria & 1.191449 & \multicolumn{2}{|c|}{ Hannan-Quinn criteria } & -3.616681 \\
\hline
\end{tabular}

\section{Discussion}

The study and analysis of the operational results complements the experience and knowledge of firefighting professionals. The monitoring of the operational sequences in the achievement of the objectives included in the attack plans allows us to monitor the results achieved and identify those other objectives that could not be achieved. The collection of variables and the behavior maintained by them during extinction operations provides a starting point to evaluate the causes that could limit the suppresion actions.

The modeling of fire suppression production functions represents a potentially useful tool to support the preparation of operational suppression plans. The methodological approach presents a comparative analysis of two production functions: the Cobb-Douglas production function and a second model following the characteristics of the Constant Elasticity of Substitution (CES) production function.

The comparison suggests that the CES model has greater predictive accuracy than the CobbDouglas model. The CES model has an adjusted coefficient of determination (adjusted $\mathrm{R}^{2}$ ) of 0.74 , compared to 0.66 corresponding to the Cobb-Douglas model. Likewise, the values obtained for the Akaike, Schwarz and Hannan-Quinn criteria of the CES function are lower than those obtained for the Cobb-Douglas function.

This method offers a promising approach for developing explanatory models of wildfire suppression efforts. Combining variables in a production model to provide information on factors that offer sufficient predictive ability can indicate the production capacity provided by a specific combination of trained teams for the extinction of forest fires.

\section{Conclusions}

The application of efficiency and productivity analysis tools is a new opportunity to study wildfire suppression operations. This provides the possibility of reducing operational uncertainty in order to obtain reliable information to support the selection and combination of extinction means according to 
operational scenarios. Improvements in suppression planning based on the production analysis presented in this paper can inform efforts to reducethe costs of wildfire suppression and net change in value of the natural resources affected by wildfires.

\section{Acknowledgements}

The authors express their gratitude to the GEPRIF project (RTA2014-00011-C06-01), and to the VIS4FIRE project (RTA2017-00042-C05-04), funded by the National Institute for Agricultural, Food Research and Technology (INIA). Likewise, they express their gratitude to the INFOCA Plan management of the Junta de Andalucía (Ministry of Environment and Territory Planning), for the access to the databases and the support in the field monitoring and research work that have been necessary to achieve the objectives of this work.

\section{References}

Aigner, D., Lovell, C., Schmidt, P (1977). Formulation and estimation of stochastic frontier production function models. J. Econ. 6, 21e37.

Alvarez Pinilla A., Arias Sampedro C., Orea Sánchez 1 (2003). Introducción al análisis empírico de la producción. Universidad de Oviedo y Universidad de León.

Arrow K., Chenery H., MInhas B., Solow R (1961). Labor Substitution and Economics Efficiency. The Reviw of Economics and Statistics. Vol 43, No 43.

Battese G, Coelli T (1992). Frontier Production Functions, Technical Efficiency and Panel Data: with Application to Paddy Farmers in India. Journal of Productivity Analysis 3(1-2): 153-169.

Battese, G.E., Coelli, T.J 1(995). A model for technical inefficiency effects in a stochastic frontier production function for panel data. Empir. Econ. 20, 325e332

Greene, W., 2003. Econometric Analysis. Prentice Hall, Upper Saddle River, New Jersey

Greene, W., 2004. Distinguishing between heterogeneity and inefficiency: stochastic frontier analysis of the World Health Organization's panel data on national health care systems. Health Econ. 13, $959 \mathrm{e} 980$.

Grimsrud, K.M., Chermak, J.M., Hansen, J., Thacher, J.A., Krause, K (2008). A twoagent dynamic model with an invasive weed diffusion externality: an application to Yellow Starthistle (Centaurea solstitialis L.) in New Mexico. J. Environ. Manag. 89, $322 \mathrm{e} 335$.

Hadri, K., Guermat, C., Whittaker, J., 2003. Estimation of technical inefficiency effects using panel data and doubly heteroscedastic stochastic production frontiers. Empir. Econ. 28, 203e222.

Holmes, T.P., Calkin, D.E (2013). Econometric analysis of fire suppression production functions for large wildland fires. International Journal of Wildland Fire 22, $246 \mathrm{e} 255$.

Huang, C.-H., Finkral, A., Sorensen, C., Kolb, T (2013). Toward full economic valuation of forest fuels-reduction treatments. Journal of Environmental Management. 130, $221 \mathrm{e} 231$.

Katuwal H., Calkin D., Hand M (2016). Production and efficiency of large wildland fire suppression effort: A stochastic frontier analysis. Journal of Environmental Management 166 (2016) 227-236. http://dx.doi.org/10.1016/j.jenvman.2015.10

Kovacs, K.F., Haight, R.G., Mercader, R.J., McCullough, D.G (2014). A bioeconomic analysis of an emerald ash borer invasion of an urban forest with multiple jurisdictions. Resour. Energy Econ. 36, $270 \mathrm{e} 289$.

Meeusen, W., Den Broeck, J. Van (1977). Efficiency estimation from Cobb-Douglas production functions with composed error. Int. Econ. Rev. 18, $435 \mathrm{e} 444$. 
Molina J.R.; Rodríguez y Silva F.; Herrera M.A.: Zamora R (2009). A simulation tool for socioeconomic planning on forest fire supression management. Libro Forest Fires: Detection, Supresión and Prevention. Nova Science Publishers. USA.

Penman, T., Collins, L., Price, O., Bradstock, R., Metcalf, S., Chong, D. (2013). Examining the relative effects of fire weather, suppression and fuel treatment on fire behaviour. A simulation study. J. Environ. Manag. 131, 325e333.

Rodríguez y Silva F (2002). Visual Forest Fire Weather Index (VVFFWI) a mathematical model for the prediction of forest fires weather danger in Mediterranean ecosystems. IV Conference on Forest Fire Research. Luso. ADAI.

Rodríguez y Silva, F., Aguado I (2004). El factor meteorológico en los incendios forestales. En nuevas tecnologías para la estimación del riesgo de incendios forestales. Editores: Emilio Chuvieco Salinero y María del Pilar Martín Isabel. Colección de Estudios Ambientales y Socioeconómicos. Consejo Superior de Investigaciones Científicas. Madrid.

Rodríguez y Silva, F (2005). Sinami, un método para el análisis económico de los planes de defensa contra incendios forestales mediterráneos, desarrollo de una aplicación desde una perspectiva provincial. $4^{\circ}$ Congreso Forestal Español. La ciencia forestal: respuesta para la sostenibilidad. Sociedad Española de Ciencias Forestales. Junta de Andalucía. Gobierno de Aragón. Zaragoza.

Rodríguez y Silva F., Molina Martínez J.R., Rodríguez Leal J (2014). The efficiency analysis of the fire control operations using the VISUAL-SEVEIF tool. Chapter 7 - Social and Economic Issues. Advances in Forest Fire Research http://dx.doi.org/10.14195/978-989-26-0884-6_210

Rodríguez y Silva, F., Molina Martínez J.R., González-Cabán A (2014). A methodology for determining operational priorities for prevention and suppression of wildland fires. 'International Journal of Wildland Fire . http://dx.doi.org/10.1071/WF13063

Rodriguez y Silva, F (1999). A forest fire simulation tool for economic planning in fire suppression management models: an application of the Arcar-Cardin. Proceedings of the Symposium on Fire Economics, Planning and Policy: Bottom lines, USDA Forest Service. Pacific Southwest Research Station. General Technical Report PSW-GTR-173. San Diego California.

Rodríguez y Silva, F. Molina JR. Herrera M. Zamora R (2007). Vulnerabilidad socioeconómica de los espacios forestales frente al impacto de los incendios, aproximación metodológica mediante sistemas de información geográficos (proyecto Firemap). IV International Wildland Fire Conference. Proceedings. Sevilla. (www.wildfire07.es).

Rodríguez y Silva F., MolinA J.R., Herrera M.A., Zamora R (2009). The impact of fire and the socioeconomic vulnerability of forest ecosystems: A methodological approach using remote sensing and geographical information systems. General Technical Report PSW-GTR-227. Pacific Southwest Research Station. Forest Service, U.S. Department of Agriculture; 151 - 168.

Rodríguez y Silva F. ;, González-Cabán A (2010). "SINAMI": a tool for the economic evaluation of forest fire management programs in Mediterranean ecosystems. International Journal of Wildland Fire 19: 927-936.

Rodríguez y Silva F.; González-Cabán A (2016). Contribution of suppression difficulty and lessons learned in forecasting fire suppression operations productivity: a methodological approach. Journal of Forest Economics. DOI: http://dx.doi.org/10.1016/j.jfe.2016.10.002 


\title{
Residents' experiences of the 2016 Fort McMurray Wildfire, Alberta
}

\author{
Tara K. McGee \\ University of Alberta. c/o 1-26 Earth Sciences Building, University of Alberta, Edmonton, Alberta, \\ Canada, \{tmcgee@ualberta.ca\}
}

\begin{abstract}
In May 2016, the urban centre of Fort McMurray in Alberta, Canada, was devastated by the Horse River wildfire (known as the Fort McMurray wildfire), which destroyed 2400 homes and caused the evacuation of 88,000 residents. I will present results from two online surveys carried out with a sample of residents who evacuated during the wildfire. The first survey, administered one month after the fire in June 2016, was designed to explore residents' initial evacuation experiences. The survey was developed using SurveyMonkey and distributed through Facebook groups devoted to the wildfire evacuation, Twitter, two First Nations and one First Nation organization. 447 residents of the Regional Municipality of Wood Buffalo (RMWB) completed the survey. Before the wildire occurred on May 1st, many survey respondents were unaware of the wildfire risk. On May 3rd, many respondents left their home or workplace at the last minute with little if any warning, and many experienced difficulties leaving their neighbourhood and Fort McMurray. Once they left Fort McMurray, evacuees who responded to the survey stayed in many towns throughout Alberta, Canada, and some returned home to international destinations. Social media served as a very important source of information for survey respondents. An enormous outpouring of support was provided to evacuees during and following the wildfire. Some evacuees were allowed to return to Fort McMurray starting at the beginning of June 2016, while others were unable to return until later in the summer. A second survey was administered in March 2018 to examine residents' decisions about when to return to Fort McMurray, challenges faced during the initial re-entry process and later in the recovery, and support received by residents. Lasting impacts of the wildfire on survey respondents are also identified.
\end{abstract}

Keywords: Experiences, wildfire evacuation, recovery

\section{Introduction}

Evacuations are used by governments in many countries to protect public safety in the event of a wildfire and other hazards. For example, between 1980 and 2007, there were 547 wildfire evacuations in Canada, resulting in the evacuation of an average of 7469 people per year (Beverly and Bothwell 2011). Evacuations may be voluntary where residents can choose whether to evacuate, or mandatory where residents are obliged to leave. Researchers have found that wildfire evacuations can cause considerable stress and anxiety for evacuees due to disruption in daily routines, loss of control, uncertainty about personal safety and the safety of their homes, and proximity of danger (Kent et al 2003; Cohn et al 2006; Afifi et al 2012). Evacuees with low levels of social support suffer more mental health issues than those with average or high levels of social support (Felix and Afifi 2015). However there is also some evidence that evacuations may have positive impacts when the residents become closer during the experience (Kent et al 2003).

On May 1st, 2016, the Horse River Fire started in the Regional Municipality of Wood Buffalo and quickly grew in size due to extremely hot and dry conditions. At 8pm, the RMWB's emergency department warned people in Centennial trailer park in the south of Fort McMurray to leave their homes, and put the neighbourhoods of Beacon Hill and Gregoire on evacuation alert. At 10pm, a state of local emergency was declared and a mandatory evacuation was ordered for residents of Centennial RV Park Campground, Prairie Creek and Gregoire. On Monday May 2nd, the fire grew and was almost $1 \mathrm{~km}$ from Highway 63, the road in and out of Fort McMurray. Overnight, the fire expanded west, away from the city. 
On the morning of Wednesday May 3rd, the smoke and flames were not visible from the city, but at $11 \mathrm{am}$ the fire chief warned that winds could shift. At noon, the fire jumped the Athabasca River and headed towards the city. Later that day, the Horse River wildfire entered Fort McMurray. The neighbourhoods of Beacon Hill, Abasand and the MacKenzie Industrial Park were impacted first. Although some residents had already evacuated, a mandatory evacuation order was issued by the RMWB for all of Fort McMurray at 16:20. Later in the evening, the wildfire entered the subdivisions of Thickwood and Waterways (MNP 2017).

People in the neighbourhoods of Waterways, Beacon Hill, and Abasand south of the Athabasca River were told to evacuate and head south on Highway 63. Due to traffic impediments as people in the Abasand, Beacon Hill and waterways were leaving and taking Highway 63 south, residents in neighbourhoods north of the Athabasca River were told to leave and go north on Highway 63. The estimated 15,000 to 20,000 evacuees who headed north stayed in work camps and Fort McKay First Nation. On May 4th, the province of Alberta declared a state of emergency and called for a mandatory evacuation of Saprae Creek at 4pm. At 10pm, a mandatory evacuation was ordered by the provincial government for Anzac, Gregoire Lake Estates, and Fort McMurray First Nation. A few days after May 3rd, the fire started heading north and supplies started to run out in the work camps, so evacuees were either flown out to Calgary or Edmonton, or drove south on Highway 63 back through Fort McMurray to stay elsewhere.

The Horse River fire destroyed 2400 homes and caused the evacuation of more than 88,000 residents, the largest wildfire evacuation in Canadian history. The fire was under control on July 4th after it has burned 589,552 hectares. The government of Alberta completed a phased re-entry process which allowed the first residents to return to RMWB on June 1st. Government responses to this wildfire have been examined in studies completed following the wildfire (KPMG 2017a, b; MNP 2017). These studies that examine how local and provincial government agencies responded to the wildfire provide important insights into the wildfire response. However, further study is needed to examine the experiences of residents who fled from their homes and neighbourhoods during this wildfire.

I will present the results of two online surveys completed with residents of RMWB who evacuated due to the May 2016 wildfire. The first survey was administered in June 2016, and was designed to explore residents' evacuation experiences from when the fire started, leaving, staying elsewhere during the evacution, and what helped and hindered the evacuation from their perspectives. The second survey was administered in March 2018 nearly two years after the wildfire, to examine residents' decisions about when to return to Fort McMurray, challenges faced during the initial re-entry process and later in the recovery, and support received by residents. Lasting impacts of the wildfire on survey respondents are also identified.

\section{Methods}

The first phase of this research project was initially conceived in May 2016 immediately after the wildfire evacuation of RMWB. The online survey method was selected to ensure that I could reach residents who were staying in a variety of places during the evacuation to learn about a variety of experiences. In addition, a very large and active Facebook Group that was set up at the time of the fire, 'Fort McMurray Evac Relocation Group', provided a way to reach evacuees using an online survey. The benefits of online surveys (Evans and Mathur 2018) were also important considerations. Use of SurveyMonkey provided flexibility in the question types, format, and response options, and convenience for participants; and the data analysis tools offered by SurveyMonkey also enabled data analysis to be completed fairly quickly. A short online survey was also appropriate for people who were very busy trying to cope during and shortly after the wildfire. 
This research was approved by the University of Alberta human research ethics board 1 . The survey administered in June 2016 was designed to learn about residents' initial evacuation experiences in the early days after the wildfire. The survey was developed by the author and a research assistant using SurveyMonkey. The survey included closed-ended and open-ended questions, and was designed to be completed within 10 minutes. A purposive sampling strategy was used to recruit survey participants. After obtaining ethics clearance, the survey was posted online on June 21, 2016. RMWB residents who had evacuated due to wildfire in May were invited to participate through invitations posted in Facebook groups devoted to the wildfire evacuation, Twitter, and by emailing information about the survey to two First Nations and one First Nation organization. 447 residents of the Regional Municipality of Wood Buffalo completed this online survey. The results of this first survey were analyzed using SurveyMonkey's data analysis capabilities.

In March 2016, I developed a follow-up survey to examine residents' decisions about when to return to Fort McMurray, challenges faced during the initial re-entry process and later in the recovery, and support received by residents. Lasting impacts of the wildfire on survey respondents two months short of the 2-year anniversary of the fire are also identified. Similar to the first survey, this second survey included both closed-ended and open-ended questions and was designed to be completed within 10 minutes. I recruited participants for the follow-up survey by emailing a link to the survey to 120 participants in the 2016 survey who indicated they would be interested in participating in further research. $62(51.6 \%)$ of these 120 residents completed the follow-up survey. The results of this followup survey were also analyzed using SurveyMonkey's data analysis capabilities.

\section{Findings}

\subsection{June 2016 Survey}

Survey respondents included those with a range of characteristics. Most survey respondents (93.8\%) were living in Fort McMurray before the fire, with the remaining 24 respondents living in other communities in the RMWB. Survey participants included those in a range of age groups, with $3.9 \% 18-24$ years of age, $25.5 \% 25-34,29.0 \% 35-44,25.5 \% 45-54,13.1 \% 55-64,2.1 \% 65-74$, and $2.1 \%$ age 75 and over. Most survey respondents $(81.2 \%)$ were women, $16.7 \%$ men, and $0.26 \%$ other. $63.5 \%$ of respondents stated that they were in full time paid employment before the wildfire, $8.5 \%$ had full time domestic/parenting duties, $8 \%$ were unemployed, $8 \%$ had part-time/casual employment, $7.7 \%$ were unemployed, $4.9 \%$ retired, and $1.8 \%$ full time students. Most survey respondents' homes had minor damage $(40.9 \%)$ or no damage at all $(35.7 \%)$, but $17.7 \%$ of respondents had lost their home in the wildfire.

A few days before the evacuation, most respondents perceived the wildfire threat to be low. This is surprising due to Fort McMurray's location in the boreal forest, past wildfires in the area, and hot and dry conditions in the weeks before the wildfire. The Residents became aware of the Horse River wildfire entering Fort McMurray and the need to evacuate because of what they could see (19.9\%), from information on the radio $(19.4 \%)$, and from a family member $(15.3 \%)$. Others learned they would have to leave from a posting on social media (9.8\%), their employer (9.1\%), a friend or neighbour $(7.9 \%)$, with a few receiving this information from an RCMP officer, TV, or from the RMWB website. Many had little or no time to prepare to leave. $11.7 \%$ had to leave immediately and did not have any time to get ready, $16.9 \%$ spent less than 15 mintes getting ready, while another $39.1 \%$ said that they spent between 15 minutes and 1 hour getting ready. $16.6 \%$ spent between 1 and 2 hours getting ready to leave, and only $8.6 \%$ said that they spent more than 2 hours getting ready. As survey respondents tried to leave their neighbourhoods and urban Fort McMurray, 52.7\% stated that they experienced difficulties. Traffic delays were common for residents trying to leave their neighbourhood and then once they were heading north or south on Highway 63. Many survey respondents ran out of gas. Some respondents said they were not sure which way to go on Highway 63. Some survey respondents were 
separated from family members and friends during their departure. Others did not have enough food or water during the drive away from Wood Buffalo. Many survey respondents also spoke of the fear they experienced as they were trying to leave while the fire was burning around them.

Evacuees stayed in many towns throughout Alberta, elsewhere in Canada, and some returned home to international destinations during the evacuation. A majority of survey respondents stayed in more than one community, which caused additional challenges. Social media served as a very important source of information for survey respondents, but $46.1 \%$ of survey respondents stated that they did not receive enough information during the evacuation. An enormous outpouring of support was provided to evacuees during and following the wildfire from local residents, elsewhere in the province of Alberta, Canada, and overseas. Evacuees received help from a vast array of sources including friends, family, strangers, aid organizations, the government of Alberta, host communities, and businesses. Financial support from the Government of Alberta and the Red Cross, and donations from other organizations were appreciated by survey respondents, however some survey respondents experienced challenges accessing funds from the Red Cross.

Some evacuees were allowed to return to Fort McMurray starting at the beginning of June 2016, while others were unable to return until later in the summer.

\subsection{March 2018 Survey}

These survey respondents had varied home loss experiences, with $21.05 \%$ losing their home, $5.25 \%$ whose home had major damage, $36.84 \%$ experiencing minor damage to their home, and $36.84 \%$ whose home was not damaged due to the wildfire. $94.74 \%$ of survey respondents stated that they had insurance for their home at the time of the wildfire. At the time of the survey, $59.65 \%$ of respondents were living in the home where they lived before the Fort McMurray wildfire, $7.02 \%$ were living in temporary accommodation until they can rebuild their home, $12.28 \%$ were living in a different home, and $21.05 \%$ were living elsewhere including a newly built home or in a house in another city where they now reside.

Most (88.5\%) of the 62 follow-up survey respondents had returned to live in the RMWB since the wildfire, but $11.48 \%$ (7) had not returned. Most survey respondents (78.18\%) returned to RMWB for the first time to their home or property after the wildfire in June, with $20 \%$ returning in July or August. Most survey respondents also returned to live permanently in RMWB in June (64.81\%), with 29.63\% returning to live permanently in July or August. A majority of survey participants (58.49\%) indicated that they were concerned about returning to live permanently in the RMWB after the wildfire, with $41.51 \%$ indicating they were not concerned. Most participants (83.3\%) faced challenges during the reentry phase. Most respondents (76.67\%) said they received help during this phase from friends and family, the Red Cross and counselling services, re-entry centres, and appreciated the welcome back from first responders and support from strangers.

After the re-entry phase, survey respondents dealt with insurance; fixed damaged homes and replaced lost furniture, apliances, and belongings; and dealt with the social and psychological impacts of the wildfire. During this stage of the recovery process, $70.37 \%$ experienced challenges related to the insurance and restoration companies, the rebuilding process, mental health impacts, and financial losses. Help received during this part of the recovery process came from friends and family, coworkers, other residents of RMWB, the Red Cross, counsellors, and the municipality, and Facebook groups. Twenty-three participants expressed that their experiences with the wildfire re-entry and recovery had lasting impacts on them or their household members, from a heightened appreciation of what they have and empathy for others going through a difficult time, to ongoing mental health impacts. As several participants explained, it was a life-altering event.

\section{Conclusions}

The results of this study highlight what can happen when a sizeable population has to evacuate at the last minute due to a wildfire. Limited road access out of neighbourhoods and away from Fort 
McMurray caused traffic delays, which resulted in residents running out of gas and in some cases food and water as they tried to get to safety. The need to evacute quickly also meant that there was some confusion about how to get out of Fort McMurray safely, and separation of families and friends also occurred. It is clear that this last minute evacuation contributed to the trauma experienced by survey respondents.

The recovery process as experienced by survey respondents in Fort McMurray follows the key steps in the disaster recovery process as identified in the SAMHSA phases of disaster collective reactions model: the heroic phase and honeymoon state, where Fort McMurray residents helped each other and were helped by others, particularly while residents were away from Fort McMurray. Importantly, the SAMHSA model indicates that the disillusionment phase occurs up to one year, then after the 1-year anniversary, residents work through their grief. The results of the follow-up survey clearly show that some survey respondents are still in the disillusionment phase, with some still waiting to rebuild their house and dealing with the mental health impacts of the wildfire.

The results of this provide important insights into the wildfire evacuation and recovery process at two points in time: 1 month after the wildfire and 22 months afterwards. The timing of the first survey enabled the collection of recent experiences which were still vivid in survey respondents' memories. The second survey followed the same respondents to learn about their experienes in the recovery process almost 2 years after the wildfire. Further follow-up study is planned to examine the recovery process as it continues over the next few years.

\section{References}

Afifi WA, Felix ED, Afifi TD (2012) The impact of uncertainty and communal coping on mental health following natural disasters. Anxiety, Stress, \& Coping: An International Journal 25(3): 329347. Doi: 10.1080/10615806.2011.603048

Beverly JL, Bothwell P (2011) Wildfire evacuations in Canada 1980 - 2007. Natural Hazards, 59(1), 571-596.

Cohn PJ, Carroll MS, Kumagai Y (2006) Evacuation behaviour during wildfires: results of three case studies. West J. Appl. For. 21(1): 39-48.

Evans JR, Mathur A (2018) The value of online surveys: a look back and a look ahead. Internet Research, https://doi.org/10.1108/IntR-03-2018-0089

Felix ED, Afifi W (2015) The role of social support on mental health after multiple wildfire disasters. J Community Psychol 43(2),156-170.

Kent B, Gebert K, McCaffrey S, Martin W, Calkin D, Schuster E, Martin I, Bender HW, Alward G, Kumagai Y, Cohn PJ, Carroll MS, Williams D, Ekarius C (2003) Social and economic issues of the Hayman fire. USDA, Forest Service Rocky Mountain Research Station, Fort Collins, Colorado. Pp. 315-396. Gen Tech Rep RMRS-GTR-114.

KPMG (2017a) Regional Municipality of Wood Buffalo Lessons Learned and Recommendations from the 2016 Horse River Wildfire. July 27, 2017, Final Report.

KPMG (2017b) May 2016 Wood Buffalo Wildfire Post-Incident Assessment Report. Prepared for Alberta Emergency Management Agency, May 2017, Final Report.

MNP (2017) A Review of the 2016 Horse River Wildfire Alberta Agriculture and Forestry Preparedness and Response.

SAMHSA (undated) Phases of Disaster. https://www.samhsa.gov/programscampaigns/dtac/recovering-disasters/phases-disaster 


\title{
Risk assessment and reflections on socio-economic perception of wildfires at the fireshed level
}

\author{
George Mitri ${ }^{1 *}$; Joseph Bechara ${ }^{2}$; Maya Nehme ${ }^{2}$ \\ $1 *$ Institute of the Environment, University of Balamand, Kelhat-Koura, Lebanon, \\ \{george.mitri@balamand.edu.lb\} \\ ${ }^{2}$ Lebanon Reforestation Initiative, Hazmieh, Lebanon, \{jbechara@lri-lb.org\}
}

\begin{abstract}
Assessing and evaluating socio-economic perceptions of fire risk and impact can help in the design and development of proper fire risk management and planning. This work aimed at assessing public perception of socio-economic impact of wildfires at the level of a fireshed in Lebanon. The specific objectives were to 1) map fire risk at the fireshed level, and 2) investigate the socio-economic perceptions of key local players involved in fire risk management. The methodology of work comprised 1) delineation of the fireshed of Nahr El Kelb, 2) assessment of fire risk and identification of priority villages/towns for intervention, and 3) development of a targeted socio-economic survey. The delineation of the fireshed started by an initial delineation of the watershed using a Digital Elevation Model. Fire risk was assessed as a product of fire hazard and fire vulnerability. A total of three maps were produced, namely fire hazard, fire vulnerability and fire risk. Identification of priority sites included first the calculation of relative area of high fire risk within each cadastral unit. Consequently, a survey on public socio-economic perception of wildfires was implemented by sampling population in the high risk fire areas. The survey results included answers from 80 respondents on public perception of fire risk, fire damage to constructions and infrastructures, and estimation of fire suppression costs. Other results reflected the public perceptions in relation to direct and indirect effects of fire events in the targeted fireshed. Additional analytical work of questionnaire data will allow for the extraction of specific conclusions and the identification of future steps.
\end{abstract}

Keywords: Fireshed, fire risk, socio-economic perception of wildland fires

\section{Introduction}

A fireshed is conceptually analogous to a watershed. Basically, it surrounds areas of similar wildfire threat where a similar response strategy could influence the wildfire outcome (Bahro et al. 2007). A fireshed assessment though is based on the premise that fuels treatments strategically located to modify fire behavior can positively affect the outcome of a wildland fire by limiting the area severely burned and reducing negative effects on communities, habitat, and watersheds (Finney 2001). Since public and private lands are often intermingled in Lebanon, developing a coordinated program of work requires close collaboration with multiple landowners and interested parties. Hence, two critical pieces must come together to change large wildfire outcomes: (1) collaboration and coordination among agencies, landowners, and the interested public, and (2) on-the-ground implementation of a program of work, which establishes spatial locations, priorities, and schedules for multiple hazardous fuel treatment projects, ideally across all land ownerships in an area. In this context, there is an urgent need to assess and evaluate the local socio-economic perceptions of fire risk and impact in order to design and develop proper fire risk management and planning. Accordingly, this work aimed at assessing public perception of socio-economic impact of wildfires at the level of a fireshed in Lebanon. The specific objectives were to 1) map fire risk at the fireshed level, and 2) investigate the socio-economic perceptions of key local players involved in fire risk management. 


\section{Methodology of work}

This work comprised 1) delineation of the fireshed of Nahr El Kelb, 2) assessment of fire risk and identification of priority villages/towns for intervention, and 3) development of a targeted socioeconomic survey.

\subsection{Study area and dataset description}

The watershed of Jeita has a total size of 40,560 ha and it is located in the center of Lebanon, starting $15 \mathrm{~km}$ northeast of Beirut, on the western exposed side of the Lebanon Mountains (Figure 1). Its central coordinates are 760907.07 and 3763476.89 (UTM zone 36, WGS 84, Zone 36). It ranges between sea level and $2400 \mathrm{~m}$ asl at Mt. Sannine. Steepest reliefs occur along the hillside of fluvial shaped valleys in the southern center of the catchment and along the catchment's northern boundary. The watershed was initially selected for existence of a dense forest cover composed of highly combustible fuel (e.g. pine forests). At the same time the watershed is strategic in providing fresh water supply to the capital city of Beirut (BRG/CDR 2008) and is highly vulnerable to degradation (including risks of fire and pollution).

\subsection{Delineation and topography}

The delineation of the fireshed started by mapping the watershed using a Digital Elevation Model (DEM). The initial area of the watershed was expanded into a fireshed by annexing external areas of administrative units crossed by the borderline of the watershed. As a result, the total area of the identified fireshed was 44,100 ha (i.e., 3,540 ha larger than the size of the initial watershed) comprising a total of 79 villages (Figure 1).

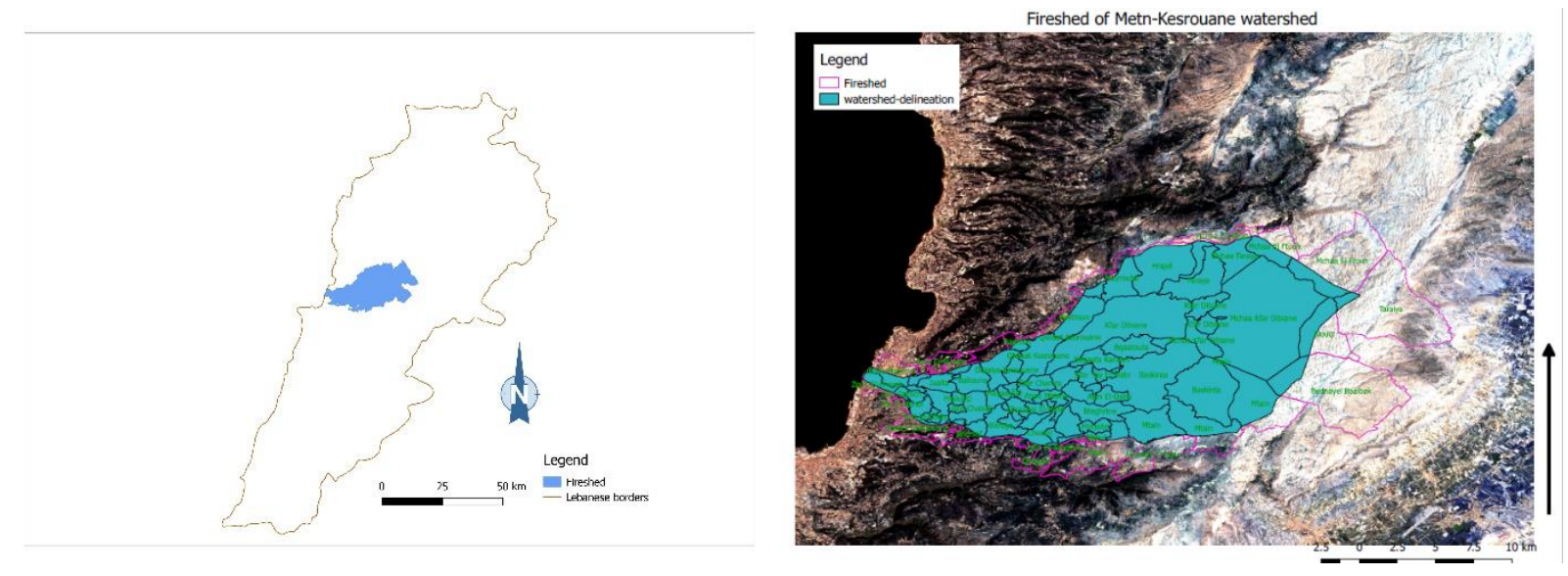

Figure 1 - Location of the fireshed on the map of Lebanon (left) and delineation of the fireshed (right)

\subsection{Fire risk assessment}

An overall wildfire risk assessment was prepared for the fireshed. More specifically, fire risk was assessed as a product of fire hazard and fire vulnerability (Mitri et al. 2015). Fire hazard assessment involved the use of data mainly related to the distribution and density of forest fuel using Landsat satellite imagery. Fire vulnerability was assessed at the cadastral unit level (i.e., village as a mapping unit) and in function of the relative area of hazard per mapping unit. Evaluating fire risk in the area involved the use of a cross mapping between hazard and vulnerability. Consequently, priority sites/villages for intervention were identified by calculating the relative area of high fire risk within each village/town (Table 1 ). 
Table 1 - Cross-tabular mapping of fire risk

\begin{tabular}{|l|l|l|l|}
\hline Hazard $\backslash$ Vulnerability & High vulnerability & Moderate vulnerability & Low vulnerability \\
\hline High hazard & High risk & High risk & Moderate risk \\
\hline Moderate hazard & High risk & Moderate risk & Moderate risk \\
\hline Low hazard & Moderate risk & Moderate risk & Low risk \\
\hline No hazard & Moderate risk & Moderate risk & Low risk \\
\hline
\end{tabular}

\subsection{Socio-economic survey}

Consequently, a survey was developed for use in field data collection. More specifically, one questionnaire was addressed to key stakeholders at the local level within the fireshed. Categorization of the survey included the following main sections:

- General questions on main existing forest activities in function of land ownership.

- Perception of wildfire risk and means of reducing hazard.

- Level of knowledge about fire occurrence and fire affected areas within the fireshed.

- Rating of resulting damages, impacts and costs of fires.

Participants to the survey included a total of 80 respondents randomly selected from priority municipalities (i.e., characterized by high risk of fires) and local service departments (e.g., Civil Defense).

\section{Results and discussion}

A total of three maps were produced (Figure 2), namely fire hazard, fire vulnerability, and fire risk (i.e., low risk, moderate risk, high risk, and very high risk).
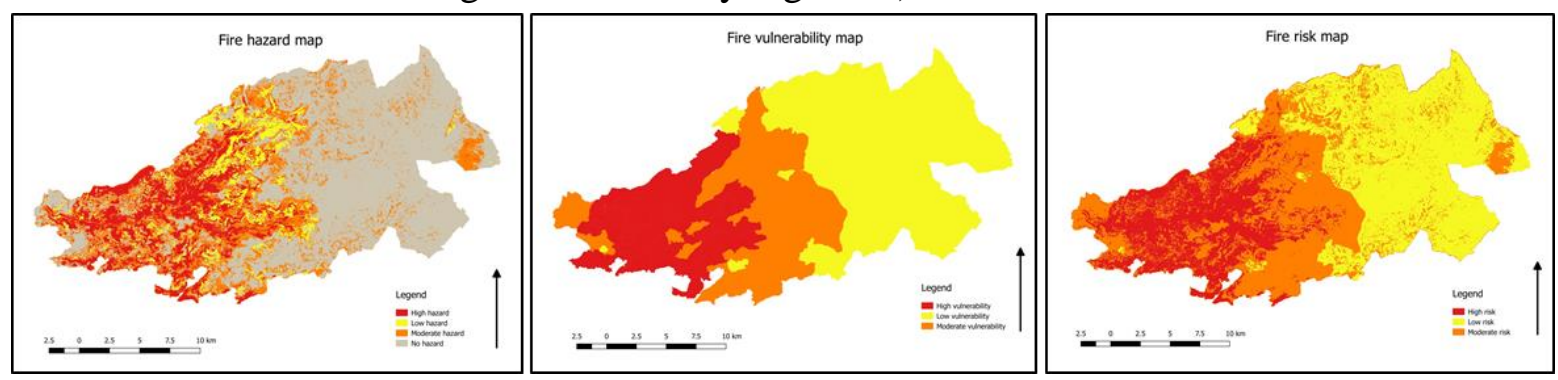

Figure 2 - Fire hazard (left), vulnerability (middle), and risk (right)

Identification of priority sites included first the calculation of relative area of high fire risk within each cadastral unit. As a result, villages including more than $80 \%$ of high risk areas were highlighted on the map. Then, the Wildland-Urban Interface (WUI), described as the area where human development intersects with wildland, was also characterized within the study area. The assessment of wildfire risk in the WUI in Lebanon was based on mapping areas of high risk of fire within a buffer zone of $100 \mathrm{~m}$ from urbanized lands, settlements, and roads. The top 20 villages (i.e., priority sites) including an area above 94 ha of WUI of high risk were identified and located on the map. As a result, a total of three clusters of towns and villages were identified for the survey (Figure 3).

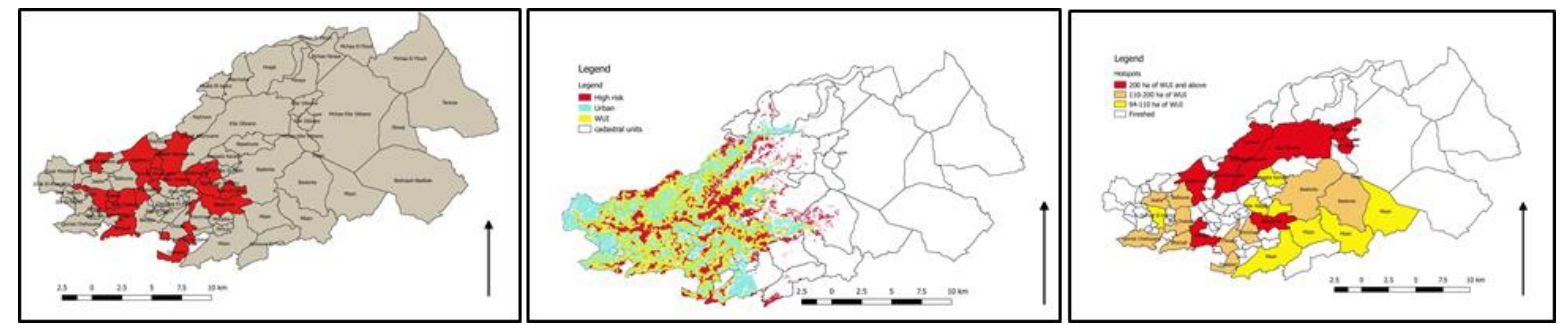

Figure 3 - Villages including more than $80 \%$ of high risk in red (left), WUI (middle), and identified top20 villages (right) 
While respondents were randomly selected from municipalities characterized by high risk of fires, the results of the survey showed that $32 \%$ of sampled population believed that their region is characterized by a high risk of fire, followed by $43 \%$ as moderate risk and $21 \%$ as low risk. Only $4 \%$ of respondents believed of no fire risk in their region. This reflected the need to raise awareness about fire risk for improving readiness and fire risk reduction measures.

Forest land was considered as most prone to fire for $73 \%$ of respondents, while grassland and cropland were considered as most prone to fire for $27 \%$ of respondents. Around $19 \%$ of sampled population indicated a potential of high risk of damage to constructions and infrastructure in case of a fire event, $44 \%$ as moderate risk of damage and $37 \%$ as low risk of damage. As a result, fire risk appeared to be underestimated in the WUI.

In the absence of proper accounting of fire suppression costs in Lebanon, 63\% of sampled population estimated an average cost range of 0-2500 USD, 23\% of respondents estimated an average cost range of 2500-5000 USD per fire intervention, and only $14 \%$ of respondents estimated an average cost higher than 5000 USD. Also, $34 \%$ believed that fire hazard can be reduced primarily by increasing awareness, while $24 \%$ believed that fire hazard can be reduced by improving management of forest fuel. This indicated people's willingness to take active measures before fire events.

Perceptions of possible damages/impacts in case of a fire occurrence in the town of respondents are presented below (Figure 4). More specifically, 39\% of respondents anticipated a high impact on wood resources in case of a fire event in their towns. Also, 39\% of respondents expected a high impact on public health in case of a fire event. As such, wood is still considered as a valuable resource to the local communities while health and air quality issues remain a concern to people given previous health incidents related to smoke emission from fires.

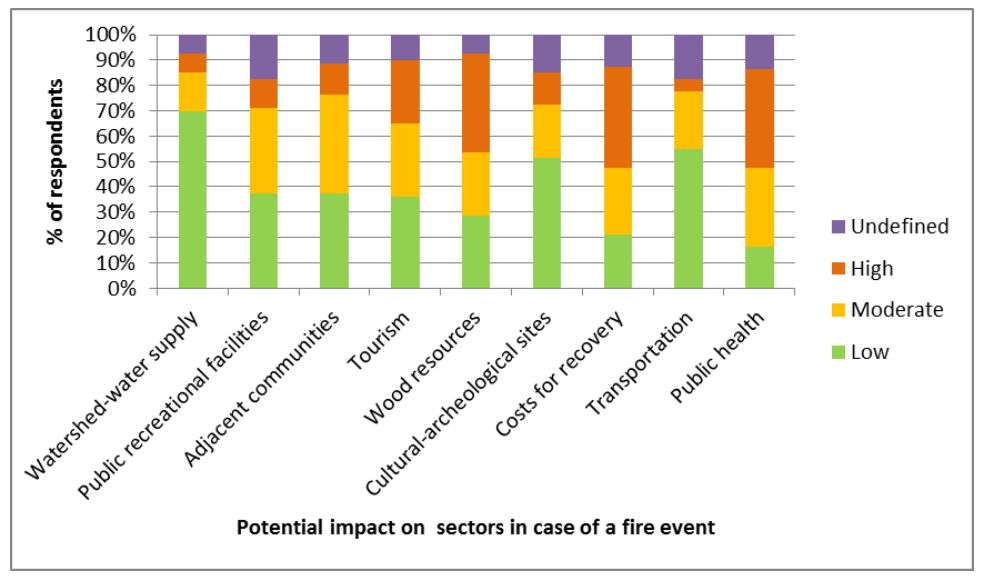

Figure 4 - Possible damages/impacts in case of a fire occurrence in the town of respondents

\section{Conclusions}

This work investigated the spatial distribution of fire risk at the fireshed level and conducted a socio-economic survey accordingly. Preliminary results showed how the public perceived fire as an environmental and socio-economic problem in areas characterized by high risk of fire. While all respondents were randomly selected from municipalities characterized by high risk of fires, the results of the survey showed that a relatively large number of people were not aware of the presence of high risk of fire in their towns. Also, surveyed people mostly expected losses and damages in wood resources in case of a fire event; while very few of them expected low impact on the watershed and water supply, knowing that the study area was part of a strategic and vulnerable watershed. In brief, the results highlighted the need to increase collaboration and coordination among agencies, landowners, and the local communities, while promoting priorities for fire risk management. Future 
work will include further analysis of the survey results for improved assessment of collected socioeconomic data.

\section{References}

Bahro B, Barber K, Sherlock J, Yasuda D (2007) Stewardship and Fireshed Assessment: A Process for Designing a Landscape Fuel Treatment Strategy. USDA Forest Service Gen. Tech. Rep. PSWGTR-203.

BGR/CDR (2008) Water Balance for the Groundwater Contribution Zone of Jeita Spring using WEAP. (Eds P Schuler, A Margane). PROJECT NO.: 2008.2162.9.

Finney MA (2001) Design of regular landscape fuel treatment patterns for modifying fire growth and behavior. Forest Science 47, 219-228.

Mitri G, Jazi M, McWethy D (2015) Assessment of wildfire risk in Lebanon using Geographic Objectbased image analysis. Photogrammetric Engineering \& Remote Sensing 81, 499-506. 


\title{
The consideration of arson for forest fires caused by traditional uses of fire prohibited in Spain. A problem under discussion.
}

\author{
José M Martínez-Navarro ${ }^{1 *}$; Carmen Vázquez-Varela ${ }^{2}$ \\ ${ }^{1}$ PhD in Geography. Independent Consultant, \{josemaria.martineznavarro@gmail.com*\} \\ ${ }^{2}$ Department of Geography, University of Castilla-La Mancha. Avenida de los Alfares, 44, 16071 \\ Cuenca, Spain, \{carmen.vazquez@uclm.es\}
}

\begin{abstract}
In some southern European countries, and in particular in Spain, the custom, rooted in the paradigm of the exclusion of fire, of considering and classifying in official statistics as intentional forest fires all ignitions generated by prohibited agricultural uses related to the traditional culture of fire, is maintained. This custom generates, from our perspective, a series of problems that deserve to be considered before deciding whether or not to maintain this criterion in our official statistical information systems. These problems are, first of all, the lack of uniformity in the classification criteria for the European EFFIS database, which means that the results for the different Member States are not comparable, thus rendering the effort made to produce and maintain a common European statistic meaningless, as well as the possibilities for analysing the problem associated with EFFIS.

Secondly, and already at the national level, this criterion distorts any rigorous analysis of the causes of ignitions, and without a rigorous initial analysis it is difficult to design effective prevention policies. Thirdly, the criterion, together with the sensationalist treatment of fires by the media, has created a monumental problem in public opinion, which does not understand what is happening, and which ends up generating a political problem. Politicians are struggling to lead this public opinion, and at present this is leading to an escalation of efforts in the police and judicial fight against a crime problem that does not really exist, to the detriment of real prevention policies based on Integrated Fire Management. It is precisely the latter that have been analysed in the European project FIREPARADOX and defended by the majority of experts and technicians today. Finally, the maintenance of this criterion is a clear disadvantage for the understanding by the non-expert public of the postulates of fire ecology, making the efforts that various organizations are beginning to make in this regard very complicated.

In relation to the sources and methodology used in this research, we have analysed the criteria for classifying the causes of forest fires used in the database of the Spanish General Statistics on Forest Fires (EGIF) and their problematic adaptation to the harmonized classification scheme of fire causes in the EU adopted for the European Fire Database of EFFIS. Subsequently, we compared the results of the current classification of the Spanish General Statistics of Forest Fires (EGIF) with those of an adaptation of the same prepared using the harmonized classification scheme of EFFIS. Finally, we have compared the results obtained from the two classifications with the data published by the Attorney General's Office on the intentionality of forest fires in judicial investigations over the last decade.
\end{abstract}

Keywords: Arson; traditional uses of fire; Spanish General Statistics on Forest Fires (EGIF); harmonized classification scheme of EFFIS; Spain.

\section{Introduction: The sterility of the causal-guilt approach}

Many of the specialists (scholars and technicians) and studies consulted for the Spanish case agree on the inevitable sterility of the approach that addresses the problem of forest fires from a causal-guilt relationship, especially in the case of the rural population and traditional/cultural uses. The results of the Socioeconomic Study of the Regions included in the Plan of Preventive Measures against Forest Fires of Castilla y León (Plan 42) (Molinero et al., 2008a) conclude that, for farmers, fire has always been used as a management tool for forests and their uses, and although it may seem contradictory in 
the context of the current abandonment of rural areas, fires for the regeneration of pastures continue to be one of the main reasons for forest fire in the fire reports of the region. In addition to this, there are conflicts with the administration over the control and use of forest products -pasture, firewood, wood, mushrooms and hunting- without forgetting the abandonment of arable land and the reduction of livestock pressure, which favour the advance of the bush, and the scarce or null interest in the exploitation of forest lands due to the sharp fall in the price of wood (Molinero et al. 2008b).

In the same vein, a previous work on Galicia by Professor Pérez Vilariño coincided with the group led by Fernando Molinero, who pointed out that the technocratic approach that does not contemplate cultural aspects is particularly sterile for understanding the phenomenon. In the opinion of the authors of the study, to attempt to explain the vast majority of forest fires by the same diffuse cause deliberateness or intentionality - is tantamount to not clarifying anything. "Resorting to armed gangs and other similar forms of conspiracies or mythical imaginaries, without being able to prosecute more than some poor man, who can hardly be accused of any fire, is the most convincing proof of the blurring of the causal-guilt model" (Pérez Vilariño and Delgado Fernández, 1995: 110).

Against this background, this contribution seeks to analyse the problem of the classification in the Spanish forest fire database (EGIF) of all cultural burns that are prohibited or carried out without a permit and that lead to forest fires, such as arson. This decision results in a problem of comparability and lack of uniformity in relation to the classification criteria of the European EFFIS (European Forest Fire Information System) database.

Secondly, and already at the national level, this criterion distorts any rigorous analysis of the causes of ignitions, and without a rigorous initial analysis it is difficult to design effective prevention policies. Thirdly, the criterion, together with the sensationalist treatment of fires by the media, has created a monumental problem in public opinion, which does not understand what is happening, and which ends up generating a political problem. Politicians are struggling to lead this public opinion, and at present this is leading to an escalation of efforts in the police and judicial fight against a crime problem that does not really exist, to the detriment of real prevention policies based on Integrated Fire Management.

To carry out this analysis we have used both national data and data from the elaboration of the Doctoral Dissertation of the first signatory of this contribution (Martínez Navarro, 2017), which focus on the region of Castilla-La Mancha. The data handled come from the regional historical database on forest fires (EGIF) for the period 1968-2013, from the national EGIF 2011-2013, and from the reports of the Attorney General's Office for the Environment for the period 2007-2015.

\section{The methodological problem of disaggregating causes and motivations}

The enumeration of a long list of causes (36 categories in the Negligence and Accidents group) prevents us from seeing the grouping and relevance of the negligence related to traditional rural culture, which constitutes one of the central nuclei to be analyzed in order to understand the causality of forest fires, as demonstrated by all the qualitative research carried out in our country (APAS and IDEM, 2004; Castro et al., 2007; Molinero et al., 2008a; Molinero et al., 2008b; Pérez Vilariño and Delgado Fernández, 1995; Priego and Lafuente, 2007; Vélez Muñoz, 1999).

The same problem can be found when analyzing the motivations of arson, which are in a database field other than the disaggregation of negligence. Since 1989, the Fire Report has received the addition of a new field associated with deliberate causation, which is labelled as "motivation" and which establishes as categories an exhaustive series of motivations that can lead a person to deliberately cause a forest fire. In 1989 this repertoire included 16 types of motivation. In the next update of the report form, i.e. from 1998 onwards, 7 new types of motivation were added, for a total of 24 (CLIF, 1997a; CLIF 1997b). 
We can see that for $73 \%$ of the deliberate forest fires included in the regional EGIF database, the motivation is unknown, a logical question if we take into account that in order to know the motivation we have to identify the arsonist. However, we believe that a thorough analysis of the remaining $27 \%$ should be carried out in order to better understand the problems behind the intentionality from a territorial point of view.

To do this, we should start by pointing out that the motivation series presents the same methodological problem as the description of cause associated with the Accidents and Negligence group: it has too many categories and many of them lack statistical significance because they are represented by a minimum number of fire reports. For this reason, it was decided to propose a model of reasoned groupings that would allow us to generate more clarifying categories from a territorial perspective and at the same time with some statistical significance. We consider that a model already exists that meets these requirements and for which the necessary recoding of the EGIF database is standardised, which is none other than the new European fire database EFFIS to which the Spanish Ministry incorporates the EGIF information with a public access protocol.

The EFFIS database is better oriented to analysis than the EGIF one because it classifies causality into three hierarchical levels instead of grouping causes into broad categories and then incorporating disaggregation for negligence and deliberate ones into separate fields, so that any fire can be analysed at any of the three levels, an issue that solves the problem of disaggregation without renouncing the detailed information contained in the EGIF.

However, it is the formally recording (harmonization) of the "Spanish" causality of EGIF in the international database that offers us some conceptual and legal doubts that we will expose in the analysis of the corresponding EFFIS categories, especially in the sense of keeping the agricultural and livestock burns without permission within groups with other criminal causes contrary to the criteria defined by the JRC (Joint Research Centre).

\section{The EFFIS database}

In 1998, the Joint Research Centre (JRC), a Directorate-General of the European Commission (EC), and the only service directly responsible for research, created a research group to work specifically on the development and implementation of advanced methods for forest fire risk assessment and mapping of burnt areas at European level. In addition, a first meeting of the "Forest Fire Experts Group" was held in the same year. All these activities led to the development of the European Forest Fire Information System (EFFIS), which became operational in 2003 to provide improved information and contribute to a common understanding of the origin of forest fires in Europe, while preserving as far as possible the historical databases of each country and exploiting the information available at the highest level of detail.

\section{The structure of the EFFIS database}

Traditionally, until the introduction of the new harmonised classification structure supervised by the JRC, the European-wide recording of the causes of forest fire has been carried out using, since 1992, a simple scheme of four main causality categories for forest fires: arson, accidental/negligent, natural and unknown. This classification system conflicted with the collection and classification model of the different European countries, which was usually much more detailed and for which there was no proposal for harmonisation, so the comparative or cross-sectional analyses were very complex.

The need for a harmonised European classification structure, more disaggregated than the original four categories, and which could be incorporated into the EFFIS fire database, was a pressing need for further progress in improving information and shared knowledge on the origin of forest fires in Europe. 
The new classification structure has been designed to be applied with limited changes to each of the data collection schemes of the different EU countries, preserving as far as possible the historical data series of each state and exploiting the level of detail of the available information. The structure is hierarchical and is constructed from 29 classes of fire causes, 8 groups and 6 major categories (see Table 1). The explicit declaration of the level of certainty in the allocation of the cause of the forest fire is also introduced as a key element in the new model. Finally, it is expected that the new causality classification scheme will be progressively adopted by the countries participating in the EFFIS network and therefore integrated into the registration system of the European forest fire database, with significant added value for the knowledge of the technical and scientific community on the origin of forest fires on the continent.

Table 1 - Classes, groups and categories of the harmonized fire causes classification scheme Source: Joint Research Centre, 2013: 10.

\begin{tabular}{|c|c|c|}
\hline CATEGORY & GROUP & CLASS \\
\hline EFFIS 1 & EFFIS 2 & EFFIS 3 \\
\hline 100 Unknown & 100 Unknown & 100 Unknown \\
\hline \multirow[t]{3}{*}{200 Natural } & \multirow[t]{3}{*}{200 Natural } & 201 Lightning \\
\hline & & 202 Volcanism \\
\hline & & 203 Gas emission \\
\hline \multirow[t]{7}{*}{300 Accident } & \multirow[t]{7}{*}{300 Accident } & 301 Electrical power \\
\hline & & 302 Railroads (Railways) \\
\hline & & 303 Vehicles \\
\hline & & 304 Works \\
\hline & & 305 Weapons (firearms, explosives, etc.) \\
\hline & & 306 Self-ignition (auto-combustion) \\
\hline & & 307 Other accident \\
\hline \multirow[t]{9}{*}{400 Negligence } & \multirow[t]{5}{*}{410 Use of fire } & 411 Vegetation management \\
\hline & & 412 Agricultural burnings \\
\hline & & 413 Waste management \\
\hline & & 414 Recreation \\
\hline & & 415 Other negligent use of fire \\
\hline & \multirow[t]{4}{*}{420 Use of glowing objects } & 421 Fireworks, firecrackers and distress flares \\
\hline & & 422 Cigarettes \\
\hline & & 423 Hot ashes \\
\hline & & 424 Other use of glowing object \\
\hline \multirow[t]{8}{*}{500 Deliberate } & \multirow[t]{6}{*}{510 Responsible (arson) } & 511 Interest (profit) \\
\hline & & 512 Conflict (revenge) \\
\hline & & 513 Vandalism \\
\hline & & 514 Excitement (incendiary) \\
\hline & & 515 Crime concealment \\
\hline & & 516 Extremist \\
\hline & \multirow[t]{2}{*}{520 Irresponsible } & 521 Mental illness \\
\hline & & 522 Children \\
\hline 600 Rekindle & 600 Rekindle & 600 Rekindle \\
\hline
\end{tabular}




\section{Re-encoding of the Spanish forest fire database to the EFFIS harmonised classification}

As noted in previous sections, we have chosen to recode the information collected in the EGIF using as classification categories, groups and classes those proposed in the new harmonised European classification system. The latter provides a simplified model for analysis and allows us to compare national data and perspectives without entering into aspects of legal liability and prosecution, which we will address later.

Thus, the set of Forest Fire Reports gathered in the regional base for the period 1968-2013 are included in one of the six pre-established categories (Figure 1) with two particularities, the first of which refers to the fact that a small part of the negligence within the EGIF, fires caused by children, would pass to the group of deliberate fires if we adhered to the EFFIS criteria, since the harmonised European base includes them in this group although the perpetrators are considered irresponsible. It is a small group of 100 fire reports that we have distinguished by maintaining the green color but adding its weight to the group of fire reports of deliberate origin.

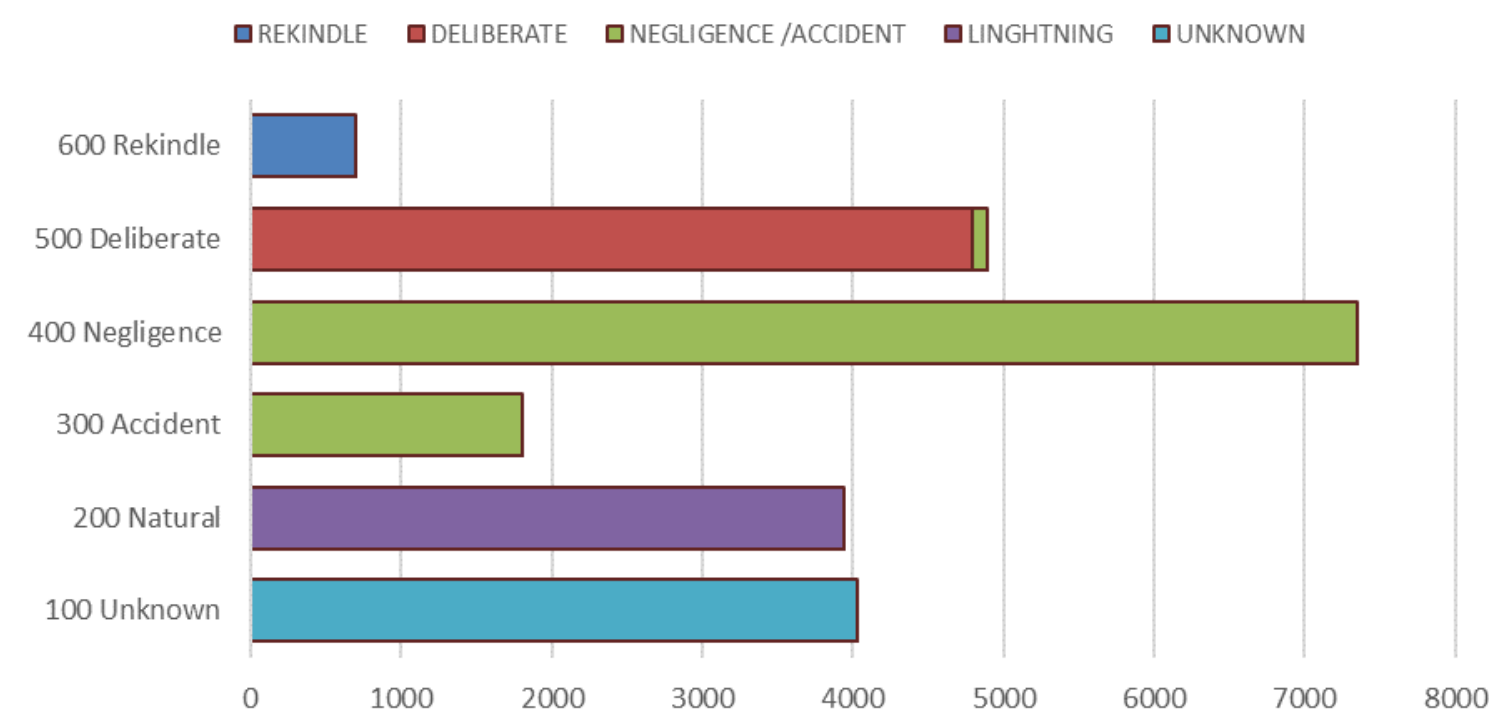

Figure 1 - Harmonisation of "Causes Group" EGIF and EFFIS1 categories. Number of fire reports. Castilla-La Mancha 1968-2013

The second particularity refers to a fact that we will develop in depth below, but which we anticipate by commenting that a large part of the fires included in the category of deliberate fires in the harmonised Spanish base, vegetation management and those derived from agricultural burnings and waste management carried out without the appropriate burn permits, are included by the rest of the countries (as can be seen from the approach of the harmonised base itself) in the category of fires due to negligence. The only two countries that include these fires of cultural origin, tied to the rural world, in the category of arson are Spain and Italy. In other words, in Spain any forest fire caused by agricultural burnings or due to vegetation management that has been carried out without the required permits or outside the limited period for this purpose is automatically considered an arson.

Having made this fine-tuning, the harmonised classification of the EFFIS3 categories would be as shown in Table 2. Continuing with the adjustments between the harmonized database and the original EGIF, it is necessary to remember that, since 2011, the group of Negligent and accidental causes appears with two labels in which the numbers 2 for negligence and 3 for accidents precede the name, as shown in the table below, a division that is perfectly consistent with the EFFIS coding. 
Table 2 - Number of Forest Fire Reports by EFFIS3 classification and EGIF Category Groups. Castilla-La Mancha 1968-2013

\begin{tabular}{|c|c|c|c|c|c|c|}
\hline & 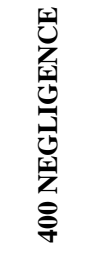 & 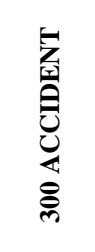 & $\begin{array}{l}z \\
\vdots \\
z \\
z \\
3 \\
8 \\
8\end{array}$ & 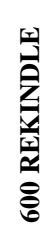 & 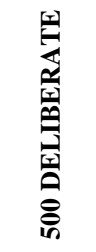 & لِ \\
\hline 100 Unknown & & & 4,033 & & & \\
\hline 201 Lightning & & & & & & 3,942 \\
\hline 301 Electrical power & & 398 & & & & \\
\hline 302 Railroads (Railways) & & 155 & & & & \\
\hline 303 Vehicles & & 149 & & & & \\
\hline 304 Works & & 1,076 & & & & \\
\hline 305 Weapons (firearms, explosives, etc.) & & 32 & & & & \\
\hline 400 Negligence (unspecified) & 779 & & & & & \\
\hline 411 Vegetation management & 1,517 & & & & & \\
\hline 412 Agricultural burnings & 2,639 & & & & & \\
\hline 413 Waste management & 1,013 & & & & & \\
\hline 414 Recreation & 703 & & & & & \\
\hline 421 Fireworks, firecrackers and distress flares & 36 & & & & & \\
\hline 422 Cigarettes & 1,218 & & & & & \\
\hline 424 Other use of glowing object & 12 & & & & & \\
\hline 500 Deliberate (unspecified) & & & & & 3,423 & \\
\hline 511 Interest (profit) & & & & & 1,002 & \\
\hline 512 Conflict (revenge) & & & & & 169 & \\
\hline 513 Vandalism & & & & & 142 & \\
\hline 514 Excitement (incendiary) & & & & & 3 & \\
\hline 515 Crime concealment & & & & & 1 & \\
\hline 521 Mental illness & & & & & 50 & \\
\hline 522 Children & 101 & & & & & \\
\hline 600 Rekindle & & & & 131 & & \\
\hline
\end{tabular}

\section{Harmonisation for the " 500 Deliberate" category of the EFFIS database}

The fires classified by the Spanish administration as deliberate occupy the second place in the region according to the cause of the fire (4,790 fire reports between 1968 and $2013-4,891$ if we add fires caused by children - which is equivalent to $21 \%$ of the total) but the first by the extent of the burned surface $(41 \%)$. These data, together with its exponential growth trend, show that arson is currently one of the biggest problems to be solved and one of the most difficult to tackle, because to set fire is easy but to prevent this type of event is far from being so. On the other hand, the classes of fire causes included in this group translate an approach to the problem from the paradigm of fire exclusion that conditions the territorial management of anthropogenic risk of ignition and is the source of numerous conflicts between the administration and the local population living in the rural world. In addition, the motivation for deliberate forest fires is particularly poorly known: only $28.5 \%$ of deliberate cases are cleared up (as can be seen from the table below, of the 4,790 fire reports in the 
arson group 3,423 appear in the "unspecified" class). Moreover, as the motivations are set out, even today, they are not always interpreted by the forest officials who elaborate the fire reports in the same way. The motives are understood according to the peculiarities of the local reality, the custom of its forestry section or simply the experience that each one has (Carracedo Martín et al., 2009). When making a complaint, it is difficult to consider this information valid because of the burden of intuition it entails. For this reason, the work of the Forest Fire Investigation Brigades (BIIF) is becoming increasingly important.

Table 3 - Harmonisation for the "500 Deliberate" category of the EFFIS database. Castilla-La Mancha 1968-2013 (in red "cultural" causes)

\begin{tabular}{|c|c|c|c|c|c|}
\hline EFFIS/Motivations & $\begin{array}{l}\text { Number of } \\
\text { fire reports }\end{array}$ & $\begin{array}{c}\text { Total area } \\
\text { burned }\end{array}$ & $\begin{array}{l}\text { Average burned } \\
\text { surface area }\end{array}$ & $\begin{array}{l}\text { Maximum area } \\
\text { burned by fire }\end{array}$ & $\begin{array}{l}\text { Standard deviation } \\
\text { of burned surface }\end{array}$ \\
\hline \multicolumn{6}{|l|}{500 Deliberate (unspecified) } \\
\hline \multicolumn{6}{|l|}{500 Deliberate (unspecified) } \\
\hline Other motivations & 71 & 282.8 & 4.0 & 74.0 & 11.8 \\
\hline Unspecified & 3,352 & $75,260.0$ & 22.5 & $14,225.0$ & 274.6 \\
\hline \multicolumn{6}{|l|}{510 Responsible (arson) } \\
\hline \multicolumn{6}{|l|}{511 Interest (profit) } \\
\hline $\begin{array}{l}\text { Earning wages in forest fire } \\
\text { fighting or restoration }\end{array}$ & 4 & 68.4 & 17.1 & 57.0 & 27.0 \\
\hline $\begin{array}{l}\text { To promote the production of } \\
\text { forestry products }\end{array}$ & 1 & 0.01 & 0.01 & 0.01 & \\
\hline To gain changes in land use & 9 & 37.5 & 4.2 & 22.4 & 7.2 \\
\hline $\begin{array}{l}\text { Triggered to scare away animals } \\
\text { (wolves, wild boars) }\end{array}$ & 25 & 60.7 & 2.4 & 33.7 & 6.6 \\
\hline Agricultural burnings & 572 & $1,505.2$ & 2.6 & 300.0 & 13.3 \\
\hline $\begin{array}{l}\text { Provoked by hunters to facilitate } \\
\text { hunting }\end{array}$ & 116 & $1,274.9$ & 11.0 & 338.0 & 39.3 \\
\hline $\begin{array}{l}\text { Caused by political groups to create } \\
\text { social unrest }\end{array}$ & 2 & 0.3 & 0.1 & 0.2 & 0.1 \\
\hline $\begin{array}{l}\text { Herders and ranchers provoke them } \\
\text { to regenerate the grass }\end{array}$ & 269 & $1,448.7$ & 5.4 & 200.0 & 19.7 \\
\hline \multicolumn{6}{|l|}{512 Conflict (revenge) } \\
\hline Opposition to reforestation & 3 & 184.1 & 61.4 & 180.0 & 102.7 \\
\hline $\begin{array}{l}\text { Disagreements on the ownership of } \\
\text { public or private forests }\end{array}$ & 1 & 0.01 & 0.01 & 0.01 & \\
\hline $\begin{array}{l}\text { To force resolutions of consortia or } \\
\text { agreements }\end{array}$ & 4 & 12.8 & 3.2 & 5.0 & 1.8 \\
\hline Incited against hunting restrictions & 70 & $2,893.6$ & 41.3 & $1,495.0$ & 184.8 \\
\hline Caused by vengeance & 79 & $11,434.0$ & 144.7 & $5,489.4$ & 709.3 \\
\hline $\begin{array}{l}\text { Opposition to the creation or } \\
\text { existence of protected natural areas }\end{array}$ & 8 & 46.1 & 5.8 & 38.3 & 13.2 \\
\hline $\begin{array}{l}\text { Retaliation by reducing public } \\
\text { investment in forests }\end{array}$ & 4 & 16.2 & 4.1 & 15.0 & 7.3 \\
\hline \multicolumn{6}{|l|}{513 Vandalism } \\
\hline Vandalism & 142 & 653.9 & 4.6 & 100.0 & 14.4 \\
\hline \multicolumn{6}{|l|}{514 Excitement (incendiary) } \\
\hline To admire the work of fire fighting & 3 & 41.1 & 13.7 & 40.0 & 22.8 \\
\hline \multicolumn{6}{|l|}{515 Crime concealment } \\
\hline $\begin{array}{l}\text { Caused by criminals, etc. to distract } \\
\text { law enforcement officials }\end{array}$ & 5 & 2.7 & 0.5 & 2.0 & 0.9 \\
\hline \multicolumn{6}{|l|}{516 Extremist } \\
\hline $\begin{array}{l}\text { Caused by political groups to create } \\
\text { social unrest }\end{array}$ & 2 & 0.29 & 0.125 & 0.2 & 0.5 \\
\hline
\end{tabular}




\begin{tabular}{|c|c|c|c|c|c|}
\hline EFFIS/Motivations & $\begin{array}{l}\text { Number of } \\
\text { fire reports }\end{array}$ & $\begin{array}{l}\text { Total area } \\
\text { burned }\end{array}$ & $\begin{array}{l}\text { Average burned } \\
\text { surface area }\end{array}$ & $\begin{array}{l}\text { Maximum area } \\
\text { burned by fire }\end{array}$ & $\begin{array}{l}\text { Standard deviation } \\
\text { of burned surface }\end{array}$ \\
\hline $\begin{array}{l}\text { Pseudo-Religious Rites and } \\
\text { Satanism }\end{array}$ & 0 & 0 & 0 & 0 & 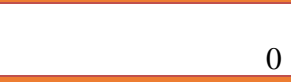 \\
\hline \multicolumn{6}{|l|}{520 Irresponsible } \\
\hline \multicolumn{6}{|l|}{521 Mental illness } \\
\hline Caused by pyromaniacs & 50 & $2,188.0$ & 43.8 & $1,392.0$ & 204.1 \\
\hline \multicolumn{6}{|l|}{522 Children } \\
\hline Negligence (Children's Games) & 101 & 206.1 & 2.0 & 60.3 & 6.7 \\
\hline Cumulative total & 4,891 & 97,617 & 20.0 & 14,225 & 247.0 \\
\hline
\end{tabular}

The EFFIS 500 Deliberate category, like the Negligence category, differentiates between two major groups, in this case on the basis of the criminal liability of the act, which presents problems of harmonisation for all the national classifications integrated into the European network, and in particular with the EGIF, as we will analyse below. This differentiation in terms of liability generates two groups of causes: the Responsible (arson) and the Irresponsible 510, which includes those fires caused by people who cannot be charged because they are not criminally responsible for their actions, minors (criminal law) and certain mentally ill people, an issue that changes depending on how nonliability is addressed in the criminal legislation of each country.

For this reason we find two EFFIS3 classes in the "Irresponsible" group, 522 Children and 521 Mentally ill. As can be seen in the table above, the "Responsible (arson)" group includes six EFFIS3 classes, the first two being 511 Interest (profit) and 512 Conflict (revenge), which group a greater number of Forest Fire Reports and EGIF motivations.

In addition, another category is included in the harmonization document that includes all those reports of deliberate fires that are not assigned a motivation and that are named as in EFFIS1: "500 Deliberate", in our case all those prior to 1989 and $67 \%$ of those that occur from that year until 2013. For the national database 2011-2013, the number of arson cases without specifying the reasons for them is $26.7 \%$, while in Castilla-La Mancha during the same period the figure rises to $43.6 \%$, a data that emphasises the idea of the damage caused by budget cuts in the investigation of causes in our region. The percentage distribution of the EFFIS classes, in which the intentionality has a known motivation, compared for Spain and Castilla-La Mancha, is as follows:
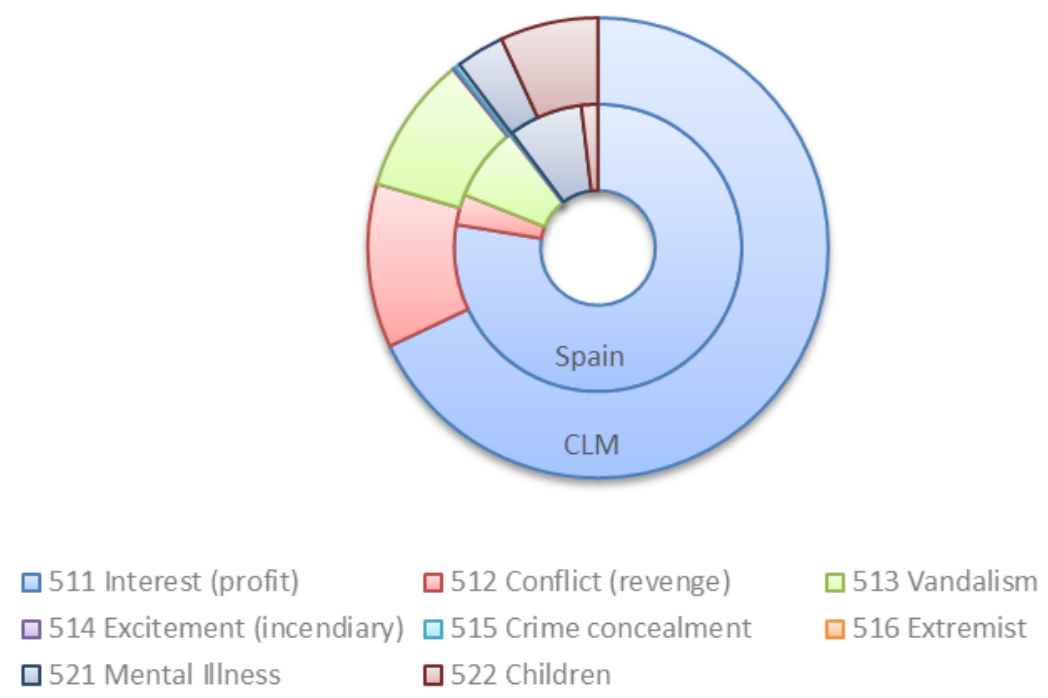

Figure 2 - Comparison of the percentage distribution of EFFIS3 classes within the category 500 Deliberate between Castilla-La Mancha (1989-2013) and Spain (2011-2013). Source EGIF. 
For both scales the main class is 511 Interest (profit), since it includes the cultural burnings carried out without a permit or outside the limited time interval for it, with a difference of ten percentage points in favour of the whole country, since the mentioned class includes almost $78 \%$ of all deliberate fires with known motivation in Spain. The EFFIS3 Conflict (revenge) class, second in terms of the number of reports in Castilla-La Mancha with $11.5 \%$, is nevertheless the fourth on a national scale with just 3.5\%, the main reason for the difference being the conflicts associated with hunting reserves, given the importance of this activity in the region. We found no significant differences in the fires caused by vandalism ( 8.5 and $9.6 \%$ respectively) while for the category 521 Mental illness the regional rate is much lower $(3.41 \%)$ than the national rate (8.35), very skewed, as we will see below, by the behavior of Galicia. Finally, the only class that changes category from Negligence to Deliberate is "Children" which is included, as we have already seen, in group 520 "Irresponsible", although with some adjustment problems.

Legally, pyromaniacs, whose number is also very marginal, should be included in the harmonisation of the Responsible (arson) group, thus separating them from the "serious" mentally ill who are not criminally responsible for their acts, which is why the harmonisation of the EGIF motivation "caused by pyromaniacs " with the EFFIS3 class 521 Mental Illnesses shows, in our opinion, a problem of conceptualization. The fact that they are included in group 520 Deliberate "irresponsible" presupposes that "pyromaniacs" are not criminally liable, an issue that is not exact in our criminal law. On the other hand, fires caused by diagnosed pyromaniacs are a small part of the events associated with this motivation, which serves, a priori, to accommodate any ignition produced by a person with a mental disorder.

In short, non-liability is established, except in anecdotal cases, on the basis of mental insanity, i.e. paranoia, schizophrenia and the Temporary Mental Disorder underlying either of these two illnesses, as well as for people with disabilities due to serious mental retardation. Pyromania, as a behavioural disorder, is considered in any case as an attenuating factor or, in interaction with other psychological problems, as a partial exonerating factor, so the harmonisation of this EGIF motivation should never be carried out in the group of "Irresponsible", since in Spain they are.

\section{The class EFFIS3 511 Interest (profit) and the problem of the criminalization of cultural burnings in Spain}

In addition to the doubts raised by the inclusion of cultural burnings without a permit within arson group, there is the fact that cultural burnings are classified in a group that includes other criminal motivations that are, although rare, in two of the cases clearly "mafia-like" (they are organized crime, some order and others execute), and all the others guilty of pursuing a direct economic benefit from the destruction of the forest, while in the case of cultural burnings - administratively illegal - such destruction may or may not take place, but in no case is the aim of who deliberately lights the fire.

As we have already pointed out, it has always been argued by the Spanish Ministry responsible for the environment that arson attacks include both 'malicious' cases (where there is wilful misconduct, the intention to burn the forest) and 'culpable' cases, where there is no intention to burn the forest but the (cultural) use of fire is normally consciously in contravention of the administrative rules on the matter and is therefore illegal. For the Ministry, the difference lies in the fact that in the deliberate ones the burning is carried out knowing that in that place and moment it is prohibited to carry out such burning, or the fires are abandoned before they are correctly extinguished, which, in its opinion, implies intentionality and not negligence in the cultural use of fire, although in both cases what underlies it is the traditional rural culture.

This criterion is certainly controversial, and in our opinion it is also not neutral, since calling arson what is not precisely 'intended' to burn the forest is a distorted picture of the problem for anyone who is not an expert in the forest. The use of the term 'forest' here is also intentional. 
The group of motivations that we have called "cultural", and which we point out in red in the table above, brings together the four sub-classes that we consider to be directly associated with cultural uses, two of which are in the majority and also generate a certain degree of confusion when compared with cultural negligence, since they have the same purpose and are almost identically named: vegetation management and agricultural burnings. If we compare the regional data with the national EGIF database for the period 2011-2013, we can see that the situation is even more problematic for the country as a whole, as can be seen in the following figure:

$\square$ To gain changes in land use
$\square$ To bring down the price of wood
$\square$ Earning wages in forest fire fighting
or restoration
$\square$ Provoked by hunters to facilitate
hunting
$\square$ Triggered to scare away animals
(wolves, wild boars)
$\square$ To promote the production of
forestry products
$\square$ Agricultural burnings
Herders and ranchers provoke them
to regenerate the grass

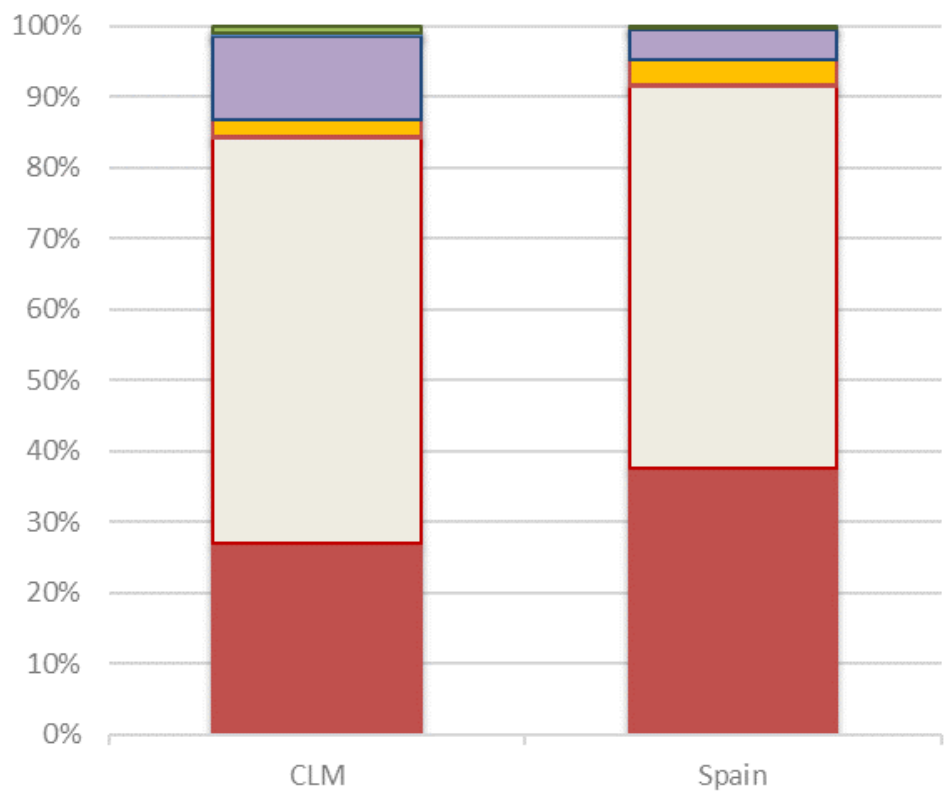

Figure 3 - Comparison of the percentage of Forest Fire Reports by motivation included in the EFFIS class 511 Interest (profit). Spain and Castilla-La Mancha. Source EGIF

In relation to the cultural uses of fire in the rural world, we cannot forget the fact that the vast majority of rural areas in our country, and specifically in Castilla-La Mancha, have a very old population, a consequence of the rural exodus experienced in recent decades. This population, when they are still able to work in agriculture, livestock or forestry, uses traditional management methods, i.e. they continue to burn to remove brushwood, brambles and plant residues or to regenerate the grass, with the consequent risk that these burns will spread to adjacent forest areas, due to their reduced physical capacity for fire control or to take all the necessary preventive measures.

As Enrique Martínez Ruiz (2001) argues, the depopulation of rural areas and the consequent abandonment of the countryside are the main cause of the serious problem of forest fires that has been dragging on in Spain for more than 35 years. However, sometimes the need for the farmer (farmer, rancher and forester) to remove vegetation is less well known, and the most expeditious and cheapest way to do this is by fire. But now the control of this fire is more difficult due to the accumulation and continuity of fuels and the uncontrolled burning due to negligent and deliberate causes provokes the majority of forest fires in Spain. In this context, public opinion, more in urban than in rural society, hardly understands the use of fire, which necessarily can and should be done by applying prescribed or controlled burning techniques (Martínez Ruiz, 2001: 6).

Illegal burning by farmers, herders and ranchers together accounts for $60 \%$ of deliberate fires of known motivation. Since the beginning of the development of agricultural activities, fire has been one of the common tools of agriculture and stockbreeding, indispensable throughout history for slashing and converting mountain areas into meadows and pastures. This has made it difficult today to observe a vegetation landscape that has not been shaped by fire (Vélez, 1986). Even today, the shepherd still uses it to limit the spread of woody plants and ensure the growth of herbaceous plants, in short, to 
maintain pastures; the farmer, in order to stop the invasion of wild plants and to favour growth conditions suitable for crops. This is the reason why the motivations that concentrate a greater number of fires are those related to the elimination of scrub or agricultural residues and the regeneration of pastures (Barroso and González, 2007), what we have called the cultural uses of fire in the rural world.

The harmonization document's own definitions specify that class 511 Interest (profit) includes (or should include) fires set to obtain a benefit, directly economic or other than monetary: fraud, insurance, liquidation of property, liquidation of business, elimination of parcels, competition for agricultural uses, hunting, pastoral or forestry use, to scare off wild animals, to obtain work in the firefighting area, change of land use, obtaining wages, etc. Whereas fires associated with class 411 Vegetation management are those used to control all types of vegetation, including regeneration of pasture, forest, pruning waste, etc. including prescribed fires and except for fires for agricultural purposes, as we know from experience reserved for class 412 and which collect all types of agricultural burnings. Both definitions show that, in the opinion of the Joint Research Centre of the European Commission, cultural burns, even illegal ones, should not be included in class 511 of deliberate fires for the sake of interest.

In short, Italy and Spain are the only two European countries that classify illegal (cultural) agroforestry/pastoral (forest) burning as a deliberate (intentional) fire, according to the internal regulations of both countries. In the absence of any other explanation, this is the reason why their arson rates are much higher, around 50\% of total fires, than those of the rest of the Mediterranean countries.

The differences in the consideration of the causes already appear in the 2008 report of the Attorney General's Office "In certain issues such as forest fires, the statistics provided by the different official bodies responsible for the matter almost necessarily have to differ, given that there is no clear and strict conceptual agreement. This undoubtedly explains the differences between the statistics provided by each of these institutions". Pursuing the divergence of data in the category of deliberate forest fires, it continues to point out that "intentionality as a parameter of voluntariness managed by the Administration may arouse some kind of suspicion, in the view of non-experts in the field, however, an example is enough to explain what we say here. An agricultural burning on a farm without asking for a permit can be an arson attack for the administration, and for penal parameters it can be unpunished" (Fiscalía General del Estado, 2008: 38).

However, the Attorney General's Office claims to use the classification of the causes of forest fires by the Ministry responsible for the Environment, and indicates that only those caused by negligence or accidents and those that are deliberate are of interest to the Attorney General's Office, according to the criteria of the Prosecutor General's Office for the Environment, establishing the definition of each of these categories. Thus, it considers accidents to be "activities in which the perpetrator could not have foreseen that a forest fire would occur. This activity is not associated with forest fires and, if they occur, is due to fortuitous causes", and negligence such as "human activities which cause a forest fire without the person involved intending to cause it, even though he has carried out an activity which may cause a fire or has omitted the safety measures which prevented it from occurring". Finally, for arson, it states that "In general, we can consider actions whose purpose is the deliberate generation of a forest fire" (Fiscalía General del Estado, 2015: 120). Therefore, the public prosecutor's office explains that it understands, like most citizens, that an arson attack is one in which the perpetrator intends to start a forest fire.

In addition to the perplexity and concern caused by the obvious lack of communication and coordination between the highest authorities responsible for forest fire risk management in our country, the General Directorate of Biodiversity and the General State Prosecutor's Office, it is clear that the criterion described above of considering "criminally negligent" fires to be arson is not in line with our general legal system. Our legislation has well defined the intentionality, the volitional element in Criminal Law, drawing a clear distinction from recklessness, which in this case also involves criminal liability but in a lessened form. In the latter case, the criminal liability of those who burn the forest due to negligence/imprudence is less than that of those who wish to burn it. 
The information provided by the Attorney General's Office in its annual reports is devastating. On the other hand, the quality of the data is necessarily higher, since it is not the prosecution that is counted, but the cases in which there are accused persons, so that the information on the specific cause or motivation that has triggered the claim is assumed to be sufficiently reliable to initiate legal proceedings with guarantees that it will succeed.

Based on the data on claims investigated with an accused person, organized by cause and compiled in the annual reports of the Attorney General's Office, we have prepared three statistics. The breakdown by cause is included from the 2009 report, with percentage data, so we first convert them to absolute numbers using a rule of three that uses the number of fires with accused/detained person offered by the same memory. Therefore, we directly use the prosecution's percentage as the intentionality rate that allows us to analyze the performance of the entire data set from the 2007 report. The evolution of causation in the statistics of the Attorney General's Office since the systematization of the data began is as follows:

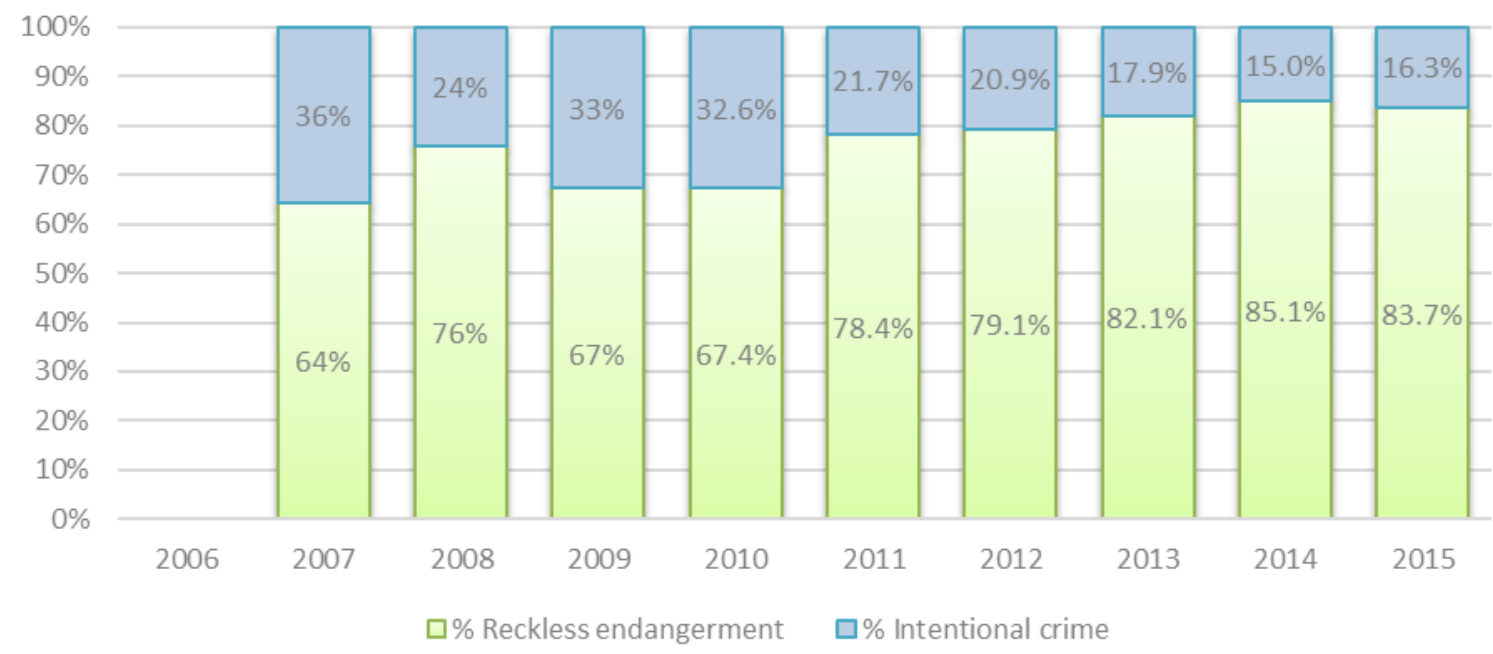

Figure 4 - Percentage of court cases with accused persons by general causality group. Spain 2007-2015. Source Proceedings of the Attorney General's Office.

Cultural burnings are also the main cause of fires investigated by the courts, along with accidents caused by machinery, power lines or vehicles. All this leads us to review the reasoning behind the inclusion of burnings without a permit in the category of arson by the General Directorate of Biodiversity. Negligence with non-compliance with administrative legislation is reckless endangerment for both pastoralists and peasants and for those responsible for railways, power lines, machinery owners or smokers who do not comply with the respective sectoral regulations. That is to say, practically any negligence should be considered arson, since the same legal elements apply as for burning without a permit. Let us remember, for example, that, in general, smoking is prohibited on forest land, all rural land not used for agriculture, inside High Risk Areas, so any accident caused by a cigarette butt should be considered legally the same as a burning of pastures without a permit. The proof of this is how the Attorney General's Office "welcomes" the accusations of several people in cases involving power lines or the accusation of mayors for fires originating in landfills.

In order to distinguish malicious cultural uses from cultural burnings included in the group of negligence, the Attorney General's Office calls this set of motivations "inappropriate traditional practices" and considers them malicious on the basis of the assessment of dolus eventualis, since, although there is no wilful intent, the intention to burn the forest, the perpetrator, due to his knowledge and experience, can reasonably understand that the most likely consequence of his act is that the fire will break out (that the fire will advance without control). The procedure thus moves from being prosecuted as a punishable offence to being prosecuted as a criminal offence, a matter which satisfies 
the interest of the Attorney General's Office in obtaining exemplary sentences. The concept of dolus eventualis is repeated in the reports of the Attorney General's Office and is not very comprehensible to a lay person in criminal law, however, we consider that its interpretation is important for understanding the criminal response as an essential mechanism for managing the risk of forest fires.

\section{Discussion and conclusions}

A revision of the criteria for classifying forest fires in the official Spanish EGIF database is urgent, adapting it to the original EFFIS criteria, as it would contribute to a better general (socio-economic, cultural and territorial) and political understanding of the problem.

This review should be applied to the entire EGIF historical series, one of the best statistics in the world for the study of forest fires, a review that according to the experience acquired in our research does not raise any serious methodological problem.

However, the general and deeper problem in Spain is the need for the paradigm of fire ecology to extend beyond the academic and technical realm to the entire population and the legal framework. In the past decades and in some countries, however, a slight and gradual change in perspective has occurred, such that for fire managers, preserves managers, researchers, and non-government organizations, the fire ecology as well as the management of fire by rural communities have been incorporated into what is now referred to as "integral fire management". This term may be defined as the fusion of firefighting and prevention with the ecological use of fire and community fire management in order to preserve nature and to make the land productive (Rodríguez-Trejo et al. 2011). Now one of the challenges is to better understand the traditional use of fire by the rural communities and to identify those that apply good use of fire and invite them to extend this practice to other communities. It is also necessary to convince more universities to participate in the ecological and social research of fire in the different regions of the countries and to educate the public to understand and support integral fire management. The final challenge is to connect in a sustainable way, classic fire management that has been centered on prevention and firefighting, with the ecological use of fire and, above all, with fire management of the rural communities (Rodríguez-Trejo et al. 2011: 49).

\section{References}

APAS (Asociación para la Promoción de Actividades Socioculturales), IDEM (Instituto de Estudios del Medio) (2004) Estado del conocimiento sobre las causas de los incendios forestales en España, Asociación para la Promoción de Actividades Socioculturales. http://www.idem21.com/descargas/pdfs/CAUSAS_IF.pdf.

Barroso Fernández J, González Bascones V, (2007) Motivaciones de incendios forestales intencionados en España. In 'Wildfire 2007, 4' Conferencia Internacional sobre Incendios Forestales', Sevilla. http://www.eufirelab.org/toolbox2/library/upload/2298.pdf.

Carracedo Martín V, Diego Liaño C, García Codrón JC, Rasilla Álvarez DF (2009) 'Los incendios forestales', Barcelona, Davinci Continental, S.L., Collection “Nueva Geoambiente XXI”, 250 p.

Castro R, Simarro ME, Priego C, Lafuente R, Sancho A. (2007) Investigación social sobre los incendios forestales en Andalucía. 'Wildfire 2007, $4^{\mathrm{a}}$ Conferencia Internacional sobre Incendios Forestales', http://decastro.ricardo.googlepages.com/Investigacinsocialeincendiosforestal.pdf.

CLIF (1997a) Apuntes para la codificación en oficina del Parte de Incendio Forestal, Madrid, Comité de Lucha Contra Incendios Forestales, DGCONA, Ministerio de Medio Ambiente.

CLIF (1997b) Instrucciones para rellenar el Parte de Incendio Forestal, Madrid, Comité de Lucha Contra Incendios Forestales, DGCONA, Ministerio de Medio Ambiente. 
Joint Research Centre (JRC) (2013): Harmonized classification scheme of fire causes in the EU adopted for the European Fire Database of EFFIS, Executive Report 25923, Luxembourg: Publications Office of the European Union, 51 p. http://publications.jrc.ec.europa.eu/repository/bitstream/JRC80682/lb-na-25-923-en-n.pdf.

Fiscalía General del Estado (2008) Memoria 2008. Memoria de la actividad desplegada por el Ministerio Fiscal a lo largo del año 2007. http://www.mapama.gob.es/es/ceneam/grupos-detrabajo-yseminarios/fiscalias-de-medio-ambiente/memo_fiscalia2008_tcm7-13294.pdf.

Fiscalía General del Estado (2012) Memoria 2012. Memoria de la actividad desplegada por el $\begin{array}{llllll}\text { Ministerio Fiscal a lo largo } & \text { del }\end{array}$ https://www.fiscal.es/fiscal/publico/ciudadano/documentos/memorias_fiscalia_general_estado/!ut /p/a0/04_Sj9CPykssy0xPLMnMz0vMAfGjzOI9HT0cDT2DDbwsgozNDBwtjNycnDx8jAwszPQ Lsh0VAQluD0w!/?selAnio=2012.

Fiscalía General del Estado (2015) Memoria 2015. Memoria de la actividad desplegada por el $\begin{array}{lllllll}\text { Ministerio Fiscal a } & \text { lo } & \text { largo } & \text { del } & \text { año } & 2014 .\end{array}$ https://www.fiscal.es/memorias/memoria2015/FISCALIA_SITE/recursos/pdf/MEMFIS15.pdf.

Martín Isabel MP, Chuvieco Salinero E, Aguado I (1998) La incidencia de los incendios forestales en España. Serie Geográfica, vol. 7, 23-36.

Martínez Navarro JM (2017) La gestión territorial del riesgo antrópico de ignición forestal en CastillaLa Mancha, PhD dissertation, Department of Geography and Spatial Planning, University of Castilla-La Mancha.

Martínez Ruiz E (2001) 'Manual de quemas controladas. El manejo del fuego en la prevención de incendios forestales'. Madrid, TRAGSA \& Ediciones Mundi Prensa, 178 p.

Molinero Hernando F, Cascos C, García A, Baraja E (2008a) Dinámica de los incendios forestales en Castilla y León., Boletín de la Asociación de Geógrafos Españoles, 48, 39-70.

Molinero Hernando F, García A, Cascos C, Baraja E, Guerra JC (2008b) La percepción local de los incendios forestales y sus motivaciones en Castilla y León. Ería, 76, 213-229.

Pérez Vilariño J (2004) Incendios forestales y reconstrucción social del monte. In 'Reflexiones sociológicas: homenaje a José Castillo Castillo’ (Ed. Díez Nicolás J et al.), pp. 651-678. (Centro de Investigaciones Sociológicas: Madrid).

Pérez Vilariño J, Delgado Fernández JL (1995) Análisis del riesgo de incendio forestal en Galicia. Agricultura y Sociedad, 77, 109-124.

Priego C, Lafuente R (2007) 'La Investigación Social como una nueva herramienta contra la lucha de los incendios forestales en Andalucía'. http://www.iesaa.csic.es/archivos/Comunicaciones/PRIEGO-Y-LAFUENTE.pdf.

Rodríguez-Trejo DA, Martínez-Hernández PA, Ortiz-Contla, H, Chavarría-Sánchez MR, HernándezSantiago F (2011) The present status of fire ecology, traditional use of fire, and fire management in Mexico and Central America, Fire Ecology, 7(1), 40-56. doi:10.4996/fireecology.0701040.

Vélez Muñoz R (1986) Incendios forestales y su relación con el medio rural. Revista de Estudios AgroSociales, 136, 195-224.

Vélez Muñoz R (1999) El período 1848-1997 en la defensa contra incendios forestales en España. In 'Incendios históricos: una aproximación multidisciplinar' (Ed. E Araque Jiménez) pp. 13-38. (Universidad Internacional de Andalucía: Jaén). 


\section{Chapter 7}

Short Contributions 
Short contribution - Fire Risk Management

A universal rate of spread index for Australian fuel types

\author{
Jason J. Sharples ${ }^{1 *}$; Mona F. Z. Bahri²; Steve Huntley ${ }^{3,4}$ \\ ${ }^{1}$ School of Physical, Environmental and Mathematical Sciences, UNSW Australia, \\ \{j.sharples@adfa.edu.au*\} \\ ${ }^{2}$ School of Physical, Environmental and Mathematical Sciences, UNSW Australia, \\ ${ }^{3}$ NSW National Parks and Wildlife Service, Office of Environment \& Heritage \\ ${ }^{4}$ Charles Sturt University
}

\begin{abstract}
Empirical fire spread models for Australian fuel types date back to the 1950s-60s, with the work of Alan McArthur, and extend through to the current day with the most recent developments in shrubland fire spread models and refinements to the curing function in grassland fire spread models. These models are designed to provide a relatively simple and timely answer to the question: "Given a specific set of environmental conditions, what is the expected forward rate of spread of a fire?" In this paper we present a critical analysis of the the current suite of fire spread models employed operationally in Australia. In particular, we explore the functional form and parametric dependence of these rate of spread models. We then introduce an alternative functional form for predicting rate of spread, which is remarkable parsimonious compared to current operational models. We further show that this single functional model can be used as a universal index for fire spread, by which we mean that the same model can be applied to different fuel types and produce numerical predictions that are practically identical to those produced by the existing suite of operational models used in Australia.
\end{abstract}

Keywords: Rate of spread, eucalypt, grassland, shrubland, model parsimony, wind, fuel moisture content

\title{
1. Introduction
}

One of the main goals of wildfire research is to provide a relatively simple and timely answer to the question: "Given a specific set of environmental conditions, what is the expected forward rate of spread of a fire?" Indeed, pursuit of such an answer has engaged some of the brightest minds in wildland fire science, and has produced a variety of fire spread models that apply across a number of common vegetation or fuel types. In Australia, these models date back to the 1950s-60s, with the work of Alan McArthur, and extend through to the current day with the most recent developments in shrubland fire spread models and refinements to the curing function in the CSIRO grassland fire spread model. In this paper we show how consider the way that meteorological factors are incorporated into the suite of existing fire spread models, which encompass a variety of different fuel types, and discuss an approach that unifies their inclusion. The utility of this unified modelling approach is demonstrated via model comparison using real meteorological data over a range of vegetation types. In particular, we demonstrate that the meteorological (i.e. non-fuel) sub-models of the current suite of operational models, which are of many and varied functional form, can be replaced by a single, unified, twoparameter model, with no appreciable loss in model performance. The unified model has the distinct advantage of being conceptually straightforward and extremely parsimonious compared to current operational approaches. The existence of a simple, yet effective, unified approach to fire spread modelling has implications for initiatives such as the National Fire Danger Rating project, as it establishes a common modelling basis that can be applied to the many different fuel types that are encountered across the nation. 


\section{Rate of fire spread models for Australian fuel types}

We consider current operational models for the following fuel types: grasslands; buttongrass moorland; temperate shrubland; South Australian mallee-heath; and dry eucalypt forest. The rate of spread models for each of these fuel types are described in detail by Cruz et al. (2015).

In this study we specifically focus on how the rates of spread derived from the models mentioned above depend on the fire weather variables: temperature, relative humidity and wind speed. Fuelrelated factors such as availability and structural descriptors (e.g. fuel height) are assumed constant for each fuel type.

It is of interest to note the number of model parameters that are associated with each of the rate of spread models for the different fuel types considered. These parameters represent degrees of freedom in the model, and have to be determined through regression-type analyses of empirical data relating to the rate of spread and environmental predictor variables. Ignoring their fuel dependent components, the grassland model has 10 parameters (Cheney et al., 1998), the buttongrass moorland model has 6 parameters (Marsden-Smedley and Catchpole, 1995), the temperate shrubland model has 9 parameters (Anderson et al., 2015), the S.A. mallee-heath model has 7 parameters (Cruz et al., 2010), and the dry eucalypt forest model has 13 parameters (Cheney et al., 2012).

\section{Universal rate of spread index}

Previous work (e.g. Sharples and McRae, 2012) has considered the utility of the following simple dimensionless index in describing fuel moisture content. The fuel moisture index is defined as:

$$
F M I=10-0.25(T-H),
$$

where $T$ is air temperature $\left({ }^{\circ} \mathrm{C}\right)$ and $H$ is relative humidity $(\%)$.

The FMI has been combined with wind speed in simple functional forms, which have been shown to provide estimates of fire danger and rates of spread that are comparable to those derived from accepted models. In this work, we extend this idea, and examine how predictions from a simple, twoparameter model for fire spread, based on wind speed $U$ and FMI, compares to those from the various models for different fuel types. The particular model, which we refer to as the spread index, is:

$$
S(\mu, p)=\left(\frac{\max (1, U)}{F M I+\mu}\right)^{p}
$$

where $\mu$ and $p$ are the two parameters defining the model.

To facilitate the comparison between current operational models and the spread index, we use halfhourly fire weather data recorded at Canberra Airport between November 2006 - March 2007; that is, approximately over the course of a fire season.

\section{Results}

In this preliminary work the spread index parameters $\mu$ and $p$ were varied by hand until a good fit was obtained between predictions of the spread index and those arising from each of the rate of spread models for grassland, buttongrass, temperate shrubland, S.A. mallee-heath and dry eucalypt forest. An example of a comparison of the predictions of the spread index compared to the predictions of the temperate shrubland model (Anderson et al., 2015) and the dry eucalypt model (Cheney et al., 2012) can be seen in Figure 1. Note that in each case the spread index values have been scaled so that their mean equals the mean of the predictions from the fuel-specific model. 
In the worst case the spread index accounts for around $94 \%$ of the variability in the dry eucalypt forest rate of spread model, while in the best case it accounts for over $99 \%$ of the variability in buttongrass moorland model.
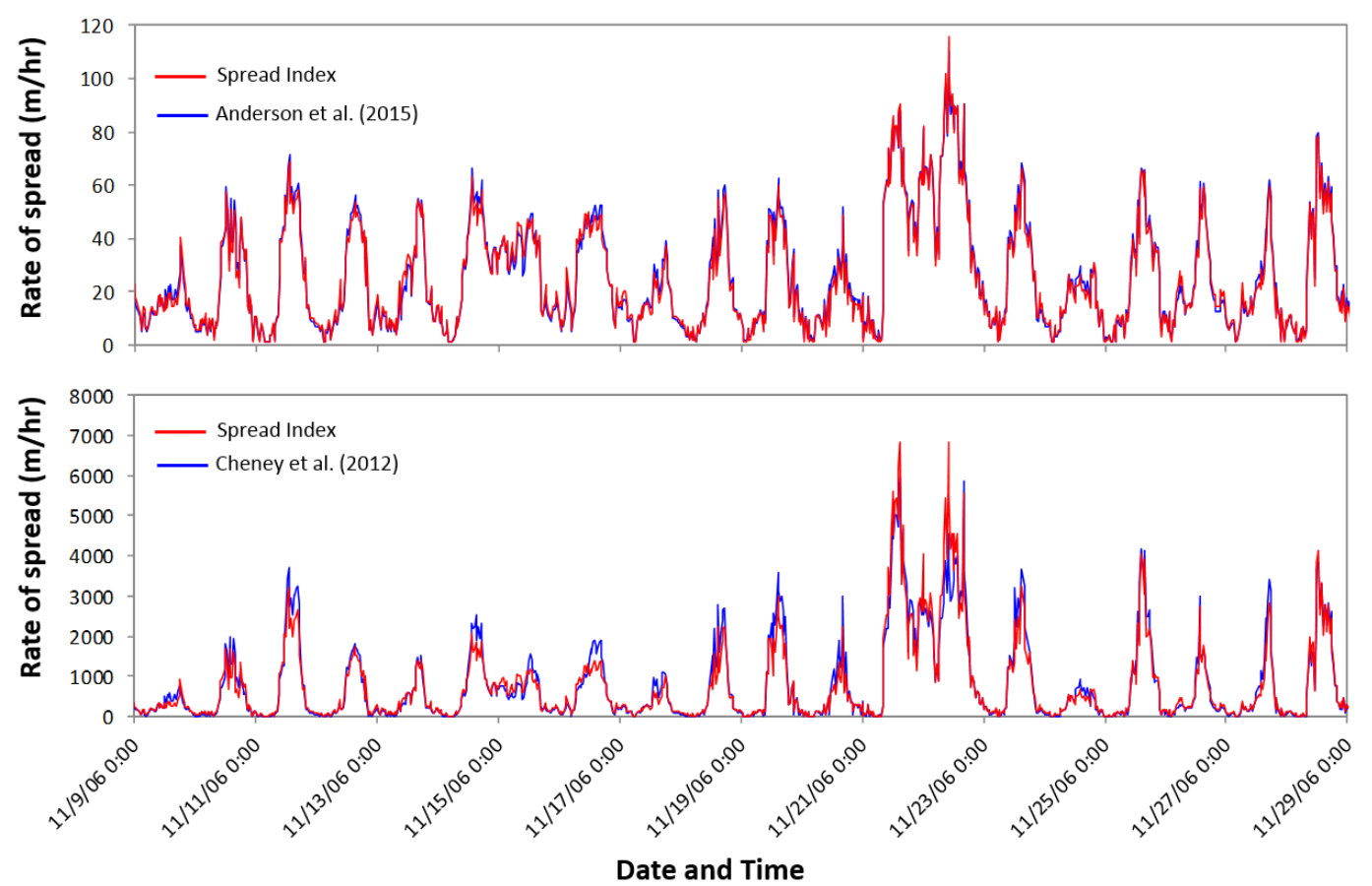

Figure 1 - Rate of spread predictions from the temperate shrubland model of Anderson et al. (2015) and the dry eucalypt forest fire model of Cheney et al. (2012) compared to those from the spread index. The spread index values have been scaled in each case so that their mean value matches the mean value of the fuel-specific model predictions.

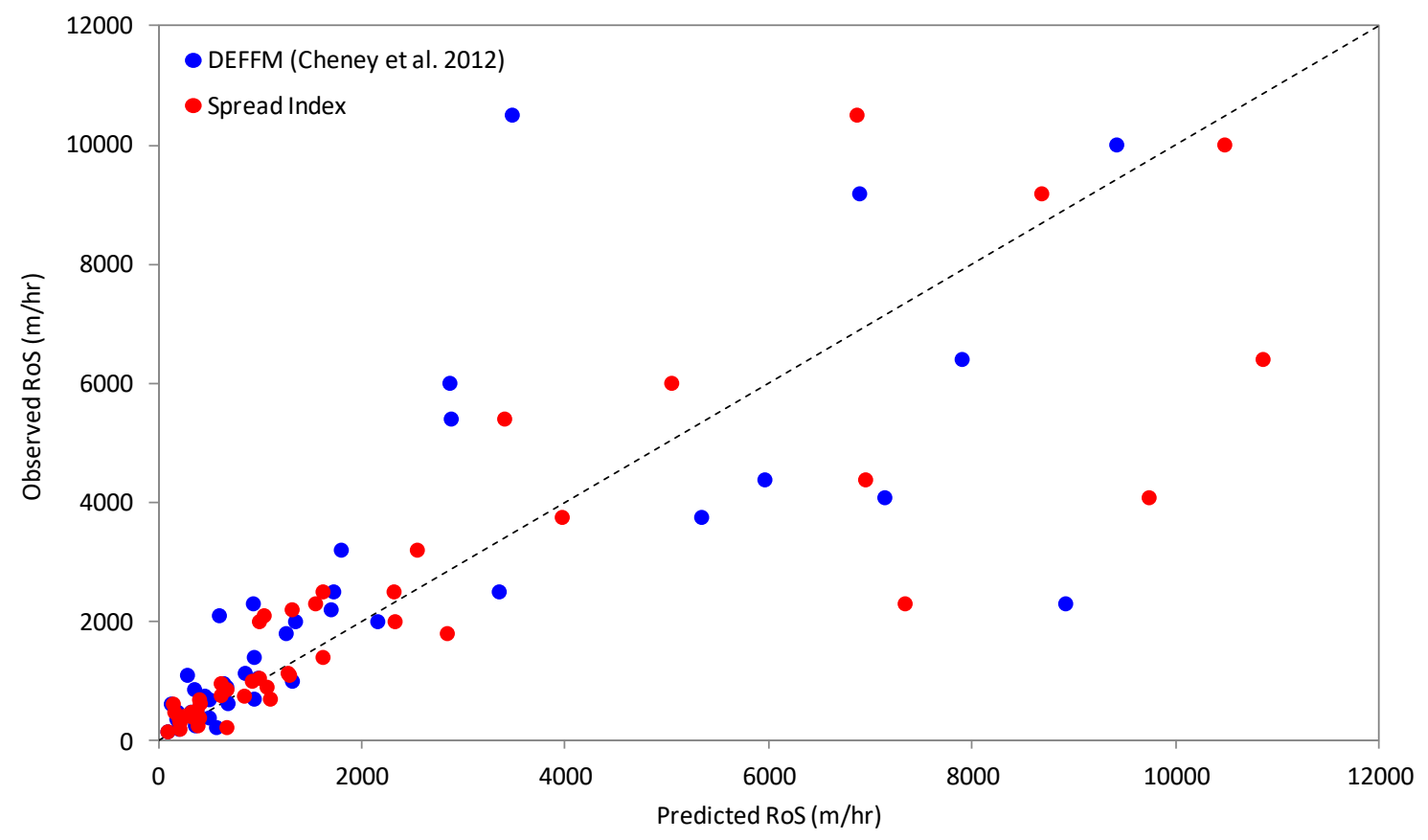

Figure 2 - Observed wildfire rates of spread plotted against predictions from the dry eucalypt forest fire model (DEFFM) of Cheney et al. (2012) and the spread index. The same scaling as utilised in Figure 1 (bottom panel) has again been applied to the spread index. 
The spread index also produces results that are comparable to existing operational models when used to predict wildfire observations. Figure 2 shows observed wildfire rates of spread plotted against predictions made using the dry eucalypt forest fire model of Cheney et al. (2012) (blue dots) and those made using the spread index (red dots). Note that the same scaling has been applied to the raw spread index values as was applied to obtain the values shown in the bottom panel of Figure 1. Overall statistics indicated that the model of Cheney et al. (2012) gave rate of spread predictions with a mean absolute error of $51 \%$ and a mean bias error of $-24 \%$, while the simple spread index gave rate of spread predictions with a mean absolute error of $44 \%$ and a mean bias error of $-24 \%$. These results suggest that the spread index provides the better guidance on assessing wildfire rate of spread. In particular, these statistics indicate that spread index offers a three-fold improvement over the model of Cheney et al. (2012) in under-predicting actual rates of spread. This result is even more remarkable when we note that no information on fuel was included in the spread index calculations, while the predictions of the model of Cheney et al. (2012) utilized detailed information on fuel via surface and near-surface fuel hazard scores and near-surface fuel height.

\section{Conclusions}

Predictions from the meteorological sub-models of five state-of-the-art fire spread models were compared with predictions derived from a single two-parameter fire spread index. The results indicated that the simple spread index was able to reproduce the predictions of the more complicated models to a remarkable degree of accuracy $\left(R^{2}=0.94-0.99\right)$. The results further suggest that the state-of-the-art models are considerably over-complicated: the predictions from models with 6-13 parameters can all be accurately emulated by a model with only two parameters (or three parameters, if a scaling/calibration factor is included). This indicates that the current suite of operational models have about 2-6 times more free parameters than necessary. Indeed, the spread index offers a far more parsimonious approach to modelling rate of spread, is far more conceptually simple, and provides a unified way of assessing rate of spread across a variety of fuel types.

\section{References:}

Anderson WR, Cruz MG, Fernandes PM, McCaw L, Vega JA, Bradstock RA, Fogarty L, Gould J, McCarthy G, Marsden-Smedley JB, Matthews S. A generic, empirical-based model for predicting rate of fire spread in shrublands. International Journal of Wildland Fire 2015; 24: 443-460.

Cheney NP, Gould JS, Catchpole WR. Prediction of fire spread in grasslands. International Journal of Wildland Fire 1998; 8: 1-3.

Cheney NP, Gould JS, McCaw WL, Anderson WR. Predicting fire behaviour in dry eucalypt forest in southern Australia. Forest Ecology and Management 2012; 280: 120-131.

Cruz MG, Matthews S, Gould J, Ellis P, Henderson M, Knight I, Watters J. Fire dynamics in malleeheath: fuel, weather and fire behaviour prediction in south Australian semi-arid shrublands. Bushfire Cooperative Research Centre, Report A; 2010.

Cruz MG, Gould JS, Alexander ME, Sullivan AL, McCaw WL, Matthews S. A Guide to Rate of Fire Spread Models for Australian Vegetation. CSIRO Land and Water Flagship and AFAC: Canberra, ACT and Melbourne, VIC; 2015.

Marsden-Smedley JB, Catchpole WR. Fire behaviour modelling in Tasmanian buttongrass moorlands. II. Fire behaviour. International Journal of Wildland Fire 1995; 5: 215-228.

1Sharples JJ, McRae RH. Evaluation of a very simple model for predicting the moisture content of eucalypt litter. International Journal of Wildland Fire 2012; 20:1000-1005. 
Short contribution - Fire Risk Management

Assessment of wildfire exposure and vulnerability factors in Alvares, Góis: integration of structural and dynamic factors at the local scale

\author{
Sandra Oliveira ${ }^{1 *}$; Ana Gonçalves ${ }^{1}$, Akli Benali ${ }^{2}$, Ana Sá ${ }^{2}$, José Luís Zêzere ${ }^{1}$, José Miguel Pereira ${ }^{2}$ \\ ${ }^{1}$ Institute of Geography and Spatial Planning, Universidade de Lisboa, Lisbon, Portugal \\ \{sandra.oliveiral@campus.ul.pt*\} \\ ${ }^{2}$ Forest Research Centre, School of Agriculture, Universidade de Lisboa, Lisbon, Portugal
}

\begin{abstract}
According to climate projections, global warming is associated with increasing temperatures and dry spells in some parts of the world, especially the Mediterranean area. This climate change has already triggered increases in wildfire danger and fire season length in Southern Europe and is expected to amplify in the forthcoming decades. However, it is quite challenging for the scientific community to assess the intensity of these changes, because (i) the trend relies on the greenhouse gases (GHG) emission scenario and (ii) fire occurrence depends on multiple factors (including climate, but not only). A proper assessment of the trend in terms of fire occurrence and of uncertainties associated with this increasing trend, still lacks, especially for the French territory.

Our study refines traditional approaches of fire risk projection under climate change on two aspects: (i) the impact of climate prediction uncertainties on the prediction of fire danger, and (ii) the translation of a danger index into a fire occurrence (per size classes).
\end{abstract}

Keywords: wildfire exposure; vulnerability factors; dynamic approach; local scale; Portugal

\title{
1. Introduction
}

Wildfires are a major threat in Portugal. The year 2017 was particularly severe, with a large burned area extent and heavy impacts on human lives and assets. The civil parish of Alvares (Góis) has been strongly affected by wildfires over the last decades and had $60 \%$ of its surface area burned by a single large wildfire in June 2017. In addition to the land cover dominated by forest fuels and the rugged topography, this area is characterized by a dispersion of small human settlements, inhabited by few elderly people, intermingling with vegetation that occupy what was once farmland and is now abandoned (Moreira et al., 2011; Oliveira, Zêzere, Queirós, \& Pereira, 2017). These particular conditions increase the exposure of this territory to wildfire occurrence and damages.

In this context, and considering the influence of the local features in the implementation of mitigation and adaptation strategies to wildfires, it becomes of high relevance to integrate exposure and vulnerability assessment at the local scale in wildfire management and risk mitigation approaches (Alcasena et al. 2015; Carroll and Paveglio 2016; Paveglio, Prato, et al. 2016).

This research focused on the assessment of exposure and vulnerability of the human settlements (small villages) within a civil parish, with the purpose of identifying settlements with greater potential for loss, where more urgent mitigation strategies and interventions to improve population safety are needed.

The civil parish of Alvares is located in a central-inner region of the Portuguese mainland, Pinhal Interior, in the southern part of Góis municipality (Fig. 1). It has an area of ca. $100 \mathrm{~km} 2$, of which forested areas occupy over $90 \%$. 


\section{Materials and Methods}

\section{Study area}

According to the last population Census survey (INE, 2012), the civil parish has 812 inhabitants, spread over nearly 40 small settlements. The three largest villages are located in the south and east, whereas the northwest corner of the civil parish is mainly occupied by a natural protected area. Almost half of the population is above 65 years old (47\%), $17 \%$ are illiterate and only $28 \%$ of residents are economically active.

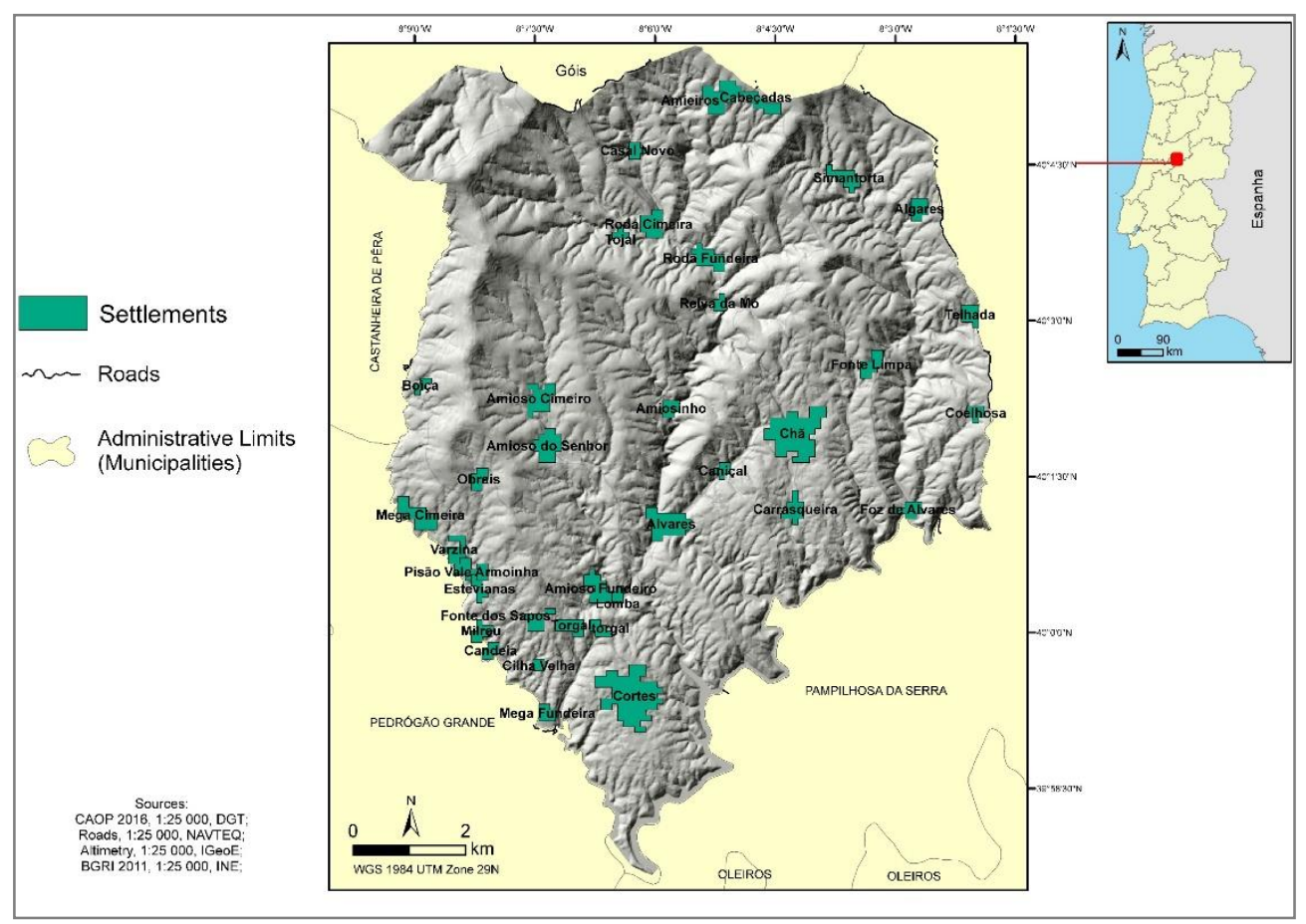

Figure 1 - Location of the human settlements in the civil parish of Alvares, Góis

\section{Exposure assessment}

Wildfire exposure was assessed at settlements scale within the civil parish. It generally represents the probability of wildfires causing damages to the population and existing assets within the settlements. The perimeters of the settlements were obtained from cartographic data available from local sources, specifically municipal institutions. To harmonize with the scale used in fire hazard modelling ( $1 \mathrm{ha}$ ), these perimeters were adjusted to include all the 1 ha pixels where human structures belonging to that settlement exist. We have then identified the exposed tangible assets located within the perimeter of each settlement, namely people, buildings and roads, retrieved from the most recent statistical and cartographic sources and at the smallest unit area possible (i.e. sub-section within the civil parish; INE 2012). Subsequently, their density was computed for the total area of the settlements and a combined index was obtained by summing the values for all the three parameters, weighted according to the municipal plans for protection against wildfires (AFN, 2012): population with higher weighting (1), followed by buildings $(0.75)$ and roads $(0.25)$. This index was then normalized into a common scale (0-100) to allow comparisons.

Afterwards, the index representing the elements exposed was combined with a burn probability measure for each unit area, applying three different approaches: i) static; ii) structural; and iii) dynamic. Static exposure considers a constant value of burn probability for the entire study area, therefore focusing on the existing assets. The structural exposure integrates historical burned area data to evaluate the number of times each settlement was affected by a fire between 1980 and 2017. The 
dynamic exposure was analyzed using burn probability simulations obtained with the FARSITE model (Finney, 1998), assuming different scenarios of land cover, extreme weather conditions and forest management options.

\section{Vulnerability assessment}

Vulnerability represents the degree of loss that can result from a wildfire, depending on the characteristics of the population and exposed assets (Aubrecht, Özceylan, Steinnocher, \& Freire, 2013; Gallina et al., 2016; UNISDR, 2009). Data on demographic and socioeconomic conditions were obtained from the 2011 Census survey (INE, 2012). Age groups, education levels, activity, building age and number of vacant houses, among other parameters, were retrieved and analyzed for each settlement.

\section{Results}

First results show that $33 \%$ of the settlements have high and very high structural exposure, corresponding generally to those settlements that were burned within their perimeter more than twice since 1980 (Fig. 2). When integrating the burn probability, considering land cover and weather conditions similar to those verified in 2017, the proportion of settlements in the higher exposure classes increased to $42 \%$.

Regarding vulnerability, about half of the settlements have more than $50 \%$ of elderly residents. Education levels are generally low, with $22 \%$ of settlements presenting more than $50 \%$ of people with only elementary education and $14 \%$ have more than $40 \%$ of illiterate people. The proportion of vacant housing is above $10 \%$ in $56 \%$ of the settlements (Fig. 3). In the settlements with high and very high structural exposure, the mean proportion of elderly people is $51 \%$ and the mean proportion of people with only elementary school level is $42 \%$.

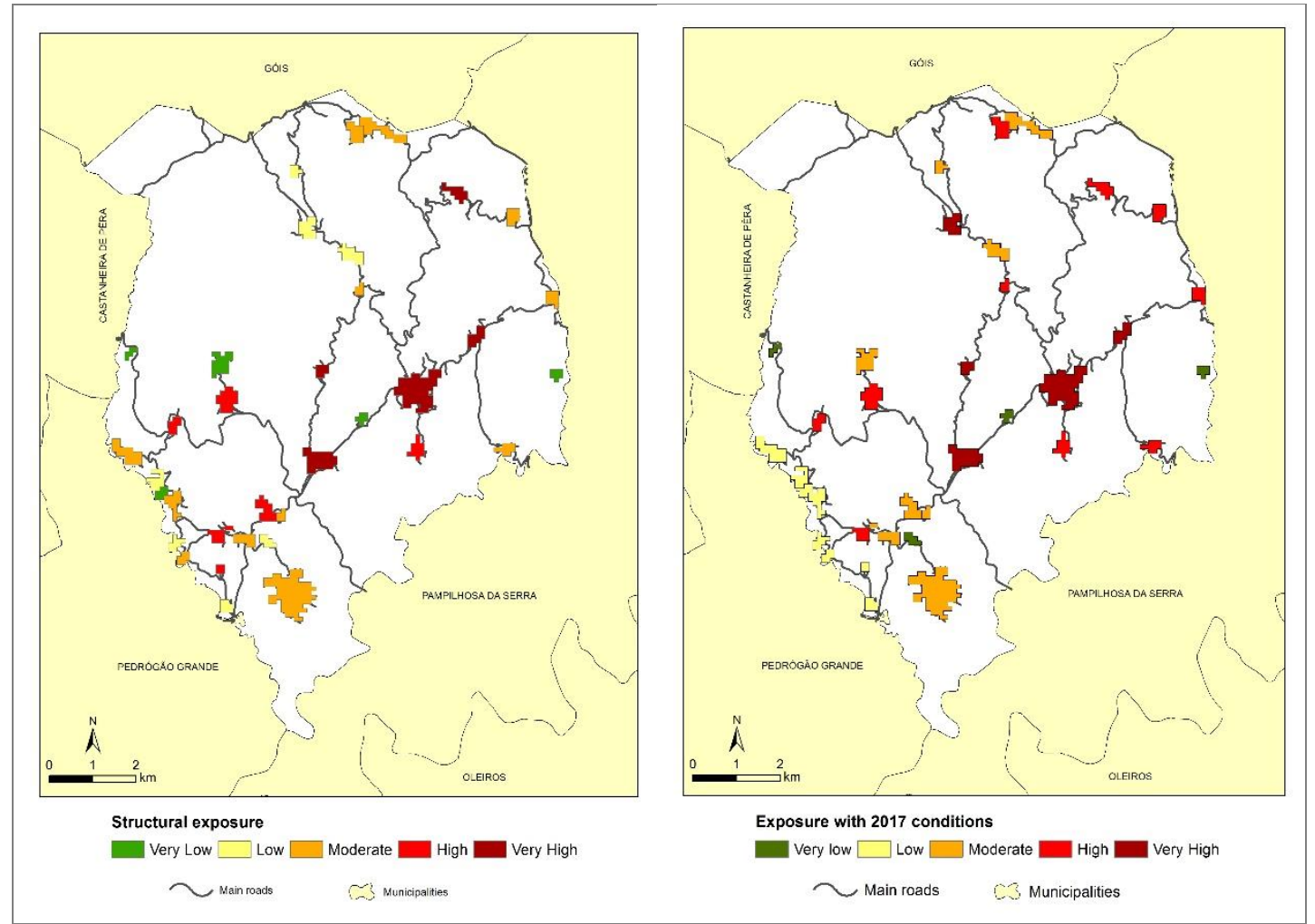

Figure 2 - Structural exposure (left) and exposure with 2017 conditions (right) for the human settlements in the civil parish of Alvares 
These conditions represent the overall social context of the local communities, which influence the coping capacity of the population and the type of protection and mitigation operational measures that could be efficiently implemented to reduce the levels of exposure and vulnerability to wildfires (Oliveira et al., 2017; Paveglio, Abrams, \& Ellison, 2016).
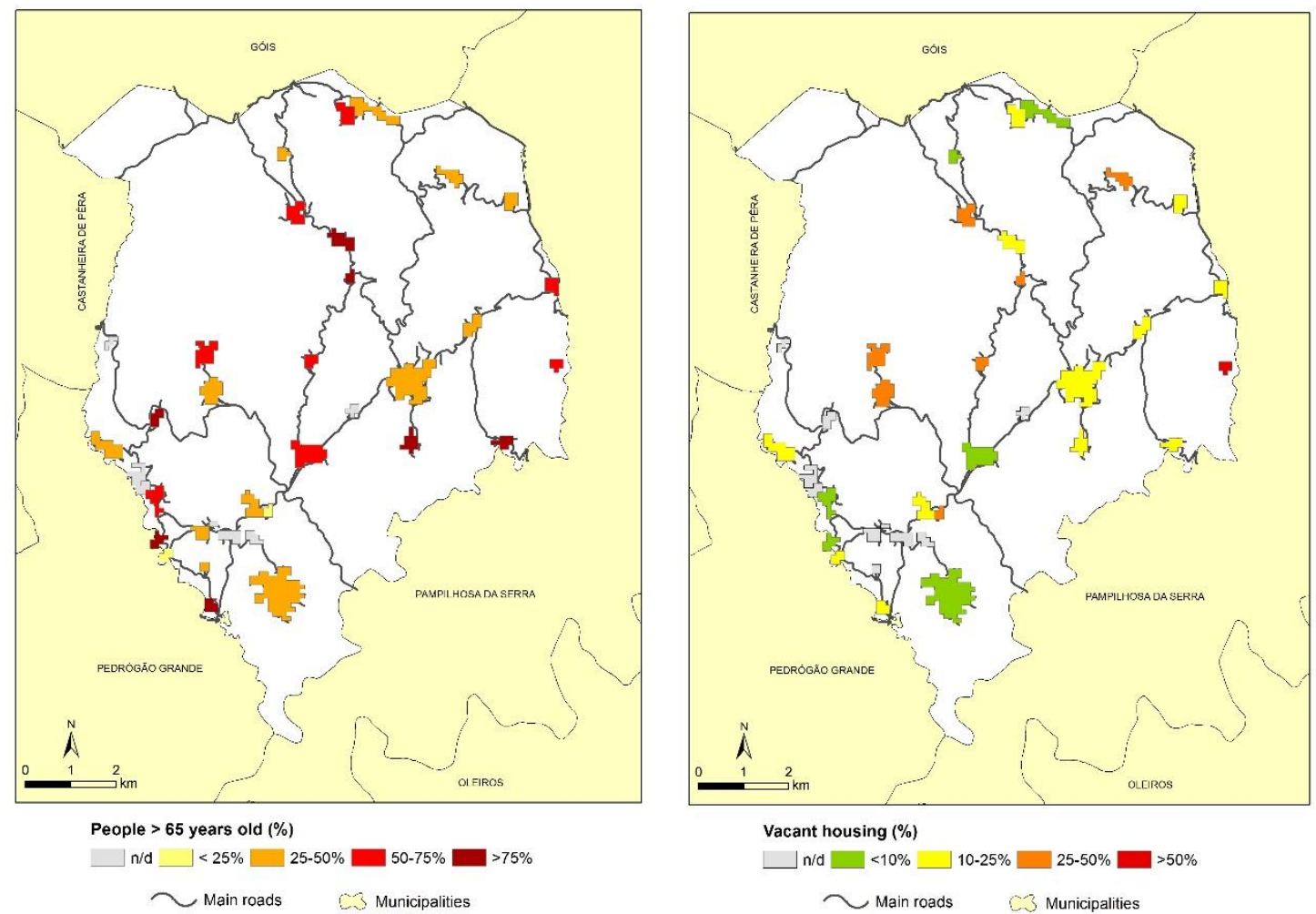

Figure 3 - Some of the vulnerability factors analyzed at settlement level: proportion of elderly people (left) and proportion of vacant housing (right)

\section{Further work}

The next step of this research will analyze the dynamic exposure, by evaluating potential changes in the exposure levels of settlements by integrating the effects of different weather and land cover conditions on burn probability simulations. Assessing these potential exposure variations will provide valuable indications regarding potential adjustments of mitigation measures to the characteristics and needs of the population, according to dynamic factors that influence wildfire exposure.

\section{References}

AFN, Autoridade Florestal Nacional (2012). Plano municipal de defesa da floresta contra incêndios (PMDFCI) - Guia técnico. Direção de Unidade de Defesa da Floresta.

Alcasena, F. J., Salis, M., Ager, A. A., Arca, B., Molina, D., \& Spano, D. (2015). Assessing Landscape Scale Wildfire Exposure for Highly Valued Resources in a Mediterranean Area. Environmental Management, 55(5), 1200-1216. http://doi.org/10.1007/s00267-015-0448-6

Aubrecht, C., Özceylan, D., Steinnocher, K., \& Freire, S. (2013). Multi-level geospatial modeling of human exposure patterns and vulnerability indicators. Natural Hazards, 68(1), 147-163. http://doi.org/10.1007/s11069-012-0389-9 
Carroll, M. S., \& Paveglio, T. B. (2016). Using community archetypes to better understand differential community adaptation to wildfire risk. Philosophical Transactions of the Royal Society of London B: Biological Sciences, 371(1696). http://doi.org/10.1098/rstb.2015.0344

Gallina, V., Torresan, S., Critto, A., Sperotto, A., Glade, T., \& Marcomini, A. (2016). A review of multi-risk methodologies for natural hazards: Consequences and challenges for a climate change impact assessment. Journal of Environmental Management, 168, 123-132. http://doi.org/10.1016/j.jenvman.2015.11.011

Moreira, F., Viedma, O., Arianoutsou, M., Curt, T., Koutsias, N., Rigolot, E., ... Bilgili, E. (2011). Landscape-wildfire interactions in southern Europe: Implications for landscape management. Journal of Environmental Management, 92(10), 2389-2402. http://doi.org/10.1016/j.jenvman.2011.06.028

Oliveira, S., Zêzere, J. L., Queirós, M., \& Pereira, J. M. (2017). Assessing the social context of wildfire-affected areas. The case of mainland Portugal. Applied Geography, 88, 104-117. http://doi.org/10.1016/j.apgeog.2017.09.004

Paveglio, T. B., Abrams, J., \& Ellison, A. (2016). Developing Fire Adapted Communities: The importance of Interactions Among Elements of Local Context. Society \& Natural Resources, 29(10), 1-16. http://doi.org/10.1080/08941920.2015.1132351

Paveglio, T. B., Prato, T., Edgeley, C., \& Nalle, D. (2016). Evaluating the Characteristics of Social Vulnerability to Wildfire: Demographics, Perceptions, and Parcel Characteristics. Environmental Management, 58(3), 534-548. http://doi.org/10.1007/s00267-016-0719-x

UNISDR. (2009). Terminology on Disaster Risk Reduction. United Nations International Strategy for Disaster Reduction (UNISDR). 
Short contribution - Fire Risk Management

\title{
Causes of forest fires in Federal Conservation Units of Brazil from 2006 to 2012
}

\author{
Aline das Graças Costa*; Fillipe Tamiozzo Pereira Torres; Gumercindo Souza Lima; Milton Ribas da Silva \\ Júnior; José Cola Zanuncio \\ Universidade Federal de Viçosa. Departamento de Engenharia Florestal, Viçosa, Minas Gerais, Brasil, \\ \{alinegrcosta@gmail.com*,fillipe.torres@ufv.br; gslima@ufv.br\}
}

\begin{abstract}
Forest fires represent the greatest threats to biodiversity in Conservation Units (UCs) in Brazil. Knowledge of the causes and regions with the highest occurrences allows the use of fire risk management. The objective of this study was to evaluate the causes of forest fires in the Federal Conservation Units of Brazil from 2006 to 2012 to help in the management of fire risks in Federal UCs of Brazil. The Registros de Ocorrências de Incêndios (ROIs) of the UCs were obtained from the database of the Sistema Nacional de Informações sobre o Fogo (SISFOGO) maintained by the Instituto Brasileiro do Meio Ambiente e dos Recursos Renováveis. The total number of records was 2259 , of which $88.2 \%$ were identified and $11.8 \%$ were unidentified. The unrecorded data evidenced failures in the identification of the fire. Unknown causes accounted for $42.2 \%$ of the records, followed by burn for cleaning (26.7\%) and incendiarys (18.5\%). The "rays" (natural causes) had low representativity $(2.8 \%)$. Minas Gerais, Rio de Janeiro, Ceará, Bahia and Piauí were the states with the highest occurrences with 19.8, 15.5, 12, 9.6 and 7.4\%, respectively, of the records for Brazil. UCs suffer burnings for pasture clearance in neighboring areas that reach these protected areas as well as on purposeful fires. The largest fire registry was in Minas Gerais, due to favorable conditions for fire and the prolonged season of drought in this state. Failures in the record of occurrence of fires and in the identification of their causes justify the great number of unknown causes. Fires recorded without identifying the causes and/or registered as unknown causes represent a failure to use the ROI tool by the UC and in the fire identification skill, making it difficult to make decisions against fire. The causes "unknown", "burn for cleaning" and "incendiaries" represent the group of major causes of fires.
\end{abstract}

Keywords: protected areas; fire; fire registry; fire prevention.

\section{Introduction}

Fire is widely used by man to transform and manage the landcapes and is one of the major agents of disturbing of ecosystems (Andreae e Merlet, 2001).

The Conservation Units (UCs) in Brazil are intended for the conservation of biodiversity and fire is a constant threat to threat to UCs (Koproski et al., 2011). The Brazilian UCs are areas with rare and endangered species, with fire-sensitive biomes or small areas and isolated by agricultural monocultures or cities (Medeiros and Fiedler, 2003). The causes of fires should be known for the prevention and reduction of fires (Soares and Santos, 2002).

The main causes of fires are anthropic, such as arsonists and improper use of fire for cleaning neighboring areas and renewal of pastures (Medeiros, 2002). The most common natural causes of fires are electric discharges (lightning) (Matos, 2004).

Brazil has 960 federal UCs of the Sistema Nacional de Unidades de Conservação (SNUC) (MMA, 2017) and knowing the location of the fires allows to determine the areas of greatest risk and to establish specific programs for each region (Soares e Santos, 2002).

The objective of this work was to evaluate the causes of forest fires in the Federal Conservation Units of Brazil from 2006 to 2012 to help in the management of fire risks in Federal Conservation Units of Brazil. 


\section{Methodology}

The data on forest fires in UCs were obtained from the Sistema Nacional de Informações sobre o Fogo (SISFOGO) of the Centro Nacional de Prevenção aos Incêndios Florestais (PREVFOGO), which is maintained by the Instituto Brasileiro do Meio Ambiente e dos Recursos Renováveis (IBAMA, 2015), where the fire occurrence records are located (Registros de Ocorrências de Incêndios - ROIs).

The ROIs provide information about the fire occurrence; terrain; meteorological data; georeferencing; combat data; spending; damages and origin and cause. In the origin and cause is the 26 possible causes and the 33 possible causal agents, being recorded the cause and causal agent corresponding to the fire.

The data were scanned in a Microsoft Excel 2010 worksheet, forming a file with the fires records of the UCs with their respective causes and state of Brazil from 2006 and 2012.

The fire causes were categorized according to the Food and Agriculture Organization (FAO) groups or categories (Santos et al., 2006). FAO does not use the category "unknown" because it considers that it induces the inclusion of other categories of fires in it (Soares, 1988). However, this category was included in this study due to its large record as being a fire cause in the ROIs reports.The causes were grouped into seven categories or groups: unknown (I), several (II), recreational fires (III), incendiary (IV), forest operations (V), burn for cleaning (VI), and rays (VII). The group of "diverse" causes includes the categories of hunting (i), high voltage cable (ii), windborne spark (iii), fireworks (iv), others (v), burn of balloon, (vi) and reignition (vii).

The means comparison of fire causes groups by the period studied considered the groups of causes as treatments and the ocurrence years as repetitions. Causes groupswith low numbers, including zero, were transformed into log values in all fire registers. The means of the treatments were compared by the SNK (Student-Newman-Keuls) test at the 5\% probability in the Excel 2010 program.

The tabulated data allowed the identification of the major fire causes in the Brazilian UCs from 2006 to 2012.

\section{Results and Discussion}

From 2006 to 2012, a total of 2259 fire events were recorded registring $1992(88,2 \%)$ ocurrences with possible cause or causal agent identified. The unrecorded data $(11,8 \%)$ evidenced failures in the identification of the fire cause and/or in the completion of the ROIs (IBAMA, 2006).

The "unknown" cause was the main one of the records in Brazil and, for this reason, was maintained in the same groups. The causes "burn for cleaning" and "incendiary" were the most important, according to Table 1. The largest were from "incendiary", "burn for cleaning" and "unknown" groups.

Table 1 - Causes of forest fires in Federal Conservation Units of Brazil from 2006 to 2012

\begin{tabular}{|c|c|c|c|c|c|c|c|c|c|c|c|c|c|c|c|c|}
\hline & \multicolumn{2}{|c|}{2006} & \multicolumn{2}{|r|}{2007} & \multicolumn{2}{|r|}{2008} & \multicolumn{2}{|r|}{2009} & \multicolumn{2}{|c|}{2010} & \multicolumn{2}{|c|}{2011} & \multicolumn{2}{|c|}{2012} & \multicolumn{2}{|c|}{ Total } \\
\hline & $\mathrm{n}^{\circ}$ & $\%$ & $\mathrm{n}^{\circ}$ & ? & $\mathrm{n}^{\circ}$ & $\%$ & $\mathrm{n}^{\circ}$ & $\%$ & $n^{\circ}$ & $\%$ & $\mathrm{n}^{\circ}$ & $\%$ & $\mathrm{n}^{\circ}$ & $\%$ & $n^{\circ}$ & $\%$ \\
\hline Unk & 439.0 & 75.0 & 243.0 & 34.9 & 47.0 & 20.0 & 34.0 & 24.1 & 46.0 & 22.5 & 17.0 & 17.9 & 15.0 & 41.7 & 841.0 & 42.2 \\
\hline $\mathrm{BC}$ & 103.0 & 17.6 & 220.0 & 31.6 & 77.0 & 32.8 & 43.0 & 30.5 & 58.0 & 28.4 & 22.0 & 23.2 & 8.0 & 22.2 & 531.0 & 26.7 \\
\hline Incen & 6.0 & 1.0 & 113.0 & 16.2 & 83.0 & 35.3 & 39.0 & 27.7 & 78.0 & 38.2 & 42.0 & 44.2 & 8.0 & 22.2 & 369.0 & 18.5 \\
\hline Div & 10.0 & 1.7 & 74.0 & 10.6 & 14.0 & 6.0 & 10.0 & 7.1 & 15.0 & 7.4 & 11.0 & 11.6 & 1.0 & 2.8 & 135.0 & 6.8 \\
\hline Rays & 19.0 & 3.2 & 18.0 & 2.6 & 6.0 & 2.6 & 10.0 & 7.1 & 1.0 & 0.5 & 0.0 & 0.0 & 1.0 & 2.8 & 55.0 & 2.8 \\
\hline $\mathrm{FO}$ & 3.0 & 0.5 & 15.0 & 2.2 & 3.0 & 1.3 & 4.0 & 2.8 & 3.0 & 1.5 & 1.0 & 1.1 & 3.0 & 8.3 & 32.0 & 1.6 \\
\hline RFi & 5.0 & 0.9 & 13.0 & 1.9 & 5.0 & 2.1 & 1.0 & 0.7 & 3.0 & 1.5 & 2.0 & 2.1 & 0.0 & 0.0 & 29.0 & 1.5 \\
\hline Total & 585 & 100 & 696 & 100 & 235 & 100 & 141 & 100 & 204 & 100 & 95 & 100 & 36 & 100 & 1992 & 100 \\
\hline
\end{tabular}

Causes: unknown (Unk); burn for cleaning (BC); incendiary (Incen); diverse (Div); forestry operations (FO); recreation fires $(\mathrm{RFi})$ 
The "unknown" cause and data not registered in the ROI are fails in the fire expertise or in the completion of the ROIs, and disagreements of this information make dificult the complete and coherent analysis of the report (IBAMA, 2006; Bontempo, 2011). The lack of regular records of forms in several UCs and/or the sending of incomplete data to PREVFOGO may be due to inadequate structure and staff, or lacking basic information such as burned area, geographic coordinates, fire cause and/or vegetation type (IBAMA, 2006).

The "rays" (natural causes) had low representativity (2.8\%). The "rays" are a uncommon cause of fires in Brazil due to rainfall occurrence increasing the humidity and reducing the amount of fuels available and, consequently, the fire development (Matos, 2004).

The group "smokers" and "railway" were allocated to the "diverse" group because the first group had no records and the second group had a low occurrence $(6.8 \%)$. The group of "diverse" causes is the group of causes with low frequency, of regional character and include causes that do not fit in the other groups (Soares, 1988).

From the 27 Brazilian states, 25 had registered fire cases, including the Federal District, and no occurrences were reported in the states of Acre and Rio Grande do Norte from 2006 to 2012. Minas Gerais (19.8\%) was the state with the highest occurrence of fires, next were Rio de Janeiro (14.5\%), Ceará (12.0\%), Bahia (9.6\%) and Piauí (7.4\%). The others ones had records below 5.9\%. The greatest fire risk in Minas Gerais was due to the large commercial forest areas in its territory, and the prolonged dry season (Soares and Santos, 2002).

The "unknown", "burn for cleaning" and "incendiaries" groups were the most important causes of fire (Table 2).

Table 2 - Number of forest fires by groups of causes by SNK test at 5\% probability level

\begin{tabular}{ccccccccccc}
\hline Cause & 2006 & 2007 & 2008 & 2009 & 2010 & 2011 & 2012 & Total & Mean & \\
\hline Unk & 6.08 & 5.49 & 3.85 & 3.53 & 3.83 & 2.83 & 2.71 & 28.32 & 4.05 & $\mathrm{a}$ \\
BC & 4.64 & 5.39 & 4.34 & 3.76 & 4.06 & 3.09 & 2.08 & 27.36 & 3.91 & $\mathrm{a}$ \\
Incen & 1.79 & 4.73 & 4.42 & 3.66 & 4.36 & 3.74 & 2.08 & 24.78 & 3.54 & $\mathrm{a}$ \\
Div & 2.30 & 4.30 & 2.64 & 2.30 & 2.71 & 2.40 & 0.00 & 16.65 & 2.38 & $\mathrm{~b}$ \\
Rays & 2.94 & 2.89 & 1.79 & 2.30 & 0.00 & 0.00 & 0.00 & 9.93 & 1.42 & $\mathrm{~b}$ \\
FO & 1.10 & 2.71 & 1.10 & 1.39 & 1.10 & 0.00 & 1.10 & 8.49 & 1.21 & $\mathrm{~b}$ \\
RFi & 1.61 & 2.57 & 1.61 & 0.00 & 1.10 & 0.69 & 0.00 & 7.58 & 1.08 & $\mathrm{~b}$ \\
\hline
\end{tabular}

Causes: unknown (Unk); burn for cleaning (BC); incendiary (Incen); diverse (Div); forestry operations (FO); recreation fires (RFi)

\section{Conclusion}

The "unknown", "burn for cleaning" and "incendiary" causes were the main occurrences of forest fires at federal UCs in Brazil from 2006 to 2012. Minas Gerais was the brazilian State with the highest number of fires. Failures in the identification and recording of fires make it difficult to assess its causes.

\section{Acknowledgements}

To the Coordenação de Aperfeiçoamento de Pessoal de Nível Superior (CAPES) for the availability of the study package and to the Departamento de Engenharia Florestal of the Universidade Federal de Viçosa.

\section{Conflicts of Interest}

The authors declare no conflicts of interest 


\section{References}

Andreae MO, Merlet P (2001) Emission of trace gases and aerosols from biomass burning. Global Biogeochemical Cycles 15, 955-966.

Bontempo GC, Lima GS, Ribeiro GA, Doula SM, Jacovine LAG (2011) Registro de Ocorrência de Incêndio (ROI): evolução, desafios e recomendações. Biodiversidade Brasileira 2, 247-263.

Instituto Brasileiro do Meio Ambiente e dos Recursos Naturais Renováveis (2006): Relatório de ocorrências de incêndios em unidades de conservação federais. Access in: http://ibama.gov.br/phocadownload/ prevfogo/relatorios/incendios_florestais/prevfogo-incendiosflorestais-relatorio_de_incendios_em_ucs_05_08.pdf

Instituto Brasileiro do Meio Ambiente e dos Recursos Naturais Renováveis (2015) Sistema Nacional de Informações Sobre Fogo. Access in: http://siscom.ibama.gov.br/sisfogo/ (acessado em 08 de Março de 2016).

Koproski L, Ferreira MP, Goldammer JG.; Batista, AC (2011) Modelo de zoneamento de risco de incêndios para unidades de conservação brasileiras: o caso do Parque Estadual do Cerrado (PR). Floresta 41, 551-562.

Matos EF (2004) Incêndios Florestais. In: Prevenção e combate aos incêndios florestais em Unidades de Conservação. (Secretaria de Meio Ambiente e Recursos Hídricos do Distrito Federal) pp.19-30.

Medeiros MB (2002) Manejo de Fogo em Unidades de Conservação do Cerrado. In: Boletim do Herbário Ezechias Paulo Heringer 10: 75-88.

Medeiros MB, Fiedler NC (2003) Incêndios florestais no Parque Nacional da Serra da Canastra: desafios para a conservação da biodiversidade. Ciência Florestal 14, 157-168.

Ministério Do Meio Ambiente (2017) Tabela consolidada das Unidades de Conservação. Access in: http://www.mma.gov.br/images/ arquivo/80112/CNUC_Agosto\%20-\%20Categorias_copy.pdf

Santos JF, Soares, RV, Batista AC (2006) Perfil dos incêndios florestais no Brasil em áreas protegidas no período de 1998 a 2002. Floresta 36, 93-100.

Soares RV, Santos JF (2002) Perfil dos incêndios florestais no Brasil de 1994 a 1997. Floresta 32, 219232.

Soares RV (1988) Perfil dos incêndios florestais no Brasil de 1984 a 1987. Floresta. 18, 94-121. 
Short contribution - Fuel Management

Climate-induced variations in global severe fire weather conditions

\author{
W. Matt Jolly*; Patrick Freeborn \\ US Forest Service, Rocky Mountain Research Station, Fire Sciences Laboratory, 5775 Hwy 10 W, \\ Missoula, MT, USA 59808, \{mjolly@fs.fed.us*, patrickhfreeborn@fs.fed.us\}
}

\begin{abstract}
Surface weather conditions are a major drivers of wildland fire size and intensity. Climatic changes over the last four decades are thought to be influencing these burning conditions both regionally and globally but few studies have comprehensively explored how climatic changes may be affecting fire weather severity during the fire season. Here we explore how these climatic changes impact two metrics of wildland fire weather: the weather-mediated length of the fire season and the within-season fire weather severity. We show that these metrics are strong predictors of burned area across the Western US, Western Europe and other fireadapted global ecosystems. We leverage these two metrics to describe changes in fire season length and severity across the globe in an effort to map areas that are experiencing significant changes in both fire weather season length and fire weather severity. These results will help us better understand how climatic variations are manifesting themselves as tangible changes in observed fire activity and it will improve our understanding of the complex interactions between long-term climatic changes, short-term weather variations and wildland fire-induced carbon cycle feedbacks.
\end{abstract}

Keywords: climate change, fire danger, fire weather severity, fire weather season length

\title{
1. Introduction
}

Wildland fire activity across the globe is heavily tied to local weather variations and over time, climatic shifts may alter the sizes and types of fires that occur. Climatic changes are occuring across the globe and these changes are lengthening global fire seasons. Regionally, tese longer fire seasons are leading to more area burned and possible higher intensity fires. However, another possibility exists that the within-season fire weather severity is changing

Here we explore how climatic shifts are influencing two aspect of global fire weather conditions: a lengthening of the fire weather season and an increase in the within-season fire weather severity.

\section{Methods}

\subsection{Meteorological data}

Three global reanalysis projects provided gridded, sub-daily surface meteorological data from 1979 to 2016. Two data sets at $\sim 210 \mathrm{~km}$ spatial resolution were obtained from NCEP, including the Reanalysis I and the DOE Reanalysis II data sets. Six-hourly data fields for $2 \mathrm{~m}$ maximum temperature, minimum temperature, specific humidity, surface pressure, precipitation rate, water equivalent of actual snow depth and $10 \mathrm{~m} \mathrm{U}$ and $\mathrm{V}$ wind components were summarized to daily data. We assumed that diurnal variations in actual vapor pressure are small and thus daily mean actual vapor pressure was calculated from the NCEP data using mean daily specific humidity and surface pressure65, and saturation vapor pressure was calculated from daily maximum and minimum temperature to calculate daily maximum and minimum relative humidity. In addition, we used the ECMWF ERA Interim Reanalysis. This data set is similar to NCEP's but at a higher spatial and temporal resolution $(\sim 78 \mathrm{~km}$ resolution). We extracted 3-hourly $2 \mathrm{~m}$ air temperature, dewpoint temperature, surface total precipitation, and $10 \mathrm{~m} \mathrm{U}$ and $\mathrm{V}$ wind components using the ECMWF GRIdded Binary Application Programming Interface (GRIB-API) and used them to derive daily maximum and minimum 
temperature, maximum and minimum relative humidity, maximum wind and total daily precipitation amount and daily precipitation duration. Daily maximum and minimum relative humidity were calculated using mean daily dewpoint temperature and minimum and maximum daily $2 \mathrm{~m}$ air temperature, respectively.

\subsection{Fire Danger Indices}

These data were used to calculate three daily fire danger indices, the US Burning Index, the Canadian Fire Weather Index and the Australian (McArthur) Forest Fire Danger Index, for each of the three ensemble datasets. A fire weather season length and a season fire weather severity metric were calculated for each index / reanalysis dataset combo.

\section{A: United States National Fire Danger Rating System}

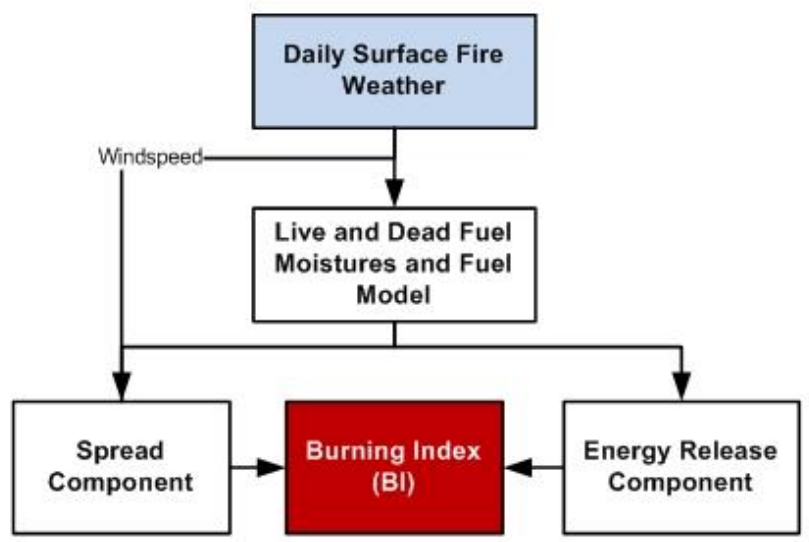

\section{B: Canadian Fire Weather Index System}

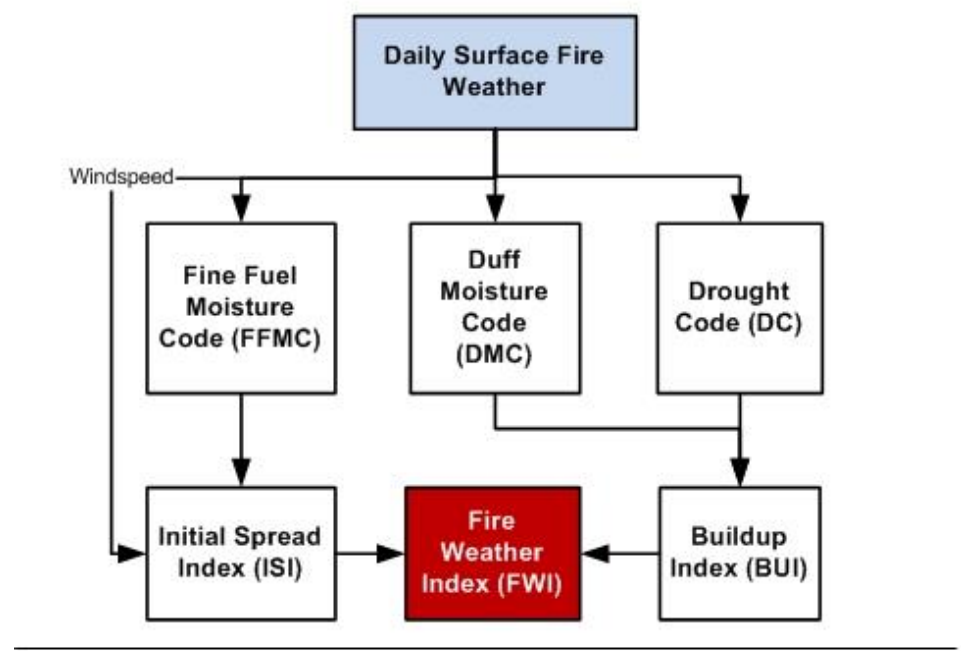

C: McArthur Australian Forest Fire Danger Index

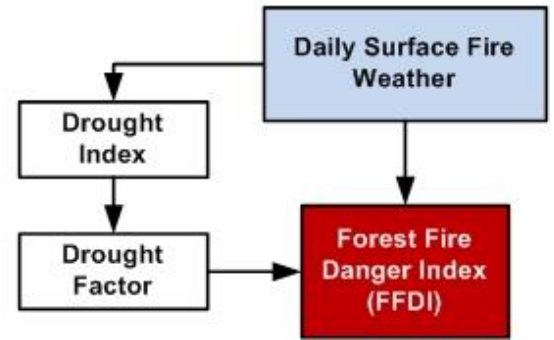

Figure 1 - Simplified diagrams of the three fire danger rating indices used for this study. 


\section{Results}

Consistent with our previous study, we found increases in fire weather season length across nearly $20 \%$ of the global vegetated land mass. Further, we found a steady increase in fire weather severity since 2000 in the adsence of any changes in season length suggesting a previous unexplored way that climatic changes may be impacting seasonal burning conditions worldwide. Mean global fire weather season severity increased abruptly after about the year 2000 and have remained high for the last one and a half decades (Figure 2). Further, across much of the Western US, fire seasons are both longer and within-season conditions are becoming more severe, while in Europe, some places are observing more severe within-season burning conditions while the lengths of the seasons are remaining relatively constant. Part of Western South America, Sub-sahelian West Africa and Western India are all observing shorter fire seasons and reduced burning condition severity.

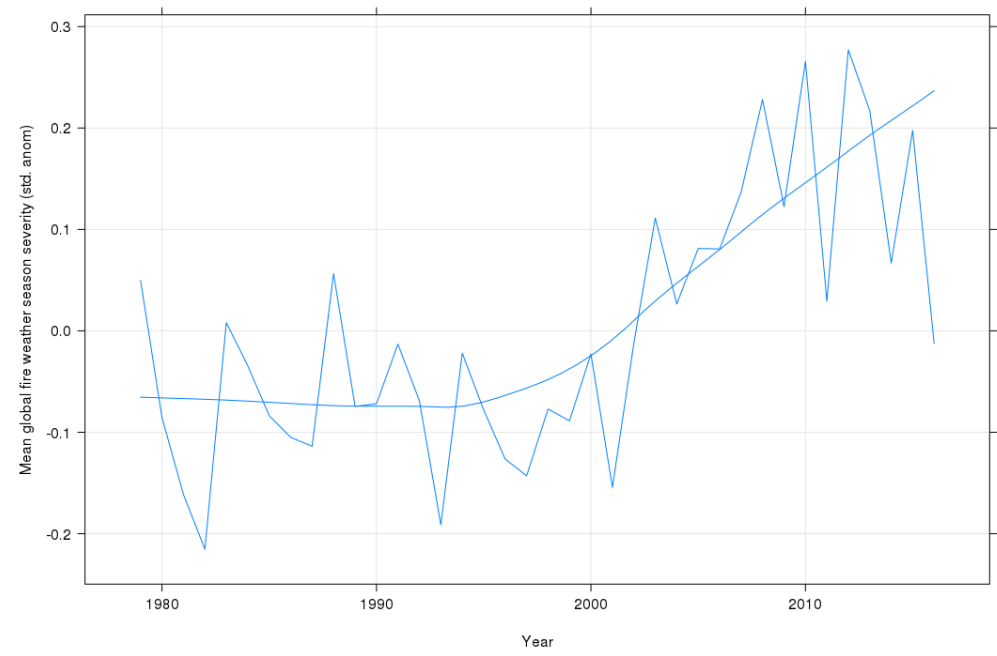

Figure 2 - Mean global fire weather severity from 1979-2016 calculated from an ensemble of fire weather season length metrics and cumulative annual fire weather severity.

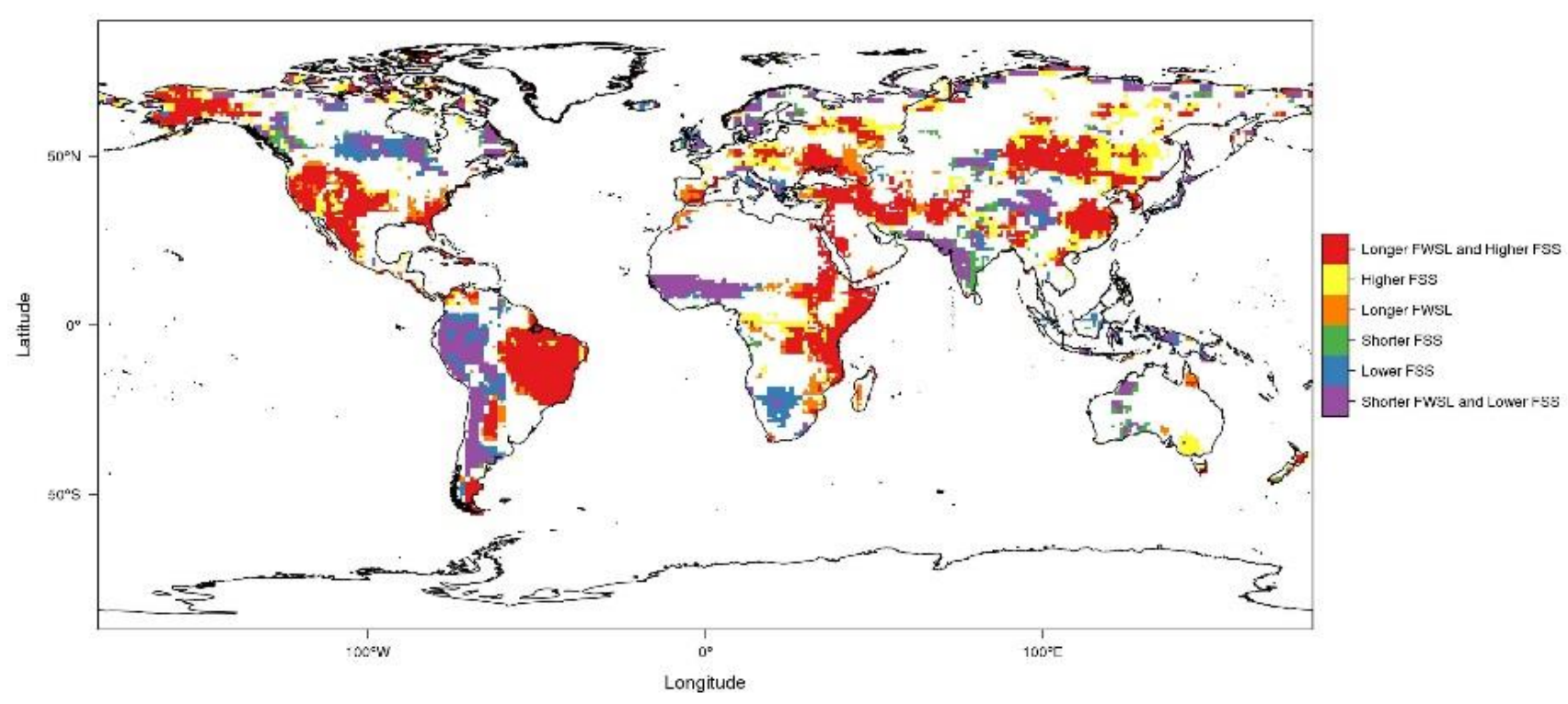

Figure 3 - Global, composite change in Fire Weather Season Length (FWSL) and Fire Season Severity (FSS). Red areas show regions where seasons are lengthening and the within-season conditions becoming more severe, yellow areas show regions where only the sesaonal severity is increasing and the FWSL is not changing. Orange areas show areas where only the FWSL is increasing, green areas show places where the seasons are getting shorter, blue areas are becoming less severe and purple areas are observing shorter seasons and less seasonal weather severity. 


\section{Discussion}

Previous work has shown that fire weather season length is increasing across nearly $20 \%$ of the vegetated surface of the Earth. These longers seasons are contributing to more days available to burn each year and these fires come at a high socio-economic cost. Extending from this previous work, we show that regional changes in within-fire season severity are also increasing, suggesting that while fire season lengthening may play a role in increased regional fire activity, more severe fire weather conditions may also be playing an important role in regulating global fire activity. These regional changes in fire season length and severity can have a strong impact on fire activity and can lead to substantial changes in atmospheric carbon feedback. 
Short contribution - Fire Risk Management

Cohesive forest fire management within an imperfect information environment: a review of risk handling and Decision Support Systems actually in use

\author{
Abílio P. Pacheco ${ }^{1 *}$, João Claro ${ }^{1}$, Paulo M. Fernandes ${ }^{2}$, Richard de Neufville ${ }^{3}$, Tiago M. Oliveira ${ }^{4}$, José G. \\ Borges $^{4}$, José Coelho Rodrigues ${ }^{5}$ \\ I* INESC TEC and Faculdade de Engenharia, Universidade do Porto, \{app@fe.up.pt*, jclaro@fe.up.pt\}. \\ ${ }^{2}$ CITAB, UTAD. Quinta de Prados, 5000-801 Vila Real, Portugal, \{pfern@utad.pt\}. \\ ${ }^{3}$ MIT Institute for Data, Systems, and Society, Massachusetts Institute of Technology. 77 Massachusetts \\ Avenue, Cambridge, MA 02139, USA, \{ardent@mit.edu\}. \\ ${ }^{4}$ Forest Research Centre, School of Agriculture (ISA), University of Lisbon. Tapada da Ajuda, 1349-017 \\ Lisboa,Portugal, \{toliveira@isa.ulisboa.pt, joseborges@isa.utl.pt\}. \\ ${ }^{65}$ INESC TEC and FEUP. Porto, Portugal, \{jpcr@fe.up.pt\}.
}

Keywords: Forest Fire Management, Decision Support Systems, Risk, Economic Efficiency, Natural Resources, Implementation Management, Fire Simulation

\title{
1. Introduction
}

Wildfire management has been struggling in recent years with escalating devastation, expenditures, and complexity. Uncertain and highly unpredictable factors, such as weather forecasts, performance of suppression resources, and fire behavior, spread and effects are the basis of fire management and policy decisions, across multiple levels and scales. Given these copious factors and the complexity of their interactions, uncertainty in the outcomes is a prominent feature of wildfire management strategies, at both policy and operational levels. Theoretical and computational progress in the last four decades has enabled the development of risk-based Decision Support Systems (DSS) that contribute to improve those decisions, namely by facilitating a structured assessment of the outcomes and costs associated with alternative policies, budgets, and suppression resource mixes. Improvements in risk handling and in risk-based decision support tools have therefore a key role in addressing these challenges. In this context, we review key systems created to support wildfire management decisionmaking at different levels and scales, and describe their evolution from an initial focus on landscapelevel fire growth simulation and burn probability assessment, to the incorporation of exposure and economic loss potential (allowing the translation of ignition likelihood, fire environment - terrain, fuels, and weather - and suppression efficacy into potential fire effects), the integration with forest management and planning, and more recently, to developments in the assessment of values at risk, including real-time assessment. This evolution is linked to a progressive widening of the scope of usage of these systems, from an initial more limited application to risk assessment, to the subsequent inclusion of functionality enabling their utilization in the context of risk management, and more recently, to their explicit casting in the broader societal context of risks and decisions, from a risk governance perspective. This joint evolution can be seen as the result of a simultaneous pull from methodological progresses in risk handling, and push from technological progress in wildfire management decision support tools, as well as more broadly in computational power. Seeking to characterize this movement, in a recent paper (Pacheco et al 2015) we identify the key benefits and challenges in the development and adoption of these systems, as well as future plausible research trends. 
level fire growth simulation and burn probability assessment, to the incorporation of exposure and economic loss potential (allowing the translation of ignition likelihood, fire environment - terrain, fuels, and weather - and suppression efficacy into potential fire effects), the integration with forest management and planning, and more recently, to developments in the assessment of values at risk, including real-time assessment. This evolution is linked to a progressive widening of the scope of usage of these systems, from an initial more limited application to risk assessment, to the subsequent inclusion of functionality enabling their utilization in the context of risk management, and more recently, to their explicit casting in the broader societal context of risks and decisions, from a risk governance perspective. This joint evolution can be seen as the result of a simultaneous pull from methodological progresses in risk handling, and push from technological progress in wildfire management decision support tools, as well as more broadly in computational power. Seeking to characterize this movement, in a recent paper (Pacheco et al 2015) we identify the key benefits and challenges in the development and adoption of these systems, as well as future plausible research trends.

\section{Materials and Methods}

In recent years, several authors have updated the state of the art on the way these challenges have been addressed, organizing and aligning the sources of uncertainty with decision support tools and methodologies, in order to facilitate cost-effective, risk-based wildfire management and planning efforts. Reviewed developments include operations research methods applicable to wildfire management, and the economic efficiency analysis theory behind the fire management measures of fire management DSSs in use in America, Australia and Europe. Some of these DSSs allow the integration of wildfires into forest planning, sometimes also addressing risk and uncertainty, but broadly they include (real-time or not) wildfire simulators (autonomous or not) which in turn, were built over some surface fire spread simulation model.

\begin{tabular}{|c|c|c|c|}
\hline \multicolumn{4}{|c|}{ Risk Management to Risk Governance } \\
\hline & \multirow{2}{*}{\multicolumn{2}{|c|}{ Risk Assessment to Risk Management }} & WFDSS (FSPro), 2007 \\
\hline & & & FireDST (PHOENIX Rapidfire), 2011 \\
\hline \multirow{2}{*}{\multicolumn{2}{|c|}{ Risk Assessment }} & \multirow{7}{*}{$\begin{array}{r}\text { SINAMI (Visual-Cardin) } 2004 \\
\text { FPA } 2 \text { (FSim), } 2011\end{array}$} & Thompson et al. (FSim), 2013 \\
\hline & & & \\
\hline & \multirow{5}{*}{$\begin{array}{r}\text { Burn-P3, } 2005 \\
\text { FSPro, } 2006 \\
\text { FSim, 2009 }\end{array}$} & & \\
\hline Assessment & & & \\
\hline (early scientific approaches) & & & \\
\hline BEHAVE, 1983 & & & \\
\hline CARDIN, 1989 & & & \\
\hline
\end{tabular}

Figure 1 - Conceptual outline of the expanded focus, from risk assessment to management to governance, with examples (DSSs and methodologies).

Our review first assesses the characteristics of the fire modeling systems in operation (BehavePlus, Visual-Cardin used by SINAMI, FARSITE, SiroFire, Prometheus used by Burn-P3, FSPro used by WFDSS with RAVAR, FSim used by FPA, and PHOENIX Rapidfire used by FireDST) which include fire behavior, fire spread, and probabilistic fire spread simulators, and then adopts a higher-level perspective to provide a broader and more complete view of the evolution of the field. Indeed, we concisely present several important risk-based decision support models for fire management, on the one hand highlighting their usefulness within the scope and the purposes that guided their development, but on the other rendering explicit a number of limitations that they present. Some of these limitations have also been discussed recently, although in a fragmented way, in the literature on challenges in the development and deployment of risk-based decision support systems.

We bring together this set of observations, and highlight what seems to us to be an important trend of broadening of concerns from risk assessment, to risk management, to risk governance (Figure 1). 
This trend frames an increasingly ambitious utilization of these systems, gradually and successively broadened to address each of those areas of concern. This overall evolution pattern is the result of simultaneous methodological progress in risk handling, as well as specific technological progress in wildfire management decision support tools, and generic technological progresses in computation.

\section{Results}

A myriad of interacting social and ecological factors influences the severity of forest fires. Thus, we need to understand the non-linear relationships between interconnected physical, biological, and cultural systems to be able to effectively reduce the vulnerability of ecosystems and human societies, through improved and proactive risk governance. Problem structuring methods, system dynamics, simulation, decision analysis, and optimization, together with qualitative methods such as expert elicitation, open interviews, questionnaires, and surveys, can help model the dynamics and advance the understanding of these complex systems, gaining insight into problem structures, and better enabling the exploration of alternative management options, in face of always present budgetary constraints.

The integration of forest fire risk concerns into forest planning processes is a significant step. However, more research is needed characterizing the impacts of alternative fire management options on market and nonmarket values at risk, and on the economic losses in goods and services triggered by the fire consequences that they try to mitigate. Risk-based analysis is required for the integration of risk handling and fire management, in order to improve the prioritization of future efforts to mitigate the risks associated with these natural and human caused disturbances. Risk assessment should also identify and characterize the importance and weight of uncertainties to improve the management of human and ecological resources at risk, in areas ranging from fuel mapping to how society values those resources.

Our review of DSSs in current use stresses the importance of the integration between risk handling and DSS development, to facilitate and improve the quality of decisions under uncertainty, and enable a cohesive fire management in an uncertain environment. It also points out the importance of understanding the institutional constraints of management programs within which forest fire mitigation programs develop, that along with ecological constraints, the need to engage multiple stakeholders, and the need for adaptation to local contexts, must be reflected in the usability and flexibility of these systems. In the case of Portugal, our work may also contribute to address the lack of a DSS in use.

\section{Acknowledgements}

This work is financed by the ERDF through the COMPETE and by National Funds through the FCT within project FIRE-ENGINE/MIT/FSE/0064/2009 and The Navigator Company; and by project UID/EEA/50014/2013. FCT also supported the research performed by Abílio Pereira Pacheco (Grant SFRH/BD/92602/2013). Our work greatly benefited from the many colleagues acknowledged in the cited paper.

\section{References}

Pacheco, Abílio Pereira, João Claro, Paulo M. Fernandes, Richard de Neufville, Tiago M. Oliveira, José G. Borges, and José Coelho Rodrigues. 2015. "Cohesive fire management within an uncertain environment: A review of risk handling and decision support systems." Forest Ecology and Management 347 (0):1-17. doi: 10.1016/j.foreco.2015.02.033. 
Short contribution - Fire Risk Management

\title{
Extreme weather conditions: The role of an heat wave on wildfires in Portugal
}

\author{
Joana Parente $^{1 *}$; Mário G. Pereira ${ }^{1,2}$; Malik Amraoui ${ }^{1}$; E. M. Fischer ${ }^{4}$ \\ ${ }^{1}$ Centro de Investigação e Tecnologias Agroambientais e Biológicas, Universidade de Trás-os-Montes e \\ Alto Douro, Quinta de Prados, 5000-801 Vila Real, Portugal. \{joanaparente@utad.pt*, gpereira@utad.pt, \\ malik@utad.pt\} \\ ${ }^{2}$ Instituto Dom Luiz, Faculdade de Ciências da Universidade de Lisboa, Lisboa, Portugal \\ ${ }^{3}$ Institute for Atmospheric and Climate Science, ETH Zurich, Switzerland, \{erich.fischer@env.ethz.ch\}
}

\begin{abstract}
Heat waves (HW) combined with periods of drought, as the one of 2017, may have a significant impact on the occurrence of extreme wildfires (EW) which, in turn, can have devastating social, economic and environmental consequences. EWs may modify (i) vegetation structure and dynamics; (ii) water quantity and quality; (iii) air quality; (iv) human health, loss of life, damage to property and infrastructures. On last decades, EWs in Continental Portugal increased in frequency and impacts. The main focus of this study is to assess the influence of HWs on the occurrence of EWs in mainland Portugal. In this study, EWs will be considered as wildfires with burnt area $\geq 5000$ ha. In a first stage, HW occurrence was assessed on the basis of Fischer and Schär (2010) definition for a recent past and different future climate scenarios. In the second stage, spatial and temporal distributions of EWs were related to the occurrence and characteristics of HWs in the recent past. Finally, projections of EWs for the future are inferred from the potential changes in the characteristics of HWs for different future climate scenarios. This study benefits from the existence of reliable fire datasets, with accurate and detailed information on the date/time of ignition/extinction, the location, the shape and the size of the burnt area, as well as consistent high resolution of meteorological datasets for recent past and future climate scenarios. Results disclose: (i) almost all EWs were active during an HW; (ii) the vast majority of total EWs days were also HW days; (iii) 4/5 of the EWs had duration completely contained in the duration of an HW; and, (iv) the same amount of the EWs occurred during and in the area affected by HW. This study aims to help the definition of strategies for adaptation and mitigation of climate change to HW as well as for fire risk management.
\end{abstract}

Keywords: Heat wave, Extreme wildfire, Extreme conditions, Climate change, Portugal

\section{Introduction}

In last decades, EWs in Continental Portugal increased in frequency and impacts which attracted the attention of researchers for these fires and their drivers. In Portugal, extreme wildfires (EW) tend to occur in association with extreme weather conditions, namely heat waves (HW) during the summer fire season and drought in the previous months. Therefore, the main aim of this study is to assess the influence of HWs on the occurrence of EWs in mainland Portugal as well as to assess the potential changes in the characteristics of the HW for future climate scenarios and periods. As an example, the results obtained for 2003 will be presented, which was one of the years with the highest annual burnt area in Portugal.

\section{Data and Methodology}

\subsection{Study Area}

Mainland of Portugal has an area of $90,000 \mathrm{~km}^{2}$, is located between Spain and Atlantic Ocean and has a temperate or Mediterranean climate. The Tagus River divides territory into two regions with 
different sub-types of climate: the north region, characterized by dry and warm summers; and, the south with dry and hot summers (Rubel and Kottek 2010). Along with other environmental characteristics (e.g., topography, land use land cover) these climatic conditions combined with extreme hot spells promote the occurrence of EWs.

\subsection{Meteorological datasets}

The meteorological datasets used in this study comprises daily maximum temperature ( $\left.\mathrm{T}_{\max }\right)$ from: (i) ERA-Interim dataset (Dee et al. 2011) for a spatial domain $\left(10^{\circ} \mathrm{W}-6^{\circ} \mathrm{E}, 36.5^{\circ} \mathrm{N}-42.5^{\circ} \mathrm{N}\right)$ centred in Continental Portugal for control period $(1981-2010)$, with a spatial resolution of $0.125^{\circ} \times 0.125^{\circ}$ latitude/longitude; (ii) CORDEX (Coordinated Regional Climate Downscaling Experiment) Adjust project dataset (http://cordex.org/data-access/bias-adjusted-rcm-data/) with $12 \mathrm{~km}$ (EUR-11) resolution for control and 5 consecutive 30-year future climatological periods (2021-2050, 2031-2060, 2041-2070, 2051-2080 and 2061-2090), for 4.5 and 8.5 representative concentration pathways (RCP), and for 3 pairs of General Circulation Model/Regional Climate Model, namely, MOHC-HadGEM2ES/RACMO22E, MOHC-HadGEM2-ES/CCLM4-8-17 and MPI-M-MPI-ESM-LR/REMO2009.

\subsection{Fire datasets}

Information from the two official fire datasets provided by the Portuguese Institute for the Conservation of Nature and Forests (www.icnf.pt), were combined to obtain an EW dataset with detailed temporal (ignition and extinction date and time) and spatial (burnt area, BA, polygon) data. In this study, EWs are defined as very large wildfires (BA $\geq 5000 \mathrm{ha}$ ). In the control period a total of 62 EWs occurred in Portugal, which corresponds to $0.02 \%$ of total number of wildfires but to almost $19 \%$ of total BA. These events tend to occur more in central and south-eastern regions of Portugal (Figure 1).

\subsection{Methodology}

The Fischer and Schär (2010) definition of HW was adopted in this study for being especially suitable to capture absolute (summer) extreme events of $\mathrm{T}_{\max }$. An HW is defined as a period of at least six consecutive days with $\mathrm{T}_{\max }>\mathrm{P} 90$. HWs occurrence in control period and for future projections were characterized by their number (HWN90), duration (HWD90), frequency (HWF90) and amplitude (HWA90). Robustness of the climate change assessment was based on Jacob et al. (2014) and Pfeifer et al. (2015). To understand the impact of HWs in EWs, spatial distribution and temporal evolution of HWD90 and HWA90 were compared with the spatial-temporal distribution of wildfire events.

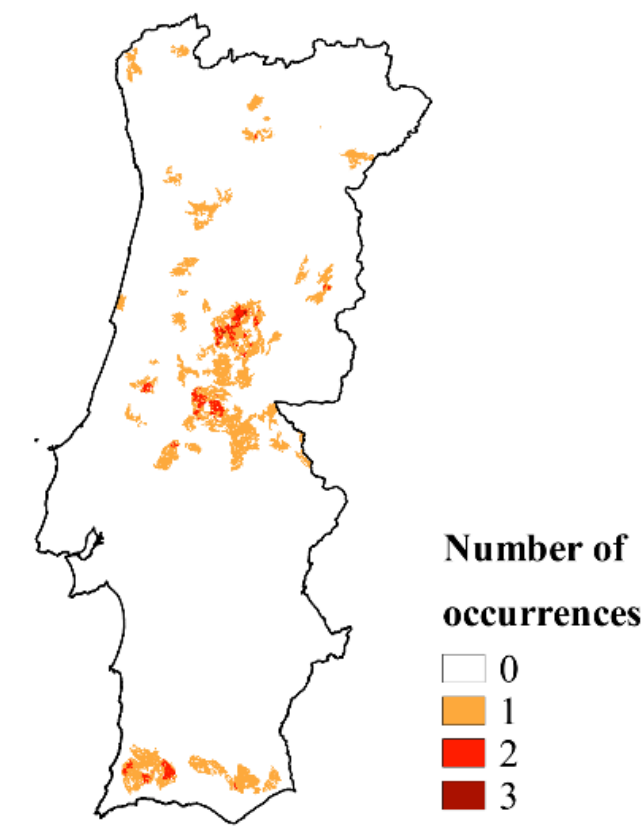

Figure 1 - Number of extreme wildfire occurrences taking in account the 62 extreme wildfires (very large wildfires with burnt area $\geq 5000$ ha) define between 1981 and 2010. 


\section{Results}

\subsection{HWs occurrence characterization}

The intra- and inter-annual variability of HWs characteristics, namely, HWN90, HWD90, and HWA90 (Figure 2) unveil: (i) that HWs tend to occur between May and October; (ii) HWD90 and HWA90 present two significant increasing trends in the 1981 - 1992 and 1993 - 2010 periods; (iii) that HWN90 present a decreasing followed by a decreasing trend in the same periods; (iv) $1981-2010$ annual HWD90 and HWA90 peak is in 1990; (v) more HWA90 doesn't mean more HWD90 or more HWN90 in every case; and, (vi) 1981 and 2009 were the years with more HWN90.
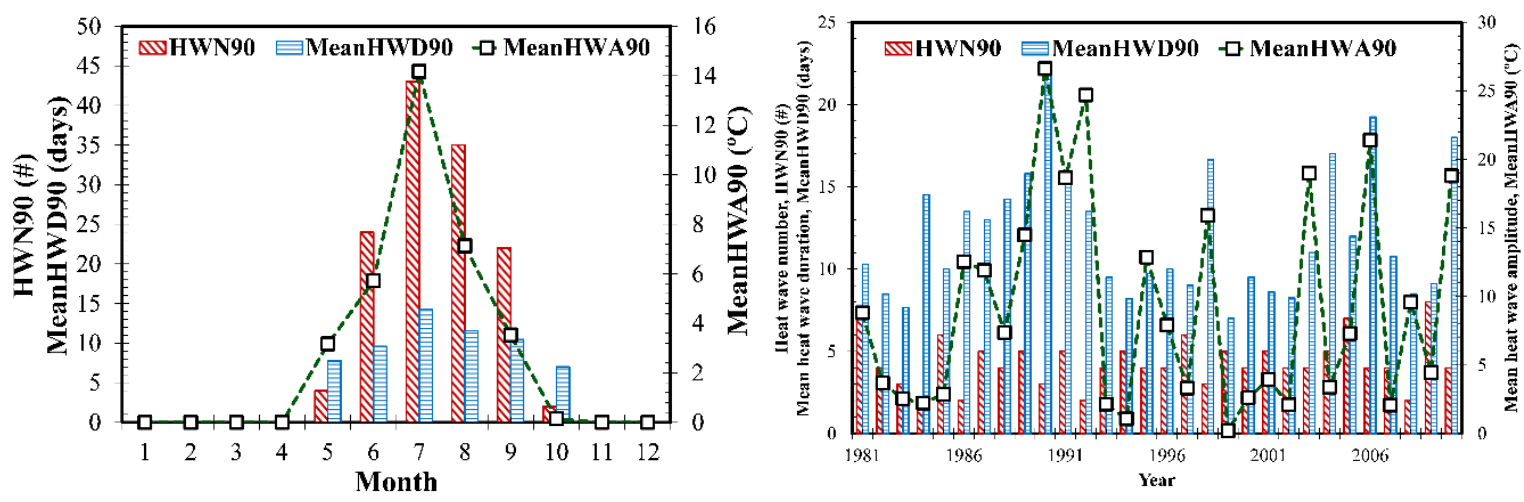

Figure 2 - Monthly and annual distributions of heat wave number (HWN90), mean heat wave duration (MeanHWD90) and mean heat wave amplitude (MeanHWA90) for control period (1981 - 2010).

Spatial distribution of annual HWN90 and HWD90 (Figure 3) reveal higher values in the northeast and in the south region with emphasis near the Spain border. In contrast, the annual spatial distribution of HWA90 is more homogeneous with high values in central region. Future projections of HWs shows that: (i) HWN, HWD and HWF are expected to increase in future for both RCP's; and, (ii) HWA is expected to decrease for both RCP's, with the exception after the second future period of RCP 8.5 where its start to gradually increase. Spatial future projections unveil that HWN for RCP 4.5 are higher in central north region, while for RCP 8.5 are higher in the interior and central north regions.
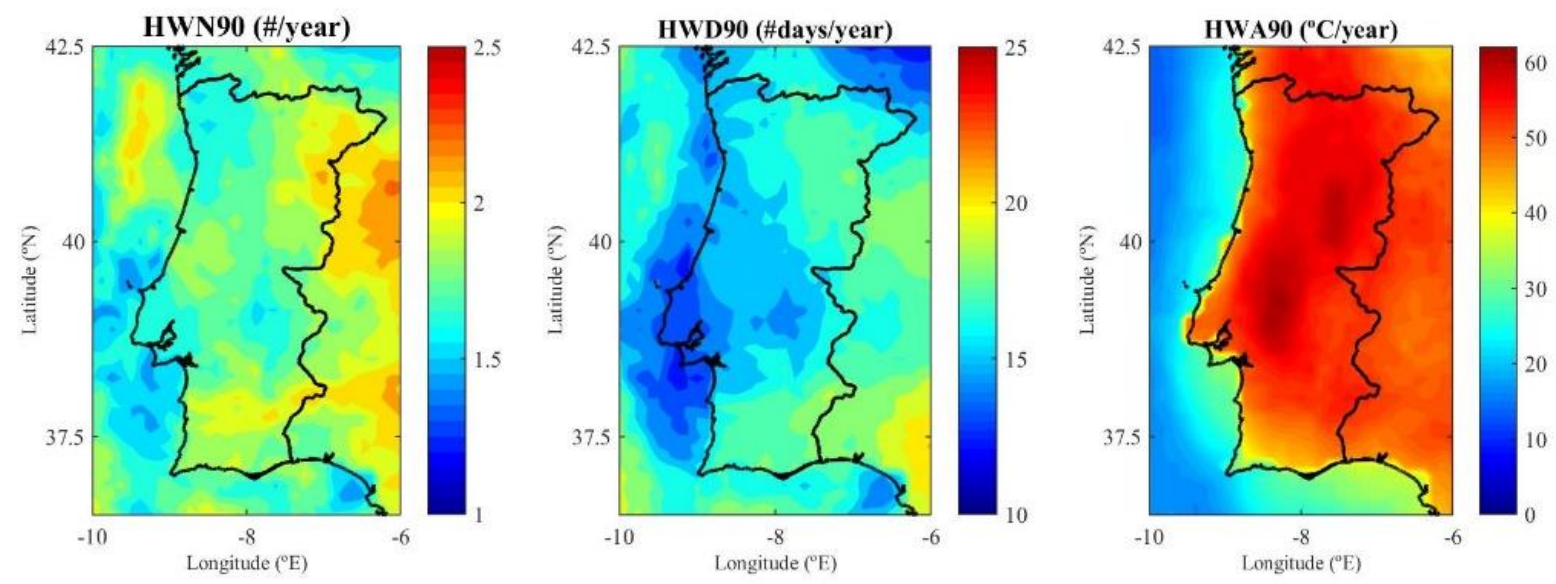

Figure 3 - Spatial distribution of annual heat wave number (HWN90), duration (HWD90) and amplitude (HWA90) for control period $(1981-2010)$. 


\subsection{The influence of HWs on EWs occurrence}

The analysis of spatial and temporal distributions of EWs, HWD90 and HWA90 (Figure 1 and Figure 3) disclose: (i) $3 \%$ of EWs were not active during an HW; (ii) only $10 \%$ of total EW days were not also HW days; (iii) more than $18 \%$ of the EWs had duration completely contained in the duration of an HW; and, (iv) $18 \%$ of EWs occurred during and in the area affected by HW. For example, Figure 4 unveils that 2003 EWs occurred on the most affected areas by 2003 HWs, even the ones on the southeastern region.
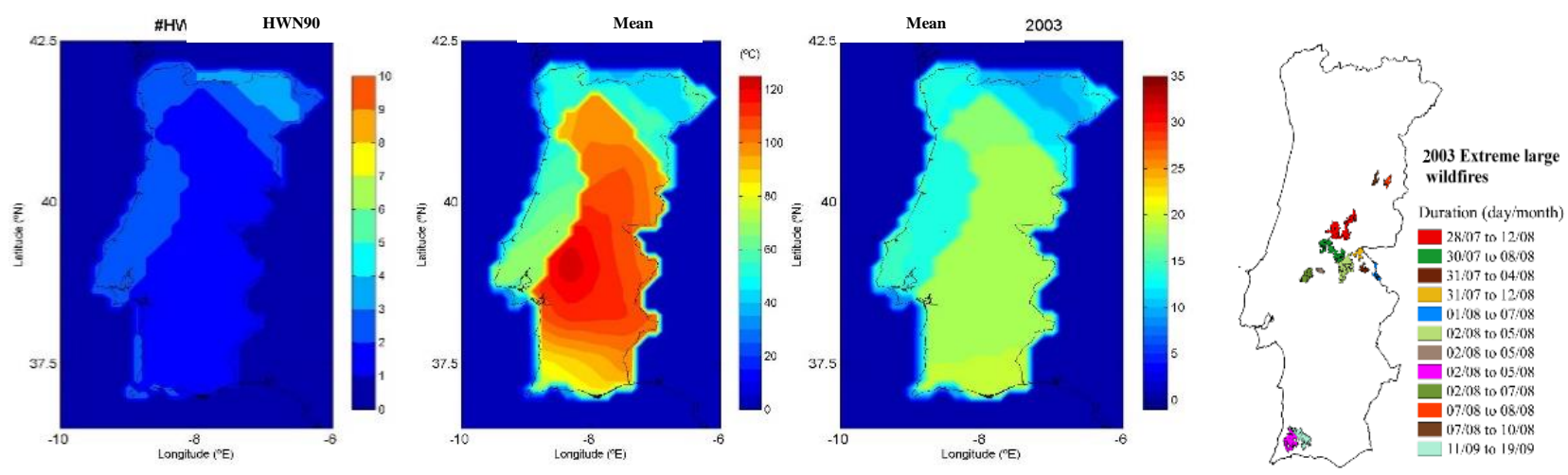

Figure 4 - Burnt area polygons of 2003 extreme large wildfires and heat wave 2003 statistics.

\section{Conclusions}

Due to the adopted definition of heat waves, this study is focused on summer extreme events of surface air temperature. This is particularly evident in the intra-annual variability of the heat wave descriptor of number, duration and amplitude. This study also disclose a high inter-annual variability of those features, with a sequence of low and high values, superimposed by increasing trends in two sub periods (1981 - 1992 and 1993 - 2010), except HWN90 which present a decreasing trend in the first sub period. The spatial distribution of the heat wave descriptors reveal the NE and south as the most affect regions, in terms of number and duration, the central region is the most affected in terms of the amplitude. Central Portugal is also the region most affected by the extreme wildfires as was clear in 2003, one of the most extreme years in terms of burnt area. Temporal and spatial analysis disclose that all the extreme wildfire events occurred during or immediately after several long intense heat waves. This study aimed to help decision makers and practitioners on the definition of strategies for fire risk management and adaptation to climate change to HW since our results suggest the HW should increase in number, duration and amplitude for different future scenarios of climate change.

\section{Acknowledgements}

This work was prepared in the frame of project FIREXTR - Prevent and prepare society for extreme fire events: the challenge of seeing the "forest" and not just the "trees", co-financed by the European Regional Development Fund (ERDF) through the COMPETE 2020 - Operational Program Competitiveness and Internationalization (POCI Ref: 16702) and national funds by FCT-Portuguese Foundation for Science and Technology (FCT Ref: PTDC/ATPGEO/0462/2014. The study was also supported by Project Interact - Integrative Research in Environment, Agro-Chain and Technology, NORTE-01-0145-FEDER-000017, research line BEST, co-funded by FEDER/NORTE 2020. 


\section{References}

Dee DP, Uppala SM, Simmons AJ, Berrisford P, Poli P, Kobayashi S, Andrae U, Balmaseda MA, Balsamo G, Bauer P (2011) The ERA-Interim reanalysis: Configuration and performance of the data assimilation system. Quarterly Journal of the royal meteorological society 137, 553-597.

Fischer EM, Schär C (2010) Consistent geographical patterns of changes in high-impact European heatwaves. Nature Geoscience 3, 398-403.

Jacob D, Petersen J, Eggert B, Alias A, Christensen OB, Bouwer LM, Braun A, Colette A, Déqué M, Georgievski G (2014) EURO-CORDEX: new high-resolution climate change projections for European impact research. Regional Environmental Change 14, 563-578.

Parente J, Pereira MG, Amraoui M, Fischer EM (2018) Heat waves in Portugal: current regime, changes in future climate and impacts on extreme wildfires. The Science of the total environment.

Pfeifer S, Bülow K, Gobiet A, Hänsler A, Mudelsee M, Otto J, Rechid D, Teichmann C, Jacob D (2015) Robustness of ensemble climate projections analyzed with climate signal maps: seasonal and extreme precipitation for Germany. Atmosphere 6, 677-698.

Rubel F, Kottek M (2010) Observed and projected climate shifts 1901-2100 depicted by world maps of the Köppen-Geiger climate classification. Meteorologische Zeitschrift 19, 135-141. 
Short contribution - Fire Risk Management

\title{
High Resolution Seasonal Forest Fire Danger mapping using WRF forecasts for Greece: A tool for forest fires prevention planning and fire risk management support
}

\author{
Vassiliki Varela ${ }^{* 1}$; Diamando Vlachogiannis ${ }^{1}$; Athanasios Sfetsos ${ }^{1}$; Stelios Karozis ${ }^{1}$; Nikolaos Gounaris \\ '; Angelos Sphyris ${ }^{2}$ \\ ${ }^{1}$ Environmental Research Laboratory, National Center for Scientific Research "DEMOKRITOS", Address: \\ Patr. Gregoriou E \& 27 Neapoleos Str, 15341 Agia Paraskevi, GREECE, \{vvarela@ipta.demokritos.gr*\} \\ ${ }^{2}$ Independent Research, Athens, Greece
}

\begin{abstract}
The Fire Weather Index, FWI, which is part of the Canadian Forest Fire Danger Rating System(CFFDRS), has been validated and recognized worldwide as one of the most trusted and important indicators for fire danger mapping. The classic use of the FWI is associated with the daily fire danger mapping, based on meteorological measurements, while in recent research papers this indicator has been used with forecasted climatic data for studying the changes in the occurrence and intensity of natural disasters. (Climate change Impacts Study Committee, 2011. The purpose of this work is the calculation of the Canadian FWI system indices combined with a state-of-the-art seasonal meteorological forecasting model, for predictive high resolution fire danger mapping, as a tool to design medium-term prevention planning. More specifically, the current paper presents a methodology for the calculation of a series of seasonal forecasted maps of meteorological fire danger, for the fire season (May-October) 2018, for Greece, using the Fire Weather Index (FWI) of the Canadian System CFFDRS. To this end, five components that account for the effects of fuel moisture and wind on fire behaviour have been calculated and investigated, namely, the Drought Code (DC), the Fine Fuel Moisture Code (FFMC), the Built Up Index (BUI), the Initial Spread Index (ISI) and FWI for the referenced period. The meteorological data needed for the calculation of FWI are produced from high resolution simulations with the WRF model (v3.5.1), for a period of six (6) months, using a grid spacing of $5 \times 5 \mathrm{~km}$ covering the whole country. The above referenced indices are calculated for every day of the fire season. Subsequently, from the mean value of the maps of seven (7) days, a weekly map is calculated, which constitutes the representative, final map for each index. The maps can be calculated at the beginning of the fire season and for its entirety. Hence, the FWI system values can be provided at three time levels as weekly, monthly and seasonal forecasts. Appropriate classification of FWI values yields estimation of the Forest Fire Danger. The calculated FWI system components and Forest Fire Danger are important to support decisions, about the prevention and management of forest fires, made by the local and central authorities of the country. Acknowledgment: The current work is carried out in the framework of project SERV_FORFIRE funded through H2020 ERA4CS ERA-NET and the European Commission (Grant Agreement 690462).
\end{abstract}

Keywords: Seasonal meteorological forecast, FWI, fire danger mapping, fire prevention

\section{Introduction}

The digital mapping of Forest Fire Danger is important to support decisions about the prevention and management of forest fires. The dynamic danger mapping at the national, regional and local level, taking into account the meteorological conditions represents a valuable tool for more effective daily effective design by the local and central authorities. The Fire Weather Index FWI, which is part of the Canadian Forest Fire Danger Rating System (CFFDRS), has been validated and recognized worldwide as one of the most trusted and important indicators for meteorological fire danger mapping. The classic use of the FWI is associated with the daily fire danger mapping, based on meteorological measurements, while in a recent research paper by the Bank of Greece, this indicator has been used with forecasted climatic data for studying the changes in the occurrence and intensity of natural 
disasters. (Climate change Impacts Study Committee, 2011). The purpose of this work is the calculation of FWI system components, combined with a state-of-the-art seasonal meteorological forecasting model, for predictive fire danger mapping, as a tool to design medium-term planning, at the beginning of the fire period.

\section{Calculation and Classification of FWI}

FWI system is comprised of six components: three fuel moisture codes and three fire behaviour indices. Calculation of the components is based on daily observations made at noon of air temperature, relative humidity, $10-\mathrm{m}$ open wind speed and 24-hour cumulative precipitation. The calculation algorithm of FWI is quite complex. (C.E. Van Wagner \& T.L. Picket, 1985). According to the literature, FWI values range from 0 to above 100 and are categorized, for operational purposes, in four (4) to six (6) classes, depending on the application area, corresponding to different meteorological fire danger levels, (JRC-EFFIS system, Dimitrakopoulos et al., 2011). In the present study, a five (5) classes categorization of FWI values, developed by NCSRD researchers, has been adopted as more effective for operational purposes for Greece. The classification approach which is based on Percentile Indices provides suitably varying FWI boundaries of classes taking into account the specific physical characteristics of the country.

\section{Methodology}

\subsection{Calculation and mapping of FWI system components}

The FWI and the Daily Severity Rating (DSR) maps have been calculated at the beginning of the fire season 2018, based on the meteorological forecast for each day of the fire season. DSR maps were grouped into the final weekly, monthly and seasonal maps, based on the respective average value of the Indices. In addition, monthly and seasonal maps were calculated for Fine Fuel Moisture Code and Drought Code components. Below, a flowchart is presented, describing the processing of forecast fire danger mapping which is proposed in this paper (Figure 1).

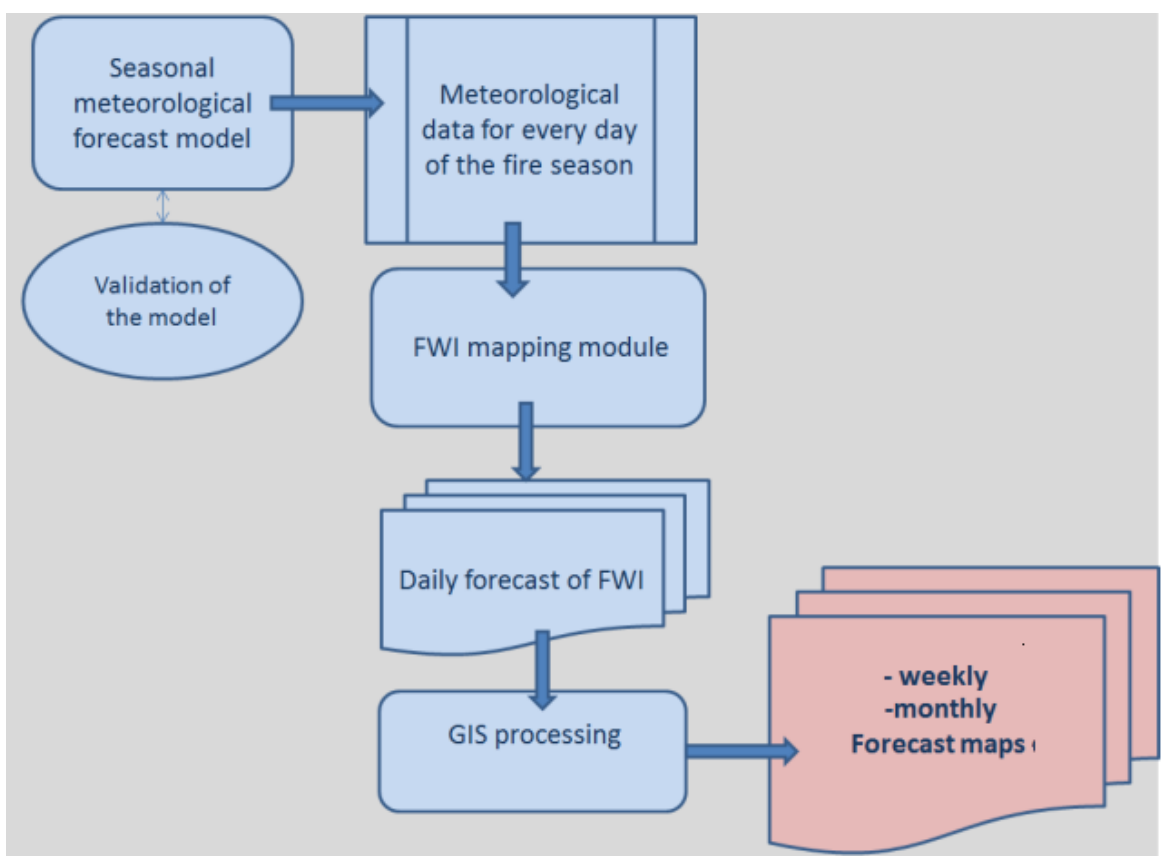

Figure 1 - Flowchart of seasonal forecast mapping of FWI system components 


\subsection{Seasonal forecast of meteorological parameters}

WRF-ARW model (version 3.5.1) has been suitably parameterized for high resolution production medium-term (seasonal) forecasts for Greece. The creation of higher resolution data was established with a one-way nesting procedure. The horizontal resolution of the spatial domain of Greece is 5 x 5 $\mathrm{km} 2$.

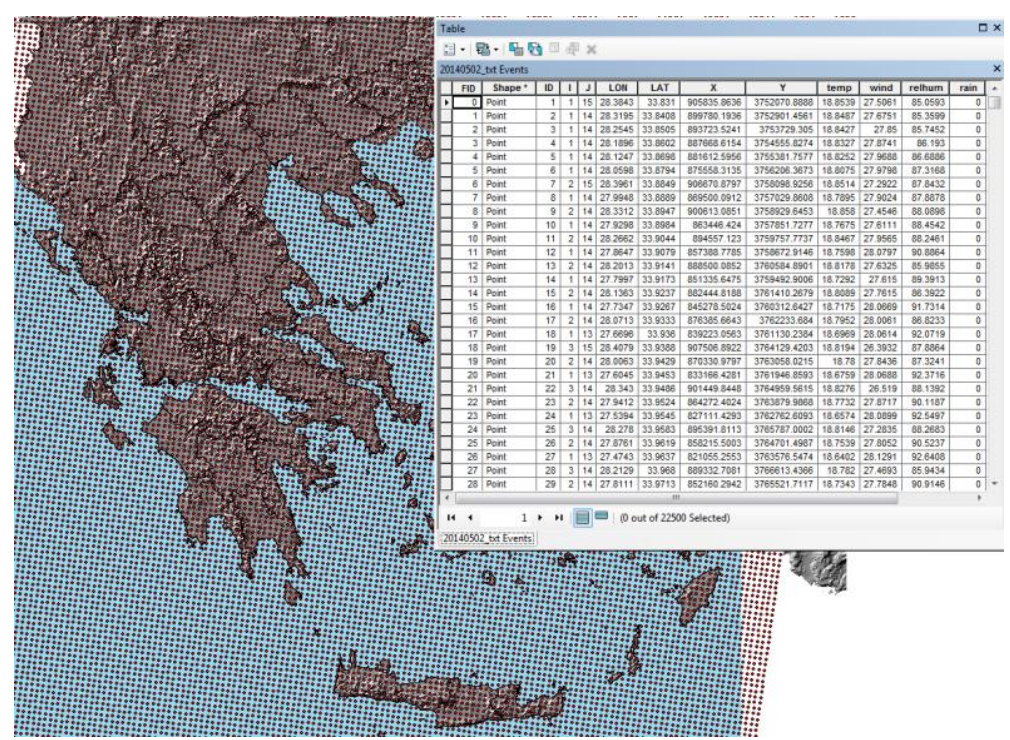

Figure 2 - High resolution grid of the Greek domain

Initial boundary conditions for the WRF model simulations, were determined by twelve-hour analyses provided by the Climate Forecast System of Environmental Prevention (National Center for CFSv2, Saha et al., 2010, 2013) at 00 and 12 UTC for the time period of interest. The ARW-WRF model was validated extensively by using data of temperature, wind and precipitation from meteorological stations all over Greece, for which data is available on the Internet (more than 100 meteo-stations http://www.metar.grhttp://weather.noaa.gov/pub/data/observations/metar/stations/).

\section{Results-Conclusions}

A series of seasonal forecasted maps of meteorological fire danger, for the fire season (MayOctober) 2018, for Greece, using the Fire Weather Index System of the Canadian System CFFDRS have been calculated and investigated. A sample of a set of weekly Severity Rating maps is presented in Figure 3.

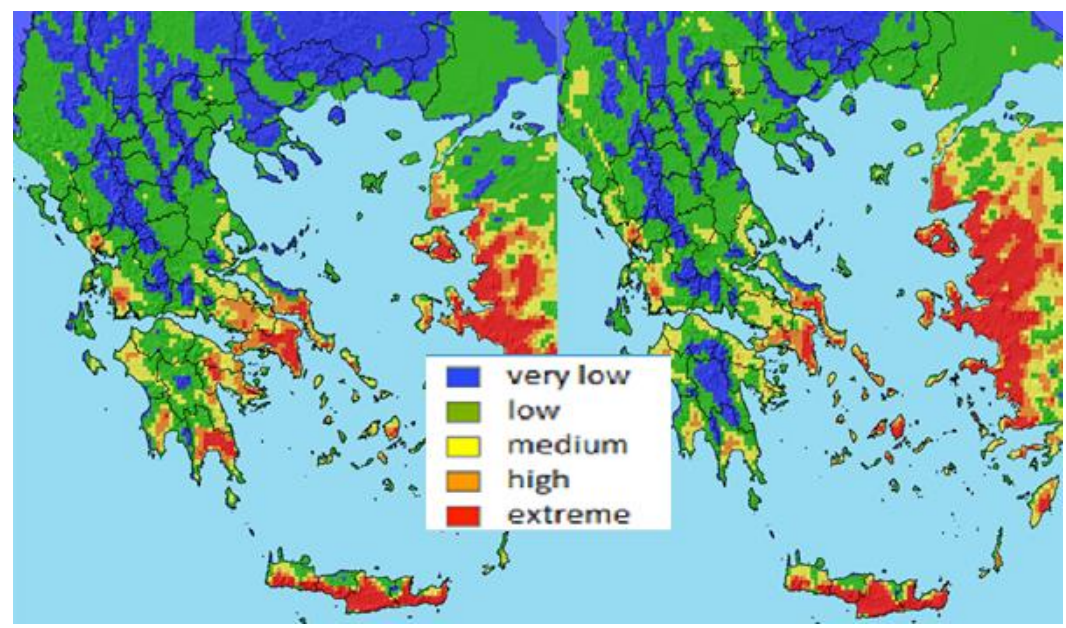

Figure 3 - Sample of a set of weekly Severity Rating maps 
Five components of FWI system that account for the effects of fuel moisture and wind on fire behaviour have been calculated, namely, the Drought Code (DC), the Fine Fuel Moisture Code (FFMC), the Built Up Index (BUI), the Initial Spread Index (ISI) and FWI. Daily Severity Rating maps were also calculated from the respective FWI maps. The weekly, monthly and fire-season maps have been estimated and investigated for the Severity Rating, FFMC and DC indices of FWI system. Appropriate classification of the value maps yield a set of forecast maps for the evaluation of Meteorological Forest Fire Danger level for the respective time level.

The features provided by the modern seasonal model ARF-WRF and the capability of the forecast mapping of meteorological parameters which are related to the forest fire danger, we believe that are important for supporting prevention of forest fires. These forecasts can be used for the seasonal mapping of the Meteorological Fire Danger at the National, regional and local scale. The resulting maps can be proved a very useful tool for the effective exploitation of fire prevention means and personnel.

\section{Acknowledgment}

The current work is carried out in the framework of project SERV_FORFIRE funded through H2020 ERA4CS ERA-NET and the European Commission (Grant Agreement 690462).

\section{References}

Dimitrakopoulos, AP, Bemmerzouk, AM, Mitsopoulos ID (2011). Evaluation of the Canadian fire weather index system in an eastern Mediterranean environment. Meteorological Applications. 18, Issue 1, 83-93.

Joint Research Centre, EFFIS, Fire danger forecast, http://forest.jrc.ec.europa.eu/effis/abouteffis/technical-background/fire-danger-forecast/

Saha, Suranjana, et al. (2010). The NCEP Climate Forecast System Reanalysis. Bull. Amer. Meteor. Soc., 91, 1015.1057. doi: 10.1175/2010BAMS3001.1

Van Wagner CE, Pickett TL (1985). Equations and FORTRAN program for the Canadian Forest Fire Weather Index. Forestry Technical Report FTR-35, Canadian Forestry Service: Chalk River,Ontario. 
Short contribution - Fire Risk Management

Simulating the effectiveness of prescribed burning at altering wildfire behaviour in Tasmania

\author{
J. M. Furlaud*, G. J. Williamson and D. M. J. S. Bowman \\ School of Biological Sciences, University of Tasmania, Hobart, Tasmania, Australia, \\ \{james.furlaud@utas.edu.au*\}
}

\begin{abstract}
Prescribed burning is a widely accepted wildfire hazard reduction technique; however, knowledge of its effectiveness remains limited. To address this, we employ simulations of a widely used fire behaviour model across the ecologically diverse Australian island state of Tasmania. We simulate three broad scenarios: (1) no fuel treatment, (2) a maximal treatment, with the most possible prescribed burning within ecological constraints, and (3) 12 hypothetically implementable state-wide prescribed-burning plans. In all simulations, we standardised fire-weather inputs to represent regionally typical dangerous fire-weather conditions. Statistical modelling showed that an unrealistically large maximal treatment scenario could reduce fire intensity in three flammable vegetation types, and reduce fire probability in almost every vegetation type. However, leverage analysis of the 12 more-realistic implementable plans indicated that such prescribed burning would have only a minimal effect, if any, on fire extent and that none of these prescribed-burning plans substantially reduced fire intensity. The study highlights that prescribed burning can theoretically mitigate wildfire, but that an unrealistically large area would need to be treated to affect fire behaviour across the island. Rather, optimisation of prescribed burning requires careful landscape design at the local scale. Such designs should be based on improved fire behaviour modelling, empirical measurement of fuels and analysis of actual wildfires. Simulation modelling showed that an unrealistically large-scale prescribedburning program across Tasmania would be necessary to reduce wildfire extent and intensity under dangerous fire-weather conditions. By contrast, more feasible, but geographically constrained, broad-scale prescribedburning plans had substantially reduced effects on area burnt and fire intensity. This study highlights the need for targeted localised fuel treatments rather than broad-scale prescribed burning.
\end{abstract}

Keywords: prescribed burning, dry eucalypt forests, eucalypt forests, fire behaviour model, fire management, fire weather, fuel reduction, fuel treatment, leverage, sedgelands, temperate rainforests, wet eucalypt forests

\title{
1. Introduction
}

Prescribed burning is a widely accepted wildfire hazard reduction technique, however, knowledge of its effectiveness remains limited. Prescribed burning is designed to reduce fuel loads in flammable areas across a landscape, which in turn reduces the probability of wildfires encountering high fuel load areas. This should result in a reduction of both the extent and intensity of wildfire across a landscape. The effectiveness of prescribed burning at reducing the extent of wildfire has been quantified using the metric leverage: defined as the reduction in area burnt by wildfires resulting from one unit increase in area treated with prescribed burning. However, prescribed burns involve numerous potential negative social and ecological side effects. Therefore, understanding the trade-offs between the effectiveness of prescribed burning, particularly among different vegetation types, and its drawbacks is crucial.

\section{Methods}

To better understand these trade-offs, we employ simulations of the Phoenix Rapidfire model, a fire behaviour model that is widely used throughout southeast Australia. It is based on the McArthur Mk5 forest and CSIRO grassland fire behaviour models and calculates fire spread as a function of fuels, weather and topography. We simulated 62 series of 11,059 fires across the ecologically diverse island 
of Tasmania. Even though the Phoenix model was built for forests and grasslands, it has been adapted to Tasmania using empirically derived fuel accumulation curves for each vegetation type. For each series of simulations, we simulated a different fuel treatment plan, each of which falls under one of three broad treatment scenarios: a) no fuel treatment, b) an unrealistic maximal treatment, with the most prescribed burning possible within ecological and social constraints, and c) 12 more realistic implementable prescribed burning plans. In all simulations, we distributed ignitions uniformly and standardised fire weather inputs to represent typical dangerous fire weather conditions (i.e. the 9999. $5^{\text {th }}$ percentile McArthur Forest Fire Danger Index (FFDI)) for each of 45 meteorological regions in Tasmania. It should be noted that, due to Tasmania's diverse climate, this fire weather simplification did not substantially constrain the range of FFDI values used in the analysis (8.7-38).

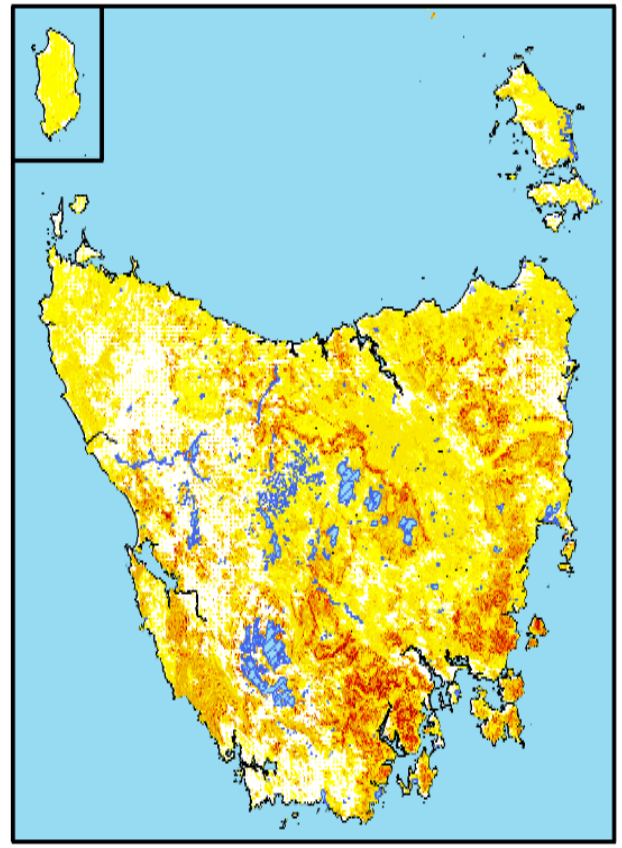

(a)

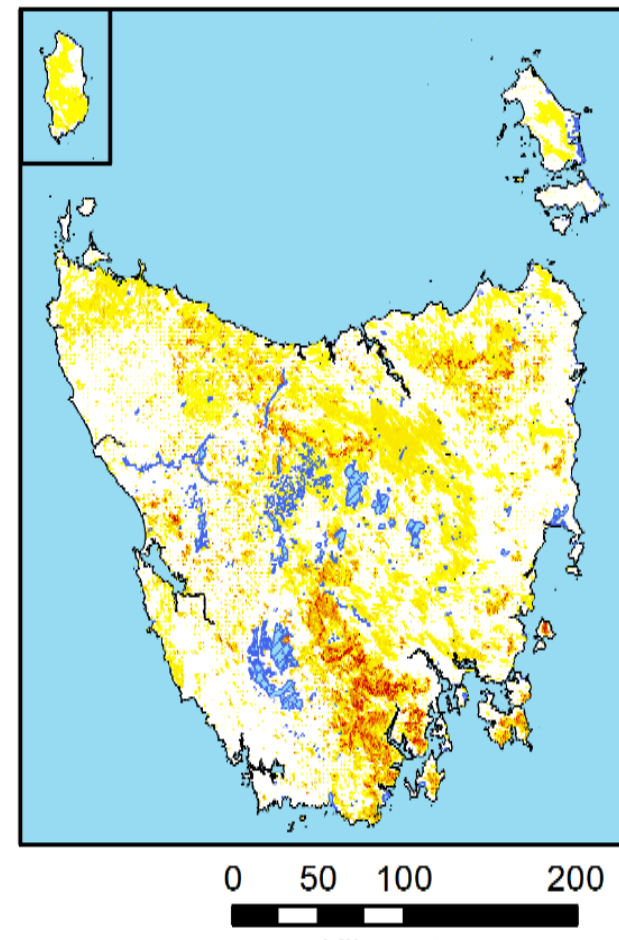

Kilometers

Figure 1 - Geographic patterns of maximum simulated fire intensities in Tasmania with (a) no fuel treatment scenario and (b) a maximal prescribed burning scenario in which all treatable fuels were burnt state-wide .

\section{Results \& Discussion}

We analysed the maximum simulated fire intensity under the null and maximal treatments qualitatively, using maps, and quantitatively, using generalised linear modelling (GLMs). The maps revealed distinct geographic patterns relating to vegetation, region, and terrain, and suggested that a maximal prescribed burning treatment substantially reduced both the extent and intensity of wildfire (Figure 1). The GLMs confirmed that maximal prescribed burning can reduce fire probability in almost every vegetation type, regardless of whether or not the vegetation was subject to treatment. However the GLMs also clarified that maximal prescribed burning can only reduce fire intensity in the three vegetation types which were extensively treated. Meanwhile, the 12 implementable prescribed burning plans were considerably less effective than the maximal scenario at reducing fire extent and intensity in all vegetation types. These implementable plans exhibited leverage in four vegetation types: dry eucalypt forest (leverage: 0.28 ), native grassland (0.3), native scrub (0.2), and non-eucalypt forest (0.24) (Figure 2), suggesting these plans were roughly half as effective as the maximal scenario. 
Additionally, our estimates of overall reductions in maximum intensity resulting from one hectare of prescribed burning (i.e. leverage intensity) were negligible for all vegetation types. These results suggest that intensive levels of prescribed burning are required to significantly alter fire activity, and that hypothetical plans that more closely reflect those implementable by fire managers will have little impact at an island-wide scale.

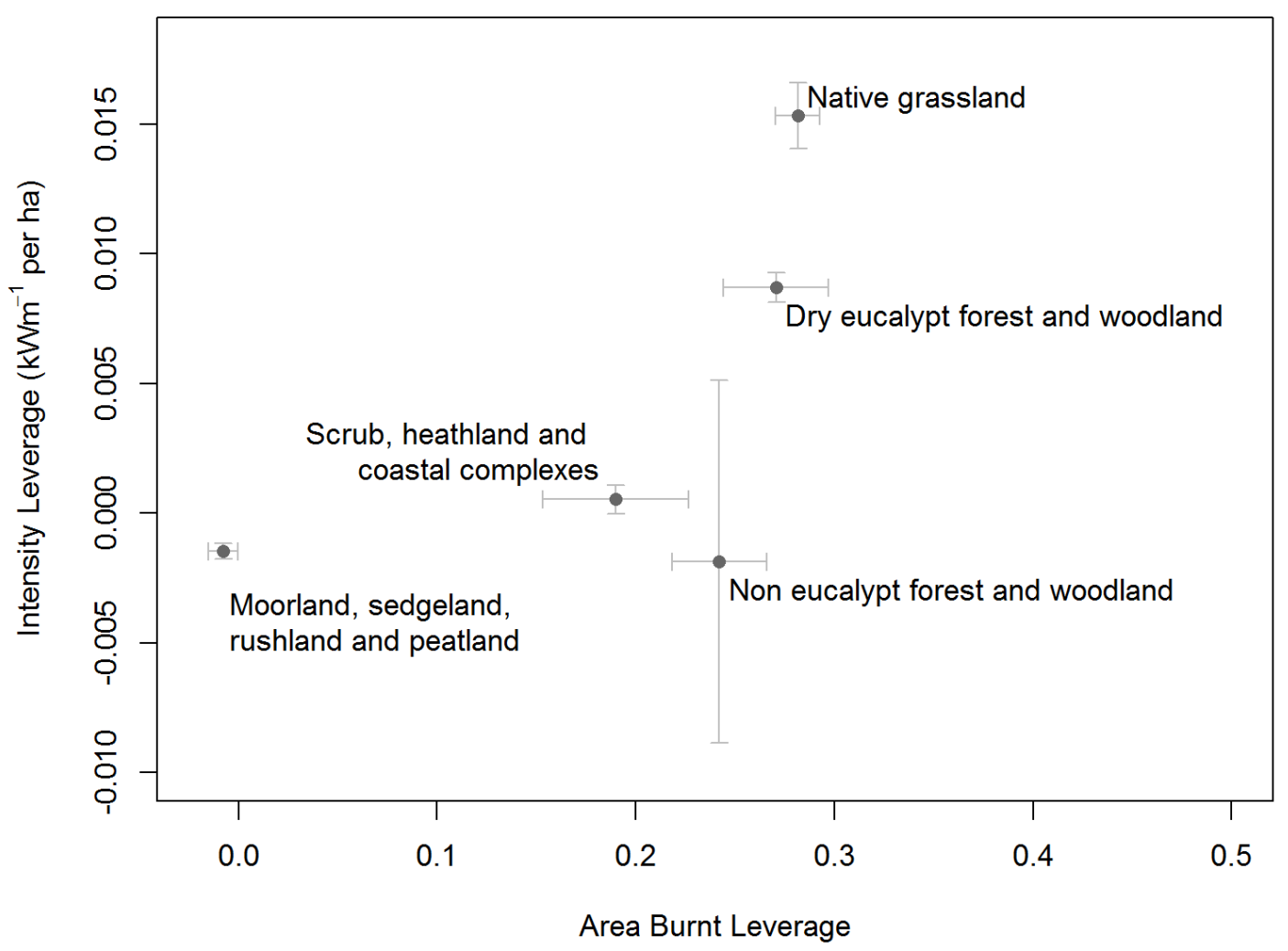

Figure 2 - Ordination of estimated area burnt leverage and intensity leverage (bars represent one standard error) for each of the Tasmanian vegetation types under the implementable prescribed burning scenarios (12 treatments). Area burnt leverage is defined as the reduction in unplanned burning resulting from one unit area treated through planned burning. Intensity leverage is defined as the reduction in intensity in $\mathrm{kW} / \mathrm{m}$ per hectare treated.

This study highlights that prescribed burning can be effective for wildfire mitigation, but suggests that an unrealistically large area would need to be treated with prescribed burning to meaningfully alter fire behaviour across the island of Tasmania. We argue that this indicates a need for intensive, but localised, fuel treatments. Therefore, we conclude that optimisation of prescribed burning requires careful landscape design based on improved fire behaviour modelling, empirical measurement of fuels, and analysis of actual wildfires. 


\title{
Short contribution - Fire Risk Management
}

\section{The role of fire size, geometry, and intensity, in "extreme" plume development}

\author{
Rachel L. Badlan ${ }^{1 *}$; Jason J. Sharples ${ }^{1}$; Jason Evans ${ }^{2}$; Rick H. D. McRae ${ }^{3}$ \\ ${ }^{I}$ School of Physical, Environmental and Mathematical Sciences, University of New South Wales, Canberra, \\ ACT 2600, Australia, \{r.badlan@adfa.edu.au*\} \\ ${ }^{2}$ Climate Change Research Centre, University of New South Wales, Kensington, NSW 2052, Australia \\ ${ }^{3}$ Australian Capital Territory Emergency Services Agency, Canberra, ACT 2600, Australia
}

\begin{abstract}
Fire spread associated with violent pyrogenic convection is highly unpredictable and is difficult to suppress. Wildfire-driven convection may generate cumulonimbus (storm) clouds which are also known as pyrocumulonimbus (pyroCb). Research into such phenomena has tended to treat the fire on the surface and convection in the atmosphere above as separate processes.

This research investigates the relationship between the characteristics of the surface (i.e. the fire) - its shape, size and intensity - and the resultant convective plume. This is achieved using a coupled fireatmosphere model (the Weather Research and Forecast model (WRF)) to identify the combined effects of the fire behaviour and atmospheric structure on the occurence of pyroCb events. This is achieved with idealised model runs with a static heat source of variable dimension and intensity in order to examine the plume's sensitivity to the size and intensity of the fire, and the stability of the atmosphere in which it develops. It also considers the role of geometry of the spatial expanse specifically, as large areal fires (so-called "deep flaming" events) have been associated with the development of pyroCb. Moisture from either the fuel or combustion is not included in the surface fluxes at this point in the study.

Initial results indicate that both the size and intensity of the fire are the variables which control the height to which the pyroconvective plume may rise. It also suggests that given a large enough fire source (deep flaming), these factors are the key determinanats of whether the plume will couple with the atmosphere and that the atmospheric stability may not be as influential in the development of violent pyroconvection if deep flaming is occuring.

One of the metrics used to determine the pyroconvective potential of a plume is the total energy released, which is a function of the fire's area across the ground and intensity of the heat. This research also takes into account the shape of the fire by investigating the ratio between the perimeter of the fire and the area it encompasses. Our research determines how the the shape of the fire affects the height of the plume by comparing circular, square and rectangular fires of varying length and width which represent the difference between firelines and areal fires. This sensitivity study of fire geometry reveals that the perimeter/area ratio influences the amount of entrainment that the plume experiences and therefore the height to which the plume rises before it loses buoyancy. These results will be used to aid in the prediction of blow-up fires and may be useful in determining where fire agencies deploy their limited resources.
\end{abstract}

Keywords: Wildfire simulation, fire-atmosphere interaction, deep flaming, pyroconvection, pyrocumulonimbus

\section{Introduction}

The prediction of violent pyroconvection is an important area of research. The particular geometric characteristics of the fire have not been a major consideration until now, and this research seeks to better understand the relationship of the fire - its shape, size and intensity - and the plume it produces.

These areal fires have been shown to occur in several instances in which a pyroCb developed, some Australian examples being; the 2003 Canberra fires (McIntyres Hut and Bendora Dam), 2006 Grose 
Valley fire, 2013 Wambelong and Aberfeldy fires. These large spatial fires are known as deep flaming (McRae and Sharples 2014), which may encompass several square kilometres and occur when wildfires experience any (or more) of the following triggers:

- Very strong winds - so the head fire advances more rapidly than the back of the flaming zone;

- Changes in wind direction - so the long flank of the fire is transformed into a fast running head fire;

- Eruptive fire behaviour - where steep slopes can cause a fire to accelerate rapidly;

- Vorticity-driven lateral spread - where strong winds and steep terrain interact to rapidly drive a fire laterally, accompanied by downwind spotting (Sharples et al. 2012, Simpson et al. 2013, Simpson et al. 2014); and

- Fire coalescence - including mass spot fires from embers and lightning or merging of fires introduced into the landscape by other means.

As deep flaming has shown itself to be associated with pyroCbs, this study intends to explore this relationship more thoroughly.

\subsection{Method}

\subsubsection{Circular heat sources}

Using the WRF, several idealised models were run using three different static cicular heat sources $\left(25 \mathrm{~kW} \mathrm{~m}^{-2}, 25 \mathrm{~kW} \mathrm{~m}^{-2}\right.$, and $100 \mathrm{~kW} \mathrm{~m}^{-2}$ ) with radii of $150 \mathrm{~m}, 500 \mathrm{~m}, 750 \mathrm{~m}, 1 \mathrm{~km}$ and $1.5 \mathrm{~km}$. All of these were then run in a stable, neutral and unstable atmospheric profile (Fig. 1).

Initial soundings
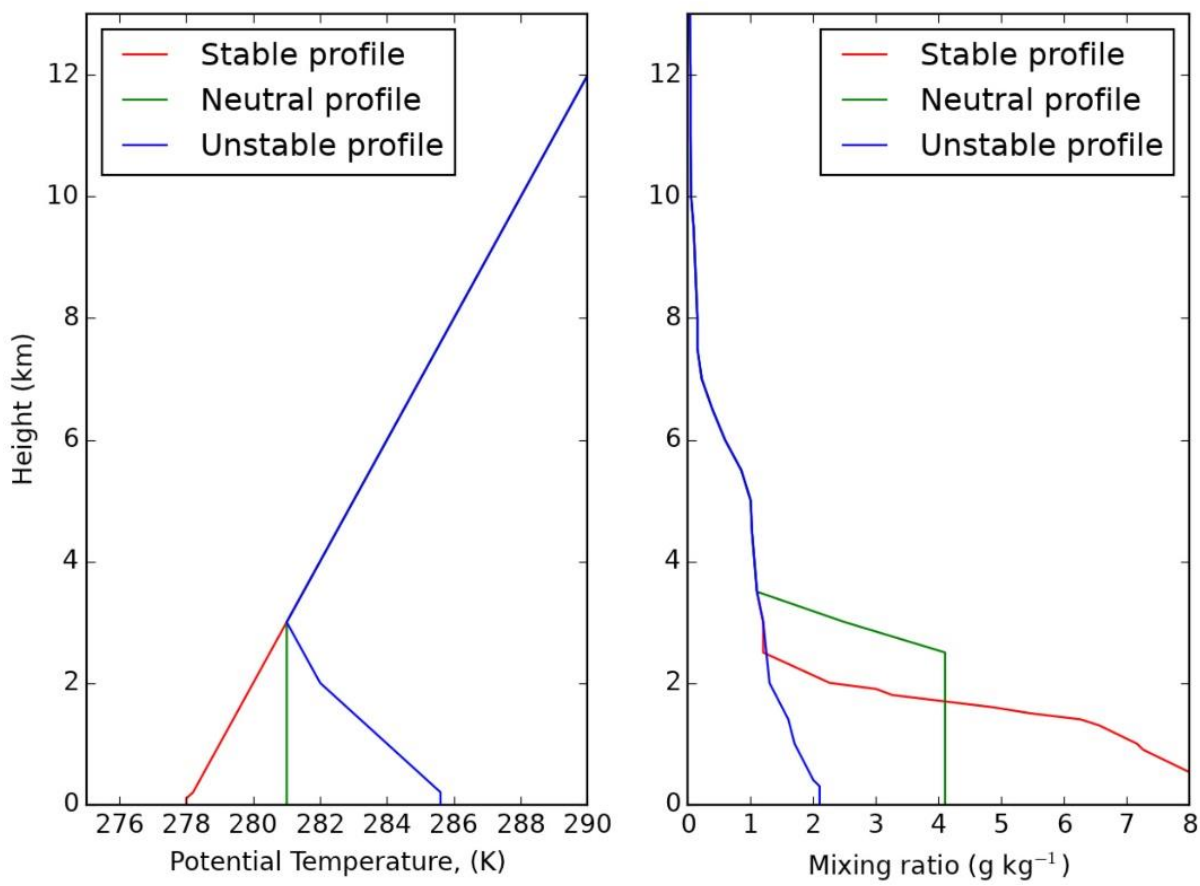

Figure 1 - Input soundings for all model runs of potential temperature in $\mathrm{K}$ (left) and mixing ratio in $\mathrm{g} \mathrm{kg}^{-1}$ (right) for the lowest $13 \mathrm{kms}$ are shown. 
Figure 2 shows cross-sections of four of the different size fires after one hour of the simulation with a neutral atmospheric stability. This profile is typical of high fire danger in Australia with a deep mixed layer. As the heat source increases, the height of the cloud base which represents the Lifting Condensation Level (LCL) increases, as does the cloud top (the level of neutral buoyancy). The larger the heat source, the less the effect of entrainment on the convective column, which means that the plume experiences less dilution and more air reaches the LCL, driving convection. It was also found that the higher intensity heat source had a similar effect on the pyroconvection due to warmer air enhancing the convection.

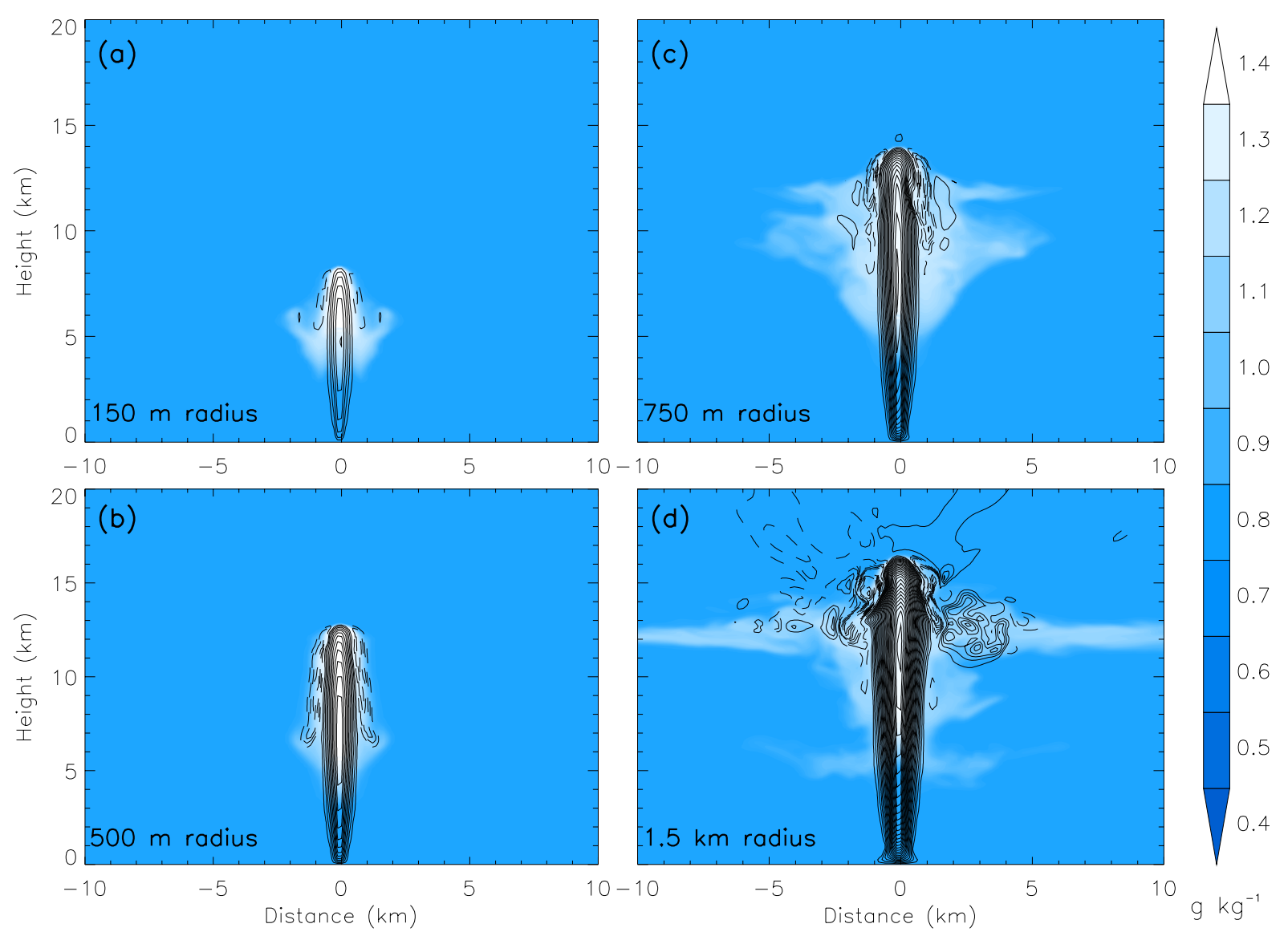

Figure 2 - Cross-sections of the plume after 1 hour simulation time for (a) $150 \mathrm{~m}$ radius, (b) $500 \mathrm{~m}$ radius, (c) $750 \mathrm{~m}$ radius and $(d) 1.5 \mathrm{~km}$ radius fires. The blue/white shading represents the cloud mixing ratio in $\mathrm{g}$ kg-1 and the solid contours are vertical velocity with solid being positive values and dashed, negative. Contours are every 5 m s-1.

\subsubsection{Other shapes}

Figure 3 shows the other shapes of the static heat source that were then run. Each of these fires encompasses the same area as the $1 \mathrm{~km}$ radius fire described in the previous section.

Results show that the geometry of the fire dramatically affects the plume height and longevity of the plume. These results have important implications for the prediction of pyroCb and/or towering pyrocumulus formation and will aid fire risk analysts in determining such dangerous conditions. 

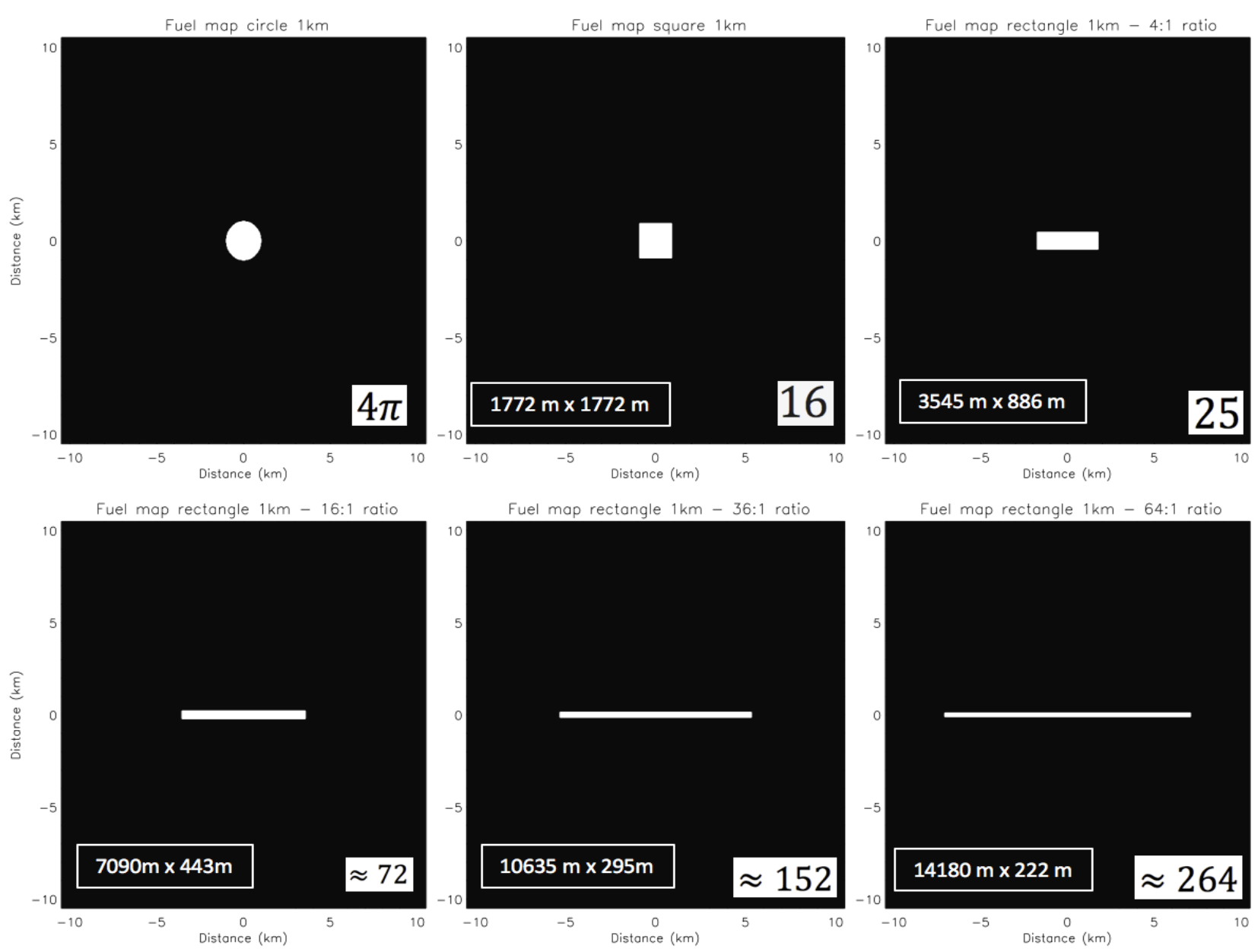

Figure 3 - The fuel maps used to determine how the ratio of the perimeter of the fire to the area affects the plume height. The approximate dimensions (to the nearest metre) are shown in the boxed area and the isoperimetric ratio is shown in the bottom right of each domain.

\section{References:}

McRae, R.H.D. \& Sharples, J.J. (2014). Forecasting conditions conducive to blow-up fire events. CAWCR Research Letters, 11, 14-19.

Sharples, J.J., McRae, R.H. and Wilkes, S.R., 2012. Wind-terrain effects on the propagation of wildfires in rugged terrain: fire channelling. International Journal of Wildland Fire, 21(3), pp.282296.

Simpson, C. C., Sharples, J. J., Evans, J. P., \& McCabe, M. F. 2013. Large eddy simulation of atypical wildland fire spread on leeward slopes. International Journal of Wildland Fire, 22(5), 599-614.

Simpson, C.C., Sharples, J.J. and Evans, J.P., 2014. Resolving vorticity-driven lateral fire spread using the WRF-Fire coupled atmosphere-fire numerical model. Natural Hazards and Earth System Sciences, 14(9), pp.2359-2371. 
Short contribution - Fuel Management

A rapid technique to quantify bark fuel hazard with smartphones

Luke Wallace; Karin Reinke; Samuel Hillman; Bryan Hally; Simon Jones

School of Science, RMIT University, Melbourne, Australia

Bushfire and Natural Hazards Cooperative Research Centre, Melbourne, Australia

\begin{abstract}
In eucalypt forests, tree bark can be a major contributor to the potential spread and behaviour of a wildfire and suppression difficulty. In particular, very fine fibres of bark found on eucalypt stringybark provides highly flammable material that can produce large quantities of embers and short distance spotting. In contrast, long strips or ribbons of bark can produce substantial spotting at distances greater than $2 \mathrm{~km}$. Due to these impacts on fire behaviour, land managers emphasise the importance of assessing bark within their hazard evaluation protocols and may target specific mitigation approaches via prescribed burning to reduce the hazard. Such hazard assessments not only inform prescribed burning priorities but also play an important role as input variables into fire spread and behaviour models. Bark type, thickness, size and shape are assessed in combination with the attachment characteristics to determine the potential hazard posed by the trees within the forest. Having accurate, reliable and quantitative information is necessary for input into these models. To date, assessment of bark hazard relies on qualitative and subjective visual assessments. In this paper we present an alternative to visual assessments by using image analysis and photogrammetry (or remote sensing) techniques applied to photographs taken using smartphones.
\end{abstract}

Keywords: Bark, Fire Behaviour, Fuel Hazard, Remote sensing,

\title{
1. Introduction
}

In eucalypt forests, tree bark can be a major contributor to the potential spread and behaviour of a wildfire and suppression difficulty. In particular, very fine fibres of bark found on eucalypt stringybark provides highly flammable material that can produce large quantities of embers and short distance spotting. In contrast, long strips or ribbons of bark can produce substantial spotting at distances greater than $2 \mathrm{~km}$. Due to these impacts on fire behaviour, land managers emphasise the importance of assessing bark within their hazard evaluation protocols and may target specific mitigation approaches via prescribed burning to reduce the hazard. Such hazard assessments not only inform prescribed burning priorities but also play an important role as input variables into fire spread and behaviour models. Bark type, thickness, size and shape are assessed in combination with the attachment characteristics to determine the potential hazard posed by the trees within the forest. Having accurate, reliable and quantitative information is necessary for input into these models. To date, assessment of bark hazard relies on qualitative and subjective visual assessments. In this paper we present an alternative to visual assessments by using image analysis and photogrammetry (or remote sensing) techniques applied to photographs taken using smartphones.

\section{Method}

\subsection{Bark Class Definitions and Inter-comparison Data}

Bark was classified using the methodology outlined in the Overall Fine Fuel Hazard Guide (McCarthy et al. 1999). This method of assessment separates bark into three main categories stringybark/fine fibrous, ribbon/candle and other bark types. Visual inspection of the bark aims to assign hazard ratings based on the thickness, size and shape of bark pieces, relative quantity of bark 
and the nature of attachment to the tree. The process results in a categorical hazard estimate for the bark on a single stem. For each tree assessed in this study the process outlined in McCarthy et al. (1999) was undertaken for inter-comparison with the remote sensing approach.

\subsection{Processing workflow}

Remote sensing techniques have been recently employed to measure the properties of bark (Othmani et al. 2013). These approaches often employ high resolution images or highly accurate laser scans of the bark surface from which measures of texture can be derived. These texture measures can be then used with machine or deep learning approaches to enable species to be accurately distinguished.

In this paper 3D bark maps are created from three to six overlapping photos captured from a single vantage points. The four photos are imported into Agisoft Photoscan which is used to generate a dense point cloud. The point clouds are then passed through a workflow (figure 1) which allows bark depth to be recovered. Following descriptor metrics are extracted for use in a random forest classifier.

In this study images 350 trees were collected with a minimum of 30 trees of each hazard type. This set of trees was divided into training and validation sets for use with the random forest classifier.

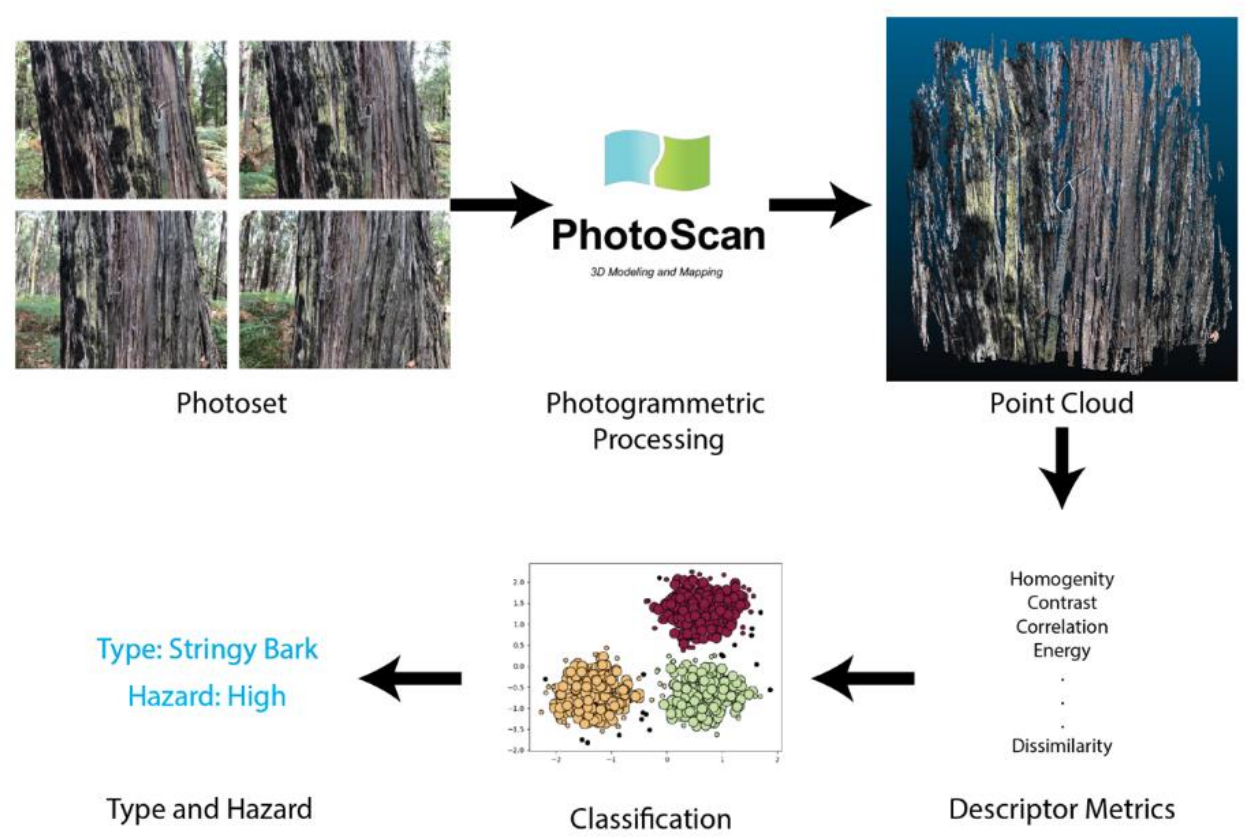

Figure 1 - Workflow used to generate bark texture metrics and the final classification.

\section{Results and discussion}

The bark classification shows a high rate of agreement with field observations of type and hazard. The process can also be run in the field on a smartphone device for real-time assessment of bark hazard.

Although this paper only addresses the bark component of fuel hazard similar methods have been previously used to provide estimates of surface and near-surface fuels with greater reliability than achieved in the visual inspection approach (Spits et al. 2017). Once the accuracy of these methods have been fully established, the metrics, which are quantifiable and objective, will enhance the input data available for use in existing and next generation fire behaviour models. 


\section{References}

McCarthy, G. J., Tolhurst, K. G., \& Chatto, K. (1999). Overall fuel hazard guide. Fire Management, Department of Natural Resources \& Environment.

Othmani, A., Voon, L. F. L. Y., Stolz, C., \& Piboule, A. (2013). Single tree species classification from terrestrial laser scanning data for forest inventory. Pattern Recognition Letters, 34(16), 2144-2150.

Spits, C., Wallace, L., \& Reinke, K. (2017). Investigating surface and near-surface bushfire fuel attributes: a comparison between visual assessments and image-based point clouds. Sensors, 17(4), 910. 
Short contribution - Fuel Management

Aliens \& Flames - a new research initiative joining fire behaviour and invasion ecology

Joaquim S. Silva* ${ }^{* 1,2}$; Ernesto Deus ${ }^{2}$; Mauro Nereu ${ }^{1}$; David A. Davim ${ }^{2,1}$; Carlos G. Rossa ${ }^{3}$

${ }^{1}$ College of Agriculture, Polytechnic Institute of Coimbra, 3040-316 Coimbra, Portugal, \{jss@esac.pt*\}

${ }^{2}$ Centre for Applied Ecology 'Prof. Baeta Neves', School of Agriculture, University of Lisbon, 1349-017

Lisbon, Portugal

${ }^{3}$ Centre for Research and Technology of Agro-environmental and Biological Sciences (CITAB), University

of Trás-os-Montes e Alto Douro, Quinta de Prados, Apartado 1013, 5001-801 Vila Real, Portugal

\begin{abstract}
Wildfires and alien plant invasions are two major environmental concerns in Portugal. Some of the most aggressive invasive plant species are fire-adapted, which causes two problems: on one hand, wildfires may aggravate the invasions and promote the expansion of these species; on the other hand, the expansion of these plant species increases the risk of wildfires. This is the case of Acacia dealbata Link. and Hakea sericea Schrader, two species native to Australia that are problematic invaders in Portugal. Aliens \& Flames is an innovative approach which unites two branches of science that have been following two separate paths and have never been explored together in fire-adapted exotic plant species in Europe: fire behaviour and invasion ecology. This work provides insights into the Aliens \& Flames project, with a particular focus on the methodology and the expected results.
\end{abstract}

Keywords: alien invasive plants; prescribed fire; plant management; wildfires; Acacia dealbata; Hakea sericea

\title{
1. Introduction
}

\subsection{Wildfires and invasive plants in Portugal}

Wildfires are perhaps the most concerning environmental threat in Portugal, the European country most affected by wildfires and one of the most affected worldwide. Over the last decades, wildfires have been aggravating, ravaging Central Portugal particularly in 2017 (ICNF 2017). Alien invasive plants are another major environmental concern in Portugal. These invasions originate direct (e.g. production loss) and indirect costs (e.g. increased fire hazard, ecosystem degradation) and, unlike other degradation processes (e.g. forest fires, soil erosion), their effects are in many cases nearly irreversible (Moreira et al. 2010). Yearly, large amounts of money are spent in the control of plant invasions, which led to the establishment of specific legislation in Portugal (Decree-Law 565/99) and in the European Union (Regulation UE 1143/2014). Some of the most problematic invasive plants are adapted to fire. Frequently, fire facilitates plant invasions which in turn may facilitate fire spread, leading to a fireinvasion cycle (Brooks et al. 2004). In Portugal, Acacia dealbata Link. stands and Hakea sericea Schrader have expanded (ICNF 2013) as a result of the current fire regime (Marchante et al. 2014). Fire acts directly on the A. dealbata soil seed bank, stimulating germination, and the plant resprouts vigorously after canopy damage (Lorenzo et al. 2010). There is also evidence of a strong relationship between $H$. sericea and fire. This serotinous obligate seeder spreads wind-dispersed seeds through fire-triggered dehiscence (Esler et al. 2009), leading to new invasion foci.

In this work, we present a new project started in 2018 that uses a new approach in Europe that addresses both problems - wildfires and plant invasions - by using fire to control fire-adapted invasive plant species. 


\subsection{Alien species and invasion dynamics}

\subsubsection{Target species}

Acacia dealbata is a tree up to 15 meters native to Southeast Australia and Tasmania. The flowers are bright-yellow. The fruits are brownish pods that mature yearly, releasing seeds between June and August (Northern hemisphere). Seeds $(4-5 \times 2.5 \mathrm{~mm}$ in size) are numerous, hard-coated and dormant, and form extensive soil seed banks that can remain viable for decades. Seeds bear an elaiosome, near half their size, highly attractive to ants that may disperse them (Montesinos et al., 2012). Although seeds are not adapted to wind dispersal, they may be transported by water and soil tillage.

Hakea sericea is a shrub or tree up to $4 \mathrm{~m}$, with irregular canopy. The leaves are green to greyishgreen needle shape $(0.5-1.5 \mathrm{~mm}$ diameter $)$, evergreen, very robust and sharp. The flowers, appearing from January to April (Northern hemisphere) are white, bland and arranged in axillary fascicles of 17 flowers. The fruits are woody follicles $(3 \mathrm{~cm})$, dark brown with a patent crest and beak, having two black winged seeds. Dehiscence occurs after tissue necrosis, allowing the release of the seeds (Marchante et al. 2008).

\subsubsection{Invasion dynamics}

Acacia dealbata is invasive in several world regions, such as Asia, South Africa, New Zealand, USA, South America and Madagascar. In Europe, it was introduced by the end of the 18th century as an ornamental species and the first evidence of invasive behaviour occurred in mid-19th century in France. It is a very problematic species in some countries of the Mediterranean Basin (Lazzaro et al. 2014), including Portugal where it was introduced mainly to stabilize soils and as an ornamental. $A$. dealbata is a fire-adapted species with a wide distribution in Portugal. Adaptations include vigorous resprouting, after fire and an extensive soil seed bank triggered by high temperatures. Therefore, after wildfires most individuals survive and new individuals normally arise, invading new areas and replacing the native vegetation. As a consequence, A. dealbata forms dense, monospecific stands, with high fuel load, potentially increasing the risk of wildfires. Management of this species is very difficult, costly, and frequently unsuccessful, because most people cut the individuals that vigorously resprout after treatment.

Hakea sericea is invasive in South Africa (Esler et al. 2009) and Portugal (Marchante et al. 2014). The invasive success of $H$. sericea can be attributed to prolific seed production and serotinous habit. Wind-dispersed seeds are massively released following the death of parent plants by fire. Seeds usually fall within tens of meters away from the parent plant (Richardson et al. 1987), but some may be reach much greater distances, establishing new foci and complicating control (Moody and Mack 1988).

\section{The project}

\subsection{Aims and scope}

Aliens \& Flames is a five-year project run by the Polytechnic Institute of Coimbra. It is an innovative approach that gathers two branches of science that have been following separate paths and have never been explored together in fire-adapted, invasive vegetation in Europe: fire behaviour and invasion ecology.

Aliens \& Flames aims to help improving the control of two of the most concerning environmental threats in Portugal: wildfires and alien plant invasions. We will explore the use of prescribed fire to control and eradicate invasions of $A$. dealbata and $H$. sericea. The main objectives are: a) to provide tools that allow reducing the risk of invasion in areas where prescribed fire is applied to vegetation management to prevent wildfires; b) to improve an alternative technique to control plant invasions; c) to improve forecasting plant invasions in burnt areas; d) to elaborate accurate fuel models for areas invaded by the target species; e) to advise forest owners about the problem of plant invasions and the 
effects of wildfires on plant invasions. The project currently focuses on the two referred target species, but has the ambition of enlarging its scope and encompass other fire-adapted plant invaders.

\subsection{Methods and expected results}

A set of experimental burns will be conducted in invaded areas in Central Portugal (Figure 34), featuring different slash-and-burn treatments corresponding to distinct fire regimes. A comprehensive set of data will be collected before, during and after the burns, including floristic composition and vegetation structure, seed bank properties, plant demography, fire behaviour parameters, soil characterization, and post-fire erosion.

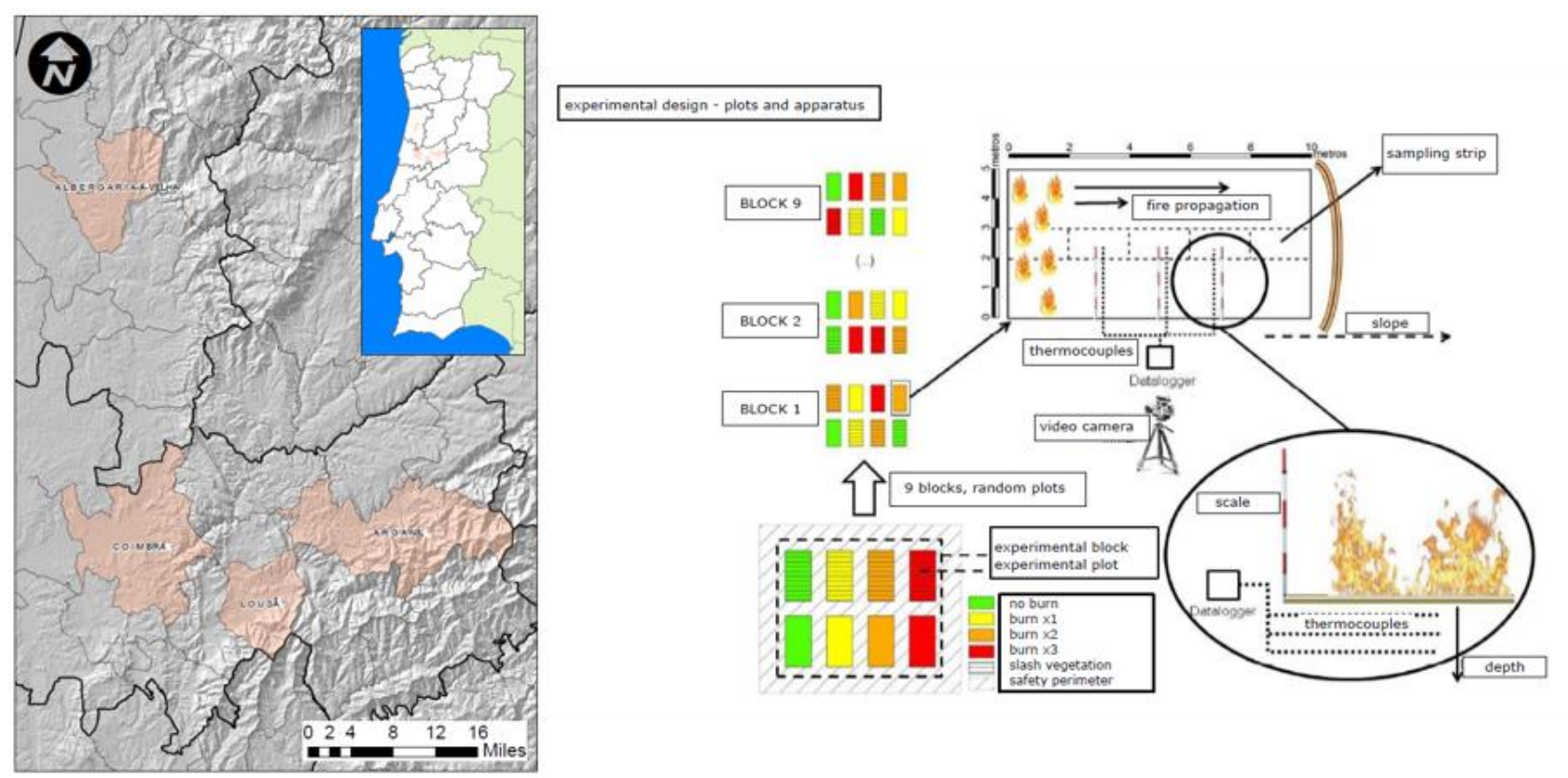

Figure 1 - Central Portugal study areas (left). Experimental design, treatment blocks and instrumentation (right).

Prior to the burns, the fuel complex at each plot will be characterized in order to develop fuel models representing the invaded areas. Plot treatments will allow testing different fire intensities and residence times, aimed at depleting the seed bank and eliminating alien plant individuals. During the burns meteorological parameters, such as air temperature and relative humidity, wind direction and speed, will be recorded using a portable weather station. Fire behaviour (e.g. rate of spread, flame geometry) will be recorded with a video camera, and the soil profile temperatures will be measured using a thermocouple array. First order fire effects will be assessed (e.g. fire severity, fuel consumption, soil heating) immediately after the burn. Plant demography (including native and alien plants), seed bank dynamics and soil erosion will be monitored along the entire project duration (five years).

Expected results, i.e. information on fire behaviour, vegetation dynamics and responses to fire, should provide the necessary knowledge to support fuel and fire management strategies and operations.

\section{Conclusion}

The results of this project will provide insights into the two-way relationships between two important invasive plant species and fire. This knowledge will allow a better management of these species through the use of prescribed burns. It will allow forecasting the extent of new invasions in burned areas and a better assessment of fire hazard in invaded areas. The infrastructure created by Aliens \& Flames through a network of experimental plots, is also an opportunity for collecting data on additional specific aspects of fire ecology, invasion ecology and fire behaviour. Therefore, we invite 
experts in these areas, interested in using this infrastructure for obtaining specific data, to contact the project leader (Joaquim S. Silva; jss@esac.pt) for future collaboration.

\section{References}

Brooks ML, D'Antonio CM, Richardson DM, Grace JB, Keeley JE, Di Tomaso JM, Hobbs RJ, Pellant M, Pyke D (2004) Effects of invasive alien plants on fire regimes. Bioscience 54:677-688.

Esler KJ, van Wilgen BW, te Roller KS, Wood AR, van der Merwe JH (2009) A landscape-scale assessment of the long-term integrated control of an invasive shrub in South Africa. Biological Invasions 12:211-218.

ICNF (2013) IFN6 - Áreas dos usos do solo e das espécies florestais de Portugal continental. Resultados preliminares. Instituto da Conservação da Natureza e das Florestas, Lisboa.

ICNF (2017) 10. ${ }^{\circ}$ Relatório Provisório de Incêndios Florestais - 2017: 01 de Janeiro a 31 de Outubro. Departamento de Gestão de Áreas Públicas e de Proteção Florestal. Instituto da Conservação da Natureza e das Florestas, Lisboa.

Lazzaro L, Giuliani C, Fabiani A, Agnelli AE, Pastorelli R, Lagomarsino A, Foggi B (2014) Soil and plant changing after invasion: The case of Acacia dealbata in a Mediterranean ecosystem. Science of the Total Environment 497:491-498. http://doi.org/10.1016/j.scitotenv.2014.08.014

Lorenzo P, González L, Reigosa MJ (2010) The genus Acacia as invader: the characteristic case of Acacia dealbata Link in Europe. Annals of Forest Science 67, 101.

Marchante E, Freitas H, Marchante H (2008) Guia prático para a identificação de plantas invasoras de Portugal Continental. Imprensa da Universidade de Coimbra, Coimbra.

Marchante H, Morais M, Freitas H, Marchante E (2014) Guia prático para identificação de plantas invasoras em Portugal. Imprensa da Universidade de Coimbra, Coimbra.

Moody ME, Mack RN (1988) Controlling the spread of plant invasions: the importance of nascent foci. Journal of Applied Ecology 25:1009-1021.

Montesinos D, Castro S, Rodríguez-Echeverría S, (2012) Invasive acacias experience higher ant seed removal rates at the invasion edges. Web Ecology 12:33-37.

Moreira F, Catry F, Silva JS, Rego F. (Eds.) (2010) Ecologia do fogo e gestão de áreas ardidas. ISAPress, Lisboa.

Richardson DM, van Wilgen BW, Mitchell DT (1987) Aspects of the reproductive ecology of four Australian Hakea species (Proteaceae) in South Africa. Oecologia 71:1432-1939. 
Short contribution - Fuel Management

Bench-scale measurement of pyrolysis products from intact live fuels

Thomas H. Fletcher ${ }^{1 *}$; Mohammad-Saeed Safdari ${ }^{1}$, Elham Amini ${ }^{1}$, David R. Weise ${ }^{2}$

${ }^{1}$ Chemical Engineering Dept., Brigham Young University. Provo, Utah, USA 84602

\{tom_fletcher@byu.edu*; saeed_safdari_1988@yahoo.com; amini.sharifuni@gmail.com\}

${ }^{2}$ USDA Forest Service, PSW Research Station. 4955 Canyon Crest Dr., Riverside, California, USA 92507,

\{dweise@fs.fed.us\}

\begin{abstract}
Prescribed burning (controlled burning) is used to decrease accumulation of combustible materials and reduce impact of uncontrolled wildland fires. Prescribed fires are often used to burn undergrowth in southern forests of the United States. In order to improve prescribed fire application, accomplish desired fire effects, and limit potential runaway fires, an improved understanding of the fundamental processes related to combustion of heterogeneous fuel beds of live and dead fuels is needed. The initial processes of combustion involve pyrolysis and ignition. During this research, fast pyrolysis of 14 live and dead (biomass) plant species which are native to the southern United States have been studied using a flat-flame burner (FFB) apparatus. The FFB apparatus enables experiments at a high heating rate $\left.\left(\sim 100^{\circ} \mathrm{C} \mathrm{s}^{-1} \text {, or } \sim 100 \mathrm{~kW} \mathrm{~m}\right)^{-2}\right)$ and moderate temperature $\left(\sim 765^{\circ} \mathrm{C}\right)$ to imitate pyrolysis during typical fire spread conditions. Pyrolysis products have been analyzed in detail using a gas chromatograph equipped with a mass spectrometer (GC-MS) for analysis of tars, and a gas chromatograph equipped with a thermal conductivity detector (GC-TCD) for analysis of permanent (light or non-condensable) gases. Differences between yields of light gas species were small between plant species. Composition of tars included aromatic compounds with 1 to 5 rings with very few attachments. The pyrolysis products observed at this temperature and heating rate appear to have experienced secondary pyrolysis. The tar composition showed some large changes with plant species. Comparison of products from pyrolysis of live vegetation and dead vegetation of the same plant species showed differences in tar, gas, and char yields, but no major changes in the types of chemical compounds observed.
\end{abstract}

Keywords: pyrolysis, live shrubs

\title{
1. Introduction
}

Wildland fire is an important component of many North American ecosystems and has been used by humans to accomplish various objectives for several thousand years. Prescribed burning in the southern United States is an important tool used by the Department of Defense and other land managers to accomplish several objectives including hazardous fuel reduction, wildlife habitat management, critical training area maintenance, ecological forestry and infrastructure protection. The vegetation on Department of Defense installations is heterogeneous, unlike the homogeneous fuel beds assumed by current operational fire spread models. These models do not contain fundamental descriptions of chemical reactions and heat transfer processes necessary to predict fire spread and energy release needed for process-based fire effects models. To improve prescribed fire application to accomplish desired fire effects and limit potential escapes, an improved understanding is needed of the fundamental processes related to pyrolysis and ignition in heterogeneous fuel beds of live and dead fuels. The objective of this project is to address several fundamental questions to improve our understanding and modeling capability of fire propagation in natural fuel beds including 1) detailed description of pyrolysis and the evolution of its products for a greater variety of southern fuels than is currently known, 2) how convective and radiative heat transfer from flames to live fuel particles influences pyrolysis and ignition at laboratory and field scales, and 3) more detailed insight into pyrolysis, combustion and heat transfer processes in wildland fire spread through the use of high- 
fidelity physics-based models. This work is part of a larger project which includes trying to measure pyrolysis products in a test bed of vegetation in a wind tunnel as well as in a small field-scale prescribed burn (Weise et al. 2018).

\section{Experimental Approach}

A flat-flame burner (FFB) apparatus was used to study pyrolysis of foliar samples from the plants, as shown in Figure 1. A horizontal fuel sample was attached to a horizontal rod and was suspended in the middle of the glass duct. The rod was attached to a Mettler Toledo XS204 scale. The flat-flame burner structure was placed on wheels which enabled the structure to be moveable. The flat-flame burner was operated under fuel-rich conditions with an equivalence ratio of 1.13, providing a gas background at $765^{\circ} \mathrm{C}$ with no $\mathrm{O}_{2}$. The pyrolysis sampling system consisted of a glass funnel connected to stainless steel tubing wrapped with heating tape and insulation with a cold trap to separate the pyrolysis products. Pyrolysis products were analyzed using (1) a gas chromatograph (GC) equipped with, a thermal conductivity detector (TCD), and (2) a GC combined with a mass spectrometer (MS). In the cold trap, the high molecular weight hydrocarbons were condensed and then analyzed by GCMS after solvent extraction using dichloromethane.

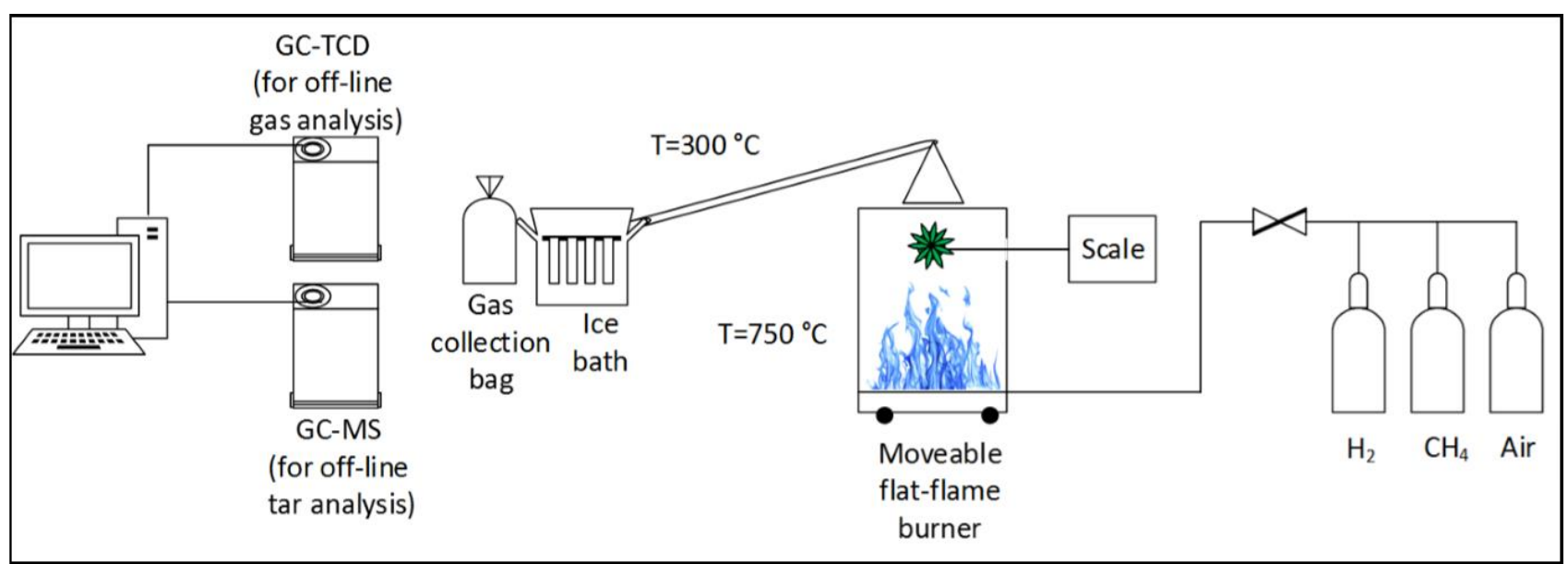

Figure 1 - Flat-flame burner setup used to measure pyrolysis products resulting from heating southern wildland fuels at a high heating rate.

\section{Results}

Figure 2 shows the yields of tar, light gas, and char from the high-heating rate pyrolysis in the FFB system. The results are the average of three experiments and are shown on a dry ash-free basis. The data showed an excellent reproducibility with the standard deviation of less than 5\%. There was not a significant difference in the observed pyrolysis product yields between live and dead samples of a specific plant species. 


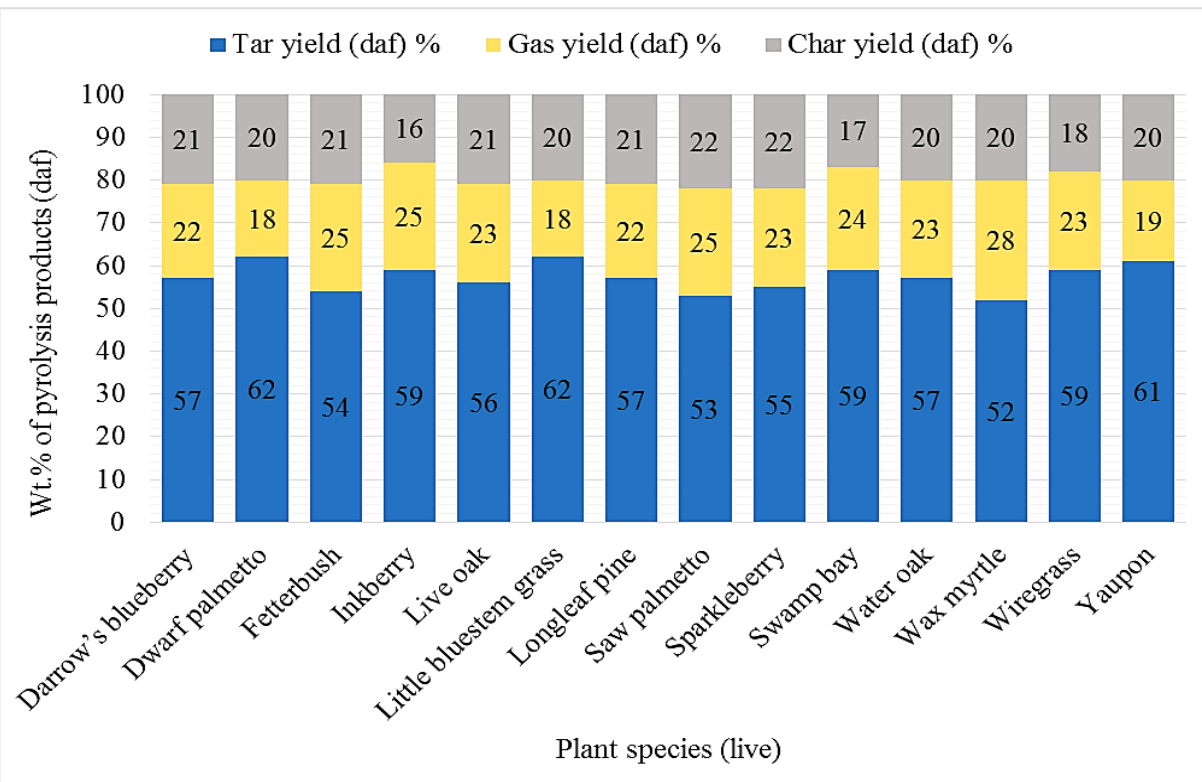

Figure 2 - Yields of tar, gas, and char produced by high-heating rate pyrolysis of live southern fuels (by weight percentage of dry ash-free plant).

Figure 3 shows the species distribution of light gas, presented as wt $\%$ of the light gas on a dry basis (with background gases from the FFB subtracted). The major light gas species for all plants was CO, followed by $\mathrm{CO}_{2}$, then $\mathrm{CH}_{4}$ and $\mathrm{H}_{2}$. The $\mathrm{CO}$ content varied the most for the Swamp Bay plant. Pyrolysis of dead plant species yielded similar results.

Phenol, naphthalene, fluorene, anthracene, phenanthrene, fluoranthene, and pyrene were the major identified tar compounds, with 1 to 5-ring aromatic compounds. Differences in tar composition were observed for each plant species. The tar species data seemed to lie in four general areas: broad leaf, pine needle, palmetto-type, and grasses. For example, in tar analysis, phenol ranged from 6 mole $\%$ in dead little bluestem grass to 36 mole\% in live saw palmetto. See Safdari, et al. (2018) for additional details.

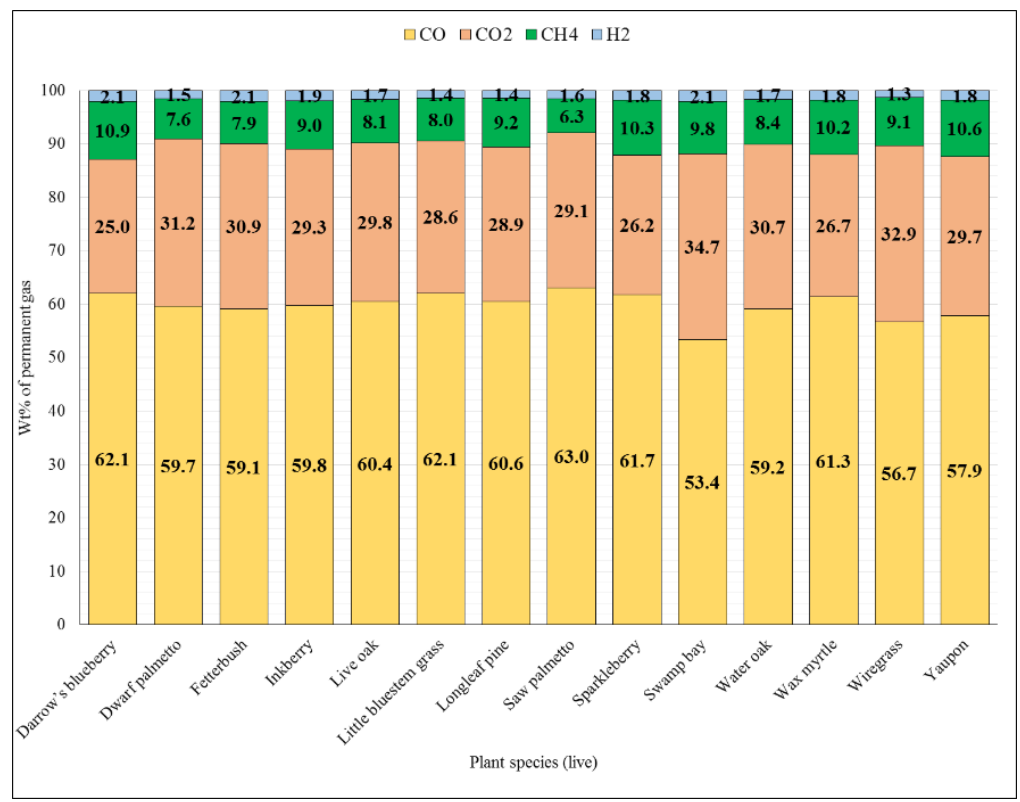

Figure 3 - Composition of permanent (i.e., non-condensable) gas from pyrolysis of live southern fuels (by weight percentage of dry permanent gas). 


\section{Conclusions}

Most plant species from the same type of plant (broadleaf, grass, or needle-like) showed only small differences in yields of the light gas species $\left(\mathrm{CO}, \mathrm{CO}_{2}, \mathrm{CH}_{4}\right.$, and $\left.\mathrm{H}_{2}\right)$. Tar compounds consisted of 1to 5-ring compounds with very few attachments on the rings. Major tar species observed included phenol, naphthalene, fluorene, anthracene, phenanthrene, fluoranthene, and pyrene. Tar compounds from different plant species exhibited a significant difference in the distribution of functional groups.

\section{Acknowledgement}

This work was supported was the DOD/EPA/DOE Strategic Environmental Research and Development Program project RC-2640.

\section{References}

Safdari M-S, Rahmati M, Amini E, Howarth JE, Berryhill JP, Dietenberger M, Weise DR, Fletcher TH (2018) Characterization of pyrolysis products from fast pyrolysis of live and dead vegetation native to the Southern United States. Fuel 229, 151-166.

Weise DR, Fletcher TH, Johnson TJ, Wao W, Dietenberger M, Princevac M, Butler B, McAllister S, O'Brien J, Loudermilk L, Ottmar R, Hudak A, Kato A, Shotorban B, Mahalingham S, Mell WE (2018) A Project to Measure and Model Pyrolysis to Improve Prediction of Prescribed Fire Behavior. In '8th International Conference on Forest Fire Research'. Coimbra, Portugal 
Short contribution - Fuel Management

Fire in wet eucalypt forests: rethinking fuel-accumulation models for Tasmania's most unique fuel type

\author{
J. M. Furlaud* and D. M. J. S. Bowman \\ School of Biological Sciences, University of Tasmania, Hobart, Tasmania, Australia, \\ \{james.furlaud@utas.edu.au*\}
}

\begin{abstract}
Keywords: fuel accumulation, fuel loads, temperate rainforests, sclerophyllous forests, wet eucalypt forests, fire hazard, chronosequence, macro-ecological gradients, climate change
\end{abstract}

\title{
1. Introduction
}

Wet eucalypt forests are potentially the most dangerous fuel type in Tasmania, an island state in Australia's temperate southeast. Their long fire return interval (sometimes hundreds of years) allows for massive accumulation of fuels that could potentially result in the highest fire intensities on Earth. However, there is little empirical data describing fuel loads in these forests, especially in Tasmania, where current fire behaviour models are calibrated using data from ecologically-distinct Victorian forests. Understanding how these fuels accumulate over time is critical for calibrating fire behaviour models for this fuel type.

\section{Methods}

We measured fuels along a chronosequence of 24 plots in southeast Tasmanian wet eucalypt forests, ranging from recently-burnt to old-growth forests. In each plot we measured the structure and finefuel biomass in tonnes per hectare ( $\mathrm{t} / \mathrm{ha}$ ) of the surface fuels, elevated fuels, and canopy bark. We also recorded qualitative fire-hazard scores and measured temperature and humidity of the understorey climate. With this data, we developed novel fuel-accumulation models to describe fuel loads and fire danger as a function of time since previous fire. We also measured fuels across the TERN Ausplots Forest Monitoring Network: 48 permanent plots in mature tall wet eucalypt forests across a variety of Australian climates. This provided insight into how the structure and fuel load might change in Tasmania's forests as the climate warms.

\section{Results \& Discussion}

In developing novel fuel-accumulation models for Tasmania's wet eucalypt forests, we found that, even though overall fuel biomass continues to increase as a forest ages, the trends in fuel hazard in the different layers are substantially more complex. In general, however, it appears that while fuel hazard increases, sometimes dramatically so, directly after a fire, it decreases as a forest matures into old growth forest (Figure 1). Additionally, after mid-successional stages, the composition of the understorey (i.e. the elevated fuel layer) shifts from moderately-flammable sclerophyllous species to less-flammable rainforest species. This shift is not represented in the hazard scores, suggesting that the reduction in flammability in mature forest understoreys is even greater than is indicated by Figure 1. Additionally, our understorey temperature and humidity measurements indicate that as the forest ages, and the canopy closes, a cooler and moister microclimate develops (Figure 2), further reducing fire hazard. Analysing the data from our continental-scale 

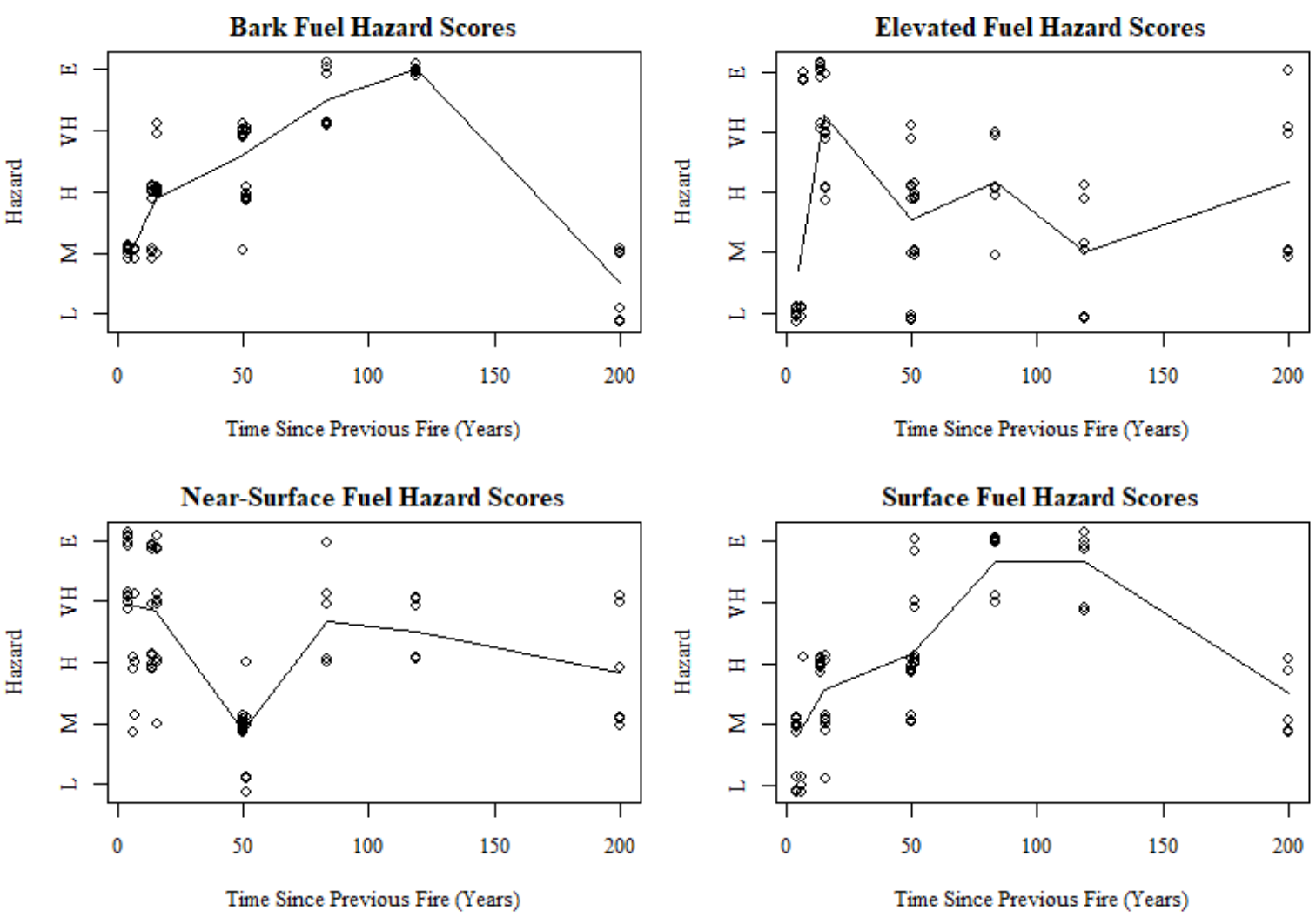

Figure 1 - Qualitative hazard scores, as used by local fire agencies, plotted against stand age, as measured by time since previous fire for each of the chronosequence plots. Hazard scores are presented for the bark, elevated, nearsurface, and surface fuel layers. The black line represents the trend for the mean hazard score for each age class.

Hazard score abbreviations are as follows: L-Low, M-Moderate, H-High, VH-Very High, E-Extreme.

(a)

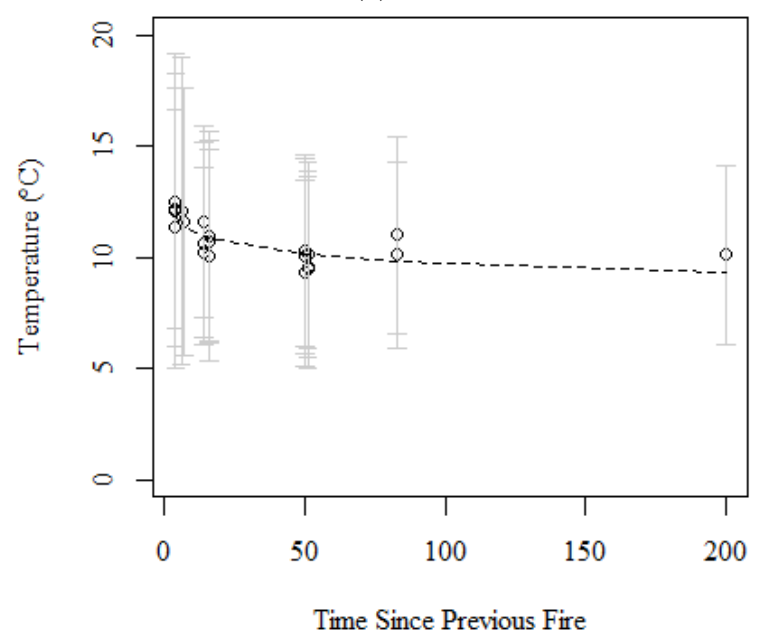

(b)

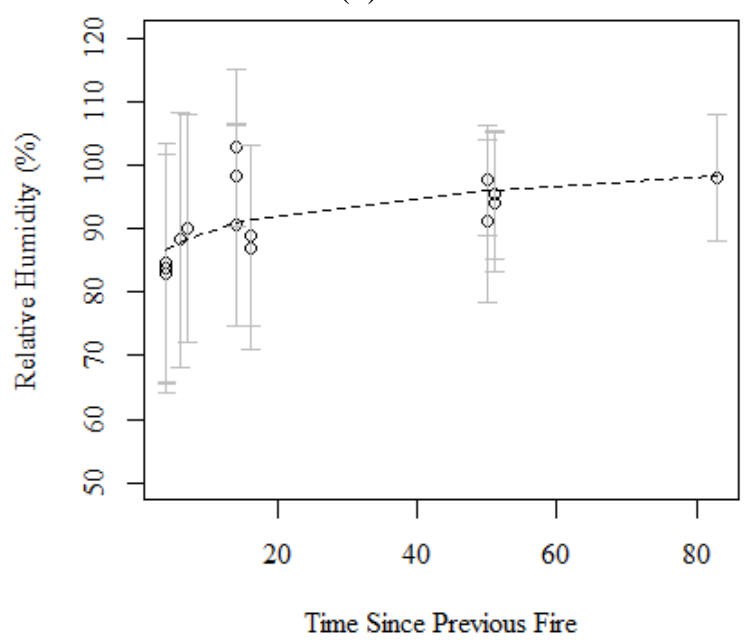

Figure 2 - Mean temperature (a) and relative humidity (b) in the understorey, as measured by data loggers, plotted against time since previous fire for each of the chronosequence plots. Dots represent the mean of measurements from the six-month period between August 2016 and January 2017. Grey bars represent one standard deviation. The dashed line represents the best-fit line according to a generalised linear model.

Ausplots Forest Monitoring Network, we found fuel biomass and structure in each layer to be highly variable across different climates. Generally speaking, warmer climates appear to support forests with 
more continuous vertical fuel structure and more regular crown fires. This could suggest that climate change will alter the structure, and hence the fire regime of Tasmania's wet eucalypt forests, however a more detailed analysis is required. Perhaps more importantly, we found the vertical structure of these fuels to be highly complex along both chronological and climatic gradients. This is problematic as fire behaviour models currently treat fuel load as a singular mass. Our research suggests a need for a more multifaceted representation of fuel loads in these models. 
Short contribution - Fuel Management

Fire, landscape pattern and biodiversity: Using fire for conservation in a south-eastern Australian ecosystem

\author{
Alan York*; Julian Di Stefano; Holly Sitters; Matthew Swan \\ School of Ecosystem and Forest Sciences, University of Melbourne. 4 Water Street Creswick VIC Australia \\ 3363, \{alan.york@unimelb.edu.au*\}
}

Keywords: animal diversity, fire mosaic, pyrodiversity, species richness

\title{
1. Introduction
}

Fire is an important process that influences species and ecosystems worldwide. By changing the availability of resources for organisms in space and time, fire has the potential to be a powerful driver of species diversity. Biodiversity conservation in fire-prone environments presents a challenge for management because it is unclear how best to apply planned burns to the landscape. It is widely assumed that spatially and temporally variable fire regimes benefit biodiversity conservation. This is based on ecological theory, which predicts positive associations between species diversity and environmental heterogeneity. A mosaic of fire histories constitutes a mosaic of environmental states, which is generated by differences in the resetting, or partial resetting, of the successional process in time and space.

Although the notion that variable fire regimes benefit biodiversity is grounded in ecological theory, it has rarely been tested. As the application of planned (prescribed) fire is becoming more common amid forecasted increases in frequency and severity of wildfire under climate change, we need to better understand the relationship between fire regimes and biodiversity. The principal aim of this research was to test the theorised relationship between native animal diversity and landscape spatial pattern complexity within a fire-prone ecosystem. We sought to examine how animal communities are influenced by (i) fire-mediated spatial pattern ('pyrodiversity'), which is subject to control by land managers, and (ii) spatial pattern in vegetation type, which remains relatively static. We differentiated between these two influences by mapping fire age class and vegetation type separately, and tested predictions about the response of animal diversity to the observed spatial patterns.

\section{Methods}

This study was conducted in a 59,000 ha section of the Otway Ranges in southern Victoria, Australia. The climate of this region is temperate with cool wet winters and warm dry summers. In response to an elevation and rainfall gradient, vegetation communities change from areas of treeless heath in the north east to tall wet forests in the south. The study area has been subject to wildfires in 1939, 1967 and 1983, with prescribed fire undertaken extensively for fire mitigation purposes over the last 10 years.

For the purposes of this study we defined 100 ha 'landscapes' as the sampling unit. Thirty-six landscapes ('mosaics') were stratified according to the composition of both fire age classes and vegetation types. Five sub-sampling sites based around $100 \mathrm{~m}$ transects were established in each mosaic (Figure 1). We surveyed diurnal birds using two 10 minute interval counts at the 20 and $80 \mathrm{~m}$ marks of each transect (Sitters et al. 2014a,b). We surveyed terrestrial mammals using a combination of trapping and wildlife camera detection (Swan 2014, Swan et al. 2015). We also quantified a number of habitat variables along each transect. We used generalized linear mixed models to investigate how animals respond to landscape diversity and configuration. We also used generalized additive mixed models to explore relationships between fire, habitat structure and animal abundance. 


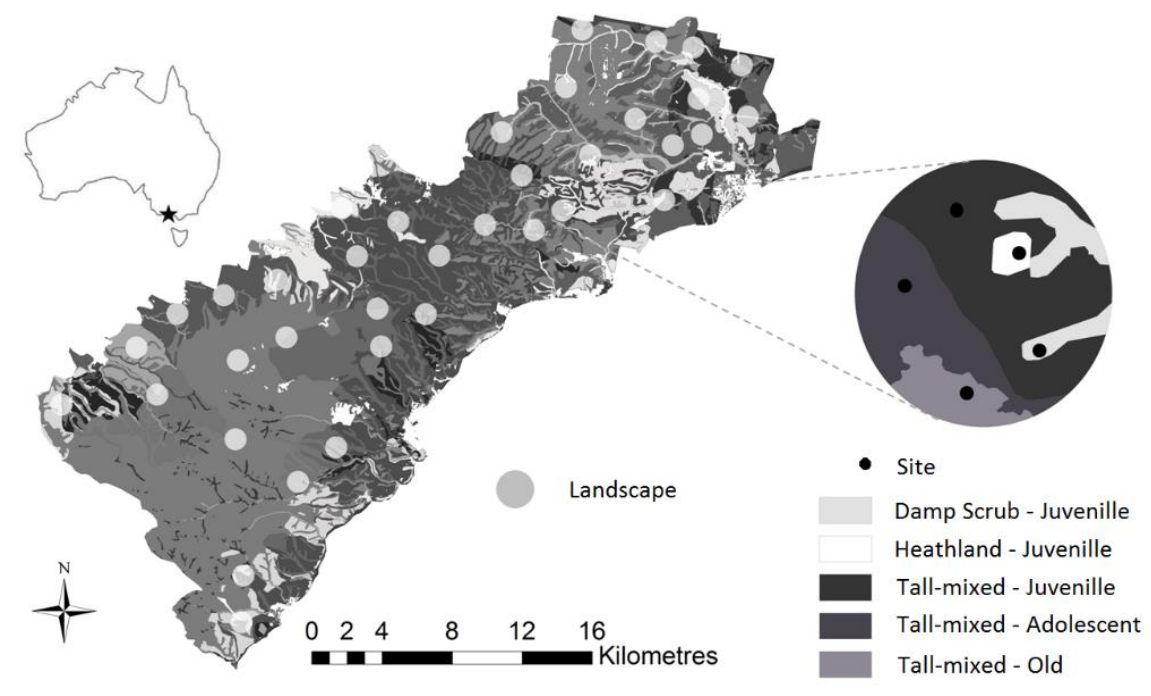

Figure 1 - Location of the study area in the Otway Ranges, southern Australia showing study landscapes. Different shades represent combinations of vegetation type and age class. The blowout shows a single landscape (mosaic) containing three vegetation types and three age classes.

\section{Results}

For birds, species richness was positively associated with fire age class diversity, age class configuration and vegetation type diversity. Habitat structure variables predicted the occurrence of 13 bird species, however overall, time since fire was a poor predictor.

We found that although time since fire was moderately correlated with habitat structure, it was a poor surrogate for mammal occurrence. Variables representing habitat structure were better predictors. Overall, terrestrial mammal diversity was not related to pyrodiversity but to spatial heterogeneity in habitat structure.

\section{Discussion}

This study is one of a few landscape-scale analyses to provide empirical support for the widely held assumption that spatially variable fire regimes can increase the diversity of faunal assemblages. Managers can potentially increase bird species richness by increasing both the diversity of fire age classes, and the complexity of age class configuration, within relatively small areas. However, our results suggest that time since last fire, a measure commonly used in management, is unlikely to be a useful surrogate for either birds or ground dwelling mammals. At landscape scales, fire can be used to positively influence mammal diversity by increasing the spatial variability in structure.

Time since fire is currently used by Australian land managers to define post-fire vegetation growth stages. The application of planned fire to create a mosaic of growth stages underpins current ecological fire strategies. However, our research has demonstrated that time since fire is, by itself, a poor surrogate for faunal occurrence in these forests. To better conserve biodiversity in these landscapes we need a improved understanding of the relationship between fire regime variables and habitat structure. More accurate mapping of fire severity and patchiness, and the inclusion of these factors in fire management planning, is likely to lead to a more sophisticated understanding of the role of fire in conserving native animal communities.

\section{References}

Sitters H, Christie F, Di Stefano J, Swan, M, Penman T, Collins P, York A (2014a) Avian responses to the diversity and configuration of fire age classes and vegetation types across a rainfall gradient. Forest Ecology and Management 318, 13-20. 
Sitters H, Christie F, Di Stefano J, Swan M, Collins P, York A (2014b) Associations between occupancy and habitat structure can predict avian responses to disturbance: implications for conservation management. Forest Ecology and Management 331, 227-236.

Swan M (2014) Interactions between fire, environmental heterogeneity and ground-dwelling mammals. Unpubl. PhD Thesis. Department of Forest and Ecosystem Science, The University of Melbourne, Australia.

Swan M, Christie F, Sitters H, York A, Di Stefano J (2015) Predicting faunal fire responses in heterogeneous landscapes: the role of habitat structure. Ecological Applications 25, 2293-2305. 
Short contribution - Fuel Management

FireCaster Decision Support System: on the need for a new fuel description approach

\author{
Y. Pérez-Ramirez Y*; JB Filippi; L Ferrat \\ UMR SPE 6134 CNRS, Université de Corse, 20250 Corte, \{perez-ramirez@univ-corse.fr*, filippi@univ- \\ corse.fr,ferrat@univ-corse.fr\}
}

\begin{abstract}
The goal of the FireCaster project is to develop a new generation national (French) scale Wildfire Decision Support System (FCDSS) to provide operational agencies more efficient ways to forecast fire danger and handle ongoing crisis. A key task of the project concerns fuel, defined as dead and live biomass. On the one hand, fuel characteristics are required inputs for fire behavior models, which are used in the framework of the project to predict the fire front evolution and to compute probabilistic risk by means of mass ensemble simulations. On the other hand, one of the objectives of the project is developing the methods and procedures to predict fuel state (e.g. live and dead fuel moisture content, fuel loading) and its spatiotemporal evolution across the landscape from an operational land surface model. To respond to these needs it is primary necessary to determine which fuel variables are needed and at which scale and level of detail, and to conceive the data structure to store the geo-referenced fuel variables. Fuel description systems have been traditionally developed to match input variables of a particular fire behavior model. However, advances in computing capabilities and simulations techniques have opened up new possibilities for modelling fire behavior and fuels at finer scales. FCDSS has been designed with genericity and evolvability in mind, in order to match a variety of uses and sources of fuel data. In order to tackle these challenges, the FireCaster Fuel Descriptions System (FCFDS) has been defined based on the review of existing fuel classification and descriptions systems, fuel input parameters of the current fire behavior models and the goals of the FireCaster project. In the FCFDS a vegetation stand is composed by one or more structurally distinct pseudo-homogeneous georeferenced fuel layers, which in turn are composed by fuel elements (i.e. individual plant of a stand, e.g. tree, shrub) or fuel particle arrangements (e.g. herb patch) sharing the same structural, physical, chemical and physiological response characteristics. Fuel elements are in turn composed by different sizes and types of fuel particles (i.e. leaves, twigs, branches). A set of fuel layer attributes has been defined to describe the state, structure and arrangement of fuel. Fuel layer attributes related to fuel state will be predicted by using the operational land surface model SURFEX, which takes into account the soil-vegetation-atmosphere interactions. Correspondingly, a set of fuel elements and fuel particles attributes has been defined. These attributes can be shared by elements and particles in fuel layers located at different positions in the landscape. The way fuel data is structured in FCFDS allows to consider different levels of detail, from the stand scale to the particle scale. Moreover, linkages to different fire behavior models will be developed by means of adaptors. This paper introduces the framework for the definition of the FCFDS, as well as the structure, the associated fuel attributes and the range of spatial scales considered. Moreover, an example of application will be presented.
\end{abstract}

Keywords: Fuel modelling, Fuel attributes, Fuel Description System, Wildfires

The goal of the FireCaster project is to develop a new generation national (French) scale Wildfire Decision Support System (FCDSS) to provide operational agencies more efficient ways to forecast fire danger and handle ongoing crisis. A key task of the project concerns fuel, defined as dead and live biomass. On the one hand, fuel characteristics are required inputs for fire behavior models, which are used in the framework of the project to predict the fire front evolution and to compute probabilistic risk by means of mass ensemble simulations. On the other hand, one of the objectives of the project is developing the methods and procedures to predict fuel state (e.g. live and dead fuel moisture content, fuel loading) and its spatiotemporal evolution across the landscape from an operational land surface 
model. To respond to these needs it is primary necessary to determine which fuel variables are needed and at which scale and level of detail, and to conceive the data structure to store the geo-referenced fuel variables.

The way fuel is described, this is the set of categories, components and fuel variables used for describing fuel, depends on the intended application (i.e. fire management, fire behavior, fire ecology), the spatial and temporal resolution scale and the available resources (i.e. data, technology, fuel sampling techniques). Fuel description systems have been traditionally developed to match input variables of a particular fire behavior model. However, it is necessary to reconsider fuel description across the landscape in order to take into account advances in the knowledge on fire behavior and its modelling, fire risk, pollutants emissions, fuel ecology and fuel dynamics (Keane, 2013). Moreover, advances in computing capabilities and simulations techniques have opened up new possibilities for modelling fire behavior and fuels at finer scales (Parsons et al., 2011). In the same way, progresses in remote sensing (e.g. LIDAR, satellite) have led to the improvement of the quality and resolution of available fuel-related data but also to the increase in the volume of data to manage and store.

FCDSS has been designed with genericity and evolvability in mind, in order to match a variety of uses and sources of fuel data. In order to tackle these challenges, the FireCaster Fuel Descriptions System (FCFDS) has been defined based on the review of existing fuel classification and descriptions systems (e.g. Ottomar et al., 2007; Hollis et al., 2015), fuel input parameters of the current fire behavior models and the goals of the FireCaster project. The FCFDS will be firstly implemented and tested in the Corsican Region by re-analysis of past fire events and mass ensemble simulations. Moreover, in the FCFDS linkages to different fire behavior models (i.e. semi-physical, physics-based) will be developed by means of adaptors (Figure 1).

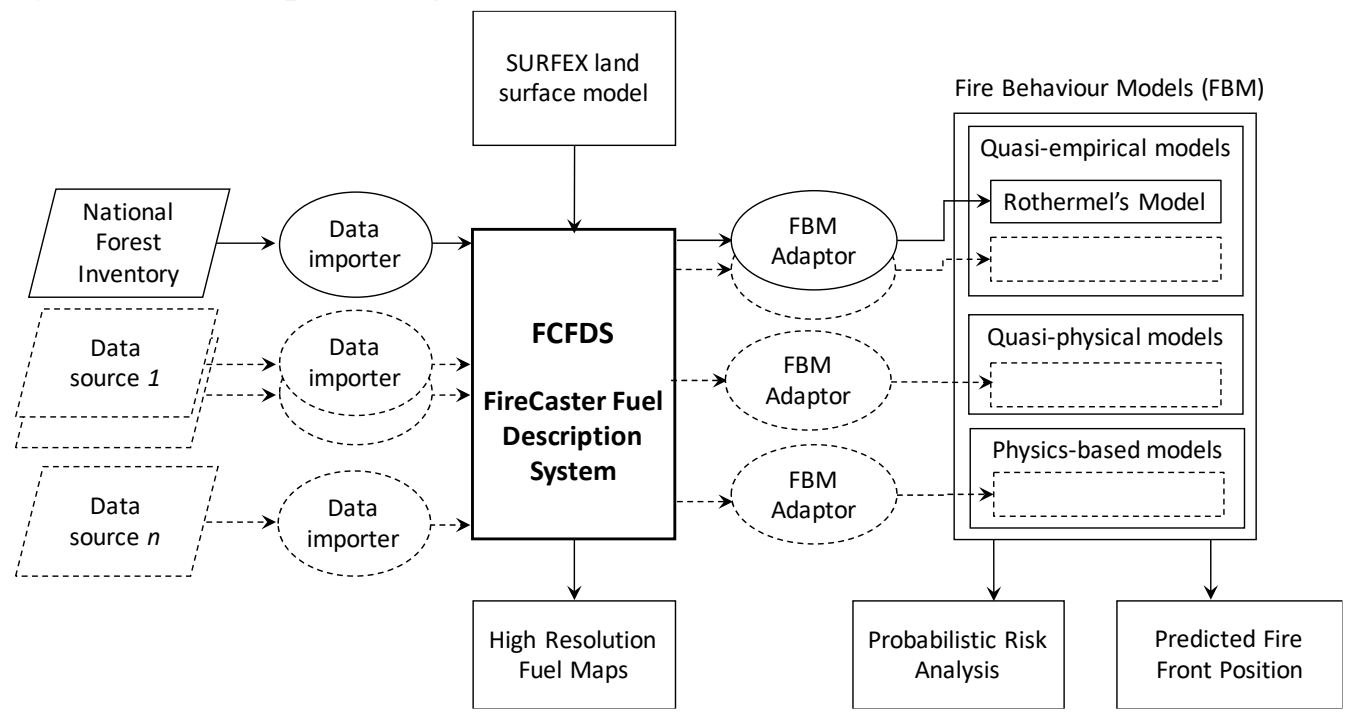

Figure 1 - Overall view of the FireCaster Fuel Description System (FCFDS) and its interactions to other elements of the Fire Caster Decision Support System (FCDSS).

This paper introduces the framework for the definition of the FCFDS, as well as the structure, the associated fuel attributes and the range of spatial scales considered. An example of application to a fuel stand will be presented as well.

The way fuel data is structured in the FCFDS allows to consider different levels of detail, from the stand scale to the particle scale. In the FCFDS a vegetation stand is composed by one or more structurally distinct pseudo-homogeneous geo-referenced fuel layers (spatial resolution ranging from $50 \mathrm{~m}$ to $5 \mathrm{~m}$ ), which in turn are composed by fuel elements (i.e. individual plant of a stand, e.g. tree, shrub) or fuel particle arrangements (e.g. herb patch) sharing the same structural, physical, chemical and physiological response characteristics (Figure 2). It is important to note that a fuel layer does not 
necessarily corresponds to a vegetation stratum, and that a vegetation stratum can be described by several fuel layers. Fuel elements are in turn composed by different sizes and types of fuel particles (i.e. leaves, twigs, branches).
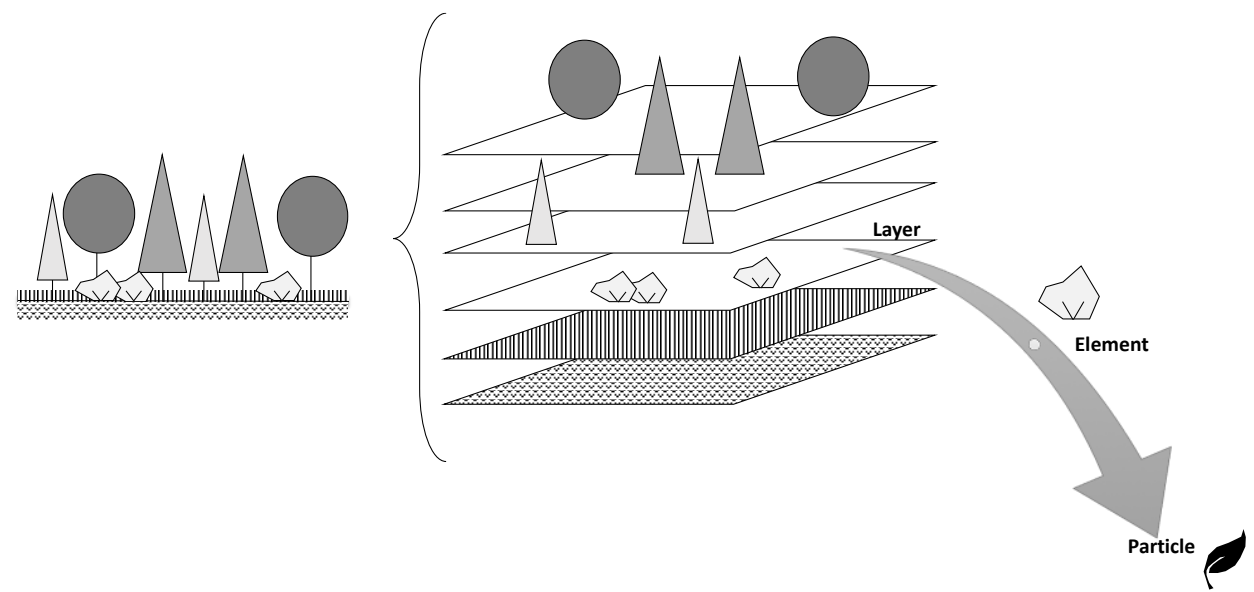

Figure 2 - Example scheme of the different layers composing a fuel stand.

A set of fuel layer attributes has been defined to describe the state, structure and arrangement of fuel. These fuel layer attributes are unique for a certain point in the landscape and time evolving. Fuel layer attributes related to fuel state will be predicted by using the operational land surface model SURFEX (Masson et al., 2013), which takes into account the soil-vegetation-atmosphere interactions. The number of fuel layers to cosider in a certain point in the landscape will be partly derived from the French Forest Inventory. Correspondingly, a set of fuel elements and fuel particles attributes has been defined. These attributes (i.e. physical, chemical and ecophysiological properties) can be shared by elements and particles in fuel layers located at different positions in the landscape. Fuel elements and fuel particle attributes, obtained from the literature, include data values, but also allometric relationships and other simple fuel models describing spatiotemporal variations of fuel attributes.

\section{References}

Hollis JJ, Gould JS, Cruz MG, McCaw WL (2015) Framework for an Australian fuel classification to support bushfire management. Australian Forestry, 78, 1 - 17.

Keane RE (2013) Describing wildland surface fuel loading for fire management: a review of approaches, methods and systems. International Journal of Wildland Fire, 22, 51 - 62.

Masson V, Le Moigne P, Martin E, Faroux S, Alias A, Alkama R, Belamari S, Barbu A, Boone A, Bouyssel F, Brousseau P, Brun E, Calvet JC, Carrer D, Decharme B, Delire C, Donier S, Essaouini K, Gibelin AL, Giordani H, Habets F, Jidane M, Kerdraon G, Kourzeneva E, Lafaysse M, Lafont S, Lebeaupin Brossier C, Lemonsu A, Mahfouf JF, Marguinaud P, Mokhtari M, Morin S, Pigeon G, Salgado R, Seity Y, Taillefer F, Tanguy G, Tulet P, Vincendon B, Vionnet V, and Voldoire A (2013) The SURFEXv7.2 land and ocean surface platform for coupled or offline simulation of earth surface variables and fluxes. Geoscientific Model Development, 6, 929-960.

Ottmar RD, Sandberg DV, Riccardi CL, Prichard SJ (2007) An overview of the Fuel Characteristic Classification System - quantifying, classifying, and creating fuelbeds for resource planning. Canadian Journal of Forest Research, 37, 2383 - 2393.

Parsons R, Mell WE, McCauley P (2011) Linking 3D Spatial models of fuels and fire: effects of spatial heterogeneity on fire behavior. Ecological Modelling, 222, 679-691. 
Short contribution - Fuel Management

\title{
Mid-term effects of thinning on canopy variables related to crown fire hazard on pure, even- aged pine stands
}

\author{
Stéfano Arellano Pérez ${ }^{1 *}$; Fernando Castedo-Dorado 2 ; Juan Gabriel Álvarez-González ${ }^{1}$; Jose Antonio \\ $\mathrm{Vega}^{3}$; Ana Daría Ruiz-González ${ }^{1}$ \\ ${ }^{1}$ Department of Agricultural and Forestry Engineering. University of Santiago de Compostela. Campus \\ universitarios/n 27002,Lugo,Spain.\{stefano.arellano@usc.es*, juangabriel.alvarez@usc.es, \\ anadaria.ruiz@usc.es\} \\ ${ }^{2}$ Departamento de Ingeniería y Ciencias Agrarias, Universidad de León, Campus de Ponferrada, 24401, \\ Ponferrada, Spain. \{fcasd@unileon.es\} \\ ${ }^{3}$ Centro de Investigación Forestal de Lourizán, PO Box 127, 36080, Pontevedra, Spain. \\ \{joseantonio.vega.hidalgo@xunta.gal\}
}

\begin{abstract}
One of the main objectives of forest stand management in Mediterranean areas of Europe is the mitigation of crown fire hazard. Effective silvicultural strategies for reducing the likelihood and intensity of crown fires include reducing surface fuels, increasing canopy base height $(C B H)$ and reducing canopy bulk density $(C B D)$. All these fuel variables depend to a certain degree on stand structure and are therefore responsive to stand density management through thinning treatments. It is well known that canopy fuel load $(C F L)$ and $C B D$ diminish and $C B H$ increases immediately after thinning from below. Nevertheless, for how long the thinning effect be sustained and whether it could be species-specific is less clear. We examined how different levels of thinning intensity altered canopy fuel variables in the mid-term in Pinus pinaster and Pinus radiata stands. We used data from 41 thinning trial locations installed in pure, even-aged stands of $P$. pinaster (22 locations) and $P$. radiata (19 locations). At each location, three rectangular plots were established and a different treatment was applied to each plot: control (unthinned), low thinning (20\% of the basal area removed), and heavy thinning ( $40 \%$ of the basal area removed). Six years after treatments, $C B D$ and $C F L$ in treated plots were still significantly lower than in untreated plots for both species. Moreover, for radiata pine, $C B D$ values were even lower than in the pre-thinning situation. On the other hand, $C B H$ did not show any change following thinning in $P$. pinaster, whereas for $P$. radiata, heavy thinning resulted in significantly lower $C B H$ values since four years after the treatment. The results highlight that thinning may only have a limited impact towards diminising the potential for crown initation, especially in maritime pine stands, altough they could significantly influence the crown fire rate of spread in stands of both species.
\end{abstract}

Keywords: Canopy fuel load, Canopy Base Height, Canopy Bulk Density, Maritime pine, Radiata pine

\section{Introduction}

Mediterranean pine stands without proper fuel management are prone to high-intensity crown fires. Effective silvicultural strategies for reducing the likelihood and intensity of crown fires are aimed to decrease quantity and continuity of forest fuels. The initiation and spread of crown fires in pine stands are strongly influenced by three canopy fuel stand variables: canopy base height $(C B H)$, canopy fuel load (CFL) and canopy bulk density (CBD) (Van Wagner, 1977). These variables are responsive to stand density management, i.e., thinning treatments (Ruiz-González et al., 2015; Hevia et al., 2018). Although it is well known that both $C F L$ and $C B D$ diminish and $C B H$ increase immediately after thinning from below, for how long that effect will be sustained is less clear. The main objective of this work was to evaluate the mid-term effects of thinning on these variables in pine stands. 


\section{Material and Methods}

The study area was located in the North-west of Spain. The dataset corresponds to 41 thinning trial locations installed in pure, even-aged stands of Pinus pinaster (22 locations) and $P$. radiata (19locations). At each location, three rectangular plots $\left(1000 \mathrm{~m}^{2}\right.$ in size $)$ were established and a different treatment was applied to each plot: control (unthinned), low thinning (20\% of the basal area removed) and heavy thinning (40\% of the basal area removed). The plots were thinned from below immediately after plot establishment in 2009-10 and were re-measured in winter 2011-12, winter 201314 and winter 2015-16.

The values of $C B H, C F L$ and $C B D$ were estimated from tree diameter, height and height to the base of live crown measurements. The 'load over depth' method', proposed by Van Wagner (1977), was used to define these variables. According to this method, $C B H$ was calculated as the vertical distance between the ground surface and the mean crown base height; $C F L$ was calculated as the biomass of needles and fine twigs (up to $5 \mathrm{~mm}$ at the thick end) which were considered as the fuel that is assumed to be consumed within the flaming front of a crown fire; and $C B D$ was calculated by dividing $C F L$ by the canopy length, estimated as the difference between the mean stand height and $C B H$. Species-specific equations were used to estimate the biomass of needles and fine twigs (DiéguezAranda et al., 2009).

Analyses of variance (ANOVA) were conducted to assess the influence of the thinning intensity on the canopy variables of each inventory. Differences between mean values were evaluated with the Tukey's HSD test $(\alpha=5 \%)$. The value of the canopy variable analysed measured on the previous inventory was included in the ANOVA as a covariate for all post-treatment comparisons.

\section{Results}

Box-plots of each canopy fuel variable, differentiating by species, thinning intensity and inventory are shown in Figure 1. The results indicate that the values of the three canopy fuel variables on both species before thinning treatments (BT, 2009-10) were not significantly different between plots assigned with different thinning intensities.

Significant differences between thinning intensities were found for $C F L$ and $C B D$ for both species in the first post-thinning remeasurement (2011-12). Moreover, the differences were maintained along the 6-year period analyzed with a similar pattern for both species. The observed $C B D$ values in the thinning plots at the end of the study period (2015-16) reached the values before thinning for maritime pine but not for radiata pine. The response of $P$. pinaster $C B D$ and $C F L$ agreed with previous results (Jiménez et al., 2016) in a 5 years study, but contradicts the results of Soler et al. (2017) who did not find change in $C D B$ seven years after thinning. It must be noted that the latter study was carried out in older stands and under Mediterranean climate with less precipitation. Fuel management to reduce $C B D$ below the empirical threshold value of $0.10 \mathrm{~kg} / \mathrm{m}^{3}$ has been recommended since the likelihood of active crown fire is strongly reduced (e.g. Scott and Reinhardt, 2001). Six year after treatments only the mean value of the radiata pine trials with heavy thinning remains below this threshold (Figure 1, red dotted line for $C B D$ ). 

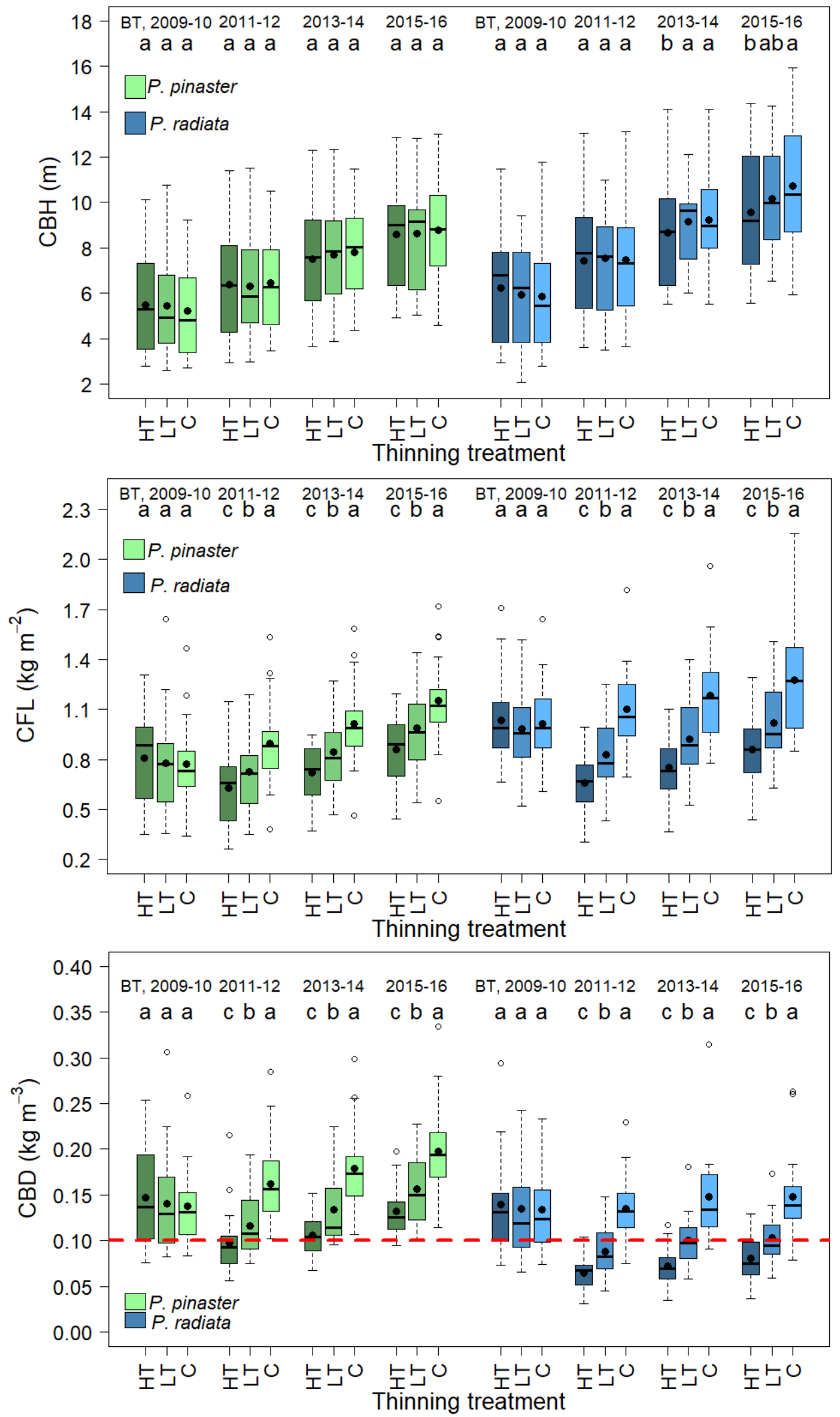

Figure 1 - Box-plots of the canopy variables for each species, inventory and thinning intensity: Control (C), Low thinning $(L W)$ and Heavy thinning $(H T)$. Different letters indicate significant differences between mean values (Tukey's HSD test. $\alpha=5 \%$ ). Black points correspond to mean values.

Significant differences between thinning intensities were found for $C F L$ and $C B D$ for both species in the first post-thinning remeasurement (2011-12). Moreover, the differences were maintained along 
the 6-year period analyzed with a similar pattern for both species. The observed $C B D$ values in the thinning plots at the end of the study period (2015-16) reached the values before thinning for maritime pine but not for radiata pine. The response of $P$. pinaster $C B D$ and $C F L$ agreed with previous results (Jiménez et al., 2016) in a 5 years study, but contradicts the results of Soler et al. (2017) who did not find change in $C D B$ seven years after thinning. It must be noted that the latter study was carried out in older stands and under Mediterranean climate with less precipitation. Fuel management to reduce $C B D$ below the empirical threshold value of $0.10 \mathrm{~kg} / \mathrm{m}^{3}$ has been recommended since the likelihood of active crown fire is strongly reduced (e.g. Scott and Reinhardt, 2001). Six year after treatments only the mean value of the radiata pine trials with heavy thinning remains below this threshold (Figure 1, red dotted line for $C B D$ ).

On the other hand, $C B H$ did not show any change following thinning in $P$. pinaster, a species with marked self-pruning. Jiménez et al. (2016) found significantly higher $C B H$ values in treated plots, probably due to its high pre-treatment stocking. For $P$. radiata, heavy thinning resulted in significantly lower $C B H$ values since four years after the treatment, likely caused by enhanced lower branch elongation linked to increased light availability.

Since $C B H$ strongly influences the likelihood of crown fire initiation, and $C B D$ the crown fire rate of spread, the results highlight that thinning treatments: (i) may only have a limited impact towards limiting crown fire occurrence, especially in maritime pine stands; and (ii) can have substantial impact in the crown fire rate of spread and in discriminating the type of crown fire, especially in radiata pine stands.

\section{References}

Diéguez-Aranda U, Rojo Alboreca A, Castedo-Dorado F, Álvarez González JG, Barrio-Anta M, Crecente-Campo F, et al. (2009). Herramientas selvícolas para la gestión forestal sostenible en Galicia. Xunta de Galicia. Santiago de Compostela, España.

Hevia H, Crabiffosse A, Álvarez-González JG, Ruiz-González AD, Majada J (2018). Assessing the effect of pruning and thinning on crown fire hazard in young Atlantic maritime pine forests. J. Environ. Manage. 205, 9-17.

Jiménez E, Vega-Nieva D, Rey E, Fernandez C, Vega JA (2016) Midterm fuel structure recovery and potential fire behaviour in a Pinus pinaster Ait. forest in northern central Spain after thinning and mastication. Eur J For Res 135: 675-686

Ruiz-González AD, Castedo-Dorado F, Vega JA, Jiménez E, Fernández-Alonso JM, ÁlvarezGonzález, JG (2015). Modelling canopy fuel dynamics of maritime pine stands in north-west Spain. Int. J. Wildland Fire 24, 92 -102.

Scott JH, Reinhardt ED (2001). Assessing crown fire potential by linking models of surface and crown fire behaviour. USDA Forest Service Res Pap RMRS-RP-29.

Soler Martin M Bonet JA, De Aragón JM, Voltas J, Coll L, Resco V (2017) Crown bulk density and fuel moisture dynamics in Pinus pinaster stands are neither modified by thinning nor captured by the Forest Fire Weather Index. Ann. For. Sci. 74: 51

Van Wagner CE (1977). Conditions for the start and spread of a crown fire. Can. J. For. Res. 7, 23-24. 


\title{
Multidisciplinary fire science research at the Sycan Marsh, Oregon
}

\author{
Russ Parsons ${ }^{1}$; Katie Sauerbrey ${ }^{2}$; Nicole Vaillant ${ }^{3}$; Lloyd Queen ${ }^{4}$; Chris Moran ${ }^{4}$; Matt Cunningham ${ }^{4}$; \\ Nancy Grulke ${ }^{5}$; Craig Bienz ${ }^{6}$; Bret Butler ${ }^{1}$ \\ ${ }^{1}$ USFS RMRS, Fire Sciences Lab. 5775 Highway 10 W, Missoula, MT, \{rparsons@fs.fed.us*, \\ bwbutler@fs.fed.us\} \\ ${ }^{2}$ Nature Conservancy, Klamath Basin Field Office \{katie.sauerbrey@tnc.org\} \\ ${ }^{3}$ USFS PNWRS, Wildland Fire Management RD\&A, \{nvaillant@fs.fed.us\} \\ ${ }^{4}$ University of Montana, National Center for Landscape Fire Analysis, \{lloyd.queen@firecenter.umt.edu, \\ chris.moran@firecenter.umt.edu,matt.cunningham@firecenter.umt.edu\} \\ ${ }^{5}$ USFS PNWRS WWETAC, Prineville, OR \{ngrulke@fs.fed.us\} \\ ${ }^{6}$ Nature Conservancy, Klamath Basin Field Office \{cbienz@tnc.org\}
}

\begin{abstract}
In October 2017, a diverse campaign of fire research was carried out in the context of management prescribed burns at the Nature Conservancy's Sycan Marsh Preserve, a relatively isolated site in south central Oregon characterized by extensive grasslands, dry ponderosa pine forests and unique wetland habitats. The overarching objective of this effort was to develop integrated and reliable fuels, weather and fire behavior datasets for evaluation of different fire models. This broad goal required an integrated methods approach to facilitate fuel mapping and fire behavior observation and measurements at multiple scales. Fuel mapping efforts, intended to provide 3D inputs for physics-based fire models, link traditional surface fuels and tree measurement sampling with new approaches that enable development of comprehensive data across larger areas. These new approaches include a mobile app-based tool for surface fuels, drone imagery-based mapping, airborne LiDAR data, and 3D modeling using the STANDFIRE prototype fuel modeling platform. Wind measurements included a large network of $3 \mathrm{~m}$ anemometer towers and a SODAR instrument to capture the vertical wind profile in 3D. Emissions data collection included vehicle and aerostat mounted sensors sampling particulates and gas concentrations. Fire behavior measurements included surface instruments and thermal imagery from fixed wing and drone platforms. This presentation will provide an overview of the overall experiment, present some preliminary data, and discuss future directions. With more research burns planned in the months and years to come, we see the Sycan Marsh Preserve as a site for collaborative fire science research, and as a test bed for new methods and approaches in mapping fuels in 3D at landscape scales, integration of fire behavior and weather data across scales, and in data and approaches for evaluating models across scales and different levels of detail.
\end{abstract}

Keywords: Fuel Mapping, Fuel modeling, Prescribed Fire, Fire Behavior, UAS, Drones, Thermal Imagery

\section{Site Description}

The Sycan Marsh Preserve is a 12,364 ha preserve in south central Oregon owned and managed by The Nature Conservancy, a non-profit dedicated to natural resource conservation. In many of its preserves, The Nature Conservancy considers active management, such as forest fuel treatments and prescribed fires, as key components in their stewardship and restoration strategies. Located on mostly flat terrain at an elevation of around $1585 \mathrm{~m}$, the Sycan Marsh Preserve is predominantly grass and wetlands but has extensive forest areas, dominated by ponderosa pine, at the margins of the marsh. Such dry forest types have undergone significant shifts in forest structure, composition and disturbance regimes over the last century, stimulating the need for restoration (Hessburg et al 2005). Management practices include cattle grazing, and forestry; a series of forest thinnings with different specifications have been carried out in the last several years, with the most recent, in 2016, having been designed to enhance spatial heterogeneity for restoration purposes following recent ecological guidelines (Larson 
and Churchill, 2012). This treated area is scheduled to be prescribed burned in fall 2018. In the present paper we describe research data collected on a prescribed fire carried out in October 2017.

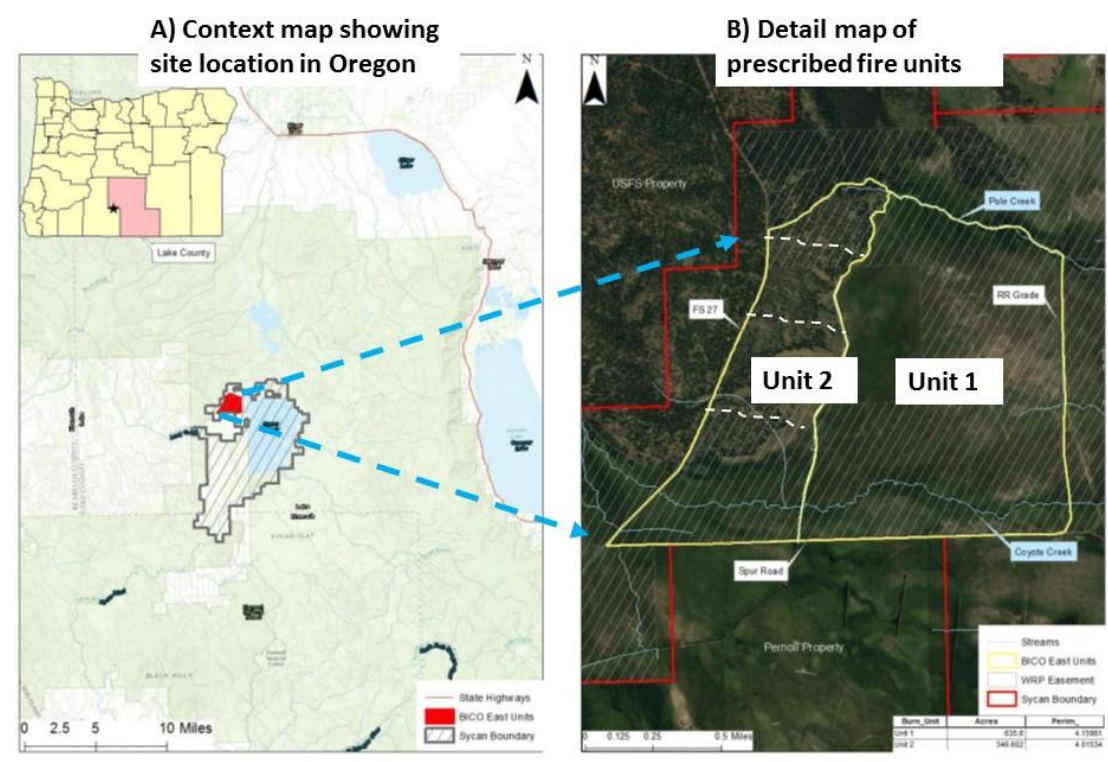

Figure 1 - Site location

\section{2017 Prescribed fire}

The 2017 prescribed fire was located in the northwestern portion of the Sycan Marsh Preserve, and totalled 398 ha, divided into a grass unit (Unit 1, 257.5 ha) and a forest unit (Unit 2, 140.5 ha) (Figure 1). Unit 1 had been previously prescribed burned in 2013, and about $80 \%$ of Unit 2 had been prescribed burned in 2006 following a conventional thinning operation. Management objectives in the grass area (Unit 1) were to reduce encroachment of lodgepole pine and juniper into the open prairie and increase native bunchgrass and forb densities, while management objectives in the forest area (Unit 2) focused on fuel reduction and maintenance of low density, resilient forest structure. Unit 1 was burned on October 11, 2017. Unit 2 was burned the following week, divided up into sub units burned in succession over a two day period (October 17 and 18). The northern portion of Unit 2 was not burned due to concerns of possible escape. In both units, ignition patterns were modified in the vicinity of focused data collection areas (described below) to permit free-burning fire conditions.

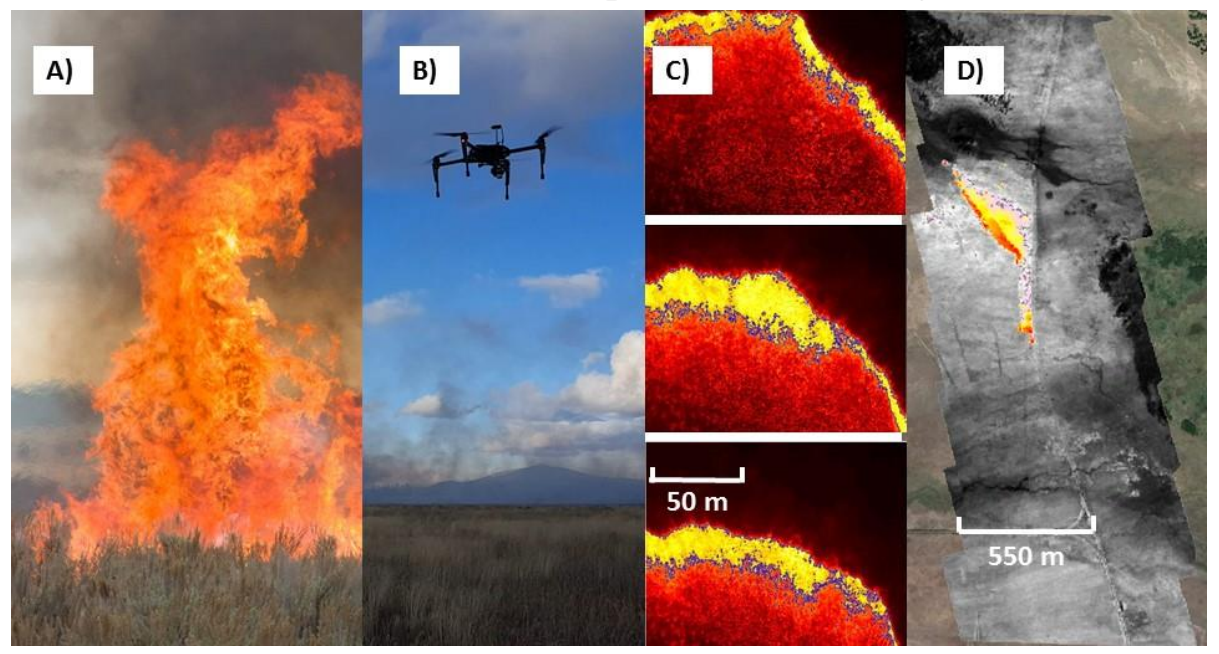

Figure 2 - Cross-platform fire behavior observations link A) surface fire behavior in sagebrush, B) drone-based thermal mapping, C) example drone plot fire progression, shown at intervals of $\sim 10$ s between selected frames, and D) airborne thermal imagery for a single flight line at a point in time. 


\section{Fire Science Research Data}

As wildland fire science continues to mature, the field is gaining new capabilities to embrace and understand complexity; rather than focusing entirely on steady state behavior and rate of spread, researchers can now explore interactions and gain insights into more fundamental physical quantities and their interactions (Yedinak et al 2018). Modeling plays a critical role in this expanding capability. While a growing body of evidence illustrates the performance of the physics-based models (e.g. Linn et al 2005b, Mell et al 2009, Pimont et al 2009, Hoffman et al 2016), model evaluation, through comparison against robust, integrated datasets is essential. Several complementary datasets were collected as part of this project, characterizing fuels, fire behavior, weather and emissions. Fuel mapping efforts, currently underway, will integrate surface and canopy fuels sampling, LiDAR and drone-based imagery. As the prescribed burn units are fairly large, fuel and fire behavior measurements centered on focused data collection areas called "drone plots" of $100 \mathrm{~m}$ x 100m, within which extensive field sampling, and drone-based fuel and fire mapping, took place. Drone-based fuel mapping was carried out with significant end- and side-lap image collection at high resolution, enabling highly detailed 3D fuels mapping through photogrammetric approaches. This data complements high quality airborne LiDAR data from 2010 and airborne imagery from 2016. A prototype 3D fuel modeling system, STANDFIRE (Parsons et al. 2018, Pimont et al 2016), will be used with this data to develop fuels inputs to the WFDS (Mell et al 2009) and FIRETEC (Linn et al 2005) physics-based fire models, providing a means for model evaluation against observed data. Fire behavior observations (Figure 2) within the drone plots included fire behavior packages (Butler et al 2004), oriented both horizontally and vertically (Unit 1 only), drone-based thermal nadir imagery (Figure 2 B and C) taken with a frequency of $\sim 1 \mathrm{hz}$, complemented by airborne thermal imagery capturing the larger burn unit with multiple images captured over the course of the burn (Figure $2 \mathrm{D}$ ). Wind data consisted of a large number of $3 \mathrm{~m}$ anemometer towers complemented by a SODAR collecting 3D wind data at $10 \mathrm{~m}$ intervals for several hundred meters of height. Finally, emissions data were collected using sensors on an aerostat (large ballon) and a vehicle. Data processing is ongoing at this time; upon completion, experimental outcomes and associated data will be published and made available to the larger fire science community.

\section{References}

Butler BW, Cohen J, Latham DJ, Schuette RD, Sopko P, Shannon KS, Jimenez D and Bradshaw LS (2004) Measurements of radiant emissive power and temperatures in crown fires. Canadian Journal of Forest Research, 34(8), 1577-1587.

Hessburg PF, Agee JA, Franklin JF (2005) Dry forests and wildland fires of the Inland Northwest USA: Contrasting the landscape ecology of the pre-settlement and modern eras. Forest Ecology and Management 211, 117-13.

Hoffman CM, Canfield J, Linn RR, Mell W, Sieg CH, Pimont F and Ziegler J (2016) Evaluating crown fire rate of spread predictions from physics-based models. Fire technology, 52(1), 221-237.

Larson AJ, Churchill D (2012) Tree spatial patterns in fire-frequent forests of western North America, including mechanisms of pattern formation and implications for designing fuel reduction and restoration treatments. Forest Ecology and Management 267, 74-92

Linn R, Winterkamp J, Colman JJ, Edminster C, Bailey JD (2005a) Modeling interactions between fire and atmosphere in discrete element fuel beds. International Journal of Wildland Fire 14, 37 48.

Linn RR and Cunningham P (2005b) Numerical simulations of grass fires using a coupled atmospherefire model: basic fire behavior and dependence on wind speed. Journal of Geophysical Research: Atmospheres, 110 (D13). 
Mell W, Maranghides A, McDermott R, Manzello SL (2009) Numerical simulation and experiments of burning douglas fir trees. Combustion and Flame 156, 2023-2041.

Parsons RA, Pimont F, Wells L, Linn RR, Cohn G, Jolly WM, de Coligny F, Rigolot E, Dupuy J-L, Mell W, and Linn RR (2018) Modeling thinning effects on fire with STANDFIRE. Annals of Forest Science 75(1), 1-11

Pimont F, Dupuy J-L, Linn RR, Dupont S (2011) Impacts of tree canopy structure on wind flows and fire propagation simulated with FIRETEC. Annals of Forest Science 68, 523-530. doi:10.1007/s13595011-0061-7

Pimont F, Parsons R, Rigolot E, de Coligny F, Dupuy J-L, Dreyfus P and Linn RR (2016) Modeling fuels and fire effects in 3D: Model description and applications. Environmental Modelling and Software 80, 225-244

Yedinak KM, Strand EK, Hiers JK, and Varner JM (2018) Embracing complexity to advance the science of wildland fire behavior. Fire 1, (2) 20 
Short contribution - Fire Management

\title{
A comparison of in-situ fire energy measurements to remote sensed thermography using Unmanned Aerial Systems (UAS)
}

\author{
Daniel Jimenez ${ }^{1}$; Bret Butler ${ }^{1}$; Lloyd Queen ${ }^{2}$; Valentijn Hoff ${ }^{2}$; Joseph O’Brien ${ }^{3}$; J. Kevin Heirs ${ }^{4}$ \\ ${ }^{I}$ Fire Science Laboratory, Rocky Mountain Research Station, USDA Forest Service, Missoula, Montana, \\ USA, \{djimenez@fs.fed.us*\} \\ ${ }^{2}$ National Center for Landscape Fire Analysis, University of Montana, Missoula, Montana, USA \\ ${ }^{3}$ Center for Forest Disturbance Science, Southern Research Station, USDA Forest Service, Athens, \\ Georgia, USA \\ ${ }^{4}$ Tall Timbers Research Station \& Land Conservancy, Tallahassee, Florida, USA
}

\begin{abstract}
Fire science and management are often limited by an inability to quantitatively measure and track the properties of wildland fire at their full spatial and temporal scales (Riggan et al. 2004). Direct experimental measurements of the energy, fluid flow, and chemical processes occurring in full-scale wildland fires over a spatial extent are difficult to obtain. Techniques exist to measure the radiant intensity or emissive power of the flame; however, the high temperature and transient nature of naturally burning wildland fires has been limited to just a few localized measurements of fire intensity (Butler et al. 2004). Recent advances in unmanned aerial systems (UAS) and light weight, high resolution sensors provide an opportunity to address the various limitations associated with previous research in landscape scale fire radiative power (FRP) estimates. A series of nine small scale $\left(0.5-1 \mathrm{~km}^{2}\right)$ field experiments were instrumented with multiple horizontal and nadir view fire behavior packages (FBPs) in order to characterize point source fire energy measurements. Additionally, in-situ and remote sensed (UAS) radiometric thermograhy data with collocated at high resolution in space and time. Coordinates of each in-situ location were acquired and pre-loaded into the UAS flight plan to ensure collocation data collects. This paper presents the experimental design and preliminary results with the intent to develop a strong correlation between highly resolved ground based fire energy measurements collacted with UAS remote sensed thermography.
\end{abstract}

Keywords: Fire behavior, thermography, remote sensing, unmanned aerial systems

\section{Introduction}

It is widely recognized that fire science and management are critically limited by an inability to quantitatively measure and track the properties of wildland fire at their full spatial and temporal scales (Riggan et al. 2004). An understanding of the variability and dynamics of fire properties is necessary at both plot and landscape scales for future physics based fire behavior models and land managers alike. Fire management requires either tracking or accurate prediction of fire spread rates, intensity, and spread direction in order to ensure firefighter safety and maintain landscape scale objectives. Accurate assessment of the ecological impacts of fire requires either active monitoring or a means to deduce them post burn.

Direct experimental measurements of the energy, fluid flow, and chemical processes occurring in full-scale wildland fires over a spatial extent are difficult to obtain. Techniques exist to measure the radiant intensity or emissive power of the flame; however, the high temperature and transient nature of naturally burning wildland fires has been limited to just a few localized measurements of fire intensity (Butler et al. 2004). Furthermore; wildland fire radiant energy (FRE) is one of the only measurements of combustion that can be made at wide spatial extents and high temporal and spatial resolutions (O'Brien et al. 2016). 
Small-scale experiments have demonstrated that FRE is linearly related to fuel combusted, but such a relationship has not been shown at the landscape level of prescribed fires (Kremens et al. 2010, Hudak et al. 2016). The difficulty in acquiring total radiative energy of a landscape scale fire requires sufficient sampling over time to integrate the radiative energy from instantaneous measures of the fires radiative power (FRP) (Johnston et al. 2017). Several experiments have attempted to classify FRP from a variety of remote sensed platforms, but lengthy return intervals (hrs - mins) and course scale pixel resolution $(\mathrm{km}-\mathrm{m}$ ) have lead to gaps in the data collection (Riggins et al. 2004, Dickenson et al. 2015, Kremens et a. 2010).

Recent advances in unmanned aerial systems (UAS) and light weight, high resolution sensors provide an opportunity to address the various limitations associated with previous research in landscape scale fire radiative power (FRP) estimates. The use of a multi-rotor UAS equipped with infrared (IR) thermography sensor systems is a unique innovation and logical advance and complement to current ground based technology, and is expected to have significant benefits in sampling efficiency, time, and cost.

\section{Methods}

Here we report the priliminary results of a series nine small scale $\left(0.5-1 \mathrm{~km}^{2}\right)$ field experiments conducted at the Tall Timbers Research Station located in Tallahassee, FL in April 2017. The fuels were representative of heterogenious southern rough that varied in age from 1 to 3 years growth since previous fire introduction. Plots were instrumented with in-situ fire behavior packages (FBPs) (Butler et al. 2010) and nadir view in-situ radiometric thermal imaging systems (O'Brien et al. 2012). Additionally, UAS remote sensed radiometric thermal images were collocated with the in-situ instrumentation to collect highly resolved $(\mathrm{cm})$ thermal data orthorecitified in space and time.

Direct experimental measurements of the radiative energy, fluid flow, and chemical processes occurring in full-scale wildland fires over a spatial extent are difficult to obtain, yet critical for understanding fire variability on the landscape. Field deployable ground-based fire behavior packages (FBPs) and digital video cameras provide point source in-situ, timeresolved measurements of radiant and convective energy transfer from the fire, horizontal and vertical air flow, air temperature, and digital video footage. Multiple horizontal and nadir view FBPs $(n=3-5)$ were deployed randomly through each of the Tall Timbers field study plots and sampled at $10 \mathrm{~Hz}$ (Figure 1). FBPs were arranged in pairs, one horizontal and one nadir view, in an effort to capture total hemispherical radiative power at each point in space and time. Sensors were positioned around coordinate ground control points to allow for georeferencing on the landscape.

Additionally, fine scale $(\mathrm{cm})$ nadir view IR radiometric data was collected in-situ from an $8.2 \mathrm{~m}$ tall tripod design (O'Brien et al. 2012). A long wave infrared (LWIR) themal imaging systems from FLIR Inc. (SC660) mounted atop the tripod systems was used to collect $1 \mathrm{~Hz}$ radiometric in-situ data (Figure 2).

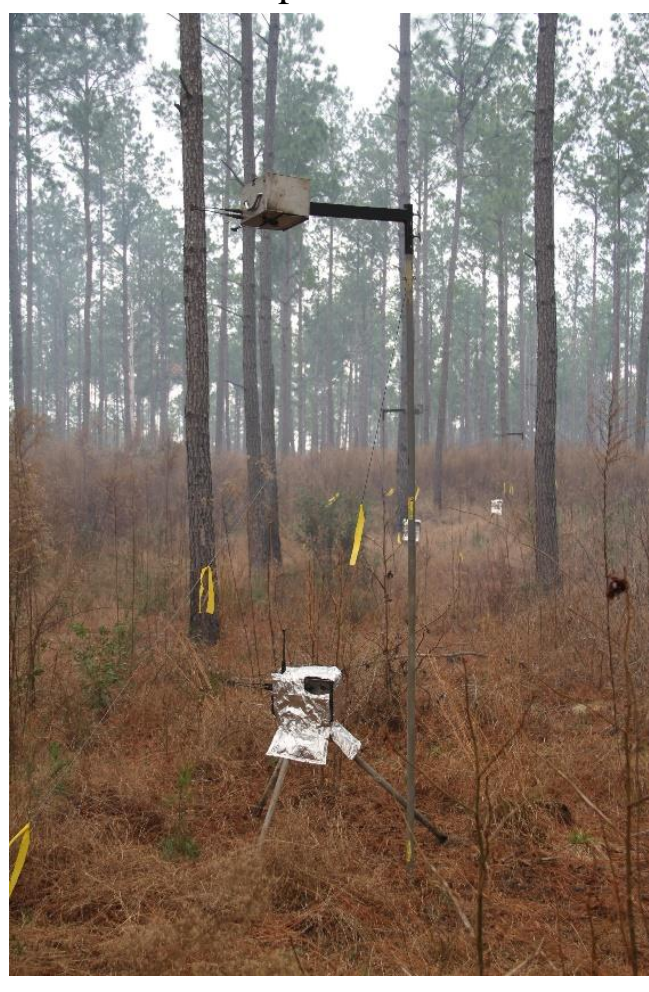

Figure 1 - In-situ horizontal and nadir view FBPs in southern rough fuel type. 
The aerial thermal imagery was acquired using the FLIR Zenmuse XT camera mounted on the DJI matrice UAS platform. The DJI/FLIR combination offers expertise in flight stability, gimbal technology, and image transmission (Figure 3). These coupled systems are a fundamental step in linking ground based measurements to nadir radiometric images.

The experimental design was to pre-select areas of interest within each burn unit that would allow for a dual collect (insitu FBP and UAS and tripod thermography) upon ignition. Areas were identified base on fuel characteristics, access, and canopy enclosure. Each in-situ set up consisted of a horizontal mount FBP at $0.5 \mathrm{~m}$ above ground level (AGL), a nadir mount FBP at $3.0 \mathrm{~m}$ AGL, a horizontal GoPro camera at $0.5 \mathrm{~m}$ AGL, a nadir view GoPro housed within the nadir FBP at 3.0 m AGL and one $8.2 \mathrm{~m}$ thermography tripod. The in-situ FBP sensor array was within the measurement footprint of the tripod IR sampling array. Coordinates of each in-situ location were acquired with Real Time Kinematic (RTK) precision positioning system and pre-loaded in the UAS flight plan to ensure collocation data collects.

This approach provided an infrared 2-dimensional temperature profile collocated with in-situ point source fire behavior measurements that are well-understood.

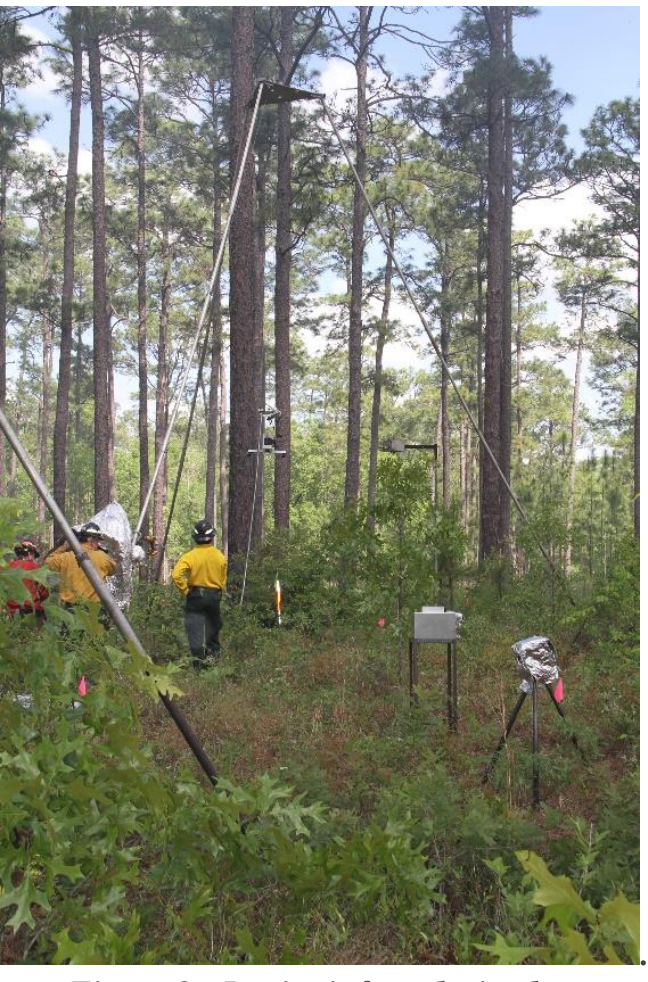

Figure 2 - In-situ infrared tripod system, FPBs and video sensors.

Furthermore, by correlating UAS imagery to in-situ fire energy release, the UAS system acquired data can be calibrated and interpreted in terms of engineering units. The significance of this correlation is that the data become objective, independent of sensor and UAS platform, and quantifiable.

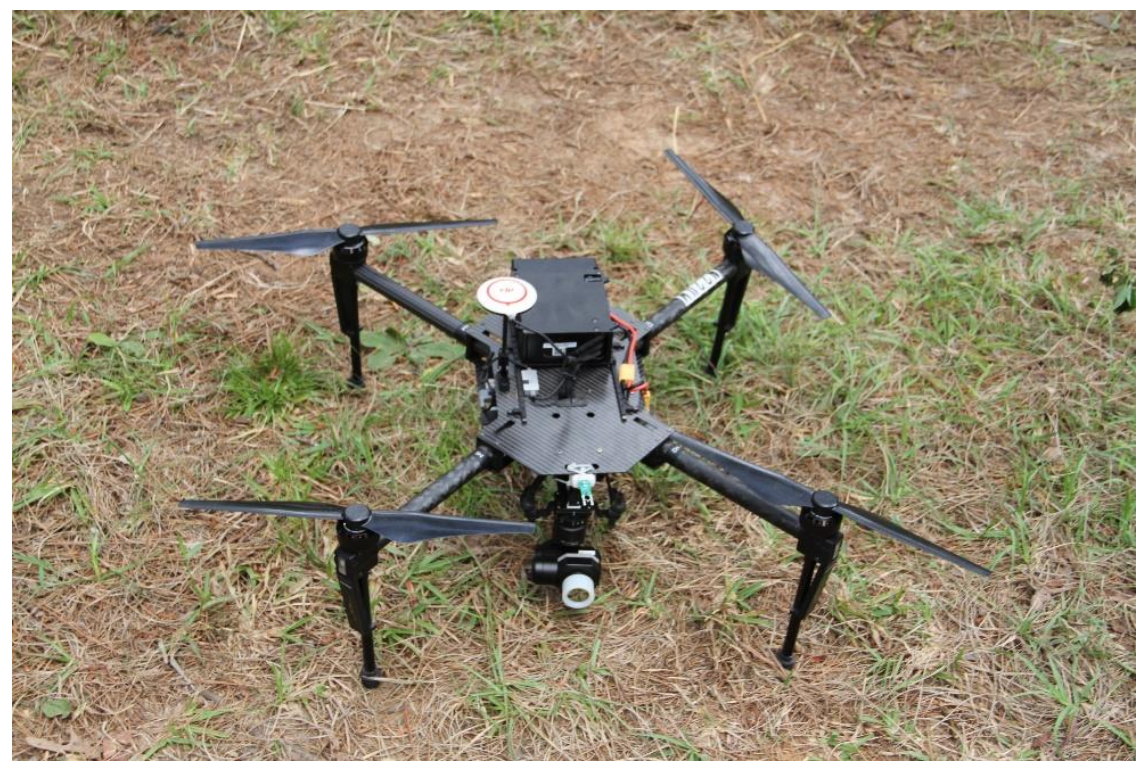

Figure 3 - DJI matrice UAS with FLIR Zenmuse XT infrared camera. 


\section{Results and Discussion}

The experiments were conducted at the Tall Timbers Research Station over the course of three separate burning periods. Physical fire behavior data was collected both in-situ and remotely from various platforms for each of the burn plots. Horizonal and nadir view FBP $10 \mathrm{~Hz}$ data collected radiant and convective heat flux ( $\mathrm{kW} / \mathrm{m} 2)$, horizontal and vertical air flow (mph), and air temperature (C). Addtionally, in-situ nadir view and remote sensed radiometric images were collected at $1 \mathrm{~Hz}$ using a tripod mounted and UAS systems (Figure 4). Ground control points and real time kinematic (RTK) precision positioning systems were used to georeference point source data in space and time.
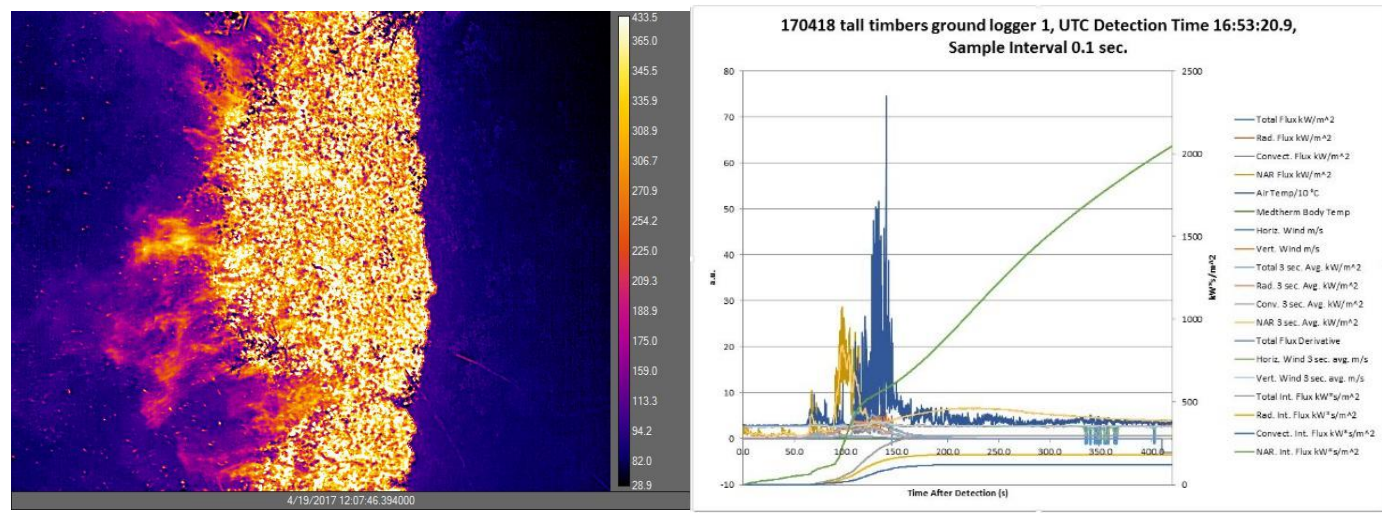

Figure 4 - Radiometric image for UAS platform(left) and data profile from in-situ FBP(right).

This presentation presents the experimental design and primilnary results with the intent to develop a strong correlation between highly resolved ground based fire behavior measurements collocated with UAS remote sensed thermography. The benefits are multifaceted, the primary being demonstrating how data acquired using UAS systems can be used by fire managers to more effectively characterize fire energy release and thereby achieve management objectives. Other benefits include cost savings through reduction in required monitoring activities. This demonstration has the ability to supplement and expand ground based monitoring programs to quantify fire effects, habitat impact, fuel reduction and other management objectives. Other benefits include reduced risk to personnel through improved situational awareness. In-situ and UAS data analysis will provide a benchmark for landscape scale management techniques that will provide natural resource specialists with quantifiable data and tools necessary to analyze pre and post burn imagery.

\section{Acknowledgements}

This work was supported through funding provided from the officeof the US Forest Service Deputy Chief for Research and the University of Montana. The gracious assistance of the Tall Timbers Research Station staff during the experiments is also appriciated.

\section{References}

Riggan, P. J., Tissell, R. G., Lockwood, R. N., Brass, J. A., Pereira, J. A. R., Miranda, H. S., ... \& Higgins, R. (2004). Remote measurement of energy and carbon flux from wildfires in Brazil. Ecological Applications, 14(3), 855-872.

Butler, B. W., Cohen, J., Latham, D. J., Schuette, R. D., Sopko, P., Shannon, K. S., ... \& Bradshaw, L. S. (2004). Measurements of radiant emissive power and temperatures in crown fires. Canadian Journal of Forest Research, 34(8), 1577-1587. 
O'Brien, J. J., Loudermilk, E. L., Hornsby, B., Hudak, A. T., Bright, B. C., Dickinson, M. B., ... \& Ottmar, R. D. (2016). High-resolution infrared thermography for capturing wildland fire behaviour: RxCADRE 2012. International Journal of Wildland Fire, 25(1), 62-75.

Kremens, R. L., A. M. S. Smith, and M. B. Dickinson. "Fire metrology: current and future directions in physics-based methods." Fire Ecology 6.1 (2010): 13-35.

Hudak, A. T., Dickinson, M. B., Bright, B. C., Kremens, R. L., Loudermilk, E. L., O'Brien, J. J., ... \& Ottmar, R. D. (2016). Measurements relating fire radiative energy density and surface fuel consumption-RxCADRE 2011 and 2012. International Journal of Wildland Fire, 25(1), 25-37.

Johnston, Joshua M., et al. "Direct estimation of Byram's fire intensity from infrared remote sensing imagery." International Journal of Wildland Fire 26.8 (2017): 668-684.

Dickinson, M. B., Hudak, A. T., Zajkowski, T., Loudermilk, E. L., Schroeder, W., Ellison, L., ... \& Bright, B. C. (2016). Measuring radiant emissions from entire prescribed fires with ground, airborne and satellite sensors-RxCADRE 2012.

Butler, BW, D Jimenez, J Forthofer, K Shannon, and P Sopko. (2010) A Portable System for Characterizing Wildland Fire Behavior. Paper presented at the Proceedings of the 6th International Conference on Forest in Fire Research.[CD-ROM]. Coimbra, Portugal: University of Coimbra.

O'Brien, Joseph J., et al. "High-resolution infrared thermography for capturing wildland fire behaviour: RxCADRE 2012." International Journal of Wildland Fire 25.1 (2016): 62-75. 
Short contribution - Fire Management

\title{
Assessing fire severity using charcoal reflectance following a recent heathland wildfire on Carn Brea, Cornwall, UK
}

\author{
Stacey L. New*, Victoria A. Hudspith \& Claire M. Belcher \\ wildFIRE Lab, Hatherly Laboratories, University of Exeter, Exeter, EX4 4PS, \\ United Kingdom \{S.New@exeter.ac.uk*\}
}

\begin{abstract}
Charcoal has recently been suggested to retain information about the fire that generated it (Belcher and Hudspith, 2016). When looking under a microscope charcoals formed by different aspects of fire behaviour indicate different ability to reflect the amount of light when studied using the appropriate technique. It has been suggested that this method might be able to provide a quantitative fire severity metric that can be used in conjunction with or instead of standard qualitative fire severity scores. We studied charcoals from a recent wildfire in Carn Brea, Cornwall and assessed whether charcoal reflectance (Ro) can be linked to standard qualitative fire severity scores for the burned area. We found that charcoal reflectance was greater at sites along the burned area that have been scored as having a higher fire severity, whilst surrounding sites with a lower severity score have a noticeably lower Ro measurement. We suggest, by measuring the reflectance of charcoals that this may be able to provide quantitative information about the spatial distribution of heat across a burned area post-fire, and that this should provide better linkages between fire behaviour, fire severity and ecosystem effects.

Synthesis: Results from the analysis of the Carn Brea charcoal suggests that variation in charcoal reflectance measurements may be due to changes in fire severity across a burn site, enabling researchers to gain information that links fire severity and fire behaviour by analysing the charcoal left behind post-fire. This is particularly useful if quantitative fire severity measurements cannot be obtained via remote sensing.
\end{abstract}

Keywords: Charcoal, Reflectance, Severity, Surface Fire, Surface Fuels

\section{Introduction}

Charcoal is a key product of wildfires that remains in abundance after wildfire events. Researchers have established that the structure of charcoal varies during creation due to a number of differing factors e.g. wood species, wood density and heating (Cohen-Ofri et al., 2006; Lowden and Hull, 2013). Experimental research has indicated that during the combustion process charcoal transitions through various phases in which cells are eventually re-ordered to a more graphite-like structure (Cohen-Ofri et al., 2006; Belcher and Hudspith, 2016). This re-ordering of cells alters the reflective properties of the charcoal i.e. there is an increase in the quantifiable amount of light reflected from the surface of the charcoal, thus, allowing researchers to study the reflectance properties of charcoal (Belcher and Hudspith, 2016).

Recently, researchers have begun to develop the use of charcoal reflectance in post-fire assessments, (e.g. Belcher and Hudspith, 2016; Hudspith et al., 2017). Here we consider the use of charcoal reflectance as a tool in post-fire assessments as a quantitative measure of fire severity. We present reflectance measurements in combination with standard qualitative fire severity scores from a recent wildfire which occurred in Carn Brea, Cornwall $\left(50.2141^{\circ} \mathrm{N}, 5.2551^{\circ} \mathrm{W}\right)$. Using both a fire severity matrix across the burn scar, acquired in the field, and charcoal reflectance, conducted in the laboratory, we consider whether charcoal reflectance may serve as a quantitative fire severity measure in place of, or in combination with the qualitative fire severity matrices. 


\section{Methods and Materials}

\subsection{Site and Material Collection}

A heathland fire in a region dominated by heather (Calluna sp.) and gorse (Ulex europaeus) occurred on the $26^{\text {th }}$ of May 2015 burning 7 hectares of a small area of Cornwall, UK (BBC, 2015). The gorse in particular had been left unmanaged, meaning that it was overgrown and dense (The Heritage Journal, 2015), this resulted in a large abundance of fuel on the heathland for the fire to burn. The fire started at the bottom of the site and travelled up the hill to the top where a footpath intersected the heathland; this may have acted as a 'natural' fire break. Charcoal samples were collected from the burn scar in June 2015, two days after the fire. A transect was taken across the axis of the fire scar and 12 sampling locations documented using a GPS and photographs. Charcoal samples were collected every $\sim 1 \mathrm{~m}$. At each sampling location a $30 \mathrm{~cm}$ by $30 \mathrm{~cm}$ quadrat was placed on the ground and charcoal collected from within that area. A mixture of heather and gorse charcoal was collected.

\subsection{Fire Severity}

Qualitative fire severity scores were assigned to each sampling location according to the table presented in Ryan and Noste (1985) but was modified for a heathland ecosystem (see Hudspith et al., 2014). Fire severity was found to be similar across the entire transect, but became higher in the denser, more overgrown gorse-dominated areas. Of the 12 sampling locations along the transect 10 were classified as having a light fire severity (severity score 3), 'surface litter, mosses and herbs charred or consumed', the two remaining sampling locations were given a moderate or severe fire severity description (severity score 4) which includes 'all understory plants charred or consumed, fine dead twigs on soil surface consumed, pre-fire soil organic layer largely consumed' (Hudspith et al., 2014).

\subsection{Reflected Light Microscopy}

Reflected light microscopy was used to assess the reflectance of the charcoals collected along the transect. All charcoal samples were oven dried at $40^{\circ} \mathrm{C}$ before preparing for analysis under the reflectance microscope. Charcoal samples, a mixture of vegetation types (heather and gorse), were embedded in polyester resin blocks and subsequently ground and polished using a MetaServ 250 with Vector Power Head grinder-polishing machine (Buehler, Neckar, Germany), with a silicon carbide disc (50 $\mu \mathrm{m}$ grain size) (Belcher and Hudspith, 2016). The type of resin used is referred to as cold-mounting epoxy resin, consisting of two components, an adhesive and a hardener. Reflectance was measured using a Zeiss Axio-Scope A1 optical microscope with a TIDAS-MSP 200 system attached. The TIDAS-MSP 200 system is calibrated using three synthetic reflectance standards, strontium titanite $(5.41 \%$ reflectance in oil (Ro)), gadolinium gallium, garnet (GGG) (1.719\% reflectance in oil (Ro)) and spinel (0.42\% reflectance in oil (Ro)) (Belcher and Hudspith, 2016). An x50 objective (with x32 eyepiece magnification) was used and the measurement of reflectance was manually taken at the cell-wall junction using MSP200 v 3.27 software (Belcher and Hudspith, 2016), 100 reflectance measurements were taken per block.

Reflected-light microscopy allows charcoal to be visually analysed whilst also being able to quantitatively measure the amount of light that is reflected back from the polished charcoal surface; the brighter the charcoal appears under the microscope the more light is being reflected back (Figure 1) (Belcher and Hudspith, 2016). 

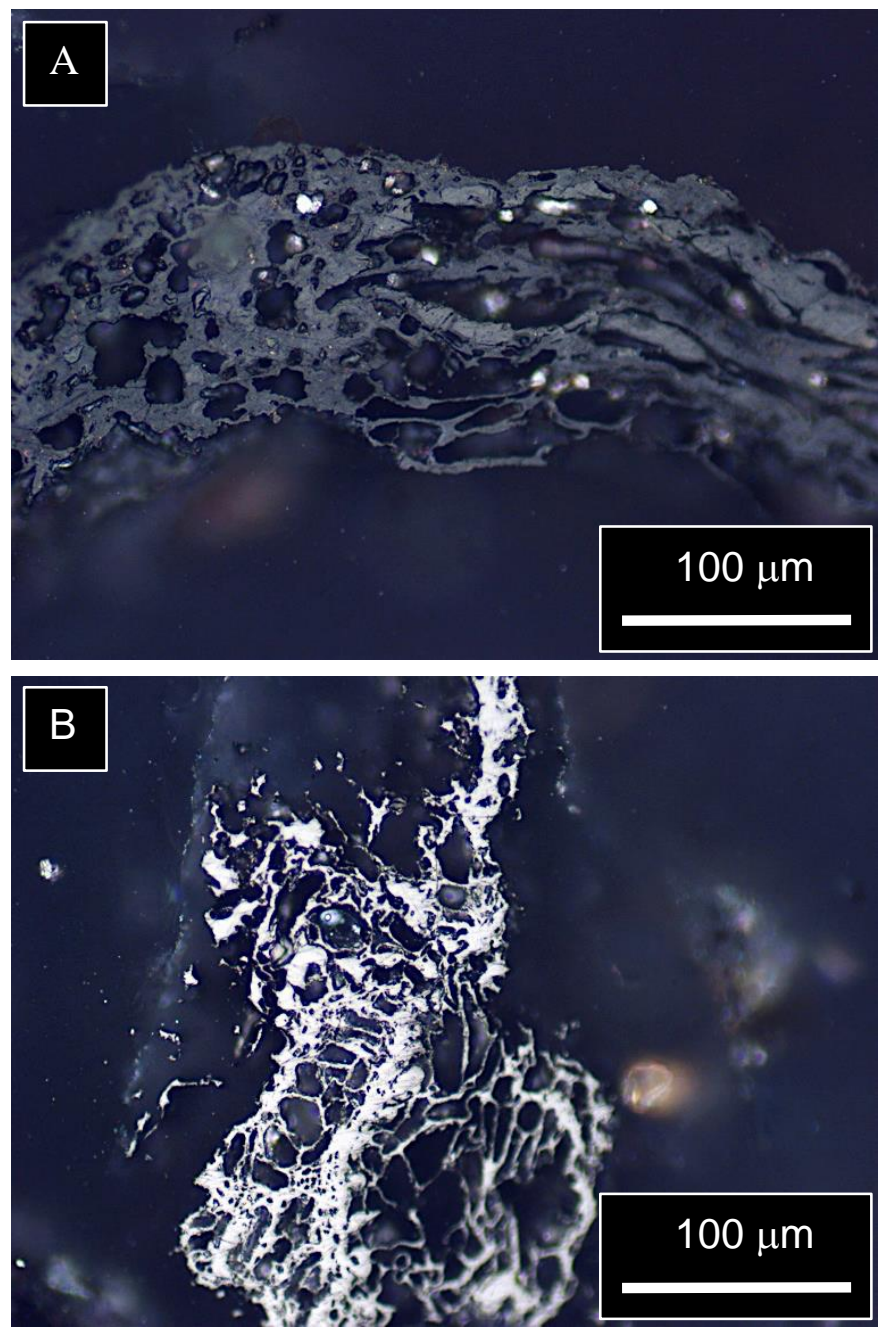

Figure 1-Images of charcoal under the reflectance microscope. A) Shows a lower reflecting piece of charcoal from a location along the burn scar given a severity score of 3 in the field, and B) shows a higher reflecting piece of charcoal from a location along the burn scar given a severity score of 4 in the field.

\section{Results and Conclusions}

We observed that locations along the burn scar that experienced higher qualitative fire severity scores produced charcoals that were more higher reflecting when compared to those at lower severity sites (Figure 2). Images of charcoal showing this visual difference in reflectance, under the reflectance microscope, are shown in Figure 1. 


\section{Carn Brea}

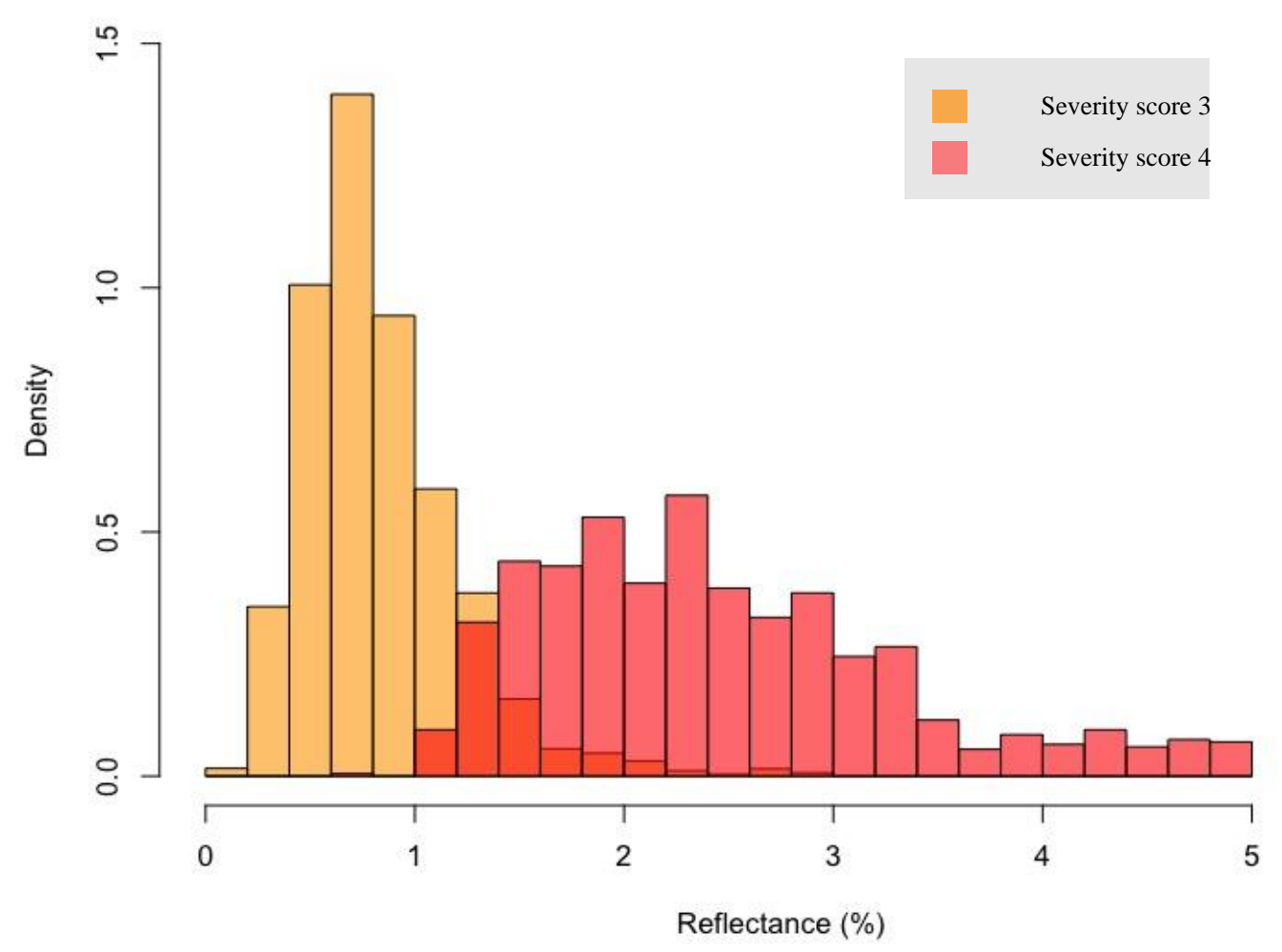

Figure 2 - Histogram showing the distribution of reflectance measurements of the Carn Brea charcoal. Bars are coloured to represent the different severity scores given to the sites along the burn scar, the red bars represent the sites given a severity score of 4 and the orange bars represent the sites given a severity score of 3.

A t-test was conducted on the reflectance values to compare all of the sites with a qualitative fire severity score of 3 , to site 7 , and then to site 8 , both of which had higher quantitative fire severity scores of 4 . There was a significant difference in the scores for all sites with a fire severity score of 3 $(\mathrm{M}=0.65, \mathrm{SD}=2.25)$ and site 7 (fire severity score 4) $(\mathrm{M}=2.56, \mathrm{SD}=0.84)$; $\mathrm{t}(499)=-45.733, p=<2.2 \mathrm{e}$ 16 , and again when testing site 8 (fire severity score 4$)(M=2.33, S D=0.88)$ to the sites which a severity score of $3 ; \mathrm{t}(499)=-37.636, p=<2.2 \mathrm{e}-16$. These results suggest that quantitative fire severity scores do correspond to quantitative charcoal reflectance measurements; where the higher the charcoal reflectance the higher the qualitative fire severity score. Therefore, we suggest that charcoal reflectance measurements taken from burned areas may provide a useful quantitative means to assess aspects of fire severity that link more directly to aspects of fire behaviour (e.g. see Belcher and Hudspith, 2016; Hudspith et al., 2017) than existing qualitative fire severity scores. We aim to continue to develop this approach in the hope that it might be able to aid our understanding of post-fire recovery following fire events.

\section{Acknowledgments}

SLN is funded by the Natural Environment Research Council [NE/L002434/1] as part of the GW4+ Doctoral Training Partnership (http://www.nercgw4plus.ac.uk/). CMB acknowledges a European Research Council Starter Grant ERC-2013-StG-335891-ECOFLAM (awarded to CMB). We thank Dr MJ Grosvenor and Dr SJ Baker for assistance in the field, and Dr CA Boulton for assistance with R. 


\section{References}

Belcher CM, Hudspith VA (2016) The formation of charcoal reflectance and its potential use in postfire assessments. International Journal of Wildland Fire, 25(7), 775-779

BBC. (2015) 'Carn Brea gorse fire land 'will take years to recover', http://www.bbc.co.uk/news/ukengland-cornwall-32955448

Cohen-Ofri I, Weiner L, Boaretto E, Mintz G, Weiner S (2006) Modern and fossil charcoal: aspects of structure and diagenesis. Journal of archaeological science, 33(3): 428-439

Hudspith VA, Belcher CM, Yearsley JM (2014) Charring temperatures are driven by the fuel types burned in a peatland wildfire. Frontiers in Plant Science, 5(December), 714. http://doi.org/10.3389/fpls.2014.00714

Hudspith VA, Belcher CM, Barnes J, Dash CB, Kelly R, Hu FS (2017) Charcoal reflectance suggests heating duration and fuel moisture affected burn severity in four Alaskan tundra wildfires. International Journal of Wildland Fire, 26(4), pp.306-316.

Lowden L, Hull T (2013) Flammability behaviour of wood and a review of the methods for its reduction. Fire Science Reviews, 2(1), 4. http://doi.org/10.1186/2193-0414-2-4

Ryan K, Noste N (1985) Evaluating prescribed fires (230-238) in: JE Lotan et al. (tech. coor) Proceedings-Symposium and Workshop on Wilderness Fire. USDA Forest Service Intermountain Forest and Range Experiment Station, General Technical Report INT-182.

The Heritage Journal (2015) 'Carn Brea blaze - an opportunity for investigation?' https://heritageaction.wordpress.com/2015/05/28/carn-brea-blaze-an-opportunity-for-investigation. 
Short contribution - Fire Management

Carbon dioxide emission on recurrent burnt peat swamp forest in Raja Musa Forest Reserve, Selangor, Malaysia

\author{
Ahmad Ainuddin Nuruddin ${ }^{1}{ }^{*}$; Nur Haifaa' Izwa Asari ${ }^{2}$, Hazandy Abdul Hamid ${ }^{1}$, and Mohd Kamil Ismail ${ }^{2}$ \\ ${ }^{1}$ Institute of Tropical Forestry and Forest Product, Universiti Putra Malaysia, 43400 Serdang, Selangor, \\ Malaysia \{ainuddin@upm.edu.my*,hazandy@upm.edu.my\} \\ ${ }^{2}$ Faculty of Forestry, University Putra Malaysia, 43400 Serdang, Selangor, Malaysia, \\ \{haifaizwan@gmail.com,mkamil1986@gmail.com\}
}

\begin{abstract}
Peatlands represent globally significant stores of soil carbon that have been accumulating for millennia and currently. However, the human activities have causes degradation which led to increase in forest fire incidences and emissions of green house gases into the atmosphere. However, not musch is known on emissions of green house gases on recurrent burnt peat forest. The objective of this study was to investigate carbon dioxide emission and its relationship with environmnetal factors on recurrent burnt tropical peat swamp forest. This study was conducted on plot which has been experiencing recurrent fires since 1996 in Raja Musa Forest Reserve, Selangor, Malaysia. The carbon diaoxide emission rates in recurrent burnt plot was in the ranges from 2.13 to $8.50 \mu \mathrm{mol} \mathrm{m}^{-2} \mathrm{~s}^{-1}$. The result also showed the variation in relationship between soil $\mathrm{CO}_{2}$ emission across time, weekly and monthly and statistically analysis showed a significant correlation between soil $\mathrm{CO}_{2}$ emission with soil temperature and soil humidity. In conclusion, soil temperature and relative humidity were the factors influencing the soil $\mathrm{CO}_{2}$ variation significantly according to different months.
\end{abstract}

Keywords: Tropical peat samp forest, Carbon dioxide, forest fire

\title{
1. Introduction
}

Tropical peat swamp forests are an important component of the world's wetlands and provide a variety of benefits in the form of timber products, fishery resources, energy, flood mitigation, water supply and groundwater recharge. However, since 1990, 5.1 Mha of the total 15.5 Mha of peatland in the southeast Asia have been deforested, drained and burned while most of the remaining peat swamp forest has been logged intensively (Miettinen and Liew, 2010).

Over the same period there is an increase of tropical peat swamp forest areas that have been converted to oil palm and plywood plantations from 2 to $15 \%$ of the total peatland area. This high rate of conversion has affect the delicate balance of ecosystem process in the area and has led to the draining of the tropical peat swamp coupled with the drought cycle has causes fires in the are Ainuddin and Goh, (2010). The impacts of these fires were haze and release of green house gasses into the atmosphere (Syaufina et al., 2004).

Hence, there is a strong interest in quantifying carbon dioxide emission from unburnt and recurrent tropical peatlands as part of the wider debate on the impacts of tropical land use change on climate change processes.

\section{Methodology}

In this study, sampling was conducted at the latitude of $03^{\circ} 26^{\prime} 08^{~ " N o r t h ~ a n d ~ l o n g i t u d e ~} 101^{\circ} 25^{\prime} 09^{\prime \prime}$ East at Raja Musa Forest Reserve (RMFR), Bestari Jaya Selangor. RMFR covers 23,000 ha and has 
annual rainfall ranged from 1336 to $2673 \mathrm{~mm}$ with high rainfall in November while the lowest in June. RMFR and together with Sungai Karang Forest Reserve are known as North Selangor Peat Swamp Forest (NSPSF).

Plots with $20 \mathrm{~m} \times 20 \mathrm{~m}$ in size that easy for accessibility were chosen in compartment 6.200 small plots were measured with an area of $1 \mathrm{~m} \times 1 \mathrm{~m}$. Three small plots were selected using the random sampling methods which were plots 68,70 and 151.

Three sampling point were established and soil collars were inserted in a certain depth and the volume of soil covered in the soil collar is determined and leave for 24 hours before data collection to create equilibrium stage and avoid soil disturbance. The offset between the ground and the top edge of the collar were taken for the total volume correction (offset). Next, the measurements of soil CO2 emission were taken using Closed Dynamic Chamber (CDC) (Li-COR 8100 Automated Soil CO 2 Flux System) with $20 \mathrm{~cm}$ sized. This newly developed fully automated system can measure soil $\mathrm{CO}_{2}$ efflux at one stop over time repeatedly. The data was recorded every five minutes for every observation. 30 seconds from 5 minutes observation is a pre-purge stage functioning to avoid overestimation of $\mathrm{CO}_{2}$ emission caused by the initial accumulation of the gas in the chamber. Other environmental factors also can be measured using Li-COR 8100 such as soil temperature and relative humidity.

\section{Results}

The total soil $\mathrm{CO}_{2}$ efflux in three months showed that there was varied at different months. The diurnal pattern of soil $\mathrm{CO}_{2}$ emission from the three months measurement from 3 August 2016 to 15 October 2016 displaced a mean of 4.26 to $5.09 \mu \mathrm{mol} \mathrm{m}^{-2} \mathrm{~s}^{-1}$ (table 1) that showed different emission from 11.40 a.m. to 2.45 p.m. with nearly similar soil temperature and relative humidity. Soil $\mathrm{CO}_{2}$ efflux showed daily rates of 2.13-5.06, 3.34-5.99 and 3.06-8.50 $\mu \mathrm{mol} \mathrm{m}^{-2} \mathrm{~s}^{-1}$ in August, September and October respectively.

Table 1 - Descriptive statistics of soil $\mathrm{CO}_{2}$ efflux $\left(\mu_{\mathrm{mol} \mathrm{m}}^{-2} \mathrm{~s}^{\boldsymbol{4}}\right)$

\begin{tabular}{cccccc}
\hline Month & Mean & $\begin{array}{c}\text { Standard } \\
\text { Deviation }\end{array}$ & $\begin{array}{c}\text { Standard } \\
\text { Error }\end{array}$ & Minimum & Maximum \\
\hline August & 4.78 & 2.71 & 1.36 & 2.13 & 8.43 \\
September & 4.26 & 0.22 & 0.61 & 3.34 & 5.99 \\
October & 5.09 & 2.97 & 1.72 & 3.06 & 8.5 \\
\hline
\end{tabular}

The result for soil $\mathrm{CO}_{2}$ effluxes is summarized in Figure 1 until Figure 11 for each subplot (subplot 68,70 and 151). The mean soil $\mathrm{CO}_{2}$ efflux in this area was $4.68 \mu \mathrm{mol} \mathrm{m}^{-2} \mathrm{~s}^{-1}$. The scatter plots showed the relationship between the soil $\mathrm{CO}_{2}$ effluxes and time which were measured from 10.40 a.m. until 02.45 p.m.. Even though there are some Figures showed negative relationship but it can be concluded that the soil $\mathrm{CO}_{2}$ flux increases gradually across time as $73 \%$ of the scatter plots showed positive relationship. In addition, soil respiration could be affected by photosynthesis process (Ekblad and Hogberg, 2001). Photosynthesis process was depending on the sunlight as the more sunlight means high in temperature will increased photosynthesis process in plant and this will affect soil respiration. As photosynthesis happen, $\mathrm{CO}_{2}$ will be released. There are some plots that show negative relationship because it probably depends on environmental factors. 


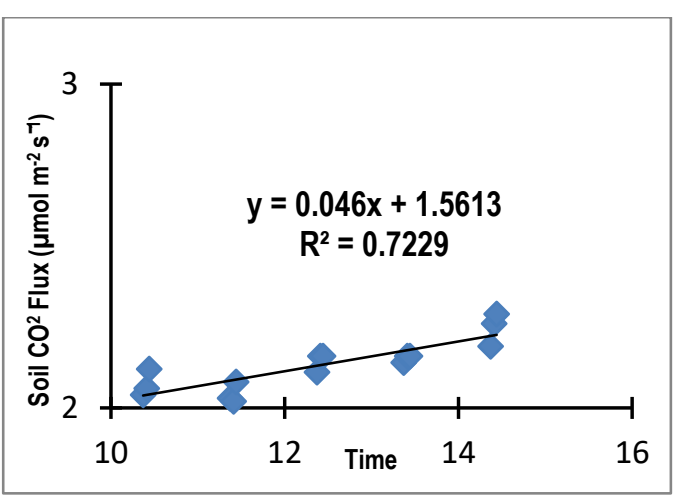

Figure 1 - Soil $\mathrm{CO}_{2}$ flux at plot 151

(03.08.2016)

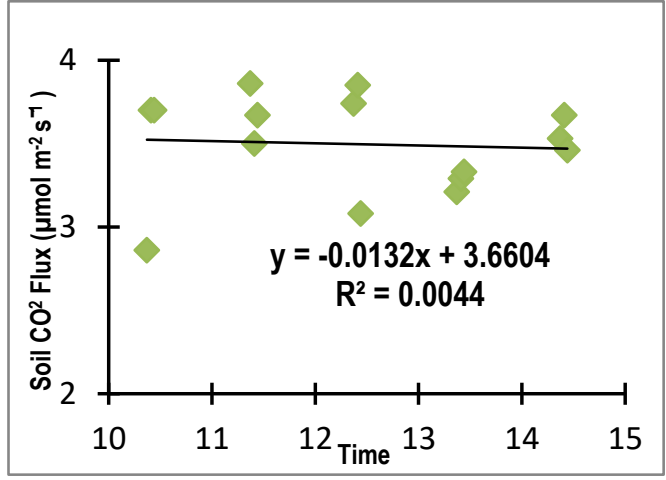

Figure 2 - Soil $\mathrm{CO}_{2}$ Flux at plot 70

$(10.08 .2016)$

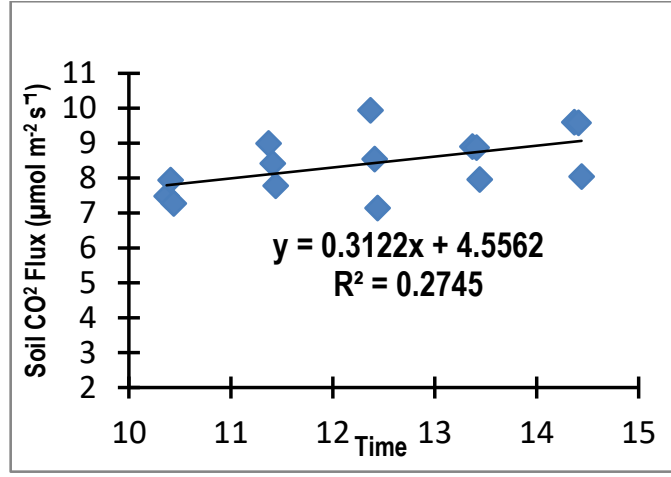

Figure 3 - Soil $\mathrm{CO}_{2}$ Flux at plot 68

(17.08.2016)

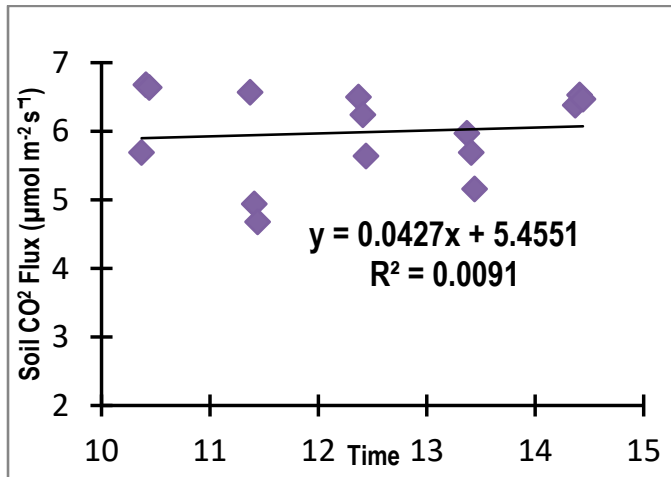

Figure 5 - Soil $\mathrm{CO}_{2}$ Flux at plot 70

(01.09.2016)

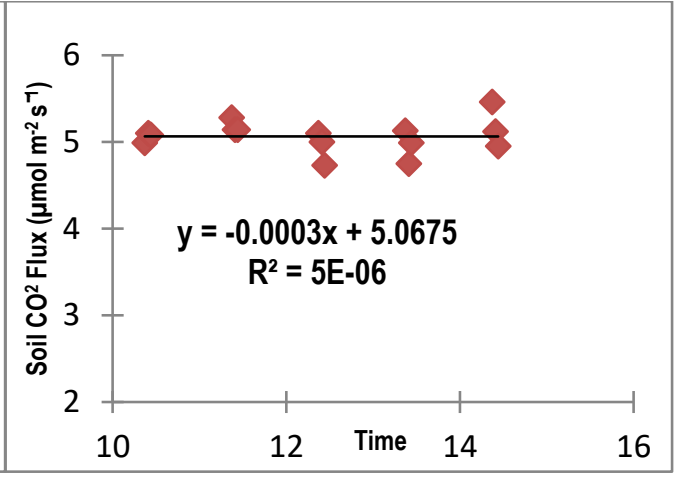

Figure 4 - Soil $\mathrm{CO}_{2}$ Flux at plot 151

(24.08.2016)

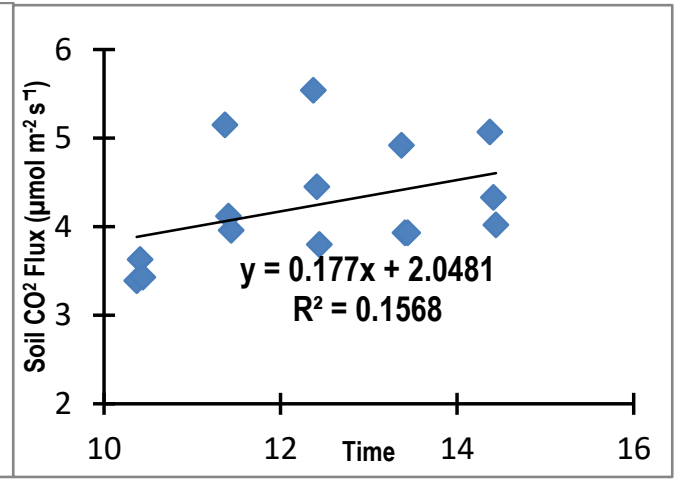

Figure 6 - Soil $\mathrm{CO}_{2}$ Flux at plot 68

$(10.09 .2016)$ 


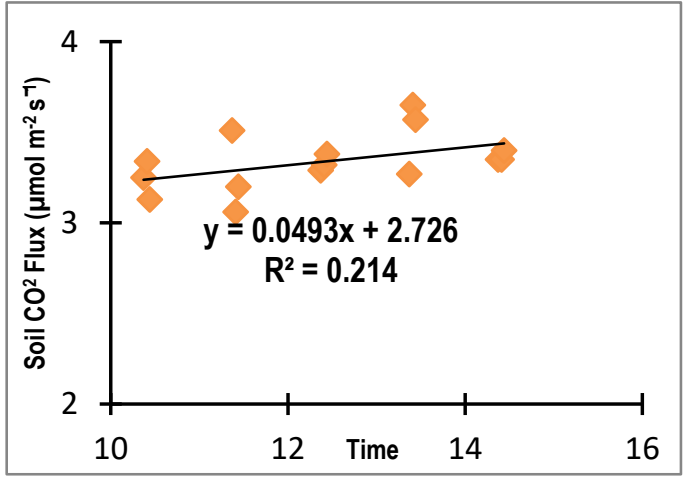

Figure 7 - $\mathrm{Soil} \mathrm{CO}_{2}$ Flux at plot 151

$(15.09 .2016)$

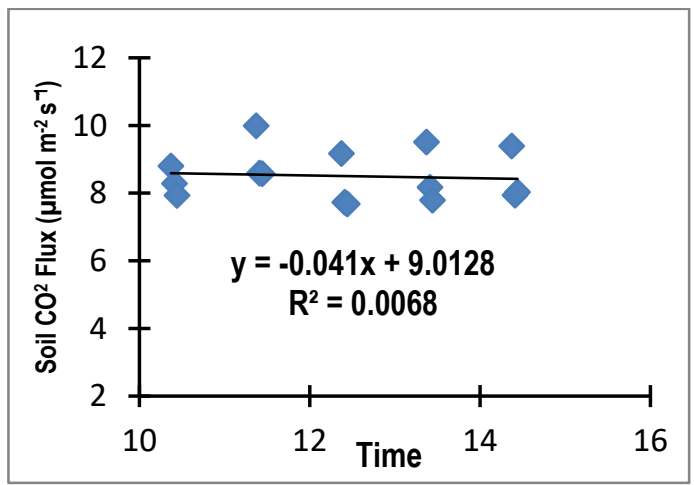

Figure 9 - Soil $\mathrm{CO}_{2}$ Flux at plot 68

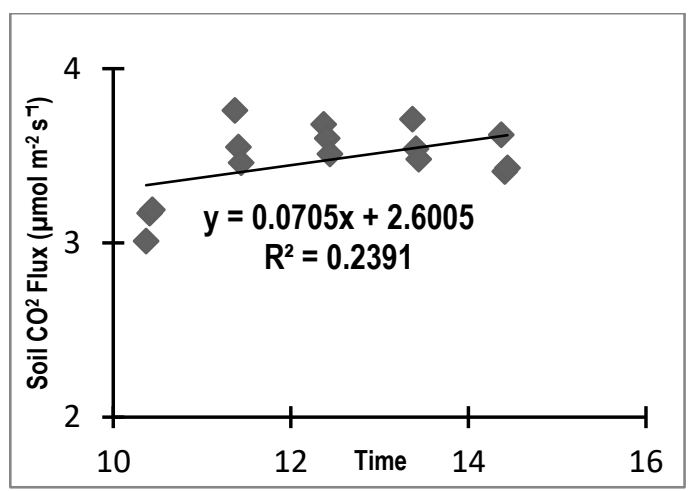

Figure 8 - $\mathrm{Soil} \mathrm{CO}_{2}$ Flux at plot 70

(24.09.2016)

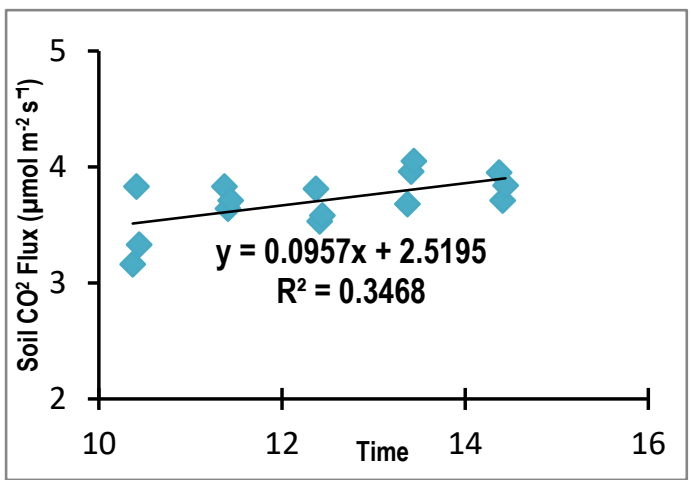

Figure 10 - Soil $\mathrm{CO}_{2}$ Flux at plot 151

$(08.10 .2016)$

$(01.10 .2016)$

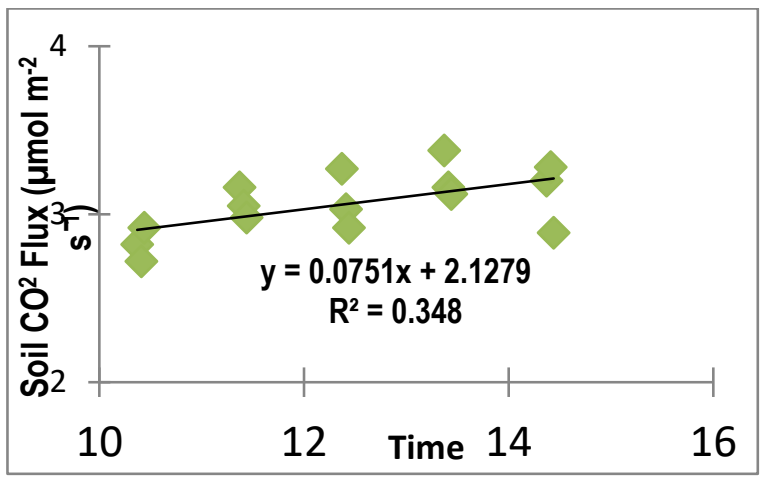

Figure 11 - Soil $\mathrm{CO}_{2}$ Flux at plot 70 (15.10.2016)

\section{Conclusion}

In conclusion, this study showed that the soil $\mathrm{CO}_{2}$ efflux varied depends on the environmental factors such as soil temperature and soil relative humidity, thus influenced the microbial activity. The average soil $\mathrm{CO}_{2}$ efflux in this area was $4.68 \mu \mathrm{mol} \mathrm{m} \mathrm{m}^{-2} \mathrm{~s}^{-1}$. Temperature started to increased and influenced the soil $\mathrm{CO}_{2}$ efflux to increase. In addition, soil temperature was the main factor that influenced soil respiration compared to soil relative humidity. 


\section{Acknowledgements}

This study was funded us MOHE FRGS grant No. 07-01-15-1748FR.

\section{References}

Ainuddin, N. A., \& Goh, K. (2010). Effect of forest fire on stand structure in raja musa peat swamp forest reserve, Selangor, Malaysia. Journal of Environmental Science and Technology, 3(1), 56-62.

Ekblad, A., \& Hogberg, P. (2001). Natural abundance of 13C in CO2 respired from forest soils reveals speed of link between tree photosynthesis and root respiration. Oecologia, 127(3), 305-308.

Miettinen, J. and Liew, S.C (2010). Degradation and development of peatlands in peninsular Malaysia and in the islands of Sumatra and Borneo, Land Degrad. Dev., 21, 285-296.

Syaufina, L., Nuruddin, A. A., Basharuddin, J., See, L. F., \& Yusof, M. R. M. (2004). The effects of climatic variations on peat swamp forest condition and peat combustibility. Jurnal Manajemen Hutan Tropika, 10(1). 
Short contribution - Fire Management

\title{
Characterization of long term retardants
}

\author{
Vicens Mans*; Sheila Espasa \\ "Budenheim Iberica, Extramuros s/n La Zaida, Zaragoza, Spain. \{vicente.mans@budenheim.com*, \\ sheila.espasa@budenheim.com\}
}

\begin{abstract}
Retardants are additives that once added to the water, the mix retards the fire spread by reducing the fuel flammability. There are two types of retardant, the so-called short term and long term. The difference among them is that short-term retardants are only effective when water is still there, while long-term retardants are effective even if water is evaporated.

The long-term retardants, retardants since now on and the base product for the presentation, modify the combustion process of the fuel, leading to a char formation instead of flammable gasses emission when it is submitted to pyrolysis. This particular aspect is well explained in the paper detailing the chemical reactions involved in the FR mechanism, such as here below case for the char formation mechanism to prevent flammable gases emission and avoid flame spread.
\end{abstract}

Keywords: retardants, forest fire fighting

\section{Characterization of long term retardants}

Besides the expected functionality of a retardant, the paper presents the regulatory aspects affecting the use of retardants, such as REACH compliance and Safety data Sheet required as per CE 1272/2008 for a safe use and application.

\section{Physical-chemicals parameters}

- Viscosity: The rheological properties of the retardants have a key influence in the air drops patterns ( $1 / \mathrm{m} 2$ left on the ground) and in the adhesion properties on the fuel (pine trees old forest needs higher viscosity than bushes). This parameter is measured for concentrated and mixed retardant (diluted with water at the end use condition) and are qualified as High (>1000 cp ), Medium ( 100$1000 \mathrm{cp})$ and Low viscosity (<100 cp)

- $\quad \mathrm{pH}$ : To determine acidity or alkalinity. Most of specs required a neutral $\mathrm{pH}$ ( 6-8 )

- Density: Retardants in form of a liquid concentrate use to have high densities $(1,4 \mathrm{Kg} / \mathrm{l})$. Mixed retardants density, typically $1,1 \mathrm{~kg} / \mathrm{l}$, is an important parameter since flying conditions are affected by the takeoff weight. Tested in both concentrate and mixed retardant.

- Freezing temperature. Temperature at which the liquid retardant concentrate turns into solid. This is an important parameter for winter storing of products. This effect is not important if the product can turn back into liquid after a temperature increase. Only tested in liquid concentrates.

- $\quad$ Active ingredient: The main component of the retardant is the responsible for the extinguishing capacity. The chemical reaction with the fuel to form char is based on an acidic dehydration of the cellulose. The acid is formed by ammonia release at $>250^{\circ} \mathrm{C}$ and the left acid is causing the dehydration. Two ammonium salts have been used for years: ammonium sulfate and ammonium phosphate/polyphosphate. Nowadays, ammonium sulfate is no longer used for two reasons: a) environmental reasons, b) Sulfates are not efficient for afterglow because SO3 is released and the catalytic effect is gone. Ammonium phosphates/polyphosphates remain because P2O5 is not volatile 
and remain on the fuel with a catalytic effect. Therefore, that characterization on the active ingredient is concentrated nowadays on the Phosphorous content, expressed as P2O5 as usual in fertilizer terminology. Tested in concentrates.

For solid FR concentrates, ammonium phosphates are used and for liquid FR concentrates, liquid ammonium polyphosphates are used.

\section{Performance characterization}

\subsection{Extinguishing efficiency.}

This is the most important parameter to qualify a retardant as such and be accepted in public tenders. Tests are run for mixed retardants according to the water dilution rate recommended by the manufacturer. There are three different tests and related assessments performed in three countries: a) Spain. A thermo-scale with a pine needles layer is $1 / 3$ treated with retardant, fire spread is measured, and ashes/residues weighted. With the test data, efficiency is rated from 0 to 4 . INIA is the responsible test institution. b) France: Two tests are performed: 1: Heat release determination. 2: Thermo-scale evaluating flame spread and weight loss. Fuel used are preconditioned wood chips. The Institution responsible for is CEREN. c) USA : For retardants that claim the $\mathrm{P} 2 \mathrm{O} 5$ concentration is above $8 \%$ in the mixed retardant, no test is required since experience proves that such concentration is enough to satisfy extinguishing properties. For concentrations below 8\%, mixed retardant are tested in a wind tunnel where pine needles on a long plate are ignited and flame spread and weigh loss is measured.

\subsection{Corrosion.}

This parameter is relevant regarding operations with retardant, mainly for aerial application. There is one common assessment method used by CEREN and USFS based on a 90 days weight loss of metal coupons immersed in the retardants. Alloys tested are: steel, copper brass, aluminum and magnesium (for helicopters with fix tank ). Testing conditions are total immersion, partial immersion, $20^{\circ} \mathrm{C}$ and $50^{\circ} \mathrm{C}$. There are some complementary methods, as inter-granular corrosion (USFS) or galvanic corrosion (CEREN). .

\subsection{Abrasion:}

Retardants contains solids in suspension, such as coloring agents, clay thickeners, that may cause abrasion problems in pumps, etc. tests method for such assessment are described in the presentation.

\subsection{Toxicity:}

The use of retardants shall not threat human health and environment. Testing and assessment described in OCDE and OPPTS ( EU and USA ) methodology.

\subsection{Impact on the flora.}

Retardants may cause seed germination problems due to the salinity concentration. The assessment method is based on the \% of germinated seeds compared with a blank sample. Typically, it is allowed a decrease of $50 \%$.

\subsection{Coloring.}

Retardants have to be identified from the air or ground in order to overlap air drops or ground applications.

Red color is the worldwide recognized color, which can be permanent (excluding rain washing) or fugitive (degraded by sun light). 
Assessment is made by visual methodologies, applied either on real vegetation or on laboratory application on white/black paper.

\subsection{Conclusions. Proposal for a EN standard for retardants assessment.}

Nowadays, technical requirements in public tenders in Spain are issued by each Autonomic Region. In France, Italy ... are issued by each of the Governments and it implies more than ten different specifications/requirements within EU. This lack of common assessments obviously imply difficulties for all stakeholders. The sector (Administrations, testing labs, suppliers,..) believe that a standardized assessment methodology would favor the competition, may avoid national trade barriers, and a common reference in the technical specification for retardants acquisition. For that purpose, the Spanish wildfire sector group in Tecnifuego, the fire safety association in Spain, that hosts the UNE/CTN23 Fire Safety technical Committee, has proposed to UNE the creation of a WG to develop such retardant assessment standard. This UNE standard will allow the different Spanish autonomies to specify according to this standard. Further, on, UNE can propose that standard as a draft to CEN to elaborate the EN standard. WG will be integrated by industry sector, institutions (INIA, UPC, UCO,..) and administrations ( Ministries,... ) 
Short contribution - Fire Management

Data mining techniques in the assessment of usability and effectiveness of forest fire video surveillance

\author{
Ljiljana Šerić $^{1 *}$; Danijela Mikuličić ${ }^{2}$, Maja Braović ${ }^{1}$ \\ ${ }^{1}$ Department for Modeling and Intelligent Systems, University of Split, Faculty of Electrical Engineering, \\ Mechanical Engineering and Naval Architecture, 21000 Split, Croatia, Ruđera Boškovića 32, \\ \{ljiljana.seric@fesb.hr*,maja.braovic@fesb.hr\} \\ ${ }^{2}$ Locastic, Lovački put 7, 21000 Split,Croatia, \{danijela@locastic.com\}
}

\begin{abstract}
Since forest fires are natural phenomenon that cannot be avoided nor their occurrence can be prevented it is of utmost importance to have a reliable fire management system at hand. Damage prevention and early fire detection are two approaches that are widely used in forest fire management today.

In the last decade, video surveillance has been recognized as useful tool for forest fire management. It helps both firefighters and forest managers in several aspects of their work, and consists of a video sensor located in the area that would otherwise be difficult to access. This sensor provides video information about the state of the surveilled forest, and can be helpful in two forest fire video surveillance phases:

- monitoring phase, that takes place before fire occurs band includes continuous monitoring of the surrounding area in search for signs of fire and smoke, and

- surveillance phase that occurs during the firefighting operative actions and that provides commanding officers with information on the severity and spread of fire.

Since there is a great demand for video surveillance for early fire detection, both in the firefighting and in the public community, this area of research is in the focus of many scientists and developers. Automatic fire detection can aid early fire response, therefore minimizing the possible fire damages while also not relying on human observers that might be suspect to inadequate experience and possible tiredness.

The work presented in this paper was motivated by the need to define quantitative measures that can help decide if a video surveillance system is effective in firefighting processes during the fire season. These measures can ultimately be used as a tool to perform cost-benefit analysis when designing and planning an integration of firefighting and forest fire management with systems for video monitoring and video surveillance.

A pilot forest fire video monitoring and surveillance system was installed in Šibenik-Knin County as the output of the HOLISTIC project at the end of 2016 fire season. In 2017 fire season the system was fully functional and used in everyday activities.

In this paper we used a variety of available data related to forest fires that took place in the area of ŠibenikKnin County in the summer of 2017. All available data was aggregated and used as the input data for the presented analysis. Several data mining techniques were used for data formatting and association. Ultimately this data was combined in a single document where the activities that were logged in the system logs were correlated with information from the operative actions reports, social media and other sources. Data mining techniques such as clustering and frequent itemset analysis were utilized for the assessment of usability of the overall video surveillance system.

The correlation of the alarms raised by the system and real operative actions in the context of media reports provide a measure of usefulness of the video surveillance system both for fire detection and for guidance during the firefighting process.
\end{abstract}

Keywords: fire monitoring, video surveillance, data mining, fire season analysis 


\section{HOLISTIC pilot forest fire video monitoring and surveillance system}

HOLISTIC pilot forest fire video monitoring and surveillance system was installed at the end of the 2016 fire season. The system is composed of three monitoring locations - Kamenar, Dubravice and Srima, and is covering the area shown in Figure 1. Each monitoring location is equipped with two PTZ (pan-tilt-zoom) cameras mounted on a column. Each camera provides $180^{\circ}$ cover of the surrounding area.

The software system that controles the total of 6 cameras can operate in two modes: automatic and manual. In the automatic mode the cameras move continuously, and each monitors one side of the column. The software searches for visible signs of fire and smoke. In case of suspicious phenomena, an alarm is raised. In the manual mode the camera is managed by the human operator that controls it manually via pan tilt zoom module. Manual mode is mostly used in the case of fire occurrence, as it can provide detailed visual inspection of the

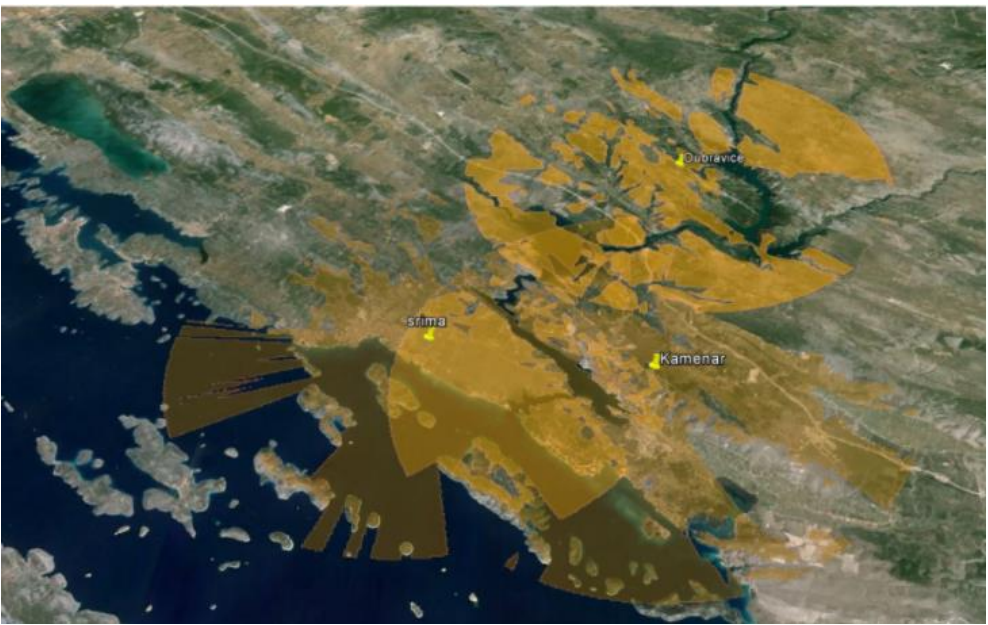

Figure 1 - Area covered by HOLISTIC pilot forest fire video system area to the operating centre.

All the alarms that are raised by the system, as well as all of the actions performed in the system by an operater, are archived and can be analyzed in the post analysis of the fireseason.

\section{2017 forest fire season in Šibenik-Knin county}

2017 summer fire season was especially hard in all Mediterranean countries, so Croatia and Šibenih-Knin county were no exception. The entire 2017 summer season was characterized by high and very high fire danger index, since the country went through very dry periods and received no rain for almost 3 months.

Total area burned during the fire season was mapped by firefighting commander who shared his personal records with authors for research purposes. Burned area overlayed with area protected by the monitoring system is shown in figure 2 . The figure indicates that fires that occurred inside protected area are extinguished sooner and affects a smaller surface.

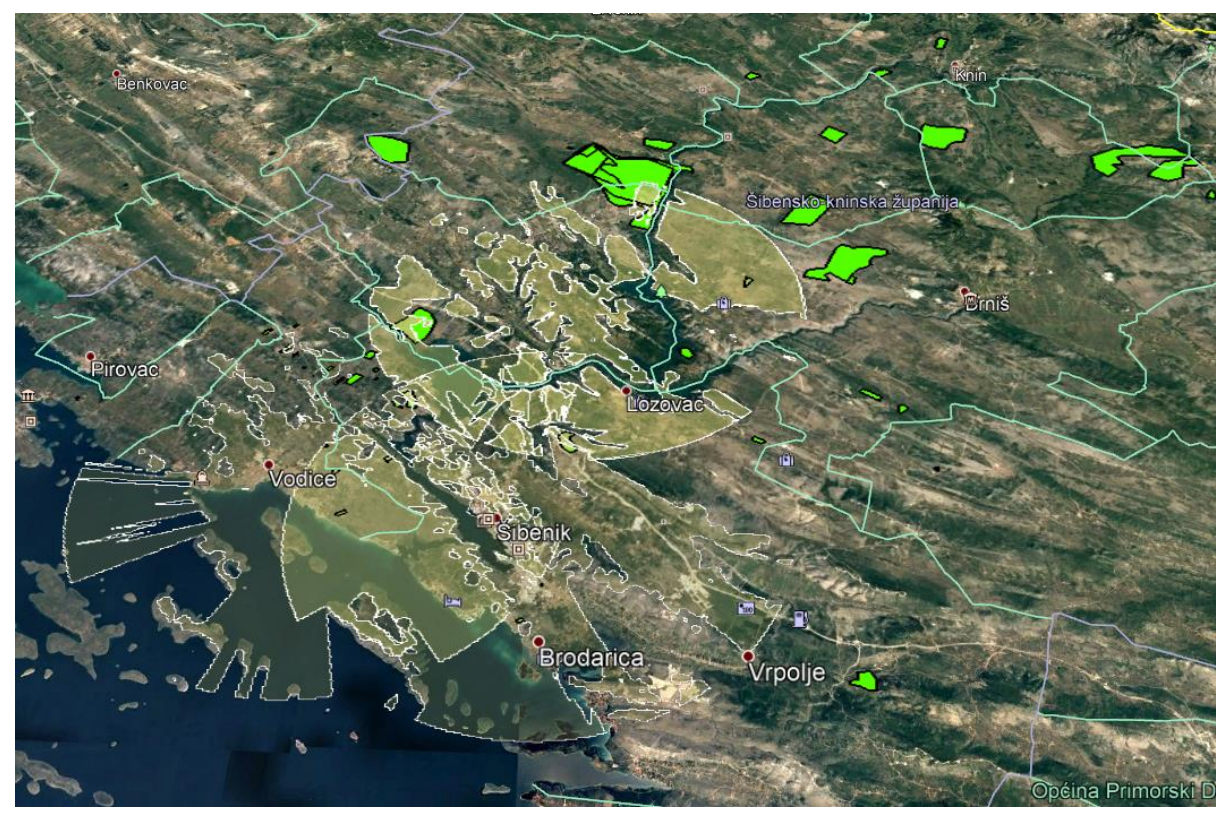

Figure 2 - Burned area during fire season overlayed with protected area of the Šibenik knin county 


\section{Assessment of usefulness based on data mining techniques}

After the 2017 fire season, which officially starts in July and lasts until September, all alarm and activity logs were collected and used in this work. The period analyzed in this work was from the month of April, since from that period the number of fires noticeably started to rise in comparison to previous years average. This analysis included the analysis of the number of alarms per camera, and the analysis of user action logs. In the analysis of user action logs special interest was given to cases where users started to use the manual camera mode, as this was considered as a sign of the true fire alarm on that camera.

In addition, the data regarding the burned area was enhanced by personal and official data collected by the firefighters and firefighting commanders, and was aggregated into the same document on which clustering and frequent itemset analysis was performed.

\section{Data Sources}

Data used in assesement of usefullnes comes from various sources, such as:

1. System logs - all user interaction with the system is stored for future analysis. These logs include login information, manual/automatic mode change, parameters change and similar.

2. Burned area records - personal records kept by firefighting commander and used for official report writing and analysis. These records consists of georeferenced polygons of burned area annotated with information about date and time of start of the firefighting operative action.

3. Image archive - personal archive kept by operators for reporting and analysis. These archive consists of images stored with information about location, date and time of the fire.

4. Media and social reports - public articles on wildfires in the area analyzed. Larger fires are usually reported in news and social media.

Additionally, weather data for the season was collected and taken into account.

The process of association and analysis of all data is shown in figure 3.

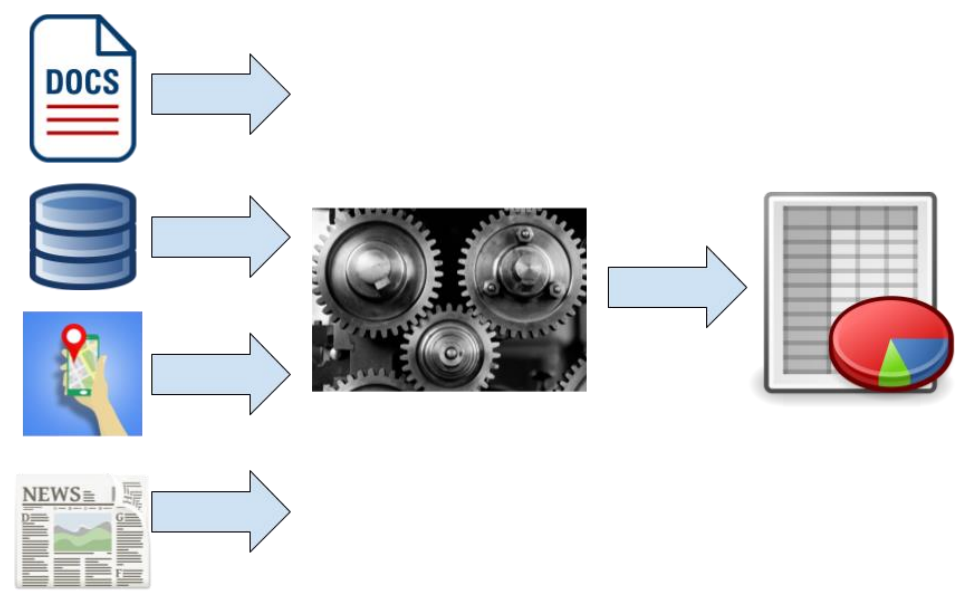

Figure 3 - Data association and processing

\section{Assessment of usefulness based on data mining techniques}

Data from various sources was collected, associated and preprocessed for application of data mining techniques. Two aspects of the system were taken into account while evaluating the usefulness:

- Automatic mode - analysis of valid alarms and number of false alarms

- Manual mode - analysis of system usage and helpfulness for inspection and surviellance of fires. 


\subsection{Analysis of automatic mode}

Final associated data was used for assesement of several conclusions.

Comparison of hourly distribution of alarms and valid alarms (Figure 4) has shown that majority of false alarms happen in the morning during sunrise due to the effects of sun on camera lense. This information is taken into account when planning future development of algorithm for automatic smoke and fire detection.

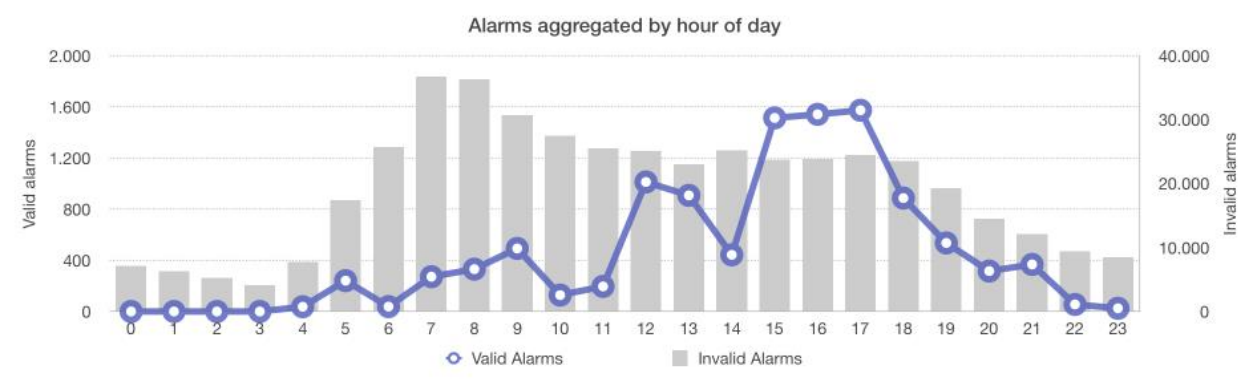

Figure 4 - Alarms aggregated by hour of day

A priori algorithm applied on collection of data shown interesting conclusions. This algorithm is used for finding frequent itemset in collection of aggregated data from various sources. We used this analysis to identify the events that often happen together. Associated events are detected using raw algorithm and later the semantics of rules for associated events are constructed.

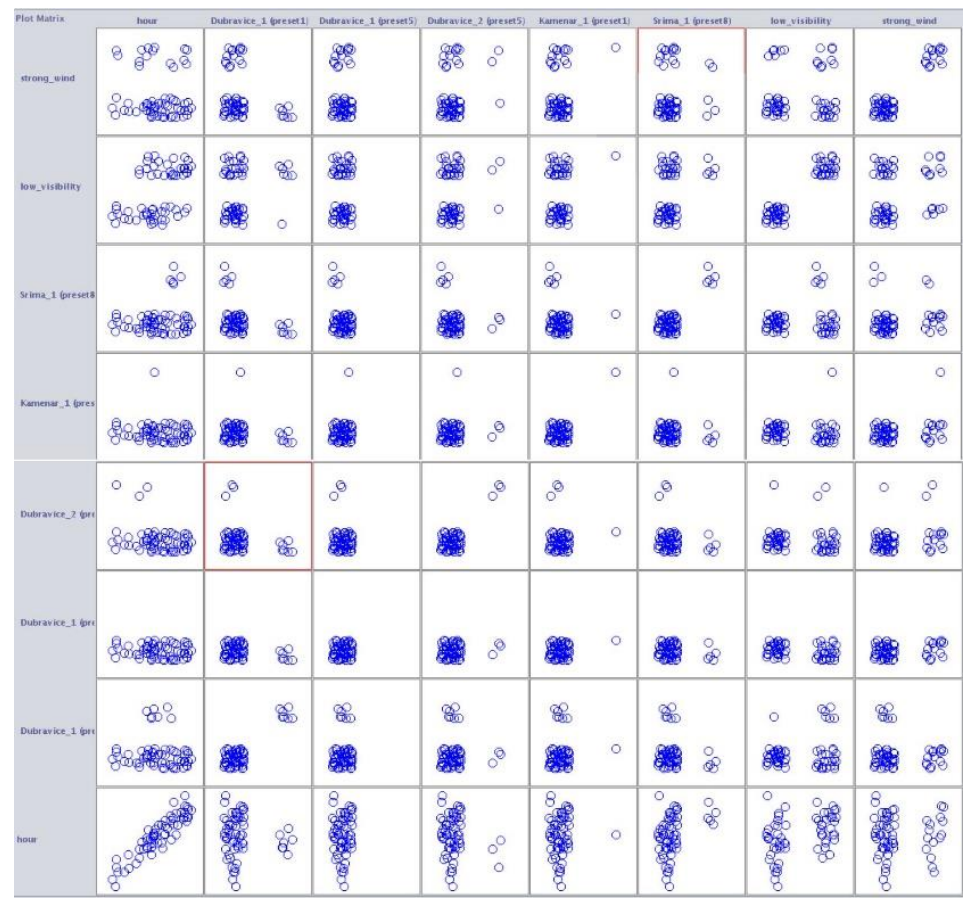

Figure 5 - Visualized results of best rules given as output from apriori analysis of valid alarms data

Figure 5 depicts the best rules calculated by apriori analysis of raised alarms after which the system was put into manual mode. The most frequent valid alarms were raised on different presets of cameras Dubravice and Srima, which in comparison with burned areas in 2017 is exactly where the fires were placed in monitored area.

Analysis was also performed on falsely raised alarms, which were very frequent in given dataset. Results of this analysis have shown that most of false alarms were raised either to bad weather conditions (low visibility or strong wind) or during the sunrise/sunset on east/west facing cameras. 
Number of valid and invalid alarms aggregated by hour of day is shown on the graph above, which shows that most of invalid alarms were raised during sunrise hours.

\subsection{Analysis of manual mode}

Besides automatic detection of forest fires another feature of the system is manual camera control and surveillance of forest fire. To assess the usefulness of this mode we analyzed system logs and determined the length of periods when camera was used in manual mode to inspect or surveilled forest fire.

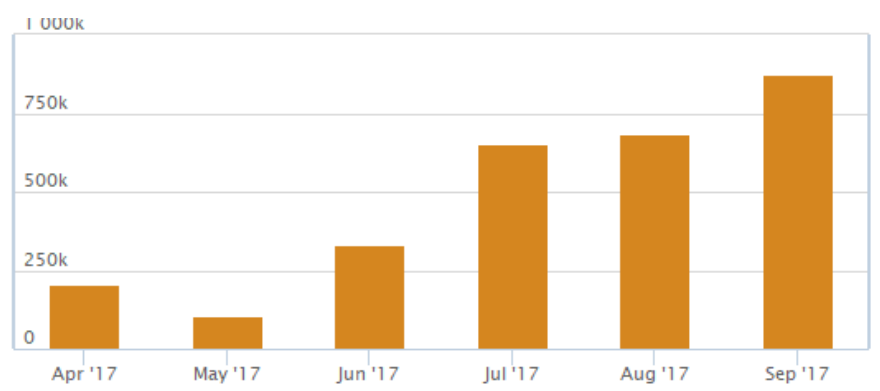

Figure 6 - Distribution of time spent in manual mode by month

During months with highest number of fires manual mode was more frequently used.

\section{Results}

A total of 479964 alarms was raised during the monitored period. The system was switched to manual mode 10917 times. Some of the factors that cause a lot of alarms to be raised are camera's azimuth, time of the day, visibility and wind. All the aforementioned factors were taken into account while performing data analysis.

Total resulting numbers are summed in the following table:

\begin{tabular}{|c|c|}
\hline Number of alarms & 479964 \\
\hline Number of manual mode swich & 10917 \\
\hline Total time in manual mode & $727 \mathrm{~h}$ \\
\hline
\end{tabular}

\section{Conclusions}

HOLISTIC pilot forest fire video monitoring and surveillance system still has a large amount of false positive results, mostly caused by weather conditions, lens flare or camera instability. On the other hand, it is apparent that the fire affected areas in the monitored area are much smaller than the fire affected areas outside the monitored area. Considering the analysis performed on given data, cameras placed in the fire affected areas were the ones which raised the largest count of valid alarms. Even with the large number of false alarms, this proves the system's use case, which is early forest fire detection, resulting in faster firefighting reaction.

\section{Acknowledgements}

Pilot system was installed with the financial assistance of the IPA Adriatic Cross-Border Cooperation Programme.

The authors would like to thank the Šibenik-Knin County Firefighting Operations Center and County firefighting commander Darko Dukić for sharing personal records on fires in 2017 season. 
Short contribution - Fire Management

\title{
Drivers of wildland fire behaviour variation across the Earth
}

Paulo M. Fernandes ${ }^{1 *}$; Ângelo Sil ${ }^{1}$; Davide Ascoli ${ }^{2}$; Miguel G. Cruz ${ }^{3}$; Martin E. Alexander ${ }^{4}$; Carlos G. Rossa $^{1}$; Jaime Baeza ${ }^{5}$; Neil Burrows ${ }^{6}$; G. Matt Davies ${ }^{7}$; Alessandra Fidelis ${ }^{8}$; James S. Gould ${ }^{3}$; Navashni Govender'; Musa Kilinc ${ }^{10}$; Lachlan McCaw ${ }^{11}$.

${ }^{1}$ Centro de Investigação e Tecnologias Agroambientais e Biológicas, Universidade de Trás-os-Montes e Alto Douro. Quinta de Prados, 5001-801 Vila Real (Portugal), \{pfern@utad.pt*\}

${ }^{2}$ Dipartimento di Agraria, University of Naples Federico II. Via Università 100, 80055 Portici, Napoli (Italy), \{davide.ascoli@unina.it\}

${ }^{3}$ CSIRO. GPO Box 1700, Canberra, ACT 2601 (Australia) \{miguel.cruz@ csiro.au\}

${ }^{4}$ Wild Rose Fire Behaviour. 180 - 50434 Range Road 232, Leduc County, AB T4X OL1 (Canada), \{mea2@telus.net\}

${ }^{5}$ Universidad de Alicante.Ap. 99, E- 03080,Alicante (Spain) \{jaime.baeza@ua.es\}

${ }^{6}$ Department of Parks and Wildlife. 17 Dick Perry Avenue, Kensington WA 6151 (Australia),

\{neil.burrows@dpaw.wa.gov.au\}

${ }^{7}$ School of Environmental and Natural Resources, The Ohio State University. Columbus, Ohio, USA

\{davies.411@osu.edu\}

${ }^{8}$ Universidade Estadual Paulista (UNESP), Instituto de Biociências. Av. 24A, 1515, 13506-900, Rio Claro, Brazil, http://orcid.org/0000-0001-9545-2285 \{afidelis@rc.unesp.br\}

${ }^{9}$ Conservation Management, Kruger National Park. Private Bag X402, Skukuza 1350, South Africa, \{navashni.govender@sanparks.org\}

${ }^{10}$ Country Fire Authority, F\&EM - Predictive Services. 8 Lakeside Drive East Burwood VIC 3151 (Australia), \{m.kilinc@cfa.vic.gov.au\}

${ }^{11}$ Science and Conservation, Dpt. Biodiversity, Conservation and Attractions. Locked Bag 2, Manjimup WA 6258 (Australia), \{lachie.mccaw@dbca.wa.gov.au\}

\begin{abstract}
Fire behaviour characteristics define the impacts on society and the environment. While wildland fire science has expanded to include the analysis of fire activity and effects across the globe, an understanding of global fire behaviour patterns and its drivers remains incomplete. We utilized the literature and unpublished datasets as sources for compiling a worldwide fire environment and fire behaviour database consisting of field-based experimental fires, planned-ignition prescribed fires and wildfires. Analysis of the database allows for the assessing of the influences of fuel and weather descriptors on fire behaviour characteristics at various scales, namely climate zone, biome, ecoregion, and vegetation type. The database comprises nearly 6000 fires. Fire spread rate and fireline intensity vary by five orders of magnitude (seven in the former case if smouldering is considered). Variation in fire-spread rate is dominated by fuel moisture content within Koppen-Geiger climates, except in tropical wet and hot desert climates, where wind speed prevails. Fuel structure is less important than weather-related variables, but it explains 35 and $30 \%$ of the variability within monsoon-influenced humid subtropical and hot summer Mediterranean climates, respectively. Fuel structure and load metrics increase in importance in regards to fireline intensity, being the dominant influence (66$91 \%$ ) in hot semi-arid, temperate oceanic, and hot summer Mediterranean climates. Fuel moisture content exerts by far the major control in the variation in fire spread rate within broad vegetation types (forest, woodland, shrubland, grassland), while fuel structure metrics dominate the variation observed in fireline intensity, except in woodlands, where fuel moisture content is slightly more influential. Efforts to understand potential fire activity and fire regime shifts in relation to global change, and the formulation of policies for adaptive fire management will benefit from the results of this study.
\end{abstract}

Keywords: fire spread rate, fireline intensity, fire modelling, fuel structure, fire weather, fire environment 


\section{Introduction}

The environmental and societal consequences of wildland fire depend on how fast it spreads, its areal extent, how much biomass it consumes, and how much and at what rate it releases energy. While wildland fire science has expanded to include the analysis of fire activity and effects across the globe (Moritz et al. 2012), an understanding of global fire behaviour patterns and their drivers remains incomplete, and is addressed by the on-going BONFIRE project.

We utilized the literature and unpublished datasets as sources for compiling a worldwide fire environment and fire behaviour database consisting of field-based experimental fires, planned-ignition prescribed fires and wildfires. Among other possibilities, analysis of the database allows for assessing the influences of fuel and weather descriptors on fire behaviour characteristics at various spatial scales, namely climate type and different levels of vegetation classification. In this study we quantify the relative importance of fuel and weather-related variables in determining fire behaviour variation at those scales.

\section{Data and Methods}

We surveyed the peer-reviewed and grey literature and identified candidate field-based experimental fires to include in the database, supplemented by unpublished data on file. We compiled information from natural or activity (slash and masticated) fuels, the former comprising both flaming and smoldering fires. Fires backing into the wind or downslope, as well as ignition patterns resulting in fire front interaction in both experimental and prescribed fires (e.g. strip-head firing) were not included in the analysis, as well as fires spreading as fronts $<2 \mathrm{~m}$ wide that do not reach their potential rate of spread even under mild weather conditions (Wotton et al. 1999). When multiple observation periods were available, wildfire data collection was limited to the period of maximum rate of spread in a given vegetation type. We retrieved as much information as possible on:

- Fire weather: wind speed, ambient temperature and relative humidity, fuel moisture contents per condition - dead or live - and size class, Canadian Forest Fire Weather Index System codes and indices (Van Wagner 1987);

- terrain slope;

- surface fuel properties and other attributes of the fuel complex (e.g. loads per size class and live or dead condition, curing or \% fine dead fuel, fuel depth or height, fuel layer cover), fuel type (defined by the existing surface fuel layer(s), e.g. litter-grass);

- type of ignition source (i.e. point or line), and ignition line length;

- characteristics of the heading fire, i.e. the forward section of the fire front: rate of spread, flame geometry, and fireline intensity (Byram 1959);

- fuel consumption by fuel layer and size class.

The resulting database is, understandably, highly heterogeneous in its completeness. Each fire was assigned a georeferenced location, Koppen-Geiger climate classification (Peel et al. 2007), mean annual temperature and rainfall (1970-2000) from the WorldClim 2 database (Fick and Hijmans 2017), biome and ecoregion (Olson et al. 2001), NCAR LSM surface type (e.g. cool broadleaved deciduous forest) (Bonan 1996), generic vegetation type (i.e. forest, woodland, shrubland, or grassland), and dominant species. Additionally we attributed reliability scores for weather, fuel and fire behaviour characteristics (Cheney et al. 2012).

Regression tree analysis of fire spread rate and fireline intensity as a function of fuel and weather variables was carried out after adjusting rate of spread for zero slope (Sullivan et al. 2014). The results are presented after discarding the influence of ignition type and ignition line length. 


\section{Preliminary Results and Conclusion}

The database comprises about 6000 fires (experimental fires - 73\%, 15\% wildfires, and 12\% prescribed burns) from 33 countries. However, $72 \%$ of the data comes from Australia, USA, Canada, and South Africa (Figure 1), reflecting their long-standing research programs and fire management policies. Proportionally, forest is the more represented vegetation type, followed by grassland, woodland, and shrubland.

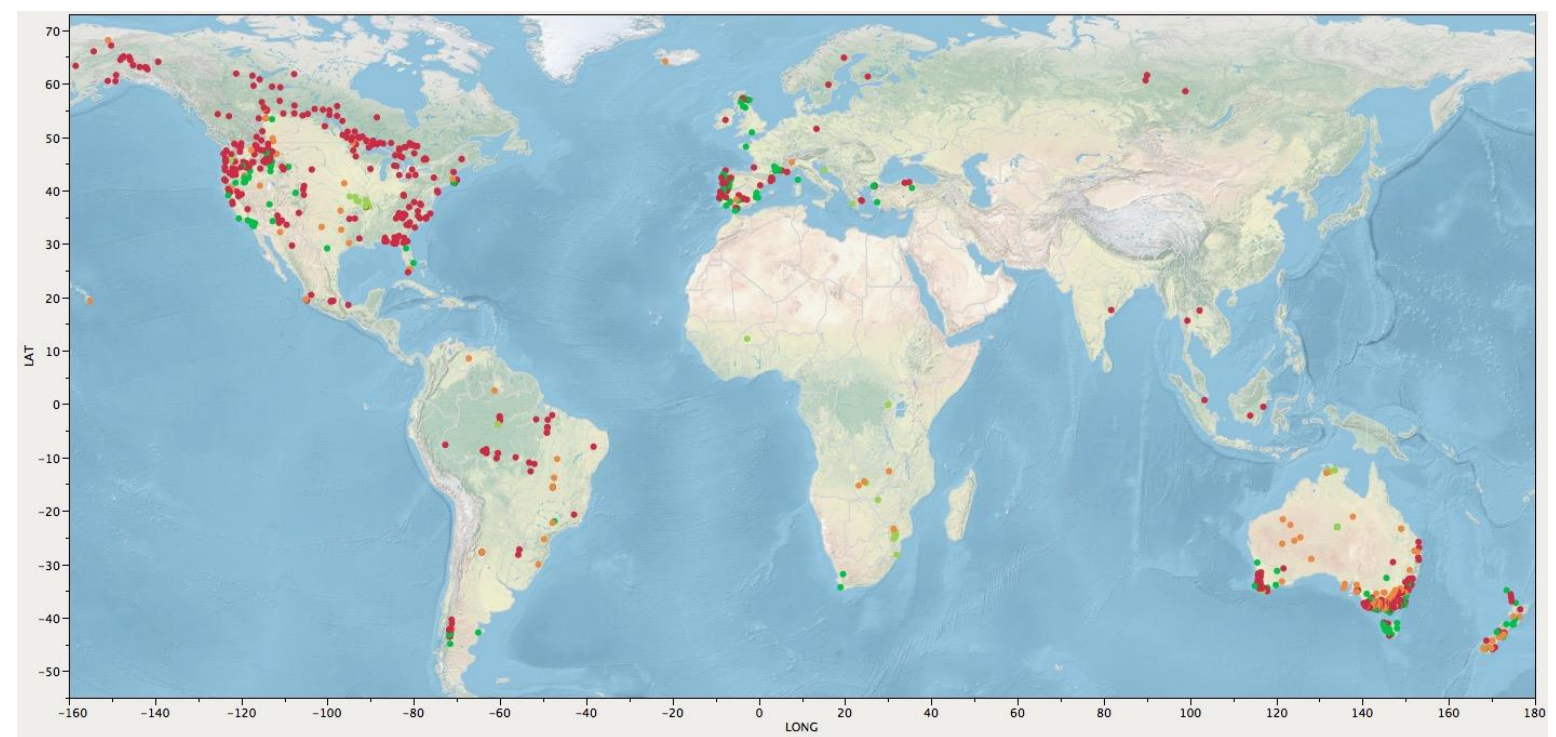

Figure 1 - Location of the fires in the database. Red = forest; orange = grassland; light green = woodland; green = shrubland. Sites in North America, temperate Australasia, and southwestern Europe are prevalent, with scarce locations in Russia, Asia and the other regions of Africa and, to a lesser degree, Central America and South America.

Fire spread rate and fireline intensity in the database vary by five orders of magnitude, and by seven in the former case if smouldering is considered. Variation in fire-spread rate is dominated by fuel moisture content within Koppen-Geiger climates, except in tropical wet and hot desert climates, where wind speed prevails. Fuel structure is less important than weather-related variables, but it explains 35 and $30 \%$ of the variability within monsoon-influenced humid subtropical and hot summer Mediterranean climates, respectively. Fuel structure and load metrics increase in importance with regards to fireline intensity, being the dominant influence (66-91\%) in hot semi-arid, temperate oceanic, and hot summer Mediterranean climates. Fuel moisture content exerts by far the greatest control on variation in fire spread rate within a given broad vegetation type (forest, woodland, shrubland, grassland), while fuel structure metrics dominate the variation observed in fireline intensity, except in woodlands, where fuel moisture content is slightly more influential.

Efforts to understand potential fire activity and fire regime shifts in relation to global change, and the formulation of policies for adaptive fire management will benefit from the results of this study. Visit https://www.researchgate.net/project/BONFIRE-gloBal-scale-analysis-and-mOdelliNg-ofFIRE-behaviour-potential-PTDC-AAG-MAA-2656-2014 to keep up with the latest project developments.

\section{Acknowledgments}

This study was funded by the Foundation for Science and Technology (FCT) and co-financed by the European Regional Development Fund (ERDF) through COMPETE 2020 - Operational Program for Competitiveness and Internationalization (POCI), in the frame of the BONFIRE project - gloBalscale analysis and mOdelliNg of FIRE behavior potential (PTDC/AAG-MAA/2656/2014). 


\section{References}

Bonan GB (1996) A land surface model (LSM version 1.0) for ecological, hydrological, and atmospheric studies: Technical description and user's guide. National Centre for Atmospheric Research, Technical Note NCAR/TN-417+STR. (Boulder, CO)

Byram GM (1959) Combustion of forest fuels. In 'Forest fire: control and use'. (Ed KP Davis) pp. 6189. (McGraw-Hill: New York, NY)

Cheney NP, Gould JS, McCaw WL, Anderson WR (2012) Predicting fire behaviour in dry eucalypt forest in southern Australia. Forest Ecology and Management 280, 120-131.

Fick SE, Hijmans RJ (2017) WorldClim 2: new 1-km spatial resolution climate surfaces for global land areas. International Journal of Climatology 37: 4302-4315.

Moritz MA, Parisien MA, Batllori E, Krawchuk MA, Van Dorn J, Ganz DJ, Hayhoe K (2012) Climate change and disruptions to global fire activity. Ecosphere 3, art49, doi:10.1890/ES11-00345.1

Olson DM, Dinerstein E, Wikramanayake ED, Burgess ND, Powell GVN, Underwood EC, D'Amico JA, Itoua I, Strand HE, Morrison JC, Loucks CJ, Allnutt TF, Ricketts TH, Kura Y, Lamoreux JF, Wettengel WW, Hedao P, Kassem KR (2001) Terrestrial ecoregions of the world: a new map of life on Earth: a new global map of terrestrial ecoregions provides an innovative tool for conserving biodiversity. BioScience 51, 933-938.

Peel MC, Finlayson BL, McMahon TA (2007) Updated world map of the Köppen-Geiger climate classification. Hydrology and Earth System Sciences Discussions 4, 439-473.

Sullivan AL, Sharples JJ, Matthews S, Plucinski MP (2014) A downslope fire spread correction factor based on landscape-scale fire behaviour. Environmental Modelling and Software 62, 153-163.

Van Wagner CE (1987) Development and structure of the Canadian Forest Fire Weather Index System. Government of Canada, Canadian Forestry Service For. Tech. Rep. 35. (Ottawa, ON)

Wotton BM, McAlpine RS, Hobbs MW (1999) The effect of fire front width on surface fire behaviour. International Journal of Wildland Fire 9, 247-253. 
Short contribution - Fire Management

Effect of moisture content and ventilation on the burning rate of porous fuel beds

Sara McAllister

USDA Forest Service - RMRS Missoula Fire Sciences Laboratory. 5775 US Highway 10 W, Missoula, MT, 59808,USA, \{smcallister@fs.fed.us\}

Keywords: burning rate, moisture content, ventilation

\section{Introduction}

The burning rate of wildland fuels is not well understood. In most operational wildland fire models, a very simplistic model of burning rate and residence time is used that does not include the effects of fuelbed geometry, particle size and arrangement, ventilation, or moisture content (Albini 1976, Anderson 1969). The variation in burning rate with moisture content is examined here, both in a quiescent environment and subjected to ventilation. The fuel beds considered are wood cribs. Cribs consist of evenly spaced sticks that are stacked in cross piles. Unlike more realistic wildland fuelbeds, the use of cribs allows for a highly repeatable fuelbed that can be arranged to produce vary different qualities. Cribs are used frequently in the fire protection engineering field, and previous work has shown that they can provide useful insights into how the parameters of the fuelbed can directly influence fire behavior (McAllister and Finney 2016). Porous fuelbeds such as cribs have long been understood to burn in two different regimes (Gross 1962). The burning rate of densely packed fuelbeds is limited by oxidizer availability, so an increase in the fuelbed porosity allows for more oxidizer inside of the fuelbed and results in an increased burning rate. The burning rate of loosely packed fuelbeds however is governed by the heat and mass transfer processes of the individual fuel elements, and so it remains constant with increases in porosity.

\section{Methods}

As shown in previous work (McAllister and Finney 2016), the crib design can have unexpected influence on the results, so a range of geometries and sizes was tested. Five different crib designs were considered here that range in porosity from the densely-packed regime to the loosely-packed regime (see Table 1). Two different stick thicknesses were used $(1.27$ and $0.64 \mathrm{~cm})$. Each crib design was tested at three moisture content levels (approximately 1\%, 3.25\%, and 6.5\%). The moisture content was attained by conditioning the cribs for several days in a conditioning chamber set to a temperature and relative humidity chosen to produce the desired moisture content $\left(35^{\circ} \mathrm{C}\right.$ and $3 \% \mathrm{RH}, 18^{\circ} \mathrm{C}$ and $17 \% \mathrm{RH}$, and $18^{\circ} \mathrm{C}$ and $\left.40 \% \mathrm{RH}\right)$. Each crib design and moisture content combination was tested in both quiescent conditions and subject to ventilation. In order to produce ventilation evenly through the crib, it was placed under a $3.66 \mathrm{~m}$ chimney. The gap between the top of the crib and the bottom of the chimney was held constant at $3 \mathrm{~cm}$ for all tests. The reduced plume entrainment due to the chimney forces ambient airflow through the fuelbed underneath. As seen in Figure 1, all cribs are placed on 3" spacers to allow for unrestricted airflow up through the bottom of the crib. For the tests with ventilation, the cribs are first lit away from the chimney. Once ignition was established, they were then rolled underneath the chimney where the burning rate measurements began. Burning rate was measured through the use of three load cells under the platform that recorded the change in mass at $10 \mathrm{~Hz}$. The slope of the curve fits through the data produces the burning rate. Sample raw mass and burning rate data is shown in Figure 2. The reported value of the burning rate was the maximum rate achieved. Three replicates of each test were conducted and the results were averaged. Thus a total of 90 tests were conducted. 
Table 1 - Crib designs tested. Cribs with porosity under $0.05 \mathrm{~cm}$ are considered densely packed.

\begin{tabular}{|c|c|c|c|c|c|}
\hline $\begin{array}{c}\text { Crib } \\
\text { design \# }\end{array}$ & $\begin{array}{c}\text { Stick } \\
\text { thickness } \\
(\mathrm{b}, \mathrm{cm})\end{array}$ & $\begin{array}{c}\text { Stick } \\
\text { length } \\
(\mathrm{l}, \mathrm{cm})\end{array}$ & $\begin{array}{c}\text { Number of } \\
\text { sticks/layer } \\
(\mathrm{n},[])\end{array}$ & $\begin{array}{c}\text { Number } \\
\text { of layers } \\
(\mathrm{N},[])\end{array}$ & $\begin{array}{c}\text { Heskestad } \\
\text { porosity } \\
(\varphi, \mathrm{cm})\end{array}$ \\
\hline 1 & 1.27 & 12.7 & 5 & 10 & 0.0215 \\
\hline 2 & 1.27 & 25.4 & 4 & 21 & 0.1202 \\
\hline 3 & 0.64 & 25.4 & 10 & 10 & 0.7246 \\
\hline 4 & 0.64 & 25.4 & 14 & 15 & 0.0213 \\
\hline 5 & 0.64 & 30.5 & 8 & 14 & 0.1210 \\
\hline
\end{tabular}

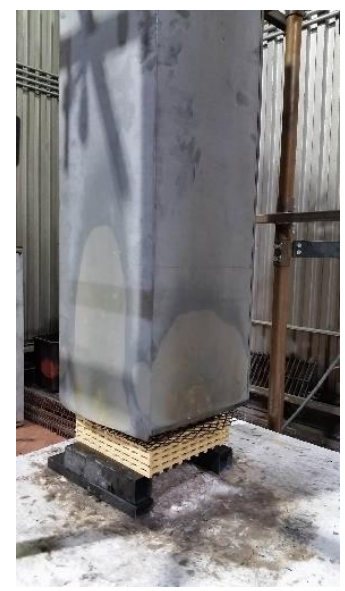

Figure 1 - Crib design \#3 under $3.66 \mathrm{~m}$ tall chimney.

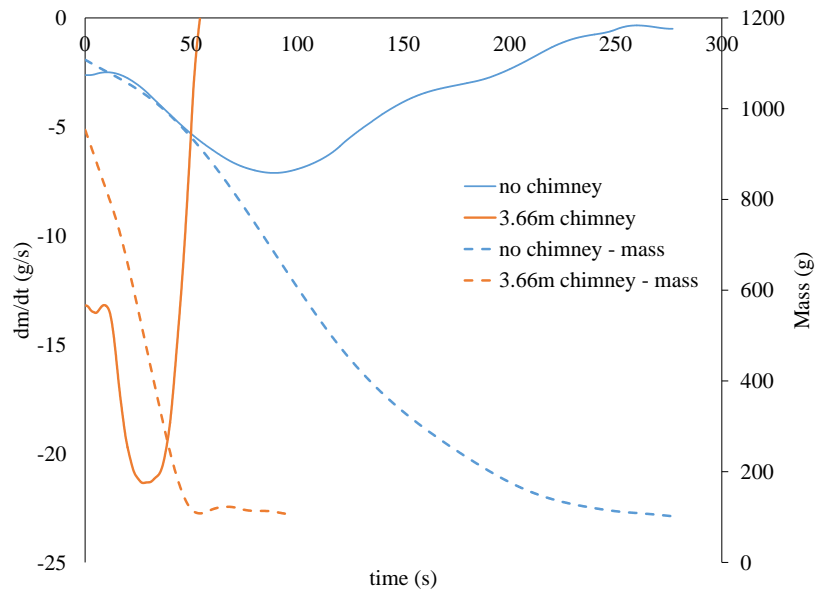

Figure 2 - Typical curves of mass loss rate (dm/dt) and mass as a function of time with and without ventilation provided by a 3.66m tall chimney.

\section{Results and Discussion}

Adding ventilation can have a dramatic effect on the burning rate. For example, the first two columns (light blue and orange) in Figure 3 show the average normalized burning rates of the five crib designs tested with $1 \%$ moisture content both with no ventilation (light blue) and the ventilation produced by the presence of a 3.66 m chimney (orange).

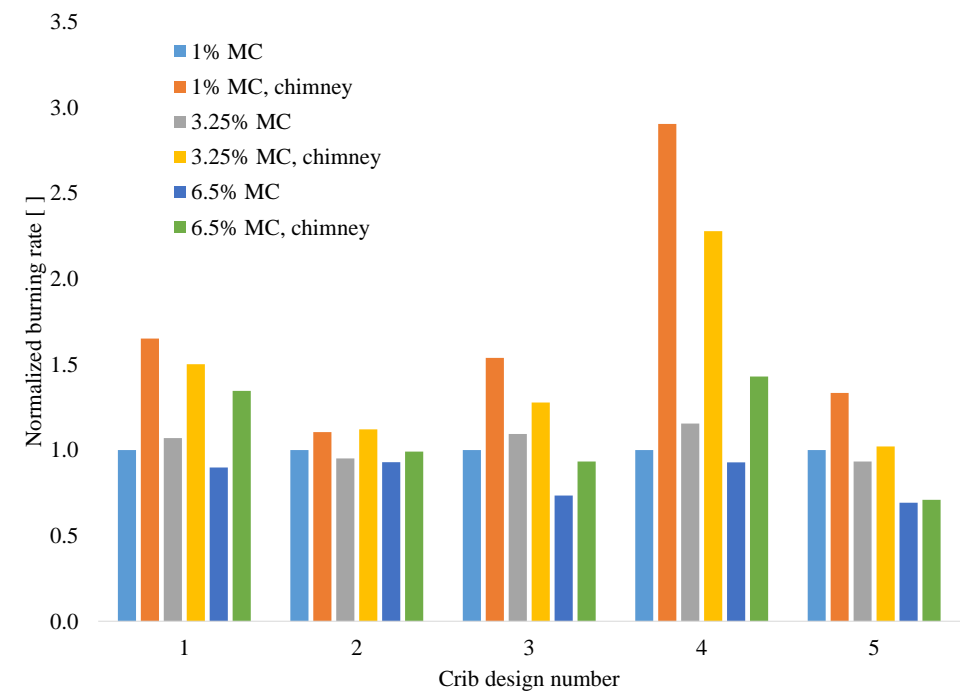

Figure 3 - Average normalized burning rate for each crib design at several MCs with and without ventilation due to the presence of a 3.66 m chimney. Burning rate normalized to $1 \%$ MC and no ventilation case. 
All results were normalized compared to the $1 \% \mathrm{MC}$ and no ventilation case. Depending on the crib design at $1 \% \mathrm{MC}$, the burning rate increased from $10.5 \%$ for crib design \#2 up to $190.5 \%$ for crib design \#4 compared to the non-ventilated cases. This increase in burning rate is caused by two mechanisms. For the densely packed cribs (designs \#1 and 4), forcing air through the fuel bed essentially reduces the local equivalence ratio for these locally rich systems. Because the cribs are built with a charring material, this forced ventilation also increases the char oxidation rate. This could have important implications for wildland fires as it could promote flaming combustion over smoldering combustion, further increasing the fire intensity.

The moisture content had an interesting effect on the burning rate. As seen by comparing the light blue, grey, and dark blue columns in Figure 3, with no ventilation a small increase in moisture content (from $1 \%$ to $3.25 \%$ ) may actually slightly increase the burning rate, such as for crib designs \#1, 3, and 4. With ventilation, however, all crib designs either had the same or reduced burning rates for the same small change in moisture content (comparing the orange and yellow columns). All crib designs and ventilation conditions showed a decrease in burning rate as the moisture content increased further to $6.5 \%$. However, the relative reduction in burning rate is more significant with ventilation. For example, crib design \#4 had a $7.2 \%$ reduction in burning rate with no ventilation between $1 \%$ and $6.5 \% \mathrm{MC}$, whereas the decrease was $50.1 \%$ between in the cases with ventilation. Some of this may be a result of the method of ventilation and the fact that the ventilation is directly tied to the burning rate. Future work will include designing a testing apparatus that will blow a fixed flow through the bottom of the crib. Future work will also include testing crib designs with thinner sticks to examine the role of thermal thickness in this process.

\section{Acknowledgements}

This work was funded by the National Fire Decision Support Center. The author wants to thank Chelsea Phillips and Sophia Vernholm for building the many cribs and help performing the experiments; Mark Finney, Jason Forthofer and Torben Grumstrup for many discussions on this idea; Randy Pryhorocki and Josh Deering for building the apparatus; and Cyle Wold for setting up the data acquisition system.

\section{References}

Albini FA (1976) Estimating wildfire behavior and effects. General Technical Report INT-30, Intermountain Research Station, USDA Forest Service. (Ogden, UT)

Anderson HE (1969) Heat transfer and fire spread. Research Paper INT-69, Intermountain Research Station, USDA Forest Service. (Ogden, UT)

Gross D (1962) Experiments on the burning of cross piles of wood. Journal of Research of the National Bureau of Standards - C. Engineering and Instrumentation 66c(2), 99-105.

McAllister S, Finney M (2016) Burning rates of wood cribs with implications for wildland fires. Fire Technology 52(6), 1755-1777. 
Short contribution - Fire Management

Evaluating ecological tipping points across levels of wildfire suppression under various climate and fuel treatment scenarios on US northern Rocky Mountain landscapes using landscape simulation

\author{
Robert E. Keane ${ }^{1 *}$; Kathy Gray $^{2}$; Brett Davis ${ }^{1}$; Lisa Holsinger ${ }^{1}$ \\ ${ }^{1}$ *US Forest Service, Rocky Mountain Research Station, Missoula Fire Sciences Laboratory, 5775 Hwy 10 \\ West, Missoula, Montana 59808. \{rkeane@fs.fed.us*\} \\ ${ }^{2}$ California State University at Chico, Department of Math and Statistics, 400 West 1st Ave, Chico, CA \\ 95929-0525, \{klgray@csuchico.edu\}
}

Keywords: ecological thresholds; wildland fire use; FireBGCv2; landscape modeling; wildfire; prescribed fire; fuel treatments

\title{
1. Introduction
}

Wildfires are becoming major concerns across the western US because, over the last 20 years, they have increased in frequency, intensity and size in many areas (Westerling 2016). The successful US fire suppression program over the last century has kept fire out of many fire-prone forests resulting in excessive buildups of wildland fuel, specifically surface and crown fuels, which has, in turn, increased the potential wildfires (Keane et al. 2002, Flannigan et al. 2009). This suppression legacy has left US fire management at a crossroads. Fire suppression costs are spiraling out of control, and if fire suppression continues, even more biomass will accumulate to create conditions that will foster even more intense fires than both their contemporary or historical counterparts (Van Wagtendonk 1985). And to make matters worse, future climates in western North America are predicted to be hotter and drier creating longer and more severe fire seasons (Flannigan et al. 2013, Abatzoglou and Williams 2016, Westerling 2016). Increasing fuels and warmer climates will mean firefighters will be asked to fight more dangerous fires, putting themselves at even higher risks for injury and fatality (Withen 2015). The solution to this fire management dilemma may be extremely complex because it must integrate comprehensive pro-active fuel treatment strategies (e.g., prescribed fire, mechanical cuttings) with wildland fire use (WFU: controlled wildfires or letting some fires burn under prescribed conditions) and wildfire suppression initial attack into a more effective fire management strategy (Zimmerman and Bummell 1999). A first step towards developing an integrated strategy may be to identify those critical thresholds of wildfire suppression efforts, primarily in initial attack, where fuels and fire conditions, and associated ecosystem health and resilience, significantly differ from historical conditions when no fires were suppressed, fire hazard was low, and ecosystems were most resilient.

In this study, we use landscape modeling implemented in a complex simulation experiment to create the data for evaluating possible tipping points in wildfire management in the US northern Rocky Mountains (NRM). Wildfire tipping points identified by our statistical techniques were then summarized to develop a set of possible management targets for NRM landscapes. The tipping point levels of fire suppression effort should provide fire managers insight into how many wildfires can be initially attacked before there are major changes in the ecological characteristics of landscapes.

\section{Methods}

The FireBGCv2 landscape ecosystem model was used in a complex simulation experiment to evaluate possible tipping points in wildfire management in the NRM (Keane et al. 2011). We used a fully factorial simulation design where a set of four factors represented different levels of fire 
suppression effort, fuel treatments, climate, and landscape setting. The primary vector of evaluation for this experiment was fire suppression level (FSL) where we simulated ten levels of increasing fire suppression: 0\% suppression (no fires suppressed; historical fire regime) to $90 \%$ suppression (estimated level of current fire suppression) by 10\% levels (e.g., 0, 10, 20, 30... 90\% suppression). Another factor was Landscape (LAN) with the three levels reflecting the three landscapes: (1) East Fork Bitterroot River (EFBR) on the Bitterroot National Forest, Montana, USA is a 128,000 ha dry mixed-conifer ecosystem with a mixed frequency and severity fire regime; (2) Crown of the Continent $(\mathrm{CROWN})$ is a 100,000 ha mesic mixed-conifer landscape with fire regimes of variable frequencies and severities; and (3) the Yellowstone Central Plateau (YCP) in Yellowstone National Park: a high elevation (>2300 m MSL) landscape with mostly infrequent stand-replacement fire regimes and composed mostly of mature lodgepole pine. We evaluated gradients of fire suppression effort in the context of two other factors. First, we evaluated if tipping points would be different if fuel treatments were employed so we simulated four levels of fuel treatment effort (FTE): (1) no treatments, (2) business as usual (BAU; 3\% landscape treated per year), (3) fully funded fuels program (FF; 10\% landscape treated per year), and (4) no holds bar (NHB; treat everything that needs treatment). All fuel treatments were a combination of cutting and removing all fire-sensitive, shade tolerant species down to $4 \mathrm{~cm} \mathrm{DBH}$ and implementing a low intensity prescribed burn ( $400 \mathrm{~kW} \mathrm{~m}-1$ fireline intensity). We also looked at two climates (CLI) -a historical climate and a future climate (RCP8.5). Each level for each of the four factors was simulated in a multifactorial design with ten replicates for a total of 1,800 simulations for each landscape. In this study, we assumed that landscapes are most resilient when they are within HRV, even in the future. As a result, the base case for each landscape is no fire suppression and no fuel treatments with historical climates that is the estimate of the historical range and variability (HRV) of landscape dynamics. We output values for response variables every 10 years to create a simulation time series of 25 observations for each simulation run. We analysed the time series using ShewhartQCC plots for all seven response variables over the gradient of fire suppression levels, but only show Shewhart QCC plots for a single variable (canopy bulk density; CBD) for both climate scenarios (HIST, RCP8.5), but only two fuels treatment scenarios (None, NHB), for brevity.

\section{Results and Discussion}

We found that approximately 40-70\% of wildfires can be suppressed leaving 30 to $60 \%$ of wildfires to be managed as WFU fires, and we also found that a fuel treatment campaign (BAU, FF, NHB) can allow 10 to $30 \%$ more fires to burn, depending on the intensity of the area treated each year (Figure 1). However, there are caveats to this finding. First, landscape setting greatly influences any potential tipping point in fire suppression level. Landscapes with historically frequent fires, such as EFBR, tend to have higher tipping points in fire suppression $(>60 \%)$ than mixed, less frequent fire regimes, such as the CROWN (>40\%), and stand-replacement, infrequent fire regimes (no tipping point), such as the YCP. This is a result of two interacting factors -fire frequency and simulation time. Landscapes with frequent fires (EFBR) have many fires in the short simulation time, so a reduction in the number of fires may not significantly impact landscape dynamics over the 200 years, but landscapes with long fire return intervals (YCP) have only a few fires during the simulation, and a reduction in these few fires may cause significant changes in landscape dynamics. Another caveat is that the variables used to assess tipping points matter. In our analysis, we used a set of variables that were selected because we thought they may be important to fire management. We found that each variable had its own specific tipping point when using HRV as reference and when we collapsed all seven variables down to two principal component axis (PC1 and PC2), we got an entirely new set of tipping points. A last concern is climate. Tipping points using historical climates are quite different from those in the future, because future climates will be hotter and drier than today. This brings up the question of whether using HRV for the baseline comparison is appropriate if future climates are significant different from historical analogs. In this paper, we assumed that HRV is a good reference because it broadly 
represents those conditions under which most of the biota on the landscapes evolved and as such provides an ecologically viable benchmark. The best way to implement findings from this effort is to simulate both HRV and a set of future ranges of variation which represent new climates and management alternatives and then search for overlaps between HRV and FRVs).

EFBR
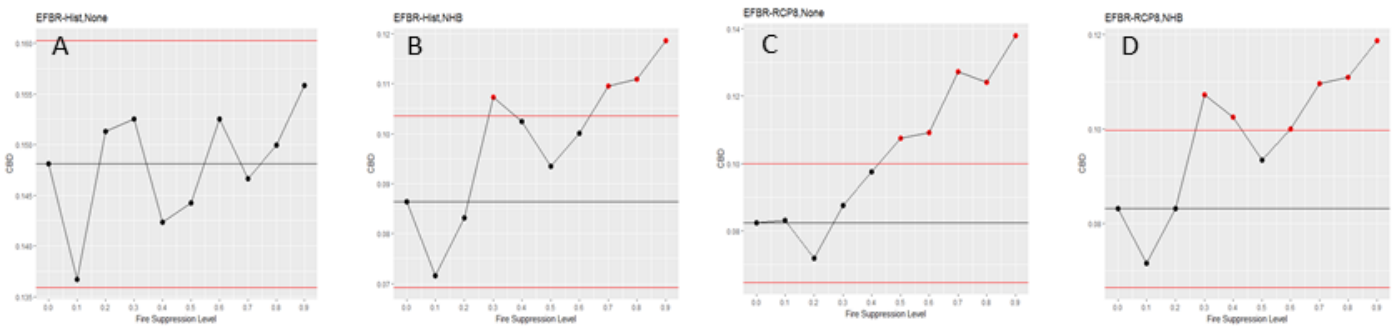

CROWN
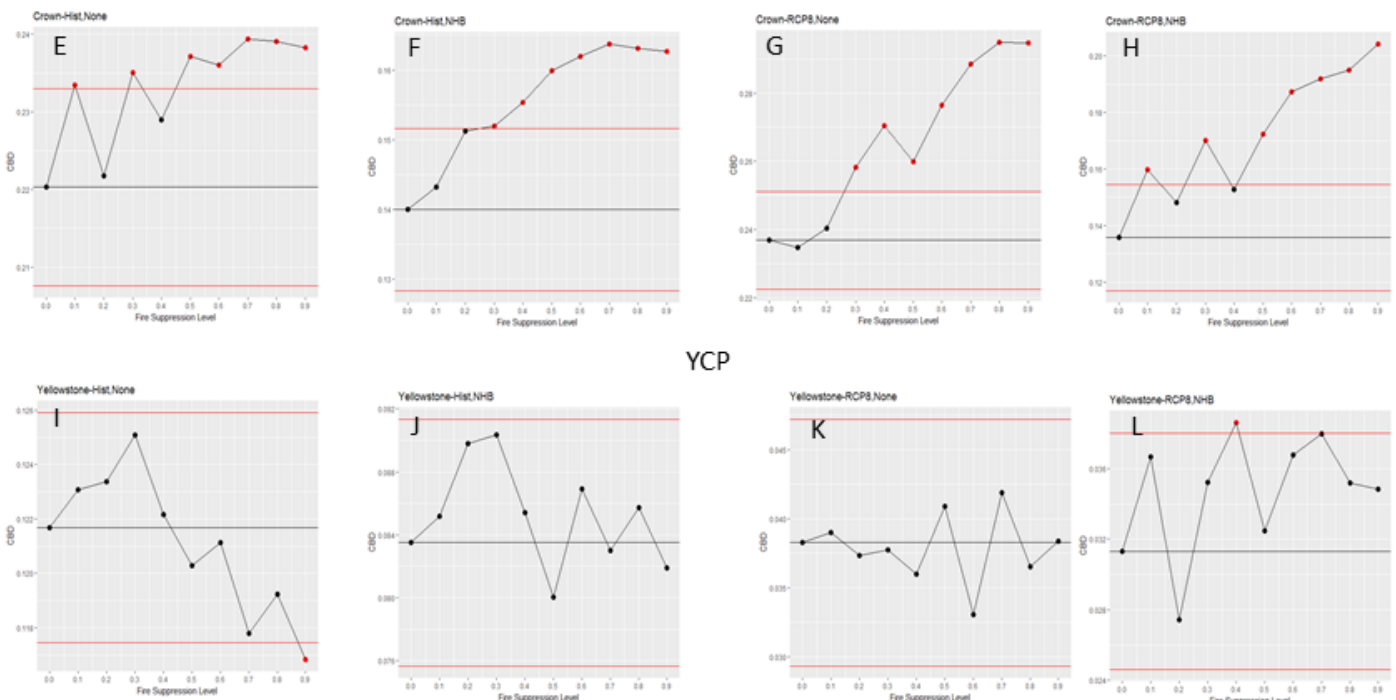

YCP

Figure 1 - Shewhart QCCs for the variable canopy bulk density (kg m-3) for all three study areas (EFBR, CROWN YCP) for both historical (hist) and future (RCP8.5) climate and for only two of the four fuel treatment scenarios (none-no fuel treatments and NHB-no holds barred). Figures arranged by landscape, climate, and fuel treatment with historical weather-no fuel treatment, historical weather-NHB, future weather-no treatments, future weather-NHB for $\operatorname{EFBR}(A, B, C, D), C R O W N(E, F, G, H)$, and $Y C P(I, J, K, L)$

\section{References}

Abatzoglou, J. T. and A. P. Williams. 2016. Impact of anthropogenic climate change on wildfire across western US forests. Proceedings of the National Academy of Sciences 113:11770-11775.

Flannigan, M. D., M. A. Krawchuk, W. J. de Groot, B. M. Wotton, and L. M. Gowman. 2009. Implications of changing climate for global wildland fire. International Journal of Wildland Fire 18:483-507.

Flannigan, M., A. S. Cantin, W. J. de Groot, M. Wotton, A. Newbery, and L. M. Gowman. 2013. Global wildland fire season severity in the 21st century. Forest Ecology and Management 294:5461.

Keane, R. E., Thomas Veblen, Kevin C. Ryan, Jesse Logan, Craig Allen, and B. Hawkes. 2002. The cascading effects of fire exclusion in the Rocky Mountains. Pages 133-153 in J. B. (Editor), editor. Rocky Mountain Futures: An Ecological Perspective. Island Press, Washington DC, USA. 
Keane, R. E., R. A. Loehman, and L. M. Holsinger. 2011. The FireBGCv2 landscape fire and succession model: a research simulation platform for exploring fire and vegetation dynamics. General Technical Report RMRS-GTR-255, U.S. Department of Agriculture, Forest Service, Rocky Mountain Research Station, Fort Collins, CO USA.

Van Wagtendonk, J. W. 1985. Fire suppression effects on fuels and succession in short-fire-interval wilderness ecosystems. Pages 119-126. Proceedings, Symposium and Workshop on Wilderness Fire, Missoula, Montana, November 15-18, 1983 : proceedings of a symposium. Ogden UT : U.S. Dept. of Agriculture Forest Service Intermountain Forest and Range Experiment Station 1985.

Westerling, A. L. 2016. Increasing western US forest wildfire activity: sensitivity to changes in the timing of spring. Philosophical Transactions of the Royal Society of London B: Biological Sciences 371.

Withen, P. 2015. Climate Change and Wildland Firefighter Health and Safety. NEW SOLUTIONS: A Journal of Environmental and Occupational Health Policy 24:577-584.

Zimmerman, T. G. and D. L. Bummell. 1999. Wildland fire management for the 21 st century: evolving applications and capabilities. Pages 316-323 in Proceedings of the Society of American Foresters 1999 national convention. SAF Publication, Portland, Oregn, USA. 
Short contribution - Fire Management

Field-tested laboratory-derived models to predict forest fire front spread rate

\author{
Carlos G. Rossa*; Paulo M. Fernandes \\ Centre for the Research and Technology of Agro-environmental and Biological Sciences (CITAB), \\ University of Trás-os-Montes e Alto Douro (UTAD). Quinta de Prados, Apartado 1013, 5001-801 Vila \\ Real, Portugal. \{carlos.g.rossa@gmail.com*,pfern@utad.pt\}
}

Keywords: fire behaviour, live fuels, empirical models, outdoors fires, fire management.

\title{
1. Introduction
}

Forest fire-spread rate models provide crucial support to decision support in fire management, such as assisting wildfire suppression operations. Most modelling studies aim at predicting fire spread rate $(R)$ for wind- or slope-driven fires (Sullivan et al. 2014). Even so, the ability to estimate basic fire spread rate $\left(R_{0}\right)$, i.e., on level ground in the absence of wind, is relevant because it provides a measure of fuel bed flammability and some modelling approaches estimate $R$ by multiplying $R_{0}$ by a wind or slope factor (e.g., Rothermel 1972). Conversely, wind-driven fire spread rate $\left(R_{\mathrm{U}}\right)$ has been the motivation of many modelling efforts. Slope has also a strong effect on fire spread. However, when the distance travelled is sizably larger than distances between landscape undulations, $R$ is satisfactorily equated to that of a fire spreading across a corresponding expanse of flat ground (Sullivan et al. 2014). As a result, the distance travelled by large fires is mainly a function of wind speed. Yet, a field-tested empirical $R_{\mathrm{U}}$ model for fire spread in generic vegetation is currently lacking.

In this work, we present results of laboratory experiments to analyse fire spread under $R_{0}$ and $R_{\mathrm{U}}$ conditions. The data are used in the development of models for fire propagation in generic fuel beds. Fuel beds were built from natural vegetation, mimicking the real-world structure of litter and shrubs. Empirical models were derived using regression analysis.

\section{Data and methods}

We conducted laboratory fire spread experiments in litter and shrub-like fuel beds. Some of the tests to measure $R_{0}(n=220)$ were immediately followed by a $R_{\mathrm{U}}$ test in an identical fuel bed $(n=108)$, to isolate and assess the effect of wind. Shrub-like fuel beds were composed of vertically-placed tree or shrub twigs, sometimes under-layered by litter (Figure 1). We used various plant species (Pinus pinaster Ait., Eucalyptus globulus Labill., Eucalyptus obliqua L'Her., Acacia mangium Willd., Quercus robur L., Pinus resinosa Sol. ex Ait.) and fuel beds were often composed of mixed dead and quasi-live vegetation. Quasi-live fuels were collected live, with fuel moisture content $(M)$ decreasing as a function of storage time. $R$ and $M$ were measured in all tests and flame length and angle were also assessed in some of the trials. All fuel beds were at least 1-m long and 1 to $1.2-\mathrm{m}$ wide.

We used regression analysis to relate $R_{0}$ with the pertinent variables. The effect of wind was assessed the same way and the wind effect and $R_{0}$ were multiplied to predict $R_{\mathrm{U}}$. The laboratoryderived relationships were then tested against independent laboratory and field fires. Independent datasets were retrieved from the literature and included fire spread in no-wind and no-slope conditions, as well as wind-driven fires. 


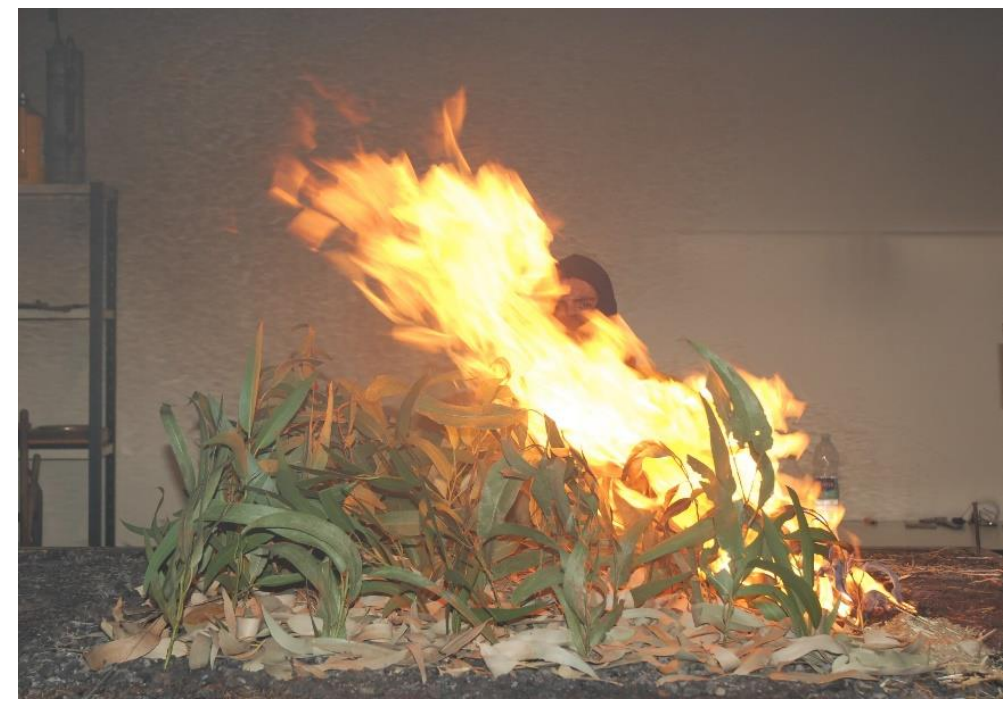

Figure 1 - Wind-driven fire spread test in a fuel bed of Eucalyptus globulus leaves litter over layered by quasi-live E. globulus twigs.

\section{Initial results and conclusion}

Our results show that both $R_{0}$ and $R_{\mathrm{U}}$ models can be predicted by a single formulation and a common set of variables, independently of vegetation type. Although the models were fitted to laboratory experiments data, they were tested against independent laboratory and field fires data, and hence justify further testing in other real-world fire spread situations for a wider range of fuel complexes.

\section{Acknowledgments}

This work was supported by Fundação para a Ciência e a Tecnologia (FCT) under post-doctoral grant SFRH/BPD/84770/2012 (financing programs POPH and FSE) to the first author. It was also supported by FCT and FEDER - Fundo Europeu de Desenvolvimento Regional (COMPETE 2020, POCI - Programa Operacional Competitividade e Internacionalização), in the framework of projects BONFIRE (POCI-01-0145-FEDER-016727, PTDC/AAGMAA/2656/2014) and CITAB (UID/AGR/04033/2013, POCI-01-0145-FEDER-006958). We are grateful to Délio Sousa, Paulo Ventura, and André Rosas (University of Trás-os-Montes e Alto Douro) for assisting in some of the laboratory experimental work. The help of Fernando Frazão, David Davim, José Silva, Nuno Marcos, Rafael Figueiredo, and Raquel Roliz in fuel collection is greatly appreciated.

\section{References}

Rothermel RC (1972) A mathematical model for predicting fire spread in wildland fuels. USDA Forest Service, Intermountain Forest and Range Experiment Station, Research Paper INT-115. (Ogden, UT)

Sullivan AL, Sharples JJ, Matthews S, Plucinski M (2014) Estimating downslope fire spread for landscape fire spread prediction. Environmental Modelling \& Software 62, 153-163. doi:10.1016/j.envsoft.2014.08.024 
Short contribution - Fire Management

Flexible design of a helipad network for forest firefighting helicopters, applied to the case of Sardinia

Hugo Torres ${ }^{1}$, Abílio Pereira Pacheco ${ }^{2 *}$, João Claro ${ }^{3}$, Michele Salis ${ }^{2}$, Matthew P. Thompson ${ }^{3}$, Crystal S. Stonesifer ${ }^{3}$, Gavino Diana ${ }^{4}$, Silvio Cocco ${ }^{4}$

${ }^{1}$ INESC TEC. Campus da FEUP, Rua Dr. Roberto Frias, 4200-465 Porto, Portugal,

\{hugo.torres@fe.up.pt; app@fe.up.pt*, jclaro@fe.up.pt\}.

${ }^{2}$ National Research Council, Institute of Biometeorologia. Traversa La Crucca n.3, Sassari (Italy); Euro-

Mediterranean Center on Climate Change Foundation, IAFES Division of Sassari. via de Nicola 9, Sassari

(Italy),\{miksalis@ibimet.cnr.it\}.

${ }^{3}$ Rocky Mountain Research Station, USDA Forest Service. Missoula, MT 59801, USA,

\{mpthompson02@fs.fed.us., csstonesifer@fs.fed.us\}.

${ }^{4}$ Sardinia Forest Service. Via Umberto I ${ }^{\circ} 414$, Jerzu, Italy,

\{gdiana@regione.sardegna.it, scocco@regione.sardegna.it\}

\begin{abstract}
Cost-effective strategic management of aerial forest fire suppression resources involves decisions about fleet location, in order to efficiently match supply and demand. In this study, we explore the flexible design of such a system, pooling the demand of several bases into a "zone", and analyzing alternative partitions of the territory into zones. Feasible partitions consider an upper limit on the distance between bases, and require their adjacency, within the same zone. Then, we classify the feasible partitions according to a set of six criteria and explore the non-dominated partitions. Finally, we select the preferred partition, with the support of several experts, by applying the AHP method. The considered criteria evaluate, for each partition, the (c1) mean and the (c2) value at risk of the number of unattended active fires, the (c3) helicopter idleness, and the (c4-6) Gini index for each of the previous three criteria.

We apply this strategy to the island of Sardinia, where helicopters play a central role in the suppression system, and compare the results with the current design, in which each base is autonomous (i.e., each zone has only one base). Sardinia has eleven helipad operational bases, where the helicopters are allocated at the beginning of the fire season, with the objective of fighting wildfires located in each base's coverage area. We used fire occurrence and helicopter flight data from 2006 to 2010, which revealed spatiotemporal asymmetries in the forest fire patterns, along the island, and thus the existence of effective opportunities to benefit from demand pooling. The AHP method was applied with Portuguese experts, and our results point towards an improvement in the values related to unattended fires, as well as the Gini indexes, if the proposed strategy of reorganizing the territory in larger zones (with more than one base) is applied.

We explore the results of our approach, and identify efficiency gains, potential fire management implications, limitations, and opportunities for additional research. In the scope of the latter, as the optimal value for helicopter idleness is naturally achieved with the current design (partition in zones with one base), this criterion provides a key trade-off with the other five, that can be used, for instance, to examine a flexible strategy for helicopter location-allocation along the years, with annual partition redefinitions, considering all data available up to that point of decision.
\end{abstract}

Keywords: helicopter basing, flexibility, territory partition, partitions, cost-effective analysis, AHP, decision factors

\title{
1. Introduction
}

Cost-effective strategic management of aerial forest fire suppression resources involves decisions about fleet location, in order to efficiently match supply and demand. Sardinia currently has eleven 
operational helicopter bases, where helicopters are allocated at the beginning of the fire season, to fight the fires located in each base's coverage area. Our goal was to optimize the usage of fire-fighting helicopters, creating a dynamic and flexible designed system for helicopter management, as opposed to the current system, a fixed design where the helicopters are allocated to each base and remain attached to the base for the whole season of fires.

The new flexible design consists in a system of partitions, where the coverage of each area can be shared with other bases, which leads to a narrower gap between the level of service and the demand of the helicopters. This work comes as the sequence of the previous work of Pacheco et al. (2014), later adapted to Sardinia by Stonesifer et al. (2015).

The results - first presented in Torres et al. (2017) - point towards an improvement in the values related to unattended fires, as well as the Gini indexes, and departing from the optimal value for helicopter idleness naturally achieved with the current configuration, this being the key trade-off that should be explored in order to find a solution that balances the multiple requirements.

\section{Materials and Methods}

Sardinia island is a territory belonging to Italy, situated on the Mediterranean Sea, west of the Italian mainland with approximately $1.7 \mathrm{M}$ inhabitants (Salis et al. 2013) and an area of approximately $24,000 \mathrm{Km} 2$ (Bajocco and Ricotta 2008) consisting mostly on shrublands (28\% of the islands' area).

In this study, we explore the flexible design of such a system, pooling the demand of several bases into a "zone", and analyzing alternative partitions of the territory into zones. Feasible partitions consider an upper limit on the distance between bases, and require their adjacency, within the same zone (please see Figure 1).
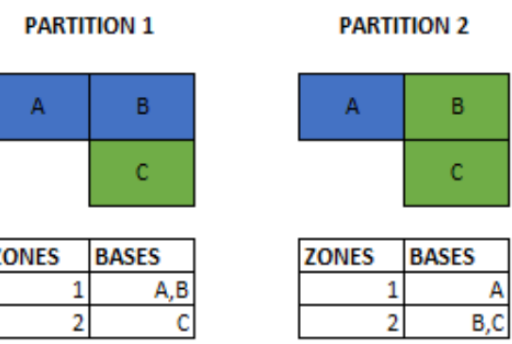

PARTITION 3
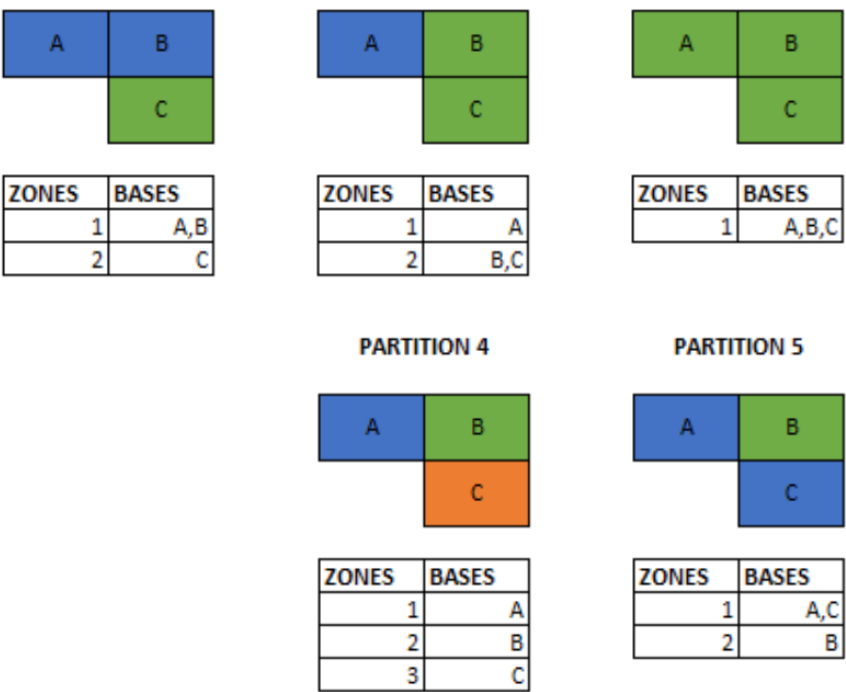

PARTITION 5
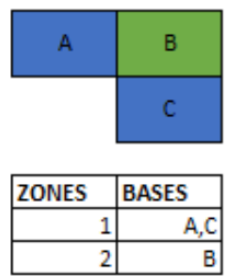

Figure 1 - An example with a territory with 3 bases $(A, B$, and $C)$; there are five possible partitions: four partitions are viable (\#1-4), and one partition is not viable (\#5) as in the "blue" zone 1, bases $A$ and $C$, are not adjacent.

To evaluate the adjacency of bases, we build a matrix in which the position $(i, j)$ is 1 if the bases $i$ and $j$ are adjacent, and 0 if not. This matrix is then subset when we evaluate the adjacency of the bases within a zone. It is important to notice that this method also represents a different restriction in this scenario. Since some of the bases are equipped to a specific type of helicopter, they cannot be included in zones where there are other types of helicopters. Given this, the adjacency matrix can indicate that two bases are not adjacent if they are not prepared for the same type of helicopters, even though they are geographically adjacent. 
After evaluating adjacency and distance, we classify the feasible partitions according to a set of six criteria and explore the non-dominated partitions. Finally, we select the preferred partition, with the support of several experts, by applying the AHP method. The considered criteria evaluate, for each partition, the (c1) mean and the (c2) value at risk of the number of unattended active fires, the (c3) helicopter idleness, and the (c4-6) Gini index for each of the previous three criteria.

We apply this strategy to the island of Sardinia, where helicopters play a central role in the suppression system, and compare the results with the current design, in which each base is autonomous (i.e., each zone has only one base). Sardinia has eleven helipad operational bases, where the helicopters are allocated at the beginning of the fire season, with the objective of fighting wildfires located in each base's coverage area. We used fire occurrence and helicopter flight data from 2006 to 2010, which revealed spatiotemporal asymmetries in the forest fire patterns, along the island, and thus the existence of effective opportunities to benefit from demand pooling.

The AHP method was applied with experts from the Portuguese authorities related to fire, suppression such as the National Authority for Civil Protection (ANPC) or the National Institute for Forest Conservation (ICNF), and our results point towards an improvement in the values related to unattended fires, as well as the Gini indexes, if the proposed strategy of reorganizing the territory in larger zones (with more than one base) is applied.

\section{Results}

We explore the results of our approach (please see Figure 2), and identify efficiency gains, potential fire management implications, limitations, and opportunities for additional research.

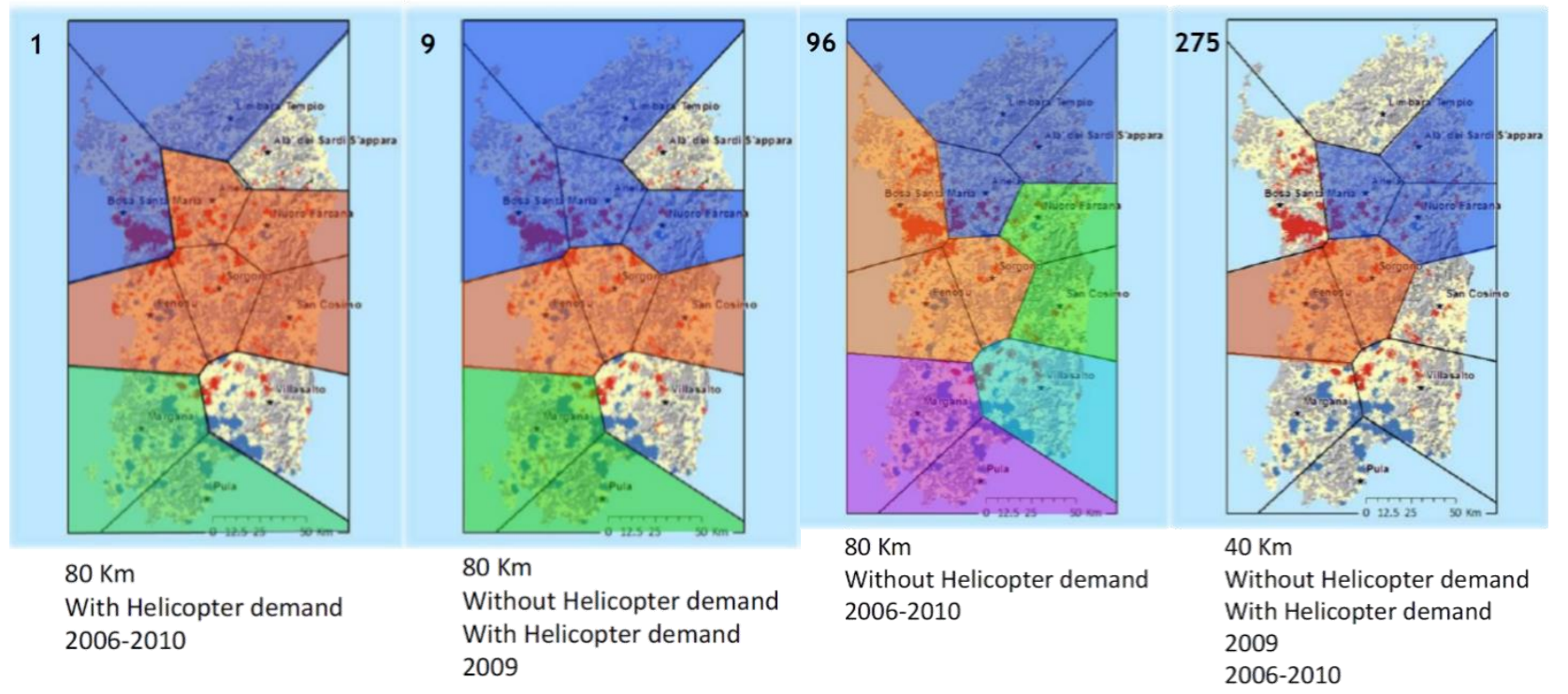

Figure 2 - Territory partitions chosen by the AHP analysis for a threshold of $80 \mathrm{Km}$ considering the demand probability (each color represents a zone).

In the scope of the latter, as the optimal value for helicopter idleness (c3) is naturally achieved with the current design (partition in zones with one base), this criterion provides a key trade-off with the other five, that can be used, for instance, to examine a flexible strategy for helicopter locationallocation along the years, with annual partition redefinitions, considering all data available up to that point of decision.

\section{Conclusion}

The results seem to indicate that pooling the demand of the system may lead to an improvement of the efficiency, favoring the flexible design over the fixed design. However, we believe that there is 
room for improvement for this algorithm. The trade-off between the helicopter idleness and the other criteria, along with the possibility of relating fires and flights and using a broader time interval, seem to be some interesting starting points for future development of this design.

\section{Acknowledgements}

This work is financed by the ERDF - European Regional Development Fund through the Operational Programme for Competitiveness and Internationalisation - COMPETE 2020 Programme within project «POCI-01-0145-FEDER-006961», and by National Funds through the FCT - Fundação para a Ciência e a Tecnologia (Portuguese Foundation for Science and Technology) as part of project UID/EEA/50014/2013. FCT also supported the research performed by Abílio Pereira Pacheco (Grant SFRH/BD/92602/2013).

The authors are deeply grateful to Eng. ${ }^{\circ}$ Rui Almeida (ICNF) and Professor Richard de Neufville (MIT, IDSS) for their enthusiasm and advice. We also thank Tenente-Coronel Albino Fernando Quaresma (GIPS), Comandante Marco Martins (B.V. de Óbidos), Doutor João Carlos Verde, Eng ${ }^{\circ}$. Paulo Mateus (ICNF), Dr. Alexandre Benigno (ANPC), and Eng ${ }^{\circ}$. Paulo Bessa (GTF Penafiel) for answering the survey used to apply the AHP model.

\section{References}

Bajocco, S, Ricotta, C (2008) Evidence of selective burning in Sardinia (Italy): which land-cover classes do wildfires prefer? Landscape Ecology 23, 241-248.

Pacheco, AP, Neufville, Rd, Claro, J, Fornés, H (2014) Flexible design of a cost-effective network of fire stations, considering uncertainty in the geographic distribution and intensity of escaped fires. In 'Advances in forest fire research'. (Imprensa da Universidade de Coimbra: Coimbra)

Salis, M, Ager, AA, Arca, B, Finney, MA, Bacciu, V, Duce, P, Spano, D (2013) Assessing exposure of human and ecological values to wildfire in Sardinia, Italy. International Journal of Wildland Fire 22, 549-565.

Stonesifer, C, Thompson, M, Salis, M, Pacheco, AP, Claro, J CMCC (Ed.) (2015) Flexible Design of Helicopter Basing Strategies: A Case Study of Sardinia, Italy, 'International Conference on Fire Behaviour and Risk'. Alghero, Italy.

Torres, HS, Pacheco, AP, Claro, J, Salis, M, Thompson, MP, Stonesifer, CS, Diana, G, Cocco, S (2017) Flexible design of a network of helipads for a fleet of forest firefighting helicopters, 2017 'World Conference on Natural Resource Modeling: Vulnerability and Resilience of Socio-ecological Systems'. Barcelona, Spain, June 6-9, 2017. Available at http://www.paucostafoundation.org/nrm2017/wpcontent/uploads/2017/06/wcnrm2017_program_doublepage.pdf 
Short contribution - Fire Management

Influence of moisture and inorganic contents on smouldering spread rates in the lateral and indepth directions

\author{
Eirik G Christensen ${ }^{1}$; Nieves Fernandez-Anez ${ }^{2}$; Guillermo Rein ${ }^{1}$ \\ ${ }^{1}$ Imperial College London SW7 2AZ, \{e.christensen16@imperial.ac.uk*, g.rein@imperial.ac.uk\} \\ ${ }^{2}$ Western Norway University of Applied Sciences, 5523, Haugesund \{Nieves.Fernandez@hvl.no\}
}

\begin{abstract}
Peat, an organic soil resulting from the accumulation of dead organic matter, is a material that can sustain smouldering fires. Annually, peat fires contribute significantly to wildfire emissions, releasing large quantities of stored organic carbon contributing to positive climate feedback and can be blow into nearby cities causing significant adverse health effects (Hinwood \& Rodriguez 2005). This paper, for the first time systematically studies the influence of moisture and inorganic content, two key soil properties, on the horizontal and indepth spread rate simultaneously. A negative linear relationship was found between spread rate and increasing moisture and inorganic contents. While moisture content apeared to increase the indepth spread rate, decreaseing the inorganic content reduces the spread rate, both tendencies are consistent with the results found in literature. These finding greatly improves the understanding of the influence of soil properties on smouldering spread in thin layers.
\end{abstract}

Keywords: Smouldering, Spread rate, Peat

\title{
1. Introduction:
}

Peatland fires have the ability to emit significant quantities of greenhouse gases (Turetsky et al. 2011; Page et al. 2002) due to the vast quantities of carbon stored in the soil (Huang et al. 2009). Peat predominately burns by smouldering, which is the slow, low temperature form of combustion that occurs in porous char forming media (Rein 2016). It propagates though organic soils in well establish stages: drying, preheating, pyrolysis and oxidation. Drying involves the removal of moisture due to heating, while preheating is the steady increase of the soil temperature, until the onset of pyrolysis whereby temperature are significantly high that the organic material begins to decompose, forming a solid char (Rein 2016). Finally, this char oxidizes - an exothermic reaction that drives the previously mentioned steps.

Smouldering is governed by two fundamental mechanisms: heat transfer and oxygen supply. These mechanisms are affected by a broad range of parameters, being the three most influential, for smouldering organic soils: moisture content, inorganic content and bulk density (Rein 2013). A negative linear relation was found for the critical combinations of moisture content and inorganic content that sucessfully supports self-sustained smouldering (Frandsen 1987). Similarly, both density and moisture content influence the horizontal spread rate of smouldering peat (Prat-Guitart et al. 2016). Notably, the influence of moisture content on the spread rate are different depending if the mode of spread is horizontal or in-depth (Huang \& Rein 2016).

The focus of this paper is to experimentally study the spread of a radial smouldering front examining both the horizontal and indepth spread rate of smouldering propagation as influenced by the fuel properties, moisture and inorganic content. 


\section{Method}

The experiments are conducted using an open top insulated reactor with internal dimensions of 400 x 400 x $15 \mathrm{~mm}$ and a small coil, providing $5 \mathrm{~W}$ for $30 \mathrm{~min}$, placed in the center to ignite smouldering in a bed of commercial peat, creating a radially propagating smouldering front. The spread is monitored using a visual and infrared camera.

Time lapse images, spaced by $60 \mathrm{~s}$, are taken from directly above the ignition and the image series are analyzed in Matlab. The center of the image is manually selected based on the location of the coil and the scale is set based on comparing the number of pixel width of the reactor and its known length. A conservative estimate of the scale error is calculated to be $2 \%$, based on repeated measurements of the pixel number. The time lapse infrared images are grayscaled and intensity profiles of 60 evenly spaced transects originating from the center of the ignition and extending outwards is retrieved. The location of the first and last value to cross a threshold value signifies the trailing and leading edge. Comparing the change in position of these edges in subsequent images and dividing by the time between images give the spread rate. The in-depth spread rate at a given position can be evaluated by dividing the depth of the sample by the time taken between the leading and trailing edge to pass the said horizontal position. This concept is presented in Figure 1, as the first instance of smouldering occurs once the char forms, after which the peat is consumed in depth; Then finally, the trailing edge marks the complete consumption of the fuel.

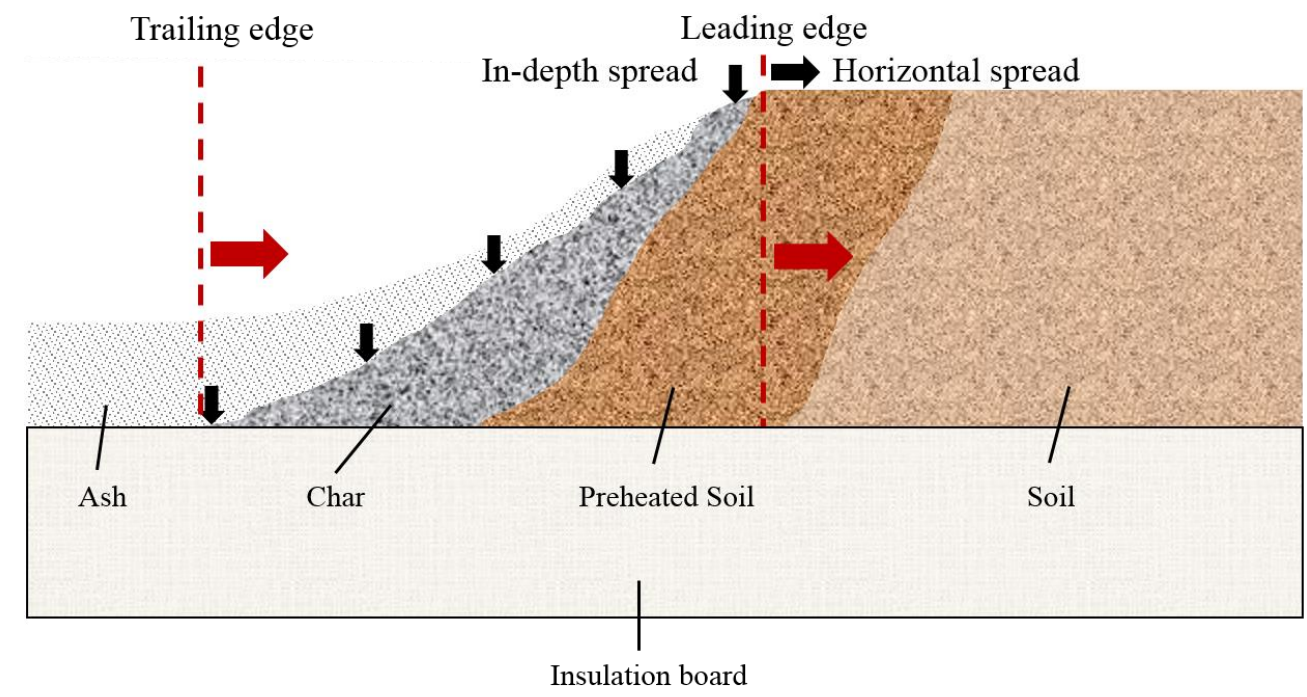

Figure 1 - Profile view of smouldering. Illustrating the how the leading edge is a function of the horizontal spread rate, while the trailing edge is determined by the horizontal spread rate and the depth of the sample.

\section{Results \& discussion:}

For the first time, this study shows the diminishing horizontal spread rate with increasing moisture content across a range of inorganic contents as well as shown a negative linear relationship between horizontal spread rate and inorganic content owed to the increasing heatlosses. Both moisture and inorganic contents have a similar influence on horizontal spread rate, but they have a diverging effect on indepth spread rate; whereby an increase in moisture content was found to increase the in-depth spread rate while inorganic content was shown to decrease the speed of spread. This finding is supported by previous researches. The influence of soil properties were also visually observable. Figure 2, is a composed image and illustrates key differences resulting from varying fuel properties. The image on the left is a sample with minimal inorganic content (2.5\% dry basis) and a moisture content close to the critical value $(110 \%)$, while the one on the right has a inorganic content near the 
critical value (70\%) with minimal moisture content $(0 \%)$. It is clear that the peak temperatures, surface coloration, thickness of smouldering wave, and spread rates of both the front and trailing edge are influenced by the fuel properties.

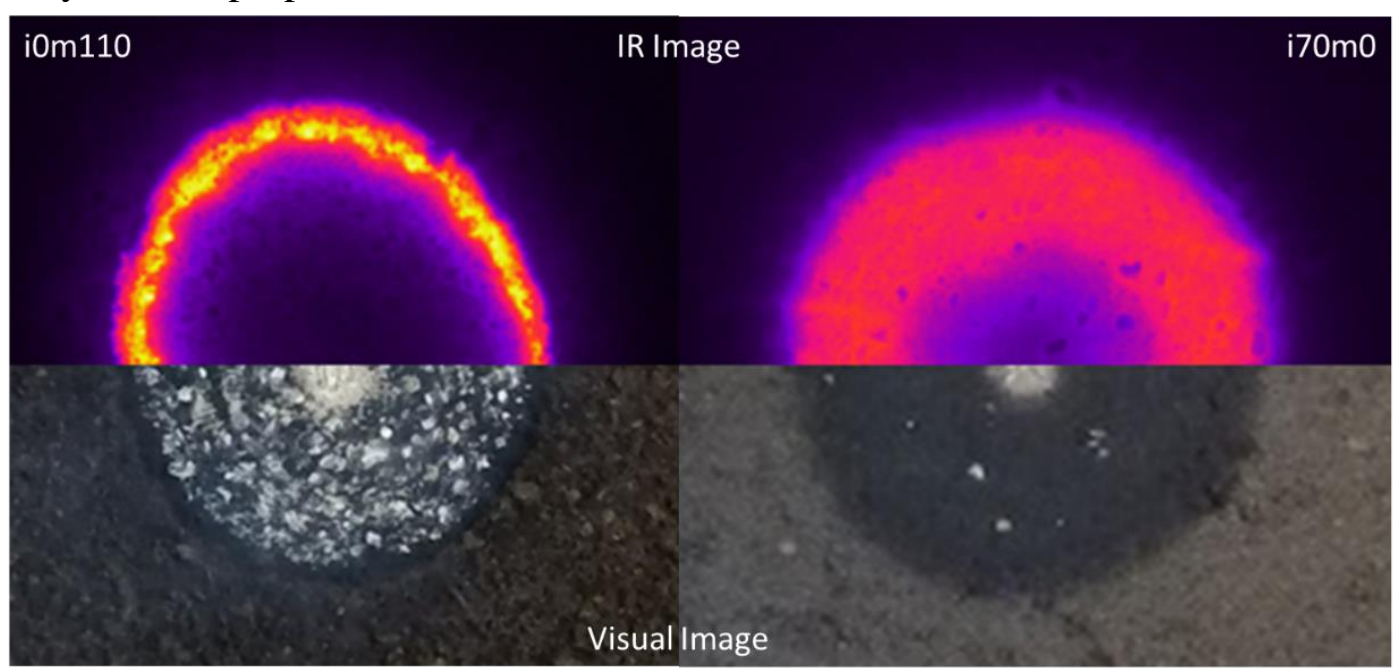

Figure 2 - Comparing infrared and visual images of smouldering samples with different fuel properties. The composite image on the left is a sample with almost zero inorganic content and $110 \%$ moisture; the image of the right has $70 \%$ inorganic content and near zero moisture content. The smouldering front is seem to be more narrow and hotter how the high moisture condition and produces more ash at the surface.

\section{Conclusion:}

The systematic study of the influence of soil properties on the smouldering spread both indepth and horizontal provide understanding for the smouldering dynamics and can inform land managers and firefighters as to the risks of smouldering fires.

\section{References}

Frandsen, W.H., 1987. The influence of moisture and mineral soil on the combustion limits of smoldering forest duff. Canadian Journal of Forest Research, 17(12), pp.1540-1544.

Hinwood, A.L. \& Rodriguez, C.M., 2005. Potential health impacts associated with peat smoke: A review. Journal of the Royal Society of Western Australia, 88(3), pp.133-138.

Huang, P.-T. et al., 2009. Classification of Organic Soils. Soil Science Society of America Journal, B7(1), p.108.

Huang, X. \& Rein, G., 2016. Downward Spread of Smoldering Peat Fire: the Role of Moisture, Density and Oxygen Supply. International Journal of Wildland Fire [under review], (Mc).

Huang, X., Rein, G. \& Chen, H., 2015. Computational smoldering combustion: Predicting the roles of moisture and inert contents in peat wildfires. Proceedings of the Combustion Institute, 35(3), pp.2673-2681. Available at: http://dx.doi.org/10.1016/j.proci.2014.05.048.

Page, S.E. et al., 2002. The amount of carbon released from peat and forest fires in Indonesia during 1997. Nature, 420(6911), pp.61-65. Available at: http://www.nature.com/doifinder/10.1038/nature01141.

Prat-Guitart, N. et al., 2016. Propagation probability and spread rates of self-sustained smouldering fires under controlled moisture content and bulk density conditions. International Journal of Wildland Fire, 25(4), pp.456-465. 
Rein, G., 2016. SFPE handbook of fire protection engineering, fifth edition. SFPE Handbook of Fire Protection Engineering, Fifth Edition, pp.1-3493.

Rein, G., 2013. Smouldering Fires and Natural Fuels. Fire Phenomena and the Earth System: An Interdisciplinary Guide to Fire Science, (1984), pp.15-33.

Turetsky, M.R. et al., 2011. Recent acceleration of biomass burning and carbon losses in Alaskan forests and peatlands. Nature Geoscience, 4(1), pp.27-31. Available at: http://dx.doi.org/10.1038/ngeo1027. 
Short contribution - Fire Management

\title{
New Zealand prescribed fire experiments to test convective heat transfer in wildland fires
}

\author{
Mark A. Finney ${ }^{1 *}$; Grant Pearce ${ }^{2}$; Tara Strand ${ }^{2}$, Marwan Katurji ${ }^{3}$, Craig Clements ${ }^{4}$ \\ ${ }^{1}$ USDA Forest Service, Missoula Fire Sciences Laboratory. 5775 Highway 10 West, Missoula MT, 59808 \\ \{mfinney@fs.fed.us*\} \\ ${ }^{2}$ Scion (NZ Forest Research Institute Ltd). 10 Kyle St, Riccarton, Christchurch 8011, New Zealand. \\ \{Grant.Pearce@scionresearch.com,Tara.Strand@scionresearch.com\} \\ ${ }^{3}$ Dept. of Geography, Centre for Atmospheric Research. University of Canterbury, Christchurch, New \\ Zealand. \{marwan.katurji@canterbury.ac.nz\} \\ ${ }^{4}$ Fire Weather Research Laboratory, San Jose State University, California, USA. \\ \{craig.clements@sjsu.edu\}
}

\begin{abstract}
The physics of bushfires has traditionally been studied through intensive modelling that requires numerous assumptions of combustion and heat transfer adapted from established structure-fire engineering relations. Renewed emphasis in experimental research has caused rethinking of some of the most basic concepts in wildland fuel particle ignition and flame spread. These findings suggest new possibilities for advancing bushfire behaviour science. Laboratory and field experiments conducted by the U.S. Forest Service's Missoula Fire Sciences Lab reveal the source of convective heating in spreading fires derives from fireinduced vorticity which forces flames downward and ahead of the combustion zone in intermittent contact with fuel particles. New laboratory techniques capture the intermittency and suggest it has predictable average frequencies familiar in studies of buoyant instabilities. Dependent only on buoyancy, these scaling relations have shown promise at field scales. A project being led by Scion's Rural Fire Research Group in New Zealand aims to test this hypothesis that heat-driven buoyancy (convection) creates a series of peaks and troughs in the flame front that drive fire spread and scale with flame size and wind speed. The theory is being tested through a series of heavily-instrumented fire experiments at sites in a range of fuel types, starting in uniform crop stubble fuels (February 2018), moving to more complex scrub fuels (2018/19) and then to wilding pine forest fuels $(2019 / 20)$.
\end{abstract}

Keywords: Convective Heat Transfer, Prescribed Fire, Remote Sensing

\section{Introduction}

The physics of bushfires has traditionally been studied through intensive modelling that requires numerous assumptions of combustion and heat transfer (Sullivan 2009) adapted from established structure-fire engineering relations. Renewed emphasis in experimental research has caused rethinking of some of the most basic concepts in wildland fuel particle ignition and flame spread. These findings suggest new possibilities for advancing bushfire behaviour science.

Laboratory and field experiments conducted by the U.S. Forest Service's Missoula Fire Sciences Lab reveal the source of convective heating in spreading fires derives from fire-induced vorticity which forces flames downward and ahead of the combustion zone in intermittent contact with fuel particles (Finney et al. 2015). New laboratory techniques capture the intermittency and suggest it has predictable average frequencies familiar from studies of buoyant instabilities. Dependent only on buoyancy, these scaling relations have shown promise at field scales.

A project being led by Scion's Rural Fire Research Group in New Zealand aims to test this hypothesis that heat-driven buoyancy (convection) creates a series of peaks and troughs in the flame front that drive fire spread and scale with flame size and wind speed. The theory is being tested through a series of heavily-instrumented atmospheric and fire experiments at sites in a range of fuel types, 
starting in uniform crop stubble fuels (February 2018), moving to more complex scrub fuels (2018/19) and then to wilding pine forest fuels (2019/20).

\section{Instrumentation and Preliminary Results}

Nine plots were established in Darfield, New Zealand to measure atmospheric turbulence structure and scales, heat transfer, and flame characteristics on spreading line-fires in cereal crops (Figure 1b). Loading and particle sizes were measured in the fields of wheat, barley, and triticale. Fires were ignited with drip torches from multiple line segments along the upwind edge to allow a linear flame zone to develop (Figure 1b).

Experimental design focussed on measuring a cascade of spatial and temporal scales starting from the fuel bed, through the flame and up to a few hundred meters in the atmosphere. Air turbulence and temperature characteristics as the fire passed were measured by a 10 meter tower near the center of each plot with sonic anemometers at $2 \mathrm{~m}, 5 \mathrm{~m}$, and $10 \mathrm{~m}$ along with array of 20 thermocouples installed at intervals along the vertical pole (Figure 2a). A 30m sonic anemometer tower was setup outside the fire perimeter to measure the near-surface atmospheric background turbulence structure. Instruments to measure heat fluxes and flame behaviors were installed at intervals along 30 meter lines oriented parallel with the anticipated wind direction. These instrument packages consisted of a heat flux package with tri-directional differential pressure disks to measure air and flame velocity (see paper by Grumstrup et al. this volume), two fine wire thermocouples (bead 5e-5m) for gas temperature variations and, and a wide angle radiometer (Figure 2b). Frequency and distance of forward flame bursts were measured using $4.8 \mathrm{~m}$ horizontal arrays of 32 fine-wire thermocouples at intervals of $0.15 \mathrm{~m}$; thermocouples were elevated to the height of the fuel particles ( . .2-.4m) and the array oriented parallel to the fire spread direction (Figure 2c). Changes in atmospheric pressure (hydrostatic) were measured using a pressure transducer with the orifice flush with a steel plate positioned on the ground (Fgiure 2d). This package also measured flame residence time with a flame ionization probe that records changes in electrical conductivity from ioniziation when immersed by flame
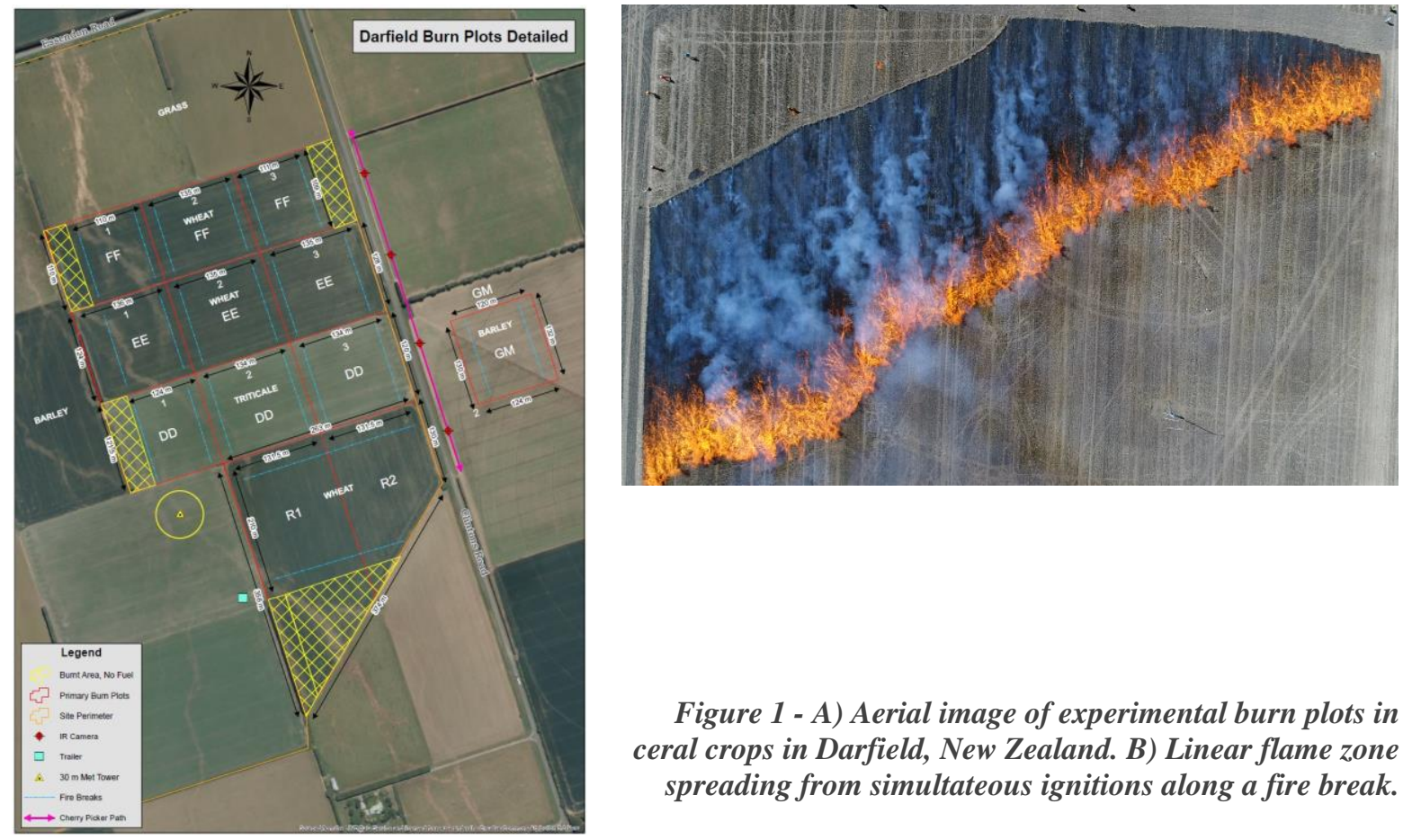

Figure 1 - A) Aerial image of experimental burn plots in ceral crops in Darfield, New Zealand. B) Linear flame zone spreading from simultateous ignitions along a fire break.

Finally, a series of in-fire cameras were installed to record passage of flame zones (60 frames per second) through the instrument arrays (Figure 2e). All of these instrument packages used a custom- 
built 8 -channel data logger (Figure $2 \mathrm{f}$ ) recording at $50 \mathrm{~Hz}$ which synchronized time-stamps and sensor location with an on-board GPS unit.
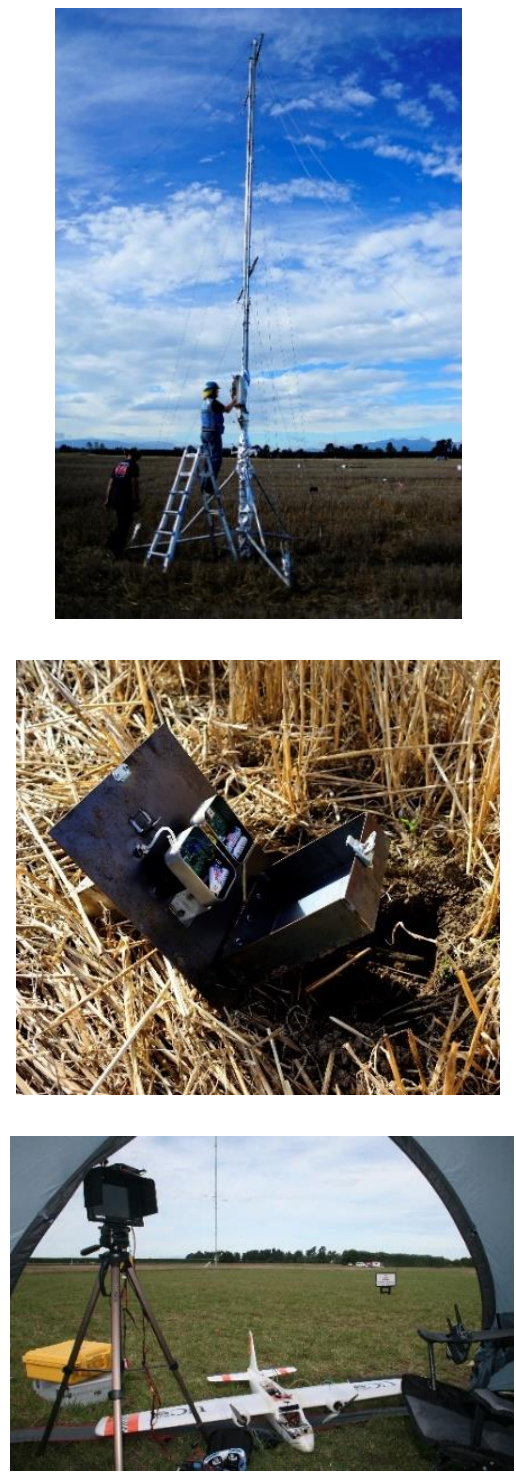
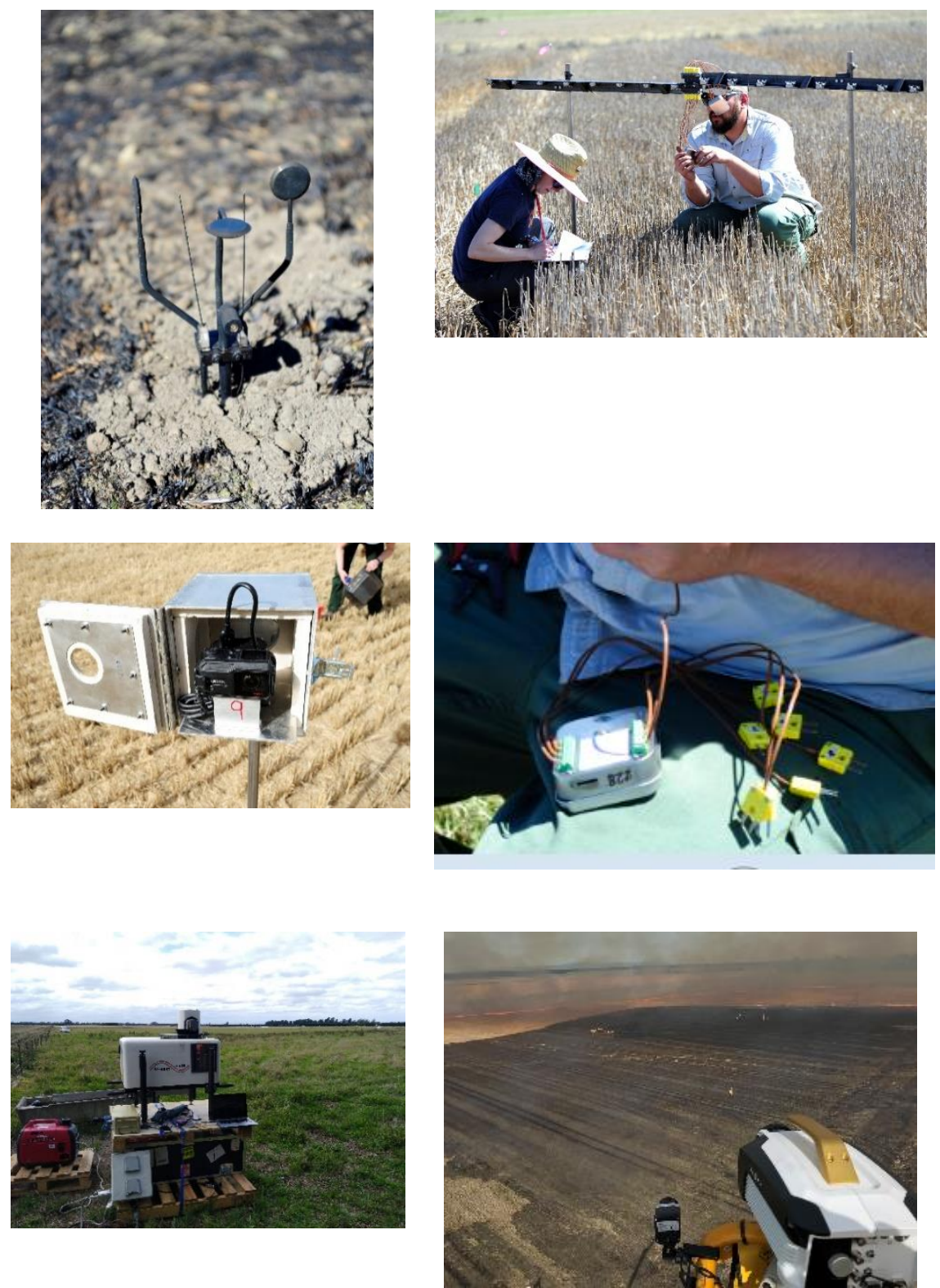

Figure 2 - Pictures of field instrumentation a) $10 \mathrm{~m}$ tower with sonic anemomenters and vertical array of fine-wire thermocouples, $b$ ) heat flux sensor with tri-directional velocity disks, radiometer, and thermocouples, $c$ ) horizontal array of fine-wire thermocouples, $d$ ) hydrostatic pressure and flame ionization detector, $e$ ) in-fire video cameras, and f) custom 8-channel data logger with GPS

Remote sensing was accomplished with a variety of tools. A LIDAR unit with $18 \mathrm{~m}$ resolution was used to scan horizontal and vertical swaths of wind velocity, while a SODAR was continuously measuring the velocity boundary layer up to 300m AGL. Three high-speed longwave infrared cameras were mounted on a $23 \mathrm{~m}$ portable lift recorded oblique video of fire spread as well as pre-fire, post-fire thermal features of the airflow across the fields. A fixed-wing unmanned aerial vehicle (UAV) flying pre-programmed traverses was used to capture changes in atmospheric turbulence before, during and after the fires. Aerial video was recorded from two UAVs flying overhead as well as obliquely to the experimental plots. High-speed thermal video was recorded of the fires from a ground-based camera (FLIR 6811) at 480fps and standard video (240 fps, 700fps) from multiple angles. 


\section{Preliminary Findings}

All fires occurred with $10 \mathrm{~m}$ winds of approximately 6 to $10 \mathrm{~m} \mathrm{~s}^{-1}$. With different fuel species (wheat, barley, triticale), wind, and fuel moisture, fire rate of spread among plots varied from about 40 $\mathrm{m} \mathrm{min}-1$ to over $100 \mathrm{~m} \mathrm{~min}^{-1}$ with flame lengths of approximately $1 \mathrm{~m}$ to $5 \mathrm{~m}$. Forecasted wind direction was accurate to within about 10 degrees and used to orient directionally-dependent instruments (thermocouple arrays, heat flux packages) with the expected spread direction. Preliminary observations and examination of the data suggest these field-scale stubble fires were consistent with laboratory results that convective heating plays the crucial role in heating fuel particles to ignition in wind-driven wildland flame spread. In-fire video (Figure 3a) revealed intermittent flame bursts contacting fuel particles 1 to 5 meters forward of the ignition interface. No pyrolysis products were visible ahead of the flame zone before flame contacts occurred. Coherency of the flame burst parcels was evidenced from synchronous spikes in temperature among thermocouples extending in a line forward of the flame zone (Figure 3c). Flame residence time was approximately 10 seconds as estimated from video as well as the ionization detectors (Figure 3d).
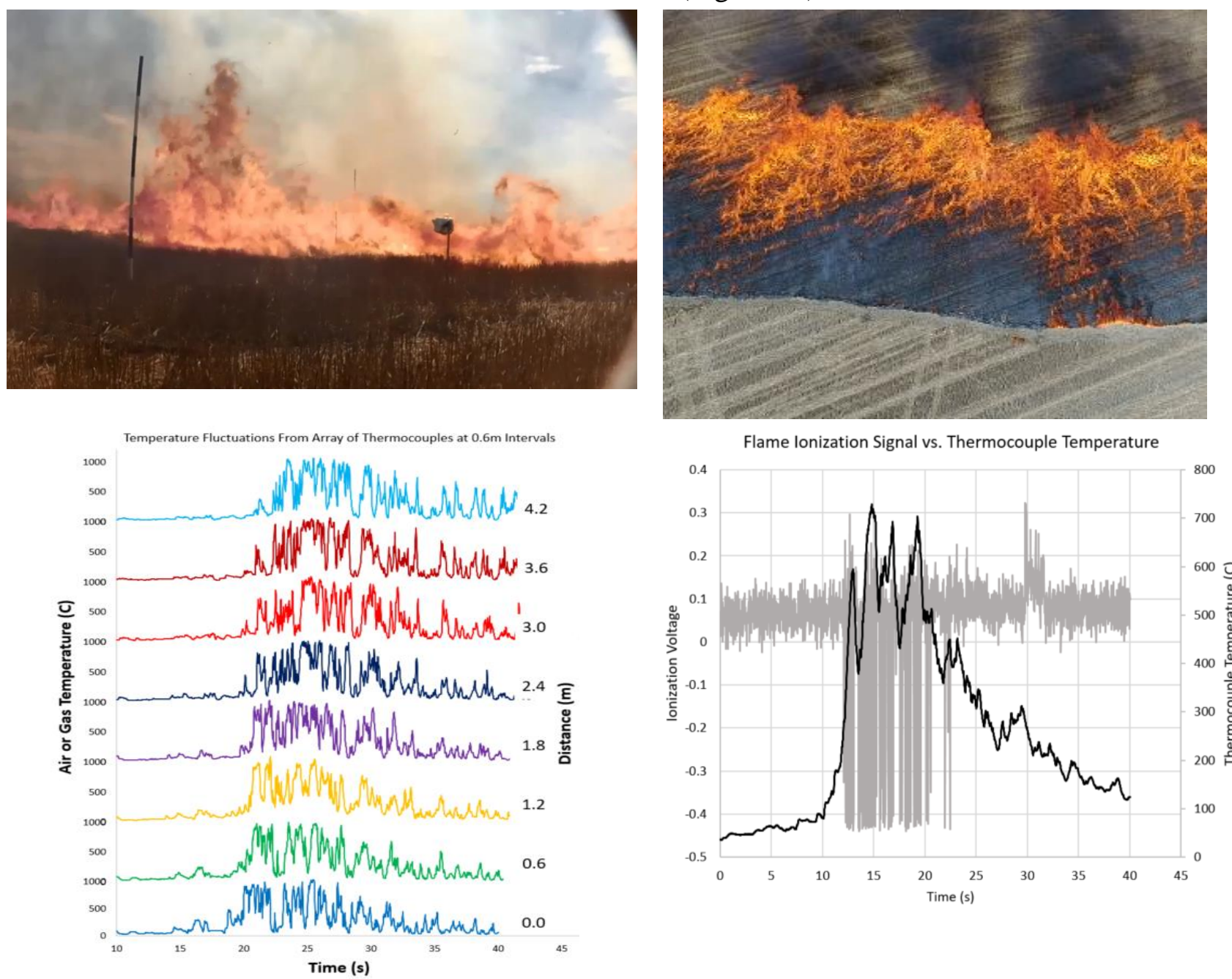

Figure 3 - Still image from in-fire video camera (a) and UAV (b) showing peak-and-trough flame structure consistent with buoyant instabilities of the flame zone. (c) Coherency of temperature data from $4.8 m$ long thermocouple array for plot DD2 (spread rate $\sim 100 \mathrm{~m} \mathrm{~min}^{-1}$ ), (d) example of flame residence time estimated at about 10 s from plot EE3 shown relative to medium-fine $1 \mathrm{e}-4 \mathrm{~m}$ diameter thermocouple temperature. 


\section{Future Work}

Full analysis of all data will be required to convective heating characteristics associated with these stubble fires. Future field research will entail application of these experimental methods to gorse shrub fuel types and to crown fire in conifer forests to test scaling of heat transfer in fires with larger flames.

\section{References}

Finney MA, Cohen JD, Forthofer JA, McAllister, SS, Gollner MJ, Gorham DJ, Saito K, Akafuah NK, Adam BA, English JD (2015) Role of buoyant flame dynamics in wildfire spread. Proceedings of the National Academy of Sciences 112(32), 9833-9838.

Sullivan AL (2009) Wildland surface fire spread modeling, 1990-2007. 1. Physical and quasi-physical models. International Journal of Wildland Fire 18(4):349-368. 
Short contribution - Fire Management

Numerical investigations of coupled fire-atmosphere feedbacks influencing fire behavior on slopes

Rodman Linn ${ }^{1 *}$; Alexandra Jonko; ${ }^{1}$ Judith Winterkamp ${ }^{1}$; Isabelle Runde ${ }^{1}$; Carolyn $\mathrm{Sieg}^{2}$; Russ

Parsons $^{3}$; Francois Pimont ${ }^{4}$

${ }^{1}$ Los Alamos National Laboratory, Los Alamos, NM, USA.

\{rrl@lanl.gov*,ajonko@lanl.gov, judyw@lanl.gov, isabelle.runde@gmail.com \}

${ }^{2}$ USDA Forest Service, Flagstaff, AZ USA, \{csieg@fs.fed.us \}

${ }^{3}$ USDA Forest Service, Missoula, MT, USA \{rparsons@fs.fed.us \}

${ }^{4}$ URFM INRA. 84914 Avignon, France, \{francois.pimont@inra.fr\}

\begin{abstract}
Numerical simulations with a coupled fire/atmosphere model, FIRETEC, were used to investigate some of the mechanisms through which fire is influenced by topographic slope in the direction of the wind. This investigation focuses its attention on the local two-way feedbacks between the fire and the atmosphere around it. Idealized configurations are used to eliminate some of the complexity associated with variable environmental slope, winds and fuels that are frequently found in real-world wildland-fire situations. The simulated fires burn through homogeneous tall grass with static prescribed upstream wind profiles. Simulations were performed on homogenized slopes in which the upstream wind is aligned parallel to the ground in the direction of maximum slope (wind blowing uphill). Simulations were also performed to investigate the influences of having winds intersect the slope at oblique angles, such as horizontal winds colliding with a slope. The results of these simulations provide insight into some of the phenomenological feedbacks that determine the nature of these macro-scale trends. By changing the local slope, the balances that control the interactions between the fire and surrounding atmosphere, thus changing both the local wind patterns and the fire behavior. Components of the wind perpendicular to the land surface also influence the fire behavior, suggesting the importance of incorporating knowledge of upstream topography into fire behavior predictions. This numerical study takes advantage of the richness of information available in process-based simulations and the control over the environmental conditions that is inherent to this type of tool, but is intended to complement laboratory and field observations, which are not subject to the simplifications or abstractions of fine-scale details in such models.
\end{abstract}

Keywords: wildfire modeling, slope effects

\title{
1. Introduction
}

In order to reliably predict wildland fire behavior, it is valuable to have a fundamental understanding of the environmental factors influencing fires, including topography. Even though numerous observations have noted that fire can be drastically influenced by topography, there are still substantial gaps in our understanding of the phenomenological mechanisms involved. There are many aspects of both local and nonlocal topography that can influence fire behavior, but one of the most commonly recognized topographic factors influencing fire behavior is the local slope of the terrain at the site of the fire. A variety of previous studies have characterized the influences of slope on fire behavior through laboratory (Silvani et al. 2012, Viegas et al. 2013, Liu et al. 2014, Rossa et al. 2015) and field observations (Raposo et al. 2015 ).

Field observations provide critical information regarding the macroscopic behavior in true wildland fire scenarios, potentially including the dynamic evolution of specific state variables at specified locations. However, it is difficult t completely characterize the ambient environment (dynamic winds and fuels), and repeatability is also a significant challenge for field observations. 
Laboratory experiments take advantage of the more tightly controlled conditions and potentially higher-density instrumentation of the laboratory setting. Unfortunately, studying the effects of slope in the presence of an ambient wind is non-trivial in traditional wind tunnels. Identifying cause and effect relationships associated with slope solely through such observations is challenging due to the complex interplay between coexisting local and nonlocal (surrounding) conditions. Process-based coupled fire/atmosphere models can be used to complement such observationally-based investigation, leveraging their repeatability, information richness and opportunity to tailor fire scenarios to investigate specific aspects of fire behavior (Linn et al. 2010, Pimont et al. 2012).

\section{Methods}

A coupled fire/atmosphere process-based wildfire model, FIRETEC, was used to investigate the cause-and-effect process interactions that result in a wildland fire's response to changes in topographic slope. The goal was to use the flexibility of numerical modeling to help isolate the effects of local slope from the those of numerous other topographic factors that can confound the clear observation of slope influences on fire behavior in the field or laboratory. Such other factors include upwind or downwind (or upslope and downslope) topographic features, topographic curvature, topographic gradients aligned with the winds, transient winds, or heterogeneous fuel loading. With this approach we were able to explore idealized combinations of wind and slope that are difficult to study in the field or wind tunnel. We use idealized configurations to eliminate some of the complexity associated with variable environmental slope, winds and fuels that are frequently found in real-world wildland-fire situations. The simulated fires burn through homogeneous tall grass with static prescribed upstream wind profiles. Simulations were performed on homogenized slopes in which the upstream wind is aligned parallel to the ground in the direction of maximum slope (wind blowing uphill) and then influences of having winds intersect the slope at oblique angles we explored, such as horizontal winds colliding with a slope.

\section{Results}

Many of the macro-scale scale trends in fire behavior of this study are consistent with trends that have been observed in previous field, laboratory and numerical investigations, including the steeper slopes causing faster spread rates and more pointed fireline shapes (figure 1). However, the nature of this exploration also provided insight into some of the phenomenological feedbacks that determine the nature of these macro-scale trends. By changing the local slope, the balances that control the interactions between the fire and surrounding atmosphere. The tower and trough fireline dynamics that exists in wind driven fires on flat ground changes as the fireline and wind direction become more aligned and the entrainment patterns near the heading and flanking portions of the fire change. As the depth of the fireline increases with the slope the penetration of the winds through the fireline is hindered, thus increasing the role of winds that are drawn towards the fire from outside the fire perimeter. Slope effects on fire behavior can also be influenced by the alignment of the ambient winds with the hillside, indicating that in nonlocal topographic effects and the position on a hillside (e.g. top of a hill or bottom of a hill) should also be considered in prediction of fire behavior in complex terrain. 

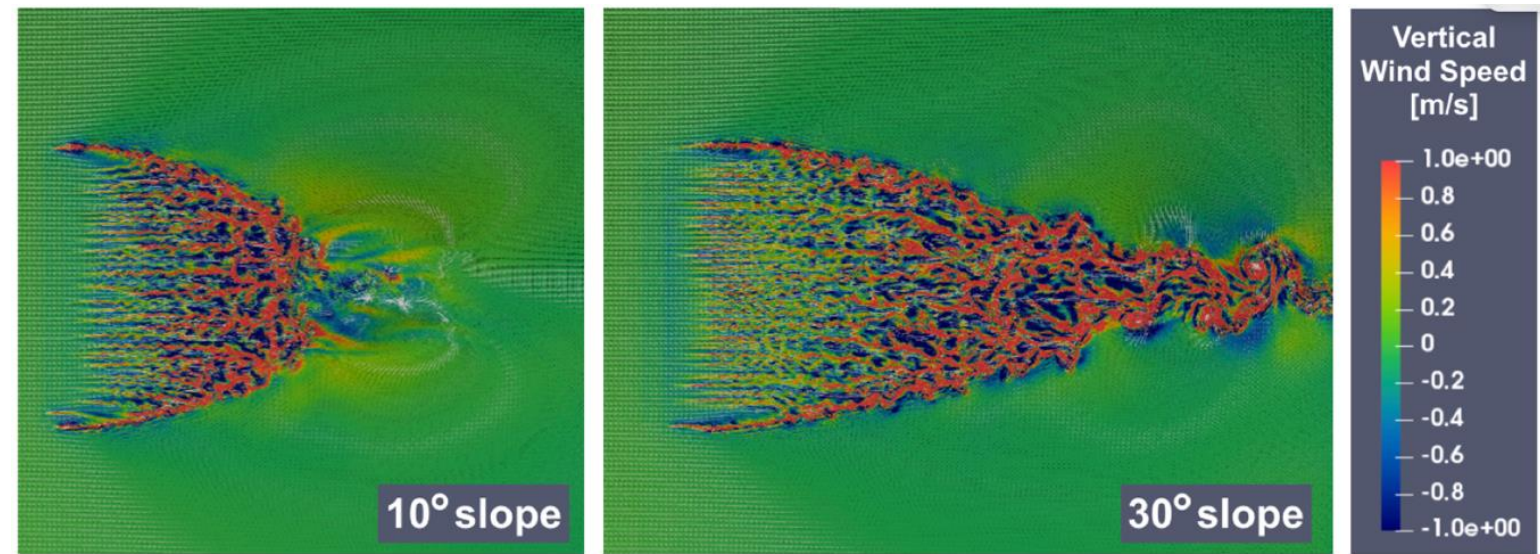

Figure 1 - Results from FIRETEC simulations of grass fires on slopes with the upstream wind aligned parallel to the slope. Colors indicate the wind velocity component that is perpendicular to the land surface.

\section{Conclusions}

Models such as FIRETEC are in continual need of validation and are not replacements for observations. However, this investigation adds perspective to the investigation of topography influences on fire behavior. Observations and experiments can subsequently be designed to test modeling-based hypotheses, supporting or refuting model results, and thus provide critical information to continually assess the validity of models and keep their applicability in perspective (Canfield et al. 2014).

\section{References}

Canfield JM, Linn RR, Sauer JA, Finney M, Forthofer J (2014) A numerical investigation of the interplay between fireline length, geometry, and rate of spread. Agricultural and Forest Meteorology 189-190, 48-59.

Linn RR, Winterkamp JL, Weise DR, Edminster C (2010) A numerical study of slope and fuel structure effects on coupled wildfire behaviour. International Journal of Wildland Fire 21 (4), 396417.

Liu N, Wu J, Chen H, Zhang L, Deng Z, Satoh K, Viegas D, Raposo J (2014) Upslope spread of a linear flame front over a pine needle fuel bed: The role of convection cooling. Proceedings of the Combustion Institute. 35. 10.1016/j.proci.2014.05.100.

Pimont F, Dupuy JL, Linn RR (2012) Coupled slope and wind effects on fire spread with influences of fire size: a numerical study using FIRETEC. International Journal of Wildland Fire 21 (7), 828842.

Raposo J, Cabiddu S, Viegas D, Salis M, Sharples J (2015) Experimental analysis of fire spread across a two-dimensional ridge under wind conditions. International Journal of Wildland Fire. 24. 10.1071/WF14150.

Rossa C, Davim D, Viegas D (2015) Behaviour of slope and wind backing fires. International Journal of Wildland Fire. 24. 10.1071/WF14215.

Silvani X, Morandini F, Dupuy JL (2012) Effects of slope on fire spread observed through video images and multiple-point thermal measurements. Experimental Thermal and Fluid Science. 41. 99111. 10.1016/j.expthermflusci.2012.03.021.

Viegas D, Raposo J, Figueiredo A (2013) Preliminary Analysis of Slope and Fuel Bed Effect on Jump Behavior in Forest Fires. Procedia Engineering. 62. 1032-1039. 10.1016/j.proeng.2013.08.158. 
Short contribution - Fire Management

Numerical simulation of low-intensity fire spread in pine litter

\author{
Eric V. Mueller ${ }^{1 *}$; Zakary Campbell-Lochrie ${ }^{1}$; William Mell²; Rory M. Hadden ${ }^{1}$ \\ ${ }^{1}$ University of Edinburgh. Edinburgh, UK \{e.mueller@ed.ac.uk*\} \\ ${ }^{2}$ USDA Forest Service. Seattle, WA, USA
}

\begin{abstract}
Detailed physics-based models of wildland fire behavior have shown utility as a tool for investigating the physical drivers of fire spread. However, the continued development of such models requires extensive testing against robust experimental measurements. This remains an outstanding challenge for the research community. A particular application space of interest is the simulation of low-intensity fires, in either stillair or backing fire conditions, as such models have been tested relatively little for such scenarios. A numerical tool capable of properly representing the relevant physics is valuable for understanding the impact of prescribed fire activities, which are often carried out in these conditions. Therefore, ongoing efforts are being undertaken to assess the ability of a detailed physics-based model to simulate fire spread in pine needle litter in still air. Particular focus is placed on the representation of flow within the fuel bed and its effect on fire behavior. The experimental data necessary for model testing was obtained from bench-scale flame spread experiments on a $1.5 \mathrm{~m}$ long table. The outputs included both global observations of fire behavior and point measurements of physical processes within the fuel bed (e.g. flow). Numerical modeling was conducted with a computational fluid dynamics model - the Fire Dynamics Simulator - using a multiphase formulation to represent the vegetation. Modifications were implemented in FDS to improve the representation of vegetation, particularly related to thermal degradation in the char oxidation stage. Maintaining a constant fuel load, fuel bed bulk densities of $11 \mathrm{~kg} \cdot \mathrm{m}-3$ and $20 \mathrm{~kg} \cdot \mathrm{m}-3$ were tested and compared. Results show that, for the same fuel loading, there is a lower limit to the bulk density, for which the fire will not spread. While this limit will exist in reality, experimental results suggest that the model over-predicts its magnitude. This appears to be linked to the flow within the bed, as the lower bulk density case resulted in more entrainment, and thus greater convective cooling ahead of the fire front. Further, flow measurements within the bed show that the model is not adequately representing the removal of fuel, and thus increased flow, behind the fire front. In order to more directly assess the numerical representation of the fluid dynamics within the fuel bed, a heated wind tunnel has been constructed. Flow and convective heating through various fuel arrangements and compositions are being tested. This is aiding in the evaluation of the limits of these submodels, particularly as they apply to low-intensity fire spread in litter fuels.
\end{abstract}

Keywords: numerical modeling, flame spread, fuel structure, flow

\title{
1. Introduction
}

Detailed physics-based models of wildland fire behavior have shown utility as a tool for investigating the physical drivers of fire dynamics (e.g. Mell et al. 2009; El Houssami 2016). However, the continued development of such models requires extensive testing against robust experimental measurements. This remains an outstanding challenge for the research community.

A particular application space of interest is the simulation of low-intensity fires, in either still-air or backing fire conditions. Detailed physics-based models have been tested relatively little for such scenarios, and the behavior of these fires can prove more difficult to predict than those which are strongly driven by the wind. A numerical tool capable of properly representing the relevant physics is valuable for understanding the impact of prescribed fire activities, which are often carried out in these conditions. Therefore, ongoing efforts are being undertaken to assess the ability of a detailed physicsbased model to simulate fire spread in pine needle litter in still air. Particular focus is placed on the representation of flow within the fuel bed and its effect on fire behavior. 


\section{Methods}

\subsection{Experimental methods}

Experimental data pertaining to flame spread in pine litter was collected under the calorimetry hood at the University of Edinburgh. Beds of pine needles were re-constructed on a $1.5 \mathrm{~m}$ long table with a vermiculite insulation board substrate. This table was instrumented with a series of thermocouples and pressure probes in order to help characterize the mechanisms contributing to flame spread.

The pine needles used were a hybrid cross of pitch pine (Pinus rigida) and loblolly pine (Pinus taeda). These dead needles had a typical moisture content of $\sim 10 \%$ at the time of testing. The work presented here focuses on two experimental configurations with an identical fuel load of $0.8 \mathrm{~kg} \cdot \mathrm{m}^{-2}$. In the first scenario the fuel bed had a height of $0.04 \mathrm{~m}$ and a bulk density of $20 \mathrm{~kg} \cdot \mathrm{m}^{-3}$. The second scenario had a bed height of $0.07 \mathrm{~m}$ and a bulk density of $11 \mathrm{~kg} \cdot \mathrm{m}^{-3}$.

\subsection{Numerical methods}

Three-dimensional simulations of flame spread in pine litter were carried out using the National Institute of Standards and Technology Fire Dynamics Simulator (FDS), version 6.6.0 (McGrattan et al. 2018). Some new modifications were implemented in the source code, specifically for this work, in order to permit the representation of vegetation through the multiphase formulation (Grishin 1997). In this approach, vegetative fuel is represented as a collection of thermally-thin subgrid-scale particles, which can be described by a set of bulk properties. The multiphase formulation has previously been implemented within an FDS-based framework, known as WFDS (Mell et al. 2009). However, this updated framework is of interest as it represents a re-integration of the vegetation models into the most current version of FDS, where they can leverage ongoing development efforts on other aspects of the code.

Aerodynamic drag within the fuel bed was represented using a Reynolds-dependent coefficient for a cylinder in cross-flow, and convective heat transfer was modeled using the maximum of a natural and forced convective coefficient for a cylinder (McGrattan et al. 2018). Radiative heat transfer was modeled using an extinction coefficient for bulk vegetation. Thermal degradation of the fuel was simulated using Arrhenius reaction models for vaporization of moisture, pyrolysis, and char oxidation (El Houssami 2016). Numerical formulations which were not specific to vegetation, for example the combustion model and radiation transport algorithm followed the default FDS configuration (McGrattan et al. 2018).

A numerical grid resolution of $1.25 \mathrm{~cm}$ was used, with the computational domain divided over 48 processors. The ratio of computational time to simulation time was approximately 600:1. In this instance, simulations were terminated after $180 \mathrm{~s}$ of simulation time.

\section{Results and discussion}

An example of the computational domain with a simulated flame front is shown in Figure 1, along with the predicted mass loss for the two different bed configurations. The higher bulk density scenario reaches a quasi-steady burning rate of $\sim 2.0 \mathrm{~g} \cdot \mathrm{s}^{-1}$. The simulated spread rate was estimated to be 0.43 $\mathrm{cm} \cdot \mathrm{s}^{-1}$, compared to $0.22 \mathrm{~cm} \cdot \mathrm{s}^{-1}$ in the experiment. The lower bulk density case briefly attains a similar burning rate but then begins to decay, and the fire is extinguished after $35 \mathrm{~s}$. This is contrary to the experiments, where the flames spread to the end of the table at a rate of $0.29 \mathrm{~cm} \cdot \mathrm{s}^{-1}$. 

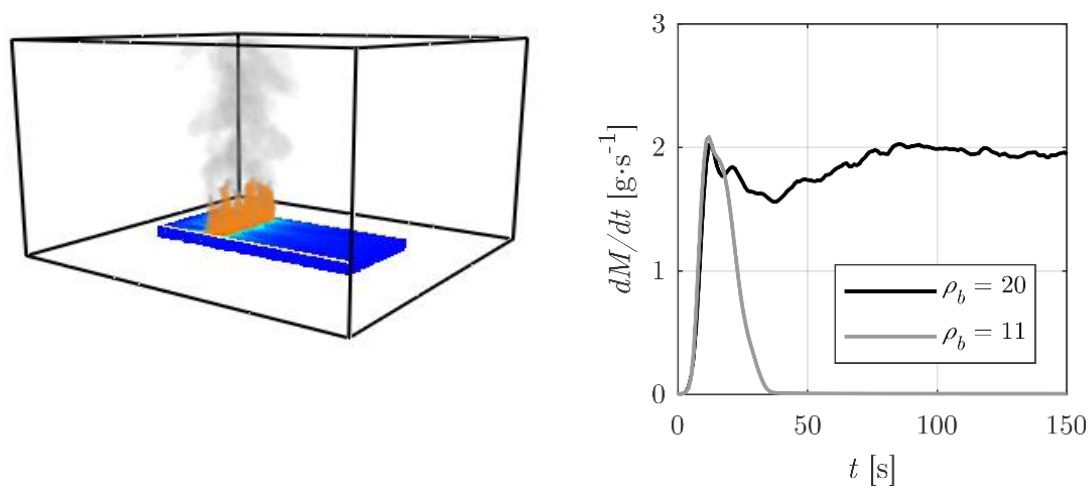

Figure 1 - (left) Example flame spread simulation for bulk density of $11 \mathrm{~kg} \cdot \mathrm{m} 3$, and (right) simulated mass loss rate for the two different fuel bed bulk densities, $\rho b$.

In order to explore this further, the entrainment flow velocity (parallel to the direction of fire spread) and the convective heat transfer coefficient are shown in Figure 2. These values were obtained within the fuel bed, at a location $0.5 \mathrm{~m}$ from the ignition line and $0.025 \mathrm{~m}$ from the table surface. The flame front passage is immediately clear in the high density case, marked by the reversal of the flow direction (as entrainment is opposed to the direction of spread ahead of the flame front, and concurrent behind it). The entrainment flow reaches a peak of $\sim 0.2 \mathrm{~m} \cdot \mathrm{s}^{-1}$ in both directions. In the experiment, the velocity peaks at $0.16 \mathrm{~m} \cdot \mathrm{s}^{-1}$ ahead of the fire, and $0.36 \mathrm{~m} \cdot \mathrm{s}^{-1}$ behind it. The model does not capture any increase behind the front, likely because it under-predicts the extent of complete fuel particle consumption following char oxidation, and the subsequent increase in porosity. In the low density case, the fire does not reach the location of interest. However, both the flow magnitude and convective coefficient are greater in the early stages of spread for this configuration. This is a result of the higher porosity, and increases convective cooling ahead of the front, which may help to explain the extinction of the fire.
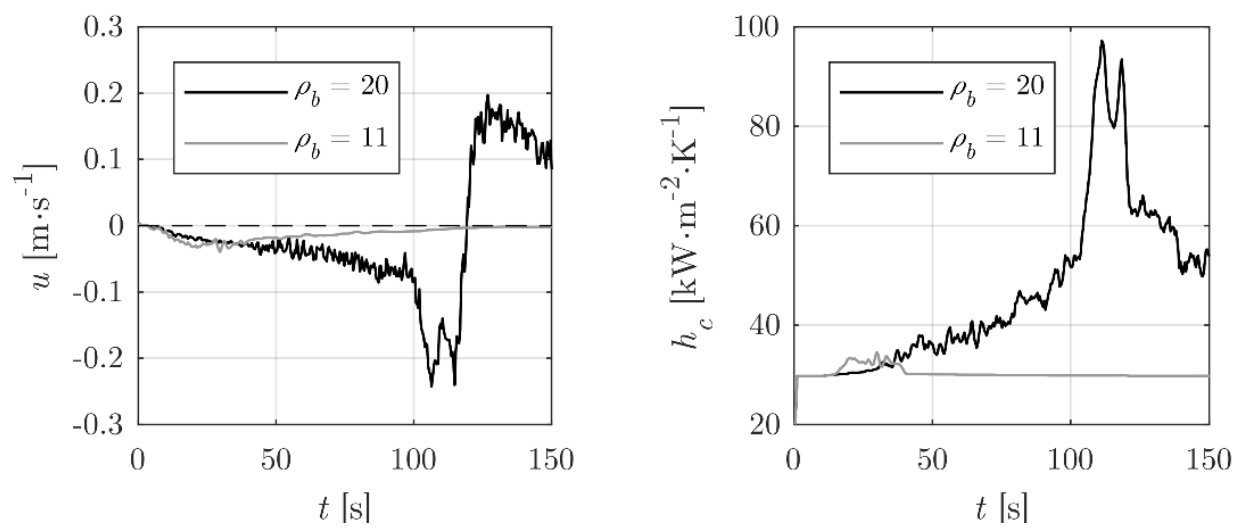

Figure 2 - (left) Entrainment flow velocity, $u$, and (right) convective heat transfer coefficient, $h_{c}$. Values are obtained within the fuel bed, $0.5 \mathrm{~m}$ from the ignition line.

\section{Summary and ongoing work}

Results from numerical simulations of low-intensity flame spread in pine litter, using the multiphase formulation, indicate that, for the same fuel loading, there is a lower limit to the bulk density, for which the fire will not spread. This will also occur experimentally, however data for the relevant values show that the model over-predicts the magnitude of this limit. The $11 \mathrm{~kg} \cdot \mathrm{m}^{-3}$ fuel bed should still support fire spread.

These results demonstrate a clear dependence of the model on bed structure which is not fully representative of experimental data. Therefore, it is desirable to directly assess the quality of both the drag and convective heat transfer models, as applied to simulations of pine litter beds. To that end, a heated wind tunnel is being used to quantify convection through various fuel arrangements and 
compositions, particularly those representative of pine litter beds. These results will allow evaluation of limits of these submodels.

\section{References}

Grishin AM (1997) 'Mathematical modeling of forest fires and new methods of fighting them.' (Publishing house of the Tomsk state university: Tomsk, Russia)

El Houssami M (2016) Development of a numerical and experimental framework to understand and predict the burning dynamics of porous fuel beds. PhD Thesis, University of Edinburgh.

McGrattan K, McDermott R, Hostikka S, Floyd J, Vanella M, Weinschenk C, Overholt KJ (2018) Fire dynamics simulator (version 6), user's guide. National Institute of Standards and Technology, 1019.

Mell W, Maranghides A, McDermott R, Manzello SL (2009) Numerical simulation and experiments of burning douglas fir trees. Combustion and Flame 156(10), 2023-2041. 
Short contribution - Fire Management

Out of context: fire background temperature and methods for its calculation

Bryan Hally ${ }^{1,2,3^{*}}$, Luke Wallace ${ }^{1,2}$, Chermelle Engel ${ }^{1,2}$, Chathura, Wickramasinghe ${ }^{1,2}$, Karin Reinke ${ }^{1,2}$ \& Simon Jones ${ }^{1,2}$

${ }^{1}$ School of Science, RMIT University, Melbourne, AU. ORCID 0000-0003-2771-492X \{bryan.hally@rmit.edu.au*\}

${ }^{2}$ Bushfire and Natural Hazards Cooperative Research Centre, Melbourne, AU

${ }^{3}$ Faculty for Geo-Information Science and Earth Observation (ITC), University of Twente, Enschede, NL

\begin{abstract}
Background temperature is an important component of any fire detection and monitoring method; the use of the contrast between the expected brightness temperature of a location and the brightness temperature associated with fire activity is the basis for most fire detection algorithms. The commonly used method for calculation of fire background temperature involves estimation of the surface characteristics using the immediately adjacent, non-occluded surrounds of the target pixel, in order to provide a contextually - based estimate of temperature. Depending upon conditions such as cloud, smoke, surface water and heterogeneity of land cover, this derivation of background temperature from the surrounding landscape can be vastly different from the measured brightness temperature of a pixel in a non-fire context. This paper examines the relationship between pixel brightness temperature and pixel context, to identify situations where the currently used contextual methods are most likely to perform below the required level of accuracy for fire detection. Initial results show that in many cases the ideal candidate pixels for estimating temperature at any one location may not be those that sit immediately adjacent spatially.
\end{abstract}

Keywords: diurnal modelling, land surface temperature, fire detection, spatial context, multi-temporal estimation

\title{
1. Introduction
}

Remote sensing techniques for detecting and attributing fire activity require the calculation of a pixel's nominal background - the temperature a pixel would display without the influence of fire - in order to determine whether a fire exists, and the magnitude of fire activity. Without the ability to directly measure this background temperature, most methods currently derive their background temperature from the image context, the area immediately surrounding the pixel spatially. This technique leverages the tendency of nearby pixels to display relatively similar characteristics due to spatial auto-correlation.

Problems arise with contextual methods when the area examined is not relatively homogeneous for surface temperature examination. Factors such as slope and aspect, weather effects, surface water, undetected cloud and smoke, and land cover changes can all affect the accuracy of results derived from the pixel context. This work looks to examine the efficacy of context for providing temperature estimation, and especially looks at the effects that occur when using contextual pixels that are further away than the immediate surrounds of a target pixel.

\section{Method}

This paper uses data from the AHI-8 sensor onboard the Japan Meterological Agency's Himawari 8 geostationary satellite. Band 7 MWIR $(4 \mu \mathrm{m})$ images were selected over a section of central Thailand for a period of 31 days, with the central day based upon the VNP14IMGLL (Schroeder et al., 2014) 
maximum fire occurrence for 2016. Images were selected for coincidence with the VIIRS overpass time for the area, which roughly corresponds to 0630 UTC. The area covered by the study in shown in Figure 1. Cloud areas were discarded using a cloud mask based upon Xu et al. (2010), and a land mask was used to eliminate lakes and oceans from examination based upon ancillary data provided by the Australian Bureau of Meteorology.

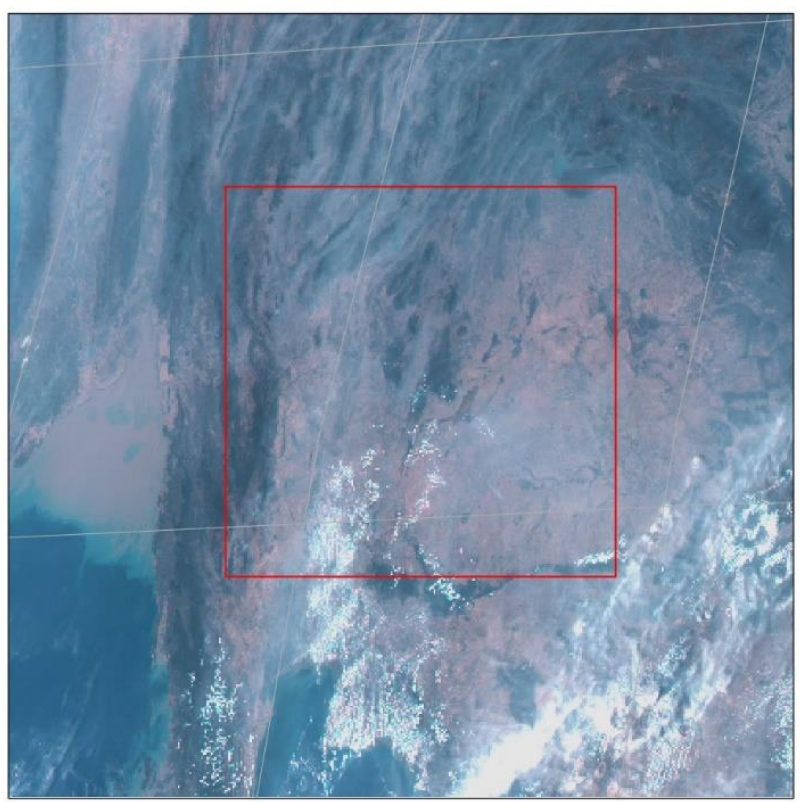

Figure 1 - The study area in central Thailand, as seen from AHI-8.

The study calculated the mean temperature of the surrounding pixel context in the area of examination for pixels in various configurations around the central pixel. Firstly estimation was based upon all available non-masked pixels in the $5 \times 5$ area surrounding the central pixel, then this area was increased to $7 \times 7,9 \times 9$ and finally $11 \times 11$ pixels. Means from these areas were then compared to the central pixel value to determine their error, and the means and variances of these differences were recorded for analysis.

The study also looks at use of pixel temporal information in comparison to pixels in the study area to provide a prediction of pixel brightness temperature. One pixel was randomly selected from the study area shown, then the root mean square error was calculated between this pixel and all other pixels in the first thirty days of the study period. The best 24 pixels for RMSE were then used as a predictive set for temperature in the target pixel for the 31st day of the study, and comparison was made to contextual pixel estimation in the $5 \times 5$ window for the same day.

\section{Results and Discussion}

Figure 2 demonstrates the spatial distribution of errors in contextual temperature estimation in the area selected. The first map shows the variation in means at the $5 \times 5$ window, which highlights areas of relief and land cover changes as edge effects. Bodies of water that have not been properly eliminated also demonstrate temperature over-estimation. As the window of examination increases, these variations in the landscape become more prominent as drivers of error for background temperature.

The distributions shown to the right of the maps describe the statistical distribution of temperature estimates. There is no skew to the results - globally the mean tends to zero, but as the window size grows the distribution of points flattens and the variance of the calculated values increases markedly. 

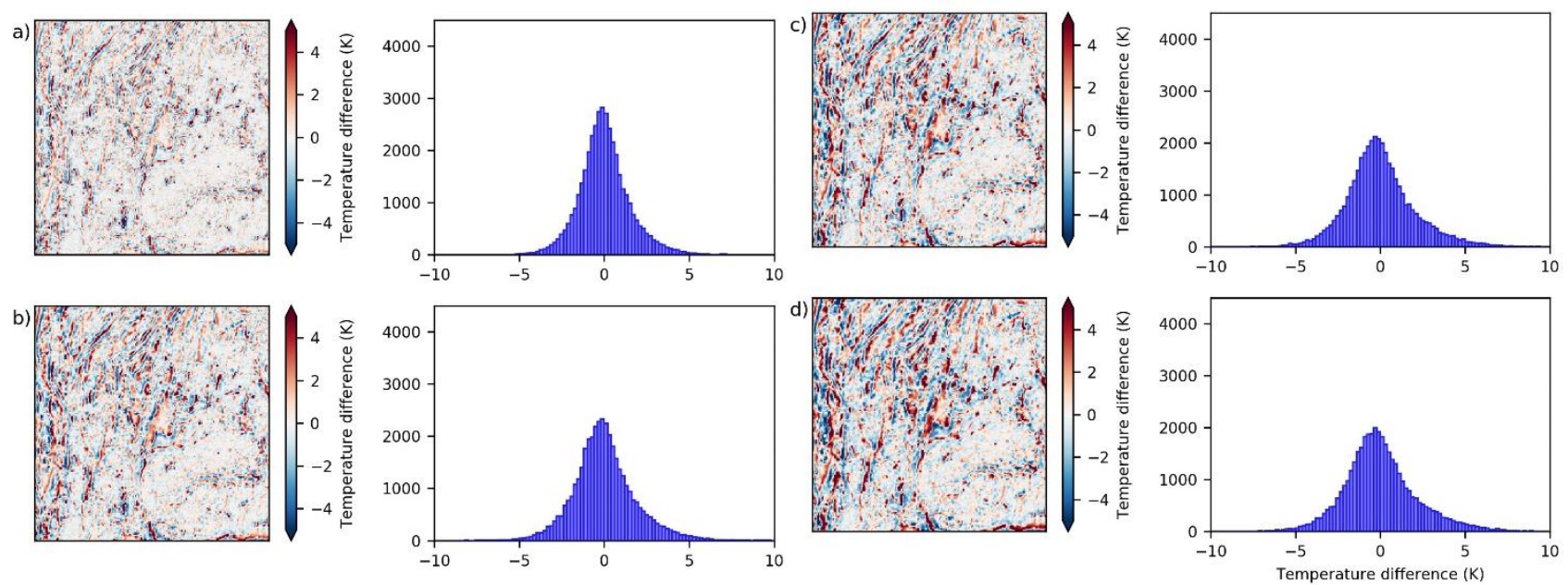

Figure 2 - Effect of expanding the contextual area of analysis on brightness temperature estimation for part of central Thailand. The spatial and statistical distributions for pixel differences from the context are shown for a) $5 \times 5$, b) $7 \times 7$, c) $9 \times 9$, and d) $11 \times 11$ contextual windows.

Figure 3 shows the spatial distribution of pixels that have the lowest root mean square error compared to the selected pixel (AHI 1836, 977). The lowest 24 RMSE values were then selected as a training series for estimation of pixel values on the 31 st day of the study period. As can be seen here, using the pixel histories in this way can provide data even when a large portion of the pixel history is cloud affected, and the resulting mean value from the selected pixels provides a better estimate of the target pixel temperature than the context in this case.
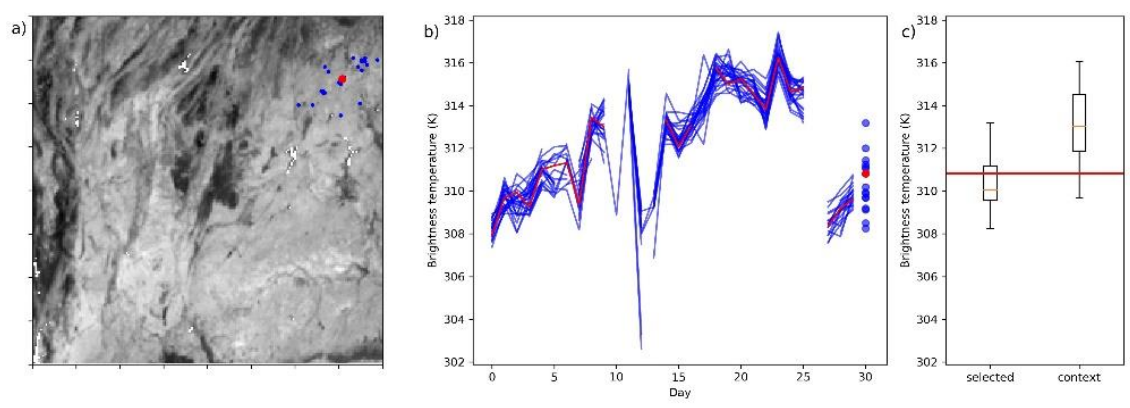

Figure 3 - Using selected pixels from the study area to provide background estimates. a) Location of pixels with lowest RMS error compared to the examined (red) pixel; b) diagram of temperatures over the thirty day period; c) plot of selected pixel values versus the pixel context.

This work shows a small sample of some of the issues that can occur with use of contextual temperature estimation. Land cover change is a particular driver of error in this area, but there are several other factors that can cause increased variance in calculated temperature values. Any method using contextual information for estimating temperature values should be aware of the specific sources of error that exist in the region of examination, as the errors seen here can easily result in misattribution of fire characteristics, and errors in both omission and commission of fire products.

\section{References}

Schroeder, W., Oliva, P., Giglio, L., Csiszar, I., 2014. The New VIIRS 375m active fire detection data product: Algorithm description and initial assessment. Remote Sens. Environ. 143, 85-96. doi:10.1016/j.rse.2013.12.008

Xu, W., Wooster, M.J.J., Roberts, G., Freeborn, P.H., 2010. New GOES imager algorithms for cloud and active fire detection and fire radiative power assessment across North, South and Central America. Remote Sens. Environ. 114, 1876-1895. doi:10.1016/j.rse.2010.03.012 
Short contribution - Fire Management

Simulations of surface fire propagating under a canopy: flame angle and intermittency

Duncan Sutherland*1,4; Jimmy Philip ${ }^{2}$; Andrew Ooi $^{2}$; Khalid Moinuddin ${ }^{3,4}$

${ }^{I}$ School of Physical, Environmental, and Mathematical Sciences, UNSW Canberra, Canberra, Australia, \{duncan.sutherland@adfa.edu.au*\}

${ }^{2}$ Department of Mechanical Engineering, Melbourne School of Engineering, University of Melbourne, Melbourne Australia., \{jimmyp@unimelb.edu.au,a.ooi@unimelb.edu.au\}

${ }^{3}$ Institute for Sustainable Industries and Liveable Cities, Victoria University, Melbourne, Australia

\{khalid.moinuddin@vu.edu.au\}

${ }^{4}$ Bushfire and Natural Hazards Cooperative Research Centre, Melbourne, Australia

\begin{abstract}
We conduct physics-based simulations of a wind driven surface fire entering, propagating through, and leaving a region of aerodynamic drag. This is an idealised model of a real-world grassfire propagating through a short canopy such as a stand of trees. The study is motivated by the need to understand how fires entering forested regions adjust to the lower wind speed inside the forest. This will lead to more informed operational modelling practices and predictions of fire spread. In these simulations the driving open $(10 \mathrm{~m})$ wind speed is varied from $1 \mathrm{~ms}^{-1}$ to $10 \mathrm{~ms}^{-1}$. For lower driving wind speeds the rate-of-spread of the fire is largely unaffected by the canopy, however, for the higher driving wind speeds the fire appears to transition from a wind-driven mode, characterised by a low flame angle to a buoyancy-driven mode, characterised by a nearly vertical flame.
\end{abstract}

Keywords: Physics-based fire simulation, surface fire, tree canopies, flame intermittency, non-quasisteady fires

\title{
1. Characterising fire spread
}

We report on a physics-based simulation study of a surface fire in a uniform fuel bed propagating through a region of aerodynamic drag. This is an idealised model of a wildfire entering, propagating through a canopy, and leaving the canopy region (c.f. figure 1). Wildfires may be broadly classified into two modes: wind-driven fires, which are dominated by the driving wind characterised by an elongated flame at an acute angle to the ground; and buoyancy-driven fires, which are dominated by the dynamics of the plume and characterised by a vertical flame. Fires can also propagate in an intermittent surge-stall manner (Dold 2011) where the fire oscillates between the wind-driven and buoyancy-driven modes. The time-averaged driving wind field associated with a fire propagating from open grassland to a forested region and eventually back to open grassland will vary in space. Simulations of flow through isolated tree canopies surrounded by clearings have been conducted in the absence of a fire (eg Kanai-Shuring and Raasch (2017)). Simulations (Keifer et al. 2015) and measurements (Seto et al. 2014) of the mean and turbulence statistics from downstream of low intensity surface fires within canopies have also been conducted. The simulations of Keifer et al. involved modelling the fire as a surface temperature anomaly instead of explicitly simulating fuel combustion as done here. Seto et al. measured the temperature and vertical velocity fluctuations as a surface fire progressed under a canopy for similar sized fires as simulated here (and detailed in section 2). The spatial development of the time-averaged wind field over an isolated canopy is relatively straightforward to simulate by modelling the tree canopy as a region of aerodynamic drag which depends on the Leaf Area Density (LAD) of the forest. We hypothesise that the fire will transition between the wind driven mode in the open grassland to a buoyancy dominated fire in the canopy, and 
then back to a wind driven mode downstream of the canopy. The surge-stall regime is expected to occur in regions around the transition between these two modes. The aim of the present study is to use full-physics-based simulations of an idealised model fire to examine how and where the fire transitions between the wind driven and buoyancy modes as the fire progresses through the canopy.

\section{Idealised Model}

A series of physics-based three-dimensional large-eddy simulations of a grass fire entering, propagating under, and leaving a short tree canopy, without burning the canopy itself are conducted varying only the magnitude of the driving wind speed. The well-known physics-based fire simulation Fire Dynamics Simulator (FDS, McGrattan et al. 2013) is used for this study. FDS uses a Large Eddy Simulation (LES) methodology for the gas phase, a mixture-fraction combustion model, a linear model of the fuel pyrolysis, and accounts for heat transfer by conduction, convection, and radiation. Mell et al. (2007) performed simulations of quasi-steady open grassfires and validated against experimental data. To reduce the computational effort and as a first approximation to a general spanwise varying fire-front, we considered a narrow domain. As such, the fire front is homogeneous in the $y$-direction, resulting quasi-two-dimensional simulation. The fuel along the ground is kept constant in the canopy and non-canopy regions and the burning of the tree canopy is not considered. Therefore, the canopy acts only as a region of aerodynamic drag. A schematic showing the simulation domain, the ignition line, and the canopy is shown in Figure 1. The idealisations in this model are used to isolate the effect of the aerodynamic drag of the canopy upon the fire dynamics. The burning of the canopy may be considered in a later study. The parameter space investigated here is variation of inlet wind speed. The inlet wind speed at $10 \mathrm{~m}$ height is varied between $u(x=0, z=10)=1,2,4,6,8$, and $10 \mathrm{~ms}^{-1}$. All other parameters are held constant.

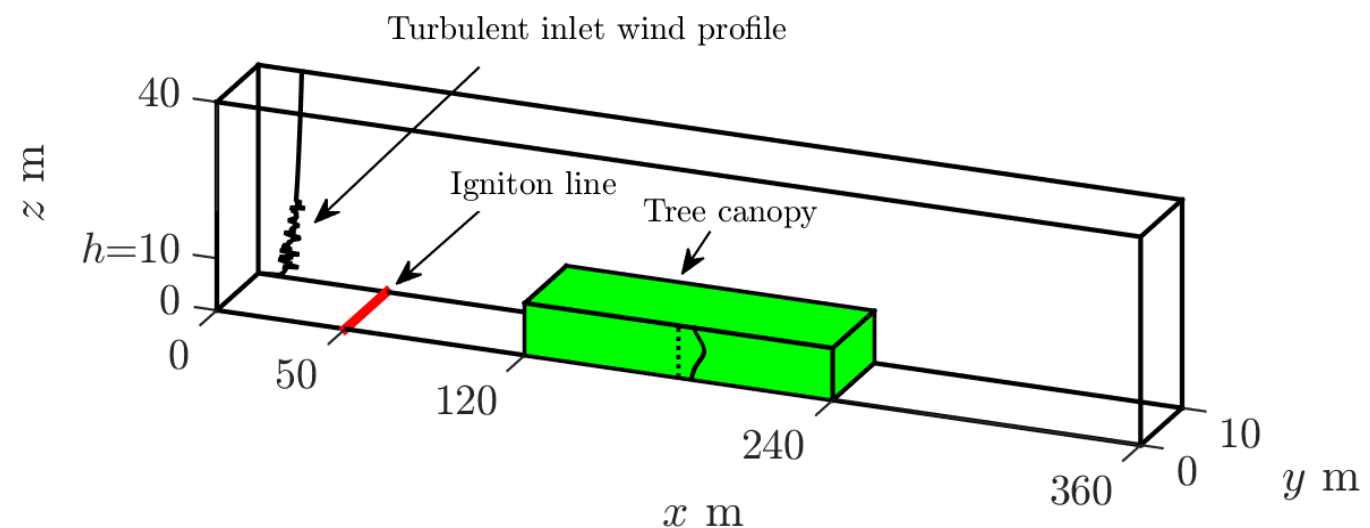

Figure 1 - Simulation domain showing the location of the model tree canopy, the turbulent inlet wind conditions, and the fire ignition line. The profile of leaf area density is also shown.

The inlet wind speed is simulated using the synthetic eddy method (SEM) similar to Jarrin et al. (2006). The SEM introduces artificial turbulence at the inlet to ensure fast development of a realistic boundary-layer flow. A mean profile, in this case a logarithmic profile, is specified at the inlet and randomly generated eddies sampled from a distribution with specified length and velocity scales are superimposed upon the mean profile. The SEM leads to the development of a realistic turbulent loglayer after approximately $50 \mathrm{~m}$. (Pavlidis et al. 2010). Contours of the $u$-velocity and $w$-velocity for the $6 \mathrm{~ms}^{-1}$ driving wind case and $u$-velocity as a function of $x$ at $z=2$ and $10 \mathrm{~m}$ for all cases are shown in Figure 2. 

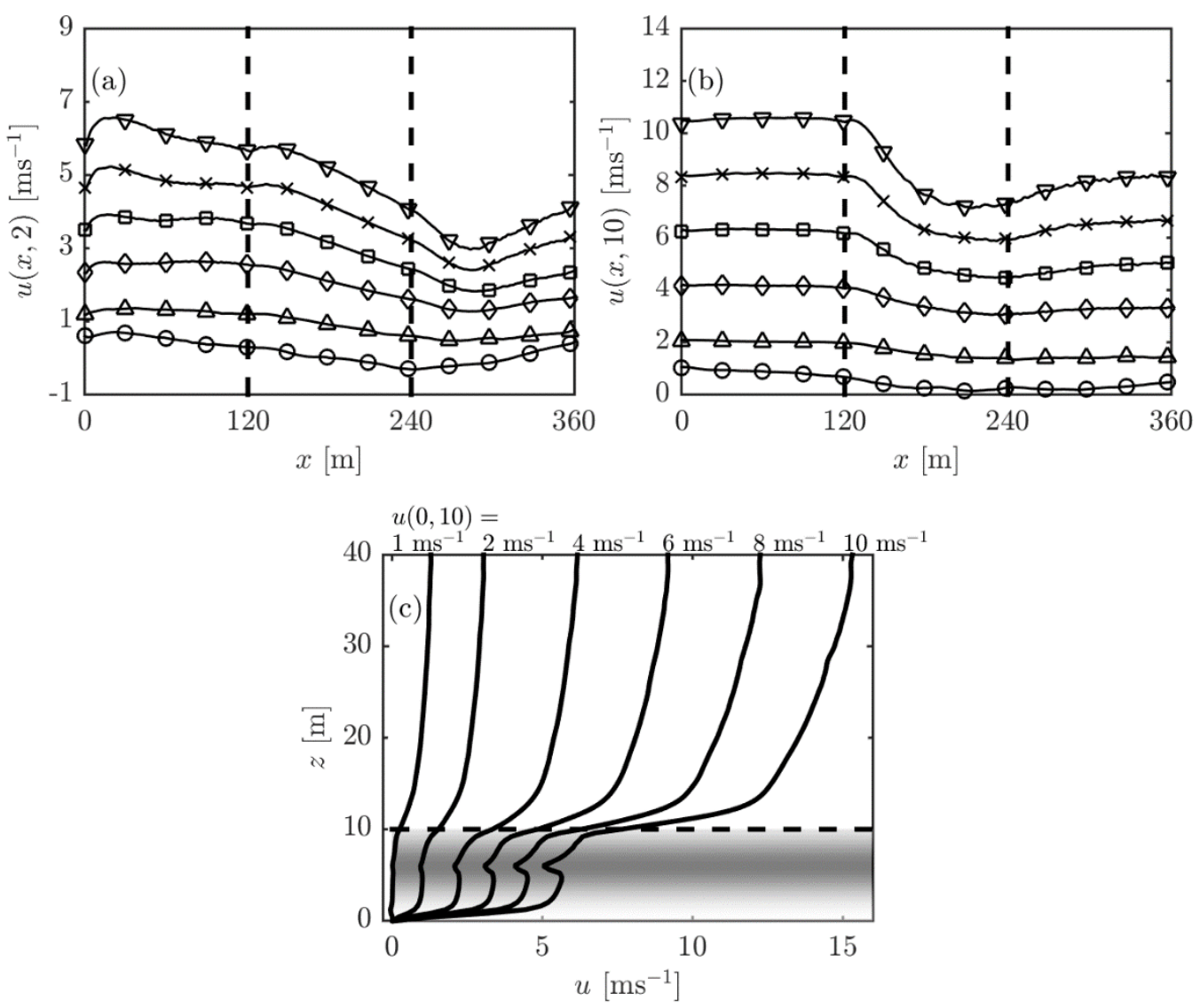

Figure 2 - (a) The $u(x, z)$ at $z=2 \mathrm{~m}(c)$ and $(b) u(x, z)$ at $z=10 \mathrm{~m}(c)$ u-velocities at $x=180 \mathrm{~m}$ all cases. The canopy edge is represented by the dotted line. The LAD profile is represented by the grey shading. Recall the height of the canopy is $10 \mathrm{~m}$ and the significant drop in velocity is due to the canopy. The minor differences between the actual and desired inlet velocity is due to the imposition of a turbulent boundary condition there.

\section{Simulated fire fronts and flame angle}

Because the fire maintains a straight front the results of the fire spread may be averaged across the domain. The contours of boundary temperature are shown in Figure 3 to visualise the fire spread. For $u(0,10)<4 \mathrm{~ms}^{-1}$ the propagation is largely unaffected by the presence of a canopy. In the higher driving velocity cases the rate-of-spread decreases suddenly near the downstream edge of the canopy. The $x$-distance of the transition point occurs later for higher driving wind speeds. The pyrolysis and drying regions narrow as the rate-of-spread of the fire decreases. The flame angle as a function of location is shown in Figure 4. For driving wind speeds $u(0,10)>2 \mathrm{~ms}^{-1}$ the flame angle undergoes a dramatic jump towards the downstream edge of the canopy and the flame angle also exhibits significant oscillations in the downstream region. In the highest driving wind speed case $u(0,10)=10 \mathrm{~ms}^{-1}$ the flame angle oscillates significantly over the whole domain. 


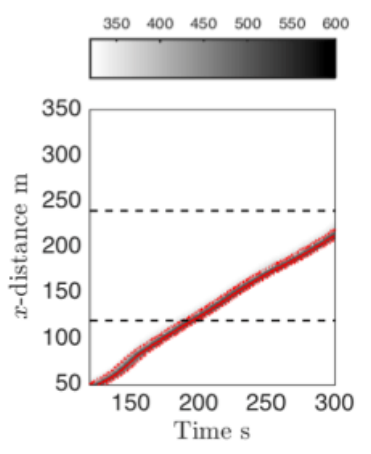

(a) $u_{10}(0)=1 \mathrm{~ms}^{-1}$

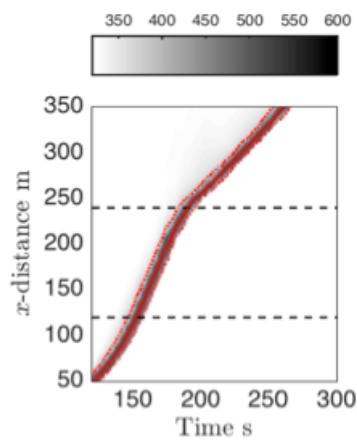

(d) $u_{10}(0)=6 \mathrm{~ms}^{-1}$

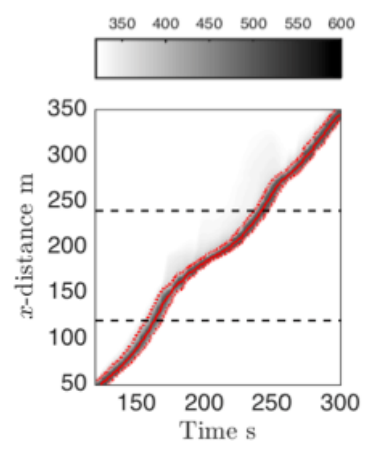

(b) $u_{10}(0)=2 \mathrm{~ms}^{-1}$

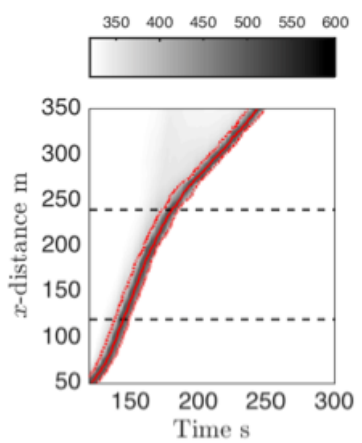

(e) $u_{10}(0)=8 \mathrm{~ms}^{-1}$

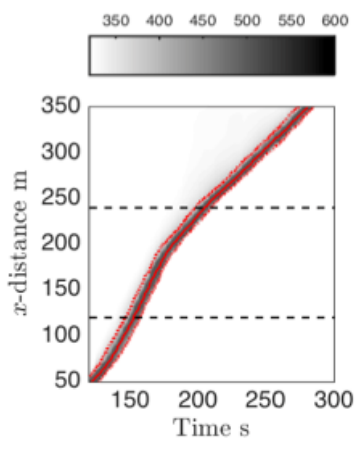

(c) $u_{10}(0)=4 \mathrm{~ms}^{-1}$

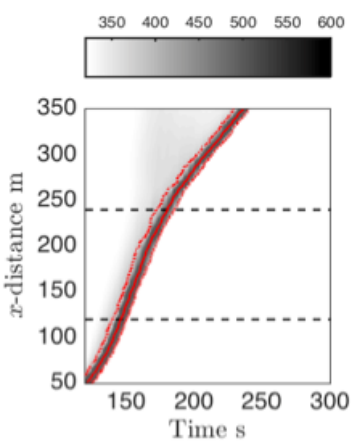

(f) $u_{10}(0)=10 \mathrm{~ms}^{-1}$

Figure 3 - Visualisations of the fire. Contours of boundary temperature (grey shading), the fire front location $x *$ (solid red), the drying region (thin red dotted), and the canopy region (horizontal thick black dashed). Pyrolysis occurs in the dark shaded region and preheating of the fuel bed is also apparent.
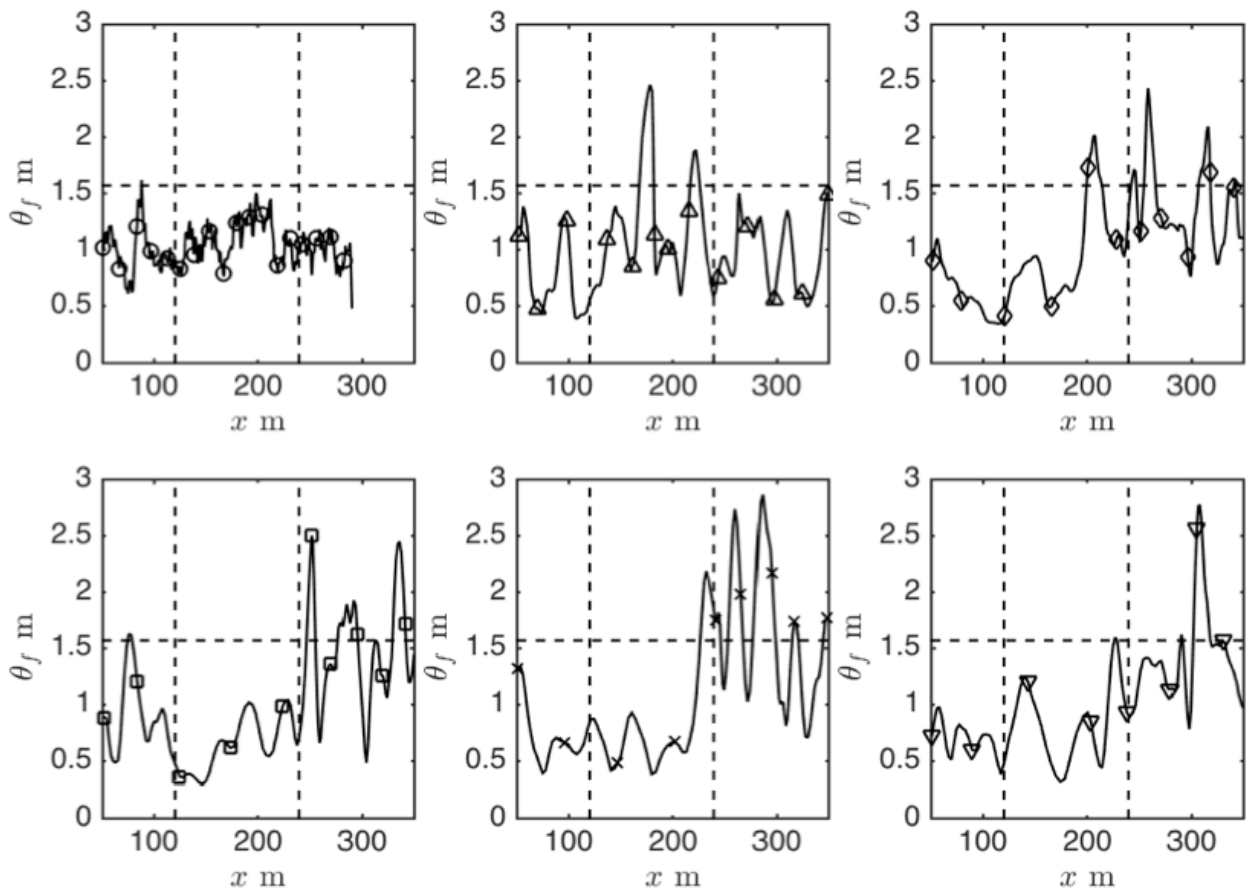

Figure 4 - Flame angle (radians) as a function of $x$-location for the cases as shown. The canopy boundaries are shown as vertical dashed lines and the horizontal line represents an angle of $90^{\circ}$. Symbols: $($ circles $) u(0,10)=1 \mathrm{~ms}$ ${ }^{1}$,(triangles) $2 \mathrm{~ms}^{-1}$,(diamonds) $4 \mathrm{~ms}^{-1}$, (squares) $6 \mathrm{~ms}^{-1}$, (crosses) $8 \mathrm{~ms}^{-1}$, (inverted triangles) $10 \mathrm{~ms}^{-1}$. 


\section{Concluding remarks}

For low driving wind speed, the fires are largely unaffected by the presence of the canopy. For higher driving wind speeds there appears to be a transition from a wind-driven mode to a buoyancy-driven model near the canopy exit. This transition is characterised by a jump in flame angle, decreased rate-of-spread and an increased flame angle variability. In future, it is desirable to simulate a considerably longer domain to gain further insight into the dynamics of the fire as it adjusts to the sub-canopy wind field.

\section{References}

Dold, J, 2011, Fire spread near the attached and separated flow transition, including surge and stall behaviour. In Proc. 19th Int. Congress on Modelling and Simulation, 200-206.

Jarrin, N, Benhamadouche, S, Laurence, D and Prosser, R, 2006. A synthetic-eddy-method for generating inflow conditions for large-eddy simulations. International Journal of Heat and Fluid Flow, 27(4), 585-593.

Kanani-Suhring, F and Raasch, S, 2017, Enhanced scalar concentrations and fluxes in the lee of forest patches: A large-eddy simulation study. Boundary-Layer Meteorology, 164(1), 1-17.

Kiefer, M.T., Heilman, W.E., Zhong, S., Charney, J.J. and Bian, X., 2015. Mean and turbulent flow downstream of a low-intensity fire: Influence of canopy and background atmospheric conditions. Journal of Applied Meteorology and Climatology, 54(1), 42-57.

McGrattan, K, Hostikka, S, and Floyd, JE, 2013, Fire dynamics simulator, users guide. NIST special publication, 1019.

Mell, W, Jenkins, MA, Gould, J and Cheney, P, 2007, A physics-based approach to modelling grassland fires. International Journal of Wildland Fire, 16, 1, 1-22.

Pavlidis, D, Gorman, GJ, Gomes, JL, Pain, CC and ApSimon, H, 2010. Synthetic-eddy method for urban atmospheric flow modelling. Boundary-layer Meteorology, 136(2), 285-299.

Seto, D, Strand, TM, Clements, CB, Thistle, H and Mickler, R, 2014. Wind and plume thermodynamic structures during low-intensity subcanopy fires. Agricultural and Forest Meteorology, 198, 53-61. 
Short contribution - Fire Management

\title{
Spontaneous ignition of vertically positioned wood samples under time-dependent heat flux
}

\author{
Alexander I. Filkov*12, Trent D. Penman ${ }^{1}$ \\ 1 *University of Melbourne. Creswick, Victoria Australia 3363, \{alexander.filkov@unimelb.edu.au*\} \\ ${ }^{2}$ Bushfire and Natural Hazards Cooperative Research Centre. Melbourne, Victoria Australia 3002, \\ \{office@bnhcrc.com.au\}
}

\begin{abstract}
Dynamic heating regimes are observed during structural and wildland fires. Most experiments to date use static/constant heating regimes, whereas ignition characteristics under time-dependent heat flux has been poorly studied. Existing apparatuses have limitations to study this effect, such as heating conditions and sample size/position. In this study, we conduct experiments on a custom-made apparatus to investigate the spontaneous ignition and convection cooling effect on this ignition of different vertically positioned wood species subjected to both static and dynamic heat fluxes. All experiments were conducted on cypress wood. Temperature, mass and ignition time were recorded during the experiment. Samples were exposed to 30 $\mathrm{kW} / \mathrm{m}^{2}$ static heat flux and increasing heat flux. Preliminary results showed that for static heat flux two out of four samples were ignited at $100 \mathrm{sec}$ and $78 \mathrm{sec}$ with mass consumption $9.5 \%$ and $8 \%$ respectively. Two other samples were smouldered intensively during the heating period without flaming ignition observed. Their consumed mass was 28 and $31 \%$ after 5 minutes. For increasing heat flux, ignition occurred at $743 \pm 7$ sec with mass consumption $20.7 \pm 0.8 \%$ for all four samples. Infrared video showed that flaming ignition for increasing heat flux started after the phase when the wood sample surface cracked into rectangular areas. This led to the heating of a deeper section of the sample, thus generating more pyrolysis gases, which was indirectly confirmed by the two times greater temperature at $3 \mathrm{~mm}$ depth for increasing heat flux. The next step of the proposed research will be to investigate the convective cooling on spontaneous ignition using various types of wood species. Different static and dynamic heating regimes will be used and their influence on the ignition process will be evaluated.
\end{abstract}

Keywords: spontaneous ignition, wood samples, time-dependent heat flux, ignition time

\section{Introduction}

Flame spread is an important parameter in the assessment of fire hazards. Ignition and combustion are dependent on the type of the heat flux, whether it is static or variable. The majority of previous research has used static heat flux, despite dynamic heating regimes being commonly observed during structural and wildland fires.

Dynamic fluxes have been tested (Peterson et al. 2015; Vermesi et al. 2016; Zhai et al. 2017) but these studies have generally been limited. Many only use one regime that is simulated to be either increasing, decreasing or parabolic. Most studies use a cone calorimeter or flame propagation apparatus, which have limitations, such as heating conditions and sample size/position (DiDomizio et al. 2016; Vermesi et al. 2016). Furthermore, the majority of the experiments are conducted for horizontally located samples, which are heated from above in contrast to "classical" fire where the flame propagates horizontally heating the fuel from the side. Chen et al. (2014) demonstrated that ignition time is strongly dependent on sample orientation.

To increase the realism of ignition experiments we experimentally test spontaneous ignition of vertically positioned wood samples subjected to both static and dynamic heat fluxes. 


\section{Methods}

The experiment was conducted using a custom-made Radiative Heat Flux Apparatus (Fig. 1).

The apparatus consists of: 1) radiative panel, 2) linear stage, 3) shutter and PC control system. The radiative panel contains infrared short wave quartz lamps producing radiative heat flux. The radiative panel was installed on a 1.5 m linear stage that allows the panel to move forward or backward, simulating variable heat flux. An infrared camera (FLIR T1050sc) was used to measure temperatures on the exposed surface with frequency $30 \mathrm{~Hz}$ and resolution $1024 \mathrm{x}$ 768. An DSLR camera (Canon EOS 600D) was used to film the experiment. Square cypress wood samples with a depth of $19 \mathrm{~mm}$ and width of $65 \mathrm{~mm}$ were used. Wood boards were cut into $65 \mathrm{~mm}$ square samples.

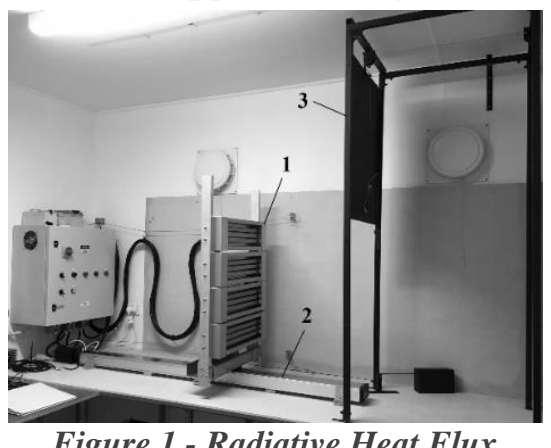

Figure 1 - Radiative Heat Flux Apparatus

One thermocouple was imbedded at a depth of $3 \mathrm{~mm}$ from the exposed surface, two thermocouples - $10 \mathrm{~mm}$ from the exposed surface and one on the back side of the sample. Samples were dried to a constant mass state using an oven at $104{ }^{\circ} \mathrm{C}$ for 48 hours (Kuznetsov and Fil'kov 2011). To avoid the influence of heterogenous wood surface properties (texture, colour etc.) on heat flux absorption, the exposed surface of the sample was coated with lampblack (Kuznetsov and Fil'kov 2011) (fig. 2a). To investigate the influence of convection cooling effect on the ignition time two sample holders were made. One with blocked sides and bottom (fig. $2 \mathrm{~b}$ ) and second without.

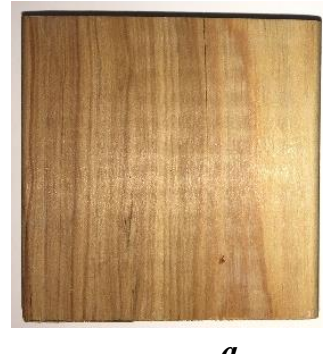

$a$

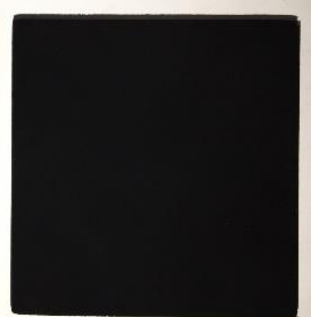

Figure 2 - a) Original (left) and blackened (right) sample; b) sample holder to block convection cooling

The sample holder was constructed of two layers of $7.5 \mathrm{~mm}$ thick cement board. To prevent the heat loss along the edges of the sample internal layer of $25 \mathrm{~mm}$ silica boards was used as an insulation. The entire sample holder was positioned on the scale. Five repetitions were conducted for each condition.

At the first stage, the samples were exposed to $30 \mathrm{~kW} / \mathrm{m}^{2}$ static heat flux during $5 \mathrm{~min}$ and increasing heat flux $12.5 \mathrm{~min}$ duration. The length of time for the increasing regime was chosen to approximate the integral of the heating function. For the dynamic regime, heat flux was increased by moving the radiative panel with a constant speed of $0.4 \mathrm{~mm} / \mathrm{sec}$.

\section{Results and discussion}

Preliminary results showed that for static heat flux $30 \mathrm{~kW} / \mathrm{m}^{2}$, two out of four samples were ignited at $100 \mathrm{sec}$ and $78 \mathrm{sec}$ with mass consumption of $9.5 \%$ and $8 \%$ respectively. Two other samples smouldered intensively during the heating period without flaming ignition. Their consumed mass was $28 \%$ and $31 \%$ after 5 minutes. For increasing heat flux all four samples were ignited at $743 \pm 7 \mathrm{sec}$ with mass consumption of $20.7 \pm 0.8 \%$. Infrared video showed that flaming ignition for increasing heat flux started after the phase when the wood sample surface cracked into rectangular areas. This led to the heating of a deeper section of the sample, thus generating more pyrolysis gases, which was indirectly confirmed by the two times greater temperature at $3 \mathrm{~mm}$ depth for increasing heat flux. For 
static heat flux, the average temperature at the time of ignition was $112{ }^{\circ} \mathrm{C}$ and for the increasing regime it was $219^{\circ} \mathrm{C}$.

The next step of the proposed research will be to investigate the effects of convective cooling and wood type on spontaneous ignition. Different static and dynamic heating regimes will be used and their influence on the ignition will be evaluated. Further investigation is also required to determine why some samples were not ignited in flaming mode under considered static heat flux.

\section{References}

Chen X, Zhou Z, Li P, Zhou D, Wang J (2014) Effects of sample orientation on pyrolysis and piloted ignition of wood. Journal of Fire Sciences 32, 483-497. doi:10.1177/0734904114534612.

DiDomizio MJ, Mulherin P, Weckman EJ (2016) Ignition of wood under time-varying radiant exposures. Fire Safety Journal 82, 131-144. doi:10.1016/j.firesaf.2016.02.002.

Kuznetsov VT, Fil'kov AI (2011) Ignition of various wood species by radiant energy. Combustion, Explosion and Shock Waves 47,. doi:10.1134/S0010508211010096.

Peterson DA, Hyer EJ, Campbell JR, D. FM, Hair JW, Butler CF, Fenn MA (2015) The 2013 Rim Fire: Implications for Predicting Extreme Fire Spread, Pyroconvection, and Smoke Emissions. Bulletin of the American Meteorological Society 96, 229-247. doi:10.1175/BAMS-D-14-00060.1.

Vermesi I, Roenner N, Pironi P, Hadden RM, Rein G (2016) Pyrolysis and ignition of a polymer by transient irradiation. Combustion and Flame 163, 31-41. doi:10.1016/j.combustflame.2015.08.006.

Zhai C, Gong J, Zhou X, Peng F, Yang L (2017) Pyrolysis and spontaneous ignition of wood under time-dependent heat flux. Journal of Analytical and Applied Pyrolysis 125, 100-108. doi:10.1016/j.jaap.2017.04.013. 
Short contribution - Fire Management

\title{
Toward safer firefighting strategies and tactics
}

S. Lahaye ${ }^{1,2 *} ;$ J. Sharples ${ }^{1}$, C. Hély ${ }^{2}$, T. Curt $^{3}$

${ }^{1}$ School of Physical, Environmental and Mathematical Sciences, University of New South Wales Canberra, ACT 2600, Australia. \{sebastien.lahaye@safecluster.com*\}

${ }^{2}$ Institut des Sciences de l'Evolution de Montpellier (ISEM), EPHE, PSL Research University, CNRS, Université de Montpellier, IRD, Place Eugène Bataillon, CC 065, 34095 Montpellier, France.

${ }^{3}$ Irstea EMAX Mediterranean Ecosystems and Risks, route Cézanne, 13182, Aix-en-Provence cedex 5, France.

\begin{abstract}
Adverse weather conditions and fuel accumulation may induce destructive fires. Therefore firefighters are highly exposed to fire entrapments, i.e. to be caught by the fire heat or smoke.

As there are several types of fires, firefighters face different types of mitigation difficulties. They also face different situations potentially leading to entrapment. We suggest here ways to implement safer mitigation practises at both strategical and tactical levels.

We analysed 103 firefighters' entrapments described in both publications and internal reports from fire agencies across Europe, Australasia and North-America. We distinguished three types of fire (i.e. topography-influenced, wind-driven and convective-dominated fires) and we assessed the potential contributions of dynamic fire behaviours in entrapments for each fire type. The dynamic fire behaviours investigated are changes in wind direction, steep slopes prone to flame attachment and leeward slopes prone to vorticity-driven lateral fire spread.
\end{abstract}

Our results show that $94 \%$ of the entrapments in topography-influenced fires have happened on steep slopes prone to flame attachment. We also point out that slopes prone to vorticity-driven lateral fire spread have been the most prominent factor associated with entrapments during wind-driven fires. Finally, in convective-dominated fires, many configurations can lead to entrapments, including those without any obvious dangerous fire behaviour characteristics.

Whereas our study took months to identify risks associated to each fire type, firefighter commanders have to take mitigation strategic decisions within few minutes. On the field, crews have an even shorter decision time to choose safe and efficient tactical options.

This is why dissemination of the fire type concept and consequent safety issues should be declined in several levels and time lags within fire agencies.

At the top level of scientific understanding, fire behaviour analysts should be able to forecast the expected fire type in relation to weather, fuel and topographic inputs. They should both produce maps of dangerous areas, trigger points, key points, and assess the expected efficiency and danger from different strategic options.

Incident commanders, division commanders and other command staff should be aware of the general behaviour of a fire regarding its type. They should also be informed of the associated areas at risk and the most efficient strategies to fight the fire regarding its type.

Finally, crews should be trained to recognise risky zones on the ground and to egress rapidly such zones before dangerous conditions get aligned.

Keywords: entrapment, fire behaviour analyst (FBAN), fire type, flame attachment, vorticity-driven lateral spread (VLS). 


\section{Introduction}

In wide areas of Northern America, Southern Australia or Mediterranean Europe, adverse weather conditions and fuel accumulation may induce very large and destructive fires. Firefighters have developed massive initial attack strategies to protect endangered population and assets as a priority. Therefore, they can become themselves highly exposed to fire entrapments, i.e. to be caught by the fire heat or smoke during their intervention.

The fire type concept (Costa et al. 2011) distinguishes several types of fires, depending on the main driver of the fire propagation, which can be the topography, the wind or the fuel. Therefore firefighters face different types of mitigation difficulties (Lahaye et al. 2014). They also face different risks of entrapment (Page and Butler 2017; Lahaye et al. 2018a).

We investigated more than 100 firefighters' entrapments across the world and we determined both the type of fire and the likely contribution of dynamic fire behaviours. Considering the results, we suggest here ways to implement safer mitigation practises at both strategical and tactical levels.

\section{Fire types and associated risks}

\subsection{Investigation of 103 entrapments}

We analysed 43 firefighters' entrapments in Australia mentioned by Lahaye et al. (2018a) and 48 entrapments in France described by Lahaye et al. (2018b). Most the cases were extracted from fire agencies' unpublished internal reports. We added to this set one entrapment in New Zealand (Pearce et al. 2004), one in Portugal (Viegas et al. 2009), one in Croatia (Viegas et al. 2009), two in Greece (Xanthopoulos 2007) and five in Spain, reported in Viegas et al. (2009), Zamora (2007) and in unpublished reports. Finally, we extended the study to two fully documented cases in the USA: Mann Gulch (Maclean 1992) and South Canyon (Butler et al. 1998). In total, we analysed 103 incidents distributed across three continents (Fig. 1). All of them correspond to fires suddenly exhibiting high rates of spread and intensity.

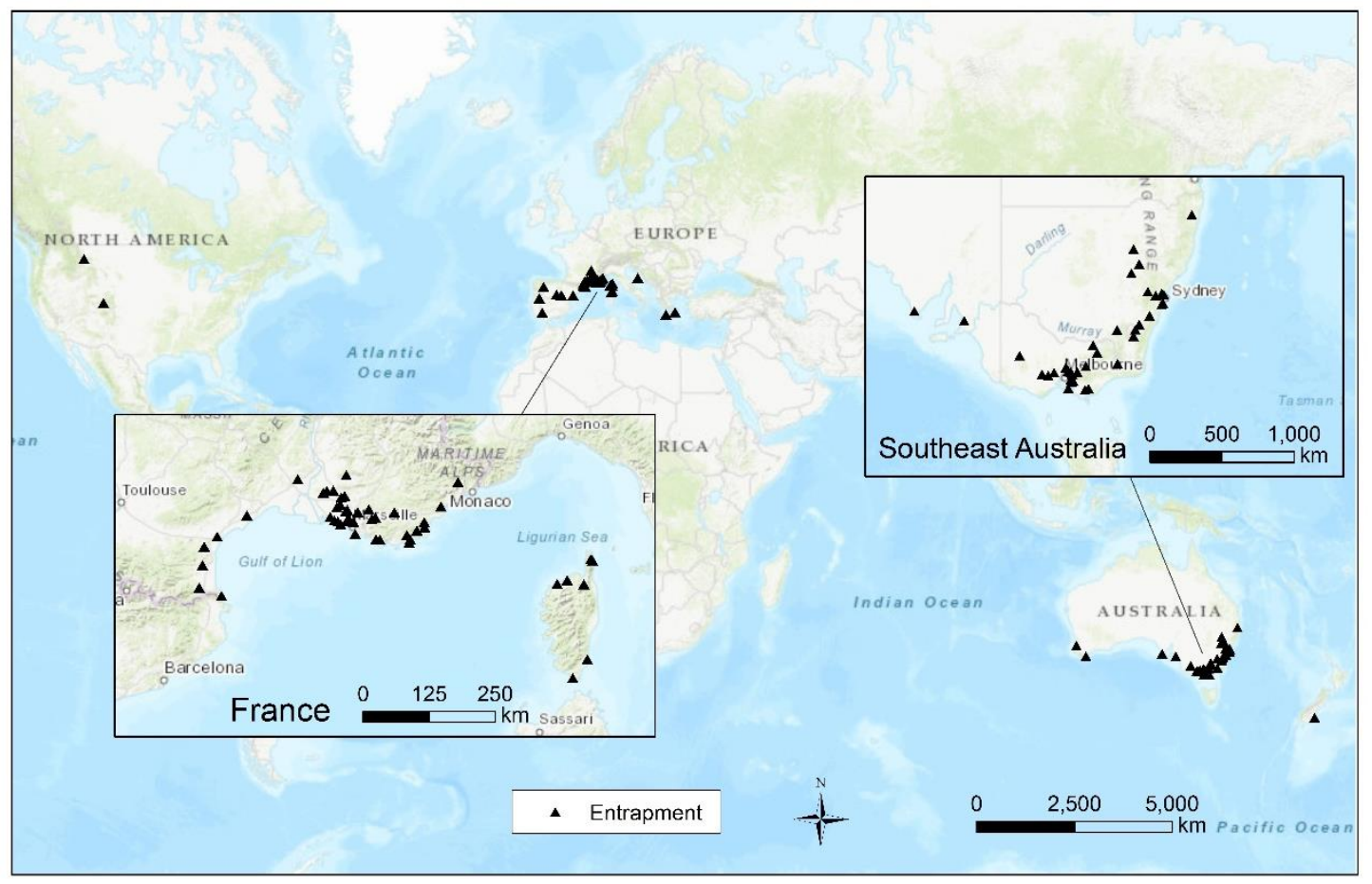

Figure 1 - Location of the entrapments investigated in this study, not representative of the global distribution of incidents. 
Several authors investigated the types of fires (Byram 1959; Rothermel 1991; Morvan 2010) and Costa et al. (2011) proposed a classification of three types: topography-influenced, wind-driven and convective-dominated. Costa et al. (2011) did not provide threshold criteria for these three types of fires but they described their environment and their behaviour so that we could assigned each of the 103 entrapping fires of our set to one of the types.

For each entrapment, we assessed the potential contributions of configurations conducive to dynamic fire, namely changes in wind direction exceeding $45^{\circ}$, upwind slopes steeper than $20^{\circ}$ prone to flame attachment, and leeward slopes prone to vorticity-driven lateral fire spread (VLS) as defined by Sharples et al. (2012). VLS prone landscapes are lee-facing slopes over $20^{\circ}$ with wind speed over $20 \mathrm{~km} \mathrm{~h}^{-1}$ (Simpson et al. 2016). VLS can induce massive spotting likely to propagate the fire over long distances (Fig. 2).

\subsection{Results}

$16 \%$ of the entrapments studied here happened during topography-influenced fires, $55 \%$ during wind-driven fires and $28 \%$ during convective-dominated fires (Table 1).

Table 1 - Distribution of firefighters' entrapments, deaths and injuries regarding the fire types.

\begin{tabular}{lccc}
\hline \multicolumn{1}{c}{ Fire type } & Entrapments & Deaths & Injuries \\
\hline Topography-influenced & 17 & 62 & 29 \\
Wind-driven & 57 & 48 & 71 \\
Convective-dominated & 29 & 34 & 27 \\
\hline
\end{tabular}

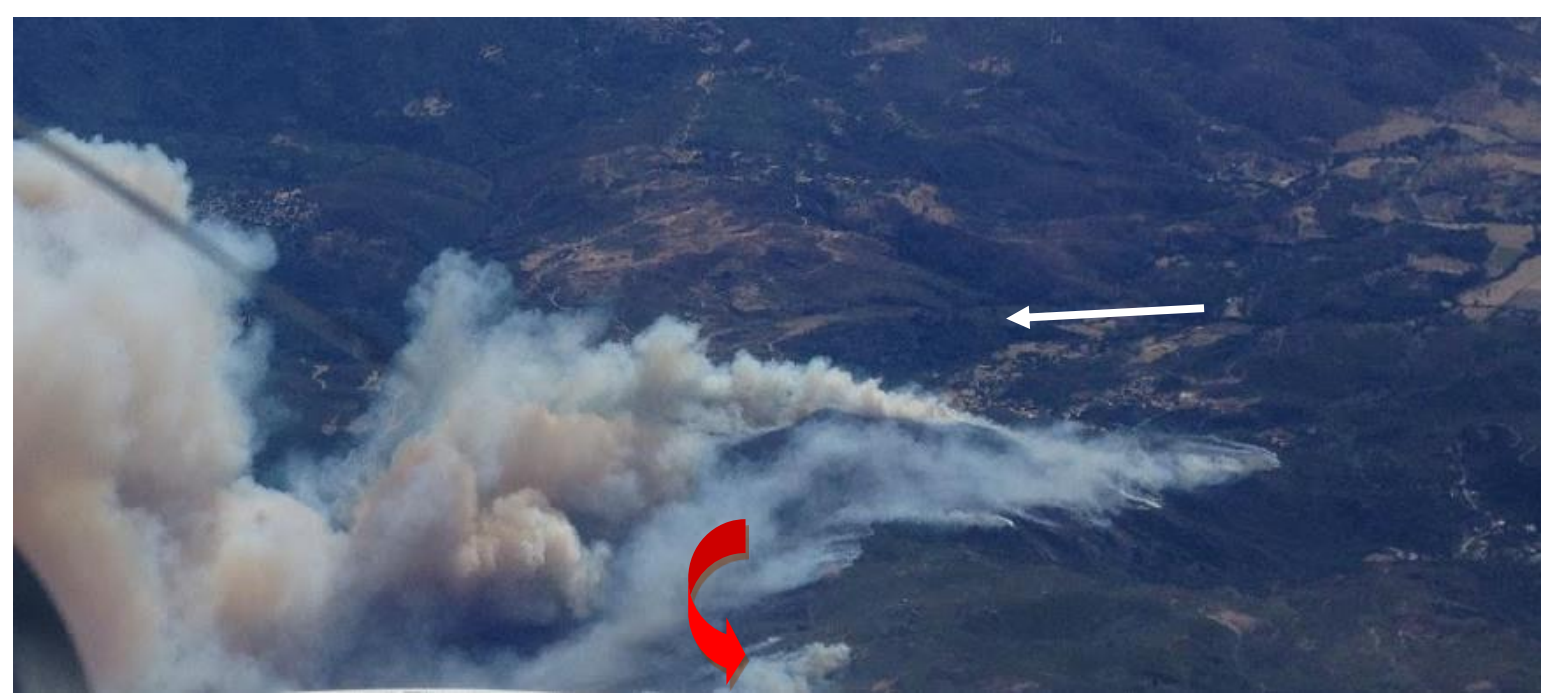

Figure 2 - A case of vorticity-driven lateral spread (VLS) in Corsica, France, 2017. The white arrow figures the main wind direction and the red arrow shows VLS. (Photo: P. Schaller)

Our results show that $94 \%$ of the entrapments in topography-influenced fires occurred on steep slopes prone to flame attachment (Table 2). We also point out that slopes prone to VLS were the most prominent factor associated with entrapments during wind-driven fires. Finally, in convectivedominated fires, many configurations can lead to entrapments, including those without any obvious dangerous or changing fire behaviour characteristics. 
Table 2 - Presence of dynamic fire configurations in entrapments regarding the type of fire. 'None' indicates entrapments where none of the investigated configurations were present.

\begin{tabular}{lcccc}
\hline \multicolumn{1}{c}{ Fire type } & $\begin{array}{c}\text { Wind } \\
\text { shift }\end{array}$ & $\begin{array}{c}\text { Flame } \\
\text { attachment }\end{array}$ & VLS & None \\
\hline Topography-influenced & $29 \%$ & $94 \%$ & 0 & $6 \%$ \\
Wind-driven & $10 \%$ & $32 \%$ & $42 \%$ & $30 \%$ \\
Convective-dominated & $38 \%$ & $24 \%$ & $24 \%$ & $48 \%$ \\
Total & $21 \%$ & $40 \%$ & $30 \%$ & $31 \%$ \\
\hline
\end{tabular}

\section{Discussion}

Whereas this study took months to identify risks associated to each type of fires, firefighter commanders have to take mitigation strategic decisions within few minutes. On the field, crews have an even shorter decision time to choose safe and efficient tactical options. This is why the fire type concept and consequent safety issues should be integrated at different stages of the fire agencies' decision process: before the fires (land management, training, and preparedness), during the fires and after the fires (lessons learned) (Fig.3).

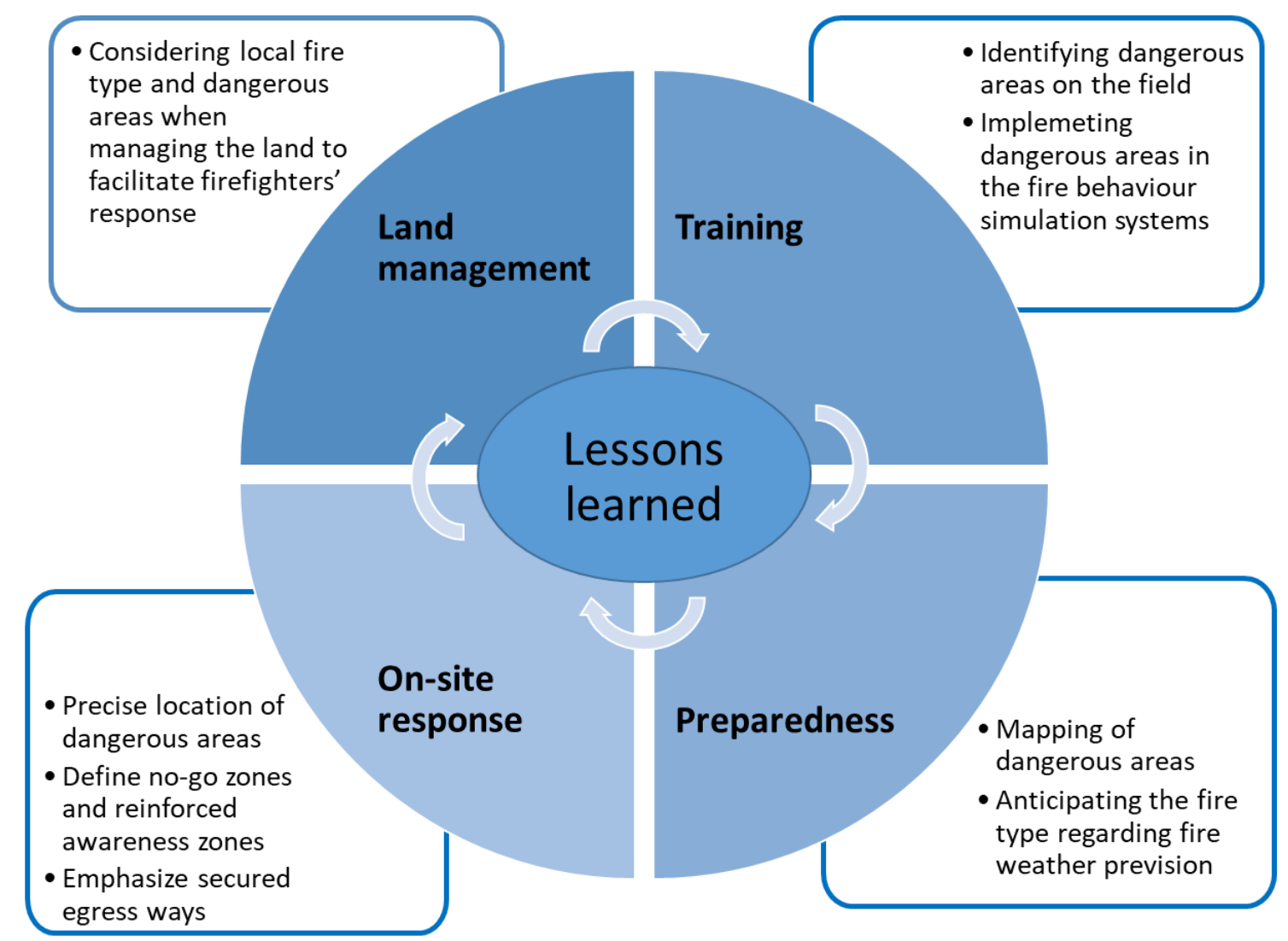

Figure 3 - Concept flow for integrating fire types and safety issues in fire agencies' decision process.

The results of this study should also be disseminated through several levels of decision-making to support the integration in the different activities of the fire agencies.

At the top level of scientific understanding, fire behaviour analysts (FBAN) should be able to forecast the type of fire regarding weather, fuel and topographic inputs. They may also produce maps of dangerous areas, trigger points, key points and assess the expected efficiency and danger of different strategic options. Whereas North American FBAN have developed knowledges and competencies for 
decades (Hardy and Hardy 2007), the situation in Australia and in Europe is more diverse. In Australia, fire agencies in the south-eastern states started to develop FBAN trainings in the late 2000's (Gibos et al. 2014) and then rapidly raised the capability. In Europe, although Molina et al. (2010) identified the key role of FBAN, few agencies have empowered them yet, with the exception of the Catalonian Fire Service (Otero and Nielsen 2017).

Besides of FBAN with strong scientific knowledges and capabilities to predict wind shifts, flame attachment, VLS or other dynamic behaviours, incident commanders, division commanders and other command staff should be aware of the general behaviour of a fire regarding its type. They should also be informed of the associated dangerous areas and the most efficient strategies to fight the fire regarding its type.

Finally, crews should be trained to recognise risky zones on the ground and to egress rapidly such zones before dangerous conditions get aligned. Standard operative procedures acknowledge these dangerous conditions in north America (National Wildfire Coordinating Group 2014) and in Australia (New South Wales Rural Fire Service 2010) but do not differentiate the types of fires. Integrating the types of fires in the procedures may make them more effective. In Europe, especially in France, agencies have focused much on protecting equipment, e.g. trucks and clothing, than on fire behaviour understanding. However, consistent European training systems and facilities may facilitate dissemination of basic procedures to avoid dangerous zones and to egress from them before it is too late.

\section{References}

Butler BW, Bartlette RA, Bradshaw LS, Cohen JD, Andrews PL, Putnam T, Mangan RJ (1998) Fire behavior associated with the 1994 South Canyon Fire on Storm King Mountain, Colorado. USDA Forest Service, Research Paper RMRS-RP-9 (Fort Collins, CO, USA)

Byram GM (1959) Combustion of forest fuels. In 'Forest fire: control and use'.(Ed. KP Davis). pp. 61-89. (New York)

Costa P, Castellnou M, Larranaga A, Miralles M, Kraus D (2011) Prevention of large wildfires using the fire types concept. Generalitat de Catalunya, Fire Paradox European Project (Barcelona, Spain) www.efi.int/files/attachments/publications/handbook-prevention-large-fires_en.pdf.

Gibos K, Slijepcevic A, Wells T, Fogarty L (2014) Building Fire Behavior Analyst (FBAN) Capability and Capacity: Lessons Learned From Victoria, Australia's Bushfire Behavior Predictive Services Strategy. InKeane R, Jolly M, Parson R, Riley K (eds) Missoula, MT, USA. 91. (USDA Forest Service: Missoula, MT, USA)

Hardy CC, Hardy CE (2007) Fire danger rating in the United States of America: an evolution since 1916. International Journal of Wildland Fire 16, 217-231.

Lahaye S, Curt T, Frejaville T, Sharples J, Paradis L, Hely C (2018b) What are the drivers of dangerous fires in Mediterranean France? International Journal of Wildland Fire 27, 155-163. doi:10.1071/WF17087.

Lahaye S, Curt T, Paradis L, Hely C (2014) Classification of large wildfires in South-Eastern France to adapt suppression strategies. InViegas DX (ed) 'Proceedings of 7 th International Conference on Forest Fire Research 17-20 Nov 2014', Coimbra, Portugal. 696-708. (ADAI: Coimbra, Portugal) doi:10.4195/978-989-26-0884-6.

Lahaye S, Sharples J, Matthews S, Heemstra S, Price O, Badlan R (2018a) How do weather and terrain contribute to firefighter entrapments in Australia? International Journal of Wildland Fire 27, 8598. doi:10.1071/WF17114.

Maclean N (1992) 'Young men and fire.' (University of Chicago Press: Chicago, USA) https://search.library.wisc.edu/catalog/999694827902121. 
Molina D, Castellnou M, Garcia-Marco D, Salgueiro A (2010) Improving fire management success through fire behaviour specialists. 'Integrated Fire Management - Outcomes of European Project Fire Paradox'. (Eds JS Silva, FC Rego, P Fernandes, E Rigolot) pp. 105-119. (European Forest Institute: Joensuu, Finland)

Morvan D (2010) Physical Phenomena and Length Scales Governing the Behaviour of Wildfires: A Case for Physical Modelling. Fire Technology 47, 437-460.

National Wildfire Coordinating Group (2014) 'Incident Response Pocket Guide.' (National Wildfire Coordinating Group. PMS 461 NFES 1077: Boise, ID, USA)

New South Wales Rural Fire Service (2010) 'Firefighters'pocket book.' (New South Wales Rural Fire Service V.1.3: Sydney, Australia)

Otero I, Nielsen JØ (2017) Coexisting with wildfire? Achievements and challenges for a radical socialecological transformation in Catalonia (Spain). Geoforum 85, 234-246. doi:10.1016/j.geoforum.2017.07.020.

Page WG, Butler BW (2017) An empirically based approach to defining wildland firefighter safety and survival zone separation distances. International Journal of Wildland Fire 26, 655-667. doi:10.1071/WF16213.

Pearce HG, Hamilton RW, Millman RI (2004) Fire behaviour and firefighter safety implications associated with the Bucklands Crossing Fire burnover of 24 March 1998. Forest Research, Rotorua, in association with the New Zealand Fire Service Commission and the National Rural Fire Authority, Forest and Rural Fire Scientific and Technical Series 4. (Wellington, NZ)

Rothermel RC (1991) Predicting behavior and size of crown fires in the northern Rocky Mountains. U.S. Department of Agriculture, Forest Service, Intermountain Research Station. Research Paper INT-RP-438, (Ogden, UT, USA)

Sharples J, McRae RHD, Wilkes SR (2012) Wind-terrain effects on the propagation of wildfires in rugged terrain: fire channelling. International Journal of Wildland Fire 21, 282-296. doi:10.1071/WF10055.

Simpson CC, Sharples JJ, Evans JP (2016) Sensitivity of atypical lateral fire spread to wind and slope. Geophysical Research Letters 43, 1744-1751. doi:10.1002/2015GL067343.

Viegas DX, Simeoni A, Xanthopoulos G, Rossa C, Ribeiro L, Pita L, Stipanicev D, Zinoviev A, Weber R, Dold J, Caballero D (2009) 'Recent Forest Fire Related Accidents in Europe.' (European Commission: Luxembourg) www.forest.jrc.ec.europa.eu/media/cms.../recent-forest-fire-relatedaccidents-in-europe.pdf.

Xanthopoulos G (2007) Forest fire related deaths in Greece: confirming what we already know. In '4th International Wildland Fire Conference 13-17 May 2007, Seville, Spain'. (Seville, Spain). https://www.researchgate.net/publication/242307152_Forest_fire_related_deaths_in_Greece_conf irming_what_we_already_know_1.

Zamora FC (2007) Guía técnica para la investigación de accidentes en la extinción de incendios forestales. Gobierno de Espana, Ministerio de medio ambiente, (Madrid, Spain) http://www.mapama.gob.es/es/desarrollo-rural/temas/politicaforestal/8.Guia_tecnica_para_la_investigaci\%C3\%B3n_de_accidentes_tcm7-336275.pdf. 
Short contribution - Fire Management

Towards more resilient and productive forest landscapes: tradeoffs in a recently burned area in Portugal

Akli Benali ${ }^{1}$; Susana Barreiro ${ }^{1}$; Ana Sá ${ }^{1}$; João Rua ${ }^{1}$; Yannick Le Page ${ }^{1}$; João Pinho ${ }^{2}$; Paulo Fernandes ${ }^{3}$; Silvia Nunes ${ }^{4}$; Miguel Mota Pinto ${ }^{4}$; Ricardo Trigo ${ }^{4}$; Carlos da Camara ${ }^{4}$; José M. C. Cardoso Pereira ${ }^{1}$

${ }^{1}$ Centro de Estudos Florestais, Universidade de Lisboa. Tapada da Ajuda, Lisboa (Portugal) \{aklibenali@gmail.com*\}

${ }^{2}$ Instituto da Conservação Natureza e das Florestas. Avenida da República, 16, Lisboa (Portugal)

${ }^{3}$ Centro de Investigação e Tecnologias Agroambientais e Biológicas - Universidade de Trás-os-Montes e Alto Douro. Quinta de Prados, 5001-801 Vila Real (Portugal)

${ }^{4}$ Instituto Dom Luiz, Universidade de Lisboa. Campo Grande, Ed. C1, Piso 1, Lisboa (Portugal)

Keywords: fire danger, forest planning, wood production, modelling

\section{Introduction}

On the $17^{\text {th }}$ of June of 2017 , two very large wildfires struck the centre of Portugal, burning over 47000 ha, causing 64 fatalities and a large amount of economic losses. The Alvares parish was largely affected by one of those wildfires, adding up to its large fire incidence over the last 40 years. Historically, this parish is part of a strong wood production area that has suffered significant depopulation and land abandonment since the middle of the $20^{\text {th }}$ century.

Within the context of the ongoing trend towards a drier and warmer climate in Iberia, it is necessary to consider a more resilient, yet productive, forest landscape that minimizes the negative impacts of wildfires and promotes economic revenue, crucial to avoid further land abandonment. Our objective is to assess the impact of specific fire and fuel management strategies at the landscape-level on potential changes in future landscape fire danger and wood production.

\section{Data \& Methods}

\subsection{Study Area}

Alvares parish, part of Góis municipality, was struck by a large wildfire in June 2017 that burned around $70 \%$ of its total area (10800 ha). Prior to the fire, $89 \%$ of the parish was forested, mainly with eucalypt (53\%) and maritime pine (30\%). Almost all of the forest is privately owned, the exception being a small area of municipal and communal lands managed by the National Forest Service. We extended the study region to a 55 by $55 \mathrm{~km}$ area centred in the parish to account for wildfire transmission.

\subsection{Fire Danger}

The fire danger arising from very large wildfires (>1000 ha) was estimated for the study region using the FARSITE fire modelling system. Associated weather conditions at the synoptic and mesoscale were simulated using the WRF model. Fuels were defined by reclassifying the 1990 and 2010 land cover maps into the Portuguese fuel model typology. Reported ignitions were used to derive a probability surface.

Fire danger was estimated running a large number of fire spread simulations (25000) that randomly combined weather, fuel and ignition location. The simulations were combined to create burn probability maps. Model calibration was performed by comparing outputs with the observed wildfire incidence since 1980, and tuning the adjustment factors accordingly. 


\subsection{Wood Production}

The first step was to engage private stakeholders in the characterization of general forest management approaches (FMAs) in the parish area, especially those related to eucalypt silviculture systems. Three types of manager were defined:

a) Professional owners have intensive sustainable management and carry out proper site establishment, use genetically improved material, fertilize, and perform intensive fuel and pest control operations (20\% of total area);

b) Semi-professional owners focus on site establishment operations, have no access to genetically improved material, carry out less fertilization and fuel control operations (30\%);

c) Near-absent forest owners that simply focus on stand regeneration operations and usually anticipate harvest (50\%).

A set of FMAs was defined for each forest owner type. StandsSIM.md was used to simulate the growth of eucalypt for a planning horizon of 90 years producing estimates of harvested volume.

Future forest and land use scenarios

Different scenarios regarding the future spatial organization of the landscape (involving land use and forest cover changes) and silviculture practices were defined and its impacts on fire danger and wood production were simulated and analysed. In practice, the different scenarios affect the the distribution of fuels acros the landscape.

The scenarios are described as follows:

i) The landscape/management is equivalent to the one existing prior to the 2017 fire (i.e the "business as usual" scenario - BAU);

ii) An extended network of fuel breaks is implemented, contributing to the increase of non-wooded open areas;

iii) Additionally to ii), an intensification in forest management in eucalypt stands.

The distribution of the fuels over Alvares was assigned to each scenario based on expert knowledge, information provided by stakeholders, and the land cover prior to the 2017 fire. The impact of such scenarios was assessed by evaluating changes in burn probability and harvested wood volume for the entire parish.

\section{Results}

The burn probability maps showed an overall good agreement with observed data

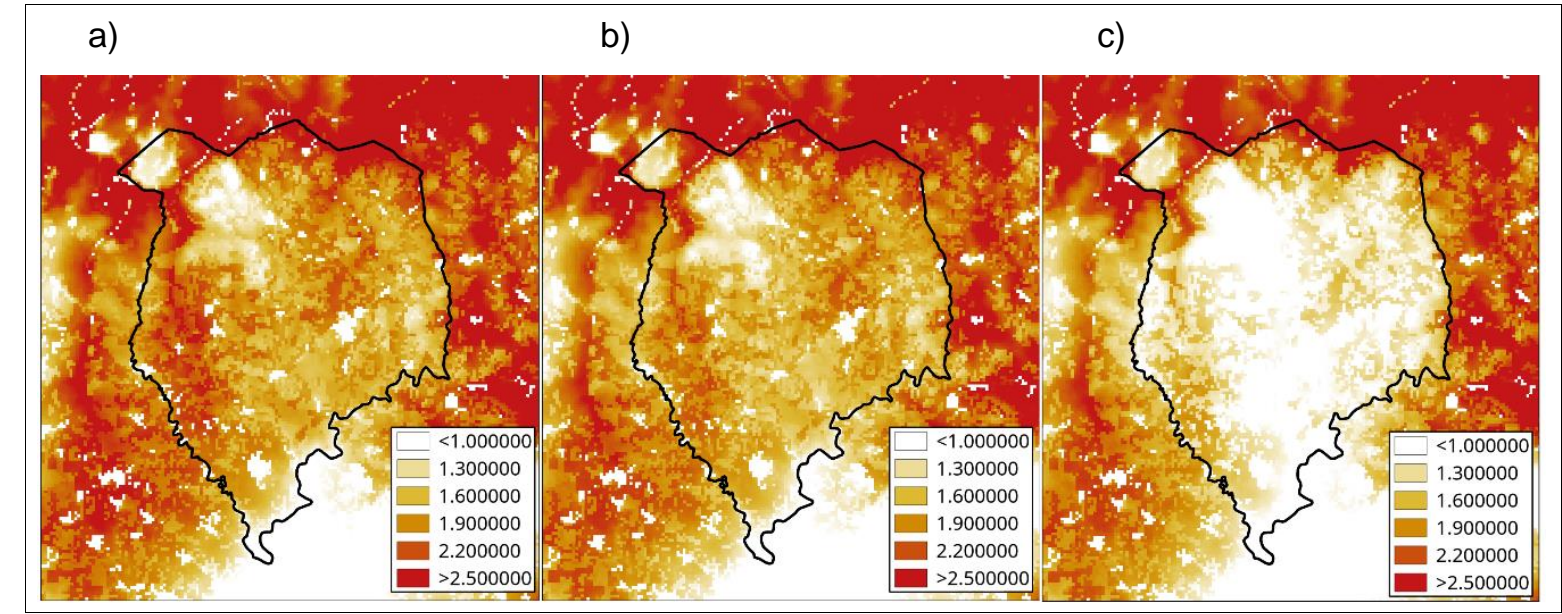

Figure 1 - Burn probability for a) Business as usual, b) Extended Fuelbreak network and c) Intensified forest management 
Results suggest that the implementation of an extended fuel break network in Alvares leads to a slight decrease in the burn probability $(\sim 10 \%)$, when compared with the BAU scenario (Fig1a,b). On the other hand, the intensification of forest management decreases burn probability by about $30 \%$ (Fig1a,c). The changes are spatially homogeneous at the parish level due to the density of the fuel break network and the large forest coverage.

The estimated harvested eucalypt volume in the BAU scenario is $1295 \times 10^{3} \mathrm{~m}^{3}$ for the 90 years horizon (Table 1). With the implementation of the fuelbreak network, eucalypt area is reduced c.a. $25 \%$, leading to an equivalent decrease in harvested volume. However, with the intensification of forest management in a small area, the reduction in harvested volume can be compensated and match 'business as usual' values.

Table 1 - Simulated Harvested wood volumes for a) Business as usual, b) Extended Fuelbreak network and c) Intensified forest management

\begin{tabular}{|c|c|c|c|c|c|c|}
\hline Scenario & \multicolumn{2}{|c|}{ Business as Usual } & \multicolumn{2}{|c|}{ Fuelbreak network } & \multicolumn{2}{|c|}{ Intensified management } \\
\hline $\begin{array}{l}\text { Type of } \\
\text { Management }\end{array}$ & $\begin{array}{l}\text { Area } \\
\text { (ha) }\end{array}$ & $\begin{array}{c}\text { Harvested } \\
\text { volume }\left(10^{3} \mathrm{~m}^{3}\right)\end{array}$ & $\begin{array}{c}\text { Area } \\
\text { (ha) }\end{array}$ & $\begin{array}{c}\text { Harvested } \\
\text { volume }\left(10^{3} \mathrm{~m}^{3}\right)\end{array}$ & $\begin{array}{l}\text { Area } \\
\text { (ha) }\end{array}$ & $\begin{array}{c}\text { Harvested } \\
\text { volume }\left(10^{3} \mathrm{~m}^{3}\right)\end{array}$ \\
\hline Professional & 1120 & 473 & 800 & 338 & 800 & 338 \\
\hline Semi-Professional & 1680 & 484 & 1200 & 346 & 3200 & 922 \\
\hline Near- Absent & 2800 & 499 & 2000 & 356 & 0 & 0 \\
\hline Total & 5300 & 1295 & 4000 & 1040 & 4000 & 1260 \\
\hline
\end{tabular}

\section{Preliminary Conclusions \& On-Going Work}

A relevant change in the landscape and associated economy is required to decrease the probability of occurrence of large wildfires. Preliminary results show that the generalized increase in forest and fuel management standards can significantly decrease fire danger and stimulate the wood-based economy, leading to a more resilient, safer and still productive landscape. Work is on-going to improve the analysis of the relation between landscape resilience and economic potential, namely:

- The extension of the wood production estimates to other tree species plantations;

- Integration of the costs of implementing and maintaining fuel management areas;

- Coupling the wood production estimates with fire danger estimates;

- Suggesting wood-production zoning and conservation areas and understanding their impacts on future fire danger and harvested volume.

\section{Acknowledgments}

We acknowledge the "Observador" journal for sponsoring the project. 
Short contribution - Fire Management

\title{
Vegetation fire management in strict nature reserves
}

\author{
Alexandra V. Volokitina ${ }^{1}$; Tatiana M. Sofronova ${ }^{2 *}$, Mikhail A. Korets ${ }^{1}$ \\ ${ }^{1}$ Sukachev Institute of Forest SB RAS, ul.Akademgorodok,50/28, Krasnoyarsk 660036, \\ Russia,\{volokit@ksc.krasn.ru,mik@ksc.krasn.ru\} \\ ${ }^{2}$ Astafiev Krasnoyarsk State Pedagogical University, ul. A. Lebedevoy,89, Krasnoyarsk 660049, Russia, \\ \{tmsofronova@gmail.com*\}
}

\begin{abstract}
The protected areas (PAs) are established to conserve biological diversity, to maintain nature complexes and objects in their natural condition. Strict nature reserves prevail in Russia by their total area. The whole nature complex is forever extracted from economic use in nature reserves. Here it is prohibited to pursue any activity which might disturb or damage the nature complexes. Even under the existing strict protection from anthropogenic ignition sources, vegetation fires do occur on their territory. Besides, lightnings - these natural ignition sources - are impossible to exclude. Since large destructive fires are impermissible in nature reserves, the latter especially need vegetation fire behavior prediction for fire management. Fire behavior prediction includes fire spread rate, development (from surface fire into crown or ground one) and effects. All this is necessary for taking optimal decisions on how to control each occurring fire and how to suppress it. The Sukachev Institute of Forest SB RAS has developed a technique of vegetation fire behavior prediction using vegetation fuel maps (VF maps).
\end{abstract}

Keywords: strict nature reserves, vegetation fire management, fire behavior prediction, vegetation fuel maps, identifier of primary fire carriers

\section{Introduction}

The protected areas (PAs) are established to conserve biological diversity, to maintain nature complexes and objects in their natural condition. Taking into account the special regime and status of the environmental conservation institutions there, various IUCN categories of PAs are distinguished: strict nature reserves or wilderness areas (including biosphere ones), national parks, habitat/ species management areas, natural monuments, etc. Strict nature reserves prevail in Russia by their total area.

Large destructive fires are impermissible todevelop in nature reserves. This issue can be resolved largely by improvement fire danger rating, fire behavior prediction and fire management. Vegetation fire management in nature reserves is quite possible on the basis of modern fundamnetal fire science elaborations which improve fire danger rating, prediction of an occurred fire depending upon weather conditions, and make it possible to control flame (or flameless) combustion spread over the area and to take optimal decisions on fire suppression.

\section{Background}

L.V. Kuleshov and V.N. Korotkov (2002) made a detailed analysis of wildfires in nature reserves of Russia. Fire dynamics was analyzed for the period from 1975 till 2000. Growth tendency was identified in the number of wildfires in nature reserves and in the number of nature reserves subject to burning in this period. What draws attention is the correlation of forest and non-forest areas. For instance, after 1990, burnt areas predominated in non-forest areas. This fact should be obligatory taken into account when developing the strategy of fire management in nature reserves. It should be noted that the US, Canada and Australia have developed guidelines for fire management on protected areas and that "similar tasks are urgent for Russia with its vast forested areas, high diversity of forest 
vegetation conditions and high forest fire danger" (Kuleshova, Korotkov, 2002).

By now, forest fire scientists of the Sukacev Institute of Forest SB RAS have developed ways to improve fire danger rating according to weather conditions and according to vegetation fuel (VF) complexes, predict burning intensity on a given vegetation plot as well as possible fire effects.

\section{Improvement of fire danger rating in nature reserves}

Two kinds of fire danger are defined in nature reserves: fire danger rating according to weather conditions and according to VF complexes. For fire danger rating according to weather conditions the following indices are used: the Nesterov Index with rough account of precipitation and the PV-1 Index with a more differentiated account of precipitation. Further improvement of the enumerated indices is realized in the index developed at the Sukachev Institute of Forest SB RAS. It takes into account humidity and hygroscpicity of the surface fuels and "works" under negative temperatures (Sofronov, Volokitina, Sofronova, 2008).

\section{Vegetation fire behavior prediction in nature reserves}

When studying fires in nature reserves they are often called forest fires. However, fires spread over non-forest areas with burnt areas larger over non-forest than forest areas. Therefore it would be better to call them vegetation fires. The term "fire management" should also be explained. This term is suggested to understand in a broad and in a narrow ways. In itsbroad meaning, it includes exclusion of anthropogenic causes of fire occurrence (fire preventive arrangement of the area), creation of favorable conditions for timely fire detection and fire suppression (airpatrol, a net of fire lookout posts, a road net), etc. In its narrow meaning, fire management should be understood as control over development of ocurring fires on the basis of their behavior and effects prediction. Methods of VF mapping and fire behavior prediction (including GIS) are developed (Volokitina, Sofronov, 2002; Volokitina, Korets, Sofronova, 2012; Korets, Volokitina, 2014, 2015). On their basis, in cooperation with the Eastern-Siberian Forest Inventory Institution an information database was created and VF maps were made for two different periods of a fire season in four nature reserves of Russia: Stolby (Krasnoyarsk krai), Sayano-Shushensky (Krasnoyarsk krai), Kuznetsky Alatau (Kemerovskaya oblst), and Ubsunurskaya kotlovina (The Republic of Tyva). Besides, pyrological characteristics of forest types was developed for the Baikalsky Nature Reserve and the Tunkinsky National Park (Sofronova, Sofronov, Volokitina, 2005; Sofronova, Volokitina, Sofronov, 2007).

\section{Vegetation fire management in nature reserves}

To manage vegetation fires in nature reserves, first of all, it is necessary to creat an information database containing VF maps. Since Russian nature reserves go through the first forest inventory type, it is quite possible to create the information database using the data of the previous forest inventory using the technique developed by the Sukachev Institute of Forest SB RAS for analysis of forest type schemes and their pyrological characteristic. If old forest inventory data are not relevant already and a new forest inventory is being planned then it would be better to create a new information database for making VF maps with more accurate pyrological characteristics on the basis of the Sukachev Institute identifier for primary fire carriers in the process of forest inventory.

Further, on the basis of a chosen fire spread model, a developed computer software is used to predict behavior of a specific fire: specific pyrological characteristics are introduced from the VF map as well as weather data on a given time. The software program calculates the surface fire edge spread in a given time periods. On the sites where a surface fire can develop into a crown or a ground one, the program shows this probability. Besides, the software reflects possible fire effects in forest stands depending upon the combustion intensity, tree species and mean tree stand dimeter in terms of tree 
mortality expressed in percent. Additionally, taking into account the length of a fire edge, the software calculates the necessary suppression forces for a given stage.

By now, the vegetation fire behavior prediction software has successfully gone through a retrospective performance test in plain conditions (the Chunsky Forest Office of the Krasnyarsky krai) and in mountain conditions (the Stolby Nature Reserve of the Krasnoyarsk krai).

\section{Conclusion}

Vegetation fire management is quite realistic in nature reserves of Russia. Most nature reserves have the necessary information database for their forest inventory is of a high forest inventory type. Nature reserves have their own weather stations and use automatic ones. The software for vegetation fire behavior prediction is pretty straightforward for the specialists of nature reserves to master. 
Short contribution - Fire Management

Wind and slope effects on flame scaling in laboratory fires

Mark A. Finney; Jason A. Forthofer; Torben P. Grustrup

USDA Forest Service, Missoula Fire Sciences Laboratory. 5775 Highway 10 West, Missoula MT, 59808. \{mfinney@fs.fed.us*\}

Keywords: Buoyancy, Flame Scaling, Wildfire Spread

\section{Introduction}

Ignition of wildland vegetation in wildfires is strongly dependent on convective heat transfer (Finney et al. 2015) because the fine size and sparse distribution of foliage and grasses enhances cooling by ambient air. This is sufficient to offset heating by thermal radiation until the flame front is close enough for impingement. Flame impingement on fuel particles occurs intermittently as flame parcels burst forward from the burning zone (Finney et al. 2015). These bursts and non-steady flame patterns are still poorly understood but may have genesis as buoyant or hydrodynamic instabilities (Tang et al. 2015, Tang et al. 2017). Prior experiments with wind-driven fires have revealed that ignition of fine fuel particles was caused by intermittent heating with average frequencies of $2-8 \mathrm{~Hz}$ [Finney et al. 2015, Tang et al. 2015). These frequencies exhibited Strouhal-Froude number scaling (Finney et al. 2015) such that lower frequencies occurred with longer flames but higher frequencies with stronger winds. This paper concerns results from additional experiments on flame characteristics in fires spreading up an inclined platform in the absence of wind.

\section{Methods}

A tilting 4 x $6 \mathrm{~m}$ platform, adjustable from $0-40^{\circ}$ slope angle was constructed for the purpose of studying upslope flame spread through engineered cardboard fuel beds (Figure 1). The surface of the platform was formed by a horizontal stack of cement-board lath (each $0.06 \mathrm{~m} 0.008 \mathrm{~m}$ ) held by a steel frame. Fuel consisted of cardboard cards with attached laser-cut particles of varying dimensions resembling a comb [4]. The spines of each comb were inserted into the slots between cement-board laths at varying spacing to produce fuel beds of vertically oriented cardboard particles.

Fires were ignited instantaneously along the downslope edge of the platform with a line of gasoline. A $60 \mathrm{~cm}$-long ramp without fuel allowed a boundary layer for induced air inflow to develop parallel to the platform before contacting the fuel bed (Figure 1). Flame fluctuations and spread rates were recorded with a linear array of 64 fine-wire thermocouples $(5 \mathrm{e}-5 \mathrm{~m})$, each separated by $0.03 \mathrm{~m}$. The thermocouple array extended upslope from a location $3 \mathrm{~m}$ from the ignition edge of the platform to allow flame spread to accelerate before measurements were taken. To measure hydrostatic pressure, a series of 16 pressure transducers were installed every $0.3 \mathrm{~m}$ with the pressure port located flush with platform surface. The pressure sensors were each co-located with a relatively large thermocouple $(0.127 \mathrm{~mm})$ to record flame arrival. Sampling frequency of all instruments was $500 \mathrm{~Hz}$ using National Instruments data logger.

\section{Results and discussion}

A total of 33 fire spread experiments on the tilting platform were conducted over a range of fuel and platform angles. Fuel properties and slope were varied to achieve different combinations of spread rate and intensity. Video showed the flame zone structure of fires on slopes to be qualitatively similar to those observed in wind driven fires (Figure 1). The leading edge of the flame zone displayed the same peak-and-tough structure produced by alternating upward and downward flows (Finney et al. 
2015). Spread rate varied from 0.004 to $0.155 \mathrm{~m} \mathrm{~s}^{-1}$. Flame length was estimated from video relative to graduated poles and varied from 0.3 to $3.0 \mathrm{~m}$, with spanwise wavelength between flame peaks 0.16 to $1.22 \mathrm{~m}$.

Flame contact with fuel particles forward of the ignition interface was seen to occur from intermittent horizontal bursts issuing forward from the trough-locations of the flame edge (Figure 1). These bursts originate from forward advection of coherent flame structures that first became visible near the rear of flaming combustion region (Figure 1a). The flame zone was composed of these concave parcels and suggests the topology of circulations in Raleigh-Benard (RB) cells for gasses. RB cells could result from the unstable stratification of low density flame gasses originating below denser ambient air. According to Koschmeider 1993, p 64, the circulation of gasses in RB cells is reversed from that of liquids, showing downward motion in the center. Thus, the visible flames trace a concave region of downflow within self-contained $\mathrm{RB}$ circulations that remain coherent as they advect forward through the flame zone. Instabilities become relevant to flame spread through their downstream bursts of flame into fresh fuels ahead of the ignition interface.

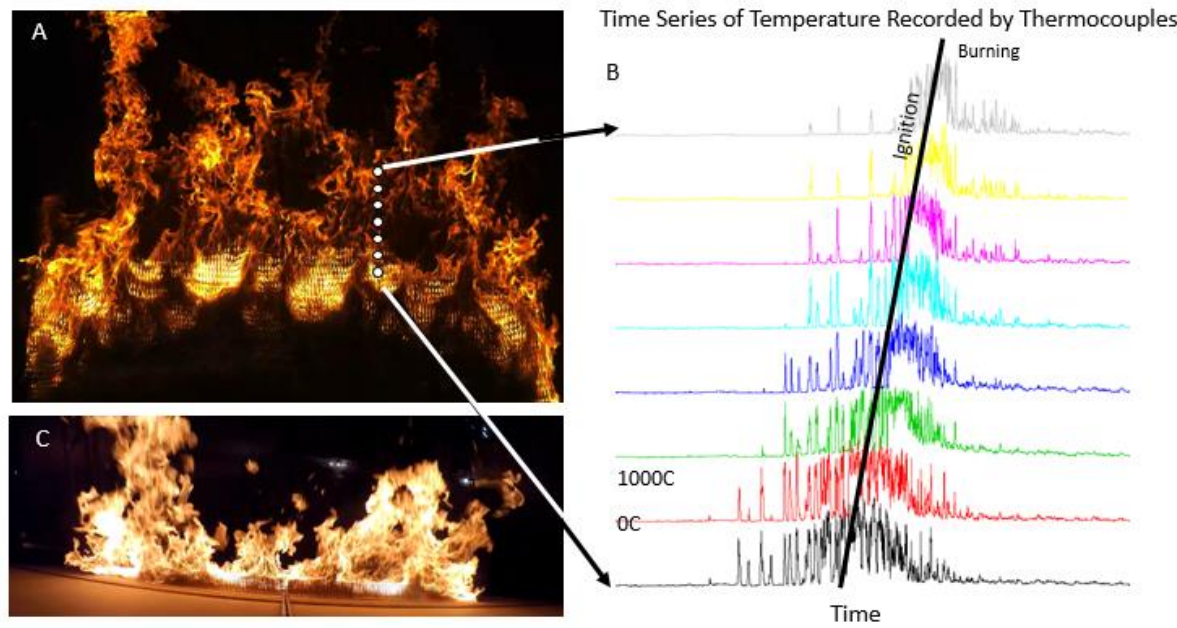

Figure 1 - Top view of flame zone for fire spreading upslope. (A) dish-shaped parcels and location of thermocouple rake that recorded temperature fluctuations from forward flame bursts as the fire approached. Temperature time series $(B)$ shows flame intermittency before ignition, $(C)$ peak and trough structure as viewed toward the advancing flame front.

The coherent structure of flame bursts was revealed by their downstream travel estimated as the "zero correlation distance" forward of the ignition interface at the point where the temperature signals among thermocouples (Figure 1b) showed no correlation. This correlation distance suggested a nearly linear relation to flame zone depth for slope burns, but wind increased the horizontal deflection of flames in wind driven fires (Figure 2).

The predictive utility of these relations is not clear because fires spreading at near steady state (as these were) require the forward convective heating, by definition, to be sufficient to ignite fuels at the same rate as fuel burnout at the rear of the flame zone. It is suspected that the steady spread condition of fires on slopes evolves through some interplay of hydrostatic pressure and induced air inflow. This is evidenced by the observation that flames are vertical soon after ignition but eventually tilt toward the upslope direction as the flow-field develops. By contrast, flames in wind-driven fires are nearly horizontal at ignition. 


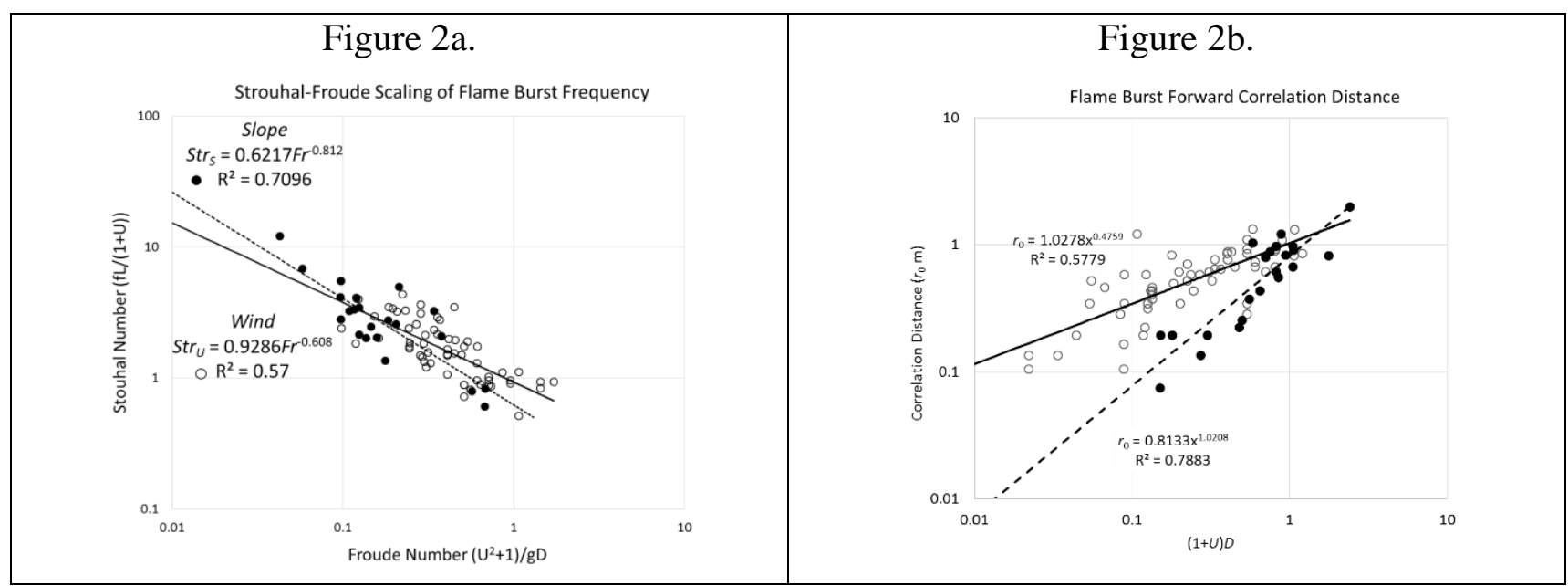

Figure 2. a) Flame burst frequency scaling between wind-driven fire and current slope-driven experimental fires using a Strouhal-Froude relation ( $f=$ frequency $(\mathrm{Hz}), \mathrm{L}=$ flame length $(\mathrm{m}), \mathrm{U}=$ wind speed $\left(\mathrm{m} \mathrm{s}^{-1}\right)$, $g=$ acceleration of gravity $\left(9.81 \mathrm{~m} \mathrm{~s}^{-2}\right)$, and $D=$ flame zone depth from ignition to trailing edge $(\mathrm{m})$. b) Forward flame burst distance (ro) reflects the length of convective heating ahead of the combustion zone (calculated as the correlation of downstream thermocouple temperatures) and depends upon flame zone depth $(D, m)$ for fires spreading upslope but also wind speed in wind driven fires $\left(U, \mathrm{~m} \mathrm{~s}^{-1}\right)$.

\section{References}

Finney MA, Cohen JD, Forthofer JA, McAllister, SS, Gollner MJ, Gorham DJ, Saito K, Akafuah NK, Adam BA, English JD (2015) Role of buoyant flame dynamics in wildfire spread. Proceedings of the National Academy of Sciences 112(32), 9833-9838.

Finney MA, Forthofer JA, Grenfell IC, Adam BA, Akafuah NK, Saito K (2015) A study of flame spread in engineered cardboard fuel beds, Part I: Correlations and Observations. Seventh International Symposium on Scale Modeling, Hirosaki, Japan, August 6-9, 2013. Pp. 71-83, In: Saito et al. (eds) Progress in Scale Modeling volume II, Springer.

Koschmieder EL (1993) Benard Cells and Taylor Vortices. Cambridge Monographs in Mechanics and Applied Mathematics. Univ. Cambridge.

Tang W., Gorham D., Gollner M., Forthofer J. Finney M. (2015) Forward pulsation behavior of winddriven line fires. 9th U. S. National Combustion Meeting.

Tang W, Gorham DJ, Finney MA, McAllister SS, Cohen JD, Forthofer JA, Gollner MJ (2017) An experimental study on the intermittent extension of flames in wind-driven fires. International Association of Fire Safety Science, https://doi.org/10.1016/j.firesaf.2017.03.030. 
Short contribution - Fire at the Wildland Urban Interface

\title{
Aerodynamic study of the airflow around a scale triangular prismatic hill for Low Reynolds
} Number

\author{
Eusébio Z. E. Conceição ${ }^{1}$, Mª Manuela J. R. Lúcio ${ }^{1}$, Domingos X. Viegas ${ }^{2}$, and Maria T. Viegas ${ }^{2}$ \\ ${ }^{1}$ FCT - University of Algarve - Campus de Gambelas - 8005-139 Faro, Portugal \\ ${ }^{2}$ FCT - University of Coimbra - Pinhal de Marrocos - Pólo II - 3030 Coimbra, Portugal
}

\begin{abstract}
In this work is made the aerodynamic study of the airflow around a scale triangular prismatic hill for low Reynolds number. The study is made experimentally and numerically. The numerical simulation is used to complement the experimental results. The study considers a scale triangular prismatic hill with $4 \mathrm{~cm}$ of side and $20 \mathrm{~cm}$ of length. The scale triangular prismatic hill is located inside a channel with a square section, with $20 \mathrm{~cm}$ of side and $100 \mathrm{~cm}$ of length. The experimental study is made in a low air velocity aerodynamic tunnel, with a square experimental section of $20 \times 20$ $\mathrm{cm}^{2}$. In the experimental test, the airflow around a scale triangular prismatic hill is obtained using the smoke visualization, a laser and a film machine. In the image acquisition a rate of 25 images per second is used. In the numerical simulation the Computational Fluid Dynamics is used. The Computational Fluid Dynamics numerical model considers the two-dimensional turbulent airflows under steady-state and non-isothermal conditions. In the study the air velocity is $0.15 \mathrm{~m} / \mathrm{s}$. The air velocity is controlled, in the experimental tunnel, using a Babuc-A and confirmed in the final using a graphic methodology. In the paper the airflow around a scale triangular prismatic hill is analysed. The vortexes dimensions in steady state conditions are numerically calculated, while the vortexes formation in transient conditions are experimentally analysed.
\end{abstract}

Keywords: Numerical Simulation, Experimental Tests, Aerodynamic Study, Airflow Around a Scale Triangular Prismatic Hill.

\section{Introduction}

The airflow around triangular prim hills was studied in the last years. The idea of this study is to evaluate the airflow amount and downstream of the triangular prismatic hill. This study is important because the airflow verified in the downstream area, in a forest fire situation, can return the fire to the triangular prismatic area.

The aerodynamic tunnel is used and applied in studies of airflow in controlled conditions. Several studies were developed in the aerodynamic of the forest fire and of the terrain topology. However, in this study the aerodynamic tunnel is used to evaluate the airflow around triangular prismatic hills.

The Computational Fluid Dynamics numerical model, developed in the last years by the authors, can be seen in detail in Conceição et al. (2008) and Conceição et al. (2016), was validated and applied in experimental chamber with controlled thermal conditions.

\section{Mathematical Model}

The Computational Fluid Dynamics numerical model, used in this work, considers the twodimensional turbulent airflows under steady-state and non-isothermal conditions. The numerical model, developed by the authors, can be seen in detail in Conceição et al. (2008) and Conceição et al. (2016). The numerical model solves the partial differential equations (using the finite volume method), namely, the mass continuity equation, moment equations (two-dimensional air velocity), energy 
equation, turbulence kinetic energy equation and turbulence energy dissipation rate equation (using the RNG turbulence model). This numerical model is used to evaluate the airflow around a hill (twodimensional components of air velocity, air temperature, air pressure, turbulent kinetic energy and turbulent energy dissipation rate).

\section{Experimental test}

The experimental study is made in a low air velocity aerodynamic tunnel with recirculation. The low air velocity aerodynamic tunnel has a height of $3 \mathrm{~m}$ and a length of $6 \mathrm{~m}$. The test section, with a transparent perspex square experimental section of $20 \times 20 \mathrm{~cm} 2$, has a length of $1 \mathrm{~m}$ (see figure 1). In the experimental test, the airflow around a scale triangular prismatic hill is obtained using the smoke visualization, a laser and a film machine. In the image acquisition, a rate of 25 images per second is used.

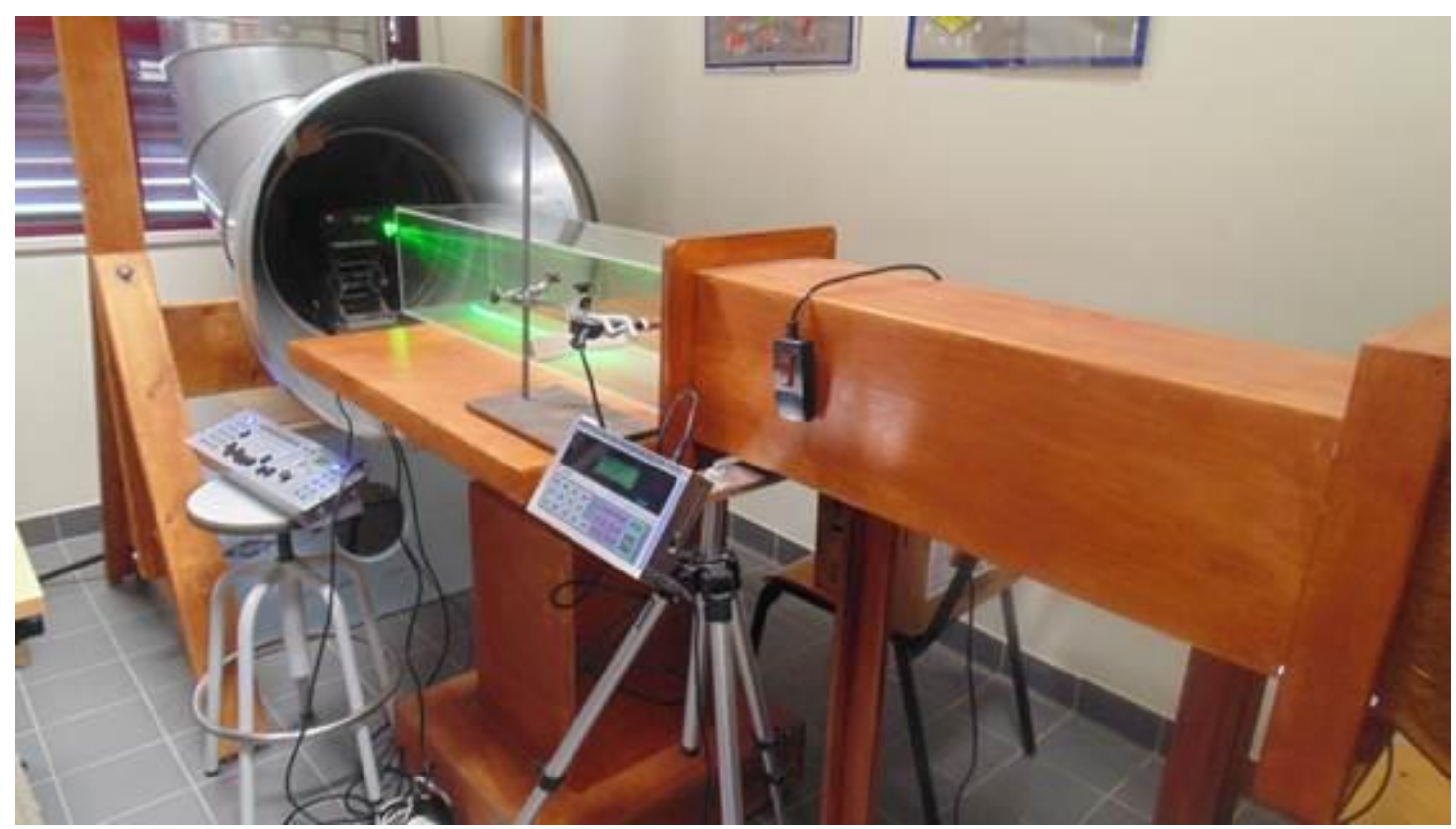

Figure 1 - Scheme of the low air velocity aerodynamic tunnel, test section and laser used in the experimental tests.

The study considers a scale triangular prismatic hill with $4 \mathrm{~cm}$ of side and $20 \mathrm{~cm}$ of length. The scale triangular prismatic hill is located inside a channel with a square section, with $20 \mathrm{~cm}$ of side and $100 \mathrm{~cm}$ of length (see figure 2).

In the study the air velocity is $0.15 \mathrm{~m} / \mathrm{s}$. During the experimental study the air velocity is controlled, using a Babuc-A. In the final, using a graphic methodology, the air velocity is confirmed.

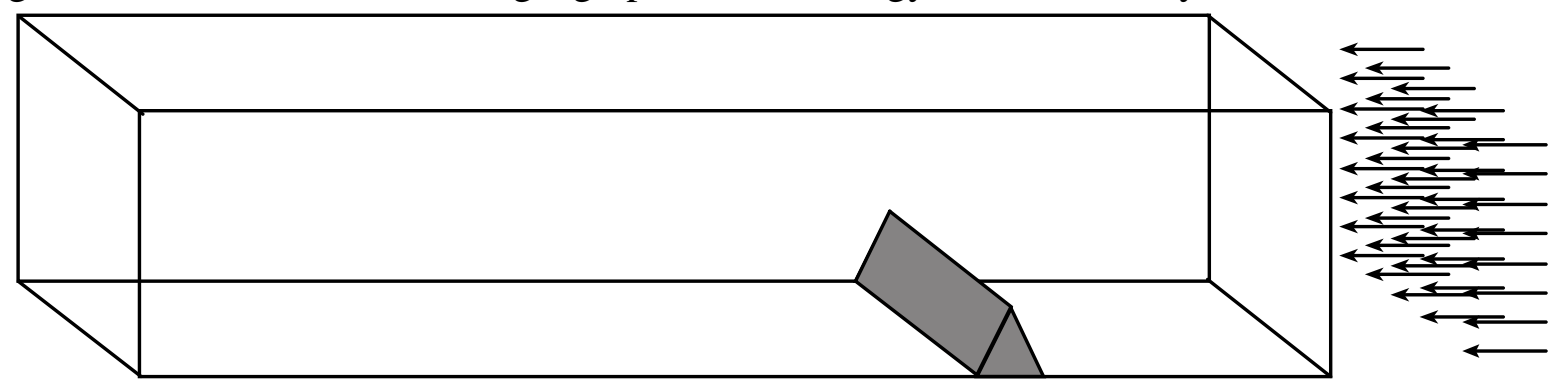

Figure 2 - Scheme of the test section equipped with a triangular prismatic hill. 


\section{Numerical Methodology}

The numerical study is made using a Computational Fluid Dynamics numerical model. The numerical simulation, using a $2 \mathrm{D}$ simulation, can be seen in figure 3 . The study considers a triangle with $4 \mathrm{~cm}$ of side. The simulation domain are $20 \mathrm{~cm}$ of height and $100 \mathrm{~cm}$ of length.

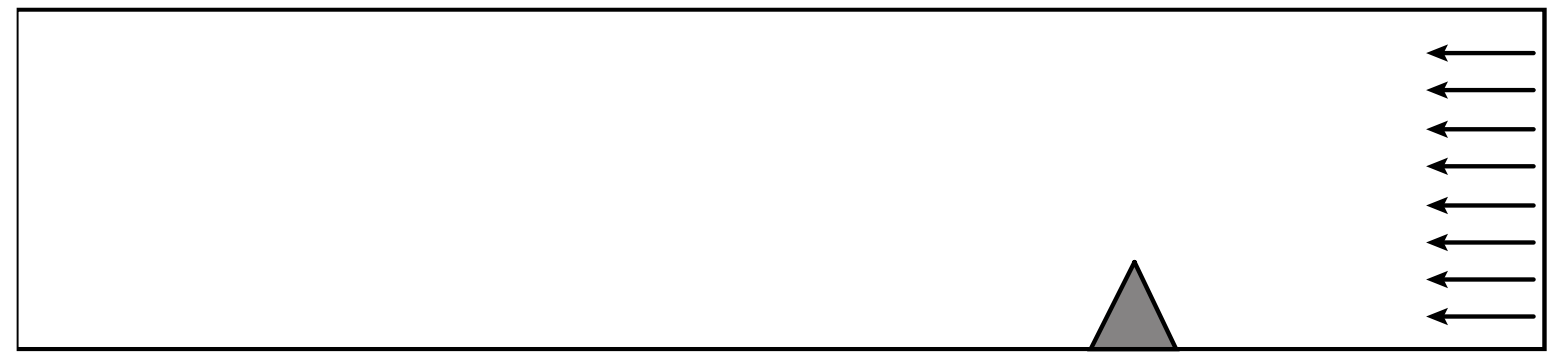

Figure 3 - Numerical simulation scheme of the section equipped with a triangular prismatic hill.

\section{Results and Discussion}

In this section the numerical and the experimental results are presented. The aerodynamic study of the airflow around a scale triangular prismatic hill for low Reynolds number is developed.

\section{Experimental test}

In the experimental tests the vortexes formation in transient conditions are presented in figure 4 . In these figures three sequences of 21 images, obtained in $2.52 \mathrm{~s}$, are presented.

In figure 5 is presented the identification of the main parts of the vortex in the downstream triangular prismatic hill. This identification is obtained in accordance to the results obtained in figure 4 .

In accordance to the obtained experimental results, the main vortex can be divided in four parts, namely, the development, the upper transport, the lower return and the recirculation.

\section{Development:}

The vortex development is made in the up downstream area of the triangular prismatic hill, in the upward direction. The development is periodic. The periodicity is around $1.9 \mathrm{~s}$.

Upper Transport:

The vortex transport is made in the upper main vortex area, in airflow direction. 

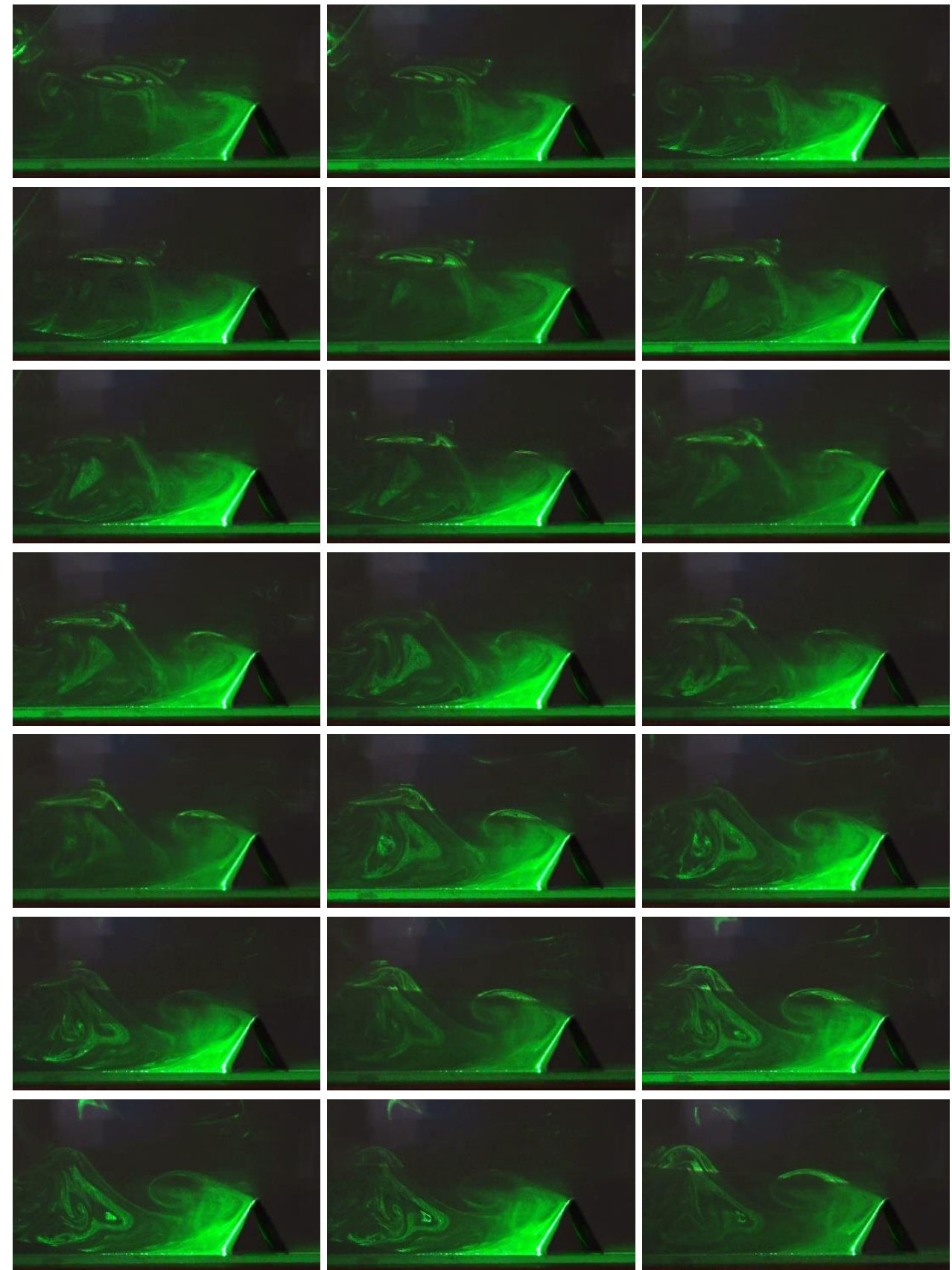

Figure 4 - Experimental tests of the airflow around a scale triangular prismatic hill. Figure integrating a sequence of 21 images.

\section{Lower Return:}

The lower return, made in the lower downstream main vortex area, is responsible to the power supply of the vortex developed (see figure 5), with the direction opposite to the airflow. 

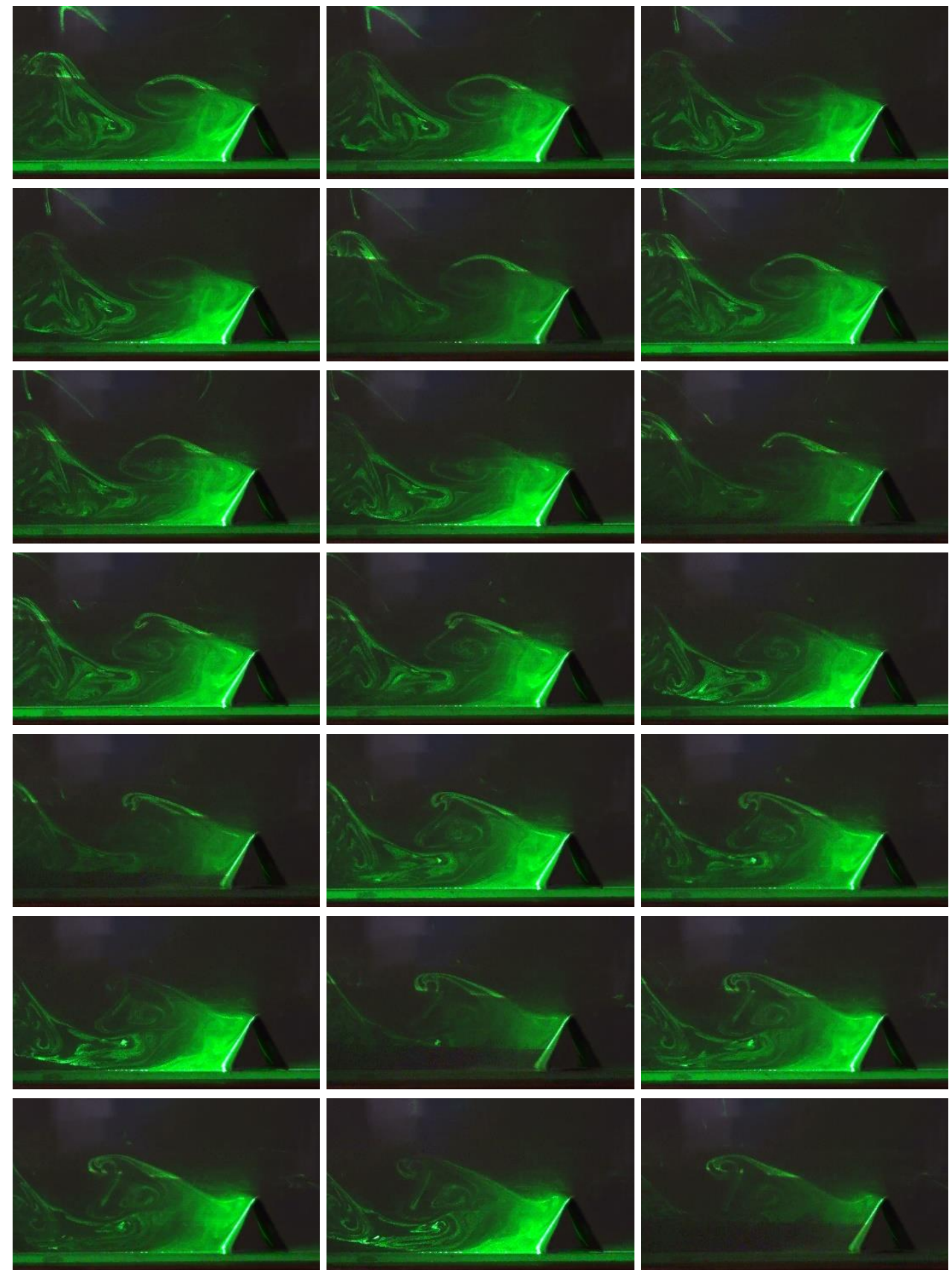

Figure 4 - Experimental tests of the airflow around a scale triangular prismatic hill. Figure integrating a sequence of 21 images (continuation).

\section{Recirculation:}

The circulation is verified in the main vortex centre. The direction of recirculation is made in the upper area in the direction of the airflow and in the lower area in the opposite direction to the airflow. 

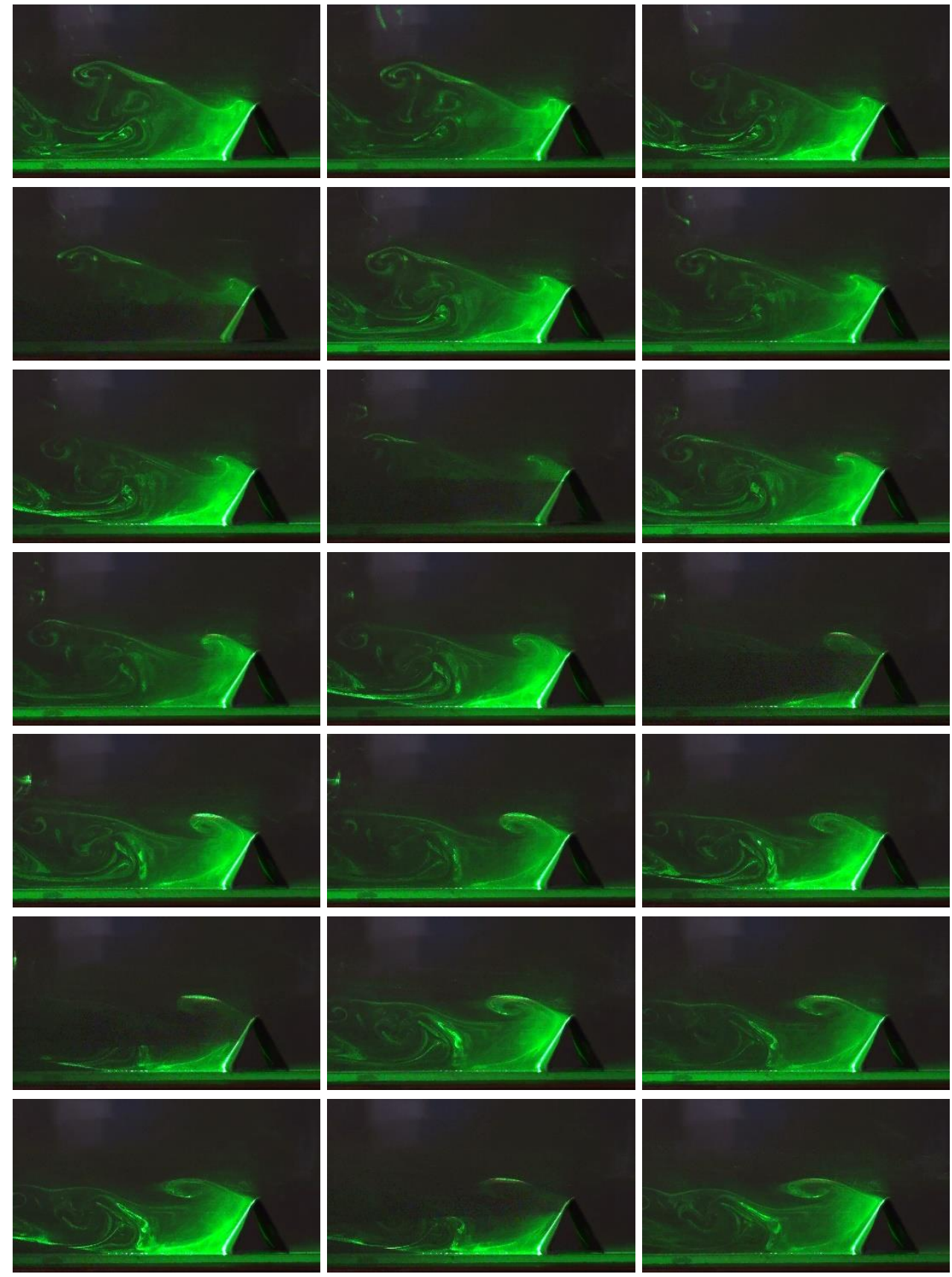

Figure 4 - Experimental tests of the airflow around a scale triangular prismatic hill. Figure integrating a sequence of 21 images (continuation).

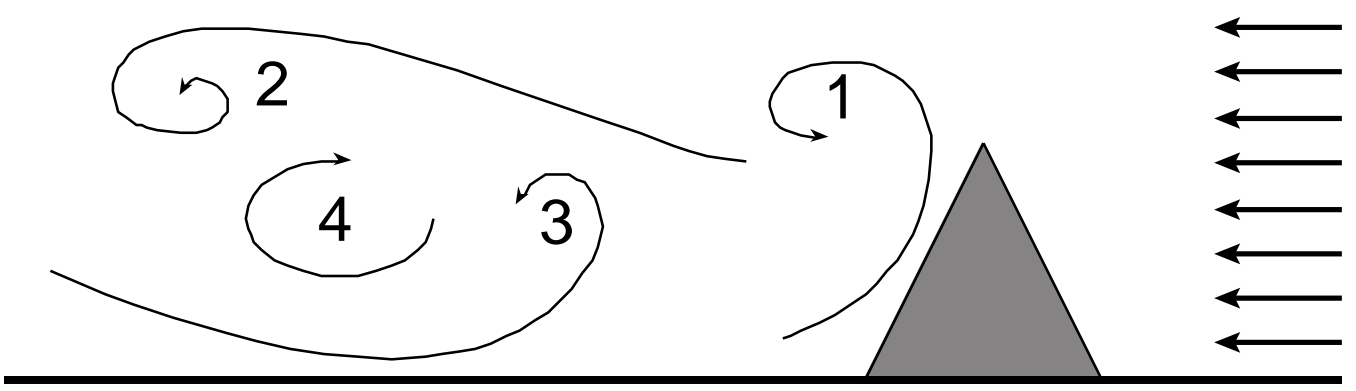

Figure 5 - Identification of the main components of the vortex in the downstream triangular prismatic hill. 


\section{Numerical simulation}

The idea of the numerical simulation, using the RNG k-epson turbulence model, is to obtain a main idea of the airflow topology around the triangular prismatic hill, namely in amount and mainly in the downstream area.

The numerical simulation results, used to obtain the vortexes dimension in steady state conditions, is presented in figure 6 . The influence of the vortexes length is function to the inlet air velocity and the triangular prismatic hill dimensions.

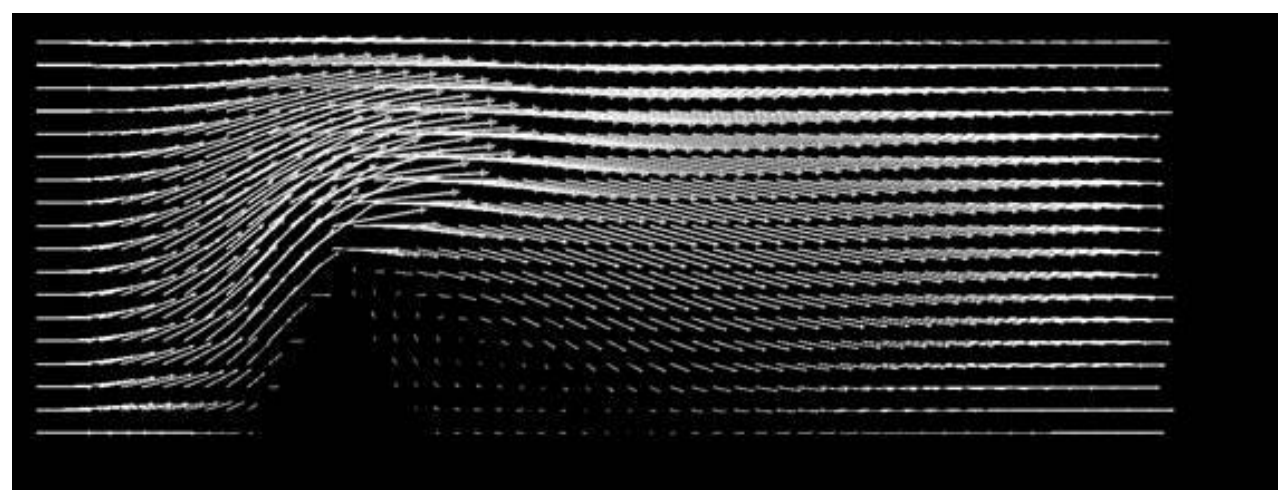

Figure 6 - Numerical tests of the airflow around a scale triangular prismatic hill.

The recirculation area in amount to the triangular prismatic hill, in accordance with the obtained results, verified in the lower area, is small. This fact is associated with the prismatic hill confutation and inclination.

In accordance with the obtained results, in the downstream area, the vortex dimension present a height equal to the triangular prismatic hill side. The vortex length is approximately 1.5 of the triangular prismatic hill side.

\section{Conclusions}

In this work is made the aerodynamic study of the airflow around a scale triangular prismatic hill for low Reynolds number. The study is made experimentally and numerically. The experimental study is made in a low air velocity aerodynamic tunnel, while the numerical study is developed using a Computational Fluid Dynamics numerical model.

The study considers a scale triangular prismatic hill with $4 \mathrm{~cm}$ of side and $20 \mathrm{~cm}$ of length. The scale triangular prismatic hill is located inside a channel with a square section with $20 \mathrm{~cm}$ of side and with $100 \mathrm{~cm}$ of length.

In the experimental test was analysed in detail vortex, in the downstream area. In acordance with the experimental obtained results, the vortex has a frequency of $0.52 \mathrm{~Hz}$. The vortex has a development in the upper triangular prismatic hill area, has a transport in the upper area in the airflow direction, has a return down downstream main vortex area with the direction opposite to the airflow and has a recirculation in the in the main vortex centre.

In the numerical tests was verified a first vortex, located amount the triangular prismatic hill, with very small dimension, and a second vortex, located downstream the triangular prismatic hill, with a height equal to the prismatic hill side and with a length approximate to 1.5 of the prismatic hill side.

In this work the two dimensional airflow study is made. In future works is suggest the three dimensional evaluation of the airflow downstream of the triangular prismatic hill. 


\section{References}

Conceição, E. Z. E., Santiago, C. I. M. and Awbi, H. B. 2016. Numerical Study of Different Ceiling Mounted Localized Air Distribution Systems For a Virtual Classroom, Indoor and Building Environment, July 19.

Conceição, E. Z. E., Vicente, V. D. S. R. and Lúcio. Ma M. J. R. 2008. Airflow inside school buildings office compartments with moderate environment. International Journal on Heating Air Conditioning and Refrigerating Research, ASHRAE, Vol. 14, N. 2, March 2008, pp. 195-207. 
Short contribution - Fire at the Wildland Urban Interface

Burnt area and the wildland-urban interface: A first approach to the Galician wildfires occurred in October 2017

\author{
E. García-Martínez ${ }^{1}$; M.L. Chas-Amil*²; J. Touza ${ }^{3}$ \\ ${ }^{1}$ Universidad de Zaragoza. Pedro Cerbuna 6. Zaragoza, Spain, edgm_73@yahoo.es \\ ${ }^{2}$ Universidade de Santiago de Compostela. Baixada Burgo das Nacións s/n, 15782 Santiago de \\ Compostela, Spain, \{marisa.chas@usc.es*\} \\ ${ }^{3}$ University of York. Heslington, York, YO10 5NG,UK, julia.touza@york.acc.uk
}

Keywords: buildings, Galicia, population, Spain, wildland-urban interface

\title{
1. Introduction
}

Last year catastrophic wildfires in the NW of the Iberian Peninsula destroyed thousands of hectares, provoking human deaths and important economic damages. The wildfire wave occurred in one week in October 2017, with the number of fire incidents reaching peak levels between 14 and 16 October. In those days, the extreme fire meteorology- dry biomass and high temperatures- were combined with storm Ophelia and a high rate of intentional ignitions, which aggravate these conditions. Wildfires occurred in close proximity to residential areas posing an extreme threat to properties and human lives. Forest fire fighters brigades had to prioritize protecting the public for a wide range of emergency situations. This wildfire wave was defined as the worst in history in terms of deaths registered and damages caused in both Portugal and the Spanish region of Galicia. In this paper we characterize the forest fires occurred during this wildfire outbreak of October 2017 in Galicia (Spain). We delimitate the area burnt and describe its spatial characteristics, quantifying the wildland-urban interface (WUI) and population at risk.

The choice of our study area is relevant because the region of Galicia has the highest wildfire incidents in Spain and nearly all fires are human-caused, which traditionally have been associated with illegal behavior through intentional ignited fires (Calviño-Cancela et al. 2016). In 2017, Galicia registered $23 \%$ of the total wildfires burning almost $35 \%$ of the total forest area affected in Spain (MAPAMA 2018), which more than 61,700 ha occurred along the year, mostly during the wildfire wave of October 2017. Our previous study (Chas-Amil et al. 2013) highlighted the vulnerability of the interface to fire in this region. Nearly three quarters of the population settlements are located in the WUI, and that fire ignitions are approximately two times more frequent in WUI than in non-WUI areas. We also found a significant spatial diversity in the vulnerability of WUI areas across this studied region. In this work, we present the first attempt to characterize wildfires occurred in October 2017 in Galicia, focusing on the delimitation of populated areas at risk on WUI areas.

\section{Data and Methods}

We defined the WUI as the area within a 50m radius around buildings at a distance of up to $400 \mathrm{~m}$ from wildland vegetation, where bush clearing is compulsory, according to the current fire-protection legislation in the region (Law 3/2007 of April 9, 2007, addressing the issues of wildfire prevention and suppression, as modified by Law 7/2012 of June 28, 2012 of Galician Forestry). The WUI was obtained combining the most updated layer of buildings from the Galician Topographic Base 1:10,000 (BTG 2016) with information about forest area given by the Fourth National Forest Inventory (IFN4).

The best cloud-free Sentinel 2A-MSI images before and after of the wildfire wave, October 12 and October 27, 2017, were selected and compared in order to determine the severity of wildfires by 
delimitating the area burnt in the studied period. The land use/land cover type (LULC) of the area burnt was determined by using information from the Fourth National Forest Inventory (IFN4). Burned area was also classified attending to their location within or outside the WUI, as well as population and buildings affected. The spatial distribution of the area burnt was also explored by municipalities, describing its spatial characteristics, quantifying the wildland-urban interface (WUI) and population at risk. Jacobs' selectivity index (Jacobs 1974) was used to measure wildfire preference by land cover types and woodland forest type (e.g., Barros and Pereira 2014, Reilly et al. 2018).

\section{Results}

Our results show a total area under WUI in Galicia of 383,711 ha, $13 \%$ of the region. The wildfire wave in October 2017 caused a burned area of 42,314 ha, affecting 97 of 313 Galician municipalities ( $1.43 \%$ of the total Galician area). Forest is the most affected land cover with 40,509 ha, representing $95 \%$ of the total area burned. According to the results from the Jacobs' selectivity index (Figure 1), shrublands was the only land cover burned more than expected based on its presence in the territory, both in the WUI and in no-WUI. These results also show that fires had a clear preference for wood forest lands within the WUI. In terms of population vulnerability to these fires events, results show that a total of 3,665 buildings, corresponding to a total built area of almost 42 ha, 51 population entities and 841 people lived within wildfire perimeters. This shows a high affectation of population and buildings, in fact, we found that 43,048 people live in 500 meters or less to the burned area. The highest relative affectation to the population is detected in forest district XVII-O Condado-A Paradanta, where more than $20 \%$ of the district was burned, and more than $18 \%$ of its population lived within fire perimeters or only $500 \mathrm{~m}$ apart. However, forest district XI-O Ribeiro-Arenteiro was the most affected in terms of burned area (28\%).
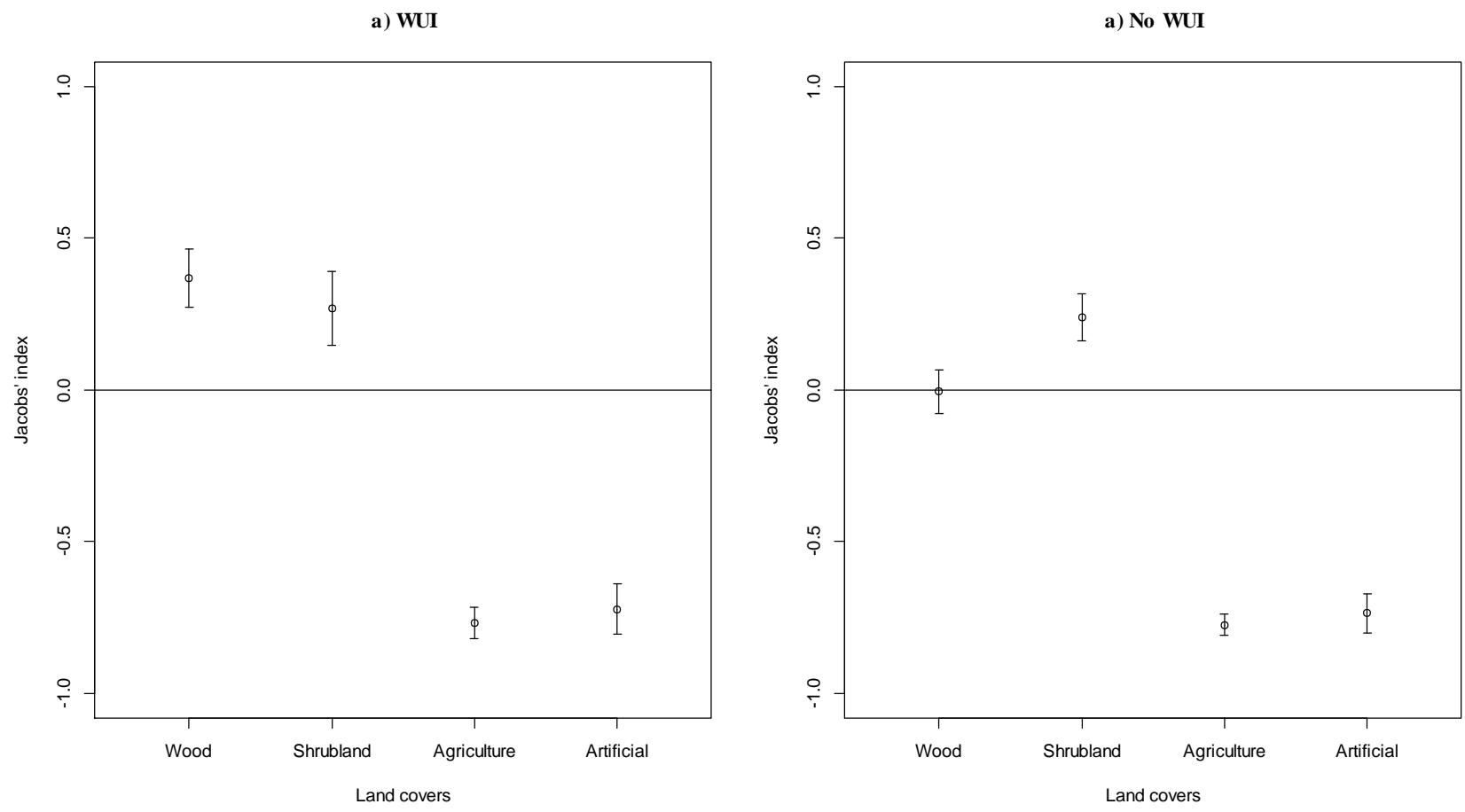

Figure 1 - Mean Jacobs' selection ratios (Ji) with 95\% confidence intervals by land cover types burned in October 2017. 


\section{Conclusions}

This first approach will give us some key element to defend the necessity of implementing an integrated land-use planning, not only based on forest but also focusing on human developments. Our work can lead to the progress of spatial delimitation policies of infrastructures particularly in high risk areas with the aim of developing and promoting plans for the protection of human life, homes and economic activity.

\section{Acknowledgments}

This research was funded in part by Project ECO2017-89274-R MINECO/AEI/FEDER, UE.

\section{References}

Barros, A.M.G., Pereira, J.M.C. 2014. Wildfire Selectivity for Land Cover Type: Does Size Matter? PlosOne, 9(1): 1-10.

Calviño-Cancela, M., Chas-Amil, M.L, García-Martínez, E., Touza, J. 2016. Wildfire risk associated with land cover types, topography and human activities within and outside wildland-urban interfaces. Forest Ecology and Management. 372: 1-9

Chas-Amil, M.L., J. Touza, E. García-Martínez. 2013. Forest fires in the wildland-urban interface: A spatial analysis of forest fragmentation and human impacts. Applied Geography, 43: 127-137.

Jacobs J. 1974. Quantitative measurement of food selection: a modification of the forage ratio and Ivlev's electivity index. Oecologia 14: 413-417.

MAPAMA. 2018. Los incendios forestales en España. 1 enero - 31 diciembre 2017 Avance Informativo Available at http://www.mapama.gob.es/es/desarrollorural/estadisticas/iiff_2017_def_tcm30-446071.pdf [Verified 4 June 2018]

Reilly, M.J., Elia, M., Spies, T.A., Gregory, M.J., Sanesi, G., Lafortezza, R. 2018. Cumulative effects of wildfires on forest dynamics in the eastern Cascade Mountains, USA. Ecological Applications, 28 (2): 291-308. 
Short contribution - Fire at the Wildland Urban Interface

Burnt severity and satellite data: a first approach in the ornemental vegetation in wildland urban interface

\author{
Marielle Jappiot *; Adeline Bellet; Fabien Guerra; Christian Travaglini; Roland Estève \\ IRSTEA. EMR, Mediterranean Ecosystem and Risks, 3275 Route de Cézanne, CS40061, 13182 Aix-en- \\ Provence Cedex 5, \{marielle.jappiot@irstea.fr*, adeline.bellet@irstea.fr, fabien.guerra@irstea.fr \\ ,christian.travaglini@irstea.fr,roland.esteve@irstea.fr\}
}

Keywords: fire, burnt severity, damage, remote sensing, Sentinel, wildland urban interface

\title{
1. Introduction
}

When a fire occurs, the knowledge on its location and the burnt area allows calculating economic loss, observing the impact on the environment, and modeling landscape evolution (Gitas et al., 2007). Fire severity assessment allows predicting post fire vegetation dynamics, and anticipating soil erosion (Allen and Sorbel, 2008). It also provides data for fire assessment models by giving information on fire frequency and fire intensity. An exhaustive knowledge of the past fires on a landscape, allows validating and improving those fire risk models.

Database on past fires are not fulfilled systematically with georeferenced contours. When contours are available, these are generally fires larger than 50 ha. Indeed, large fires causing damage to buildings are generally very well described: contours are made from satellite images available quickly, with field checks at the margin. For fires $<50$ ha, there is no rule for the systematic collection of information. In fact, databases are heterogeneous from one department to another. However, knowledge on these "small fires" is important, especially since they often occur in wildland urban interface (WUI) areas where forest fire risk management poses particular problems. With the aim of building accurate and comprehensive fire history databases, satellite imagery is a very interesting source of information as it provides information on vegetation over large areas.

\section{Objectives}

We will use the complementarity of these different satellite sources (spatial and temporal resolution, different wavelength channels) to develop a method of fire severity mapping. Severity levels were related to levels of damage observed in the field. Damage assessment methods based on vegetation indices and before / after event image comparisons were developed to characterize the severity of fires. However, there are few studies on fire behavior in the ornemental vegetation, and none on the characterization of the damage in WUI, which requires the development of a specific methodology. It is a first approach that is developed here.

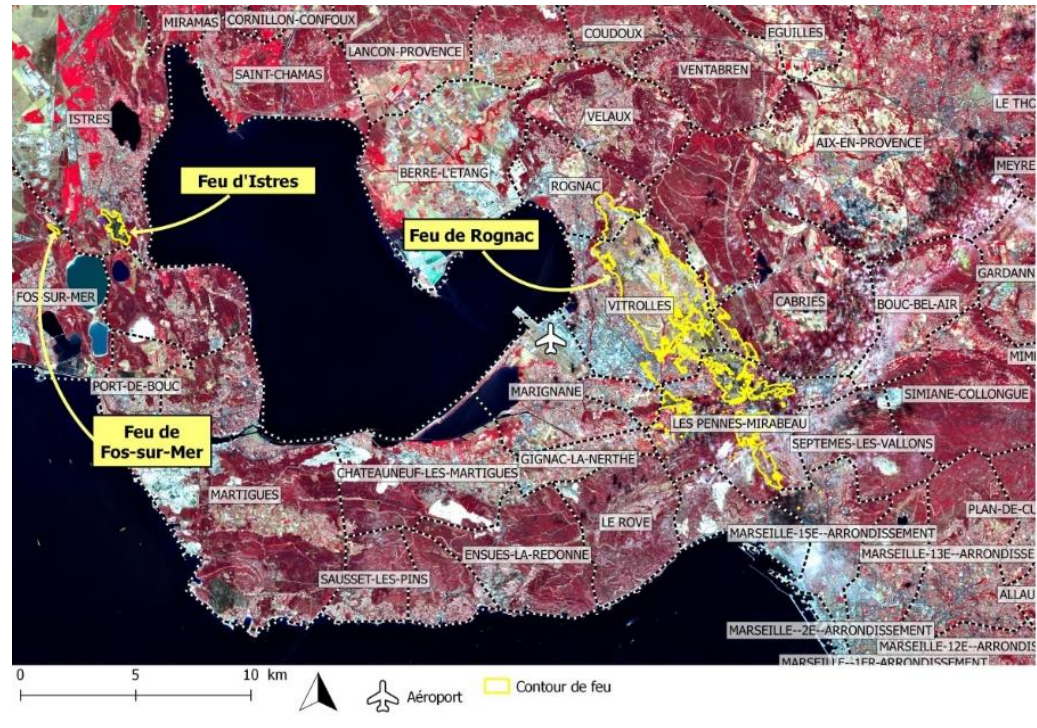

Figure 1 - Location map of the past fires. Source image Sentinel 2, Données Copernicus Sentinel-2 2017 traitées au niveau 2A par le Cnes pour le centre de données Theia 


\section{Material and methods}

The study area is situated in southern France, and focused on three past fire (Fig. 1).

In France, within the framework of the THEIA data center (www.theia-land.fr), the GEOSUD team aims to develop a national satellite imagery infrastructure for environmental and landscape research, and its applications to management and public policies. This scientific and technical structure aims to facilitate the use of these images. Thus, the Pleiades and SPOT6 / 7 images are available for research as well as Sentinel-2 images.

For this study, several complementary satellite sources were used on 3 fires in the south of France: Rognac 2016, Fos 2017 and Istres 2017, of variable size, which affected the WUIs

Damage surveys were carried out in the field, in the natural zone and in the interface zone, adapting the protocol. Scores of damage were calculated. Vegetation indices were calculated from satellite data, including Gdnvi which has been classified in severity level. The indices were then correlated to the field scores.

Fig 2. Gives an illustration of the plots (yellow) in the WUI.

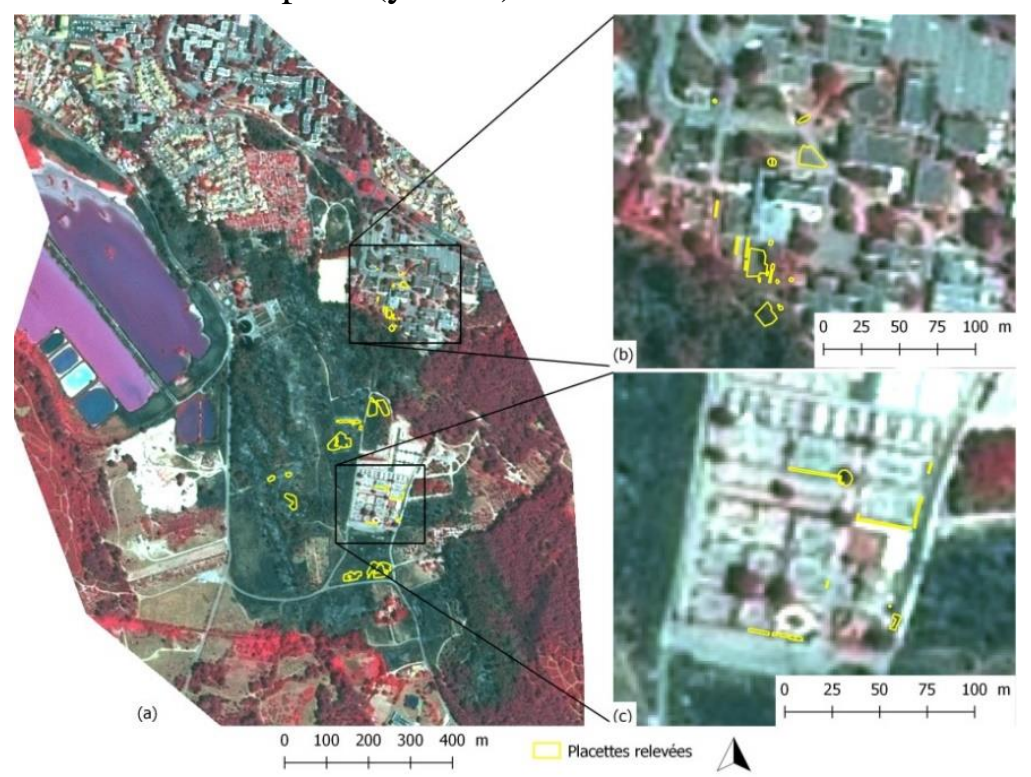

Figure 2 - Plots on the Istres Fire. a : General map of the fire ; $b$ : location 1 ; $c$ : location 2. Source image : SPOT 6 (C) AIRBUS DS 2017

\section{First results}

Ornemental vegetation is precisely geolocalized on Pleiades, and SPOT6 as well (Fig 3).

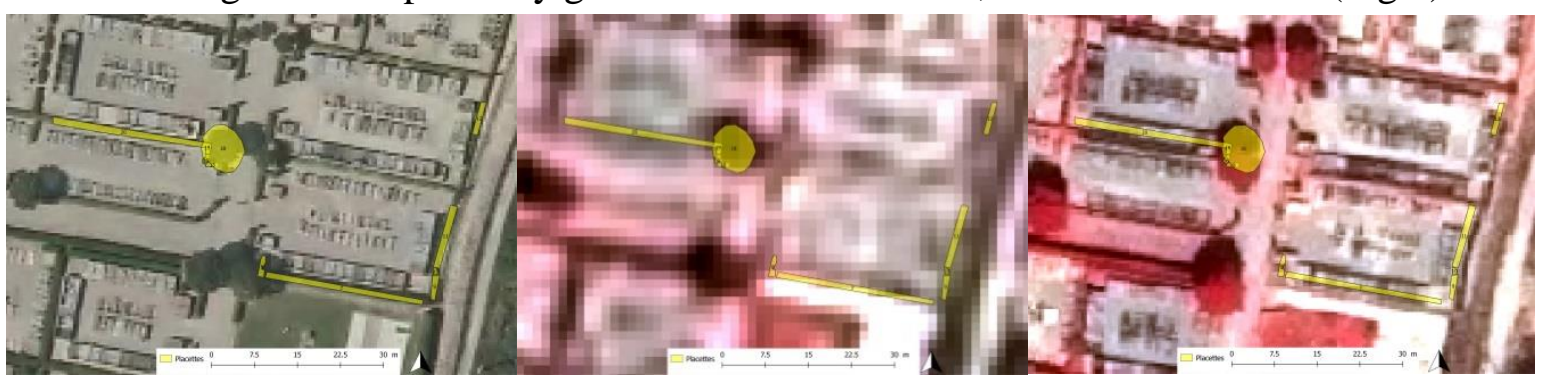

Figure 3 - Source images from left to right : Orthophoto 2011 IGN / Image Pléiades @ CNES 2017, Distribution Astrium Services / Spot Image S.A., France, tous droits réservés. Usage commercial interdit / SPOT 6 C AIRBUS DS 2017 
We can observe correlations between GNDVI level and field score according to the type of vegetation in the wildland urban interface (Fig. 4).

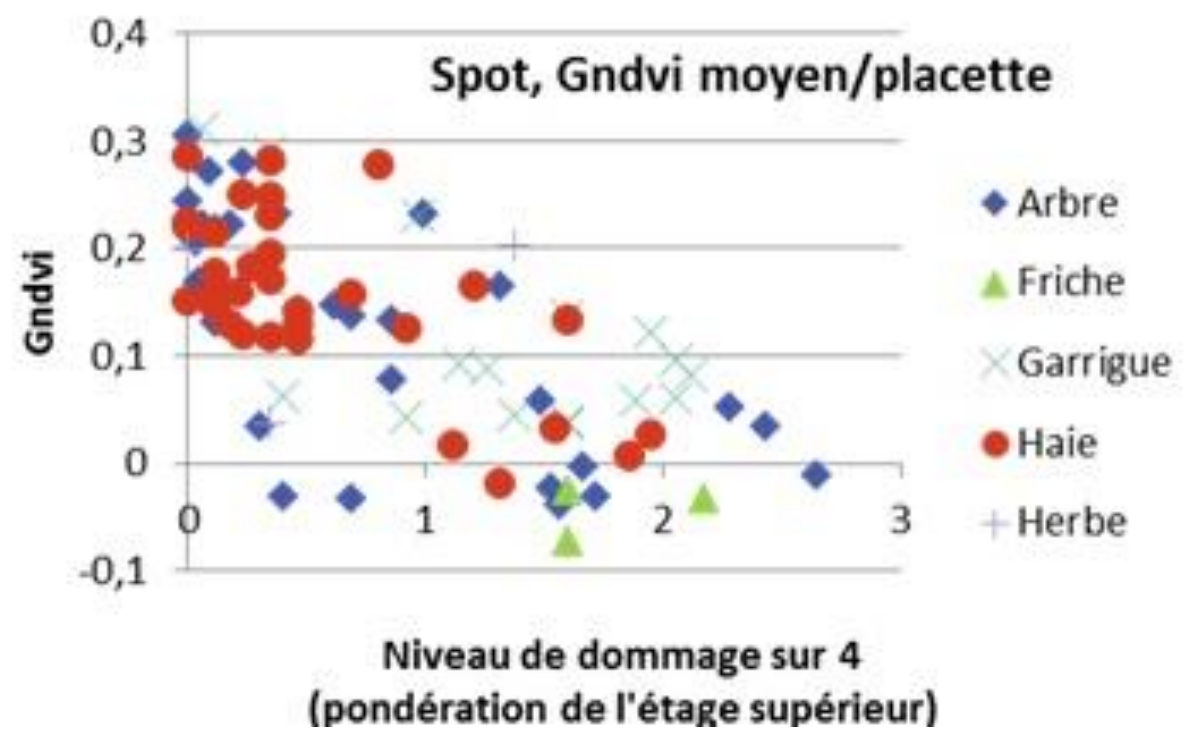

Figure 4 - relationship between Gndvi and field damage score 
Short contribution - Fire at the Wildland Urban Interface

Critical conditions for the ignition of cedar needle fuel bed as a result of firebrands accumulation

\author{
D. P. Kasymov ${ }^{1 *}$, M. V. Agafontsev ${ }^{1}$, V. N. Fateev ${ }^{1}$, V. V. Reyno ${ }^{2}$, A. I. Filkov ${ }^{3}$ \\ ${ }^{1}$ National Research Tomsk State University, Tomsk, Russia, \{denkasymov@gmail.com*\} \\ ${ }^{2}$ V.E. Zuev Institute of Atmospheric Optics SB RAS, Tomsk, Russia, \{reyno@iao.ru\} \\ ${ }^{3}$ University of Melbourne, School of Ecosystem and Forest Sciences, Melbourne, Australia, \\ \{alexander.filkov@unimelb.edu.au\}
}

Keywords: glowing firebrands, accumulation, spotting, critical conditions, ignition time

\title{
1. Introduction
}

It is well known that flaming and glowing particles are a major cause of wildland and wildlandurban interface fires (Cohen 2000; Caton et al. 2017). In recent years, many papers devoted to this problem have studied firebrands trajectory in the airflow, their transportation distance (Ellis 2000; Anthenien et al. 2006; Kortas et al. 2009; Song et al. 2017) and evaluated firebrands ability to ignite fuel beds(Manzello et al. 2008; Filkov et al. 2016; Kasymov et al. 2016; Fateev et al. 2017). However, the problem still requires further research, as, in particular, critical conditions for the ignition of the fuel bed as a result of firebrands accumulation depending on their size, shape and ambient conditions remain largely unknown.

It should be noted that the interaction of flaming (burning) and glowing particles with the fuel bed is a complex process, which requires both theoretical and experimental approaches. The earlier papers demonstrated that flaming particles ignite the fuel bed more easily; for this reason, our research has been focused on glowing particles.

As was shown in(El Houssami et al. 2016), during surface fires tree bark and twigs turn into glowing particles. Therefore, the aim of the present work is to study accumulation of glowing twigs of various sizes and their interaction with forest fuel beds. In particular, the paper studied fuel bed ignition time, flaming and smouldering duration times and the heat flux critical value sufficient to ignite the fuel bed.

\section{Methods and Results}

An experimental setup was created which permitted to simulate firebrands accumulation with a controlled number of firebrands being dropped and which in turn allowed to evaluate their effect on the fuel bed (fig. 1).
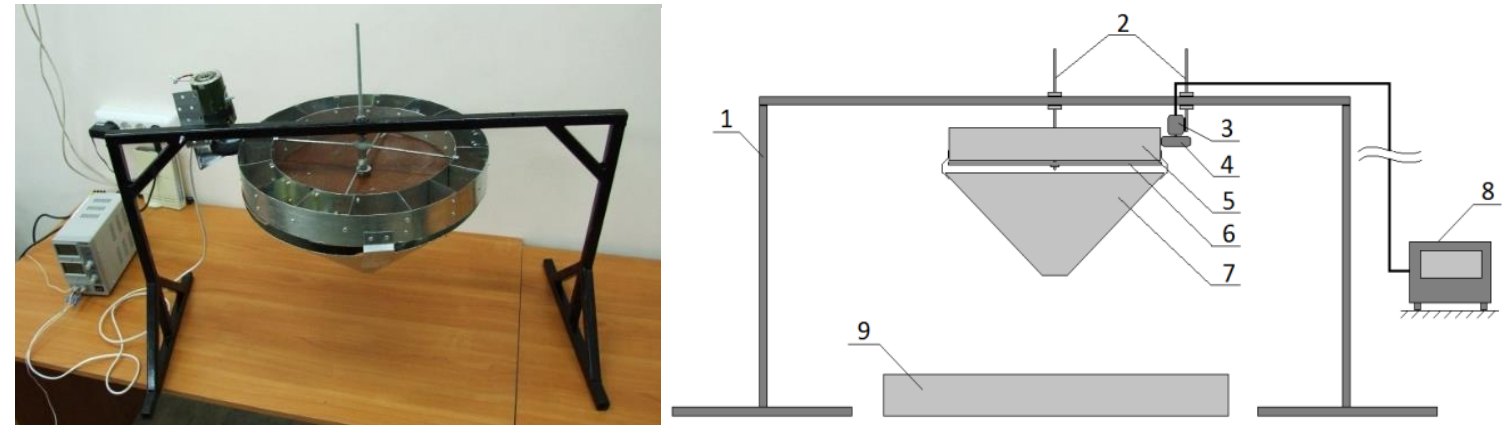

Figure1 - Experimental setup to simulate firebrand accumulation on the fuel bed: 1 - rack; 2 -adjusting bolts; 3 electric motor; 4 -driving wheel; 5 -box; 6 -disk-separator; 7 -funnel; 8 -adjustable power supply; 9 -tray 
Birch tree cylindrical samples with diameters of $6 \mathrm{~mm}, 8 \mathrm{~mm}, 10 \mathrm{~mm}, 12 \mathrm{~mm}, 14 \mathrm{~mm}$ and $50 \mathrm{~mm}$ in length were used as firebrands (fig.2).

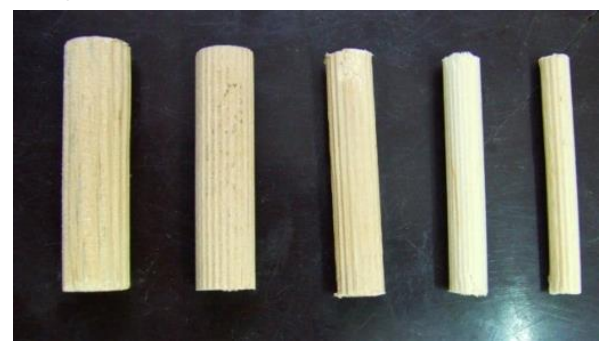

Figure 2 - Firebrand samples

The moisture content was determined using the AND MX-50 moisture analyser. The firebrands moisture content and the fuel bed (cedar needles) were $4.2 \pm 0.2 \%$ and $6 \pm 0.2 \%$ respectively. A thermal imaging camera JADE J530SB and a video camera Canon HF R88 were used to evaluate the firebrands temperature and to record the experiment. Three repetitions were performed for each experiment, three successful or unsuccessful ignitions in a row. In case of one or two ignitions, the number of experiments was increased to reach at least three ignitions for statistical analysis.

The following procedure was used for the experiment: cedar needles were placed on the tray (9) to simulate a fuel bed with a density of $56 \mathrm{~kg} / \mathrm{m}^{3}$; firebrands were put into the box (5) and heated with gas burners until they began to glow while the whole process was controlled with the thermal imaging camera. The frequency of firebrands drops on the tray (9) was regulated by changing the electric motor rotation speed (3). The precision of firebrands drops was regulated by adjusting the height of the setup over the fuel bed using adjusting bolts (2) and funnel (7). The experiment started with dropping one particle every 4 seconds until reaching a total of 10 particles. If the fuel bed was ignited, fresh cedar needles were put on the tray (9). Three repetitions were carried out for each experiment. Fuel bed ignition was defined as a moment when a flame appeared over the fuel bed, which was recorded with a video camera. Fuel bed smouldering was defined as continuous uninterrupted smouldering caused by firebrands and which lasted for $5 \mathrm{~min}$.

A preliminary series of experiments was carried out in the absence of wind and with wind speed of $1.5 \mathrm{~m} / \mathrm{s}$ with firebrands $50 \mathrm{~mm}$ long and with diameters of $6 \mathrm{~mm}$ and $8 \mathrm{~mm}$. Under the chosen experimental parameters in the absence of wind fuel bed ignition did not occur except on a few occasions when fuel bed smouldering was initiated and lasted for $3 \mathrm{~min}$ after the firebrands had stopped burning. With the wind speed of $1.5 \mathrm{~m} / \mathrm{s}$, the fuel bed ignition was observed and was started by eight $6 \mathrm{~mm}$ particles, or by six $8 \mathrm{~mm}$ particles, i.e. an increase in the firebrands diameter led to a decrease of the number of firebrands required ignition to occur.

During the next experimental stage, particles with diameters of $10 \mathrm{~mm}, 12 \mathrm{~mm}$ and $14 \mathrm{~mm}$ and the wind speed of $1-1.5 \mathrm{~m} / \mathrm{s}$ will be used to evaluate both the probability of ignition depending on the number of particles and the critical heat flux produced by them.

\section{Acknowledgements}

This study was supported by the Russian Foundation for Basic Research (project No. 18-07-00548).

\section{References}

Anthenien RA, Tse SD, Carlos Fernandez-Pello A (2006) On the trajectories of embers initially elevated or lofted by small scale ground fire plumes in high winds. Fire Safety Journal 41, 349363. doi:10.1016/j.firesaf.2006.01.005. 
Caton SE, Hakes Raquel SP, Gorham DJ, Zhou A, Gollner MJ (2017) Review of Pathways for Building Fire Spread in the Wildland Urban Interface Part I: Exposure Conditions. Fire Technology 53, 429-473. doi:10.1007/s10694-016-0589-z.

Cohen JD (2000) What is the Wildland Fire Threat to Homes? https://www.fs.fed.us/rm/pubs_other/rmrs_2000_cohen_j003.pdf.

Ellis PF (2000) The aerodynamic and combustion characteristics of eucalypt bark—a firebrand study. Ph.D. Dissertation, Australian National University, Canberra.

Fateev V, Agafontsev M, Volkov S, Filkov A (2017) Determination of smoldering time and thermal characteristics of firebrandsunder laboratory conditions. Fire Safety Journal 91, 791-799.

Filkov AI, Kasymov DP, Zima VP, Matvienko OV (2016) Experimental investigation of surface litter ignition by bark firebrands. In 'AIP Conf. Proc. 1698', 060004

El Houssami M, Mueller E, Filkov A, Thomas JC, Skowronski N, Gallagher MR, Clark K, Kremens R, Simeoni A (2016) Experimental Procedures Characterising Firebrand Generation in Wildland Fires. Fire Technology 52, doi:10.1007/s10694-015-0492-z.

Kasymov DP, Filkov AI, Baydarov DA, Sharypov OV (2016) Interaction of smoldering branches and pine bark firebrands with fuel bed at different ambient conditions. In 'Proc. SPIE 10035', 100356H doi: $10.1117 / 12.2249083$.

Kortas S, Mindykowski P, Consalvi J, Mhiri H, Porterie B (2009) Experimental validation of a numerical model for the transport of firebrands. Fire Safety Journal 44, 1095-1102. doi:10.1016/J.FIRE SAF.2009.08.001.

Manzello SL, Cleary TG, Shields JR, Maranghides A, Mell W, Yang JC (2008) Experimental investigation of firebrands: Generation and ignition of fuel beds. Fire Safety Journal 43, 226-233. doi:10.1016/j.firesaf.2006.06.010.

Song J, Huang X, Liu N, Li H, Zhang L (2017) The wind effect on the transport and burning of firebrands. Fire Technology 1-14. doi:10.1007/s10694-017-0647-1. 
Short contribution - Fire at the Wildland Urban Interface

Institutional engagement and public involvement at the WUI. A case study in the South of Spain: Córdoba municipality

\author{
Clara Quesada-Fernández ${ }^{1 *}$; Daniel Quesada-Fernández ${ }^{2 *}$ \\ ${ }^{1}$ University of Córdoba. Spain. \{claraquesada@gmail.com*,z32quefec@uco.es*\} \\ ${ }^{2}$ Consultant, \{danielquesadafernandez@gmail.com\}
}

Keywords: Córdoba, civic engagement, fire regulation, fire prone areas, fuels management, institutional and public involvement, safety, perception, risk, safety, Spain, social involvement, wildland firefighters, wildland fires, wildland urban interface.

\title{
1. Introduction \& antecedents
}

There are numerous cases of emergencies in fire prone and wildland urban interface areas that have occurred during the last years, with an exponential growth as time goes by. In Spain most of the situations were declared level 2 (out of a maximum of 3), fires endangering seriously people and properties- according to the current Basic Civil Protection Directive.

Some close examples, all of them recognizable by the media, occurred last year. We must remember numerous cases where populations were affected and dramatic fire situations with thousands of evacuated and killed people (Doñana National Park-Spain, Encinedo-León, Galicia, Tejeda-Gran Canaria, Pedrógão Grande, Coimbra, Valença, Sertã, Viana do Castelo, Braga, Viseu and Monção in Portugal; Var, Bouches-du-Rhone and Vaucluse, Corsica, Marseille and Nice in France; Campania, Calabria, Puglia, Tuscany, Lazio, Sicily and Sardinia, Enna, Messina and Palermo in Italy; Athens, in Greece; Valparaíso, in Chile; Los Angeles, in California).

\section{Córdoba municipality}

The reality of the municipality of Córdoba was included in 2011 in the PYROSUDOE European Project focused on forest fire risk perception in the southeast and southwest of the Mediterranean WUI areas. The general objective was the characterization of WUI municipalities of Córdoba and Almodóvar del Río. It consisted on a preliminary study on fire reality at WUI areas and their associated risks reflecting, analyzing and proposing a basic action guide for preventive purposes and future applications. The specific objective of the work was to review the territory In order to characterize and distribute the WUI situations in the municipalities of Córdoba and Almodóvar del Río, and their risks associated with forest fires.

A diagnosis of the urban-forest interface was obtained in relation to the risk associated with forest fire episodes, identifying the types of interfaces, estimating the associated vulnerability, obtaining cartography of the distribution and incorporating this study into the calculation of fire risk.

The municipality of Córdoba (Spain) is one of the largest municipalities in the homonymous province. Within its area of $1,255 \mathrm{~km} 2$ the presence of areas of wildland urban interface is widespread and the occupation of the territory with this spatial

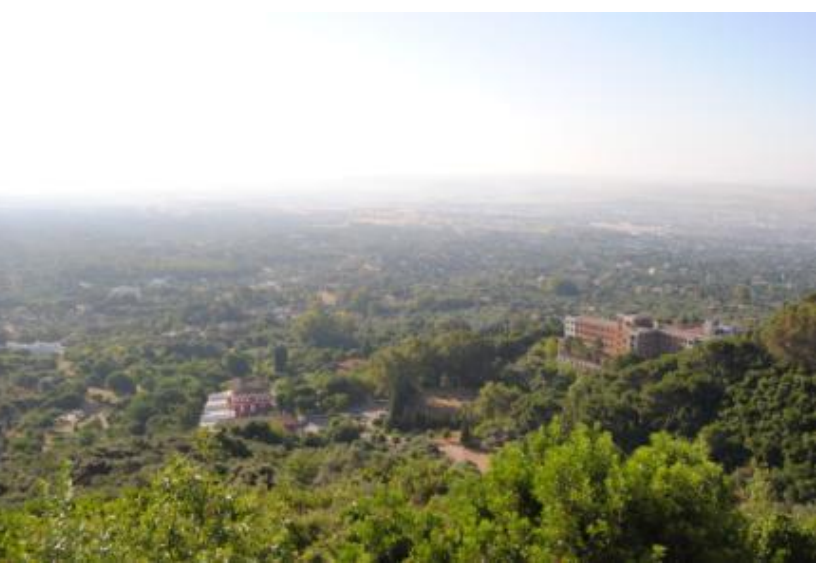

Figure 1 - The reality of the municipality of Córdoba (Spain) seen from the North of the city. 
structure is extensive. In recent years, urban expansion in forestry has entailed a new problem such as the scenario of forest fires near or within urban settlements. To the techniques and protocols for fire suppression, often limited in these environments, it is necessary to add those of civil protection since people, properties and infrastructures are threatened and affected by fires and smoke.

\section{The forest, territorial and fire regulations}

The present fuels, which are no longer only of a vegetable / forestry nature, as well as the infinite combination of possible situations, make it difficult to work in these areas. The suppression in the WUI needs, therefore, an adaptation of the current Incident Command System, that is, the way in which the operation is organized in the intervention. It must be adapted to the emergency in which it is not possible to rely on the generalized application of the same attack techniques that have been used until in previous interventions. From current legislation it is necessary to question whether the current texts are sufficient and are updated enough to deal with the problem in a practical and problem solving way. In this sense we should consider the validity of the current Basic Guideline for Emergency Civil Protection Planning for Forest Fires, (RD 893/2013), the current State Forestry Law 43/2003, the autonomous articles as well as the provincial and municipal ones.

\section{Institutional engagement and public involvement at the WUI.}

The Consejería de Medio Ambiente y Ordenacion del Territorio, Regional and local forest Administration, are trying to communicate to people a forest culture in terms of the proper use of fire and its implication in a context of urban-based communites in the areas of the municipality. Fuels management in WUI zones (private and public lands) and public information (residents and scholars) are considered as essential matter and they are welcomed by residents and general public.

\subsection{Fuels management in WUI zones.}

This action it is focused on three types of action areas depending on the distance between structures and fuels. It is considered location of the structures as well as actual fuels structure versus future structure after management of them and the type of action in each one.

Fuels management depends on priority zones Priority $1(0-10 \mathrm{~m})$, Priority $2(10-30 \mathrm{~m})$ and Priority $3(30-100 \mathrm{~m})$. It is also regulates the fuels management around power lines

Fuels elimination is made following fire regulations and it depends on the moment of year in which they are generated. The can be eliminated using fire under and strict observance of weather and public occupation in forest areas.

\subsection{Public information}

Residents in WUI areas in the municipality of Córdoba receive annual information regarding to fire uses, fire regulations and which implications of a bad use of fire could affect (more) negatively on wildland firefighters operations and safety.

In early ages the "Crece con tu árbol" ("Growing up beside your tree") andalusian programme has created and educational context in terms of culture of fire use by visiting primary and secondary schools in the municipality.

\section{Conclusions}

Forest forestry and forest fire prevention in forestry so far had been based on the so-called classical forestry actions such as the repopulation of areas affected by fires or diseases, the repair of roads and hydrological correction combined with a reasonable use of fire in order to use and exploit the forest biomass generated and available in the forest. 
However, changes in social uses have compromised the maintenance of our forests and the involvement of the population with them. In these new realities and from the operational point of view and intervention in the emergency, new strategies and new tactics of struggle and suppression are imposed. In that sense we should consider what happens with territorial planning policies, why we have reached these realities and what solution(s) can be given. The combination of possibilities and territorial variety of forms of the problem leave numerous examples that illustrate the problem of an emergency in the urban-forest interface. It is in these moments in which one considers how to act in such a reality.

We highly appreciate the collaboration of Consejería de Medio Ambiente y Ordenación del Territorio (Junta de Andalucía, España (Regional Forest Administration) through Centro Operativo Provincial (Córdoba) (Province Wildland Fire Operations Center) and we would like to thank all its members.

\section{Bibliography}

Luque Marín, Iván: 2015. Experiencias en municipios con aplicación de regulaciones y recomendaciones: Córdoba. I Seminario sobre actuaciones de prevención, seguridad y extinción en zonas de interfaz urbano-forestal. Colegio de Ingenieros Técnicos Forestales. San Fernando de Henares, 12 de noviembre de 2015.

Quesada Fernández, Clara: "Formación y capacitación técnica de profesionales para la gestión de la interfaz y la prevención” I Seminario sobre actuaciones de prevención, seguridad y extinción en zonas de interfaz urbano-forestal. Colegio de Ingenieros Técnicos Forestales. San Fernando de Henares, 12 de noviembre de 2015.

Quesada Fernández, Clara: Oportunidades, limitaciones y retos de seguridad en la defensa y combate de incendios en la interfaz urbano forestal. V Congreso Científico de Investigadores en Formación de la Universidad de Córdoba, "Creando redes", 2016.

Quesada Fernández, Clara y Quesada Fernández, Daniel: Fire Culture and Environmental Education in Fire Prone Communities' Areas: State of the Art and Case Study in Spain en Proceedings of the the IV International symposium on fire economics, policy and planning: climate change and fire. 5-11 de noviembre de 2012, Ciudad de Méjico (Méjico), USDA Forest Service, Riverside, California, 2012.

Quesada Fernández, Clara y Quesada Fernández, Daniel: Perception du risque dans les petites communautés sujette au feu. Etude de cas (Fire risk perception in fire prone communities. Case of Cerro Muriano, (Córdoba, Spain) en International Conference on forest fire risk modelling and mapping. 30 de septiembre - 2 de octubre de 2013. Aix en Provence (Francia), IRSTEA, 2013.

Quesada Fernández, Clara y Quesada Fernández, Daniel: Safety at the WUI: a firefighters view en Viegas (ed.), Advances in Forest Fires Research, Coimbra, Press Coimbra, 2014.

Quesada Fernández, Clara y Quesada Fernández, Daniel: Residents perception of forest fire risk at WUI. Study cases in northwestern Andalusia (Spain) en II International Conference on Fire Behaviour and Risk (Alghero, Cerdeña, Italia), 2015.

Quesada Fernández, Clara y Quesada Fernández, Daniel: Wildland urban interface: Wildland Firefighting Safety Problems and Challenges en $14^{\text {th }}$ International Summit on Fire Safety. Barcelona (España), 2017.

Quesada Fernández, Clara, Quesada Fernández, Daniel, Luque, I. 2016. Opportunities to prevent fires in early ages. A mediterranean example: "Crece con tu Árbol" ("Growing up beside your tree”) andalusian programme. FORESTFIRE 2016. International Conference on Forest Fires and WUI Fires. Aix en Provence (Francia), IRSTEA, 2016. 
Short contribution - Fire at the Wildland Urban Interface

\title{
Mapping Canadian Interface Areas
}

\author{
Lynn Johnston $^{1 *}$; Mike Flannigan ${ }^{2}$ \\ ${ }^{1}$ Canadian Forest Service. 1219 Queen Street E., Sault Ste Marie, Ontario, Canada, P6A 2E6, \\ \{lynn.johnston@canada.ca*\} \\ ${ }^{2}$ University of Alberta. 7-13A General Services, University of Alberta, Edmonton, Alberta, Canada, T6G \\ 2H1, \{mike.flannigan@ualberta.ca\}
}

\begin{abstract}
Abstrac t
This project aimed to produce the first map of the Canadian wildland-urban interface (WUI). We also extended the interface concept beyond the traditional "urban" structures of the wildland-urban interface to include industrial structures (e.g. oil and gas structures) or infrastructure values (e.g. roads, powerlines, railways); producing two additional national maps to reflect the interface areas of these potentially vulnerable industrial structures (i.e. the wildland-industrial interface) or infrastructure values (i.e. the wildland-infrastructure interface) with wildland fuels. The interface maps provide a baseline for future work but also have a variety of practical applications such as fire management planning and decision support, insurance, municipal planning, and fire mitigation.
\end{abstract}

Keywords: wildland-urban interface, wildland fire, fire risk, GIS

\section{Introduction}

Recent Canadian wildfire disasters such as Slave Lake (2011) and Fort McMurray (2016) are examples of the destructive power of wildfires to human structures. Fires that have the potential to cause this kind of destruction are referred to as "interface" fires (Figure 1).

The wildland-urban interface (WUI) is where homes or other human structures meet with or intermingle amongst burnable wildland fuels. Canadian fire management agencies actively mitigate and suppress fire in these interface areas in order to protect human life and structural values.

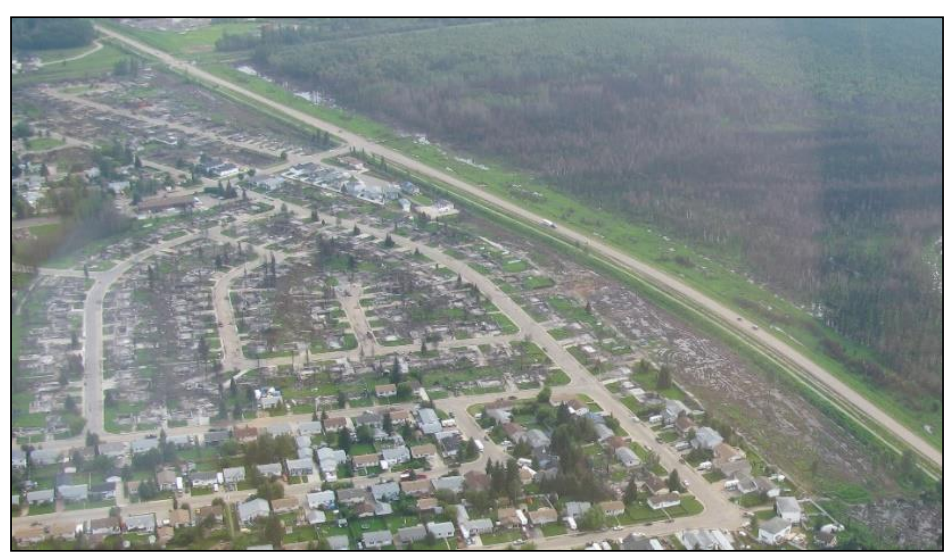

Figure 1 - Destruction of homes in Slave Lake, Alberta, Canada in 2011 due to an interface fire (photo credit: Mike Flannigan/University of Alberta).

For effective fire management in the interface, we need information on what areas are at risk.

In Canada, a national examination of risk was not available and represented a large gap in wildfire research. The WUI has been mapped and studied in other areas of the world, with many studies additionally quantifying interface fire risk (e.g. Radeloff et al. 2005; Theobald and Romme 2007; Vadrevu et al. 2010; Haas et al. 2013; Chuvieco et al. 2014; Thomas and Butry 2014; Fox et al. 2015; Radeloff et al. 2018). However, in Canada there is very limited information on this topic despite the potential for destructive impacts of wildfire on human structures.

The objective of this project is to map the interface areas of Canada that are at risk to wildland fires. There are two main components to this project: 1) mapping interface areas and 2) quantitatively assessing the amount of fire risk in those interface areas.

Part one of this project is complete (Johnston and Flannigan 2018); it resulted in the production of 
the first national map of the interface in Canada. This map includes the more traditional interface of wildland fuels with homes, commercial buildings, and public structures, forming the wildland-urban interface (WUI). We also extended the interface concept to industrial (e.g. oil and gas or mining structures) and infrastructure (e.g. roads, railways, powerlines) areas to provide a more comprehensive view of potential impacts to buildings and essential infrastructure.

\section{Methods}

There are a wide variety of ways of defining and mapping interface areas (see Mell et al. 2010; Platt 2010). This study selected a fuels-focused definition of the interface areas, using a buffer around each potentially vulnerable feature. A brief summary of the methods used to map the interface will be covered here, but for full methods see Johnston and Flannigan (2018).

Mapping the interface areas required data on both structures/infrastructure and on wildland fuels (Fig. 2a). Structure/infrastructure locations were taken from the CanVec+ (Natural Resources Canada 2015a) dataset, and each potentially vulnerable feature was included in calculations of the wildlandurban interface, wildland-industrial interface, or infrastructure interface, depending on if they were primarily urban/community features, industrial features, or infrastructure.

Relevant fuels data was extracted from the Land Cover circa 2000 (Natural Resources Canada $2015 \mathrm{~b}$ ) dataset. The fuels were then classified according to their relative fire hazard they may impart to structures (similar to the methods of Theobald and Romme (2007)). A "high hazard" fuel would be something like a continuous conifer forest, which can have potentially extreme fire behaviour and could impart extreme fire risk to nearby structures. A "low hazard" fuel would be something like a sparsely vegetated shrubland, which has low potential for fire spread and would impart a much lower risk to nearby structures.

To produce the actual interface areas, a buffer of vegetated areas was calculated around each potentially vulnerable structure. The size and shape of the buffer was dictated by the type and arrangement of surrounding fuels. A large buffer (and therefore a large interface area) would be produced with higher hazard fuels, and smaller buffers (small interface areas) where there is less fuel or lower hazard fuels. The maximum distance the buffer could extend was limited to $2400 \mathrm{~m}$. Nonfuel areas were removed from the buffered areas, resulting in the final interface areas (Fig. 2b).

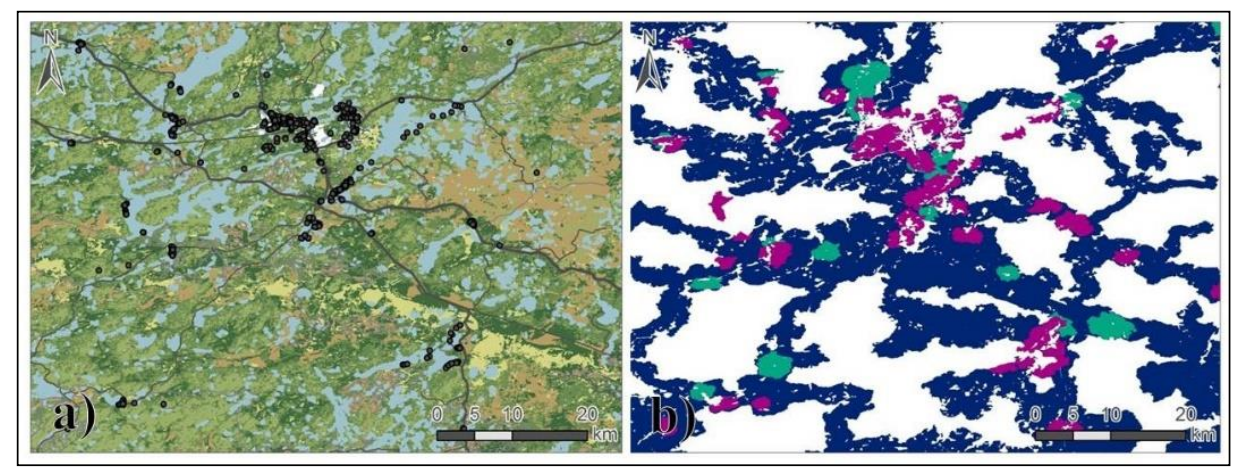

Figure 2 - Example maps showing a) the data inputs: the fuels (greens, browns) and values (black), and also water is shown in blue, and then image b) shows the wildland-urban interface (magenta), wildland-industrial interface (cyan), and infrastructure interface (navy).

\section{Results}

Nationally, these interface maps (Figure 3) show that Canada has 32.3 million ha of WUI (3.8\% of total national land area), 10.5 million ha of wildland-industrial interface (1.2\%), and 109.8 million ha of infrastructure interface (13.0\%). This is a widespread issue affecting large areas of Canada. 


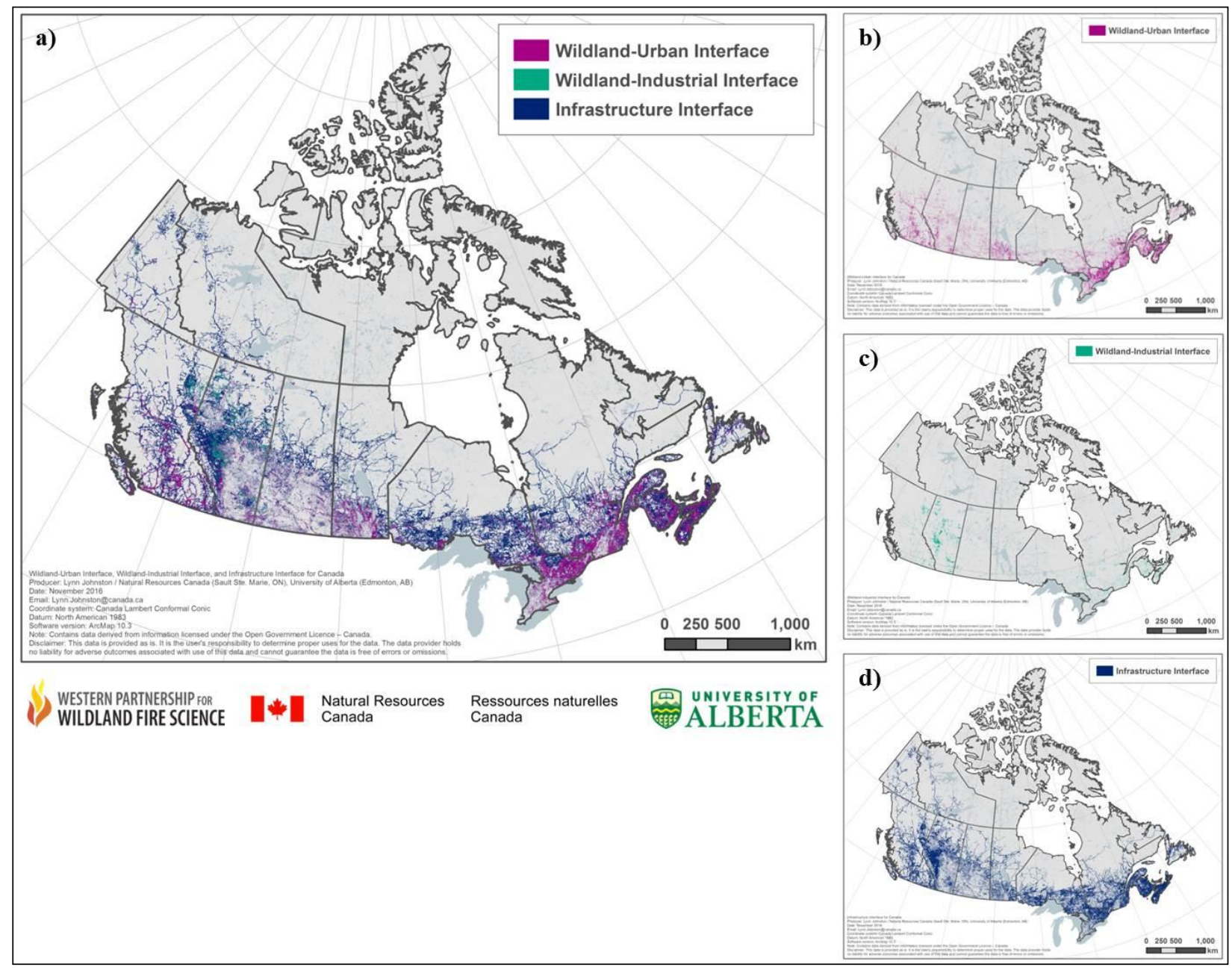

Figure 3 - Canadian national interface maps, with a) showing all three interface "types" together, b) showing the wildland-urban interface (WUI), c) showing the wildland-industrial interface (WII), and d) showing the infrastructure interface.

Overall, $60 \%$ of all cities, towns, settlements, and reservations across Canada were found to have a significant amount of WUI (defined as those with more than 500 ha of WUI within a $5 \mathrm{~km}$ radius). There have been many recent fires near or within the interface areas; $40 \%$ of recent fires would be considered an "interface fire". For more information on the production and initial analysis of these maps, see Johnston and Flannigan (2018).

\section{Discussion}

Maps of the interface may imply that there is a fire risk, but a map of the interface alone is not a proxy for risk. Mapping the interface is the first step in assessing interface fire risk and provides the locations of where fire risk to interface areas may be possible (i.e. where wildland fuels and human structures intermix or meet). A multitude of additional factors need to be considered to fully quantify risk. Fire risk is inconsistently defined in wildfire literature, but the traditional natural hazards risk definition assesses fire risk as the conditional probability of an event occurring multiplied by the expected consequence when the event occurs (Finney 2005; Hardy 2005; Thompson et al. 2011; Haas et al. 2013; Miller and Ager 2013; Chuvieco et al. 2014). We can further refine the definition of fire risk to the function of likelihood, intensity, and effects (e.g. Thompson and Calkin 2011; Miller and Ager 2013). Assessment of these three risk components can use variety of potential approaches and 
quickly develops into a complex paradigm with many elements, as visualized in the word cloud in Figure 4.

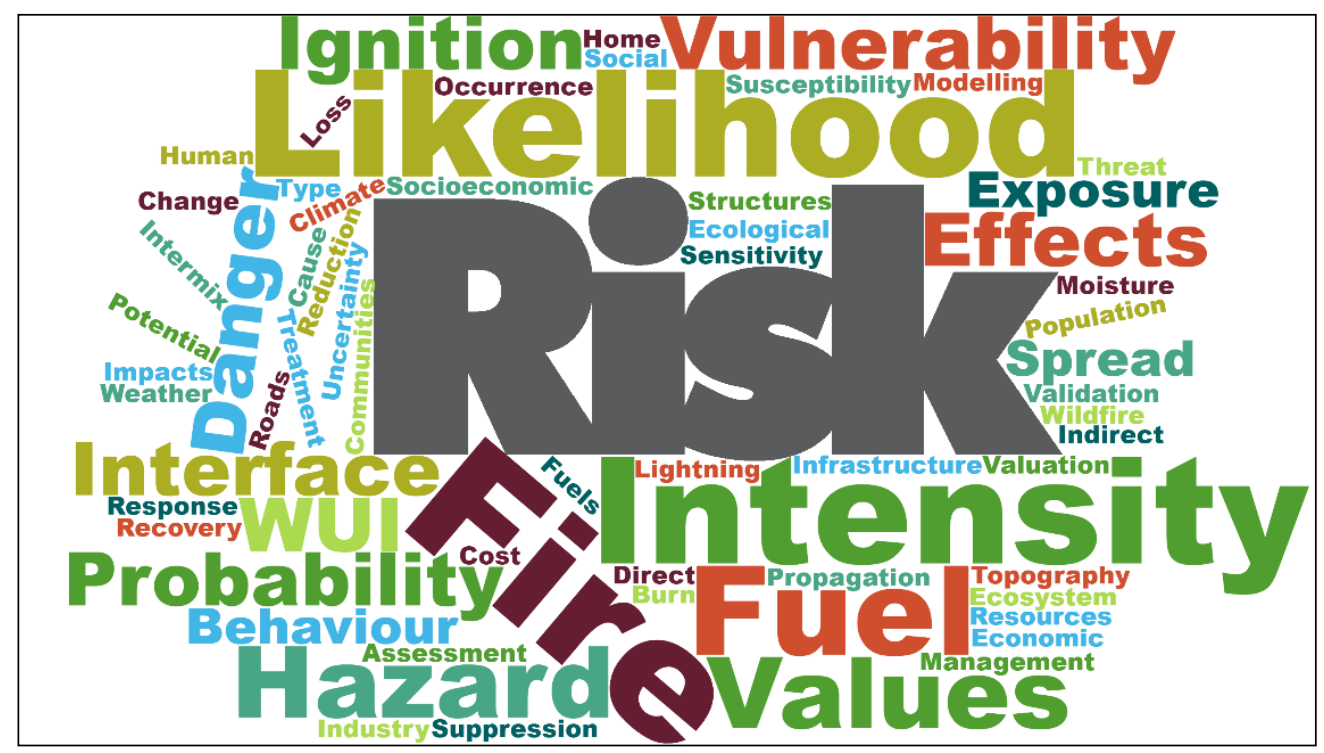

Figure 4 - Word cloud of terms related to fire risk.

The results of this project will be used for the second component of this project, i.e. quantifying fire risk within interface areas. In addition, we will investigate fire activity within these interface areas to characterize fire intensity, seasonality, and ignition sources. Additionally, these maps will be used to detect recent growth patterns in the interface and to model future interface areas.

The interface maps are available for practical applications, and if used for the correct application and at the correct spatial scale, can be useful for a variety of applications, for example: fire insurance, municipal planning, fire suppression, values protection, fuel management, and forestry.

\section{References}

Chuvieco, E, Aguado, I, Jurdao, S, Pettinari, ML, Yebra, M, Salas, J, Hantson, S, de la Riva, J, Ibarra, P, Rodrigues, M, Echeverria, M, Azqueta, D, Roman, MV, Bastarrika, A, Martinez, S, Recondo, C, Zapico, E, Martinez-Vega, FJ (2014) Integrating geospatial information into fire risk assessment. International Journal of Wildland Fire 23, 606-609. 10.1071/WF12052.

Finney, MA (2005) The challenge of quantitative risk analysis for wildland fire. Forest Ecology and Management 211, 97-108. 10.1016/j.foreco.2005.02.010.

Fox, D, Martin, N, Carrega, P, Andrieu, J, Adnès, C, Emsellem, K, Ganga, O, Moebius, F, Tortorollo, $\mathrm{N}$, Fox, E (2015) Increases in fire risk due to warmer summer temperatures and wildland urban interface changes do not necessarily lead to more fires. Applied Geography 56, 1-12. 10.1016/j.apgeog.2014.10.001.

Haas, JR, Calkin, DE, Thompson, MP (2013) A national approach for integrating wildfire simulation modeling into Wildland Urban Interface risk assessments within the United States. Landscape and Urban Planning 119, 44-53. 10.1016/j.landurbplan.2013.06.011.

Hardy, CC (2005) Wildland fire hazard and risk: problems, definitions, and context. Forest Ecology and Management 211, 73-82. 10.1016/j.foreco.2005.01.029.

Johnston, LM, Flannigan, MD (2018) Mapping Canadian wildland fire interface areas. International Journal of Wildland Fire 27, 1-14. 10.1071/WF16221. 
Mell, WE, Manzello, SL, Maranghides, A, Butry, D, Rehm, RG (2010) The wildland-urban interface fire problem - current approaches and research needs. International Journal of Wildland Fire 19, 238-251. 10.1071/WF07131.

Miller, C, Ager, AA (2013) A review of recent advances in risk analysis for wildfire management. International Journal of Wildland Fire 22, 1-14.

Natural Resources Canada (2015a) CanVec+. Dataset. Used under the Open Government Licence Canada. (EaSS GeoGratis Client Services. Natural Resources Canada, Canada Centre for Mapping and Earth

Observation). ftp://ftp2.cits.rncan.gc.ca/pub/canvec/archive/canvec+_archive_20151029/doc/CanVec+_en_relea se_notes.pdf.

Natural Resources Canada (2015b) Land Cover, circa 2000 - vector. Dataset. Used under the Open Government Licence - Canada. (EaSS GeoGratis Client Services. Natural Resources Canada, Canada Centre for Mapping and Earth Observation). www.GeoGratis.gc.ca.

Platt, RV (2010) The Wildland-Urban Interface: Evaluating the Definition Effect. Journal of Forestry 108, 9-15.

Radeloff, VC, Hammer, RB, Stewart, SI, Fried, JS, Holcomb, SS, McKeefry, JF (2005) The wildlandurban interface in the United States. Ecological Applications 15, 799-805.

Radeloff, VC, Helmers, DP, Kramer, HA, Mockrin, MH, Alexandre, PM, Bar-Massada, A, Butsic, V, Hawbaker, TJ, Martinuzzi, S, Syphard, AD (2018) Rapid growth of the US wildland-urban interface raises wildfire risk. Proceedings of the National Academy of Sciences 115, 3314-3319. 10.1073/pnas. 1718850115.

Theobald, DM, Romme, WH (2007) Expansion of the US wildland-urban interface. Landscape and Urban Planning 83, 340-354. 10.1016/j.landurbplan.2007.06.002.

Thomas, DS, Butry, DT (2014) Areas of the US wildland-urban interface threatened by wildfire during the 2001-2010 decade. Natural Hazards 71, 1561-1585. 10.1007/s11069-013-0965-7.

Thompson, MP, Calkin, DE (2011) Uncertainty and risk in wildland fire management: a review. Journal of Environmental Management 92, 1895-1909.

Thompson, MP, Calkin, DE, Finney, MA, Ager, AA, Gilbertson-Day, JW (2011) Integrated nationalscale assessment of wildfire risk to human and ecological values. Stochastic Environmental Research and Risk Assessment 25, 761-780. 10.1007/s00477-011-0461-0.

Vadrevu, KP, Eaturu, A, Badarinath, K (2010) Fire risk evaluation using multicriteria analysis - a case study. Environmental monitoring and assessment 166, 223-239. 
Short contribution - Fire at the Wildland Urban Interface

Risk analysis of LPG tanks at the wildland-urban interface: are regulated safety distances large enough?

E. Pastor ${ }^{1}$; D. Caballero ${ }^{2}$; J. Martín ${ }^{1}$; E. Planas ${ }^{1}$

${ }^{1}$ Department of Chemical Engineering, Centre for Technological Risk Studies, Universitat Politècnica de Catalunya·BarcelonaTech, Eduard Maristany 10-14, E-08019 Barcelona, Catalonia, Spain. \{elsa.pastor@upc.edu*,jmartin93upc@gmail.com,eulalia.planas@upc.edu\}

${ }^{2}$ Freelance International Consultant, Consultant: \{davidcaballero@europe.com\}

Keywords: WUI fires, WUI microscale, propane tank, domino effect, fire safety.

Forest fires affecting urban and rural communities represent a rising problem throughout the world. They pose tremendous management challenges in terms of civil protection and fire mitigation. These emergencies often exceed fire-fighters capacities due to its multi-risk nature: they usually involve wildfire suppression, structures protection and communities' evacuation and they can even trigger natech-type events when interfacing industrial infrastructure.

The WUI (wildland-urban interface) fire problem is inherently complex, as it is characterized by the interaction of multiple phenomena of diverse nature occurring at different observation scales: the macroscale or landscape scale, the mesoscale or development/settlement scale and the microscale or home/plot scale. It is at the microscale where the specific events that jeopardize residents and assets can be observed and where prevention actions at home-owner level must be undertaken. The WUI microscale is quite often characterized by the presence of all sorts of combustible elements around structures (ground fuels, ornamental vegetation, stored material, etc.) whose hazard is poorly characterized and thus remarkably disregarded by residents.

In this sense, it has to be highlighted the hazard associated with domestic LPG (liquefied petroleum gas) storage tanks, used as energy source for heating, hot water production or cooking in WUI developments. This type of infrastructure can be seriously threaten by a fire nearby, particularly in those cases where negligences or regulatory gaps allow a very close exposure of these tanks to flames coming from nearby fuels.

In countries where this type of domestic LPG infrastructure is currently used, regulations specify, among others, safety distances from the LPG supply unit to vulnerable elements, operation conditions, fire extinguishing systems and other protection measures that have to be taken into account. However, there exist serious deficiencies in the overall regulation framework, particularly in those areas where WUI fires usually occur. For instance, Spanish Standard UNE 60250 (Aenor, 2008) for the set-up, exploitation and manteinance of LPG vessels indicates that for 1 to $5-\mathrm{m}^{3}$ tanks, safety distances should be of $2 \mathrm{~m}$ from the walls of the tank and $3 \mathrm{~m}$ from the service orificies (figure 1). This distances can be reduced by a $50 \%$ for smaller tanks. The Standard also states that the LPG unit should be provided with an enclosure made of metal mesh or other materials with very limited contribution to fire, to avoid manipulation from non qualified personel. Nevertheless, this measure can be ignored in case the tank is located inside a single-family house plot with perimeter elements delimiting the property and preventing the access to outsiders. The Standard has no explicit mention to natural fuels (e.g. ornamental vegetation), usually present at the WUI microscale, that can be located inside the plot and be eventually ignited in case of WUI fire. With these provisions, an scenario of a 5- $\mathrm{m}^{3}$ LPG tank closely (i.e. $3 \mathrm{~m}$ ) surrounded by a fence made of a higly flammable ornamental hedgerow (e.g. Cupresus arizonica) is permitted to be set and obviously entails an inherent risk. 


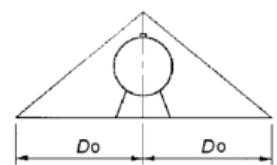

Sección A-A
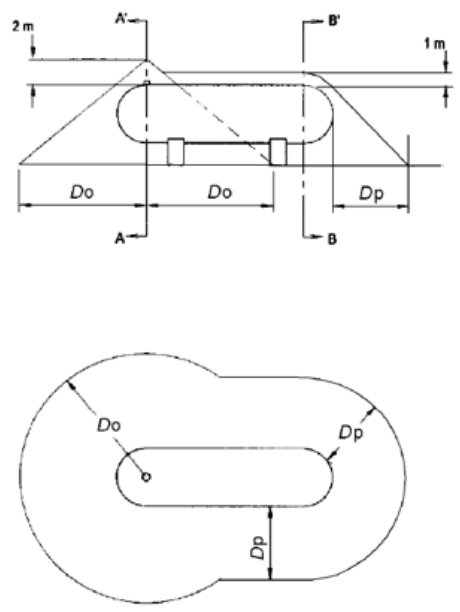

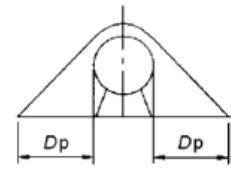

Sección B-B'

Figure 1 - Distances between walls (Dp) and orificies (Do) as indicated by UNE 60250

In recent WUI fire events (e.g. Benitatxell, Spain, 2016; Madeira, Portugal, 2016; Calabassas, California, 2016) these type of infrastructures were dangerously involved in the emergency (figure 2). The lack of an effective safety distance between the LPG tank and the surrounding fuels, provoked undesired overpressure inside the tanks leading to the opening of the safety relief valves. Released gas immediately ignited giving rise to intense jet fires. Although explosions did not occur, the magnitude of the consequences in case of a BLEVE (Boling Liquid Expanding Vapour Explosion)-Fireball event could have been devastating, given the high population and assets density that usually characterize WUI areas.
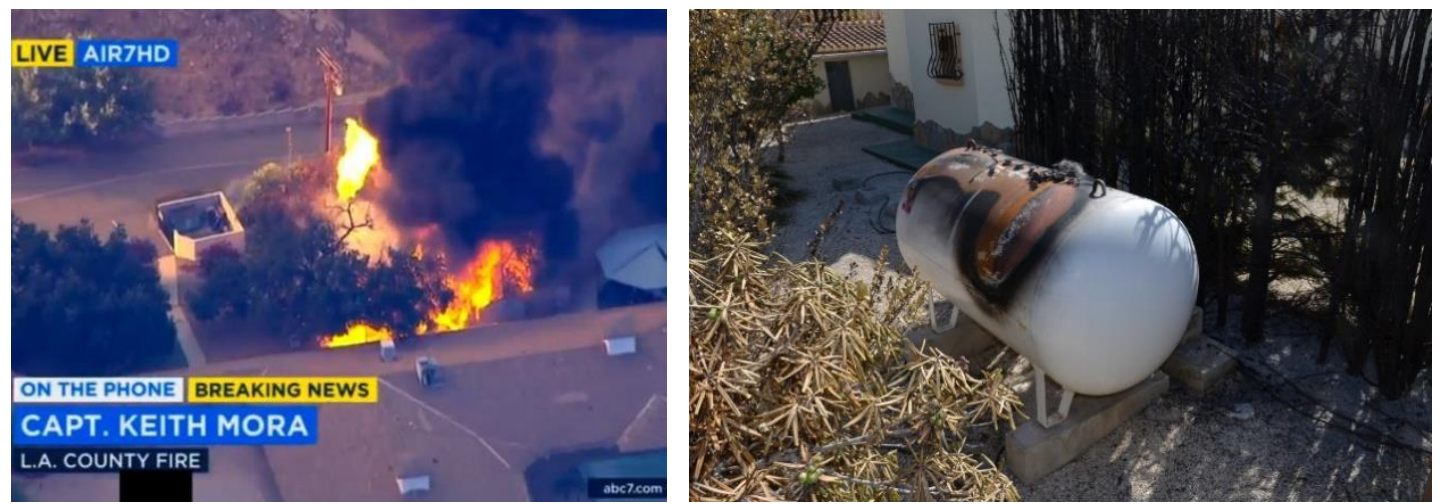

Figure 2 - Left: Calabassas fire (California, 2016). A propane jet fire can be observed at the centre of the image.

Flames from ornamental fuels are close to the tank. LPG infrastructure was inside a kindergarten facility. Right: Domestic LPG tank in Benitatxell (Spain, 2016) damaged by the jet fire. The tank was surrounded by an ornamental hedgerow that ignited by spotting.

The scientific community has already been aware of these type of hazardous scenarios and has started tackling the problem either by an experimental approach (Heymes et al., 2013a) or by physical modelling of the processes involved (Heymes et al., 2013b; Scarponi et al., 2018). However, the scenarios studied have not been reflecting the WUI micro-scale reality. Rather, a less realistic scenario of a distant fully developped crown-fire, which might be developpping at the meso-scale, has been considered as the one threatening an LPG tank.

This paper presents a comprehensive overview by which we identify both technical and scientific needs that have to be addressed to minimize the fire/explosion risk of LPG domestic tanks at the WUI. We analyse the existent regulations in WUI-fire prone countries dealing with domestic LPG services to detect gaps, deficiencies and inconsistencies between countries and regulation bodies. We review the state of the art of experimental and modelling studies dealing with LPG infrastructure risk 
assessment, highlighting the main findings and available methods, and finally, we explore a CFD modelling framework that shall be of help to establish effective safety distances.

\section{References}

Aenor, Asociación Española de Normalización y Certificación. (2008). UNE 60250: instalaciones de almacenamiento de gases licuados del petróleo (GLP) en depósitos fijos para su consumo en instalaciones receptoras. Retrieved from https://temariosformativosprofesionales.files.wordpress.com/2012/11/une_602502008.pdf

Heymes, F., Aprin, L., Birk, A.M., Slangen, P., Jarry, J.B., François, H., Dusserre, G. (2013). An experimental studyof an LPG tank at low filling level heated by a remote wall fire. Journal of Loss Prevention in the Process Industries, 26, 1484-1491.

Heymes, F., Aprin, L., Forestier, S., Slangen, P., Jarry, J.B., François, H., Dusserre, G. (2013). Impact of a distant wildland fire on an LPG tank. Fire Safety Journal, 61, 100-107.

Scarponi, G. E., Landucci, G., Heymes, F., Cozzani, V. (2018). Process Safety and Environmental Protection, 114, 251-270. 
Short contribution - Decision Support Systems and Tools

Analysis of the temporal behaviour of the number of forest fires in some countries of Europe

\author{
J.L. Legido ${ }^{3 *}$, M.M. Mato ${ }^{4}$, S. García-Garabal ${ }^{5}$, T. Carballas ${ }^{2}$, M.I. Paz Andrade ${ }^{1}$ \\ ${ }^{1}$ Department of Applied Physics, Microcalorimetry, Faculty of Physics, University of Santiago de \\ Compostela. Campus Universitario Sur, E-15782 Santiago de Compostela, Spain, \\ \{inmaculada.paz.andrade@usc.es\} \\ ${ }^{2}$ Agrobiological Research Institute of Galicia (IIAG-CSIC), Campus Universitario Sur, Section 122, E- \\ 15780 Santiago de Compostela, Spain, \{tcf@iiag.csic.es\} \\ ${ }^{3 *}$ Department of Applied Physics, University of Vigo. Campus Lagoas-Marcosende. 36310 Vigo, Spain, \\ \{xllegido@uvigo.es*\} \\ ${ }^{4}$ Department of Applied Physics (FCCED), University of Vigo. Campus de Pontevedra. 36005 Pontevedra, \\ Spain, \{fammmc@uvigo.es\} \\ ${ }^{5}$ Department of Physics, University of A Coruña, A Coruña, Spain, \{Sandra.garcia-garabal@udc.es\}
}

\begin{abstract}
This paper presents a temporal analysis of the number of forest fires that occurred in different European countries between 1990 and 2016. The countries studied were Bulgaria, Croatia, France, Finland, Germany, Greece, Hungary, Italy, Latvia, Lithuania, Norway, Poland, Portugal, Romania, Slovakia, Spain, Sweden, Switzerland and Turkey.

In these countries, the data from the studied time interval indicated an annual average of 74000 forest fires where more than $75 \%$ occurred in countries of southern Europe, such as Portugal, Spain, France, Italy, Greece, Croatia and Turkey. In addition, most of them occurred in Portugal (30\%) and Spain $(23 \%)$ which indicates that the fires occurred in the Iberian Peninsula exceeded $50 \%$.

On the other hand, Portugal had the highest rate of number of fires per area and population with 0.24 annual fires per $\mathrm{km}^{2}$ and 2.04 annual fires per thousand inhabitants, whereas Germany has a rate of 0.0031 annual fires per $\mathrm{km}^{2}$ and 0.014 annual fires per thousand inhabitants.

Clustering data of annual number of fires for the studied countries were fitted to a variable degree polynomial function. According to this fitting, the number of fires increased up to the year 2000 and decreased afterwards.
\end{abstract}

Keywords: forest fires, number, europe, mathematical functions

\title{
1. Introduction
}

European countries, as well as other countries in the world, are suffering the tremendous scourge of forest fires, which were devastating in recent decades. Their direct destructive effects are the main cause of the enviroment degradation and the destruction of the forest ecosystem and provoke important socio-economic losses. The use of mathematical models and systems based on knowledge is of paramount importance to develop efficient tools for the forest fire fighting.

\section{Methods}

This paper presents a temporal study of forest fires that occurred in different European countries between 1990 and 2016 (Forest Fires in Europe, Middle East and North Africa 2016 (JRC, 2016)). The countries studied, shown in figure 1, are: Bulgaria, Croatia, France, Finland, Germany, Greece, Hungary, Italy, Latvia, Lithuania, Norway, Poland, Portugal, Romania, Slovakia, Spain, Sweden, Switzerland and Turkey. 
An analysis of the number of forest fires with the area or the population for each studied country was made.

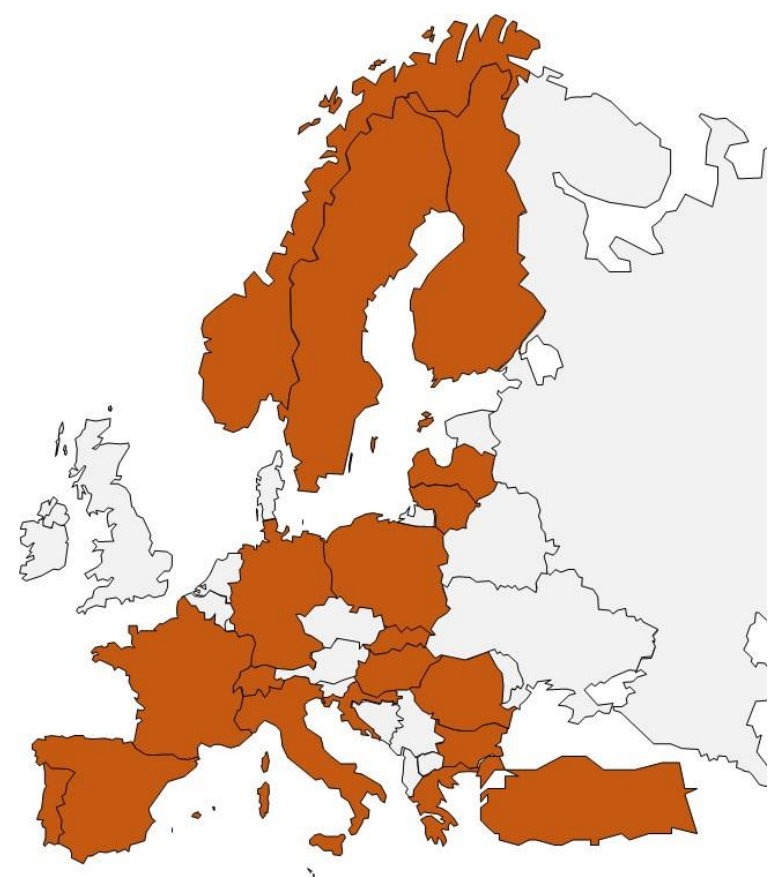

Figure 1 - Studied area

\subsection{Mathematical functions}

In this work, data on the number of annual forest fires registered in the studied countries were normalized [2] to weighted functions $(g(x))$ between 0 and 1 .

$$
g(x)=\left(f(x)-f_{\min }\right) /\left(f_{\max }-f_{\min }\right)
$$

where $f(x)$ is the number of annual forest fires, $f_{\min }$ the minimum of fires and $f_{\max }$ the maximum in the interval of the study and where $x=y-y_{0}+1$ ( $y$ is the year and yo.the initial year of the study).

The clustering function $(h(x))$ was determined [3]

$$
h\left(z_{k}\right)=\left(\sum_{j=1}^{m} g\left(x_{j}\right)\right) / m \quad z_{k}=\left(\sum_{j=1}^{m} x_{j}\right) / m
$$

where $\mathrm{m}$ is the clustering coefficient (number of years grouped).

The clustering functions were fitted to polynomials of the form:

$$
h(z)=\sum_{i=0}^{n} A_{i}(z)^{i}
$$

where $A_{i}$ are the regression coefficients and $n$ the degree of the polynomials.

\section{Results}

Figure 1 shows the fittting functions, h (z), for all studied countries compared with those of Portugal and Germany and in figure 2 Spain, France and Finland are compared. The best correlations for the functions $h(z)$ were obtained for cubic functions $(n=3)$. 
Portugal shows an increase in the evolution of the number of fires (by means the proposed functions) until 2000, while in Germany decreased throughout the studied time interval. The set of countries Europe shows a growth rate in the number of fires between 1990 and 1998.

In figure 2 it can be seen that Spain presents a behavior very similar to that of Portugal. The maximum number of fires in Spain occurred in 1999 while in Finland it was in 2006.

The data from the studied time interval indicated an annual average of 74000 forest fires where more than 75\% occurred in countries of southern Europe, such as Portugal, Spain, France, Italy, Greece, Croatia and Turkey. In addition, of all the studied countries, Portugal had the highest annual average of forest fires, with $30 \%$ of the total as well as Spain reached $23 \%$ of the total, which indicates that the fires occurred in the Iberian Peninsula exceeded 50\%.

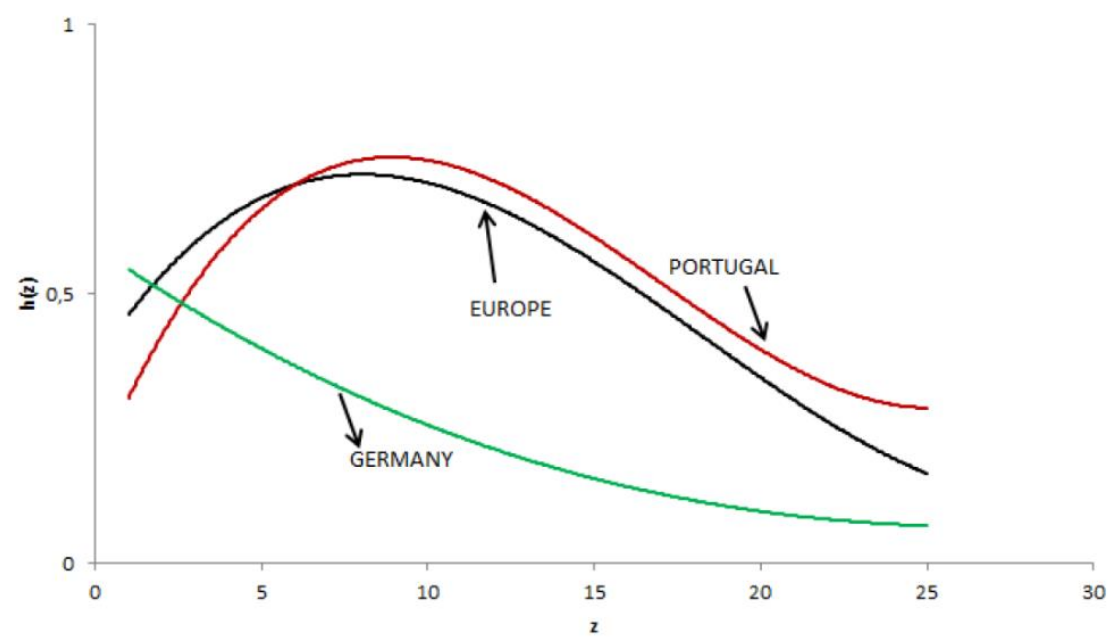

Figure 2 - Functions $h(z)$ for all the studied countries compared with those of Portugal and Germany

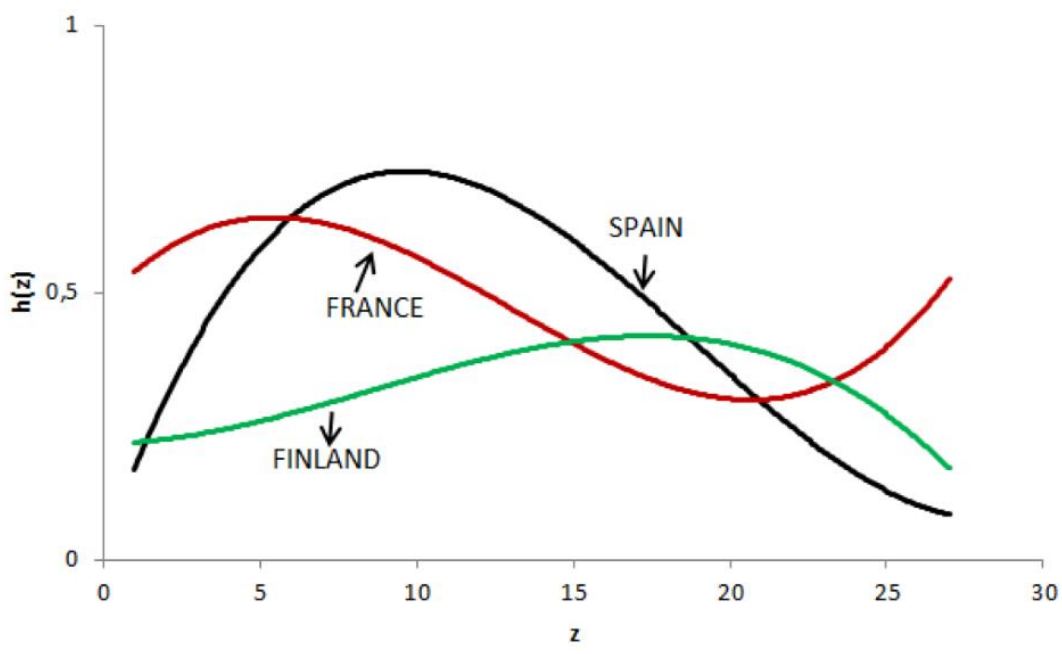

Figure 3 - Comparison of the h(z) functions for Spain, France and Finland.

Table 1 shows the results obtained, using the equations 2 and 3, for all the countries studied in this work. The values of the clustering coefficients, m, vary between 3 and 5 .

The annual average data from Bulgaria, Finland, Germany, Latvia, Lithuania, Slovakia, Sweden, Switzerland, France, Spain, and Greece were clustered each five years $(m=5)$. The value $m=4$ was used for the data from Croatia, Hungary, Lithuania, Norway, Romania, Turkey, and Italy. Finally, the fire data from Poland were clustered with $\mathrm{m}=3$. 
The countries that obtain correlation coefficients higher than 0.9 were Germany, Italy and Spain. On the other hand, poor correlations, with correlation coefficients below 0.7 , were obtained, for Italy, Croatia and Denmark.

Table 1 - Clustering coefficients, $m$, from eq. 2, regression coefficients, $A_{i}$, from eq. 3 and the correlation coefficients, $R^{2}$, of the fitting curves, $h(z)$, for all estudied countries.

\begin{tabular}{|c|c|c|c|c|c|c|}
\hline COUNTRIES & m & $A_{0}$ & $A_{1}$ & $A_{2}$ & $A_{3}$ & $R^{2}$ \\
\hline BULGARIA & 5 & 0.4724 & -0.0985 & 0.0103 & -0.00028 & 0.5624 \\
\hline CROATIA & 4 & 0.4212 & -0.0140 & 0.0018 & 0.000067 & 0.4788 \\
\hline FINLAND & 5 & 0.2835 & -0.0272 & 0.0047 & -0.00015 & 0.9471 \\
\hline GERMANY & 5 & 0.6808 & -0.0481 & 0.0012 & -0.0000094 & 0.9922 \\
\hline HUNGARY & 4 & 0.1820 & -0.0406 & 0.0035 & 0.000065 & 0.6066 \\
\hline LATIVIA & 5 & 0.4668 & -0.0488 & 0.0060 & -0.00020 & 0.9998 \\
\hline LITHUANIA & 4 & 0.5310 & -0.0376 & 0.0035 & -0.00011 & 0.7765 \\
\hline POLAND & 3 & 0.0352 & 0.0402 & -0.00041 & -0.000016 & 0.8252 \\
\hline NORWAY & 4 & 0.2239 & 0.0199 & 0.00055 & -0.00054 & 0.6028 \\
\hline ROMANIA & 4 & 0.1289 & -0.0208 & 0.0034 & -0.000099 & 0.4500 \\
\hline SLOVKIA & 5 & 0.30594 & 0.21517 & -0.01709 & 0.00037 & 0.9862 \\
\hline SWEDEN & 5 & 0.0749 & -0.0040 & 0.0052 & -0.00021 & 0.6939 \\
\hline SWITZERLAND & 5 & 0.2826 & 0.01585 & -0.0020 & 0.000042 & 0.8239 \\
\hline TURKEY & 4 & 0.2168 & 0.0261 & -0.0029 & -0.000098 & 0.8008 \\
\hline PORTUGAL & 4 & -0.1791 & 0.2081 & -0.0138 & 0.00025 & 0.8682 \\
\hline SPAIN & 5 & 0.1435 & 0.2120 & -0.0155 & 0.00030 & 0.9954 \\
\hline FRANCE & 5 & 0.1644 & 0.1764 & -0.0176 & 0.00045 & 0.7547 \\
\hline ITALY & 4 & 1.201 & -0.1430 & 0.0085 & -0.00018 & 0.9924 \\
\hline GREECE & 5 & 0.6110 & 0.0050 & 0.0013 & -0.000076 & 0.9195 \\
\hline
\end{tabular}

Table 2 shows the annual average number of fires per unit area and the annual average number of fires per thousand inhabitants. The average number of annual fires per unit area in Europe was 0.022 fires $/ \mathrm{km}^{2}$, obtaining the highest value Portugal with a rate of 0.24 fires $/ \mathrm{km}^{2}$. Related to the average annual number of fires per thousand inhabitants, the European average was 0.32 fires per thousand inhabitants and the average of Portugal was 2.04 fires per thousand inhabitants. Germany, in contrast, reached a rate of 0.003 annual fires per $\mathrm{km}^{2}$ and 0.014 annual fires per thousand inhabitants.

Once again, Portugal has the highest rate of annual average fires per $\mathrm{km}^{2}$, reaching $58 \%$ of the total and the highest rate of annual average fires per thousand inhabitants, about $33 \%$ of total.

Table 2 - Annual average number of fires per unit area and annual average number of fires per thousand inhabitants from each one studied countries, from the sum of all of them (almost all of Europe ${ }^{1}$ ) and from average of them ${ }^{2}$

\begin{tabular}{|c|c|c|c|c|c|c|c|}
\hline COUNTRIES & BULGARIA & CROATIA & FINLAND & GERMANY & HUNGARY & LATIVIA & LITHUANIA \\
\hline Fires $/ \mathrm{km}^{2}$ & 0.0047 & 0.0048 & 0.0037 & 0.0031 & 0.0060 & 0.0115 & 0.0084 \\
\hline Fires/1000 inh. & 0.069 & 0.059 & 0.23 & 0.014 & 0.056 & 0.31 & 0.16 \\
\hline
\end{tabular}




\begin{tabular}{|c|c|c|c|c|c|c|c|}
\hline COUNTRIES & POLAND & NORWAY & ROMANIA & SLOVKIA & SWEDEN & SWITZERLAND & TURKEY \\
\hline Fires/km $\mathbf{k m}^{\mathbf{2}}$ & 0.0013 & 0.0263 & 0.00087 & 0.0079 & 0.0090 & 0.0026 & 0.0028 \\
\hline Fires/1000 inh. & 0.010 & 1.7 & 0.0097 & 0.071 & 0.43 & 0.014 & 0.20 \\
\hline COUNTRIES & PORTUGAL & SPAIN & FRANCE & ITALY & GREECE & EUROPE & $\begin{array}{c}\text { Avg. } \\
\text { EUROPE }^{2}\end{array}$ \\
\hline Fires/km $\mathbf{2}$ & 0.24 & 0.033 & 0.0078 & 0.028 & 0.012 & 0.41 & 0.022 \\
\hline Fires/1000 inh. & 2.04 & 0.36 & 0.067 & 0.14 & 0.13 & 6.1 & 0.32 \\
\hline
\end{tabular}

\section{Conclusions}

The annual average data of the studied countries were clustering with $m=3$ to 5 .

The best correlations for the functions $\mathrm{h}(\mathrm{z})$ were obtained for $\mathrm{n}=3$.

The average number of annual fires per unit area in Europe is 0.022 fires $/ \mathrm{km}^{2}$, obtaining the highest value Portugal with a rate of 0.24 fires $/ \mathrm{km}^{2}$.

Europe shows a growth rate in the number of fires between 1990 and 1998, which decreases from 2001.

\section{References}

Forest Fires in Europe, Middle East and North Africa 2016. Joint report of JRC. European commission. Luxembourg. doi:10.2760/17690 ISBN 978-92-79-71292-0 (2017)

J.L. Legido, M.M. Mato, E. Miguez, V. Caselles, E. Jiménez, T. Carballas, M.I. Paz Andrade. II International Conference on Fire Behaviour and Risk. Alghero, Sardinia, Italy, 26-29 May (2015)

M.M. Mato, J L Legido, E. Miguez, V. Caselles, E. Jiménez, T. Carballas, M.I. Paz Andrade. Advances in Forest Fire Research editor Domingos Xabier Viegas. Chapter 6 - Forest Management. 16281633. http://dx.doi.org/10.14195/978-989-26-0884-6_179 (2014) 
Short contribution - Decision Support Systems and Tools

Building the prototype for a new National Fire Danger Rating System for Australia

Stuart Matthews ${ }^{1}$; Paul Fox-Hughes ${ }^{2}$; Saskia Grootemaat ${ }^{1}$, Simon Heemstra ${ }^{1}$, Jennifer Hollis ${ }^{1}$, Belinda

Kenny $^{1}$, Samuel Sauvage ${ }^{2}$, Corey Shackleton ${ }^{1}$, Lew Short ${ }^{3}$, Deb Sparkes ${ }^{3}$

${ }^{I}$ NSW Rural Fire Service, Lidcome, NSW 2142, Australia, \{stuart.matthews@rfs.nsw.gov.au*\}

${ }^{2}$ Bureau of Meteorology, Melbourne, VIC 2001, Australia

${ }^{3}$ Australasian Fire and Emergency Services Authorities Council

Keywords: fire danger; fuel; fire behavior; fire weather;

\section{Introduction}

In July 2014, Senior Officers and Ministers agreed that the development of a new National Fire Danger Rating System is a national priority. The current system is based on science and research developed in the 1960s and does not meet the needs of emergency service authorities or the community in achieving the best emergency management outcomes in a bushfire event.

The National Fire Danger Rating System Program (the Program) will implement significant improvements to the system in the areas of fire weather prediction, fire ignition and behaviour potential and the impact on life, assets and the environment. The Program is designed to strengthen the ability of fire authorities to accurately communicate bushfire risk to the community, enhance agency readiness and preparedness and contribute to risk management.

It was decided to take a phased approach to maintain momentum and provide a better understanding of what needs to be done and funded to achieve a new system for Australia. This includes the development of a research prototype fire danger rating system, which the NSW Rural Fire Service (RFS) and Bureau of Meteorology (BoM) built and tested in the 2017/18 southern fire season.

\section{Improving ratings}

Fire danger is a general term, representing many factors culminating in a fire environment; ignition potential or fire hazard, risk, rate of spread, difficulty of control, fire impact(s) and safety. Fire danger rating is a valuable tool in communicating bushfire risk to the community, not only by increasing public awareness but also as a trigger for notification of potential threats. Importantly, because of its foundation in fire weather and behaviour, fire danger rating also provides a tool for decision making in fire suppression operations as well as supporting prescribed burning. It is for operational fire management that we aim to address how the fire danger ratings will be defined as well as the thresholds between categories.

To establish these fire danger ratings and to ensure they are accurate, relevant, clear and useful, we sought input from fire managers around Australia through a workshop and series of exercises considering fire danger in a range of fuel types. There was general consensus that the consequences of potential fires escalate with fire danger. At the lower end of the scale, fires are easily controlled and prescribed burns can be conducted safely. At the next level of danger fire suppression becomes challenging and may sometimes fail, but losses are expected to be limited due to successful property protection. Initial attack is expected to mostly succeed. At higher FDR levels, community and firefighter safety become the top priority with decreasing effectiveness of suppression activities and the probability of losses is expected to increase. At these higher FDR levels initial attack will only succeed in the very early stages with significant suppression resources. 


\section{Fire behaviour models and metrics}

The research prototype system uses the best fire spread models that are available and ready for application at present, as presented in Table 1. As outputs, all fire behaviour models provide a measure of rate of spread $\left(\mathrm{m} \mathrm{hr}^{-1}\right)$, and some provide a measure of spotting $(\mathrm{m})$ or flame height/length $(\mathrm{m})$. Another measure we took into account as part of the research prototype is fireline intensity $\left(\mathrm{kW} \mathrm{m}^{-1}\right)$.

Most available fire spread models assume fully dry conditions and a fully developed head fire. A simple measure of fuel availability was used to account for recent rainfall, since use of fully developed head fire spread equations for a fire in damp fuels during the initial attack phase would be inappropriate.

Table 1 - Fire behaviour models used in the research prototype rating system.

\begin{tabular}{lll}
\hline Fire behaviour model & Reference & Fuel type \\
\hline CSIRO Grassland fire spread model & Cheney et al. (1998) & Continuous grasslands \\
\hline CSIRO for northern Australia & Cheney et al. (1998) & Woodlands and savannah \\
\hline Desert spinifex model & Burrows et al. (2015) & Hummock grasslands \\
\hline Buttongrass moorlands model & $\begin{array}{l}\text { Marsden-Smedley and } \\
\text { Catchpole (1995) }\end{array}$ & Buttongrass moorlands \\
\hline $\begin{array}{l}\text { Dry Eucalypt Forest Fire Model } \\
\text { (DEFFM or "Vesta") }\end{array}$ & Cheney et al. (2012) & Dry sclerophyll forests \\
\hline Mallee heath model & Cruz et al. (2013) & Semi-arid mallee heath \\
\hline Heathland model & Anderson et al. (2015) & Temperate shrublands \\
\hline Adjusted pine plantation model & Cruz et al. (2008) & Pine plantations \\
\hline
\end{tabular}

\section{Other metrics}

The behaviour of the head fire is the core of the research prototype rating. However, this is not the whole story. Other things which need to be considered are e.g. atmospheric stability, wind change and spotting potential. In the research prototype we consider:

CHaines index, as a measurement of atmospheric stability,

- A wind change danger index, since wind change can increase the fire danger (a) by causing instability and turbulence in the vicinity of the wind change, or (b) by dramatically increasing the fire size when a fire flank becomes the head fire,

- Bark characteristics and spotting models, for forests with bark fuels.

These aspects were not incorporated into the determination of the rating directly, but were included as 'red flag' warnings.

\section{Fuel classification and data}

Nationally consistent fuel data were used to drive the selection of fire behaviour models and provide the model parameters. Fuel data were derived from national and state datasets classified to common fuel types and aggregated to $1.5 \mathrm{~km}$ resolution to match the scale of the weather forecast and reanalysis grids used to calculate the ratings.

Fuel types have been defined at the level required for selection of appropriate fire behaviour models (Figure 1). There are many vegetation types for which fire behaviour models have not been developed, as they are generally less flammable than the fuel types in Table 1. Classifying these vegetation types into fuel types based on their fuel structure was the best available approach. However, there are often factors limiting the flammability, fuel availability or connectivity, or potential rate of spread in these vegetation types; hence the structurally equivalent models are likely to overestimate fire behaviour. These limitations were treated as modifiers to the model calculation. The fuel attributes included in 
the research prototype were determined based on the requirements of the fire behaviour models. Where local fuel attribute data was not available, a set of generic fuel attributes was applied.

Fire history was used as a modifier to adjust fuel attributes from their peak state using only fires larger than 100 ha.

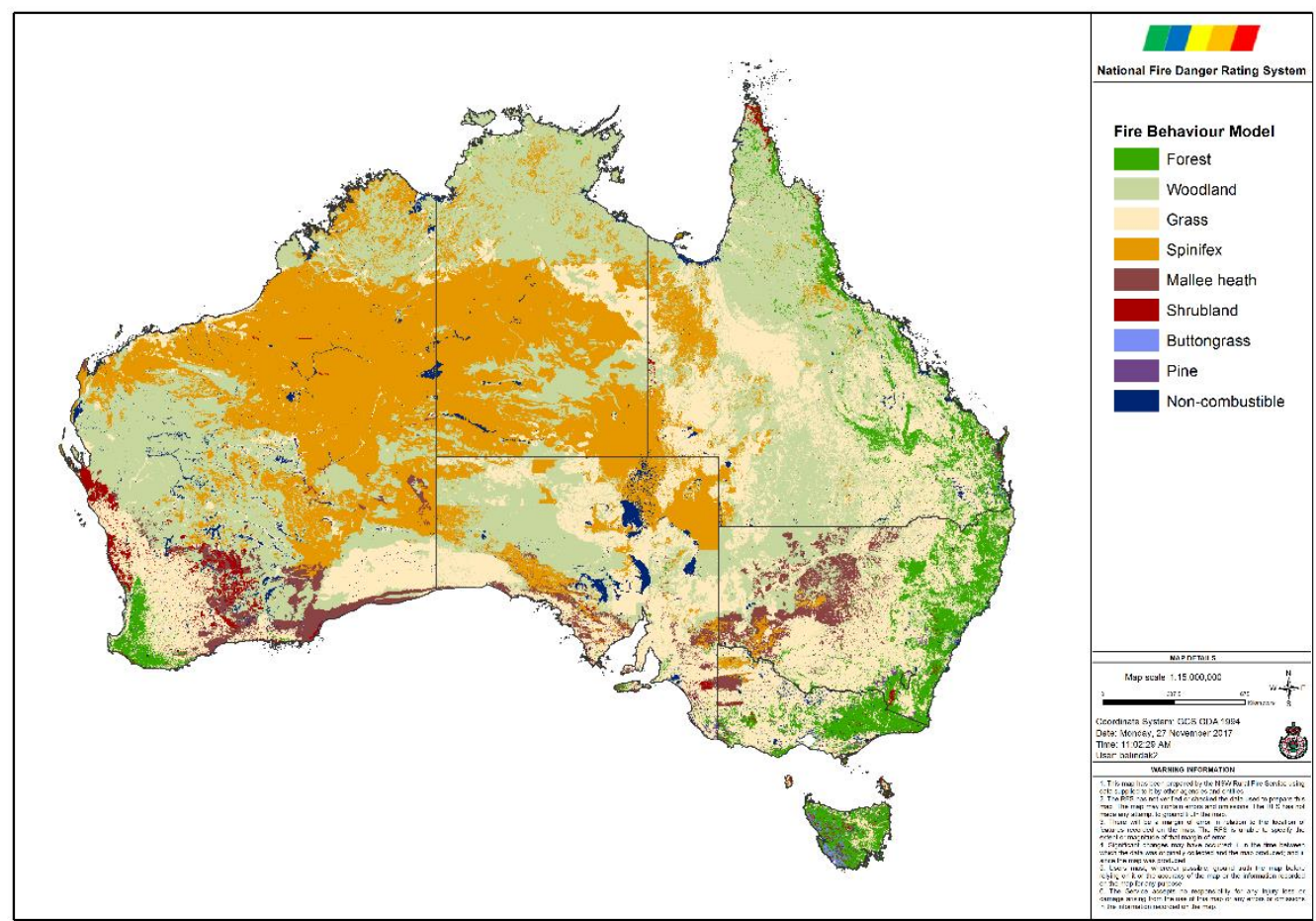

Figure 1 - Map of major fuel types across Australia classified by fire behaviour model

\section{Implementation}

The research prototype system was implemented by the Bureau of Meteorology ahead of the live trial of the system in October 2017. The research prototype algorithms were applied to weather forecasts and base layers (fuels, topography, etc.) using Python scripts. The system produces maps and tables of daily fire danger rating, as well as binary data files containing hourly outputs. Rating products were distributed through existing channels such as a registered user web page and the BoM's FTP service.

\section{Live trial and evaluation}

Ratings from the research prototype system were available during a live trial running from October 2017 to March 2018. Participants compared forecast ratings with the outcomes of incidents using the rating definitions. Historical incidents from agency fire databases and reanalysis weather data were also used in the development and evaluation of the research prototype.

\section{References}

Anderson, W. R., et al. (2015). "A generic, empirical-based model for predicting rate of fire spread in shrublands." International Journal of Wildland Fire 24(4): 443-460.

Burrows, N. D., Liddelow, G. L. \& Ward, B. (2015). A guide to estimating fire rate of spread in spinifex grasslands of Western Australia (Mk2v3). Science and Conservation Division, Department of Parks and Wildlife. 
Cheney, N. P., et al. (1998). "Prediction of fire spread in grasslands." International Journal of Wildland Fire 8(1): 1-13.

Cheney, N. P., Gould, J. S., McCaw, W. L., \& Anderson, W. R. (2012). Predicting fire behaviour in dry eucalypt forest in southern Australia. Forest Ecology and Management, 280, 120-131.

Cruz, M. G., McCaw, W. L., Anderson, W. R., \& Gould, J. S. (2013). Fire behaviour modelling in semi-arid mallee-heath shrublands of southern Australia. Environmental Modelling \& Software, 40, 21-34.

Cruz, M. G., Alexander, M. E., \& Fernandes, P. A. (2008). Development of a model system to predict wildfire behaviour in pine plantations. Australian Forestry, 71(2), 113-121.

Gould and Cruz (2015) Bushfire Fuel Classification. Consultation draft report. Australasian Fire and Emergency Service Authorities Council, Melbourne.

Marsden-Smedley, J. B. and W. R. Catchpole (1995). "Fire modelling in Tasmanian buttongrass moorlands II. Fire behaviour." International Journal of Wildland Fire 5(4): 215-228. 
Short contribution - Decision Support Systems and Tools

Fire and land use in recent years in the Atlantic Forest in Brazil

\author{
Manoel Cardoso \\ Centro de Ciência do Sistema Terrestre, Instituto Nacional de Pesquisas Espaciais (CCST/INPE), São José \\ dos Campos - SP, 12227-010 Brazil, \{manoel.cardoso@inpe.br\}
}

\begin{abstract}
The biome Atlantic Forest in Brazil covers a substantial portion of the country, where a large population and major cities are located. In spite of the substantial influence from human activities, the region is one of the most diverse tropical ecosystems with a large number of endemic species, and it is one of the world's biodiversity hotspot. In this region, fire is an important disturbance, due to its strong effects on nutrient cycling, vegetation succession, and atmospheric emissions of gases and aerosols, and therefore must be considered when planning on the sustainability of the region. This study presents a set of initial analyses of fire occurrence in respect to different types of land cover and use as classified by the recent Brazilian MapBiomas Project (http://mapbiomas.org). Information on fire occurrence are from remote-sensing with the Moderate Resolution Imaging Spectroradiometer (MODIS) Collection 6 Active Fire Product. The data on fires data are for years 2001-2017. The selected data on land cover and use are for year 2010, nearly at the middle of the fire data time spam. For analyses, both datasets were aligned in space and aggregated at 0.5deg spatial resolution. It was found that in average, most of fires happened during months with less precipitation, as expected. In comparison with other major biomes in Brazil, the density of fire detections in the Atlantic Forest was lower, but still a substantial number of fires are detected yearly in the region. Perhaps, more important is the fact that despite of most of fires detected with MODIS occurred in areas dominated by agriculture and pasture (as expected by the general relations between fire and land use in Brazil), a relatively high number of fires were detected in areas dominated by natural forests. That has important consequences for the high interest in conservation of the study region, based on its marked biodiversity and potential for providing relevant ecosystem services to society.
\end{abstract}

Keywords: Fire, land use, Atlantic Forest, Brazil

\title{
1. Introduction
}

The biome Atlantic Forest in Brazil covers a substantial portion of the country, where a large population and major cities are located. Most of its primary forests have already been converted to agricultural and pasture areas, but important forest and other natural vegetation formations still remain (Ribeiro et al. 2011). Despite substantial influence from human activities (Joly et al. 2014), the region is one of the most diverse tropical ecosystems with a large number of endemic species, and it is one of the world's biodiversity hotspot (Myers et al. 2000).

In this region, fire is one of the important disturbances, with implications for maintenance of its natural resources (Joly et al. 2014, Clemente et al. 2017). Fires have several important effects on nutrient cycling, vegetation succession, and atmospheric emissions of gases and aerosols and must be considered in plans of the sustainability of the region. In this study, it is presented a set of initial analyses of fire occurrence for years 2001-2017 in respect to different classes of land cover and use based on recent information from the Brazilian MapBiomas Project (http://mapbiomas.org).

\section{Methods}

Given the large extent of the study region (Fig. 1a), the majority of information on fire activity comes from satellites, because of their ability to cover large regions in relatively short periods of time. In this study, the data on fire occurrence are from remote-sensing with the Moderate Resolution 
Imaging Spectroradiometer (MODIS) Collection 6 Active Fire Product (Giglio et al. 2016), for years 2001-2017.

The information on land use were recently produced by the Brazilian MapBiomas Project, which is a multi-institutional initiative to generate annual land cover and use maps using automatic classification processes applied to satellite images (complete description at http://mapbiomas.org). From the most recent version of the dataset (2.3), it was selected data for year 2010, nearly at the middle of the fire data time spam. For analyses, all datasets were aligned in space and aggregated at 0.5-deg spatial resolution.

\section{Results}

As shown in Figs 1a and 1b, several active fires were detected in the Atlantic Forest region in the years from 2001 and 2017. The spatial distribution of the fire detections in the study region (Fig. 1a) shows that generally coastal areas had relatively lower fire activity, and interior portions of the states of São Paulo, Bahia, Paraná and Santa Catarina had relatively more fires. In time (Fig.1b), most of fires were detected between the months of July to October, when precipitation is lower in most of the region. In comparison with the country as a whole, and other major biomes in Brazil, the Atlantic Forest presented lower fire activity density.
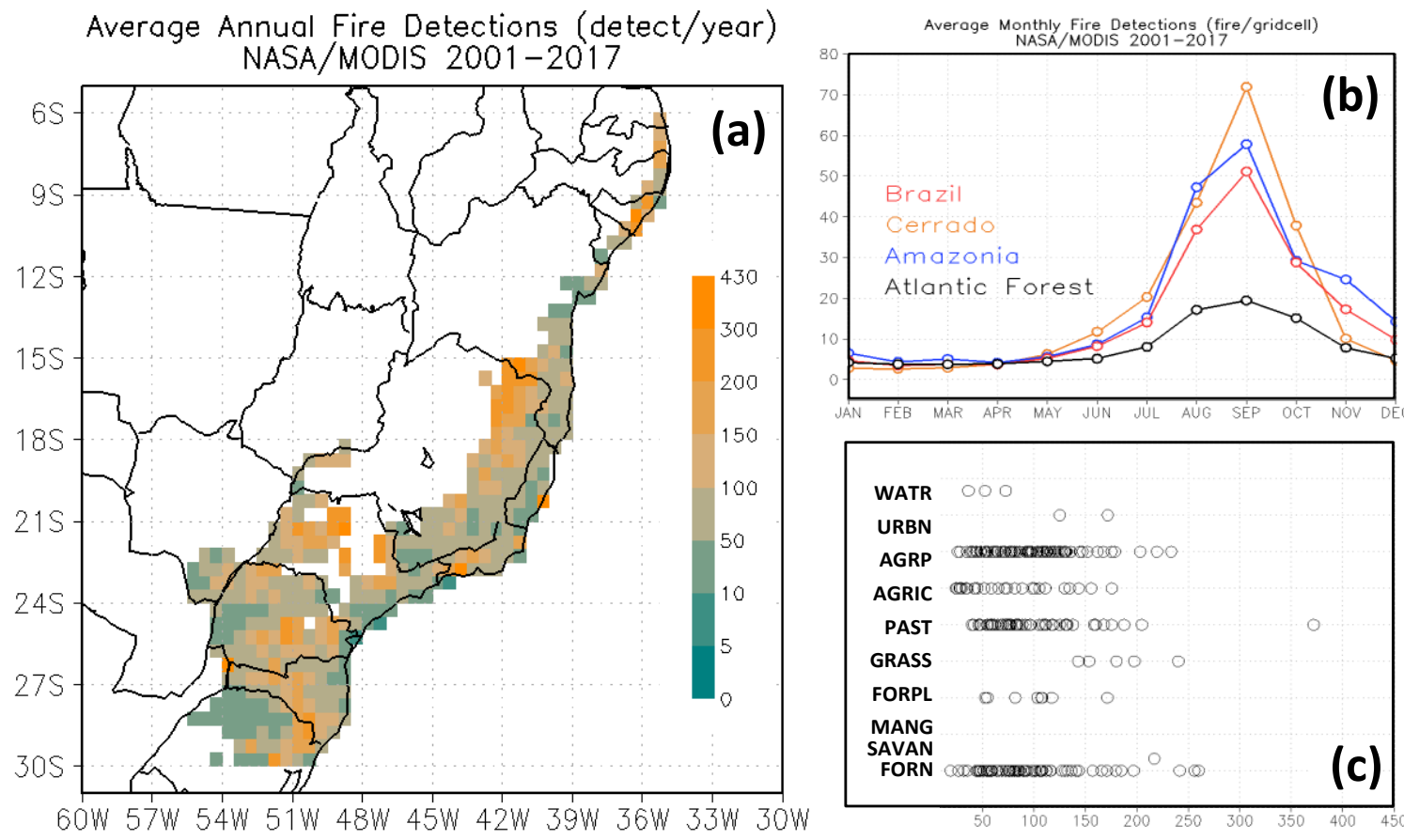

Figure 1 - Characteristics of fire occurrence and land use in recent years in the Brazilian Atlantic Forest. In (a), the spatial distribution of MODIS average fire detection density in the Atlantic Forest in Brazil from 2001 to 2010 , in units of detections per year within each 0.5-deg gridcell of the study region. In (b), the average monthly fire detection density in the Atlantic Forest (black), in the whole Brazil (red), and in the biomes Cerrado (orange) and Amazonia (blue). In (c), the distribution of average annual fire detections per type of the land cover and use in the Atlantic Forest, where the classes correspond to the dominant land cover and use within each gridcell: WATR are grid cells dominated by water bodies, URBN are gridcells mostly covered by urban surfaces, and AGRP corresponding to agriculture or pasture, AGRIC corresponds to agriculture, PAST corresponds to pastures, GRASS to grasslands, FORPL planted forests, MANG mangroves, SAVAN natural savannas, and FORN to natural forests.

Figure 1c shows the distribution of the average annual fire detections per type of the land use in the studied region. As shown, the majority of fires were detected in areas related to agriculture and pastures 
(classes AGRP, AGRIC, and PAST), but a substantial number of detections were in areas of natural forests (FORN).

\section{Conclusions}

In this study, it is presented an initial set of analyses of fire occurrence for years 2001-2017 in the Atlantic Forest in Brazil, in respect to different classes of land cover and use as described by the Brazilian MapBiomas Project (http://mapbiomas.org). It was found that in average, most of fires happened during months with less precipitation, as expected. In comparison with other major biomes in Brazil, the density of fire detections in the Atlantic Forest was lower, but still a substantial number of fires are detected yearly in the region. Perhaps, more important is the fact that despite of most of fires being detected with MODIS occurred in areas dominated by agriculture and pasture (as expected by the general relations between fire and land use in Brazil) a relatively high number of fires were detected in areas dominated by natural forests. That has important consequences for the high interest in conservation of the study region, based on its marked biodiversity and potential for providing relevant ecosystem services to society.

\section{References}

Clemente SS, de Oliveira Júnior JF, Louzada MAP (2017) Focos de Calor na Mata Atlântica do Estado do Rio de Janeiro. Revista Brasileira de Meteorologia 32(4), 669-677. doi: http://dx.doi.org/10.1590/0102-7786324014.

Joly CA, Metzger JP, Tabarelli M (2014) Experiences from the Brazilian Atlantic Forest: ecological findings and conservation initiatives. New Phytologist 204, 459-473 doi: 10.1111/nph.12989.

Myers N, Mittermeier RA, Mittermeier CG, Da Fonseca GA, Kent J (2000) Biodiversity hotspots for conservation priorities. Nature 403, 853-858. doi: 10.1038/35002501.

Giglio L, Schroeder W, Justice CO (2016) The collection 6 MODIS active fire detection algorithm and fire products. Remote Sensing of Environment 178, 31-41. https://doi.org/10.1016/j.rse.2016.02.054.

Ribeiro MC, Martensen AC, Metzger JP, Tabarelli M, Scarano F, Fortin MJ (2011) The Brazilian Atlantic Forest: A Shrinking Biodiversity Hotspot. In 'Biodiversity Hotspots' (Eds F Zachos, J Habel) pp. 405-434. Springer: Berlin, Heidelberg. 
Short contribution - Decision Support Systems and Tools

FireCCI50: a global burned area mapping algorithm based on MOD09GQ within Fire_cci project

Joshua Lizundia-Loiola*; Gonzalo Otón; Ruben Ramo; Emilio Chuvieco

Geography Department, Universidad de Alcalá.Colegios 2, 28801, \{joshua.lizundia@uah.es*, gonzalo.oton@uah.es,ruben.ramo@uah.es,emilio.chuvieco@uah.es\}

Keywords: Burned area, MODIS, Hotspots, algorithm

\section{Introduction}

Fire is one of the main contributors of greenhouse gases and aerosol emissions and it is closely related to carbon cycle (van der Werf et al., 2010). It has a clear influence in global vegetation dynamics and its effect in human property and lives is increasing.

As a result, it is considered an Essential Climate Variable (ECV) and it is deeply investigated within the Fire_cci Project. In the aim of contributing to the Global Climate Observing System (GCOS) programme, the European Space Agency (http://cci.esa.int/, las accessed March 11, 2018) created the Climate Change Initiative (CCI), in which 13 ECV are studied (Fire Disturbance, among others).

\section{Methods}

\subsection{Framework}

Hybrid algorithms have proved to be appropriate to burned area (BA) detection. Two of the most used global BA products, MCD64 (Giglio et al., 2009) and MERIS Fire_cci v4.1 (FireCCI41) (AlonsoCanas y Chuvieco, 2015), have been generated based on this approach. They combine the effectiveness of thermal anomalies products to detect active fire position, with the reflectance changes that affect to the complete BA. The algorithm presented on this paper continues the steps of its predecessor, FireCCI41, using a two steps hybrid approach, with a seed detection phase and a growing phase.

\subsection{Input images and spectral indices}

This algorithm is based on the sensor MODIS (MOderate Resolution Imaging Spectroradiometer) MOD09GQ product (250 m resolution) and the MCD14ML active fire product. The former one is distributed in tiles of $1200 \times 1200 \mathrm{~km}$ and the second one globally. In addition to the MODIS NIR band, Global Environmental Monitoring index (GEMI) was computed (Pinty y Verstraete, 1992) which proved being a good index to BA detection among the spectral indices based on NIR-Red bands (Alonso-Canas y Chuvieco, 2015). GEMI was computed as follows:

$$
\begin{aligned}
& G E M I=\eta \cdot(1-0.25 \cdot \eta)-\frac{\left(\rho_{R}-0.125\right)}{\left(1-\rho_{R}\right)} \\
& \eta=\frac{2 \cdot\left(\rho_{N I R}{ }^{2}-\rho_{R}{ }^{2}\right)+1.5 \cdot \rho_{N I R}+0.5 \cdot \rho_{R}}{\left(\rho_{R}+\rho_{N I R}+0.5\right)}
\end{aligned}
$$

where $\rho_{\text {NIR }}$ is the Near Infrared (MODIS band 2) and $\rho_{R}$ is Red (band 1).

As auxiliary data, Land Cover CCI product v1.6.1 (https://www.esa-landcover-cci.org/, last accessed March 11, 2018) was also used, which permitted obtaining a burnable - unburnable mask. 


\subsection{Composite}

Due to the strong BRDF (Bi-Directional Reflectance Distribution Function) effect of MODIS sensor (swath dimension of $2330 \mathrm{~km}$ ) several approaches to reduce it were tested. However, the smoothing effect of those techniques weakened too much the temporal trend of burned pixels. So temporal compositing is used to reduce that angular effect and enhance burned signal.

Two criteria were used to perform the composites: the assignation of the nearest active fire (also called hotspots or HS) date based on Thiessen matrix (Brassel y Reif, 1979) and the selection of the proper minimum NIR value. As BA is expected to have very low NIR values (Alonso-Canas y Chuvieco, 2015) 3 minimum values are searched within a given month. However, this low NIR values can be caused by other events like cloud, topographic shadows or flooding. To avoid possible noise caused by the mentioned events the algorithm selects the first minimum after the date of HS detection. If all the minimums are found on previous dates, the second minimum is selected. At the same time, maximum GEMI of the month (GEMI_max) and GEMI of the selected days for the composite (GEMI_month) are calculated.

\subsection{Seed phase}

The objective of this first phase was to reduce commission errors by selecting those HS that have high probability of being burned. Therefore, Cumulative Distribution Function was computed for unburned class using those pixels of the composite that are further than a $41 \mathrm{x} 41$ window around each HS. The first decile (10\%) is selected as threshold (TH_NIR) and NIR values have to be below it. Second, pixels have to show NIR value decrease between $t$ and t-1. Finally, the third condition is that 5 of the 8 surrounding pixels ( $3 \times 3$ window) has to fulfil the two previous conditions to ensure spatial correlation of burned tendencies. The statistic were performed for each tile, biome and month.

\subsection{Growing phase}

Although active fire products have accurate location of fire, this position is related only with those areas that were burning during satellite pass, so many authors have used contextual algorithms for BA mapping (Bastarrika et al., 2011; Alonso-Canas y Chuvieco, 2015) in order to reduce omission error.

An additional variable is used to stop the region growing process (Zhang et al., 2005). To do so, difference between GEMI_max of the previous month and GEMI_month of the current month (difGEMI) were used. The first one represents the maximum greenness of the immediate pre-fire conditions, while the second one shows the vegetation after burning date. The higher the difference was the higher probability of being burned.

To estimate an adequate threshold for difGEMI, CDFs for burned and unburned classes are calculated. The mean of the decil 9 of unburned class and decil 1 of burned class is computed and used as difGEMI threshold (TH_difGEMI).

A pixel is classified as burned if (1) it has a NIR value below TH_NIR, (2) a difGEMI value over TH_difGEMI, (3) a lower NIR value than the previous month (t-1) and (4) at least one pixel seed or burned pixel in a $3 \times 3$ window.

\section{Results}

A new global BA product has been generated based on this algorithm (ftp://anonftp.ceda.ac.uk/neodc/esacci/fire/data/burned_area/, last accessed March 11, 2018). The time series goes from 2001 to 2016 improving the existing ESA BA products and also the spatial resolution offered by the current BA products (500 m MCD64 and $300 \mathrm{~m}$ FireCCI41).

The product assessment was performed using a set of global validation sites, taking into account the diversity of fires all over the world and also through the time series. In addition, an intercomparison 
with other existing products has been developed. FireCCI50 product shows a reduction of $10 \%$ in both commission and omission errors in comparison to FireCCI41, but 10\% higher than MCD64 c6. The estimation of burned area is similar to the trends observed in other products.

\section{Bibliography}

Alonso-Canas, I. \& Chuvieco, E. 2015. Global Burned Area Mapping from ENVISAT-MERIS data Remote Sensing of Environment, 163, 140-152.

Bastarrika, A., et al 2011. Mapping burned areas from Landsat TM/ETM+ data with a two-phase algorithm: balancing omission and commission errors. Remote Sensing of Environment, 115, 1003-1012.

Brassel, K. E. \& Reif, D. 1979. A procedure to generate Thiessen polygons. Geographical Analysis, 11(3), 289-303.

Giglio, L., et al 2009. An active-fire based burned area mapping algorithm for the MODIS sensor. Remote Sensing of Environment, 113(2), 408-420.

Pinty, B. \& Verstraete, M. M. 1992. GEMI: a non-linear index to monitor global vegetation from satellites. Vegetatio, 101, 15-20.

Van Der Werf, G. R., et al 2010. Global fire emissions and the contribution of deforestation, savanna, forest, agricultural, and peat fires (1997-2009). Atmospheric Chemistry and Physics, 10, 1170711735 .

Zhang, Q. F., et al 2005. A semi-automatic segmentation procedure for feature extraction in remotely sensed imagery. Computers \& Geosciences, 31(3), 289-296. 
Short contribution - Decision Support Systems and Tools

Global generation of long-term burned area with AVHRR-LTDR data

Gonzalo Otón*, Rubén Ramo, Joshua Lizundia-Loiola, Emilio Chuvieco

Department of Geology, Geography and the Environment, Universidad de Alcalá, Spain

\{gonzalo.oton@uah.es*; ruben.ramo@uah.es; joshua.lizundia@uah.es; emilio.chuvieco@uah.es\}

Keywords: Remote sensing, Burn area, AVHRR-LTDR, Multitemporal, Random Forest.

\section{Introduction}

Burned Area (BA) information is critical to assess the impacts of biomass burning, both in atmospheric emission models and dynamic vegetation studies (Hantson et al. 2016). Therefore, the fire variable is inside of Essential Climate Variables (ECV) for the Global Climate Observing System (GCOS) program. This work is part of the Fire Disturbance project, part of the Climate Change Initiative (CCI) Programme of the European Space Agency (ESA).

Current global burned area products expand from 1995 to 2017, being consistent as of 2001 (Mouillot et al. 2014). Longer time series would be desirable to analyze the impacts of climate cycles. This paper presents the development of a global BA algorithm adapted to Land Long-Term Data Record (LTDR, version 5, Pedelty et al. 2007), a time series based on NOAA-AVHRR (Advanced Very High Resolution Radiometer) data that covers the period from 1982 to 2017.

\section{Methods}

\subsection{LTDR and composites}

The LTDR is based on images acquired by the AVHRR sensor on board the NOAA satellites from 1981 until the present. This dataset includes atmospheric and geometric corrections of AVHRR data and provides daily thermal and reflectance values at $0.05^{\circ}(\approx 5 \mathrm{~km})$ of spatial resolution (Pedelty et al. 2007). As LTDR daily images present noise, radiometric instability and distortion, monthly composites were created by selecting the most adequate observation for burned area discrimination. The criterion to create the composites was based on selecting the maximum temperature of the period. This method improves the burn pixel separability and avoids clouds and shadows. (Chuvieco et al. 2005).

In addition, the unburnable areas were masked out with information derived from the CCI Land Cover product (Version 1.6.1., Kirches et al. 2013).

\subsection{MCD64}

The MCD64A1 dataset (Collection 6, Giglio et al. 2009) is generated through MODIS images from it is available (2001-present). The dataset is used to train the model as reference BA.

\subsection{Synthetic index}

A synthetic index was created from the different spectral bands (RED, NIR, Temperature channel 5), derived indices (GEMI, BAI) and temporal differences. The input bands were selected based on their spectral discriminability between burned and unburned areas. The final formula is: 


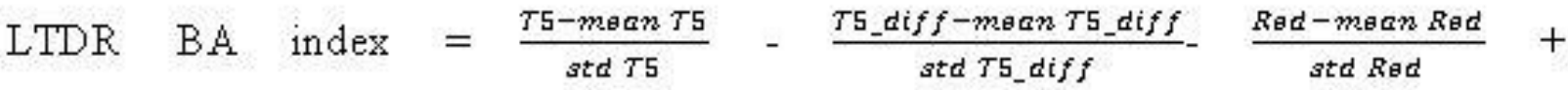

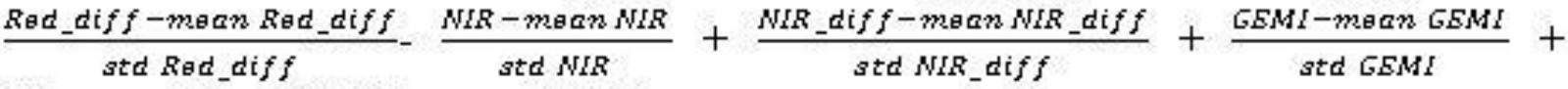

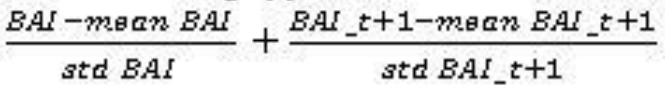

where

T5 = TOA brightness temperature of Channel $5(\sim 11.5-12.5 \mu \mathrm{m})$

$\mathrm{T} 5 \_$diff $=\mathrm{T} 5 \mathrm{t}-1-\mathrm{T} 5 \mathrm{t}$, where $\mathrm{t}=$ monthly composite being analyzed, and $\mathrm{t}-1=$ composite of the previous month.

Red $=$ Surface reflectance of Channel $1(0.5-0.7 \mu \mathrm{m})$

Red_diff $=\operatorname{Red}_{\mathrm{t}-1}-\operatorname{Red}_{\mathrm{t}}$

$\mathrm{NIR}=$ Surface reflectance of Channel $2(0.7-1.0 \mu \mathrm{m})$

NIR_diff $=$ NIR $1-1-$ NIR

GEMI (Global Environmental Monitoring Index, Pinty and Verstraete 1992) as:

$$
G E M I=\eta^{*}(1-0,25 \eta)-\frac{\rho_{R}-0,125}{1-\rho_{R}} \quad \eta=\frac{2^{*}\left(\rho_{I R}^{2}-\rho_{R}^{2}\right)+\left(1,5^{*} \rho_{I R}\right)+\left(0,5^{*}, \rho_{R}\right)}{\rho_{I R}+\rho_{R}+0,5}
$$

Being $\rho_{R}$ and $\rho_{I R}$ the reflectivity in the red and infra-red bands, respectively.

BAI, the Burned Area Index (Martín and Chuvieco 1998):

$$
B A I=l /\left(\left(\rho_{R}-\rho_{R}\right)^{2}+\left(\rho_{R}-\rho_{R}\right)^{2}\right)
$$

where $\rho_{\mathrm{cIR}}$ and $\rho_{\mathrm{cR}}$ are the convergence values for burned vegetation (defined for AVHRR as 0.06 and 0.1 , respectively).

$\mathrm{BAI}_{t+1}$ is the BAI of the posterior monthly composite.

Seasonal variation of this index was also taken into account for training. So, the phenology variability is represented in the different months.

\subsection{Random Forest}

Random Forest (RF, Breiman 2001) is a machine-learning classifier based on a random combination of decision trees. RF classified each pixel by the number of times that pixel is assigned to a particular category. In our case, 600 trees were created for each monthly model (from January to December). RF models were created combining monthly composites of LTDR data from several years (both with high and low fire occurrence). Then, they were applied to the full-time series, a different model for each month of the year.

The output of RF was a probability per month and year.

\subsection{Sensitivity analysis and percentage}

Since the burned pixels are much more unlikely than the unburned, we performed a sensitivity analysis to obtain the appropriate cut-off threshold to improve overall accuracy and balance between omission and commission errors. Uncertainty information was estimated from the probability of RF.

A temporal analysis of the pixels that are burned in the MCD64 every month and its percentage was made. A value was assigned to each pixel per month and applied to the result of the sensitivity analysis. 


\subsection{Validation}

The final classification was compared with the most recent NASA BA product (MCD64A C6) for common years (2001 - 2016). The confusion matrix of each model was calculated, in addition to commission and omission error and Dice coefficient.

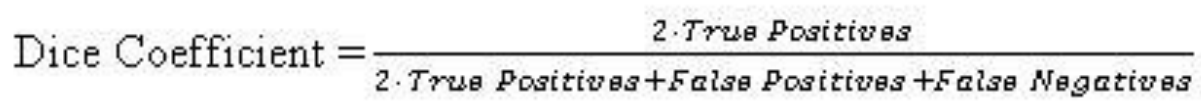

\section{Results}

The algorithm has been applied for whole time series (1981 - 2017). The product generated is the first to consistently offer such long time series, doubling the current time series. The study of sensitivity and percentage improve significantly the results.

The months with high fire occurrence obtained better results than months with low fire occurrence. Omission and commission errors were found quite balanced in the time series. Specifically, commission and omission error for the month of January is 0.53 and 0.47 and July is 0.49 and 0.57 , respectively. Average Dice coefficients were 0.50 for January and 0.46 for July. The best month was December (Figure 1) with 0.41 in commission error, 0.43 as omission error and a Dice coefficients of 0.58 .

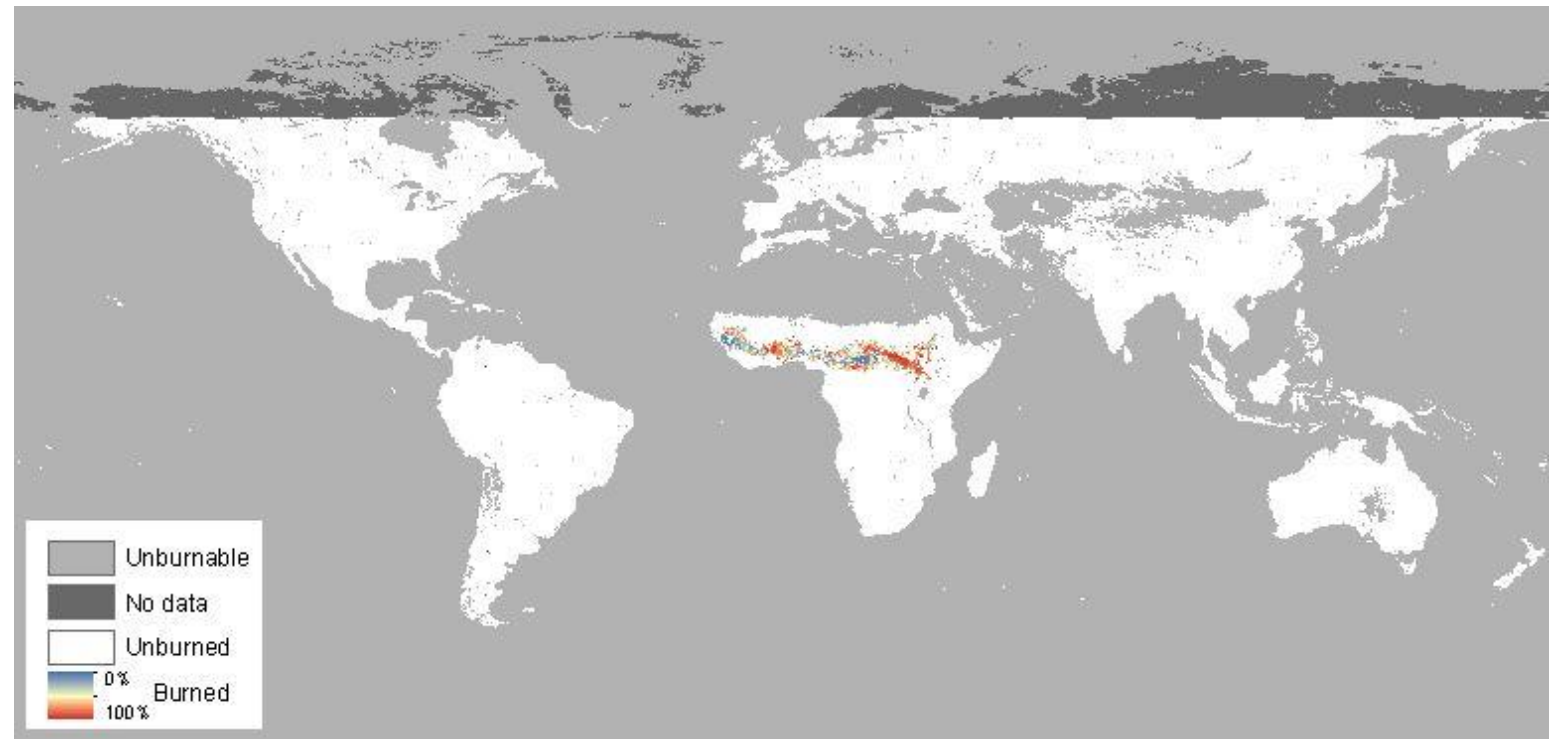

Figure 1 - Percentage BA of LTDR data for December 2008

\section{Bibliography}

Breiman, L. (2001). Random forests. Machine learning, 45, 5-32

Chuvieco, E., Ventura, G., Martín, M.P., \& Gomez, I. (2005). Assessment of multitemporal compositing techniques of MODIS and AVHRR images for burned land mapping. Remote Sensing of Environment, 94, 450 - 462

Giglio, L., Loboda, T., Roy, D.P., Quayle, B., \& Justice, C.O. (2009). An active-fire based burned area mapping algorithm for the MODIS sensor. Remote Sensing of Environment, 113, 408-420

Hantson, S., Arneth, A., Harrison, S.P., Kelley, D.I., Prentice, I.C., Rabin, S.S., Archibald, S., Mouillot, F., Arnold, S.R., \& Artaxo, P. (2016). The status and challenge of global fire modelling. Biogeosciences, 13, 3359-3375 
Kirches, G., Krueger, O., Boettcher, M., Bontemps, S., Lamarche, C., Verheggen, A., Lembrée, C., Radoux, J., \& Defourny, P. (2013). "Land Cover CCI: Algorithm Theoretical Basis Document Version 2.". Land_Cover_CCI_ATBDv2_2.3., Louvain, Belgium, 191 pp. Available at http://www.esa-landcovercci.org/?q=documents\#.

Martín, M.P., \& Chuvieco, E. (1998). Cartografía de grandes incendios forestales en la Península Ibérica a partir de imágenes NOAA-AVHRR. Serie Geográfica, 7, 109-128

Mouillot, F., Schultz, M.G., Yue, C., Cadule, P., Tansey, K., Ciais, P., \& Chuvieco, E. (2014). Ten years of global burned area products from spaceborne remote sensing-A review: Analysis of user needs and recommendations for future developments. International Journal of Applied Earth Observation and Geoinformation, 26, 64-79

Pedelty, J., Devadiga, S., Masuoka, E., Brown, M., Pinzon, J., Tucker, C., Vermote, E., Prince, S., Nagol, J., \& Justice, C. (2007). Generating a long-term land data record from the AVHRR and MODIS instruments. In, Geoscience and Remote Sensing Symposium, 2007. IGARSS 2007. IEEE International (pp. 1021-1025): IEEE

Pinty, B., \& Verstraete, M.M. (1992). GEMI: a non-linear index to monitor global vegetation from satellites. Vegetatio, 101, 15-20 
Short contribution - Decision Support Systems and Tools

High resolution weather forecasting applied to forest fire behaviour simulation

\author{
Jean-Baptiste Filippi ${ }^{1 *}$; Yolanda Perez ${ }^{1}$; Frédéric Allaire ${ }^{2}$; Aurelien Costes $^{3}$; Mélanie Rochoux ${ }^{4}$; Vivien \\ Mallet $^{2}$; Christine Lac ${ }^{3}$. \\ ${ }^{1}$ SPE - UMR 6134 CNRS. Université de Corse, France. \{filippi@univ-corse.fr*,perez-ramirez@univ- \\ corse.fr \} \\ ${ }^{2}$ Team ANGE (Inria, CEREMA, UPMC, CNRS). 2 rue Simone Iff, CS 42112, 75589 Paris Cedex 12, France \\ and Sorbonne Universités, UPMC Univ. Paris 06, \\ Lab. Jacques-Louis Lions UMR CNRS 7598, 75005 Paris, France \\ \{frederic.allaire@inria.fr,vivien.Mallet@inria.fr\} \\ ${ }^{3}$ CNRM, Météo-France-CNRS,Toulouse,France.\{aurelien.costes@meteo.fr, christine.lac@meteo.fr\} \\ ${ }^{4}$ CECI, Université de Toulouse, CNRS, CERFACS, 42 Avenue Gaspard Coriolis, 31057 Toulouse cedex 01, \\ France.\{melanie.rochoux@cerfacs.fr\}
}

Keywords: simulation ; wildfire ; high performance computing ; fire meteorology

Availability of supercomputers and new high-resolution data sources (such as Sentinel satellites or airborne LIDAR) are providing new ways of forecasting fire behaviour, opening the opportunity to have better fuel description, perform large probabilistic forecasts and data assimilation of fire/weather coupled models. Several codes using high performance computing already exist, with methods and services for forest fire forecasting developed through major innovations such as a coupled highresolution fire/atmosphere model (Coehn et al, 2013; Mandel et al, 2011; Filippi et al, 2013) data assimilation (Zhang et al 2017) of fire front positions, and ensemble simulations for uncertainty evaluation (Finney et al 2011).

High-resolution (sub km)/high-frequency ( $<10$ minutes) weather forecasting is in particular among the major enhancements allowed by the availability of such tools because it does reach relevant scales for day-to -day forest fire behavior wind forcing, but also fuel moisture and data assimilation.

FireCaster simulation chain is an experiment to develop integrated tools, methods, datasets and uses of such tools in an operational context. Its core is a daily computer intensive batch run of the mesoscale weather forecasting model Meso-NH (Lac et al 2017) initialized with the French national service Arome model, parameterized with a $600 \mathrm{~m}$ horizontal resolution, with outputs every 2 minutes for a $42 \mathrm{~h}$ forecasting duration.

One of the major use of such kind of run providing fire-specific zoomed view of a high risk area, then, in case of fire, specific input parameters for different kinds of interactive (controlled by an operator) fire behavior simulations (fire propagation, ensemble-based fire probability), taking into account fuel moisture and specific high resolution land use of the fire area (fig. 1) to test fighting scenarios with sub minute simulation time.

The other decision support tool for on-demand simulation is the fire weather forecast, deterministic $\mathrm{H}+6$ to $\mathrm{H}+12$ front positions, smoke and micro-meteorology forecasts every hour to be able to alert population and derive precise estimate of the front advance. Fire/weather simulation is using here Meso-NH code coupled with ForeFire (Filippi et al, 2013). This coupled code is run on-demand, in case of an effective fire ignition to estimate specific fire weather event (fire effect on local winds, coupled effect on fire), very local pollution and smoke dispersion. 


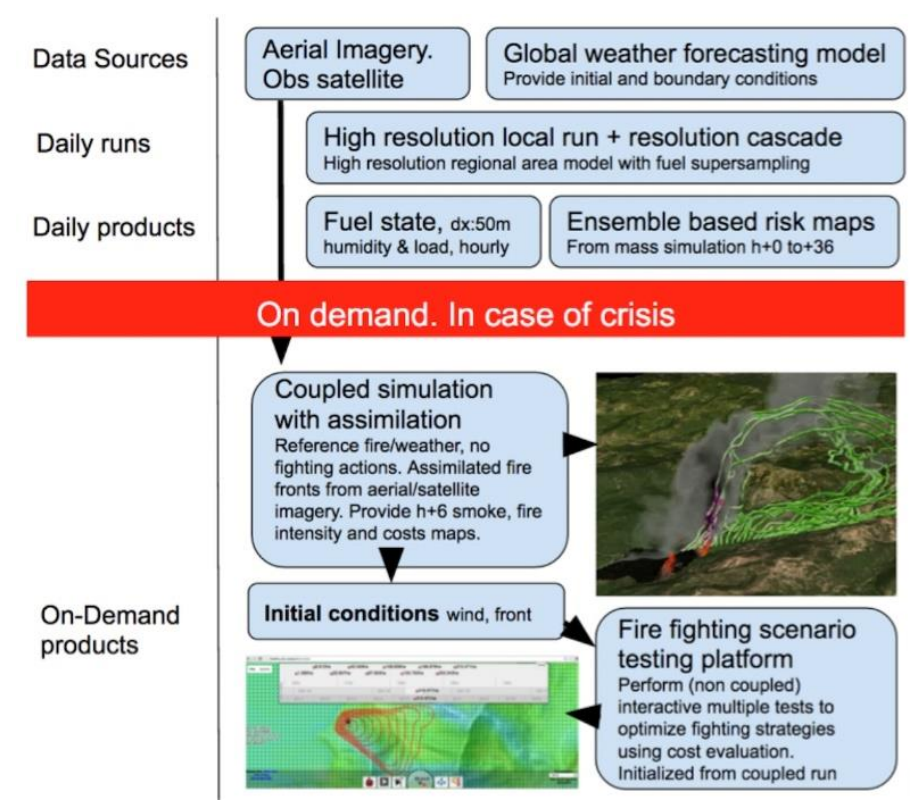

Figure 1 - Overall view of the components in the tool-chain

The main challenge is that both day-to-day and crisis, on-demand models share the same components (fuel model, ensembles) that have been developed to be interoperable, implying that not only the data format but also the physical fields (resolutions, definitions) were coherently designed.

The tool-chain aims to reach a national scale and has been running experimentally since May 2017 over the island of Corsica. Several large fires occurred in this fire season giving the opportunity to tests the tool-chain by operational services, requiring to develop specific new ways of delivering the information of the high-resolution weather forecasting model (online video with focus on specific areas) and a rapid response web-based fire propagation tool. While the user interface and information delivery are still requiring enhancement, it appeared to be a relevant decision support system in specific complex terrain or meteorological situations where quick local effects are of major importance.

An example is the Calenzana Fire where a sudden wind shift allowed a strong foehn wind and quick rise in temperature, well captured by the model (Figure 2), with the animation and high resolution of the meteorological fields providing a different understanding of the situation.
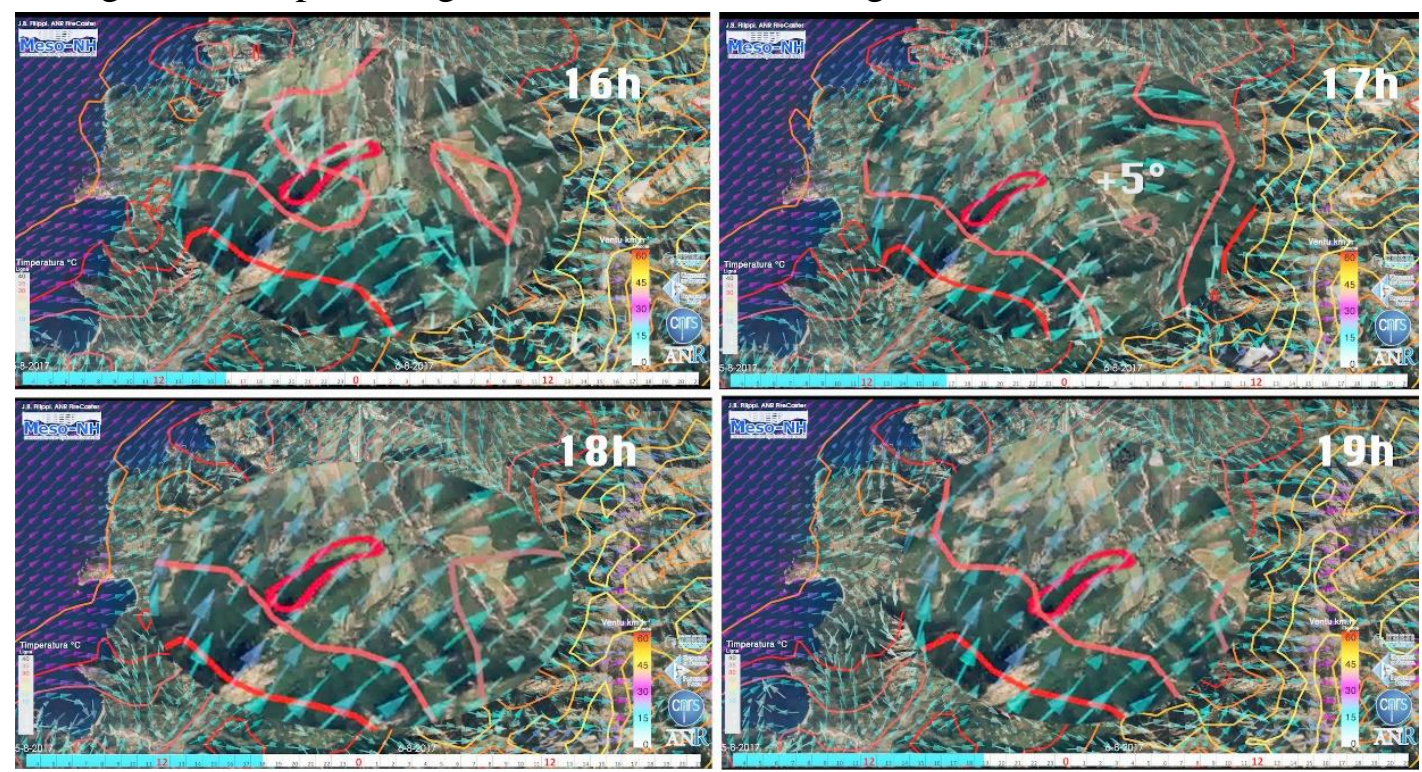

Figure 2 - Calenzana Fire, Aug. 6. 2017. Fire Area in Red. Temperatures as isolines, wind as vectors. Fire Caster forecast view at 16:00 (top left), 16:00 (top right), 18:00 (bottom left), 19:00 (bottom right). 
Rapid response web simulation of the fire (available in less than 2 minutes) also allowed to guess the possible extent of the fire (figure 3), and evolution. Fortunately this fire was very quickly handled by firefighters and almost stopped in less than 2 hours.

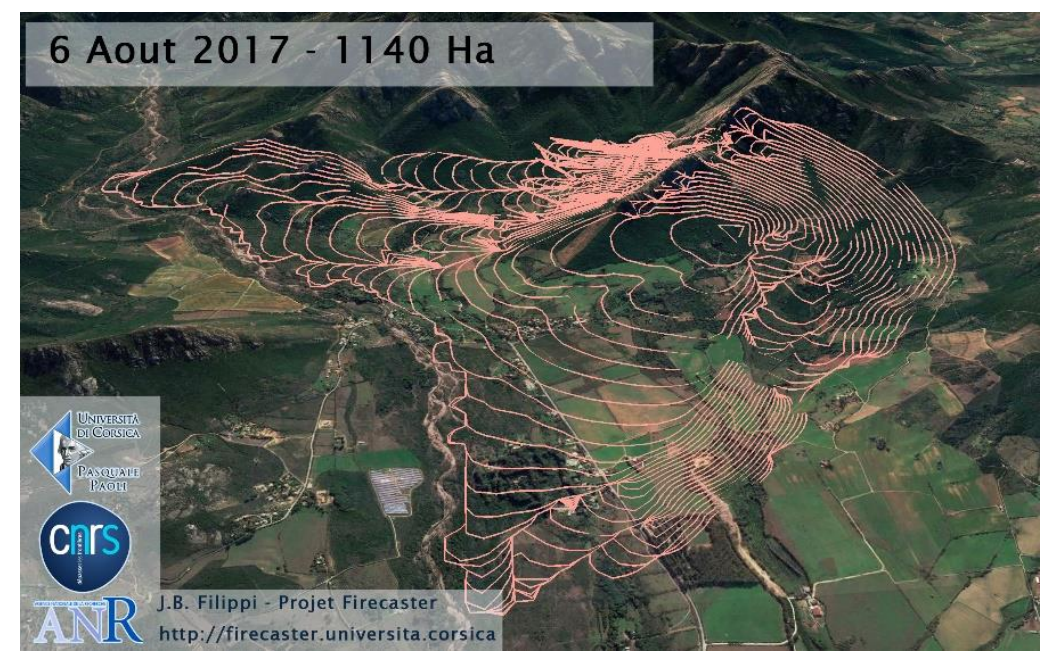

Figure 3 - Rapid-Response simulation, without firefighting actions. Fire as pink 20 minutes isolines, 7 hours total duration.

Rapid response smoke dispersion model is still only experimental and will be tested in the summer 2018 in case of fire. Overall, FireCaster tool-chain requires 240 processors 8 hours and 120 Go of data archiving per day. In 2017, six 2 minutes online videos were produced daily and more than 800 simulation performed, with all simulation tools and results available on the program website.

\section{References}

Coen, J. L., Cameron, M., Michalakes, J., Patton, E. G., Riggan, P. J., \& Yedinak, K. M. (2013). WRFFire: Coupled Weather-Wildland Fire Modeling with the Weather Research and Forecasting Model. Journal of Applied Meteorology and Climatology, 52(1), 16-38. https://doi.org/10.1175/jamc-d-12-023.1

Mandel, J., Beezley, J. D., \& Kochanski, A. K. (2011). Coupled atmosphere-wildland fire modeling with WRF 3.3 and SFIRE 2011. Geoscientific Model Development, 4(3), 591-610. https://doi.org/10.5194/gmd-4-591-2011

Assessment of ForeFire/Meso-NH for wildland fire/atmosphere coupled simulation of the FireFlux experiment, J.-B. Filippi, X. Pialat, C.-B. Clements, Proceedings of the Combustion Institute, Volume 34, Issue 2, Pages 2633-2640, 2013.

C. Zhang, M.C. Rochoux, W. Tang, M.J. Gollner, J.-.B. Filippi \& A. Trouvé: Evaluation of a datadriven wildland fire spread forecast model with spatially-distributed parameter estimation in simulations of the FireFlux I field-scale experiment, Fire Safety Journal, vol. 91, pp. 758767, doi: 10.1016/j.firesaf.2017.03.057

Finney, Mark A., McHugh, Charles W., Grenfell, Isaac C., Riley, Karin L., Short, Karen C., 2011. A simulation of probabilistic wildfire risk components for the continental United States. Stochastic Environmental Research and Risk Assessment. 25, 973-1000.

Lac, C., Chaboureau, J.-P., Masson, V., Pinty, J.-P., Tulet, P., Escobar, J., ... Wautelet, P. (2018). Overview of the Meso-NH model version 5.4 and its applications. Geoscientific Model Development Discussions, 1-66. https://doi.org/10.5194/gmd-2017-297 
Short contribution - Decision Support Systems and Tools

\title{
Laboratory measurement of transient carbon emissions from controlled smouldering peat fire
}

\author{
Yuqi Hu; Eirik Christensen; Guillermo Rein* \\ ${ }^{1}$ Imperial College London,SW7 2AZ,UK, \{y.hu15@imperial.ac.uk, e.christensen16@imperial.ac.uk, \\ g.rein@imperial.ac.uk*\}
}

\begin{abstract}
Smouldering peat fires, the largest fires on Earth in terms of fuel consumed per unit surface, contribute a large fraction of greenhouse gas emissions to the atmosphere on an annual basis and are responsible for regional haze episodes. Haze induces severe air quality deterioration, vast economic losses and poses a threat to health of local communities. Emissions from smouldering peat fire have become an international concern, however, knowledge on this topic is poor. Remote sensing detection technologies developed from studies of flaming combustion become ineffective when applied to smouldering peat fires. This work investigates the transient emissions from smouldering peat fire through small-scale laboratory experiments. Oven dried moss peat sample was burned in an open-top horizontal reactor open-top reactor with a dimension of $20 \mathrm{~cm}$ by 20 $\mathrm{cm}$ in cross section and $10 \mathrm{~cm}$ in depth. The mass loss rate (MLR), the evolutions of mass flux and emission factors (EFs) of the most prominent carbon species $\left(\mathrm{CO}_{2}, \mathrm{CO}\right.$ and $\left.\mathrm{CH}_{4}\right)$ were measured. Three combustion stages identifying from the two MLR peaks were used to describe the varying combustion dynamics as the fire progresses. $\mathrm{CO}_{2}$ and $\mathrm{CO}$ are mainly emitted from char oxidation and present a similar emission evolution, whose mass fluxes peaked at the same time with the $2^{\text {nd }}$ MLR peak ( 120 min). Transient EFs of these two species increase monotonically as the proportion of char being oxidised increases throughout the experiments. In contrast, $\mathrm{CH}_{4}$ is mainly emitted from peat pyrolyis and it shows a different emission behaviour: both its mass flux and EFs peaked at the same time as the $1^{\text {st }}$ MLR peak $(\sim 30 \mathrm{~min})$, a time when intensified pyrolysis ends. This study helps understand the link between the smouldering peat combustion dynamics and the transient emissions. Recognising the different emission mechanisms among gas species can be useful in developing effective remote sensing and smouldering peat fire mitigation strategies to tackle this global challenge.
\end{abstract}

Keywords: remote sensing; wildfire; smouldering combustion; in-depth burning; transient emission

\section{Introduction}

Peatlands, the terrestrial ecosystems resulting from the accumulation of partially decayed vegetation, are vulnerable to smouldering combustion - the slow, low-temperature, flameless burning of porous fuels (Rein 2015). A smouldering fire can be initiated with a weak ignition source and sustained for very long periods despite firefighting attempts, extensive rains or weather changes (Rein 2013). Smouldering peat fire propagates both vertically and horizontally, rendering it a multidimensional burning phenomenon (Rein 2013; Huang et al. 2016). Carbon emissions derived from peat fires are equivalent to $15 \%$ of anthropogenic emissions, creating large perturbations to the global carbon cycle (Rein 2013). These fires are also responsible for regional haze (Rein 2013; Hu et al. 2018). Haze causes air quality deterioration, transportation disruption, and impaired visibility and induces surges of health emergencies in the population (Hu et al. 2018). The study of these fires using remote sensing is an important topic (Figure 1). Despite the diverse effects of emissions from smouldering peat, knowledge on this topic is limited to a handful of studies (Rein et al. 2009; Hu et al. 2018). This work provides insights into transient peat fire carbon emissions through controlled smallscale smouldering experiments. 


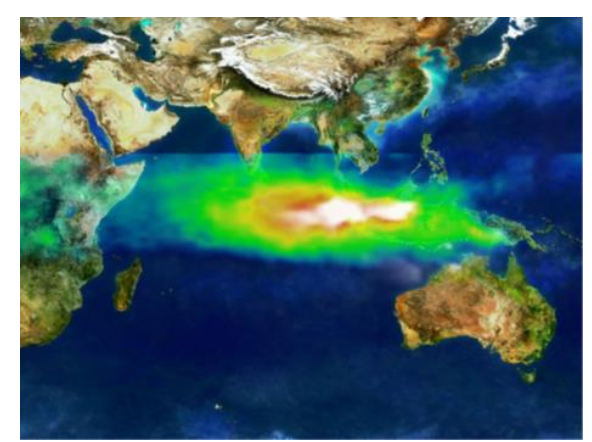

Figure 1 - Aerosol imaging by NASA satellite in October 1997 shows the vast smoke haze released by smouldering peat fires in Southeast Asia.

\section{Experimental method}

Roughly $0.88 \mathrm{~kg}$ of oven dried commercial peat was burned in an open-top reactor which has been used in previous smouldering study (Huang et al. 2016). A coil heater was used to ignite the peat by applying $100 \mathrm{~W}$ of power to the coil for $30 \mathrm{~min}$. Emissions from the smouldering peat were collected by an extraction hood under a steady extraction rate. The mass loss of the peat was recorded by a balance; the transient concentration of $\mathrm{CO} 2, \mathrm{CO}$ and $\mathrm{CH} 4$, the most prominent carbon emission species from peat fire, were measured by a Fourier-transform infrared spectroscopy (FTIR). The spread of the smouldering peat was monitored by a visual and an infrared camera. Each experiment lasted 8 hours and was repeated for 3 times.

\section{Results and discussion}

Mass flux and emission factor (EFs), the two most widely used parameters for biomass emission quantification, were calculated and discussed. Figure 2 shows the MLR of the dry peat as a function of the mass flux and EFs of $\mathrm{CO}_{2}$. It is seen that the combustion dynamics of the smouldering peat vary as the fire progresses.

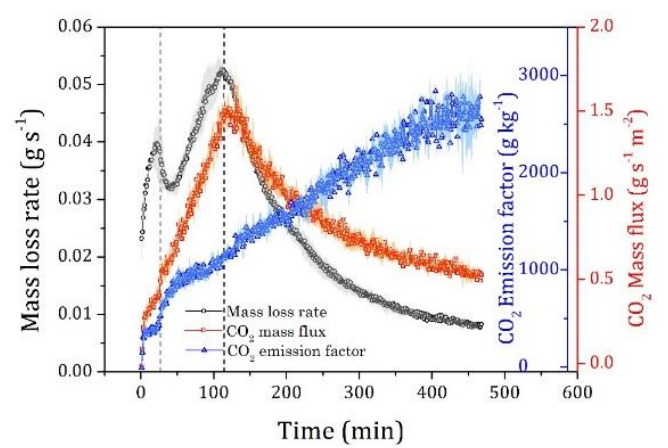

Figure 2 - Evolution of MLR, transient $\mathrm{CO}_{2}$ mass flux and EFs. The means and range (appeared as cloud) from all the 3 repeated smouldering peat experiments are shown. Vertical dashed line indicates the $1^{\text {st }}$ and $2^{\text {nd }}$ peak of the MLR of dry peat.

The MLR reached its first peak when the ignitor was turnt off $(\sim 30 \mathrm{~min})$. The $\mathrm{CO}_{2}$ mass flux peaked $\left(1.5 \mathrm{~g} \mathrm{~s}^{-1} \mathrm{~m}^{-2}\right)$ simultaneously with the the second peak of the MLR $(\sim 120 \mathrm{~min})$, a time when the surface peat was observed to spread to the far end of the reactor. $\mathrm{CO}_{2} \mathrm{EF}$ was found to increase monotonically throughout the experiments, reaching $\sim 2,500 \mathrm{~g} \mathrm{~kg}^{-1}$ at the end of the experiment. The comparison between MLR, mass flux and EFs indicates that the transient $\mathrm{CO}_{2}$ emissions are related with the combustion dynamics of the peat. 
Figure 3 shows the transient $\mathrm{CO}$ and $\mathrm{CH}_{4}$ emissions from smouldering peat. $\mathrm{CO}$ presents a similar evolution of mass flux and EFs with $\mathrm{CO}_{2}$, while for $\mathrm{CH}_{4}$, both the mass flux and EFs peaked at $\sim 30$ min, indicating a different emission mechanism.
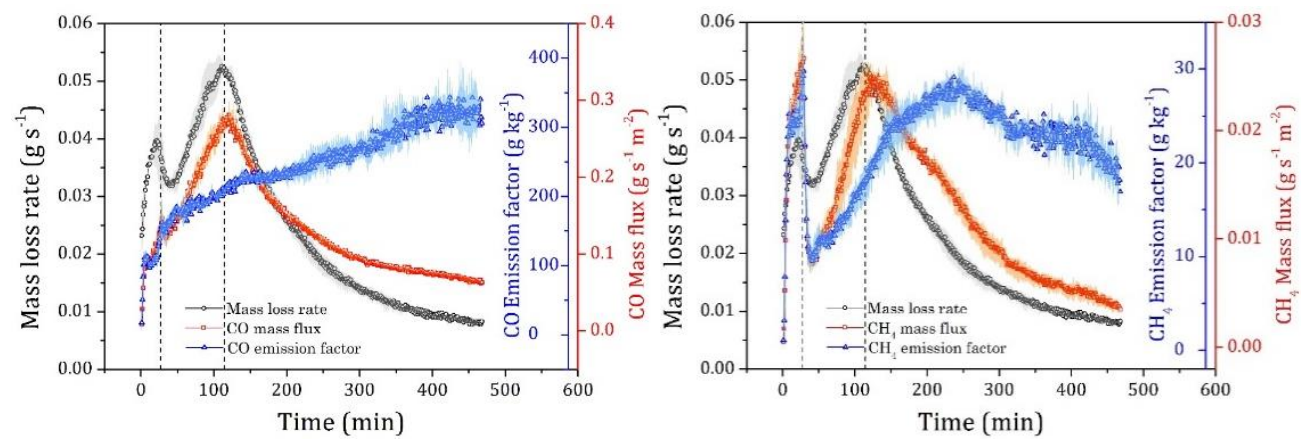

Figure 3 - Evolution of MLR, transient mass flux and EFs for $\mathrm{CO}$ (left) and $\mathrm{CH}_{4}$ (right).

\section{Conclusions}

Controlled laboratory experiments were carried out to investigate the transient emissions from smouldering peat. The results show that the mass loss rate, mass flux and emission factors of $\mathrm{CO}_{2}, \mathrm{CO}$ and $\mathrm{CH}_{4}$ vary as the fire progresses. The transient emissions are found to be related with the combustion dynamics. This work indicates that smouldering peat has different emission mechanisms among species, identifying these transient emissions could serve to develop effective remote sensing and mitigation strategies for peat fire.

\section{Acknowledgements}

The research has been funded by the China Scholarship Council (CSC) and the European Research Council (ERC) Consolidator Grant HAZE (682587).

\section{Reference}

G .Rein. "Smouldering Combustion,” In “The SFPE Handbook of Fire Protection Engineering”, 2016, (Ed Hurley MJ, pp. 581-603. Springer: New York).

G .Rein. "Smouldering Fires and Natural Fuels," In "Fire Phenomena and the Earth System: An Interdisciplinary Guide to Fire Science”, 2013, (Ed Belcher CM, pp. 15-34. John Wiley \& Sons: Oxford).

X. Huang, F.Restuccia, M.Gramola, G. Rein. "Experimental study of the formation and collapse of an overhang in the lateral spread of smouldering peat fires", Combustion and Flame, 2016, 168, 393 402.

Y. Hu, N. Fernandez-Anez, T. E. L. Smith, and G. Rein. "Review of emissions from smouldering peat fires and their contribution to regional haze episodes", International Journal of Wildland Fire, 2018.

G. Rein, S.Cohen, A. Simeoni . "Carbon emissions from smouldering peat in shallow and strong fronts", Proceedings of the Combustion Institute, 2009, 32, 2489-2496. 
Short contribution - Decision Support Systems and Tools

Management of an infrared imaging system for wildfire monitoring

\author{
Christian Mata*; Mario M. Valero ${ }^{1}$, Elsa Pastor, Eulàlia Planas \\ Center for Technological Risk Studies, Universitat Politècnica de Catalunya-BarcelonaTech. \\ c/Eduard Maristany, 10-14(EEBE),08019 Barcelona, Spain, \{christian.mata@upc.edu*\}
}

\begin{abstract}
In this work, we present our system architecture developed to manage and pre- and post-process TIR (thermal infrared) imagery for wildfire applications. It is mainly composed of an acquisition module, a preprocessing unit and a GUI graphical interface for post-processing analysis. Specific data-acquisition software was developed to overcome the limitations of the applications provided by camera manufacturers. This data acquisition module was complemented with a pre-processing unit to deal with those videos already recorded using manufacturers' software. This pre-processing unit converts stored IR raw files (which are unreadable) into a standard format that allows an overall thermal analysis. Our system can handle video formats used by two of the most important IR camera providers, i.e. Optris and FLIR. Finally, a graphical interface application provides the user with a user-friendly menu to detect fire edge position at desired time steps (i.e. automated perimeter tracking), to calculate rate of spread maps and interact with the data-driven forecasting module. This system makes two important contributions. First, it constitutes a common framework that ensures coherence in the treatment of IR data, no matter how it was obtained. Additionally, it incorporates the image processing algorithms in which we have been working for the past years, making them easy-to-use and facilitating the analysis of their results.
\end{abstract}

Keywords: infrared imaging system, GUI graphical interface, wildfire monitoring

\title{
1. Introduction
}

Technological innovations are being increasingly developed in recent years by the wildfire research community to provide managers with fire monitoring and simulation tools. During the past five years, we have been developing an integrated system for tactical monitoring and data-driven spread forecasting, with the aim of making available an affordable and fully automated tool for tactical wildfire decision-making. Infrared imagery- based fire edge detection and inverse modelling shortterm fire spread prediction algorithms have already been published elsewhere (Rios et al. 2016, Valero et al, 2017, Rios et al., 2018 and Valero et al, 2018). Those are the core elements composing our system, envisaged to couple meaningful automated wildfire monitoring with accurate fire spread forecasting.

Concretely, we can summarize the purpose conducted along two different lines. Firstly, the fire spread simulator published in (Rios et al. 2014, Rios et al. 2016). Secondly, the observed isochrones needed for calibration, which had been provided manually, can now be detected automatically using thermal aerial infrared (TIR) imaging and the algorithms presented in (Valero et al. 2017b, Valero et al. 2018). In this sense, the fact of using TIR imagery as the only source of optical data reduces the system complexity, weight and cost in comparison with hyperspectral imagers. However, an acquisition system and pre-processing stage with a high quality management is required in order to perform the post-processing analysis.

In this work, a system architecture developed to acquire, manage and pre- and post-process TIR imagery is presented. An acquisition module, a pre-processing unit and a GUI graphical interface for post-processing analysis mainly compose this architecture. The importance to record and store IR raw files (e.g. .RAVI) are input in the pre-processing unit. Specific software has been developed and implemented to convert these original (and unreadable) data into a standard format that allows an 
overall thermal analysis. Finally, a graphical interface application provides the user with a userfriendly menu to detect fire edge position at desired time steps (i.e. automated perimeter tracking), to calculate rate of spread maps and interact with the data-driven forecasting module.

\section{Management of the Infrared Imaging System Architecture}

\subsection{Acquisition}

Data acquisition hardware consists in an affordable computer programmed to obtain IR data from an IR camera. Specific software was developed to acquire IR data from Optris cameras. Optris produces some of the smallest and most affordable IR cameras available at present, but their software is totally opaque and it has critical limitations. The main idea is to develop a generic software to be adapted tom other common IR imaging systems. An overview of data management in the image acquisition and pre-processing module is depicted in Figure 1. The data acquisition module is defined as following: acquisition device, camera control platform, supported operating systems and export data system (using different data formats).

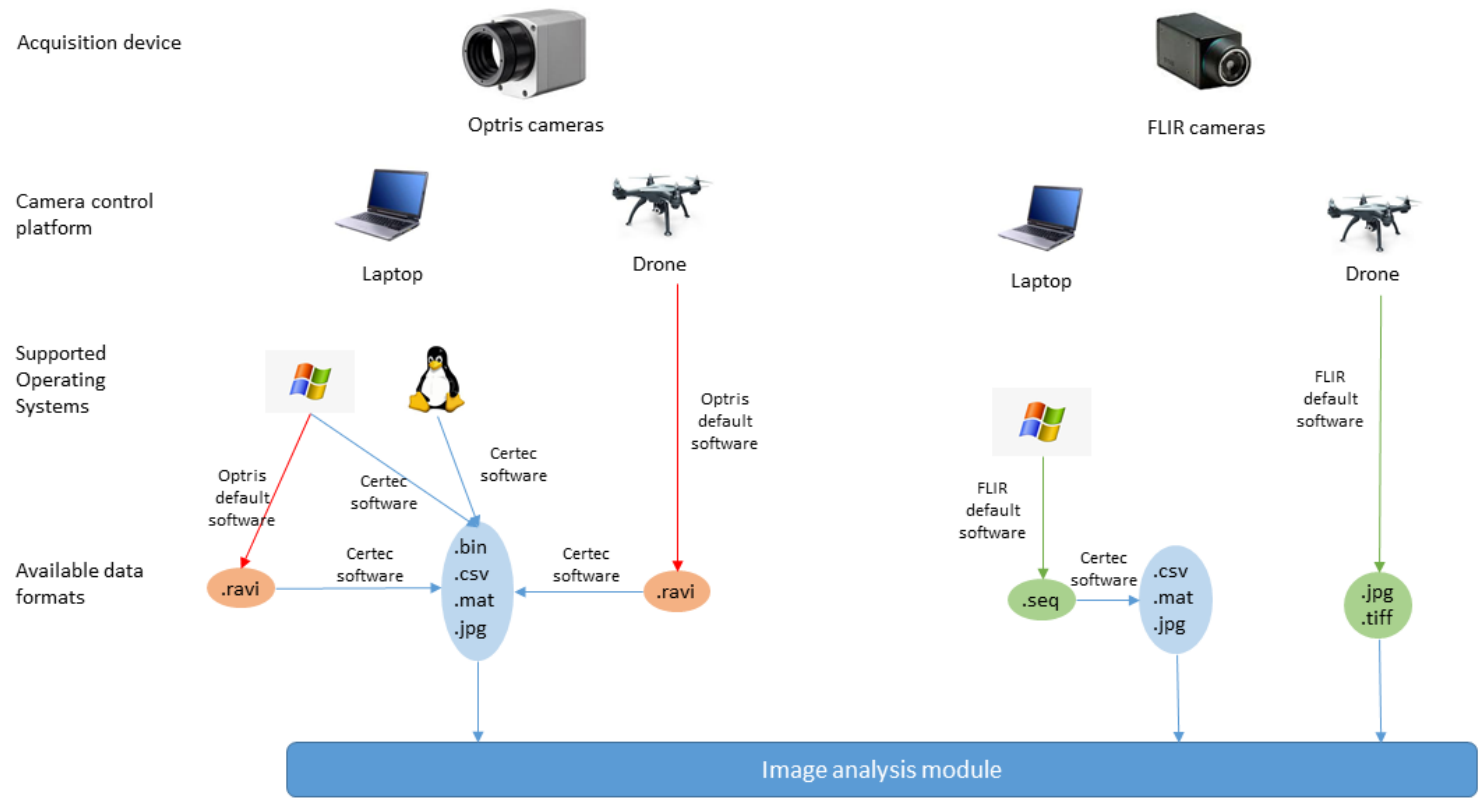

Figure 1 - Overview of data management in the image acquisition and pre-processing module

One of the advantages of this acquisition system is the integration of different IR cameras such as Optris PI and FLIR into the software. Furthermore, this software could be adapted to other cameras if needed in the future. The fact of using TIR imagery as the only source of optical data reduces the system complexity, weight and cost in comparison with other commercial systems. Moreover, this software was implemented in different versions depending of the supported operating systems such as Microsoft and Linux products. After the acquisition, the main problematic step is to export the record data into an easy format to be post-processed.

\subsection{Pre-processing Management}

CERTEC software was implemented in order to convert recorded video into a readable format for the image analysis module. As can be seen in Figure 1, .RAVI is the format extension used for the Optris PI camera and .SEQ is the specific format for the FLIR cameras. All the recorded files using the Optris PI camera are stored in IR raw files. In this case, the IR camera uses a specific format such as . $R A V I$ extension. These files has the inconvenient that should open them using the software provided 
for the same company called PI-Connect. Then, it exists limited options to extract the data in other formats but should be manually performed and it is not useful for a long records.

For this reason, specific software has been developed and implemented to convert these original (and unreadable) data into a standard format that allows an overall thermal analysis. This application was implemented using libirimager library. This library grants you access to all cameras featured in the Optris PI series. In order to obtain a human-interpretable representation, the SDK supports several conversion functions. This developer library enables your software team to process a camera's data stream with $\mathrm{C} / \mathrm{C}++$.

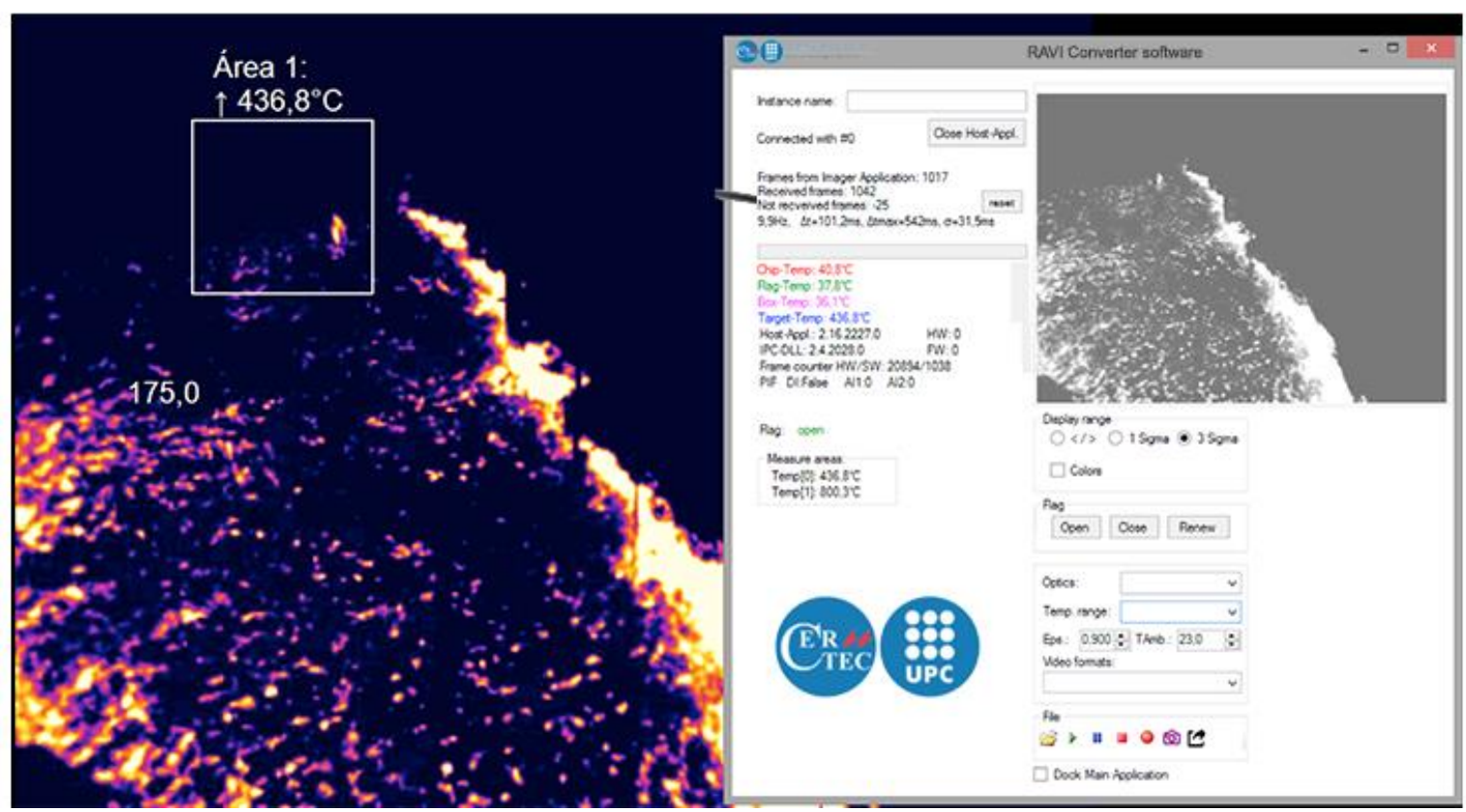

Figure 2 - Sample screenshot of the video format converter developed for Optris .RAVI files

One of the advantages of this library is the representation of the IR data with different colouring palettes so that the user can get a false colour representation of their data takes. Figure 2 depicts an example of the implemented graphical interface that allows loading a thermal record, visualizing it and using different options to extract into your workspace all the frames (thermal images), and information related with the image such as frame time, geo-reference position, etc. Notice that this software was implemented in Windows environment integrated in an EXE file, then it is not necessary a previous installation neither an additional library packages.

\subsection{Post-processing analysis}

The importance to acquire thermal data from a robust system for a post-processing analysis was the main objective of this work. According to this analysis, the proposed system architecture and preprocessing management were designed. We also incorporated our previous work on the coupling of remote sensing data with fire spread simulation (Valero et al. 2017a). Figure 3 displays sample screenshots of the graphical user interface and their knowledge distribution. The methodology proved able to automatically detect the location of the fire perimeter and emit a reliable forecast of its future evolution. It is based on edge detection algorithms applied to TIR aerial imagery and data assimilation techniques, fire rates of spread being computed through an on-line-adjustable Rothermel model. 


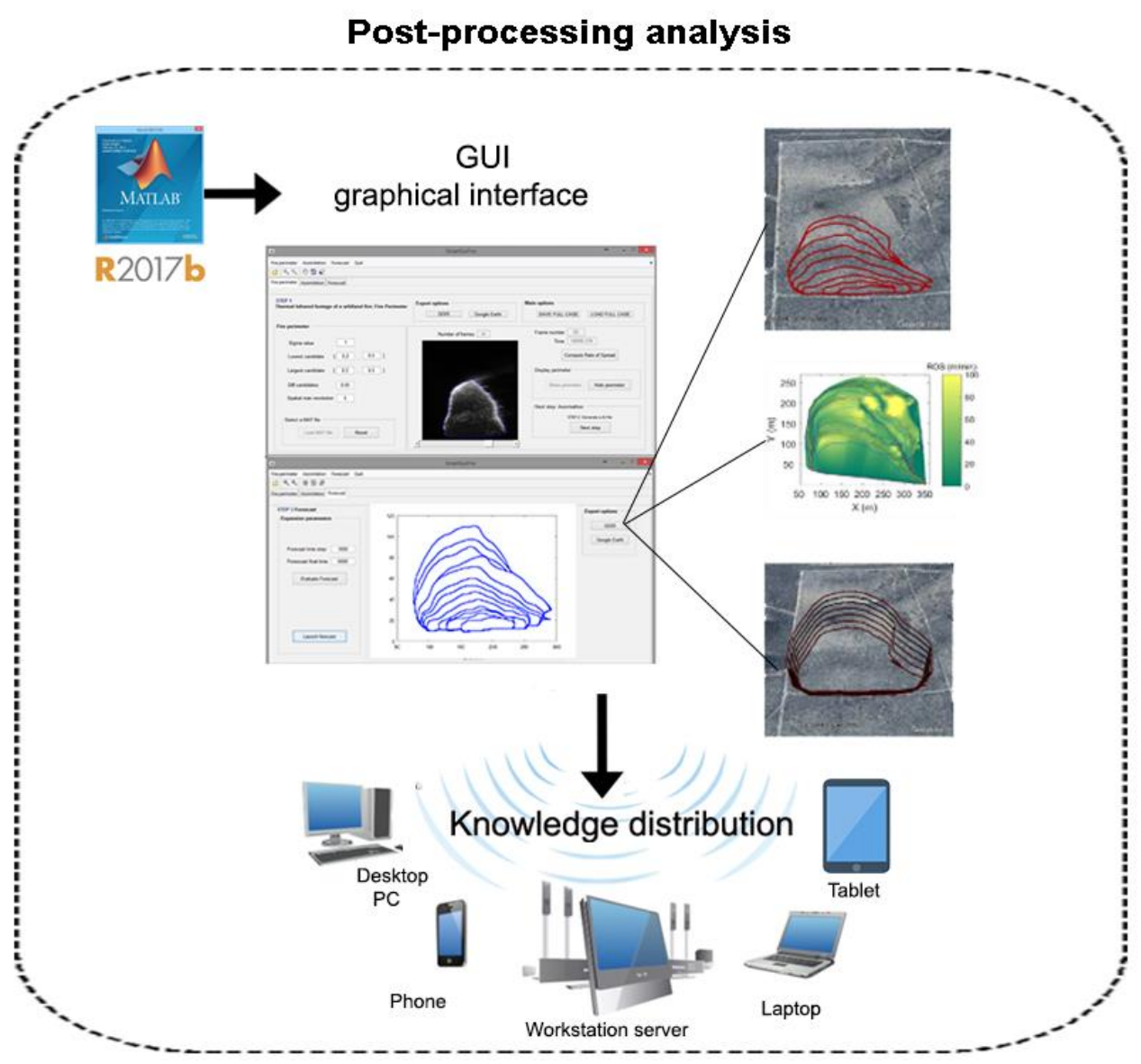

Figure 3 - Benchmark design of the proposed architecture system. Three different tasks are described in this architecture within the scope of this work

\section{Conclusions}

We hereby presented the management of an infrared imaging system for wildfire monitoring. One of the important advantages of this management is the contribution to develop a suitable system architecture promoting a decision support system for a wildfire emergency. Firstly, an acquisition task of the benchmark design of the proposed architecture system was detailed. It is adaptable to IR imagery of different formats and sources and it can be coupled with different pre- and post-processing modules.

This architecture is easily adaptable to other low cost IR monitoring cameras and to our knowledge is the most cost-effective solution for wildfire monitoring. According to a specific format of the infrared camera used, a pre-processing software is also presented in order to export thermal sources. In case that uses want to use another camera, it should be implemented a new pre-processing software according to their features.

Finally, the post-processing task allows an easy analysis of the IR data, facilitating the extraction of fire information.

\section{Acknowledgements}

This research was partially funded by the Spanish Ministry of Economy and Competitiveness (project CTM2014-57448-R and, CTQ2017-85990-R, co-financed with FEDER funds). 


\section{References}

Rios O, Jahn W, Rein G (2014). Forecasting wind-driven wildfires using an inverse modelling approach, Nat. Hazards Earth Syst. Sci. 14, 1491-1503.doi: 10.5194/nhess-14-1491.

Rios O, Pastor E, Valero MM, Planas E (2016). Short-term fire front spread prediction using inverse modelling and airborne infrared images. International Journal of Wildland Fire 25, 1033-1047.

Valero MM, Rios O, Mata C, Pastor E, Planas E (2017a). An integrated approach for tactical monitoring and data-driven spread forecasting of wildfires. Fire Safety Journal 91, 835-844. doi: 10.1016/j.firesaf.2017.03.085.

Valero MM, Verstockt S, Rios, O, Pastor, E, Vandecasteele, F., Planas, E (2017b). Flame filtering and perimeter localization of wildfires using aerial thermal imagery, in: Proc. SPIE 10214. Thermosense: Thermal Infrared Applications XXXIX. https://doi.org/10.1117/12.2262100

Rios, O., Jahn, W., Pastor, E., Valero, M.M., Planas, E., 2018. Interpolation framework to speed up near-surface wind simulations for data-driven wildfire applications. Int. J. Wildl. Fire 27, 257-270. https://doi.org/10.1071/WF17027

Valero MM, Rios O, Pastor E, Planas E. (2018). Automated location of active fire perimeters in aerial infrared imaging using unsupervised edge detectors. Int. J. Wildl. Fire 27, 241-256. https://doi.org/10.1071/WF17093. 
Short contribution - Decision Support Systems and Tools

\title{
Modeling fuels and treatment effects in 3D with STANDFIRE
}

\author{
Russ Parsons ${ }^{1 *}$; Francois Pimont ${ }^{2} ;$ Lucas Wells $^{3}$; Greg Cohn ${ }^{4} ;$ W. Matt Jolly ${ }^{1}$; Brett Davis ${ }^{1} ;$ Francois de \\ Coligny"; William "Ruddy" Mell'; Rod Linn"; Eric Rigolot'; Jean-Luc Dupuy ${ }^{2}$ \\ ${ }^{1}$ USFS RMRS, Fire Sciences Lab. 5775 Highway 10 W, Missoula, MT, \{rparsons@fs.fed.us*\} \\ ${ }^{2}$ URFM INRA. 84914, France, \{francois.pimont@inra.fr\} \\ ${ }^{3}$ Department of Forest Engineering, Resources and Management, College of Forestry, Oregon State \\ University, 210 Snell Hall, Corvallis, OR 97331, USA, \{lawells36@gmail.com\} \\ ${ }^{4}$ Department of Forest Ecosystem and Society, College of Forestry, Oregon State University, 321 \\ Richardson Hall, Corvallis, OR 97331, USA, \{cohng@oregonstate.edu\} \\ ${ }^{5}$ AMAP, INRA, Cirad, CNRS, IRD, Univ Montpellier, France \{francois.decoligny@inra.fr\} \\ ${ }^{6}$ U.S. Forest Service Pacific Wildland Fire Sciences Lab, $400 \mathrm{~N} 34^{\text {th }}$. Street, Suite 201, Seattle, WA 98103, \\ USA, \{wemell@fs.fed.us\} \\ ${ }^{7}$ Environmental Sciences Division, Los Alamos National Laboratory, Mail stop: D401, Los Alamos, NM \\ 87544,USA, \{rrl@lanl.gov\}
}

\begin{abstract}
Fuel treatments offer a means of proactively mitigating risks to firefighters and communities, and to restore altered ecosystems. The cost and potential impacts associated with implementing such treatments, however, suggest a need for thorough understanding of their effectiveness before they are carried out on the ground, particularly at landscape scales. At present, modelling tools used to evaluate fuel treatments are often limited in their ability to represent fuels or fuel/fire interactions. This is particularly true for treatments that seek to modify forest spatial patterns; most modelling frameworks are not spatially explicit and thus can only describe forests in terms of overall average characteristics. Here, we describe STANDFIRE, a prototype 3D platform for modeling wildland fuels and fire behavior at stand scales. Leveraging a powerful core modeling architecture, STANDFIRE links a forest growth model to physics-based fire behavior models, providing a means by which detailed fuels data can be used as input in physics-based fire behavior simulations which can explore fuel treatment effectiveness or sensitivity to environmental conditions. We describe the model, some applications, and plans for continuing development.
\end{abstract}

Keywords: Fuel modeling, Fuel treatments, Fire behavior, Physics-based, WFDS, FIRETEC, FuelManager

\section{Introduction}

Fuel treatments such as thinning are used to alter fire behavior, mitigate risks to firefighters and communities and reduce fire severity. Many forest ecosystems have undergone significant changes in composition and structure over the last several decades (Hessburg et al 2005), often resulting in conditions that threaten their long term viability (Fulé et al 2014). Recent studies suggest that fuel treatments that create spatially heterogeneous fuel patterns can restore ecosystem resilience by creating diverse post-fire conditions (Larson and Churchill 2012). However, at present, forest modeling systems used to evaluate fuel treatments are not spatial, and employ very simple fire models that cannot accommodate fuel heterogeneity at an appropriate level of detail (Rothermel 1972). Given the scale at which ecosystem restoration and associated fuel treatments are needed, there is a substantial need for modeling that can be used to explore fuel treatment effectiveness in a spatially explicit manner. In this paper, we discuss STANDFIRE, a 3D prototype modeling platform for fuels, fire behavior and effects, that may offer new capabilities in this arena. 


\section{Model Overview}

STANDFIRE is a prototype system for spatially explicit, 3D modeling of fuels, fire behavior and effects. STANDFIRE's modular architecture links four main components (Figure 1): 1) forest growth and biomass modeling with the Forest Vegetation Simulator (FVS, Crookston and Dixon 2005) 2) a core 3D fuel modeling architecture (see below) producing fuels inputs for 3) physics-based fire behavior models WFDS (Mell et al 2009) and FIRETEC (Linn et al. 2005) and 4) post-processors that calculate fire behavior and effects results from simulation outputs. More detail regarding these components can be found in a recent publication (Parsons et al 2018).

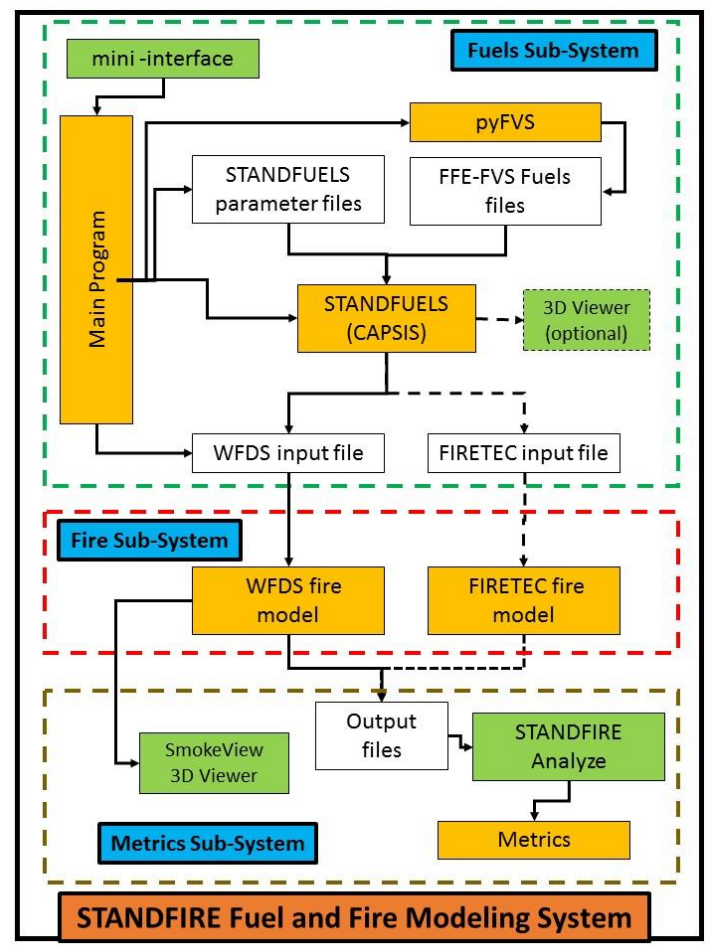

Figure 1 - Flow chart of STANDFIRE components

A particularly important component in STANDFIRE is STANDFUELS, a module of the CAPSIS platform (Dufour-Kowalski et al 2012) which provides critical capabilities for detailed but scalable fuel modeling. This module is based on the FireLib library -also used in FuelManager (Pimont et al 2016)- and models vegetation either as Plants, such as large trees or shrubs, or as LayerSets, which are irregular (user specified) polygons (Figure 2A) associated with sets of attributes and which can contain other spatially explicit entities. For example, a LayerSet could contain multiple shrub species (as separate Layers) each of different sizes and properties. These basic, but powerful building blocks provide flexibility to model nearly any kind of wildland fuel. FireLib also carries out the non-trivial numerical task of converting fuel elements with specific geometries (like a tree crown) to voxels, in a file format which serves as inputs in the physics-based models. Additional detail regarding these capabilities is provided in Pimont et al (2016).

\section{Applications}

STANDFIRE has many potential applications. The primary application that it was designed for is to facilitate more in-depth analysis of fuel treatment effectiveness. Unlike empirical or semi-empirical models which significantly abstract fuels, weather and fire, enabling rapid calculations but limiting the insights they can provide (Andrews et al., 2003, Cruz et al., 2005), physics-based fire models can model fire in situations closer to what occurs in the real world (i.e. complex topography, time and space varying ignition, and dynamic weather conditions). One of the key aspects that such models 
bring to evaluation of fuel treatments is their capacity to model fire in discontinuous and heterogeneous fuels, which are often the result (indeed, the intent) of fuel treatments (e.g. thinning). A growing body of work has examined fuel heterogeneity in a number of situations including discrete fuel beds (Linn et al., 2005), and beetle killed trees (Linn et al., 2013, Hoffman et al., 2015). Recent applications in fuel treatments highlight the importance of spatial relationships in fuels (Linn et al., 2005, Parsons 2006, Pimont et al., 2011, Contreras et al., 2012, Parsons et al., 2017, Ziegler et al., 2017). While much more work is needed, the successes of these efforts so far justify continued exploration of fuel treatments with systems such as STANDFIRE.

STANDFIRE's use of FVS for forest growth and biomass enables simulations for most forest types in the U.S. Fuel treatment effectiveness assessments are carried out through fire simulations with physics-based fire models, and through subsequent calculations of fuel and fire behavior metrics. Figure 2B shows fire behavior at the same point in time for an untreated forest (Fig. 2B upper left) and three different crown space thinning treatments. Surface fire spread rates increased with increasing levels of thinning due to reduced canopy drag effects on the wind field.

\section{Ongoing Development}
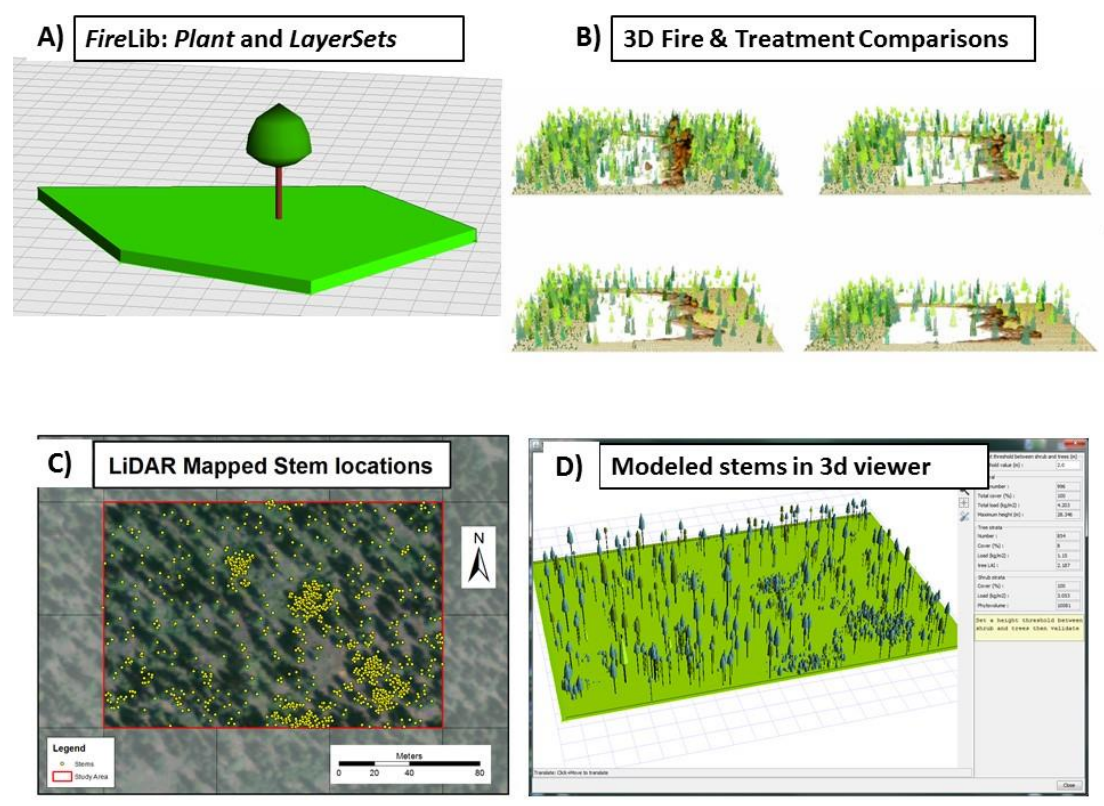

Figure 2 - Various aspects of STANDFIRE: A) example Plant and LayerSet fuel modeling approaches, B) example fire behavior comparison of different fuel treatments, C) example LiDAR mapped forest data D) example LiDAR mapped forest, as modeled fuels in STANDFIRE's $3 D$ viewer

In addition to application in fuel treatment analysis, development is currently underway to expand STANDFIRE to facilitate simulation of prescribed fires. Current efforts are focused on expanding capabilities to read in real world fuels data such as from airborne LiDAR (Figure 2C, D). At present this import is carried out through a spatial shapefile derived from LiDAR point clouds rather directly through the point cloud itself. As LiDAR data become more available, and improved approaches for automated data processing are developed (Cabo et al., 2018), the capacity to rapidly develop very detailed 3D fuels inputs will be increasingly useful. Other ongoing development includes import of different raster datasets including topography, as well as spatial and temporal data associated with real world fires, such as ignition GPS tracks and wind data. These new features will expand STANDFIRE's capabilities to rapidly develop input datasets for physics-based fire behavior models for simulations of prescribed fires, providing new capabilities for training and for examination of numerous factors that can affect prescribed fire outcomes, including fuel conditions, ignition patterns, and sensitivity to 
weather conditions, among others. These developments will also facilitate efforts to evaluate fire models with real world fire data, a key objective of several large scale fire data collection efforts in recent years.

\section{References}

Andrews PL, Bevins CD, and Seli RC (2003). BehavePlus fire modeling system, version 2.0: user's guide. USDA For Serv. Gen. Tech. Rep. RMRS-GTR-106WWW

Cabo C, Ordóñez C, López-Sánchez CA and Armesto J (2018) Automatic dendrometry: Tree detection, tree height and diameter estimation using terrestrial laser scanning. International Journal of Applied Earth Observation and Geoinformation, 69, 164-174.

Contreras MA, Parsons RA and Chung W (2012) Modeling tree-level fuel connectivity to evaluate the effectiveness of thinning treatments for reducing crown fire potential. Forest Ecology and Management, 264, 134-149.

Crookston NL, Dixon GE (2005) The forest vegetation simulator: a review of its structure, content, and applications. Comput Electron Agric 49, 60-80.

Cruz MG, Alexander ME and Wakimoto RH (2005) Development and testing of models for predicting crown fire rate of spread in conifer forest stands. Canadian Journal of Forest Research, 35(7), 16261639.

Dufour-Kowalski S, Courbaud B, Dreyfus P, Meredieu C, De Coligny F (2012) Capsis: an open software framework and community for forest growth modelling. Ann. For. Sci. 69, 221-233.

Fule' PZ, Swetnam TW, Brown PM, Falk DA, Peterson DL, Allen CD, Aplet GH, Battaglia MA, Binkley D, Farris C, Keane RE, Margolis EQ, Grissino-Mayer H, Miller C, Hull Sieg C, Skinner C, Stephens CL, Taylor A (2014) Unsupported inferences of high severity fire in historical western United States dry forests: response to Williams and Baker. Global Ecology and Biogeography 23, 825-830. doi:10.1111/GEB. 12136

Hessburg PF, Agee JA, Franklin JF (2005) Dry forests and wildland fires of the Inland Northwest USA: Contrasting the landscape ecology of the pre-settlement and modern eras. For. Ecol. Manag. 211, 117-13.

Hoffman CM, Linn R, Parsons R, Sieg C, Winterkamp J (2015) Modeling spatial and temporal dynamics of wind flow and potential fire behavior following a mountain pine beetle outbreak in a lodgepole pine forest. Agricultural and Forest Meteorology 204, 79-93.

Larson AJ, Churchill D (2012) Tree spatial patterns in fire-frequent forests of western North America, including mechanisms of pattern formation and implications for designing fuel reduction and restoration treatments. Forest Ecology and Management 267, 74-92.

Linn R, Winterkamp J, Colman JJ, Edminster C, Bailey JD (2005) Modeling interactions between fire and atmosphere in discrete element fuel beds. International Journal of Wildland Fire 14, 37-48.

Linn RR, Sieg CH, Hoffman CM, Winterkamp JL and McMillin, J.D (2013) Modeling wind fields and fire propagation following bark beetle outbreaks in spatially-heterogeneous pinyon-juniper woodland fuel complexes. Agricultural and Forest Meteorology 173, 139-153.

Mell W, Maranghides A, McDermott R, Manzello SL (2009) Numerical simulation and experiments of burning douglas fir trees. Combustion and Flame 156, 2023-2041.

Parsons RA (2006) FUEL3-D: a spatially explicit fractal fuel distribution model. In 'Fuels Management - How to Measure Success: Conference Proceedings', 28-30 March 2006; Portland, OR, USA. (Eds PL Andrews, BW Butler) USDA Forest Service, Rocky Mountain Research Station, Proceedings RMRS-P-41, pp. 253-272. (Fort Collins, CO, USA) 
Parsons RA, Linn RR, Pimont F, Hoffman C, Sauer J, Winterkamp J, Sieg, CH and Jolly WM (2017) Numerical investigation of aggregated fuel spatial pattern impacts on fire behavior. Land, 6(2), 43.

Parsons RA, Pimont F, Wells L, Linn RR, Cohn G, Jolly WM, de Coligny F, Rigolot E, Dupuy J-L, Mell W, and Linn RR (2018) Modeling thinning effects on fire with STANDFIRE. Annals of Forest Science 75(1), 1-11

Pimont F, Dupuy J-L, Linn RR, Dupont S (2011) Impacts of tree canopy structure on wind flows and fire propagation simulated with FIRETEC. Annals of Forest Science 68, 523-530. doi:10.1007/s13595011-0061-7.

Pimont F, Parsons R, Rigolot E, de Coligny F, Dupuy J-L, Dreyfus P and Linn RR (2016) Modeling fuels and fire effects in 3D: Model description and applications. Environmental Modelling and Software 80, 225-244

Rothermel RC (1972) A Mathematical Model for Predicting Fire Spread in Wildland Fuels; General Technical Report INT-115; U.S. Department of Agriculture, Forest Service, Intermountain Research Station: Ogden, UT, USA

Ziegler JP, Hoffman C, Battaglia M, Mell W (2017) Spatially explicit measurements of forest structure and fire behavior following restoration treatments in dry forests. Forest Ecology and Management $386,1-12$ 
Short contribution - Decision Support Systems and Tools

Modeling the probability of impact from wildland fires: a near real-time approach

\author{
Adrián Cardil $^{1 *}$; Joaquin Ramirez ${ }^{2}$; Santiago Monedero ${ }^{1}$ \\ I* Tecnosylva. Parque Tecnológico de León. 24009 León, Spain, \{acardil@tecnosylva.com*. \\ smonedero@tecnosylva.com\} \\ ${ }^{2}$ Technosylva.UCSD Calit2 Qualcomm Institute.La Jolla, CA 92037, \{jramirez@technosylva.com\}
}

Keywords: fire behaviour, fire modelling, Wildfire Analyst,

\title{
1. Background
}

Large and intense wildfires burn millions of hectares annually, impacting societies worldwide. Environmental, social, and economic impacts can reach catastrophic levels, particularly where human populations interact closely with natural and managed landscapes in fire prone areas. While fire professionals work to protect ecological and human resources during fire suppression, personnel are frequently challenged by the complexity of controlling wildfires in wildland urban interfaces. Protecting firefighters, citizens, homes, environmental, and economic resources is made more complex by the need to prioritize limited wildfire suppression personnel and equipment to address changing wildfire conditions. The ability to estimate the probability of a wildfire reaching an area where these resources are most vulnerable is critically important to preventing loss of human life and property, and damage to ecological and economic assets. We present an innovative probabilistic fire simulation mode of the Wildfire Analyst (WFA) software; an approach to simulate fire inversely while considering uncertainties in input data.

\section{Exposure probabilistic analysis}

This mode can assess the time and probability that a fire will reach a vulnerable area, by simulating (n) evacuation simulations with varying input data according to a potential range of deviations added by the analyst in near real-time. The evacuation method is also available in WFA software and is based on a fire inverse travel time mode technique that allows computing $\mathrm{x}$-hour firesheds around valuable assets or evacuation points. This propagation mode computes the time that a standard simulation starting at any point of the landscape would take to spread to a set of user defined points (for instance, a wildland urban interface area, infrastructures, assets, etc), instead of showing the usual time a fire would take to spread from a set of user defined ignition points to any point of the landscape.

\section{Where has this method implemented?}

The method is already implemented in WFA software and can be applied by fire analysts worldwide. We show in Figure 1 the Vall d'Ebo Fire (Spain, 2015) using Rothermel's model as the underlying surface fire spread engine. Simulated fire growth showed a good agreement with the final fire perimeter as shown in Figure 1a. To test the exposure probabilistic method in this fire, we run 100 evacuation simulations considering weather predictions before starting the fire, dealing with weather uncertainties by using a range of variation of those more influencer input variables on fire behavior and spread. We used a range of variation of $3 \mathrm{~km} / \mathrm{h}$ for wind speed, $30^{\circ}$ for wind direction and $1 \%$ for fine fuel moisture content (1h). 
a)

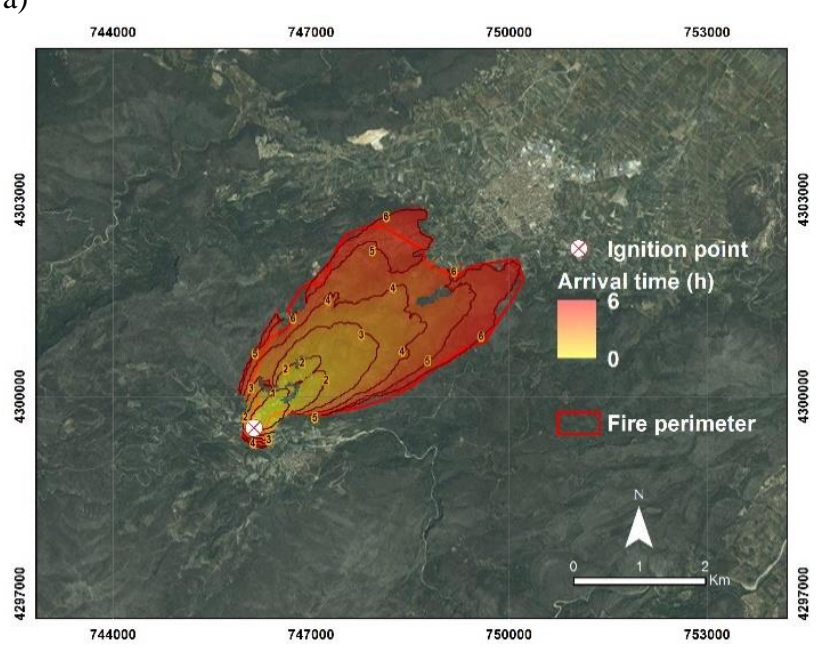

b)

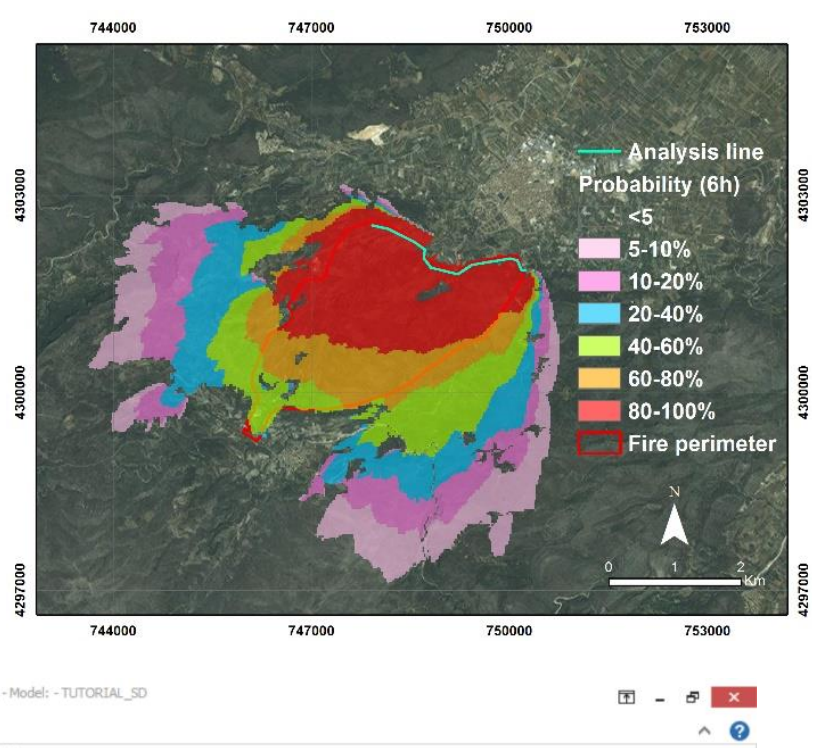

c)

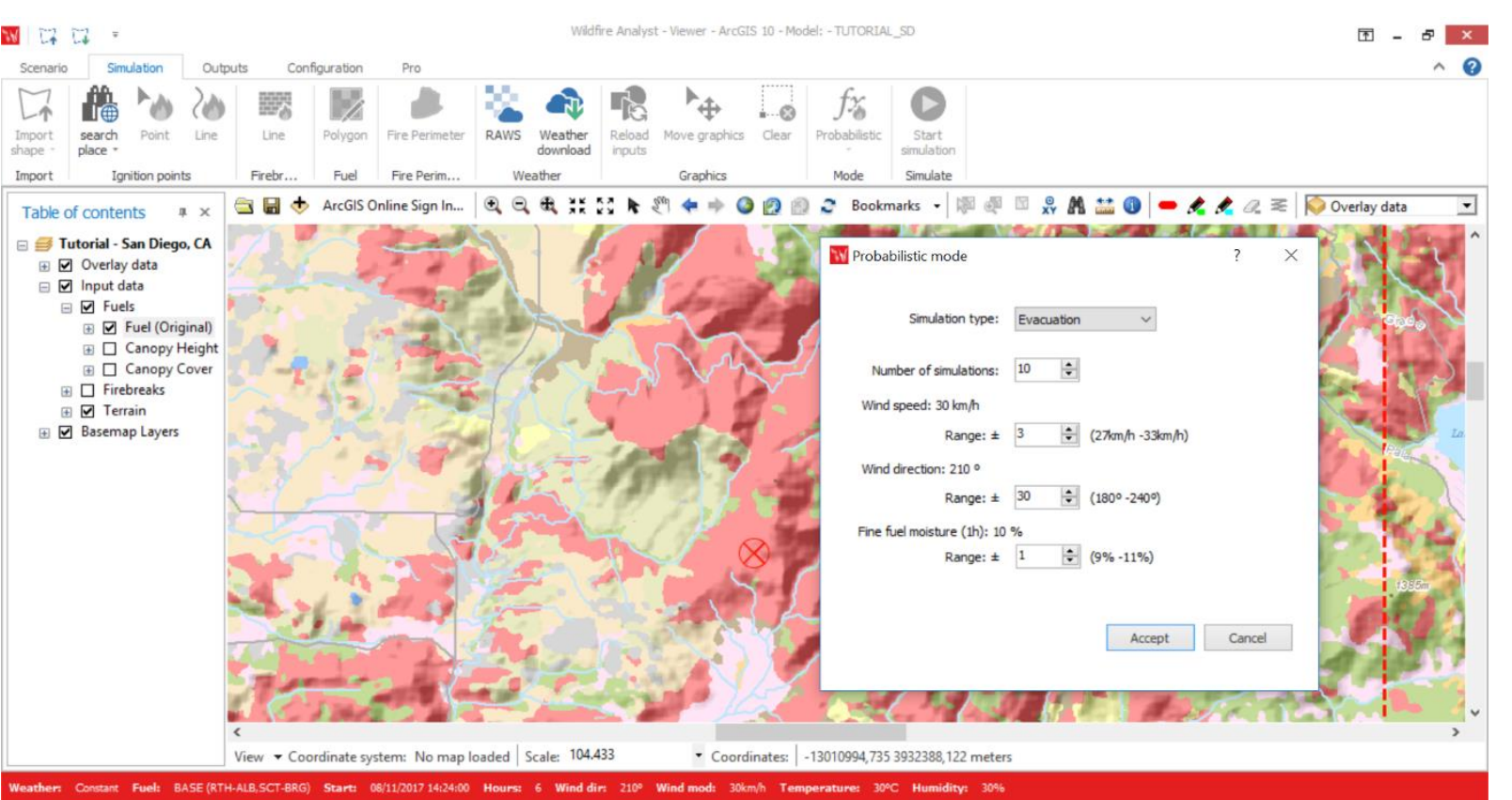

Figure 1 - Vall d'Ebo (Spain, 2015) fire simulation and real fire perimeter in the first 6 hours. a) Forward in time fire simulation from the ignition point; $b$ ) Probabilistic evacuation mode considering the edge of the wildland urban interface (Analysis line), running 100 fire evacuation simulations with a duration of 6 hours; $c$ ) WFA interface for the Exposure Probabilistic Analysis

\section{Implications for fire management.}

The exposure probabilistic mode provides a perfect framework for analyzing suppression actions in the WUI or vulnerable assets in near real-time and allows decreasing the uncertainty of the fire analyst in the decision-making process. By identifying structures or wildland communities this mode will determine the time of arrival for each location identified providing a baseline for evacuation planning. Additionally, this tool can be used to optimize fuel treatment location and quantify fire behavior and spread near WUIs. Towards the development of effective fire management operational systems, the use of fire behavior and spread models and decision support planning modules such as FireResponse (Technosylva, Inc) plays an important role. Today's firefighting needs demand systems that are able to conduct on a timely manner fire behavior predictions for several different users of fire suppression and civil protection agencies involved. In this sense, both the algorithms and methods that we show in this work can be solved in real-time in a few seconds and the mode is already implemented in WFA. 
Short contribution - Decision Support Systems and Tools

Modelling the performance of forest and wildland fire aerial detection systems

\author{
David L. Martell ${ }^{1 *}$; Colin McFayden ${ }^{2}$; Robin Santiago ${ }^{3}$; Douglas G. Woolford ${ }^{4}$; B. Mike Wotton ${ }^{5}$ \\ ${ }^{1}$ University of Toronto, \{david.martell@utoronto.ca* ${ }^{*}$ \} \\ 2Ontario Ministry of Natural Resources and Forestry, \{colin.mcfayden@ontario.ca\} \\ ${ }^{3}$ University of Toronto, \{robin.santiago@mail.utoronto.ca\} \\ ${ }^{4}$ University of Western Ontario, \{dwoolfor@uwo.ca\} \\ ${ }^{5}$ Canadian Forest Service, \{mike.wotton@utoronto.ca\}
}

Keywords: Forest and wildland fire detection, Detection patrol aircraft tracking, Detection probability model

\title{
1. Introduction
}

Detection and initial attack systems are important components of response systems for forest and wildland fires, the primary objective of which is to contain potentially destructive fires quickly at a small size at a reasonable cost. Modern forest fire detection systems are large complex systems that often include fixed towers, fixed-wing aerial detection patrol aircraft and "loaded" patrols by rotarywing aircraft carrying fire fighters and their equipment that can be dropped off to combat any fires that are detected along their flight path. Most forest fire management agencies also rely heavily on the public to report fires at little or no cost in or near heavily populated areas or along heavily used transportation corridors. Additionally, satellites sometimes serve as the first detector of fires in remote areas.

Decisions concerning when what detection patrol aircraft should fly what routes should be informed by estimates of the probability that the on-board detection observers will detect any undetected fires near their planned flight paths. The estimation of that probability is complicated by the fact that although we know about a detection observer's successes - the fires he or she found and reported, it's impossible to identify all the fires the fires that were "missed". In this paper we describe how we used aircraft tracking technology and lightning stroke counter technology to overcome that obstacle.

\section{Methods}

In order to develop an aerial detection probability model we had to identify a set of fires, their locations, when they were ignited, when aerial detection patrol aircraft flew nearby and whether or not the detection observer detected and reported the fire. We used lightning-caused fire ignition data on the assumption that it's difficult to confirm the ignition time of many human-caused fires.

We began by compiling a sample of lightning-caused fires that occurred in the province of Ontario, Canada during the years 2008-2015. We used lightning strike data collected by Ontario's lightning strike counter system, along with fire report dates, to estimate which day each lightning ignited fire occurred and when during the day that lightning occurred. Fire report data was used to determine when each of those fires was detected and reported and the detection agent.

The detection aircraft tracking system data is available in the form of a single "heartbeat" file for each aircraft. Each heartbeat datum includes the registration, datetime, latitude and longitude of the aircraft that was recorded every two minutes while the aircraft was powered up. Unfortunately, the raw heartbeat data for each detection aircraft does not indicate the type of flight that was taking place at that time. A subset of the heartbeat data might for example, be associated with a planned detection patrol, a training flight, or the ferrying of the detection aircraft from one airport to another. 
We developed heartbeat parsing rules to partition each aircraft's stream of heartbeat data into discrete flights and estimated the purpose of each of those flights. Our parsing rules were based on flight durations and patterns (e.g., the number and magnitudes of turning angles) that are characteristic of planned detection flights that have been flown in Ontario in the past.

Our next task was to identify "looks", namely points in time and space when a detection aircraft on a planned patrol flew a straight line segment of a patrol near an undetected fire. We began by creating a $25 \mathrm{~km}$ radius circle around each fire and identified all the flight segments that passed through that circle between the time the fire was estimated to have been ignited and when it was known to have been detected. Each such look was characterized with respect to several attributes including the shortest distance from the flight segment to the fire, the type of fuel in which the fire was burning, the fire danger rating conditions and whether or not each look was successful. A successful look is defined as the fire being detected and subsequently reported.

Logistic regression methods were then used to relate the probability that an aerial detection observer will detect an undetected fire to the distance of the fire from the detection aircraft's flight path, the fire weather conditions, the time of day the look took place and the forest fuel in which the undetected fire was burning.

\section{Potential use of the detection probability model}

Each day the detection planner must decide upon the departure time and the flight path of each of his or her detection patrol aircraft. Suppose the fire management area has been partitioned into a large number of small grid cells and that he or she has access to a spatially explicit fire occurrence prediction system that provides probabilistic predictions of the number of undetected fires burning in each cell. One can then specify a proposed detection patrol route, determine when and how close that flight will pass by each grid cell and estimate the expected number of fires detected by that patrol. If one can estimate the values at risk in each cell one can estimate the expected values at risk "found" by that patrol.

The routing of aerial detection patrols is a very complex variant of the traditional vehicle routing problem with multiple vehicles, multiple depots with uncertainty concerning the time required to complete each flight due in part to uncertainty concerning the time required to asses any fires detected en route. Our plan is to eventually incorporate our new detection probability model in a detection patrol routing optimization model.

\section{Acknowledgements}

This research was supported by the Ontario Ministry of Natural Resources and Forestry and the Natural Sciences and Engineering Research Council of Canada. 
Short contribution - Decision Support Systems and Tools

Rate of spread in coupled models: fireline curvature and pyrogenic potential

C.M. Thomas ${ }^{1 *}$; J.J. Sharples ${ }^{1}$; J.E. Hilton ${ }^{2}$; J.P. Evans ${ }^{3}$

${ }^{1}$ Applied and Industrial Mathematics Research Group, School of Physical, Environmental and

Mathematical Sciences, UNSW, Canberra, ACT 2600,Australia, \{christopherthomas@cmt.id.au*\}

${ }^{2}$ Data61, CSIRO, Clayton South, VIC 3169, Australia

${ }^{3}$ Climate Change Research Centre and ARC Centre of Excellence for Climate System Science, UNSW, Sydney, NSW 2052, Australia

Keywords: Dynamic fire behaviour, fire-spread models, curvature, coupled atmosphere-fire modelling, pyrogenic potential

\section{Introduction}

The inclusion of curvature-dependent rate of spread has been proposed as a computationally efficient way of improving simple fire-spread models. Such a technique has been used to model the behaviour of laboratory-scale junction fires (Sharples et al. 2013) and field-scale grass fires (Hilton et al. 2016), where it is shown that the inclusion of curvature dependence in a simple model improves the predictions of the model. Thomas et al. (2017) used a coupled atmosphere-fire model, WRF-Fire (Coen et al. 2013), to model junction fires (Viegas et al. 2012; Raposo et al. 2018). They did not find a relationship between local fireline curvature and local rate of spread in the model output, but they noted that, if it exists, such a relationship is unlikely to be local and should be searched for in some kind of mean sense. We report on more comprehensive numerical experiments in which we search for such a relationship using WRF-Fire. We also make a preliminary comparison of some fire-spread simulations using WRF-Fire and the pyrogenic potential model (Hilton et al. 2018).

\section{WRF-Fire simulations of junction fires}

Thomas et al. (2017) compared the local curvature at a point on the fireline with the instantaneous rate of spread at that point; no relationship was found. However, pyroconvective processes driving fire-induced winds are not local, and the computation of fireline curvature is a discrete approximation which is susceptible to small-scale variability (noise). This is illustrated in the left-hand panel in Figure 1. To increase the scale of the analysis, we compute an average fireline curvature by forming centred, moving averages of the pointwise fireline curvature, the averaging window having length $2 r$ where $r$ is arc length. Figure 1 shows the results for $r=50 \mathrm{~m}$, and is typical of all averaging lengths considered. There does not appear to be a relationship between fireline curvature, averaged in this way, and rate of spread for the WRF-Fire modelled junction fires.

\section{WRF-Fire simulations of arc fires}

Another way of increasing the scale of the analysis is to consider the curvature and the rate of spread of some notion of a mean fireline. To do this, we model fires that are ignited along a circular arc. At least for a period of time, these fires continue to burn in an approximately circular arc with radius $r$ which decreases as the fire evolves. This provides a systematic way of defining average curvature, $1 / \mathrm{r}$, and average rate of spread, $-\mathrm{d} r / \mathrm{d} t$. Figure 2 shows the results for three configurations. In each panel the initial fireline has the same curvature, and in each case the curvature increases (becomes more negative) as the fire evolves. 

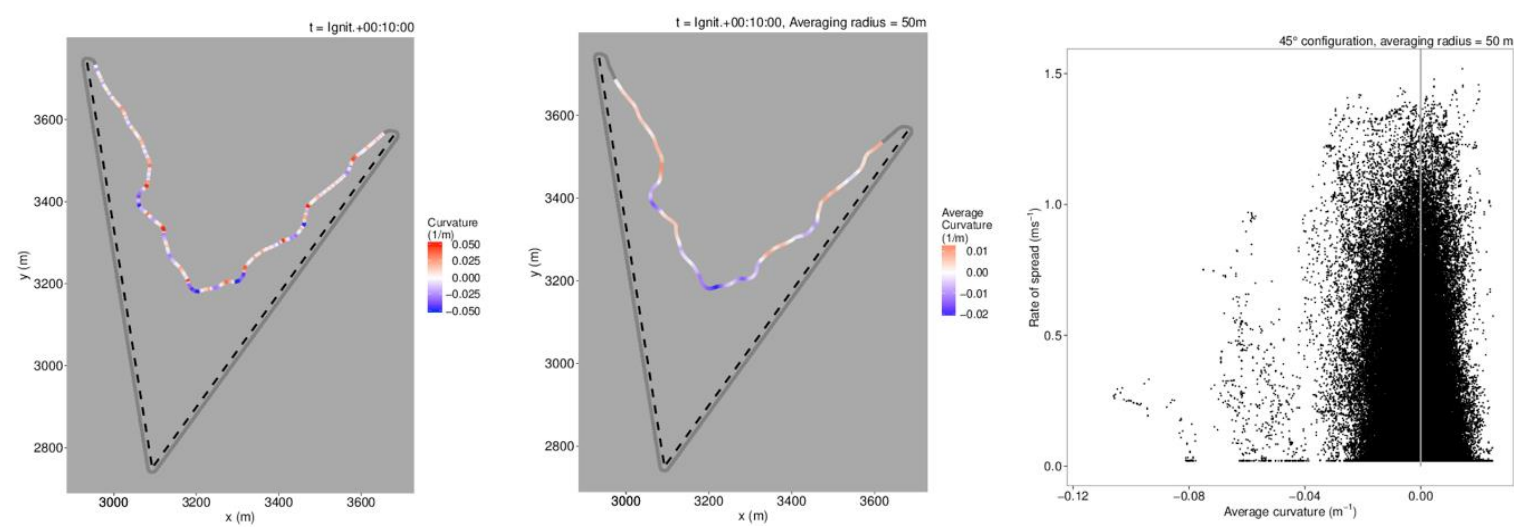

Figure 1 - Output of coupled atmosphere-fire model (WRF-Fire) for junction fires. Left panel: variability of pointwise fireline curvature in model output. Centre panel: average fireline curvature using $50 \mathrm{~m}$ radius moving window. Right panel: rate of spread versus average fireline curvature.

The distances between the isochrones indicate that the rate of spread increases with increasing length of initial arc, and does not increase as the fire evolves and the curvature increases.
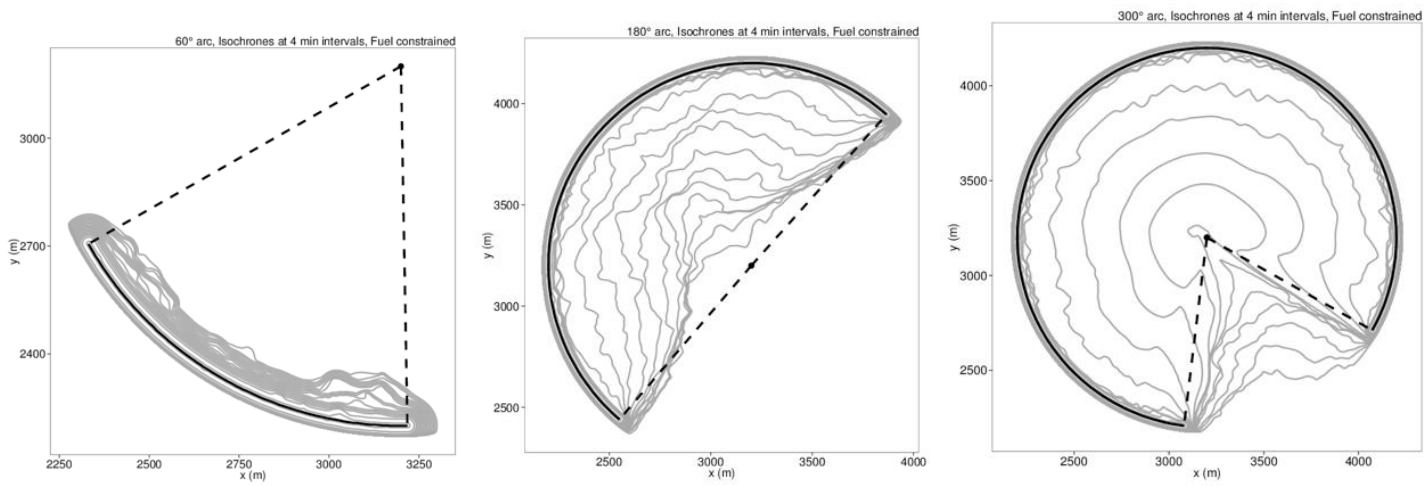

Figure 2 - Arc fire evolution simulated using WRF-Fire. Grey lines are isochrones at 4 minute intervals of simulated fires ignited along arcs of $60^{\circ}, 180^{\circ}$ and $300^{\circ}$, with radius $1000 \mathrm{~m}$. The distance between the isochrones indicates rate of spread.

\section{Comparison with arc fires modelled using pyrogenic potential}

Hilton et al. (2018) described a new model that simulates pyrogenically-induced bulk surface flow; the flow is assumed to be irrotational and divergence free, and when restricted to the surface can be described using Poisson's equation. Based on the assumption that surface winds are entrained into the plume, firelines represent sinks in the source term of the Poisson equation. The equation can be solved very efficiently, and the computed surface winds can be input into a fire-spread model, which then calculates fireline spread. The strength of the sinks are assumed to be related to fire intensity. This process can be iterated to provide a simple and very computationally efficient coupled model. Figure 3 shows isochrones at 4 minute intervals for preliminary modelling of arc fires using the pyrogenic potential model. It exhibits similar features to Figure 2. In particular, rates of spread increase with increased initial arc length, and do not increase with increasing curvature as the fireline evolves. 


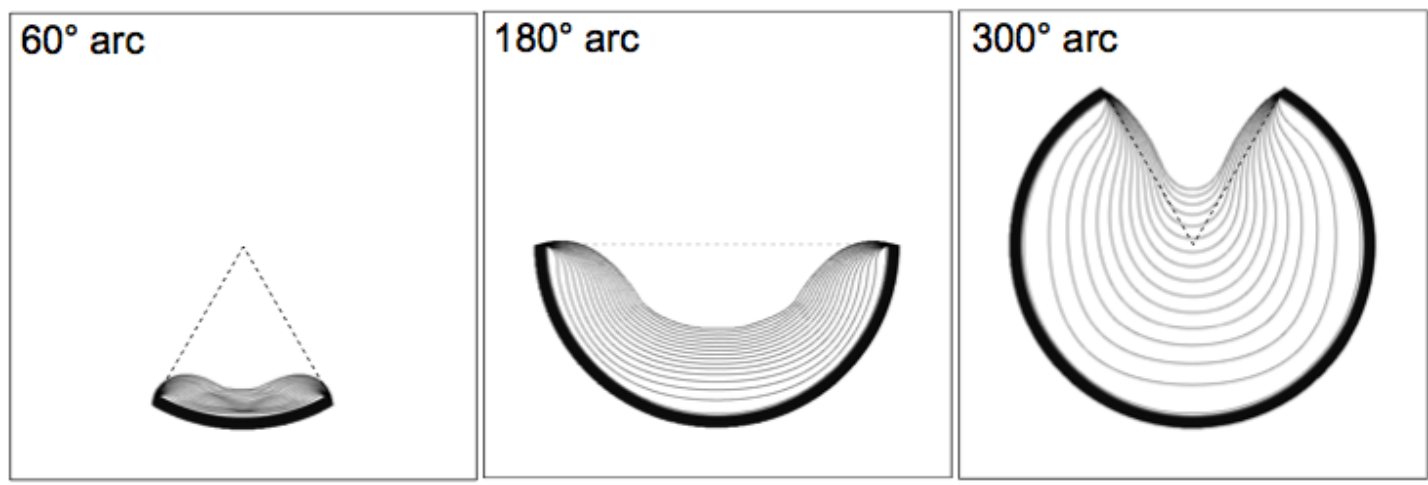

Figure 3 - Arc fire evolution simulated using the pyrogenic potential model. The lines are isochrones at 4 minute intervals.

\section{Conclusion}

Simulations of junction fires and arc fires using WRF-Fire do not indicate a relationship between fireline curvature and rate of spread at any scale. However, simulations of arc fires using WRF-Fire and the pyrogenic potential model exhibit similar features. This indicates the possibility that the results of a coupled atmosphere-fire model may be approximated, to first order, by a simple potential model. Future work may include comparison of these results with experiments, and exploration of the relationshipe between fire intensity and the strength of the sinks in the Poisson equation.

\section{References}

Coen, Janice L, Marques Cameron, John Michalakes, Edward G Patton, Philip J Riggan, and Kara M Yedinak. 2013. "WRF-Fire: Coupled Weather-Wildland Fire Modeling with the Weather Research and Forecasting Model." Journal of Applied Meteorology and Climatology 52 (1): 16-38. https://doi.org/10.1175/JAMC-D-12-023.1.

Hilton, J. E., C. Miller, J. J. Sharples, and A. L. Sullivan. 2016. "Curvature Effects in the Dynamic Propagation of Wildfires." International Journal of Wildland Fire. https://doi.org/10.1071/WF16070.

Hilton, J. E., A. L. Sullivan, W. Swedosh, J. Sharples, and C. Thomas. 2018. "Incorporating Convective Feedback in Wildfire Simulations Using Pyrogenic Potential." Environmental Modelling \& Software 107 (September): 12-24. https://doi.org/10.1016/j.envsoft.2018.05.009.

Raposo, J. R., D. X. Viegas, X. Xie, M. Almeida, A. R. Figueiredo, L. Porto, and J. Sharples. 2018. "Analysis of the Physical Processes Associated with Junction Fires at Laboratory and Field Scales." International Journal of Wildland Fire 27 (1): 52-68. https://doi.org/10.1071/WF16173.

Sharples, J J, I N Towers, G Wheeler, V.-M. Wheeler, and McCoy. 2013. "Modelling Fire Line Merging Using Plane Curvature Flow." In 20th International Congress on Modelling and Simulation, edited by J Piantadosi, R S Anderssen, and J Boland, 256-62. Adelaide, Australia.

Thomas, C. M., J. J. Sharples, and J. P. Evans. 2017. "Modelling the Dynamic Behaviour of Junction Fires with a Coupled Atmosphere-Fire Model." International Journal of Wildland Fire 26 (4): 331 44. https://doi.org/10.1071/WF16079.

Viegas, Domingos X, Jorge R Raposo, David A Davim, and Carlos G Rossa. 2012. "Study of the Jump Fire Produced by the Interaction of Two Oblique Fire Fronts. Part 1. Analytical Model and Validation with No-Slope Laboratory Experiments." International Journal of Wildland Fire 21: 843-56. 
Short contribution - Decision Support Systems and Tools

RPI Engine: Visualization in a web environment of post-fire regeneration using Landsat time series

\author{
Eduardo Moreno Gil ${ }^{1}$; Alba Viana-Soto* ${ }^{1}$; Inmaculada Aguado ${ }^{1}$; Susana Martínez ${ }^{2}$; Julia Clemente ${ }^{3}$ \\ ${ }^{1}$ Department of Geology, Geography and Environment, University of Alcala, Calle Colegios, 2, 28801 \\ Alcalá de Henares, Spain; \{eduardomorenogil@gmail.com,albal3vs@gmail.com*, \\ inmaculada.aguado@uah.es\} \\ ${ }^{2} 3$ edata Ingeniería Ambiental. Complejo El Palomar, Palacio de Ferias y Congresos, s/n-27004 Lugo, \\ Spain; \{campoxurado@gmail.com\} \\ ${ }^{3}$ Department of Automatic, University of Alcala, University Campus, Alcalá de Henares, Spain; \\ \{julia.clemente@uah.es\}
}

\begin{abstract}
The main objective of this study is to develop a web visualization tool which allows recognizing post-fire vegetation regeneration at pixel level from selected burned areas. Regeneration trajectories are based on the variations that spectral indices have (NDVI, NBR and TCW). Making use of the Earth Engine Code Editor platform (EECE) has been possible through the use of JavaScript language and other algorithms already integrated in Google Earth Engine (GEE), to generate those indices from Landsat time series (1984-present). Moreover, we implemented the graphical interface with which the user can visualize and interact with results and additional information.
\end{abstract}

Keywords: post-fire regeneration, Large Forest Fires, remote sensing, Google Earth Engine, Landsat time series, temporal trajectories.

\title{
1. Introduction
}

Wildfires are one of the most important environmental problems at present. In the European context, Spain is among the countries with the highest fire incidence in number and burned surface (SanMiguel-Ayanz et al. 2017). The analysis of Large Forest Fires (LFF, according to the statistics in Spain, $>500$ hectares) has special interest despite its low frequency as they are the ones that cause the greatest environmental and social damage. In the 90s in Spain, the year 1994 was the second worst in the statistical time series regarding the area affected by fire, with a total of 437,602 burned hectares. From these, almost $80 \%$ of were burned in $93 \mathrm{LFF}$.

Over the last few years several studies have underlined the importance of remote sensing to analyse ecological dynamics following fire and study post-fire vegetation regeneration (Lentile et al. 2006, Bartels et al. 2016). Satellite images provide broader information of burned areas by obtaining biophysical variables of wildfires. Due to the open data availability, new advances and challenges have arisen in remote sensing applied to fires (Gitas et al. 2014). Large time series of Landsat have been widely used to assess post-fire vegetation regeneration (Röder et al. 2008, Pickell et al. 2016, Chu et al. 2017, Martínez et al. 2017). At present the GEE cloud processing platform is available for open use. This tool enables us to study regeneration dynamics by using large time series and auxiliary data optimizing processing time, and also provides a visualization of results in a web environment (Gorelick et al. 2017).

This research is part of the Spanish scientific project SERGISAT, which main objective is to study the vegetation recovery dynamics from a selection of LFF that occurred in 1994 in the Spanish Mediterranean biogeographic region. In this context, this research aims to develop a tool which allows 
analysing regeneration trajectories and disseminating the results through the visualization in a web environment.

\section{Materials and Methods}

This research utilizes the free and open access to imagery available from the Landsat satellite programme and auxiliary data in order to explain the regeneration dynamics (fire perimeters, fire severity and forest cover). Six burned areas with comparable biophysical characteristics were selected as study areas in the SERGISAT project. We present here the case of the Uncastillo fire occurred on July 161994 where 16,442 hectares were burnt (Figure 1).

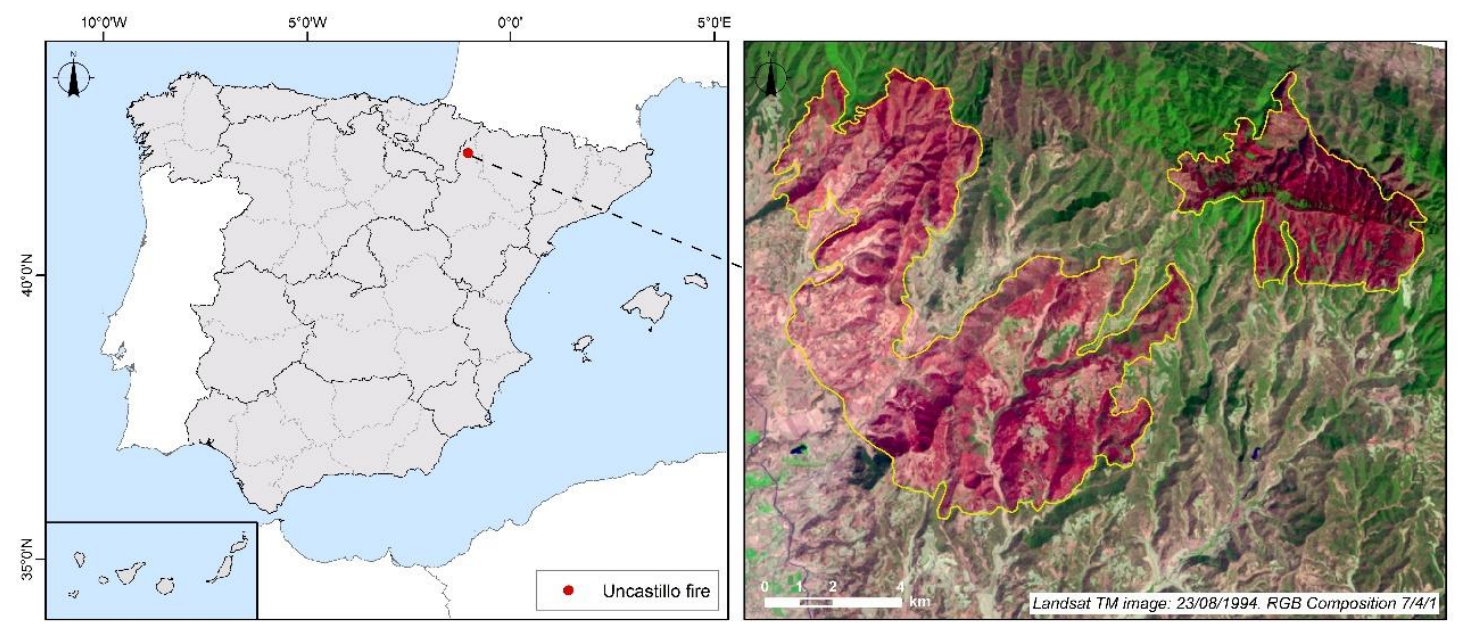

Figure 1 - Location of the study area and false colour image after the Uncastillo fire.

A time series from 1984 to present was generated by using the Landsat collection provided by GEE. We used the Collection Surface Reflectance Tier 1 from Landsat TM, ETM+ and OLI. This dataset is the atmospherically corrected surface reflectance which contains 4 visible and near-infrared (VNIR) bands and 2 short-wave infrared (SWIR) bands processed to orthorectified surface reflectance. These data have been atmospherically corrected using LEDAPS, Landsat Ecosystem Disturbance Adaptive Processing System (Masek et al. 2006) and include a cloud, shadow, water and snow mask produced using CFMASK, C Function of Mask (Foga et al. 2017), as well as a per-pixel saturation mask. A total of five WRS-2 scenes were used selecting images between June and September of each year with less than $1 \%$ cloud coverage. All the Landsat products used have been generated by Google using a "Docker" image provided by the USGS (Gorelick et al. 2017).

Additionally, an annual forest cover map of the year 2016 generated by the Space Observation Center of the Japanese agency (JAXA) is avaliable on GEE, which represent in one band the Forestry and Non Forestry coverage (FNF) for the entire terrestrial globe. Moreover, we used information generated in the SERGISAT project as the fire perimeters obtained by using Burned Area Algorithm Software (BAMS) (Bastarrika et a. 2014), fire severity images measured by GeoCBI (De Santis and Chuvieco 2009), and a vegetation cover map created combining the Corine Land Cover 1990 and the Second National Forest Inventory of Spain $(1986$ - 1995) to identify the pre-fire vegetation recovery.

The programming was done through JavaScript language using the EECE algorithms to calculate three spectral index: the Normalized Difference Vegetation Index (NDVI) (Rouse et al, 1984), Normalized Burn Ratio (NBR) (Key \& Benson 2003), and Tasseled Cap Transformation-Wetness (TCW) (Crist 1985, Huang et al. 2002, Baig et al. 2014).

In order to detect areas where regeneration is continued over time, we used the algorithm ee.ImageCollection.formaTrend integrated in GEE. This algorithm makes a search of trends around a main condition to define thresholds from the indices values. Values within the thresholds represent 
areas were regeneration was continuous. Results are filtered to isolate positive values (continuous trend) and final values are presented in a range between 0 and the maximum for each index. Finally, a graphical interface has been implemented in which the user can visualize and interact with the results, establishing the desired graphic style from the CSS code.

\section{Results and Discussion}

The web tool developed has been called RPI Engine (Post-Fire Engine Regeneration) and is accessible at the following link: https://code.earthengine.google.com/cca8d5a8403c91ff1e06118ccb42c67f

The main screen of RPI Engine (Figure 2) shows the different options with which the user can interact at the beginning of the application. It is organized in three sections: the central one corresponds to the map (result display area), the right part consists of a panel in which different burned areas can be selected, spectral indices can be calculated, and auxiliary information can be added. Finally, the left section shows graphics that represent spectral trajectories.

The algorithm implemented has allowed to extract continuous regeneration trends that have occurred in each fire. Figure 2 shows the results obtained for the Uncastillo fire. The user could evaluate the regeneration trends comparing visually the actual vegetation cover with the forest map of 2016 created by JAXA. In this context, in spite of its ecological interest we have not considered areas with a discontinuous trajectory, because this was not considered in the SERGISAT project. That is why there are gaps within the burned area perimeter.

Compared to other viewers, the RPI Engine has fewer functions to interact with, being a viewer exclusively designed to extract trajectories of the programmed indices. Other similar examples as Climate Engine viewer (http://climateengine.org/, last accessed on 27 June 2018, Huntington et al. 2017) have more capacity allowing the user to generate statistics of pixel data from numerous layers of climate information, or GFW (https://www.globalforestwatch.org/, last accessed on 27 June 2018), designed to see changes in forest cover and offers the possibility of reproducing the images. It is our intention to continue our line of work to include in the RPI Engine some of the capabilities of these viewers.

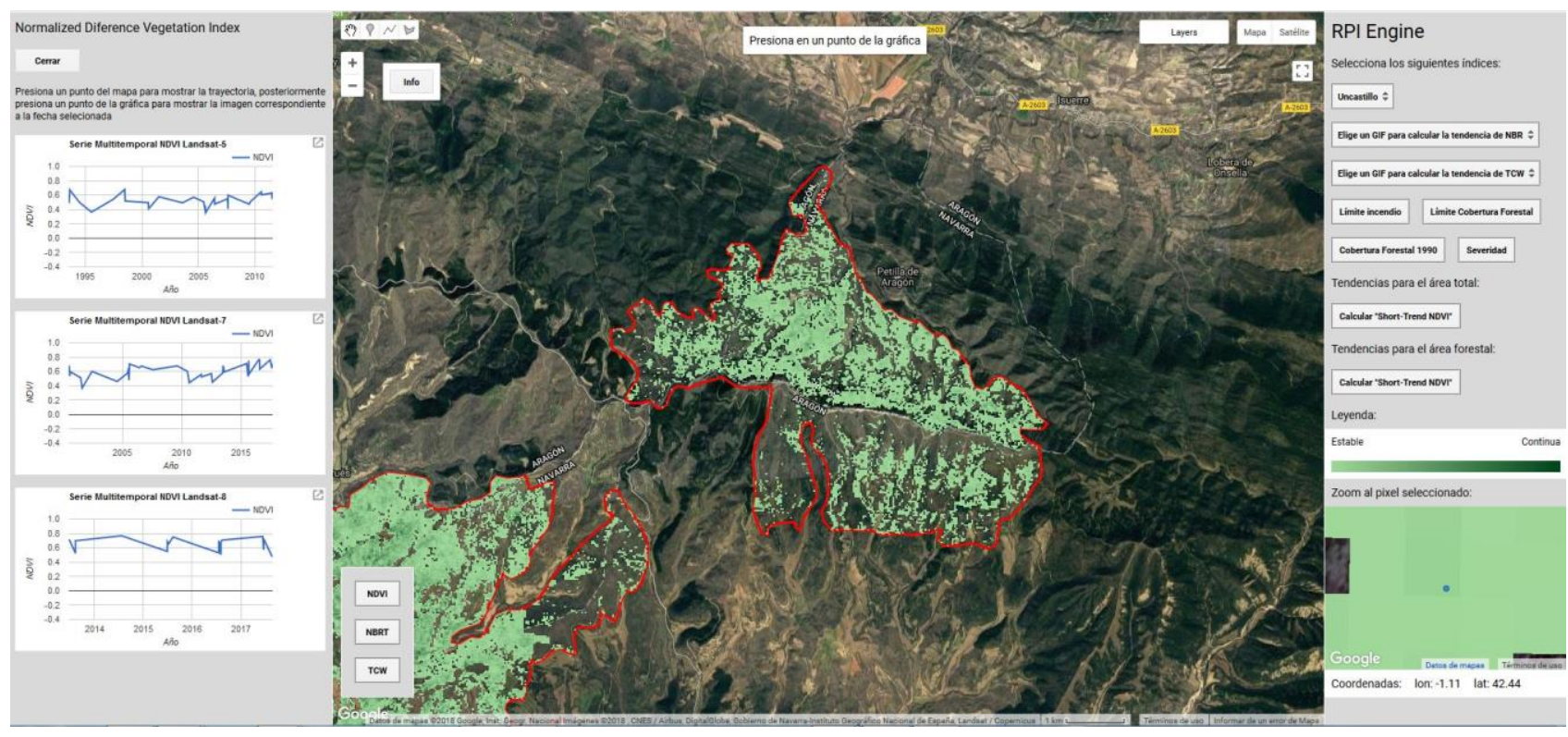

Figure 2 - RPI Engine user interface. Areas with continuous regeneration trends, using NDVI and Spectral trajectories for each Landsat Collection. 


\section{Conclusion}

The GEE platform has provided a framework to evaluate forest fires regeneration from a large amount of satellite data available. In this study we have taken advantage of this platform to exploit a long time series of Landsat satellite data.

Advances in this research could help decision-makers in determining which forest areas would have difficult regenerating processes after LFF, thus requiring the implementation of specific restoration programmes. In addition, web visualization will provide users with information and other spatial analysis functions necessary for studies in similar areas.

\section{Acknowledgements}

This study was supported by the Project "Severity and Regeneration after large forest fires from satellite remote sensing and geographic information systems" (SERGISAT; http://www.sergisat.es/), funded by the Ministry of Economy, Industry and Competitiveness of Spain.

\section{References}

Baig MHA, Zhang L, Shuai T, Tong Q (2014) Derivation of a Tasselled Cap Transformation Based on Landsat 8 at-Satellite Reflectance. Remote Sensing Letters 5 (5), 423-431.

Bartels SF, Chen HYH, Wulder MA, White JC (2016) Trends in post-disturbance recovery rates of Canada's forests following wildfire and harvest. Forest Ecology Management 361, 194-207.

Bastarrika A, Alvarado M, Artano K, Martinez M, Mesanza A, Torre L, et al. (2014) BAMS: A tool for supervised burned area mapping using Landsat data. Remote Sensing 6, 12360-12380

Chu T, Guo, X, Takeda K (2017) Effects of burn severity and environmental conditions on post-fire regeneration in Siberian Larch forest. Forests, $8,76$.

Crist EP (1985) A TM Tasseled Cap Equivalent Transformation for Reflectance Factor Data. Remote Sensing of Environment 17 (3), 301-306.

De Santis A, Chuvieco E (2009) GeoCBI: A modified version of the Composite Burn Index for the initial assessment of the short-term burn severity from remotely sensed data. Remote Sensing of Environment 113, 554-562.

Foga S, Scaramuzza PL, Guo S, Zhu Z, Dilley RD, Jr Beckmann T, Schmidt GL, Dwyer, JL, Joseph Hughes M, Laue B (2017) Cloud Detection Algorithm Comparison and Validation for Operational Landsat Data Products. Remote Sensing of Environment 194, 379-390.

Gitas IZ, San-Miguel-Ayanz, J, Chuvieco E, Camia, A (2014) Advances in remote sensing and GIS applications in support of forest fire management. International Journal of Wildland Fire 23(5), 603-605.

Gorelick N, Hancher M, Dixon, M, Ilyushchenko S, Thau, D, Moore R (2017). Google Earth Engine: Planetary-scale geospatial analysis for everyone. Remote Sensing of Environment 202, 18-27.

Huang C, Wylie B, Yang L, Homer C, Zylstra G (2002) Derivation of a Tasselled Cap Transformation Based on Landsat 7 at-Satellite Reflectance». International Journal of Remote Sensing 23 (8), 1741-1748.

Huntington JL, Hegewisch KC, Daudert B, Morton CG, Abatzoglou JT, McEvoy DJ, Erickson T (2017) Climate Engine: Cloud Computing and Visualization of Climate and Remote Sensing Data for Advanced Natural Resource Monitoring and Process Understanding. Bulletin of the American Meteorological Society 98 (11), 2397-2410.

Key CH, Benson NC (1999) The Normalized Burn Ratio (NBR): A Landsat TM radiometric measure of burn severity. United States Geological Survey, Northern Rocky Mountain Science Center. (Bozeman, MT).

Lentile LB, Holden ZA, Smith AMS, Falkowski MJ, Hudak AT, Morgan P, Lewis SA, Gessler PE, Benson NC (2006) Remote sensing techniques to assess active fire characteristics and post-fire effects. International Journal of Wildland Fire 15, 319-345. 
Martínez S, Chuvieco E, Aguado, I, Salas J (2017). Burn severity and regeneration in large forest fires: an analysis from Landsat time series. Revista de Teledetección 49, 17-32.

Masek JG, Vermote EF, Saleous NE, Wolfe R, Hall FG, Huemmrich KF et al. (2006). A Landsat surface reflectance dataset for North America, 1990-2000. IEEE Geoscience and Remote Sensing Letters 3(1), 68-72.

Pickell PD, Hermosilla T, Frazier RJ, Coops NC, Wulder MA (2016) Forest recovery trends derived from Landsat time series for North American boreal forests. International Journal of Remote Sensing 37, 138-149.

Röder A, Hill J, Duguy B, Alloza JA, Vallejo R (2008) Using long time series of Landsat data to monitor fire events and post-fire dynamics and identify driving factors. A case study in the Ayora region (eastern Spain). Remote Sensing of Environment 112, 259-273.

San-Miguel-Ayanz S, Durrant T, Boca R, Libertà G, Branco A, de Rigo D, Ferrari D, Maianti P, Vivancos TA, Schulte E, Loffler P (2017) Forest Fires in Europe, Middle East and North Africa 2016. (Publications Office of the European Union: Luxembourg) DOI: 10.2760/17690. 
Short contribution - Decision Support Systems and Tools

\title{
The determinants of crown fire runs during extreme wildfires in broadleaf forests in Australia
}

\author{
Alexander I. Filkov ${ }^{1,2 *}$, Luke Collins ${ }^{3,4}$, Anthony Rawlins ${ }^{1}$, Thomas J. Duff ${ }^{1}$, Brett Cirulis ${ }^{1}$, Trent D. \\ Penman $^{1}$ \\ ${ }^{1}$ University of Melbourne. Creswick, Victoria Australia 3363, \{alexander.filkov@unimelb.edu.au*\} \\ ${ }^{2}$ Bushfire and Natural Hazards Cooperative Research Centre. Melbourne, Victoria Australia 3002, \\ \{office@bnhcrc.com.au\} \\ ${ }^{3}$ La Trobe University. Bundoora, Victoria, Australia 3086 \\ ${ }^{4}$ Arthur Rylah Institute for Environmental Research, Department of Environment, Land, Water and \\ Planning, Heidelberg, Victoria, Australia 3084
}

\begin{abstract}
Crown fires in forest ecosystems can pose a major threat to life and property due to their high intensities and rapid rates of spread. However, research into the prediction of crown fire dynamics in the Eucalyptus forests of Australia is limited. Previous studies have focused on coarse temporal scales, utilised low resolution weather based predictors, and disregard the spatial nature of crown fires. Our study aimed to use observations from large wildfires in eucalypt forests to develop an empirical model to predict the likelihood of crown fire events using environmental predictors at an hourly scale. Our study was conducted in south-eastern Australia using data from fifteen large wildfires that occurred between 2009 and 2015. Fire severity maps were created for each fire at a $30 \mathrm{~m}$ resolution using Landsat imagery from which we calculated the proportion of $30 \mathrm{~m}$ pixels experiencing crown fire within a $150 \times 150 \mathrm{~m}$ window (2.25 ha). Predictor variables were chosen to represent the four key environmental drivers of fire behaviour, namely fuel moisture (i.e. live and dead fuel), fuel load and structure (i.e. surface, elevated and bark fuels, tree height), fire weather (i.e. vapour-pressure deficit, wind speed, relative wind direction) and topography (i.e. slope and ruggedness). Random Forests were used to model the effect of environmental drivers on the proportion of crown fire. Fuel moisture content variables were the best predictors of probability of crown consumption. Topographic variables and fire weather had only an intermediate influence and fuel load and structure had the lowest influence. Crown fire runs largely occurred when thresholds in vapour-pressure deficit $(<4 \mathrm{kPa})$ and dead fuel moisture content $(<7 \%)$ were exceeded. Predictions from the model showed a high degree of agreement with the raw fire severity maps. The proposed models have the potential to provide guidance on the likelihood of crown fire during fire events.
\end{abstract}

Keywords: crown fires; risk; prediction; modelling; wildfire

\section{Introduction}

Extreme fire behaviours are responsible for a disproportionate level of damage (Cruz et al. 2012; Peace et al. 2016). However, there is limited research regarding their prediction. In part, this is due to the challenges with observing rare complex phenomena and difficulty in measuring behaviours that occur under dangerous conditions. Crown fires are a relatively common type of extreme fire behaviour in forest ecosystems, which are characterised by high fire intensities, rapid rates of spread and high release of radiant heat. Consequently, crown fires reduce the likelihood of successful suppression and can threaten lives of suppression crews (Alexander and Cruz 2011). Crown fires may have negative impacts on some ecosystems services such as biodiversity and water quality (Nyman et al. 2011; Smith et al. 2011).

The occurrence and nature of crown fires has been extensively studied in conifer systems, with a 
large number of models having been developed (Cruz and Alexander 2013). Crown fires have been found to be a function of crown fuel properties (such as dead fuel moisture content, bulk density and the gap from the surface fuels to the canopy), surface fuel properties, topography and weather conditions (such as wind speed, temperature). Despite its importance, published research into crown fire dynamics in the broadleaved Eucalyptus forests of Australia is limited.

Remotely sensed maps of fire severity have been used to study environmental drivers of crown fires occurrence. Fire severity is a retrospective measure of the environmental impact of a fire (Keeley 2009). Severity maps can be used to determine the influence of factors including fuels, weather, terrain and disturbance history on fire severity, as a surrogate of crown fire behaviour. Previous studies examining crown fire occurrence in eucalypt forest have used coarse scale measures of fire weather indices, which combine information on fire weather and moisture availability into one value (Price and Bradstock 2012; Taylor et al. 2014; Storey et al. 2016). These indices do not disentangle the contribution of fire weather and fuel moisture. Furthermore, past studies have considered the likelihood of crown fire at a single point (Price and Bradstock 2012; Collins et al. 2014; Storey et al. 2016) whereas from a management perspective the prediction of large patches of crown fire, or crown fire runs is desirable as they have larger impacts and are a greater threat to fire suppression activities.

In this study, we aimed to examine the relative importance for drivers of crown fires in Eucalypt forests. We use satellite derived fire severity mapping from 15 large wildfires in south-eastern Australia to develop a model to predict the likelihood and extent of crown fire events using spatially derived environmental predictors and a range of weather measurements.

\section{Methods}

A key objective of our study was to model crown fire occurence in eucalypt forests using fine temporal scale (i.e. sub daily) fire weather data. Therefore, in our study we only considered fires that had reconstructed perimeter isochrones of progression and linescans at a sub-daily resolution ${ }^{1}$ and burnt predominantly within eucalypt forests. Fifteen case study fires between 2009 and 2015 met the criteria to be suitable for analysis. These fires all have several progression isochrones each day with an average interval of 4 hours.

Fire severity maps were created for the study fires using Landsat imagery (30 m resolution) and a Random Forest (RF) classifier, following the approach outlined in Collins et al. (Collins et al. 2018). Fire severity maps were generated using the Google Earth Engine platform (Gorelick et al. 2017). The mapping approach used numerous spectral indices derived from pre- and post-fire Landsat imagery as predictor variables for a RF classifier. Five fire severity classes were classified in the mapping unburnt, crown unburnt, partial crown scorch, crown scorch and crown consumption. We reclassified each pixel as either experiencing crown fire (i.e. crown consumption) or not. In our study we quantified crown fire runs by calculating the proportion of pixels experiencing crown fire within a $5 \times 5$ pixel moving window $(150 \mathrm{~m} \times 150 \mathrm{~m})$.

Predictor variables were selected to represent the four key drivers of fire severity included in existing crown fire models and fire severity studies - fuel moisture, fuel load, fire weather and topography. Eleven predictor variables were used in the analysis, each representing different aspects of the four drivers: i) Live and dead fuel moisture content (fuel moisture); ii) Surface, elevated and bark fuels and tree height (fuel load); iii) Vapour-pressure deficit, wind speed and relative wind

\footnotetext{
${ }^{1}$ Linescans are images from high altitude aircraft mounted Infrared linescan systems
} 
direction (fire weather); and iv) Slope and topographic ruggedness (topography). We used Random Forests ${ }^{1}$ to model the effect of these environmental drivers on (i) crown fire occurrence and (ii) the proportion of pixels experiencing crown fire.

\section{Results and discussion}

Assessment of the importance of predictor variables, based the Gini scores ${ }^{2}$, indicates that variables reflecting air and fuel moisture were most influential in determining crown fire runs, with fire weather and topography having intermediate influence and fuel load and structure having the lowest influence (Fig. 1).

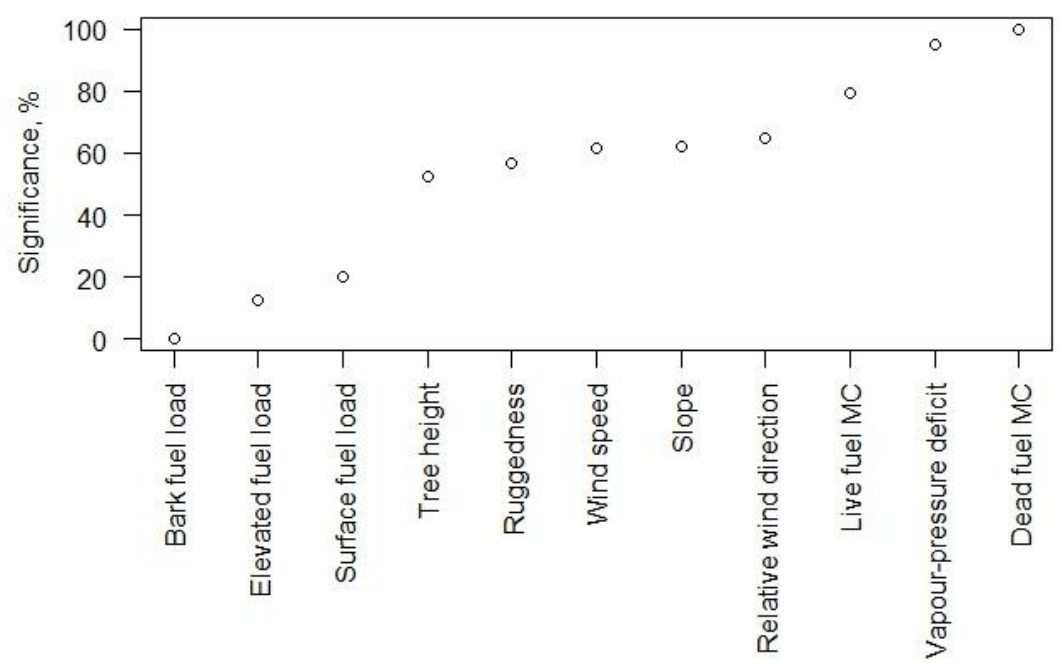

Figure 1 - Importance of predictor variables for the prediction of crown fire runs. Relative significance of variable is dimentionless value changing from 0 to $100 \%$.

Dead fuel moisture content and vapour-pressure deficit. had clearly identified thresholds, below which crown fires rarely occurred. These threshold values for vapour-pressure deficit and dead fuel moisture content were $4 \mathrm{kPa}$ and $6.9 \%$ respectively. A decrease of dead fuel moisture content less of one percent (below 6.9\%) and an increase of vapour-pressure deficit from $4 \mathrm{kPa}$ to $7 \mathrm{kPa}$ led to growth of the proportion of pixels where crown fires occurred by 3 times (Fig. 2). These findings highlight the fact, that moisture plays an important role in the ignition and combustion process (Zhou et al. 2005; Weise et al. 2016). Even low changes of it can vital increase the crown fire likelihood.

For the predictor variables; tree height, surface fuel load, wind speed and relative wind direction, the number of pixels where crown fires occurred doubled. This occured at threshold values of: $>35 \mathrm{~m}$ tree height, $<18 \mathrm{t} / \mathrm{ha}$ load, $>40 \mathrm{~km} / \mathrm{h}$ speed, $45^{\circ}-90^{\circ}$ and $135^{\circ}-180^{\circ}$ wind direction. The rest of predictor variables changed within $10 \%$ of pixels without showing any consistent patterns.

${ }^{1}$ Random forests or random decision forests are an ensemble learning method for classification, regression and other tasks, that operate by constructing a multitude of decision trees at training time and outputting the class that is the mode of the classes (classification) or mean prediction (regression) of the individual trees.

2 The Gini coefficient measures the inequality among values of a frequency distribution. 

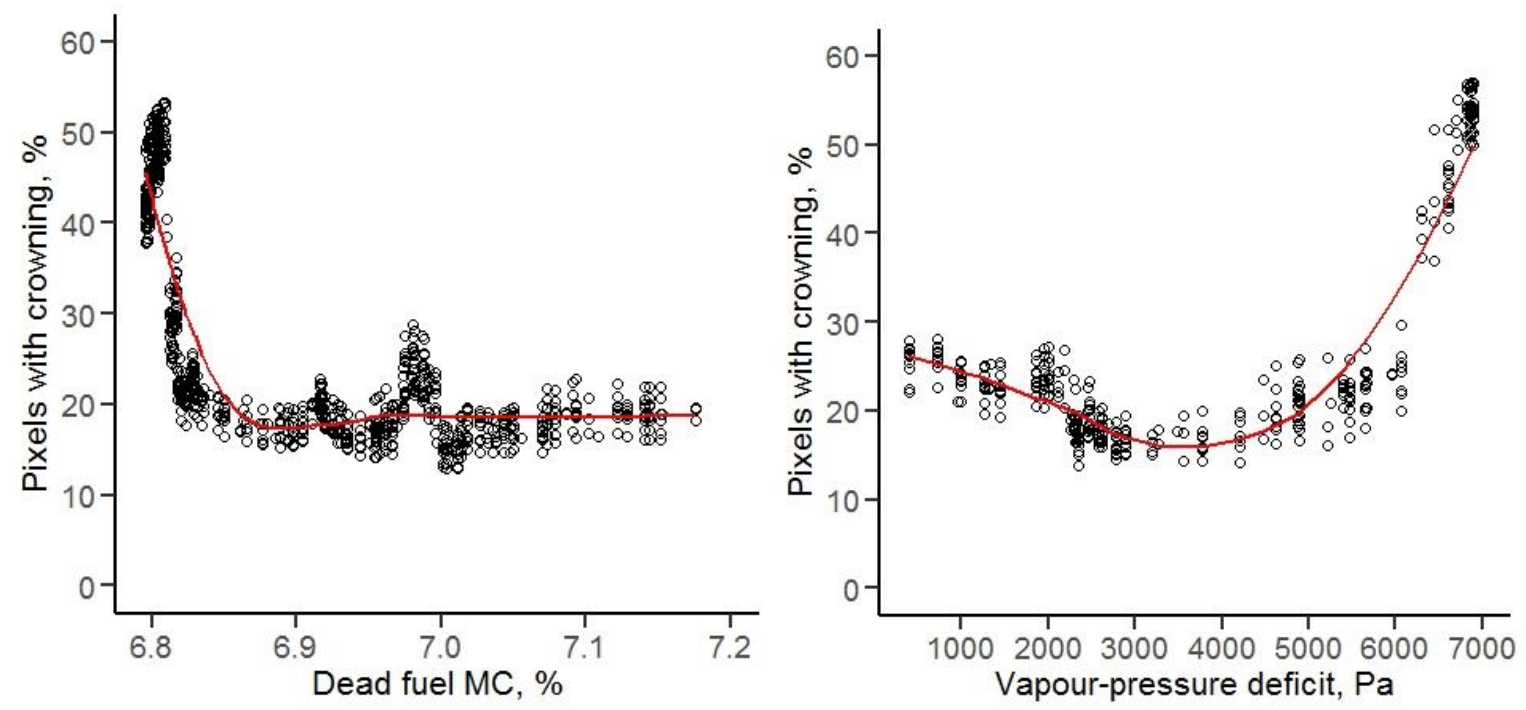

Figure 2 - Influence of dead fuel moisture content and vapour-pressure deficit on the percent of pixels effected by crown fire. Proportion is changing from 0 to $100 \%$, which is corresponding to total amount of pixels.

Mapped predictions from the Random Forest model show a high degree of agreement with the mapped fire severity observations suggesting it could be useful tool for decision support (Fig. 3).

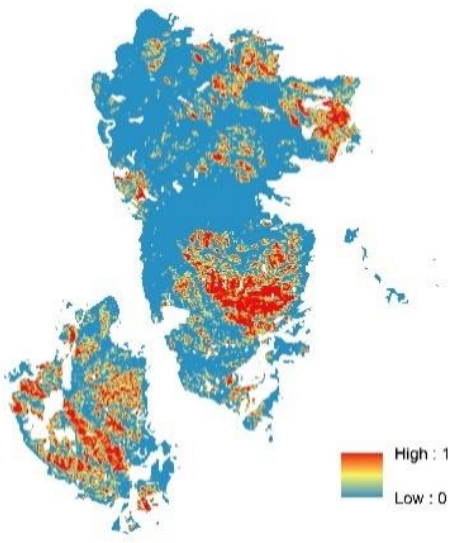

Observation

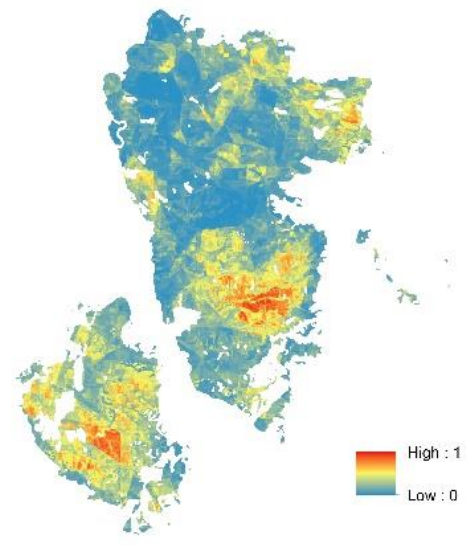

Prediction

Figure 3 - Comparison of observed proportion of pixels with crowning vs predicted. Proportion of pixels with crowning is changing from 0 (no crowning) to 1 (all pixels with crowning).

As the weather variables can be forecast into the future, Random Forest predictions could be used to forecast the likelihood of crown fire runs while fires are occurring. In the study area, potential fire runs could be forecast at an hourly temporal resolution for up to 7 days into the future. This could provide managers with a rapid means of assessing the likely fire impacts and risks to personnel. Such information would be invaluable for fire managers in terms of allocating fire suppression resources and issuing public warnings

\section{Acknowledgements}

This study was funded by the Bushfire and Natural Hazards Cooperative Research Centre 'Determining threshold conditions for extreme fire behaviour' project. 


\section{References}

Alexander M, Cruz M (2011) What are the safety implications of crown fires? InFox R (ed) 'Proceedings of 11th International Wildland Fire Safety Summit', Missoula. 1-16. (International Association of Wildland $\quad$ Fire: https://publications.csiro.au/rpr/download?pid=csiro:EP114880\&dsid=DS2.

Collins L, Bradstock RA, Penman TD (2014) Can precipitation influence landscape controls on wildfire severity? A case study within temperate eucalypt forests of south-eastern Australia. International Journal of Wildland Fire 23, 9-20. doi:10.1071/WF12184.

Collins L, Griffioen P, Newell G, Mellor A (2018) The utility of Random Forests for wildfire severity mapping. (in Review).

Cruz MG, Alexander ME (2013) Uncertainty associated with model predictions of surface and crown fire rates of spread. Environmental Modelling and Software 47, 16-28. doi:10.1016/j.envsoft.2013.04.004.

Cruz MG, Sullivan AL, Gould JS, Sims NC, Bannister AJ, Hollis JJ, Hurley RJ (2012) Anatomy of a catastrophic wildfire: The Black Saturday Kilmore East fire in Victoria, Australia. Forest Ecology and Management 284, 269-285. doi:10.1016/j.foreco.2012.02.035.

Gorelick N, Hancher M, Dixon M, Ilyushchenko S, Thau D, Moore R (2017) Google Earth Engine: Planetary-scale geospatial analysis for everyone. Remote Sensing of Environment 202, 18-27. doi:10.1016/j.rse.2017.06.031.

Keeley JE (2009) Fire intensity, fire severity and burn severity: A brief review and suggested usage. International Journal of Wildland Fire 18, 116-126. doi:10.1071/WF07049.

Nyman P, Sheridan GJ, Smith HG, Lane PNJ (2011) Evidence of debris flow occurrence after wildfire in upland catchments of south-east Australia. Geomorphology 125, 383-401. doi:10.1016/j.geomorph.2010.10.016.

Peace M, Mattner T, Mills G, Kepert J, McCaw L (2016) Coupled fire-atmosphere simulations of the Rocky River fire using WRF-SFIRE. Journal of Applied Meteorology and Climatology 55, 11511168. doi:10.1175/JAMC-D-15-0157.1.

Price OF, Bradstock RA (2012) The efficacy of fuel treatment in mitigating property loss during wildfires: Insights from analysis of the severity of the catastrophic fires in 2009 in Victoria, Australia. Journal of Environmental Management 113, 146-157. doi:10.1016/j.jenvman.2012.08.041.

Smith HG, Sheridan GJ, Lane PNJ, Nyman P, Haydon S (2011) Wildfire effects on water quality in forest catchments: A review with implications for water supply. Journal of Hydrology 396, 170 192. doi:10.1016/j.jhydrol.2010.10.043.

Storey M, Price O, Tasker E (2016) The role of weather, past fire and topography in crown fire occurrence in eastern Australia. International Journal of Wildland Fire 25, 1048-1060. doi:10.1071/WF15171.

Taylor C, Mccarthy MA, Lindenmayer DB (2014) Nonlinear Effects of Stand Age on Fire Severity. Conservation Letters 7, 355-370. doi:10.1111/conl.12122.

Weise DR, Koo E, Zhou X, Mahalingam S, Morandini F, Balbi JH (2016) Fire spread in chaparral - A comparison of laboratory data and model predictions in burning live fuels. International Journal of Wildland Fire 25, 980-994. doi:10.1071/WF15177.

Zhou X, Mahalingam S, Weise D (2005) Modeling of marginal burning state of fire spread in live chaparral shrub fuel bed. Combustion and Flame 143, 183-198. doi:10.1016/j.combustflame.2005.05.013. 
Short contribution - Decision Support Systems and Tools

The relative contributions of climate drivers on extreme Australian fire weather

\author{
Sarah Harris ${ }^{1 *}$; Chris Lucas ${ }^{2}$ \\ ${ }^{1}$ Monash University, Melbourne, Australia, 000-0002-2807-9529, \{sarah.harris@monash.edul*\} \\ ${ }^{2}$ Bureau of Meteorology, Melbourne, Australia, \{chris.lucas@bom.gov.au\}
}

Keywords: El Nino Southern Oscillations, Forest Fire Danger Index, spatiotemporal variability

Modes of climate variability have been linked to fire weather around the globe. Variations in sea surface temperatures alter atmospheric circulations, resulting in a change to global distributions of temperature and rainfall. Australia has a high degree of interannual climate variability, modulated by several modes of climate variability. Logically, these modes should also impact the variability of Australian fire weather, but the relationships are not entirely clear. The mechanisms behind this influence, its spatiotemporal variability and the relative contributions of the different climate drivers remain to be understood. Understanding the interactions between climate drivers and Australian fire weather are a step towards improved seasonal forecasts of fire weather, potentially resulting in more effective fire planning and resource management.

In this study, we examine the seasonal relationships of three climate drivers that are known to affect Australia - El Niño Southern Oscillation (ENSO), Southern Annular Mode (SAM) and Indian Ocean Dipole (IOD) with the annual cumulative and seasonal 90th percentile McArthur Forest Fire Danger Index (FFDI) at 39 locations across Australia. We also determine the relative contributions of the climate drivers using partial correlations and regression analysis, as well as considering the seasonal lag effect.

We find that the relationship between ENSO and extreme fire weather ( $90^{\text {th }}$ percentile of FFDI) is significant and widespread across most of the country throughout winter, spring and summer. The strongest values occur in spring and the relationship with cumulative fire weather is stronger than with the concurrent seasonal extreme fire weather. Considering the lag relationships with ENSO indices there is high predictability for both one- and two- seasons in advance for the summer FFDI for most of the country. This is important because this is a critical high fire danger period for southern and eastern states. Additionally, predicting spring FFDI is possible for many parts of the country up to one season in advance. This is particularly important for early season fires that have been found to occur in New South Wales(NSW)/Australian Captial Territory (ACT), although ENSO was not statistically significant along the coast. For the autumn period there is some ability to predict fire weather in the NSW/ACT region; this may be useful for prescribed burning planning or for late season bushfires. Finally, in the winter months there is some predictability of FFDI for northern parts of Australia, which is useful as this is the beginning of their peak burning period. Overall, the relationship between cumulative FFDI and ENSO was stronger than for those found between seasonal extreme fire weather.

For SAM, extreme fire weather in the inland areas across Australia is significantly related to SAM in autumn, however for all other seasons SAM is strongly related to fire weather across the eastern states, with the peak occurring in spring and highest values across coastal NSW. When lag seasons are considered for SAM there appears some advanced predictability for the summer fire weather for the south east of the country, particularly Victoria, Tasmania and South Australia (AS). A negative SAM in the spring results in higher FFDI values in summer. Additionally, for the spring fire weather there is some predictability one season in advance for northern NSW and southern Queensland (QLD). Overall seasonal SAM is more strongly related to seasonal extreme fire danger than to cumulative fire danger. 
The relationship between IOD and extreme fire weather in winter is patchy across southern Australia with the strongest relationships in the west. In spring the relationship is stronger and concentrated in the south east of the country including stations from NSW/ACT, Victoria, SA and Tasmania. There are both one- and two-season lag relationships with summer extreme fire danger in Tasmania. The cumulative fire danger is not as strongly related with IOD as extreme fire danger but there are more stations with cumulative FFDI significantly related to IOD in Western Australia (WA) during spring than was found for extreme fire weather during the same period.

When the combined effect is considered to identify the dominant drivers we find in autumn SAM dominates central and western stations and ENSO has a weak relationship with stations in the south east of NSW/ACT. In winter the IOD is the dominant driver over the south of SA, the south and western coast of WA along with northern Australia. ENSO dominates much of north central Northern Territory (NT) and QLD along with NSW, whereas SAM dominates some of the stations in southern QLD, parts of NSW and Tasmania. In spring, the whole of QLD extending across central Australia is dominated by ENSO whereas SAM dominates NSW, and IOD dominate in parts of SA and Tasmania. In spring for Victoria there are no dominant drivers when the combined effect are considered suggesting that extreme fire weather requires the combined effect of IOD and ENSO to predict the variability. However, the relationship between IOD in winter with winter FFDI values is stronger than ENSO for WA and SA (with the exception of the inland SA site of Woomera). In summer, for the combined drivers, (noting that IOD is not included during this period) ENSO dominates most of the country particularly the Eastern coastal regions with the exception of SAM, which is the dominant driver in north east of NSW. When considering the lag effect of spring climate drivers on summer extreme fire weather ENSO dominates the entire eastern coast extending up to Darwin and also dominates in central WA. Whereas, SAM is the dominant climate driver across SA, Victoria and Tasmania. Their annual results also indicate that other drivers dominate over the rest of the state, which corresponds to the locations of the two Tasmania stations in this study, particularly IOD and ENSO in the north of the state (where Launceston AP is located). Overall, SAM and ENSO have little impact on the independent relationships with fire weather, however ENSO and IOD are not independent of each other in relation to fire weather.

Our study demonstrates that using a varying combination of climate drivers throughout each season there is considerable potential for producing long-range seasonal forecasts of fire weather. This advanced warning of fire weather may be useful for fire agencies making decisions around resource allocation and risk management. 
Short contribution - Decision Support Systems and Tools

Understanding fire, weather and land cover interactions from long-term terrestrial observations and satellite data on a transect from Europe to North Africa

\author{
N. Koutsias ${ }^{12 *}$, G.B. Pezzatti ${ }^{1}$, A. Madoui ${ }^{3}$, F. Mouillot ${ }^{4}$ and M. Conedera ${ }^{1}$ \\ ${ }^{1}$ Community Ecology, WSL Swiss Federal Institute for Forest, Snow and Landscape Research, a Ramél 18, \\ Cadenazzo, CH-6593, Switzerland, \{nkoutsia@upatras.gr*\} \\ ${ }^{2}$ Department of Environmental and Natural Resources Management, University of Patras, G. Seferi 2, GR- \\ 30100 Agrinio, Greece. \\ ${ }^{3}$ Université du Québec à Montréal, C.P. 8888, Succursale, Centre-ville, Montréal, QC H3C 3P8, Canada. \\ ${ }^{4}$ UMR CEFE 5175, CNRS, Université de Montpellier, Université Paul-Valéry Montpellier, EPHE, IRD, \\ 1919 route de Mende, 34293 Montpellier Cedex 5, France
}

Keywords: Long-term documented historical fire records, Switzerland, Greece, Algeria, Tunisia, remote sensing, generalized linear models, extremes, climate, fire selectivity.

\title{
1. Project overview
}

Long-term historical time series records of fire activity (number of fires and total area burned) extending back to the late 1800s, that are very rare worldwide, were found and used within the GRADIENT project that correspond to (i) Switzerland, central Europe (1900-2014), (ii) Greece, south Europe (1897-2014), (iii) Algeria, north Africa (1870-2014) and (iv) Tunisia (1902-2015), north Africa which together with the spatial-explicit reconstruction of recent fire history from Landsat satellite images (1984-2016), gave a unique and excellent opportunity to understand fire, weather and land use/land cover (LULC) interactions in a north to south transect. The Tunisia study case was added during the implementation period of the project since in the original proposal only the first three study cases were proposed.

Differences in bio-geographical characteristics provided by the four selected study areas, located on a large geographical gradient covering two continents gave the opportunity to document the role of fire in different biomes, to explore cross-scale issues and assess how fire-weather-LULC interactions vary across different scales, especially under a climate change context. GRADIENT project consisted of three topics that correspond mainly to three different scales. The specific objectives were: (i) the identification of trends, patterns and relationships between forest fires, weather, land cover and socioeconomic variables from long-term observations, (ii) the reconstruction of recent fire history and the assessment of burning patterns and fire selectivity on an annual basis from satellite images, and (iii) the exploration of post-fire vegetation dynamics and recovery for selected large fire events using time series satellite images.

\subsection{Trends, patterns and relationships between forest fires, weather, land cover and socio- economic variables}

There are similarities and non-similarities among the four study area that compose the gradient from north to south. In principle there is a characteristic fire activity in all four study areas defined by the general pyro-environment with certain peaks occurred at specific years associated to physical and social factors. The role of precipitation is different in the gradient from the wet to dry areas. Moisture is more evident as an underlying explanation mechanism in the wet study area while temperature is more evident in the dry study areas. It was evident that there is a clear and different role of precipitation from promoting to discouraging fire activity across the north to south gradient together with the social aspects and the role of human dimension. 
Concerning the extremes, two clear patterns were observed according to the two discrete roles of the explanatory variables recognized previously; the first pattern where the role of the explanatory variable is to promote fire activity as the example of Switzerland with the effect of precipitation or dry period area promoting fires (e.g. drought) and the second pattern where the role of the explanatory variable is to discourage fire activity as the example of Greece or Algeria with the effect of precipitation or dry period area discouraging fires (e.g. wet conditions). We recognize a gradient from north (Switzerland) to south (Algeria) where the role of explanatory variable to fire activity is changing from promotion to discourage.

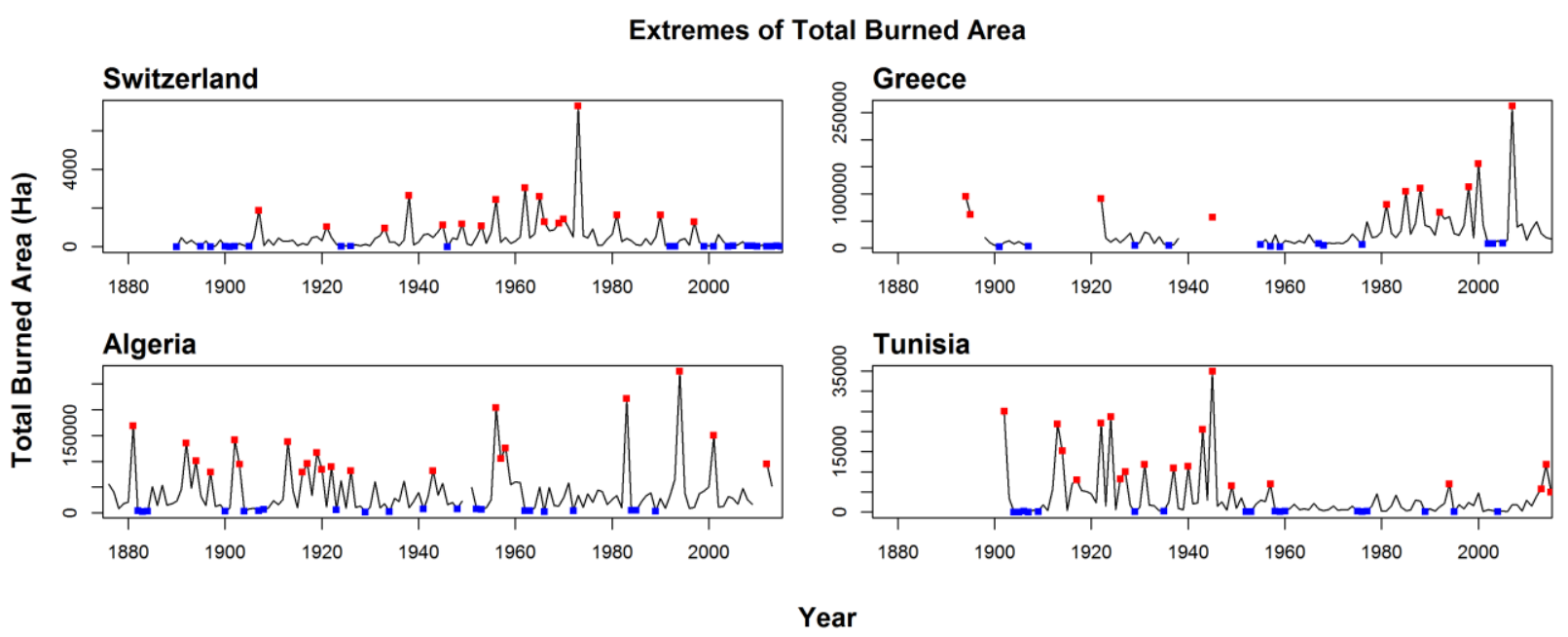

Figure 1 - The extreme years for both high and low fire activity at yearly basis for the four study areas. The distribution of the extreme years is similar to all countries: $14-18 \%$ is the range of the high extremes, $12-17 \%$ is the range of the low extremes and $67-74 \%$ is the range of the non-extremes which is the majority.

\subsection{Reconstruction of recent fire history and the assessment of burning patterns and fire selectivity}

The interactions of landscape components and fire were analyzed by comparing the relative proportions of what the fires burnt during the period 1984-2015 against what is available to burn across the landscape (e.g. CORINE, or other available global land cover data, e.g. ESA global land cover data), considering a random model that accounts for spatial autocorrelation. To determine whether the wildfires burn significantly different proportions of LULC classes than what is available to burn we applied a Monte Carlo randomization test considering spatial autocorrelation on the basis of randomizing the fire events using however the exact fire shape to account for the spatial autocorrelation. For all study area selective burning is evident that also depends on the available to burn landscape. Frequent fires were also observed that burn mainly grassland and shrublands.

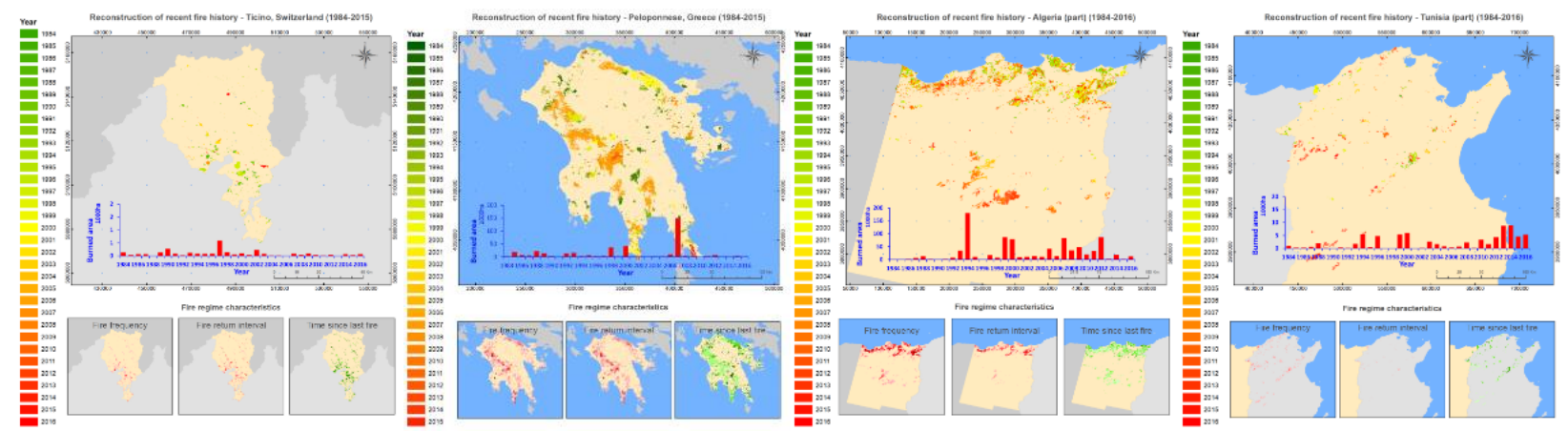

Figure 2 - Fire regime characteristics depicting (i) fire scar maps that include patterns of burned and unburned patches, (ii) fire frequency, (iii) fire return intervals, and (iv) time since last fire event. 


\subsection{Exploration of post-fire vegetation dynamics and recovery using time series satellite images}

Satellite remote sensing data from MODIS and LANDSAT satellites in the period from 1984 to 2016 were acquired and processed to extract the temporal profiles of the spectral signal for selected areas within the fire-affected areas. This dataset and time period analyzed together with the time that these fires occurred gave the opportunity to create temporal profiles for almost half years before and half years after the fire. The different scale of the data used gave us the chance to understand how vegetation phenology and therefore the recovery patterns are influenced by the spatial resolution of the satellite data used.

Within the GRADIENT project vegetation phenology and time series statistics proved very useful not only to study vegetation recovery in fire affected areas but also to identify the time period where the fire or fires occurred and define also the vegetation phenology before the fire. This is very useful first for integrating this concept into a burned land mapping approach and second for identifying what type of vegetation is burned.
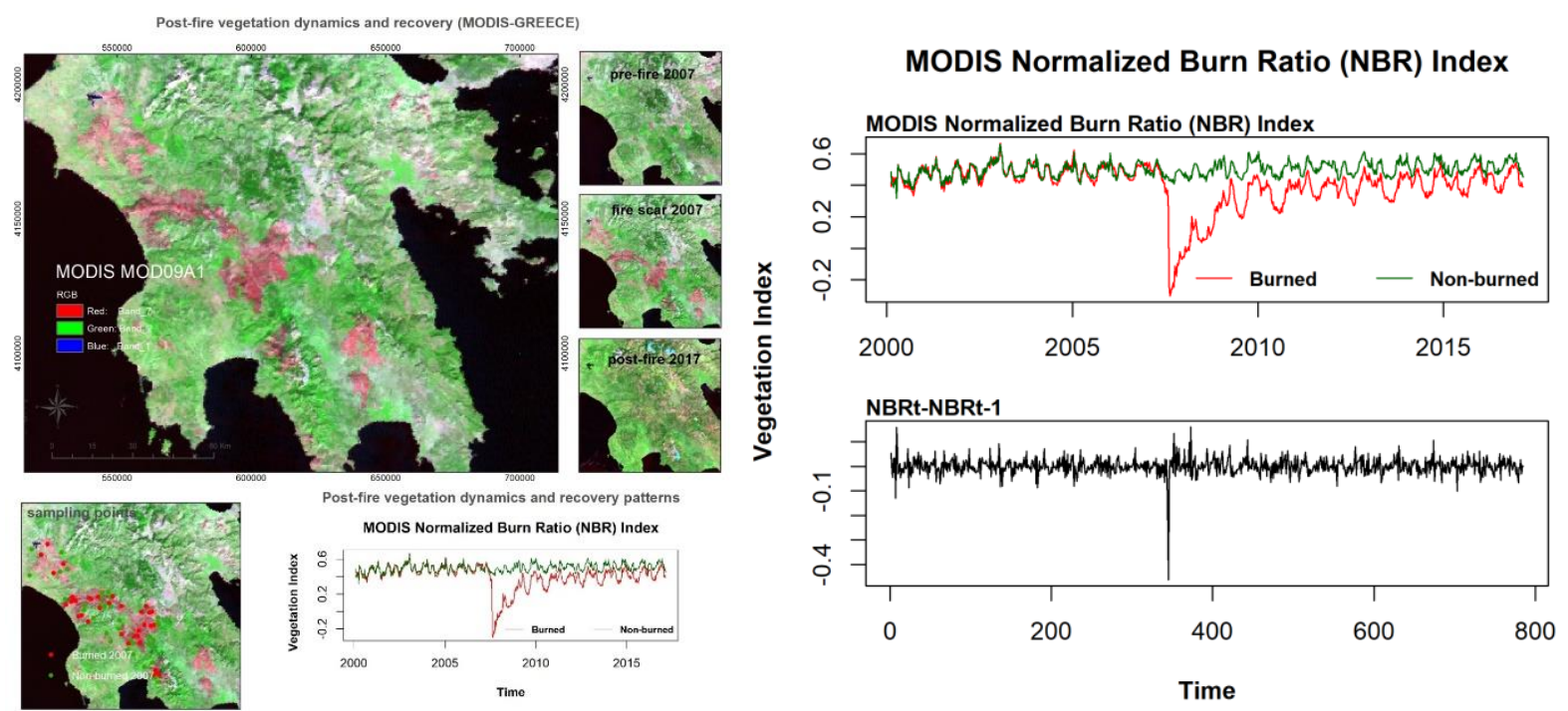

Figure 3 - Vegetation recovery of a 2007 fire in Greece using the phenology from the time series MODIS data. The date of fire occurrence is very well defined in the NBRt-NBRt-1 time series. 
Short contribution - Socio Economic Issues

Does it pay to invest in better suppression resources? - policy analysis of alternative scenarios with simulation

\author{
Abílio P. Pacheco** ${ }^{1}$, David Pereira da Silva ${ }^{1}$, João Claro ${ }^{1}$, Tiago M. Oliveira ${ }^{4}$ \\ ${ }^{1 *}$ INESC TEC and Faculdade de Engenharia, Universidade do Porto. Campus da FEUP, Rua Dr. Roberto \\ Frias, 378, 4200-465 Porto,Portugal, \{app@fe.up.pt*,ee10290@fe.up.pt, jclaro@fe.up.pt\} \\ ${ }^{2}$ Centro de Estudos Florestais, Instituto Superior de Agronomia, Universidade de Lisboa. Tapada da Ajuda, \\ 1349-017 Lisboa, Portugal, \{toliveira@isa.ulisboa.pt\}
}

\begin{abstract}
Rekindles (RKD) and false alarms (FA) are unusually high in the Portuguese wildfire management system. Together they represent a high burden on suppression resources in particular, and fire management resources in general. Indeed, e.g., during 2010, according to data provided by the Portuguese Institute for Nature Conservation and Biodiversity (ICNF), in 20,049 occurrences that the suppression system handled in the summer, $12.5 \%$ were FA and $15.0 \%$ were RKD.

During the fire season, it is usual to have large usage of suppression resources to combat wildfires and on peak days, firefighters are in a tight spot due to the pressure to move incessantly from one fire to the next one. In such occasions the system may not be able to effectively meet the needs, getting out of control. If there are fires waiting to be fought, suppression crews are pressured to prematurely abandon mop-up operations (moving towards the initial attack of new fires), without the needed time to use the appropriate tools to effectively carry out mop-up. When one of these fires with a bad mop-up rekindles, it is one more to join the other new ignitions or primary fires, and they are generally more aggressive than the latter.

We first developed a discrete-event simulation model of a wildfire suppression system, designed to analyze the joint impact of primary fires, RKD and FA on the system performance. Recently (unpublished), we explicitly closed the causal loop between primary fires and RKD, and modeled the suppression resources in greater detail, by distinguishing standard crews of volunteer firefighters (with and without training) from expert crews of professional firefighters. Using a Portuguese district as case study, with a set of scenarios, we analyzed the cost-effectiveness of investing in the training of standard and/or expert crews, considering different dispatch policies.

We found that reducing FA and RKD to benchmark values would significantly reduce pressure on firefighting teams, enabling more effective suppression operations, and that it pays to invest in better suppression resources.
\end{abstract}

Keywords: Forest Fire Suppression, Rekindles, False Alarms, Cost-Effective Analysis, Discrete Event Simulation

\title{
1. Introduction
}

Rekindles (RKD) and false alarms (FA) are unusually high in the Portuguese wildfire management system. Together, they represent a high burden on suppression resources in particular, and fire management resources in general. Indeed, e.g., during 2010, according to data provided by the Portuguese Institute for Nature Conservation and Biodiversity (ICNF), in 20,049 occurrences that the suppression system handled in the summer, $12.5 \%$ were FA and $15.0 \%$ were RKD (Pacheco et al. 2014c).

During the fire season, it is usual to have large usage of resources (human and material) to combat wildfires and, on peak days, firefighters are in a tight spot, due to the pressure to move incessantly from one fire to the next one (Beighley and Hyde 2009). In such occasions, the system may not be able 
to effectively meet the needs, getting out of control. If there are fires waiting to be fought, they keep spreading, becoming harder to extinguish, with an increased likelihood of becoming mega-fires, reaching people, homes and animals, besides destroying the forest landscape (Lourenço and Rainha 2006). Consequently, pressured suppression crews prematurely abandon mop-up operations (moving towards the initial attack of new fires), without the needed time to use the appropriate tools to effectively carry out mop-up. When one of these fires with a bad mop-up rekindles, it is one more to join the other new ignitions or primary fires, and they are generally more aggressive than the latter (Pacheco et al. 2012; Pacheco et al. 2014a).

\section{Materials and Methods}

We first developed a discrete-event simulation model (implemented in ®ARENA) of a wildfire suppression system, designed to analyze the joint impact of primary fires, RKD and FA on the system performance (Pacheco et al. 2014b).

\begin{tabular}{|c|c|c|c|c|c|c|}
\hline & \multicolumn{2}{|c|}{$\begin{array}{l}\text { STD crews } \\
\text { (not specialized) }\end{array}$} & \multirow{2}{*}{$\begin{array}{l}\text { EXPRT crews } \\
\text { (specialized) }\end{array}$} & \multirow{2}{*}{ K } & \multirow{2}{*}{$\mathbf{P}$} & \multirow{2}{*}{ Description } \\
\hline & $\begin{array}{l}\text { without extra } \\
\text { training (as is) }\end{array}$ & $\begin{array}{l}\text { with more } \\
\text { training }\end{array}$ & & & & \\
\hline Scenario 0 & $100 \%$ & & $0 \%$ & 1 & not applicable & 100 crews \\
\hline Scenario 1 & $100 \%$ & & $0 \%$ & 1 & not applicable & $\begin{array}{l}\text { Variation in the } \\
\text { number of crews }\end{array}$ \\
\hline Scenario 2 & $0 \%$ & $100 \%$ & $0 \%$ & ]0,1[ & not applicable & $\begin{array}{l}100 \text { crews } \\
\text { (with training) }\end{array}$ \\
\hline Scenario 3 & $80 \%$ & & $20 \%$ & 1 & $1 \%, 3 \%$, and $6 \%$ & $\begin{array}{l}\text { Variation of the } \\
\text { failure probability (p) of EXPRT crews }\end{array}$ \\
\hline $\begin{array}{l}\text { Scenario } 3 \\
\text { (composition) }\end{array}$ & $\begin{array}{l}80 \% \\
60 \% \\
40 \% \\
\ldots\end{array}$ & & $\begin{array}{l}20 \% \\
40 \% \\
60 \% \\
\ldots\end{array}$ & 1 & $3 \%$ & Variation in the crew composition \\
\hline Scenario 4 & $z \%$ & & $1-z \%$ & 1 & $3 \%$ & $\begin{array}{l}\text { With different " } z \% \text { ", three dispatch } \\
\text { policies tested }\end{array}$ \\
\hline
\end{tabular}

Figure 1 - Battery of tests performed with the ®ARENA simulation model.

This model contributes to fill a research gap concerning that impact, and features a novel application of simulation to suppression systems, as screening tools to support more holistic analyses. Recently (unpublished paper), we explicitly closed the causal loop between primary fires and RKD, and modeled the suppression resources in greater detail, by distinguishing standard crews of volunteer firefighters (with and without training) from expert crews of professional firefighters.

We use a Portuguese district as case study, and with a set of scenarios (please see Figure ), we analyzed the cost-effectiveness of investing in the training of volunteer firefighters and/or using expert crews of professional firefighters, under different dispatch policies.

Our model aims to support the analysis of the impact of different forest fire suppression policies on rekindles and false alarms and these policies are based on the existence of different types of suppression crews: volunteers (as-is), volunteers with more training, and professional firefighters.

\section{Results}

We found that reducing FA and RKD to benchmark values would significantly reduce pressure on firefighting teams, enabling more effective suppression operations, and that it pays to invest in better suppression resources (Pacheco et al. 2014b). 


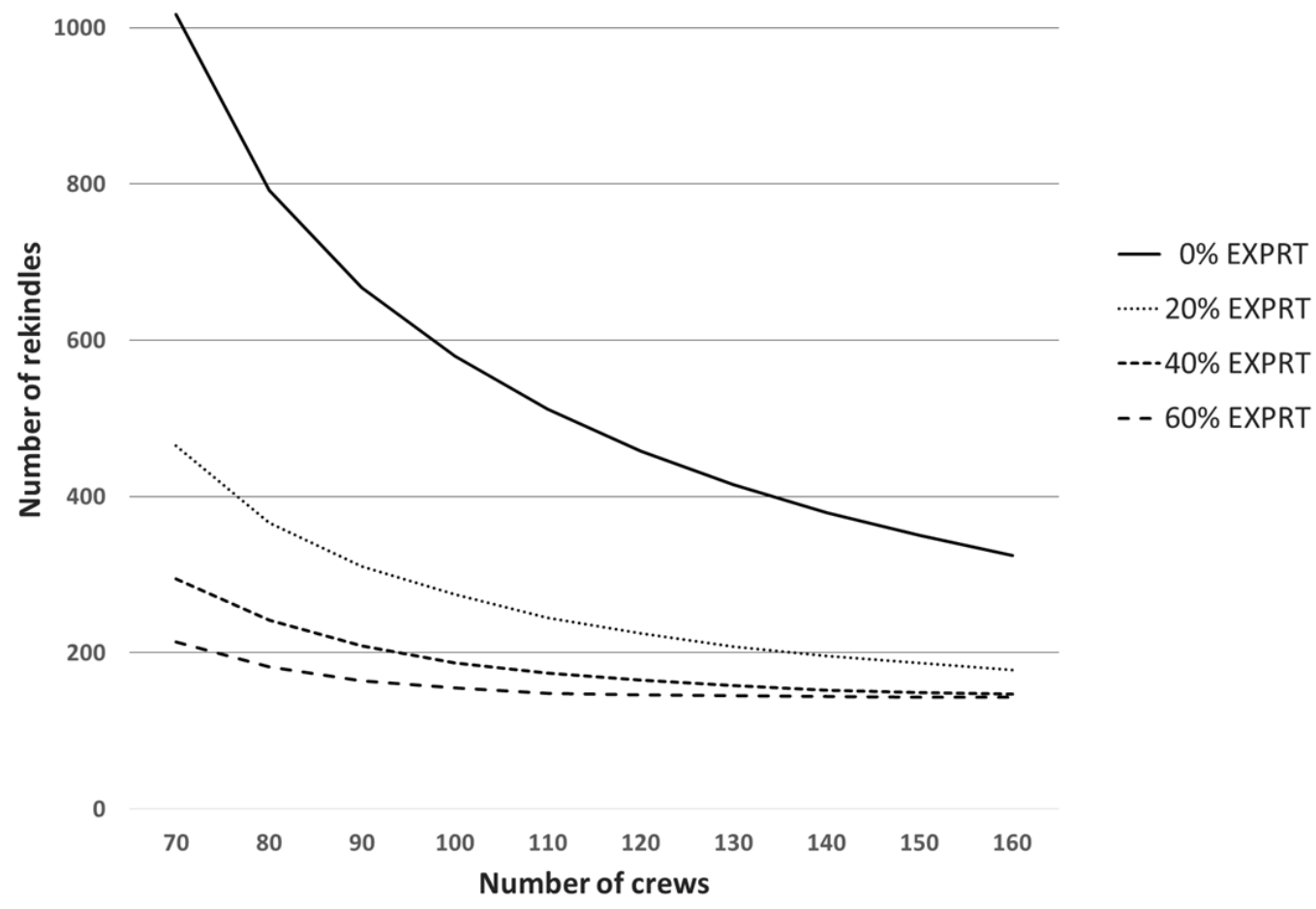

Figure 2 - Example of the kind of results obtained, in this case the evolution of rekindles by changing the percentage of professional crews (EXPRT).

The results of the cost assessment (e.g., Figure ) for the different scenarios (in Figure ) indicate that modifying the current (fixed) system design to a more flexible one, mixing volunteer firefighters with professional firefighters and/or investing in the training of the volunteers, appears to offer good prospects in terms of improving forest fire management, as more fires with a good mop-up will lead to fewer rekindles and thus, less pressure over the firefighting crews.

Finally, the eventual increase in the system costs must be weighed against the benefits resulting from the damage avoided with fewer rekindles.

\section{Acknowledgements}

This work is financed by the ERDF - European Regional Development Fund through the Operational Programme for Competitiveness and Internationalisation - COMPETE 2020 Programme within project «POCI-01-0145-FEDER-006961», and by National Funds through the FCT - Fundação para a Ciência e a Tecnologia (Portuguese Foundation for Science and Technology) as part of project UID/EEA/50014/2013. FCT also supported the research performed by Abílio Pereira Pacheco (Grant SFRH/BD/92602/2013).

The authors are deeply grateful to Rui Almeida and Manuel Rainha (ICNF) and Paulo Bessa (GTF Penafiel) for their enthusiasm and advice.

\section{References}

Beighley, M, Hyde, AC (2009) Systemic Risk and Portugal's Forest Fire Defense Strategy: An Assessment of Wildfire Management and Response Capability. 
Lourenço, L, Rainha, M (2006) As mediáticas 'mãos criminosas dos incendiários'e algumas das 'lições dos fogos florestais de 2005 em álbum fotográfico. Contributo para a desmistificação dos incêndios florestais em Portugal. Territorium 71-82.

Pacheco, AP, Claro, J, Oliveira, T (2012) Rekindle dynamics: validating the pressure on wildland fire suppression resources and implications for fire management in Portugal. In 'Modelling, Monitoring and Management of Forest Fires III.' Vol. 3 pp. 258. (Wessex Institute of Technology: Ashurst, Southampton, UK)

Pacheco, AP, Claro, J, Oliveira, T (2014a) Rekindles or one- $\sigma$ quality in forest fire fighting: validating the pressure on firefighters and implications for forest fire management in Portugal. In 'Advances in forest fire research.' (Imprensa da Universidade de Coimbra: Coimbra)

Pacheco, AP, Claro, J, Oliveira, T (2014b) Simulation analysis of the impact of ignitions, rekindles, and false alarms on forest fire suppression. Canadian Journal of Forest Research 44, 45-55.

Pacheco, AP, Claro, J, Oliveira, T (2014c) Waste in non-value-added suppression activities: simulation analysis of the impact of rekindles and false alarms on the forest fire suppression system. In 'Advances in forest fire research.' (Imprensa da Universidade de Coimbra: Coimbra) 
(Página deixada propositadamente em branco) 

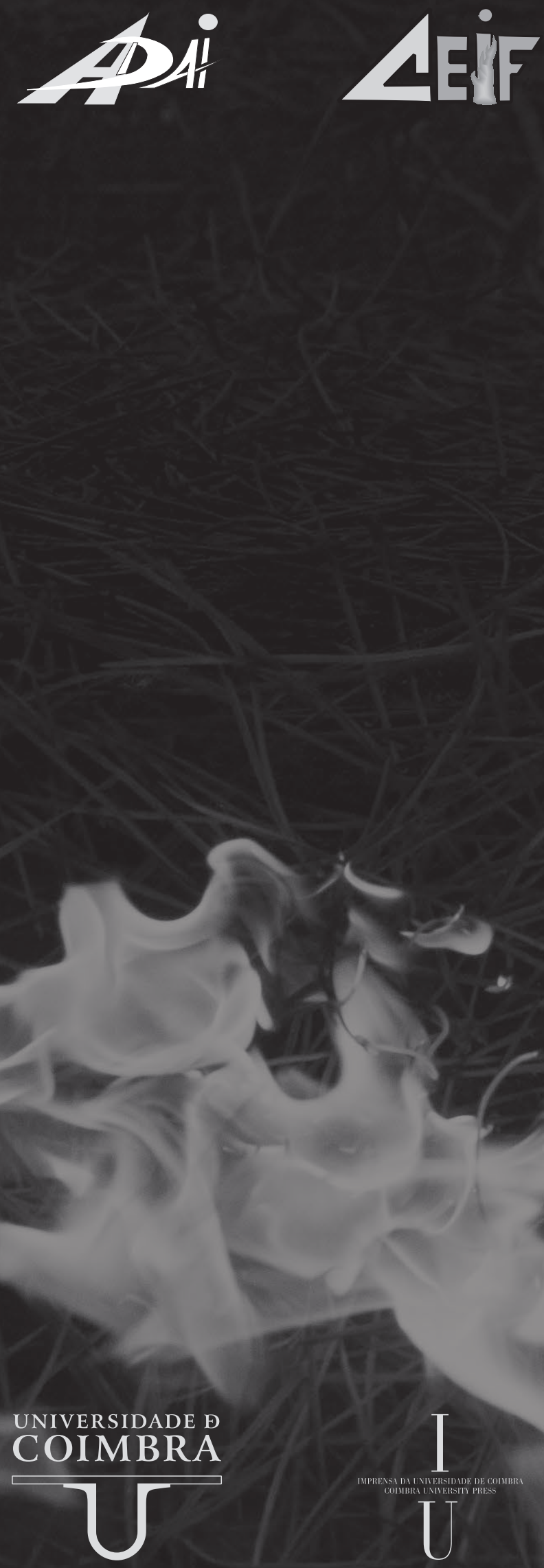